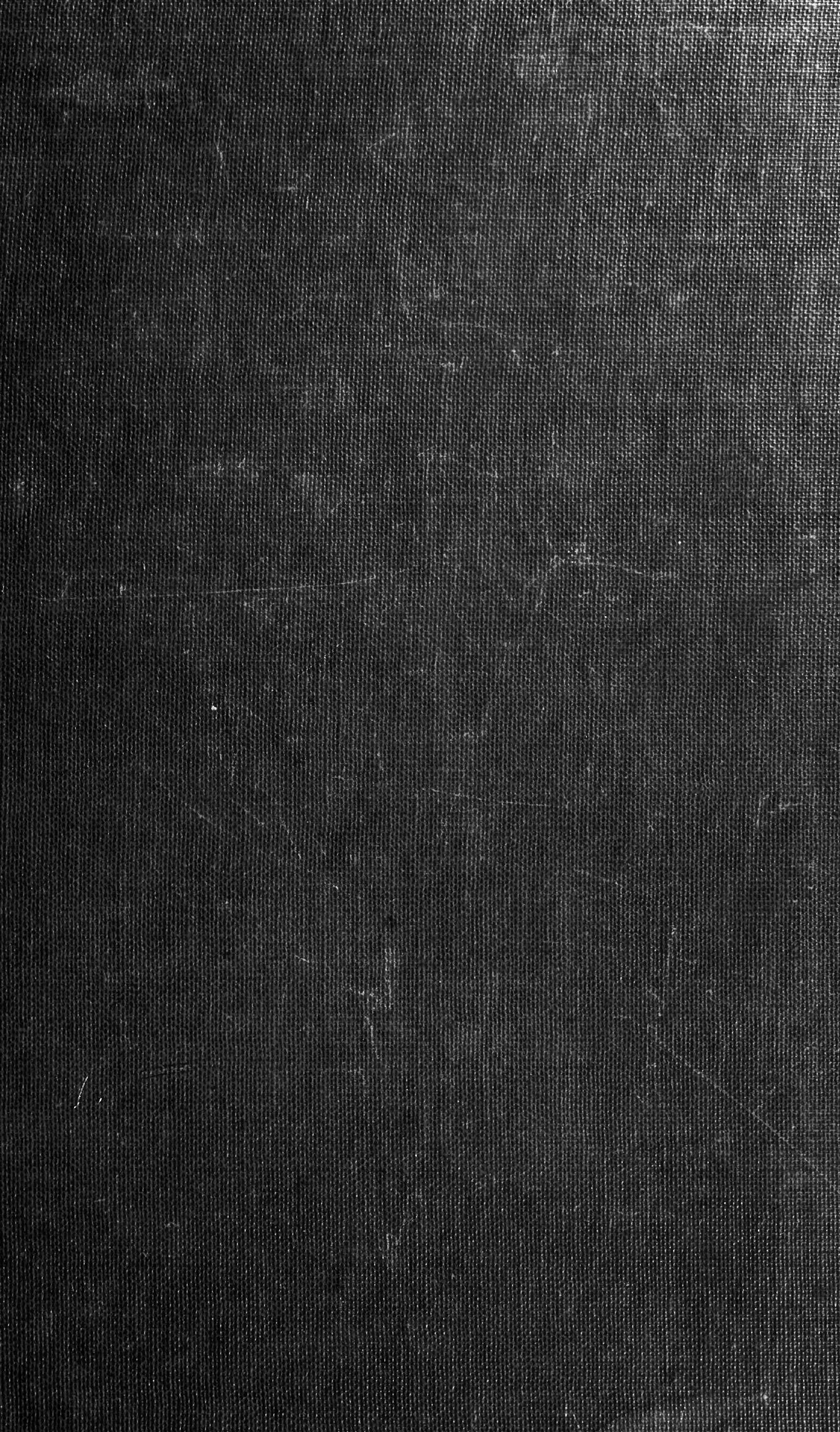






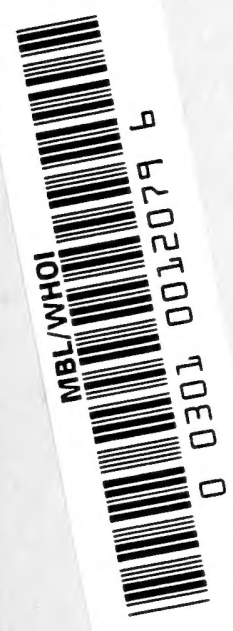


$+4$

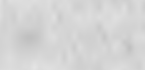

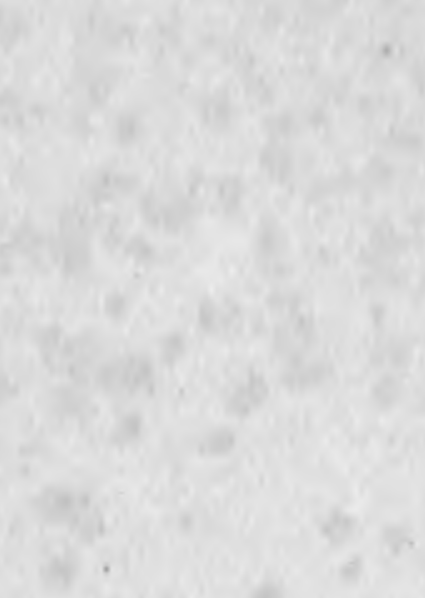

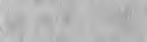


F UNDAMENTAL ASPECTS OF NORMAL AND MALIGNANT GROWTH 
$x^{2}+x^{2}$

y

4

t

.

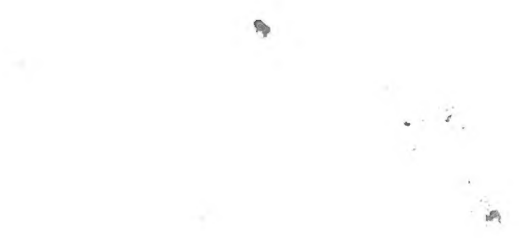

, 


\title{
FUNDAMENTAL ASPECTS OF NORMAL AND
}

\section{MALIGNANT GROWTH}

\author{
edited by \\ WIKTOR W. NOWINSKI \\ Associate Professor of Biochemistry, \\ Director of Tissue Metabolism Research Laboratory, \\ University of Texas, Medical Branch, \\ Galveston, Texas
}
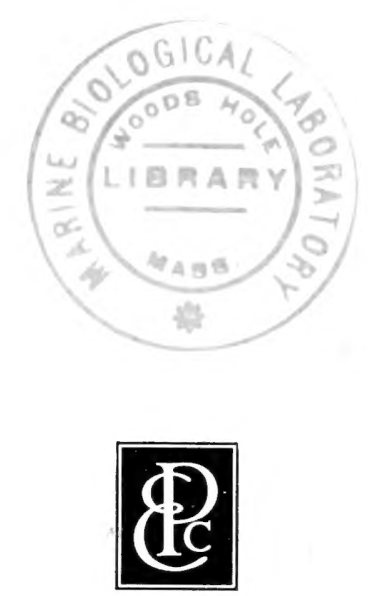

ELSEVIER PUBLISHING GOMPANY AMSTERDAM, LONDON , NEW YORK, PRINGETON 1960 


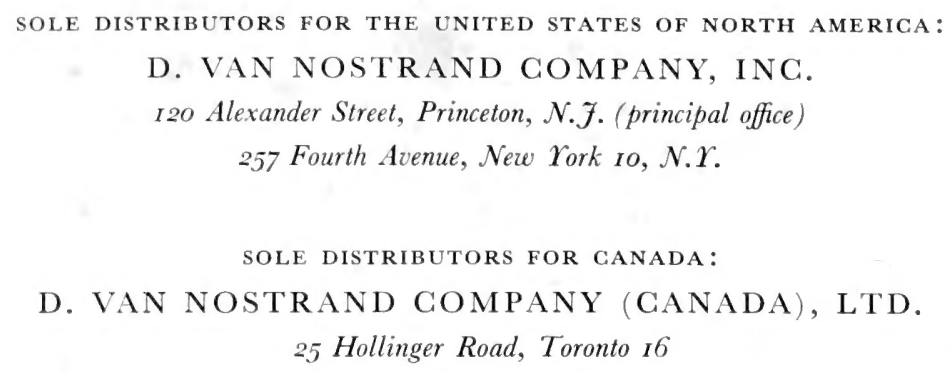

SOLE DISTRIBUTORS FOR THE BRITISH COMMONWEALTH EXCLUDING CANADA:

D. VAN NOSTRAND COMPANY, LTD.

$35^{8}$ Kensington High Street, London W. I4

With 339 illustrations and 66 tables

Library of Congress Catalog Card Number 59-8944

ALL RIGHTS RESERVED. THIS BOOK OR ANY PART THEREOF MAY NOT BE REPRODUGED IN ANY FORM (INCLUDING PHOTOSTATIC OR MICROFILM FORM) WITHOUT WRITTEN PERMISSION FROM THE PUBLISHERS

PRINTED IN THE NETHERLANDS BY JOH, ENSCHEDÉ EN ZONEN, HAARLEM 


\section{PREFACE}

The present interest that is shown in the problem of growth extends not only to theoretical aspects but also to problems of basic research in cancer. It seems therefore strange that since I 942 when Joseph Needham's monumental Biochemistry and Morphogenesis appeared, no work giving a comprehensive survey of the present state of our knowledge on this subject has been published; the excellent books Chemical Embryology by Jean Branchet and Analysis of Development edited by Hamburger, Weiss and Willier cover embryonic growth only. In the past few years a series of highly important and interesting symposia on growth have been published, but a symposium is primarily directed to the specialist and only covers specific problems; it does not pretend to serve as an introduction to the study of a subject.

Because of these reasons for a long time we have been planning to edit a book on growth, written by prominent specialists. Our intention was that it should be an introduction into various aspects of the growth problem, a kind of stepping stone from which-on the basis of ample references - the reader would be able to go deeper into details of the problems in which he is particularly interested. We hope that the present book will be of interest not only to the biologist who works principally in other fields, but also to all those interested in cancer research and who look for an up-to-date survey of the results in basic cancer research and allied fields.

The size of this book, which was decided at the start, did not allow all the problems of growth to be included, since such a complete survey would require several volumes. However, in this book the reader will find the most important aspects of this fascinating problem. Owing to lack of space, the contribution of the Editor could not be included, but it will appear as a separate book, also published by Elsevier.

A new feature is included in the present book: because morphological growth problems are becoming increasingly bound up with biochemistry, which is manifested in practically every chapter, the book starts with a comprehensive summary of intermediary metabolism, written by Dr. S. Kit. This chapter can be either read as a whole, or used as a source of reference to recall certain metabolic pathways or enzymic reactions, referred to in other chapters.

We wish to express our deep gratitude to Dr. Chauncey D. Leake, Professor of Pharmacology, Ohio University Medical School, and President of the American Association for the Advancement of Science, who-while he was Dean and Executive Director of the University of Texas Medical Branch-brought us into personal contact with the executives of Elsevier, thus contributing to the realization of our plans.

Our sincere thanks are due to our friend, Dr. John G. Sinclair, Professor of Embryology at the University of Texas Medical Branch, who with his wise council often helped us to make the right decision. He also took over the editorial duties for five months, when the Editor of this book was lecturing as Visiting Professor at the University of Buenos Aires. We also wish to thank our collaborator, Mr. William C. Mahaffey, M. Sc., for his help in compiling the Index, and 
to express our gratitude to the publishers whose kindness and co-operation were such that all our (i.e. the Authors' and the Editor's) wishes were taken into consideration (though some of them were far from simple!).

Finally, I would like to thank my former secretary Mrs. Leedia Greve Hanrahan and my present secretary, Mrs. Dorothy Rohde, who contributed so effectively towards making the task of the Editor much easier.

January 1960

WIKTOR W. NOWINSKI 
Warren Andrew, Chairman, Department of Anatomy, Indiana University School of Medicine, Indianapolis, Indiana.

Ludwig von Bertalanffy, Sloan Visiting Professor, The Menninger Foundation, Topeka, Kansas.

John 7. Biesele, Professor of Zoology, The University of Texas, Austin, Texas; Associate Scientist, Sloan-Kettering Institute for Cancer Research, New York. Jean Brachet, Professor at the Faculty of Sciences and Director, Laboratory of Animal Morphology, Université Libre de Bruxelles, Belgium.

Hugh Clark, Associate Professor of Zoology; Chairman, Executive Committee, Institute of Cellular Biology, University of Connecticut, Storrs, Connecticut. Albert M. Dalcq, Professor of Anatomy and Director, Institute of Anatomy, Faculty of Medicine, Université Libre de Bruxelles, Belgium.

A. Clark Griffin, Chairman, Departments of Biochemistry, The University of Texas M. D. Anderson Hospital and Tumor Institute and Baylor University College of Medicine, Houston, Texas.

Heinz Herrmann, Professor of Zoology, Institute of Cellular Biology, Department of Zoology, University of Connecticut, Storrs, Connecticut.

Arthur Kirschbaum*, late Professor of Anatomy and Chairman, Department of Anatomy, Baylor University School of Medicine, Houston, Texas.

Saul Kit, Associate Biochemist and Head, Section of Nucleoprotein Metabolism,

Department of Biochemistry, The University of Texas M. D. Anderson Hospital and Tumor Institute, Houston, Texas.

A. E. Needham, University Demonstrator in Zoology, Department of Zoology and Comparative Anatomy, University Museum, Oxford, England.

Kenneth $V$. Thimann, Professor of Biology, Harvard University, Cambridge, Massachusetts.

Wesley W. Washburn, Fr., Instructor, Plastic and Maxillo-Facial Service, Department of Surgery, University of Texas Medical Branch, Galveston, Texas. Charity Waymouth, Staff Scientist, Roscoe B. Jackson Memorial Laboratory, Bar Harbor, Maine.

* deceased 


\section{CONTENTS}

Chapter I. Intermediary Metabolism of Building Blocks Involved in Growth, by Saul Kit

I. Introduction .

II. Energy Yielding Processes

A. Introduction

B. The Catabolism of Glucose and Fatty Acids .

I. The Embden-Meyerhof glycolytic sequence, $3-2$. The hexose monophosphate shunt, $4-3$. The oxidation of pyruvate to acetyl-CoA, $5-4$. The fatty acid spiral, $6-5$. Tricarboxylic acid cycle (Citric acid cycle: Krebs cycle), $7-6$. The formation of $\mathrm{CO}_{2}, 8-7$. Oxidative steps, $9-$

C. Terminal Hydrogen and Electron Transport System .

I. "Dismutation" reactions and pyridine nucleotide dehydrogenases, I $_{5}$ -

2. Reoxidation of reduced pyridine nucleotides via the cytochrome system, I 6 - 3. The oxidation of succinate, fatty acids, choline and xanthine, 2 I 4. Autooxidizable flavoproteins, $2 \mathrm{I} \sim 5$. Glycolate as a hydrogen carrier, $22-$ 6. Nitrate as a hydrogen acceptor, $22-7$. Quinone reductase and plant respiration, $24-8$. Glutathione and ascorbic acid as respiratory catalysts, 25

D. Localization of Enzymes within Cells .

E. Generation of Energy-Rich Phosphate Bonds . . . . . . . I. Substrate phosphorylation, $27-2$. Electron transport phosphorylation, $29-3$. Photosynthetic phosphorylation, $32-4$. The storage of high-energy phosphate bonds, 33 -

III. The Utilization of ATP and Related Nucleotides

A. Group I Reactions

B. Nucleotide Transfer Reactions

C. Uridyl Transferase Reactions .

D. The Activation of Fatty Acids and Cholic Acid

E. The Activation of Sulfate .

F. $\mathrm{CO}_{2}$ Fixation

G. Ammonia Fixation

H. Activation Reactions in Nucleic Acid Synthesis

I. The Peptide Bond of Pantothenate

J. Peptide Bond Formation in the Incorporation of Amino Acids into Protein

K. Glutathione Synthesis .

IV. The Synthesis of Protoplasmic Building Blocks

A. Pentose Formation

I. Hexose monophosphate shunt, 47-2. Transketolase-transaldolase pathway, $4^{8-3}$. Evaluation of the Embden-Meyerhof and phosphogluconate pathways of glucose oxidation, $49-4$. Evaluation of the transketolasetransaldolase pathway and pentose formation, $5 \mathrm{I}-5$. Deoxyribose synthesis, 52 -

B. The Biosynthesis of Amino Acids .

I. Distribution of free amino acids in tissues, 53-2. Amino acid relationships, 54-3. Essential amino acids, 55-4. The synthesis of the carbon skeletons of amino acids, 56 -

C. Fixation of Inorganic Nitrogen and Transfer .

I. Nitrogen assimilation, $81-2 . \mathrm{NH}_{3}$ fixation, $82-3$. Amino transfer reactions and transaminations, $8_{3}-4$. The transfer of the amide group of glutamine, 85-5. Aspartic acid and the transfer of $\alpha$-amino nitrogen, $87-$

D. Porphyrin Synthesis 
E. Nucleotide Synthesis

1. Occurrence of nucleotides in tissues, $91-2$. "De novo" purine synthesis, $9 \mathrm{I}-3$. The utilization of free purines, $95-4$. "De novo" pyrimidine synthesis, $98-5$. Utilization of uracil, cytosine and thymine, $99-6$. The amination of uracil compounds to cytosine and the deamination of cytidine, $100-7$. Trans-N-glycosidation, IOI-8. Phosphorylation of cytidine and uridine, IO2-9. Nucleoside monophosphate and diphosphate kinase, IO2- Io. Polynucleotide and ribonucleic acid synthesis, I03-II. The mechanism of DNA-thymine (DNA-T) synthesis, Io3-

F. Phospholipid Biosynthesis

I. Ethanolamine and choline formation, ro6 - 2. The enzymatic synthesis of lecithin and kephalin, $107-$

G. Vitamin Biosynthesis

I. Cholesterol and vitamins $A$ and $D_{3}$, I $08-2$. Ascorbic acid, I I I - 3. Thiamine, I $12-4$. Riboflavin, I I3-5. Pyridoxal phosphate, I I4-6. Biotin, I I47. Myoinositol, I I $5-8$. Niacin, $\mathrm{DPN}^{+}$and TPN ${ }^{+}$, I I $5-9$. Coenzyme A synthesis, I I - I0. p-Aminobenzoic acid and folic acid compounds, Ir 9 -

V. Summary.

VI. Addendum

Literature

Chapter 2. Principles and Theory of Growth, by Ludwig von Bertalanfy .

I. Introduction .

(a) Definition of growth, I37 - (b) Various aspects of the problem of growth, I 39 - (c) Growth and differentiation, I 4 I - (d) Limitations of growth, I4 I -

II. The Organism as an Open System

(a) Introduction, I43 - (b) Definitions, I44 - (c) Kinetics of open systems, I 45 - (d) Turnover of building materials of the organism, I 47 - (e) Energy requirements of protein synthesis, I49 - (f) Energy requirements of the maintenance of the steady state of the organism, I5I - (g) Thermodynamics of open systems, I5I - (h) Principles of irreversible thermodynamics, I52 (i) Entropy and the living world, I55-(j) Dynamic morphology, I56-

III. Cell Growth and Multiplication .

(a) Nuclear growth and nuclear series, 156 - (b) Interaction between nucleus and cytoplasm, I 58 - (c) Energy sources of mitosis, I59-(d) Mathematical theory of cell growth, $161-$

IV. Growth of Tissues

(a) Cell renewall, I63- (b) Cell constancy, I66 - (c) Mathematical theory of growth of tissue cultures, I66 -

V. Growth in Time of the Total Organism .

(a) Definitions, I68 - (b) General considerations on models and laws of growth, I 70 - (c) Empirical growth formulas, I 74-(d) Quantitative theory of animal growth, I 77 - (e) Dependence of metabolic rate on body size, I8 I (f) Explanations of the size-dependence of metabolic rate, 185-(g) Metabolic types and growth types, I90 - (h) Application of the Bertalanffy growth equations in fishery biology, 193 - (i) Metabolic types and growth types (continued), I96-(j) Verification of the constants of the growth equation, $198-(k)$ Deductive derivation of the system of growth equations, $200-$ (l) Further discussion of the growth model, 204-(m) Growth model of Weiss and Kavanau, 208 - (n) Summary of models of growth, 2 I2 - (o) Equifinality of growth, $213-(\mathrm{p})$ Growth and temperature, 2 I6 - 
VI. Growth of Mammals .

(a) Postembryonic growth, 218-(b) Growth and hormones, 219-(c) Embryonic growth, 223 -

VII. Relative Growth .

(a) The allometric equation, 224 - (b) Morphological applications of allometry, 229- (c) Physiological competition, 230- (d) Biochemical applications, 232 - (e) Physiological applications, 232 - (f) Physiological and growth gradients, $233-(\mathrm{g})$ Growth of organs in time, $234-$

VIII. Growth and Evolution - (a) Transformation, $238-$ (b) Evolutionary allometry, $240-$ (c) Changes of the organism-as-a-whole as growth problem, 245 - (d) The uniqueness of man as a problem of growth, $247-$ (e) Retardation, $248-$ (f) The evolution of the brain, $250-$

Literature

Chapter 3. Nucleic Acids and Growth, by 7. Brachet . . . . . . . 260

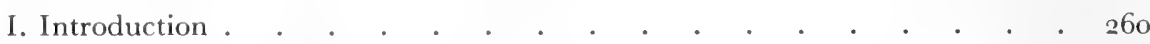

II. Brief Survey of Nucleic Acid Chemistry and Cellular Localization . . . $\quad 260$ (a) Chemical composition, 260 - (b) Physical properties and molecular structure, 26I - (c) Gytochemical techniques for detection, 262 -

III. DNA and Growth: Mitosis

(a) DNA content per cell nucleus, $265-$ (b) Metabolic stability of DNA, $266-$

(c) Genetic activity of DNA, 268 - (d) DNA synthesis during mitosis, $269-$

(e) Effects of physical and chemical agents on DNA and its synthesis, 27 I -

IV. RNA and Growth: Protein Synthesis

(a) Cytochemical and quantitative evidence, 273-(b) The role of RNA in plantvirus multiplication, $276-$ - (c) Direct evidence for a role of RNA in growth and protein synthesis, 277 - (d) The mechanisms of protein synthesis, $284-$

V. Nucleic Acids and Embryonic Development .

(a) Synthesis of DNA and RNA during development, $285-$ (b) RNA and morphogenesis, 288 - (c) The effects of an abnormal nuclear composition on nucleic acids and development, 292 -

VI. Final Remarks

(a) Independence of DNA synthesis and protein synthesis, $293-$ (b) The role of the cell nucleus in growth and protein synthesis, 295-(c) Nucleic acids and regulation of growth, 297 -

Literature

Chapter 4. Germinal Organization and Induction Phenomena, by Albert M. Dalcq

I. Organizing Aspects of Gametogenesis

A. The cytoplasm of the gametes.

B. Growth and polarization of the oocytes

C. Symmetrization during oogenesis .

II. Intrinsic Morphochoresis .

A. General trend and goal of intrinsic morphochoresis

B. Fertilization and morphochoretic pattern 
C. Cleavage and morphochoretic pattern

D. Kinetic and causative aspects of intrinsic morphochoresis .
(a) The factors at work, $34^{1}-$ (b) Enumeration of the problems, $34^{2}-$

(c) The localisation of the blastopore in Anamniots. An operative approach, 342 - (d) Interpretation of kinematics in amniots, 347 - (e) Some biochemical information, $34^{8}$ - (f) Modifying intrinsic morphochoresis by operative and biochemical means, $35^{0}-(\mathrm{g})$ Intradermic inductions, 35 $\mathrm{x}$ -

E. Mechanisms of intrinsic morphochoresis. A discussion

III. Neurogenic Induction, the Main Aspect of Extrinsic Morphochoresis (with some data on non-neurogenic inductions)

A. Observations

B. Experimental data relative to primary induction .

(a) Operations on the whole embryo, 366 - (b) Explantations of unmodified territories, 396 -

C. Experimental modifications of morphochoresis

(a) Physical means, 4I $\mathrm{I}$ - (b) Biochemical modification of inductors and reactors, $419-$

D. Xeno-inductions

(a) Fresh foreign tissues, 427 - (b) Killed foreign tissues, 428 - (c) Regional influence of the host, $43 \mathrm{I}-$ (d) First approaches to the nature of the xenoinductors, 43 I - (e) Fractionation of the xeno-inductors, 433 - (f) Interpretative remarks, 443 -

E. Secondary and minor inductions .

(a) The cephalic placodes, 449 - (b) Neural crest and derivatives, $454-$ (c) Limb buds, $45^{6}-$ (d) Some epiblast derivatives, $459-$ (e) The induction of cartilages and somites, 46 I - (f) Other in vitro inductions, $463-$ (g) Gonadic inductions, $466-$

F. General remarks on induction

(a) About definition, 468 - (b) On the evolution of inductive processes, 468 - (c) On the appreciation of the data, 469 - (d) On the morphogenetic effects of induction, $469-$ (e) On the nature of the inducing agents, $474-$

(f) On the intimate changes in the reactor, $479-$

IV. Final Considerations ,

Literature

Chapter 5. Molecular Mechanisms of Differentiation. An Inquiry into the Protein Forming System of Developing Cells, by Heinz Herrmann.

I. Introduction .

II. The Utilization of Protein Precursors in the Differentiating Cell . . . . (a) Precursors larger than amino acids, 497 - (b) Amino acids as precursors, 498 - (c) Pathways of precursor utilization, 505 -

III. The Protein Forming System (PFS) of Differentiated and Embryonic Cells . (a) Nucleic acids as components of the PFS, 509- (b) Structural aspects of the PFS : Protein formation in microsomes and mitochondria, $512-$ (c) Nucleocytoplasmic interactions related to protein formation, $5^{1} 4^{-}$

IV. Regulating Factors of the PFS During Development.

(a) Apparent qualitative changes in the PFS (Induction), 519- (b) Apparent quantitative aspects of protein formation in embryonic cells, $522-$ (c) The relation of proliferation and differentiation, $523-$

V. Conclusions: The Convergence of Concepts .

Literature 
Chapter 6. Growth in Tissue Culture, by Charity Waymouth . . . . 546

I. Effects of Environment on Growth in Tissue Culture _ . . . . . 549 (a) Cell autonomy and interdependence. Single cell clones, 549- (b) Chemical factors, $554-$

II. Physical Factors - Temperature, Irradiation, pH, Tonicity, Physical Nature of Substrate . . . . . . . . . . . . . . . . (a) Effects of temperature on growth in tissue culture, $556-$ (b) Effects of visible light, $55^{8}-$ (c) Effects of ultraviolet irradiation, $55^{8}$ - (d) Effects of ionizing radiations, $55^{8}$ - (e) Effects of $\mathrm{pH}, 559$ - (f) Effects of tonicity, 559 (g) Effects of the physical nature of the substrate, $560-$

III. Relationship of Growth in vitro to Maintenance, Differentiation and Function. Conditions for Growth, Survival and Differentiation. . . . . . . 56 I

IV. Definitions of Growth in Tissue Culture . . . • . . . . . 566

V. Methods of Assessing Growth in Tissue Culture . . . . . . . 568

(a) Linear or areal measurements, $569-$ (b) Mitotic counts, 57 I - (c) Possibility of subdivision, $572-$ (d) Cell and nuclear counts, $572-$ (e) Tissue mass, 574 - (f) Cell or culture volume, 574 - (g) Nephelometry, 575 - (h) Metabolic measurements, 575 - (i) Glucose utilization, $576-$ (j) Nucleic açids, $576-$ (k) Protein, $577-$

VI. Recording Growth and Behaviour of Cells in Tissue Culture . . . $\quad 578$

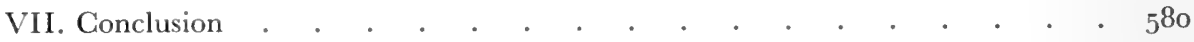

Literature . . . . . . . . . . . . . . . 58 I

Chapter 7. Regeneration and Growth, by A. E. Needham . . . . $\quad 588$

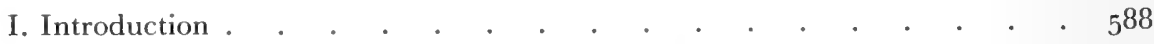

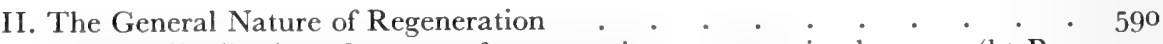

(a) The distribution of powers of regeneration among animals, $590-$ (b) Regeneration and other growth processes, 591 -

III. The Stages in an Act of Regeneration . . . . . . . . . 595

IV. Quantitative Aspects of Regeneration . . . . . . . . . . 602

V. Metabolic Changes during Regeneration. . . . . . . . . . 608

(a) Protein metabolism, 6I I - (b) Metabolism of nucleic acids, 6I 2 - (c) Activities of phosphatases, 6I I $_{4}$ - (d) Metabolism of sulphur compounds, 6I5 (e) Respiration and carbohydrate metabolism, 6I8 - (f) Fat metabolism, 619(g) Water metabolism, 620 - (h) Inorganic cations, $622-$

VI. The Effect of Extrinsic Factors on Regenerative Growth .

(a) Temperature, 623 - (b) Short-wave radiation, 624 - (c) Electrical forces, 624 - (d) Extraneous chemicals, 625 -

VII. Internal Factors in the Control of Regeneration .

(a) Nutrition and regeneration, $627-$ (b) Hormones in the control of regeneration, $631-$ (c) Chemical factors produced by regenerating tissues, $636-$ (d) Nerve-supply and regeneration, 640 - (e) The vascular system and regeneration, 644 - (f) Diffusing factors, 645 - (g) Local factors, 646 -

VIII. Conclusion 
Chapter 8. Wound Healing as a Problem of Growth, by Wesley $W$. Washburn $\mathrm{Fr}$.

I. Introduction .

II. The Stimulus for Wound Healing

III. Wound Closure

(a) Contracture, 669 - (b) Intussusceptive growth, 67I - (c) Growth from the wound edge, $67 \mathrm{I}-$

IV. Epithelialization .

(a) Retraction of the epidermal edge, 675-(b) Detachment of the epidermis from the basement membrane, 675 - (c) Migration of epithelium over the wound surface, $675-$ (d) Cessation of cell movement and reattachment, $680-$

V. Chemical Changes Associated with Epithelialization.

VI. Source of Granulation Tissue

VII. Fibroblastic Proliferation .

VIII. The Chemistry of Granulation Tissue

IX. Alkaline Phosphatase .

$\mathrm{X}$. Role of Sulfur in Wound Healing

XI. Summary.

Chapter 9. Nitrogen Metabolism and Growth, by Hugh Clark

I. Introduction

(a) Growth and development, 7 I2 - (b) Metabolism of nitrogen compounds, $715-$

II. Nitrogen Metabolism in Relation to Aspects of Development

(a) Gametogenesis and fertilization, 7I 7 - (b) Cleavage and blastulation, 72 I - (c) Observations of protozoa, $725-$ (d) Gastrulation, $727-(\mathrm{e})$ Organogenesis, 727 - I. Protein Synthesis, 728 - a. Chemical limitations, 728 b. Cytological mechanisms, 729 - c. Relationship with yolk storage, $729-$ 2. Growth Regulation, $730-a$. Differential growth, $730-b$. Cessation of growth, 73 I - c. Genetic control of growth, 733 - 3. Energy Sources of Development, 735 - a. General, $735-b$. Succession of energy sources, $736-$ c. Nitrogen metabolism in cleidoic eggs, $738-$

III. General Summary

Acknowledgements

Literature

Chapter I0. Plant Growth, by Kenneth V. Thimann . . . . . . 748

I. General Characteristics of Plant Growth . . . . . . . . . 748

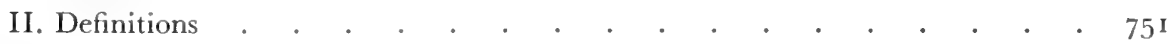

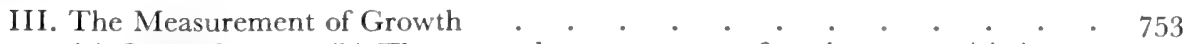

(a) General, 753 - (b) The use and measurement of auxins, 755 - (c) Assays for other growth factors, $75^{8-}$

IV. Chemistry of the Growth Substances . 
V. Cell Division .

(a) Apical meristems, $766-$ (b) Secondary meristems, $767-$ (c) Morphology of cell division, $767-$ (d) Physiology of cell division, $769-$

VI. Cell Enlargement

(a) The elongation of tissues in an axis, 775 - (b) Control by auxin, $776-$ (c) Control by oxygen, $779-$ (d) Control by gibberellin, $780-$ (e) The effects of nutrients and ions, $780-$ (f) Specific inhibitors, $782-(\mathrm{g})$ Relation between cell enlargement and metabolism, $787-(\mathrm{h})$ Osmotic inhibition, $792-$ (j) Concepts of the mode of auxin action and the nature of cell enlargement, 793-

VII. Growth of Specific Organs

(a) Growth of leaves, 797 - (b) Growth of roots, 80I - (c) The growth of fruits, $807-(d)$ Interrelations between buds; integration of the plant, 8I I -

VIII. Conclusion

Literature

Chapter I I. The Carcinogenic Stimulus, by Arthur Kirschbaum

I. Hormonal Factors in Carcinogenesis .

(a) Pituitary and thyroid neoplasms induced by alteration in thyroid function, 824 - (b) Hormonal imbalance per se as a factor in inducing neoplasia, 829 (c) Endocrine tumors following imbalance induced by estrogenic hormone, $83 \mathrm{I}-$ (d) Neoplasms of tissues whose normal growth is stimulated by estrogen, 835 - (e) Neoplasms of "ton-target" tissues induced by estrogen, 837 (f) Tumor-promoting activity of steroids other than estrogenic, $840-$ (g) Inhibition of tumorigenesis by steroid hormones, $840-$ (h) Effect of steroid hormones on tumor growth, 842 - (i) The influence of the pituitary gland on the genesis, growth and secretion of tumors, $844-(\mathrm{j})$ Influence of thyroid secretion on genesis and growth of tumors, $846-(\mathrm{k})$ Diabetes and the growth and development of neoplasms, $847-$

II. Ionizing Radiations and Cancer .

(a) Local action of ionizing radiations, 847 - (b) Somatic mutation and carcinogenesis, 848 - (c) Neoplasms following whole body irradiation, 848 -

III. Viruses and Cancer
(a) Chicken and rabbit tumors, $849-$ (b) Mouse mammary cancer, $850-$

(c) Mouse leukemia, $853-$ (d) Parotid and skin tumors of the mouse, $855-$

(e) Nature of the tumor viruses, 855 -

IV. Carcinogenic Chemicals .

(a) Polycyclic hydrocarbons, $858-$ (b) Azo dyes, 86 I - (c) Amino compounds, 862 - (d) Halogenated hydrocarbons, $863-$ (e) Urethane, $863-$

V. Nutrition and Cancer

VI. Genetic Studies

VII. Summary.

Literature

I. Introduction .

II. Carbohydrates and Lipids
(a) Composition, $880-$ (b) Respiration and glycolysis, $8 \dot{8}_{\mathrm{I}}-\dot{ }_{\text {(c) }}$ Citric cycle, 888 - (d) Phosphorylation, 89I - (e) Electron transport, $892-$ (f) Fatty acid metabolism, 894 - 
III. Proteins and Amino Acids

896

(a) Composition, 896 - (b) Protein metabolism, 897 - (c) Amino acid metabolism, 899 -

IV. Nucleic Acids and Nucleoproteins

902

(a) Composition, $902-$ (b) Metabolism, $906-$

V. Other Metabolic or Compositional Features of Neoplastic Tissues

913

(a) Enzymes, 913 - (b) Minerals, 915 - (c) Other metabolic features, 915-

VI. Summary.

9 I 6

Literature

Chapter 13. Action of Certain Antimetabolites as Mitotic Poisons, by John 7. Biesele

I. Introduction .

II. Preprophasic Inhibition

(a) Antifolic acids, 929 - (b) Antiglutamines, $930-$ (c) Purine and pyrimidine analogues, 930 - (d) Nucleosides, 935 - (e) Nucleotides, 936 - (f) Amino acids and analogues, 936 -

III. The Poisoning of Chromosomes

(a) Amino acids, $93^{8}$ - (b) Antifolic acids, 939 - (c) Purines and pyrimidines, 939 -

IV. Poisoning of the Spindle

$94^{\mathrm{I}}$

(a) Antifolics, 943 - (b) Nucleic acid components and analogues, $944-$

V. Conclusion $94^{6}$

Literature

Chapter I4. Growth and the Aging Process, by Warren Andrew . . . $95^{2}$

Conclusion . . . . . . . . . . . . . . . 969

Literature . . . . . . . . . . . . . . 971

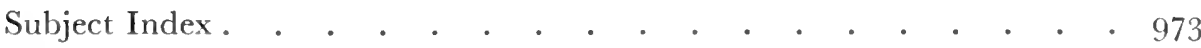




\section{ABBREVIATIONS}

(used in Chapter $\mathrm{r}$ )

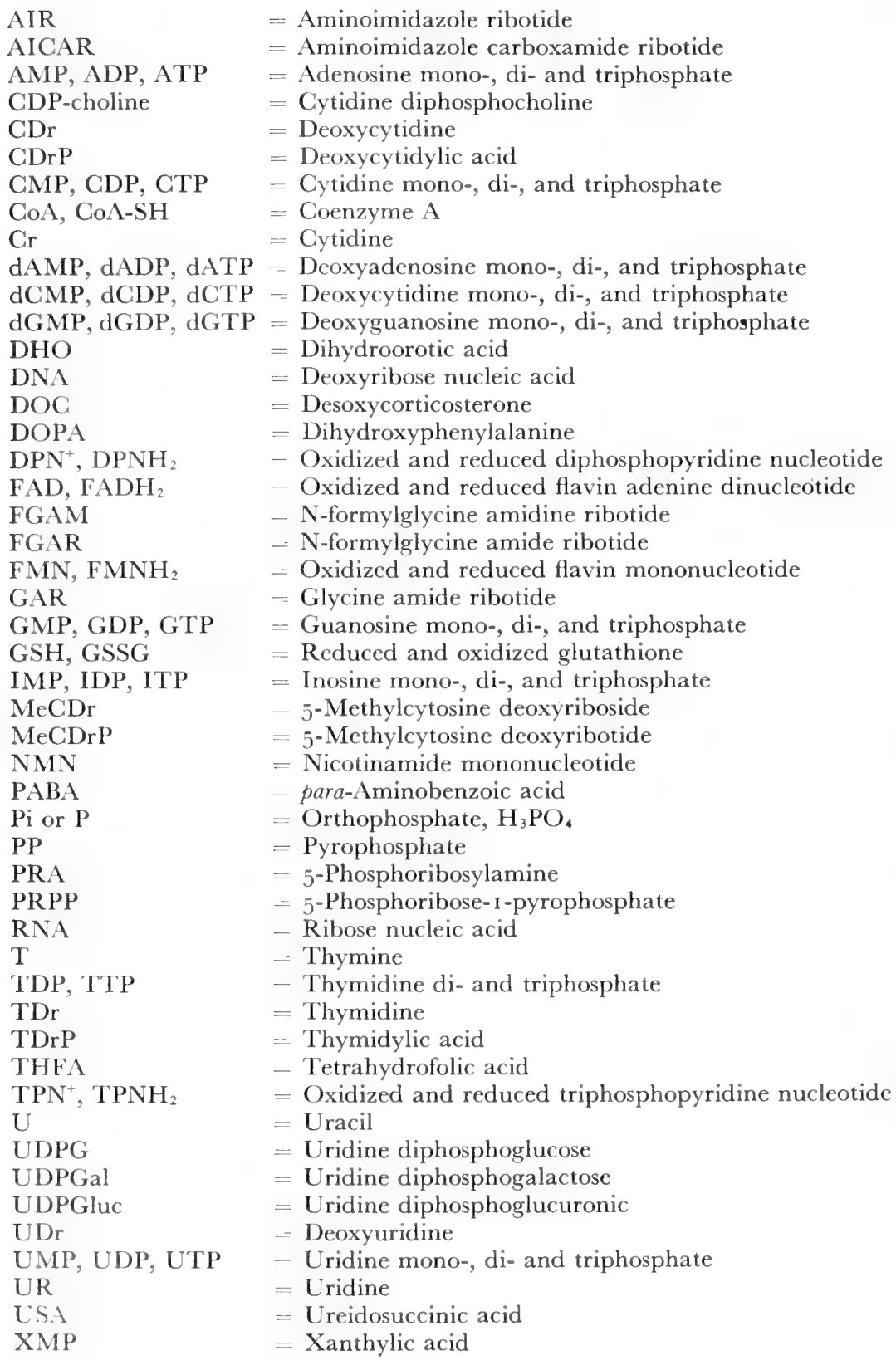




\title{
Chapter 1
}

\section{INTERMEDIARY METABOLISM OF BUILDING BLOGKS INVOLVED IN GROWTH ${ }^{1}$}

\author{
Saul Kit
}

I. INTRODUCTION

For growth to take place, an adequate intracellular pool of amino acids, purine and pyrimidine derivatives and vitamin coenzymes must be available. The requirement for these building blocks of protoplasm may be satisfied in many microorganisms and plants entirely by the synthetic activities of the organism. Thus, autotrophic organisms can synthesize all of the protoplasmic constituents from inorganic nitrogen and sulfur compounds and from carbon dioxide. On the other hand, most organisms, including the vertebrates, cannot synthesize many of the building blocks of protoplasm which hence must be supplied with the food. Organisms differ with respect to their biosynthetic potentialities and therefore their nutritional requirements. The same applies to the tissues and cells of a given multicellular organism. These differences reflect the enzymatic accoutrement of the cells in question. For example, the inability to synthesize a given amino acid, reflects the absence of a key enzyme which catalyzes the synthesis of that amino acid.

It is thus of interest to inquire into the mechanisms by which protoplasmic building blocks are assembled. One of the striking results of biochemical investigation is the recognition that the metabolic pathways, along which chemical substances are utilized, are basically similar in microorganisms, fungi, plants, and animals.

A map summarizing these metabolic pathways will be found in the pocket on the inner side of the back cover. This map depicts the orderly sequence of chemical reactions which leads to the synthesis of amino acids, hormones, vitamins, or other cellular metabolites and shows the relationships between these substances. It is to be emphasized that each of the chemical reactions shown on the metabolic map is catalyzed by an enzyme. However, the metabolic map summarizes the reactions which take place in a generalized or idealized rather than any actual cell. The metabolic

1 The author would like to express his appreciation to the American Cancer Society and the Leukemia Society, Inc. for research support, to Mrs. Beth Rafferty for painstaking assistance in the preparation of this manuscript, and to Dr. D. N. Ward for helpful criticism. Literature survey completed, January, 1957. 
map of any cell differs from this general map in that certain areas of the map are deleted and certain arrows are interrupted. For example, the reactions leading to the synthesis of thyroxine and of steroid hormones take place in endocrine tissues but not in the other tissues of the animal; the steps leading to the synthesis of the amino acids, arginine and tyrosine, take place readily in liver cells but only to a very limited degree in most of the other cells of the body. The mold, Neurospora, synthesizes lysine, by the aminoadipic acid pathway whereas many bacteria synthesize this amino acid by the diaminopimelic acid pathway, and some microorganisms and also mammalian cells cannot synthesize lysine at all. In other words, cells also differ from one another in that, I) a particular metabolic pathway is quantitatively more important in one type of cell, and 2) alternative solutions may be found for the same metabolic problem, as in the case of synthesis of lysine.

Since growth is an endergonic process, the metabolic activities of cells must be organized in such a way that the free energy derived from catabolic processes is available for the growth process. In this chapter, we will first examine the varied biochemical processes as a result of which free energy becomes available for cellular anabolism; secondly, the coupling devices for shunting the free energy into the growth process will be discussed; and thirdly, we will examine the chemical processes which lead to the synthesis of the building blocks of protoplasm.

The subject of intermediary metabolism deals with one of the most dynamic branches of biochemistry. Thus, the research of tomorrow will undoubtedly render obsolete some of the tentative formulations of today. However, we may confidently build upon the solid foundation of factual knowledge which has been amassed.

Much of the information discussed in this chapter has been summarized recently in a series of monographs, on intermediary metabolism. These include Chemical Pathways of Metabolism (Greenberg, I954), and a number of Symposia held under the auspices of Johns Hopkins University (McElroy and Glass, I95 I-I 952, I 953, I 954, I 955). Excellent reviews on intermediary metabolism have also appeared in the annual volumes of Advances in Enzymology and the Annual Reviews of Biochemistry.

\section{ENERGY YIELDING PROGESSES}

\section{A. Introduction}

It is convenient to begin a discussion of cellular metabolism by directing attention to the biochemical sequences referred to as glycolysis and the tricarboxylic acid cycle. These chemical reactions catalyzed by enzymes may properly be considered the backbone of the chemical activities of the cell and are shown in detail in Fig. I. It is to be noted that certain metabolites, namely, glucose, glyceraldehyde phosphate, ribose phosphate, acetyl-CoA, pynuvate, phosphoenolpyruvate, oxalacetic acid, $\alpha$-ketoglutaric acid, and succinyl-CoA, have been emphasized as key intermediates. Each may serve as the substrate for several different enzymatic reactions. For example, pyruvate may be reduced by a dehydrogenase to lactate, carboxylated to oxalacetate, aminated by a transaminase to alanine, or it may be oxidatively decar- 


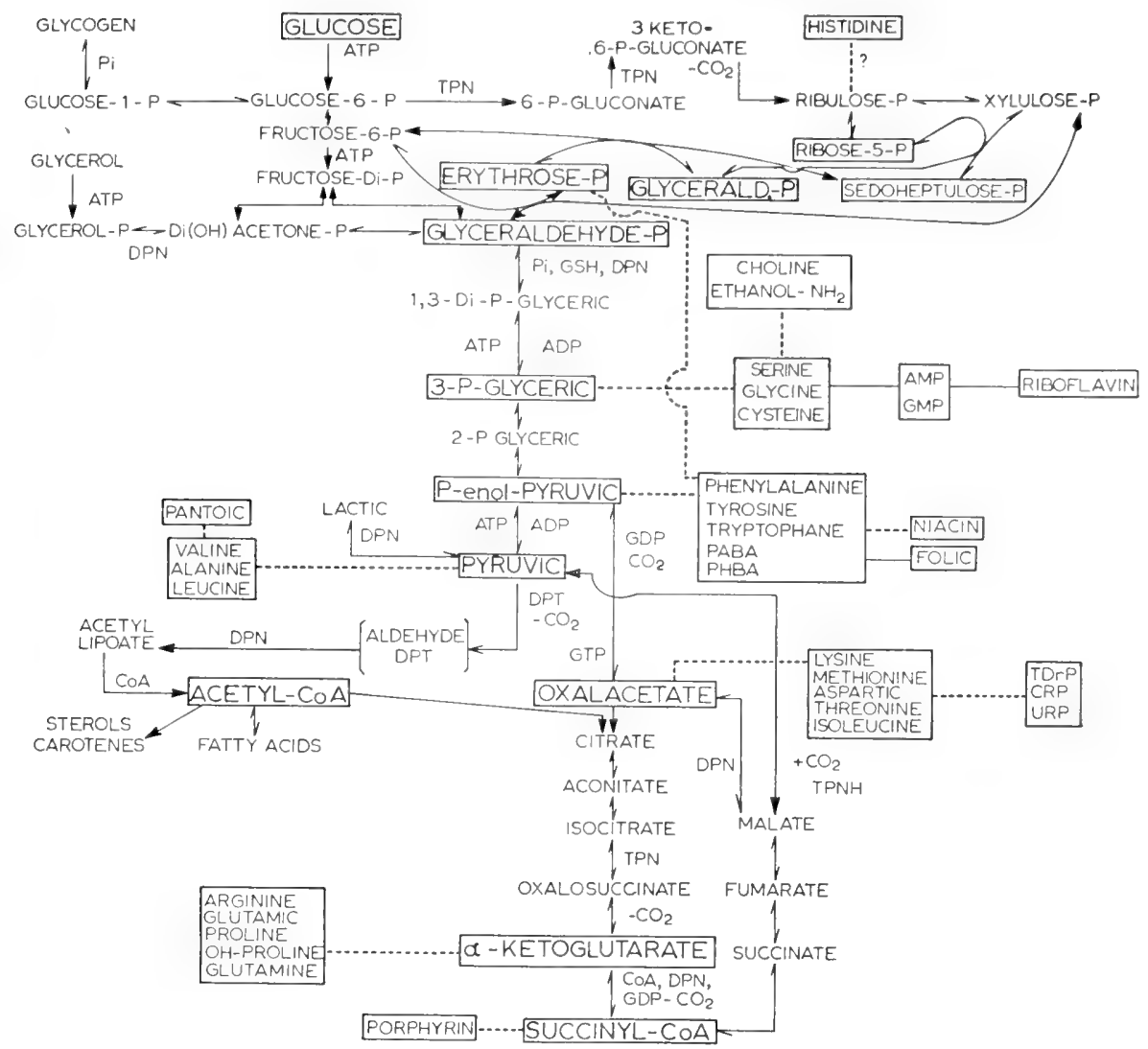

Fig. I. Relationship between glycolytic, pentose and tricarboxylic acid cycles and amino acids, vitamins and nucleotides.

boxylated to acetyl-CoA. Consequently, the level in the metabolic sequence designated by pyruvate and the other already mentioned metabolites, may be thought of as important points of bifurcation in the metabolic highways. At each of these points the compound in question may be metabolized by the cell along catabolic pathways or the metabolism may be diverted along metabolic pathways which lead to growth.

The relation between pyruvate, $\alpha$-ketoglutarate, the other key metabolic intermediates and various amino acids, vitamins, and nucleic-acid constituents, is also shown in Fig. I. This relationship will be discussed in more detail later.

\section{B. The Catabolism of Glucose and Fatty Acids}

\section{The Embden-Meyerhof glycolytic sequence}

The substances normally catabolized by a given cell vary with the inherent capabilities of the cell and the exigencies of the food supply. Since glucose and its 
polymers, starch and glycogen, represent primary sources of energy for the tissues of animals and for many lower organisms, we shall begin by outlining the pathways of glucose catabolism.

The first of these is the Embden-Meyerhof glycolytic sequence (Fig. I). In the presence of the enzymes hexokinase and hexose isomerase, glucose is phosphorylated to glucose-6-phosphate and isomerized to fructose-6-phosphate. The fructose-6-phosphate is then phosphorylated to fructose diphosphate and the latter substance is cleaved in the presence of enzyme, aldolase, to two triose phosphate molecules. The triose phosphates are oxidized to phosphoglyceric acid which is in turn converted in a number of steps to pyruvic acid. Under anaerobic conditions, many cells reduce pyruvic acid to lactic acid. Alternatively, the pyruvic acid may be decarboxylated by yeast cells to acetaldehyde which is in turn reduced to ethanol. The overall result is the conversion of one molecule of glucose to two molecules of lactate or, in the case of the yeast cells, to two molecules of ethanol and two molecules of $\mathrm{CO}_{2}$. Carbons one and six of the original glucose molecule are transformed to the beta carbons while carbons three and four give rise to the carboxyl groups of the lactate molecules. Tumor tissues and many embryonic tissues manifest an extremely high anaerobic glycolysis. Warburg (1956) has pointed out that tumors derive a far greater proportion of their energy from the glycolytic breakdown of carbohydrate than do normal tissues (see Chapter I2).

Glucose-6-phosphate may also be derived from glycogen or starch. The enzyme, phosphorylase, catalyzes the breakdown of glycogen and starch to glucose-Iphosphate. The glucose-I-phosphate may then be transformed to glucose-6phosphate, in a reaction catalyzed by the enzyme, phosphoglucomutase.

\section{The hexose monophosphate shunt}

Alternatively, glucose may be catabolized via the "direct oxidative" or "hexose monophosphate shunt" cycle (Racker, I954; Horecker and Mehler, I955). The glucose must initially be phosphorylated to glucose-6-phosphate, as in the EmbdenMeyerhof sequence. However, the glucose-6-phosphate is then oxidized to 6phosphogluconic acid rather than isomerized to fructose-6-phosphate.

Ribose-5-phosphate, acts as a catalyst in this metabolic cycle. As a result of the cycle, one molecule of glucose is catabolized to three molecules of $\mathrm{CO}_{2}$ and to one molecule of triose phosphate. The carbon dioxide molecules are derived from carbon atoms one to three of the glucose molecule while carbon four of glucose is converted to the aldehyde carbon of glyceraldehyde phosphate. The cycle is shown in Fig. I and is summarized by the equations on page 5 . Steps a) and b) are mediated by $\mathrm{TPN}^{+}$requiring dehydrogenases. The product of step $\mathrm{b}$ ) is a pentose phosphate (Srere et al., I 955; Stumpf and Horecker, I956). Steps d) and e) are catalyzed by the enzymes, transketolase and transaldolase respectively (Horecker et al., I956a, b; Horecker and Smyrniotis, I955; Racker, I954; Horecker and Mehler, I 955). Step g) is also catalyzed by the enzyme, transketolase, while steps h) and f) are mediated by isomerase enzymes. The glyceraldehyde-phosphate which arises from carbons 4-6 of the original glucose molecule may be further transformed by glycolytic enzymes to pyruvate or lactate. 
$\mathrm{TPN}^{+}$

a) Glucose-6-phosphate $\longrightarrow$ 6-phosphogluconic acid

$\mathrm{TPN}^{+}$

b) 6-Phosphogluconic $\longrightarrow$ ribulose-5-phosphate $+\mathrm{CO}_{2}$ (carbon atom I of glucose)

c) Ribulose-5-P $\longrightarrow$ xylulose- $-5-\mathrm{P}^{\text {P }}$

transketolase

d) Xylulose-5-P + ribose-5-P $\longrightarrow$ sedoheptulose-7-P + glyceraldehyde-3-P transaldolase

e) Sedoheptulose-7-P + glyceraldehyde-3-P —— fructose-6-P + erythrose-P

f) Fructose-6-P $\longrightarrow$ glucose-6-phosphate

Repeat steps a,b,c) glucose- $6-\mathrm{P} \rightarrow$ xylulose $-5-\mathrm{P}+\mathrm{CO}_{2}$ (carbon atom 2 of glucose)

transketolase

g) Xylulose-5-P + erythrose- $\mathrm{P} \longrightarrow$ fructose- $6-\mathrm{P}+$ glyceraldehyde- $\mathrm{P}$

Repeat steps $\mathrm{f}, \mathrm{a}, \mathrm{b})$ fructose-6-P $\rightarrow$ ribulose $-5-\mathrm{P}+\mathrm{CO}_{2}$ (carbon atom 3 of glucose)

h) Ribulose-5-P — ribose-5-phosphate

Sum: glucose-6-P + (ribose-5-P) $\rightarrow 3 \mathrm{CO}_{2}+$ glyceraldehyde- $\mathrm{P}+$ (ribose- 5 -phosphate)

\section{The oxidation of pymuate to acetyl-CoA}

Under aerobic conditions, pyruvate may be oxidized to acetyl-CoA. Four vitamins are involved in this transformation, thiamine, lipoic acid, niacin, and pantothenic acid (Horecker and Mehler, I 955; Hager and Gunsalus, r 953). The mechanism is shown in Fig. 2.

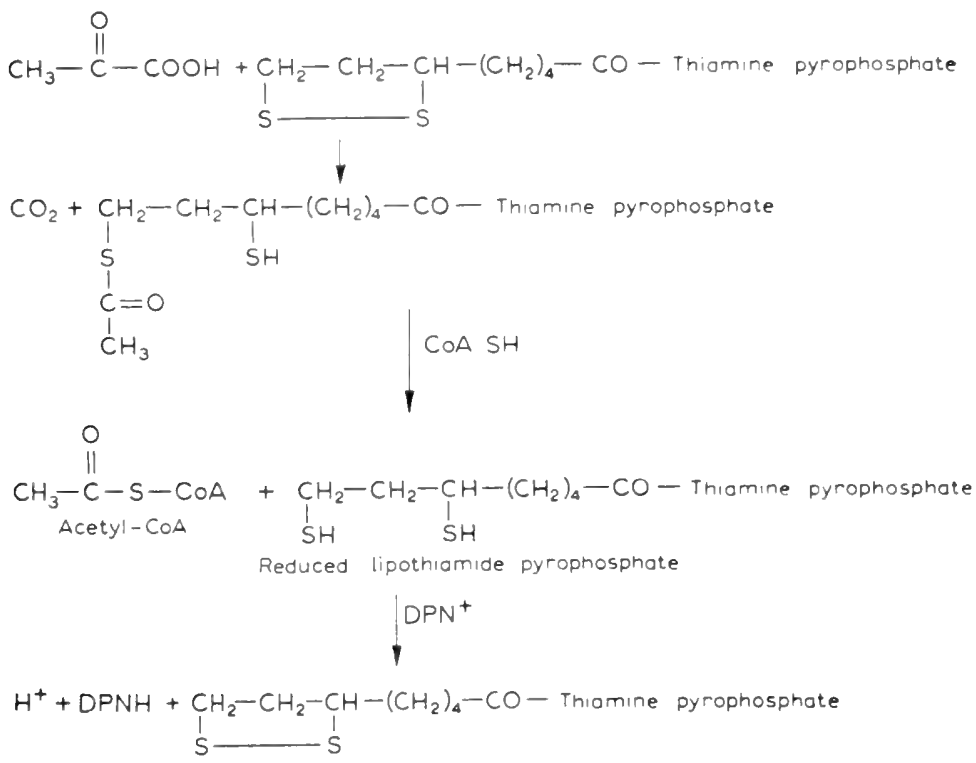

Fig. 2. Formation of acetyl-CoA from pyruvate. 
Acetyl-CoA is a key intermediate of metabolism. The acetyl unit of the molecule may either be converted to citrate (see section 5), or utilized for the synthesis of fatty acids and sterols, or for the acetylation of certain amines or alcohols, such as glucosamine or choline.

\section{The fatty acid spiral}

Neutral fats are hydrolysed to fatty acids and glycerol prior to further catabolism. The glycerol moiety of the molecule may be phosphorylated to $\alpha$-glycerol phosphate, which can then undergo oxidation to dihydroxyacetone phosphate, a glycolytic intermediate.

The fatty acids are oxidized by way of the "fatty acid spiral" (Lynen, I955). Fatty acids must be activated to acyl-CoA-thioesters prior to oxidation. ATP is required in the activation reaction which is catalyzed by thiokinase enzymes

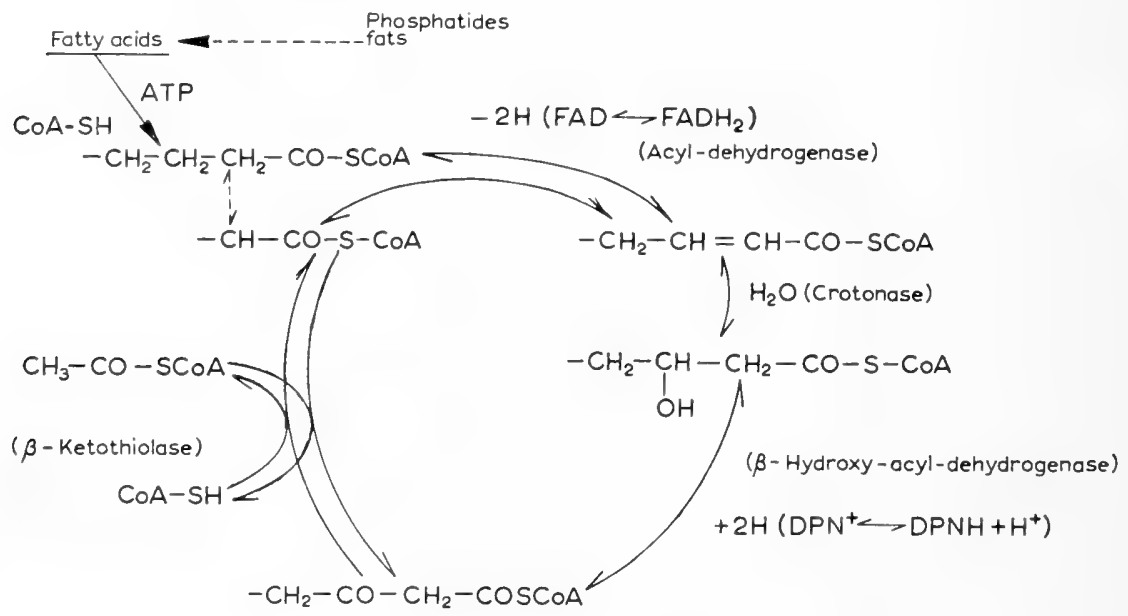

Fig. 3. Fatty acid spiral.

(Fig. 3). At least three kinds of thiokinases occur in liver (Kornberg and Pricer, I 953a; Mahler et al., I953). The first is specific for fatty acids of chain length of 2,3 , or 4 carbon atoms, the second for fatty acids of intermediate chain length $\left(\mathrm{C}_{4}-\mathrm{C}_{12}\right)$, and the third for long chain fatty acids $\left(\mathrm{C}_{14}-\mathrm{C}_{18}\right)$.

The enzyme which activates fatty acids of chain length, $\mathrm{C}_{5}-\mathrm{C}_{12}$ is found in the soluble portion of liver cytoplasm. Activity has also been demonstrated in heart and kidney. On the other hand, the enzyme concerned with the activation of higher fatty acids is found in the particulate portion of liver tissue. Mono-, di-, or trienoic acids also serve as substrates for the latter thiokinase.

The activated fatty acid is next oxidized by an acyl-dehydrogenase. Several acyl-dehydrogenases have been noted. One of these, a copper containing flavoprotein, oxidizes butyryl-CoA and fatty acid esters of CoA of chain length up to eight carbons. Another, so called " $\mathrm{Y}_{1}$ " enzyme, contains iron as well as flavin and oxidizes fatty acids of chain length $\mathrm{C}_{4}-\mathrm{C}_{16}$. A third iron containing flavoprotein, the " $\mathrm{Y}_{2}$ " enzyme, desaturates acyl esters of CoA of chain length of $\mathrm{C}_{14}$ or longer 
(Green and Beinert, I955; Crane and Beinert, I954; Mahler and Green, I954).

The unsaturated fatty acid ester is next converted to a hydroxyacyl fatty acid ester due to the action of enoyl-hydrase enzymes (Stern et al., I 956c). One of these, crotonase, which has been crystallized from ox liver, has been demonstrated in most animal tissues, bacteria and in spinach. The crystalline enzyme-hydratesCoA esters of chain length to at least $\mathrm{C}_{4}-\mathrm{C}_{9}$. It also acts upon $\beta$-methyl-crotonylCoA and hydrates cis as well as trans isomers.

The beta hydroxy fatty acid ester is next dehydrogenated to the $\beta$-keto fatty acid ester by a pyridine nucleotide-dehydrogenase. It is of interest that the $\beta$ hydroxyacyl dehydrogenase of liver or heart is specific for the L-hydroxy fatty acid esters of $\mathrm{CoA}$ whereas the enzyme which attacks free $\beta$-hydroxy-butyric acid acts upon the D-diasterioisomer.

The $\beta$-keto acid is next cleaved in the presence of coenzyme $\mathrm{A}$ by the enzyme, $\beta$-ketoacylthiolase, to acetyl-CoA and to a fatty acid ester of having two less carbon atoms than the original fatty acid. Pig heart thiolase is highly specific for acetoacetyl-CoA whereas ox liver thiolase acts upon $\beta$-keto fatty acid-CoA derivatives of chain length of from 4-12 carbon atoms. The latter enzyme preparations have been studied in crude form only so that it is likely that there exist a number of thiolases with different chain length specificity.

It is significant that the fatty acid esters derived from the action of thiolase are already in the activated coenzyme $\mathrm{A}$ form. The process of oxidation, hydration, oxidation, and thiolytic cleavage may thus be repeated until the entire fatty acid molecule has been converted by beta oxidation to acetyl-CoA units.

Although the activation of fatty acids by liver enzymes requires the presence of ATP and CoA, heart, kidney, adrenal gland and skeletal muscle enzymes can activate acetoacetic acid by a somewhat different mechanism (Stern et al., I956a). This entails the action of soluble enzymes known as thiophorases and involves the transfer of CoA from succinyl-CoA as shown in the following equation:

$$
\text { Succinyl-CoA }+\mathrm{CH}_{3}-\mathrm{CO}-\mathrm{CH}_{2}-\mathrm{COOH} \longrightarrow \text { succinate }+\mathrm{CH}_{3}-\mathrm{CO}-\mathrm{CH}_{2}-\mathrm{CO}-\mathrm{SCoA}
$$

In the presence of coenzyme $\mathrm{A}$, the acetoacetyl-CoA may next be cleaved by the thiolase enzyme to two molecules of acetyl-CoA. As a result, acetoacetate, which is produced in liver in excess under certain conditions, may be activated and oxidized in peripheral tissues at the sacrifice of the thioester linkage of succinylCoA. The enzyme found in heart muscle is capable of transferring CoA from succinyl-CoA to $\beta$-ketovalerate and $\beta$-ketocaproate, as well as to acetoacetate (Stern et al., I956b). A thiophorase enzyme also occurs in Clostridium kluyveri. However, the bacterial enzyme has a somewhat different specificity in that it catalyzes the reversible transfer of CoA from acetyl-CoA to saturated fatty acids of chain length $\mathrm{C}_{3}-\mathrm{C}_{8}$, to vinylacetic acid and lactic acid, but not to acetoacetic acid.

\section{Tricarboxylic acid cycle (Citric acid cycle: Krebs cycle)}

The acetyl-CoA, generated by the oxidation of fatty acids or pyruvate, is oxidized to $\mathrm{CO}_{2}$ and water through the citric acid cycle. The cycle begins with the condensation of acetyl-CoA with oxalacetate to form citrate. The citrate is transformed 
to isocitrate and the isocitrate is oxidized to oxalosuccinic acid. The latter is decarboxylated to $\alpha$-ketoglutaric acid. The oxidative decarboxylation of $\alpha$-ketoglutaric acid is analogous to that of pyruvate. Lipothiamide pyrophosphate, $\mathrm{DPN}^{+}$, and coenzyme A are required and succinyl coenzyme $\mathrm{A}$ is formed as a product of the overall reaction. The succinyl-CoA is further metabolized to fumarate, and malate. The malate is next dehydrogenated, thereby regenerating oxalacetate. The oxalacetate may react with a second molecule of acetyl-CoA so as to start the cycle once again. With each turn of the cycle, two molecules of $\mathrm{CO}_{2}$ are formed.

It is apparent that the rate at which acetyl-CoA can be oxidized through the tricarboxylic acid cycle will depend in part on the concentration of available oxalacetate. It is therefore of interest to inquire as to the origin of the latter substance. Two general sources may be mentioned. First, aspartic acid may readily be converted to oxalacetic acid, by transamination. Likewise; tricarboxylic acid cycle intermediates or closely related compounds such as $\alpha$-ketoglutarate or glutamate may be utilized for the synthesis of oxalacetic acid. Secondly, oxalacetic acid may be generated from the glycolytic intermediates, pyruvate and phosphoenolpyruvate. The synthesis of dicarboxylic acids from pyruvate and phosphoenolpyruvate is shown in equations I) and 2):
I) Pyruvate $+\mathrm{CO}_{2}+\mathrm{TPNH}_{2} \stackrel{\mathrm{Mn}^{++} \text {or } \mathrm{Mg}^{++}}{\longrightarrow}$ malate $\stackrel{\mathrm{DPN}^{+}}{\longrightarrow}$ oxalacetate

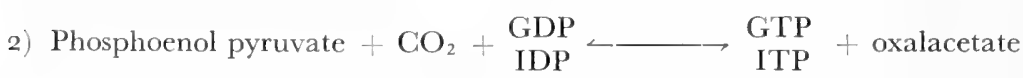

Reaction I) is catalyzed by "malic enzyme" (Ochoa et al., I950; Ochoa and Kaufman, 195I), and involves a reductive carboxylation of pyruvate to malate; the malate may then be oxidized to oxalacetate.

Reaction 2) is catalyzed by the mitochondrial enzyme, oxalacetic carboxylase. (Bandurski and Lipmann, I956; Utter and Kurahashi, I954a, I954b; Utter et al., 1954). Inosine and guanosine pyrophosphate function as acceptors of the phosphate group of phosphoenolpyruvate during this reaction.

\section{The formation of $\mathrm{CO}_{2}$}

The Embden-Meyerhof glycolytic sequence and the tricarboxylic acid cycle constitute pathways for the complete combustion of glucose to $\mathrm{CO}_{2}$ and water. Likewise, the fatty acid spiral and the tricarboxylic acid cycle provide pathways for the complete oxidation of fatty acids. However, the release of $\mathrm{CO}_{2}$ from organic compounds ordinarily takes place at only three points on these metabolic sequences. These are:

I) Pyruvate $\rightarrow \mathrm{CO}_{2}+$ acetyl-CoA

2) Oxalosuccinic $\rightarrow \mathrm{CO}_{2}+\alpha$-ketoglutarate

3) $\alpha$-Ketoglutarate $\rightarrow \mathrm{CO}_{2}+$ succinyl-CoA

Equation I) refers to the generation of acetyl-CoA from the pyruvate which is formed during glycolysis while equations 2) and 3) refer to steps of the citric acid cycle which account for the formation of $\mathrm{CO}_{2}$ from the acetyl-CoA. The latter is generated either via glycolysis or from the oxidation of fatty acids. Under 
conditions of ketogenesis in the liver, $\mathrm{CO}_{2}$ may also be formed by the decarboxylation of acetoacetate:

4) Acetoacetate $\rightarrow \mathrm{CO}_{2}+$ acetone

The oxidative decarboxylation of 6-phosphogluconate represents a fifth reaction in which $\mathrm{CO}_{2}$ is generated:

5) 6-Phosphogluconate $\rightarrow$ ribulose phosphate $+\mathrm{CO}_{2}$

These then are the principal chemical reactions which account for the formation of $\mathrm{CO}_{2}$ in animal tissues. It may be noted however that some $\mathrm{CO}_{2}$ may be formed as a result of the decarboxylation of a number of amino acids and metabolites of amino acids. Pyridoxal phosphate is a coenzyme for these amino acid decarboxylases. Some of the amino acid decarboxylation reactions occur primarily in bacteria while those which take place in mammalian tissues are of importance in the terminal steps of hormone synthesis.

\section{Oxidative steps}

As shown in Fig. I, there are two oxidative steps in the direct oxidative pathway of glucose metabolism. Likewise, the conversion of glyceraldehyde phosphate to I, 3 -diphosphoglyceric acid, $\alpha$-glycerophosphate to dihydroxyacetone phosphate, lactate to pyruvate, acetyl lipothiamide to acetyl-CoA and lipothiamide, all involve oxidation-reduction reactions. There are four oxidation steps in the tricarboxylic acid cycle and two for each turn of the fatty acid spiral.

In all but two of the examples cited above, pyridine nucleotides participate as cofactors. The two exceptions are the acyl dehydrogenase of the fatty acid spiral and the succinic dehydrogenase of the tricarboxylic acid cycle. It should first be emphasized that oxidation-reduction reactions involving pyridine nucleotides are extremely common. In Table I, there are collected over 70 pyridine nucleotide linked oxidation-reduction reactions. Although in most of these, the nicotinic acid derivative is diphosphopyridine nucleotide $\left(\mathrm{DPN}^{+}\right)$, many triphosphopyridine nucleotide $\left(\mathrm{TPN}^{+}\right)$reactions are also known. These include the oxidation of isocitric acid, glucose-6-phosphate, and 6-phosphogluconate.

The reactions of Table I are classified as suggested by Racker (I955). Pyridine nucleotides are involved in the oxidation of alcohols to aldehydes, aldehydes to acids, hydroxy acids to keto acids, hemiacetals to aldonic acid lactones, and in the oxidation of cyclic compounds, mercaptols, and other compounds. Many of these oxidation reactions are essential steps in biosynthetic sequences. For example the oxidation of dihydroorotic acid to orotic acid is one of the steps of pyrimidine synthesis, while the reduction of aspartic semialdehyde to homoserine is a step in the synthesis of the amino acids, methionine and threonine. The pyridine nucleotide dehydrogenases are so numerous and the substrates so varied that pyridine nucleotide enzymes have rightfully been called the "work horses" of biological oxidations. Pyridine nucleotide dehydrogenases also function in amino acid oxidation, sterol synthesis, and in the maintenance of the redox-potential of the cell.

The oxidation process involves the transfer of two electrons and a hydrogen atom to the nicotinamide portion of the coenzyme while the second hydrogen atom is released as an ion in solution (Fig. 4, p. I4). 
TABLE 1

PYRIDINE NUCLEOTIDE DEHYDROGENASES

\begin{tabular}{|c|c|c|}
\hline Reaction & Source & Cofactor \\
\hline \multicolumn{3}{|l|}{ I. Enzymes oxidizing alcohols } \\
\hline I. ethanol $\rightarrow$ acetaldehyde & yeast, liver & DPN \\
\hline 2. D-sorbitol $\rightarrow$ fructose & $\begin{array}{l}\text { liver, seminal } \\
\text { vesicles }\end{array}$ & DPN \\
\hline $\begin{array}{l}\text { 3. D-mannitol- I-phosphate } \rightarrow \text { fructose- } 6 \text { - } \\
\text { phosphate } \\
\text { 4. } \mathrm{N}^{10} \text {-hydroxymethyl-THFA } \rightarrow \mathrm{N}^{10} \text {-formyl- }\end{array}$ & E. coli & DPN \\
\hline THFA & liver & TPN \\
\hline 5. vitamin $\mathrm{A} \rightarrow$ retinene & retina & DPN \\
\hline $\begin{array}{l}\text { 6. } \alpha \text {-glycerophosphate } \rightarrow \text { dihydroxy- } \\
\text { acetone phosphate }\end{array}$ & muscle & DPN \\
\hline $\begin{array}{l}\text { 7. } 3 \alpha, \text { I } 7 \alpha, 20 \beta, 2 \text { I-tetrahydroxypregnane-I I- } \\
\text { one } \rightarrow 3 \alpha, \text { I } 7 \alpha, 2 \text { I-trihydroxypregnane-I I, } \\
\text { 2o-dione ( } \beta \text {-cortolone) }\end{array}$ & kidney, liver & TPN \\
\hline 8. UDP-glucose $\rightarrow$ UDP-glucuronic & liver & DPN \\
\hline 9. L-histidinol $\rightarrow$ L-histidinal & $\begin{array}{l}\text { E. coli, yeast, } \\
\text { Arthobacter }\end{array}$ & DPN \\
\hline ı. UDP-glucose $\rightarrow$ UDP-galactose & liver & DPN \\
\hline I I. xylitol $\rightarrow$ L-xylulose & liver & TPN \\
\hline I2. xylitol $\rightarrow$ D-xylulose & liver & DPN \\
\hline I3. ribitol $\rightarrow$ D-ribulose & liver & DPN \\
\hline I4. sorbitol $\rightarrow$ D-glucose & seminal vesicles & TPN \\
\hline I5. sorbitol $\rightarrow$ sorbose & Aerobacter & $\mathrm{TPN}$ \\
\hline I6. iditol $\rightarrow$ L-sorbose & liver & DPN \\
\hline I 7. threitol $\rightarrow$ L-erythrulose & liver & DPN \\
\hline \multicolumn{3}{|l|}{ II. Enzymes oxidizing cyclic compounds } \\
\hline I8. myoinositol $\rightarrow$ 2-ketoinositol & Aerobacter & DPN \\
\hline 19. shikimic $\rightarrow$ 5-dehydroshikimic & Aerobacter & TPN \\
\hline 20. quinic $\rightarrow$ 5-dehydroquinic & Aerobacter & DPN \\
\hline 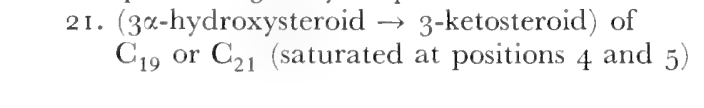 & $\begin{array}{l}\text { liver, kidney, } \\
\text { testes, } \\
\text { Pseudomonas }\end{array}$ & DPN (TPN) \\
\hline$*_{2}$ ra. $3 \beta$-hydroxysteroid $\rightarrow 3$-ketosteroid & Pseudomonas & DPN \\
\hline I $7 \beta$-hydroxysteroid $\rightarrow$ I 7 -ketosteroid & $\begin{array}{l}\text { Pseudomonas, } \\
\text { yeast, liver }\end{array}$ & DPN \\
\hline 22. inosinic $\rightarrow$ xanthosinic & Aerobacter & DPN \\
\hline $\begin{array}{l}\text { * (skeletons of androstane, etiocholane, } \\
\text { cholane, pregnane). }\end{array}$ & & \\
\hline \multicolumn{3}{|l|}{ III. Enzymes oxidizing hydroxyacids } \\
\hline 23. L-lactic $\rightarrow$ pyruvic & ubiquitous & $\mathrm{DPN}$ \\
\hline $24 . \mathrm{L}$-malic $\rightarrow$ oxalacetic & ubiquitous & $\mathrm{DPN}$ \\
\hline 25. L-malic $\rightarrow$ pyruvic $+\mathrm{CO}_{2}$ & ubiquitous & TPN \\
\hline 26. glycolic $\rightarrow$ glyoxylic & spinach leaves & DPN \\
\hline $\begin{array}{l}\text { 27. } \text {-hydroxyisobutyric } \rightarrow \alpha \text {-methylmalonic } \\
\text { semialdehyde }\end{array}$ & ubiquitous & DPN \\
\hline 28. L-gulonate $\rightarrow$ D-glucurono- $\gamma$-lactone & liver & TPN \\
\hline 29. $\alpha$-acetolactate $\rightarrow \alpha$-ketoisovalerate & yeast & TPN \\
\hline
\end{tabular}


Table I, continued

Reaction

30. $d$-glyceric $\rightarrow$ hydroxypyruvic

3I. D(-) $\beta$-hydroxybutyric $\rightarrow$ acetoacetic

32. D-isocitric $\rightarrow$ oxalosuccinic

33. phosphogluconic $\rightarrow$ ribulose-5-phosphate

$$
+\mathrm{CO}_{2}
$$

34. $\mathrm{L}(+)$ - $\beta$-hydroxybutyryl-CoA $\rightarrow$ acetoacetyl-CoA

$35 \cdot \beta-\mathrm{OH}-\beta$-methylbutyryl-CoA $\rightarrow$ $\beta$-methylacetoacetyl-CoA

36. homoserine $\rightarrow$ aspartic- $\beta$-semialdehyde

\section{$I V$. Hemiacetal dehydrogenases}

37. D- $\beta$-glucose $\rightarrow$ gluconolactone

38. D-glucose-6-phosphate $\rightarrow$ 6-phosphogluconic

\section{$V$. Other aldehyde oxidases}

39. acetaldehyde $\rightarrow$ acetic

40. acetaldehyde $\rightarrow$ acetyl-CoA

4I. betaine aldehyde $\rightarrow$ betaine

42. formaldehyde $\rightarrow$ formic

43. formic $\rightarrow \mathrm{CO}_{2}+\mathrm{H}_{2} \mathrm{O}$

44. L-histidinal $\rightarrow$ L-histidine

45. glyceraldehyde phosphate $\rightarrow$ I,3-diphosphoglyceric

46. glyceraldehyde phosphate $\rightarrow$

I, 3-diphosphoglyceric

47. pyruvic $\rightarrow$ acetyl-CoA

48. $\alpha$-ketoglutaric $\rightarrow$ succinyl-CoA

49. $\beta$-aspartyl semialdehyde $\rightarrow \beta$-aspartyl

phosphate

50. 5-hydroxyindole acetaldehyde $\rightarrow$ 5-hydroxyindole acetic

\section{Dehydrogenases leading to unsaturation}

$5 \mathrm{I}$. glutamic $\rightarrow \alpha$-ketoglutaric $+\mathrm{NH}_{3}$

52. proline $\rightarrow$ pyrroline-5-carboxylic

53. dihydroorotic $\rightarrow$ orotic

$54 \cdot 5,6,7,8$-tetrahydrofolic $\rightarrow$ 7, 8-dihydrofolic

55. butyryl-CoA $\rightarrow$ crotonyl-CoA

56 . dihydrouracil $\rightarrow$ uracil

57. dihydrothymine $\rightarrow$ thymine

58. L-gulonate $\rightarrow$ L-ascorbate

$\begin{array}{ll}\text { Source } & \text { Cofactor } \\ \text { plant leaves } & \text { DPN } \\ \text { liver } & \text { DPN } \\ \text { ubiquitous } & \text { TPN } \\ \text { ubiquitous } & \text { TPN } \\ \text { liver } & \text { DPN } \\ \text { liver, heart } & \text { DPN } \\ \text { yeast } & \text { DPN }\end{array}$

liver

ubiquitous

DPN (TPN)

TPN

liver, (yeast)

E. coli,

C. kluyveri

DPN (TPN)

liver

DPN $(\mathrm{CoA})$

liver, yeast

pea seeds

yeast, $E$. coli,

(DPN) (GoA)

DPN (GSH)

DPN

Arthobacter

plant leaves

TPN

ubiquitous

DPN $(\mathrm{GSH})$

ubiquitous

DPN (CoA), lipothiamide

ubiquitous

DPN (CoA)

yeast

TPN

soluble fraction

of liver, kidney

DPN

liver, etc.

liver

DPN (TPN)

(Neurospora)

microorganisms

DPN (TPN)

DPN

liver

TPN

mitochondria TPN

liver TPN

liver TPN

liver TPN 
Table I, continued

\section{Reaction}

Source

Cofactor

59. saturation of double bond of $\Delta^{4}-3$-ketosteroids (cortisone, cortisol, DOC, compound $\mathrm{B}$, compound $\mathrm{S}$, aldosterone, progesterone, I I-ketoprogesterone, I 7-hydroxyprogesterone, testosterone, adrenosterone, cholestenone) liver

TPNH

VII. 2 RSH $\rightarrow$ RSSR

60. 2 GSH $\rightarrow$ GSSG

6 I. 2 cysteine $\rightarrow$ cystine

62. lipoic $($ reduced) $\rightarrow$ lipoic (oxidized)

$\begin{array}{ll}\text { ubiquitous } & \text { TPN } \\ \text { yeast } & \text { DPN } \\ \text { ubiquitous } & \text { DPN }\end{array}$

VIII. Transhydrogenase

63. $\mathrm{TPNH}+\mathrm{DPN} \rightarrow \mathrm{TPN}+\mathrm{DPNH}$

Pseudomonas, liver, muscle, kidney, heart

IX. Hydroxylating enzymes

64. L-kynurenine $\rightarrow$ 3-hydroxykynurenine

liver mito-

chondria

65. 2 I-hydroxylations

adrenal

I $7 \alpha$-hydroxyprogesterone $\rightarrow$

I 7, 2 I-dihydroxyprogesterone (compd. S)

progesterone $\rightarrow$ DOC

II $\beta$-hydroxyprogesterone $\rightarrow$ corticosterone

I I $\beta$, I $7 \alpha$-dihydroxyprogesterone $\rightarrow$ compd. F

66. phenylalanine $\rightarrow$ tyrosine (hydroxylation)

67. I I $\beta$-hydroxylation (deoxycortisol $\rightarrow$ cortisol, DOC $\rightarrow$ corticosterone)

68 . squalene $\rightarrow$ lanosterol

69. p-hydroxyphenylpyruvic $\rightarrow$ homogentisic

70. imidazole acetic $\rightarrow$ formiminoaspartic

71 . nonspecific aromatic hydroxylase (produce phenols)

microsomes +

supernatant

TPNH, $\mathrm{O}_{2}$

TPNH, $\mathrm{O}_{2}$

soluble fraction

of liver

TPNH, $\mathrm{O}_{2}$,

adrenal

$\mathrm{Fe}^{++}$

mitochondria

TPNH, $\mathrm{O}_{2}$

soluble fraction

of liver

liver

TPNH, $\mathrm{O}_{2}$

Pseudomonas

TPNH?

DPNH, $\mathrm{O}_{2}$

72. dealkylating enzymes

(3-methyl-4-monomethylaminoazobenzene $\rightarrow$

3-methyl-4-aminoazobenzene),

(methylamphetamine, meperidine, methyl-

aniline, quinacrine, diacetylmorphine,

methadone, codeïne)

73. deaminating enzymes

(amphetamine $\rightarrow p$-hydroxyamphetamine)

74. estradiol $\rightarrow 6$ \%-hydroxyestradiol

liver microsomes TPNH, $\mathrm{O}_{2}$

liver microsomes TPNH

liver

TPNH, $\mathrm{O}_{2}$, $\mathrm{Fe}^{++}$

liver microsomes TPNH, $\mathrm{O}_{2}$ 
TABLE 2

FLAVIN ENZYMES

\begin{tabular}{|c|c|c|}
\hline Reaction & Source & Cofactor \\
\hline I. glycolic $+\mathrm{O}_{2} \rightarrow$ glyoxylic $+\mathrm{H}_{2} \mathrm{O}_{2}$ & plants & FAD \\
\hline 2. nitrate $+\mathrm{TPNH}(\mathrm{DPNH}) \rightarrow$ nitrite $+\mathrm{TPN}^{+}\left(\mathrm{DPN}^{+}\right)$ & $\begin{array}{l}\text { Neurospora, } \\
\text { Aspergillus, } \\
\text { soy bean leaves }\end{array}$ & FAD-Mo \\
\hline 3. nitrite + DPNH $(\mathrm{TPNH}) \rightarrow$ hydroxylamine $+\mathrm{DPN}^{+}$ & $\begin{array}{l}\text { Neurospora, } \\
\text { soy bean leaves }\end{array}$ & FAD (Mn?) \\
\hline 4. hydroxylamine $+\mathrm{DPNH}(\mathrm{TPNH}) \rightarrow \mathrm{NH}_{3}+\mathrm{DPN}^{+}$ & $\begin{array}{l}\text { Neurospora, } \\
\text { soy bean leaves }\end{array}$ & FAD (Mn?) \\
\hline $\begin{array}{l}\text { 5. DPNH }+\mathrm{H}^{+}+2 \text { cyt. C-Fe } \mathrm{De}^{+++} \rightarrow \mathrm{DPN}^{+}+ \\
2 \text { cyt. c-Fe }{ }^{++}+2 \mathrm{H}^{+}\end{array}$ & $\begin{array}{l}\text { heart, muscle } \\
\text { mitochondria }\end{array}$ & FAD-Fe \\
\hline $\begin{array}{l}\text { 6. } \mathrm{TPNH}(\mathrm{DPNH})+\mathrm{H}^{+}+2 \text { cyt. } \mathrm{c}-\mathrm{Fe}^{+++} \rightarrow \mathrm{TPN}^{+} \\
\left(\mathrm{DPN}^{+}\right)+2 \mathrm{H}^{+}+2 \text { cyt. c-Fe } \mathrm{Fe}^{++} \\
\text {7. } \mathrm{TPNH}+\mathrm{H}^{+}+2 \text { cyt. c-Fe } \mathrm{Fe}^{++} \rightarrow \mathrm{TPN}^{+}+\end{array}$ & microsomes & FAD \\
\hline $\begin{array}{l}\text { 2 cyt. c-Fe }{ }^{++}+2 \mathrm{H}^{+} \\
\text {8. benzoquinone }+\mathrm{TPNH}_{2}\left(\mathrm{DPNH}_{2}\right) \rightarrow \text { catechol }\end{array}$ & yeast & FMN \\
\hline$+\mathrm{TPN}^{+}$ & $\begin{array}{l}\text { bacteria, } \\
\text { animal tissues }\end{array}$ & FAD \\
\hline $\begin{array}{l}\text { 9. quinone }+\mathrm{DPNH}_{2} \rightarrow \text { hydroquinone }+\mathrm{DPN}^{+} \\
\text {10. menadione quinone }+(\mathrm{TPNH}) \mathrm{DPNH} \rightarrow\end{array}$ & pea seeds & FAD \\
\hline menadione $+\mathrm{DPN}^{+}$ & $\begin{array}{l}\text { animal and } \\
\text { plant tissues, } \\
\text { bacteria }\end{array}$ & FAD \\
\hline $\begin{array}{l}\text { I I. succinic }+2 \text { cyt. b-Fe } \mathrm{Fe}^{+++}(?) \rightarrow \text { fumaric }+ \\
\quad 2 \text { cyt. b-Fe }\end{array}$ & mitochondria & FAD \\
\hline $\begin{array}{l}\text { 12. }(\mathrm{G}) \text { acyl-CoA }\left(\mathrm{C}_{4}-\mathrm{C}_{8}\right)+\mathrm{ETF} \rightarrow \text { unsaturated } \\
\text { acyl-CoA }+ \text { ETFH } \\
\text { I2. }\left(\mathrm{Y}_{1}\right) \text { acyl-CoA }\left(\mathrm{C}_{4}-\mathrm{C}_{16}\right)+\mathrm{ETF} \rightarrow \text { unsaturated }\end{array}$ & mitochondria & FAD-Cu \\
\hline $\begin{array}{l}\text { 13. }\left(\mathrm{Y}_{1}\right) \text { acyl-CoA }\left(\mathrm{C}_{4}-\mathrm{C}_{16}\right)+\mathrm{ETF} \rightarrow \text { unsaturated } \\
\text { acyl-CoA }+ \text { ETFH } \\
\text { 14. }\left(\mathrm{Y}_{2}\right) \text { acyl-CoA }\left(\mathrm{C}_{14--}\right)+\text { ETF } \rightarrow \text { unsaturated }\end{array}$ & mitochondria & FAD \\
\hline acyl-CoA $+\mathrm{ETFH}_{2}$ & mitochondria & FAD \\
\hline $\begin{array}{l}\text { 15. } \mathrm{Y}_{2} \mathrm{FADH}_{2}+2 \mathrm{cyt} . \mathrm{c}-\mathrm{Fe}^{+++} \stackrel{\mathrm{G}}{\longrightarrow} \mathrm{Y}_{2} \mathrm{FAD}+2 \text { cyt. } \\
\text { 16. choline }+2 \text { cyt. c-Fe } \mathrm{Fe}^{+++} \rightarrow \text { betaine aldehyde }+\end{array}$ & $\begin{array}{l}\mathrm{c}-\mathrm{Fe}^{++} \text {mito- } \\
\text { chondria }\end{array}$ & FAD \\
\hline 2 cyt. $\mathrm{c}-\mathrm{Fe}^{++}$ & mitochondria & FAD \\
\hline $\begin{array}{l}\text { I7. aldehyde }+\mathrm{O}_{2} \rightarrow \text { acid }+\mathrm{H}_{2} \mathrm{O}_{2} \\
\text { 18. xanthine }+\mathrm{O}_{2} \rightarrow \text { uric }+\mathrm{H}_{2} \mathrm{O}_{2} \text { (aldehydes, }\end{array}$ & mitochondria & FAD-Mo \\
\hline purines, pterins, DPNH) & $\begin{array}{l}\text { milk, bacteria, } \\
\text { liver }\end{array}$ & FAD-Mo \\
\hline I 9. D-amino acid $+\mathrm{O}_{2} \rightarrow \mathrm{NH}_{3}+$ keto acid $+\mathrm{H}_{2} \mathrm{O}_{2}$ & $\begin{array}{l}\text { liver and kidney, } \\
\text { mitochondria }\end{array}$ & FAD \\
\hline 20. L-amino acid $+\mathrm{O}_{2} \rightarrow \mathrm{NH}_{3}+$ keto acid $+\mathrm{H}_{2} \mathrm{O}_{2}$ & $\begin{array}{l}\text { kidney, } \\
\text { snake venom }\end{array}$ & FMN \\
\hline 21. glycine $+\mathrm{O}_{2} \rightarrow \mathrm{NH}_{3}+$ glyoxylic $+\mathrm{H}_{2} \mathrm{O}_{2}$ & $\begin{array}{l}\text { kidney, liver, } \\
\text { microorganisms }\end{array}$ & FAD \\
\hline 22. monoamine $+\mathrm{O}_{2} \rightarrow \mathrm{NH}_{3}+$ aldehyde $+\mathrm{H}_{2} \mathrm{O}_{2}$ & various tissues & FAD \\
\hline $\begin{array}{l}\text { 23. diamine }+\mathrm{O}_{2} \rightarrow \mathrm{NH}_{3}+\text { aldehyde }+\mathrm{H}_{2} \mathrm{O}_{2} \\
\text { 24. } \mathrm{H}_{2}+\mathrm{E}-\mathrm{FAD} \rightarrow \text { E-FADH }\end{array}$ & $\begin{array}{l}\text { various tissues } \\
\text { Clostridrium, } \\
\text { Micrococcus }\end{array}$ & $\begin{array}{l}\text { FAD } \\
\text { FAD-Mo }\end{array}$ \\
\hline
\end{tabular}




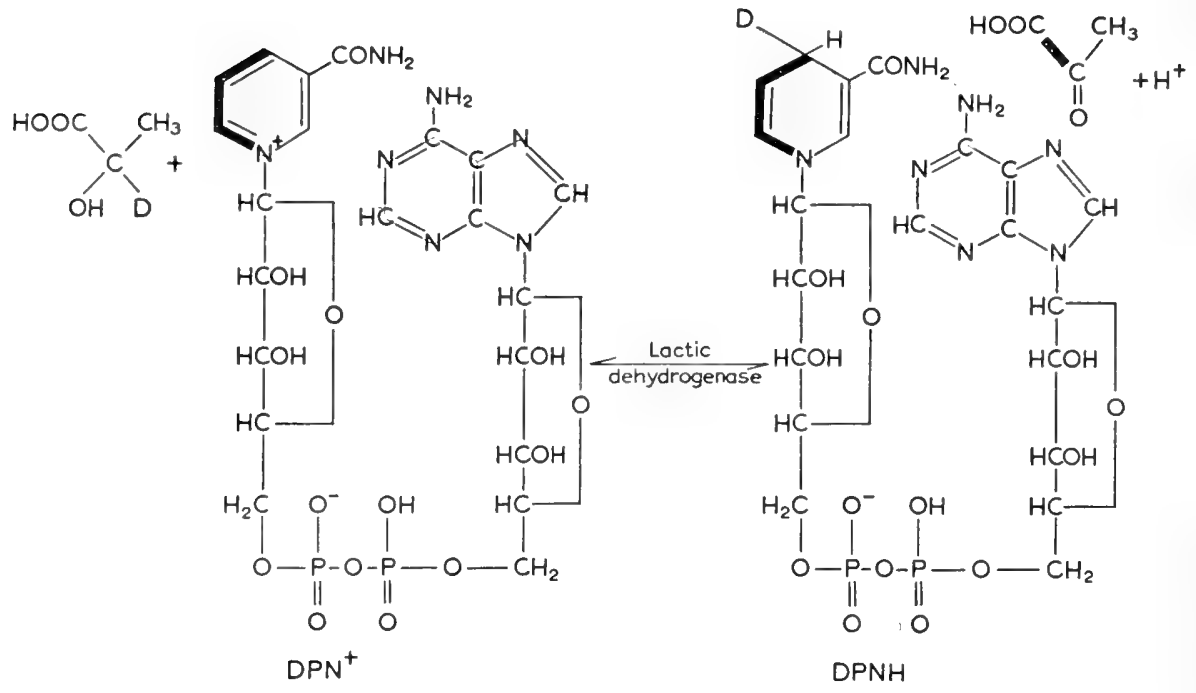

Fig. 4. Mechanism of oxidation of $\mathrm{DPN}^{+}$by lactic acid.

Let us now consider the acyl dehydrogenases and succinic dehydrogenase enzymes which have been purified from mitochondria. It has been ascertained that at least three acyl dehydrogenases exist. These are the $G, Y_{1}$, and $Y_{2}$ enzymes (Crane et al., i 956). The G enzyme is a cuproflavoprotein. The $Y_{1}$ and $Y_{2}$ flavoproteins contain small amounts of iron (Table 2).

The mechanism of dehydrogenation is shown in Fig. 5. It is to be noted that both of the hydrogen atoms are transferred from the substrate to the FAD portion of the dehydrogenase. Presumably, the metal component of the enzyme mediates

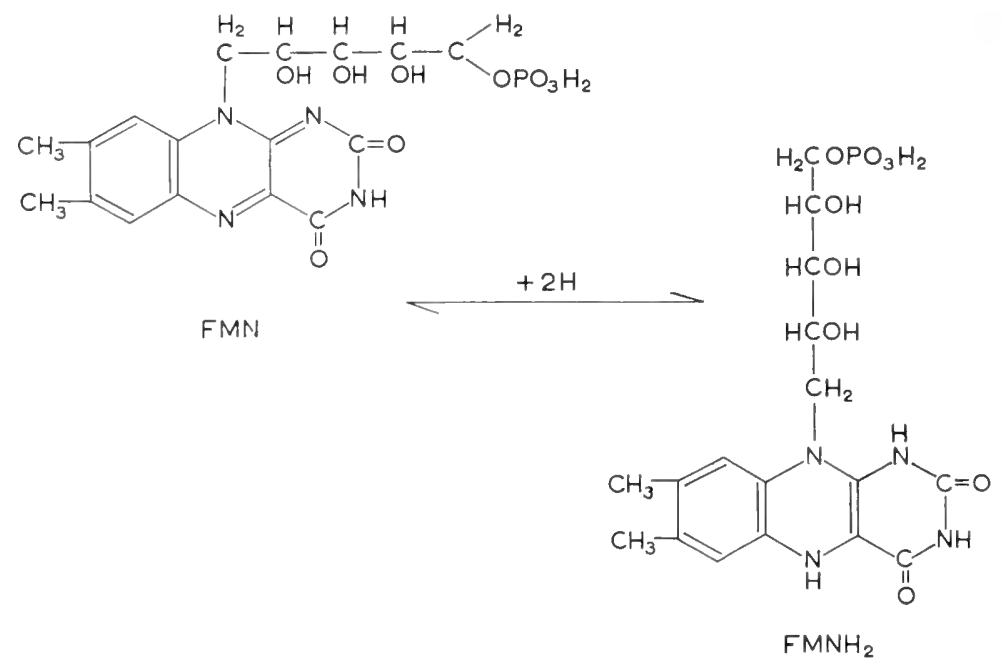

Fig. 5. Oxidized and reduced forms of flavin mononucleotide. 
a stepwise one electron transfer reaction in what would otherwise be a two electron transfer reaction.

A particulate form of succinic dehydrogenase obtained from beef heart mitochondria has been purified IO-I5 fold over that of the starting mitochondrial suspension. Flavin, heme, and non-heme iron, are present in the succinic dehydrogenase complex in a ratio of $1: 4: 16$ respectively. Io to $30 \%$ of the flavin is in the form of flavin adenine dinucleotide. (Green et al., i955). A soluble form of succinic dehydrogenase has been obtained (Singer et al., 1956) by extracting rat liver or beef heart mitochondria with an alkaline buffer (Kearney and Singer, I956). Although the enzyme cannot interact with cytochrome c or ferricyanide, the activity can be followed by means of the reduction of phenazine methosulfate. The purified enzyme contained iron and flavin.

As in the case of pyridine nucleotide dehydrogenases, many other FAD dehydrogenases are known. Some of these are listed in Table 2. Of particular significance in cellular metabolism is the enzyme, DPNH oxidase. It has been shown that DPNH oxidase is a ferroflavoprotein. Several examples of molybdenum containing flavoprotein enzymes are also given in Table 2 (nitrate reductase, aldehyde oxidase, xanthine oxidase).

\section{Terminal Hydrogen and Electron Transport System}

As indicated above, many cell metabolites are oxidized by pyridine nucleotide or flavin dehydrogenases. As a result of these oxidations, $\mathrm{DPNH}_{2}, \mathrm{TPNH}_{2}$ and $\mathrm{FADH}_{2}$ are generated. If the oxidation of the substrate is to continue, the reduced coenzymes must be reoxidized. A number of devices have been developed by cells to accomplish this.

\section{I. "Dismutation" reactions and pyridine nucleotide dehydrogenases}

Whenever the oxidation or reduction of two substrates is catalyzed by dehydrogenases requiring the same coenzyme, the oxidation of the first substrate may be coupled with the reduction of the second substrate. Muscle cells have recourse to this mechanism under anaerobic conditions as do fermenting yeast cells (Fig. 6).

The $\mathrm{DPNH}_{2}$ generated in reaction I, Fig. 6, is presumably released from the glyceraldehyde-phosphate dehydrogenase and is then bound to the alcohol or lactic dehydrogenase. Following reduction of pyruvate or acetaldehyde (Fig. 6, reactions 2 and 3 ), the oxidized $\mathrm{DPN}^{+}$is then released from the latter dehydrogenases and may recombine with glyceraldehyde-phosphate dehydrogenase to continue the cycle. The "dismutation" mechanism for the reoxidation of TPNH also may take place under certain circumstances:

glucose-6-P

dehydrogenase

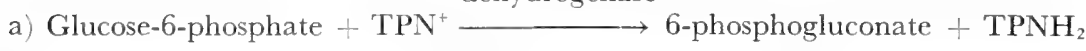

malic

enzyme

b) $\mathrm{CO}_{2}+$ pyruvate $+\mathrm{TPNH}_{2} \longrightarrow$ malate $+\mathrm{TPN}^{+}$

Literature p. 124 


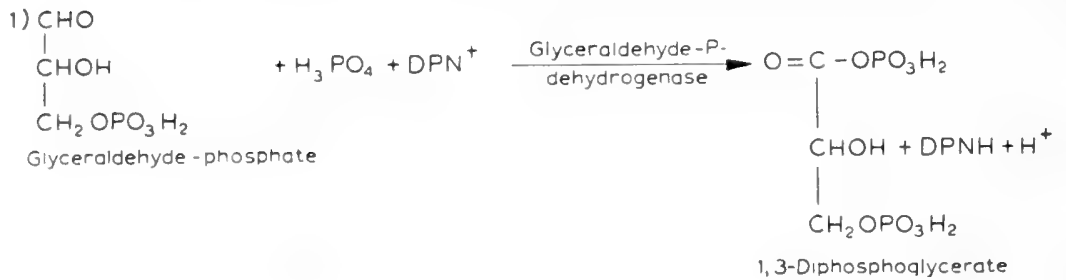

2)

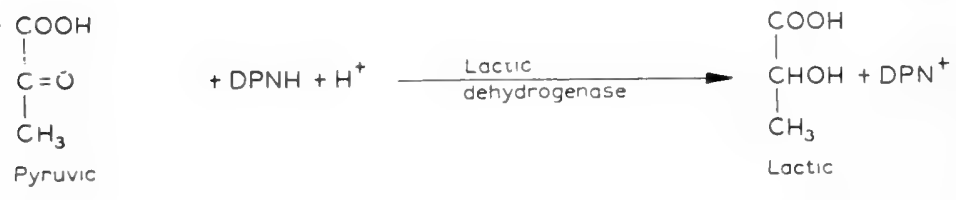

3)
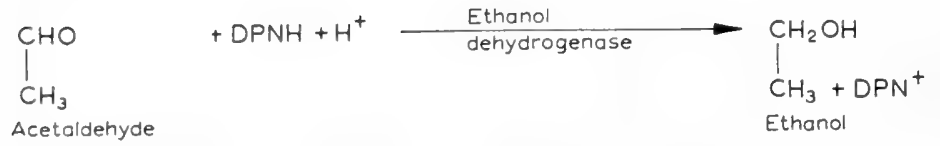

Fig. 6. Dismutation reactions involving $\mathrm{DPN}^{+}$.

\section{Reoxidation of reduced pyridine nucleotides via the cytochrome system}

It will be noted that in the "dismutation" system described above, pyruvate, acetaldehyde, or "pyruvate $+\mathrm{CO}_{2}$ " function as the hydrogen and electron acceptors from $\mathrm{DPNH}_{2}$. In most cells, however, the ultimate hydrogen and electron acceptor is oxygen, and the final product formed is water. The hydrogen and the electrons are not transferred directly from the substrate and $\mathrm{DPNH}_{2}$ to oxygen. Instead, the transfer is mediated by a series of auxiliary carriers, which consist of flavoproteins and several iron-porphyrin enzymes, the cytochromes. The iron moieties of the cytochromes are reduced from the ferric to the ferrous state by electrons derived from the substrates and are reoxidized from the ferrous to the ferric state by oxygen. The cytochrome pigments have highly characteristic absorption spectra which may be used for the purposes of identification (Chance, I953-1954). The wave lengths at which maximum absorption occurs are shown in Table 3 , which also shows the standard electrode potentials of the components of the electron transport chain and of certain other metabolites which are of interest in connection with electron transport.

Two points are worthy of note in connection with the electrode potentials of Table 3 . First, the standard oxidation-reduction potential of riboflavin is approximately $-0.185 \mathrm{~V}$ while that of xanthine oxidase flavoprotein is -0.350 , that of the liver mitochondrial flavoproteins, approximately -0.060 , and that of the DPNH-cyt. $\mathrm{c}$ reductase is about 0.00 . Thus, the oxidation-reduction potential of a substance is greatly modified by combination with proteins. Similar considerations apply with respect to the oxidation-reduction potentials of the iron-porphyrin pigments.

Secondly, the actual oxidizing power of a given couplet depends on the ratio of the concentrations of the components of the couplet within the cell. Thus, the standard electrode potentials of Table 3 do not reflect the actual oxidizing or reducing power of the substances within the cell. The ratio of the oxidized to reduced forms of the components of the electron 
TABLE 3

REDOX POTENTIALS OF BIOLOGICAL INTEREST AND

ABSORPTION MAXIMA OF COMPONENTS OF ELECTRON TRANSPORT SYSTEM

Component

$\begin{array}{lll}E_{0}^{\prime}{ }^{1}(V) \text { Oxidized } 2 / \text { reduced } & E_{h} & \text { Absorption maxima }\end{array}$

\begin{tabular}{|c|c|c|c|c|c|c|}
\hline $\begin{array}{l}\mathrm{H}_{2} \mathrm{O} / 1 / 2 \mathrm{O}_{2} \\
\mathrm{NH}_{2} \mathrm{OH} / \mathrm{NH}_{3} \\
\text { nitrate/nitrite }\end{array}$ & $\begin{array}{l}+0.815 \\
+0.800 \\
+0.540\end{array}$ & $p \mathrm{O}_{2}$ (o.I atmosphere) & +0.800 & & & \\
\hline $\begin{array}{l}\text { cytochrome } \mathrm{a}_{3} \\
\text { hydroquinone/quinone } \\
\text { cytochrome a }\end{array}$ & $\begin{array}{l}+0.300 \\
+0.290\end{array}$ & $\begin{array}{l}\text { very large } \\
\text { I9 }\end{array}$ & +0.370 & $\begin{array}{l}6000 \\
6050\end{array}$ & - & 4500 \\
\hline $\begin{array}{l}\text { cytochrome c } \\
\text { FAD (Y-acyl-CoA- } \\
\text { dehydrogenase) } \\
\text { ascorbic/dehydro- } \\
\text { ascorbic }\end{array}$ & $\begin{array}{l}+0.260 \\
+0.250 \\
+0.080\end{array}$ & I6 & +0.330 & $\begin{array}{l}5500 \\
4650\end{array}$ & 5210 & 4160 \\
\hline $\begin{array}{l}\text { cytochrome e }\left(c_{1}\right) \\
\text { cytochrome m } \\
\quad \text { (solubilized) } \\
\text { succinate/fumarate }\end{array}$ & $\begin{array}{c}+0.020 \\
0.0\end{array}$ & & & $553^{\circ}$ & 5230 & $4 \mathrm{I} 8 \mathrm{o}$ \\
\hline $\begin{array}{l}\text { FAD (cyt. c-reductase) } \\
\text { menadione/menadione } \\
\text { quinone } \\
\text { cytochrome b }\end{array}$ & $\begin{array}{c}0.0 \\
-0.005 \\
-0.040\end{array}$ & 5.2 & 0 & $5^{6} 4^{\circ}$ & 5300 & 4300 \\
\hline $\begin{array}{l}\text { flavoprotein } \\
\text { (mitochondria) } \\
\text { glycolate/glyoxylate } \\
\text { cytochrome m }\left(b_{5}\right) \\
\text { suspensions }\end{array}$ & $\begin{array}{l}-0.060 \\
-0.087 \\
-0.120\end{array}$ & 4.0 & -0.030 & $\begin{array}{l}4650 \\
5560\end{array}$ & 5270 & $424^{\circ}$ \\
\hline $\begin{array}{l}\text { glutathione/GSSG } \\
\text { lactate/pyruvate } \\
\text { ribollavin phosphate }\end{array}$ & $\begin{array}{r}-0.130 \\
-0.180 \\
-0.219\end{array}$ & & & & & \\
\hline $\begin{array}{l}\mathrm{DPN}^{+} \\
\mathrm{TPN}^{+} \\
\text {(FAD xanthine } \\
\quad \text { oxidase) } \\
\alpha \text {-ketoglutarate } / \mathrm{CO}_{2} \\
\quad \text { succinate }+\mathrm{CO}\end{array}$ & $\begin{array}{l}-0.320 \\
-0.324 \\
-0.350 \\
-0.600\end{array}$ & I.I3 & -0.318 & $\begin{array}{l}3400 \\
3400\end{array}$ & & \\
\hline
\end{tabular}

${ }^{1} E_{\circ}^{\prime}=\mathrm{V}$ at $25^{\circ}, \mathrm{pH} 7$ when concentrations of oxidized and reduced forms are equal.

${ }^{2}$ Liver mitochondria under conditions of high ADP, substrate, and oxygen concentrations (Chance and Williams, 1956). 
transport chain of liver mitochondria are shown in the third column of Table 3 (Chance and Williams, 1956). It will be noted that although $\mathrm{DPN}^{+}$is half oxidized and half reduced when substrate and ADP are present at high concentration, cytochrome $\mathrm{c}$ is almost entirely oxidized. Thus, as Table 3, column 4 shows, the potentials which actually obtain in the mitochondria in many cases differ considerably from the standard potentials.

The total content of $\mathrm{DPN}^{+}$and $\mathrm{DPNH}_{2}$ of various tissues and tumors and the ratio of oxidized to reduced $\mathrm{DPN}^{+}$have been measured by Jedeikin and Weinhouse (Jedeikin and Weinhouse, I955). Ratios of $\mathrm{DPN}^{+} / \mathrm{DPNH}$ were found to be greater than I, ranging from I.2 in rat liver to over 20 in skeletal muscle. Tumors displayed intermediate values of this ratio of 2.5 to 4.5 . Likewise, the total DPN ${ }^{+}$ content varied from tissue to tissue. It was high in liver, kidney, and muscle and lower in spleen and brain. The $\mathrm{DPN}^{+}$content of a variety of transplanted tumors were in the range of spleen and brain.

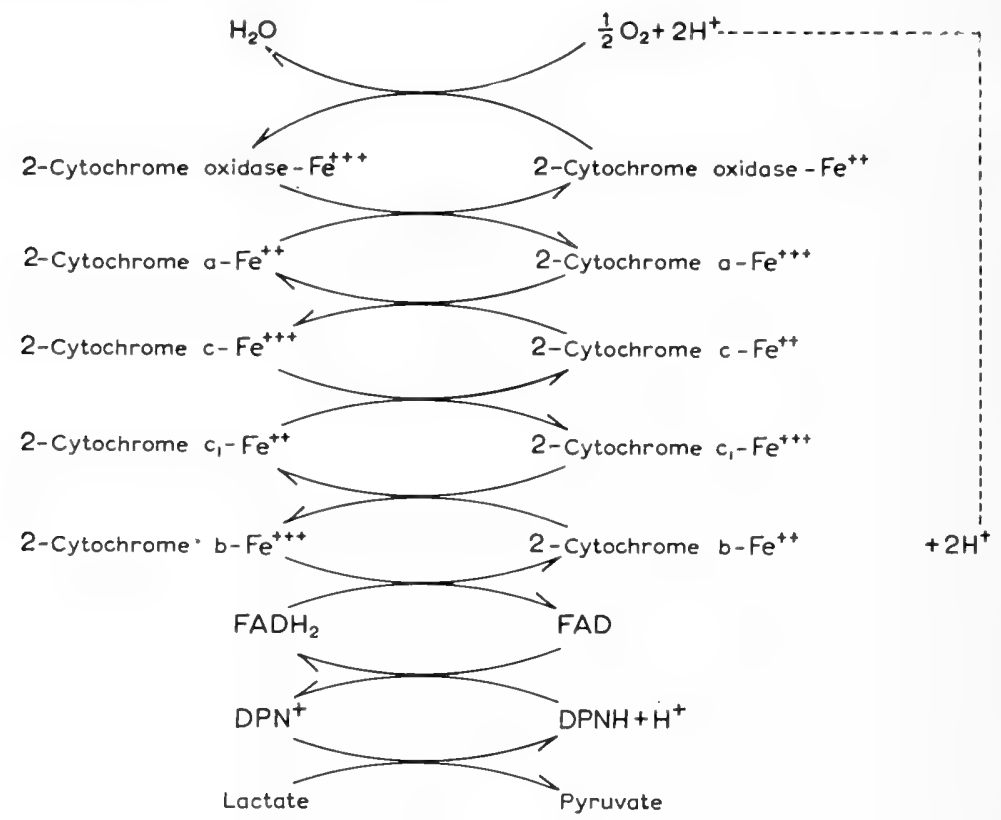

Fig. 7. Electron transport chain.

The participation of pyridine nucleotides, flavoproteins, and cytochrome pigments in the respiratory activities of intact yeast cells and ascites tumor cells has been elegantly demonstrated by Chance and collaborators (Chance, I953-I954; Chance and Williams, I956). Likewise, the above mentioned components have been implicated in the oxidative metabolism of liver mitochondria. Fig. 7 illustrates present concepts of electron transport.

It is quite probable that the electron transport system contains other components in addition to the flavoproteins and cytochrome pigments. In purified preparations of rat skeletal muscle, $d$ - $\alpha$-tocopherol functions as an activator in the enzymatic reduction of cytochrome c by DPNH or succinate (Nason and Lehman, 1955). Isooctane extraction of the particulate enzyme fraction with the concommitant removal of only $10 \%$ of the 
enzyme lipid results in a $75-95 \%$ decrease in cytochrome c reductase activity. Enzymatic activity can be completely and specifically restored upon the addition of the lipid cofactor which is removed by the isooctane extraction or by the addition of tocopherol (Nason and Lehman, 1956). Whether tocopherol (or the unknown lipid cofactor) serves in some indirect fashion or undergoes alternate oxidation and reduction during the course of electron transport-possibly to the free radical or to the tocopheroxide - has, however, not as yet been established. The tocopherol activation of DPNH-cytochrome c reductase has also been observed in yeast, Neurospora, soy bean leaves, rat liver, and beef heart preparations. ${ }^{1}$

Vitamin $\mathrm{K}$ has also been implicated in the electron transport scheme. It has been reported that the oxidation of phosphogluconate, glucose-6-phosphate, and ribose phosphate by yeast autolysates is promoted by the vitamin $\mathrm{K}$ analog, menadione, (Strength and Seibert, I955a). Enzymes capable of reducing menadione are widely distributed in nature (Wosilait and Nason, 1954a). Pyridine nucleotides function in the reduction of menadione in $E$. coli while in $S$. faecalis, ro $\mathrm{Cl}$, either FAD or FMN is required for menadione reductase activity. Menadione at a concentration of $10^{-5}-10^{-4} M$ (Dolin, I954) also causes an appreciable stimulation of the nitrate reductase system of E. coli (Wainwright, 1955).

Chance has shown by spectroscopic measurements that the cytochrome b content of several tumors is very low (Chance and Castor, 1952). The addition of cytochrome b and menadione to preparations of a lymphosarcoma markedly increases the rate of cytochrome c reduction by DPNH while cytochrome b or menadione alone are ineffective (Strength and Siebert, 1955a, 1955b). The vitamin $\mathrm{K}$ antagonist, dicumarol, inhibits cytochrome $\mathrm{c}$ reduction by the tumor preparations and the inhibition is reversed by the addition of menadione. The site of the stimulation of cytochrome c reduction by menadione and the inhibition by dicumarol has been localized between cytochrome b and c. The reduction of cytochrome $c$ is also inhibited by BAL (dimercaptopropanol), 2-alkyl-3-hydroxy napthoquinone, (Ball et al., 1947) and antimycin A. Since the reduction of cytochrome $\mathrm{c}$ is enhanced by vitamin $\mathrm{K}$, or menadione, it does not appear unlikely that the various inhibitors already mentioned exert their effect on the same component of the electron transport system. It may be noted that the standard potential of the menadione couple lies between that of cytochrome $b$ and cytochrome c.

Evidence for an additional cytochrome component between cytochrome b and c has also been presented (Wainio and Cooperstein, 1956). This pigment has been referred to as cytochrome $c_{1}$ and as cytochrome e. It is characterized by an absorption maximum at $553^{\circ} \AA$. Cytochrome $c_{1}(e)$ is reduced during the oxidation of DPNH or succinate by purified skeletal or heart muscle preparations.

\section{Electron transporting particle}

It was observed during the I930's that soluble enzyme preparations from muscle which catalyzed pyridine nucleotide linked dismutation reactions were unable to react with cytochrome $\mathrm{c}$, with $\mathrm{O}_{2}$, or with dyes such as methylene blue. The addition of a particulate cell fraction restored the ability of the so called "mutases" to reduce methylene blue or to consume oxygen. The active component of the particulate fraction was termed the "coenzyme factor" by Green. In I939, Straub isolated a soluble flavoprotein from heart muscle which could interact with dyes (diaphorase activity) and showed that the prosthetic group was a flavin-adenine-dinucleotide, while Corran et al., (Corran, Green and Straub, I939), tested the catalytic properties of the enzyme and concluded that it was identical with the "coenzyme factor".

The problem of the reoxidation of $\mathrm{DPNH}_{2}$ has recently been reinvestigated by Green and associates. A particulate enzyme system was extracted from beef heart mitochondria which catalyzed the oxidation of $\mathrm{DPNH}_{2}$ by moleculąr oxygen but which catalyzed at a

\footnotetext{
${ }^{1}$ See Addendum to this Chapter, Note I, p. 123.
} 
much lower rate only the reduction of cytochrome c by $\mathrm{DPNH}_{2}$ or the oxidation of reduced cytochrome c by molecular oxygen (reaction I) (Green et al., 1954; Mackler et al., 1954; Green and Beinert, 1955).

I) $\mathrm{DPNH}_{2}+1 / 2 \mathrm{O}_{2} \rightarrow \mathrm{DPN}^{+}+\mathrm{H}_{2} \mathrm{O}$

Reaction I) is completely blocked by low levels of antimycin A. When this purified particulate system was treated with desoxycholate, the capacity to catalyze the overall oxidation of $\mathrm{DPNH}_{2}$ by molecular oxygen was greatly reduced whereas the capacity to catalyze the oxidation of $\mathrm{DPNH}_{2}$ by cytochrome c, (cytochrome reductase activity) or the oxidation of reduced cytochrome c by molecular oxygen (cytochrome oxidase activity) increased 25 fold (reactions 2 and 3 ):

2) $\mathrm{DPNH}_{2}+2 \mathrm{cyt} . \mathrm{c}-\mathrm{Fe}^{+++} \rightarrow \mathrm{DPN}^{+}+2$ cyt. c-Fe $\mathrm{Fe}^{++}+2 \mathrm{H}^{+}$

3) 2 cyt. $\mathrm{c}-\mathrm{Fe}^{++}+2 \mathrm{H}^{+}+1 / 2 \mathrm{O}_{2} \rightarrow 2 \mathrm{cyt}$. c-Fe $\mathrm{Fe}^{+++}+\mathrm{H}_{2} \mathrm{O}$

The desoxycholate treated enzyme, though devoid of succinic dehydrogenase or the oxidizing enzymes of the citric acid cycle showed absorption peaks when in the reduced state at $5250 \AA, 555^{\circ} \AA$, and $6070 \AA$. A broad bifurcated Soret band at $4300 \AA$ and $4450 \AA$ was also observed.

These observations have been interpreted as follows (Green and Beinert, 1955). $\mathrm{DPNH}_{2}$ oxidase exists as an enzymatic unit in which electrons are transferred from the primary prosthetic group to the final electron acceptor (molecular oxygen) by way of a series of intermediary electron carriers such as heme groups and metals. This unit has the character of an impenetrable "closed" unit in the sense that electrons can only enter by way of $\mathrm{DPNH}_{2}$ and leave by way of oxygen. There is thus little possibility of interaction with electron acceptors like cytochrome c or dyes. When the unit is exposed to desoxycholate, the conducting circuit is interrupted in the sense that a barrier is raised to the flow of electrons at some point, possibly by the loss of some component. Such an "opened" $\mathrm{DPNH}_{2}$ oxidase unit can now interact with acceptors like cytochrome $\mathrm{c}$, which then undergoes a cycle of reduction by $\mathrm{DPNH}_{2}$ in the reducing moiety of the unit and of oxidation by molecular oxygen in the oxidizing moiety. In other words, the opened $\mathrm{DPNH}_{2}$ oxidase behaves as if it were a complex of two enzymes, $\mathrm{DPNH}_{2}-\mathrm{cytochrome}^{\mathrm{c}}$ reductase and cytochrome oxidase which are no longer in electronic communication with each other. According to this interpretation, both $\mathrm{DPNH}_{2}$ cytochrome c reductase and reduced cytochrome $\mathrm{c}$ oxidase are, in this case at least, artifactual. The opening of the DPNH oxidase unit has created an artificial situation in which exogenous cytochrome $c$ is closing the opened circuit again. It should be pointed out that the highly purified particulate $\mathrm{DPNH}_{2}$ oxidase contains no detectable cytochrome $\mathrm{c}$ and that cytochrome $\mathrm{c}$ is not liberated in consequence of the treatment with desoxycholate.

By treating the particulate $\mathrm{DPNH}_{2}$ oxidase with trypsin in the presence of cholate, and subsequent ammonium sulfate fractionation, two heme types having different catalytic activities can be obtained. One contains a green heme group and copper and manifests cytochrome oxidase activity; the other contains a red heme group and non-heme iron and manifests $\mathrm{DPNH}_{2}$ dehydrogenating activity with dyes as electron acceptors. Cytochrome reductase activity is lost during the treatment necessary for separation.

A $\mathrm{DPNH}_{2}$ oxidizing enzyme system which does reduce cytochrome $\mathrm{c}$ has been isolated in highly purified form from heart muscle (Mahler and Elowe, I953, 1954). This enzyme system contains flavin and non-heme iron, in a ratio of $4: 1$ but no heme. Both the iron and the flavin can be reduced by the substrate and oxidized by the acceptor. Prolonged dialysis against 8-hydroxy-quinoline results in a marked lowering of the non-heme iron of the ferroflavoprotein enzyme and concomitantly, the capacity of the enzyme to interact with cytochrome $\mathrm{c}$ is lost. On the other hand, the enzyme with reduced iron content is still capable of reacting with dyes such as 2,6-dichlorophenolindophenol (diaphorase activity). Preparations of Straub's diaphorase are also very low in iron content. Full enzyme activity can be restored by the addition of ferric iron at a concentration of $5 \cdot 10^{-4} \mathrm{M}$ : 


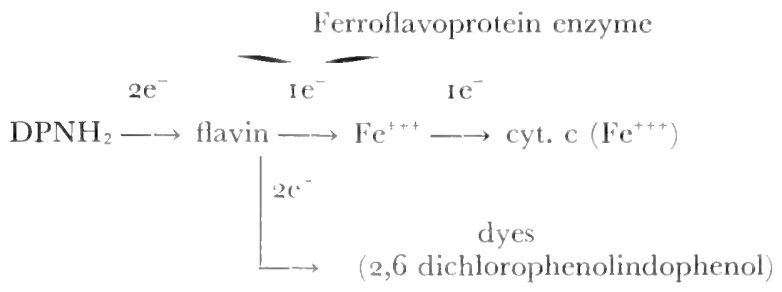

To summarize, the $\mathrm{DPNH}_{2}$ dehydrogenase exists in at least three different forms: a) as the "closed" complex, $\mathrm{DPNH}_{2}$ oxidase, which reacts with molecular oxygen, b) as the form which contains a heme group and which is obtained by tryptic digestion of the former enzyme, and c) as a soluble ferroflavoprotein. The last named form of the enzyme can be further degraded to a simple flavoprotein without iron which is similar to Straub's diaphorase.

\section{The oxidation of succinate, fatty acids, choline, and xanthine}

Electron transport from substrate to the cytochrome pigments need not necessarily be mediated by pyridine nucleotides. Thus, some of the flavin enzymes shown in Table 2 transfer electrons, directly from substrate to the cytochrome system. Succinate is one of the substrates oxidized in this way. An abbreviated electron transport system illustrating the oxidation of succinic acid looks as follows:

$$
\text { Succinate } \rightarrow \text { ferroflavin } \rightarrow \text { cyt. b } \rightarrow \text { cyt. } c_{1} \rightarrow \text { cyt. c } \rightarrow \text { cyt. a } \rightarrow \text { cyt. a } \rightarrow \mathrm{O}_{2}
$$

The three known acyl-dehydrogenases are also flavoproteins. The transfer of electrons to the cytochrome pigments in this case is mediated by a fourth flavoprotein, the electron transferring flavoprotein (ETF) (Crane and Beinert, I954; Crane, 1956; Crane and Beinert, 1956). The ETF flavoprotein, in contrast to the $\mathrm{G}, \mathrm{Y}_{1}$ and $\mathrm{Y}_{2}$ acyl dehydrogenases, interacts with cytochrome $\mathrm{c}$ :

$$
\mid \begin{aligned}
& \mathrm{Y}_{1} \\
& \mathrm{Y}_{2} \\
& \mathrm{G}
\end{aligned} \rightarrow \text { ETF } \rightarrow \text { cytochromes } \rightarrow \mathrm{O}_{2}
$$

Flavoproteins are also involved in the oxidation of choline to betaine aldehyde and of xanthine and various aldehydes (but not betaine aldehyde). The oxidation of choline by rat liver mitochondria may be linked with the reduction of cytochrome c (Rothschild et al., 1954). The flavoprotein, aldehyde oxidase (Hurwitz and Cooperstein, I955) of liver is capable of reducing either cytochrome b or cytochrome c. However, in the system studied, the evidence indicated that the flavoprotein interacted with cytochrome oxidase and oxygen without the necessary participation of cytochrome c. A xanthine oxidase occurs in milk, which is exceedingly unspecific as to substrate. It oxidizes aldehydes, $\mathrm{DPNH}_{2}$, pterins, and hypoxanthine as well as xanthine (Mahler and Green, 1954). It is worth noting that milk xanthine oxidase, liver aldehyde oxidase, and nitrate reductase require molybdenum for their activity.

\section{Autooxidizable flavopioteins}

Several flavoprotein enzymes have been isolated which lack a metal component, which do not interact with the cytochromes, but which instead mediate the transfer of hydrogen between substrate and molecular oxygen. The amino acid oxidases, 
the amine oxidases, glucose oxidase, and glycolic oxidase are examples of this class of enzymes (Table 2). The oxidation of leucine by amino acid oxidase is shown in equations $\mathrm{I}$ and 2.

\section{FAD enzyme}

I) Leucine $+\mathrm{O}_{2} \longrightarrow \alpha$-ketoisocaproic acid $+\mathrm{NH}_{3}+\mathrm{H}_{2} \mathrm{O}_{2}$

2) $\mathrm{H}_{2} \mathrm{O}_{2} \stackrel{\text { catalase }}{\longrightarrow} 1 / 2 \mathrm{O}_{2}+\mathrm{H}_{2} \mathrm{O}$

It will be noted that hydrogen peroxide is one of the products of the reaction. The enzymes catalase or peroxidase, catalyze the subsequent breakdown of the hydrogen peroxide to water and oxygen.

\section{Glycolate as a hydrogen carrier}

Plant tissues contain two types of dehydrogenases which utilize glycolic acid as substrate. One of these, glycolic acid reductase requires $\mathrm{DPN}^{+}$or $\mathrm{TPN}^{+}$as a prosthetic group while the other, glycolic acid oxidase, functions in the presence of FAD. The glycolic oxidase can be oxidized by $\mathrm{O}_{2}$. Both enzymes have been highly purified from spinach leaves (Zelitch and Ochoa, I953; Zelitch, I953). It has been suggested that glycolate may function in conjunction with the above two enzymes in a novel hydrogen carrier system from reduced pyridine nucleotides to molecular oxygen. The system may be depicted as follows:

I) Substrate- $\mathrm{H}_{2}+\mathrm{DPN}^{+} \stackrel{\text { dehydrogenase }}{\longrightarrow} \mathrm{DPNH}+\mathrm{H}^{+}+$substrate

glycolic reductase

2) $\mathrm{DPNH}+\mathrm{H}^{+}+$glyoxylate $\longrightarrow$ glycolate $+\mathrm{DPN}^{+}$ glycolic oxidase-FAD

3) Glycolate $+\mathrm{O}_{2} \longrightarrow$ glyoxylate $+\mathrm{H}_{2} \mathrm{O}_{2}$

4) $\mathrm{H}_{2} \mathrm{O}_{2} \stackrel{\text { catalase }}{\longrightarrow} \mathrm{H}_{2} \mathrm{O}+1 / 2 \mathrm{O}_{2}$

Sum: substrate $-\mathrm{H}_{2}+1 / 2 \mathrm{O}_{2} \longrightarrow$ substrate $+\mathrm{H}_{2} \mathrm{O}$

\section{Nitrate as a hydrogen acceptor}

In the above sections, we have considered hydrogen and electron transport systems in which $\mathrm{O}_{2}$ is the terminal acceptor. Many bacteria utilize $\mathrm{O}_{2}$ as the terminal hydrogen acceptor under aerobic conditions and alternatively substitute $\mathrm{HNO}_{3}$ as the acceptor under anaerobic conditions, or under conditions when the oxygen available to cells is in short supply. Anaerobic conditions and an adequate nitrate supply favor the adaptive development of nitrate reductase enzymes. In some instances, as in Pseudomonas and Micrococcus

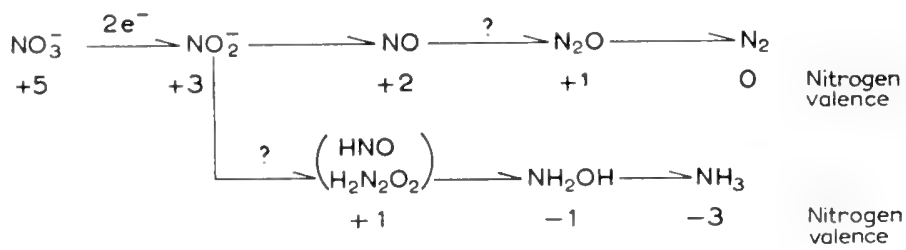

Fig. 8. Nitrate reduction. 


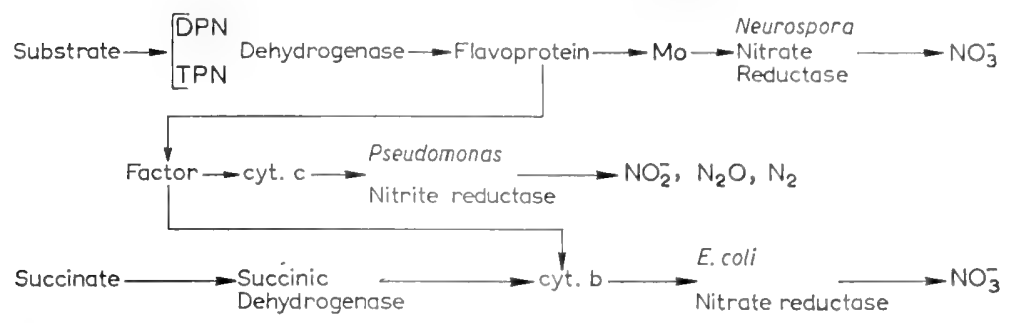

Fig. 9. Nitrate and nitrite as hydrogen acceptors.

denitrificans, the reduction of nitrate may proceed in a stepwise manner to the production of $\mathrm{N}_{2}$ and $\mathrm{N}_{2} \mathrm{O}$; in other instances, as in the case of $B$. licheniforms, B. subtilus or $E$. coli, much of the nitrate may be reduced to $\mathrm{NH}_{3}$ (Delwiche, 1956). These alternatives are depicted in Fig. 8.

Pyridine nucleotides, flavoproteins, and cytochrome pigments participate in hydrogen transport to nitrate as well as in hydrogen transport to oxygen. The hydrogen transport system of Neurospora and $E_{\text {. coli }}$ which terminates in nitrate reduction is shown in Fig. 9.

Fig. 9 also depicts a similar system for the reduction of nitrite in Pseudomonas (Verhoeven, 1956 ).

Enzyme preparations capable of reducing nitrate have been purified from E. coli, Pseudomonas, fungi, and soy bean leaves. Reduction of nitrate by $E$. coli occurs in the presence of $\mathrm{TPNH}_{2}$ or $\mathrm{DPNH}_{2}$ (Wainwright, I955). FMN or FAD is required for maximal activity. Menadione at a concentration of ${ }^{10^{-5}} M$ causes appreciable activation while $\mathrm{Fe}^{++}$ $(M / 80)$ still further activates when preincubated with the enzyme prior to addition of substrates (Nason, 1956). Dicumarol, which inhibits menadione reductase, is a potent inhibitor of $E$. coli nitrate reductase.

Molybdenum probably act as an electron acceptor since the enzymatic oxidation of $\mathrm{FMNH}_{2}$ by molydate takes place under anaerobic conditions in the absence of nitrate. Likewise, chemically reduced molybdate enzymatically reduces nitrate to nitrite.

The TPNH-nitrate reductase of Neurospora is a molybdenum flavoprotein (Nason and Evans, 1953). A metal and a flavin also mediate the reduction of nitrite to hydroxylamine and of hydroxylamine to ammonia by pyridine nucleotide linked reductases of Neurospora: (Nason et al., I954; Zucker and Nason, 1955).

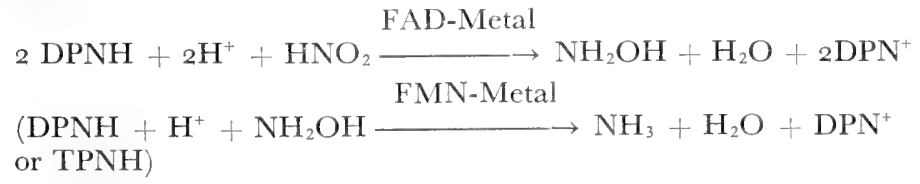

The studies of Sato and collaborators have implicated the cytochrome pigments in the reduction of nitrate by $E_{\text {. coli }}$ and other bacteria (Taniguchi et al., 1956; Sato, 1956). The $E$. coli enzyme is found in the particulate matter of the cell and may be sedimented by centrifuging cell free extracts at $22,000 \mathrm{~g}$ for $30 \mathrm{~min}$. The partially purified enzyme catalyzes the anaerobic reduction of nitrate or nitrite by reduced methylene blue, formate, or DPNH. Succinate, lactate, $\alpha$-glycerophosphate, or formate are also effective hydrogen donors. Spectroscopic observations indicate that the addition of nitrate to cell free extracts (in the absence of oxygen) causes the characteristic bands of reduced cytochrome b to fade. This anaerobic oxidation of cytochrome is prevented by cyanide at concentrations which inhibit the reduction of nitrate by the same extracts. Likewise, 2-heptyl-4-hydroxyquinoline-N-oxide (HOQNO), a substance which inhibits electron transport between 
cytochrome $\mathrm{b}$ and $\mathrm{c}$ in the heart muscle succinoxidase system, markedly reduces the reduction of nitrate by hydrogen donors. Instead of cytochrome b, as in E. coli, cytochrome c is oxidized during the anaerobic reduction of nitrate in $M$. denitrificans. Cytochrome $\mathrm{a}_{3}$ may also be involved in the electron transport system in this organism.

Other microorganisms, such as Neurospora, nitrate adapted Bacillus subtilus, and Azotobacter vinelandi do not show changes in the absorption spectra of the cytochrome bands during the course of nitrate reduction. These latter organisms are however all strongly aerobic. A third group of microorganisms which utilize nitrate in the electron transport system may be distinguished. These are the obligate anaerobes, such as Clostridium welchii, which lack cytochrome pigments.

Cytochrome pigments have also been implicated in bacterial nitrite reduction. A nitrite reductase has been purified (Verhoeven and Takeda, 1956) from Pseudomonas aeruginosa.

In the presence of nitrite and the purified enzyme, bacterial cytochrome c was oxidized. In the obligate anaerobic sulfate bacteria, sulfate may be utilized in place of $\mathrm{O}_{2}$ as an electron and hydrogen acceptor with the production of $\mathrm{H}_{2} \mathrm{~S}$. Reduced cytochrome $\mathrm{c}$ is oxidized by sulfate in the sulfate reducing bacteria.

\section{Quinone reductase and plant respiration}

Various benzoquinone and napthoquinone derivatives are widely distributed in nature. Oxidases for the phenolic forms of these compounds are also known (polyphenol oxidase, tyrosinase, laccase). Copper rather than iron undergoes valency changes in the course of the oxidations catalyzed by the phenol oxidases. It has been suggested that these enzymes function as terminal respiratory catalysts in plants (Mason, 1955).

In the presence of quinone reductases of animal or plant tissues, pyridine nucleotides can reduce quinones (Kertesz and Albano, I955; Wosilait et al., I954; Wosilait and Nason, I954a, I 954b), (Fig. Io).

Hydroquinone has been used as an intermediary carrier in coupling experiments with laccase while I,2-naphthoquinone has been similarly used with polyphenol oxidase (Wosilait et al., I954). DPNH is rapidly oxidized by the enzymatically produced oxidation products of hydroquinone or catechol.

1)

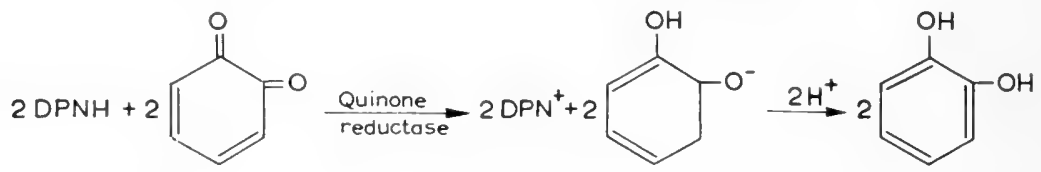

2)
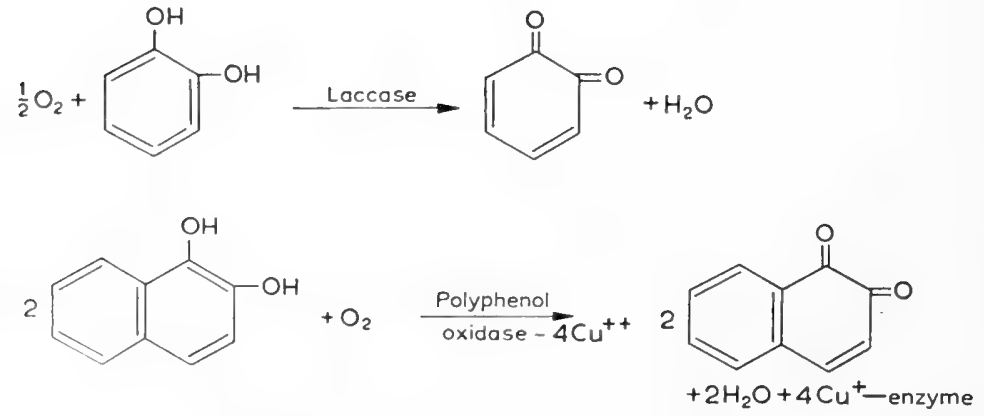

Fig. I0. Quinone mediated oxidation of $\mathrm{DPN}^{+}$. 
Plant polyphenol oxidase has been employed as a terminal oxidase in experimental studies with animal neoplasms (Kertesz and Albano, 1955). Thus, the respiration of Ehrlich tumor cell homogenates in the presence of glucose is increased by the addition of a terminal electron transport system consisting of DOPA in catalytic amounts $(50 \gamma)$ and plant polyphenol oxidase. Concommitantly, the aerobic glycolysis of the tumor cells is markedly inhibited.

\section{Glutathione and ascorbic acid as respiratory catalysts}

Glutathione and ascorbic acid may also function as components of electron and hydrogen transport systems in plants. Pyridine nucleotide linked-glutathione reductase enzymes have been observed in various plant and animal tissues (Racker, I 955b; Conn and Vennesland, I95 I; Rall and Lehninger, 1952).

Many plant enzymes also catalyze the reduction of dehydroascorbic acid by reduced glutathione. A variety of possible pathways exist for the aerobic oxidation of ascorbic acid. For example, plants contain ascorbic acid oxidases in which copper functions as the prosthetic group (Reactions I-3).

I) $\mathrm{TPNH}+\mathrm{H}^{+}+\mathrm{GSSG}$ glutathione reductase

$$
\stackrel{\text { glutathione reductase }}{\longrightarrow} 2 \mathrm{GSH}+\mathrm{TPN}^{+}
$$

dehydroascorbic reductase

2) $2 \mathrm{GSH}+$ dehydroascorbic $\longrightarrow$ GSSG + ascorbic

3) Ascorbic $+1 / 2 \mathrm{O}_{2} \stackrel{\mathrm{Cu}^{++} \text {-oxidase }}{\longrightarrow}$ dehydroascorbic $+\mathrm{H}_{2} \mathrm{O}$

Sum: TPNH $+\mathrm{H}^{+}+1 / 2 \mathrm{O}_{2} \longrightarrow \mathrm{TPN}^{+}+\mathrm{H}_{2} \mathrm{O}$

The significance of the ascorbic acid-glutathione respiratory pathway in pea seedlings was recently studied (Mapson and Moustafa, 1956). Dehydroascorbic reductase, glutathione reductase and ascorbic oxidase were readily demonstrated in pea seeds which had germinated for 1.5 or 7 days. An oxidase catalyzing the oxidation of ascorbic acid was absent from mitochondria prepared from one day old seedlings but was present in those particles prepared from seven days old seedlings. However, even in the latter case, it accounted for but $10 \%$ of the total oxidase activity. It was inferred that approximately $25 \%$ of the respiration of pea seedling cotyledons pass over the glutathione-ascorbate system. However, the suggestion also has been made that glutathione reductase functions primarily in the maintenance of the reduced state of sulfhydryl containing protein enzymes, rather than as a component of an electron transport chain.

\section{Localization of Enzymes within Cells}

Mention should be made of the distribution of energy yielding enzymes within the cell. The enzymes concerned with glycolysis and the hexose monophosphate shunt are to be found in the soluble cytoplasm of tissues. It is possible, however, that these enzymes also occur in cell nuclei (Stern and Timonen, I952). The nuclei of wheat germ manifest vigorous glycolytic activity. Glucose-6-phosphate dehydrogenase activity was also found in the nuclei of thymus cells (Stern and Mirsky, I 954). However, the enzymes concerned with hydrogen transport, cytochrome c, cytochrome reductase, cytochrome oxidase, and succinoxidase, appear to be absent from nuclei and the concentration of glutathione reductase is but a fraction of that of the cytoplasm (Stern and Timonen, 1952). The present evidence therefore suggests that the metabolic organization of the nucleus is anaerobic in 
character. The dehydrogenases, if actually present in the nucleus, presumably function as mutases.

Mention has been made in the previous section of the postulated electron transport system of plants mediated by glutathione reductase, dehydroascorbic dehydrogenase, and ascorbic oxidase. These enzymes are apparently also localized in the soluble cytoplasm of the cell (Mapson and Moustafa, I 956; Rall and Lehninger, I 952). The flavin enzymes, xanthine oxidase and aldehyde oxidase, are found in the soluble cytoplasm of liver.

Microsomes represent an active site of protein and cholesterol synthesis. The oxidation of certain drugs has been observed in liver microsomes (Brodie et al., I 955) and the enzymatic steps for the 2 I -hydroxylation of I $7-\alpha$-hydroxyprogesterone have been shown in beef adrenal microsomes (Ryan and Engel, I956). Interestingly enough liver microsomes actively reduce exogenous cytochrome c via TPNH or DPNH linked reactions, although cytochrome $\mathrm{c}$ and cytochrome oxidase are absent from microsomal particles (Hogeboom et al., I953; Strittmatter and Ball, I 954). However, a cytochrome pigment, designated as cytochrome $m$, with reduced absorption spectra showing peaks at 5570,5270 , and $4240 \AA$ is to be found in liver microsomal suspensions (Strittmatter and Ball, I954). The $E_{0}^{\prime}$ of cytochrome m was estimated to be - $0.12 \mathrm{~V}$. This low oxidation-reduction potential might favor the participation of cytochrome $m$ in reductive synthetic reactions.

Liver cytochrome $m$ has recently been solubilized with pancreatic lipase and purified (Strittmatter and Velick, i 956a). The molecular weight was estimated to be 16,900 and the concentration of flavin not significant. The standard potential of the purified cy tochrome $\mathrm{m}$, calculated on the basis of equilibrium measurements, was $+0.02 \mathrm{~V}$, in contrast to the value estimated for the cytochrome $\mathrm{m}$ of the microsomal suspension (Velick and Strittmatter, I956). In the presence of either TPNH or DPNH, microsomal suspensions are capable of reducing cytochrome $\mathrm{m}$ (Strittmatter and Velick, I 956b). A reductase specific for both DPNH and for microsomal cytochrome $m$ which was completely inactive with TPNH as electron donor or with cytochrome $\mathrm{c}$ as electron acceptor has been isolated. Exogenous cytochrome $\mathrm{c}$ is however reduced by the reduced microsomal cytochrome $\mathrm{m}$, thereby completing a DPNH-cytochrome c transfer. The microsomal cytochrome $m$ reductase contains flavin but the role of this nucleotide in electron transfer has not as yet been established. The cytochrome $m$ reductase is capable of reducing 2,6-dichlorophenolindophenol, thereby exhibiting the so called diaphorase activity.

Several of the energy yielding cycles are localized in the mitochondria. Thus, the enzymes concerned with tricarboxylic acid cycle oxidations, the enzymes of the fatty acid oxidative spiral, the succinic oxidase system, the electron transport particle (Green and Beinert, I 955) all are found in the mitochondria fraction of cell homogenates (Hogeboom et al., I 953). Isocitric dehydrogenase and aconitase, are, however, notable examples of tricarboxylic acid cycle enzymes which have been found in high concentration in the soluble cytoplasm of the cell. Mitochondria particles manifest dehydrogenase activity towards various other substrates including choline, betaine, D-amino acids, and amines. Catalase activity is also found associated with the mitochondria. The nitrate reductase system of microorganisms is localized in the particulate matter of the cell. 


\section{E. Generation of Energy-Rich Phosphate Bonds}

\section{Substrate phosphorylation}

Part of the energy liberated as a result of catabolic processes is conserved in the form of nucleotide compounds containing energy-rich phosphate bonds. These compounds differ from most of the esterified phosphorus compounds in that approximately $10-16 \mathrm{kcal}$ of free energy rather than $2-5 \mathrm{kcal}$ are liberated as a result of the hydrolysis of the phosphate linkage.

Mechanisms for the generation of high-energy phosphate bonds may be classified into three categories: I) substrate phosphorylation, 2) oxidative phosphorylation, and 3) photosynthetic phosphorylation. Substrate phosphorylation and oxidative phosphorylation occur in most organisms; photosynthetic phosphorylation takes place in the chloroplasts of plants. In the case of oxidative phosphorylation, the formation of the energy-rich bond is coupled with the oxidation of DPNH or reduced flavin through the mitochondrial electron transport system. We shall discuss oxidative phosphorylation and photosynthetic phosphorylation in the next section. Let us first direct attention to some examples of substrate phosphorylation.

(a) The oxidation of glyceraldehyde phosphate to 3-phosphoglyceric acid

There are two substrate phosphorylation reactions in the glycolytic sequence.

a) The first of these occurs during the oxidation of glyceraldehyde phosphate to I,3-diphosphoglycerate: (Fig. I I Krimsky and Racker, I955). In the proposed

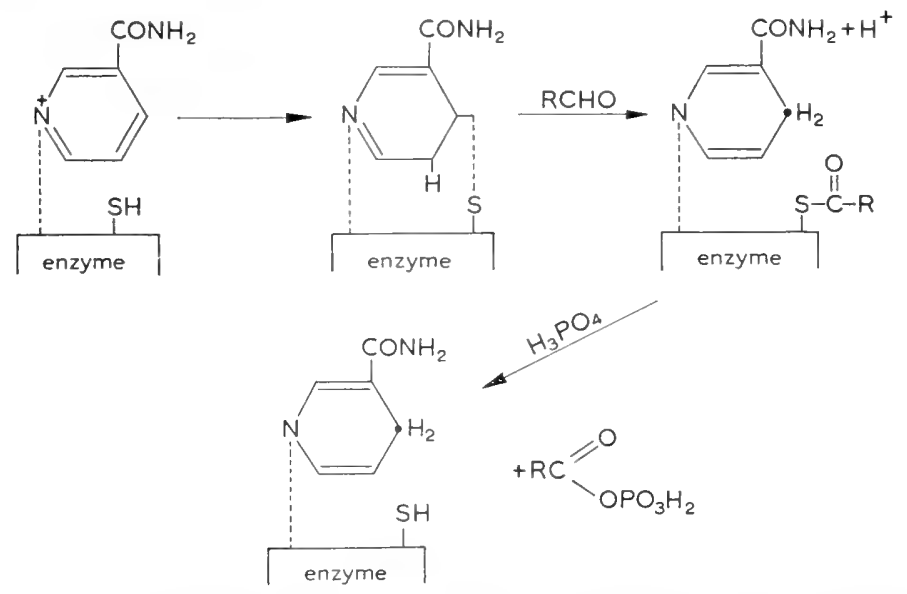

Fig. I I. Postulated mechanism for the glyceraldehyde phosphate dehydrogenase reaction.

sequence of events, a DPN ${ }^{+}$-enzyme compound interacts with the aldehyde substrate to yield reduced $\mathrm{DPN}^{+}$and an acyl enzyme, in which the acyl moiety of the oxidized substrate is attached to a sulfur atom of the protein; this thiol ester is split by inorganic phosphate to yield the regenerated SH-enzyme and I,3-diphosphoglycerate, a compound containing the energy-rich acyl phosphate bond. Glutathione is a cofactor of glyceraldehyde phosphate dehydrogenase. One mole 
of "reduced" muscle glyceraldehyde-phosphate dehydrogenase contains three moles of $\mathrm{DPN}^{+}$, and two moles of glutathione. The glutathione may be liberated from the enzyme by tryptic digestion. If prior to tryptic digestion, the enzyme is treated with the sulfhydryl binding agent, iodoacetate, no free glutathione is formed. Iodoacetate is also an inhibitor of this enzyme.

As shown in reaction $\mathrm{b}$ ), the reaction of 1,3 -diphosphoglycerate with ADP results in the formation of ATP:

b) 1,3-Diphosphoglycerate $+\mathrm{ADP} \stackrel{\mathrm{Mg}^{++}}{\longrightarrow}$ 3-phosphoglycerate $+\mathrm{ATP}$

The $\mathrm{DPNH}_{2}$ generated by reaction a) may be reoxidized in dismutation reactions or by $\mathrm{DPNH}_{2}$ oxidase. When one mole of $\mathrm{DPNH}_{2}$ is reoxidized by mitochondrial DPNH oxidase enzymes, three additional moles of ATP are generated by electron transport phosphorylation (see below).

\section{(b) Phosphoenol pyruvate}

The second substrate phosphorylation of the glycolytic sequence takes place at the level of phosphoenolpyruvate. The dehydration of 2-phosphoglycerate, results in the formation of phosphoenol pyruvate in which part of the energy of the molecule is distributed in the labile phosphate linkage (Fig. I2).

In the event that phosphoenol pyruvate next reacts with ADP, ATP and pyruvate are formed.

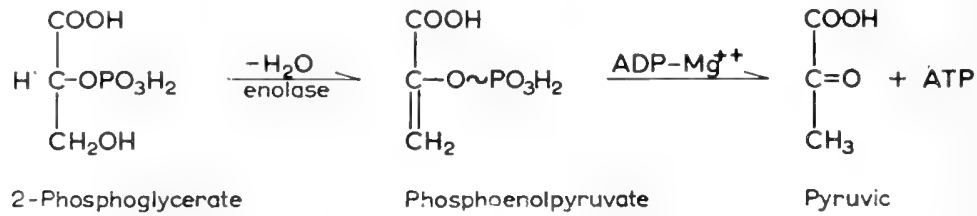

Fig. 12. Phosphoenolpyruvate and substrate phosphorylation.

(c) Phosphorylation coupled to the oxidation of $\alpha$-ketoglutarate

A substrate phosphorylation occurs during the oxidative decarboxylation of $\alpha$-ketoglutaric acid to succinic acid (Reactions a-c).

a) $\alpha$-Ketoglutarate $+\mathrm{DPN}^{+}+\mathrm{CoASH} \rightarrow$ succinyl-CoA $+\mathrm{DPNH}+\mathrm{H}^{+}+\mathrm{CO}_{2}$

b) Succinyl-CoA $+\mathrm{GDP}+\mathrm{P}_{\mathrm{i}} \stackrel{\mathrm{Mg}^{++}}{\longrightarrow}$ succinate $+\mathrm{CoASH}+\mathrm{GTP}$

c) $\mathrm{GTP}+\mathrm{ADP} \stackrel{\mathrm{Mg}^{++}}{\longrightarrow} \mathrm{GDP}+\mathrm{ATP}$

The enzymes of this phosphorylation system have been purified from beef heart mitochondria (Sanadi et al., 1956). GDP or IDP rather than ADP is required in the phosphorylation step b). ATP is then generated from the GTP by a nucleotide transphosphorylation reaction ( $\mathrm{step} \mathrm{c}$ ). 
It should be noted that succinyl-CoA may also be generated from acetoacetyl-CoA by a thiotransferase reaction. Thus, a substrate phosphorylation may be effected as a result of the following reactions:

a) 2 Acetyl-CoA $\rightarrow$ acetoacetyl-CoA $+\mathrm{CoA}$, or

$$
\mathrm{DPN}^{+}
$$

b) $\beta$-Hydroxybutyryl-CoA $\longrightarrow$ acetoacetyl-CoA

c) Acetoacetyl-CoA + succinate $\rightarrow$ acetoacetate + succinyl-CoA

d) Succinyl-CoA $+\mathrm{GDP}+\mathrm{P}_{\mathrm{i}} \rightarrow \mathrm{GTP}+\mathrm{CoA}+$ succinate

Acetyl-CoA is generated as a result of the oxidative decarboxylation of pyruvate or during the oxidation of fatty acids. The following additional mechanism for substrate phosphorylation occurs in those microorganisms which contain the enzymes, coenzyme A-transferase and phosphotransacetylase (Lynen, 1955):

a) Acetyl-CoA + phosphate $\rightarrow$ acetyl phosphate + CoA

b) Acetyl phosphate $+\mathrm{ADP} \rightarrow \mathrm{ATP}+$ acetate

\section{Electron transport phosphorylation}

Table 3 shows that the average potential between oxygen and a pair of average substrate hydrogens is I.2 V;0.25 V corresponds to approximately $\mathrm{I} 2 \mathrm{kcal}$, the energy content of the pyrophosphate bond of ATP. Therefore a total of four highenergy phosphate bonds theoretically could be generated as a result of the oxidation of substrates and the transport of a pair of hydrogens to oxygen through the mitochondrial electron transport system. The generation of $2-3$ high-energy phosphate bonds per atom of oxygen utilized $(\mathrm{P}: \mathrm{O}=3)$ has in fact been demonstrated during the oxidation of $\beta$-hydroxybutyrate by intact mitochondria (Lehninger, I953-I954; Chance, I953-1954) and by mitochondrial fragments obtained by exposing freshly prepared mitochondria to digitonin. In order to demonstrate phosphorylation reactions with exogenous DPNH as substrate (Cooper and Lehninger, I956a, I956b; Devlin and Lehninger, 1956) instead of $\beta$-hydroxybutyrate, it is necessary to increase mitochondrial permeability to DPNH by pretreatment with ice cold hypotonic $\mathrm{KCl}$ or sucrose solutions for a short period of time. Following the restoration of isotonicity, $\mathrm{P}: \mathrm{O}$ ratios of as high as 2.6 have been observed accompanying the oxidation of exogenous $\mathrm{DPNH}_{2}$ by oxygen. These experiments provided the first direct proof that phosphorylations occur beyond the substrate level during the transport of electrons from $\mathrm{DPNH}_{2}$ to oxygen via the respiratory chain of mitochondria (Lehninger, I953-I 954).

It is to be recalled that four dehydrogenation reactions of the tricarboxylic acid cycle and two reactions of fatty acid oxidation are localized in the mitochondria. However, many of the dehydrogenation reactions which result in the generation of $\mathrm{DPNH}_{2}$ occur externally to the mitochondrion. Moreover, as in the case of glyceraldehyde-phosphate dehydrogenase, the dehydrogenases of the soluble cytoplasm are of particular importance in the energy economy of the cell. In view of the selective permeability of the mitochondrial membrane, there is some question as yet as to the manner of interaction of externally generated $\mathrm{DPNH}_{2}$ and mitochondria $\mathrm{DPNH}_{2}$-oxidase. 
Another interesting question relates to the oxidation of $\mathrm{TPNH}_{2}$. As yet, no decisive demonstration of a coupling of $\mathrm{TPNH}_{2}$ oxidation with phosphorylation has been shown, although such coupling presumably exists. Possibly, phosphorylation can be obtained with $\mathrm{TPNH}_{2}$ oxidation only after electron transfer to $\mathrm{DPN}^{+}$ via the pyridine nucleotide transhydrogenase (Kaplan et al., 1956b). Although isocitrate plus $\mathrm{TPN}^{+}$is oxidized by liver mitochondria which have been depleted of pyridine nucleotides, no phosphorylation is observed. However, when supplemented with $\mathrm{TPN}^{+}$plus $\mathrm{DPN}^{+}$they oxidize isocitrate with a $\mathrm{P}: \mathrm{O}$ ratio of 2.3 . It is possible that the prime function of $\mathrm{TPNH}_{2}$ is to serve as a reducing agent, while $\mathrm{DPNH}_{2}$ is primarily oxidized and thereby gives rise to utilizable energy in the form of energy-rich phosphate bonds. It has been shown that $\mathrm{TPNH}_{2}$ can act as a reducing agent in the conversion of crotonyl-CoA to butyryl-CoA; $\mathrm{DPNH}_{2}$ cannot replace $\mathrm{TPNH}_{2}$ in this reaction. According to this hypothesis, pyridine nucleotide transhydrogenase serves as part of a regulatory mechanism for the conservation of oxidative energy. As shown below, the shifting of electrons from $\mathrm{DPNH}_{2}$ to the $\mathrm{TPN}^{+}$system would result in a lowering of phosphorylation; a shift in the direction of the $\mathrm{DPN}^{+}$system would increase the level of phosphorylation (Kaplan et al., r 956b):

Fast

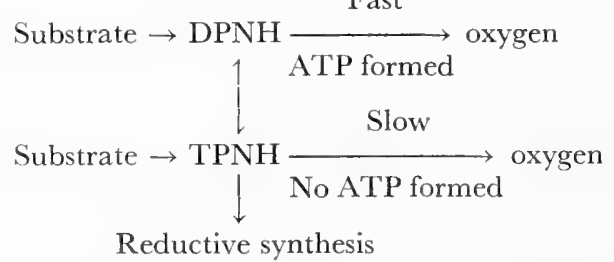

The experiments of Lehninger and collaborators, and Maley and Lardy (1954) have done much to clarify the precise localization of the three phosphorylation reactions which occur between $\mathrm{DPNH}_{2}$ and $\mathrm{O}_{2}$, which may be schematically written as follows:

I) $\mathrm{DPNH}+\mathrm{H}^{+}+\mathrm{FAD}+\mathrm{ADP}+\mathrm{P}_{\mathrm{i}} \rightarrow \mathrm{DPN}^{+}+\mathrm{FADH}_{2}+\mathrm{ATP}$

2) 2 cyt. b-Fe $\mathrm{Fe}^{++}+2$ cyt. c-Fe $\mathrm{F}^{+++}+\mathrm{ADP}+\mathrm{Pi}_{\mathrm{i}} \rightarrow 2$ cyt. b-Fe $\mathrm{F}^{+++}+2$ cyt. c-Fe $\mathrm{F}^{++}+\mathrm{ATP}$

3) 2 cyt. a-Fe $\mathrm{Fe}^{++}+2 \mathrm{H}^{+}+1 / 2 \mathrm{O}_{2}+\mathrm{ADP}+\mathrm{P}_{\mathrm{i}} \rightarrow 2$ cyt. a-Fe $\mathrm{Fe}^{+++}+\mathrm{H}_{2} \mathrm{O}+\mathrm{ATP}$

In the presence of cyanide, which inhibits cytochrome oxidase, the freshly prepared mitochondria or the mitochondrial fragments catalyze the reduction of ferricytochrome $\mathrm{c}$ at the expense of $\beta$-hydroxybutyrate with a $\mathrm{P}: \mathrm{O}$ ratio approaching two. Since no phosphorylation occurs during the transfer of the electrons and hydrogens from $\beta$-hydroxybutyrate to $\mathrm{DPNH}_{2}$, this implies that there are two phosphorylation sites between $\mathrm{DPNH}_{2}$ and cytochrome c. The phosphorylations are uncoupled by dinitrophenol and gramicidin. Likewise, the oxidation of the substrate by ferricytochrome $\mathrm{c}$ and the phosphorylation reactions are completely blocked by antimycin A, an agent which is believed to act between cytochrome b and cytochrome c. With ferricyanide as electron acceptor instead of ferricy tochrome $\mathrm{c}$, the $\mathrm{P}: \mathrm{O}$ ratio is about one, indicating that one phosphorylation takes place very early in the chain. 
As indicated previously, succinate is oxidized to fumarate and choline is oxidized to betaine aldehyde by flavoproteins rather than by pyridine nucleotide dehydrogenases. When these substrates are oxidized by liver mitochondria, a $\mathrm{P}: \mathrm{O}$ ratio of about two is observed (Chance, I953-1954; Rotschild et al., 1954). Thus, there are two phosphorylation sites between $\mathrm{FADH}_{2}$ and oxygen.

The span between cytochrome $\mathrm{c}$ and oxygen was studied by utilizing cytochrome c reduced chemically by hydrogen (palladium catalyst) or by ascorbate or adrenalin (Maley and Lardy, 1954). In this case, a P:O ratio of 0.9 is observed. Since the standard oxidation-reduction potential of cytochrome $\mathrm{c}$ is about 0.26 and that of cytochrome $\mathrm{a}$ is about 0.29 , and a difference of $0.25 \mathrm{~V}$ is required for one phosphorylation, it can be inferred that the third phosphorylation is localized between cytochrome a and oxygen.

The rate of aerobic phosphorylation is depressed in rat liver mitochondria by dicumarol without affecting respiration (Martius and Nitz-Litzow, I953). The uncoupling effect of dicumarol $\left(\mathrm{IO}^{-6} \mathrm{M}\right)$ may be shown either on the overall span, $\beta$-hydroxybutyrate to oxygen, or on the span between hydroxybutyrate and cytochrome c, or cy tochrome $\mathrm{c}$ and $\mathrm{O}_{2}$ (Cooper and Lehninger, I956a, I956b). This supports the hypothesis that vitamin $\mathrm{K}$ is either an electron carrier in the electron transport chain or is a component of the phosphate transferring enzyme system responsible for the formation of ATP from the primary high energy linkage at the three phosphorylating sites in the respiratory chain.

Some of the factors which control the rate of oxidative reactions have been demonstrated by Chance (Chance, 1953-I954). Using intact liver mitochondria, it was shown that the rate of oxygen consumption is low in aerobic resting mitochondria. When substrate is added in excess, an increased reduction of $\mathrm{DPN}^{+}$and the cytochrome pigments is observed, although the rate of oxygen consumption remains low. If, however, both ADP and substrate are added to aerobic mitochondria, a rapid increase in the respiratory rate takes place concomitant with a reoxidation of $\mathrm{DPNH}_{2}$ and the cytochrome pigments and the phosphorylation of ADP to ATP. As the ADP is phosphorylated to ATP, the rate of respiration falls and an increased reduction of $\mathrm{DPN}^{+}$, flavin, and cy tochrome $\mathrm{b}$ is again observed. Under conditions where substrate and oxygen are not limiting, the rate of respiration within the mitochondria is therefore dependent upon the availability of ADP and phosphate.

The requirement for phosphate acceptors in mitochondrial oxidations has also been demonstrated in experimental systems in which glucose and the enzyme hexokinase have been used to regenerate ADP from ATP:

\section{I) ATP + glucose $\stackrel{\text { hexokinase }}{\longrightarrow}$ glucose-6-phosphate $+\mathrm{ADP}$}

Under these conditions, stimulation of oxygen uptake has been noted. The capacity of dinitrophenol to stimulate respiration while completely inhibiting oxidative phosphorylation has been attributed to the fact that "ATPase activity" is stimulated by dinitrophenol. As a result, the $\mathrm{ADP}$ and $\mathrm{H}_{3} \mathrm{PO}_{4}$ required for maximal respiration is maintained (Chance and Williams, I956). The action of dinitrophenol is depicted schematically in Fig. I 3. In Fig. I 3, X I represents the labile compound formed during oxidative phosphorylation. 


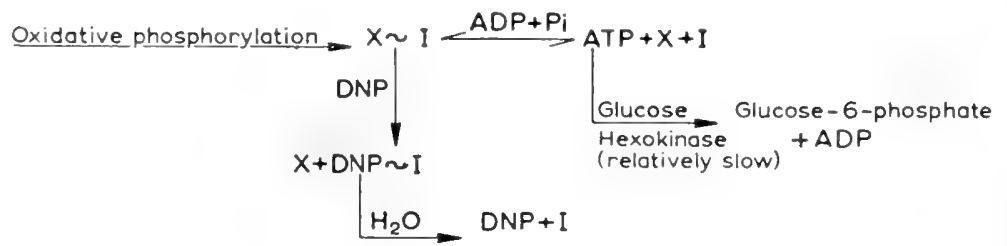

Fig. I3. Postulated mechanism of dinitrophenol action.

The breakdown of glucose is inhibited by oxidative cellular processes. This effect is known as the Pasteur effect. The inverse of this, that is, the inhibition of respiration by glycolysis, has also been demonstrated in tissues which manifest an unusually rapid glycolysis (Chance and Williams, I956). Thus, the addition of glucose to respiring ascites tumor cells is followed by an increase in oxygen consumption and an oxidation of the steady state level of cytochrome b. This may be attributed to the increase in the ADP pool due to the hexokinase reaction as indicated in the scheme shown above. In about 40 sec., however, the above effects are reversed; the cytochrome b is reduced once again, and the ADP level falls, due to the competition for ADP between glycolytic enzymes and mitochondria. As a result, the respiration rate is once again inhibited.

\section{Photosynthetic phosphorylation}

Plant chloroplasts contain multienzyme systems which catalyze three distinct light dependent reactions: I) photolysis of water, 2) photosynthetic phosphorylation, and 3) $\mathrm{CO}_{2}$ fixation (Arnon, 1955; Arnon et al., 1956):

$$
\begin{aligned}
& \text { I) } \mathrm{H}_{2} \mathrm{O} \longrightarrow 2(\mathrm{H})+(\mathrm{O}) \\
& \text { 2) } \mathrm{ADP}+\mathrm{P}_{\mathrm{i}} \stackrel{\text { light }}{\longrightarrow} \mathrm{ATP} \\
& \text { 3) } \mathrm{CO}_{2}+\mathrm{H}_{2} \mathrm{O} \longrightarrow\left(\mathrm{CH}_{2} \mathrm{O}\right)+\mathrm{O}_{2}
\end{aligned}
$$

There is an increasing order of complexity for the three photochemical reactions. Photolysis can occur in preparations incapable of photosynthetic phosphorylation and $\mathrm{CO}_{2}$ fixation. In turn, photosynthetic phosphorylation is found to proceed unimpaired in preparations which can not fix $\mathrm{CO}_{2} \cdot \mathrm{CO}_{2}$ fixation, however, occurs only in chloroplast preparations capable of photolysis of water and of phosphorylation.

In the presence of appropriate acceptor compounds, the light catalyzed photolysis of water may be coupled with reductive processes in a reaction first studied by $\mathbf{R}$. Hill. Benzoquinone, ferricyanide, cytochrome c, flavins, menadione, and $\mathrm{TPN}^{+}$, or $\mathrm{DPN}^{+}$can be reduced by illuminated chloroplast grana under anaerobic conditions. Oxygen is simultaneously evolved (Jagendorf, I956; San Pietro and Lang, 1956)

$$
\begin{aligned}
& \text { 4) } 2(\mathrm{H})+(\mathrm{O})+\text { benzoquinone } \underset{\text { chloroplasts }}{\longrightarrow} \text { hydroquinone }+1 / 2 \mathrm{O}_{2} \\
& \text { 5) } 2(\mathrm{H})+(\mathrm{O})+\mathrm{TPN}^{+}\left(\mathrm{DPN}^{+}\right) \stackrel{\text { chloroplasts }}{\longrightarrow} \mathrm{TPNH}_{2}\left(\mathrm{DPNH}_{2}\right)+1 / 2 \mathrm{O}_{2}
\end{aligned}
$$

The $(\mathrm{H})$ generated in reaction $\mathrm{I})$ may be utilized by the chloroplasts for the reduction of $\mathrm{CO}_{2}$ into organic linkage (reaction 3 ), or the $(\mathrm{H})$ may be reoxidized concomitant with photosynthetic phosphorylation (reaction 2). The competition for $(\mathbf{H})$ between the 


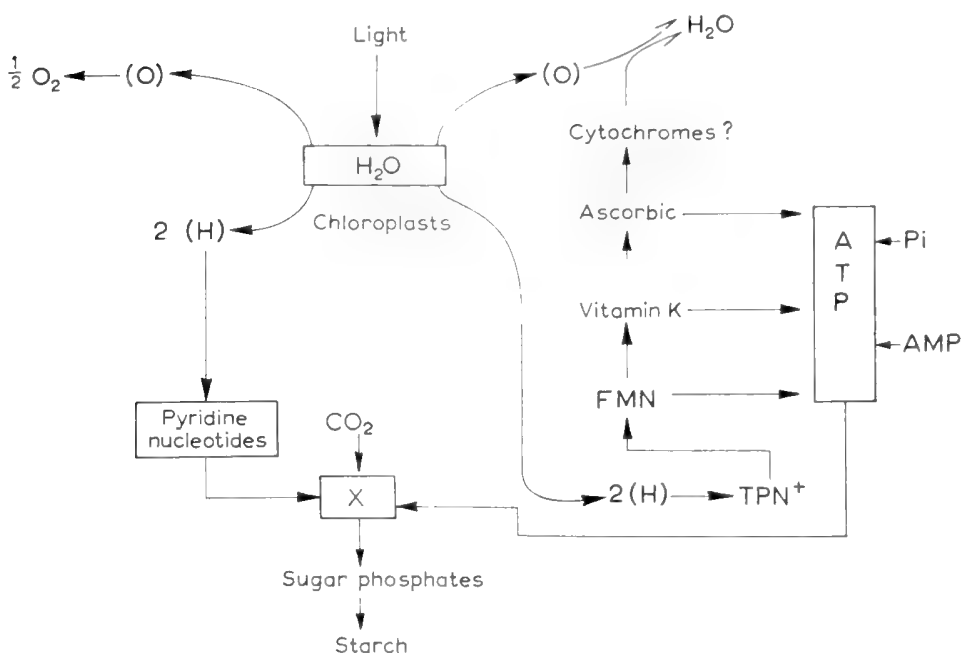

Fig. 14. Scheme for photosynthesis by isolated chloroplasts. Photolysis of water (center) leading either to ATP synthesis and the reconstitution of water (right) or to $\mathrm{CO}_{2}$ reduction (below) linked to oxygen evolution (left).

enzymes of photosynthetic phosphorylation and the enzymes of reductive carboxylation is shown in Fig. I4. In broken chloroplast preparations, $\mathrm{CO}_{2}$ fixation is greatly stimulated by the addition of pyridine nucleotides, ATP, phosphorylated sugars, and a soluble enzyme system which is obtained by treating intact chloroplasts with water (Whatley et al., I956).

The components of the chloroplast phosphorylation system have not as yet been fully established. However, it is known that flavin mononucleotide, ascorbate, $\mathrm{Mg}^{++}$, and vitamin $\mathrm{K}$, when added in catalytic amounts, greatly stimulate photosynthetic phosphorylation. Possibly, the cytochrome pigments also participate in the process (Arnon, 1955). $\mathrm{TPN}^{+}$is a component of the chloroplast phosphorylation system.

Photosynthetic phosphorylation may be distinguished from oxidative phosphorylation in the following respects:

I) Oxidative phosphorylation occurs in mitochondria in the dark; photosynthetic phosphorylation takes place in illuminated chloroplasts.

2) The latter process is anaerobic whereas the former occurs in an oxygen atmosphere and oxygen is consumed in the process.

It is to be emphasized that aerobic phosphorylation also takes place in plant tissues and is indispensable for the maintenance of cellular function during periods of darkness.

Indeed, a light dependent reduction of $\mathrm{DPN}^{+}$or $\mathrm{TPN}^{+}$by plant chloroplasts can be coupled with the reoxidation of the $\mathrm{DPNH}_{2}\left(\mathrm{TPNH}_{2}\right)$ by plant mitochondria with the resulting generation of ATP (Vishniac and Ochoa, 1952) or with other reductive processes of plant cells (Jagendorf, I956).

\section{The storage of high-energy phosphate bonds}

ATP generated by the mechanisms discussed above may react with creatine in the presence of the enzyme, creatine kinase:

I) Creatine $+\mathrm{ATP} \longleftarrow$ creatine phosphate $+\mathrm{ADP}$ 
TABLE 4

REACTIONS INVOLVING HIGH-ENERGY PHOSPHATE BONDS

Source Reaction

\section{Kinases}

I. Yeast, Brain liver

3. Muscle, liver

4. Liver, (E.coli? $)$, muscle Fructose

5. E. coli

6. Muscle, etc. Muscle, etc.

7. Muscle, yeast

8. Liver, E. coli Liver

9. Bacteria, liver

I0. Liver, etc.

II. Spinach

12. E. coli, Yeast, brain Streptococcus, liver

13. Liver, yeast

I4. Liver

15. Yeast, liver

I6. Yeast, liver

I7. Yeast, liver

18. Liver

19. Liver

20. Various tissues

21. Various tissues

22. Liver

23. Yeast

24. E. coli

25. Pseudomonas

26. Pseudomonas

27. Acetobacter

28. Liver

29. Liver

30. Liver, heart, muscle

31. Various tissues

32. E. coli
Glucose

Glucosamine

Fructose

Mannose

Galactose

Galactosamine

Glucose

Glucose (mannose)

Fructose-6-phosphate

Fructose-1-phosphate

Glucose-I-phosphate

Ribose

Desoxyribose

Xylulose

Glycerol

Ribulose-5-phosphate

Pyridoxal

Pyridoxamine

Pyridoxine

Thiamine

Thiamine phosphate

Choline (ethanolamine)

Glyceric

Nicotinamide riboside

Riboflavin

Panthetheine

4-Aminoimidazolecarboxamide riboside

Adenosine

Inosine

Dephospho-CoA

DPN

Thymidine

Gluconate

2-Ketogluconate

Dihydroxyacetone

Uridine

Adenosine phosphosulfate

"Inactive phosphorylase" 3-Phosphoglyceric

L-Rhamnulose
+ ATP $\rightarrow$ glucose-6-phosphate

+ ATP $\rightarrow$ glucosamine-6-phos.

+ ATP $\rightarrow$ fructose-6-phosphate

+ ATP $\rightarrow$ mannose-6-phosphate

+ ATP $\rightarrow$ galactose-I-phosphate

+ ATP $\rightarrow$ galactosamine-I-phos.

+ ATP $\rightarrow$ glucose-6-phosphate

$+\mathrm{ADP}$

ADP

$-\mathrm{ADP}$

- ADP

- ADP

+ ADP

$+\mathrm{ADP}$

$+\mathrm{ATP} \rightarrow$ fructose-I-phosphate

- ADP

$+\mathrm{ATP} \rightarrow$ ?

+ ATP $\rightarrow$ fructose diphosphate

ADP

- ADP

+ ATP $\rightarrow$ fructose diphosphate

$+\mathrm{ADP}$

+ ATP $\rightarrow$ glucose-I,6-diphosphate + ADP

$+\mathrm{ATP} \rightarrow$ ribose-5-phosphate

$+\mathrm{ADP}$

+ ATP $\rightarrow$ desoxyribose-5-phos.

+ ATP $\rightarrow$ xylulose-5-phosphate

+ ATP $\rightarrow \alpha$-glycerol phosphate

$+\mathrm{ATP} \rightarrow$ ribulose-I, 5-diphos. pyridoxal phosphate

$+\mathrm{ADP}$

$+\mathrm{ADP}$

$+\mathrm{ADP}$

$+\mathrm{ADP}$

$+\mathrm{ADP}$

+ ATP $\rightarrow$ pyridoxamine phos. + ADP pyridoxine phosphate + ADP

+ ATP $\rightarrow$ thiamine pyrophos. $\quad+$ AMP

$+\mathrm{ADP} \rightarrow$ thiamine pyrophos. $\quad+\mathrm{AMP}$

$+\mathrm{ATP} \rightarrow$ choline phosphate $\quad+$ ADP

+ ATP $\rightarrow$ 3-phosphoglyceric + ADP

$+\mathrm{ATP} \rightarrow \mathrm{NMN}+\mathrm{ADP}$

$+\mathrm{ATP} \rightarrow \mathrm{FMN}$

+ ATP $\rightarrow$ phosphopantetheine

+ ADP

+ ADP

$+\mathrm{ATP} \rightarrow 4$-AICA ribotide $+\mathrm{ADP}$

$+\mathrm{ATP} \rightarrow$ adenosine-5-phosphate + ADP

+ ATP $\rightarrow$ inosine-5-phosphate + ADP

$+\mathrm{ATP} \rightarrow$ coenzyme-A $\left(3^{\prime}\right) \quad+$ ADP

$+\mathrm{ATP} \rightarrow \mathrm{TPN}\left(2^{\prime}\right)$

+ ATP $\rightarrow$ thymidylic

+ ATP $\rightarrow$ 6-phosphogluconate

$+\mathrm{ADP}$

+ ADP

+ ADP

+ ATP $\rightarrow$ 2-keto-6-phosphogluconate

$+\mathrm{ADP}$

$+\mathrm{ATP} \rightarrow$ dihydroxyacetone phos. + ADP

$+\mathrm{ATP} \rightarrow \mathrm{UMP}$

+ ATP $\rightarrow$ adenosine-3'-phospho-

5'-phosphosulfate + ADP

$+\mathrm{ADP}$

$+\mathrm{ATP} \rightarrow$ "active phosphorylase" + ADP

$+\mathrm{ATP} \rightarrow$ diphosphoglycerate + ADP

+ ATP $\rightarrow$ L-rhamnulose - I-phos. + ADP

\section{Other phosphate transfer reactions}

33. Glutamic

34. Oxalacetate
+ ATP

$+\operatorname{ITP}(\mathrm{GTP})$ $\longleftrightarrow$ ADP

$+\gamma$-glutamyl phosphate

IDP (GDP) $+\mathrm{CO}_{2}+$ phosphoenol pyruvate 


\section{Reaction}

36. 3-Phosphoglyceric + ATP

37. Creatine

38. $\mathrm{CO}_{2}+\mathrm{NH}_{3}$

39. Succinic

40. Aspartate

4I. Adenosine deoxyriboside-5-P

42. Inosine diphosphate

43. GMP

44. UMP

45. AMP

46. UDP

48. AMP

49. UMP

50. GMP

5. AMP

52. Deoxycytidine phos.

53. Acetate

55. UDP

56. UDP

57. dADP
35. Pyruvic + ATP

47. GDP

54. AMP
+ ATP

+ ATP

+ GTP

$+\mathrm{ATP}$

$+\operatorname{ATP}(\mathrm{dATP})$

+ ATP

+ ATP

+ ATP

+ ATP

+ ATP

+ ATP

+ UTP

+ UTP

+ GTP

+ ITP

+ ATP

+ ATP

+ GTP

+ GTP

+ ITP

$+\mathrm{ATP}$

$$
\begin{aligned}
& \longleftrightarrow \text { ADP } \\
& \longleftrightarrow \text { ADP } \\
& \longleftrightarrow \text { ADP } \\
& \longleftrightarrow \text { ADP } \\
& \longleftrightarrow \text { GDP } \\
& \longleftrightarrow \text { ADP }
\end{aligned}
$$

$\longleftrightarrow \mathrm{ADP}(\mathrm{dADP})+$ deoxyadenosine diphosphate

$\longleftrightarrow$ ADP

$\longleftrightarrow$ ADP

$\longleftrightarrow$ ADP

$\longleftrightarrow$ ADP

$\longleftrightarrow$ ADP

$\longleftrightarrow \mathrm{ADP}$

$\longleftrightarrow \mathrm{UDP}$

$\longleftrightarrow$ UDP

$\longleftrightarrow$ GDP

$\longleftrightarrow$ IDP

$\longleftrightarrow \mathrm{ADP}$

$\longleftrightarrow$ ADP

$\longleftrightarrow$ GDP

$\longleftrightarrow$ GDP

$\longleftrightarrow$ IDP

$\leftrightarrow \mathrm{ADP}$

$\longleftrightarrow$ AMP

$\longleftrightarrow$ AMP

$+\mathrm{ATP}$

$+\mathrm{ATP}$

58. Ribose-5-ph
59. Thymidylic

(dAMP, dGMP, dGMP)

IV. XMP transfer reactions

6o. $\alpha$-D-Xylose-I-phos. + UTP

61. Phosphopantetheine

62. NMN

63. FMN

+ ATP

$+\mathrm{ATP}$

$+\mathrm{ATP}$

64. Phosphocholine

$$
\left(\text { Et-NH }{ }_{2}-\mathrm{P}\right)+\mathrm{CTP}
$$

65. Mannose-I-phosphate + GTP

66. Glucose- I-phosphate + UTP

67. N-acetylglucosamineI-phosphate

$+\mathrm{UTP}$

68. Sulfate

+ ATP

+ ATP

$+\mathrm{ATP}$

70. Acetate (propionate

71. Butyrate

72. Octanoate

+ ATP

+ ATP

$+\mathrm{UTP}$

+ ATP

$+\mathrm{ATP}$

$+\mathrm{ATP}$

74. Pantoate

+ UTP

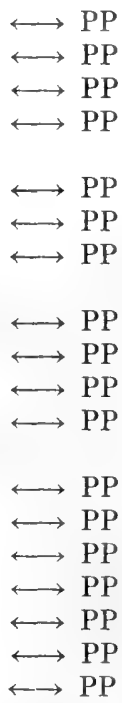

+ UDP-xylose

+ dephospho-CoA

+ DPN

+ FAD

+ CDP-choline

+ GDP-mannose

+ UDPG

+ UDP (N-acetylglucosamine)

+ adenosine phosphosulfate

+ adenyl $\left(\mathrm{CO}_{2}\right)$

+ adenyl acetate (adenyl propionate)

+ adenylbutyrate

+ adenyloctanoate

+ UDP-galactose

+ adenylpantoate

+ adenylmethionine

+ adenylamino acids

+ UDP-arabinose 
Table 4, continued

\section{Reaction}

78. $\alpha$-L-Arabinose-I-phos. + UTP

79. Selenate

8o. Luciferin

+ ATP

81. Glucosamine-I-phos. + U'TP

82. Benzoate

83. Lipoate

+ ATP

$+\mathrm{ATP}$

\section{$V$. Activating reactions}

84. Fatty acid $\left(\mathrm{C}_{12}-\mathrm{C}_{22}\right)$ $+\mathrm{CoA}+\mathrm{ATP}$

85. Acetoacetate $+\mathrm{CoA}+\mathrm{ATP}$

86. Cholic + CoA + ATP

87. Pantothenic + cysteine

+ ATP

88. Methionine

$+\mathrm{ATP}$

89. Xanthosinic $+\mathrm{NH}_{3}+\mathrm{ATP}$

9o. Aspartic + citrulline $+\mathrm{ATP}$

$V I$. Other activating reactions

91. $\mathrm{CO}_{2}+$ propionyl-CoA $+\mathrm{ATP}$

92. Glutamic + cysteine + ATP

93. $\gamma$-glutamyl cysteine

+ glycine + ATP

94. Aspartic + inosinic + GTP

95. Xanthosinic +

$$
\text { glutamine }+ \text { ATP }
$$

96. $\mathrm{NH}_{3}+\mathrm{UTP}+\mathrm{ATP}$

97. Glutamine + FGAR + ATP

98. Aspartic + AIR +

$$
\mathrm{H}_{2} \mathrm{CO}_{3}
$$

$+\mathrm{ATP}$

99. FGAM

$+\mathrm{ATP}$

ıoo. Formate + THFA

$+\mathrm{ATP}$

VII. PRPP reactions

IOI. Uracil + PRPP

I02. Glutamine + PRPP

$\begin{aligned} & \longleftrightarrow P P \\ & \longleftrightarrow P P \\ & \longleftrightarrow P P \\ & \longleftrightarrow P P \\ & \longleftrightarrow P P \\ & \longleftrightarrow P P \\ \text { PPPP } & \longleftrightarrow P P\end{aligned}$

I03. Orotic + PRPP

I04. Guanine + PRPP

105. Adenine + PRPP

106. Hypoxanthine + PRPP

107. Anthranilic + PRPP

I 8 . Nicotinamide

+ PRPP

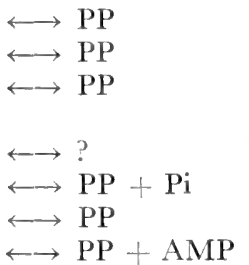

$+\mathrm{AMP}+$ acyl-CoA

$+\mathrm{AMP}+$ acetoacetyl-CoA

$+\mathrm{AMP}+$ cholyl-CoA

+ pantothenylcysteine

+ S-adenosylmethionine

$+\mathrm{AMP}+\mathrm{GMP}$

+ arginosuccinic

$\longleftrightarrow \mathrm{ADP}+\mathrm{Pi}+$ succinyl-CoA

$\longleftrightarrow \mathrm{ADP}+\mathrm{Pi}+\gamma$-glutamylcysteine

$\longleftrightarrow \mathrm{ADP}+\mathrm{Pi}+$ glutathione

$\longleftrightarrow \mathrm{GDP}+\mathrm{Pi}+$ adenylosuccinic

$\longleftrightarrow \mathrm{ADP}+\mathrm{Pi}+$ guanyloglutaric

$\longleftrightarrow \mathrm{ADP}+\mathrm{Pi}_{\mathrm{i}}+\mathrm{CTP}$

$\longleftrightarrow \mathrm{ADP}+\mathrm{Pi}+$ glutamic + FGAM

$\longleftrightarrow \mathrm{ADP}+\mathrm{Pi}+5$-amino-4-imidazole ( $\mathrm{N}$-succinylo-carboxamidribotide)

$\longleftrightarrow \mathrm{ADP}+\mathrm{Pi}+\mathrm{AIR}$

$\longleftrightarrow \mathrm{ADP}+\mathrm{Pi}+\mathrm{N}^{10}$-formyl-THFA

VIII. Polynucleotide phosphorylase 109. $\mathrm{n}(\mathrm{XRPP}) \longleftrightarrow \mathrm{nPi}+(\mathrm{X}-\mathrm{R}-\mathrm{P})_{\mathrm{n}}$ เ เо. $\mathrm{n}(\mathrm{TTP}+\mathrm{dCTP}+\mathrm{dGTP}+\mathrm{dATP})$

$$
+ \text { DNA }
$$

+ UMP (Lactobacillus bulgaricus)

+ 5-phosphoribosamine + glutamic

$+\mathrm{UMP}+\mathrm{CO}_{2}$

+ GMP

+ AMP

+ IMP

+ indole-3-glycerol phosphate

$+\mathrm{NMN}$

$\mathrm{X}=\mathrm{A}, \mathrm{I}, \mathrm{C}, \mathrm{U}, \mathrm{G}$ $\longleftrightarrow 4 \mathrm{n}(\mathrm{PP})+\underset{\mathrm{dGMP}, \mathrm{dAMP})}{\mathrm{DNA}-(\mathrm{TMP}, \mathrm{dCMP},}$ 
The guanidine-phosphate bond of creatine phosphate is also a "high-energy" phosphate bond since approximately if kcal of free energy are liberated upon the hydrolysis of one mole of phosphocreatine. Creatine phosphate is a major storage form of "high-energy phosphate", especially in muscle and liver of animal tissues. When the concentration of ATP declines, reaction I) is reversed and ATP is regenerated from the creatine phosphate. In invertebrates, phosphoarginine replaces phosphocreatine as the storage compound for high-energy phosphate bonds.

\section{THE UTILIZATION OF ATP AND RELATED NUGLEOTIDES}

High-energy phosphate bonds of ATP are required in a variety of chemical reactions which are of significance in biosynthetic processes. Table 4 lists some of these reactions involving ATP and other compounds containing "energy-rich" phosphate bonds. The reactions have been grouped into seven classes.

Group I and Group II include those reactions in which there is a transfer of the terminal phosphate of ATP, GTP, ITP, or UTP; the corresponding diphosphate is the product of the reaction. The two reactions of Group III illustrate the transfer of pyrophosphate to acceptor compounds; in this case adenylic acid is a product. The reactions of Group IV and probably, Group V involve the transfer of an adenylic acid, guanylic acid, cytidylic acid, or uridylic acid moiety to an acceptor compound; here, pyrophosphate is a product of the reaction. The reactions of Group VI have been placed in a separate category since the mechanism has not been precisely defined. It is likely that the Group VI reactions involve at least two steps and that these reactions will later turn out to belong to either Groups I, II, or III.

Group VII includes the growing list of reactions requiring 5-phosphoribose pyrophosphate rather than ATP. They are of interest in that 5-phosphoribose is transferred to an acceptor compound while pyrophosphate is a product of the reaction. The polynucleotide phosphorylase reactions shown in Group VIII require nucleotide diphosphates or triphosphates and as in the case of the Group IV reactions, nucleotide monophosphate groups are transferred to acceptor compounds.

\section{A. Group I Reactions}

Let us now inquire as to the function of the reactions of Table 4. Many of the reactions of Group I are catalyzed by kinase enzymes and result in the formation of phosphate ester bonds. It will be noted that a number of the latter are sugar phosphate compounds. A phosphorylation of a sugar to the sugar phosphate is in almost every case a necessary preliminary step for the further metabolism of the sugar. Likewise, the phosphorylations of glycerol, glyceric acid, or gluconic acid convert these compounds to substances which are readily metabolized by cells.

The kinase reactions shown in Group I also include steps in the synthesis of coenzymes and nucleotide intermediates of nucleic acid synthesis. The phosphorylation of nicotinamide riboside, thiamine, pyridoxal, or pantetheine are examples of the former while the phosphorylation of thymidine, adenosine, or uridine are examples of the latter. Reactions which are of importance in the synthesis of polynucleotides and of coenzymes are also to be found in Groups II to VIII. The 
products of the reactions catalyzed by choline (ethanolamine) kinase are intermediates in phospholipid synthesis.

The free energy of hydrolysis of the phosphate esters which are the products of the Group I reactions is approximately $2-4 \mathrm{kcal} / \mathrm{mole}$. Consequently, the reactions of Group I are essentially irreversible.

\section{B. Nucleotide Transfer Reactions}

The reactions of Group II, are reversible reactions. Reactions 35, 36, 39, and 53, when written from right to left, will be recognized as the substrate phosphory]ation reactions discussed previously. Reactions 4 I to 52 and 54 to 56 are nucleoside monophosphate and nucleoside diphosphate kinase reactions. As a result of these reactions, the entire series of nucleotide mono-, di-, or tri-phosphates may readily be generated. Reactions $33,34,38$, and 40 will be discussed later.

\section{Uridyl Transferase Reactions}

Reaction 66 is catalyzed by the enzyme, uridyl transferase, which has been observed in yeast, spinach leaves, and various animal tissues (Leloir and Cardini, r 956; Mills et al., I 954). In liver tissue, the enzyme is apparently localized in the nuclei of the cells. UDP-acetyl glucosamine may substitute for UDPG as a substrate for this enzyme (Reaction 67). A similar yeast enzyme catalyzes the formation of UDP-galactose from galactose-I-phosphate (Reaction 73) (Kalckar et al., 1953) while a guanylic transferase functions in the synthesis of GDP-mannose from mannose-I-phosphate (Reaction 65) (Munch-Peterson, I955a, I955b). A uridyl transferase enzyme of mung bean seeds catalyzes the formation of UDPxylose from $\alpha$-D-xylose-I-phosphate (Reaction 6o) (Ginsberg et al., i 956).

Purified uridyl transferase of yeast catalyzes an exchange of ${ }^{32} \mathbf{P}-{ }^{32} \mathrm{P}$ with UTP in the absence of glucose- I-phosphate (Munch-Peterson, I 955a). The enzyme also catalyzes an exchange of the phosphate of glucose- $\mathrm{I}$-phosphate ${ }^{32} \mathrm{P}$ - or ${ }^{14} \mathrm{C}$ glucose-I-phosphate with UDPG in the absence of pyrophosphate. On the other hand, no exchange of ${ }^{14} \mathrm{C}$ of $\mathrm{UMP}-{ }^{14} \mathrm{C}$ takes place in the presence of UTP or UDPG. The following mechanism is consistent with the experimental observations:

a) UDPG + enzyme $\longleftarrow$ UMP $\sim$ Enzyme + glucose- - -32P

$$
\begin{aligned}
& \int^{32} \mathbf{P}-{ }^{32} \mathbf{P} \\
& \text { UTP + enzyme }
\end{aligned}
$$

The same mechanism is involved in the ${ }^{32} \mathrm{P}-{ }^{32} \mathrm{P}$ exchange reactions with GTP and ATP which are catalyzed by crude yeast enzyme extracts.

The reactions mentioned above are of significance in connection with the synthesis of hyaluronic acid and other structural polysaccharides. Considerable progress has been made recently in the elucidation of the biosynthetic pathways of hyaluronic acid synthesis. Let us digress for a moment to summarize the results of this research. 
Hyaluronic acid is a linear polymer of alternating $\mathrm{N}$-acetyl-D-glucosamine and D-glucuronic acid residues joined in $\beta$-glycosidic linkage. The molecular weight may vary between 300,000 and 1,500,000, depending on the source. Cell free extracts of the Rous sarcoma synthesize oligosaccharides of the hyaluronic acid chain when incubated with labelled substrates (Glaser and Brown, I955) as shown in reactions b) and c):

b) UDP-N-acetyl $\left({ }^{14} \mathrm{C}\right)$-glucosamine + UDP-glucuronate $\rightarrow{ }^{14} \mathrm{C}$-oligosaccharide

c) UTP + N-acetyl $\left({ }^{14} \mathrm{C}\right)$-glucosamine-6-phosphate + UDP-glucuronate $\rightarrow{ }^{14} \mathrm{G}$-oligosaccharide

UDPG and $\mathrm{DPN}^{+}$can replace UDP glucuronate in reactions b) and c) due to the presence of the dehydrogenases required for UDP glucuronate synthesis (Strominger et al., I954):

Liver, tumor

d) $\mathrm{UDPG}+2 \mathrm{DPN}^{+} \longrightarrow$ UDP-glucuronate $+2 \mathrm{DPNH}_{2}$

The carbon skeletons of glucuronate and $\mathrm{N}$-acetyl glucosamine are both derived from glucose without prior fission of the carbon chain. The synthetis of glucuronate will be discussed in connection with ascorbic acid biosynthesis. $\mathrm{N}$-acetylglucosamine may be derived from glucose as follows:

e) Hexose-6-phosphate + glutamine $\rightarrow$ glucosamine-6-phosphate + glutamic

f) Fructose-6-phosphate $+\mathrm{NH}_{3} \longrightarrow$ glucosamine-6-phosphate

g) Glucosamine-6-phosphate + acetyl-CoA $\rightarrow \mathrm{N}$-acetyl-glucosamine-6-phosphate $+\mathrm{CoA}$

h) N-acetylglucosamine-6-phosphate $\stackrel{\text { mutase }}{\longleftarrow} \mathrm{N}$-acetylglucosamine- I-phosphate

i) Glucosamine + ATP $\rightarrow$ glucosamine-6-phosphate + ADP

PP-uridyltransferase

j) N-acetylglucosamine-I-phosphate + UTP $\longrightarrow$ UDP-N-acetylglucosamine

Reactions e) and g) have been observed in Neurospora (Leloir and Cardini, 1953, I956) while reaction $\mathrm{f}$ ) takes place in kidney, liver, and other animal tissues. Reaction $\mathrm{h}$ ) occurs in the Rous sarcoma, Neurospora, and animal tissues. Reaction i) is catalyzed by hexokinase of yeast or brain (Leloir, I95 I ; Harpur and Quastel, 1949). Enzymes which phosphorylate galactosamine to galactosamine- $\mathrm{I}$-phosphate also occur in liver and yeast (Leloir and Cardini, 1953).

In connection with UDP galactose, mention should be made of another uridyl transferase enzyme of yeast and liver (Maxwell, 1956):

k) galactose-I-phosphate + UDPG

GP-uridyl transferase

Reaction $\mathrm{k}$ ) is non pyrophosphorolytic, in contrast to reaction a).

Bacteria, yeast, and many animal tissues also contain the enzyme galactowaldenase thereby providing a third mechanism for generating UDP galactose:

1) UDP-galactose $\stackrel{\text { galactowaldenase }}{\longleftarrow}$ UDP-glucose

Mammary gland tissue from lactating rats and brain tissue from $2-3$ week old rats contain galactowaldenase and the P-P uridyl transferase (reaction 73, Table 4, p. 35) but not GPuridyl transferase. The livers from these animals contained all three enzymes. Galactowaldenase has been purified from liver. $\mathrm{DPN}^{+}$is required, suggesting that the inversion at carbon 4 occurs by an oxidation and subsequent reduction of the carbohydrate (Maxwell, 1956). 


\section{The Activation of Fatty Acids and Cholic Acid}

Fatty acids must be activated to the corresponding CoA thioester derivatives prior to oxidation. ATP is required in the activation process. Several examples of fatty acid activation reactions are shown in Group IV (reactions 70-72), Group V (reactions 84,85 ).

Likewise, cholic acid must be activated to cholyl-CoA prior to utilization for the synthesis of taurocholic acid or glycocholic acid. An enzyme which catalyzes the activation of cholic acid has been observed in liver microsomes; ATP is required as shown in reaction 86 (Siperstein and Murray, I956; Elliott, I955, 1956). A second enzyme of the supernatant fraction of liver catalyzes the condensation of cholyl-CoA with taurine:

Cholyl-CoA + taurine $\rightarrow$ taurocholic + CoA

The microsome enzyme is also capable of activating the formation of other bile acid-CoA derivatives. Deoxycholyl-CoA and lithocholyl-CoA have been prepared in micromole amounts in this way.

An enzyme capable of activating acetic acid, but not the longer chain fatty acids, has been obtained from yeast (Hilz and Lynen, I953), animal tissues, plants, and rhodospirillum (Berg, I 956a). The mechanism of the activation process has been clarified by Berg (Berg, I 956b, c). The yeast enzyme catalyzes an acetate or propionate dependent exchange of ${ }^{32} \mathrm{P}-{ }^{32} \mathrm{P}$ with ATP. On the other hand, Coenzyme A inhibits the exchange of ATP with ${ }^{32} \mathrm{P}-{ }^{32} \mathrm{P}$. Acetohydroxamic acid is formed from ATP, acetate, and hydroxylamine in the absence of added CoA. The exchange of AMP $-{ }^{14} \mathrm{C}$ with $\mathrm{ATP}$ is dependent on the presence of acetate and $\mathrm{CoA}$ and the exchange of acetate- ${ }^{14} \mathrm{C}$ with acetyl-CoA requires both AMP and PP. These facts support the following mechanism for acetate activation:
I) $\mathrm{AMP} \sim \mathrm{PP}+$ acetate $\stackrel{\mathrm{Mg}^{++}}{\longrightarrow}$ adenyl $\sim$ acetate $+{ }^{32} \mathrm{P}-32 \mathrm{P}$
2) Adenyl $\sim$ acetate + CoA-SH $\longleftrightarrow$ acetyl-CoA + AMP

Sum: 3) ATP + acetate + CoA-SH $\longleftrightarrow$ acetyl-CoA $+\mathrm{AMP}+\mathrm{P}-\mathrm{P}$

Adenylacetate, which is postulated as a product of reaction $\mathrm{I}$, has been synthesized chemically by Berg. In the presence of ${ }^{32} \mathrm{P}-{ }^{32} \mathrm{P}$, adenylacetate, and the yeast enzyme, ATP labelled with ${ }^{32} \mathrm{P}$ is formed. Likewise, acetyl-CoA is formed from adenylacetate and coenzyme $\mathrm{A}$; while in the presence of oxalacetate and the condensing enzyme, citrate is formed. Coenzyme A, by competing for adenylacetate, inhibits ATP formation from adenylacetate and ${ }^{32} \mathrm{P}-{ }^{32} \mathrm{P}$.

It seems likely that the same mechanism underlies the activation of other fatty acids. Jencks has demonstrated a CoA independent acyl activation of octanoate by pig liver enzymes in the course of which pyrophosphate is formed from ATP (Jencks, 1953). It has also been shown that in the presence of the butyrate activating enzyme, butyryl-CoA can be formed from synthetic adenylbutyrate and CoA while ATP can be formed from the adenylbutyrate and pyrophosphate. 


\section{E. The Activation of Sulfate}

(Table 4, reactions 29 and 68, pp. 34, 35). Liver and yeast contain soluble enzymes which catalyze the activation of sulfate and the transfer of the "active sulfate" to nitrophenol or to $m$-aminophenol (Hilz and Lipmann, I 955; Segal, I 956; Wilson and Bandurski, I 956). In the presence of the liver enzymes, 3.3-3.8 moles of inorganic phosphate are liberated per mole of sulfate ester formed, showing that more than

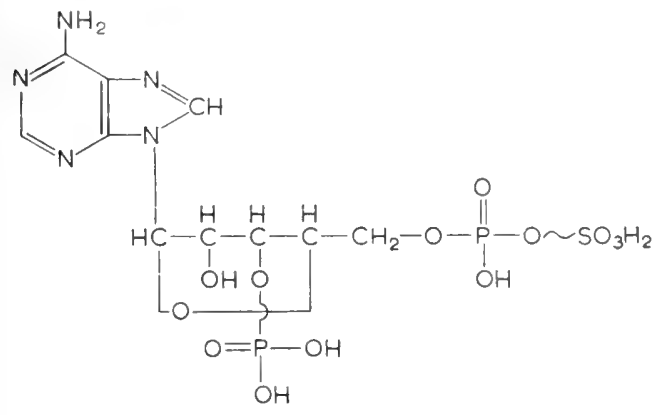

Fig. 15. "Active sulfate". one mole of ATP is involved in the reactions. A sulfate activating enzyme is also present in Neurospora extracts but in this case, the enzyme needed for the esterification of nitrophenol is absent (De Meio and Witzerkanuik, 1956). "Active sulfate" has been shown to be adenosine - $3^{\prime}$ - phosphate $-5^{\prime}$ - phosphosulfate (Robbins and Lipmann, I 956), Fig. I 5 .

The sulfate activating enzyme catalyzes a sulfate dependent exchange of ${ }^{32} \mathrm{P}-{ }^{32} \mathrm{P}$ with ATP. Pyrophosphate inhibits the esterification of $m$-aminophenol by sulfate. The following mechanism is consistent with these observations:

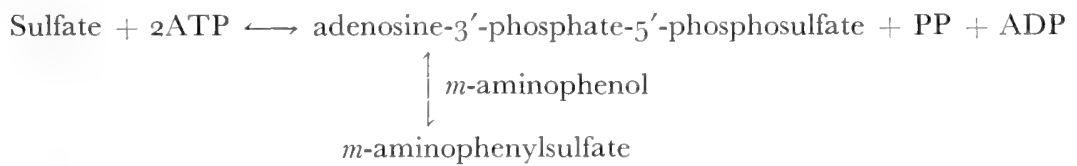

"Active sulfate" is probably utilized by various transferring enzymes for the synthesis of the sulfate esters of amino sugars, and steroid phenols and alcohols.

\section{F. $\mathrm{CO}_{2}$ Fixation}

Reactions illustrating the role of high energy phosphate bonds in the fixation of $\mathrm{CO}_{2}$ into organic linkage are shown in Table 4 , reactions $34,38,69,9 \mathrm{I}$, and 98 . These include the reactions which lead to the synthesis of the tricarboxylic acid cycle catalysts, oxalacetate and succinate, of carbamyl phosphate, and of 5-amino4-imidazole-N-succinylo-carboxamide ribotide (Jones et al., r 955; Marshall et al., I955; Lowenstein and Cohen, I954). Carbamyl phosphate is required for the synthesis of the amino acids, citrulline and arginine, and for the intermediate in pyrimidine synthesis, ureidosuccinic acid, while the carboxamide ribotide derivative is an intermediate in purine biosynthesis.

Oxalacetic carboxylase, the enzyme which catalyzes reaction 34, Table 4(p. 34), occurs in liver mitochondria and has also been observed in rat kidney (Bandurski and Lipmann, I 956; Utter and Kurahashi, I954a, I 954b; Utter et al., I 954). The related enzyme "malic enzyme" also catalyzes the formation of a tricarboxylic 
acid cycle intermediate from $\mathrm{CO}_{2}$ and pyruvate. The differences between oxalacetic carboxylase and the malic enzyme are worth noting (Fig. I6) (Ochoa et al., I950; Ochoa and Kaufman, I95I; Veiga-Salles and Ochoa, I950). Whereas pyruvate is a required substrate for the malic enzyme, phosphoenol pyruvate is the substrate for the oxalacetic carboxylase enzyme. IDP or GDP are required for $\mathrm{CO}_{2}$ fixation in the reaction catalyzed by oxalacetic carboxylase but TPNH is required in the malic enzyme reaction. Oxalacetic carboxylase facilitates the synthesis of

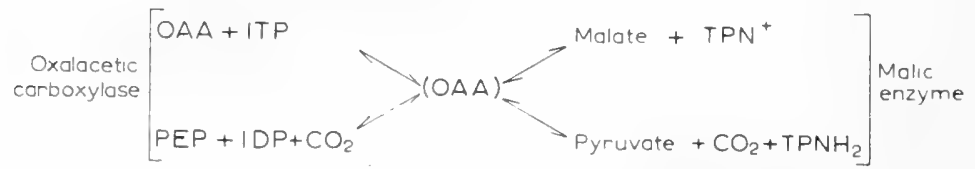

Fig. I6. Postulated relationship between oxalacetic carboxylase and malic enzyme.

phosphoenol pyruvate from oxalacetate or pyruvate by a series of reactions that do not require the participation of pyruvate kinase. This may be advantageous to the organism at a time when the requirements of gluconeogenesis are increased:

$$
\begin{aligned}
& \text { 1) Pyruvate }+\mathrm{CO}_{2}+\mathrm{TPNH}_{2} \stackrel{\text { malic enzyme }}{\text { malic dehydrogenase }} \text { malate }+\mathrm{TPN}^{+} \\
& \text {2) Malate }+\mathrm{DPN}^{+} \stackrel{\text { oxalacetate }+\mathrm{DPNH}_{2}}{\longrightarrow} \text { 3) Oxalacetate }+\mathrm{ITP} \stackrel{\mathrm{OA} \text { carboxylase }}{\longrightarrow} \mathrm{IDP}+\mathrm{CO}_{2}+\text { phosphoenol pyruvate }
\end{aligned}
$$

Reactions $\mathrm{I}$ to 3 explain the finding that the hexose of glycogen formed by liver slices from pyruvate- $2-{ }^{14} \mathrm{C}$ is almost equally labelled in carbon atoms $\mathrm{I}, 2,5$ and 6 although acetyl groups formed from pyruvate show no such randomization.

The enzymes concerned with the fixation of $\mathrm{CO}_{2}$ into succinic acid have recently been obtained in soluble form from acetone powders of rat liver mitochondria, (Lardy and Adler, I956; Friedberg et al., I956), kidney mitochondria, and bacteria (Whiteley, I 953). Although propionyl-CoA is more effective than propionate, ATP is still required in the liver mitochondrial system:

$$
\text { Propionyl-CoA }+\mathrm{CO}_{2} \underset{\mathrm{Mg}^{++} \text {or } \mathrm{Mn}^{++}}{\longrightarrow} \text { succinyl-CoA }
$$

Conceivably, isosuccinate rather than succinate is the primary product (Katz and Chaikoff, I955). In the event that this is the case, the mechanism of isomerization of isosuccinate to succinate remains to be elucidated.

Biotin has been implicated in the carboxylation reactions noted above. Oxalacetic carboxylase contains bound biotin at a higher concentration than that found in the entire liver (Bandurski and Lipmann, 1956). Malic enzyme activity is decreased in livers from biotin deficient turkeys although highly purified pigeon liver malic enzyme is essentially free from biotin. However, biotin in vitro does not activate the enzyme from biotin deficient turkey livers (Blanchard et al., 1950; Korkes et al., 1950). Malic enzyme is an adaptive 
enzyme in Lactobacillus arabinosus. When the bacteria are harvested and then exposed to malate, much less enzyme is formed if the microorganisms have been grown in a biotin deficient medium. The evidence seems to suggest that the biotin is concerned with the formation of the malic apoenzyme and that it does not directly function as a coenzyme in $\mathrm{CO}_{2}$ fixation.

Preparations of propionyl-CoA carboxylase from biotin deficient rats are also much less effective than those from normal rats in catalyzing the formation of succinate. The metabolic defect is repaired by biotin. Tissues from biotin deficient animals also manifest an impaired capacity to fix $\mathrm{CO}_{2}$ into citrulline or purines, (Lardy and Peansky, I953).

Isovaleryl-CoA is an intermediate in the biosynthesis of terpenes and steroids. The carboxylation of isovaleryl-CoA, one of the steps in the catabolism of leucine is another $\mathrm{CO}_{2}$ fixation reaction which requires ATP.

I) $\mathrm{ATP}+\beta$-hydroxyisovaleryl-CoA $+\mathrm{CO}_{2} \rightarrow \beta$-hydroxy- $\beta$-methyl-glutaryl-CoA +

Enzymes for reaction I) have been found in heart and liver (Bachhawat et al., 1956a). It now appears that reaction I) may be resolved into two steps (Robinson et al., I955; Bachhawat et al., I956b):

2) $\mathrm{ATP}+\mathrm{CO}_{2} \rightarrow \mathrm{AMP} \sim \mathrm{CO}_{2}+$ pyrophosphate

3) $\mathrm{AMP} \sim \mathrm{CO}_{2}+\beta$-hydroxyisovaleryl-CoA $\rightarrow \mathrm{AMP}+\beta$-hydroxy- $\beta$-methylglutaryl-CoA

The primary reaction may consist of the formation of the new "active $\mathrm{CO}_{2}$ " compound, adenyl- $\mathrm{CO}_{2}$. An enzyme purified 60 fold from heart tissue liberates pyrophosphate from ATP but does not accomplish the carboxylation of hydroxyisovaleryl-CoA unless a preparation of hydroxyisovaleryl carboxylase is added. Recently Bachhawat and coworkers (1954, I956a) have synthesized a compound believed to be adenosine phosphoryl-carbonate. This compound is capable of carboxylating hydroxyisovaleryl-CoA in the presence of carboxylase and in the absence of the activating enzyme.

It is also appropriate to mention at this point a key carboxylation reaction of photosynthesis. The work of Calvin and coworkers had established that in algae 3-phosphoglycerate is an early product of the "dark" carboxylation reaction (Wilson and Calvin,

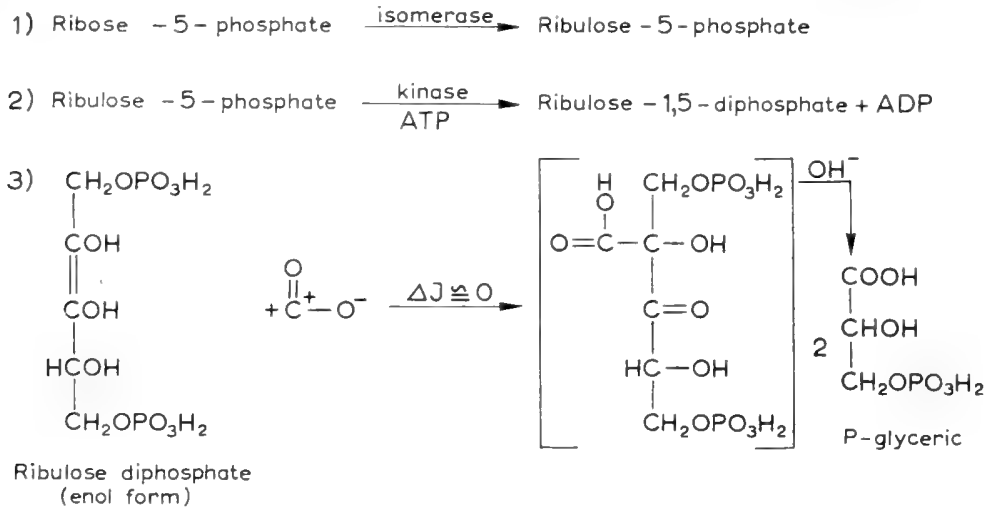

Fig. 17. Carboxylation of ribulose diphosphate. 
1955). This reaction has also been demonstrated in soluble preparations from spinach leaf (Jacoby et al., 1956; Weissbach et al., 1956), and the chemosynthetic bacterium, Thiobacillus denitrificans (Trudinger, 1955). It is possible that this carboxylation reaction also takes place in mammalian lymphatic tissues and tumors (Guzman-Barron et al., I955). In the presence of non-labelled ribose-5-phosphate, ATP, $\mathrm{Mg}^{++},{ }^{14} \mathrm{CO}_{2}$, and the crude enzyme system, phosphoglycerate- $\mathrm{I}-{ }^{14} \mathrm{C}$ is formed, whereas when ribose- 5 -phosphate- $\mathrm{I}-{ }^{14} \mathrm{C}$ is the substrate, phosphoglycerate- $3^{-14} \mathrm{C}$ is formed. Ribose- 1,5 -diphosphate can replace ribose- 5 -phosphate and ATP. With purified ribulose diphosphate carboxylase, ribulose diphosphate- ${ }^{-1}{ }^{14} \mathrm{C}$, and $\mathrm{CO}_{2}$, two moles of beta labelled phosphoglycerate are formed (Fig. I7). Divalent metal ions are required for full activity (Wilson and Calvin, 1955; Weissbach et al., 1956). The enzyme phosphoribulose kinase has been purified by Horecker and coworkers (Hurwitz et al., 1956; Horecker et al., 1956a).

\section{G. Ammonia Fixation}

High energy nucleotides are required for the fixation of $\mathrm{NH}_{3}$ into organic compounds. Two $\mathrm{NH}_{3}$ fixation reactions are shown in Table 4 (reactions 38 and 96) (Jones et al., I955; Lieberman, 1955).

\section{H. Activation Reactions in Nucleic Acid Synthesis}

"Energy-rich" phosphate bonds function in the activation of intermediates in the synthesis of purine nucleotides. A number of these reactions are shown in Group VI of Table 4. They will be discussed in more detail later. The synthesis of 5-phosphoribose pyrophosphate is also mediated by a nucleotide dependent reaction (Group III, reaction 58). 5-phosphoribose pyrophosphate is required for the anabolic reactions shown in Group VII of Table 4.

\section{The Peptide Bond of Pantothenate}

Extracts of $E$. coli catalyze a pantoate dependent exchange reaction between ${ }^{32} \mathrm{P}-{ }^{32} \mathrm{P}$ and ATP. Significantly, $\beta$-alanine does not promote the equilibration between ATP and pyrophosphate (Lipmann, I954). In view of the similarities of the pantoate dependent exchange reaction with other pyrophosphate exchange reactions, it seems possible that the mechanism is as follows:

I) $\mathrm{ATP}+$ pantoate $\longleftrightarrow$ adenyl $\sim$ pantoate $+{ }^{32} \mathrm{P} \sim 32 \mathrm{P}$

2) Adenyl $\sim$ pantoate $+\beta$-alanine $\longleftrightarrow$ AMP + pantothenic

\section{Peptide Bond Formation in the Incorporation of Amino Acids into Protein}

The enzymatic incorporation of labelled amino acids into the proteins of liver homogenates was demonstrated by Winnick and coworkers and Borsook and coworkers (Winnick et al., I948; Borsook et al., I949).

Subsequently it was shown that amino acid uptake could take place in particulate fractions of liver homogenates. Uptake by rat liver particles was accelerated by ATP, $\alpha$-ketoglutarate or citrate, and a mixture of non-labelled amino acids (Peterson et al., I 95I ; Peterson and Greenberg, I952, Kit and Greenberg, I952). Later Siekevitz demonstrated that when DL-alanine- ${ }^{14} \mathrm{C}$ was incubated 
with a rat liver homogenate, the highest specific activity in the homogenate protein, was to be found in the microsome fraction (Siekevitz, I 952). Significant incorporation did not occur when either isolated microsomes or mitochondria were incubated with radioactive alanine but when mitochondria and microsomes were incubated together, active incorporation was observed. When mitochondria were incubated with $\alpha$-ketoglutarate or with succinate and cofactors, a soluble factor was formed which permitted the anaerobic incorporation of labelled alanine into microsome protein. Amino acid incorporation into microsomal protein in the absence of mitochondria was studied by Zamecnik and Keller (I954). Incorporation proceeded under anaerobic conditions provided that a soluble, non-dialyzable liver fraction, ATP, and an ATP generating system such as ATP and phosphocreatine were added. The formation of labelled microsomal protein thus seemed to involve two main steps. The first step, catalyzed by the soluble cytoplasmic proteins, constituted the carboxyl-activation of the amino acids. The second step involved the condensation of the carboxyl activated amino acids to form polypeptide chains and took place most actively on ribonucleoprotein particulates of the microsomes. With regard to the second step, it was found that after incorporation of labelled amino acids into the cell free liver system, the ribonucleoprotein particles contained a large part of the total labelled protein. The same was found for the incorporation into rat liver proteins in vivo at two to three minutes after the intravenous injection of a labelled amino acid. In addition to the activating enzymes, the microsomes, ATP, and the ATP generating system, GTP was required (Keller and Zamecnik, I 956). The role of GTP has not as yet been clarified.

The amino acid activating system could be precipitated from the soluble cytoplasmic proteins by the addition of acetic acid to $\mathrm{pH}$ 5.2. The precipitated, "pH 5.2 proteins", catalyzed an amino acid dependent exchange of ${ }^{32} \mathrm{P}-{ }^{32} \mathrm{P}$ with ATP (Hoagland, I955). A similar amino acid dependent exchange of ${ }^{32} \mathrm{P}-$ ${ }^{32} \mathrm{P}$ has been described in bacterial extracts (De Moss and Novelli, I955).

The exchange catalyzed by the liver enzyme was dependent both on the total concentration and number of amino acids present. The microsome fraction of the cell also catalyzed a pyrophosphate exchange reaction but in this case, the exchange was not influenced by amino acids. AMP failed to inhibit the amino acid dependent exchange; and ${ }^{14} \mathrm{C}$-AMP did not itself exchange with ATP. These results suggested that the amino acids were activated as amino acid-AMP compounds. This possibility was given further support by the finding that $\alpha$-amino hydroxamic acids were formed in the presence of high hydroxylamine concentrations, with concomitant loss of ATP. One mole of pyrophosphate was formed per mole of hydroxamate but no orthophosphate. Since the amino acids did not cause a net splitting of ATP unless hydroxylamine was present, it was proposed that AMP was bound on the enzyme surface and dissociated from the enzyme to only a small extent (Hoagland et al., I 956). The L-amino acid effect on exchange and on hydroxamic acid formation was additive with different amino acids while D-amino acids were inert in this system ${ }^{1}$ :

1 See Addendum, Note 2, p. 123. 
I) "pH 5.2 enzyme" + ATP $\longrightarrow$ enzyme-AMP $\sim \mathrm{PP}$

2) Enzyme-AMP $\sim \mathrm{PP}+\mathrm{RCH}\left(\mathrm{NH}_{2}\right) \mathrm{COOH} \longleftarrow$ enzyme-AMP $\sim \mathrm{C}-\mathrm{CH}\left(\mathrm{NH}_{2}\right) \mathrm{R}$

$\mathrm{O}$

3) Microsome-ribonucleoprotein + enzyme-AMP-C- $\mathrm{CH}\left(\mathrm{NH}_{2}\right) \mathrm{R} \underset{\mathrm{R}}{\text { microsome-ribonucleoprotein-amino acid-14 } \mathrm{C}+\mathrm{AMP}+\text { " } \mathrm{pH} 5.2 \text { enzyme" }}$

4) $\mathrm{ATP}+\mathrm{L}$-methionine $\longleftrightarrow$ adenyl-L-methionine + PP

A protein which catalyzed a methionine dependent pyrophosphate exchange with ATP was also obtained by ammonium sulfate fractionation of the soluble cytoplasmic liver proteins. A similar enzyme system has been reported in yeast (Berg, I956c). In the presence of methionine, AMP- ${ }^{14} \mathrm{C}$ does not exchange with ATP in the yeast enzyme system. The liver fraction that catalyzes the acyl activation of methionine is similar to the one which also synthesizes "active methionine" (S-adenosylmethionine), see Table 4 reaction 88, p. $3^{6}$ (Cantoni, 1953).

\section{K. Glutathione Synthesis}

Enzymes from extracts of acetone dry powders of pigeon liver catalyze the synthesis of $\gamma$-glutamyl cysteine from glutamic acid and cysteine and of glutathione from $\gamma$-glutamyl cysteine and glycine. ATP is required in both reactions (Snoke and Bloch, 1952; Snoke et al., I953):

I) Glutamic + cysteine $+\mathrm{ATP} \rightarrow \gamma$-glutamyl cysteine $+\mathrm{ADP}+\mathrm{H}_{3} \mathrm{PO}_{4}$

2) $\gamma$-Glutamylcysteine $+\mathrm{ATP} \rightarrow \mathrm{GSH}+\mathrm{ADP}+\mathrm{H}_{3} \mathrm{PO}_{4}$

ADP will not substitute for ATP in the above reactions. Both reactions are accelerated by $\mathrm{K}^{+}$and $\mathrm{Mg}^{++}$. Similar enzymes have been found in wheat germ (Webster and Varner, I955). The purified enzyme required for the synthesis of glutamyl cysteine does not catalyze an exchange of orthophosphate- ${ }^{32} \mathrm{P}$ with ATP but does catalyze an exchange of $\mathrm{AD}^{32} \mathrm{P}$ with $\mathrm{ATP}$. This is consistent with the concept that an enzyme-phosphate compound is an intermediate:

3) Enzyme $+\mathrm{ATP} \rightarrow$ enzyme-P $+\mathrm{AD}^{32} \mathrm{P}$

4) Enzyme-P + glutamic $\rightarrow$ enzyme-glutamic + phosphate

The enzyme will however catalyze an exchange of orthophosphate in the presence of glutamate. Cysteine reduces the glutamate induced exchange. The enzyme also catalyzes the exchange of cysteine- ${ }^{35} \mathrm{~S}$ with $\gamma$-glutamyl cysteine in the absence of ATP:

5) Enzyme-glutamate + cysteine $\rightarrow$ enzyme + glutamylcysteine

The mechanism of formation of glutathione from glutamylcysteine and glycine is similar to that for the synthesis of glutamyl cysteine: 
6) Enzyme + ATP $\rightarrow$ enzyme-P + ADP

7) Enzyme-P + glutamyl cysteine $\rightarrow$ enzyme-glutamyl cysteine $+\mathrm{P}_{\mathrm{i}}$

8) Enzyme-glutamyl cysteine + glycine $\rightarrow$ glutathione + enzyme

The glutathione synthesizing enzyme fails to catalyze any significant exchange of phosphate residues between orthophosphate and ATP unless glutamyl cysteine is present. This exchange is slightly decreased by glycine. No experiments on the exchange of $\mathrm{AD}^{32} \mathrm{P}$ with ATP have been performed as yet with the latter enzyme. It is therefore possible that an adenosine diphosphate glutamylcysteine compound may be an intermediate in the reaction instead of the enzyme-phosphate compound. Glutamic and aspartic acid derivatives of ADP have been reported in extracts of chick liver and mammary tissue (Hansen and Hageman, 1956).

IV. THE SYNTHESIS OF PROTOPLASMIG BUILDING BLOGKS

\section{A. Pentose Formation}

\section{Hexose monophosphate shunt}

The pentose sugars, ribose and deoxyribose, are required for the biosynthesis of RNA, DNA, and various coenzymes and nucleotides. Although an enzyme which phosphorylates free ribose and deoxyribose has been described in calf liver (Agranoff et al., I954) free pentose is rather poorly utilized by mammalian tissues. Certain microorganisms are, however, capable of fermenting pentose compounds, particularly after periods of adaptation (Stumpf and Horecker, I 956). It seems likely, that most organisms synthesize their pentose requirements from glucose when an adequate supply of the latter compound is available.

Two mechanisms are known which can account for a net synthesis of ribose from glucose. The first of these, the direct oxidative pathway of glucose metabolism has already been referred to (see p. 3). As a result of the direct oxidative pathway, one mole of glucose is converted to one mole of pentose and one mole of $\mathrm{CO}_{2}$. The dehydrogenase enzymes of the hexose monophosphate shunt have been observed in plants, yeast, bacteria (Lanning and Cohen, I954; Hauge et al., 1955) fungi (Newburgh et al., 1955) aphids (Newburgh and Cheldelin, 1955) animal tissues and tumors (Racker, I 954; Glock and McLean, i 954; Williams-Ashman, i 953; Villavicencio and Guzman-Barron, I955). Rather high levels of the dehydrogenase enzymes are found in adrenal cortical tissues (Kelly et al., I955) and in lymphatic tissues (Glock and McLean, I954), while the enzyme content of skeletal or cardiac muscle is very low. The levels of both dehydrogenases increase rapidly in lactating mammary tissue from the end of pregnancy to the end of lactation and then fall to very low levels in the involuting mammary gland (Glock and McLean, I953). The dehydrogenases are markedly reduced in livers of diabetic or fasting animals but increased in the livers of thyroxine treated rats (Glock and McLean, I 956; Glock et al., 1956b). Glucose-6-phosphatase activity is also increased in the livers of diabetic or thyroxine treated rats. 
(a) Mechanism

\section{Transketolase-transaldolase pathway}

A second pathway of pentose formation exists as a result of which three moles of pentose can be formed from two and one half moles of glucose:

I) 1/2 Fructose-6-phosphate $\rightarrow$ glyceraldehyde phosphate

transketolase

2) Fructose-6-phosphate + glyceraldehyde- $\stackrel{\longrightarrow}{\longrightarrow}$ xylulose-P + erythrose- $\mathrm{P}$

3) Fructose-6-P + erythrose-P $\stackrel{\text { transaldolase }}{\longleftarrow}$ sedoheptulose- $\mathbf{P}+$ glyceraldehyde-P transketolase

4) Sedoheptulose- $\mathrm{P}+$ glyceraldehyde-P $\longrightarrow$ xylulose- $\mathrm{P}+$ ribose $-\mathrm{P}$

pentose epimerase

5) 2 Xylulose-P 2 ribulose- $\mathrm{P}$

pentose isomerase

6) 2 Ribulose-P 2 ribose- $P$

Sum: $21 / 2$ Fructose $-6-\mathrm{P} \rightarrow 3$ ribose $-5-\mathrm{P}$

The fructose-6-phosphate is, of course, derived from glucose as a result of the reactions catalyzed by hexokinase and hexose isomerase, while the glycolytic enzymes, phosphofructokinase, aldolase, and triose isomerase, catalyze the formation of glyceraldehyde-P from fructose-6-phosphate. Enzymes catalyzing reactions 2 to 6 are widely distributed in nature. Phosphoribose isomerase has been demonstrated in yeast, alfalfa, spinach leaves (Hurwitz et al., I 956) and lymphatic tissues (Villavicencio and Guzman-Barron, I955); phosphoketopentose epimerase in spinach, yeast (Srere et al., I 955), spleen (Ashwell and Hickman, I954) muscle, and Lactobacillus pentosus (Horecker et al., i 956a; Stumpf and Horecker, 1956). Transketolase has been crystallized from yeast and both transketolase and transaldolase occur in spinach leaves, yeast, algae, liver and most of the tissues which contain the glucose-6-phosphate and the 6-phosphogluconic acid dehydrogenases (Horecker and Mehler, I 955). Thiamine pyrophosphate is a cofactor for transketolase. The enzymes concerned with pentose formation are not sedimented when the supernatant cytoplasmic proteins of rabbit liver and kidney homogenates are centrifuged at $105,000 \times g$ for $2 \mathrm{~h}$. The enzymes are, however, sedimented after centrifugation at $144,000 \times g$ for $16 \mathrm{~h}$. (Newburgh and Cheldelin, I956).

\section{(b) "Active glycoladehyde" donors and acceptors in transketolase reactions}

A number of substances serve as "active glycolaldehyde" donors. They include Dsedoheptulose-7-phosphate, D-fructose-6-phosphate, D-xylulose-5-phosphate, Lerythrulose, and hydroxypyruvate. It is noteworthy that the orientation of the hydroxyl group on the third carbon atom of the first three compounds listed above is trans to that of carbon four. "Active glycolaldehyde" acceptors include: glycolaldehyde, DL-glyceraldehyde, D-glyceraldehyde-3-phosphate, L-glyceraldehyde-3phosphate, erythrose-4-phosphate and ribose-5-phosphate (Horecker and Mehler, I955). 
(c) Donors and acceptors in transaldolase reaction

Sedoheptulose-7-phosphate or fructose-6-phosphate function as "active dihydroxyacetone" donors in the transaldolase reaction. D-glyceraldehyde-3phosphate or D-erythrose-4-phosphate function as acceptors.

\section{(d) Aldolase reaction}

In the presence of the enzyme aldolase, dihydroxyacetone phosphate condenses with D-glyceraldehyde-3-phosphate or with D-erythrose-4-phosphate. (Smyrniotis and Horecker, 1956). In the first case, fructose diphosphate is the product while in the second case, sedoheptulose diphosphate is formed (Ballou et al., I 955). Muscle aldolase also catalyzes a condensation between dihydroxyacetone phosphate and glycolaldehyde phosphate to give xylulose diphosphate (Byrne and Lardy, I954). The configuration of the newly formed secondary hydroxy groups are trans with respect to one another in each case. Although L-glyceraldehyde-3-phosphate can substitute for D-glyceraldehyde phosphate in an aldolase catalyzed reaction, the latter substrate reacts much more rapidly than the former (Tung et al., I954). In the presence of crystalline muscle aldolase D- or L-glyceraldehyde or glycolaldehyde may also condense with dihydroxyacetone phosphate to yield D-fructose-Iphosphate, L-sorbose-I-phosphate, or D-xylulose-I-phosphate, respectively.

\section{Evaluation of the Embden-Meyerhof and phosphogluconate pathways of glucose oxidation}

When glucose- $-{ }^{14} \mathrm{C}$ or glucose- $-6-{ }^{14} \mathrm{C}$ are incubated with tissue slices or cell suspensions, it is observed that considerably more ${ }^{14} \mathrm{CO}_{2}$ is derived from glucose$\mathrm{I}-{ }^{14} \mathrm{C}$ than from glucose- $6-{ }^{14} \mathrm{C}$. A preferential oxidation of glucose- $\mathrm{I}-{ }^{14} \mathrm{C}$ over uniformly labelled glucose- ${ }^{14} \mathrm{C}$ is also found. The preferential oxidation of the first carbon of glucose to ${ }^{14} \mathrm{CO}_{2}$ constitutes evidence for the participation of the phosphogluconate pathway in glucose metabolism. The ratio of the yield of ${ }^{14} \mathrm{CO}_{2}$ from glucose $-\mathrm{I}-{ }^{14} \mathrm{C}$ to glucose- $6-{ }^{14} \mathrm{C},(G-\mathrm{I}) /(G-6)$, depends upon the tissue, the physiological state of the tissue, the concentration of glucose employed, the duration of the experiment, the ionic constitution of the medium, and the capacity of a given tissue to oxidize to completion the pyruvate or acetyl-CoA formed from glucose via glycolysis. It is to be anticipated that in neoplastic tissues in which the aerobic formation of lactate is observed or in liver slices from diabetic animals in which ketone bodies are formed, the oxidation of glucose- $6-{ }^{14} \mathrm{C}$ to ${ }^{14} \mathrm{CO}_{2}$ will be retarded. Experimentally, the observed $(G-1) /(G-6)$ ratios have ranged from $I . I$ in mammary tissue at the end of pregnancy to 15.7 at IO-I 8 days of lactation, and to 2.I at the beginning of involution of the mammary tissue (Glock et al., I 956a). The ratios for mammary carcinomas are much lower than for lactating mammary tissue (Abraham et al., 1956). $(G-\mathrm{I}) /(G-6)$ ratios varying from 2-5 have been observed with normal liver, regenerating liver, and hepatomas (Bloom and Stetten, I955; Abraham et al., 1955; Agranoff t al., I 954; Bloom et al., 1953 and Katz et al., I 954, I955), lymphatic tissues and lymphatic tumors (Kit, 1956), various other tumors, (Emmelot et al., I955), and yeast cells (Blumenthal et al., I954). On the other hand, the ratios of $(G-\mathrm{I}) /(G-6)$ approach one in rat diaphragm 
(Wenner and Weinhouse, I956a), consistent with the low levels of the direct oxidative pathway dehydrogenases in that tissue (Bloom and Stetten, 1953). The ratio is also about one in brain, heart, or kidney slices (Wenner and Weinhouse, I956a) and in the respiratory $\mathrm{CO}_{2}$ collected after glucose labelled at various positions is injected into the intact rat (Bloom et al., I953).

The radioactivity of the fatty acids, acetoacetate, acetate, lactate, or alanine has also been measured after tissues have been incubated with labelled glucose. In this case, more radioactivity is observed in the metabolite after incubation with glucose $-6-14 \mathrm{C}$ than with glucose- $-{ }^{14} \mathrm{C}$. Radioactivity from glucose- $\mathrm{I}-{ }^{14} \mathrm{C}$ can reach the former compounds primarily as a result of the glycolytic pathway of glucose metabolism since the label is lost when glucose is metabolized via the direct oxidative pathway. On the other hand, radioactivity derived from glucose$6-{ }^{14} \mathrm{C}$ can reach the fatty acids, alanine, or lactate as a result of the metabolism of glucose via the glycolytic pathway and also the 6-phosphogluconate pathway, provided that pentose phosphate, formed from the shunt oxidations is further degraded to the pyruvate level. A preferential labelling of triose phosphate derived from glucose- $6-{ }^{14} \mathrm{C}$ compared with glucose- $-{ }^{14} \mathrm{C}$ may also be anticipated to the extent that the transketolase-transaldolase pathway of pentose formation is operating (Reactions 2 to 4 ; Section IV-A-2-a p. 48). The excess of radioactivity found in the three compounds or the fatty acids thus provides a means of estimating the percentage of three carbon compounds derived via the shunt or via the glycolytic pathway. On this basis, it has been estimated that $0-17 \%$ of the ethanol of Saccharomyces cerevisiae (Blumenthal et al., 1954) and $13-23 \%$ of the alanine of spleen and tumor cells are derived from glucose via the shunt (Kit, I956). The corresponding values for the fatty acids and lactate derived via the shunt were 25-58\% for hepatocarcinomas and lactating mammary tissues (Abraham et al., I 956 ), and $12-25 \%$ for mouse ascites tumors (Wenner and Weinhouse, I956b). In liver slices or homogenates of the rat, approximately $25-33 \%$ of the acetoacetate units were derived from glucose via the shunt pathway (Wenner and Weinhouse, I 956a; Bloom and Stetten, I955; Ashmore et al., 1956). The conversion of glucose to acetyl units via the shunt is increased in liver homogenates by adding $\mathrm{TPN}^{+}$to the experimental vessels.

The injection of insulin into diabetic rats greatly increases the conversion of labelled glucose to $\mathrm{CO}_{2}$ and to fatty acids by liver slices. Felts and co-workers have observed that the effect of hormone treatment on the conversion of glucose-6- ${ }^{14} \mathrm{C}$ to fatty acids is considerably more pronounced than the effect on glucose- $\mathrm{I}^{-14} \mathrm{C}$, while the opposite is true of the effect of insulin on the oxidation of glucose- ${ }^{-14}{ }^{14} \mathrm{C}$ and glucose- $6-{ }^{-14} \mathrm{C}$ to ${ }^{14} \mathrm{CO}_{2}$ (Felts et al., 1956). Glock and associates found an increase in the $(G-1) /(G-6)$ ratio after insulin administration to alloxan diabetic rats (Glock et al., 1956b). After thyroxine treatment, the formation of ${ }^{14} \mathrm{CO}_{2}$ from glucose- $6-{ }^{-14} \mathrm{C}$ by liver slices is significantly increased whereas that from glucose- $\mathrm{I}-{ }^{-14} \mathrm{C}$ is slightly increased.

The difference between the micromoles of ${ }^{14} \mathrm{CO}_{2}$ formed from glucose- ${ }^{14}{ }^{14} \mathrm{C}$ and glucose- $6-{ }^{14} \mathrm{C}$ can be taken as a first approximation of the amount of glucose not oxidized via the citric acid cycle. This value also approximates the total micromoles of pentose formed via the direct oxidative pathway since a pentose phosphate is the product of the decarboxylation of glucose- ${ }^{14}{ }^{14} \mathrm{C}$ to ${ }^{14} \mathrm{CO}_{2}$. On the 
basis of these inferences, Kit has estimated that twice as much exogenous glucose is utilized for pentose formation via the direct oxidative pathway by lymphomas as by normal lymphatic tissues (Kit, I 956).

\section{Evaluation of the transketolase-transaldolase pathway and pentose formation}

The significance of the transketolase-transaldolase pathway of pentose formation has been investigated by Bernstein (1953, I 956). Labelled acetate or formate was administered to chicks and labelled bicarbonate to rats which had been fasted for $48 \mathrm{~h}$. Tissue glycogen and the ribose of ribonucleic acids were isolated and degraded. The results may be illustrated by the experiments with rats. Labelled bicarbonate is converted to carbons three and four of the glucose of glycogen by the rat but there is very little isotope to be found in the other glucose carbons. It was therefore anticipated that pentose carbons two and three should have been labelled, if the pentose had been formed from glucose via the direct oxidative pathway of glucose metabolism. It was found, however, that ribose carbons three and four contained by far the highest specific activity and that the specific activity of carbon three of ribose was twice as great as that of carbon four. This pattern of labelling was therefore indicative of the formation of ribose by a " $\mathrm{C}_{3}$ and $\mathrm{C}_{2}$ " condensation rather than of ribose formation by the direct oxidative pathway. However, Agranoff has shown that the ratio of ${ }^{14} \mathrm{C}$ derived from glucose- $-{ }^{14} \mathrm{C}$ compared with glucose- $-{ }^{14} \mathrm{C}$ is reduced in the livers of rats fasted for $72 \mathrm{~h}$. (Agranoff et al., I954) while as indicated previously, Glock and McLean, I956) reported diminution in the dehydrogenases of the direct oxidative pathway in liver tissue of fasting rats. It is therefore possible that the nutritional status of the animals in part accounts for the results of Bernstein.

In $E$. coli $B$, ribose is synthesized predominantly by the loss of hexose carbon-I (Lanning and Cohen, I954; Bernstein, 1956b). The distribution of radioactivity from labelled glucose in the ribityl moiety of riboflavin has been measured in the growing mold, Ashbya gossypii (Plaut and Broberg, I956). The following results were obtained:

TABLE 5

Counts per min. per $\mathrm{mmol} \cdot 1 \mathrm{O}^{-3}$

\begin{tabular}{lcc}
\hline Compound recovered & Glucose $-I-{ }^{14} C$ & Glucose $-6-{ }^{-14} C$ \\
\hline Riboflavin & 220 & 398 \\
Carbon I' ribityl & 66 & 8 \\
Carbon 5' ribityl & 30 & 158 \\
\hline
\end{tabular}

When glucose-2-14 $\mathrm{C}$ was the substrate, there was twice as much radioactivity in carbons $\left(2^{\prime}-4^{\prime}\right)$ of the ribityl molecule as in carbon $I^{\prime}$ and very little label in carbon $5^{\prime}$. These results are consistent with the possibility that pentose is derived both by the direct oxidative pathway and by the transaldolase-transketolase pathway. If both processes were operating to an equal extent, one might expect with glucose-2-14 $\mathrm{C}$ as substrate, that the molar specific activity of carbons $2^{\prime}$ to $4^{\prime}$ portion of the ribityl molecule would be about $I^{1 / 2}$ times as great as that of carbon I'; this is fairly consistent with the results obtained. 
(a) Deoxyribose aldolase

\section{Deoxyribose synthesis}

The enzyme, deoxyribose aldolase, which catalyzes reaction I, has been observed in E. coli, C. diphtheriae, S. faecalis, liver and thymus (Racker, I95I, I952).

1) Acetaldehyde + glyceraldehyde-phosphate $\longleftrightarrow$ deoxyribose phosphate

This reaction constitutes a possible mechanism for deoxyribose synthesis from two and three carbon compounds. Consistent with this mechanism is the observation that when $E$. coli cells, or $E$. coli cells infected with bacteriophage were grown with glucose- $\mathrm{I}-{ }^{14} \mathrm{C}$ as the sole carbon source, they converted glucose- $\mathrm{I}-{ }^{14} \mathrm{C}$ to deoxyribose of the DNA of the bacteria or the phage (Lanning and Cohen, r 955). In these experiments, the deoxyribose of $E$. coli had $20-26 \%$ and the deoxyribose of phage $4 \mathrm{I}-59 \%$ of the molar specific activity of the original glucose. It was estimated that deoxyribose phosphate synthesized by the combination of acetaldehyde and glyceraldehyde should have had an activity of 76 to $100 \%$ of that of the reference glucose. It is therefore apparent that the functioning biosynthetic mechanism was not correctly represented by the enzymatic condensation alone.

\section{(b) Ribose as a deoxyribose precursor}

A second possible source of deoxyribose is ribose or a derivative of ribose. In growing $E$. coli cells, approximately $37 \%$ of the glucose which is metabolized is degraded via the phosphogluconate pathway. The ribose which is required for ribonucleic acid synthesis is derived principally from this pathway. However, the participation of the transaldolase-transketolase pathway for ribose synthesis is also indicated since the ribose of RNA contained about $25 \%$ of the molar specific activity of the glucose- $-{ }^{14} \mathrm{C}$, in experiments in which the latter substance was the sole carbon source (Lanning and Cohen, I954, I955). In phage infected cells, ribonucleic acid synthesis is reduced and the activities of the bacteria are directed to the synthesis of viral constituents. It is conceivable that an increase in the transaldolase-transketolase pathway of pentose synthesis is one of the alterations in $E$. coli metabolism incident to the synthesis of viral deoxyribose. Thus, the observed ratio of the deoxyribose to glucose radioactivity in growing $E$. coli cells and in phage infected $E$. coli cells which were quoted in the last section are as plausible on the basis of a $\mathrm{C}_{3}$ plus $\mathrm{C}_{2}$ condensation involving the transaldolase-transketolase system as on the basis of the deoxyribose aldolase reaction. There is evidence to suggest that a ribose to deoxyribose conversion can occur at the nucleoside or nucleotide level. Rose and Schweigert (I953) administered uniformly labelled cytidine $-{ }^{14} \mathrm{C}$ to rats and isolated the cytidine and deoxycytidine from the nucleic acids of the rat tissues. It was observed that the molar ratio of the radioactivity of the pyrimidine alone to that of the entire nucleoside was approximately one in both cases, indicating that the original nucleoside had been incorporated into the nucleic acids without a rupture of the sugar-pyrimidine linkage. Similar experiments have been performed with labelled cytidylic and adenylic acids (Roll et al., I 956b). On the other hand, with uridylic acid or guanylic acid, some cleavage of the glycosidic linkage occurs during deoxynucleoside synthesis (Roll et al., I $956 \mathrm{a})$. 
Experiments have also been carried out with $E$. coli $B$ cells (Rose and Schweigert, I953). In this organism, free cytosine and cytidine are essentially equivalent as sources of RNA pyrimidines. It was observed that extensive cleavage of the glycosidic linkage occurred during the incorporation of uniformly labelled cytidine into both the RNA-pyrimidine nucleosides and the DNA-pyrimidine nucleosides. The ratio of radioactivity of pyrimidine to sugar was essentially the same for the RNA-cytidine and uridine and the DNA-deoxycytidine and thymidine although in each case considerably greater than that for the cytidine substrate. Vitamine $\mathrm{B}_{12}$ may be involved in the de noz'o synthesis of deoxyribose (Downing and Schweigert, 1956).

\section{(c) Phosphogluconic dehydrogenase pathway}

Pseudomonas saccharophila degrades glucose to pyruvate and D-glyceraldehyde3-phosphate by the metabolic pathway shown in Fig. I 8 (McGee and Doudoroff,

1) Glucose $\stackrel{\text { ATP }}{\longrightarrow}$ Glucose -6 -phosphate $\stackrel{\text { TPN }^{+}}{\longrightarrow}$ 6-Phosphogluconate

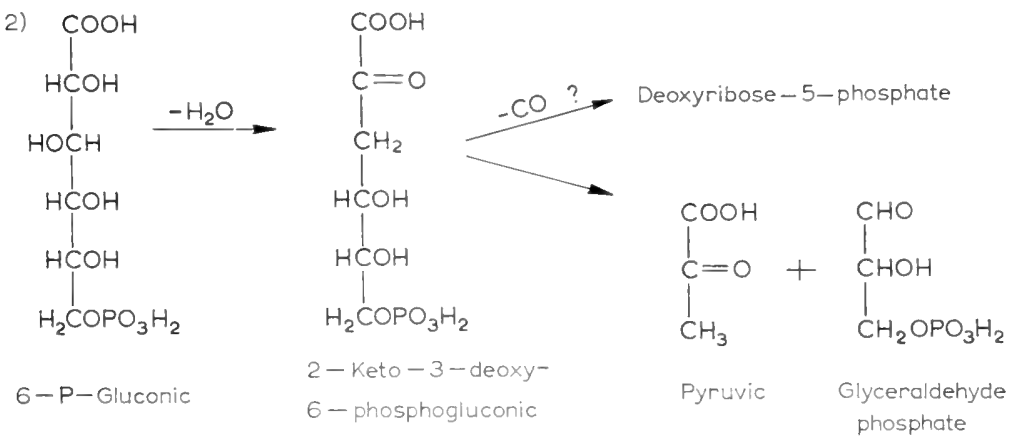

Fig. 18. Metabolism of 6-phosphogluconate by Pseudomonas saccharophila.

I 954). 2-Keto-3-deoxy-6-phosphoghuconate has been identified as an intermediate in the reaction. The latter compound is extremely interesting in that a decarboxylation of this compound would result in the formation of deoxyribose-5phosphate. However, evidence for such a decarboxylation is still lacking. This intermediate is also of interest in that it is produced from 6-phosphogluconate by a dehydration reaction, a reaction similar to one which may take place during the conversion of cytidine to deoxycytidine.

\section{B. The Biosynthesis of Amino Acids}

\section{Distribution of free amino acids in tissues}

Animal tissues, plants, and many microorganisms contain intracellular pools of free amino acids (Kit and Awapara, I953, I 954; Kit, I953; Awapara et al., I 950; Roberts and Tanaka, I956; Schurr et al., I950; Solomon et al., I95I; Spiegelman et al., I 955). The concentrations of the free amino acids differ from tissue to tissue 
but are highly characteristic for a given tissue (Roberts and Frankel, I949; Kit, I 953; Kit and Awapara, I 954). Following a prolonged fast, a decrease is observed in the concentrations of amino acids related to the glycolytic and tricarboxylic acid cycle intermediates and an increase in some of the essential amino acids (Kit and Awapara, I954; Wu, I954). However, the relative constancy of the free amino acid concentration of most tissues is noteworthy.

The patterns of the free amino acids of tumors differ from those of related normal tissues in several respects. The concentrations of free alanine, glycine, and proline are rather high in neoplastic tissues although the concentrations of ethanolaminephosphate, aspartate and glutamine may be rather low (Roberts and Frankel, 1949; Kit and Awapara, 1953). The interesting observation has been made that although the glutamine pool is low in growing tumor tissues, this pool increases to the level found in normal tissues when the tumors undergo regression (Roberts and Tanaka, 1956). The low concentrations of glutamine in tumors may reflect a high rate of utilization of the latter substance in anabolic processes. Likewise, the glycine pool of tumor tissue may be high due to the fact that the beta carbon of the glycine precursor, serine, is utilized at a considerably faster rate than the glycine moiety. The high concentration of alanine is in all probability related to the rapid aerobic glycolysis of tumors.

The concentration of the essential amino acids has been measured in various animal tissues (Schurr et al., I950; Solomon et al., I95 I). These amino acids and proline are generally found in concentrations ranging from about $0.2-1.5 \mu \mathrm{mol} / \mathrm{g}$ wet weight of tissue. The concentration of free methionine is very low. On the other hand, the concentrations of glutamic, aspartic, glycine, serine, alanine, glutamine, ethanolamine phosphate, glutathione, and taurine are usually much higher, and in some cases may be as high as seven micromoles per gram wet weight of tissue. $\gamma$-aminobutyric acid occurs at relatively high levels in brain tissue, while low levels of glycerolphosphorylethanolamine are also frequently encountered in tissue extracts.

\section{Amino acid relationships}

Information as to the metabolic relationships among the amino acids and between the amino acids and intermediates of glycolysis and the tricarboxylic acid cycle may best be illustrated by citing the experiments of Abelson and coworkers (Abelson, I954; Abelson and Vogel, I955). E. coli cells or yeast cells were grown in the presence of glucose $-{ }^{14} \mathrm{C}_{1}{ }^{14} \mathrm{CO}_{2}$, or some other labelled substrate. At the end of the experiment, the cell proteins were hydrolyzed and the radioactivity of each of the amino acids was determined. In parallel experiments, exogenous unlabelled amino acids or other metabolites were added to the culture medium. Certain of the exogenous amino acids, depressed the incorporation of the labelled glucose or carbon dioxide into related amino acids. The results obtained provide a measure of the relative utilization of competing exogenous and endogenous amino acids. For example, in the presence of non-labelled exogenous aspartate, the incorporation of labelled glucose into the following amino acids of $E$. coli cell protein is depressed: aspartate, threonine, isoleucine, methionine, and lysine. There was also a lesser reduction of the incorporation of label into the glutamic acid and proline of the protein. One may thereby infer that a metabolic relationship exists 
between the above mentioned amino acids. This method of establishing biochemical relationships is called the "Isotope Competition" method, and will be referred to frequently below. While the interpretation of isotope competition experiments may sometimes be rather complicated, the studies are a welcome supplement to those based on an analysis of biochemical mutants, conventional isotope studies, or enzyme studies.

Some of the metabolic relationships among the amino acids are illustrated in Fig. I. It will be observed that six groups may be distinguished:

(a) Histidine. The carbon of histidine is probably derived from a pentose derivative.

(b) Serine family. When yeast cells were incubated with labelled serine, the protein serine, glycine, and cysteine, had the same specific activity. However, tracer glycine experiments with Torulopsis utilis, showed that cysteine and serine had only $15 \%$ of the specific activity of protein glycine. (Abelson and Vogel, 1955). In E. coli, exogenous threonine contributed to the serine family and serine contributed to the pyruvate family (Abelson, 1954).

(c) Aromatic family. A relationship exists between the aromatic amino acids, phenylalanine, tyrosine, and tryptophane, and the vitamin, $p$-aminobenzoic acid. Much of the carbon of these amino acids is derived from the phosphoenolpyruvate and erythrose phosphate.

(d) Pyruvate family. In T. utilis, labelled alanine is incorporated into protein bound alanine, valine, and leucine. Non-labelled pyruvate reduces the incorporation of glucose-14 $\mathrm{C}$ in to the above amino acids (Abelson, 1954).

(e) Aspartic family. In yeast cells, labelled aspartate is incorporated into a number of protein bound amino acids. On the basis of relative specific activity, these amino acids fall into two groups. Aspartate, threonine, isoleucine, and methionine have approximately the same specific activity. Glutamic, arginine, proline, and lysine have less than half the specific activity of the former amino acids.

( $f$ ) Glutamic family. Glutamate- $1-14 \mathrm{C}$ is incorporated into the following protein bound amino acids of yeast cells: glutamic, proline, arginine. $\alpha$-Ketoglutarate reduced the incorporation of labelled glucose into these amino acids and into amino acids of the aspartic family. Glutamic semialdehyde depressed the incorporation of glucose- ${ }^{14} \mathrm{C}$ into glutamate and arginine of yeast cell protein.

\section{Essential amino acids}

Although many microorganisms and plants synthesize all of the amino acids which are commonly found in protein, mutant strains are known which are incapable of synthesizing particular amino acids. An exogenous source of these amino acids must be available if growth is to occur. Likewise, animal organisms manifest nutritional requirements for particular amino acids, due to their inability to synthesize these amino acids from the materials ordinarily available at a speed commensurate with the demands for normal growth. The amino acids, histidine, isoleucine, leucine, lysine, methionine, phenylalanine, threonine, tryptophane, and valine must be supplied in the diet to promote the growth of the young mouse. Arginine, as well as the former nine amino acids, is essential for the young rat, while in the chick, glycine supplements are required along with the ten amino acids required by the rat.

In adult animals, the nutritional requirements of the animal may be investigated by determining which amino acids are necessary for the maintenance of nitrogen equilibrium. In man, eight amino acids are essential for nitrogen equilib- 
rium (Rose, 1949), namely isoleucine, leucine, lysine, methionine, phenylalanine threonine, tryptophane, and valine. Histidine is also required for nitrogen equilibrium in the adult dog. Although arginine is not needed for nitrogen equilibrium in man, spermatogenesis is reduced in diets lacking in arginine. Sperm cells are rich in arginine.

The biosynthetic potentiality of the entire animal should be distinguished from that of the individual tissues. The nutritional requirements of two strains of mammalian cells have been studied by tissue culture techniques (Eagle et al., I956a). Mouse fibroblast cells (Strain L) and human uterine carcinoma cells (HeLa) require $13 \mathrm{~L}$-amino acids for growth. These are arginine, cysteine, histidine, tyrosine, glutamine and the eight amino acids required by the adult for nitrogen equilibrium. In the presence of ammonium ions, glutamic acid can replace glutamine in HeLa but not in mouse fibroblast cells. However, ro-20 times as much glutamic acid as glutamine is required. Probably, few mammalian tissues other than liver effect a net synthesis of arginine, cysteine, and tyrosine.

The biosynthetic activities of normal and malignant mouse tissues have been studied with ${ }^{14}$ C-labelled substrates. With labelled glucose or acetate (Kit and Graham, 1956b; Busch et al., I956) radioactivity is incorporated into free alanine, glycine, serine, glutamate, aspartate and proline. The glutamine of diaphragm, liver and brain; and the $\gamma$-amino butyric acid of brain tissue were also labelled (Kit and Graham, 1956a). In liver tissue, the conversion of labelled acetate to arginine also takes place (Greenberg and Winnick, 1949). These results complement those obtained in nutritional studies.

\section{The synthesis of the carbon skeletons of amino acids}

\section{(a) Histidine}

The terminal steps in the biosynthesis of histidine are shown in Fig. 19. The following evidence may be cited in support of this scheme:<smiles>O=P(O)(O)CC(O)c1c[nH]cn1</smiles>

D-Erythroimidazole glycerol phosphate<smiles>NC(Cc1c[nH]cn1)C(=O)O</smiles>

Histidine.
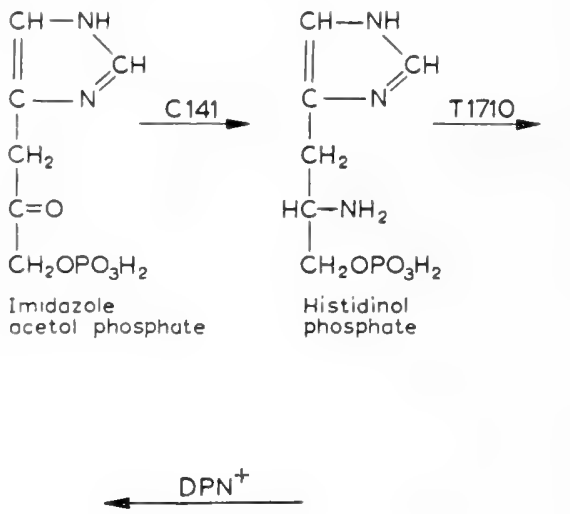

Fig. 19. Histidine biosynthesis.

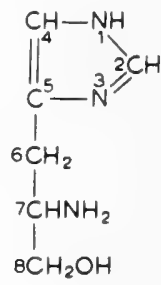

Histidinol

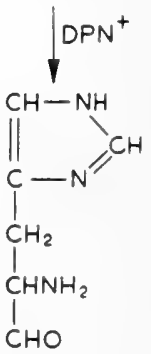

Histidina

I) Imidazoleglycerol phosphate, imidazoleacetol phosphate, and histidinal phosphate accumulate in the culture medium of Neurospora mutants. Likewise, histidinol has been 
isolated from the culture medium of E. coli and Neurospora mutants which require histidine. The histidinol may be utilized for growth by a second histidineless E. coli mutant (Davis, 1955a).

2) An enzyme exists in cell free preparations of Neurospora which converts imidazoleglycerol phosphate to imidazoleacetol phosphate and a second enzyme which catalyzes the transamination of the latter to histidinal phosphate (Ames and Mitchell, 1955). Glutamic acid and pyridoxal phosphate are required for the transamination reaction (Ames and Horecker, 1956). The phosphate esters are hydrolyzed by crude potato phosphatase. Since free imidazole compounds have been found in the mutant media, it would seem that the hydrolysis of histidinal phosphate to histidinol is not improbable. It is to be noted that the configuration of the terminal carbon atoms of D-erythroimidazoleglycerol phosphate is similar to that of D-ribose phosphate. This suggests that ribose phosphate might be a precursor of the former compound.

3) Soluble enzymes obtained from E. coli, yeast or Arthobacter, catalyze the conversion of histidinol to histidine. Two moles of $\mathrm{DPN}^{+}$are reduced per mole of histidinol oxidized (Adams, I955a, b). L-histidinal is active in the enzyme system which catalyzes the above conversion. Histidinal is reduced to histidinol by DPNH. In those E. coli mutants which are incapable of forming histidine from histidinol, histidinal is not active as a histidine precursor.

In an effort to throw light on the precursors of imidazoleglycerol phosphate, E. coli mutants which accumulated histidinol were incubated with glucose- $\mathrm{I}^{-14} \mathrm{C}$ or glucose- $6-{ }^{14} \mathrm{C}$. The histidinol was isolated and degraded (Westley and Ceithaml, 1956). In each case, the radioactivity was almost entirely confined to carbons 2,6 , and 8 of the histidinol molecule. This pattern of labelling is consistent with a mechanism involving a pentose compound formed by a condensation of glycolytic intermediates but not on the basis of a pentose compound derived by way of the hexosemonophosphate shunt. The results obtained in experiments with $E$. coli cells are however at variance with these found in other experiments with yeast cells, in which the histidine of the protein was isolated and degraded. In this case, approximately half of the label from glucose- $-1+{ }^{-1} \mathrm{C}$ was attributable to carbon four of the histidine molecule while the remainder of the radioactivity was distributed between carbons $5^{-8}$ and carbon two of the imidazole ring (Levy and Coon, 1954). Non-labelled glutamic acid depressed the incorporation of labelled acetate but not labelled glucose into histidine which constitutes further evidence that the intermediates in histidine synthesis are not derived directly from tricarboxylic acid cycle metabolites.

In lactic acid bacteria as in yeast, formate is a precursor of the $\mathrm{C}_{2}$ of the imidazole ring of histidine (Mitoma and Snell, r955). It is probable that glucose- $-_{-14} \mathrm{C}$ and glucose- $6-{ }^{14} \mathrm{C}$ are precursors of $\mathrm{C}_{2}$ by virtue of the fact that they are potentially convertible to "active formate" compounds. It is of interest that in certain bacteria, carbon two of guanine and the amino group attached to carbon two are precursors, respectively, of carbon two and nitrogen one of histidine. Aminoimidazolecarboxamide ribotide is a product of this reaction (Mitoma and Snell, 1955; Magasanik, 1956; Magasanik et al., 1956).

The transfer of the second carbon and the amino group of guanine may represent an example of a formimino group $(-\mathrm{CH}=\mathrm{NH})$ transfer reaction. Folic acid derivatives are apparently coenzymes in formimino group transfers, two examples of which have been studied. Formimino glutamic acid (FGA), an intermediate in

1) $\mathrm{FGA}+$ THFA $\longleftrightarrow \mathrm{N}^{10}$-(formimino)-THFA + glutamic

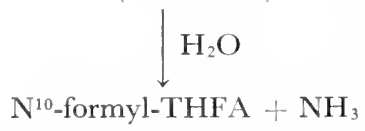

histidine catabolism, accumulates in the livers of folic acid deficient rats. Liver enzymes from normal rats catalyze the transfer of the formimino group from FGA to tetrahydrofolic acid. The same enzymes in the presence of THFA catalyze 
an exchange of glutamic-2- ${ }^{14} \mathrm{C}$ with the glutamyl moiety of FGA (Miller and Waelsch, I956). A similar exchange reaction involving the intermediate in purine degradation, formiminoglycine, and glycine- $2-{ }^{14} \mathrm{C}$ is catalyzed by Clostridium enzymes (Sagers et al., i 956).

2) Formiminoglycine- ${ }^{14} \mathrm{C}+$ THFA $\longrightarrow$ glycine-2-14 $\mathrm{C}+$ formimino THFA

$$
\text { formate }+\mathrm{NH}_{3}+\mathrm{THFA} \longleftarrow \mathrm{H}_{2} \mathrm{O}
$$

In E. coli, the amide nitrogen of glutamine is also an efficient precursor of nitrogen one of the imidazole ring of histidine (Neidle and Waelsch, r 956).

The second carbon of histidine is also a precursor of "active formate". Thus, when histidine- $2-{ }^{14} \mathrm{C}$ is injected into rats, the label can be detected in the following products of formate metabolism: a) the choline methyl groups of liver or hepatoma, b) the methyl group of creatine, and c) urinary allantoin, and uric acid (Reid and Landefeld, I 95 I; Reid et al., I952).

\section{(b) Serine and glycine biosynthesis}

Interconversion of glycine and serine. A relationship between the amino acids, serine and glycine, was demonstrated by Shemin. When serine labelled with ${ }^{15} \mathrm{~N}$ in the amino group and with ${ }^{13} \mathrm{C}$ in the carboxyl group was fed to rats, labelled glycine could be isolated with no change in the ratio of ${ }^{15} \mathrm{~N}$ to ${ }^{13} \mathrm{C}$. This suggested that the conversion of serine to glycine involved the splitting out of the beta carbon of serine (Shemin, I 946). The reverse reaction, the formation of serine from glycine

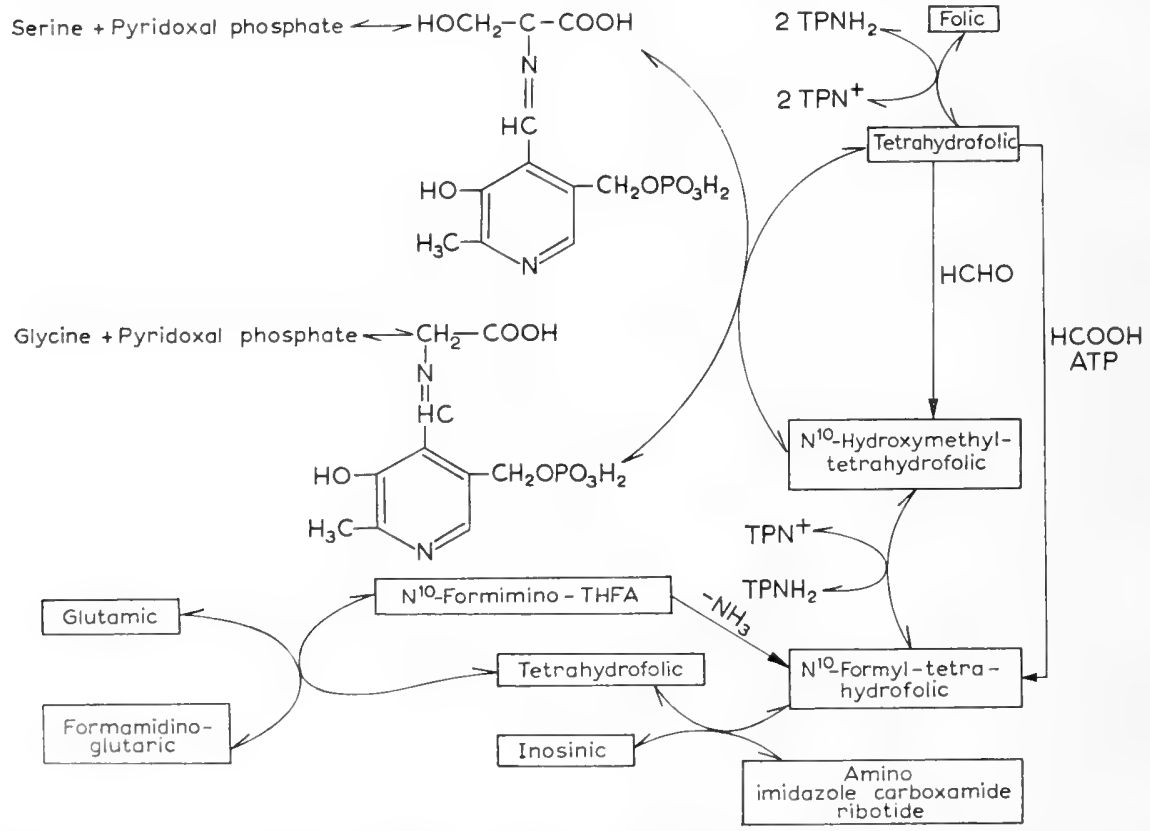

Fig. 20. Role of pyridoxal phosphate and folic acid derivatives in serine and glycine interconversion. 
was thereafter demonstrated by Sakami and by Greenberg and coworkers. The latter investigators showed that rat liver slices converted glycine- $2-{ }^{14} \mathrm{C}$ to both the alpha and the beta carbon of serine. Likewise, beta labelled serine could be formed from formate- ${ }^{14} \mathrm{C}$ and unlabelled glycine in liver slices or in the intact rat (Siekevitz and Greenberg, I 949; Sakami, 1948). Pyridoxal phosphate and tetrahydrofolic acid derivatives are cofactors in the interconversion of glycine and serine (Kisliuk and Sakami, 1955; Alexander and Greenberg, I 955, 1956). It has been postulated that pyridoxal phosphate forms a Schiff base with glycine, thereby activating the methylene carbon atom of glycine (Fig. 20). Also, tetrahydrofolic acid functions as an acceptor of formate or $\mathrm{HCHO}$ in numerous one carbon transfer reactions including the synthesis of serine from glycine and formate (Kisliuk and Sakami, I955; Blakeley, I954). Reactions one to eight have been demonstrated with enzymes of pig liver (Jaenicke, I955):

\section{ATP}

I) Formate- ${ }^{14} \mathrm{C}+\mathrm{THFA} \longrightarrow \mathrm{N}^{10-F o r m y l-14} \mathrm{C}(\mathrm{THFA})$

2) Serine-3-14 $\mathrm{C}+\mathrm{THFA} \rightarrow \mathrm{N}^{10}$-Formyl-14 $\mathrm{C}($ THFA $)+$ glycine

$3 \mathrm{TPNH}_{2}$

3) ${ }^{14} \mathrm{C}$-Formyl (folic) + glycine $\longrightarrow$ serine-3-14 $\mathrm{C}+$ THFA

$\mathrm{DPNH}_{2}$

4) $\mathrm{N}^{10}$-Formyl- ${ }^{14} \mathrm{C}$ (THFA) + glycine $\longrightarrow$ serine-3-14 $\mathrm{C}+$ THFA

5) THFA + serine $\rightarrow \mathrm{N}^{10}$-hydroxymethyl (THFA) + glycine

6) $\mathrm{N}^{10}$-Formyl (THFA) $+\mathrm{TPNH}_{2} \rightarrow \mathrm{N}^{10}$-hydroxymethyl $(\mathrm{THFA})+\mathrm{TPN}^{+}$

7) N10-Formyl (THFA) + THFA $\stackrel{\mathrm{TPN}^{+}}{\longrightarrow}$ Dihydrofolic $+\mathrm{N}^{10}$-(hydroxymethyl) THFA ATP

8) Formate + glycine $\underset{\mathrm{DPNH}_{2}}{\longrightarrow}$ serine

THFA

ATP is required in reaction $\mathrm{I}$ ) and reaction 8 ) in order to activate the formate. Reduced TPN is needed to convert folic acid to tetrahydrofolic acid in reactions 3) and to convert $\mathrm{N}^{10}$-formyl tetrahydrofolic acid to $\mathrm{N}^{10}$-hydroxymethyltetrahydrofolic acid (reaction 6). $\mathrm{N}^{10}$-formyltetrahydrofolic acid serves as an "active formyl" group donor in the formation of inosinic acid or in the synthesis of carbon two of the imidazole ring of histidine (Jaenicke, 1955). See Fig. 20.

There is reason to believe that the natural coenzyme is a polyglutamate rather than a monoglutamic acid derivative of folic acid. Polyglutamates of folic acid act catalytically in stimulating the conversion of serine to glycine by Clostridium enzymes under conditions where monoglutamyl folic acid compounds are either inactive or active only at substrate concentrations (Wright, I955, I956, Wright and Stadtman, 1956).

Serine synthesis from phosphoglycerate. Since glycine and serine are both nutritionally non-essential amino acids in animal tissues and many microorganisms, it is necessary to inquire next as to the origin of the carbon skeletons of these amino acids. The evidence now indicates that serine can be synthesized prior to glycine in certain 


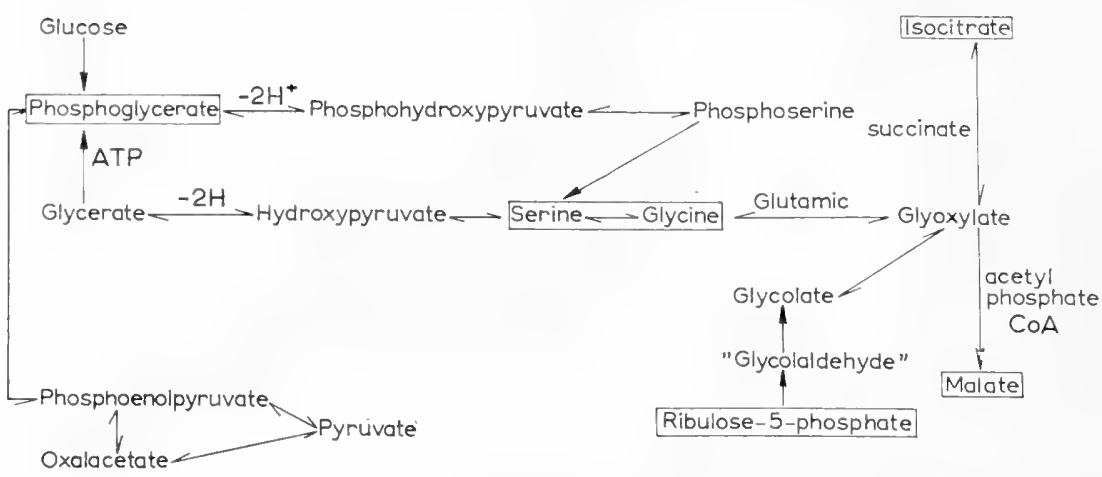

Fig. 21. Proposed pathways of serine and glycine biosynthesis.

tissues by a pathway in which the glycolytic intermediate, phosphoglycerate is a proximal precursor (Fig. 2I) (Black et al., 1955). When uniformly labelled glucose was fed to young rats, it was found that both the serine and the glycine became labelled (Arnstein and Keglevic, I956; Arnstein and Stankovik, I956). The feeding of glucose $-\mathrm{I}^{14} \mathrm{C}$ led to the labelling of serine but not of glycine, a result to be anticipated, since the first carbon of glucose is converted via glycolysis to the beta carbon of phosphoglycerate and to the beta carbon of serine. The latter carbon atom is lost when serine is converted to glycine. However, when labelled glucose was fed to folic acid deficient rats, the labelling of glycine was greatly inhibited while serine radioactivity was not reduced. Since the deficiency of folic acid inhibits the interconversion of glycine and serine, it was evident that the serine was formed prior to glycine from the labelled glucose. Radioactive alanine was a much poorer precursor of rat serine and glycine than of alanine, aspartic, and glutamic acids, suggesting that the pyruvate which is derived from alanine was not the immediate precursor of serine.

The synthesis of the beta carbon of serine from glucose- $\mathrm{I}-{ }^{14} \mathrm{C}$ and from glucose$6-{ }^{14} \mathrm{C}$ was also demonstrated in vitro in tumors and in various normal tissues of the mouse and rat (Kit, I 955). From the results with glucose- ${ }^{14} \mathrm{C}$ the conclusion could be drawn that serine could be synthesized by a mechanism which did not necessitate the direct oxidative pathway of glucose metabolism since the labelled carbon would have been lost as $\mathrm{CO}_{2}$ if glucose- $-{ }^{14} \mathrm{C}$ had been metabolized via the latter pathway. When glucose $-2-{ }^{14} \mathrm{C}$ or uniformly labelled glucose were used as substrates, radioactivity was found in both glycine and serine. This result is also consistent with the postulated pathway via phosphoglycerate since phosphoglycerate- $2-{ }^{14} \mathrm{C}$ and serine- $2-{ }^{14} \mathrm{C}$ are presumably formed from glucose- $2-{ }^{14} \mathrm{C}$ and, therefore, the label is retained in the glycine which is formed from serine. The formation of serine and of glycine from glycerol- $\mathrm{I}^{14} \mathrm{C}$ was also demonstrated in lymphatic tissues and tumors (Kit and Graham, r956b).

Scrine and glycine biosynthesis was not inhibited when fluoride and non-labelled pyruvate were added to the incubation flasks, although the labelling of alanine, aspartate, und glutamate was greatly inhibited. This result supports the idea that phosphoglycerate is a more likely serine precursor than phosphoenolpyruvate or pyruvate. Serine was also formed prior 
to glycine in the tumor cells since the addition of non-labelled serine to the medium reduced the conversion of labelled precursors to glycine but increased the radioactivity of serine.

Enzymes effecting the conversion of glyceric $-3^{-14} \mathrm{C}$ to serine have been partially purified from rat liver (Ichihara and Greenberg, 1955). $\mathrm{ATP}^{\mathrm{T}}, \mathrm{DPN}^{+}$, and an amino donor such as glutamic acid or alanine, stimulate serine synthesis. The conversion of phosphoglycerate and phosphohydroxypyruvate to serine could also be shown in the same enzyme system. The formation of labelled phosphoserine and serine from glycerol or glyceric acid has been demonstrated in experiments with intact tumor cells and with tumor homogenates (Kit, unpublished).

The above results are all consistent with a pathway of serine and glycine biosynthesis via phosphoglycerate, phosphohydroxypyruvate, and phosphoserine. It is also possible, however, that serine may be formed by a pathway involving hydroxypyruvate and D-glycerate as direct precursors. Free glycerate occurs in tobacco leaves and in cress seedlings (Palmer, I956; Isherwood et al., r954b). Free hydroxypyruvate and also glyoxylate have been observed in rabbit kidney, liver, and tumor tissue (Linko and Virtanen, I 955). Plant tissues contain an enzyme which catalyzes the reduction of hydroxypyruvate by DPNH to D-glycerate (Stafford et al., I 954). The hydrolysis of phosphoglycerate to glyceric acid as well as of phosphoserine to serine is apparently catalyzed by non specific enzymes. The transamination of hydroxypyruvate and alanine to serine and pyruvate by enzymes from liver and kidney has also been shown (Sallach, I 954). However, hydroxypyruvate- $2-{ }^{14} \mathrm{C}$ is a relatively poor serine precursor in the green algae, Scendesmus (Milhaud et al., I956) possibly due to the fact that the exogenous hydroxypyruvate is rapidly catabolized (Schweet et al., I95I; Horecker, I 954). In yeast cells, glycine and serine biosynthesis has been studied with pyruvate-2${ }^{14} \mathrm{C}$ as substrate (Wang et al., I954). Although the alanine which was isolated was labelled primarily on the alpha carbon atom, it was observed that serine and glycine were equally labelled on the carboxyl and alpha carbons. This result suggested that pyruvate was not directly converted to serine and glycine, but that pyruvate- $2-{ }^{14} \mathrm{C}$ was first carboxylated to malate- $2-{ }^{14} \mathrm{C}$ by the malic enzyme. As a result of the oxidation of the latter substance through the tricarboxylic acid cycle, the malate- $2-{ }^{14} \mathrm{C}$ would be converted to oxalacetate- $1,2-{ }^{14} \mathrm{C}$ which in turn could be decarboxylated by the enzyme, oxalacetic carboxylase, to phosphoenolpyruvate $-\mathrm{I}, 2-{ }^{14} \mathrm{C}$. This substance could then give rise to the serine and glycine compounds with the observed pattern of labelling.

The formation of glycine from pentose. A "serineless" E. coli mutant is known in which the serine requirements cannot be supplied by glycine. When this strain is

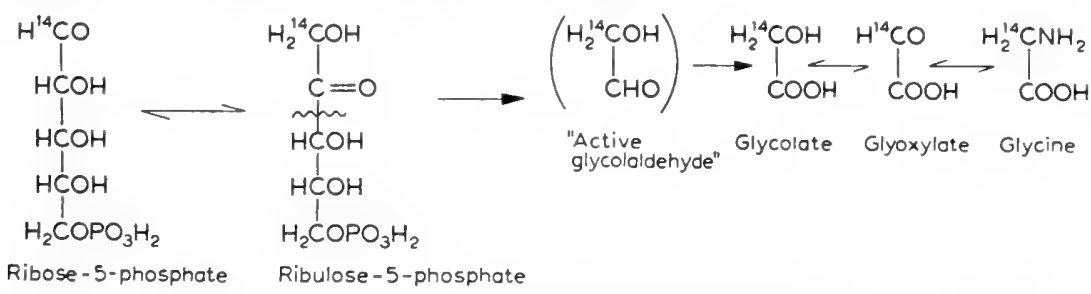

Fig. 22. Postulated mechanism of glycine synthesis from ribose-5-phosphate. 
grown in the presence of uniformly labelled glucose, glycine but not serine becomes labelled (Meinhart and Simmonds, I955). Since this mutant is still capable of synthesizing glycine, it is apparent that mechanisms of glycine formation must exist in which serine is not an obligatory precursor of glycine. The probable glycine precursors in this instance are glyoxylate, glycolate, and glycolaldehyde. Since the latter substances are rapidly converted to glycine by animal and plant tissues (Weinhouse and Friedmann, I95 I ; Friedmann et al., I956), it is of interest to inquire as to the possible sources of these two carbon compounds (Friedmann et al., I 956; Tolbert and Cohan, i953).

The "active glycolaldehyde" donor, ribulose phosphate is an interesting potential precursor of glycine. Weissbach and Horecker have demonstrated glycine formation from $\mathrm{I}^{14} \mathrm{C}$-ribose-5-phosphate in spinach leaf extracts (Fig. 22; Weissbach and Horecker, I955).

It was observed that $80 \%$ of the glycine radioactivity was attributable to the alpha carbon. Little label was found in serine. The distribution of radioactivity ruled out the possibility that fructose-6-phosphate and phosphoglycerate were intermediates, even though the conversion of $\mathrm{I}^{14} \mathrm{C}$-ribose-5-phosphate to $\mathrm{I}, 3^{-}$ ${ }^{14} \mathrm{C}$-fructose-6-phosphate, occurs in spinach extracts. The latter substance would have been metabolized to glycine- ${ }^{14} \mathrm{C}$ instead of to glycine- $2-{ }^{14} \mathrm{C}$.

Isocitric lysase ${ }^{1}$. The tricarboxylic acid cycle intermediate, isocitric acid is also a potential glycine precursor. The following aldolose type of reaction is catalyzed by enzymes from yeast, Aspergillus, Rhizopus, and Pseudomonas (Smith and Gunsa-

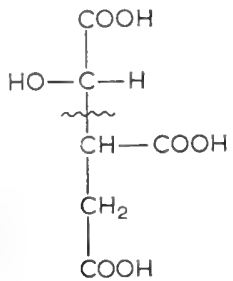

Isocitric

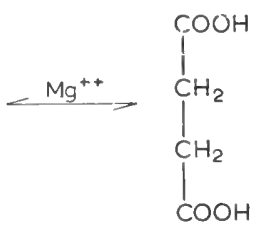

Succinic

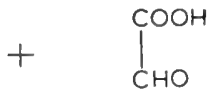

Glyoxylate

Fig. 23. Isocitric lysase reaction.

lus, 1954; Olson, 1954; Saz and Hillary, 1956) (Fig. 23). It is to be noted that since this reaction is reversible, it also represents an alternative mechanism for the formation of the tricarboxylic acid cycle intermediate, the isocitrate.

Glyoxylate from malate ${ }^{1}$. An enzyme purified from $E$. coli G-26 catalyzes the following reaction (Wong and Ajl, I956) :

$$
\text { Acetyl phosphate }+ \text { glyoxylate } \underset{\substack{\mathrm{Mg} \\ \text { cysteine }}}{\stackrel{\mathrm{CoA}}{\longrightarrow}} \text { malate }
$$

1 See Addendum, Note 3, p. 123. 
Threonine aldolase. Liver and kidney enzymes catalyze the breakdown of threonine to glycine and acetaldehyde (Braunstein and Vilenkina, I 949; Lin and Greenberg, I 954) (Fig. 24):

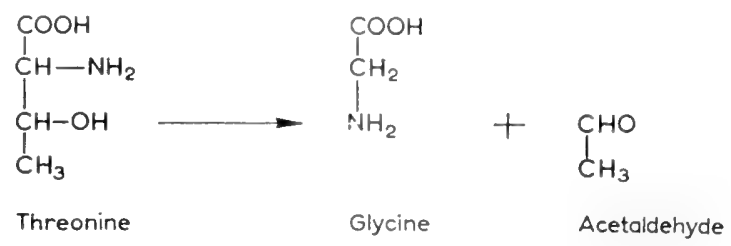

Fig. 24. Threonine aldolase reaction.

It is possible that this reaction is of some significance in mammalian tissues. Thus, after administering threonine $-4{ }^{-14} \mathrm{C},{ }^{15} \mathrm{~N}$ to rats, ${ }^{15} \mathrm{~N}$ glycine and ${ }^{14} \mathrm{C}$ acetate were isolated (Meltzer and Sprinson, I952) while with threonine- $1,{ }^{2-}{ }^{14} \mathrm{C}$, glycine $-{ }^{14} \mathrm{C}$ was obtained (Lien and Greenberg, I952).

\section{(c) Cysteine}

The carbon atoms of cysteine are derived from serine in microorganisms and in mammals. Thus, in $E$. coli, non-labelled serine suppresses the incorporation of labelled glucose to cysteine (Abelson, I954) while in Neurospora (Abelson and Vogel, I955), serine- $3-{ }^{14} \mathrm{C}$ is incorporated into protein cysteine. The nitrogen is also derived from serine since in the rat, serine ${ }^{15} \mathrm{~N}$ is converted to cysteine $-{ }^{15} \mathrm{~N}$ (Stekol, r 954).

Methionine is a potential source of the sulfur needed for cysteine synthesis. Homocysteine, and cystathionine are intermediates in this process:

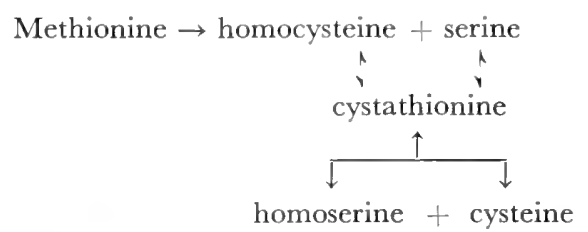

The evidence is as follows: a) methionine, homocysteine, and L-cystathionine can replace cysteine in the diet of the rat, $b$ ) the feeding of methionine plus bromobenzene increases the excretion of mercapturic acids, c) the feeding of methionine increases the urinary cysteine of cystinurics, $d$ ) methionine ${ }^{35} \mathrm{~S}$ is converted to cysteine- ${ }^{35} \mathrm{~S}$ of rat hair and to L-cystathionine- ${ }^{35} \mathrm{~S}$ of rat liver (Tabachnick and Tarver, 1955), $e$ ) when methionine labelled with sulfur- 34 and carbon-13 on the beta and gamma carbons, respectively, was administered to rats, only the labelled sulfur was converted to cysteine. L-cystathionine $-{ }^{35} \mathrm{~S}$ was also converted to cysteine in the rat but the ${ }^{14} \mathrm{C}$ of cystathionine was converted to cysteine only when the label was present on the aminopropionic acid portion of the cystathionine molecule, $f$ ) cysteine formation from homocysteine and serine or from L-cystathionine takes place in rat liver homogenates while the reverse reaction, the formation of cystathionine from serine and homocysteine is also catalyzed by liver enzymes. Pyridoxal phosphate is required in the synthesis of cysteine from serine and homocysteine (Stekol, 1954).

The conversion of sulfate to cysteine. The intermediary steps in the conversion of sulfate to cysteine have been investigated by Hockenhull (Hockenhull, I949). 
Cystineless mutants of the mold, Aspergillus nidulans were grown in the presence of various inorganic sulfur compounds. Some strains grew on sulfite but not on sulfate. Others grew on thiosulfate but not on sulfate or sulfite (Fig. 25):

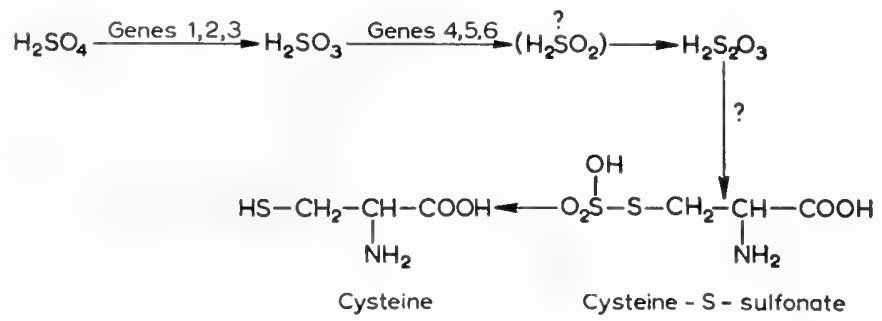

Fig. 25. Postulated pathway of cysteine formation from sulfate.

It is not clear at what level of reduction inorganic sulfur is converted to organic sulfur. The thiosulfate of serine (cysteine-S-sulfonate) has been suggested as an intermediate in cysteine synthesis. Cysteine-S-sulfonate supports good growth in the "thiosulfateless" strains of Aspergillus while growth is luxuriant on thiosulfate plus serine. All known mutants of Aspergillus can utilize thiosulfate for growth.

Animal organisms are also capable of fixing sulfate into organic linkage. Cysteine sulfinic acid and taurine rather than cysteine are however the principal products of these reactions (Machlinet al., I 953, I 955; Dziewiatkowski, I 954; Lowe and Roberts, I 955; Block et al., I 95I ). Acetone powders of rabbit kidney catalyze the synthesis of cysteine sulfinic acid from sulfite, pyruvate, and glutamic acid (Chapeville and Fromageot, I954; Chapeville et al., i956). The reduction of cysteine sulfinic acid to cysteine has also been demonstrated recently. Thus, sulfite-35 was incorporated into the cysteine, cysteine sulfinic acid, and taurine of eviscerated rabbit tissues. The specific activity measurements of the three compounds suggested that cysteine sulfinic acid was the precursor of the other two. Under the same conditions, labelled sulfate was not incorporated into cysteine. The enzymes concerned in these transformations are probably the same as those which also participate in the catabolism of cysteine to sulfite and sulfate. The conversion of cysteine to sulfate has been shown in liver and kidney slices. Cysteine sulfinic acid is itself converted to sulfate and alanine by liver mitochondria or Proteus vulgaris enzymes (Fig. 26; Froma-

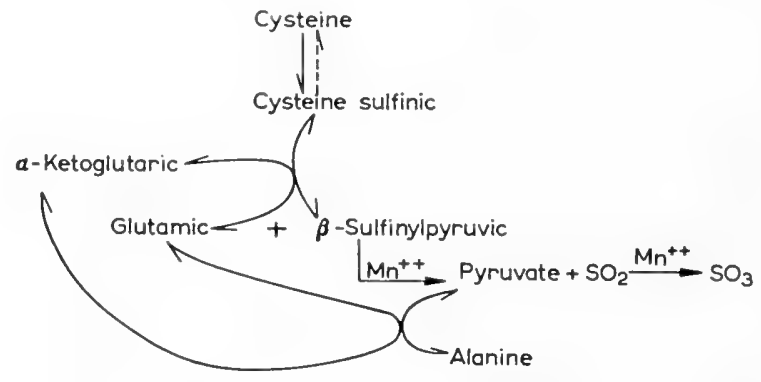

Fig. 26. Metabolism of cysteine sulfinic acid. 
geot, I953-1954; Singer and Kearney, I956). The cysteine sulfinic acid transaminates with $\alpha$-ketoglutaric acid to form glutamic acid and $\beta$-sulfinylpyruvic acid. A cysteine sulfinic acid transamination reaction with $\alpha$-ketoglutarate has also been observed in brain tissue. Cysteine sulfinic acid is readily desulfinated to sulfite and to pyruvate. Presumably, $\beta$-sulfinylpyruvate is an intermediate in sulfite formation. The sulfite may then be further oxidized to sulfate. The pyruvate formed above reacts with the glutamic acid to regenerate the $\alpha$-ketoglutarate while alanine accumulates under the experimental conditions used. In mammalian tissues, cysteine sulfinic acid may also be decarboxylated and oxidized to hypotaurine and to taurine.

\section{(d) Phenylalanine, tyrosine and tryptophane (Fig. 27)}

The benzene rings of phenylalanine, tyrosine, and tryptophane are derived from a series of precursors whose recognition was made possible by the isolation of a number of "aromatic polyauxotrophs" of E. coli, Aerobacter aerogenes and Neurospora (Davis, I955a, b). These mutants required the three aromatic amino acids for growth and in addition most of them required traces of three other benzenoid compounds: $p$-aminobenzoic acid, $p$-hydroxybenzoic acid, and an unknown sixth compound. Shikimic acid, a substance previously found in plants, substituted for all of the above factors (Davis, I95I). Another group of $E$. coli mutants, blocked at later stages in aromatic synthesis, accumulated shikimic acid in the culture filtrates. These results suggested that shikimic acid was a common precursor of the aromatic metabolites.

By similar observations, dehydroshikimic acid and dehydroquinic acid were shown to be on the same metabolic sequence prior to shikimic acid. E. coli strains blocked immediately before shikimic acid accumulated dehydroshikimic acid. A Neurospora mutant (Y $7655^{\mathrm{a}}$ ), on the other hand, accumulated some dehydroshikimic acid but mostly protocatechuic acid (3, 4-dihydroxybenzoic acid; Tatum and Gross, I 956). Further support for the shikimate pathway was obtained by the demonstration of an enzyme in $E$. coli extracts which dehydrated 5-dehydroquinic acid to 5-dehydroshikimic acid (Mitsuhashi and Davis, 1954a). A second enzyme found in E. coli, plants, yeast, and Aerobacter cells, catalyzed the reduction of dehydroshikimic to shikimic acid (Mitsuhashi and Davis, I954b; Yaniv and Gilvarg, I955). This enzyme was absent from those $E$. coli mutants which were blocked between dehydroshikimic and shikimic acid. Reduced $\mathrm{TPN}^{+}$was a cofactor for the reaction.

Certain mutants which accumulated shikimic acid, also accumulated 5-phosphoshikimate and an unknown compound, $Z_{1}$, (Weiss and Mingioli, r 956). The unknown compound, $Z_{1}$, is apparently a conjugate of shikimate with pyruvate. Extracts of a mutant blocked after $\left(Z_{1}\right)$ synthesize this substance $\left(Z_{1}\right)$ from phosphoshikimate and phosphoenol pyruvate, but not from shikimate. Phosphoshikimate therefore appears to be an intermediate between shikimate and compound $Z_{1}$.

Knowledge as to the origin of shikimic acid was derived from enzymatic and isotope studies. E. coli mutants which accumulated shikimic acid were grown in the presence of radioactive glucose. The distribution of radioactivity in the carbons of shikimic acid 
66

INTERMEDIARY METABOLISM AND GROWTH

1

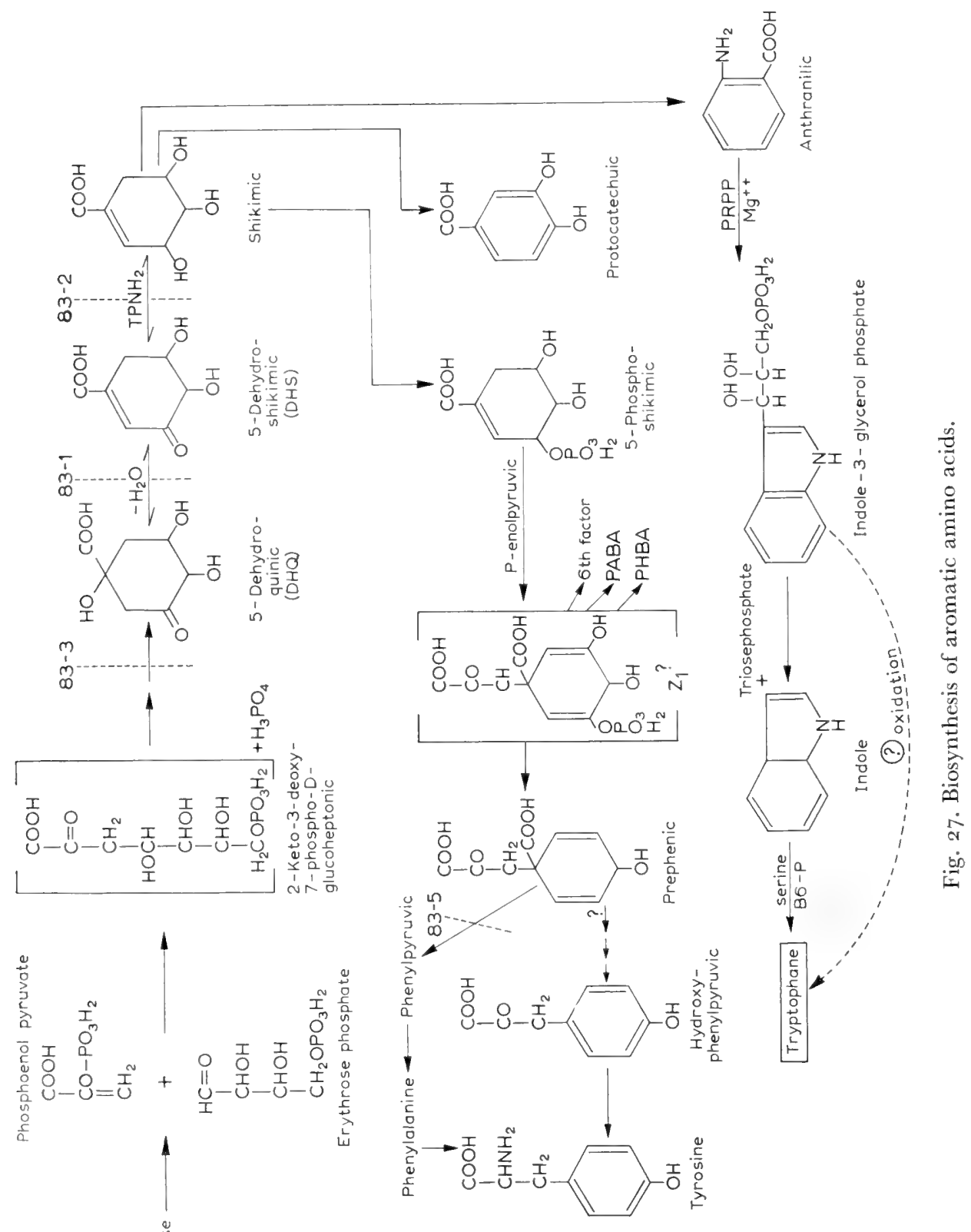


indicated that the carboxyl carbon and carbons one and two of shikimic acid were derived from a three carbon intermediate of glycolysis while the remaining carbon atoms probably originated from a tetrose which in turn was formed by the action of transketolase and transaldolase on fructose-6-phosphate (Srinivasan et al., 1956). In the presence of nonlabelled glucose, labelled bicarbonate, acetate, formate, or pyruvate were poorly incorporated into shikimic acid.

Cell free extracts of an $E$. coli mutant were next obtained which converted fructose-6phosphate or fructose diphosphate to dehydroshikimic or shikimic acid in $5 \%$ yield but converted sedoheptulose diphosphate to shikimic acid almost quantitatively (Srinivasan et al., 1955). Dehydroshikimic was also formed from erythrose-4-phosphate and phosphoenol pyruvate by this enzyme system. Phosphoenolpyruvate and erythrose phosphate were shown to be the more immediate precursors, since fluoride and iodoacetate, which block the formation of phosphoenolpyruvate, inhibited the formation of shikimic from sedoheptulose diphosphate but not from phosphoenol pyruvate and erythrose phosphate, and since $\mathrm{DPN}^{+}$was required when fructose diphosphate was used as substrate but not when phosphoenolpyruvate and erythrose phosphate were the substrates.

The experiments with the Neurospora mutants which accumulated protocatechuic acid were also consistent with the above mechanism (Gross et al., 1956; Tatum and Gross, 1956).

The next step in the synthesis of phenylalanine and tyrosine from compound $Z_{1}$ involves the formation of prephenic acid. This substance accumulates in the medium of a "phenylalanineless" E. coli mutant. It may be converted non enzymatically in acid solution to phenylpyruvic acid and enzymatically by the wild type of $E$. coli to phenylalanine (Weiss et al., I 954). It is possible that the terminal steps in the conversion of prephenic acid to phenylalanine and to tyrosine are different. Thus, enzymes from an E. coli mutant $(83-5)$ which is blocked in the conversion of prephenic to phenylpyruvic catalyzed the formation of $p$-hydroxyphenyl lactic acid from prephenic acid. However mutants blocked in the synthesis of both phenylalanine and tyrosine did not form $p$-hydroxyphenyllactic acid from prephenic acid (Ghosh, I956).

The pathway for phenylalanine and tyrosine synthesis (Fig. 27) is also consistent with isotope experiments carried out with yeast cells (Gilvarg and Bloch, I950; Thomas et al., I953). Glucose-I- ${ }^{14} \mathrm{C}$ and pyruvate-2 $-{ }^{14} \mathrm{C}$, were shown to be the precursors, respectively, of the $\beta$ - and $\alpha$-carbons of the tyrosine and phenylalanine side chains (Gilvarg and Bloch, I952).

Animal tissues, although incapable of synthesizing phenylalanine, are able to form tyrosine from dietary phenylalanine. Tyrosine can spare the dietary requirements of phenylalanine. Direct evidence for the conversion of phenylalanine to tyrosine was obtained by Udenfriend and Mitoma (I 955). These investigators fed dogs phenylalanine- $-3^{-14} \mathrm{C}$ and isolated labelled tyrosine from the serum albumin. The synthesis of tyrosine by a soluble rat liver enzyme has also been demonstrated (Mitoma, I 956). Two protein fractions are required. The first protein fraction occurs only in liver whereas the second fraction could also be obtained from kidney or heart. Reduced TPN+, oxygen, and ferrous iron were required for maximum tyrosine synthesis.

Terminal steps in tryptophane synthesis. It is probable that anthranilic acid and indole are terminal intermediates in the synthesis of tryptophane. "Tryptophaneless" E. coli and Neurospora mutants have been obtained which accumulate 
these substances while other mutants can utilize anthranilic or indole for growth (Yanofsky, I955a, I 956). The indole-anthranilic pathway is also consistent with the results of isotope experiments.

Glucose is a more direct precursor of the protein tryptophane of Aerobacter aerogenes than is acetate. Glucose- $-3{ }^{14} \mathrm{C}$ is converted to the carboxyl carbon of tryptophane and to several ring carbon atoms (Rafelson, I955a, b).

In Neurospora, the amino-nitrogen is retained and the carboxyl group is lost during the conversion of anthranilic acid to indole (Yanofsky, i955a). Isotope experiments with labelled glycerol or labelled glucose suggested that carbons two and three of the indole ring were derived respectively from carbons one and two of a ribose derivative. On the basis of studies with E. coli extracts, 5-phosphoribosylI-pyrophosphate (PRPP) was implicated as the actual donor of the two carbon atoms (Yanofsky, I955b). An $E$. coli enzyme was obtained which catalyzed the condensation of the former substance with anthranilic acid to give indole-3-glycerol phosphate. The latter substance was isolated from the incubation medium (Yanofsky, I956). Indole-3-glycerol is accumulated by several tryptophane requiring strains of $E$. coli. A second enzyme converted indole-3-glycerol phosphate to indole and triose phosphate.

The final step in tryptophane synthesis is the pyridoxal phosphate catalyzed condensation of indole and serine (Tatum and Shemin, I954). By using serine labelled with ${ }^{14} \mathrm{C}$ in the beta carbon, ${ }^{15} \mathrm{~N}$ in the amino group, and deuterium on the alpha and beta carbons, it was shown that in Neurospora the condensation involves an intramolecular dehydration of serine and a condensation of the alpha aminoacrylic acid-pyridoxal phosphate intermediate thereby formed with indole. This follows from the fact that one deuterium atom is lost in the condensation reaction. Had the condensation resulted from an intermolecular dehydration reaction, no deuterium would have been lost.

\section{(e) Alanine, valine, leucine, and isoleucine}

Alanine. Alanine is formed by transamination from the glycolytic intermediate, pyruvate. The conversion of labelled pyruvate, lactate, glucose, or glycerol to free alanine has been demonstrated both in vivo and in vitro in a variety of animal tissues (Busch et al., I956; Kit and Greenberg, I95 I Kit and Graham, I956a). Neoplastic tissues are particularly active in converting labelled substrates to alanine.

Valine and isoleucine. Isotope competition experiments with E. coli and Neurospora suggested that a metabolic relationship exists between valine and leucine and that both amino acids belong to the "pyruvate family" (Abelson, I954; Abelson and Vogel, I955). Thus, the incorporation of glucose $-{ }^{14} \mathrm{C}$ into protein valine and leucine is reduced by non-labelled pyruvate, $\alpha$-ketoisovalerate and valine. Evidence as to the intermediates involved was derived initially from nutritional experiments with microorganisms. A Neurospora mutant was isolated which required both valine and isoleucine for growth. This mutant accumulated $\alpha, \beta$-dihydroxy- $\beta$ methy] valeric acid and $\alpha, \beta$-dihydroxyisovaleric acid in the culture medium. The mutant was able to grow, however, if $\alpha$-ketoisovaleric acid and $\alpha$-keto- $\beta$-methyl 
valeric acid were supplied. A nutritionally similar double auxotroph of E. coli accumulated the keto analogs of valine and isoleucine (Umbarger and Magasanik, I95I; Umbarger and Mueller, I95I; Adelberg et al., I955). On the other hand, Neurospora mutants which were blocked earlier in the valine and isoleucine biosynthetic pathway, were able to utilize the dihydroxy analogs of valine and isoleucine for growth.

The origin of the keto- and the dihydroxyanalogs was clarified by isotope experiments. Fig. 28 depicts the mechanism for valine biosynthesis thereby deduced
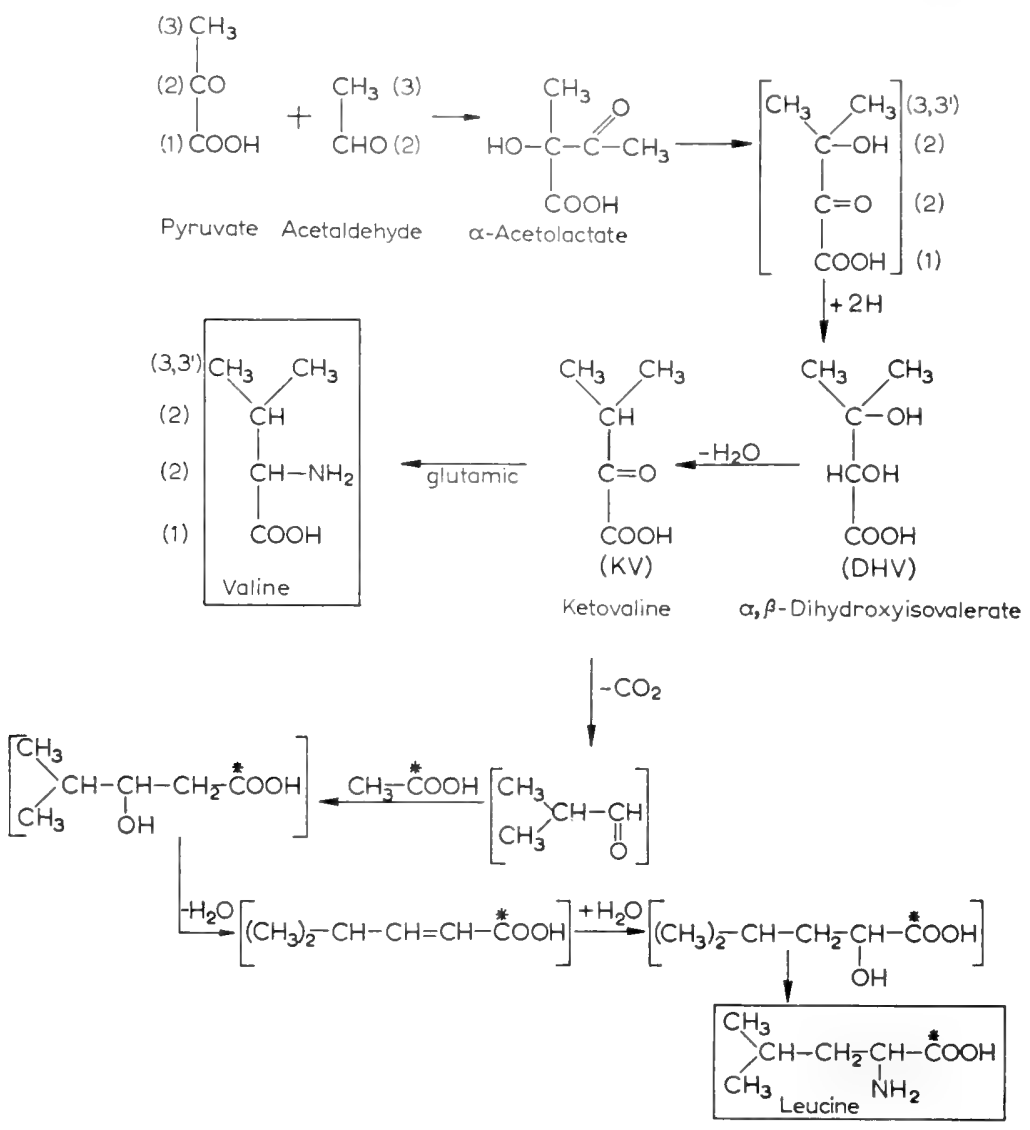

Fig. 28. Biosynthesis of valine and leucine.

by Weinhouse and coworkers (Strassman et al., I953). A somewhat similar mechanism was proposed independently by Adelberg (I954).

It will be observed that a ketol condensation of acetaldehyde with pyruvate to acetolactate is proposed. The latter substance then undergoes a pinacol rearrangement to an intermediate which may be reduced to the dihydroxy analogue of valine. Upon dehydration, $\alpha$-ketoisovalerate is formed and the valine is synthesized from the latter by transamination. The following evidence from isotope experiments performed with yeast and with Aerobacter cells is consistent with the mechanism: 
I) The carboxyl group of acetate, lactate, glycine and the third and fourth carbons of glucose are precursors of the carboxyl group of valine (carbon I), (Strassman et al., 1953, 1955; McManus, 1954; Rafelson, 1955a), 2) the alpha carbon atoms of the above compounds are precursors of carbons two and carbon three of valine, 3) glucose- ${ }^{1-}{ }^{14} \mathrm{C}$ is converted to the methyl groups of valine. As a result of the reactions of glycolysis glucose $-3,4^{-14} \mathrm{C}$ gives rise to the carboxyl carbon of pyruvate while glucose- $-{ }^{14} \mathrm{C}$ is a precursor of the beta carbon of pyruvate and of the alpha carbon of acetaldehyde.

The synthesis of isoleucine takes place by a similar pinacol rearrangement except that $\alpha$-ketobutyrate replaces pyruvate as the compound which condenses with acetaldehyde (Strassman et al., 1954, 1956; Fig. 29).

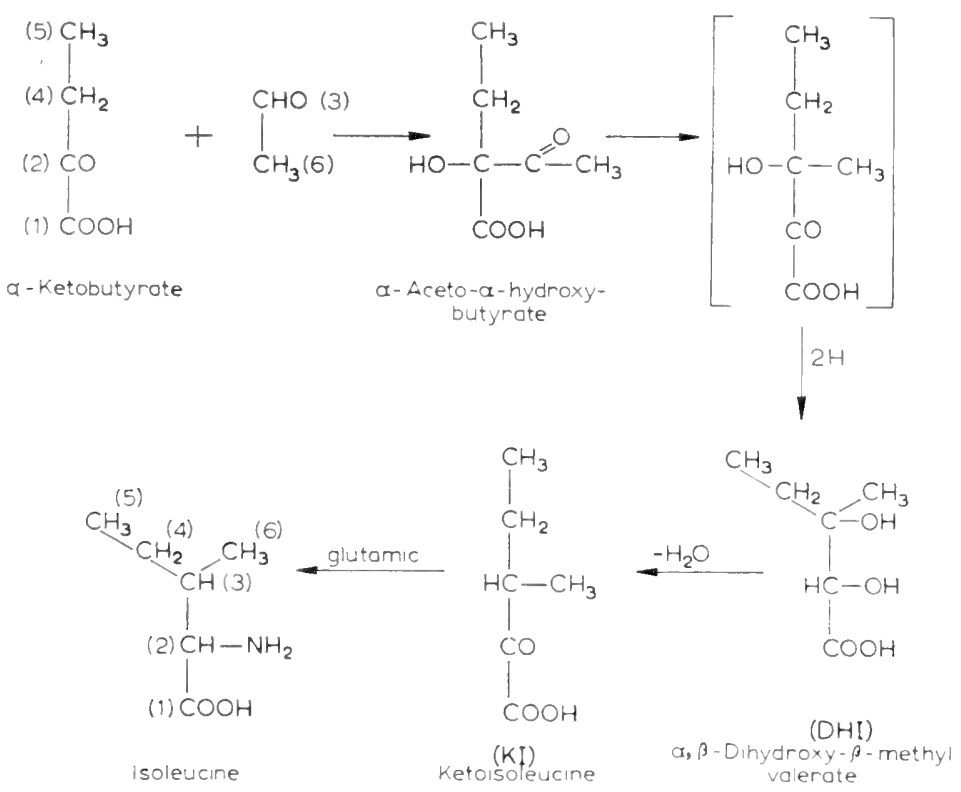

Fig. 29. Biosynthesis of isoleucine.

The $\beta$-ketobutyrate, which is required for isoleucine synthesis, is derived from threonine or homoserine. These substances are in turn formed from aspartic acid. The reactions of Fig. 29 are consistent with the results of the isotope competition experiments of Abelson and coworkers. Thus, non-labelled aspartate, homoserine, threonine, $\alpha$-ketobutyrate, $\alpha$-aminobutyrate, $\alpha$-keto- $\beta$-methyl-valerate, and isoleucine markedly reduced the incorporation of labelled glucose into the isoleucine of $E$. coli protein. Likewise, non-labelled $\alpha$-ketobutyrate, and $\alpha$-keto- $\beta$-methylvalerate reduced the conversion of labelled glucose and homoserine, threonine, and isoleucine suppressed the incorporation of ${ }^{14} \mathrm{CO}_{2}$ into the isoleucine of $\mathrm{Neu}$ raspora protein (Abelson and Vogel, I955; Abelson, 1954).

Threonine $1,2{ }^{14} \mathrm{C}$ was also converted by Neurospora mutants to the carboxyl and alpha carbons of the dihydroxy analog of isoleucine with $73 \%$ of the specific activity of the threonine precursor (Adelberg, 1954; Adelberg et al., 1955). However, 
negligible radioactivity was found in dihydroxyisovalerate, the valine precursor. Non-labelled threonine prevented the incorporation of labelled acetate into carbons I, 2,4 and 5 of $\alpha, \beta$-dihydroxy- $\beta$-methylvalerate.

The following further evidence has been obtained by Weinhouse and coworkers (Strassman et al., I 954, I956): (I) the carboxyl group of lactate is only slightly incorporated into yeast isoleucine, (2) the alpha carbon of lactate contributes to carbons I, 5, and carbon 3, (3) the lactate beta carbon gives rise to isoleucine carbon atoms $\mathrm{I}, 2,4,5$, and carbon $6,(4)$ label from acetate- $\mathrm{I}-{ }^{14} \mathrm{C}$ is present exclusively in carbons $\mathrm{I}$ and 5 of isoleucine, (5) label from acetate-2- ${ }^{14} \mathrm{C}$ is found in isoleucine carbons 2 and 4 with a specific activity twice that of carbons $\mathrm{I}$ and 5 .

These are the results to be anticipated if acetate or lactate is metabolized through the tricarboxylic acid cycle to form oxalacetate, a precursor of aspartate, threonine, and $\alpha$-ketobutyrate. It is also to be recalled that the carboxy ]carbon of lactate is lost during the conversion of that substance to acetaldehyde. Fig. 30 summarizes

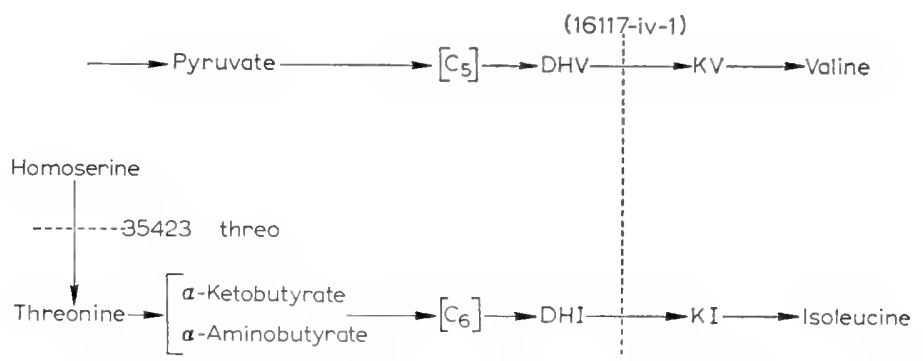

Fig. 30. Relationship between valine aud isoleucine biosanthesis in Neurospora.

the relationship between valine and isoleucine in Neurospora. The accumulation of DHI and DHV by mutant I6I I 7-iv-I is due to the inability of that organism to effect the conversion of the dihydroxy acids to the keto acids. In an interesting experiment, strain I6I 77 -iv-I was genetically combined with the threonine requiring mutant (35423 threo). The washed mycelial pads of the double mutant and the single mutant were then compared for DHI and DHV accumulation in the presence and the absence of threonine (Adelberg et al., I955). As predicted in the diagram, the introduction of threonine increased the accumulation of DHI but did not cause a parallel increase in DHV.

Leucine. By isotope competition experiments, it was shown that non-labelled pyruvate markedly reduced the incorporation of uniformly labelled glucose into the leucine of Neurospora and E. coli proteins (Abelson, I 954; Abelson and Vogel, 1955). Furthermore, $\alpha$-ketoisovalerate, valine, and $\alpha$-ketoisocaproate reduced the labelling of $E$. coli leucine. Moreover, alanine-2- ${ }^{14} \mathrm{C}$ and valine-2 $-{ }^{14} \mathrm{C}$ were incorporated into the leucine of $E$. coli and Neurospora respectively. Acetate- ${ }^{14} \mathrm{C}$ is incorporated exclusively into the carboxyl group of the leucine of yeast and bacterial protein (Reed et al., I 954). This suggests that leucine is synthesized by a condensation of acetate with the $\alpha$-ketoisovalerate (Fig. 28).

Yeast cells were also grown in the presence of pyruvate- $2-{ }^{14} \mathrm{C}$ and the radio- 
activity of the protein leucine was determined. Radioactivity was observed in the carboxyl group of leucine and in the beta and gamma carbon atoms. This is in accord with the pathway postulated above since pyruvate- $2-{ }^{14} \mathrm{C}$ would be expected to form labelled $\alpha$-ketoisovalerate- $2,3-{ }^{14} \mathrm{C}$ in the course of valine synthesis. Likewise, pyruvate-2 $-{ }^{14} \mathrm{C}$ would give rise to acetate- $\mathrm{I}-{ }^{14} \mathrm{C}$ by oxidative decarboxylation. As indicated above, the latter substance is the precursor of the leucine carboxyl group.

\section{(f) Aspartate family (aspartic, methionine, threonine, isoleucine)}

\section{Aspartate}

Aspartic acid is formed from oxalacetic acid by transamination:

$$
\text { Glutamic }+ \text { oxalacetic } \longleftrightarrow \text { aspartic }+\alpha \text {-ketoglutarate }
$$

\section{Homoserine-threonine-methionine}

\section{Aspartate to homoserine and threonine}

Aspartic acid is the precursor of threonine and methionine. There is increasing evidence that homoserine is an intermediate in these transformations. Homoserine can replace the methionine and threonine (Teas et al., I 948) which is needed for the growth of a Neurospora mutant. A mutant blocked in methionine synthesis alone was found to accumulate homoserine as well as threonine. The results of isotope competition experiments are consistent with these findings. Thus, in $E$. coli and Neurospora, non-labelled homoserine prevents the incorporation of glucose $-{ }^{14} \mathrm{C}$ into the threonine and methionine of the protein. Labelled aspartic acid is converted to labelled threonine in both these organisms (Abelson, I954; Abelson and Vogel, I 955). Threonine and lysine partially spare the aspartic acid requirements of certain Lactobacilli (Ravel et al., I954).

The conversion of aspartic acid to homoserine has been demonstrated with partially purified yeast enzymes (Black and Wright, I955a, b, c). The steps are as follows:

a) Aspartate $+\mathrm{ATP} \rightarrow \mathrm{ADP}+\beta$-aspartyl phosphate

b) $\beta$-Aspartyl phosphate $+\mathrm{TPNH}_{2} \rightarrow$ aspartic semialdehyde $+\mathrm{TPN}^{+}+$phosphate

c) Aspartic semialdehyde $+\mathrm{DPNH}_{2}\left(\left(\mathrm{TPNH}_{2}\right) \rightarrow\right.$ homoserine $+\mathrm{DPN}^{+}\left(\mathrm{TPN}^{+}\right)$

The conversion of aspartate to homoserine and to threonine has also been studied in cell suspensions and extracts obtained from E. coli mutants (Hirsch and Cohen, 1954). In the presence of glucose and phosphate, washed suspensions of a mutant, which was blocked in threonine synthesis, aerobically synthesized L-homoserine from L-aspartate. Suspensions of another mutant as well as of a wild type strain utilized L-homoserine for threonine synthesis. Extracts obtained from a mutant capable of activating aspartic acid in the presence of ATP were incapable of reducing the activated compound. Extracts from a second mutant reduced aspartate in a $\mathrm{TPNH}_{2}$ dependent reaction but no homoserine was formed. A third mutant yielded an extract which with ATP and $\mathrm{TPNH}_{2}$ formed homoserine from aspartate. These results parallel those obtained by Black and Wright with yeast cells. One difference was however noted. The activation of aspartate by $E$. coli cell extracts was stimulated by coenzyme A as well as by ATP. It is therefore possible 
that aspartyl-CoA is an intermediate in homoserine synthesis by $E$. coli. Pyridoxal phosphate and ATP are required in the conversion of homoserine to threonine. The activation of homoserine may therefore precede the utilization of this substance.

The relationship between aspartate and threonine in $E$. coli and in yeast cells is supported by isotope experiments. It has been observed that when these organisms are grown in the presence of pyruvate- $2-{ }^{14} \mathrm{C}$ or labelled acetate, the distribution of label in threonine corresponded to that in aspartate as predicted by the above pathway (Wang et al., i 955 ).

\section{The conversion of homoserine to methionine}

Cystathionine and homocysteine are intermediates in the formation of methionine from homoserine. Experiments by Horowitz showed that a Neurospora mutant which required methionine and could respond to homocysteine accumulated cystathionine in the mycelium while another mutant responded only to methionine. A third mutant, blocked earlier in the biosynthetic sequence, responded to cysteine as well as to methionine, homocysteine, or cystathionine (Horowitz, I 947). A series of mutants with similar nutritional requirements were found in $E$. coli.

Cystathionine is probably formed by a condensation of homoserine with cysteine. The cystathionine may then be cleaved to homocysteine and serine. Evidence for the reverse of these reactions has already been described in connection with the formation by animal tissues of cysteine from methionine and homocysteine (Binkley and Okeson, I950; Rachele et al., I950; Tabachnick and Tarver, 1955).

\section{Methionine formation from homocysteine}

Neogenesis of methyl groups. The following substances are potential precursors of the methyl groups of methionine: formate, formaldehyde, the beta carbon of serine, the alpha carbon of glycine, the methyl groups of sarcosine, and dimethylglycine (McKenzie and Abeles, 1956). Vitamin $B_{12}$ and folic acid are required for the neogenesis of methyl groups from these substances (Johnson et al., I955). The conversion of formate- ${ }^{14} \mathrm{C}$ or serine $-3-{ }^{14} \mathrm{C}$ to methionine or choline methyl groups is increased in weanling rats when cobalamine and homocysteine is added to the diet (Arnstein and Neuberger, I953). The conversion of serine $-3-{ }^{14} \mathrm{C}$ or $\mathrm{H}_{2}{ }^{14} \mathrm{CO}$ and homocysteine to methionine has been demonstrated at the enzyme level with sheep liver extracts (Nakao and Greenberg, I955). Leucovorin, ATP, $\mathrm{Mg}^{++}$and $\mathrm{DPN}^{+}$stimulate the conversion while deoxypyridoxine inhibits methionine synthesis. The inhibition is partially reversed by pyridoxal phosphate.

The methyl groups of dimethylglycine and sarcosine, are probably oxidized to the level of formaldehyde before being used for the synthesis of the methyl groups of methionine or choline (McKenzie and Abeles, I956). When sarcosine, completely labeled with deuterium in the methyl group, was incubated with liver mitochondria, it was observed that the beta carbon of serine and free formaldehyde contained deuterium having the same atom per cent excess as the substrate. The conversion of the beta carbon of serine to the methyl groups of choline and thymine without loss of its bonded hydrogen atoms has also been demonstrated (Elwyn et al., 1955). These conversions may be schematically represented as follows: 


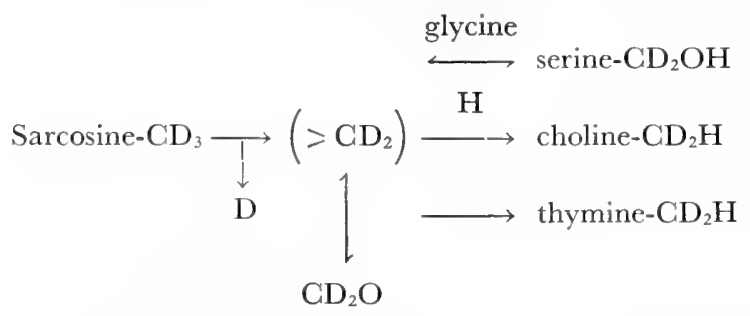

It is possible that S-hydroxymethylmethionine is an intermediate in the synthesis of methionine from homocysteine and "formaldehyde". Consistent with this hypothesis is the fact that when methionine- $\mathrm{S}-{ }^{14} \mathrm{CH}_{2} \mathrm{OH}$ is administered to rats, radioactivity is observed in the methyl group of the methionine (Stekol, I954).

Betaine transmethylase. In the presence of the enzyme, transmethylase, one methyl group of betaine may be transferred directly to homocysteine:

$$
\text { Betaine }+ \text { homocysteine } \rightarrow \text { methionine }+ \text { dimethylglycine }
$$

In 1939, Du Vigneaud showed that betaine permitted growth of rats with homocysteine as the only sulfur containing amino acid. Choline which was also active in this respect, was active by virtue of the fact that it could be converted to betaine (Du Vigneaud, 1952). Only organisms possessing an active choline oxidase could utilize choline instead of betaine as a methyl donor for methionine synthesis. Choline, which serves as a methyl donor in crude liver enzyme systems only under aerobic conditions is converted to dimethylglycine and not to dimethylethanolamine when incubated with homocysteine and rat liver homogenates. The betaine-homocysteine transmethylase enzyme has been purified from rat and pig liver (Ericson et al., 1955a).

\section{Methyl group transfer from methionine}

The methyl group of methionine is also utilized in transmethylation reactions for the synthesis of the methyl groups of choline, creatine, and other methylated compounds. These transmethylation reactions are not inhibited in animals lacking adequate vitamin $B_{12}$ or folic acid. As in the case of betaine, the methyl group of methionine is transferred as a unit, without loss of bonded hydrogen atoms (Du Vigneaud et al., I 956). However, the methionine must be activated prior to methyl group transfer (Cantoni and Vignos, 1954). Evidence has been presented that active methionine is identical with S-adenosyl methionine (Cantoni, I953; Fig.3I).

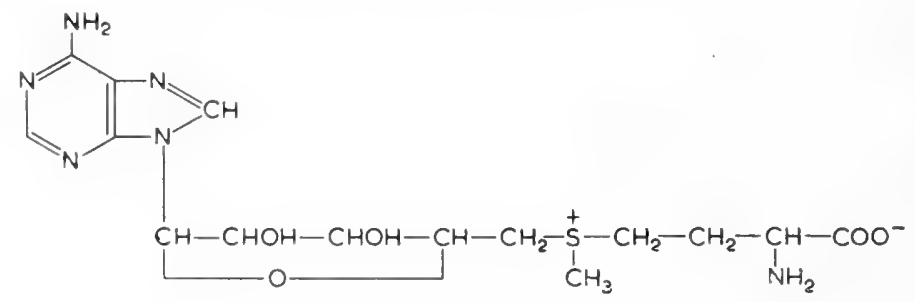

Fig. 3I. S-Adenosylmethionine. 
The latter substance was isolated after a purified enzyme preparation was incubated with methionine and ATP. With methionine- ${ }^{35} \mathrm{~S}$ or with methionine-2${ }^{14} \mathrm{C}$ as substrate, radioactivity was found in $\mathrm{S}$-adenosyl methionine.

A soluble enzyme of liver catalyzes the transfer of the methyl group of Sadenosyl methionine to nicotinamide or to guanidoacetic acid (Cantoni and Vignos, I954; Fig. 32). S-adenosylhomocysteine, the product of the above reaction, may then be hydrolyzed to adenosine and homocysteine.

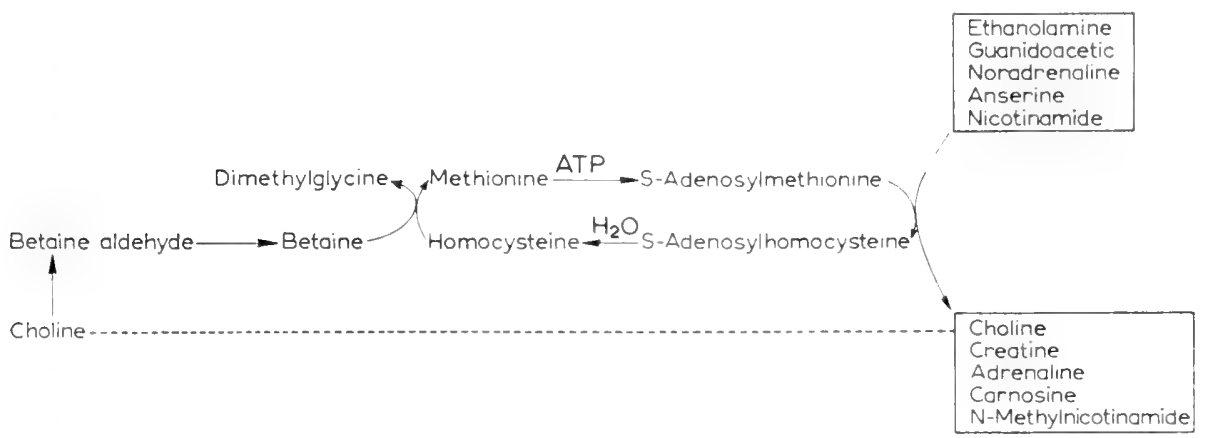

Fig. 32. Transmethylase reactions.

Adenosine thiomethylriboside, a compound related to S-adenosylmethionine, is accumulated by yeast cells when methionine is added to the culture medium (Schlenk and Smith, 1953). Methionine doubly labelled with ${ }^{35} \mathrm{~S}$ and with ${ }^{14} \mathrm{C}$ in the S-methyl group of the molecule is incorporated into adenosine thiomethyl riboside. The latter substance is probably formed by hydrolysis of S-adenosylmethionine:

\section{$\mathrm{H}_{2} \mathrm{O}$ \\ S-adenosylmethionine $\longrightarrow$ homoserine + adenosine thiomethylriboside}

The fact that thiomethyladenosine can be utilized by yeast cells for the synthesis of methionine emphasizes the close relationship between these compounds. However, in the transmethylase system of liver which functions in the synthesis of methionine, homocysteine rather than $S$-adenosylhomocysteine is the substrate (Ericson et al., I 955a). S-adenosylhomocysteine is only $40 \%$ as effective as homocysteine as a substrate in this reaction (Ericson et al., I955b) in crude enzyme systems and has negligible activity in the purified enzyme system. The crude enzyme extracts are capable of cleaving S-adenosylhomocysteine to homocysteine and adenosine (Fig. $32)$.

\section{Homoserine to $\alpha$-ketobutyric}

As mentioned earlier, the biosynthesis of isoleucine by microorganisms involves the condensation of acetaldehyde and $\alpha$-ketobutyrate to a six carbon compound which by pinacol rearrangement is converted to the isoleucine precursors. Methionine and homoserine are potential precursors of the $\alpha$-ketobutyrate required for isoleucine synthesis. DL-methionine-2- ${ }^{14} \mathrm{C}$ can also be converted to homoserine and to $\alpha$-aminobutyric acid in animal tissues (Matsuo and Greenberg, 
I955). It has been shown that rat liver extracts (Matsuo et al., I956) convert homoserine-2- ${ }^{14} \mathrm{C}$ to labelled $\alpha$-ketobutyrate, $\alpha$-hydroxybutyrate, $\alpha$-aminobutyrate, and propionic acid (Fig. 33).

Partially purified enzyme preparations, however, formed $\alpha$-ketobutyrate as virtually the only radioactive product of the reaction. The formation of $\alpha$-aminobutyric acid is apparently attributable to a transamination reaction, since the presence of glutamic acid greatly increased the radioactivity of the former substance. An $\alpha$-aminobutyric transaminase also occurs in heart muscle.

\section{The conversion of threonine to $\alpha$-ketobutyrate}

$\alpha$-Ketobutyrate can also be formed from threonine. The conversion of threonine to $\alpha$-aminobutyrate and of the latter to $\alpha$-ketobutyrate and propionate has been demonstrated in liver extracts (Kinnory et al., 1955) (Fig. 33).

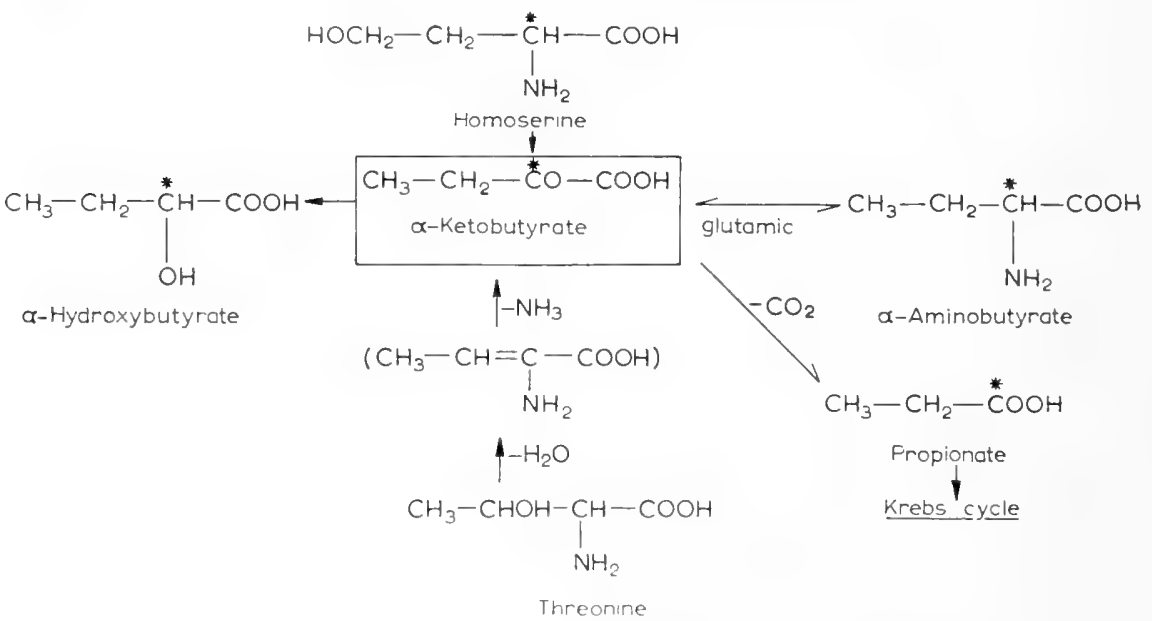

Fig. 33. Metabolism of homoserine, $\alpha$-ketobutyrate, and threonine.

\section{(g) Lysine biosynthesis}

Two pathways exist for the biosynthesis of lysine. In Neurospora and Torulopsis, $\alpha$-aminoadipic acid is an intermediate in lysine synthesis, while in $E$. coli and possibly other bacteria, diaminopimelic acid is the lysine precursor.

Aminoadipic acid pathway. $\alpha$-aminoadipate, a constituent of corn protein, is a growth factor for certain lysine auxotrophs of Neurospora (Windsor, I 95I). This substance, labelled with ${ }^{14} \mathrm{C}$ on the epsilon carbon atom, is incorporated into the lysine of Neurospora protein.

$\alpha$-Aminoadipate is probably derived from intermediates of the tricarboxylic acid cycle. It has been shown that uniformly labelled glutamic acid but not glutamic- $\mathrm{I}-{ }^{14} \mathrm{C}$ is incorporated into the lysine of Neurospora and Torulopsis protein (Abelson and Vogel, I955). Glutamic- ${ }^{14} \mathrm{C}$ is better incorporated than aspartic- ${ }^{14} \mathrm{C}$. As in the case of leucine, acetate is a precursor of the carboxyl and alpha carbons of lysine (Strassman and Weinhouse, I953). These results suggest that acetate may condense with $\alpha$-ketoglutarate to form homocitrate and 


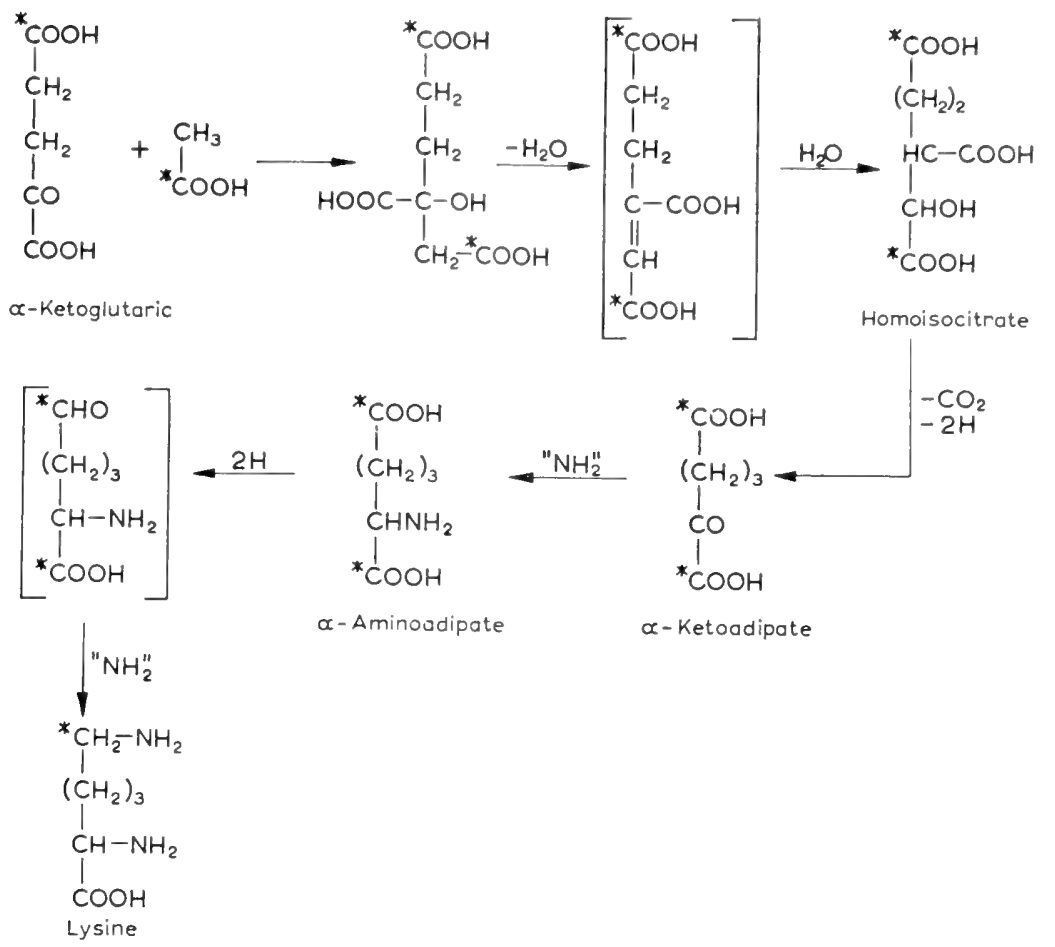

Fig. 34. Postulated pathway of lysine biosynthesis.

that $\alpha$-ketoadipic acid and $\alpha$-aminoadipic acid may be formed from homocitrate by a series of reactions analogous to those which take place in the citric acid cycle (Fig. 34). Not inconsistent with this scheme is the observation that acetate- $1-{ }^{14} \mathrm{C}$ is a precursor primarily of carbons one and six of Torulopsis lysine whereas acetate-2-14 $\mathrm{C}$ is incorporated into carbons $2-5$ of lysine. The radioactivity of carbon 2 is however nearly twice as great as that of carbons $3-5$ and that of carbon 6 is about two thirds of carbon I. The radioactivity of carbons 3-6 resemble closely that to be expected from the succinyl moiety of $\alpha$-ketoglutarate. Neurospora extracts contain an enzyme which catalyzes a transamination reaction between $\alpha$-aminoadipic acid and $\alpha$-ketoglutarate (Ames and Horecker, 1956).

Diaminopimelic pathway of $E$. coli. $\alpha, \varepsilon$-Diaminopimelic acid occurs in the protein of $E$. coli (Dewey et al., I 954). A pyridoxal phosphate requiring enzyme exists in this organism and in Aerobacter which catalyzes the decarboxylation of

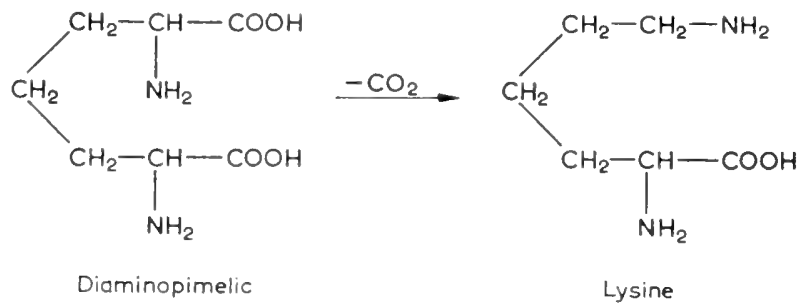

Fig. 35. Decarboxylation of diaminopimelic acid to Iysine. 
diaminopimelic acid to lysine (Fig. 35). This enzyme is lacking in those lysine auxotrophs of $E$. coli which accumulate diaminopimelic acid. Lysine requiring mutants of Neurospora do not respond to diaminopimelic acid nor do E. coli mutants respond to $\alpha$-aminoadipic acid (Dewey and Work, I952; Davis, 1952).

Although aspartate does not seem to be a direct aminoadipic acid precursor in molds, it would seem that a closer relationship exists between lysine biosynthesis and aspartate in bacteria. Non-labelled aspartate reduces the conversion of glucose- ${ }^{14} \mathrm{C}$ to lysine in $E$. coli (Abelson, 1954). Certain incompletely blocked diaminopimelic mutants can respond to threonine, diaminopimelic, or lysine (Davis, 1952), but not to aspartate. Aspartic, asparagine, proline, tyrosine, pyridoxal, inositol, and choline can substitute for the lysine requirements of Streptococcus faecalis (6057) (McClure et al., 1954). On the other hand, lysine and threonine can spare aspartic acid in those Lactobacillus arabinosus cells whose growth is inhibited by the antimetabolite of aspartate, cysteic acid (Ravel et al., 1954).

(h) Glutamic family: Glutamic, proline, hydroxyproline, arginine

Glutamic. The formation of glutamic acid from $\alpha$-ketoglutarate and aspartate is catalyzed by the enzyme, transaminase. Glutamic acid may also be formed by the reductive amination of $\alpha$-ketoglutarate (reactions $\mathbf{I}$ and 2 ) or as a result of the catabolism of histidine, proline, arginine, and ornithine.
transaminase
I) Aspartic $+\alpha$-ketoglutaric $\longleftrightarrow$ glutamic + oxalacetic
glutamic dehydrogenase
2) $\alpha$-Ketoglutaric $+\mathrm{TPNH}_{2}+\mathrm{NH}_{3} \longleftrightarrow$ glutamic $+\mathrm{TPN}^{+}$

Proline. Glutamic $-{ }^{14} \mathrm{C}$ is a precursor of both the proline and arginine of rat or Neurospora protein (Stetten, I955; Abelson, I954). Glutamic- $\gamma$-semialdehyde reduces the incorporation of labelled glucose into proline. An enzyme which catalyzes the reduction of glutamic to glutamic semialdehyde exists in Neurospora. Glutamic semialdehyde spontaneously cyclizes to $\Delta \mathrm{I}$-pyrroline-5-carboxylate which can be reduced to proline (Yura and Vogel, 1955) (Fig. 36). In the presence of glutamate, a mutant strain of $E$. coli accumulates pyrroline carboxylate (Strecker and Mela, I955).

Ornithine is also a proline precursor in mammals, fungi and bacteria. When ornithine labelled with ${ }^{15} \mathrm{~N}$ on the $\alpha$-amino group was fed to mice, the proline, hydroxyproline, glutamate, and aspartate of the proteins contained isotopic nitrogen (Stetten, I95I). The gamma $\mathrm{N}$ of ornithine contributed to a greater degree to glutamic acid than to proline. Ornithine labelled with deuterium was also converted to proline and to glutamate in mice. The loss of the terminal amino group from ornithine would result in the formation of glutamic semialdehyde, the proline precursor. An enzyme which catalyzes the formation of glutamic semialdehyde from ornithine occurs in mammalian liver preparations and in fungi (Meister, I 954; Fincham and Boulter, I956, Fig. 36). Some ornithine- ${ }^{14} \mathrm{C}$ is also incorporated into the proline, glutamate, and arginine of $E$. coli protein (Vogel, I956) by the metabolic pathway shown above. However, this metabolic link appears to be relatively unimportant in this organism.

Hydroxyproline. The feeding of proline ${ }^{15} \mathrm{~N}$ to rats results in the labelling of the hydroxyproline of the rat proteins (Stetten, I949). The reverse reaction does not 


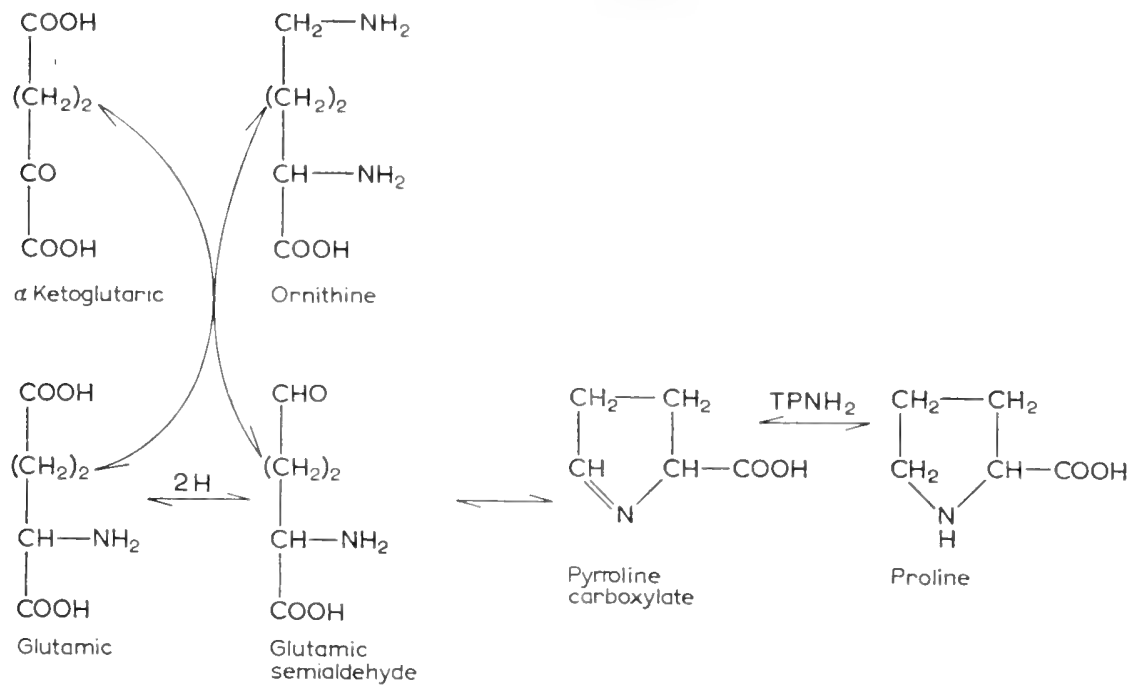

Fig. 36. Conversion of glutamic acid to proline.

take place to a significant degree. Hydroxyproline ${ }^{15} \mathrm{~N}$ is a poor precursor of the hydroxyproline of rat proteins. More isotope is found in protein hydroxyproline after feeding ${ }^{15} \mathrm{~N}$-proline than ${ }^{15} \mathrm{~N}$-hydroxyproline, likewise, the nitrogen of ${ }^{15} \mathrm{~N}$-hydroxyproline is converted to a greater extent to protein glutamate than to protein hydroxyproline. It is possible that proline is oxidized to hydroxyproline while present in peptide linkage. Hydroxyproline occurs in appreciable amounts in gelatin, collagen, and elastin but is present at very low concentrations in other proteins.

Ornithine synthesis. Isotope competition experiments suggest the following pathway of ornithine synthesis from glutamic acid in E. coli cells:

$$
\begin{gathered}
\text { Glutamic } \rightarrow \text { N-acetyl glutamate } \rightarrow \text {-acetyl glutamic semialdehyde } \underset{\alpha \text {-ketoglutaric }}{-\frac{\text { glutamic }}{\mathrm{H}_{2} \mathrm{O}}} \\
\text { N-acetyl ornithine } \longrightarrow \text { ornithine }
\end{gathered}
$$

An enzyme exists in $E$. coli which catalyzes the acetylation of glutamate. The latter substance may be converted to acetyl ornithine by reduction and transamination. Acetyl ornithinase has been partially purified from E. coli extracts (Vogel and Bonner, 1956) and acetyl ornithine and free ornithine occur in certain plants (Virtanen and Linko, 1955). It is significant that $\mathrm{N}$-acetyl glutamic semialdehyde rather than glutamic semialdehyde is an intermediate in ornithine synthesis, in that the acetylated compound, unlike free glutamic semialdehyde, cannot cyclize to pyrroline carboxylate. Proline $-^{15} \mathrm{~N}$ is converted to protein arginine in the mammal (Stetten, I95I). The postulated intermediate, glutamic semialdehyde, is however a relatively poor ornithine precursor, suggesting that the acetyl glutamate pathway also exists in animal tissues. On the other hand, in Neurospora which lacks an acetyl ornithinase, glutamic semialdehyde is a better ornithine precursor. Here, glutamic semialdehyde inhibits the conversion of glucose- ${ }^{14} \mathrm{C}$ to Neurospora arginine (Vogel and Bonner, 1956). 
Ornithine to arginine. Both the $\alpha$ - and the $\varepsilon$-amino nitrogens of ornithine are incorporated into tissue arginine in the rat (Stetten, 195I). The role of ornithine and arginine in the urea cycle has been recognized for many years. It was noted early that a small amount of ornithine catalyzed the formation of large amounts of urea in liver slices (Ratner, I95.5). Citrulline is an intermediate in this conversion. The ornithine-citrulline-arginine cycle takes place in Neurospora, Penicillium, E. coli, and Tetrahymena as well as in mammalian liver tissue. The first step in arginine synthesis is the formation of citrulline from ornithine. This reaction requires carbamyl phosphate and has been demonstrated in liver and in microorganisms (Fig. 37; Lowenstein and Cohen, I956; Reichard et al., I955; Marshall et al., I 955; Jones et al., 1955). The citrulline then reacts with aspartic acid to form an intermediate, argininosuccinic acid. The latter substance is then cleaved by a third enzyme to fumarate and arginine.

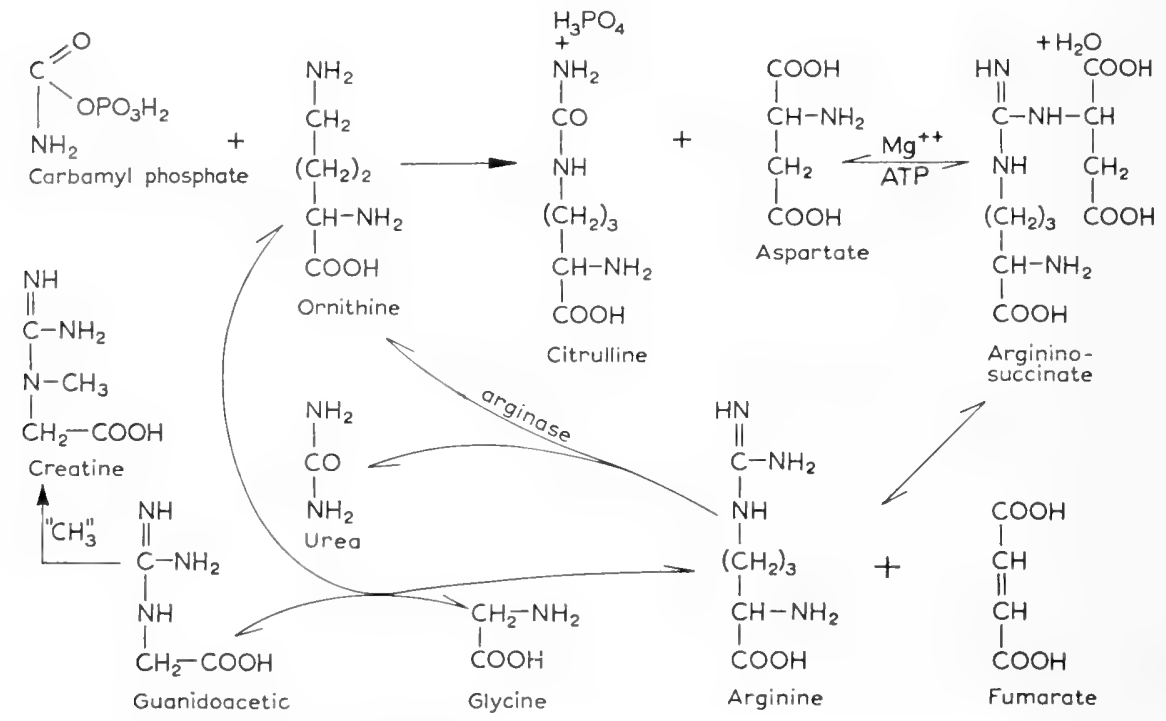

Fig. 37. Arginine biosynthesis and the formation of creatine.

Urea, the end product of nitrogen metabolism in the mammal, is formed from arginine as a result of the action of the enzyme, arginase; at the same time, ornithine is regenerated.

Creatine synthesis and transimidation. Creatine phosphate functions as a storage form of "high-energy phosphate bonds" in mammalian tissues. Guanidoacetic acid the intermediate in creatine synthesis is formed by a condensation of the guanido group of arginine with glycine. This is followed by the methylation of guanidoacetic acid to creatine by the transfer of a methyl group from methionine (Fig. 37). The amidine group of arginine $(=\mathrm{N}-\mathrm{C}=\mathrm{N}-$ ) is transferred as a unit (Stetten and Bloom, I956) during guanidoacetate synthesis. The reaction is reversible; labelled arginine can be formed from guanidoacetic acid labelled 


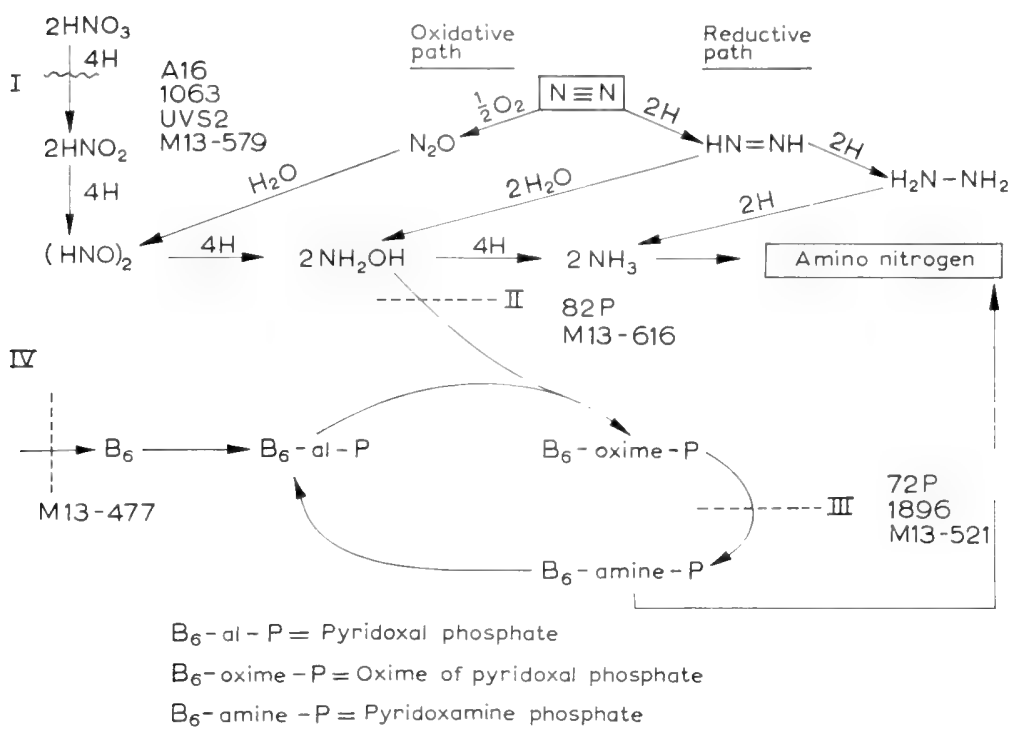

Fig. 38. Proposed pathways of nitrogen assimilation.

with ${ }^{14} \mathrm{C}$ in the amidine group (Horner et al., I 956). Kidney enzymes also catalyze an amidine group transfer reaction between ornithine and arginine (Walker, I956).

I) Arginine + ornithine-2-14 $\mathrm{C} \longleftrightarrow$ ornithine + arginine-2-14 $\mathrm{C}$

2) Guanidoacetic ${ }^{14} \mathrm{C}+$ ornithine $\longleftarrow$ arginine- ${ }^{14} \mathrm{C}+$ glycine

\section{Fixation of Inorganic Nitrogen and Transfer}

\section{Nitrogen assimilation}

We have previously considered the process of denitrification in connection with the role of inorganic nitrogen compounds as alternative hydrogen and electron acceptors in microorganisms and plants. These reductive processes are, however, of particular significance for the growth process, in that molecular nitrogen or inorganic nitrate is reduced to compounds which can be combined in organic linkage. The ability to fix or reduce molecular nitrogen is apparently restricted to microorganisms, the only exception being the remarkable system represented by the root nodule bacteria and their plant symbionts. The free living nitrogen fixers appear to be dispersed at random throughout the microbial kingdom from the photosynthetic algae and bacteria to heterotrophic Clostridia with complex nutritional requirements. The capacity to reduce nitrates and nitrites is, however, shared by many microorganisms and plants. The reduction of nitrogen and of nitrate proceeds through a number of intermediate steps, some of which are still admittedly speculative, until hydroxylamine or $\mathrm{NH}_{3}$ are formed (Fig. $3^{8}$ ).

It is not as yet certain whether the form of nitrogen which is first fixed into organic linkage is $\mathrm{NH}_{3}$ or hydroxylamine. An analysis of the nutritional requirements of Neurospora mutants has led to the suggestion that organic oxime compounds 


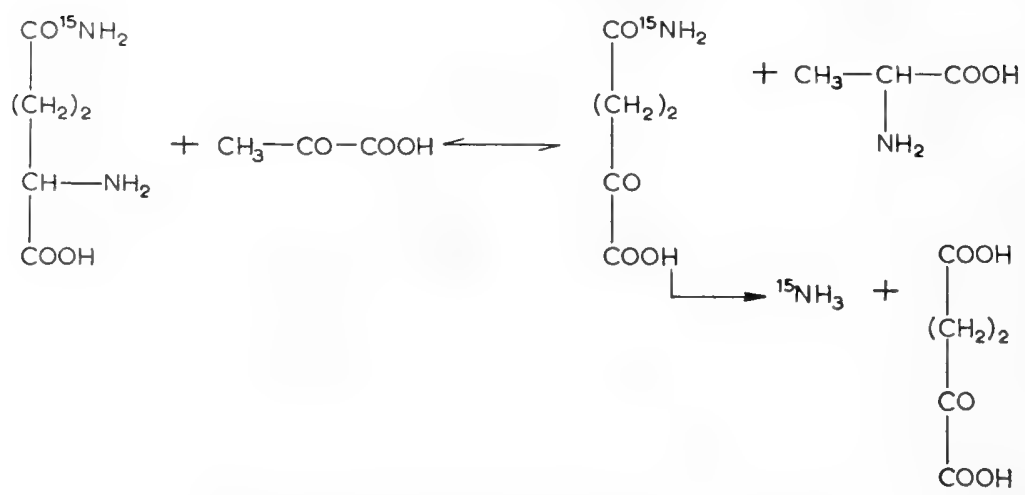

Fig. 39. Glutamine as a transaminating agent.

are intermediates in amino acid synthesis (Silver and McElroy, I954). As shown in Fig. 38, four mutants were isolated which lacked nitrate reductase and required nitrite for growth (Block I, Fig. 38). Eight $\mathrm{NH}_{3}$ requiring strains (Block II, Fig. 38) were also found. Most of these contained the enzymes, nitrite reductase and hydroxylamine reductase although two strains accumulated nitrite and hydroxylamine in the medium. One strain (Block IV, Fig. 38) required pyridoxal for growth. If pyridoxal was lacking in the medium, nitrite accumulated and nitrite- and hydroxylaminereductase were reduced in concentration. In the presence of pyridoxal, the mutant grew on nitrate or nitrite suggesting that vitamin $\mathrm{B}_{6}$ is directly involved in nitrite utilization. Strains blocked at III also require pyridoxal and accumulate nitrophenyl hydroxylamine when tested for the ability to reduce dinitrobenzene. Strains blocked at II do not accumulate nitrophenylhydroxylamine.

The possibility that oxime compounds are intermediates in amino acid synthesis is also suggested by experiments with Torulopsis (Virtanen and Saris, 1955). Torulopsis cells grown in the presence of nitrite or nitrate generate bound oxime derivatives of glyoxylate, oxalacetate, pyruvate, and $\alpha$-ketoglutarate. Upon chemical reduction, glycine, aspartate, alanine, and glutamate, respectively are formed.

\section{2. $\mathrm{NH}_{3}$ fixation}

Enzymatic reactions resulting in the fixation of $\mathrm{NH}_{3}$ are widely distributed in living cells. At the present time, seven ammonia fixation reactions may be distinguished:

(I) Glutamic dehydrogenase. The enzyme, glutamic dehydrogenase, which occurs in liver, kidney, muscle, brain, and heart, catalyzes the following reaction:

$$
\mathrm{TPNH}_{2}+\mathrm{NH}_{3}+\alpha \text {-ketoglutarate } \longleftarrow \text { glutamic }+\mathrm{TPN}^{+}
$$

The concentration of the enzyme is several fold higher in leukemic than in normal leukocytes (Waisman et al., I956).

(2) Glutamine synthetase. The synthesis of glutamine from glutamic acid and $\mathrm{NH}_{3}$ requires $\mathrm{Mg}^{++}$and ATP. Glutamine synthetase occurs in liver, kidney, muscle, 
brain, heart, pea seeds, and Staphylococcus aureus (Elliott and Gale, 1948; Speck, I 949; Levintow and Meister, I954; Elliott, I 953). The purified enzyme catalyzes the exchange of ${ }^{32} \mathrm{PO}_{4} \mathrm{H}_{3}$ with ATP and this exchange is greatly increased in the presence of glutamate. The following mechanism for glutamine synthesis has been proposed:

I) Enzyme $+\mathrm{ATP} \longrightarrow$ Enzyme-P $+\mathrm{ADP}$

2) Enzyme-P + glutamate $\longrightarrow$ Enzyme-glutamic $+\mathrm{P}$

3) Enzyme-glutamic $+\mathrm{NH}_{3} \longrightarrow$ glutamine + Enzyme

All known enzymes which catalyze the synthesis of glutamine also catalyze a glutamyl transfer reaction with hydroxylamine. The converse is also true:

Glutamine $+\mathrm{NH}_{2} \mathrm{OH} \longrightarrow \mathrm{NH}_{3}+$ glutamohydroxamate

Phosphorus or arsenate, $\mathrm{Mn}^{++}$, and trace amounts of ATP are required. The ATP acts catalytically here in contrast to the glutamine synthesis system.

(3) Asparagine synthesis. The formation of the amide bond of asparagine has been less studied than the synthesis of glutamine. The reaction is, however, of primary importance in plants. It is not unlikely that the mechanism of synthesis is similar to that of glutamine.

(4) Carbamyl phosphate synthesis. The formation of carbamyl phosphate from $\mathrm{NH}_{3}$ and $\mathrm{CO}_{2}$ has been discussed previously (Table 4 , reaction $38, \mathrm{p} .35$ ).

(5) Cytidine triphosphate formation. An enzyme purified 45 fold from $E$. coli extracts catalyzes the amination of UTP or UDP (Table 4, reaction 96, p. 36 ; Lieberman, 1955).

(6) Glucosamine-6-phosphate synthesis. The following reactions occur in kidney, liver, brain, lung or intestine (Leloir and Cardini, r956):

a) N-acetylglucosamine-6-phosphate $\rightarrow$ fructose-6-phosphate $+\mathrm{NH}_{3}+$ acetate

b) Glucosamine-6-phosphate $\longrightarrow$ fructose-6-phosphate $+\mathrm{NH}_{3}$

Catalytic amounts of $\mathrm{N}$-acetylglucosamine-6-phosphate are required for reaction b). Reaction b) is reversible, thereby providing a mechanism for ammonia fixation. Although fructosylamine-6-phosphate is probably an intermediate, it isomerizes to glucosamine-6-phosphate and the latter substance is isolated.

(7) Aspartase. The enzyme, aspartase, of many bacteria and plants catalyzes the following reaction:

Fumaric $+\mathrm{NH}_{3} \longleftrightarrow$ aspartic

\section{Amino transfer reactions and transaminations}

Many alpha keto acids can substitute for the corresponding essential amino acids in the diet of animals and microorganisms. It is therefore apparent that the facile 
synthesis of amino acids from keto acids must take place (Meister, I 954). Isotope competition experiments (Abelson, I954) are consistent with the possibility that in E. coli, isoleucine, leucine, valine, alanine, glutamic, aspartic, and phenylalanine are formed from their keto acids. The transfer of the nitrogen atom of amino acids in animal tissues was demonstrated by Schoenheimer and associates: the ${ }^{15} \mathrm{~N}$ of dietary leucine or glycine was rapidly transferred to almost all of the amino acids of the tissue protein except threonine and lysine (Schoenheimer, I 942).

In I930, Needham demonstrated that muscle "brei" catalyzed the anaerobic disappearance of glutamic acid without formation of free $\mathrm{NH}_{3}$. When it was found that aspartic acid and $\alpha$-ketoglutarate were products of this reaction, it became apparent that amino acids were in many cases synthesized from keto acids by transamination:

$$
\text { I) Glutamic + oxalacetic } \stackrel{\text { pyridoxal-P }}{\longrightarrow} \alpha \text {-ketoglutaric }+ \text { aspartic }
$$

Pyridoxal phosphate and pyridoxamine are cofactors in transamination reactions (Meister, I954, I955). It is probable that the amino group is transferred from amino acids to pyridoxal phosphate and that the pyridoxamine phosphate which is thereby formed, next transfers the amino group to a keto acid, thereby regenerating the pyridoxal phosphate. The transfer of ${ }^{15} \mathrm{~N}$ from pyridoxamine-phosphate and from amino acids to $\alpha$-ketoglutarate has been demonstrated in pig heart homogenates (Tanenbaum, I956).

The activity of transaminase enzymes varies with the amino and keto acid substrates. The most active transaminases of the cell are those which catalyze reaction I) and reaction 2 ):

2) Glutamic + pyruvate $\longleftarrow \alpha$-ketoglutaric + alanine

Less active transamination reactions can be demonstrated in most tissues for the following substrates:

3) $\left\{\begin{array}{ll}\text { Leucine }+\alpha \text {-ketoglutarate } \longleftrightarrow \text { glutamic } & +\alpha \text {-ketoisocaproate } \\ \text { Isoleucine } & \alpha \text {-keto- } \beta \text {-methylvalerate } \\ \text { Valine } & \alpha \text {-ketoisovalerate }\end{array}\right\}$

The close relationship between alanine and the dicarboxylic amino acids and the glycolytic and tricarboxylic acid cycles should be noted.

Transamination reactions involving other pairs of amino acids and keto acids have also been found. $\alpha$-Ketoglutarate functions as an amino acceptor in transamination reactions with practically all of the amino acids. Reactions are also known in which monoamino monocarboxylic acids and $\alpha$-keto monocarboxylic acids are the substrates and products:

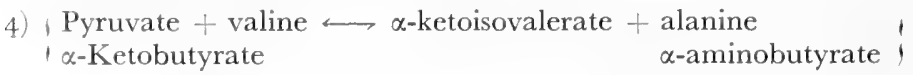

Reactions between glutamic acid and the aldehydes, glyoxylate, glutamic semialdehyde, and succinic semialdehyde also occur. There is an interesting reaction between ornithine and glyoxylate, in which aldehydes are substrates and products of the reaction: 


\section{5) Ornithine + glyoxylate $\stackrel{\text { liver }}{\longleftarrow}$ glutamic-\%-semialdehyde + glycine}

Glutamine and asparagine also are amino donors in transamination reactions. The keto analogues of over thirty amino acids are capable of reacting with glutamine. The keto analogues of isoleucine, valine, lysine and ornithine are exceptions and do not react. In these reactions, the alpha nitrogen of glutamine or asparagine is transferred, not the amide nitrogen. This has been demonstrated in experiments with glutamine-amide ${ }^{15} \mathrm{~N}$ (Meister, I955; Fig. 39).

Several apoenzymes catalyze transaminase reactions. The pig heart apoenzyme for the glutamine--oxalacetic reaction has been separated from the apoenzyme for the glutamic-pyruvate reaction. Different transaminase apoenzymes are present in $E$. coli and in Neurospora (Fincham and Boulter, 1956). From the former, three classes of transaminases have been separated: I) enzyme fraction A catalyzes reactions between $\alpha$-ketoglutarate and aspartate, phenylalanine, tyrosine, or tryptophane, 2) enzyme fraction B catalyzes the reaction of $\alpha$-ketoglutarate with isoleucine, valine, and leucine (Meister, 1955), 3) enzyme fraction C is specific for valine and pyruvate or $\alpha$-ketobutyrate. Table 6 summarizes the principal transamination reactions which are of importance in amino acid synthesis.

\section{The transfer of the amide group of glutamine}

The amide group of glutamine ${ }^{1}$ is utilized in a number of extremely important biosynthetic reactions:

(I) Phosphoribosylamine $(P R A)$. Liver and yeast enzymes catalyze the synthesis of the purine intermediate, 5-phosphoribosylamine (Goldthwait et al., I 955):

$$
\text { Glutamine }+\mathrm{PRPP} \rightarrow \mathrm{PRA}+\mathrm{PP}+\text { glutamic }
$$

(2) $\mathcal{N}$-formylglycineamidine ribotide $(F G A M)$. Glutamine-nitrogen is also required in the synthesis of FGAM from formylglycineamide ribotide (FGAR) (Levenberg et al., 1956).

$$
\text { FGAR }+ \text { glutamine } \underset{\begin{array}{c}
\text { ATP } \\
\text { pigeon }
\end{array}}{\stackrel{\text { FGAM }}{\text { liver }}} \text { Flutamic }
$$

(3) Guanylic acid synthesis. The conversion of xanthosinic acid to guanylic acid is catalyzed by enzymes from bone marrow cells (Abrams and Bentley, 1955a) and pigeon liver (Lagerkvist, 1955).

$$
\text { Xanthosinic acid }+ \text { glutamine } \stackrel{\mathrm{Mg}^{++}}{\longrightarrow} \text { guanylic + glutamic }
$$

(4) Glucosamine-6-phosphate. Kidney and liver enzymes catalyze the formation of glucosamine-6-phosphate from fructose-6-phosphate and $\mathrm{NH}_{3}$. A second mechanism of glucosamine-6-phosphate synthesis which has been observed in Neurospora, involves the transfer of the amide nitrogen from glutamine (Leloir and Cardini, I 953).

Hexose-6-phosphate + glutamine $\rightarrow$ glucosamine-6-phosphate + glutamic

1 See Addendum, Note 4, p. I 23. 
TABLE 6

TRANSAMINATION AND AMINO ACID SYNTHESIS

\begin{tabular}{|c|c|}
\hline Amino Acid & Reaction \\
\hline I. Glycine & glyoxylate + glutamic $\longrightarrow$ glycine $+\alpha$-ketoglutaric \\
\hline 2. Serine & $\begin{array}{l}\text { hydroxypyruvate }+ \text { alanine } \longleftarrow \text { serine }+ \text { pyruvate } \\
\text { phosphohydroxypyruvate }+ \text { glutamic } \longleftarrow \text { phosphoserine }+ \\
\alpha \text {-ketoglutaric }\end{array}$ \\
\hline $\begin{array}{l}\text { Cysteine } \\
\text { Glycine } \\
\text { Tryptophane }\end{array}$ & \\
\hline 3. Cysteine & $\beta$-sulfinylpyruvate + glutamic $\longleftrightarrow$ cysteinesulfinic + -ketoglutaric \\
\hline 4. Alanine & pyruvate + glutamic $\longrightarrow$ alanine $+\alpha$-ketoglutaric \\
\hline 5. Valine & $\alpha$-ketoisovalerate + glutamic $\longrightarrow$ valine $+\alpha$-ketoglutaric \\
\hline 6. Leucine & $\alpha$-ketoisocaproate + glutamic $\longleftrightarrow$ leucine $+\alpha$-ketoglutaric \\
\hline 7. Isoleucine & $\begin{array}{l}\alpha \text {-keto- } \beta \text {-methyl-valeric }+ \text { glutamic } \longrightarrow \text { isoleucine }+ \\
\alpha \text {-ketoglutaric } \\
\alpha \text {-ketobutyric }+ \text { glutamic } \longleftrightarrow \alpha-\mathrm{NH}_{2} \text {-butyric }+\alpha \text {-ketoglutaric }\end{array}$ \\
\hline $\begin{array}{l}\text { 8. Aspartic } \\
\text { Isoleucine } \\
\text { Threonine-glycine } \\
\text { Methionine } \\
\text { Homoserine }\end{array}$ & oxalacetic + glutamic $\longrightarrow$ aspartic $+\alpha$-ketoglutaric \\
\hline $\begin{array}{l}\text { 9. Glutamic } \\
\text { Proline } \\
\text { Ornithine-arginine } \\
\text { Hydroxyproline }\end{array}$ & oxalacetic + glutamic $\longrightarrow$ aspartic $+\alpha$-ketoglutaric \\
\hline ro. Ornithine-arginine & $\begin{array}{r}\text { glutamic- } \gamma \text {-semialdehyde }+ \text { glutamic } \longrightarrow \text { ornithine }+ \\
\alpha \text {-ketoglutaric }\end{array}$ \\
\hline I1. Arginine & $\begin{aligned} \mathrm{N} \text {-acetyl glutamic semialdehyde } & + \text { glutamic } \longleftrightarrow \mathrm{N} \text {-acetyl- } \\
& \text { ornithine }+\alpha \text {-ketoglutaric }\end{aligned}$ \\
\hline 12. Histidine & 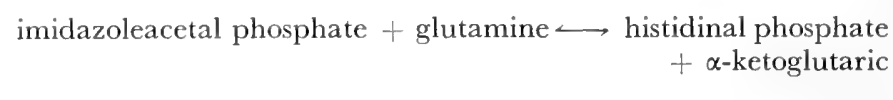 \\
\hline 13. Lysine & $\alpha$-ketoadipic + glutamic $\longrightarrow \alpha$-aminoadipic $+\alpha$-ketoglutaric \\
\hline I4. Tyrosine & $p$-hydroxyphenylpyruvic + glutamic $\longleftarrow$ tyrosine $+\underset{\alpha \text {-ketoglutaric }}{+}$ \\
\hline I5. Phenylalanine & phenylpyruvic + glutamic $\longleftrightarrow$ phenylalanine $+\alpha$-ketoglutaric \\
\hline
\end{tabular}


Glucosamine-6-phosphate is required for the synthesis of hyaluronic acid and other amino polysaccharides. The synthesis of the glucosamine moiety of hyaluronic acid has been studied in Streptococcus hemolyticus (Lowther and Rogers, 1956). Washed bacterial suspensions synthesize hyaluronate from glucose, provided that glutamine or ammonium glutamate is present. Cell free extracts, however, require glutamine rather than glutamate. In the presence of ammonium glutamate, synthesis is much more sensitive to methionine sulfoxide than when glutamine is added. Methionine sulfoxide inhibits glutamine synthesis from ammonia and glutamate. The transfer of the amide group of glutamine in glucosamine synthesis is also indicated by the fact that ${ }^{15} \mathrm{NH}_{3}$ is incorporated efficiently into the glucosamine of hyaluronate in the presence of a large pool of unlabelled glutamate.

(5) Imidazole nitrogen of histidine. Experiments with glutamine-amide- ${ }^{15} \mathrm{~N}$ have shown that in $E$. coli cells, the amide group of glutamine is a more efficient precursor of nitrogen I of the imidazole ring than $\mathrm{NH}_{3}$, glutamate, or asparagine (Neidle and Waelsch, I956). It is not known whether the amide group of glutamine participates directly in histidine synthesis by primary formation of an amino sugar or an amino aldehyde or indirectly by group transfer from an intermediate such as guanine. The amide-nitrogen of glutamine also contributes to nitrogen 3 of the imidazole ring, but less efficiently than to nitrogen I. Possibly, the $\alpha$-amino group of glutamate is the precursor of both nitrogen 3 of the imidazole ring and of the $\alpha$-amino group of histidine.

\section{Aspartic acid and the transfer of $\alpha$-amino nitrogen}

Four reactions are now known involving the transfer of the alpha amino nitrogen of aspartate. Mention has already been made of I) the transamination reaction with glutamic acid and, of 2) the condensation of aspartate with citrulline to form argininosuccinic acid, the arginine precursor. The other two reactions are steps in the synthesis of purine nucleotides. The first of these is the conversion of inosinic acid to adenosine-5-phosphate. The reaction has been studied in yeast (Carter and Cohen, 1955) E.coli (Lieberman, I 956) and bone marrow cells (Abrams and Bentley, I $955 \mathrm{a}, \mathrm{b}, \mathrm{c})$. Adenylosuccinate is an intermediate in the conversion:

I) Inosinic + L-aspartate $+\mathrm{GTP} \rightarrow$ adenylosuccinate $+\mathrm{GDP}+\mathrm{P}_{\mathrm{i}}$

2) Adenylosuccinate $\rightarrow$ adenosine-5-phosphate + fumarate

It was shown with aspartate- $-{ }^{14} \mathrm{C}$ and with inosinic acid-8- ${ }^{14} \mathrm{C}$ (Carter and Cohen, 1955) that the former compounds are incorporated into adenylosuccinate and into adenosine-5-phosphate without dilution of the radioisotope. It should be noted that GTP is required in this process (Lieberman, I956). The second reaction is the synthesis of 5-amino-4-imidazole ( $\mathrm{N}$-succinyl carboxamide) ribotide from amino-imidazole ribotide (AIR): (Lukens and Buchanan, I956).

\section{ATP}

3) $\mathrm{AIR}+$ aspartate $+\mathrm{HCO}_{3}{ }^{-} \longrightarrow 5$-amino-4-imidazole $(\mathrm{N}$-succinyl-carboxamide $)$ ribotide This reaction accounts for the fact that the nitrogen of aspartic acid is the source of nitrogen I of the purine rings of adenylic and guanylic acids (Sonne et al., i 956; 


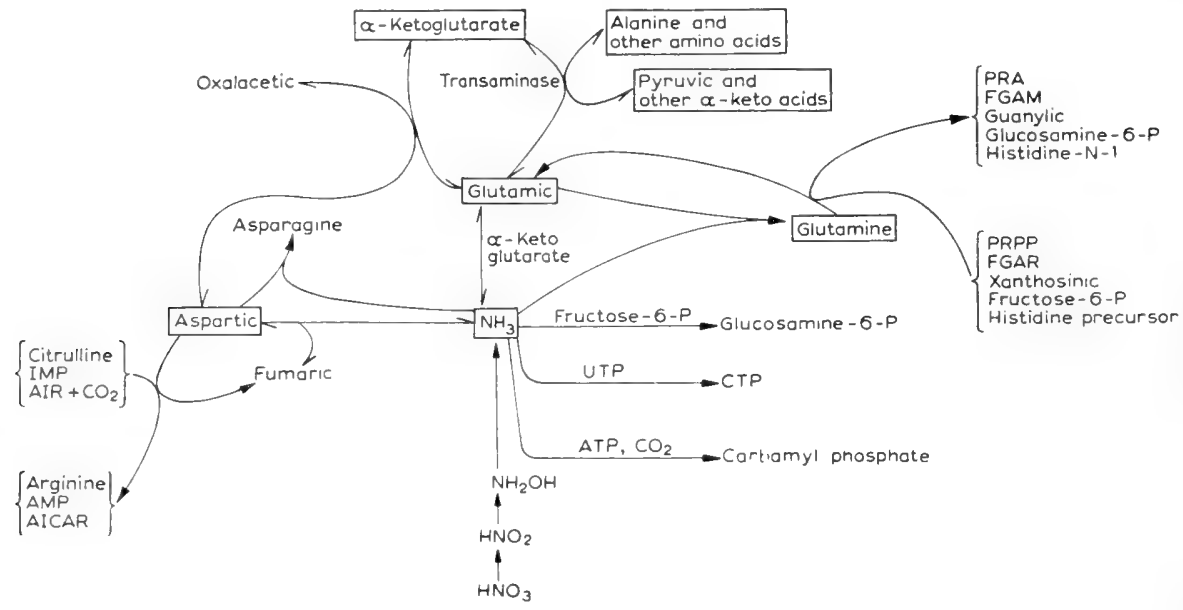

Fig. 40. $\mathrm{NH}_{3}$ utilization and nitrogen transfer reactions.

Levenberg et al., I956). Mention should also be made of the fact that aspartic acid is a precursor of $\beta$-alanine and of pyrimidines. The former involves the decarboxylation of aspartate. The first step in the latter process is the reaction of aspartate with carbamyl phosphate to give ureidosuccinic acid (Lowenstein and Cohen, 1956). Fig. 40 summarizes the $\mathrm{NH}_{3}$ fixation and nitrogen transfer reactions discussed in this section.

\section{Porphyrin Synthesis}

As a result of tracer experiments it was inferred that the 34 carbon atoms and the four nitrogen atoms of protoporphyrin of duck erythrocyte (Fig. 4I) are derived from labelled acetate and labelled glycine (Shemin and Wittenberg, I95 I) in the following manner:

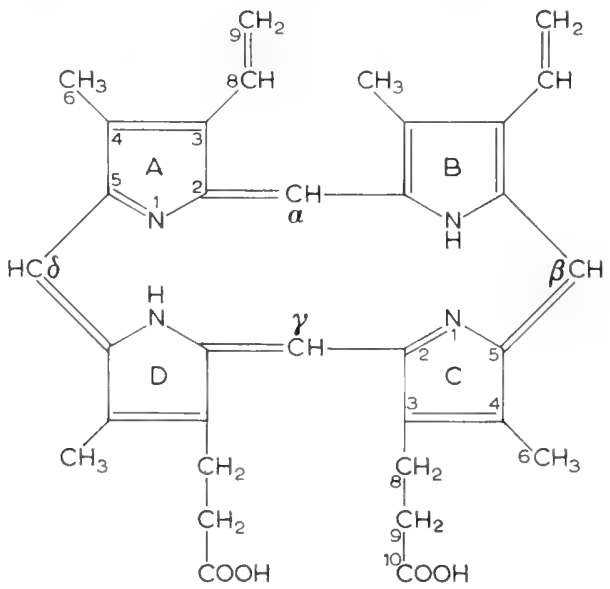

Fig. 4I. Protoporphyrin IX. 

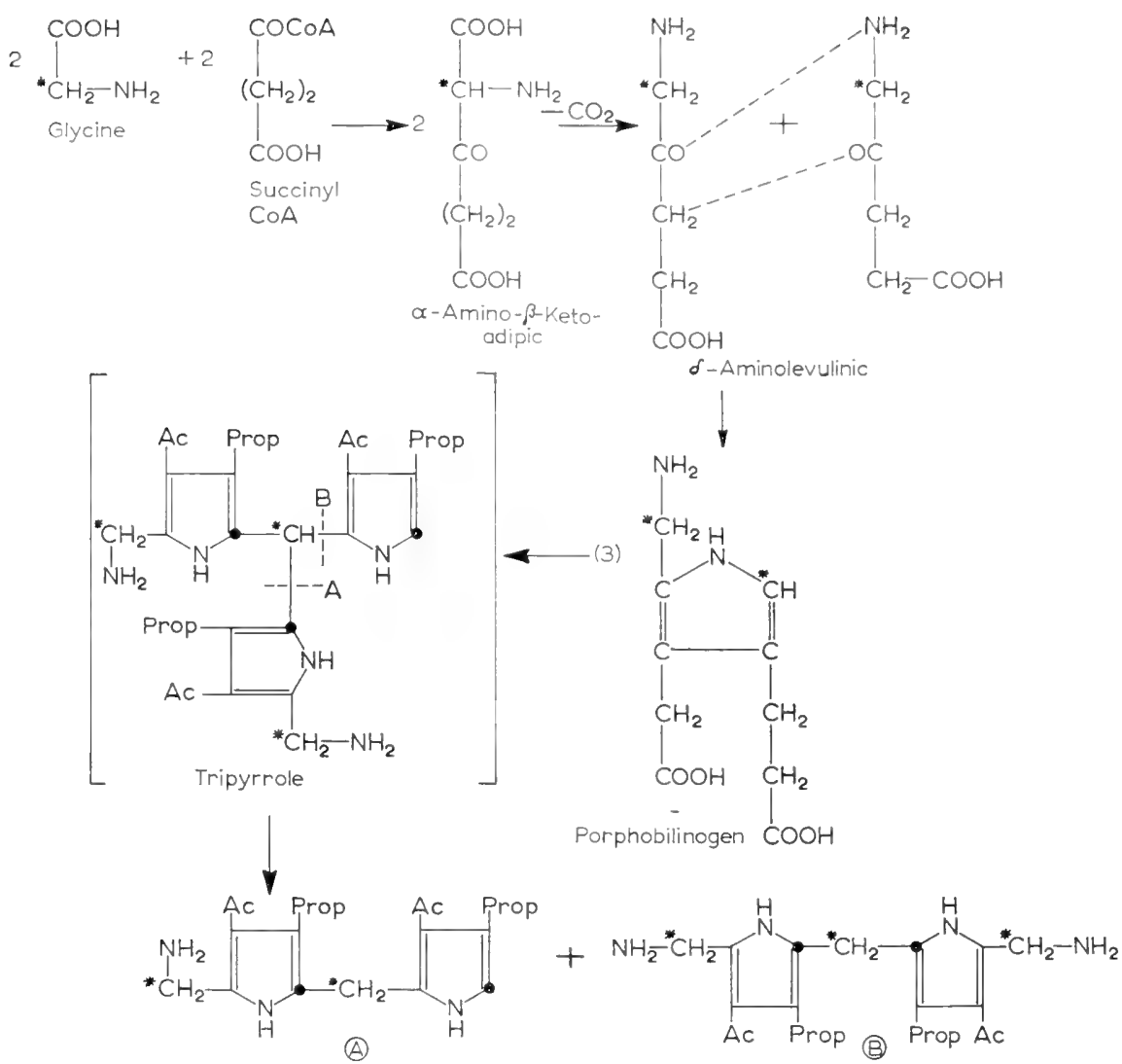

Fig. 42. Proposed pathway of porphyrin synthesis.

Twenty six carbon atoms are derived from acetate. The methyl group of acetate is the precursor of carbons 3 to 6 , and 8 and 9 of each of the pyrrole rings. The two carboxyl groups are derived from the carboxyl group of acetate. Carbon 2 of the pyrrole rings and the methylene bridge carbons originate from the $\alpha$ carbon of glycine while the glycine amino group is the precursor of all of the nitrogen of the molecule. The carboxyl group of glycine is not incorporated into protoporphyrin.

The distribution of radioactivity in the protoporphyrin molecule suggests that all four pyrrole rings are derived from a common precursor pyrrole; furthermore, that carbon groupings $9,8,3$, and $6,4,5$ probably come from the same compound which contains a carboxyl group, from which carbon to is derived but which is lost from all four carbon- 6 atoms during the synthesis and from two of the carbon- 9 atoms. A condensation between glycine and succinyl-CoA seemed involved in the synthesis of the precursor pyrrole. Experiments with hemolyzed duck erythrocytes supported this hypothesis. It was shown that $\alpha$-ketoglutarate $-5-{ }^{14} \mathrm{C}$ or citrate- $\mathrm{I}, 5^{-14} \mathrm{C}$, was incorporated into carbon $\mathrm{Io}$ of rings $\mathrm{C}$ and $\mathrm{D}$, carbon atoms previously shown to originate from acetate- $\mathrm{I}-{ }^{14} \mathrm{C} \cdot \alpha$-ketoglutarate- $\mathrm{I}, 2-{ }^{14} \mathrm{C}$ was 
incorporated into carbon atoms 3 and 5 of the pyrrole rings. Malonate inhibited the labelling of protoprophyrin when acetate-2- ${ }^{14} \mathrm{C}$ was the substrate but not when acetate- $\mathrm{I}-{ }^{14} \mathrm{C}$ was the substrate (Wriston et al., I955).

The product of the condensation of succinyl-CoA and glycine is $\alpha$-amino- $\beta$-ketoadipic acid. The latter substance is converted to $\delta$-aminolevulinic acid by loss of the $\alpha$-carboxyl group, the carboxyl atom of the glycine substrate (Fig. 42). When glycine- $2-{ }^{14} \mathrm{C}$ was incubated with the hemolyzed duck erythrocytes, $\delta$-aminolevulinic acid $-5-{ }^{-14} \mathrm{C}$ could be isolated from the medium. Labelled $\delta$-aminolevulinic acid was also obtained when ${ }^{14} \mathrm{C}$-succinyl-CoA was the substrate. Furthermore, non-labelled $\delta$-aminolevulinic acid markedly inhibited the incorporation of labelled glycine-or succinyl-CoA into protoporphyrin (Shemin and Russell, I 953).

Particle free extracts of the erythrocyte hemolysates contained the enzymes for the synthesis of protoporphyrin from $\delta$-aminolevulinic acid labelled with either ${ }^{14} \mathrm{C}$ in the fifth carbon or with ${ }^{15} \mathrm{~N}$ (Schmid and Shemin, I955). Furthermore, extracts from chicken erythrocytes, spinach, Chlorella cells or a Tetrahymena strain were also capable of effecting this conversion. The precursor pyrrole was identified as porphobilinogen, a compound isolated from the urine of patients with acute porphyria (Granick, I 954). $\delta$-aminolevulinic acid itself was also isolated from the urine of men with acute porphyria and from normal men (Mauzerall and Granick, I 956; Fig. 42). The synthesis of labelled porphobilinogen from labelled $\delta$-aminolevulinic acid or the diethylester of $\alpha$-amino- $\beta$-ketoadipic acid was also demonstrated in experiments with ox liver or duck erythrocyte enzymes. Moreover, the porphobilinogen had twice the molar specific activity of the precursor $\delta$-aminolevulinic acid. Labelled porphobilinogen was in turn incorporated into protoporphyrin by erythrocyte enzymes or by colorless extracts of Chlorella cells. The enzymes for the synthesis of porphobilinogen from $\delta$-aminolevulinic acid were also found in mature human erythrocytes, cells which are incapable of effecting the complete synthesis of heme in vitro.

The side chains of pyrrole rings $\mathrm{A}$ and $\mathrm{B}$ of protoporphyrin differ from those of rings $\mathrm{C}$ and D (Fig. 4I). The mechanism by which the side chains are decarboxylated in the synthesis of protoporphyrin is not known as yet. There are indications that uroporphyrin, coproporphyrin, and protoporphyrin are formed independently from porphobilinogen (Schwartz, r955). Shemin et al. have suggested that a tripyrrole methane compound is an intermediate in the synthesis of the tetrapyrroles (Fig. 42) (Shemin et al., i 955). Cleavage of the tripyrrole methane at $\mathrm{A}$ or $\mathrm{B}$ would produce a dipyrrole methane compound of structure (A) or (B) respectively and a monopyrole. The condensation of two molecules of dipyrrole methane (A) would produce a tetrapyrrole of the porphyrin I series whereas a tetrapyrrole of the porphyrin III series would arise by a condensation of one molecule of (A) with one molecule of dipyrrole methane (B). It may also be seen that the above mechanism necessitates the loss of one of the three aminomethyl side chains in the formation of a protoporphyrin III compound from the condensation of the A and B dipyrroles. Consistent with the suggested mechanism was the finding that radioactive formaldehyde was formed as a result of conversion of porphobilinogen labelled with ${ }^{14} \mathrm{C}$ on the aminomethyl group to porphyrins either by heating under acid conditions or enzymatically in cell free extracts. 


\section{E. Nucleotide Synthesis}

\section{Occurrence of mucleotides in tissues}

Inosinic acid and the mono-, di-, and triphosphates of adenosine, guanosine cytidine, and uridine have been demonstrated in the cytoplasm of yeast, various animal tissues and tumors of mice and rats (Smith and Mills, I954, I954a; Schmitz, I954a, b; Schmitz et al., I 954a, b, I955a, b; Bergkvist and Deutsch, I954a, b) and probably occur in all tissues. Nucleotide compounds such as uridine diphosphate glucose, (UDP-glucose), UDP-acetylglucosamine, UDP-glucuronic acid, UDP-acetyl galactosamine, UDP-galactose, UDP-xylose, and UDP-arabinose have also been reported.(Ginsberg et al., I956). The concentrations of these substances vary from one tissue to another. The concentration of uridine diphosphoglucuronic acid is very low in sarcoma 37, while the oviduct of the hen contains relatively high concentrations of a number of uridine compounds, including UDP-acetylglucosamine phosphate and UDP-acetyl-galactosamine sulfate, compounds not hitherto demonstrated in other tissues (Strominger, I955). Guanosine diphosphomannose occurs in yeast and in oviduct tissue. On the other hand, the concentration of ATP is rather low in hen oviduct tissue. Cytidine diphosphoribitol and cytidine diphosphoglycerol have been found in Lactobacilli (Baddiley et al., I956) while CDP-choline and CDP-ethanolamine occur in liver and yeast (Kennedy and Weiss, I956). Liver extracts contain adenosine diphosphoribose, adenosine diphosphoglutamic, and adenosine diphosphoaspartic acids (Hansen and Hageman, I956).

Of the deoxyriboside nucleotides, only the thymidylic and the deoxycytidylic acid series of compounds have so far been reported. The mono-, di-, and triphosphates of these compounds occur in thymus tissue and thymidylate has also been reported in E. coli (Potter and Schlesinger, I955). Low concentrations of deoxyuridine, deoxycytidine, and thymidine have been found in many animal tissues (Schneider, I 955; Schneider and Brownell, I956). The concentration of deoxyribonucleosides is somewhat higher in lymphatic tissues and tumors than in most normal animal tissues (Schneider, 1955; Lansford and Shive, I955). The total amount of deoxyribosidic compounds is about Io $\mathrm{Y} / \mathrm{g}$ of liver or Novikoff tumor but is increased by over $50 \%$ in the liver of a partially hepatectomized rat before actual regeneration occurs ( 24 hours post operatively). In normal lever, $90 \%$ of the deoxyribosidic compounds can be accounted for as deoxycytidine whereas in the hepatoma, $50 \%$ of the deoxyribosidic compounds are attributable to deoxycytidine, deoxyuridine, and thymidine in the approximate ratio of $(5: \mathrm{I}: \mathrm{I})$ while the rest are presumably due to nucleotides. About $25-30 \%$ of the deoxyribosidic compounds of the liver $24 \mathrm{~h}$. after partial hepatectomy are also found to have nucleotide like properties (Schneider and Brownell, I956).

\section{2. "De novo" purine synthesis}

The precursors of tissue purines have been elucidated as a result of studies on the incorporation of labelled metabolites into the uric acid of the pigeon and rat, and the nucleic acid purines of various animal tissues and microorganisms (Buchanan, 
I95I). Carbon dioxide is the precursor of carbon 6 of the purine ring; the carboxyl- and $\alpha$-carbons of glycine give rise to carbons 4 and 5 respectively, while formate and the $\beta$-carbon of serine are the precursors of carbons 2 and 8 (Buchanan, I95I; Elwyn and Sprinson, 1954) (Fig. 43). By comparing the incorporation of glycine- $\mathrm{I}-{ }^{14} \mathrm{C}$ and the ${ }^{15} \mathrm{~N}$ of ${ }^{15} \mathrm{~N}$-labelled compounds into the hypoxanthine synthesized by extracts of pigeon liver, organic substrates which are the precursors of nitrogen atoms of the purine ring base have also been determined. One mole of glycine nitrogen, two moles of the amide nitrogen of glutamine, and one mole of aspartic acid nitrogen are incorporated for every mole of glycine- ${ }^{1}-{ }^{14} \mathrm{C}$ (Levenberg et al., I 956; Sonne et al., I956). The nitrogen of glycine is the precursor of

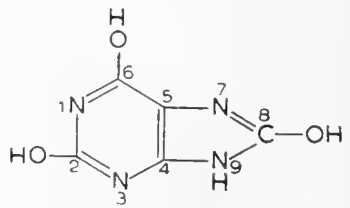

Fig. 43. Uric acid. purine atom number 7 , the amide nitrogen of glutamine gives rise to nitrogen 9 and 3 , while aspartic acid is the precursor of nitrogen I of the purine ring.

The incorporation of labelled glycine, formate, or serine into the nucleic acid purines or the purines of acid soluble nucleotides (Elwyn and Sprinson, I954) has been readily demonstrated in vivo (Edmonds and LePage, r 955; Bennett et al., I955, I956; Harrington and Lavik, I955; Drochmans et al., I952) and in vitro (Mannell and Rossiter, 1955; Totter, I954; Abrams and Goldinger, I95I) in normal tissues and tumors (LePage, I 953) and yeast cells (Abrams, I952).

As a result of experiments with pigeon liver extracts, it became apparent that ribose phosphate derivatives are intermediates in the synthesis of purine nucleotides (Greenberg, I954; Korn et al., I955; Levenberg and Buchanan, I956). The under-mentioned reaction: the formation of 5-phosphoribose pyrophosphate may be taken as the starting point of purine nucleotide synthesis (Kornberg et al., I 955a; Goldthwait et al., I 955).

$$
\text { Ribose-5-phosphate + ATP } \rightarrow \text { AMP + 5-phosphoribosepyrophosphate }
$$

The enzyme catalyzing this reaction has been partially purified from pigeon liver and has also been demonstrated in mammalian liver and in microorganisms. The succeeding steps are outlined in Fig. 44. In the presence of labelled glycine,PRPP, ATP, and glutamine, liver enzymes catalyze the formation of GAR (Goldthwait et al., I955; Goldthwait, I956). Azaserine is a competitive antagonist of glutamine in this reaction. Presumably, PRA is an intermediate in GAR synthesis although this substance has not as yet been isolated from the reaction mixtures. It is known, however, that this intermediate can be readily degraded to ribose-5-phosphate. Chemically synthesized PRA can replace glutamine and PRPP in GAR synthesis. However, ATP is still needed, presumably, for the activation of glycine. In the presence of a pigeon liver extract which contains all of the enzymes needed for the ncorporation of labelled glycine into inosinic acid, azaserine inhibits inosinic acid synthesis and at the same time, labelled GAR and FGAR accumulated (Hartman et al., r 955, I 956). The formation of GAR and FGAR in the absence of azaserine has also been demonstrated (Goldthwait et al., I 956). The FGAR also is labelled when formate $-{ }^{14} \mathrm{C}$ is used as substrate, but in this case, the GAR in unlabelled. Either GAR $-{ }^{14} \mathrm{C}$ or FGAR- ${ }^{14} \mathrm{C}$ can be converted by the liver enzymes to inosinic- 

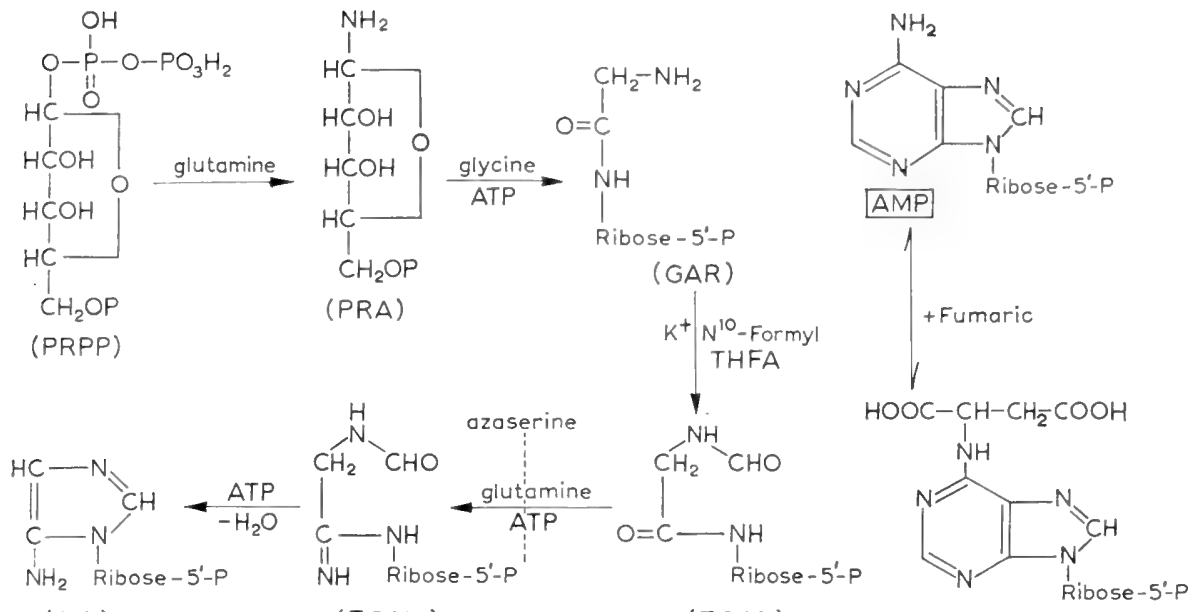

$(A \mid R)$

(FGAM)

(FGAR)

Adenylosuccinate
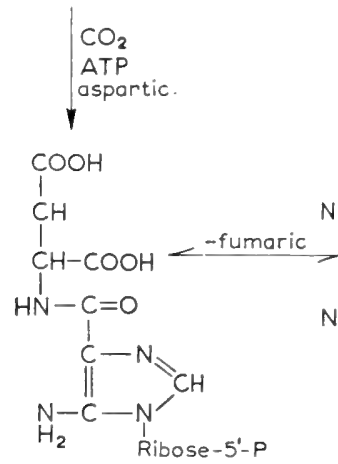

5-Amino- 4 imidazole ( $N$-Succinylocarboxamide) ribotide

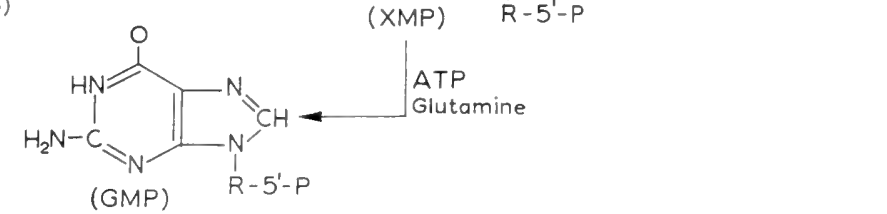

Fig. 44. Pathway of de novo purine nucleotide synthesis.

${ }^{14} \mathrm{C}$, when the enzyme system is supplemented with bicarbonate, formate, glutamine, aspartate, and 3-phosphoglycerate. Non-labelled glycine does not inhibit this process. With FGAR, labelled with ${ }^{14} \mathrm{C}$ on the formate group, inosinic acid-8- ${ }^{14} \mathrm{C}$ is obtained. The $\beta$-carbon of serine can also function as a precursor of the formyl group of FGAR (Warren and Flaks, I956). High levels of glutamine reverse the inhibitory effect of azaserine on de novo synthesis of inosinic acid. Tetrahydrofolic acid (THFA) or the citrovorum factor is required for the synthesis of FGAR from its precursors (Goldthwait et al., I 954, I956).

Using enzymes from pigeon liver extracts fractionated with ethanol, it was shown that FGAR reacts with glutamine to yield an arylamine which on incuba- 
tion with aspartate and $\mathrm{CO}_{2}$ yields 5-aminoimidazole carboxamide ribotide (AICAR) (Levenberg and Buchanan, I956). The amine was identified as 5 -aminoimidazoleribotide (AIR). By fractionating the enzymatic components, a second compound, an intermediate in the conversion of FGAR to AIR, was isolated and it was identified as $\alpha-\mathrm{N}$-formylglycineamidine ribotide (FGAM).

The conversion of FGAM and of AIR to inosinic acid also takes place in the presence of liver enzymes supplemented with bicarbonate, aspartate, ATP and formate. ${ }^{14} \mathrm{CO}_{2}$ is fixed into these ribotides. Azaserine does not affect the conversion of AIR to AICAR and IMP but does prevent the formation of FGAM from FGAR in the enzyme system, confirming the primary locus of inbibition by this substance. The enzymes of pigeon liver which convert AIR to AICAR were separated into two fractions. Fraction I catalyzed the conversion of AIR, aspartate, and bicarbonate, to an intermediate, 5-amino-4-imidazole ( $\mathrm{N}$-succinylocarboxamide) ribotide. (Lukens and Buchanan, 1956). In experiments in which aspartate$3^{-14} \mathrm{C}$ was used, the intermediate became labelled. Enzyme fraction II converted this intermediate to AICAR and fumarate.

An active formyl-THFA derivative was also required for the formation of inosinic acid from AICAR (Warren and Flaks, I956). With serine as the donor of the formyl group, potassium ions, leucovorin and pyridine nucleotides were needed for IMP formation or for FGAR formation by chicken or pigeon liver enzymes. The transformylation reactions were found to be reversible:

leucovorin

a) $\mathrm{IMP}+$ glycine $+\mathrm{TPNH}_{2} \longleftrightarrow$ AICAR + serine $+\mathrm{TPN}^{+}$

b) $\mathrm{IMP}+\mathrm{GAR} \underset{\mathrm{DPN}^{+}, \mathrm{K}^{+}}{\stackrel{\text { leucovorin }}{\longrightarrow}} \mathrm{FGAR}+\mathrm{AICAR}$

An enzyme which catalyzed exchange of formate- ${ }^{14} \mathrm{C}$ with IMP, stimulated by leucovorin, has also been demonstrated (Flaks and Buchanan, I954). In the presence of THFA, pigeon or pig liver enzymes catalyze the formation of IMP from formate $-{ }^{14} \mathrm{C}$ and AICAR (Greenberg et al., I 955). ATP is required to activate the formate. $\mathrm{N}_{10}$-formyl-tetrahydrofolic acid can substitute for formate and ATP in IMP synthesis.

The above formulation for the terminal steps in IMP biosynthesis is strengthened by earlier studies with microorganisms. Sulfanilamide, a metabolic antagonist of paraaminobenzoic acid (PABA), interferes with the synthesis of the active coenzyme form of folic acid. In sulfanilamide inhibited $E$. coli cells, aminoimidazolecarboxamide (AICA) and the riboside of this compound accumulate. AICA riboside is also formed by a PABA requiring mutant of $E$. coli (Greenberg and Spilman, 1956). It is reasonable to assume that the riboside arises from the ribotide, AICAR, by phosphatase action and is then excreted into the medium. Small amounts of AICAR can also be isolated from E. coli grown under sulfanilamide bacteriostasis (Greenberg, 1956).

The formation of adenosine- 5 '-phosphate (AMP) from inosinic acid requires aspartate, and GTP (Lieberman, 1956; Carter and Cohen, 1956). E. coli enzymes catalyzing AMP synthesis have been purified 40 fold. Adenylosuccinate is an intermediate in this conversion. Aspartate- $\mathrm{I}, 4^{-14} \mathrm{C}$ is incorporated into adenylosuccinate without dilution as is inosinic acid-8 $-{ }^{14} \mathrm{C}$. The requirement for aspartate in the conversion of inosinic acid to AMP has also been demonstrated in bone marrow extracts (Abrams and Bentley, 1955a). 
The reverse of the condensation of IMP and aspartate, the condensation of fumaric acid with AMP to form adenylosuccinate, takes place in yeast autolysates.

The synthesis of guanylic acid (GMP) requires the prior oxidation of IMP to xanthylic acid (XMP). In this oxidative step it has been shown in experiments with bone marrow (Abrams and Bentley, I955a, b, c), Aerobacter, and E. coli enzymes (Gehring and Magasanik, I955) that $\mathrm{DPN}^{+}$is necessary. The amination of XMP to GMP has been studied with bone marrow (Abrams and Bentley I 955 b) and liver enzymes (Lagerkvist, I955). The amide group of glutamine is the source of the 2-amino group of GMP. ATP is required in the conversion. By analogy with the adenylosuccinate intermediate in AMP synthesis, a guanyloglutaric acid derivative has been suggested as an intermediate in GMP synthesis. In the case of the bacterial enzymes, ammonia is required instead of glutamine:

I) $\mathrm{XMP}+\mathrm{ATP}+\mathrm{NH}^{+}{ }_{4} \rightarrow \mathrm{GMP}+\mathrm{AMP}+\mathrm{PP}$

An Aerobacter mutant which requires guanine and excretes xanthosine is unable to aminate XMP (reaction I). The conversion of guanine to nucleic acid adenine apparently does not take place by a reversal of these reactions. During the conversion of guanine to adenine in Aerobacter mutants, the amino substituted carbon 2 of guanine is probably transferred to THFA. The products of this reaction are AICAR and $\mathrm{N}_{10}$-formimino-THFA. The transformylation of AICAR results in the formation of IMP, the AMP precursor (Magasanik et al., i956).

\section{(a) Labelled purines}

\section{The utilization of free purines}

Experiments with adenine-8- $-{ }^{14} \mathrm{C}$ or adenine $-{ }^{15} \mathrm{~N}$ have shown that this substance is rapidly incorporated in vivo into nucleic acid purines of normal tissues and tumors (Furlong et al., I 955; Bennett et al., I 955, I956; Brown, I950, I 95 I ), the ribonucleic acid or acid soluble nucleotide purines of tissue slices or homogenates (Goldwasser, I953, I954; Lajtha et al., I 954; Abrams and Goldinger, I 95 I) and into Lactobacilli and yeast nucleic acids (Abrams, I952; Bennett and Kruekel, I955; Marrian, I954). On the other hand, free guanine is rather poorly incorporated into the purines of the mouse, hamster, or rabbit bone marrow cells (Brown, I950, I95I). Adenine is converted to guanine and guanine to adenine by rabbit bone marrow cells, (Abrams and Goldinger, 195 I). Guanine is a very poor nucleic acid precursor in human or rat tumors (Bennett and Skipper, I955; Bennett et al., I 955, I 956).

In contrast to the higher animals, Tetrahymena, in which de novo purine synthesis is apparently blocked (Kidder et al., 1950), requires guanine for growth. Adenine or hypoxanthine can spare but not replace the guanine requirements of this organism.

Hypoxanthine-8 ${ }^{14} \mathrm{C}$ is a moderately effective precursor of the nucleic acid purines of the hamster but not of the rat or mouse (Bennett and Skipper, I955). Neither hypoxanthine nor xanthine are well utilized by a number of tumor tissues (Bennett et al., 1956) nor is xanthine utilized significantly by normal rat tissues.

Some free AICA can be utilized as a precursor of nucleic acid purines by animals and microorganisms. It is converted to uric acid by man (Seegmiller et al., 1955) and by pigeons (Miller et al., 1950) and to allantoin, nucleotide purines, and nucleic acid purines in the rat, to nucleotide and nucleic acid adenine in yeast, and tissue nucleic acid purines in mice. Pigeon livers convert AICA to hypoxanthine. 
PRPP reactions. Two mechanisms are known for the utilization of free purines. The first, involving the reaction of the purine base with PRPP, has been studied in liver and yeast extracts (Kornberg et al., i 955b). Nucleotides are the products of these reactions:

$\begin{aligned} & \text { Adenine } \\ & \text { Guanine } \\ & \text { Hypoxanthine }\end{aligned}+\mathrm{PRPP} \underset{\text { yeast }}{\longrightarrow} \mathrm{PP}+\underset{\mathrm{GMP}}{\mathrm{AMP}}$

Nucleoside phosphorylase. A second mechanism for purine utilization is mediated by nucleoside phosphorylase enzymes. Purine nucleosides are formed as a result of these reactions (Korn and Buchanan, 1956) (Table 7).

TABLE 7

NUCLEOSIDE PHOSPHORYLASE

\begin{tabular}{|c|c|c|}
\hline Substrate & Product & \\
\hline $\begin{array}{l}\text { Guanine } \\
\text { Hypoxanthine liver, E. coli } \\
\text { Xanthine } \\
\text { Adenine (beef liver) } \\
\text { AICA }\end{array}$ & $\begin{array}{l}\text { Guanosine } \\
\text { Inosine } \\
\text { Xanthosine } \\
\text { Adenosine } \\
\text { AICA riboside }\end{array}$ & $+\mathrm{H}_{3} \mathrm{PO}_{4}$ \\
\hline Hypoxanthine & $\begin{array}{l}\text { Hypoxanthine- } \\
\text { deoxyriboside }\end{array}$ & \\
\hline+ deoxyribose-I-phosphate $\stackrel{\text { liver, E. coli, thymus }}{\longrightarrow}$ & $\begin{array}{l}\text { Guanine- } \\
\text { deoxyriboside } \\
\text { Xanthine- } \\
\text { deoxyriboside }\end{array}$ & $+\mathrm{H}_{3} \mathrm{PO}_{4}$ \\
\hline
\end{tabular}

A nucleoside phosphorylase, specific for inosine and guanosine, occurs in erythrocytes and in brain tissue (Huennekens et al., i 956). Hypoxanthine, guanine or xanthine, but apparently not adenine, may also react with deoxyribose-I-phosphate in the presence of nucleoside phosphorylase, in which case deoxyribonucleosides are formed (Friedkin and Kalckar, I950; Hoff-Jorgenson et al., I950; Manson and Lampen, I95I, I953; Friedkin, I953; Lampen, I952; Kalckar, I 955).

\section{(b) Mutase enzymes}

The ribose-I-phosphate which is required for the synthesis of the nucleosides, is formed from ribose-5-phosphate in a reaction catalyzed by phosphoribosemutase. Ribose diphosphate is a cofactor in the reaction (Reissig, I956; Klenow and Emberland, I955; Guarino and Sable, I956; Klenow, 1953).

a) Ribose-5-phosphate $\frac{\text { ribose diphosphate }}{\mathrm{Mg}^{++}}$ribose-I-phosphate

deoxyribose diphosphate (?)

b) Deoxyribose-5-phosphate deoxyribose-I-phosphate 
Deoxyribose-5-phosphate may sulsstitute for ribose-5-phosphate so that deoxyribose-I-phosphate is formed (Manson and Lampen, I95I). Phosphoglucomutase and the phosphoribomutases of yeast and muscle are apparently different enzymes.

\section{(c) Kinase enzymes}

In the presence of kinase enzymes, purine ribosides are converted to nucleotides. The enzyme, adenosine kinase, has been observed in liver, kidney, and yeast (Kornberg and Pricer, I95I; Ostern et al., 1938; Sable, I950; Caputto, I 95I; Greenberg, I 956).

I) Adenosine $+\mathrm{ATP} \rightarrow \mathrm{ADP}+\mathrm{AMP}$

2) $\mathrm{AICA}$ riboside $+\mathrm{ATP} \rightarrow \mathrm{ADP}+\mathrm{AICAR}$

It is to be noted that reactions I) and 2) provide a third pathway for AMP formation and a second pathway for the formation of aminoimidazole carboxamide ribotide. It is probable that inosine and xanthosine kinase enzymes also exist in tissues:

3) Inosine $+\mathrm{ATP} \rightarrow \mathrm{ADP}+\mathrm{IMP}$

4) Xanthosine $+\mathrm{ATP} \rightarrow \mathrm{ADP}+\mathrm{XMP}$

The conversion of inosine $-{ }^{14} \mathrm{C}$ to labelled adenine and guanine compounds and to inosinic acid takes place in bone marrow cells under conditions where hypoxanthine- ${ }^{14} \mathrm{C}$ is not utilized. Xanthosine- ${ }^{14} \mathrm{C}$ is also converted to guanylic acid by bone marrow cells (Abrams and Bentley, 1955a). Xanthosinic acid is probably an intermediate in this conversion. Inosine $-{ }^{14} \mathrm{C}$ as well as adenosine- ${ }^{14} \mathrm{C}$ is readily incorporated into the acid soluble and ribonucleic acid purines of Ehrlich tumor cells (Edmonds and LePage, 1956).

\section{(d) Deaminase enzymes}

It is known that adenine-8- ${ }^{14} \mathrm{C}$ can be utilized for the synthesis of nucleic acid guanine as well as nucleic acid adenine in many tissues (Brown, I950, I95I). Probably, the free adenine, is deaminated after it is converted to adenosine or adenylic acid (Krebs and Hems, I 955). Enzymes which catalyze the deamination of adenosine or of adenylic acid occur in most animal tissues (Goldwasser, I953) and in bacteria (Friedkin, I953; Abrams and Bentley, I955a; Kornberg et al., I 955 a).

r) Adenosine $\rightarrow$ inosine $+\mathrm{NH}_{3}$

2) Adenylic $\rightarrow \mathrm{IMP}+\mathrm{NH}_{3}$

\section{(e) Isotope competition experiments}

Evidence concerning purine interconversions has also been obtained by isotope competition experiments. ${ }^{14} \mathrm{CO}_{2}$ is incorporated into both the adenine and guanine of $E$. coli cell nucleic acids. The incorporation of ${ }^{14} \mathrm{CO}_{2}$ into nucleic acid-guanine is reduced by exogenous adenine, adenosine, guanine, or guanosine, but the labelling of nucleic acid adenine is reduced by adenine or adenosine only (Bolton, 1954). The effect of exogenous purine compounds on the incorporation of labelled glycine into purines of the acid soluble pool and of the nucleic acids has been studied in mouse liver, the Gardner tumor and the Ehrlich tumor (LePage, 1953). The following exogenous compounds decreased the 
conversion of glycine-2-14 $\mathrm{C}$ to nucleic acid adenine or guanine and to the adenine of the acid soluble pool: adenine, adenosine, adenylic, hypoxanthine, inosine, and IMP. Guanine, guanosine, guanylic, inosine, IMP, and hypoxanthine decreased the conversion of glycine to nucleic acid guanine only while guanosine and GMP suppressed the radioactivity of the guanine of the acid soluble pool. The incorporation of inosine- ${ }^{14} \mathrm{C}$ to $\mathrm{RNA}$-adenine was reduced by exogenous $\mathrm{AMP}$ but the conversion of $\mathrm{AMP}-{ }^{14} \mathrm{C}$ to $\mathrm{RNA}$-adenine was not reduced by non-labelled inosine, indicating that inosine was active as a result of its conversion to AMP. Inosine- ${ }^{14} \mathrm{C}$ and $\mathrm{IMP}-14 \mathrm{C}$ were effective precursors of nucleic acid adenine and guanine of the Ehrlich tumor (Edmonds and LePage, 1956).

\section{4. "De novo" pyrimidine synthesis}

(a) Tissue slice and in vivo experiments

The mechanism of pyrimidine synthesis from aspartic acid, $\mathrm{CO}_{2}$, and $\mathrm{NH}_{3}$ is shown in Fig. 45. Evidence for this biosynthetic pathway has been obtained
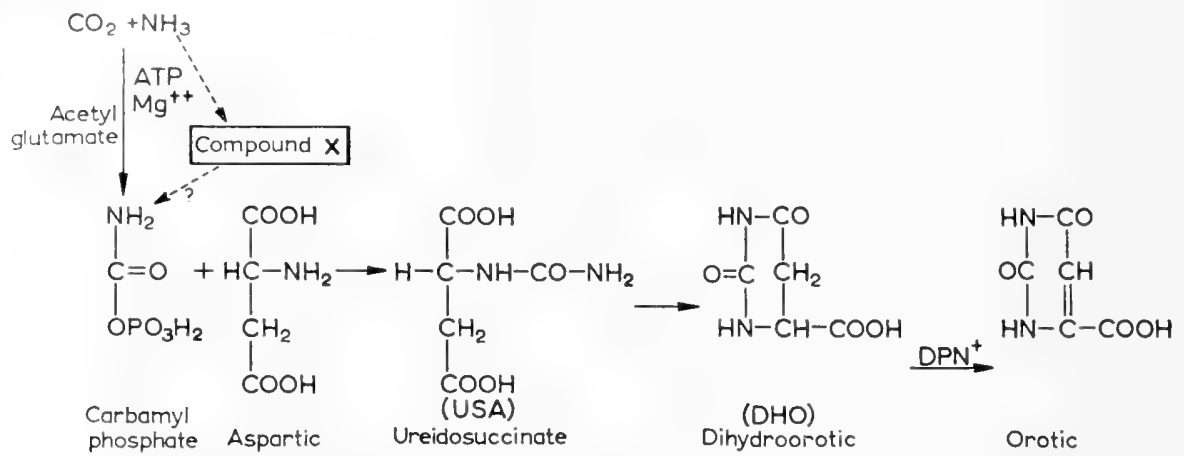

Fig. 45. Biosynthesis of orotic acid.

by isotope studies with microorganisms, animals in vivo, and slices or homogenates from normal and malignant tissues. ${ }^{15} \mathrm{NH}_{4} \mathrm{Cl}$ is incorporated into pentose nucleic acid (PNA) uracil and cytosine of the Ehrlich tumor cells and of regenerating liver slices (Lagerkvist et al., I 955; Reichard, I 952). The incorporation is inhibited by non-labelled orotic acid or dihydrorotic acid. Simultaneously, these compounds become labelled with ${ }^{15} \mathrm{~N}$. The incorporation of ${ }^{15} \mathrm{NH}_{4} \mathrm{Cl}$ into the orotic acid of liver slices is reduced by non-labelled ureidosuccinate (USA). ${ }^{14} \mathrm{CO}_{2}$ is incorporated into the pentose nucleic acid pyrimidines by liver slices or by $E$. coli cells (Bolton, I 954). The incorporation of aspartic acid labelled with ${ }^{15} \mathrm{~N}$ or with ${ }^{14} \mathrm{C}$ into nucleic acid pyrimidines or orotic acid of Ehrlich tumor cells, liver slices, or $E$. coli cells has also been demonstrated. In liver slices, aspartate- ${ }^{14} \mathrm{C}$ is converted to USA- ${ }^{14} \mathrm{C}$. Finally, USA, labelled with either ${ }^{15} \mathrm{~N}$ or ${ }^{14} \mathrm{C}$ is in turn a precursor of orotic acid, or pentose nucleic acid pyrimidines in normal tissues, tumors and microorganisms (Werkheiser and Visser, I955; Weed and Wilson, I 954; Lagerkvist et al., I 955; Anderson et al., 1955). Exogenous dihydrorotic acid and orotic acid can reduce the labelling of nucleic acid pyrimidines from USA- ${ }^{14} \mathrm{C}$. A compound related to USA, aminofumaric acid, is apparently a pyrimidine precursor in. Neurospora and in rat liver slices (Fairley, I954; Cooper et al., I955). 


\section{(b) Homogenate and enzyme experiments}

A labile compound formed from $\mathrm{CO}_{2}, \mathrm{NH}_{3}$, and $\mathrm{ATP}$ is an intermediate in the synthesis of USA (Fig. 45). This labile compound may be carbamyl phosphate (Reichard and Hanshoff, 1955; Lowenstein and Cohen, 1955; Jones et al., 1955; Marshall et al., 1955), or it may be an unknown complex form of carbamyl phosphate, "Compound X" (Grisolia et al., I955). Acetyl glutamate stimulates, but is not essential, for the formation of carbamyl phosphate or "Compound X". The synthesis of carbamyl phosphate has been demonstrated so far only in liver mitochondria, kidney and in Streptococci, but the subsequent carbamylation of aspartic acid is catalyzed by enzymes present in most rat organs, and in many organisms. "Compound $\mathrm{X}$ " can also be formed in liver tissue or in microorganisms from the ureido group of citrulline by the reversal of the steps involved in the formation of citrulline from ornithine, $\mathrm{CO}_{2}, \mathrm{NH}_{3}$, and ATP (Reichard and Smith, 1955). This explains the observations that carbamyl labelled citrulline is incorporated into carbon atom 2 of Neurospora nucleic acid cytosine and uracil (Heinrich et al., I954), the nucleic acid pyrimidines of pigeon liver (Schulman and Badger, I954), and orotic acid of rat liver (Smith and Stetten, I954).

Liver homogenates convert ureidosuccinic acid to dihydroorotic acid and both these compounds to orotic acid (Cooper et al., 1955). Enzymes catalyzing the interconversion of USA, DHO, and orotic acid have been purified from the orotic acid fermenting bacterium, Zymobacterium oroticum (Lieberman and Kornberg, 1955) and from E. coli $B$ cells (Yates and Pardee, 1956). The enzyme, dihydroorotic dehydrogenase is lacking, however, from an $E$. coli mutant which cannot produce orotic acid but which can utilize it as a growth factor. Another mutant, which uses uracil and cytosine as growth factors, excretes USA, DHO, and orotic acid.

\section{(c) Orotic acid utilization}

Orotic acid is readily utilized for the synthesis of acid soluble nucleotide and nucleic acid pyrimidines by normal and tumor tissues of the rat in vivo, by bacteria (Hurlbert and Potter, I952; Spicer et al., I 952; Reichard and Lagerkvist, I 953) yeast (Edmonds et al., I 952) rat spleen, rat liver, and tumor tissue slices (Lagerkvist et al., I955; Reichard, I952; Weed and Wilson, I95I). Soluble preparations obtained from pigeon liver, $E$. coli cells, rat liver, intestine, and the Flexner-Jobling rat tumor incorporate labelled orotic acid into uridine nucleotides. In the case of rat liver and tumor, the largest amount of label is found in UTP (Heidelberger and Harbers, I956). The conversion of labelled orotic to UDP, UTP, and UDPG by a pigeon liver enzyme system is greatly diminished by the addition of unlabelled $\mathrm{UMP}$ while the radioactivity of $\mathrm{U}$ MP is increased. Although some radioactive uracil and uridine are also formed, the labelling of these substances can be attributed to the action of hydrolytic enzymes. Further evidence against the role of uridine as an intermediate in UMP synthesis in this system is the fact that non-labelled uridine does not alter the conversion of orotic- ${ }^{14} \mathrm{C}$ to UMP (Hurlbert and Reichard, I 954). Two enzymes mediate the conversion of orotic acid to UMP (Lieberman et al., I 955a), an enzyme catalyzing the condensation of orotic with PRPP and an enzyme which is concerned with the decarboxylation of orotidine- 5 -phosphate.

$$
\text { Orotic }+ \text { PRPP } \underset{\text { liver }}{\longrightarrow} \text { orotidine-5'-phosphate } \stackrel{-\mathrm{CO}_{2}}{\longrightarrow} \text { UMP }
$$

\section{Utilization of uracil, cytosine and thymine}

\section{(a) Introduction}

Exogenous uracil may be utilized for the synthesis of nucleic acid pyrimidines in bacteria (Moore and Boylen, I 955; Rose and Schweigert, I 953; Wright and Miller, 
I 952; Bolton and Reynard, I 954; Bolton et al., I952) and in Tetrahymena (Kidder et al., 1950). In the case of the protozoa cells, neither cytosine nor orotic acid can replace uracil, although nucleotides of cytosine or uridine are utilized. Labelled uracil is poorly incorporated into nucleic acid pyrimidines of normal rat tissues in vivo (Rutman et al., I953) but is more effectively incorporated by regenerating liver, rat hepatomas (Cantarow et al., I 956), the Flexner-Jobling tumor, or rat intestine (Heidelberger and Harbers, I 956). The incorporation of uracil- $-2-{ }^{14} \mathrm{C}$ into the RNA of rat liver slices is a function of the uracil concentration of the medium. At low uracil concentrations, the decomposition of the substrate is virtually complete and incorporation does not take place. The incorporation is greatly increased when the proportion of substrate oxidized is diminished (Canellakis, 1955). In contrast to the rat, uracil is fairly well incorporated into mouse intestine and liver nucleic acids in vivo and into the nucleic acids of the Ehrlich tumor of the mouse in vivo or in vitro (Lagerkvist and Reichard, I954).

Although thymine or cytosine may be utilized by certain microorganisms, these substances are rather poorly utilized for nucleic acid synthesis by the rat (Reichard, 1955; Reichard and Estborn, I95I; Holmes et al., 1954). Thymine is also poorly utilized by chick embryo tissue (Friedkin et al., 1956) or by mouse tumors in vitro (Kit, 1957).

\section{(b) Pyrimidine nucleoside phosphorylase}

Pyrimidine nucleoside phosphorylase probably functions in the limited utilization of free pyrimidines, manifested by certain tissues. The action of uracil phosphorylase, is illustrated in reaction I) (Cardini et al., I950). In the presence of ATP and a liver enzyme system containing uracil phosphorylase, the incorporation of uracil-2- ${ }^{14} \mathrm{C}$ into UMP, UDP, and UTP has been demonstrated (Canellakis, I956a). Nucleoside phosphorylase enzymes have also been found in $E$. coli (Paege and Schlenk, I952) and in soil bacteria (U-I) (Wang and Lampen, I 952). The latter enzymes catalyze reactions 2-4:

I) Uracil + ribose-I-phosphate $\longleftrightarrow$ uridine + phosphate

2) Cytosine + ribose-I-phosphate $\longleftrightarrow$ cytidine + phosphate

3) Thymine + deoxyribose-I-phosphate $\longleftrightarrow$ thymidine + phosphate

4) Uracil + deoxyribose-1-phosphate $\longleftarrow$ deoxyuridine + phosphate

Active thymidine phosphorylase enzymes have been reported in liver, thymus, kidney, bone marrow (Manson and Lampen, I95 I ; Friedkin, r953), Tetrahymena, and $E$. coli cells (Manson and Lampen, 1953).

An alternative mechanism of uracil utilization is suggested by the observation that enzymes from two strains of Lactobacillus bulgaricus convert free uracil to UMP as shown in reaction 5 ):

5) $\mathrm{Uracil}+\mathrm{PRPP} \rightarrow \mathrm{UMP}+\mathrm{PP}$

\section{The amination of uracil compounds to cytosine and the deamination of cytidine}

Although it is known that cytosine compounds are readily formed from derivatives of orotic acid or uracil in vivo, the mechanism of cytosine formation has not been satisfactorily established as yet ${ }^{1}$. In $E$. coli $B$ cells, CDP or CTP is formed from UDP or UTP, respectively (Lieberman, I955):

1 See Addendum, Note 4, p. 123. 
I) $\mathrm{UTP}+\mathrm{NH}_{3} \stackrel{\mathrm{ATP}}{\longrightarrow} \mathrm{CTP}+\mathrm{ADP}+\mathrm{P}_{\mathrm{i}}$

The reverse process, the formation of uracil compounds from cytosine derivatives, has been readily demonstrated in most animal tissues, microorganisms, and yeast (Wang et al., 1950; Wang and Lampen, 1952; Friedkin, 1953):

2) Cytidine $\longrightarrow$ uridine $+\mathrm{NH}_{3}$

$$
\text { E. coli }
$$

3) Deoxycytidine $\longrightarrow$ deoxyuridine $+\mathrm{NH}_{3}$

4) 5-Methylcytosine $\longrightarrow$ yeast $\longrightarrow$ thymine $+\mathrm{NH}_{3}$

yeast, $E$. coli

5) Cytosine $\underset{\text { soil bacteria }}{\longrightarrow}$ uracil $+\mathrm{NH}_{3}$

It is to be noted that the combined action of cytidine (or deoxycytidine) deaminase and uridine (or deoxyuridine) phosphorylase on cytidine would result in the formation of uracil, thereby simulating "cytidine phosphorylase" activity (Manson and Lampen, 1953). Deoxycytidine deaminase may be of importance in connection with the formation of deoxyuridine, a thymidine precursor.

\section{Trans- $\mathcal{N}$-glycosidation}

During a study of pyrimidine metabolism in Lactobacillus helveticus McNutt discovered a group of enzymes which catalyze the following type of reaction (McNutt, I952; Lampen, I 952):

Purine deoxyriboside + pyrimidine $\longleftarrow$ purine + pyrimidine deoxyriboside

The reaction takes place between two purines, two pyrimidines, or a purine and a pyrimidine and provides a mechanism whereby purines or pyrimidines can be incorporated into deoxyribosyl linkage. Nucleoside phosphorylase, on the other hand, is not detectable in this organism. Table 8 shows some of the compounds which react with adenine deoxyriboside or thymidine. The enzymes are also present in other lactic acid bacteria.

TABLE 8

FORMATION OF DEOXYRIBOSIDES BY TRANS-N-GLYCOSIDATION

Adenine deoxyriboside $\left\{\begin{array}{l}+ \text { guanine } \\ + \text { hypoxanthine } \\ + \text { xanthine } \\ + \text { thymine } \\ + \text { uracil } \\ +5 \text {-methyluracil }\end{array}\right.$ Thymidine $\left\{\begin{array}{l}+ \text { adenine } \begin{array}{l}+ \text { guanine deoxyriboside } \\ + \text { hypoxanthine deoxyriboside } \\ + \text { adenine } \\ + \text { guanine } \\ + \text { hypoxanthine deoxyriboside }\end{array} \\ + \text { thymidine } \\ + \text { deoxyuridine } \\ +5 \text {-methyl cytosine deoxyriboside }\end{array}\right.$




\section{Phosphorylation of cytidine and uridine}

Gytidine and to a lesser extent, uridine, are utilized for RNA and DNA pyrimidine synthesis by the rat (Hammarsten et al., I950; Reichard, I955), Tetrahymena (Kidder et al., I950), and bacteria (Rose and Schweigert, 1953; Bolton, I954). Cytidine- $4-{ }^{14} \mathrm{C}$ is incorporated into the RNA of both the nucleus and cytoplasm by rat liver slices (Grossman and Visser, I954). It is therefore likely that kinase enzymes exist which catalyze the phosphorylation of the nucleosides to nucleotides. Evidence has also been reported for the phosphorylation of uridine by rat liver enzymes (Canellakis, I956a).

\section{Nucleoside monophosphate and diphosphate kinase}

Enzymes catalyzing the formation of ADP, UDP, GDP, and adenine deoxyribose diphosphate from the corresponding monophosphates occur in yeast (Lieberman et al., I 955b). These reactions are shown in Group II of Table 4 (p. 35). Various phosphate transfer reactions in which the triphosphates may be formed from the diphosphates are also shown in Table 4 and it is to be noted that inosine diphosphate and inosine triphosphate reactions (Table 4, reactions 42 and $5 \mathrm{I}, \mathrm{p} .35$ ) are also included. Enzymes catalyzing reactions $4^{2}$ and $5^{1}$ have been found in brain and liver tissue (Krebs and Hems, I 955) while reactions $43,5^{1}$, and $54-5^{6}$ occur in kidney and heart mitochondria (Gibson et al., 1956). The triphosphates of adenine, guanine, or inosine, can also be generated directly from the diphosphates during substrate or electron transport phosphorylation reactions.

Formation of the di- and triphosphates of the purine and pyrimidine ribosides is extremely rapid in rat liver. Following the injection of radioactive inorganic phosphate, the terminal phosphates of the di- and triphosphates become radioactive and approach the specific activity of inorganic phosphate in 15 minutes. The monophosphates, are however, much less radioactive and do not approach the specific activity of inorganic phosphate even at 60 min. (Brumm et al., i956).

Rat liver mitochondria phosphorylate any of the nucleoside diphosphates but lack nucleoside monophosphate kinases, or enzymes similar to adenylate kinase for phosphorylating the monophosphates other than AMP. It is significant, however, that the mitochondria do contain adenylate kinase and that this enzyme does not act on CMP, UMP, or GMP in mitochondrial preparations. Recent studies on ${ }^{32} \mathrm{P}$ incorporation and turnover suggest that adenylate kinase may be present at two sites in rat liver mitochondria. One site is the "outer zone" which is in contact with the surrounding medium. These two "zones" are separated by some sort of permeability or enzymatic barrier so that the phosphorylated form of adenosine which most readily penetrates this barrier to the site of oxidative phosphorylation, is AMP. If this barrier behaves in a similar manner to uracil, cytosine or guanine nucleotides, then the phosphorylation of CDP, UDP, or GDP must occur chiefly in the "outer zone" by a reaction with ATP rather than with donors in the hydrogen transport system. The ratio of adenine nucleotides to other kinds of nucleotides is much greater in mitochondria than in other components of the cell. The ATP subsequently may be released to the supernatant fluid where 
transphosphorylations with GMP, CMP, and UMP take place to give the corresponding diphosphates (Herbert and Potter, I956).

Adenine deoxyribose phosphate can be phosphorylated in place of adenylic acid to the di and triphosphate by enzymes of muscle and kidney (Sable et al., I 954).

\section{IO. Polynucleotide phosphorylase and ribonucleic acid synthesis}

The experiments of Ochoa and his collaborators suggest that purine and pyrimidine nucleoside diphosphates may be proximal precursors of ribonucleic acid (Grunberg-Manago and Ochoa, I955; Grunberg-Manago et al., I955). These investigators prepared partially purified extracts from Azotobacter vinelandii which catalyzed the exchange of $\mathrm{H}_{3}{ }^{32} \mathrm{PO}_{4}$ with the terminal phosphates of ADP, IDP, UDP, or CDP, and at a less rapid rate with GDP. Upon incubating the enzyme extracts with the nucleoside diphosphates, a water soluble, non dialyzable polymer was formed which was precipitated by trichloroacetic acid or ethanol. Solutions of this product were highly viscous, strongly acidic and had a UV spectrum characteristic of nucleotides. The reaction may be depicted as follows:

$$
\mathrm{n}(\mathrm{XRPP}) \longleftrightarrow(\mathrm{X}-\mathrm{R}-\mathrm{P})_{\mathrm{n}}+\mathrm{n} \mathrm{P}_{\mathrm{i}},
$$

where XRPP is a purine or pyrimidine nucleoside diphosphate and $\mathrm{X}-\mathrm{R}-\mathrm{P}$ is the corresponding nucleotide. Polymers having a molecular weight of 570,000-800,000 have been obtained. Enzymatic studies demonstrated that the nucleotides were present in the polymer as $3^{\prime}, 5^{\prime}$-diesters. Upon digestion with ribonuclease or alkali, $2^{\prime}$ and $3^{\prime}$ nucleotides were obtained, as in the case of ribonucleic acid. The $\mathrm{X}$-ray diffraction patterns of the AMP biosynthetic polymer closely resembled those produced by native RNA fibers. By incubating ADP, GDP, UDP, and CDP in a ratio of $1: 0.5: I: I$, mixed polymers containing all four bases were obtained.

Extracts prepared from Micrococcus lysodeikticus also contain polynucleotide phosphorylase enzymes (Beers, 1956). The incorporation of $5^{\prime}-\mathrm{UMP}^{-4}{ }^{-14} \mathrm{C}$ and adenosine $-5^{\prime}{ }^{-32} \mathrm{P}$, into cytoplasmic RNA of rat liver homogenates (Potter et al., i956; Heidelberger et al., 1956) and of $\mathrm{AMP}-4,6-{ }^{14} \mathrm{C}$ or $\mathrm{AM}^{32} \mathrm{P}$ into the RNA of pigeon liver homogenates (Goldwasser, 1955) has also recently been demonstrated. In the experiments with the rat liver homogenates, mitochondria and oxidizable substrates were used to generate ATP but the mitochondria could be omitted if phosphoglyceric acid was added. Since the cytoplasmic proteins which were used in these experiments contained nucleoside monophosphate kinase enzymes, it is possible that nucleoside diphosphates were the proximal RNA precursors, as in the case of the experiments with bacterial enzymes.

\section{The mechanism of $D \mathcal{N} A$-thymine $(D \mathcal{N} A-T)$ synthesis}

Formate (Elwyn and Sprinson, I954; Harrington and Lavik, I955; Herrmann et al., I955; Totter et al., I95I) the $\beta$-carbon of serine, the $\alpha$-carbon of glycine (Elwyn and Sprinson, I 954) or the methyl group of methionine (Herrmann et al., I955) are precursors of the methyl group of the DNA-thymine in normal tissues and tumors (Mannell and Rossiter, 1955; Prusoff et al., I 956; Prusoff and Lajtha, I 956; Kit, I 957; Totter and Best, I 955; Totter, I 954). Folic acid derivatives function 
as cofactors in the transfer of the "one carbon groups" to the methyl group of DNA-T. Incorporation of formate $-{ }^{14} \mathrm{C}$ or $2{ }^{14} \mathrm{C}$ uracil deoxyriboside into DNAthymine is inhibited by aminopterin and the inhibition is partially reversed by leucovorin (Friedkin and Roberts, I955, I956; Prusoff et al., I956; Totter and Best, I955). Incorporation of radioactive thymidine into DNA-thymine is not inhibited by aminopterin. In the synthesis of the methyl group of DNA-thymine from ${ }^{2} \mathrm{H}^{14} \mathrm{COONa}$ or L-serine- $3{ }^{-14} \mathrm{C}^{2} \mathrm{H}_{2} \mathrm{OH}, 0.9$ and $\mathrm{I} .5$ atoms of deuterium, respectively, accompany the labelled carbon. On the other hand, during the conversion of the $\beta$-carbon of serine and of formate to carbon atoms 2 and 8 of purines, an extensive labilization of deuterium takes place. These differences are probably attributable to the fact that a hydroxymethylfolic acid derivative is required for thymine biosynthesis and a formyl-folic acid derivative for purine synthesis.

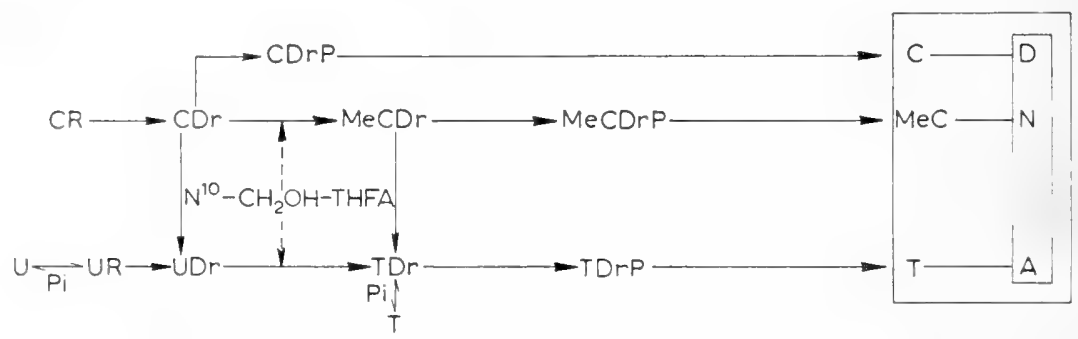

Fig. 46. Deoxyribonucleoside metabolism and DNA-thymine synthesis.

Uracil deoxyriboside and cytosine deoxyriboside are acceptors of the $\beta$-carbon of serine or of formate (Fig. 46) $\cdot 2-{ }^{14} \mathrm{C}$ uracil deoxyriboside is converted to thymidine and to DNA-thymine by bone marrow cells and by the tissues of the rat (Friedkin and Roberts, I 955, I 956; Reichard, I955). Deoxyuridine is not, however, a precursor of DNA-cytosine or of RNA pyrimidines. Likewise, in the rat, deoxycytidine ${ }^{15} \mathrm{~N}$ is converted to DNA thymine and cytosine but not to RNA pyrimidines. (Reichard and Estborn, I95I). The presence of non-labelled deoxyuridine or deoxycytidine stimulates the incorporation of formate- ${ }^{14} \mathrm{C}$ or of serine- $3^{-14} \mathrm{C}$ into free thymidine and in some cases DNA-thymine by normal lymphatic tissues and tumors (Kit, I957) and into the DNA-thymine of bone marrow and Ehrlich ascites tumor cells (Prusoff et al., i 956; Prusoff and Lajtha, I956). On the other hand, free uracil, uridine, cytosine, or thymine do not stimulate the formation of thymidine or DNA-thymine by normal and malignant lymphatic tissues (Kit, I957), although uridine does stimulate the conversion of formate ${ }^{14} \mathrm{C}$ to DNA thymine by Ehrlich tumor cells (Prusoff and Lajtha, I956). Free uracil- $2-{ }^{14} \mathrm{C}$ is a DNA-thymine precursor in the Ehrlich tumor cells (Kit, 1957; Lagerkvist et al., I 955).

It is unlikely that 5-methyluridine and free thymine are direct precursors of DNA-thymine in the rat. The administration of labelled thymine or 5-methyluridine to the rat resulted in very little labelling of the DNA-thymine (Reichard, 1955). Deoxyuridine may be an intermediate in the conversion of deoxycytidine to thymidine. However, to a minor degree, deoxycytidine may also react directly 
with the "one carbon compound" to produce 5-methyl deoxycytidine (or a derivative thereof) and the latter compound may then be deaminated to thymidine. The presence of deoxycytidine increases the conversion of formate to DNA-5-methylcytosine in lymphatic tissues and tumors (Kit, I957; Fig. 46).

The deamination of cytosine compounds to uracil compounds has already been discussed. Three further points concerning DNA-thymine synthesis should be mentioned. First, a hydroxymethyl derivative of thymine may well be the primary product of the reaction of deoxyuridine with the hydroxymethyl derivative of tetrahydrofolic acid. Hydroxymethyl cytosine is a component of the DNA of $E$. coli phage. Secondly, it is possible that phosphorylated, or dihydroderivatives of deoxyuridine rather than the free nucleosides are the physiological acceptor compounds of formate. Thirdly, there is the question of the origin of deoxycytidine and deoxyuridine. These compounds possibly are derived from cytidine and uridine Labelled cytidine and (at high concentrations) labelled uridine are precursors of DNA cytosine and thymidine in the rat (Reichard, I955; Grossman and Visser, I 954; Hammarsten et al., I 950). The presence of non-labelled uridine and cytidine stimulate the conversion of formate- ${ }^{14} \mathrm{C}$ to DNA-thymine by Ehrlich tumor cells suspensions (Prusoff and Lajtha, I956) while the presence of cytidine greatly increases the conversion of formate- $-{ }^{14} \mathrm{C}$ or serine- $3-{ }^{14} \mathrm{C}$ to DNA-thymine and increases somewhat the labelling of thymidine of normal lymphatic tissue and tumor cell suspensions (Kit, I957).

The conversion of cytidine to the deoxycytidine and thymidine of DNA occurs in the rat without the cleavage of the pyrimidine-sugar linkage (Roll et al., I956a; Rose and Schweigert, I953). There is some cleavage of the pyrimidine-sugar linkage of uridine, however, during its transformation to DNA-thymine (Roll et al., 1956a). In $E$. coli $B$ cells, which can utilize free cytosine or uracil for growth, cytidine and uridine are not obligatory intermediates in nucleic acid synthesis (Rose and Schweigert, I 953; Bolton, I 954).

It is to be noted that orotic acid is well utilized for the synthesis of DNA-cytosine and thymine in vivo and in vitro by normal tissues and tumors (Harrington and Lavik, I955; Hurlbert and Potter, I952; Lagerkvist and Reichard, I954; Weed and Wilson, I954). Thymidine is in rapid equilibrium with the proximal DNAthymine precursors. Thymidine- $2-{ }^{14} \mathrm{C}$ is readily incorporated into DNA thymine but not into other nucleic acid pyrimidines (Reichard, I955). The utilization of labelled thymidine for DNA-thymine synthesis has been demonstrated in bacteria (Downing and Schweigert, 1956) plant tissues, protozoa, and animal tissues in vivo and in vitro (Friedkin et al., I956; Friedkin and Wood, I956). The presence of non-labelled thymidine inhibits the incorporation of $2-{ }^{14} \mathrm{C}$-uracil deoxyriboside (Friedkin and Roberts, I 956) formate- ${ }^{14} \mathrm{C}$, or serine- ${ }^{14} \mathrm{C}$ into the DNA thymine of bone marrow cells and tumors (Prusoff et al., I956; Kit, I957).

Several enzyme fractions have been partially purified from $E$. coli $B$ cells which convert ${ }^{14} \mathrm{C}$-thymidine to thymidylate, thymidine triphosphate, and a polydeoxyribonucleotide having the properties of DNA. The polynucleotide is acid insoluble, destroyed by deoxyribonuclease, alkali stable, and resistant to ribonuclease. The conversion of $2{ }^{14} \mathrm{C}$ thymidine to the polynucleotide is reduced $50^{\circ} \%$ by the addition of non-labelled thymidylate, which in turn becomes radioactive. 
${ }^{32} \mathrm{P}$-thymidylate is also incorporated into the polynucleotide (Kornberg et al., I $956 \mathrm{a}, \mathrm{b})$.

$$
\text { Thymidine } \stackrel{\text { ATP }}{\longrightarrow} \text { thymidylate } \longrightarrow \text { thymidine triphosphate } \stackrel{\text { ATP }}{\longrightarrow} \text { DNA }
$$

Polymerization of thymidine triphosphate (TTP) requires ATP, a heat stable DNA fragment, provisionally regarded as a primer, and two enzyme fractions which have been purified more than I oo fold. TDP can replace TTP in the reaction but a decision as to the more immediate precursor requires further purification of the system (Kornberg et al., I 956b). The polyphosphates of adenine, cytosine and guanine deoxyribosides are also used for polynucleotide synthesis by the crude enzyme preparations but the rates are appreciably slower than that for the polymerization of TPP by the purified enzymes, suggesting the presence of different enzymes for each of the deoxyribonucleoside triphosphates. Mixtures of the triphosphates give additive rates, further suggesting different enzymes.

The enzymatic formation of the di- and tri-phosphates of deoxycytidine and thymidine from deoxycytidylate and thymidylate takes place in the cytoplasmic fraction of rat liver homogenates (Hecht et al., I954) while the phosphorylation of deoxyadenylic acid to the di- and tri-phosphates occurs in kidney and muscle (Sable et al., r954).

\section{F. Phospholipid Biosynthesis}

\section{Ethanolamine and choline formation}

The inability of the rat to form labelled phospholipid-choline from glycine- ${ }^{-14} \mathrm{C}$, whereas both glycine-2- ${ }^{14} \mathrm{C}$ and serine- $-3-{ }^{14} \mathrm{C}$ yield choline which is labelled in the two carbon moiety (Arnstein, I 95I) supports the view that this latter compound is formed by a decarboxylation of serine to ethanolamine (Du Vigneaud et al., I 946) and the subsequent methylation of the ethanolamine. A similar mechanism apparently occurs in plant leaves (Bregoff and Delwiche, 1955).

The role of ethanolamine as a choline precursor is supported by experiments on the nutritional requirements of Neurospora mutants (Horowitz, I946; Fig. 47).

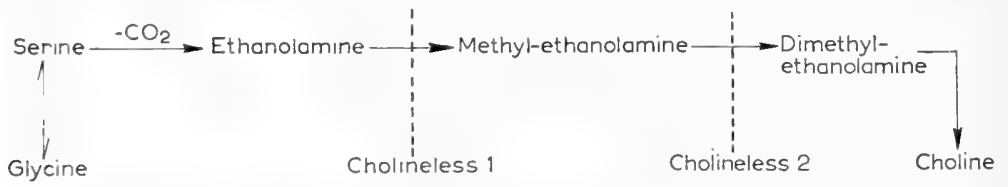

Fig. 47. Choline biosynthesis from serine and ethanolamine.

Monomethylethanolamine accumulates in the culture medium of one cholineless mutant (\#2) which is active in supporting the growth of another cholineless mutant $(\# \mathrm{I})$. Direct proof was obtained by demonstrating that in rats, ethanolamine $-{ }^{15} \mathrm{~N}$, ethanolamine-I, $2-{ }^{14} \mathrm{C}$, or deuterium labelled monomethylethanolamine, are readily incorporated into phospholipide choline (Pilgeram et al., I 953; Du Vigneaud et al., I 946). The methyl groups of choline are derived from the methyl group of methionine, from formate, or from other one-carbon donors (Bregoff and Delwiche, 1955; Arnstein, I95I; Elwyn et al., I 955; Du Vigneaud, I 952 ; Section IV, B4, p. 56). 


\section{The enzymatic synthesis of lecithin and kephalin}

The enzymatic synthesis of lecithin and kephalin has been clarified by recent experiments from the laboratories of Kornberg and of Kennedy. Cytidine diphosphocholine (CDP-choline) and cytidine diphosphoethanolamine (CDP-ethanolamine) function as coenzymes in the process. The condensation of these "activated" forms of choline and ethanolamine with $\alpha, \beta$-diglycerides are the final steps in phosphatide synthesis (Kennedy and Weiss, I 955, 1956).

\section{(a) CDP-choline formation}

Phosphorylcholine and phosphorylethanolamine occur in the acid soluble extracts of a variety of tissues. The formation of these compounds from the free bases has been demonstrated in yeast and animal tissues (Wittenberg and Kornberg, I953).

I) Choline (ethanolamine) $+\mathrm{ATP} \rightarrow$ phosphorylcholine (phosphoryl-ethanolamine)

Phosphorylcholine doubly labelled with ${ }^{32} \mathrm{P}$ and ${ }^{14} \mathrm{C}$ is incorporated into the phospholipids of liver mitochondria with practically unaltered ratios of the isotopes (Kornberg, I954). Enzymes which carry out the synthesis of CDP-choline and CDP-ethanolamine from phosphorylcholine and phosphorylethanolamine are also widely distributed in nature.
2) CTP + phosphorylcholine $\stackrel{\mathrm{Mg}^{++}}{\longrightarrow}$ CDP choline + PP
3) CTP + phosphorylethanolamine $\stackrel{\mathrm{Mg}^{++}}{\longrightarrow}$ CDP ethanolamine + PP

CDP-choline next reacts with $\alpha, \beta$-diglycerides to form lecithin:

PC-cytidyl transferase

4) CDP choline $+\alpha, \beta$-diglyceride $\longleftarrow$ lecithin + CMP

An analogous reaction occurs with CDP-ethanolamine.

\section{PE-cytidyl transferase}

5) CDP-ethanolamine $+\alpha, \beta$-diglyceride $\longrightarrow$ phosphatidyl ethanol-amine + CMP

The CMP which is formed in reactions 4) and 5) can be rephosphorylated to CTP at the expense of ATP in reactions catalyzed by enzymes which occur in the cytoplasm of tissues (Herbert and Potter, I 956).

\section{(b) Phosphatidic acid formation}

The $\alpha, \beta$-diglyceride is probably formed by the dephosphorylation of phosphatidic acid, a substance which is present in fairly high concentration in plant tissues (Kornberg, I954; Kennedy and Weiss, 1956). Phosphatidic acid is in turn formed as follows (Kornberg and Pricer, I953a, b):

6) Fatty acids $+\mathrm{ATP}+$ coenzyme $\mathrm{A} \rightarrow$ fatty acyl-CoA $+\mathrm{AMP}+\mathrm{PP}$

7) 2 Fatty acyl-CoA $+\alpha$-glycerol phosphate $\rightarrow$ phosphatidic acid $+\mathrm{CoA}$

The role of $\alpha$-glycerolphosphate as an intermediate in phospholipid synthesis is supported by the following evidence. The incorporation of $\mathrm{H}_{3}{ }^{32} \mathrm{PO}_{4}$ into phospholipides of liver mitochondria is accelerated by glycerol but inhibited by non-labelled $\alpha$-glycerol phosphate 
(Kennedy, I953). At the same time, the $\alpha$-glycerol phosphate becomes labelled. Moreover, $\alpha$-glycerol phosphate-32 $\mathrm{P}$ is itself incorporated into phosphatidic acid and mitochondrial phospholipids. The mechanism of lecithin and phosphatidyl ethanolamine formation may be a general one, applicable to the synthesis of serine phosphatides and acetal phosphatides. Sphingomyelin, although not a glycerophosphatide, has a phosphoryl choline moiety like that of lecithin and it is possible that CDP-choline may be a precursor of this portion of the sphingomyelin molecule.

Phosphoglycerol choline and phosphoglycerol ethanolamine also occur in aqueous extracts of tissues. However, these compounds are probably degradation products rather than precursors of phosphatidyl choline or ethanolamine (Dawson, I955).

\section{G. Vitamin Biosynthesis}

\section{Cholesterol and vitamins $A$ and $D_{3}$}

Carotenoids are present in virtually all plants. It is probable that the carotenoids are formed by the polymerization of an "isoprene-like precursor" compound. The distribution of the carbon atoms in vitamin A conforms to that expected from the polymerization of four isoprene units. The five carbon compound, $\beta$-methylcrotonyl-CoA, is probably closely related to the "isoprene-like" precursor. This compound is an intermediate in the catabolism of leucine and can also be formed from acetyl-CoA and acetoacetic acid by animal tissues (Fig. 48).

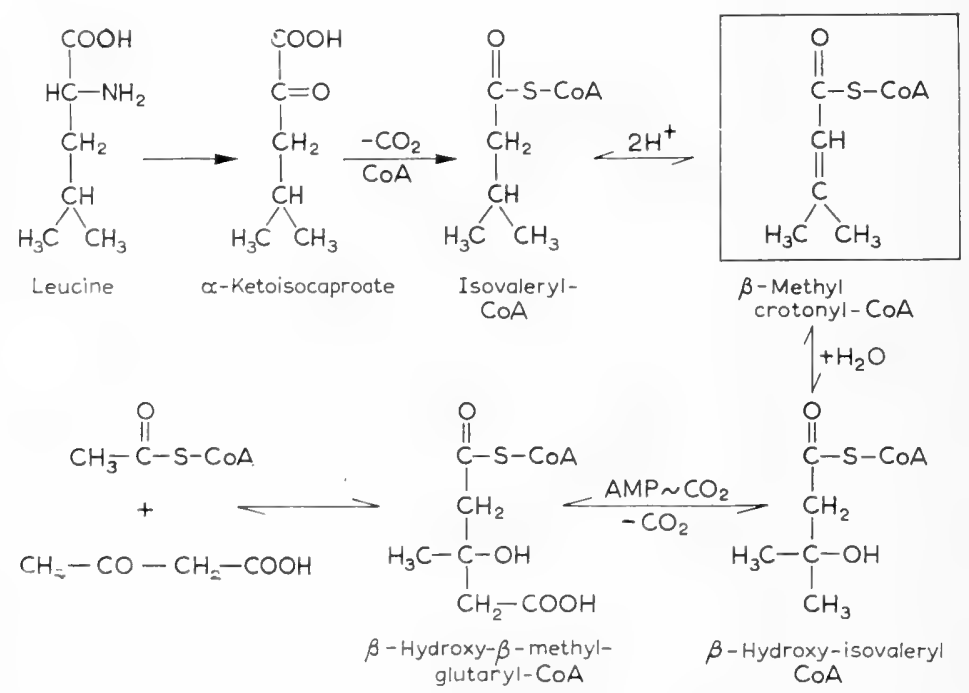

Fig. 48. Conversion of leucine and acetyl-CoA to $\beta$-methylcrotonyl-CoA.

The synthesis of $\beta$-methyl- $\beta$-OH-glutaric acid and $\beta$-methyl crotonic acid from acetate $-{ }^{14} \mathrm{C}$ or pyruvate- $\mathrm{I}-{ }^{14} \mathrm{C}$ is catalyzed by rat liver enzymes (Rabinowitz and Gurin, I954; Rabinowitz, I 954). The $\beta$-methyl crotonic acid is formed by the dehydration of $\beta$-hydroxy-isovaleric acid, a reaction catalyzed by the enzyme crotonase (Stern et al., I 956a). Enzymes have been purified from heart tissue which catalyze the formation of $\beta$-methyl- $\beta$-hydroxyglutaryl-CoA from ${ }^{14} \mathrm{CO}_{2}$ (Bachhawat et al., I 956a). A high energy adenyl- $\mathrm{CO}_{2}$ compound is apparently an inter- 
mediate in these reactions. The enzyme catalyzing the activation of the $\mathrm{CO}_{2}$ has been separated from the one concerned with the $\mathrm{CO}_{2}$ fixation reaction. The heart enzyme system also effects the breakdown of $\beta$-hydroxy- $\beta$-methylglutaryl-CoA to acetoacetate and acetyl-CoA (Bachhawat et al., I954).

Consistent with the possibility that $\beta$-methyl-crotonyl-CoA is a carotene precursor is the fact that the alpha carbon of acetate is incorporated into the lateral methyl groups of carotene; on the other hand, the vicinal quaternary carbon atoms of the aliphatic chain are derived from the carboxyl carbon of acetate (Grob and Butler, 1954). The synthesis of carotene has been studied in the mold, Phycomyces blakesleeanus. In the presence of $\beta$-ionone, both leucine and asparagine stimulate carotene synthesis. Although leucine- ${ }^{-14} \mathrm{C}$ is not incorporated into the carotene of this organism, leucine-2-14C is incorporated. This is consistent with the fact that the carboxyl group of leucine is lost during the conversion of the amino acid to $\beta$-methylcrotonyl-CoA (Goodwin and Lijinsky, I95I; Chichester et al., 1955).

A condensation of two "isoprene-like" molecules would give rise to a Io carbon compound having an isoprene chain. A 20 carbon compound could be formed by the condensation of two Io carbon compounds or by the condensation of a 5 carbon compound with a 15 carbon compound. In animal tissues, $\beta$-carotene is cleaved to two molecules of vitamin A.

Evidence exists which indicates that the 30 carbon trihydroterpene, squalene, an intermediate in the synthesis of cholesterol and other steroids, is also probably formed by a condensation of six "isoprene" units. Rat liver extracts which incorporate acetate- $\mathrm{I}-{ }^{14} \mathrm{C}$ into $\beta$-methylcrotonate also form labelled cholesterol. The conversion of acetoacetate $-{ }^{14} \mathrm{C}$ to cholesterol takes place without the preliminary formation of two carbon compounds (Brady and Gurin, I 95 I). The incorporation of $\mathrm{I}-{ }^{14} \mathrm{C}$-acetyl-CoA into liver cholesterol is lowered to less than half by the addition of $\beta$-methylcrotonate or $\beta$-hydroxy- $\beta$-methylglutarate (Rabinowitz and Gurin, I 953) ${ }^{1}$.

Squalene occurs naturally in shark liver oil, yeast, human dermatoid cysts, and has been isolated in trace amounts from rat liver and intestine (Langdon and Bloch, I953a). Biosynthetically labelled squalene is efficiently converted to cholesterol in mouse tissue in vivo (Langdon and Bloch, I953b). The observed distribution of the methyl and carboxyl carbons of isotopically labelled acetate in tissue cholesterol is also consistent with the role of squalene as a sterol precursor (Langdon, 1954; Fig. 49). It will be noted that the hypothesis predicts that the two central carbons of squalene shall be derived from the carboxyl carbon of acetate. In accord with this hypothesis, carbons 11 and 12 of the $\mathrm{G}$ ring of ergosterol (which correspond to the central carbon atoms of the squalene) of yeast grown in the presence of acetate- $1-{ }^{14} \mathrm{C}$ were equally labelled (Langdon and Bloch, I953a; Dauben and Hutton, 1956). Both the carboxyl and methyl groups of acetate are converted to squalene in the rat.

Acetate ${ }^{14} \mathrm{C}$ is also converted to the $\mathrm{C}_{30}$-steroids, lanosterol and agnosterol by rat liver homogenates (Clayton and Bloch, 1956a). The biosynthetically labelled lanosterol is in turn converted to cholesterol by the same enzyme system (Clayton and Bloch, 1956b). Both lanosterol and agnosterol occur in trace amounts in liver. In experiments with yeast, acetate- ${ }^{14} \mathrm{C}$ gave rise to labelled ergosterol, squalene and zymosterol. When labelled zymosterol was administered to rats, cholesterol became labelled (Schwenk et al., 1955). It could be formed from lanosterol by the loss of three methyl groups. It will be noted that the double bonds of zymosterol and lanosterol are in corresponding positions in the two molecules (Fig. 49). The cyclization of squalene to lanosterol by rat liver enzymes

\footnotetext{
1 See Addendum, Note 5, p. I23.
} 

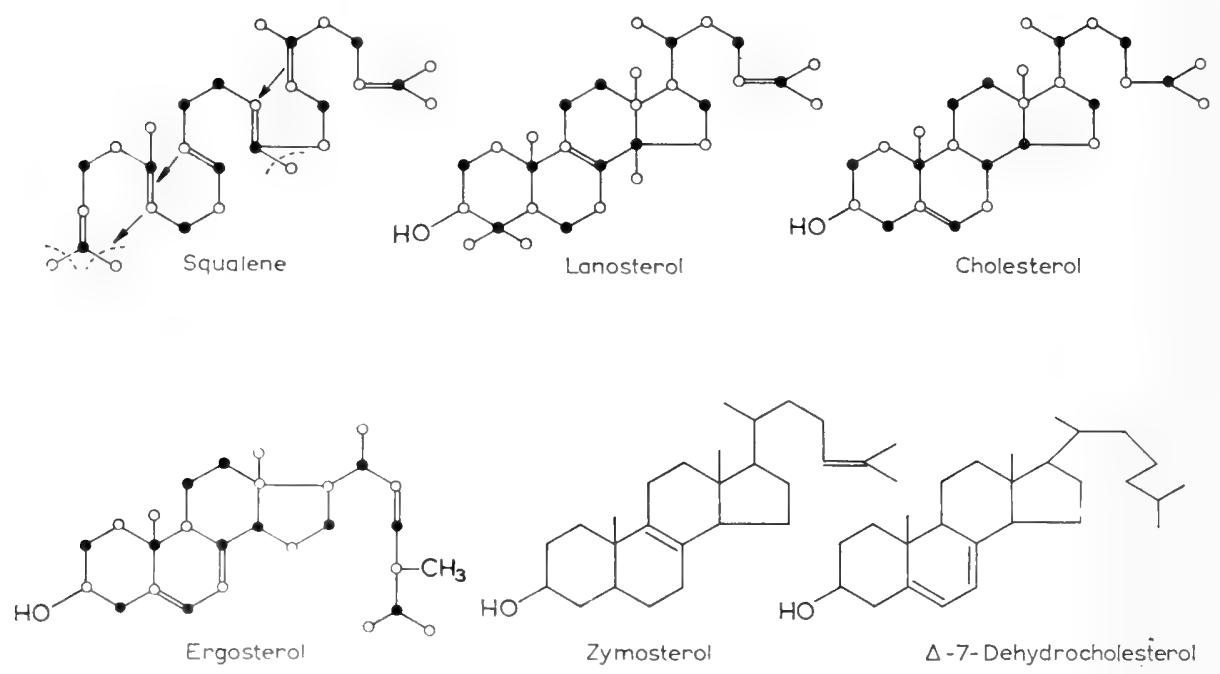

- = Carboxyl carbon of acetate

$\circ=$ Methyl carbon of acetate

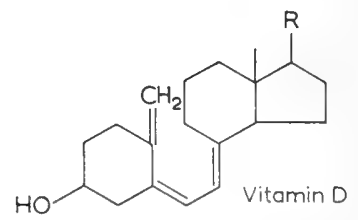

Fig. 49. Structure of cholesterol and related compounds. The distribution of acetate carbon atoms found experimentally in cholesterol, the hypothetical distribution of acetate carbon in squalene and a possible mechanism of squalene cyclization is denoted by the closed and open circles.

takes place with the introduction of ${ }^{18} \mathrm{O}_{2}$ into the lanosterol molecule but with little or no addition of $\mathrm{D}_{2} \mathrm{O}$ (Tchen and Bloch, 1956). 7-dehydrocholesterol may be a late intermediate in cholesterol synthesis. In feeding experiments, this steroid behaves in a manner very similar to that found for cholesterol and $\Delta-7$-cholestenol. It causes an increase in hepatic cholesterol concentration and a decreased rate of sterol synthesis by liver. However, the ingestion of squalene by rats results in the accumulation in liver of $\Delta$ - 7 -cholestenol, but not of 7-dehydrocholesterol (Langdon, 1954). The incorporation of acetate-14 $\mathrm{C}$ into squalene and of squalene into liver cholesterol has been demonstrated in vitro. $\beta$-hydroxy$\beta$-methylglutarate is also incorporated into squalene by liver enzymes. Non-labelled farnesol or farnesenic acid, compounds having i5 carbon atoms, can reduce the incorporation of acetate $-14 \mathrm{C}$ into squalene. At the same time, the recovered farnesenic acid becomes radioactive (Dituri et al., 1956) suggesting that farnesenic acid or a related compound may be an intermediate in squalene synthesis.

For the biosynthesis of squalene and cholesterol from acetate- ${ }^{14} \mathrm{C}$ to occur in vitro, both microsomes and soluble cell constituents are required, along with a glycolytic substrate, $\mathrm{ATP}$, and $\mathrm{DPN}^{+}$. Under anaerobic conditions, radioactivity accumulates in squalene whereas in an oxygen atmosphere, cholesterol is formed. Thus, oxygen is apparently required for the conversion of squalene to cholesterol 
rather than for the synthesis of squalene from acetate. The need for the soluble fraction in cholesterol synthesis is probably attributable to the requirement for glycolytic enzymes and acetate activating enzymes. The microsomes, which contain over $80 \%$ of the total cytoplasmic cholesterol, also contain most of the newly synthesized cholesterol. Over $90 \%$ of the newly formed liver cholesterol obtained at short time intervals after the administration of labelled acetate to rats is also in the microsomal fraction (Bucher and McGarrahan, I956). When ergosterol or $\Delta 7$-dehydrocholesterol is exposed to ultraviolet light, a complex series of reactions takes place involving the rupture of the $\mathrm{B}$ ring and leading to the formation of vitamin $\mathrm{D}_{2}$ and vitamin $\mathrm{D}_{3}$, respectively.

\section{Ascorbic acid}

The synthesis of ascorbic acid is markedly accelerated in rats treated with chloretone. The administration of labelled D-glucose to such rats is followed by the excretion of labelled $\mathrm{L}$-ascorbate in the urine. When D-glucose- $\mathrm{I}-{ }^{14} \mathrm{C}$ is the substrate, the ascorbate is labelled on carbon 6 , whereas with $\mathrm{D}$-glucose- $6-{ }^{14} \mathrm{C}$, the Lascorbate is labelled on carbon one. Thus, carbon 6 enters the ascorbate molecule by the same molecular pathway as that followed by carbon I. The conversion is apparently direct, with the maintenance of the steric configuration around carbons 2 and 3 of glucose (carbons 5 and 4, respectively, of ascorbate; Horowitz and King, I953b). D-glucose- $-{ }^{14} \mathrm{C}$ is also converted to ascorbate- $6-{ }^{14} \mathrm{C}$ in the normal rat (Burns and Mosbach, 1956).

On the other hand, there is evidence against the possibility that L-sorbose is a direct $\mathrm{L}$-ascorbate precursor (Isherwood et al., I 953). L-sorbose-6-14 $\mathrm{C}$ is converted to ascorbic acid labelled equally on carbons 1 and 6 and appreciable label is observed in carbons 2 and 5 of ascorbate. Thus, the conversion of $\mathrm{L}$-sorbose to ascorbic acid follows the fragmentation of the L-sorbose to three carbon compounds (Burns et al., I955).

D-glucuronic acid and L-gulonic acid derivatives may be intermediates in the formation of ascorbate from glucose. Uniformly labelled D-glucuronolactone- ${ }^{14} \mathrm{C}$ is more efficiently converted to ascorbate in the chloretone treated rat than is D-glucose itself (Horowitz and King, I 953a). D-galacturonic acid or L-galactono$\gamma$-lactone derivatives may also be ascorbate precursors (Fig. 50; Isherwood et al.,

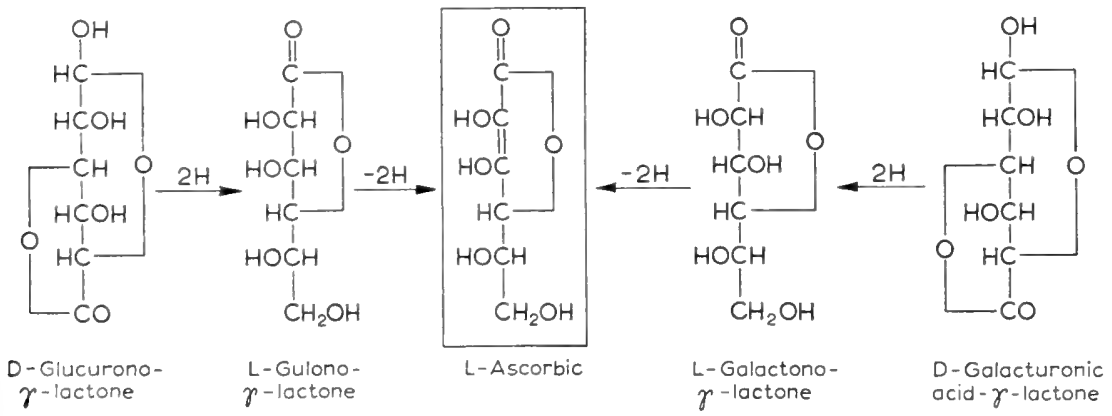

Fig. 50. Postulated precursors of ascorbate. 
I 954a). The feeding of D-glucurono- $\gamma$-lactone, L-gulono- $\gamma$-lactone, D-galacturonic methyl ester, and L-galactono- $\gamma$-lactone to cress seedlings or the administration of these compounds to rats is followed by increased ascorbate synthesis. No other sugar acid $\gamma$-lactone, of many tested, behaves in this way.

Ascorbate synthesis differs somewhat in mung bean seeds from cress seedlings and the rat. Mitochondria of mung bean seeds convert L-galactono- $\gamma$-lactone and the methyl ester of galacturonic acid to ascorbate but neither L-gulono- $\gamma$-lactone, D-glucuronic acid- $\gamma$ lactone nor free galactonic acid were converted to ascorbate.

The oxidation of glucose to glucuronic acid takes place in the particle free supernatant of liver tissue (Strominger et al., I 954). UDP glucose rather than free glucose is the substrate of this oxidation:

I) $\mathrm{UDPG} \stackrel{2 \mathrm{DPN}^{+}}{\longrightarrow} \mathrm{UDP}$-glucuronic $+2 \mathrm{DPNH}+2 \mathrm{H}^{+}$

This suggests that in the rat, ascorbate synthesis may take place as follows:

ATP UTP

2) Glucose $\longrightarrow$ glucose-6-P $\longrightarrow$ glucose-I-P $\longrightarrow$ UDPG

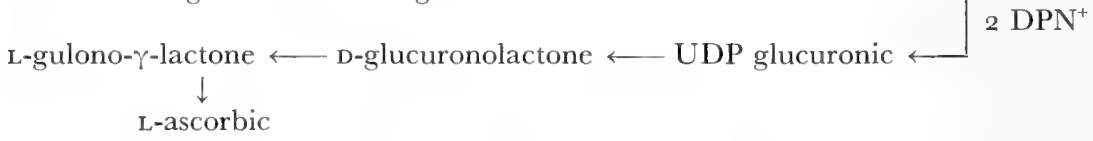

\section{Thiamine}

On the basis of nutritonial experiments with Neurospora and $E$. coli mutants, it has been inferred that two pathways of thiamine synthesis exist (Harris, 1955). These are shown in Fig. 51. Neurospora mutant (18558) requires thiamine for growth and accumulates "pyrimidine" (P) in the culture medium. Thiazole $(\mathrm{T})$ can substitute for thiamine in this organism, but not in mutants, 9185 and 85902 . The latter organisms require thiamine and accumulate both $\mathrm{P}$ and $\mathrm{T}$ in the culture medium. Thus, one pathway of thiamine synthesis involves the condensation of $\mathrm{P}$ with $\mathrm{T}$ to yield thiamine. The growth requirement of two other Neurospora mutants (I7084 and 5650I) can also be met by thiamine or by $P$ plus $T$. However, these organisms accumulate an unknown compound, P-t in the mycelium. Moreover, P-t can replace thiamine as a nutrient in strains 9185 and 18558 .
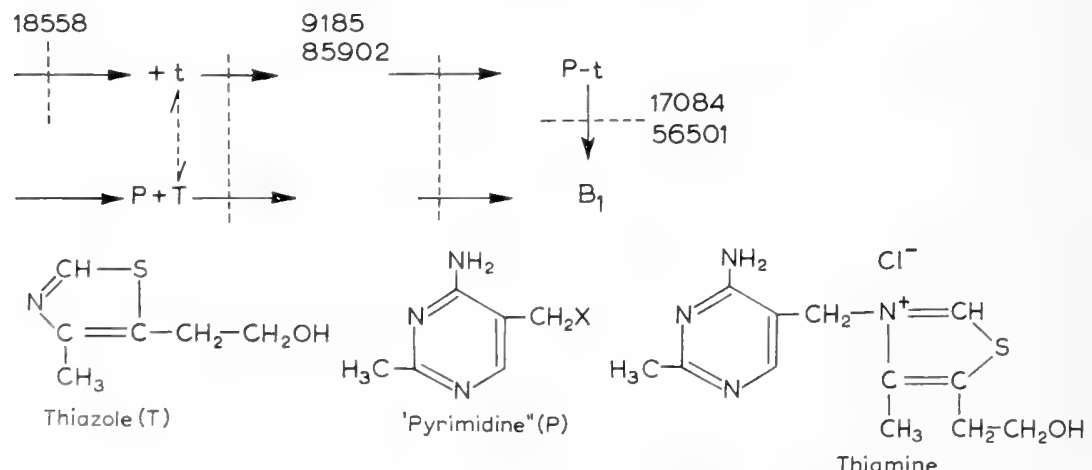

Fig. 5I. Proposed pathways of thiamine synthesis. 
Interestingly enough, strains 9185 and 5650 I can grow on $\mathrm{P}$ and large amounts of exogenous $\mathrm{T}$ even though $\mathrm{T}$ is normally excreted into the medium. This is best explained as due to a competition of $\mathrm{T}$ with its precursor, $\mathrm{t}$, for the pyrimidine compound $\mathrm{P}$. It is believed that the P-t pathway of thiamine synthesis is the major one in Neurospora.

Although animal tissues are incapable of synthesizing thiamine, enzymes occur in liver which catalyze the phosphorylation of the vitamin to its pyrophosphate (Bessey et al., I953):

$$
\text { Thiamine }+\mathrm{ATP} \underset{\text { liver, yeast }}{\stackrel{\mathrm{Mg}^{++}}{\longrightarrow}} \text { thiamine pyrophosphate }+ \text { AMP }
$$

Thiamine pyrophosphate, is active as a coenzyme in the decarboxylation of pyruvate and $\alpha$-ketoglutarate (Reed, I953) and in the transketolase reaction. Lipothiamide pyrophosphate, a conjugate of thiamine with lipoic acid, functions as the active coenzyme for the decarboxylation of $\alpha$-keto acids in certain microorganisms.

\section{Riboflavin}

Isotope experiments with yeast have established that the $\alpha$ and carboxyl carbons of glycine are precursors of carbons $4 \mathrm{a}$ and $9 \mathrm{a}$, respectively, of the riboflavin molecule (Plaut, I954a; Fig. 52). The nitrogen of glycine is also incorporated into

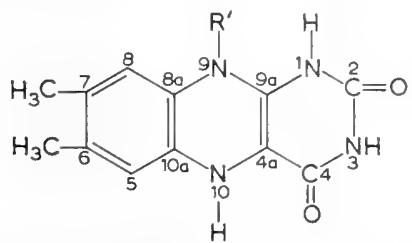

Fig. 52. Structure of riboflavin. riboflavin. Moreover, formate is a precursor of carbon 2 while $\mathrm{CO}_{2}$ gives rise to carbon 4 of riboflavin. This pattern of labelling is reminiscent of that observed in the biosynthesis of purines. Moreover, it is to be noted that the ribityl group of riboflavin is in the same position as that of the ribose of adenosine. This suggests that purines are riboflavin precursors in yeast. Consistent with this concept is the fact that purines or their ribosides or ribotides enhance riboflavin synthesis by yeast under conditions where growth is unaffected (Brown et al., I 955). The amino acids, L-threonine, L-serine and L-tyrosine are also stimulatory, the stimulatory effects of the amino acids and purine compounds being cumulative (Goodwin and Pendlington, 1954).

Uniformly labelled adenine $-{ }^{14} \mathrm{C}$ is efficiently incorporated into the pyrimidine portion of riboflavin but adenine-8- ${ }^{14} \mathrm{C}$ is not a precursor (McNutt, I 954, I 956). Thus, carbon 8 of the adenine is lost during the condensation of the pyrimidine portion of the riboflavin molecule with the dimethyl benzene precursor. The origin of the dimethyl benzene end of the molecule has not as yet been fully clarified. Acetic- $1-{ }^{14} \mathrm{C}$ is a precursor of carbons 6 and 7 and $8 \mathrm{a}$ and roa. A more random pattern of labelling is observed, however, with acetic $-2-{ }^{14} \mathrm{C}$ as substrate. Most of the label is found in the methyl groups and in carbons 5 and 8 but there is also significant radioactivity in carbons 6 and 7 , and $8 a$ and roa, the carbon atoms derived from acetate $-1-{ }^{14} \mathrm{C}$. This suggests that a tricarboxylic acid cycle metabolite is an intermediate in the incorporation of acetate into the aromatic ring of riboflavin (Plaut, I 954a; Klungsoyr, I 954). The labelling of the aromatic ring with glucose- $\mathrm{I}-{ }^{14} \mathrm{C}$ or glucose- $-6-{ }^{14} \mathrm{C}$ as substrate is similar to that 
found with acetate- $2-{ }^{14} \mathrm{C}$ as substrate. Glucose is also a precursor of the ribityl portion of riboflavin as indicated in the discussion of ribose synthesis (Plaut and Broberg, 1956).

The coenzymes, Flavin Adenine Dinucleotide (FAD) and Flavin Mononucleotide (FMN), are important in various oxidative reactions in the cell (Snell, I953). These substances can be synthesized from riboflavin by animal tissues, plants, and microorganisms. The synthesis of FAD from riboflavin has been demonstrated with enzymes of yeast and erythrocytes (Schrecker and Kornberg, 1950).

I) Ribollavin + ATP $\longrightarrow$ FMN + ADP

2) $\mathrm{FMN}+\mathrm{ATP} \longleftrightarrow \mathrm{FAD}+\mathrm{PP}$

\section{Pyridoxal phosphate}

Pyridoxal is synthesized by green plants and many microorganisms. The precursors of the vitamin have not, however, been defined. The conversion of pyridoxine, to the active coenzyme, pyridoxal phosphate, has been demonstrated in yeast extracts (Hurwitz, I952). Pyridoxine, pyridoxamine, and the antimetabolite deoxypyridoxine, are also phosphorylated by the same enzyme system.

$$
\text { Pyridoxal }+ \text { ATP } \rightarrow \text { ADP }+ \text { pyridoxal phosphate }
$$

Pyridoxal phosphate serves as a coenzyme in several reactions involving amino acids as substrates. These include $I$ ) transamination reactions (Meister, I955), 2) the decarboxylation of amino acids to amines (Snell, I953), 3) the serine and threonine dehydrase and the cysteine desulfhydrase reaction, 4) the formation and the cleavage of cystathionine, 5) the conversion of glycine to serine, 6) the formation of tryptophane from serine and indole, and 7) the breakdown of tryptophane to indole, pyruvate, and ammonia by bacterial tryptophanase.

\section{Biotin}

Pimelic acid and desthiobiotin may be intermediates in biotin synthesis (Fig. 53). Desthiobiotin satisfies the biotin requirements of some microorganisms, is inactive with others, and is an antimetabolite with still others. Growing yeast or Aspergillus niger cells can convert desthiobiotin to biotin. In the presence of pimelic acid, desthiobiotin accumulates in the medium during the growth of a biotin requiring
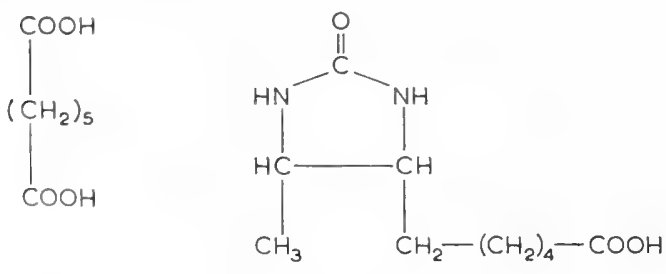

Pimelic

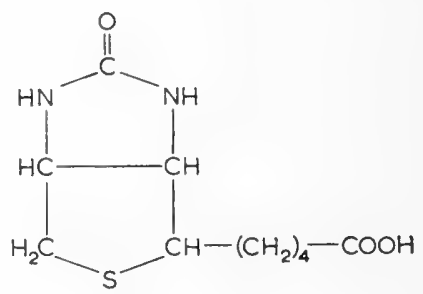

Biotin

Fig. 53. Structure of biotin, desthiobiotin, and pimelic acid. 
mutant that does not utilize desthiobiotin (Wright and Driscoll, I954). Biotin has been implicated in a number of reversible carboxylation reactions (Lardy and Peansky, I953; Plaut, I95I).

\section{Myoinositol}

Myoinositol has been shown to be essential for the survival and growth of a number of microorganisms, and the proliferation of human normal and malignant cells in tissue culture (Eagle et al., I 956b). A deficiency of this factor results in alopecia in mice and rats. Myoinositol is also known to exert a lipotrophic effect independent of that of choline. Glucose is a precursor of myoinositol. The biosynthesis of myoinositol from glucose $-{ }^{14} \mathrm{C}$ has been demonstrated in the internal organs of the young rat and in the chick (Daughaday et al., I 955). The capacity of the adult rat for myoinositol synthesis is however, restricted (Halliday and Anderson, I955).

\section{Niacin, $D P \mathcal{N}^{+}$and $T P \mathcal{N}^{+}$}

\section{(a) Nicotinic acid synthesis}

Tryptophane and metabolites derived from it are converted to nicotinic acid derivatives in intact animals and in liver slices. Kynurenine is an intermediate in the conversion. This metabolic pathway has been studied by nutritional, isotopic, genetic, adaptive enzyme, and enzyme experiments; thus, the pathway is well documented in animals, bacteria, Neurospora, and insects (Knox and Mehler, I950; Mehler, 1954) (Fig. 54). Some of the evidence may be summarized as follows: $I$ ) the enzyme tryptophane peroxidase, which converts the amino acid to formyl kynurenine occurs in the cytoplasm of liver. 2) Formyl kynurenine is hydrolyzed to kynurenine by the enzyme, formylase. Formylase activity has been observed in animal tissues, bacteria, and Neurospora. Formyl kynurenine accumulates when tryptophane is oxidized by liver enzyme systems lacking formylase (Mehler and Knox, I950).3) The pyridoxal phosphate requiring enzyme, kynureninase, has also been demonstrated in bacteria, Neurospora, and in liver (Mehler, I 954). Kynureninase can attack 3-hydroxykynurenine as well as kynurenine. 4) After tryptophane is fed to rats, quinolinic acid, N-methylnicotinamide, kynurenic acid and xanthurenic acid, metabolic products of kynurenine and 3-hydroxykynurenine, are found in the urine (Henderson and Hankes, I956). 5) Liver mitochondria catalyze the aerobic conversion of L-kynurenine to 3-hydroxykynurenine; TPNH is required for this hydroxylation (DeCastro et al., I 956).6) 3-hydroxykynurenine can replace niacin in certain Neurospora mutants and it accumulates as a product of tryptophane metabolism in cultures of another niacin requiring mutant. A human tubercular patient given tryptophane, excreted 3 -hydroxyanthranilic acid in the urine. Rat liver preparations convert 3 -hydroxyanthranilic acid to quinolinic acid and ferrous ions and ascorbate stimulate this conversion (Miyake et al., I954), with the formation of a quinone intermediate. Only the synthesis of the intermediate is catalyzed by an enzyme; the subsequent formation of quinolinic acid is spontaneous. An enzyme which does attack the intermediate has been concentrated from extracts of liver. This enzyme converts the inter- 

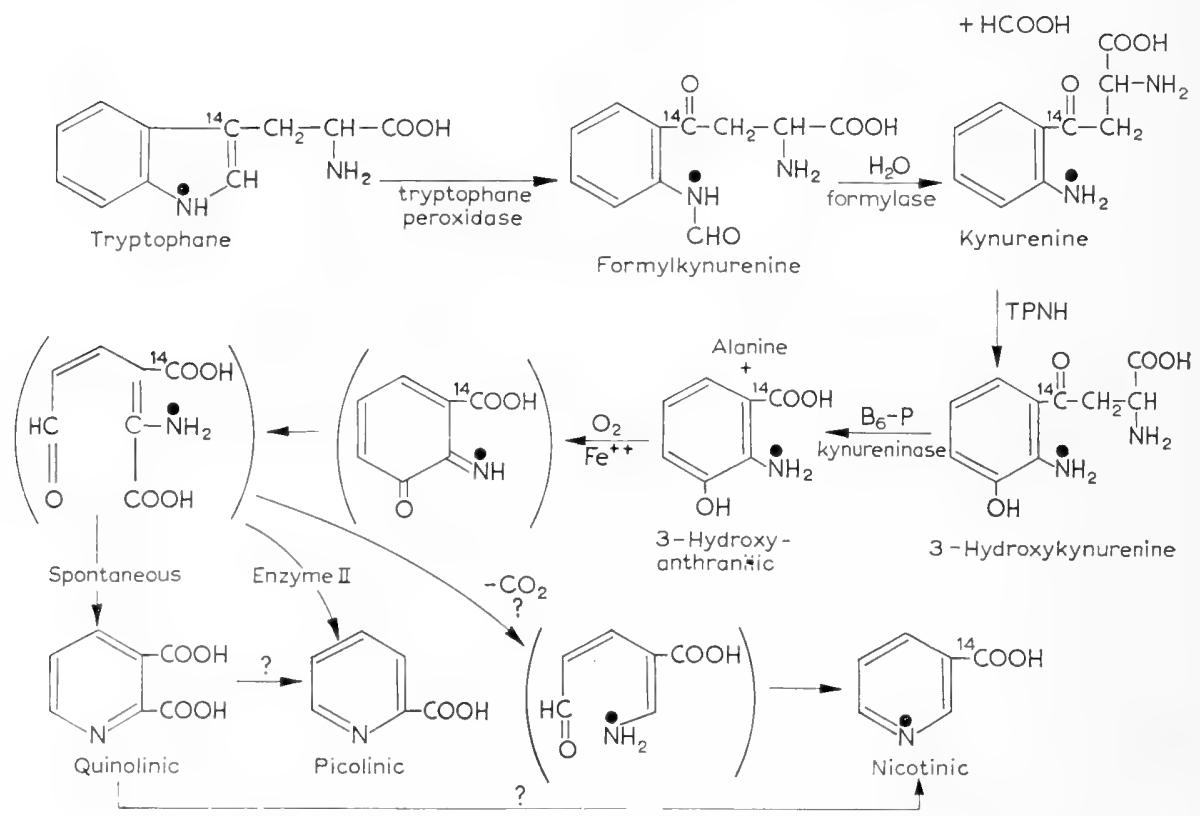

Fig. 54. Metabolic pathway of conversion of tryptophane to nicotinic acid.

mediate to quinolinic acid (Mehler, I956). The significance of picolinic and quinolinic acids is not as yet clear, the latter being poorly converted to nicotinic acid. It is possible that under physiological conditions, the amide bond of nicotinamide is formed prior to the closure of the pyridine ring. By isotope experiments, it has been shown that the side chain of tryptophane is lost during the formation of nicotinic acid. The $\beta$-carbon of the indole ring becomes the carboxyl group of nicotinic acid and the indole nitrogen becomes the pyridine nitrogen (Fig. 54). These observations are consistent with a mechanism involving the opening of the ring of 3 -hydroxyanthranilic acid and cyclization of the presumed intermediate aldehyde to form a pyridine ring.

Although niacin is synthesized from tryptophane in Neurospora and rat liver, it appears that neither tryptophane nor indole function as niacin precursors, in $E$. coli and in $B$. subtilis. The niacin precursors in these bacteria are as yet unknown (Yanofsky, 1954).

\section{(b) Formation of $D P \mathcal{N}^{+}$and $T P \mathcal{N}^{+}$}

The administration of nicotinamide to mice results in a ten fold rise in the DPN ${ }^{+}$ content of liver. Increases are also found in spleen, kidney, and neoplastic tissues (Kaplan et al., I956a). Although nicotinic acid and tryptophane also promote an increase in liver $\mathrm{DPN}^{+}$, the magnitude is much smaller than that with nicotinamide.

Erythrocytes can form $\mathrm{DPN}^{+}$from nicotinamide and glucose in vitro. It is not possible to demonstrate an increase in nicotinamide riboside or the ribotide, suggesting that the conversion of the latter substances to $\mathrm{DPN}^{+}$is very rapid; however formation of nicotinamide riboside by partially purified hog liver enzyme has been demonstrated (Rowen and Kornberg, r95I). 
a) Nicotinamide + ribose-I-phosphate $\stackrel{\text { phosphorylase }}{\longleftarrow}$ nicotinamide riboside $+\mathrm{P}_{\mathrm{i}}$

ATP

b) Nicotinamide riboside $\longrightarrow \mathrm{NMN}+\mathrm{ADP}$

In the presence of ATP, nicotinamide mononucleotide was also formed (reaction b). Liver and yeast enzymes catalyze the formation of $\mathrm{DPN}^{+}$from $\mathrm{NMN}$ and of $\mathrm{TPN}^{+}$from $\mathrm{DPN}^{+}$(Kornberg, I $\left.950 \mathrm{a}, \mathrm{b}\right)$.

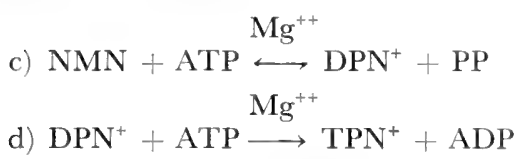

The reduced form of $\mathrm{DPN}^{+}$may substitute for $\mathrm{DPN}^{+}$in reaction c) and d). The third phosphate of $\mathrm{TPN}^{+}$is esterified at the $2^{\prime}$-position of the adenosine moiety of the $\mathrm{TPN}^{+}$molecule (Heppel et al., I955).

\section{(a) Pantoate}

\section{Coenzyme $A$ synthesis}

In Bacterium linens, 456, pantothenic acid is required for growth. PABA can however replace pantothenic acid. The growth of PABA requiring bacteria is inhibited competitively by sulfanilamide or salicylate. Since pantoate and ketopantoate as well as pantothenate reverse the sulfanilamide inhibition non-competitively, it seems possible that ketopantoate and pantoate are pantothenate precursors in this organism. This concept is supported by the fact that ketopantoate and pantoate are equivalent to pantothenate as growth factors in the absence of PABA.

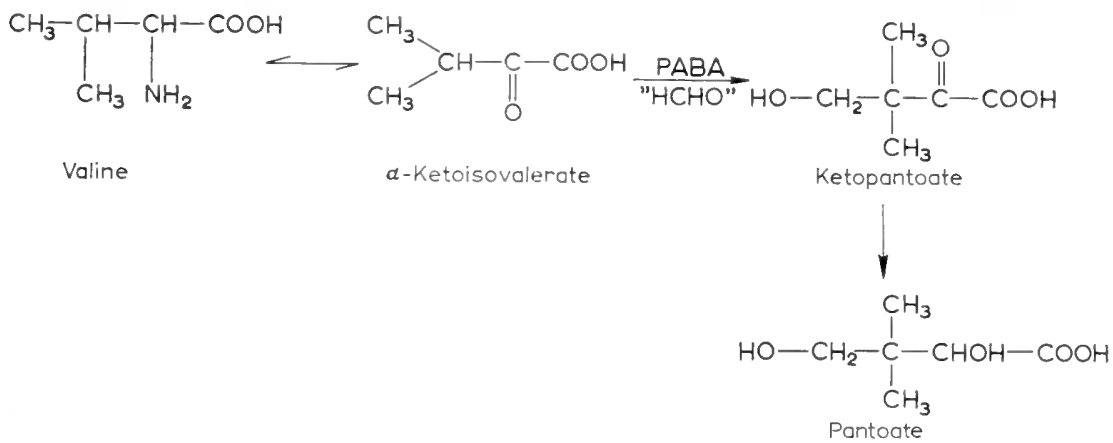

Fig. 55. Pantoate synthesis from valine.

With limiting concentrations of PABA, $\alpha$-ketoisovalerate, the keto-analog of valine, enhances the growth of Bacterium linens (Purko et al., I 954). $\alpha$-ketoiso* valerate is also converted to pantoate in E. coli. Presumably, the PABA is required for the hydroxymethylation of $\alpha$-ketoisovalerate (Fig. 55). Extracts of acetone dried $E$. coli cells are active in synthesizing pantothenate from pantoate and $\beta$-alanine (Maas and Novelli, I 953).

$$
\beta \text {-Alanine }+ \text { pantoate }+\mathrm{ATP} \rightarrow \text { pantothenate }+\mathrm{PP}+\mathrm{AMP}
$$




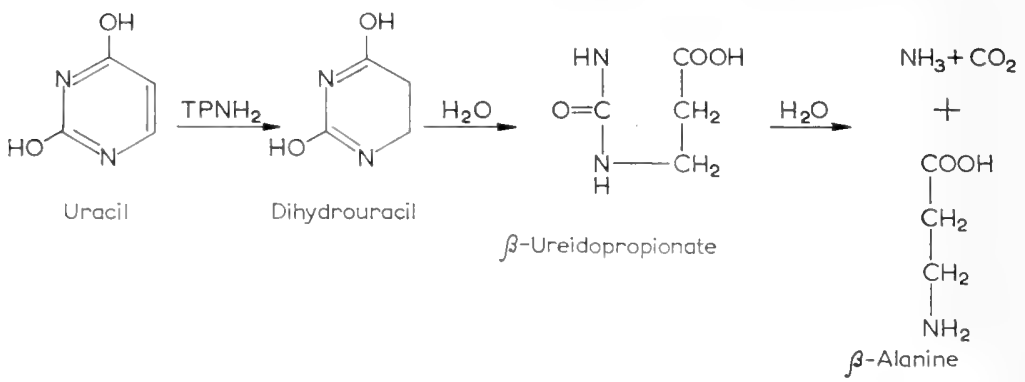

Fig. 56. Conversion of uracil to $\beta$-alanine.

\section{(b) Source of $\beta$-alanine}

Two sources are now known for the $\beta$-alanine.

a) $\beta$-alanine may be formed in certain bacteria by the $\alpha$-decarboxylation of aspartate (David and Lichstein, I950):

$$
\text { Aspartic } \rightarrow \beta \text {-alanine }+\mathrm{CO}_{2}
$$

b) Evidence for the formation of beta alanine from uracil has been obtained in microorganisms, the intact rat, in rat liver slices, and extracts of rat liver (Fink et al., I953, 1956; Fink, I956; Canellakis, I956a, b; Fig. 56). The degradation of thymine takes place by an analogous mechanism.

Thymine $\rightarrow$ dihydrothymine $\rightarrow \beta$-ureidoisobutyric $\rightarrow \beta$-aminoisobutyric $+\mathrm{NH}_{3}+\mathrm{CO}_{2}$

(c) Formation of coenzyme A from pantothenate.

All of the enzymes required for the synthesis of coenzyme A from pantothenate have been demonstrated in pigeon liver extracts (Hoagland and Novelli, I954; Reactions I-5):

I) Pantothenate + cysteine $+\mathrm{ATP} \stackrel{\mathrm{Mg}^{++}}{\longrightarrow}$ pantothenyl cysteine

2) Pantothenyl cysteine $\longrightarrow$ pantetheine $+\mathrm{CO}_{2}$

$$
\mathrm{Mg}^{++}
$$

3) Pantetheine $+\mathrm{ATP} \longrightarrow$ 4-phosphopantetheine + ADP

4) 4 -Phosphopantetheine $+\mathrm{ATP} \stackrel{\mathrm{Mg}^{++}}{\longrightarrow}$ dephospho-CoA $+\mathrm{PP}$

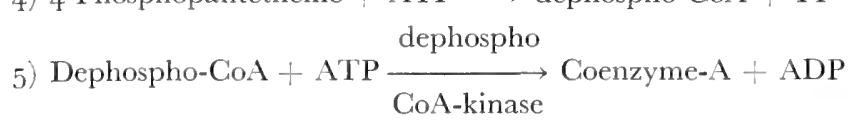

In the liver enzyme system, pantothenylcysteine can replace ATP, pantothenate, and cysteine in the formation of pantetheine. $\beta$-mercaptylethylamine cannot substitute for cysteine in reaction I). The decarboxylation of pantothenylcysteine has also been demonstrated in Acetobacter suboxydans (Brown and Snell, I953; Levintow and Novelli, I954). The structure of coenzyme A is shown in Fig. 57. The sulfhydryl group of the $\beta$-mercaptylethylamine portion of the molecule is the active group in coenzyme A function. Acyl-CoA derivatives are important intermediates in fatty acid synthesis and oxidation, cholesterol synthesis, citrate synthesis, and the synthesis of N-acetyl-amino compounds (Novelli, I953). 
<smiles>CC(COP(=O)(O)OP(=O)(O)OCC1COCCC(n2cnc3c(N)ncnc32)C1O)C(O)C(=O)NCCC(=O)NCCS</smiles>

Fig. 57. Coenzyme A.

\section{Io. $p$-Aminobenzoic acid and folic acid compounds}

Folic acid compounds function in "one carbon compound" transfer reactions leading to the synthesis of the methyl group of thymine, the $\beta$-carbon of serine, carbons 2 and 8 of the purines and other building blocks of protoplasm. All forms of folic acid contain a $p$-aminobenzoic acid (PABA) residue. The structural formulas of PABA, folic acid, and certain related compounds are shown in Fig. $5^{8}$.

It is probable that $\mathrm{N}^{10}$-formyl tetrahydrofolic acid $\left(\mathrm{N}^{10}\right.$-formyl-THFA) and $\mathrm{N}^{10}$-hydroxymethyltetrahydrofolic acid $\left(\mathrm{N}^{10}-\mathrm{HOCH}_{2}-\mathrm{THFA}\right)$ or closely related derivatives are the actual coenzyme forms of folic acid in the cell. The postulated relationship between some of the above compounds is shown in Fig. 59.

Shikimic acid is a precursor of PABA. Certain E. coli and Aerobacter aerogenes<smiles>[R10]Oc1ccc(C(=O)O)cc1</smiles><smiles>Nc1nc(O)c2nc(CNc3ccc(C(=O)NC(CCC(=O)O)C(=O)O)cc3)cnc2n1</smiles>

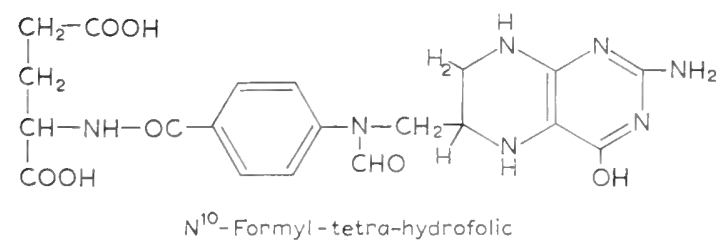

Fig. 58. Structure of folic acid and related compounds. 


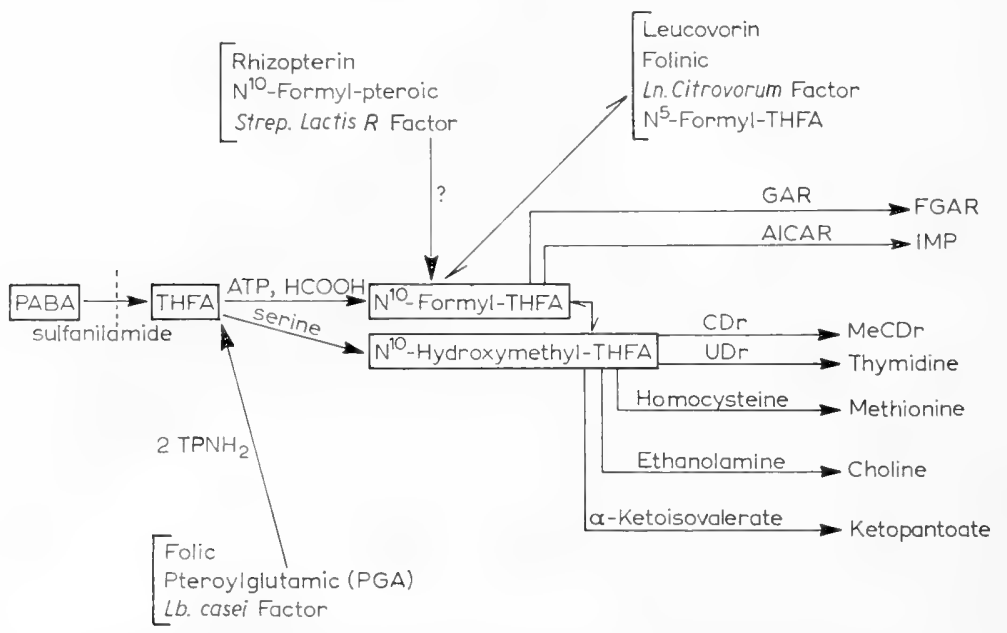

Fig. 59. Scheme showing possible metabolic relationship between p-aminobenzoic acid and some members of the folic acid group.

mutants manifest a multiple requirement for PABA, $p$-hydroxybenzoic acid, tyrosine, phenylalanine, tryptophane, and under certain conditions, a sixth factor (Davis, I 955a, b). The synthesis of aromatic compounds via shikimic acid has been discussed in connection with tyrosine synthesis. The synthesis of folic acid from glutamic acid, PABA, and glucose, has been demonstrated using Lb. plantarum ${ }_{5} S$ and $L n$. mesenteroides $P$-6o (Woods, 1954). No preformed pteridine needs to be added to growing cultures of these bacteria to effect folic acid synthesis. Sulfonamide compounds inhibit this synthesis by competition with PABA.

Folic acid can replace PABA as a growth factor and it can non-competitively overcome the inhibition of bacterial growth due to the sulfonamides in some microorganisms. However, for others, folic acid is either inactive or is less active than PABA itself. Likewise, folinic is not appreciably more active than folic acid as a substitute for PABA except for $L n$. mesenteroides P-6o. This latter organism grows equally well on PABA or folinic but not at all on folic acid. These facts cast doubt on the concept that folic acid and folinic acid are direct intermediates in the synthesis of the folic acid coenzyme. Similarly, pteroic acid and its $\mathrm{N}^{10}$-formyl derivative, rhizopterin are probably not direct products of PABA utilization. These substances are inactive or are competitive rather than non-competitive in overcoming sulfonamide inhibition with $L b$. plantarum and other organisms for which folic acid is effective in rendering the organism insensitive to the drug. $p$-Aminobenzoylglutamate, another possible intermediate in the synthesis of the folic acid coenzyme, in the few cases where it is active, always shows a competitive relation with the sulfonamides.

Certain strains of bacteria are unable to synthesize folic acid derivatives from PABA. Thus, $L b$. casei requires folic acid or folinic as a growth factor, $L n$. citrovorum grows on folinic acid but not folic acid, while $S$. faecalis $R$ requires rhizopterin.

Tetrahydro derivatives of folic acid or pteroic acid or polyglutamate derivatives may be the direct intermediates in the synthesis of the coenzyme. $\mathrm{N}^{10}$-formyl tetrahydrofolic acid has been implicated in the formation of inosinic acid (Jaenicke, I 955; Greenberg et al., I955). Folinic acid is active to the degree that it can be 
converted to $\mathrm{N}^{10}$-formyl THFA (Greenberg, 1954). Tetrahydrofolic acid activates the interconversion of glycine and serine by pigeon liver under conditions where folic or folinic acids are inactive (Kisliuk and Sakami, I955). $\mathrm{N}^{10}$-formyl THFA is $3^{0}$ times as active as folic acid in reversing the toxicity of folic acid antagonists to S. faecalis. A series of polyglutamyl-folic acid derivatives $(\mathrm{CoG}$ ) are active in catalyzing the conversion of serine to glycine in Clostridium HF (Wright and Stadtman, I 956; Wright, I 956). Folic acid, $N^{5}$-formyl folic acid, and Anhydroleucovorum were inactive while folinic and THFA were active only at much higher concentrations than was necessary for CoC.

\section{SUMMARY}

Our discussion began with an outline of the energy yielding metabolic cycles of the cell and the various mechanisms by which the energy generated in biochemical reactions is conserved in compounds such as ATP, which contain "energy-rich bonds". The many reactions in which ATP and related nucleotides participate were next described. Thirdly, we examined the metabolic pathways which are of significance in connection with the biosynthesis of the major building blocks of protoplasm. It was noted that most of the latter substances are formed from metabolites of the glycolytic sequence and the citric acid cycle or from the amino acids which are closely related to these sequences. Thus, the catabolic cycles of the cell were shown to be of significance not only to generate the energy needed for growth but also as a source of the carbon fragments out of which the essential metabolites are constructed. Likewise, it was shown that ATP and related nucleotides are required in various activation reactions and as constituents of coenzymes of numerous transfer reactions, but also as building blocks of the nucleic acids. Finally, the double function of glutamine, glutamic acid, and aspartate as building blocks of proteins and substrates of many nitrogen transfer reactions was indicated.

Clearly, enormous progress has been made during the last two decades in charting the metabolic sequences of the cell, in isolating the intermediates, and in purifying the enzymes of these sequences. We may anticipate that this knowledge will serve as a springboard for further advances in related fields. Certainly, there should be accelerated progress in connection with $a$ ) the regulation of intracellular metabolism, b) the control of enzyme synthesis, and c) the terminal mechanisms of macromolecular biosynthesis, and $d$ ) the pathways of vitamin synthesis.

Cells are equipped with remarkably efficient mechanisms for providing for an economical operation and integration of their biosynthetic pathways. The experiments of Chance and others have clarified the mechanisms by which the rate of respiration and glycolysis are controlled. As B. D. Davis has emphasized, the mechanisms regulating the synthesis of protoplasmic building blocks are operative against the background of the capacity to carry out various of the biosynthetic reactions at rates far out of proportion to the rest of the metabolism of the cell. Many auxotrophic mutant strains of microorganisms excrete a precursor of a blocked reaction, or sometimes the end product of a different biosynthetic chain, in large amounts. Indeed, in some instances, the amount of the precursor accumulated may even exceed the dry weight of the microorganisms themselves. Yet, the ac- 
cumulation of precursors can be prevented by adding to the culture medium an excess of the required metabolite. Apparently, a later product of a biosynthetic sequence tends to inhibit one or more steps in the formation of earlier members of the same sequence or metabolites of parallel chains. Examples of this negative feedback mechanism have been observed in connection with amino acid, purine, and pyrimidine synthesis (Yates and Pardee, 1956).

The experiments of Ochoa, Kornberg, Roseman, and Zamecnik and their associates are prototypes of studies which will enhance our understanding of nucleoprotein and mucoprotein synthesis. In the past, heterogeneous mixtures of these macromolecular substances have been investigated. As time goes on, there will be an increasing tendency fruitfully to investigate the regulation of the biosynthesis of specific proteins having catalytic functions.

The elucidation of metabolic pathways and the purification of enzymes will continue; however, we may anticipate an accelerated application of the facts of intermediary metabolism to rational chemotherapeutics and to problems of hereditary metabolic diseases, including cancer. 
I) The lipid cofactor can be replaced by a group of 2,3-dimethoxy-5-methylbenzoquinone compounds which are substituted at position 6 with homologous isoprenoid chains. The latter compounds (Coenzymes $Q_{6}$ to $Q_{10}$ ) have been isolated from lipides of beef heart mitochondria and microbial sources and undergo cyclic oxidation and reduction during substrate oxidation ( $R$. L. Lester, F. L. Crane and Y. Hatefi, (i 958), 7. Am. Chem. Soc., 8o, 475I).

2) A soluble RNA residing in the same cellular fraction which activates amino acids reversibly binds amino acids in the presence of ATP. Since the amino acid so bound can be subsequently transferred to microsomal protein in the presence of GTP, the RNA-amino acid may be an intermediate in microsomal protein synthesis (M. B. Hoagland, M. L. Stephenson, J. F. Scott, L. I. Hecht and P. C. Zamecnik, (1958), 7. Biol. Chem., 23I, 241).

3) Recent evidence indicates that isocitric lysase and the malate condensing enzyme function in a "glyoxylate cycle", thereby providing a route for the net conversion of fat to carbohydrate by plants (H. L. KornberG AND H. BeEvers, (1957), Biochim. et Biophys. Acta, 26, 53 I):
a) Fat $\rightarrow$ acetyl-CoA $\longrightarrow$ malate $\rightarrow$ carbohydrate
b) 2 Isocitrate $\rightarrow \alpha$-ketoglutarate + glyoxylate + succinate $+\mathrm{CO}_{2}$
c) $\alpha$-Ketoglutarate + succinate $\longrightarrow 2$ oxalacetate $+\mathrm{CO}_{2}$
d) 2 Oxalacetate +2 acetyl-CoA $\longrightarrow 2$ isocitrate

4) Glutamine functions as an amino donor in the conversion of uridine nucleotides to cytidine nucleotides by rat tumor enzymes (H. O. Kammen AND R. B. Hurlbert, (1958) Biochim. et Biophys. Acta, 30, I96). Glutamine- ${ }^{15} \mathrm{~N}$ is also a precursor of asparagine- ${ }^{15} \mathrm{~N}$ in human cells in tissue culture (L. LEvintow, (1957) Science, I26, 6I I).

5) Recent evidence suggests that mevalonic acid ( $D L-\beta, \gamma$-dihydroxy- $\beta$-methylvaleric acid), 5-phosphomevalonic acid, 5-pyrophosphomevalonic acid, and $\Delta^{3}$-isopentenol pyrophosphate are intermediates in squalene synthesis. New carbon-carbon bonds between two isoprenoid units are formed by the interaction of two methylene groups while the carboxyl groups of mevalonic acid are lost in a concerted process with simultaneous elimination of the tertiary hydroxyl groups, and the formation of a double bond between $\mathrm{C}_{2}$ and $\mathrm{C}_{3}$ ( $\mathrm{S}$. Chaykin, J. Law, A. H. Phillips, T. T. Tahen and K. Blogh, (1958) Proc. Natl. Acad. Sci., U.S., 44, 998.

Carbon 28 of yeast ergosterol is formed by the transmethylation of the methionine methyl group to $\mathrm{C}_{24}$ of an intermediate beyond squalene. In experiments with ${ }^{14} \mathrm{CH}_{3}$-methionine, it was shown that ergosterol but not zymosterol nor lanosterol were labelled (G. J. Alexander, A. M. Gold and E. Schwenk, (i 958) 7. Biol. Chem., 232, 599). 


\section{LITERATURE}

Abelson, P. H., (1954) J. Biol. Chem., 206, 335.

Abelson, P. H. And H. J. Vogel, (1955) 7. Biol. Chem., 213, 355.

Abraham, S., P. Cady and I. L. Chaikoff, (1956) Proc. Am. Assoc. Cancer Research, 2, 89.

Abraham, S., R. Hill and I. L. Chaikoff, (1955) Cancer Research, 15, 177.

Abrams, R., (1952) Arch. Biochem. Biophys., 37, 270.

Abrais, R. and M. Bentley, (i955a) 7. Am. Chem. Soc., 77, 4i 79.

Abrais, R. and M. Bentley, (1955b) Arch. Biochem. Biophys., 56, I84.

Abrams, R. and M. Bentley, (1955c) Arch. Biochem. Biophys., 58, iog.

Abrams, R. and J. M. Goldinger, (195I) Arch. Biochem., 30, 26 I.

Adaus, E., (1955a) 7. Biol. Chem., 2I7, 3 I 7.

Adams, E., (1955b) 7. Biol. Chem., 217, 325.

Adelberg, E. A., (1954) F. Am. Chem. Soc., 76, 424I.

Adelberg, E. A., C. A. Coughlin and R. W. Barratt, (ig55) 7. Biol. Chem., $216,425$.

Agranoff, B. W., R. O. Brady and M. Colodzin, (i954) J. Biol. Chem., 21 1, 773.

Alexander, N. and D. M. Greenberg, (1955) F. Biol. Chem., 214, 821 .

Alexander, N. and D. M. Greenberg, (1956) F. Biol., Chem., 220, 775.

Ames, B. N. And B. L. Horecker, (1956) J. Biol. Chem., 220, i i 3.

Ames, B. N. And H. K. Mrtchell, (1955) F. Biol. Chem., 212, 687.

Anderson, E. P., C. Y. Yen, H. G. Mandel and P. K. Smith, (i955) 7. Biol. Chem., 213,625 .

Arnstein, H. R. V., (I951) Biochem. F., 48, 27.

Arnstein, H. R. V. and D. Keglevic, (1956) Biochem. F., 62, 199.

Arnstein, H. R. V. And A. Neuberger, (1953) Biochem. 7., 55, 259.

Arnstein, H. R. V. and V. Stankovic, (I956) Biochem. F., 62, I90.

Arnon, D. I., (1955) Science, I22, 9.

Arnon, D. I., M. B. Allen and F. R. Whatley, (1956) Biochem. et Biophys. Acta, 20, 449.

Ashmore, J., J. H. Kinoshita, F. B. Nesbett and A. B. Hastings, (i956) 7. Biol. Chem., 220, 6 I 9 .

Ashwell, G. and J. Hickman, (r954) 7. Am. Chem. Soc., 76, 5889.

Awapara, J., A. J. Landua and R. Fuerst, (1950) Biochem. et Biophys. Acta, 5, 457.

Bachhawat, B. K., W. G. Robinson and M. J. Coon, (i954) 7. Am. Chem. Soc., 76, 3098.

Bachhawat, B. K., J. F. Woessner and M. J. Coon, (I956a) Federation Proc., I5, 214.

Bachhawat, B. K., W. G. Robinson and M. J. Coon, (1956b) 7. Biol. Chem., 219, 539.

Baddiley, J., J. G. Buchanan, B. Carss and A. P. Mathias, (1956) Biochim. et Biophys. Acta, 2I, I9I.

Ball, E. G., G. B. Anfinsen and O. Cooper, (1947) 7. Biol. Chem., 168, 257.

Ballou, C. E., H. O. L. Fischer and D. L. McDonald, (1955) J. Am. Chem. Soc., 77, 2658.

Bandurski, R. S. and F. Lipmann, (1956) 7. Biol. Chem., 219, 74I.

BeERs, R. F., (1956) Nature, $177,790$.

Bennett, E. L. and B. J. Krueckel, (1955) Biochim. et Biophys. Acta, I7, 5 I 5 .

Bennett, L. L. And H. E. Skipper, (1955) Arch. Biochem. Biophys., 54, 566.

Bennett, L. L., Jr., H. E. Skipper, C. C. Stock and C. P. Rhoads, (i955) Cancer Research, 15,485 .

Bennett, L. L., Jr., H. E. Skipper, H. W. Toolan and C. P. Rhoads, (1956) Cancer Research, 16, 262.

Berg, P., (1956a) 7. Am. Chem. Soc., 77, 3163.

BerG, P., (1956b) 7. Biol. Chem., 222, 99 I.

Berg, P., (1956c) F. Biol. Chem., 222, 1025.

Bergivist, R. And A. Deutsch, (I954a) Acta Chem. Scand., 8, 1880.

Bergivist, R. And A. Deutsch, (1954b) Acta Chem. Scand., 8, i889.

Bernstein, I. A., (1953) 7. Biol. Chem., 205, 3 I 7.

Bernstein, I. A., (1956a) Biochim. et Biophys. Acta, 19, I79.

Bernstein, I. A., (1956b) 7. Biol. Chem., 221, 873.

Bessey, O. A., H. J. Lowe and L. L. Salomon, (1953) Ann. Rev. Biochem., 22, 545. 
Binkley, F. And D. Okeson, (1950) 7. Biol. Chem., I82, 273.

Black, A. L., M. Kleiber and C. F. Baxter, (i955) Biochim. et Biophys. Acta, I7, 346.

Black, S. and N. G. Wright, (1955a) J. Biol. Chem., $213,27$.

Black, S. ANd N. G. Wright, (1955b) 7. Biol. Chem., 213, 39.

Black, S. and N. G. Wright, (1955c) 7. Biol. Chem., 213, 5 I.

BLAKLEY, R. L., (1954) Nature, 173, 729.

Blanchard, M. L., S. Korkes, A. del Campillo and S. Ochoa, (1950) J. Biol. Chem., I 87,875 .

Block, R. J., J. A. Stekol and J. K. Loosli, (195I) Arch. Biochem. Biophys, 33, 353.

Bloom, B. and D. Stetten JR., (1953) 7. Am. Chem. Soc., 75, 5447.

Bloom, B. And D. Stetten Jr., (I955) 7. Biol. Chem., 2I2, 555.

Bloom, B., M. R. Stetten and D. Stetten Jr., (1953) F. Biol. Chem., 204, 681.

Blumenthal, H. J., K. F. Lewis and S. Weinhouse, (i954) 7. Am. Chem. Soc., 76, 6o93.

Bolton, E., (1954) Proc. Nall. Acad. Sci. U.S., 40, 764.

Bolton, E. T. And A. M. Reynard, (1954) Biochim. et Biophys. Acta, I3, 38 I.

Bolton, E. T., P. H. Abelson and E. Aldous, (1952) 7. Biol. Chem., 198, 179.

Borsook, H., C. L. Deasy, A. J. Haagen-Smit, G. Keighley and P. H. Lowy, (i949) J. Biol. Chem., I79, 689.

Brady, R. O. and S. Gurin, (1951) F. Biol. Chem., I89, 37 I.

Braunstein, A. E. and G. Y. Vilenkina, (1949) Doklady Akad. Nauk SSSR, 66, 243.

Bregoff, H. M. and C. C. Delwiche, (1955) 7. Biol. Chem., 2I7, 8 ig.

Brodie, B. B., J. Axelrod, J. R. Cooper, L. Gaudette, B. N. LaDu, C. Mitoma and S. Udenfriend, (I955) Science, I2I, 6o3.

Brown, E. G., T. W. Goodwin and S. Pendlington, (i955) Biochem. F., 6I, 37.

Brown, G. B., (1950) Federation Proc., 9, 517.

Brown, G. B., (195I) 7. Cellular Comp. Physiol., 38, 12 I.

Brown, G. M. And E. E. SNell, (1953) F. Am. Chem. Soc., 75, 2782.

Brumm, A. F., V. R. Potter and P. Siekevitz, (1956) F. Biol. Chem., 220, 7 I 3.

Buchanan, J. M., (195I) F. Cellular Comp. Physiol., 38, I 43.

Bucher, N. L. R. and K. MaGarrahan, (1956) 7. Biol. Chem., 222, I.

Burns, J. J. and E. H. Mosbach, (1956) F. Biol. Chem., 221 , I07.

Burns, J. J., E. H. Mosbach, S. Schulenberg and J. Reichenthal, (i955) 7. Biol. Chem., $214,507$.

Busch, H., M. H. Goldberg and D. G. Anderson, (1956) Cancer Research, I6, I75.

Byrne, W. L. And H. A. Lardy, (1954) Biochim. et Biophys. Acta, I4, 495.

Canellakis, E. S., (1955) Federation Proc., 14, 324.

Canellakis, E. S., (I956a) Federation Proc., I5, 229.

Canellakis, E. S., (1956b) F. Biol. Chem., 22I, 3 I 5 .

Cantarow, A., T. L. Williams and K. E. Paschkis, (1956) Federation Proc., 15, 30.

Cantoni, G. L., (1953) J. Biol. Chem., 204, 403.

Cantoni, G. L. And P. J. Vignos, (ig54) F. Biol. Chem., 209, 647.

Caputto, R., (195I) F. Biol. Chem., 189, 80 I.

Cardini, C. E., A. C. Paladini, R. Caputto and L. F. Leloir, (i950) Acta Physiol. Latinoam., 1,57 .

Carter, C. E. and L. H. Cohen, (i955) 7. Am. Chem. Soc., 77, 499.

Carter, C. E. and L. H. Cohen, (1956) J. Biol. Chem., 222, i 7.

Chance, B., (1953-1954) Harvey Lectures, 49, 145.

Chance, B. and L. N. Gastor, (1952) Science, iI6, 200.

Chance, B. and G. R. Williams, (1956) Advances in Enzymol., 17, 65.

Chapeville, F. and P. Fromageot, (1954) Biochim. et Biophys. Acta, I4, 415.

Chapeville, F., P. Fromageot, A. Brigelhuber and M. Henry, (i956) Biochim. et Biophys. Acta, 20, $35 \mathrm{I}$.

Chichester, C. O., T. Nakayama, G. Mackinney and T. W. Goodwin, (i955) F. Biol. Chem., 214, 515.

Clayton, R. B. and K. Bloch, (1956a) 7. Biol. Chem., 218, 305.

Clayton, R. B. and K. Bloch, (1956b) 7. Biol. Chem., 218, 319. 
Conn, E. E. and B. Vennesland, (1951) F. Biol. Chem., 192, i 7.

Cooper, C. and A. L. Lehninger, (1956a) 7. Biol. Chem., 219, 489.

Cooper, C. and A. L. Lehninger, (i956b) 7. Biol. Chem., 219, 519.

Cooper, C., R. Wu and D. W. Wilson, (1955) J. Biol. Chem., 216, 37.

Corran, H. S., D. E. Green and F. B. Straub, (1939) Biochem. F., 33, 793.

Crane, F. L. and H. Beinert, (1954) 7. Am. Chem. Soc., 76, 4491.

Crane, F. L. and H. Beinert, (1956) F. Biol. Chem., $218,717$.

Crane, F. L., S. Mit, J. G. Hauge, D. E. Green and H. Beinert, (i956) 7. Biol. Chem., 218,701 .

Dauben, W. G. and T. W. Hutton, (i956) 7. Am. Chem. Soc., 78, 2647.

Daughaday, W. H., J. Larner and C. Hartnett, (1955) F. Biol. Chem., 212, 869.

David, W. E. and H. C. Lichstein, (1950) Proc. Soc. Exptl. Biol. Med., 73, 2 i6.

Davis, B. D., (I95I) 7. Biol. Chem., 19I, 3 I 5.

Davis, B. D., (1952) Nature, 169, 534.

Davis, B. D., (1955a) Federation Proc., 14, 69 I.

Davis, B. D., (1955b) Advances in Enzymol., 16, 247.

Dawson, R. M. C., (1955) Biochem. 7., 59, 5 .

DeCastro, F. T., J. M. Price and R. R. Brown, (i956) J. Am. Chem. Soc., 78, 2904.

Delwiche, C. C., (1956) in Inorganic Nitrogen Metabolism, Johns Hopkins Press, Baltimore, p. 233.

De Meio, R. H. and M. Wizerkaniuk, (1956) Biochim. et Biophys. Acta, 20, 428.

De Moss, J. A. And G. D. Novelu, (I955) Biochim. et Biophys. Acta, I8, 592.

Devlin, T. M. And A. L. Lehninger, (1956) 7. Biol. Chem., 219, 507.

Dewey, D. L. And E. Work, (1952) Nature, 169, 533.

Dewey, D. L., D. S. Hoare and E. Work, (1954) Biochem. 7. 58, 523.

Dituri, F., F. A. Cobey, J. V. B. Warms and S. Gurin, (1956) 7. Biol. Chem., 22 , i8 I.

Dolin, M. I., (I954) Biochim. et Biophys. Acta, I5, I53.

Downing, M. and S. Schweigert, (I956) 7. Biol. Chem., 220, 521.

Drochmans, P., D. H. Marrian and G. B. Brown, (1952) Arch. Biochem. Biophys., 39, 3 Io.

DuVigneaud, V., (1952) A Trail of Research, Cornell University Press, Ithaca.

DuVigneaud, V., J. P. Chandler, S. Simmonds, A. W. Moyer and M. Cohn, (ig46) 7. Biol. Chem., 164,603 .

DuVigneaud, V., J. R. Rachele and A. M. White, (i956) 7. Am. Chem. Soc., 78, 5 I 3 I. Dziewiatkowski, D. D., (1954) 7. Biol. Chem., 207, i81.

Eagle, H., V. I. Oyama, M. Levy, C. L. Horton and R. Fleischman, (i956a) J. Biol. Chem., 218, 607 .

Eagle, H., V. I. Oyama, M. Levy and A. Freeman, (1956b) Science, I23, 845.

Edmonds, M. P. and G. A. LePage, (1955) Cancer Researeh, 15, 93.

Edmonds, M. P. and G. A. LePage, (I956) Cancer Research, i6, 222.

Edmonds, M. P., A. M. Delluva and D. W. Wilson, (1952) 7. Biol. Chem., 197, 25 I.

Elliott, W. H., (1953) 7. Biol. Chem., 201, 66r.

Elliott, W. H., (1955) Biochim. et Biophys. Acta, I7, 440.

Elliott, W. H., (1956) Biochem. J., 62, 427.

Elliott, W. H. and E. F. Gale, (I948) Nature, I6I, I29.

Elwyn, D. And D. B. Sprinson, (I954) F. Biol. Chem., 207, 467.

Elwyn, D., A. Weissiach, S. S. Henry and D. B. Sprinson, (1955) 7. Biol. Chem., 213, 28 I.

Emmelot, P., L. Bosch and G. H. Van Vals, (1955) Biochim. et Biophys. Acta, I7, 45 I.

Ericson, L. E., J. N. Williams and C. A. Elvehjem, (i955a) Acta Chem. Scand., 9, 859.

Ericson, L. E., J. N. Williams and C. A. Elvehjem, (1955b) 7. Biol. Chem., 212, 537.

Fairley, J. L., (1954) F. Biol. Chem., $210,347$.

Felts, J. M., R. G. Doell and I. L. Chaikoff, (i956) F. Biol. Chem., 219, 473.

Fincham, J. R. S. And A. B. Boulter, (I956) Biochem. F., 62, 72.

Fink, K., (I956) F. Biol. Chem., 218, 9.

Fink, R. M., K. Fink and R. B. Henderson, (1953) 7. Biol. Chem., $201,349$.

Fink, R. M., C. MaGaughey, R. E. Cline and K. Fink, (i956) F. Biol. Chem., 218 , I. Flaks, J. G. and J. M. Buchanan, (1954) 7. Am. Chem. Soc., 76, 2275. 
Friedberg, F., J. Adler and H. A. Lardy, (1956) J. Biol. Chem., 219, 943.

Friedkin, M., (1953) J. Cellular Comp. Physiol., 4I, 26 I.

Friedkin, M. and H. M. Kalckar, (1950) 7. Biol. Chem., I84, 437.

Friedkin, M. And D. Roberts, (1955) Federation Proc., 14, 215.

Friedkin, M. ANd D. Roberts, (1956) F. Biol. Chem., 220, 653.

Friedkin, M. ANd H. Wood, (1956) F. Biol. Chem., 220, 639.

Friedkin, M., D. Tilson and D. Roberts, (i956) 7. Biol. Chem., 220, 627.

Friedmann, B., H. W. Levin and S. Weinhouse, (1956) F. Biol. Chem., $221,665$.

Fromageot, C., (1953-1954) Harvey Lectures, 39, I.

Furlong, N. B., A. F. Wania and A. C. Griffin, (1955) Proc. Soc. Exptl. Biol. Med., 90, 7.

Gehring, L. B. and B. Magasanik, (1955) 7. Am. Chem. Soc., 77, 4685.

Ghosh, J. J., (1956) Federation Proc., 15, 261.

Gibson, D. M., P. Ayengar and D. R. Sanadi, (1956) Biochim. et Biophys. Acta, 2I, 86.

Gilvarg, C. And K. Bloch, (1950) 7. Am. Chem. Soc., 72, 579 I.

Gilvarg, C. and K. Bloch, (1952) F. Biol. Chem., I99, 689.

Ginsberg, V., E. F. Neufeld and W.Z. Hassid, (1956) Proc. Natl. Acad. Sci. .U.S, $42,333$.

Glaser, L. and D. H. Brown, (1955) Proc. Natl. Acad. Sci. U.S., 41, 253.

Glock, G. E. And P. McLean, (1953) Biochim. et Biophys. Acta, 12, 590.

Glock, G. E. And P. McLean, (1954) Biochem. 7., 56, I 7 I.

Glock, G. E. and P. McLean, (1956) Biochem. F., 6I, 390.

Glock, G. E., P. Mclean and J. K. Whitehead, (i956a) Biochem. F., 63, 520.

Glock, G. E., P. McLean and J. K. Whitehead, (1956b) Biochim. et Biophys. Acta, 19, 547.

Goldthwait, D. A., (1956) J. Biol. Chem., 222, 1051.

Goldthwait, D. A., G. R. Greenberg and R. A. Peabody, (1955) Biochim. et Biophys. Acta, I8, 148.

Goldthwait, D. A., R. A. Peabody and G. R. Greenberg, (i954) \%. Am. Chem. Soc., 76, 5258.

Goldthwait, D. A., R. A. Peabody and G. R. Greenberg, (1956) F. Biol. Chem., 22I, 569.

Goldwasser, E., (1953) Nature, I7I, I26.

Goldwasser, E., (I954) Biochim. et Biophys. Acta, 13, 34 I.

Goldwasser, E., (1955) 7. Am. Chem. Soc., 77, 6083.

Goodwin, T. W. And W. Lijinsky, (I95I) Biochem. J., 50, 268.

Goodwin, T. W. and S. Pendlington, (1954) Biochem. J., 57, 63 I.

Granick, S., (1954) Science, I20, I 105.

Green, D. E. and H. Beinert, (I955) Ann. Rev. Biochem., 24, I.

Green, D. E., B. Mackler, R. Repaske and H. R. Mahler, (1954) Biochim. et Biophys. Acta, 15,435 .

Green, D. E., S. Mir and P. M. Kohout, (1955) J. Biol. Chem., 217, 55 I.

Greenberg, D. M., (1954) Chemical Pathways of Metabolism, 2 Vols., Academic Press, New York.

Greenberg, D. M. And T. Winnick, (1949) Arch. Biochem., 2I, i66.

Greenberg, G. R., (I954) Federation Proc., 13, 745.

Greenberg, G. R., (1956) 7. Biol. Chem., 219, 423.

Greenberg, G. R. and E. L. Spilman, (1956) J. Biol. Chem., 219,4 I.

Greenberg, G. R., L. Jaenicke and M. Silverman, (1955) Biochim. et Biophys. Acta, I7, 589.

Grisolia, S., H. J. Grady and D. P. Wallach, (1955) Biochim. et Biophys. Acta, I7, 277.

Grob, E. C. ANd R. Butler, (1954) Experientia, Io, 250.

Gross, S. R., R. D. Gafford and E. L. Tatum, (1956) 7. Biol. Chem., 219, 78 I.

Grossman, L. and D. W. Visser, (I954) F. Biol. Chem., 209, 447.

Grunberg-Manago, M. and S. Ochoa, (1955) J. Am. Chem. Soc., 77, 3165.

Grunberg-Manago, M., P. J. Ortiz and S. Ochoa, (1955) Science, 122, 907.

Guarino, A. J. And H. Z. Sable, (1956) Biochim. et Biophys. Acta, 20, 20 I.

Guzman-Barron, E. S., M. Villavicencio And D. W. King, (1955) Arch. Biochem. Biophys., 58,500 .

Hager, L. P. and I. C. Gunsalus, (1953) 7. Am. Chem. Soc., 75, 5767.

Halliday, J. W. and L. Anderson, (1955) F. Biol. Chem., 217, 797. 
Hammarsten, E., P. Reichard and E. Saluste, (1950) 7. Biol. Chem., 183, 105.

Hansen, R. G. and E. Hageman, (1956) Arch. Biochem. Biophys., 62, 5 I I.

Harrington, H. and P. S. Lavik, (1955) Arch. Biochem. Biophys., 54, 6.

Harris, D. L., (1955) Arch. Biochem. Biophys., 57, 240.

Harpur, R. P. and J. H. Quastel, (1949) Nature, I64, 693.

Hartman, S. C., B. Levenberg and J. M. Bughanan, (i955) 7. Am. Chem. Soc., 77, 50 i. Hartman, S. C., B. Levenberg and J. M. Buchanan, (1956) J. Biol. Chem., 221 , 1057.

Hauge, J. G., T. E. King and V. H. Cheldelin, (i955) J. Biol. Chem., 214 , iI.

Hecht, L. I., V. R. Potter and E. Herbert, (i954) Biochim. et Biophys. Acta, I5, I34.

Heidelberger, C. H. and E. Harbers, (I956) Federation Proc., I5, 27 I.

Heidelberger, C. H., E. Harbers, K. C. Leibman, Y. Tagagi and V. R. Potter, (i956)

Biochim. et Biophys. Acta, 20, 445.

Heinrich, M. R., V. C. Dewey and G. W. Kidder, (i954) 7. Am. Chem. Soc., 76, 3102.

Henderson, L. M. and L. V. Hankes, (1956) J. Biol. Chem., 222, 1069.

Heppel, L. A., P. R. Whitfeld and R. Markham, (1955) Biochem. J., 6o, 9.

Herbert, E. and V. R. Potter, (i956) J. Biol. Chem., 222, 453.

Herrmann, R. L., J. L. Fairley and R. U. Byerrum, (1955) 7. Am. Chem. Soc., 77, igoz.

Hilz, H. and F. Lipmann, (i955) Proc. Natl. Acad. Sci. U.S., 4I, 880.

Hilz, H. and F. Lynen, (1953) 7. Am. Chem. Soc., 75, 3285.

Hirsch, M. and G. N. Cohen, (1954) Biochim. et Biophys. Acta, 15, 560.

Hoagland, M. B., (1955) Biochim. et Biophys. Acta, I6, 288.

Hoagland, M. B. and G. D. Novelli, (1954) 7. Biol. Chem., 207, 767.

Hoagland, M. B., E. B. Keller and P. C. Zamecnik, (i956) F. Biol. Chem., 218, 345.

Hockenhull, D. J. D., (1949) Biochim. et Biophys. Acta, 3, 326.

Hoff-Jorgensen, E., M. Friedkin and H. M. Kalckar, (i950) F. Biol. Chem., i84, 46 I.

Hogeboom, G. H., W. C. Schneider and M. J. Streibich, (I953) Cancer Research, 13, 6 I 7.

Holmes, W. L., W. H. Prusoff and A. D. Welch, (1954) F. Biol. Chem., 209, 503.

Horecker, B. L., (i954) Federation Proc., 13, 7 I I.

Horecker, B. L. and A. H. Mehler, (1955) Ann. Rev. Biochem., 24, 207.

Horecker, B. L. and P. Z. Smyrniotis, (i955) 7. Biol. Chem., 212, 8 i I.

Horecker, B. L., J. Hurwitz and A. Weissbach, (1956a) 7. Biol. Chem., 2i8, 785.

Horecker, B. L., J. Hurwitz and P. Z. Smyrniotis, (i956b) J. Am. Chem. Soc., 78, 692.

Horner, W. H., I. Siegel and J. Bruton, (1956) F. Biol. Chem., 220, 861.

Horowitz, N. H., (1946) f. Biol. Chem., I62, 413.

Horowitz, N. H., (1947) 7. Biol. Chem., r71, 255.

Horowitz, H. H. and C. G. King, (i953a) 7. Biol. Chem., 205, 815.

Horowitz, H. H. and G. G. King, (1953b) F. Biol. Chem., 200, I 25.

Huennekens, F. M., E. Nurk and B. W. Gabrio, (i956) 7. Biol. Chem., 22I, 97 I.

Hurlibert, R. B. and V. R. Potter, (1952) F. Biol. Chem., 195, 257.

Hurlbert, R. B. and P. Reichard, (I954) Acta Chem. Scand., 8, 701.

Hurwitz, J., (1952) Biochim. et Biophys. Acta, 9, 496.

Hurinitz, J. and S. J. Cooperstein, (1955) 7. Biol. Chem., 212, 77 I.

Huriwitz, J., A. Weissbach, B. L. Horecker and P. Z. Smyrniotis, (i956) 7. Biol. Chem., 218,769 .

Ichimara, A. and D. M. Greenberg, (1955) Proc. Nall. Acad. Sci. U.S., 4I, 605.

Isherwood, F. A., Y. T. Chen and L. W. Mapson, (I953) Nature, I7I, 348.

Isherwood, F. A., Y. T. Chen and L. W. Mapson, (1954a) Biochem. F., 5 6, I.

Isherwood, F. A., Y. T. Chen and L. W. Mapson, (1954b) Biochem. 7., 56 , 15.

Jacoby, W. B., D. O. Brummond and S. OchoA, (1956) 7. Biol. Chem., 2i8, 8 I I.

Jaenicke, L., (1955) Biochim. et Biophys. Acta, I7, 588.

Jagendorf, A. T., (1956) Arch. Biochem. Biophys., 62, I4I.

Jedeikin, L. A. and S. Weinhouse, (1955) F. Biol. Chem., 213, 27 I.

Jencks, W. P., (1953) Federation Proc., 12, 703.

Johnson, B. C., J. Firth, And S. P. Mistry, (i955) Arch. Biochem. Biophys., 54, 467.

Jones, M. E., L. Spector and F. Lipmann, (i955) 7. Am. Chem. Soc., 77, 8 i 9.

Kalckar, H. M., ( 1955 ) in Mechanism of Enzyme Action, Johns Hopkins Press, Baltimore, p. 675. 
Kalckar, H. M., B. Braganca, E. Gutolo and A. Munch-Peterson, (r953) Nalure, 172, I036.

Kaplan, N. O., A. Goldin, S. R. Humphreys, M. M. Giotti and F. E. Stolzenbach, (1956a) 7. Biol. Chem., 219, 287.

Kaplan, N. O., M. N. Swartz, M. E. Frech and M. M. Ciotti, (i956b) Proc. Natl. Acad. Sci. U.S., 42, $48 \mathrm{I}$.

Katz, J. and I. L. Chaikoff, (i955) F. Am. Chem. Soc., 77, 2659.

Katz, J., S. Abraham, R. Hill and I. L. Chaikoff, (1954) 7. Am. Chem. Soc., 76, 2277.

Katz, J., S. Abraham, R. Hill and I. L. Chaikoff, (1955) J. Biol. Chem., 2i4, 853.

Kearney, E. B. and T. P. Singer, (I956) 7. Biol. Chem., $219,963$.

Keller, E. B. and P. C. Zamecnik, (1956) F. Biol. Chem., 22I, 45.

Kennedy, E. P., (1953) F. Biol. Chem., 201, 399.

Kennedy, E. P. and S. B. Weiss, (1955) F. Am. Chem. Soc., 77, 250.

Kennedy, E. P. And S. B. Weiss, (1956) 7. Biol. Chem., 222, I 93.

Kelly, T. L., E. D. Nielson, R. B. Johnson and C. S. Vestling, (i955) 7. Biol. Chem., $212,545$.

Kertesz, D. and A. Albano, (1955) Cancer Research, 15, 394.

Kidder, G. W., V. G. Dewey, R. E. Parks and M. R. Heinrich (i950) Proc. Natl. Acad. Sci. U.S., 36, 43 I.

Kinnory, D. S., Y. Takeda, and D. M. Greenberg, (1955) Biochim. et Biophys. Acta, I7, $56 \mathrm{I}$.

Kisliuk, R. L. And W. Sakami, (1955) J. Biol. Chem., 214, 47.

KIт, S., (I953) Texas Repts. Biol. and Med., II, 685.

KIT, S., (1955) Cancer Research, 15, 715.

Kit, S., (1956) Cancer Research, 16, 70.

Kit, S., (1957) Cancer Research, I7, 56

Kit, S. And J. Awapara, (1953) Cancer Research, I3, 694.

Kit, S. and J. Awapara, (i954) J. Biol. Chem., 210 , il.

Kit, S. And D. M. GreenberG, (I95I) Cancer Research, II, 79 I.

Kit, S. and D. M. GreenberG, (1952) 7. Biol. Chem., $194,377$.

Kit, S. and O. L. Graham, (1956a) Cancer Research, i6, i I7.

Kit, S. and O. L. Graham, (1956b) Proc. Am. Assoc. Cancer Research, 2, 125.

Klenow, H., (1953) Arch. Biochem. Biophys., 46, 186.

Klenow, H. and R. Emberland, (1955) Arch. Biochem. Biophys., 58, 276.

Klungsoyr, L., (1954) Acta Chem. Scand., 8, 723.

Knox, W. E. And A. H. Mehler, (1950) 7. Biol. Chem., I87, 4I9.

Korkes, S., A. Del Campillo and S. Ochoa, (1950) 7. Biol. Chem., I87, 89 I.

Korn, E. D. And J. M. Buchanan, (1956) J. Biol. Chem., 217 , 183.

Korn, E. D., C. N. Remy, H. C. Wasilejko and J. M. Buchanan, (i955) F. Biol. Chem., 217,875 .

Kornberg, A., (I950a) 7. Biol. Chem., I82, 779.

Kornberg, A., (1950b) F. Biol. Chem., I82, 805.

Kornberg, A., (1954) in Fat Metabolism, Johns Hopkins Press, Baltimore, p. 150.

Kornberg, A. And W. E. Pricer, (1951) F. Biol. Chem., 193, 48 I.

Kornberg, A. and W. E. Pricer, (1953a) F. Biol. Chem., 204, 329.

Kornberg, A. and W. E. Pricer, (I953b) F. Biol. Chem., 204, 345.

Kornberg, A., I. Lieberman And E. S. Simms, (i955a) 7. Biol. Chem., 215, 389.

Kornberg, A., I. Lieberman and E. S. Simms, (I955b) 7. Biol. Chem., $215,417$.

Kornberg, A., I. R. Lehman and E. S. Simms, (I956a) Federation Proc., 15, 29 I.

Kornberg, A., I. R. Lehman, M. J. Bessman and E. S. Simms, (1956b) Biochim. et Biophys. Acta, 21, 197 .

Krebs, H. A. and R. Hems, (I955) Biochem. 7., 6r, 435.

Krimsky, I. And E. Racker, (I955) Science, I22, 3 I9.

LAgerkvist, (1955) Acta Chem. Scand., 9, 1028.

Lagerkvist, U. and P. Reichard, (1954) Acta Chem. Scand., 8, 36 I.

Lagerkvist, U., P. Reichard, B. Carlsson and J. Grabosz, (I955) Cancer Research, 15, i64. 
Lajtha, L. G., R. Oliver and F. Ellis, (I954) Brit. 7. Cancer, 8, 367.

Lampen, J. O., (1952) Symposium on Phosphorus Metabolism, 2, 363.

Langdon, R. G., (I954) in Fat Metabolism, Johns Hopkins Press, Baltimore, p 162.

Langdon, R. G. and K. Bloch, (1953a) F. Biol. Chem., 200, I29.

Langdon, R. G. and K. Bloch, (1953b) 7. Biol. Chem., 200, I35.

Lanning, M. C. and S. S. Cohen, (1954) J. Biol. Chem., 207, 193.

Lanning, M. C. and S. S. Cohen, (i955) 7. Biol. Chem., 216, 413.

Lansford, E. M., Jr. And W. Shive, (1955) Antimetabolites in the Study of Cancer, Am. Assoc.

Advancement Science, Washington, D. C.

Lardy, H. A. and J. Adler, (1956) 7. Biol. Chem., 219, 933.

Lardy, H. A. And R. Peansky, (r953) Physiol. Revs., 33, 560.

Lehninger, A. L., (I953-1954) Harvey Lectures 39, I 76.

Leloir, L. F., (195I) Symposium on Phosphorus Metabolism I, 67.

Leloir, L. F. and C. E. Gardini, (1953) Biochim. et Biophys. Acta, I2, I5.

Leloir, L. F. and C. E. Cardini, (1956) Biochim. et Biophys. Acta, 20, 33.

LePAGe, G. A., (1953) Cancer Research, 13, I 78.

Levenberg, B. and J. M. Buchanan, (1956) J. Am. Chem. Soc., 78, 504.

Levenberg, B., S. C. Hartman and J. M. Buchanan, (i956) 7. Biol. Chem., 220, 379.

Levintow, L. And A. Meister, (1954) F. Biol. Chem., 209, 265.

Levintow, L. and G. D. Novelli, (1954) 7. Biol. Chem., 207, 76 I.

Levy, L. And M. J. Coon, (1954) F. Biol. Chem., 208, 69 I.

Lieberman, I., (1955) F. Am. Chem. Soc., 77, 266 I.

Lieberman, I., (1956) 7. Am. Chem. Soc., 78, 25 I.

Lieberman, I. and A. KornberG, (1955) 7. Biol. Chem., 212, 909.

Lieberman, I., A. Kornberg and E. S. Simms, (1955a) 7. Biol. Chem., $215,403$.

Lieberman, I., A. Kornberg and E. S. Simms, (1955b) 7. Biol. Chem., 215, 429.

Lien, O. G., JR. and D. M. Greenberg, (I952) Fु. Biol. Chem., I95, 637.

Lin, S. C. and D. M. Greenberg, (i954) F. Gen. Physiol., 38, i81.

Linko, P. And A. I. Virtanen, (i955) Acta Chem. Scand., 9, 855.

Lipmann, F., (r954) in Mechanism of Enzyme Action, Johns Hopkins Press, Baltimore, p. 599.

Lowe, I. P. and E. Roberts, (1955) F. Biol. Chem., $212,477$.

Lowenstein, J. M. and P. P. Cohen, (r954) J. Am. Chem. Soc., 76, 557 I.

Lowenstein, J. M. and P. P. Cohen, (1955) 7. Biol. Chem., 213, 689.

Lowenstein, J. M. and P. P. Cohen, (1956) F. Biol. Chem., 220, 57.

Lowther, D. A. and H. J. Rogers, (1956) Biochem. J., 62, 304.

Lukens, L. N. And J. M. Buchanan, (1956) Federation Proc., 15, 305.

Lynen, F., (1955) Ann. Rev. Biochem., 24, 653.

Maas, W. K. And G. D. Novelli, (I953) Arch. Biochem. Biophys., 43, 236.

Mackler, B., R. Repaske, P. M. Kohout and D. E. Green, (1954) Biochim. et Biophys. Acta, 15, 437.

Machlin, L. J., P. B. Pearson, G. A. Denton and H. R. Bird, (i953) J. Biol. Chem., 205, 213.

Machlin, L. J., P. B. Pearson and C. A. Denton, (1955) F. Biol. Chem., 212, 469.

Magasanik, B., (1956) f. Am. Chem. Soc., 78, 5449.

Magasanik, B., H. S. Moyed and D. Karibian, (1956) J. Am. Chem. Soc., 78, 1510.

Mahler, H. R. and D. G. Elowe, (1953) 7. Am. Chem. Soc., 75, 5769.

Mahler, H. R. and D. G. Elowe, (1954) 7. Biol. Chem., 210 , i65.

Mahler, H. R. and D. E. Green, (I954) Science, I20, 7.

Mahler, H. R., S. J. Wakil and R. M. Bock, (1953) 7. Biol. Chem., 204, 453.

Maley, G. F. and H. A. Lardy, (1954) F. Biol. Chem., $210,903$.

Mannell, W. A. And R. J. Rossiter, (I955) Biochem. 7., 6I, 4 I8.

Manson, L. A. and J. O. Lampen, (I95I) 7. Biol. Chem., 19I, 95.

Manson, L. A. and J. O. Lampen, (1953) F. Biol. Chem., 193, 539.

Mapson, L. W. and E. M. Moustafa, (1956) Biochem. 7., 62, 248.

Marrian, D. H., (1954) Biochim. et Biophys. Acta, I3, 282.

Marshall, R. O., L. M. Hall and P. P. Cohen, (1955) Biochim. et Biophys. Acta, I7, 279. 
Martius, C. and D. Nitz-Litzow, (1953) Biochim. et Biophys. Acta, I2, 134.

Mason, H. S., (1955) Advances in Enzymol., 16, 105.

Matsuo, Y. and D. M. Greenberg, (I955) F. Biol. Chem., 215, 547.

Matsuo, Y., M. Rothstein and D. M. GreenberG, (1956) 7. Biol. Chem., 221, 679.

Mauzerall, D. and S. Granick, (i956) J. Biol. Chem, $219,435$.

Maxwell, E. S., (1956) F. Am. Chem. Soc., 78, i074.

McClure, L. E., R. E. Neuman and T. A. McCoy, (1954) Arch. Biochem. Biophys., 53, 50.

McElroy, W. D. and B. Glass, (1951-1952) Editors, Symposium on Phosphorus Metabolism, 2 Vols., Johns Hopkins Press, Baltimore.

MaElroy, W. D. ANd B. Glass, (1953) Editors, Mechanism of Enzyme Action, Johns Hopkins Press, Baltimore.

McElroy, W. D. and B. Glass, (1954) Editors, Amino Acid Metabolism, Johns Hopkins Press, Baltimore.

McElroy, W. D. And B. Glass, (1955) Editors, Inorganic Nitrogen Metabolism, Johns Hopkins Press, Baltimore.

MaGee, J. And M. Doudoroff, (1954) 7. Biol. Chem., 210, 6 I 7.

McKenzie, C. G. and R. H. Abeles, (1956) 7. Biol. Chem., 222, I45.

McManus, I. R., (1954) 7. Biol. Chem., 208, 639.

McNutt, W. S., (1952) Biochem. F., 50, 384 .

McNutt, W. S., (1954) F. Biol. Chem., 210, 5 Ir.

McNutt, W. S., (1956) 7. Biol. Chem., 219, 365.

Mehler, A. H., (1954) in Amino Acid Metabolism, Johns Hopkins Press, Baltimore, p. 882.

Mehler, A. H., (I956) 7. Biol. Chem., 218, 24 I.

Mehler, A. H. and W. E. Knox, (1950) 7. Biol. Chem., 187, 43 r.

Meinhart, J. O. and S. Simmonds, (1955) 7. Biol. Chem., 213, 329.

Meister, A., (1954) Science, I20, 43.

Meister, A., (1955) Advances in Enzymol., I6, 185.

Meltzer, H. L. And D. B. Sprinson, (1952) F. Biol. Chem., I97, 46 r.

Milhaud, G., A. A. Benson and M. Calvin, (1956) F. Biol. Chem., 218, 599.

Miller, A. And H. Waelsch, (I956) Arch. Biochem. Biophys., 63, 263.

Miller, C. S., S. Gurin and D. W. Wilson, (i950) Science, $112,654$.

Mills, G. T., R. Ondarza and E. E. B. Smith, (I954) Biochim. et Biophys. Acta., I4, I 59.

Miтoma, C., (1956) Arch. Biochem. Biophys., 6o, 476.

Mrtoma, C. And E. E. Snell, (1955) Proc. Natl. Acad. Sci. U.S., 4I, 89 I.

Mitsuhashi, S. And B. D. Davis, (1954a) Biochim. et Biophys. Acta, 15, 54.

Mitsuhashi, S. And B. D. Davis, (1954b) Biochim. et Biophys. Acta, I5, 268.

Mryake, A., A. H. Bokman and B. S. Schweigert, (1954) J. Biol. Chem., 21 , 39 I.

Moore, A. M. And J. B. Boylen, (1955) Arch. Biochem. Biophys., 54, 312.

Munch-Peterson, A., (1955a) Acta Chem. Scand., 9, I523.

Munch-Peterson, A., (1955b) Arch. Biochem. Biophys., 55, 592.

Nakao, A. and D. M. Greenberg, (1955) F. Am. Chem. Soc., 77, 6715.

Nason, A., (1956) Inorganic Nitrogen Metabolism, Johns Hopkins Press, Baltimore, p. Iog.

Nason, A. and H. J. Evans, (1953) 7. Biol. Chem., 202, 655.

Nason, A. And I. R. Lehman, (I955) Science, I22, I9.

Nason, A. and I. R. Lehman, (1956) 7. Biol. Chem., 222, 5 I I.

Nason, A., R. G. Abraham and B. C. Averbach, (1954) Biochim. et Biophys. Acta, I5, I59.

Neidle, A. And H. Waelsch, (i956) J. Am. Chem. Soc., 78, i 767.

Newburgh, R. W. and V. H. Cheldelin, (1955) F. Biol. Chem., 214, 37.

Newburgh, R. W. and V. H. Cheldelin, (1956) F. Biol. Chem., 218, 89.

Newburgh, R. W., C. A. Cluaridge and V. H. Cheldelin, (1955) 7. Biol. Chem., 214 , 27.

Novelli, G. D., (I953) Physiol. Revs., 33, 525.

Ochoa, S. and S. Kaufman, (1951) F. Biol. Chem., I92, 3 I 3.

Ochoa, S., J. B. Veiga-Salles and P. J. Ortiz, (i950) F. Biol. Chem., r87, 863.

Olson, J. A., (1954) Nature, I74, 695.

Ostern, P., J. Terszakowic and S. Hubl, (1938) Z.physiol. Chem., Hoppe-Seyler's, 255, i04.

Paege, L. M. and F. Schlenk, (1952) Arch. Biochem. Biophys., 40, 42. 
Palmer, J. K., (1956) Science, 123, 415.

Peterson, E. A. and D. M. Greenberg, (1952) 7. Biol. Chem., I94, 359.

Peterson, E. A., T. Winnick and D. M. Greenberg, (i95i) 7. Am. Chem. Soc., $73,503$.

Pilgeram, L. O., E. M. Gal, E. N. Sassenrath and D. M. Greenberg, (1953) 7. Biol. Chem., 204, 367.

Plaut, G. W. E., (1951) Proc. Soc. Exptl. Biol. Med., 78, 769.

Plaut, G. W. E., (1954a) 7. Biol. Chem., 208, 513.

Plaut, G. W. E., (1954b) F. Biol. Chem., 21 I, I I I.

Plaut, G. W. E. and P. L. Broberg, (1956) 7. Biol. Chem., 219, i3 I.

Potter, R. L. and S. Schlesinger, (i955) 7. Am. Chem. Soc., 77, 67 I4.

Potter, V. R., L. I. Hecht and E. Herbert, (i956) Biochim. et Biophys. Acta, 20, 439.

Prusoff, W. H. and L. G. Lajtha, (1956) Federation Proc., 15, 33 I.

Prusoff, W. H., L. G. Lajtha and A. D. Welch, (1956) Biochim. et Biophys. Acta, 20, 209.

Purko, M., W. O. Nelson and W. A. Wood, (r954) 7. Biol., Chem., 207, 5 I.

Rabinowitz, J. L., (i954) J. Am. Chem. Soc., 76, 3037.

Rabinowitz, J. L. and S. Gurin, (1953) Biochim. et Biophys. Acta, io, 345.

Rabinowitz, J. L. and S. Gurin, (i954) 7. Am. Chem. Soc., 76, 5 I68.

Rachele, J. R., L. J. Reed, A. R. Kidwai, M. F. Ferger and V. Du Vigneaud, (i950) 7. Biol. Chem., I85, 8 I 7 .

RACKer, E., (I95I) Nature, I67, 408.

Racker, E., (1952) 7. Biol. Chem., 196, 347.

RACKer, E., (1954) Advances in Enzymol., 15, 141 .

Racker, E., (I955a) Physiol. Revs., 35, I.

Racker, E., (1955b) F. Biol. Chem., 217, 855.

Rafelson, M. E., (1955a) 7. Am. Chem. Soc., 77, 4679 .

Rafelson, M. E., (1955b) 7. Biol. Chem., 213, 479.

Rall, T. W. and A. L. Lehninger, (1952) J. Biol. Chem., I94, i ig.

Ratner, S., (1955) in Amino Acid Metabolism, Johns Hopkins Press, Baltimore, p. 23 I.

Ravel, J. M., L. Woods, B. Felsing and W. Shive, (i954) J. Biol. Chem., 206, 39 I.

Reed, L. J., (1953) Physiol. Revs., 33, 544.

Reed, D. J., B. E. Christensen, V. H. Cheldelin and C. H. Wang, (i954) J. Am. Chem. Soc., 76, 5574.

Reichard, P., (1952) F. Biol. Chem., 197, 39 I.

Reichard, P., (I955) Acta Chem. Scand., 9, 1275.

Reichard, P. and B. Estborn, (195I) 7. Biol. Chem., I88, 839.

Reichard, P. and G. Hanshoff, (1955) Acta Chem. Scand., 9, 519.

Reichard, P. and U. Lagerkvist, (1953) Acta Chem. Scand., 7, I 207.

Reichard, P. And L. H. Smith, (I955) Acta Chem. Scand., 9, I94.

Reichard, P., L. H. Smith and G. Hanshoff, (1955) Acta Chem. Scand., 9, io io.

Reid, J. C. And M. O. Landefeld, (I95I) Arch. Biochem. Biophys., 34, 219.

Reid, J. C., M. O. Landefeld and J. L. Simpson, (I952) F. Natl. Cancer Inst., I2, 929.

Reissig, J. L., (I956) F. Biol. Chem., 219, 753.

Robbins, P. W. and F. Lipmann, (1956) 7. Am. Chem. Soc., 78, 2652.

Roberts, E. And S. Frankel, (I949) Cancer Research, 9, 645.

Roberts, E. And T. TANaka, (1956) Cancer Research, 16, 204.

Robinson, W. G., B. K. Bachhawat and M. J. Coon, (i955) Federation Proc., I4, 270.

Roll, P. M., H. Weinfeld, E. Carroll and G. B. Brown, (I956a) J. Biol. Chem., 220, 439.

Roll, P. M., H. Weinfeld and E. Carroll, (1956b) 7. Biol. Chem., 220, 455.

Rose, I. A. And B. S. Schweigert, (1953) 7. Biol. Chem., 202, 635.

Rose, W. C., (1949) Federation Proc., 8, 546.

Rothschild, H. A., O. Cori and E. S. Guzman-Barron, (i954) F. Biol. Chem., 208, 41.

Rowen, J. W. and A. Kornberg, (I951) 7. Biol. Chem., I93, 497.

Rutman, R. J., A. Cantarow, K. E. Paschis and B. Allanoff, (1953) Science i17, 282.

Ryan, K. J. And L. L. Engel, (1956) 7. Am. Chem. Soc., 78, 2654.

Sable, H. Z., (1950) Proc. Soc. Exptl. Biol. Med., 75, 215. 
Sable, H. Z., P. B. Wilber, A. E. Cohen, and M. R. Kane, (1954) Biochim. et Biophys. Acta, I3, I 56 .

Sagers, R. D., J. V. Beck, W. Gruber and I. G. Gunsalus, (i956) F. Am. Chem. Soc., 78, 694 .

Sakami, W., (1948) J. Biol. Chem., I76, 995.

Sallach, H. J., (I954) in Amino Acid Metabolism, Johns Hopkins Press, Baltimore, p. 782.

Sanadi, D. R., D. M. Gibson, P. Ayengar and M. Jacob, (1956) J. Biol. Chem., $218,505$.

San Pietro, A. and H. M. Lang (1956) Science, 124, 118.

SAto, R., (1956) in Inorganic Nitrogen Metabolism, Johns Hopkins Press, Baltimore, p. I63.

Saz, H. J. And E. P. Hillary, (1956) Biochem. 7., 62, 563.

Schlenk, F. And R. L. Smith, (1953) 7. Biol. Chem., $204,27$.

Schmid, R. And D. Shemin, (1955) 7. Am. Chem. Soc., 77, 506.

Schmiтz, H., (i954a) Biochim. et Biophys. Acta, I4, I60.

Schmitz, H., (1954b) Biochem. Z., 325, 555.

Schmitz, H., V. R. Potter, R. B. Hurlbert and D. M. White, (i954a) Cancer Research, I4, 66.

Schmitz, H., V. R. Potter and R. B. Hurlbert, (i954b) 7. Biol. Chem., 209, 4I.

Schmitz, H., W. Hart and H. Ried, (I955a) Z. Krebsforsch., 6o, 30 I.

Schmitz, H., V. R. Potter and R. B. Hurlbert, (1955b) Z. Krebsforsch., 6o, 4 ig.

SChNeider, W. C., (I955) 7. Biol. Chem., $216,287$.

Schneider, W. C. and L. W. Brownell, (1956) Federation Proc., I5, 349.

Schoenhermer, R., (I942) The Dynamic State of Body Constituents, Harvard University Press, Cambridge.

Schrecker, A. W. and A. Kornberg, (1950) J. Biol. Chem., I82, 795.

Schulman, M. P. And S. J. Badger, (1954) Federation Proc., I3, 292.

Schurr, P. E., H. T. Thompson, L. M. Henderson, J. N. Williams and C. A. Elvehjem, (1950) 7. Biol. Chem., I82, 39.

Schwartz, S., (1955) Federation Proc,, I4, 7 I 7.

Schweet, R. S., M. Fuld, K. Sheslock and M. H. Paul, (r95 I) Phosphorus Metabolism I, 246.

Schwenk, E., G. J. Alexander, C. A. Fish and T. H. Stoudt, (i955) Federation Proc., I4, 752.

Seegmiller, J. E., L. Laster and D. Stetten, (i955) F. Biol. Chem., 2i6, 653.

SEGAl, H. L., (1956) Biochim. et Biophys. Acta, 2I, I94.

Shemin, D., (1946) 7. Biol. Chem., I62, 297.

Shemin, D. and C. S. Russell, (1953) J.Am. Chem. Soc., 75, 4873.

Shemin, D. And J. Wittenberg, (I95I) J. Biol. Chem., 192, 3 I 5.

Shemin, D., C. S. Russell and T. Abramsky, (1955) 7. Biol. Chem., 215, 6 I 3.

Siekevitz, P., (1952) 7. Biol. Chem., 195, 549.

Siekevitz, P. And D. M. Greenberg, (1949) F. Biol. Chem., r8o, 845.

Silver, W. S. and W. D. McElroy, (1954) Arch. Biochem. Biophys., 5I, 379.

Singer, T. P. And E. B. Kearney, (1956) Arch. Biochem. Biophys., 6I, 397.

Singer, T. P., E. B. Kearney and V. Massey, (i956) Arch. Biochem. Biophys., 6o, 255.

Siperstein, M. D. And A. W. Murray, (I956) Science, I23, 377.

Smith, E. E. B. and G. T. Mills, (1954) Biochim. et Biophys. Acta, 13, 386.

Smith, E. E. B. And G. T. Mills, (1954a) Biochim. et Biophys. Acta, 13, 587.

Smith, L. H. And D. Stetten, (1954) 7. Am. Chem. Soc., 76, 3864.

Smith, R. A. and I. G. Gunsalus, (1954) 7. Am. Chem. Soc., 76, 5002.

Smyrniotis, P. Z. And B. L. Horecker, (1956) J. Biol. Chem., $218,745$.

Snell, E. E., (1953) Physiol. Revs., 33, 509.

Snoke, J. E. And K. Bloch, (1952) 7. Biol. Chem., 199, 407.

Snoke, J. E., S. Yanari And K. Blogh, (I953) F. Biol. Chem., 20I, 573.

Solomon, J. D., G. A. Johnson, A. L. Scheffner and O. Bergeim, (195 I) 7. Biol. Chem., $I 89,629$.

Sonne, J. C., I. Lin And J. M. Buchanan, (i956) F. Biol. Chem., 220, 369.

Speck, J. F., (1949) F. Biol. Chem., I79, 1405. 
Spicer, D. S., K. U. Liebert, L. D. Wright and J. W. Huff, (i952) Proc. Soc. Exptl. Biol. Med., 79, 587 .

Spiegelman, S., H. O. Halvorson and R. Ben Ishai, (1955) in Amino Acid Metabolism, Johns Hopkins Press, Baltimore, p. 124.

Srere, P. A., J. R. Cooper, V. Kybas and E. Racker, (1955) Arch. Biochem. Biophys., 59, 535.

Srinivasan, P. R., M. Katagiri and D. B. Sprinson, (i955) 7. Am. Chem. Soc., 77, 4943.

Srinivasan, P. R., H. T. Shigeura, M. Spregher, D. B. Sprinson and B. D. Davis, (I956) 7. Biol. Chem., 220, 477 .

Stafford, H. A., A. Magaldi and B. Vennesland, (1954) F. Biol. Chem., 207, 62 I.

Stekol, J. A., (1954) in Amino Acid Metabolism, Johns Hopkins Press, Baltimore, p. 509.

Stern, H. And A. E. Mirsky, (1954) J. Gen. Physiol., 38, 4 I.

Stern, H. and S. Timonen, (1952) 7. Gen. Physiol., 36, i8 I.

Stern, J. R., M. J. Coon and A. Del Campillo, (1956a) F. Biol. Chem., 221 , I.

Stern, J. R., M. J. Goon, A. Del Campillo and M. G. Schneider, (1956b) F. Biol. Chem., $221, \mathrm{I} 5$.

Stern, J. R., A. Del Campillo and I. Raw, (1956c) 7. Biol. Chem., 218, 97 I.

Stetten, M. R., (I949) 7. Biol. Chem., I8I, 3 I.

Stetten, M. R., (195I) F. Biol. Chem., I89, 499.

Stetten, M. R., (1955) in Amino Acid Metabolism, Johns Hopkins Press, Baltimore, p. 277.

Stetten, D., JR. And B. Bloom, (1956) J. Biol. Chem., 220, 723.

Strassman, M. and S. Weinhouse, (1953) 7. Am. Chem. Soc., 75, i68o.

Strassman, M., A. J. Thomas and S. Weinhouse, (1953) 7. Am. Chem. Soc., 75, 5135.

Strassman, M., A. J. Thomas and S. Weinhouse, (1955) 7. Am. Chem. Soc., 77, 126 I.

Strassman, M., A. J. Thomas, L. A. Locke and S. Weinhouse, (1954) J. Am. Chem. Soc., 76,4241 .

Strassman, M., A. J. Thomas, L. A. Locke and S. Weinhouse, (1956) J. Am. Chem. Soc., $78,228$.

Strecker, H. J. And P. Mela, (1955) Biochim. et Biophys. Acta, I7, 580.

Strength, D. R. And M. A. Seibert, (1955a) Proc. Natl. Acad. Sci. U.S., 4I, 609.

Strength, D. R. and M. A. Seibert, (1955b) Proc. Am. Assoc. Cancer Research, 2, 49.

Strittmatter, C. F. and E. G. Ball, (1954) 7. Cellular Comp. Physiol., 43, 57.

Strittmatter, P. And S. F. Velick, (i956a) J. Biol. Chem., 22I, 253.

Strittmatter, P. and S. F. Velick, (i956b) 7. Biol. Chem., 22I, 277.

Strominger, J. L., (1955) Biochim. et Biophys. Acta, 17, 283.

Strominger, J. L., H. M. Kalckar, J. Axelrod and E. S. Maxwell, (i954) F. Am. Chem. Soc., 76, 64I I.

Stumpf, P. K. And B. L. Horecker, (1956) 7. Biol. Chem., $218,753$.

Tabachnick, M. and H. Tarver, (I955) Arch. Biochem. Biophys., 56, I 5.

Tanenbaum, S. W., (1956) F. Biol. Chem., $218,733$.

Taniguchi, S., R. Sato And F. Egami, (1956) in Inorganic Nitrogen Metabolism, Johns Hopkins Press, Baltimore, p. 87.

Tatum, E. L. and S. R. Gross, (1956) F. Biol. Chem., 219, 797.

Tatum, E. L. And D. Shemin, (1954) 7. Biol. Chem., 209, 67 I.

Tchen, T. T. and K. Bloch, (1956) 7. Am. Chem. Soc., 78, i 517.

Teas, H. J., N. H. Horowitz and M. Fling, (I948) J. Biol. Chem., I72, 65 I.

'Thomas, R. C., V. H. Cheldelin, B. E. Christensen and C. H. Wang, (i953) F. Am. Chem. Soc., 75, 5554 .

'Tolbert, N. E. And M. S. Cohan, (i953) 7. Biol. Chem., 204, 649.

'Totter, J. R., (1954) 7. Am. Chem. Soc., 76, 2196.

Totter, J. R. And A. N. Best, (1955) Arch. Biochem. Biophys., 54, 318.

Totter, J. R., E. Volkin and C. E. Carter, (i95 I) F. Am. Chem. Soc., 73, 1521.

Trudinger, P. A., (1955) Biochim. et Biophys. Acta, I8, 58 I.

Tung, T. C., K. H. Ling, W. L. Byrne and H. A. Lardy, (I954) Biochim. et Biophys. Acta, I4, 488.

Udenfriend, S. and C. Mrtoma, (1955) in Amino Acid Metabolism, Johns Hopkins Press, Baltimore, p. 876 . 
Umbarger, H. E. and B. Magasanik, (195 I) 7. Biol. Chem., i89, 287.

Umbarger, H. E. and J. H. Mueller, (195I) 7. Biol. Chem., 189, 277.

Utter, M. F. and K. Kurahashi, (1954a) 7. Biol. Chem., 207, 787.

Utter, M. F. and K. Kurahashi, (1954b) 7. Biol. Chem., 207, 82 I.

Utter, M. F., K. Kurahashi and I. A. Rose, (1954) 7. Biol. Chem., $207,803$.

Veiga-Salles, J. B. and S. Ochoa, (i950) 7. Biol. Chem., I87, 849.

Velick, S. F. and P. Strittmatter, (1956) 7. Biol. Chem., $221,265$.

Verhoeven, W., (1956) in Inorganic Nitrogen Metabolism, Johns Hopkins Press, Baltimore, p. 6 I.

Verhoeven, W. And Y. Takeda, (1956) in Inorganic Nitrogen Metabolism, Johns Hopkins Press, Baltimore, p. I59.

Villavicencio, M. and E. S. Guzman-Barron, (1955) Federation Proc., I4, 297.

Virtanen, A. I. and P. Linko, (i955) Acta Chem. Scand., 9, 53 I.

Virtanen, A. I. And N. E. Saris, (i955) Acta Chem. Scand., 9, 337.

Vishniac, W. and S. Ochoa, (1952) F. Biol. Chem., 198, 5 O I.

Vogel, H. J., (1956) J. Am. Chem. Soc., 78, 2631.

Vogel, H. J. and D. M. Bonner, (1956) J. Biol. Chem., $218,97$.

Wainio, W. W. and S. J. Cooperstein, (1956) Advances in Enzymol., I7, 329.

Wainwright, S. D., (I955) Biochim. et Biophys. Acta, I8, 583 .

Waisman, H. A., C. Monder and J. N. Williams, Jr., (1956) Cancer Research, i6, 344.

Walker, J. B., (1956) J. Biol. Chem., 22I, 77 I.

Wang, T. P. and J. O. Lampen, (1952) J. Biol. Chem., 194, 775.

Wang, C. H., B. E. Christensen and V. H. Cheldelin, (i 955 ) 7. Biol. Chem., $213,365$.

Wang, C. H., J. W. Davis, V. H. Cheldelin and B. E. Christensen, (1954) Nature, I74, I 145 .

Wang, T. P., H. Z. Sable and J. O. Lampen, (1950) 7. Biol. Chem., I84, I7.

Warburg, O., (I956) Science, I23, 309.

Warren, L. and J. G. Flaks, (I956) Federation Proc., 15, 379.

Webster, G. C. And J. E. Varner, (I955) Arch. Biochem. Biophys., 55, 95.

Weed, L. L. And D. W. Wilson, (I95 I) F. Biol. Chem., I89, 435.

Weed, L. L. ANd D. W. Wilson, (I954) J. Biol. Chem., 207, 439.

Weinhouse, S. and B. Friedmann, (1951) 7. Biol. Chem., I9I, 707.

Weiss, U. and E. S. Mingioli, (1956) F. Am. Chem. Soc., 78, 2894.

Weiss, U., C. Gilvarg, and E. S. Mingioli and B. D. Davis, (1954) Science, i19, 774.

Weissbach, A. And B. L. Horecker, (r955) in Amino Acid Metabolism, Johns Hopkins Press,

Baltimore, p. 741 .

Weissbach, A., B. L. Horecker and J. Hurwitz, (i956) 7. Biol. Chem., 2i8, 795.

Wenner, C. E. And S. Weinhouse, (i956a) F. Biol. Chem., 219, 69i.

Wenner, C. E. and S. Weinhouse, (1956b) F. Biol. Chem., 222, 399.

Werkheiser, W. C. And D. W. Visser, (1955) Cancer Research, 15, 644.

Westley, J. and J. Ceithaml, (1956) F. Biol. Chem., 219, i39.

Williams-Ashman, G., (1953) Cancer Research, 13, 72 I.

Whatley, F. R., M. B. Allen, L. L. Rosenberg, J. B. Capindale and D. I. Arnon, (1956)

Biochim. et Biophys. Acta, 20, 462.

Whiteley, H. R., (1953) F. Am. Chem. Soc., 75, I 518.

Wilson, A. T. and M. Calvin, (1955) 7. Am. Chem. Soc., 77, 5948.

Wilson, L. G. and R. S. Bandurski, (1956) Arch. Biochem. Biophys., 62, 503.

Windsor, E., (195I) 7. Biol. Chem., I92, 607.

Winnick, T., F. Friedberg and D. M. Greenberg, (i948) 7. Biol. Chem., I75, i i 7.

Wittenberg, J. And A. Kornberg, (1953) F. Biol. Chem., 202, 43 I.

Wong, D. T. O. And S. J. AJL, (1956) 7. Am. Chem. Soc., 78, 3230.

Woods, D. D., (1954) Chemistry and Biology of Pteridines, Little, Brown and Co., Boston, p. 220.

Wosilait, W. D. and A. Nason, (1954a) f. Biol. Chem., 208, 785.

Wosilait, W. D. and A. Nason, (1954b) 7. Biol. Chem., 206, 255.

Wosilait, W. D., A. Nason and A. J. Terrell, (1954) J. Biol. Chem., 206, 27 I. 
Wright, B. E., (1955) Biochim. et Biophys. Acta, 16, I65.

Wright, B. E., (1956) 7. Biol. Chem., 219, 873 .

Wright, B. E. and T. C. Stadtman, (1956) F. Biol. Chem., 219, 863.

Wright, L. D. and C. A. Driscoll, (i954) J. Am. Chem. Soc., 76, 4999.

Wright, L. D. And C. S. Miller, (1952) Proc. Soc. Exptl. Biol. Med., 81, I3I.

Wriston, J. C., L. Lack and D. Shemin, (1955) J. Biol. Chem., $215,603$.

Wu, G., (1954) J. Biol. Chem., 207, 775.

Yaniv, H. and C. Gilvarg, (1955) Fै. Biol. Chem., $213,787$.

Yanofsky, C., (1954) in Amino Acid Metabolism, Johns Hopkins Press, Baltimore, p. 930.

Yanofsky, C., (1955a) Biochim. et Biophys. Acta, 16, 594.

Yanofsky, C., (1955b) J. Biol. Chem., 2I7, 345.

Yanofsky, C., (1956) Biochim. et Biophys. Acta, 20, 438.

Yates, R. A. and A. B. Pardee, (I956) 7. Biol. Chem., $221,743$.

Yura, T. And H. J. Voget, (1955) Biochim. et Biophys. Acta, I7, 582.

Zamecnik, P. C. And E. B. Keller, (i954) F. Biol. Chem., 209, 337.

Zelitch, I., (1953) F. Biol. Chem., 20I, 719.

Zelitch, I. and S. OchoA, (1953) F. Biol. Chem., $201,707$.

Zugker, M. and A. Nason, (1955) J. Biol. Chem., $213,463$. 


\section{Chapter 2}

\section{PRINGIPLES AND THEORY OF GROWTH}

\section{Ludwig von Bertalanffy}

\section{I . INTRODUGTION}

(a) Definition of growth

The term, Growth, denotes those changes of a living system that are manifest as measurable changes, and particularly as an increase in size. It is, however, obvious that such definition needs qualification. Increase of body weight, for example, by mere intake of water or deposition of fat is not considered as growth. A better delimitation is reached if growth is connected with a basic characteristic of living systems, namely, metabolism. Every living system maintains itself in a continuous exchange, anabolism and catabolism of its components. Hence we may define: Growth is the quantitative increase of a living system which results from the prevalence of anabolism of building materials over catabolism.

Building materials of the organism are defined as components which-in contradistinction to low-molecular compounds like water, sugar, etc.-not only pass the living organism but are produced in anabolic processes as complicated compounds specific to the organism in question. These, in turn, give rise to a wealth of biological structures above the chemical level. Hence growth is the increase in size of living systems which results from assimilation, i.e. production of materials which are specific for the living system in question, and are produced by multiplication of already existing protoplasm.

In this sense, growth of a living system, being a quantitative increase, is distinguished from differentiation, that is, increase in organization and heterogeneity within a living system.

Although these definitions are clear in the abstract, their implementation in the concrete case is by no means unequivocal. This is the reason why there is considerable argument regarding the definition of growth (e.g. Weiss, I 949, I 955a; Linzbach, 1955).

Assimilation is the transformation of non-living nutritive materials into living protoplasm and its derivatives. This is essentially meant by the term, "building material" of a living system. In this sense, growth may be defined (Rössle, I 923) as "increase of structurally and functionally complete biomass" (or protoplasm).

In so far as normal physiology is concerned, we shall exclude pathological changes and accept as growth an increase in size only in so far as it remains within "normality", as is implied in Rössle's definition. We do not consider the notion 
of "normality" so introduced a scientifically inadmissible value judgment; for we may define as "normal" that coordination of parts and processes in the organism which guarantees its maintenance (Bertalanffy, I946). This is not a subjective point of view, but an objective criterion. However, in no way is this criterion easily applicable.

Protoplasm is a notion whose extent cannot easily be delimited. For example, a high content of water (about 80 percent) belongs to the living protoplasm. As a rule, water content decreases during embryonic and post-embryonic development. There are, however, instances to the contrary. Thus the increase in weight of a toad's larva is essentially based upon water intake. At the time of hatching, dry substance represents 42 percent of total weight; up to the 32 nd day of larval development, it decreases to less than 4 percent; and only in metamorphosis does it increase again to about 14 percent, with a concurrent loss of total weight (Wurmbach, after Harms, I955). The relation of these changes to hormonal factors in amphibian metamorphosis (thyroxin) is obvious. Changes in water content are considered to be generally characteristic for the development of vertebrates (Linzbach, I955; Harms, I955). In the example mentioned, the considerable intake of water is part of the "normal" life cycle of the toad larva, and "biologically complete biomass" is formed. It depends solely on the viewpoint taken whether total weight increase is considered as growth and hence water is included; or whether we are interested in growth as a process chemically defined, say, as protein synthesis, in which case we have to exclude change in water content and will choose protein content as standard.

Similar considerations apply to the accumulation of depot material. It is plausible not to consider the deposit of fat in a fattened swine as legitimate growth. But a certain amount of depot materials, such as fat, glycogen, etc., and its proportionate increase during growth is a prerequisite for normal function of the organism, as it is indispensable for continuous activity with intermittent import of nutrition. There is no possibility, however, to delimitate precisely the "normal" amount of such materials ( $f f$. p. 222). Similarly, gross-weight increase passes from protein to fat in the older rat, and in the ageing human fat also is deposited. These phenomena are within the "normal" life cycle of the species concerned, and the biomass so formed is as "functionally complete" as it can be in the age concerned. Again it depends on our viewpoint whether we include or exclude such changes when we speak of growth, depending upon whether the organism as a whole, certain chemical mechanisms, or adipose tissue is the object of study.

The difficulties of an exact delimitation of "biomass" are made particularly clear by the results of isotope experiments. These show that depot materials such as fat and seemingly inactive components such as bone are far from being stable substances. Rather they participate in the continuous breaking down and regeneration within the organism, showing an amazingly high rate of turnover.

Considerations of this kind make it difficult to give the definition of growth a precise chemical meaning. There is no doubt that the growth of living systems is essentially based upon units capable of self-reproduction which, on the basis of present knowledge, can be defined as nucleoproteins. But it is hardly possible to identify growth with the self-reproduction of these units since there are non- 
reproductive structures, intracellular as well as intercellular (fibers, membranes, shells, etc.) that grow. A more restricted definition was proposed by Linzbach (1955), according to whom growth is any change-in-size of an organism "which goes along with an identical self-reproduction of structured nucleoproteins". Although there is little factual criticism of this definition, it will hardly be of help for better delimitation in dubious cases such as those mentioned, and it introduces a hypothetical element, in so far as changes in nucleoprotein content would have to be determined in any special case.

It is obvious that growth cannot be equated with cell multiplication although, as a rule, it is effectuated by it. There is cell multiplication without growth, as for example, in cleavage and gastrulation; and growth without cell multiplication, as in cell-constant animals and tissues (p. I 66).

Furthermore, if growth and differentiation are contrasted, this is possible in the abstract but not in the concrete case. In a crude approximation, it may be said that during the embryonic period of an animal the development of its organization, the histological, physiological, and biochemical differentiation of organs, tissues and cells take place; in post-embryonic life the organization thus established increases in size. However, there is no sharp borderline. Embryonic development also is a period of rapid increase in size, and processes of differentiation continue over the post-embryonic life cycle.

Thus, a definition of growth is meaningful only if it is clearly understood that in general growth is not an isolable phenomenon but rather a certain aspect of the process of life. A definition of growth can only be of an "operational" nature, and its value is exactly equal to what it may offer for a specific problem of research. Growth is an abstraction; only in this sense can one discuss foundations, theory and laws of growth.

\section{(b) Various aspects of the problem of growth}

Every living organism represents a hierarchy of organized systems. Hence, the problem of animal growth must be envisaged from different viewpoints and at various levels. Consequently, what is termed "growth" from a certain viewpoint need not be termed so from another viewpoint.

For example, if the growth of a tumor is investigated, this naturally is different from normal growth of the organism as a whole. The adipose tissue of an ageing human "grows" but not the individual. In a starving larva or fish, growth in length may continue while the body loses weight. A tissue standing in constant cell renewal like the epidermis "grows" during the full lifespan of the organism, but the adult organism in which this process takes place is in a steady state.

Growth in the biological sense is intimately connected with metabolism and with the hierarchic organization of living systems in self-reproducing units (Linzbach, I955). If the molecule of a chemical compound increases in size, it changes into another chemical compound. An organism, however, represents a system consisting of units (proteins, nucleoproteins, cells, etc.) which may increase in number but remain of the same kind within their respective species. Only this fact allows for growth as increase in size of a system which remains specifically identical. 
The more important aspects of the problem of growth can be enumerated as follows:

I. Growth as synthesis of species-specific substances. Growth signifies, first, the synthesis of high-molecular organic compounds, especially of proteins. This does not merely mean that proteins are synthesized, but proteins are produced in such way that their species, tissue, cell, and even individual specificity is maintained.

2. Growth as identical reproduction. Among the components of the cell, there are certain systems that show a characteristic not found in inanimate ones, namely, the capability of self-reproduction. These may be termed as "elementary biological units"; genes and viruses are the most important representatives. Besides selfreproduction, these systems show the further characteristic that they direct cell processes and, so far as genes are concerned, particularly direct protein synthesis in the way of the specificities mentioned. According to modern research, selfduplication is essentially connected with nucleoproteins.

3. Growth as a cellular process. The growth of an organism takes place as a cellular process by cell multiplication (mitosis), increase in cell size, and formation of intercellular substances. The surplus of anabolism over catabolism leads first to an increase of cell size. However, in spite of continuous potency for synthesis, a cell cannot surpass a certain size so that cell division takes place after this size has been reached.

4. Growth of the organism as a whole. Surveying the development of a multicellular organism, a considerable number of isolable component processes can be distinguished (J. Needham, I942). For the present consideration the following are the more important: (a) Formative movements, i.e. migrations and displacement of cells (gastrulation, invaginations in the formation of germ layers, etc.);(b) Segregation, i.e. progressive determination and separation of embryonic areas and organ anlagen;-(c) Differentiation, i.e. specialization of the structure and function of cells;-(d) Growth, i.e. enlargement and multiplication of cells, and consequent increase of body size. To a certain extent these processes are independent or can be isolated in experiment. Early embryonic processes (gastrulation, neurulation) take place without increase in size as the embryo does not have an intake of nutrition during this period. Segregation may take place during or late in segmentation (regulation ova) or may have taken place before segmentation (mosaic ova). Cell multiplication may or may not go along with simultaneous differentiation, etc.

5. Growth as a morphogenetic process. Animal development leads to the establishment of certain shapes or forms. In early developmental stages, morphogenetic changes take place essentially by way of formative movements. In later stages they are mainly caused by relative growth, i.e. different growth rates exhibited in various directions or by various components of the body. In this sense the problem of organic form is to a wide extent a problem of growth.

This viewpoint applies not only to ontogenetic, but also to phylogenetic changes. Evolutionary changes to a wide extent are changes in proportion, i.e. morphogenetic changes accomplished by change of the rates of relative growth. Hence, many evolutionary changes can be considered as problems of growth.

6. Factors of growth. Animal growth is determined or influenced by a great number of factors which are either in the organism itself, or in its environment. 
To the first category belong genetic factors, hormones, processes of ageing and differentiation, etc.; to the second, nutrition, vitamins, temperature, life space, density of population, etc. A detailed discussion of the factors influencing growth would widely surpass this presentation. Some of them, however, will be considered at the adequate place.

\section{(c) Growth and differentiation}

There is an apparent antithesis between growth and differentiation of the organism. Embryonic and undifferentiated cells, as e.g. mesenchymal elements, as a rule manifest the largest growth capacity, while highly differentiated elements such as nerve and muscle cells are incapable of division in post-embryonic life. Similarly, it is a general rule, although with many exceptions, that regenerative potencies decrease with progressive differentiation and evolutionary progress. According to an often-stated theory (Minot and others), progressive differentiation sets a limit to cell division and hence to growth: Undifferentiated protoplasm grows at a constant rate, but differentiation continually diminishes its amount, and so leads to a progressive decrease of growth rate (Schmalhausen, I93 I).

Notwithstanding the general antagonism which, as a rule, exists between growth and differentiation, this viewpoint is evidently incorrect (Bertalanffy, i95ia; Linzbach, I955). Many undifferentiated cells in the organism do not multiply although they have the ability to reproduce. Such is the case, e.g. in a hydra or planarian whose undifferentiated cells do not divide but immediately start to do so in regeneration after an injury. The same is true of many vertebrate tissus, cells of which do not divide in the adult organism but do divide in tissue culture after explantation. Thus the growth of a tissue or organ does not simply vary with the ratio between reproductive and non-reproductive cells. Conversely, the degree of differentiation of a cell is no measure of its ability to grow and divide. Cells of cell-constant animals or organs can show a vast increase of size without division ( $c f$. p. 166 and Fig. 4, p. I60). The cells of the liver are rather highly specialized and do not divide in normal life. However, after extirpation of the larger part of the rat liver compensatory hyperplasia takes place with a growth rate surpassing that of malignant tumors. The same is shown by quantitative considerations. For example, the heart is a highly differentiated and probably cell-constant muscle, but it retains a high allometry constant $(\alpha=0.95, c f$. Fig. 4) from early developmental stages to maturity. The highly differentiated skeletal musculature of man grows faster than the rest of the body. Heart and liver are post-mitotic tissues (cf. p. I63), but they show higher relative growth than the spleen which remains mitotic during the whole life. Examples of this kind which can easily be multiplied, demonstrate that notwithstanding the general antagonism between growth and differentiation, the latter by itself is not a causative factor entailing the disappearance of the ability to grow and reproduce in the cells, or the cessation of growth in the organism as a whole.

\section{(d) Limitations of growth}

The size limit of an organism is determined genetically and, at least in vertebrates and insects, by way of gene-dependent hormones. 
The maximum size in a species or taxonomic group is determined by the plan of organization and environmental conditions. Well-known examples are the insects, the heavy chitin skeleton of which prohibits surpassing a relatively small size limit, or the whales which, because of the density of the aquatic medium, can reach a far greater size and body weight than terrestrial animals.

An excellent survey of minimum and maximum sizes in the various taxonomic groups as caused by organization and environment was given by Rensch (1954). Rensch discusses in detail that allometric growth (p. 246), with change of body size, leads to complex changes of proportion of organs and physiological functions which become intolerable beyond a critical order of magnitude, thus setting a lower and upper limit of body size.

In this connection only one point will be mentioned which is closely related to theoretical concepts to be presented later. In many physiological activities absolute body size is the predominant factor governing the rate of processes, in spite of otherwise enormous anatomical, physiological, and ecological differences.

This applies, for example, to total metabolism or pulse and respiratory frequency. The latter maintain the same relation to body size over seven orders of magnitude, from a dwarf bat weighing $4 \mathrm{~g}$ to an elephant of 2,00o kg (Fig. 35, p. 233). The predominant role of body size is particularly well illustrated by the fact that arctic as well as tropical animals fit into the "mouse-elephant curve" of basal metabolic rate (proportionality to $W^{3 / 4}, c f$. p. 2 I 8 ), so that metabolic rate is essentially determined by its relation to body weight and is not adaptive (Scholander et al., I950). This corresponds to the fact that, as shown by measuring of models (Krumbiegel, I933), absolute size plays a much more inportant role in the surface-volume ratio (and hence the heat economy of warm-blooded animals) than the shape of the body.

In the allometric equation (p. 224) the constant $\alpha$ (slope of the allometric regression line) indicates the size-dependence of the process under consideration. The constant $b$ (the validity of allometric relationship presupposed) expresses taxonomical, physiological, ecological, etc. peculiarities of the group of species investigated. Benedict's (1938) objection that logarithmic plots may obscure species-specific differences is therefore unfounded because such plots rather lead to recognizing deviations from the general trend and so indicate such peculiarities if present.

In many respects surfaces of the body appear to be a limiting factor of body size. This is particularly clear in the relations between intestinal surface and body size (Hesse, I 927; Bertalanffy, I 940a, I951a; Harms, I955). For example, among coelenterates species with a smooth cylindrical gut are small, while tubularians and anthozoa, with the development of a larger intestinal surface by invaginations, reach considerable sizes. In intraspecific comparison, the relation between intestinal surface and growth was demonstrated in planarians. The same principle even applies to mammals: Dogs of larger races have a greater development of intestinal surface than smaller ones. It appears that in lower metazoa such as coelenterates and flatworms resorbing surfaces act as growth-limiting factor, while in higher animals correlations between respiration and growth can be established (p. 180). 


\section{(a) Introduction}

A modern investigation of the problem of growth must start from the fact that any living organism, as well as its components, is an open system which, as a rule, approaches a steady state.

Conventional physical theory (kinetics and thermodynamics) is limited to closed systems, i.e. systems that do not exchange matter with environment. However, the elementary fact of metabolism shows the organism to be an open system, maintaining itself in continuous exchange, import and export, building up and breaking down of its components. The laws of open systems are specific and peculiar; their investigation has led to an important expansion and generalization of physical theory.

Up to recent times there was no theory applying to systems of such kind. Biology and physiology rather tried to employ the principles and laws of closed systems to processes in the living organism. This is true of chemical kinetics which succeeded in applying the laws of reversible reactions and chemical equilibria to certain partial systems or, speaking more precisely, to certain fast reactions within the living organism. For example, the transport of oxygen from the lungs to the blood and the tissues is based upon reversible reactions and chemical equilibria between hemoglobin, oxyhemoglobin, and oxygen. In contrast, the laws of chemical equilibrium are, in principle, inapplicable to the state of the organism as a whole. This is shown by the trivial fact that the chemical equilibrium of proteins in the presence of proteolytic enzymes as found in every cell, is the nearly complete breakdown of proteins into component amino acids. However, so long as the organism is alive, it remains approximately constant in a state far distant from equilibrium, in the way that degradation of high-molecular compounds is balanced by a corresponding synthesis. As research with isotope tracers has shown, both groups of processes proceed at relatively high rates (p. I47ff.). Approach to equilibrium means death of the organism.

Thermodynamically, the living organism appears to violate one of the fundamental laws of nature. According to the second principle of thermodynamics, the general trend of physical events is toward increasing entropy, eventually leading to states of equilibrium with maximum entropy. Entropy, according to statistical theory, is a measure of probability, and hence the trend toward increasing entropy is toward a most probable distribution. The most probable distribution, however, of a mixture, e.g. of red and blue glass beads or similarly of molecules with different velocities, is a state of complete disorder; having separate all red beads on one hand and all blue ones on the other or having, within a compartment, all fast molecules, that is, high temperature, on the right side, and all slow ones and low temperature, at the left, is a highly improbable state of affairs. Hence, entropy increases in all irreversible processes, and the trend toward increasing entropy is identical with the trend toward most probable states, leveling down of existing differentiations, and states of maximum disorder.

In contrast, the living organism is thermodynamically a system of fantastic improbability. In spite of irreversible processes which are continually going on, 
the organism is maintained in a state of high order and it even advances, in ontogenesis and evolution, toward states of higher order and heterogeneity.

Hence, for a considerable time physicists have been puzzled by the apparent violation of laws of physics in living nature. For "how is it possible to understand life when the whole world is governed by such a law as the second law of thermodynamics, which points toward death and annihilation?" (Brillouin, 1949). The answer to be given (Bertalanffy, I 932, I 934; Brillouin, I 949; Schrödinger, 1944) is that an expansion of physics is necessary in order to deal adequately with the phenomena of life. It may well happen "that the discovery of new laws and some new principles in biology could result in a broad redefinition of our laws of physics and chemistry, and produce a complete change of point of view" (Brillouin, I 949). This is essentially what has taken place in recent developments of the theory of "open systems".

By necessity, conventional physical theory, i.e. the physics of closed systems, is inadequate for dealing with living systems. A true biophysics needs a theory of open systems and steady states, which amounts to an extension and generalization of conventional theory. For it is easy to see that the theory of open systems is the more general one; it is always possible to come from open to closed systems by equating the transport terms to zero, but not vice versa.

The concept of "open system" was first introduced in biology by Bertalanffy (1932) who indicated several kinetic principles of open systems (1940b, 1950a). Independently, similar considerations were advanced by Burton (1939). Only later was it noticed that the concept of open system had already been used in thermodynamics by Defay (1929). Thermodynamics of open systems is part of the modern expansion of thermodynamic theory known as Irreversible Thermodynamics, founded and developed by Meixner (1954), Onsager (193I), Prigogine (1947, I955), De Groot (195I), Haase (1952), and others.

The following discussion is limited to some properties of open systems which are of interest in connection with biological problems of metabolism and growth; for a more detailed discussion of the theory of open systems from the biological standpoint, of. Bertalanffy (1953), Bray and White (I954, I957).

\section{(b) Definitions}

Before continuing, a definition of terms is in place. A system is called isolated if it exchanges neither matter nor energy with environment; closed if it exchanges energy but not matter; and open if it exchanges both. Conventional kinetics and thermodynamics are limited to isolated and (to a certain extent) closed systems which do not exchange matter with environment. An isolated system must eventually attain a time-independent state, called equilibrium, where all macroscopic magnitudes remain constant and all macroscopic processes stop. Non-isolated systems may, certain conditions presupposed, attain a time-independent state where also all macroscopic magnitudes remain constant, but macroscopic processes of import and export continue. This is called a stationary state and, in open systems with import and export of matter, a steady state (Fliessgleichgewicht in German: Bertalanffy, I942a). Chemical equilibria are based upon reversible reactions. Steady states are irreversible as a whole, and partial reactions taking place may also be irreversible. 
(c) Kinetics of open systems

A simple open system is presented in Fig. I. Let there be transport of material A into the system which is proportional to the difference of its concentrations outside and inside the system. This material may form, in a monomolecular and reversible reaction, a component B. On the other hand, A may be catabolized,

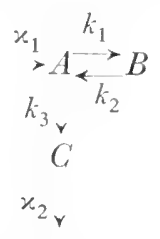

Fig. I. Simple open system. See text.

in an irreversible reaction, into $\mathrm{C}$, and $\mathrm{C}$ may be removed from the system with a rate proportional to its concentration. As can be seen from the simultaneous differential equations for this system and their solution for the steady state, the ratio of the concentrations of the reactants in the steady state is:

$$
c^{*}{ }_{\mathrm{A}}: c^{*}{ }_{\mathrm{B}}: c^{*}{ }_{\mathrm{C}}=\mathrm{I}: k_{1} / k_{2}: k_{3} / x_{2}
$$

This model is not purely imaginary. It applies to isotope experiments where turnover rate of proteins is determined (p. I48f.). Then A stands for amino acids introduced into the organism; $\mathrm{B}$ for proteins; and $\mathrm{C}$ for products of excretion.

Some interesting consequences can immediately be seen. $I$. The composition of the system in the steady state remains constant, although the ratio of the components is not based upon a chemical equilibrium of reversible reactions but the reactions continue and are in part irreversible. 2 . The steady-state ratio of the components depends only on the system constants of reaction and transport, not on the external conditions as represented by the outside concentration of $\mathrm{A}$. 3. We find $c^{*}{ }_{A}=x_{1} c_{e \mathrm{~A}} /\left(x_{1}+k_{3}\right)\left(c_{e \mathrm{~A}}=\right.$ outside concentration of A). Let us suppose that a disturbance from outside raises the rate of catabolism, which amounts to an increase of constant $k_{3}$. Then $c_{\mathrm{A}}$ must decrease. But since import is proportional to the difference of the concentration of $\mathrm{A}$ outside and inside the system, i.e. $c_{e \mathrm{~A}}-c_{\mathrm{A}}$, influx must increase. Hence the system develops forces directed against a disturbance of the steady state. Speaking biologically, the system shows "adaptation" to changes of the environment. Speaking in the language of physical chemistry, the system shows a behavior corresponding to the principle of Le Chatelier.

These, however, are the general characteristics of the self-regulation of metabolism of living systems, namely,

I. Maintenance of a constant ratio between the components which is not due to an equilibrium but maintained in a steady flow of matter;

2. Independence of the composition of the system from the varying import of material, corresponding to the fact that the composition of the organism remains constant even if imported nutritive materials vary; and

3. Reestablishment of the steady state after disturbance or, biologically speaking, a 'stimulus'. 
4. A closed system in equilibrium neither needs energy for its maintenance nor can energy be obtained from it. To perform work, a system must be not in equilibrium, but tending to attain it. In order to go on this way, the system must be maintained in a steady state. Therefore, the character of an open system is the necessary condition for the continuous working capacity of the living organism.

5. A further characteristic of open systems can also be easily seen. In conventional physical systems, the final state eventually attained is defined by the initial conditions. For example, in a closed chemical reaction system the final concentrations trivially depend on the initial ones. If either the initial conditions or the course of the process is altered, the final state also will change. In open systems, however, the steady state eventually reached is independent of the initial conditions and of the course the process has taken [as, for example, can be seen from equation (2.I)] and determined only by the system parameters of reaction and transport. Hence, the same final state can be reached from different initial conditions or in different ways. This characteristic of open systems is called equifinality, and is an important characteristic of living qua open systems ( $c f$. p. 2 I $3 \mathrm{ff}$.).

6. Closed chemical systems asymptotically approach a state of equilibrium. In open reaction systems, in contrast, phenomena of false start and overshoot appear under certain mathematically definable conditions (Burton, 1939; Denbigh et al., 1948; Bertalanffy, I 953). That is, the process first takes place in a direction opposite

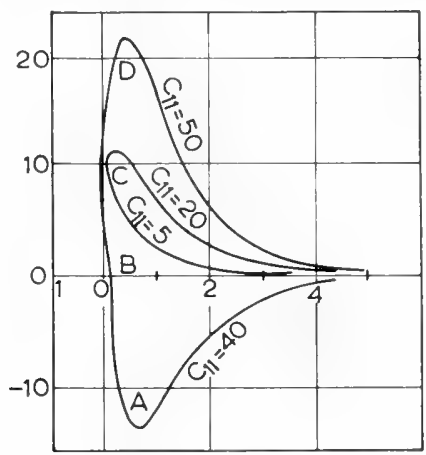

Fig. 2. Overshoot and false start in open systems. Under certain mathematical conditions (cf. Burton, 1939; Denbigh et al., 1948) phenomena of overshoot $(A)$ and false start $(C, D)$ appear even in the simplest open reaction systems, while in conventional closed systems the approach to chemical equilibrium is simply asymptotic (type of curve $B$ ). The figure represents approach to the steady state in a system of 2 reactants, the concentration of one of which passes overshoot or false start $(A, C, D)$ while the other $(B)$ asymptotically approaches the steady state which is assumed to be zero. If the final steady-state concentrations are higher than the initial concentrations, the curves are to be rotated around the $t$-axis. The curves are drawn for different arbitrary values of the constants in the differential equations describing the open system. Overshoot and false start can experimentally be reproduced in suitable open reaction systems. After Denbigh et al., r 948.

to that eventually leading to a steady state (Fig. 2). Phenomena of overshoot and false start are not encountered in conventional kinetics, but are possible in the kinetics of open systems and found in many physiological phenomena. 
7. The theory of open systems has been applied, in various forms, to quantitative study and theory in fields such as isotope experiments, calculation of the energy requirement of the steady state of the organism, growth, excitation, nerve conduction, radiation biology, pharmacology, ecological systems, and others. In a general way the basic physiological phenomena can be considered as consequences of the fact that organisms are open systems. Metabolism essentially is the maintenance of the organism in a steady state. Irritability and autonomous activities are smaller waves of processes superimposed upon the continuous flow in a steady-state system. Growth, development, senescence, and death represent an approach to, and slow changes of the steady state. It may be said that the basic fields of physiology, such as metabolism, growth, excitation, etc. today begin to be united by a common theoretical conception, namely, a theory of open systems.

8. It is worthwhile to mention that open-systems characteristics of organisms are precisely those which often have been considered to be "vitalistic" and in contradiction to basic laws of physics. For example, equifinality, the attainment of the same final state from different initial conditions or in different ways, was the first so-called "proof of vitalism" advanced by the German zoologist and philosopher, Driesch (I929; $f f$. p. 2I 4). This is, however, a general characteristic of open systems in so far as they attain a steady state. Driesch's "second proof of vitalism" was based upon the self-multiplication of biological systems (chromosomes, etc.) which probably is a consequence of their being metabolizing systems. Similarly, the apparent contrast between the direction of events in inanimate and in living nature was considered to be a proof of vitalism as was expressed, among others, by Lecomte du Noüy:

"When science declares that all phenomena of the universe must obey the principle of Carnot-Clausius, and we discover phenomena which do not seem to do so, we have a proof that the science in question does not cover all reality, and this restricts its universality. This is the case of natural evolution, which unfolds in a direction forbidden by science, i.e. toward more and more improbable states. We conclude therefore that our science is not universal as yet and only governs inanimate matter" (1949, p. I37).

However, development toward states of higher order and complexity is quite legitimate within the generalized thermodynamics of open systems (p. I 55f.).

The vitalists were quite correct in pointing out the fact that there are discrepancies between the behavior of living systems and conventional physical theory, i.e. physics of closed systems. These discrepancies, however, do not mean that organisms violate the laws of "physics" but they disappear with the expansion of physical theory, and they are, as a matter of fact, quantifiable consequences of generalized physical theory including open systems ( $c f$. Bertalanffy, I952, p. I 44 ff.).

\section{(d) Turnover of building materials of the organism}

Modern isotope methods have presented impressive demonstration of the "dynamic state" of the organism (e.g. Schoenheimer, I942; Rittenberg, I 948). By introduction of compounds marked by isotopes, the turnover rate of building materials of an organism can be estimated. The labeled molecules are synthesized 
TABLE 1

PROTEIN TURNOVER DETERMINED BY INTRODUGTION OF GLYGINE LABELLED WITH ${ }^{15} \mathrm{~N}$

After Sprinson and Rittenberg, I949b

Turnover

Half-life time

rate $(r)$

$\left(t_{1 / 2}\right)$ in days

Rat: Total protein

$\begin{array}{lc}0.04 & \text { I } 7 \\ 0.12 & 6-7 \\ 0.033 & 21\end{array}$

Proteins of liver, plasma and internal organs

0.033

Man: Total protein

$0.0087 \quad 80$

Proteins of liver and serum

Protein of musculature and other organs

0.0693

80

$0.0044 \quad 158$

into organic compounds; these eventually are catabolized and the end-products excreted. The isotope method has shown that the stream of matter which passes and at the same time maintains the living organism takes place at an unexpectedly high rate. Proteins, carbohydrates, lipids, and even seemingly inert material such as the mineral substances of dentine and the bone, are in a continuous process of degeneration and regeneration. Some estimates of turnover rates are presented in Table $\mathbf{I}$. Calculation of turnover rate is a special case of the kinetics of open systems.

For example, the calculation of protein turnover according to Sprinson and Rittenberg (I949a, b; Table I): Empirically it is found that the excretion of labelled N decreases exponentially with time:

$$
\lambda_{E}=\lambda_{0} A\left(\mathrm{I}-e^{-B t}\right)
$$

where $\lambda_{E}$ is the amount of ${ }^{15} \mathrm{~N}$ (in mequiv.) excreted up to time $t$ after an amount $\lambda_{0}$ of ${ }^{15} \mathrm{~N}$ was fed as amino acid at $t=0$, and $A$ and $B$ are constants. Theoretically, if $\lambda$ is the amount of ${ }^{15} \mathrm{~N}$ left in the metabolic pool at time $t$, it can be assumed that

$$
-\mathrm{d} \lambda=\frac{\lambda}{P} E \mathrm{~d} t+\frac{\lambda}{P} S \mathrm{~d} t-Q \mathrm{~d} t
$$

where $P$ is the metabolic pool, and $E, S$, and $Q$ are coefficients for excretion, synthesis, and deliverance into the metabolic pool, respectively. $\lambda_{0}$ is small compared to $P$ and hence also the change of $P, Q \mathrm{~d} t$, is negligible. This gives

and

$$
\frac{-\mathrm{d} \lambda}{\lambda}=\frac{E+S}{P} \mathrm{~d} t
$$

$$
\lambda=\lambda_{0} \mathrm{e}^{-[(E+S) / P] \ell}
$$

According to (2.3) the amount of ${ }^{15} \mathrm{~N}$ excreted is

$$
\mathrm{d} \lambda_{E}=\frac{\lambda}{P} E \mathrm{~d} t
$$

Insertion of (2.5) and integration gives:

$$
\lambda_{E}=\lambda_{0} \frac{E}{E+S}\left(\mathrm{I}-\mathrm{e}^{[(E+S) / P] t}\right)
$$


which, compared with (2.2) shows that

$$
A=\frac{E}{E+S}, B=\frac{E+S}{P}
$$

From this $S$ and $P$ can be calculated. Under consideration of the $N$ content per kg tissue, $r$ (turnover rate of protein per unit body weight and day) can be calculated, giving the figures of Table $\mathbf{I}$.

This is a somewhat simplified version of the model Fig. I. The equation for $c_{A}$ in this model is, import being assumed constant:

$$
\mathrm{d} a / \mathrm{d} t=a_{e}-k_{1} a+k_{2} b-k_{3} a
$$

if concentrations for simplicity are written by simple letters $\left(c_{A}=a\right.$ etc. $)$.

Suppose the administered amino acid was labeled; that tracer is incorporated in the body proteins and the metabolic pool in ratios $a / a^{\prime}$ and $b / b^{\prime}$ and that the supply of tracer was stopped at $t=0$. Then the rate of disappearance of tracer will follow an equation:

$$
\text { - } \mathrm{d} a / \mathrm{d} t=k_{1} a / a^{\prime}-k_{2} b / b^{\prime}+k_{3} a / a^{\prime}
$$

which is identical with Sprinson and Rittenberg's expression (2.4)

$$
-\mathrm{d} \lambda / \mathrm{d} t=S \lambda / P+E \lambda / P
$$

under the assumption that $k_{2}$ is small, i.e. breakdown of proteins into amino acids is negligible in comparison to the other terms.

Reiner (1953) has solved the more complete system of simultaneous equations, the first of which is (2.9) and found that, under the given conditions, the approximation is within the experimental error.

\section{(e) Energy requirements of protein synthesis}

The synthesis of high-molecular compounds and the maintenance of the organism in a steady state require energy. For this reason, even abstracting from the complication of the processes in detail and taking it as an overall balance of processes, an open system like Fig. I is only a partial model. It does not take into account the energetics of anabolic processes which deserve consideration.

Protein synthesis cannot be conceived of as an equilibrium reaction based upon the reversal of hydrolysis of proteins into amino acids and water. For in the reaction amino acids $\rightleftarrows$ proteins $+\mathrm{H}_{2} \mathrm{O}$ the equilibrium is almost completely on the left hand side, that is, the breakdown of proteins into amino acids. Therefore it is easy to break down proteins into component amino acids by hydrolytic agents, but difficult or rather at present impossible to unite amino acids into proteins in vitro.

According to G. V. Schulz (1950), the number $n^{*}{ }_{p}$ of moles of a polymer of a degree of polymerization $P$ in equilibrium (per mole of monomer) is expressed by the equation:

$$
n_{p}^{*}=\left(\frac{K}{n_{w}}\right)^{P-1}
$$

with $K=$ equilibrium constant, $n_{w}=$ number of moles of water per mole of monomer and polymer. $K$ can be calculated from the enthalpy and entropy of the reactions concerned. Using appropriate empirical values in equation (2.I2), the equilibrium concentration of proteins vs. amino acids can be calculated (Table 2). Under conditions resembling those in the organism, the concentration even of the dimer is very small and the appearance of higher polymers corre- 
TABLE 2

EQUILIBRIUM CONCENTRATIONS OF POLYPEPTIDES

After Schulz, I950

\begin{tabular}{cl}
\hline Degree of polymerization $P$ & Mole polymer per liter $n^{*}{ }_{p}$ \\
\hline 2 & $\mathrm{IO}^{-7}$ \\
$\mathrm{IO}$ & $\mathrm{IO}^{-63}$ \\
$\mathrm{IOO}$ & $\mathrm{IO}^{-693}$ \\
$\mathrm{I} 000$ & $\mathrm{IO}^{-6993}$ \\
\hline
\end{tabular}

sponding to protein-that is, with a polymerization well over 1,000 - is of a fantastically low order of magnitude.

Hence the synthesis of high polymers needs energy which must be provided by way of coupled reactions. In general, the energy yielded by cell respiration or fermentation is stored in high-energy phosphate bonds, particularly of the adenylic-acid system, and so available for non-spontaneous processes in the cell. It is a fair inference, supported by considerable evidence from isotope experiments with cell homogenates, that the energy for protein synthesis is provided by ATP or related compounds, and that the ribonucleic acids localized in the microsomes that line the endoplasmic reticulum, in some way act as templates for the production of specific proteins characterized, in each particular case, by a specific arrangement of amino acids. How protein synthesis is effectuated in detail is unknown although there is a considerable number of proposed models $(e . g$. Northrop, I949; Caldwell and Hinshelwood, I950; Haurowitz and Crampton, I 952; Dounce, I 953; Lipmann, I954; Lockingen and Debusk, I 955; Zamecnik, I 958). Irrespective of the way in which protein synthesis takes place, the energetic conditions can be indicated.

According to the calculations mentioned above (G. V. Schulz, I950, I95 I) the energy requirement for peptide bonds is $-\Delta G \approx 7 \mathrm{kcal} /$ mole for a polymerization $P=$ I0,000. To the energy requirement of polymerization "chain entropy" has to be added: Protein synthesis is not a mere polymerization but rather a formation of chains of definite length and with a specific arrangement of amino acids within the macromolecule. The additional amount of free energy which is necessary for the synthesis of macromolecules of defined structure can be calculated from Boltzmann's principle. For proteins consisting of $c a .20$ different amino acids, chain entropy results in an amount of additional $-\Delta G \approx 2 \mathrm{kcal} / \mathrm{mole}$. Since free energy of the high energy phosphate bonds of ATP is $\approx \mathbf{I} 2 \mathrm{kcal} / \mathrm{mole}$, it is sufficient to cover the energy requirements of protein synthesis, irrespective of the mechanism of this process in detail.

Isotope experiments with homogenates (Siekewitz, I952; Zamecnik and Keller, I 954; Pollister, I 954; Ehrensvärd, I955; Zamecnik, I958; and others) suggest at least two steps in protein synthesis: one requiring energy furnished by oxidative "phosphorylation and taking place in the mitochondria; and a second one requiring less energy input, taking place in the microsomes, and yielding specific protein chains. In other terms, it would appear that the first steps of protein synthesis 
which require the highest input of energy (formation of dipeptides) take place in the mitochondria which, containing the cyclophorase and phosphorylation systems, can provide this amount of energy by oxidative or fermentative processes, energy being stored in high-energy phosphate bonds which are transferred to amino acids; the subsequent formation of long chains which, for thermodynamic reasons, requires less energy, takes place in the microsomes, the RNA of which may act as template.

\section{(f) Energy requirements of the maintenance of the steady state of the organism}

The maintenance of the body proteins in a steady state distant from equilibrium and maintained in continuous degradation and regeneration requires energy. Even the cell not performing external work needs continuous import of energy as shown by the fact that under lack of oxygen all vital functions of an (aerobic) cell cease. The maintenance work of the cell is partly physicochemical such as maintenance of structures, of osmotic pressure, ion concentrations, etc., different from equilibrium; partly it is chemical, that is, maintenance of the body components in a steady state distant from chemical equilibrium (i.e., for proteins, practically complete dissociation into amino acids). These energy requirements can be calculated (Bertalanffy, I 942a; G. V. Schulz, I950; Bertalanffy, 1953). Starting from the Van 't Hoff equation for the work necessary to bring I mole of a substance from the equilibrium concentration $c^{*}$ to another concentration $c$ :

$$
A=R T \ln \left(c / c^{*}\right)
$$

some calculations lead to the approximative formula:

$$
\mathrm{d} a / \mathrm{d} t=r \cdot 93 \mathrm{cal} / \mathrm{g} \text { protein }
$$

for the maintenance work of the organism, assuming an average polymerization $P=\mathrm{I}$ ooo for proteins, an average molecular weight of $\mathrm{I}$ oo for animo acids, and $r$ being the turnover rate per day. Inserting the values known from isotope experiments (Table I), it appears, somewhat surprisingly, that only about I to 2 per cent of basal metabolic rate of an adult human or rat are required for the maintenance of proteins in a steady state. Estimates from protein metabolism with somewhat different assumptions (Borsook, I950) and from the duplication of the number of cells in regenerating rat liver (Lang, I952) lead to a similar result.

\section{(g) Thermodynamics of open systems}

As already mentioned, the apparent gulf between inanimate and living nature with respect to the second principle of thermodynamics has embarrassed physicists and philosophers for a long time. For example, the American philosopher and historian, Henry Adams ( I920), pointed out what he called "the violent contradiction between Lord Kelvin's degradation and Darwin's elevation, between the law of dissipation in physics and the law of evolution in biology". According to the second principle of thermodynamics, the general trend of physical events is toward states of increasing disorder and leveling down of differences. High forms of energy are irreversibly degraded to heat and heat gradients progressively disappear. In this way the universe "runs down" and eventually approaches heat 
death when all energy is dissipated into uniformly distributed heat of low temperature, and the world process comes to a stop. In contrast, living nature shows a transition toward higher order, heterogeneity, and organization. This is obvious in embryonic development, when the ovum, a nearly homogeneous droplet of protoplasm, eventually yields an organism of enormous complexity. It is equally true of evolution which proceeds, in geological periods, from simple creatures to ever higher forms of organization.

In order to evaluate this problem, the limitations of conventional or "classical" thermodynamics have to be contemplated. It almost exclusively deals with states of equilibrium, processes in isolated systems, and transitions from one equilibrium state to another. According to definition, the first and second principle of thermodynamics apply to closed systems, stating that energy is constant and entropy increases in such systems. They tell nothing about systems which are open, that is, where there are processes of flow across the boundaries of the system. Classical thermodynamics, therefore, should more adequately be denominated as thermostatics. It is inadequate where states of non-equilibrium, processes of material flow, and irreversible processes are concerned.

This situation has led to a modern development and generalization known as Irreversible Thermodynamics (monographs: Prigogine, I947; De Groot, I95I; Denbigh, I 95 I; Haase, I 95 I-53, I 952).

\section{(h) Principles of irreversible thermodynamics}

While a detailed presentation of this field is beyond the scope of the present study, the basic concepts of irreversible thermodynamics will be briefly outlined in view of the crucial problem of organic development indicated above.

$I$. The first basic concept of irreversible thermodynamics is the introduction of generalized thermodynamic functions which are not restricted to closed systems, but take account of the energy transport in open systems. In every closed system where irreversible processes take place, entropy must increase according to the function:

$$
\mathrm{d} S \geqq 0
$$

In an open system, entropy can change for two reasons: by transfer of entropy from outside into the system, and by production of entropy within the system. This is expressed in the generalized entropy function of Prigogine:

$$
\mathrm{d} S=\mathrm{d}_{e} S+\mathrm{d}_{i} S
$$

$\mathrm{d}_{e} S$ is the change of entropy by transfer, $\mathrm{d}_{i} S$ the production of entropy due to irreversible processes in the system, such as chemical reactions, diffusion, heat conduction, etc. Entropy production, $\mathrm{d}_{i} S$, is always positive according to the second principle. Entropy transfer, $\mathrm{d}_{e} S$, however, may be positive, zero, or negative. It is negative when, e.g., materials rich in free energy are introduced into the system. Consequently, change of total entropy, $\mathrm{d} S$, in an open system can-depending on the sign and quantity of $\mathrm{d}_{e} S$-be negative as well as positive.

2. A second basis of irreversible thermodynamics is the supposition of the so-called phenomenological lawes. Irreversible processes such as heat flow, diffusion, 
electrical currents, chemical reactions, etc.-called fluxes-are assumed to be caused by generalized forces such as temperature gradients, concentration gradients, electrical potentials, chemical affinities, etc. Such irreversible process can, as a rule, be expressed by proportionalities between the rate of flow and the corresponding force. Examples are Fourier's law for heat flow and temperature gradient, Fick's law for diffusion and the concentration gradient, Ohm's law for electrical current and electromotoric force, etc. This can be written in a general form:

$$
f=L X
$$

where $\mathcal{J}$ is the flux, $X$ the force, and $L$ a scalar parameter such as heat or electrical conductivity, diffusion coefficient, the chemical "drag" coefficient, etc.

Considering two fluxes and two forces, we find so-called reciprocal phenomena such as the Soret effect, i.e. the formation of a flow of matter due to a temperature gradient (thermodiffusion) and the Dufour effect, i.e. a flow of heat due to a concentration gradient (diffusion-thermo effect). Suppose there is a flow of energy the rate of which is $\mathcal{F}_{1}$, and a flow of matter, with a rate $\mathcal{J}_{2}$. If these processes take place independently, they will follow the phenomenological relations and can be considered as being due to the thermal force $X_{1}$, and the diffusion force $X_{2}$, respectively, with the appropriate parameters $L$. If, however, both processes take place simultaneously, it may be assumed that each flux depends on both forces present. Retaining the assumption of linearity, we have:

$$
\left.\begin{array}{l}
\mathcal{J}_{1}=L_{11} X_{1}+L_{12} X_{2} \\
\mathcal{J}_{2}=L_{21} X_{1}+L_{22} X_{2}
\end{array}\right\}
$$

3. Now we come to the third and decisive step, the Onsager reciprocal relations. The coefficients $L_{12}$ and $L_{21}$ indicate the coupling or the interaction of both processes. The Onsager relations state an equality of these coupling coefficients, so that in the particular case mentioned:

$$
L_{12}=L_{21}
$$

That is, there is a symmetry of the effect of the diffusion force on heat flow, and the effect of the thermal force on diffusion.

These formulations can easily be generalized from the consideration of two to any number of interacting forces and fluxes.

The reciprocal relations apply provided that the thermodynamic forces are suitably chosen. This, according to Onsager, means that the forces are chosen in such way that if any flux $\mathcal{J}$ is multiplied by the corresponding force $X$, the sum of the products is equal to entropy production multiplied by absolute temperature:

$$
T \sigma=\mathcal{J}_{1} X_{1}+\mathcal{J}_{2} X_{2}+\ldots=\sum_{i} \mathcal{F}_{i} X_{i}
$$

For example, in a chemical reaction entropy production per unit volume and unit time is defined by:

$$
T_{\sigma}=\sum_{i} A_{i} v_{i}
$$


where the $v$ 's are the reaction rates, i.e. the fluxes, the $A$ 's the chemical affinities (De Donder), i.e. the thermodynamic forces.

4. The reciprocal relations are based upon the principle of microscopic reversibility stating that in a system in equilibrium the fluctuations of particles away from equilibrium have the same frequency as those toward equilibrium. This principle imposes restrictions upon the possible ways in which a system remains in equilibrium, i.e. macroscopically unchanged, from which the Onsager relations can be derived.

From these principles a thermodynamics of irreversible processes and (with certain restrictions) of open systems and steady states can be derived as a consistent theory. As may easily be seen, this theory is particularly useful in phenomena where there is an interaction of two or several processes: thermodiffusion, thermoelectricity, thermoosmosis, electrochemistry, the fountain effect of Helium II, etc. The theory leads to a solution of many problems where classical theory was insufficient.

Here only some consequences of a general nature will be indicated which elucidate the new aspects opened up by irreversible thermodynamics. From the standpoint of the theory discussed above, Prigogine ( I 947; Prigogine and Wiame, 1946) has summarized these general consequences as follows:

a. Open systems tend toward a steady state which is defined by minimum entropy production.

$b$. During the development of an open system toward the steady state, the entropy of the system may decrease.

c. Steady states are, in general, stable. Hence if one of the system variables is altered, the system manifests changes in the opposite direction. The principle of Le Chatelier, therefore, is valid not only for closed systems but also for open systems which are the site of irreversible processes, provided that these systems are in a steady state.

While statements $b$ and $c$ appear to be generally valid for open systems, statement $a$, known as Prigogine's theorem, was subject to considerable discussion (e.g. Haase, I951; Denbigh, I952; Bertalanffy, I953; Jung, I956; Haase, I957; Foster, Rapoport and Trucco, I 957, with survey of literature). It can be shownand this was in no way unknown to Prigogine - that steady states correspond to states with minimum entropy production if and only if several rather severe restrictions apply, namely $I$. Linearity of the phenomenological laws; 2. Validity of the Onsager reciprocal relations for all fluxes and forces; 3. Constancy of the Onsager coefficients.

For this reason, Prigogine's theorem applies to transport processes such as, e.g. the Knudsen effect: An ideal gas is enclosed in two compartments which are kept at different temperatures and connected by a small opening. Then eventually a steady state is reached where macroscopic mass transport disappears and the pressures are different in the two compartments. Thus the final steady state (unequal pressures and inhomogeneous distribution of the molecules) shows a higher order than the initial state (equal pressures and homogeneous distribution). Calculation shows that entropy of the gas decreases in the process, and that entropy production is a minimum in the steady state. 
This, however, is in no way generally the case. In particular, it does not hold for the important case of open chemical reaction systems. The assumption that reaction rate is a linear function of the thermodynamic force (difference of the chemical potentials of reactants and end products of the reaction) is approximately true only if the system is close to chemical equilibrium. In general, however, reaction rate depends on concentrations rather than chemical potentials. It has been shown by Denbigh (1952) that the discrepancy between concentrations giving minimum entropy production and those of the steady state is considerable. Hence, minimum entropy production does not define the steady state of an open reaction system, and at present no simple thermodynamic definition of the latter is available. The approach of an open chemical system toward a steady state is determined rather by kinetic factors, namely, concentrations, than by thermodynamic forces.

Therefore, similarly as thermostatics has been generalized in (now conventional) irreversible thermodynamics on the basis of the above-enumerated principles further generalizations appear to be necessary. This applies $I$. to elimination of the above-mentioned restrictions hitherto assumed in irreversible thermodynamics, but also 2 . to a deep-reaching paradox inherent in thermodynamic theory. Entropy is, in Eddington's expression, the "Arrow of Time", the basic function indicating the unidirectional flow of physical events. However, time does not appear explicitly in the equations of thermodynamics, classical or irreversible.

An interesting attempt toward such generalization was made by Reik (I953). Reik introduces a "time axiom" which appears to be the most general expression the second principle has found hitherto. Reik's time axiom $I$. gives the classical laws for thermodynamic equilibrium if time approaches infinity; 2 . If the equations of the time axiom are developed into series for the Onsager forces and the equilibrium state and only the linear terms are retained, the phenomenological laws and reciprocal relations follow as a first approximation; 3. The time axiom is, however, more general because it is not restricted to near-equilibrium states, but also is applicable to states of non-equilibrium, and indicates the time-laws for irreversible processes.

\section{(i) Entropy and the living world}

Irrespective of these problems, it can be stated that, as previously mentioned, irreversible thermodynamics has been elaborated for numerous problems in physics. In the more difficult and fascinating problems of biophysics, several attempts to apply thermodynamic considerations to open systems have been made, such as in the energetics of metabolizing systems (p. I49ff.); the active transport by the living cell in contrast to passive penetration based upon diffusion, distribution, or osmotic equilibria; bioelectric potentials, etc.

Bypassing such special problems, the apparent contradiction to physical law in the living world can be given a precise answer ( $c f$. Bertalanffy, I 95 Ia, I953; Haase, I95I, I 957; Jung, I956). The alleged contradiction between entropy and evolution and the seeming violation of the second principle in the living world does, in fact, not exist or rather, it disappears with the generalization of physical theory. Entropy must increase in all irreversible processes. Therefore, the change 
in entropy in a closed system is always positive or, as we may also say, order is continually and increasingly destroyed. But in an open system and a living organism in particular, there is not only entropy production owing to irreversible processes, but the organism feeds, as Schrödinger (I 944) has said, on negative entropy. It imports complex organic molecules with high free energy, breaks them down, and gives the simpler end products back to environment, partly using the free energy so gained for maintaining and building up higher order. In this way, living systems can avoid the increase of entropy and loss of order and maintain themselves at a constant entropy level of the steady state. They may even advance, in development and evolution, toward states of higher order and differentiation.

The classical second law is not violated but holds true for the organism plus its environment. This, however, does not obviate but rather emphasizes the necessity of a thermodynamics of open living systems. The statement that there is an overall increase of entropy tells nothing about the functioning of any particular system. As it is necessary to develop the thermodynamics of heat engines, refrigerators or chemical reactions in each particular case, the same is true of open systems and living organisms, taken as a whole or in their component processes. This is a difficult and important task which today is in its beginnings.

\section{(j) Dynamic morphology}

Every living system is maintained in continuous flow, degeneration and renewal of its components. What appears to be a persistent structure at one level is maintained in a continuous exchange, production, growth, and death of systems at the next lower level: of chemical compounds in the cell components, of cell components in the cell, of cells in the multicellular organism. Hence the postulate of a dynamic morphology (Bertalanffy, i 941a) arises: Living systems, forms, and structures have to be considered the result and expression of an ordered flow of processes, the laws of which are to be discovered. Modern experience justifies the classical dictum of Claude Bernard (1865): "The synthetic activity by which the organism maintains itself is of the same nature basically as that by which it regenerates after a lesion or reproduces and multiplies. Organic synthesis, reproduction, regeneration, integration, wound healing" (and, as we may add, growth) "are only different aspects of a single phenomenon."

\section{GELL GROWTH AND MULTIPLICATION}

The growth of organisms takes place by growth and multiplication of cells (generally in the way of mitosis), and formation of metaplasmic and paraplasmic products. A review of these topics in their biochemical, physicochemical and morphological aspects is beyond the scope of the present study. Only a few quantitative or quantifiable relationships in cell growth will be discussed briefly.

\section{(a) Nuclear growth and nuclear series}

The average DNA content of the nucleus is constant in diploid somatic cells 
TABLE 3

DNA CONTENT PER NUCLEUS IN BEEF TISSUES

After Vendrely, I955

\begin{tabular}{lccc}
\hline Organ & $\begin{array}{c}\text { DNA in picograms } \\
\left(\mathrm{IO}^{-12} \mathrm{~g}\right)\end{array}$ & $\begin{array}{c}\text { Number of } \\
\text { nuclear types }\end{array}$ & $\begin{array}{c}\text { Probable chromosome } \\
\text { number }\end{array}$ \\
\hline Thymus & 6.6 & $\mathrm{I}$ & diploid \\
Liver & 6.4 & 2 & diploid \\
Pancreas & 6.9 & 6 & diploid \\
Kidney & 5.9 & IO & diploid \\
Sperm suspension & 3.3 & I & haploid \\
\hline
\end{tabular}

of a species. Haploid cells show half the amount (Table 3; Vendrely, 1955). This has been established by chemical analysis (Schmidt-Tannhauser and Schneider methods), as well as UV spectrophotometry and quantitative photometry in visible light (Feulgen preparations). In contrast to the RNA content of the cell which shows considerable variation with functional factors (type of tissue, nutrition, hormones, mitotic activity, etc.), the DNA content of the nucleus is a species-specific constant, remaining unaltered by such factors. This constancy of DNA content even applies to many tumor cells (in spite of pyknosis, etc. of nuclei), and appears to change only with mitosis in dividing cells and the polyploidy appearing in many malignancies. Because of the constancy of DNA per nucleus, DNA content can be used as a measure of the number and multiplication of cells within an organ. Data on DNA and RNA content of normal and tumor cells are surveyed, from own observations and survey of literature, by Sandritter (I958).

Duplication of DNA and chromatin preceding mitosis takes place during interphase and prior to the visible stages of mitosis, in a stage suitably denominated as antephase. This is shown by the methods mentioned as well as the turnover of DNA determined by introduction of $32 \mathrm{P}$ (Stevens, Daoust and Leblond, I953a; Daoust, F. D. Bertalanffy and Leblond, I954).

While somatic cells of an organ in general show the same size of the nucleus due to the same diploid chromosome set and DNA content, in many organs nuclear series are found (Jacobj, I925; Wermel et al., 1932-35; Helweg-Larsen, 1952; Fankhauser, I954; Linzbach, 1955).

In many tissues nuclear volumes appear as multiples of a unit. Hence, in graphical representations a simple binomial distribution is found in tissues containing only one nuclear class, while in organs with several nuclear classes a curve with several peaks is found, the maxima showing a ratio $1: 2: 4 \cdots$ For example, the nuclei of all (36) observed organs in man, the guinea pig and the rabbit are distributed among nine classes, their volumina showing a ratio I $: 2: 4: 8:$ I $6: 32: 64:$ 1 $28: 256$. Erythroblast nuclei belong to the smallest class, those of spinal ganglia to the largest (Jacobj, I935; Freerksen, I933; Körner, 1937; and others).

The increase of nuclear volume is paralleled by a corresponding multiplication of DNA content (Fig. 3). On the other hand, in pituitary dwarf mice (with a hereditary lack of eosinophilic cells in the hypophysis and corresponding lack of somatotropin, $c f$. p. 220), the nuclear series are absent but do appear after 
administration of somatotropin; this is paralleled by a corresponding multiplication of DNS as measured by microspectroscopy (Leuchtenberger, Helweg-Larsen, and Murmanis, 1954).

The nuclear series are essentially based upon "inner nuclear division" (Heidenhain, I923) which subsequently was elucidated as endomitosis by Geitler (1948);

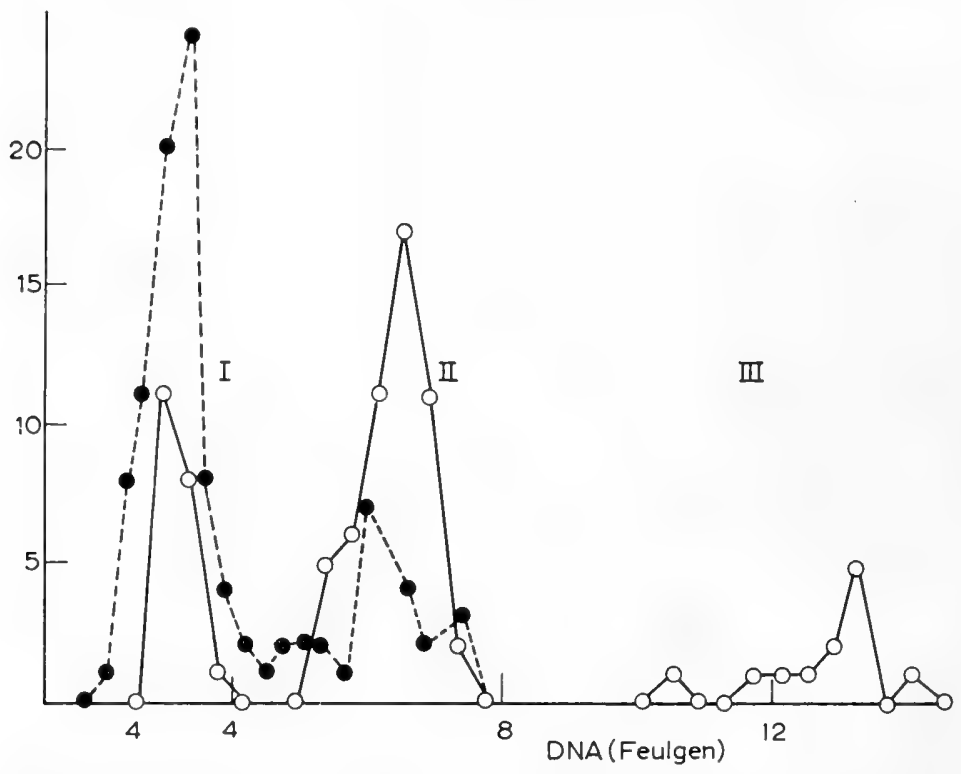

Fig. 3. Amounts of DNA, in arbitrary units, in individual mouse liver nuclei, as indicated by the Feulgen reaction. adult; ...-, to days old. After Pollister, Swift and Alfert, $195 \mathrm{I}$.

that is, duplication of the chromatin material without consecutive spindle formation so that either polytene giant chromosomes (as in the salivary glands of dipters) or else nuclear classes with multiple nuclear volume result. Hence, as a general rule, nuclear series represent polyploidy series with a corresponding multiplication of chromosome number and DNA content. The most important limitation of this rule is in the fact that nuclear size may be changed by variation of protein content (Alfert, Bern and Kahn, 1955). Besides polyploidy, this is another of the appearance of classes with different nuclear volume.

\section{(b) Interaction between nucleus and cytoplasm}

Protein synthesis and hence cell growth appear to be controlled by the genes or, chemically speaking, the DNA of the nucleus, via the ergastoplasm or RNA of the cytoplasm, although the details of this process are hypothetical. This is shown by a wealth of studies reviewed in other parts of this volume. The main steps in this argument are: $I$. Continued life and protein synthesis of the cell are possible only in the presence of the nucleus. -2 . The specificity of proteins, interspecifically as well as intraspecifically in different mutants, blood groups, etc. is determined genetically, as demonstrated by biochemical genetics which shows 
that individual genes determine definite steps of biosyntheses such as formation of certain enzymes.-3. Characteristic changes of the nucleus parallel protein synthesis. As a general rule, cells with high protein synthesis show large nucleoli and increased RNA content.-4. Tissues with high protein synthesis (such as vividly growing, secreting, embryonic, regenerative, viable malignant, etc. cells) are distinguished biochemically by a high RNA content, and morphologically by a high content of basophilic, RNA-rich inclusions of the cytoplasm. It may be briefly mentioned that the high RNA content characteristic of proliferating malignant cells has been developed by the present author and his coworkers (Bertalanffy and Bickis, I 956; Bertalanffy, Masin and Masin, I958) into a clinical method of cytological cancer diagnosis using fluorescence microscopy. - 5. The intimate interaction between nucleus and cytoplasm is expressed by the fact that, notwithstanding the controlling function of the nucleus, cytoplasmic changes (increased RNA content and basophilia) appear to precede, at least morphologically, changes of the nucleus (pyknosis, pleomorphism, hyperchromasia, abnormal mitoses, etc.) (Pirozynski and Bertalanffy, I 955; Bertalanffy, Masin and Masin, I958). The DNA content of the nucleus remains constant even in many malignancies and in advanced stages of cell degeneration where the content of the nucleus in RNA, proteins, and other compounds has materially changed (Vendrely, I955).

The nuclear control of the cytoplasm or perhaps rather the interaction and balance between nuclear and cytoplasmic systems finds a quantitative expression in the nucleocytoplasmic ratio. In a qualitative way, this is manifest in the increase of cell size in haploid, diploid, tetraploid, etc. mutants. Quantitatively, the relation between nucleus and cytoplasm (or cell size) follows the allometric equation (p. $224 \mathrm{ff}$.). As a rule, there is a constant relation of the nuclear volume to the $2 / 3$ power of cell volume, that is, to cell surface (Sinnott and Trombetta, I936; Nozawa, I940; Teissier, I94I) (Fig. 4). Pathological changes (e.g. Addison's disease, heart atrophy: Linzbach, I950; giant nuclei in malignant cells) are connected with shifts of the normal nucleocytoplasmic ratio.

\section{(c) Energy sources of mitosis}

The question of how the energy requirement for mitosis is provided appears to be unsettled at present. According to Warburg's classical theory (1926), the energy expense for cell division is essentially yielded by processes of fermentation. Growing tissues are characterized by high glycolysis and so under anaerobic conditions show high lactic acid formation. In tumors the respiratory system is damaged. Hence they are not capable of oxidizing the products of glycolysis and have high lactic acid formation. On the other hand, their high glycolytic activity permits infiltrative growth regardless of oxygen supply as is necessary for normal cells.

It appears that at present hardly a general rule can be stated ( $c f$. the recent discussion, Weinhouse, Warburg, Burk and Schade, I956). Experiments with fats and sugars labeled with radioactive carbon (Weinhouse, 1955) show that tumor cells are capable of completely oxidizing these compounds to $\mathrm{CO}_{2}$ by mechanisms corresponding to those in normal cells.

Animal ova which present the simplest conditions, show all possible differences 


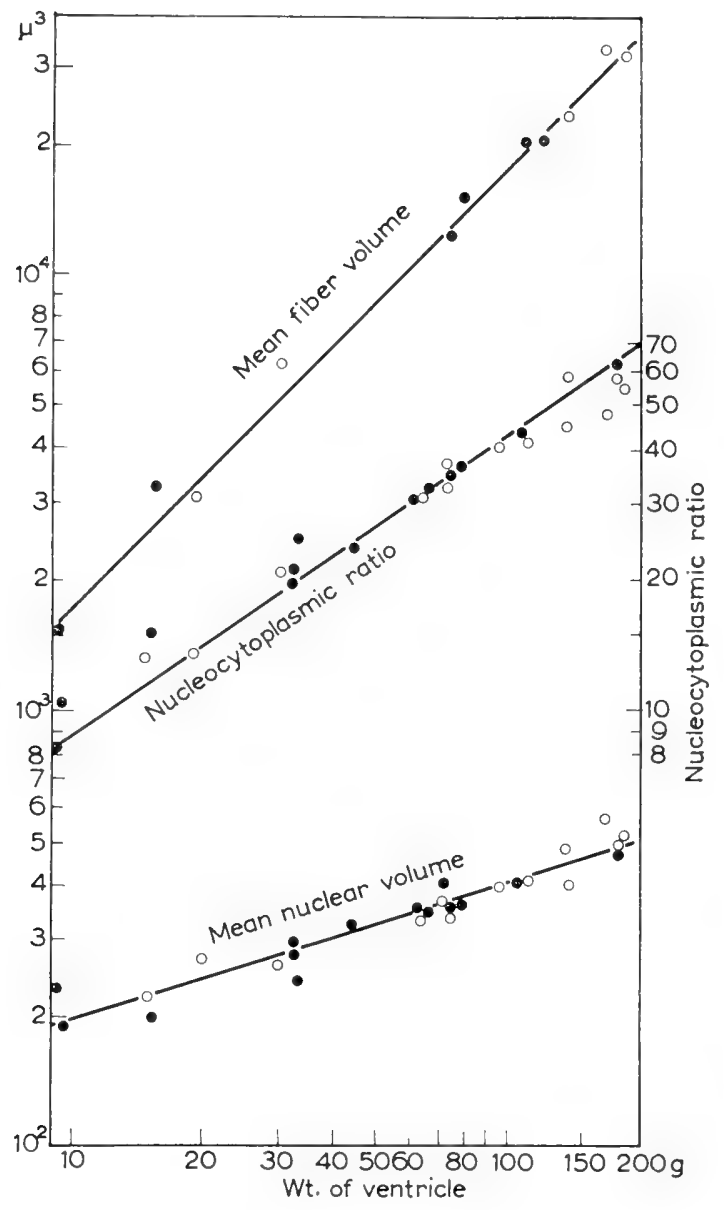

Fig. 4. Allometric growth of heart muscle fibres in man. With increasing ventricle weight, fibre volume increases isometrically $(\alpha \approx \mathrm{I})$, nuclear volume linearly (with the cubic root of ventricle volume, $\alpha \approx 0.3)$, the nucleocytoplasmic ratio is surface-proportional $(\alpha \approx 0.7)$.

o left, - right ventricle. After Linzbach, 1950.

as to whether or not mitosis can be carried through under anaerobic conditions, depending on the species concerned and its particular adaptive conditions. The increase of respiration during mitosis is small and has a maximum in metaphase and early anaphase, whereas cell division coincides with decreased respiration (review in Bullough, I952). In adult epidermis, mitosis is connected with the Krebs cycle; hence it takes place only under aerobic conditions and if glucose or the metabolites of the Krebs cycle are provided. Supply of glucose is necessary in the antephase which, in various respects, appears to be a decisive phase in mitosis (Bullough, I 952). In other tissues such as the embryonic brain of chicken aerobic glycolysis appears to be the energy-yielding process. During the decrease of mitotic rate in the third to the eighth day of development, there is no change of respiration but decrease of glycolysis. Block of glycolysis by Na-fluoride and 
Na-iodoacetate leads to disturbance of mitosis (pyknosis) (O'Connor, I 950a, b). Nowinski (1955) investigated the connection between cell metabolism and multiplication by comparing homologous cell populations which grow rapidly or slowly (leg and wing of the embr yonic chicken). According to expectation, there is an absolute increase of $\mathrm{O}_{2}$ consumption and $\mathrm{CO}_{2}$ production with the increase in mass but $Q_{\mathrm{O}_{2}}$ (calculated on the basis of $\mathrm{I} 00 \mu \mathrm{g}$ DNA) remains constant, and there is no significant difference between leg and wing. In contrast, anaerobic glycolysis shows a continuous decrease and a significant drop between the fifth and sixth day. A significant difference between wing and leg is found on the sixth and seventh day. It is concluded from the parallelism between high growth rate and anaerobic glycolysis that the latter is connected with multiplicative (exponential) growth.

\section{(d) Mathematical theory of cell growth}

It is a basic question why $I$. a cell does not surpass a certain limiting size in spite of its continuous assimilation (principle of constant cell size) and 2 . which factors lead to division when this limiting size is reached. According to Linzbach ( I 955), the cause "why cells during growth do not surpass a certain size, as well as the causes which lead to mitosis, are unknown". Even though no explanation in detail is possible at present, some model conceptions can be advanced in this respect.

Some answer to the first question can be given on the basis of the consideration that the cell is an open system maintaining itself in continuous degradation and synthesis of building materials. The growth of micro-organisms presents arguments for the thesis that growth is regulated by a surface-volume ratio. There is a basic difference in the growth curve of rodlike and spherical microorganisms (Schmalhausen and Bordzilowskaja, I930; Bayne-Jones and Adolph, I932; Bertalanffy,
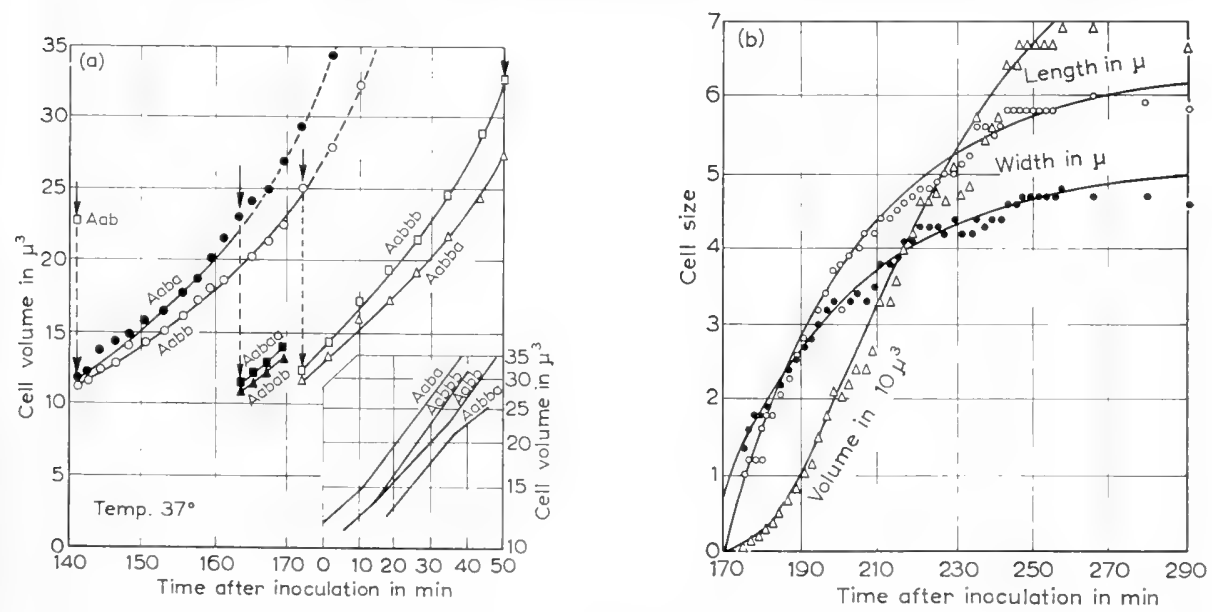

Fig. 5. Growth of micro-organisms. a Growth of individual cells of Bac. megatherium. $b$ Growth of yeast cells. Rod-like bacteria (a) show simple exponential growth (Type II); in spheroid micro-organisms $(b)$ (yeast, micrococci) growth is determined by the surfacevolume ratio (Type I). From the micro-cinematographic measurements of Bayne-Jones and Adolph (1932) calculated by Bertalanffy, I95 ra. 
I 934, r 95 Ia; Fig. 5). Rodlike bacteria grow with almost constant specific growth rate, and hence show simple exponential growth. In contrast, specific growth rate decreases in spherical bacteria and in yeast, the growth curve presenting a decaying exponential for the radius and a sigmoid curve for the volume, respectively.

If growth rate is conceived as the difference between anabolic processes dependent on import and hence on surface, and catabolic processes depending on mass or volume, then for rodlike bacteria the equations:

$$
\begin{aligned}
& \mathrm{d} v / \mathrm{d} t=\eta v-x v=c v \\
& v=v_{0} \mathrm{e}^{c t} \\
& l=l_{0} \mathrm{e}^{c t / 3}
\end{aligned}
$$

$(v=$ cell volume; $\eta, x=$ constants of anabolism and catabolism, respectively; $c=$ growth constant; $l=$ length; $v_{0}, l_{0}$ initial volume, length) will apply because rodlike bacteria grow almost exclusively in length and hence surface responsible for import of nutrients increases nearly proportional to volume. Hence, specific growth rate will be nearly constant, and the growth curve a simple exponential.

In contrast, in a spherical microorganism the surface-volume ratio is shifted in disfavor of surface. Then the growth equation:

$$
\mathrm{d} v / \mathrm{d} t=\eta v^{2 / 3}-x v
$$

will apply indicating that specific growth rate decreases. This gives for linear growth (radius $=r$ ) the decaying exponential:

$$
r=r^{*}-\left(r^{*}-r_{0}\right) \mathrm{e}^{-k t}
$$

and for growth of the volume the sigmoid curve:

$$
v=\left[\sqrt[3]{ }^{*}-\left(1^{3} \overline{v^{*}}-1^{3} \overline{v_{0}}\right) \mathrm{e}^{-k t}\right]^{3}
$$

with $r^{*}, v^{*}=$ final radius, volume, $r_{0}, v_{0}$ initial radius, volume, and $k=x / 3$.

These equations explain the characteristic differences between the growth curves of rodlike and spherical microorganisms, and between the curves of linear and volume growth of the latter, and give excellen't fit of empirical data (Schmalhausen and Bordzilowskaja, I930; Bertalanffy, I934, I951a), so showing that the model concept is essentially correct.

The theory also explains the temperature dependence of growth usually found in microorganisms, with the exception of certain phenomena in the growth of yeast (p. 205).

Furthermore, the theory gives a certain insight into the basis of the principle of constant cell size. If a growth equation of the kind of equation (3.4) applies, the growing system is equifinal, i.e. it will attain the same final size independent of initial size. Under the simple condition:

$$
v^{*}=(\eta / x)^{3}=2 v_{0}
$$

the steady state is reached when the initial volume is doubled. However, the final volume $\left(v^{*}\right)$ depends only on the constants of building up and breaking down $\left(\eta\right.$ and $x$ ), not on the initial size $\left(v_{0}\right)$. That is, the system may divide into parts 
of varying number or size (as in unequal division, formation of spores, etc.), and the final size will remain constant. The model naturally does not explain the details (such as different size of cells of different tissues, occasional appearance of giant cells, etc.), but seems to elucidate the principle of limitation of cell growth.

With respect to the physical prerequisites of division after reaching a critical size, Rashevsky ( 1948 ) has indicated a mathematical cell model consisting of a droplet where import and export, anabolism and catabolism of materials take place. Within such system concentration gradients of substances produced and flowing out, and substances flowing in and consumed will appear, and with these gradients forces tending to disrupt the system or to counteract division, respectively. It can be shown that the system will grow up to a certain critical value where metabolic forces prevail and lead to spontaneous division. The model admittedly neglects all details of mitotic division, etc. which are obvious from the cytological and cell-physiological viewpoints. It is, however, remarkable that a number of characteristics regarded as specifically "vital" can be derived from this highly simplified model: Growth, periodic division, the impossibility of a "spontaneous generation" of such droplet systems, and an order of magnitude (if appropriate physical forces are assumed) that corresponds with the average size of living cells.

IV. GROWTH OF TISSUES

(a) Cell renewal

Similar to the steady state exhibited by the living organism with respect to its chemical components, the organism is in a dynamic state with respect to its component cells. It grows if production of cells prevails, and eventually attains a steady state when wearing-out and renewal of cells balance each other.

The classification of tissues with respect to cell renewal goes back to Bizzozero (1894). He distinguished three classes of tissues: $r$. tissues with labile cells which multiply during the whole life span; 2 . tissues with stable cells which differentiate in certain directions and divide post-embryonically but not in the adult organism; 3. tissues in which mitotic activity ceases at an early period of embryonic growth and which lack physiological as well as restitutive regeneration.

The classification of tissues with and without cell renewall has been refined by Cowdry (1942, 1957). Two types with two sub-groups each are distinguished:

I. Intermitotic cells, retaining the capability of division: A. Vegetative intermitotic cells, which continually divide in the adult organism and so serve as a cell reservoir (e.g. basal epithelial cells, spermatogonia, hematoblasts); $B$. Differentiated mitotic cells which by differentiation and division yield cells of type $C$ or $D$ (e.g. spermatocytes, spermatides, immature leucocytes).

2. Postmitotic cells, i.e. highly differentiated cells which do not normally divide: C. Reversibly postmitotic cells which normally remain undivided till death, but are capable of division in hyperplasia and regeneration (e.g. liver cells, kidney epithelium, endothelia of blood vessels); D. Fixed postmitotic cells showing highest differentiation and lack of division (e.g. ganglion cells, fibers of heart and skeletal muscle, erythrocytes). 
TABLE 4

TURNOVER OF CELLS IN RAT TISSUES

After C. P. Leblond and F. D. Bertalanffy. From L. von Bertalanffy, I 953

\begin{tabular}{|c|c|c|c|}
\hline & $\begin{array}{l}\% \text { cells } \\
\text { formed } \\
\text { daily }\end{array}$ & $\begin{array}{c}\text { Renewal } \\
\text { time } \\
\text { (days) }\end{array}$ & References \\
\hline \multicolumn{4}{|l|}{ I. Tissues with no cell addition } \\
\hline Nervous tissue & 0 & & 1 \\
\hline Adrenal medulla & o & & 1 \\
\hline \multicolumn{4}{|c|}{ II. Tissues with cell addition, but no renewal } \\
\hline Adrenal cortex & 0.2 & & 1 \\
\hline Liver (cell counts) & 0.2 & & 1 \\
\hline (DNA tracer studies) & 0.7 & & 2 \\
\hline Kidney & & & \\
\hline proximal tubules & 0.3 & & 1 \\
\hline distal tubules & 0.4 & & 1 \\
\hline glomerular tufts & 0.4 & & 1 \\
\hline Thyroid & 0.3 & & 1 \\
\hline \multicolumn{4}{|l|}{ III. Tissues with cell renewal } \\
\hline Intestinal epithelium & 70.4 & I.4 & 3 \\
\hline \multicolumn{4}{|l|}{ Stomach: body } \\
\hline surface epithelial cells & $35 \cdot 4$ & 2.8 & 4 \\
\hline mucous neck cells & 15.6 & 6.4 & 4 \\
\hline \multicolumn{4}{|l|}{ Stomach: pylorus } \\
\hline surface epithelial cells & 51.6 & I.9 & 4 \\
\hline gland cells & 56.4 & 1.8 & 4 \\
\hline \multicolumn{4}{|l|}{ Epidermis: plantar } \\
\hline Malpighian layers & 5.2 & 19.2 & 5 \\
\hline stratum corneum & $3 \cdot 3$ & 30.0 & 5 \\
\hline \multicolumn{4}{|l|}{ Epidermis: ear } \\
\hline Malpighian layers & 2.9 & $34 \cdot 5$ & 1 \\
\hline Sebaceous glands & 13 & 8.0 & 8 \\
\hline \multicolumn{4}{|l|}{ Epidermis: ventral abdomen } \\
\hline Malpighian layers & 5.2 & 19.2 & 1 \\
\hline Bladder epithelium & 1.5 & 66.5 & 6 \\
\hline Tracheal epithelium & 2.1 & 47.6 & 1 \\
\hline \multicolumn{4}{|l|}{ Lymph node of lung } \\
\hline (lymphocytes) & I 4.6 & 6.9 & 7 \\
\hline \multicolumn{4}{|l|}{ Lung } \\
\hline vacuolated alveolar cells & $3 \cdot 4$ & 29.6 & 7 \\
\hline non-vacuolated alv. cells & 12.3 & 8.2 & 7 \\
\hline
\end{tabular}

1 Unpublished results.

2 Stevens, C. E., et al., (1953b) 7. Biol. Chem., 202, 177.

3 Leblond, G. P., and C. E. Stevens, (1948) Anat. Record, Ioo, 357.

4 Stevens, C. E., and C. P. Leblond, (1953) Anat. Record, 115, 23 I.

5 Storey, W., and C. P. Leblond, (I95I) Ann. N. Y. Acad. Sci., 53, 537.

6 Leblond, C. P., M. Vulpe and F. D. Bertalanffy, (i955) F. Urol., 73, 3 I I.

7 Bertalanffy, F. D., and C. P. Leblond, (1953) Anat. Record, 115, 5 I 5.

8 Bertalanffy, F. D., (1957) Anat. Record, 129, 23 I. 
Experimental study supports this histological classification. Tissues with cell renewal are characterized by a relatively high mitotic rate in the adult organism which is balanced by a corresponding loss of cells, so that the total cell number remains unchanged and neither positive nor negative growth takes place. A frequently used technique (Leblond and Stevens, I 948; Storey and Leblond, I95 I ; F. D. Bertalanffy and Leblond, I953; Leblond and Walker, I956) is the application of colchicine which stops mitosis and allows counts of mitoses and hence determination of mitotic rate over a period of time. A survey of cell renewal in the principal mammalian organs is presented in Table 4 showing that tissues such as intestinal epithelium, epidermis, and lung have a surprisingly high mitotic rate and correspondingly rapid turnover and short time of renewal. It is worth mentioning that although most investigations were carried out with mammalian organs, the same principle applies to invertebrates. The adult hydra, for example, remains in a steady state by continuous cell loss at the poles and corresponding physiological regeneration by production of cells from the undifferentiated I-cells. Amputation entails an increased cell production by the I-cells and so restitution of the steady state (Tardent, I954).

Obviously there are connections between the type of a tissue with respect to its cell renewal, mitotic activity, turnover rate as determined by isotope labelling, autolysis, and susceptibility to malignant transformation. It does not seem to be possible, however, to reduce these correlations to a hard and fast rule.

Protein turnover as determined by isotopes and other methods is only loosely correlated with cell renewal of the tissue concerned. Corresponding to the high renewal rate, high protein turnover is found in the intestinal mucosa, the bone marrow, and in embryonic and malignant as compared to normal tissues (Borsook, I950). However, functional activities must also be taken into consideration as, for example, in the liver, which shows mitotic activity only in regeneration (compensatory hyperplasia) but has a high protein turnover; or in nervous tissue which represents a cell-constant organ with no mitotic activity, but has a high content in RNA (Nissl bodies) and a rather high protein turnover (Weiss and Hiscoe, I948).

A good correlation is found between classification of tissues in terms of presence or absence of cell renewal, and experiments with 32P (Smellie, I955). With respect to incorporation of radiophosphorus into $D \mathcal{N} A$, two main classes of tissues can be distinguished: Bone narrow, spleen, thymus, and intestinal mucosa are proliferating tissues where correspondingly incorporation of the isotope is high; liver, kidney, and brain are organs with minimal or no cell division in the adult organism, and correspondingly minimal $32 \mathrm{P}$ incorporation into DNA. According to expectation, increased incorporation is also found in regenerating rat liver, in hepatoma, carcinomas, and the mammary gland during pregnancy.

In order to maintain the steady state, there must be a harmonization between cell loss and cell renewal. This is found experimentally: In the starved mouse, mitotic rate of the epidermis is reduced by 25 per cent, although neither the thickness of the epidermis nor the size of the sebacious glands is reduced. Obviously the desquamation of the stratum corneum and the secretion of sebum must be regulated accordingly (Bullough, 1952). 
From the chemical viewpoint, a harmonization between protein synthesis and degeneration is to be expected: In the steady state, cells and tissues with high protein synthesis should present high protein degradation, and in the non-steady state (growth) high protein degradation should be paralleled by a high capacity of growth and regeneration. In a crude approximation, a correlation between content of proteolytic enzymes (expressed, for example, in the autolysis of surviving organs), protein turnover and growth capacity can be found (Bertalanffy, I 942 and I951a; Pollister, I 954). At one end of the series are cells undergoing fast renewal, showing strong autolysis and partly playing a predominant role in wound healing and regeneration, such as leucocytes, mucous membranes, gland cells, and the regenerating layers of the epidermis. At the other end are cells with a low content of proteolytic enzymes and little or no capability of division and regeneration, such as muscle and nerve cells. Similarly, newborn animals compared to older ones, and animals treated with thyroid hormone show increased autolysis, corresponding to increased protein metabolism.

Correlations between cell renewal and susceptibility to malignant growth as expressed by the frequency of carcinoma in the various organs can also be found (Leblond, Storey and F. D. Bertalanffy, I95I).

\section{(b) Cell constancy}

The extreme case of cells incapable of division is found in cell-constant animals (rotatoria, nematodes, tardigrades, appendicularias) where after embryonic development no cell renewal, physiological or restitutive regeneration takes place. For example, the rotator Hydatina senta consists of 950 somatic cells distributed in exact numbers among the various organs. Cell constancy does not, however, exclude cell growth. For example, the cells of the intestinal epithelium of Ascaris grow to a size that corresponds to the total size of the freshly hatched larva. In the mammalian organism, heart muscle fibers and ganglion cells are incapable of division post-embryonically. These organs are probably cell-constant (heart: Linzbach, I 950). Nevertheless, there is a continuous renewal of cytoplasm in the nerve fiber (Weiss and Hiscoe, 1948); nerve cells still grow, their nuclei belonging to the highest class of the nuclear series; and the nerve fiber is highly capable of regeneration. Similarly, considerable cell growth takes place in the fibers of the heart muscle (Linzbach, I955; Fig. 4, p. I60).

\section{(c) Mathematical theory of growth of tissue cultures}

The quantitative aspects of the growth of organs within the organism will be considered later (p. $234 \mathrm{ff}$.). Here only the growth of tissues isolated from the organism and grown in vitro will be briefly reviewed. Several formulas for the growth of tissue cultures have been proposed of which the following appear to have the best theoretical background, and are special cases of a general model and equations of growth to be discussed in the following (p. I $77 \mathrm{ff}$.).

Buch Andersen and Fischer (1929) empirically found that for the growth of the radius of a tissue culture an equation of the type of equation (5.27) and (5.29) applies. It can be assumed that synthesis and consequent cell multiplication depend on the surface, i.e. diffusion of nutrients into the culture, while growth 
is limited by catabolic loss depending on volume. Then, omitting the initial radius $\left(r_{0}\right)$ of the culture as small and negligible, an equation:

$$
r=\frac{E}{k}\left(\mathrm{I}-\mathrm{e}^{-k t}\right)
$$

will apply as found in experiment (Fig. 6).

In contrast to assertions often made, Buch Andersen and Fischer emphasize that the growth of tissue cultures is not unlimited; growth rate decreases and

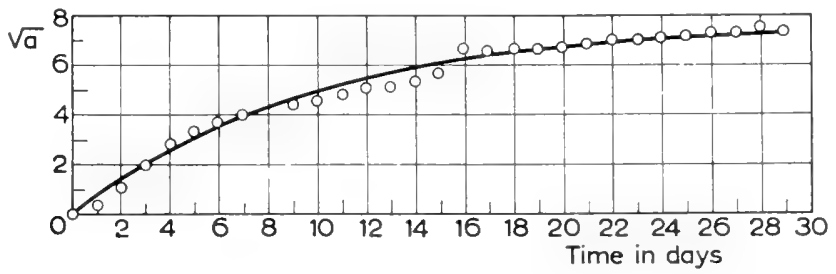

Fig. 6. Growth of a tissue culture. After Buch Andersen and Fischer, 1929.

eventually becomes zero if the experiment is sufficiently prolonged. In its first part, however, a growth curve according to equation (4.I) is nearly a straight line, the curvature becoming apparent only later. That is, growth rate of the radius is approximately constant at first:

$$
\mathrm{d} r / \mathrm{d} t \approx E
$$

hence

$$
r=r_{0}+E t
$$

Therefore, the area reached by short-living cultures at time $=t$ can be expressed by:

$$
a=(E t)^{2}
$$

if the small initial radius $r_{0}$ is neglected.

Hence, the growth curve of the area of a tissue culture in this seemingly "unlimited" growth is approximately a parabola. This is easily understood under the assumptions mentioned because surface and hence intake of nutrients is large at first compared to volume and loss; hence increase of the radius will be nearly linear $(E)$, and only later on the term $(-k r)$ will become manifest. This case is important for two reasons: $I$. The first phases show that the growth rate of the radius of a circular tissue culture would be constant if it were not eventually counteracted by degradative volume-dependent processes. The fact that growth of a tissue culture is a "marginal phenomenon" (Buch Andersen and Fischer, I 929) is a confirmation of the assumption that synthetic processes depend on a surface. 2. In growth curves where only the first part is envisaged, a similar constancy of the increase of linear dimensions and thus seemingly "unlimited" growth may appear; therefore, equation (4.3) for the growth of tissue cultures is identical with equation (6.I) for embryonic growth (p. 223).

For residual growth of tissue cultures, i.e. growth without nutritive extract, an equation (Ephrussi and Teissier, I932) applies which can be written in the form: 


$$
\mathrm{d} a / \mathrm{d} t=k a^{1 / 2}\left(a^{*}-a\right)
$$

$\left(a^{*}=\right.$ final size of area). In this case, the initial area $\left(a_{0}\right)$ enters into the parameter $a^{*}: a_{0} \cdot E^{\prime}=a^{*}$, i.e. the maximal area eventually reached is proportional to the initial area. This is obvious because in this case growth is limited by the resources lying in the explant itself. Introducing new constants, equation (4.5) can be written:

$$
\mathrm{d} a / \mathrm{d} t=\eta r^{2}-x v
$$

which is a modification of equation (5.24). Equation (4.6) implies $I$. that the growth of volume of a tissue culture is nearly equal to growth in surface because growth in thickness is negligible; 2. it differs from equation (5.24) insofar as according to the latter, total surface is responsible for growth while according to the first, only the periphery is responsible because mitoses are confined to it. Equation (4.6) is written in a somewhat different way by Ephrussi and Teissier (1932). The slight modification made here shows that the growth equation proposed by these authors is a special case of the general growth equation and model used in the present study.

\section{GROWTH IN TIME OF THE TOTAL ORGANISM}

\section{(a) Definitions}

The course of growth in time is expressed by the growth curve where the magnitudes investigated, such as weight, length, etc. are plotted against time. Some frequently used measures require definition.

Absolute growth rate is the increase of size per unit of time $(\mathrm{d} y / \mathrm{d} t)$ if $y$ is a measure of body size such as weight $(w)$ or length $(l)$.

Specific growth rate is the increase in size per unit of time and unit of size, hence $\mathrm{d} y / y \mathrm{~d} t$.

The following measures of specific growth rate are frequently used:

$I$. Minot's formula. If $y_{0}$ and $y$ are two sizes (weights, lengths) at times $t_{0}$ and $t$, the average increase per time unit is $y-y_{0} / t-t_{0}$, or if the interval $\left(t-t_{0}\right)$ is equal to $\mathrm{I}: y-y_{0}$.

Hence, specific growth rate according to Minot is:

$$
\frac{\mathrm{d} y}{\mathrm{~d} t} \cdot \frac{\mathrm{I}}{y}=\frac{y-y_{0}}{y}
$$

i.e. increase in size divided by initial size. If specific growth rate is indicated in per cent, this value is to be multiplied by $\mathrm{s} 00$.

This formula is inexact because the increase in size is related to a constant value at the beginning of the time period under consideration. In fact, body size increases continually. The error committed by calculation of growth rates after Minot's formula is the greater the larger is growth rate and the longer the intervals. 
2. The mathematically simplest assumption to take into account the continuous increase in size is to assume specific growth rate to be constant:

$$
\frac{d y}{\mathrm{~d} t} \cdot \frac{\mathrm{I}}{y}=c
$$

Integrated, this expression gives:

$$
y=y_{0} \mathrm{e}^{c t}
$$

from which specific growth rate can be calculated as:

$$
\frac{\mathrm{d} y}{\mathrm{~d} t} \cdot \frac{\mathrm{I}}{y}=\frac{\log y-\log y_{0}}{\log \mathrm{e}\left(t-t_{0}\right)}
$$

( $\mathrm{e}=$ base of natural logarithms). This formula is a better approximation than (5. I) but also is a frequently inexact approximation. It presupposes that growth is exponential according to equation (5-3). This, and the corresponding assumption that specific growth rate is constant, is in general untrue; for as a rule growth rates decrease during development. The error committed is considerable, so that equations (5.I) and (5.4) have only limited applicability.

In general, absolute and specific growth rates decrease during animal growth, and eventually the organism attains a steady state or is "adult". There are, however, also other types of animal growth (p. I 80). Apart from these cases it is a general rule that the curve of weight growth is a sigmoid. This implies that absolute growth rate $\mathrm{d} w / \mathrm{d} t$ first increases to a maximum and then decreases. Specific growth rate $\mathrm{d} w / w \mathrm{~d} t$ or, what amounts to the same, logarithmic growth rate $\mathrm{d} \log w / \mathrm{d} t$ is a monotonically decreasing function which implies that increase per cent and per unit time decreases, or that the time needed for duplication of weight continually increases.

In the case that the growing body remains geometrically similar during its increase in size, growth is called proportional. In this case, the surface $s$ can be obtained by multiplication of the square of any linear dimension $l$ with a constant; similarly the volume $v$ (or the weight $w$, if specific weight is constant) is obtained by multiplication of the third power with a constant:

$$
s=p l^{2}, v=q l^{3}
$$

In the case of proportional growth, a simple relation between growth in length, in surface, and in volume obtains:

$$
\begin{aligned}
& \frac{\mathrm{d} v}{\mathrm{~d} t} \cdot \frac{\mathrm{I}}{v}=\frac{\mathrm{d}\left(l^{3} q\right)}{\mathrm{d} t} \cdot \frac{\mathrm{I}}{l^{3} q}=\frac{3 \mathrm{~d} l}{\mathrm{~d} t} \cdot \frac{\mathrm{I}}{l} \\
& \frac{\mathrm{d} s}{\mathrm{~d} t} \cdot \frac{\mathrm{I}}{\mathrm{s}}=\frac{\mathrm{d}\left(l^{2} q\right)}{\mathrm{d} t} \cdot \frac{\mathrm{I}}{l^{2} p}=\frac{2 \mathrm{~d} l}{\mathrm{~d} t} \cdot \frac{\mathrm{I}}{l} \\
& \frac{\mathrm{d} l}{\mathrm{~d} t} \cdot \frac{\mathrm{I}}{l}=\frac{\mathrm{I}}{2} \frac{\mathrm{d} s}{\mathrm{~d} t} \cdot \frac{\mathrm{I}}{\mathrm{s}}=\frac{\mathrm{I}}{3} \frac{\mathrm{d} v}{\mathrm{~d} t} \cdot \frac{\mathrm{I}}{v}
\end{aligned} \mid
$$

That is, specific growth rate of a surface is twice, that of volume or weight three times the specific growth rate of a linear dimension. 
(b) General considerations on models and laws of growth

Because of the possibility quantitatively to express the relevant variables, growth belongs to those biological phenomena which for a considerable time have invited mathematical treatment. In these attempts, a lack of understanding the meaning of theory and laws in science can often be noticed. Hence a brief epistemological discussion appears to be in place.

Biologists are apt to expect too much and too little from a mathematics of growth: too much when looking forward to a "wonder formula" which would represent any and all observed growth data, and being disappointed if such prodigy cannot be produced; too little if correct approximation of a smaller or larger set of data is considered sufficient proof that a "law of growth" has been discovered. Furthermore, many biologists, believing in a somewhat mythical ritual called "the experimental method", are still unfamiliar with the procedure applied in theoretical science, even though this is commonplace in physics and since has diffused into the behavioral and social sciences.

The approach toward mathematical analysis of empirical data may be illustrated by a fictitious example. Suppose the problem is to establish a mathematical expression to describe the relation between time and distances passed by freefalling bodies. Two investigators may proceed in two essentially different ways. The one will let fall different objects such as cannon-balls, stones, feathers, cats and dogs; will measure the distances travelled; and tabulate or plot the results. Then he may develop distance as a function of time by way of a series:

$$
S=a_{0}+a_{1} t+a_{2} t^{2}+\ldots
$$

and will calculate the coefficients. This procedure is infallible and will allow description of any observation with any precision desired, if an arbitrary number of terms is permitted. However, the terms and coefficients have no physical meaning, and will be different from one case to another.

The other investigator who, in history, was Galileo and his followers, proceeds in an essentially different fashion. By way of some intuitive process he has arrived at the concepts of "force "and "acceleration", constant force producing constant acceleration. If acceleration is constant, the velocity reached by the falling body will be proportional to the time passed: $v=c t$, and the distance traveled will be average velocity during the time interval $t$ multiplied by time elapsed, i.e. $S=c t^{2} / 2$, which is Galileo's law.

Our investigator does not bother to fit all observations made or feasible, but has discovered a fundamental "law of nature". This law represents observations where the relevant relation is well isolated from others as, according to legend, in the cannon-balls Galileo dropped from the leaning tower of Pisa. The law is a poor approximation of many other observed phenomena but this does not disturb our physicist who answers, with Galileo's student, Torricelli: "If balls of lead, iron and stone do not obey the law, so much the worse for them; then we say that we do not speak of them".

However, it turns out that this seemingly arbitrary procedure is eminently successful. If Galileo's law is taken for granted, seeming deviations eventually 
fall in line by taking into account complicating factors, as for example air resistance, which in turn become amenable to exact law. Furthermore, Galileo's law leads to Newton's law, the derivation of Kepler's laws of planetary motion to the development of modern astronomy and mechanics in general, and from classical mechanics eventually to relativity theory and modern physics.

What Galileo and his colleagues did was to establish a mathematical model, that is, a conceptual construct representing allegedly "essential" factors in the process in question, and allowing deductions which can be tested in experiment or observation. The law refers to an "ideal" case, approached in certain experimental situations (cannon-balls and, still better, bodies falling in vacuo), but may be very unsatisfactory in others. Galileo's law does not provide a very satisfactory mathematical description for falling leaves, feathers, or cats. The same applies to any law of physics. There is, strictly speaking, no ideal gas, no absolutely rigid body, etc., and the respective laws refer to "ideal" cases or cases which experimentally have been made nearly ideal. The essential feature of a model is that it forms the basis of a hypothetico-deductive system yielding "dividends" (to use an illustrative phrase of Rapoport, I 955b), that is, results mathematically implied in the model but not ascertainable and amenable without the logical machinery. By mathematical reasoning and insertion of appropriate conditions consequences can be derived which explain a range of observed phenomena and even predict still unobserved ones. (The classic exposition on the nature of scientific theory is still: Kraft, I 926.)

A first consequence from the nature of scientific theory is that the relation between observation and law is by no means simple. If the alleged law is contradicted by observation, it naturally has to be discarded. However, mere fit of observed data does not prove a mathematical expression to be a law of nature. Particularly in complicated phenomena (p. 174), good fit may be obtained by formulas which are without meaning, or by different formulas. On the other hand, unsatisfactory fit of certain observations does not necessarily disprove the model and law. It may be that complicating factors have to be introduced as in the case of Galileo's law and air resistance; that the original model was too simple and needs amendment, as in the case of Bohr's model of the atom which allowed deduction of the spectrum of hydrogen but was not adequate for more complicated atoms; or that the initial theory eventually becomes a special case in a more general one as was the case with classical mechanics compared to the theory of relativity. In such instances, the original theory is not exactly refuted but rather turns out to be a special case, valid under certain restrictive conditions, of some more general theoretical framework. It is indeed the criterion of a good scientific theory that it leaves room for further developments, in contrast to "coverall" concepts or panchresta (Hardin, I956) such as the entelechy of the vitalists, natural selection ( $c f$. Bertalanffy, I932, p. 72; 1952, p. 89f.), etc. which "explain" everything and for precisely this reason nothing.

The transition from simple to more complicated cases and corresponindgly to more elaborate theories is the progress of science. Here, like the beginner's luck in a gamble, accident often plays an important role. Mendel was able to state his basic laws because he happened to cross peas which represented a schematic 
case; he was defeated in his later Hieracium experiments; and if he had started with some complicated case in drosophila, involving sex linkage, coupling, crossing over, position effect, etc., genetics would never have been founded by him.

Another important aspect of scientific theory is the unification of previously disparate facts it provides. To the naive observer, the revolution of planets, free-falling bodies, bodies rolling on an inclined plane, the swing of a pendulum, ebb and tide, etc. have nothing in common. Yet these phenomena are united by the laws of mechanics, and so are mechanical and thermal phenomena in statistical mechanics, optical and electrical phenomena in electrodynamics, crossing experiments and cytological facts in cytogenetics, etc.

A further "dividend" is the verification of calculated constants by independent experiment. For example, when Loschmidt calculated the number of molecules in a gas per unit volume and under standard conditions, Loschmidt's number $\mathcal{N}$, so long as this calculation stood isolated, could still be considered as a sort of freak and the atomic structure of matter as fictitious, as was actually the case with Mach, Ostwald, and other physicists. When, however, Loschmidt's number came out the same in calculations so different as from kinetic theory, Brownian movement, radioactivity, etc., doubt in the calculated value of $\mathcal{N}$ and the underlying theory became meaningless.

In the above examples we have assumed that the initial model and theory were well-chosen and productive of further development. There is, of course, no guarantee for this being the case. History of science if full of misconceived theories which did not stand the proof and were discarded. However, there is little need to worry about wrong theories; they are quickly eliminated in the progress of science. As a rule it may be said that a wrong theoretical model is better than no model at all, as can be evidenced by many examples from the history of science. A biological case to the point is Weismann's theory of "determinants" and "unequal nuclear division" which was thoroughly wrong but formed the stepping stone toward modern cytogenetics.

One more aspect of scientific theory deserves emphasis in this connection. It is often maintained that theory, models, and laws are impossible or premature because the phenomena in question are too complicated and the underlying processes too little known to allow them. This particularly applies to biological phenomena such as growth which obviously are the outcome of innumerable and largely unknown component processes.

The answer is that nothing in nature is "simple"; more or less "simple" are only the conceptual models we devise to represent certain aspects of natural phenomena.

This question is intimately connected with the hierarchical stratification of nature and what has been termed, in psychology, "molecular" and "molar" theories. "Molecular" theory tries to advance analysis into components, "molar" theory to state overall laws. In this respect, two general rules can be stated.

$I$. Scientific progress, as a rule, is in the direction of resolving overall phenomena into phenomena at lower levels, that is, from "molar" to "molecular" research. This is evident, for example, in the development from "macro" to 
"microphysics", from classical thermodynamics to statistical mechanics, from classic Mendelism to the theory of the gene, etc.

2. On the other hand, the fact that a "macroscopic" phenomenon (at any arbitrary level) is the result of complex, numerous, unaccountable or even unknown component processes, does not preclude the statement of overall models and laws. Historically, "molar" theories and laws often precede "molecular" ones; and "molar" and "molecular" descriptions complement each other.

These statements also are evidenced by examples from all fields of science. For instance, the so-called mechanistic attitude in biology demanding that all phenomena in the living world be reduced to laws of physics and chemistry implies the assumption that the latter are fundamental and simpler than those at the biological level. This was a sound working hypothesis so long as it was expected that the total of physical phenomena could be reduced to the model of Laplacean mechanics. It turned out, however, that the postulate of reductionism is far from being attained even in physics. The organization of an atomic nucleus is almost as complex and problematic as that of a living cell or organism; there are, at present (1958) some 28 fundamental particles found in nature or produced by the modern accelerators which are not accounted for by theory. However, the fact that they are not "reduced" to elementary laws of ultimate entities (as was supposed in the mechanistic doctrine expecting all natural phenomena to be resolvable into mechanical mass-points governed by a Laplacean formula) does not detract from the value of the various fields of "macrophysics", mechanics, electrodynamics, thermodynamics, etc. which remain perfectly valid so far as they go.

The same principle can be seen in the various branches of science. In thermodynamics, it is impossible to account for the individual behavior of the enormous number of molecules in a gas; but the overall result is expressed by statistical laws. In biochemistry, phenomena such as photosynthesis or respiration are the outcome of some dozens of enzymatic step reactions many of which are insufficiently known; but this does not obviate the overall or "molar" equation, $\mathrm{C}_{6} \mathrm{H}_{12} \mathrm{O}_{6}+$ $6 \mathrm{O}_{2}=6 \mathrm{H}_{2} \mathrm{CO}_{3}+674 \mathrm{kcal}$. Even inorganic reactions such as the formation of halogen hydrates which are deceivingly simple are, in fact, a balance or system of many partial reactions (Skrabal, I 949, I95 I). In physiology, basal metabolic rate is the outcome of innumerable and largely unknown processes of intermediary metabolism; nevertheless, it follows simple relations such as the surface rule (p. $18 \mathrm{I}$ ) and is used in routine diagnostic procedure. In economics, happenings are the result of innumerable factors, activities of individuals, machines, social groups, etc.; but these can be expressed in balance sheets, and economic laws may fairly express, and allow prediction of the overall outcome.

It is, therefore, not a naive oversimplification to seek overall organismic laws in processes that result from a complex multitude of component processes. This is only a special case of the fact that "laws of nature" are, in principle, possible at any level of observation and reality, and of the paradox that a multitude of component processes may obey rather simple laws as a whole. As in any case, the sole criterion is that the model works, that is, yields "dividends" in the sense explained above. 
(c) Empirical growth formulas

The above considerations apply to the topic of the present monograph, and allow quickly to dispose of a considerable number of formulas proposed in the study of animal growth.

What is called a growth curve is in fact a set of usually scattered points representing observed measurements. Curves of higher degree, even if they are rigidly fixed, often can be fitted by quite different mathematical expressions. A classical example is Kavanagh and Richard's demonstration (I934) that the logistic curve can well be approximated by the probability integral. Such consideration applies even more when a set of points is smoothed more or less arbitrarily into a continuous curve. Hence, approximation of empirical growth data is a necessary but by no means sufficient condition that an equation proposed represents a "law" of growth. Good approximation may be obtained with formulas which are meaningless, or with quite different formulas.

I. The two basic approaches discussed above apply to the phenomenon of growth. The first possibility is a purely empirical approach, that is, mathematical expressions are sought which describe observations as closely and simply as possible. The most general procedure is application of series which can represent any set of observations with any degree of approximation desired, breaking the series after the quadratic or cubic term being usually satisfactory. Such series can be used either in the straightforward form of the Taylor series (equation 5.7), or in some modified form.

Some equations frequently used for describing growth are series either in $y$ :

$$
\mathrm{d} y / \mathrm{d} t=k_{0}+k_{1} y+k_{2} y^{2}+\ldots
$$

or in $t$ :

$$
\mathrm{d} y / \mathrm{d} t=k_{0}+k_{1} t+k_{2} t^{2}+\ldots
$$

The exponential is the Taylor series retaining one term:

$$
\mathrm{d} y / \mathrm{d} t=k_{1} y
$$

$\left(k_{0}=0\right.$ as otherwise $\mathrm{d} y / \mathrm{d} t$ would not vanish with $\left.y=0\right)$; the logistic retaining two terms:

$$
\mathrm{d} y / \mathrm{d} t=k_{1} y+k_{2} y^{2}
$$

Equation (5.9) was first proposed by Enriques (1909). The parabola (Schmalhausen, I 929)

$$
y=k t^{a}
$$

is another simplification of this series. Similarly, Backman's (1943) formula is a series in the logarithms:

$$
\log y=k_{0}+k_{1} \log t+k_{2} \log t^{2}+\ldots
$$

Pearl's (p. I 76 ) formula is a logistic with a power series in $t$, etc.

Mathematical description of growth data by formulas of this kind can be useful for purposes of intrapolation and, if necessary caution is used, also of extra- 
polation; but is has no theoretical significance. The constants appearing in such formulas have no physiological meaning, and are different in each particular calculation. Fitting of data is obtained not by virtue of the particular equation used, but of the number of arbitrary constants admitted.

Instead of superimposing terms by applying series, complicated curves can also be fitted by subdividing them into segments that can be approximated by a simple equation; or the equation used can be transformed into a linear form, and the curve then subdivided into a sequence of straight lines. As a rule, such subdivision is gratuitous, and therefore fitting of data in this way only an expression of the trivial fact that any curve can be approximated by cutting it into a sufficient number of straight-line sections.

TABLE 5

FUNGTIONS USED FOR DESGRIBING GROWTH GURVES

\begin{tabular}{|c|c|c|c|c|c|c|c|}
\hline Function & $\begin{array}{l}\text { Growth rate } \\
\text { (Weight) } \\
\mathrm{d} w / \mathrm{d} t\end{array}$ & $\begin{array}{l}\text { Weight } \\
w\end{array}$ & $\begin{array}{c}\text { Inflexion } \\
(w ; t)\end{array}$ & $\begin{array}{l}\text { Growth rate } \\
\text { (length) } \\
\mathrm{d} l / \mathrm{d} t\end{array}$ & $\begin{array}{l}\text { Length } \\
\quad l\end{array}$ & $\begin{array}{c}\text { Inflexion } \\
(l, t)\end{array}$ & Authors* \\
\hline I. Exponential & $k w$ & $w_{\circ} \mathrm{e}^{k t}$ & None & $\frac{k}{3} l$ & $l_{\mathrm{o}} \mathrm{e}^{k t / 3}$ & None & \\
\hline $\begin{array}{l}\text { 2. Decaying } \\
\text { exponential }\end{array}$ & $k\left(w^{*}-w_{)}\right)$ & $w^{*}(\mathrm{I}-b \mathrm{e}-k t)$ & None & $\frac{k}{3}\left(\frac{l^{* 3}}{l^{2}}-l\right)$ & $\cdots$ & None & Brody (1945) \\
\hline 3. Logistic & $k w\left(w^{*}-w\right)$ & $\begin{array}{c}w^{*} /\left(\mathrm{I}+b \mathrm{e}-k^{\prime} t\right) \\
\left(k^{\prime}=k w^{*}\right)\end{array}$ & $0.5 w^{*} ; \frac{\ln b}{k^{\prime}}$ & $\frac{k q l}{3}\left(l^{*}-l^{3}\right)$ & $\cdots$ & $\begin{array}{c}0.63 l^{*} ; \frac{\ln (b / 3)}{k^{\prime}} \\
\left(k^{\prime}=k w^{*}\right)\end{array}$ & $\begin{array}{l}\text { Robertson } \\
\quad(1926)\end{array}$ \\
\hline 4. Parabola & $k w / t$ & $\left(w_{1} w_{1} w_{t=1}^{t k}\right)$ & None & $\mathrm{kl} / 3 \mathrm{t}$ & $\left(l_{1}=l_{1^{t}} l_{t=1}\right)$ & None & $\begin{array}{c}\text { Schmalhausen } \\
\text { (1929) }\end{array}$ \\
\hline 5. Gompertz & $w k b e^{-k t}$ & $w^{*} e^{-b e}-k t$ & $0.37 w * ; \frac{\ln b}{k}$ & $\frac{l}{3} k b \mathrm{e}-k t$ & $\begin{array}{c}l^{*} \mathrm{e}-b^{\prime} \mathrm{e}-k t \\
\quad\left(b^{\prime}=b / 3\right)\end{array}$ & $\begin{array}{l}0.371^{*} ; \frac{\ln b^{\prime}}{k} \\
\left(b^{\prime}=b / 3\right)\end{array}$ & Winsor (1932) \\
\hline
\end{tabular}

$w=$ weight; $l=$ length; $w_{0}, w^{*}=$ initial, final weight; $l_{0}, l^{*}=$ initial, final length; e $=$ basis of natural logarithms; $k, k^{3}, b=$ constants; $q=$ proportionality factor $\left(w / l^{3}\right)$.

Proportional growth $\left(w=q l^{3}\right)$ is presupposed for the above relations between growth in weight and in length.

* References selected for detailed discussion of the functions concerned and application to observed growth data.

Table 5 summarizes the more important equations applied to growth. It appears unnecessary to enter into a discussion of the theoretical ideas brought forward in connection with these formulas, as it can be shown that none of them is physiologically well-founded (Bertalanffy, I95 Ia), and it is sufficient to show that these functions, for mathematical reasons, are ill-suited to represent growth curves as empirically found.

I. The exponential is most easily tested since it gives a straight line in semilogarithmic plot. This applies in certain cases (bacteria, insects, Helicidae, p. I 96ff.), but is inapplicable in all cases where growth is limited and approaches a steady state.

2. The decaying exponential (sometimes called the "monomolecular" function) applies in certain cases, namely, for growth of linear dimensions in certain animal groups (Type I, p. I goff.). It is not suited for growth in weight which usually presents a sigmoid curve. For dealing with the latter Brody (1945) subdivided weight curves into a "self-accelerating phase", obeying the exponential or rather consisting of a sequence of exponentials of different slopes; and a "self-inhibiting 
phase", to which the decaying exponential was applied. This splitting into pieces of a very characteristic and universal curve is gratuitous, and no physiological credentials can be given.

3. The logistic has been most favored in quantitative analysis of growth. It is likely to give a correct interpretation of growth in higher green plants ( $c f$. footnote, p. 200). In the case of animal growth, however, the logistic is misplaced. The logistic has a point of inflexion at $1 / 2$ the final weight. This contradicts observed growth curves which usually show inflexion at about $1 / 3$ the final weight. The logistic is ruled out by consideration of growth in length. If the logistic is to apply for weight growth, the curve of growth in length should have an inflexion at 0.63 of final length. Such curve is never found in growth of linear dimensions.

To adjust the logistic to actual growth curves, more complicated expressions have been used. Thus, Robertson ( 1926) used an asymmetric logistic:

$$
\log \frac{w+B}{w^{*}-w}=K(t-T)
$$

with an accessory constant $B$ as compared to the solution of the logistic:

$$
\log \frac{w}{w^{*}-w}=K(t-T)
$$

$\left(T=\right.$ time for $\left.w=w^{*} / 2\right)$.

However, to fit empirical data, Robertson had to pile up superimposed curves. For the calculation of the growth of white mice he used no less than four curves (two symmetric, one asymmetric logistic, and a linear equation) (Fig. 27, p. 22 I) with I I arbitrary constants. Another way to overcome the rigidity of the logistic was used by Pearl and Reed (1925) by introducing, in place of the constant $k$, a power series of time. For example, Pearl's formula for the growth in rats reads:

$$
w=7+\frac{243}{\mathrm{I}+\mathrm{e}^{4.3204-7.2196 t+30.878 t^{2}+0.5291 t^{3}}}
$$

That complications of this sort yield better approximation is, of course, no merit of a special equation but due to the profusion of arbitrary constants to which no physiological meaning can be attached. Robertson's data, fitted by one single formula according to (5.28) are shown in Fig. 27 (p. $22 \mathrm{I}$ ). Examples calculated by Pearl are indicated in Figs. I I (p. I 9I) and 26 (p. 22I), and we see that these data are well represented by equations $(5.28,5.29)$.

4. The parabola has no steady-state solution or inflexion. Since empirical growth curves, in general, have, the parabola is unfit to describe growth in time. Approximation of empirical data by means of parabolic equations is possible only by subdividing the curve into approximable segments which is arbitrary since no physiological reason can be given. Furthermore, any function for specific growth rates which contains $t$ as the only independent variable, is a prior $i$ unfit to describe growth. For growth rates are in general a function not of time, but of the size attained (p. 21 3). In other words, formulas containing $t$ as independent variable do not allow for regulation as it does occur in many phenomena of growth.

5. The Gompertz equation has a point of inflexion at 0.37 of final weight, which corresponds tolerably well to empirical curves of weight growth, but does not 
apply to many curves of growth in linear dimensions. A physiological meaning can hardly be attached to the Gompertz equation which originally was introduced to describe death rates within a population.

As this survey shows, none of these expressions is apt to reproduce the essential and basic characteristics of the usually observed curves of growth. It is important to note that this criticism is not based upon the consideration that some particular sets of growth data are not well fitted by a certain formula. Rather it has been shown that none of these formulas is concordant with the trend and characteristics generally found in empirical growth curves.

II. The way to arrive at a rational theory of growth is outlined in the preceding paragraph. One has to start with a model, that is, plausible and testable hypotheses about this phenomenon. These have to be mathematically formulated. The supposed law must then be put to empirical test, that is, it has to be shown that it allows calculation of the process in question. In doing so, one has to start with cases that are as simple and lucid as possible. Subsequently, analysis will proceed to more complicated cases which may entail consideration of interfering factors and complication, revision, generalization, or abandonment of the original theory. A theory of growth should not only give satisfactory approximation of observed growth data, but should also allow deduction, qualitatively but preferably quantitatively, of consequences which can be tested and verified in independent experiments. Of particular importance is verification of the constants appearing in the growth equations, that is, comparing the constants calculated frow a growth curve with values independently established in physiological determinations. If such verification is successful, it may be inferred that the model under consideration is basically correct, although it may need further improvement and correction with the inclusion of additional observations and the progress of research. Eventually the theory should lead to a unification of previously separate fields, e.g. metabolism and growth.

\section{(d) Quantitative theory of animal growth}

An attempt toward a quantitative theory of growth must first emphasize an essential fact. There is no general growth curve which could be expressed in a universal formula. Rather the course of growth is different in different organisms, in different dimensions, in the organism as a whole and its parts, etc. This is self-evident because the physiological basis of growth is different in each case. As growth ultimately depends on metabolism, it will be the scope of a theory of growth to derive these differences from the comparative physiology of metabolism.

So far as the author knows, there is at present only one quantitative theory which, based upon a rational hypothesis on the mechanism of growth, allows deduction of quantitative laws, covers a considerable amount of empirical data, and allows for theoretical predictions which were confirmed, often in a surprising way, by experiment. This is the theory and equations of animal growth which, in the basic ideas, was stated by Pütter (I 920) and further was developed by the present author and his coworkers (Bertalanffy, I934, I948, I949, I95 I b, I957). 
To arrive at a rational theory of growth, we can start with the following considerations.

$I$. Every living organism and living system in general is an open system, maintaining itself in continuous import and export, building up and breaking down of component materials. This is evidenced by the fact that metabolism is a basic characteristic of living systems and, more particularly, by isotope experiments which dramatically show that the turnover of the building materials of the animal body takes place at a rate hardly expected in classical physiology (p. I47f.).

2. Growth, that is, the increase of body mass in time, is not unlimited. As a general rule, there is first a rapid increase which gradually slows down until the organism reaches a steady state or is "adult". The limitation of growth is not, in principle, due to a decline of the growth potency (whatever this may be) of the component cells and tissues; rather it is regulated by factors lying in the organism as a whole. Witness the fact that cells which have stopped growth and multiplication in the adult organism may resume it if the restrictions imposed are removed, as in regeneration, compensatory hyperplasia, tissue culture, etc.

3. What is measured as "growth" is the outcome of processes of immeasurable complexity, whether envisaged from the biochemical, cytological, physiological, or morphological viewpoints. Not only the mechanisms of synthesis of proteins, nucleoproteins, and other components, as well as the physicochemical basis of mitosis are unknown; even if we knew, the individual chemical and physicochemical events would not give a clue as to how myriads of processes are interacting in the organism in a way surpassing possible analysis. Furthermore, the growing organism undergoes changes in many respects, such as chemical composition, content of water, proteins and other compounds, the ratio between protein and fat, changes of the shape of the body, different relative growth of organs, progressive differentiation of tissues, influence of hormones and consequent physiological changes, and many others.

4. Notwithstanding this complexity, processes in the organism as a whole can be expressed by relatively simple quantitative expressions. As a rule, physiological processes obey the allometric equation (p. $232 \mathrm{f}$.), i.e. their rate can be expressed as a power function of body weight. The allometric equation was found to apply to all physiological processes hitherto investigated, at least as a first approximation, and it is unlikely that others follow a radically different type of equation; for if this would be the case, they would be incompatible with those where the allometric equation holds true (Adolph, I949). This applies even when the phenomenon under consideration is the gross result of many unknown component processes. For example, the rate of metabolism follows the allometric equation, often in the simple form of the surface rule (p. 18I), although it is the outcome of innumerable and largely unknown processes of intermediary metabolism, and the growing organism to which such relation applies undergoes many changes at the biochemical, physiological, cellular, and morphological levels. The same applies to any number of physiological processes.

5. Animal growth can be considered the result of a counteraction of processes of anabolism and catabolism, degradation and regeneration of the building materials of the body. There will be growth so long as building up prevails over breaking 
down; the organism reaches a steady state if and when both processes are equal. The overall result of both kinds of processes may, according to physiological evidence, be expected to be expressed by some power function of the size of the organism. This is expressed in the basic equation:

$$
\mathrm{d} w / \mathrm{d} t=\eta w^{m}-\varkappa w^{n}
$$

In words: The change of body weight $w$ is given by the difference between the processes of building up and breaking down; $\eta$ and $x$ are constants of anabolism and catabolism respectively, and the exponents $m$ and $n$ indicate that the latter are proportional to some power of the body weight $w$.

The degradative processes, expressed by the constant $x$, are represented by the "wearing out quota" (Rubner, I 902), that is, the continuous loss of building material as it takes place in every living organism. Cytologically, this means the continuous loss of cells and cell parts (e.g. replacement of epidermis, squamous tissue, etc., gland secretion, perishing of cells in inner organs, of blood cells), and the corresponding cell renewal found in many tissues and organs, often at an unexpectedly high rate (p. I64f.; Leblond and Walker, I956). Biochemically, it means the continuous degradation of building materials, measured by isotope techniques, nitrogen excretion, or protein loss during starvation. In the adult steady state this degradation is compensated by regeneration so that the catabolic rate equals turnover rate. In a first approximation, the catabolic constant $(x)$ of the growth equation (or Rubner's wearing-out quota) can be equated to the turnover rate of total protein ( $r$, p. I 9gf.). Naturally, however, turnover is not limited to proteins. The constant of the equation rather means the resultant of all growth-limiting factors including, beside manifest protein loss, factors such as progressive differentiation (products of which more or less withdraw from turnover), changes in water content, factors of ageing, etc. However, in a number of cases (Table I I, p. 200) protein loss appears to be the limiting factor determining the course and eventual stopping of growth.

In a physiologically plausible approximation (Bertalanffy, r95 ra) and with particular consideration of the fact that the loss of weight in starvation is proportional to body weight:

$$
-\frac{\mathrm{d} w}{\mathrm{~d} t}=c w ; w=w_{0} \mathrm{e}^{-c t}
$$

and $\mathrm{N}$ or protein content remains nearly constant in starving animals, the rate of catabolism can be assumed to be directly proportional to body weight. On the other hand, mathematical analysis of equation (5.16; $c f . \mathrm{p}$. I $99 \mathrm{ff}$.) shows that it is rather insensitive to smaller deviations of the exponent $n$ from unity. Hence, $n$ can be equated to I without considerable loss of generality, thus reducing the number of parameters and facilitating mathematical treatment:

$$
\mathrm{d} w / \mathrm{d} t=\eta w^{m}-x w
$$

The solution of this general growth equation is: 


$$
w=\left\{\frac{\eta}{x}-\left[\frac{\eta}{x}-w_{0}^{(1-m)}\right] \mathrm{e}^{-(1-m) x t}\right\}^{1 / 1-m}
$$

with $w_{0}=$ weight at time $t=0$.

Equation (5.19) is isomorphic with equation (7.13) which will be discussed later (p. 236ff.):

$$
y=\left(\mathrm{I}-c \mathrm{e}^{-k t}\right)^{a}
$$

Hence the discussion given there applies. The curve has no inflexion (for positive $t$ ) if $\mathrm{I} / \mathrm{I}-m=a \leqq \mathrm{I}$, but is sigmoid for $a>\mathrm{I}$, the point of inflexion lying between $\mathrm{o}$ and $\mathrm{I} / \mathrm{e}$ ( 0.37 of final size), and shown as function of $a(>\mathrm{I})$ by the curve for $\chi_{1}(a)$ in Fig. 39, p. 237. It can be seen that weight-growth curves will always show an inflexion because $(\mathrm{I}-m)<\mathrm{I}$, but curves of length growth may or may not have an inflexion (see below).

Anabolism of building materials, i.e. synthesis of high-molecular cell compounds needs, on the one hand, building blocks such as amino acids, sugars, phosphates,

\section{TABLE 6}

METABOLIC TYPES AND GROWTH TYPES

A. Anabolic processes depend on resorption (resorbing surfaces). (a) Micro-organisms: (I)Proportional growth: linear and volume growth curves as B, I; spherical bacteria, yeast. (2) Growth almost exclusively in length: linear and volume growth curves exponential; rod-like bacteria. (b) Probably turbellarians.

\begin{tabular}{|c|c|c|c|}
\hline Metabolic type & Growth type & Growth equations & Examples \\
\hline $\begin{array}{l}\text { I. Respiration } \\
\text { surface- } \\
\text { proportional }\end{array}$ & $\begin{array}{l}\text { (a) Linear growth curve: } \\
\text { attaining without inflexion } \\
\text { a steady state. } \\
\text { (b) Weight growth curve: } \\
\text { sigmoid, attaining, with } \\
\text { inflexion at } c a \text {. I } 3 \text { of } \\
\text { final weight, a steady } \\
\text { state }\end{array}$ & $\begin{array}{l}\mathrm{d} w / \mathrm{d} t=\eta w^{2 / 3}-x w \\
\text { a) } l=l^{*}-\left(l^{*}-l_{0}\right) \mathrm{e}^{-x t / 3} \\
\text { b) } w=\left[1 / w^{*}-\left(\sqrt[3]{w^{*}-}\right.\right. \\
\left.\left.-1 / \overline{w_{0}}\right) \mathrm{e}^{-x t / 3}\right]^{3}\end{array}$ & $\begin{array}{l}\text { Lamellibranchs, } \\
\text { fish, mammals }\end{array}$ \\
\hline $\begin{array}{l}\text { II. Respiration } \\
\text { weight- } \\
\text { proportional }\end{array}$ & $\begin{array}{l}\text { Linear and weight growth } \\
\text { curves exponential, no } \\
\text { steady state attained, } \\
\text { but growth intercepted } \\
\text { by metamorphosis or } \\
\text { seasonal cycles }\end{array}$ & $\begin{array}{l}\mathrm{d} w / \mathrm{d} t=\eta w-x w=c w \\
\text { a) } l=l_{0} \mathrm{e}^{c t / 3} \\
\text { b) } w=w_{0} \mathrm{e}^{c t}\end{array}$ & $\begin{array}{l}\text { Insect larvae, } \\
\text { Orthoptera, } \\
\text { Helicidae }\end{array}$ \\
\hline $\begin{array}{l}\text { III. Respiration } \\
\text { intermediate } \\
\text { between surface- } \\
\text { and weight- } \\
\text { proportionality }\end{array}$ & $\begin{array}{l}\text { (a) Linear growth curve: } \\
\text { attaining with inflexion } \\
\text { a steady state. } \\
\text { (b) Weight growth curve: } \\
\text { sigmoid, similar to I (b) }\end{array}$ & $\begin{array}{c}\mathrm{d} w / \mathrm{d} t=\eta^{m}-x w \\
2 / 3<m<\mathrm{I} \\
\mathrm{d} l / \mathrm{d} t=\frac{\eta^{\prime}}{3} l^{3 m-2}-\frac{\varkappa}{3} l\end{array}$ & Planorbidae \\
\hline
\end{tabular}

$B$. Anabolic processes depend on respiration.

$w, l$ : Weight, length at time $t ; w_{0}, l_{0}$ : initial weight, length; $w^{*}, l^{*}$ : final weight, length; $\eta, \%$ : constants of anabolism and catabolism. 
etc.; on the other hand, energy which, in aerobic animals, is provided by oxidative processes. Both can be contemplated as limiting factors. Experimental results indicate that the first factor is effective in unicellulars and primitive metazoa while in higher animals, in connection with the development of inner surfaces and a circulatory system providing the body with nutrients, there are correlations between respiration, anabolism, and growth.

These considerations lead to the establishment of several "metabolic" and "growth types" as summarized in Table 6. In this respect, the relations between metabolic rate and body size have first to be discussed.

\section{(e) Dependence of metabolic rate on body size}

The relation between metabolic rate and body size belongs to the classical topics of physiology. It goes back over more than a hundred years to the time when Sarrus and Rameaux (1837-39), Bergmann and Leuckart (1855), and Richet (1883) noticed that the intensity of metabolism systematically decreases with increasing body size.

According to Rubner's classical surface rule (I902), weight-specific metabolic rate, that is, the intensity of metabolism as measured by oxygen consumption or calorie production per kilogram, decreases with increasing body weight. If, however, metabolism is calculated per unit of body surface, approximately constant values appear. Rubner explained the surface rule in terms of homeothermy. Since all warm-blooded animals heat their bodies to a temperature of $c a .37^{\circ} \mathrm{C}$ and heat output takes place through the body surface, the same number of calories ( $c a$. I ooo $\mathrm{kcal} / \mathrm{m}^{2} /$ day) must be produced per unit surface in order to maintain the body temperature constant.

It is difficult to measure exactly the outer surfaces of animals, and it is questionable whether these are responsible for the reduction of metabolic rate with increasing body size. If, however, two bodies are geometrically similar, any surface can be expressed as the $2 / 3$ power of weight multiplied by a constant because the cubic root of the volume or weight is a linear dimension, and therefore its square has the dimension of a surface. Hence, the surface areas of geometrically similar bodies can be obtained by multiplying the $2 / 3$ power of the weight by a suitable constant. This is expressed in the formula of Meeh:

$$
s=b w w^{2 / 3}
$$

The surface rule of metabolism accordingly states that basal metabolic rate is proportional to the $2 / 3$ power of weight. In the case of man where determination of basal metabolism is clinical routine, the somewhat more complicated Dubois formula is applied. Dimensionally, however, the Dubois formula is identical with the surface rule. The Dubois formula is: $s=k w^{0.425} \times l^{0.725}$. As, presupposing geometrical similarity, length $l=c w^{1 / 3}$, this can be written: $s=k w^{0.425} \times$ $c w^{0.725 \cdot 0.33}=b w^{2 / 3}$.

The relation between metabolic rate and body size can be studied either interspecifically, i.e. comparing adult animals of different species and body size; or intraspecifically, i.e. comparing animals of the same species and different size, 
in general in different developmental stages. The present considerations are mainly concerned with intraspecific comparison.

Even in more recent surveys (Brody, I945; Kleiber, 1947; Krebs, 1950; Lehmann, 1956) homeothermic animals are solely taken into consideration. It is, however, necessary to consider the problem on the broader basis of comparative physiology. Furthermore, the case of mammals is by no means simple, but rather is intricate (p. 218), and many familiar conclusions and explanatory hypotheses fall flat if not only mammals but also poikilothermic vertebrates and invertebrates are taken into consideration.

Such investigation as carried through in the author's and other laboratories led to the following results:

I. The surface rule also holds for poikilothermic vertebrates and certain invertebrates. The rule is, therefore, of a wide application; but the explanation given by Rubner is too restricted, for in poikilothermic animals there is no thermoregulation, and thus the latter cannot be the basic factor in the relation between body size and metabolic rate.

2. On the other hand, there are many classes of animals in which the surface rule does not hold.

3. Thus there are different metabolic types with respect to the relation between metabolic rate and body size. Three such types can be distinguished, this classification applying, as was emphasized, to intraspecific allometry, that is, to individuals of different sizes or to growing animals within one species.

The dependence of metabolic rate on body size is a special case of the allometric equation which has a wide range of application in morphological, biochemical, physiological, and evolutionary phenomena (p. 224ff.). The dependence of metabolic rate on body size can be expressed by the equation:

$$
\left.\begin{array}{l}
M=b w^{\alpha}, \text { or } \\
\log M=\log b+\alpha \log w
\end{array}\right\}
$$

where $M$ is metabolic rate per unit time, $w$ the body weight, and $\alpha$ and $b$ constants.

Therefore, if metabolic rate is plotted against body weight logarithmically, a straight line is obtained, the slope of which indicates the constant $\alpha(\tan a=\alpha)$. If $\alpha=2 / 3$ or $a=33^{\circ} 4 \mathrm{I}^{\prime}$, metabolic rate is proportional to surface. If $\alpha=\mathrm{I}$ or the slope is $45^{\circ}$, metabolic rate is proportional to weight. With $\mathrm{I}>\alpha>2 / 3$, an intermediary case obtains.

If weight-specific values are taken, the equation becomes:

$$
\frac{M}{w}=b w^{\alpha-1}
$$

Hence weight-specific metabolic rates decrease with increasing weight, and the slope of the logarithmic plot is negative. 
TABLE 7

$\mathrm{CO}_{2}$ PRODUCTION OF Armadillidium pallasii $\left(2 \mathrm{I}^{\circ}\right)$

After Müller, I943b

\begin{tabular}{|c|c|c|c|c|c|}
\hline weight in $\mathrm{mg}$ & I 5 & 33 & 50 & 100 & 160 \\
\hline $\mathrm{ml} \mathrm{CO}_{2} / \mathrm{h}$ & 3.0 & $5 \cdot 2$ & 7.2 & I1.2 & ${ }^{1} 5.2$ \\
\hline per g/h. & 200 & 174 & 144 & II 2 & 94 \\
\hline per unit surface $w^{2 / 3} / \mathbf{h}$. & 48.5 & $54 \cdot 2$ & 53.0 & 49.8 & 5 I.6 \\
\hline
\end{tabular}

The metabolic types mentioned are:

$x$. In the first type, metabolic rate is proportional to a surface or the $2 / 3$ power of weight. Representatives of this type include fish and mammals but also certain invertebrates, such as crustaceans, clams, and ascaris. Table 7 presents one example, the metabolic rate in the sowbug, Armadillidium. As can be seen, oxygen consumption per unit weight decreases with increasing body size, but remains constant per unit surface.

TABLE 8

oXygen CONSUMPTION OF Dixippus morosus $\left(20^{\circ}\right)$

After Müller, i943a

\begin{tabular}{lccclll}
\hline weight in mg & 8 & I 30 & 250 & 450 & 630 & 850 \\
$\mathrm{ml} \mathrm{O} / \mathrm{h}$. & 2.0 & 30.6 & 60.7 & $\mathrm{II}_{3} .2$ & $\mathrm{I} 54.8$ & 206.6 \\
per $\mathrm{g} / \mathrm{h}$. & 250 & 236 & 243 & 252 & 245 & 242 \\
\hline
\end{tabular}

2. In the second type, metabolic rate is proportional not to surface but to weight itself, so oxygen consumption in an animal of double size is doubled, in an animal four times as large is quadrupled, etc. Direct proportionality of metabolic rate to weight is found in growing insect larvae and hemimetabolic insects, and interspecifically in the comparison of imagos of different related species. Table 8 shows metabolic rates in the walking stick, Dixippus morosus. Oxygen consumption per gram and hour appears to be constant over a wide range, covering all body sizes and the entire development. Other groups belonging to this type are land snails of the family Helicidae, intraspecifically as well as interspecifically, and annelids such as the earthworm.

TABLE 9

oxygen CONSumption of Planorbis spec. $\left(23^{\circ}\right)$

After Bertalanffy and Müller, I943

\begin{tabular}{|c|c|c|c|c|c|}
\hline weight in $\mathrm{mg}$ & $30-35$ & $5^{8-62}$ & $90-100$ & 140 & $190-200$ \\
\hline $\mathrm{ml} \mathrm{O}_{2} / \mathrm{h}$ & 2.3 & 3.9 & $5 \cdot 4$ & $7 \cdot 3$ & 9.5 \\
\hline per $\mathrm{g} / \mathrm{h}$ & 69 & 65 & $5^{6}$ & $5^{2}$ & 48 \\
\hline per unit surface $\left(w^{2 / 3}\right) / \mathrm{h}$. & 22.9 & $25 \cdot I$ & 26.1 & 27.0 & 28.2 \\
\hline
\end{tabular}

3. In the third type, metabolic rates are intermediate between proportionality to weight and proportionality to surface. To this type belong pond snails such as Planorbis and Lymnaea and flatworms like Planaria. Table 9 shows that metabolic rates decrease with respect to weight, but increase with respect to surface. 
TABLE 10

RELATION BETWEEN METABOLIG RATE AND BODY SIZE

\begin{tabular}{|c|c|c|}
\hline Species & Reference & $\begin{array}{l}\text { Respiration proportional to } \\
w^{2 / 3} \text { (surface), w(weight), } \\
\text { or intermediate }\end{array}$ \\
\hline \multicolumn{3}{|l|}{ Platyhelminthes } \\
\hline Dugesia gonocephala & Bertalanffy and Müller, I 943 & Intermediate \\
\hline \multicolumn{3}{|l|}{ NeMathelminthes } \\
\hline Ascaris lumbricoides & Krüger, I940 & Surface \\
\hline \multicolumn{3}{|l|}{ ANNELIDA } \\
\hline Lumbricus sp. & Müller, 1943b & Weight \\
\hline Eisenia foetida & Krüger, 1952 & Surface? \\
\hline \multicolumn{3}{|l|}{ Mollusca } \\
\hline \multicolumn{3}{|l|}{ Lamellibranchiata } \\
\hline Anodonta cygnaea & Weinland, I919 & Surface \\
\hline Dreissensia polymorpha & Ludwig and Krywienczyk, I950 & Surface \\
\hline \multicolumn{3}{|l|}{ Prosobranchia } \\
\hline $\begin{array}{l}\text { Lithoglyphus, Paludina } \\
\text { fasciata and } P \text {. vivipara }\end{array}$ & Krywienczyk, 1952a & Surface \\
\hline \multicolumn{3}{|l|}{ Pulmonata } \\
\hline Lymnaea stagnalis & Bertalanffy and Müller, I943 & Intermediate \\
\hline Lymnaea stagnalis & Füsser and Krüger, I95 I & Intermediate \\
\hline Lymnaea auricularia & Krywienczyk, I952b & Weight? \\
\hline Planorbis sp. & Bertalanffy and Müller, I 943 & Intermediate \\
\hline Planorbis corneus & Füsser and Krüger, I95I & Intermediate \\
\hline Planorbis corneus & Krywienczyk, 1952b & Intermediate \\
\hline Isidora proteus & Krywienczyk, i952b & Intermediate \\
\hline $\begin{array}{l}\text { Pulmonata and Oper- } \\
\text { culata, } 15 \text { species intra- } \\
\text { and interspecific }\end{array}$ & v. Brand, Nolan and Mann, $194^{8}$ & $\begin{array}{l}\text { Surface [high tempera- } \\
\text { ture }\left(30^{\circ} \mathrm{C}_{0}\right) \text { ?] }\end{array}$ \\
\hline \multicolumn{3}{|l|}{ Helicidae } \\
\hline $\begin{array}{l}\text { Helix, Chilotrema, and } \\
\text { Cepaea (interspecific) }\end{array}$ & Liebsch, I 929 & Weight \\
\hline Cepaea vindobonensis & Bertalanffy and Müller, I 943 & Weight \\
\hline Helicella candicans & Kienle and Ludwig, 1956 & Weight \\
\hline
\end{tabular}

The relations mentioned are typical and characteristic of the species concerned. Table Io shows the distribution of these types within the various classes of animals. A few discrepancies need elucidation, but in general it can be said that the metabolic type, i.e. the relation of metabolic rate to body size, under standardized conditions, is a physiological constant characteristic of the species or group of species concerned.

In the investigations reviewed in Table Io resting metabolism (i.e. $\mathrm{O}_{2}$ consumption under conditions of greatest possible exclusion of muscular activity) was chosen as measure for two reasons: I. The so-called basal conditions of metabolism (muscular rest, postabsorptive condition, and thermoneutrality of environment) are adapted to determination of metabolic rate in mammals and the latter two conditions are difficult to fulfil in experiments with lower and poikilothermic animals; 2. Basal metabolic rate is a useful conventional measure because it provides the least scattering in the values observed; but it is an artefact because the conditions mentioned do not correspond to the natural biological situation. 
TABLE 10 (continued)

$\begin{array}{cc}\text { Species } & \text { Respiration proportional to } \\ \text { Reference } & w^{2 / 3} \text { (surface), w(weight), } \\ \text { or intermediate }\end{array}$

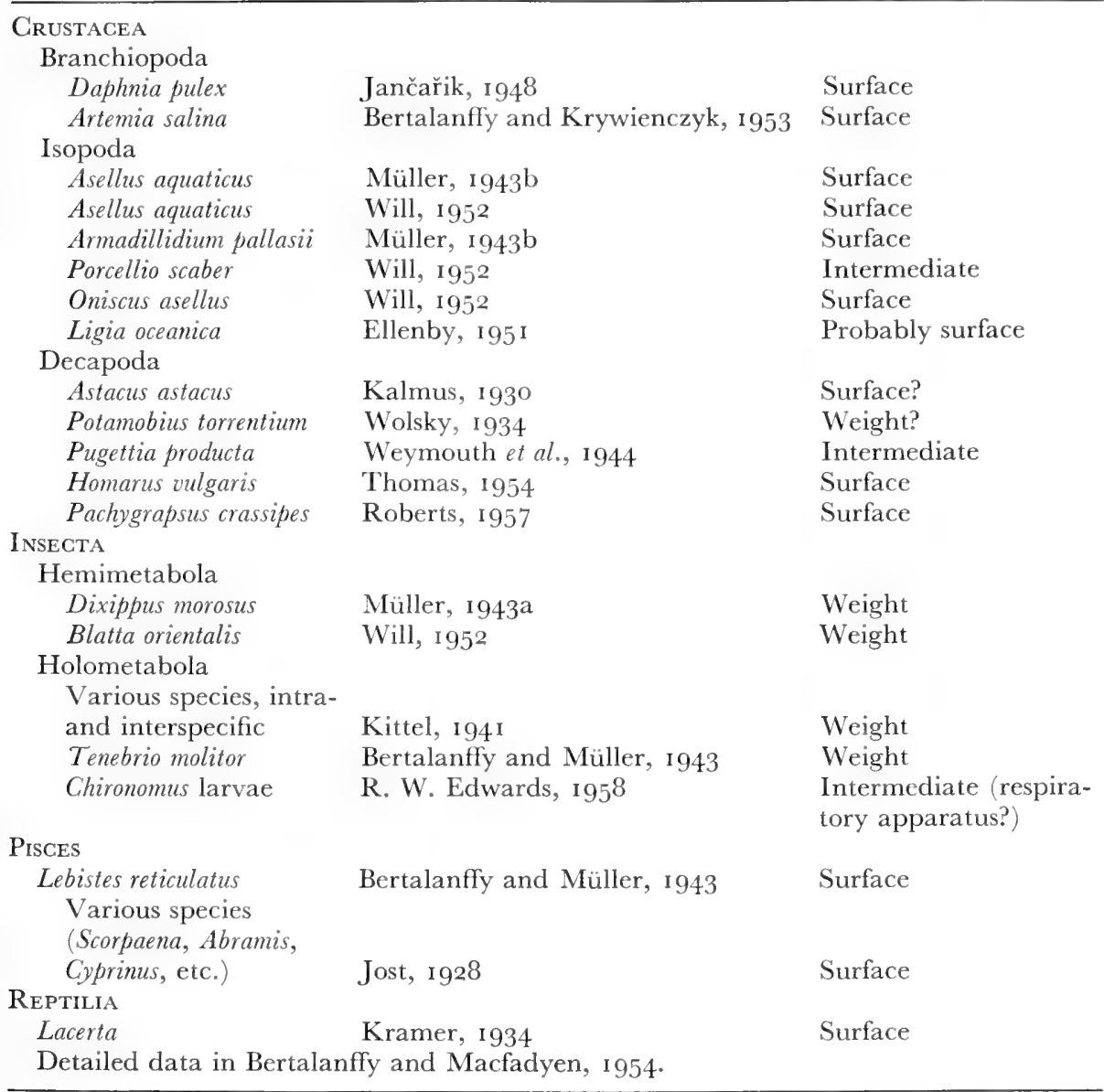

\section{$(f)$ Explanations of the size-dependence of metabolic rate}

A number of hypotheses have been advanced for explaining the size dependence of metabolism and, in particular, the surface rule. None of these explanations is satisfactory (Kleiber, I 947; Bertalanffy, 1942 and I951a; Bertalanffy and Pirozynsky, 1953). The reduction of weight-specific metabolic rate $\left(\mathrm{O}_{2} / \mathrm{g} / \mathrm{h}\right.$. $)$ with increasing body size can be due to either $(a)$ cellular or $(b)$ organismic factors. It may be based upon intrinsic differences in the metabolism of cells of smaller and larger individuals; or it may be regulated by factors lying in the organism as a whole.

(a) If the reduction of specific metabolic rate is due to intracellular factors, a corresponding decrease should be found in the respiration of isolated tissues. That 
is, $\mathrm{O}_{2}$ consumption of tissues as measured by the Warburg technique $\left(Q_{\mathrm{O}_{2}}=\right.$ $\mu \mathrm{l} \mathrm{O}_{2} / \mathrm{mg}$ dry weight/h.) should decrease with increasing body size; a similar decrease is to be expected in the concentration of respiratory enzymes and catalyzers.

$I$. A considerable amount of work in this respect has recently been done. The question is still somewhat controversial, but the statements to follow are a fair presentation of available experience.

In interspecific comparison of mammalian species of different sizes, ranging from the mouse to the horse, a decrease of $Q_{\mathrm{O}_{2}}$ with increasing body size is generally found (Kleiber, I94I ; Weymouth, Field, and Kleiber, I 942; Weymouth et al., I 944; Krebs, I950; Martin and Fuhrmann, I 955). This decrease, however, is not parallel in the various organs and, as a general rule, is less than would correspond to the $3 / 4$ power rule (p. 2 I 8 ) of metabolism. In a corresponding way, a decrease with increasing body size was found in enzymatic systems connected with respiration, such as in the concentration of glutathione (Gregory and Goss, I933; Patrušev, I 937), of cytochrome c (Rosenthal and Drabkin, I 943), of cytochrome oxidase (Kunkel and Campbell, I 952), of succinoxidase and malic dehydrogenase (Fried and Tipton, I953).

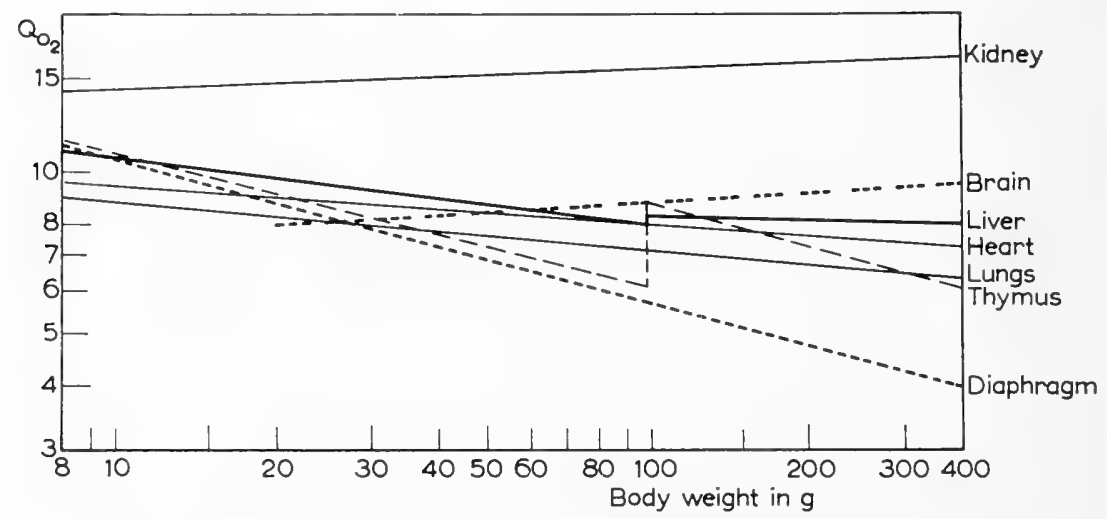

Fig. 7. $Q_{\mathrm{O}_{2}}\left(\mathrm{ml} \mathrm{O}_{2} / \mathrm{mg}\right.$ dry wt./h. $)$ of rat tissues. Only the regression lines are reproduced; for individual data and statistical analysis see original. After Bertalanffy and Pirozynski, I 953.

However, in intraspecific comparison, as between rat tissues from growing animals of different body size and age, no systematic decrease of $Q_{\mathrm{O}_{2}}$ paralleling that of specific metabolic rate was found (Bertalanffy and Pirozynski, I 95 I, I 953; Bertalanffy and Estwick, 1953). As Fig. 7 illustrates, no significant decline of average $Q_{\mathrm{O}_{2}}$ with increasing body size is found in brain, lung, and kidney; a slight decline in skeletal muscle, liver, and heart; and a marked decline in the diaphragm. Hence there is no systematic decrease of $Q_{\mathrm{O}_{2}}$ in the various organs consistent with, and responsible for the decrease of weight-specific metabolic rate with increasing body size. These results have been essentially confirmed by other workers and with other materials such as liver and kidney in growing chicken (Crandall and Smith, I952); heart muscle of the guinea pig (Wollenberger and 
Jehl, 1952); teleost brain (Vernberg and Gray, I953); and rat testes (Homma, I953; with a characteristic break in the allometric line at puberty; $c f$. p. 2I 8 f.). Similarly, Fried and Tipton (I953) did not find a decrease in the content of respiratory enzymes. The data, in intraspecific comparison of rats, on cytochrome oxidase (Kunkel and Campbell, I952) correspond well with the $Q_{\mathrm{O}_{2}}$ data (Bertalanffy and Pirozynski, 1953; Bertalanffy and Estwick, I953). There is a slight positive allometry (increase of values per $\mathrm{mg}$ ) of both cytochrome oxidase activity and $Q_{\mathrm{O}_{2}}$ in brain and kidney and slight decrease of both with body size in the liver; a discrepancy is found in skeletal muscle of larger rats $(\alpha=-0.24$ for cytochrome oxidase as against - 0.065 for $Q_{\mathrm{O}_{2}}$ ), but here Kunkel and Campbell's data have the least statical significance $(p=-0.69$ as against near unity in the other series).

It appears, therefore, that differences in enzymatic activity and $Q_{\mathrm{O}_{2}}$ of tissues are determined genetically, and are species-specific; differences in animals of the same species and different weight vary in different tissues or are inconspicuous.

Extensive investigations on the relations between $Q_{\mathrm{O}_{2}}$, body size, and temperature were conducted by Locker (1958a) on frogs (intraspecifically: 1.5-70 g, and winter and summer frogs), and including other amphibian and reptilian species (interspecifically). $Q_{\mathrm{O}_{2}}$ of frog liver $v s$. body size shows a break in the allometric regression line at $c a .25 \mathrm{~g}$ body weight; with increasing temperature, the difference of $\alpha$ for smaller and larger animals disappears. In contrast, $Q_{\mathrm{O}_{2}}$ of frog epidermis vs. body weight shows a continuous regression, $\alpha$ being $\approx-0.33$ (surface rule). The dependence of $Q_{\mathrm{O}_{2}}$ on body size further varies with different bases of comparison (dry weight or mg N), and glycogen content (Locker and Doneff, I 958). The activation energy $(\mu)$ of tissue respiration of both frog liver and epidermis (Locker, I958a) and liver of mammals (Locker, I958c) decreases systematically with body size. Locker's investigations show that the relations between body size, $Q_{\mathrm{O}_{2}}$, temperature, substratum of respiration, etc., are by no means simple. Bertalanffy's "metabolic types" (p. I goff.) express certain master reactions in the complex correlations between metabolism and growth.

2. Similar considerations apply to the hypothesis (Krebs, 1950) that the reduction of metabolic rate with increasing body size is due to a decrease of $Q_{\mathrm{O}_{2}}$ of the musculature. In intraspecific comparison of rats, $Q_{\mathrm{O}_{2}}$ of skeletal muscle only slightly declines with increasing body size (Bertalanffy and Estwick, 1953), although in mice the reduction of muscular $Q_{\mathrm{O}_{2}}$ is similar to that of weightspecific basal rate (Estwick, I953). Interspecifically, this value is similar in adult mice and rats (Bertalanffy and Estwick, I953).

(b) So we have to assume that the reduction of metabolic rate in intraspecific comparison is due to organismic factors which, within the intact organism, regulate the respiration of the tissues the sum total of which is the metabolism of the entire animal, but do not necessarily show up in isolated tissues as used for Warburg determinations (Bertalanffy and Pirozynski, I95I; Schmidt-Nielsen, Bertalanffy, and Pirozynski, I95I). The nature of these limiting factors is not definitely established and the factors usually contemplated do not offer a satisfactory explanation.

3. The most familiar one has already been mentioned, namely, thermoregulation. Energy expense for thermoregulation forms a considerable part of total metabolism in homeothermic animals. However, this explanation cannot be general 
since the surface rule also applies, and in fact is more accurately established, in cold-blooded vertebrates and even in certain invertebrates where there is no thermoregulation (Table 7).

4. Another interpretation assumes that the surface rule is based upon the anatomy and physiology of the circulatory system. The supply of oxygen and nutritive materials to the tissues is a function of the intensity of the blood current. The latter depends on factors such as the size and stroke volume of the heart, the frequency of heart beat, the diameter of the blood vessels, the degree of capillarization, etc. There are rather strict quantitative relations between body size, metabolic rate, pulse rate, and other characteristics of the circulatory system. For example, in interspecific comparison "from the mouse to the elephant", pulse rate decreases approximately proportional to the $3 / 4$ power of the weight (Fig. 35, p. 233). So does basal metabolic rate in the interspecific comparison of mammals, if adult specimens of corresponding species are plotted (Brody, I945; Kleiber, I 947). However, hemodynamics cannot offer a general explanation. Examples to the contrary are clams, where the circulatory system is completely different from that found in vertebrates, or ascaris, which has no blood circulation at all-animals whose metabolic rate nevertheless follows the surface rule.

5. Still another explanation of the reduction of metabolic rate is based upon anatomical or chemical changes in composition with increasing body size. "Metabolically active" organs such as the viscera, the brain, etc. are relatively larger in small as compared to large animals. So it has been assumed that they consume relatively more oxygen and are responsible for the higher weight-specific metabolic rate in smaller organisms. However, the relative growth of inner organs is different from one organ to the other, allometry constants being irregular and often higher than $2 / 3$ or $3 / 4$ as would correspond to the reduction of metabolic rate (Table I 6 , p. 242 ; Figs. 32,33 , p. $230,23 \mathrm{I}$ ). So it is improbable that this can yield the simple relation of the surface rule of metabolism ( $c f$. Bertalanffy, i 95 ra). A quantitative estimate (Bertalanffy and Pirozynski, I 953) shows that this factor is not sufficient to account for the actual variations of metabolic rate.

6. Recent investigations of the Ludwig laboratory (Ludwig, I956; Kienle and Ludwig, I 956; Sattel, I 956) give support to the hypothesis proposed by Ludwig and by Bertalanffy (I95Ia, p. 252f.) that the "metabolic types" are connected with types of respiratory apparatus. Gill-breathing and lung-breathing animals appear to follow the surface rule; hence its validity in fish, certain invertebrate classes, and mammals. On the other hand, the surface of tracheas in insect larvae develops proportional to body weight, as was shown by Sattel (1956) in Bombyx mori; hence the proportionality of metabolic rate to weight. With this corresponds the fact that not only respiration (Table 8) but also transpiration in insects (Gryllus domesticus: Jakovlev and Krüger, 1954) is weight-proportional. Intermediate cases would result from the presence of two types of respiratory apparatus, as in the case of fresh-water pulmonates, belonging to the intermediate type and possessing two types of respiratory mechanisms ("lungs" and cutaneous respiration).

The explanation of the reduction of metabolic rate with increasing body size is unsatisfactory at present. That the factors usually contemplated give no sufficient explanation is shown by an example like Ascaris (Krüger, 1940) which, 
being an intestinal parasite, anaerobic and poikilothermic, nevertheless follows the surface rule. The earthworm, on the other hand, respires through the body surface, but shows weight-dependence of respiration (Müller, r 943b).

Apparently regulatory factors, insufficiently known at present, are involved. Such regulation is shown by the action of hormones in mammals. For example, it is easy to induce an increase of metabolism of the entire animal by injection of thyroxin in vivo; but, in spite of many efforts made, this effect could not be reproduced by administration of thyroxin to an isolated tissue in vitro. On the other hand, chronic hormonal changes entail changes both of basal metabolism and tissue $Q_{\mathrm{O}_{2}}$, as has been shown in hypophysectomized animals and pituitary dwarf mice (Bertalanffy and Estwick, I954, of. p. 220). Such examples show the possibility of organismic factors regulating metabolic rate and at the same time demonstrate that differences in metabolic rate of the entire animal may or may not be reflected in the $Q_{\mathrm{O}_{2}}$ of isolated tissues. The most plausible interpretation appears to be that with increasing body size the metabolic activity of tissues is bridled, as it were, by some regulatory mechanism so that the systemic reduction of weight-specific metabolic rate takes place; that the same is true of the "growth potency" of tissues and organs; and that both factors are connected (Bertalanffy and Pirozynski, I953).

At present the metabolic type in the sense defined has to be taken as an
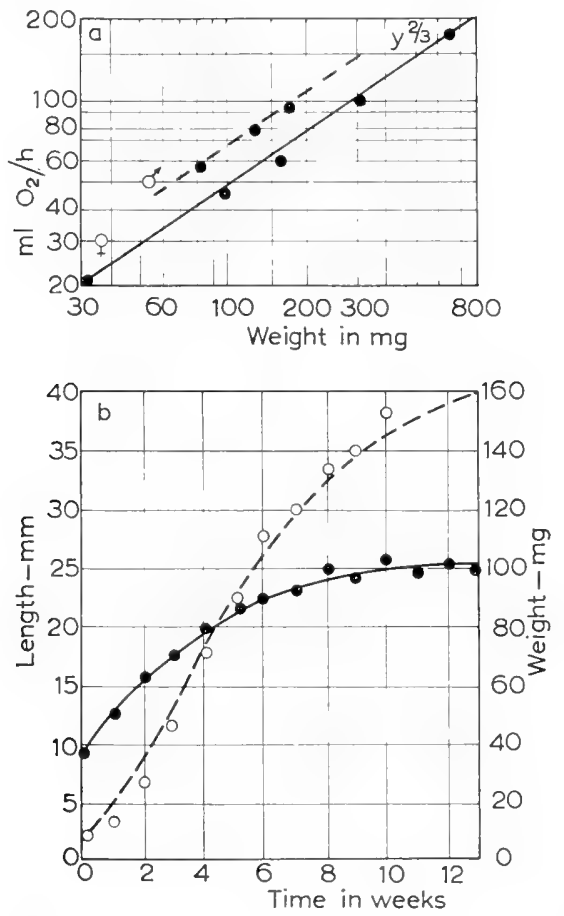

Fig. 8. First metabolic and growth type: Lebistes reticulatus. a Dependence of metabolic rate on body size. Note the ratio of I : I $\frac{1}{2}$ of oxygen consumption in $q$ and $\sigma . b$ Growth curves; — length growth, - - - weight growth $\left(\sigma^{*}\right)$. After Bertalanffy, I94I b. 
empirical characteristic of the species. It determines, however, the pertinent growth type as will be shown in the following.

\section{(g) Metabolic types and growth types}

As there are different metabolic types with respect to the relation of metabolic rate and body size, there are also several growth types distinguished by the growth curves of the species concerned. It appears that definite connections between metabolic and growth types can be established.

If for $m$ in the basic equation (5.18) (denoting the dependence of anabolism of building material on body size) the value is inserted which is experimentally found for the size dependence of resting metabolism, the growth laws for the organism in question follow.

In this way three metabolic and growth types can be distinguished.

First type. In the first type (Fig. 8) respiratory rate is proportional to the $2 / 3$ power of body weight, corresponding to the surface rule. In this case, the basic growth equation becomes:

$$
\mathrm{d} w / \mathrm{d} t=\eta w^{2 / 3}-x w
$$

Solution of this equation gives for growth in weight:

$$
w=\left[\frac{\eta}{x}-\left(\frac{\eta}{x}-w_{0}\right) \mathrm{e}^{-x t / 3}\right]^{3}
$$

with $w_{0}=$ weight at the time $t=0$. For growth in linear dimensions, proportional growth presupposed, we obtain:

$$
\begin{gathered}
\mathrm{d} l / \mathrm{d} t=E-k l \\
l=\frac{E}{k}-\left(\frac{E}{k}-l_{0}\right) \mathrm{e}^{-k t}
\end{gathered}
$$

with $l_{0}=$ initial length and the new constants $k=x / 3$ and $E=n p / 3 q$ for linear

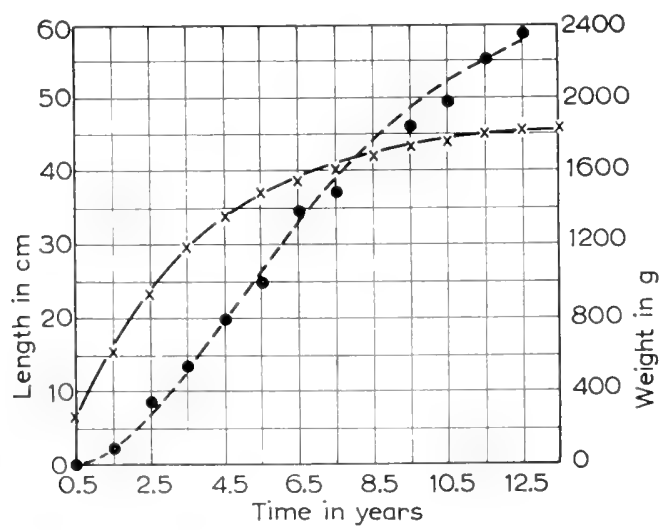

Fig. 9. Growth of Abramis brama. - length growth, - - weight growth.

Growth equations: $l=46.84-(46.84-6.8) e^{-0.278 t}$

After Bertalanffy, I934.

$$
w=\left[13.93-(13.93-2.08) e^{-0.235 t}\right]^{3}
$$


dimensions in general, and $E=\eta / 3$ for the linear dimension $w^{1 / 3}$. These equations can be written in the following forms:

$$
\begin{gathered}
w=\left[1^{3 / \overline{w^{*}}}-\left(\mathrm{J}^{3 / \overline{w^{*}}}-\mathrm{J}^{3} / \overline{w_{0}}\right) \mathrm{e}^{-k t}\right]^{3} \\
l=l^{*}-\left(l^{*}-l_{0}\right) \mathrm{e}^{-k t}
\end{gathered}
$$

with $w^{*}$ and $l^{*}=$ final weight and length, respectively. As can be seen, these equations contain only empirically testable parameters, namely, the final values which can be determined from the growth curves, and the catabolic constant which can be determined by physiological experiment.

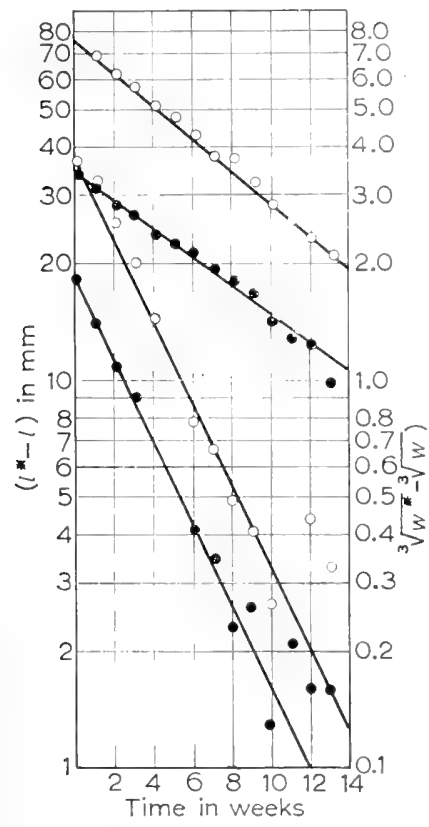

Fig. 10. Growth of Lebistes reticulatus in semilogarithmic plot. See equation (5.3 I). ○ weight, - length. Upper lines: o, lower lines: 0 . Constants:

\section{९: Calculated}

from weight: $x=0.285, \eta=2.64$

from length : $x=0.256, \eta=2.3^{8}$

o: Calculated

from weight: $x=0.723, \eta=4.06$

from length : $x=0.708, \eta=4.04$

After Bertalanffy, 1938 .

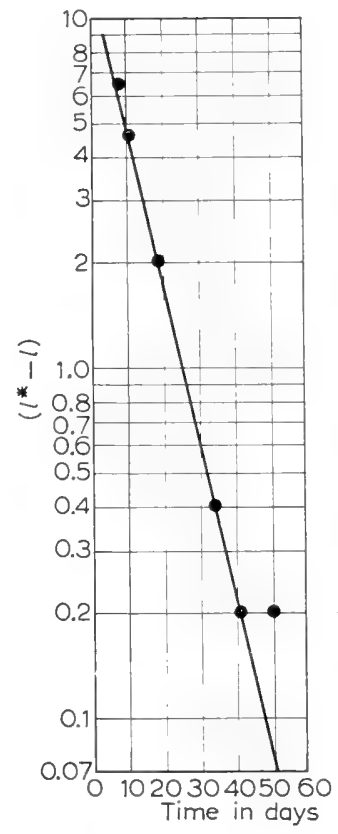

Fig. I I. Regeneration of the tadpole tail; Durbin's data. This example was one calculated by Pearl and Reed (1925) with their cumbersome formula ( $c f$. p. I 76 ). The figure shows (I) that graphical calculation according to the simple equation $(5.29)$ gives satisfactory approximation (the discrepancy of $0.13 \mathrm{~mm}$ in the last point would be experimentally undetectable); (2) that regeneration may be considered as a re-establishment of steady state, following the equations discussed in the text. After Bertalanffy, I934.

The growth curves resulting by insertion of $m=2 / 3$ into equation (5.18) have the following main characteristics: $I$. Growth rates are decreasing and growth eventually attains a steady state. 2. The curves for weight growth and linear growth are 
characteristically different. The curve of weight growth is sigmoid, with a point of inflexion at about one-third (exactly: 8/27) of the final weight. The curve of linear growth is a decaying exponential without a turning point (Figs. 8, 9).

This characteristic course of growth is easily understood. If a body, with not too much change of shape, increases in size, its surfaces increase approximately with the second power of the length, but its volume and mass with the third power. Hence, the ratio between surface and weight is continually shifted in disfavor of the surface. Consequently, so long as the animal is small, surfaceproportional anabolism prevails over weight-proportional catabolism, and the animal grows. The larger it grows, the more the surplus remaining for growth is reduced, and eventually a steady state will be reached where anabolism and catabolism balance each other, and growth comes to an end.

This is the most common form of growth curves, found in fish, in a number of invertebrate classes and also, with certain restrictions, in mammals. The validity of these growth equations has been shown in many examples (e.g. Pütter, I920; Bertalanffy, I934, I95 Ia) of the animal classes indicated in Table 6 (Figs. 8-I4). In well analyzed cases, the numerical approximation of data is so exact that it would be satisfactory even for a law of physics.

Methods to calculate growth data according to equations (5.28) and (5.29) include:

I. Graphical (Fig. IO). The linear form of equation (5.29) is:

$$
\log \left(l^{*}-l\right)=\log \left(l^{*}-l_{0}\right)-k t \log \mathrm{e}
$$

Hence, if the final value $\left(l^{*}\right)$ is properly chosen, semilogarithmic plot of $\left(l^{*}-l\right)$ gives a straight line.

If $l^{*}$ is not correctly chosen, a concave curve is obtained if the assumed $l^{*}$ is too large, and a convex curve if it is too small. Examination of the plotted growth data and some trial, if necessary, usually gives the desired result in calculations of linear growth. Calculated $\left(l^{*}-l\right)$ can be directly read from the semilogarithmic plot (Fig. Io); $k$ can be calculated from:

$$
\begin{gathered}
k=\log \left(l^{*}-l_{0}\right)-\log \left(l^{*}-l_{\text {calc. }}\right) / t \log \mathrm{e} ; \\
E=l^{*} k .
\end{gathered}
$$

2. Least-square method. The coefficients in equation $(5.26)$ can be calculated from the normal equations:

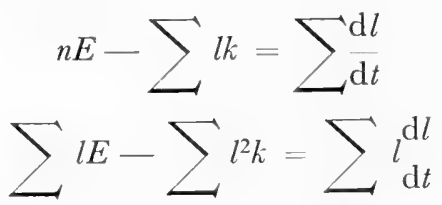

( $n=$ number of observations). For weight, $w^{1 / 3}$ instead of $l$ is taken. This method is recommended particularly for calculation of weight-growth curves as the taking of cubic roots increases the error committed, and hence the graphical method $(I)$ often gives unsatisfactory results.

Further methods and details for calculations according to equations $(5.25,5.27)$ can be found in Buch Andersen and Fischer (1929), Bertalanffy (1934), Brody (1945), and Beverton and Holt (1957). 
(h) Application of the Bertalanffy growth equations in fishery biology

The Bertalanffy equations have been extensively used in fisheries biology, and this material provides valuable evidence for both theoretical verification and practical application (Beverton, I954; Graham, I956; Beverton and Holt, 1957).

Based upon the large material of the Fisheries Laboratories of the British Ministry of Agriculture, Fisheries and Food as well as that of the Fisheries Biology Branch of the FAO (Food and Agriculture Organization of the United Nations), Beverton and Holt state:

"The equation we shall use throughout... is that due to Von Bertalanffy; not only does this give a growth curve closely similar to that shown in Fig. I3 (as reproduced in this paper, Von B.), but the underlying concepts are, in our opinion, much the most satisfactory of those which have so far been put forward... From an examination of the published growth data for a number of species of fish, it would seem that the von Bertalanffy growth equation has a wide application. In most cases the fit to the data is ... good .... while in the remainder the departures are not greater than might be expected from sampling errors or biological factors" (in Graham, 1956, p. $3^{81}$; Beverton and Holt, I957, p. 288).

Not only the wide applicability of the growth equations under consideration is important; it is of crucial significance that, if deviations from the typical curves

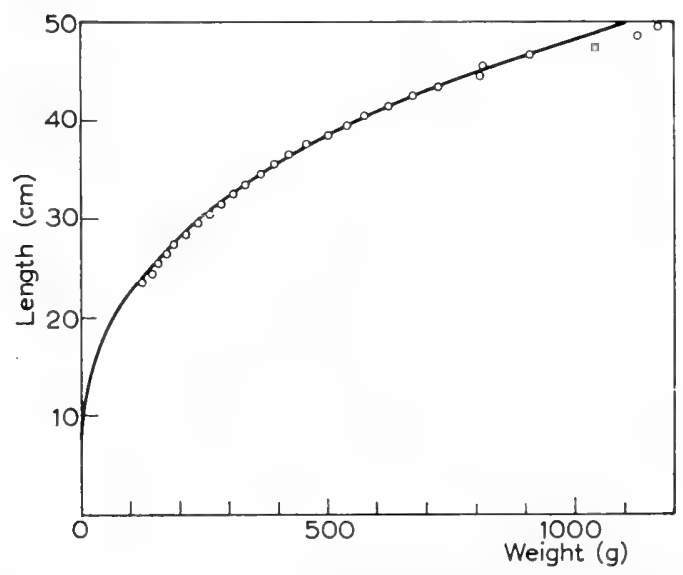

Fig. 12. Relationship between weight and length in plaice. After Beverton and Holt, 1957. Weight is expressed by the equation $w=0.00892 l^{3}$ showing that equation $(5.5)$ is approximately true and hence the relation between weight growth and length growth expressed in equations $(5.28,5.29)$ applies in fair approximation. Deviations from equation $(5.5)$ are expressed in differences in the $\%$ values as calculated from length and growth data (Figs. 9, Io). It can be seen, however, that these differences are small; if the constant $x$ be considered a physiological magnitude, the difference of calculated values would hardly exceed experimental errors.

are encountered, they can be explained by interfering factors, detailed analysis of which leads to the disappearance of the seeming discrepancies. Cases such as exemplified by Figs. I2, I3, I4 are of especial interest as verification of the theory and equations.

The Bertalanffy growth equations are used by the British researchers as part of a dynamical model of exploited fish populations which takes into account 
recruitment, growth, capture, and natural mortality within such populations. This analytical treatment was prompted by practical considerations of the fishing industry in view of problems of overfishing and conservation. Discussion of the complete model accounting for the primary factors mentioned is beyond the scope of the present paper, even though it is connected with the concepts of "open systems", "steady states", and "general system theory" (Bertalanffy, I 95ob) as applied to populations. It is, however, worth emphasizing that a theoretical model of fishery as an important branch of applied biology includes the equations under discussion as one of the fundamental factors. It appears that these equations are also introduced in Japanese fisheries (Kubo and Yoshihara, I 957; personal communication of Dr. Yoichi Yoshida).
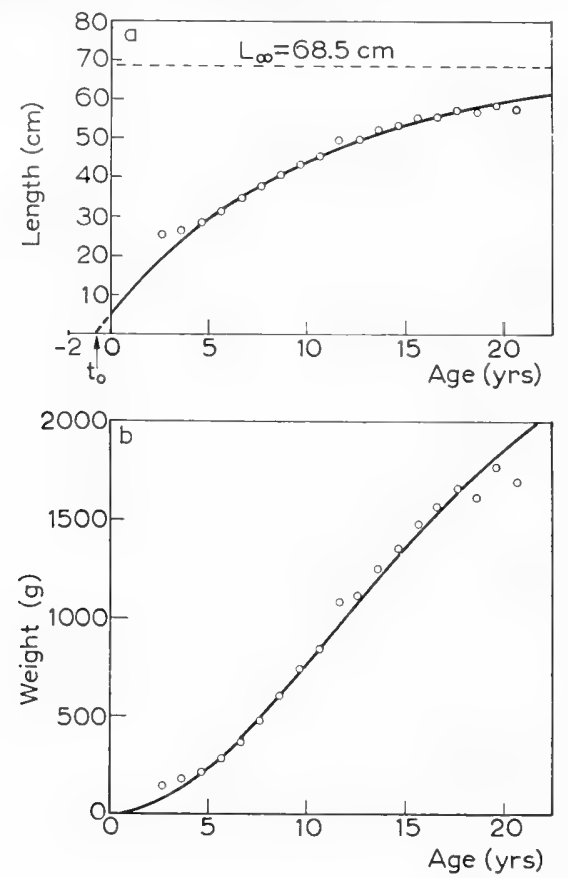

Fig. 13. Growth in length $(a)$ and in weight $(b)$ of plaice. After Beverton and Holt, 1957. Curves are fitted by the Bertalanffy equations (5.28 and 5.29), with $q=0.00892$ according to Fig. I2. This example shows the sensitivity of the equations for demonstrating complicating factors. Fit of the observed data by the equations is satisfactory, except for the youngest age groups. For explanation, see Fig. I4.

A relation similar to that derived by Bertalanffy et al. from the surface dependence of respiration was found by Yoneda and Yoshida (1955; Yoshida, 1956) in food intake. The quantity of plankton consumed by the sardine is proportional to the square of body length, and the same appears to be true for assimilating organs, such as the gill-rakers and the gut.

Yoshida (1956) interprets these results in terms of energy. If $M_{1}=$ energy intake, $M_{2}=$ maintenance metabolism, $M_{3}=$ working metabolism, $M_{4}=$ reproductive metabolism, and $M_{5}=$ growth metabolism, and if these terms are partly proportional to $w^{2 / 3}$, partly to $w$, the balance: 


$$
\frac{\mathrm{d} w}{\mathrm{~d} t}=\frac{M_{5}}{\delta}=\frac{\mathrm{I}}{\delta}\left(M_{1}-M_{2}-M_{3}-M_{4}-\ldots\right)=\frac{\mathrm{I}}{\delta}\left(\beta w^{2 / 3}-\gamma w\right)
$$

will obtain ( $\beta, \gamma$ arbitrary constants, $\delta$ energy required, per unit volume, for building up living mass).

This can be associated with the considerations given above. According to Yoneda and Yoshida, $M_{1}$ (intake) is proportional to $w^{2 / 3} \cdot M_{2}$ (maintenance $=$ turnover) will be approximately proportional to $w \cdot M_{3}$ and $M_{4}$ (muscular work and reproduction) are excluded under conditions of experimental determination of resting metabolism, and also $M_{2}$ (corresponding to - xw) can be neglected

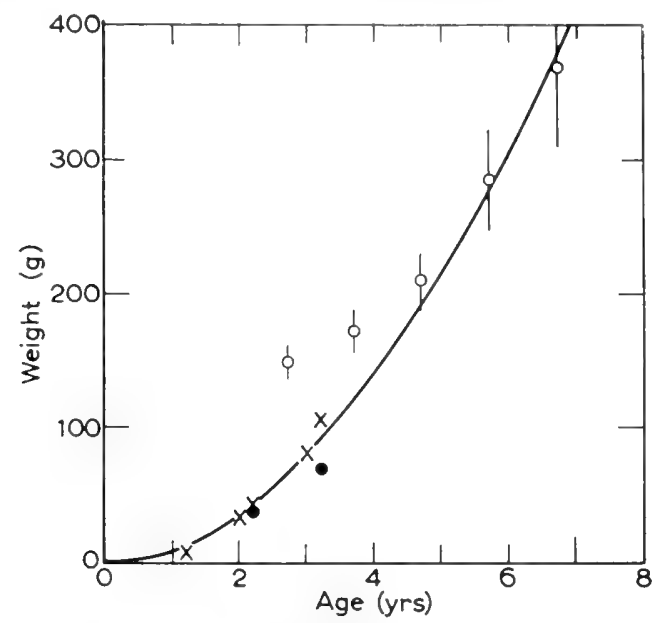

Fig. 14. Growth in weight of young plaice. The deviation of the youngest age groups from the theoretical curve, as apparent in Fig. I3, was due to mesh selection in the material used (Lowstoft and Grimsby market samples). The discrepancy disappears when material more representative of these age groups (samples from stations of the Leman-Haaks line) is used. $\mathrm{o}=$ points shown in Fig. I3, $\mathrm{X}=$ data of Wallace, $\bullet=$ data of Thursby-Pelham.

After Beverton and Holt, 1957.

in short-term experiments because energy expense for maintenance of body material in the steady state is probably but a small fraction of total (basal) metabolic rate (p. I 5 I). Under these conditions, equation (5.34) reduces to:

$$
\begin{aligned}
\text { Energy intake } & =\text { energy output in resting metabolism } \\
& + \text { energy stored in tissues }
\end{aligned}
$$

It appears that all terms in equation (5.35) are proportional to $w^{2 / 3}$, so that the simplest possible rule of dimensionality applies. Energy intake is measured by food consumed and is proportional to $w^{2 / 3}$, according to Yoshida. Energy output is measured by $\mathrm{O}_{2}$ consumption and is proportional to $w^{2 / 3}$, according to the evidence presented (p. I 83 ff.). Energy stored corresponds to the term of anabolism of the growth equation and is, by virtue of the validity of the latter, proportional to $w^{2 / 3}$. Hence, in terms of the symbols as previously defined:

$$
\beta w^{2 / 3} \approx b w^{2 / 3}+\eta w^{2 / 3}
$$


(i) Metabolic types and growth types (continued)

Second type. A second metabolic and growth type is found in insects ( $c f$. also G. A. Edwards, I953), Helicidae, etc., where (Table 8) metabolic rate does not follow the surface rule but rather is proportional to weight itself (Fig. I 5). Here the allometric regression line of metabolic rate against body weight has a slope of $45^{\circ}$. Inserting $m=$ I into equation (5. I 8) we obtain:

$$
\frac{\mathrm{d} w}{\mathrm{~d} t}=\eta w-x w=c w
$$

or integrated:

$$
w=w_{0} \mathrm{e}^{c t}, \quad l=l_{0} \mathrm{e}^{c t / 3}
$$

Hence, an entirely different growth curve is to be expected. In the first type, there is an asymptotic approach to a steady state because surface-proportional anabolism is caught up by volume-proportional catabolism and eventually a balance is reached. In the second type, anabolism and catabolism, both being weight-proportional, run at the same pace. Hence growth rate does not decrease but always increases, growth is not limited but exponential, and no steady state is reached. Growth rather is stopped by essentially different mechanisms. In
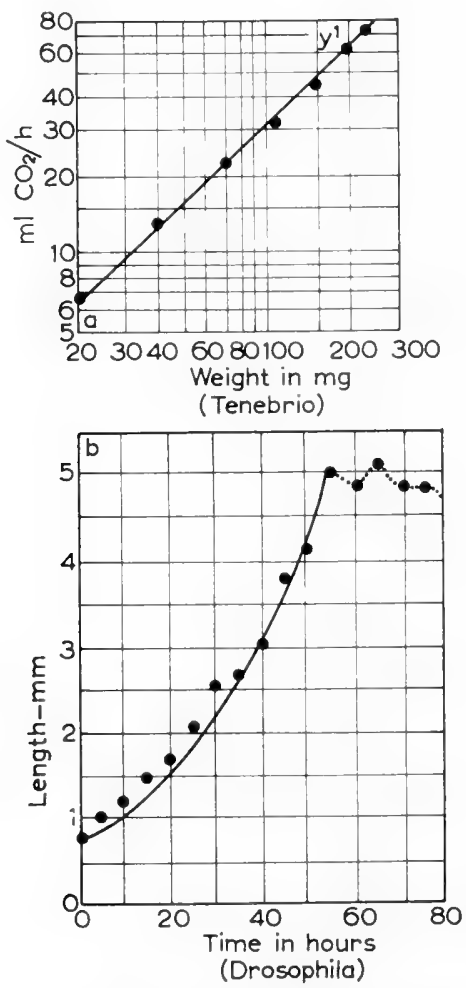

Fig. 15. Second metabolic and growth type: Insects. $a$ Dependence of metabolic rate on body size. $b$ Growth curve. After Bertalanffy and Müller, I943. 


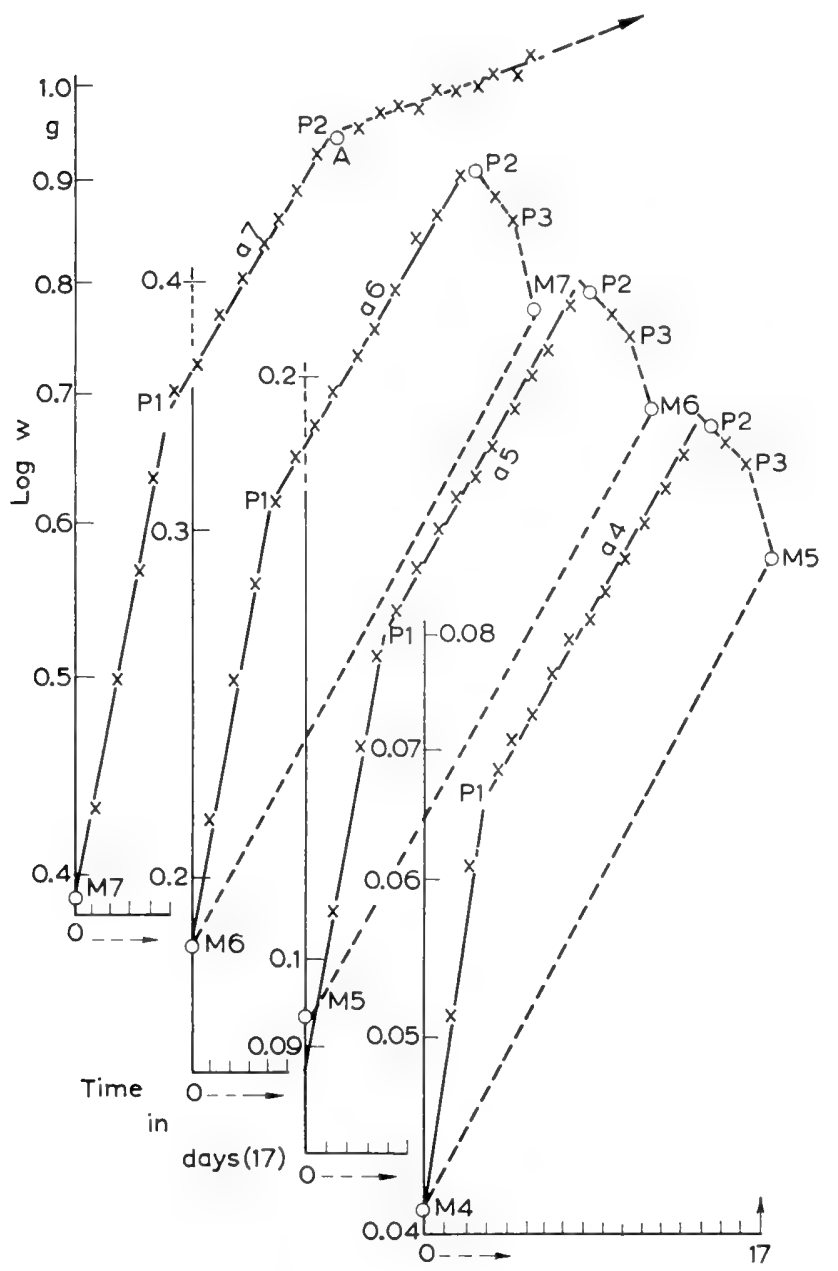

Fig. 16. Weight growth in Blatta orientalis in semilogarithmic plot. The subsequent moltings $\left(M_{4} \rightarrow M_{5}, M_{5} \rightarrow M_{6}, M_{6} \rightarrow M_{7}, M_{7} \rightarrow \mathrm{A}=\right.$ adult $)$ are juxtaposed showing that each cycle consists of 3 phases (rapid, less rapid exponential growth, and loss of weight in molting). The parallelism of the lines for each cycle shows the general exponential type of growth. After Voy, I95I.

insect larvae, metamorphosis abruptly intercepts the exponential increase, even causing a loss in body weight as large amounts of tissue are broken down in order to develop the imago. The same metabolic and growth type also applies to hemimetabolic insects (Table 8, Figs. I6, I 7) where no metamorphosis occurs. This at first glance appears perplexing but is in fact what is to be expected because there seems to be no fundamental difference between holometabolic and hemimetabolic insects in the basic developmental processes and hormonal mechanisms. In land snails which also belong to this type, exponential growth appears to be intercepted by seasonal cycles. 

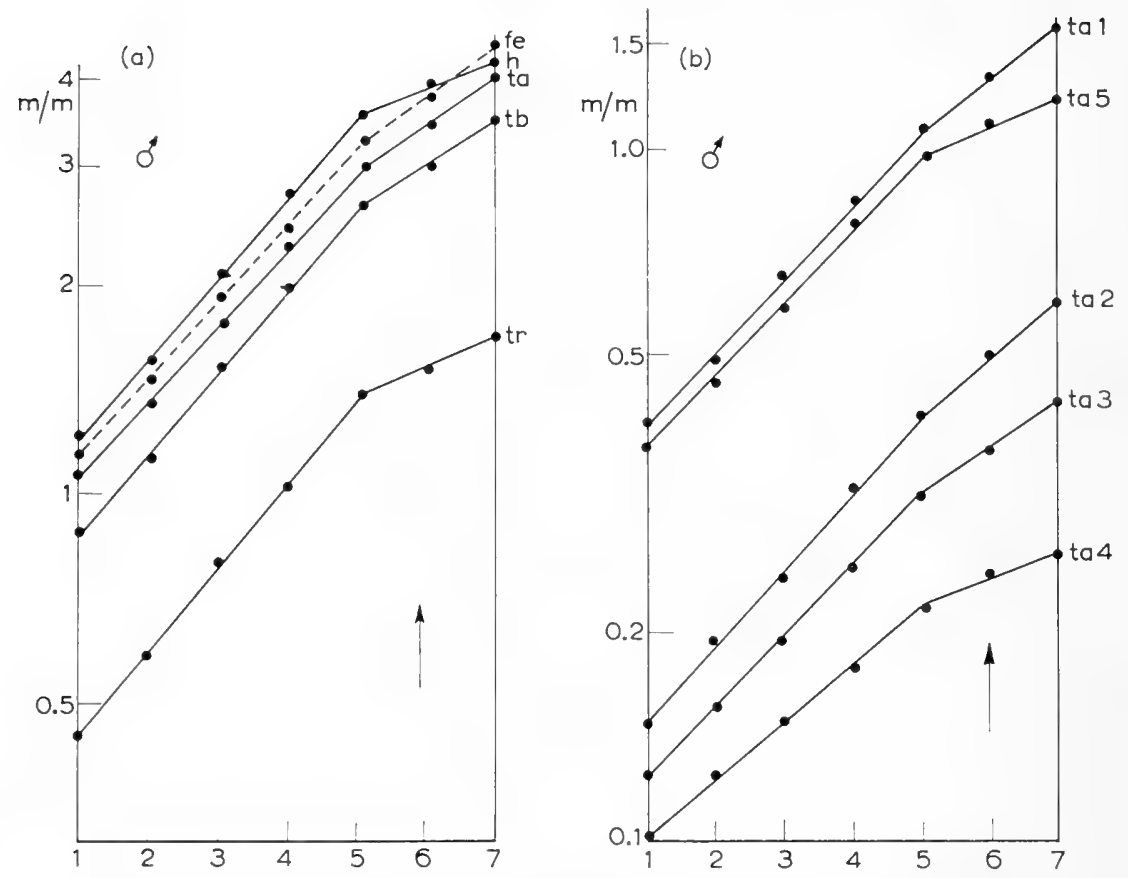

Fig. I 7. Length growth of the tarsi of Blatta orientalis 0 . Semilogarithmic plot showing exponential growth decreasing with attainment of the adult state; abscissa indicates the successive moltings. $a$ First leg: hip $(h)$, femur $(f e)$, tibia $(t b)$, trochanter $(t r)$, tarsus $(t a)$; $b$ first tarsus: $t a_{1}$ etc., the consecutive segments. After Voy 1951 .

Third type. While terrestrial pulmonate snails show weight- dependence of metabolic rate and exponential growth, fresh-water pulmonates represent a third type where metabolic rate is intermediate between surface and weight proportionality. Inserting a value $2 / 3<m<$ I into equation (5.18), it follows that growth should obey a third type. The curve of weight growth does not differ much from the first type, but the curve of linear growth is characteristic, being sigmoid with an inflexion. This prediction also is verified (Fig. I8).

So in different animal classes there are different metabolic types and different types of growth, agreeing with theoretical expectation. Table 6 (p. I8o) gives a survey of examples investigated, and may be considered as a first draft for a new chapter in physiology, namely, a comparative physiology of growth. The growth type of an animal can thus be predicted from its metabolic type. This prediction has proved to be correct in a large number of cases, often in an unexpected way.

\section{(j) Verification of the constants of the growth equation}

In well-analyzed cases, the constants of anabolism and catabolism calculated from the growth curve can be verified in physiological experiment. This is of decisive importance for the theory as it shows that the formulas applied are not solely descriptive of empirical data but reproduce the physiological basis of growth.

With regard to the anabolic constants $(\eta)$ it follows from theory that the ratio 
of these constants calculated from different growth curves should correspond to the ratio of rates of resting metabolism $\left(\mathrm{O}_{2}\right.$ consumption) of the organisms concerned. The aquarium fish, Lebistes reticulatus (Guppy), is characterized by a large sexual dimorphism in size, the females reaching double the length and a multiple of weight of the males. Accordingly, a ratio of the constants i $I: I \frac{1}{2}$ in females and males is calculated from the growth curves (Fig. 10, p. I9I). According to theory, the rates of respiration in females and males also show a ratio $\mathrm{I}: \mathrm{I} \frac{1}{2}$ (Fig. 8, p. I89) (Bertalanffy, I938, r94I b).
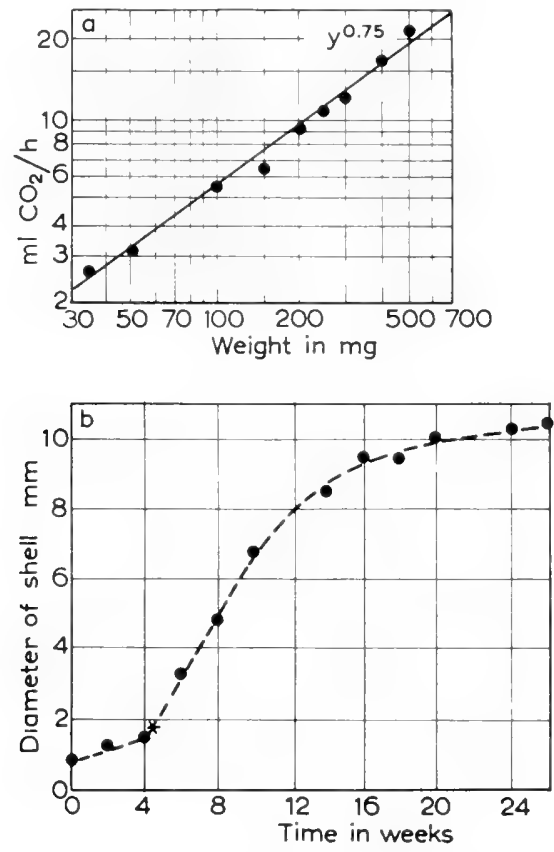

Fig. I8. Third metabolic and growth type: Planorbis $s p$. a Dependence of metabolic rate on body size. $b$ Growth curve (linear growth; diameter of shell). After Bertalanffy and Müller, i 943 .

Regarding the catabolic constant $(x)$ it follows from equations $(5 \cdot 28,5 \cdot 29$, p. I 9 I $)$ that the higher the value of this parameter, the faster the steady state is reached. That is, an animal showing high catabolism of building materials (high protein turnover) will grow fast, compared to one with sluggish turnover. This corresponds to experience. Quantitatively, good agreement is found between the values calculated from the growth curve and those determined in physiological experiment (Table I I). In view of the simplicity of the model and the errors inevitable in turnover determinations, the agreement is remarkable. For example, from the growth curve of man a daily protein turnover of I.165 g/ $\mathrm{kg}$ body weight was calculated (Bertalanffy, I938), while determination with glycine marked with ${ }^{15} \mathrm{~N}$ gave a value of $1.3 \mathrm{~g} / \mathrm{kg}$ body weight (Sprinson and Rittenberg, 1949a, b). 
TABLE 11

RATES OF CATABOLISM DETERMINED IN EXPERIMENT AND CALCULATED FROM GROWTH DATA

\begin{tabular}{|c|c|c|c|}
\hline Species & Method & $\begin{array}{l}\text { Protein turnover } \\
\quad(r) \text { per year }\end{array}$ & $\begin{array}{c}\text { Estimated rate of } \\
\text { catabolism }(x) \text { from } \\
\text { growth equation } \\
\text { per year }\end{array}$ \\
\hline $\begin{array}{l}\text { Anodonta } \\
\text { Margaritana }\end{array}$ & $\begin{array}{l}\text { Weight loss in } \\
\text { starvation }\end{array}$ & $0.0497^{1}$ & $0.045^{1}$ \\
\hline Homarus & $\begin{array}{l}\text { Weight loss in } \\
\text { starvation }\end{array}$ & $0.368^{1}$ & $0.285^{1}$ \\
\hline Pleuronectes & $\begin{array}{l}\text { Weight loss in } \\
\text { starvation }\end{array}$ & 0.624 (flesus) $)^{1}$ & $0.546{\text { (platessa })^{1}}^{1}$ \\
\hline Cyprinus & $\begin{array}{l}\text { N-excretion in } \\
\text { starvation }\end{array}$ & $0.45^{6^{1}}$ & $0.495,0.573^{1}$ \\
\hline Esox & $\begin{array}{l}\text { N-excretion in } \\
\text { starvation }\end{array}$ & $0.43^{8^{1}}$ & $0.298^{1}$ \\
\hline Rat & $\begin{array}{l}{ }^{15} \mathrm{~N} \text {-labeled } \\
\text { glycine }\end{array}$ & $0.04 /$ day $^{2}$ & $\begin{array}{c}\text { o.045/day }{ }^{3} \\
\text { (Donaldson's data) }\end{array}$ \\
\hline Man & $\begin{array}{l}{ }^{15} \mathrm{~N} \text {-labeled } \\
\text { glycine }\end{array}$ & $\begin{array}{c}0.2 \mathrm{~g} \mathrm{~N}=0.2 \times 6.25= \\
\mathrm{r} .3 \mathrm{~g} \text { protein } / \mathrm{kg} \\
\text { body wt. } / \text { day }^{2}\end{array}$ & $\begin{array}{l}\text { I. } 65 \mathrm{~g} \text { protein } / \mathrm{kg} \\
\text { body wt. } / \text { day }^{3}\end{array}$ \\
\hline
\end{tabular}

1 Bertalanffy, I934.

2 Sprinson and Rittenberg, i949b.

3 Bertalanffy, $193^{8}$.

(k) Deductive derivation of the system of growth equations

Given one almost self-evident principle and a small number of empirical facts, the family of growth equations can be derived by way of deductive mathematical reasoning (Bertalanffy, I $94 \mathrm{I}$ b).

Since the living organism is a metabolizing system maintaining itself by import and export, building up and breaking down of component materials, it may be expected that an organism grows when synthesis of building material exceeds its degradation, in whatever way these processes are defined biochemically or physiologically ${ }^{1}$. The rate of synthesis and degradation is related to body mass

1 The above statement applies to unicellular and animal organisms where, as a general rule, the products of catabolism are exported and hence appear as a negative term in the balance of metabolism and growth. It does not apply to higher plants where there is no excretion comparable to that in animals, and living mass once formed persists. Consequently, the model of growth discussed is applicable to unicellular organisms and multicellular animals. It does not apply to plant growth which rather follows the principle that increase is proportional to the living mass present, i.e. follows the logistic equation. The good approximation of growth in plants by the logistic has been shown in numerous examples. 
by some mathematical function. Experimental evidence shows that, as a rule and in first approximation, the rate of physiological processes can be expressed as a function of body mass by the simplest possible expression, namely, as a power function of the body mass present. Hence the equation:

$$
\mathrm{d} w / \mathrm{d} t=\eta w^{m}-x w^{n}
$$

hardly contains a hypothesis beside the assumption that anabolic and catabolic processes can be summated in two overall terms.

For the two exponents applies:

$$
\left.\begin{array}{l}
0<m<\text { I } \\
0<n<\text { I }
\end{array}\right\}
$$

for it would be absurd to assume that metabolic processes are inversely proportional to body mass, and physiologically un-understandable that they are proportional to a power $>$ I of body mass.

If the above assumptions are accepted, the family of growth equations follow by mathematical considerations. Growth may be $I$. unlimited, or 2 . limited.

I. Unlimited growth. In this case, growth in time gives a straight line in the semilogarithmic plot, as found in many empirical cases.

If exponential growth is found, there must be $m=n=\mathrm{I}$ and

$$
\frac{\mathrm{d} w}{\mathrm{~d} t}=c w
$$

because no other value of $m$ and $n$ yields the exponential.

2. Limited growth. In this case, there must be $(a)$ a steady-state value $w^{*}$ which is approached in the process; $(b)$ growth rates $\mathrm{d} w / \mathrm{d} t$ must be positive between $w=0$ and $w=w^{*}$. Regarding $(a)$, the steady-state value is obtained by equating (5. I6, p. I 79) to zero:

$$
w^{*}=\left(\begin{array}{l}
\eta \\
x
\end{array}\right)^{\frac{\mathbf{I}}{n-m}}
$$

According to $(b), w^{*}$ must be a maximum; otherwise, there would be negative growth, that is, not increase but decrease of $w$ between $w_{0}$ and $w^{*}$. For $w^{*}$ being a maximum, the second derivative must be negative:

It follows :

$$
w^{\prime \prime}=\eta m w^{(m-1)}-x n w^{(n-1)}<0
$$

$$
\begin{aligned}
& \eta m w^{*(m-1)}<x n w^{*(n-1)} \\
& w^{*(n-m)}<\frac{n}{m} w^{*(n-m)} \\
& m<n
\end{aligned}
$$

If, therefore, equation (5.I6) is to interpret growth approaching a final maximum value, it follows for mathematical reasons that $m<n$. 
Experience shows that in many cases (Growth Type I), there is a characteristic difference between time growth in linear dimensions and time growth in weight: the first shows monotonic leveling, the second a sigmoid curve. The conditions necessary for this characteristic, that is, the disappearance of inflexion in the curve of linear growth, can easily be ascertained.

In the case of proportional growth,

$$
w=q l^{3}
$$

Therefore we obtain (omitting the proportionality constant):

$$
\begin{gathered}
\frac{\mathrm{d} w}{\mathrm{~d} t}=\eta w^{m}-x w^{n}=\frac{\mathrm{d}\left(l^{3}\right)}{\mathrm{d} t}=\frac{3^{2} \mathrm{~d} l}{\mathrm{~d} t}=\eta l^{3 m}-x l^{3 n} \\
\frac{\mathrm{d} l}{\mathrm{~d} t}=\frac{\eta}{3} \cdot l^{(3 m-2)}-\frac{x}{3} \cdot l^{(3 n-2)}
\end{gathered}
$$

In order that this curve has no inflexion, it is necessary that $l$ vanishes in one of the two terms in the second derivative. This is possible only if

$$
\begin{gathered}
(3 m-2)=0 \\
m=2 / 3
\end{gathered}
$$

This is the necessary and sufficient condition for the difference between the curves of length growth and weight growth as observed.

It is not possible to set $n=2 / 3$ so that the second term disappears in the second derivative. For, since $m<n$, we would have, in this case, $m<2 / 3$. But then the exponent $(3 m-2)$ in the first term would be negative, that is, the rate of anabolism would be inversely proportional to length and hence to weight, which is absurd.

If $m=2 / 3$, it further follows that the inflection of the weight curve is between $\mathrm{o}$ and $\mathrm{I} / e$, and for $n=\mathrm{I}$, at $8 / 27$. That is, the sigmoid curve of weight growth is asymmetrical, with an inflexion at about $\mathrm{I} / 3$ of final weight. This is the shape of the curve of weight growth found in the organisms concerned.

While in our previous considerations we have inferred the several growth types from physiological data on the size dependence of metabolism, we now see that, given the differences of curves, these physiological relations follow for mathematical reasons. If we find the characteristic difference mentioned between weight growth and length growth, then the anabolic processes must be surfaceproportional. This deduction is verified by physiological evidence showing that the surface rule of metabolism applies precisely in those organisms where the curve of length growth shows no inflexion, and the length and weight curves are characteristically different.

3. For mathematical convenience, we have used equation (5.16) in the simplified form of equation (5.I8), assuming catabolism to be proportional to weight. This is an approximation which is not necessarily correct. There are few data on size dependence of catabolism and in particular of protein turnover. It has to be borne in mind that, apart from the objections against using $\mathrm{N}$-excretion as a measure of growth-inhibiting factors (p. 207), the methods for determining 
turnover rates are much less standardizable and hence estimates obtained show a greater variation than those of respirometry and calorimetry, owing to factors such as diet preceding the experiment, nutritive status, etc. There are also theoretical difficulties involved in the determination of turnover rates (Reiner, I953). The scarce data available on size dependence of N-excretion (Brody, i 945; Table I4b) show hardly more than that the intraspecific allometry exponent for N-excretion is somewhere not far from unity. Further experimentation on size dependence of catabolic processes, particularly with isotope labeling, is urgently needed.

It is advisable, therefore, to examine to what extent deviations of $n$ from $\mathrm{I}$ influence the shape of growth curves.

The necessary condition for limited growth and for the difference between curves of length and weight growth is:

$$
2 / 3=m<n
$$

There cannot be

$$
m=n=2 / 3
$$

for in this case, equation (5.I6) would become:

$$
\begin{gathered}
\frac{\mathrm{d} w}{\mathrm{~d} t}=n w^{2 / 3}-x w^{2 / 3}=c^{\prime \prime} w^{2 / 3} \\
w^{1 / 3}=C+c^{\prime} t \\
l=l_{0}+c t
\end{gathered}
$$

That is, the curve for length growth would be a straight line which is certainly not true for postembryonic growth.

Hence, there must be $n>2 / 3$, and the consequences of deviations of $n$ from I have to be investigated. It can be seen that such differences do not substantially alter the shape of the curves. In the First Growth Type, the curve for length growth retains its characteristic shape (no inflexion) in any case if $m=2 / 3$; for under this condition, $l$ always disappears in the first term of the second derivative of the equation for growth in linear dimensions. Hence the monotonic leveling of the curve of length growth, and the difference of this and the sigmoid curve of weight growth are not altered if $2 / 3<n<$ I. The question remains to what extent such deviations alter the point of inflexion in the weight curve. This is determined by equating to zero the second derivative. Expressed in terms of the final value, the weight at the inflexion of the growth curve is:

$$
\frac{w_{i}}{w^{*}}=\left(\frac{m}{n}\right)^{n-m}
$$

For $m=2 / 3, n=\mathrm{I}$, the inflexion is at $w_{i}=8 / 27 w^{*}=0.296 w^{*}$. If, e.g., $m=2 / 3, n=3 / 4$, we obtain $w_{i}=(8 / 9)^{12}=0.243 w^{*}$. Therefore, variations of $n$ between $2 / 3$ and $I$, that is, deviations of catabolism from weight proportionality do not substantially alter the shape of the curves. 
Similar considerations apply to the Third Growth Type $(2 / 3<m<$ I) for $m<n \neq \mathrm{I}$. Also in this case, the point of inflexion would be somewhat shifted, but the characteristic of this type, namely, a sigmoid curve of length growth, remains unchanged.

Variations of the point of inflexion within the limits calculated above (e.g. between $0.24 w^{*}$ and $0.29 w^{*}$ ) are hardly detectable in the usually scattered growth data of observation. Hence, barring further analysis based upon future experimentation, the approximation of the general growth equation (5.18, p. I 79) is satisfactory and it would at present be inadvisable to complicate the formulas.

The above derivations show that, assuming the principle that animal growth is the result of a counteraction of synthetic and degradative processes, the family of growth equations indicates the necessary as well as sufficient conditions for the variety of growth curves found in observation. This, of course, is not to say that the formulations given are final and do not allow for further analysis, approximation, and refinement which would find their expression in a resolution of the overall terms used into components, introduction of yet unconsidered factors which would appear as new terms in the growth equations, etc. It does sav, however, that the general characteristics of empirical growth curves (as well as a considerable number of related facts, p. 212) are consequences of the general model of growth described.

\section{(l) Further discussion of the growth model}

There is a rather extensive literature on the Bertalanffy growth equations, the underlying experimental results and theory, extending over the fields of physical chemistry (e.g. Bray and White, 1954, 1957; Precht, Christophersen and Henzel, I955); biochemistry (Duspiva, I 955); theoretical biophysics (Rapoport, I955a); cell physiology (Crandall and Smith, r952; Kunkel and Campbell, 1952; Schmidt-Nielsen, Bertalanffy and Pirozynski, I95I); cytology (Weiss and Kavanau, I957); microbiology (Christophersen and Precht, I954); comparative physiology (Duyff and Bruins, I942; Füsser and Krüger, I95I; Kienle and Ludwig, 1956; Krywienczyk, 1952a, b; Ludwig and Krywienczyk, 1950; Roberts, I957; Sattel, 1956; Will, 1952; Zeuthen, I955); zoology (Harms, I955; Klatt, I949; Medawar, 1945); anatomy (Haardick, 1956; Linzbach, 1950, 1955); physiology of nutrition (Mayer, 1949; L. and T. F. Zucker, 1942); anthropology (Keiter, 195 I/52); fisheries biology (Beverton, 1954; Beverton and Holt, 1957; Graham, I956; Yoshida, I956); theory of evolution (Rensch, 1954); etc.

Hence, the discussion is fairly comprehensive as an evaluation of facts and theory from different viewpoints. Objections on theoretical grounds have been raised (e.g. Duyff and Bruins, 1942; Linzbach, I955; Mayer, 1949; Medawar, 1945; Weiss and Kavanau, 1957; Zeuthen, 1955) which, so far as they deserve attention, are taken into account in the present review. However, with the exception of the data reviewed below, no factual evidence contradictory to the theory has been advanced.

On the other hand, there is a large volume of confirmatory evidence. For example Ludwig, whose laboratory with that of Bertalanffy, has done the most extensive research on comparative physiology of body size-metabolism relations, states that "no contradictions were found in the experiments to Bertalanffy's theory" (Ludwig and Krywienczyk, 1950). As already mentioned (p. 1 93ff.), the large material of the British Fisheries Laboratory and the Food and Agricultural Organization on growth of fish and other aquatic animals has led to the acceptance of the equations discussed as a general standard in this branch of applied biology. Careful examination of the various growth equations proposed led to the conclusion that "von Bertalanffy's growth equation is the most satisfactory of any that 
have hitherto been developed" (Beverton and Holt, 1957). Newly investigated organisms automatically fall into the categories (growth type) predicted by the theory even though the latter is unknown to the investigator (e.g. Fig. 16, p. 197). Sometimes (crustaceans: Roberts, 1957) a contradiction to theory was stated, but examination of the data seems to indicate that the case in question actually falls into the growth type predicted.

It is not to be expected, however, that the theory in its present state can do justice to every phenomenon of growth observed. Specifically, the following facts have to be mentioned in criticism of the theory.

I. The yeast Saccharomyces cerevisiae, being a spherical microorganism, was subsumed under Type I. This corresponds to the observed growth curves (Fig. 5, p. 164) (Schmalhausen and Bordzilowskaja, I930; Bayne-Jones and Adolph, 1932; Bertalanffy, 1934). According to the considerations indicated (p. 216), it was further to be inferred that cell size would decrease with increase of temperature as is usually the case in unicellular organisms. However, according to Christophersen and Precht (1954) energy metabolism (fermentation) of the yeast, Torulopsis kefyr, is proportional to the dry weight of cells, thus referring yeast into Type II. The growth curves of the cells of the latter species were not investigated but average cell volume increases with increasing temperature. Thus Bertalanffy's classification does not apply to this species which, however, is aberrant also in view of the fact that it does not follow the usual rule of decrease in cell size with increasing temperature. Christophersen and Precht emphasize that import of nutrient material (sugar) probably is not to be considered a process of diffusion but rather as active transport due to enzymatic reactions. Hence, Bertalanffy's supposition of a lower temperature coefficient for import and assimilation would be invalid in this case.

2. The results of different investigators on the dependence of metabolic rate on body size show, in general, good agreement (p. $184 \mathrm{f}$.) so that this relation appears to be a group- or species-specific characteristic. There are, however, some exceptions. Fish (Table 10, p. I 85 and Figs. 8-I0, I2-I4, p. I 8gff.) have been subsumed under Type I, corresponding to many experiments on metabolism and growth curves. However, in Salvelinus fontinalis a metabolic exponent between 0.8 and 0.9 was found (Job, I955), i.e. near to Type II. Plot of growth data for this species (Carlander, 1953, p. 298f.) appears to show simple exponential increase similar to Type II, different from the curve of length growth otherwise typical of fish. This, if correct, would be a confirmation of theory. It is, however, an indication that the classification summarized in Table 6 (p. I 8o) possibly is too generalized and that there may be different metabolic and growth relations even within one taxonomic group.

3. Zeuthen (1953, 1955) states that the curve of size-dependence of metabolism, interspecifically and intraspecifically, is composed of three segments. Interspecifically, the constant $\alpha \approx 0.7$ in unicellulars, $\alpha \approx 0.95$ in small metazoa (ova, larvae, and animals up to I mg $\mathrm{N}$ content), and $\alpha \approx 0.75$ for larger poikilothermic and homeothermic animals. Intraspecifically, Zeuthen contends a similar tripartite curve from the ovum to the adult which would present a special case of the rule of ontogenetic recapitulation of evolution. The physiological meaning of this broad comparison, ranging from bacteria to mammals, is difficult to understand. Zeuthen disregards, however, the various types of size-dependence of metabolism in postnatal development which are emphasized in Bertalanffy's theory. He is certainly correct in emphasizing (1955) that "there are many more 'metabolic types' which should be related to phenomena of growth than the three suggested by Von Bertalanffy". On the other hand, the correlations between metabolic and growth types, for which no alternative explanation is proposed, cannot be overlooked.

4. It has to be asked to what extent the shape of growth curves, as assumed to be typical in Bertalanffy's model, corresponds to the actual curves of growth. This concerns $(a)$ the initial, and $(b)$ the late, phase of growth.

(a) Backman (after Duspiva, I955) objects that a point of inflexion is found 
also in curves of length growth; only, e.g. in fish, it is so close to the origin of the coordinates that the remaining curve can be approximated by Bertalanffy's equation. The answer to this objection is that obviously the theory is applicable only insofar as its assumptions, i.e. an increase in size without far reaching changes of anatomic and physiological organization, hold true as a gross approximation. This is not the case in embryonic and larval development.

(b) The growth equations of Bertalanffy (and similarly almost all other equations proposed) state that the final value be reached asymptotically, i.e. "in infinite time". This is a simplification which does not strictly correspond to the facts, because many animals terminate growth within finite time. An illustration is the comparison of the mouse which terminates growth soon, and the rat, which grows during the whole life span, at least insofar as growth is defined as increase in weight. In other terms, the equations assumed disregard a factor of ageing which, at least in certain cases, is not negligible. The reason is essentially a mathematical one: for functions asymptotically tending to a limiting value are simpler and easier to handle than functions reaching such value in finite time.

There is a contrast of animals with indeterminate (e.g. fish) and terminate growth (e.g. mammals and also, based upon a different mechanism, insects), i.e. such which are growing during their whole life span and such where growth stops at a certain time. Two alternative hypotheses are possible, namely, that there is an essential difference between these two types, or that the course of growth is essentially the same and secondary factors such as puberty and senescence lead to termination of growth. At present there seems to be no physiological basis for deciding between these alternatives, and for reasons of mathematical expedience the second was adopted. In this we are in agreement with D'Ancona (1952), according to whom the distinction between indeterminate and terminate growth, as in fish and mammals

"is more apparent than real... This difference essentially depends on a different relation between life span and period of growth proper, upon which sexual maturation certainly has a preeminent influence. Thus it can be maintained at present that for fish and other species with indeterminate growth, little is known about the effective potential duration of life, and true phenomena of senescence are rarely encountered."

It is well possible that the "constants" of the growth equations which express the interplay of growth-promoting and growth-inhibiting factors actually are not constant but change during the life cycle. Physiologically, this would mean that anabolic and catabolic factors change, and processes of ageing should be taken into account. The mathematical expression of this would be that $\eta$ and $x$ are functions of time and/or additional terms should be introduced into the growth equations. However, inasmuch as the simple growth model covers a wide range of data and accessory hypotheses would be gratuitous, it is of little value to complicate the mathematical structure so long as this is not based upon physiological and experimental evidence. It would not be difficult to account for senescence and termination of growth by introducing terms in time into the growth equation (5.18); but the resulting complication of formulas and introduction of new constants would make the improvement so obtained somewhat specious, and 
differences like that between mouse and rat, as mentioned, would remain as unexplained as they are otherwise on the basis of available physiological knowledge.

The simplest assumption for growth reaching a terminal value in finite time is that the exponent $k$ is not constant but decreases monotonically with time (Haardick, 1956):

$$
k=\frac{K \mathcal{T}}{T-t}
$$

with $K=k$ at the beginning of growth, and $T=$ duration of growth. This gives for length growth:

$$
\frac{\mathrm{d}\left(l^{*}-l\right)}{\mathrm{d} t}=-\frac{\kappa T}{T-t}\left(l^{*}-l\right)
$$

which is a modification of equation $(5.26,5.29)$ :

$$
\frac{\mathrm{d}\left(l^{*}-l\right)}{\mathrm{d} t}=-k\left(l^{*}-l\right)
$$

By integration we obtain:

$$
l=l^{*}-l^{*}\left(\mathrm{I}-\frac{t}{T}\right)^{K T}
$$

which, for $T \rightarrow \infty$, gives equation (5.29) as a special case.

This equation takes account of the terminal length $\left(l^{*}\right)$ as well as the finite duration of growth $(\mathcal{T})$.

A case calculated with the generalized formula (5.47) is length growth of vertebrae in the rabbit (Haardick, I 956). It appears that application of equation $(5.29)$ is not satisfactory because the value of $k$ changes, but equation (5.47) with $K T=3$ gives satisfactory fit. However, numerical calculation according to equation (5.47) is somewhat elaborate, and decrease of $k$ according to this equation has no physiological proof at present.

5. The most severe oversimplification in Bertalanffy's model is the lumping together of growth-promoting and growth-inhibiting processes under terms of "anabolism" and "catabolism of building materials". This is justified insofar as the model yields considerable "dividends", the mathematical structure remaining simple enough for easy implementation; combined with the principle of Occam's razor that no factors should be introduced into a theory that cannot be empirically tested. However, this does not preclude but rather implies that the model should be further elaborated both by refining the mathematical structure and further analysis of the overall terms used, if and when complicating factors appear and are amenable to experimentation.

This criticism particularly applies to the identification of the parameter of "catabolism of building material" $(x)$ with protein metabolism. Experience shows that this simplification applies to a range of "model organisms" where the growth curves as well as the numerical value of the parameter in question are predicted by the theory with considerable accuracy. There is no doubt, however, and it has been strongly emphasized (Bertalanffy, I 934, I95 Ia; Racine, I953) that this is a first approximation. 
In cases available at present, the prediction from theory was verified to a surprising extent. Even discrepancies previously assumed, disappeared with improvement of experimental technique. Thus it was noted (Bertalanffy, I942a, p. I87f.; I95Ia, p. 239) that the value of $\varkappa_{\text {day }} \approx 0.045$ calculated from the growth curve of the rat, disagreed with protein loss calculated from minimum $\mathrm{N}$ excretion after Terroine (0.0028); but isotope experiments (Table I I, p. 200) showed precise agreement. This obviously goes far beyond accidental correspondence.

However, protein turnover does not cover the total of degradative processes in the organism. Not only are components like fat, bone, dentine, etc. in continuous renewal as shown by isotope experiments; but especially the factor of differentiation has to be taken into account.

Gross living mass may be subdivided into "metabolically active" and "inert body mass" (Benedict, I938), the latter represented by components which, although they do not leave the body, more or less cease to be involved in turnover and growth ${ }^{1}$. Furthermore, during development a transition from "active" to "differentiated body mass" takes place which is concealed in the "constant" $x$ and will presumably be significant in animals with rapid development and growth.

By its very structure, the growth model and equations derived therefrom refer to the "metabolically active" (Benedict, 1938), "essential" (Dunn, Murphy and Rockland, I 947), or "generative mass" (Weiss and Kavanau, I957). However, gross body weight also includes "inert" or "reducible" mass. This refers, first, to the shift of weight increase from protein to fat as in ageing mammals (Mayer, I 949). Secondly, the transition from "generative" to "differentiated" body mass is not reflected in the model.

Unfortunately, there is at present no quantitative measure for progressive differentiation. Possibly investigation of turnover (p. I $47 \mathrm{ff}$.) and mitotic rates (p. I65) of tissues could offer a way to distinguish between "active" and "differentiated mass" and to determine the transition from the first to the latter; but no study in this line appears to have been made.

\section{(m) Growth model of Weiss and Kavanau}

A much more elaborate model of growth has recently been proposed by Weiss and Kavanau (1957). The basic assumptions of this model are:

$I$. "Living mass" has the ability to reproduce and, in the absence of restraining factors, reproduces exponentially.

2. A living system consists of two components, "generative mass" which remains in reproductive activity, and "differentiated mass", derived from the former and consisting of terminal products and other secondary derivatives that do not possess the ability to reproduce. In development there is a gradual tran-

1 Weiss (1955a) has stated this very lucidly: "We certainly would not collect secretions, such as slime, urine, sweat and sebum, over a measured period and add them to the growth record. Yet, we do customarily include the bulk of cartilage and bone and other connective tissues, which consists of residues of cellular secretions, just like those other ones, but incidentally deposited, instead of extruded, hence accruing to the measured mass. Thus what we measure is related not so much to the process of production as to the accident of the disposal of the products. If they persist, we count them; if they drop out, we miss them." 
sition from "generative" to "differentiated mass", and progressive differentiation is one factor checking unlimited growth.

3. Protoplasmic reproduction or growth is essentially based upon cell-specific catalysts or "templates" which reproduce living mass of the same kind. Besides, each cell also produces specific, freely diffusibly compounds antagonistic to the former ("antitemplates") which can block and thus inhibit the reproductive activity of the corresponding "templates".

4. Hence, a second growth-regulating and limiting factor is a negative feedback in the way that an increasing population of "antitemplates" checks a corresponding proportion of "templates", so leading to a decline of growth rate.

5. Attainment of a terminal size is an expression of a steady state between incremental and decremental growth components, and between self-reproducing, intracellular "templates" and inhibiting, freely diffusible "antitemplates".

6. Both "generative" and "differentiated mass" (including "antitemplates") undergo continual metabolic degradation and replacement, but catabolic loss of generative mass is, in first approximation, negligible in comparison with the other growth-inhibiting components.

Hence, the model is indicated by the scheme (Fig. I9). A system of differential equations expressing these processes and interrelationships was formulated and solved.

\section{Growth Component}

$\mathrm{d} G=\left[\begin{array}{l}\text { Change in } \\ \text { generative } \\ \text { mass }\end{array}\right]=\frac{\left[\begin{array}{l}\text { Basic rate of } \\ \text { reproduction } \\ \text { of generative } \\ \text { mass }(\mathrm{IA})\end{array}\right]\left[\begin{array}{l}\text { Generative } \\ \text { feedback } \\ \text { term }(\mathrm{I} B)\end{array}\right] \mathrm{d} t}{\text { Generative mass formed }\left(\mathrm{d} G_{g}\right)}$

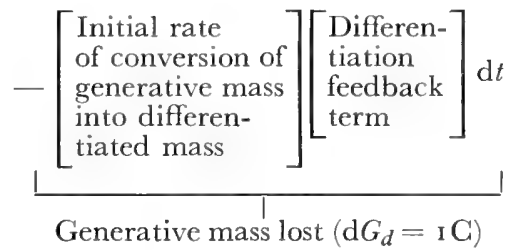

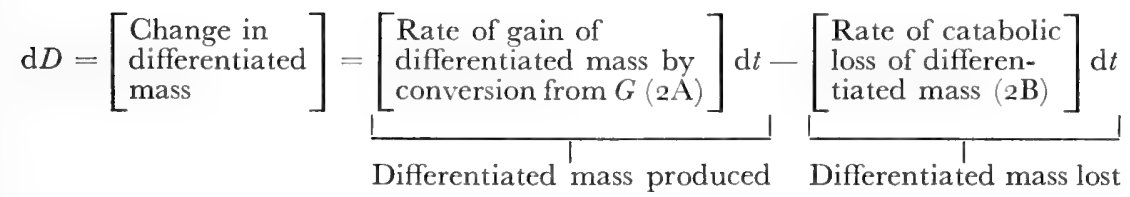

\section{Feedback Component}

$$
\mathrm{d} I=\left[\begin{array}{l}
\text { Change in number } \\
\text { of inhibitor } \\
\text { molecules }
\end{array}\right]=\underbrace{\left[\begin{array}{l}
\text { Rate of } \\
\text { production of } \\
\text { inhibitors }(3 \mathrm{~A})
\end{array}\right] \mathrm{d} t-\left[\begin{array}{l}
\text { Rate of cata- } \\
\text { bolic loss of } \\
\text { inhibitors }(3 \mathrm{~B})
\end{array}\right] \mathrm{d} t}_{\text {Inhibitors formed }}
$$

Fig. 19. Growth model after Weiss and Kavanau, 1957. The numbers (IA, etc.) refer to a system of growth equations expressing the individual terms. 
The Weiss-Kavanau model underlies objections of which the authors are by no means unaware, and which concern I. the basic assumptions, and II. the mathematical procedure in elaborating the model.

\section{The following assumptions are at present unverified:}

I. The assumption that "generative mass" in the absence of inhibition by differentiation or "antitemplates" grows exponentially does not account for the growth of microorganisms (p. I6 If.). There appears to be no difference as to differentiation or antitemplates between rod-like and spherical microorganisms; but only the first grow exponentially, while the second show a sigmoid growth curve. This shows that even in the simplest case of single cells there are growth-limiting factors different from those assumed in the model. This objection is not overruled by the fact that growth of populations, i.e. multiplication of unicellular organisms (and of organisms in general: Malthus' law) is exponential in the absence of limiting factors; for the homology is between individual organisms, unicellular or multicellular, rather than between a multicellular individual and a population of mutually independent cells. On the other hand, the growth curve of many higher organisms (Type I) is empirically similar to that of spherical microorganisms. This suggests that the mechanism producing the characteristic course of growth, although different in the underlying biochemical processes, is isomorphic in its fundamental structure (surfacevolume relations after Bertalanffy's theory).

2. "Generative" and "differentiated mass" and the transition from the first to the latter cannot, at present, be defined quantitatively. The relations between differentiation and loss of growth potential are obscure and differentiation as estimated by histological, cytological, biochemical, etc. standards is no measure of the loss of the ability to reproduce (p. I 4 I).

3. The template hypothesis is well supported with respect to nucleoproteins (WatsonCrick model of DNA and its more recent developments). However, the mechanism of protein synthesis is unknown even though a number of template models were proposed. Thus a hypothesis is introduced which, in its own field, i.e. the biochemistry of organic syntheses, so far is not proved.

4. Similar considerations apply to the "antitemplate" hypothesis. There is some supporting evidence (see below) but also this factor cannot be inserted in the model as a proved mechanism of growth limitation.

The basic assumption in the model is that catalysts or "templates" responsible for growth (e.g. nucleoproteins, proteins, myoproteins, etc.), being high-molecular compounds, are restricted to the cell while growth-inhibiting factors produced by them, being smaller molecules, are freely diffusible and hence can act in the feedback loop. The experimental evidence for this hypothesis is reviewed by Weiss (1955b) and discussed by Ebert (1954). The following facts are presented in support of the theory:

(a) The phenomenon of compensatory growth after removal of part of the organ. This, as can be shown by experiments in prefunctional stages (e.g. compensatory hyperplasia of the embryonic chick metanephros when the mesonephros is still functioning), is not necessarily due to functional overload imposed upon the remaining rest of the organ, but rather is an expression of an "equilibrium of the organs" ( $c f$. p. $229 f f$. ). One possible (although not the only) explanation is by way of a decrease of specific growth-inhibiting compounds whose concentration is lowered by removal of part of the organ producing them, with consequent compensatory spurt of growth in the rest.

(b) Supposing specific growth-regulating compounds, implants, cell extracts, etc. should have different effects, depending on the ratio between "templates" and "antitemplates". If the implanted cells survive, this may lead to an increase of "templates" or building blocks thereof, with a resulting increase of growth of the host. It may also lead to an increase of "antitemplates" produced by these cells and hence growth rate of the host would decrease. Disintegrated cells, on the other hand, may add to the pool of "templates" and/or trap circulating "antitemplates" with the effect of a spurt of growth. Transmission of organ-specific influences has been shown in various experiments: $\alpha$. En- 
largement of embryonic chick spleen and liver after implant of grafts of the homologous organ; $\beta$. Increase of mitotic activity of the kidney by injection of kidney brei, and decrease by liver brei; $\gamma$. Specific transfer of radioactivity from the graft (chicken spleen) to the homologous host tissue, and lack of this transfer to non-homologous tissue (chicken kidney $\rightarrow$ chicken spleen) and tissue of different species (mouse spleen $\rightarrow$ chicken spleen); $\delta$. Since embryo extract contains cell debris of all organs, promotion of growth of any explanted tissue in it is to be expected and is generally found. Using extract in which a certain organ was omitted gave the following results. In full embryo extract, the frequency of differentiation of tubules of embryonic kidney was greatly reduced as compared to cultures in extract without kidney tissue. Similarly, in full extract, only two of 333 heart fragments showed pulsation (indicative of differentiation of myofibrils) after the fourth day, compared to I 29 among 349 when heart extract was omitted from the medium. Because of the general antagonism between proliferation and differentiation, inhibition of the latter is interpreted as stimulation of growth by the homologous extract.

Thus, there appear to be specific influences on growth and differentiation issuing from homologous tissues and active by way of chemical communication. However, the interpretation of this evidence is not unequivocal, and explanation in terms of cell-bound "templates" and freely circulating "antitemplates" (as was emphasized by Weiss, 1955b) is to be considered as a working hypothesis. The extrapolation that an antagonism between self-multiplying and growth-inhibiting compounds is a basic and general principle in growth is not proved experimentally.

5. For these reasons, also assumption (5) is not experimentally proved.

6. The omission of catabolic loss of generative mass in the model and consequent growth equations appear not to correspond with isotope experiments (p. I48), other physiological determinations of protein turnover (Table I I, p. 200), cell turnover (Table 4, p. I64), etc., which show that this factor is by no means negligible.

II. The laws governing the rate of the processes summarized in Fig. I9 are unknown. Hence, even if these are taken for granted as growth-regulating factors, any particular function expressing these terms is necessarily arbitrary.

From the rather elaborate system of equations of the Weiss-Kavanau model, two experimentally verifiable consequences were derived, namely

I. A calculation of the growth in chickens;-2. The qualitative prediction that after experimental or pathological disturbances there will be a spurt of growth, and later on restitution of the equilibrium by an oscillatory process with first overshooting the steady-state level.

With regard to $I$, Weiss and Kavanau justly emphasize that calculation of one growth curve is no proof for the underlying model, but fitting can be achieved by different equations if a sufficient number of arbitrary parameters is permitted.

With regard to 2 , overshoot phenomena (if these can be found experimentally in compensatory growth) are a general characteristic of reestablishment of steady states under certain mathematical conditions (p. I 46). Hence, overshoot is not a consequence of the particular model and equations, but will appear in any open-system model provided these mathematical conditions are fulfilled.

The value of the Weiss-Kavanau model (as its authors emphasize) is in calling attention to certain possible mechanisms in the intricate process of growth by mathematically formulating them. Thus, malignant growth may be interpreted by a loss of control of cell multiplication exerted by cellular feedback (Weiss) and/or "higher" regulatory centers (Druckrey, I 959). Further experimentation suggested by the model may lead to more detailed analysis of the overall process of growth. 
(n) Summary of models of growth

As compared with the Weiss-Kavanau model, the Bertalanffy model has yielded a considerable number of experimentally verified "dividends" which may be briefly summarized:

I. Calculation and satisfactory approximation of a large number of empirical growth data representative of the major animal classes;-2. Derivation of a correlation between metabolic and growth types, verified in many examples;3. Prediction, from experimental determination of metabolic rate, of the existence of the Third Growth Type which was subsequently verified by the growth curves of the species concerned (Bertalanffy, I94I b; Bertalanffy and Müller, I943);4. Correct prediction of interfering factors from aberrations in the growth curve, verified by more detailed analysis (e.g. plaice, Figs, I 3, I 4, p. I 94f.); - 5. Correct prediction of ratios of anabolic constants (p. 199);-6. Correct prediction, in a number of cases, of the numerical values of the catabolic constants $(x)$, confirmed by independent physiological determinations (p. I 99f.);-7. Derivation of equifinality of growth (p. 2I3);-8. Prediction of the major characteristics of temperature dependence of growth corresponding to ecological and experimental evidence (p. 2 I 6ff.);-9. Prediction, from the calculation of $\varkappa$ values, of two growth cycles in mammals which is confirmed $(a)$ by inspection of empirical growth rates; $(b)$ by investigation of basal metabolism (Fig. 23), and (c) by numerous other changes occurring at this critical period. In consequence, correct calculation of growth in mammals (p. 2i 8ff.) by simple mathematical expressions with a consistency and precision not obtained with formulas containing a much larger number of constants (P. I 76);-IO. Correct prediction of the difference of growth curves in spherical and rod-like microorganisms (p. I6 If.);-II. Empirical equations for embryonic growth appearing as special cases of the general growth equation (p. 223f.);-I2. Interpretation of the growth of tissue cultures within the framework of the theory (p. I66ff.);-I3. Derivation of equations for time growth of organs which qualitatively correspond to observed growth curves and are capable of numerical calculation (p. $236 \mathrm{ff}$.). Correct prediction of the form of organ-growth in time if the organism grows exponentially, and if the organs show positive or negative allometry (Figs. 37,$38 ;$ p. 238);-I4. Deductive derivation of the family of growth equations from a general scheme and few empirical characteristics (p. 20off.).

Many of these items are verified not by one or a few isolated examples, but an often considerable array of empirical instances. Beside individual examples, the merit of the theory is in its successful application to seemingly unrelated experiences and fields.

In conclusion, it may be said that the theory doubtless is a first approximation, to be refined and corrected with further analysis of the factors involved and with consideration of more complicated cases. The large number of verified consequences and deductions indicates, however, that the basic model and theory are correctly chosen. 
(o) Equifinality of growth

An important consequence of the growth laws discussed is the equifinality encountered in animal growth.

It is well known and frequently found that the same species-characteristic final size may be reached $I$, after temporary suspension of growth, and 2 , from different initial sizes at the start. The first applies to cases when diet was temporarily insufficient in quantity, vitamins, etc.; there are, in particular, many experiments on rats showing this effect (Kopeč, I 938; Clarke and Smith, I 938; Jackson, I939, etc.) (Fig. 20). The second holds true in litters of varying size: in large litters weight at birth of the newborn is lower, but the same final weight is eventually reached in animals both large and small at birth (Kopeč, 1932). Naturally this equifinality of growth only prevails if no lasting damages (e.g. of bone growth in humans) remain after the period of malnutrition.

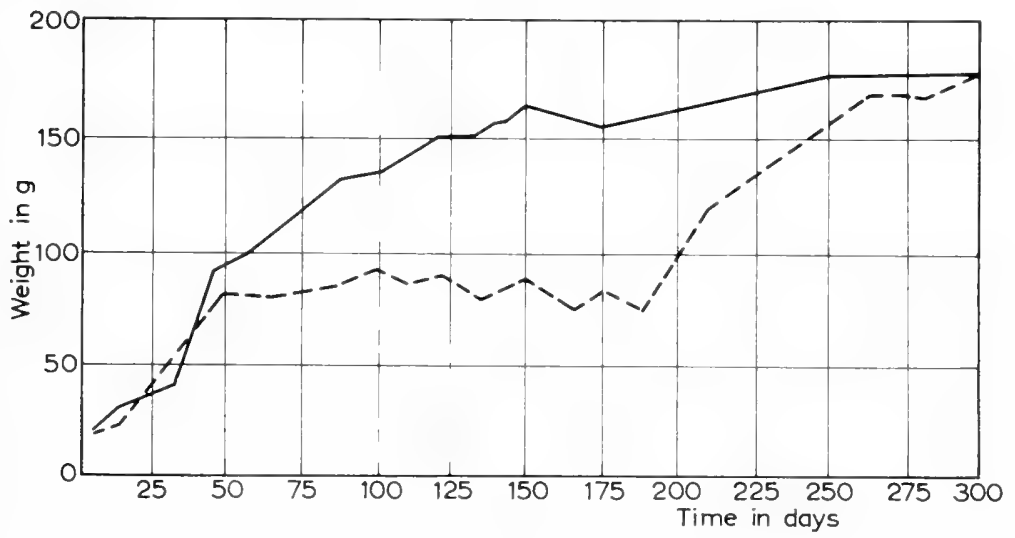

Fig. 20. Equifinality of growth. Heavy curve: normal growth of rats. Broken curve: at the 5oth day, growth was stopped by vitamin deficiency so that there was no increase but only maintenance of the body weight. When normal regime was re-established, the animals reached the normal final weight, growth rates approximating values corresponding to body size, not to age. After Höber from Bertalanffy, i 95 га.

Equifinality appears to be paradox if it is envisaged from the conventional causal viewpoint; for it appears as if events were determined by states to be reached only in the future. Nevertheless equifinality is a general characteristic of open systems in so far as they approach a steady state (p. 146). In such systems, in contrast to conventional closed systems, the time-independent final state is not determined by the initial conditions but only by the system parameters of transport and reaction. Hence, the final state is independent of the initial state as well as of the course the process has taken. It can easily be seen that any process of growth described by equation (5. I6), except for the case $m=n=$ I (i.e. growth according to types I or III), must show the property of equifinality.

Further it follows from theory that growth rate is not a function of time, i.e. does not automatically decrease as age increases, but rather is a function of the body size reached. Hence, after an inhibition is lifted, growth is resumed at a rate which corresponds to body size and not to age (Fig. 20). In a similar way, specific growth 
rate, e.g. in mice, is less in animals born in small litters and therefore having higher weight at birth, than in animals born in larger litters and with a smaller birth weight (Kopeč, I932).

The principle of equifinality is also applicable to the balance of organs and of chemical constituents in the animal organism (p. 229ff.). Apparently a certain ratio between the size of the individual organs and the size of the body must prevail if the organism is to function properly. However, different species are born in widely different stages of development. In order eventually to reach the functional balance of organs and constituents, relative growth of organs has to be different in different species. This implies that the same final result, that is, a balance of organs as expressed in interspecific allometry, will be reached in different ways, that is, by allometric growth differing intraspecifically $(c f$. p. 24Iff.; Bertalanffy and Pirozynski, I952).

Equifinalities which cannot be quantized but apparently are based upon similar principles, are found in many processes of development. For example, the stages of the gastrula, that is, a two-layered embryonic stage with ectoderm and endoderm, and of the neurula with its characteristic arrangement of archenteron, notochord, neural tube, and mesoderm, are found universally and appear to be fixed points which may be reached in quite different ways. The process of segmentation is different in the several animal classes and orders; owing to the yolk content of the ovum, equal, unequal, discoidal, etc., segmentation may take place. A coeloblastula or a sterroblastula may result. Gastrulation may occur by the ways of invagination, epiboly, immigration or delamination of cells, or a combination of these processes; but nevertheless a similar two-layered gastrula stage is eventually established. This can be seen, for example, in the various types of gastrulation found in scyphozoa or in polychetes; or experimentally, if the normal unequal segmentation of amphibian eggs is changed into discoidal segmentation by centrifugation, without this having an influence upon subsequent development. Similarly, early development of ova of Amphioxus, selachians, amphibia, and reptiles is widely different; but the neurula eventually developing in these classes is surprisingly similar. Again, the development of the vertebrate liver takes place in very different ways, and no less than 12 different ways of development were found in 30 species investigated (Elias, 1955). In striking contrast, no vertebrate organ shows more uniformity of structure in the adult stage than the liver. As justly stated by Elias, this is in contradiction both to Von Baer's law according to which embryos of different species are similar and diverge later on in the adult stage; and to Haeckel's biogenetic law since the embryonic liver does not recapitulate the structure of ancestral forms in phylogeny.

Phenomena of regulation, considered by Driesch (1929) as a "proof of vitalism", appear to fall into the same category. The same final stage, a normal and complete larva, can develop from a complete normal ovum of the sea urchin, from each half of an ovum divided in two, from two ova fused into one, etc. The same applies to regulation in ova in general and to early organ anlagen such as those of the limb, eye, and heart. According to Driesch, this equifinality of development can only be explained by the intervention of a vitalistic entelechy which directs the process in order to establish a complete organism. However, attaining the same final state from different initial conditions is by no means a vitalistic feature exhibited in processes of this sort; rather it is a general characteristic of open systems.

Similar considerations apply to regeneration. Here we find the paradox that seemingly lack of a part which was lost acts as a releasing stimulus for restitution of that part (A. E. Needham, I952). How can something that is non-existent be a causative agent? However, the paradox disappears and there is no need to hypostatize hypothetical regenerationinhibiting substances in the intact organism if it is realized that the organism, by regeneration, tends to reestablish an equifinal steady state. We are far from being able to give 
any explanation in physico-chemical terms. Essentially, however, restitutive regeneration is of the same nature as physiological regeneration by which the organism is maintained in a steady state in a continuous breaking-down and replacement of components. Physiological and restitutive regeneration are governed by the same principles, the difference only being that component parts are "worn out" and replaced as part of the normal life process in the first case, and accidentally or experimentally removed in the second. A good example of equifinality in regeneration is presented by experiments on earthworms: the number of regenerated segments is independent of the number originally present so that regeneration comes to a stop when a number of 90 segments is reestablished, irrespective of whether 5o, 40, 30, etc., segments were amputated (Moment, 1946).

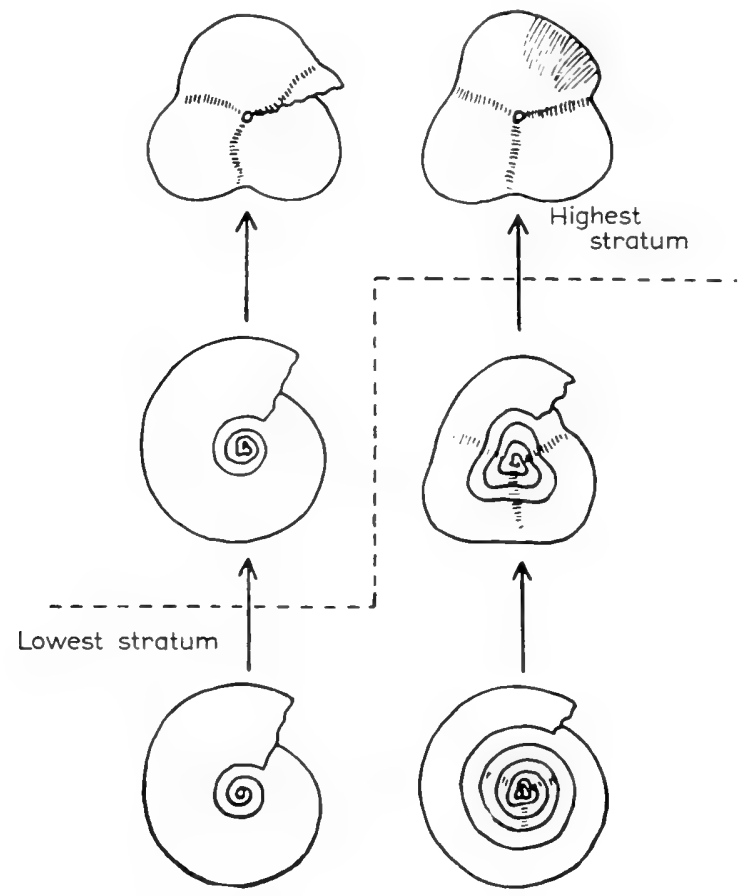

Fig. 21. Evolutionary equifinality. Convergence (evolution of three-lobed species) in independent series of ammonites. Left: Paraclymenia abeli, Wocklumeria sphaeroides, Epiwocklumeria applanata. Right: Kamptoclymenia endogena, $\hbar$. trivaricata, Parawocklumeria paradoxa.

After Schindewolf from Rensch, 1954.

It appears that similar rules even apply to evolution. It has been emphasized by Schindewolf that similar final stages may be reached in independent evolutionary series, as found e.g. in corals and the irregular types of ammonites (Fig. 2I). Since a particular adaptive value can hardly be attributed to the formations eventually reached in these evolutionary series, it may be surmised that evolutionary equifinality represents a tendency toward establishment of singular states of genic balance which may be reached if different ways.

Many of such phenomena are, of course, not amenable to quantitative treatment. Possibly, however, they can be envisaged as different expressions of the same basic principles. 


\section{(p) Growth and temperature}

Owing to the possibility of measurement and physicochemical interpretation, effects of temperature are of particular significance for the theory of growth.

According to the classical Bergmann rule (I847), animals of the same species are, in general, larger in cold climates than in warm ones. Bergmann interpreted the temperature rule in terms of the heat economy in homeothermic animals. Loss of heat is proportional to body surface; the latter decreases in its ratio to body weight with increasing size; therefore less heat per unit weight has to be produced in a larger animal in order to keep body temperature constant. Thus increase in body size saves energy and is adaptive in cold climate. Conversely, smaller body size and relatively increased surface facilitate heat output in warmer surroundings. In this way, Bergmann's geographical rule for body size is a precursor of Rubner's physiological rule for metabolism (p. I 8I ).

It is found, however, that Bergmann's rule also applies in poikilothermic animals where Bergmann's (and Rubner's) explanation evidently is not applicable. Two cases of the relation between temperature and body size can be distinguished. The first is genotypical, that is hereditary differences in body size which presumably can be explained in the way proposed by Bergmann, i.e. selective advantage of larger body size in cold, and of smaller size in warm climates. The second case is phenotypical variations, that is direct influence of temperature on growth. As a crude rule of thumb it may be stated that the first especially applies to differences in body size as found in geographical races of warm-blooded animals; the second, to many observations and experiments with invertebrates and poikilothermic vertebrates. This, however, is by no means a clear-cut distinction; geographical races with varying body size are also found in poikilothermic animals, and conversely direct effects of temperature on growth in mammals. Only the second case will be discussed here.

In the sense of the theory presented, the following inferences can be drawn. The catabolic processes can be considered as being, in the last resort, of chemical nature; therefore they will have a high temperature coefficient as characteristic of chemical reactions according to Van 't Hoff's rule. The anabolic processes, on the other hand, lastly depend on the import of materials regulated by factors of permeation, diffusion, etc., i.e. physical processes likely to have a low temperature coefficient. When, with increasing temperature, the catabolic constant $x$ of the growth equation is strongly increased, but the anabolic constant $\eta$ undergoes little change, two consequences follow: $I$. Growth rate will increase, and 2 . final size will decrease. That is, with increased temperature the organism will grow faster toward a smaller final size. This is, as a general rule, found in observation.

The complexity of the relationships between tissue respiration, body size, temperature, fasting (Locker, 1958d), pharmacological agents such as dinitrocresol (Locker and Hofer, 1958; Locker, 1958b), etc., have already been mentioned (p. 187).

The above conclusions correspond, as a rule, with experience in unicellular organisms. Cell size in general decreases with increasing temperature (Table 12; similar results with Frontonia, Stylonychia: Popoff; Paramecium: Rautmann; Foraminifera: Rhumbler; survey of literature in Bëlehrádek, 1935; Margalef, 1954). An exception is the yeast, Torulopsis kefir (Christophersen and Precht, i 954; cf. p. 205).

In invertebrates and fish, the above rules are confirmed by many examples in animal 
TABLE 12

CELL SIZE AND TEMPERATURE IN PROTOZOA

After R. Hertwig from Bertalanffy, 195 ra

\begin{tabular}{|c|c|c|c|}
\hline Temperature & $\begin{array}{r}D i \\
\text { length }\end{array}$ & $\begin{array}{l}\text { tus anser, } \\
\text { width in } \mathrm{mm}\end{array}$ & $\begin{array}{l}\text { Actinosphaerium, } \\
\text { diameter of a cyst in } \mathrm{mm}\end{array}$ \\
\hline $8^{\circ}$ & 0.510 & O.II 2 & 0.090 \\
\hline $25^{\circ}$ & $0.3^{8} 4$ & 0.096 & 0.065 \\
\hline
\end{tabular}

geography and ecology showing that growth rate increases and final size decreases with increasing temperature (Fig. 22). This was found, for instance, in the mussel, Mya arenaria (Newcombe, 1936). Broch (1933) found in hydroids that individuals of the same species grow larger in colder than in warmer regions; corresponding to the considerations given above, although without quantitative analysis, he concluded that body size is determined by a steady state between anabolic and catabolic processes which are both, though to a different extent, dependent on temperature. Exceptions from the rules mentioned are apparently due to the fact that physiological processes follow an optimum curve rather than Van 't Hoff's rule. The mechanism described will therefore be modified by optimum conditions characteristic of the species concerned. This is the case, for example, in marine snails: Increased size in cold waters was found in 64 percent of the species investigated, while larger varieties in warm oceans were found in the rest, i.e. species with a high temperature optimum (Chen-Ya-Shih, 1937).

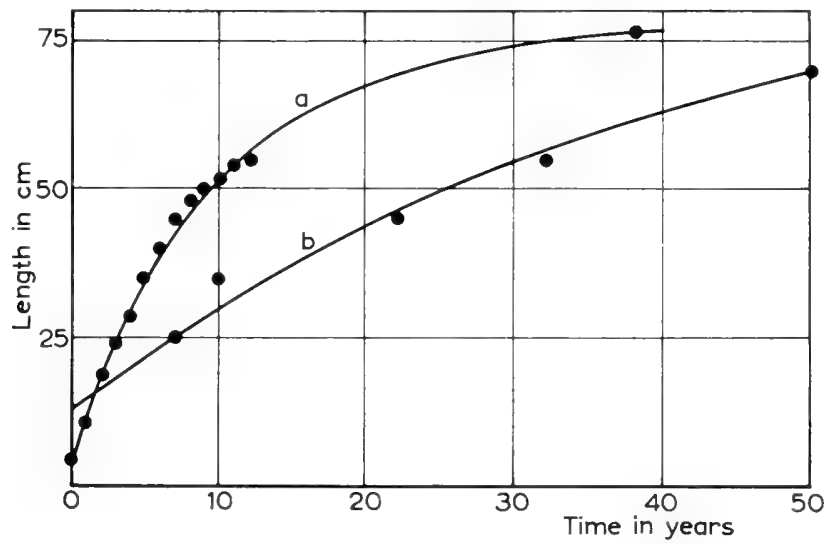

Fig. 22. Effect of temperature on growth in plaice (Pleuronsctes platessa). Heincke's data. $a$ from the Southern North Sea (summer temperature $\left.15^{-1} 6^{\circ}\right) ; b$ from the Barents Sea (summer temperature $0-4^{\circ}$ ). At higher temperature growth is faster and final size smaller.

After Bertalanffy, i95ia.

\section{TABLE 13}

BODy Weight AND TEMPERATURE in Planaria maculata

After Abeloos from Bertalanffy, I95 I a

\begin{tabular}{cc}
\hline Temperature & Final weight in $\mathrm{mg}$ \\
\hline $7^{\circ}$ & 53 \\
$12^{\circ}$ & 35 \\
$18^{\circ}$ & 25 \\
\hline
\end{tabular}


Laboratory experiments show the same dependence of body size on temperature ('Table I3). No experimental postembryonic growth curves in dependence of temperature have been found in the literature, including the extensive monograph by Precht, Christophersen and Henzel ( 1955). The only available data apparently are those of Gibson and Hirst (1955) on the Guppy (Lebistes reticulatus) which are incomplete because they only reach to the time of sexual differentiation, and growth of males and females (strikingly different in the Guppy, $c f$. p. I g8f.) was not further followed. However, the description given reveals the characteristics mentioned: while a water temperature of $32^{\circ} \mathrm{C}$ is noxious (all fish develop crooked spines) and one of $25^{\circ} \mathrm{C}$ is optimal, $I$. growth rate at $30^{\circ} \mathrm{C}$ is faster than at $20^{\circ} \mathrm{C}$; and 2. females developing at $30^{\circ} \mathrm{C}$ are much smaller than those at $20^{\circ} \mathrm{C}$.

\section{GROWTH OF MAMMALS}

\section{(a) Postembryonic growth}

Growth in mammals shows a number of complications, both with respect to metabolic and growth rates.

Rubner's surface rule of metabolism was first stated for mammals. However, more recent investigation has shown that the relation between body size and metabolic rate is more complex. In intraspecific comparison of the best-investigated laboratory animal, the rat, a crude overall relation of respiration to the $2 / 3$ power of weight can be found. Detailed study, however, shows a break in the allometric regression line so that up to approximately I $00 \mathrm{~g}$ body weight metabolic rate increases more, and afterward less than would correspond to the surface rule (Fig. 23) (Racine, 1953). The break corresponds to the onset of sexual maturation. In interspecific comparison of mammals of different species, metabolic rate varies with the $3 / 4$ power of body weight, rather than with the $2 / 3$ power as would correspond to the surface rule (Brody, I945; Kleiber, 1947).

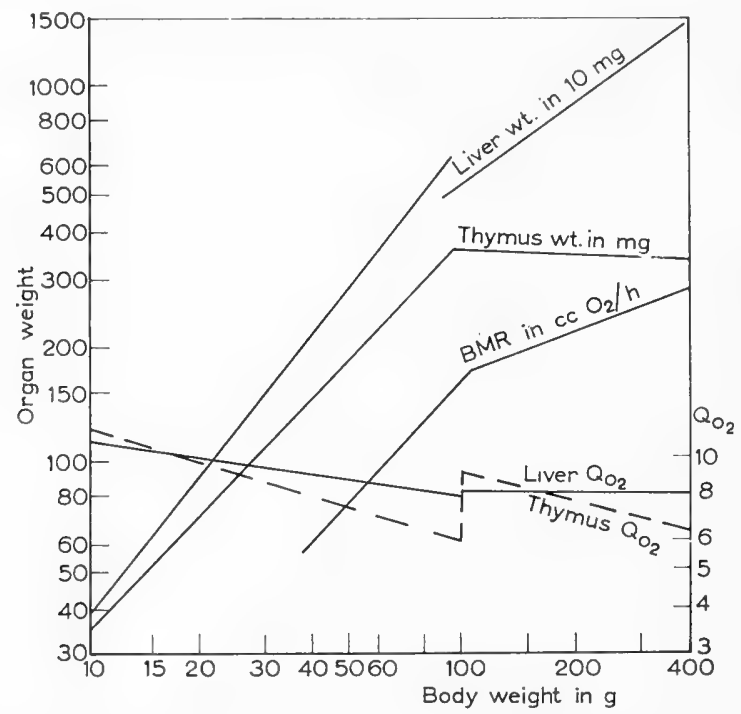

Fig. 23. Discontinuities in the allometric curve. The figure shows some phenomena of relative growth in the albino rat. All discontinuities occur at a body weight of approx. $100 \mathrm{~g}$, i.e. before puberty. A corresponding discontinuity is found in the growth of the entire animal (Fig. 26). The figure only gives regression lines; complete data and statistical analysis in: Bertalanffy and Pirozynski, 1952, (relative growth of liver and thymus); Racine, 1953 (basal metabolic rate for males); Bertalanffy and Pirozynski, I953 $\left(Q_{\mathrm{O}_{2}}\right)$. 
TABLE 14

ABSOLUTE AND RELATIVE GROWTH RATES IN WEIGHT GROWTH OF MALE WHITE MICE

After Bertalanffy, 1938

Stieve, i923 Robertson, rg16 Robertson, $1926 \quad$ Saller, 1932 Kopeć, 1933

Age in $w_{n}^{-} w_{n-1} \underline{\operatorname{IOO}\left(w_{n}-w_{n-1}\right)} w_{n}-w_{n-1} \underline{\operatorname{IOO}\left(w_{n}-w_{n-1}\right)} w_{n}-w_{n-1} \underline{\operatorname{IOO}\left(w_{n}-w_{n-1}\right)} w_{n}-w_{n-1} \underline{\operatorname{IOO}\left(w_{n}-w_{n-1}\right)} w_{n}-w_{n-1} \operatorname{IOO}\left(w_{n}-e_{n-1}\right)$ weeks

\begin{tabular}{|c|c|c|c|c|c|c|c|c|c|c|}
\hline 20 a & & $w$ & & $w$ & & $w$ & & $w$ & & $w$ \\
\hline I & I. 25 & 71.4 & 2.08 & I69. I & I. 52 & 103.4 & 1.72 & I 37.6 & 2.78 & 222.4 \\
\hline 2 & 1.33 & $44 \cdot 3$ & I. 83 & $55 \cdot 3$ & 2.10 & 70.2 & 1.67 & 56.2 & $2.5 \mathrm{I}$ & 62.3 \\
\hline 3 & 0.77 & 17.8 & $(3.62)$ & 60.4 & 2.90 & 57.0 & I. 23 & 26.5 & 2.22 & $33 \cdot 3$ \\
\hline 4 & 1.70 & $33 \cdot 3$ & $(3.62)$ & $4 I \cdot 3$ & I. 52 & I9.0 & 2.16 & 36.8 & $3 \cdot 37$ & 38.5 \\
\hline 5 & 1.03 & I 5.1 & 0.07 & 00.6 & 1.76 & I 8.5 & 2.75 & 34.2 & $2.8 \mathrm{I}$ & 23.2 \\
\hline 6 & 1.09 & I 3.9 & 3.13 & $25 . \mathrm{I}$ & 1.70 & I $5 . \mathrm{I}$ & 2.07 & 19.2 & 2.76 & I 8.5 \\
\hline 7 & I. 28 & I 4.3 & $2.5^{\circ}$ & I 6.0 & I. 94 & I 5.0 & $(\mathrm{I} .04)$ & 8. I & 2.46 & 14.0 \\
\hline 8 & 0.90 & 8.8 & I. 28 & 7.1 & I. 74 & I I. 7 & - & - & I. 53 & 7.6 \\
\hline 9 & 11.40 & I 2.6 & 1.27 & 6.6 & 1.40 & 8.3 & - & - & 0.83 & 3.8 \\
\hline IO & 0.80 & 6.0 & 0.56 & 2.7 & 0.63 & $3 \cdot 5$ & - & - & 0.67 & 3.0 \\
\hline
\end{tabular}

Bibliography $c f$. original. Second peak of growth rates is indicated by italics.

Growth curves of mammals show segments or cycles. These are not arbitrary subdivisions as assumed by many authors to fit growth curves with a preconceived formula. Rather these breaks in the growth curve are real and manifest in a decrease and subsequent new increase of growth rates (Table 14). This is in contrast to the monotonic decrease of specific growth rates in simpler growth curves, e.g. of fish (analysis of growth cycles in the mouse: Saller, 1927, I932; mouse and rat: Bertalanffy, 1938).

The break in the growth curve coincides with sexual maturation which further is expressed in many physiological changes. In the rat, all these changes, such as transition from the first to the second growth cycle, the change in the dependence of basal and resting metabolism on body size, the involution of the thymus, the changes in relative growth and in $Q_{\mathrm{O}_{2}}$ of certain tissues, etc., occur at approximately $100 \mathrm{~g}$ body weight, thus showing deep-reaching changes in metabolism connected with a shift of hormonal balance. Naturally the sharp break assumed in these calculations is a mathematical simplification of a process which, in reality, is uninterrupted.

\section{(b) Growth and hormones}

The characteristic course of growth in mammals is regulated by hormones ( $c f$. Gaunt, I954; Wolff in the present volume). Only a few remarks relevant to the present discussion will be made.

The growth hormone of the hypophysis (somatotropin) is the most important stimulator of growth in mammals. Thyroxin, with its effect of increasing metabolic rate, is essential for normal growth and development. The androgenic steroid hormones are, to an extent, growth stimulants in male but also female mammals, even though their effect may be lacking or reversed in certain doses and conditions. The glucocorticosteroids (cortisone), controlled by ACTH, have a growthinhibiting effect. 


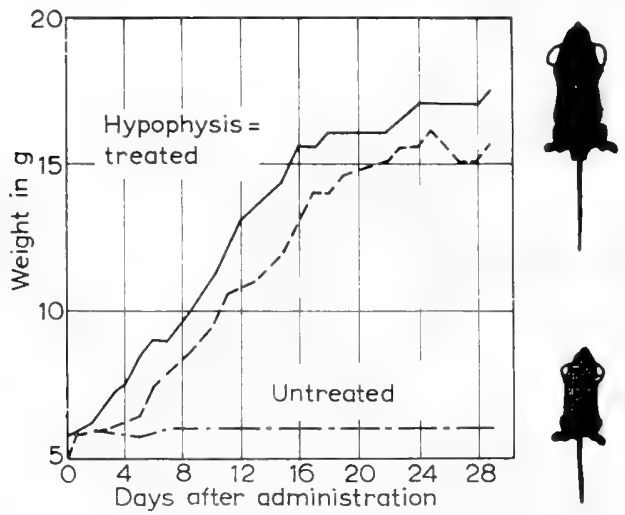

Fig. 24. Growth of pituitary dwarf mice without and with administration of growth hormone. After Smith and McDowell from Bertalanffy, r95ıa.

A classic example of hormonal control of growth are the pituitary dwarf mice whose growth is inhibited by a recessive gene. This leads to lack of eosinophilic cells in the anterior lobe, and consequent lack of growth hormone; injection of growth hormones stimulates growth so that the dwarfs reach almost normal weight (Fig. 24). The fundamental metabolic aberrations caused by the dwarf gene and the lack of growth hormone is indicated by the reduction of basal as well as tissue metabolism (Bertalanffy and Estwick, 1954) and the absence of higher nuclear classes in the dwarfs (Leuchtenberger et al., 1954). Similar changes are found in hypophysectomized animals with respect to changes in basal and tissue metabolism (Bertalanffy and Estwick, I954), decrease of kathepsin activity (Mathies, Palm and Gaebler, I 95I), and in RNA content of cells (Leslie, I955). Further investigation is needed to determine to what extent such effects are due to the absence of the somato-

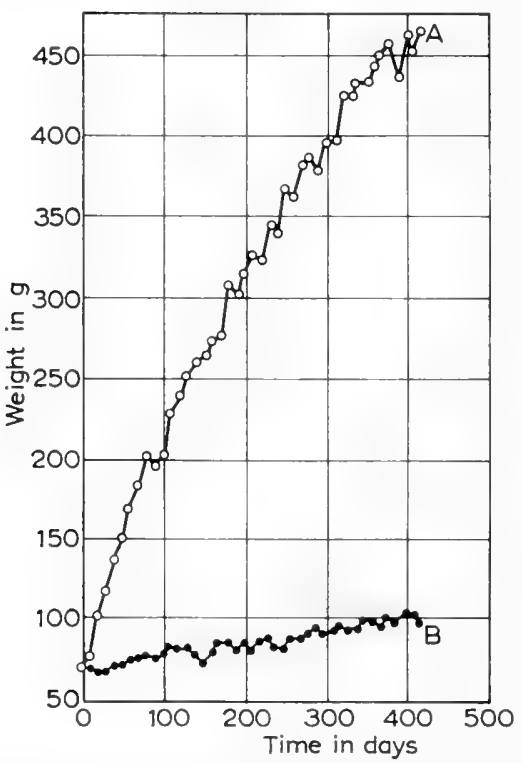

Fig. 25. Growth of hypophysectomized rats (Q). A Daily injections of growth hormone (o.1 mg, from the 14 oth day $0.2 \mathrm{mg}$ daily); $B$ Control. After Li and Evans from Harms, I955. 


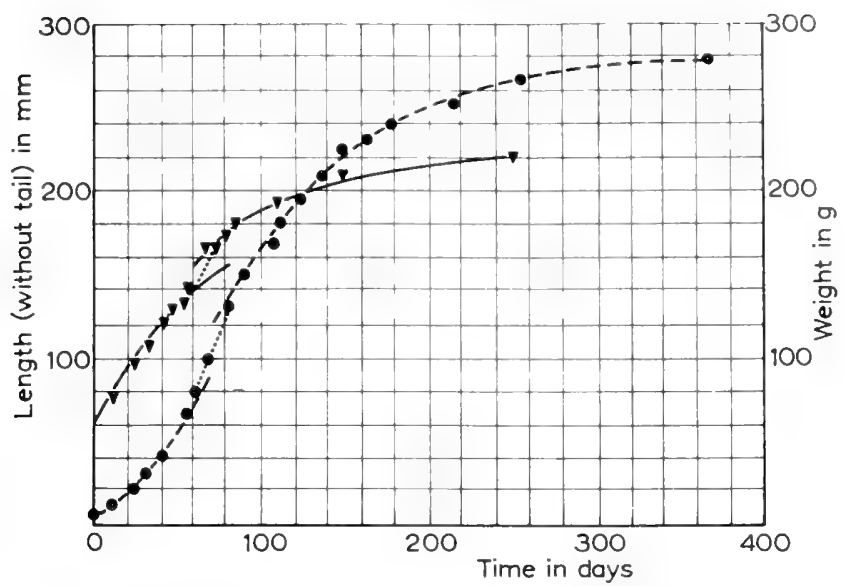

Fig. 26. Growth of the albino rat (ठ). Donaldson's data. Length growth weight growth - - C. Calculation according to equations $(5.28,5.29)$ shows two growth cycles with a shift of the values of the constants at approximately $100 \mathrm{~g}$ body weight.

After Bertalanffy, I $94 \mathrm{I}$ b.

tropic or other hormones of the hypophysis. This is indicated by the fact that hypophysectomy, i.e. removal of the whole organ, entails more drastic changes of metabolism than the dwarf gene of mice which causes lack of the eosinophilic cells and presumably of somatotropin. The deficiency in growth hormone in dwarf mice inhibits development of skin cancer by 20-methylcholanthrene (Bickis, Estwick and Campbell, 1956). Hypophysectomized rats given growth hormone apparently do not show the inflexion in the S-shaped curve of weight growth which is found in normal growth (Fig. 25).

If the growth cycles mentioned are taken into account, the growth e.g. of the mouse and rat can be reproduced with excellent approximation by equations $(5.28)$ and $(5.29)$ (Figs. 26, 27).

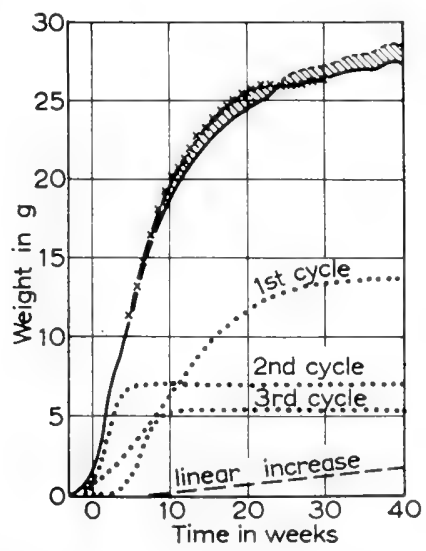

Fig. 27. Growth of the white mouse (ठ). Robertson's data. The solid line indicates mean values, the shaded zone the double probable error. This growth curve was approximated by Robertson by superposition of not less than 4 curves with a profusion of arbitrary constants. It can be fitted as well by equation (5.28), p. r 90 , only. After Bertalanffy, $193^{8}$ and 195 Ia. 
Apart from humans, full data as required for such analysis are available almost only for the small laboratory animals. Even here wide discrepancies in empirical growth data exist. For example, there is a large discussion as to what is to be considered the "typical" growth curve of the rat (L. Zucker et al., I94Ia, b; T. F. Zucker et al., I 94I, I 942; Dunn, Murphy and Rockland, I 947; Murphy and Dunn, I948; Mayer, I 948). The classical data of Donaldson (1924) (used in Fig. 26) are not considered to be satisfactory because increased growth rate and change of the shape of the growth curve can be obtained by use of improved laboratory diets. Thus, each of the mentioned groups of investigators proposed a different empirical equation for the growth of the rat which, however, does not fit data of other observers. These controversial observations and calculations show that the growth curve of rats can be widely modified by different diets. In addition the more basic problem mentioned on p. 138 arises as to whether "optimal" growth is identical with "normal" growth. If Donaldson's data presumably represent a "suboptimal" course of growth, it has to be considered, on the other hand, whether a synthetic diet yielding maximum weight increase, does not lead to "supernormal" growth, particularly with respect to deposition of fat.

Thus, even more than in the cases discussed hitherto, quantitative analysis of growth in mammals must be considered as provisional. The presence of a prepubertal and postpubertal growth cycle, and the overall classification of mammalian growth as belonging to the First Type may be considered as well established. However, the growth formulas used can only be considered as a crude first approximation. This is not changed by the fact that they fit the data well and even better than more complicated formulas.

Based upon the growth theory discussed, the following deductions were derived from mathematical analysis of growth in mammals (Bertalanffy, 1938, 1942a):

I. Protein turnover takes place at a much faster rate than presupposed in classical physiology; 2. There is a synthesis and re-synthesis of amino acids and proteins from ammonia and nitrogen-free chains; 3. Under a diet low in protein, nitrogen is retained from catabolized organic compounds and is used for regeneration of protein. This explains the protein-sparing effect of carbohydrates, that is, the fact that under a diet rich in carbohydrates, the organism needs less protein than would correspond to the nitrogen excretion in starvation.

Qualitatively, these deductions proved to be excellent predictions, later confirmed by isotope experiments (Sprinson and Rittenberg, I949a, b). The isotope method has demonstrated the rapid protein turnover, the regeneration of proteins from the metabolic pool, and the utilization of ammonia for protein synthesis under a low-protein diet.

Quantitatively, however, it has to be considered that equating the growthinhibiting factors contained in the constant $x$ with manifest protein turnover is a crude oversimplification. This simplification is confirmed, as a first approximation, in simple grow th curves and when metabolic processes, differentiation, changes in chemical composition (e.g. content of dry substance, protein-fat ratio) are relatively slow (Table I I, p. 200). However, these complicating factors cannot be overlooked, but there is no way at present to quantify them. Beside manifest 
protein loss, transition from metabolically active mass to metabolically inactive differentiations has to be taken into account (Bertalanffy, r95 ra; $c f$. p. 208).

(c) Embryonic growth

For the reasons discussed previously (p. I 40), growth as quantitative increase of the organism and its organs is a rather superficial aspect of embryonic development which proceeds with determination and segregation of embryonic areas,

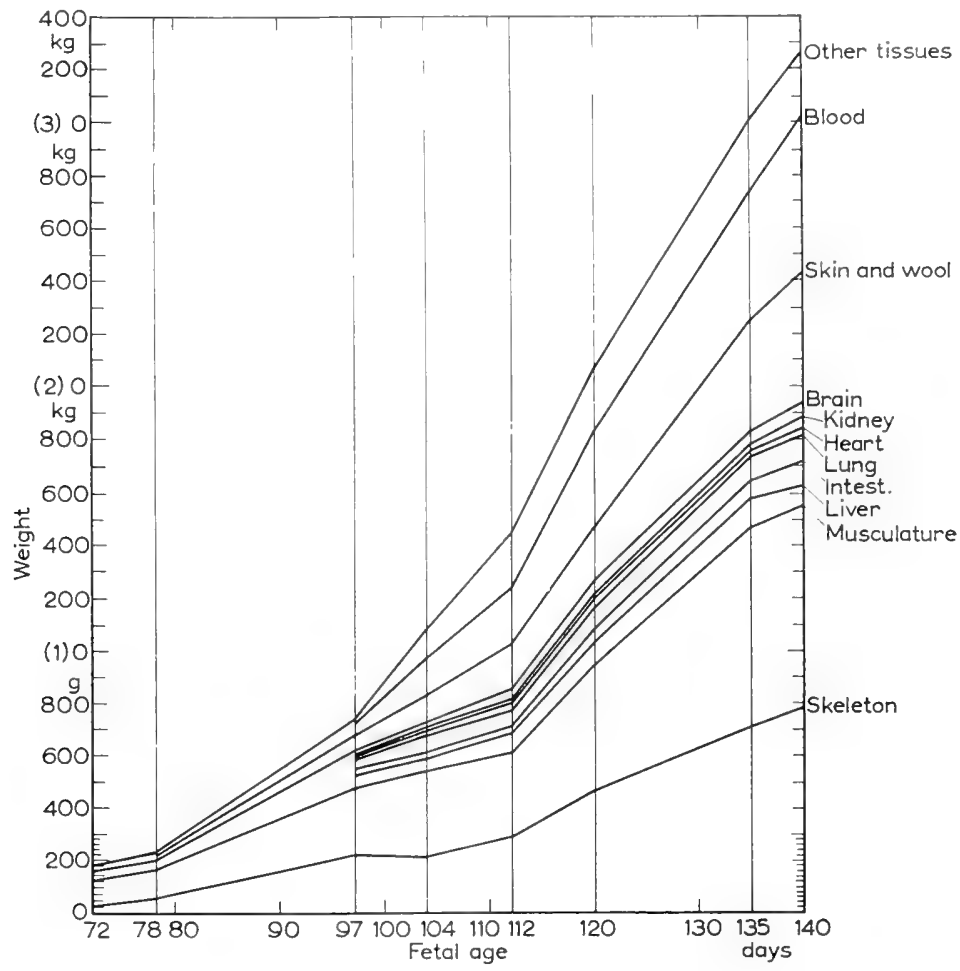

Fig. 28. Growth of organs in the sheep embryo. Weight of subsequent organ was added to the prior one; highest line represents total weight. After Barcroft from Bertalanffy, I957.

formative movements, elaboration of organs and histological differentiations, etc. Thus it is easy mathematically to fit embryonic growth by suitable formulas, but these hardly have much physiological significance.

Frequently embryonic growth curves can be approximated by simple exponential curves (Brody, I945). If plotted semilogarithmically, breaks in the straight lines representing exponential growth appear simultaneously in the various organs (Fig. 28), so showing that physiological changes take place in these periods.

According to Hugget and Widdas (I95I) embryonic growth frequently can be fitted by the empirical equation:

$$
w^{1 / 3}=c t
$$


$c$ being a species-specific constant; that is, the cubic root of weight increases proportional to time. Then

$$
\mathrm{d} w / \mathrm{d} t=\eta w^{2 / 3}
$$

i.e. growth in weight is proportional to a surface, or embryonic growth follows equation (5.24), with catabolism being negligible.

\section{RELATIVE GROWTH}

\section{(a) The allometric equation}

Organic form appears to be that problem in biology which is least amenable to quantitative treatment but it can be approached in several ways.

Two works have been pioneering in this respect: D'Arcy Thompson's On Growth and Form (1942) introducing the Theory of Transformations, and Huxley's Problems of Relative Growth (1932) which established the Principle of Allometry.

Morphogenetic changes in a growing animal chiefly take place by relative growth, that is, certain components increase at a higher or lower rate than others or, as also may be said, growth rate is different in different spatial dimensions. In the growth of the organism as a whole as discussed, time and body size are the variables under consideration. Relative growth concerns the ratio of growth rates in several components of the organism. A relation found to be of wide applicability is the allometric equation.

The relationship now termed allometry was first used by Snell (189r) for expressing the ratio between brain size and body size in mammals of different species or individuals of the same species but of different body size. E. Dubois (1898) and Lapicque (1898) applied the equation for the same problem in mammals and in birds, respectively. Klatt (1919) was probably the first to realize the general significance of this rule, applying it to the relation between heart size and body size. The equation has found general application following Huxley's work. After some terminological controversies (Huxley, J. Needham and Lerner, I94I; Reeve and Huxley, 1945) the term "allometric growth" was generally accepted. Relations of the same form are also found in social phenomena (Naroll and Bertalanffy, I956).

The principle of allometry is expressed by the equation:

$$
y=b x^{\alpha}, \quad \text { or } \log y=\log b+\alpha \log x
$$

Hence, if a variable (e.g. size of an organ, rate of a physiological process, etc.) is plotted logarithmically against another variable (e.g. size of the organism as a whole), a straight line is obtained. This simple procedure shows whether the allometric relation obtains, and allows estimate of the constants. $\alpha$ is the slope of the allometric line (tangent of the angle it forms with the abscissa; $\alpha=\tan a$ ); $b$ is the value of $y$ for the extrapolated value, $x=\mathrm{I}$. If $\alpha>\mathrm{I}, y$ grows faster than $x$, or shows positive allometry. If $\alpha=\mathrm{I}$, both magnitudes grow at the same rate or isometrically. If $\alpha<\mathrm{I}$, the relative increase of $y$ is smaller than that of $x$, or $y$ shows negative allometry.

If the variable $y$ is expressed per unit size of $x$,

$$
y / x=b x^{\alpha-1}
$$

i.e. the slope of the allometric line is negative. This is the case, for example, in the 
relation of $Q_{\mathrm{O}_{2}}$ (oxygen consumption/mg dry weight) to body weight (Fig. 7, p. I 86).

Statistical calculation of the constants $\alpha, b$, the standard deviation $S_{\log y} \cdot \log x$, and the correlation coefficient $(\rho)$ can be made by application of least-square methods. There are certain mathematical difficulties (Sholl, I948) inasmuch as application of the least-square method to $y=b x^{\alpha}$ or to $\log y=\log b+\alpha \log x$, i.e. supposition of a normal distribution either of the numeri or the logarithms, yields different values for $\alpha$ and $b$. This discrepancy appears to arise when the values for $y$ are widely scattered on a short interval of $x$; in general, the error committed by application of the least-square method to the logarithms is insignificant (Brody, I945).

The meaning of the allometric equation is that the specific growth rate of a component or process, $y$, stands in a constant ratio to the specific growth rate of another component or the total organism, $x$ :

$$
\frac{\mathrm{d} y}{\mathrm{~d} t} \cdot \frac{\mathrm{I}}{y}: \frac{\mathrm{d} x}{\mathrm{~d} t} \cdot \frac{\mathrm{I}}{x}=\alpha
$$

integrals of this equation being (7.1).

In view of criticisms of the principle of allometry (Sholl, 1954) it should be mentioned that the allometric relation is not trivial. That the specific growth rate

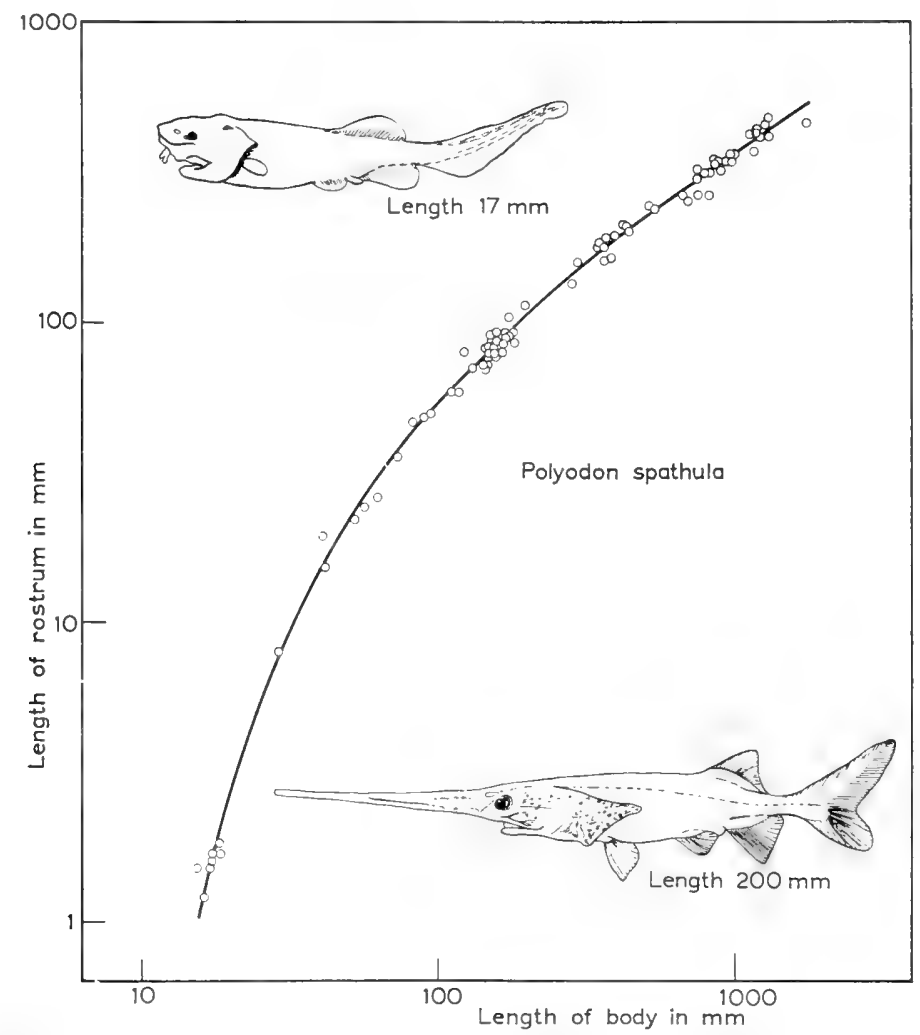

Fig. 29. Relative growth of rostrum in the spoonbill cat, Polyodon, as an example where the allometric equation does not apply. After Thompson from Hersh, I94I. 
of a part stands in a constant ratio to that of another part or the whole, is in no way obvious. In view of the tremendous complexity of the processes involved in growth, it would rather be expected that parts start and cease to grow in some irregular manner, without our being able to represent this process by a simple or even a continuous function. That, at least in many cases, the simple allometric relation applies is an indication that it is based upon general and fundamental principles. It could also be objected that, owing to logarithmic plotting and scale which maximize differences in small numbers and minimize them in large numbers, the allometric relation is spurious and that almost any data plotted in this way, can be fitted by straight lines. However, this objection is contradicted by the facts. There are cases where data cannot be fitted by straight lines in the $\log -\log$ plot and hence the allometric relation is not applicable (Fig. 29); but such cases are encountered only rarely. Conversely, if such approximation obtains, it cannot be considered an artefact of logarithmic plotting.

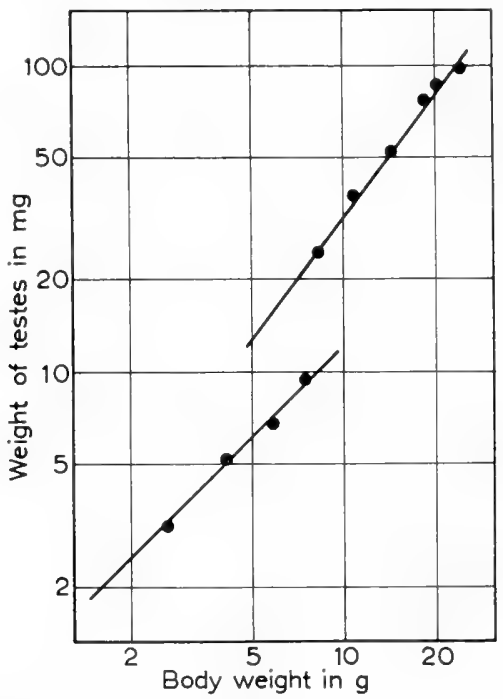

Fig. 30. Relative growth of testes in the mouse. The jump to a higher constant $b$ takes place at a body weight of about $7 \mathrm{~g}$, corresponding to the growth cycles apparent in growth-in-time of the body as a whole (Table 14). This shows the connection between growth-in-time, relative growth of the testes, and change in hormonal balance (sexual maturation). Teissier's data from Bertalanffy, I95 Ia.

Breaks and jumps are frequently found in allometric plots so that all data cannot be fitted by a single regression line (Figs. 23, 30). Then detailed analysis usually shows that these discontinuities are not accidental but connected with definable changes in the underlying process. If, for example, such discontinuities are found in a number of processes in rats at a weight of $c a$. I oo g (i.e. at the start of sexual maturation), this is easy to understand in consideration of the changes of hormonal balance at this period.

A more serious objection against the allometric equation is of a mathematical nature. If allometric equations apply to parts of an organ (e.g. the segments of 
an insect extremity), an equation of the same form cannot apply to the organ as a whole because a sum

$$
y_{1}+y_{2}+y_{3}+\ldots=b_{1} x^{\alpha_{1}}+b_{2} x^{\alpha_{2}}+b_{3} x^{\alpha_{3}}+\ldots
$$

is not of the form $b x^{\alpha}$. Hence the allometric equation can only be considered a first useful approximation (Reeve and Huxley, r 945). This objection does not apply to the size dependence of physiological processes and similar phenomena.

Although understood to be in the nature of an approximation, the principle of allometry can be given a rationale and interpretation from different viewpoints.

I. Physiologically, the mechanism underlying allometric growth becomes clear if the allometric equation $(7 \cdot 3)$ is written in a slightly different form:

$$
\frac{\mathrm{d} y}{\mathrm{~d} t}=\alpha \cdot \frac{\mathrm{d} x}{\mathrm{~d} t} \cdot \frac{y}{x}
$$

That is, the part $y$ receives from the increase of the total system $(\mathrm{d} x / \mathrm{d} t)$ a share which is proportional to its ratio to the total system $(y / x)$. The allometry coefficient, $\alpha$, is a distribution coefficient indicating the capacity of $y$ to appropriate a certain share of the total increase.

Allometric growth therefore is an expression of the competition of parts of the living organism for available resources. Although particular allometry constants can hardly be explained in this way, physiological competition is well documented by observations and experimental facts (p. 23off.).

2. Functionally, allometry can be conceived of as being an expression of the principle that the organism must remain functioning and self-maintaining in spite of variations of absolute size: principle of biological similarity (Günther and Guerra, I955; Guerra and Günther, I957).

It is a well-known engineering principle that any machine (in the broadest sense) requires changes in proportion of component parts or in rates of processes in order to remain functional, when enlarged from a small-scale model to actual size. Any magnitude $Q$ of the machine can be expressed, in the cm-g-sec system, as

$$
Q=L^{\beta} M^{\gamma} T^{\delta}
$$

( $L=$ length, $M=$ mass, $T=$ time). If $q, l, m, t$ are the corresponding measures in the model, and $L / l=\lambda, M / m=\mu, T / t=\tau$, the general reduction coefficient is:

$$
\text { and } \quad \begin{aligned}
& \chi=\lambda^{\beta} \mu \tau^{\gamma} \tau^{\delta} \\
&
\end{aligned}
$$

Since dimensionally $\mu=\lambda^{3} d\left\langle d=\right.$ density) and $\tau=\lambda^{\frac{1}{2}}$, equation (7.7) can be written (for constant $d$ ) as:

$$
\%=\lambda^{\beta+3 \gamma}+\delta / 2
$$

This is known as Newton's reduction coefficient for mechanical similarities. For electrodynamics, another relation between $\lambda$ and $\tau$ applies, namely $\tau=\lambda$, so that the electrodynamic reduction coefficient is

$$
\chi^{\prime}=\lambda^{\beta+3 \gamma+\delta}
$$

Similarly, any biological function $(y)$ will be related to that of a unit system, e.g. I $\mathrm{kg}$ by $W=\lambda^{3}(W=$ weight of the actual system) and

$$
y=b W+\left(\beta+3 \gamma+\delta^{\prime}\right)
$$

Lilerature p. 253 
where $\delta^{\prime}$ is the value for the biological similarity concerned. Equation (7.II) is isomorphic with the allometry formula:

$$
y=b x^{\alpha}
$$

with $x=$ body weight, and $\alpha$ corresponding to the exponent in equation (7.I I).

It can further be argued that the exponent of biological similarity will be somewhere between the exponents of mechanic $(\chi)$ and electrodynamic $\left(\chi^{\prime}\right)$ similarity, i.e. between $(\beta+3 \gamma+\delta / 2)$ and $(\beta+3 \gamma+\delta)$. It then appears that, considering the dimensionality of the biological function in question, the allometry constants of physiological functions such as those of the cardiovascular and renal system, heart and respiratory rate, etc. are within, and in close agreement to the theoretical values (Günther and Guerra, I955). The general principle is that, in order to remain functional, an organism cannot remain geometrically similar, i.e. increase isometrically, but must increase by way of physiological similarity, i.e. with various allometries, positive and negative, in respect to its functions and parts.

Further illustrations of the same principle are presented in Fig. 35 (heart rate in relation to body weight) and in the considerations of p. 242 (allometry constants of various organs). A functional analysis of the allometric growth of birds' wings was given by Meunier ( $195 \mathrm{I}$ ). Although it is, in general, not possible numerically to deduce the allometry constants of organs and biological functions, these can be understood qualitatively as guaranteeing "biological similarity", i.e. proper functioning in spite of variations of absolute body size. Furthermore, they are within the limits drawn by physical (mechanical and electrodynamic) similarity.

3. In the last resort, relative growth, that is, the specific capacity of organs to appropriate a certain share of available resources, is determined genetically. It is a quantitative expression of the principle of harmonized reaction rates (Goldschmidt, I 927, I938), known from genetics and physiology of development. This principle states that genes determine chains of reactions which go on simultaneously and whose quantitative ratio determines which of them will eventually gain the lead (for a discussion of Goldschmidt's principle $c f$. Bertalanffy, r 952). On the other hand, changes in the genome can lead to a displacement of this balance, that is, to changes of relative growth rates and consequently to changes in proportion manifest as evolutionary changes (p. 240ff.).

The principle of allometry can be applied either intraspecifically, i.e. in comparison of individuals of the same species but of different sizes, or interspecifically, i.e. in comparison of animals (usually adult) of different races, species, etc. (allomorphosis after Huxley, Needham and Lerner, I 94 I). Intraspecific allometry is mostly applied to different stages of development: ontogenetic allometry (heterauxesis after Huxley et al.). When species compared in interspecific allometry belong to a phylogenetic series we can speak of evolutionary allometry.

The principle of allometry was found to apply to numerous phenomena in morphology, cytology, biochemistry, pharmacology, evolution, etc. (for a review of earlier publications $c f$. Huxley, I 932 ; for more recent work Bertalanffy, I95 Ia). In the following only a few examples are given. 
The range of the allometric equation is illustrated by examples such as the interspecific comparison of heart rate (Fig. 35); the oxygen consumption in Artemia salina, which follows the surface rule over more than 5 orders of magnitude in weight (Bertalanffy and Krywienczyk, 1953); or the relative growth of the human heart (Linzbach, 1955). The heart maintains its allometry constant $(\alpha=0.95)$ over a range of 5 orders, from $1 / 100 \mathrm{~g}$ in the embryo weighing I $\mathrm{g}$ up to $300 \mathrm{~g}$ in the adult weighing $75 \mathrm{~kg}$, while it increases from the size of a pinhead to that of a fist, and undergoes all kinds of changes in shape, structure and composition.

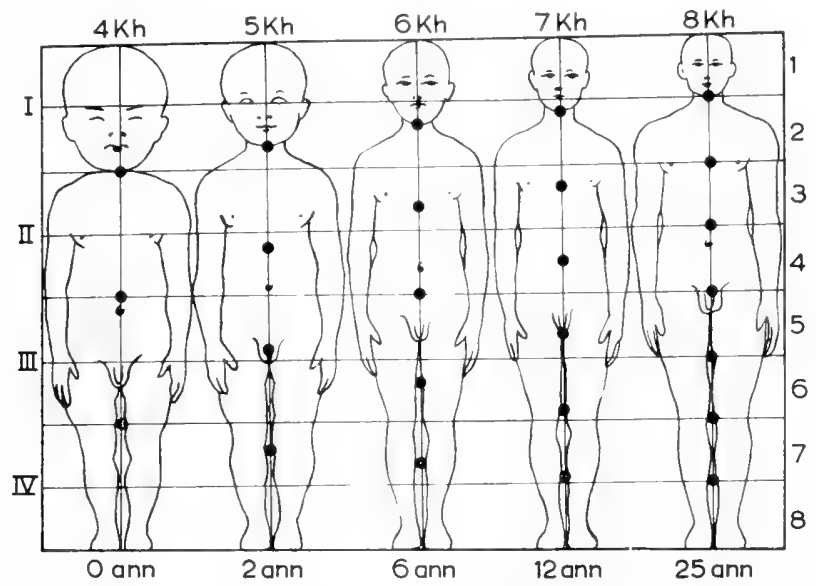

Fig. 3I, Relative growth in man. Changes in proportion are expressed in head lengths $(K h)$. After Stratz, 1928.

\section{(b) Morphological applications of allometry}

In a newborn child the length of the head is approximately I/4 of the total length of the body; in an adult, it is only $\mathbf{I} / 8$. Conversely, the legs are comparatively much shorter in the baby than in the adult (Fig. 3I). The proportions of the adult human are established in the way that the head grows relatively slower and the legs faster than the rest of the body. Thus relative growth, i.e. the ratio of growth rates of the various parts of the body, leads to changes in proportion and hence is a preeminent morphogenetic factor.

The changes of form exhibited by a developing organism are, to a large extent, changes in proportion determined by relative growth. In many cases relative growth follows the equation of simple allometry, over the entire development or at least over physiologically defined cycles of growth. The validity of the allometric equation has been shown in many hundreds of cases from all animal classes. Figs: 32 and 33 are illustrative examples.

The theoretical implications of the principle of allometry require a brief discussion. Classical morphology has spoken of a "budget law" (Goethe) or loi de balancement (Geoffroy St. Hilaire) meaning that in an organism there is a characteristic and constant "equilibrium" between the organs and, as we may add, the chemical components. Thus overdevelopment of one part will be connected with lack in another part; if the "equilibrium of the organs" is disturbed by removal of a part, compensatory growth will set in, etc. This balance of organs, intuitively recognized by the classical morphologists as modification of an ideal "type" or "bauplan" 
finds at least a partial, physiological confirmation and explanation in the principle of allometry.

There is a competition of organs for material as expressed in equation (7.5) which also indicates that the "struggle of the parts" (Roux) can be represented by a simple mathematical law. Thus the principle of allometry gives a physiological and quantitative basis to the morphological principle of the "balance of organs".

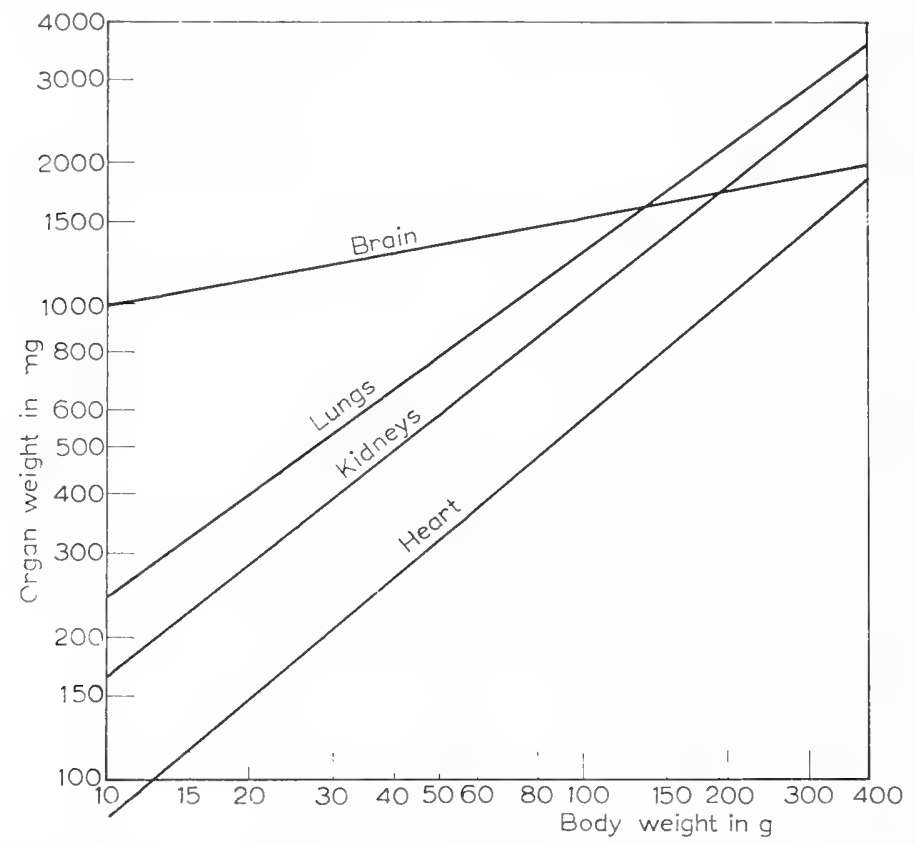

Fig. 32. Relative growth (postnatal) of some organs of the albino rat. The figure shows only the regression lines; observed values and statistical analysis see original. After Bertalanffy and Pirozynski, I952.

\section{(c) Physiological competition}

The competition for material, which is also important, in evolutionary changes, as compensation of organs (p. 246f.), can be interpreted biochemically to a certain extent. One critical factor determining mitotic rate is supply of energy in the form of glucose. Tissues apparently are competing for the available glucose supply, especially under conditions of carbohydrate deficiency (Bullough, I952). Such competition exists between adjacent as well as distant tissues. An example of the first is that a wave of hair growth induces inhibition of the mitotic activity in the epidermis, particularly involution of warts; similarly, activity of the mammary glands during the lactation period induces a reduction of mitotic activity in hair formation. The second is exemplified by embryos and tumors which have the highest priority in the competition for nutritive material and continue to grow even when the rest of the body is almost reduced to a skeleton. According to Bullough the greatest competition to mitotic activity is probably 


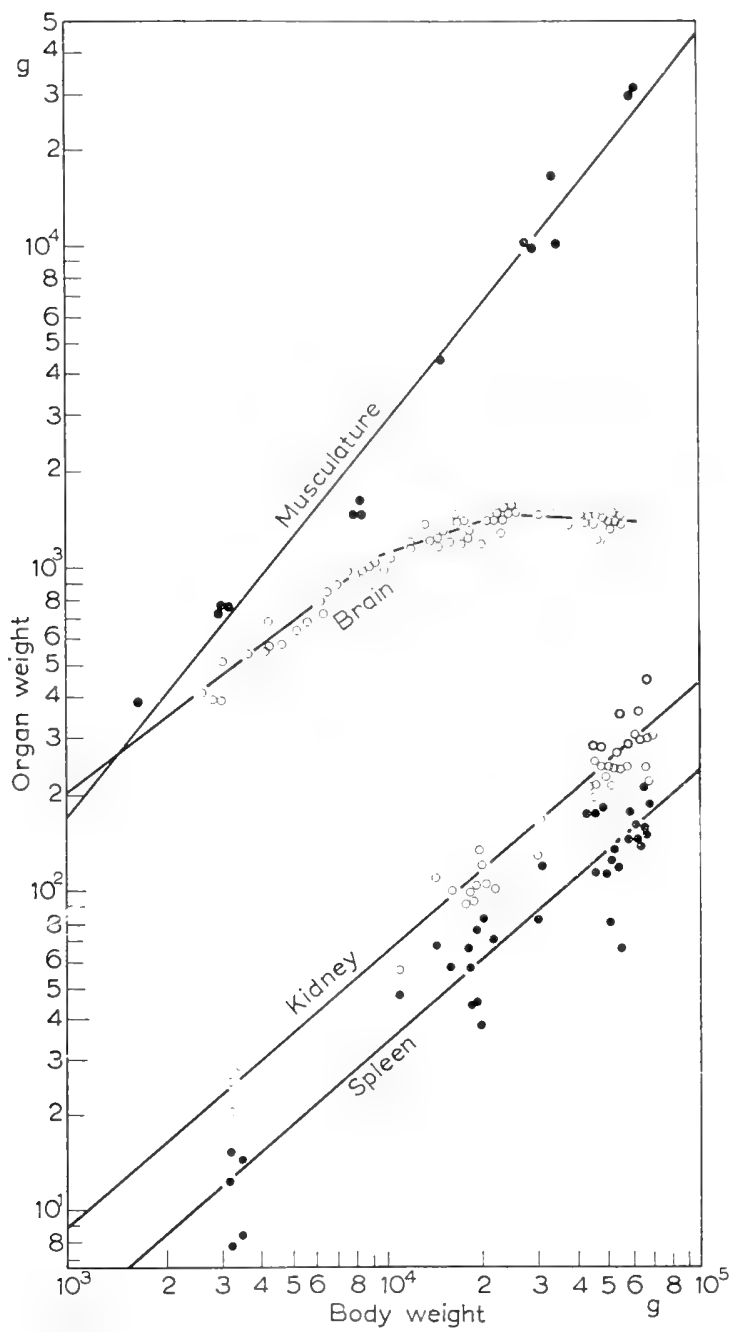

Fig. 33. Relative growth (postnatal) of some organs of man (ठ)). After Linzbach, 1955.

exerted by musculature. If available glucose is used for heavy muscular work, mitotic rate is reduced to such extent that it does not increase immediately even after rest. The well-known influence of dietetic factors on tumor growth follows a similar pattern. Cancer growth is often suppressed in a diet close to the minimum maintenance level.

Competition for nutritional factors provides certainly a partial explanation only. It illustrates, however, the general principle that every tissue apparently has a specific capability of appropriating material, which is expressed by the allometry constant and determines its relative growth.

Experimental investigations of physiological competition were made by F. E. Lehmann and coworkers. If, in the regeneration of legs in Xenopus, the available material is quantita- 
tively insufficient, regenerates are not harmonically reduced in size; rather toes of approximately normal size but reduced number develop. This is the case in treatment with colchicine which, as a mitotic poison, causes reduction of the regenerative blastema. Toes gradually disappear following an order of priority in the way that the toes formed latest in normal development are reduced most because building materials are already appropriated by those parts which have developed first. This experimental reduction in the number of toes can be compared with phenomena of rudimentation of toes in evolution (Bretscher, I947, I949; Tschumi, I953, I954).

\section{(d) Biochemical applications}

The principle of allometry further applies to changes in the chemical composition of the animal body during development. If, for example, ash content is plotted against dry weight, the slope of the allometric line (constant $\alpha$ ) is the same in animals as different as the chick, selachians and cephalopods (Fig. 34). Similar relations are found in other groups of organic compounds. The species compared

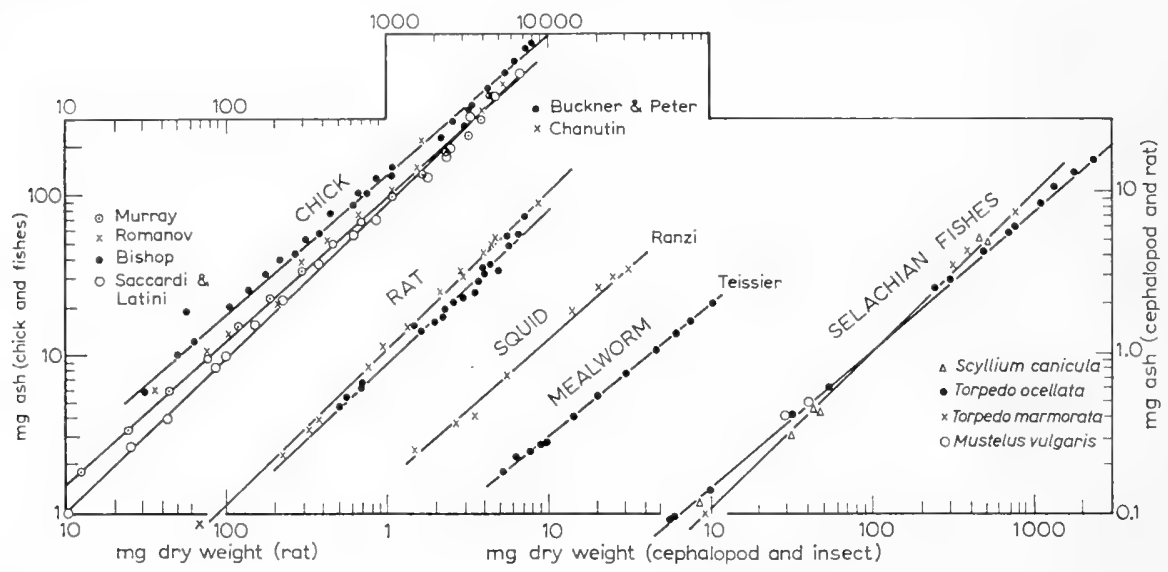

Fig. 34. Chemical allometry: Changes in ash content in various animal classes. After J. Needham, I934.

are different with respect to morphological form, absolute size, nutritional factors and time required for the changes in composition. The fact that the law governing such changes and expressed by the allometry constant is the same under so diverse conditions indicates a "chemical groundplan" (J. Needham, 1934) of the organism transcending taxonomic borders. The chemical constituents of the organism appear to tend toward a state of equilibrium or rather steady state which is based on the nature of the "living mass" and hence independent of taxonomic position and differences.

\section{(e) Physiological applications}

All physiological phenomena hitherto investigated follow the allometric relation in their dependence on body size (Adolph, 1949). That is, plotting metabolic rate, respiratory or pulse frequency (Fig. 35), excretion, enzyme content, or any other physiological magnitude against body size, an allometric line is obtained, which either covers the total range or, if breaks occur, these are related to 


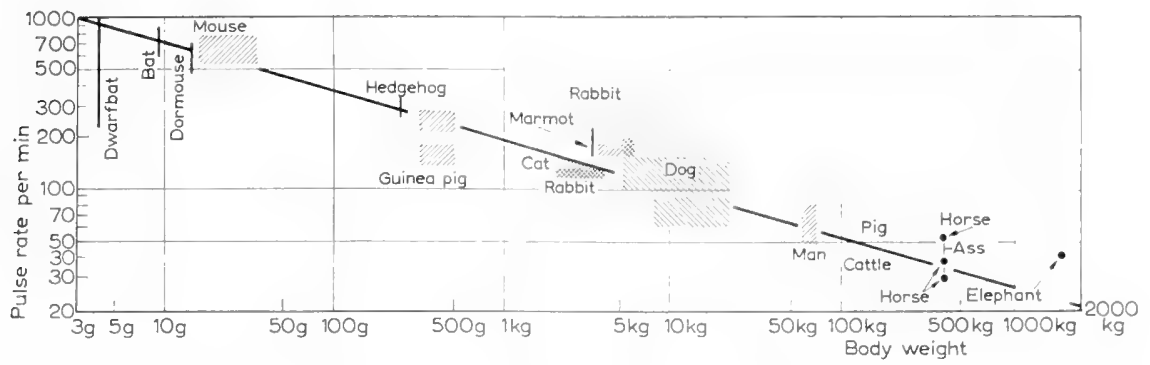

Fig. 35. Pulse rate and body size in mammals. It may be assumed that the rate of blood transported per minute is proportional to basal metabolic rate, as the oxygen consumed must be transported by the blood. This volume is equal to stroke volume $(S) \times$ pulse rate $(F)$. In a first approximation, $S$ may be taken as proportional to body weight $(w)$. Basal metabolic rate of mammals follows interspecifically the $3 / 4$ power rule (p. 218).

$$
\text { Hence: } \quad S \cdot F=C_{w}^{3 / 4} \text { and } F=\frac{C_{w}^{\prime 3 / 4}}{w}=C_{w}^{\prime} w^{-1 / 4}
$$

The figure shows that the allometry constant of pulse rate, $\alpha \approx-0.28$. Notwithstanding the gross oversimplification which neglects anatomical, physiological, ecological, etc., differences, absolute body size is the dominating factor determining pulse rate, in the enormous range from the dwarf bat $(4 \mathrm{~g})$ to the elephant $(2000 \mathrm{~kg})$. Modified after Bertalanffy, I942a, I951a.

physiological changes. The general validity of the principle of allometry for physiological phenomena renders it unlikely that phenomena not yet investigated should follow a basically different pattern because otherwise they would be incompatible with the physiological phenomena observed and their dependance on body size (Adolph, I949). Important physiological rules, such as the surface rule of metabolism (p. i $8 \mathrm{I}$ ), are special cases of the principle of allometry.

\section{(f) Physiological and growth gradients}

Another developmental principle emphasized by Huxley (1932) is that of growth gradients. The allometric equation only expresses total growth of the organ concerned. However, organs seldom grow uniformly in all dimensions but there are one or more growth centres around which growth rates vary in the form of gradients. If parts of an organ growing allometrically as a whole are examined, the allometry constants of the parts usually show a gradient. For example, in the growth of an extremity, the distal parts show the highest negative allometry. If growth gradients are plotted against the axis of the organ or organism, growth profiles are obtained which indicate the pattern of growth upon which morphogenetic changes are based.

The principle of the growth gradients is closely related to the polarity of the animal body and its "physiological gradients". According to Child (I94I) axial gradients of a quantitative nature represent the basis of the various manifestations of polarity. Such gradients appear as susceptibility gradients (different susceptibility of regions of the body toward noxious agents); as metabolic gradients (differences in respiration and cognate processes); and as morphogenetic gradients (differences in specific potency of forming organs such as the graded head-regeneration potency of different regions of the body in planarians). According to Child, these physiological gradients are, in principle, uniform 
and a general physiological differential is the common cause for the differences in susceptibility, metabolism and morphogenetic potencies. However, Child's theory is oversimplified and neglects morphological as well as cytological and physiological characteristics. Even in Child's classical example, the planaria, not only growth gradients and Child's gradients have an opposite direction but even in the latter differences are found (Fig. 36). Hence even in simple organisms such as planarians, there is not a uniform gradient but rather a bundle of polar gradients that are only loosely correlated. (Bertalanffy, 1942b; Bertalanffy, Hoffmann-Ostenhof and Schreier, I946; Hoffmann-Ostenhof, Bertalanffy and Schreier, I 948; Schreier, I949). In hydroids, the growth gradient can be interpreted cytologically; it coincides with the distribution of undifferentiated I-cells and biochemically, with the distribution of RNA which is connected with protein synthesis (Tardent, 1954).

A mathematical theory of differentiation based on the concepts of open systems, physiological dominance, competition, and gradients was developed by Spiegelman (1945).

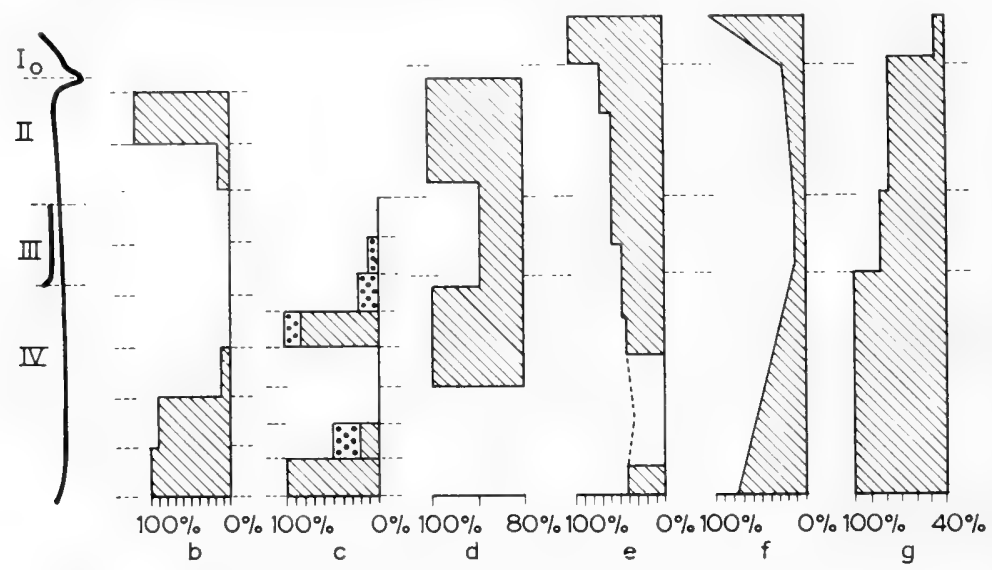

Fig. 36. Comparison of gradients in Planarians. $a$ Schema of body regions. $b$ Gradient of regenerative potency (head formation and total restitution) of the body when experimentally cut into 8 pieces; after Child. $c$ Gradient of regenerative potency of eights of the postpharyngeal region; after Child. $d$ Gradient of $\mathrm{O}_{2}$-consumption; after Hyman. $e$ Gradient of susceptibility to distilled water; after Buchanan. $f$ Gradient of susceptibility to alcohol; after Bertalanffy. $g$ Growth gradient constructed from allometry constants of growth of body regions; after Bertalanffy. After Bertalanffy, I942b.

\section{(g) Growth of organs in time}

The growth-in-time of organs (except in a special case to be mentioned presently) does not follow the formulas valid for the growth of the organism as a whole, both for biological and mathematical reasons. Biologically, the growth of an organ whose existence and development depends on the body as a whole, cannot be compared with that of an independent organism. Mathematically, a sum of equations is formally identical with the summated terms only if these are linear, which obviously is not the case either for growth of the total organism or of its parts.

Donaldson (1924) classified the organs of the rat in 3 groups showing a different course of growth: organs with early rapid growth (brain, spinal cord, eyes); organs with nearly constant increase following a phase of rapid growth (heart, kidneys, liver, lungs, digestive tract, etc.); and organs the growth of which shows 
a sigmoid curve with sharp rise preceding puberty (testes, ovaries, thymus). Donaldson's classification appears to be correct on the whole, and is exemplified in Fig. 37 for organs of the rat and in Fig. $3^{8}$ for human organs.

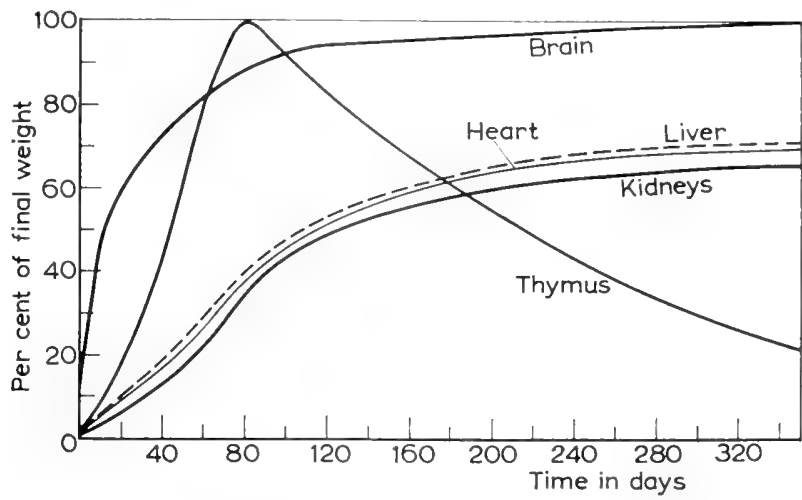

Fig. 37. Growth-in-time of organs in the rat. Curves are based on Donaldson's (1924) Tables I45, I5I and I57, expressed in \% of final weight. Donaldson's Table I 45 extrapolates organ weights to an (experimentally unobservable) final weight. It therefore appears in the figure as if the final weight of heart, kidneys and liver were not reached by far at the age of $35^{\circ}$ days. Since the figure is only to show the shape of the curves, this probably incorrect extrapolation was not adjusted. The figure shows the difference in the shapes of growth curves of organs, probably depending on their allometry constants ( $c f$. Table 16 , p. 242): The brain, having strongly negative allometric growth, shows simple flattening of the curve of growth-in-time; kidneys, heart and liver, having a higher allometry constant, show sigmoid growth curves. The thymus ( $f f$. Fig. 23, p. 218) has the usual S-shaped curve up to puberty and then undergoes involution.

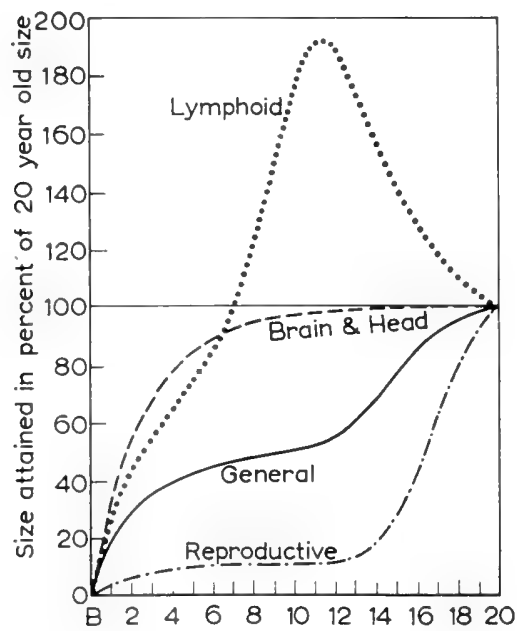

Fig. 38, Growth-in-time of human organs, showing four main types of growth. After Scammon, redrawn by Tanner, I 955 . 
A theory of growth-in-time of organs must combine the best available expressions for time growth of the entire organism with the allometric equation as the best available expression for the relative growth of organs. This can be done for both cases of $(I)$ unlimited and (2) limited growth.

I. Unlimited growth (Second Growth Type). If the growth of the organism is exponential:

$$
x=x_{0} \mathrm{e}^{c t}
$$

$(x=l, w)$, and an organ obeys the allometric equation:

$$
y=b x^{\alpha}
$$

( $y=$ length, weight of the organ), growth-in-time of the organ must follow the equation:

$$
y=b^{\prime} \mathrm{e}^{c^{\prime} t}
$$

with $b^{\prime}=b x_{0}{ }^{\alpha}$ and $c^{\prime}=\alpha c$. That is, if the body as a whole increases exponentially, so also does an allometrically growing organ. This has been found in the hemipterous insect, Notonecta (Clark and Hersh, I 939), and in Blatta, (Voy, I 95 I ; Figs. I6, I 7, p. I 97f.). Equation (7.I2) is, however, to be considered a first approximation because a sum of exponentials (growth of the individual parts) is not itself an exponential (growth of the entire body).

2. Limited growth (First Growth Type). Combining the Bertalanffy equations with the allometric formula, time growth of an organ can be derived as follows:

Equations (5.29) and (5.28), see p. 19 I, can be written in normalized form, i.e. equating final size $\left(l^{*}, w^{*}\right)$ to unity:

$$
\begin{aligned}
l & =\left(\mathrm{I}-c \mathrm{e}^{-k t}\right) \\
w & =\left(\mathrm{I}-c \mathrm{e}^{-k t}\right)^{3}
\end{aligned}
$$

with $c=\left(I-l_{0}\right)$ or $\left(I-w_{0}^{I / 3}\right)$, respectively. Similarly, the allometric equation for an organ $y$ can be written in normalized form:

$$
y=x^{\alpha}
$$

with $b$ equated to $\mathrm{I}$, i.e. organ size expressed as a multiple of its size when $x=\mathrm{I}$. Time growth of the organ then follows the equation:

$$
y=\left(1-c \mathrm{e}^{-k t}\right)^{a}
$$

with $a=\alpha$ for $x=l$ and $a=3 \alpha$ for $x=w$. This equation describes organ growth without introduction of new constants; that is, the growth of organs is calculable from that of the whole organism by insertion of the allometry constants. The growth curves according to (7.13) have the following properties. Denoting

$$
\varphi=\left(\mathrm{I}-c \mathrm{e}^{-k t}\right),
$$

the first two derivatives are:

$$
\begin{gathered}
\varphi^{\prime}-c k \mathrm{e}^{-k t}>0 \\
\varphi^{\prime \prime}=-c k^{2} \mathrm{e}^{-k t}<\mathrm{o}
\end{gathered}
$$

Hence for $y$ :

$$
\begin{gathered}
y^{\prime}=a \varphi^{a-1} \varphi^{\prime} \\
y^{\prime \prime}=a(a-1) \varphi^{a-2} \varphi^{\prime 2}+a \varphi^{a-1} \varphi^{\prime \prime}=a \varphi^{a-2}\left\{(a-1) \varphi^{\prime 2}+\varphi \varphi^{\prime \prime}\right\}
\end{gathered}
$$

with

$$
(a-\mathrm{I}) \varphi^{\prime 2}+\varphi \varphi^{\prime \prime}=(a-\mathrm{I}) c^{2} k^{2} \mathrm{e}^{-2 k t}-\left(\mathrm{I}-c \mathrm{e}^{-k t}\right) \cdot c k^{2} \mathrm{e}^{-k t}=c k^{2} \mathrm{e}^{-k t}\left(a c \mathrm{e}^{-k t_{-} \mathrm{I}}\right)
$$


The sign of $y^{\prime \prime}$ corresponds to the sign of $\left(a c \mathrm{e}^{\left.-k t_{-} \mathrm{I}\right)}=\psi(t) \cdot \psi(t)\right.$ is a monotonically decreasing function passing, between $t=0$ and $t=\infty$, the values from $(a-\mathbf{I})$ to $-\mathbf{I}$. Hence, for $a<\mathrm{I}, y^{\prime \prime}$ is always negative, i.e. the curve has no inflexion for positive values of $t$ and is qualitatively not much different from the curve for $y=\varphi(t)$. For $a>\mathrm{I}$, the curve has an inflexion. Here the second derivative of $y$ disappears:

$$
a c \mathrm{e}^{-k t_{1}}-\mathrm{I}=\mathrm{o}
$$

or, setting

$$
\begin{gathered}
c \mathrm{e}^{-k t_{1}}=\mathrm{I}-\varphi\left(t_{1}\right) \\
\varphi\left(t_{1}\right)=\mathrm{I}-\frac{\mathrm{I}}{a} \\
\varphi^{\prime}\left(t_{1}\right)=\frac{k}{a}
\end{gathered}
$$

Hence:

$$
\begin{gathered}
y\left(t_{1}\right)=\left(\mathrm{I}-\frac{\mathrm{I}}{a}\right)^{a}=\chi_{1}(a) \\
y^{\prime}\left(t_{1}\right)=k\left(\mathrm{I}-\frac{\mathrm{I}}{a}\right)^{a-1}=k \chi_{2}(a)
\end{gathered}
$$

The course of the functions $\chi_{1}(a), \chi_{2}(a)$, for $a>1$ continually variable, is apparent from:

$$
\begin{gathered}
\chi_{1}(a)-\frac{(a-1)^{a-1}}{a^{a}}(a-1) \\
\chi_{2}(a)=\frac{(a-1)^{a-1}}{a^{a}} a
\end{gathered}
$$

with the limiting conditions:

$$
\begin{gathered}
\lim _{a \rightarrow 1}(a)=0 ; \quad \lim \chi_{2}(a)=\mathrm{I} \\
a \rightarrow \mathrm{I}+\mathrm{o} \\
\lim _{\mathrm{a} \chi_{1}(a)=\lim _{\chi_{2}}(a)=\mathrm{e}^{-1}} \quad \mathrm{a} \rightarrow \infty
\end{gathered}
$$

The curves of $\chi_{1}, \chi_{2}$ (Fig. 39) determine the ordinate of the point of inflexion and the slope of the tangent at this point, respectively. The abscissa of the point of inflexion is determined by:

hence

$$
\begin{gathered}
\psi\left(t_{1}\right)=a c \mathrm{e}^{-k t_{1}}-\mathrm{I}=0 \\
t_{1}=\frac{\ln (c a)}{k}
\end{gathered}
$$

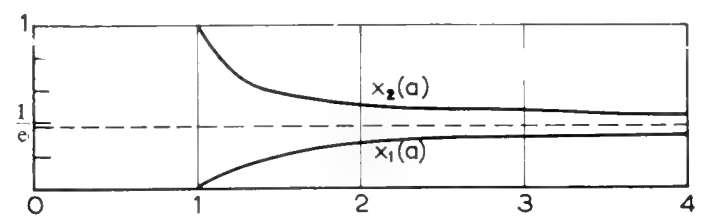

Fig. 39. Explanation see text.

Hence, $t_{1}$ is positive for $c a>1$, and negative for $c a<\mathrm{I}$. The latter case gives a curve which is qualitatively similar to the monotonic asymptotic rise of $\varphi=\mathrm{I}-c \mathrm{e}^{-k t}$ for positive $t$.

In a growth curve starting at an early age, $x_{\circ}$ will be small compared to $x^{*}$, and hence $c \approx \mathrm{I}$. Depending on the value of $a$, the curve of time growth of an organ will either show 
monotonic asymptotic approach to the final value or will be S-shaped, the point of inflexion lying between $o$ and 0.37 of final size, and the higher the greater the value of $a$. Length growth in time of an organ will show no inflexion if the relative growth of the organ is negatively allometric or isometric, but will have an inflexion in the case of strong positive allometry $(\alpha>1)$. Weight growth where the allometry constant $\alpha$ is multiplied by 3 will be sigmoid with an inflexion, except in the case of strong negative allometry of the organ $(\alpha<\mathrm{I} / 3)$ where the curve of time growth will be qualitatively similar to a decaying exponential.

Figs. 37 and $3^{8}$ illustrate the validity of this derivation, the brain as an organ with strong negative allometry ( $c f$. Table I 6, p. 242) showing monotonic decrease of growth rates, while other organs with higher allometry constants present S-shaped curves. Numerical calculation would be easy but time-consuming except when using an electronic computer.

Similar considerations apply to regressive changes. In first approximation, the weight loss in a starving animal appears to follow the simple exponential:

$$
w=w_{0} \mathrm{e}^{-x t}
$$

Hence, organs should decrease according to:

$$
y=C \mathrm{e}^{-x \alpha t}
$$

According to this, organs should decrease the more in starvation the higher their allometry constants are in progressive development, and the ratio of organs in the starving animal should be shifted in favor of those which grow slowly. If there are no irreversible differentiations, as is the case in some lower organisms, the starving animal will seemingly regress to an earlier developmental stage and appear "rejuvenated". This is well-known for the body proportions of starving planarians and can be shown quantitatively for the digestive tract (Table 15). Quantitative measurements of progressive and regressive changes, applying equations (7.13) and (7.14) would allow another experimental verification of the constants $\gamma_{\text {and }} \alpha$ and their relation.

TABLE 15

INTESTINAL SURFACE IN NORMAL AND STARVING PLANARIANS

\begin{tabular}{|c|c|c|c|}
\hline & Weight in $g$ & $\begin{array}{l}\text { Intestinal surface } \\
\text { in } \mathrm{mm}^{2}\end{array}$ & $\begin{array}{l}\text { Ratio Intestinal } \\
\text { surface/body } \\
\text { surface in } \%\end{array}$ \\
\hline $\begin{array}{l}\text { Initial weight } c a .20 \mathrm{mg}, \\
\text { starved } 6 \text { weeks at } c a .19^{\circ} \mathrm{C}\end{array}$ & $3 \cdot 9$ & 19.5 & 71 \\
\hline Unstarved animals & 4.0 & 20.9 & 76 \\
\hline $\begin{array}{l}\text { Initial weight } c a .20 \mathrm{mg} \text {, } \\
\text { starved } 9 \text { weeks at } c a . \mathrm{I}^{\circ} \mathrm{C}\end{array}$ & $9 \cdot 4$ & 40.3 & 82 \\
\hline Unstarved animals & 9.0 & 40.4 & 82 \\
\hline
\end{tabular}

After Bertalanffy, I940a

\section{GROWTH AND EVOLUTION}

(a) Transformation

Changes in proportions represent a considerable part of evolutionary changes within a given type or groundplan. Two approaches permit analysis of such 


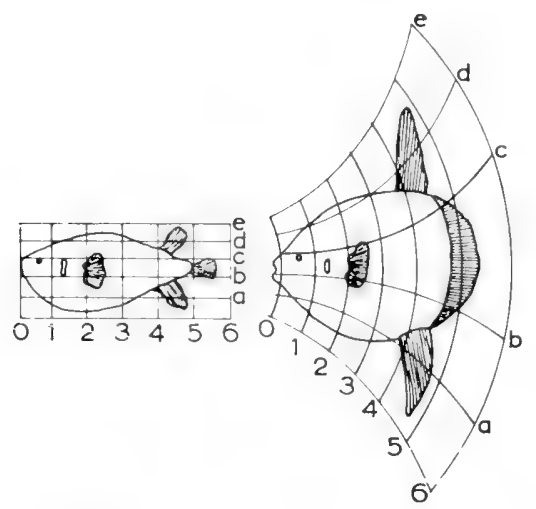

Fig. 40. Coordinate transformation of the outline of the fish Diodon into the moon fish, Orthagoriscus. After Thompson, 1942.

changes: the coordinate transformation according to D'Arcy Thompson (1942) and the principle of allometry according to Huxley (1932).

Thompson observed that, if the projection of an organ or a whole organism is entered into a system of coordinates, it can frequently be transformed into another related form by mere deformation of the coordinate system. For instance, the shape of the fish, Diodon, drawn in rectangular coordinates, gives the bizarre moonfish Orthagoriscus, if the coordinates are transformed into concentric circles in the vertical axis, and hyperbolas in the horizontal (Fig. 40).

Similarly, when the skull of Hyracotherium (Eohippus) is drawn in a Cartesian coordinate system, it can be transformed into that of the modern horse, intermediate stages appearing which correspond to evolutionary intermediates in the equine series. Applied with care, Thompsonian transformation is a suitable method for tracing evolutionary series and for excluding sidelines, as was shown by Kummer's (r953) analysis of the evolution of the human skull (cf. p. 249).
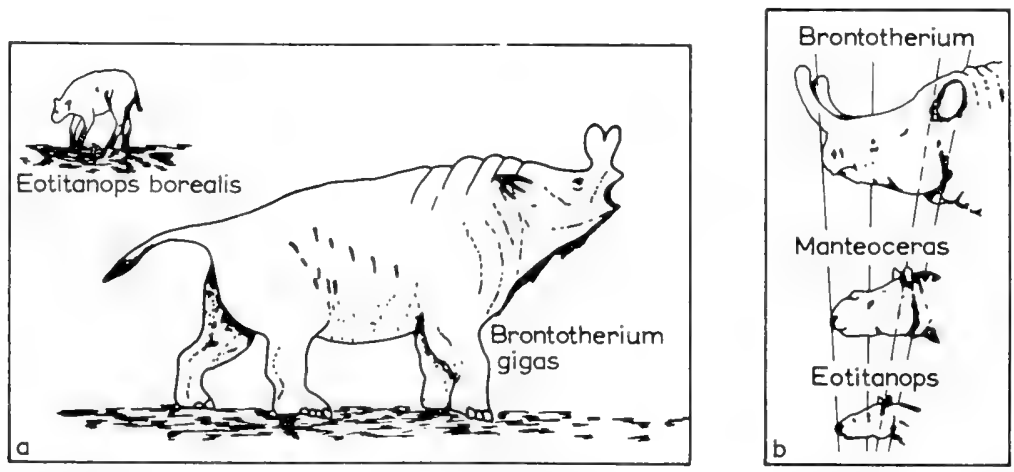

Fig. 41. Evolution in Titanotheria. a Comparison of size of Eotitanops (eocene) and Brontotherium (lower oligocence), as an example of Cope's rule. $b$ Progressive change in skull shape and orthogenetic increase of horns with increasing body size. After Osborn and Robb from Bertalanffy, i 95 Ia. 


\section{(b) Evolutionary allometry}

Thompsonian transformation is essentially typological and does not indicate the law of the evolutionary process. However, many important evolutionary series are governed by the quantitative law of allometry. In the case of positive allometry, the allometric equation states that the larger the body becomes, the larger is the allometrically growing organ. As long as body size is small, the organ will be rudimentary; it will become more prominent with increasing size. Hence any factor increasing body size will entail enlargement of the organ, not only in absolute size, but also in ratio to the body. The converse applies to negative allometry.

One classical example is the evolution in titanotheres (Hersh, 1934; Fig. 4r). The earliest and smallest forms did not have horns which only appear in later forms. In each of the groups into which the titanotheres split, increasingly larger horns appeared independently, eventually to reach monstrous dimensions. Below a certain body size, an allometrically growing organ such as the horn does not appear, as is the case in the earliest and smallest titanotheres. However, although these were too small to develop horns, they transmitted hereditary factors of horn formation to the several descendant groups, which developed horns when a certain body size was reached.

Evolutionary allometry in the ancestry of the horse was studied by Robb (1935-1937). Skull and foot show interesting differences. The progressive elongation of the skull (Fig. 42) follows simple allometry; moreover, evolutionary transformation corresponds to the ontogenetic transformation in foetal and postnatal development of the modern horse. Thus, the skull of the 5-month old foetus shows the proportions of Eohippus; that of the ro-month old those of Merychippus; in the newborn the proportions are those of Pliohippus, and in the one-year old horse, those of Equus scotti. In such way, some 60 million years of evolution appear to be governed by a simple formula, evolutionary increase in body size entailing changes in proportion of the skull. Furthermore, the evolution of the equine skull is a quantitative expression of the biogenetic law, ontogenetic development of the modern horse recapitulating the ancestral series. The latter represents a prolongation of the ontogenetic development of the ancestors inasmuch as, so to speak, new stages are added to the latter in the development of modern horses.
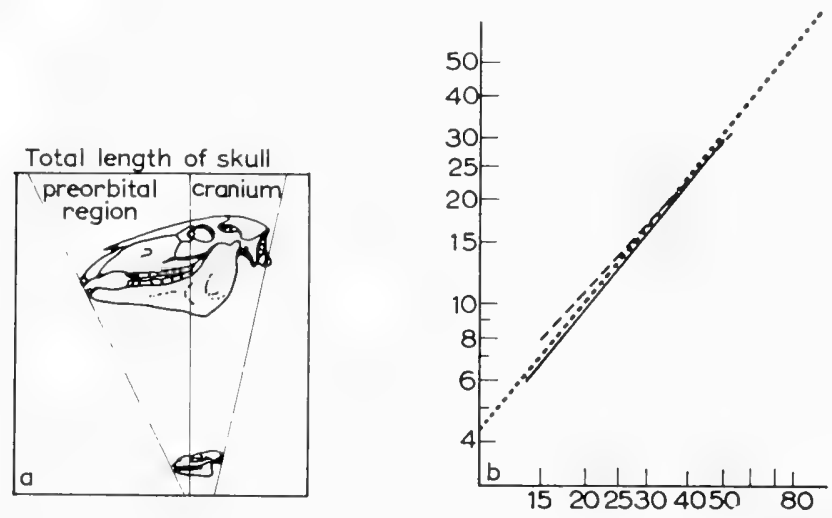

Fig. 42. Evolution of the equine skull. $a$ Relative elongation of the preorbital region with increasing body size. Below Eohippus, above Equus. $b$ Allometric plot: correspondence of phylogeny and ontogeny. —— evolution from Hyracotherium to Equus; - — - ontogenetic development in Equus caballus. After Robb and Simpson from Bertalanffy, I95Ia and Rensch, 1954. 

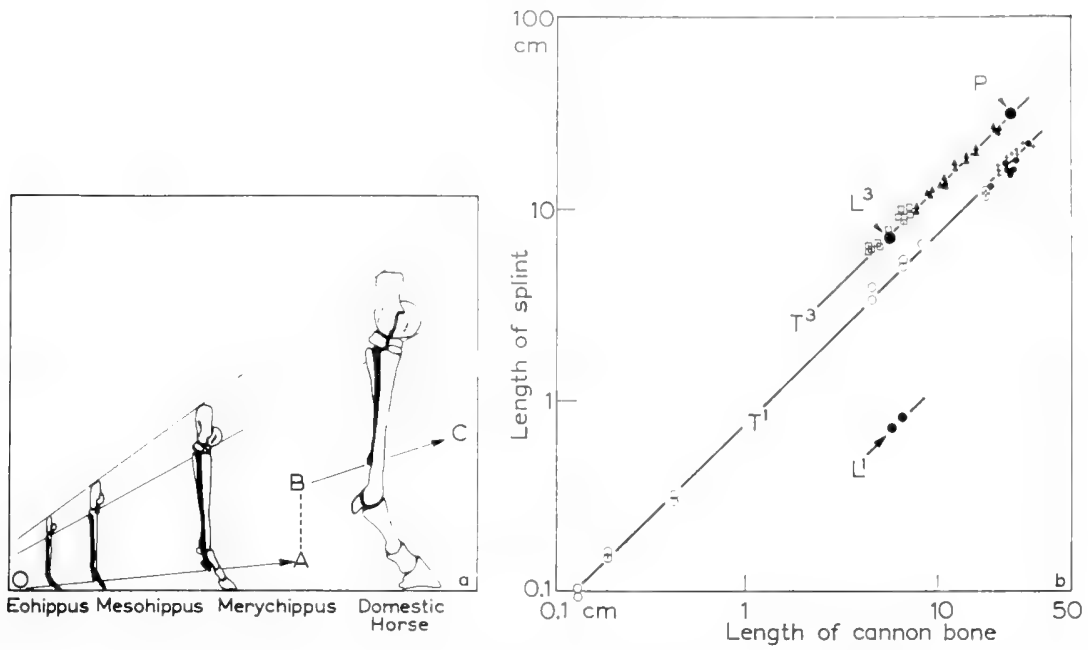

Fig. 43. Evolution of the equine foot. $a$ In the tridactyl series from Eohippus to Merychippus, the lateral digits become progressively removed from the ground and functionless $(\mathrm{O} \rightarrow A)$; this is followed by their abrupt rudimentation $(B \rightarrow C)$. In the ontogeny of the modern horse, the phylogenetic way $O \rightarrow A$ is eliminated. $b$ Allometric plot. The line $T^{3}$ represents the evolution of tridactyl horses ( $\square$ Hyracotherium and Eohippus, $\triangle$ Mesohippus and Merichippus, $P$ a modern polydactyl horse; the line also includes $L^{3}$, the extinct horse-like camel, Lithopterna). $T^{\prime}$ represents development and evolution in unidactyl horses (o fetal recent, - adult recent, + extinct unidactyl horses). $L^{1}$ is a parallel mutation in a unidactyl Lithopterna. After Robb, I937.

The case is different in the equine foot (Fig. 43). Here the evolutionary trend is toward elongation of the third toe, while the second and fourth become functionless and rudimentary. Within the tridactyl series, simple allometry and continuous transformation are found, as with increasing body size the third toe increases, the second and fourth toes are progressively reduced. In contrast, the transition from tridactyl to unidactyl horses is a discontinuous jump, indicating a deviation, a new turn taken in evolution. Consequently, there is no recapitulation in the ontogenesis of the foot of the modern horse which parallels only the unidactyl but not the tridactyl ancestral series.

These are examples of evolutionary allometry, i.e. of the comparison within well-established ancestral series whose number is naturally small. Further insight can, however, be obtained by including interspecific allometry, i.e. comparison of related recent species of different sizes which do not represent evolutionary series. To a certain extent, it may be assumed that similar relationships as found in such interspecific comparison also apply for true evolutionary series.

Such comparison shows, first, that ontogenetic and evolutionary allometry frequently do not coincide. In quantitative expression, this means that intra- and interspecific allometry constants are not identical (Table I6). A rat is not simply an allometrically enlarged mouse (Rensch, I954).

The explanation can be sought in the fact (Bertalanffy and Pirozynski, 1952) that the adult organism requires a certain balance of its organs (p. 229f.) for proper functioning, the size-dependence of the organs being expressed by the interspecific constant. 
TABLE 16

INTRASPECIFIG AND INTERSPECIFIC ALLOMETRY (CONSTANTS $\alpha$ ) IN ORGANS OF MAMMALS

After Bertalanffy and Pirozynski, 1952

\begin{tabular}{|c|c|c|c|c|c|c|c|c|}
\hline & $\begin{array}{c}\text { Rat } \\
B \text {. and } P \text {. }\end{array}$ & Brody & Cat & Dog & Monkey & Cattle & Horse & $\begin{array}{c}\text { Adult } \\
\text { mammals } \\
\text { interspecifio }\end{array}$ \\
\hline Brain & 0.20 & O.I 7 & & 0.25 & 0.62 & 0.30 & 0.24 & $\begin{array}{l}0.66 \\
0.69 \\
0.5^{8} \\
0.54\end{array}$ \\
\hline Heart & 0.82 & 0.80 & $\begin{array}{l}0^{+} 0.92 \\
\text { ᄋ } 0.82\end{array}$ & $\begin{array}{l}1.00 \\
0.86 \\
0.93\end{array}$ & 0.69 & 0.93 & & $\begin{array}{l}0.83 \\
0.82 \\
0.85 \\
0.84 \\
0.98\end{array}$ \\
\hline Lungs & 0.73 & 0.75 & & 0.82 & 0.92 & & $0.5^{8}$ & $\begin{array}{l}0.98 \\
0.99\end{array}$ \\
\hline Liver & $\begin{array}{l}\text { 1. Cycle: } \\
\text { I.26 } \\
\text { 2. Cycle: } \\
0.67\end{array}$ & $\begin{array}{l}\text { I. Cycle: } \\
\text { I. } 14 \\
\text { 2. Cycle: } \\
\text { 0.68 }\end{array}$ & & 0.71 & & 0.70 & $0.6 \mathrm{I}$ & $\begin{array}{l}0.87 \\
0.88 \\
0.92\end{array}$ \\
\hline Kidneys & 0.80 & 0.82 & $\begin{array}{l}0.65 \\
\text { 우 } 0.61\end{array}$ & 0.70 & & & 0.66 & $\begin{array}{l}0.85 \\
0.87 \\
0.76\end{array}$ \\
\hline
\end{tabular}

Bibliography $c f$. original.

For example, basal metabolic rate in the series of adult mammals "from the mouse to the elephant" is proportional to the 3/4-power of body weight (Brody, I945; Kleiber, 1947). Hence organs such as the kidneys, liver and heart will increase corresponding to the demands of metabolism including a safety factor, i.e. with an allometry exponent above 0.75 . In the lungs gas exchange takes place. Their enlargement must be approximately weightproportional if respiratory surfaces (which, neglecting structural changes, would increase as a 2/3-power of weight) are to increase corresponding to the demands of oxygen consumption. Actually the lungs do have an allometry constant $\alpha \sim$ I. With respect to the brain, E. Dubois' concept may be remembered that information from the environment is chiefly received on the body surface; or (Quiring, I94I) that the brain regulates the heat production of the organism, and heat output takes place on the surface. In both cases an approximate surface-proportionality of brain size is to be expected and also is found in interspecific comparison. Similar functional considerations can be applied to the wings of birds (Meunier, I95I) which show positive ontogenetic allometry and negative interspecific allometry.

On the other hand, different species are born in different stages of maturation. Hence, in order eventually to establish the required balance of organs in the 
adult organism, organs in different species will grow at different rates; that is, intraspecific allometry constants of organs of different species will, in general, be different from the interspecific constants of these organs. Such relation is apparent in the brain. As ganglion cells do not multiply in post-embryonic development, the relative size of the brain is high at birth. In adult organisms, proportionality to the 2/3-power of body weight is functionally required; hence ontogenetic allometry of the brain is much more negative than interspecific allometry (approximately 0.2 as against 0.66 ).
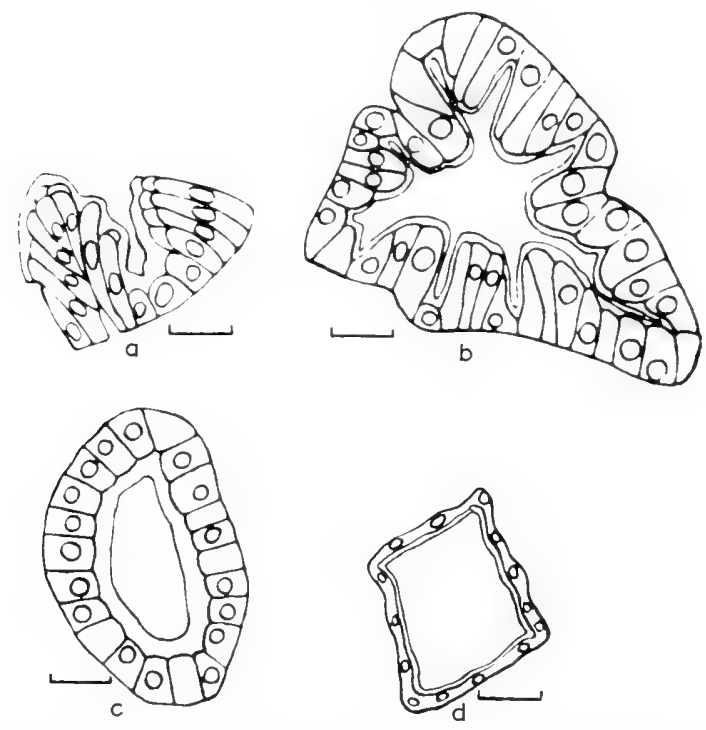

Fig. 44. Changes in the structure of the mesogastric epithelium with increasing body size in insects. Note the height of the epithelium and increasing folding. a Ctenophor $a, b$ Theobaldia, c Lycoria, $d$ Contarinia (gnats). After Partmann, i948.

On the basis of allometric relationships numerous rules governing changes of proportions with increasing body size can be stated (Rensch, I948b, I954). Larger species have relatively shorter ears, tails, legs, wings and beaks (Allen's rule); relatively smaller brains, eyes (Haller's rule), hearts, livers, kidneys, lungs, thyroids, adrenals, pancreas and hypophyses; a relatively longer gut (this in contrast to the negative ontogenetic allometry of the intestine); relatively heavier bones, etc. The rules of changes in proportion going along with evolutionary changes of body size are known sufficiently well to allow for prediction, with a 70-80 per cent probability, as to what properties an unknown species of a certain body size (say a rat in the size of a beaver) would exhibit (Rensch, I954).

A further important consequence is in the fact that allometric increase remains not confined to quantitative changes; it further entails histological and cytological differentiations (Rensch, I 948a). This was investigated by Rensch and co-workers in numerous organs and species: brain of rodents (C. Schulz, I95I), of lizards 
(Rose, I 956), of urodeles (Homeyer, I95 I ; Nolte, I 953), of insects (Goossen, I 949); liver and other organs (Rensch, I 948b); pancreas (Padour, I950); eyes of urodeles (Möller, I950); various organs of insects (Partmann, I948). Pictures of the mesogastric gland of insects of different body size are reproduced as an example (Fig. 44).

For example, the eyes of larger vertebrates have more retina cells than those of related smaller species (Möller, I950); similarly, larger insect species have more ommatidia (Partmann, 1948); increase in the number of photoreceptors makes possible a finer resolution of the visual image. If the brain increases in absolute size, it contains, in insects, a greater number of ganglion cells, and in mammals, larger ganglion cells. In both cases, more dendrites are provided, and consequently a number of synaptic junctions and

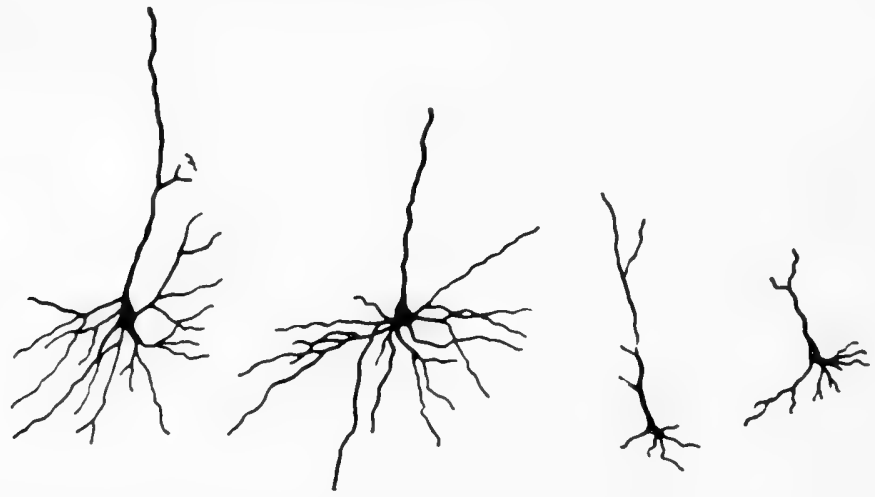

Fig. 45. Pyramid cells from homologous parts of the cortex of two rodents of different size. Left, water hog (Hydrochoerus capybara), right, guinea pig (Cavia cobaya). Same magnification. After Spina Franca Netto from Rensch, 1954.

more complicated functions (Fig. 45). Furthermore, it is particularly the neencephalic parts of the brain and among these, the most complicated regions which increase in relative size with evolutionary increase of body size. Hence increase of body size makes possible more elaborate behavior patterns, both innate and learned. Larger races of domestic fowl are able to learn a greater number of visual problems than smaller ones (Altevogt, I95I); although white mice learn faster than white rats, the latter remember longer (Boxberger, I953); memory in the larger Xiphophorus is better than in the small Lebistes (Rensch, 1954), etc. The elephant as a mammal with especially large absolute brain size, "understands" up to 20-24 verbal commands, and is able to discriminate between some 20 pairs of visual stimuli; it also shows a well-developed capacity to abstract by distinguishing, e.g., numbers of dots irrespective of their position, variations of a cross pattern against a circle, etc. (Rensch, 1956, 1957). In summary it can be stated that there are differences in learning capacity between related races or species correlated with body size. The speed of learning possibly depends on the intensity of metabolism (small forms learn faster), while larger forms have a longer memory (Rensch, 1954, 1958).

The physiological consequences of changes in body size are apparent from the facts discussed previously: Reduction of weight-specific metabolic rate with increasing body size (in mammals following the 3/4-power rule); reduction of pulse and respiratory frequency; lower blood sugar level in connection with the reduction of metabolic rate; prolonged development, etc. (Rensch, 1954). An example of the evolutionary effect of these physiological factors is provided by the giant forms appearing in glacial periods (elephants, Megaceros, large felines); decrease of temperature favored increase in size in the sense of Bergmann's rule. 
(c) Changes of the organism-as-a-whole as growth problem

The principle of allometry gives some insight into the most difficult problems of evolution, those of coadaptation, harmonious transformation of the organism as a whole and orthogenesis-problems that are difficult to reconcile with the conventional doctrine regarding evolution as a chance product of undirected mutations (Bertalanffy, I942a). Changes in body size lead automatically to changes in proportion by way of allometric growth. If, for example, an increase in body size has selective advantage, this will lead to a transformation of the body as a whole. Such change therefore does not influence one characteristic only but to a greater or lesser degree the entire organism. Furthermore, changes in one part will also have effects upon other parts by way of developmental correlations as is known of "superordinate" genes. For example, the development of the human spine (Fischer, I93I) is controlled by a pair of alleles, the dominant inducing a craniad, and the recessive a caudad shift. Under the influence of the respective genes, in the first case ribs or rib rudiments develop at the last cervical vertebrae, and in the second case, the first thoracic vertebrae do not have ribs. This change does not affect the spine only, but is accompanied by a corresponding shift in the dorsal musculature, the nerve stem with its plexus, the sternum and the position of the diaphragm, etc. In a similar way, allometric changes provide a possible mechanism of harmonious transformation and change of the organism as a whole.

Furthermore, hereditary changes of growth processes are caused in many cases by hereditary changes of the hormonal balance. These, of course, affect the organism as a whole. For example, many characteristics of man in comparison to the anthropoids can be considered as effects of changed hormonal balance, particularly with respect to the hypophysis (Versluys et al., 1939): the reduction of hair, the gnathic index, the retardation of growth, of sexual maturation and of closure of the sutures of the cranium. Conversely, an ape-like condition can reappear in the case of hormonal dysfunction (hirsutism, acromegalia, prognathism, pubertas praecox, etc.). Sometimes phenotypical changes can be induced by administration of hormones which, if they were genotypical, would represent major evolutionary steps. An example is presented by experiments with Periophthalmus, a fish occasionally stalking on land: Prolonged administration of thyroxin leads to adaptations corresponding to those of terrestrial animals, such as cornification of the epidermis, transformation of the pectoral fins into extremities used lever-fashion, etc. (Harms, I 935).

Lastly, cases of evolutionary allometry such as the evolution of the titanotheres, of the brain in mammals, or Megaceros, are paradigms of apparent directedness or orthogenesis in evolution. Such cases show, however, that orthogenesis is not a mysterious "force" or "purposiveness" but follows definite laws. Orthogenetic enlargement of organs may eventually lead to excessive formations and "blind alleys" in evolution as was probably the case in the titanotheres and in Megaceros. It may also lead to differentiations which are at first not required in the way of adaptation, but may be employed later on for higher performances. This is probably the case in the evolution of the human brain, primitive races having the same degree of cephalization as man in high cultures but apparently not making complete use of it (Versluys et al., I939). 
Rensch (1954) has advanced similar viewpoints, based upon a large amount of factual evidence, and his discussion of evolutionary allometry deserves particular attention. The general conclusions are that no evolutionary factors beside those known today need to be presupposed in macro-(transspecific) evolution. It ought to be considered, however, that the characteristics of organisms essentially are system properties resulting from interactions within the organism. Explanation, in principle, of evolutionary changes of the organism as a whole is possible by taking into account especially the following factors:

I. Pleiotropic gene action, "constructive" genes acting in the way of harmonious transformations.

2. Among such constructive genes are especially those influencing body size and hence, by way of allometric growth, the proportions of the body. Any phylogenetic change of body size may entail complex effects the analysis of which facilitates causal insight into the holistic and harmonious character of phylogenetic changes. If, according to Cope's rule which is valid in most taxonomic groups, a progressive increase in body size takes place in an evolutionary series, these changes in proportion, though only quantitative at first, may eventually lead to changes in the groundplan entailing qualitatively new characteristics. This is especially evident in eyes of arthropods where image-perception is only possible if a few hundreds of ommatidia are present; the central nervous system where more complex instinctual or learning behavior becomes possible with increased number of neurons; or the teeth which gain new functions with excessive size.

Quantitative increase of certain parts in consequence of their positive allometry may provide regions which subsequently can be used for new functions. Thus the positive allometric growth of the forebrain in comparison to the brain as a whole makes possible the progressive shift of higher functions to the forebrain as found in the vertebrate series. In particular, the increase of the cortex provides regions which eventually become Broca's motor speech center and hence an essential factor in the evolution of man.

In extreme cases changes of proportions lead to excessive formations or, conversely, to rudimentation. This interpretation is confirmed by the fact that orthogenetic excessive formations almost always appear in giant forms of the respective groups (horns of titanotheres, antlers of Megaceros, excessive appendices in beetles, etc.). Histological differentiations and basic physiological functions are altered with changes of morphological proportions showing that hereditary factors are "constructive genes" effectuating transformations of the organism as a whole.

3. A third factor is compensation due to competition for space or building materials. It may result in excessive development of certain organs and becoming rudimentary of others. Phenomena of experimental and phenotypical compensation ( $c f$. p. 232) can serve as a model for evolutionary and genotypical ones. Thus, extirpation of an organ can lead to hyperplasia of another; conversely, additional requirements for material in a regenerating part may entail reduction of others. Corresponding phenomena of competition and compensation are found in evolution; as, e.g. in sabre-toothed cats where extreme increase of the canines 
goes along with lack of the first premolars, or in horses where increase of the third toe is accompanied by reduction of the second and forth.

Hence, harmonious and holistic transformations in evolution can be explained by factors such as pleiotropic gene action, changes in proportion by allometric growth and its complex effects, orthoselection in changes of body size, compensation owing to competition for building materials, and constructive strains appearing when an upper or lower size limit is approached. There is no need to hypostatize unknown autonomous evolutionary forces, such as a "tendency toward perfection" or the like but, on the other hand, there are laws of organization often manifest as "evolutionary constraint". As opposed both to a vitalistic "autogenesis" and "ectogenesis" considering evolution as being determined solely from outside by chance factors in the environment during geological history, Rensch aptly speaks of bionomogenesis, i.e. evolution as the result of complex causal relationships of the environment as well as in the organism itself.

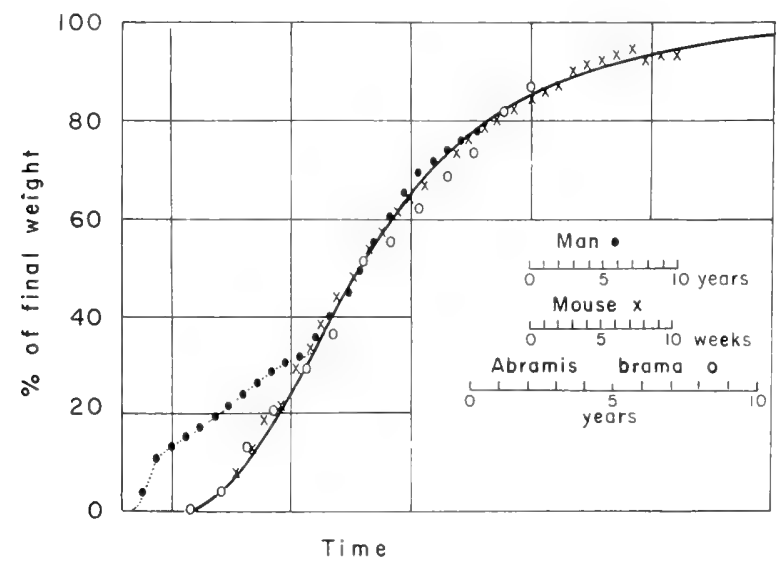

Fig. 46. The uniqueness of the human growth curve. After Bertalanffy, 1951a.

\section{(d) The uniqueness of man as a problem of growth}

The unique characteristics of man can be envisaged, to a large extent, as a problem of growth, many peculiarities found only in man being consequences of growth phenomena both in ontogenesis and evolution.

This applies, first, to the human growth curve. The general shape of the growth curve being essentially the same within vertebrates, growth curves of different species can be plotted on the same normal curve, if the scales for time and body size are appropriately chosen (Fig. 46). Even the growth cycles found in mammals (p. 2igff.) do not essentially change the shape of the growth curve. Only one organism is exceptional, namely, man.

The second part of the human growth curve, beginning at puberty, follows the general pattern. The first part, however, is different. Growth is protracted in infancy and childhood, a new growth cycle being added as it were, to the typical curve. Hence, an "adolescent spurt" (Tanner, I955), i.e. a second rise of 
growth rates after they have levelled off, appears at puberty. Although this change is heralded in the growth cycles of lower mammals (Fig. 26, p. 22I), only in man does it lead to a unique shape of the growth curve. The "abnormal" growth curve of man is of course connected with changes in the hormonal balance; this is shown in pathological conditions such as gigantism and pubertas praecox in pituitary dysfunction, when puberty takes place at an early age, as it does in other mammals and still in apes. The protraction of the juvenile period gives man a long period of somatic and mental maturation and so is a prerequisite of learning, accumulation of experience, and of human culture in general.

The cycles of human growth are, of course, much more complex than is apparent from the overall curve of weight growth. Successive periods of repletion and elongation are to be distinguished (Stratz, 1928): Fetal growth is followed by the early infantile period and a first increase of growth in length; growth at puberty is accompanied by a second increase of length growth; after the 40th year, usually weight increase by deposition of fat takes place.

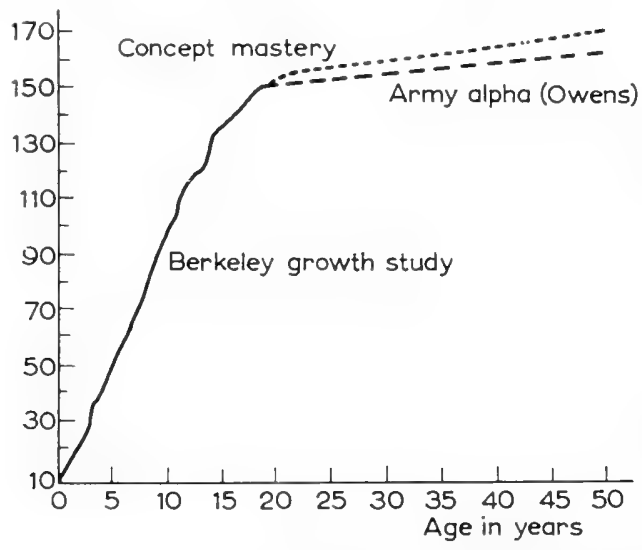

Fig. 47. A proposed age curve of intelligence from birth to 50 years. Based on data from the Berkeley Growth Study, the Terman Gifted Study and Owen's Iowa Study. After Bayley, I955.

A comparison between somatic and mental growth in man would be of considerable interest, although little has been done in this respect. Figs. 38 (p. 235) and 47 are presented as being suggestive of possible parallelisms. Brain growth follows a curve resembling a decaying exponential rather than an S-curve. This is probably connected with the strong negative allometry of the brain (p. 238). The "growth curve of intelligence", based upon certain psychological tests, appears to follow a similar pattern. A detailed analysis, taking account of the various periods of somatic growth, the adolescent spurt, critical phases, somatotypes (e.g. types of Kretschmer, Sheldon, autonomic patterns, blood chemistry), and their development in different "channels" (Wetzel, 1947), etc., and comparing characteristics of somatic growth with psychological counterparts (Bühler, I930), would be rewarding.

\section{(e) Retardation}

The peculiar curve of growth and prolongation of the growth period in man is a quantitative expression of a more general phenomenon, namely, retardation, stated by Bolk (1926) as being a specific human characteristic. According to 
Bolk's somewhat exaggerated formulation this is a "fetalization", that is, a neoteny-like retardation of development and sexual maturation providing man with his long period of youth, the capability of learning and, owing to the necessity of a long period of parental care for the children, with an essential basis for formation of social units.

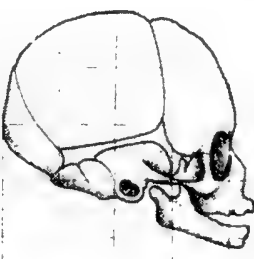

A
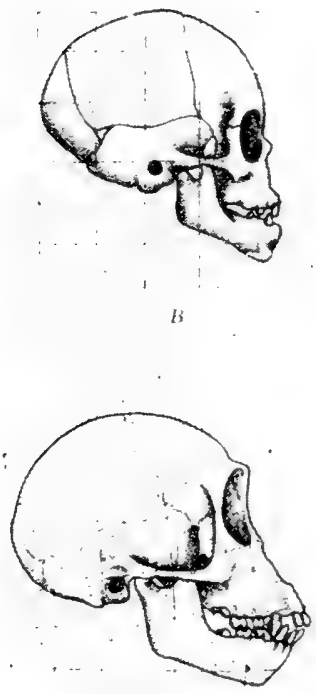

$c$

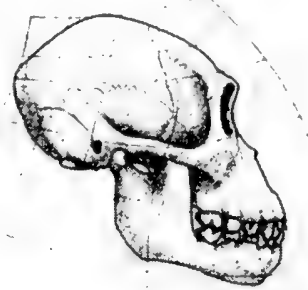

D

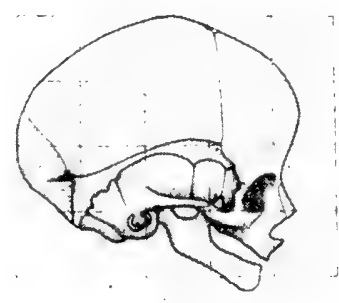

l.

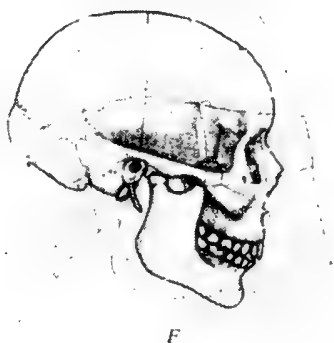

Fig. 48. Comparison of the development of the chimpanzee $(A-D)$ and man $(E-F)$. $A$ foetus, $B$, 74 days old, $C$ young animal, $D$ young $\&$ chimpanzee. $E$ human foetus 9 th month, $F$ adult. The transformation shows that the adult human skull does not correspond to a developmental stage of the chimpanzee, but ontogenesis in man has taken a course different from that in the chimpanzee. After Kummer, 1953.

It is a well-known fact that the cranium of, e.g., the baby chimpanzee is much more "human" than that of the adult. Hence human development can be envisaged as a growth problem in a second aspect, namely, to what extent human morphology can be considered a continuation of that of anthropoids. Kummer (1953) studied this problem by the method of coordinate transformation. The conclusion is that ontogenetic development of the human skull is not a mere continuation of, but marks a deviation from that in the chimpanzee (Fig. 48). Another inference which can be drawn from coordinate transformation and 
corresponds to conclusions arrived at otherwise, is that Neanderthal man is not in the evolutionary series leading to modern man. According to Kummer, the concept of "fetalization" is to be refused with respect to the ontogeny and evolution of the human skull; for this is not the result of an arrest of growth at an early phase but of a differentiation in changed direction, manifest in the propulsive growth of the neurocranium owing to the enlargement of the forebrain, the change of the viscerocranium, the skoliosis of the skull base, etc. Hence we may correctly speak of "retardation" but not of "fetalization" in human development.

TABLE 17

BRAIN WEIGHT IN MAMMALS

After Portmann, I944

\begin{tabular}{|c|c|c|c|c|}
\hline \multirow[t]{2}{*}{ Type of development } & \multirow[t]{2}{*}{ Species } & \multicolumn{2}{|c|}{ Brain weight } & \multirow[t]{2}{*}{ Factor of increase } \\
\hline & & at birth & adult & \\
\hline \multirow[t]{3}{*}{ Nidicolous } & Rat & 0.28 & 2.36 & 8.4 \\
\hline & Rabbit & I. I 8 & I0.5 & 8.9 \\
\hline & Cat & 6.5 & $3^{I}$ & $4 \cdot 7$ \\
\hline \multirow[t]{9}{*}{ Nidifugous } & Domestic pig & 33 & 90.94 & 2.7 \\
\hline & Muntjak (stag) & 50 & 125 & 2.5 \\
\hline & Mane stag & 103 & 230 & 2.2 \\
\hline & Mane sheep & $9^{2}$ & 209 & 2.2 \\
\hline & Macaque & 44 & 90 & 2.0 \\
\hline & Monkey & 32 & 70 & 2.2 \\
\hline & Chimpanzee & I 30 & $39^{\circ}$ & 3.0 \\
\hline & Orang & I 30 & 400 & 3.0 \\
\hline & Gorilla & I 30 & 470 & 3.6 \\
\hline \multirow[t]{2}{*}{ Secondary nidicolous } & Man & 370 & I $35^{\circ}$ & 3.6 \\
\hline & & & I $45^{\circ}$ & $3 \cdot 9$ \\
\hline
\end{tabular}

The table shows that, in nidicolous as compared to nidifugous species, the brain is much more immature at birth and hence shows larger increase postembryonically. Man's position as a "secondary nidicolous" is apparent.

\section{(f) The evolution of the brain}

A third problem pertaining to growth is the probably most important of all orthogenetic series: The increase in brain size eventually cuiminating in man (cf. Table I 7 ).

In the way of a first crude approximation, the interspecific increase of brain size in mammals in relation to body size follows the allometric equation, with an allometry constant $\alpha=2 / 3$ (i.e. surface-proportionality) and a high correlation coefficient $(\rho=0.9$ ) (Snell, I891; Bonin, I937; Jerison, I955). The more exact form of the evolutionary allometry of the brain is disputed.

According to E. Dubois ( 1930 ), brain size in the series of mammals is controlled by two independent factors: $I$. The relation between the size of the brain and the body, a value $\alpha=5 / 9$ (i.e. slightly less than surface-proportionality) being assumed in interspecific comparison of related species; 2. Cephalization, i.e. a different degree of development of the brain in ratio to body size which is assumed 
to increase in the evolutionary series not continually but by jumps. This corresponds to different values of $b$ of the allometric equation in the several orders of mammals. In this way, relative size of the brain (reduced to standard body size) in the series of shrews, moles, primitive ungulates (Tragulidae), higher ungulates, anthropoids, Pithecanthropus, and man stands in a proportion $1: 2: 4$ : $8:$ i $6: 32: 64$. Dubois' theory has been severely criticized both for statistical reasons because his data (as well as the similar ones of Brummelkamp, 1939) do not justify the generalizations drawn (Sholl, I948; Geiger, I956); and from the anatomical viewpoint because Dubois' theory neglects the changes in the architecture of the brain (Portmann, I948; C. Schulz, I951).

Other mathematical formulations of the evolutionary allometry of the brain are those of Brummelkamp (I939) according to which, in the transition of a mammalian order to a higher one, brain size increases not with an integer exponent but rather by 1 - 2 -jumps of cephalization; and that of Count (I947) who proposed an empirical equation:

$$
y=a x^{b \pm \log x}
$$

However, the good approximation of data by this formula is rather due to the increased number of constants than to a particular significance of the equation for which no theoretical explanation is given (Count and Jerison, I955). In contrast to Count's formulation, the simple allometric equation well applies in birds but, in contrast to Dubois, with a wide variation of the allometry constant in the different orders (Portmann, I 946-47). Intraspecifically, a wide variation of the allometry constants $(\alpha=0.23-0.74)$ of brain growth is found in teleosteans; brain size is connected with ecological factors and activity, rather than dependent on the phylogenetic rank (Dubois' cephalization factor) of the species concerned (Geiger, I956).

At present, hardly more can be stated than that interspecific increase of brain size in mammals is roughly proportional to the 2/3-power of body weight, and that intraspecific, ontogenetic allometry of the brain shows, as a rule, a much smaller allometry exponent ( $c f$. Table i6, p. 242).

The cycles in human growth and the unique characteristics of the human brain can also be seen in the allometric growth of the brain. While the relative growth of, e.g., the rat brain, is represented by an unbroken allometric line (Fig. 32, p. 230), that of the human brain follows a curve that can be approximated by 3 allometric lines; the allometric curve being steep in early childhood, subsequently flattening and showing a further break at about $30 \mathrm{~kg}$ body weight (Fig. 33, p. 23 I).

Another interesting pecularity of considerable significance for the unique position of man, concerns postembryonic development. In mammals and birds, nidifugous and nidicolous species can be distinguished; i.e. such where the young are born relatively mature and capable of independent activity, and such born immature and hence dependent on parental care. This distinction is connected with the index of cerebralization, i.e. the ratio between hemispheres and brain stem. According to Portmann (1944), man represents the unique case of a secondary nidicolous (Table I 7). Corresponding to his high cerebralization and owing to a long gestation period, he is born at a stage that would anatomically 
correspond to that of a nidifugous species; behaviorally, however, he is nidicolous, remaining under parental care for a long time and so having the opportunity of a unique psychological development.

According to the principle mentioned (p. 243f.), the quantitative increase in brain size is connected with basic qualitative, anatomical and histological, changes (Rensch, i 958). This particularly applies to the development of Broca's center (motor speech center), lacking in subhuman species and granting to man his unique abilities, language, concept formation, and symbolism (Bertalanffy, r 956).

This example shows again that analysis of growth may lead to basic problems and insight in biology and beyond. It is hardly an exaggeration to state that the problem of growth is a focussing point where the most fundamental problems of biology, from the biophysical bases of the phenomena of life up to the questions of human behavior, converge and intersect. 


\section{TERATURE}

Adams, H., (1920) The Degradation of the Democratic Dogma, MacMillan, New York.

Adolph, E. F., (1949) Science, 109, 579.

Alfert, M., A. Bern and R. H. Kahn, (1955) Acta Anat., 23, 185.

Altevogt, R., (I95I) Z. Tierpsychol., 8, 75 .

Backman, G., (1943) Wachstum und organische Zeit, Bios 15, J. A. Barth, Leipzig.

Bayley, N., (1955) Am. Psychologist, ro, 805.

Bayne-Jones, St. and E. F. Adolph, (1932) 7. Cellular Comp. Physiol., I, 387.

BĚLehrádek, T., (1935) Temperature and Living Matter, Protoplasma-Monogr. 8, Borntraeger, Berlin.

Benedict, F. G., (r 938 ) Vital Energetics, Carnegie Institution of Washington, Public. No. 503.

Bernard, CL., (1865) Introduction à l'étude de la médicine expérimentale, Baillière, Paris.

Bertalanffy, F. D., (1957) Anat. Record, I29, 23 I.

Bertalanffy, F. D. And C. P. Leblond, (1953) Anat. Record, II5, 5 I5.

Bertalanffy, L. von, (I932, I942a, I95 Ia) Theoretische Biologie, Vol. I, Borntraeger, Berlin; Vol. II, Borntraeger, Berlin; 2nd ed., Francke, Bern.

Bertalanffy, L. von, (1934) Arch. Entwicklungsmech. Organ., I3I, 6 I3.

Bertalanffy, L. von, (1938) Human Biology, Io, i81.

Bertalanffy, L. von, (I940a) Arch. Entwicklungsmech. Organ., I40, 81.

Bertalanffy, L. von, (1940b) Naturwiss., 28, 521.

Bertalanffy, L. von, (I94ia) Biol. Generalis, 15, I. $^{2}$

Bertalanffy, L. von, (194i b) Biol. Zentr., 6I, 5 Io.

Bertalanffy, L. von, (1942b) Biol. Generalis, 15, 295.

Bertalanffy, L. von, (1946) Biologie und Medizin, Springer, Wien.

Bertalanffy, L. von, (I948) Experientia, 4, 225.

Bertalanffy, L. von, (1949) Nature, $163,156$.

Bertalanffy, L. von, (1950a) Science, III, 23.

Bertalanffy, L. von, (I950b) Brit. J. Phil. Sci., I, I39.

Bertalanffy, L. von, (I95 I b) Am. Naturalist, 85, i I I.

Bertalanffy, L. von, (I952) Problems of Life, Wiley and Watts, New York and London.

Bertalanffy, L. von, (I953) Biophysik des Fliessgleichgewichts, Übersetzt von W. H. Westphal, Vieweg, Braunschweig.

Bertalanffy, L. von, (1956) Sci. Monthly, 82, 33 .

BertalanfFy, L. von, (1957) Wachstum, Kükenthals Handbuch der Zoologie, 8. Bd., 4 (6), De Gruyter, Berlin.

Bertalanffy, L. von, and I. Bickis, ( I956) F. Histochem. Cytochem., 4, 48I.

Bertalanffy, L. von, and R. R. Estwick, (1953) Am. 7. Physiol., 173, 58.

Bertalanffy, L. von, and R. R. Estwick, (1954) Am. F. Physiol, i77, i6.

Bertalanffy, L. von, O. Hoffmann-Ostenhof and O. Schreier, (1946) Nature, I58, 948.

Bertalanffy, L. von, and J. KrywienczyK, (i953) Am. Naturalist, 87, 107.

Bertalanffy, L. von, And A. Macfadyen, (1954) In: Standard Values in Nutrition and Metabolism, ed. by E. G. Albritton, Saunders, Philadelphia, Table 137.

Bertalanffy, L. von, M. Masin and F. Masin, (i958) Cancer, I1, 873.

Bertalanffy, L. von, and I. Müller, (1943) Riv. biol. (Perugia), 35, 48.

Bertalanffy, L. von, and W. J. Pirozynski, (i 95 I) Science, I13, 599.

Bertallanffy, L. von, and W. J. Pirozynski, (I952) Evolution, 6, 387.

Bertalanffy, L. von, and W. J. Pirozynski, (1953) Biol. Bull., io5, 240.

Beverton, R. J. H., (1954) Notes on the Use of Theoretical Models in the Study of the Dynamics of Exploited Fish Populations, Misc. Contrib. 2. U. S. Fishery Laboratory, Beaufort, N. C.

Beverton, R. J. H. And S. J. Holt, (1956) In: Sea Fisheries, Their Investigation in The United Kingdom, edited by M. Graham, Arnold, London, p. 372.

Beverton, R. J. H. And S. J. Holt (1957) On the Dynamics of Exploited Fish Populations, Fishery Investigations, Ser. II, vol. XIX, Her Majesty's Stationery Office, London.

Bickis, I., R. R. Estwick and J. S. Campbell, (i 956) Cancer, 9, 763.

Brzzozero, G., (r894) Wien. med. Wochschr., 696, 744. 
Bolk, L., (1926) Das Problem der Menschwerdung, Fischer, Jena.

Bonin, G. von, (1937) 7. Gen. Psychol., 16, 379.

Borsook, H., (1950) Physiol. Revs., 30, 206.

Boxberger, F. von, (1953) Z. Tierpsychol., 9, 433.

Brand, Th. von, M. O. Nolan and E. R. Mann, (1948) Biol. Bull., 95, 199.

Bray, H. G. and K. White, (1954) New Biology, Vol. i6, Penguin, p. 70.

Bray, H. G. AND K. White, (I957) Kinetics and Thermodynamics in Biochemistry, Academic Press, New York.

Bretscher, A., (1947) Rev. suisse zool., 54, 273.

Bretscher, A., (1949) Rev. suisse zool., 56, 33.

Brillouin, L., (1949) Am. Scientist, 37, 554.

Broch, K., (1933) Naturwiss., 21, 844 .

Brody, S., (1945) Bioenergetics and Growth, Reinhold, New York.

Brummelkamp, R., (1939) Acta néerl. morphol., 2, 188, 260, 268.

Buch Andersen, E. And A. Fischer, (1929) Arch. Entwicklungsmech. Organ., 114, 26.

Bühler, CH., (1930) Kindheit und Fugend, 2nd ed., Hirzel, Leipzig.

Bullough, W. S., (1952) Biol. Revs. Cambridge Phil. Soc., 27, 133.

Burton, A. C., (1939) 7. Cellular Comp. Physiol., I4, 327.

Caldwell, P. C. and C. Hinshelwood, (1950) 7. Chem. Soc., IV, 3156.

Carlander, K. D., (I953) Handbook of Fresh Water Fishery Biology, with the First Supplement, W. C. Brown, Dubuque, Iowa.

Chen-YA-Shin, (I937) Sitzber. Ges, naturforsch. Freunde Berlin.

Child, C. M., (I94I) Patterns and Problems of Development, Univ. of Chicago Press, Chicago.

Christophersen, J. and H. Precht, (1954) Zentr. Bakteriol. Parasitenk., Abt. II, io8, I.

Glark, L. B. and A. H. Hersh, (I939) Growth, 3, 347.

Clarke, M. F. and A. H. Smith, (1938) 7. Nutrition, I5, 245.

Count, E. W., (1947) Ann. N. Y. Acad. Sci., 46, 995.

Count, E. W. and H. J. Jerison, (I955) Science, I22, 647.

Cowdry, E. V., (1942) Problems of Ageing, 2nd ed., Williams and Wilkins, Baltimore.

Cowdry, E. V., (I957) In: W. A. D. Anderson, Pathology, 3rd ed., Mosby, St. Louis, p. 4.

Crandall, R. R. And A. H. Smith, (1952) Proc. Soc. Exptl. Biol. Med., 79, 345.

D'Ancona, U., (1952) Rend. accad. nazl. Lincei, 28, I3.

Daoust, R., F. D. Bertalanffy and G. P. Leblond, (1954) 7. Biol. Chem., 207, 405.

Defay, R., (1929) Acad. Roy. Belg., Cl. Sci., 5e Sér., I5, 678.

De Groot, S. R., (195I) Thermodynamics of Irreversible Processes, Interscience Publishers, New York and Amsterdam.

Denbigh, K. G., (195I) The Thermodynamics of the Steady State, Methuen and Wiley, London and New York.

Denbigh, K. G., (1952) Trans. Faraday Soc., 48, 389 .

Denbigh, K. G., M. Hicks and F. M. Page, (I948) Trans. Faraday Soc., 44, 479.

Donaldson, H. H., (1924) The Rat, Memoirs Wistar Inst., 6, and ed., Philadelphia.

Dounce, A. L., (1953) Enzymologia, 15, 25 I.

Driesch, H., (1929) The Science and Philosophy of the Organism, Black, London.

Drugkrey, H., (I959) Ciba Foundation Symposium on Carcinogenesis, I Io.

Dubois, E., (1898) Arch. Anthropol., 25, D 423.

Dubois, E., (1930) Biol. Generalis, 6, 247.

Dunn, M. S., E. A. Murphy and L. B. Rockland, (1947) Physiol. Revs., 27, 72.

Duspiva, F., ( I 955) In: Handb. Allgem. Pathologie, Vol. 6/1, ed. by F. Büchner, E. Letterer AND F. Roulet, Springer, Berlin-Göttingen-Heidelberg, p. 307.

DuYfF, L. W. AND E. M. BRuins, (1942) Arch. néerl. physiol., 26, I 79.

Ebert, J. D., (1954) In: Aspects of Synthesis and Order in Growth, ed. by D. Rudnick, Princeton University Press, Princeton, N. J., p. 69.

Edwards, G. A., (I953) In: Insect Physiology, ed. by K. D. Roeder, Wiley, New York, p. 96.

Edwards, R. W., (1958) 7. Exptl. Biol., 35, 383.

Ehrensvärd, G., (1955) Ann. Rev. Biochem., 24, 275.

Elias, H., (I955) Anat. Anz., IOI, 153. 
Ellenby, G., (1951) J. Exptl. Biol., 28, 492.

Enriques, P., (I 909 ) Biol. Zentr., 29.

Ephrussi, B. and G. Teissier, (1932) Arch. exptl. Zellforsch. Gewebezücht., 13.

Estwick, R. R., (I953) Studies on the Regulation of Tissue Respiration, Thesis, Univ. of Ottawa, Canada.

Fankhauser, G., (1954) In: Dynamics of Growth Processes, ed. by E. G. Boell, Princeton University Press, Princeton, N. J., p. 68.

Fischer, E., (1931) Verh. Ges. phys. Anthropol., 5.

Foster, C., A. Rapoport and E. Trucco, (I957) General Systems, Yearbook Soc. Gen. Systems Research, Vol. II, U. of Michigan, Ann Arbor, p. 9.

Freerksen, E., (1933) Z. Zellforsch. u. mikroskop. Anat., 18, 362.

Fried, G. H. And S. R. Tipton, (i953) Proc. Soc. Exptl. Biol. Med., 82, 53 I.

Füsser, H. ANd F. KRÜGer, (195I) Z. vergleich. Physiol., 33, I4.

Gaunt, R., (I954) In: Dynamics of Growth Processes, ed. E. J. Boell, Princeton University Press, Princeton, N. J., p. 183.

Geiger, W., (1956) Acta Anat., 26, 121; 27, 324.

Geitler, L., (1948) Öster. Botan. Z., 9, 277.

Gibson, M. B. And B. Hirst, (1955) Copeia, 241 .

Goldschmidt, R., (1927) Physiologische Theorie der Vererbung, Springer, Berlin.

Goldschmidt, R., (1938) Physiological Genetics, McGraw-Hill, New York and London.

Goossen, H., (1949) Zool. Fahrb. Abt. Physiol., 62, 1.

Graham, M. (ed.) (1956) Sea Fisheries, Their Investigation in the United Kingdom, Arnold, London.

Gregory, P. W. and H. Goss, (1933) 7. Exptl. Zool., 66, I55.

Guerra, E. And B. Günther, (1957) Acta Physiol. Latinoam., 7, I.

Günther, B. And E. Guerra, (I955) Acta Physiol. Latinoam., 5, I69.

HaARdick, H., (1956) Die Gestaltung der Körperproportionen durch begrenztes Wachstum der Skeletelemente, Acta Anat. Suppl. 26.

HAASE, R., (I95I) Z. Elektrochem. angew. physik. Chem., 55, 566.

HAASE, R., (I95I-53) Z. Naturforsch., 6a, 420;6a, 522;8a, 729 .

HaAse, R., (1952) Ergebn. exakt. Naturwiss., 26, 56.

HAASE, R., (I957) Naturwiss., 44, 4 o9.

Hardin, G., (I956) Sci. Monthly, 82, I 12.

Harms, J., (1935) Z. wiss. Zool., I46.

Harms, J., (1955) In: Handb. Allgem. Pathol., Vol. 6/1, ed. by F. Büchner, E. Letterer AND F. Roulet, Springer, Berlin-Göttingen-Heidelberg, p. I39.

Haurowitz, F. and Ch. F. Crampton, (1952) Exptl. Cell Research, Suppl. 2, 45.

Heidenhain, M., (1923) Formen und Kräfte in der lebendigen Natur, Rouxs Vorträge, Vol. 32, Springer, Berlin.

Helweg-Larsen, H. F., (1952) Nuclear class series, Acta Pathol. Microbiol. Scand., Suppl. 92, Copenhagen.

Hersh, A. H., (1934) Am. Naturalist, 68, 537.

Hersh, A. H., (194I) 3rd Growth Symposium, I 13.

Hesse, R., (1927) Über Grenzen des Wachstums, Fischer, Jena.

Hoffmann-Ostenhof, O., L. von Bertalanffy and O. Schreier, (1948) Monatsh. Ch:m., 79, 61.

Homeyer, B., (1951) Zool. Fahrb. Abt. Physiol., 63, 25.

Homma, K., (1953) Nihon Chikusan Gakkai, 24, 49.

Hugget, A. G. And W. F. Widdas, (I95I) F. Physiol., II4, 306.

Huxley, J., (1932) Problems of Relative Growth, Methuen, London.

Huxley, J., J. Needham and I. M. Lerner, (I94I) Nature, I48, 225.

Jackson, G. M., (1939) Am. 7. Anat., 64, 4I.

JAсовJ, W., (1925) Arch. Entwicklungsmech. Organ., I06, I 24.

JACOBJ, W., (1935) Z. mikroskop.-anat. Forsch., 38, г6 г.

Jakovlev, V. And F. KrüGer, (1954) Z. vergleich. Physiol., 37, 57.

Jančařik, A., (I948) Publ. Fac. Sci. Univ. Masaryk, Ser. M1, No. 305. 
Jerison, H. J., (I955) Science, I2I, 447.

Joв, S. V., (I955) The Oxygen Consumption of Salvelinus fontinalis, Publ. Ontario Fisheries Res. Lab., No. 73, Univ. Toronto Biol. Ser., No. 6r.

Jost, H., (1928) In: Handbuch Normale und Pathologische Physiologie, Vol, 5.

Jung, F., (1956) Naturwiss., 43, 73.

Kalmus, H., (1930) Z. vergleich. Physiol., 12, 725.

Kavanagh, A. J. and O. W. Richards, (1934), Am. Naturalist, 68, 54.

Keiter, F., (I95I-52) Kölner Z. Soziologie, 2, I65.

Kienle, M.-L. And W. Ludwig, (I956) Z. vergleich. Physiol., 39, 102.

Kittel, A., (1941) Z. vergleich. Physiol., 28, 533.

Klatt, B., (1919) Biol. Zentr., 39, 406.

Klatt, B., (1949) Biol. Generalis, 19, 5 I.

Kleiber, M., (194I) Proc. Soc. Exptl. Biol. Med., 48, 4 I9.

Kleiber, M., (I947) Physiol. Revs., 27, 5 II.

Körner, F., (1937) Z. mikroskop.-anat. Forsch., 42, 8 I.

Kopeč, St., (1932) Arch. Entwicklungsmech. Organ., 126, 575.

Kopeč, St., (1938) Z. vergleich. Physiol., 26, 85.

Kraft, V., (1926) Die Grundlagen der wissenschaftlichen Methoden, Sitzber. Akad. Wiss. Wien, 203.

Kramer, G., (1934) Z. vergleich. Physiol., 20, 600.

Krebs, A. H., (1950) Biochem. et Biophys. Acta, 4, 249.

KRÜGER, F., (1940) Z. wiss. Zool., 152, 547.

Krüger, F., (1952) Z. vergleich. Physiol., 34, I.

Krumbiegel, J., (1933) Biol. Zentr., 53.

Krywienczyk, J., (1952a) Z.vergleich. Physiol., 34, 6.

Krywienczyk, J., (1952b) Z. vergleich. Physiol., 34, I4.

Kubo, I. And T. Yoshinara, (1957) Suisan Sigen Gaku, Kyoritsu Shuppan, Tokyo.

Kummer, B., (1953) Untersuchungen über die Entwicklung der Schädelform des Menschen und einiger Anthropoiden, Abh. z. exakten Biologie, 3, Borntraeger, Berlin.

Kunkel, H. O. and J. E. Campbell, Jr., (I952) 7. Biol. Chem., Ig8, 229.

LANG, K., (1952) In: Mikroskopische und Chemische Organisation der Zelle, Kolloquium Moosbach, i95I, Springer, Heidelberg.

LAPicQue, L., (I898) Compt. rend. soc. biol., 5o, 62.

Leblond, G. P. and C. E. Stevens, (1948) Anat. Record, 10o, 357.

Leblond, C. P., W. F. Storey and F. D. Bertalanffy, (I95i) Acta Unio Intern. contra Cancrum, 7, 692.

Leblond, C. P., M. Vulpe and F. D. Bertalanffy, (1955) J. Urol., 73, 3 II.

Leblond, C. P. and B. E. Walker, (1956) Physiol. Revs., 36, 255.

Lecomte du Noüy, P., (1949) Human Destiny, Signet, New York.

Lehmann, G., ( I956) Das Gesetz der Stoffwechselreduktion, Kükenthals Handbuch d. Zool., 8. Bd. 4(5), De Gruyter, Berlin.

Leslie, I., (1955) In: The Nucleic Acids, ed. by E. Chargaff and J. N. Davidson, Vol. II, Academic Press, New York, p. I.

Leuchtenberger, C., H. F. Helweg-Larsen and L. Murmanis, (i954) Lab. Invest., 3, 245.

Liebsch, W., (1929) Zool. Jahrb. Abt. Physiol., 46, i6r.

Linzbach, A. J., (I950) Arch. path. Anat. u. Physiol., Virchow's, 318, 575.

Linzbach, A. J., (1955) In: Handb. Allgem. Pathol., Vol. 6/1, ed. by F. Büchner, E. Letterer And F. Roulet, Springer, Berlin-Göttingen-Heidelberg, p. I8o.

Lipmann, F., (1954) In: The Mechanism of Enzyme Action, ed. by W. D. McElroy and B. Glass, The Johns Hopkins Press, Baltimore, p. 599.

Locker, A., (1958a) Z. vergleich. Physiol., 4I, 249.

LOCKer, A., (1958b) Experientia, 14, 226.

Locker, A., (1958c) Experientia, I4, 326.

LOCKer, A., (1958d) Z. ges. expll. Medizin, r30, 396.

Locker, A., And D. Doneff, (1958) Z. vergleich. Physiol., 4I, 242.

Locker, A., And R. Hofer, (1958) Experientia, 14, 225. 
Lockingen, Ll. S. and A. Gib Debusk, (1955) Proc. Natl. Acad. Sci. U.S., 4I, 925.

Ludwig, W., (1956) Z. vergleich. Physiol., 39, 84.

Ludwig, W. and J. Krywienczyk, (1950) Z. vergleich. Physiol., 32, 464.

Margalef, R., (1954) Biol. Abstracts, 28, 23439 (abstract).

Martin, A. W. and E. A. Fumrman, (I955) Physiol. Zoöl., 28 , I8.

Mathies, J. C., L. Palm and O. H. Gaebler, (1951) Endocrinology, 49, 57 I.

MAYer, J., (1948) Growth, 12, 34I.

Mayer, J., (1949) Nature, ${ }^{6} 64,65$.

Medawar, P. B., (1945) In: Essays on Growth and Form, ed. by W. E. LeGros Clark and P. B. Medawar, Clarendon Press, Oxford, p. 157.

Meixner, J., (1954) Thermodynamik der irreversibelen Prozesse, Univ. Aachen.

Meunier, K., (I95I) Biol. Generalis, 19, 403.

Möller, A., (1950) Zool. Jahrb. Abt. Physiol., 62, 138.

Moment, G. B., (1946) 7. Exptl. Zool., 103, 487.

Müller, I., (I943a) Z. vergleich. Physiol., 30, I39.

Müller, I., (I943b) Biol. Zentr., 63, 446.

Murphy, E. A. ANd M. S. Dunn, (I948) Growth, I2, 3 I I.

Naroll, R. and L. von Bertalanffy, (1956) General Systems, Yearbook Soc. General Systems Research, Vol. I, U. of Michigan, Ann Arbor, p. 76.

Needham, A. E., (1952) Regeneration and Woundhealing, Methuen, London and New York.

Needham, J., (1934) Biol. Revs. Cambridge Phil. Soc., 9, 79.

Needham, J., (1942) Biochemistry and Morphogenesis, Cambridge University Press, London and New York.

Newcombe, C. L., (1936) Ecology, I7, 418.

Nolte, A., (1953) Zool. Fahrb. Abt. Physiol., 64, 538.

Northrop, J. H., (I949) In: The Chemistry and Physiology of Growth, ed. by A. K. PARpart, Princeton University Press, Princeton, N. J., p. 3.

Nowinski, W. W., (1955) Texas Repts. Biol. and Med., I3, 65 I.

Nozawa, K., (1940) Fapan. F. Zoöl., 9 .

O'Connor, R. J., (1950a) Brit. F. Exptl. Pathol., 3I, 390.

O'Connor, R. J., (1950b) Brit. F. Exptl. Pathol., 3I, 449.

Onsager, L., (1931) Phys. Rev., 37, 405; 38, 2265.

Padour, L., (1950) Zool. Fahrb. Abt. Physiol., 62, 102.

Partmann, W., (1948) Zool. Fahrb. Abt. Physiol., 69, 507.

PAtrušev, V.J., (I937) Compt. rend. acad. sci. U.R.S.S., N.S., I4, 573.

Pearl, R., and J. Reed, (I925) Proc. Natl. Acad. Sci. U.S., II, I6.

Pirozynski, W. J. and L. von Bertalanffy, (1955) Exptl. Med.Surg., 13, 26 r.

Pollister, A. W., ( I954) In: Dynamics of Growth Processes, ed. by E. G. Boeld, Princeton University Press, Princeton, N. J., p. 33.

Pollister, A. W., H. Swift And M.Alfert, (1951) 7. Cellular Comp. Physiol., 38, Suppl. I, i i .

Portmann, A., (1944) Biologische Fragmente zu einer Lehre vom Menschen, Schwabe, Basel.

Portmann, A., (1946-47) Alauda, 14, 2; 15, I.

Portmann, A., (1948) Rev. suisse zool., 55, 257.

Precht, H., J. Ghristophersen and H. Henzel, (1955) Temperatur und Leben, Springer, Berlin-Göttingen-Heidelberg.

Prigogine, I., (I947) Étude thermodynamique des phénomènes irréversibles, Dunod, Paris.

Prigogine, I., (I955) Introduction to Thermodynamics of Irreversible Processes, Thomas, Springfield, Ill.

Prigogine, I., and J. M. Wiame, (I946) Experientia, 2, 45I.

Pütter, A., (1920) Arch. ges. Physiol., Pflüger's, I80, 298.

Quiring, D. P., (I94I) Growth, 5, 30 I.

Racine, G. E., (1953) A Statistical Analysis of the Size-Dependence of Metabolism, Thesis, Univ. of Ottawa, Canada.

Rapoport, A., (1955a) Bull. Math. Biophys., I7, I5.

RApoport, A., (I955b) Utility and Application of Mathematical Models, mimeograph, Center for Adv. Study in the Behav. Sciences, Stanford, Calif. 
Rashevsky, N., (1948) Mathematical Biophysics, 2nd ed., Chicago University Press, Chicago.

Reeve, E. C. R. And J. S. Huxley, (1945) In: Essays on Growth and Form, ed. by W. E.

LeGros Clark and P. B. Medawar, Clarendon Press, Oxford, p. i 2 I.

Reik, H. G., (1953) Ann. Physik, (6) II, 270; (6) II, 407; (6) II, 420; (6) I3, 73.

Reiner, J. M., (1953) Arch. Biochem. Biophys., 46, 53, 80.

Rensch, B., (1948a) Evolution, 2, 2 I 8.

Rensch, B., (1948b) Zool. Fahrb. Abt. Physiol., 6I, 337.

Rensch, B., (1954) Neuere Probleme der Abstammungslehre, Die transspezifische Evolution, 2nd ed., Encke, Stuttgart.

Rensch, B., (1956) Am. Naturalist, 9o, 8I.

Rensch, B., (1957) Sci. American, February, 44.

Rensch, B., (1958) Naturwiss., 45, I45; I 75.

Rittenberg, D., (1948) $\mathcal{7}$. Mt. Sinai Hosp. $\mathcal{N}$. Y., I4, 89 I.

Rовв, R. C., (1935-37) 7. Genet., 31, 39, 47; 33, 269; 34, 477.

Roberts, J. L., (1957) Physiol. Zool., 30, 232.

Robertson, T. B., (1926) 7. Gen. Physiol., 8, 463.

Rössle, R., (1923) Wachstum und Altern, München.

Rose, F., (1956) Zool. Fahrb. Abt. Anat., 75, 433.

Rosenthal, O. and D. L. Drabkin, (1943) F. Biol. Chem., r5o, i3 I.

Rubner, M., (1902) Die Gesetze des Energieverbrauchs bei der Ernährung, Springer, Berlin und Wien.

Saller, K., (I927, I932) Arch. Entwicklungsmech. Organ., III, 453; I26, 6 I 3.

Sandritter, W., D. Müller, H. Schäfer and H. G. Schiemer, (1958) Frankfurter Z. Pathol., 68, 7 iо.

Sattel, M., (1956) Z. vergleich. Physiol., 39, 89.

Schmalmausen, J., (1929) Arch. Entwicklungsmech. Organ., II6, 567.

Schmalmausen, J., (I93 I) Arch. Entwicklungsmech. Organ., 123, I52.

Schmalmausen, J. and N. Bordzilowskaja, (1930) Arch. Entwicklungsmech. Organ., I21, 726.

Schmidt-Nielsen, K., L. von Bertallanffy and W. J. Pirozynski, (i95 I) Science, 114, 306.

Schoenheimer, R., (1942) The Dynamic State of Body Constituents, and ed., Harvard U. Press, Cambridge, Mass.

Scholander, P. F., R. Hock, Vl. Walters and L. Irving, (1950) Biol. Bull., 98, 259.

Schreier, O., (I949) Österr. Zool. Z., 2, 7o.

Schrödinger, E., (I944) What is Life?, Cambridge University Press, New York.

Schulz, C., (195I) Zool. Fahrb. Abt. Physiol., 63, 64.

Schulz, G. V., (1950) Naturwiss., 37, I96, 223.

Schulz, G. V., (195I) Z. Elektrochem. u. angew. physik. Chem., 55, 569.

Sholl, D., (1948) Proc. Roy. Soc. (London) B, 135, 243.

Sholl, D., (1954) In: Dynamics of Growth Processes, ed. by E. J. Boell, Princeton University Press, Princeton, N. J., p. 224.

Siekewitz, P., (1952) F. Biol. Chem., 195, 549.

Sinnott, E. W. And V. V. Trombetta, (1936) Am. 7. Botany, 23, 602.

Skrabal, A., (1949) Monatsh. Chem., 80, 21.

Skrabal, A., (I95I) Monatsh. Chem., 82, 107.

Smellie, R. M. S., (I955) In: The Nucleic Acids, Vol. II, ed. by E. Chargaff and J. N. Davioson, Academic Press, New York, p. 393.

Snell, O., (1891) Arch. Psychiat., 23, 436.

Spiegelman, S., (1945) Quart. Rev.Biol., 20, 121.

Sprinson, D. B. And D. Rittenberg, (1949a) F. Biol. Chem., i8o, 707.

Sprinson, D. B. and D. Rittenberg, (1949b) 7. Biol. Chem., i8o, 715.

Stevens, C. E., R. Daoust and C. P. Leblond, (1953a) Can. 7. Med. Sci., 3I, 263.

Stevens, G. E., R. Daoust and C. P. Leblond, (r953b) J. Biol. Chem., 202, i 77.

Stevens, G. E. And G. P. Leblond, (1953) Anat. Record, 115, 23 I.

Storey, W. F. and C. P. Leblond, (i95I) Ann. N. Y. Acad. Sci., 53, 537.

Stratz, H., (1928) Der Körper des Kindes, I I th ed., Encke, Stuttgart.

Tanner, J. M., (1955) Growth at Adolescence, Thomas, Springfield (Ill.). 
Tardent, P., (1954) Arch. Entwicklungsmech. Organ., 146, 593.

Teissier, G., (I94I) Compt. rend. soc. biol., 135, 662.

Thomas, H. J., (I954) 7. Exptl. Biol., 3I, 228.

'Thompson, D'Arcy, W., (1942) On Growth and Form, 2nd ed., Cambridge U. Press, Cambridge, England.

Tschumi, P., (1953) Rev. suisse zool., 6o, 496.

Tschumi, P., (1954) Rev. suisse zool., 6r.

Vendrely, R., (I955) In: The Nucleic Acids, Vol. II, ed. by E. Chargaff and J. N. Davidson, Academic Press, New York, p. I55.

Vernberg, I. J. And I. E. Gray, (I953) Biol. Bull., IO4, 445.

Versluys, J., O. Pötzl and K. Lorenz, (1939) Hirngrösse und hormonales Geschehen bei der Menschwerdung, Springer, Wien.

Voy, A., (195I) Bull. biol. France et Belg., 85.

Warburg, O., (1926) Über den Stoffwechsel der Tumoren, Springer, Berlin.

Weinhouse, S., (1955) Advances in Cancer Research, 3, "269.

Weinhouse, S., O. Warburg, D. Burk and A. L. Schade, (1956) Science, 124, 267.

Weinland, E., (I9I9) Z. Biol., 69, I.

Weiss, P., (1949) In: The Chemistry and Physiology of Growth, ed. by A. K. PArPart, Princeton U. Press, Princeton, N. J., p. I 35.

Weiss, P., (1955a) In: The Hypophyseal Growth Hormone, ed. by R. W. Smith, O. H. Gaebler and G. N. H. Long, Blackiston, New York, p. 3.

Weiss, P., (1955b) In: Biological Specificity and Growth, ed. by E. G. Butler, Princeton U. Press, Princeton, N. J., p. I95.

Weiss, P. and H. B. Hiscoe, (1948) f. Exptl. Zool., IO7, 3 I 5.

Weiss, P. and J. Lee Kavanau, (1957) 7 . Gen. Physiol., 4I, I.

Wermel, E. M. and co-workers, (1932-35) Z. Zellforsch. u. mikroskop. Anat., 16, 674, 689; $I 7,476,505 ; 20,36,43,54,459,467 ; 21,749 ; 22,185$.

Wetzer, N. C., (I947) In: Medical Physics, ed. by O. Glasser, Year Book Publishers, Chicago, p. 513 .

Weymouth, F. W., J. Field II and M. Kleiber, (1942) Proc. Soc. Exptl. Biol. Med., 49, 367.

Weymouth, F. W., J. M. Crismon, V. E. Hall, H. S. Belding and J. Field II, (I944)

Physiol. Zool., 17, 50 .

Will, A., (1952) Z. vergleich. Physiol., 34, 20.

Winsor, C. P., (1932) Proc. Natl. Acad. Sci., U.S., I8, I.

Wollenberger, A. and J. Jehl, (1952) Am. F. Physiol., I7o, i 26.

Wolsky, A., (1934) Arb. Ungar. biol. Forsch.-Inst., 7.

Yoneda, Y. and Y. Yoshida, (1955) Bull. Japan. Soc. Sci. Fisheries, 2i, 62, 467.

Yoshida, Y., (1956) Bull. Japan. Soc. Sci. Fisheries, 21, 1007.

Zamecnik, P. C., (I958) Sci. American, I98, i 18.

Zamecnik, P. C. And E. B. Keller, (1954) 7. Biol. Chem., 209, 337.

Zeuthen, E., (1953) Quart. Rev. Biol., 28, I.

Zeuthen, E., (1955) Ann. Rev.Physiol., 17, 459.

Zucker, L., L. Hall, M. Young and T. F. Zucker, (I941a) Growth, 5, 399.

Zucker, L., L. Hall, M. Young and T. F. Zugker, (194I I) Growth, 5, 4I 5 .

Zucker, L. And T. F. Zucker, (1942) F. Gen. Physiol., 25, 445.

Zucker, T. F., L. Hall, M. Young and L. Zucker, (I94I) F. Nutrition, 22, I23. 


\title{
Chapter 3 \\ NUGLEIG ACIDS AND GROWTH
}

\author{
7. Brachet
}

\section{INTRODUGTION}

Cytochemical observations and biochemical experiments have, during the past I 5 years, brought considerable evidence in favour of an important role of nucleic acids in growth processes. The subject and the literature are so vast that only the most important facts will be reviewed here, and reference will occasionally be made to a number of recent comprehensive treatises where a host of additional details can be found.

After a brief introductory survey of nucleic acid chemistry and cellular localization, we will deal with the role of the two main types of nucleic acids: deoxyribonucleic acid (DNA) and ribonucleic acid (RNA). While the former is important in chromosome reduplication and genetic activity, RNA is, according to an increasing number of biologists and biochemists, playing an essential role in protein synthesis. The two types of nucleic acids are thus apparently involved in two essential, but different, growth processes: increase in cell number and increase in the most important constituents of the living matter, the proteins.

This discussion of the role of DNA in mitosis and of RNA in protein synthesis will be followed with a study of the part played by the nucleic acids in one of the best known and most spectacular manifestations of growth: embryonic development.

Finally, the relationships existing between DNA and RNA metabolism, as well as the importance of the cell nucleus in the growth of unicellular organisms, will be discussed in the concluding remarks.

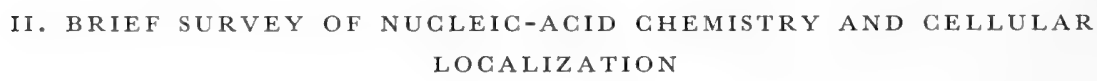

\section{(a) Chemical composition}

The considerable amount of knowledge which has accumulated on the chemistry, localization and biological role of the nucleic acids has been excellently summarized in the recent treatise edited by Davidson and Chargaff, I955. The essential facts only will be briefly reviewed here.

Two main types of nucleic acids, deoxyribonucleic acid (DNA) and ribonucleic acid (RNA) are found in every cell, whatever its function or origin. These two 
types of nucleic acids are built on the same pattern, being both made of a large number of subunits, called nucleotides; everyone of these nucleotides can be broken down, on hydrolysis, to orthophosphoric acid, a pentose or deoxypentose sugar and a purine or pyrimidine base.

Hydrolysis of a nucleic acid usually yields 4 different bases, 2 purines (guanine, adenine) and 2 pyrimidines (cytosine and thymine or uracil according to the nature of the nucleic acid). It is for this reason that the view that nucleic acids are composed of 4 nucleotides (and are thus tetranucleotides) has held so long; but such a simple hypothesis is no longer tenable, since we now know for certain that many DNA's contain a fifth base (5-methylcytosine). Furthermore, the molar proportions of the various bases vary considerably from one nucleic acid to another (Chargaff, I95I). There is excellent evidence, mainly adduced and discussed by Chargaff (I95I), that DNA's extracted from different species are distinct entities; and there is even increasing evidence that one and the same organ, calf thymus for instance, contains at least 2 different DNA's (Chargaff et al., i953; Brown and Watson, I953; Bendich, I952) distinguished by the molar ratios of their constituent bases and their metabolic stability.

The situation is not quite so clear in the case of RNA, because this type of nucleic acid is much more labile and difficult to isolate in a pure and native state than DNA : recent evidence from Chargaff's laboratory (Elson, Trent and Chargaff, I 955) indicates little difference in the composition of the bases between RNA's isolated from different organs and even from different species.

From a straight chemical viewpoint, two important differences have now been found between DNA and RNA: while RNA contains the usual pentose $d$-ribose, DNA is made of a deoxypentose, namely $d$-2-deoxyribose.
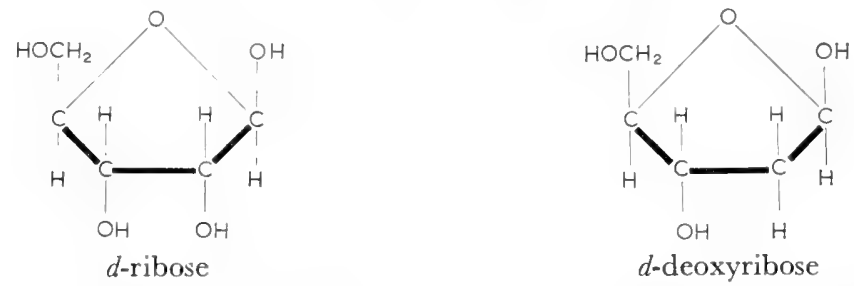

Secondly, whereas RNA possesses uracil as one of its main pyrimidine bases, DNA contains instead one of its methyl derivatives, thymine.

The rest of the two molecules (i.e. $\mathrm{H}_{3} \mathrm{PO}_{4}$, guanine, adenine and cytosine) is common to both nucleic acids and the resemblance between them is further increased by the fact that many DNA's and RNA's present striking regularities in their molar base composition: for instance, the total of the keto compounds (guanine + thymine or uracil) is equal to the sum of the amino compounds (adenine + cytosine; Elson and Chargaff, 1955). Such regularities are, as we shall soon see, of importance in assessing a structure to the nucleic acids.

\section{(b) Physical properties and molecular structure}

Physical studies have shown that nucleic acids are macromolecular compounds consisting of a very large number of individual nucleotides. In the case of DNA 
and in the best preparations of RNA (for instance RNA isolated from crystalline tobacco mosaic virus), the native molecule is extremely elongated, almost like a thread; the average molecular weight of the thymus DNA fibre is very large (around 6,000,000)

The chemical and physical data which have just been summarized, as well as

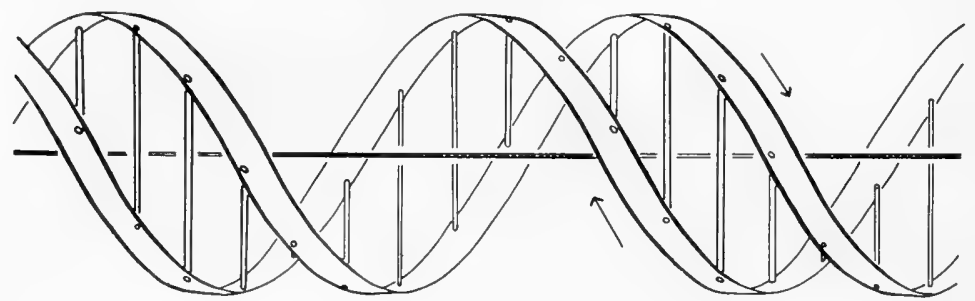

Fig. I. Watson and Crick's structure for DNA.

crystallographic evidence, have recently led Watson and Crick (1953a) to the idea that the fundamental DNA unit contains two helically intertwined chains. As seen in Fig. I, these chains are united by hydrogen bonds between pairs of bases in a specific way: adenine with thymine and guanine with cytosine. The sequence of the bases on one chain can be irregular: but whenever a certain nucleotide occurs on one chain, its partner on the other chain is specifically determined.

Such a structure is of considerable interest to biologists, because, as pointed out by Watson and Crick (I953b) themselves, this "complementary" structure of DNA readily suggests a self-duplicating mechanism: if the two chains unwind and separate, each can serve as a template for the formation of its complement. Later we will return to this important point when we discuss the biological role of DNA; meanwhile let us note that, while the DNA structural model proposed by Watson and Crick (I953a) fits in very well with a number of experimental facts, it is still hypothetical.

\section{(c) Cytochemical techniques for detection}

Nucleic acids have aroused much interest among biologists because they are unusually easy to detect in ordinary histological sections by cytochemical procedures. These procedures can be based on the properties of any of the three main constituents of nucleic acids, i.e. $\mathrm{H}_{3} \mathrm{PO}_{4}$, sugar and bases.

A remarkable property of deoxyribose, which is not shared by ribose, is the ability to give the Schiff aldehyde reaction with fuchsin bleached with $\mathrm{SO}_{2}$. This property is the basis of the extremely valuable Feulgen reaction for DNA (Feulgen and Rossenbeck, I 924): the tissue sections are subjected to mild acid hydrolysis in order to remove the DNA purines; the rest of the DNA molecule, which stays at its initial site, stains strongly violet when the hydrolyzed section is dipped into the Schiff reagent (Fig. 2, a).

The presence of phosphoric acid in the nucleic acid molecule is of course responsible for their acidic character, and therefore their affinity for basic dyes. In the case of tissue sections, the nucleic acids are thus basophilic and are easily stained with 
basic dyes (e.g. toluidine blue). Since the sections may contain acidic constituents other than nucleic acids, the reaction is not specific unless we can find a way of removing only one nucleic acid. This can easily be done by digesting away one of the nucleic acids with a specific nuclease (Brachet, r r4ra) and comparing under the microscope sections stained before and after the enzymatic digestion (Fig.2, b, c). The technique used for the cytochemical detection of RNA with basic dyes (usually Unna's mixture of methyl green and pyronine, which has the advantage of staining DNA green and RNA red) and ribonuclease has been discussed in detail (Brachet, 1953). The utilization, in the same way, of deoxyribonuclease for the detection of DNA is not so widespread, because of the existence of the much simpler Feulgen reaction.

Finally, the very high ultraviolet absorption at around $2600 \AA$ of the purines and pyrimidines has been utilized very elegantly by Caspersson (1940, I950) for the detection of nucleic acids: the principle, of course, is to take

Fig. 2. Sections of intestinal mucosa (mouse); a: Feulgen reaction; b: Stained with methyl greenpyronine; $\mathrm{c}$ : same as $\mathrm{b}$, but treated with ribonuclease before staining: cytoplasmic basophilia has disappeared.

Lilerature p. 299
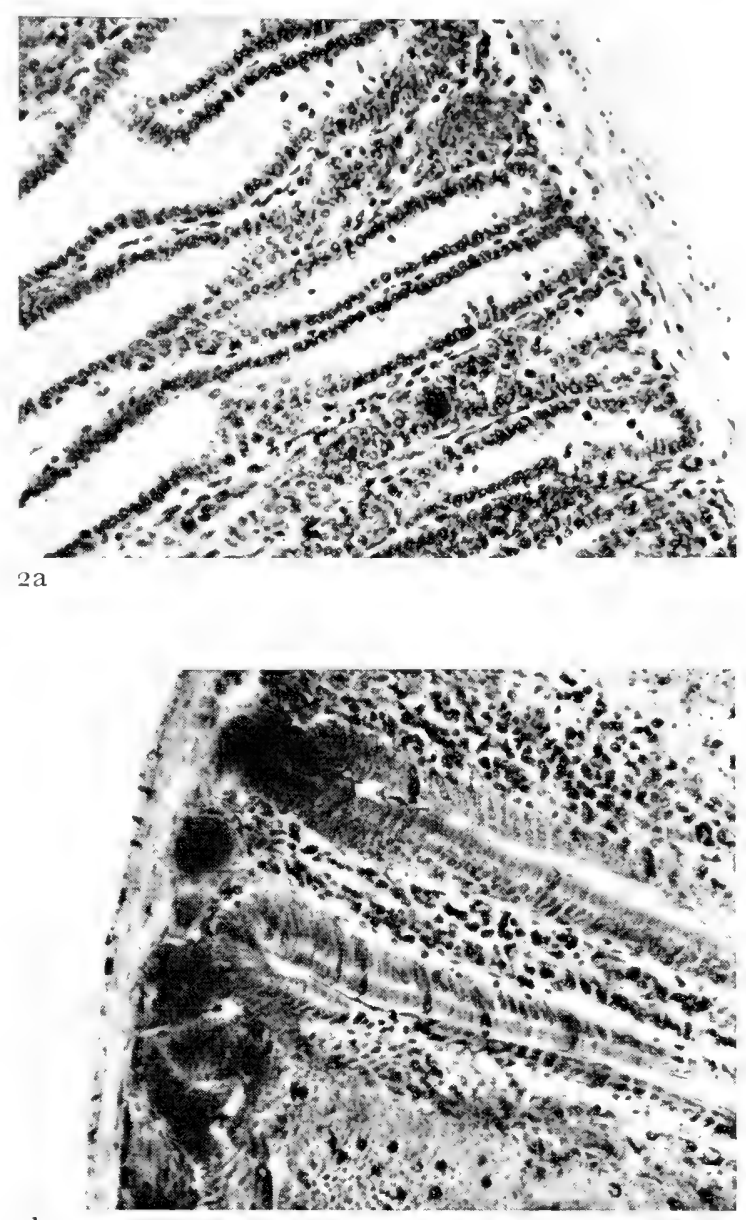

$2 \mathrm{~b}$

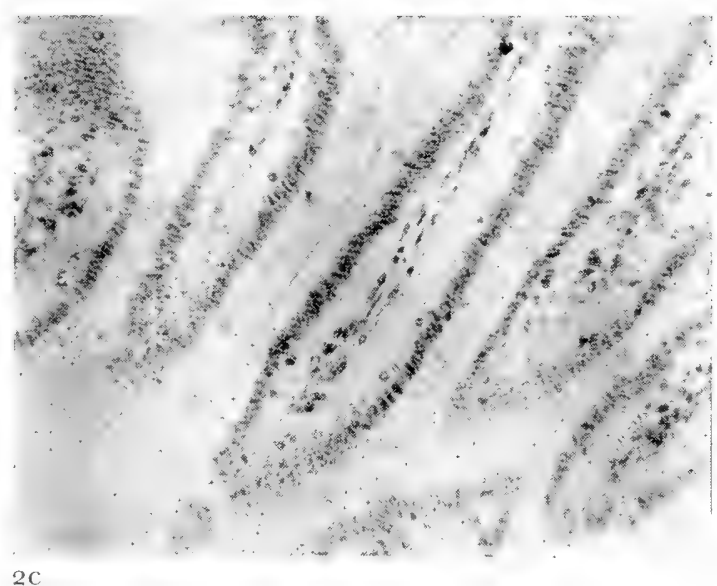


photographs of cells at this wavelength, using a quartz or reflecting microscope (Fig. 3, a). The technical difficulties have been overcome by Caspersson (1940, I 950), who has been able to measure the complete absorption spectrum of very small parts of the cell. Since the method does not distinguish between the two types of nucleic acids, it is necessary either to detect DNA with the Feulgen reaction

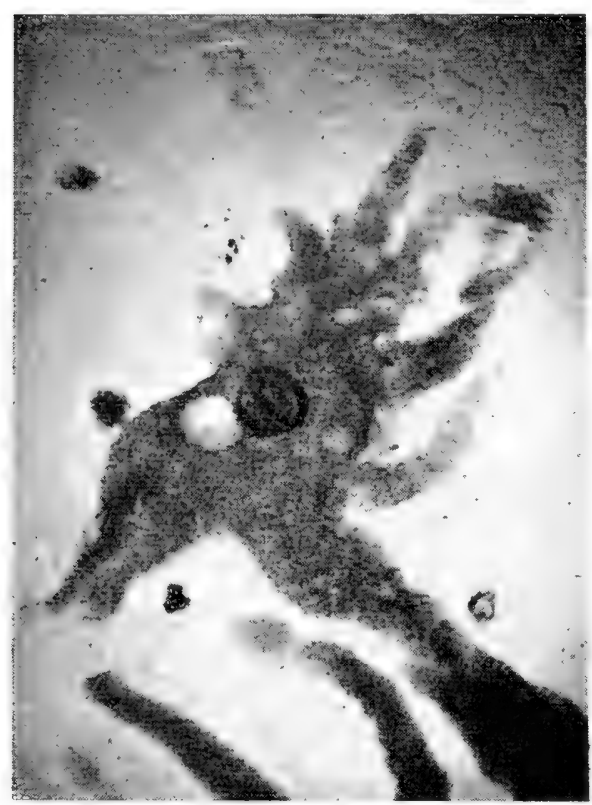

$3 a$

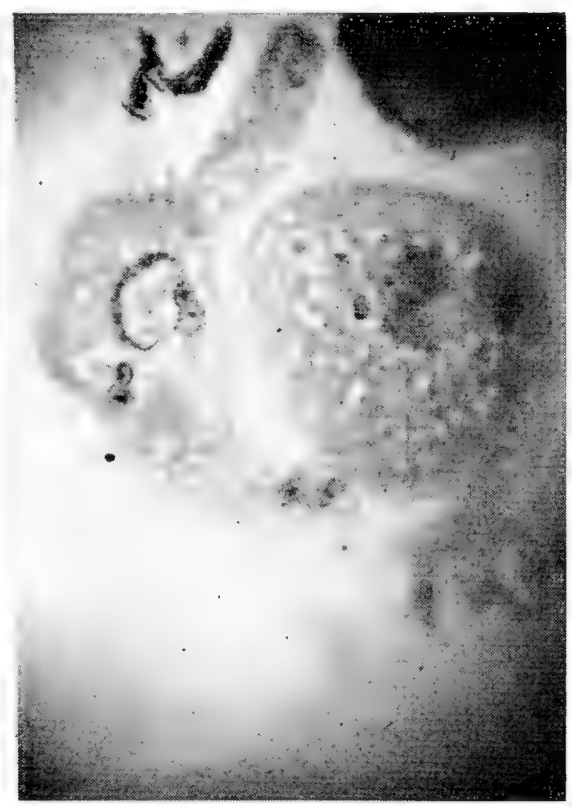

$3 \mathrm{~b}$

Fig. 3. a: U.V. photograph $(2,650 \AA)$ of an amoeba; b: same after ribonuclease treatment: nuclei of ingested flagellates still absorb the U.V., because of their DNA content.

or to remove RNA with ribonuclease in the usual way in order to follow the distribution of the two nucleic acids. (Fig. 3, b).

The results yielded by these different methods will be discussed in greater details in subsequent sections of this chapter. Here is, however, a very brief outline of the main conclusions which have been reached. DNA, as shown by the Feulgen test, is usually strictly localized in chromatin or, in dividing cells, in the chromosomes; there is no great variation in the DNA content of the nuclei in different tissues of the same species. On the other hand, RNA is mainly concentrated in the nucleolus and in small basophilic, cytoplasmic particles (the microsomes). Chromatin contains some RNA, however, in amounts varying according to the cell type. Furthermore, the RNA content of the nucleolus and the cytoplasm varies considerably from one cell type to another, in the same organism: it is always present in large amounts in cells synthesizing proteins.

We will now discuss these general conclusions in more detail and try to find out their significance for growth problems. 


\section{(a) DNA content per cell nucleus}

As already mentioned before, the Feulgen reaction gives unequivocal proof that the chromatin and the chromosomes contain large amounts of DNA; in the chromatin this DNA is associated with basic proteins (usually histones, which may be replaced by the much simpler protamines in the sperm of various species). Mirsky and Ris (195I) have shown conclusively the presence, in the chromosomes, of more complex proteins, often called chromosomine or residual proteins, because they constitute a residue when interphase chromosomes, obtained from broken nuclei, are thoroughly extracted with concentrated $\mathrm{NaCl}$ in order to remove DNA and the histones. Apparently associated with these residual proteins also are variable amounts of RNA; this represents $10 \%$ of the DNA content in liver cells, but can reach similar concentrations to that of DNA in rapidly growing tissues like wheat germs (Stern and Mirsky, 1952).

Usually, the Feulgen reaction is positive in the chromatin of resting nuclei or in the chromosomes only, and there is no evidence, except for the case of unfertilized eggs (which will be discussed in section V, p. 286), that measurable amounts of DNA exist in the cytoplasm. Since we know, from cytological evidence that chromosomes are self-duplicating units, and from classical genetics that they contain the genes, it may be supposed, as was first suggested by Boivin, Vendrely and Vendrely (1948), that all the nuclei of resting cells from the same organism have the same DNA content. In this hypothesis, it is assumed that all cells of a given organism have the same genetic composition, an assumption which is not accepted by all cytogeneticists (see for instance Sengün, I 947; Pavan and Breuer). Nevertheless, if the Boivin et al. hypothesis is correct, one could expect to find the DNA content approximately constant for each set of chromosomes in the various cells of the organism: for instance, the DNA content of a sperm cell should be one-half of the amount found in a diploid somatic cell.

This question of DNA constancy has been the subject of several recent reviews (Swift, I953; Alfert, I 954; Allfrey et al., I 955; Vendrely, I955), which should be read by all those further interested in the matter. In the writer's opinion, the question is still controversial. Early work by Vendrely and Vendrely ( 1948 ) and by Mirsky and Ris (I949), later confirmed by Leuchtenberger et al. (I95I), consisted of an analysis of the DNA content of a given number of isolated cells $(e . g$. sperm, liver cells, erythrocytes, leukocytes): these experiments have clearly shown that the DNA content of a haploid sperm cell is approximately one half the value obtained for somatic cells.

Difficulties arise, however, in the case of the latter, because not all somatic cells are always diploid: in liver, for instance, a relatively large proportion of polyploid cells is to be found. In order to study these more complex cell types, extensive use has been made of quantitative microphotometric observations of the Feulgenstaining material, as a measure of DNA content (Ris and Mirsky, I 949). Following Ris and Mirsky's lead, many cytologists have performed a large number of DNA estimations in several types of nuclei by this method (reviews by Swift, I 953; Alfert, I954; Allfrey et al., I955). There is, unfortunately, wide disagreement in 
the interpretation of the results: while most of the American authors (Swift, Alfert, etc.) firmly believe that the DNA content of the nucleus is very closely related to the degree of ploidy, a group of Belgian investigators (Pasteels and Lison, i 950; Fautrez. and Fautrez-Firlefyn, I953; Roels, I 954; etc.) strongly disagree. According to them, the DNA content of the nucleus can vary, not only in relation with the mitotic cycle and chromosome reduplication (see below), but also with the physiological activity of the tissue. For instance, according to Pasteels and Bullough (1953), great variations in the DNA content occur in the cells of the skin when the latter is rubbed with croton oil. Most striking perhaps among these exceptions is Pisi and Cavalli's (1955) recent finding, in Fautrez' laboratory, that hypertrophia of the kidney produced by thyroxin injection does not modify the DNA content of resting nuclei, while stimulation by nephrectomy increases this content. Also, according to Roels (1954), the DNA content of the nuclei varies during secretory cycles in the thyroid.

All these variations in the DNA content of resting nuclei are considered by American authors, like Swift and Alfert, to be due to technical errors or to an increase of DNA content prior to mitosis or endomitosis. A more cautious position has been recently taken by Allfrey et al. (1955), who report that, according to recent experiments by Lowe and Foley (1955), the DNA content of individual resting nuclei can vary under the influence of cortisone.

The author has unfortunately no personal experience of the methods used in these and similar experiments; as a matter of fact, he feels inclined to view most of the work done on the DNA content of individual nuclei, when estimated by the Feulgen reaction, with a certain scepticism, because there is no truly convincing evidence that this reaction follows Beer's law closely enough for quantitative purposes. On the whole, the present impression is that the DNA content of the cells is really linked to their degree of ploidy, but that one should not be too dogmatic about it: the possibility of changes in the DNA content of cells, related to surrounding physiological conditions, is by no means eliminated.

Finally, it should be added that, for general biochemical purposes, estimation of the DNA content of a tissue, when the average content of the nuclei in this tissue is known, remains a very useful method for the study of changes in cell number (Davidson and Leslie, I950; Mandel and Bieth, I95I): as a matter of fact, such estimations are still the best way we have to decide, with biochemical techniques, if growth is due to an increase in cell number or not.

\section{(b) Metabolic stability of $D \mathcal{N A}$}

The fact that there is a definite tendency for a constancy in the DNA content of the various somatic cells in the same species is insufficient to carry conviction that DNA is of genetic importance: it is also to be expected, in view of the well-known stability of the genes, that DNA is a very stable molecule.

Considerable evidence, which has been recently reviewed by Brown and Roll (1955), shows this to be the case; however, interesting work by Daly et al. (I952) indirates that the metabolic stability of DNA is not complete and that the physiological activity of the considered organ has an effect on its DNA metabolism. Their main evidence is as follows: they have studied the uptake of glycine- ${ }^{15} \mathrm{~N}$ by 
components of cell nuclei, comparing tissues with high metabolic rates (mammalian liver, kidney and pancreas) with cells endowed with a rather low rate of metabolism (avian erythrocytes and Echinoderm sperm). The experiment showed that the ${ }^{15} \mathrm{~N}$ uptake is much faster by the nuclear constituents of liver, kidney and pancreas than by those of erythrocytes and sperm. When uptake was compared with DNA, histone and residual proteins in the liver, kidney and pancreas, it was found that the uptake is low in DNA, higher in histone and much higher in residual proteins. Of great interest is the finding that, in liver, pancreas and kidney (where cells could not be dividing during Daly et al.'s short experiments), incorporation in DNA is much less in kidney than in liver and pancreas. Since the DNA content of the nucleus is the same in the three tissues, what varies is the activity of the DNA. Furthermore, in the pancreas, the ${ }^{15} \mathrm{~N}$ uptake into DNA of a well fed animal is 50\% higher than in a fasting organism. We may thus conclude, with Daly et al. (1952), that chromosomal "activity" varies in different cells and also in the same cell depending upon its over-all activity.

The situation for DNA metabolic stability is therefore much the same as for the constancy of DNA content per nucleus: this stability is considerably greater than for any other known constituent of the nucleus, but DNA is not an absolutely inert molecule.

An important fact, which is not yet completely understood, is that, according to Bendich (1952), it is possible to separate DNA from several organs in at least two fractions showing significant metabolic differences. In experiments with labelled precursors of the bases, one of the fractions is more active than the other; it has been suggested by Sparrow (1952) that one of the fractions might be equivalent to heterochromatin and the other to euchromatin. Such a suggestion is of great interest, because it might explain two facts: first that the DNA content per nucleus is not absolutely constant, and second that the metabolic stability of DNA is not absolute. We know, from the cytological work of Darlington and La Cour ( 1940 ), that the intensity of the Feulgen reaction may alter considerably in the heterochromatic segments of plant chromosomes under the influence of external factors like temperature changes. Thus the heterochromatin, which genetically is relatively inert, is apparently metabolically more active than the genetically active euchromatin. Variability in the DNA content of heterochromatin, under external and physiological influences, might then very well explain the results of those workers who, like Pasteels and Lison (I950), find that DNA content and ploidy do not always run exactly parallel. Such a hypothesis would of course also explain Bendich's (1952) results, if we assume that the metabolically active DNA is located in heterochromatin. This hypothesis could easily be tested by autoradiography methods, which should help in ruling out an alternative explanation, i.e. that the two metabolically distinct DNA's of Bendich (1952) are localized in different histological cell types in the same organ.

To summarize the various hypotheses which have just been discussed, present evidence indicates that DNA is a satisfactory candidate for genetic activity, certainly better than any of the other known biochemical constituents of the chromosomes. It is in the field of bacterial genetics, however, that the best evidence for a genetic role of DNA can be found. 
(c) Genetic activity of $D \mathcal{N A}$

Since the problem of the genetic functions of DNA lies somewhat outside the scope of the present book, a short summary only of the main evidence will be given here; the whole subject has recently been excellently reviewed by Hotchkiss (1955a).

Of special importance in the problem of the genetic role of DNA are the experiments of Hershey and Chase (I952) on the $T$ coli phages, because they deal with one very essential growth process: the duplication of DNA-containing structures, so characteristic of mitosis. Recent work on the structure and chemical composition of the $T$ phages, shows them to consist chiefly of a protein membrane, representing about $60 \%$ of the virus, surrounding a central core of DNA $(40 \%)$. On infection, the phage particles become adsorbed by their tails on the sensitive bacteria.

It has been found by Herriott (I95Ia) that the membranes can be emptied of their DNA core when they suffer osmotic shock: these protein "ghosts" can still infect the cells, but no phage is reproduced in the absence of DNA, suggesting that DNA is essential for phage formation.

In Hershey and Chase's (I952) elegant experiments, the DNA is specifically labelled with ${ }^{32} \mathrm{P}$, while the protein ghosts are labelled with ${ }^{35} \mathrm{~S}$. The result, after infection, is that the ${ }^{32} \mathrm{P}$ labelled DNA of the phage enters into the cells, while the protein (marked with ${ }^{35} \mathrm{~S}$ ) remains attached on the outside surface; most of it can afterwards be removed from the cell membrane by subjecting the infected bacteria to the action of a blender. The complete phage thus appears to function as a syringe which injects the genetically important material, DNA, into the cell. Phage reproduction, which involves synthesis of new DNA and new protein membranes, is thus a consequence of the initial introduction of phage DNA into the cell.

While these experiments strongly support the view that in phage reproduction DNA plays an extremely important role, they do not prove that DNA is equivalent to genes. This last assumption is demonstrated by the study of bacterial transformations: the famous experiments of Avery et al. (1944) have shown that DNA extracted from an encapsulated (smooth) strain of pneumococcus will transform unencapsulated (rough) bacteria into encapsulated cells. Since the transformed cells will multiply indefinitely with a capsule, the property of forming a capsule has been genetically transmitted.

The DNA extracted under these conditions behaves thus very much like a gene in inducing a heritable change and in being capable of self-duplication. As shown by Avery et al. (1944) and by Hotchkiss (1949), the biological activity of the pneumococcal DNA is lost after digestion with deoxyribonuclease, while ribonuclease and proteolytic enzymes have no effect. Although it is impossible completely to exclude the possibility that proteins contaminate DNA, it has been shown that the protein content of the purified factor is less than $0.02 \%$.

Since Avery et al.'s (I 944) pioneer work, many other bacterial transformations have been discovered and studied in detail: in all cases, the transforming activity has been found to be present in the purified DNA fraction, while the enzyme deoxyribonuclease always inactivated the transforming principle. 
There is no doubt that the evidence in favour of the view that transforming agents are identical with genes is now exceedingly strong and that there is little reason to doubt that the genes are DNA particles. Let us now go back to the cells of more complex organisms and try to find out what happens to DNA during mitosis.

\section{(d) DNA synthesis during mitosis}

We have already seen that DNA is a relatively stable substance metabolically and that there is a tendency for a constancy of the DNA content per nucleus (or rather per set of chromosomes). In view of the delicate mechanism of chromosome and gene reduplication during mitosis, it is to be expected that the whole situation will change considerably when a cell undergoes division. DNA must be synthesized, in order to be equally divided into the two daughter cells, so that the DNA content will obviously double at some point of the mitotic cycle. Furthermore, DNA synthesis will occur at the expense of various precursors: if we add them to the cells in a labelled form, we should find rapid incorporation in the newly formed DNA molecules. Therefore, in dividing cells, we should expect to find variations of the DNA content and a considerable increase in the incorporation of various precursors into the DNA molecule. A large number of experiments have definitely shown that both these expectations are actually fulfilled.

Already the pioneer experiments of Hevesy and Ottesen ( I 943) and of Andreasen and Ottesen (1945) have clearly shown that incorporation of ${ }^{32} \mathrm{P}$ into DNA is much greater in actively dividing tissues, such as bone marrow and intestinal mucosa, than in resting tissues. These early findings have been confirmed many times, with greatly improved techniques. Very recently, for instance, Smellie et al. (1955) found that DNA turnover, studied with ${ }^{32} \mathrm{P}$, is faster in appendix and bone marrow than in intestinal mucosa, spleen and thymus; on the contrary, renewal of DNA is very small in kidney. As would be expected, if the incorporation of ${ }^{32} \mathrm{P}$ into DNA is linked to synthesis during the mitosis, inhibition of incorporation by $\mathrm{X}$-irradiation is greater in bone marrow and appendix than in thymus; whereas in kidney, X-irradiation has little effect.

Similar results have been obtained when precursors other than ${ }^{32} \mathrm{P}$ (for instance formate, glycine or adenine) have been used; much experimental data supporting this contention will be found in recent reviews by Brown and Roll (I 955) and by Leslie (I955). Particularly striking is the considerably increased incorporation of all precursors into the DNA of regenerating liver $(c f$. Furst and Brown, I95I): such findings obviously mean that DNA synthesis is linked to the process of mitosis.

There is a general contention that DNA synthesis occurs prior to mitosis, i.e. during interphase; however, the exact timing of this synthesis remains a subject for hot discussion. While Lison and Pasteels (I95I) found, by quantitative use of the Feulgen reaction, that DNA synthesis occurs at the beginning of interphase, Swift (1950) believes it to happen at the end of interphase. Walker and Yates (1952) and Grundmann and Marquardt (1953) found DNA synthesis to occur slowly and progressively during most of the interphase.

In recent years, it seems that the workers who have studied DNA synthesis by 
means of the Feulgen reaction have come to the conclusion that these discrepancies are less a matter of technique than of materials: as pointed out by Fautrez and his co-workers (1953, I 954), by Leuchtenberger (1954) and by Dalcq and Pasteels (I955) in recent papers, the synthesis of DNA does not necessarily always take place at the same stage of the mitotic cycle, but the point may be different for various tissues (Leuchtenberger, I954).

A different and interesting approach to the same problem has been made by Pelc and Howard (1952) who studied the incorporation of ${ }^{32} \mathrm{P}$ into DNA of bean root cells with an autoradiography technique. These experiments have led to the following main conclusions: DNA synthesis occurs during the first part of interphase and there is neither synthesis nor loss of DNA in differentiating cells, which can no longer divide. It is well worth adding that Pelc and Howard (I952) also studied the incorporation of inorganic ${ }^{35} \mathrm{~S}$ into the proteins of the same cells and found that protein synthesis in the nuclei occurs at approximately the same time as DNA synthesis. It would certainly be interesting to repeat these observations on other material, in order to find out if they have general significance.

Recent work by Stevens et al. (1953) and Daoust et al. (1954), related to the same problem, has also produced much discussion: on the basis of extensive mitotic counts, measurements of incorporation of ${ }^{32} \mathrm{P}$ into DNA and a number of calculations, they concluded that twice as much DNA is synthesized as is needed: therefore, in order to explain their results, they made the assumption that the preexisting DNA is broken down at the time of mitosis: this means that the parental DNA ${ }^{32} \mathrm{P}$ atoms are completely replaced during division.

Such conclusions are in opposition to the hypothesis expressed by Watson and Crick (I953a, I953b) on chromosome reduplication: as we have already seen, these authors suppose that DNA is formed of two complementary strands which can unwind and separate, each serving as a template for the formation of its complement (see Fig. I, p. 262). In this case, DNA should be metabolically very stable: we have seen that this has usually been found to be the case, except for the previously mentioned claims of Daoust et al. (1954) and Stevens et al. (1953). Although it is too early to draw final conclusions, it does not seem that the work of Daoust and Stevens really carries conviction: it has been seriously critized on technical grounds by Hotchkiss (1955b) and contradictory results have recently been published by Kihara and Sibatani (1955). According to these Japanese workers, there is no turnover of DNA during growth, whether DNA is being synthesized or not, and they obtained no evidence that preexisting DNA disappears during mitosis.

If, as it now seems, we can disregard Daoust and Stevens et al.'s conclusion, there is no doubt that Watson and Crick's (1953a, I 953b) model of DNA structure and their hypothesis of chromosome reduplication remains exceedingly useful. It should be added, however, that the hypothesis presents a few weaknesses and that attempts have already been made by Delbrück (1954) and by Dekker and Schachman (I954) to improve Watson and Crick's (I953a, 1953b) scheme. However it is too early to discuss further these hypotheses, since their value has not yet been experimentally tested. 
(e) Effects of physical and chemical agents on DNA and its synthesis

Further evidence, although of a more indirect nature, for the important role of DNA in genetics and cell division can be found in many studies on the effects of various agents on mutational rate and mitotic activities: all of them have been found to act on DNA or its synthesis.

Of greatest interest in this respect is probably ultraviolet (U.V.) radiation as it has been shown that the action spectrum of $U$. $V$. radiation in inducing mutations in various organisms (i.e. its effectiveness at various wavelengths) is similar to the absorption spectrum of nucleic acids (Hollaender and Emmons, I 94 I ; Stadler and Weber, I942). It should be pointed out, however, that the absorption spectra of DNA and RNA are almost identical; but since the mutations studied were truly chromosomal, and since DNA is usually present in much larger amounts than RNA in chromosomes, there seems to be little reason left to doubt that the U.V., in these experiments, was really acting on DNA. Furthermore, it has been clearly shown by several workers (especially Hollaender et al., I94I, and Errera, I952) that prolonged U.V. irradiation modifies the physical properties of DNA, probably by producing some kind of depolymerization.

However, DNA itself is less sensitive to U.V. radiations than DNA synthesis: as found by Kelner (I953) and by Kanazir and Errera (I954), low doses of U.V. are enough to inhibit DNA synthesis in E. coli; RNA and protein synthesis still go on in the irradiated bacteria, although cell divisions are completely inhibited. Thus, in this case, growth proceeds at an almost normal rate in the absence of nuclear division. The result is the formation of filamentous bacteria containing only a few nuclei (Errera, I 954). Other instances, where DNA synthesis can be dissociated from growth, RNA and protein synthesis, will be examined in section IV, p. 275 .

A similar situation is found in the case of other well-known mutagenic agents, the mustards: sulfur mustard was the first chemical substance found to be capable of inducing mutations in Drosophila (Auerbach and Robson, I946); according to Herriott (I948), sulfur mustard is much more active on DNA than on protein or RNA. In the case of nitrogen mustard, as of U.V., DNA depolymerization occurs at relatively high concentration (Butler et al., I 950), while DNA synthesis is much more sensitive than RNA synthesis: this has been found to be the case both with amphibian eggs (Bodenstein and Kondritzer, I948) and bacteria (Herriott, I 95 I b).

Another interesting case is presented by the methylated purines, caffeine and theophylline, which are mutagenic for bacteria, and it is remarkable that their effect is counteracted by nucleosides of the normal bases (Novick and Szilard, I952). These results might well mean that the methylated purines are incorporated in the nucleic acids instead of the normal purines: this would lead to the synthesis of abnormal nucleic acids which, in turn, might be at the origin of the observed mutations. However, such a simple explanation appears to be of doubtful value, since Zamenhof and Griboff ( I 954) have found that incorporation of $17-19 \%$ of the abnormal base 5-bromouracil in the DNA of $E$. coli has no effect on the growth rate; nor does it lead to mass-mutations.

However the possibility of a slight or moderate mutagenic effect of 5 -bromouracil is not ruled out by Zamenhof and Griboff's ( I 954) experiments; furthermore, we do not know yet whether the incorporation of the abnormal base occurs in all 
the DNA molecules of the cell nucleus or only in a few and, finally, we should not forget the following very sensible remarks of Hotchkiss (1955b) "there is little reason to hold, for example, that all of a pneumococcus-transforming DNA preparation is made up of genetic determinants, whether identified or not identified. It may very likely be as unjustified as assuming all proteins to be enzymes." Hotchkiss (1955b) also reminds us of the existence of heterochromatin, the significance of which has been discussed earlier in this chapter: it might well be that the incorporation of 5-bromouracil, in Zamenhof and Griboff's (1954) experiments, occurs mostly - or perhaps entirely - in this metabolically more unstable fraction.

One last, but very important, mutagenic agent and well-known mitotic inhibitor is of course X-radiation: once more, X-irradiation produces depolymerization of DNA both in vitro (Taylor et al., I948) and in vivo (Errera, I947). And again, DNA synthesis is exceedingly sensitive to X-irradiation (Mitchell, 1942; Hevesy, I949; Holmes, I 949 ; etc.).

Summing up, it can be said that most of the evidence reviewed in this section strongly supports the view that DNA is of the utmost importance in chromosome duplication and in genetic activity. If we take into account the existence of heterochromatin and admit that its role is different from the genetically more active euchromatin, the evidence becomes even more convincing. We should therefore think of DNA as a very essential growth factor, whenever growth is closely linked to cell division.

Fresh and important evidence in favour of this view has just come from recent work by Miller et al. (I 955), who have succeeded in isolating a new growth substance for plant cells. This substance, which has been called kinetin and which seems to be identical with the "wound hormone" of the plant physiologists, is a derivative of DNA. While freshly prepared DNA is inactive in stimulating mitosis, kinetin activity is present in all preparations or in DNA which has been degraded by autoclaving for half an hour at $\mathrm{pH} 4.3$. Kinetin is biologically active at very low concentrations $(0.0 \mathrm{I} \%$ ); further work on its chemical nature will be awaited with great interest. But, whatever its nature, the close association of kinetin with DNA brings additional support for the view that DNA is an essential factor for cell division and growth.

\section{RNA AND GROWTH: PROTEIN SYNTHESIS}

Growth is, of course, not necessarily a consequence of mitotic activity: we have just seen that, according to Kelner (I954) and to Kanazir and Errera (1954), growth and protein synthesis continue in U.V. irradiated bacteria, although DNA svnthesis has stopped. Other similar cases will be discussed later. In animal cells, striking instances of growth without cell division may be found, for instance, in the oocytes and the neurones. The tremendous increase in size and dry weight of the growing oocyte is of course very well known; in the neurones, elegant ligation experiments of the axones by Weiss and Hiscoe (1948) have shown that continuous synthesis of fresh cytoplasm occurs in the cell body.

During the last 5 years, considerable evidence has accumulated in favour of 
the view that RNA plays an essential part in protein synthesis-obviously an essential process in growth. This evidence has been recently reviewed in detail by Brachet (1955a); therefore, only the main points need to be examined here. We shall successively deal with the cytochemical and quantitative facts in favour of the hypothesis, the indirect evidence based mainly on the study of plant viruses and finally the more direct experiments of the past two or three years.

\section{(a) Cytochemical and quantitative evidence}

The hypothesis that RNA plays an essential part in protein synthesis has been proposed simultaneously and independently by Caspersson (I94I) and Brachet (I94Ia) in order to explain their cytochemical findings. Using two different methods (U.V. microspectroscopy, and staining with basic dyes combined with ribonuclease digestion), both workers came to the conclusion that only the cells which are synthesizing large amounts of proteins are rich in RNA. For instance, the exocrine part of the pancreas, the pepsin-producing cells in the gastric mucosa, liver cells, nerve cells, growing oocytes and embryos, and silk glands in silk worms, all have a very high RNA content. On the contrary, certain tissues (such as kidney, heart and muscle) possess a very high physiological activity without synthesizing proteins; these are always poor in RNA. The tissues which show marked mitotic activity, like spleen, lymph glands or testis, have an intermediary RNA content.

A considerable number of investigations have confirmed these early findings and we can only refer to a few of the more recent papers dealing with the subject: in pituitary, for instance, stimulation of hormone synthesis is linked with a marked increase in RNA content (Desclin, I940; Herlant, I943; Abolins, 1952). The same relationship holds in the salivary glands of Drosophila larvae (Lesher, I95I) and in seminal glands of rats, where protein synthesis can be stimulated with testosterone (Rabinovitch et al., I95I). According to Schrader and Leuchtenberger (I950), protein and RNA synthesis are closely linked together during spermatogenesis, while DNA synthesis is independent of protein increase. Inhibition of growth and protein synthesis in the unicellular alga Acetabularia mediterranea by suppression of light (Stich, I 95 I) or metabolic poisons (Brachet, I952a) leads to a marked decrease of the RNA content of the nucleolus.

Still more recently it has been shown by Ficq and Brachet (1956) that the incorporation of a labelled amino acid (phenylalanine) into the various tissues of the mouse runs exactly parallel with cytoplasmic basophilia, i.e. RNA content: incorporation is highest in the pancreas (except in the Langerhans islets, which are 8 times less active than the exocrine part), and very active in the basophilic parts of the intestinal mucosa, liver, spleen and uterus. There is very little incorporation in tissues which are poor in RNA, like muscle, heart and lung for instance (Fig. 4a, b).

These few examples should be sufficient to show that the relationship existing between RNA and protein synthesis is a very general phenomenon, occurring in all living organisms, and under very different physiological conditions.

The cytochemical observations which have just been briefly summarized have been confirmed by a large number of quantitative biochemical estimations of the RNA content of various tissues. As shown by Brachet (I94Ib) and by Davidson ( $\mathrm{I}$ 947), there is no doubt that the protein-synthesis activity of a tissue is altered 
by experimental means: in liver, for instance, fasting is followed by a decrease in basophilia and in the RNA content (Davidson, I947), while DNA remains unaffected (Mandel et al., I 949; Campbell and Kosterlitz, I 950).

The close correlation between RNA content and protein synthesis is especially

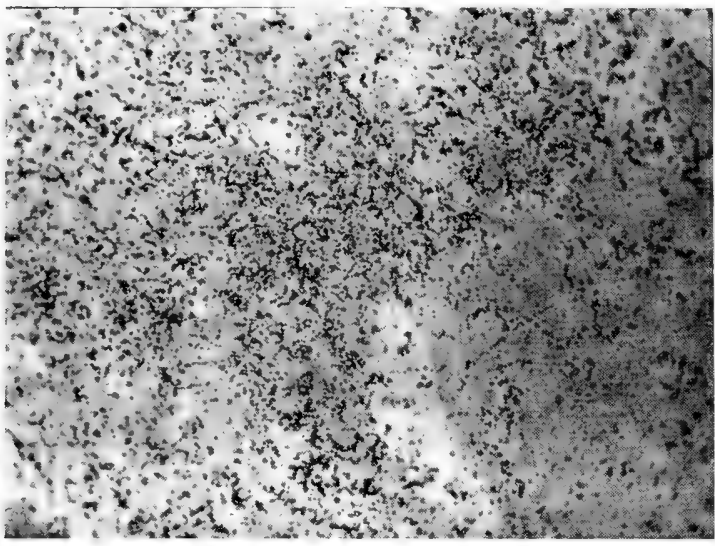

$4 a$

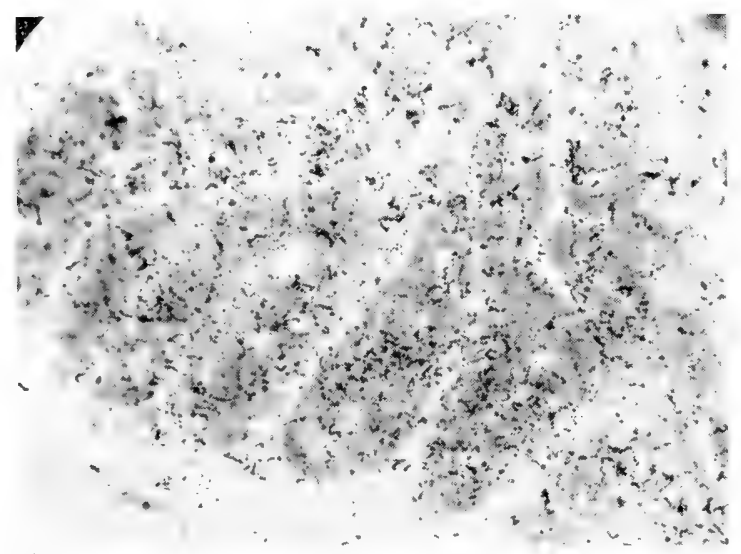

$4^{\mathrm{b}}$

Fig. 4. Autoradiographs after incorporation of labelled phenylalanine into mouse tissues. a: pancreas: much stronger activity in the exocrine part (right) than in a Langerhans islet (left). b: intestine: activity is strongest in the Lieberkuhn crypts. Very little activity in the musculosa (below). impressive in growing cultures of microorganisms: early estimations of the RNA content of growing bacteria by Vendrely ( I 946) have shown that these cells, which undergo extremely rapid protein synthesis during growth, may contain as much as I I. $5 \%$ RNA (dry weight). The cytochemical observations of Malmgren and Hedén (1948), using Caspersson's ultraviolet absorption technique, demonstrated that the RNA content is low in resting cells; there is a moderate increase in RNA during the lag phase and a considerable synthesis of U.V.absorbing substances during the logarithmic phase of growth. The RNA content reverts to the original value just at the time when all cell divisions cease: in this case, cell division seems to be very closely linked to the RNA content.

The correlation between the synthesis of RNA and the synthesis of protein is especially striking when microorganisms are analyzed during the logarithmic phase of growth: in experiments by Caldwell et al. (1950), the RNA content of bacteria remained proportion-

al to the growth rate under a variety of experimental conditions (addition of inhibitors, utilization of slow or fast growing mutants and modifications of the nutrient medium). These results have been confirmed by Northrop (I953) and by Wade (1952), on other bacteria. The importance of the culture conditions in such experiments is indicated by Jeener's (I953) experiments on the flagellate Polytomella coeca: a close relationship between the RNA content and protein 
synthesis is found only in continuous cultures, where growth maintains itself at a constant rate, which can be controlled at will by the investigator.

Later work by Gale and Folkes (I953) has also shown that, in Staphylococcus, there exists a strong correlation between the nucleic acid content of the cell and the rate of protein synthesis; however, antibiotics like chloromycetin, aureomycin and terramycin can dissociate the two processes: protein synthesis is inhibited, while RNA synthesis is increased.

Mention should also be made of a paper by Price (1952), who obtained similar results on Staphylococcus muscae: in his experiments, the increase in proteins is accompanied by a parallel increase in RNA, in contrast to DNA. Furthermore, the RNA/protein ratio is proportional to the growth rate. While the RNA content per cell depends on the growth rate during early logarithmic phase, the DNA content per cell remains fairly constant irrespective of the growth rate of the cells.

An important finding of Price (I952) is that, while the cells adapt to lactose, there is never an increase of protein without a simultaneous increase in RNA. This observation is of interest because it raises an important question: is the synthesis of a new enzyme linked to the synthesis of new RNA molecules?

This interesting problem has been receiving more and more attention lately: for instance, Pardee (I 954, I 955) has found that purine- and pyrimidine-requiring mutants of $E$. coli can synthesize adaptive enzymes only when these bases are added to the medium. The synthesis of induced enzymes stops as soon as the purines and pyrimidines are removed from the culture medium. The obvious conclusion is that continuous production of new RNA molecules is necessary for specific protein synthesis and that the bulk of bacterial RNA is metabolically inert.

Comparable results have been described by Gale and Folkes (1955a), who obtained stimulation of the induced synthesis of glucozymase on addition of a mixture of purines and pyrimidines to disintegrated Staphylococci.

But the most complete and recent analysis of the phenomenon is that of Spiegelman et al. (1955): they have found that strong interference with DNA synthesis has no striking effect on enzyme formation, whereas a $50 \%$ inhibition of RNA synthesis completely suppresses the enzyme-synthesizing capacity. Uracilless and adenineless mutants of $E$. coli are unable to synthesize an adaptive enzyme, unless the needed metabolite is added to the medium (in accordance with Pardee's [ I 954] results). Addition of purine and pyrimidine analogues, which interfere with nucleic-acid synthesis, immediately stop the adaptive enzyme synthesis of $\beta$ galactosidase in $E$. coli.

This last result of Spiegelman et al. (1955) has been confirmed by Creaser ( $1955^{\mathrm{a}}$, I955 b), who obtained inhibition of induced synthesis of $\beta$-galactosidase, catalase and glucozymase in Staphylococcus, on the addition of purine analogues (azaguanine and 2,6-diaminopurine); he also obtained some indications of a synthesis of new DNA and RNA molecules during adaptive synthesis of the enzyme.

Pardee (1954, 1955), Spiegelman et al. (1955) and Creaser (1955a, 1955b) arrive at the same conclusion: synthesis of new RNA is a compulsory concomitant of the continued formation of new enzyme molecules.

Confirmations of this conclusion can be found in recent papers by Reiner and Goodman (I955) and Chantrenne (1956): according to Reiner and Goodman 
(1955), cells of $E$. coli adapted to gluconate contain substances which accelerate the synthesis of the enzyme gluconokinase. These substances are a mixture of ribosepolynucleotides. Chantrenne's (1955) experiments are of an even more direct nature: when non-respiring yeast cells are synthesizing catalase under the inducing action of oxygen, new RNA molecules are built up. Under Chantrenne's ( 1955 ) experimental conditions, adenine is being incorporated in RNA at a faster rate than in non-adapting cells and this incorporation occurs preferentially in one particular cell fraction.

Present evidence, in the case of microorganisms, is thus very strongly in favour of the idea that the synthesis of a specific enzyme protein is associated with the synthesis of a new, presumably specific, $R \mathcal{N} A$.

It is unfortunately impossible to say whether this statement is also valid for protein synthesis in animal cells. However, cytochemical (Brachet, I952b; Thorell, I 947) and biochemical (Davidson, Leslie and Waymouth, I949) evidence suggests that a high RNA content always precedes protein synthesis. More conclusive is Hammarsten's (I95I) opinion, drawn from his work on the incorporation of labelled precursors into RNA and proteins, that nucleoprotein synthesis not only precedes protein synthesis, but is even necessary for the latter to occur. This conclusion is entirely confirmed by recent work done in the author's laboratory: comparative studies on the incorporation of adenine into RNA and of phenylalanine into proteins with an autoradiography method has shown that, in starfish oocytes as well as in mouse liver, the incorporation of adenine is always much faster than that of phenylalanine (Ficq, I955; Ficq and Errera, I955a; Moyson, I 955).

\section{(b) The role of $R \mathcal{N} A$ in plant-virus multiplication}

Plant viruses are extremely important in a discussion of the role of RNA in growth and protein synthesis, because of their chemical simplicity: all the plant viruses which have been purified and often obtained in crystalline form contain only RNA (6\% to $35 \%$ ) and proteins. Nevertheless, these simple nucleoproteins are able to reproduce themselves and are thus endowed with genetic continuity: they represent therefore an ideal material for the study of protein synthesis and the genetic function of RNA. In this respect, they should be compared with the DNA containing $T$ phages of $E$. coli, which have been discussed earlier in this chapter.

Regarding the role of RNA in plant virus multiplication, Markham and Smith's (I949) important experiments have shown that leaves infected with turnip yellow mosaic virus contain two main components: the virus, and a protein serologically identical to the virus, but devoid of RNA and of infectivity. The fact that only the complete, RNA containing, virus is capable of multiplication has led Markham ( I 953) to the conclusion that RNA apparently is the substance controlling virus multiplication.

These findings were soon extended to the well known tobacco mosaic virus by Jeener and Lemoine (I 952), Jeener, Lemoine and Lavand'homme (I954), Takahashi and Ishii (1952) and Commoner et al. (I953): they isolated from the infected leaves a crystallizable protein, free of RNA, immunologically identical with the virus, but non-infective (soluble antigen of Jeener).

Confirmatory evidence of the essential role of RNA in tobacco mosaic virus 
multiplication has been obtained from an entirely different type of experiment: substances which interfere with RNA metabolism (for instance thiouracil which may be regarded as a competitive analogue of uracil) inhibit the synthesis of the virus (Commoner and Mercer, I 95I). However, an analysis of the phenomenon by Jeener and Rosseels (I 953) disclosed a remarkable fact: thiouracil does not act as a competitive inhibitor, but it is incorporated into the virus RNA. The result is the synthesis of an abnormal RNA and a decrease in infectivity. Identical results have been obtained by Matthews (1954, 1955) with 8-azaguanine: here again, the abnormal base is incorporated in the virus RNA, with a simultaneous loss of infective power.

The obvious conclusion is that removal of the virus RNA or alteration of its composition by introduction of abnormal bases always leads to an inhibition of virus multiplication, i.e. to the suppression of growth. The very recent and spectacular experiments of Fraenkel-Conrat and Williams (1955) confirm this statement in a more direct way: by proper treatment of tobacco mosaic virus, FraenkelConrat and Williams (I955) have succeeded in separating the protein and the RNA moieties and found that neither of them are infectious. But if the two fractions are mixed together, partial resynthesis of the virus occurs and infectivity is restored to a certain extent. There is no longer any doubt that integrity of both RNA and protein, and their proper combination are essential for full virus activity.

A last and very interesting point should be discussed about the plant viruses: as we already know, they show genetic continuity, i.e. they are reproduced by the infected cell without any change in their specific molecular structure. We have seen earlier in this chapter that DNA plays the leading part in phage genetics: does RNA play a similar role in plant viruses? The question has been raised recently by Jeener (I956) in a very thoughtful paper, in which he reports experiments showing that, in infected leaves, the incorporation of radioactive $\mathrm{CO}_{2}$ is higher in the non-virulent antigenic protein (soluble antigens) than in the virus; it is lowest in the normal protein of the leaves. The experimental data are compatible with the hypothesis that the soluble antigens are precursors of the virus. This hypothesis is supported by the additional finding that soluble antigens accumulate in the leaf as soon as the synthesis of the virus is complete.

Jeener (1956) draws the conclusion that RNA and proteins are synthesized independently in the virus-infected cell: just as in the phage, multiplication of the virus is not due to the division of preexisting particles. If so, RNA could play the same role in plant viruses as DNA in phage and it might represent a genetical model for protein synthesis ${ }^{1}$.

\section{(c) Direct evidence for a role of $R N A$ in growth and protein synthesis}

The evidence which has been reviewed so far lends strong support to the idea that RNA is directly concerned in growth and protein synthesis: however, the evidence has so far been only circumstantial.

The first successful attempt to show that nucleic acids are directly involved in protein synthesis has been made by Gale and Folkes (1954, 1955a), who studied

I This conclusion has now been confirmed by Gierer and Schramm's (1956) important finding that pure tobacco mosaic virus RNA is infective. 
the protein metabolism of Staphylococci disrupted by ultrasonics. They found that the disrupted cells are still capable of amino-acid incorporation into proteins, and even of net protein synthesis, provided that energy sources (adenosinetriphosphate (ATP) and hexosediphosphate) and an adequate mixture of amino acids are present. Removal of the nucleic acids by various treatments, including digestion with specific nucleases, greatly inhibits protein synthesis; addition of a mixture of DNA and RNA from Staphylococci restores the activity of the system. Treatment with either ribonuclease or deoxyribonuclease inactivates these nucleic-acid preparations; nucleic acids from other sources are inactive. These findings suggest, of course, that specific nucleic acids are needed to promote incorporation of amino acids into proteins: however, breakdown products of the specific staphylococcal nucleic acids, after they have been digested with nucleases, are also active. The experiments further show that incorporation of amino acids into proteins is different from true protein synthesis, although both processes have some features in common.

As already mentioned, Gale and Folkes (1954, r955a) found that a mixture of pyrimidines and purines stimulates adaptive synthesis of glucozymase in their system, provided that amino acids are present. When the disrupted cells are depleted of their nucleic acids, addition of nucleic acids stimulates the induced enzyme synthesis; RNA is much more effective for catalase than DNA, while a mixture of purines and pyrimidines promotes best the $\beta$-galactosidase synthesis.

The results can be accounted for, according to Gale and Folkes (I954, I955a), by the following hypothesis: DNA, perhaps associated with a protein, is the initial organizing structure; it is incapable of synthesizing proteins itself, but it acts as an organizer for the synthesis of RNA; once RNA has been synthesized, protein synthesis can take place at a rate dependent upon the amount of specific RNA present.

Gale and Folkes' ( I 954, I 955a) results on the incorporation of amino acids into proteins have been quickly confirmed by Lester (I 953) and by Beljansky (I954); they worked on bacteria lysed with lysozyme (so called protoplasts) and afterwards treated with nucleases.

Similar results have also been obtained with extracts from animal cells: Allfrey, Daly and Mirsky (1953), and Zamecnik and Keller (1954) have found that treatment with ribonuclease strongly inhibits the incorporation of labelled amino acids into the proteins of the microsomes present in liver homogenates. Incidentally, it is worth mentioning that these small cytoplasmic particles, which are very rich in RNA, incorporate amino acids into their proteins in vivo and in vitro much more rapidly than any other cell fraction, including mitochondria and nuclei (Hultin, I 95I; Siekevitz, I952; etc.).

Recently, Gale and Folkes (I955b) and Gale (I955) have reported on extremely interesting developments of their work on disrupted Staphylococci. While, as mentioned earlier, non specific RNA's isolated from yeast or liver are unable (1) restore incorporation of amino acids into the proteins, ribonuclease digests of these nucleic acids are active. This observation led Gale and Folkes (I955b) to an extensive fractionation of digests of staphylococcal RNA by chromatography and ionophoresis: single fractions could be obtained from the digest, which promoted the incorporation instead of RNA, the active fraction being different for each 
amino acid. The separated fractions were at least oo times as effective as staphylococcal RNA. Gale's (I955) conclusion is "that the whole RNA complex is not necessary for the incorporation of any particular amino acid, and that RNA can be replaced by small fragments obtained by ribonuclease digestion of the whole RNA structure". The significance of these important observations for present
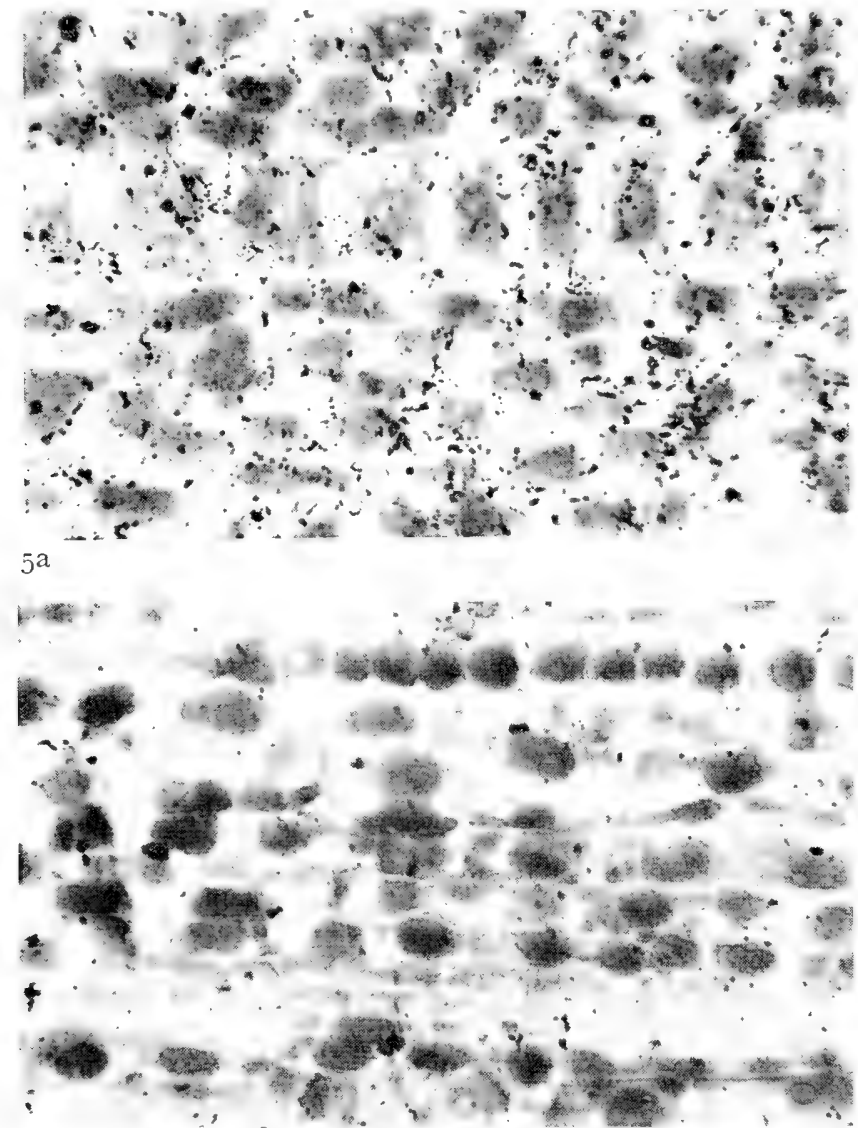

$5 \mathrm{~b}$

Fig. 5. Autoradiograph after incorporation of labelled phenylalanine into onion roots. a : normal root; b : root treated in vivo during $3 \mathrm{~h}$. with ribonuclease ( $\mathrm{I} \mathrm{mg} / \mathrm{ml}$ ).

theories on the mechanisms of protein synthesis will be discussed later (p. 285).

The experiments of Gale and Folkes (1954, i 955a, I955b) raise a new and important question for the biologist: is it possible to inhibit growth and protein synthesis in living cells or organisms by ribonuclease treatment?

This problem has been extensively studied in the author's laboratory during the past two years: starting from the observation by Kaufmann and Das (1954, I955), that mitotic abnormalities occur in onion root-tips immersed in ribonuclease solutions, it was decided to study the biochemical effects of ribonuclease on various cells. Only the main results will be summarized here. 
In onion root-tips (Brachet, I954a), ribonuclease ( $\mathrm{Img} / \mathrm{ml}$ ) produces a $90 \%$ inhibition of amino-acid incorporation into proteins within $3 \mathrm{~h}$. Fig. 5 illustrates the results obtained, with an autoradiography technique, in the case of phenylalanine. The inhibition of the incorporation of the amino acids into the proteins is faster and stronger than that of the penetration of the free amino acids; this suggests that ribonuclease does not act primarily on cellular permeability. Furthermore, ribonuclease strongly inhibits growth (Fig. 6): the inhibition is

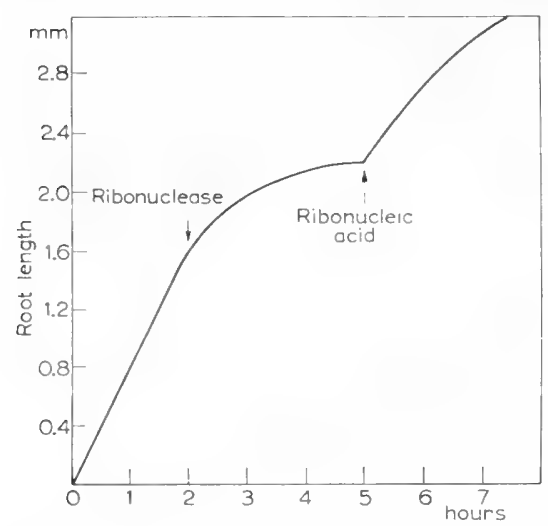

Fig. 6. Inhibition of growth in onion roots by ribonuclease; partial recovery on addition of RNA. almost complete within $3 \mathrm{~h}$. and is usually irreversible (Brachet, I 955b). Chemical estimations of the protein content have shown, as might be expected, that net protein synthesis is completely inhibited in the ribonuclease-treated onion roots. Another interesting finding is shown in Fig. 6: addition of yeast RNA to the ribonuclease-treated roots partially restores growth, at least for a few hours.

RNA is only broken down to a limited extent in the ribonuclease-treated roots; but RNA metabolism is certainly deeply affected: for instance, adenine incorporation in RNA is first stimulated, then inhibited, and free nucleotides increase. It seems that, in the treated roots, RNA becomes abnormal in constitution and unable to fulfill its role in protein synthesis; or that RNase combines with RNA to produce an inactive complex.

The inhibition of protein synthesis in ribonuclease-treated roots is not due to an indirect action of the enzyme on energy producing reactions, since the oxygen consumption and the ATP content remain essentially normal after $3 \mathrm{~h}$. treatment.

Still more interesting perhaps is the case of the amoeba A. proteus (Brachet, I 955c): low concentrations of ribonuclease (0.I $\mathrm{mg} / \mathrm{ml}$ ) are sufficient to produce loss of locomotion followed by cytolysis after $2-3 \mathrm{~h}$. If the amoebae are returned to their normal medium after I h., they show poor motility and ultimately die, even if they are well fed. But when the normal medium is fortified with yeast RNA ( $1.4 \mathrm{mg} / \mathrm{ml}$ ), complete recovery occurs in a given percentage of the amoeba population (20-50\% according to experimental conditions): shape, locomotion and ability to multiply if fed are almost normal in the amoebae which have recovered after the RNA treatment.

Cytochemical observations and chemical micro-analyses have conclusively shown that ribonuclease produces a marked drop $(20-45 \%)$ in the RNA content of the amoebae, the nucleoli and the cytoplasm being both affected (Fig. 7); simultaneously, free nucleotides increase; as in the onion root-tips, the oxygen consumption and the ATP content undergo little, if any, change. The RNA content of the a moebae which have recovered from ribonuclease treatment in an RNAcontaining medium, practically returns to normal. 
Thus the investigator is able, with amoebae, to modify almost at will the RNA content by suitable treatments with ribonuclease and RNA. What are the consequences of these changes in the RNA content on growth and protein metabolism?

Growth, as we have already seen, is directly related to the RNA content of the amoebae: the organisms which have had their RNA store depleted are unable to divide; after restoration of the RNA content, on the addition of external RNA, normal multiplication proceeds. Autoradiography observations on the incorporation of labelled phenylalanine into the proteins of the treated amoebae entirely support this view: as can be seen on Fig. 8, ribonuclease produces a considerable inhibition (about $90 \%$ ) of the incorporation; recovery of basophilia, on the addition of RNA, is usually followed by a parallel recovery of incorporation.

Very similar results have been obtained, in our laboratory, by Ficq and Errera (I955b) on starfish oocytes: treatment with ribonuclease produces a considerable drop of basophilia and a parallel inhibition of the incorporation of labelled phenylalanine, especially in the RNA-rich nucleolus and cytoplasm. However, in the starfish oocytes, the RNA-RNase complex breaks down if the treated cells are simply washed and left in normal sea-water: simultaneously, the incorporation of the labelled amino acids returns to normal.

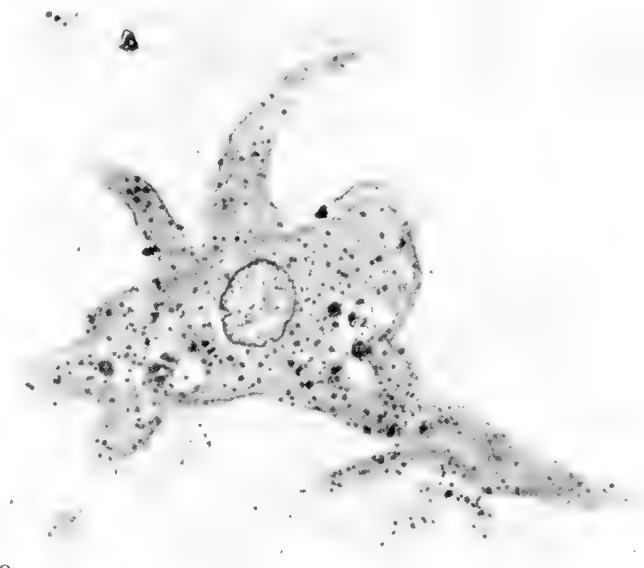

$7 a$

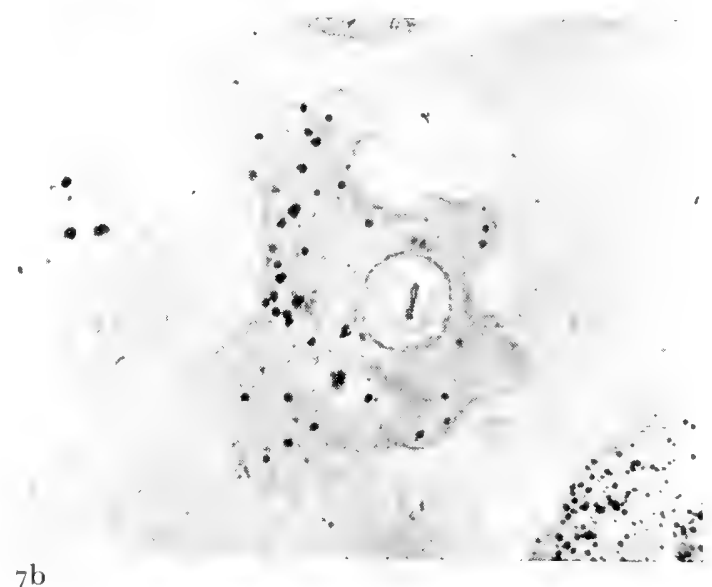

$7 \mathrm{~b}$

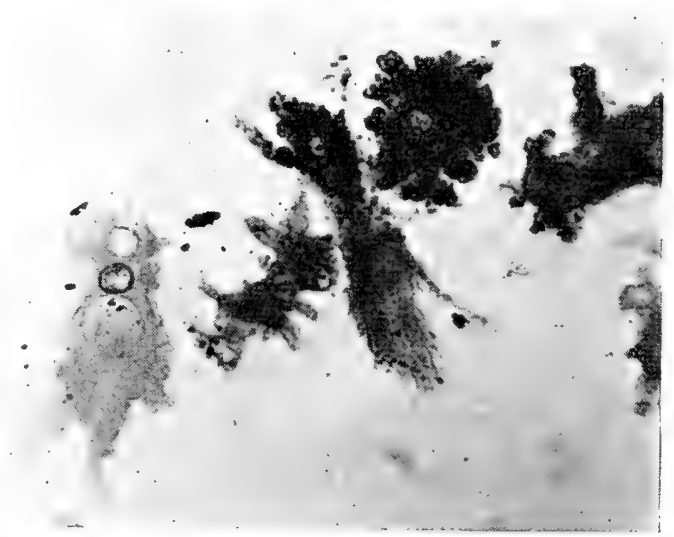
$7 \mathrm{C}$

Fig. 7. a: control amoeba; b: ribonuclease treated amoeba (I h., o.I $\mathrm{mg} / \mathrm{ml}$ ); c: recovery of the ribonuclease treated amoebae upon addition of RNA (Unna). 


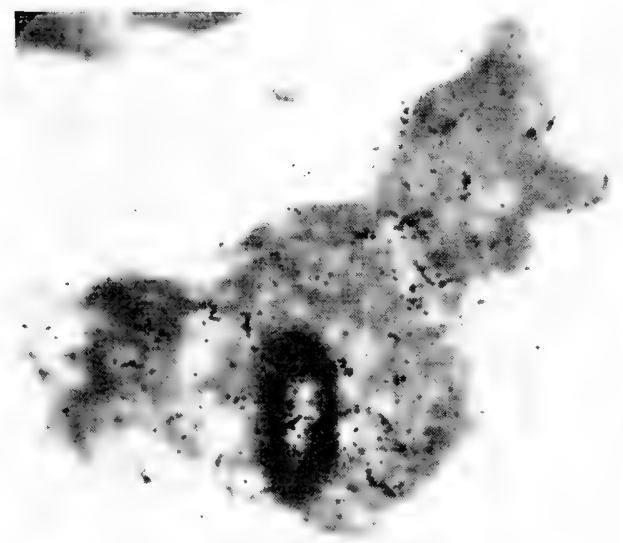

$8 a$

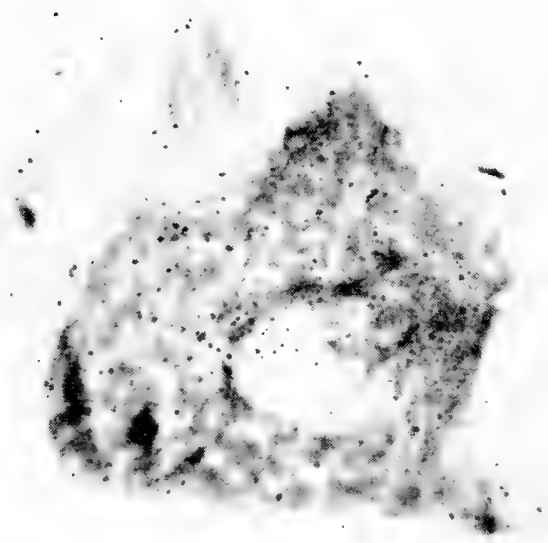

$8 \mathrm{~b}$

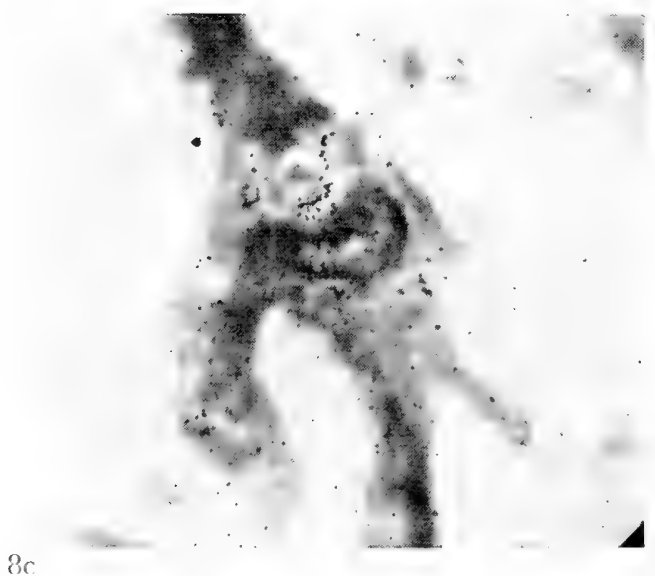

Comparable findings have also been made on frog oocytes (unpublished observations of A. Ficq) and on ascites tumour cells (Ledoux and Baltus, I954; Ledoux and Revell, I955); in the latter, ribonuclease induces an initial synthesis of RNA, followed by a rapid breakdown; but, even during the first synthetic phase, incorporation of the amino acids into the proteins is strongly inhibited.

These results of Ledoux et al. (I 954, I955) might very well have considerable importance for the chemotherapy of cancer: if growth is linked to protein synthesis and RNA metabolism, one might expect ribonuclease-if it enters the malignant cells - to inhibit the growth of tumours. In fact, recent experiments by Ledoux, ( 1955a, I 955b) show that the survival time of tumour-bearing mice is markedly increased when they are injected intraperitoneally with ribonuclease.

Ledoux's (I 954, I955b) experimental results obviously suggest that the antimitotic activity of ribonuclease, first discovered by Kaufmann and Das (1954, 1955), might be a fairly general phenomenon. Such an effect can very easily be demonstrated in amphibian eggs (Brachet and Ledoux, I 955). Simple immersion of cleaving urodeles eggs in ribonuclease ( 0.5 $\mathrm{mg} / \mathrm{ml}$ ) solutions is sufficient to quickly bring cell division to a standstill. Ribonuclease is most effective during early cleavage

Fig. 8. Autoradiographs after incorporation of phenylalanine into amoebae. a : control amoe$\mathrm{ba}$; b: ribonuclease treated amoeba (loss of basophilia and of incorporation); $\mathrm{c}$ : recovery of basophilia and incorporation in the ribonuclease-treated amoebae upon addition of RNA. 
Fig. 9. Block of mitotic activity in ribonuclease-treated morula of Pleurodeles (note marked swelling of the arrested interphase nuclei).

Fig. Io. Partial blastula after 4 h. ribonuclease treatment and culture in normal medium during $64 \mathrm{~h}$.

Fig. I I. Partial neurula after treatment with a ribonuclease-RNA mixture $(4 \mathrm{~h}$.) and culture in normal medium during $64 \mathrm{~h}$.

stages, probably because the enzyme can no longer penetrate into the eggs at later stages. As shown in Fig. 9, mitoses are blocked in interphase, probably because ribonuclease interferes with centrosomes or spindle development. Penetration of ribonuclease into morulae is slow; if the eggs, after a few hours treatment, are returned to a normal medium, mitosis is resumed in the internal cells which surround the blastocele. These cells may, after a few days, migrate through the irreversibly blocked external blastomeres and form an atypical ectoderm (Fig. Io). If the ribonuclease treatment is made less effective by the addition of RNA, one can observe the formation of a neural tube, laying on large, uncleaved and undifferentiated blastomeres (Fig. I I). In amphibian eggs, as in other cells, ribonuclease produces a strong $(85 \%)$ inhibition of the incorporation of labelled precursors into the proteins: it is worth mentioning that, according to the autoradiography observations, protein synthesis is chiefly limited to the nuclei during early cleavage. This synthesis of nuclear proteins is therefore very sensitive to ribonuclease.

Results, which entirely confirm our observations on amoebae, have

Literature p. 299
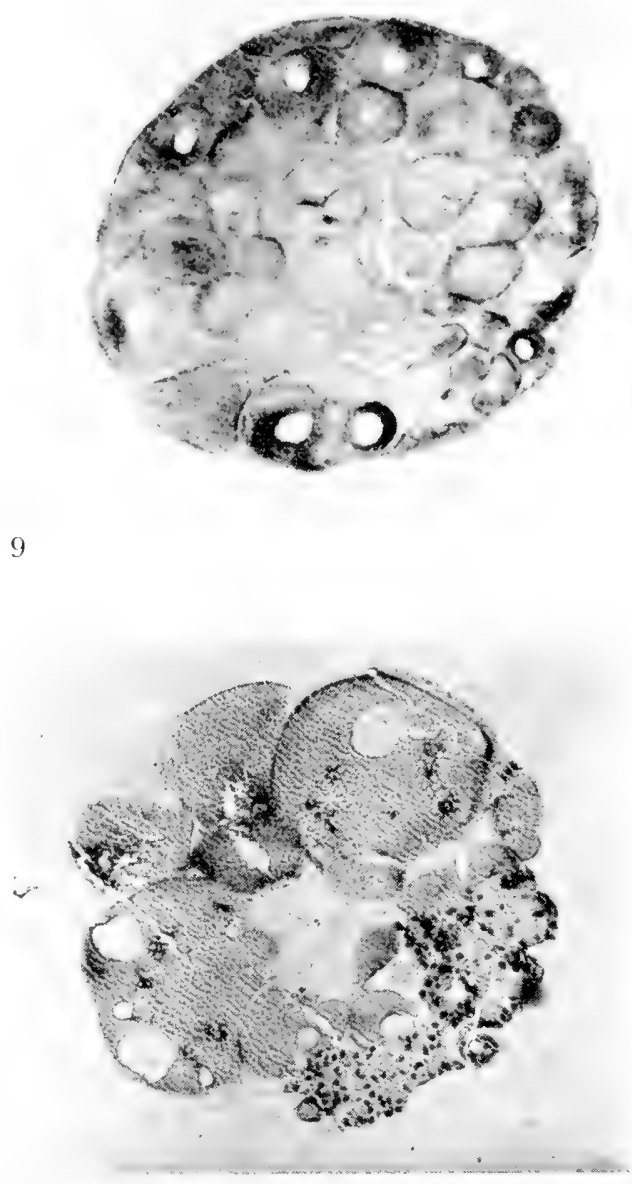

I O

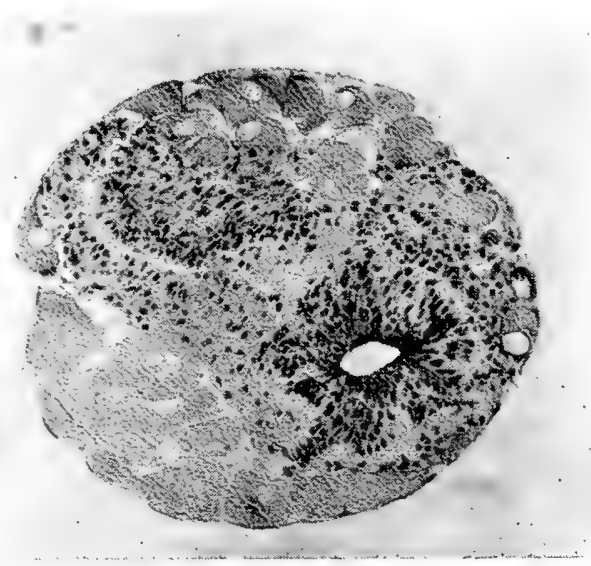


recently been reported by Chèvremont and Chèvremont-Comhaire (I 955) on tissue cultures of fibroblasts: it had already been found by Chèvremont and Firket ( 1952 ) that RNA stimulates the growth of slow-growing tissue cultures. In their recent experiments, Chèvremont and Chèvremont-Comhaire (r955) observed an inhibition of growth in cultures treated with ribonuclease (I/300-I/I,00o); as with the amoebae, addition of RNA restores the growth capacity of the ribonuclease treated cells. An interesting point in these investigations is that, according to quantitative cytochemical observations, ribonuclease induces a drop in the RNA content of the cytoplasm and the nucleoli, but that it does not interfere with DNA synthesis: the cells are blocked in early prophase and the DNA content of their nuclei is 4 times that found for spermatozoa.

Finally, valuable results have also been obtained with ribonuclease in the case of two RNA-containing viruses: tobacco mosaic virus (Casterman and Jeener, I 955) and influenza virus (Le Clerc, I 956). In both cases, multiplication is inhibited when the host cells (tobacco leaves and chorioallantoic membrane, respectively) are treated with ribonuclease prior or shortly after infection. Such findings lend additional support to Jeener's hypothesis on the genetic role of RNA: apparently, ribonuclease interferes with the virus multiplication at a stage of its development when RNA and protein are being synthesized independently.

To summarize, ribonuclease can penetrate in a number of living cells, without interfering with the energy producing mechanisms; the enzyme deeply modifies RNA metabolism. In all cases so far studied, growth, mitotic activity, protein synthesis and incorporation of amino acids into proteins are drastically inhibited. Addition of RNA, in many instances, alleviates to a certain extent the effects of ribonuclease. The experimental facts, which require further and deeper analysis, strongly indicate that RNA integrity is essential for growth and protein synthesis.

\section{(d) The mechanisms of protein synthesis}

It is beyond the scope of this chapter to discuss at length the many hypotheses which have been proposed to explain protein synthesis; a very short summary of what appears to be the most likely hypothesis at present will be given and the reader is referred for further details to two recent reviews (Brachet, I955a; Borsook, I 955).

The main problem, for the biologist, is the mechanism of formation of specific proteins; only the so-called template hypothesis, for which there is no substitute so far, can achieve this aim. The hypothesis postulates the existence of a model (template) under the influence of which the building blocks are arranged in the right order. The template would form a mould, constituting the counterpart of the protein to be formed.

In most of the recent versions of the template hypothesis, the template is identified with RNA and the building blocks with free amino-acids. According to the hypothesis, a great number of specific RNA's exist, each one being the counterpart of the specific protein which is to be synthesized.

The reasons why the hypothetical template might be identical with RNA are obviously based on the belief that RNA is essential for protein synthesis; there is, of course, no point in discussing them further now. The best available facts in favour of the identity of RNA with the template lay in Gale and Folkes' (r955b) 
observations, which have already been mentioned (p. 278): as will be remembered, they have definitely proved that small fragments (oligonucleotides), obtained by ribonuclease digestion of RNA, promote the incorporation of different amino acids in a relatively specific way. It has been suggested by Gale (1955) that "the factors isolated from ribonuclease digests represent specific groupings forming loci for the combination of specific amino acids, and bringing about exchange of those amino acids with corresponding residues in proteins of the preparation". Such an explanation might very well also be valid in the cases we have studied, where addition of RNA restored amino-acid incorporation in ribonuclease-treated living amoebae.

Recent work on microorganisms, especially the experiments of Spiegelman, Halvorson and Ben-Ishai (1955), provides strong evidence in favour of the view that free amino acids are the building blocks for protein synthesis: induced enzyme synthesis occurs de novo, directly at the expense of the free amino-acid pool of the cell; there is no indication for peptides intermediates in such cases. Most of the work done on animal tissues supports this contention [for instance that of Heimberg and Velick (I954) and of Askonas et al., (1955)]; however, experiments by Anfinsen and Steinberg (195 I, I952) on unequal labelling of proteins synthesized in vitro by tissue slices incubated in the presence of ${ }^{14} \mathrm{CO}_{2}$ are difficult to reconcile with this view. Their results might, of course, be due to the unphysiological in vitro conditions they adopted. On the whole, present evidence is very much in favour of the identification of the building blocks with the free amino acids.

The mere presence of RNA and amino acids is not, however, enough to synthesize proteins: as pointed out repeatedly by many biochemists, especially Borsook (1955), Lipmann (1949), Chantrenne (1953) and Siekevitz (1952), an energy source, presumably ATP, should be available to the template. How and when this energy source comes into the picture is not clear, and only hypotheses can be made: one possibility, which has been proposed by Chantrenne (1953) and discussed recently in detail by Borsook (I 955), is that amino acids are activated prior to their fixation on the template. This activation might well be a phosphorylation at the expense of ATP, since we know from Chantrenne's ( I953) work that synthetic phosphorylated amino acids easily react with ordinary amino acids to form peptides. Another possibility, which has been suggested by Dounce ( 1952), is that the template itself, i.e. RNA, is phosphorylated and activated by ATP: the usual free amino acids would attach themselves on the activated template, their position being determined by specific nucleotide arrangements in the RNA molecule. Further discussion of this question, in view of the scantiness of experimental evidence, seems to be fruitless for the time being ${ }^{1}$.

\section{NUCLEIC AGIDS AND EMBRYONIC DEVELOPMENT}

(a) Synthesis of DNA and RNA during development

Early work by Brachet (I933) has shown that unfertilized eggs always contain a store of RNA and that a marked DNA synthesis occurs during development;

1 More recent developments in this field will be found in a paper by Hoagland, Zamecnik and Stephenson (1957). 
since, in experiments on sea urchin eggs, the RNA content seemed to decrease when DNA was synthesized, it was concluded that part of the RNA store is converted into DNA. Such a conclusion should now be rejected, because later work by Schmidt et al. (I948), Villee et al. (I949) and Elson et al. (I954) has demonstrated, with improved methods, that the RNA content of sea urchin eggs remains essentially constant during early development. The analytical data obtained by Brachet (I933) were probably inaccurate because RNA was estimated as furfural after acid hydrolysis; it was not realized at the time that the jelly coats, which surround the unfertilized eggs and which dissolve at later stages of development, produce large amounts of furfural on hydrolysis.

According to Elson et al.'s (I954) most recent estimations for sea urchin eggs, the nucleotide distribution in RNA does not change during development, even when the eggs are treated with $\mathrm{LiCl}$ in order to produce morphogenetic abnormalities. The RNA content, however, varies markedly during morphogenesis: an initial rapid drop occurs after fertilization, then a first rise is observed during cleavage; a second rise is found just before the onset of gastrulation, and might be associated with protein synthesis: the importance of gastrulation, as the initial stage of new protein synthesis, has been emphasized by several workers (Brachet, I 949a; Perlmann and Gustafson, I948; Gustafson and Hasselberg, I 95 I).

According to our co-worker Steinert (I 95 I, I 953), the RNA content of Amphibian eggs remains essentially constant until gastrulation: it then steadily increases, presumably in connection again with increased protein synthesis. Inhibition of development by metabolic poisons like dinitrophenol or usnic acid is accompanied with a block in RNA synthesis; both phenomena are simultaneously reversible.

Isotope experiments by Villee et al. (I949) and by Abrams (I95I) have further indicated that, in sea urchin eggs, RNA cannot be the precursor of DNA. According to Abrams (I95I), the DNA purines synthesized during development have Io times the specific activity of those of RNA, which are therefore ruled out as precursors. Abrams (I95I) finds, however, that the great majority of the DNA synthesized by sea urchin eggs $(70-81 \%$ ) comes from a precursor pre-existing in the egg; this precursor might or might not be RNA. It might even be that all of the synthesized DNA comes from pre-existing precursors, because insufficient caution has been taken in Abrams' ( $195 \mathrm{I}$ ) experiments to avoid contamination of the embryos by other organisms (bacteria and unicellular algae). Growth of these organisms, which are difficult to separate from the eggs, can be extremely rapid as soon as even a small fraction of the eggs are cytolysed during culture: it is entirely possible that all of the tagged DNA found by Abrams (I 95 I) comes from such contaminations.

If we exclude RNA as a precursor of DNA, from where does the latter originate? Recent experiments by Hoff-Jorgensen (1954) and by Elson et al. (I954) indicate that the unfertilized sea urchin eggs contain a DNA reserve in the cytoplasm: the microbiological techniques used by these workers indicate that the sea urchin eggs contain some excess DNA, amounting to $5^{-15}$ times the diploid DNA content. According to Hoff-Jorgensen (I 954), the DNA content in the sea urchin embryo remains constant from the unfertilized egg to the 16 -cell stage.

Similar results have been obtained, again with sea urchin eggs, by Marshak and 
Marshak (1953), who used an isotope dilution technique; bu1, after further analysis, Marshak and Marshak (1954a, I 955) have arrived at very different conclusions. Taking into account the presence of contaminating ovarian cells and polar bodies, they come to the rather unexpected view that sea urchin and starfish eggs contain no DNA at all! A startling consequence of these findings is that DNA cannot have a primary genetic role. According to Marshak and Marshak ( I 955), DNA probably plays a regulating role and competes with RNA in cellular metabolism. Marshak and Marshak (1954b) find further evidence for the absence of any DNA in sea urchin eggs in the fact that ripe unfertilized eggs contain $96.5 \%$ of their thymine in an acid-soluble form: there apparently is a breakdown of the ovarian DNA at the time of maturation. Finally, Marshak and Marshak (i 954a, I 955) have repeatedly emphasized the fact that the egg nucleus, i.e. the female pronucleus, is Feulgen negative and therefore contains no DNA.

It is difficult at the present time to draw definite conclusions from such conflicting evidence; there is no doubt that Marshak and Marshak (I954a, I955) are right when they emphasize the importance of contaminating ovarian cells and polar bodies. As a matter of fact, the same point was made long ago by Brachet (1944a) when criticizing an early claim by Blanchard (1935) that DNA is present in large amounts in unfertilized sea urchin eggs. On the other hand Marshak and Marshak's (I 954a, I 955) conclusions are based on calculations assuming that 3 polar bodies are eliminated, without giving cytological evidence for this opinion: in many species, only 2 polar bodies are eliminated, the first one remaining uncleaved. Finally, the statement that the female pronucleus is Feulgen negative cannot remain unchallenged: it is true that no Feulgen staining material could be found by Brachet (1933), nor by Mirsky and Ris (1949). But recent unpublished observations have convinced the author that the nucleus of unfertilized sea urchin eggs actually contains a shell of Feulgen positive material, just under the nuclear membrane; the centre of the nucleus remains unstained. These observations have been made repeatedly on two different species, using the best available conditions for fixation, staining and microscopical observation. Similar findings, on another species of sea urchin, have also been recently reported by Burgos (i 955 ).

In conclusion, the author is convinced that sea urchin eggs contain DNA in their nucleus, just as any other cell, and that Marshak and Marshak's (I954a, I955) sweeping conclusion about the genetic role of DNA should be regarded with considerable scepticisnı. On the other hand, their work clearly indicates that sea urchin eggs contain very little, if any, DNA store.

The situation is also very puzzling when other species are investigated for the presence of a DNA store in unfertilized eggs: in frog eggs (Hoff-Jorgensen and Zeuthen, I 952; Hoff-Jorgensen, I 954), the DNA content is said to remain constant until the late blastula stage, where rapid synthesis occurs. According to the Danish authors, the unfertilized egg contains enough DNA, as an initial reserve, for about 5,000 cells. This reserve is much larger in eggs of hens (Hoff-Jørgensen, I 954), since no DNA is synthesized until the fourth day of incubation, when the embryo contains $c a \cdot 5^{\circ} \mathrm{I}^{7}$ diploid cells.

It is obvious that the true significance of these results entirely depends on the 
absolute specificity of the methods used: the larger the egg, the more difficult becomes the analytical problem of estimating very small amounts of DNA in the presence of enormous quantities of foreign substances. In our own experience (Brachet, I $954^{\mathrm{b}}$ ), eggs of frogs contain small amounts of substances which give colour reactions for deoxypentose and yield small quantities of thymine on hydrolysis: but these substances appear to be bound to the yolk platelets, which become digested at a late stage of development only: it is difficult to visualize how they could be utilized as DNA precursors during early cleavage. In the egg of the hen, it is also somewhat perplexing to find such a large store of DNA, when we know from the work of Spratt (1948) that the embryo can grow and differentiate in a medium containing only glucose as a carbon source. It is certain that Spratt's explanted embryos undergo an extensive DNA synthesis: it is probable that they are capable of a total DNA synthesis and that they are not dependent on a preexisting DNA reserve (Solomon, I957).

It should be added that recent work by Levenbook et al. (I 955) has conclusively shown that Drosophila eggs contain large amounts of free purines and pyrimidines, ribosides, deoxyribosides (especially uracil deoxyriboside) which might be effective precursors of DNA (grasshopper eggs contain important amounts of DNA [Durand, r 955]). Insect eggs might, however, prove to be a special case, because of the absorption of nurse-cells, including their nuclei, by the growing oocyte.

Much more experimental work is obviously needed before any conclusion can be drawn: recent investigations suggest the presence, in unfertilized eggs, of relatively large amounts of DNA precursors. They may range in complexity from free purines, especially hypoxanthine, (Steinert, I952, for frog eggs and Hultin, I 953, for sea urchin eggs) to large DNA-resembling molecules. It certainly will be of considerable interest to know whether kinetin, the DNA derivative which promotes cell division in plant tissues according to Miller et al. (I 955), is also present in unfertilized eggs.

\section{(b) $R \mathcal{N A}$ and morphogenesis}

This problem will just be outlined here, since the all-important question of organizers will be treated by Dalcq (see Chapter 4); furthermore, the question of the role of RNA in morphogenesis has been discussed at some length by Brachet (I952) in a fairly recent monograph.

Cytochemical observations on the distribution of RNA during development of Amphibia show a distinct polarity gradient in unfertilized, fertilized and cleaving eggs: the amount of RNA progressively decreases from the animal to the vegetal pole.

At gastrulation, a secondary RNA gradient, decreasing from dorsal to ventral superimposes itself on the primary animal-vegetal gradient. The interaction of the two, during gastrulation and neurulation, leads to the appearance of welldefined cephalo-caudal (in the nervous system and the notochord) and dorso-ventral (chordomesoblastic) RNA gradients.

At still later stages, just before differentiation of the organ begins, a marked increase in basophilia is conspicuous.

The existence of the early vegetal-animal, dorso-ventral and cephalo-caudal 
gradients in the blastulae, gastrulae and neurulae has been confirmed by quantitative estimations (Brachet, I94Ib; Steinert, I95I; Takata, I953; Chantrenne and Brachet, 1946).

It should be added that these gradients are strikingly parallel to the hypothetical morphogenetic gradients of the experimental embryologists; it would, however, be a mistake to believe that morphogenetic gradients are nothing else but RNA gradients, since a similar distribution has been found for many other substances: SH-groups linked to proteins (Brachet, I940), reducing power (Fischer and Hartwig, 1936; Piepho, I938; Child, I948) alkaline phosphatase (Krugelis, I 947), oxygen consumption (Sze, I 953), dipeptidase (Barth and Sze, I 953). On the other hand, there is no dorso-ventral gradient, at the gastrula stage, for total proteins and lipids (Barth and Sze, I953; Gregg and Lovtrup, 1950). Since we know that RNA and SH-groups are associated mostly with small cytoplasmic granules, the microsomes, while respiratory enzymes are concentrated in the mitochondria, we can hardly escape one conclusion: that the biochemical gradients which are parallel to the morphogenetic gradients are, in fact, gradients in the distribution of cytoplasmic particles.

If RNA synthesis and distribution in gradients are important morphogenetic factors, one would expect experimental alterations of RNA metabolism to interfere with morphogenesis: this has been found to be the case in all instances studied so far.

It has been shown, for instance, that chemical analogues of purines and pyrimidines, which slow down nucleic-acid synthesis, completely stop morphogenesis at stages which are characteristic for each compound (Brachet, 1944a, I946; Bieber, I954, for amphibian eggs; Fox and Goodman, I 953; Waddington et al., I 955, for chicken embryos). In most cases, the effect is reversible when the embryos are placed in normal medium; but, in the chicken, inhibition by the purine analogue azaguanine is only reversed on the addition of hypoxanthine. Furthermore, the regions of the embryo which are most sensitive to the inhibitors are generally those where incorporation of labelled amino acids into the proteins is most active (Waddington et al., I955). Similar results have been obtained with acriflavine (Brachet, I946), which precipitates nucleic acids in vitro: reversibility of development is improved by addition of RNA or adenylic acid. (For further data on analogues and growth see Chapter 5 ).

As already mentioned before, poisons of oxidative phosphorylations like dinitrophenol and usnic acid inhibit RNA synthesis and morphogenesis simultaneously in Amphibians; if development is allowed to proceed by removal of the inhibitor, RNA synthesis is resumed, and it runs parallel with morphogenesis (Steinert, I 953).

Another interesting case is that of female sex hormones, which, according to Cagianut (1949), affect the RNA distribution in amphibian eggs; the RNA gradients are altered during early cleavage and, as a consequence, development becomes highly abnormal and leads to asymmetrical embryos. It is noteworthy that addition of yeast RNA definitely improves the development.

Physical agents are no less interesting, in this respect, than chemical substances: for instance, centrifugation of just fertilized eggs strongly modifies the initial RNA polarity gradient and leads to more or less complete failure of development 
(hypomorphic embryos). On the other hand, centrifugation of blastulae leads to the formation of double embryos; again the duplication can be linked to abnormal distribution and synthesis of RNA (Brachet and Pasteels, in Brachet, I952b).

The study of effects of heat shocks on morphogenesis and RNA synthesis also indicates a close correlation between the two processes: heating frog gastrulae at $3^{6.6^{\circ}}$ for I h. irreversibly stops development, although the embryos do not cytolyze until 2 or 3 days later; heating at a slightly lower temperature $\left(36.2^{\circ}\right)$ produces only a temporary block of development, followed by the appearance of morphogenetic abnormalities (Brachet, I 948, I949a). Cytochemical observations (Brachet, I948) and chemical quantitative estimations (Steinert, I953) show that RNA synthesis is completely stopped after an irreversible heat shock; when the effects of heating are reversible, RNA synthesis is resumed as soon as development starts again. Furthermore, if a fragment of an irreversibly blocked gastrula is grafted into a normal embryo, it differentiates again after a lag period; simultaneously the basophilia of the heated cells begins to increase. It seems that heating destroys substances necessary for morphogenesis and that these substances could diffuse from the normal host to the graft. Biochemical observations (Brachet, I949b) have shown that the RNA-containing microsomes are especially sensitive to heating: in the heated gastrulae, a large proportion of the RNA is no longer bound to the microsomes and is found in the supernatant fraction after ultracentrifugation.

These observations clearly indicate that morphogenesis is closely linked to RNA distribution and synthesis in developing embryos. But it would be, as already mentioned, an error to believe that RNA is the only factor involved in the experiments which have just been mentioned: it should be emphasized once more that RNA is only a constituent of complex cytoplasmic particles, the microsomes; their main function, as far as we know, is protein synthesis (Brachet and Jeener, 1944; Allfrey et al., I 953; Pollister, I 953). RNA alone is unable to synthesize proteins; similarly RNA alone can not induce morphogenesis, i.e. act as an organizer.

The erroneous view that RNA plays the leading role in the induction of nervous systems by abnormal inductors has been put forward by Brachet (1944a, I945) on the basis of the following experimental findings: ribonucleoproteins, especially after alcohol treatment, are very good inducing agents when they are grafted into amphibian gastrulae; this inducing activity is considerably reduced if they are treated with ribonuclease prior to implantation. Later work by Kuusi (1953), Brachet, Gothié and Kuusi (1952) and, more recently, by Yamada and Takata (1955) has confirmed that ribonucleoproteins (microsomes or tobacco mosaic virus) are excellent inducing agents; but, in these new experiments, it was found impossible to abolish the inducing activity by ribonuclease treatment (see also Engländer et al., I953). Since the ribonuclease used by Brachet in his early experiments (1944a, I 945) was a rather crude preparation and since Toivonen (1949) and Yamada and Takata (1955a) have found that a slight digestion of the protein part of the ribonucleoprotein with trypsin or chymotrypsin is enough to completely inhilit the induction, there is little doubt that the effects observed earlier were due to partial proteolytic degradation of the implants, rather than to removal 
of the RNA. Confirmation of this conclusion can be found in recent papers from Yamada's laboratory (I 955, I 955a, I 955b) : they definitely show that, in kidney, trypsin, but not ribonuclease, abolishes the inducing activity, while RNA itself is inactive.

One can conclude that, in the case of abnormal inductors, RNA is not necessary for the activity: the sensitivity of these inductors to proteolytic digestion, and the fact that their activity disappears when $\mathrm{NH}_{2}$-groups are blocked with formalin or ketene (Lallier, 1950; Kuusi, I953; Smith and Schechtman, I954) strongly suggests that the inducing activity is linked to the proteins rather than to RNA.

How far conclusions drawn from abnormal organizers, killed tissues for instance, are valid for the normal, living organizer is a very debatable question. There is no doubt that, as shown by Holtfreter (1947), any agent which induces a condition of sublethal cytolysis in the ectoderm promotes its spontaneous neuralization. But we do not know yet whether the sublethal agents and the normal organizer produce the same biochemical changes in the ectoderm. Recent experiments by Holtfreter (1955) indicate that killed tissues liberate substances, which can diffuse through cellophane membranes and perhaps act on the ectoderm as cytolyzing agents; such a diffusibility is quite different from what has been found in the normal inductors whose action is stopped by a cellophane membrane (Brachet, I950; McKeehan, I 95I).

These findings suggest that, in contrast to what happens in abnormal inductors, normal induction is mediated by large molecular aggregates, perhaps cytoplasmic granules like microsomes and mitochondria.

Recent work by Niu and Twitty (1953) lends some support to the view that, in the case of the normal organizer, nucleoproteins play indeed an important part: they found that explanted chordomesoderm produces in the medium a neuralizing agent. Niu and Twitty (1953) exclude the possibility that the neuralizing activity is due to mere toxicity, since no signs of sublethal cytolysis can be detected. If ectoderm is cultivated in the medium in which chordomesoderm has been explanted for a few days, it differentiates nerve fibers and pigment cells. An interesting hint is that the culture medium shows a strong U.V. absorption typical of nucleic acids.

A somewhat comparable finding has been reported by Levi-Montalcini and Hamburger (I953): mouse sarcoma contains a diffusible agent that produces hyperplasia of sympathetic ganglia. When this agent is purified, it is found to be entirely concentrated in the microsomes; like other ribonucleoproteins, it is precipitated by streptomycin and it strongly absorbs U.V. (Cohen, Levi-Montalcini and Hamburger, r954).

It is obvious that much more experimental work is needed before we can assess the real place of RNA in morphogenesis. While RNA is certainly not necessary for induction by abnormal inductors, there is a considerable amount of circumstantial evidence for a role of ribonucleoproteins, probably microsomes, in normal induction by the living organizer.

It is unlikely that it will ever be possible to demonstrate a role of RNA in normal induction by direct experiments, such as treatment of the living organizer with ribonuclease: the author has performed many experiments of that type, but the 
results have been so disappointing that they have not yet been published. Ribonuclease has no visible effect on gastrulae or explanted dorsal lips in many species; apparently the enzyme does not get into the cells at that stage. With some Urodeles, ribonuclease dissolves the surface coat (Holtfreter, I 943) which unites the cells: the ectoderm is progressively pealed off and induction is of course deficient or absent in these ectodermless embryos. But the failure of induction is due to the destruction of the reacting ectoderm rather than to a direct action on the inductor, since chorda and somites can differentiate normally in the treated embryos. An interesting point, however, is that the disintegrating action of ribonuclease on the surface coat is highest in the regions which are richest in RNA: a clear differential susceptibility is evident in this case.

\section{(c) The effects of an abnormal nuclear composition on nucleic acids and development}

Abnormalities of nuclear composition (hybridization, haploidy, polyploidy, etc.) lead to interesting abnormalities of development. Since the whole subject has recently been reviewed in detail by Moore (I 955), we shall deal only with the very few cases where nucleic-acid metabolism has been studied.

Of greatest interest are the diploid lethal hybrids in Amphibians: in the lethal combinations Rana esculenta $\$ \times$ Rana fusca 5 (Brachet, I944b) and Rana pipiens $\$$ $\times$ Rana sylvatica (Moore, I 946, I 947, I 948), the introduction of the foreign sperm nucleus is followed by normal cleavage; but development stops entirely at the onset of gastrulation and the blocked embryos cytolyze a few days later.

Just as in the heat-treated eggs, the dorsal lips of the lethal hybrid amphibian gastrulae resume further development and show inducing activity if they are grafted into normal embryos (even from a remote species, such as a Urodele). The inducing ability of the lethal hybrid's organizer seems, however, to be somewhat reduced (Brachet, I 944a; Moore 1946, I 947, I 948). It looks as if the presence of a foreign nucleus made it impossible for the hybrid to produce substances necessary for further development; these substances easily diffuse from the host to the graft which is "revitalized".

Cytochemical observations (Brachet, I 944a) have shown that RNA synthesis does not occur in the arrested lethal hybrids, although abnormal amounts of RNA may accumulate in their nuclei (Brachet, I954b). When a piece of the blocked gastrula is grafted in a normal host, the basophilia of the nucleoli and the cytoplasm markedly and steadily increases when morphogenesis is resumed: the close link between RNA synthesis and development is especially obvious in this case. These cytochemical observations have been entirely confirmed, in the lethal combination $R$. esculenta $\$ \times R$. fusca by the quantitative estimations of Steinert (195I): there is almost no RNA synthesis in the blocked embryo, while the controls steadily increase their RNA content.

Comparable results, in the Urodele combination Triton palmatus $\$ \times$ Salamandra atra 5 , have been reported by Shen (1954), who even found a drop in the RNA content of the lethal embryos at the time lethality sets in. This drop might be due, as shown by cytochemical observations of Baltzer and Schönmann (I95I) and Baltzer (I 952), to the fact that many cells die at this stage in the Triton $\times$ Salamandra hybrids; this is not the case in the Rana hybrids we studied. 
The problem of DNA synthesis in lethal hybrids is obviously of even greater interest; but it is a difficult one, because of the already mentioned difficulties in estimating accurately the DNA content during early development.

DNA synthesis in Rana fusca has been studied in eggs fertilized with sperm previously treated with nitrogen mustard; they display exactly the same type of lethality as the hybrids (Brachet, I 954b). The arrested blastulae have a $34 \%$ lower DNA content than the control; afterwards, although development is entirely blocked, a measurable DNA synthesis $\left(24^{\circ}\right.$ increase) occurs during 2 days. In the normal controls, which reach the early neurula stage, DNA synthesis is more pronounced $(33 \%)$. Similar results have been obtained by Shen (1954) on his Triton $\times$ Salamandra hybrids: DNA synthesis in the lethal hybrid is definitely reduced, but not entirely stopped.

All these data come from experiments where DNA was estimated with colorimetric methods, which are unspecific and not very sensitive; but it is interesting to note that the same conclusions have recently been reached by Gregg and Lovtrup (I 955), who used the microbiological method which enabled Hoff-Jorgensen (I954) to study DNA synthesis during normal development of frog eggs. Their experiments show that the DNA content of normal Rana pipiens embryos remains apparently constant until the end of segmentation, at which point synthesis occurs at a constant rate. In the lethal Rana pipiens $q \times$ Rana sylvatica hybrids, synthesis of DNA is practically normal, even though development has stopped in the gastrula stage; the DNA content of the hybrids ceases to increase further when it has reached the value characteristic for controls at the neural fold stage.

It is clear, from the experimental data, that one simple hypothesis of the mechanism of lethality has been disproved: if the eggs contain, as Hoff-Jorgensen ( 1954 ) believes, a store of DNA or DNA precursors, one might have expected the lethal embryos to stop development when their DNA reserves are exhausted. This is obviously not the case, since DNA synthesis continues in the blocked embryos of all the lethal combinations so far studied.

These experiments further show that, while $R \mathcal{N A}$ synthesis and morphogenesis are closely linked together, there is a dissociation between DNA synthesis and development: DNA synthesis still proceeds when development has stopped. Other instances of dissociation between DNA synthesis and growth, obtained on very different material, will now be examined.

\section{VI, FINAL REMARKS}

\section{(a) Independence of DNA synthesis and protein synthesis}

We already know that RNA is directly involved in the formation of proteins and that, in microorganisms at least, synthesis of new RNA molecules is a compulsory concomitant of induced enzyme synthesis (Pardee, I 954, I 955; Spiegelman et al., I955; Creaser, I 955a, I955b; Chantrenne, I956). It is clear that the same questions may be asked for DNA as for RNA: is synthesis of DNA necessary for protein synthesis? Does DNA play a direct role in protein synthesis?

The answer to these questions comes entirely from biochemical studies on microorganisms, except for the already mentioned fact that, in blocked lethal 
hybrids, DNA synthesis can go on in the absence of protein synthesis, RNA synthesis and growth. Present evidence for micro-organisms indicates that the converse situation is possible, i.e DNA synthesis can be inhibited without interference of protein synthesis, RNA synthesis and growth; furthermore, inhibition of protein and RNA synthesis without any effect on DNA synthesis, as in the lethal hybrids, can be obtained when bacteria are treated with a chemical analogue of phenylalanine (Pardee and Prestidge, 1955).

Cytological observations by Jeener and Jeener (1952) have shown that, in Thermobacterium acidophilus, suppression of DNA in the culture medium stops nuclear multiplication, but not growth: the result is the formation of filamentous bacteria, containing only a small number of nuclei. When uracil is absent from the medium, synthesis of RNA is stopped: the result, this time, is an inhibition of growth.

A more complete biochemical analysis of a comparable case has been made by Cohen and Barner (I954), who studied the effects of thymine deficiency on a thymine-requiring mutant of Escherichia coli: the organism loses the power to form colonies, and this type of sterilization is accompanied by a marked increase in bacterial length and girth and doubling of the RNA content; but there is almost no synthesis of DNA.

Of special interest is the fact that these bacteria, which lack an appreciable DNA synthesis, are nevertheless capable of induced enzyme synthesis (synthesis of xylose isomerase in response to the presence of xylose in the medium). These experiments clearly show that cytoplasmic and induced enzyme synthesis are possible in the absence of appreciable DNA synthesis.

Similar conclusions are reached when the effects of various physical and chemical agents on bacteria cultures are studied : for instance, Kelner ( I 953) and Kanazir and Errera ( I 954) have independently shown that low doses of U.V. light have little effect on RNA synthesis and growth, although they completely stop DNA synthesis. As in the case studied by Jeener and Jeener (1952), the result is the appearance of filamentous bacteria.

It should however be pointed out that, according to Swenson ( I950), U.V. light inhibits the induced synthesis of galactozymase in yeast, the action spectrum being similar to a nucleic acid absorption spectrum. This finding, together with that of Kelner (1953) and Kanazir and Errera (1954) on the immediate inhibition of DNA synthesis by U.V. light, might point toward a direct nuclear control of induced galactozymase synthesis. But more recent investigations of Halvorson and Jackson (I954) indicate that the U.V. doses used by Swenson were too high to obtain a dissociation between DNA synthesis and growth; they further found that marked effects on protein synthesis are obtained when slight alterations occur in the RNA molecules. The conclusion of Halvorson and Jackson (I 954) is, again, that suppression of DNA synthesis does not inhibit protein synthesis; the latter is, on the other hand, strongly dependent on the integrity of the RNA molecules.

It is a well established fact that DNA and DNA synthesis are much more sensitive than RNA to such agents as X-rays and mustards: it is therefore not surprising that Baron et al. (1 953) found X-rays to be ineffective in inhibiting induced enzyme synthesis and that Sher and Mallette (1954), Pardee (I954) and Gros et 
al. (I955) came to similar conclusions in the case of sulfur and nitrogen mustards. The evidence which has just been reviewed shows conclusively that protein synthesis is possible in the absence of D.VA synthesis; RNA synthesis is also independent of DNA synthesis. But RNA and protein synthesis, on the other hand, are very closely linked together.

We now come to the second question: does DNA play a direct role in protein synthesis? As compared to the wealth of information we have regarding the role of RNA in protein synthesis (see section IV of this Chapter, p. 272), the present evidence is still meagre.

Definite indications in favour of a direct role of DNA in protein synthesis come from the work of Gale and Folkes (1954, I955a); they found that either RNA or DNA are able to restore the ability to incorporate amino acids into the proteins of disrupted Staphylococci whose nucleic acids had previously been removed. As a matter of fact, DNA is even more effective than RNA in promoting amino-acid incorporation into the proteins of the nucleic acid-depleted cells, regarding both the rate and the final amount of incorporation attained. It is for this reason that Gale and Folkes (1954) believe that DNA, while not capable of synthesizing proteins itself, might bring about exchange reactions in the proteins; DNA would act as an organizer for RNA synthesis, which in turn would catalyse protein synthesis.

A different type of experiment, by Allfrey (1954) and by Mirsky (I956), leads to similar results and conclusions: thymus nuclei isolated in sucrose are able to incorporate labelled amino acids into their proteins, the process requiring the presence of oxygen. The important fact is that the incorporation reaction is dependent on the presence of DNA, since breakdown of DNA by the enzyme deoxyribonuclease (DNase) strongly inhibits the amino acid uptake. Addition of thymus DNA to the DNase-treated nuclei restores the activity to some extent. These interesting observations lead Mirsky (1956) to the conclusion that protein synthesis in the cytoplasm depends upon the presence of RNA, while it depends on DNA in the nucleus.

The validity of this conclusion will be discussed after reviewing what is known about the role of the nucleus in growth and protein synthesis.

\section{(b) The role of the cell nucleus in growth and protein synthesis}

We shall chiefly deal with experiments performed on nucleated and non-nucleated fragments of unicellular organisms. The most interesting case is perhaps that of Acetabularia mediternanea, because of its unusual ability to regenerate in the absence of the nucleus (Hämmerling, I934). Hämmerling's (I934) experiments clearly show that non-nucleated fragments of this giant unicellular alga are capable of prolonged survival and considerable regeneration: there is no question that, in this rather exceptional case, growth and even morphogenesis are possible for quite a long time in the absence of the nucleus. According to our own observations (Brachet, Chantrenne and Vanderhaeghe, I955), it takes 3 weeks at least before the regenerative power disappears from the non-nucleated pieces.

Extensive biochemical observations performed in this laboratory have led to the following main conclusions (Brachet, Chantrenne and Vanderhaeghe, r 955): non-nucleated pieces of Acetabularia are capable of incorporating labelled precur- 
sors (amino acids, orotic acid) into their proteins and RNA for several weeks. Their activity is normal during two weeks; afterwards, it begins to decrease and becomes inferior to that of the nucleated pieces. More important still is the fact that net synthesis of R. $\mathrm{VA}$ and proteins occurs in the non-nucleated halves; as a matter of fact, this synthesis is even faster than in the nucleated fragments during the first IO-I 5 days following the operation. However, synthesis steadily decreases after this period.

This finding is important because two popular hypotheses can now be ruled out: first, it is obvious that since independent RNA synthesis can occur in the cytoplasm, nuclear RNA is not necessarily a precursor of cytoplasmic RNA. Secondly, the fact that growth, RNA synthesis and protein synthesis occur in non-nucleated cytoplasm demonstrates that DNA is not directly concerned with these processes (no DNA could be detected in the non-nucleated pieces, even when using an isotope dilution technique).

The case of Acetabularia clearly shows that Gale and Folkes' explanation for the role of DNA in protein synthesis is not entirely correct: DNA can obviously not act as an organizer for the synthesis of RNA in non-nucleated Acetabularia fragments, since they contain no DNA. Nevertheless RNA and protein synthesis are very extensive. However, the possibility remains that the slowing down of growth, RNA and protein synthesis, which occurs when the fragments have been separated from the nucleus for more than ro days, is in some way linked to DNA. A comparable situation to that just described for Acetabularia is found in the reticulocytes, i.e. immature red blood cells which have lost their nuclei, but retained some of their RNA store. As shown by Borsook et al. (1 952), Holloway and Ripley (1952), Koritz and Chantrenne (I954), reticulocytes still incorporate amino acids into their proteins, as well as glycine into their RNA (Kruh and Borsook, (1955). They are even capable of synthesizing hemoglobin (Nizet and Lambert, I953) and various enzymes (Koritz and Chantrenne, I954). During the maturation of the reticulocytes, their RNA content steadily drops and an excellent correlation between RNA content and ability to incorporate amino-acids into the proteins has been found (Holloway and Ripley, I952, Gavosto and Rechenmann, I954).

Removal of the nucleus, in unfertilized eggs of the sea urchin (Malkin, I954) and the newt (Tiedemann and Tiedemann, I954) has no measurable effect on the incorporation of precursors into RNA and proteins. Net protein synthesis probably does not occur in unfertilized eggs: but, at any rate, the experiments demonstrate that the turnover of RNA and proteins does not require the presence of the nucleus in egg cells. However, it should be noted that the experiments of Malkin ( I954) and Tiedemann and Tiedemann (1954) do not necessarily rule out an intervention of DNA in the non-nucleated egg fragments, in view of the possible existence of a DNA store in the cytoplasm of unfertilized eggs (see section v, p. 286).

The only organism which has protein metabolism under close nuclear control is Amoeba proteus. According to Mazia and Prescott (I955), removal of the nucleus produces a six-fold decrease of the incorporation of methionine into the proteins, within only a few hours. After 2-3 days, non-nucleated halves are 20 times less efficient in this respect than nucleated fragments. Our own observations (Brachet, 1955d) on the same material, but with phenylalanine as a precursor, indicate a similar trend. But the differences are much smaller than in Mazia and 
Prescott's (1955) experiments: the activity of the nucleated halves is only twice that of the non-nucleated halves when the amoebae have been cut from $\mathrm{I}-6$ days. It can be concluded that, even in amoebae, protein metabolism is not under a direct control of the nucleus.

Amoebae are different from the other cells studied in that the non-nucleated fragments are unable to feed; perhaps as a consequence of this starvation, the RNA content of the cytoplasm drops rapidly after removal of the nucleus (Brachet, i $955^{\mathrm{a}}$; James, I 954).

To summarize: in all the organisms and cells studied so far, the nucleus only exerts a remote control on protein metabolism. RNA and protein, as should be expected, always run parallel: the extreme cases are $A$. proteus, where RNA content and protein metabolism quickly decrease, and Acetabularia, where net synthesis of RNA and proteins occurs in the absence of the nucleus.

The nucleus can thus no longer be considered as the only center of protein metabolism; but this does not mean that protein anabolism is negligible in the nucleus. On the contrary, the autoradiography observations of Ficq (1955), Ficq and Errera (1955a), Moyson (1955) and Sirlin (1955) clearly show that in growing oocytes (starfish, frog), in developing embryos and in mammalian liver, incorporation of amino acids is faster in the nucleus than in the cytoplasm. We do not know yet how general these findings are : but there is no doubt that in rapidly dividing cells and in protein-synthesizing cells, protein anabolism in the nucleus itself is far from negligible.

Among the constituents of the nucleus, it is the nucleolus which shows the highest activity: in young starfish oocytes, according to Ficq (I955), incorporation of amino acids into the nucleolar proteins can be $\mathrm{I}$ oo times greater than in any other part of the cell. In the nucleolus, as elsewhere, RNA is intimately concerned with protein anabolism: if the living oocytes are treated with ribonuclease, inhibition of amino-acid incorporation is strongest in the nucleolus (Ficq and Errera, r 955 b).

This very high activity of the nucleolus in protein metabolism probably explains why removal of the nucleus in Acetabularia accelerates protein and RNA synthesis: it is likely that the very large and basophilic nucleolus, in this alga, efficiently competes with the cytoplasm for the uptake of the precursors required for the synthesis of ribonucleoproteins.

A consequence of this important role of the nucleolus in protein synthesis is that Mirsky's (1956) suggestion that protein synthesis depends on DNA in the nucleus possibly is an oversimplification: Mirsky's ( 1956) statement might well be true for the thymus nuclei he studied, because their nucleoli are inconspicuous. It is very unlikely that in protein-synthesizing cells where, as emphasized by Caspersson ( $194^{\circ}$, I 950), the nucleoli are always very large, RNA is not also of great importance in protein synthesis. It seems more probable that, if DNA is directly concerned with protein synthesis, its role is limited to the production of the proteins which are closely associated to chromatin and the chromosomes.

\section{(c) Nucleic acids and regulation of growth}

It should now be clear that nucleic acids are deeply and directly involved in growth processes: additional evidence, if necessary, can be found in various reports 
of stimulating effects of RNA or nucleoproteins on the regeneration of planarians (Brondsted and Brondsted, 1953) and on the growth of tissue cultures (Kutsky, I 953; Maganini et al., I 953). But very little is known about the possible existence of natural inhibitors of growth, acting on the nucleic-acid system.

One possible factor for such a role is obviously ribonuclease, since we already know that this enzyme inhibits mitosis and stops growth in many biological systems: onion roots (Kaufmann and Das, I954, I955; Brachet, I954a, I955b), amoebae (Brachet, I 955c), tumour cells (Ledoux and Baltus, I 954; Ledoux, I 955a, I955b; Ledoux and Revell, I955), amphibian eggs (Brachet and Ledoux, I955), tissue cultures of fibroblasts (Chèvremont and Chèvremont-Comhaire, I955). Unfortunately, very little is known about ribonuclease concentration and activity in resting and growing cells, a question which deserves a thorough study. Estimations of the ribonuclease content of developing frog eggs (Finamore, 1955, and unpublished personal observations) do not indicate great changes in ribonuclease content during development; but no conclusion can be drawn until more is known about the state of activity in which the enzyme is present within the cell. Such a warning is especially necessary in view of the recent report by Roth (I 955) of the existence of a natural ribonuclease inhibitor in liver.

One could also speculate about deoxyribonuclease as a natural growth regulator: but the investigations of Allfrey and Mirsky (I 952) indicate that the function of this enzyme is not limited to some simple role in cell division. Furthermore, according to Allfrey and Mirsky (1952), the bulk of the enzyme is located in the cytoplasm, and not in the nucleus where DNA is localized. It is interesting, however, that the deoxyribonuclease content of liver cells is strongly dependent on the physiological conditions: the enzyme concentration more than doubles on severe fasting.

Another possibility is that the activity of the nucleic acids might be inhibited by basic proteins, such as the normally occurring protamines and histones. Recent work by Fischer and Wagner (1954) and Fischer and Brandis (I954) lends some support to this contention: they found that protamines and histones readily penetrate into many living cells and that they inhibit the multiplication of phage $\mathrm{T}_{1}$. Inhibitory effects on cell division (Kaufmann and Das, I955), growth and protein synthesis (Brachet, I955b) have also been reported for living onion roottips and cancer cells (Zbarskič and Perovschchikova, 1954). It has recently been suggested by Mirsky (1956) that the well-known replacement of histones by protamines during spermatogenesis may be the reason for the genetic inactivity of mature sperm.

Obviously, much more experimental work is needed before the existence and the role of normal inhibitors of nucleic acid metabolism are proved; the problem might, however, well be of the utmost importance for an understanding of normal and malignant growth.1

1 The author wishes to thank Dr. J. B. Solomon, who considerably improved the text of the present Chapter. 


\section{LITERATURE}

Abolins, L., (1952) Exptl. Cell Research, 3, I.

Abrams, R., (I95I) Exptl. Cell Research, 2, 235.

Alfert, M., (1954) Intern. Rev. Cytol., 3, 13 I.

Allfrey, V. G., (1954) Proc. Natl. Acad. Sci. U.S., 4o, 83 I.

Allfrey, V. G., M. Daly and A. E. Mirsky, (1953) J. Gen. Physiol., $37,157$.

Allfrey, V. G. and A. E. Mirsky, (1952) 7. Gen. Physiol., 36, 227.

Allfrey, V. G., A. E. Mirsky and H. Stern, (i955) Advances in Enzymol., 38, 405.

Andreasen, E. And J. Ottesen, (1945) Acta Physiol. Scand., io, 258.

Anfinsen, C. B. And D. Steinberg, (1951) 7. Biol. Chem., I89, 739.

Askonas, B. A., P. N. Campbell, C. Godin and T. S. Work, (1955) Biochem. J., 6I, I05.

Auerbach, C. and J. M. Robson, (1946) Nature, 157, 302.

Avery, O. T., C. M. MacLeod and M. MaCarty, (1944) F. Exptl. Med., 79, 137.

Baltzer, F., (1952) Symposia Soc. Exptl. Biol., 6, 230.

Baltzer, F. and W. Schönmann, (I95 I) Rev. suisse zool., 58, 459.

Baron, L. S., S. Spiegelman and H. J. Quastler, (1953) F. Gen. Physiol., 36,63 r.

Barth, L. G. and L. C. Sze, (r953) Physiol. Zool., 26, 205.

Beljanski, M., (1954) Biochim. et Biophys. Acta, 15, 425 .

Bendich, A., (1952) Exptl. Cell Research, Suppl. 2 , 182.

Bieber, S., (r954) 7. Cellular Comp. Physiol., 44, i I.

Blanchard, K. C., (1935) F. Biol. Chem., io8, 25 I.

Bodenstein, D. And A. A. Kondritzer, (1948) 7. Exptl. Zool., io7, iog.

Boivin, A., R. Vendrely and C. Vendrely, (1948) Compt. rend., 226, 106.

Borsook, H., (1955) Congr. intern. biochim., ze Congr., Brussels, p. 54.

Borsook, H., C. L. Deasy, A. J. HaAgen-Smit, O. Keighly and P. H. Lowy, (1952)

7. Biol. Chem., I96, 669.

Brachet, J., (I933) Arch. biol. (Liège), 44, 5 I9.

Brachet, J., (1940) Arch. biol. (Liège), 5I, I67.

Brachet, J., (r941a) Arch. biol. (Liège), 53, 205.

Brachet, J., (I94I I) Enzymologia, ro, 87.

Brachet, J., (1944a) Embryologie chimique, Desoer, Liège; Masson, Paris.

Brachet, J., (1944b) Ann. soc. roy. zool. Belg., 75, 49.

Brachet, J., (I945) Bull. acad. roy. Belg., 29, 707.

Brachet, J., (1946) Compt. rend. soc. biol., I40, I I 23.

Brachet, J., (1948) Experientia, 4, 353.

Brachet, J., (1949a) Pubbl. staz. zool. Napoli, 21, 77.

Brachet, J., (I949b) Bull. soc. chim. biol., 3I, 724.

Brachet, J., (1950) Experientia, 6, 56.

Brachet, J., (1952a) Experientia, 8, 347.

Brachet, J., (1952b) Le rôle des acides nucléiques dans la vie de la cellule et de l'embryon, Masson, Paris.

Brachet, J., (1953) Quart. 7. Microscop. Sci., 94, I.

Brachet, J., (1954a) Nature, I74, 879.

Brachet, J., (1954b) Arch. biol. (Liège), 65, I.

Brachet, J., (1955a) in Nucleic Acids, Academic Press, New York, p. 475.

Brachet, J., (1955b) Biochim. et Biophys. Acta, i6, 6I I.

Brachet, J., (1955c) Nature 175, 851.

Brachet, J., (1955d) Biochim. et Biophys. Acta, I8, 247.

Brachet, J., H. Chantrenne and F. Vanderhaeghe, (1955) Biochim. et Biophys. Acta, I8, 544 .

Brachet, J., S. Gothié and T. Kuusi, (1952), Arch. biol. (Liège), 63, 429.

Brachet, J., AND R. Jeener, (1944) Enzymologia, II, 196.

Brachet, J., And L. Ledoux, (1955) Exptl. Cell Research, Suppl. 3, 27.

Brøndsted, A. And H. V. Brondsted, (1953) F. Embryol. Exptl. Morphol., I, 49. 
Brown, G. B. And P. M. Roll, (1955) in Nucleic Acids, Academic Press, New York, Vol. 2, pp. 34I, 393 .

Brown, G. L. ANd M. Watson, (1953) Nature, I72, 339.

Burgos, M. H., (1955) Exptl. Cell Research, 9, 360.

Butler, J. A. V., L. A. Gilbert And K. A. Smith, (1950) Nature, 165, 714.

Cagianut, B., (1949) Z. Zellforsch. u. mikroskop. Anat., 34, 47 I.

Caldwell, P. C., E. L. Meckor and Sir Gyril Hinshelwood, (i950) F. Chem. Soc., 3 I 5 I.

Campbell, R. M. and H. W. Kosterlitz, (1950) Endocrinology, 6, 308.

Caspersson, T., (1940) 7. Roy. Microscop. Soc., 6o, 8.

Caspersson, T., (194:I) Naturwissenschaften, 28, 33.

Caspersson, T., (1950) Cell Growth and Cell Function, Norton, New York.

Casterman, C. and R. Jeener, (1955) Biochim. et Biophys. Acta, 16, 433.

Chantrenne, H., (1953) Symposium Soc. Gen. Microbiol., 2nd, I.

Chantrenne, H., (I956) Nature, 177, 579.

Chantrenne, H. and J. Brachet, (1946) Compt. rend. soc. biol., i4o, 892.

Chargaff, E., (I95I) F. Cellular Comp. Physiol., 38, suppl. I, 4 I.

Chargaff, E., C. F. Crampton and R. Lipshitz, (1953) Nature, 172, 289.

Chèvremont, M. and S. Chèvremont-Comhaire, (I955) Nature, I76, I075.

Chèvremont, M. and H. Firket, (1952) Compt. rend. assoc. anat., 39.

Child, C. M., (1948) 7. Exptl. Zool., rog, 79.

Commoner, B. and J. Mercer, (I95I) Nature, I68, i I3.

Commoner, B., Y. Yamada, S. D. Rodenberg, Tung-Yue Wang and E. Basler, JR., (1953) Science, I18, 529.

Cohen, S. S. and H. D. Barner, (1954) Proc. Natl. Acad. Sci. U.S., 40, 885.

Cohen, S. S., R. Levi-Montalcini and V. Hamburger, (i954) Proc. Natl. Acad. Sci. U.S., 40,1014 .

Creaser, E. H., (1955a) Nature, I75, 899.

Creaser, E. H., (I955b) Nature, $176,556$.

Dalce, A. And J. Pasteels, (1955) Exptl. Cell Research, Suppl. 3, 72.

Daly, M. M., V. G. Allfrey and A. E. Mirsky, (1952) F. Gen. Physiol., 36, i 73.

Daoust, R., F. D. Bertalanffy and G. P. Leblond, (1954) F. Biol. Chem., 207, 454.

Darlington, C. D. and L. La Cour, (i 940 ) J. Genet., 40, i85.

Davidson, J. N., (1947) Cold Spring Harbor Symposia Quant. Biol., I2, 50.

Davidson, J. N. and E. Chargaff, (I955) in Nucleic Acids, Academic Press, New York.

Davidson, J. N. And I. Leslie, (i950) Nature, $165,49$.

Davidson, J. N., I. Leslie and C. Waymouth, (1949) Biochem. 7., 44, 5.

Dekker, C. A. and H. K. Schachman, (1954) Proc. Natl. Acad. Sci. U.S., 4o, 894.

Delbrück, M., (1954) Proc. Natl. Acad. Sci. U.S., 40, 783.

Desclin, L., (1940) Compt. rend. soc. biol., 133, 457.

Dounce, A. L., (1952) Enzymologia, 15, 25 I.

Durand, M. C., (1955) Compt. rend., 24I, 1340.

Elson, D. and E. Chargaff, (1955) Biochim. et Biophys. Acta, I7, 367.

Elson, D., T. Gustafson and E. Chargaff, (i954) F. Biol. Chem., 208, 285.

Elson, D., L. W. Trent and E. Chargaff, (1955) Biochim. et Biophys. Acta, I7, 362.

Engländer, H., A. G. Johnen and W. Vahs, (1953) Experientia, 9, IOo.

Errera, M., (1947) Cold Spring Harbor Symposia Quant. Biol., 12, 60.

Errera, M., (1952) Thesis, I I8pp.

Errera, M., (1954) Brit. 7. Radiol., 27, 76.

Fautrez, J. And N. Fautrez-Firlefyn, (I953) Nature, I72, i i 9.

Fautrez, J. and H. Roels, (1954) Arch. biol. (Liège), 65, 460.

Feulgen, R. And H. Rossenbeck, (1924) Z. physiol. Chem. Hoppe-Seyler's, 135, 203.

Fice, A., (1955) Arch. biol. (Liège), 66, 509.

Fice, A. And J. Brachet, (1956) Exptl. Cell Research, II, I35.

Fice, A. And M. Errera, (I955a) Biochim. et Biophys. Acta, 16, 45.

Fice, A. ANd M. ERrera, (1955b) Arch. intern. physiol., 63, 259.

Finamore, F. J., (1955) Exptl. Gell Research, 8, 533. 
Fischer, F. G. And H. Hartwig, (I936) Z. vergleich. Physiol., 24, I.

Fischer, H. ANd H. Brandis, (I954) Naturwissenschaften, 4I, 533.

FischeR, H. AND L. WAGNER, (1954). Naturwissenschaften, 4I, 532.

Fox, J. J. And I. Goodman, (1953) Biochim. et Biophys. Acta, IO, 77.

Fraenkel-Conrat, H. and R. G. Williams, (1955) Proc. Natl. Acad. Sci. U.S., 4I, 690.

Furst, S. S. And G. B. Brown, (1951) 7. Biol. Chem. 191, 239.

Gale, E. F., (1955) Congr. intern. biochim., 3e Congr., Brussels, p. $7 \mathrm{r}$.

Gale, E. F. and J. P. Folkes, (1953) Biochem. F., 53, 483, 493.

Gale, E. F. ANd J. P. Folkes, (1954) Nature, I73, I 223.

Gale, E. F. And J. P. Folkes, (1955a) Biochem. F., 59, 675.

Gale, E. F. And J. P. Folkes, (1955b) Nature, 175, 592.

Gavosto, F. and R. Rechenmann, (1954) Biochim. et Biophys. Acta, 13, 583.

Gierer, A. and G. Schramm, (1956) Z. Naturforsch., il B, I38.

Gregg, J. R. and S. Lovtrup, (1950) Compt. Rend. Trav. Lab. Carlsberg, Ser. chim., 27, 307.

GregG, J. R. And S. Lovtrup, (1955) Biol. Bull., I08, 29.

Gros-Doulget, F., F. Gros and S. Spiegelman, (1955) Congr. intern. biochim., ze Congr., Brussels, p. 74 .

Grundmann, E. And H. Marquardt, (1953) Naturwissenschaften, 40, 557.

Gustafson, T. and I. Hasselberg, (195I) Exptl. Cell Research, 2, 642.

Halvorson, H. O. and L. Jackson, (i954) Bacteriol. Proc. Soc. Am. Bacteriologists, 54, i i i 7.

Hammarsten, E., (195I) Ciba Conference on Isotopes in Biochem., London, p. 203.

Hämmerling, J., (1934) Arch. Entwicklungsmech. Organ., I3I, I.

Heimberg, M. and S. F. Velick, (1954) F. Biol. Chem., 208, 725.

Herlant, M., (I943) Arch. biol. (Liège), 54, 225.

Herriott, R. M., (1948) 7. Gen. Physiol., 32, 22 I.

Herriott, R. M., (I95Ia) 7. Bacteriol., 6I, 752.

Herriott, R. M., (195Ib) 7. Gen. Physiol., 34, 76r.

Hershey A. D. and M. Chase, (I952) F. Gen. Physiol., 36, 39.

Hevesy, G., (1949) Nature, 163, 869.

Hevesy, G. and J. Ottesen, (1943) Acta Physiol. Scand., 5, 237.

Hoagland, M. B., P. C. Zamecnik and M. L. Stephenson, (1957) Biochim. et Biophys. Acta, 24, 215.

Hoff-Jorgensen, E., (1954) Proc. Symposium Colston Research Soc., 7, 79.

Hoff-Jorgensen, E. And E. Zeuthen, (1952) Nature, I69, 245.

Hollaender, A. And C. W. Emmons, ( I94 I) Cold Spring Harbor Symposia Quant. Biol., 9, 179.

Hollaender, A., J. P. Greenstein and W. V. Jenrette, (i 94 I) J. Natl. Cancer Inst., 2, 23.

Holloway, B. W. and S. H. Ripley, (1952) J. Biol. Chem., 196, 695.

Holmes, B. E., (1949) Brit. J. Radiol., 22, 487.

Holtfreter, J., (1943) 7. Exptl. Zool., 93, 25 I.

Holtfreter, J., (1947) F. Exptl. Zool., io6, 197.

Holtfreter, J., (1955) Exptl. Cell Research, Suppl.3, i88.

Hotchkiss, R. D., (i 949) Colloq. intern. centre natl. recherche sci. (Paris), 8, 57.

Нотснкiss, R. D., (I955a) in Nucleic Acids, Academic Press, New York, p. 435.

Нотснкiss, R. D., (1955b) 7. Cellular Comp. Physiol., 45, Suppl. 2, I I4.

Hultin, T., (1951) Exptl. Cell Research, I, 376; 599.

Hultin, T., (1953) Arkiv Kemi, 6, I95.

James, T. W., (1954) Biochim. et Biophys. Acta, 15, 367.

Jeener, R., (1953) Arch. Biochem. Biophys., 43, 38 I.

JeEner, R., (I956) Advances in Enzymol., 17, 477.

Jeener, R. And H. Jeener, (1952) Exptl. Cell Research, 3, 675.

Jeener, R. and P. Lemoine, (1952) Arch. intern. physiol., 6o, 547.

Jeener, R., P. Lemoine and C. Lavand'homme, (1954) Biochim. et Biophys. Acta, I4, 32 I.

Jeener, R. And J. Rosseels, (1953) Biochim. et Biophys. Acta, II, 438.

Kanazir, D. And M. Errera, (I954) Biochim. et Biophys. Acta, I4, 62.

Kaufmann, B. P. and N. K. Das, (1954) Proc. Natl. Acad. Sci. U.S., 40, 1052.

Kaufmann, B. P. And N. K. Das, (I955) Chromosoma, 7, I9. 
Kelner, A., (1953) F. Bacteriol., 62, 252.

Kihara, H. K. and A. Sibatani, ( I955) Biochim. et Biophys. Acta, 17, 579.

Koritz, S. B. and H. Chantrenne, (i954) Biochim. et Biophys. Acta, I3, 209.

Krugelis, E.g (1947) Biol. Bull., 93, 2 I 5.

KRUH, J. AND H. BorsooK, (1955) Nature, I75, 386.

Kutsky, R. J., (1953) Proc. Soc. Exptl. Biol. Med., 83, 390.

Kuusi, T., (1953) Arch. biol. (Liège), 64, 189.

Lallier, R., (1950) Experientia, 6, 92.

Le Glerc, J., (I956) Nature, I77, 578.

Ledoux, L., (1955a) Nature, 175, 258.

Ledoux, L., (1955b) Nature, $176,36$.

Ledoux, L. AND E. Baltus, (1954) Experientia, Io, 50 I.

Ledoux, L., And S. H. Revell, (I955) Biochim. et Biophys. Acta, I8, 4 I6.

Lesher, S., (1951) Exptl. Cell Research, 2, 577.

Leslie, I., (I955) in Nucleic Acids, Academic Press, New York, Vol. 2, p. I.

Lester, R. L.g (1953) 7. Am. Chem. Soc., 75, $544^{8}$.

Leuchtenberger, C., (I954) Statistics and Mathematics in Biology, Iowa State College Press.

Leuchtenberger, C., R. Vendrely and G. Vendrely, (i95i) Proc. Natl. Acad. Sci. U.S., 37,33 .

Levenbook, L., E. K. Travaglini and J. Schultz, (1955) Congr. intern. biochim., 3rd Congr., Brussels, p. 70 .

Levi-Montalcini, R. and V. Hamburger, (i953) F. Exptl. Zool., I23, 233.

Lipmann F., (1949) Federation Proc., 8, 597.

Lison, L. And J. Pasteels, (195I) Arch. biol. (Liège), 62, I.

Lowe, C. U. and J. F. Foley, (I955) Federation Proc. I4, 4I I.

Maganini, H., A. W. Schweitzer and G. M. Hass, (1953) Federation Proc., I2, 453.

Malkin, H. M., (1954) J. Cellular Comp. Physiol., 44, I05.

Malmaren, B. And C. G. Hedén, (i948) Acta Pathol. Microbiol. Scand., 24, 437, 448, 472, 496.

Mandel, P. And R. Bieth, (I95I) Experientia, 7, 343.

Mandel, P., R. Bieth and R. Stoll, (1949) Bull. soc. chim. biol., 3I, I 335.

Markham, R., (I953) Advances in Virus Research, I, 3 I 5.

Markham, R. and K. M. Smith, (1949) Parasitology, 39, 330.

Marshak, A. and C. Marshak, (1953) Exptl. Cell Research, 5, 288.

Marshak, A. and C. Marshak, (1954a) Nature, I74, 9 I 9.

Marshak, A. And C. Marshak, (1954b) Biochim. et Biophys. Acta, I5, 584.

Marshak, A. and C. Marshak, (1955) Exptl. Cell Research, 8, I 26.

Matthews, R. E. F., (1954) F. Gen. Microbiol., 10, 52 I.

Matthews, R. E. F., (1955) Virology, I, I65.

Mazia, D. And D. M. Prescott, (1955) Biochim. et Biophys. Acta, I7, 23.

McKeehan, M. S., (I95I) F. Exptl. Zool., II7, 3 I.

Miller, C. O., F. Skoog, M. H. von Saltza and F. M. Strong, (1955) 7. Am. Chem. Soc., 77, 1392.

Mirsky, A. E., (1956) Congr. intern. biochim., 3e Congr., Brussels, Academic Press, New York, p. 345 .

Mirsky, A. E. And H. Ris, (1949) 7. Gen. Physiol., 34, 45 I.

Mirsky, A. E. And H. Ris, (I95 I) F. Gen. Physiol., 34, 475.

Mitchell, J. S., (1942) Brit. F. Exptl. Pathol., 23, 309.

Moore, J. A., (1946) J. Exptl. Zool., Ior, I73.

Moore, J. A., (1947) F. Exptl. Zool., 105, 349.

Moore, J. A., (1948) 7. Exptl. Zool., 108, 127.

Moore, J. A., (1955) Advances in Genet., 7, I39.

Moyson, F., (1955) Arch. biol. (Liège), 66, 149.

Niu, M. G. and V. G. Twitty, (1953) Proc. Natl. Acad. Sci. U.S., 39, 985.

Nizet, A. And S. Lambert, (1953) Bull. soc. chim. biol., 35, 77 I.

Northrop, J. H., (1953) F. Gen. Physiol., 36, 58 I.

Novick, A. ANd L. Szilard, (1952) Nature, I7o, 926. 
Pardee, A. B., (1954) Proc. Natl. Acad. Sci. U.S., 40, 263.

Pardee, A. B., (1955) 7. Bacteriol., 69, 233.

Pardee, A. B. and L. S. Prestidge, (I955) Federation Proc., I4 262.

Pasteels, J. and W. S. Bullough, (I953) Arch. biol. (Liège), 64, 27 I.

Pasteels, J. And L. Lison, (1950) Compt. rend., $230,780$.

Pavan, C. And M. E. Breuer, cited by Allfrey et al., 1955.

Pelc, S. R. And A. Howard, (1952) Exptl. Cell Reseurch, Suppl. 2, 269.

Perlmann, G. and T. Gustafson, (1948) Experientia, 4, 48 I.

Piepho, H., (1938) Biol. Zentr., 24, I.

Pisi, E. And G. Cavalli, (1955) Arch. biol. (Liège), 66, 439.

Pollister, A. W., (1953) Dynamics of Growth Processes, Princeton University Press, Princeton, N. J., p. 33 .

Price, W. H., (1952) 7. Gen. Physiol., 35, 741.

Rabinovitch, M., L. C. Jungueira and H. A. Rothschild, (I95I) Science, II4, 55 I.

Reiner, J. M. and F. Goodman, (1955) Arch. Biochem. Biophys., 57, 475.

Ris, H. and A. E. Mirsky, (1949) Jै. Gen. Physiol., 33, I25.

Roels, H., (1954) Nature, 174, 5 I4.

Roth, J. S., (1955) Federation Proc., I4, 272.

Schmidt, G., H. Hecht and S. J. Thannhauser, (I948) J. Gen. Physiol., 3I, 203.

Schrader, F. and C. Leuchtenberger, (1950) Exptl. Cell Research, I, 42 I.

SEngün, A., (1947) Rev. fac. sci. univ. Istanbul, Sér. B, I2, 289.

Shen, P. S., (1954) Experientia, 10, 212.

Sher, H. I. And M. F. Mallette, (1954) Arch. Biochem. Biophys., 52, 33 I.

Siekevitz, P., (I952) 7. Biol. Chem., 195, 549.

Sirlin, J. L., (1955) Experientia, II, II2.

Smellie, R. M. S., G. F. Humphrey, E. R. M. Kay and J. N. Davidson, (1955) Biochem. 7., 6o, 177 .

Smith, A. E. S. and A. M. Schechtman, (1954) J. Exptl. Zool., 125, 265.

Solomon, J. B., (1957) Biochim. et Biophys. Acta, 25, 69.

Sparrow, A. H., (1952) Exptl. Cell Research, Suppl. 2, 199.

Spiegelman, S., H. O. Halvorson and R. Ben-Ishai, (1955) Symposium on Amino Acid Metabolism, Johns Hopkins Univ. Press, Baltimore, I 24.

Spratt, N. J., (1948) F. Exptl. Zool., I07, 39.

Stadler, L. J. and F. M. Weber, (1942) Genetics, 27, 84.

Steinberg, D. and C. B. Anfinsen, (I952) J. Biol. Chem., 199, 25.

Steinert, M., (I95I) Bull. soc. chim. biol., 33, 549.

Steinert, M., (1952) Bull. soc. chim. biol., 34, 923.

Steinert, M., (1953) Biochim. et Biophys. Acta, IO, 427.

Stern, H. And A. E. Mirsky, (1952) 7. Gen. Physiol., 36, i8 I.

Stevens, G. E., R. Daoust and C. P. Leblond, (1953) F. Biol. Chem., 202, 177.

Sтісн, H., (I95I) Z. Naturforsch., 6b, 3 I 9.

Swenson, P. A., (1950) Proc. Natl. Acad. Sci. U.S., 36, 699.

Swift, H. H., (1950) Physiol. Zool., 23, I69.

Swift, H. H., (1953) Intern. Rev. Cytol., 2, I.

Sze, L. C., (1953) Physiol. Zool., 26, 2 1 2.

Takahashi, W. N. And M. Ishir, (1952) Phytopathology, 42, 690.

TAKata, A., (1953) Biol. Bull., I05, 348.

Taylor, B., J. P. Greenstein and A. Hollaender, (1948) Arch. Biochem., i6, ig.

Thorell, B., (1947) Cold Spring Harbor Symposia Quant. Biol., I2, 247.

Tiedemann, H. And H. Tiedemann, (I954) Naturwissenschaften, 4I, 535.

Torvonen, S., (1949) Arch. Soc. Zool. Botan. Fennicae, "Vanamo", 4, 28.

Vendrely, R., (I946) Symposium sur les Protéines, Masson, Paris.

Vendrely, R., (1955) in Nucleic Acids, Academic Press, New York, p. I 55.

Vendrely, R. And G. Vendrely, (1948) Experientia, 4, 434; 5, 327.

Villee, C. A., M. Lowens, M. Gordon, E. Leonard and A. Rich, (ig49) J. Cellular Comp. Physiol., 23, 93 . 
Waddington, C. H., M. Feldman and M. M. Perry, (1955) Exptl. Cell Research, Suppl. 3,366 .

WADE, H. E., (1952) F. Gen. Microbiol., 7, 24.

Walker, P. M. B. And H. B. Yates, (1952) Proc. Roy. Soc. (London), B $40,274$.

Watson, J. D. and F. H. C. Crick, (I953a) Nature, I7I, 737.

Watson, J. D. and F. H. C. Crick, (I953b) Nature, I7I, 964.

Weiss, P. and H. B. Hiscoe, (i948) F. Exptl. Zool., IO7, 3 I 5.

Yamada, T. And K. Takata, (I955a) Exptl. Cell Research, Suppl. 3, 402.

YAMADA, T. AND K. TAKATA, (1955b) F. Exptl. Zool., I28, 29 I.

Yamada, T., K. Takata and S. Osawa, (1955) Embryologia, 2, I 23.

Zamecnik, P. C. and E. B. Keller, (i954) 7. Biol. Chem., 209, 337.

Zamenhof, S. And G. Griboff, (1954) Nature, 174, 307.

Zbarskič, J. B. and K. A. Perovschchikova, (i954) Byull. Eksptl. Biol. Med., 38, 6 i. 


\section{Chapter 4

\author{
GERMINAL ORGANIZATION AND
} \\ INDUCTION PHENOMENA ${ }^{1}$}

Albert M. Dalcq

To the Memory of my son André [I926-I958]

In this volume whose purpose it is to discuss the multiple aspects of growth, a study of germinal organization is a prerequisite, especially if it examines the constituents involved and their spatial and dynamic relations. At definite moments in development, these relations, granted a favorable milieu, allow growth to occur and flourish to various extents, and insure the prosperity and resistance of the organism, while their deficiency sometimes causes its more or less tragic disorganization.

According to current ideas, growth makes its appearance only when the various types of cells have been formed by cytodifferentiation or histodifferentiation, but this is probably too radical a distinction. In development, a clearcut division into periods devoted to distinct tasks does not exist; in spite of most textbook presentations, growth, the most imperious urge of living beings, begins insidiously at early stages. Moreover, the production of eggs and spermatozoa results from intense growth processes. It is the characteristic feature of sexual reproduction that growth in both kinds of gametes enters a sort of blind alley if fertilization does not take place. Male gametes, so far as is known, are never able to develop by themselves, and female ones do it only exceptionnally; parthenogenesis is always either secondarily acquired or experimentally achieved. In most cases, the inertia of the egg makes fertilization necessary for the release of the new life cycle.

Our task is here to examine the main data necessary for comprehension of the general conditions reigning in a young organism until the moment when growth becomes conspicuous. This means that we should consider gametogenesis and early development as processes which gradually build up the organized whole, and that we should analyse the special features progressively acquired by the cells composing first the presumptive areas, and later the germ layers, and the several blastemas. To understand the fate of these primordia, induction phenomena

1 I would like to express my thanks to Miss Martha Brice, Fulbright student in my Laboratory, for the linguistic revision of this manuscript. Mr. Karl Enselberg, Univ. or Pennsylvania, also diligently helped me to include, in 1958 , several recent results. 
deserve close attention, for they are indeed among the most characteristic events of early development and exercise a definite effect on ultimate growth processes.

Naturally, in treating an extensive succession of intricate events, I shall be forced to be quite selective. The following account presupposes a rather advanced knowledge of the basic events of development. This condition will permit us to focus our attention on events and results most significant for our present purpose, to treat with brievity classical information, while explaining and illustrating in some detail special aspects insufficiently appreciated or too recent to be well known.

One of the servitudes of Embryology is that we are rarely able to get complete information by studying only the groups or species in which we are, for various reasons, directly interested. We often have to use results of research performed on distant forms, implicitly assuming that, if data are related to events of general occurrence, they may have a general application. This is the reason why we shall feel justified in wandering from one animal group to another, without treating one particular form with completeness. We suppose that the reader will be sufficiently aware of the special aspects of the various materials, for which he can easily refer to elementary textbooks or more extensive treatises (Schleip, 1928; Huxley and De Beer, I934; Dalcq, I938b; Weiss, r939; Dalcq, I94 Ib; Raven, I954; Willier, Weiss and Hamburger, I955; Kühn, I 955; Waddington, I 956; Gallien, I 958).

Therefore, in this chapter, the preparatory events of gametogenesis will be considered first, and with them fertilization, which affords a landmark between egg and sperm formation and the unfolding of germinal potentialities. Then, in order to follow the course of development, a distinction will be made, for the sake of analysis, between the intrinsic aspects of organization and the processes of induction. The interest of these will justify a rather extensive treatment, which will throw some light on the nature of differentiation and the onset of growth.

To my deep regret, it will be often impossible to refer to the promotor of a given piece of research or to the earlier works of an author. Quotations are mostly chosen for their applicability and as a source of reference.

\section{ORGANIZING ASPECTS OF GAMETOGENESIS}

The formation of male and female gametes is usually considered from the point of view of chromosomal transformations, which are, of course, of primary importance. But, since we are striving to understand morphogenesis, which mobilises the whole resources of the germ, we must also especially draw our attention to the cytoplasm.

\section{A. The cytoplasm of the gametes}

The existence of the germinal lineage being taken for granted, we have to remember, however trivial it may be, that the cytoplasm of oogonia or spermatogonia is continuous with the cytoplasm of primary gonocytes, and, through these, the cytoplasm of the parent egg and of its blastomeres. So far no one can make a thorough inventory of the implications of this continuity. But thanks to 
our knowledge of general cytology, so considerably increased in recent years by electron microscopy, we may guess that this descendance implies the transmission of (a) mitochondria, with some kind of internal partitioning and a complex enzymatic equipment, (b) the cytoplasmic reticulum, mostly phospholipidic (analogous to the ergastoplasm of secretory cells), and the attached Palade's granules which bear most of the RNA, both these structures corresponding to the fraction obtained by centrifugation which has been called microsomes, (c) the host of Golgi vesicles mostly grouped around or near the nucleus, (d) the centriole(s) whose significant complexity has been described in several mouse and chicken adult cells (De Harven and Bernhardt, 1956). Moreover, less individualized macromolecules of the cytoplasm, especially proteins, including enzymes, may be in material continuity with the same components of the parent cells, as are also possibly the mucopolysaccharides and other specialized compounds. Of these numerous plasmatic constituents, the centrioles do in fact reproduce themselves by division, but for all others, such division has not, in the best cases, been proved with certainty. Some or most of these organelles or even the macromolecules may either reduplicate, be replaced thanks to some recombination or growth, or be synthesized from more minute elements. How much these events, whatever they may be, depend upon the nucleus, or, more specifically, upon its genes, remains an open problem. As a result of several observations (cf. pp. $33^{2}, 480$ ) we have some reason to conclude that they are dependent on nuclear activities, especially on the nucleoli, which implies definite activities of the chromosomes. However, to assert that all cytoplasmic features depend on genes would be based only on the conviction that the manifestation of certain hereditary characters proves that genes influence mitochondria, or ergastoplasmic structures, or the Golgi system. This would be merely a postulate. Therefore, we should leave the problem of the relations between genes and the cytoplasmic organelles, a task for further exploration.

Consequently, we cannot simply assert that the hereditary endowment of spermato- or oo-gonia is located in the genes. They are indeed the carriers of many characters, but neither the rest of the nucleus nor the fundamental constituents of the cytoplasm, with their species-inherent properties, can be ignored without risk of an error. The whole system must be taken into account when we try to describe the morphogenetic organization of the egg.

\section{B. Growth and polarization of the oocytes}

We shall pass rapidly over the period of multiplication without discussing the problem of the stem cells, which has been solved recently in some spermatogeneses (Cleland, I95I; Leblond and Clermont, I952; Roosen-Runge and Barlow, 1953), but consider the period of growth in the young auxocytes. Here we must mention the evident link between the male or female gene complex and the respectively limited $(\widetilde{\zeta})$ or complete (o) growth of the auxocytes.

In all groups, excepted sponges and nematods, the male gamete, when having performed its meiosis and attained the stage of spermatid, elongates and builds up an elaborate system of fibrillar and granular differentiations, which insures 
the motility of the spermatozoon. This equipment of the male gamete implies polarization and even the acquisition of a bilateral symmetrical structure, and testifies the presence of the mechanisms necessary for cyto-differentiation. However
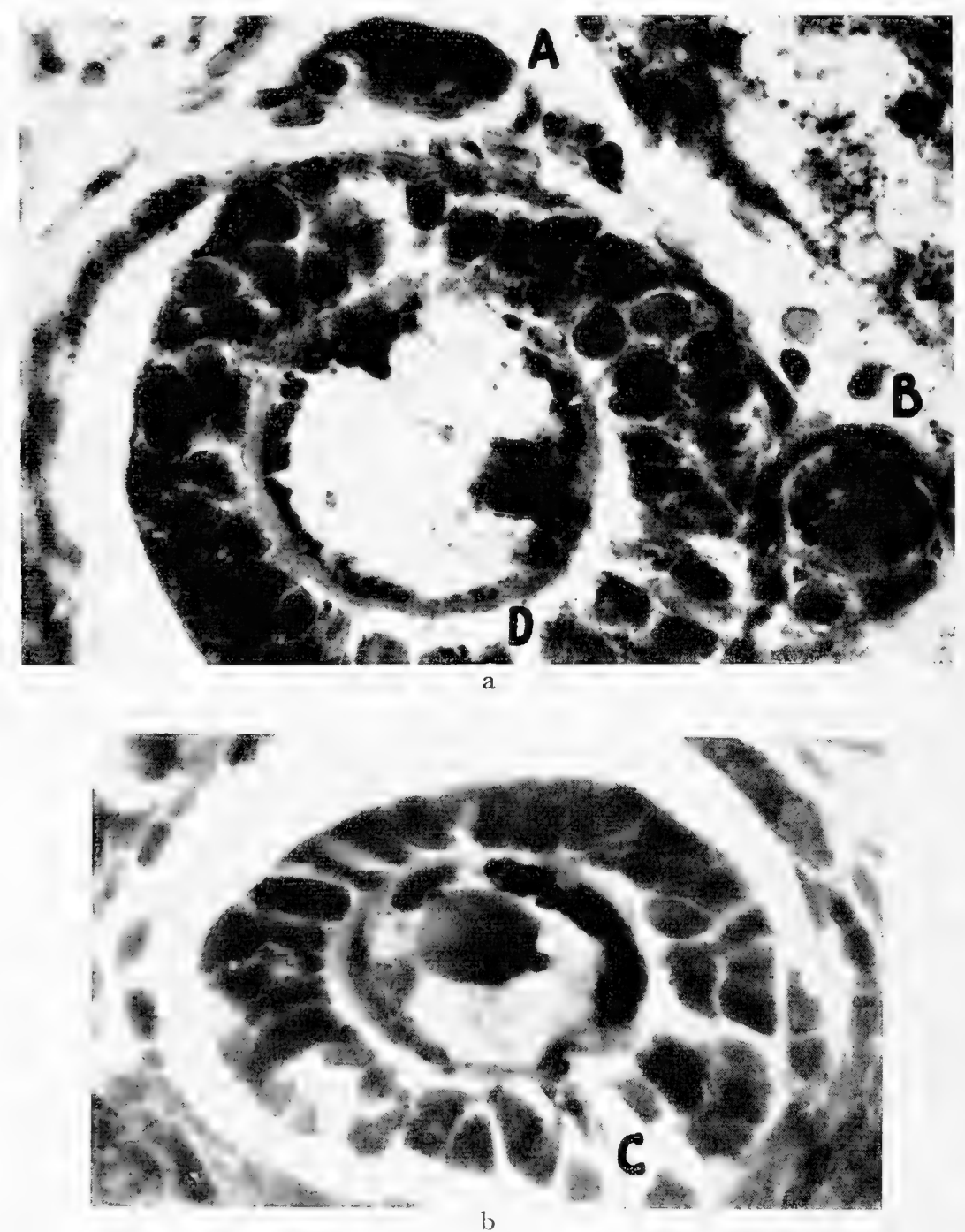

Fig. I. Conspicuous mitochondria in growing oocytes of the hamster. Altmann technique. Four stages are presented, stages A B D in Fig. ra, stage C in Fig. Ib. A. Stage of incipient growth: the cytoplasm is made completely opaque by mitochondria; the clear spot is a vesicle of nuclear sap expelled from the nucleus. B. young primary follicle: the mitochondria of the egg are somewhat more dispersed; nucleus below, polarization already distinct. G. Secondary follicle: a rather large amount of cytoplasm has been formed; mitochondria, mainly peripheral, are still quite abundant; the clear vesicle to the right of the nucleus is also an emission of nuclear sap. D. More advanced secondary follicle, with the mitochondria beginning to disperse (Dalcq and Van Egmond, I953). 
interesting these processes may be ( $c f$. Mulnard, 1956) we must refrain from dwelling on them. Only the events essential to the oocytes must retain our attention.

During their growth, emissions of nuclear material have often been described, sometimes with a disputable but highly probable validity. Concerning the eggs, aspects observed in the hamster ovary may be mentioned here. In the young oocytes, the mitochondria assume the extraordinary configuration of a compact

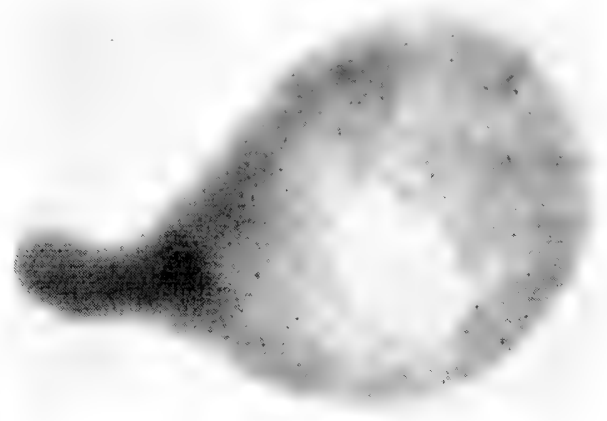

Fig. 2. Immature oocyte of Gryphaea aculeata. Serra-Unna. The basophilic stalk indicates the previous attachment to the ovarian epithelium. Original slide, courtesy of Prof. J. Pasteels.
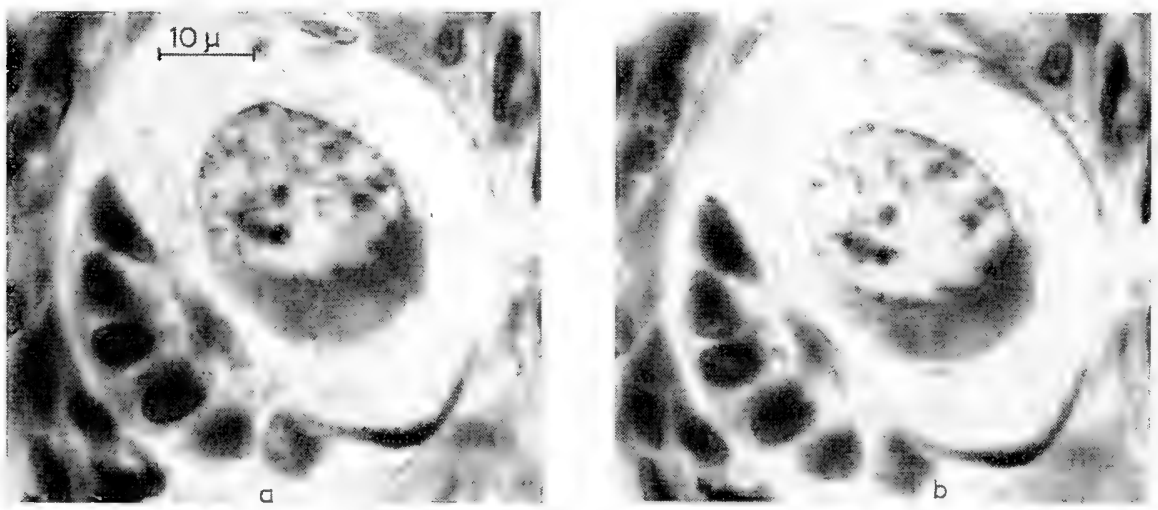

Fig. 3. Two levels of the same human primary follicle. (Bouin, sectioned by congelation, hemalun). In (a), inside a large mitochondrial crescent, a clear centrosphere with its centriole is visible. In (b), several spherical bodies (proteins?) can be seen in the same region. Such a centrosome has already been described by Vanderstricht (1923). Photogr., courtesy of Prof. Cordier.

mass, located on one side of the nucleus (see infra); under this mass, an emission of nuclear sap is frequently caught up by the fixative (Fig. I, $c f$. Dalcq and Van Egmont, I953, p. 365-369). Soon after that, the mitochondrial mass dissociates and apparently plays a role in the growth of the cytoplasm. Similar observations of nuclear contributions to cytoplasmic growth have been recorded on amphibian eggs (Wittek, I952), and on the small viviparous teleost Lebistes (Vakaet, I955), 

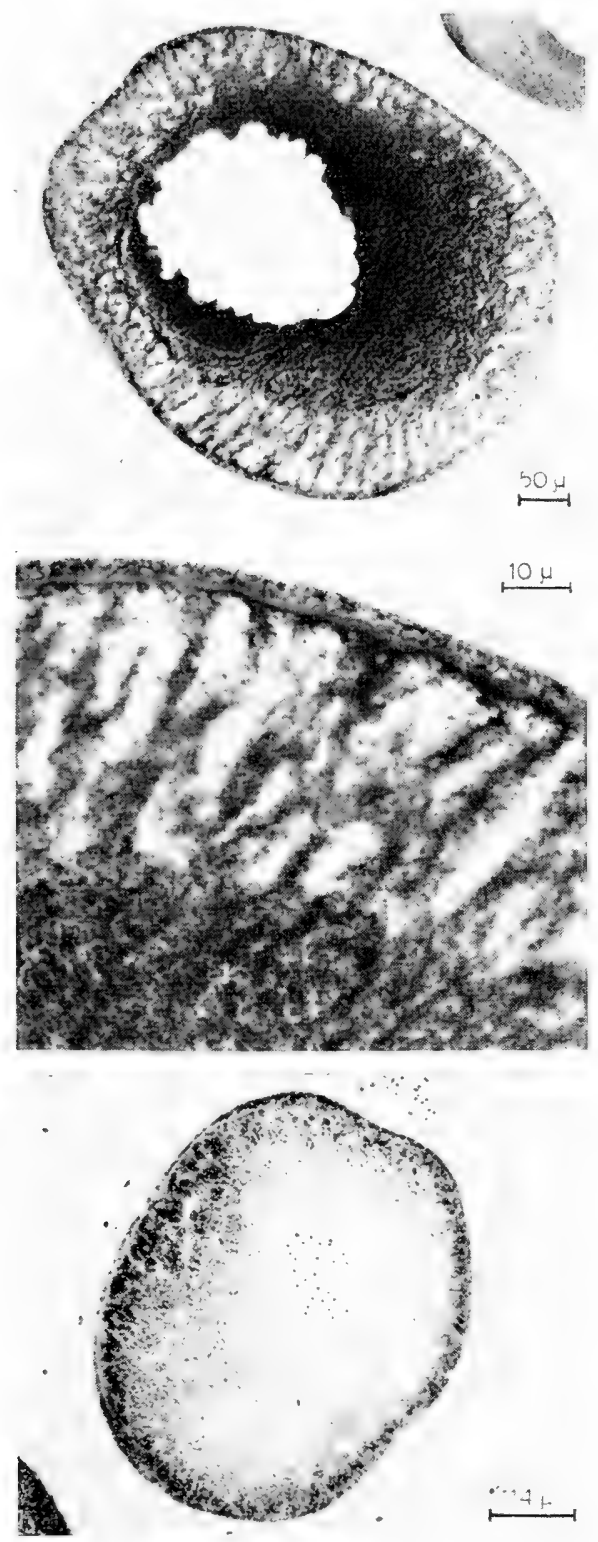

Fig. 4. Previtellogenesis in Xenopus oocytes. Zenker fixation. (a) Young oocyte cut along its axis of polarity (and possibly along its plane of symmetry) and stained with toluidine blue; the dark zone around the nucleus is the RNA-rich cytoplasm; vitellogenesis takes place at the periphery and dissociates the cytoplasm as shown in $(b) ;(b)$ Detail of the animal pole showing, from top to bottom, the unstained chorion (scarcely visible), the granular layer of cortical cytoplasm (see Figs. 6 , b and c), a homogeneous subcortical layer (see Fig. 6 a, under the cortical granules c), some pigment granules (darker spots) sticking to this layer, clear columns of young platelets piling up from the periphery to the center, dark radial strands of basophilic cytoplasm, dissociated by the vitellogenesis, and the granular structure of the central cytoplasm; (c) somewhat more advanced oocyte cut along its polar axis (and possibly along its plane of symmetry) and stained with hemalun-eosin; only yolk platelets are stained, making conspicuous the centripetal progression; some pigment has appeared at the animal pole (upper part); numerous nucleoli are dispersed in the nucleus. (From Wittek, I952). 


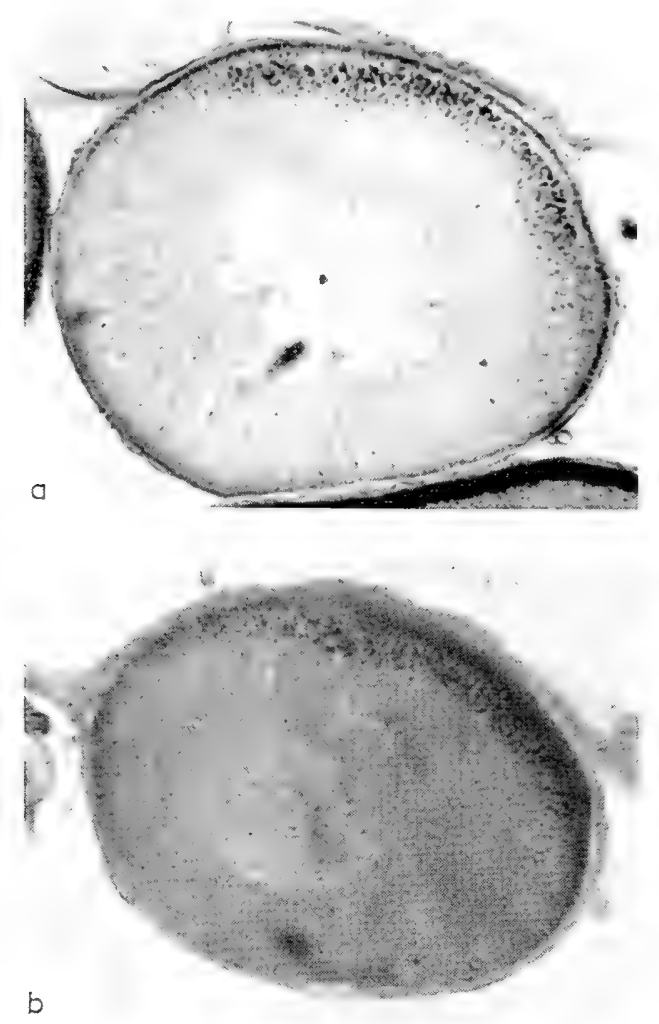

Fig. 5. Melanogenesis at the animal pole of Xenopus eggs fixed in a picro-formol mixture. (a) PAS reaction; (b) Hale reaction. Both reactions selectively stain the granules located at the animal pole. In (a) the cords of peripheral vitellogenesis are visible (clear). Courtesy of Wartenberg, 1956.

to mention only some of the recent data. These observations fall in line with others of general cytology, which show that the nucleus is an organ of active elaborations.

It is during their time of growth that eggs acquire the properties of organization which endow them with a morphogenetic pattern. In all groups, they become polarized; in several, they acquire some differential property on one side of the axis, and eventually attain a bilaterally symmetrical organization (symmetrization).

Polarization of the egg cell can no longer be considered as a primary structural property inherent to the whole cytoplasm. It has been recognized that, in ovaries with a tubular structure, the eggs preserve the polarity which they had while arranged in an epithelium. In the case of the snail Limnea, the young egg cells detach themselves from the wall, wander in the lumen by amoeboid movement, and settle down again: their region of adherence becomes the antipole (traditionally called vegetal or vegetative pole) and, most probably, the polarity axis is established in that way (Bretschneider and Raven, I95I). In the Portuguese 

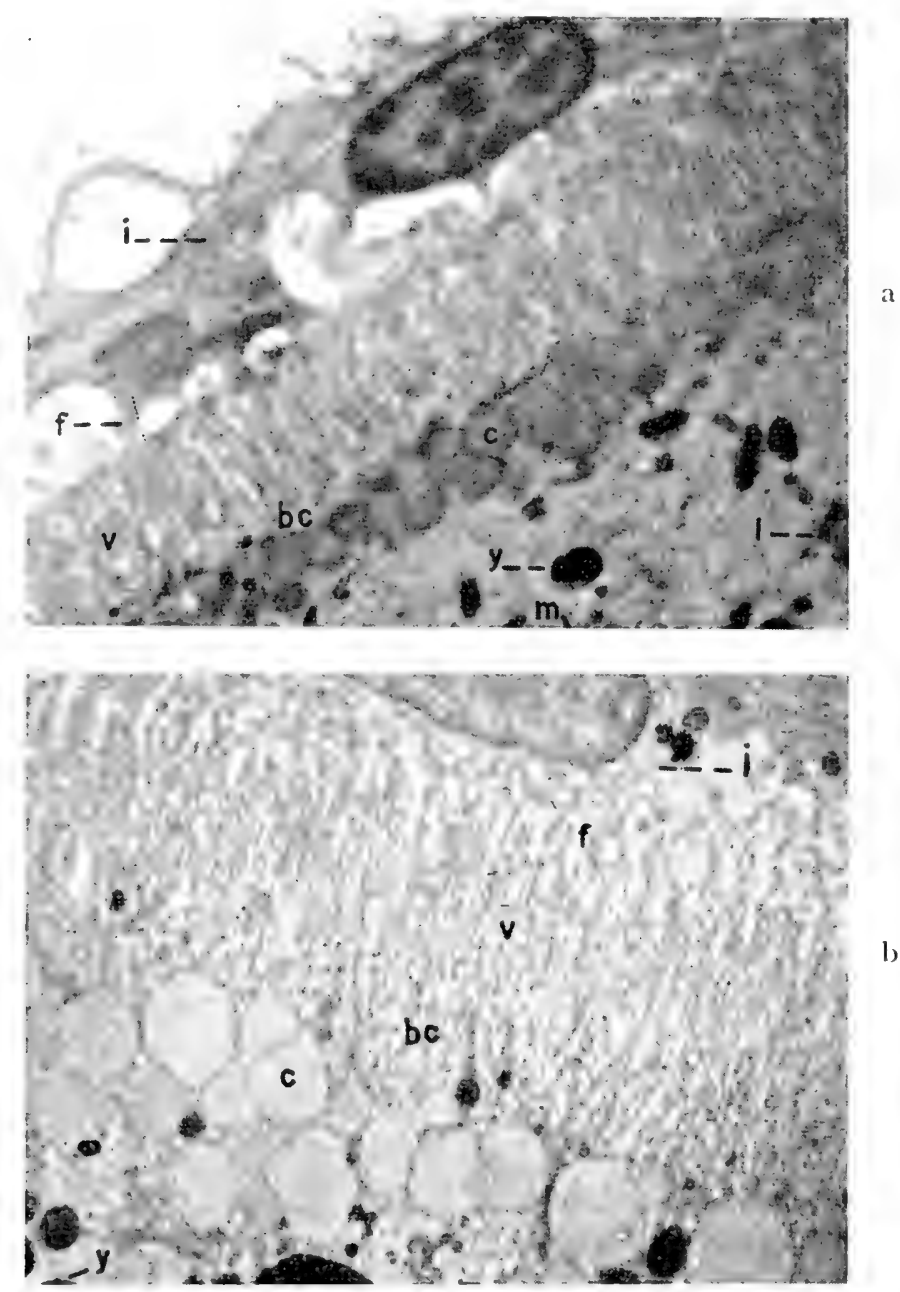

b)

Fig. 6. Electron microscopic pictures $\left(\times 5^{8} 30\right)$ of the cortex of frog oocytes. (a) at the appearance of the first yolk platelets $(y)$ : the cortex contains the layer of cortical granules $(c)$, the basal cortical cytoplasm (bc) and the zone of microvilli $(\mathrm{v})$ which correspond to the zona striata and have protruded gradually; protoplasmic follicular cells (i) extend toward the microvillous layer. 1 , lipochondria, $\mathrm{m}$, mitochondria; (b) similar region at a later stage: the cortical granules have enlarged, the basal cortical cytoplasm is now folded into alternating ridges and valleys, and the microvilli extend from the peaks of the ridges. Note also that the follicular processes (f) traverse the space between oocyte and inner follicle cells (i). Courtesy of Kemp, 1956.

oyster, the stalk of the pear-shaped oocyte is indicative of the former implantation on the gonadic epithelium. Moreover, this region remains free of yolk and forms an antipolar plasm filled with ribosenucleic acid (or RNA); no chorion deposit appeared there, so the membrane preserves a definite landmark (Fig. 2) of the antipole (Pasteels and Mulnard, i957). In non-tubular ovaries, such a relation 


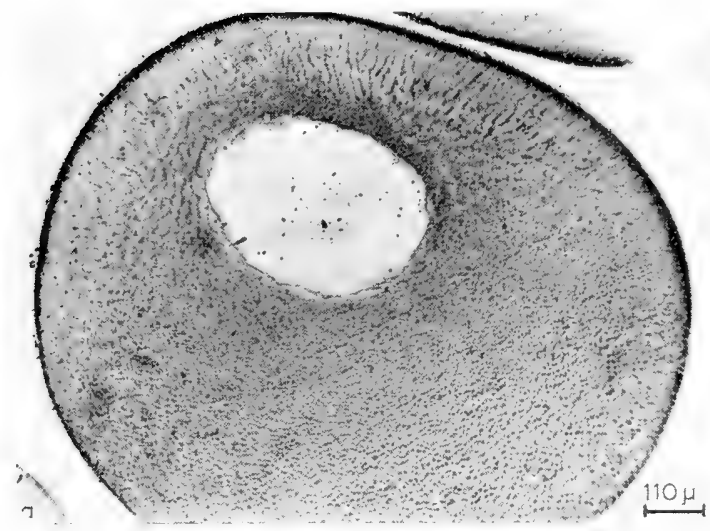

a

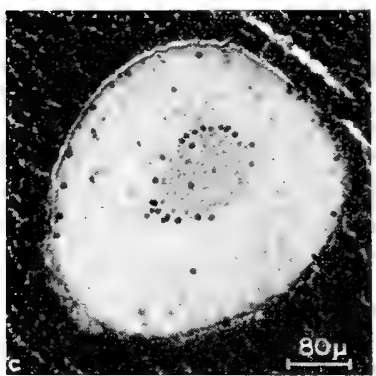

c

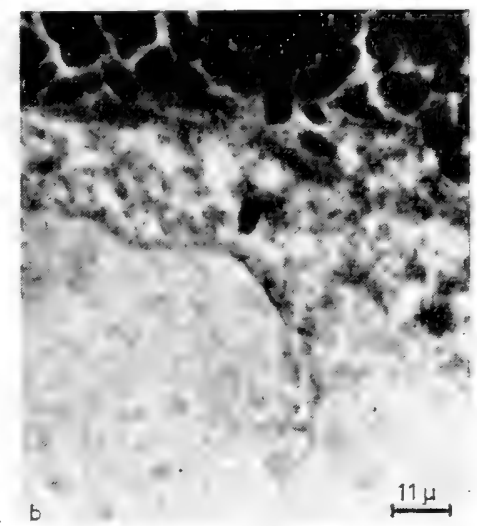

b

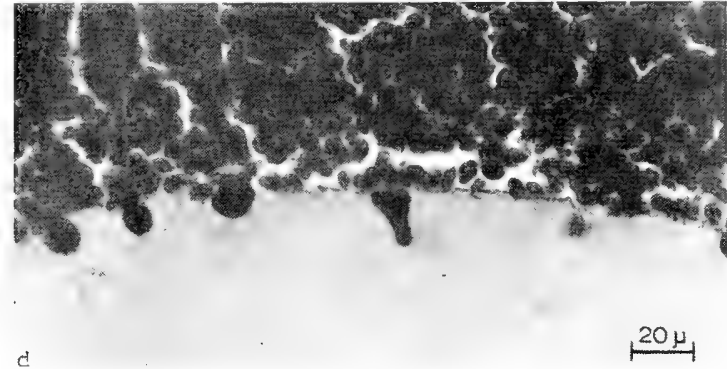

d

Fig. 7. Secondary vitellogenesis in Amphibians. (a) Xenopus oocyte (Zenker, Unna-Brachet stain): the section is probably sagittal, on the animal side of the nucleus; a basophilic zone, where secondary vitellogenesis begins is visible; (b) Part of a frog oocyte (Bouin, trichrome) showing from bottom to top the nuclear content, the deeply folded nuclear membrane, the perinuclear production of small platelets, and the primary yolk; (c) the nuclear zone of a newt oocyte (Zenker, Azan): the large nucleus appears clear in the dark background of yolk; in the center, chromosomes (grey) and a swarm of central nucleoli; at the periphery, other nucleoli against the membrane; (d) Same material and technique: detail of the scattered nucleoli inside the nuclear membrane. From Wittek, 1952.

cannot, of course, be found. Polarization results from the location of paraplasm or deutoplasm on the side of the nucleus where the centriole and Golgi system are combined with much RNA (Fig. 3). This complex called the yolk nucleus, is less an organ of yolk elaboration than a structure controlling the location of yolk (Chandhry, r952). In the teleost Lebistes, a definite change of polarity takes place during the growth of the oocyte. The germinal vesicle first lies in the region farthest from the ovarian hilus; during the late period of vitellogenesis, the vesicle gradually moves nearer to the hilus and consequently the axis turns through an angle of $90^{\circ}$ (fig. 9). The cause seems to be the unilateral formation of a special plasm containing large granules (Vakaet, I955). For amphibian oocytes ${ }^{1}$, it has

1 The relative content of newt oocytes in RNA and proteic components at the successive stages of growth has been estimated by Osawa and Hayashi (1953). The young cell is first loaded with RNA, but the synthesis of ribonucleoproteins is soon largely surpassed by the formation of new cytoplasm and yolk. 

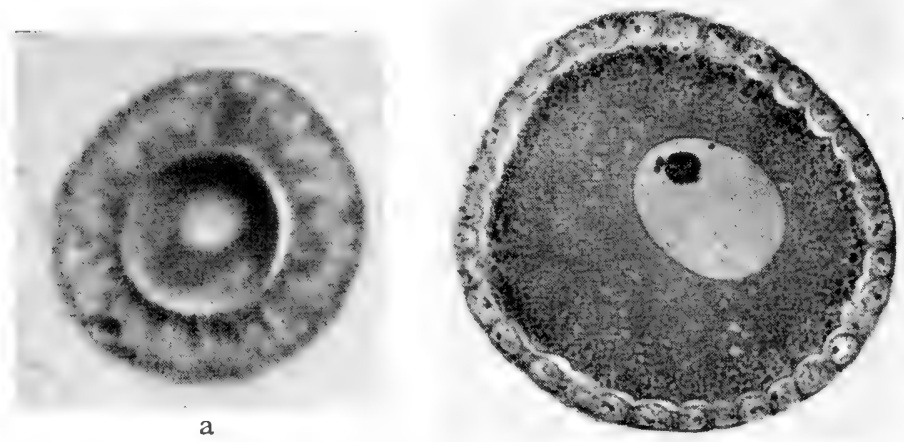

b

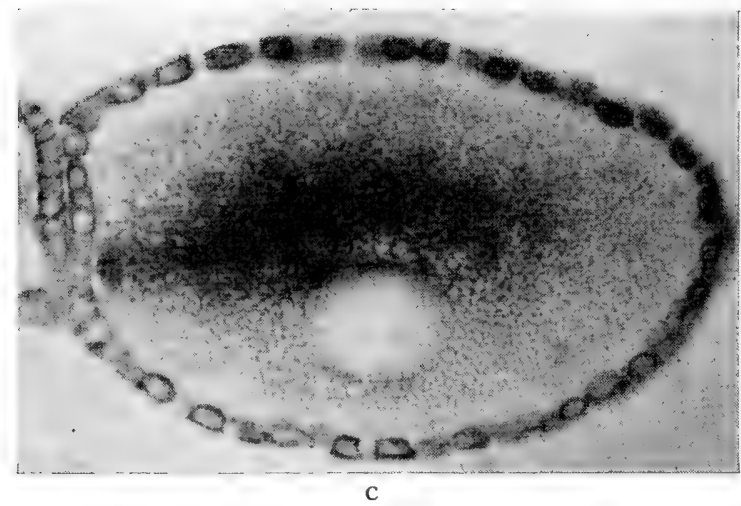

Fig. 8. Oogenesis in a bean-weevil, (a) young oocyte (Serra, Unna-Brachet) with much RNA at the animal pole and the beginning of peripheral vitellogenesis in other parts; (b) more advanced follicle (fixed in Bouin-Allen; transverse section; stained with ironhematox.) showing the peripheral yolk granules (dark); (c) longitudinal section of an oocyte in full vitellogenesis (ethanol, Unna-Brachet) with its central remnant of RNA, extending to the posterior pole. From Mulnard, i954.

been established that the first sign of polarity is the "yolk nucleus" and that the location of this structure has no constant relation to the axis of the ovary. But the "yolk nucleus" elaborates only lipids, and stimulates the growth of the antipolar cytoplasm, while vitellogenesis first takes place under the cortex and progresses centripetally ${ }^{1}$ (Fig. 4). In this early period of vitellogenesis granules are produced which crowd the cortex of the pole, and which are detected by several tests used for mucopolysaccharides (Fig. 5); they seem to be prepigment granules (Voss and Von Wartenberg, 1955; Von Wartenberg, 1956). Simultaneously, an elaborate structure appears in the cortex. Studies with electron microscope (Kemp, I956) show that the aspect of the zona striata known in the chorion is due to microvilli of the cytoplasm interdigitating with the finger-like processes of the follicular cells ${ }^{2}$ (Fig. 6). This increase of contact evidently favors assimilation and growth of the

1 This is also true in fish eggs ( $c f$. Konopacka, 1937; Yamamoto, 1956-7).

2 Similar villi also exist in invertebrate oocytes (Rebhun, 1958). 
egg. The filling of the cytoplasm with yolk is completed by a special perinuclear process of secondary vitellogenesis (Fig. 7). In arthropods, the earliest suggestion of polarity seems to be the greater amount of RNA on the side of the still spherical oocyte nearest the germinal vesicle (Mulnard, I954). The antipole sometimes shows a definite "yolk nucleus" (chilopods). This region grows intensely, while yolk begins to be elaborated around the entire periphery and to accumulate centripetally. The characteristic elongation of the egg occurs only secondarily, in the hind part of the ovigerous tubes. The nuclear pole generally corresponds to the dorsal side of the mother (Fig. 8).

From these examples, it can be concluded that polarity is nothing transcendental, and is not the expression of some intimate property which could be related to a crystalline structure or to a uniform orientation of macromolecules. Polarity is both inherited and acquired. It is inherited in the sense that it results either from the structure of the ovary - a feature of the preceeding generation-or from some attraction which associates the centriole, the Golgi vesicles, and the RNA rich granules in a complex system. It is acquired in the sense that trophic, extrinsic relations certainly play their role, as do also the physical conditions of equilibrium among the substances elaborated in the cytoplasm.

Also, polarity is the direct consequence of the formation of a giant cell in which the nucleus and the centro-Golgian complex remain unique. It depends upon the direction of the nutritive flow into the egg. It must be realized that the origin of these nutritive materials may vary: they may be synthesized in the egg with the help of the adjacent cells, which eventually form a follicle; or they may be directly transformed into yolk by specialized vitelline cells; or they may be elaborated at a distance, as the yolk nucleoproteins of birds and fishes which are synthesized in the liver ${ }^{1}$ (Clavert, I952; Clavert and Zahnd, I956, 1957).

\section{Symmetrization during oogenesis}

In several instances it must be admitted that a bilateral symmetrized structure is present in the unfertilized egg. There are at least four cases, as far as I can see, in which the acquisition of symmetry can be followed in the growing oocytes. The first case concerns the insect egg, where the correspondence between the cephalo-caudal axis of mother and embryo has long been known (Hallez' rule). Here the symmetry certainly depends upon the form of the ovarian cavity and oviduct, and it is confirmed by the general existence of the posterior polar plasm and sometimes by other details of cytoplasmic structure, recorded in Drosophila and in Acanthoscelides (Fig. 8, c). In the viviparous teleost Lebistes, the eggs of which are fertilized in the ovary, and develop in situ, a granular plasm, already mentioned to explain the change of polarity, develops quite early in oogenesis on one side of the nucleus, and endows the oocyte with a visible bilateral organization (Fig. 9). According to Fautrez and Vakaet (1954), this early established

1 See also the investigations, based on isotopic and serological methods, of Flickinger and Rounds (1956). 
plane of symmetry is preserved during the whole development ${ }^{1}$. In the growing oocytes of amphibians, a bilateral symmetry is often, but not always visible, owing to the distribution of yolk, pigment and RNA (Fig. 7, a). In this case, it seems to be caused, at the origin of the oocyte, by some excentricity of the "yolk nucleus".

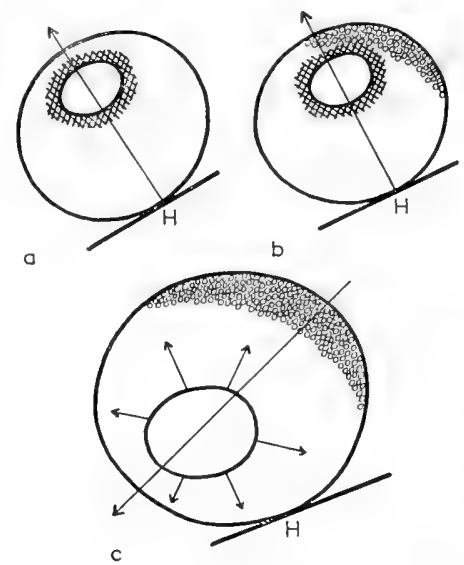

Fig. 9. First steps of growth in the oocyte of the guppy fish, Lebistes retic. $\mathrm{H}$ is the hilum of the ovary given as a point of reference. In a, the nucleus is already excentric, and a primary polarity is established (arrow). While this continues (b), a plasm which is proteic in nature (circles) appears near the primary "animal" region. In (c), growth has proceeded by synthesis of cytoplasm around the nucleus (small radiating arrows) while the germinal vesicle has migrated to a new "animal" region, and the definitive polarity (long arrow) has appeared. From Vakaet, 1955.

Finally, in several mammalian eggs, symmetry is acquired along with polarity. Although a preferential direction of growth is provided by the so-called "yolk nucleus" complex - a still more inappropriate designation, since practically no yolk will be formed - some deviation from this direction seems to be caused by the location of the one or two primary follicular cells. As a result, the primary follicle is distinctly more active on one side of the polarity axis (Fig. Io). In this way, the growing oocyte appears to acquire a denser, more RNA-rich cytoplasm on one side, while the other side is more areolar, and will, in certain species $(e . g$. guinea pig, mole) become loaded with lipids.

The remarks made in connection with polarity could be repeated here. This bi-axial organization is not the expression of any intimate structure of the cytoplasm. It is only a consequence of the anatomical or histological features of the mother organism. But, once impressed upon the egg, it is likely to have important consequences. At this point, let us consider the degree of stability of this primary egg pattern, and where it might be most firmly established in the egg cytoplasm. All the results of centrifugation agree on the stability of the cortical structure of the unfertilized egg. This ectoplasmic layer (or cortical layer, or cortex), which

1 Attention has, however, been drawn (Clavert and Filogamo, 1958) to the fact that the orientation of the polarity axis mentioned above (p. 313 ) is not valid for full grown oocytes, and that the location of the blastodisc itself can be modified by inverting for a day or so the normal position of the mother fish. So, this problem is not quite settled. 

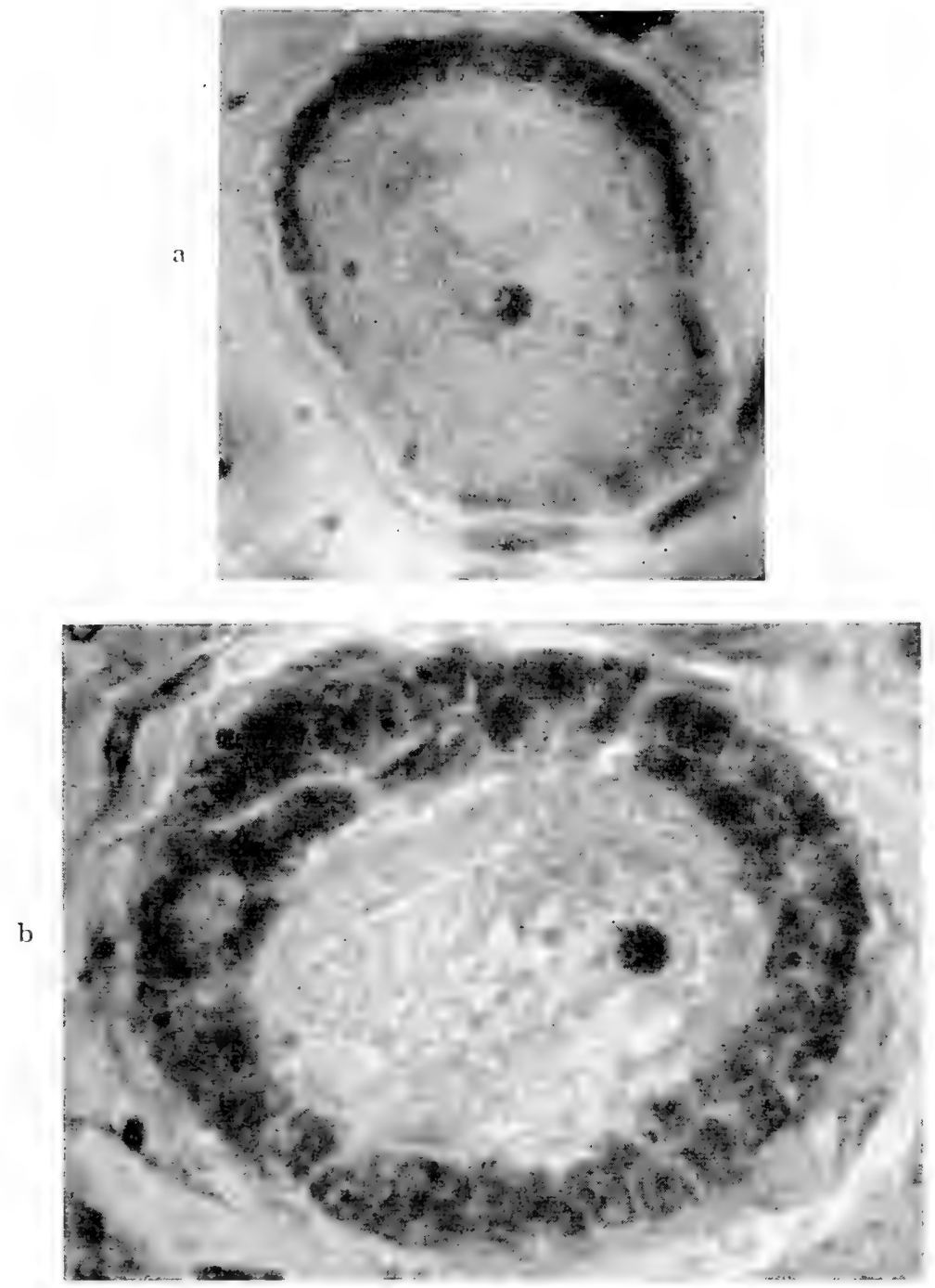

Fig. 10. Early oogenesis in the chimpanzee Pan paniscus. Serra fixation, Unna-Brachet stain. (a) Primary follicle in which the follicular cells are enveloping the oocyte (see also the primary unilateral location of the follicular cells in Fig. 3, p. 309). Polarity is still undistinct. The region near the thicker follicular layer is slightly more basophilic. (b) A young secondary (2-layered) follicle showing the corresponding thicker zone; polarity is acquired, the cytoplasm being slightly denser and more basophilic at the animal pole and on the side of the thicker follicle wall. Orig. material gathered in the Belgian Congo by Professor G. Vandebroek.

is of variable thickness and is often crowded with mitochondria, should not be confused with the plasmolemma; the latter is the thin, homogeneous hyaline layer, enveloped more or less closely by the chorion, which is a secretion of the egg cell. Under higher power (electron microscope) the plasmolemma often shows 
a villous appearance (Fig. 6, b, p. 3I2) which is probably the expression of the intense activity of this critical interface. The cortical layer includes this film plus a variable underlying layer, partly homogeneous, partly crowded with granules and quite difficult to displace by centrifuging. It seems that this cortical layer is the most stable agent of polarity. Not only does it explain why a centrifuged egg is able, after a sufficient lapse of time, to restore the typical distribution of its materials, but it also accounts for the special segregation which takes place in certain eggs with spiral cleavage. Among the species (Schleip's Spiralia, I928) which show this characteristic feature, the one which has perhaps been the most

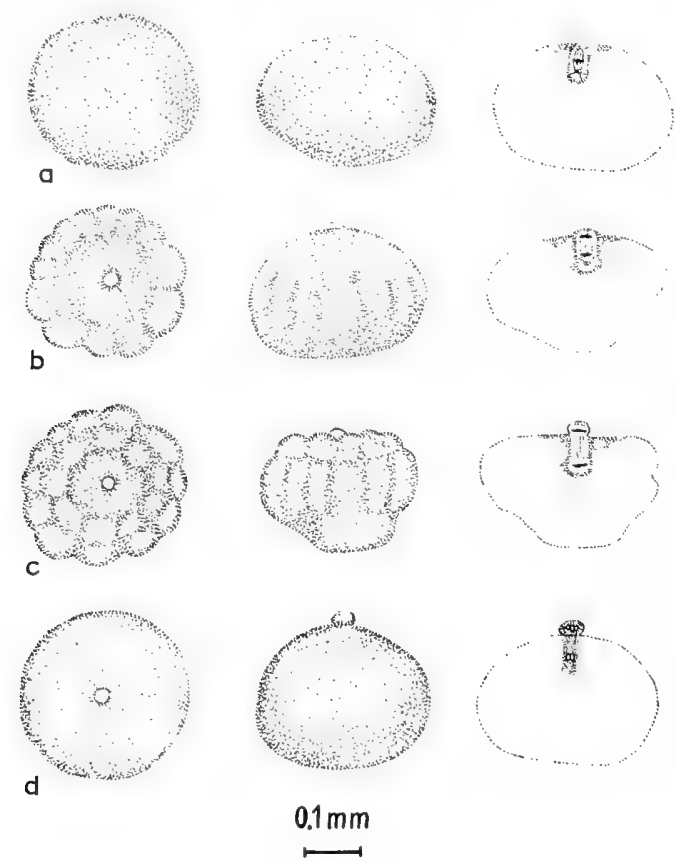

Fig. I I. Ordered and polarized changes of form in the egg of Tubifex, during the formation of the first polar body. (a) Metaphase of the first maturation spindle; (b) early anaphase with the outer pole bulging at the surface; (c) advanced anaphasis with the pinching off of the polar body; (d) telophase. First vertical row: the whole egg, seen from its animal pole at the corresponding stages. Second vertical row, lateral view of the same. Third row shows the progress of maturation. From Rötheli, I950.

thoroughly investigated is Limnea; for this mollusk egg also, the only recognised basis of a possible explanation is that polarity is in some way inscribed in the cortex (Raven, 1948, I958). A quite simple observation reveals this stabilizing influence of the cortex. If a time lapse film is taken of the rupturing nucleus in the transparent oocyte of the bivalve Barnea candida (records of Pasteels and Mulnard, 1957), such a complete whirl of the internal cytoplasm appears that it is impossible to imagine any definite structure or stratification which could exist there and be preserved. And yet, maturation and all further events take place according to the preexisting polarity of the oocyte. That the formation of 
the polar bodies is not an activity limited to the pole, but a process involving the whole egg cell, is immediately apparent in case of the Tubifex egg (Fig. I I).

The unfertilized eggs, according to the species considered, may remain in the state of an oocyte with an unruptured germinative vesicle, may enter maturation (Fig. I 9, p. 328 ), or may expel one, or even both polar bodies before being fertilized. These differences, more important than it may seem, need not be considered here. It should only be emphasized that maturation does somewhat affect the cytoplasmic organization, with biochemical consequences and appreciable modifications of the cortex.

\section{INTRINSIC MORPHOGHORESIS}

Scientific progress sometimes necessitates a renovation of vocabulary. For several reasons (Dalcq, I 949), it seems indispensable to include in one word the processes of cell-division, cell-movements, internal and general transformation which take place before the period in which cells or tissues effectively become different in their inner structure, metabolism and growth. The word morphochoresis will be used here to describe these processes. The term is more or less equivalent to "early morphogenesis", which will be reserved to more general situations. For any particular part of the germinal system, morphochoresis may either result mainly from inherent conditions, or be due to influences of other parts, often by the mechanisms of induction. These different circumstances necessitate the distinction-again somewhat relative-between intrinsic and extrinsic morphochoresis. A discussion of the latter is mainly reserved for our third subchapter (p. 357).

For the sake of clarity, it seems advisable to use a terminology different from the usual one, but somewhat more precise.

The conventional designations of animal and vegetal or vegetative poles should, whenever possible, be replaced by pole and antipole. The three primary layers of vertebrate embryos will be called ectoblast, chordomesoblast and endoblast, instead of ectoderm, mesoderm and endoderm, which should be reserved for advanced or adult stages. During the pregastrulation of birds and mammals, the two layers will be called ectophyll and endophyll, instead of epiblast or primary ectoderm and hypoblast or primary endoderm. During neurulation, the ectoblast will become subdivised into neuroblast and epiblast; thus, the use of these terms immediately tells that stages beyond gastrulation are being considered.

In the analysis of experimental modifications of morphochoresis the words normogenesis and paragenesis will also be used. The first term will suggest that the fate of a germinal part has not been changed by the operation considered. The second term will indicate the opposite result. Paragenesis may, or may not be, oriented toward regulation.

\section{A. General trend and goal of intrinsic morphochoresis}

The realization of morphochoresis evidently results from some unfolding of the properties acquired by the organized system. It is also pertinent that this unfolding is not necessarily stereotyped, and that the system is able to cope with situations imposed upon it by accident or by experiment. On the other hand, 
this ability to adapt efficiently comes to an end just when the active period of kinematics and layer formation is over and cytodifferentiation is about to take place. This change is currently referred to as determination, a concept which needs some immediate comment. Determination, sensu stricto, means the impossibility of changing the fate of cells which have developed beyond a certain critical stage. It has been recognised that, although "determined" cells breed true, they still possess a rather limited potential repertoire of modulations (Weiss, I 953) when exposed to different conditions. It is also well known that in an embryo the decisive stage is not attained simultaneously throughout the whole system. Instead, there arise differences, so to speak, in physiological age. Secondary fields of morphochoresis, like limb buds, tail buds, imaginal discs, echinoid rudiments and similar systems, which are formed later in development, are still capable of regulation or reorganization when other parts of the embryo appear to be fully determined.

Now, there is a theoretical possibility (Harrison, 1933) that an adequate medium could change the characteristics of cells beyond the recognized limits of modulation. There is even one group of recent experiments which apparently show that such may be the case if an amphibian neurula with closing neural folds is submitted to appropriate antisera (Inoue ${ }^{1}$ and Ishikawa, I956). Under this influence, presumptive neural, chordal, somitic, and pronephritic cells regress to a "lower" level of differentiation. This means that in the case of the amphibian neurula, the time of "determination" is now assigned to a sensibly later period than previously reported. Consequently and however antinomic it may seem, determination must be considered only as a relative concept. Nevertheless, the fact remains, and is duly established by numerous experiments, that in the normal inner embryonic milieu, or in equivalent culture conditions, intrinsic morphochoresis necessarily leads to a decisive change, which suppresses the capacity for regulation.

Finally, it is important to state that (relative) determination must be considered at the supra-cellular level more than in the individual cells. Stability is better for large communities of cells (Grobstein and Zwilling, I953; Muchmore, I956). Moreover, again in the case of the amphibian neurula, if the embryo is submitted to cell dissociation by a controlled digestion of intercellular cements, cells of the same strain are able to join together again; these re-associated groups then move over each other until they form a layer-like, nearly normal topographical arrangement (Fig. I2) which at least resembles the normal advanced neurula (Townes and Holtfreter, I955). According to Steinberg (1958), this amazing property reveals the existence on the cell surface of a lattice of ionised acidic groups having some affinity for $\mathrm{Ca}$ and $\mathrm{Mg}$ cations. The spacing of such groups would be different according to the layer or tissue, which would permit cells wandering at random to recognise their likes and make reaggregation selective. Such an enticing interpretation suggests, as does also the para-determination by antisera, that organ specificity is primarily linked with surface changes, whose nature could well orientate cytodifferentiation (see also Spiegel, i954).

This is nearly the way we can actually conceive determination, which looks, in a certain sense, like the goal of morphochoresis. Let us now examine how the

1 According to an oral account at the Providence Conference. 
general pattern sketched in the mature egg can orientate the events of the early stages, and how far these events have already been understood. We have to consider therefore which changes may occur at fertilization, the relation of cleavage to the primary egg pattern, and how and why cell movements appear and result in a discrete diversification of the general system.

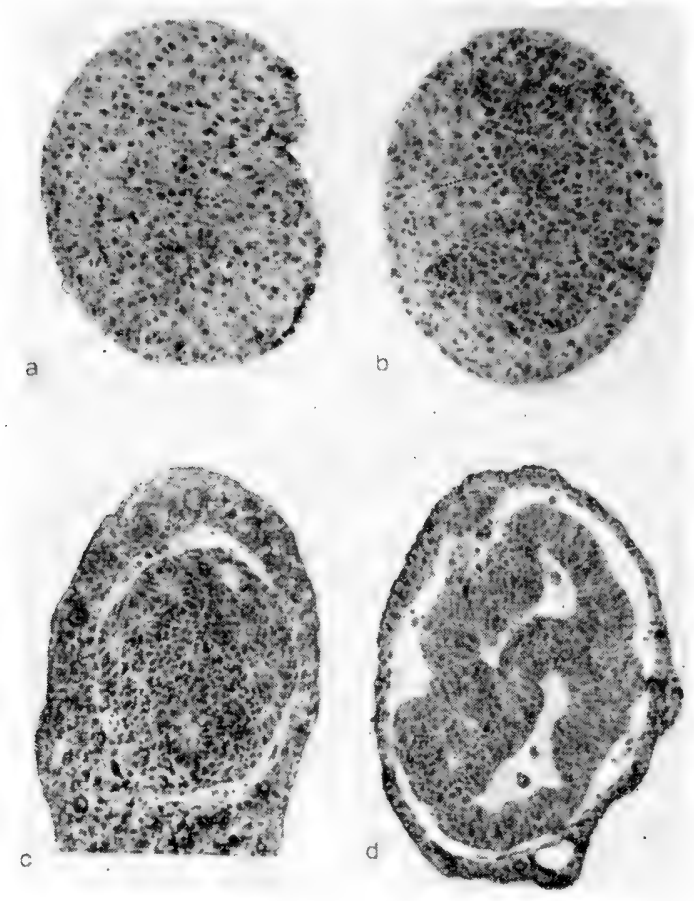

Fig. 12. Successive stages (stained sections of fixed specimens) of "sorting out" occurring in intermingled epiblast and neuroepithelial cells in an Amphibian. A piece of neural plate (without archenteric roof) and one of epiblast have been excised, and disaggregated by means of alkali. Under readjusted conditions, the cells reaggregate (a) and subsequently segregate (b, c, d) so that the surface of the explant becomes epiblastic and a brainlike mass is reformed inside. From Townes and Holtfreter, I955.

\section{B. Fertilization and morphochoretic pattern}

As strange as it may seem, the influence of fertilization upon the egg pattern is only known - and not yet exhaustively-for one animal group, the amphibians. There exist also some cases in which the ordinary point of penetration is known, as in Ascidians or in Insects, but in which there is no indication, so far, of any effect on the pattern.

Amphibian eggs have been known for a long time to show, shortly after fertilization, a semi-macroscopical picture which permits recognition of the future dorsal side. This is due to a general "upwards" movement of the cortical layer and its covering pellicle in this region, which pulls the pigment up after it (Fig. I3). Simultaneously, a slight ascension of the somewhat modified yolk mass takes place 
in the same direction (Fig. I4, b). The result of this rather complex change is a crescent shaped zone which is either of a lighter tint or distinctly grey, because there is an inner yolk wall covered by a thin veil of melanic pigment. The region endowed with this grey or lighter crescent will be the site of the blastopore, and is called dorsal. In Urodeles, such a "clear" crescent is simply due to a general reaction of the cytoplasm, without any apparent relation to the place of fertili-

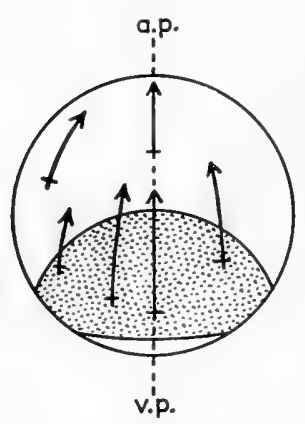

Fig. 13. Displacement of tiny electrolytic marks placed on the pellicle of a frog egg. The dotted area is the region where the grey crescent will later appear. For each of the marks, the cross indicates the initial location, the arrow, the point attained. Note the direct upward movement of the marks placed in the mediosagittal plane, the convergence of the others toward this plane. a.p., animal pole; v.p., vegetal pole. From Ancel and Vintemberger, 1948.

zation. In frog eggs which have been activated by pricking, the same absence of correlation holds true. However, in normally fertilized frog eggs, the grey crescent is always opposite to the point where the sperm has penetrated. This point is consequently medio-ventral. Moreover, it can be demonstrated that if fertilization is experimentally localized, the spermatozoon may penetrate at any point either in the polar or in the equatorial region, and the grey crescent will be just opposite (Ancel and Vintemberger, I 948). In this case, bilateral symmetry is determined by the experimenter, and may be said to be acquired by epigenesis (Fig. I4).

However, the question is whether or not this conclusion holds for eggs fertilized under normal conditions, i.e. when the female lays her egg in a pool. It is not impossible that some part of the cortex would offer more favorable conditions for the adhesion or incorporation of the spermatozoon, either by the chorion being richer in fertilizin or being simply softer or thinner. No one has so far found a way for testing this hypothesis. Pointing in favor of a non-random place of penetration is the fact that indices of bilateral organization have been observed in the growing oocyte (p. 316). The discrepancy existing, in this problem, between some anurans and the other amphibians, suggests that the primary state of affairs is the existence in the egg of a tendency to acquire its own, more or less preformed bilateral organization, and that the epigenetic situation is a secondary acquisition of some groups.

Whatever the issue may be, the mechanism of symmetrization, in itself, has been remarkably elucidated. The development of the spermatic aster is the primum movens. Being somewhat eccentric, it repels the big yolk platelets of the subequatorial region towards the opposite side of the egg. The yolk wall formed in this way has, for some unknown reason, an effect on the cortical layer and the hyaline pellicle. It produces in these layers a movement which begins dorsally, continues polewards, proceeds ventralwards, and stops against the yolk mass (Figs. I 3-I 4 ). 

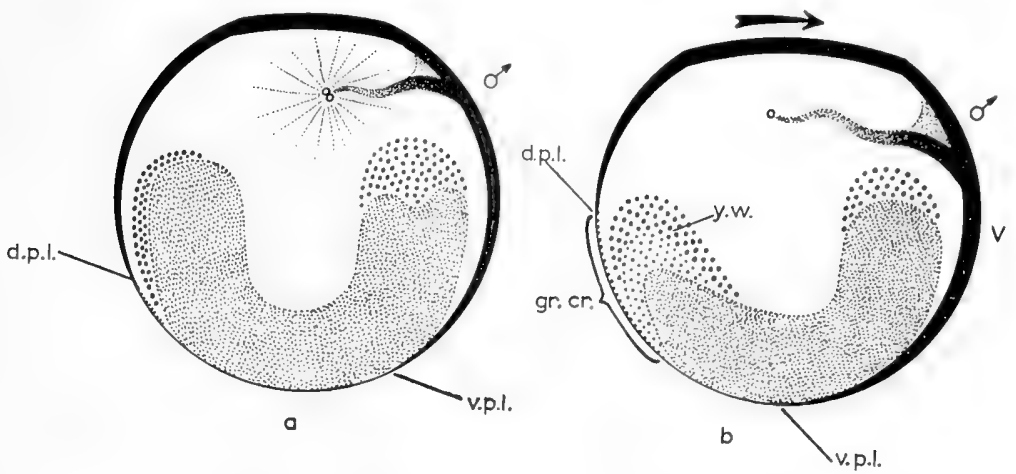

Fig. 14. Modification of the internal structure of a frog egg after fertilization. (a) The egg was oriented animal pole up, fertilized by a tiny drop of sperm suspension between the animal pole and equator, fixed $\mathrm{I} h$. later and cut along its axis and in the plane of the pigmented spermatic track $\left(\delta^{\circ}\right)$. The spermatic aster has developed and the two pronuclei have joined in its central area. The vegetal cupule formed of big platelets and due to primary vitellogenesis ( $c f$. p. 3 I4 and Fig. 4-7) begins to disaggregate both near the spermatic track and opposite to it. The inferior limit of the pigment, primarily horizontal, has begun to move, the side opposite to the sperm-entrance point elevating (d.p.l.), the other, lowering (v.p.l). (b) Egg treated the same way but fixed $2 \mathrm{I} / 2 \mathrm{~h}$. after localized fertilization: The spermatic aster has faded, the spermatic track leads to the fused pronuclei, the yolk cupule has modified even more, especially in the part farthest from the sperm entrance point, and the yolk wall (y.w.) has been formed there. Its presence under a shallow layer of cytoplasm and some remnants of melanic pigment result in the aspect of the grey crescent (gr. cr). From now on the dorsal side (d) and the ventral side (v.) are fixed, if the polarity is not experimentally modified (see p. 343). From Ancel and Vintemberger, $194^{8 .}$

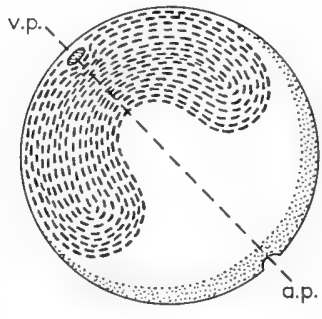

a

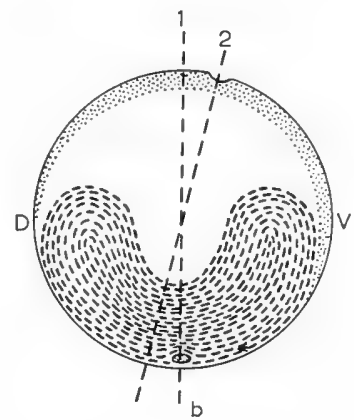

Fig. 15. Schematic representation of the changes occurring in a frog egg submitted to a symmetrizing rotation. (a) The egg in its initial position (Fig. 30, a) with its pigment (dots) and yolk cupule (dashes) quite symmetrical relative to the polar axis. The animal pole (a.p.) bears the maturation depression; the vegetative pole (v.p.) has a small pigmented dot serving as a landmark. (b) The gravitational rotation of the egg inside its somewhat viscous perivitelline fluid has first brought the polar axis to a vertical position (interrupted line I) but the gliding of the cortex, shown by the pigment, has transferred the maturation depression toward the side opposite the direction of the rotation (broken line 2). This side will be ventral and the yolk wall and grey crescent will appear opposite (under D). From Ancel and Vintemberger, $194^{8 .}$ 
The remarkable fact is that the situation acquired in this way is still modifiable for a certain lapse of time. Up until the fusion of the pronuclei, adequate manipulations, consisting in reversing the egg and thus causing it to rotate by gravity in a chosen direction (Fig. 3o, a, b, p. 343), will change the plane of symmetry. In this simple but clever experiment the main axis, from pole to antipole, remains unchanged, but the dorso-ventral axis can be displaced at will (Fig. I5). The reason lies in a diffuse traction exerted by the chorion on the pellicle and the associated cortical layer, through the viscous perivitelline fluid; this movement stretches the yolk of the antipole which modifies the original yolk wall or produces a new one. Thus, a reciprocal interaction exists between the cortical layer and the underlying endoplasm. Until premitotic activity more or less fixates the situation, the same general effect may be obtained by a process starting either in the endoplasm (normal course of events) or in the cortex (orientated rotation).

What importance are we to conceed to these results? Are they simply experimental findings, of limited interest because they concern only the amphibians, or do they reveal of a more general mechanism at work in normal development? This second possibility holds true at least for birds, especially for the pigeon. In these eggs, the probable orientation of the dorso-ventral axis is given by the Van Baer's rule. This prognosis is based on the form of the shell (round and pointed end) which corresponds inside to a definite torsion of the chalaziae. The torsion suggests that, during the time when the egg descends in the oviduct and is gradually covered by the layers of albumen, it could be normally submitted to a prolonged rotation somewhat similar to the conditions in amphibians. This
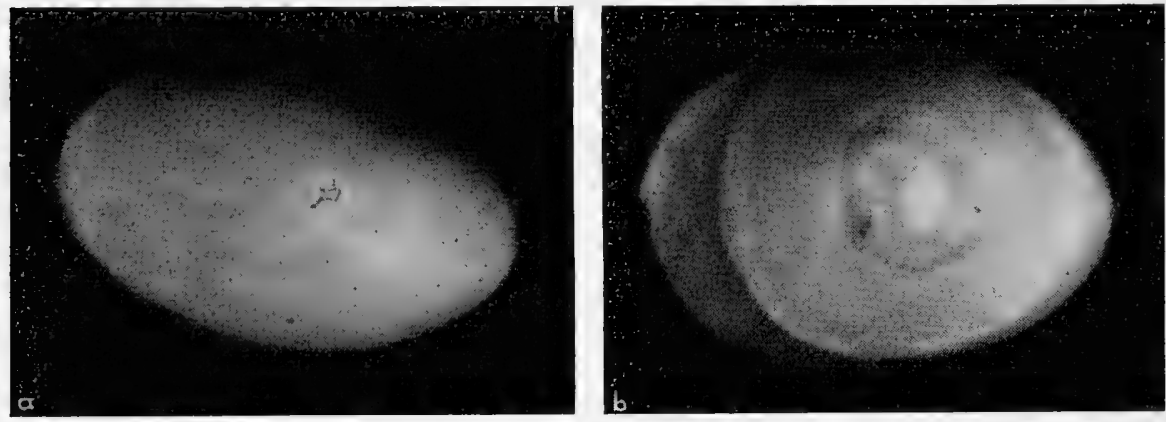

Fig. I6. Cleavage and gastrulation in Camaesura anguina (Lacertilian). (a) The early blastoderm divided into approximately 6 blastomeres (good example of partial segmentation); (b) the expanded blastoderm nearly covers the upper half of the yolk; in its center a large area pellucida can be seen with aspects of yolk desintegration at the periphery and the embryonic shield in the center. The immigration process, accompanied by medio-dorsal convergence and axial, cephalo-caudal stretching is already nearly completed. The limit of the homogeneous spreading mesoblast is perceptible outside the medullary plate; the transverse fold of the brain is emerging, limited anteriorly by an amniotic groove (posterior to the proamniotic crescent). The axis of the embryo is perpendicular to the axis of the egg, as if v. Baer's rule for birds were valid for reptiles, which appears more and more likely (see p. 325). Courtesy of Prof. J. Pasteels. 
hypothesis has received experimental support, and also explains the puzzling exceptions to the v. Baer's rule (Clavert and Vintemberger, I954, I957, 1958; Vintemberger and Clavert, I958a, b). A similar line of investigation has been started (Fig. I6) on reptile eggs (Pasteels, I955b, Clavert and Zahnd, I 955). The results although still limited, are in agreement with the preceding views. It may seem astonishing that such processes of reorganization take place in these amniotes, in eggs which are not only fertilized, but already divided into several blastorneres. This is indeed a difficulty, but we must remember that in these telolecithic eggs there exists, during the first steps of cleavage, a zone of cytoplasmic continuity under the blastomeres, a device which might explain the general effect of the orientated rotation.

In mammals, as stated above, symmetrization can be demonstrated in several species before fertilization. In spite of thorough studies (De Geeter, I954; Dalcq, I955, I956a) no modification of the pattern has so far been detected at fertilization; however, the method used was not experimental, and perhaps not completely adequate. It has been claimed (Ancel and Reiss-Brion, I956) that in the mouse conditions existing in the oviduct and uterus could influence the orientation of the embryo. The problem is certainly not yet solved.

If a conclusion may be drawn from this too limited inquiry, it seems probable that the acquisition of a polarized and symmetrized pattern is generally realized during oogenesis, although terminal growth may more or less veil these features. In some cases, the site of fertilization may modify the previous organization and impose a new plane of symmetry. In the same cases, subsequent manipulations may again re-transpose the dorso-ventral axis. This mechanism acts normally in birds and probably also in reptiles in order to impose the final symmetry of the embryo. An analysis of these processes discloses a reciprocal influence between the cortex and the endoplasm. This is one of the important consequences of fertilization (see p. 343).

\section{Cleavage and morphochoretic pattern}

It has long been recognized that to a variable degree and according to groups cleavage can be affected by the morphogenetic organization of the fertilized egg. Among chordates, this correlation is most evident in tunicates; it was later found to exist also in the Amphioxus (Conklin, I932) so that it is considered a characteristic of protochordates. In amphibians, the relations are much looser, but in cases where the first division is in the frontal plane, the dorsal blastomere is usually smaller, which means that the first spindle was located more dorsally, due to some influence of the light (or grey) crescent. If the first furrow is sagittal, the second will also be displaced dorsalwards. In reptiles, and also in monotremes (Flynn and Hill, I939), cleavage may also show some symmetry, but the correlations are not so clear. In the rat ${ }^{1}$, the orientation of the first and second furrow

1 cf. Dalcq, I954b, r955. A cytochemical reaction has now (1959) detected a cortical differentiation with a field pattern, the maximum intensity corresponding to the dorsal (embryonic) half of the egg.

Literature p. $4^{8} 3$ 


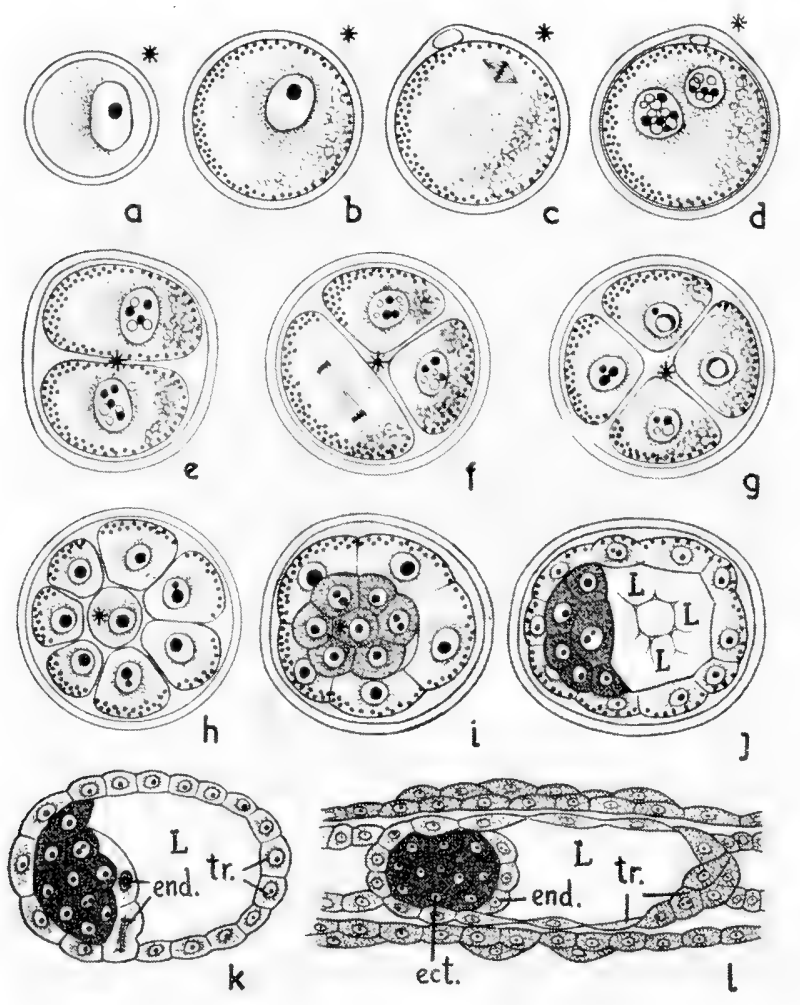

Fig. I 7. Patterns of basophily due to RNA corpuscules, in the first week of the development of the rat; an asterisk labels the animal pole; (a-d) seen obliquely, (e-i) along the primary axis, $(\mathrm{j}-\mathrm{k})$ more or less along the primary axis. Big dots signify cortical mitochondria, small dots mainly internal mitochondria ; in (b-g) right, the lacunar appearance of the "ventral" cytoplasm is suggested. (a) Young ovarian oocyte, from a primary graafian follicle; (b) nearly ripe oocyte from a tertiary follicle; (c) ripe unfertilized egg, with second maturation spindle; (d) fertilized egg aged about I 2h.; (e) 2-cell stage (second day, from 30-45h. old); (f) 3-cell stage (third day, about $56 \mathrm{~h}$.), with two recently formed blastomeres, the other cell in anaphase; $(\mathrm{g})$ 4-cell stage (about 60-70h.) where there is a distinct symmetry pattern; (h) 8-cell stage (fourth day, $75^{-80 h}$.) with the blastomeres arranged nearly in one plane and the same symmetry pattern; (i) young blastocyst $\left(85^{-90} \mathrm{~h}\right.$.) with the first appearance of a lecithocoele $(\mathrm{L})$ and the distinction between an enveloping layer, formed by the four large cells of $(\mathrm{h})$, and an inner 8-cell mass; tiny RNA granules have just appeared here; ( $\mathrm{j}$ ) somewhat elongated blastocyst of the fifth day, with a larger blastocoele (or lecithocoele), a continuous enveloping layer of decreasing thickness, the inner-cell mass intensely packed with RNA granules; ( $\mathrm{k}$ ) young cylinder stage (sixth day), still free in the uterus, with the migrating endophyll (end.), while the rest of the enveloping layer becomes the trophoblast (tr.); (1) cylinder stage (eighth day) which has penetrated a uterine gland, its trophoblastic part ahead, and is preparing to implant; increasing basophily of the whole embryo. For the general appearance of further stages, see Fig. 37, e-i, p. 364 . RNA is then abundant in the mesoblast and in the induced parts of the ectoblast. From 

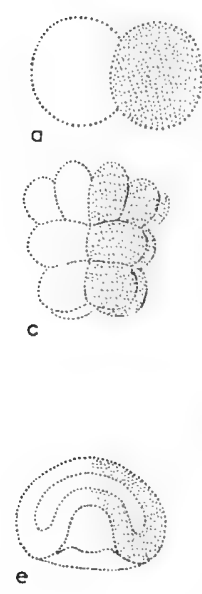

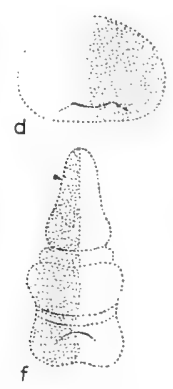

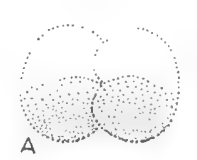

$$
\text { A }
$$

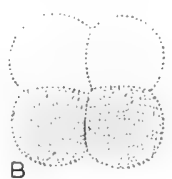

$B$
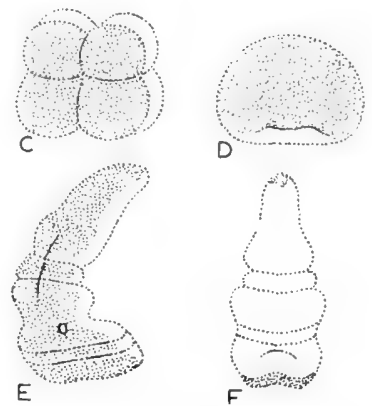

Fig. 18. Coincidence of the first cleavage with the plane of symmetry in the egg of Saccoglossus kow. (enteropneust). (a) Nile blue vital staining of the right $1 / 2$ blastomere; (b) to $(f)$ further cleavage, gastrulation, ventral view of the embryo. $A, B$, Nile blue staining of the $2 / 4$ blastomeres; $C, D$, further cleavage and gastrulation; $E, F$, dorso-lateral and ventral view of the larva. From Colwin and Colwin, $195 \mathrm{I}$.

relative to the plane of symmetry is as variable as in amphibians, but at the 8-cell stage most of the eggs, if they are not of the tetrahedral type, appear as a slightly oblong cupule in which the four dorsal smaller cells (which divide immediately) contain most of the embryonic material. The four larger cells, which divide more slowly, will become the trophoblast and the endophyll (p. 3I9): the cytochemical activities make the distinction quite clear-cut (Figs. I 7, 20-22). In other mammals such a differential cleavage probably also exists, but the disposition of the cells in a morula makes the distinction less schematic ${ }^{1}$. Among invertebrates, the sea urchin eggs show their micromeres at the antipole, just where the experimental analysis locates the more powerful "vegetalizing" activities. In the enteropneusts (Colwin and Colwin, I95I), a constant coincidence between the first plane of cleavage and the plane of symmetry is again encountered (Fig. I8), as clear as in the protochordates. In the numerous worms, mollusks and related groups commonly refered to as Spiralia, the successive spindles receive a definite location and orientation which, by a special gearing of the processes, results in the acquisition of a relatively bilateral cell-distribution.

It is currently admitted that polarity corresponds to yolk accumulation towards the antipole, and that this mechanical factor plays a role in the orientation of the

1 This is the case for the rabbit egg, in which the careful inquiry of De Geeter (1954) could nevertheless recognize a cytoplasmic organization conforming to the above account. His conclusion finds support in Seidel's recent experiments (1956). The suppression of one $\mathrm{I} / 2$ rabbit blastomere, followed by re-implantation, has been ascertained to yield two types of results. In one type, regulation takes place; in the other, a blastocyst deprived of embryonic knot or primitive streak is obtained. The explanation is that both blastomeres are not equipotent. Only one of them contains the embryonic area of the egg, in full agreement with the cytochemical data concerning the rat (see Figs. I 7 and 20). 

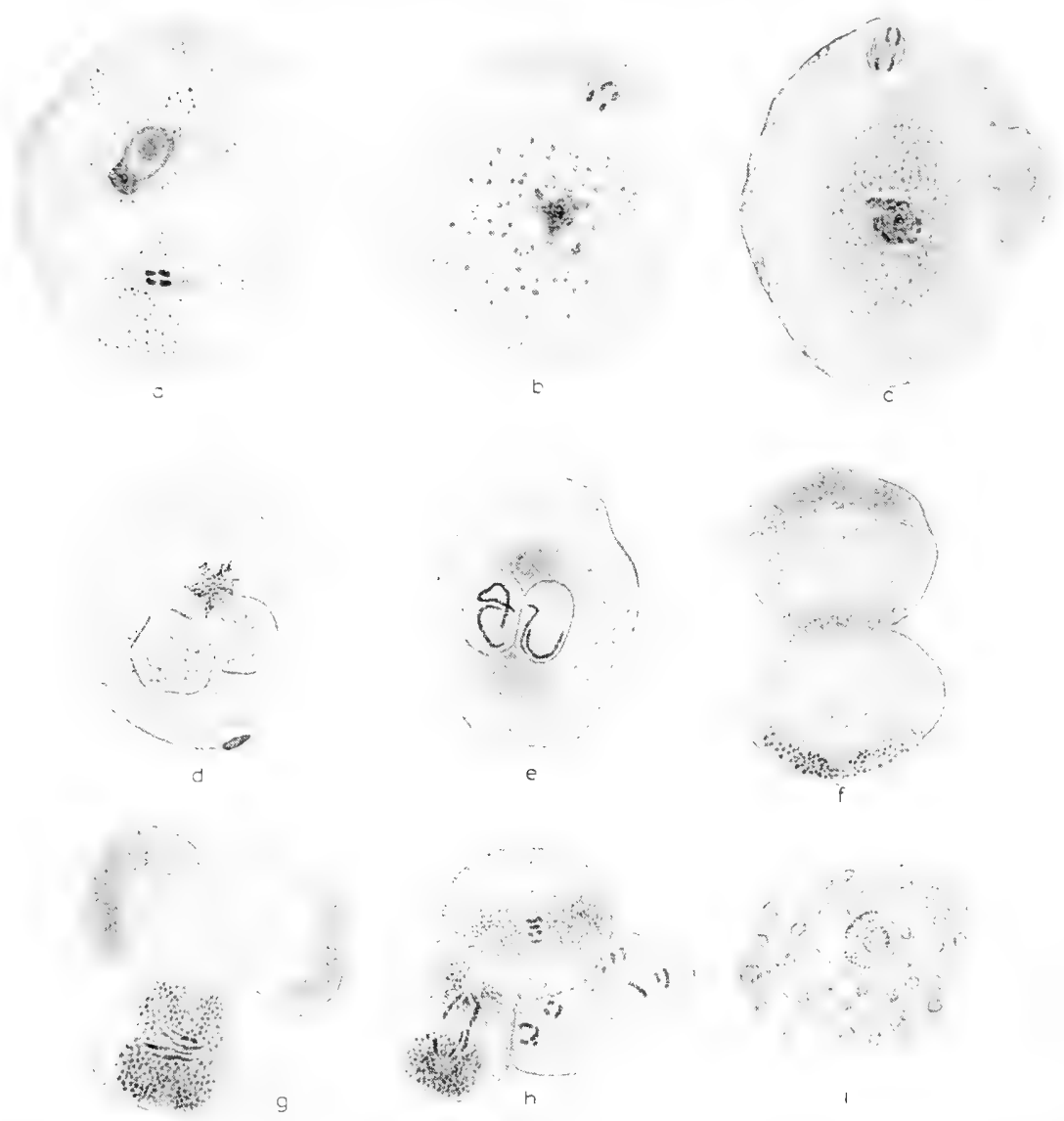

Fig. 19. Chromosome diminution and RNA distribution in Parascaris eq. (var. bivalens) eggs (fixed in Carnoy, sections stained with Unna-Brachet). (a) Shortly after fertilization; the ist maturation spindle is in anaphase with 2 pairs of dyads; the incorporated spermatozoon is hollowed out by a vesicula still containing the refingent sphere (in grey), the cytoplasm is filled with RNA (darker gray), the nucleus is still compact (black); the egg cytoplasm has only some dispersed RNA granules. (b) During telophasis of the same maturation mitosis; more RNA has been formed in the sperm cytoplasm, which disperses progressively; the male nucleus has grown, but still is homogeneous; the RNA of the egg cytoplasm is located in larger granules. (c) Ana-telophase of the 2nd maturation mitosis. The paternal chromosomes disperse in the RNA-rich central area, while the egg RNA granules gather in the surrounding territory. (d) Pronuclei stage, with the 2nd polar body sticking to the egg (below); RNA granules are localized in a starlike structure near the pronuclei. (e) Prophase of first cleavage showing the two centrospheres and the two long chromosomes; each centrosphere contains the same quantity of RNA granules. (f) Telophase of the first cleavage, the larger upper cell $(\mathrm{AB})$ contains the animal pole; the smaller, vegetative cell (P) has more RNA granules in its centrosphere. (g) The AB cell has divided, with diminution of its 4 chromosomes ( 3 visible); the $\mathrm{P}$ cell, in metaphasis, shows asters congested with RNA, especially in the vegetative one. (h) Further stage of cleavage, with complete chromosomes in the vegetative daughter cell of $\mathbf{P}$, where the asters are again RNA-congested, especially the vegetative one. (i) The cell protected by the $R \mathcal{N} A$ granules against diminution of the chromosomes becomes the initial cell of the germline, and is recognizable by its larger nucleus and RNArich cytoplasm. After Pasteels, i 948. 

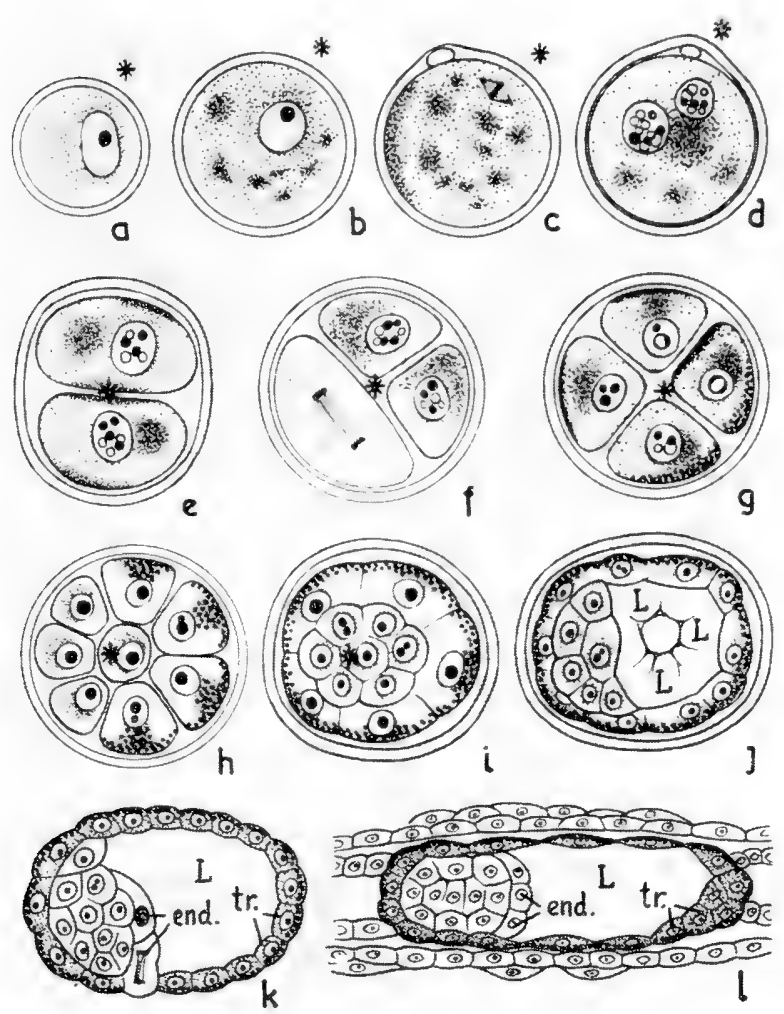

Fig. 20. Distribution of mucopolysaccharides (MPS) as shown by metachromasia in vivo and by other tests. The seriation and presentation of the stages is the same as in Fig. I 7 . The specimens are shown as stained in vivo for $15 \mathrm{~min}$. in a $2 \cdot 10^{-5}$ solution of toluidine blue, at $\mathrm{pH} 7 \cdot 5^{-8.0}$. The young oocyte (a) has only a few scattered granules. The fully grown oocyte (b) has some discrete groups of MPS granules. The maturing unfertilized egg (c) shows metachromasia in scattered granules and in large groups of reddish granules. At the pronuclei stage (d) the reaction is especially intense in the vicinity of the pronuclei. At the 2-cell stage, a large ball of positive granules accompanies each nucleus; MPS are also present in the cortex and between the cells. At the 3 -cell stage (fugace), the dividing cell is distinctly less positive (f). At the 4-cell stage ( $\mathrm{g}$ ) more and larger MPS-loaded granules are found, grouped together near the nuclei and at the surface of the blastomeres, especially in the two larger ones. With the 8-cell stage $(\mathrm{h})$, the blastomeres are arranged in two distinct groups, the 4 larger ones strongly metachromatic, containing columns of large positive mitochondria. The symmetry becomes quite distinct. When these four large cells have enveloped the smaller ones $(i, j, k)$ the enveloping layer remains characterized by an intense metachromasia. Nidation (l) does not noticeably change the picture. From Dalcq, I955.

first spindles (O. Hertwig rule). But this is only a partial view of the picture; the true relations are more complex.

Although these observations are only sporadic, they suggest that segmentation is not independent of the morphochoretic pattern as is generally believed. In all the cases so far mentioned, the two first furrows cut through the primary axis. However, there is a remarkable exception to the rule in the case of the nematodes, 

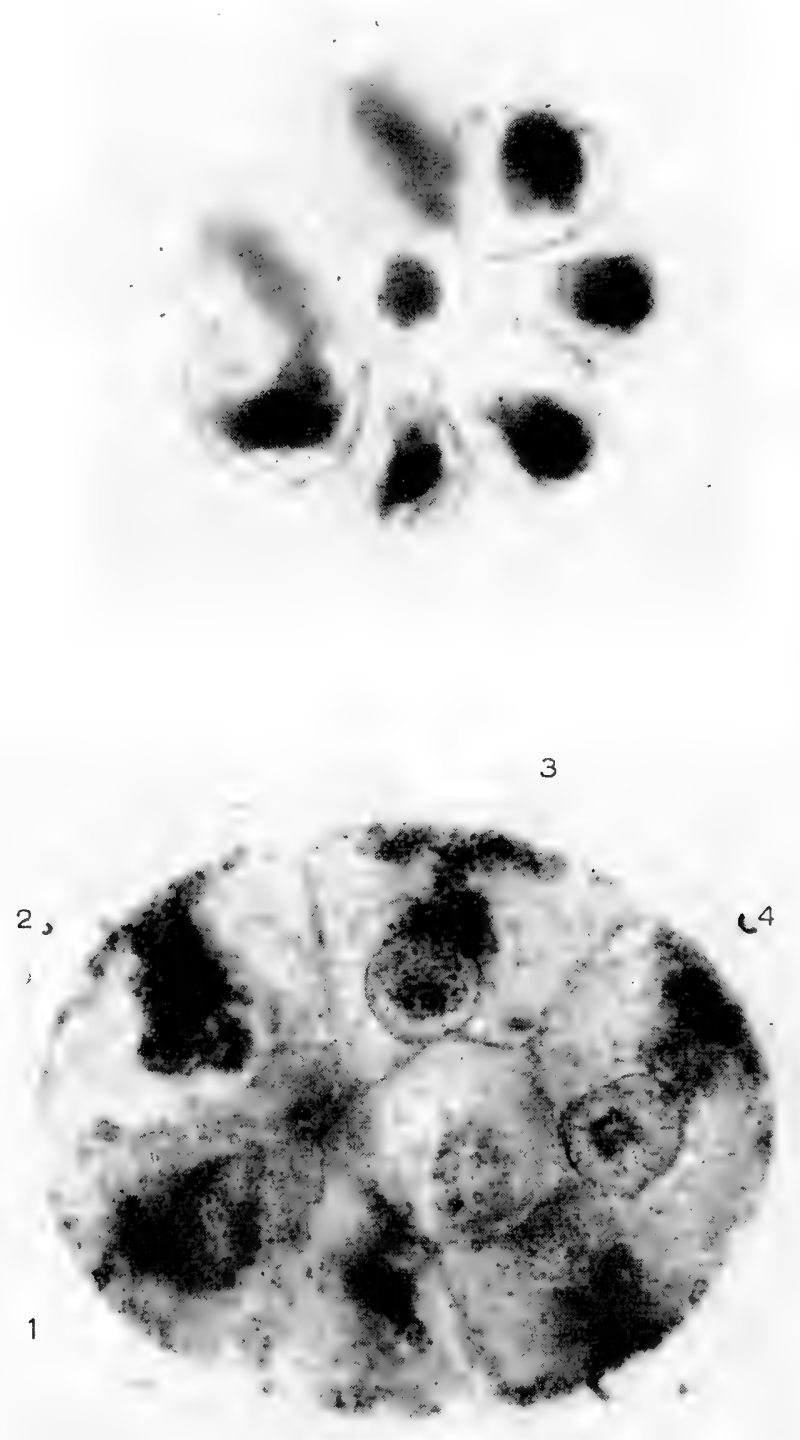

Fig. 21. Toluidine blue metachromasia in the 8-cell stage of the rat. The eggs stained in vivo have been fixed by a special mixture (Izquierdo, 1955) which preserves the stain. (a) A placula-like specimen, with the larger cells below and left, the smaller ones above and right ( 2 out of focus). See the dark masses of MPS-loaded granules, especially developed in the large cells. The symmetry pattern is apparent. (b) A less flattened specimen. The large, enveloping cells are numbered I to 4 ; their columns of metachromatic granules are conspicuous. The smaller, embryonic cells (only 3 visible, I out of focus) contain fewer of these organelles. Photographs of Izquierdo, I955. 
where the eggs divide into polar and antipolar halves. This is really a differential cleavage, for the antipolar blastomere contains more RNA granules, which insure the preservation of the full length chromosomes; while, in the other cell, they undergo "diminution". The cleavage goes on with some spiral deviation, and again one daughter cell of the antipolar half is favored in the same way as in the preceding cycle; this goes on until the primordial germ cells are formed, with their abundant RNA granules and long, whole chromosomes (Fig. I9; Pasteels,

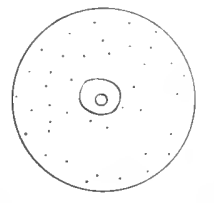

1

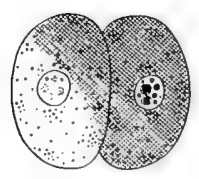

3b

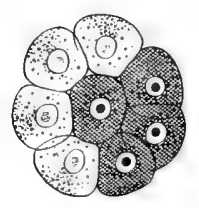

5

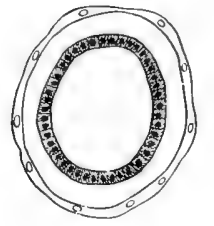

8

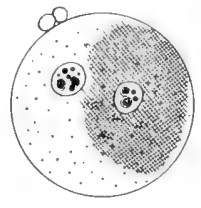

2

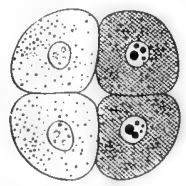

40

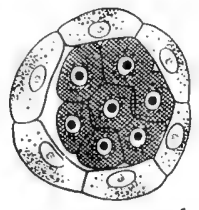

6

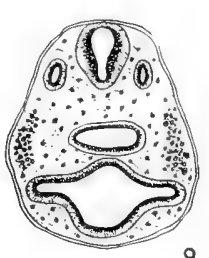

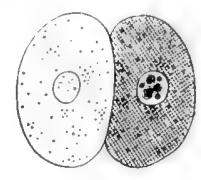

$3 a$

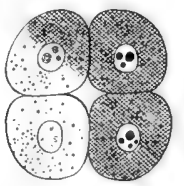

4b

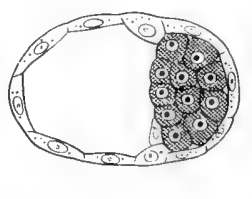

7

Fig. 22. Localization of acidic phosphatases in the rat egg. The oocyte contains only a few scattered granules. After fertilization, the enzyme is present in two forms, a diffuse one (crossed areas) and a granular one (dots). Generally speaking, the diffuse reaction appears in the areas more rich in RN $\Lambda$ (compare with Fig. I 7) while the granular reaction is linked with the MPS-rich organelles. 2-5, cleavage stages; $6-7$, a young and a more advanced blastocyst; 8 , cylinder stage; 9 , young embryo. From 2 to 8 the dorsal part is orientated toward the right of the figure. From Mulnard, 1955.

I948). In Cyclops eggs, a differential distribution of RNA granules can also be shown; this probably paves the way for differentiation of the primary gonocytes (Stich, I950).

It is well-known that in the so-called mosaic eggs, specialized zones of cytoplasm are formed by ooplasmic segregation (Costello, I 945) and are displaced after fertilization and during cleavage. These processes have been most accurately considered by Lehmann and his students on Tubifex eggs (Lehmann and Biss, 
1949; Lehmann, I956; Weber, I956); the recent investigation of Weber (1958) on the submicroscopic organization and on the biochemical features of these eggs has clearly shown that the differences related to the distribution of the classical plasm can be attributed to the relative frequency of mitochondria, ultramicrosomes and other active particles. This conclusion is also valid for molluscan eggs, e.g. Ilyanassa (Clement, I956; Clement and Lehmann, I956). Thereby, cleavage acquires a definitely constructive value.

This positive role of cleavage in the preliminary steps of morphochoresis is also made plain by another, less known trend of research, an account to which will now be given.

In I950, Kelly described in vivo metachromasia in Chaetopterus eggs that has been stained with toluidine blue. This author believed this metachromasia was due to the presence of heparinlike substances, and insisted that this treatment did not in anyway alter the development.

Independently, it was found that when staining rat eggs (Ist to $5^{\text {th }}$ day) with toluidine blue $-\mathrm{IO}^{-5}$, there is a striking appearance of metachromatic granules, which show distinct differences at various stages (Dalcq, I95I). Extended to other mammalian eggs (mouse, rabbit, guinea pig), this procedure has proved a most useful method for revealing various cytochemical processes. Special groups of chromotrope granules, which at first were thought to be mitochondria, display metachromasy, apparently because they are activated by nuclear emissions. Their distribution corresponds to some organization of the cytoplasm (Fig. 20, 21; Dalcq, I954c; Izquierdo, I955). They are more abundant in the ventral half of the egg, and their number and importance increase during the first steps of cleavage (with an apparent reduction during active mitosis). These metachromatic granulations were shown to contain mucopolysaccharides (Fig. 2 I ; Mulnard and Dalcq, I955) and were shown to synthetise acid phosphatase in increasing quantities (Mulnard, I955). In the hyaloplasm lying between these granules an excess of acetalphosphatides (true plasmalogene) could be demonstrated (Dalcq, I956a). More recently, it was shown by using brillant cresyl blue combined with Janus green B, and recourse to centrifugation, that the chromotrope metachromatic granules are distinct from the mitochondria. (Dalcq and De Greef, 1958).

Since i 955, a similar analysis has been performed on various invertebrate eggs, with the advantage that some basic metachromatic dyes behave as truly vital stains in most species ${ }^{1}$. So far the enquiry has now concerned the eggs of sea urchins (Pasteels, I955a, I958; Mulnard, I958; Vercauteren, I958; Brice, I959), of two bivalve mollusks, the clam Barnea and the oyster Gryphaea (Pasteels, I955a; Pasteels and Mulnard, I 957), the worm Chaetopterus (Mulnard, I958) and finally ascidians (Dalcq, I957c, I 959). Admitting some restrictions for the last group, in which cytochemical analysis has to be continued, the chromotrope and metachromatic equipment of these eggs show rather uniform features.

The prerequisite for good vital staining is that the eggs are exposed for a few minutes to the penetration of the dye-the less toxic one being brilliant cresyl blue $\left(3 . \mathrm{IO}^{-4}\right)$ - and

1 Marthasterias glacialis excepted (Brice, r959a). 
then thoroughly washed in sea-water. They immediately show a gradual metachromasy, due to the staining of numerous, just microscopically visible, granules which Pasteels has labelled $\alpha$. In the sea-urchin Paracentrotus these granules also stain by neutral red. A cortical film, corresponding to the vitelline membrane, i.e. the plasmolemma of the cell, is always perfectly unstained. In some eggs (Spiralia) a layer of larger granules which are also seen in the cortex at early stages are metachromatic. They correspond to F. R. Lillie's cortical granules and remain unchanged during cleavage. Their metachromasy is due to mucopolysaccharides.

The true $\alpha$-granules are evenly distributed in the cytoplasm, except in Ascidiella where at first they are mostly located under the plasmolemma of the unfertilized or just fertilized egg. In this special case, they flow towards the antipole, next to the "yellow" plasm, and soon are caught in the rays of the spermaster (Fig. 29, a-d).

Everywhere, centrifugation shows that the $\alpha$-granules (certainly not an artefact produced by the vital staining) are relatively heavy, sometimes even heavier than the yolk platelets (Fig. 27, a, b, c). After fixation, they stain by the PAS technique, even after enzymatic digestion, but not by alcian blue (at a low $\mathrm{pH}$ ) unless the sections have previously been submitted to a strong oxydation (Fig. 28, a, b) ; RNA seems to be absent or scarce; reactions for acid phosphatases (Fig. $28 \mathrm{~d}$ ) are positive except for the case of Paracentrotus eggs. Thus these granules appear to be mostly built up of neutral or feebly acidic mucopolysaccharides, and a protidic moiety which acts as a phosphomonoesterase on the acidic side of the $\mathrm{pH}$ scale.

After a certain delay, a second category of granules, Pasteels' $\beta$, becomes apparent. They are distinctly larger (often more than $I \mu$ ) and less regular, also less numerous, mostly located in the perinuclear or perifusorial area (Fig. 23; 28, c) where their presence in unstained eggs (Barnea) can be ascertained by phase contrast examination. In Spiralia eggs, where the staining can be performed on oocytes, some granules already exist around

(text continued on $p .336$ )
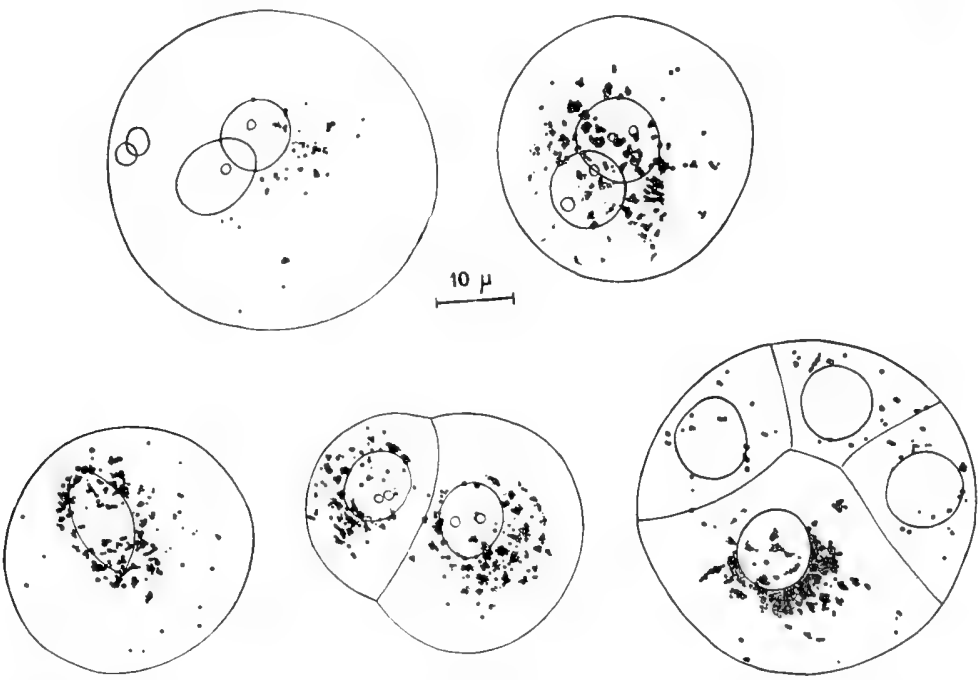

Fig. 23 Metachromasia in vivo in a Barnea cand. egg (Lamellibranch). The fertilized eggs were dipped for $5 \mathrm{~min}$. in toluidine blue $1 / 30,000$ in sea water, then carefully washed and the development observed. Specimens were fixed at successive moments in Izquierdo's fluid and mounted. These drawings show only the $\beta$-granules, appearing mainly near the pronuclei and increasing in number until the first cleavage, where they gather inside the asters. In the 2-cell stage, the larger cell (CD) contains more of these granules. The cycle begins again at each cleavage, the diffuse $\alpha$-granules transmitting the dye to the still more numerous $\beta$ bodies. The $\mathrm{D}$ cell is the richest in $\beta$-granules. The test for acidic phosphatase is positive at any of these stages, in these granules and also in the yolk. From Pasteels and Mulnard, 1957. 

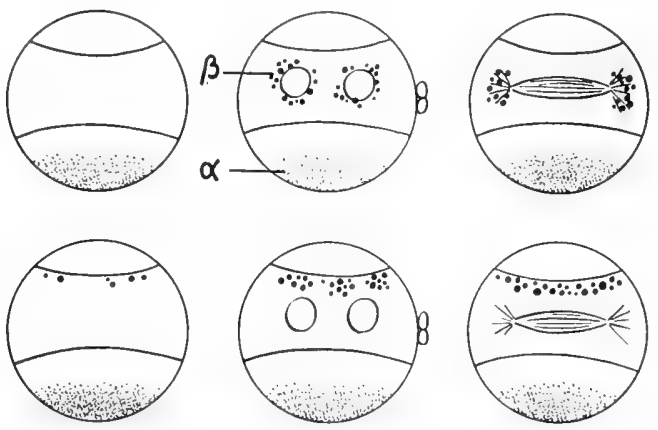

Fig. 24. Effect of centrifugation on Barnea eggs stained in vivo by toluidine blue. In each egg, the 3 zones obtained contain, from top to bottom, lipids, clear cytoplasm, yolk and $\alpha$ granules (small dots). Upper row, moderate centrifugation. Lower row, stronger centrifugation. Left, the egg during maturation; strong centrifugation reveals some $\beta$-granules, lighter than the cytoplasm; center, moderate centrifugation (above) leaves the $\beta$-granules near the pronuclei, while stronger force draws them between the cytoplasm and fat; right, the same difference for the $\beta$-granules embedded in the astral rays. From Pasteels and J. Mulnard, I957.
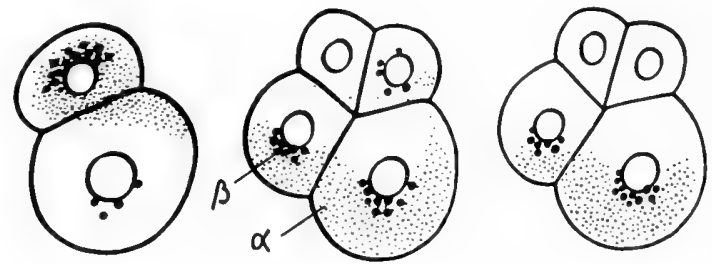

Fig. 25. Experimental demonstration of the relation between the $\alpha$-and $\beta$-granules in the egg of Barnea. The initial staining was done as in the preceding case. Centrifugation was applied shortly before the first division. At the II-or IV-cell stage, the blastomeres show a variable amount of $\alpha$-granules (small dots). The amount of $\beta$-granules formed (large dots)

is roughly proportional to the amount of the $\alpha$-granules present in each cell.
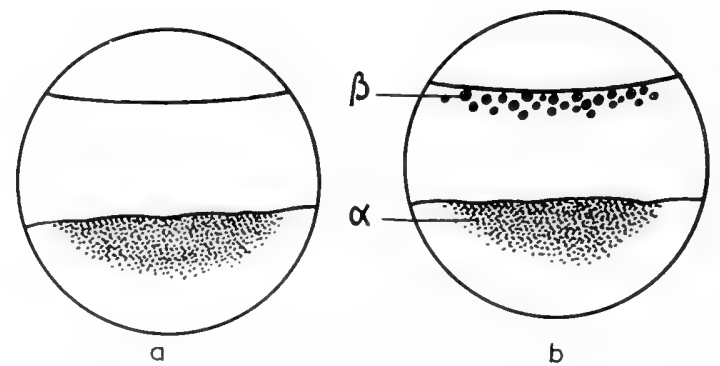

Fig. 26. Eggs of portuguese oyster (Gryphaea angulata) stained as in the preceding cases and submitted to centrifugation. (a) during maturation, with the $\alpha$-granules driven to the centripetal level of the yolk mass, and (b) during the pronuclei stage, with the $\beta$-granules which have been formed in the meantime and are driven, as in Barnea, to the interface between the clear cytoplasm and the fat globules. From Pasteels and Mulnard, 1957. 

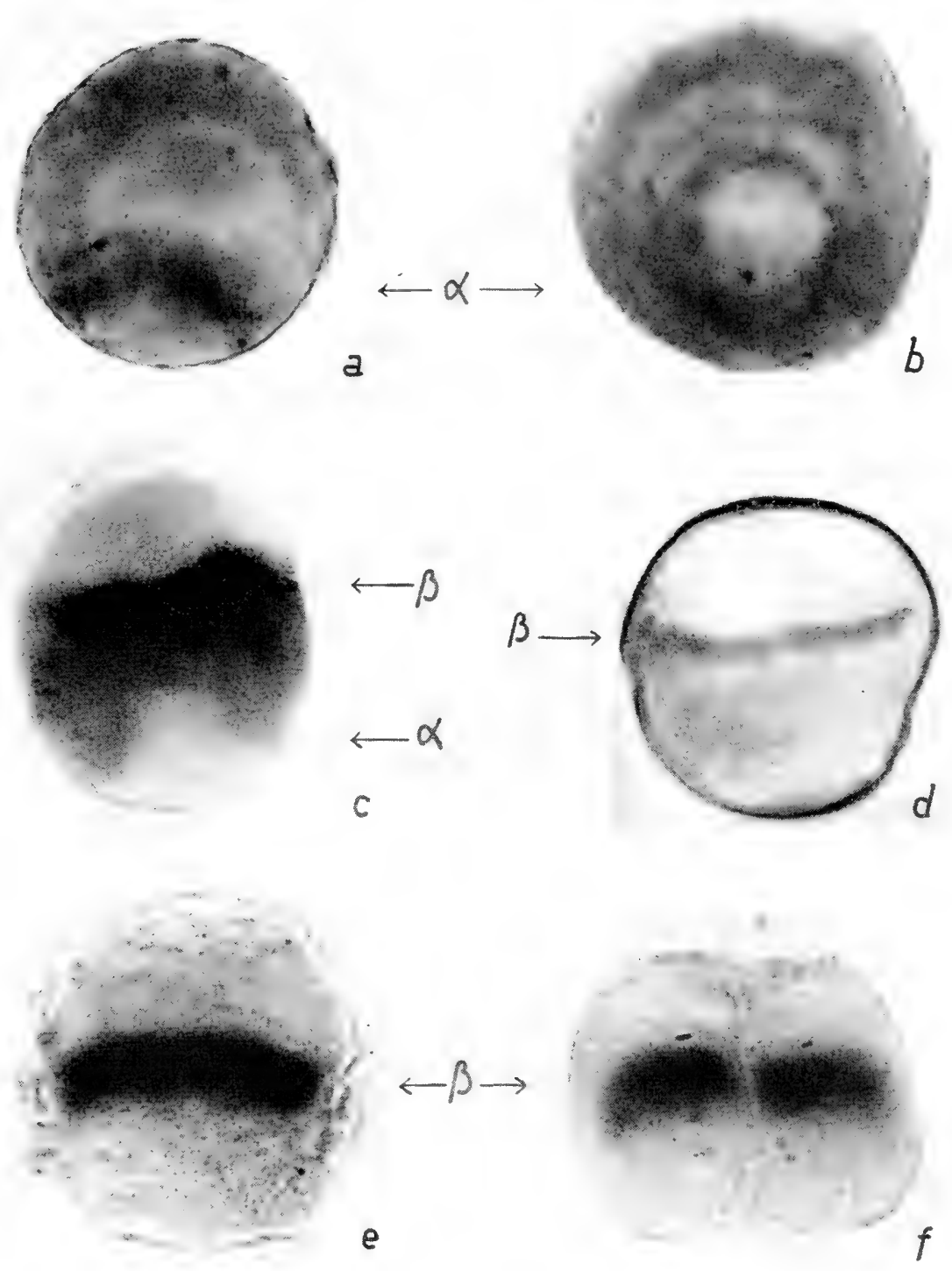

Fig. 27. The $\alpha-\beta$ granules of sea-urchin eggs, revealed by centrifugation. a, b, c: eggs stained in vivo, centrifuged, fixed in Izquierdo's mixture and mounted in toto; a, b, eggs centrifuged some minutes after the staining, and showing the $\alpha$-granules; in a, centripetal side on top, centrifugal down; in b, the egg is seen from the centrifugal side. The clear centrifugal cap is extremely rich in RNA and contains the majority of mitochondria. The $\alpha$-granules form a girdle between this cap and the yolk platelets; in c, the egg has been centrifuged at the streak stage, the lower cap and the $\alpha$-granules, scattered among the yolk, are visible, while the $\beta$-granules form a dense layer under the hyaline layer with the pronuclei; $d, a$ section of similar eggs, fixed in formol saline and stained with Alcian blue at $\mathrm{pH}$ i ; selective staining of $\beta$-granules; $e, f$, acidic phosphatase revealed in the $\beta$-granules (whole mounts) at the $\mathrm{I}$ - and 2-cell stages. From Pasteels, 1958. 
the first maturation spindle (Fig. 24, left) and may increase in number if unfertilized eggs linger at maturation stages. In sea-urchin eggs stained just after their fertilization, the secondary granules appear at the streak stage. If eggs are stained at later stages, only $\alpha$-granules will be seen during the mitotic cycle concerned, but as soon as a new cleavage has taken place, the $\beta$-granules will be observed (Fig. 25). Their number increases during the three or four first steps of cleavage (Fig. 23); then, a gradual fading excludes further appreciation. In clam and oyster eggs, $\beta$-granules are seen to move

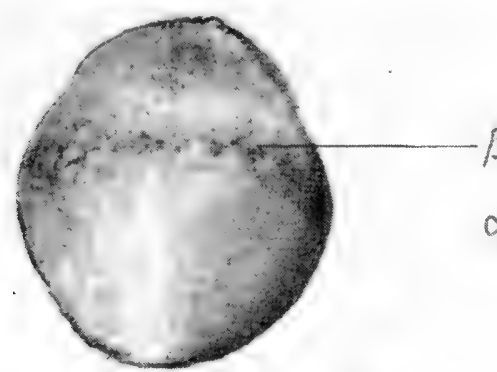

a

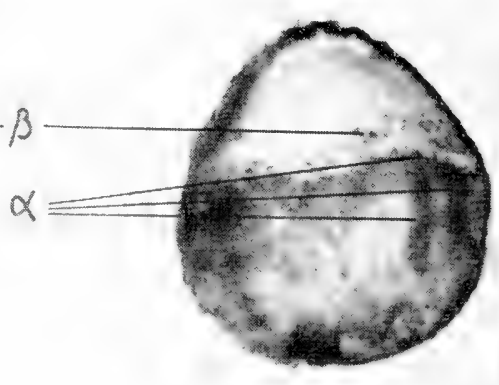

$b$
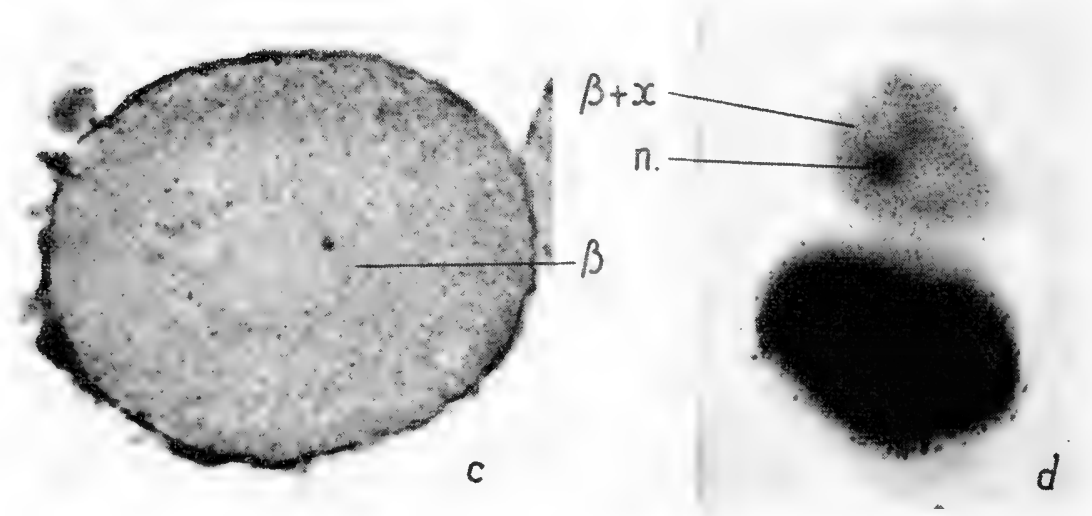

Fig. 28. (a) Section of a pressurised centrifuged Arbacia $p$. egg at the pronuclei stage. Alcian blue technique after picric-formalin fixation showing a selective staining of the $\beta$-granules sedimented in the lower hyaline band.

(b) same technique, but applied after oxidation by sulfuric permanganate. In addition to the $\beta$-granules, Alcian blue now stains the $\alpha$-material, concentrated in the upper-vitelline band, part of it being still mixed with the yolk. In a and b, centripetal side up (with lipid droplets on top), centrifugal side down (with yolk and pigment). From Mulnard, Auclair and Marsland, 1959.

(c) a section of a Chaetopterus $p$. egg at metaphase of first mitotic cleavage, fixed by picric formalin and stained by Alcian blue. Selective staining of $\beta$-granules scattered in one of the astral zones.

(d) an egg of Chaetopterus $p$. centrifuged at the pronuclei stage, mounted in toto after formol saline fixation and revealing acid phosphatase. Positivity of the yolk mass and of the $\alpha$-granules (below, mainly due to $\alpha$-material) and of the $\beta$-granules and diffused material ( $\mathrm{x}$ ) around the pronuclei ( $\mathrm{n}=$ one nucleolus) in the upper hyaline zone. From Mulnard, I $95^{8}$. 

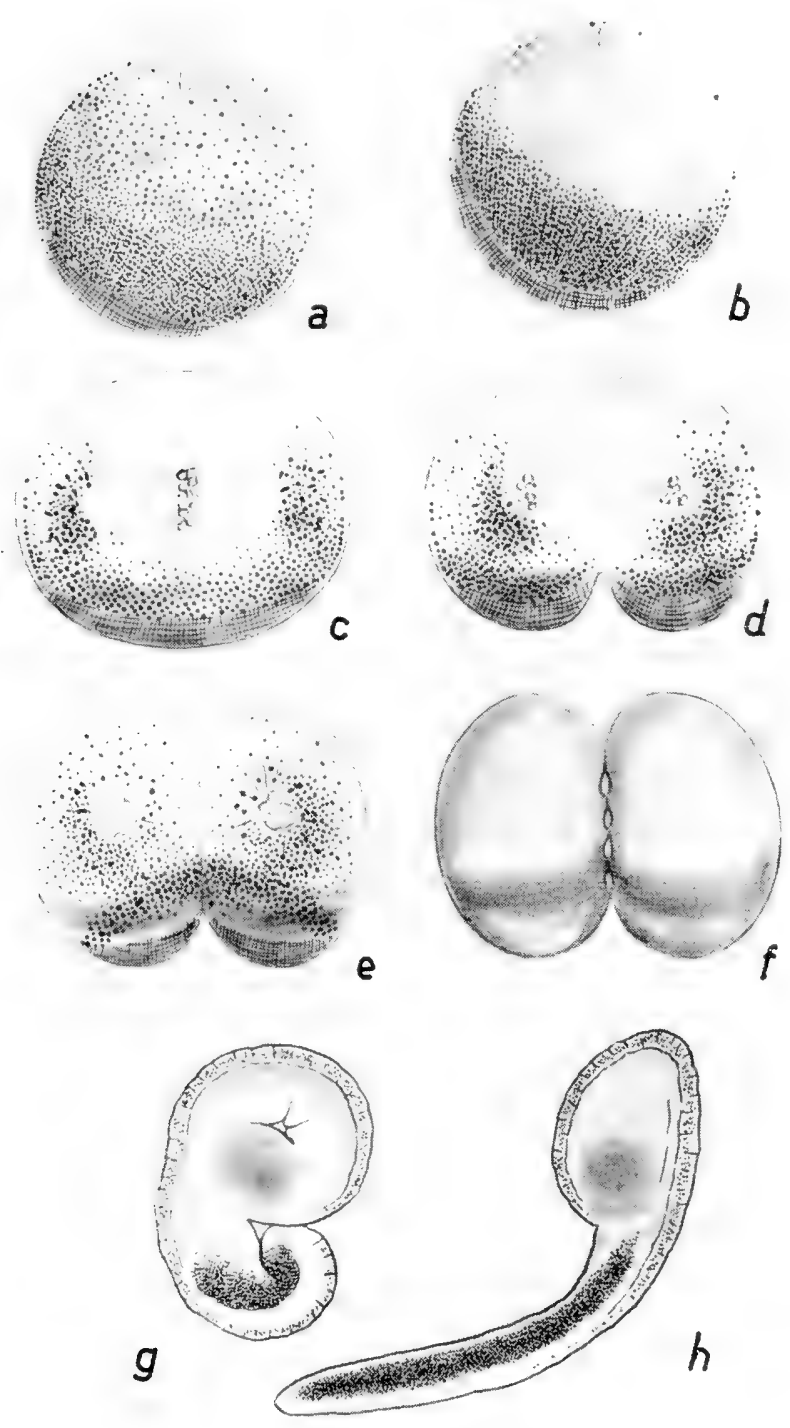

Fig. 29. In vivo metachromasy in eggs of Ascidiella scabra. Large grey dots: yolk platelets; black dots : the metachromatic granules; crossed hatchings : the yellowish plasm (myogenic); diffuse cloud, the metachromatic plasm. (a, b) first modifications after fertilization, while maturation proceeds; the cortical $\alpha$-granules flow towards the vegetal pole; (c,d) metaphase and telophase of first cleavage; a lot of granules have been caught in the rays of the asters; (e, f) a two-cell stage; (f) from outside with hyaline droplets expelled in the furrow (e) in optical section; $(\mathrm{g}, \mathrm{h})$ young and older tadpole, with granules in the myoblasts and the diffuse plasm in the caudal part of the endoblast (Dalcq, unpublished). 
over short distances. Pasteels could establish a relationship between the amount of $\alpha$ - and $\beta$-granules (Fig. 25), and he has good reason to believe that the cations of the basic metachromatic dyes are at first combined with the $\alpha$-granules and then detach from these, taking along some part of them in their migration towards the $\beta$-granules, upon the surface of which they become concentrated. If stained eggs are deprived of $\mathrm{O}_{2}$, they discolour by liberating the dye cations. Vercauteren could show, on $\beta$-granules isolated in vitro (although not yet purified), that this was due to an oxydo-reduction process. It is also interesting to quote that by centrifugation of clam eggs, blastomeres can be obtained which are apparently free of granulations; however these granulations are seen to reappear somewhat later.

The $\beta$-granules of sea-urchins and Spiralia eggs are lighter than the yolk and than the $\alpha$ 's. (Fig. $24 ; 27, \mathrm{c}, \mathrm{d} ; 28, \mathrm{a}$ ). In fixed eggs, they are PAS positive, even after enzymatic digestion, stain directly by Alcian blue at a low pH (Fig. 28, a) and are apparently not rich in RNA, whereas they exhibit a strong acid phosphatase activity. Thus, they appear as mainly consisting of frankly acid mucopolysaccharides, with a protidic part acting as a phosphomonoesterase in an acidic medium.

It has not yet been settled whether the metachromatic granules encountered in ascidians and mammals eggs behave exactly in the way just described. In Ascidiella, two categories probably exist, and the secondary one contains acidic phosphatase, but it seems to consist of minute bodies, and to be directly stainable. In any event, it is a fascinating picture to observe the purplish $\alpha$-granules (also stainable by neutral red, as in the case of Para-centrotus) mostly associated with the yellow myogenous plasm, surround and penetrate the aster rays (Fig. 29). In mammals, only one category of granules could be detected so far. They stain directly and have not as yet been followed in successive stages, probably owing to a reduction process. But it was just established that they belong to a more complex system, formed of heavier and lighter elements impregnable by osmium, thus most probably of Golgian nature.

A third manifestation of vital metachromasy has been observed in two instances. In Ascidiella scabra, a diffuse plasm has been detected in the subequatorial region of the recently fertilized egg, pervading the region where the $\alpha$-granules gather above the myogenous plasm. This new component could be traced into the caudal part of the larval enteron (Fig. 29; Dalcq, unpublished). In Chaetopterus, Mulnard also observed a rather continuous area, his x-component (Fig. 28, d), which is probably microgranular and could be an intermediate between the $\alpha$ - and $\beta$-granules.

What kind of organelles are the metachromatic granules? At first sight, they were supposed to form a special category of mitochondria, but this initial interpretation of mine lost more and more ground. As mentioned above, in rat and mouse eggs it could be shown that we are dealing with corpuscules which are neither Nadi-positive nor stainable by Janus green. In sea-urchin eggs, neither the $\alpha$-nor the $\beta$-granules settle out on the same level as the mitochondria. Electronmicroscopic studies performed by Pasteels, Castiaux and Vandermeerssche ( I 958a, b) have not revealed structures corresponding to the sought after $\beta$-granules ${ }^{1}$. At the level where these granules settle in centrifuged eggs, there is an abundance of whole of Golgi vesicles, and it could well be that these granules are a part of the Golgi apparatus ${ }^{2}$. The $\alpha$-granules seem to have the same E.M. aspect as small yolk platelets. Also, perhaps, the $\alpha$ - and $\beta$-granules are not properly fixed by the buffered $\mathrm{OsO}_{4}$ mixture used. In the eggs of another bivalve, Spisula, Rebhun (I957)

1 This enquiry had shown that a large amount of cytoplasmic RNA, which litterally fills, mixed with mitochondria, the centrifugal cap of the centrifuged eggs (Fig. 27, a, b, c) is certainly not bound to ultramicrosomes (Palade bodies). A similar conclusion has been reached by Ruthmann (1958) in a E.M. study of crayfish spermatocytes. Thus, it might well turn out that in gametes a large part of RNA is not included in the ultramicrosomes. 2 In 1959 , hypothesis received full confirmation. 
revealed the $\beta$-granules by vital staining with methylene blue, but concluded from his E.M. studies that he was dealing with mitochondria severely modified by the staining treatment. However, one knows it is always a delicate problem to establish a correspondance between microscopic and ultra-microscopic appearances.

Finally, we could ask what is the possible function of the metachromatic particles. At first sight, there are several hints of some relation between the distribution of $\beta$-granules and early morphogenesis. In mollusk eggs, an excess of $\beta$-granules endow the D-quadrant or eventually the polar lobe. In sea-urchin eggs, the micromeres when they appear, as well known, at the antipole during the $4^{\text {th }}$ cleavage, are practically devoid of $\beta$-granules. Later on, these bodies surround the nuclei of the morula, except in the micromere derivates. In the swimming blastula, they form a continuous layer at the base of the cilia, possibly in coincidence with the parabasal bodies. In the gastrula and young pluteus, the stain is liberated and condensed into vacuoles. This process of clearance first takes place in the mesenchyme, then in the ectoblast, except its thicker parts which prepare the arms and the oral plate, still later in the archenteron. In rat eggs, metachromatic granules become most conspicuous at the 8-cell stage and are more abundant in the 4 large "ventral" cells, which will envelop the 4 smaller ones and form the enveloping layer (Fig. 2I), devoted partly to the endophyll (p. 319) and mostly to the trophoblast. In Ascidians, the metachromatic granules show a tendency to be associated with the myogenic plasm (Fig. 29, a-f), and indeed, in the tadpole, the myoblasts of the tail remain the more distinctly metachromatic (Fig. 29, g, h). The diffuse metaplasm is linked with the hind part of the enteron (Fig. 29, a-h).

Considering these relations more closely, it may be remarked that the abundance of $\beta$-granules in the D-quadrant of Spiralia-eggs may depend on the well-known inequality of the mitotic poles at the first and second cycles. If this is the case, the distribution of the $\beta$-granules would thus be secondary and depend on the fundamental organization pattern of the egg. It may however be linked with the recognized abundance of mitochondria in the somatoblasts $(c f$. p. $33 \mathrm{I})$. In seaurchin eggs, the scarcity of metachromasia in the micromeres may simply be a consequence of the rather deep location of the $\beta$-granules. This is contrary to the idea of a positive influence by the $\beta$-granules on the course of morphochoresis, and, thereby, on morphogenesis, for it has indeed been established by Hörstadius that the micromeres possess the highest degree of "vegetalizing" activity". Moreover, Brice ( $1959 \mathrm{~b}$ ) has observed that, in the same eggs, metachromatic staining interfers with neither animalization nor vegetalization. Again, stained eggs remain capable of regulation after separation of the first blastomeres. If the $\alpha$ - and $\beta$ granules had a positive role in these processes, it seems likely that their becoming loaded with dye cations would not have an indifferent effect. The sequence of clearance in the young plutei may be related to the size of the cells, their location, a.s.o. Thus, in sea-urchin eggs, the evidence is not in favour of a direct morpho-

1 There are definite hints that mitochondria are involved in the egg pattern of gradients. In the morula and blastula, they are distinctly more numerous in the animal half than in the vegetative one (Hörstadius, 1952; Gustavson and Lenique, 1952; Lenique, Hörstadius and Gustavson, 1953).

Literature p. $4^{83}$ 
genetic role, a conclusion well in accordance with the penetrating views of Lallier ( 1957) who insists on the prevailing role of basic proteins, left untouched by our stains. For mammalian eggs, the available data also speaks against a primary role of the metachromatic granules in morphogenesis. They are precisely prevalent in the part of the egg devoted to mainly trophic functions, especially to the relations with the maternal tissues. In ascidians, the link with morphogenesis is somewhat less loose, and the fate of cells seems to depend largely on the relative proportions of hyaloplasm, mitochondria - the role of which is emphasized by Reverberi ( 1956 , a, b; I957a, b) and Berg (1956-7) - metachromatic granules and metachromatic plasm. But the anlagen of the top organs, e.g. notochord and neural plate, are definitely the less metachromatic.

The above cited facts lead us to think that these $\alpha-\beta$ granules have more to do with cellular life than with morphogenesis, which they only help by promoting cleavage and early differentiation. The combined synthesis of acid mucopolysaccharides and acid phosphatase is probably their most significant feature; this enzymatic equipment, which requires further investigation, is suggestive of "lytic" as well as of "synthetic" functions. It is also interesting to point out that numerous works on cellular localization of the non-specific acid phosphomonoesterase in the cells or tissues have led their authors to the conclusion that the enzyme must play a role in the "general metabolic processes of the cell" rather than in the more specific cellular activities. In the actual state of knowledge, a role in the digestion of yolk would be so far plausible; in the eggs of mammals, which are deprived of yolk platelets, the same organelles would be mostly concerned with the assimilation of maternal nutrient.

Finally, when searching for a representation of the granules at later stages, or even in adult tissues, one is bound to think of de Duve's lysosomes, to which the author also attributes a role in intracellular digestion (1958). But there are appreciable differences, e.g. the size of the elements, their intimate relationship with mitosis. This interesting suggestion needs further exploration at both levels.

The most evident feature of the chromotrope granules of the egg is their relationship to the initiation of development and the beginning of cleavage. Thus, they must be linked in some way with the progressive synthesis of DNA. Thanks to histophotometric measurements, for which work on mammals is also advantageous, we have begun to learn the steps in the synthetic process (Alfert, I950; Dalcq and Pasteels, 1955; Vandekerckhove, 1959). If the mean somatic DNA content of the nuclei is assumed to be 2 units, the oocyte nucleus has 4 , the first polar body and the chromosome set of the second spindle (as far as measurable) have 2 , the second polar body and the young pronuclei have I each. Thus, at the conclusion of the events of fertilization and maturation, the egg has a typical diploid value of 2 units. These simple relationships have also been ascertained in a bean wevil (Mulnard, 1954) and in a liver fluke (Govaert, I957).

Now, in rat and mouse eggs, it could be shown that from the I- to the I6-cell stage, there is necessarily a doubling of the amount of DNA. This takes place quite early, that is immediately after the telophase. The cells preserve their 4 -unit value during the entire intercinesis. Thus, some other process must influence the onset of mitosis, and must prepare the quantity of DNA precursor which has to be available if there is to be a recuperation of DNA. It is at this point that the $\alpha-\beta$ system of chromotrope granules could possibly play the role of a mobiliser of metabolic resources by its cooperation in digestive activities. It has not been settled as to whether the chronological and spatial patterns remain unchanged during the remainder of cleavage, or if there is a change in DNA 
turnover in the various territories. These questions have been partially solved for Paracentrotus liv., where considerable differences have been detected between ectoblast, mesenchyme, endoblast (Lison and Pasteels, I95I). In Lytechinus $v$. the measurements performed by McMaster (1955) are only in partial agreement. Nevertheless, the results of this author indicate that at the i6-cell stage, there are differences in the rate of DNA assimilation according to the tiers of blastomeres.

\section{Kinetic and causative aspects of intrinsic morphochoresis}

In the intimacy of its events, mitosis is fundamentally a kinetic process, not only concerning the dividing of chromosomes but also the distribution of the various cytoplasmic organelles. However, the prevailing usage is to speak only of kinetics when the involved animal germs cease to be a spherical mass of cells in their development toward a more complicated form, or when blastoderms of reptiles or birds elongate along their main axis with the concomitant appearance of an embryonic shape. At this critical stage, when the germ charges from its spherical form, general and local forces are simultaneously at play.

In the first part of this century, appropriate methods which revivalised experimental embryology have revealed active deformation and characteristic shifting of the various territories. These strictly ordinated and harmonious movements are the core of morphochoresis. This kinetics is described in all modern books on development ${ }^{1}$. We have only to consider here the general aspects and theoretical interpretation of these characteristic events.

\section{(a) The factors at work}

Developmental kinetics is of course quite a progressive process, which begins within the cells some time before becoming externally visible. One necessary condition for its appearance is certainly a sufficient reduction of the cell size; another prerequisite is the existence of forces capable of overcoming the cohesion progressively established between the cells. On the other hand, one important factor, although antikinetic, is the existence of an extracellular covering layer. Embryologists of the past century had already become acquainted with the hyaline layer of the seaurchin eggs. Much later the coat was discovered in amphibians ${ }^{2}$ and masterfully analysed by Holtfreter (1943). In recent years its existence was recognized in birds (Malan, I 953) and in mammals (Dalcq, I 954c). The intrinsic mechanism of the morphochoretic movements was so far only explained by some indirect but nevertheless interesting inferences. The tentative idea is analogous to that concerning amiboid movements; that is, contractile proteins coupled with an energetic system producing $\mathrm{ATP}^{3}$ and responsible for dephosphorylation ( $c f$. Mulnard, 1956).

It may be assumed that they are apparently due to a modification or perhaps some diffuse contraction (Lewis, I947), taking place in the non-coated part of the cell. This change takes place almost simultaneously, (and with a similar and definite character) in the cells of each presumptive territory which, at this very

1 Recently a valuable contribution to the movements taking place in the primitive streak of birds, especially at the level of the Hensen node, has been added by Spratt (1957). 2 For the physico-chemical properties of this coat, see Bell (1958).

$3 \mathrm{H}$. Tiedemann (1957) has drawn arguments from the study of nucleotides in embryos submitted to aerobiosis and anaerobiosis in favor of the role of ADP and ATP in gastrulation. 
moment, expresses in this way its individuality. Of course, there is no sharp distinction, at the beginning, between the types of deformation exhibited by different groups. However, as mapping by vital staining shows, the final and total result is that discontinuities appear, because - at the limit of two territoriescells on one side go one way, and those on the other side, the other way. The individuality of the primordial organs is soon strengthened by the acquisition of new surface properties, which cause either adhesiveness or non-adhesiveness of cells. Since, in the main trend of kinetics, we have to do with a conflict of divergent forces, it is no wonder that the presumptive territories each have a definite shape ${ }^{1}$, limited by straight or curved lines (Vogt, I929; Pasteels, I936, I937, I940; Nakamura, I938; Spratt, I942; Malan, I953). It is no wonder either, that the movements accomplished by the various primordia not only transpose the cellgroups as such, but also deform and remould them in different directions. The real picture depends on the intrinsic properties and general configuration of the germinal material at the onset, and on the more or less condensed character of the cell streams. Patient and exact work has now resulted in valuable descriptive accounts for all groups; they may serve as a basis for comparisons which reveal the uniform dominants of kinetics: epiboly, invagination or immigration, dorsal convergence, and cephalo-caudal stretching. For our present purpose, this general sketching of the processes seems sufficient.

\section{(b) Enumeration of the problems}

What we are particularly concerned with here is a possible explanation of the very fact that kinematics begins, follows a definite course, achieves the separation of the layers and of the primordial organs. Let us consider the blastula stage of a fish or an amphibian. Why does a blastopore appear, underlined by a groove progressively deepening into an archenteron? Why does the middle layer soon produce the chorda, the somites, the nephrotomes and the lateral plate, all names which imply a typical fate? If we consider the reptile egg, why does not gastrulation begin at the margin of the blastoderm but in some inner, slightly eccentric region? If we examine a bird egg, why does an endophyllic layer at first creep under the superficial layer, and then the primitive streak does arise along a radius of the oblong blastoderm? If we follow a mammalian blastocyst, of a rodent for convenience, why do the enveloping cells ( $c f$. Fig. I 7 k, p. 326) first provide an endophyll layer by immigration, so that a twolayer stage is attained, which will proceed in a manner similar to bird development?

We are far from having formed definite answers to these querries. For the last three, so far no experiment has been attempted and we have no better resource than comparative embryology, which is necessarily conjectural. However, it seems plausible that if the case of some Anamniota could be solved, we would have a clue to a more general interpretation.

1 It has been claimed that in eggs of Xenopus the typical pattern of dorso-marginal areas does not exist, an exception capable of invalidating the above considerations. However, the contention of Nieuwkoop and Florschütz (1950) is based only on the study of sections. Sirlin (1956) supported the conclusions of these authors on the basis of labelled grafts, but this argument is also questionable. Damas (1957) has applied vital staining to the same eggs and maintains that a superficial chordo-mesoblastic area exists, as in all other species. 
(c) The localisation of the blastopore in Anamniots. An operative approach

This gives value to my earlier suggestions of $1935 \mathrm{a}$ and to the experiments which have been done on the eggs of frog, newt, axolotl, trout, minnow, gold-fish, pike and others. Again, for historical reasons, amphibians have to be considered first. For more than half a century, it has been known that if frog (or newt) eggs are put upside down when still undivided or while at the 2- or even 4-cell stage, and are maintained in that reversed position until cleavage is fairly advanced, gastrulation will be deeply affected (Fig. 30, 3I). From the analysis of innumerable results, two relations have come to light. (I) A blastoporal groove will appear (Penners and Schleip, 1928; Penners, 1929; Pasteels, 1938) at any place where a packet of big yolk platelets have been incorporated in the egg cortex; around these few large yolk cells, an intermediate brownish ring (frog) of less loaded cells will be formed, and at least part of this ring will invaginate with the customary aspects of bottle-necked cells. (2) A yolk packet is not the only prerequisite for gastrulation. When the mass of deutoplasm is a bit large, it can be observed that gastrulation prevails on one side; there, morphochoresis begins earlier and more cells invaginate and stretch than on the other side. If an embryo can be formed, its somites, pronephros and even its brain and spinal cord are more strongly built on the favored side. The difference has been demonstrated to be caused by the previous location of the grey (or light) crescent (Fig. 32), and it could be established that the whole cortex may be considered as a gradient field, with activity decreasing from the grey crescent in all directions. The observed asymmetry indicates that at the border of a transposed or reduced yolk mass, a kind of activating reaction takes place between yolk derivates and the cortex (Dalcq and Pasteels, 1937, 1938). These two notions have been the core of a theory that in a normal egg a similar interaction is responsible for the starting of morphochoresis. The resultant, in a given territory, of this interaction is meant to endow this area with a given morphogenetic potential, an expression also used, in the same period, by Yamada (I940, his p. I95). In a fertilized egg which has
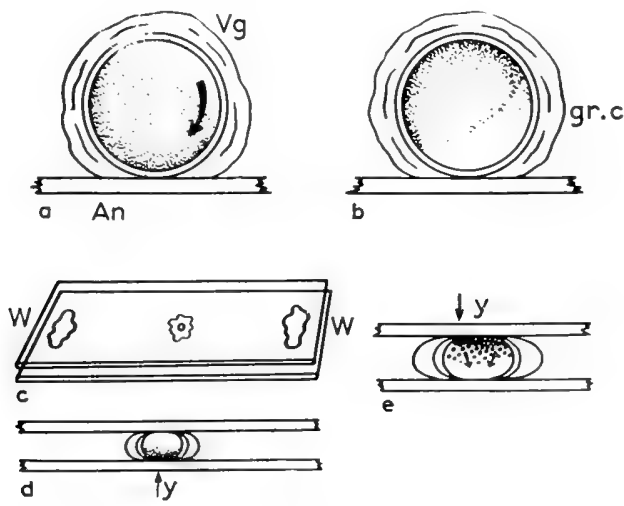

Fig. 3o. Experiments of oriented rotation (a, b) and of complete inversion ( $\mathrm{c}, \mathrm{d}, \mathrm{e})$ on the fertilized frogg egg. (a) The egg is oriented on a slide, to which it adheres by its jelly, in such a way that the vegetal pole $(\mathrm{Vg})$ is uppermost and a little to one side $(\mathrm{An}=$ animal pole). The egg is free to rotate within its vitelline membrane and the greater density of the yolky vegetal pole causes it to move in the direction shown by the arrow. The meridian of rotation will become the plane of bilateral symmetry (b) and the grey crescent (gr.c.) will appear ( $c f$. p. 324 for explanation) on the side where the vegetative pole was originally placed. (c-e) Technique for keeping a fertilized frog's egg in a fixed inverted position (O. Schultze). The egg, on the point of dividing, is placed between two slides, kept a certain distance apart by small pieces of modelling wax (W); (c) general view of the arrangement; (d) vertical section before reversal; the pressure distorts the perivitelline space, and prevents the egg from returning to its normal position; (e) the same after reversal; only the yolk (y) is affected by gravity (indicated by arrows). From Dalcq, I952. 
attained the pronuclei stage, there exists $(a)$ the cortical field possibly reinforced by a greater thinness of the pellicle (or coat) on the dorsal side ${ }^{1}$ (Dalcq and Dollander, 1948; Dollander, 1953), and (b) a certain distribution of yolk in the endoplasm, not exactly a true yolk gradient, but graded differences among the polar, equatorial and antipolar zones. If interactions take place between these two sets of factors ${ }^{2}$, as was already suggested for the earliest stages by the orientated rotations ( $c f . p .324)$, the results would vary according to the proportional value of the two involved sets of factors. It can be conceived
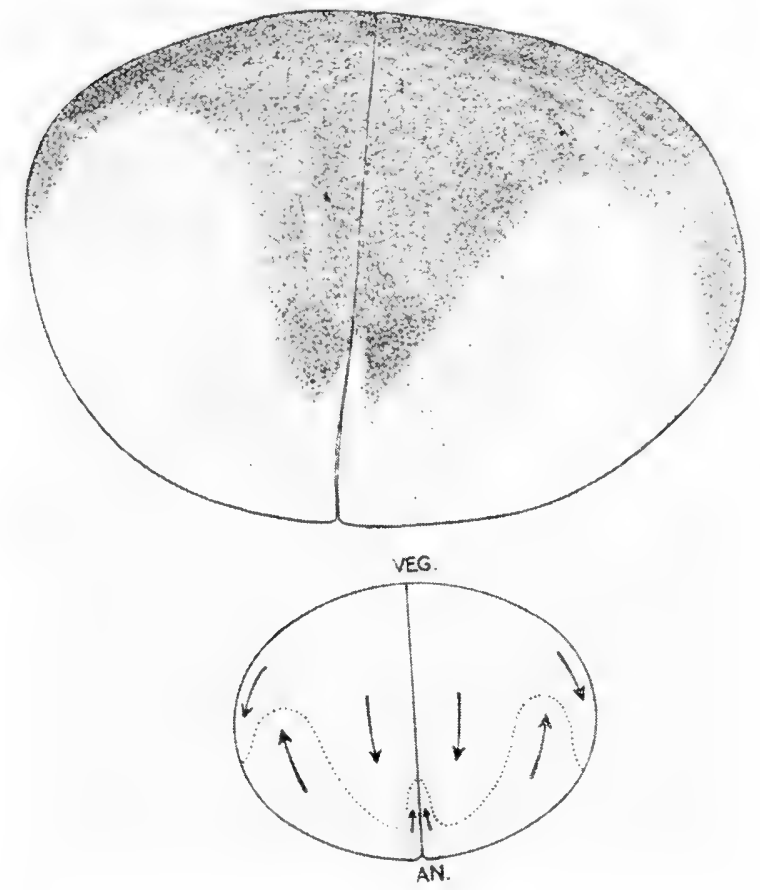

Fig. 3r. Vertical section of an inverted frog egg, during the descent of the yolk. The egg is now divided into two blastomeres. The platelets are indicated by dots, heavier or lighter according to their mean size. The white part is the animal cytoplasm. The yolk glides along the cell walls from the vegetative (VEG) to the animal pole (AN). From Pasteels, I95I.

1 It has been recently assumed (Lovtrup, 1956) that the resulting facilitation of oxygen supply to this presumptive dorsal region could explain its leading role in early development; indeed, an experimental demonstration, however somewhat indirect, was given that an unilateral restriction to the oxygen supply in fertilized eggs is able to cause a displacement of the blastoporal groove (Lovtrup and Pigeon, I958). On the other hand, Dollander, (1958) has produced arguments in favor of a higher permeability in the region of the "grey" crescent. This author has even shown that in eggs submitted to inversion, as seen in Fig. 3o, c, d, e, this property remains unchanged.

2 The assumption that yolk is not only, as generally considered, nutritive material, biologically inert, but plays a more dynamic role, is supported by two recent results. After exploring the distribution of cathepsin, Deuchar concludes: "it has been specially interesting to demonstrate that ventral tissues, which are so often placed at the bottom of gradients in amphibian embryos that one has come to believe they are in some way inferior, have in fact a higher catheptic activity for cell than dorsal tissues" (I958b, her p. 234).

On the other hand, E.M. studies on chick blastoderm have shown that yolk platelets apparently include, and later liberate, mitochondria, plus microparticles which could form RNAgranules and circular bodies which could contribute to the endoplasmic reticulum (Bellairs, 1958). In Ranzi's laboratory Lanzavecchia and Coultre (I 958) have made on frog embryos E.M. observations suggesting the formation of mitochondria from yolk platelet material. 
that a given threshold will be surpassed earlier in the region of maximal interaction, and that the process will be gradually extended in a preferential direction to nearby territories. It would also explain why the lines of disjunction, i.e. the limits of the presumptive areas, display on the blastula or nearly gastrula such a geometrical design.

This proposal has not been generally accepted since it has been considered too speculative (Rotmann, I94I). This criticism is no longer completely justified, for each set of factors has been elaborately analyzed and explored as far as now possible by the authors of the theory. Their opponents were forced to propose an alternative explanation of the experimental results, so they have claimed that the organizer is in some way present in the dorsal marginal zone, and that the forced inversions only draw this active plasm into abnormal locations (Lehmann, r $942 \mathrm{~b}$ ). This conception is hardly compatible with a sound epigenetic interpretation. In support of the original theory, an appropriate experiment of compulsory inversion was performed whose results could not be explained by the transfer of previously formed material (Pasteels, 1946). It has also been suggested that the patch of yolk cells would only provide a resistant basis for the folding and movement of the adjacent sheet of brown cells (see Holtfreter and Hamburger, I955, their p. 234). This mechanical explanation would not account for the striking effect of the relation with the grey crescent.

A more real difficulty lies in the fact that there is at least one case in which a blastoporal lip arises de novo and for which an interaction between yolk and cortex cannot be advocated.
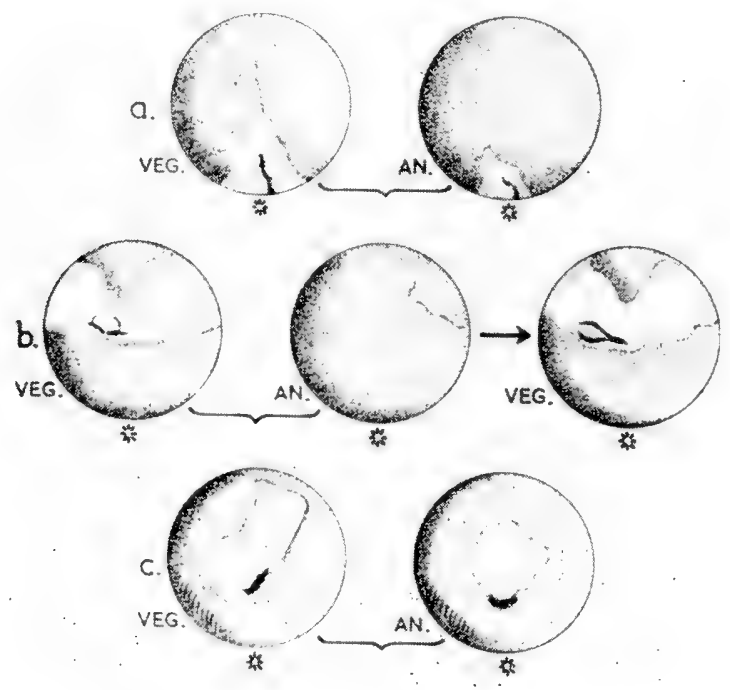

Fig. 32. Gastrulation in previously inverted frog eggs. After the manoeuvre shown in Fig. 30 (c-e) the location of the grey crescent was marked on the slides by glass pencil (indicated here by an asterisk). VEG and AN refer to the connotations before inversion (as in Fig. 30, c). The pigmented zones are dotted and the areas where yolk is located are white. (a) The yolk has flowed toward the grey crescent; the blastoporal lip appears in the middle of the yolk mass. (b) The yolk forms a girdle, widest on the vegetative side, with a denser central area; the lip appears on the side of this mass toward the grey crescent. (c) The yolk has partly adhered to the animal region and partly attained the vegetative part, each time with a central mass. The lip appears simultaneously near both masses, each time on the side turned toward the grey crescent. The rule is thus that morphochoresis begins in relation with the grey crescent, therefrom in relation to the cortical field, centred on the grey crescent and not affected by inversion. From Pasteels, $193^{8}$. 
When a newt blastula or a young gastrula is submitted (Fig. 33) to a meridian ligature (sagittal or frontal) a new blastoporal lip immediately appears at the lesion where the egg wall has been split and more or less crushed, and this lip will be the beginning of a small secondary embryonic system (Dalcq and Huang, 1 948; Brice, 1958). At least three factors may be involved here: $(I)$ in spite of the drastic deformation, the new lip appears in marginal material which has been intrinsically prepared for ingression, quite possibly by the above postulated mechanism; (2) lesion of the coat has liberated the superficial part of most cells, and facilitates their migration; $(3)$ the wound has apparently some kind of stimulating effect which can increase the metabolism. This is also suggested by the suddenness of the modification. Such a view is in agreement with the fact, observed by Piepho (1938), that the reduction of brillant cresyl blue is accelerated at the level of a slight wound and occurs there as rapidly as in the organizer territory. Thus it seems that in this ligature experiment we have a specific process, which does not use the same mechanism as the progressive formation of the normal blastopore lip.

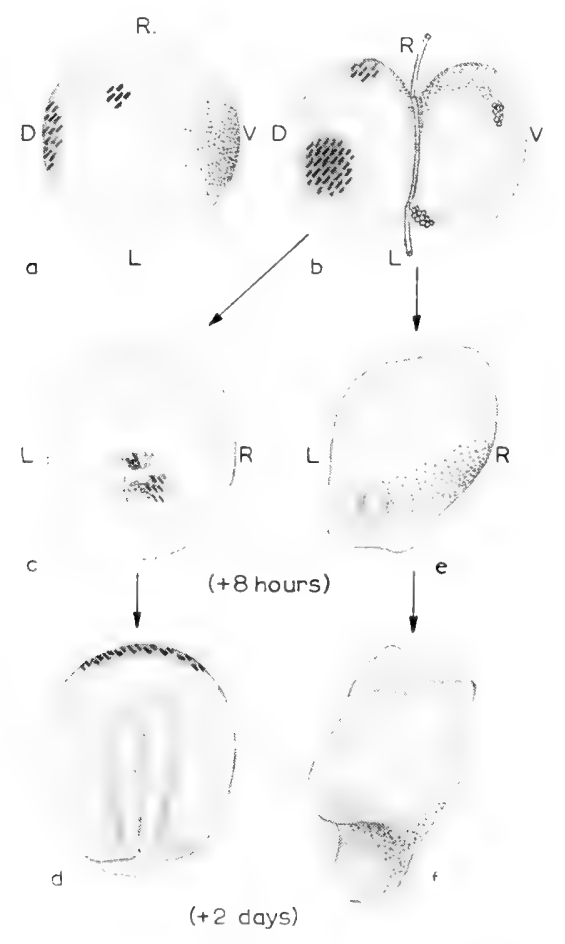

Fig. 33. A case of frontal ligature in a blastula, with regulation of both halves. (a) The germ seen from its vegetal aspect, with marks of Nile blue (coarse hatching) and neutral red (dots). D, dorsal; L, left; $\mathrm{R}$, right; V, ventral. (b) The ligature is made with a hair, at the price of two small wounds (circles). The two parts are now viewed from the side of separation $(\mathrm{c}, \mathrm{d})$ the dorsal half; $(\mathrm{e}, \mathrm{f})$ the ventral half. (c) The blastopore of the dorsal half, which has been immediately formed at the place wounded by the ligature; the marks have converged toward the blastopore and have partially migrated inside. (d) The resulting "dorsal" embryo with a still-open and defective neural plate; the Nile blue stain is seen beneath the surface; (e) the blastopore suscitated on the ventral half at the place wounded; (f) the "ventral" embryo, with an elongating tail bud, but a defective head. From coloured drawings by Huang (1948) ( $f f$. Dalcq and Huang, 1948). 
Nevertheless, the lips obtained by ligature may be quite efficient and typical morphochoresis can develop there, with the production of notochord, somites, etc. This could suggest that the kinematic processes, once initiated in a given center, are by themselves sufficient to initiate the ultimate production of the axial organs without any former prolonged elaboration. This idea, however, does not hold in all cases. Other experiments performed on Discoglossus eggs, at the same stage, have shown that the presumptive areas are not only a topographic expression of the results obtained by vital staining, but that the main territories are endowed with intrinsic, although labile, properties, analogous to Vogt's Bahnung or "predispositions" (Dalcq, I 940, I941 a). Thus, in the case of the ligatures of Huang, the operation seems to have produced a local increase of morphogenetic potential in the presumptive marginal material which is involved.

Results obtained in teleost eggs would be too complicated to describe in detail. Let us recall here the elementary, but important fact, that the embryogenic area is at first a limited cap of pure cytoplasm, and that it rapidly increases by a flow of material squeezed from the yolk. In certain eggs, such as the zebra fish (Lewis and Roosen-Runge, 1943), this flow is repeated alternately with mitosis. Latitudinal sections, which isolate the blastodisc at various stages, give results which differ according to the species, to the importance of the material already incorporated in the blastodisc, and to the nature of the culture medium used (Devillers, r949; Trinkhaus, I 953; Tung, I955).

There are hints that active materials, originating from the yolk, become located in a definite part of the periblast, and cause this side to become dorsal. Such interpretation has been proposed by Tung et al. (1945) for the gold-fish egg, and tested on the trout by Devillers (1952). This rather complex experiment consisted of exchanging dorsal and ventral half of blastodiscs; the results were in general agreement with Tung's hypothesis. Thus, these data are favorable to the general idea of an effective interaction between the cortex and the yolk material.

\section{(d) Interpretation of kinematics in amniots}

How far this concept can be applied to reptiles or birds has not been ascertained; it can only be said that the similarity, existing in later processes, suggests that a similar mechanism may be operating at the youngest stages. In placentar mammals, the situation is apparently different. No real yolk is available, and therefore no definite interaction between a cortex and an endoplasm can be hypothetized. The first steps of cleavage, already mentioned, have secondarily acquired a special modification, the meaning of which, as an adaptation to placental viviparism, has been examined elsewhere (Dalcq, I 954b). Why an endophyllic layer is produced by the enveloping layer (at least in rodents) remains enigmatic. We can only remark that this pregastrular kinematics is accompanied by an appearance in the ectophyllic material of a considerable amount of RNA and alkaline phosphatase, both located in similar granules (Fig. I 7, p. 326 and Fig. 37, p. 364). During this period, the germ is already in full growth, and the assimilated metabolites are largely used to build up the 2-layer stage, especially the ectophyll. It remains difficult to guess the cause of the appearance of the primitive streak, and why it should be located along a given radius. An inducing action of the endophyll can be suggested, but the idea remains conjectural.

In spite of their incompleteness, the information available on the appearance, causation, and realization of morphochoresis justifies the general idea that the field-gradient system of the fertilized egg gradually transforms and matures during cleavage. In this way, in a vertebrate egg, a region of special importance gradually becomes the so-called marginal zone, which includes mainly the material destined to become notochord ${ }^{1}$, mesoblast and part of the endoblast. 
In this zone, there is a continuity of properties or activities which are still labile but tentative towards specialization. We can imagine, as the simplest explanation a purely quantitative variation, with a tendency to preserve the level reached by each territory, differences in physiological age providing simultaneously various stages of one process; or, if a more complex explanation is necessary, it may be two or more processes varying at a different rate, or even in opposite directions, or a variation in the relative number of several organelles.

\section{(e) Some biochemical information}

Another clue toward the understanding of developmental kinetic is the search for biochemical differences between more and less active parts of the germinal system, mostly considered during gastrulation. Only a few of these important investigations need be mentioned here.

In amphibians especially, the impressive bulk of results (see Gregg and Ornstein, 1952, I953; Lovtrup, I955) must be reduced here to essential information. Numerous instances of regional differences concerning the general trend of metabolism have been brought to light. There have been discovered variations of enzymatic activities along the main axis (Barth and Sze, I953), and differences between dorsal and ventral half in the respiratory quotient (J. Brachet, I939; Sze, I953), in their capacity to reducing brillant cresyl blue (Piepho, I938), in their adenosine triphosphate and apyrase content (Fujii et al., I95I), in their protease activity (D'Amelio and Ceas, I956), and in capacity to incorporate tagged amino-acids (Eakin et al., I951; Waddington, I954; Flickinger, I 954) and labelled $\mathrm{CO}_{2}$ (Tencer, I958).

An extensive chromatographic study of free amino acids in Xenopus embryos and larvae has been published by Deuchar (1956). For the early stages which interest us here, it is remarkable that the whole content in free aspartic and glutamic acids is higher in the morula than in the gastrula; also that in the early gastrula the dorsal lip, compared to the presumptive neural area and to the ventral parts, has the highest content in free amino acids, the neural area being second in quantity; similar differences are found for both aspartic acid and valine, while glutamine is more concentrated in the whole dorsal than in the ventral half. Several of these amino acids definitely vary during cleavage: glutaminic acid increases from the 2-cell stage to the 32 -cell stage, glutamic acid only from 2- to 8-cell stage, while the aspartic acid content falls from the 8 -cell stage to the blastula (Deuchar, I958a).

Other measurements have yielded valuable information, although not to be correlated at this point with morphochoresis. Changes in tyrosinase activity occur very early in the frog egg. They seem to be a direct consequence of fertilization. This enzymatic activity increases up to the beginning of gastrulation, then drops slowly (Stearns and Goldstein, I956).

1 For an accurate study of the cytological changes taking place in the cells of the notochordal area, the progressive increase of their mutual contact, their secondary arrangement in disks and coins, the conversion of their content into cell membranes containing large sap vacuoles, their behavior in disaggregating media, see Mookerjee, Deuchar and Waddington, (1953). 
A chromatographic analysis has detected numerous nucleotides in the unfertilized eggs, and recourse to the incorporation of ${ }^{32} \mathrm{P}$ has shown that adenosine monophosphate and adenosinetriphosphate are also present and active in these cells (Finamore and Grouse, 1958). The effects of developmental inhibitors on the phosphorus balance of gastrulae have been studied (Gregg and Kahlbrock, I957); by measuring the respiration of homogenised hybrid embryos (Gregg and Ray, 1957) it has appeared that no synthesis of new enzymes occurs, but that the changes in the early stages mostly concern the accessibility to the existing enzymes.

In sea-urchin eggs probable predominance of glycolytic processes in the animal half and of proteolytic ones in the vegetative (antipolar) half (Lindahl, I936), or at least, differences in the nature of the proteins have been found, as shown by the recent studies of $R$. Lallier (1956a) concerning the action of zinc and sulfonic stains (especially chloro-azol sky blue).

For more elaborate information concerning germ proteins, immunological methods have proved most useful. The gradual formation of new antigens which are probably related to the synthesis of new proteins have been established in amphibians (Flickinger and Nace, I952; Clayton, I953; Spar, I953; Nace, I956) and birds (Ebert, I 954). In the chicken blastoderm thoroughly explored by Ebert, serological tests for cardiac myosin have shown that it begins to be detectable during the appearance of the primitive streak, when the antigen is quite widely distributed; when the streak is established, the antigen is present in the whole ectophyll, but not in the endophyll; at the head process stage, it becomes restricted to the lateral cephalic territories shown in explantations by Rawles (I943) to be cardiogenic ${ }^{1}$; at this stage, at least one of the antigens, corresponding to cardiac actin, is present (see Chapter 5). This often-quoted result is indeed reminiscent of the old views which admitted a progressive restriction (Driesch, I896) or a segregation (F. R. Lillie, I929) of potentialities. The possibility has been repeatedly suggested that several or even most enzymes could be present as seeds in the germ; this could also be true for macromolecules. The point is indeed important. If such biochemical preformism must be admitted, it would mean that the impression of novelty in each further step of development would not hold at the molecular level. However, the case of cardiac proteins may well be a unique one, in which an important synthesis at first takes place in an entire layer, and only subsequently is utilized differently according to the region; this would be only a form of epigenesis. Cytochemistry of the mammalian egg also speaks strongly in favor of a biochemical epigenesis, as far as the microscopical level is concerned (Mulnard, I955).

Results of considerable interest will probably come to light in the near future, thanks to the serological method, a still recent but very efficient tool. As an encouraging example, let us quote again (p. 320) the recent experiment ${ }^{2}$ which consists of preparing very active rabbit antisera against the main parts of neurulae

1 For the metabolic characteristics of these heart forming areas, see Duffey and Ebert (1957).

2 See note p. 320. 
(grosso modo dorsal, middle, ventral parts) and testing the effect of these antisera on the cultivation of different marginal territories of the gastrula (Inoue and Ishikawa, 1956). They generally yield structures corresponding to a degradation of a more ventral type. This result fits in well with the idea that the pieces used as antigens were already on the way toward building up the specialized components of notochord, myotoms, pronephros, lateral plate and hematoblasts. When cultivated in the presence of the corresponding antibodies, they were prevented from building up the proteins corresponding to their presumptive level, but remained able to form the proteins appropriate to the more ventral organ. This seems to show that the more dorsal parts of the middle layer have a larger repertoire of hierarchized syntheses than the ventral parts, and that the richness of this repertoire corresponds to a high, middle or low morphogenetic potential.

\section{(f) Modifying intrinsic morphochoresis by operative and biochemical means}

The results of the preceeding biochemical measurements and of serological techniques are, indeed, purely descriptive. Their meaning will be made clearer by the consideration of some other experiments able to influence greatly later events of morphochoresis.

Analysis would clear the striking correspondence between vertebrates and echinoderms. Concerning the latter ( $c f$. Rünnström, I928, I954), all possible combinations between blastomeres occupying the various tiers along the main axis have been explored (Hörstadius, I 935, I950). The well-known hypothesis of the two opposite and interwoven gradients has been carefully tested. No doubt there are two "scales" of animalizing and vegetalizing tendencies, and that by juxtaposition of different levels, a new equilibrum can be attained. A reciprocal influence of cells of various origin is certain, and thus, for simplicity, we can speak of induction. In this case, it appears to be a purely experimental process, not active in the whole morula, or only as a conservative device. Its effective mechanism is not known. At first view, a transfer of some agent seems probable. At closer inspection, a regrouping of the "tendencies" towards both poles would also be plausible; this could mean either transfer, or simply inhibition in the intermediate zone. On the other hand, attention has been repeatedly drawn to the immediate rearrangement of the combined cells. If we think of the production of $\alpha$ - and $\beta$-granules (p. 332) and similar events, the new shape acquired by the cells, their respective conditions of respiration and other interactions could well be important. However, in spite of these suggestions, it seems difficult to escape the possibility of a transfer of some active agents.

Another group of results concerns the effects of various chemicals on the same two tendencies. The two classical agents are the vegetalizing $\mathrm{LiCl}$ and the animalizing NaCNS. Not only are they able, if adequately used, to counterbalance each other, but their action can be combined with well-planned operations in the most amazing way.

In vertebrates, we may recall first the action of these two chemical agents. The fundamental difference in organization is so deep, however, that it would not be reasonable to search for a close correspondence with the two preceding effects. LiCl acts on both the cephalo-caudal and the dorso-ventral hierarchy 
of the organs. It decreases the inductive ability of the prechordal plate ( $c f \cdot$ p. 372 ) and causes presumptive notochordal material to become somitic, and somitic to become pronephritic. The most cephalic parts of these primordia are affected first. The picture of these hypomorphoses is remarkably coherent. They show all grades of what can be called a lowering of the morphogenetic (or morphochoretic) potential, in both cephalo-caudal and dorso-ventral directions. An extensive biochemical study (Lallier, I954) has largely elucidated the effects of $\mathrm{Li}^{+}$; it affects mainly the utilization of glucides and the synthesis of proteins. If the suggestion expressed supra has some value, $\mathrm{Li}^{+}$would depress the syntheses to the highest grade, according to the duration and strength of its action. NaCNS enhances the formation of chordal material ( $c f$. Corti, 1950), but its antagonism to $\mathrm{LiCl}$ does not seem to be as precise as in the sea-urchin. However, the intimate action of certain chemicals on cell metabolism and the physical properties of cytoplasm may be suspected to be of the same nature and probably concern proteins ( $c f$. Ranzi and Citterio, 1954).

There is another remarkable parallelism in the fact that in vertebrate eggs, effects similar to these of hypermorphic and hypomorphic chemicals may be attained by appropriate operations. These results of microsurgery have in fact been gathered as a consequence of the discovery of the organizer, and mostly from investigations concerned with neurogenic induction. This problem is reserved for our last subchapter, but, assuming that our readers are acquainted with the general trends of modern embryology, it will do no harm to go on with the experimental modifications of the inductive substratum. This will lead us to an immediate consideration of the inconspicuous but important variety of induction which occurs inside the middle layer (see also p. 467).

\section{(g) Intradermic inductions}

The reciprocal influence exerted in sea-urchin morulae by blastomeres tiers on their neighbour cells could be accounted by an induction process (p. 350). But it is mainly in the middle layer of amphibian gastrulae, neurulae and young embryos that such processes of induction between parts of the same layer, i.e. intradermic induction, have been recognized. Their prospection has so far not been as exhaustive as for interdermic induction. Nevertheless, their interest is considerable, and their thorough analysis could well be necessary to discover the key to the essence of induction.

When working on a blastula or young gastrula of an amphibian, it is much easier to demonstrate the mutability of the marginal areas than their relative stability. Most operations on the dorsal region show two kinds of effects. When the lowest (presumptively, the most cephalic) part of the notochordal area is extirpated, it is easily replaced in all its capacities by somitic material which moves inwards to close the wound. If the extirpated piece is grafted on the ventral marginal zone of another gastrula (fundamental organizer experiment) two sets of events may occur. ( $I$ ) In the first, but less known possibility, the graft is too small, or has been a little damaged, or comes from a slightly deficient egg. Its morphochoretic potential is not maintained, and the graft sinks deeply into the marginal zone: it may form some somites and pronephros, with an induced spinal chord, or, more often, it may migrate along the lateral plates of the host (unelucidated 
process) to join its pronephros or its myotomes ${ }^{1}$. (2) In the second, quite famous possibility (Spemann's organizer transplantation), the graft sinks or invaginates, incorporates itself into the ventral middle layer and not only develops into notochordal material and some somitic parts, but also causes the lateral plates of the host to become notochord, somites and pronephros. The reaction is generally completed by the induction of a neural plate, which will be considered later.

Other operations performed on the same early stages, e.g. rotations of the animal half (Fig. 51, p. $3^{83}$ ), or of a large dorsal piece (Figs. 49, 5o, pp. $3^{8} \mathrm{I}-3^{82}$ ); cause juxtaposition of the median (chordo-somitic) material and the more ventral parts of the mesoblastic layer (Dalcq, I940, I941a, I942). If they are realised early enough, the chances are that a typical series of notochord-somites-nephrotome and lateral plate will be established. This phenomenon is accomplished, as Nileblue vital staining has ascertained, by some recasting of the tendencies present in the graft and an elevation of potential in the adjacent material.

These results, and several others, attest the reality of an inductive influence taking place inside the middle layer. However, these are only experimental data. One might ask whether a similar mechanism plays a role in normal development. We do find such a process in the awakening action exerted by the duct of the pronephros on the mesonephritic blastema. There the necessity of a contact or at least of a narrow proximity (p. 464) has been demonstrated (Cambar and Gipouloux, I 956) and its immediate consequence is the enrichment of the cells in RNA, their proliferation, and their subsequent arrangement in canaliculi (O'Connor, I939; Van Geertruyden, I 946; and, for the exception found in axolotl embryos, Nieuwkoop, I 948). This shows that intra-mesoblastic induction is not only experimental. It may well be thought to exist inside the middle layer, as a conservative safeguard of the hierarchized potentials.

We should now inquire how this induction is accomplished. Here, we do not know any details concerning the immediate consequences of the relevant operations. Other types of induction, as we shall see, have been studied more accurately. Although it has not been demonstrated directly, it is very likely that some agent is transmitted from the inductor to the reacting cells.

A considerable change in the fate of mesoblastic material can also be obtained at a later stage, when the neural plate is already beginning to form. In grafting and isolation experiments, it has been shown that somitic material has a tendency to transform into pronephros, especially if it is adjacent to lateral plate material; the presence of notochord favors the persistence of myogenic cells (T. Yamada, I937, I940). Recently, a rather large amount of somitic material (about five presumptive somites) has been cultivated, wrapped in ectoblast. It yields an appreciable quantity of myoblasts, pronephros, limb bud, lateral plates, blood cells ${ }^{2}$

1 As far as I know, this migration of a homograft toward the corresponding dorsal material of the host has been described in works made in my laboratory first by Minganti (1949) and then, more explicitely, by Benoit (1952). This enigmatical process is probably equivalent to the "homing instinct" which insures recolonization of the bone marrow of irradiated animals by injecting a suspension of normal lymphoid and red blood cell-forming tissue (see Mitchison, 1958).

2 For an experimental analysis of the conditions necessary for the differentiation of the crythroblasts, see Finnegan, 1953. 
and even pulsating cardiomyoblasts. This is a considerable diversification, which has been interpreted as the production of new fields potentially contained in the presumptive somites ${ }^{1}$ (Muchmore, I956).

Another interpretation seems possible. If it is admitted that the fate of a given amount of mesoblast depends on certain products of metabolism, whose concentration corresponds to a definite potential (notochordal, somitic, pronephritic, etc.) and that these products, or some of their antecedents, can be transmitted to other cells by diffusion or otherwise, juxtaposition of cell groups belonging to different levels may result in a new equilibrium. At first this new equilibrium expresses itself in a continuous gradient; secondarily, owing to a threshold effect, it takes the form of a scalar distribution, yielding adjacent correlative organs. From the new results of Muchmore, it might be supposed that, at the periphery of the explanted somitic lump, slight lesion and especially dispersion of cells could result in some dilution of the morphogenetic products. This would be the first step towards the secondary production of adjacent fields with their own type of organization. It must be added that at least one striking experiment strongly suggests that the institution of regional fields is linked with the elaboration of definite products inherent to organs such as notochord, neural cord, myotomes, pronephros, which are also transmissible to receptive cells. Reference is made here to the experiment in which lumps of ectoblast of a newt gastrula are superimposed upon several organs of advanced neurula or young tailbud stages and form chordal, nervous, muscular, pronephritic tissue, according to the organ to which they are juxtaposed or to the field in which they are integrated (Holtfreter, I933a). If this result really reveals the gradual elaboration of organ-specific substances, it falls well in line with the previous data.

Quite recently, it has been found that the moment of axial organ stabilisation actually occurs somewhat later during neurulation than was believed. If Triturus neurulae, at the stage of a well formed neural plate are exposed during 30 to $60 \mathrm{~min}$. to a temperature of 30 to $40^{\circ} \mathrm{C}$, their development is momentaneously stopped and then recommences, but with a twinning effect. This process is accompanied by a rapid enlargement of the neural plate (Takaya and Watanabe, 1957). This is a further encroachment on the idea of sudden determination.

In this issue as in many others, the difference of opinion revolves around preformation versus epigenesis. The current conception admits that, in the early neurula, fields of pronephros, limb and heart exist potentially already, broader than the organ will be, and overlapping each other. This concept (Holtfreter, I938b, his Fig. 68) is probably acceptable for stages immediately preceding the appearance of the new organs, but to reflect these ideas back on the young neurula ${ }^{2}$ or gastrula may seem inadequate. This interpretation necessarily postulates latent potencies, and forces us to imagine an exceedingly complex organization. However, a simpler solution is possible (p. 474).

1 For the problem of meristic segmentation, see Dalcq (1947a), Waddington and Deuchar (1954).

2 An experimental analysis of determination and differentiation of the mesoblastic structures of the neurula has been made in Urodeles by Chuang and Pai (1956). They are opposed to the idea of overlapping fields and favorable to the concept of a general induction field. 


\section{E. Mechanisms of intrinsic morphochoresis. A discussion}

At the end of this second section, it is perhaps fair to contrast the two main theoretical positions preferred by embryologists today. These concepts are not exclusively based on the study of what we have called intrinsic morphochoresis, but they necessarily include ideas relative to extrinsic morphochoresis, mainly the processes of induction.

For the first position we may refer to the "general conclusion" expressed by Ten Cate (1956, his p. 84) as the result of a most clear and attentive introduction summarizing many investigations concerning early development, and including one of the best accounts ever given of the gradient field concept and its intricate implications;

" $a$. First, it should be emphasized that nuclear, genetic factors have a predominant influence on embryonic development. This appears to be demonstrated by the intensive growth of the oocyte nucleus and by other phenomena.

$b$. As demonstrated by many experiments, a restricted portion, the grey crescent, appears to be highly important for the development of the entire embryo; even entodermal differentiations were absent in ventral halves. Although some experiments show deviations from this rule, it may be assumed that in this stage, the morphogenetic factors are mainly concentrated in the grey crescent where all kinds of cytoplasmic constituents, including the residual nuclear substance, appear to be present or at least in the near vicinity.

c. In the subsequent stage, a distribution of morphogenetic factors throughout the embryo appears to occur, whereby some of them become demonstrable in wide regions; consequently, these factors become highly mixed with each other. Perhaps, during this period of expansion, the factors may be transferred from one cellular complex to an adjacent one by means of induction.

In terms of embryonic fields, i.e. self-organizing systems of morphogenetic factors, it may be stated that in this period the fields are extending over increasingly larger regions and therefore will largely overlap each other. The spreading of morphogenetic factors is somewhat similar to the extensive cytoplasmic displacements which are so clearly visible in ascidian eggs and by which large complexes become segregated.

In the amphibian embryo, such displacements may be less extensive and definitive, as is shown by the longer-lasting possibility of extensive regulations. Therefore, the effect of gastrulation might be more important than in those species where a mosaic stage of differentiation is reached as early as the late cleavage stages (mosaic types).

$d$. The following period may be characterized by segregation of morphogenetic factors, leading to their concentration in organogenetic areas. Segregation may be accompanied by induction phenomena if the factors make their way, not only through intimately coherent cellular complexes, but also from one complex (germ-layer or organ-part) to another, partly via cytoplasmic bridges which are established during a certain period of time. For example, in this stage, the prosencephalic factors residing in the early dorsal blastopore-lip, after invagination may be transferred to the overlying pres. neural ectoderm which itself already contains some of these factors also derived from the grey crescent and arrived there through the continuous layer of micromeres of the blastula or the ectoderm of the gastrula. Now, the embryonic fields come into the fore-ground; these build up stronger potencies to develop a specific part by concentrating the available factors and by reproducing them in a larger quantity.

$e$. As a result of the activity of the fields, in the subsequent period, the mosaic stage of differentiation is reached in which the embryo contains the segregated organogenetic areas.

The effects of negative and positive affinity might underly the whole complex of occurrences; spreading might be either a simple diffusion of factors into the remainder of the egg or be stimulated by positive affinities of homologous and by negative affinities of heterologous factors.

These properties might be expressed in the general cytoplasmic displacements in the egg as well as in the movements of cell-complexes and cells in subsequent stages of development." 
It is unnecessary to describe the alternative position at length, since it has induced the preceding sections. The difference lies first in a more accurate consideration of the cytoplasmic organization in the oocyte and of its acquisitions; second, in a more precise appreciation of the properties of the grey crescent, which are deduced from definite observations and experiments, and not from the abusive projection back on to grey crescent material of the activities recognized in the organizer; third, in a more strict use of the gradient field concept, which is only licit when positive arguments permit, that is in the cortex of the fertilized egg and the cleavage stages, in the mesoblastic layer of the archenteric roof, in the induced neural plate, and (perhaps) around the presumptive areas of some secondary organs; fourth, in a reluctance to admit that induction processes exist in cases where they are not actually demonstrable, as in the grey crescent region or along the epithelial layers; and fifth, in the recognition that regional differences are responsible for what has been described above as morphogenetic $\operatorname{potential}(\mathrm{s})$.

To be quite clear, it will be useful to submit the above quotation to further criticism; this will be done without any intention to depreciate Ten Cate's highly valuable contribution. The discussion will concern only the conclusion which a most skillful and competent worker has felt authorized to draw from an extensive revision of the embryological literature.

To item $a$. This general statement must be admitted. For $\S 2$, the intensive growth of the oocyte nucleus is certainly important from several points of view, but whether or not it expresses a special intervention of genetic factors, we do not know. The fluid content of the germinal vesicle is not necessarily different from that of any somatic nucleus.

To item $b$. Although one can agree with the general sense of this statement, the grey crescent region is not as priviledged as presented by Ten Cate. Absence of differentiation in isolated ventral halves is a classical assertion which, as was shown by Dollander (1950), lacks definite proof. Careful experiments in which ligature was combined with vital staining have demonstrated that the ventral halves of Triton helveticus eggs are able in a large proportion of cases to form an embryo, if they are not smaller (preferably a bit larger) than the dorsal halves. Holtfreter (195 I his p. I I9, note I) has attempted to invalidate Dollander's experiment with the objection that his ligatures were not really frontal. Although this criticism was a priori met in Dollander's original research, he nevertheless made new vital staining experiments which showed that no error could have been committed (1952). Accordingly, the opinion that "morphogenetic factors are mainly concentrated in the grey crescent" is consistent neither with the regulation of isolated ventral halves, nor with the production of new blastoporal lips in inverted eggs (p. 343). Whether or not the "residual nuclear substance" plays a role, seems impossible to decide owing to lack of experimental possibilities to check it.

To item $c$. The redistribution of morphogenetic factors and their secondary mixing is an assumption resulting from the two exclusive roles attributed to the grey crescent region. If we are concerned with pregastrula stages, it is quite unnecessary to imagine active inductions from one blastomere to another. Of course, for the sea-urchin egg, such processes do seem to occur (p. 350) but only under experimental conditions. It has been claimed that in ascidian eggs, the induction of the neural territory takes place during cleavage (Vandebroek, I938; Rose, I939; Reverberi and Minganti, 1949); without questioning the accuracy of the experimental data, I suspect that this induction is of a special kind and need not to be discussed here.

To item $c \S 2$. As far as pregastrula stages are concerned-and the allusion to displacements of cytoplasm in Ascidians indicates that they are-the assumption of several overlapping fields is invalid, since it is based only upon the erroneous "expansion of 
morphogenetic factors". From fertilization to incipient gastrulation, the whole egg cannot, in my opinion, represent more than one field. In passing, let us note the danger of using such expressions as "self-organizing systems of morphogenetic factors". We have as much reason to protest against such illusory expressions as our predecessors of fifty years ago had against entelechy.

To item $c \S 3$. This statement is more satisfactory, although it oversimplifies the explanation of regulation. The problem is, why blastomeres of certain eggs, and not of others, are able to retrace the earlier steps of development. This probably depends on the gradual distribution of the various kinds of organelles. Regulation is a potential ability of all eggs; it is only the period during which they retain this capacity which varies. Let us mention here that induction has a special significance. As Medawar (I954) rightly hinted, induction adds to development an additional degree of freedom. As such, induction is an endowment of eggs belonging to the highly evolved classes, i.e. vertebrates, echinoderms, mollusks (see Raven, I958), insects.

To item $d$. If morphogenetic factors have been mixed, and if, thereby, a complex situation of overlapping fields has arisen, it becomes necessary to postulate a segregation of these factors. But this return to a workable simplicity is again illusory. Development proceeds really from apparent simplicity towards evident complexity. Gastrulation, if we think of vertebrates, is a period of complication, not one of simplifying segregation of imaginary potencies. The period when the egg was still one unitary field comes to an end at this time, because, as a consequence of activities inherent to the primary field, kinematics has started. From now on at least two fields exist, one pervading the invaginated material, derived from the primary field, and one resulting from the induction exerted by the inner material upon the external layer. It is likely that very soon a third field will express the induction of the mesoblast on the endoblast; the results of T. S. Okada (1953, I956, I957) afford an experimental basis for this assertion.

Whether or not the prosencephalic factors as such reside in the blastoporal lip will be discussed later. The possibility that some special properties proceeding from the dorsal half of the normal egg exist in the neurectoblast is not excluded, although these properties are not indispensable to the formation of a normal nervous system. However, it is certainly unnecessary to imagine that these properties originate in the grey crescent and have climbed towards the pole through the micromeres or the ectoblast. If there does exist some very slight influence of the neurectoblast, it simply owes its existence to the primitive unitary field.

And now, says Ten Cate, "the embryonic fields come into the fore-ground ...", and, the advantage of the system he has adopted is that he immediately attains an explanation of the biochemical specificity of organs. Behind his "segregation of morphogenetic factors" is concealed the idea of representative particles, which have only to multiply in order to yield specificity. But this is, in fact, a purely hypothetical representation, and indeed, too easy a solution. How the establishment of secondary fields could be more preferably explained will however be more easily examined after a study of neurogenic induction (p. 472).

To item $e \S I$. is only a statement of facts, and avoids the difficult problem of explaining the so-called determination. We have already met this same enigma (p. 320), with only a relative success.

To item $e$ 92-3. This passage refers to the new data concerning cell adhesiveness and reciprocal affinities, which have been extended still further in the last three years, and are among the most striking of Holtfreter's numerous investigations. The statement that a "simple diffusion of factors" can explain the "spreading" is indeed questionable. It is more essential to appreciate the real importance of cell affinities in the general picture of early development, without committing the frequent error of seeing in the latest discovery, whether it be regulation, germinal localizations, genes, organizers, or affinities, the key to all the enigmas of ontogenesis. Surface properties of the blastomeres and of their derivatives do not exist a priori; they are acquired progressively, and are thus relevant to epigenesis. As far as the eggs of the anamniots are concerned, especially those in these investigations, the trend of ideas which considers reactions between endoplasmic components and the cortex fits in well with the concept of gradual transformation of surface properties. 
These do evidently play some role in the installation of kinematics or the appearance of a blastoporal lip. Later on, the surface specialization of cells belonging to the various layers and regions (neural plate, young somites, pronephros, endoblast, etc.) is to be considered one aspect of their respective morphogenetic potential. This is the evidence which Townes and Holtfreter (1955, p. 95) fail to consider. In discussing the facts already mentioned, they put the concept we advocate under the heading: "Unsupported hypotheses on morphogenesis". These authors write: "Dalcq and Pasteels (Dalcq, I94 I b) have postulated that invagination of the blastoporal material is caused by an interplay of two hypothetical concentration gradients. It would require much imagination to find support of this hypothesis in the present results". The least that can be said is that such a statement is completely irrelevant to their paper. On the contrary, a simple objective consideration of observations and experimental data obtained from successive stages by all efficient methods, shows a logical and still more satisfactory picture, although it evidently is not yet definitive nor complete.

III. NEUROGENIG INDUGTION, THE MAIN ASPEGT OF

EXTRINSIC MORPHOCHORESIS

(with some data on non-neurogenic inductions)

The classification of processes of induction as processes of extrinsic morphogenesis is perhaps a bit unusual, yet it helps to give them their exact importance in development. Their significance would only be a subsidiary or complementary if the organs, owing their existence to induction were not so essential to life.

There are several types of induction, and they may be distinguished according to chronology or space. One distinguishes first between primary, secondary, and tertiary inductions. ( $I$ ) The primary ones occur either inside the middle layer, a process which has already been considered (p. 35I) or from this layer on the endoblast (p. 356) or from the middle layer on the ectoblast, the phenomenon which will receive our special attention in this section. At both ends of the gut, the endoblast also apparently acts on the epiblast to form the stomodoeum and the proctodaeum and these relatively late events in morphochoresis can also be classified as primary inductions. (2) Secondary inductions are mainly dependent on the neural tube, mostly on brain parts or their derivates, acting on the epiblast before its determination as epiderm. Sometimes, two or three inductors may act either successively or simultaneously on one "Anlage", to obtain a definite organ in its full complexity (Holtfreter's coordinated inductor systems, Lehmann's Kombinative Einheitsleistung). (3) Tertiary inductions are known, or at least suspected, in the differentiation of the cornea, the teeth, the dorsal fin, and probably many other instances. Some manifestations of rather advanced stages, e.g. the development of the apical cap of limb buds, the experimental induction of a tympanic membrane, and the organizing effect of mesenchyme on the salivary gland, are difficult to classify, but are probably relevant to tertiary inductions.

A certain fascination is attached to the neurogenic induction. When Spemann, in I92 I, recognized this entirely new phenomenon, he used the word "Organisationszentrum" (see also Spemann and Mangold, I924) with its broad implications. Further investigations on the "organizer" placed emphasis on the reactor as much as on the inductor. The obstinate search for a biochemical explanation has not yet been successful. Numerous ways of imitating induction by various means have been discovered, but their common factor, if it exists, still escapes the 
perspicacity of workers. It remains difficult to interpret normal development in the light of the numerous experimental results. Therefore, an attempt to summarize actual data and ideas concerning neurogenic induction must be undertaken as a heuristic enterprize. In order to give it the best objectivity, I think it advisable not to follow a chronological order, but to group at least the larger part of the relevant data according to the methods by which they have been obtained: first, the observations sensu stricto; second, the strictly microsurgical experiments, with a distinction between those carried out on the whole egg and those concerning isolation, or combination of fragments or large pieces, e.g., explantations, sandwiches; third, the modification of development by use of physical and chemical agents; fourth, the recourse to foreign (xeno-) inductors. A more limited inquiry will be devoted to secondary and minor inductions.

When we have gathered all this information, we should, if this field of research has already been sufficiently explored, be able to perceive a clue towards an explanation of the neurogenic induction. A restriction however, must be immediately brought to this extensive program. It is neither advisable nor required, to present here an extensive review of the five categories of enquiries. The results of the pre-war period have been summarized in several books (Huxley and De Beer, I934; Spemann, I938; P. Weiss, I 939; Dalcq, I 935b, I938b, I 94 I b), and are largely to be considered as classical knowledge. More recently, comprehensive reviews have been written by Pasquini (1949), Bautzmann (1951), Raunich (1953), Holtfreter and Hamburger (1955) on amphibians in general, by Mangold (1958) on the head of Urodeles, and by Rudnick on fish and birds (1955); moreover, extensive reports have been written by Woerdeman (I952, I955a, b) on the use of serological methods, while Waddington (1952b) has devoted a book to experimental results concerning bird eggs. Therefore it will suffice to consider only research which is relatively recent and apparently significant for the understanding of neurogenic induction.

Before entering this inventory, let us add first that we intend to consider for each methodological category the results available not only concerning amphibians but any vertebrate, second, we are not interested in unravelling all kinds of subtleties relative to any particular method of investigation, but only in gathering all the available information which may help to answer the following question: how does neurogenic induction work during the development of a vertebrate embryo?

\section{A. Observations}

How the cells of the ectoblast progressively change during induction with respect to their shape, the structure of their cytoplasm and their mutual arrangement (Fig. 34) has been observed a long time ago by embryologists, using classical methods. From sections, one gets the impression of a shape-giving growth; the occurrence of numerous mitoses is always observed and also the active utilisation of the yolk. In the young Pleurodeles gastrula, the ectoblastic cells are rich in yolk platelets, and the dorsal region is thicker than the ventral (Fig. 34, A). In the young neural plate, already made distinct by a pigmented hem, the upper part of the cells is largely free of platelets; in the corresponding thinner ventral epiblast, the yolk resorption is less pronounced. This difference is made more 

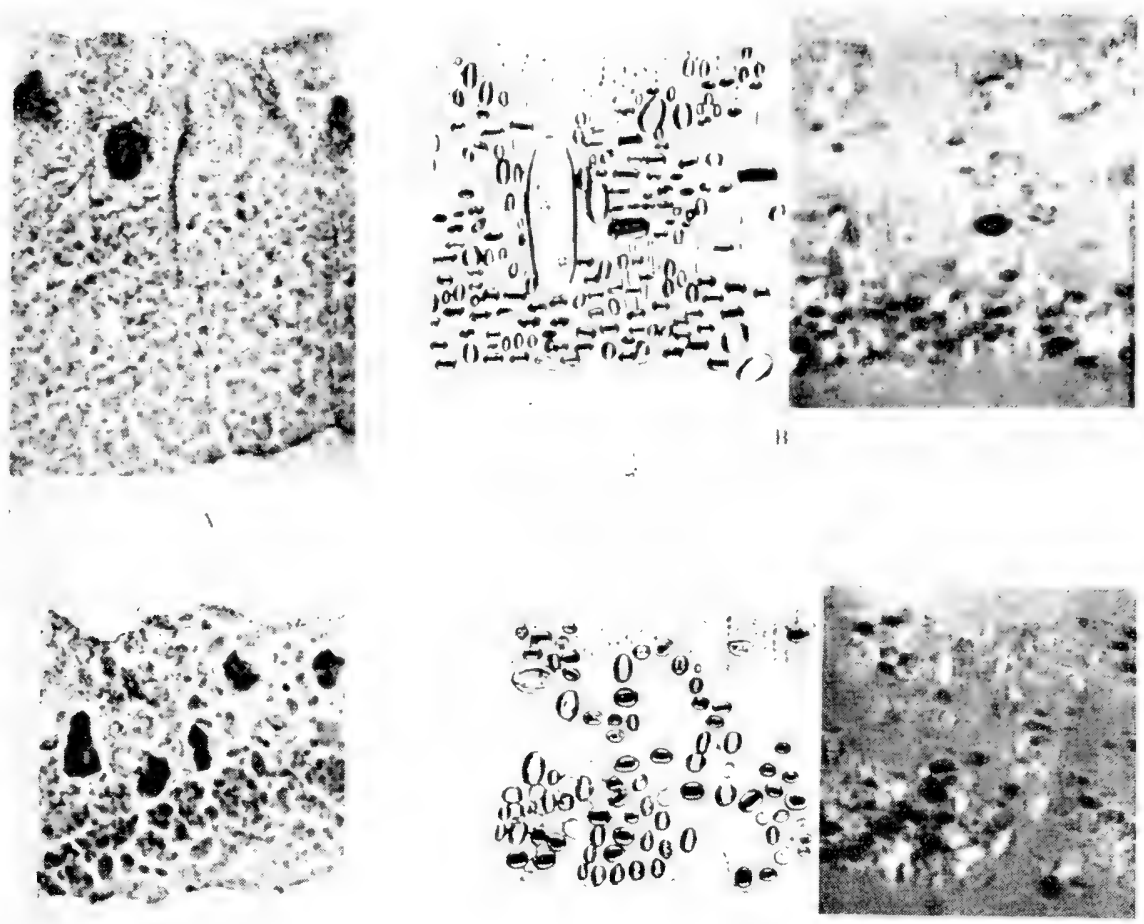

1)

6

Fig. 34. Structure of the ectoblast and neurectoblast in normal Pleurodeles embryos (A-C) and in embryos submitted to urea (D). A. Thin section of the dorsal ectoblast in a young gastrula, stained with pyronin-methyl green. Phase contrast. High columnar cells filled with pigment and yolk. B. Neuro-epithelium of a young neurula. Thin unstained section, polarized light. The nucleus is elongated, the platelets are less abundant, are elongated horizontally and are concentrated at the base of the cells; large "empty" areas of orientated cytoplasm (see schema, left). C. The ventral epiblast at the same stage, under the same conditions. Similar but less marked differentiation in more cubic cells. D. The neuro-epithelium of an embryo treated by urea. Same conditions as in A. The epithelium is thicker than in a normal neurula $(70 \mu$ rather than $40 \mu$ ) and is undifferentiated. The polarization microscope does not reveal a structure comparable to B and C. The enlargement of A and

D is about half of B and C. From Gallera and Baud, I954.

conspicuous (Fig. 34, B, C) under polarizing microscope (Gallera and Baud, I 954). Our information concerning the ultrastructural changes occurring during neurogenic induction is quite recent. We are endebted to Eakin and Lehmann ( I957) for an electron-microscopic study of the aspects observable in the amphibian ectoderm, mainly in Xenopus, at various stages from the early gastrula on. The effect of induction on the presumptive neurectoblast is described as a densification of the cytoplasmic reticulum, a reduction of size in the various inclusions and a definite numeric increase of the mitochondria. The intimacy and complexity of the limit between the archenteric roof and the external layer suggest easy transfers of macromolecules. At later stages, a further differentiation of the mitochondria 
is apparent in the young epidermis, where they become slender, s-shaped and quite numerous.

A parallel approach was carried on in the laboratory of ' $T$. Yamada. The photographs just published by Karasaki ( $1959 \mathrm{a}, \mathrm{b}$ ) demonstrate considerable modifications, from the blastula to the neurula stage, both in the cytoplasm and in the nucleus. Among the six or seven plasmatic constituents which can be distinguished, the mitochondria show the most conspicuous change. In the ectoblast they are ellipsoid and of a rather simple inner structure and they do not change much in the epiblast. In the neurectoblast they are now found more numerous, filamentous $(3 \mu)$, and often branched, with a highly complex inner structure. The appearance of numerous ultramicrosomes around the vesicles of the endoplasmic reticulum is in agreement with the increased RNA content. The changes in the nuclei are the appearance of the nucleolus at the gastrula stage (in accordance with Brachet's earlier descriptions), the gradual increase in density of the nucleoplasm and a more distinct separation of the two layers forming the nuclear membrane. Various aspects revealing cytonuclear interactions have also been described.
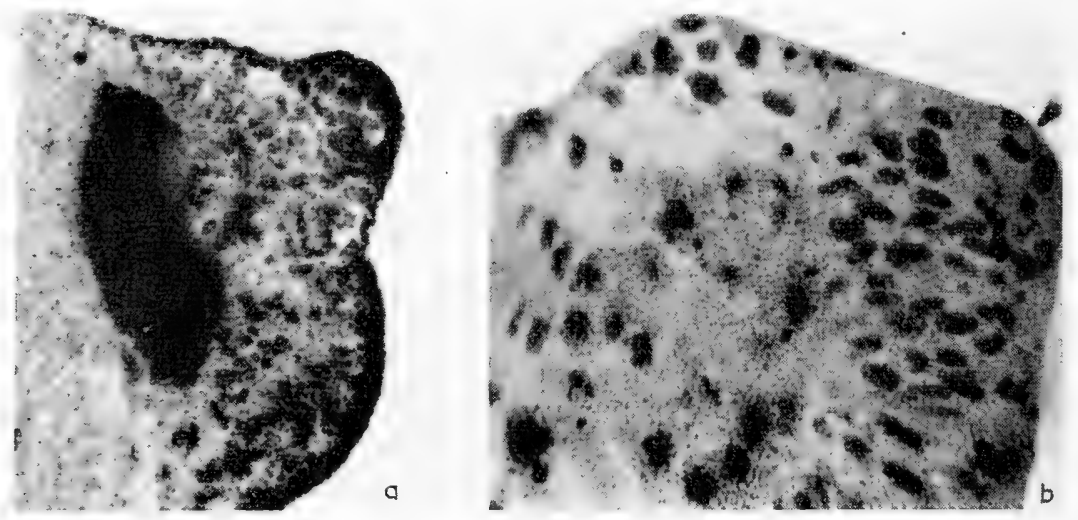

Fig. 35. Effects of inductors labelled with glycine-2-14 $\mathrm{C}$. Autoradiographs, (a) Piece of brain and eye embedded and introduced into the blastocoele. The induction is limited to a thickening of the ectoblast. Note dense autoradiographs of graft and induction, and faint autoradiographs of the internal endoblast. (b) Labelled piece of organizer used in a sandwich. Note very dense autoradiographs on the graft, particularly on the nuclei. The autoradiographs on the palisade are much stronger than on the epiblast in the upper part of the figure. From Sirlin, Brahma and Waddington, I 956.

Important biochemical changes have been progressively detected in the induced neurectoblast, mostly thanks to advances in cytochemistry and in the use of isotopes.

When Brachet (1940, his p. I9o) elucidated the meaning of the Unna procedure (pyronine and methyl green) he immediately observed in the neurectoblast of amphibian and bird embryo that induction was accompanied by an apparent increase in ribonucleoproteins. This change remains one of the most characteristic and general manifestations of primary-and also secondary-induction, and reveals some change in intimate metabolism; the first signs of it can be detected in the margino-dorsal region, and especially in the blastoporal lip. This early change is quite apparent in the prechordal area of Xenopus (Pasteels, 1949). Brachet also showed that the $\mathrm{SH}$-protein complexes had roughly the same distribution as the RNA. Fischer and Hartwig (I936) in their work on the speed of 
reduction of brillant cresyl blue found that the dye was most rapidly reduced in the neural plate. The same high activity was observed in the presumptive neural territory and, still earlier, in the dorso-marginal zone of earlier stages (Piepho, I $93^{8}$ ).

Another body of significant data concerning the RNA has just been published by Rounds and Flickinger (1958). Using frog embryos at six stages, from the early gastrula to the neurula with closing tube (Shumway's stages io to I5, Fig. $36, \mathrm{~A})$, they chilled them and cleanly separated the inducing and reacting material. The pooled fragments from a dozen embryos were homogenized and submitted to successive centrifugations with appropriate saline dilution. Three fractions were obtained and precipitated by trichloroacetic acid in order to estimate together RNA, DNA and protein nitrogen content in: $(a)$ the soluble fraction, containing proteins soluble in such media, lipids and some of the microsomes; $(b)$ the yolk fraction, formed of the dissolved yolk platelets; (c) the residual fraction made up of the insoluble cellular debris, such as fragments of cell membranes, pigment granules, mitochondria and some of the microsomes. The method is thus characterised by measuring protein and RNA relatively to the DNA content, which affords a cellular basis of reference.

The protein nitrogen content is constant for fractions $a$ and $c$ both in inductor and reactor, but for fraction $b$ there is a decrease in the inductor during its invagination and "the rate of decrease becomes pronounced just prior to the appearance of the neural plate" (Fig. $36, \mathrm{~B}$ ). In the reactor the same fraction is relatively constant with the exception of a slight decrease at the time when the cells of the neural tube are beginning to differentiate (Fig. 36, C).

The RNA content of fraction a remains constant both in inductor and in reactor. For fraction $b$, a regular decrease is observed in the inductor, while in the reactor a fall only occurs, as for protein, in the plain neurula. For fraction $c$, the RNA level of the inductor increases slightly during invagination, then decreases significantly ( 6 consistent determinations) during the most active induction period, to increase again while the neural plate is appearing and soon closing. In the reactor, at the same time, a great increase is observed (Fig. 36, D, E). The total levels of RNA in the inductor and reactor (Fig. 36, F) are not less impressive. Clearly, the amount of this substance is first reduced in the inductor just when the archenteric roof becomes subjacent to the ectoblast. Then an increase takes place in both layers, more intensely in the reacting ectoblast.

These data suggest that yolk platelets progressively break down in the chordomesoblast during invagination with a subsequent release of RNA to the cytoplasmic granules. The RNA is then used in some way during the incipient period of induction, but is apparently not immediately recuperated, at least not as such, by the reacting ectoblast. As soon as morphological changes are perceptible in the neural plate and in the underlying archenteric roof the level of RNA increases in both layers, however not at the same rate, but distinctly more in the ectoblast. This bulk of new information is especially valuable.

Important cytological observations have also been made by means of the isotopes. In fact, they have been obtained by operative techniques (Eakin et al., I 95 I Ficq, I 954 b; Sirlin, Brahma and Waddington, I 956), but the interest of their results lies 

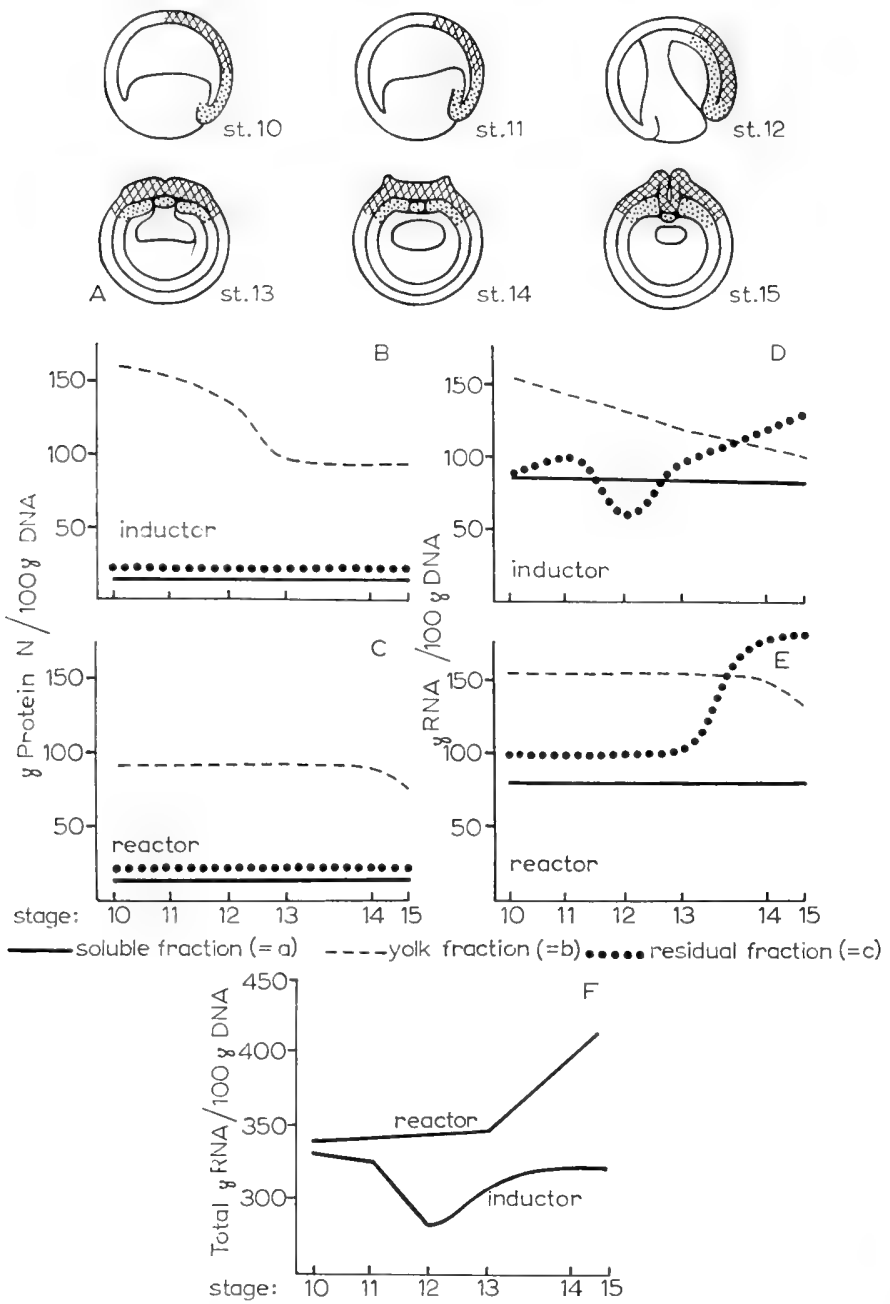

Fig. 36. Changes in protein and RNA content relatively to the DNA content during gastrulation in Rana pipiens. A. The stages used, IO, I I, I2 in sagittal section, I3, I4, I5 in transverse section; inductor stippled, reactor crosshatched. B, C, D, E, relative levels of the protein nitrogen and RNA in the three fractions (see text) of the homogenised inductor and reactor. F. Total relative levels of RNA in inductor and reactor. From Rounds and Flickinger, 1958 (modified).

in $(I)$ the dispersion of the tagged compounds around the labelled inductor and in the induced structures, and (2) the incorporation of the chosen compounds radioactive glycine and adenine), in the host nuclei (Fig. 35). Several aspects strongly suggest an active participation of the nuclei in the induction processes.

Some data obtained on chicken embryos favor the idea that the macromolecules responsible for the induction process include sulfhydril groups. The distribution of such groups bound to proteins follows a characteristic sequence 
(Katayama, 1956). The reaction first appears in the anterior node, in which the material for the notochord is incorporated, then in the neural plate and finally in the somites.

Recourse to isotopic tracer techniques helps further analysis but again implies an experimental procedure which, however, remains, by its aim, strictly observational. The most useful tracer has been so far DL-methionine- ${ }^{35} \mathrm{~S}$. According to Waddington and Mulherkar (1957), this isotope is incorporated in the induced structures, mainly in the nucleoles, the associated chromatine and in the cytoplasm. The transmission of inductor to reactor seems to be performed together under the form of amino acid and of a SH-protein, which could be the real inducing factor. In the same laboratory, Pantelouris and Mulherkar (1957) have cultivated the embryos in albumen (New's method) added with radioactive methionine until the IO-I 4 somites stage. They found that "the neural tube and the notochord show a higher energy uptake than any other structure". By grafting a labelled organizer into a normal embryo, it can be ascertained that the inducing notochord and mesoblast proceed from the implant, but the secondary neural plate or tube shows a substantial activity uptake. This phenomenon becomes negligible if unlabelled methionine is added "as a trap" to the culture medium. "This is evidence for diffusion of free molecules of the labelled amino acid. It also proves that the transferred unit is the amino-acid molecule and not the labelled radical only." On the other hand, by grafting more lateral ectophyll, it may happen that the labelled implant develops in neural tissue adjacent to the primary neural tube, and that the latter shows a gradient of activity. This proves that migration of the radioactive molecules may take place irrespective of whether an induction process occurs or not. "The conclusion seems inevitable, therefore, that induction is not correlated to a transfer of methionine-containing macromolecules from the graft on any large scale. But, as Waddington and Sirlin (1955) pointed out, this does not exclude the transfer from the graft to the inductively activated cells of a few molecules able to trigger off a chain reaction. If the results of these experiments provide no positive answer to the question of the nature of the inducing factor, they exclude at least the possibility of a large-scale transfer of macromolecules, and perhaps make more likely the alternative of the transfer of small quantities of the effective agent". This work is typical of the intricacies of the induction process and shows how necessary it is to consider all its aspects before drawing any definite conclusion.

In the paper quoted above, Rounds and Flickinger report several experiments in which chordomesoblast previously incubated in $\mathrm{Na}_{2}{ }^{14} \mathrm{CO}_{3}$ was wrapped in non-radioactive competent ectoblast (and reciprocally). After 8 h. contact, both parts, and due controls of these, were homogenised and the radioactivity checked in the TCA-insoluble and the nucleic fraction. The induced ectoblastic fraction showed a much higher level of activity. This result suggests a passage of labelled nucleoprotein molecules from the inductor into the reactor.

A similar conclusion has been reached, by the same authors, by a serological procedure. A Rana $p$. inductor was combined with Taricha $t$. ectoblast. After $4^{-5}$ days culture the Taricha tissue was separated and tested by serum of rabbits prepared by injections of Rana $p$. embryos. The tests performed with convenient 
controls pointed in favor of the passage of frog antigens into the Taricha tissue.

Recently, the study of mammalian embryos has called attention to a relation between induction and alkaline phosphatases. As soon as the neurectoblast is induced, it actively elaborates this enzyme (Fig. 37); the same holds true for all further induced structures, such as neural crest, large and small placodes
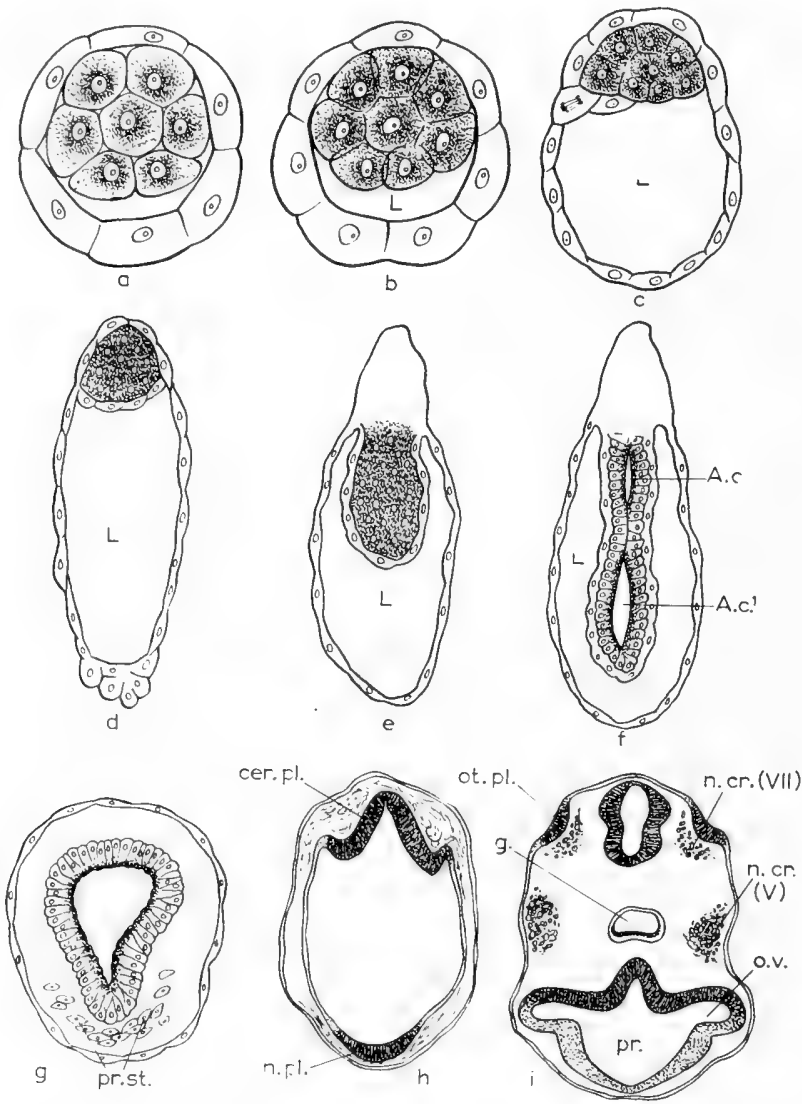

Fig. 37. Alkaline phosphatase in the early stages of the rat. This enzyme is not present before the sixteen cell stage (a). It appears at this time in tiny granules around the nuclei of the inner cell mass, and soon extends to the whole cytoplasm of these cells (b). (c)

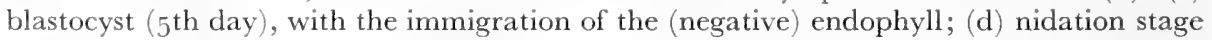
(6th day) with endophyll(negative) and still compact ectophyll; (e) young cylinder stage ( 7 th day) with the elongating embryonic region; (f) 2-layer stage (8th day) with the formation of the amniotic cavity; the reaction fades appreciably at this stage; only the inner part of the cells contains the enzyme; (g) primitive streak stage ( $9^{\text {th }}$ day), in transverse section, showing the same reaction in the ectophyll; ( $h$ ) neurula stage (I oth day) with the neural plate strongly positive; (i) young embryo of the I I th day, with alk. phosphatase in all induced structures and to some extent in the gut. A. c., amniotic cavity, in the amniogenic region; $\mathrm{Ac}^{1}$, the same, in the embryogenic region; cer. pl., cerebral plate; g., gut; n. cr V-VII, neural crest of the trigeminal and facial nerve; n. pl., neural plate; ot. pl., otic placode; o.v., optic vesicle. After Mulnard's documents, I955. 
(Mulnard, I955). As already pointed out, the enzyme first appears in the enveloped group of cells which soon transform into the ectophyll. During the movements of the streak, the positive reaction partially decreases and parallels the utilization of RNA; the immigrated mesoblast is quite feebly positive, with a decrease from the presumptive somitic material towards the future lateral plate.
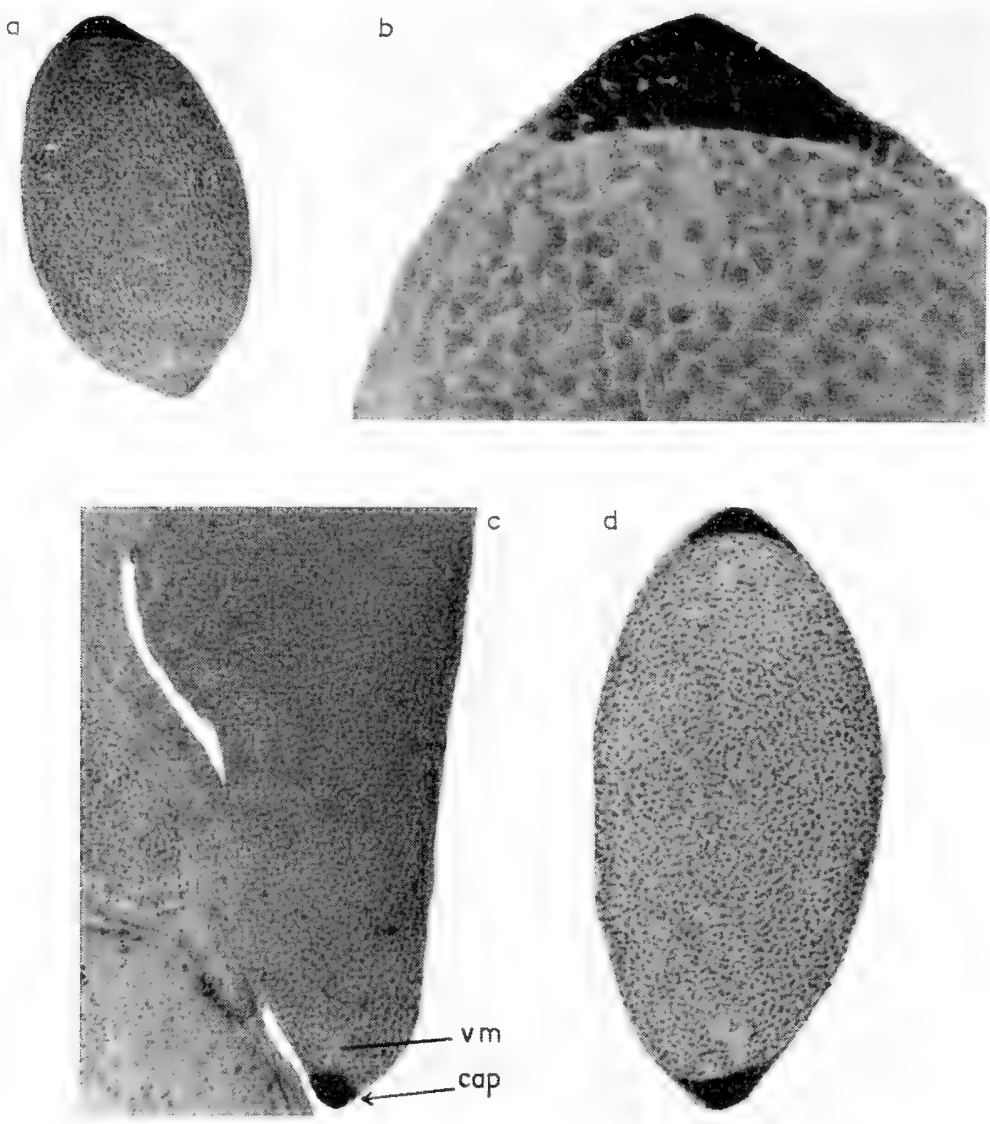

Fig. 38. Synthesis of alkaline phosphatase in the apical ridge of the limb buds of the rat $(a, b)$ I $3^{\text {th }}$ day; the enzyme has only appeared in the caudal part of the ridge; none existed at the preceding stage. The marginal veinous sinus runs just under the ridge; $b$ ) detail of the positive region; (c, d) I4th day; longitudinal (c) and transversal (d) section. The whole ridge now produces the enzyme. From Milaire, 1956.

The ectoblast (i.e. the non-immigrating part of the ectophyll) becomes for a while moderately positive. Then, the enzyme seems to disappear from the epiblast (future epiderm), while it becomes quite abundant in the neural plate, which is also known to elaborate ribonucleoproteins.

One might ask why such a striking difference has not been perceived in 
earlier investigations, such as those of J. Brachet (I944) and Osawa (I95I) on amphibians embryos, and of Moog (1944) on the bird embryo. Indeed a positive reaction of neural structures has been described there, but not especially strong when compared with the underlying material. It seems that, at least in amphibians, the blurring of the difference depends on the presence of the yolk, which is more abundant in the mesoblastic cells than in the external layer. The yolk platelets and the cytoplasm which surround them seem also to possess this enzyme. In chicken embryos, an improved technique entirely confirms, in spite of a more positive mesenchyme, the aspects observed in mammals (Milaire, unpubl. 1959).

It seems advisable to correlate this richness in phosphatase of the induced material with the positivity of the apical cap which develops on the tip of limb buds of mammals. Immediately after being formed, this cap acquires (Fig. 38) a positive reaction (McAlpine, I 956; Milaire, I956). This is also true for chicken limb buds (Milaire, unpubl.). I have suggested that this fact means that the apical cap has also been induced, an idea which seems to throw some more light on the differentiation of the limb buds (see p. 456).

\section{B. Experimental data relative to primary induction}

To put this intricate field into some order, it seems advisable to deal first with the primordial event which invokes the formation of the neural organ, and to classify the experiments in order of complexity. The most simple attack is certainly the purely surgical one, performed aseptically on the whole egg in a well-balanced physiological saline solution. Isolation of fragments, and their eventual combinations, are farther from normality, and not necessarily easier to interpret. The conditions of environment are less normal, and the territory which is being investigated does not benefit from the instalment of circulation as it would in the complete embryo. The objection has been raised that uncontrolled factors present in the whole embryo could viciate the experiment. Yet this inconvenience must not be exaggerated, and can be avoided by appropriate controls.

\section{(a) Operations on the whole embryo}

From the numerous experiments performed on amphibian eggs by Spemann and his followers, the type of result obtained by grafting, exchanging or transposing the various territories of the egg can be formulated in general terms. The maximum plasticity is encountered in the blastula and the young gastrula, and a restriction gradually occurs during gastrulation.

In the young, more malleable stages, many operations remain without appreciable consequence for the embryo. The perturbation is neutralized because the extraneous material becomes assimilated into its new surrounding ( $c f$. p. $35 \mathrm{I}$ ). Only territories of the dorso-marginal region, that is presumptive pharyngeal endoblast, prechordal, chordal and somitic mesoblast, show a distinct tendency to overcome the uniformizing action of the surrounding and to keep their own individuality.

In order to follow the fate of a graft or of any transposed territory, it has 
become customary to stain it in vivo, usually with Nile blue, or sometimes with neutral red. In a vast majority of operations, this tagging is absolutely innocuous and without appreciable effect on the morphological fate of the material. However, a few recent results obtained on eggs of fishes and birds show the contrary. In the exchanges of ventral and dorsal halves of trout blastodiscs performed by Devillers ( $c f$. p. 347) staining of the ventral half by neutral red causes it to gastrulate if the dorsal half is removed; the vacuolizing effect of the dye can be observed in vivo. In the chick blastoderm at the primitive streak stage, the unstained Hensen node, when isolated and grafted, yields ectoblast and eventually a neural tube, while when stained with Nile blue it develops into notochord and mesoblast (Grabowski, 1956, I957), or even into a whole minute embryo (Grabowski, 1958). It could well be suspected that in amphibian eggs also, the staining would either raise or lower the morphogenetic potential in some special cases. However, a critical examination of the various experiments in which this useful method was applied does not make it appear objectionable.

Among the many modifications obtained by operation on blastulae or young gastrulae, the impressive phenomenon remains, more than 35 years after its discovery, the evocation of a secondary embryo. In amphibians, this can be realized either by the famous graft of a blastoporal lip into the ventral side of a host; or by insertion of the inducing piece into the blastocoele (Mangold's Einsteckungsmethode); or, at least in anurans, by the $180^{\circ}$ rotation of the animal half on the vegetal half, i.e., a subequatorial translocation. Induction can also be obtained in fish (trout, lamprey) by similar methods. Among amniots, only birds have been properly investigated, although technical difficulties have limited the enquiry. Waddington (1933a, b), working in vitro, obtained a secondary primitive streak by superimposing two ectophylls (primary ectoderms) in opposite directions; Woodside (I937) confirmed this result by the direct insertion of a primitive streak between the two layers. Also, rotating the ectophyll i $80^{\circ}$ relatively to the endophyll, produces induction of a secondary streak (Waddington, r933a, b). A few attempts by this author have shown that a rabbit ectophyll reacts to the contact of a chicken primitive streak, and vice versa.

These operations, as such, demonstrate only the existence of a neurogenic induction; its causal analysis must be approached by other techniques. Beyond the crude fact of induction immediately appears the problem of how the new system is organized, and especially, why it shows the distinction between the head and the rest of the body. The answer to this query could only be searched for in amphibians, and since Spemann (I93I) recognized the possibility of inducing separately a head, a trunk, or a tail, it has remained a central topic of experimental embryology. For sake of brevity, it must be stated beforehand that it has been admitted, during the last ten years, that the relevant distinction is not between head and body, but between the prechordal and chordal parts of the organism. This assumption has made necessary the use of new terms $e . g$. archencephalon, deuterencephalon, spino-caudal structures (Lehmann, I94I, I942a, b), and acrencephalon, chordencephalon (Dalcq, r 947b) but they are no longer sufficient to describe easily and without ambiguity the various situations. To face this terminological difficulty, I have proposed (I957a) the solution shown in Fig. 39, which 
mostly uses proposals of ancient descriptive embryologists (Hatschek, Hubrecht, Assheton, A. Brachet, DeLange). The corresponding terms acrogenesis, notogenesis, deutogenesis, cormogenesis, ourogenesis may be suggested to designate the constitution of induced structures. This terminology will be consistently followed in the present work.

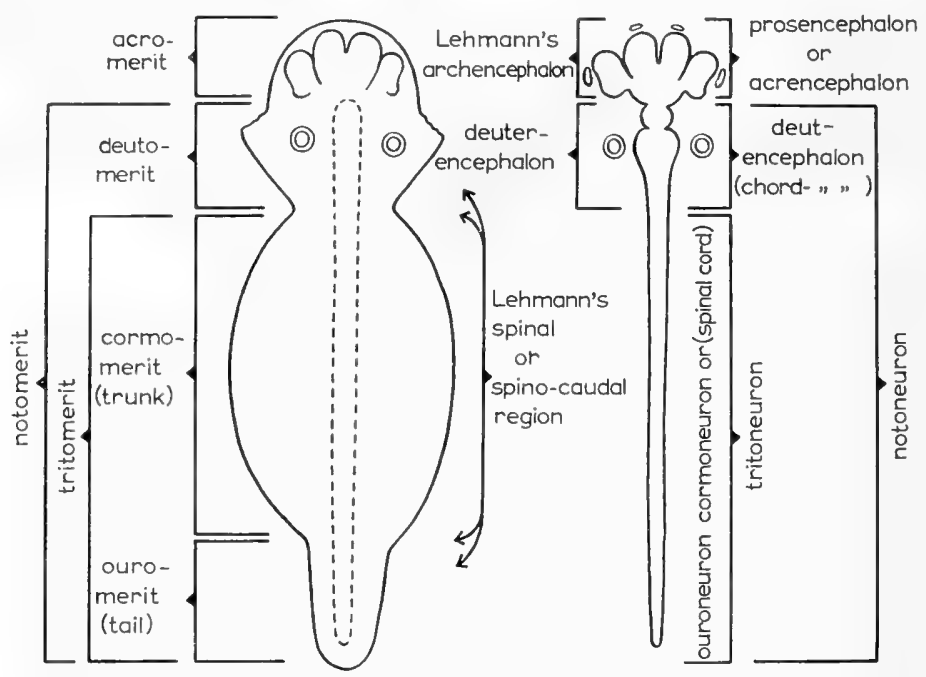

Fig. 39. General terminology utilizable for the description of induction phenomena. To depict the appearance of a whole region, such as the acromerit, deutomerit, etc., corresponding compound words using -genesis can be employed. Acrogenesis and notogenesis will be frequently used here. Orig.

In order to enter this exploration, we should first consider the operations performed on the neurula ${ }^{1}$, where territories can be chosen with sufficient precision, and then discuss the earlier stages. To detect the inducing capacity of the archenteron roof, Mangold (I933) carefully dissected parts of this roof from the overlying ectoblast, and inserted these pieces into the blastocoele of young gastrulae. In these favorable surroundings the grafts preserve their full vitality, are pushed by the movements of gastrulation against the ventral ectoblast, and induce significant structures in this fully receptive reactor, while they themselves produce the same tissues as they would have in their normal situs. The only inconvenience in this procedure is that the implant may be located too close to the organs of the host and may be submitted to some influence exerted by these. In the numerous experiments done by Mangold (1933, I936), one result especially

1 It might be useful to recall that in the neural plate the hind part of the neuro-epithelium will form the posterior somites of the trunk and all those of the tail ( $c f$. Bijtel, 1956, I958). This potency is even controlled by an induction exerted by the underlying chordomesoblast (Spofford, I 945, 1948). 
draws our attention: parts of the anterior brain and corresponding sense organs were practically never induced by the prechordal endomesoblast, but were easily obtained by inserting a transverse slice containing the anterior fourth of the chorda.

There is, clearly, a discrepancy with the expected results, for other investigations have shown (see Mangold, I958) a close relationship between deficience of the prechordal plate and a reduction or even an imparity of the brain hemispheres, eye, vesicles, and nasal pits.
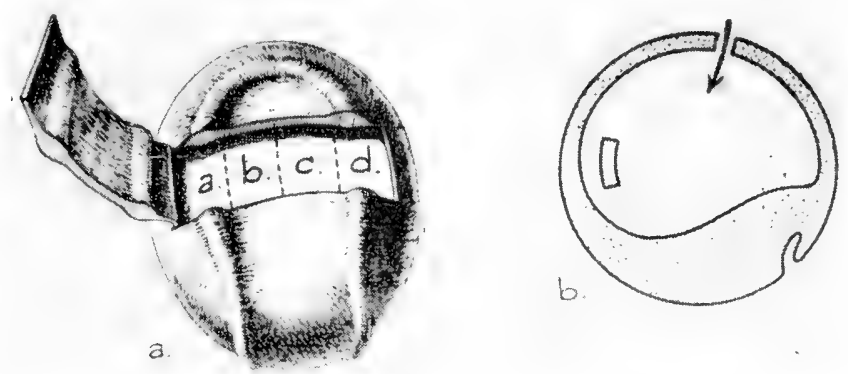

Fig. 40. Difference of inductive power between median and lateral parts of the archenteron. The chordomesoblast of the deutomerit (Fig. 39) has been divided into 2 median and 2 lateral pieces, which were inserted in the blastocoele. From Raven and Kloos, 1945.

Operations of the same type, but concerning younger donor neurulae and only the anterior chordal region of the archenteric roof were performed by Raven and Kloos ( I 945). A transverse strip of dorsal mesentoblastic material just behind the prechordal area (Fig. 40) was divided into four pieces (two median and two lateral) which were inserted separately in the blastocoele of early gastrulae. The resulting structures were rather polymorphous. Notochord and muscle were provided by the graft itself, while many structures were induced in the host ectoblast: neural tissue, ganglion cells, cartilage (possibly from the graft), pigment cells, mesenchyme, lateral line, sense organs, ear vesicle, nasal pit, mouth with teeth (in two cases only), and atypical epiblast thickenings. Notochord was only provided by the inserted median pieces. These also had the privilege of inducing masses of nervous tissue, which were never a medullary tube, but mostly a rather posterior brain, and only exceptionally a rudimentary diencephalon (with paraphysis but no eyes). Two nasal pits were described, but one was dubious and the other was related to a partial reduplication of the primary forebrain. Induction of ear vesicle and lateral line organs conformed to the nature of the grafts. The striking result was the difference between the median and lateral pieces and concerned their capacity of differentiation (the lateral ones produced only some recognizable myoblasts) and their inductive power (less frequent and poor in structure in the lateral fragments). This was the pertinent demonstration that a dorso-lateral gradient exists inside the young archenteric roof, with some earlier "determination" of the presumptive noto- 

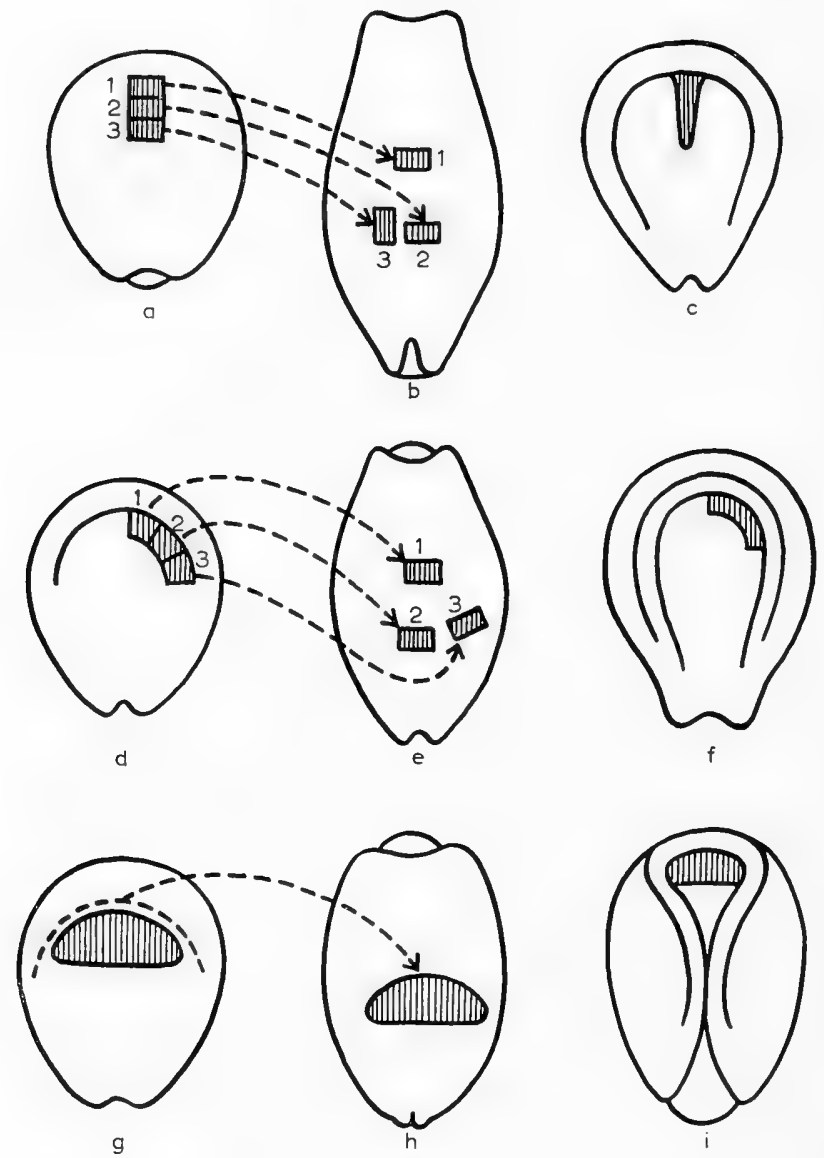

Fig. 4I. Grafts of prechordal neuroepithelium, not including prechordal plate, on the abdomen of a tail-bud stage. Left row $(\mathrm{a}, \mathrm{d}, \mathrm{g})$, the young neurulae used as donors. Middle row, the hosts with their grafts; right row the donor in the process of healing. From Gallera,

1947.

chordal material. This observation had already been made in several earlier investigations, and was utilized by Pasteels and myself to coin the theory of morphogenetic potentials (p. 343). The dorso-lateral decrement of such properties can be recognized when working on the corresponding presumptive areas of the gastrula and even of the blastula, and also when depressing the scales of potentials by toxic substances (p. $35^{\circ}, 422$ ). It will not be necessary in this review to give more arguments in support of this well-established notion.

In 1947 , when I still thought it likely that the obtaining of an acrencephalon was due more to the intensity and precocity of the induction than to some specific cause, Gallera and Damas simultaneously studied in my laboratory the consequences of depriving small neurectoblast pieces of the inducing action exerted on them by the archenteric roof (Fig. 4I). The pieces were carefully detached 
and grafted to the ventral region of a host. Gallera (1947) observed that the structures obtained from prechordal brain fragments of Pleurodeles $W$. and of axolotls were generally less complex than they would have been in situ. The degree of regression was less accentuated for large pieces than for small ones; when small adjacent fragments were compared, the median and anterior ones preserved their individuality better than lateral or posterior fragments. Presumptive ocular material could form a piece of brain with paraphysis or epiphysis. Regularly, a large part of the graft transformed into neural crest. However, the transformation of a graft into a spinal cord was not observed. Working on the spinal cord level with young neurulae of Axolotl and Xenopus, Damas (I947) also described the partial transformation of the graft into neural crest and its derivates ${ }^{1}$. The residue remained of spinal type, but its differentiation was distinctly hindered.

In a later investigation, Gallera (1949) reexamined in newt embryos the inducing ability of the prechordal chordomesoblast. He took the grafts from three stages: young neurulae, gastrulae with circular blastopore, and young gastrulae. Care was taken to insert the fragments in the most ventral part of the blastocoele with their superficial side adhering to the presumptive ectoblast of the host. No vital staining was necessary to recognize the fate of the grafts and thus these data are all the more reliable. The results of this extensive experiment ( 57 grafts) were far from schematic but afford valuable information.

The prechordal territory of the neurula (Fig. 42, a) was used either entire, halved, or divided into one median and two lateral fragments. The latter had a strong tendency to disperse into the mesenchyme ( 35 out of 40 cases), and in the few cases where an induction was exerted, it always produced balancers, but only once induced a brain (anterior); a modelling of the endoblast into a pharynx (twice including teeth) was observed. Thus, the graft behaved like cephalic mesoblast and pharyngeal endoblast, and not properly like prechordal material. With the whole fragment or its halves, individuality was better preserved (44 out of 54 operations) and manifested itself in producing muscles and indefinite cartilages and by inducing, in order of decreasing frequency, pharynx (39 cases), stomodoeum ( 36 cases, eventually with teeth in 27 cases), balancers ( 28 cases), anterior brain (rather poor, 26 cases), nasal pits (simple or double, i9 cases), and optic vesicles (unique or double, 18 cases). These sense organs were always accompanied by brain. The size of the neural structures was compared to the normal dimensions of these organs and was found to be more reduced for the brain parts (maximum $10 \%$ of the normal) than for the eyes or nasal pits (nearly half of the normal). Thus, the neural inductions were definitely of an acromeritic character, but the optic vesicles were exaggerated at the expense of the brain, (Fig. 42, b, c) and the induction of the olfactory pits from the epiblast stronger than expected.

When the preneurula stage was used (small yolk plug, Fig. 43, a), an anterior and a posterior fragment were dissected and inserted in the blastocoele. All the anterior ones (20) were assimilated and could be considered as purely endoblastic. Out of i 8 posterior fragments, 3 only ( 1 entire, I median part, I lateral half) caused inductions. In all of these cases the inductions were strictly prosencephalic: brain, eyes, nose, balancers. The lateral part also induced a small otocyst, probably because the dissection had encroached on parachordal mesoblast (Fig. 43, b, c). The frequency of assimilations is probably due to the loose structure of this still migrating material. This difficulty was considerably reduced

1 The experiment of Damas has been repeated, with the same results, by Denis (I957) and by Gallera (1958b). It remains however possible that the implantation of the graft on the abdomen may cause in it some stretching which could partially inhibit neuralisation (cf. Denis, 1958). 
when the donor was a young gastrula (Fig. 44, a). With the fragment presumed to be prechordal (or mostly so), ro of 35 grafts were assimilated, while most others produced complex structures attributable to the head, the trunk or even the tail. In two cases, however, the induction was exclusively of a prosencephalic nature. One of these complexes was well isolated on the belly, and contained a small brain, two large nasal placodes, and two large eyes. A third specimen showed only a rather complete head with a predominant eye, plus one otocyst (Fig. 44, b, c, d). When the superior (larger) fragment was used as a transplant, cases of assimilation were few, and most secondary systems produced a whole embryo amputated at the level of its otocystes. In 3 out of I I successful implantations, however, an anterior head was formed, sometimes more normal than the one produced by the inferior graft of the same donor (Fig. 44, e, f).

These data have a double meaning. No doubt a "differentiated" prechordal territory exists not only at the end of gastrulation, but also during and even at the beginning of this process. The fact that most grafts do not work so specifically is due $(I)$ to some encroaching on the next territory and (2) to a regulative completing by the host. On the other hand, it is plain that sometimes the nonprechordal presumptive territory becomes also capable, when grafted, of performing the inductive functions of the prechordal material by regulation. This is an important modification which is definitely provoked by this type of operation. We shall meet it again, and try to discuss it in detail.

For the moment, let us state that the paradoxical results of Mangold are at least partially solved. It has been clearly established that the concept of a prechordal territory, responsible for the induction of the acrencephalon, is valid.
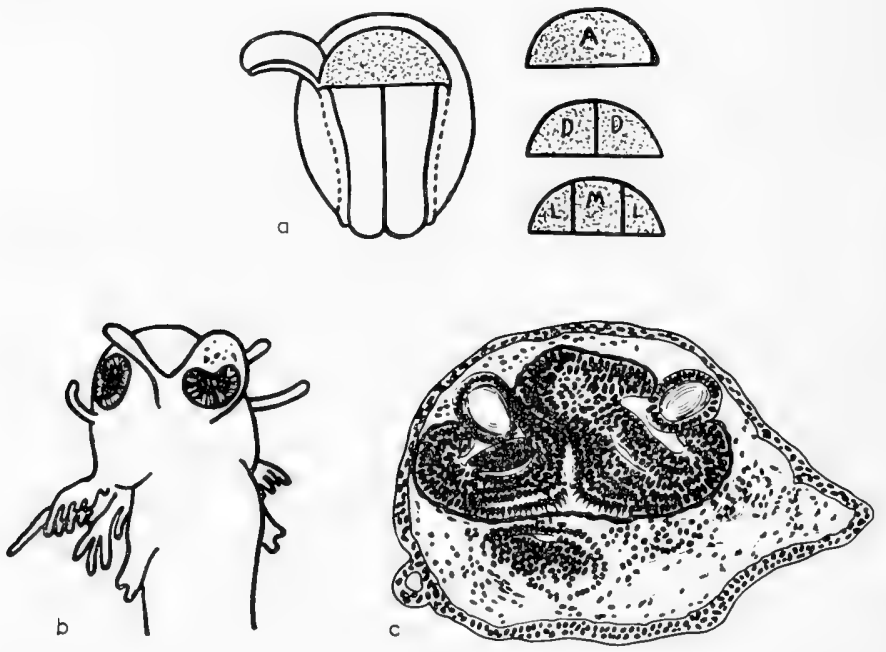

Fig. 42. Insertion of the prechordal endomesoblast of a newt neurula into the blastocoele (close to the ventral wall of the cavity) of a gastrula. (a) Schema of the operation for obtaining the grafts whole (A) or halved (D) or a middle (M) and two lateral (L) pieces. (b) Secondary acromerite with eyes and balancers induced on the ventral part of the head by an M implant. (c) Section of this specimen, showing a rather limited brain (below) and two large optic cupules with lenses. Note the predominent utilization of the neuroepithelium for the visual organs. An olfactory pit was also present. An example of definite although somewhat limited acrogenesis. From Gallera, 1949. 

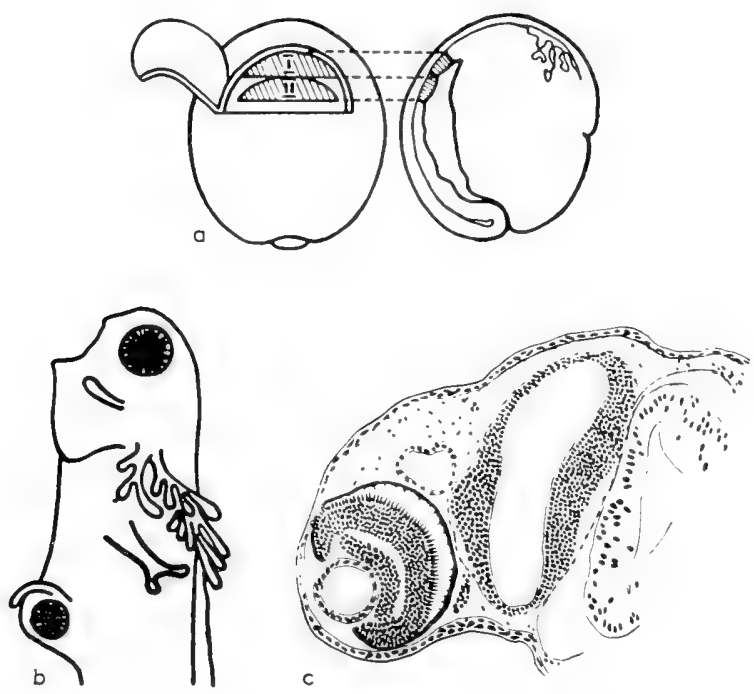

Fig. 43. Comparison of inductive capacity of the anterior and posterior halves of the prechordal endo-mesoblast taken from an advanced gastrula. Same technique as in Fig. 42; (a) cutting the implants from a yolk plug stage; (b) induction of an anterior head by the median part of the zone II ; an eye and one balancer are present. (c) section through these structures, showing from right to left the host gut, a large brain ampulla, a small thinwalled otocyst, and a fine cyclopic eye with lens; an olfactory pit was also present. The presence of the otocyst is probably due to some encroaching of the operation on the parachordal region. An example of definite acrogenesis, although accompanied by cyclopia.

From Gallera, I949.

However, there remains the fact that the chordal parts of the archenteric roof in neurulae can exert this same induction plus the production of hindbrain and trunkal structures, etc. Gallera suggests that this depends on the excessive age difference between the donor and the host in Mangold's experiment. Yet we may remark that this appearance of prechordal abilities seems quite similar to the sporadic observations made on the chordomesoblastic territory of the young gastrula (Fig. 44, see also Figs. 52-3, pp. 384, 385).

It may be also added here that in $195^{2}$ Gallera reexamined the effects of extirpating the anterior part of the archenteric roof (i.e., prechordal territory, with some encroaching on the chordal one) while leaving the neurectoblast intact. These operations were performed on neurulae and gastrulae with a circular blastopore. They caused deficiencies which conformed to expectation, such as tubular prosencephalon, synophthalmy and cyclopy, and monorhyny, but the point must be stressed that for such an extensive deficiency there was an appreciable degree of regulation. This could mean either that a reconstitution of the prechordal inductor takes place at the expense of the adjacent chordomesoblast, or that the neurectoblast early acquires an ability to autonomous progress. The latter contention would agree with the results of some other "folding" experiments (p. $3^{87}$ ), but this neurectoblast auto-organization does not manifest itself in "ordinary" transplantations nor in explantations (p. 397). It seems to me more likely that a relative morphological and dynamic reconstitution of the prechordal plate occurs, with an increase of morphogenetic potential. Thus, in these events disclosed by Mangold and by Gallera, 
we are faced with an intrinsic change in the morphogenetic potential of the graft, in the sense of an elevation or even of a qualitative change. The remarkable fact is that this ability seems to persist beyond gastrulation.

Various other experiments also performed on the neurula stage (Gallera, I95I, I952; Okada and Hama, I945a) have shown that the archenteric roof is an inducing system which gradually evolves and is plainly established when the urodele neural plate is surrounded by a pigmented hem. At this stage, each region
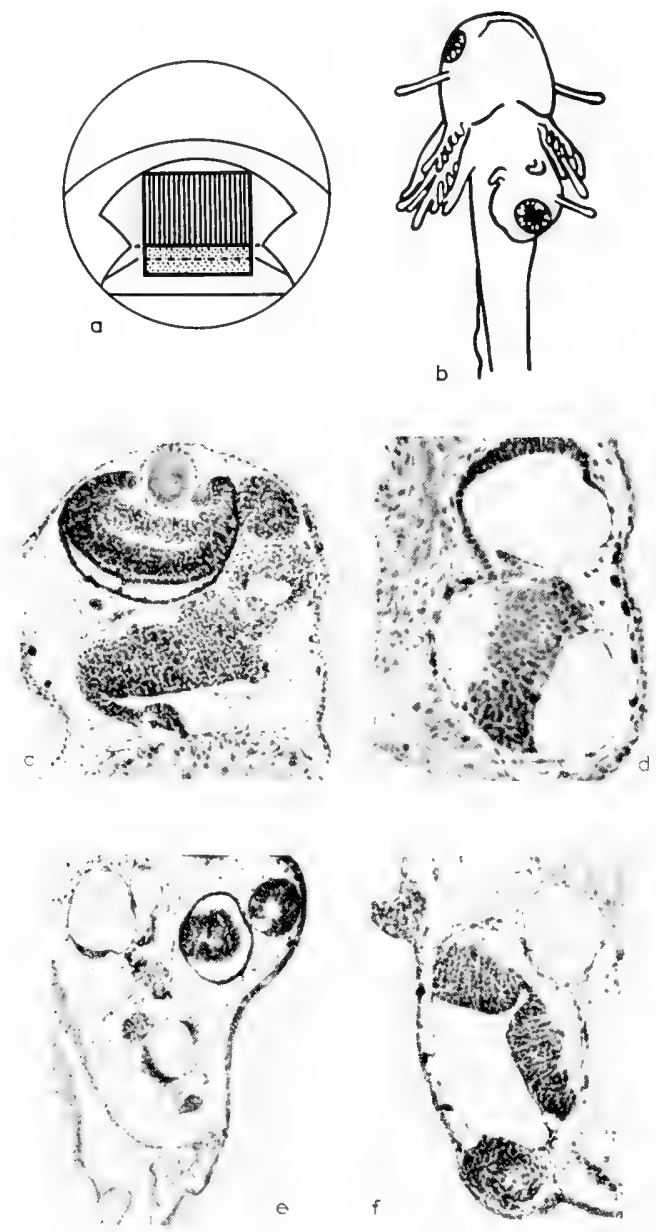

Fig. 44. Comparison of the inductive capacity of prechordal endomesoblast and chordal material taken from an early gastrula. (a) Limitation of the grafts, prechordal area stippled, chordal area hatched. (b) An extensive induction caused by the prechordal graft. (c, d) Anterior (c) and posterior (d) sections of this structure, which proves to be a cyclopic acromerit continued by a deutomerit, with a fine otic vesicle. (e, f) Result of implanting chordal territory, which has induced a whole head and even a part of the notomerit. (e) Shows a paraphysis (dorsal to the brain) and two otic vesicles; in (f) a small limb bud has grown to the right of the rhombencephalon. An olfactory pit was also present. From Gallera, I949. 
of the roof is clearly responsible for the fate of the overlying neurectoblast ${ }^{1}$. Parts of the neuroepithelium isolated or transplanted at this moment show an ability to form brain, eyes, spinal cord, etc., autonomously, according to their origin. On the other hand, the normal duration of this process of primary induction can be somewhat shortened. If, in a late gastrula, the neurectoblast is completely replaced by ectoblast of the young gastrula, a normal embryo may be obtained (Waddington and Deuchar, 1952): "There was no question but that the presumptive ectoderm was able to develop into a regionally differentiated neural axis, even though it had never experienced archenteron roof actually invaginating beneath it" (ibid., their p. 502).

For several springs, Gallera continued his analysis, performing operations that were suggested to him by the investigations of Nieuwkoop, which will soon be extensively considered (p. 389, 390). The attention of Gallera was now focussed on the legitimacy of Nieuwkoop's conceptions of the induction process as consisting of two successive phases, activation followed by transformation. These new experiments, combining several territories of three embryos, sometimes of different age, and using in vitro cultivation together with grafting on the ventral region of hosts, are rather complex, but indicate the following two points. On one hand, Gallera (I958a) considered whether ectoblast having undergone induction by the anterior chordal part of the archenteron roof (and consequently quite able to proceed by itself along the ways of deutogenesis by yielding a rhombencephalon) can, if temporarily grafted over a prechordal plate, still be induced into an acrencephalon; such was the case, which would mean, in Nieuwkoop's interpretation, that activation could still prevail on transformation. On the other hand, Gallera (I958b) sought for the incipient inducing effect of this anterior chordal part of the archenteron roof on the corresponding presumptive ectoblast, but considered at successive ages from middle aged gastrula to young neurula; the author found that acrencephalic structures are only obtained when an age discrepancy exists between the inductor and the reacting ectoblast, a conclusion important for appreciating Nieuwkoop's position.

With the last group of experiments we have begun to consider operations made on the gastrula. Several other results relative to this crucial stage must also be examined. All of them bear more or less directly on our main problem: what is the mechanism responsible when an acromerit rather than a notomerit (Fig. 39) is obtained?

In I942, Okada and Takaya, also working on newt embryos (Triturus pyrrhogaster), discovered that the same presumptive material did not have an identical inductive capacity when dissected before or after its invagination. The method, borrowed from Schechtman (1938) (Fig. 45), consisted of isolating a piece of tissue from just above the blastoporal groove of a young gastrula and dissecting a fragment of the same size from the wall of the blastocoele. Both pieces were for a short period intimately superimposed on the agar bottom of the dish; then the complex was used to close the gap created in the blastocoele wall. A variant

1 For a recent examination of the regulative aptitude of the archenteron roof after regional rotations and exchanges, with deviation or reversal of the axes, $f f$. Sládecék, 1953, 1955. 
method called affixation was also used (Hama, I949). With either method, a close adherence between inductor and reactor is obtained. The purpose of the experiment can be understood (Okada and Hama, I945b) by considering Fig. 46.

At the appearance of the blastopore, the material of the lip $(a)$ is shown by combined staining (coarse stippling, Nile blue; fine stippling, neutral red) to contain material which at the large yolk plug stage $(c)$ will form the anterior half of the archenteric roof $(c$, coarse and fine stippling). This territory is already known, from Gallera's experiments (p. 375), to contain endoblast anteriorly, endo-mesoblast laterally, prechordal plate in the intermediate median part. This area corresponds roughly to Spemann's head organizer. If the lip is stained a few hours later when the blastoporal groove has deepened $(b)$, the material there will be devoted to the hind part of the roof (coarse stippled in $c$ ) and is equivalent to Spemann's trunk organizer. Once this topography had been established, 27 transplantations of the stained fragment shown in (a) uniformly resulted "not only in the absence of a single instance in which the induced structure could be identified unmistakably as a head, but also in the occurrence of many instances in which a trunk or a tail was induced" (their p. 507). Indeed, this result was the same as when the stained material of $(c)$ was transplanted. Then, at this second stage and at a somewhat later one, the roof of the blastoporal groove was dissected, and its anterior and posterior parts were used for the same kind of grafting. The percentage and size of inductions was reduced, which is in agreement with Gallera's observations. "Grafts of anterior regions stimulated the formation of head organs regardless of their position in the host... while grafts of posterior regions were always limited to the induction of a tail" (also their p. 507). Further, "it can be said with certainty that neither a tail was formed by grafts of the anterior half nor a head by grafts of the posterior half" (ibid). This clear-cut result hinted at the possibility of a difference existing between the performances of the same material taken before and after invagination. Consequently, an accurate comparison was done between the inductive ability of grafts formed respectively of corresponding invaginated and uninvaginated material between stages $(a)$ and $(c)$. "The results were very clear: as often as the uninvaginated portion of the dorsal lip of each of the gastrula stages affected induction, regardless of the site of the transplant, the induced body was without exception either trunk or tail, or at times both ... In contrast to this, the portion which had already invaginated to form the dorsal wall of the archenteron always stimulated the development of a head" (p. 509). It is remarkable that this was already true for the narrow invaginated portion of the shallow blastopore at stage $(a)$ as shown in Fig. 47, a. Naturally, when invaginated material is obtained from stage $(c)$, just under the lip, it induces only structures of trunk or tail type. The acquisition, as the result of invagination, of the new property of inducing the acrencephalon only concerns material which travels over the blastoporal lip before stage $(c)$. The change is also revealed in sections by differential staining with thionin: the uninvaginated part stains blue, the invaginated part red (Hama in Okada and Hama, 1948, their p. 34). Finally, the authors examined whether other conditions would have the same effect. They found that culturing the uninvaginated fragment of stage $(a)$ in vitro for more than $20 \mathrm{~h}$. incited the power of inducing head organs. The same result can be obtained by cultivation in the blastocoele of a blastula, although only after a longer delay ( 24 to $26 \mathrm{~h}$.). Accordingly, the authors exclude the idea that the kinematic activities could be responsible for the acquisition of the new inducing properties; an intrinsic maturation process would be the real cause, the two phenomena being parallel in time. This is an opinion to which we shall have to return.

Extending their enquiry to the whole dorso-marginal region, Okada and Takaya found that the same modification could be obtained, by in vitro cultivation, in a larger territory than the young blastoporal lip, though only in its immediate vicinity. This means that this territory is predisposed to exhibit this important property. 


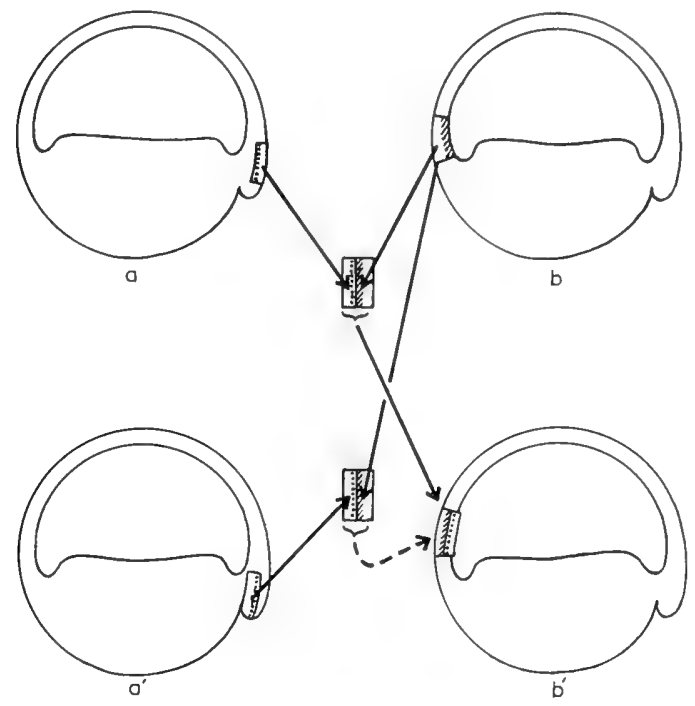

Fig. 45. Method used to insert an implant in the blastocoele in such a way that it will stay in the chosen location. ( $\left.a, b, b^{1}\right)$ An uninvaginated piece of the dorso-marginal zone is dissected from an early gastrula (a) and a fragment of the same size is cut from the ventral wall of the blastocoele in another gastrula (b). Both pieces are apposed, inner sides together, for $2-3 \mathrm{~min}$; ; as soon as they adhere together, they are used to fill the hole created in (b), as shown in $\left(b^{1}\right) \cdot\left(a^{1}, b, b^{1}\right)$ The same process is used in the case of an invaginated part of the blastoporal lip. From Schechtman, I938, modified. The Japanese authors also used a simplified method of affixation, consisting in scratching the inner side of the blastocoele wall and placing the inner side of the graft against this modified region.
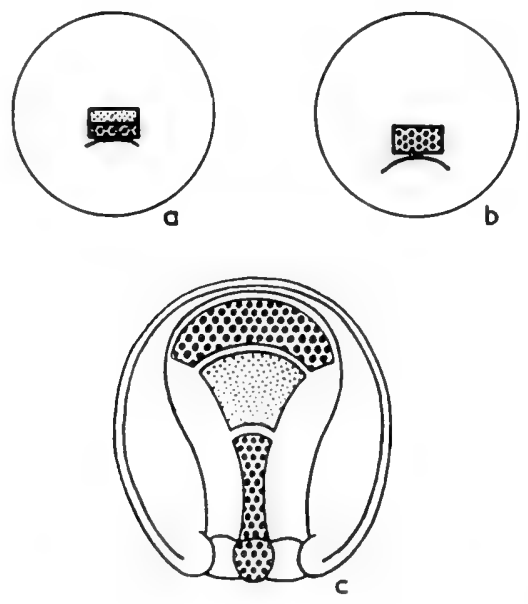

Fig. 46. Mapping the fate of the early (a) and somewhat more advanced (b) newt gastrula by vital staining. In (c) the embryo at the early neurula stage is seen from its dorsal side with the neuroepithelium removed. See text. From Okada \& Hama, 1945b. 
As the authors express it (their p. 512); "with regard to inductive capacity, the median dorsal lip, though similar in general to the other parts, possesses in a latent condition an additional characteristic, which is lacking elsewhere and which cannot be other than the quality that later causes this section to be imbued with the head-organizing effect" (expression apparently used with the acrencephalon in mind). Finally, this fundamental paper mentions that in frog gastrulae, uninvaginated material of the early blastoporal lip is already capable of inducing the forebrain when inserted in the wall of newt gastrulae. In Anurans, this property appears earlier than in Urodeles. To be complete, let us mention that in a further investigation Okada and Takaya (1942) examined whether the combination of the two inductors could produce a normal embryonic axis. Such was the result in several cases, but in others, the notomeritic inductor had a debasing effect on the acromeritic one. It is also useful to record that the potencies of the uninvaginated part of the young

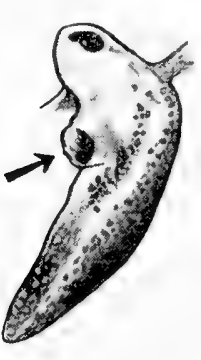

a

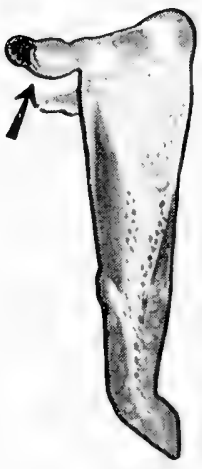

c
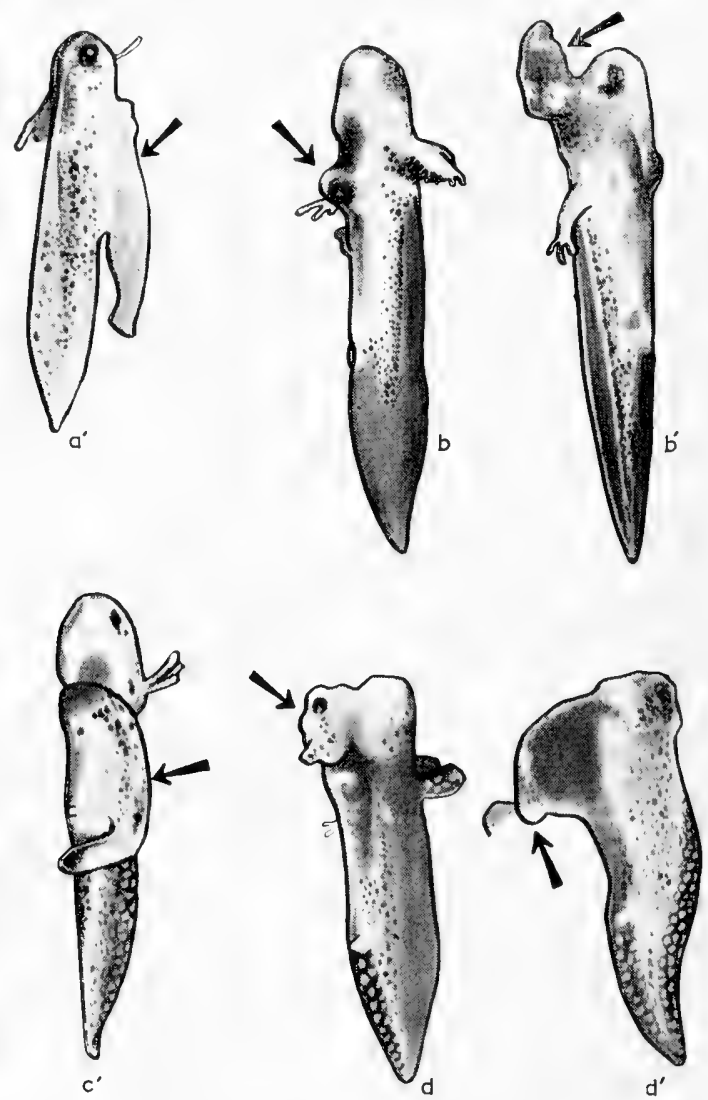

Fig. 47. Modification of the inductive capacity of a given presumptive area, considered either before or after its invagination. $a-a^{1}$ to $c-c^{1}$ concern nezet embryos. (a) Induction of a head by the invaginated material at stage (a) of Fig. 45, i.e., the inner part of the quite young blastoporal lip. ( $\left.\mathrm{a}^{1}\right)$ Induction of trunk and tail by the uninvaginated material of the same stage; (b-b ${ }^{1}$ ) same difference relative to invaginated and unvaginated material of stage (b), Fig. $45 ;\left(c-c^{1}\right)$ induction caused respectively by the anterior and posterior part of the archenteric roof as shown in fig. $45(\mathrm{c})$; $\left(\mathrm{d}^{-} \mathrm{d}^{1}\right)$ induction by invaginated and uninvaginated material of a frog gastrula; here, a head is obtained in both cases. From Okada and Takaya, 
blastoporal lip were tested by an other method. The pieces were grafted to a neurula and covered with ectoblast of a late gastrula. They formed mostly notochord, and induced hind brain and sometimes an ear vesicle (Okada and Hama, 1948).

Thus, the situation which this investigation reveals is not without variations according to the species considered, the moment where the change takes place, the factors able to cause it, etc. These nuances explain, for example, the apparent discrepancy between the above results and some of Gallera. The species were not the same and the technique was not identical. In Gallera's cases, where the ability to induce a complete head was recognized in the chordomesoblast, it probably did not exist there beforehand, but was acquired, as suggested, under the experimental conditions.

At this stage of our analysis, we may keep in mind that two distinct inductors are operating to form a normal neural organ, but that, at least in Urodeles, both are not effectively present before gastrulation. This ripening or emergence of the acrencephalic inductor seems to be more precocious in Anurans, and this may be, after all, the essential difference between their eggs and those of the Urodeles.

Epigenesis of the acrencephalic inductor may be quite a useful concept in reconsidering the results I obtained, from 1933 to I947, on anuran eggs, first Rana temporaria and then mainly Discoglossus pictus. These experiments were begun at a time when the distinction between head and trunk organizer (Spemann, I93I) had barely been realized; they were planned as a further step in the exploration of the dorso-marginal zone, the recognized site of the organizer.

The first operations performed were of limited extent. However, since they were of a new type and were mostly concerned with the blastula, they afforded some valuable information.

By a temporary opening through the roof of the blastocoele, the inner material of the dorso-marginal zone was detached and transported to the ventral part of the cavity. As a result, the primary embryo remained normal, but secondary structures were obtained and induced in its ventral side, including parts of definite acrencephalic character (Fig. 48). It thus seems that this kind of implant not only contained presumptive chorda, somites and pronephros, but also prechordal plate, or else was able to produce the acrencephalic inductor itself. Yet, the dorsal part of the egg was capable of compensating for the loss inflicted upon it. This regulative capacity was also observed after an excision of a rectangle including the endoblast above the blastopore, the prechordal plate and some mesoblast. This remarkable ability had been previously observed by several workers in Spemann's group ( $c f$. Schenk, 195I), but it is worth stating that this is also true for the Discoglossus egg. Another limited but interesting operation consisted of subjecting this same central region to a $180^{\circ}$ rotation (Fig. 49). The expected result would have been the formation of a blastoporal lip at the upper border of the rotated fragment, and a downward invagination. However, instead of this, the upper part progressively sank and migrated toward the animal pole in such a position that the pharyngeal endoblast and the prechordal region led the way as they would have done normally. This change may be described as a true kinematic regulation, a process also observed more recently in newt gastrulae ${ }^{1}$ (p. $3^{86}$ ). The space corresponding to the graft was covered by the expanding ectoblast, which was submitted to induction. Acrencephalic structures did appear, although they were not always normal (Fig. 50). No doubt their inductor was present in the rotated material. This result agrees with the contention of Okada and Takaya that in Anurans the prechordal properties exist before gastrulation.

1 Waddington and Yao ( 1950 ) have also performed the same operation on the newt. Regulation occurred in the majority of cases, while other embryos were affected with spina bifida. 

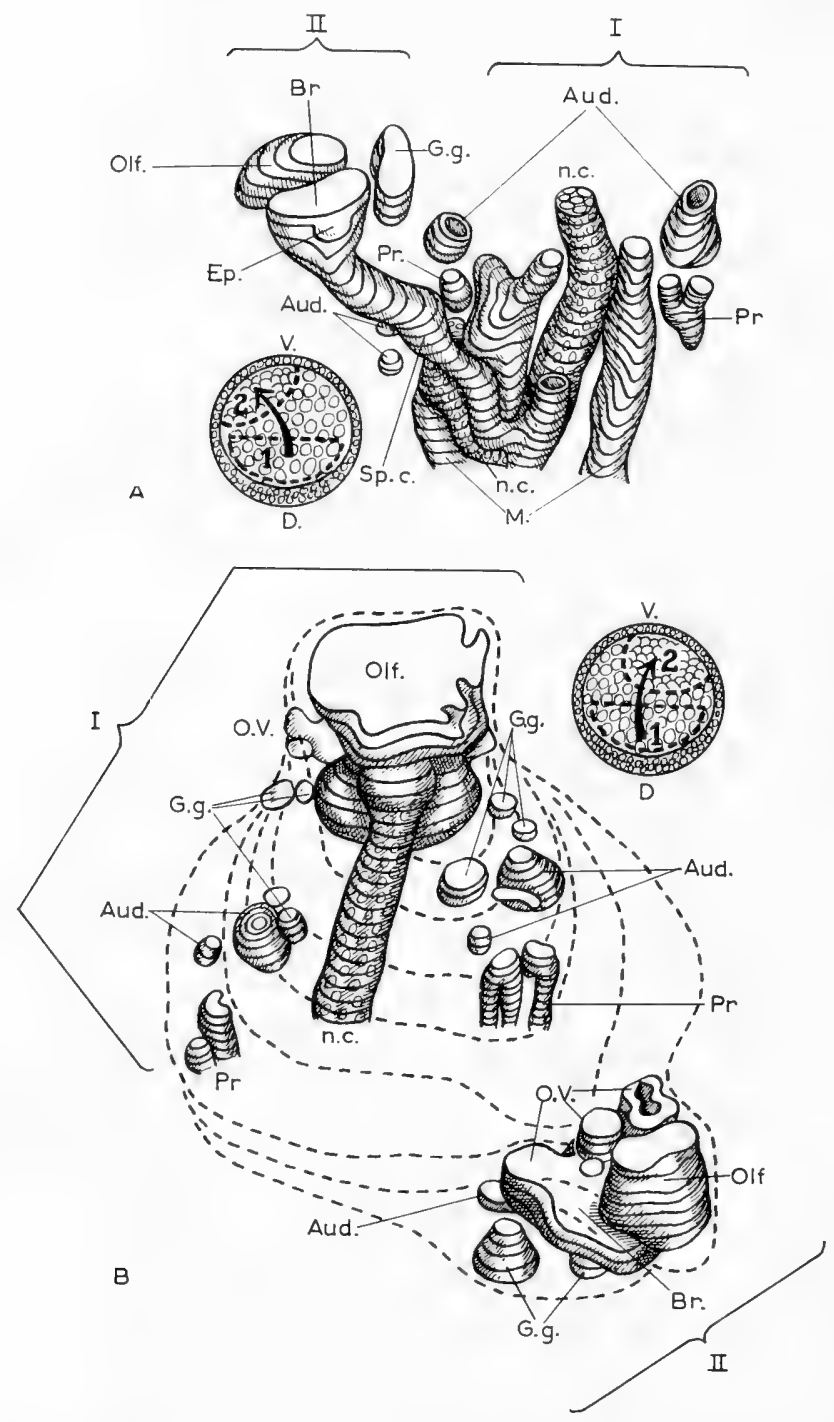

Fig. 48. Effect of transposing the inner dorsal part of the blastocoele floor in an early Discoglossus gastrula to the ventral part of this same cavity (insets: the blastocoele floor is seen from above; I, 2: successive positions of the displaced material). In (A), this internal translocation has been done to the left. A secondary embryo has been obtained there. The graphic reconstruction shows the primary (I) and secondary systems (II) as seen from their dorsal aspects. The anterior part of the nervous system of I has been omitted. The secondary system is rather complex, except for eyes. In (B) the transfer has been done to the ventral right part. The secondary induction (II) is limited to an acrencephalon (perhaps the whole acromerit?) plus a small otocyst and a pair of ganglia. The primary embryo (I) is somewhat deficient. The reconstruction is seen from the ventral side of I. Its somites have been omitted. Aud.-otocyst; Br.-brain; D.-dorsal; Ep.-epiphysis; Gg.-ganglio; M.-somites; n.c.-notochord; Olf.-olfactory placode; O. v.-optic vesicle; $\mathrm{Pr}$.-pronephros; sp. c.-spinal cord; V.-ventral. Such operations reveal that appreciable factors of organization exist in the yolky cells located in the dorsal part of the blastocoele floor, and that the dorso-marginal field extends fairly deeply into this area. From Dalcq, I940. 
A more extensive operation was then endeavoured, to which the Discoglossus eggs resisted pretty well. It consisted of a thorough modification of the topography in the whole marginal zone, by the technique called (sub-)equatorial translocation. The blastula or young gastrula is cut horizontally at the level of the blastocoele floor, the upper part turned through $180^{\circ}$ and replaced on the vegetal half. Spemann (1918) was the first to use this technique on newt eggs, but owing to the low position of their dorso-marginal zone and possibly to some other reason, he did not obtain double embryos. This negative result was, however, very important, for it suggested to Spemann that an extrinsic factor was responsible for obtaining the neural plate, and led him as a next step to the crucial experiment of transplanting the blastoporal lip. It occurred to me that frog eggs would not react in the same way. As long as this technique divided the dorso-marginal zone, it resulted in double embryos, which exhibited a correspondance worth of a more extensive study, which could be best done on Discoglossus eggs, whose consistency permitted this rather drastic operation without appreciable loss of material (Fig. 5I). This fast-developing material shows most convincingly that as early as the late blastula stage the dorso-marginal zone is no more a continuum (with graded properties), but that the notochordal, somitic, and pronephritic territories have acquired some properties of their own. These tendencies can express themselves in small parts of the presumptive area when isolated from their normal connections. Such intrinsic and rather stable qualities explain the striking correspondance between left and right parts, respectively, of the two axial systems obtained. This interpretation can even be extended to the production of the two groups of cells responsible for the primary induction of the otocystes, located in the parachordal head mesoblast (Dalcq, I933). Moreover, in the majority of cases, both embryos possess a forebrain. The degree of its perfection and

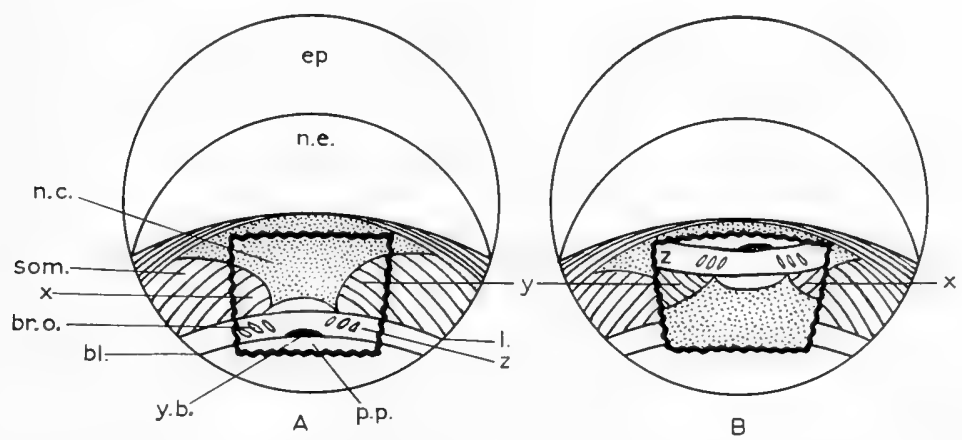

Fig. 49. Computation of the results produced by an in situ $180^{\circ}$-rotation of the central part of the dorso-marginal zone, in an early Discoglossus gastrula. (A) Map of presumptive areas with the heavy line indicating the extent of the cut. Note that the cut has been slightly asymmetrical, shifted from 10 to $20^{\circ}$ to the right; bl., line which will be followed by the blastopore, now limited to its youngest part, y.b.; br. o., branchial openings; ep., epiblast; l., limit between endoblast (below) and chordomesoblast (above); n.c., notochord; n.e., neurectoblast; p.p., prechordal plate; som., somites; $x, y, z$, three particular areas indicated to draw attention to their change in position (see legend of Fig. $50, c$ ). (B) the pattern of presumptive areas after the rotation. From Dalcq, I94Ia. 


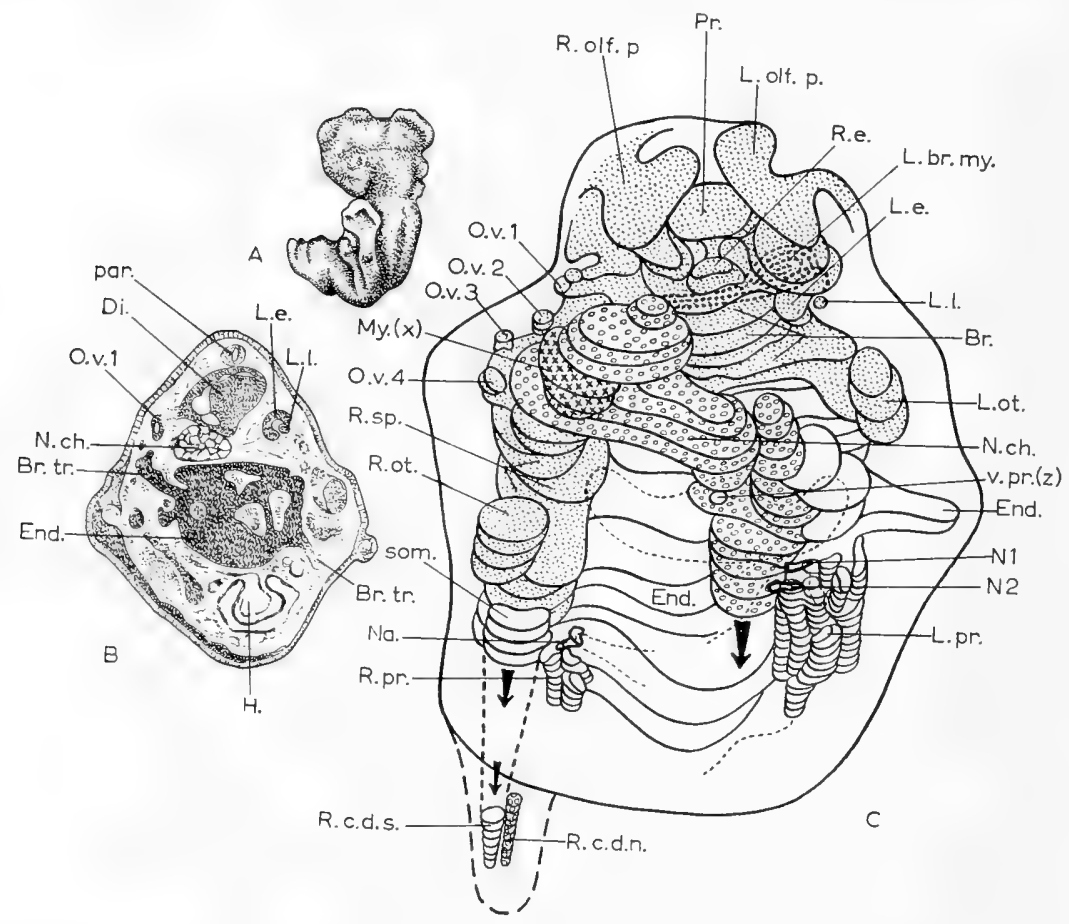

Fig. 50. An example of the effects of the in situ rotation of the central part of the mediodorsal zone, in the case of the slightly asymmetrical cut shown in Fig. 49, a. (A) The embryo seen from its right side; there exists a dorsal hiatus (spina bifida) and the tail is curved dorsalwards; the cephalogenesis has taken place, but the head is distinctly atypical; (B) shows a section through the middle level of the head; $(\mathrm{C})$ is a graphic reconstruction of the main organs as seen from their ventral side. The remarkable points are that (I) the notochord (circles) deviates to the left, owing to the asymmetrical cut; (2) while the left notochord is uninterrupted to the end of the tail (which is suggested by an arrow), the right notochord is lacking in the whole trunkal region, but reappears in the right wing of the tail (inset, below); (3) a packet of myoblasts (crosses) is found inserted to the right of the chorda, and corresponds to the $\mathrm{x}$ zone (Fig.48, b) of presumptive somites; (4) the right pronephros is strongly reduced, with only one nephron and its nephrostom $\mathrm{Na}$, while the left one is much larger, with two nephrostoms ( $\mathrm{NI}_{1}, \mathrm{~N}_{2}$ ); a slight pronephritic vesicle has been transposed near the left notochord (v.pr.), corresponding to the material z; (5) the right somitic material y (Figs. $49, \mathrm{a}, \mathrm{b})$ has been combined with the left somites; (6) the anterior brain has induced two nearly equal olfactory placodes, has formed two optic vesicles, the left one with a lens, the "right" one nearly median, and, moreover, four slender optic vesicles (O.v. I, 2, 3, 4); thus the inductive pattern of the acromerit has been considerably modified. Such operations demonstrate that the rotated material dives directly towards the animal pole, without turning around a blastoporal lip (kinematic regulation), and that the various areas of the dorso-marginal zone are endowed with a high degree of predisposition, usually blurred in similar operations on Urodeles.

Br-brain; Br. tr.-branchial tractus; Di.-diencephalon; End.-endoblast; H.-heart; L. br. My.-left branchial myoblasts; L. e.-left eye; L. l.-left lens; L. olf. p.-left olfactory placode; L.ot.-left otocyst; L. pr.-left pronephros; $\mathrm{My}(\mathrm{x})$-transposed myoblasts ( $\mathrm{x}$ area); N. ch.notochord; $\mathrm{Na}, \mathrm{N}$ I, N2-nephrostomes; O.v. I, 2, 3, 4-supplementary right optic vesicles; par.-paraphysis?; Pr-prosencephalon; R. c.d. n.-right caudal notochord; R. c.d. s.-right caudal spinal cord; R.e-right eye; R. olf. p.-right olfactory placode; R. ot.-right otocyst; R.pr.-right pronephros; R. sp.-right spinal cord; Som.-somites; V. pr. (z)-pronephric vesicle. From Dalcq, ig4ra. 
also the dimension and conformations of the optic vesicles, the olfactory placodes, and the epiphysis vary considerably.

As for these acrencephalic structures, the features are distinctly different from those displayed by the chordomesoblast and the parts of the neural axis which it induces. Of course, according to the level of the cut, we may have either two normal or nearly normal equal embryos, or one normal and the other defective; this defective embryo may be located either on the primary dorsal side, or on the other. Moreover, no correspondence is found between the homonymous sides; for example, one does not find a large right optic vesicle and a small left one in one embryo and the reverse in the other embryo. Again, the translocations performed on blastulae or very young gastrulae reveal a strong tendency to form two embryos, if the section is made about half way through the dorso-marginal zone. With translocations performed on more advanced gastrulae, a small acrencephalic complex can be evoked on the ventral side by selective transfer of the already invaginated prechordal material, but the principal embryo can nevertheless be complete.

For a long time, I have attempted to understand the results on the basis of a minute pre-existing prechordal primordium, which the cutting could include to a variable degree. However, no coherent interpretation could be attained with this hypothesis. Today, the more dynamic representations suggested by Okada and Takaya's work seems to afford the clue which I lacked. Most probably, in

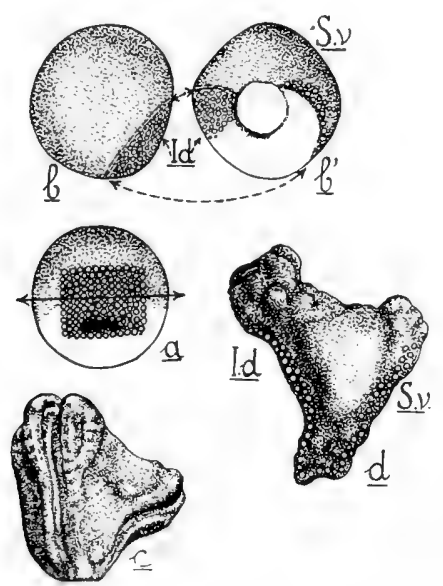

Fig. 5I. An example of equatorial translocation on a Discoglossus blastula. (a) A large Nile blue mark (circles) is placed upon the grey crescent, which is quite distinct in this species; the black dots indicate the level where the blastoporal lip will appear; an horizontal cut has been made, and the upper part rotated $\mathrm{I}^{\circ} 0^{\circ}$. (b, $\left.\mathrm{b}^{1}\right)$ The embryo seen at the yolk plug stage, from the animal and from the vegetal pole. I. d., the infero-dorsal part, S.v. the supero-ventral part (rotated). (c) The double neurula, with open folds. (d) The double embryo, showing the places where the stain was re-found in the sections. The I. d. system is practically normal, while the S.v.system is only feebly cephalised. Other cases have given two complete heads. It is now understood that a new prechordal primordium must be re-formed from chordo-mesoblastic material (from Dalcq, 1947b). 
the Discoglossus as in the frog egg, the acquisition of the prechordal prerogatives is on the way before this presumptive territory migrates around the blastoporal lip. The appearance of two acrencephalic regions becomes easy to explain, without postulating a division of the pre-existing prechordal area. If the section cuts through the chordomesoblast higher than the prechordal material, the upper part would become able to form by its own activity a new prechordal region when transferred to the ventral side. The lower the section, the more easy will be this regulation and the more typical the new prechordal territory produced, and vice versa. This would explain why the secondary (ventral) acrencephalon is as symmetrical as the dorsal one, for its inductors do not partake in the system of predisposed areas.

A similar interpretation seems to be valid for the induction of a forebrain in the half-embryos obtained by sagittal ligature of newt blastula or young gastrula ( $c f$. p. 346 ). It is a striking fact that in these half-embryos the forebrain appears symmetrical and forms two optic vesicles. This can be readily understood if the prechordal material has been formed from the chordomesoblast and does not proceed, as generally assumed, from a pre-existing prechordal "plate".

Another aspect of my enquiry concerning this essential prechordal material consisted in repeating the classical grafts of the blastopore lip. The main difference with Spemann's original work was that vital staining was used in an especially
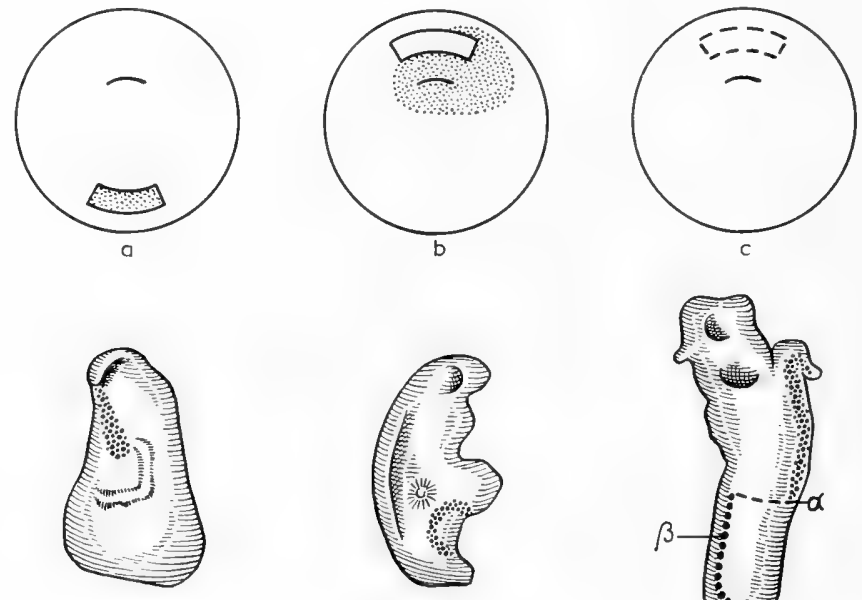

$a^{\prime}$
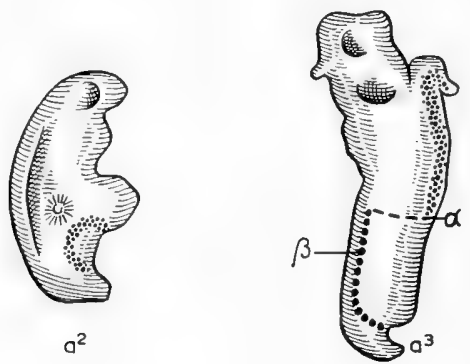

Fig. 52. A graft of a dorso-marginal area, with the control of vital staining. (a) The young gastrula with the graft (dots) inserted in its ventro-marginal zone; the graft has been cut out of the stained area (dots) of the (b) embryo; the empty place has been filled with the corresponding material of a third gastrula (c). The distribution of the stain in the fixed (b) embryo will check the presumptive value of the grafted area. $\left(a^{1}, a^{2}, a^{3}\right)$ Successive stages of the host embryo. In $\left(a^{1}\right)$, the orientation is the same as in (a), the mark has been invaginated and a neural plate is forming. In $\left(a^{2}\right)$, where the orientation is reversed, the secondary system visibly forms a head. In $\left(a^{3}\right)$, a considerable stretching has occurred, from the point $\alpha$, the stained cells have been drawn to the right of the primary axis, in $\beta$. The degree of cephalization is seen in Fig. 53 (From Dalcq and Lallier, 1948). 


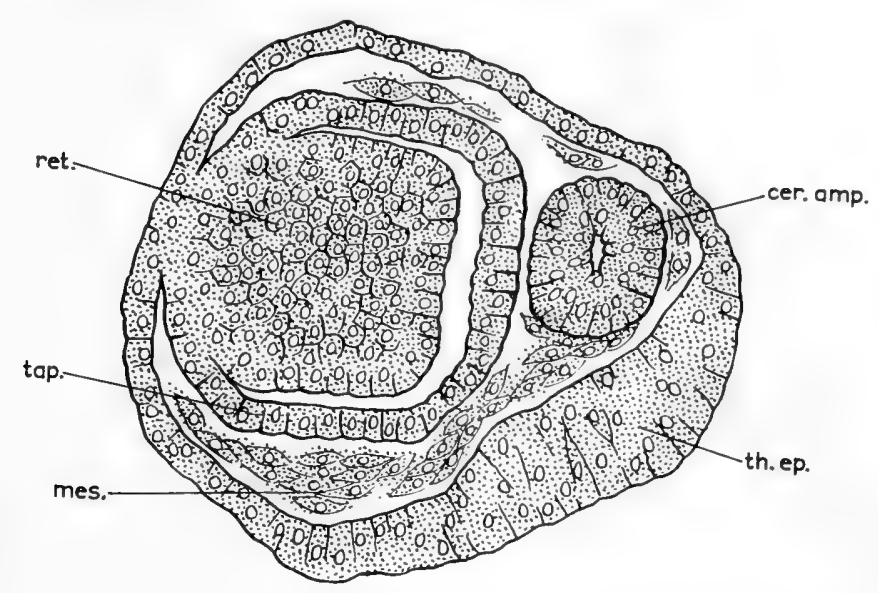

Fig. 53. Transversal section through the head of the secondary embryo obtained by the operation of Fig. 52. A brain of reduced side is accompanied by a large optic vesicle. Thus, a graft taken in the notochordal area has definitely caused acrogenesis; cer. amp.-cerebral ampulla; mes.-mesenchyme; ret.-retinal layer; tap.-tapetal layer; th.ep.-thickened epiblast. From Dalcq and Lallier, $194^{8 .}$

discriminative way, including the detection of the stain in sections of the operated embryos. This method allowed an accurate comparison between the presumptive value of the grafts and the structures they yielded and induced.

Many results of the typical grafts studied by Lallier (Dalcq and Lallier, 1948) and by Minganti (1949) are only of a confirmative nature. However, it remains worth recording that a purely chordomesoblast graft, free of prechordal material, can sometimes induce a head well-provided with prosencephalic structures (Figs. 52, 53). Moreover, an extraordinarily narrow fragment, taken from the upper blastoporal lip and consisting only of presumptive pharyngeal endoblast with at most some presumptive prechordal material, produces a complex containing practically all components of an embryonic system excepting optic vesicles and nasal placodes (Dalcq and Lallier, 1948, their p. 347). In this last case, the use of the word "organizer" is certainly defendable. Although this term has ceased to be appropriate for a general designation of induction phenomena, we must not lose sight of the fact that sometimes such an organizing influence is obvious. In both the results obtained by Lallier, the elevation of morphogenetic potential is patent; the first operation mentioned is especially interesting since, as in Gallera's experiment ( $\mathrm{p} .374$ ) notochordal material is partly transformed into prechordal plate.

Minganti grafted in the ventro-marginal region vitally stained lateral pieces of the organizer, i.e. strictly presumptive mesoblast. With axolotl as with newt embryos, the most frequent cases showed assimilation into the host neighboring tissues, sometimes in situ, more often after an active migration (p. 35 I) into the mesoblast of the host. Inductions caused by such mesoblastic grafts (with either concordant or inversed polarity) were mainly homoeogenic and, if neurogenic, only evoked a slender spinal cord, never with a cerebral swelling (see also Mookerjee, I954).

Benoit (1952) investigated in details the effects of similar grafts, but with $180^{\circ}$ reversal of the cephalo-caudal axis. This operation is somewhat analogous to my own rotations in situ of the Discoglossus medio-dorsal zone (p. $3^{8 \mathrm{r}}$ ) except that in Benoit's experiment, the implant was put independently either in or above the ventral zone, and he worked with the Urodele egg. The surface of the graft was stained with Nile blue towards the pole (caudal material) and with neutral red toward the antipole, so that the fate of both parts could be easily ascertained by frequent observations. Two results must be mentioned here. 

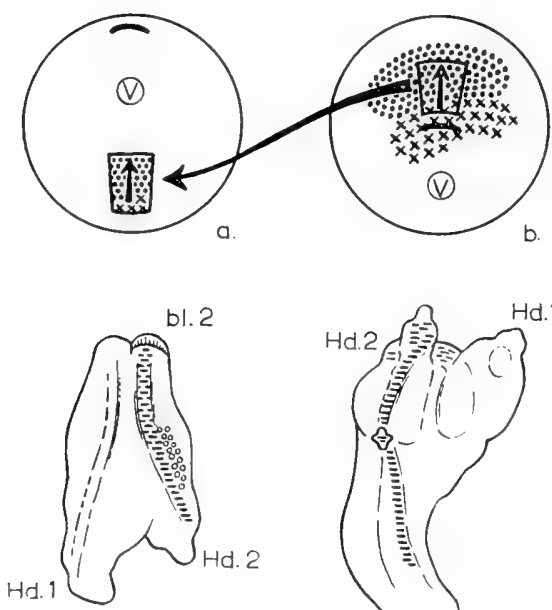

g.
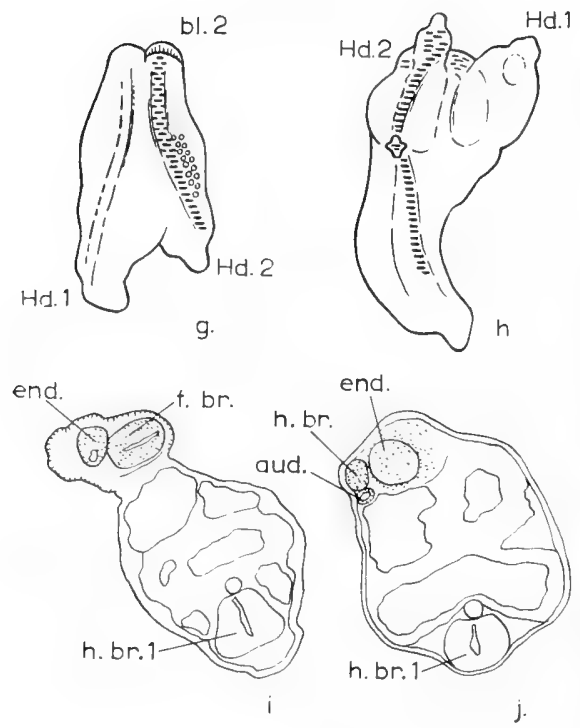

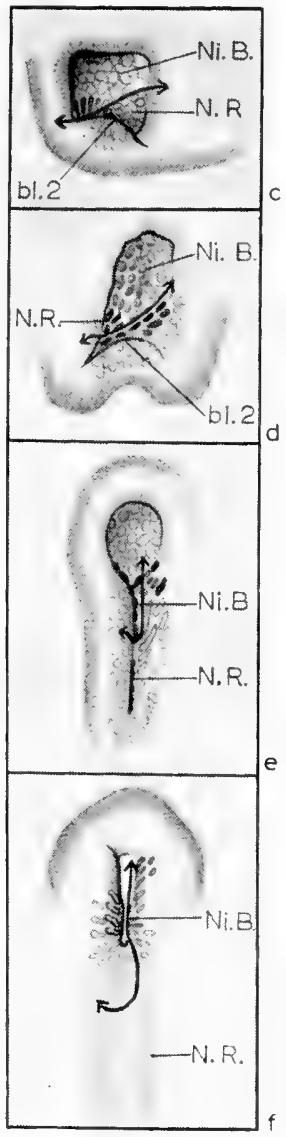

Fig. 54. A graft of organizer with reversal of the cephalo-caudal axis. The young gastrula donor (b) has received a combined mark of Nile blue (dots) and neutral red (crosses) in the blastoporal region; $\mathrm{V}=$ vegetative pole. The central area of this patch is grafted above the ventro-marginal zone, with reversal relative to the animal-vegetative axis (arrow). (c to $\mathrm{f}$ )

Aspects of the grafted region at successive stages; $(\mathrm{g}-\mathrm{h})$ dorsal views of the obtained embryo; (i-j) two sections through its cephalic part. In $\mathrm{c}$ to $\mathrm{f}$, the upper part is stained with Nile blue (Ni. B.), the inferior part with neutral red (N.R.); the arrowed line separates these territories. The host is seen from its ventro-marginal aspect, i.e. the same orientation as in (a). The whole grafts sinks progressively, and is gradually surrounded by a sort of blastoporal $\operatorname{lip}(b l .2)$ formed by the host ectoblast $(\mathrm{c}, \mathrm{d})$. The endo-prechordal part of the implant (stained in red) migrates straight toward the animal pole, while the blue part is covered by the lateral lips $(e, f)$. In $g$, the embryo at the 6 th day (at $\pm 10^{\circ} \mathrm{C}$ ) is orientated in the same way; the hatching corresponds to red parts visible under the surface, the circles are the blue material, also under the surface.

In $\mathrm{h}$, (8th day) the orientation has been reversed, the Nile blue was no longer perceptible in vivo. The sections (i-j) show that the secondary head was formed of an isolated and rudimentary fore-brain ampulla $\left(f . b r_{0}\right)$ accompanied by a small endoblastic tube (end.); more caudally, a hind-brain ( $h . b r$.) with a small otocyst appears, followed by a spinal cord. In these structures and in the surrounding mesenchyme the stain is preserved, but the distinction between Nile blue and neutral red is not possible. Thus a complete, but hypomorphic system has been formed. The depressive effect is related to a retardation of kinematics and, correlatively, to a decrease in induction.

Aud.-otocyst; bl.2-secondary blastopore (atypical); end.-endoblast; f.br.-fore-brain (secondary); h. br.-hind-brain (secondary); h. br. ${ }^{1}$-primary hind-brain; Hd ${ }^{1}$-primary head;

$\mathrm{Hd}^{2}$-secondary head. From Benoit, $195^{2}$. 
The kinematic behaviour of the graft confirmed what has been seen in Discoglossus gastrulation. The original antipolar material of the transplant migrates directly through the depth of the ventral zone toward the pole, without tumbling over a blastoporal lip, and is followed by the previously polar part. An atypical blastoporal lip nevertheless appears in the host mesoblast or ectoblast, and its movement helps to cover the implant (Fig. 54,a-f). By this kinematic regulation (p. 379) both parts of the graft nevertheless file in a normal sequence, but the migration is slowed down and the obtained embryonic axis remains slender and often atypical. Especially the forebrain is poorly developed (Fig. $54, \mathrm{~g}, \mathrm{j}$ ) and in no case has the prechordal plate expressed its full inductive capacity.

One general observation in these experiments, as in the above mentioned translocations, has been the greater activity of the medio-dorsal material in contrast to the more lateral one. Grafts containing presumptive notochord material are undoubtedly superior in all aspects of activity to purely somitic ones (compare Lallier's and Minganti's results). This remark, which will also be supported, for bird embryos, by Mulherkar's results (p. 4Io), refutes the criticism of Holtfreter an Hamburger who write (1955, their p. 243) . . "it is furthermore arbitrary to assume that the prospective notochord possesses a higher concentration of this substance (organisine) hence a higher 'morphogenetic potential' than the prospective somites." (for the concept of organizin, see p. 478).

Before passing to operations of a quite different type, a reference must be made to a paper of Waechter (1953). It is an extensive description of experiments largely initiated by Mangold ( 1936 ) and consisting of heteroplastic insertions (in the blastocoele) of fragments cut from the central part of the neural plate. The intrinsic development of the implants varied according to their origin, as did also, more or less, the inductions they evoked. From both points of view, a medio-lateral gradient was manifest.

We now come to a different, quite original trend of experiments. They were conceived by the Dutch embryologist Nieuwkoop and performed, from 1947 on, by himself and a series of guests at the Hubrecht Laboratory (Utrecht).

Three different types of experiments have been studied by this team of workers: a first group on whole embryos, a second group with normal explants reared in ectoblast sandwiches, and a third with killed inductors, also inserted in sandwiches. According to our plan, we shall be forced to dissociate these three groups, a procedure which I think will be beneficial.

Nieuwkoop's principal operations (1952) have consisted of implanting a folded piece of still indifferent ectoblast in the neural field of various Urodeles (Fig. 55). Xenoplastic combinations were frequently used. When performed on young gastrulae, this addition does interfere with gastrular morphochoresis; the superior part of the chordomesoblastic territory becomes neurectoblastic, while the upper part of the neural territory forms only epiblast (Fig. 56). The essential cause is that the immigration of the dorso-marginal material is reduced and the archenteron shortened. The graft does not persist for a long time in this flat folded state. Its base swells and its attachment expands or contracts depending on the case. The ultimate transformation depends upon the location of the fold and the age of the host.

It is considered certain that all implants made outside the neural field yield only atypical ectoblast, eventually with one or more balancers at their root (Fig. 57, a). 

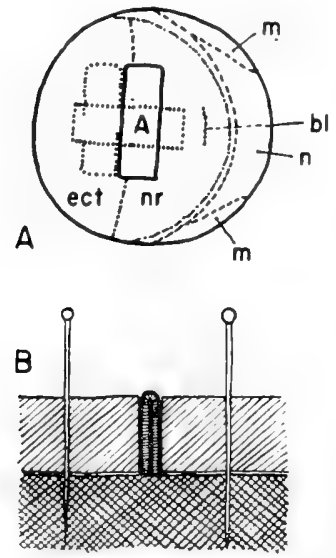
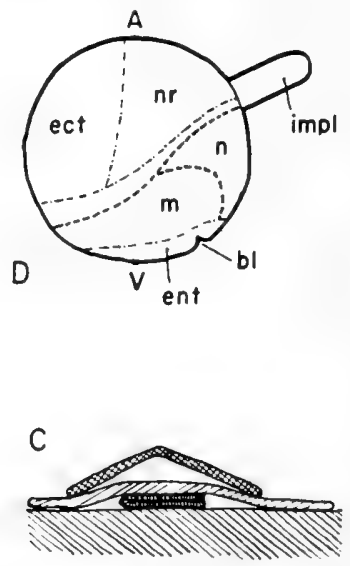

Fig. 55. Realization of a dorsal ectoblast fold, according to Nieuwkoop. A. A rectangular piece of ectoblast is isolated from the animal pole region of a young gastrula. B. Folding and pressing of this piece between two blocks of agar, fixed with needles to wax-bottom of operation dish C. Similar folding and pressing between two layers of agar weighted by a small glass bridge. D. Implantation of fold into an early gastrula in the dorsal midline, near the border line between the prospective marginal zone and the neural area.

A. animal pole; bl.-blastopore; ect.-ectoblast; m.-somitic area; n.-notochordal area; nr.-neural area: V. vegetative pole. From Nieuwkoop et al., 1952.

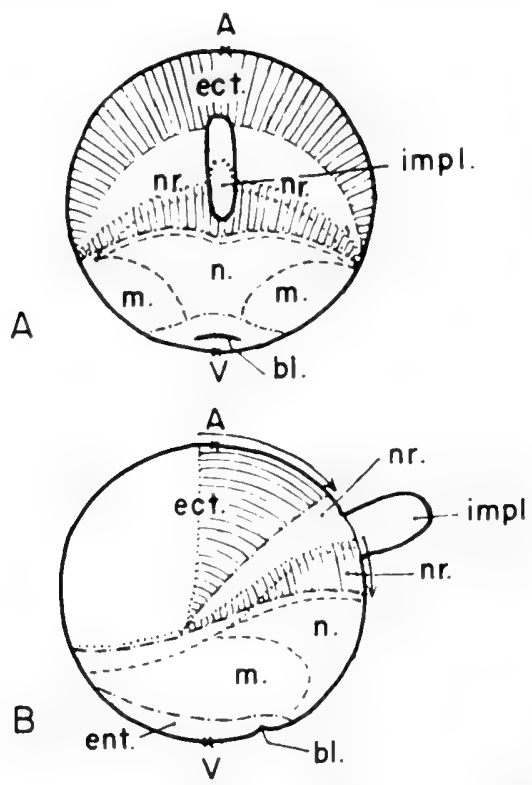

Fig. 56. Diagram giving the displacement of border lines between prospective marginal zone, neural area and anterior ectoblast. This displacement is an effect of the implanted fold on the kinematics. The areas over which displacement has taken place are roughly hatched. Same abbreviations as in Fig. 55, plus: ent.-endoblast; impl.-implant. From Nieuwkoop et al., 1952. 

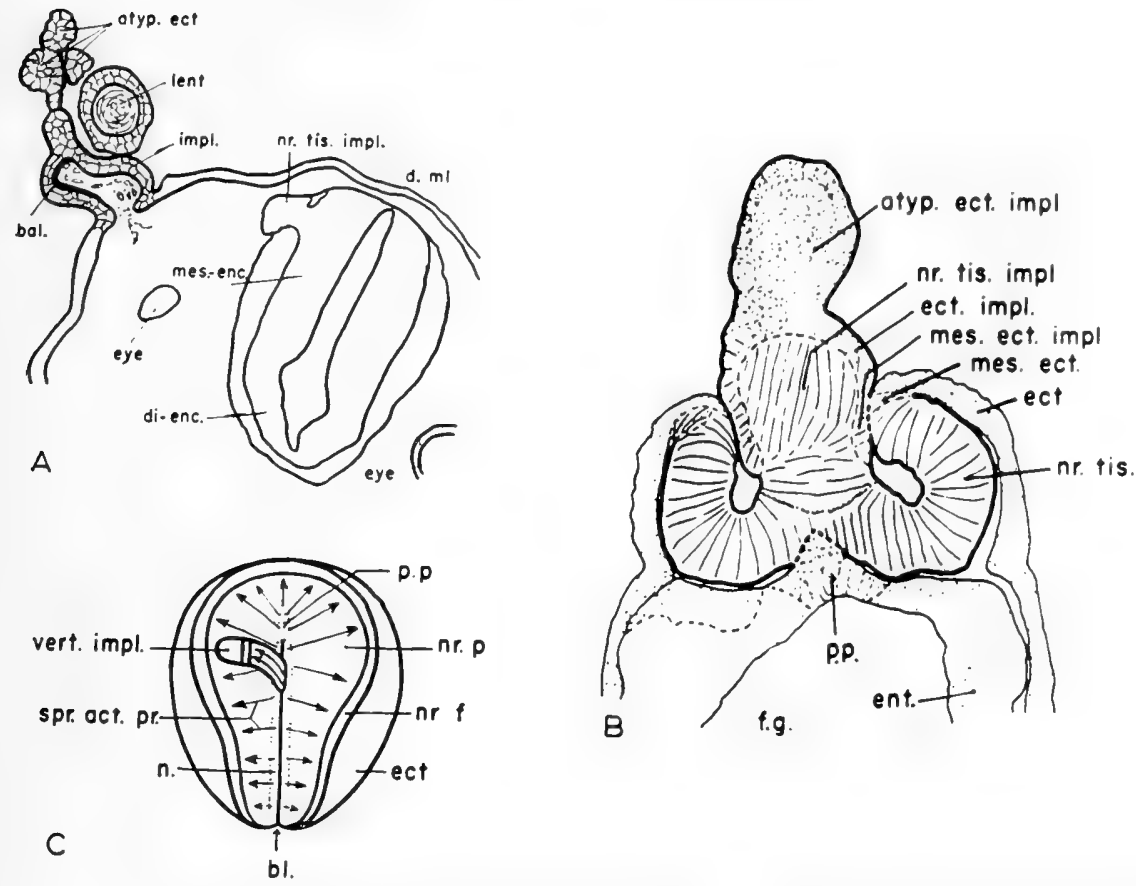

Fig. 57. Fate of an ectoblast fold implanted as in Fig. 54. (A) an implant probably grafted outside the neural area, or at the limit of this area; other cases yield atypical epiblast. (B) an attachment to the prechordal region of an axolotl neurula, with the differentiation in three zones, neural, mesectoblastic, ectoblastic. (G) hypothetical spreading of the activating principle through the prospective neural and mesectoblastic areas of host and implanted fold. atyp. ect., atypical ectoblast; bal., balancer; di-enc., diencephalon; d.m.l., dorsal middle line; ect., ectoblast; ent., endoblast; f.g., foregut; impl., implant; lent., lentoid; mesect., mesectoblast; mes. enc., mesencephalon; n., neural groove; n.r.f, neural fold; n.r.p., neural plate; nr. tis., neural tissue; p.p., prechordal plate; spr. act. pr., spreading activity of the prechordal material; vert. impl., vertical implant. From Nieuwkoop et al., I 952.

When the implantation happens to be located in the forebrain area, the base soon takes on the same appearance as the neural plate, while the distal end may remain atypical ectoblast (Fig. 57b). Sometimes the two zones are separated by some ectomesoblast. The interpretation of these early aspects is that some "activating" principle spreads "through the ectodermal layer, mainly emanating from the dorso-median zone of the ectoderm where this ectoderm is in close contact with the median portion of the underlying archenteron" (their p. 25). This assumption (Fig. 57c) would explain why lateral implants are less modified than median ones. The territory which is normally exposed to the activating influence, and in which there is a maximum zone with a decrement in all directions is called the neural field (Nieuwkoop et al., I952).

In 1954, the same author gave complementary information on the early behavior of the folded transplants (Nieuwkoop and Nigtevecht, I 954, their p. I 78). Using axolotl gastrulae as donors, he implanted the graft with a sagittal orientation on the presumptive rhombencephalon. Nieuwkoop insists that modifications of the external aspects take place and adds 
that "there were no indications of a displacement of cells inside the fold". The implant takes the shape of a toadstool, with three perceptible regions. The basal one contains "neural material only", the transitional region consists of neural crest and epidermal material, and the distal region is composed of atypical epidermis.

When the $195^{2}$ embryos were reared a sufficient time to show discernable brain parts and sense organs, the fate of the implant followed a definite rule. In cases where it is finally connected with the fore-brain, most of the grafted tissue takes part in the formation of the prosencephalon or, more frequently, builds acrencephalic parts supplementing the normal ones. When the graft shows final connections with the hind-brain, the proximal part of the implant is of rhombencephalic character, but the distal part sometimes contains parts of the acrencephalon. It should be added that several cases in which the implant combined with the brain show a striking reduction in, or even absence of, the host prosencephalon. When using the middle yolk plug- or preneurula stages, implantations in a more trunkal site could be obtained. Sometimes, a reduced acromerit appeared together with deutomeritic structures. The results remained about the same for implantations on the early- or even middle-neurula stages. One or two cases are described in which implants which were connected with the hind part of the spinal cord seemed to have formed a small acro-deutomerit complex.

Complementary results have just been published (Nieuwkoop, 1958) concerning the same experiment, repeated on axolotl embryos, a species with a specially sensitive ectoblast. Presumptive cranio-ventral ectoblast at various stages has provided folds always grafted in the deutomerit territory of young neurulae. Planimetry of organs has allowed some quantitative appreciation of the inductive effect that was obtained. As soon as the blastopore of the donnor becomes circular the competence of the ectoblast abruptly falls, with the interesting feature that the acrencephalic structures still appear in the tip of the fold but soon regress into mesenchyme. Even this labile reaction vanished at the next small yolk plug stage (Harrison's stage I2) and only rhombencephalic structures, if any, are then observed. Thus, the diffusion of some inducing factor(s) through the basis of the fold seems granted but, at the critical stage, the apex of the fold is still submitted to "activation" but not stabilized by the "transformation" process. The author invokes a fall of the competence threshold but discards Gallera's idea ( $c f . p .423$ ) of a gradual impermeabilisation of the reacting ectoblast.

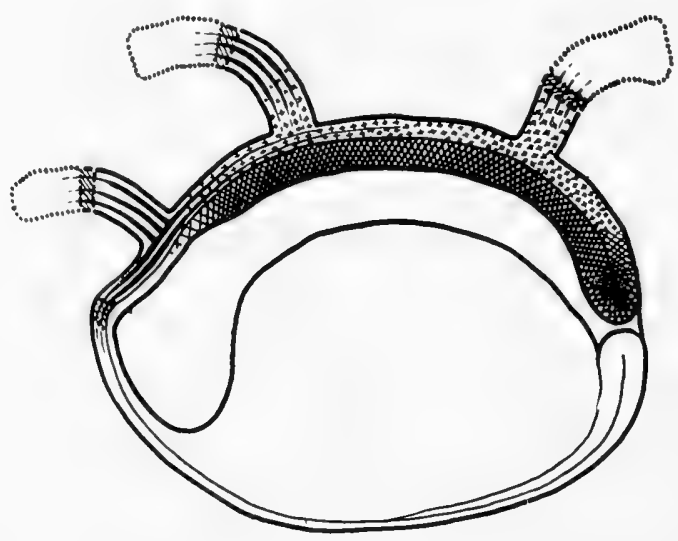

Fig. 58. Diagrammatic representation of the primary activation and subsequent interaction of transforming mesoblastic influences, in folds of competent ectoblast attached to acromerit, deutomerit, cormomerit. Hatched, the archenteric roof; plain pointed lines, the activating factor penetrating the folds; crosses, the transforming factor, most dense near the blastopore. From Nieuwkoop et al., 1952. 


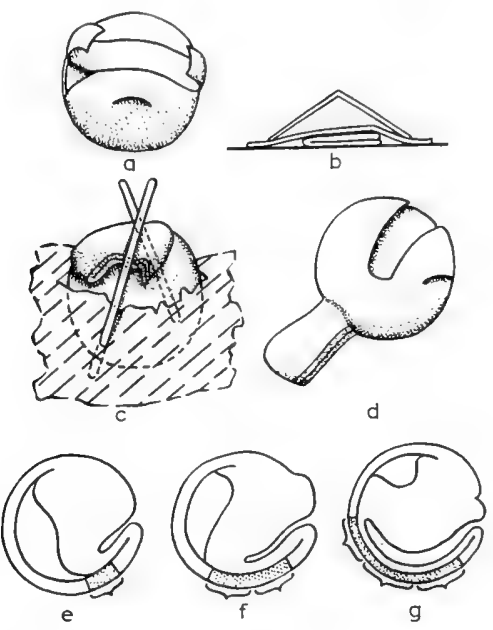

Fig. 59. Ventral implantation of a fold formed of presumptive "activated" neurectoblast of Urodele gastrula. (a-d) The operation procedure; (e-g) sagittal sections of successive stages to show the areas utilizable for this exploration. (a) A lengthy piece is cut from the dorsal part of the gastrula, above the probable limit of invagination. (b) The piece is folded under the weight of a sheet of agar and a glass bridge. (c) The same egg is placed in an appropriate cavity, a slit is made on its medio-ventral side, and the fold caused to adhere by the weight of glass rods. (d) The operated embryo, before healing of the dorsal wound (a variable event). (e) The extent of the zone of an early gastrula which had contact with the immigrating archenteron; only one location can be used for the fold at this stage; (f) middle gastrula, with curved blastopore, a stage where contact allows cutting the fold at two different levels; (g) advanced gastrula, with a yolk plug, a stage which authorizes cutting the fold at three different levels, with corresponding tissues of "activating" and "modulating" contacts. From Eyal-Giladi, 1954.

An interpretation of these data, which certainly strike out a new line in this field, has been influenced by results obtained with explantations and abnormal inductors, experiments to be considered later (p. 396 and 419). The induction of acrencephalic structures is attributed to an activation of the ectoblast, i.e., to the "liberation of prosencephalic differentiation tendencies" (1952, his p. 86). This activation is considered as the first step of induction; a second step is imagined to be a "counteraction", according to suggestions presented by Lehmann (I950) and by Yamada (I95ob). The counteraction expresses the inductive action of the chordomesoblast and is responsible for the notoneuron (Fig. 39, p. 368). Such an application of the experimental data to the normal course of morphogenesis leads Nieuwkoop to represent the differentiation of the implants like in Fig. 58. Thus the two inductive factors are not only assumed during the process of gastrulation, but are also implied in the later influence of the archenteric roof on the implanted folds. The figure shows the activating principle expanding from the neural plate, and probably being transmitted along the ectoblastic layer. However, in I 954, Nieuwkoop (in: Nieuwkoop and Nigtevecht, I 954, their p. I87) seems to see the activating source in the chordomesoblast, with which the foot of the implanted fold necessarily comes in contact. 
More should be said concerning the theoretical implications of this interpretation, but these comments will be reserved for the final part of this section. The factual contribution of these experiments is evident, and acromerits have been obtained in conditions which could not have been predicted beforehand.

Another aspect of the same phenomenon is found in the work of Eyal-Giladi ( 1954$)$. Here, similar folds were used, but were implanted on the ventral territory of the same embryo (Fig. 59,a, b, c). The latter shows a variable deficit in its dorsal zone (Fig. 59,d), and the author has attempted to compare the structure appearing in the folds with those lacking in the main embryo. The analysis is however complicated by the fact that the operation on the dorsal side interferes with morphochoresis.

We may immediately dispose of the folds obtained on neurulae, on gastrulae with a slit blastopore, and even in the majority of cases concerning gastrulae with middle to small yolk plug. In these the author asserts a satisfactory agreement between the deficiencies observed in the main embryo and the structures obtained in the folds. The absent brain parts, eyes, nasal pits, ear vesicles seem to be re-found in the ventral protuberance derived from the implant. For stages following the blastopore closure, this balancing only means that induction was already well on the way, and that the structures could be formed, at least qualitatively, in the physiologically-isolated neurectoblast. Their development was probably favored by the fact that, since the lengthy symmetrically-cut piece was exactly folded, each potency could find its equivalent vis-à-vis, without undue interference.

As a second group, we shall consider the operations performed on relatively "early" gastrulae (Fig. 59, e). Taking into account table I of Mrs. Giladi's paper and the description, the events can be pictured in the following way. In "some" cases, most of which are not tabulated, the fold became atypical epidermis. In two Pleurodeles embryos of this category, which were not discarded because they were considered as controls, the brain of the main axis was entirely missing. The author considers that all these negative results were due to strips "lying in front of the cranial border of the archenteron roof" (Fig. 60, a). This, however, does not sound so convincing; it may well be that strips of this stage often remain negative in spite of some contact with the archenteron roof. This suspicion is confirmed, in my opinion, by an analysis of her Table I. Among 9 of the Pleurodeles embryos, 4 show only "mesectoderm", while others yield: $(I)$ dubious telencephalon and paraphysis; $(2)$ teland diencephalon, 2 olfactory placodes, epiphysis, ganglion; (3) telencephalon, rudimentary

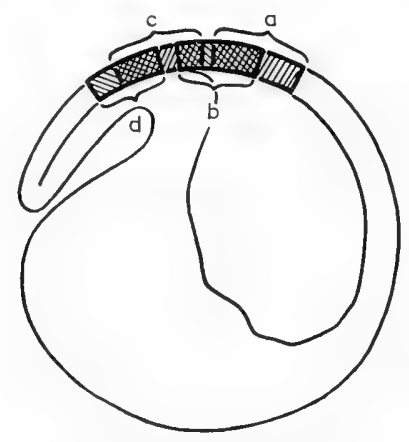

Fig. 6o. Further possibilities for cutting the strip for a fold in a rather young axolotl gastrula. (a) Anterior to the archenteron pouch; (b) above the anterior part of the same pouch; (c) entirely above the pouch; (d) in the zone of plain contact with the roof. Modified from Eyal-Giladi, I954. 
diencephalon, paraphysis; (4) telencephalon, I olfactory placode, paraphysis, ganglion; (5) telencephalon, complex nasal placode, paraphysis, ganglion. Among the I 3 Axolot embryos, 4 should be discarded here, for one strip carried some "mesoderm cells", and the two others produced tail-like formation, indicating that the strip was cut too low and included presumptive tail material. Among the Io others, we read: $(I)$ atypical neural mass; (2) the same; (3) prosencephalon, I nasal placode, a rudimentary retina; (4) a telencephalon, nasal placodes, paraphysis; (5) the same; (6) diencephalon, I eye, I lens; (7) badly-infected neural mass; ( 8 ) tel-and diencephalon, I nasal placode, 2 paraphyses, eye, lens, epiphysis; (9) prosencephalon, I nasal placode, I atypical eye; (Io) prosencephalon, 2 nasal placodes, 2 eyes. Altogether, this makes out of 19 valid cases, 13 with ascertained manifestations of prosencephalon. If the untabulated experiments are taken into consideration, it appears that an appreciable number of these early transplants have not shown induction in the folds. Of course, the main interest lies in the positive cases, but we must insist here on a consideration of the negative ones to avoid the impression, probably false, that the "activation" is quite a sudden process. The above enumeration follows Giladi's order, in which the strips she presumed the more caudal are placed last. It can be seen, especially for the axolotl, that these inductions are the best ones. This could mean that they had time enough to get the best from the inductory flow. On the other hand, if the assumed location is exact, the contact with the migrating archenteric roof, and especially with its cephalic part, could only be transitory. This is indeed a remarkable fact, and worth looking into the question of how such a process begins. "In the early gastrulae (p. 187 ) the implanted folds remain unchanged for 2 or 3 days, after which period the basis of the fold began to thicken" (Fig. 6r, b). Such an appendix was very fragile, and often remained unchanged for I-2 days, then progressively disintegrated from top to base. However, in 2 cases which were fixed on the third day after an early operation, at the moment disintegration began, an irregular mass of neural tissue, with

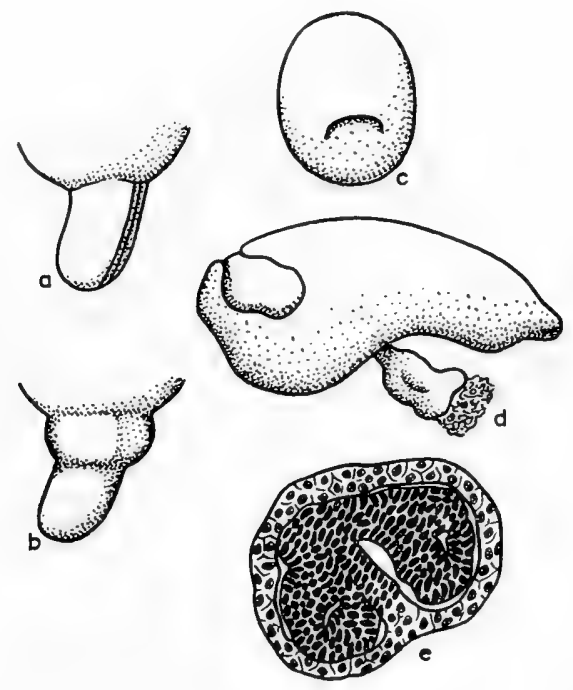

Fig. 6r. Two examples of a ventral fold created in the most reactive species, the axolotl. (a, b) First case, (c-e) second case. (a) Shortly after the operation; (b) two days later, with the characteristic swelling at the base of the fold; (c) the young gastrula before the operation; (d) the embryo three days after the operation, still showing a dorsal defect; the tip of the thickened fold begins to desintegrate; (e) section through the base of the fold fixed at this stage; the inner cells have a distinct neural appearance, even forming a mass with two cavities, suggesting a brain (Eyal-Giladi, 1954). 
some cavities, was found (Fig. 6I, e). From this picture, it can be admitted that these reactions might represent a young prosencephalon, but it can also be remarked that it is not formed as a small neural plate which closes by a converging movement of its borders, but by a process of delamination and neuralization of the inner layer. From this point of view, the two to three days delay is certainly of importance. In my opinion, there may be something more than the simple induction exerted by the passing archenteric roof.

Leaving this intimate aspect of the process, we may come to the third group, in which the same meaningful manifestations will again be met. This group concerns the "middle" gastrulae, and probably some of the yolk plug stages. Here the region from which one or two strips were cut (Fig. 59, f) is the one which, if the embryo had remained intact, would have formed notoneural parts. This situation is not always reflected by considerable deficiencies observed in the main neural system, because high cuts interfere with the forward migration of the archenteric roof and, the gap having a tendency to expand, no anterior brain has been formed. Giladi believes that there is a closer correspondance between implant and defect because, I think, she did not realize that the presumptive value of the strip could not be deduced from the deficiencies due to its ablation. This slight rectification, however, only makes the morphogenetic activities of the graft more significant, as appears from the somewhat simplified presentation in Table I of the results concerning Pleurodeles middle gastrulae. It is patent that in 8 of the I I cases, acrencephalic structures have been found in the fold.

They are, however, not of a uniform type, and the author has noted these variations, in this series and in others, without proposing an explanation. The three non-prosencephalic results merit a brief comment. $I$ is simply negative or unreadable; $\mathbf{F}$ has only produced a rhombencephalic neural mass, which seems to fit exactly with its origin; $\mathrm{K}$ has formed a segment of spinal cord, while the main axis has lost only the hind part of its medulla and the anterior of its spinal cord. One may be surprized that in such cases, where according to the author's and Nieuwkoop's ideas the "transforming" action has just replaced the "activation", no prosencephalic structure is apparent. However, Giladi states that in embryo L, and also in one of her axolotl embryos, "the graft behaved very strangely, forming a "neural mass which showed developmental tendencies in both prosencephalic and rhombo-spinal directions at the same time". This bare assertion is however not too precise, for in $\mathrm{L}$ no rhombencephalon, spinal cord or ear vesicle is recorded present, and, in the axolotl, the only result mentioned, is "mixed brain structure".

In her discussion, this author has raised many aspects of the kinematic and dynamic activities of amphibian morphochoresis. These points have either already been considered in this chapter or will be discussed later on. But we cannot leave Giladi's important paper without quoting her clearly-expressed view of Nieuwkoop's theory:

"The invaginating anterior part of the archenteron roof in which the inducing factor plays the chief role, on establishing contact with the neuro-ectoderm, causes therein a wave of activation (Nieuwkoop et al., 1952) which, when exceeding a certain treshold, enables the neural competence of this area to realize itself. This wave of activation right from the beginning takes the form of a neural field with a very definite spatial configuration, and leads to the formation of a prosencephalon. This field has a very dynamic character, and is constantly moving in an anterior direction during the period of invagination. Consequently, every cell in the presumptive neural area constantly changes its relative position in the field, and at the same time its potency is altered. As invagination advances, the most posterior part of the presumptive neural plate comes to lie above more caudal parts of 
TABLE 1

GILADI'S OPERATIONS ON MIDDIE GASTRULAE OF PLEURODELES

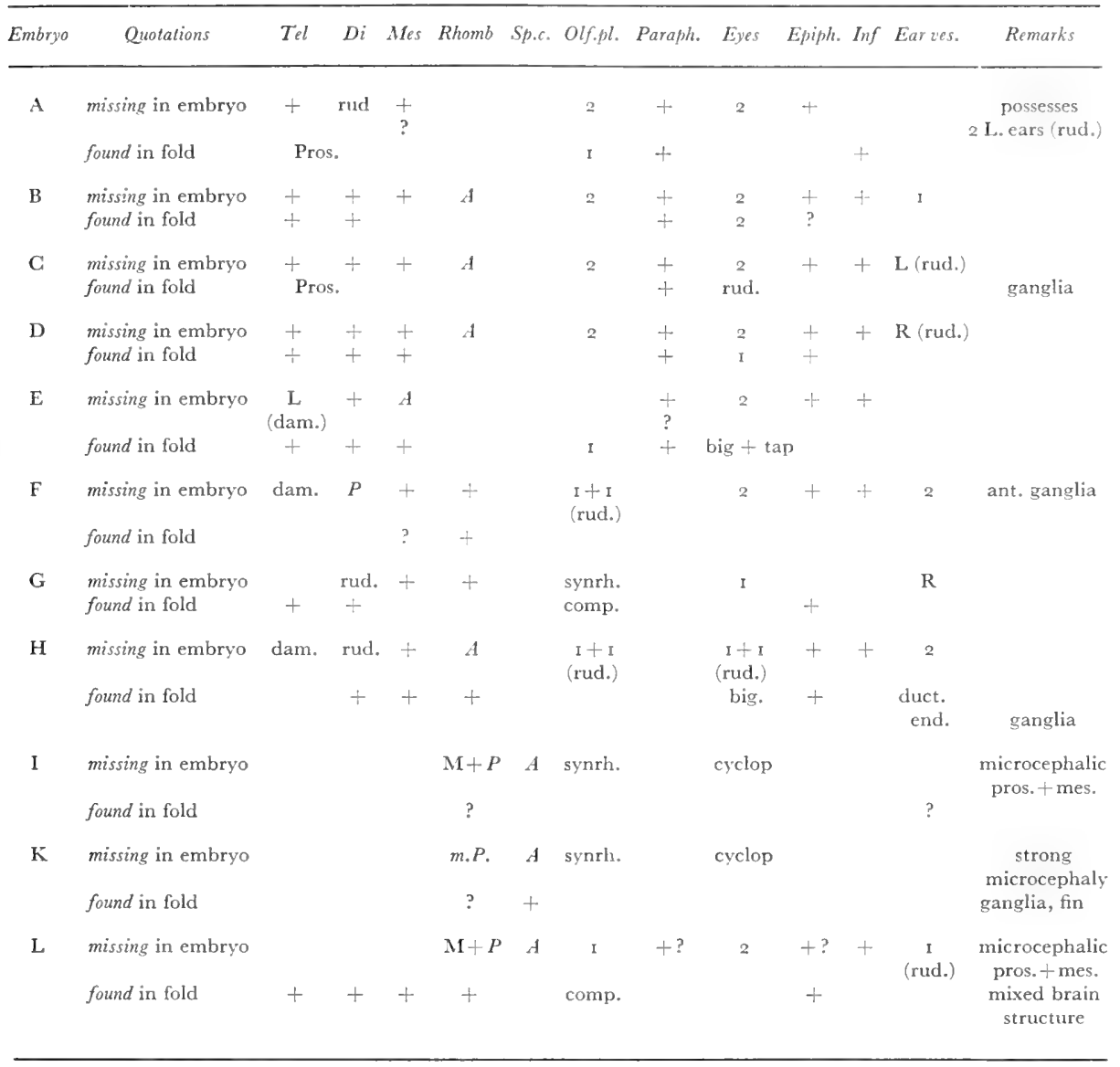

\section{Explanation of Table 1}

Operations on middle gastrulae of Pleurodeles.

For each embryo, the two superimposed horizontal rows tell which organs are missing in the embryo as a consequence of the ablation of a neurectoblast strip, and which ones are present in the fold made in this strip and grafted on the ventral side of the same gastrula. In the first row, + means the absence of the organ, in the second + means the presence of an organ of moderate size. $A=$ anterior; comp. = complex; dam. = damaged; duc. end. $=$ ductus endolymphaticus; Inf. $=$ infundibulum; $\mathrm{L}=$ left $\mathrm{M}=$ median; $m P=$ most posterior; $P=$ posterior; Pros. = prosencephalon; $\mathrm{R}=$ right; rud. = rudimentary; Sp.c. = spinal cord; synrh. = synrhinie; tap. = tapetum; ves. = vesicle. Except for embryo L, the strip used is the most caudal part stippled on Fig. 59, f. The author has listed the embryos according to the probable location of the excised strip (deduced from the defects in the neural axis), those with more cerebral presumptive value placed first. Compiled from Table II of Eyal-Giladi, I954. 
the archenteron roof, in which the relative proportion between the inductive and the transformative factor is changing steadily in favour of the latter. So, the differentiation tendency of the most posterior part of the presumptive neural plate changes during the period of gastrulation under the influence of the invaginating archenteron roof from prosencephalic through rhombencephalic to spinal cord, and finally probably into tail mesoderm. More anterior parts of the presumptive neural plate are not transformed as far as that; the process of transformation stops with the differentiation tendency to spinal cord. Still more anterior parts, also first activated to prosencephalic differentiation tendencies, are only transformed as far as the rhombencephalic level, while the most anterior part of the presumptive neural plate continues its further differentiation in the original prosencephalic direction, since no transformative influences are exerted upon it" (p. 235).

Such is the new proposal. After gathering the full amount of available information, we shall have to make our final conclusion on this theory (p. 473). Let us state here that the activation of the ectoblast by the archenteron roof which passes beneath it seems to be an established fact. In the implanted folds used to reveal this activation, it expresses its influence by producing diverse prosencephalic structures, perhaps due to some factor inherent in this experimental technique. I also agree that the inductive action of the chordomesoblast differs from that of the prechordal mesoblast, that the structures of the hind-brain have partly a transitional character, and that the chordomesoblastic type becomes purer in more caudal parts. However, whether or not the succession of activation and transformation is real and has a physiological meaning remains, I think, open to discussion. Let us look at other techniques which are already helping us to solve this complicated problem ${ }^{1}$.

\section{(b) Explantations of unmodified territories}

A quarter of century ago, there was a hope that the in vitro cultivation of cell groups would immediately reveal the true potencies of the chosen area. This expectation has not been realized either in amphibians or in birds.

In urodeles and anuran eggs, all parts of the very early gastrula have been systematically and most skilfully isolated and aseptically cultivated by Holtfreter (1938b) in a saline solution (which is since that time recognized as the most appropriate). Hardly any region shows typical morphogenesis. If the cells disperse on the bottom of the dish, as often happens, they generally remain "indifferent". If the explanted fragment preserves its cohesion and form, it generally yields more than would be expected from its presumptive value. The fate of the marginal zone is the most interesting. Most frequently, parts of the cells flatten and stretch over the surface, covering the fragment with a kind of epiblastic layer. Inside, tissues or groups of cells differentiate remarkably. The degree of paragenesis is moderate for pieces explanted from the medio-ventral region of the marginal zone, more accentuated for the lateral region, and still more for the dorsal one. Here, the notochordal and somitic areas behave similarly. Notochord and muscu-

1 This account has been entirely devoted to amphibian embryos, on which most of the recent experiments have been performed. Chick blastoderms have however been used also. Abercrombie and Bellairs (I954) have exchanged the anterior node of primitive streak stages with the posterior part of this streak. The results are essentially variable, but in some cases at least the unity of the embryo is restored. Grafts labelled with ${ }^{32} \mathrm{P}$ were used to show that the notochord, often V-shaped, was formed from host material. 


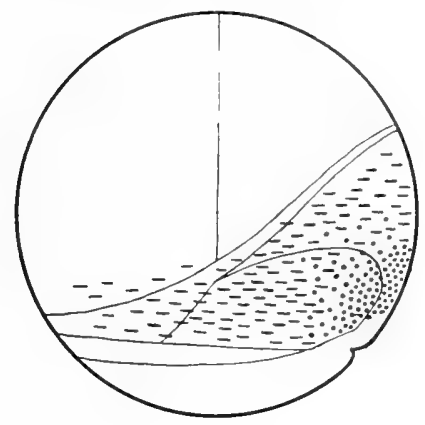

a

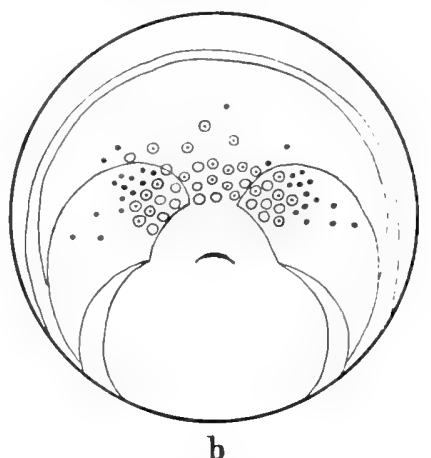

b

Fig. 62. A graphic summary of the results obtained from the explantation of Urodele dorsomarginal material. For the meaning of the map areas, see Fig. 49, p. $3^{81}$. In (a) the dots indicate the areas which yielded brain parts, and the lines, the areas which produced tail and fin structures. Thus, a rough distinction between cephalo-and ourogenesis (Fig. 39, p. 368). In (b), similar observations for olfactory placodes (empty circles, optic vesicles (dotted circles) and otic placodes (dots) are given. This distribution corresponds to the distinction between acro- and deutogenesis. From Holtfreter, I938 b.

lature are regularly obtained, and often also pronephric tubules. The mesoblastic structures are frequently accompanied by a neural axis, which may show a brainlike swelling at one end ${ }^{1}$. It seems that such an ectoblast formed by cells of the marginal territory is more easily induced than the normal ectoblast. In cases where some cephalization takes place, the relation to the intrinsic axes (or gradients) of the explant has not been strictly ascertained. However, a strong probability that such a relation exists is suggested by the fact that the explants which yield olfactory placodes and optic and otic vesicles, are obtained from the region where the existence of the corresponding inductors is assumed (Fig. 62).

Owing to technical difficulties involved in obtaining typical explantations, many authors have prefered to wrap the fragments in a piece of ectoblast taken from a young gastrula. This envelope also serves as a detector of the inducing ability possessed by the explant. A further modification has been to insert the investigated piece of embryonic tissue between two ectoblastic sheets (sandwich technique).

The development of the newt explants wrapped in young ectoblast of anurans has already been examined by Holtfreter, in i 936 . The xenoplastic method allowed him to discern which structure originated from the explant and which from the enveloping tissue. A clear-cut difference was observed between cases in which the explant was a young blastoporal lip, and those in which it was the hind part of the neurula archenteric roof. In this last instance, trunk-tail com-

1 When spinal cord or brain parts appear in such explants, they do not result necessarily from a process exactly similar to the normal course of events. Except when relatively large amounts of material are available, no neural plate is observed, and a fortiori no closing in a neural tube. Concurrently with the distribution of the inner material into a notochordal and a myogenic anlage, a certain amount of inner cells of the ectoblastic covering apparently detach themselves and form a mass or cord which becomes neuralized. 
plexes were obtained, while with the blastoporal lip the cephalization was obvious. The analysis was yet still guided at that time by the bare opposition between the head and trunk-tail organizers.

A few years later, Okada's laboratory produced some papers mainly concerning the possible differences between the non-invaginated and invaginated parts of the dorso-marginal material ( $c f$. p. 376$)$.

In the first group of explantations, performed on Triturus pyrrhogaster, Okada and Hama ( 1943 ) compared the fate and the inducing ability of the blastoporal lip excised at six different stages, and the archenteric roof often divided into two or three parts, anterior, middle, posterior. For the uninvaginated material of the younger stages up until the formation of the semicircular blastopore, a marked difference was found between these results and those from affixation in the blastocoele (p. 377). Trunk organs were still obtained, but they were completed in numerous cases by typical acrencephalic structures. The proportion of olfactory placodes and optic vesicles was highest when the uninvaginated part of the crescent-shaped blastoporal lip was used, a fact that may be interpreted by the presence, at this very level, of the prechordal territory. At this same stage, the invaginated portion was distinctly less active from the same point of view. From the semicircular blastopore stage on, a decline in the inducing activity of the mostly prechordal material was observed. It seems that no case, either using still un- or invaginated material, provided only acrencephalic inductions.

In 1945, the same authors made another comparison concerning the regions of the archenteric roof from the semicircular to the circular blastopore stages. The fragments were either inserted in a young neurula and covered with ectoblast of an advanced gastrula, or wrapped in the same tissue. The inductions of acrencephalic type were only few, and were obtained, as in Mangold's experiments, from explants more posterior than had been expected. Somites and other mesoblastic manifestations of induction were regularly missing. Otic vesicles were often induced, especially by trunk chordomesoblast, even quite posterior.

At about this time, Ter Horst (I948) reinvestigated the fate of explants taken from the still open neural plate. Parts of the neurectoblast and of its chordomesodermal substratum were cultivated separately in heteroplastic sandwiches. Most of the results are now easily understandable, and do not need a detailed account. Two of them, however, deserve to be mentioned. (I) An alpestris sandwich provided with the anterior fourth of a cristatus neural plate formed a complete brain; this organ had been formed by the explant, except for its telencephalon and olfactory placodes which had been induced in the alpestris material: a classical case of complementary induction. (2) A purely mesoblastic part (without notochord), taken about mid-length of the archenteric roof, developed partly into a neural tube, while the rest mostly failed to differentiate, except for some muscle cells (Fig. 63). This is a typical case of direct neuralization of mesoblastic material. The same event has been observed also by Töndury (I936) and by Dalcq and Lallier (1948). In these last cases, which concerned transplantations of blastoporal lips, diagnosis of the graft was possible thanks to vital staining and it cannot be excluded that the Nile blue could have played a role. This objection does not hold for the observation of Ter Horst, for she only used a heteroplastic technique.

Gallera used the sandwich method (1948) for a control in his transplantation experiments. He inserted the prechordal neurectoblast between pieces of neurula epiblast. The development of the explants was slower than for grafts made on embryos. With young donors only, neuroidal differentiation took place. With more 


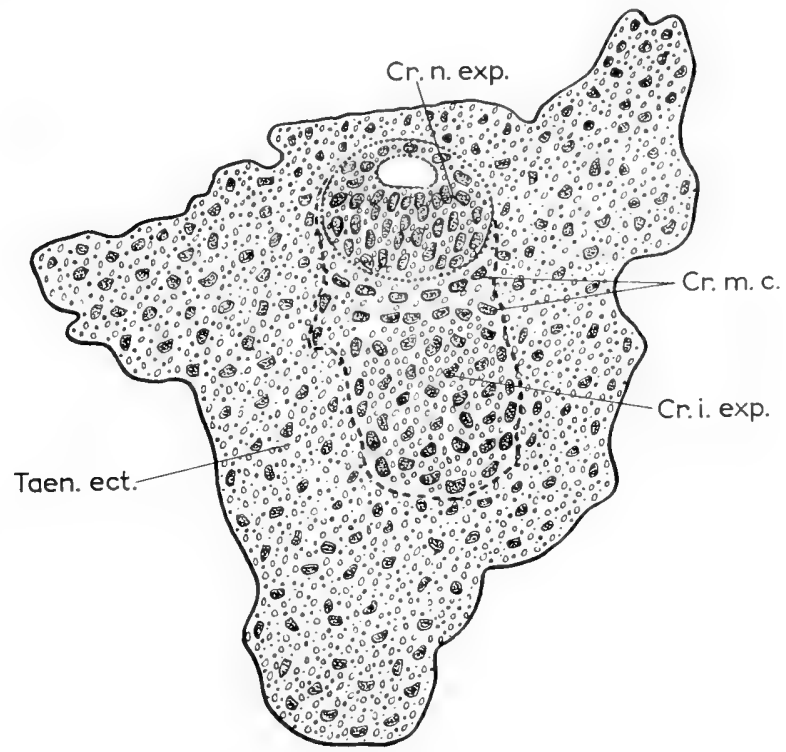

Fig. 63. A case of direct neuralization in a heteroplastic transplantation. The middle part of the archenteric roof was dissected from a T. cristatus neurula and wrapped in T. toeniatus ectoblast. It provided a small neural tube $\left(C r . n\right.$. exp.), some myoblasts $\left(G r_{0} m . c_{0}\right)$ and undifferentiated cells (Cr. i. exp.) Taen. ect., the enveloping ectoblast of T. toeniates. From Ter Horst, 1948.

advanced stages, brain ampullae and optic vesicles were obtained. Clearly, this method is less favorable than grafting on to the ventral region of host neurulae (see Denis' data, p. 412); this can be easily understood owing to more normal environmental conditions to which the incipient blood circulation soon contributes.

Better technical success was attained by Von Woellwarth (1952) with his pure explantations (not sandwiches) of the newt brain territory plus some adjacent epiblast, at 9 successive stages.

The youngest one was a gastrula with a circular blastopore, the latest a neurula with recently closed neural tube. There was no attempt toward a strict topographical limitation of the neurectoblastic area; on the contrary, a variable portion of the hind-brain was always involved. The mesoblastic substratum was carefully avoided. Thus, the scope of the work was nearly the same as Gallera's transplantations (p. 371): disclosing the consequences of suspending the inductive action. A progression in the quality and complexity of the structures formed was of course observed and accurately demonstrated. Whe shall only refer to the results of the first stages investigated. Of 26 pieces isolated from gastrula's with medium or small yolk plug, ig formed only atypical indifferent epithelium, 5 some mesenchyme, melanocytes and ganglia, 2 the same components plus brain parts, in I case rudimentary, in the other barely recognizable as prosencephalic structures. At the slit blastopore stage, half of the 20 explants were either undifferentiated or abortive, while Io showed well-closed vesicles containing distinct but rather rudimentary prosencephalic structures; about 4 of these also formed hind-brain parts. In view of the fact that at these two stages contact had already been made with the anterior part of the middle layer, it is intriguing that such a large proportion of the explants remained undifferentiated. This negative result does not quite fit with Nieuwkoop's thesis of a primary and rather intense activation. The positive aspect is that induction is not an "all or none" reaction but passes through a neural crest level before full neuralization. 
During the next year, Deuchar (I 953) needed controls for her dissociation experiments (p. 422), so she wrapped in young ectoblast the first, second, and last third, respectively, of the archenteron roof of the late gastrula, the neurectoblast having been peeled off. A valuable innovation in her experiment was that she looked for a comparison between the inducing effect of I explant and 3 others of the same origin. The main results are summarized in Table 2.

TABLE 2

INDUCING EFFECT OF THE ARCHENTERON ROOF

Each piece simple or triplicated

\begin{tabular}{|c|c|c|c|c|c|c|c|}
\hline Region & $\begin{array}{l}\text { Total of } \\
\text { experiments }\end{array}$ & $\begin{array}{l}\text { Total number } \\
\text { of inductions }\end{array}$ & Brain & Eye $(s)$ & $\begin{array}{c}\text { Neural } \\
\text { tube }\end{array}$ & Neuroïd & $\begin{array}{l}\text { With tail } \\
\text { outgrowth }\end{array}$ \\
\hline I anterior third & 30 & $8(26.7 \%)$ & 5 & 3 & I & I & 2 \\
\hline 3 anterior thirds & 13 & $3(23.1 \%)$ & 2 & o & $\mathbf{I}$ & o & 2 \\
\hline I middle third & 39 & I6 $(4 \mathrm{I} \%)$ & 7 & 8 & 3 & 0 & 8 \\
\hline 3 middle thirds & 13 & $9(69.2 \%)$ & 9 & 8 & I & o & 4 \\
\hline I posterior third & 29 & $16(55.2 \%)$ & 6 & 3 & I0 & 0 & 3 \\
\hline 3 posterior thirds & 9 & $9(100 \%)$ & 3 & I & 6 & o & 2 \\
\hline
\end{tabular}

Statistically, significant differences appear in the number of inductions of each type produced by the successive thirds, especially when 3 pieces were used together. The general inducing activity is the highest for posterior parts of the roof, but the acrencephalic character, revealed by the eye frequency, is more often manifested by the middle parts, in agreement with Mangold's and Ter Horst's results. Trunk and tail characteristics are reinforced with posterior parts. However, no group specifically induces the acrencephalon or the suprachordal cord. This absence of definite regionality must be applied with caution to the normal roof of the archenteron. First, conditions in explants are, as already noted (p. 399) somewhat different from blastocoele insertions. Second, the division of the inducing system may be important in itself. The author expresses the interesting suggestion that "the anterior third of the organizer normally depends upon the two other thirds for supplies of essential substrates". Whe shall come back to this idea (p. 473).

In I953, Takaya re-examined the modification of potency correlated with invagination. His new experiment differed from the one of Okada and Hama only in the fact that he did not use the blastoporal lip itself but two neighboring areas, one uninvaginated, the other already incorporated in the roof of the archenteron. They were wrapped in competent ectoblast and reared for 3 more weeks. The interest lies in the early stages of gastrulation, previous to the large yolk plug stage.

The results were very clear cut. All surface fragments, even if they included the presumptive prechordal plate, yielded notochord, which is not astonishing, but remarkably they induced chordal parts of the neural axis, except in I case. The same material, when recently invaginated did not differentiate into notochord (only in I case out of 26 ) and induced a forebrain in 9 of the 26 cases, twice including eye; most other cases formed a neural mass or a spinal cord. This result is in good agreement with the insertions in the blastocoele (p. 375). As in the latter, the influence of age was examined. Explants 
cultivated in vitro for some hours began to elongate and, if left for sufficient time, became club shaped, and eventually formed a pigmented streak. In this state, they were again wrapped in ectoblast and reared. Their ability to form notochord considerably decreased and they often induced forebrain. Thus, they had been modified as if they had performed invagination.

A similar inquiry has now been realized in axolotl gastrulae (Hoessels, 1957). The area of the blastopore lip, representing the prechordal plate, was dissected at successive stages and reared in ectoblast sandwiches. At the youngest stages of gastrulation, the induced structures were of notogenic character, but, as soon as the material had invaginated, the character became either acrencephalic or neuroid. It was also found that the roof of the incipient archenteron, i.e. presumptive pharyngeal endoblast, induced acrencephalon. On the other hand, it seemed impossible, by this technique to isolate from invaginated tissue a purely notochordal explant; prechordal material always appeared simultaneously.
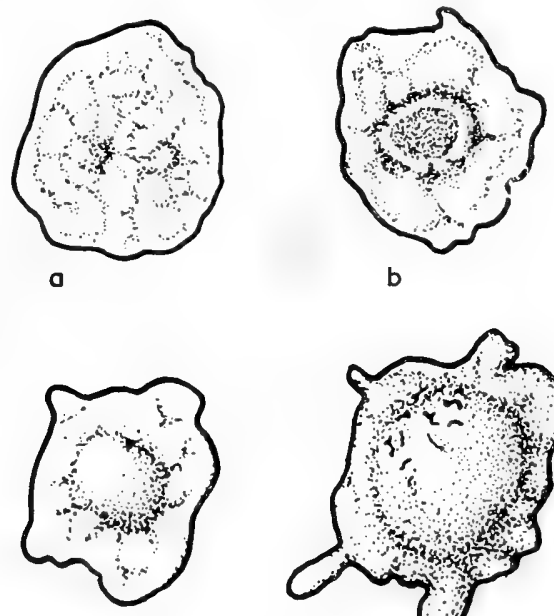

c
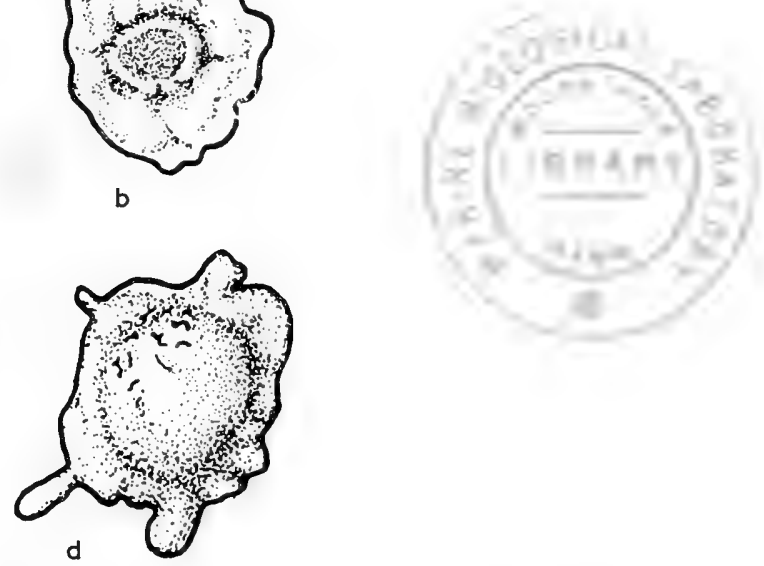

Fig. 64. In vivo aspects of sandwiches formed of the anterior part of the neurula notochord, between two pieces of ectoblast. The species used were newt and axolotl. (a) 2 I h., (b) 70 h.,

(c) 96 h., (d) i I days after the operation. From Nieuwkoop and Nigtevecht, I 954.

Thus, there is good agreement between implantations and explantations in showing that the organizing and inducing abilities of the dorso-marginal zone change in Urodeles during the transit through the blastoporal lip. The prechordal potency does not preexist, but effectively appears during the first steps of gastrulation. In my opinion, this change is not simply brought about by an aging process, but by the more special process of the accomplishment of kinematic performances. These are not suppressed during cultivation in vitro, although they may be appreciably reduced.

But an other relation has been recently disclosed. The fate of this material does not only depend on its age and location, but also on the tissue in which it is wrapped. If one uses, in place of gastrular ectoblast, neurular epiblast, no chorda is formed by the uninvaginated blastoporal lip, but simply endoblast (Kato and T. S. Okada, I957.) Would it be because it could not release its inducing substances? 


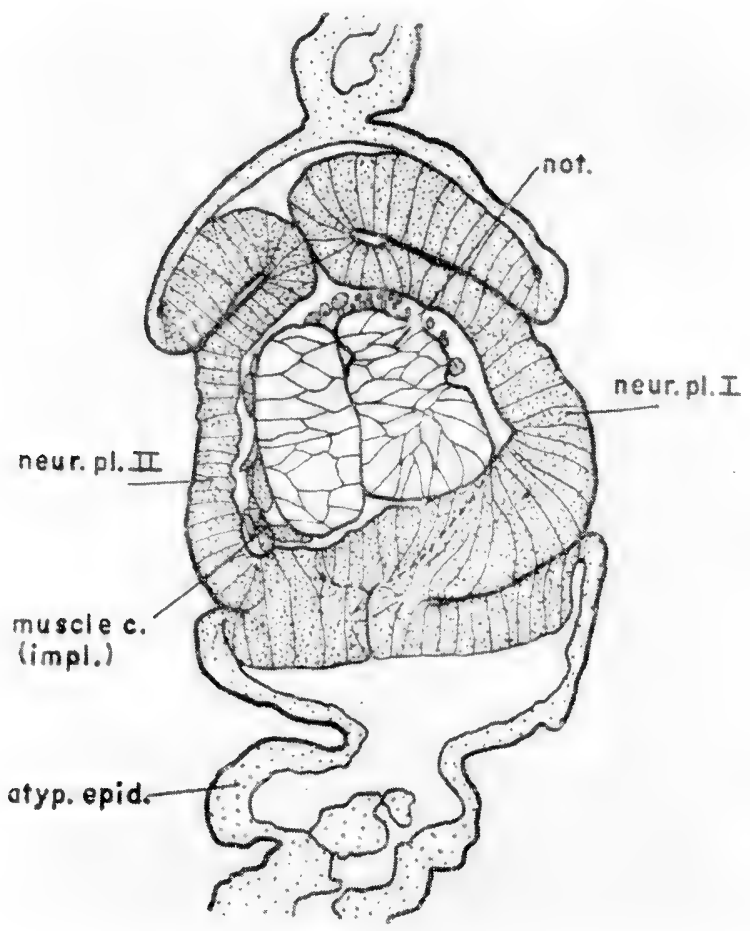

Fig. 65. Transverse section (schematic) through the 7 days newt explant. The two neural plates (neur. pl. I, II) are still open. The notochord (not.) is accompanied by a few muscle cells (muscle c.) from the implant (impl.) and small necrotic elements. Atyp. ep.- atypical epidermis. From same paper as Fig. 64.

An unexpected effect has been disclosed as a result of inserting the cephalic part of the notochord in a sandwich made of early gastrula ectoblast. The complex remains apparently quiescent for $2-3$ days; then, an induced neural plate becomes apparent on both sides. The surface soon smoothes out, and the central part swells (Fig. 64). Full differentiation takes a long time: 2 weeks in the axolotl, 4 weeks for the newt material. In the latter, one or two plates are still present after 7 days (Fig. 65). When they are closed, thin brain ampullae are formed in which the authors (Nieuwkoop and Nigtevecht, 1954) recognize a rhombencephalon and sometimes prosencephalic parts, including retinal and tapetal regions. Near the surface of these ampullae, small otocysts, nasal placodes and balancers may be formed. The proposed interpretation is that the implant first exerts a general activating action which spreads as far as the periphery of the sandwich. Secondarily, this field is modified by a "transformative action" which causes the material surrounding the chordal implant to become brain, while the periphery only attains the level of mesectoblast and, farther outside, may either remain as indifferent epiblast or produce nasal placodes (Fig. 66). 

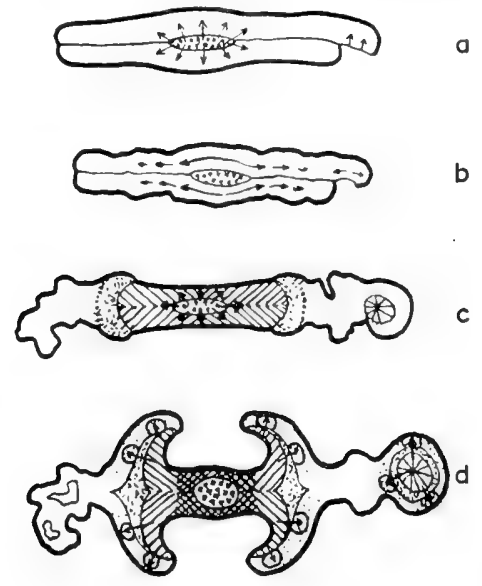

Fig. 66. Schematic representation of the processes in the sandwich considered in Figs. $64-65$; (a) the arrows suggest "activation" by the implant (center) and by the external medium acting on the exposed inner layer of the ectoblast; (b) spreading of these activating processes; (c) formation of areas with different differentiation tendencies; central arrows, the "transforming" action; oblique hatching, central neural area; dots, mesectoblast and activated epiblast; (d) neuralization phase, with the full complex of results, with rhombencephalon inside, acrencephalon outside, olfactory placodes (circles) surrounding the organizer center; outside the undifferentiated epiblast an olfactory placode (wheel, right) takes its origin from the independent activation mentioned in (a). From the same authors as Figs. 64-65.

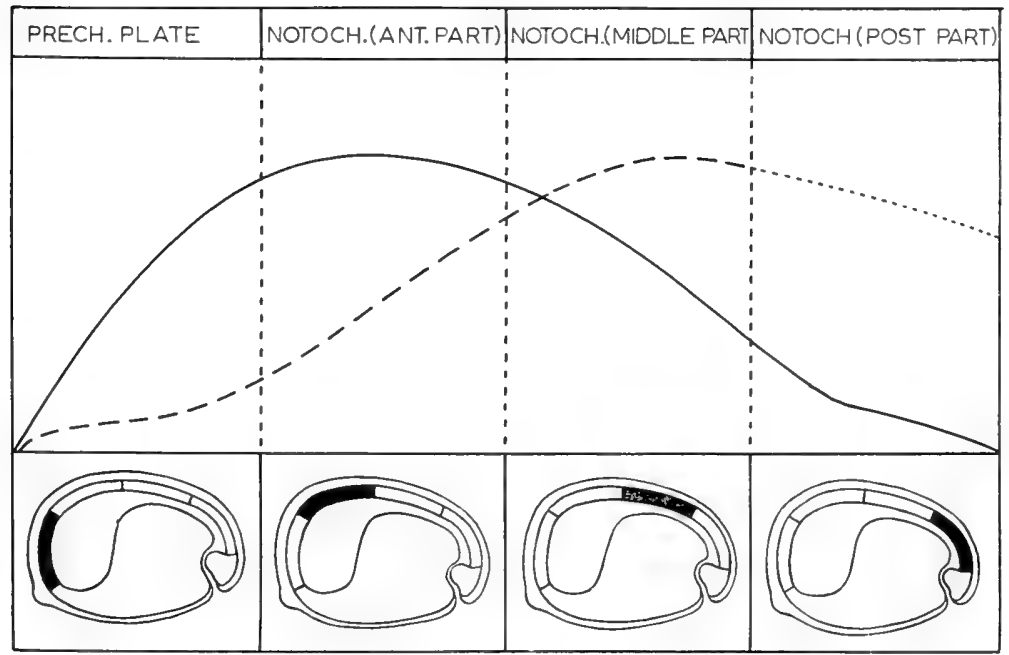

Fig. 67. Conventional representation of the "activating" and "transforming" effects exerted by explants of the successive medial parts of the archenteric (lower row) roof. Plain line, "activation"; broken line, "transformation". From Sala, I954. 
The same problem has been reexamined by Sala (I954, I955, I956); he did not only use the anterior part of the notochord, but explored the whole median strip of the archenteric roof, which was divided into 4 regions (Fig. 67, p. 403).

TABLE 3

INDUCTIONS PRODUCED BY THE SUCCESSIVE PARTS OF THE MEDIO-SAGITTAL STRIP OF THE ARCHENTERON ROOF

\begin{tabular}{|c|c|c|c|c|c|c|c|}
\hline $\begin{array}{c}\text { Part of } \\
\text { mediosagittal } \\
\text { strip }\end{array}$ & $\begin{array}{l}\text { Total } \\
\text { number }\end{array}$ & $\begin{array}{c}\text { Acrencephalic } \\
\text { structures }\end{array}$ & $\begin{array}{c}\text { Deutencephalic } \\
\text { structures }\end{array}$ & $\begin{array}{l}\text { Cranial } \\
\text { ganglia }\end{array}$ & $\begin{array}{l}\text { Spinal } \\
\text { cord }\end{array}$ & $\begin{array}{l}\text { Spinal } \\
\text { ganglia }\end{array}$ & $\begin{array}{c}\text { Melanocytes } \\
\text { or neural crest }\end{array}$ \\
\hline \multirow[t]{2}{*}{ Ist fourth } & 22 & 18 & & I & & & 3 \\
\hline & & 4 & 4 & I & 2 & & 2 \\
\hline \multirow[t]{2}{*}{$2 \mathrm{~d}$ fourth } & 20 & $\begin{array}{c}I \\
I 4\end{array}$ & $\begin{array}{r}\mathrm{I} 4 \\
I\end{array}$ & $\begin{array}{l}7 \\
\text { I }\end{array}$ & I & 2 & $\begin{array}{l}\text { I } \\
8\end{array}$ \\
\hline & & & 4 & 4 & 4 & 2 & 4 \\
\hline $3 \mathrm{~d}$ fourth & 22 & 7 & $\begin{array}{r}7 \\
I O\end{array}$ & $\begin{array}{l}4 \\
4\end{array}$ & $\begin{array}{l}5 \\
9 \\
\end{array}$ & $\begin{array}{l}4 \\
5\end{array}$ & $\begin{array}{r}7 \\
10 \\
\end{array}$ \\
\hline \multirow[t]{3}{*}{$4^{\text {th }}$ fourth } & 16 & $I$ & $I$ & I & $\begin{array}{c}5 \\
I\end{array}$ & 1 & $\begin{array}{l}3 \\
I\end{array}$ \\
\hline & & $I$ & 2 & & I & I & $\begin{array}{l}\text { I } \\
2\end{array}$ \\
\hline & & & & & 8 & 3 & $\begin{array}{l}3 \\
4\end{array}$ \\
\hline
\end{tabular}

For sake of simplification, negative cases and some dubious diagnoses have been omitted; the mesencephalic structures have been left aside, considering them as having a transitional value. The author's distinction between main series and transitional groups (plausible but established a posteriori) has been suppressed. In each group, the numbers at the right of the first qualitative quotation (heavy type) refer to observations made in the same sandwiches. Compiled from Sala, I954.

Fully established neurulae of axolotl were used, and the sandwich cultured as long as 3 weeks. The results given in several extensive tables have been summarized in Table 3 . In his conclusions, the author states that the prechordal explants only induce prosencephalic structures, while the successive parts of the notochord induce respectively the anterior half of the deutencephalon, then its posterior half, more posteriorly the spinal cord and derivates of the neural crest, sometimes including the mesenchyme of the dorsal fin. The inductions of maximal size are produced by the second fourth (anterior part of the notochord), and decrease in size anteriorly and posteriorly. Taking into account the cephalocaudal stretching, Sala admits the existence of two factors which he calls activating and caudogenic influences. The first one is assumed to diffuse more easily, while the other would exert on the already activated ectoblast an action causing more and more caudal structures to appear. The variation of these two factors is tentatively represented by two discordant curves (Fig. 67).

The following remark can be made when considering Sala's results, as they are presented in Table 3. For the $2 \mathrm{~d}$ fourth, one piece had an exclusively acrencephalic effect, while five pieces produced no more than deutencephalic structures. These "exceptions" are difficult to explain by assuming the fragments to be 
overriding the usual limits. In the first case, the notochord must have been present without exerting its transforming effect. In the five others, it must be accepted that the activated territory was integrally transformed, without any evidence of an activation phase.

I do not wish to suggest that the idea of two factors acting in these complexes is without foundation. The criticism bears more on the assumption of the two successive influences acting from the notochordal explant by a kind of impregnation into the surrounding ectoblast. It is worthwhile to recall that a rather long delay here preceeds the appearance of neuralization. Do not some unsuspected events take place inside the sandwich during the early period?
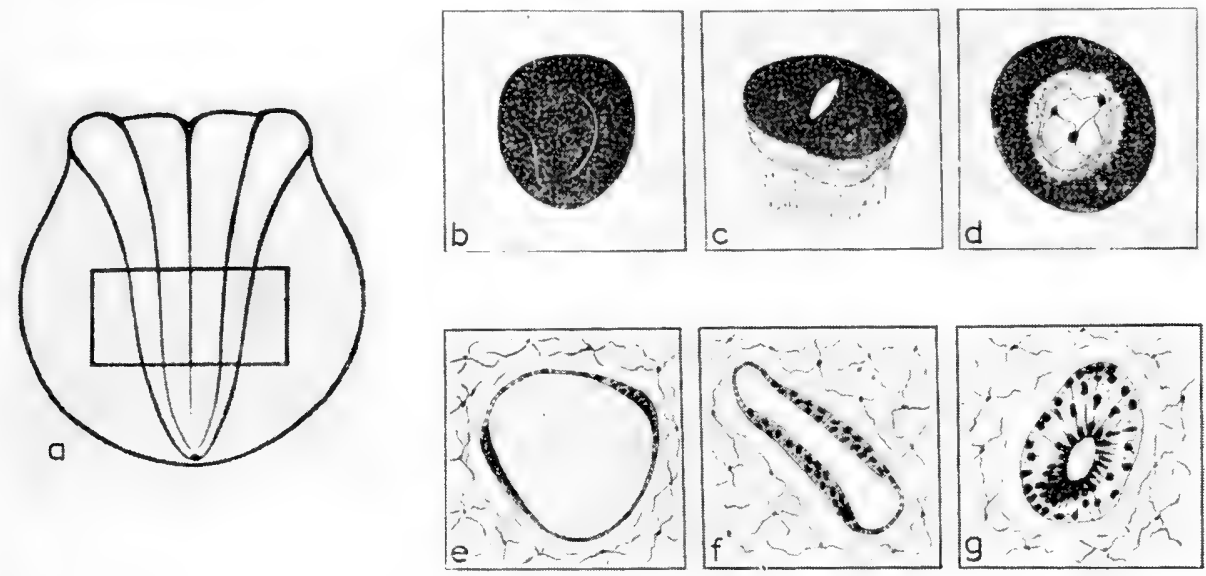

Fig. 68. Influence of the neighboring tissue on the differentiation of the neuro-epithelium in Urodeles and Anurans. (a) the explanted area of a neurula with elevated neural folds; (b) the neuro-epithelium in the absence of mesoblast; (c) accompanied by myoblasts and fibers (white matter) at the contact zone; (d) surrounding a notochord piece, also with fibers near this tissue; $(e, f, g)$ in simple mesenchyme resulting in three degrees of epithelial differentiation and distension. From Takaya, 1956b.

The influence of neighboring tissue on the morphology of the neural axis was recognized by Holtfreter (1934a), but has now been explored further by Takaya ( $1955^{\mathrm{a}}$, I956a, b, c). This author has isolated from urodele and anuran neurulae (with a distinct neural fold) the region of ectoblast including the brachial ${ }^{1}$ part of the neural plate (Fig. 68, a). If the chordomesoblastic substratum is included, typical normogenesis takes place, as expected. Considerable differences occur, however, if the mesoblastic lining is wholly or partly removed. At first, Takaya was struck by the brain-like appearance of certain explants, but he also observed considerable difference in the growth of the neural tissue, in the differentiation of fibers (white matter), and in the degree of swelling, according to the nature of the adjacent material. By expanding the views expressed by Holtfreter ( 934 a, p. 294), he has now attained a satisfactory interpretation.

1 i.e. the level which will furnish the innervation of the upper limb. 
In the absence of any mesoblastic component, the explant of neural tissue always forms "a solid or globular mass, containing a large number of neuroblasts" (Fig. 68, b) ordered in strata originating from a germinative layer surrounding a narrow ependymal lumen. The picture is the same, whether the mass is naked or surrounded by epiblast. With the simultaneous explantations of some notochordal or somitic material, some white matter may appear, preferably near muscle cells (Fig. 68, c, d). An association of neural tissue and mesenchyme only is often encountered in explants cut through the whole archenteric roof and secondarily deprived of the median strip of notochordal tissue. In these cases, the proliferation of neuroblasts is considerably reduced and a variable expansion of the ependymal layer takes place (Fig. 68, e, f, g).

A significant change occurs when the spinal cord happens to be accompanied by a mixture of mesenchyme and other mesoblastic derivatives, especially myoblasts. In this
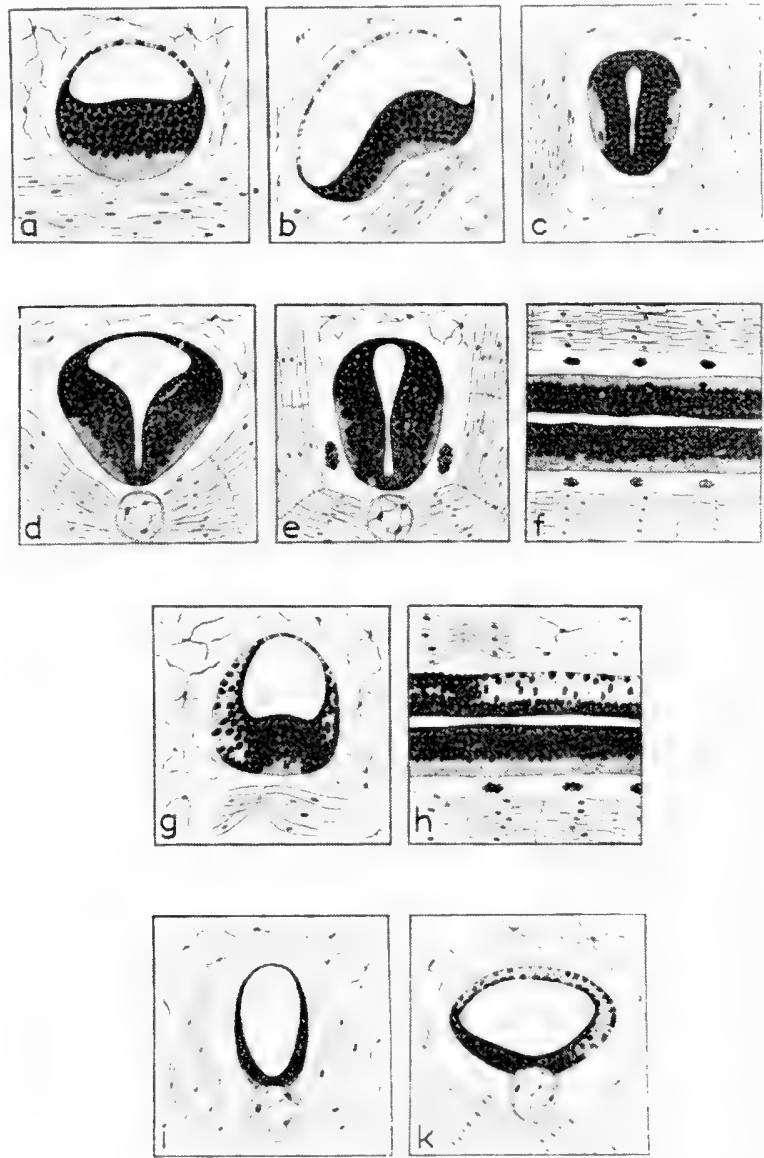

Fig. 69. Effects of the neighboring tissues on the shape and differentiation of a neural tube. $(\mathrm{a}, \mathrm{b}, \mathrm{c})$ Associated influence of myoblasts and mesenchyme; (d, e, f) association with notochord, segmented musculature, mesenchyme; (d) is of a rhombencephalic type; $(e, f)$ is more similar to spinal cord; $(g, h)$ various degrees of neural proliferation resulting from contact with different quantities of myoblasts; $(i, k)$ direct contact with notochord in a surrounding medium of mesenchyme and some myoblasts; the notochord alone does not cause appreciable proliferation. From Takaya, i 956a. 
case, growth and fiber production are found in relation with the myoblasts (Fig. 69, a, b, c). This effect is exerted either by unsegmented or by segmented musculature (the last type being formed if notochord is present). Even a small number of myoblasts has a definite influence on the proliferation of the neuroblasts and the thickness of the wall (Fig. 69, g, h). Combination between the influence of the mesenchyme and of the myoblasts explains the resulting form of an atypical spinal cord and the graded distribution of the neuroblasts. The same simple technique produces cases where notochord is located quite near the neural tube, while the myoblasts are either scarce and dispersed or maintained at some distance. These instances teach us that the notochord exerts practically no effect on the growth of the neural wall (Fig. 69, i, k). The relative inertness of the notochordal material was already noted by Holtfreter; this factor, combined with the positive action of the somites, plays an obvious part in the typical structuration of the normal spinal cord (Fig. 69, d, e, f).

Takaya's recent contributions reveal a new step in the transformation of the inducing system: the two derivatives of the somite, the myoblasts and the mesenchyme, have a quite different action. The dispersion and transformation into stellar cells goes hand in hand with the loss of the growth-promoting induction and with some effect of the permeability of the neuroepithelium. These ideas allow us to reconsider the formation of the brain, and especially of its prechordal part. If mesenchyme and myoblasts play such a conspicuous role in the modellins of the cord, are we not permitted to infer that similar conditions are active inside the head? That the mesenchyme has the combined property of limiting the proliferation of the neuroblasts and favorizing the expansion of the ependymal cavity is especially attractive. The thinning of the dorsal brain wall and the existence of the two membranae tectoriae suggest the participation of especially active mesenchyme. Its mode of action might well be influenced by the neural crest cells which are known to contribute to the leptomeninge; the association of these meningeal components might be responsible for the formation of the choroid plexus and the accumulation of fluid in the future ventricles ${ }^{1}$. On the other hand, the basis of the brain is characterized by zones of intense neuroepithelial growth, in the wall of the rhombencephalon, the optic vesicles, and the telencephalic lobes, for which the inducing role of the parachordal and prechordal mesoblast has been recognized. Takaya's work must remind us that these inducing anlagen are mesenchymous only in their appearance, for many of their cells possess myogenic potency (tongue and eye musculature). We can understand the general picture of brain morphogenesis by admitting that these growth-promoting elements assume a definite pattern under the anterior half of the neural plate, while later, the predominance of the complex cephalic mesenchyme around several dorsal parts of the primary brain vesicle influences their fate. In the basal region of the vesicle, the parachordal mesenchyme seems to acquire two areas of special inducing activity, responsible for the auditory placodes. Similarly, the prechordal mesenchyme can be imagined to concentrate into two areas which induce the optic vesicles and two responsible for the telencephalon. The intermediate spaces would be filled by an inert, growth-inhibiting

1 At early stages, no mesenchyme is, however, present against the thin roof of the rombencephalon. It seems that the epiblast must play the role of inhibiting cell division and making the epithelium impermeable to the ependymal fluid. 
mesenchyme, which would explain the constriction zones between the five basic brain parts. This hypothesis does not need further development. Let us only add that indeed a brain is not as specifically distinct from a spinal cord as we have the tendency to assume; the prosencephalon is only remarkable in the size it can acquire and in the refinement of its differentiations. The characteristics of brain morphogenesis can be reduced to: an initially larger amount of neuro-epithelium; an exceptional capacity of growth in certain territories; a gradual thinning of definite areas of the wall, and a correlated expansion of the ventricles. All these features could be explained by an interaction between myogenic and nonmyogenic mesenchyme, with a possible collaboration of the ectomesenchyme.

In view of this hypothesis, we feel entitled to raise the question: is any part of the neurectoblast endowed with a capacity of self-organization (Holtfreter, I95 I, p. I 40; Nieuwkoop et al., I 952, p. 99; I 955, p. 272) or is the evolving of structures from a neural anlage always dependent on a combination of inducing factors? Could the hypothesis outlined above be applied to all cases in which an organized prosencephalon appears? A continuation of this analysis will show that certainly the true mesenchyme cannot be considered as the agent always present in such cases. Thus, if we feel reluctant to admit self-organization, some other approach has to be found to solve the riddle (see p. 444).

Before leaving the consideration of purely microsurgical experiments, we should mention the remarkable results first obtained by Niu and Twitty (1953) and further studied by Niu (1956) under conditions which, although concerning in vitro cultures, seem the most direct approach for the analysis of the agents responsible for normal induction. In this new technique, the reactor is still the young ectoblast, but it is divided into pieces of about 20 or 30 cells; the inductor is not strictly an adjacent piece of tissue, but a modified Holtfreter solution especially "conditioned" by having contained, for several days, an explant of some actively inducing material. The advantage of using tiny explants of ectoblast is that they possess only a limited amount of coat, just enough to cover their previously outer surface; when they round up, more than half of the sphere surface remains naked, thus permitting any agent in the medium to act directly upon the exposed cells. It is obvious that in case of a positive result obtained with a fluid inductor, the way would be opened for a biochemical analysis. Naturally, with such small reacting pieces, no proper morphochoresis can be achieved, but only cell differentiation. With proper controls available, if the ectoblast differentiates into neuroblasts or pigmentocytes or myoblasts, an inducing effect of the medium can be demonstrated.

This in fact did happen. An explant of the dorso-marginal zone of a newt early gastrula was cultivated for 7-Io days in a drop of the medium, thereby conditioning it; the tiny reactor was then introduced at a fair distance from the inductor, to be reared there some 10-20 days more and a positive induction effect was observed. The posterior (myogenic) part of the neural plate (Fig. 7o) was also used as an active explant for such trials. The presence of the inductor in the culture is not necessary. The fluid in which it has been cultivated may act in the same way, and homoeogenic induction in vitro may be demonstrated. Substances having diffused from the posterior neural plate cause the differentiation of myoblasts (up to $73 \%$ of the cells), or of neuroblasts and pigment cells if the duration of previous cultivation has been longer. In all these results, comparison with the controls 
shows that cell division occurs practically at the same rate in both cases. The difference between a fresh saline medium and a conditioned one does not depend upon the presence of growth-promoting, but of differentiating agents.

Further analysis has shown that $\mathrm{pH}$ change or other precytolytic factors could be excluded, since the reaction differed among species, being the most active in Ambystoma, less in Triturus, $T$. rivularis being preferable to torosus. In the case of $T$. rivularis, 24 -h. time in the conditioned medium is sufficient to obtain induction. The relative efficiency of the inductors has been tentatively expressed as follows:

dorsal lip $\geqq$ posterior region of medullary plate $>$ notochord $\geqq$ medullary plate and fold $>$ neural fold $>$ somites $>$ endoblast. The nature of the change introduced in the saline medium by the cultivation of the explant will naturally be considered when we examine the intimate nature of induction (p. 476).
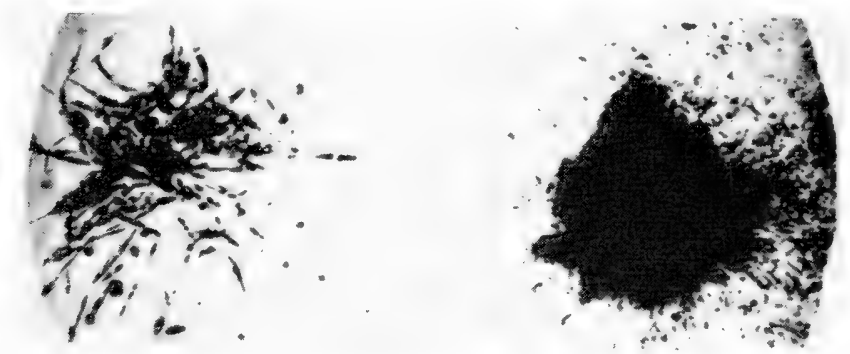

Fig. 70. Effect of conditioned medium. See text. From Niu, I956.

Some operations of explantation and grafting have been performed on bird blastoderms. In this material, the typical culture in vitro of isolated fragments is still less favorable than in amphibians. Preference had to be given to the chorioallantoic grafts which have been extensively studied (Hunt, Rawles, Rudnick, literature in Rudnick, I955). The same general process of paragenesis is observed, with a maximum of complexity for pieces originating from the mediodorsal area, if they are of a sufficient size.

The nutritional requirements of bird blastoderms have been established thanks to the extensive work of Spratt (I952). Working on the duck egg, Lutz and Ostertag (1955) have observed that non-incubated blastoderms are unfavorable for explantation but that when placed in contact with a piece of endoblast (taken from primitive streak stages) lccal morphochoresis is induced in them with all its consequences. This is a confirmation of the older data of Waddington concerning the inductive activity of the endophyll.

The difficulty of cultivation has now been overcome by the very practical method of New (1955) in which the blastoderm is simply deposed on a stretched piece of the vitelline membrane and covered with albumen. Using this technique, Mulherkar (1958) has methodically explored the inductive capacity of minute regions surrounding the anterior node at the stage where the definite primitive streak becomes perceptible. The results, represented in Fig. 7I, show a maximum of induction capacity in the node itself, and a decrease not only posteriorly and anteriorly, but also laterally. With regard to the laterality, there is a remarkable asymmetry in favor of the left side. 


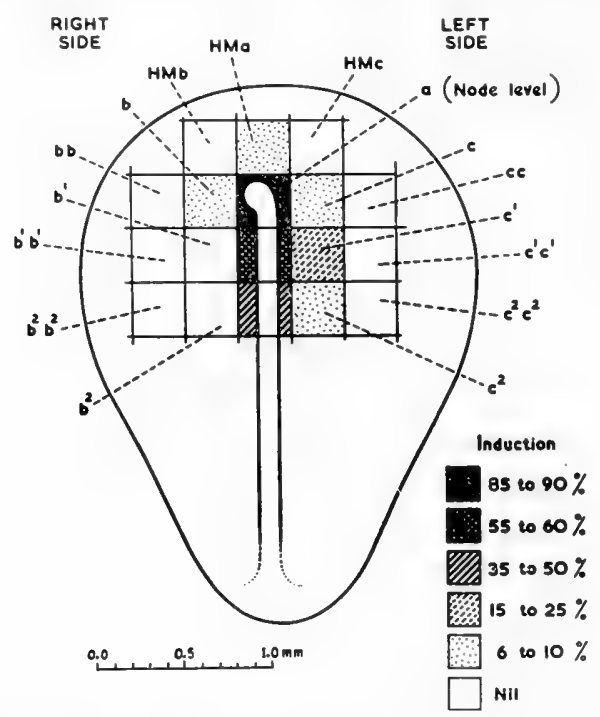

Fig. 71. A map of induction frequency of the perinodal area at the primitive streak stage of the chick. The various pieces have been isolated and inserted between two layers of a host blastoderm cultivated in vitro. From Mulherkar, $195^{8}$.

At the end of this rather extensive, but not exhaustive survey of comparable experiments, a few points may be stressed. Before gastrulation, the most active region of the Urodele egg is only able, as such, to notogenesis. The Anuran egg has probably passed through a similar stage but has already developed a prechordal anlage with specific potency. This step is made by the Urodele egg while it begins to gastrulate, and could well be correlated with the kinematics of invagination. Gradually, the anterior part of the archenteric roof acquires the morphological and dynamic features of the prechordal plate. At this stage the main system of inductive processes may be considered double: headward, the prechordal endoblast and mesoblast; behind, the chordomesoblast. Several results show the formation of acrencephalic organs in conditions which suggest the neoformation of prechordal material from adjacent chordomesoblast. Other experiments cause the same brain parts and sense organs to appear by an apparently different mechanism, called "activation".

During the final steps of morphochoresis, when mesoblastic mesenchyme is produced, its cells acquire a new type of influence on the spinal cord and probably on the whole neural axis, i.e., their presence limits the proliferation of the neuroblasts located in the germinative layer and controls, perhaps indirectly, the expansion of the ependymal lumen. For those who know the advances which have been made in the study of induction, the striking innovation is the idea that the inductive system is not a preformed complex capable of simply unfolding itself but a device which becomes more complicated along with the course of morphochoresis. This chronological transformation is the discovery of $\mathrm{Y} . \mathrm{K}$. Okada and his students. 


\section{Experimental modifications of morphochoresis}

Many of the possible ways to modify morphochoresis have been used on the whole egg, on isolated parts of the marginal zone, or on the ectoblast before it normally reacts. It is not necessary here to consider separately general treatments on the germinal system, transplantations, and explantations; however, the distinction between physical and chemical procedures will be helpful.

\section{(a) Physical means}

The three accessible properties of physical systems-distribution in space, modification by time, and energetic changes by temperature or radiations-have been explored.

The influence of age on the reacting ability of the ectoblast has been recognized by Holtfreter (1938a); however, he thought, wrongly, that the ability to form a brain disappeared first, and was practically suppressed when the explants have been cultivated during the period of time necessary for the controls to attain the blastopore stage. Gallera (1952) using newt gastrulae and a technique (Fig. 72)

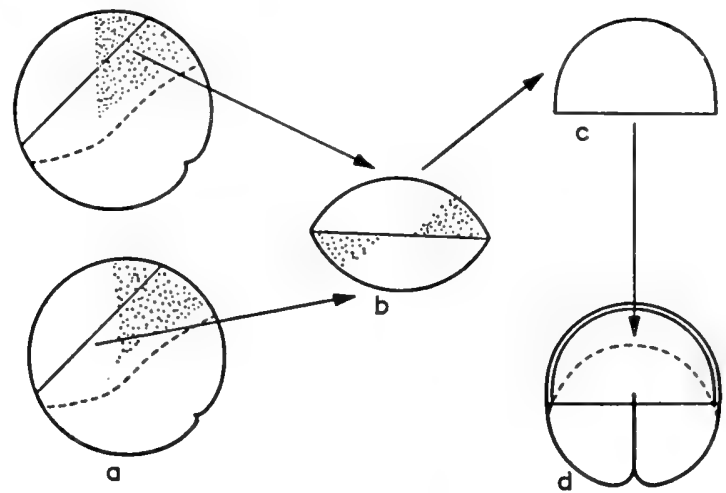

Fig. 72. Technique for testing the maintenance of competence of isolated ectoblast. (a) Two early gastrulae have received a large mark of Nile blue. (b) Their ectoblastic areas are then dissected and combined with their inner sides together. (c) After due cultivation, a piece of this vesicle is used (d) to replace the anterior part of the neural field in a young neurula. From Gallera, 1952.

avoiding the unfavorable action of the saline medium on the inner side of the explant, has been able to maintain brain competence until a stage corresponding to incipient neurulation in the control. Just a few hours later, when a pigmented hem marks the limit of the plate, the competence for acrencephalon inductions briskly disappears, while balancers and stomodaeum are still produced. During the period of declining competence, the ability to form optic material, often only one vesicular expansion, is more resistant than telencephalon production. This result again shows how important is the technique used to determine the stage at which a change of potency occurs in a given layer or territory. Let us remember that with the fold technique the ability to form neural structures vanishes distinctly earlier (p. 390). 
The time factor was also considered by Johnen (in Nieuwkoop et al., I952) when she repeated the insertion of anterior notochordal material in a sandwich and extirpated the implant after various delays. A prolonged contact produces "rather big inductions with central rhombencephalic and peripheral prosencephalic structures". With short exposures, it is possible to obtain an exclusively acrencephalic induction. The necessary time of contact is much shorter in the axolotl (even $5 \mathrm{~min}$.) than in the newt (at least $4 \mathrm{~h}$.). Longer exposures cause deutencephalic structures to appear. In both cases, neural crest derivations are often present and sometimes are the sole sign of induction (Johnen, 1956). A variant of Damas' experiment (p. 371) has been recently executed in his laboratory on Pleurodeles embryos (Denis, 1957). Pieces of (non-induced) gastrular neurectoblast were implanted in the flat neural plate just behind the cerebral region. After 3 h., 6 h., ... 24 h., they were again isolated and either reared in sandwiches, or implanted in the abdomen of advanced gastrulae. In both cases, the sole signs of induction have been either neural crest derivates or a piece of neural tissue of the spinal cord type (never cerebral) or often both. The differentiation is much better in the grafts than in the explants. With short exposures, a gradation clearly related to time does not appear in these conditions. After $3 \mathrm{~h}$. of induction, in some cases neuralization can be as manifest as when the sojourn in the neural tube has been longer.

Ultraviolet irradiation of the dorso-marginal zone, with a variety of localizations, has been actively used from I933 to I94I (Brandes, I 940, I94I) with appreciable results. Brandes, particularly, has made an extensive study on several species and at various stages. An effect on morphochoresis is evident, but it varies according to the irradiated territory and to the duration of the treatment. The most

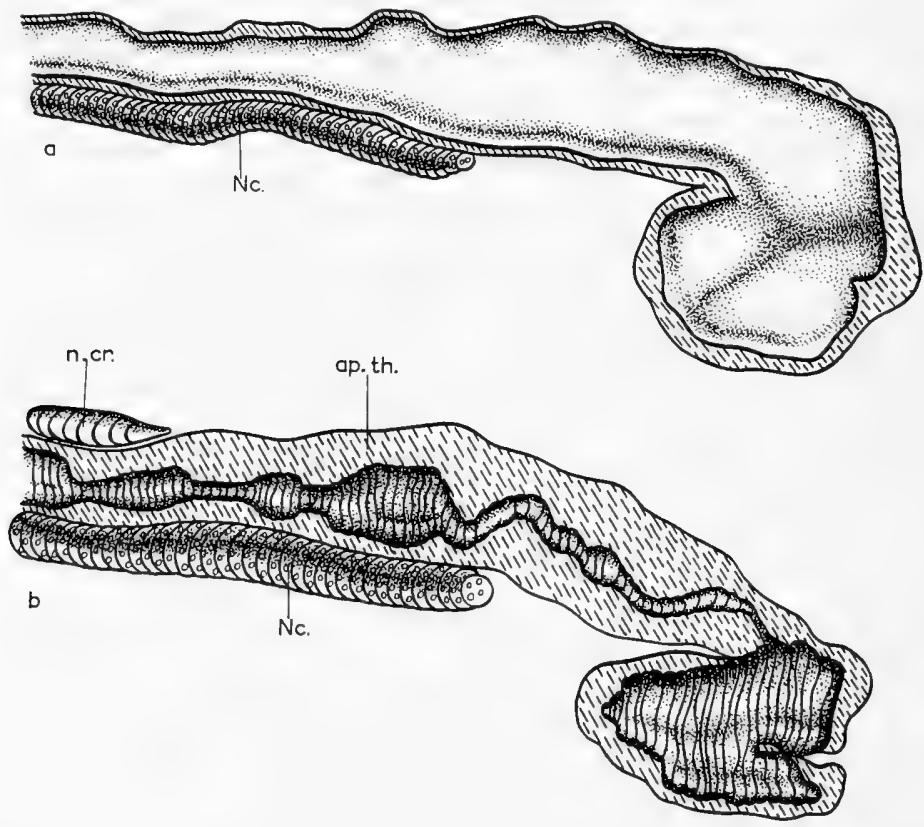

Fig. 73. Effect of U. V. irradiation of the organizer on the conformation and differentiation of the neural organ. (a) Reconstruction of a normal axolotl embryo, the neural organ seen in median sagittal section; (b) the neural organ of the irradiated specimen, with the irregular lumen and dorsal thickening. From Brandes, I94I. 
interesting feature is one which has so far remained completely enigmatical: the formation of supplementary neural material on the dorsal side of the spinal cord or even of the brain. Inspired by Takaya's results (p. 405), I have re-examined the most striking example of this anomaly (Fig. 73). The zone of contact between the dorsal part of the hind-brain and the epiblast is definitely atypical. More than the normal amount of mesenchyme is present ( $c f \cdot$ p. 407 , note I), and it vaguely surrounds large oblong ampullae, which were certainly full of fluid in vivo; they are interposed between the neural tissue and the covering epiblast. The cause of the inward proliferation of the neuroepithelium could well lie there.

Centrifugation $^{1}$ is a classical procedure for changing the relations of the cellcomponents without interrupting the course of the vital activities. In amphibian eggs, it has been used on a large scale (literature in Schleip, I928) and several investigations of the prewar period analysed the interesting effects exerted on morphochoresis in eggs centrifuged during the earliest stages (literature in Pasteels, 1940). It must be remembered here that this technique, especially when applied to undivided frog eggs, hampers, according to the action intensity, the appearance (or at least the utilization) of the acrencephalic factors of induction, and thereby provokes hypomorphosis of the notomerit strikingly parallel to the effects of lithium (see p. 423).
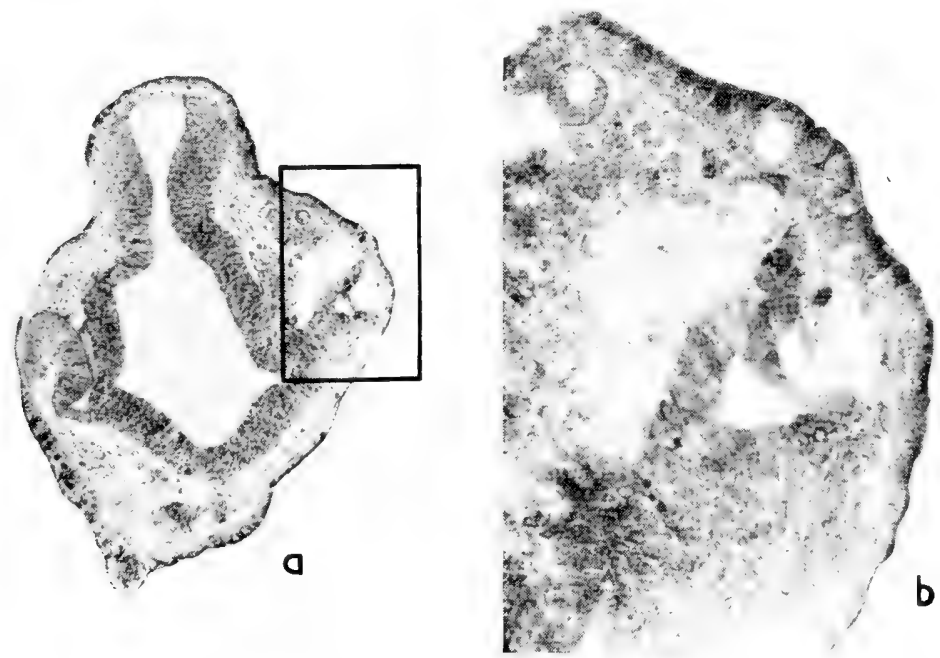

Fig. 74. An effect of centrifugation of frog gastrulae on the differentiation of the primary acromerit. The right optic vesicle is largely transformed into notochordal cells. One small otocyst has even been formed. This is a real interpenetration between acro- and deutomerit.

From Pasteels, I953.

These results of Pasteels speak in favor of a primary unity in the inducing factors. By exploring the successive stages, a remarkable manifestation was discovered: the formation of a secondary axial system originating from the collapsed part of the blastocoele roof.

1 The data briefly summarized in this section could be advantageously compared with the results obtained in invertebrate eggs, especially in Limnaea (Raven and Beenakkers, I 955; see also Raven, I958). 
This is, however, only one among the many interesting effects of this simple treatment. They are obtained (Pasteels, 1953, 1954) with a rather moderate centrifugation (460 g for I to Io min.). The typical reaction of an autonomous secondary embryo which develops on the ventral side of the "host" is best encountered in Rana temporaria after centrifugation of the blastula (age of ig h. at $20^{\circ} \mathrm{C}$ ). The effect practically fails with "middle" blastulae ( $\mathrm{I} 5 \mathrm{~h}$.) and disappears as soon as the blastopore becomes crescent shaped. The phenomenon is indeed complex. It has been studied in 3 Anuran and 4 Urodele species; it is present in all of them except the newt, although there are considerable differences in susceptibility. It also varies according to the stage treated and the duration of application. Even the maximal frequency of the various organs produced, chorda, somites, pronephros, neural axis is not attained simultaneously, but varies with slightly different treatments. There are also conditions in which the modification of the ectoblast expresses itself only by inconspicuous anomalies. In certain species, like the axolotl, the anomaly concerns only the structure of the brain which, moreover, may be complicated by supplementary parts, of either acrencephalic or chordencephalic nature. Chordalizations of the neuroepithelium often take place, even inside acrencephalic organs, e.g. the optic vesicle (Fig. 74).

When a definite secondary system is evoked, it often shows connections with the primary one; in many cases, action at a distance has been observed, which strongly suggests a diffusion of active "substances" impregnating or imbueing the intermediate region. The transformation of the prechordal anlage into notochord could even be demonstrated in two cases in which, correlatively, the brain has lost any acrencephalic character. These observations prove that features normally characteristic of the notomerit may easily be evoked from the prechordal region, which means either that their factors are potentially present, or that a reconversion of the acrogenetic factors is easily obtainable.

There are three important aspects of this experiment. First, when an autonomous secondary system is obtained, it is cephalized in the same direction as the normal system (causal relation, as demonstrated by transplantations). Secondly, the additional brain never shows any of the acrencephalic structures; the secondary system is strictly notomeritic. Frequently, the predominance of this tendency makes that many cells of the spinal cord become vacuolized, as if of notochordal nature (Fig. 75). Clearly the conditions causing neuralization and chordalization are intimately related.

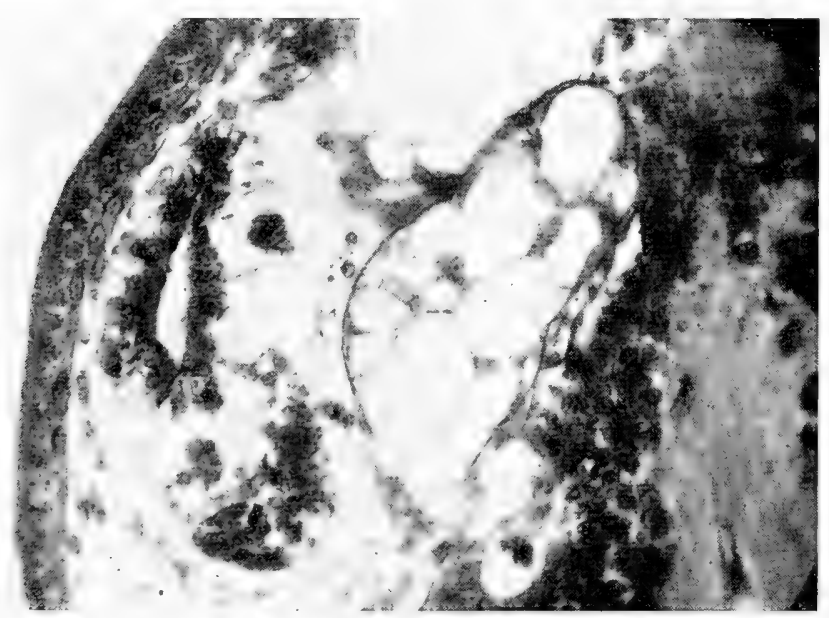

Fig. 75. Abnormal structures caused by centrifugation of a frog blastula. The secondary system here is to the right of the rhombencephalon. It consists of an enlarged notochord and a brain part in which numerous cells are "chordalized". From Pasteels, 1953. 
Thirdly, the cause of the aberrant morphochoresis lies in the collapse of the blastocoele roof (Fig. 75-77). At the susceptible stages, the rumpling and folding of this region recedes only slowly, and causes a distinct increase of true basophily (Fig. 78, a, b, c, d). During gastrulation, a pancake-shaped mass of tissue is found there (Fig. 78 , b) which finally splits into a mesoblastic basophilic thickening and a more moderate thickening of the epiblast (Fig. 78, c). Neurogenic induction soon begins but is somewhat retarded relative to the main system (Fig. 78, d).

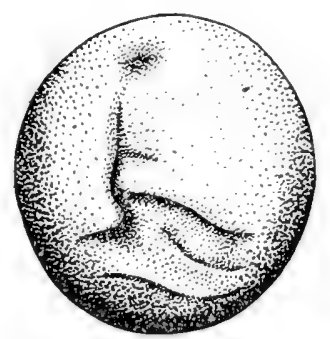

Fig. 76. Immediate effect of centrifugation on the frog blastula. The roof of the blastocoele is deeply folded. From Pasteels, I953.

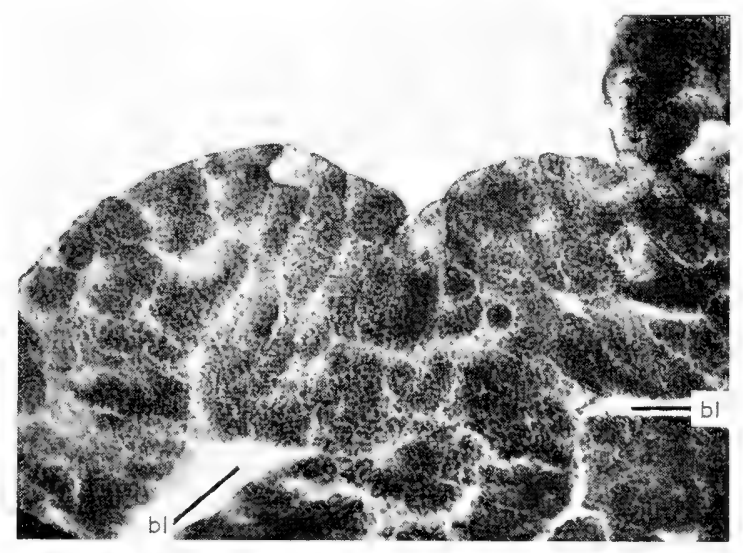

Fig. 77. Section of the collapsed roof of the blastocoele seen in Fig. 76 . Only parts of the cavity remain free (bl.). From Pasteels, I953.

It is impossible to escape the idea that local enrichment in ribose nucleic acid (free or combined with a protein), as a consequence of a mild lesion in the collapsed ectoblast, is responsible for secondary morphochoresis. If the treatment is applied at non-susceptible stages, the collapsing is transitory, the tissue unfolds, and the effect is deleted, which shows the role of the mechanical consequences of the centrifugation and the way they affect the ectoblast. This is important for it has been claimed that Karasaki and Yamada (I955) repeated and confirmed Pasteels' experiment, but did not obtain any result when using a piece of ectoblast isolated before centrifugation. No reaction whatever was obtained and therefore some authors were tempted to dispose of Pasteels' results by assuming that they were due to induction by the yolk mass and the inner marginal zone with which the ectoblast comes into contact (Toivonen, I954b). 

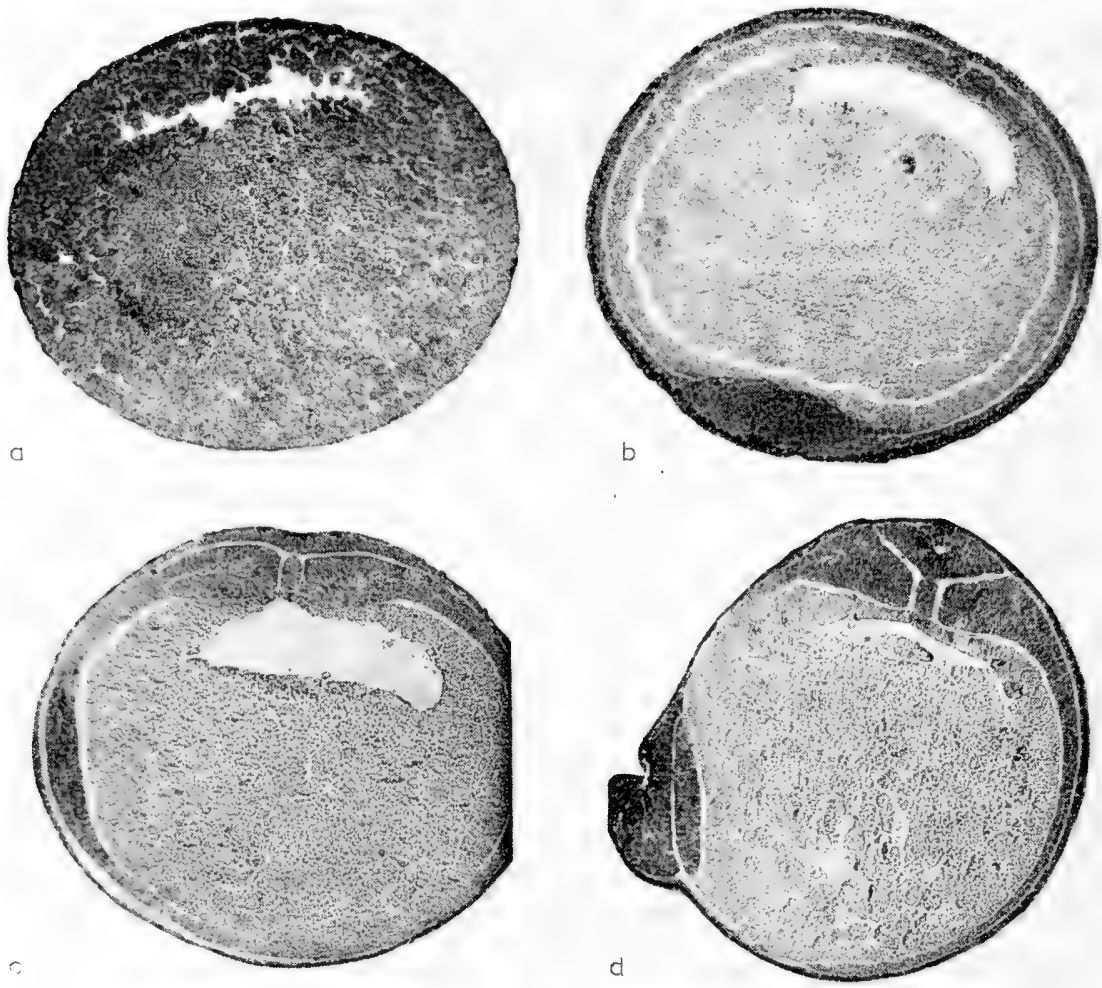

Fig. 78. Early consequences of centrifugating a Xenopus blastula. The embryos have been fixed in Zenker and stained with toluidine blue. The dark parts are rich in RNA. (a) An axial section of the blastula; (b, c, d) successive neurula stages. In each case, the primary system with its archenteron is above. See text. From Pasteels, I 953.

Pasteels has met this objection with several arguments. The localization of secondary formations does not always fit the induction hypothesis; the period of effectiveness is much more limited than the induction processes; the secondary embryos are not of the type obtained by induction, since they regularly lack an acrencephalon, they show irregularities or desequilibria between the components, and they form mixtures of tissues more similar to the results of xeno-inductions. The explanation of Yamada's failure with centrifuging isolated pieces of ectoblast is probably that the tissue is not submitted to the same physical conditions as when it falls onto the resilient floor or the blastocoele. Consequently, it is legitimate to stress that this thorough investigations has provided us with valuable informations. On the one hand, it teaches us that the inherent autonomous potency of the ectoblastic territory is to perform notogenesis, but not acrogenesis, which needs some complementary condition (p. 472). On the other hand, the initial steps of the process show that a thick sheet of ectoblast may produce mesoblastic structures by delamination. This is a kind of pseudogastrulation, a possibility which must not be ignored.

For the problem of induction, as for other biological analyses, abnormal temperatures have been used, at extra- and intra-liminal levels.

Extra-liminal cold or warmth have proved not only to stop life processes, but often also to modify inducing capacity. It was early recognized that killed tissues, no 
matter by what means, became inductors even if they did not possess that property while living. This discovery suscitated a systematic enquiry of the change produced by cooling or warming definite territories, especially those of the dorsomarginal zone. Studies of this type have been done mostly on the neurula rather than the gastrula.

Holtfreter's fundamental work (I934b, c) showed that heating to $60^{\circ}$ or $100^{\circ} \mathrm{C}$, in or out water, did not make any difference, nor did the exact origin of the embryonic fragments which he inserted in the blastocoele. In 1943, Barth and Graff used freeze-dried anterior and posterior parts of the neurula axis, which they inserted into explants of ectoblast. They found that while no mesoblast induction took place, neural tubes or masses appeared; in the delay of culture used by them, they did not acquire a definite structure.

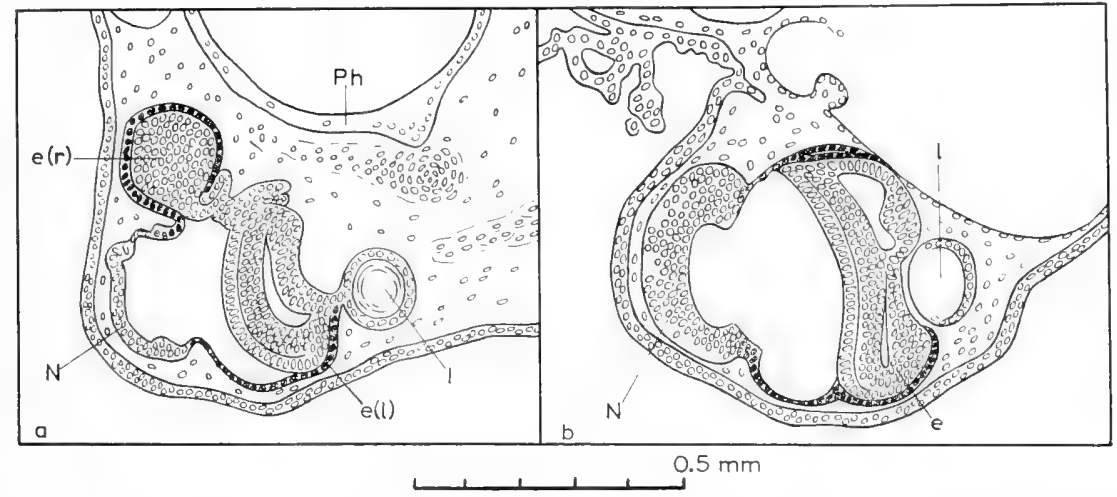

Fig. 79. Induction produced on the ventral side of the host Triturus pyrrh. by a boiled piece of organizer. (a) A reduced diencephalon with two optic vesicles. (b) A larger diencephalon with cyclopic eye. e.-optic vesicle; e(l).-pigment layer of optic vesicle; $\mathrm{e}(\mathrm{r})$.-retinal part of optic vesicle; 1.-lens; N.-cerebral tissue; Ph.- pharynx. From Okada and Hama, I944.

Okada and Hama (1944) fixed their attention on the uninvaginated part of the blastopore lip, at the stage of the crescent shaped blastopore, and compared what it yielded and induced $(a)$ when affixed in the blastocoele, $(b)$ when inserted in the same cavity after previous boiling for io to $15 \mathrm{sec}$. (Fig. 79), (c) when explanted, in the living state, in ectoblast. Their results are summarized in Table 4 .

Although the numbers are rather small, it is plain that the heat treatment has profoundly modified the inducing capacity of the investigated material. Its ability to induce notogenetic structures has completely disappeared; its ability for acrencephalic induction, only exceptional in vivo, has become generalized. Explantation of the living material confirms that its inducing ability takes in vitro a more acrencephalic character, but the change is only of an intermediate nature. With the boiled inductor, the reaction often tends to produce a minimum of brain tissue with an exaggerated optic vesicle (Fig. 79); the absence of true telencephalon is suggested by the absence of olfactory placode ( 9 of the I I cases). This predominance of the optic vesicles recalls Gallera's observations (p. 37 I). It also recalls an older result of Lopashov (I935) who obtained such an organo-specific effect by using boilecl oftic vesicles as an inductor. Unfortunately. the Japanese authors do not relate the localization of the reaction with the dead implant. Whatever may be this relation, the diference in results after boiling can be exploim in several ways, ranging from differences of thermostability to the transformation of the motngenetic inductor into an acrenceshalic one. 
TABLE 4

INDUCTION BY THE UNINVAGINATED PART OF THE BLASTOPORE LIP

(Compiled from Okada and Hama, I944, Table I, p. 37)

\begin{tabular}{|c|c|c|c|c|c|c|c|c|c|c|c|}
\hline $\begin{array}{l}\text { Cate- } \\
\text { gory } \\
\text { (see } \\
\text { text })\end{array}$ & $\begin{array}{c}\text { Number } \\
\text { of } \\
\text { cases }\end{array}$ & Brain & $\begin{array}{l}\text { Eye } \\
\text { ves. }\end{array}$ & $\begin{array}{c}\text { Olf. } \\
\text { placode }\end{array}$ & $\begin{array}{l}\text { Bal- } \\
\text { anc- } \\
e r\end{array}$ & $\begin{array}{l}\text { Oto- } \\
\text { cyst }\end{array}$ & $\begin{array}{l}\text { Spinal } \\
\text { cord } \\
\text { Noto- } \\
\text { chord } \\
\text { Somites }\end{array}$ & $\begin{array}{c}\text { Pro- } \\
\text { nephros }\end{array}$ & $\begin{array}{l}\text { Endo- } \\
\text { blast }\end{array}$ & Tail & Observations \\
\hline a & 22 & I 2 & 3 & 2 & 3 & 10 & 20 to 22 & 4 & 20 & I 6 & $\begin{array}{l}3 \text { prosen- } \\
\text { cephalic } \\
\text { inductions, } \\
\text { explained } \\
\text { by regional } \\
\text { effect (near } \\
\text { host brain) }\end{array}$ \\
\hline b & I I & 10 & I I & 2 & o & o & 0 & 0 & o & o & \\
\hline c & I 6 & 13 & 8 & 7 & 3 & I I & I 2 to 16 & o & I 6 & 8 & $\begin{array}{l}4 \text { cases with } \\
\text { a free lens }\end{array}$ \\
\hline
\end{tabular}

A similar change has been recorded by Rollhäuser-Ter Horst (1953). She has repeated Mangold's experiment (p. 368) after a $90^{\circ} \mathrm{C}$ heating of the fragments. All of them became acrogenic. Her figures are interesting because of the evident absence of any mesenchyme and the formation of the thickest part of the brain at the closest contact of the implant. When this part was sufficiently thickened (Fig. 8o) it could be diagnosed as a rudimentary retina.

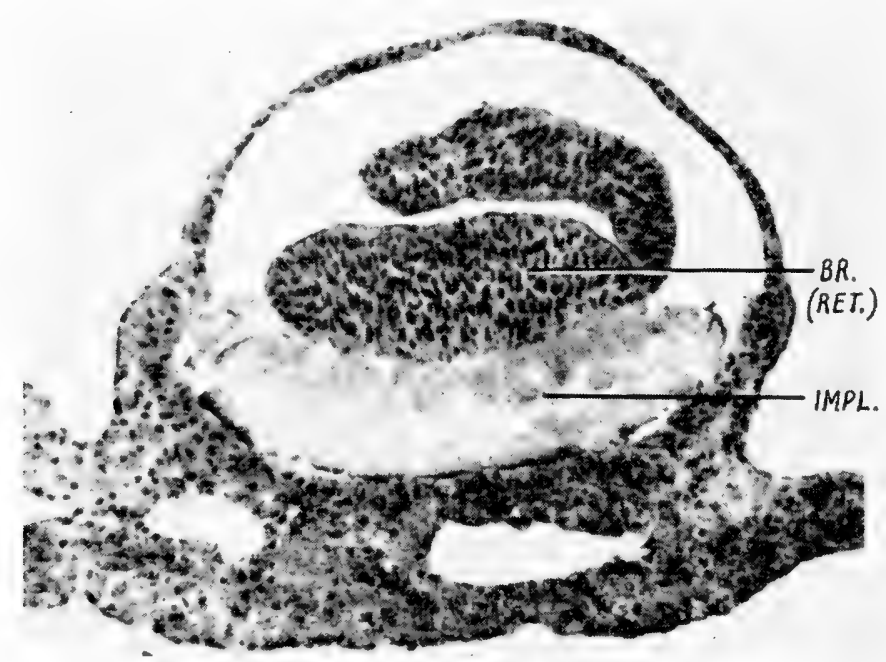

Fig. 80. Section of a sandwich in which acrogenic induction has been caused by a heated part of the archenteron roof. See text. Br. (ret).- retine-like part of the brain; impl.- the dead implant. From Rollhäuser, 1953. 
Waddington (I952a) has attempted to find out how far reactions evoked by a dead inductor are related to its origin, or different, or simply chaotic. He used newt stages from neurula to tail bud stage. The embryos were immersed not more than $5 \mathrm{sec}$. in $90^{\circ} \mathrm{C}$ water; then, the anterior and posterior parts of the axis were used in sandwiches, the ectoblast being taken from gastrulae with sickle-shaped blastopores. There was some regional effect in the sense that eyes were induced only by the anterior pieces, in $30 \%$ of the cases. However, close attention was devoted to earlier stages, where the situation appeared less clear.

"In general, the earlier the stage, the more chaotic the induced tissues are likely to be We frequently find the whole interior of the explant full of a complex of neural vesicles and tubes, with larger plate-like areas lying against the more extended surfaces of the dead implant. At very early stages one can sometimes find a symmetrically folded neural groove, but in the immediately succeeding stages it is rare to find any formation which bears much resemblance to the normal neural system of similar age" (Waddington, 1952, p. 492). This is an important statement. It leads the author to assume that "if some region of the mass happens to have a shape roughly like that of some part of the neural system, forces arise within it which cause it to approach that part in its development. This would amount to the spontaneous self-individuation of particular organs, arising on the basis of chance approximations to some normal arrangement". Waddington also stresses the fact that in these forebrain-like inductions no "mesoderm" is formed, while it is well-represented in cases where a trunk neural tube appears. He insists on the "mesodermal-to-neural ratio" and we have seen already how well-founded this idea is. However, I doubt that we may feel satisfied with an explanation based only on resemblance attained by chance. It has already been emphasized that the difference between two types of primary induction is of a more fundamental nature; this will become still more evident in the discussion of chemically-modified inductors and of xeno-inductors.

As far as I know, only one author has examined the effect of intraliminal temperatures $^{1}$. Takaya (1955b) did this on fragments cut above the blastoporal lip at five successive stages, and subjected them to temperatures of $30^{\circ}$ to $38^{\circ} \mathrm{C}$ for one hour. The ability to cause notogenesis was not suppressed. There was however some shifting of the induction toward the acrogenetic type.

It is also necessary to recall that, according to Penners ( 1936 ), warming the ventro-marginal zone of an egg while cooling the dorsal part may cause the formation of an embryo on the ventral side. This isolated experiment would be worth confirmation and a more detailed analysis.

\section{(b) Biochemical modification of inductors and reactors}

A considerable number of definite chemical variations and different alterations of the standard medium have been tried on several species. We shall consider here only the instances which bear on the primary induction problem; all of them will concern amphibians.

The ionic equilibrium of such a medium as Holtfreter's solution has been recognized to be of importance, especially in certain urodeles groups, Ambystoma and Triturus. Barth (I94I) first observed the aberrant neuralization of isolated Ambystoma ectoblast ${ }^{2}$. Holtfreter (1944a; I945, 1947a) submitted this puzzling

1 In the molluscan egg Limnea, an elaborate study of the morphogenetic effects of heat shock has been performed (Raven, de Roon and Stadhouders, r 955; see also Raven, I 958). ${ }^{2}$ A similar process may be observed by culturing ectoblast isolated from Rana pipiens gastrulae; the differentiation of muscular and neural cells is observed (Barth and Barth, 1958). 
phenomenon to experimental analysis and found that he could control this kind of neuralization by temporarily submitting the ectoblast to media of an increased pH; the lowering of $\mathrm{Ca}^{++}$concentration also acts in the same way. In addition, these conditions if not duly controlled tend to dissolve the coat and the intercellular cement and to cause cytolysis. The type of neuralization obtained in the various types of experiments, some on whole embryos and others on explants, showed a wide variation. In explants, however, the thickness of the neural epithelium and its folding often resembled brain, and exceptionally, acrencephalic structures were recognizable. Some of the illustrations show mesenchyme, and the organization looks better in such cases. The author insisted on a close relation between such abnormal conditions and the cytolytic effect exerted on cells. $\mathrm{He}$ showed that mechanical lesions could sometimes exert a similar effect and that abnormal behavior of ectoblast was more frequent in the presence of dead cells and debris. He even went so far as to attribute the inducing effect of various chemicals to their toxicity. The question is whether the autonomous neuralization
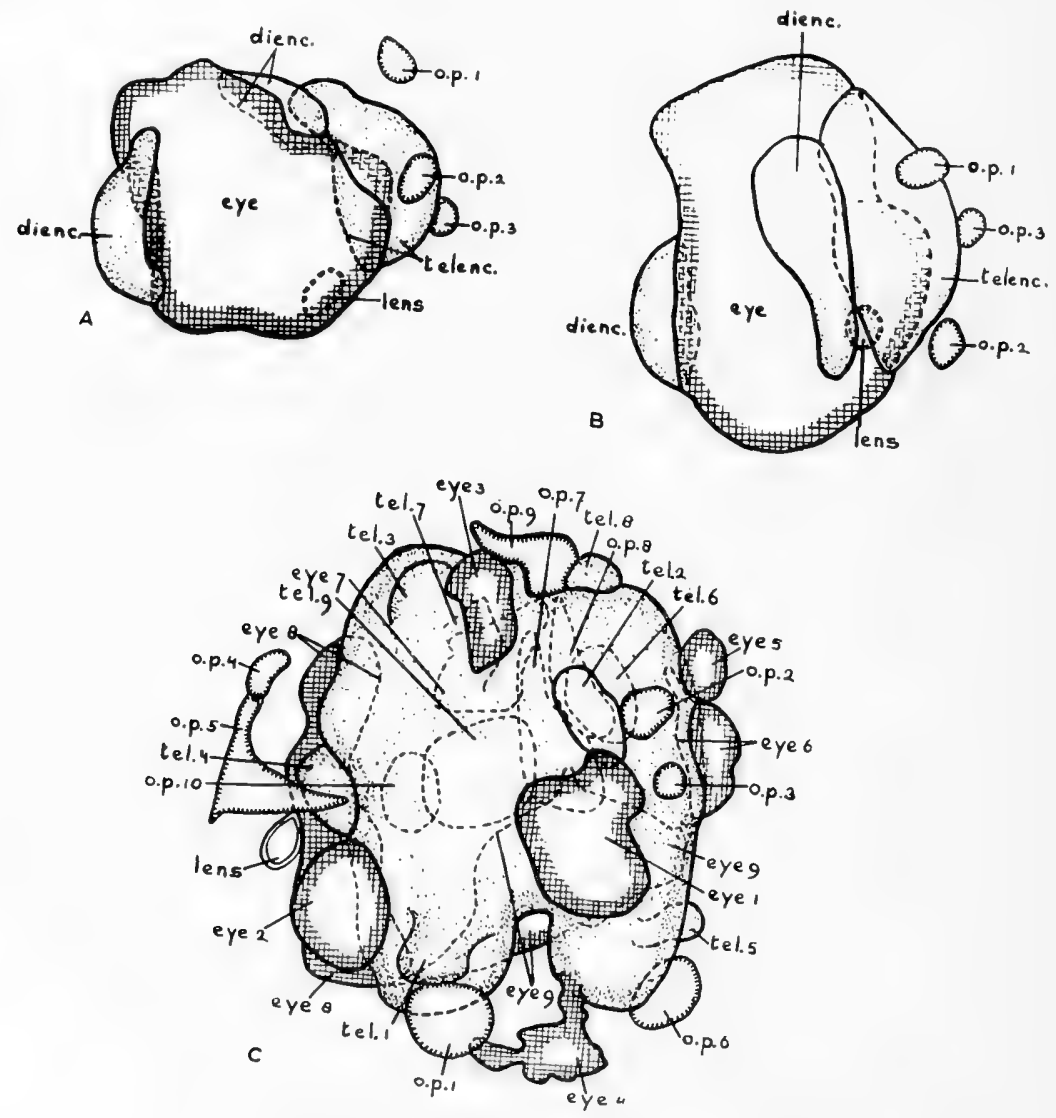

Fig. $8 \mathrm{I}$. Results of the reaggregation of the anterior (mostly prechordal) part of the neural plate of a newt neurula. (a, b) Cases with relatively simple pattern; (c) case displaying a complex pattern; o. p. - olfactive placodes; tel. - telencephalic parts. From Boterenbrood, $\mathbf{1 9 5 8 .}$ 
depends upon only one or several mechanisms. The investigated ionic inequilibria are indeed related to cytolysis, but other factors may be at play.

The reactivity of ectoblast to precytolytic agents already appears 20 hours before gastrulation (Chuang Hsiao-Hui, I 955). This information might have some bearing on explaining the results of experiments where the reactor is considerably younger than the inductor, as those of Nieuwkoop (p. 402) and Johnen (p. 412).

A notorious consequence of this research was a methodical investigation (Holtfreter, I944b, I947b) of the ability of disintegrated germs or germ parts to reorganize $e^{1}$ Even when thoroughly mixed, the cells of the various presumptive territories seem able, as mentioned above, to reassociate according to their nature. This is still true for neurula stages (Townes and Holtfreter, 1955), including their neuro-epithelium. Thus, this layer has acquired the definite surface organization which is necessary for selective cell adhesiveness (Fig. I 2, pag. 32 I), due probably to some correspondence or complementarity between the molecules of the plasmolemma ( $c f$. p. 3 I 7). It seems that during morphochoresis these properties gradually evolve by the acquisition of new devices for reciprocal linkage.

It could however be erroneous to think that reaggregation amounts to a regulation. The pattern of the reformed organs may on the contrary be very abnormal. This is clearly shown by Fig. 8I. The prosencephalic area of the neural plate may reaggregate in some cases in a rather simple group of organs, in which, however, the optic part is considerably exaggerated, reminding us of some of Gallera's results (p. 372, Fig. 42). In other cases, brain parts, eye rudiments and olfactory placodes may be scattered and multiplied in a most puzzling manner (Boterenbrood, I958).

The relationship of dissociation and reaggregation to inductive capacity has been investigated by Deuchar (1953). She used the three successive thirds of archenteric roofs obtained from late gastrulae; in each piece of young ectoblast she wrapped three reaggregated pieces from the same level joined together. The results are presented in Table 5 in a way comparable to the controls already used in Table 2 (p. 400).

TABLE 5

EFFEGT OF DISSOCIATION, FOLLOWED BY REAGGREGATION, ON INDUCTION

\begin{tabular}{|c|c|c|c|c|c|c|c|}
\hline Region & $\begin{array}{l}\text { Total of } \\
\text { experiments }\end{array}$ & $\begin{array}{l}\text { Total } \\
\text { number of } \\
\text { inductions }\end{array}$ & Brain & Eyes & $\begin{array}{c}\text { Neural } \\
\text { tube }\end{array}$ & Neuroid & $\begin{array}{c}\text { With } \\
\text { tail } \\
\text { outgrowth }\end{array}$ \\
\hline 3 ant. thirds & 23 & I $5(65.2 \%)$ & 9 & 4 & 8 & I 2 & 7 \\
\hline 3 middle thirds & 26 & I $8(69.2 \%)$ & IO & 5 & 8 & I 5 & 5 \\
\hline 3 post. thirds & 22 & $\operatorname{Ig}(86.4 \%)$ & 7 & I & 9 & I 2 & 3 \\
\hline
\end{tabular}

Contrary to what appeared in the controls (Table 2, p. 40o) the numerical differences are no more significant. A sort of uniformity has appeared, however, the anterior thirds induce optic structures (which they did not in the controls) while the posterior pieces

1 See also Stearns (1955).

Lilerature p. $4^{83}$ 
have not acquired any apparent acrogenetic ability. The proportion of neural tubes, neuroids, and even tails, has been appreciably increased. Thus, the uniformity goes hand in hand with some alterations of the morphogenetic potential.

The effect of disaggregation on early dorsal lips was also tested, with the resulting impression that the controls - which showed only modest reactions- "did not feature any more complete or successful inductions than the experiments" (her p. 3I). Two reaggregated cell masses from the dorsal lip were also successfully implanted into the ventral half of a young gastrula. One induced only a spinal cord, the other a notomerit.

It may be concluded that disaggregation does not suppress the inducing capacity of the blastoporal lip (as already seen by Holtfreter, I944b) nor of the archenteron roof but interferes to a certain extent with the quality of the process. Except for the anterior third of the roof-where the existence of acrogenetic factor(s) is not surprising-this treatment does not produce the change so characteristic of heat (supra) and other agents (infra).

A close relation exists between the preceding results and the effects which Yamada (I950a) described after "shock-treatment" by ammonia on explants of the ectoblast and of the ventro-marginal zone; his experiments practically amount to a temporary disaggregation of the cells, including appreciable cytolysis. Consequently, the neuralization of ectoblast explants has only a confirmative value. However, it can be emphasized that the differentiation of the ventral marginal zone is distinctly enhanced. Instead of blood islands, blood vessels, mesothelium and pronephric tubules, it yields notochord, muscle and the same kidney tubules.

A further analysis of the "dorsalization" exerted by ammonium on the ectoblast and on ventral marginal zone has lead Karasaki (I957) and Ôgi (I958a, b) to consider other cytolytic and disaggregating agents. The former author has shown that disaggregation does not necessarily bring forth dorsalization nor acrencephalic induction. For example, mild treatment with ethanol disaggregates the cells, but does not exert any morphogenetic effect. To get this action, it seems necessary that the agent should cause solation of the cell endoplasm.

The second author has first used a drug known for its activity on morphochoresis, sodium thyocyanate (p. 349). It causes acrencephalic structures to appear in the ectoblast, a rather banal and perhaps precytolytic effect. In isolated ventral mesoblast, NaCNS suscitates notochord, muscles even otocysts. Such a deutogenic modification may also be obtained by lactic acid, sodium iodide, urea, hydrochloric acid, zinc chloride. Ôgi now explored which kind of induction would exert on normal ectoblast fragments of the ventral marginal zone submitted to these various reagents to the point where disaggregation begins. It turned out that most of them induced deutomerit structures, except $\mathrm{HCl}$ and $\mathrm{ZnCl}_{2}$, who are acrogenic. Thus, both kinds of inducing "potencies" may be suscitated in the same presumptive ventral mesoblast, by using appropriate means.

As other inorganic substances which exert appreciable effects on morphochoresis, we must mention: boric acid, which perturbates the formation of the notochord in toad eggs (Takenchi, I 958); lithium chloride, important enough to deserve the next paragraphs; acidification of the standard (Holtfreter's) solution (Kawakami and Okano, I955); formalin, which combines with amine groups and thereby reduces the activity of proteolytic enzymes (Lallier, 1950); zinc salts which cause expansions of the ectoblast cytoplasms and increase sensibility to the same enzymes (Lallier, I955); sodium azide, a depressive agent which seems to dissociate metabolism of maintenance from that of differentiation and growth (Hall and Moog, I 948). 
The general effect ${ }^{1}$ of $\mathrm{LiCl}$ on morphochoresis has been studied by Lehmann (1937) and especially by Pasteels (1945). The depressive result progressively affects the cephalo-caudal and dorso-ventral gradients, without exhibiting any difference of susceptibility between the acrencephalic part and the rest of the embryo; on the contrary, some of the anomalies obtained override the limit of the two regions.

A further experimental analysis has been done, mostly in Urodeles, by three authors. Hall (1942) cultivated in association parts of gastrulae obtained from normal and Litreated eggs. He concluded that the chordo-mesoblast has a higher susceptibility than the reacting ectoblast. Gallera (1949) repeated the experiment described on p. $37 \mathrm{r}$ using Li-treated donors. In the 8 cases where the action of both grafts (Fig. 42, p. 372) could be compared, a general depression of induced structures was apparent. Especially grafts B, containing the prechordal area, induced a rudimentary fore-brain only once, with a slight optic expansion. The other cases produced only trunkal structures of a rather low level, myotomes, pronephros, and limb buds. This result is in close agreement with the effects obtained on whole embryos (supra) and means a real conversion of the inducing system. Grafts containing chordo-somitic material at the exclusion of the prechordal territory formed only a notochord of reduced size and a slender spinal cord. No explant disclosed any acrogenetic tendency, a noticeable difference from similar, non-treated explants (p. 374).

Lombard (1952) re-studied the Li-effects on Xenopus and several U rodeles. The novelty in his work was that in Ambystoma and Pleurodeles he compared the effect of exchanging the neurectoblastic areas of normal and Li-treated young gastrula. Both combinations, either a normal gastrula provided with treated neurectoblast, or vice versa, behave remarkably better than whole embryos. While these were effected with cyclopy, the operated animals were only slightly micrencephal. Moreover, the degree of micrencephaly was slightly more severe in the first combination, and the author asserts that this difference is statistically significant. It would, however, be difficult to accept his conclusions that "the lithium effect is to a greater extent caused by the action of the lithium ion on the ectoderm, viz. the presumptive neural plate, than by its action on the archenteron roof" (Lombard, $195^{2}$, p. $5 \mathrm{I}$ ). The main disorders have indeed been observed in the chordomesoblast; these phenomena of recovery, although interesting, cannot prevail against this fact ${ }^{2}$.

The morphogenetic effect of urea must be considered, although it does not directly concern primary induction. The addition of this chemical to the medium causes essentially a chordalization of neighboring organs, e.g., the neural axis, sometimes the somites, and more frequently the endoblast. A thorough analysis by Fautrez (I95I) concludes that a process takes place later than primary induction, and probably consists in a diffusion of organ-specific substance(s) elaborated by the diffcrentiating notochord. This effect could be due to the rather ordinary permeabilizing effect of urea. This idea would be consistent with the prolongation of the period of competence which Gallera (I953) has provoked in the aging ectoblast by this means.

1 By using labelled $\mathrm{Li}^{+}$, it has been shown that the cation accumulates especially in the dorsal part of the egg, and concentrates into the pigment granules (Ficq, I954a).

${ }^{2}$ For LiCl, see also Kawakami (1953, 1955) and Ohtsuka (1956). This author has shown that the hypomorphic effect of $\mathrm{Li}^{+}$is lessened by a subsequent treatment with 2, 4-dinitrophenol. This can however not be explained by an inhibition of glycolysis, for the concentration of the inhibitor is too low for that. Its favorable action seems due to the arrest of development, during which the absorbed $\mathrm{Li}^{+}$has time to abandon the egg by diffusion. 
The procedure used by Barth and Graff (1943) is an attempt to separate and concentrate the inducing substance. They gathered parts of the whole archenteric roof, extracted with $5 \% \mathrm{NaCl}$ at $5^{\circ} \mathrm{C}$, filtered the extract and either added Holtfreter's solution or dialized. In both cases they obtained a precipitate which they injected into the blastocoele. Moreover, the material first extracted by $\mathrm{NaCl}$ was re-extracted by $0.05 \mathcal{N} \mathrm{NaOH}$ and the filtered product precipitated by acetic acid. This preparation was also injected into the blastocoele. Its inductive activity proved largely superior to the saline extract. It consisted sometimes of short neural tubes, sometimes in multi-layered, brainlike vesicles. The result is supposed to reveal the importance of the protein component of the archenteric roof.

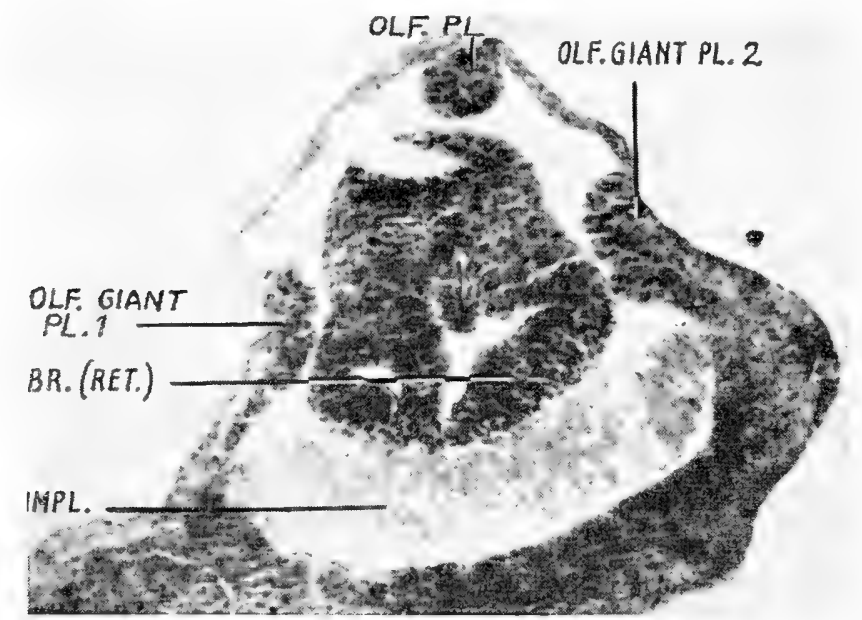

Fig. 82. Same experiment as in Fig. 80, but with an inductor killed with ethanol. The relation of the brain to the inductor, its retina-like structure in the zone of closer contact, and the absence of mesenchyme are still more striking. The acrencephalon is completed by two olfactory placodes, a giant, half-circle one which is cut twice, and a small one, at the tip of the brain. $B r(r e t)$. - the diencephalon partly transformed into a retina; impl. - the dead implant; olf. $p l$. - the apical olfactory placode; olf. giant $P l .(\mathrm{I}, 2)$ the two sections of the same giant placode. From Rollhäuser, 1953.

Ethanol has been used by several authors to stop life processes in the "organizer" (Holtfreter, I934b). The morphogenetic effect of this treatment is similar to that of heat, and especially pertinent (Fis. 82) in Rollhäuser's work (I053). It has become customary to state that "denaturation" of the proteins contzined in the

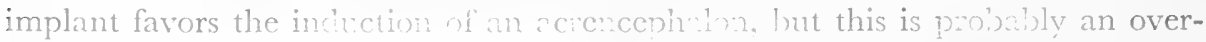

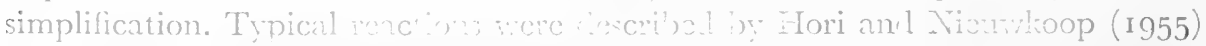

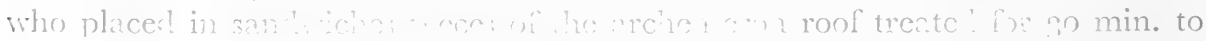

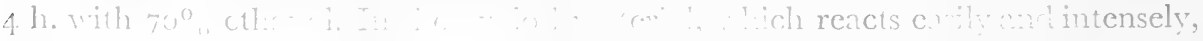

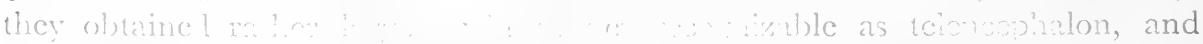

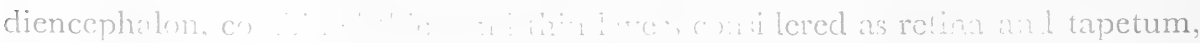

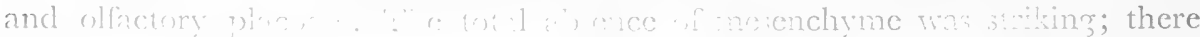


was not even a trace of neural crest derivates. They considered these results as typical "activations" ( $c f$. p. 391).

The technique was approximatively the same in Okazaki's operations (1955). He used Triturus pyrrhogaster middle and posterior pieces of the archenteric roof (neurectoblast included), treated for hours with ethanol at two temperatures; $-18^{\circ} \mathrm{C}$ and $5^{\circ} \mathrm{C}$, and cultivated in sandwiches. The interest does not lie so much in the nature of the inductions, which were all, as has been expected, of the acrencephalic type, as in their numerical incidence. Treatment at $-18^{\circ} \mathrm{C}$ provokes only a few inductions ( 5 to $12 \%$ ), while at $5^{\circ} \mathrm{C}$ they are numerous $(72 \%)$. The question now is whether the first procedure simply inactivates an inducing property resistant to death, or whether ethanol suppresses this property which existed in vivo, but also causes, at temperature above $0^{\circ} \mathrm{C}$, a secondary inducing property to appear de novo. The author is in favor of the second alternative, his main argument being that the succession of the two treatments causes the inducing property to reappear. Moreover, the inductive ability obtained by ethanol at $5^{\circ} \mathrm{C}$ is not annihilated by being placed for a time in ethanol at - $8^{\circ} \mathrm{C}$. As a corroborating argument, it has been shown that treatments which do not appreciably denature the proteins, such as freezing by solid $\mathrm{CO}_{2}$-ether mixture (plus I day storage at $-\mathrm{I} 8^{\circ} \mathrm{C}$ ), or freezing $3^{-5} \mathrm{~min}$. in a $\mathrm{CO}_{2}$-ethanol mixture (plus thawing in Holtfreter's solution), only suscitate weak inductive capacity, if any. Thus, the cold $\left(-\mathrm{I} 8^{\circ} \mathrm{C}\right.$ ) ethanol is thought to annihilate the power of induction which existed in the living explant; warmer $\left(5^{\circ} \mathrm{G}\right)$ alcohol evokes a new inducing property.

This conclusion is perhaps too narrow. The possibility exists that refrigeration, used alone or combined with ethanol treatment, only reduces the inductive power by some condition which prevents the inducing agent from leaving the cells. Relative re-heating or longer alcoholization could remove such a physical block, but evidently can also evoke a supplement of inducing factors. Whatever it may be, it is not in the interest of our analysis to assert, without pertinent reasons, the absence of a relation between normal inducers and those which are revealed by an alteration of some inductor.

Several other chemicals have been found to modify the induction process or to cause neuralization. In 1944, Brachet mentioned (p. 389) that, although numerous substances were inactive (cresyl blue, dinitrophenol, uric, hippuric, lactic, phenacetic, and oleic acids), a variable amount of positive results could be obtained by using methylene blue, several polycyclic hydrocarbons, benzopyrène, methylcholanthrene, auxin and heteroauxin, 1, 2, 5, 6-dibenzenthracene-endosuccinate. This substance ${ }^{1}$ has been again shown by Shen (1942) to be active when added simply to the standard medium: it elicits the ectoblast brain-like masses, probably acrencephalic, without any trace of mesoblast. Trypanblue, now largely used to cause cardiac and other anomalies in mammalian embryos, is effectively teratogenic on amphibian eggs, and somewhat analogous in its action to $\mathrm{LiCl}$ (Waddington and Perry, 1956).

The effects of tenso-active substances, especially sodium oleate and linoleate have been explored by Gothié (1950), but the rather limited results may be accounted for by a cytolyzing effect.

1 For the absorption of carcinogenes in Urodeles embryos, see Waddington and Goodhart (1949). 
In relation with the cytochemical results mentioned p. 360 , the action of compounds bearing $\mathrm{SH}$ - or SS-groups is especially interesting. Brachet (in Rapkine and Brachet, I95I) insisted on the efficiency of reductors such as cysteine and thiomalic acids for the neuralization of ventral explants. He consistently answered the criticism expressed by Holtfreter (I945) and maintained that the results were not caused by cytolysis. The opposite action of such oxidants as alloxan was also emphasized, against Holtfreter. These experimental results are in agreement with the observation data mentioned p. 360 and favor the idea that macromolecules bearing SH groups are especially important in the process of induction. This hypothesis is supported, although indirectly, by Lallier's results (1954) who stained the blastopore lip with dyes thought to combine with ribosenucleic acid (acridine, orange, toluidine blue, acriflavine), and nevertheless obtained positive results.

It would be tempting to introduce here the one known case of induction in vitro by a definite organic substance. It could be permissible to present under this aspect the discovery of Wilde ( $1955^{\mathrm{a}}, \mathrm{b}$ ) who demonstrated certain neural crest characters in the ventral epiblast of advanced gastrulae, or of neurulae, by addition of phenylalanine to the medium. Remarkably enough, the chordomesoblast of the archenteron roof could be shown to act as the normal source of this amino-acid (Wilde, I956). However, if the neural crest primordium is indeed a result of primary induction, the author does not explicitly refer to this process, but more to the later appearance of the various neural crest derivatives in correlation with the presence of endoblast or true mesoblast. This differentiation falls more into the category of secondary inductions and will be considered in this connection. Moreover, the experiment consisting of submitting ectoblast of the early gastrula to the same conditions mentioned here does not seem to have been performed.

Thanks to the physical and chemical means just discussed, some more information has been revealed concerning the process of primary induction. The young (indifferent) ectoblast can be influenced in two opposite ways. It can be caused either to perform notogenesis, or it can be caused to build up, often in the absence of any mesenchyme or mesoblast, a thick-layered brain piece, eventually acquiring the characters of an acrencephalon.

The first transformation can be obtained only by a moderate centrifugation of the fully segmented egg. The second may be attained by various means: cultivation in a medium of high $\mathrm{pH}$ and/or low in $\mathrm{Ca}^{++}$; short contact with the anterior part of the notochord; or more prolonged contact with marginal material or archenteron roof parts previously submitted to heating or to alcohol.

It is important to decide which of these results expresses the tendencies nearest to the normal ectoblast. In my opinion, it is the shift to notogenesis, for this tendency is evidently present in the marginal zone, and this territory is in obvious continuity with the ectoblast. The delimitation has only a "presumptive" value and can, by micro-surgery, be shifted up or down. Also, centrifugation, at the speed which has been used, is not traumatic, but only concentrates components of equal or subequal density, a condition which probably allows them to exert, as Pasteels 
thinks, a mass effect. On the contrary, the means which evoke an acrencephalon are cytolytic (Holtfreter) or even lethal, and their consequence is not only denaturation of the proteins, as currently admitted, but probably the production of an extra-normal inductor. If we think therefore in terms of latent potency in the ectoblast, it can only be a tendency toward notogenesis.

As revealed by micro-surgery, the region where the factors conditioning notogenesis are at their peak is able to evolve into the prechordal inductor. This process can be hampered by lithium and by early centrifugation, while it can be favored by several different operations.

The possibility of eliciting characteristic aspects of neural crest differentiation by the introduction of definite aminoacids allows the best prospect for further elucidation of primary induction.

\section{Xeno-inductions}

When it had been recognized in Spemann's laboratory (Bautzmann, Holtfreter, Mangold and Spemann, I932) that the inductive property was not limited to the "organizer" but could be evoked in other parts of the germ by life-stopping treatments, the way was opened for a more sensational generalization which included practically all tissues or organs of animals, vertebrates or even invertebrates, directly or after appropriate alteration (Holtfreter, I 934 b, c).

This discovery made possible a new approach to the nature of the inducing process and provoked a movement of investigations of a still-growing interest (compare with the accounts of Brachet, I944, I950). Their results are of considerable variety and intricacy, but, in the recent period, they decidedly tend towards clarification.

\section{(a) Fresh foreign tissues}

The use of fresh, untreated inductors is rather limited since many of them exert a deleterious effect on the reacting material (inserted in the blastocoele or wrapped in a piece of ectoblast from a young amphibian gastrula). Moreover, the implanted tissue is likely to undergo aseptic autolysis which may cause undesirable complications. Nevertheless, pieces of fresh organs have been used successfully in several cases:

Salamandra liver, inducing a lens (Holtfreter, 1934b); lizzard (Lacerta agilis) liver, inducing a tail (Holtfreter, I934b); kidney of the same animal, inducing some nervous cells (Holtfreter, 1934b); mouse heart, inducing, in clear relation with the implant, a brain and nervous masses, or a hind-brain with otocysts, or neuroid parts, or a small tail (Holtfreter, I934b); mouse liver, inducing a notomerit (Holtfreter, I934b); mouse kidney, inducing the same complex (Holtfreter, I934b) or, sometimes, an acrencephalon (Chuang, I940); newt liver, inducing either acrencephalon or notomerit structures (Chuang, 1940); perch and plaice heart muscle, inducing a lens (Toivonen, 1945); frog abdominal skin, inducing chordomesoblast only (Okada 1948); mouse bone marrow, inducing myotomes, myoblasts, and notochord, sometimes with a spinal cord (Kawakami and Mifune, I955); mouse spleen stroma, inducing either an acrencephalon and olfactory placodes or myoblasts and notochord (Kawakami and Mifune, 1955); cortical parts of mouse kidney, inducing hind-brain, 
otocysts and truncal structures ${ }^{1}$ (Kawakami and Mifune, 1955); mouse liver, inducing an acrencephalon (Kawakami and Mifune, 1955); various organs of irradiated mice, which show a more acrogenic tendency (Kawakami and Mifune, 1957); bone marrow and kidney medulla of mice injected with nitrogen-mustard- $\mathcal{N}$-Oxide, which induce less mesoblast and more neural structures, with a shift towards acrencephalic organs (Mifune, 1957); and finally crude extract of 9-days chicken embryos, inducing mostly deutencephalic structures, accessorily truncal ones (Von Woellwarth, 1956).

These instances suffice to authenticate the existence in vivo of these inducing properties and their wide distribution as well as their variability, even concerning one kind of implant. In the case of the mouse kidney, the discrepancies are due to the exact origin of the tissue, the outer layer of the cortex having an acrencephalic effect, while the inner layer of the cortex and the medulla provoke notogenesis (Mifune, I955). In spite of these variations, probably due to the complexity and physiological state of the organs used, the distinction emerges between the two familiar types of induction, with a hint that another more limited category exists: that of isolated lenses, evidently related to the acrencephalon.

\section{(b) Killed foreign tissues}

The same two groups of results appear, but more consistent, when we consider the effects of the same organs used after boiling or dipping in ethanol, as done by Holtfreter (1934b), Chuang (I 939, I 940), Toivonen (I940) and Rotmann (1942). A change, however, is produced by these treatments, whereby most inductions take on an acrencephalic character. Chuang had the merit of recognizing that boiling the mouse kidney for increasing periods up to one hour causes progressive increase of the inductive ability which becomes mostly acrogenic; with longer boiling, up to $2 \mathrm{~h}$., this property gradually fades and practically disappears. Newt liver also loses its notogenic effect by boiling, after only a few minutes. With $5 \mathrm{~min}$. or more boiling it exerts a stronger acrogenic action, which disappears after $15 \mathrm{~min}$. treatment.

In his extensive investigations of I940, Toivonen always used as implants specimens treated with ethanol. He obtained acrencephalic structures when implanting either liver pieces of perch, or viper, guinea pig, and snake kidney, while kidney pieces of perch, shrike and guinea pig were consistently notogenic. Guinea pig thymus was found to induce lenses, while Rotmann (I942) observed only tail formation; this odd discrepancy turned out to depend on the age of the donor and also on the length of the ethanol action. Engländer, Johnen and Vahs (1953) indeed established that this factor causes a shifting from notogenesis to acrogenesis in the cases of guinea pig kidney, parotis, heart, and thymus (Fig. 83, A, B). They also showed that food deficiency ( $c f$. also Toivonen, I95I) works in the same way, but the frequency of rhombencephalic structures is not appreciably modified (Fig. 83, C), a feature also noticed with alcoholization of the parotis (see also Engländer and Johnen, 1957).

Among inductions obtained with other heated and ethanol-treated organs are

1 According to Mifune (1955), this effect is only obtained when using the inner part of the renal cortex and the medulla; the superficial part of the cortex is more acrogenic. 
the following: by Holtfreter [1934c, boiled ovary and muscles of the stickleback (acrogenic), boiled heart and brain of the european salamander (acrogenic), boiled liver and thyroid of man (notogenic)]; by Rotmann (1942), guinea pig mediastinal lymphnodes, treated by ethanol, acetone, chloroform boiled (noto-
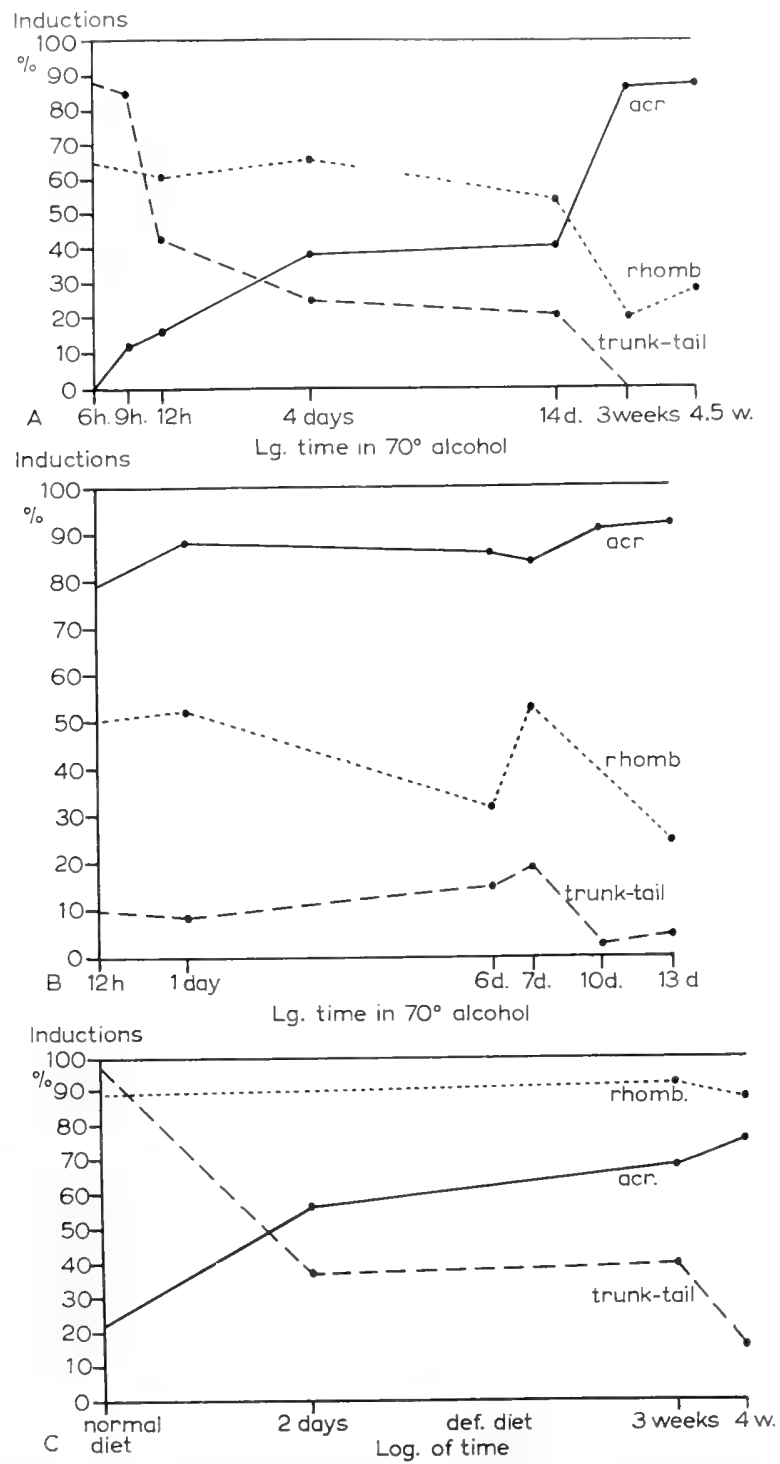

Fig. 83. Experimental modifications of xeno-inductors. A. Changes in the inductive capacity of mouse kidney implanted in newt gastrula after various lengths of time in ethanol. B. Same experiment with guinea pig heart C. Changes in the parotidis of a guinea pig submitted to starvation. acr. - acrencephalic structures; rhomb. - deutencephalic manifestations; trunktail, cormomeritic and ouromeritic (Fig. 39, p. 368) structures. From Engländer, Johnen and Vahs, 1953. 
genic effect, suppressed by methanol); by Toivonen (I945), ethanol-treated heart muscle of fishes (lenses); by Toivonen and Kuusi (I95I), ethanol-treated ox liver or kidney (both together acrogenic and notogenic); by Okada (I948) boiled frog ventral skin, which induces neural structures, perhaps acrencephalic; by V'ahs ( I955), mouse kidney boiled I 5 minutes (only balancers); by Kuusi (I953), pigeon muscles treated with ethanol (acrogenic); by Kriegel (1956), ethanoltreated germinal vesicles (acrogenic); by Vainio (1958), a coagulum of entire blood (homo, cavia) or of its various parts, which contain together notogenic and acrogenic factors, thus present in our circulation.

Let us add that Holtfreter (1955b) obtained some slight induction with dead or dying pieces of mouse liver or kidney which were coated with agar, in conditions which excluded intimate contact between graft and reactor.

Forelimb rudiments
Fin
Notochord
Myotomes
Pronephros
Mesenchyme
Proctodaeum
Neural cells?

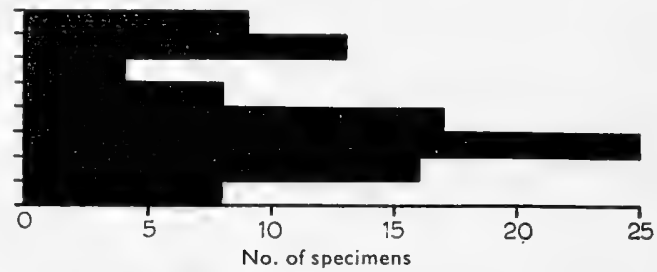

Fig. 84. A histogram of the inductions obtained with ethanol-treated bone marrow of guinea pig. Implantation in the blastocoele of newt gastrulae. From Toivonen, I953.

A special interest is attached to the guinea pig bone marrow (from the thigh) which Toivonen (1953, 1954a, b) used after $24 \mathrm{~h}$. in ethanol and described as a purely (or nearly pure) mesodermal inductor (Fig. 84). If first boiled and then treated with ethanol, the same tissue, implanted in the blastocoele, induces lenses and balancers while, when explanted in sandwiches, its action is limited to lenses or lentoïds, with neither balancers (or at the most very poor ones) nor neural crest derivatives. This is a new instance of a shifting by heat in the acrencephalic direction. Toivonen and Saxén (1955a) then proceeded to combine the inducing effects of the two tissues of the guinea pig which, when submitted to ethanol, exert "complementary" induction, i.e. liver and bone marrow, acrogenic and notogenic respectively. In this case, the effect of liver tissue has been reexamined with the appreciable rectification that, especially if originating from well-fed animals, it does not only induce an acrencephalon and its related sense organs, but also a chordencephalon with or without its otocyst(s). The possibility of obtaining combined fore- and hind-brain inductions, without any trunk component, appears already in the results (see infra) of Kuusi (I95I). Bonemarrow on the other hand is not completely notogenic, because a neural tube is practically lacking. The two tissues, previously fixed in $70 \%$ ethanol, were implanted together either in the blastocoele or in an ectoblast sandwich. In both case;, the characteristic effect appeared centred on the individual inductor, but with distinctly more trunk or tail spinal cord than could be obtained in the controls with each inductor separately (Fig. 85). Thus, "the spinal cord can only be induced when the inductors contain agents capable of inducing both hind- 
brain and axial mesoderm" (Kuusi, I95I, p. 356). This statement needs, however, a slight attenuation (p. 436). On the other hand, the same xeno-inductor will, by appropriate fractionation, provide us with valuable information (p. 44r).

\section{(c) Regional influence of the host}

Before examining in more detail the nature of the xeno-inductors, it is worth mentioning that their study provided some more information concerning the influence of the primary embryo on the induced structures. This is the classical problem of regionality, especially important with implantations in the blastocoele.

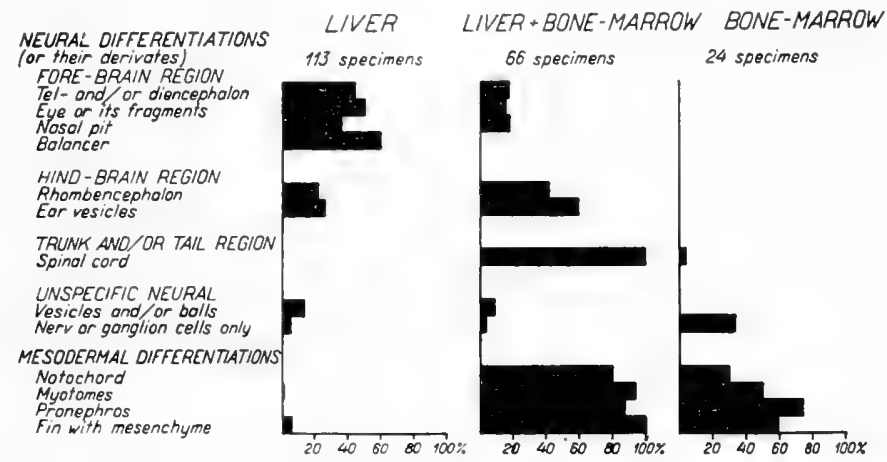

Fig. 85. Histograms of the inductions obtained with liver, bone marrow and the tissues together. The implants were taken from guinea $\operatorname{pig}(\mathrm{s})$ and implanted in the blastocoele of newt gastrulae. From Toivonen and Saxén, 1955b.

Chuang ( I 939) recognized that this host influence was as real with xeno-inductions as with organizer grafts. Vahs (I 956) led a broader and statistically-based enquiry for which a pool of 1327 individual cases of xeno-inductions was examined, without regard to the inductors used. $263 \mathrm{I}$ induced structures were available; they were all classified according to the number of each type located in (I) the head region, (II) the cardiac region, (III) the fore part and (IV) the hind part of the trunk. The results (Fig. 86) confirm that an influence from the primary system exists, but only concerning acrencephalic structures. In my opinion, the source of this influence must be in the prechordal anlage of the young archenteric roof. The fact that other parts do not exert such a control or harmonization, not even for organs as cephalic as the otocysts, fits well with the idea that notogenesis is a more autonomous process and implies a close hierarchy of the involved structures.

\section{(d) First approaches to the nature of the xeno-inductors}

Attempts to disclose the nature of the induction process by a biochemical analysis of some very active inductors, began with the discovery of xeno-induction, and the passionate work first accomplished in this direction by Fisher, Needham, Waddington, Barth, and their coworkers ( $c f$. Brachet, I950) deserves a tribute of admiration. This long and arduous exploration has resulted during the last 
five or six years in focussing on proteins and nucleoproteins as the most likely answers to this enigma.

Without really attempting a classification of the methods used, let us begin with the techniques which have succeeded in isolating a fraction endowed with some definite inductive effect, and continue with those which, moreover, apaparently bear on known biochemical entities.

For practical reasons, much work has been done with guinea-pig liver and kidney, which, as we have seen, possess rather opposite inductive properties.
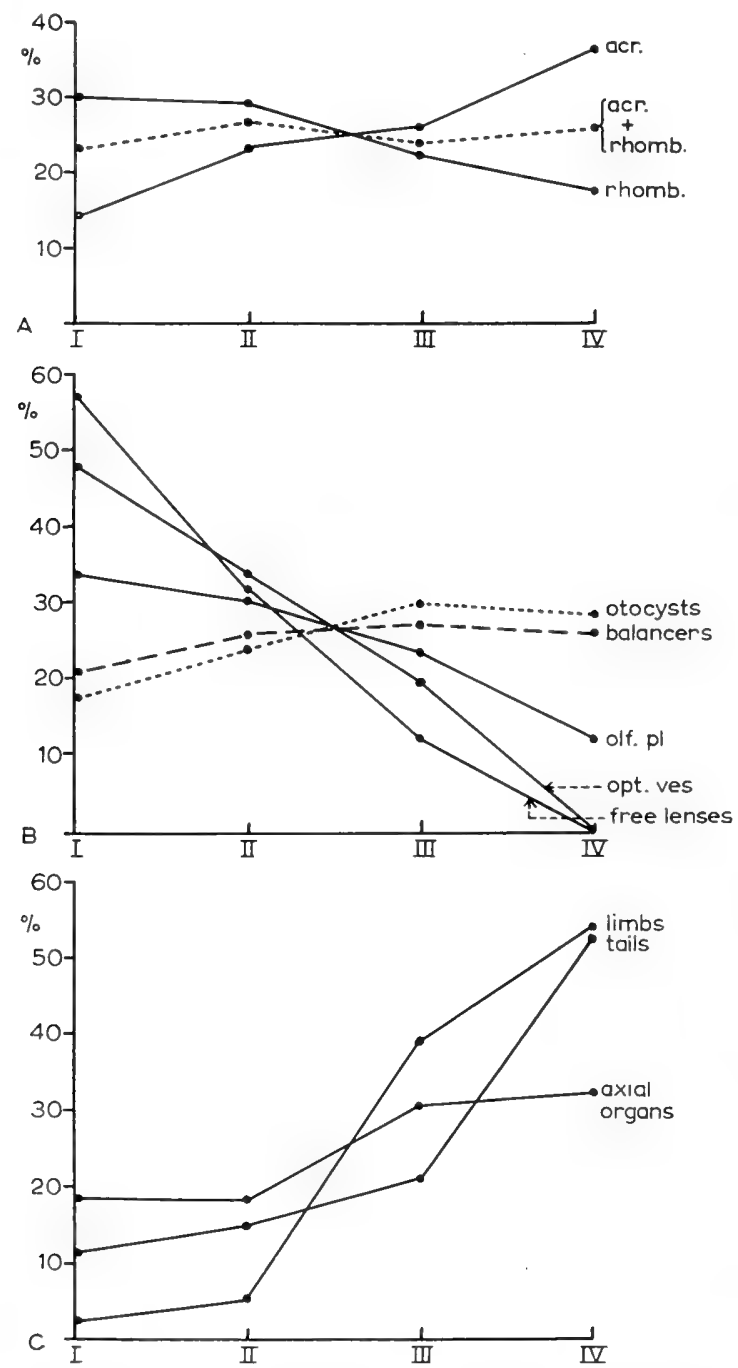

Fig. 86. Influence of host region on the nature of xeno-inductions. As abscissae, the four main regions: I, acromerit; II, deutomerit; III, cormomerit; IV, ouromerit (cf. Fig. 39). A. the distribution of brain parts; B. sensory organs. C. structures of the tritomerit. From Vahs, 1956. 
Dialysis alone is, of course, a mild procedure. When applied to the liver, it deprives the tissue of its acrogenic property and leaves a mainly notogenic inductor (Toivonen, I949; Toivonen and Saxén, 1955a, b). The same result is obtained (Toivonen, r949) by extraction with petroleum ether; however, the evaporated residue does not show any activity; saponification of this residue yields an inactive sterol fraction and a fatty acid fraction with slight acrogenic activity, but probably through cytolytic action. Similarly, the kidney tissue preserves its notogenic influence after extraction by petroleum ether, though practically no spinal cord appears. These results were then tested on the corresponding ox organs (Toivonen and Kuusi, 1948), which do not, after simple treatment with ethanol, have distinct inductive effects. No qualitative change could be registered after dialysis or after petroleum ether extraction, but only a general lessening of inductive power. The evaporated residue gave results analogous to those of guinea pig extracts. Consequently, a dissociation of these inductors seems to be obtainable only when starting from organs having a priori a different action.

The lipids may be disposed of as possible induction agents. Their inactivity has already been made evident by H. Lehmann (I938) and confirmed by Fujii (I944). It may also be stated, tentatively, that of the two main inductors, the notogenic one, especially its mesoblastic part, is less thermostable, but more firmly bound to the tissue stroma, from which it cannot be separated by dialysis nor by petroleum ether extraction. In contrast, the acrogenic inductor is more thermostable, resistent to ethanol, and extractable by dialysis or by petroleum ether (Toivonen, I950). With many organs, the question arises if the more acrogenic effect observed after the action of heat or of ethanol is due to the selective destruction of the notogenic inductor or to the conversion of the latter into an acrogenic factor.

\section{(e) Fractionation of the xeno-inductors}

Attempts to dissociate the xeno-inductors into several fractions, with the hope of detecting the responsible components, have been numerous and of variable efficiency. Holtfreter (1934b) tried chick embryo extract, which is indeed a complex, and observed mixed effects, with a serious predominance of notogenic structures. Recently, Von Woellwarth (1956) has reared ectoblastic explants in a supernatant of embryonic extract sap of 9 days embryos, centrifuged at $25,000 \mathrm{~g}$, this supernatant being used at various dilutions. Only pieces of sufficiently large size react positively. They swell and elongate in a rather extraordinary way. The induction consists of abundant mesenchyme, pigment and neural structures ressembling spinal cords or even hind-brains with otocysts, thus, a picture of notogenesis. This result is reminiscent of the acrogenesis obtained by Shen with a water-soluble hydrocarbone. There is no doubt in such case that the action has been exerted by substances dispersed in the medium, and that the organization power is in the reactor itself. Further separations have been performed on the embryo extract, but they will be considered later.

It is somewhat difficult to localize correctly the inductions obtained by blastocoele implantation of dry extract pellets (Shering) of oxen prepituitary (Mangold, 
Tiedemann and Von Woellwarth, I956). The effect was intense but somewhat variable, with a dominant deutogenic tendency.

From the true fractionations which have been tried with increasing success during the last five or six years, two orientations have appeared as possible solutions at the biochemical level: proteins and ribonucleoproteins.

A definite hint in favor of the role of proteins was contained in the extensive work of Kuusi (I95I). Using guinea pig liver and kidney, she elicited characteristic inductions with nucleoprotein and protein extracts. As these effects were not suppressed by previous incubation of the inductors with ribonuclease, it was insisted that proteins were of greater importance. This opinion was supported by the considerable reduction of inductive power caused in ox liver and kidney after digestion with pepsin or tripsin (Toivonen and Kuusi, 1948).

In the same period, Yamada (1950b) used an extract of ox muscle. The ground tissue was kneaded for Io min. in $0.25 \% \mathrm{NaCl}$. The mixture was added with two volumes $\mathrm{H}_{2} \mathrm{O}$, then filtered and the residue macerated for some hours in $0.1 \mathrm{M} \mathrm{Na}_{2} \mathrm{HPO}_{4}$. Centrifugation yielded a supernatant which coagulated at $80^{\circ} \mathrm{C}$, and dessicated by heating. The reactions obtained in ectoblast sandwiches were exclusively of acrencephalic type. Since iron powder was added to most of the explants, a participation of cytolysis can be suspected. However, in one series without this noxious ingredient, the reactions, though less numerous, remained of the same type. It may be remarked that the extract used was far from containing exclusively proteins, and especially that ribonucleoproteins were certainly also present. This paper is, however, quite important owing to the biochemical orientation and to its theoretical discussion, which included the concept of dorso-ventral and cephalo-caudal potentials and of their mediators (p. 47i).

An important advance in the same direction is represented by the attempt of Yamada and Takata (1955) to explore the successive steps of fractionation of the guinea-pig kidney. Samples were obtained by fundamentally following the technique of Kerr and Seraidarian (1949). The homogenate was extracted at low temperature in $0.14 \mathrm{M} \mathrm{NaCl}$, centrifuged, either redissolved at neutral $\mathrm{pH}$ or partly precipitated by acidification, and, according to the case, finally either coagulated by heat or precipitated (from supernatants) by $70 \%$ ethanol. This last step was always followed by a thorough washing in standard saline solution. Due attention was given to the time of preservation $\left(\right.$ at $5^{\circ} \mathrm{C}$ ) of the specimens, which turned out to be a rather important factor. This procedure has been paralleled by manipulating as much of the same incipient extract as necessary to separate the ribonucleoprotein fraction (see infra). Leaving out some infrequent manifestations not attributable to a definite region, the results are summarized in Table 6.

This complex but valuable enquiry brings us several pieces of new information. Firstly, it makes apparent a previously undescribed modality of experimental induction i.e. a quite elongated fragment containing a spinal cord and rather abundant mesenchyme (Fig. 87). The authors state that in Io cases they obtained a spinal cord without either somites or notochord, but do not mention to what extent mesenchyme was present; this striking reaction seems to appear sporadically. They insist that a deutencephalon was often observed in the absence of notochord and somites, which, in my opinion, does not mean the absence of any cephalic mesoblast. They also confirm the presence of notochord and somites without a neural tube as obtained in the case of bone marrow. We shall interprete these experimental dissociations on p. 444. Secondly, the results show that the ribonucleoprotein ( $=\mathrm{RNP}$ ) content of the inductor does not by itself command the quality of the reaction; specimens 4 and 9 of Table 6 exhibit the primarily 
TABLE 6

MAIN INDUCTIVE ACTIVITY OF SUCGESSIVE FRACTIONS OF GUINEA PIG KIDNEY

Compiled from Yamada and Takata, 1955

\begin{tabular}{|c|c|c|c|c|c|}
\hline $\begin{array}{l}\text { Fractionation procedure } \\
(\vec{\downarrow}=\text { centrifugation, } \\
\downarrow=\text { precipitation })\end{array}$ & $\begin{array}{l}R \mathcal{N} A-P \mathrm{mg} \\
\text { pro } m g \mathcal{N}\end{array}$ & $\begin{array}{l}\text { Acren- } \\
\text { cephalon }\end{array}$ & $\begin{array}{c}\text { General } \\
\text { notogenesis }\end{array}$ & $\begin{array}{l}\text { Chorden- } \\
\text { cephalon }\end{array}$ & Remarks \\
\hline
\end{tabular}

I. Blocks of tissue + ethanol
(a) preserved 2 h. to 5 days
(b) preserved i 7 h. to 45 days

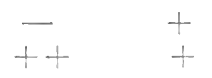
with 45 days acren. +++

2. (a) Homogenate in $0.14 M \mathrm{NaCl}$ supernatant + ethanol I 8.2

(Preserved I-Io days, and washed)

(b) idem + heat

3. Id. preserved 37 days at $5^{\circ} \mathrm{C}$.

id.?

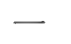

4. Supernatant of $2 \mathrm{a}, \downarrow \mathrm{pH}_{4.2}$,

$\rightarrow$, reextracted, $\rightarrow$

redissolved $\mathrm{NaCl}, \downarrow$ ethanol,

washed

31.7

$+\quad ?$

(unpubl. data mentioned

in discussion)

5. Supernatant of $2 \mathrm{a}, \downarrow \mathrm{pH}_{4} .2, \quad \pm 3 \mathrm{I} .2 \quad+++$ reextracted, $\rightarrow$ redissolved

$\downarrow 3 M \mathrm{NaCl}(36 \mathrm{~h}$.)

$\downarrow$ ethanol

6. precipitate of 5 resuspended ca. 10.6 in $3 M \mathrm{NaCl}, \downarrow$ ethanol (variable)

7. suspension of 6 filtered, pH $4.2+$ ethanol

г $3.9+++\quad+$

8. suspension of $5, \downarrow \mathrm{pH}_{4.2}$ + ethanol

? $\quad+++\quad+$

9. Supernatant of $2 \mathrm{a}, \downarrow \mathrm{pH}_{4} \cdot 2$, $\rightarrow$, supernatant $\downarrow$ by ethanol

I0. (2b) supernatant $\downarrow$ by heat (like 2?)

optic vesicle atypical

The repetition of the sign + has only a meaning inside the horizontal line concerning one single specimen; it is intended to show the numerical predominance of one type of structure over another. 
notogenic action of the tissue used, although the richness in ribonucleoproteins is much reduced in the $3 \mathrm{~d}$ specimen; also, specimens 4 and 5,7 and 9 reveal opposite actions in spite of their approximately equal RNA content. This, however, does not exclude a role of the ribonucleoproteins which could be based, as we shall soon learn, on more specific properties. Thirdly, the concept of the main acting inductors - whether two or three in number - is much clarified. It may be added to the characteristics mentioned on p. 432 that extraction with concentrated $\mathrm{NaCl}$ (specimens 6, 7, 8) favors acrogenesis. Since the frequency of inductions remains approximately constant, it is unlikely, as the authors rightly say, that we have to do with a selection of preformed substances. A transformation of the primary notogenic inductor (and of its deutogenic part, if it is worth being distinguished) into the acrogenic one appears nearly certain. These remarks lead the authors to the interesting opinion that the available data do not exclude the fundamental singleness of the inductive agent:

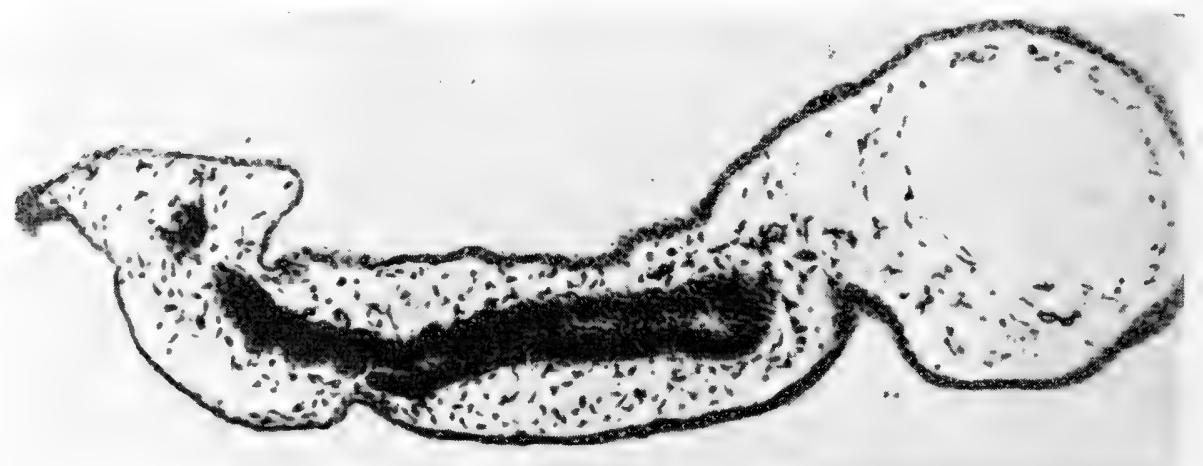

Fig. 87. Remarkable xeno-induction by a proteinic extract. Elongation of the explant and induction of a spinal cord and ectomesoblast without presence of chordomesoblast. See text. From Yamada and Takata, I955.

"A protein or protein complex present in the tissue in its native, unstable state induces spino-caudal structures; this state is easily and irreversibly transformed into a stable one in which the same protein or its complex induces archencephalic structures. During the transitional phase of this transformation the same protein gives rise to deuterencephalic induction", and further ... "the so-called spinocaudal, deuterencephalic and archencephalic factors of the tissue all would belong to one chemical category or to a group of closely related proteins and form one series of structural transformations, which can be released easily by different treatments".

This statement is in close agreement with some of our preceding remarks (p. 40I, 4IO) and will be an essential argument in our general interpretation.

The last step will be devoted to a reconsideration of the role of ribonucleoprotein complexes. After his discovery of the so strongly suggestive relation between RNA and induction (p. 36o), Brachet looked for experimental evidence in favor of this relation. He found ( 1944 , p. 4I8) that the higher the RNA content of a xenoinductor, the higher was the percentage of positive results. The most efficient 
material tested was the tobacco-mosaic virus, which, as was expected, lost much of its activity after treatment with ribonuclease. When Kuusi (I95I) observed that this enzyme did not appreciably reduce the inductive capacity of several active parts of xeno-inductors, she repeated the experiment with the same virus, hydrolyzing the RNA with perchloric acid, but did not observe a decrease of its inductive power (Kuusi, I953). The discrepancy was attributed to possible proteolytic impurities in the ribonuclease previously used by Brachet.

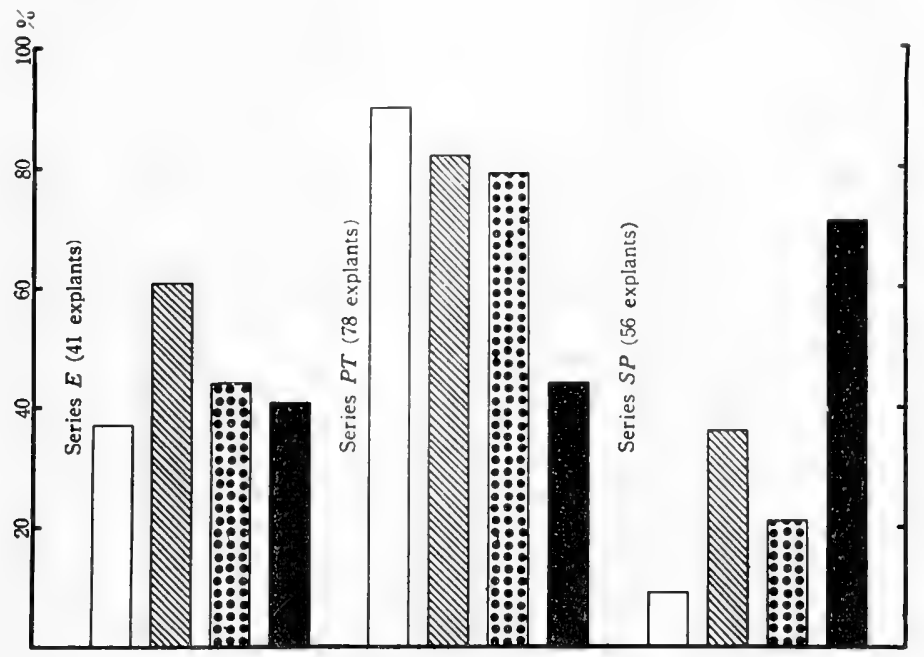

Fig. 88. Comparison of the induction frequencies obtained with the ribonuclein (RNP) extract of guinea pig liver. In series $\mathrm{E}$ the inductor was the homogenate precipitated by ethanol. In series PT, the extract was submitted to precipitation by streptomycin sulfate and further purifications to obtain the RNP precipitate. In series SP the supernatant obtained after precipitation by streptomycin was dialyzed under definite conditions (рн 7.6) and precipitated by ethanol. In each group: white column, frequency of optic vesicles; hatched column, acrencephalon; dotted, olfactory placodes; black, mesenchyme. The greater frequency of acrencephalic structures in series PT is manifest. From Hayashi, 1956.

Other results, however, still hinted that nucleic acids played a positive role: H. Lehmann (1938) obtained inductions with the DNA-containing fraction of pancreas, thymus, and liver; Brachet, Kuusi and Gothié (1952) got inductions, mainly acrencephalic, with cell-nuclei of rat, guinea-pig, pigeon liver, and thymus nucleo-histone, but were for the most part unsuccessful with injections of fresh liver microsomes, due probably to the small quantity of inductor. Kuusi (I953) obtained positive results with nucleoli of star-fish oocytes, commercial RNA, RNA from rabbit liver microsomes and from guinea pig kidney. Still other results pleaded in the opposite direction. Yamada, Takata and Osawa (I954) proceeded to the isolation of the RNA contained in guinea-pig liver homogenates but did not obtain really positive results, except in cases when cytolysis was suspected. Saxén and Toivonen (1957) compared the inductive effect of rat liver either normal or taken in phase of full regeneration, when much richer in RNA. Both samples have the same activity, qualitatively (acrogenic) and quantitatively. 
Further investigations soon ascertained the following two facts: $(I)$ ribonuclease (or deoxyribonuclease) digestion of xeno-inductors from vertebrates does not suppress induction (Engländer et al., I 953; Okazaki and Osawa, I 954; Tiedemann, 1955. Hayashi, I955); Engländer even observes, in certain experiments, an intensification of induction, with an increased acrencephalic tendency; Vahs (1957a) confirms this point for mouse kidney treated with ethanol (Einsteck methode); and (2) proteolytic enzymes (i.e. pepsin, trypsin, and chemotrypsin) do attack the inducing agents (Toivonen and Kuusi, 1948; Tiedeman, I955; Yamada and Takata, I955). The agreement in these results does not, however, preclude the possible role of ribonucleoproteins: the protein part of these may be

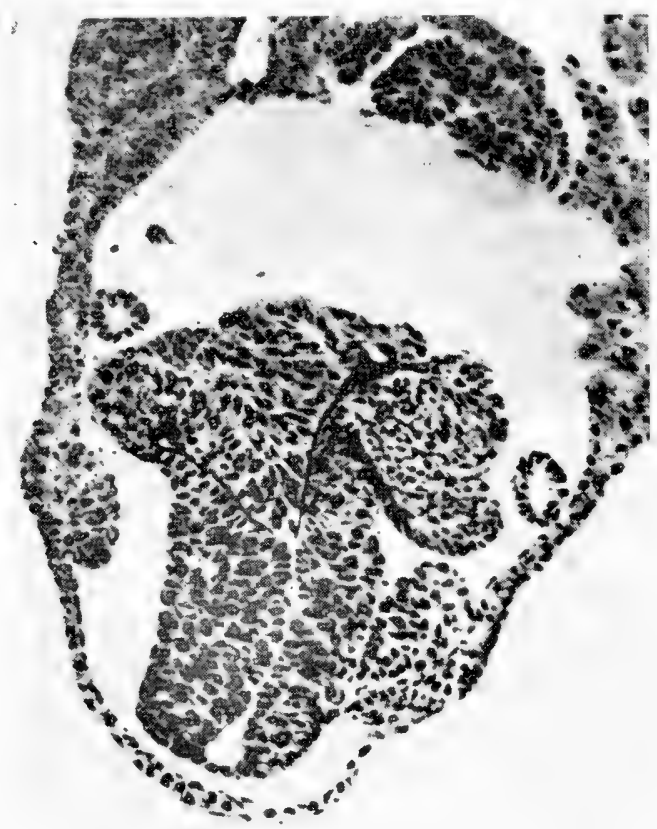

Fig. 89. Typical induction of an acrencephalon by the PT inductor (see legend, Fig. 88). Note the relation with the implant (grey mass); the presence of optic vesicles, lenses, forebrain, olfactory placodes, and the absence of mesenchyme. Compare with Figs. 80, p. 418, and 82 , p. 424. From Hayashi, 1956.

important enough to be inactivated by proteolytic digestion, while ribonuclease may liberate parts of the RNA nucleosides, which would not exclude recombinations with the aid of the enzymes contained in the living reactor cells. Brachet has indeed insisted on the observation that any implant is immediately submitted to the activity of the host enzymes, with results which cannot yet be elucidated.

These considerations are sufficient to appreciate the full meaning of the last results to be quoted.

Two of them are intimately correlated, for the fractions described have been obtained by practically the same method: one series from guinea pig liver 
(Hayashi, I956), the other from kidney (Yamada and Takata, 1956). In both cases, the fractionation procedure was described by Kutzky (1953), and consisted roughly in extracting the homogenate with $0.14 M \mathrm{NaCl}$ for $\mathrm{I} h$., centrifuging, taking out the supernatant, and precipitating the ribonucleoproteins by streptomycin sulfate. This precipitate was repeatedly redispersed in a stronger $\mathrm{NaCl}$ solution, and collected by centrifugation, then dialyzed against the same solution, and finally precipitated by ethanol. All operations were run at low temperatures. For comparison, the supernatant left after precipitation by streptomycin was treated approximately in the same manner. The essential results are summarized

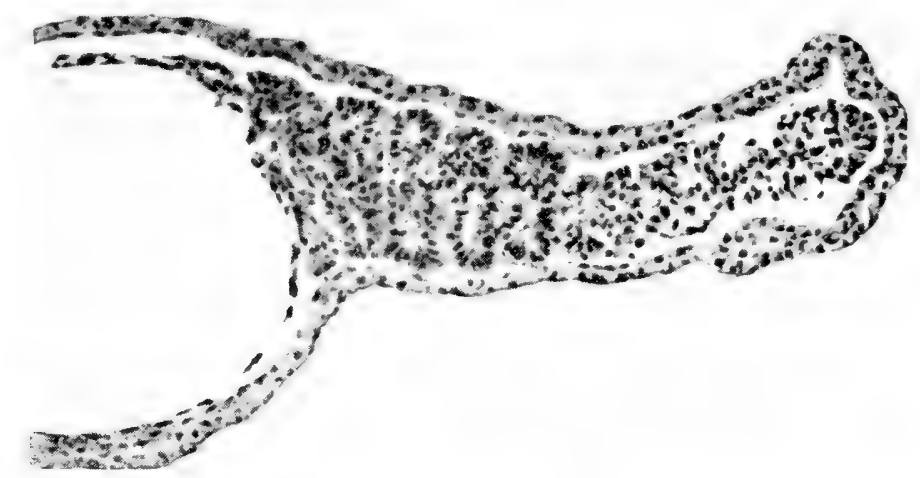

Fig. 90. Typical induction of somites by a purified RNP extract of guinea pig kidney. From Yamada and Takata, 1956.

in Table 7, which includes some analytical data. The ribonucleoprotein fraction used seems quite homogeneous, more than in any other known instance. It contains a large amount of RNP, about 8 times more than the richest extracts considered in Table 6. This fraction seems to contain the principles responsible for the most characteristic inductions. This is especially true for the liver extract (Fig. 88), with which some implants develop a remarkably typical effect (Fig. 89). The kidney extract shows a more varied picture, though also typical in the majority of cases (Fig. 90, 9I). For some structures, like somites and spinal cord, it seems that all the active factors have not yet been extracted. Thus, in these two joint experiments, the role of ribonucleoproteins is firmly established, but it remains to be seen if the ribosenucleic acid-or the protein- moiety would be the active part of the isolated compound, and if the RNP is also the prevailing factor in other xeno-inductors. Here we are again endebted to the Nagoya laboratory for important results, which we shall still have to consider in the light of bacterial xeno-inductions obtained at Köln.

Examining more closely his guinea-pig liver extract, Hayashi (1958) has found that exhaustive defatting of the sample almost preserved its morphogenetic activity, as did also its treatment with ribonuclease, removing most of the RNA. Treatment of the RNP sample with $0,1 \%$ pepsin at $\mathrm{pH} 4.0$ during $30 \mathrm{~min}$. reduced the eye frequency and increased the mesenchyme frequency. With 60 and 120 min. treatments, a reduction or a suppression 
TABLE 7

DIFFERENCE IN INDUCTIVE POWER BETWEEN THE RIBONUCLEOPROTEINS FROM LIVER AND FROM KIDNEY

Compiled from Hayashi (1956) and Yamada and Takata (1956)

\begin{tabular}{|c|c|c|c|c|c|c|}
\hline \multirow[t]{2}{*}{ Aspects considered } & \multicolumn{3}{|c|}{ Liver } & \multicolumn{3}{|c|}{ Kidney } \\
\hline & $\begin{array}{l}\text { Whole } \\
\text { extract }\end{array}$ & $\begin{array}{c}\text { Non preci- } \\
\text { pitable } \\
\text { fraction }\end{array}$ & $\begin{array}{c}\text { Ribonucleo- } \\
\text { protein } \\
\text { fraction }\end{array}$ & $\begin{array}{l}\text { Whole } \\
\text { extract }\end{array}$ & $\begin{array}{l}\text { Non preci- } \\
\text { pitable } \\
\text { fraction }\end{array}$ & $\begin{array}{l}\text { Ribonucleo- } \\
\text { protein } \\
\text { fraction }\end{array}$ \\
\hline $\begin{array}{l}\text { Electrophoresis } \\
(\text { pH } 8.5 \text { and } 7.5)\end{array}$ & $?$ & complex & I component & ? & ? & I component \\
\hline Absorption spectrum & ? & ? & $\begin{array}{l}\text { of nucleo- } \\
\text { protein }\end{array}$ & ? & ? & $\begin{array}{l}\text { of nucleo- } \\
\text { protein }\end{array}$ \\
\hline RNA-P $\mu g r$ pro mgr N & ? & $9 \cdot 5$ & 264.0 & 18.2 & 9.0 & \pm 245.0 \\
\hline $\begin{array}{l}\text { Number of available } \\
\text { explants }\end{array}$ & $4^{I}$ & 56 & 78 & 71 & 60 & 69 \\
\hline $\begin{array}{l}\text { Number of explants } \\
\text { with any induction }\end{array}$ & $4 \mathrm{I}$ & $5^{6}$ & 78 & 65 & 45 & 62 \\
\hline $\begin{array}{l}\text { Acrencephalic } \\
\text { inductions }\end{array}$ & 29 & 23 & 76 & 0 & 6 & o \\
\hline $\begin{array}{l}\text { Deutencephalic } \\
\text { inductions }\end{array}$ & - & - & - & 13 & 17 & 34 \\
\hline Otocysts & 一 & - & - & 16 & 5 & 17 \\
\hline Somites & - & - & - & 54 & 7 & $2 \mathrm{I}$ \\
\hline Spinal cord & - & - & - & 14 & 2 & 8 \\
\hline Notochord & - & - & - & 3 & o & 3 \\
\hline Mesenchyme & I 7 & 40 & 34 & 26 & I9 & 21 \\
\hline Non-specifiable brain & 7 & Io & 6 & 2 & 4 & I \\
\hline
\end{tabular}

of the inductory power was observed. A similar experiment was tempted with cristalline trypsin $(0.01 \%$ at $\mathrm{pH} 7.6)$, with the important addition that this enzyme was inactivated in the sample, before implantation, by heating at $85^{\circ} \mathrm{C}$ for $5 \mathrm{~min}$. Strictly comparable controls were used. Again with $30-, 60-$ and 120-min. trypsic digestion a progressive suppression of induction was caused. With the longer exposure, only mesenchyme or melanophores appeared. Thus, in this case, it is consistently established that the xenoinductor works through the proteic moiety of the ribonucleoprotein extracted from the liver.

Further tests have however revealed that, in spite of its apparent homogeneity, the ribonucleoproteins extracted from guinea-pig liver are not always purely acrogenic and may contain some deutogenic factors. In an attempt for further dissociation, Hayashi and Takata (1958) have submitted the ribonucleoprotein extract, obtained as above, to high 
speed centrifugation, and have compared the effects of successive precipitates and evaporated supernatants. The former are strictly deutogenic, while the latter still show a mixed inductive action. In discussing the various results concerning this problem, the authors admit that only the protein moiety is responsible, and that the difference between the two factors only concerns the degree of stability. The deutogenic effect would be caused by a protein in an unstable state, the acrogenic effect, by the same molecules in a stable state. Physical or chemical differentiation between the samples has been searched for by the most sensitive procedures, flow birefringence, electrophoresis and chromatography, but so far in vain.

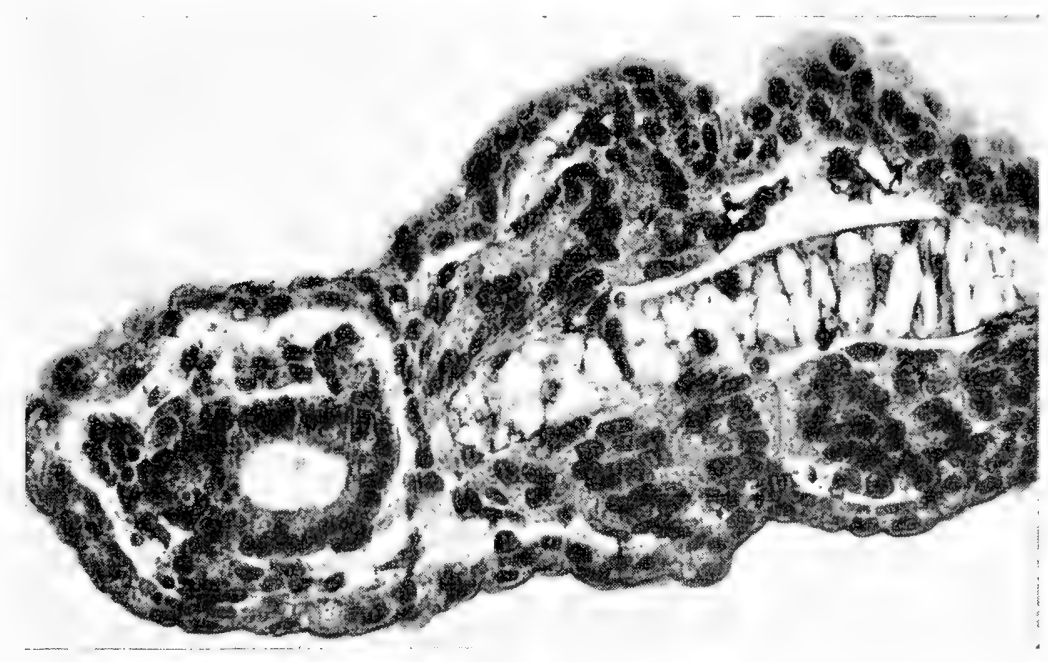

Fig. 9I. Inductive effect of a subfraction of pentose nucleoprotein from guinea pig kidney on the isolated ectoderm, somites, notochord, ear vesicle, mesenchyme and melanophores. From Yamada, 1958b.

That the active protein is bound with RNA cannot be extended to another potent inductor obtainable from the same animal, namely from its bone marrow. Indeed, this potent mesoderm inducer discovered by Toivonen has been submitted to a close examination by Yamada ( $1958 \mathrm{~b}$ ). It is, as stated, typically notogenic, the spinal chord being yet mostly absent. Streptomycine treatment permitted the separation of the RNP fraction and a non-precipitable proteic fraction. The former was nearly inactive, while the latter preserved the characteristic original effect. It is interesting to quote again that warming the sample from 25 to 50 sec. progressively yielded typical notogenesis (with spinal chord), then deutogenesis, and finally acrogenesis ${ }^{1}$.

Thus, from these valuable investigations, it appears that the substance responsible for the xeno-induction exerted by organs of guinea pig might well be a protein but, in the cose of liver (and probably also of kidney, see supra) it is extractable as a ribont:conrotein, while in the case of bone marrow, it is obtained as an apparently frce protein.

1 After a closer study the existence of one in: heat treatment, Yamada (I959) reasserts his preference for §actor, but transformable by adequate means (sce p. 434 
However, this clear-cut exclusion of the RNA as the real inductor is not so far supported by a study of Vahs (I957b), which bears on a quite different source of xeno-inductors, bacteria.

Gram-positive bacteria (Staphylococcus, Thermobacterium, Corynebacterium) were compared to gram-negative ones (Escherichia, Proteus, Chromobacterium). Pure cultures were agglomerated by ethanol, washed, and implanted in the blastocoele. Gram-negative bacteria are deprived of any induction power, while the gram-positive ones induce, in order of frequency, balancers, free lenses, lentoids, and epidermal thickenings, i.e., inductions of an acrencephalic type, but of limited importance. The positivity of staining is due to a Mg-ribonucleoproteic complex and the corresponding bacteria were submitted to agents capable of suppressing this component, e.g., extractions by bile-salt solutions or by $\mathcal{N ~ H C l}$, treatment by ribonuclease, irradiation with bactericidal ultraviolet doses. Bacteria rendered gramnegative by any of these procedure also proved to have lost their ability to induce.

Striking as it is, this result is not yet sufficiently analysed to draw a definite conclusion. The ribonucleic complex has not been isolated, tested for itself and subsequently submitted to enzymatic digestion. As we have seen for other xenoinductors, ribonucleoproteins may appear to play the leading role, but the analysis finally turns in favor of the protein moiety.

To complete the picture, a few data concerning some other xeno-inductors should be added.

In the paper quoted p. 433 concerning the pituitary powder, it is mentioned that a fractionation performed by the Tiedemann's has isolated a ribonucleoprotein with a mainly deutencephalon effect-an agent already manifest in the whole powder-and a protein fraction inducing trunks and tails (tritogenic)

The work of the same authors (Tiedemann and Tiedemann, I956 and 1957) on the extract of chick embryos, a material presumably nearer to the true physiological agent of induction, is especially interesting. It has first been found possible to isolate two protein fractions, one acrogenic and the other tritogenic. Then, 7-9 day embryos have been homogenized and submitted to successive centrifugations, precipitations and extractions. In certain samples, RNA could be eliminated by phenol extraction, while in others, the nucleoproteins were precipitated by using streptomycinsulfate. From the published results and according to a personal communication, it appears that three different inducing agents exist in this embryo extract. The first one exerts a tritogenic induction of mesoblastic character, or nearly; when submitted to high temperature in alcaline conditions, its action shifts towards the exclusive induction of a spinal cord; this agent is a protein, not bound to a nucleic acid, and of a relatively low molecular weight. The second agent is specially deutogenic; it is obtained from the fraction containing the cell nuclei; its activity is distinctly reduced by hydrolysis; this is also essentially a protein, but its action is probably influenced by the presence of nucleic acids. The third agent is precipitable by streptomycine from the supernatant of high speed centrifugation; this ribonucleoprotein induces a deutencephalon, but, if warmed shortly, becomes acrogenic. More detailed information is expected in a near future.

From the standpoint of the morphologist, the novelty in these recent data is the likelihood of dissociating the three organization levels included in notogenesis. 
Definite fractions will perhaps be isolated which would specifically invoke deuto-, cormo- or ourogenesis. The induction of mesoblast and of neural tissue would also be controlled by different factors ${ }^{1}$. Would this experimental dissociation really mean qualitatively different inductors? Is it justifiable to transpose such a notion to normal development? These are two more questions to be solved by future research.

From the standpoint of the biochemist, it appears that these recent investigations are in agreement with the idea that the active inducing agent existing in xenoinductors is a protein, but that in frequent instances it is bound with a nucleic acid.

A similar conclusion has been attained in a rather special case of xeno-induction, discovered by Montalcini, Meyer and Hamburger (1954). The hyperplasia of spinal and sympathetic ganglia caused in chicken embryos by extracts of mouse sarcoma was first found to depend on a ribonucleoprotein contained in the ultramicrosomes of a tumor. Further analysis demonstrated that the activity was bound to the protein moiety (Cohen and Montalcini, I 956, I 957; see also Hamburger, 1956; Levi-Montalcini, I956) which is also present in snake venom, and even in mouse salivary glands.

\section{(f) Interpretative remarks}

In relating this experimental analysis of a most intriguing phenomenon, our interest has been mainly orientated by the search for the active substance(s) which could be sufficiently ubiquitous and biologically active to account for the numerous facts observed. These extensive investigations have however revealed much more than just the biochemical aspects of the problem. As a supplementary reward, they give a new insight into the transformation evoked in the induced ectoblast when the reaction consists either in notogenesis or in acrogenesis. They suggest, in certain cases at least, a plurality of agents, not only corresponding to these two main types of reaction, but also causing either deutogenesis or tritogenesis, or inducing the chordomesoblast separately from the neural organ, and reciprocally. They rise the question of whether these different agents, their authenticity being supposed granted, are convertible in one or both directions, or whether they simply coexist and are able to be selectively inhibited or excluded by various treatments. Let us consider first the morphological processes at play in the notogenic and acrogenic reactions, then the problems of plurality of agents and of their possible conversion, and finally their biochemical nature.

Notogenesis, either complete or under its more regional aspects of deuto-, trito-, cormo-, ourogenesis, is often obtained by implanting normal or foreign inductors, usually via the sandwich method. It is time to stress the fundamental difference between these processes and the induction normally exerted by the roof of the archenteron. The latter process presupposes that the conditions necessary to build a notochord and somites exist before this stage. They are the endowment of the dorsomarginal zone, and we have tried to explain how they have been acquired (p. 343). Notogenic induction implies that the ectoblast is provided

${ }^{1} \mathrm{Niu}$ (1956, p. 162) reports that he used "fixed" adult tissues and various preparations of nucleoproteins and nucleic acids. He often obtained differentiation in neural tissues and pigment cells, but he failed so far to observe the appearance of myoblasts. 
with a factor which it lacks to become chordomesoblast. Since it has been established by several observations ( $c f$. p. 43o, Holtfreter's data and also Grobstein's, p. 463) that induction does not require intimate contact, one may assume, in satisfactory agreement with the studies using isotopes and antigens, that a transport of organic macromolecules occurs. Before penetrating into the offered ectoblast, these molecules are integrated, at least for a while, in the cortex of its cells. Regardless of resulting intimate changes in metabolism, visible early manifestations of induction are: synthesis of new cytoplasm, cell multiplication, and special arrangement of the young cell units. The initial difference between (chordo-) mesoblast formation and pure neuralization apparently concerns the rate of mitosis and the reciprocal arrangement of the resulting cells. Urodele ectoblast, the usual reactor, is a practically one-layered cylindrical epithelium (Fig. 34, p. 359). The first step in induction is a local initiation of numerous mitoses and the formation of a pluri-layered epithelium with more cuboidal inner cells. If both reactions are to take place, the deeper elements stop their proliferation earlier; then, their cells arrange themselves in a rather loose pavimentous epithelium. The upper cells, on the contrary, divide further, mainly from the surface downward, and remain closely adherent, their elongated nuclei suggesting a columnar arrangement inside a nearly continuous matrix. Clearly, the surface properties have much to do with these differences. As often mentioned ( $c f$. Fujii, I944), the same reactor cells may easily differentiate in one direction or another.

Acrogenesis takes place, at least in some instances, as a purely neural induction, limited to prosencephalon structures. In such cases, the xeno-inductor, or its specific isolated fraction, is unable to induce the ectoblast to form the equivalent of the prechordal mesoblast, it cannot cause a sufficient rate of inward proliferation nor adequate surface properties in the reacting epithelium. In this, its primary activity is like the neuralizing agent present in notogenesis, but its effects are more powerful. Given a sufficient concentration, it causes the maximum possible thickening of the ectoblast. This mode of reaction directly attains the structural level of the primary optic vesicle, which is normally the earliest definite primordium to appear in the whole neural organ. Its production expresses the most intense action of the neighboring inductor, as apparent in Fig. 8o (p. 4r 8), as already observable in Toivonen's illustrations (I940), as admitted by Gallera and by myself, also by Yamada (r 950b) and by Hayashi (1956). The complement of the neuralized epithelium, i.e. the part which does not bulge out as an optic vesicle, forms the prosencephalon, with its moderate power of elongation and its ability to induce olfactory placodes. Sometimes, acrogenesis elicits prosencephalic structures without optic vesicle(s). No result, however, suggests the existence of distinct inducing agents; thus, the difference seems to be related to quantitative conditions of the one acrogenic agent.

In notogenesis as in acrogenesis, relations of symmetry are especially important, for they intervene largely in the striking impression of ressemblance with the corresponding regions of the normal embryo. In the whole notomerit, bilateral symmetry results from the differentiation of the central part of the new formed middle-layer into notochord, and from the position of the neural tube. In an 
experimentally induced acrencephalon, symmetry does not seem to appear spontaneously, but if the explant is conveniently shaped, it can elicit a symmetrical fore-brain. Thereby, it approaches the influence of the normal prechordal plate, although the derivatives of the latter control symmetrization more strictly, thanks to their orderly morphochoresis. For, by its kinematic behavior, the prechordal plate behaves typically as a part of the middle layer.

How many distinct agents of induction have been shown to exist in the xenoinductors? This evaluation can only be made by reference to the qualitative, morphogenetic effect registred; this does not necessarily imply-we shall come back to this point-biochemical identity. It is a striking result of these investigations that the number of agents distinguishable on this basis is remarkably small. An early discovery, with amphibians, was that a neural tube induced its like in competent ectoblast (Mangold and Spemann, 1927), that notochord, somitic mesoblast and pronephros primordium all respectively induce their like (Holtfreter, I933a). These results seemed to suggest the possibility of organo-specific xenoinductions, brain by brain, eye by eye, lens by lens, kidney by kidney, but this hypothesis had to be abandoned. The embryonic origin of the organs used does not afford a valuable clue. If such mesoderm derivatives as kidney, bone-marrow, spleen (see p. 446 , note 2 ) are mostly notogenic, the acrogenic activity of the liver does not correspond to any developmental relation, and moreover the inductive effect of one same organ may vary if taken from different species. After the elimination of unadequate ideas, the distinction between an acrogenic and a notogenic agent has become generally accepted, a concept in excellent agreement with the experiments on the major developmental inductors. In addition, some fractionations performed on material rich in the latter agent succeeded in isolating samples which induces electively either deutomerit structures or the (chordo-) mesoblast, or the neural tube. Here the correlation with normal development must be considered with caution, for direct work on the embryos has not yet permitted such dissociations. On the contrary, observational as well as experimental data speak for a close relation, if not for a complete identity, between the inducing factors acting inside the middle layer and the ones evocating the neural plate. In the instances where opposite results were obtained with the xeno-inductors, we may have been dealing with some kind of experimental artefact, which could be explained in several ways. It could well be, for example, that the deutogenic fraction would be a variety of notogenic agent completely absorbed by a relatively small territory of ectoblast, in which its action would be maximal. For the selective induction of (chordo)mesoblast, the explanation could be that the agent is completely incorporated in the deeper layer of proliferating ectoblast and is not transmitted to its surface layer. In the case of exclusive neural plate induction, no inwards proliferation would be provoked, and the epithelial layer would, therefore, be directly neuralized. Of course, these ideas are guesswork, only proposed to show that the multiplicity of specific agents is not necessarily transposable to the normal mechanisms.

For the relation between the notogenic and the acrogenic agent, there are several instances where the former can be converted into the latter by an appropriate denaturation. The opposite conversion has not been obtained by physical 
or chemical means. Yet, in two cases, a biological treatment apparently realizes such a reverse transformation in the guinea-pig liver, which is known as typically acrogenic after the ordinary treatment with ethanol. A sufficient starvation of the donor causes its liver to become notogenic (Vahs, i957a) a modification thus related to a longrun change in the liver cells. A more rapid reverse takes place when a piece of liver (from a well-fed animal) is preserved overnight at $4^{\circ} \mathrm{C}$ in organo-specific rabbit antiserum to be treated $3 \mathrm{~h}$. at $+4^{\circ} \mathrm{C}$ in ethanol, and finally carefully rinsed. A distinct modification of the inductive action is then observed in comparison with pieces treated with normal rabbit serum (Vainio, I958). Both kind of pieces, indeed, become more notogenic than when used without any previous sejourn in rabbit serum, but the shift to notogenesis, perceptible by the higher frequency of fin, pronephros, myotomes, notochord, is more marked after the antiserum treatment. Parallel experiments with bone marrow of the same donors only reveal a reduction of the notogenic effect, and this is also partly true for kidney. The author is of the opinion that these results are due to an adsorption by the liver of a notogenic agent present in the rabbit serum ${ }^{1}$, and apparently more abundant in the antiserum.

It is not necessary to insist that these two exceptions to the ordinary conversion are obtained in complex conditions and do not mean that the acrogenic agent is effectively transformed into the notogenic one.Thus, one is not bound to consider that the transformation is possible both ways.

For the regular shifting from noto- to acrogenesis, it is of course difficult to exclude the interpretation that the latter agent is present beforehand, but inhibited by the former one, which has simply to be eliminated by some procedure, in order to obtain the opposite effect. Toivonen and his group, as well as Engländer and Johnen (1957) and Vahs (1957a), are favorable to this interpretation. Their thesis is newly supported by two accurate investigations. Kuusi (1957) has proceeded to fractionation of the guinea-pig bone marrow and compared the inductive capacity of the various parts. The notogenic-the author speaks of mesoderm inductor-component is predominent, but there is some weak acrogenic action, which the author calls neural. Preserving the bone marrow in ethanol for seven months, in a refrigerator, practically eliminates all notogenic reaction. Fractionation in butanol completely suppresses the same agent, both in the soluble fraction and in the tissue residue; some acrogenic reactions, especially sensory placodes, are maintained. Saline extraction works in the same direction ${ }^{2}$. For several reasons, none of which appears as really cogent, Kuusi considers that "the mesodermal and neural inductors are more or less independent of each other". She adds: "Since these two different types of inactivation exist",-an allusion to the effects of brief heating and of ethanol treatment-_ "it seems improbable that the mesoderm inductor is transformed into neural inductor". Pentinen, Saxén, Toivonen and Vainio (I958) have described a new method fror changing the inducing properties of cultivated cells. HeLa cells are cultivated

1 The existence of inducing factors in blood has been confirmed by Vainio, Toivonen and Saxén (1958).

${ }^{2}$ The same paper mentions that the spleen is also definitely notogenic, the white and red pulps acting the same way; this organ is, like bone-marrow, a strict derivate of the mesoderm. 
either in "active" human serum, or in the same serum inactivated by heating it thirty minutes at $65^{\circ}$. In the first case, the cell-material, fixed by ethanol, shows a predominating notogenic effect, in the second, the acrogenic inductions become predominant. The difference could be due to a notogenic agent present in the active serum and adsorbed on the HeLa cells, so that a maskage would occur. The Finnish authors have a tendency ${ }^{1}$ to equate the two main groups of inductions to a mesodermal and a neural inductor, but there is no firm basis in favour of this oversimplification. It is by far preferable to maintain the concept of the two main types of induction, with the complexity inherent to them, which does not exclude that the corresponding agents can be relatively simple.

What do we know concerning the biochemical nature of the agents responsible for xeno-inductions? It is certainly a noteworthy achievement to have discovered that they are so far uniformly proteins, but, it is scarcely necessary to stress the vagueness of this conclusion. We are ignoring whether or not the agent contained in different organs, and causing the same type of induction is the same protein, whether the efficiency is bound to some definite amino acids, or to sulfhydril groups, whether some structural property is linked with the type of induction. In addition, the relation with nucleic acids remains puzzling. It is certainly of consequence that several remarkable inductors can be obtained as (ribo)nucleoproteins, even if a further fractionation turns in favor of the proteic moiety. There is also the possibility that one agent would be more complex than the other. This eventuality, as seen above, is not excluded by Tiedemann. For Vahs (I957a), the notogenic complex is fundamentally represented by proteins - a contention also admitted by Yamada (I958a, b) -but is possibly associated with enzymes or RNA. $\mathrm{He}$ also assumes that the acrogenic agent is a most ubiquitous ribonucleoprotein which would be primary in the egg, although often masked by the notogenic complex. What it may be, we do not know whether the active macromolecules originate from the epithelial cells of the organ, or from its connective tissue and vascular epithelia, whether they are related to the differentiated structures of some cells, or whether they work in these as metabolites or as enzymes.

In presence of these and other persisting difficulties, it is probably not an idle task to push the discussion in the direction of the relation to normal mechanisms of induction. The first question to consider is that of a possible identity or relationship between the substances extracted from organs and the normal agents of induction. Such an assumption cannot be accepted without caution. It is of course a possibility that the normal inductors subsist in the cells of most tissues, either in their primitive form, or modified, and even that they play during the whole life some unknown but important role. But it is also possible that the xeno-inductors simply

1 This point of view is definitely expressed in a still more recent contribution of Saxén and Toivonen (1958) concerning a further analysis of the inductive power of HeLa cells. They insist that the M-principle, mesodermalizing, is dependent on external factors, i.e., in their experiment, on the nature of the human serum used for cultivation, while the N-principle, neuralizing, is "more stable". It is implicite in their conviction that the inductive agents present in various xeno-inductors and exerting the same effect are necessarily of the same nature, an assumption which, in my opinion, cannot be granted (see below). 
afford macromolecules able to penetrate into the cells of the ectoblast offered to them, and to perturbate their inner metabolism. It is plain that a sheet of competent ectoblast can only behave in a limited number of ways. It can stretch in an undifferentiated epiblast layer; or proceed along the above described events of notogenesis; or adopt the activities leading to acrogenesis; or - not to neglect more exceptional eventualitiesdevelop either into a deutomerit, either into a (chordo) mesoblast layer or a spinal chord. Each of these potencies needs, for its realisation, quite an array of reactions among metabolites and enzymes. Any kind of macromolecules which are apt to penetrate into the ectoblast cells, not being destroyed in this new milieu, and interfering with some of the reactions, will be likely to shift the trend of biochemical processes along one of the two or three progressive ways open to them. If this speculation has some value, it would explain the astonishing extension of the inductive properties, their frequent variability from species to species, their occurrence even in vegetal tissues. The patient search for xeno-inductors, which has absorbed such a huge work, would amount to detecting the macromolecules able to penetrate the cells of the reactor and perturbate their inner equilibria in a non-lethal but profitable way, with the resulting haphasard choice of destinies. From the available data, it appears that such macromolecules would be preferably either (ribo)nucleoproteins or simple proteins. It is likely that the normal inducing agents appertain to the same biochemical categories, but this does not imply any real identity.

These remarks mean that the biochemical problem of induction has certainly benefitted a lot from the study of xeno-inductors, but imperiously needs a direct attack at the embryological level. Personally, I am inclined to admit, as a common feature of xeno- and auto-inductors, the one-way transformation of the notogenic into the acrogenic agent $(\mathrm{s})$. In my opinion this process is the characteristic event which takes place in the normal prechordal primordium. I consider that agents isolated by the Nagoya workers from the kidney and the liver of well-fed guineapigs are the best available models of the two normal inductors. On one hand, they preexist in the organs as ribonucleoproteins, and are probably synthetized as such, on the other hand, it has recently appeared that their active protein moiety shifts from the noto- to the acrogenic effect by becoming more stable, reducing its molecular size and acquiring more ability for cell-to-cell diffusion. Even if the intimate nature of the xeno- and auto-agents is different, these properties seem to be immediately transposable to the normal inductor as present in the archenteron roof. Before trying to make a further and last step in this theoretical discussion, we must acquire additional knowledge of the secondary and minor inductions.

\section{E. Secondary and minor inductions}

idering the primary aspect of neurogenic induction, I have tried to conrecent data and especially on the results which he? is to understand :tion of the normal neural plate. This procedure ould a fortion be " inductions of a secondary character. From this selece ive and restricted ve shall briefly review the main advances in this acive licld of research. 
(a) The cephalic placodes

The head epiblast produces major and minor placodes characterized by their topography and their individual features. Two pairs of macroplacodes and a single one are typical of the acromeritic region, while the chordal part acquires one pair of macroplacodes and a host of microplacodes.

The diagnosis of an acrencephalon could be doubtful if the hind part of it was not soon flanked by the lenses, and the forepart completed by olfactory placodes. Their localization is caused respectively by the optic rudiments and the telencephalon, which thus appears primarily as the olfactory part of the brain. The lens first buds from the epiblast, then detaches itself, its cells elongating moderately without building up fibrillae, their close packing within a tough hyaluronic membrane excluding any vascularization. The olfactory placode behaves more like a small bit of neural plate which would remain incorporated in the epiblast, for its cells elongate centripetally in fibers which characterize them as neurones.

The early specialization of both structures immediately suggests that their inductive agent cannot be identical. Contrary to what happens with homoeogenic induction of which, for exemple, the spinal cord is capable, the acrogenic agent undergoes some transformation when it is incorporated in the optic expansions or in the telencephalic lobes. However, the kinship remains close, for some xenoinductors have been found to elicit both isolated nasal placodes and lenses while others specifically induce the one or the other structure. It seems that some "degradation" of the acrogenic agent gives this result ${ }^{1}$. On the other hand, the problem can be raised as to whether or not the agents inducing the whole optic vesicle, and the one produced by this organ to induce the lens are identical, related, or different. A result of Sasaki, Kawakami, Mifune and Tamanoi (I957) strongly speaks in favor of an intimate kinship. A crude protein extract from rabbit bone marrow induces brain with optic vesicles from Hynobius gastrular ectoblast, while, applied to epiblast of the caudal bud stage, the same agent evokes a lens.

The one odd placode forms the anterior lobe of the hypophysis, and was thought to depend on a conjugated induction by the prosencephalon and the pharyngeal endoblast. But, according to Eyal-Giladi (1958) its main inductor would be the anterior part of the notochord (see also Etkin, I958) acting on the stomodoeal part of the ectoblast, thus on an epithelium already induced by the pharyngeal endoblast.

The neural lobe may form independently of the adeno-hypophysis (Eakin and Bush, 1957), but it exerts a definite secondary induction on the anterior lobe. In Anurans, it has been seen, by cultivating embryos in sucrose solutions, that the intermediate part does not differentiate in the absence of contact with the neural lobe (Driscoll and Eakin, I 955). In Pleurodeles larvae, the bud of cells proceeding from the placode only acquires the characteristics of the various groups of the anterior pituitary lobe if a sufficient contact with the pituitary part of the infundibulum is established. If the neuro-hypophysis is removed or displaced before the first tokens of differentiation appear in the anterior part, it becomes assimilated in the pharyngeal epithelium to form mucous cells or even neurogemmas (Pasteels Jr., I 954, I 957). Moreover, inductive relationships seem to operate in the differentia-

1 The problem of free lenses was also examined by Okada (1949). 
tion itself, for the territory nearest to the neuro-pituitary acquires the basophilic cells, i.e. the thyrotropic and the gonadotropic elements, while the eosinophilic cells, which are shown in this work to be corticotropic, appear farther from the neuro-hypophysis, and remain present for the longest time when there is no longer contact with the posterior lobe (Pasteels Jr., I954, I957). It is pertinent that the important endocrine functions of the pituitary owe their existence to a tertiary induction exerted by the medio-ventral part of the young thalamo-encephalon, being itself the hind-median part of the acrencephalon.

The normal induction of the olfactory placodes has been thought to depend on the forebrain and the endoblast. However, after a re-examination of the problem, Haggis (1956) concludes that the anterior part of the prosencephalon plays the exclusive role. Nevertheless, olfactory placodes have been obtained in the absence of prosencephalon in some of Gallera's experiments (p. 373); they were associated with a rhombencephalon, and their presence seems related to the presence of the anterior part of the archenteron roof.

The causal correlation between retinal layer and lens was the first to be postulated in the study of Entwicklungsmechanik, and the first to be experimentally demonstrated. The discovery of this logical link marked the beginning of the finest chapter in the history of developmental biology ( $c f$. Woerdeman, r 955b). The often-quoted difficulty which arose from experimental cases of apparently independent lens formation elicited much research with far-reaching consequences ( $c f$. Ten Cate, I 956). The sensibility of serological methods has permitted workers to prove (Ten Cate \& Van Doorenmalen, I950, Flickinger et al., I955) the existence of organ-specific protein ${ }^{1}$. Woerdeman (1950, $c f$. I 955 b, p. 43) could even use this opportunity to demonstrate what seems to be chemical induction in vitro. $\mathrm{He}$ first tested saline extracts of the head epiblast of young axolotl neurulae before the appearance of lens placodes, and found that they did not react with antiserum to adult lenses. Then he tested extracts of young isolated optic vesicles in the same way, and found them to be also inactive. Finally, he mixed equal parts of both saline extracts, incubated the mixture at $37^{\circ} \mathrm{C}$ for 24 hours, and got a positive reaction, while the separate extracts incubated for the same length of time remained negative. The clear meaning of this remarkable experiment is that the ingredients for the synthesis of the specific lens proteins are located in the two ectoblastic primordia, optic vesicle and epiblast, and simply need to be brought together to produce the normal result of this induction. A relatively similar experiment has been performed by De Vincentiis (I954) on the frog; this author obtained a positive precipitine reaction between an anti-lens rabbit serum and a homogenate of lens presumptive epiblast, but only when this extract was mixed with an extract of otocysts.

The intimate nature of the action exerted by the retinal layer on the epiblast

1 Recently, Van Doorenmalen (1957) has been able, by combining anti-lens sera with a fluorescent dye (isocyanide), to detect a fixation of the antibodies on the germinative zone of the lens. In the same Amsterdam laboratory, Langman (1957) cultivated primary optic vesicles with the lens forming epiblast in the presence of anti-lens sera and obtained definite alterations of the lens area. Other results also suggest that during the period of induction the cells possess free groups which are able to react with the antisera. 
has been frequently investigated (see Stone and Dinnean, I943) and the necessity of an intimate contact with the reacting epiblast much discussed (Weiss, i 949a, b; Mc Keehan, I 95 I), without reaching a final conclusion. From culture and transplantation experiments, it has appeared that lens induction is not a trigger-like process, but a continuous, gradual one (Mc Keehan, I954; Liedke, I955). A precise quantitative study by Mc Keehan (1956) has compared the variation of RNA content in the retinal layer and the lens of chicken optic vesicles. He concludes that the nearest interpretation is that the retinal RNA is broken up into smaller molecules and transmitted to the lens, a process already admitted by Gallera and Oprecht (1948). It is however likely that these cytochemical modifications are only an expression of phenomena concerning the ribonucleoproteins. The factors determining the size of the lens have been examined by several authors, especially by Balinsky ( 1957 ).
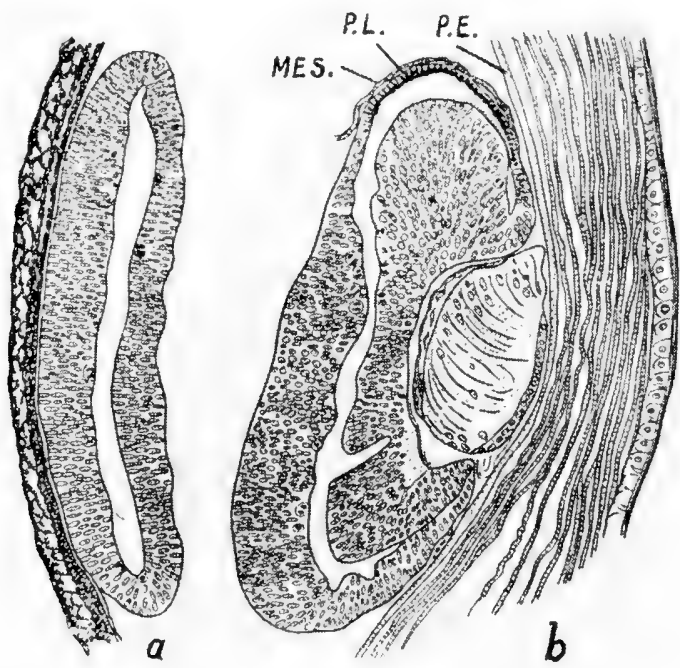

Fig. 92. Modification of the retinal layer of a rat optic vesicle when implanted in the anterior eye chamber of an adult. (a) The implant was a foetal optic vesicle (I I I/2 days) still deprived of lens, and liberated of its mesenchyme; after 6 days in the host, it has become uniformly retinal; left, the host iris; (b) implant was a vesicle with a lens (12 I/2 days), with most of its mesenchyme pealed off; no pigmented layer has differentiated, except in a small zone of contact with the mesenchyme (above); right, the host cornea; mes. - mesenchyme; p.1. pigment layer; p.e. - posterior epithelium of the cornea. From Stroeva, 1956b.

The distinction between the retinal pigmented layers of the optic vesicle has been known for a long time to be experimentally modifiable. An overproduction of retinal parts has been obtained in Ambystoma by grafting the vesicle into contact with the otocyst (Detwiler and Van Dyke, I953; Detwiler I954b). The most efficient part of the otocyst appeared to be the region of the lateral canal with its crista, and the macula utriculi. Let us remark that these active regions of the epithelium are also quite rich in RNA. Stroeva ( $1956 \mathrm{a}$ ) reports that a partial transformation of the pigmented layer into retinal islands is obtainable by rearing frog tadpoles 
in a solution of indophenol $\left(2 \cdot 10^{-6}\right)$, which interferes with the metabolism of melanin. In the rat, implantation of young optic vesicles in the anterior eye chamber of adults also causes overdevelopment and thickening (Fig. 92) of the outer layer (Stroeva, I956b). Contact with mesenchyme favors pigmentation, but it is certainly not the sole factor.

These new data remain in agreement with my interpretation of $194 \mathrm{I}$, mainly based on the relation of the optic vesicle with the prechordal substrate and its asymmetrical mode of folding. The most induced part, with the highest morphogenetic potential, expands more powerfully and invaginates to form the retinal layer, while the upper part, less induced, remains thinner and produces melanin, not without resemblance to neural crest cells. This concept is still valid today. It explains why any supplement of induction causes a retinization of the outer layer. It also implies that a particular middle value is attributed to the peripheral, transitional part of the retina. This is the region which has been shown by Stone (1944, cf. I 948, I953, I957; see also Lopashov, I955a, b) to be capable of regenerating the retina of amphibians throughout life.

Another implication is that the tissues from which the upper and outer part of the optic cup are derived possess a singular potential, for it is the part of the inner layer which has been the least induced, although still sufficiently to become retinal. This singularity explains the phenomenon, ascertained by the many cleverly performed operations of Sato ( $c f$. I953; see also Stone, I953; Reyer, I $954 \mathrm{a}, \mathrm{b}$ ), namely that the upper sector of the iris exhibits the potency of regenerating the lens, if the latter has been extirpated. The bud formed in these conditions by the newt is astonishingly rich in RNA (Takata, 1952). The idea that this means a transfer of material (RNP?) from the retina is supported by the fact that, in newt eyes, isolating the dorsal iris from the neural retina inhibits the regeneration of the lens (Stone, r 958).

The formation of the choroid has been recently restudied by Lopashov (I 956), who considers it to be due to a mixed contribution of the neural crest and of true mesenchyme, both helped by the optic vesicle itself.

In the prechordal part of the head, all vertebrates possess two kinds of sense organs, the most elaborate of which perceives light waves, the lesser receiving chemical stimuli. Similarly, the chordal part of the head is equipped with two kinds of prominent sense apparatus, the most complicated reacting to "static" conditions and sound, the other registering chemical sensation. An intricate system of nerves and perception organelles for general cutaneous and other perceptions completes the chordal part.

The otocyst assumes a special rank among induced structures owing to the fact that although it depends normally on a primary inductor, it is produced by the epiblast after the neural plate formation. The mechanisms at play in obtaining the typical inner ear are necessarily complex, and I have tried recently (I954a) to review the main points of the available information. The primary inductor ${ }^{1}$ is part of the parachordal mesenchyme. It can perhaps be related to the mes-

${ }^{1}$ For the numerous experimental inductions of otocysts, see supra, passim and also Kawakami (1950). 
enchyme forming the myoblasts of the heart. By vital staining of salamander neurulae, Wilens (I 955) has observed that the heart-forming mesenchyme is at first located on both sides of the brain; when it migrates into the mesobranchial region ${ }^{1}$, it is replaced by another mesenchymatous group from nearby, which remains in the otic region. This substitution, if confirmed, could well explain the elusiveness of the primary inductor, and the obstinacy with which the otocyst appears even in the rudimentary embryos.

The action of the primary inductor is immediately relayed by a secondary one represented by the lateral masses of the rhombencephalon ${ }^{2}$ (Yntema, I950). It seems also that the mesenchyme plays a role quite early, as suggested by the discussion on p. 407. This influence may explain why the epithelium remains relatively thin and insures the retention of the endolymph fluid, evidently analogous to the ependymal liquid. These combined factors, however, account only for the appearance of the otic vesicle as an almost spherical or ellipsoidal turgescent body, for the budding of the ganglionic cells, and perhaps also for the first bulging of the endolymphatic canal.

The main division into an utricular and a saccular part, the modelling of the folds initiating the semicircular canals, the stretching and growth of the lagena or cochlea, and many other more delicate features lack an explanation. On the basis of some too elementary cytochemical aspects concerning RNA and glycogen, I suggested ( I 953) that the vascular pattern may be instrumental in this modelling. In the neighborhood of the young vessels, especially of the veins, it can be seen that trophic substances are abundantly furnished to the mesenchyme and that, with its cooperation, definite parts of the otocyst epithelium become more prosperous and bulge or deform in the expected way.

An elaborate investigation of the distribution of several metabolites and enzymes in the cephalic mesenchyme and epithelial structures of mouse embryos has been performed by Milaire. The pattern of glycogen distribution depends at first on the dorsal aorta, later on the veins. The general picture substantiates my previous hypothesis, but many details and complementary relations have now appeared ${ }^{3}$. One experimental although indirect argument for this trophic epigenesis is that in ectopic otocysts, as are formed in non-vascularized grafts of the embryonic shield of Fundulus, the semi-circular canals do not appear (Oppenheimer, I955). This role of trophic epigenesis ought to be explored further experimentally ${ }^{4}$.

The formation of the cartilaginous capsule of the ear is known to be a reaction of true mesenchyme to the presence of the otic vesicle. Experiments of J. A. Benoit (1955,-6,-7a, b) on chick embryos have confirmed this general statement by showing that the mesenchyme does not react if the otocyst is replaced by a lens or by a piece of paraffin. Moreover, they have revealed that all parts of the surrounding mesenchyme do not have an equal tendency toward chondrogenesis. These differences are probably related to the pattern of various territories which cytochemical tests can detect in the mesenchyme of the head.

1 The pharyngeal endoblast could be a secondary inductor for the heart (see Mangold, I956).

${ }^{2}$ According to Nieuwkoop ( $195^{8}$ ) the neural crest of the rhombencephalon may by itself induce an otic placode.

3 This paper will be published in Vol. 70 of the Arch. biol. (Liège).

${ }^{4}$ Walder (1950) has described avascular explants. 
Of the other manifestations which appear in the brain, epiphysis and paraphysis must be mentioned. Both are derivatives of the prosencephalon and are to be considered as delayed inductions. In Discoglossus at least, an isolated epiphysis may be obtained. A detailed analysis is due to Van de Kamer (1949) by experiments on the neural plate stage. This author considers the paraphysis as a "relatively lower grade of segregation" compared to epiphysis.

\section{(b) Neural crest and derivatives}

The limited space unables us to discuss some important contributions as those of Twitty (I 944, I 945), of Twitty and Bodenstein (I 944), of Rawles (1955), of H. Lehmann (1952). Lehmann and Young (1952) and of Niu (1954). Let us restrict ourselves to two aspects of direct theoretical interest for the understanding of induction. The first concerns the biochemical factors permitting and even causing the realization of the neural crest potencies as demonstrated by the previously mentioned (p. 426) experiments of Wilde (1955a, b). The demonstration proceeded in three elaborate steps.

(I) An appropriate nutrient "basal" medium, free of exogenous proteins, was chosen, composed of half-strength Holtfreter's solution, glucose, sodium nucleinate, agar and ultrafiltrated serum. The lateral rim of the cerebral plate of Ambystoma mac., i.e., the material containing the neural crest cells, was excised and cultivated in this medium. The type of cells which differentiated was recorded for these tissues explants either alone or combined with the following tissues: true mesoblast, stomodoeal epiblast and foregut endoblast taken together; foregut endoblast alone or stomodoeal epiblast and true mesoblast together. These histiotypic cultures established first that the explanted neuro-epithelium by itself produced neuroblasts and pigmented ectomesenchyme. With the adjunction of foregut endoblast, procartilage and cartilage proceeded from the same neuro-epithelium. ${ }^{1}$ If stomodoeal epiblast was added to this, teeth were obtained. With these combinations, some striated muscle may also appear. True mesoblast "has no effect on the appearance of differentiated head organs and tissues in vitro, beyond an increase in the amount of striated muscle" (p. 588). In short, all the known derivatives of the neuro-epithelium ${ }^{2}$ are obtained in this basal medium, which means that its composition is completely reliable (Wilde, I955a).

(2) Starting from the suggestion of Willmer (I95I) that neural crest cells may have a characteristic metabolism concerned with tyrosine, and considering that in vertebrates tyrosine is normally replaceable by phenyl-alanine, the influence of analogues of the latter was studied (Wilde, $195^{6 \mathrm{a}}, \mathrm{b}$ ). The addition of $\mathrm{r}$.o mmol/1 of phenyl-alanine (or tyrosine) to the basal medium did not alter the histiotypic

1 This is in good agreement with other works bearing on the branchial region, for inst. T. S. Okada (1956, 1957), Mangold (1957). Mangold has also found that endoblast is an inductor of the branchial structures, and that the anterior part of the endoblast induces the heart.

2 Seno and Nieuwkoop (1958) have re-examined the autonomous and dependent differentiation of neural crest in newt embryos. Corium is obtained if epiblast is available. No leptomeninx nor ganglions differentiate if no central nervous system is present. Nerves are accompanied by Schwann cells. Cartilage may appear in presence of the pharyngeal gut and also of the stomachal region. 


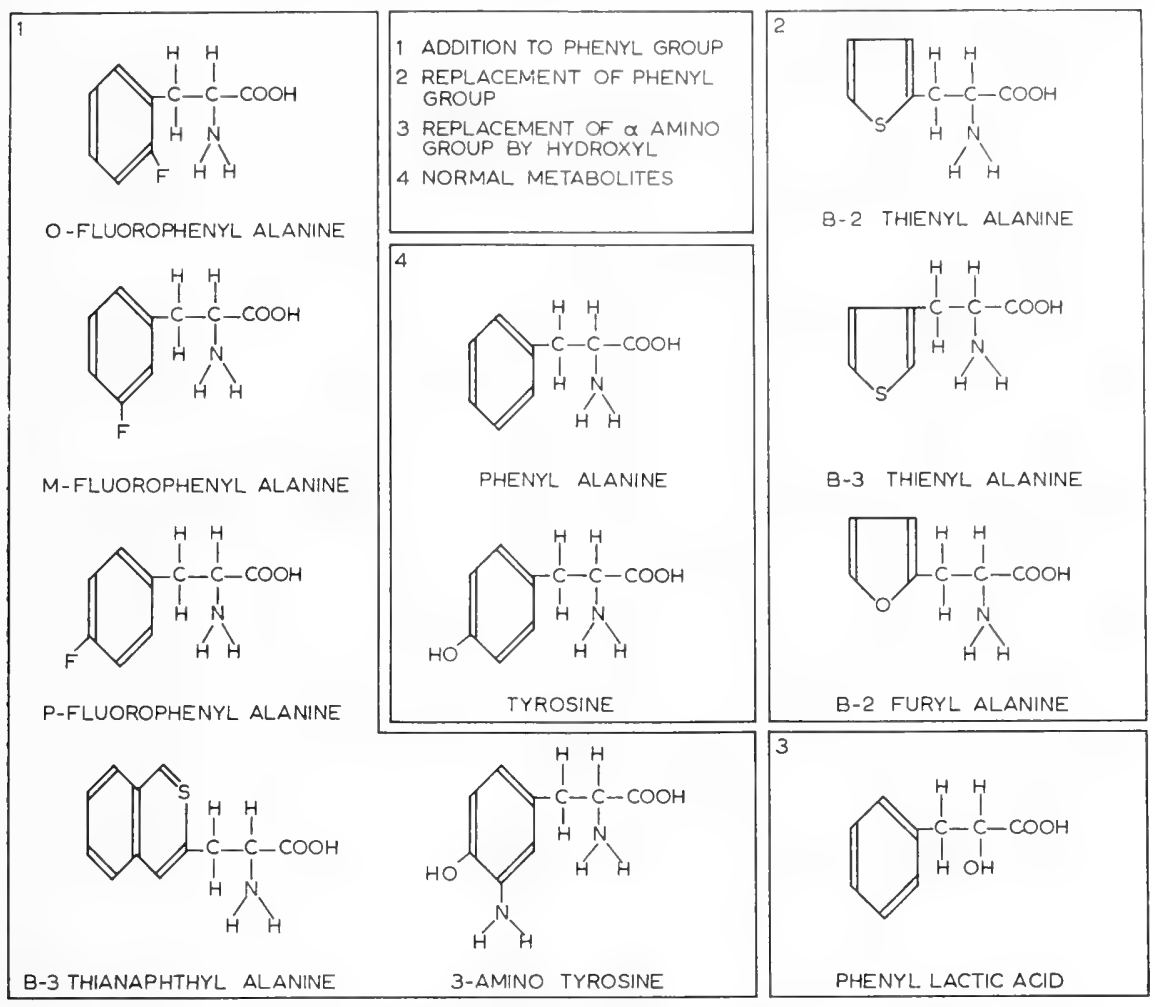

Fig. 93. Synoptic table of the amino-acid analogues utilized to modify the medium in which the explants of neural crest have been reared. From Wilde, 1956b.

differentiation. The replacement of the amino acid by its analogues (Fig. 93) was tested. The recurrence of certain types of cells and the inhibitory exclusion of others could be correlated with the presence of the phenyl ring and/or of the amino group of the molecule. Within the framework of the anti-metabolite theory, admitting that each analogue stops some stage of metabolism by competing with a specific enzyme, it becomes plausible that the mild structural difference introduced "leads to immobilization of each enzyme which acts upon it." Accordingly, melanogenesis and the pigment load in the cells depends on "the availability of sufficient $\alpha$-amino groups on molecules of the configuration of phenyl alanine or tyrosine." Alternatively, "unchanged phenyl groups appear to be essential to chemical processes of ecto-mesenchyme differentiation".

(3) Ventral epiblast of postgastrular stages was cultivated (ibid.) in the basal medium, supplemented by phenyl alanine (I.0-8.o mmols/l). Under this influence, the explant ceases to expand "as a broad sheet of mutually adherent cells". The cells disperse in a typical ectomesenchyme fashion; after some I-Io days spent as amoeboid cells, they become spindle-shaped or stellate "with slender filopodia", and melanogenesis occurs. Such transformation of the ventral epiblast, formerly thought to be nearly determined, means that it possesses beforehand the enzymatic 
equipment capable of utilizing amino-acid molecules with the two phenyl-and $\alpha$-amino groups. On this basis, this supplementary metabolite is necessary and sufficient to induce differentiation of neural crest by mobilizing this pre-existing machinery. This fine analysis is now being extended with reference to the possible activity of precursors of phenyl alanine (Wilde, I956a).

The second aspect of neural crest activities concerns the induction exerted by it and is especially worth consideration, for it discloses a so far unnoticed modality of induction. It has been long known that the mesenchyme of the dorsal fin formed by amphibian tadpoles is a derivative of the neural crest, and is even the inductor of this epiblastic, keel-shaped fold. In I 94 I, Twitty and Bodenstein already insisted that in this complex the epiblast was not passive, but cooperated actively in the modelling of the small organ. This idea has been worked out by Bodenstein ( $195^{2}$ ) in a series of transplantation experiments which are really exemplary. His simple but important conclusion is that, when the inductor is utilized to build op the organ, which is not the rule for earlier inductions, the neural crest first influences the epiblast to grow into a fold, and then creeps into the pocket formed. In addition, by this very process, the epiblast acquires the capacity to influence the included neural crest cells to adopt their special arrangement and become turgescent. The same mechanism of interplay is valid for the formation of balancers, by assuming some more general conditions inherent to the head region. This is indeed reciprocal induction, a concept which can be used now in considering the mechanisms of the formation of the paired limbs.

\section{(c) Limb buds}

A score of problems emerges when we try to understand the limbs of walking vertebrates. How did these appendages evolve out of the paired fins of swimming vertebrates? Why, in practically all vertebrates, does a so-called Wolffian crest of mesenchyme form all along the flanks at a definite dorso-ventral level, near the ventral boundary of the somites? Why do the major parts of these crests rapidly disappear only to leave four small heaps of mesenchymatous cells, two anterior and two posterior ones, adherent to the most dorsal part of the somatopleure? Why do the regions of the trunk, surrounding each young bud, acquire and preserve during the whole life of the vertebrate the potencies of a limb-forming field? Why does the epiblast covering the young bud rapidly thicken into a domeshaped or acuminated apical cap? Why does the growing bud show a distal paddle, evidently announcing the formation of a hand or foot? Which factors do cause the earliest differentiations inside the mesenchymatous filling? Why does the paddle, and soon also the narrowed parts, elongating in arm and fore arm, or in thigh and leg, early acquire a characteristical asymmetry? Which conditions do provoke the ordered bulging of the finger buds? How is the location and shape of the cartilage rudiments, including the girdles, determined? How do the muscles creanize in such a characteristic fashion? How are other anatomical features, such as joints, blood vessels, and nerve tracts, to be explained? Why are the details of these processes different in the upper and lower limbs? What are the mechanisms acting in regeneration of the formed limbs? How are their other so variable morphological features to be explained? 
Most of these questions have still only a programmatic value, while two or three of them have been extensively investigated. The analysis of asymmetry by Harrison ( $c f$. I 945) and his students has been one of the great embryological accomplishments of the interwar period, while the recent post-war years have brought the partial elucidation of induction processes in the limb bud formation.

The greater part of this work has been done on the chick embryo, first by Hamburger (r 938) who opened the experimental way by grafting the limb buds, and later by Saunders and by Zwilling. More recently, descriptions of the cytochemical changes in the rat and mouse limb-buds have added valuable information. The basis of the general interpretation recently formulated is a mechanism of reciprocal induction. The existence of the initial mesenchyme heaps, in their four sites, being assumed as a starting point, these cell concentrations induce the covering
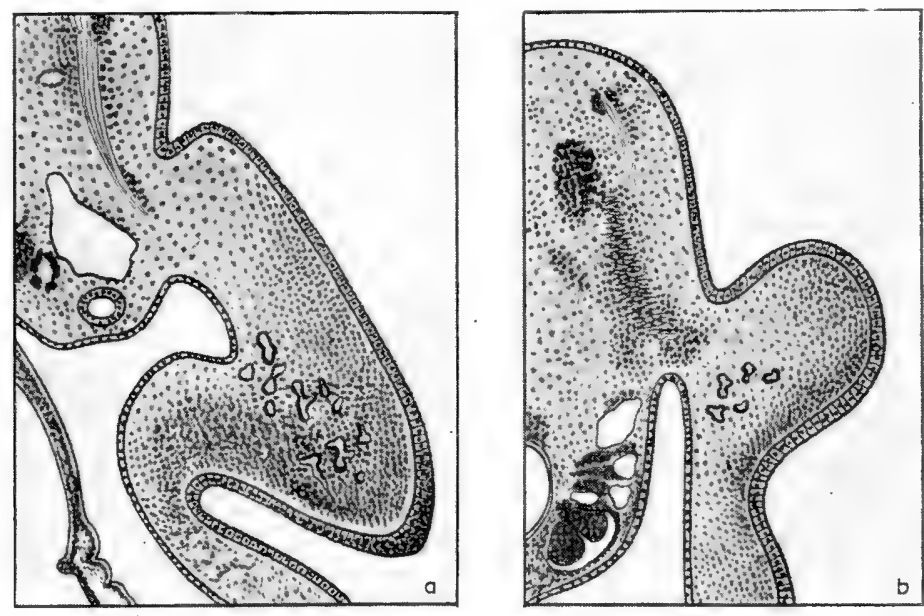

Fig. 94. Role of apical ridge in the formation of the limb bud. Left, section of a normal 4-day chick-embryo, with the wing bud covered by the typical, acuminated epiblast cap. Right, a wingless embryo of the same age. After Zwilling, I949.

epiblast, in which the alkaline phosphatase synthesis (Fig. 38, p. 365) characteristic of induced epithelium can soon be detected. Then the leading role passes to the apical ridge, which influences the mesenchyme, orients its first cytochemical steps toward differentiation, and promotes its growth. (Milaire, I956; Hinrichsen, I 955). The installment of the blood vessels and their local effect on cell nutrition (Milaire, I 956) combines its effects with the slight differences induced in the mesenchyme to promote effective differentiation.

The delicate role played by the apical ridge has been demonstrated by Saunders ( 1 948) and by Saunders et al. (1957, 1958) through methodical experiments performed on the chicken embryo. The non-differentiation of an apical ridge in embryos of a strain affected with a wingless syndrome (Zwilling, I 949) confirms (Fig. 94) the role attributed to this structure.

A further advance has been made possible thanks to Zwilling's method (1955) of interchanging the epiblast and mesoblast of the limb buds. This skillful technique has been 
used for: (a) performing $180^{\circ}$ and $90^{\circ}$ rotations of the epiblast on the mesenchyme mound at the third day (Zwilling 1956a); (b) covering a part of this mesenchyme with an apical sliver, or applying two or even three apical slivers on one mesenchyme mound (Zwilling, 1956b); (c) exchanging the epiblast and mesenchyme between buds of normal and polydactylous strains (Zwilling 1956c); and (d) effecting the same exchange between normal and wingless strains. The results can be interpreted along the lines traced just above, but naturally add some details to the general interpretation. The reality of an interaction between apical ridge and mesenchyme appears from the fact that in expt. $a$, the $180^{\circ}$ rotation gives an asymmetry conforming to the mesenchyme, while a $90^{\circ}$ rotation results in an asymmetry conforming to the epiblast. This difference is interpreted as a function of the deeper perturbation of the system in the second case, so that the tendency already inherent in the epiblast can express itself. The existence of an influence exerted by the apical ridge is also made apparent by the fact that in expt. $b$, a considerable reduction of the mesenchyme mass allows the formation of a normal limb, while covering a whole mesenchyme mound with two apical ridges causes reduplication; however, grafting three apical ridges in a tandem-like fashion does not change the limb unity, which shows that the lateral parts of the mesenchyme are not as reactive as the distal part. This influence is expressed by Zwilling as "growth inducing factor" and the first indications of this action can be noticed, in the rat limb bud, by the peripheral accumulations of RNA, the appearance of the peripheral veinous sinus, and the synthesis of glycogen around this vessel. Finally, expt. $c$ and $d$ show that the mesenchyme continues to exert an action on the apical epiblast even after the 3 rd day of development. This is shown by the fact that if a normal apical sliver is grafted on the mesenchyme mound of a polydactylous limb ${ }^{1}$, this apical ridge will extend more than usual on the pre-axial side, and its growth-promoting action will thereby cause polydactyly. Reciprocally, when a normal epiblast is combined with "wingless" mesenchyme, the apical ridge begins to form, but soon recedes and the growth of the bud stops.

These delicate interrelations between mesenchyme and apical epiblast of the limb bud have been submitted to further investigation. Amprino and Camosso (I958a, b) have used once more the insertion of Carbon particles for a precise mapping of the presumptive territories. There is no doubt that the distal parts of the mesenchyme arise progressively by the expansion of a tiny matrix formed of a few cells, and that, on the way, the topographical relations of the mesenchyme with the apical ridge change gradually. By experiments of section, rotation, reimplantation, these authors have obtained reduplications which cannot be explained by a perturbation of the interactions between mesenchyme and apical ridge; they reveal another influence exerted by the mesenchyme present at the basis of the bud.

Saunders, Cairns and Gasseling (1957) have transplanted mesenchyme of the thigh bud (stage 20) into the wing bud (stage $2 \mathrm{I}$ ) in various locations, realizing different relations of the graft with the apical ridge. From the nature of appendices and feathers obtained, "it is concluded that the apical ridge exercises an inductive action on the subjacent limb mesoderm, bringing about the formation of terminal limb parts, and that this influence affects the regional character of morphological differentiation and inductive specificity of the mesoderm with respect to the proximo-distal axis of the limb but does not affect its specific wing or leg quality".

Thus, it remains admitted that this wing or leg quality is inherent to the mesenchyme and concerns the territory of the body (Guyénot, 1927) in which this mesenchyme has been formed. Taking advantage of trypsin dissociations, Saunders, Gasseling and David (1958) have methodically explored the consequences of rotating the apical cap and recognized a predominant role of its caudal region. By a remarkable correlation, this is also the territory of the cap which is, at least in the bud of the fore limb of the rat, the richest in alkaline phosphatase (Fig. 38, p. 365).

1 The mound of a limb which will become polydactylous is not increased in size. 
This conception of limb development is not only valid for amniots. Tschumi (1956) has extended it to Amphibians by clever experiments performed on Xenopus tadpoles. The general interactions are of the same type, with the complement that the peripheral vessel is induced by the apical cap, exactly as it has appeared to me from the cytochemical aspects obtained by Milaire in the rat.

In the realm of experiments on amphibians, one of the recent interesting results has been the production of supernummerary anterior limbs in the neck when the whole archenteric roof of this region had been replaced by epiblast. The mechanism of the anomaly has now been elucidated (Fautrez, I956). It depends on the sub-division of the limb bud and on the migration of its dorsal half into the region where notochord and rhombencephalon have been suppressed. How this local disturbance causes the doubling of the bud remains to be explained ${ }^{1}$.

Generally speaking, the problem of limb formation is certainly approaching a solution valid for all tetrapods. The mesenchyme provides the epiblast with some agent which, according to data on rodents, produces in this layer a limited RNA synthesis, and a more evident production of cytoplasmic proteins, as well as alkaline phosphatase $(\mathrm{s})$. The mutants considered in the chick interfere with the quantity of this agent secreted by the mesenchyme. Polydactylous strains form too much of it, wingless strains too little. It is also interesting that the asymmetry of a normal limb can be related to an unequal production of this agent in the mesenchyme, at first probably distributed along a decreasing cephalo-caudal gradient, and later also along a dorso-ventral one.

Finally, let us mention that induction in the limb is not only a striking example of reciprocal induction, but also represents, in the complex picture of induction processes, an uncommon mode: the apical ridge regresses as soon as the first steps of morphochoresis are over and simply has the value of a transitory organ with a necessary role in a phase of embryonic life.

\section{(d) Some epiblast derivatives}

Our rapid survey must be limited to two examples of present interest, teeth and feathers.

Teeth primarily proceed from mesenchyme mounds in the location of which the regional endoblast and perhaps the branchial cartilages play a role. The neural crest derivates included in these cartilages and probably also in the palate could explain such inductive activities. This reaction of the epithelium covering such primordia is probably also of inductive nature. As soon as the clock-shaped rudiment begins to form, RNA becomes abundant in the cylindrical epiblast as well as in the peripheral cells, which are the future odontoblasts of the mesenchyme mound. The intensity of basophily at successive stages, and the variations in its pattern (Dalcq, I953b; Hermann, I956) again suggest a role of the ribonucleoproteins whose activity is rapidly followed by the synthesis of alkaline phosphatase (Dalcq and Mulnard, I953).

Feathers and scales, the two kinds of phanera present in birds, are evidently

${ }^{1}$ For other work too specialized to be considered here, $c f$. for example, Balinsky, 1934; Perri, 1952; Dei, 1954; Holtfreter, 1955a. In regeneration, the necessary presence of nerve fibers ( $c f$. Butler and Schotté, 1949) may amount to an induction. The role of the field surrounding the limb has been especially studied in Guyénot's laboratory ( $c f$. Kiortsis, 1953). For the cytochemistry of regeneration, of. Brachet, 1946a. 
formed by the association of dermal mesenchyme with epiderm, like the limb bud. The size of each complexed unit is indeed much more reduced, but it is repeated many times, and the feather germ tracts are disposed according to a distinct pattern, the determinism of which has been explored by Saunders and Gasseling (1957). The possibilities offered by trypsin dissociation and in vitro cultivation have enabled Sengel (1958) to analyse the mechanisms at play in these processes. The method consists in combining epiderm and mesenchyme of different ages and origins. It reveals a succession of four phases.

The first one is of a banal character and concerns the induction of ectoblast in epiblast under the influence of the peripheral mesenchyme. In a second phase, this mesenchyme, which is thus a mixture of mesoblast and ectomesoblast, becomes arranged into distinct mounds, which may be presumed heterogeneous. In 7-day embryos, a medio-dorsal crest appears along the spine, as far as the root of the tail, and soon becomes fragmented in 22 small masses of mesenchyme cells (Holmes, 1935) which are as many germs of feathers appearing as low skin elevations. During the end of the same day and the 8th, new mounds appear successively, in dorso-ventral direction, along parallel rows, but disposed in quincunx and perfectly equidistant. This regularity of pattern suggests the competitive utilization of some trophic material. When Sengel isolated at $63 / 4$ days a rectangular piece of back skin cut on one side of the spine and already endowed with three median rows of papillae, he observed in culture the regression of these germs and, after one to two days, the reappearance of a new row along the median line of the fragment and the gradual differentiation of other parallel rows. Thus, the pattern of feather germ is apt to complete regulation under new trophic conditions. Consequently, the acquisition of the normal pattern depends on a general factor present under the skin, and I should prefer, contrary to the author, not to consider this second phase as an induction. By contrast, the third phase is definitely inductive. The mesenchymal condensations cause the epiderm to bulge in buds which will be determined as feathers or scales according to the nature of the mesenchyme, the latter differentiation being specific of the tarso-metatarsal region. During the fourth phase the epiderm exerts a way-back induction which has a double role: it fixes the direction of the feathers or scales according to the cephalo-caudal polarity of the epiderm and it causes the dermal cells to colonise the epidermal sheath of the feathers.

Compared to the formation of limb bud, the acquisition of phanera-the above analysis may be presumed to be largely valid for hairs-shows a general similarity of mechanisms, but the way-back induction plays a minor role, and only in the terminal phase of the process. This difference relates evidently to the epidermal differentiation of the apical region in the limb bud.

In the formation of feathers, the cytochemical aspects observable in the young buds are remarkable. The dermal concentrations of mesenchyme are rich in both RNA and in alkaline phosphatase. When the shaft has grown upward, an interesting dissociation appears. The parts richer in RNA are in the epiderm, and their variations are paralleled in the mesenchyme by the alkaline phosphatase (Koning and Hamilton, I954). It seems that the metabolite and the enzyme cooperate intimately. Cultures of embryonic skin fragments have recently been grown, in ronditions which permit the introduction of the Beryllum basic acetate $\left[\mathrm{BeO}_{4}\right.$ $\left.\mathrm{C}_{2} \mathrm{H}_{3} \mathrm{O}_{2}\right)_{6}$ ], which is considered to be a specific inhibitor of the phosphatases Hamilton and Koning, I956). The effects are quite striking because, at a given level of inhibition, the feathers resemble hairs. This is indeed very suggestive of the physological importance of these biochemical factors. Of course, every biologist is conscious that these are only two entities among many molecular populations 
which are operative in the process. Nevertheless, the association of RNA and alkaline phosphatase(s), eventually combined with mucopolysaccharides and acidic phosphatase $(s)$ too frequently happens to be deprived of significance. To quote these same authors: "induction or morphogenetic activity at any level of organization involves a chain of reactions, with alkaline phosphatase providing molecular units for the synthesis of RNA and the latter mediating the synthesis of proteins and morphogenesis proper" (1956, p. 70).

Similar remarks could be presented concerning the cytochemistry of the hair buds, which has just been accurately studied by Achten (1959) in human and rat embryos.

The uropygial gland of the duck recently has been submitted to a parallel investigation, both in vivo and in vitro (Lutz and Gomot, I956; Gomot, I956a, b). The folding of the epiblast in the sacral region of the caudal bud is preceded by the appearance of alkaline phosphatase and RNA in a subjacent layer of mesenchyme. When the epiblastic furrows produce buds, the same indices of overactivity are found in their cells. Trypsic dissociations of the caudal bud and culture of its components, isolated or combined, have shown that the inductor is the outer layer of the mesenchyme, only limited by the phosphatase line. This layer of mesenchyme is endowed with the special glandulogenic activity, which is absent either in pectoral or in feather buds mesenchyme. The epiblast, as ordinarily, is unspecialized; it may be transplanted from any part of the body, and even be taken from chicken or rabbit. With toad, frog or trout epiblast, first steps of uropygial differentiation are obtainable (Gomot, 1958).

\section{(e) The induction of cartilages and somites}

As already mentioned, the cartilages encapsulating the sense organs (p. 453) and those sustaining the branchial arches (p. 454) are known to be induced. The same is true for the vertebral arches, as shown by operations performed by Watterson (1952) on Fundulus embryos, by H. Holtzer and Detwiler (1953) and by Detwiler (r954a) on salamander embryos, and by Strudel (I955) on the chick.

In the case of amphibians ${ }^{1}$ at the tail bud stage, removal of the spinal cord and of parts of the somites and the grafting of the neural tube, with or without notochord, in the somite region, demonstrate that the neuraxis acts by establishing somite $^{2}$ growth centers and inducing the cartilages.

The same mechanism works also in chick embryos. According to Lash and Holtzer ( $1957 \mathrm{a}, \mathrm{b}$ ) the inductive influence of the spinal chord passes through a millipore membrane; the material transmitted is not a pure fluid but contains Bodian positive particles. Notochord does not play any role for chondrogenesis in amphibians, but in birds, this organ, although not indispensable for somite genesis (Fraser, I958), is necessary to obtain the vertebral bodies. The chondrogenic influence of the chick notochord has been confirmed by the investigations of Avery, Chow and Holtzer ( I956). It manifests itself when somites of appropriate stages (I4 to I6) are cultured along with notochord. Contrary to what happens when using spinal cord, the cartilage appears in contact with the inductor. According to Strudel, the spinal cord not only exerts a chondrogenic role, but also controls the produc-

${ }^{1}$ See also Muchmore (1958).

2 If somatopleural material of frog embryos is grafted in contact with the hind-brain, muscle cells are obtained (Liedke, 1958). 
tion of separate neurarcuals. This contention should perhaps be checked, since it looks much more plausible that the primary metamerisation (that of the somites) is responsible for the appearance of separate vertebrae.

This point being reserved, the inductive correlation between the spinal cord and vertebral column has been proved for fish, amphibians, and birds, and we shall see that it is also valid for mammals. The possible role of the neural crest has, however, not been excluded. This same correlation is even valid for the regeneration of the Urodele tail (Holtzer, 1956). In order to take place, this process needs the presence of the neural axis; the cord can be shown to induce the new vertebral arches, but only by its ventral motor part. The notochord forms only if it attains the blastema, while myoblasts arise either from the former muscles or from blastema cells.

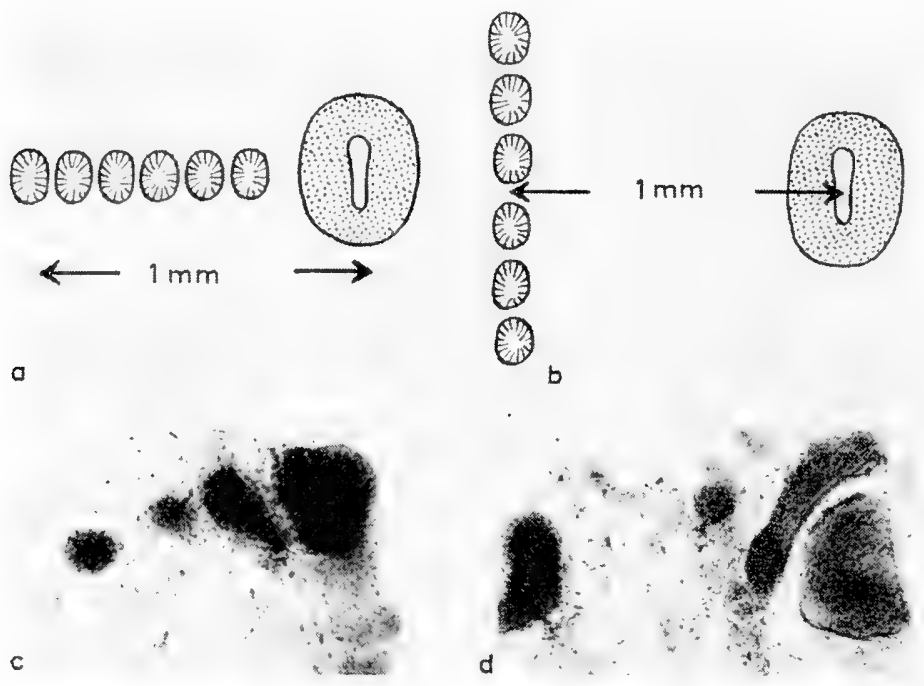

Fig. 95. In vitro combinations of somites and spinal cord to check the importance of proximity. (a) A row of 6 somites starting from the spinal cord fragment, used as inductor. (b) A row placed tangential to the cord, at I mm distance. In both cases, a continuous sheet of mesenchyme is first produced. The (b) controls remain completely negative, unless a fortuitous displacement accidently provokes contact. (c, d) Two positive results of (a). The induction takes place earlier and is stronger in the mesenchyme adjacent to the cord, but islands of chondrogenesis appear at a distance, and not necessarily at the place where a somite was placed. (c, d) from Grobstein and Holtzer, I955.

Axial chondrogenesis has been studied in mammalian embryos by means of in vitro culture of the anterior region of mouse embryos (less than 25 somites) either alone or with pieces of spinal cord; these induce chondrogenesis, and once more the ventral parts are the most efficient (Grobstein and Parker, I954). In a further analysis, Grobstein and Holtzer (1955) checked the necessity of contact for induction to occur. The result of this experiment (Fig. 95 ) is interesting but not decisive. On one hand, chondrogenesis only occurs if at least one somite has been placed near the piece of cord; on the other hand, if this neighboring mesenchyme forms a piece of cartilage, the same impulse toward differentiation may be transrnitted through the sheet of stellate cells to some condensation existing at a distance of I $\mathrm{mm}$ or so. It seems to us as if the inductive stimulus from the cord is not transmissible without contact, but a center of recently-induced chondrification, i.e. early precartilage, would let diffuse an assimilative stimulus at a distance. Observations of Milaire (r956, p. 347) concerning the limb bud suggest that alkaline phosphatase could well be present in the sheet and help in the transmission of this stimulus. 


\section{(f) Other in vitro inductions}

Grobstein (1955a) has chosen to study mammalian organ rudiments in his investigation of induction processes. He has examined three instances of interaction between epithelial and mesenchyme cells of the mouse: chondrogenesis in the vertebral column (see supra), the formation of the submaxillary gland, and the development of the metanephros. The analysis is made possible, in the last two cases, by the use of trypsin as a means of precise separation of two embryonic tissues.

The proliferation and especially the normal arrangement of the salivary epithelium is the most exacting of these processes (Grobstein, I953). It needs the presence of the capsular mesenchyme itself, to the exclusion of mesenchyme of other origin (general mesenchyme, or mesenchyme of lung, or of metanephros). In the case of the kidney (Grobstein, I955b), the interaction is reciprocal; the mesenchymatous blastema of the mesonephric cord favors the proliferation of the ureteral bud epithelium, while these cells cause the activation of the mesenchyme and its organization into tubules. This last effect is not so specific. It is also obtainable with the salivary parenchyme and the dorsal spinal cord ${ }^{1}$. It cannot be considered, however, as a reaction indifferent to the nature of the inductor. Indeed, a rather broad enquiry has been led with embryonic epithelia (salivary glands, lung, stomach, prepituitary, pancreas, Wolffian duct), embryonic nervous tissues (parts of the spinal cord, medulla, fore-brain, spinal ganglia), early embryo parts (head fold ectoblast, neural plate, primitive streak, ectoplacental trophoblast), and adult tissue (iris, cornea, retina, liver, kidney medulla, one kidney tubule). All these living inductors, except the three cited as efficient, were negative. Similarly, negative results were obtained with dead tissues, such as heat-killed or frozen spinal cord, heat-killed liver and heat-killed kidney medulla. This is strikingly different from the xeno-inductors which act on early stages (p. 427).

Since spinal cord may be used for both vertebral chondrogenesis and metanephric tubule formation, a difference between the two cases must be stressed. In the first case, the ventral part is more efficient, the dorsal one practically inactive; in the second induction, it is just the reverse (Fig. 69, b). Thus, pointing to the involvement of some ordinary cell constituent would be an oversimplification.

It has often been thought that interposing some permeable membranes would solve the dilemma between diffusion or contact influence. Grobstein has used this technique for the submaxillary gland and for the metanephros, and got an ambiguous but interesting answer. Using several "Millipore" membranes of 20 or $30 \mu$ thickness, with pores evaluated to be $0.5 \mu-1 \mu$ diameter, he could show that the inductive stimulus passes through the membrane. The salivary epithelium or the metanephric blastema is cultivated on the upper surface of the membrane, the activating tissue on the under surface, and it is observed that the reactor organizes much better, if not always typically, than it does by itself. Sections of the membrane do not show any penetration, or only occasionally in the case of the salivary mesenchyme (Fig. 96, a) while with the complex spinal cord-filter-kidney a penetration of cytoplasmic threads is the rule (Fig. 96, b); if the pores are large enough, the threads can be traced across the filter. By fixing in alcohol-formalin and scratching the tissue out, it

1 For the latter, the activity persists after dissociation and reaggregation (Auerbach \& Grobstein, 1958). 
can be seen that the filter is locally attacked by penetration of organic material containing mucoproteins (Fig. 96, c) and RNA. Correlatively, trans-inductions are more successful with filters possessing the larger pores. On the other hand, the cytological character of the two inductions, which are sharply localized, cannot be readily explained by a long-range movement of freely dissociated molecules. Moreover, such a diffusion process would not be restricted by barriers of the lowest porosity range used, which give less favorable results. Thus, this hypothesis must be excluded, but an opposition of large areas of the cell surfaces must also be discarded. Therefore "something intermediate between full cellular contact and free diffusion is suggested". In this way, the author is led to propose the intervention of an intercellular matrix (Fig. 96, d), an idea worth consideration from a more general standpoint. Part of this cautious interpretation may be modified today. Indeed, in the case of metanephrogen mouse tissue submitted to spinal chord, with interposition of filters, it was now convincingly shown that contact is not necessary to obtain a clearcut induction. The use of T-V filters of low porosity, $20 \mu$ thick, authorizes this conclusion (Grobstein, 1957). The range of maximal distance attains $80 \mu$, and is in good agreement with the measurements made by Cambar in amphibian embryos (p. 352). In a further
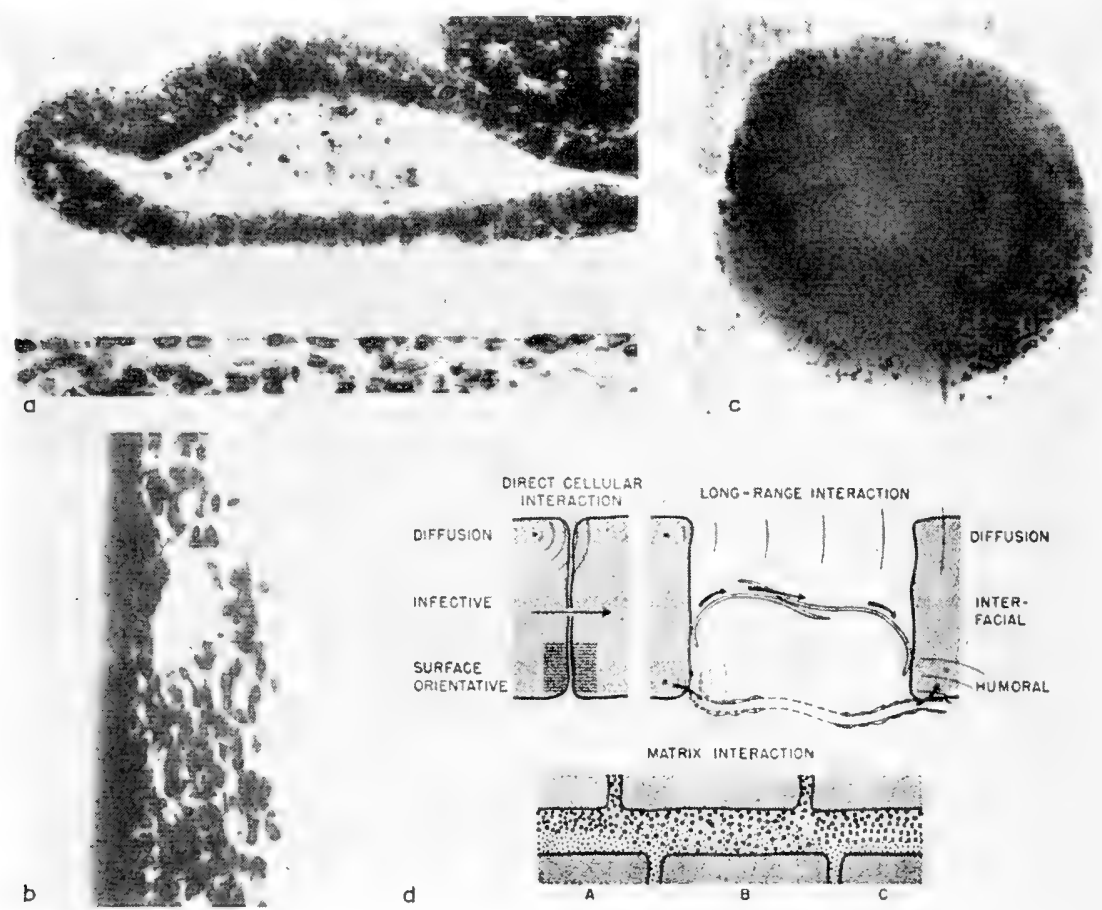

Fig. 96. Role of mesenchyme in the differentiation of epithelium. (a) A section through a culture in which autogenous mesenchyme was placed under a filter (clear space) and the salivary epithelium above. (b) Section stained with toluidine blue through a filter (left) supporting a culture of spinal cord. The stain penetrates through the surface layer of the filter, and ceases abruptly at the limit of the explant (not shown). (c) Surface view of the spot on a filter where cultivation took place. The PAS reaction gives a purplish color, while adhering fragments of tissue are stained deep blue. (d) Schematic representation of assumed or demonstrated mechanisms of tissue interaction and induction in development; A, B, C,

three hypothetical cases of matrix interaction. From Grobstein, 1955a. 
experiment, Grobstein and Dalton (1957) compared the transmission of the inductive activity through three kinds of filters:

a. TA, with pore diameters of $0,8 \mu$, and a flow rate of $363 \mathrm{ml} / \mathrm{min} / \mathrm{cm}^{2}$

b. THA, with pore diameters of $0,45 \mu$ and a flow rate of $67,2 \mathrm{ml} / \mathrm{min} / \mathrm{cm}^{2}$

c. TV, with pore diameters of $0,1 \mu$ and a flow rate of $I 1,5 \mathrm{ml} / \mathrm{min} / \mathrm{cm}^{2}$

$a$ permits a plain induction of tubules; $b$, a definite induction, although any direct contact is practically excluded; $c$, a lesser induction but still clearly positive, while electron-microscope exploration fails to show any contact of cytoplasmic expansions.

There is, however, a new rebound to this multiface question: the formation of definite metanephric tubules has been observed in pieces of the blastem taken at the I I th day before any contact with the Wolffian duct and grafted in the anterior chamber of the eye (Grobstein and Parker, 1958). Would the "milieu intérieur", in the case of the aqueous humor of the eye, contain the necessary inductor? An answer will probably not be long to be found to this new puzzle.

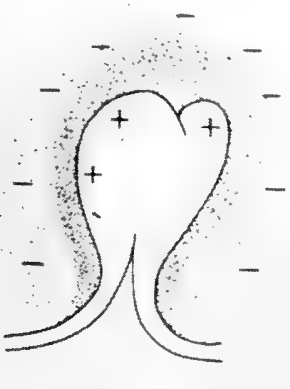

A

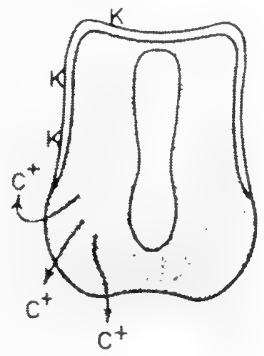

B

Fig. 97. A tentative explanation of induction phenomena in the case of the salivary epithelium (A) and the spinal cord (B).For the latter organ two different properties are considered, the ability to provoke chondrogenesis $\left(\mathrm{C}^{+}\right)$and to induce the formation of kidney tubules

(K). See text. From Grobstein 1955a.

Finally, Grobstein's interpretation of the two phenomena which he was able to analyse most thoroughly is shown in Fig. 97 . For the salivary system $(A)$, three tissues interact: $I$. the epithelium, whose tendency to form dichotomously branching tubules can be disrupted by direct exposure to a surface; 2 . the "condensed" specific or autogenous capsular mesenchyme immediately surrounding the epithelium bud, which at least protects and may promote its morphogenetic tendency; 3. the general mesenchyme, which inhibits the morphogenetic tendency of the epithelium, possibly because of an overly strong anti-spreading effect. It is added that the protection exerted by the capsular mesenchyme may depend on a different, more gelated matrix. For the inducing activity of the spinal cord $(B)$, attention is drawn to the glycoprotein matrix which covers the more dorsal region. 


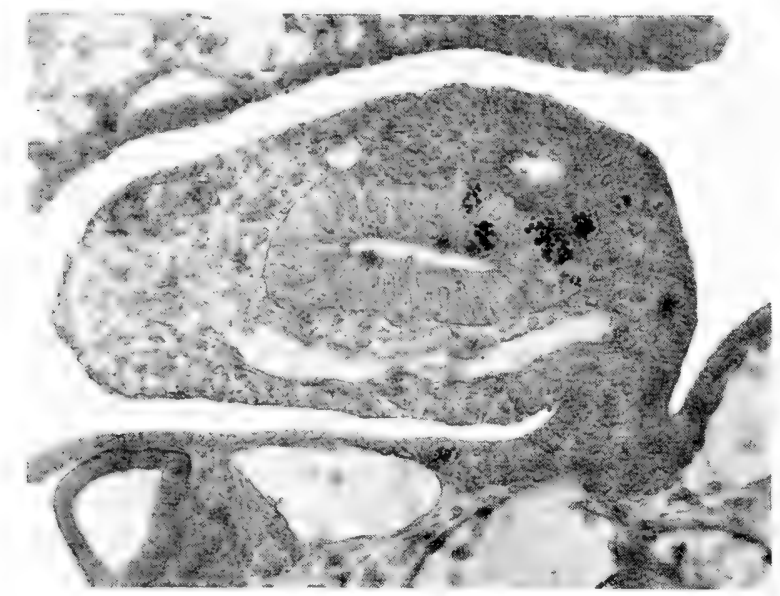

Fig. 98. Gonocytes in the hind-gut of a tortoise, Pseudemys. In this species the gonocytes remain in the endoblast long enough to be incorporated in the epithelium. They are here made evident by a periodic acid-Schiff reaction, preceded by digestion with saliva to eliminate the glycogen. Some of the gonocytes are shown abandoning the epithelium, and one is already in the somatopleura at the left of the mesentery. Photogr. courtesy of J. Milaire.

This material is "involved in the induction of kidney tubules ${ }^{1}$ but impenetrable to the passage of the mobile cartilage-inducing factor produced in the ventral regions".

\section{(g) Gonadic inductions}

At least, an allusion must be made to inductive mechanisms at work in the gonad. They have been discovered by Witschi ( $c f$. 1950) in amphibians, the group where they are now best known. This knowledge, however, is limited to the bare fact that induction occurs, but its content illustrates more clearly than anywhere else the relation of sexual differentiation with genes on the one hand, with hormones on the other hand.

We have already met induction in the formation of the urinary tract where the pronephric duct activates the mesonephric blastema. This is a typical case of intradermic induction, which is convenient to express in terms of morphogenetic potential, the anterior and physiologically older material having the initiative. The events occurring in the gonads of amphibians appear as a further step in the same embryonic area: part of the activated cell cords of the metanephric blastema glide into the young genital ridge.

${ }^{1}$ Grobstein rightly remarks that this function of the dorsal cord region has no obvious in vivo function. He mentions, however, that at the stage of the open neural plate this material was superposed on the nephrotome. This amounts to a suggestion that a normal induction of the nephrotomes by the lateral part of the neural plate takes place. This idea is, I think, incompatible with several experimental results with amphibians. The nephrotomes are not induced, but express a given level of morphogenetic potential inside the middle layer ( $\mathrm{p} .35 \mathrm{I}$ ). 
How and why this ridge is formed, and receives the phosphatase-loaded primary gonocytes is partly known, and probably also implies induction. Indeed, the somatopleural epithelium is activated along the root of the dorsal meso of the gut by two different influences: anteriorly, by the migration of a massive cord of neural crest cells, yielding the "medullary" part of the adrenal complex (not unified in amphibians); and more posteriorly, by the arrival of the gonocytes (Fig. 98), which cause the proliferation of the coelomic epithelium known as the cortex of the gonad.

At this level, the attraction of some of the urogenital cords is possibly due to another induction, but more certainly the penetration of these elements into the forming gonad exerts an inductive action on the cortex. This results from the experimental analysis performed in Gallien's laboratory ( $c f$. I 954, 1956, I958), especially by Houillon (I957) on Pleurodele larvae. The chain of events can be cut at its root by hindering the backward elongation of the pronephric duct. If the mesonephric blastema remains quiescent, no presumptive medulla cells enter the tiny cavity of the genital ridge and the presumptive cortex also remains quiescent.

At this stage, the genetic constellation of factors enters the scene, and its intervention is as decisive as brief.

If the combination of chromosomes is $o$ ( $\mathrm{zw}$ in amphibians), the cortex proliferates and organizes into an inner-secretory gland which rapidly influences the whole soma. From what is known of the process in fish and birds (p. 3I5) it can be admitted by interpolation that the liver of the young o elaborates vitellins and ribonucleoproteins which allow the gonocytes to grow intensely and to perform vitellogenesis.

If the chromosomal combination is 0 ( $\mathrm{zz}$ in amphibians), the medulla is more activated than the cortex and mobilizes the gonocytes to be colonized. This process seems to imply a modification of the surface properties and activities of the gonocytes, which may be rather similar to an induction. The rudiment of the male gonad, enclosed by the quiescent cortex, begins to produce the corresponding sexual hormones, and, sooner or later, the Wolffian and Mullerian duct will be influenced by these hormones. The liver is less sollicitated, at least not in the same sense as in o embryos, growth of the auxocytes remains limited, and yolk is completely lacking.

When sexual hormones are administred at the appropriate period and in due quantity, they can reverse the balance between cortex and medulla. It seems that they act by a differential action, depressing one part in favor of the other. However, it is not excluded that they might awaken some inductive processes, like the drawing into the medulla of the gonocytes, but definite information is lacking on this point.

Considering these events, it appears that the balance of the genes acts clearly on the hormonal setting, but less explicitly on inductive processes. In the organogenesis of the gonads, which, so to speak, closes the circle and achieves the work of the morphochoretic mechanisms, induction seems indifferent to the chromosomal setting, as in all preceding phases, and apparently only needs healthy nuclei.

\section{F. General remarks on induction}

After this rather complete-though not exhaustive-survey of our up-to-date knowledge of induction processes, we should try to take a not too distorted birdseye view of them, to see how thoroughly we understand their general picture. 


\section{(a) About definition}

Typical induction, as embryologists understand it, exists whenever the fate of a cell-layer or group is influenced by another adjacent cell population. The nature of the influence, being still disputable, cannot be taken as the criterion for a more strict definition. There are doubtful cases ${ }^{1}$, in which the older expression of correlation seems sufficient, as when perichordal mesenchyme condenses into the chordal sheath. In apparently similar cases, however, such as around sensory organs, where the condensing mesenchyme is the precursor of the cartilage capsule, this differentiation is more than simply a correlation since an induction is responsible for it. The change in the fate of the reactor also implies that it is still malleable, or, as Waddington says, competent. After so-called "determination" has taken place, some possibility of modulation in the mode of cytodifferentiation may still remain (Weiss, I 952), as when, in an eye grafted with lung tissue, cornea cells proliferate into epiderm nodules which become keratinized (May et al., I957). Such an event is necessarily exceptional: inductive changes of structure are not observed in nonembryonic tissues. The existence of the xeno-inductors could at first sight be interpreted as if the inducing principle(s) subsist in the various organs throughout life, but this idea, as we did see above, does not resist to critical examination. The phenomenon of xeno-induction can be interpreted otherwise (p. 447).

Thus, manifestations of induction are numerous and manifold, with many normal, experimental, teratological and even pathological aspects, but the process remains fundamentally a developmental mechanism. Beyond a certain stage, the induced tissue acquires full autonomy, evident by the fact that in pure culture it will generally "breed true". Nevertheless, the possibility is not excluded that some structures could depend for their maintenance upon the presence of their inductor or even of an organ which they have induced, as for example, the large power of regeneration inside the embryonic nervous axis or the lens-retina relation. As expressed by Bautzmann (I95I), the inductor and the reactor form a functional couple.

\section{(b) On the evolution of inductive processes}

From the evolutionary point of view, inductive mechanisms appear to be a secondary acquisition. Chordates most probably descended from certain Metazoa in which these devices may have been present (or potentially prepared), but not morphologically active. The protochordate ancestors did use them to some extent (p. 355) but their intensive utilization can be thought to have appeared progressively in vertebrates. This progression is patent in the fact that the reproductive cycle itself has rapidly become dependent upon induced structures, and only a few lower groups still show direct shedding of eggs by a genital pore. Repeated improvement of inductive mechanisms, with their extension to organs of circulation (heart), excretion (meso- and metanephros), respiration (branchial

1 Such are also several interactions between neural plate, notochord, somites. It has been considered that the neural plate or the spinal cord plays a role in the segmentation of the mesoblast (Ragozina, I946), that the notochord has a similar influence (Takaya, 1956a), and that the same organ limits the dispersion of the neural crest cells (Tada, cited from Fujii, 1944) 
clefts), feeding (stomodeum and proctodeum), hormonal integration (hypophysis, adrenals, gonads, and probably other endocrine glands), motility (limbs), nervous transmission and sensory perception, and reproduction, has undoubtedly played a considerable role in evolution. Advantages capable of having a selective value were attached to these improvements, which favored, refined, and amplified definite functions of structures, and also increased the degree of freedom in development (Medawar, I 954) and its opportunities for inventing efficient modifications.

Thus, processes of induction were a powerful instrument in the creative interplay between the new modes of development tentatively initiated by the various chordates, especially vertebrate lineages, and the unavoidable struggle for life. Vertebrates may be said to owe their existence to the invention and extensive utilization of developmental inductions. Such considerations have some value for other animal groups, which have also adopted mechanisms of induction, although they are apparently different. For example, in insects induction acts mainly from ectoderm on mesoderm and from mesoderm on endoderm (Bock, 1942; Haget, 1953; Krause, 1958); in echinoderms, induction probably helps the processes of metamorphosis analyzed by Mc Bride (I9I9) while for younger stages it is more a possibility than a working mechanism; and in flat-worms, induction has been demonstrated to be active in regeneration (Lender, I952; Wolff, I953).

\section{(c) On the appreciation of the data}

Part of the fascination in the study of the essential aspects of life is due to their elusiveness. In the last three decades investigations on induction have proceeded in all imaginable ways without attaining a really decisive result. While the picture has been considerably clarified, it has constantly become more complex, without producing the evidence of one unequivocal solution. Confronted with such a riddle, we have no choice but to estimate the relative value of the results and see if they can be organized into a coherent interpretation.

In this task, priority should undoubtedly be given to accurate observation, mainly of normal embryos, enriched by comparison, and sometimes completed by reference to suggestive experiments. This basic information will build up a framework into which experimental data will have to be integrated. The classification adopted in this review and essentially based on methodology, helps this appreciation, but not less weight should be given to investigations described in the last categories; this particularly so if the conditions of the experiment, although quite remote from the normal trend of ontogenesis, are more favorable for a thorough analysis.

\section{(d) On the morphogenetic effects of induction}

Induction, as we have seen, performs several other tasks besides endowing the embryo with a nervous system. In normal development, induction is responsible for regional features of the uro-genital tract, the limbs, probably also the digestive and respiratory tracts. Experimentally, the various parts of the organism may be obtained with a relative selectivity, especially for the acromerit and the notomerit or its hind parts. It is essential to note that their order of succession is rigid. As Rotmann (1949) noticed, it seems impossible to get either an acromerit followed by a 
tail without transitional structures, or a deutomerit appearing at the end of a trunk. In spite of some fascinating accomplishments, the experimenter remains subordinated to law inherent to vertebrate organization.

The part played by induction in the construction of the nervous system is an accomplishment which legitimately deserves our due attention. In the normal course of events, the neural plate appears as a progressive cephalo-caudal transformation involving a characteristically delimited field, at first uniform at any transverse level. The process looks continuous, but the shape of the young plate, with its gradual restriction of breadth behind the cerebral region and the rather uniform width of the spinal part, suggests some duality in the subjacent inductor.

In this first step of neuralization, we may speak with Waddington of an evocation of the neuroepithelium. All cell functions are apparently reinforced, and ribonucleoproteins and alkaline monophosphoesterase are produced. These cytochemical data suggest many other changes in metabolites and enzymatic equipment. The cells grow either by utilization of their yolk or thanks to imbibition by a nutrient flow, processes which imply further syntheses of proteins. The DNA metabolism is soon involved, cell proliferation takes place, and a columnar arrangement of the epithelium appears, probably due to the orientation of macromolecules inside the cytoplasm and to special surface affinities which draw the elongated cells toward the medio-sagittal plane with a convergence-extension movement. It will be useful to state here that this initial set of events is fundamentally the same at the beginning of later, less extensive inductions.

Evocation of the neural plate leads to a mass transformation, in close correlation with morphochoresis of the inner materials. These independent events correspond to Waddington's concept of individuation. The concentration of cell toward the medio-sagittal plane combines with an active elongation of the whole plate, which also begins to fold, more rapidly around the head-trunk limit. The cerebral region takes form as a whole and its closure proceeds slowly toward the neuropore, while the presumptive medulla already shows more individuation by forming the powerful cephalic neural crests. While the cerebral vesicle is still closing anteriorly, the anterior half of its floor partly bulges into optic vesicles, the rest becoming the diand telencephalon. During the relatively late stages of this process, more of the neuroepithelial cells begin to throw off the thin elongating extensions which characterize them as neuroblasts.

Thus, cytodifferentiation follows a prolonged phase of participation in morphochoresis, during which the neural axis acquires its typical shape. However, cytodifferentiation does not necessitate the previous completion of these more general individuation processes. Induction in vitro (Niu) clearly shows that after due time, neuroblasts, ectomesenchyme, and also myoblasts (the latter by paragenesis) can be obtained directly without global morphochoresis. Thus, induction endows the cells with the complements necessary and sufficient for their cytodifferentiation. This statement may be considered of general value. Of course, the question whether all kinds of cells need to be induced in order to differentiate remains unanswered. One could be tempted to decide that chordal, somitic, and endrblastic cells differentiate by their own resources. However, the fact that any explant from the corresponding areas, no matter how tiny it may be, develops 
into several kinds of mixed cells, calls for caution, as does the interdependance of rudiments inside the middle layer. It might well be that, in the beginning, differentiation always depends on exchanges with the intercellular medium, itself conditioned by neighboring cells, while later a metabolic specialization calls forth the so-called determination.

Whatever it may be, typical induction leads to differentiation not only in the neuro-epithelium, or in its secondary derivatives, retina and tapetum, but also in the lens, the olfactory and auditive epithelia, lateral line organs, limbs, mesonephros, metanephros, and all kinds of glands. Thus normal induction provides the conditions not only for the shaping of the rudiments but also for their functional differentiation, which is the real aim of the process.

Clearly conscious of these effects of induction we may now attack the core of the problem as it stands today: the individuation of the neural axis into a prechordal and chordal part. By the preceding remarks, and also by those on p. 408, we are prepared to admit that the differences are not abrupt, but gradual, mostly related to quantitative variations in the concentration of cells, the intensity of proliferation the extent of mass movements, the progression of cyto-differentiation, the dispersal into ectomesenchyme, and the amount of melanin produced. Even such remarkable layers as the retina and tapetum are not so different from other neuroepithelial derivatives: at the onset, the retina makes an impression only by the number of neuroblast layers and the orderliness of their concentric differentiation, while tapetum behaves nearly as neural crest cells. On the other hand, inspection of neural development and the course of many experiments testify that the characteristics of the various regions are not acquired at once, as if released by some trigger mechanism, but evolve progressively.

Although practically all authors concerned with induction have expressed more or less personal views on this problem, nowadays three or four main positions may be distinguished and considered without reference to their preliminary or side aspects.

The one proposed by Yamada (I950b) and repeatedly advocated by its author and his coworkers admits the distinction between a dorso-ventral morphogenetic potential $(P . d v)$ and a caudo-cephalic one $(P . c c)$, decreasing as suggested by these expressions. It was an original and important step to consider the second gradient as increasing from head to tail, in opposition with the current assumption. The same idea must also be accredited to F. E. Lehmann (I950). To represent the factors which confer to each gradient the values attained at each level during the successive phases of early development, Yamada invokes mediators (a dorso-ventral and a caudo-cephalic one, $M . d v$ and $M . c c$ ) which reflect, translate or sum up the normal or the experimental conditions ruling individuation. The representation is certainly supple and suggestive but implies a fundamental duality in the morphogenetic tendencies of the normal egg. The factors represented by $P . d v$ and $M . d v$ are supposed to be inherent to the presumptive ectoblast, the $P . c c$ and $M$. cc agents to the chordomesoblast.

The interpretation proposed by Nieuwkoop et al. (1952) stresses the auto-organizing tendency of the activated ectoblast. In his opinion, this process is a release mechanism, which is necessarily the first step of all neuralization. Left to itself, the neuroepithelium essentially proceeds to build up a prosencephalon. The formation of other parts of the neural axis depends on transformation agents which are normally produced by the chordomesoblast. Thus, normal neurogenic induction is due to the succession of activation and transfor- 
mation. The author of this fascinating theory insists more and more on the dynamic character of this two-step process.

Toivonen, on his side, takes an intermediate position, (I958) well represented by Fig. 99. Not convinced of the validity of Nieuwkoop's theory, he simply advocates the double action of the normal inductor. One action is neuralizing, and the other is mesodermalizing. These gradients are in opposition to one another.

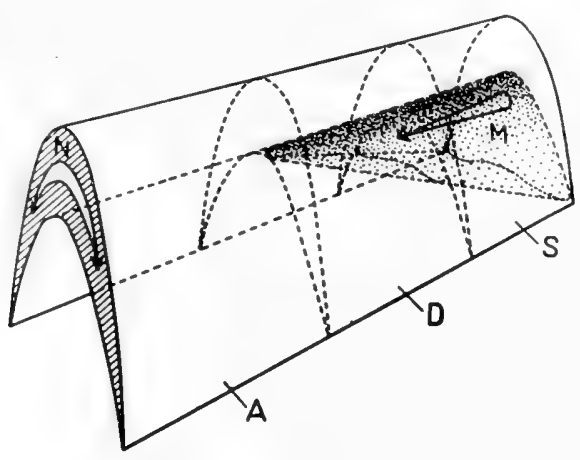

A

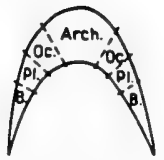

D

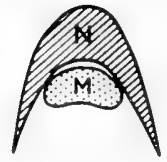

S

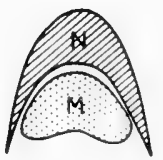

Fig. 99. Scheme illustrating the gradients of the two inducing principles in normogenesis. N. - neuralizing principle; M. - mesodermalizing principle; A. - archencephalic region; D. deuterencephalic region; S. - spinocaudal region; Arch. - archencephalon; Oc. - eye; Pl. placodes; B - balancer (Toivonen and Saxén, 1955b).

About seven years ago, in order to cope with the results which have brought to light the existence in gastrula stages, of the two distinct agents of induction, I have modified (1952) the proposal primarily formulated in collaboration with Pasteels. In my opinion, Nieuwkoop's activation is an experimental result which does not reveal the true nature of the agents initially operating in the normal germinal system. To conceive of the egg as a morphogenetic unity seems to me a logically cogent condition of a coherent representation of these events. Also, without adhering too closely to a principle of embryonic recapitulation, I think it preferable to adopt a view which can easily agree with evolution, whose progression has in any case taken place through the fertilized eggs; it is unsatisfactory to imagine that the primitive equipment of vertebrate eggs would have directly aimed at building up a fore-brain with its highly specialized sense organs. On the contrary, the primary purpose must have been alimentary and motoric, still evident in the predominantly muscular organization of inferior groups. Therefore, I think it plausible to attribute more value to the results of operations and centrifugations, which reveal the primary tendency to notogenesis. This tendency exists inherently in the dorso-marginal zone, which is also the source of the normal inductive agents; in order to stress its primitive, archaic character, I prefer to call it protochordal $(P)$. It is not totally restricted to the "organizer" but extends to the whole ectoblast, where normally it remains latent. In the normal course of events, the protochordal tendency implies that in the area where these agents reach their maximum concentration, either a spontaneous "maturation" occurs (anurans), or morphogenetic kinematics comes to the rescue (urodeles), thus causing the acrogenic conditions $(A)$ to evolve. This acquisition 
of two different but genetically related inductors is a progressive, time related event. The conversion process $P$ to $A$ explains the origin of the powerful prechordal plate, but it is necessarily not limited to this structure. Several experiments suggest that the cephalic part of the dorsal chordomesoblast or even the whole roof of the archenteron produces the acrogenic agent, but at a decreasing rate from head to tail; therefrom the apparently caudo-cephalic gradient $P$ as a secondary feature. It could even be imagined that a headward flow of $A$ products gradually reinforces or feeds the inductive yield of the prechordal anlage, but positive data for this idea are not yet available. Its only real justification is that it would give a functional meaning to the nearly generalized $P \rightarrow A$ transformation. In any case, a score of experiments demonstrate the relatively labile state of the $P$-tendency and its inclination to shift to the more stable $A$-condition, while no pertinent example of the opposite process has been observed. Only early centrifugation and Li-action could be thought to cause this reversal; however, it is much more satisfactory to consider that these treatments either inhibit, stop, or moderate the transformation $P \rightarrow A$, an interpretation supported by their depressive effect on the $P$-tendency itself.

It is immediately apparent that these four theses have several points in common. The difference between the proposals of Yamada and Nieuwkoop is mainly that the former does not insist so strongly upon the succession of two neuralizing agents. On the other hand, his conception could easily be made compatible with ours, since a dorso-ventral decrease of potential is also at the basis of my interpretation. I also agree with his "caudocephalic" gradient but emphasize in addition that it is established secondarily. However, I do not refer to abstract mediators, but rather attribute acrogenesis to the secondarily evolved prechordal plate, and notogenesis to the primarily present chordo-mesoblast.

At present, the main difficulty is to decide whether or not the necessity of the two successive steps of activation and transformation in early neuralization can be admitted. For the simple formation of the neural axis, it can be easily agreed that secondary individuating influences are exerted on the one hand by the prechordal plate and on the other hand by the derivatives of the chordo-mesoblast. Concerning the cause of the initial change, I do not adhere to the two-waves theory of induction, for the following reasons: (I) the appearance of the neural plate is a continuous event; (2) the fold experiments can be interpreted by hypothetizing a continuous production of the A-agent in the whole chordomesoblast, with graded space-time variations; (3) the activation-transformation idea is contrary to the bulk of evidence relative to the exclusive modification of $P$ into $A$; 4 ) the fact that in certain sandwiches acrencephalic structures appear earlier and farther from the inductor than notogenic ones may be explained by the probable smaller molecular size of the $A$-agent; (5) the necessity of a preliminary activation does not manifest itself in the Yamada's latest experiments, where the $P$-agent seems to have been better isolated than ever before; and (6) although the course of events in amphibians is favorable to the idea of an activation by the foreward migration of the prechordal endo-mesoblast, the same process is not evident in birds, where the presumptive prechordal plate, included in the head process, does not come into contact with the truncal part of the presumptive neurectoblast.

The unitary interpretation which I advocate is also in agreement with our knowledge concerning the genesis of the dorso-marginal zone. It still allows us to consider the normal course of events in terms of morphogenetic potential, a preliminary expression which should be translated into biochemical terms when possible or advisable. We should also be conscious that this term includes substances which are not intimately the same at all successive stages. Before the blastoporal lip appears, differences between areas are, in my conception, purely quantitative within a single field, the substrates of which so far escape analysis. When gastrulation is put into action, a qualitative difference emerges, little by little. Morphogenetic potentials should be compared not only within the general field ordered around the presumptive notochord, but also in the more restricted region devoted to the acromerite ( $\mathrm{p} .452)$. For this stage, we have at least indirect evidence concerning the nature of the inducing agents (see infra). With neurulation, the picture changes again. In the notogenic part, the middle layer distinctly shows three levels of potential, still able to merge with each other (p. 352), while around the spinal cord and medulla the role of the mesenchyme complicates the situation (p. 406). In the prechordal part, relations of potentials 
become especially useful to explain transformation in the visual organs. Again, in this restricted field, the biochemical substrate of these relations is certainly modified, but any precision in our knowledge is lacking.

Our point of view also makes intelligible the close relation between myogenic and neurogenic induction. Except in some exceptional cases discussed p. 445, the same agents which induce the neuroepithelium at the surface evoke mesoblast and even chordo-mesoblast in the deeper layers (p. 445). The conditioned medium of an organizer culture induces myoblasts as well as neuroblasts (p. 409). Various experiments cause a local chordalization of the neuro-epithelium. This can even happen with a prechordal derivative such as retina (Fig. 74, p. 413), which is a good sign that there is a basic affinity between these apparently distinct differentiations. It is not too audacious a guess to consider that in the archenteron roof, in an implantation- or in a sandwich experiment, much depends on the amount of inducing agent that the (chordo-) mesoblast reserves for its own differentiation in comparison with the amount which is allowed to escape, apparently after some transformation, and to exert neurogenic induction. The prechordal mesoblast is, so to speak, the least greedy, while the caudal mesoblast even absorbs a supplement of inducing agent (p. 368 , note). Experimentally, the balance may be shifted nearly completely either in favor of the spinal cord (Fig. $87, \mathrm{p} .436$ ) or in favor of the chordomesoblast, which is formed without a trace of neuroepithelium (Fig. 9I, p. 44I).

A word should be added about the concept of morphogenetic fields. In early stages, the expression of morphogenesis in terms of fields is easy to grasp; it simply designates a certain area characterized by homogeneous but varying properties. At later stages, the concept becomes less clear, for it points to the territory surrounding a forming rudiment, e.g. lens, ear, or limb and suggests the existence of ambiguous latent potencies (see p. 353). A solution has been proposed by Kawakami ( $195^{2}$ ) in an interesting discussion of several operations yielding secondary inductions. In his own terms, "mesoderm having strong inductive capacity operates as the inductor of the sensory organs, while the same, with weak inductive capacity, determines the field of these organs". Other conditions like cell dispersion and competitive relations, could also be considered, but progress toward a logical explanation is sufficiently indicated.

\section{(e) On the nature of the inducing agents}

As soon as it was recognized that induction was not necessarily restricted to living parts of the organizer, the opinion prevailed that the process was caused by substances released by the inductor, and several workers tried to find out their biochemical nature. They soon realized the complexity of the problem, and the importance the part played by secondary elaborations inside the reacting tissue. However, there was no doubt that a substantial flux was at work, until Weiss ( I949a) drew attention to the probable necessity of contact between the protagonist tissues. He suggested that transmission was questionable and could be tentatively replaced by an occurrence of physico-chemical changes due to mutual influences between the cell surfaces causing consequences within the molecular population of the cytoplasm. This proposal deserved and received consideration, but was not (c) far confirmed. Its merit has been to emphasize the undeniable importance of the surface properties of cells, but the modification of these surfaces without penetration of extraneous molecules seems entirely theoretical, at least for induction processes. In induction, the available evidence remains favorable to an ab- 
sorption of active substances, i.e. molecules with definite appropriate configurations. Some cases of induction at a distance or through a fluid medium (p. 409) have been observed. With dead inductors, the topographical relations may often be best interpreted by imagining a diffusion gradient from the implant (Fig. 89, p. 438). With living inductors, a similar relation can be sometimes observed in vivo, as Pasteels did when studying the influence of the primary embryo on a secondary one obtained by centrifugation of the blastula (p. 4I4). The crucial test of transmission at a distance was effectively satisfied, at least for a definite secondary induction, ${ }^{1}$ by the use of adequate filters, and the objection could be eliminated (p. 465) that a contact by thin cytoplasmic filaments penetrating the pores was necessary.

The appreciable benefit from this discussion is a better understanding of the different possible mechanisms for the transmission of an inductive agent (Fig. 96, p. 464). My impression is that a transfer of organic molecules may be considered certain, but that their transmission through a fluid medium is only experimental, the normal process taking advantage of close contact and probably of the cellular intercellular matrix.

The biochemical nature of the transmitted agents is, at least for many workers, the goal of these investigations. Fractionation of xeno-inductors has been much used in this purpose but, as we have seen in the discussion of these investigations, it is doubtful that they could reveal the nature of the agents at play in normal induction. For this, the normal process should be studied in itself, and this has been done at least in some instances.

Cytochemical methods have been accurately applied to primary and secondary inductions, and they have uniformly drawn the attention to two components, the ribonucleoproteins and the alkaline phosphatase. The active synthesis of this enzyme can be shown, on adequate material, as occurring in the reactor, and is thus more a consequence than a cause of induction. By contrast, changes in the RNA reaction are observed as well in the inductor as in the reactor, as first recognized by $\mathrm{J}$. Brachet. Of course, in xeno-inductions, it has been repeatedly demonstrated that RNA is not the active fraction, but Brachet was right when he remarked (Brachet, Kuusi and Gothié, 1952, p. 439) that these criticisms do not invalidate the relations he had formerly stressed in normal development. Moreover, the variations in RNA content may be thought to concern, in the living cells, ribonucleoproteins.

The RNA of reactor and inductor at various stages has been measured, as related above (Fig. $3^{6}$, p. 362 ) by Rounds and Flickinger. Their results agree with the preceding idea, except that the increase in the reactor does not really coincide with the decrease in the inductor. It seems thus that induction is not only a transfer of definite agents ${ }^{2}$, but also the awakening of various syntheses in the reactor cells, according to my concept of metabolic contagion ( 1940). The introduction of foreign macromolecules in the induced cells would not be simply an enrichment by addition, but the release of new syntheses, an idea also advocated by Nieuwkoop,

${ }^{1}$ For primary induction, a new tentative by Brahma (1958) of interposing a filter failed.

2 Possibly in very small amounts (see p. 363). 
but only for his initial activation. Such a release mechanism probably implies enzymatic activities, of which we perceive some signs by the detection of alkaline phosphatases. In the idea that some ribonucleoproteins could be reponsible for induction, Flickinger (I956) has attempted to explain their origin. This author draws the attention on the relation of phosphoprotein-phosphatase activity to yolk platelet utilization in amphibian eggs. He suggests that livetin and its associated RNA are released from the yolk platelets to afford the inductive agents. We did see here that several arguments, as well experimental (p. 343) as biochemical (p. 36r) favor this idea of the participation of yolk in morphochoresis. If such is the case, mammals, where yolk is not longer present, should be studied in order to find the biochemical devices which they have substituted to the use of yolk derivatives. The remarkable increase of RNA in the embryonic knot of mammals, and the coincident appearance of alkaline phosphatase, further the gradient field of RNA in the ectophyll are to be kept in mind.

A direct study of the primary inducing agent(s), presumably without alteration of these, has been attempted by Niu (1956) by mass cultures of small pieces of organizer-tissue, explanted with a minimum of coat. The active macromolecules are presumed to pass into the "conditioned" medium (p.408), and its content was used to culture competent ectoblast and was submitted to biochemical examination. A sufficient quantity of such media could not have been gathered from hanging drop cultures, but was obtained by cultivating 20-30 pieces of organizer-tissue in the well of depression-slides. "Seventy such slides prepared at one time will yield, after 7-10 days, 5-6 $\mathrm{ml}$ of the conditioned medium. . . The advantage of this method is that after withdrawal fresh salt solution may be added to the culture and 7-10 days later the medium may be withdrawn again. This process is repeated 4-6 times". In this way, conditioned media from the ist to the 6th order could

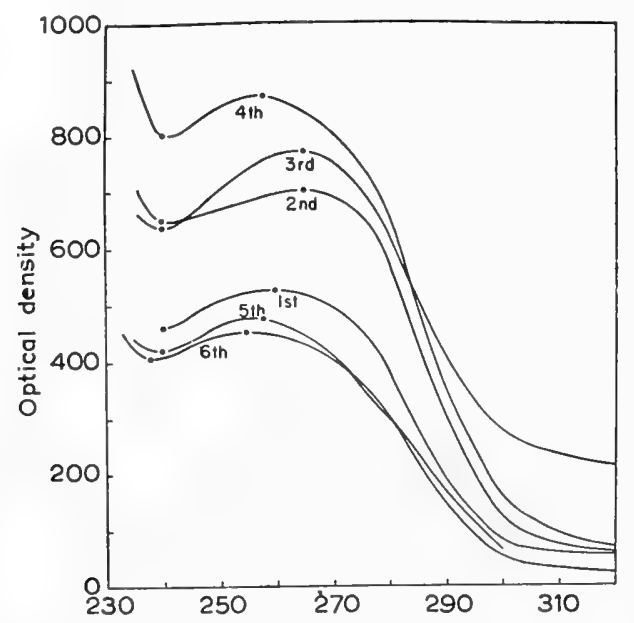

Fig. 100. A chart of U. V. absorption spectra of the conditioned media collected from deep-well cultures of $A$. tigrinum dorsal lip. Ist to 6 th, the conditioned media from the first through the sixth order (see text). From Niu, 1956. 


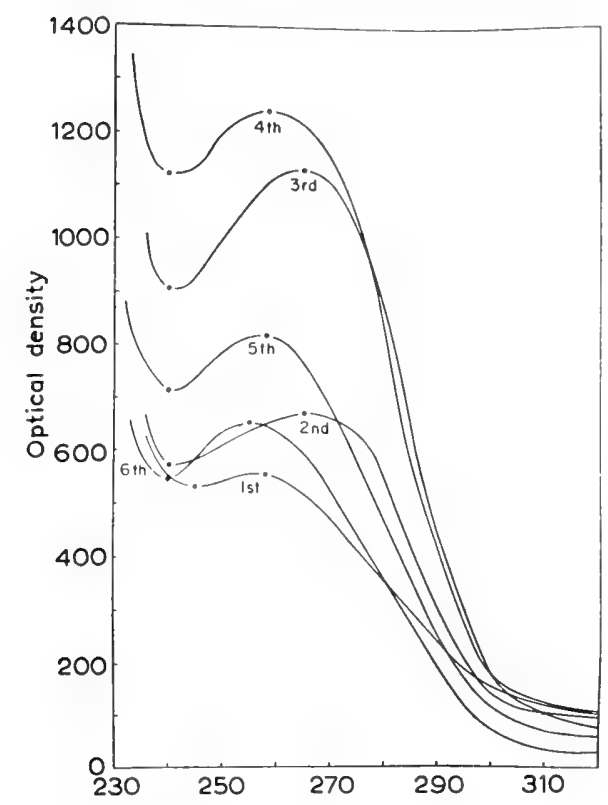

Fig. IоI. The same document as in Fig. Ioo, but concerning the posterior part of the neural plate, in T. torosus. From Niu, 1956.

be submitted to analysis. It could easily be ascertained that the induction was not due to cytolysis caused in the reactor by a change of $\mathrm{pH}$, for this remains $7.8 \pm 0.15$. The main interest lies in the spectrophotometric results. Two sets of measurements, made on Triturus tigrinum and T.torosus (Fig. I0o, IOI) both show a minimal absorption at 2400 or $2450 \AA$, a maximal at 2580 or $2650 \AA$. In both cases, there is a difference between the Ist and $2 \mathrm{~d}$ order media, the maximal absorption shifting from around $2580 \AA$ to around $2650 \AA$. Niu comments on the intrinsic differences between the two cultures, in relation to normal development, and suggests that the first medium contains mainly the neural inductor, the second one mainly an homoeogenetic inductor, causing myoblast differentiation. Accordingly, there would be some difference between these two inductors. This is contradictory to our suggestions concerning retention versus liberation of the inductive agent. Its storage in the middle layer cells may well imply some further transformations of the molecular structure. Assuming this step to be reversible, it could explain the chordal islets sometimes included in the neuroepithelium (p.4I3). Whether or not the inducing agent is simple or double, spectrophotometric study points to a nucleoprotein. This diagnosis agrees with the results of dialysis, which show that the inducting substances are non-dyalizable, macromolecular compounds. They are also precipitable by ethanol and, according to color tests, contain DNA and RNA, in the ratio I/6. The presence of DNA can be attributed to a certain degree of cytolysis occurring in the deep well cultures. Finally, the effect of enzymes on the conditioned media was tested, but with the difficultly that they could also 
act on the reactor (Brachet [ I 955, I 957] for example has demonstrated the degree of the disturbance caused by ribonuclease). Generally speaking, trypsin, chymotrypsin, and both nucleases reduce or suppress the inductive power. "A conclusion that might be drawn from these experiments in that induction in torosus and axolotl by the conditioned medium is due mainly to ribonucleic acid, somewhat to desoxyribonucleic acid, but not to protein". This conclusion has led his author (Niu, I958) to isolate from veal kidney, liver and thymus their ribonucleoproteins and their RNA, and to test the influence of this RNA on component ectoblast. To obtain a plain differentiation, the cultures were grafted on the flank of older embryos. In 25 to $40 \%$ of cases, a pattern of differentiation corresponding to the origin of RNA was observed. The frequency was increased if some protein was added to the culture medium.

Coming back to the nature of the primary inductor, our representation remains, after this elaborate discussion, that it is linked with a gradient field of some ribonucleoproteins endowed with notogenic properties. With the final period of segmentation they undergo, in the areas of greater concentration, a gradual conversion into smaller, more easily transmissible and more stable molecules, a conversion taking place at the highest rate in the primordium of the prechordal plate, at a lesser and decreasing rate in the primordia of the notomerit. Owing to the definite characters of the various secondary inductions, and organs, it may be assumed that the primary agents undergo a further modification, adapted to each case, in order to become the secondary and tertiary inductors.

It is also not excluded that these populations of specialized molecules would persist in the organs throughout the life of the individual, but, so far, they did escape detection. The idea is neither new nor obsolete that such components, influencing cell proliferation, differentiation and normal growth could, through abnormal transformation, be at the origin of malignant growth (see Holtfreter, I 948). This is of course a prominent reason to wish further advances in this intriguing field.

Assuming that these views are sound, a few comments may be introduced here in favor of the concept or organizin ${ }^{1}$ which Pasteels and myself proposed twenty years ago. If the primary morphogenetic field of pregastrular stages is actually due to some ribonucleoprotein which is partly transformed during gastrulation into a somewhat different compound according to the scheme developed above, and if other modulations of the molecular structure soon follow, it seems convenient to use the term "organizin" which so clearly suggests an inductive action. Even if the exact nature of some induction were soon disclosed, e.g. of the acrogenetic or notogenetic one, the name of the substance will presumably be a complex one, and its formula extremely complicated. It would certainly be easier to speak of acro-organizin and noto-organizin, or, for more specific cases, of a neuro-, or myo-, or lens-organizin, There are sufficient examples of cases where substances with definite effects have been named according to their origin or function, for example, adrenalin, thyroxin, secretin, insulin, folliculin, etc., before their chemical identity

1 Lender currently used this concept in his research on the induction of eyes under the influence of brain in regenerating flat-worms ( $c f .1952)$. 
could be established. "Organizin" now appears as a convenient general designation for the variety of ribonucleoproteins, which are the main agents of induction. This situation still conforms to the general idea we had in mind when we coined the term.

\section{(f) On the intimate changes in the reactor}

Induction, like hormonal excitation, is primarily a trophic process. Cells with a limited range of activity receive a stimulus which suddenly raises their metabolism to an unforeseen level and causes differentiations of definite structural and functional character. The primum movens in these changes remains unknown. If we can accept the suggestion that a ribonucleoprotein is introduced, it is probably attacked first by a proteinase, then by the cell ribonuclease, and the various fragments of the molecule introduced into chains of unknown but imaginable reactions. It is known that one of the earliest consequences is a considerable increase of the RNA-rich granules, and that this change persists in the daughter cells. Simultaneously, the alkaline phosphatase synthesis takes place. These changes are so intense and so stable that they suggest, as I already mentioned above, what I have called (1940) a metabolic contagion. This expression, although vague, at least emphasizes the rapid repetition of some reactions, perhaps the multiplication of discrete particles, which may, or may not be similar to plasmogenes. In such a scheme, the first step would be accomplished by a proteolytic enzyme, which conforms to the hypothesis formulated by Lallier (I 955) on the basis of indirect but convincing arguments. A permanent treatment of newt eggs by zinc acetate, from cleavage on, causes thickenings and expansions of the neurula epiblast, especially in the fields of active induction. The abnormality may be considered as a result of an effect upon the competence of the reacting epiblast. On the other hand, formaline which combines with amine groups and reduces the activity of proteolytic enzymes, is known to suppress induction. The author suspects that the link between this blockage, the $\mathrm{Zn}$ effects, and the autonomous neuralization of ectoblast in abnormal conditions concerns proteolytic enzymes. In my opinion, this interesting hypothesis should be applied to the events occurring inside the reactor more than to the activity of the inductor itself.

For a deeper comprehension of these changes, the most important fact that certain inimical conditions are sufficient to cause autonomous, brain-like neuralization cannot be neglected, and is not antinomic with the last remarks.

\section{FINAL GONSIDERATIONS}

This survey has brought the analysis to the stage when conditions are prepared for further differentiation of the embryonic rudiments and for the growth of tissues and organs. Beginning with the formation of gonocytes, it has led us to an organism possessing young gonads, so that the reproductive cycle is virtually complete.

During the days or weeks when a new organism is being created, no stage is insignificant and no part of the system can be lost of view without inconvenience. 
Each phase is intensely constructive, first in the synthesis and storage of the materials and energetic resources, secondly in the production of devices necessary to morphogenesis, and finally in the mutual interplay of such devices in the successive steps of induction. Similarly, all organelles present in the nucleus or in the cytoplasm are in constant activity, and if the most conspicuous changes take place in the cytoplasm, it does not mean that the nucleus is passive. On the other hand, if disturbances in the nucleus are less easily compensated for than those of the cytoplasm, it does not mean that genes necessarily have the leading role throughout development, as specialized geneticists would be too tempted to assert. Although nuclear factors are probably active in the successive phases of ontogenesis, as is suggested by experiments with hybridization (c.f. Baltzer, I 950), by transplantations of nuclei (King and Briggs, 1954) and by the existence of many mutations which interfere with different processes at various stages ( $c f$. GlücksohnWaelsch, I954; Grüneberg, I 948, I 950, I957; Smith, I956, etc), it would be exaggerated to consider genes as the operating staff. Of course, their role for the selective adoption of the specific or individual characters cannot be questioned. However, during gametogenesis and the morphogenetic phase of development, control of genes seems less essential than the general functions of the nucleus (Dalcq, r 956b, r 957b). These functions are accomplished through continuous interaction with cytoplasmic components which are an integral part of the endowment inherited from the parent organisms, especially from the female. The cytoplasm of the egg comes from the one of the primary gonocytes of the female gonads, and it is now fairly well established that the yolk originates from general metabolic processes which are hormonally controlled. Thus, the link between the successive generations is both nuclear and cytoplasmic, and even related to sex hormones, a relation which is quite plausible. The differentiation of the chromosomes into multiple loci is balanced, or nearly so, by the numerous metabolites and enzymes intermingled in the cytoplasm. Both are an integral part of the basic material of heredity.

The tasks of development are accomplished by the utilization of biochemical systems and by organelles which are fundamentally the same as those existing in the adult cells. However, there are special ontogenetic mechanisms which deserve attention. In gametogenesis, we observe growth of cytoplasm typical to each species, since it is free of any additions other than yolk, which is even sometimes lacking; the nucleus performs functions and acquires a composition which is never observed at other phases. After fertilization and during the mitotic phase of morphochoresis, the cytonuclear interplay is probably maximal, and the cyclic elaboration of the $\alpha$ - and $\beta$ - granules systems at least accompanies the establishment of the morphogenetic pattern, which is mainly cortical. This intriguing activity seems one of the most specifically embryonic ones. Thereafter, the striking process of kinematic morphogenesis introduces the mechanisms of induction, which are of gradually increasing complexity. Induction regulates morphochoresis and controls most, and perhaps all of the differentiations.

Investigators have succeeded in disclosing some substances which have appreciably extended our biochemical understanding of the processes involved in the highly complex transformations from gamete to young embryo. These key-substances are the nucleic acids and their proteic compounds, the mucopolysaccharides and 
the phosphatases. Their association clearly points to the general involvement of high-energy phosphorus bonds; adenosine di- and triphosphate and similar compounds have remained here in the background because of our inability to detect and localize them in the cells or blastomeres ${ }^{1}$. However, a recent theoretical paper devoted to movements in embryonic cells (Mulnard, I956) has shown how many reasons we have for suspecting the role of the high-energy phosphorus compounds and the corresponding enzymes in all the processes with which we have been concerned.

The finality of development is obviously the construction of associated organs and tissues with their appropriate cytodifferentiations ${ }^{2}$. The individualization of organs is clearly the result of morphochoresis, while cytodifferentiations proceed from more varied causes: $(I)$ the intrinsic constitution of some cells, e.g. in derivatives of chordo-mesoblast or endoblast; a concept perhaps open to revision since it is not demonstrated yet that cultured cells of these embryonic anlagen will produce a completely homogeneous lineage; (2) inductive processes which may (a) primarily influence the form of the cells, as in neuroblasts; (b) evoke, probably with the collaboration of the mitochondria, definite thread-like structures due to chains of macromolecules for example neurofibrils at a later stage of the neuroblasts, myofibrils (in the experimentally induced myoblasts), and the fibrils of cilia, such as those in the epidermis of tadpoles and in the sensory patches of the inner ear; (c) provoke the synthesis of specialized proteins such as lens protein, myosin and actomyosin, etc.; (3) possible activity of the centriolar apparatus, which may well be more extensive than the production of cilia or flagellae, and whose associations with the Golgi apparatus may be more frequent than has been observed (as suggested by the origin of the acrosomal substance); (4) hormonal influences clearly apparent in the reproductive organs and directly demonstrated in vitro for vitamin A (Fell and Mellanby, i 953; Fell, I 954; Weiss and James, I 955). Thus, it may be said that differentiation is mostly provoked, and even that its occurrence as an autonomous or "spontaneous" process could be questioned.

The above schematic enumeration is merely a glance upon the vast and promising problem of differentiation. For additional recent information, the constructive essay of Weiss (1953) and the proceedings of the Providence conference (1956) should be consulted (Rudnick, I958).

It is important to emphasize that, in an impressive number of cases, cytodifferentiation can now be produced by introducing definite substances of the organizin or vitamin type into the cell milieu. It should be stressed also that some puzzling processes occurring in adult organisms will probably benefit from studies conducted with the basic mechanisms of development in mind. One good example is osteo-

1 I have recently been able to detect cytochemically adenosine-triphosphatase in mouse, rat and mole eggs. The activity of this powerful enzyme, probably located in the mitochondria, is far from being constant throughout early development. This new approach seems quite promising.

${ }^{2}$ For the elaborate character of the properties acquired by differentiation, observations by Weiss and Moscona (1958) are especially striking. If the mesenchymes derived from the sclerotic and from the limb bud are submitted to dissociation and reaggregation, they preserve their respectively bi- and tridimensional arrangement. 
genesis, for the explanation of which Lacroix (I946, I 95 I) has suggested the existence of a substance which he has called osteogenin ${ }^{1}$. More recently, Danis (I957) has carefully studied the production of a bone shell by pieces of bone marrow grafted into the anterior chamber of the eye. A positive result always occurs with autotransplants, frequently with homotransplants, and never with heterotransplants. His experiments included grafts of killed bone marrow, which proved ineffective and the author felt entitled to doubt the existence of osteogenin. From the standpoint of an embryologist, however, this objection does not seem too valid. The embryologist emphasizes that bone marrow is a powerful xeno-inductor and he observes that during the first five days, the ocular graft goes through a process of partial cytolytic decay and dedifferentiation to become a blastema-like mass; this rapidly redifferentiates into osteogenic areas mixed with hematopoietic marrow and finally reorganizes into a peripheral bone sheath containing a core of marrow. Clearly, bone is produced by the implant and not by the host tissue which would function as a reactor. Reciprocally, it is known that grafts of reparative fibrocartilage can develop into a small bone containing marrow. Thus, bone and marrow form a morphogenetic system; when one of the components is excluded, the other reorganizes to privide the complementary structure. One may infer that this process is quite analogous to the reorganization of an organizer fragment, or to the regeneration of a lens from the retina. We also have some reason to suspect that such processes are concerned with liberation, displacement, transfer and other activities of organizins.

In the majority of cases, true growth only takes place after the establishment of differentiation, since most germinal systems possess reserves, which need only to be converted into active protoplasm. However, embryonic cells are capable of assimilation at earlier stages, as is clearly apparent in mammals.

All mechanisms disclosed by the study of development are more or less correlated with specific growth processes, and this is particularly true for organizins. Students of normal and pathological growth should be aware that the organisms possess, at the beginning of their development, powerful growth devices which may well have some still-unknown equivalent in adult cells.

1 The existence of an osteogenic factor in rabbit bones is supported by recent studies of Moss (1958). 


\section{LITERATURE}

Abercrombie, M. and R. Bellairs, (1954) 7. Embryol. Exptl. Morphol., 2, 55.

Achten, G., (1959) Arch. biol. (Liège), 70, I.

Alfert, M., (1950) 7. Cellular Comp. Physiol., 36, 38 I.

Amprino, R. and E. Camosso, (1958a) Experientia, 14, 24 I.

Amprino, R. and E. Gamosso, (1958b) Arch. Entwicklungsmech. Organ., 150, 509.

Ancel, P. And M. Reiss-Brion, (1956) Compt. rend., $243,1380$.

Ancel, P. And P. Vintemberger, (1948) Bull. biol. France et Belg., Suppl. 3I, I.

Auerbach, R. and C. Grobstein, (1958) Exptl. Cell Research, 15, 384.

Avery, G., M. Chow and H. Holtzer, (1956) F. Exptl. Zool., 132, 409.

Balinsky, B. I., (1934-1935) Zool. Fahrb., 54, 328.

Balinsky, B. I., (1957) F. Exptl. Zool., I35, 255.

Baltzer, F., (1950) Rev. suisse zool., 57, 93.

BARth, L. G., (I941) 7. Exptl. Zool., 87, 37 I.

Barth, L. G. and L. J. Barth, (I958) Anat. Record, I3I, 530.

Barth, L. G. and S. Graff, (1943) Proc. Soc. Exptl. Biol. Med., 54 , 1 i 8.

Barth, L. G. And L. C. Sze, (1953) Physiol. Zoöl., 26, 205.

Bautzmann, H., (1951) Anat. Anz., Erganzungsheft, 97, 6.

Bautzmann, H., J. Holtfreter, O. Mangold and H. Spemann, (1932) Naturwiss., 20, 5 I.

Bell, E., (1958) Anat. Record, I3I, 532.

Bellairs, R., (1958) 7. Embryol. Exptl. Morphol., 6, I49.

Benoit, J. A. A., (1952) Arch. biol. (Liège), 64, I.

Benoit, J. A. A., (1955) Compt. rend. soc. biol., 149, 908.

Benoit, J. A. A., (1956) Compt. rend. soc. biol., I50, 240.

Benoit, J. A. A., (1957a) Compt. rend. soc. biol., I5I, 1298.

Benoit, J. A. A., (1957b) Année biol., [3], 33, 385 .

Berg, W. E., (1956) Biol. Bull., IIO, I.

BERG, W. E., (I957) Biol. Bull., I13, 365.

Bijtel, J. H., (1956) Experientia, I2, 259.

Bijtel, J. H., (1958) 7. Embryol. Exptl. Morphol., 6, 466.

Bock, E., (I942) Arch. Entwicklungsmech. Organ., I4I, I59.

Bodenstein, D., (1952) 7. Exptl. Zool., I20, 213.

Boterenbrood, E. C., (1958) Koninkl. Ned. Akad. Wetenschap., Proc., Ser. C, 6I, 47 I.

Brachet, J., (1939) Arch. biol. (Liège), 50, 233.

Brachet, J., (1940) Arch. biol. (Liège), 51, i67.

Brachet, J., (1944) Embryologie chimique, Desoer, Liège, and Masson, Paris.

Brachet, J., (I946a) Experientia, 2, I 43.

Brachet, J., (I950) Chemical Embryology, translated by L. G. BARTH, Interscience Publ., New York and London.

Brachet, J., (I955) Nature, $175,85$.

Brachet, J., (1957) ye Fournées cyto-embryol. holl. belges, Liège, (unpublished).

Brachet, J., T. Kuusi and S. Gothié, (I952) Arch. biol. (Liège), 63, 429.

Brahma, S. K., (1958) 7. Embryol. Exptl. Morphol., 6, 4 I8.

Brandes, J., (1940) Arch. biol. (Liège), 5I, 219.

Brandes, J., (1941) Arch. biol. (Liège), 53, 149.

Bretschneider, L. H. And Chr. P. Raven, (195I) Arch. néerl.zool., Io, I.

Brice, M., (I958) Arch. biol. (Liège), 69, 57 I.

Brice, M., (I959a) Arch. biol. (Liège), 70, I 2 I.

Brice, M., (1959b) Arch. biol. (Liège), 70, 126.

Butler, E. G. ANd O. E. Schotté, (1949) 7. Exptl. Zool., II2, 36 I.

Cambar, R. and J. D. Gipouloux, (1956) Compt. rend., 242, 2862.

Chandhry, H. S., (1952) Z. Zellforsch. u. mikroskop. Anat., 37, 455.

Ghuang, H. H., (I939) Arch. Entwicklungsmech. Organ., I39, 556.

Ghuang, H. H., (1940) Arch. Entwicklungsmech. Organ., I40, 25.

Ghuang, H. H., (r955) 7. Acad. Sinica, 4, I5I. 
Chunang, H. H. and T. H. Pai, (1956) Acta exptl. biol. sinica, 5, 289.

Clavert, J., (1952) Arch. Anat. Microscop., 4I, 209.

Clavert, J. and G. Filogamo, (I958) Compt. rend. soc. biol., i5I, i 740 and 2187.

Clanert, J. and P. Vintemberger, (1954) Compt. rend. soc. biol., I48, i 122.

Clanvert, J. and P. Vintemberger, (1957) Compt. rend. soc. biol., 15I, 2183.

Clanvert, J. and P. Vintemberger, (I958) Compt. rend. soc. biol., I52, I 49.

Clanert, J. and J. P. Zahnd, (i955) Compt. rend. soc. biol., I49, I650.

Gilavert, J. And J. P. Zahnd, (I956) Compt. rend. soc. biol., I50, I26..

Clavert, J. And J. P. Zahnd, (I957) Compt. rend. soc. biol., I5I, i 234.

Glayton, R. M., (1953) 7. Embryol. Exptl. Morphol., I, 25.

Cleland, K. W., (195I) Australian F. Sci. Research, Ser. B, 4, 344.

Clement, A. G., (1956) 7. Exptl. Zool., r32, 426.

Clement, A. C. and F. E. Lehmann, (1956) Nature, $43,478$.

Cohen, S. and R. Levi-Montalcini, (1956) Proc. Natl. Acad. Sci., U.S., 42, 57 I.

Cohen, S. and R. Levi-Montalcini, (I957) Cancer Research, I7, I 5.

Colwin, A. L. and L. Hunter Colwin, (1951) 7. Exptl. Zool., iI7, i i I.

Conklin, E. G., (1932) 7. Morphol., 54, 69.

Corti, G., (1950) Riv. biol. (Perugia), 42, 443.

Costello, D. P., (1945) 7. Elisha Mitchell Sci. Soc., 61, 277.

Dalce, A., (1933) Arch. Anat. Microscop., 29, 388.

DAlce, A., (1935a) Compt. rend. soc. biol., I19, I $42 \mathrm{I}$.

DalcQ, A., (I935b) L'organisation de l'oeuf chez les Chordés, Gauthier-Villars, Paris.

DAlcQ, A., (1938a) Arch. biol. (Liège), 49, 397.

Dalce, A., (1938b) Form and Causality in Early Development, Cambridge University Press, Cambridge.

DalcQ, A., (1940) Arch. biol. (Liège), 5I, 387 .

DalcQ, A., (1941a) Arch. biol. (Liège), 52, 2.

DAlcQ, A., (194I I) L'oeuf et son dynamisme organisateur, Albin Michel, Paris.

Dalce, A., (1942) Arch. biol. (Liège), 53, 2.

Dalce, A., (1947a) Proc. Intern. Congr. Exptl. Cytol., 6th Congr., Stockholm, 1947, 483.

DalcQ, A., (1947b) 6th Growth Symposia, 85, I 19.

DalcQ, A., (1949) Actes soc. helv. sci. nat., I2ge Réun., Lausanna, 37.

Dalce, A., (195I) Verhandl. Kon. Ned. Akad. Wetenschap., Ser. C, 54, 35I, 469.

DAlcQ, A., (1952) Initiation à l'embryologie générale, Masson, Paris, and DeSoer, Liège.

DAlcQ, A., (1953a) Bull. acad. roy. Belg., Cl. Sci., I1 28.

Dalce, A., (1953b) Compt. rend. soc. biol., I47, 2038.

Dalce, A., (r 954 a) in R. Cordier and A. Dalco, Traité de zoologie, P. Grassé, Ed., Masson, Paris.

DalcQ, A., (1954b) Bull. soc. zool. France, 79, 240.

DAlcQ, A., (1954c) Compt. rend. soc. biol., I48, 1332.

Dalce, A., (1955) Proc. Soc. Study Fertility, 7, i I 3.

Dalce, A., (1956a) Exptl. Cell Research, 10, 99.

Dalce, A., (1956b) Rev. univ. Bruxelles, 2, I.

Dalce, A., (1957a) Acta Anat., 30, 242.

DalcQ, A., (1957b) Initiation to General Embryology, translated by J. Medawar, Glarendon Press, Oxford.

Dalce, A., (1957c) Bull. soc. zool. France, 82, 296.

Dalce, A. And M. De Greef, (1958) Compt. rend. assoc. anat., 45 (in the press).

Dalce, A. And A. Dollander, (1948) Compt. rend. soc. biol., 142, 1307.

Dalce, A. And A. C. Huang, (1948) Compt. rend. soc. biol., I42, I3 I 2.

Dalce, A. and R. Lallier, (1948) Arch. biol.(Liège), 49, 267.

Dalce, A. And J. Mulnard, (1953) Compt. rend. soc. biol., I47, 2040.

Dalce, A. and J. J. Pasteels, (1937) Arch. biol. (Liège), 48,669.

Dalce, A. and J. J. Pasteels, (1938) Bull. acad. roy. méd. Belg., [6], 3, 26 I.

Dalce, A. and J. J. Pasteels, (I955) Exptl. Cell Research, Suppl.3, 72.

Dalce, A., J. J. Pasteels and J. Mulnard, (1956) Bull. acad. roy. Belg., Cl. Sci., 42, 77 I. 
Dalco, A. and M. van Egmond, (1953) Arch. biol. (Liège), 64, 312.

Damas, A., (1947) Arch. biol. (Liège), 58, 15.

Damas, A., (1957) ze Journée cyto-embryol. holl. belge, (unpublished).

D'Amelio, V. ANd M. P. CeAs, (1956) Experientia, 13, I 52.

DAnis, A., (1957) Acta med. belgica, Thesis, 7.

De Duwe, Chr., (1958) Bull. acad. roy. méd. Belg., 23, 608.

De Geeter, L., (1954) Arch. biol. (Liège), 65, 363.

De Harven, E. and W. Bernhard, (I956) Z. Zellforsch. u. mikroskop. Anat., 45, 378.

DeI, L., (1954) Riv. biol. (Perugia), 46, 193.

Denis, H., (1957) Ann. soc. roy. zool. Belg., 87, 50 I.

Denis, H., (1958) 7. Embryol. Exptl. Morphol., 6, 444.

Detwiler, S. R., (1954a) Bull. Hosp. Foint Diseases, 15, Oct. 1954.

Detwiler, S. R., (1954b) 7. Exptl. Zool., I26, I 35.

Detwiler, S. R. and R. H. Van Dijke, (1953) 7. Exptl. Zool., I22, 367.

Deuchar, E. M., (1953) J. Exptl. Biol., 30, i8.

Deuchar, E. M., (1956) 7. Embryol. Exptl. Morphol., 4, 328.

Deuchar, E. M., (I958a) Exptl. Cell Research, I4, 84.

Deuchar, E. M., (1958b) 7. Embryol. Exptl. Morphol., 6, 223.

Devillers, CH., (1949) Journées cyto-embryol. holl. belges, 67.

Devillers, Ch., (I952) Compt. rend. assoc. anat., 38e Réun., 4 I8.

De Vincentis, M., (I954) Boll. Zool., 21 (2), 379.

Dollander, A., (1950) Arch. biol. (Liège), 6I, I.

Dollander, A., (1952) Compt. rend. soc. biol., I46, I607.

Dollander, A., (1953) Arch. Anat. Microscop., 42, 185.

Dollander, A., (1958) Compt. rend. soc. biol., I52, 982.

Driesch, H., (I896) Arch. Entwicklungsmech. Organ., 4, 75.

Driscoll, W. T. and R. M. Eakin (1955) 7. Exptl. Zool., I29, I 49.

Duffey, L. And J. Ebert, (i957) 7. Embryol. Exptl. Morphol., 5, 325.

EAkin, R. M. ANd F. Bush, (1957) Anat. Record, I29, 279.

Eakin, R. M., Ph. B. Kutsky and W. E. Berg, (I95I) Soc. Exptl. Biol. Med., 78, 502.

Eakin, R. M. and F. Lehmann, (1957) Arch. Entwicklungsmech. Organ., 150, I 77

Eвert, J. D., (1954) in: Aspects of Synthesis and Order in Growth, ed. D. Rudnick, University

Press, Princeton, p. 69.

Engländer, H. and A. G. Johnen, (1957) 7. Embryol. Exptl. Morphol., 5, i.

Engländer, H., A. G. Johnen and W. Vahs, (1953) Experientia, 9, 100.

EtKin, W., (1958) Anat. Record, I3I, $54^{8}$.

Eyal-Giladi, H., (1954) Arch. biol. (Liège), 65, I80.

Eyal-Giladi, H., ( $\left.195^{8}\right)$ Koninkl. Ned. Akad. Wetenschap., Proc., Ser. C, 6I, 224.

Fautrez, J., (I95I) Arch. biol. (Liège), 62, 195.

Fautrez, J., (1956) Arch. biol. (Liège), 67, 47 I.

FAUtrez, J. And L. VAKAET, (I954) Compt. rend. assoc. anat., 4I, 465.

Fell, H. B., (1954) 7. Embryol. Exptl. Morphol., 2, 348.

Fell, H. B. and E. Mellanby, (1953) F. Physiol. (London), i19, 470.

Fice, A., (1954a) 7. Embryol. Exptl. Morphol., 2, 204.

Fice, A., (1954b) 7. Embryol. Exptl. Morphol., 2, I94.

Finamore, F. J. and G. T. Grouse, (1958) Exptl. Cell Research, I4, i6o.

Finnegan, G. V., (I953) 7. Exptl. Zool., 123, 37 I.

Fischer, F. G. and H. Hartwig, (1936) Z. vergleich. Physiol., 24, I.

Flickinger, R. A., (1954) Exptl. Cell Research, 6, 172.

Flickinger, R. A., (1956) 7. Exptl. Zool., 13I, 307.

Flickinger, R. A., E. Levi And A. E. Smith, (I955) Physiol. Zoöl., 28, 79.

Flickinger, R. A. And G. W. NAce, (1952) Exptl. Cell Research, 3, 395.

Flickinger, R. A. And D. E. Rounds, (I956) Biochim. et Biophys. Acta, 22, $3^{8}$.

Flynn, T. T. And J. P. Hill, (1939) Proc. Zool. Soc. (London), 24, 445.

Fraser, R. G., (I958) Anat. Record, I3I, 555.

FujIr, T., (r944) 7. Fac. Sci. Univ. Tokyo, Sect. IV, 6, 45 I. 
Fujil, T., S. Utida, T. Ohnishi and T. Yanagissawa, (1951) Annotationes Zool. Fapon., 24, II 5 .

Gallera, J., (1947) Arch. biol. (Liège), 58, 22 I.

Gallera, J., (1948) Rev. suisse zool., 55, 295.

Gallera, J., (I949) Arch. Anat. Histol. Embryol., 32, I 2 I.

Gallera, J., (195 I) Arch. Entwicklungsmech. Organ., 145, I43.

Gallera, J., (1952) Arch. Entwicklungsmech. Organ., I46, 21.

Gallera, J., (1953) Rev. suisse zool., 6o, 547.

Gallera, J., (1958a) Acta Anat., 35, 47.

Gallera, J., (1958b) Compt. rend. assoc. anat., 45, in the press.

Gallera, J. and G. A. Baud, (1954) J. Embryol. Exptl. Morphol., 2, 106.

Gallera, J., and H. Oprecht, (1948) Rev. suisse zool., 55, 243.

Gallien, L., (1954) Bull. biol. France et Belg., 88, I.

Gallien, L., (1956) Bull. biol. France et Belg., 90, i63.

Gallien, L., (1958) Problèmes et concepts de l'embryologie expérimentale, Gallimard, Paris.

Gluecksohn-Waelsch, S., (1954) Cold Spring Harbor Symposia Quant. Biol., I9, 9 I.

Gомот, L., (1956a) Compt. rend. soc. biol., I50, 9 Iо.

Gомот, L., (1956b) Compt. rend., 243, 2142.

Gомот, L., (1958) 7. Embryol. Exptl. Morphol., 6, 162.

Gothié, S., (1950) Thesis, Paris.

Govaert, J., (I957) Arch. biol. (Liège), 68, I65.

Grabowski, C. T., (1956) Anat. Record, I24, 296.

Grabowski, G. T., (1957) Am. 7. Anat., IOI, IOI.

Grabowski, C. T., (1958) Anat. Record, I3o, 412.

GreGG, J. R. AND M. Kahlbrock, (1957) Biol. Bull., I13, 376.

GregG, J. R. And N. Ornstein, (1952) Biol. Bull., IO2, 22.

GregG, J. R. And N. Ornstein, (I953) Biol. Bull., io5, 466.

GregG, J. R. And F. L. RAY, (I957) Biol. Bull., II3, 382.

Grobstein, CL., (1953) 7. Morphol., 93, I9.

Grobstein, Cl., (1955a) Ann. N. Y. Acad. Sci., 6o, 1095.

Grobstein, Gl., (1955b) 7. Exptl. Zool., 130, 3 19.

Grobstein, Gi., (1957) Exptl. Cell Research, 13, 575.

Grobstein, Cl. and A. Dalton, (i957) 7. Exptl. Zool., 135, 57.

Grobstein, Cl. And H. Holtzer, (i955) 7. Exptl. Zool., I28, 333.

Grobstein, Cl. and G. Parker, (1954) Proc. Soc. Exptl. Biol. Med., 85, 477.

Grobstein, Cl. and G. Parker, (1958) J. Natl. Cancer Inst., $20,107$.

Grobstein, Cl. and E. Zwilling, (I953) 7. Exptl. Zool., 22, 259.

Grüneberg, H., (1948) in: Growth in Relation to Differentiation and Morphogenesis, Cambridge University Press, p. I 55 .

GrüneberG, H., (1950) Rev. suisse zool., 57, I29.

GrüneberG, H., (1957) 7. Genetics, 55, 181 .

Gustavion, T. and P. Lenique, (1952) Exptl. Cell Research, 3, 25 I.

Guyénot, E., (1927) Rev. suisse zool., 34, I.

Haget, A., (1953) Bull. biol. France et Belg., 37, 125.

HagGis, A. J., (1956) 7. Embryol. Exptl. Morphol., 4, I 20.

Hall, Th. S., (1942) 7. Exptl. Zool., 89, I.

Hall, TH. S. and F. Moog, (1948) F. Exptl. Zool., Iog, 339.

Hama, T., (1949) Proc. Japan. Acad., 25, 4.

Hamburger, V., (1938) 7. Exptl. Zool., 77, 379.

Hamburger, V., (1956) Cellular Mechanisms in Differentiation and Growth, p. 191.

Hamilton, H. L. and A. L. Koning, (1956) Am. 7. Anat., 99, 53.

Harrison, R. G., (i933) Am. Naturalist, 67, 306.

Harrison, R. G., (1945) Trans. Conn. Acad. Arts Sci., 36, 277.

HAYASHI, Y., (1955) Embryologia, 2, I45.

Hayashi, Y., (1956) Embryologia, 3, 57.

Hayashi, Y., (1958) Embryologia, 4, 33. 
Hayashi, Y. And K. Takata, (1958) Embryologia, 4, I49.

Hermann, R., (1956) Z. Zellforsch. u. mikroskop. Anat., 45, 176.

Hinrichsen, K., (1955) Z. Anat. Entwicklungsgeschichte, 119, 350.

Hoessels, E. L., (I957) Thesis, Utrecht.

Holmes, A., (1935) Am. 7. Anat., 56, 5 I3.

Holtfreter, J., (1933a) Arch. Entwicklungsmech. Organ., 127, 619.

Holtfreter, J., (1933b) Arch. Entwicklungsmech. Organ., I27, 591.

Holtfreter, J., (1934a) Arch. exptl. Zellforsch. Gewebezücht., 15, 28 I.

Holtfreter, J., (1934b) Arch. Entwicklungsmech. Organ., I32, 225.

Holtfreter, J., (1934c) Arch. Entwicklungsmech. Organ., 132, 307.

Holtfreter, J., (1936) Arch. Entwicklungsmech. Organ., I34, 465.

Holtfreter, J., (1938a) Arch. Entwicklungsmech. Organ., I38, I63.

Holtfreter, J., (1938b) Arch. Entwicklungsmech. Organ., $13^{8}, 522$.

Holtfreter, J., (I943) 7. Exptl. Zool., 93, 25 I.

Holtfreter, J., (1944a) 7. Exptl. Zool., 95, 307.

Holtfreter, J., (1944b) Rev. can.biol., 3, 220.

Holtfreter, J., (1945) 7. Exptl. Zool., 98, i62.

Holtfreter, J., (1947a) 7. Exptl. Zool., I06, I97.

Holtfreter, J., (1947b) 7. Morphol., 80, 25, 57.

Holtfreter, J., (1948) Growth in Relation to Differentiation and Morphogenesis, Cambridge University Press, p. 17.

Holtfreter, J., (I95I) Growth Symposia, io, i I 7.

Holtfreter, J., (1955a) 7. Exptl. Zool., I29, 623.

Holtfreter, J., (1955b) Exptl. Cell Research, 3, i88.

Holtfreter, J. and V. Hamburger, (I955) in: B. H. Willier, P. A. Weiss and V. HamBUrger, Analysis of Development, Section VI, Chapter I, Amphibians, p. 23o. Saunders Cy., Philadelphia and London.

Holtzer, H. and R. Detwiler, (i953) F. Exptl. Zool., I23, 335.

Holtzer, S. W., (1956) 7. Morphol., 99, I.

Hori, R. And P. D. Nieuwkoop, (1955) Koninkl. Ned. Akad. Wetenschap., Proc., Ser. C, 58, 266.

Hörstadius, Sv., (1935) Pubbl. staz. zool. Napoli, I4, 25 I.

Hörstadius, Sv., (1950) Année biol., 26, 381 .

Hörstadius, Sv., (1952) 7. Exptl. Zool., I20, 421.

Houillon, CH., (1957) Bull. biol. France et Belg., 9o, 360.

Huxley, J. And G. R. DE BeER, (1934) The Elements of Experimental Embryology, Cambridge University Press.

Inoue, K. And T. Ishikawa, (1956) Intern. Congr. Devel. Biol., Brown University (non published).

IzQuierdo, L., (1955) Arch. biol. (Liège), 66, 403.

Johnen, A. G., (I956) Koninkl. Ned. Akad. Wetenschap., Proc., Ser. C, 59, 652.

KARASAKI, S., (1957) Embryologia, 3, 31 7, 335.

Karasaki, S., (r959a) Embryologia, 4, 247.

KaraSAKI, S., (I959b) Embryologia, 4, 273.

Karasaki, S. ANd T. Yamada, (I955) Experientia, II, I9I.

Katayama, H., (1956) Japan. F. Zoöl., 12, 39.

Kato, K. And T. S. Okada, (1957) Mem. Coll. Sci. Univ. Kyoto, Ser. B, 23 , I.

Kawakami, I., (1950) Mem. Coll. Sci. Univ. Kyoto, Ser. B., I9, No. 3, 20.

Kawakami, I., (1952) Annotationes Zool. Fapon., 25, 97.

Kawakami, I., (1953) Mem. Fac. Sci. Kyushu Univ., Ser. E, r, I 25.

Kawakami, I., (1955) Acta Pathol. Japon., 5, 475.

Kawakami, I. and S. Mifune, (1955) Mem. Fac. Sci. Kyushu Univ., Ser. E, 2, 21.

Kawakami, I. and S. Mifune, (1957) Mem. Fac. Sci. Kyushu Univ., Ser. E, 2, I4I.

Kawakami, I. and H. Okano, (1955) Mem. Fac. Sci. Kyushu Univ., Ser., E, 2, I.

Kelly, J. W., (1950) Protoplasma, 39, 386.

Kemp, N. E., (1956) 7. Biophys. Biochem. Cytol., 2, 28 r.

Kerr, S. E. and K. Seraidarian, (1949) 7. Biol. Chem., I80, i203. 
King, T. L. AND R. BRiggs, (1954) 7. Embryol. Exptl. Morphol., 2, 73.

KioRTsis, V., (1953) Rev. suisse zool., 6o, 301 .

Koning, A. L. and H. L. Hamilton, (i 954) Am. F. Anat., 95, 75.

Konopacka, B., (1937) Pubbl. staz. zool. Napoli, I6, 327.

Kuusr, T., (1951) Ann. Zool. Soc. Zool. Botan. Fennicae Vanamo, I4, I.

Kuusi, T., (1953) Arch. biol. (Liège), 64, I89.

Kuusi, T., (1957) Arch. Soc. Zool. Botan. Fennicae Vanamo, II, I36.

Krause, G., (1958) Ergeb. Biol., 20, 159.

Kriegel, H., (1956) Naturwissenschaften, 43, I 85.

KüHN, A., (1955) Entwicklungsphysiologie, Springer, Berlin.

Kutzky, R. J., (1953) Proc. Soc. Exptl. Biol. Med., 83, 390.

Lacroix, P., (1946) Arch. biol. (Liège), 57, 99.

Lacroix, P., (195I) The Organization of Bones, Churchill, London.

LALlier, R., (1950) Experientia, 6, 92.

Lallier, R., (1954) 7. Embryol. Exptl. Morphol., 2, 323.

Lallier, R., (1955) Compt. rend., 24I, I 5 IO.

Lallier, R., (1956a) Compt. rend., 242, 2772.

Lallier, R., (I956b) Exptl. Cell Research, $11,648$.

Lallier, R., (1957) Pubbl. staz. zool. Napoli, 30 , I85.

Langman, J., (1957) 7e Journ. cyto-embryol. holl.-belges, Liège, janvier 1957 (unpublished).

Lanzavecchia, G. And A. L. Coultre, (1958) Arch. ital. anat. e embriol., 63, 445.

Lash, J. and S. Holtzer, (1957a) Anat. Record, I27, 323.

Lash, J. And S. A. H. Holtzer, (1957b) Exptl. Cell Research, I3, 292.

Leblond, C. P. and Y. Clermont, (1952) Ann. N. Y. Acad. Sci., 55, 548.

Lemiann, F. E., (1937) Arch. Entwicklungsmech. Organ., I36, I 12.

Lehmann, F. E., (1941) Schweiz. med. Wochschr., 7I, 485 .

Lehmann, F. E., (1942a) Naturwissenschaften, 30, 515.

Lehmann, F. E., (1942b) Rev. suisse zool., 49, 223.

Lehmann, F. E., (1950) Rev. suisse zool., 57, I41.

Lehmann, F. E., (I956) Naturwissenschaften, 43, 289.

Lehmann, F. E. And R. Biss, (1949) Rev. suisse zool., 56, 264.

Lehmann, H., (1938) Dissert. inaug., Friburg i. Br.

Lehmann, H., (1952) 7. Elisha Mitchell Sci. Soc., 68 (no page).

Lehmann, H. and L. M. Young, (1952) 7. Exptl. Zool., I2I, 419.

Lender, Th., (1952) Bull. biol. France et Belg., 86, 140.

Lenicque, P., S. Hörstadius and T. Gustavson, (1953) Exptl. Cell Research, 5, 400.

Levi-Montalcini, R., (1958) in: Symposium on Chemical Basis of Development, Baltimore, p. 646.

Lewis, H., (1947) Anat. Record, 97, 139.

Lewis, W. H. and E. C. Roosen-Runge, (1943) Anat. Record, 85, 326.

LiedKe, K. B., (1955) 7. Exptl. Zool., I30, 353.

LiedKe, K. B., (1958) Anat. Record, I3I, 97.

Lillie, F. R., (1929) Arch. Entwicklungsmech. Organ., II8, 499.

Lindahl, P. E., (1936) Acta Zool. (Stockholm), 17, I79.

Lison, L. and J. Pasteels, (I95I) Arch. biol. (Liège), 52 , I.

Lombard, G. L., (1952) Thesis, Utrecht.

Lopashov, G., (1935) Biol. Zentr., 55, 606.

Lopashov, G., (1955a) Doklady Akad. Nauk, S.S.S.R., 105, 599.

Lopashov, G., (1955b) Doklady Akad. Nauk, S.S.S.R., Io9, 653.

Lovtrup, S., (1955) Compt. rend. Lab. Carlsberg, Sér. chim., 29, 26 г.

Lovtrup, S., (1956) 7. Embryol. Exptl. Morphol., 6, I 5.

Lovtrup, S. and A. Pigeon, (1958) 7. Embryol. Exptl. Morphol., 6, 486.

Lutz, H., (r955) 7. Embryol. Exptl. Morphol., 3, 59.

Lutz, H. And L. Gomotт, (1956) Compt. rend. soc. biol., 150, 2055.

Lutz, H. and Y. Ostertag, (1955) Compt. rend. assoc. anat., 42, 972.

McAlpine, R.J., (I956) Anat. Record, I26, 8 I.

McBride, E. W., (19i9) Proc. Roy. Soc. (London), B, 9o, 323. 
McKeehan, M. S., (1951) 7. Exptl. Zool., II7, 3 I.

MaKeehan, M. S., (1954) 7. Exptl. Zool., 126, 157.

MaKeehan, M. S., (1956) Am. 7. Anat., 99, I3 I.

McMaster, R., (1955) 7. Exptl. Zool., I30, I.

Malan, E., (1953) Arch. biol. (Liège), 64, I 49.

Mangold, O., (1933) Naturwissenschaften, 21, 76 I.

Mangold, O., (1936) Naturwissenschaften, 24, 753.

Mangold, O., (1956) Naturwissenschaften, 43, 287.

Mangold, O., (1957) Naturwissenschaften, 44, 289.

Mangold, O., (1958) Anat. Anz., Io4, Erganzungsheft 3.

Mangold, O. and H. Spemann, (1927) Arch. Entwicklungsmech. Organ., III, 342.

Mangold, O., H. Tiedemann and G. von Woellwarth, (1956) Arch. Entroicklungsmech. Organ., I49, 45 .

May, R. M., P. Ganter and R. Kourilsky, (1957) 7e Fourn. cyto-embryol.holl.-belges, Liège, janvier 1957 (unpublished).

Medawar, P. B., (1954) 7. Embryol. Exptl. Morphol., 2, 172.

Mifune, S., (1955) Mem. Fac. Sci. Kyushu Univ., Ser. E, 2 , I3.

Mifune, S., (1957) Mem. Fac. Sci. Kyushu Univ., Ser. E, 3, I49.

Milaire, J., (1956) Arch. biol. (Liège), 47, 297.

Minganti, A., (1949) Arch. biol. (Liège), 6o, 25 I.

Mitchison, N. A., (1958) New Biol., 27, 39.

Montalcini, R., H. Meyer and V. Hamburger, (1954) Cancer Research, I4, 49.

Moog, F., (r944) Biol. Bull., 86, 5 I.

Mookerjee, S., (1954) 7. Embryol. Exptl. Morphol., 124, 505.

Mookerjee, S., E. M. Deuchar and G. H. Waddington, (i953) F. Embryol. Exptl. Morphol., I23, 399.

Moss, M. L., (1958) Science, 127, 755.

Muchmore, W. B., (1956) Intern. Congr. Devel. Biol., Providence (unpublished).

Muchmore, W. B., (1958) Anat. Record, I3I, 58 I.

Mulherkar, L., (1958) 7. Embryol. Exptl. Morphol., 6, I.

Mulnard, J., (1954) Arch. biol. (Liège), 65, 26 I.

Mulnard, J., (1955) Arch. biol. (Liège), 66, 525 .

Mulnard, J., (1956) Ann. soc. roy. zool. Belg., 86, 309.

Mulnard, J., (1958) Arch. biol. (Liège), 69, 645.

Mulnard, J., W. Auclair and D. Marsland, (1959) 7. Embryol. Exptl. Morphol., 7.

Mulnard, J. And A. Dalco, (I955) Compt. rend. soc. biol., I49, 836.

NACE, G. W., (1956) Intern. Congr. Devel. Biol., Providence (unpublished).

Nakamura, O., (1938) Zool. Mag. (Tokyo), 5o, 443.

New, D., (1955) 7. Embryol. Exptl. Morphol., 3, 326.

Nieuwkoop, P. D., (1947) 7. Exptl. Biol., 24, 145.

Nieuwkoop, P. D., (1948) Experientia, 4, 391 .

Nieuwkoop, P. D., (1950) Rev. suisse zool., 57, 23.

Nieuwkoop, P. D., (1955) Exptl. Cell Research, suppl. 3, 262.

Nieuwkoop, P. D., (1958) Actu Embryol. Exptl., 2, 13.

Nieuwkoop, P. D. et al., (1952) 7. Exptl. Zool., I20, I.

Nieuwroop, P. D. et al., (1955) Koninkl. Ned. Akad. Wetenschap., Proc., Ser. C, 58, 2 I9, 355.

Nieumkoop, P. D. And P. A. Florschütz, (1950) Arch. biol. (Liège), 5 I, I I 3.

Nieuwkoop, P. D. and G. V. Nigtevecht, (1954) 7. Embryol. Exptl. Morphol., 2, 15.

Niu, M. G., (1954) 7. Exptl. Zool., I25, i99.

Niu, M. C., (1956) Cellular Mechanisms in Differentiation and Growth, ed. D. Rudnick, Princeton University Press, p. I55.

Niv, M. G., (1958) Anat. Record, 13I, 585.

Niu, M. C. and V. C. Twitty, (I953) Proc. Natl. Acad. Sci., U.S., 39, 985.

O'Connor, R., (1939) 7. Anat., 74, 34.

Ogi, K. I., (1958a) 7. Embryol. Exptl. Morphol., 6, 412.

OGI, K. I., (1958b) Embryologia, 2, I6r. 
Ohtsuka, E., (1956) Sieboldia, I, 323.

Okada, T. S., (1953) Mem. Coll. Sci. Univ. Kyoto, Ser. B, 20, I57; 21, I, 7.

Okada, T. S., (1956) Mem. Coll. Sci. Univ. Kyoto, Ser. B, 23, 27.

OKada, T. S., (1957) 7. Embryol. Exptl. Morphol., 5, 438.

Okada, Y. K., (1948) Proc. Japan. Acad., 24, 21.

Okada, Y. K., (1949) Proc. Japan. Acad., 25, 45.

Okada, Y. K. and T. Hama, (1944) Proc. Imp. Acad. (Tokyo), 20, 36.

Okada, Y. K. And T. Hama, (1945a) Proc. Japan. Acad., 2I, 240.

Okada, Y. K. and T. Hama, (I945b) Proc. Japan. Acad., 21 , 34 I.

Okada, Y. K. and T. Hama, (1948) Proc. Fapan. Acad., 24, 29.

Okada, Y. K. and H. Takaya, (1942) Proc. Imp. Acad. (Tokyo), I8, 505, 514.

Okazaki, R., (1955) Exptl. Cell Research, 9, 579.

Okazaki, R. and S. Osawa, (1954) Zool. Mag. (Tokyo), 63, I 74.

Oppenheimer, J., (1955) Exptl. Cell Research, 9, 579.

Osawa, S., (1951) Embryologia, 2, I.

Osawa, S. And Y. Hayashi, (1953) Science, I18, 84.

Pantelouris, E. M. and L. Mulherkar, (1957) F. Embryol. Exptl. Morphol., 5, 5 I.

Pasquini, P., (1949) Pubbl. staz. zool. Napoli, Suppl. 21, I06.

Pasteels, J. J., (1936) Arch. biol. (Liège), 47, 205.

Pasteels, J. J., (1937) Arch. biol. (Liège), 48, I05, 381.

Pasteels, J. J., (1938) Arch. biol. (Liège), 49, 629.

Pasteels, J. J., (I940a) Biol. Reus. Cambridge Phil. Soc., 15, 59.

Pasteels, J. J., (1940b) Arch. biol. (Liège), 5I, 335.

Pasteels, J. J., (1945) Arch. biol.(Liège), 56, I05.

Pasteels, J. J., (1946) Acta Anat., 2, I.

Pasteels, J. J., (1948) Arch. biol. (Liège), 59, 405.

Pasteels, J. J., (1949) Arch. biol. (Liège), 6o, 235.

Pasteels, J. J., (195I) Bull. soc. Zool. France, 76, 23 I.

Pasteels, J. J., (1953) F. Embryol. Exptl. Morphol., I, 5, I25.

Pasteels, J. J., (I954) $\mathcal{F}$. Embryol. Exptl. Morphol., 2, I22.

Pasteels, J. J., (I955a) Bull. acad. roy. Belg. Cl. Sci., 4I, 76r.

Pasteels, J. J., (1955b) Arch. Anat. Histol. Embryol., 37, 4.

Pasteels, J. J., (1958) Arch. biol. (Liège), 69, 59I.

Pasteels, J. J., P. Gastiaux and G. Vandermeerssche, (1958a) Arch. biol. (Liège), 69, 627.

Pasteels, J. J., P. Castiaux and G. Vandermeerssche, (i958b) 7. Biophys. Biochem. Cytol., 4, 575 .

Pasteels, J. J. and J. Mulnard, (i957) Arch. biol. (Liège), 68, i 15.

Pasteels, J. L., (1954) Compt. rend. soc. biol., I48, 939.

Pasteels, J. L., (1957) Arch. biol. (Liège), 68, 65.

Penners, A., (1929) Arch. Entwicklungsmech. Organ., im6, 63.

Penners, A., (1936) Z. wiss. Zool., r48, 189.

Penners, A. And W. Schleip, (I928) Z. wiss. Zool., I-4, 305; 5-6, I.

Penttinen, K. E., E. Saxén, S. Torvonen and T. Vainio, (1958) Ann. Med. Exptl. et Biol., Fenniae (Helsinki), 36, 27.

Perri, T., (1952) Riv. biol. (Perugia), 44, I8I.

Piepho, H., (1938) Biol. Zentr., 58, 90.

Ragozina, M. N., (I946) Doklady Akad. Nauk S.S.S.R., 5 I, 245.

Ranzi, S., (1958) Acta Embryol. Exptl., 2, 102.

Ranzi, S. and P. Citterio, (1954) Pubbl. staz. Zool. Napoli, 25, 201.

Rapkine, L. And J. Brachet, (I95I) Bull. soc. chim. biol., 33, 427.

Raunich, L., (i953) Arch. ital. anat. e embriol., 57, 400.

Raven, Ch. P., (1948) Biol. Revs. Cambridge Phil. Soc., 23, 333.

Raven, Gh. P., (1954) An Outline of Developmental Physiology, Pergamon Press, London.

Raven, Cir. P., (1958) Morphogenesis, The Analysis of Molluscan Development, Pergamon Press, London.

Raven, Ch. P. And A. M. Th. Beenakkers, (1955) 7. Embryol. Exptl. Morphol., 3, 286. 
Raven, Ch. P., A. G. de Roon and A. M. Stadhouders, (1955) 7. Embryol. Expol. Morphol., $3,142$.

Raven, Gi. P. and J. Kloos, (1945) Acta Neerl. Morphol., 5, 348.

Rawles, M. E., (1943) Physiol. Zoöl., I6, 22.

Rawles, M. E., (1955) Am. F. Anat., 97, 79.

Rebhun, L. I., (1957) Soc. Cell Biol., St. Andrewes Meeting, unpublished.

Rebhun, L. I., (1958) Anat. Record, I30, 362.

Reverberi, G., (1956a) Experientia, I2, 5 I.

Reverberi, G., (1956b) Experientia, I2, 55.

Reverberi, G., (I957a) Scientia (Milan), (6), 5 I, I.

Reverberi, G., (1957b) Pubbl. staz. Zool. Napoli, 29, i87.

Reverberi, G. And A. Minganti, (1949) Riv. biol. (Perugia), 4I, i 25.

Reyer, R. W., (1954a) Quart. Rev.Biol., 29, r.

REYer, R. W., (I954b) F. Exptl. Zool., 125, I.

Rollhäuser, J., (I953) Arch. Entwicklungsmech. Organ., I46, 183.

Rose, S. M., (1939) Biol. Bull., 77, 2 I6.

Roosen-Runge, E. C. And F. D. Barlow, (1953) Am. 7. Anat., 93, 43.

RöTHeli, A., (1950) Z. Zellforsch. u. mikroskop. Anat., 35, 62.

Rotmann, E., (I941) Fortschr. Zool., N. F., 7, i67.

Rotmann, E., (1942) Naturwissenschaften, 30, 60.

Rotmann, E., (1949) Arztl. Forsch., 3, 209.

Rounds, D. E. And R. A. Flickinger, (1958) 7. Exptl. Zool., 137, 479.

Rudnick, D., (i955) in: B. H. Willier, P. A. Weiss and V. Hamburger, Analysis of Development, Section VI, Chapter 2, p. 297. Saunders Cy, Philadelphia and London.

Rudnick, D., (1958) Cytodifferentiation, a Report from the Developmental Biology Conference Series, 1956, The University of Chicago Press.

Runnström, J., (1928) Arch. Entwicklungsmech. Organ., I13, 555.

Runnström, J., (1954) Verhandl. deut. zool. Ges., p. 32.

Ruthmann, A., (1958) 7. Biophys. Biochem. Cytol., 4, 267.

Sala, M., (1954) Pontif. Acad. Sci., Commentationes, I6, 24I.

Sala, M., (1955) Koninkl. Ned. Akad. Wetenschap., Proc., Ser. C, $58,635$.

Sala, M., (1956) Koninkl. Ned. Akad. Wetenschap., Proc., Ser. C, 59, 66 I.

Sasaki, N., I. Kawakami, S. Mifune and I. Tamanoi, (1957) Mem. Fac. Sci. Kyushu Univ., Ser. C, 3, I59.

SAto, T., (1953) Arch. Entwicklungsmech. Organ., I46, 487.

SAunders, J., (1948) F. Exptl. Zool., Io8, 363 .

Saunders, J. W., J. M. Cairns and M. T. Gasseling, (1957) J. Morphol., IOI, 57.

Saunders, J. W. and M. T. Gasseling, (1957) 7. Exptl. Zool., 135, 503.

Saunders Jr., W., M. T. Gasseling and S. M. David, (1958) F. Exptl. Zool., r37, 39.

SAXÉn, L. AND S. Toivonen, (1957) Embryologia, 3, 353.

Saxén, L. And S. Toivonen, (I958) 7. Embryol. Exptl. Morphol., 6, 6 I6.

Schechtman, A. M., (1938) Proc. Exptl. Biol. Med., 38, 430.

Schenk, R., (195I) Rev. suisse zool., 58, 529.

Schleip, W., (1928) Die Determination in der Primitiventwicklung, Akademische Verlagsgesellschaft, Leipzig.

SeIdel, F., (I 956) Naturwissenschaften, 43, 306.

Sengel, P., (1958) Année biol., 34, 30.

Seno, T. and P. D. Nieuwkoop, (1958) Koninkl. Ned. Akad. Wetenschap., Proc. Ser. C, 6I, 489.

Shen, S. C., (1942) 7. Exptl. Biol., 19, 5.

Sirlin, J. L., (I956) Arch. Entwicklungsmech. Organ., I48, 489.

Sirlin, J. L., S. Brahma and C. Waddington, (1956) F. Embryol. Exptl. Morphol., 4, 248.

SlÀdecek, F., (1953) Acta Soc. Zool. Bohemoslovenicae, I6, 322.

Slàdecek, F., (1955) Acta Soc. Zool. Bohemoslovenicae, r9, I38.

Sмiтн, L. J., (1956) 7. Exptl. Zool., 132, 5 I.

SPAR, I. L., (1953) J. Exptl. Zool., I23, 467 .

Spemann, H., (1918) Arch. Entwicklungsmech. Organ., 43, 448. 
Spemann, H., (193I) Arch. Entwicklungsmech. Organ., I23, 389.

Spemann, H., (1938) Embryonic Development and Induction, Yale University Press, New Haven, Conn.

Spemann, H. And H. Mangold, (1924) Arch. mikroskop. Anat. u. Entwicklungsmech., roo, 599.

Spiegel, M., (1954) Biol. Bull., I07, I30.

SPOFFORD, W. R., (1945) 7. Exptl. Zool., 99, 35.

SPOFFORD, W. R., (1948) F. Exptl. Zool., 107, 123.

Spratt, N. T., (1942) 7. Exptl. Zool., 89, 69.

Spratt, N. T., (1952) Ann. N. Y. Acad. Sci., 55, 40.

Spratt, N. T., (1957) 7. Exptl. Zool., 135, 319.

Stearns, R. N., (I955) Anat. Record, I22, 442.

Stearns, R. N. and L. Goldstein, (i956) Physiol. Zoöl., 39, 337.

Steinberg, M. S., (1958) Am. Naturalist, 92, 65.

Sтісн, H., (1950) Arch. Entwicklungsmech. Organ., I44, 364 .

Stone, L. S., (1948) Ann. N. Y. Acad. Sci., 49, 856.

Stone, L. S., (1953) Am. 7. Ophtalmol., (3), 36, 3 I.

Stone, L. S., (1957) 7. Exptl. Zool., I36, 75.

Stone, L. S., (1958) Anat. Record, I3I, 15 I.

Stone, L. S. and F. L. Dinnean, (I943) 7. Biol. Med., I6, 3 I.

Stroeva, O. G., (1956a) Doklady Akad. Nauk S.S.S.R., I08, 562.

Stroeva, O. G., (1956b) Doklady Akad. Nauk S.S.S.R., Iog, 657.

Strudel, G., (1955) Arch. Anat. Microscopy, 44, 208.

Sze, L. C., (1953) Physiol. Zoöl., 26, 2.

Takenchi, T., (1958) Sci. Repts. Tôhoku Univ., Fourth Ser., 24, 33.

TAKATA, K., (1952) Experientia, 8, 2 I 7.

TAkaya, H., (1953) Proc. Fapan. Acad., 29, 374.

Takaya, H., (1955a) Proc. Japan. Acad., 3I, 360.

Takaya, H., (1955b) Proc. Fapan. Acad., 3I, 366.

Takaya, H., (1956a) Annotationes Zool. Fapon., 29, 133.

Takaya, H., (1956b) Proc. Japan. Acad., 32, 282.

Takaya, H., (1956c) Proc. Japan. Acad., 32, 287.

Takaya, H. and T. Watanabe, (1957) Annotationes Zool. Fapon., 3o, i38.

Ten Cate, G., (1956) Verhandl. Koninkl. Ned. Akad. Wetenschap., Sect. II, 5I, 6 I.

Ten Cate, G. and W. J. Van Doorenmalen, (1950) Verhandel. Kon. Ned. Akad. Wetenschap., Sect. II, 53, 3 .

Tencer, R., (1958) F. Embryol. Exptl. Morphol., 6, 117.

Ter Horst, J., (1948) Arch. Entwicklungsmech. Organ., I43, 275.

Tiedemann, H., (1955) Naturwissenschaften, 42, 560.

Tiedemann, H., (i 957) Biochim. et Biophys. Acta, 23, 385 .

Tiedemann, K. and H. Tiedemann, (1956) Z. physiol. Klinik, 36, 7.

Tiedemann, K. and H. Tiedemann, (1957) Experientia, 13, 320.

Toivonen, S., (1940) Ann. Acad. Sci. Fennicae, Ser. A II, No. 55, I.

Toivonen, S., (1945) Ann. Zool. Soc. Zool. Botan. Fennicae Vanamo, II, I.

Toivonen, S., (1949) Arch. Soc. Zool. Botan. Fennicae Vanamo, 4, 28.

Torvonen, S., (I950) Rev. suisse zool., 57, 41 .

Toivonen, S., (1951) Arch. Soc. Zool. Botan. Fennicae Vanamo, 6, 63.

Torvonen, S., (1953) 7. Embryol. Exptl. Morphol., I, 97.

Tolvonen, S., (1954a) Ann. Sci. Fennicae, Ser. A., 22, 3.

Torvonen, S., (1954b) 7. Embryol. Exptl. Morphol., 2, 239.

Tolvonen, S., (1958) 7. Embryol. Exptl. Morphol., 6, 479.

Torvonen, S. And T. Kuusi, (1948) Ann. Zool. Soc. Zool. Botan. Fennicae Vanamo, I3, I.

Toivonen, S. and L. Saxén, (1955a) Ann. Acad. Sci. Fennicae, A IV, No. 3o, I.

Torvonen, S. and L. Saxén, (1955b) Exptl. Cell Research, Suppl. 3, 346.

Töndury, G., (1936) Arch. Entwicklungsmech. Organ., I34, I.

Townes, P. L. And J. Holtfreter, (i955) 7. Exptl. Zool., I28, 54.

Trinkhaus, P., (1953) Anat. Record, 115, 375. 
Tsснumi, P. A., (1956) Rev. suisse zool., 63, 707.

Tung, T. C., (1955) Acta Soc. Biol. Exptl. Sinica, 4, 107, 146, 365.

Tung, T. C., G. Y. Chang and Y. F. Tung, (I945) Proc. Zool. Soc. (London), II5, 75.

Twitty, V. C., (1944) 7. Exptl. Zool., 95, 259.

Twitty, V. C., (1945) 7. Exptl. Zool., roo, I44.

Twitty, V. C. and D. Bodenstein, (1941) F. Exptl. Zool., 86, 343.

Twitty, V. C. And D. Bodenstein, (1944) 7. Exptl. Zool., 95, 214.

VAHS, W., (1955) Z. Naturforsch., I06, 412.

Vahs, W., (1956) Biol. Zentr., 75, 360.

VAHs, W., (1957a) Arch. Entwicklungsmech. Organ., 149, 339.

VAHS, W., (1957b) Embryologia, 3, 201.

Vainio, T., (1958) Exptl. Cell Research, I5, I84.

Vainio, T., S. Toivonen and L. Saxén, (1958) Ann. Med. Exptl. et Biol. Fenniae (Helsinki), 36,285 .

VAKaet, L., (1955) Arch. biol. (Liège), 66, I.

Vandebroek, G., (1938) Natuurw. Tijdschr. (Ghent), 20, 234.

VAN de Kamer, J. G., (1949) Thesis, Utrecht.

Vandekerckhove, D., (1957) Nature, (in the press).

Van der Stricht, O., (I923) Arch. biol. (Liège), 33, 229.

Van Doorenmalen, W. J., (r957) je Journ. cyto-embryol. holl.-belges, (unpublished).

Van Geertruyden, J., (1946) Arch. biol. (Liège), 57, I45.

Vercauteren, R., (1958) Arch. biol. (Liège), 69, 620.

Vintemberger, P. And J. Clavert, (1958a) Compt. rend. soc. biol., I52, I46.

Vintemberger, P. and J. Clavert, (1958b) Compt. rend., 247, 512.

VOGT, W., (I929) Arch. Entwicklungsmech. Organ., I20, 384.

Von Wartenberg, H., (1956) Acta Histochem., 3, 25.

Von Woellwarth, C., (1952) Arch. Entwicklungsmech. Organ., I45, 582.

Von Woellwarth, G., (I956) Arch. Entwicklungsmech. Organ., I48, 504.

Voss, H. and H. Von Wartenberg, (i955) Wiss. Z. Friedrich-Schiller-Univ. Jena, Math.-

Naturwiss. Reihe, 4, $4 \mathrm{I} 3$.

Waddington, C. H., (1933a) Arch. Entwicklungsmech. Organ., 128, 502.

Waddington, C. H., (1933b) F. Exptl. Biol., 1o, 38.

Waddington, C. H., (r952a) 7. Exptl. Biol., 29, 489.

Waddington, G. H., (1952b) The Epigenesis of Birds, University Press, Cambridge.

Waddington, C. H., (1954) 7. Embryol. Exptl. Morphol., 2, 340.

Waddington, C. H., (1956) Principles of Embryology, G. Allen and Unwin, London.

Waddington, C. H. And E. M. Deuchar, (1952) 7. Exptl. Biol., 29, 496.

Waddington, C. H. And E. M. Deuchar, (1954) 7. Embryol. Exptl. Morphol., I, 349.

Waddington, G. H. and B. Goodhart, (I 949) Quart. F. Microscop. Sci., 9o, 209.

Waddington, C. H. and L. Mulherkar, (i957) Proc. Zool. Soc. (London), (Mookerjee

Memorial Volume), I4I.

Waddington, G. H. and M. M. Perry, (1956) F. Embryol. Exptl. Morphol., 4, i io.

Waddington, C. H. and J. L. Sirlin, (1955) Proc. Roy. Soc. (Edinburgh), B, 24, 28.

Waddington, G. H. ANd T. YaO, (I950) 7. Exptl. Biol., 27, I26.

Waechter, H., (1953) Arch. Entwicklungsmech. Organ., I46, 202.

Walder, P., (1950) Acta Zool. (Stockholm), 3I, 187.

Watterson, R., (1952) Biol. Bull., 103, 310.

Weber, R., (1956) Rev. suisse zool., 63, 277.

Weber, R., (1958) Arch. Entwicklungsmech. Organ., I50, 495.

Weiss, P., (1939) Principles of Development, Holt, New York.

Weiss, P., (1949a) Exptl. Cell Research, Suppl. I, 475.

Weiss, P., (I949b) Differential Growth in: A. K. Parpart, The Chemistry and Physiology of Growth, Princeton University Press.

WEIss, P., (1952) Arch. néerl. zool., IO, Suppl. I, I65.

Weiss, P., (1953) 7. Embryol. Exptl. Morphol., I, i81.

Weiss, P. and R. James, (1955) Exptl. Cell Research, Suppl. 3, 381. 
Weiss, P. and A. Moscona, (1958) 7. Embryol. Exptl. Morphol., 6, 238.

WildE, C. E., (1955a) 7. Exptl. Zool., I30, 573.

Wilde, C. E., (I955b) 7. Morphol., 97, 3 I3.

WILde, C. E., (1956a) Anat. Record, 124, 382.

Wilde, C. E., (1956b) 7. Exptl. Zool., 133, 409.

Wilens, S., (1955) 7. Exptl. Zool., 129, 579.

Willier, B. H., P. A. Weiss and V. Hamburger, (1955) Analysis of Development, W. B. Saunders Co.

Willmer, E. N., (195I) Some Aspects of Evolutionary Cytology, in: G. A. Bourne, Cytology and Cell Physiology, 2nd ed., Chapter I I, p. 444, Oxford.

Witschi, E., (1950) Arch. Anat. Microscopy, 39, 2 I 5.

Wittek, M., (1952) Arch. biol. (Liège), 63, I34.

Woerdeman, M. W., (1952) Arch. néerl. zool., io, Suppl. I, I43.

Woerdeman, M. W., (1955a) Immunological Approach to some Problems of Induction and Differentiation in: D. Rudnick, ed., Biological Specificity and Growth, Princeton University Press.

Woerdeman, M. W., (I955b) Ned. Tijdschr. Geneesk., roo (II), 24.

WolfF, E., (1953) Rev. suisse zool., 60, 542.

Woodside, G. L., (1937) 7. Exptl. Zool., 75, 359.

Yamada, T., (1937) Arch. Entwicklungsmech. Organ., 137, I5I.

Yamada, T., (1940) Folia Anat. Fapon., 19, I3 I.

Yamada, T., (r950a) Biol. Bull., 98, 98.

Yamada, T., (I950b) Embryologia, I, I.

Yamada, T., (1958a) Année biol., 62, 167.

YAMADA, T., (1958b) Experientia, I4, 8I.

YAMADA, T., (I959) Embryologia, 4, I 75.

Yamada, T., K. TaKata AND S. Osawa, (1954) Embryologia, 2, i 23.

Yamada, T. and T. Takata, (1955) Exptl. Cell Research, Suppl. 3, 402.

Yamada, T. AND K. TAKata, (1956) Embryologia, 3, 69.

Yамамото, K., (1956) Bull. Fac. Fisheries, 7, 208.

Yамамото, K., (1957) Ann. Zool. Fap., 3o, 33.

YNTEMa, C. L., (1950) 7. Exptl. Zool., II3, 21 I.

Zwilling, E., (r949) 7. Exptl. Zool., 111, I 75.

Zwilling, E., (1955) 7. Exptl. Zool., I28, 423.

Zwilling, E., (1956) F. Exptl. Zool., 132, I41, 157, 173, 219. 


\title{
Chapter 5
}

\author{
MOLEGULAR MEGHANISMS OF DIFFERENTIATION
}

AN INQUIRY INTO THE PROTEIN FORMING SYSTEM OF DEVELOPING CELLS

\section{Heinz Herrmann}

\section{INTRODUCTION}

The basic concepts of experimental embryology such as induction, determination, competence and regulation remained closed for a long time to an interpretation in terms of other fields of biology, of chemistry, or of physics. Consequently the bulk of experimentation in embryology appeared to be largely a verification and extension of the initial observations on different organ systems and in diverse species. From this work arose a body af data which was systematized according to the empirically defined phases of embryonic development. Even more recent comprehensive texts of embryology follow in part this type of organization e.g. "Analysis of Development" (Willier, Weiss and Hamburger, 1955).

While many phenomena of embryology have at least a well-defined empirical content, cellular differentiation is lacking in any sharp delimitation. It is conventionally treated as the elaboration of specific cell characteristics during later embryonic or fetal development. Defined in this sense, differentiation and some of its chemical aspects generally and in several organ systems specifically has been covered by recent reviews (Boell, I 948; Weiss, I 950; Moog, I 952; Ten Cate, I 953; Herrmann, I953a; Gustafson, I954; Spratt, I954; Boell, r955; Duspiva, I 955; Ebert, Tolman, Mun and Albright, I955; Flexner, I 955; Needham, i 955; Shen, r 955 and Waelsch, 1955). To add another similar survey of this topic would seem unnecessary. It may be justified, however, to explore the extent to which the facts of differentiation in early and in late embryogenesis can be subsumed under a more general, conceptually fruitful, frame of reference.

From the reviews of differentiation cited above, it follows that cellular differences are based on the formation of new types of proteins. Even the elaboration of such specific cell characteristics as the deposition of ground substances, or of a lipoidal myelin sheet, initially depends on the presence of proteins with enzymatic activity which synthesize these substances. As a first approach one can consider that the appearance of any new structural or functional cell property is the final expression of a change in the protein pattern of a cell due to the formation either of structural proteins like lens proteins, collagen or keratin, or of enzymes and the products of their activity. It should be stated that the mere chemical assay of a protein per se 
is not an end in itself and has no more significance for the understanding of differentiation than a descriptive account of the change of any other morphological or physiological property. Ultimately, explanatory value can be attributed to these chemical measurements only insofar as they contribute directly to the interpretation of developmental phenomena, not in terms of temporal or spatial correlations, but in terms of a chain of concrete molecular interactions which eventually produces the classically described changes in form and function. This requirement would seem to be met most directly by an attempt to define the changes in the protein forming apparatus itself of the developing cell and its control by cellular and extracellular chemical factors. That the analysis of embryonic development is beginning to turn to this problem is borne out by the contents of the two volumes, Butler, Ed. (I 955) and Rudnick, Ed. (I 954) of the Symposia of the Society of Growth and in particular of the article by Ebert (I 954b) contained in the latter.

The merit of a presentation in which differentiation is regarded as a problem of protein formation ultimately stands or falls with the understanding of the cellular and molecular mechanisms of protein synthesis. Only a few years ago the problem of protein synthesis seemed so untractable that it precluded such an interpretation. This situation has changed rapidly. Recent explorations of some phases of protein formation in the fully differentiated cell have advanced so significantly that it would seem highly desirable to follow some of these leads in differentiating tissues ${ }^{1}$. With only a little optimism one could actually ask whether an investigation of the embryonic cell with its presumably diversified types of protein formation may provide exceptionally favorable material for a comparative study of the problems of protein synthesis.

In discussing the data on differentiation as a problem of protein formation, the available material was organized in the following way. To a large extent the properties of the protein forming system (PFS) have been inferred from measurements not pertaining directly to the constituents of the PFS. One of the first, and still widely used, methods of obtaining information about the activity of the PFS consists of measuring the utilization of protein precursors. Therefore, we will be concerned first with a summary of recent data on utilization of amino acids and other protein precursors in embryos. As the next step in the elucidation of the PFS, attempts to identify the actual molecular composition of this system will be considered. A comparison of such work, carried out with differentiated and embryonic cells, thus forms the second section of this chapter.

The relationship of embryonic differentiation to protein biosynthesis is dealt with in a discussion of the control of the PFS. These problems will be considered in section III.

1 The rapid progress in this field is documented in the sections on "Amino Acid Activation and Protein Synthesis" and "Protein Synthesis: Microsomes, RNA, and Templates" in Federation Proc., 17, 1958, and in several Symposia (in particular, the Symposium and numerous papers presented at the Fourth International Congress of Biochemistry of Morphogenesis, Vol. 6, I 959). Pertinent also in the present context is the Symposium on "Chemical Basis of Development", (W. D. McElroy and H. B. Glass, Johns Hopkins Press, 1958), The "Symposium on Amino Acid Activation", Proc. Natl. Acad.Sci., U.S., 44 (1958) 667, and the article, "Antigens and Antibodies as Cell Phenotypes", by Schultz (I959). 
In the concluding section an attempt will be made to discuss the main problem of embryonic differentiation in the light of concepts and results developed recently in some related fields of biology such as microbiology and immunology.

II. THE UTILIZATION OF PROTEIN PREGURSORS IN THE

DIFFERENTIATING CELL

\section{(a) Precursors larger than amino acids}

In the study of the nature of protein precursors and of their utilization in embryonic cells two different, but not necessarily mutually exclusive hypotheses have been explored. One line of research has been designed to test the possibility that precursors of a molecular size larger than amino acids, as large perhaps as protein molecules, give rise to the protein molecules of the differentiating embryonic cell. Schechtman (1955) who, with his associates, contributed much experimental work to this problem, has recently summarized evidence that proteins can actually be transferred through cellular membranes and taken up as undegraded molecules into embryonic cells ${ }^{1}$. Indeed, uptake of immunoproteins and serum proteins tagged with fluorescent dyes has been shown to occur in the rapidly growing cells of neoplastic tissues (Busch and Greene, I955). However, uptake of, at least small protein molecules into mature cells is also indicated by observations of experimentally produced effects of ribonuclease on various intracellular structures (Kaufmann and Das, I955) and by the demonstration of the uptake of homologous antibodies into adult tissues (Humphrey and McFarlane, 1954). Indications of an incorporation of protein precursors larger than amino acids were also obtained in interesting work with cells in vitro (Francis and Winnick, I953 and Gerarde and Jones, I 953). An effect of the intact proteins in cell growth in vitro is indicated by the gradual adaptation of cells to heterologous media (Langman, i953a, b) and the reactions to antibodies (Nace, I955).

Actual utilization of large molecular cell components taken up into embryonic cells was demonstrated by the observation that organ implants accelerate specifically the growth of the homologous organs in the host embryo. Weiss (1947) postulated that large molecular material released from these transplants is taken up specifically by the cells of the homologous host organ and acts catalytically as a "template" for the protein forming apparatus of these cells. As in so many other instances in embryological research, the failure to perform a quantitative analysis led to this erroneous assumption. By labelling the proteins of the donor tissue with methionine $-{ }^{35} \mathrm{~S}$, Ebert could compare quantitatively the amount of label lost from the donor with the amount of label taken up specifically by the homologous host organ (Ebert, I954a). He found that within the range of experimental accuracy the observed increase in the host organ could be accounted for by the amount lost from the donor tissue. These experiments did not support the assumption of a catalytic role of the transferred high molecular material which was apparently

1 An increased permeability of embryonic cells to large molecules is also indicated by the distribution of inulin in muscle tissue of ten to twelve day chick embryos (Herrmann, White and Cooper, 1957). 
used as a precursor for protein formation in the host organ either directly, or after intermediary degradation to amino acids. Complete absence of activity in other host tissues would have indicated that no amino acids were liberated from the proteins of the graft and that these proteins or their polypeptide degradation products were taken up specifically by the homologous host organ. The fact that small amounts of activity did appear in non-homologous host tissues implies that either some donor protein was incorporated as such even in non-homologous tissues or that amino acids liberated in the breakdown of the graft proteins enter the circulation and are incorporated non-specifically into cellular proteins of heterologous host organs Recent experiments by Walter, Allman and Mahler ( I956) seem to corroborate Ebert's findings. The relationship between this specific uptake of homologous proteins and the stimulation of mitosis by homologous tissue suspensions (Andres, I 955) remains, as yet, to be explored.

It is noteworthy to add that substantial amounts of peptides can be identified in protein free extracts of sea urchin and amphibian embryos. Published photographs of chromatograms seem to show in the case of Hyla regilla an increase in the intensity of these spots from stages $2-7$ to stage 18 , while in Rana pipiens the intensity of the peptide spots decreases during the same progression of developmental stages (Berg, I950; Eakin, Berg and Kutsky, I950; and Kutsky, Eakin, Berg and Kavanau, I953). Recently, large amounts of peptides were identified in extracts of Triton alpestris (Chen, I956a). Peptides with growth promoting activity were also described by Gustafson and Hjelte (I95I) and Gustafson, Hjelte and Hasselberg (1952), Kavanau, et al. (1954) and Hjelte, et al. (r955) in extracts of sea urchin embryos. Since in fully differentiated cells peptides seem to be present only in much smaller quantities than are found in embryonic tissues, the question arises as to whether peptides play a more significant role in protein metabolism of at least some embryonic tissues.

\section{(b) Amino acids as precursors}

Amino acids are probably used as precursors of proteins throughout embryogenesis. To what extent utilization of amino acids or of larger protein building blocks supplement each other and under what condition utilization of the one or the other precursor predominates is not known. The presence of free amino acids in extracts of sea urchin and amphibian embryos was detected chromatographically by Berg (I950); Eakin, Berg and Kutsky (1950); Kutsky, Eakin, Berg and Kavanau (1953) and Chen (1956a), but little progress has been made in correlating the observed changes in the amino acid content of these extracts with protein formation. On the other hand the question of the free amino acid content of sea urchin embryos was investigated by Gustafson and Hjelte (I95I), who found rather striking changes occurring during the mesenchyme blastula and gastrula stage; they suggested that these changes are correlated to the apparently increased synthesis of proteins as manifested by the appearance of new enzymes (Gustafson and Hasselberg, I 95 I and Gustafson, I954) and new antigenic proteins (Perlmann and Gustafson, I948 and Perlmann, I953).

A systematic investigation of the amino acid content and its possible correlation to protein formation in the developing sea urchin embryo was attempted by 
Kavanau (I953 and I954). The results of these analyses were interpreted as indicating periodic phases of yolk degradation followed by new synthesis of embryonic proteins. The accumulation of non-protein amino acids, following the decrease in yolk protein content, was assumed to indicate that the yolk proteins are first degraded into amino acids and peptides which serve as the precursors for new synthesis of embryonic proteins.

The utilization of amino acid storage depots has been approached by widely different lines of investigation. The complex structure of yolk granules and the changes occurring during the degradation of this material have been recorded in the electron microscope by Bellairs (1958). An extensive study of the changes in the yolk proteins of the frog egg and stimulating suggestions concerning the regulation of their breakdown by a phosphorylation mechanism have been published by Barth and Barth (I954) and the relation of phosphatase activity to yolk platelet utilization was also investigated by Flickinger (1956). Studies of the amino acid content of chick embryos (Williams, DaCosta, Newman and Marshall, i954) and of the disappearance of the proteins from the yolk and the egg white (Rupe and Farmer, r 955) have been initiated.

Other interesting approaches to the problem of amino acid utilization in embryos are the explorations of genetic control of the free amino acid content in Drosophila and Ephestia (Hadorn, r955; Chen, I956b; and Chen and Kühn, I956) and experiments designed to elucidate in chick embryos the relationship of hormonal control (cortisone) of the synthesis of specific proteins (collagen) to the blood level of one of the specific amino acid components (hydroxyproline) of collagen ( $\mathrm{Ro}-$ berts, Karnofsky and Frankel, I95I).

As an alternative method, amino acid utilization in protein formation can be measured by adding labelled amino acids to the amino acid pool and following the incorporation of the label into the proteins. The advantage of this method is the relative directness with which it indicates simultaneously both the rates of protein formation and amino acid utilization.

Using labelled alanine and glycine Hultin (1952) found, working with sea urchin embryos, that the incorporation of these amino acids into a protein fraction insoluble in isotonic $\mathrm{KCl}$ increases rapidly after fertilization and reaches a maximum rate at about 12 hours of development (end of the blastula stage). During the first eight hours of development the $\mathrm{KCl}$ soluble fraction exhibits a much slower rate of incorporation than that of the insoluble one. After this period a sharp increase in the rate of incorporation occurs in the soluble fraction up to about the rath hour of development, resuming thereafter the original slow rate of incorporation, at least, until the 3 oth hour of development. This discrepancy in the incorporation rates of the two fractions is attributed to the rapid development of cellular particulate matter i.e. microsomes, mitochondria (insoluble fraction) beginning at fertilization and continuing during the first stages of ontogenesis. Later, a temporary period of more rapid formation of soluble enzymes ensues.

Utilization of small molecular precursors in the formation of proteins in amphibian embryos could be demonstrated indirectly in experiments with ${ }^{14} \mathrm{CO}_{2}$ which penetrates rapidly into the embryo and is incorporated first into amino 
TABLE 1

INCORPORATION OF GLYCINE-I $-{ }^{14} \mathrm{C}$ INTO TISSUES OF II-I 3 SOMITE CHICK EMBRYOS EXPLANTED FOR 4 h ON AGAR-EGG EXTRACT MEDIUM CONTAINING LABELLED GLYCINE

\begin{tabular}{|c|c|c|c|}
\hline Tissues & $\begin{array}{l}\text { No. of } \\
\text { organs } \\
\text { per } \\
\text { sample }\end{array}$ & $\begin{array}{l}\text { Mean specific activity }{ }^{1} \\
\frac{\mu g \text { glycine-1 }-{ }^{14} C \times 1 \mathrm{O}^{3}}{\mu g \text { total glycine }}\end{array}$ & Standard error ${ }^{2}$ \\
\hline Primitive knot & Io & 7.02 & \pm 0.900 \\
\hline Neural tube & Io & 6.20 & $\pm \mathbf{1} .260$ \\
\hline Somites & 250 & $4 \cdot 99$ & \pm 0.824 \\
\hline Segmental plate & 20 & 4.14 & \pm 0.549 \\
\hline Brain & I0 & 3.62 & \pm 0.500 \\
\hline Heart & 10 & 1.97 & \pm 0.219 \\
\hline Notochord & Io & I.I 8 & $\pm 0.18 \mathrm{I}$ \\
\hline
\end{tabular}

${ }^{1}$ Derived from analyses of glycine isolated chromatographically from the tissue proteins.

${ }^{2}$ Derived from nine separate determinations.

acids which are then incorporated into the proteins of the embryo. Flickinger (1954) demonstrated that in the neurula of Rana pipiens, for example, the major activity is found in the neural folds, notochord and somites, but much less in the remaining embryo. Cohen (I954) showed, in addition, that the rate of incorporation of labelled $\mathrm{CO}_{2}$ into the protein moiety of developing amphibian embryos increased considerably from the blastula to the neurula stage. Since labelled $\mathrm{CO}_{2}$ can be incorporated into proteins only by first forming a part of the amino acid molecules, these experiments demonstrate that amino acid incorporation is actually taking place and that the rates of amino acid synthesis and incorporation vary in different organs and at different developmental stages of the amphibian embryo. Although labelled amino acids are not well suited for incorporation studies in amphibians because of a limited permeability of the ectodermal coat of the embryo, a higher uptake in the dorsal half of the gastrula could be demonstrated (Friedberg and Eakin, I949 and Eakin, Kutsky and Berg, I95I) using bisected embryos.

During the early development of the chick embryo, the uptake of labelled amino acids can be followed advantageously in explants using Spratt's technique (Spratt, I947 and I948). In recent experiments in our laboratory, embryos at $5-7$, or I I-I 3 somite stages were explanted on an agar medium containing, in addition to the egg extract, labelled glycine. By isolating chromatographically, after acid hydrolysis, the tracer glycine incorporated after varying times of incubation $(\mathbf{I}-\mathbf{I} 2 \mathrm{~h}$.), it was possible to follow its uptake into the proteins of the whole embryo and of the isolated embryonic organs. As indicated in Table I, neural tube and somites are among the embryonic tissues with a higher rate of tracer incorporation while in the heart and notochord incorporation occurs at a much slower rate (Schultz and Herrmann, I958).

In another series of experiments the uptake of tracer amino acids was allowed to proceed for a limited time $(e . g .2 h$. ). After this period the embryos were transferred tr) an agar-medium plate without labelled amino acid and the release of the tracer from the proteins and from the free amino acid pool was measured. It was found 
that the free glycine equilibrates rapidly (within one hour) with the external medium while the proteins lose their label only at a very slow rate, if at all. The protein fraction does not lose statistically significant amounts of the label during a six-hour incubation period. This shows that there is no immediate extensive protein breakdown of the proteins of the explant. With the establishment of conditions for rapid and slow growth of the explants (Britt and Herrmann, 1959), possibilities for further explorations of the relation of tracer turnover to protein accumulation seem indicated.

Measurements of the uptake of tracer amino acids into isolated proteins have been carried out so far in chick embryos only at a more advanced stage of development (Herrmann, Lerman and White, I 958). In embryos at I 4 days of incubation the proteins collagen and actomyosin could be isolated from the leg musculature at varying times after injection of glycine- $\mathrm{I}^{14} \mathrm{C}$ directly into the embryonic circulation. The amounts of total and of tracer glycine were determined in the blood, in the free amino acid pool of the cells, and in the two proteins. Unexpectedly, it was found that the incorporation curves for the two proteins seemed to differ qualitatively and quantitatively although the net accumulation of the two proteins was found to be of the same order of magnitude. In attempting to explain this discrepancy, it was suggested that in the formation of collagen and myosin, the tracer amino acid is first rapidly incorporated into the proteins at the site of their initial synthesis (microsomes). After the protein molecules are formed and deposited as part of the extra- or intracellular structures, their rate of equiliberation with the amino acid pool decreases markedly and may become neglibly small. In order to account for the differences in the incorporation curves for the two proteins, it seemed possible to assume that in case of the collagen determination, the active precursor was lost in the course of our preparation; while in case of the actomyosin fraction, the active portion of the incorporation portion of this protein fraction was included in our preparation. Continuation of such analyses seemed to open possibilities for a further study of the different phases of protein formation and of the relation of tracer incorporation and net protein accumulation in growing tissues.

In the preceding paragraphs data have been summarized dealing with the utilization of amino acids and of larger building blocks (peptides, proteins) in the formation of the cellular proteins of the embryo. The main line of evidence for the utilization of large molecular precursors was the selectivity with which such precursors are taken up by homologous organs. The mere presence of high concentrations of peptides in cell extracts suggests, possibly, that they play a more prominent role in protein formation in the embryo than in adult tissues. The utilization of amino acids was demonstrated by qualitative and quantitative variations of the amino acid pool and by the incorporation of labelled amino acids into the proteins of various embryos. A sharper, qualitative and quantitative, definition of the protein precursors used at various stages of development is, in fact, of considerable interest. Utilization of larger precursors will give the formed proteins a certain structural relationship to such precursors and the energy requirements for the formation of proteins from peptides are probably considerably lower than for the synthesis de novo from individual amino acids. 
Synthesis of proteins which are highly specific cell products (e.g collagen, lens proteins) presupposes in many instances an alignment of amino acids in a rather unique sequence. Such proteins would have to be built up from either small peptides or free amino acids. On the other hand, during early embryogenesis, some high molecular precursors could possibly be utilized for energetically "inexpensive" protein formation in the rapidly proliferating cells. Also, in some species no net synthesis of proteins occurs during long periods of development (sea urchin, amphibian). In these instances conversion of yolk proteins into different cytoplasmic protein must take place and the possibility of utilization of higher protein precursors for the formation of some cell proteins would seem advantageous for rapid protein formation.

The investigation of the types of precursors which are utilized in protein synthesis is greatly aided by the use of amino acid analogs. These compounds interfere primarily with the utilization of amino acids in protein formation and are supposed to be indifferent with respect to protein formation from larger precursors and lead, therefore, to the distinction of these two types of protein precursors. The usefulness of amino acid analogs has been demonstrated in the field of microbiology. Some of the experiments which are of particular relevance in the context of this discussion have been carried out by Halvorson and Spiegelman (1952) and have been reviewed by Spiegelman, Halvorson and Ben-Ishai (I955). These authors found a considerable amino acid pool in yeast cells. Just as in the case of the sea urchin embryo, the amino acid content of yeasts proved to be a sensitive index of the state of protein forming activity of the cell. Upon the stimulation of growth as well as during induced enzyme synthesis, the quantity of this amino acid "pool" diminished. Neither overall protein formation (growth), nor formation of specific proteins (induced enzymes), nor a depletion of the amino acid pool would take place in the presence of amino acid analogs (Halvorson and Spiegelman, I952). From this analysis it can be concluded that growth and the formation of induced enzymes are dependent upon a supply of free amino acids.

Amino acid analogs were introduced into the field of morphogenesis by Lehmann, Bernhard, Hadorn and Lüscher (1945) and by Erlenmeyer and Lehmann (1949). In testing the effects of aminoketones as analogs of leucine it was found that these substances inhibit the regeneration of the tail of amphibians. While the mitotic activity and the histogenetic phase of the regenerative process were found to be undisturbed, the formation of the blastema itself was impaired presumably by an inhibition of cell migration (Erlenmeyer and Lehmann, I949; Lehmann and Dettelbach, I 952; Lehmann and Bretscher, I 952 ; Lehmann, I954a, b; Lehmann, Weber, Aebi, Baumler and Erlenmeyer, I954). Further analysis of this effect suggested a possible correlation of this inhibition with an increased proteolytic activity (Lehmann and Løvtrup, I956). Such a correlation seems to be corroborated by our experiments on the chick embryo.

Testing the effects of amino acid analogs on explanted chick embryos it was frund that different analogs of the same amino acid gave similar abnormalities; bit, analogs of different amino acids gave characteristically different effects (Hermann, I953b; Rothfels, I954; Herrmann, Konigsberg, and Curry, I 955). With ethionine, the analog of methionine, no abnormal morphogenesis could be 
observed in explanted embryos. Even concentrations of $2.0 \mathrm{mg} / \mathrm{ml}$ of the medium caused only a slight retardation of growth. Thienylalanine, the analog of phenylalanine, however, gave rise to distinct anomalies. The main deviations observed were: a malformed brain, open and frequently distorted neural tubes, and small, pale staining somites which showed cavitation in the sclerotomes. These abnormal features of the early developmental period were accompanied by a marked retardation of overall growth. The latter effect could be prevented, at least in part, by the addition of the normal amino acid, phenylalanine, but the formation of abnormalities was irreversible.

The analogs of valine and leucine caused very little growth retardation. Their interference with development was restricted almost entirely to a disturbance of somite development. Somites appearing about two to three hours after explantation were no longer separated from each other but retained the structure of an uninterrupted plate. The justification for referring to this unsegmented plate as an abnormal somite development is derived from the observation that at least with some of the leucine-valine analogs formation of the dermomyotome and of a sclerotome could be observed. Thus, the abnormal plate formed is not completely devoid of somite characteristics. The effect of the leucine analogs could be reversed not only by leucine and valine but, to a greater or lesser extent, by other amino acids which contain a chain of more than three carbon atoms. Serine and alanine were found to be much less effective in alleviating the abnormality.

In view of the absence of any effect by ethionine, it seemed desirable to test this analog in systems where the mechanism of its effect on protein synthesis could be explored more readily. In using the older chick embryo, it was found that following an injection of ethionine into the yolk or onto the chorioallantoic membrane, growth, measured in terms of body weight, was not retarded until after the $7_{\text {th }}$ day of development. These findings were corroborated by the experiments carried out independently by Karnofsky, et al. (I955). After the 7 th day of chick development, the increase of bodyweight was markedly reduced and at later stages certain pathological symptoms developed which are of less importance in the present context.

These results seemed to present some rather perplexing contradictions. If it is assumed that the effects of amino acid analogs are due to interference with protein formation, one would have expected that any one of the tested analogs would inhibit synthesis of all proteins with the result of a general growth inhibition but without specific effects in any particular tissue. Instead, the one analog (ethionine) was found to be practically ineffective in explants tested while other analogs exhibited rather specific effects on various organ systems.

As an explanation of these discrepancies several possibilities can be considered. First, it is possible that various analogs inhibit the rate of formation of different proteins to a different extent depending, possibly, upon the number of molecules of the corresponding normal amino acid in the respective protein. This could lead to a change in the proportion of different proteins in different cell types resulting in the abnormal reactions of various cells in this change.

In exploring the mechanism of the effect of the analogs leucine and methionine, the incorporation of labelled glycine into proteins of explanted chick embryos 
was investigated. Preliminary measurements indicate that the amount of labelled glycine taken up does not change on addition of the leucine analog. However, the total protein glycine content of the somites after explantation with the analog was found to be smaller than in the controls. This may be regarded as an indication of an enhanced protein breakdown and parallels the observations made with aminoketones discussed previously. Ethionine was found to be without gross effect on the development of explanted embryos under the conditions of cultivation used in these experiments. However, it inhibited the incorporation of tracer glycine into the embryonic proteins ( $\mathrm{I}-\mathrm{I} 3$ somite stage) by about twenty per cent. In these cases the effect of the analogs on morphogenesis is not correlated to the inhibition of amino acid incorporation into the embryonic proteins (Schultz and Herrmann, 1958). As a second alternative, it could be assumed that during embryogenesis some tissues or cells depend to a larger extent upon protein formation from large molecular precursors than others and, therefore, are less sensitive to amino acid analogs. Also, it should be considered that different analogs will be incorporated as such into some proteins, as has been observed in the case of ethionine (Levine and Tarver, I95I). In this way, certain proteins would be altered more than others, thus producing the specific effects. Preliminary experiments exploring this alternative did not provide evidence for the presence of leucine analog in paper chromatograms obtained from protein hydrolyzates of either early embryos or of muscle tissue from older animals.

A series of studies on the effect of amino acid analogs on the early development of amphibian and chick embryos were also carried out by Waddington and Sirlin (1954) and Feldman and Waddington (1955). The embryos in situ were exposed to solutions of labelled amino acids and amino acid analogs and an evaluation of the uptake of labelled material was attempted by radioautographs ${ }^{1}$ of the embryos (the number of silver grains was determined as an index of the incorporation rate). In other experiments the protein activity was also determined by a conventional Geiger counting procedure. Xenopus embryos were kept for 2 I hours in a solution of glycine- $\mathrm{I}-{ }^{14} \mathrm{C}$. It was shown radioautographically that a distinct maximum of radioactivity occurred in the epidermis and in the somites. Notochord and neural tube showed only one-half of the activity and the endoderm only slightly more than one tenth. Measurements obtained by counting techniques demonstrated that the incorporation of glycine into whole amphibian embryos was decreased to about one-half when the analog para-fluorophenylalanine was added.

A similar study was carried out with early chick embryos between stages seven and nine, corresponding to the formation of the first somites. Evaluation of radioautographs seemed to indicate that the neural fold showed the highest incorporation of labelled methionine- ${ }^{35} \mathrm{~S}$. In the early stages, the ectoderm seemed to be more active than the axial mesoderm and the reverse condition prevailed in the later stages. However, the differences observed in chick embryos were much smaller

\footnotetext{
1 Rates of protein synthesis can, at best, be derived from time curves for the specific activities of tissue proteins. Since radioautographs do not lend themselves readily to the establishment of such curves their value for measurements of rates of protein synthesis is limited at the present time.
} 
than in amphibian embryos. Similar to the results obtained in amphibians, amino acid analogs diminished the incorporation of methionine $-{ }^{35} \mathrm{~S}$.

Effects of amino acid analogs on sea urchin development have also been reported in a preliminary note (Gustafson and Hörstadius, I 955). The results of the reported experiments show that the amino acid analogs utilized (ethionine, thienylalanine, phenyllactic acid, allylglycine, methionine sulfoxide and d-leucine and d-norleucine) have a "vegetalizing" effect. From the reported data it is uncertain whether the observed effects are actually due to interference with amino acid utilization in protein formation. This can only be demonstrated by a reversal of the abnormalities by addition of the corresponding normal amino acids, and by measurements of the incorporation rates of labelled amino acids with and without amino acid analogs. Actually "non-metabolic" effects observed with other groups of apparent anti-metabolites were suggested earlier by Hörstadius and Gustafson (1954). Assuming that the anomalies are actually due to an impaired amino acid utilization, the appearance of a "vegetalizing" effect might indicate that the formation of specialized proteins is suppressed. This suggestion is based on the fact that other "vegetalizing" agents, like lithium, prevent the production of enzymatically and serologically specific proteins which appear in the course of sea urchin development.

\section{(c) Pathways of precursor utilization}

Assays of the changing quantities of amino acids occurring in the different tissues of the developing embryo, determinations of the incorporation rates of tracer amino acids, or the observations of the utilization of large molecular protein precursors into embryonic cells, become meaningful only when such studies are directed toward an understanding of the reactions by which these precursors are transformed into the proteins of the embryo. Up to the present time the two main lines of research dealing with the pathways of amino acid utilization in differentiated tissues have hardly been considered in work with embryonic cells.

One of these approaches comprises investigations of the mechanism by which amino acids are activated preliminary to the formation of peptide bonds, the primary reaction in protein synthesis. This work is of great importance because it points to the mechanism by which metabolic energy is utilized in protein synthesis.

Stimulated by earlier ideas of Lipmann ( 1954), the result of the work of several investigators (Hoagland, I 955 and Hoagland, Keller and Zamecnik, I956) led to the recognition that peptide bond synthesis involved the activation of amino acid by a reaction involving ATP leaving the amino acid bound to an adenosinemonophosphate radical. Specific enzymes catalyze this reaction for each individual amino acid tested so far. Presumably in this intermediary form the amino acid becomes attached to the site where peptide bond formation is catalyzed. Observation of a transient adsorption of free amino acids may be related to this step in amino acid utilization (Britten, Roberts, French, I955).

Nothing is known, as yet, about the activating mechanism of amino acids in embryonic tissues during proliferation and differentiation. The importance of the recognition of this mechanism for the problem of protein synthesis during differen- 
tiation lies in the possibility that the production of different proteins is, at least in part, controlled by the selective activation of the different enzymes which catalyze the utilization of the individual amino acids. The activation of these enzymes again may depend on the total metabolic energy available, or upon the specific metabolite which acts as the carrier of the energy supply. It is apparent that this aspect of the problem of protein synthesis in embryonic cells links it to possible changes in intermediary metabolism of embryonic tissues.

A second approach to the problem of protein formation has emerged from studies of the patterns of enzymatic reactions by which amino acids are linked to give the specific sequence which is characteristic for each protein species. The work in this field has been summarized recently in three lucid reviews which provide a valuable basis for the following discussion (Borsook, I956a, b; Fruton, 1955 and Steinberg, Vaughan and Anfinsen, I956).

Following Fruton's line of thought, peptide chain formation is brought about by a stepwise addition of individual amino acids to form peptides of gradually increasing chain length. The energy for the initial steps in this process is derived from ATP, the end product of metabolic oxidation, and is utilized for the production of certain key substances such as glutamine. Without further energy being required, the amide group of glutamine can be replaced by amino acids resulting in the formation of a dipeptide. In subsequent transpeptidation, formation of larger peptides is achieved. Intracellular proteinases such as cathepsin are supposed to be the catalysts for these reactions. Although conclusive evidence for the biological significance of the enzymatic reactions indicated in this scheme is still wanting it could give meaning to the appearance of glutamine and of high concentrations of peptides in embryonic tissues mentioned in the earlier part of this section. To which extent the association of a high activity of proteinase and peptidases in centers of active growth (Urbani, I955), the appearance of the enzyme glutamotransferase (Rudnick and Waelsch, I955) in embryonic tissues, or the rapid rate of amidation in sea urchin embryos (Hultin, r953d) can be associated with a scheme of protein synthesis similar to Fruton's remains to be explored.

Other experiments designed to investigate the mechanism of protein synthesis were based on the following considerations. If proteins are synthesized by the simultaneous formation of peptide bonds between all of the amino acids necessary for a particular protein molecule, addition of a labelled amino acid to the amino acid pool of a cell should result in a uniform distribution of the label in the different portions of this molecule. If, however, individual peptides are synthesized at different rates and then put together to form a complete protein molecule the proportions of labelled amino acids in the various portions of the protein molecule would be expected to be different. Anfinsen and Steinberg (I95 I); Steinberg and Anfinsen (1952); Peters (r953); Flavin and Anfinsen (1954) and Vaughan and Anfinsen (1954) using in vitro systems of liver, chick oviduct and pancreas tissue, isolated serum albumin, ovalbumin, ribonuclease and insulin from the tissue preparations incubated in the presence of the labelled amino acid. The analysis of these isolated proteins showed a highly unequal distribution of the labelled amino acid in the various peptides obtained from these proteins. In contrast, 
hemoglobin (Muir, Neuberger and Perrone, I 952), various muscle proteins (Simpson and Velick, 1954), and milk proteins (Askonas, Campbell and Work, I954), isolated after the injection of labelled amino acids into the intact animal, gave no indication of an unequal distribution of the tagged amino acid in the peptides derived from these proteins. Whether these two sets of results actually indicate the operation of two different mechanisms has been questioned by Steinberg et al. ( $195^{6}$ ). He points out that for reasons of kinetics unequal rates of incorporation of labelled amino acids into "intermediate conjugates" would be maintained only for a short time. Correspondingly the unequal labelling of the parts of the protein molecule which are made up from these conjugates would be detectable only during a limited time span after addition of the tracer. However, Borsook ( $956 \mathrm{a}, \mathrm{pp} .62-65$ ) contends that non-uniform labelling could be explained as well by a rapid exchange of amino acids after adsorption on the "template", but prior to peptide link formation. The investigation of this problem in embryos may prove to be laborious and difficult since it presupposes work with substantial amounts of highly purified proteins. However, exploration of this mechanism in proliferating and in differentiating tissues may provide a comparative approach of considerable interest both for the embryologist and the protein chemist.

III. THE PROTEIN FORMING SYSTEM (PFS) OF DIFFERENTIATED AND

EMBRYONIC CELLS

In the preceding section the PFS was discussed from the standpoint of the rate and the possible mechanisms of the utilization of protein precursors. For an understanding of the changes in the utilization of protein precursors and the production of new types of cell proteins, which occur during development, a more direct examination of the components of the PFS and of their mode of operation at different stages of development, is required. As yet, too little knowledge is available about the PFS in the developing cell to even consider such an account. Most of the work carried out so far with embryonic tissues has followed such divergent methodological approaches which were applied under such different conditions that no coherent picture can be given of the changes of even a single parameter of the PFS throughout the main phases of development. Incorporation studies with microsomes in sea urchin eggs, the use of purine analogs combined with radioautography in the chick embryo, and measurements of uptake of ${ }^{32} \mathrm{P}$ in amphibian development have yielded so far only a loose patchwork of observations concerning the PFS in embryonic cells. However, considerable advances have been made during the past few years in elucidating the nature of the PFS of adult cells, and it may be most profitable to use the data obtained from differentiated animal and microbial cells to indicate some fruitful approaches to an analysis of the PFS in developing tissues.

Decisive progress in the analysis of protein synthesis has resulted essentially from two lines of research on differentiated cells. The one deals with measurements 
of protein formation ${ }^{1}$ in the isolated structural elements of the cell (nucleus, mitochondria and microsomes) and with the dependence of the synthetic process in these structures on the presence of nucleic acids and on the supply of energy in the form of specific metabolic reactions.

Representing the second approach, electron microscopy has contributed greatly to an appreciation of the organization and the possible interactions of the isolated sites of protein synthesis within the cell. The results of this work must be taken into consideration in any discussion of protein formation in intact cells. The advances in the analysis of the PFS in mature cells are summarized in the following paragraphs as an essential prerequisite for a discussion of measurements and observations obtained thus far with embryonic cells and an orientation of future investigations.

The results of the investigations on the PFS of differentiated cells can be summarized at the present time as follows:

(I) Ribonucleic acid (RNA) is an important component of the PFS.

(2) In certain instances the rate of protein formation is related to the actual content of cellular RNA. These results were obtained in cells (i.e. liver, pancreas) in which the protein forming system may be regarded as being stabilized with respect to protein formation. In more labile cells during synthesis of new types of proteins the rate of protein formation seems to depend on the rate of synthesis of new RNA molecules rather than on RNA content alone. The possible implications of each of these aspects of RNA metabolism to protein synthesis in a developing system seems evident. In certain phases of embryonic development differentiation may be marked by the appearance of new types of proteins; at other phases a cell type may differentiate by the preferential accumulation of only certain specific proteins and a corresponding change in the dependence on the metabolism of RNA may be expected. For fully developed tissues such a possibility has been pointed out by Mazia and Prescott (1955).

(3) In differentiated cells RNA is associated with discrete cytoplasmic structures (microsomes, mitochondria) each of which plays a specific role in protein formation. In embryonic cells RNA may exist unassociated with cell particulates (Brachet, I952). The absence of structural association during development may make this "free" RNA more adaptable in its function as a component of the PFS or it may be indicative of other unknown differences in its function.

(4) Considerable evidence has been gathered relative to the site of synthesis of the cytoplasmic RNA involved in protein synthesis. If, as one line of evidence suggests (Brachet, I952; Taylor et al., I 955), cytoplasmic RNA is synthesized in the nucleus, an approach to the role of the gene in development could be envisioned. Moreover, other experiments indicate that protein synthesis in the nucleus is affected by cytoplasm activities (Allfrey, Daly and Mirsky, 1955). These facts and conjectures suggest possible approaches to the intriguing problem of reciprocal effects of the differentiating cytoplasm on an originally equipotent genome.

\footnotetext{
${ }^{1}$ Protein formation is used as an inclusive term in those instances where no sharp distinction has been made between (I) net synthesis of proteins, (2) protein formation representing one component of protein turnover, (3) exchange of amino acids without total synthesis of protein molecules.
} 
(5) In interpreting these findings, it was frequently pointed out that RNA may act as a "template" or "mold" in the assembling process of amino acids into whole protein molecules (Borsook, I956a, b). Whether RNA merely orders the sequence of amino acids in the peptide chain or whether it is also involved in the folding of the peptides into the sterically specific configuration of the protein molecule is still a matter of conjecture.

\section{(a) Nucleic acids as components of the PFS}

The role of RNA as a key component of the PFS has become more evident from studies of protein formation in microbes in general and of adaptive enzymes in particular. Gale and Folkes (1953a, I953b, 1955a and 1955b) observed that in Staphylococci the amount of cellular protein increases more rapidly if nucleic acid precursors such as purines and pyrimidines are added to the amino acid mixture which maintains bacterial growth. At the same time, the RNA content of the bacteria increases. It is noteworthy that not only does omission of purines and pyrimidines from the medium lead to a marked drop in the accumulation of proteins, but the omission of a single essential amino acid also prevents the accumulation of RNA. It is apparent that in this instance formation of proteins and RNA are closely interlinked processes and impairment of either one results in impairment of the other.

In disrupted staphylococcus cells, formation of enzymes can be greatly stimulated by the addition of nucleic acids or purines and pyrimidines. The degree of stimulation by DNA, RNA, or purine-pyrimidine mixtures differs for each of the investigated enzymatic activities. The decisive point in this investigation is the finding that no incorporation of RNA precursors into RNA in preparations of disrupted cells could be observed unless protein synthesis e.g. formation of adaptive enzymes was occurring. The latter finding suggests again a close interdependence of the synthesis of proteins and RNA (see also review by Gale, I 956). It must be pointed out, however, that it is not clear from these experiments whether RNA acts in this system directly in some catalytic role or indirectly as a reservoir of co-factors needed in the enzymatic production of metabolic energy.

The importance of RNA in protein formation is further emphasized by the results of investigations carried out with yeast mutants requiring purines for growth (Spiegelman, Halvorson and Ben-Ishai, I $955^{1}$ ). It was found that the pool of purines and pyrimidines could be exhausted by rapid protein synthesis indicating again a close connection between purine and protein metabolism. In addition, it was demonstrated that in these "purine free" yeast cells protein synthesis, as measured by the rate of formation of induced enzymes, could be reactivated by replenishing the purine pool in the cell by adding the required compound to the medium.

Similarly, in mutants of $E$. coli which can not synthesize certain precursors of RNA, depletion of the pool of purines and pyrimidines in the bacteria causes a marked delay in the onset of enzyme formation while in "normal" strains the formation of adaptive enzymes starts immediately after addition of the inducing

1 Full data unpublished. 
substrate. In the mutant the RNA necessary for the initiation of protein synthesis has to be manufactured before protein synthesis can begin. Similar results were obtained by Pardee (1954).

After irradiation with ultraviolet light, RNA synthesis in E. coli as measured by uptake of ${ }^{32} \mathrm{P}$ is impaired (Spiegelman, Halvorson and Ben-Ishai, I955). Under these same conditions the production of adaptive enzymes, such as galactosidase was almost completely abolished while overall growth persisted at the rate observed in the non-irradiated cells. When the synthesis of RNA was inhibited by a purine analog, 5-hydroxy-uridine, again no effect on the overall growth rate was observed but the formation of adaptive enzymes was again abolished. Even after enzyme synthesis had started the analog caused an abrupt cessation of the synthetic activity. This again may indicate that it is the process of RNA synthesis and not merely the presence of RNA which is responsible for the formation of the adaptive enzyme, while the formation of proteins necessary for overall growth is unaffected by the analog and does not seem to depend on the synthesis of RNA. This interpretation is not fully conclusive since it has not been established that 5 -hydroxyuridine inhibits RNA synthesis only.

These experiments provide evidence for the close relationship between the formation of nucleic acid and the synthesis of proteins (adaptive enzymes). In this instance it is not the mere presence of nucleic acids in high concentration which is the essential factor for synthesis of new types of proteins, but a concomitant rapid synthesis of the RNA molecule is required.

The importance of nucleic acids for protein formation in cells of more highly organized animals has become apparent in recent years. As is well known, a connection between RNA and protein formation in animal cells had long been postulated by Brachet (1950) and Caspersson (1950). These authors based their assumption on rather tenuous and circumstantial evidence such as the occurrence of large quantities of RNA in cells with a rapid rate of protein formation.

These initial interpretations have now been substantiated in various ways. Without exception it has been found that enzymatic removal of nucleic acids from the protein forming centers, discussed above, abolished their capacity to continue protein synthesis.

From the microsome moiety, a fraction can be separated which is very rich in RNA and which shows an incorporation of labelled amino acids into its protein fraction which is several times higher than the incorporation into the non-RNA containing microsomal proteins. Again the incorporation can be abolished by pretreatment of the microsome fraction with RNase (Hultin, I $95^{\circ}$ and Littlefield, Keller, Gross and Zamecnik, I955). Hultin (1955) points out that in the case of liver cells from newly hatched chicks the incorporation patterns of the soluble and of the microsomal RNA proteins are contrary to what might be expected. The RNA component obtained from microsomes shows the lower and the soluble RNA the higher rate of incorporation while the reverse holds true for the corresponding protein fractions. The implications of this interesting observation are obscure.

Important data concerning the relationship of the RNA to protein synthesis were obtained by a comparison of the protein forming capacities of pancreas, liver and kidney (Allfrey, Daly and Mirsky, I953). In comparing the composition 
of the microsomal fraction of these organs an RNA phosphorus content of 2.3, I.I, and $0.6 \%$ was found, respectively. The corresponding figures for the incorporation of glycine- ${ }^{15} \mathrm{~N}$ into cellular proteins after injection into the intact animal were found to be $0.183,0.107$, and 0.046 atom per cent excess, respectively. This appears to indicate a remarkable parallelism between RNA content and the rate of incorporation of amino acids into the proteins of the microsomal fraction. On the other hand no parallelism was found between the uptake of the microsomal proteins and the uptake of RNA (0.006 and $0.03^{8}$ atom per cent excess in the RNA of pancreas and liver, respectively). The authors recall results of data presented by Abrams ( I95I) which show that irradiation of the intestinal epithelium does not impair protein synthesis whereas RNA synthesis is severely inhibited. In contrast to the findings in microbial cells these data indicate that, in these organs, protein formation is related to RNA quantity and not to RNA turnover. As Kihara, et al. (1955) suggest, acceptance of this dual role of RNA already mentioned earlier eliminates the apparent disagreement of the conclusions of Allfrey, et al. (1953) and Hokin and Hokin (I953).

The exploration of the role of RNA in differentiated cells has been described in detail in order to indicate the large number of experimental approaches which have been used in establishing its significance in protein synthesis. In contrast, there is almost a complete lack of systematic information about the relation of RNA quantity and RNA synthesis to protein formation during proliferation and during elaboration of specific cell products during later phases of differentiation in embryonic tissues. Indirectly, a relationship between RNA and protein synthesis in early embryonic development is suggested, perhaps, by experiments with purine analogs. The conclusiveness of these experiments is limited since analogs were used which are antagonists of both RNA and DNA. Using the purine analogs 8-azoguanine and benzimidazole, Waddington, Feldmann and Perry (1955) studied the effect upon the development of the chick and the newt Triturus Alpestris. In discussing possible explanations of the abnormal forms of development caused by the analogs, Waddington dismisses interference with cell division since none of the sensitive tissues such as the gastrula or the sensitive mesodermal derivatives (somites) show a particularly high mitotic rate normally. The hypothesis is put forward that the greater sensitivity of certain tissues at certain phases of development is due to an interference with a relatively higher protein synthesis in these cells. In support of this hypothesis, Waddington points out that methionine- ${ }^{35} \mathrm{~S}$ is incorporated at an apparcntly increased rate in the primitive streak region of the chick blastoderm (gastrulation) while the invaginated tissues showed much less activity in radioautographs. The same intense incorporation was noted in the neural fold which also proved sensitive during the earlier exposure time to azoguanine (I8-2 I h.). At later stages methionine accumulated particularly in the anterior portion of the neural tube and at still later stages again in the somites of the chick embryo, these also being the regions with the most marked purine analog sensitivity. Waddington points out that the effects of purine analogs are not paralleled in detail by the effects of ethionine. Since ethionine is supposed to inhibit protein synthesis directly, this discrepancy requires further investigation. Objections to an interpretation of radioautographs in terms of rates of protein synthesis 
have been stated earlier. In spite of these reservations Waddington's results are of interest because they raise the question as to whether the embryonic cells are particularly sensitive to the action of nucleic acid analogs at the time when production of new types of proteins might be expected. If further research leads to a corroboration of these results by the use of more specific purine analogs and a quantitative detailed analysis of protein formation in different organs, some progress in the clarification of the role of nucleic acids in protein synthesis in embryonic tissues could be expected. Some explorations, described later on, of the relationship of RNA content and protein synthesis, have been carried out in connection with erythropoiesis and regeneration of the liver and an extended examination of these tissues should prove to be a valuable approach in exploring this problem. Other experimental material suggesting a relationship of RNA to the control of protein formation in induction is more fully discussed in Chapter 4.

\section{(b) Structural aspects of the PFS: Protein formation in microsomes and mitochondria}

In electron microscope studies mitochondria appear as separate units with a distinct ultra-structure whereas particulate elements of the size of microsomes apparently are part of a fine network which has been designated as the endoplasmic reticulum. The microsomes are apparently released from this cytoplasmic network by the conventional homogenization processes (Palade and Siekevitz, I 956; Novikoff, I 956)

The investigation of protein formation in isolated cytoplasmic particles was initiated by Siekevitz (I 952) who measured the uptake of labelled amino acids in to these cell components ${ }^{1}$. The most rapid uptake was found to take place in the microsome fraction while mitochondria and nuclei showed less activity. Results similar to those of Siekevitz were obtained by Zamecnik and his group who could demonstrate that a rapid incorporation into microsomal proteins occurs in vivo (Keller, Zamecnik and Loftfield, I954). Incorporation into microsomes in vitro will proceed even anaerobically if the non-particulate fraction and ATP are added to the preparation as the sole source of energy (Zamecnik and Keller, 1954; Zamecnik et al., I 956). Aerobically, a sufficiently high ATP level can also be maintained by the addition of a more complex source of metabolic energy such as mitochondria and some utilizable substrate.

The role of microsomes in the net synthesis of the specific proteins of differentiating cells was elucidated in a series of interesting experiments with pancreas cells where formation of the enzymatic cell proteins such as the pancreatic enzymes can be influenced by various drugs and by fasting and feeding (Allfrey, Daly and Mirsky, I953). These authors found that the same stimuli which enhance the quantity of secreted enzymes also increase the rate of amino acid incorporation into the proteins of the microsome fraction. Time curves of the uptake into the micro-

1 From nuclei, at least active enzyme proteins are lost during their isolation unless special precautions are taken. Because of the possibility of similar losses it is not certain whether the differences in the activities of the cell particles, when isolated according to more conventional techniques, give a correct picture of the tracer amino acids in the total protein moiety. 
somal and unbound (supernatant) proteins of the pancreas cell showed that the incorporation into the microsomal material took place more rapidly and reached a higher maximal value than the tissue proteins. Accordingly, the microsomal proteins could have been the precursors of the pancreatic enzymes but this expectation was not borne out by the analysis of the purified proteins trypsinogen and chymotrypsinogen (Daly et al., I 955). However, in organs in which feeding or stimulation by pilocarpine does not cause increased protein formation, no increased incorporation into the microsomal protein fraction was observed. Therefore the microsomal activity still seems essential for the formation of the secretory products.

Rather far reaching differences in the state of the structural elements of the PFS in the embryonic cell may be indicated by the observation that the amount of free cytoplasmic RNA (RNA unattached by particulate material sedimented below $105,000 \mathrm{~g}$ ) is relatively large in embryos of the mouse and of amphibians; which diminishes with ensuing development and is practically absent in the fully differentiated tissues of the adult animal (Brachet and Chantrenne, I942). This may mean that parallel with the decrease of unattached RNA there is an increase of cytoplasmic, RNA containing, particles. While no direct quantitative measurements of the microsomal fraction have been carried out in the experiments cited above, in the sea urchin embryo it was found that particulates of the size and the staining properties of mitochondria become morphologically apparent at the late blastula stages (Gustafson and Lenicque, I952; Lenicque, Hörstadius and Gustafson, I 953; Gustafson and Lenicque, r 955; Shaver, I956). As mentioned previously the mitochondria in mature cells are the primary sites of the metabolic organization of the energy supply needed for the formation of proteins and therefore are only indirect indices of the overall capacity of protein formation in a cell. However, the appearance of mitochondria may be indicative of the progressing organization of other particulate elements. In this connection it is of interest that, similar to RNA, cytochrome oxidase accumulates first in the cell matrix and becomes incorporated only secondarily into mitochondria (Boell and Weber, I955). The cytochrome oxidase activity was found actually to increase if calculated per unit quantity of mitochondrial material.

Somewhat more information has become available about the protein forming capacity of the different particulate fractions of embryonic cells as indicated by measurements of amino acid incorporation into their proteins (Hultin, I 953a). During cleavage of the sea urchin egg the incorporation of alanine- $\mathrm{I}-{ }^{14} \mathrm{C}$ into proteins of a cell fraction collected at $105,000 \mathrm{~g}$ (microsome), is considerably higher than the incorporation into either the fraction which is brought down at a speed as low as at $20,000 \mathrm{~g}$ (mitochondria), or the fraction which cannot be sedimented at higher speeds (supernatant). With reference to the discussion of the pathways of protein synthesis in the previous section the suggestion of an extensive transamidation during this early phase of sea urchin development is of interest (Hultin, I953b). Beginning with the development of the mesenchyme blastula, the incorporation of amino acids into the mitochondrial proteins increases rapidly and reaches the level of the microsomal fraction. Evidence for an increase of mitochondria has been mentioned above. An increase in the incorporation of $\mathrm{NH}_{3}$ into RNA and into the soluble proteins (Hultin, I952; 
Hultin, I953a) precedes slightly the mesenchyme blastula stage. A distinct elevation of acetate utilization in RNA synthesis coincides with mitochondrial development (Hultin, I953d).

In evaluating his results, Hultin (I953e,f) suggests that the more rapid incorporation of labelled amino acid into the microsome fraction in the early period of development reflects the rapid production of microsomes. Similarly, an increase in the rate of incorporation into the mitochondrial fraction at a somewhat later stage of development was correlated to the increase in the number of mitochondria mentioned before. However, it should be pointed out that coinciding with the increase in mitochondrial activity there is also an increase in the incorporation of amino acids into the non-centrifugable non-particulate proteins (particularly in a fraction regarded as non-sedimentable protein). In attempting to ascribe to these results some morphogenetic meaning, Hultin points out that the early increase in microsomal activity may be brought about by a rapid multiplication of these particles and that this phase is closely correlated to the onset of determination in the sea urchin embryo. The later increase in the net incorporation rates of the mitochondrial and soluble fractions would be due to a corresponding increase in the quantity of these fractions. This is apparently corroborated by direct counts of mitochondria by Gustafson and Lenicque (1952) and by a sudden increase in the enzymes usually found to be associated with mitochondria as opposed to those enzymes occurring freely in the cytoplasm (Gustafson and Hasselberg, I 95 I). It should be mentioned, however, that these claims have been questioned recently (Shaver, I955). In view of these uncertainties it should be pointed out that a study of the changes in cell particulates during development of mosaic eggs (Rattenbury and Berg, I 954; Berg, I 956; Reverberi, I 956) may be more rewarding and better accessible to further analysis since here particulates with distinct properties become located in readily separable cells.

These data, as they stand, indicate significant changes in the structural organization of the PFS during various phases of development. It is noteworthy, for example, that, although the rate of protein synthesis must be very high during the earliest phases of embryogenesis, the populations of neither the microsomal nor mitochondrial particles seem to be present in large numbers and the constituents of the particulate elements, as $e . g$. RNA, are found mainly in the cytoplasmic matrix. Is the high rate of protein formation during this phase of development due then, at least in part, to synthetic processes which occur independently of these organized centers and is it possible that "templates" occur, unattached to particulate elements? As the number of protein species increases during the first phases of differentiation do individual particles (e.g. microsomes) become diversified in their protein synthesizing "repertoire" or does synthesis of a new protein require organization of a new particulate center? Is each particle responsible for production of one or several proteins? For the elucidation of such questions, embryonic tissues may very well offer favorable and interesting material.

\section{(c) Nucleo-cytoplasmic interactions related to protein formation}

The far-reaching role which the consideration of nucleo-cytoplasmic interactions has played both in experimental embryology and in physiological genetics has 
been cogently pointed out in a recent review by Schultz (1953). Investigations of nucleo-cytoplasmic relations of particular pertinence from an embryological point of view have been summarized by Fankhauser (1952 and 1955) and by Briggs and King (1955). Beginning with the work of Roux, Weisman, Boveri and continuing with the investigations of Baltzer and Hadorn and leading up to the present cytochemical studies of Caspersson and others, the effects of cytoplasm on the nucleus have received alternatingly greater and lesser emphasis. The role of the nucleus in embryonic development is borne out by the interesting experiments carried out by King and Briggs ( 1955 ). Nuclei were isolated from cells of amphibians (Rana pipiens) at early and late gastrula stages and implanted into enucleated eggs. While early gastrula nuclei maintain development through larval stages, implantations of late gastrula nuclei lead to a developmental arrest at the blastula, gastrula, or neurula stage. The nuclear differentiation accompanying spermatogenesis has been explored recently with elegant histochemical methods by Alfert ( I 956).

The bulk of chemical evidence for a nucleo-cytoplasmic interaction has centered upon the demonstration of a transfer of RNA from the nucleus to the cytoplasm. The speculation seemed attractive that RNA might act as a mold in transferring structural properties of genetic determinations.

More recently direct evidence for the transfer of nuclear RNA to the cytoplasm has been established (Goldstein and Plaut, I955). Cell constituents of amoebae are labelled with ${ }^{32} \mathrm{P}$ by feeding them Tetrahymena containing this tracer. Under the conditions of the experiment (arrested proliferation) the nucleus of the amoeba incorporates the label only into its RNA molecules. A labelled nucleus was removed and transferred to another amoeba and the distribution of the label from the nucleus of the donor into the cytoplasm of the host was followed radioautographically. For five hours no label left the nucleus but after 12 hours a considerable portion of the labelled material had penetrated into the cytoplasm of the host amoeba and was still present in the form of RNA (RNase digestible). Since an unlabelled nucleus placed into the cytoplasm together with the labelled nucleus did not show any uptake of the label, the transport of the RNA seems to be possible only from the nucleus into the cytoplasm and not in the opposite direction. Transfer of nuclear material has been observed in the electron microscope in the form of characteristic nuclear blebs which are released into the cytoplasm coincident with secretory activity in salivary gland cells of Drosophila (Gay, I955 and I956).

To what extent protein formation in the cytoplasm of different cell types depends on the supply of nuclear RNA is still undecided. The early decrease in incorporation into cytoplasmic proteins observed by Mazia and Prescott (1955) immediately after enucleation of amoebae would seem to suggest strongly that the nucleus does participate in the maintenance of the protein forming capacity of the cytoplasm. Similar experiments in Acetabularia have led to a decay of cytoplasmic protein formation only after a longer period of enucleation (Brachet, Chantrenne and Vanderhaeghe, I955).

Mazia and Prescott also suggest the possibility that protein formed in the nucleus may become associated with nuclear RNA and be transferred to the cytoplasm when RNA is released from the nucleus. Some protein forming capacity of the 
nucleus in situ has been frequently demonstrated by the incorporation of amino acids into nuclear proteins. In these investigations it remained undecided to which extent this uptake depended on the presence of the cytoplasm. However, in a recent investigation protein formation in isolated nuclei could be demonstrated (Allfrey, I 954; Allfrey, Mirsky and Osawa, I 955). Using an isolation procedure which precludes, loss of nuclear proteins, the observed uptake of alanine $-{ }^{14} \mathrm{C}$ into the nuclear protein fractions was found to be much higher than in previously studied nuclear preparations. It became evident that the energy supplied by metabolic reactions occurring in the nucleus itself was utilized for protein formation since metabolic inhibitors prevented incorporation. Fractionation of the nuclear proteins showed highest incorporation in a non-histone protein which was bound to deoxyribonucleic acid. A soluble non-histone protein fraction also showed considerable activity while the histone fraction contributed only a small portion of the total activity. The observed differences between the activities of different protein fractions were essentially in line with activities obtained in protein fractions of nuclei obtained after injection of labelled glycine- ${ }^{15} \mathrm{~N}$ into the intact animal and subsequent separation of the nuclear protein fractions from liver, pancreas and kidney cells (Allfrey, Daly and Mirsky, I955). ${ }^{1}$ The significance of the differences in the incorporation rates of these proteins is obscure at the present time. Rapid formation of all fractions is certainly necessary during marked proliferative activity of cells. Whether the incorporation into one of the fractions, e.g. histones, follows more directly the proliferative rate while the other nuclear protein fractions may retain a high rate of incorporation when cytoplasmic proteins are being formed, is again a question which awaits a systematic study in a tissue during its transition from the predominately proliferative to a predominately differentiating and eventually to a mature adult state. The embryonic development of the lens, of muscle, or of connective tissue may yield valuable information on this point.

The importance of the undisturbed maintenance of nucleo-cytoplasmic interactions in embryonic development has become apparent from studies of lethal hybrids (Briggs and King, I 955, p. 2 I 9-224; P.S. Chen, I954; Gregg and Lovtrup, I955). By an original analysis of careful analytical work on merogonic hybrids of Triturus cristatus (sperm) x Triturus palmatus (egg cytoplasm) Zeller ( I956) could demonstrate the occurrence of an interesting deviation of the RNA content in these embryos. In following the RNA content in eggs and embryos of either Triturus palmatus or cristatus it was observed that the scattering of the values changed in a characteristic fashion during development. In the early part of development the range between the RNA values of different clutches contributes more than one half of the total scattering, the rest of the scattering being due to variations between eggs from the same clutch. As development continues the

1 These incorporation rates cannot necessarily be regarded as a direct quantitative index of the rate of formation of the respective proteins. If the investigated proteins differ greatly in their composition, for example, in their alanine content and if alanine is used as the labelled tracer, the protein with lowest alanine content would show the lowest activity per protein nitrogen. Only expression of the data as labelled alanine per total protein alanine, would give values which would make incorporation data of different proteins directly comparable. 
scattering between eggs from one clutch reaches the magnitude of scattering observed between different clutches. The mean RNA value rises during gastrulation, accumulation coming to a standstill during neurulation. In the merogonic hybrids (palmatus sperm $\mathrm{x}$ cristatus egg cytoplasm) the initial RNA content is lower than in the controls, but in the course of development the rate of accumulation in the merogonic hybrids reaches the magnitude of that observed in the controls. Also, the accumulation of RNA in the hybrids continues past the stage of development during which RNA accumulation in the controls has come to a standstill. Therefore, the hybrids acquire, with time, a higher RNA content than the controls. Throughout the observed course of development the scattering of the RNA values in the hybrids was found to be restricted to a narrower range than in the controls. As an interesting interpretation of these results, Zeller suggests that the RNA, present in the egg before fertilization, is degraded in the hybrid more rapidly, giving rise to the initially lower RNA values and also to a lower scattering. The newly synthesized RNA, produced under the control of the cristatus nucleus cannot be degraded in the palmatus cytoplasm and therefore the accumulation continues longer than in the controls. It is concluded that hybridization, in this case, interferes mainly with the breakdown of the newly synthesized RNA. Further investigation seems warranted to examine the possibility that the final lethal effect of this hybridization is due to the absence of RNA turnover and a subsequent inhibition of new protein formation which depends upon a constant replenishment of RNA as in the case of induced enzymes in bacteria.

In considering nucleocytoplasmic relations, experiments should be mentioned which show that that caryoclastic agents like nitrogen mustard exert two different effects; the one, consisting in a direct interference with mitosis; the other becoming manifest in cells at an early state of differentiation such as neural tissue shortly after induction (Nieuwkoop and Lehmann, I 952). The two types of action have been sharply differentiated by Bodenstein and Kondritzer (1948) in experiments with Drosophila. In the former case one finds inhibition of mitosis with continuation of cytoplasmic synthesis, continued RNA synthesis, and formation of giant cells (Bodenstein and Kondritzer, I 948). In the other case interference with the nuclear activity leads to a complete degeneration of the cell. Similarly, Gelfant et al. (1955) could show that under conditions of mitotic arrest by aminopterin and nitrogen mustard the response of the uterine epithelium (increase in cell size) to hormonal stimulation is not inhibited. In contrast, the growth of the muscular layer after hormonal stimulation seems to depend on nuclear proliferation and is inhibited by the antimitotic agents investigated. Increased incorporation of methionine- ${ }^{35} \mathrm{~S}$ (Sirlin, I955) and glycine-I $-{ }^{14} \mathrm{C}$ (Sirlin and Waddington, I954) into nuclei of the dorso-axial structures and of the induced neural plate was observed radioautographically and has been interpreted as an indication of a higher nuclear metabolism which is possibly related to the nucleo-cytoplasmic interactions discussed above.

Although in cytological and chemical work more attention is given, at the present time, to the effect of nuclear factors upon cytoplasmic activities, the reverse relationship must also be considered in view of the experimental evidence cited above. Chemical evidence for cytoplasmic effects on the nucleus during embryonic development seem to be lacking and for this reason a recent suggestion made by 
Allfrey, Daly and Mirsky (1955), concerning chemical evidence for such a relationship in mature cells, seems of particular interest. In studying the incorporation of amino acids into nuclear, microsomal, and cytoplasmic proteins of the pancreas, liver, and the kidney, it was found that feeding of previously fasted animals stimulated the incorporation of amino acids not only into the proteins of the cytoplasm but also into the proteins of the nucleus. The striking effects led the authors to conclude: "The experiments described in this paper show clearly that under certain conditions uptake and retention of ${ }^{15} \mathrm{~N}$ by cytoplasmic and chromosomal proteins are correlated. These experiments do not disclose just how the correlation is accomplished. In a general way, however, it may be said that feeding mice brings about changes first of all in the cytoplasm of pancreas, liver and kidney cells and that the changed conditions of the cytoplasm in some way produce a change in the nucleus. Modifications in chromosomal proteins may, therefore, be regarded as a response to an altered cytoplasm. Since it is well known that chromosomes influence activities in the cytoplasm it may be supposed that modifications in chromosomal proteins specially those combined with DNA will affect the way in which the chromosomes influence the cytoplasm. There can be little doubt that we are here dealing in a fragmentary way with interactions between cytoplasm and chromosomes and that the pattern of interaction here considered, the cytoplasm, changed by external conditions, producing modifications in the chromosomes and these modifications reacting upon the cytoplasm-holds for the physiological changes in non-dividing cells and also for the differentiation that occurs in the course of development." From these conclusions it is evident that the investigation of this hypothesis would be of paramount importance for the embryologist. It would give a clue as to how a constant genome could give rise to the manifestations of differentiation under the influence of some cytoplasmic control.

\section{REgulating FACTORS OF THE PFS DURING DEVELOPMENT}

It is assumed that, as a general rule, during the earliest phases of development the specific cell products of the differentiated cell (lens proteins, collagen, trypsin) are found at most in small quantities, if at all. In some forms of development the potential capacity to form such cell products may be found to be a fixed property of the embryonic cell at the very onset of development (cleavage). In this case the different components of the PFS must be spatially separated and functionally differentiated in the egg or in the early cleavage stages already. In another type of development the cells retain for a longer time a certain flexibility of development and can respond to different environmental conditions with the formation of different cell products. Later in development this flexibility is lost. Such a distinction is commonplace to embryologists who have called the first form of development mosaic development, the other regulative development. In the latter case the transition from a labile to a stable state has been called determination. It seems important to recall here this distinction because it must be clear that in approaching the problem of the control of the PFS from an embryological point of view, one may or may not be inquiring into two different processes. One group of processes may involve formation and stabilization of the PFS in regulative 
development. The second set of processes may concern the selective control of the bulk production of a given set of cell proteins (and consequently of other cell products) characteristic of the cell types.

Before accepting this dichotomy as a basis for a rigid classification of developmental processes and for a hypothetical distinction between mechanisms involved, the limitations of such a classification have to be clearly recognized.

It is well known that at very early stages of development the PFS even of "mosaic" eggs will exhibit some degree of lability. On the other hand, in regulative development some properties of the cells of embryos may become stabilized while others remain labile for a longer time. This eliminates the distinction between these two types of development in principle.

Due to the limitations of our analytical methods, it has not been established beyond any doubt that apparent qualitative differences found between embryonic cells are not merely the expression of very large quantitative differences. For example, during early amphibian development the undifferentiated mesodermal cell can respond to environmental factors by forming either hemoglobin or muscle protein (Yamada, I 940 and Muchmore, I95I). At a critical period in the development of the primitive mesoderm the cells initiate bulk production of only one of these proteins and become either blood or muscle cells. Once this change has occurred, these cells do not any longer respond with production of the alternate protein type to the same environmental conditions which elicited formation of such proteins during an earlier stage of development. However, it cannot be said, with absolute certainty as yet, that reversibility of this change is excluded under all experimental conditions or that the fraction of the PFS which can be induced during early phases of development to form an alternate protein species (e.g. hemoglobin) has become completely and irreversibly inactivated and does not merely function at a rate too low to allow detection of its product without special methodology.

Only with these reservations in mind have we discussed separately those factors which seem to control the development of the qualitative aspects of the PFS and the factors which control quantitatively the production of protein. It may remain impossible, for reasons of the limitations of analytical procedures, to distinguish between these aspects.

\section{(a) Apparent qualitative changes in the PFS (Induction)}

Long before the nature of the PFS was, or could be, considered as a key to the mechanism of differentiation, the factors influencing qualitatively the formation or function of the PFS had become the main issue of experimental embryology. With Spemann's analysis of the "Organizer" it became apparent that some forms of interactions between mesodermal cells and the ectoderm lead to the transformation of the latter into the parts of the brain and neural tube (summarized by Spemann, I938). This discovery started an intense search for the biochemical mechanism of the phenomenon of induction. Initially it was assumed that a single specific substance was produced by the mesodermal cells which was responsible for the transformation of an undifferentiated ectodermal layer into the highly specific neural tissue. Reports that inductions had been obtained with 
more or less purified chemical compounds were hailed with considerable enthusiasm. However, as these investigations continued it became apparent that a great variety of chemically unrelated and rather simple substances would elicit a response in the primitive ectoderm. But these observations did not provide further insight into the nature of the actually occurring embryonic inductive processes. A role of unspecific cytoplasmic agents in induction was suggested by Holtfreter (1948b and 1955). It is difficult to say at the present time whether the effects of various chemical fractions obtained during this valiant search are actually without biological significance. However, even if the failure of such a search for an "Organizer" substance was only an apparent one, it gave the problem of induction the appearance of even greater inaccessibility than it had before the start of this biochemical Odyssey.

In view of the pivotal significance of induction, several lines of research have been recently initiated to establish a sounder basis for a renewed attack on this knotty problem. Most important seems the progress made in the biological analysis of the induction phenomenon. Through the ingenious experimentation of Nieuwkoop and his associates (Nieuwkoop et al., 1952; Eyal-Giladi, I954; Nieuwkoop and Nigtevecht, I 954; Nieuwkoop et al., I 955; Nieuwkoop, I 955) it could be shown that the transformation of the primitive ectoderm cell into a highly specialized cell can be divided into two phases. First, a stimulus emanating from the mesoderm underlying the primitive ectoderm elicits a process in the ectoderm itself which, without further control, leads to the development of pro-encephalic structures in all parts of the ectoderm that react to this initial stimulus (activation).

The second principle supplied by the mesodermal primordium becomes effective in controlling the response of the ectoderm resulting in the formation of structurally and functionally different components of the central nervous system (Nieuwkoop's "transforming principle"). The two principles contributed by the mesoderm can be experimentally distinguished. During the development of the mesoderm the appearance fo the "activating" principle precedes the appearance of the "transforming" principle and also the spatial distribution of the two factors is different in the mesodermal primordium. Also the activating factor is heat stable, while the "transforming" principle is strictly specific and heat labile.

Apart form this exemplary re-examination of the biological characteristics of neural induction, several other biological approaches have been pursued which may eventually provide a fruitful basis for chemical investigations. Notable are the advances of Grobstein ( I 955 a, b, I956 and I957) and Grobstein and Holtzer ( I 955) in establishing conditions for very striking induction phenomena in vitro. Zwilling's ( 1955 ; I956a, b, c, and d) successful experiments in removing and transplanting the apical ectoderm of the limb bud may provide possibilities for the study of the mechanism of the effect of this tissue on the limb bud mesoderm. Interactions between neural tube tissue and somite mesoderm have been analyzed in greater detail by Holtzer and Detwiler (I953 and I954); Watterson et al. (I954); Avery, Chow and Holtzer (1956) and S. W. Holtzer (1956) and the dependence of mesodermal differentiation in amphibians upon the cellular environment demonstrated experimentally by Yamada (I 937 and 1940) was elaborated by Muchmore (I95I). The intriguing phenomenon of production of lens proteins by iris cells of adult sala- 
manders in response to the removal of the lens from the eye has been given continued consideration by Stone (1955) and by Reyer (1954) (Reyer and Stone, 1955). These systems may prove to be advantageous since they provide tissues at more advanced stages of development and are more easily accessible to the necessary manipulations in the exploration of inductive mechanisms.

In pursuing the question of the chemical nature of the factors responsible for the apparent qualitative changes of the protein forming system Brachet has repeatedly stressed the possible importance of RNA as a controlling factor. A summary by Brachet of the formidable body of suggestive observations is found in another chapter of this volume. Recently Yamada and Takata (1956) and Hayashi (1956) demonstrated morphogenetic effects of nucleoproteins obtained from the liver and the kidney. It should be pointed out, however, that some RNA proteins lose their activity after tryptic digestion and remain active after treatment with RNase (Yamada and Takata, I955; Hayashi, I955) suggesting that it may be the protein component which is responsible for the inducing activity.

Leads as to the nature of the mesodermal activating factors can be found in the work of Niu and Twitty (I953) and Niu (I956) and in the experiments by Toivonen (1950, I952) and Toivonen and Saxen (1955) which demonstrate a mesodermal induction with bone marrow extracts. As Nieuwkoop (I955 p. 270-27I), points out, the action itself (of the principle obtained from bone marrow) may not be the same as the physiological "transforming principle" of the mesoderm. However, the use of the bone marrow principle may be advantageous in studying the process of transformation itself (Yamada, I958).

For a long time, changes in the metabolic patterns of the developing cells have been regarded as important factors in the regulation of developmental process. Various gradient theories, referring to gradients of basic metabolic activities such as oxidation-reduction processes (Gustafson, I 954; Hörstadius, I 953, I 955; Lindahl, I942; Pease, I941; 1942a and I942b), were considered to control the development of the animal and vegetal structures of the sea urchin embryo and of characteristic products of differentiation in many other lower organisms. Metabolic intermediates resulting from the oxidative degradation of glucose phosphate were found to promote development of ectodermal structures (animalization) (Hörstadius and Gustafson, I948 and Hörstadius, r949).

An interesting transformation in vitro of squamous, keratinizing epithelium into cuboidal mucus-producing epithelium depending upon the vitamin A content of the medium has been observed by Fell (r953) and Fell and Mellanby (I953), and by Weiss and James (1955). Although a metabolic role of vitamin A has not been established the results of experiments by Ernster et al. (1950) on the role of vitamin $\mathrm{A}$ as coenzyme, may justify the inclusion of a vitamin A effect among instances of metabolic control of differentiation.

A transformation in vitro of the chorion epithelium into keratinizing cells was observed by Moscona (i 958 ).

Intercellular metabolic reactions (Herrmann and Hickman, I948b) and their possible role in differentiation of more complex tissues (Flexner, I939; Flexner and Stiehler, I 938) have been discussed in a previous review (Herrmann, I 952).

Exactly how, qualitative or quantitative, differences in energy metabolism can 
become translated into production of different protein patterns has hardly been formulated as a tangible problem. Changes of the state of proteins by metabolic reactions are discussed on p. 536 and it may be that similar alterations of the PFS would lead to corresponding changes in the proteins produced.

\section{(b) Apparent quantitative aspects of protein formation in embryonic cells}

In discussing the quantitative aspects of protein formation in embryos, factors have to be considered which can usually be disregarded in similar work with fully differentiated tissues. As was pointed out in the preceding section there is little evidence to support or to reject the assumption that proteins having apparently similar functions at the beginning and at the end of a developmental period consist actually of identical types of molecules. Certainly in the case of hemoglobin it has been recognized for some time that the fetal protein differs in many respects from the protein in the mature erythrocyte (Haurowitz and Hardin, I 954). Obviously, similar differences could occur in the case of any enzyme or nonenzymatic protein. For example, the substrate concentration curves for alkaline phosphatase of chick muscle may indicate some change during the transition from the embryonic to the more differentiated state of the tissue (Konigsberg and Herrmann, 1955). Generally speaking, for proteins with enzymatic activity, a first approach towards demonstration of such changes could be made by measurements of the Michaelis-Menten constant of the enzyme preparation. With non-enzymatic proteins the identity might be established immunologically by obtaining quantitative precipitation curves. The establishment of the identity of proteins at different stages of development with either enzymatic and quantitative immunological methods must, in any event, serve as a necessary prerequisite for subsequent quantitative analysis.

Whatever methods are used (enzymatic, immunological, etc.), the measurements of the changes in the actual quantities of protein are not necessarily indices of the protein forming capacity of the respective cells. The accumulation of proteins measured in this way is merely the balance of synthesis and breakdown of the investigated protein. Changes in the accumulation rate of a protein can be due to any one of the several possible combinations in the magnitudes of these two processes. An increased rate of accumulation can be due to increased synthesis, decreased breakdown or both and conversely a decreased accumulation can be brought about by decreased synthesis, increased breakdown or both. Factors controlling the accumulation rates could thus act on either of these two processes. An approximate estimate of the relationships of synthesis and breakdown to the accumulation of proteins can be obtained by an analysis of the incorporation of labelled amino acids into proteins. In order to obtain interpretable results certain methodological prerequisites must be observed. (I) Since the state of proliferation and differentiation is different in different organs of an embryo, data obtained on whole embryos are of limited value. (2) The incorporation measurements should be carried out on individual isolated proteins. During differentiation the rates at which different proteins accumulate change markedly and incorporation data on a protein mixture which shows no apparent change may be the result of an increased rate of incorporation in one protein and a decreased rate in another. 
(3) The amount of labelled amino acids present in the protein and in the pool of free amino acids in the cell must be measured over a time span which includes both the increasing and decreasing phase of specific activity.

Applying these prerequisites the incorporation of radioactive glycine into the glycine moiety of actomyosin and collagen, obtained from the leg musculature of the I4-day chick embryo and the 7-day chick indicates, as discussed previously, (p. 50I) that at least a large part of the increase in the specific activity in the proteins is a measure of actual net synthesis (Herrmann, Lerman and White, I $\left.95^{8}\right)$.

For a meaningful interpretation of quantitative measurements it has to be kept in mind that the formation of increasing amounts of a specific cell protein can be the result of entirely different situations relative to overall protein production. The appearance and the increase of new types of proteins may be concomitant with, and merely an expression of, an increase in the capacity of the entire protein forming apparatus. One might think of the possibility that the centers of protein production increase in total number or that the energy supply required for protein synthesis increases. Under these circumstances it would be more likely that a greater number of new protein ty pes would appear and accumulate at an increased rate $(e . g$. the simultaneous appearance and accumulation of enzymes in the mesenchyme blastula of the sea urchin). If the appearance and accumulation of these new protein types represents a true overall increase in the productivity of the entire PFS, this would lead to an increase in cell proliferation, in the protein content per cell, or in both.

An alternative situation would be the synthesis of specific proteins with an unchanged rate of total protein production. In this case, appearance and accumulation of new proteins would be possible only if the formation of other protein types would be reduced to a correspondingly lower rate. This reduction could be an expression of a reduced growth rate, i.e. reduced formation of new cells.

The appearance of new proteins could occur possibly during a period when overall synthesis is reduced either by a cessation of the proliferative growth or by the decrease of protein production by the individual cell. There is, of course, no reason to assume that the one or the other of these possibilities is a generally valid scheme for the differentiation of all tissues of every species. To the contrary, it must be expected that in the course of differentiation several of the proposed mechanisms will be operating.

\section{(c) The relation of proliferation and differentiation}

It has been generally assumed that in rapidly proliferating cells differentiation is suppressed. In the absence of quantitative data it seemed that during phases of rapid proliferation the specific cell products were not formed and only when proliferation was retarded or completely abolished a rapid accumulation of specific cell products could occur. Complete incompatibility could be defined more precisely in the development of ascidians. In these organisms Berrill (I935) determined for each cell type the numbers of cell divisions which precede the first cytological appearance of the differentiation products in the developing buds. For example, at an early stage of development when the thoracic mesenchyme 
of the developing Distaplia bud has formed roo cells, mitosis comes to a complete standstill in 6 of these cells and from that time on they begin to develop the morphological characteristics of muscular cytodifferentiation. In the completely developed zooid these 64 muscle cells appear in the form of eight strands of four cells on either side of the thorax. In the remaining mesodermal cells, proliferation ceases at a later stage with subsequent formation of blood and other mesenchymal tissues. Similarly sharp separations of multiplication and cytodifferentiation can be found in almost all cell types of ascidians.

In higher forms, e.g. vertebrates, an incompatibility of proliferation and differentiation was demonstrated more directly in tissue culture experiments. Beginning with the experiments of Fischer and Parker (I 929) it was found again and again that under conditions of rapid proliferation little differentiation took place, while in slowly growing cultures manifestations of differentiation could be clearly observed. The classical examples are: the pigment formation in slowly growing cells of iris epithelium (Doljanski, I930-I931a) or the formation of glycogen granules in slowly growing liver cells (Doljanski, I930-I93 Ib). Both of these cell products do not form in rapidly growing cultures. The possibility that apparent oscillations of embryonic growth curves indicate alternating periods of more or less rapid differentiation has been suggested by Schmalhausen (I926) and Schmalhausen and Stepanova (I926) and is discussed by Needham (I93I). However, statistical validity of these oscillations would have to be re-examined.

A possible relationship of proliferation and differentation in early embryogenesis can be explored in the embryos of sea urchins and of amphibians where the two parameters have been defined to some extent in chemical terms. After the initial cleavage stages of sea urchin embryos, an increase in cell number is paralleled by a corresponding increase in DNA while the RNA and protein contents remain unchanged ${ }^{1}$. Neither the increase in the quantity of DNA, nor the rate of uptake of ${ }^{32} \mathrm{P}$ into the RNA molecule indicates a period of declining proliferative activity during development of the larvae (Villee, Lowens, Gordon, Leonard and Rich, I949). In spite of this uniformity in the rate of proliferation, a distinct period of differentiation can be recognized in the later blastula stage which is indicated by the appearance of mitochondria and of new mitochondrial enzymes (Gustafson and Lenicque, I 952; Gustafson and Lenicque, I 955; Shaver, 1956). This phase of differentiation coincides with the rapid increase in the incorporation of amino acids into mitochondrial proteins, a high rate of incorporation observed in the microsomal fractions, and an increase in the utilization of acetate for RNA synthesis (Hultin, 1953d). Thus, the investigations of early sea urchin development do not seem to reveal an incompatibility of proliferation and differentiation with respect to the indicated parameters.

\footnotetext{
1 Apparently, total RNA content remains constant, while RNA incorporation increases at certain periods. Determinations of DNA as a measure of proliferation during early phases of development of sea urchin or amphibian embryos have to be carefully evaluated in view of the findings of Hoff-Jorgensen and Zeuthen (1952) and Marshak and Marshak (I953). Also a certain error is introduced by the fact that a change in the rate of mitosis will affect the DNA figures to some extent.
} 
In amphibians the relationship of proliferation, measured as an increase of the quantity of DNA, to differentiation, measured as formation of specific enzymes, has been investigated repeatedly. This material has been subjected to a careful re-examination by Løvtrup (I955) who found a rapid increase in the DNA content of the embryo beginning after segmentation and continuing until the onset of the larval stage indicating a considerable proliferative activity during this period. During the first part of the larval period (Ambystoma mexicanum, I 2 days, stage $37-40$ ) no increase in the DNA content occurs and therefore cell proliferation would seem to have abated (however, one can not exclude the possibility that cell increase is compensated to some extent by cell degeneration). During the phases in which considerable DNA accumulation occurs there is only an increase in $\mathrm{O}_{2}$ uptake, alkaline phosphatase, and amylase activity, and during the later tailbud stage there is an increase in cathepsin activity and RNA content. This RNA increase is regarded as being preparatory to the formation of enzymes during the larval period ${ }^{1}$.

The period of depressed proliferative activity during the larval period is distinguished by the appearance and rapid accumulation of numerous cytoplasmic enzyme proteins: cholinesterase, adenosinetriphosphatase, cytochrome oxidase, succinic dehydrogenase, acid phosphatase and tripeptidase. Also catalase and dipeptidase show a slight increase. At earlier phases of development, throughout neurulation and the first half of the tailbud stage, none of the investigated enzymes showed an increase. These results may actually indicate a complementary relationship of proliferation and differentiation at least during some phases of the development of certain amphibians. It should be pointed out, however, that Krugelis et al. (1952) do not find a similar break in their DNA curve for amphibian development (A. punctatum) and further investigation is needed to obtain more conclusive material.

The relationship of proliferation and differentiation has found further consideration in connection with Goldschmidt's theory of gene action. According to this theory, genes control the rates of developmental processes and abnormal forms of development are caused by quantitative changes in the rates of proliferation of the organ primordia. Interesting examples are the developmental abnormalities which are obtained in Drosophila larvae after treatment with such inhibitors of proliferation as nitrogen mustard or colchicine. In the wild type last instar larvae it was found that the normal development of arista was altered to a development of aristapedia (Bodenstein and Abdel-Malek, I949) while in a mutant which normally forms aristapedia treatment with colchicine led to the appearance of arista

1 The data on RNA accumulation deserve closer examination. With development taking place at $18{ }^{\circ} \mathrm{C}$ or $15{ }^{\circ} \mathrm{C}$ the increase in RNA is shifted from stage $18-20$ to stage 26 , respectively. It might be expected that further lowering of the temperature to $13{ }^{\circ} \mathrm{C}$ would bring the onset of RNA accumulation to stage 30 or 32 . This would bring the onset of RNA accumulation close to the beginning of the period of DNA constancy and would emphasize the possibility of a competitive formation of RNA and DNA and of the dependent protein forming processes at this phase of development. In this connection it would be of interest to see whether enzyme formation would be dependent upon the change in the onset of RNA accumulation at different temperatures. 
(Vogt, I 947). This suggests that a decrease in the rate of proliferation leads to a divergent course of differentiation.

Fascinating objects for the study of differentiation in general (Raper, 1956) and of the relation of differentiation and proliferation in particular are theslime molds. Recent studies (Bonner and Frascella, i 952; Sussman, I 956) have shown that the late manifestations of differentiation, like formation of the stalk wall, or of the fruiting body occur after the cessation of proliferation. However, histochemical indices of differentiation like absence or presence of PAS staining material can be detected while proliferative activity still occurs (Bonner, Chiquoine, and Kolderie, I 955). From the latter study it is also apparent that the process of differentiation in slime molds can be reversed during a remarkably long period of development.

Quantitative data on the relationship between proliferation and differentiation have become available in the course of a systematic survey of the transition of the undifferentiated mesoderm into fully differentiated muscle cells (Herrmann, I 952). The rate of proliferation during early mesodermal differentiation may be represented by measurements of protein nitrogen, RNA, and DNA, in somites of the chick embryo. Before the 24-somite stage the values found for these parameters double in IO-I $8 \mathrm{~h}$. and by the time the full complement of somites is formed doubling time has increased to $24 \mathrm{~h}$. (Herrmann, Schneider, Neukom and Moore, I 95I). About the same value is still found for the growth rate of the total leg muscle from the 8th to the I I th day of development (Herrmann, White and Cooper, I957). Data on the weight of the entire leg of the chick embryo from the $5^{\text {th }}$ to the I I th day of development show only a slight increase in doubling time from 19-26 h. and for the DNA content of the leg a varying value of $25-33 \mathrm{~h}$. is found for a I $00 \%$ increase (Nowinski and Yushok, I953). After the I I th day the rate of accumulation of the dry weight and the DNA of the leg muscle begins to diminish and a sharp drop occurs after the $4_{4}$ th day. DNA accumulation comes practically to a standstill after the I 8 th day. On the other hand, beginning at about the I 2 th day of development the proteins, actomyosin and collagen, differentiation products of the myoblast and the fibroblast respectively, become detectable with analytical methods and the total quantity of these proteins continues to increase at a high rate. For collagen the doubling time remains $24 \mathrm{~h}$. up to the $\mathrm{I} 6 \mathrm{th}$ day and diminishes rapidly afterwards, reaching the rate of the overall protein accumulation. In the case of actomyosin the doubling time is somewhat less than $24 \mathrm{~h}$. on the 12 th day but this rate decreases less abruptly than in case of collagen and even at one month after hatching actomyosin accumulates faster than the remaining bulk of cellular proteins (Herrmann and Barry, i 955; Csapo and Herrmann, I 95 I ; Herrmann, White and Cooper, 1957).

These results seem to indicate that even in expressing the degree of differentiation as accumulation of individual specific proteins, at least in the case of muscle, a certain complementarity of proliferation and differentiation can be observed. It is rather remarkable, however, that the rate at which the specific proteins continue to accumulate after cell proliferation diminishes, is of the same order of magnitude as the accumulation of the bulk of proteins during the more rapid phases of proliferation. Considering the three alternative relations between total protein 
formation and formation of specific cell proteins, the present case would seem to conform most closely to the situation of a decreasing total protein formation with the specific proteins maintaining a high rate of accumulation. This could actually mean that small quantities of specific proteins are formed even during the early phases of development. Since, during this period, the production of proteins necessary for cell proliferation is so rapid, the capacity of the PFS to form larger amounts of specific proteins remains restricted to a very small fraction of the total protein production. In fact, with enzymatic methods, very small quantities of such proteins have been detected in very early phases of development which would support this suggestion (Boell and Shen, I944). Following this reasoning, differentiation would consist not in the formation of protein species completely de novo but merely in an increased rate of their production relative to the formation of other protein species. Parallel observations from the field of microbiology are discussed below. It is not suggested that such a mechanism operates in all types of cytodifferentiation. Although it could hold for a number of cell types, the possibility of a true de novo synthesis of certain proteins in some organs (lens proteins) can not be dismissed (Ten Cate and Van Dooremaalen, I950; Edds, I958).

For a study of the relationship between proliferation and differentiation in mammalian tissues the rat liver seems to offer interesting possibilities (Eliasson, Hammersten, Richard, Aqvist, Thorell and Ehrensvärd, I95 I). After removal of about two-thirds of the liver of the adult rat, regeneration to about the original size of the organ takes place within 6-8 days. At the end of the first day of regeneration there is a large increase in mitotic activity and growth of the organ is primarily due to cell proliferation. At the end of the second day mitotic activity has fallen almost to its initial value and growth must be primarily due to production of cytoplasmic material without significant proliferative activity. Within this regenerative period there is a rapid transition from an actively proliferating tissue to one which is growing by elaboration of specific cell products. The first proliferative period in liver regeneration is accompanied by a marked increase in the quantities of both DNA and RNA (in absolute figures as well as relative to dry weight) and in the incorporation of labelled glycine into nucleic acids. The rate of incorporation of labelled amino acids into proteins increases rapidly during the first day. From the 2nd day on the increase in incorporation is slower and reaches a peak on the third day followed by a slow decrease in the incorporation rate.

In contrast, the concentration of apyrase and rhodanase per $g$ protein begins to fall when the total protein of the regenerating liver increases (Rosenthal, Rogers, Vars and Ferguson, 195I). A similar fall was also observed (Novikoff and Potter, 1948) for the enzymes succinoxidase, malic dehydrogenase and cytochrome reductase. Since all five enzymes are involved in specific functions of the differentiated tissue these findings may mean that in regeneration actually a part of the specific cell products (enzymes) is lost during the proliferative phase of growth.

Another promising object for a study of the relation of growth and differentiation is the red blood cell (Thorell, I 947; Thorell, I953; Hammarsten, Thorell, Aqvist, Eliasson and Akerman, I 953). In the mature erythrocyte a large fraction of the total protein moiety consists of hemoglobin which represents the specific 
functional product of this cell. Due to its characteristic absorption spectrum, hemoglobin can be determined quantitatively in single cells and in this way its accumulation can be followed in the maturing erythrocyte at different stages of development. By measurements of cell number, total cell volume, and protein content per unit cell volume, a comparison could be made between the rate of total protein production (growth) and hemoglobin formation (differentiation). It was found that only in a relatively late phase of ery throcyte development, in the polychromatic erythrocyte, does hemoglobin formation take place. At this stage total protein production has come practically to a standstill and the RNA content of the cells has reached a minimum. These results seemed to indicate that in this cell type a very distinct complementarity of growth and differentiation exists. However, the possibility was considered that perhaps globin, the protein moiety of hemoglobin, was formed at an earlier proliferative phase of erythrocyte development while the formation of the hemin component took place only at the later stage. An attempt was made to decide this question by comparing the incorporation rates of labelled glycine into the hemin and the globin components of the hemoglobin molecule in immature erythrocytes formed after phenylhydrazin hemolysis.

Although the results of these experiments seem to support this assumption, further extension of these data would add to their conclusiveness ${ }^{1}$. Even if the interpretation is accepted the fact that hemin formation during later development presupposes activation and presumably formation of an enzyme protein required for this synthesis. Again, it may be that increased turnover of at least part of the RNA and not RNA quantity in itself is a decisive factor in this process. Borsook (1956a, p. 56) concludes that in rabbit reticulocytes rapid protein synthesis coincides with a low RNA content and turnover of only a small RNA fraction.

In the course of development the survival of certain embryonic cells soon becomes dependent on numerous extracellular factors. To cite one example, innervation becomes an essential condition for regeneration (Singer, I952; Singer, Flinker and Sidman, I956) and for the maintenance, growth and differentiation of muscle tissue (Eastlick, I 943) and in turn the development and growth of the nervous system become dependent on its interaction with peripheral organ rudiments $^{2}$. The actual isolation from a sarcoma and from snake venom ${ }^{3}$ of an interesting protein fraction which stimulates proliferation and neuron growth of sensory and sympathetic ganglia has been accomplished (Cohen, Levi-Montalcini and Hamburger, 1954).

1 The factors which give these results a certain degree of uncertainty are the following: For each experimental condition only a single measurement was made for each time period. The two animals used for the entire experimental series showed large differences in the output of erythrocytes and a wide variation was observed in the ratios of glycine incorporation in the hemin and globin where a constant ratio should have been expected (Exp. II, a and b). Finally, it is not evident just why the total amount of glycine incorporated into hemin after $38 \mathrm{~h}$. is smaller than after $6 \mathrm{~h}$. If this would be due to a rapid turnover, and equilibration with decreasing activities of the glycine pool, the rate of hemin synthesis would have to be corrected by this factor.

2 Work on this phase of development has been reviewed by Hamburger (I955).

3 Personal communication. 
In later stages of development the endocrine glands exert characteristic effects on the processes of the development of certain tissues. Some of these processes, such as metamorphosis in insects and amphibians, are among the most widely known phenomena of embryonic development. Many of the endocrine glands affect growth of organisms in general or stimulate growth in specific target organs by an increase in protein anabolism (White, I956). In certain organs it could actually be demonstrated that hormone stimulated growth is equivalent to an activation of general protein synthesis (Kochakian and Tillotson, I956) or an increased formation of specific proteins in one cell type (Csapo, I950; Scow, i 95 I, I 953; Mueller, I953; Hurley and Herrmann, I 955; Harkness and Harkness, r 956). However, the mechanism of these effects has remained surprisingly obscure. There is, in most instances, little information relative to the step or steps in protein synthesis acted upon by those hormones which stimulate growth. At the same time it must be realized that elucidation of the mechanism of hormonal action would yield information on the control of the PFS which could be applied to earlier phases of development. The possibility cannot even be entirely excluded that hormone-like substances, e.g. steroids, may occur rather ubiquitously, possibly located in the cell surfaces, in many cells of the early embryo, and exert at this time an effect by transmission from cell to cell. The relationship between hormonal effects and embryonic induction and the pertinence of studies of hormonal mechanisms for the understanding of embryonic development is particularly evident in studies on sex differentiation (Chang and Witschi, I 956) and has been well stated by Moog. In one of her papers (Moog, I 953) dealing with the control of phosphatase production in the intestinal epithelium of the chick and the mouse embryo (Moog, I950, I 95 I ; Moog and Richardson, I955) she expresses her thoughts as follows: "Classical studies in experimental embryology have long since established the fact that early embryonic development involves the interactions of series of inductive stimuli and correlated competences. Now it appears that in later stages similar situations obtain, with hormones constituting the inductors and acting on target tissues that pass through transient phases of sensitivity, or competence."

In attempting to come to a better understanding of the relationship between hormonal stimulation and the formation of the protein moiety of a highly specialized differentiating tissue, investigations were carried out on the hormonal control of the formation of the muscle proteins in the leg musculature of the chick embryo. In view of the fact that a marked increase in dry weight of the chick leg musculature as well as an increase in activity of several endocrine organs of the chick takes place at about the I2th day of development, the possibility was considered that development of muscle after this time is, at least in part, regulated hormonally. As the first step in this investigation the effect of thyroid activity on muscle development was examined (Konigsberg, I958). Thyroid activity was depressed by a carefully established single dose of thiourea administered intravascularly on the tenth day of development. Histological examination of the thyroid glands of the experimental animals showed very marked suppression of thyroid activity. For the present discussion the most significant finding was that the increase of protein and of RNA per cell (DNA) was delayed to the i 6 th day of development while in controls an increase in both parameters occurs after the 
I $4^{\text {th }}$ day. After the 6 th day RNA accumulation per DNA was found to occur at the same rate as in controls but the rate of protein accumulation was only $52 \%$ of the accumulation rate of the controls. In pituitary deficiency RNA and protein accumulation is affected to the same extent. However, DNA accumulation continues for a longer period of time than in control embryos (Love and Konigsberg, I 958).

Two points of interest emerged from these experiments. The first is the same restriction of responsiveness of muscle tissue to hormones which Moog compares with embryonic competence (absence of growth stimulation in muscle by thyroxin before the I $4^{\text {th }}$ day). However, while in embryology the designation of competence is as yet merely a name for an observed phenomenon, several concrete alternatives can be proposed as experimentally verifiable mechanisms for the changes in reactivity of muscle tissue to thyroid and pituitary hormones. Secondly, in developing muscle tissue the disturbances in protein formation are associated with alterations in RNA production and indicate profound effects on the PFS. The delayed accumulation of both RNA and protein per cell may actually indicate that protein synthesis is impaired by a failure of RNA formation.

\section{CONCLUSIONS: THE GONVERGENCE OF CONCEPTS}

In the present chapter the protein forming system of the cell was made the center of the problem of differentiation during embryonic development. The intention in following this approach was to show that the conceptual and experimental isolation, which has plagued embryology hitherto, has ceased to exist. Some of the points of convergence with other fields of biology have been touched upon in preceding sections of this chapter. It remains, then, for this concluding section to summarize the manifold relationships between embryonic differentiation and other fields of biology.

As pointed out previously, in studying the changes in the amino acid content of embryonic tissues, in measuring the uptake of labelled amino acids into the proteins of the embryos, and by exploring the effects of amino acid analogs, the approaches to the question of precursor utilization in protein formation in embryonict issues, in unicellular organisms and in adult tissues of higher animals have found at least common methodological denominators. However, the relatively high concentration of peptides in the tissues of some embryos and the observation of an uptake of presumably high molecular cell constituents into embryonic cells suggests the possibility that proteins or larger protein breakdown products, as such, can be utilized as protein precursors and that embryonic cells may be in some way unique, in this respect.

Another question concerns the maintenance of the amino acid pool. In experiments with yeast Halvorson and Spiegelman (1953) and Halvorson, Fry and Schwemmin (1955) have shown that the amino acid pool can be replenished by the breakdown of cellular proteins and that the amino acids supplied in this way can be utilized for the synthesis of specific proteins such as the induced enzyme galactosidase. Apparently, the proteins which represent the source of the amino acids are unessential for the maintenance of the yeast cell during this period and 
are distinguished by a metabolic lability which leads to their preferential breakdown by an unknown mechanism.

In contrast to yeast, in other microbial forms like $E$. coli, no indication was found of any degradation of proteins into amino acids and the utilization of these degradation products of labile proteins for new protein synthesis under conditions of rapid growth (Hogness, Cohn and Monod, 1955). However, later experiments with $E$. coli showed enzyme induction in the alssence of growth, suggesting conversion of some bacterial proteins into enzyme proteins (Rickenberg and Lester, I955; Lovtrup, i 956). Correspondingly, it would be of considerable interest to find out whether in differentiating embryonic tissues different cellular proteins are broken down at differential rates and to what extent this protein breakdown is compatible with or requisite to the further development of the cell. For example, in the case of the replacement of embryonic erythrocytes into adult red blood cells the fetal hemoglobin is presumably liberated in the spleen after destruction of the embryonic erythroblasts. Whether the fetal hemoglobin has to be completely degraded into amino acids or whether larger peptides can be utilized for the resynthesis of the hemoglobin molecule of the mature organism has not been investigated. Also, it is not known as yet for other proteins such as myosin, lens protein, etc. whether there exist chemically distinct embryonic and mature forms as in the case of hemoglobin; whether in such instances the conversion of the embryonic protein into the adult protein form requires complete degradation to amino acids; and whether formation of the adult form occurs in the same cell or in subsequent cell generations. Possibly, the degradation of yolk proteins to amino acids for utilization in the synthesis of cytoplasmic proteins may provide excellent material for an understanding of the interconversion of cellular proteins in general.

The similarities of the PFS in embryonic cells, in microbial forms and in mature animal and plant tissues have become apparent from the study of its constituents, in particular of the RNA moiety and of the structural organization of the protein forming system itself. It may be recalled that the first suggestion of an importance of RNA in protein synthesis came from the investigations of Caspersson and of Brachet on rapidly growing, essentially embryonic, tissues. The initially tenuous correlation between RNA and protein formation has become more firmly established through microbiological studies and through the investigation of protein synthesis in such highly differentiated cells as the pancreas or the pea seedling root (Webster and Johnson, I955).

Although little is known about the exact role of nucleic acids, and particularly RNA, in protein formation it has been found that they form an essential part of organized centers in the synthesis of proteins. In differentiated cells the biochemist has associated microsomal activity with protein formation in the cytoplasm. Recent electron microscopic studies have shown these particles to be part of the highly organized endoplasmic reticulum from which the microsomes are presumably liberated on homogenization. In contrast, during early embryonic development part of the RNA seems to occur unassociated with any cytoplasmic particle. If this "free" RNA is just as important for protein formation as the microsomal RNA, it may present a much better opportunity to investigate its role in the syn- 
thetic process than the microsome bound RNA, since fractionation of RNA may be facilitated in this free state.

In view of the great interest of the embryologists in the mechanisms of differentiation by the interaction of embryonic cells, experimentally induced protein formation in microbial organisms has become a model system of great significance for the study of inductive processes. A possible relationship between enzyme induction in unicellular organisms and embryonic differentiation was anticipated by microbiologists about ten years ago (Monod, 1947; Spiegelman, I 948). Experimental advances made since then are discussed by Monod and by Cohn in contributions to a symposium on "The Origin of Enzymes." Together with papers by Ephrussi (Enzymes in cellular differentiation), Gale (Nucleic acids and enzyme synthesis), and Spiegelman (On the nature of the enzyme forming system) and the penetrating discussions following these papers, this symposium (Gaebler, ed. I956) can be expected to open numerous approaches to a fundamental analysis of embryonic cells. Studies of the kinetics of enzyme induction in bacteria and of its inhibition indicate that this process is the result of an interaction of inducing substances with some presumably large molecular cell component of the protein forming system, which in a modified form catalyzes synthesis of an apparently new protein type. Such inducing mechanisms were postulated by Cohn and Monod (1953) for the induction of the enzyme galactosidase in E. coli and by Pollock ( I 953) for the formation of the enzyme penicillinase, which catalyzes the breakdown of penicillin in Bacillus cereus. In the latter case it was found that contact of the bacterial cell with penicillin for one minute was sufficient to induce enzyme formation when the bacillus was grown after this period in penicillin-free medium. It should be emphasized that this is an instance where an induced enzyme persists even after removal of the inducer. Tests with ${ }^{35} \mathrm{~S}$ labelled penicillin showed that an amount of about 100 molecules of penicillin per cell suffices for penicillinase production to proceed at maximum speed. The discrepancy in the quantities of inducer needed and enzyme produced suggested that the penicillin reacted with or acted upon a catalyst which is involved in the synthesis of the enzyme. Again the assumption was made that the production of new enzyme (penicillinase) is initiated by a modification of a part of the PFS by the inducer. From the results of their experiments Monod ( $195^{6}$ ) and Cohn (1956) conclude that induction of an enzyme like beta-galactosidase in Escherichia coli involves a transient exposure of the cell to the inducing substance at a concentration sufficient for the formation of a system that concentrates the inducing chemical (e.g., galactoside) in the bacterial cell and leads subsequently to formation of an adaptive enzyme. Once formed, the concentrating system persists even in the presence of a minimal concentration of the inducer which would not be sufficient to induce either the concentrating mechanism or the adaptive enzyme. In many instances embryonic induction depends on at least transient close proximity of the inducing and the responding tissues. It may be that during this short period the inducing material reaches a concentration threshold in the vicinity of the responding cell which is sufficient for the establishment of a concentrating mechanism. With the subsequent accumulation of the inducing material (it may be a multiplicity of chemical entities) in the cell, formation of new types of proteins would be elicited. After the transient interac- 
tion of the tissues a minimal and possibly ubiquitous concentration of the inducing material would suffice to maintain the concentrating mechanism and consequently enzyme induction. Possibilities for stabilization of such a system are indicated in some of the articles and in the discussions included in the symposium referred to above (Gaebler, I956).

Although in part speculative, the conceptual background of this scheme seems to be of great significance for certain problems of differentiation since it suggests that inducing substances may modify the PFS either with or without becoming part of it, bringing about the formation of a new type of protein. What is important in the parallelism between this scheme and embryonic induction is not the nature of the inducing agent which in embryology can be expected to be an intermediary metabolite, a nucleic acid, a protein, or even a surface rearrangement after cellular contact. What is important is the suggestion of a mechanism for the modification of the PFS itself which in microbes and embryonic cells may exhibit, in principle, similar properties irrespective of the possibly dissimilar nature of the modifying stimulus.

Further analysis of the penicillinase system ${ }^{1}$ has led to findings which seem of importance for the elucidation of another aspect of differentiation, primarily because it has become apparent that bacterial strains without minimum quantities of penicillinase do not respond to penicillin with enzyme formation. For example, in the inducible strain Bacillus cereus 569 the enzyme quantity increases from a minimum level of 75 molecules of penicillinase per bacterial cell to 25,000 molecules per cell after maximal induction and in this instance induction is, therefore, not absolutely de novo formation of a protein.

The relationship of these observations to the problem of differentiation is suggested by the work of Sawyer (1943a, b); Boell and Shen (1944); Boell, Greenfield and Shen (1955); Shen, Greenfield and Boell (1955), the results of which define quantitatively the onset and progress of neural differentation by one specific biochemical parameter. Since the differentiated nerve cell is distinguished by a high cholinesterase activity, the rapid increase of this enzyme following induction can be regarded as a quantitative index of the induction process. Therefore, one consequence of induction of the central nervous system must be the activation of that portion of the PFS which produces cholinesterase. From the data presented it is likely that cholinesterase is present in very low concentration in most embryonic tissues and also in preinduced ectoderm. If the enzymes found before and after induction are identical it would have to be inferred, in the case of this particular enzyme, that the induction process initiates merely a quantitative acceleration of cholinesterase accumulation and that the intercellular loci for the formation of this enzyme are already preformed at this time. A somewhat comparable situation seems to prevail in the case of heart formation where heart myosin is found very widely distributed in low concentrations before the formation of the heart actually begins (Ebert, I 953, I954a, Ebert et al., I 955). A biochemical site of heart myosin production in the PFS of many primordial cells is thus present before bulk production of the protein is initiated. Histogenesis of the heart muscle cell may merely involve a relative acceleration of myosin accumulation in the heart primordia.

1 M. R. Pollock, personal communications. 
Myosin formation in the cells of the other primordia is eliminated. In other instances there is no evidence that the cell specific proteins are formed in small quantities before induction of the respective organ (e.g. lens) (Ten Cate and Van Dooremaalen, I950). Although the sensitivity of enzymatic and immunological methods may not be high enough to determine amounts of protein species of only a few hundred individual molecules, still, the possibility cannot be excluded that induction may require the new formation of a component of the PFS which was completely lacking before induction took place.

One disadvantage of an interpretation of differentiation based purely on quantitative differences in protein production may lie in the difficulty of explaining the apparently irreversible nature of the differentiation process. If the formation of either a brain or a lens cell would be merely a matter of adjusting rates of formation, e.g. of brain or lens proteins, the reversal of this adjustment should be readily possible. Only if accelerated formation of one specialized protein would lead to an irreversible deterioration of the centers for formation of other proteins, the basis of an irreversibility of differentiation would become apparent. Experimentally these possibilities would lead to the following testable conditions. Before the onset of differentiation minimal or near minimal quantities of a series of proteins could be detected in the same cell type. In a primitive mesenchyme cell there would be small quantities of myosin ATPase, (premuscle condition), slight amounts of enzyme for formation of chondroitinsulfuric acid (prebone condition) and some enzymes specific for the differentiated erythrocyte or kidney tissue cell. With the onset of differentiation each cell should lose all the minimal activities found in the undifferentiated cell except the one which is specific for the respective organ. A certain ambivalence of protein forming capacity in differentiated organs such as lens formation from some amphibian iris cells (Wolffian regeneration) may be due to an incomplete eradication of the lens forming apparatus in these cells. Actually, by careful analysis this capacity can be found in iris cells of a wider variety of species than was previously assumed to be the case (Sato, 1952/54).

The postulate of the irreversibility of differentiation invites further comments. Its validity has been questioned by Trinkaus (1956) and by Ephrussi (1956), the latter pointing out that all of the evidence that could be arrayed in its support was of a negative nature. At the same time Ephrussi (1956, p. 33) concludes that whether complete irreversibility is assumed or not, the high stability of the states of restricted potentiality poses a problem to which neither the geneticist nor the enzymologist concerned with enzyme synthesis can remain indifferent. To account for irreversibility Ephrussi considers nuclear changes such as deformations of the nucleic acid helices, alterations in the intimate structure of chromosomes, changes resembling bacterial modifications introduced by phage, and the possible role of cytoplasmic self-reproducing units such as plasmagenes or kinetosomes (reviewed by Ephrussi, I953). Interesting experiments of several authors are rliscussed by Pollock (1953) suggesting that irreversibility of differentiation is associated with the loss of cytoplasmic particles as indicated in the production of the "petite" colony variants of yeast induced by acridine compounds and associated with loss of particles involved in the formation of cytochrome oxidase.

Aspects of microbiology which are possibly more closely related to differentia- 
tion of animal cells than enzymatic adaptation are represented by investigations of spore germination as well as similar investigations on spore formation. Spore formation (for review see Foster, 1956) for example, is initiated when vegetative proliferation ceases. Antigenically new material is formed in the course of sporulation and once sporulation is induced the bacterial cells cease to react to an inducing substance with formation of adaptive enzymes, indicating a restriction of the potentialities of the PFS similar to that observed in embryonic determination.Also, overall acceleration of protein synthesis by the addition of glucose to the medium leads to a suppression of sporulation. This may be regarded as an example of metabolic regulation of protein formation.

Hachisuka et al. (1956) recently have shown that the enzymes of the glucose oxidizing system of Bacillus subtilis do appear in a step-wise fashion with the development of distinct morphological phases in the transformation from a spore to the vegetative cell. The possibility that the failure to detect, in bacterial spores, enzymes which occur in the vegetative forms of the same bacterium is actually due to the insensitivity of the analytical methods used has been considered (Church, Halvorson and Halvorson, I954).

For a considerable time before adaptive enzyme formation in bacteria became known, one type of induction of protein formation in metazoan tissues had been demonstrated; viz. antibody production in response to a foreign protein. The relevance of antibody production as a model for the activity of the PFS of developing cells has been fully appreciated by Tyler (1947, I 955) and Weiss (I947, I955). The essential points in interpreting antibody formation in this sense can be stated as follows. Injection of an antigen elicits in certain cells of the mature organism formation of a new protein, the antibody. This protein is structurally related to the antigen, giving rise to a highly specific interaction between the two proteins in the formation of an antigen-antibody complex. Antibody formation may continue for many years after the transient exposure to the antigen. This must mean that the initial response to the antigen becomes more or less permanently fixed in the PFS of the responding cell. In explaining this persistence, two hypotheses have been suggested. Either the antigen itself becomes a part of the antibody-forming system of the cell or it changes, in a specific way, the structure of this system so that production of proteins, structurally related to the antigen, becomes irreversibly established. These steps in antibody formation are analogous to the production of new proteins in the process of induced enzyme formation and possibly in differentiation where the inducing conditions provide the trigger and in part the control for the PFS of the responding cell. That a further elucidation of this alternative was achieved by an analysis of the properties of embryonic tissues is an important additional demonstration of the interaction of research in embryology and immunology. The remarkable phenomenon first recognized in its significance by Burnet and Fenner (I 949) consists in the failure of embryos to form antibodies against certain heterologous proteins and to remain unresponsive to immunological stimulation during post-natal life ${ }^{1}$. This absence of immune response can be

${ }^{1}$ Experiments in which this phenomenon could not be confirmed were published by Bauer et al. (1956). 
elicited experimentally not only with cells and grafted tissues but also with purified proteins as the antigenic material. From the latter studies it was concluded that this phenomenon was based on a modification of the embryonic PFS, possibly without persistence of the antigenic material itself in the embryonic cell (Cinader and Dubert, i 956). The wide ramifications of the elucidation of this problem for an understanding of the control of the PFS are discussed in a stimulating symposium on "Immunological Tolerance" (Medawar et al., i956). A modification of the PFS by addition of heterologous proteins to tissue cultures has been claimed by Langman (1953a, b). Confirmation of his results would amount to a demonstration in vitro of a cellular reaction closely related to immune tolerance. A wide array of immunological approaches to the problems of differentiation has been summarized by Nace (I955) and Woerdeman (I955).

Before concluding this discussion it should be pointed out that in many instances the protein molecule does not assume its functional role in the cell as an individual entity but rather in combination with other protein molecules of the same or of a different species or as a part of a complex of proteins, nucleic acids, mucopolysaccharides or lipids. Participating in such a molecular aggregation the protein molecule becomes a building block in the formation of those larger structural units which become visible, at least in the electron microscope, as the smallest distinct morphological units. Thus, in contributing to the formation of supramolecular complexes the protein molecule participates directly in morphogenetic phenomena. Some factors involved in the aggregation process of molecules of the same protein species were explored in a morphogenetic model system, consisting of the aggregation of dissolved collagen molecules with the subsequent formation of collagen fibers. More recent progress in this investigation has been reported in an exemplary paper by Jerome Gross (1956). Of importance from the perspective of morphogenesis are the recent investigations of Nickerson and his associates (Nickerson, r 954 a) concerning the metabolic control of the state of polymerization of cell-wall proteins.

The starting point of this series of investigations was the observation that nutritional conditions determine whether yeast will grow as a rapidly budding cellular form or as filamentous mycelium without budding. Further analysis showed that the presence of cysteine in the medium maintains budding and eliminates the occurrence of filamentous forms (Nickerson and Mankowski, I953). The dependence of this transformation upon definite metabolic reactions could be established by the analysis of a divisionless yeast mutant in which budding and cell division did not take place but which grew rapidly in a filamentous condition (Nickerson and Chung, 1954).

Investigations carried out to explain this difference showed that in the parent strain a metal flavoprotein is coupled to an acceptor which initiates cytokinesis, whereas in the mutant this coupling is blocked and substrate generated reducing capacity is diverted to non-specific dye reduction (Nickerson, I954b). Since cytoplasmic division is maintained and filament formation prevented by the addition of cysteine the possibility of a shift in the reduction of SS to SH groups as a controlling mechanism in cytokinesis and as a receptor for the metal flavoprotein system was postulated. In supporting this Falcone and Nickerson (1956), 
Nickerson and Falcone (1956a, b) showed that the normal yeast possesses an enzyme system capable of reducing disulfide bonds in a cell-wall mannanprotein complex whereas the divisionless mutant is deficient in this activity. Budding and subsequent proliferation occurred only if the cell-wall protein was depolymerized subsequent to reduction of its SS groups. The noteworthy series of investigations on the metabolic control of morphogenesis in Blastocladiella carried out by Cantino (1955) and his associates as well as the exploration of the mechanism of spore formation in bacteria (reviewed by Foster, 1956) may point to similar relations of protein aggregation and the metabolic activity of the cell.

From the point of view of animal morphogenesis these findings are potentially significant in two respects. They may be directly pertinent to an understanding of protein polymerization in metabolically controlled morphogenetic processes such as formation of ciliae in the apical tuft of sea urchin larvae, which is regarded as index of "animalization" and known to depend on the metabolic state of the animalized cell. It cannot be excluded as yet, however, that metabolic processes, similar to those which affect the state of proteins involved in these polymerizations, also affect the enzymatic units of the PFS, such as enzymes similar to the proteases, or influence the structure of "templates" and in this way modify indirectly the structure of the processed proteins. Ranzi and his associates (Ranzi, I953; Ranzi, 1955; Ranzi and Citterio, 1955) have initiated a series of studies aimed at defining the state of aggregation of embryonic proteins and their alteration after exposure of the embryos to agents, such as lithium, which are known to influence markedly the course of development.

Obviously, the interaction of proteins with each other, with nucleic acids, mucopolysaccharides, or lipoids is essential in the formation of larger structural units such as the endoplasmic reticulum, mitochondria, and the cell surface. The role of the first two entities in embryonic differentiation has been repeatedly touched upon in this chapter. The significance of the cell surface as a large molecular aggregate should be given added emphasis in this context. First it should be pointed out that the identification of protein as a structural unit in the cell surface has recently been followed by a remarkable variety of methodological approaches. Such are the use of proteolytic enzymes for the separation of tissues (Herrmann and Hickman, I 948a) and of cells (Moscona, I 952 and Moscona and Moscona, I952) implicating proteins as the main component in the system maintaining the coherence of cells, localization of enzymes in the cell surface (Rothstein and Maier, I949), the analysis of surface groups of bacterial cells responsible for the attachment of virus particles (Tolmach, I957), and the immunological analysis of bacterial cell surfaces (Vennes and Gerhardt, I956). Apart from this purely structural consideration of the cell surface, Christensen ( $1955 \mathrm{a}, \mathrm{b}$ ) suggests that the cell surface is not only the site of amino acid activation connected with their transfer into the interior of the cell but represents possibly a site of protein synthesis. Such a possibility may seem plausible in view of electron microscopic observations revealing a continuity of the endoplasmic reticulum, with its microsomal derivatives, and the cell cortex. Since microsomes are apparently the main site of protein synthesis the inclusion of microsomal material in the cell cortex could indicate a similar function. One is also tempted to speculate 
about the possible relationship of such a cortical organization to the RNA coat of gram positive bacteria (Henry and Stacey, I943) and of animal and plant cells (Lansing and Rosenthal, I952). The significance of the cortical structure in embryonic development in general has been emphasized more recently by Lehmann (1945) and Runnström (1952). Weiss (r947) has pointed to the possible role of surface interactions in the induction process and several investigators (Holtfreter, I948a; Spiegel, I 955; Gregg, I956; Moscona, I956) have attributed primary importance to specific forms of surface interactions in the aggregation of cells in the course of embryogenesis.

The preceding discussion suggests a more generalized view of the mechanism of differentiation. From the examples drawn from different fields of study it has become apparent that all approaches are confronted with the same crucial question of the molecular mechanism by which a chemical, a hormone, a microbial transforming principle, an enzyme substrate analog, a hapten, some tissue proteins or chordamesoderm, can leave an imprint on the protein forming system of the cell. This should not be interpreted to mean that one could or should expect that further progress in this field will lead to a single comprehensive theory of differentiation. The numerous consecutive reactions in the transformation of a rapidly proliferating tissue to one which exhibits all the characteristics of structurally and functionally differentiated organs will remain complex and highly specific for each tissue and each species investigated. The individual steps in this process and the interaction of the parts of the developing embryo are too specific to be resolved by a single expression describing the overall process (which undoubtedly could be done) without consideration of the components of the system (e.g. "laws of growth"). In the disciplines of the physicist where the components of the total system are of sufficient qualitative similarity, comprehensive theory construction is more satisfactory both as descriptive and as explanatory schemes of thought. In biology and in particular in embryonic development the interaction of qualitatively different components is of primary importance in an explanation of the observed changes of the total system. The first step toward an explanation seems to consist in an attempt to demonstrate the mechanism of these individual interactions (Herrmann, I953c). In this sense embryonic development appears as a picture embroidered in a broad canvas with highly irregular shapes and with the entire spectrum of colors, and not as the regular geometric patterns of a physical theory. Embryology will be written as an epic rather than as a sonnet. In the full account of embryogenesis the general theories of physics will be merely tools to exhibit and to appreciate the diversity of the individual phases of the developing systems ${ }^{1}$.

1 Work from this laboratory reported in this Chapter was made possible by grants from the U.S.Public Health Service, Am. Cancer Soc., Assoc. Aid Crippled Children, and Muscular Dystrophy Assoc. 


\section{LITERATURE}

Abrams, R., (195I) Arch. Biochem. Biophys., 30, 90.

Alfert, M., (1956) J. Biophys. Biochem. Cytol., 2, 109.

Allfrey, V. G., (1954) Proc. Natl. Acad. Sci. U.S., 40, 88 I.

Allfrey, V. G., M. M. Daly and A. E. Mirsky, (1953) F. Gen. Physiol., 37, 157.

Allfrey, V. G., M. M. Daly and A. E. Mirsky, (1955) J. Gen. Physiol., 38, 415.

Allfrey, V. G., A. E. Mirsky and S. Osawa, (1955) Nature, 176, 1042.

Anfinsen, C. B. and D. Steinberg, (i95I) 7. Biol. Chem., r89, 739.

Andres, G., (1955) 7. Exptl. Zool., I30, 221.

Askonas, B. A., P. N. Gampbell and T. S. Work, (1954) Biochem. J., 58, 326.

Avery, G., M. Chow and H. Holtzer, (1956) J. Exptl. Zool., 132, 409.

Barth, L. G. And L. J. Barth, (1954) Energetics of Development: A Study of Metabolism in the

Frog Egg, Columbia University Press, N.Y.

Bauer, J. A., P. E. Peckman and A. G. Osler, (1956) Proc. Soc. Exptl. Biol. Med., 94, 7 I 4.

Bellairs, R., (1958) 7. Embryol. Exptl. Morphol., 6, I49.

Berg, W. E., (1950) Proc. Soc. Exptl. Biol. Med., 75, 30.

Berg, W. E., (1956) Biol. Bull., Ito, I.

Berrill, N. J. (I935) J. Morphol., 57, 353.

Bodenstein, D., (1948) 7. Exptl. Zool., 108, 93.

Bodenstein, D. and A. A. Kondritzer, (1948) J. Exptl. Zool., 107, iog.

Bodenstein, D. and A. Abdel-Malek, (i949) F. Exptl. Zool., iII, 95.

Boell, E. J., (1948) Ann. N. Y. Acad. Sci., 49, 773.

Boelt, E. J., (I 955 ) Analysis of Development, B. H. Willier, P. A. Weiss and V. Hamburger,

Eds., W. B. Saunders Company, Philadelphia and London.

Boell, E. J., P. Greenfield and S. G. Shen, (1955) F. Expll. Zool., 129, 415.

Boell, E. J. and S. C. Shen, (1944) F. Exptl. Zool., 97, 2 I.

Boell, E. J., and R. Weber, (1955) Exptl. Cell Research, 9, 559.

Bonner, J. T., A. D. Ghiquoine and M. Q. Kolderie, (i955) F. Exptl. Zool., izo, i 33.

Bonner. J. T., and E. B. Frascella, (1952) F. Exptl. Zool., i21, 561.

Borsook, H., (1956a) 7. Cell. Comp. Physiol., 47, Suppl. I, 35.

Borsook, H., (1956b) Proc. 3rd Int. Congr. Biochem., 92.

Brachet, J., (I950) Chemical Embryology, Interscience Publishers, New York.

Brachet, J., (1952) Symp. Soc. Exptl. Biol., 6, i 73.

Brachet, J. and H. Chantrenne, (I942) Acta Biol. Belg., 2, 45 I.

Brachet, J., H. Chantrenne and F. Vanderhaeghe, (1955) Biochim. et Biophys. Acta, I8, 544 .

Briggs, R. And T. J. King, (1955) Biological Specificity and Growth, E. G. Butler, Ed., Princeton University Press, Princeton, N. J.

Britt, L. G. and H. Herrmann, (i959) 7. Embryol. Exptl. Morphol., in the press.

Britten, R. J., R. B. Roberts and E. F. French, (1955) Proc. Natl. Acad. Sci. U.S., 4I, 863.

Burnet, F. M. And F. Fenner, (1949) The Production of Antibodies, Macmillan, Melbourne.

Busch, H. And H. S. N. Greene, (1955) Yale 7. Biol. and Med., 27, 339.

Butler, E. G., Ed., (1955) Biological Specificity and Growth, Princeton University Press, Princeton, N.J.

Cantino, E. C., (i955) Quart. Rev. Biol., 30, i38.

Caspersson, T., (1950) Cell Growth and Cell Function: A Cytochemical Study, W. W. Norton, New York.

Chang, C. Y. and E. Witschi, (1956) Proc. Soc. Exptl. Biol. Med., 93, i40.

Chen, P. S., (1954) Experientia, Io, 2 I2.

Chen, P. S., (1956a) Exptl. Cell Research, Io, 675.

Chen, P. S., (1956b) Rev. suisse zool., 63, 2 I6.

Ghen, P. S. And A. KüHn, (1956) Z. Naturforsch., $1 I-b, 305$.

Christensen, H. N., (I955a) Amino Acid Metabolism, W. D. MaElroy and H. B. Glass, Eds., The Johns Hopkins Press, Baltimore.

Christensen, H. N., (I955b) Amino Acid Biogenesis and Protein Synthesis, University of California, Los Angeles. 
Church, B. D., H. Halvorson and H. O. Halvorson, (1954) J. Bacteriol., 68, 393.

Cinader, B. and J. M. Dubert, (1956) Proc. Roy. Soc. London, B, i46, i8.

Cohen, S., (1954) J. Biol. Chem., 21 II, 337.

Cohen, S., R. Levi-Montalcini and V. Hamburger, (i954) Proc. Natl. Acad. Sci. U.S., 40,1014 .

Cohn, M. And J. Monod, (1953) Symposium Soc. Gen. Microbiol. 3rd, I32.

Cohn, M., (1956) In: Enzymes: Units of Biological Structure and Function, O. H. Gaebler, Ed., Academic Press, New York.

Csapo, A., (1950) Am. F. Physiol., 162, 406.

Cisapo, A. and H. Herrmann, (i95i) Am. 7. Physiol., 165, 70 i.

Daly, M. M., V. G. Allfrey and A. E. Mirsky, (1955) F. Gen. Physiol., 39, 207.

DolJAnski, L., (1930-31 a) Compt. rend. soc. biol., ro5, 343.

DolJanski, L. (1930-3I Ib) Compt. rend. soc. biol., 105, 504.

Duspiva, F., (1955) Naturwissenschaften, 42, 305.

Eakin, R. M., W. E. Berg and P. B. Kutsky, (1950) Proc. Soc. Exptl. Biol. Med., 75, 32.

Eakin, R. M., P. B. Kutsky and W. E. Berg, (195I) Proc. Soc. Exptl. Biol. Med., 78, 502.

Eastlick, H. L., (1943) J. Exptl. Zool., 93, 27.

Ebert, J. D., (r953) Proc. Natl. Acad. Sci. U.S., 39, 333.

Ebert, J. D., (I954a) Proc. Natl. Acad. Sci. U.S., 40, 337.

Ebert, J. D., (1954b) Aspects of Synthesis and Order in Growth, D. Rudnick, Ed., Princeton University Press, Princeton, N.J.

Ebert, J. D., R. A. Tolman, A. M. Mun and J. F. Albright, (ig55) Ann. N. Y. Acad. Sci., 6o, 968 .

Edds, M. V., Jr., (I958) Proc. Natl. Acad. Sci. U.S., 44, 296.

Eliasson, N. A., E. Hammarsten, P. Reichard, S. Aqvist, B. Thorell and G. Ehrensvärd, (1951) Acta Chem. Scand., 5, 43 I.

Ephrussi, B., (1953) Nucleo-Cytoplasmic Relations in Microorganisms, Oxford University Press.

Ephrussi, B., (1956) Enzymes: Units of Biological Structure and Function, O. H. Gaebler, Ed., Academic Press, New York.

Erlenmeyer, H. and F. E. Lehmann, (1949) Experientia, 5, 472.

Ernster, L., R. Zetterstrom and O. Lindberg, (1950) Exptl. Cell Research, I, 494.

Eyal-Gilad, H., (I954) Arch. biol. (Liège), 64, I79.

Falcone, G. and W. J. Nickerson, (1956) Science, I24, 272.

Fankhauser, G., (1952) Intern. Rev. Cytol., r, i6 5 .

Fankhauser, G., (i955) Analysis of Development, B. H. Willier, P. A. Weiss and V. HamBurger, Eds., W. A. Saunders Company, Philadelphia and London.

Fell, H. B. and E. Mellanby, (1953) 7. Physiol. (London), 119, 470.

FELL, H. B., (1953) F. Embryol. Exptl. Morphol., I, 287.

Feldian, M. and C. H. Waddington, (1955) 7. Embryol. Exptl. Morphol., 3, 44.

Fischer, A. and R. C. Parker, (1929) Proc. Soc. Exptl. Biol. Med., 26, 585.

Flavin, M. and C. B. Anfinsen, (i954) F. Biol. Chem., 21 I 375.

Flexner, L. B., (1939) J. Biol. Chem., 13I, 703.

Flexner, L. B., (1955) Ann. N. Y. Acad. Sci., 6o, 986.

Flexner, L. B. and R. D. Stiehler, (i938) 7. Biol. Chem., i26, 6 i 9.

Flickinger, R. A., (1954) Exptl. Cell Research., 6, I 72.

Flickinger, R. A., (1956) 7. Exptl. Zool., 131, 307.

Foster, J. W., (1956) Quart. Rev. Biol., 3I, 102.

Francis, M. D. And T. Winnick, (I953) 7. Biol. Chem., 202, 273.

Friedierg, F. and R. M. Eakin, (ig49) 7. Exptl. Zool., iIo, 33.

Fruton, J. S., (1955) Aspects of Synthesis and Order in Growth, D. Rudnick, Ed., Princeton University Press, Princeton, N.J.

Gaebler, O. H., Ed., (1956) Enzymes: Units of Biological Structure and Function, Acad. Press, Inc., N.Y.

Gale, E. F., (1956) Enzymes: Units of Biological Structure and Function, O. H. Gaebler, Ed., Academic Press, New York.

Gale, E. F. and J. P. Folkes, (1953a) Biochem. 7., 53, 483. 
Gale, E. F. And J. P. Folkes, (1953b) Biochem. J., 53, 493.

Gale, E. F. and J. P. Folkes, (I955a) Biochem. J., 59, 675.

Gale, E. F. and J. P. Folkes, (1955b) Biochem. 7., 59, 66 I.

Gay, H., (1955) Proc. Natl. Acad. Sci. U.S., 4I, 370.

GAY, H., (1956) 7. Biophys. Biochem. Cytol., 2, Suppl., 407.

Gelfant, S., R. K. Meyer and H. Ris, (1955) F. Exptl. Zool., I28, 219.

Gerarde, H. W. and M. Jones, (1953) 7. Biol. Chem., 201, 553.

Goldstein, L. and W. Plaut, (I955) Proc. Natl. Acad. Sci. U.S., 4I, 874.

Gregg, J. H., (1956) 7. Gen. Physiol., 39, 813.

GregG, J. R. And S. Lovtrup, (1955) Biol. Bull., 108, 29-34.

Grobstein, C., (I955a) Aspects of Synthesis and Order in Growth, D. Rudnick, Ed., Princeton University Press, Princeton, N.J.

Grobstein, C., (1955b) 7. Exptl. Zool., I30, 3 19.

Grobstein, C., (1956) Exptl. Cell Research., 10, 424.

Grobstein, C., (1957) Exptl. Cell Research, 13, 575.

Grobstein, C. and H. Holtzer, (1955) J. Exptl. Zool., 128, 333.

Gross, J., (1956) J. Biophys. Biochem. Cytol., 2, Suppl., 26 I.

Gustafson, T., (1954) Intern. Rev. Cytol., 3, 277.

Gustafson. T. and I. Hasselberg, (195I) Exptl. Cell Research, 2, 642.

Gustafson, T. and M. B. HJelte, (195I) Exptl. Cell Research, 2, 474.

Gustafson, T. M. B. Hjelte and I. Hasselberg, (i952) Exptl. Cell Research, 3, 275.

Gustafson, T. and S. Hörstadius, (1955) Exptl. Gell Research, 9, Suppl. 3, i 70.

Gustafson, T. and P. Lenicque, (1952) Exptl. Cell Research, 3, 25 I.

Gustafson, T. and P. Lenicque, (i955) Exptl. Cell Research, 8, i I4.

Hachisuka, Y., N. Asano, M. Kaneko and T. Kanabe, (1956) Science, I24, 174.

Hadorn, E., (1955) Letalfaktoren, Georg Thieme Verlag, Stuttgart.

Halvorson, H. O. and S. Spiegelman, (1952) 7. Bacteriol., 64, 207.

Halvorson, H. O. and S. Spiegelman, (i953) F. Bacteriol., 65, 496.

Halvorson, H., W. Fry and D. Schwemmin, (1955) 7. Gen. Physiol., 38, 549.

Hamburger, V., (1955) Biochemistry of the Developing Nervous System, H. Waelsch, Ed., Academic Press, New York.

Hammarsten, E., B. Thorell, S. Agvist, N. Eliasson and L. Akerman, (1953) Expil. Cell Research, 5, 404.

Harkness, M. L. R. and R. D. Harkness, (1956) J. Physiol. (London), I32, 492.

Haurowitz, F. and R. L. Hardin, (i954) The Proteins, Vol. II, Part A, H. Neurath and K. Bailey, Eds., Academic Press, New York.

HAYAShi, Y., (1955) Embryologia, 2, 145.

HaYashi, Y., (1956) Embryologia, 3, 57.

Henry, H. ANd M. Stacey, (1943) Nature, I5I, 67 I.

Herrmann, H., (i952) Ann. N. Y. Acad. Sci., 55, 99.

Herrmann, H., (I953a) Arch. néerl.zool., Io, Suppl. I, I32.

Herrmann, H., (I953b) 7. Embryol. Exptl. Morphol., I, 29 I.

Herrmann, H., (1953c) Philosophy of Science, 20, I 49.

Herrmann, H. and S. R. Barry, (1955) Arch. Biochem. Biophys., 55, 526.

Herrmann, H. and F. H. Hickman, (1948a) Bull. Johns Hopkins Hosp., 82, 182.

Herrmann, H. and F. H. Hickman, (1948b) Bull Johns Hopkins Hosp., 82, 225.

Herrmann, H., U. R. Konigsberg and M. F. Curry, (1955) 7. Exptl. Zool., 128, 359.

Herrmann, H., L. Lerman and B. N. White, (1958) Biochim. et Biophys. Acta, 27, i6 I.

Herrmann, H., M. J. B. Schneider, B. J. Neukom and J. A. Moore, (i95i) J. Expll. Zool., 118,243 .

Herrmann, H., B. N. White and M. Cooper, (1957) F. Cellular Comp. Physiol., 49, 227.

Hjelte, M. B., T. Gustafson and I. Thoren, (1955) Exptl. Cell Research, 8, 76.

Hoagland, M. B., (I955) Biochim. et Biophys. Acta, 16, 288.

Hoagland, M. B., E. B. Keller and P. C. Zamecnik, (1956) 7. Biol. Chem., 2i8, 345.

Hörstadius, S., (1949) Pubb. staz.zool. Napoli, 21, Suppl., I3I.

Hörstadius, S., (1953) 7. Embryol. Exptl. Morphol., I, 257. 
Hörstadius, S., (1955) F. Exptl. Zool., I29, 249.

Hörstadius, S. And T. Gustafson, (1948) Symposia Soc. Exptl. Biol., 2, 50.

Hörstadius, S. and T. Gustafson, (1954) F. Embryol. Exptl. Morphol., 2, 216.

Hoff-Jorgensen, E. And E. Zeuthen, (1952) Nature, I69, 245.

Hogness, D. S., M. Cohn and J. Monod, (1955) Biochim. et Biophys. Acta, I6, 99.

Hokin, L. E. And M. R. Hokin, (1953) Biochim. et Biophys. Acta, II, 59 I.

Holtfreter, J., (i948a) Ann. N.Y. Acad. Sci., 49, 709.

Holtfreter, J., (1948b) Symposia Soc. Exptl. Biol., 2, i 7.

Holtfreter, J., (1955) Exptl. Cell Research, 9, Suppl. 3, 188.

Holtzer, H. and S. R. Detwiler, (i 953 ) F. Exptl. Zool., I23, 335.

Holtzer, H. And S. R. Detwiler, (1954) Anat. Record, II8, 390.

Holtzer, S. W., (1956) F. Morphol., 99, I.

Hultin, T., (1950) Exptl. Cell Research, I, 376.

Hultin, T., (1952) Exptl. Cell Research, 3, 494.

Hultin, T., (1953a) Arkiv Kemi, 5, 559.

Hultin, T., (1953b) Arkiv Kemi, 5, 543.

Hultin, T., (I953C) Arkiv Kemi, 5, 267.

Hultin, T., (1953d) Arkiv Kemi, 6, i95.

Hultin, T., (1953e) Arch. néerl. zool., io, 76.

Hultin, T., (1953f) Studies on the Structural and Metabolic Background of Fertilization and Development, Stockholm.

Hultin, T., (1955) Exptl. Cell Research, 9, Suppl.3, 2 Iо.

Humphrey, J. H. and A. S. McFarlane, (1954) Biochem. 7., 57, 195.

Hurley, L. S. and H. Herrmann, (1955) Am. F. Physiol., I83, 67.

Karnofsky, D. A., C. P. Dagg and C. R. Lacon, (I955) Federation Proc., I4, 439.

Kaufmann, B. P. And N. K. Das, (1955) Chromosoma, 7, I9.

Kavanau, J. L., (1953) 7. Exptl. Zool., 122, 285.

Kavanau, J. L., (1954) Exptl. Cell Research, 7, 530.

Kavanau, J. L., T. Gustafson and Z. G. Banhidi, (1954) Exptl. Cell Research, 6, 567.

Keller, E. B., P. C. Zamecnik and R. B. Loftfield, (1954) F. Histochem. Cytochem., $2,378$.

Kihara, H. K., M. Amaro, H. Inemota and A. Sibatani, (I955) Biochim. et Biophys. Acta, I7, I 43 .

King, T. J. and R. Briggs, (1955) Proc. Natl. Acad. Sci. U.S., 4I, 321.

Kochakian, C. D. and C. Tillotson, (1956) Hormones and the Aging Process, G. Engle and

E. T. Pincus, Eds., Academic Press, New York.

Konigsberg, I. R., (1958) 7. Cellular Comp. Physiol., 52, I.

Konigsberg, I. R. and H. Herrmann, (1955) Arch. Biochem. Biophys., 55, 534.

Krugelis, E. J., J. S. Nicholas and M. E. Vosgian, (1952) J. Exptl. Zool., i2I, 489.

Kutsky, P. B., R. M. Eakin, W. E. Berg and J. L. Kavanau, (i953) F. Exptl. Zool., $124,263$.

Langman, J., (1953a) Koninkl. Ned. Akad. Wetenschap., Proc. Ser. C, 56, 6, I 7, 2 I 4.

Langman, J., (1953b) Koninkl. Ned. Akad. Wetenschap, Proc. Ser. C, 56, 219.

Lansing, A. I. and T. B. Rosenthal, (1952) 7. Cellular Comp. Physiol., 40, 337.

Lehmann, F. E., (1945) Einführung in die physiologische Embryologi, pp.81-89, Birkhäuser Verlag.

Lehmann, F. E., (1954a) Rev. suisse zool., 6I, 428.

Lehmann, F. E., (I954b) Arch. Julius Klaus-Stift. Vererbungsforsch. Sozialanthropol. u. Rassenhyg., 19, 359.

Lehmann, F. E., W. Bernhard, H. Hadorn and M. Lüscher, (1945) Experientia, I, 232.

Lehmann, F. E. And A. Bretscher, (1952) Helv. Physiol. et Pharmacol. Acta, ro, 20.

Lehmann, F. E. and H. R. Dettelbach, (1952) Rev. suisse zool., 59, 253.

Lemmann, F. E. And S. Lovtrup, (1956) Helv. Physiol. et Pharmacol. Acta, in press.

Lehmann, F. E., R. Weber, H. Aebi, J. Baumler and H. Erlenmeyer, (1954) Helv. Physiol. et Pharmacol. Acta, I2, I 47.

Lenicque, P., S. Hörstadius and T. Gustafson, (1953) Exptl. Cell Research, 5, 400-403.

Levine, M. and H. Tarver, (195I) 7. Biol. Chem., 192, 835.

Lindahl, P. E., (1942) Quart. Rev. Biol., I7, 213. 
Lipmann, F., (1954) Mechanism of Enzyme Action, W. D. MaElroy and H. B. Glass, Eds. The Johns Hopkins Press, Baltimore.

Lipmann, F., et al., (1958) Proc. Natl. Acad. Sci. U.S., 44, 667.

Littlefield, J. W., E. B. Keller, J. Gross and P. C. Zamecnik, (i955) F. Biol. Chem., 217 , II I.

Love, D. S. And I. R. Konigsberg, (1958) Endocrinology, 62, 378.

Lovtrup, S., (1955) Compt. Rend. Lab. Carlsberg, Sér. Chim., 29, 262.

Lovtrup, S., (1956) Biochim. et Biophys. Acta, 19, 247, 433.

Marshak, A. and C. Marshak, (1953) Exptl. Cell Research, 5, 288.

Mazia, D. and D. M. Prescott, (1955) Biochim. et Biophys. Acta, 17, 23.

Medawar, P. B. et al., (I956) Proc. Roy. Soc. (London), B, I46, I.

Monod, J., (1947) Growth, II, 223.

Monod, J., (I956) Enzymes: Units of Biological Structure and Function, O. H. Gaebler, Ed., Academic Press, New York.

Moog, F., (1950) 7. Exptl. Zool., I15, rog.

Moog, F., (195I) 7. Exptl. Zool., I18, 187.

Moog, F., (1952) Ann. N.Y. Acad. Sci., 55, 57.

Moog, F., (1953) 7. Exptl. Zool., I24, 329.

Moog, F. and D. Richardion, (1955) 7. Exptl. Zool., 130, 29.

Moscona, A., (1952) Exptl. Cell Research, 3, 535.

Moscona, A., (1956) Proc. Soc. Exptl. Biol. Med., 92, 410.

Moscona, A., (1958) Proc. Soc. Exptl. Biol. Med., 98, 757.

Moscona, A. and H. Moscona, (1952) J. Anat., 86, 287.

Muchmore, W. B., (1951) 7. Exptl. Zool., I18, 137.

Mueller, G. C., (I953) F. Biol. Chem., 204, 77.

Muir, H. M., A. Neuberger and J. C. Perrone, (i952) Biochem. 7., $52,87$.

NaGe, G. W., (1955) Ann. N. Y. Acad. Sci., 6o, 1038.

Needham, J., (I93I) Chemical Embryology, Vol. I, Cambridge University Press, Cambridge, England, p 440 .

Needham, J., (1955) Ann. Rev. Physiol., I7, 37.

Nieuwroop, P. D. et al., (1952) F. Exptl. Zool., r20, I.

Nieuwkoop, P. D. et al., (1955) Koninkl. Ned.Akad. Wetenschap. Proc. Ser. C, $58,219,356$.

Nieuwkoop, P. D., (1955) Exptl. Cell Research, 9, Suppl. 3, 262-273.

Nieuwroop, P. D. and F. E. Lehmann, (1952) Rev. suisse zool., 59, I.

Nieuwkoop, P. D. and G. V. Nigtevecht, (1954) F. Embryol. Exptl. Morphol., 2, i 75.

Nickerson, W. J., (1954a) Ann. N. Y. Acad. Sci., 6o, 50.

Nickerson, W. J., (1954b) F. Gen. Physiol., 37, 483 .

Nickerson, W. J. and Z. Mankowski, (I953) Am. F. Botany, 4o, 584 .

Nickerson, W. J. And C. W. Chung, (1954) Am. F. Botany, 4I, i i 4 .

Nickerson, W. J. And G. Falcone, (1956a) Science, 124, 3 I8.

Nickerson, W. J. and G. Falcone, (1956b) Science, I24, 722.

Niv, M. G., (I956) Cellular Mechanisms in Differentiation and Growth, D. Rudnick, Ed., Princeton University Press, Princeton, N. J.

Niu, M. G. And V. C. Twitty, (I953) Proc. Natl. Acad. of Sci. U.S., 39, 985.

Novikoff, A. B., (1956) 7. Biophys. Biochem. Cytol., 2, Suppl., 65.

Novikoff, A. B. And V. R. Potter, (1948) 7. Biol. Chem., I73, 223.

Nowinski, W. W. And W. D. Yushok, (1953) Biochim. et Biophys. Acta, II, 497.

Palade, G. E. and P. Siekevitz, (1956) 7. Biophys. Biochem. Cytol., 2, i 7 I.

Pardee, A. B., (1954) Proc. Natl. Acad. Sci. U.S., 40, 263.

Pease, D. G., (1941) F. Exptl. Zool., 86, 38 I.

Pease, D. C., (1942a) 7. Exptl. Zool., 89, 329.

Pease, D. C., (1942b) 7. Exptl. Zool., 89, 347.

Perlmann, P., (1953) Exptl. Cell Research, 5, 394.

Perlmann, P. and T. Gustafson, (1948) Experientia, 4, 48 I.

Peters, T., (1953) 7. Biol. Chem., 200, 461.

Pollock, M. R., (1953) Symposium Soc. Gen. Microbiol., 3rd, I50. 
Ranzi, S., (1953) Arch. néerl. zool., io, Suppl. I, 92.

Ranzi, S., (1955) Le Macromolecole dei Viventi, Instituto Lombardo: Convegno Baselli.

Ranzi, S. and P. Citterio, (1955) Exptl. Cell Research, 9, Suppl. 3, 287.

RAPER, K. B., (1956) Mycologia, 48, I69.

Rattenbury, J. C. and W. E. Berg, (i954) F. Morphol., 95, 393.

Reverberi, G., (1956) Experientia, I2, 55.

Reyer, R. W., (1954) Quart. Rev. Biol., 29, I.

Reyer, R. W. and L. S. Stone, (1955) F. Exptl. Zool., I29, 257.

Richenberg, H. V. and G. Lester, (I955) F. Gen. Microbiol., 13, 279.

Roberts, E., D. A. Karnofsky and S. Frankel, (1951) Proc. Soc. Exptl. Biol. Med., 76,289 .

Rosenthal, O., C. S. Rogers, H. M. Vars and C. C. Ferguson, (195I) J. Biol. Chem., I80, $83 \mathrm{I}$.

RothFels, U., (1954) F. Exptl. Zool., I25, I 7.

Rothstein, A. And R. Meier, (1949) 7. Cellular Comp. Physiol., 34, 97-i i 4.

Rudnick, D., Ed., (1954) Aspects of Synthesis and Order in Growth, Princeton University Press, Princeton, N. J.

Rudnick, D. And H. Waelsch, (1955) 7. Exptl. Zool., I29, 309.

Runnström, J., (1952) Modern Trends in Physiology and Biochemistry, E. S. G. Barron, Ed., Academic Press, New York.

Rupe, G. O. and G. J. Farmer, (i955) 7. Biol. Chem., $213,899$.

SAto, T., (1952/54) Arch. Entwicklungsmech. Organ., I46, $4^{87}$.

Sawyer, C. H., (1943a) F. Exptl. Zool., 92, I.

Sawyer, C. H., (1943b) 7. Exptl. Zool., 94, I.

Schechtman, A. M., (I955) in: E. G. Butler, Ed., Biological Specificity and Growth, Princeton University Press, Princeton, N. J.

Schmalmausen, I., (1926) Arch. Entwicklungmech. Organ., I08, 322.

Schmalhausen, I. and J. Stepanowa, (1926) Arch. Entwicklungmech. Organ., IO8, 72 I.

Schultz, J., (1953) Exptl. Cell Research, 4, Suppl. 2, I 7.

Schultz, J., (I959) Science, I29, 937.

Schultz, P. W. and H. Herrmann, (1958) 7. Embryol. Exptl. Morphol., 6, 262.

Scow, R. O., (I95I) Endocrinology, 49, 64I.

Scow, R. O., (1953) Am. J. Physiol., I73, 199.

Shaver, J. R., (I955) Experientia, II, 35I.

Shaver, J. R., (1956) Exptl. Cell Research, I1, 548.

Shen, S. C., (1955) Biological Specificity and Growth, E. G. Butler, Ed., Princeton University Press, Princeton, N. J.

Shen, S. C., P. Greenfield and E. J. Boell, (1955) 7. Comp. Neurol., 102, 7 I 7.

Siekevitz, P., (1952) 7. Biol. Chem., 195, 549.

Simpson, M. V. and S. F. Velick, (I954) F. Biol. Chem., 208, 6 I.

Singer, M., (1952) Quart. Rev. Biol., 27, I69.

Singer, M., D. Flinker and R. L. Sidman, (i956) F. Exptl. Zool., I3I, 267.

Sirlin, J. L., (1955) Experientia, II, II2.

Sirlin, J. L. And C. H. Waddington, (1954) Nature, I74, 309.

Spemann, H., (1938) Embryonic Development and Induction, Yale University Press, New Haven.

Spiegel, M., (i 955) H. C. Dalton, Ed., Ann. N. Y. Acad. Sci., 6o, 1056.

Spiegelman, S., (I948) Symposia Soc. Exptl. Biol., 2, 286.

Spiegelman, S., H. O. Halvorson and R. Ben-Ishai, (1955) Amino Acid Metabolism,

W. D. McElroy and H. B. Glass, Eds., The Johns Hopkins Press, Baltimore.

Spratt, N. T.Jr., (1947) 7. Exptl. Zool., I06, 345.

Spratt, N. T. Jr., (1948) 7. Exptl. Zool., I07, 39.

Spratt, N. T. Jr., (1954) Physiol. Revs., 34, I.

Steinberg, D. And C. B. Anfinsen, (1952) 7. Biol. Chem., 199, 25.

Steiniberg, D., M. Vaughan, and G. B. Anfinsen, (1956) Science, 124, 389.

Stone, L. S., (1955) J. Exptl. Zool., 129, 505.

Sussman, M., (1956) Biol. Bull., i Io, 9 I. 
Taylor, J. H., R. D. McMaster and M. F. Caluya, (1955) Exptl. Cell Research, 9, 460. Ten Cate, G., (1953) Arch. néerl. zool., Io, Suppl. I, 108.

Ten Cate, G. and W. J. van Dooremanlen, (1950) Koninkl. Ned. Akad. Wetenschap. Proc., Ser. $C, 53,894$.

Thorell, B., (1947) Acta Med. Scand., Suppl., 200, I.

Thorell, B., (1953) J. Embryol. Exptl. Morphol., 1, 235.

Toivonen, S., (1950) Rev. suisse zool., 57, Suppl. I, 41.

Toivonen, S., (1952) Experientia, 8, I20.

Toivonen, S. and L. Saxen, (1955) Exptl. Cell Research, 9, Suppl. 3, 346.

Tolmach, L. J., (1957) Advances in Virus Research, 4, 63.

Trinkaus, J. P., (1956) Am. Naturalist, 9o, 273.

Tyler, A., (I947) Growth, Io, Suppl. 7.

Tyler, A., (1955) Analysis of Development, B. H. Willier, P. A. Weiss and V. Hamburger, Eds., W. B. Saunders, Co., Philadelphia.

Urbani, E., (1955) Experientia, II, 209.

Vaughan, M. and C. B. Anfinsen, (1954) J. Biol. Chem., 21 I, 367.

Vennes, J. W. and P. Gerhardt, (1956) Science, I24, 535.

Villee, C. A., M. Lowens, M. Gordon, E. Leonard and A. Rich, (ig49) J. Cellular Comp. Physiol., 33, 93 .

Vogt, M., (1947) Experientia, 3, 156.

Waddington, C. H. and J. L. Sirlin, (I954) J. Embryol. Exptl. Morphol., 2, 340.

Waddington, C. H., M. Feldman and M. M. Perry, (1955) Exptl. Cell Research, 9, Suppl. $3,366$.

Waelsch, H., Ed., (1955) Biochemistry of the Developing Nervous System, Academic Press, New York.

Walter, H., D. W. Allmann and H. R. Mahler, (1956) Science, I24, I25I.

Watterson, R. L., I. Fowler and B. J. Fowler, (1954) Am. J. Anat., 95, 337.

Webster, G. G. and M. P. Johnson, (I955) 7. Biol. Chem., 217, 641 .

Weiss, P., (1947) Yale 7. Biol. and Med., 19, 235.

Weiss, P., Ed., (1950) Genetic Neurology: Problems of the Development, Growth, and Regeneration of the Nervous System and of its Functions, Univ. Chicago Press, Chicago.

Weiss, P., (1955) Biological Specificity and Growth, E. G. Butler, Ed., Princeton University Press, Princeton, N.J.

Weiss, P. And R. James, (1955) Exptl. Cell Research, 9, Suppl. 3, 38 I.

White, A., (1956) Hormones and the Aging Process, E. T. Engle and G. Pincus, Eds., Academic Press, New York.

Williams, M. A., W. A. DaCosta, L. H. Newman and L. M. Marshall, (1954) Nature, I73, 490 .

Willier, B. H., P. A. Weiss and V. Hamburger ( I955), Eds., Analysis of Development, W. A. Saunders Co., Philadelphia and London.

Woerdeman, M. W., (i955) Biological Specificity and Growth, E. G. Butler Ed., Princeton Univ. Press, Princeton, N.J.

Yamada, T., (1937) Arch. Entwicklungsmech. Organ., I37, I5I.

Yamada, T., (1940) Folia Anat. Japon., 19, 13 I.

YAMADA, T., (1958) Experientia, I4, 81.

Yamada, T. and K. Takata, (1955) Exptl. Cell Research, 9, Suppl. 3, 402.

Yamada, T. And K. Takata, (I956) Embryologia, 3, 69.

Zamecnik, P. C. And E. B. Keller, (1954) 7. Biol. Chem., 209, 337.

Zamecnik, P. G., E. B. Keller, J. W. Littlefield, M. B. Hoagland and R. B. Loftfield, (1956) 7. Cellular Comp. Physiol., 47, Suppl. 1, 81.

Zeller, C., (1956) Arch. Entwicklungsmech. Organ., I48, 3 II.

Zwilling, E., (1955) 7. Exptl. Zool., I28, 423.

Zwilling, E., (1956a) 7. Exptl. Zool., 132, I 57.

Zwilling, E., (1956b) 7. Exptl. Zool., 132, I 73.

Zwilling, E., (1956c) F. Exptl. Zool., 132, 219.

Zwilling, E., (1956d) 7. Exptl. Zool., I32, 241. 


\section{Chapter 6}

\section{GROWTH IN TISSUE CULTURE}

\section{Charity Waymouth}

The title of this chapter contains traps for the unwary, and it is well to examine and avoid these. What is "tissue culture"? A tissue (L. texere, to weave) in the histological sense, denotes an integrated assemblage of cells, the whole being a recognizable entity with an architecture which makes it more than merely a random aggregate of the component cells. Tissue culture (L. cultura, the original feminine of the future participle of colere, to till), insofar as it is etymologically related to "colony" (L. colonia, a band of husbandmen), perhaps connotes a means whereby, with skilful husbandry, a crop can be harvested from a tissue. The crop, of course, is cells. By general standards of good husbandry, it is to be expected that the harvest will be greater than the seed. And so the idea of "growth", in the sense of producing a crop appears to be implicit in the term "tissue culture". The traps are three. One is that, as it is practiced, tissue culture often involves the use of cells not integrated in the histological sense - that is, the technique covers a broader field than this, and is in reality a method for studying populations of cells. Secondly, the methods of tissue culture do not invariably result in an increased crop of cells. The colony studied may decline in numbers; it may survive; differentiation may occur; it may remain stable by reaching an equilibrium between cell death and cell proliferation; or, the colony may indeed increase. For the purpose of compiling their comprehensive bibliography of research in tissue culture, Murray and Kopech (I953) adopted the definition of tissue culture as "the maintenance of isolated portions of multicellular organisms in artificial containers outside the individual for considerable periods of time". This is the current usage. The third trap lies in the term "growth". In a volume devoted to this topic, it may be out of place here to labour the fact that "growth" is a hydra-headed monster. Let it suffice to say that increases of various kinds - in number, length, volume, mass, surface area-are comprised in this term, and that every use of the word should be examined, to find out which manifestation or combination of manifestations is alluded to in the case in point.

Tissue culture is a valuable method for the study of populations of cells, organized or unorganized, away from the parent organism. Interactions of the cells with each other and with the environment can be investigated and observed. It must not be lost sight of that a tissue culture consists of more than the explanted cells: the culture includes also the medium. Cells and medium form a system the 
parts of which are mutually dependent. As with the organism, so with a tissue culture, "Les conditions de la vie ne sont ni dans l'organisme ni dans le milieu extérieur, mais dans les deux à la fois." (Bernard, 1865). The principal advantages of tissue culture are two: (I) an advantage over fixed sections or smears, that the cells can be examined in the living state; and (2) an advantage over in situ studies, that, the cells being isolated from the parent organism, complicating factors introduced by the numerous regulatory and secretory mechanisms of the host are excluded. The environment can be controlled.

For many years, and perhaps this phase has not wholly been outgrown, tissue culture and its exponents were surrounded by a certain aura of fascination. Partly this was traceable to the fact, so surprising to physiologists of $5^{\circ}$ years ago, that cells taken from their host could not only remain alive but could grow and multiply. The wonderment of the achievement of potentially unlimited growth by the excised part so enthralled many investigators that, not content with potential immortality of the cells, they have most commonly used maximum growth as a principal criterion of success in tissue culture. The more neutral term "explantation", introduced by Roux and also used by Oppel ( $c f$. Craciun, I93 I) was swept away in the enthusiasm for perpetual proliferation. Tissue culture today comprises what was originally understood by "explantation" (survival in vitro) and "true" tissue culture (multiplication of cells). Lewis and Lewis (1924) were freer than many workers in the tissue culture field of preoccupation with "growth" per se. They stated that "the first factor which makes tissue culture possible is the ability of cells to survive for varying lengths of time after they have been removed from the organism" ... "Tissue culture depends primarily upon two factors: (I) the ability of the various types of cells to survive... and (2) the ability of the cells to migrate away from the explant into positions where their behavior and structure can be observed under favorable conditions. A third factor, the multiplication of cells, plays an important role in many investigations". From this it is evident that multiplication was not considered an essential factor for success by these authors. It should in fairness be said, however, that from the beginning there were many who used the new technique for detailed observations of the behaviour and structure of living cells. Tissue culture, with Harrison (I go6-07; 1906-08), and with the Lewises (Lewis and Lewis, I 924; Lewis, 1936) was a method making possible for the first time exact descriptions of cells during life. Though Carleton ( 1923 ) predicted that "the histological researches of the future will be based more on the physiology and dynamics of the cell than on descriptive morphology", there are still today histologists and cytologists whose familiarity with cells is based only on fixed preparations. Concepts of the cell built up from such studies are sometimes hard to reconcile with the picture seen in the living cell, and it is still not unknown for the observations made on living cells to be rejected in favour of the more familiar static picture of textbook histology.

The preoccupation with proliferation, to the neglect of other manifestations of the living cells in vitro, can be partly attributed to fascination with growth as a process, but also partly to the apparent ease with which "growth" could be measured. The potentialities of the method for studies on metabolism, and on persistence or differentiation of functions such as muscular contraction, ciliary 
motion, endocrine secretion became evident only later, when methods became available which were sufficiently refined to make quantitative studies in some of these fields possible. So, while interest in proliferative growth has produced a large literature, the reader is invited to keep in mind that proliferation is only one facet of the concept of "growth" in tissue cultures. Survival without overall increase in mass, in volume or in the number of the cell population (zero growth), or even decrease in population size (negative growth) may occur in cultures maintained over long periods of time. Their value as experimental material is not necessarily lessened because they are not increasing in size.

Tissue cultures have been made in many ways since the first simple beginning in a study of a fragment of frog nervous tissue in clotted lymph(Harrison, I 906-07; I 906-08; I 908). The embryonic chick was found to be a most convenient source of tissues, having the advantages that cells can be readily obtained aseptically from the egg, and that much activity was quickly evident. The use of clotted chicken blood plasma as a nutrient and support for the cells was introduced by Burrows (I9II); and Carrel (I9I3) produced a nutrient much superior for proliferation and active migration of chick embryonic fibroblasts by adding to the plasma an extract of chicken embryonic tissues.

Many variants of the now classical plasma-embryonic extract mixture have since been introduced, e.g. the use of heterologous plasma, serum and tissue extracts; of extracts from embryos of different ages, from adult tissues and from different organs and cells; of ascitic fluid; of ultrafiltrates and other fractions from both plasma and tissue extracts ( $c f$. review by Stewart and Kirk, I954). In addition, a variety of artificial media, some fully defined in chemical terms (e.g. White, I 946, I949; Morgan, Morton and Parker, I950; Healy, Fisher and Parker, I 954b, I 955; Trowell, I 955; Evans, Bryant, McQuilkin, Fioramonti, Sanford, Westfall and Earle, 1956; Waymouth, 1955), some serum-free (e.g. Baker and Ebeling, 1939; Waymouth, I956b), and some still supplemented with serum or other protein-containing fluids (e.g. Wilson, Jackson and Brues, I942; Fischer, 1948; Eagle, I 955a, b), have been contrived. The use of a plasma clot, long thought essential for the success of cultures of both organized and unorganized tissues, has recently become less common. Fluid nutrient media can now be used even for organized cultures, when the cultures are supported upon paper (Chen, I954; Trowell, I 954), agar (Wilde, I 948; Trowell, I952) or other inert material. Plasma clots still have an important place in tissue culture technique, but the possibility of growing many types of culture without them broadens the scope of possible experiments which can be made with the cells. For example, separation of the cells from the nutrient is difficult when the cells are embedded in a coagulum. Cells grown in a fluid medium can readily be separated from the nutrient and used for quantitative studies. The large scale cultivations of cells as hosts for viruses are now almost exclusively made in fluid culture media.

Tissue cultures can be made in a number of ways, and special techniques selected for different types of experiment. Very simple preparations made by placing a fragment of tissue in a drop of plasma on a coverslip sealed over a depression slide constitute the classical "hanging drop" cultures (Carrel, I929). Variation and elaborations on this method include the Maximow double coverslip technique 
and the use of perfusion chambers. Cultures made in Carrel or Earle type flasks, or in other flasks, bottles or tubes of various shapes, can be maintained for prolonged periods in the same vessel, and may be modified by agitation or rotation or by the introduction of "flying coverslips" into flasks or tubes. Descriptions of the principal technical procedures for tissue culture are to be found in various excellent handbooks (e.g. Strangeways, I924a; Fischer, 1930; Parker, 1950; Cameron, I950; White, I954; Hanks, Scherer, Fawcett, Leighton and Porter, I955).

Great experimental variability is possible with the tissue culture technique. Variations can be made in (a) the physical substrate (plasma, cellophane, paper, glass); (b) in the type of cell population ("pure"[clonal]lines of cells, cells of one type or mixed cell cultures, organized cultures); and (c) in the nutrient medium. The possibility of manipulation of all these factors makes for a very flexible experimental system. This very flexibility makes necessary a number of safeguards against unintended variations. It also necessitates the adoption of special criteria for evaluating each type of culture. Tissue culture techniques are so diverse that certainly no single set of rules can be set up for determining success in all possible cell systems. Growth in one of its manifestations is in many cases an important criterion of successful cultivation, but, as has already been mentioned, and will be further discussed below, this is a far from simple criterion. Cells in vitro, as in vivo, are engaged in a multitude of activities; a few or many of these may contribute to the overall process called "growth".

\section{EFFECTS OF ENVIRONMENT ON GROWTH IN TISSUE CULTURE}

\section{(a) Cell autonomy and interdependence. Single cell clones}

In tissue culture one can study either populations of similar cells, mixtures of cells as they come from the living host, or different cell types deliberately cultivated together. As has been stressed, the cells and their environment together form the tissue culture, both contributing to the behaviour of the cells, including their growth. The complete environmental needs of most types of cells still elude our understanding, and therefore our full control. The basic knowledge is gradually being built up which will enable a variety of cell types to be maintained or grown under fully standardized conditions, so that they may be submitted to exact and reproducible experimental procedures. Even now it is possible to manipulate the environment in many ways which affect the growth and behaviour of the cells.

One of the most interesting kinds of study which these techniques make possible deals with the mutual interaction of the cell and its environment. In the parent animal, the environment of the cell is composed of its "milieu extérieur" or "milieu extra-organique", and its "milieu intérieur" or "milieu intra-organique" of Claude Bernard (1865). Tissue culture makes possible a defined and controlled "milieu extérieur" for the study of growth.

Consideration of the course of cellular differentiation in embryogenesis makes it no surprise that one type of cell should affect the growth and development of 
another. The question of the excitatory and inhibitory effects of one tissue upon another, which was already a subject of much study before tissue culture was extensively practiced, led Carleton (I923) to conclude that "many of the phenomena of tissue culture would seem to fall into line with this conception of the mutual dependence of tissues". It is not quite so obvious from analogy with the events of normal tissue behaviour in vivo that, not only unlike cells making up distinct tissues, but also like cells, may interact. There is abundant evidence that such mutual interaction plays an important part in the survival and growth of cells in culture. With the question of mutual dependence is bound up the question whether a tissue culture should be considered simply as a population of single cell units, or whether the colony itself is more than the sum of its parts, i.e. whether it can be thought of as a primitive "organism". There are those who regard the cell as having no true autonomy, but as being inevitably dependent upon other cells in its environment. Fischer ( 1946 ) has argued against the theory that the cell is an elementary organism, and the organism a cell colony, because it had proved impossible to induce cell division in single metazoan cells isolated in a medium which, in amount and quality, was fully adequate for proliferation of a larger number of similar cells. In his view, tissue cultures "must be regarded as regenerating tissue fragments, primitive cell states, or organism-like systems with strong correlations, and not as colonies of independent cell individuals". There are those, on the other hand, like Sinnott (1939), who emphasize the autonomy of the cell which "on its own level ... displays a unity of organization independent of the organ above or the smaller units below". Both of these apparently contrary views contain part of the truth. Cell clones can now be obtained from single cells, as will be described below, but these are "colonies of independent cell individuals" only in the sense that each cell can become, under very stringent conditions, the parent of a new colony. Independence is relative - there is no doubt that a single isolated cell requires much more exacting conditions in order to survive or divide than the same cell in the presence of a large number of other cells. If, as Weiss has argued, cells can live only in the presence of the products of their own (or of other adjacent cell) secretions, the independence of the cell is to that extent limited.

Homogeneous suspensions, not dissimilar in behaviour to yeast or protozoan cultures, can be cultivated from metazoan cells. The most uniform cultures of this type are those derived as clones from single cells. But explants of most kinds of tissue, particularly those containing more than one cell type, are apt to have some degree of overall organization in culture. Abercrombie and Heaysman ( I 953) considered the colony obtained from fresh explants of chick embryo heart as an "organism" and, taking the cell as the unit, studied the "social behaviour" of the cells, in particular the question of how cell movement is influenced by the proximity of other cells. Individual cell velocity was found to fluctuate considerably during an observation period of several hours. Cells in established contact with others have a reduced velocity, and detachment of one cell from another is associated with acceleration of movement. A positive "contact-inhibition" was also postulated (Abercrombie and Heaysman, I954a), causing the fibroblasts to move preferentially in to unpopulated areas rather than over the surface of other cells. This 
hypothesis provides an explanation for the radial pattern of outgrowth which is typical in tissue fragments freshly explanted in a plasma clot. It should not be thought, however, that mutual repulsion is a general phenomenon. Holtfreter (1947) showed that cells from amphibian embryos adhere to and grow over each other in preference to growing on a glass surface; and Abercrombie and Heaysman (r954b) themselves have demonstrated that certain tumour cells (Sarcoma 37 and Crocker mouse sarcoma), grown together with neonatal mouse cells (skeletal muscle, bone, heart or liver), do not show "contact-inhibition" with respect to the normal cells, but penetrate among them in a manner which, it is suggested, is analogous to the invasiveness of malignant tumours in vivo.

Almost from the earliest time that successful tissue culture has been practiced, the idea of growing colonies from a single cell has been an attractive possibility. Attempts to realize this possibility were made as early as I 9 I 6 by Rous and Jones, and repeatedly since that time. Important questions about cellular differentiation and about cellular responses to different stimuli have been awaiting answers which depend upon a knowledge of cell lineage, or answers which become more meaningful when the cell population is more uniform than can be obtained from a mixed population of cells. The long period of failure to derive clones from single cells gave rise to many speculations on its cause. Burrows (I 923-24; I 924; 1926) was among the first to discuss the problem. He recognized that growth "may be a property of any fixed tissue cell when placed in the proper environment", and concluded that a certain density of cell population was a necessary part of this environment. The situation in tissue cultures was compared with that in yeast, and in some bacterial cultures, where it had been shown that a few cells in a large volume of fluid would not divide. Burrows suggested that, as in these examples, some material formed by the cells, which he named "archusia", had to accumulate in a certain concentration before growth could occur. He suggested, but apparently did not actually try, restricted volumes of medium. Fischer (1923) and Barnard (1925) had unsuccessfully made attempts to produce cultures from isolated cells, and in I 928 Harrison was still puzzled by the "inexplicable fact that single somatic cells isolated in tissue culture media do not proliferate".

A number of attempts was made to determine in a broader way the relationships between size of explant, volume of medium and successful cultivation (Fischer, I 923; Earle and Thompson, I930; Buchsbaum, I932; Ephrussi and Teissier, I932-33; Olivo, I 932). Buchsbaum, using fresh explants from 6-day embryonic chick heart in hanging drop cultures, cxamined the "growth" of cells in these cultures in the light of the hypothesis that, by analogy with the phenomenon of "allelocatalysis" applicable to protozoa, multiplication of one cell may be accelerated, in a restricted volume of fluid, by contiguity with other cells. These experiments were made with explants of $0.1-2.5 \mathrm{~mm}^{2}$, and with volumes of media of $0.004-0.12 \mathrm{ml}$. Within the limits of these variables, smaller explants increased in area relatively more than larger ones, in a $48 \mathrm{~h}$. period. Size of explant was of greater influence than volume of medium, though equal explants "grew" (in the sense of area increase) more in larger than in smaller volumes of nutrient. Buchsbaum concluded that the hypothesis that cells are mutually beneficial or that cell crowding was favorable to growth was not supported by these results. However, his explants 
were above the size which others have shown to be limiting for proliferation and moreover most of the expansion which he observed could have been due to migration rather than proliferation.

Earle, Sanford, Evans, Waltz and Shannon (195I) made a study of capacity for proliferation and survival in relation to inoculum size (using strain $\mathrm{L}$. mouse connective tissue cells), and to volume of nutrient (using a medium containing chick embryonic extract, horse serum and a balanced salt solution). These cells are capable of rapid and continuous proliferation in this medium, if large enough inocula are used. A minimum of $100,000-500,000$ cells $/ 2 \mathrm{ml}$ of this nutrient was found to be necessary to ensure $100 \%$ survival of the cultures; with inocula of $10,000-50,000$ cells, only $64 \%$ of the cultures proliferated, and with much smaller inocula (6oo cells), no cultures survived. Hollomon and Fisher (I950) have considered the limiting factors in such systems in terms of the geometry of the container, critical colony size and the concentration of a hypothetical substance produced by the cells themselves.

The first successful production of clones from single, isolated cells was achieved by Sanford, Earle and Likely in I $94^{8}$. They based their method on the old hypothesis that heavily populated cultures can modify or "condition" the medium, to make it adequate for growth. The same strain of mouse cells (strain L), which had been cultivated in liquid medium under perforated cellophane, was selected for the isolations. Cells from this strain were taken up in capillary tubes, ioo $\mu$ internal diameter, containing a film of plasma. Plasma and embryonic extract were drawn in, the capillary tubes sealed at both ends and incubated at $37.5{ }^{\circ} \mathrm{C}$ for I 5-20 h. Portions of capillary containing a single cell near the middle of a 4-5 mm length were selected, the ends cut open, and each tube placed in a $\mathrm{D}_{3.5}$ Carrel flask containing $0.5 \mathrm{ml}$ plasma and a total of $1.5 \mathrm{ml}$ of a "conditioned" medium (fortified with $100 \mathrm{mg} / \mathrm{I} 00 \mathrm{ml}$ glucose) from an actively growing culture. The proportion of cells which survived and grew into colonies was only about $4 \%$. This, however, was sufficient to enable several lines of cells, which have become important laboratory tools, to become established. For example, progeny from a single mouse subcutaneous tissue cell have given rise to several cell lines with different capacities to induce sarcomas on injection into animals of the parent strain $\left(\mathrm{C}_{3} \mathrm{H}\right)$ (Sanford, Likely and Earle, I954).

Another study of growth in relation to number of cells and amount of medium was made by Harris (I 955), who used freshly isolated rat fibroblasts brought into suspension by controlled treatment with trypsin. For his experiments he used a culture chamber enclosing a space for medium between two coverslips. The cells grew on one coverslip, and serial counts were made in selected, marked areas, to determine changes in cell number. Over short periods of time, he found that growth rates were independent of inoculum size (above a minimum size), though the total amount of growth was proportional to inoculum size. Inocula of $4500-5000$ cells in $0.43 \mathrm{ml}$ medium, corresponding to $16-\mathrm{s} 8 \mathrm{cells} / \mathrm{mm}^{2}$, evenly distributed on the coverslip, usually failed to survive. Use of medium (diluted serum) taken from cultures which had been multiplying for some hours did not permit growth of single cells or of small inocula. The concept of "adaptation" is therefore regarded by Harris as, if not untenable, at least an incomplete explanation of the facts. It is 
arguable that the period of "several hours" contact of medium with multiplying cells was too short to produce the hypothetical critical changes in the medium. But the most plausible kind of change would appear to consist of the production by the cells at their surface of a diffusible material depending for its effectiveness upon high concentration. This is compatible with Harris' observation that inocula smaller than I6-i 8 cells $/ \mathrm{mm}^{2}$ could grow if the distribution was uneven. Small clusters with a density of 20 cells $/ \mathrm{mm}^{2}$ or greater would proliferate. From this finding Harris concluded that the "minimum viable inoculum size appeared to be more closely related to the density of the cell population than to the ratio of the total population to the volume of medium". The hypothesis that "adaptation" occurs at the cell surface rather than in the medium is further supported by Harris' observations on the effect of repeated changes of the nutrient upon rapidly growing cultures. No interruption of growth occurred, either by suspension of cells already in the course of division, or by prevention of others from entering upon the process. The growth curve (cell number with time) was unchanged. This, as Harris states, "would appear to provide strong evidence against the view that when a growing culture of connective tissue cells is transferred to fresh medium the medium has to be "adapted" before growth can continue". Harris did, moreover, succeed in obtaining growth from single cells, either in "adapted" or fresh medium, by reducing the volume of fluid surrounding the cell to capillary dimensions, similar to those in the capillary tubes used by Sanford. Single, or a few, cells were placed between a coverslip and a serum-agar slab (resembling the arrangement described by Pulvertaft, I952, for microscopic study of bacteria) and colonies were successfully grown.

The first isolation of a clone, made by Sanford, Earle and Likely (1948), was a considerable technical feat. The proportion of successful isolations was low, but was nevertheless followed by isolations from several other strains. More recently it has been reported (Puck and Marcus, I955; Puck, Marcus and Cieciura, I 956), that clones of cells, from certain cell strains, can be obtained in $103 \%$ yield from single cells. The methods used are technically relatively simple. In the first method, a suspension of cells, prepared by the use of trypsin as a disaggregating agent in essentially the manner of Rous and Jones (I 916 ), is allowed to settle in a layer on the bottom of a Petri dish. This layer of cells is irradiated with X-rays in a dose sufficient to render the cells incapable of multiplication, but not sufficient seriously to affect their metabolism. Into this layer of immobilized "feeder" cells, the cells for clonal isolation are sceded. They are found to divide and give rise to colonies with (in the case of strain HeLa human cervical carcinoma) 100\% efficiency. The second method dispenses with the "feeder" cell layer, and is similar in principle to the Pulvertaft-Harris method. The cells are allowed to settle on glass and are overlaid with a soft agar gel. The agar acts in two ways (i) by increasing the viscosity of the medium and so decreasing loss of metabolites from the cells by diffusion and (ii) by depressing the tendency of the cells to migrate. These techniques open up for the future possibilities of isolating "mutant" clones from amongst a large population of cells, and for obtaining easily cultures of known cell lineage hitherto available only to those with much patience and elaborately equipped laboratories. 
(b) Chemical factors

Tissue culture started as a highly empirical practice, and has only gradually moved towards being a technique under much fuller experimental control. Nowhere is this more clearly exemplified than in the development of the media used. The complex biological fluids used at first-lymph, serum, plasma, tissue extracts - still form the nutritional basis for most cultures. But great advances have been made, through the early steps from homologous to heterologous sera and extracts, through the stages of simplification of the biological media by the use of fractionation procedures and through the building up of defined nutrient solutions, to a much fuller understanding of many specific nutritional requirements of living cells for growth.

Nutrition is a term nearly as elusive as growth when exact definitions are sought. The best that can be done is to define it as the act of supplying whatever is necessary for the growth, formation or proper condition of (in the present context) the cultures. Perhaps because of the indefiniteness attached to both "growth" and "nutrition", there has been some confusion between the two concepts. Growth does depend upon the adequacy of the environment to support it; growth is closely associated with, and in some cases is synonymous with, synthesis of new cellular substance; and the principal environmental factors upon which this synthesis depends are, by definition, nutritional. In other words, proper nutrition is a necessary, but not a sufficient condition for growth. With this confusion has been associated the somewhat unspecific and often very unhelpful concept of the "growth promoting substance". Assuming a set of conditions sufficient for maximum growth (and for any cell type there may be several different systems providing this possibility), alteration or omission of one single factor may make the environment less, or not at all, able to support growth. This missing or altered condition (which may be, for example, a single nutrient substance, or a change in some physical factor such as temperature) may then be called a "growth promoting factor". The search for specific growth promoting factors in biological nutrients has been fraught with many difficulties, and these have largely arisen because the multivariant nature of the tissue culture system has not been clearly enough appreciated. So the search for the variously named "trephones", "embryonin" etc. has been little more fruitful than the search for the philosophers' stone. Because it has not been understood that any one of many factors could be a limiting one, apparently contradictory statements have appeared; e.g. here, that heated embryonic extract is non-growth promoting; there, that it is active in this respect. Or, that growth depends on a high molecular fraction from embryonic extract and a low molecular one from serum; and that the high molecular fraction from embryonic extract is inactive, whereas the low molecular fraction from embryonic extract and the high molecular fraction from serum is active. These statements are all probably true in relation to the rest of the systems used in each case. It is not appropriate to speak of "the" requirement of any cell culture for any single nutrient, without defining the rest of the system, nor is it proper to search in a biological nutrient for "the" growth promoting factor.

Much work aimed towards producing an environment optimal in chemical terms for the growth of cells has been based on the concept that simulating the 
"natural" environment, namely (so it is assumed) blood plasma, represents the end to be achieved. It was thus that the "physiological" saline solutions were built up, in imitation of the ionic composition of plasma. The amino acid mixture recently adopted by Evans, Bryant, Fioramonti, McQuilkin, Sanford and Earle (1956) was very carefully modelled upon the pattern of amino acids found by analysis in an ultrafiltrate from embryonic extract and horse serum, a biological mixture which had been found to be an active growth supplement. This may be a useful step, but it may be questioned whether such thinking does not somewhat underestimate the synthetic and selective capacities of the cells themselves. It may even turn out that the number and amounts of amino acids found in biological fluids are not at all equivalent to the optimum mixture for growth. The activity associated with such a mixture may well represent a balance between necessary and unnecessary or even inhibitory factors.

Nevertheless, much useful qualitative information about factors which contribute to cell nutrition and growth has accrued from attempts to imitate natural media. But sometimes too close adherence to this idea has proved misleading. For example, almost all the physiological salt solutions in common use, one or another of which forms the inorganic basis for the composition of most artificial or synthetic nutrients which have been devised, are, as we have seen, imitations of the inorganic composition of blood plasma. It is of course well known that cells have a highly developed capacity for active uptake of certain ions; that the ionic composition within a cell is very different from that of its normal environment; and that moreover most ions, both anions and cations, are not free within the cell, but exist there in the form of complexes. It should not, therefore, be surprising to find that, for example, although cells have a capacity to concentrate phosphate from a medium low in phosphate ions, growth in an otherwise adequate medium can be stimulated markedly by increasing the external concentration of inorganic phosphate to a value considerably above that in normal serum (Waymouth, I954a). It is probable that one of the important contributions of the classical embryonic extract to the growth of cells lies in the fact that such extracts contain a high concentration of bound but easily available phosphate. This is a particular case; the reasoning is undoubtedly of general application.

With the above reservations in mind, we may briefly summarize the requirements, in chemical terms, of cells in tissue culture for growth. Detailed information is available in the reviews of Stewart and Kirk (1954) and of Waymouth (1954b). It appears to be generally accepted that growth will take place in media approximately isotonic with blood plasma (avian or mammalian), but a fairly wide range of hypo- and hypertonicity can be tolerated by some cells. The composition of individual ions can also be varied, and for some cells at least, and under certain conditions, considerably higher concentrations of certain ions than are found free in plasma are tolerated, or prove actually stimulatory (e.g. Owens, Gey and Gey, I956). Glucose is the usual carbohydrate supplied, and forms the principle source of energy for the cells. The most important low-molecular weight components other than salts and sugar appear to be amino acids, and several of the B group of vitamins which are important as precursors of the metabolically active coenzymes. In some instances, a purine derivative may be stimulatory; 
there is some evidence that peptides, and a suggestion that whole protein molecules (Winnick, 1952) may be assimilated by the cells. In brief, the requirements for grow th are probably relatively simple, and consist chiefly of (I) substances which act as energy sources; (2) those which act as building blocks for the synthesis of new protoplasm (and some substances, e.g. glucose and the amino acids may fall into both categories); and (3) components or activators of varous biocatalysts, e.g. the vitamins and some ions (see Chapter I).

\section{PHYSICAL FACTORS-TEMPERATURE, IRRADIATION, PH, TONICITY, PHYSICAL NATURE OF SUBSTRATE}

\section{(a) Effects of temperature on growth in tissue culture}

It is generally assumed that the optimum temperature for survival and growth of cells from a warm-blooded animal is the body-temperature of the donor animal. Quite early in the history of the tissue culture technique (Lambert, I9I2), the tolerance of tissue from the chick embryo heart to a wide range of temperatures was established. At $44^{\circ} \mathrm{C}$ diffuse outgrowth occurred for several days, though less than at $38{ }^{\circ} \mathrm{C}$ : at $46^{\circ} \mathrm{C}$ no growth took place, though subjection of the cells to $46{ }^{\circ} \mathrm{C}$ for $45 \mathrm{~min}$. did not prevent subsequent growth at $38^{\circ}$. Very severe inactivation took place at $50^{\circ}$, and complete suppression of all activity at $55^{\circ}$. These results were corroborated and extended by Pincus and Fischer (I93I), who found that the maximum duration of exposure to a supranormal temperature consistent with survival of chick osteoblasts fell with increasing temperature; e.g., even a prolonged sojourn at $42^{\circ}$ or $44^{\circ}$ was not lethal to these cells, but they were killed by $105 \mathrm{~min}$. at $47^{\circ}, 6 \mathrm{~min}$. at $50^{\circ}$ and $3.5 \mathrm{~min}$. at $52^{\circ}$. Lambert (19I2) also studied the sub) physiological range of temperatures. Chick heart fragments in hanging-drop beat more regularly and for longer at $29^{\circ}$ than at $38^{\circ}$; outgrowth was slower at $21^{\circ}-27^{\circ}$ than at $38^{\circ}$ and not so extensive, though it continued for a longer time. A culture kept at $26^{\circ}$ for 3 days and then cooled to $-\mathrm{I}^{\circ}$ for $\mathrm{I} 2 \mathrm{~h}$. resumed beating $45 \mathrm{~min}$. after return to $26^{\circ}$. Lambert's cultures survived $-4^{\circ}$ for $4^{8} \mathrm{~h} .,-10^{\circ}$ for I o min. and $-18^{\circ}$ for $2 \mathrm{~min}$., though $15 \mathrm{~min}$. at $-18^{\circ}$ was lethal. In a further study, Lambert (I9I3) stored fragments of chick or rat embryonic tissue, embedded in a plasma clot in hanging drop preparations, at various temperatures $\left(-7^{\circ}\right.$ to $\left.+21^{\circ}\right)$ for different periods of time (2-6 days). The cultures were then returned to $37^{\circ}$ or $37.5^{\circ}$ and the effects upon subsequent outgrowth of cells observed. The freezing point of chicken plasma is $-7^{\circ}$, and those of the chick cell cultures kept in the range $-7^{\circ}$ to $-6^{\circ}$ which froze did not recover, though unfrozen cultures showed occasional outgrowth up to 8 days. Viable cells were found after maintenance at $-\mathrm{I}^{\circ}$ to $\mathrm{O}^{\circ}$ for $\mathrm{I} 0$ days; at $+6^{\circ}$ to $+7^{\circ}$ for $\mathrm{I} 6$ to $\mathrm{I} 8$ days; at $+\mathbf{I} 2^{\circ}$ to $+14^{\circ}$ for I 2 days; and at $+18^{\circ}$ to $+2 \mathrm{I}^{\circ}$ for II days. Rat tissues were more sensitive to freezing than those of the chick. Lake (I916, I9I7) made a study similar to Lambert's, with similar results. Later studies on storage of cells at very low temperatures have shown the importance for viability of (i) the nature of the suspension medium (Schere" and Hoogasian, I954; Taylor and Gerstner, 1955; Parshley and Deterling, 1956) and (ii) the rates of freezing and thawing (Smith, I952; Smith and 
Parkes, 1954; Pomerat and Moorhead, I 956). The most effective media are those in which ice crystal formation is minimized. Cooling and thawing in saline solutions, or in sucrose, permits little or no survival. Serum is a better protective medium, but the most satisfactory suspension fluids contain glycerol or ethylene glycol. Luyet and Gonzales (I95I) obtained abundant outgrowth from chick heart which had been frozen rapidly to - I $95^{\circ}$ in $30 \%$ ethylene glycol, and rapidly thawed. Ovarian granulosa cells survived slow cooling better than rapid freezing: $50-70 \%$ of these cells survived slow freezing to $-79^{\circ}$ in serum; the survival was even better in serum plus glycerol (Smith, I952).

Different types of cell respond differently to brief exposure to low temperatures (thermal shock). Pomerat and Lewis ( I 953) exposed chick skin and skeletal muscle to temperatures ranging from $-3.4^{\circ}$ to $-39.2^{\circ}$ by rapid cooling and immediate thawing, and prepared cultures from the treated fragments of tissue. The cells frozen either in Gey's balanced salt solution or in serum showed different lower temperature limits for survival. Muscle fibres emigrated after exposure to $-\mathrm{I} 8^{\circ}$ or $-20^{\circ}$; skin epithelium tolerated $-30^{\circ}$, and spindle cells resisted temperatures of near $-40^{\circ}$. Similarly, there was a difference, though perhaps not very significant, in resistance to low temperatures of human newborn epithelium $\left(-24 \cdot 4^{\circ}\right)$ and adult human skin $\left(-20.5^{\circ}\right)$. Human spindle cells, like those of the chick, could withstand lower temperatures $\left(-30^{\circ}\right)$.

Mitosis can apparently persist at much reduced temperatures. Spear (1928) observed that the duration of mitosis in chick cells was doubled by a $10^{\circ}$ drop in temperature $\left(3^{\circ}-28^{\circ}\right)$, and was still further delayed at lower temperatures. Nuclear divisions were still seen by Bucciante $(1927-28)$ at $0^{\circ}$, though without cytoplasmic division, so that multinucleate cells were formed. The fact that cultures kept at temperatures a few degrees below physiological (e.g. $\left.30^{\circ}-34^{\circ}\right)$ have a lower metabolic activity and mitotic rate has been put to practical use by Gey, Hanks and Barrett (1948) for maintaining cultures with a minimum of care. In an appropriate medium, chick cells were kept for 6 o days at $30^{\circ}$ without renewal of the medium (Hanks, I 948).

Tissues from cold-blooded animals can grow at rather a wide range of temperatures, and an interesting study has been made of the effect of variations in temperature on the growth of cultures of frog kidney carcinoma by Lucké, Berwick and Nowell (I953). Using Ebeling's (I92 I) area measurement method, the total new growth area was plotted against time, in four temperature ranges $\left(20^{\circ}-35^{\circ}\right)$. The temperature coefficient of growth, $Q_{10}$, was computed from the formula:

$$
\log Q_{10}=\frac{\left(\log K_{1}-\log K_{2}\right)}{\Delta t} \times \text { Iо }
$$

where: $K_{1}=$ rate of growth at the higher temperature

$K_{2}=$ rate of growth at the lower temperature

$\Delta t=$ difference of temperature between two measurements.

$Q_{10}$ was found to decrease slightly with time over a $2-7$ day growth period. The average value of $Q_{10}$ was $2.5^{\circ}$ (range I.9-3.9). 


\section{(b) Effects of visible light}

Normal connective tissue cells are not greatly affected by exposure to visible light of the intensities necessary for ordinary observation or even for high power photomicrography. Goodrich and Scott (1922) were among the first to study this question, and they compared hanging drop cultures of chick heart fibroblasts grown in the dark with similar cultures grown under four days continuous exposure to polychromatic light of an intensity of 270 foot candles. They observed no detectable difference. A careful study of the effects of light upon living cells was made by Earle (1927, I 928). Leucocytes responded by increased motility, followed by cellular degeneration. Chick fibroblasts suffered rapid degeneration in the presence of red blood corpuscles (which themselves respond by baemolysis), but irradiation of the fibroblasts for periods of up to $24 \mathrm{~h}$. in the absence of haemoglobin caused only slight damage. There appears to have been no systematic study of the effects of visible light on a variety of cells, which might reveal differences in sensitivity of different cells to different intensities and different wavelengths. Roffo (r 933 ) detected a greater sensitivity in cells from a rat sarcoma than in normal chick fibroblasts. The effects of visible light of various wavelengths at constant intensity ( 90 foot candles) upon cells from the chick embryo was studied by Frédéric (1954b). Degeneration began after $30 \mathrm{~min}$. exposure to short wavelengths $(436 \mathrm{~m} \mu)$. With increasing wavelengths, the response was slower, and at $55^{6} \mathrm{~m} \mu$ the effect might be reversible up to $60 \mathrm{~min}$. At 57 I $\mathrm{m} \mu$, the changes took three times as long, and exposure to $623 \mathrm{~m} \mu$ appeared to produce no effect, even after $10 \mathrm{~h}$. illumination, except acceleration of the mitochondrial movements. The intensity used was about 20 times that required for high power photomicrography, so it is concluded that yellow-green light is perfectly safe for such purposes.

\section{(c) Effects of ultraviolet irradiation}

Levaditi and Mutermilch (I9I3b) found differences in response to general ultraviolet radiation between chick fibroblasts and amoeboid cells from the chick spleen; the former were damaged by 20-30 min. irradiation, which had no effect on the motility of the latter. The effects of UV upon chick mesenchyme cells were studied by Mayer and Schreiber (1934), who grew their cultures upon quartz coverslips and exposed them to ultraviolet radiation of $366 \mathrm{~m} \mu-230 \mathrm{~m} \mu$ at varying intensities and for varying lengths of time. The longer wavelengths (366 $\mathrm{m} \mu)$ had no effects even after very high dosages. With decreasing wavelengths, the sensitivity increased. This is in agreement with the general proposition ( $c f$. Loofbourow, I948; Giese, I950) that it is the range 200-310 m $\mu$ that most readily kills or injures cells, whereas the near UV is innocuous.

\section{(d) Effects of ionizing radiations}

Cells growing in tissue culture provide excellent material for studying the effects of irradiation upon various cellular functions. Different cell types have very different sensitivities to radiation, and the response varies with the dose and with environmental conditions such as $\mathrm{pH}$, temperature and oxygen tension (Patt, I 955 . Relatively small doses may slow down the cell multiplication rate, produce mitotic alonormalities or completely inhibit cell division. With increasing doses, 
the onset of cell degeneration is accelerated. Cell migration and metabolism may proceed practically unimpeded after low dosages, and cells which are prevented from dividing may continue to grow in size (Price-Jones and Mottram, I9I4; Halberstaedter and Doljanski, I937; Paterson, I942; Brues and Reitz, I95 I Reid and Gifford, I 952). For example, cells of the HeLa strain of human cervical carcinoma, subjected to $1000 \mathrm{r}$, can yield a population consisting solely of giant cells, and even after $10,000 \mathrm{r}, 5-20 \%$ of the irradiated cells develop into giant cells with a volume about is times that of the normal HeLa cell (Puck and Marcus, i 956). Very high doses, of the order of $100,000 \mathrm{r}$, may be required to cause immediate cessation of all cell activity, though $2000-4000 \mathrm{r}$ are sufficient to cause irreversible injury and ultimate complete elimination of a cell population. A review of the effects of radiations upon cells in tissue cultures has been made by Stroud and Brues ( 1954 ).

\section{(e) Effects of $p H$}

As with the composition of nutrient solutions, so with $\mathrm{pH}$, it has been usual to imitate host blood. Therefore media for mammalian and avian tissues have usually been prepared at about $\mathrm{pH} 7 \cdot 4-7.6$. However, as the range of $\mathrm{pH}$ which most cells will tolerate is quite wide, at least for short periods of time ( $c f$. Lewis and Felton, 1922) and as cells actively metabolizing glucose rapidly lower the $\mathrm{pH}$ of their environment unless the medium is very well buffered, it is common practice to start with a medium of rather higher $\mathrm{pH}($ e.g. $\mathrm{pH} 7.8-8.0)$. Phenol red, which in concentrations of up to about $2 \mathrm{mg} / \mathrm{I} 00 \mathrm{ml}$ is not toxic to living cells, is customarily incorporated into tissue culture media, so that changes in $\mathrm{pH}$ can be easily detected visually.

Not much evidence is yet available about the true $\mathrm{pH}$ optima for the maintenance and growth of different cell types, though these will undoubtedly be found to vary. Adult skin epithelium has been shown (Parshley and Simms, 1950) to grow best in a medium containing one part of serum or plasma diluted with two

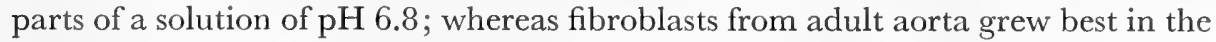
range $\mathrm{pH} 7.3-7.9$ and were somewhat inhibited at $\mathrm{pH}$ 6.9. Growth of a mammary tumour was achieved at the unusually high $\mathrm{pH}$ of 9.3 (Pikovski, 1954); normal mammary gland or connective tissue cells were severely damaged or destroyed at this $\mathrm{pH}$. Femurs from 7 day chick embryos develop $19 \%$ more bone in 14 days at $\mathrm{pH} 7.0-7.2$ than at $\mathrm{pH}$ 7.8-8.0 (Paff, I948).

\section{(f) Effects of tonicity}

Here again it has been the usual practice to simulate the "natural" environment of the cells, namely plasma. And here also there is evidence that cells can tolerate environments of ionic strengths, and therefore of tonicities, which vary widely froin the norm. Reports of the use of slightly hypotonic solutions appear in the literature from the time of Carrel and Burrows ( I I I ). It has been common practice to incorporate hypotonic solutions-e.g. Pannett and Compton's (1924) solution which has a total ionic strength approximately one half of that of Ringer's or Tyrode's solutions - into tissue culture media. It should not, however, be inferred from this that hypotonicity is the optimum condition for cells in vitro. The 
principal reason for the employment, especially in cultures of small volume, of hypotonic media is that in such cultures some evaporation is apt to take place, which, if the medium was isotonic at the start, would leave the cells in a hypertonic environment. Hypotonicity is in general less traumatic to cells than hypertonicity. Brues and Masters (I936) observed reversible swelling of fibroblasts in media down to 0.2 and $0.3 \% \mathrm{NaCl}(34-5 \mathrm{I}$ m.eq. $/ 1)$, with maximum migration at $0.86 \% \mathrm{NaCl}$ ( I $50 \mathrm{~m}$. eq. $/ \mathrm{l}$ ) and irreversible damage at $2.00 \%(342 \mathrm{~m}$. eq. $/ \mathrm{l})$.

\section{(g) Effects of the physical nature of the substrate}

For a long time the idea was firmly entrenched that all cells, but particularly connective tissue fibroblasts and various kinds of epithelium, required a particular type of supporting substrate. A coagulum of lymph or plasma appeared to be ideal, because cells could migrate throughout the fibrous meshwork or on the surface of the clot. Such a substrate does indeed effectively support the migration of many types of cells, though others are quite unable to grow in such an environment. Strains of cells have been isolated which will actually not tolerate a plasma clot, but which grow luxuriantly in fluid media (Evans, Earle, Wilson, Waltz and Mackey, I 952). The use of a clot has the additional disadvantages that it is composed of materials which the cells may metabolize, and that its composition is variable and indeterminate. Many efforts have been made to find truly inert substrates for various kinds of cells. Agar is rather better in this respect than plasma, though it may carry nutrient substances adsorbed upon it, and has been used for diverse types of tissue cultures by Smyth (I9I4-I5), Wolff and Haffen (1952), Trowell (1952) and Puck, Marcus and Cieciura (I956). Hotchin (1955) has successfully substituted methylcellulose for agar. Fibrous supports of several kinds have been tried, e.g. spider's web (Harrison, I9I4), silk and cotton fibres, lens paper (Beard and Rous, I934; Trowell, I954; Chen, I954), cellulose fibres and films (Pearce and Paterson, I952; Moscona and Moscona, I953), nylon cloth (Goossens, I953, I954), and glass fibres (Warner, Hanawalt and Bischoff, I 949; Frisch, 1952). Many, but not all, cells can spread upon a glass surface and are able to proliferate there. A few cell types have been grown in fluid suspension, being maintained so by methods of agitation. Among inert substrates other than glass which permit cell attachment and migration is cellophane, first used by Fischer (1930), and widely adopted in the form of perforated sheets since the introduction of these by Evans and Earle (1947). Other plastic surfaces, e.g. Formvar (Wirth and Barski, I947) and Araldite (Barski, I954) are satisfactory.

All these substrates can be used in conjunction with a fluid nutrient. This is not to claim that all types of cultures can best be grown in fluid media, though it is often a great advantage to be able to separate the cells from the medium, and the possibilities of doing so have been widened to include many types of unorganized and organized tissues, among the latter being organ rudiments (Chen, I954), whole lymph nodes (Trowell, I952, I954) and tumours (Leighton, I95 I, I 954). A three dimensional type of growth is made possible by the use of pieces of cellulose or gelatin sponge (Leighton, I 95 I, I 954; Leighton and Kline, I 954; Leighton, Kline, Belkin and Tetenbaum, I $95^{6}$ ), in the interstices of which a high degree of tissue organization is possible, so that the growth characteristics of certain tumours 
in vitro are recognizably similar to the histological sections made from the tumours as they grow in vivo.

Particularly promising substrates for growing cells appear to be the bacterial cellulose membranes introduced by Moscona and Moscona (1953) and the sheets of reconstituted collagen of Ehrmann and Gey (1956). The use of collagen, a normal substrate for many cells in vivo, enables cells to be studied on a "natural" surface.

Under certain conditions, some cells will proliferate in suspension, unattached to each other or to a solid substrate. Owens, Gey and Gey (I954) have achieved rapid growth (four-fold increase in five days) of the MB III lymphoblasts with the cells and medium kept in a state of constant agitation, by end-over-end rotation of the culture tube at $3^{8} \mathrm{rpm}$ (2280 rph). Measurements have been made by Earle, Schilling and Bryant ( 1954 ) of the rates of proliferation of strain L mouse cells in conventional roller tubes (rotating round the long axis of the tube) at speeds from less than io rph up to $1200 \mathrm{rph}$. Using the regular medium of horse serum and embryo extract diluted with balanced salt silution, and estimating growth by nuclear counts, it was found that the cell populations at the end of three weeks in cultures rotated at speeds between 150 and $1200 \mathrm{rph}$ were $50 \%-70 \%$ greater than in tubes rotated at the more usual rate of 6-9 rph. But even at the higher velocities, most of the cells remained adherent to the glass of the tubes. To maintain the cells in suspension, Earle, Schilling, Bryant and Evans (1954) slightly increased the viscosity of the medium by adding methylcellulose. In this type of suspending fluid, and at a velocity of $2400 \mathrm{rph}$, none of the strain L cells remained attached to the glass. Most of them were also unattached to each other, though groups of up to about io cells could be found. At lower rates of rotation, though most of the cells were detached from the tube, they formed free floating aggregates, the size of which appeared to be approximately proportional to the velocity of rotation. Clumps $0 . \mathrm{I}-0.5 \mathrm{~mm}$ were formed at $300 \mathrm{rph}$. Still more effective cell suspensions have been made by shaking the cultures on a rotary shaker at 210 or $230 \mathrm{rpm}$ in the methylcellulose-containing medium (Earle, Bryant, Schilling and Evans, I956). Under these conditions, strain L mouse fibroblasts, HeLa human cervical carcinoma and clone 1469 mouse liver epithelium proliferate very actively. Slower rates permit cell aggregation, impede oxygenation and give poorer growth. This type of system allows efficient utilization of nutrients, e.g. $2 \mathrm{~g}$ of new cells could be produced in 4 days with the expenditure of $325 \mathrm{ml}$ of medium.

\author{
III. RELATIONSHIP OF GROWTH IN VITRO TO MAINTENANGE, \\ DIFFERENTIATION AND FUNGTION \\ GONDITIONS FOR GROWTH, SURVIVAL AND DIFFERENTIATION
}

It is found empirically that certain conditions can be created, of which the principal ones are adequate nutrition and appropriate temperature, in which cells can proliferate rapidly over an indefinite period. In general, the more vigorous the proliferative growth, the less readily will the cells in a particular population mani- 
fest those properties which distinguish them from other cells. Appropriate choice of culture conditions can determine whether the cells either multiply rapidly, or survive without proliferation, or manifest or develop their functions and differentiated characteristics.

Survival of cultures, usually with a very low rate of proliferation, results when the total metabolic activity of the cells is lowered, either by creating a partial nutritional deficiency with respect to that necessary to maintain growth, or by reducing the temperature. The pioneers in attempts to create a system in which the cells would survive in dynamic equilibrium were Fischer and Parker (Fischer, I 926; Fischer and Parker, I 928-29, I 929). Parker (I936) was able to cultivate chick fibroblasts in a healthy but non-multiplying state for over a year, by using a medium consisting of diluted serum. Addition of embryonic extract to such cultures immediately stimulated proliferation. Brues, Rathbun and Cohn (I944) also restricted growth by use of a nutritionally incomplete medium. Among others whose aim was to maintain cells at a very low rate of proliferation, sufficient only for renewal, and so to set up conditions similar to those obtaining in the adult organism, were Gey, Hanks and Barrett (I948) and Hanks (1948). Their cells were kept in a plasma-embryonic extract medium at a reduced temperature $\left(32^{\circ}-\right.$ $28^{\circ}$ ) for several months, and required only occasional replenishment of the medium. This method has practical advantages for maintaining stocks of cells without frequent attention.

Tissue culture techniques afford possibilities for studying both growth and differentiation of cells and cell populations, and the interdependence of the two processes. The literature on tissue culture contains, in various forms, statements that "cells growing in tissue culture dedifferentiate" and that "growth and differentiation are incompatible". In evaluating these assertions, one has first to clarify the meaning attached to the word "differentiation" by individual authors. The review of Bloom ( 1937 ) contains a discussion of these problems, preceded by careful definitions of terms. Cellular differentiation is defined as "the process which results in specialization of the cell as measured by its distinctive actual and potential functions", and is "sharply distinguished from those cellular changes, no matter how striking they may be, which are only temporary reflections of the reaction of the cell to new stimuli". Differentiation is really a relative term, for there are all degrees of difference between the totipotent cell and one which can be recognized by various criteria as highly specialized. It is a common experience to see, in cells in tissue culture, a loss of characteristic morphological features, and this has often been loosely termed "dedifferentiation". But, as Strangeways ( 1924 b) said: "It should be clearly understood...that the term 'dedifferentiation" is here used only to imply the latency, not the loss, of those potentialities which enable cells to assume the specific structure and histological architecture of the organ of which they form part". It is best, unless change of potencies can actually be demonstrated, to assume that cells in tissue culture retain the inherent capacities which their parents had at the time of explantation, and to beware (Weiss, I $949 \mathrm{a}, 1)$ ) of equating visible changes of form with irreversible changes in character. Very striking changes of morphology can take place in cultivated cells, so that for example Teiss and Fawcett (I 953) were able to see, in cultures of chicken leuco- 
cytes, cells resembling fibroblasts, mesothelium and even epithelium. The appearance of cells may in some instances be more a function of the medium which surrounds them than of the origin of the cells themselves. Parker (1950) commenting upon Carrel's observations, remarks that "blood serum impresses upon leucocytes the physiological and pathological peculiarities of the individual providing the serum". Weiss applies the term "modulation" to the "loss of some actual functions without change of potencies" (Bloom, 1937; Weiss, I939), in contrast to true dedifferentiation, which is the "loss of some actual functions and an increase in potential functions". It is in fact perhaps better to think of the variable in cellular differentiation, not as the cell as a whole, but as the particular cellular function or functions which can be demonstrated. As Harrison (I 940) said: "That the isolated cells often achieve so little is no indication of limitation in what they can do, since the conditions under which they are isolated are too restricted. Until such cells are tested under a great variety of conditions, or better still all such as are possible, their full capacity will not be known".

Persistence of function, and the retention of characteristics peculiar to the explanted tissue, are common features in cultures, even after many generations of rapid proliferation and even though the cells undergo drastic morphological changes which make it at first sight improbable that the specific properties are still inherent in the new generations of cells. For example, immunological specificities are retained (Landsteiner and Parker, I940); ability to produce hormones persists (Anderson and Haymaker, I935-36); and liver cells are still able to store glycogen (Westfall, Evans, Shannon and Earle, I953). The most numerous and convincing examples of the persistence of specific characters are the tumour cells. After many generations in tissue culture such cells may still produce, on inoculation into susceptible hosts, tumours histologically indistinguishable from the parent neoplasms. There exist exceptions to this generalization. In the first place, in a number of cases, normal cells after prolonged cultivation in vitro, have undergone alteration and acquired the capacity to produce tumours (Earle, I 943; Gey, Gey, Firor and Self, I949; Goldblatt and Cameron, I953; Landschütz, I953). Secondly, the ability of some cultures of tumour cells to grow in appropriate host animals has been lost or greatly attenuated (Sanford, Likely and Earle, I954). However, the great majority of tumour cell cultures retain for months or years the capacity to induce tumours like those from which the cultivated cells originated.

While most cell strains, grown in tissue culture over a period of years, have remained stable in specific properties, e.g. in ability to produce specific substances (hormones by parathyroid, glycogen by liver cells), or in ability to produce tumours in homologous animals, others, kept continuously multiplying, have altered in their growth behaviour and have taken on new characteristics (Gey and Bang, I949; Parker, I955; Gey, I956), e.g. ability to multiply rapidly in fluid suspensions, or ability to produce tumours. Acquisition of a new character (e.g. malignancy) does not exclude the possibility of loss by the same cells of some other property which might be examined (e.g. ability to produce fibres). In summary it may be said that stability is more common than change of potencies in vitro; that in most cases little or nothing is known about factors determining 
stability or change, though it is to be hoped that in the future these may come under experimental control.

Bloom (I937) and Willmer (1954) conclude that, although cellular multiplication and cellular differentiation are distinct processes, each usually takes place only in the absence of the other. Histodifferentiation, or the process by which cells become organized into tissues, as distinct from cellular differentiation, may also take place in growing cultures. Fell (195I) has reviewed this topic, to which she has been a major contributor for more than 20 years. From the pioneer work of Champy (I9I4), Rienhoff (I922), Drew (I923a, b) and others on the interrelations of epithelium and mesenchyme has developed the more precise studies of recent years, notably of Fell and Mellanby (I 952, I953) and of Grobstein (1953a, b, c; I 955). Great possibilities are beginning to be seen and exploited in manipulating the environment to control or alter the direction of histogenetic activity in vitro. Spratt (1948), for example, made a study of the effects of some specific nutrients on the development of early chick embryo in vitro, and demonstrated the overriding importance of an energy source (carbohydrate) for morphogenesis and growth. Differences between cell types in quantitative and qualitative substrate utilization and especially in ability to incorporate the carbon skeleton of glucose into various amino acids, have been studied and discussed by Markert (1955). Limb bone rudiments from the chick, explanted by the classical technique of Fell and Robison (1929), grow and differentiate in a manner closely similar to normal development in vivo. When exposed to an excess of vitamin A, they undergo degenerative changes quite similar to those seen in hypervitaminotic animals, suggesting that the effect of vitamin A in vivo may be directly on the bones. Perhaps even more striking is the effect of vitamin A on tissue (containing both dermis and epidermis) from the body wall of the 6-7 day chick embryo. In a normal medium, feather germs and melanocytes develop and keratinization of the epidermal epithelium occurs (Fell and Mellanby, I953). With excess of vitamin A, the epithelium assumes the character of a secretory membrane. Ciliated cells also develop, and the skin tissue in this medium takes on a remarkable histological similarity to the normal mucus-secreting ciliated membrane of the nasal cavity. On withdrawing the excess vitamin A, the explants (after a brief acceleration of differentiation of the secretory layer) resume their differentiation into squamous epithelium. It was shown by Weiss and James ( 1955) that only brief and intermittent exposures to vitamin A were necessary to swing the differentiation in the direction of mucus-secreting epithelium. Vitamin A appears to exert upon cultures of chick fibroblasts a purely proliferation-stimulating effect, as judged by a significant increase in mitotic rate (Lasnitzki, I955).

The variables growth, survival and differentiation have been studied from the viewpoint of their responses to nutritional factors by Wolff and his school in cultures of syrinx and gonads. Certain individual amino acids favour all three variables in cultures of chick embryo syrinx, whereas other amino acids, which stimulate growth, diminish the proportion of differentiation and survivals (Em. Wolf, I 955). Similarly, Stenger-Haffen (1955) found that certain amino acids specifically encourage differentiation of chick embryo gonads. Wilde (I955) has studied the functions of phenylalanine in the chemical processes involved in the 
differentiation of amphibian neural crest cells in tissue culture. The evidence indicates that the $\alpha$-amino group of this amino acid is necessary for the differentiation of pigment cells, and the phenyl ring for differentiation of ectomesenchyme. The character of growth in some cultures may be altered, without varying the composition of the medium, by simply changing the $\mathrm{pH}$. Under conventional conditions in vitro, the mouse mammary tumour studied by Pikovski (I954) lacks organization and produces a strong growth of connective tissue. If the tumour cultures are maintained at $\mathrm{pH} 9.0-9 \cdot 3$, ducts and acini develop, and the resulting highly differentiated cultures of glandular tissue can be maintained, with transplantation every three or four weeks, for many months. They retain their malignancy.

Part of the environment of any cell is the other cells in the vicinity, and important studies have been made on the interactions of cell types in tissue culture. When two cell types are grown in apposition, one culture may affect the growth of the other. Normal and malignant fibroblasts have been shown to be stimulated by leucocytes; normal mouse fibroblasts were inhibited by several sarcomas but stimulated by carcinomas (Ludford, I940; Ludford and Barlow, I 944). On the other hand, the maintenance of some types of tumour cell in vitro requires the presence in the culture of a supporting population of connective tissue cells, perhaps corresponding to the stroma of tumours in vivo. Thus De Bruyn, Korteweg and Van Waveren (I 949) and De Bruyn (I949a, b) maintained over a period of years cultures of a mouse lymphosarcoma, and inoculation of these cells into suitable mice produced tumours. It was found possible to make separate cultures of the two types of cells found in the cultures of this tumour, namely lymphoblasts on the one hand and the associated fibroblasts on the other. The fibroblasts produced no tumours. For the first few months in tissue culture, the lymphoblasts were capable of producing tumours, but thereafter this capacity was lost and the cells became "altered" and non-malignant. Recombination of the two strains of cells did not result in restoration of the tumour-producing capacity of the cultures. Another kind of mutual dependence of normal and tumour cells was demonstrated by Bichel (1952). In this case the growing leukaemic cells destroyed the normal cells. The Yoshida ascites tumour of the rat is dependent, not merely for maintenance of neoplastic character but for survival upon the presence of normal cells (Schleich, I956). Tissue cultures prepared from this tumour after subcutaneous passage contained a mixed population of tumour cells, macrophages and fibroblasts. Tumour cells which lost contact with fibroblasts did not survive; if cultivated independently they ceased to divide within Io days, and began to degenerate. A dramatic revival occurred if normal rat fibroblasts were then added to the cultures, even if the normal and tumour cells were not in contact. Extracts of fibroblasts were ineffective. A single tumour cell inoculated into a normal fibroblast culture was able to give rise to a luxuriant growth of tumour cells. The interaction and interdependence of normal cells in tissue cultures in relation to tissue differentiation is a field of growing importance. As early as I9I4 Champy had observed that the presence of mesenchyme "prevented dedifferentiation" of organized epithelia. Drew's (1923a, b) experiments in this area were discussed by Strangeways (I924b) and he already at that time concluded that: "although a 
group of cells may possess the necessary potentialities, may be grown in favourable body fluids or other media, and may be in a state of reproductive quiescence, they are, nevertheless, incapable of differentiation into their specific tissue if this is of a complex type. A fourth factor is required, viz., interaction with cells of the other tissues composing the organ". The thorough investigations of Grobstein (I953a, b, c; 1955) have beautifully demonstrated and more fully substantiated this statement. Normal morphogenesis of the mouse salivary gland depends upon the presence of both mesenchymal and epithelial elements from the rudiment being explanted together. In cultures of submandibular epithelium placed together with mesenchyme from other parts of the embryo, morphogenesis does not occur. Metanephric rudiments, as was first observed by Rienhoff and by Drew, show a similar dependence-i.e. no morphogenesis takes place if either epithelium or mesenchyme is cultivated alone, though in this case several non-metanephric epithelia can induce tubule formation by metanephric mesenchyme.

That histodifferentiation of explants in tissue culture is not merely the result of development and growth of cells already spatially oriented is shown particularly well by the experiments of Moscona (1952) and Moscona and Moscona (1952). Cells from trypsin-disaggregated tissues of the early chick embryo were found to regroup into structures analogous to the normal organization of these tissues. A suspension of cells from chick mesonephros gave rise to tubules similar to normal urinary tubules. Weiss and James (1955) have shown that the dissociated cells of chick skin, reduced to a suspension of predominantly single cells, will reassociate and by 16 days in vitro develop typical feather germs.

The study of the interactions between cells growing in tissue cultures, at the morphological and at the chemical levels, is already giving form to the not yet clearly defined concepts of induction and differentiation, and will undoubtedly continue to uncover the basic mechanisms responsible for these processes (see Chapter 4).

IV. DEFINITIONS OF GROWTH IN TISSUE GULTURE

As at other biological levels, so in tissue culture, it is possible to define growth in terms of a number of parameters. To estimate something corresponding to an agreed definition of growth, it is necessary to select one or more of these parameters for measurement. The nature of the variability and multiple character of the growth process in tissue cultures has been discussed and given due emphasis by Tompkins, Cunningham and Kirk (1947); Weiss (1949a, b) and Waymouth (1949, 1952, 1954b).

For a long time (about 1910-1940) the method of explanting small fragments of tissue into a plasma clot containing embryonic tissue extract, and observing the radial outgrowth of cells which took place approximately in one plane, was the predominant tissue culture technique. "Growth" of such cultures was usually assessed by the methods of Ebeling (I9I4, I92I) (see below), which consist in making daily measurements of the radius or area of the areola of cells. These methods are very simple to apply. They do not, however, measure a simple 
quantity. As Lewis and Lewis (1924) reported of the hanging drop type of culture, "If the culture is successful the cells migrate from the explant... The mass of migrant cells, which may or may not increase in number by division, are termed growth or outgrowth." This statement draws attention to a general problem which will appear in the discussion below of the different methods of measuring "growth" -namely that "growth" sometimes refers to the end and overall result of a number of distinct processes. On the other hand, the same term is sometimes explicitly stated to apply to the measurement of a single variable. In the zone of cell outgrowth from a hanging drop culture, the relative contributions of migration and of cell division cannot be judged from an area measurement alone, and in fact many such cultures increase markedly in area without undergoing any appreciable increase in total cell population. Willmer and Jacoby ( 1936 ) showed that increasing concentrations of embryonic extract raised the rate of migration as well as the rate of mitosis of fibroblasts in hanging drop cultures. A most important experiment was made by Doljanski and Goldhaber (1945) to demonstrate the effect of embryonic extract on migratory, as distinct from mitotic, activity. The separate effects of such extracts upon the two cell functions were studied by allowing cells which had been made incapable of mitosis to migrate. Chicken fibroblasts, in hanging drop cultures, were irradiated with X-rays, and then transplanted to Carrel flasks. Growth curves were made, using the surface area method. The dose of X-rays applied was $25,000 \mathrm{r}$, i.e. 2.5 times the amount thought necessary to inhibit cell multiplication completely. The irradiated culture was divided into two equal halves. The fragment placed in chicken plasma diluted with Tyrode's solution increased in three days to about 8 times the initial area and then remained constant up to about 6 days. The sister half placed in a similar medium supplemented with embryonic extract to $30 \%$ had increased to about 14 times at 3 days, and continued to increase steadily to about 20 times at 6 days. Expressed another way "the surface of cultures grown in a medium to which embryo extract was added exceeded by $200 \%$ (average of I 7 experiments) the growth area of cultures developed in plasma alone". Besides being a clear demonstration of the separability of the processes of migration and cell division, this was also the first unequivocal refutation of the previously widely accepted idea that the principal effect of embryo extract in a tissue culture medium was upon cell division.

For each type of experiment, the appropriate method of assessing some aspect of the growth complex must be chosen. A great diversity of cell and tissue types can be cultivated, so it is not surprising that particular procedures demand special methods. Some of these methods measure one parameter, some the resultant of several. The principal criteria of growth are: (I) linear change, change in surface area, mass or volume of cell population; (2) change in number of cells or of cell nuclei; (3) number of mitoses per unit area, per unit volume or per Iooo cells; (4) ability to subdivide the culture at regular intervals without diminution of population density, from which growth can be inferred; and (5) change in some component of the cells, e.g. total $\mathrm{N}$, nucleic acid $\mathrm{P}$, rate of oxygen consumption or glucose utilization.

Possible methods of measuring growth will be discussed below under the general heading of (I) physical methods and (2) chemical methods. 


\section{METHODS OF ASSESSING GROWTH IN TISSUE GULTURE}

A great amount of effort and ingenuity has been put into the task of designing methods for following quantitatively the course of a growing culture. Different methods, based on different criteria of "growth", have been devised for cells cultivated in different ways. One of the technical limitations restricting the kinds of methods available to the earlier students of growth in tissue culture was the almost universal use of a plasma coagulum as a support for the cells. Methods of growth measurement had therefore to be contrived to take this into account, that is, to be suitable for application to cells embedded in a plasma substrate. Much less frequently, separation of the cells from the clot was attempted. This was generally considered impracticable, and the majority of methods was therefore based on acceptance of the inseparability of the cells from the medium.

It is significant that a textbook on tissue culture published in I93 I Craciun, I93 I), when the technique was being widely practiced, particularly for studies on proliferation, mentions only three methods of growth measurement (by area measurement, cell counts and tissue weight). Craciun points out that only strains of cells maintained for a long time in standard conditions give replicate area measurements reproducible within $\mathrm{IO} \%$; that errors can occur because growth is not planar in the plasma-clot cultures, and because the thickness of the culture and the degree of cell crowding in the peripheral zone are not taken into account.

Nevertheless, area measurement was apparently the only method regarded as practical by Craciun; cell counts, and weighing the newly migrated cells, were dismissed as too difficult. Even as recently as I950, the same three methods were still the only ones considered feasible by one authority (Cameron, I950), though she also mentions the possible use of estimations of the physiological activity of the living cells. At the same time (Signorotti, Hull and Kirk, I950) it was still being lamented that none of the customary methods of tissue culture fulfilled the criteria necessary for strictly quantitative work. These authors considered reducing the plasma clot, still generally thought to be essential for supporting fibroblast cultures, but they did not entertain the possibility of dispensing with it altogether, though this had already become a fairly usual practice (White, I946; Evans and Earle, 1947). Earle and his collaborators were amongst the first to make determined attempts to improve conditions for quantitative study by employing a fluid medium and an inert substrate, either perforated cellophane or simply the glass of the culture vessel (Evans and Earle, I 947; Earle, Evans and Schilling, I950; Schilling, Earle and Evans, 1950). Once the fluid nutrient could be easily and completely separated from the cells, several good methods of evaluating growth became possible. Many cell types have now been grown in this way, in fluid media directly on glass (White, I946, I949, 1955; Earle, Evans, Sanford, Shannon and Waltz, I95 I Ehrmann and Gey, i 953) or in fluid suspension (Earle, Schilling, Bryant and Evans, I954; Owens, Gey and Gey, 1954), and the range of quantitative methods has been correspondingly extended.

Some methods of growth measurement can be used upon the undisturbed living cultures; some require disturbance of the system or even its total destruction, so that serial measurements upon a single culture are not possible. Each method has its 
merits in particular circumstances and choice has to be made in the light of these. The methods discussed below are broadly divided into the physical methods, many of which involve little or no disturbance of the living cells; and chemical methods, most of which require the death of the cells before the estimation can be made.

\section{(a) Linear or areal measurements}

The outgrowth from an explanted fragment in a hanging drop or a flask culture can be estimated by measuring one or more diameters of the roughly circular zone of cells (Ebeling I9I4), or by measuring the superficial area covered by the cells (Ebeling, I 92 I). Ebeling himself recognized some of the limitations of his method, and stated his belief that "the only means of measuring the growth of a fragment of tissue accurately would be to measure the increase in its weight". Ebeling emphasized the necessity of ensuring that the thickness of the cultures was as uniform as possible, so that the increases of area might be approximately proportional to increases in mass. However, this condition is extremely difficult to fulfil in practice, and moreover, even if equal thickness could be achieved at the outset, the thickness seldom remains constant. Cultures thin out at the centre as migration takes place (Davidson, Leslie and Waymouth, I949), and the rate at which this occurs may not be constant or comparable in pairs of cultures set up for comparison. The limitations of area measurement for assessing growth have been very fully discussed, e.g. by Parker (I950) and by Willmer (I954), but still need constant emphasis if the method, which has its uses under certain strictly defined conditions, is not to be abused. Ebeling adopted the measurement of radius (by ocular micrometer) or area (by projectoscope and planimetric measurement) because he regarded weight measurements as impossible. Two fragments can be compared by means of area measurements, and various forms of "relative growth index" derived. If $A_{c}$ and $A_{e}$ are the initial areas of the fragments of tissue, and $B_{c}$ and $B_{e}$ the areas of the cultures at the end of the growth period, the ratio

$$
\frac{B_{e} A_{c}}{B_{c} A_{e}}
$$

may be derived. This is the growth index used by Ebeling. A somewhat different relative growth index was introduced by Fischer (1923), namely:

$$
\frac{\left(B_{e}-A_{e}\right) A_{c}}{\left(B_{c}-A_{c}\right) A_{e}}
$$

Both of these methods of comparison were regarded by Mayer (I939) as unsatisfactory, because they gave a distorted picture of growth if, as is usually the case, there is a difference in size between the original fragments. In Mayer's (I 939) comprehensive survey of data up to that time on the growth of cell colonies in vitro, attention is drawn to the fundamental differences between fresh explants of fibroblasts and passage cultures, particularly the greater uniformity of growth of the latter. Six factors contributing to the "balance of growth" which is estimated by increase in area are named: 
(i) centrifugal migration of cells

(ii) mitotic cell division

(iii) increase in cell size

(iv) centripetal migration

(v) death of cells

(vi) decrease in cell size

The contribution of each to the "growth" of an individual culture is variable and the relative contributions of each are extremely unequal. Recently Ehrmann and Gey (I953) have used area measurements for studying the growth of an established strain of rat fibroblasts which grows in a monocellular layer on the glass of a roller tube. Though they are here still dealing with the "balance of growth" resulting from these six factors, in this case the objections raised by differences in thickness are absent.

Several studies have been made on the effect of initial size upon the growth (area) of fibroblast cultures. Earle and Thompson (1930), using hanging drop cultures, studied variations in culture area with size of explant. With fresh explants, division of a fragment $\mathrm{I} \times \mathrm{I} \times \mathrm{I}$ mm into $4-24$ fragments made little difference to the final size. Fragments divided into two halves gave greater final areas; divided into 36-64 fragments, the growth of each was severely reduced; and when each culture was less than $\mathrm{I} / 64$ th of the original, only a few cells $(\mathrm{I}-4 \mathrm{0})$ migrated out in $48 \mathrm{~h}$. Passage cultures gave a larger final area than fresh explants when subdivided below I/36th of the colony size, but division into 64 or more pieces also in this case greatly limited the outgrowth area. Earle and Thompson's conclusion was that the absolute increase in the area of a culture varies more or less directly to the size of the explant. More recently, Firket (I954) has analyzed the growth of fresh explants and of established cultures of chick fibroblasts in relation to size of explant. He showed that, whereas with fresh explants the relative growth (in terms of superficial area, $S$ ) $\log S_{48} / S_{\text {。 }}$ was well correlated with initial area, for cultures established for several passages in vitro, there appeared to be complete independence between initial and final size.

Other factors were taken into consideration in a study by Ephrussi and Teissier (1932-33). According to these findings, the final sizes of colonies of fibroblasts, grown in Carrel flasks in media without embryonic extract, are proportional to the initial size, and small colonies stop growing sooner than large. By contrast, with colonies in media containing embryonic extract, those of smaller size catch up the larger during the first day in vitro, and the final sizes are nearly independent of the initial size. The final size was found to be greater, the higher the concentration of embryonic extract in the medium. Fischer (1946), using ample embryonic extract in the medium, found that the growth of small fragments ceased earlier than that of large fragments. However, Hull and Kirk (1950b) recalculated Fischer's data on the basis of increase in area per unit initial area, and failed to agree that the final area was proportional to the surface area at the beginning. Migration being so important a contributor to final area, it is pertinent to recall that Willmer (1927) found the amount of migration of cells from the eleven day 
chick intestine to be proportional to the glucose concentration, up to about I $000 \mathrm{mg} / \mathrm{I} 00 \mathrm{ml}$. It is evident from this kind of consideration that the nature of the entire system (size and origin of tissue, type and concentration of medium) must be carefully taken into account in evaluating area measurements, which are by no means simple to interpret.

Meaningful measurements of length can be taken of certain kinds of organ culture, e.g. the chick limb-bone rudiments of Fell and Robison (1929) and the rat femora of Ray, Mosiman and Schmidt (I954), since migration is here limited to connective tissue cells surrounding the bone rudiment itself, and increase in linear dimensions of the bone represent a useful measure of total tissue.

\section{(b) Mitotic counts}

Apart from area measurements, the commonest technique employed over the years for evaluating growth in unorganized cultures, e.g. hanging drop and flask cultures of fibroblasts, epithelial cells and macrophages, has been the mitotic count. This method can, like area measurements, be applied repeatedly to individual cultures without disturbing their growth.

The presence in a culture of cells in mitosis would appear to be incontrovertible evidence of proliferative increase. It is, however, by no means easy to convert this evidence into a quantitative criterion of growth. Cunningham and Kirk (1942) and Tompkins, Cunningham and Kirk (1947) analyzed the use of mitotic counts in growth measurements, and they stressed the many variables which make it nearly impossible to estimate the true rate of increase of a cell population from such counts. For example, the rate of cell division varies widely with time (Willmer, I $\left.933^{a}, \mathrm{~b}\right)$, so that there may be cycles of high and low mitotic activity. And it is necessary, in order to convert mitotic counts into accurate information on the rate of formation of new cells, to know the mitotic rhythm, and the duration of mitosis and of intermitosis, all of which may fluctuate. These factors have been studied, e.g. by Gaillard (I 935) and by Neukomm and Richard (I952), and serve chiefly to emphasize that in interpreting growth measurements based on mitotic counts, the multiple factors involved must be kept in mind. Nevertheless, many studies of growth have been based on the use of some type of "mitotic index" (Champy, I9I4), usually a comparative figure relating the proportion of mitoses in a total population of cells to a similar ratio in a control culture. Perhaps the most satisfactory type of index of this sort is based on the kind of evidence obtained from serial photography, at short intervals, of selected areas, as practiced by Willmer ( $933 \mathrm{a}$ ) and Willmer and Jacoby ( 1936 ) on colonies of fibroblasts and by Jacoby ( 1937 ) on chicken macrophages. This method involves taking photographs at 5 or $6 \mathrm{~min}$. intervals for periods of several days of areas containing initially soo or more cells. A growth index is derived, which takes into account the fact that the cell population increases as a result of both cell division and emigration of cells from the explant. The number of cells $X$ which underwent mitosis during the time $t$ in hours is subtracted from the number of cells $b$ in the field at the end of the experiment. The mean is taken between this number and the number of cells $a$ in the field at the start of the period. The number of mitoses is expressed as a percentage of this 
mean figure, and the whole divided by the duration $t$. The "growth index" is thus:

$$
\frac{\frac{x}{a+(b-x)}}{2} \times \frac{100}{t}
$$

According to Willmer (I933a, b), the concentration of embryonic extract affects migration rate, but appears to have little influence on the mitotic index of heart fibroblasts. The mitotic index of cultures of periosteal fibroblasts, on the other hand, is more affected by the concentration of embryo extract. It is important to bear in mind that treatments which arrest mitosis (e.g. radiation, colchicine) may increase the mitotic index at a particular time, without this increase being in any way correlated with growth by increase in the cell population ( $c f$. Stroud and Brues, 1954).

An attempt to achieve a more complete picture of the growth of explants in hanging drop cultures than is given by mitotic counts alone was made by Tompkins, Cunningham and Kirk (I947), who measured, as well as the number of dividing cells and the number of resting cells per unit area, the culture thickness, average cell size and number of cells per unit volume of the culture. Their most important observations were that (a) mitosis is more prevalent near the periphery in young $(24-48 \mathrm{~h}$.) cultures; (b) average cell size in the interior may be $\mathrm{I} / 2-\mathrm{I} / 4$ that at the periphery; (c) the maximum number of cells per unit volume occurs half way between the centre and the periphery and (d) thickness increases markedly from periphery to centre.

\section{(c) Possibility of subdivision}

A culture which can be divided into two or more parts, each of which is capable of reaching the size of the parent part within a given period, is evidently growing. A quantitative measure can be derived from information on the time intervals between subdivisions, because a rapidly growing culture, unless the rate of cell destruction is unusually high, can be subdivided more frequently than a slowly growing one ( $c f$. Perry, Evans and Earle, I955). Over fairly long periods of time (i.e. of the order of months) this can be a very accurate measure of the nett rate of growth of cells in tissue culture.

\section{(d) Cell and nuclear counts}

Where the size of the cell population is taken as the basis for growth measurements, the method of counting whole cells or cell nuclei is applicable. It is not often technically possible to count the total number of cells in a culture, though this has been done by Puck, Marcus and Cieciura (1956) as a means of evaluating the rate of growth of clonal colonies derived from single cells. Usually, sampling from a uniform cell suspension is necessary. Success therefore depends upon being able to make such a suspension, and the recent technical advances have led to the now common routine practice of harvesting, suspending and sampling cells for inoculating new cultures as well as for enumeration (Evans, I95 I ; Evans, Earle, Sanford, Shannon and Waltz, r95I).

The method of isolating cell nuclei by treatment with citric acid of cells from a 
culture grown in a fluid medium, was devised by Sanford et al. (Sanford, Earle, Evans, Waltz and Shannon, I95I; Sanford, I95I). The culture is incubated for I h. at $37^{\circ} \mathrm{C}$ with o. I $M$ citric acid. This releases the cells from the glass and strips the cytoplasm from the nuclei. The nuclei are then collected by centrifugation, washed, and resuspended in a fluid whose viscosity is increased by addition of methylcellulose. The suspension is well shaken to ensure uniform distribution during sampling, and the nuclei, stained with crystal violet, are counted in a haemocytometer. This method is admirable for rapidly growing and healthy cultures, but has its disadvantage: nuclei from dead as well as living cells may be included in the count. The Sanford method has been widely applied, occasionally with some modifications. For example, Eagle ( I955a, b) cut down the treatment with citric acid to so or $15 \mathrm{~min}$., and further shortened the method by omitting washing and centrifugation of the cells after treatment with citric acid. Parker, Healy and Fisher (I954) improved the yield of nuclei by a brief pre-treatment with $\mathrm{I} \%$ tannic acid, to coagulate the nuclei before adding the citric acid. This group of workers (Healy, Fisher and Parker, I954a, b) found excellent agreement between nuclear count (Earle's strain L cells) and deoxyribonucleoprotein phosphorus measurements in growing cultures. Eagle, Oyama, Levy, Horton and Fleischman ( 1956) later adopted the principle of Zwilling's (1954) technique for facilitating dispersal of the cells by use of sodium versenate (disodium ethylenediamine tetraacetate). The cells so dispersed were suspended in citric acid containing crystal violet and samples taken for counting in a haemocytometer. In a similar way, Ginsberg, Gold and Jordan (I955) have used versene acid (ethylenediamine tetraacetic acid) as an aid to dispersing cells from roller tube cultures, in preparation for counting in a haemocytometer.

A number of investigators has made use of the observation of Rous and Jones (1916) that living cells are relatively resistant to digestion by trypsin, to obtain suspensions of cells, free from dead cells and from intercellular protein. Among those who have recently published data depending on cell counts from trypsindispersed cultures are Rinaldini ( 1953, I 954); Scherer, Syverton and Gey (1953); Syverton and Scherer (1954); Cailleau and Kirk (1954); Neuman and McCoy (1955) and Puck, Marcus and Cieciura (I956). Harris (I955) has made a critical study of the effect of trypsin upon living connective tissue cells from the rat. He concluded that the resistance of this type of cell to trypsin was relatively low, and found that considerable damage could be caused by prolonged treatment with trypsin. The changes produced were reversible in the early stages, but continued action produced irreversible effects, culminating in complete cell disintegration. The method should therefore be applied with caution and not indiscriminately; the resistance of particular cell types should be studied carefully. Harris suggests that the conditions used by some investigators might result in $50 \%$ of non-viable cells. In his own experiments, he limited the concentration $\left(5^{\circ} \mu \mathrm{g} / \mathrm{ml}\right.$ of crystalline trypsin) and the time for which the enzyme acted (I o min.), to avoid damage to the rat fibroblasts. He counted freshly isolated samples of the cells from trypsinized suspensions in a haemocytometer, cultivated them in special small culture chambers permitting oxygenation of the cells over a period of several days, and made serial counts of all the cells in several marked areas of the cultures as a 
means of assessing growth. Cell suspensions from chick embryo tissue have been prepared by simply bringing the $\mathrm{pH}$ to 9.0-9.2 (Mookerjee, I953); and rat liver cells can be readily dispersed without trypsin by incubating the tissue at $37^{\circ}$ for Io min. in mildly acid solution (Longmuir and apRees, I956). These methods might be applicable, with suitable modifications, to the dispersal of other cells.

Cells grown in the suspended state, e.g. in the "tumbling tube" cultures of mouse lymphosarcoma MB III (Owens, Gey and Gey, I954, I956) or the strain of monkey kidney cells which multiplies in suspension (Graham and Siminovitch, I955), can be sampled directly for enumeration.

\section{(e) Tissue mass}

The direct measurement of tissue mass is one of the older methods by which attempts were made to assess changes in the amount of tissue in a growing culture. Several investigators who made respiration and other chemical measurements on growing cultures, tried weighing in order to be able to express their results in terms of wet or dry weight.

Wet weight estimations were made by Lipmann (I933), after freeing the tissue from plasma by treatment with $0.25 \mathcal{N}$ sodium hydroxide. Such estimations when applied to tissue cultures are open to the objections which apply generally to wet weight measurements - namely the difficulty on the one hand of freeing the tissue from adherent fluid, and on the other of avoiding undue desiccation of the tissue.

The determination of dry weight also involves difficulties. Because water makes up $80 \%$ or more of the total tissue mass, the absolute mass of the conventional type of tissue culture is rather small to give precise measurements of dry weight. Moreover, precautions must be taken to maintain a moisture equilibrium during weighing.

Cultures have been separated from their plasma substrate for dry weight estimations by use of dilute alcohol (Meier, I 93 I) or pepsin digestion (Laser, I 932). The magnitude of the quantities handled may be judged from the fact that Meier estimated the initial dry weight of his cultures to be 0.0 I $5 \mathrm{mg}$. Laser used the dry weight for comparison with area measurements in fibroblast cultures, and showed that, whereas area stopped increasing after 4 days, dry weight continued to go up until at least Io days, and that the shapes of the two types of growth curve were quite different.

Gemmill, Gey and Austrian (1940) worked with tissue in roller tubes, and measured both wet and dry weights. In 45 determinations on rat sarcoma cells, they found that the dry weight averaged I $1.7 \%$ of the wet weight, with a range of $6 . \mathrm{I}-\mathrm{I} 8.2 \%$ 。

Mass cultures of the kind now regularly grown in agitated flasks by Earle, Bryant, Schilling and Evans (1956), in which a single flask may contain nearly 6. I $0^{9}$ cells $(23.6 \mathrm{~g}$ wet weight) make it possible to apply wet weight measurements to whole cultures or to samples taken from individual large flasks.

\section{(f) Cell or culture volume}

Because of the technical difficulties of weighing the cells, Gemmill, Gey and Austrian (1940) tried substituting measurement of the total volume of cells in 
their cultures, by centrifuging them in haematocrit tubes. This method has many advantages for cells which can be freely obtained in suspension, either by simple mechanical means, or by treatment with trypsin, versene or other aids to dispersion. It has been found particularly suitable for estimating increases in cell population in rapidly growing cells in fluid media (Waymouth, 1956a) and for comparing the effects of different nutrient media on cell proliferation (Waymouth, 1956b). The VanAllen microhaematocrit (VanAllen, 1925) is of suitable dimensions for measuring the volume of the cell population from a $\mathrm{D}_{3} .5$ Carrel flask culture.

\section{(g) Nephelometry}

Where cells can be kept in a state of uniform suspension, nephelometry can be used, as with bacterial cultures. This method has been applied to embryonic chick heart cells by Rinaldini (I954), and to monkey kidney cells by Youngner (1954). Comparison between optical density and counts of cell nuclei by the Sanford method, made by Youngner, revealed that the optical density values were in general below those expected. This was attributed to varying degrees of aggregation. A correction could be applied for this.

\section{(h) Metabolic measurements}

Attempts have been made to use respiration and glycolysis as measures of growth. The principal difficulty encountered in trying to correlate metabolism with growth is that the maintenance metabolism forms a large and variable proportion of the total, and to make proper corrections for it is extremely difficult. Major fluctuations in metabolic activities may result from a number of variables which may or may not be independent of growth, e.g. in response to changes in available oxygen, in concentrations of certain enzymes and coenzymes, or concentration of glucose or other substrates. Since isolated particles from cell cytoplasm are capable of respiring, the existence of measurable activity is not even a reliable indication that viable cells are responsible for the process measured. Metabolic measurements cannot therefore be recommended as a means of assessing proliferative activity. Early attempts to correlate glycolysis with growth (Meier, I93I ; Laser, I932; Lipmann, I933; Lipmann and Fischer, 1932) are of historic interest only. However, these and later measurements of the metabolism of cells in tissue cultures (e.g. by Gemmill, Gey and Austrian, I 940; Danes and Leinfelder, i 95 I ; Danes, Christiansen and Leinfelder, I 953; Danes, I955a, b; Prop, I 954; Harris and Barclay, I955) are of interest in demonstrating differences in overall metabolism of various cell types, and the relative capacities of certain cells in vitro for aerobic and anaerobic glycolysis, though they bear no valid relation to growth measurements. Thus for example, Harris and Barclay (1955) compared glucose utilization and lactic acid production by rabbit macrophages under aerobic and anaerobic conditions. They calculated the oxygen uptake of the cells from measurements based on a logarithmic relationship between oxygen tension in the medium and the current produced between a platinum cathode and a non-polarisable (lead) anode. Changes of $1-2 \mu$ moles/l could be recorded without amplification. This appears to be a sensitive means of measuring respiration in tissue cultures. 


\section{(i) Glucose utilization}

Closely related to determinations of glycolysis depending upon respirometric techniques are the methods involving estimation of glucose utilization (or lactate production) in the medium from growing cultures. Such measurements have been made by Gemmill, Gey and Austrian (1940), by Wilson, Jackson and Brues (I 942) and by Willmer (I 942). Lactate production is, by and large, correlated with utilization of glucose, but neither is a function of growth alone.

\section{(j) Nucleic acids}

On the assumption of proportionality between the nucleic acid content of the cell and the amount of cellular protoplasm (Berenblum, Chain and Heatley, r 939), Willmer (1942) introduced the method of determining the nucleoprotein phosphorus content of tissue cultures, as a measure of the "total number of cells present, together with some estimate of their size". The method of Willmer was adopted by Davidson and Waymouth (I 943, I 944, I 945, I946) and later extended (Davidson, Leslie and Waymouth, I949) to measurement of both ribonucleic acid phosphorus (RNAP) and deoxyribonucleic acid phosphorus (DNAP) in the same cultures (Waymouth, I95I). Hull and Kirk (I95Oa), taking as a working definition of growth the "increase in metabolizing protoplasm over the amount implanted", made RNAP and DNAP estimations, after Schmidt-Thannhauser fractionation, on roller tube cultures of chick heart tissues, in a manner essentially similar to that of Davidson, Leslie and Waymouth ( 1949). They found also (Hull and Kirk, I 95ob) that incorporation of ${ }^{32} \mathrm{P}$ into DNA was most active at the time of maximum mitotic rate and that ${ }^{32} \mathrm{P}$ uptake into the cultures as a whole was similar to the uptake into the nucleic acid fractions (Hull and Kirk, I950c). Gerarde, Jones and Winnick (1952b) studied ${ }^{32} \mathrm{P}$ uptake and turnover in the DNA and RNA of growing cultures, and reported high turnover of DNA during growth. Incorporation of adenine-8- ${ }^{14} \mathrm{C}$, free or in labelled nucleic acids, into growing tissue cultures was studied by Lu and Winnick ( $1954 \mathrm{a}$ ). The labelled adenine was used for synthesis of both adenine and guanine, of both RNA and DNA. In a subsequent report, Lu and Winnick (I954b) found that incorporation of adenine into the nucleic acids could be abolished by respiratory inhibitors, and they claimed that conditions which stimulate active growth also stimulate active uptake of adenine and thymidine. However, it cannot be excluded that conditions of active metabolism without growth might favour uptake of adenine and thymidine. In the system of Lu and Winnick, the two appear not be to separated.

Barski and Manigault (I95I) used a photometric method for measuring the amounts of nucleoprotein in cultures stained in a quantitative manner with toluidine blue. The method as described estimates total nucleoprotein, though the authors suggest that the principle could be extended to differential determination of RNA and DNA, using specific stains for each. Variations between replicates were high $( \pm 25 \%)$, but the method was nevertheless applicable to crmparison of the effects of various media on growth (Barski, Maurin, Wielgosz and Lépine, r 95I).

Ribonucleic acid is known to be present in most cells in larger amounts than DNA, and to be subject to variations under different physiological conditions. 
However, much evidence has been put forward in support of the hypothesis that the deoxyribonucleic acid content of the interphase nucleus is constant in amount. In large samples, as in a tissue culture, the average value for a particular cell type (and for all the somatic cells in any one species) appears sufficiently constant that it can be used to calculate the total cell number in the sample (Davidson and Leslie, I950). Davidson and Leslie (195I) deduced cell number in roller tube cultures of chick heart fibroblasts from DNAP measurements, assuming the average figure of $2.35 \cdot 10^{-10} \mathrm{mg}$ DNAP per nucleus. Changes in other components of the cells during growth $\left(e_{.} g\right.$. in protein and non-protein nitrogen, and in $\mathrm{P}$ fractions other than nucleic acid $\mathrm{P}$ ) were expressed in relation to the DNAP and so in terms of weight per cell.

Healy, Fisher and Parker (1954a) have successfully used DNAP measurements for assessing growth in tissue cultures. They introduced a modification of the Schmidt-Thannhauser technique, namely the use of insulin and MacFadyen's reagent to ensure better recovery and separation of the RNA and DNA fractions. With this technique, excellent correlations were obtained between DNAP and nuclear counts in growing cultures.

\section{(k) Protein}

Measurements of total protein, usually as nitrogen, can be used to give an estimate of total cellular material. There are however, two difficulties associated with this procedure. The first is that of making a clear separation of cells from a protein-containing nutrient solution, especially in the case of cultures grown in a plasma clot. This is more easily overcome than the second and related problem, which arises from the fact that extracellular protein may be associated with the cells, either produced by the growing cells themselves (e.g. collagen) or adsorbed to the cell surface from the medium. It may be difficult in practice to separate this closely associated protein from the cells, and it is indeed sometimes open to argument whether such protein ought or ought not to be regarded as "cellular protein". In some cases it seems clear that it ought not; for example, collagen has been found to increase even in cultures in which RNA and DNA were decreasing (Gerarde and Jones, I953), but the distinction cannot always be clearly made.

To circumvent some of the problems associated with the use of a clot, Warner, Hanawalt and Bischoff ( I 949) grew heart tissue from chick and rat on a glasscloth substrate in a fluid medium. They encountered the difficulty that nitrogen-containing material, probably protein, accumulated on the glasscloth, even when the cloth alone, in the absence of any tissue, was immersed in a protein-containing nutrient solution. Moreover the accumulation started within the first hour of incubation, and increased up to $2 \mathrm{I}$ days. It was therefore necessary to use a uniform and minimum amount of glasscloth, and to run control blanks without tissue at each time interval. This experience draws attention to the need for similar controls when other substrates are used.

Davidson and Leslie (I95I) measured protein nitrogen on the trichloroacetic acid-precipitated and lipid-extracted residue from roller tube cultures. The protein material was dissolved in I $\mathcal{N}$ sodium hydroxide by incubation at $37^{\circ} \mathrm{C}$ and the nitrogen estimated by a micro-Kjeldahl method. Instead of determining 
total protein nitrogen, Gerarde, Jones and Winnick (r952a) in an otherwise similar method, isolated the protein and weighed it. They used relatively large amounts of tissue in their cultures (20-40 $\mathrm{mg}$ wet weight of tissue per roller tube), and pooled the contents of three roller tubes in a tared centrifuge tube for precipitation with trichloroacetic acid, extraction with ethanol-ether, drying at $105{ }^{\circ} \mathrm{C}$ and final weighing. Similar protein residues, dissolved in $3 \%$ sodium hydroxide, were used by Stewart and Kirk (1952) for micro-Kjeldahl measurements and by Boyer and Kirk (1952) for a biuret estimation. Good agreement was reported between these two methods.

A method has been reported (Oyama and Eagle, I 956) based on the Folin-Ciocalteau reagent for phenols. The cells from four T-I 5 flasks (Earle and Highhouse, 1954) were pooled, dissolved in an alkaline copper reagent and treated with the Folin-Ciocalteau solution. Crystalline bovine albumin was used as a protein standard and results expressed in terms of a "bovine albumin equivalent". With each of three strains of cells (Osgood's No. I I I human leukemia, Gey's HeLa and Henle's intestinal epithelium), the increases of protein measured between the first and fourth days after explantation were approximately three-fold.

\section{REGORDING GROWTH AND BEHAVIOUR OF GELLS IN TISSUE CULTURE}

The technique of time-lapse cinephotomicrography has been applied to both quantitative and descriptive studies of cells in tissue culture. Moving pictures of organ rudiments were first made by Braus ( I 9 I I ) and of living cells by Comandon (Comandon, Levaditi and Mutermilch, igr3; Comandon and Jolly, I913). Since that time a great deal has been learned from cinephotomicrographs about the behaviour and growth of both cells and tissue rudiments; about changes in cell form and the mode of locomotion of the whole cell; about differentiation and cellular interactions; and about many intracellular activities particularly those concerned in the mitotic cycle.

The early observations of Comandon and his group were upon migration and "growth" (Comandon, Levaditi and Mutermilch, I9 I3). Levaditi and Mutermilch (1913a) recorded contractions in cultures of chick heart fragments. The first films of mitosis in living cells were made by Comandon and Jolly (I913; I9I 7-18).

The locomotion of chick fibroblasts was the subject of a study by Carrel and Ebeling (1926). They concluded that the cells move in straight lines with a uniform speed of approximately $33.3 \mu / \mathrm{h}$. Lymphocytes progress much more rapidly, at an average rate of $\mathrm{I} 9 \mu / \mathrm{min}$ (Lewis, I 930). More recently, Enterline and Coman ( $195^{\circ}$ ) studied the amoeboid movements of various normal and neoplastic cells. All were found to be actively amoeboid, but these authors recorded that the movements are intermittent and vary in speed from fractions of $I \mu / \mathrm{min}$. to $6 \mu / \mathrm{min}$. (average $\mathrm{I}-2 \mu / \mathrm{min}$. i.e. 60-120 $\mu / \mathrm{h}$.). Characteristic movements were described for the different types of blood leucocyte (Rich, Wintrobe and Lewis, I939; Lewis and Lewis, I94Ia). An important characteristic of living cells, the process by which large droplets of fluid are enfolded and assimilated, was discovered 
(Lewis, I931b; I936-37; I937) from a study of moving pictures and named "pinocy tosis".

Comparison of tumours with normal cells in moving pictures has been a fruitful field. Among those who studied the movements and the mode and rate of proliferation of normal and neoplastic cells, and made observations which are important for the differential diagnosis of certain types of tumour, especially those of the nervous system, have been Canti (I 932); Lewis and Lewis (1 933); Bland, Russell and Canti (1936-37); Bland and Russell (1938); Lewis (1948, r950); Pomerat ( I95 I, I 952); Costero et al. (1955a, b). Gey, Gey, Firor and Self (I 949) and Gey, Bang and Gey ( I 954) besides making such comparisons, also studied cells which underwent a transformation in vitro from normal to neoplastic.

The growth and organization of capillaries and endothelium in explants from subcutaneous tissue of seven-day chick embryos was observed in films by Lewis (I931a, I941a). Similarly, organized growth in rudiments of limb (Canti and Fell, I933a, b; Fell and Canti, I934) and sternum (Fell, I936-37) has been continuously recorded in moving pictures.

Movements of parts of cells have been followed in films, e.g. cilia (Danes, I 949a, b); mitochondria (Frédéric, 1950, 195I; Chèvremont and Frédéric, I95I; Frédéric and Chèvremont, I952; Frédéric, 1952, I954a); pulsatile activity in oligodendroglia (Canti, Bland and Russell, I935; Pomerat, I95I ; Lumsden and Pomerat, I95I); and rotation of nuclei (Lewis, I937; Pomerat, I953a, b). Some of these types of movement have been the subjects of quantitative measurements, e.g. the rate of contraction of the oligodendroglia is about I contraction $/ 5 \mathrm{~min}$., and nuclear rotations in human nasal mucosa take an average of 280 sec. per revolution, the fastest rotation seen being one which was complete in $75 \mathrm{sec}$.

Numerous studies have been made, with various refinements in technique such as the introduction of dark-ground (Lewis, I923; Carrel and Ebeling, I926; Strangeways and Canti, I 926, I927-28; Canti, I929) and phase-contrast illumination (Gey, Gey, Firor and Self, I 949; Hughes and Swann, I948; Lewis, Pomerat and Ezell, 1949) upon the mitotic process and other intracellular events (Pomerat, Lefeber and Smith, I954). Some of the finest films, however, were made with direct illumination by Lewis (r930, г93 гa, b, I936-37, г940, 1941a, b, I948). Careful studies have been undertaken to elucidate the length of each phase of the mitotic cycle in both normal and tumour cells (Lewis and Lewis, I933; Lewis, I939). The different phases of mitosis in chick embryo fibroblasts were found to vary considerably in different cells observed. Prophase, which is hard to recognize (even with phase contrast, which Lewis did not have) lasts about $30-60$ min.; metaphase I-I $5 \mathrm{~min}$. (average $3 \mathrm{~min}$.); anaphase $2-3 \mathrm{~min}$. and telophase $\mathrm{I}-2$ min. According to Lewis, fibroblasts from adult rat and mouse take about twice as long in metaphase, anaphase and telophase as the embryonic chick cells, and malignant fibroblasts twice as long as the normal adult rodent cells. Exact timing is complicated by the difficulty in deciding, even in normal cells, just when prophase begins. Some malignant cells have nuclei which, in interphase, look like normal prophases (Lewis and Lewis, I 94I I). Extensive studies, with the aid of phase contrast, have been made by Hughes (Hughes and Preston, I 948; Fell and Hughes, I949; Hughes and Swann, r 949; Hughes, 1952). His data on chick cells 
differ somewhat from Lewis', especially in a longer estimate for the telophase, viz. prophase 19-25 min.; metaphase, 4-7 min.; anaphase, 3.5-6 min. and telophase 7.5-14 min. Hughes' ( 1952) times for mouse spleen cells are, in agreement with Lewis, nearly twice as long as for the chick cells. The effects of mitotic inhibitors have been examined in living cells by phase contrast cinephotomicrography (Bucher, I950; Lettré, I954).

\section{CONCLUSION}

Growth, thought of as a process affecting a whole multicellular organism, can be broken down into a number of distinct but interrelated concepts. One of the advantages of applying the methods of tissue culture to the study of growth is that several of these components of "growth" can be experimentally, as well as conceptually, separated. The cells themselves grow in size and complexity; populations of cells proliferate and interact. These changes can be seen and followed in explanted cell cultures. The cells are very sensitive to changes in their environment, both the non-cellular environment and the adjacent cells. The external environment of the cells can be quite closely controlled and the effects of varying it made the subjects of quantitative and qualitative experiments. The still very incompletely understood process of mitosis may be studied, and methods of promoting and inhibiting cell division devised. Some of these studies are intimately concerned with questions such as the differences and similarities between normal and neoplastic growth. Growth is one of the responses of the cell or group of cells to environmental factors. In reality, growth, metabolism, differentiation, and special and general cell functions, are all closely connected processes. Tissue culture methods are designed to place the environmental factors under experimental control, so that specific effects at the cellular level can be recognized.

The environment can be varied, or the type of cell. Insights into the properties of individual cell types, separated from the parent organism, are gained by growing cells originating from different tissues, normal and neoplastic, in cultures. The dynamic cytology and histology made possible by tissue culture methods supplement and amplify studies made on fixed cells. Modern methods for isolating clones from single cells make feasible studies of the interactions of cells of known lineage, of stability and variation within particular cell strains, and of variations in metabolic patterns. Methods are available for growing, not only cultures from a single cell, but cultures containing $6 \cdot 10^{9}$ cells in a single vessel. In fact, the methods of tissue culture and their applications are so diverse that no attempt could be made to cover here all of those relevant to the study of growth. It is hoped that the ground traversed and the examples given may suffice to demonstrate some of the achievements and indicate some of the potentialities of this field. 


\section{LITERATURE}

Abercrombie, M. and J. E. M. Heaysman, (1953) Exptl. Cell Research, 5, i I I.

Abercrombie, M. and J. E. M. Heaysman, (1954a) Nature, 174, 697.

Abercrombie, M. and J. E. M. Heaysman, (1954b) Exptl. Cell Research, 6, 293.

Anderson, E. and W. Haymaker, (1935-36) Proc. Soc. Exptl. Biol. Med., 33, 313.

Baker, L. E. And A. H. Ebeling, (I939) 7. Exptl. Med., 69, 365.

Barnard, J. E., (1925) Brit. J. Exptl. Pathol., 6, 39.

BARSKI, G., (1954) Bull. microscop. appl., 4, II2.

Barski, G. and P. Manigault, (195I) Ann. Inst. Pasteur, 81, 33.

Barski, G., J. Maurin, G. Wielgosz and P. Lépine, (I951) Ann. Inst. Pasteur, 81, 9.

Beard, J. W. and P. Rous, (1934) 7. Exptl. Med., 59, 577; 593.

Berenblum, I., E. Ghain and N. G. Heatley, (1939) Biochem. 7., 33, 68.

Bernard, C., (1865) Introduction à l'étude de la médecine expérimentale, Paris.

Bichel, J., (1952) Acta Pathol. Microbiol. Scand., 3I, 4 IO.

Bland, J. O. W. and D. S. Russell, (1938) F. Pathol. Bacteriol., 47, 29 I.

Bland, J. O. W., D. S. Russell and R. G. Canti, (1936-37) Arch. exptl. Zellforsch. Gewebezücht., 19, $5 \mathrm{I} \mathrm{I}$.

Bloom, W., (1937) Physiol. Revs., 17, 589.

Boyer, H. K. And P. L. Kirk, (I952) F. Cellular Comp. Physiol., 39, 95.

Braus, H., (I9I I) Münch. med. Wochschr., 58, 2237.

Brues, A. M. and C. M. Masters, (1936) Am. F. Cancer, 28, 3 I4.

Brues, A. M., E. N. Rathbun and W. E. Cohn, (1944) J. Cellular Comp. Physiol., 24, i 55.

Brues, A. M. and I. Reitz, (I95I) Ann. N. Y. Acad. Sci., 5I, I 497.

Bucciante, L., (1927-28) Arch. exptl. Zellforsch. Gewebezücht., 5, I.

Bucher, O., (1950) Le Sang, 2I, 382.

Buchsbaum, R., (1932) 7. Exptl. Zool., 63, 483.

Burrows, M. T., (I9I I) 7. Exptl. Med., Io, 63.

Burrows, M. T., (1923-24) Proc. Soc. Exptl. Biol. Med., 21, 97.

Burrows, M. T., (1924) Southern Med. F., I7, 233.

Burrows, M. T., (1926) 7. Cancer Research, Io, 239.

Cailleau, R. and P. L. Kirk, (1954) J. Natl. Cancer Inst., 15, 295.

Cameron, G., (1950) Tissue Culture Technique, 2nd Ed., Academic Press, New York.

Cantr, R. G., (1929) Arch. exptl. Zellforsch. Gewebezücht., 8, I33.

Cantr, R. G., (1932) Trans. Ophthalmol. Soc. United Kingdom, 52, 478.

Canti, R. G., J. O. W. Bland and D. S. Russell, (1935) Research Publs. Assoc. Research Nervous Mental Disease, I6, I. Quoted by Pomerat (I 951).

Canti, R. G. And H. B. Fell, (I933a) Arch. exptl. Zellforsch. Gewebezücht., I4, 346.

Canti, R. G. And H. B. Fell, (1933b) Arch. exptl. Zellforsch. Gewebezücht., 15, 461.

Carleton, H. M., (r923) F. Exptl. Biol., I, I3I.

Carrel, A., (I913) 7. Exptl. Med., I7, I4.

Carrel, A., (1929) Compt. rend. soc. biol., 102, 742.

Carrel, A. and M. T. Burrows, (I9I i) F. Exptl. Med., I3, 562.

Garrel, A. and A. H. Ebeling, (1926) 7. Exptl. Med., 44, 26 I.

Champy, G., (1914) Compt. rend. soc. biol., 76, 31. Quoted by Lewis and Lewis, (1924).

Chen, J. M., (1954) Exptl. Cell Research, 7, 518.

Ghèvremont, M. And J. Frédéric, (1951) Compt. rend. soc. biol., I45, 1245.

Comandon, J. And J. Jolly, (1913) Compt. rend. soc. biol., 75, 457.

Comandon, J. and J. Jolly, (1917-18) F. physiol. et pathol. gén., I7, 573.

Comandon, J., C. Levaditi and S. Mutermilch, (igi3) Compt. rend. soc. biol., 74, 464.

Costero, I., G. M. Pomerat, R. Barroso-Mognel and A. Z. Chevez, (1955a) J. Natl. Cancer Inst., 15, I34I.

Costero, I., C. M. Pomerat, I. J. Jackson, R. Barroso-Mognel and A. Z. Chevez, (1955b) 7. Natl. Cancer Inst., I5, 13 I9.

Craciun, E. C., (I93I) La culture des tissus en biologie expérimentale, Masson et Cie., Paris.

Cunningham, B. And P. L. Kirk, (1942) 7. Cellular Comp. Physiol., 20, 343. 
Danes, B. S., (1949a) Anat. Record, 103, 590.

Danes, B. S., (1949b) F. Exptl. Zool., II2, 417 .

Danes, B. S., (1955a) Compt. Rend. Lab. Carlsberg, Sér. chim., 29, 209.

Danes. B. S., (1955b) Exptl. Cell Research, 8, 543.

Danes, B.S., G. S. Christiansen and P. J. Leinfelder, (1953) Exptl. Cell Research, 5, 234.

Danes, B. S. and P. J. Leinfelder, (I95I) 7. Cellular Comp. Physiol., 37, 427.

Davidson, J. N. And I. Leslie, (1950) Nature, $165,49$.

Davidson, J. N. And I. Leslie, (I95I) Exptl. Cell Research, 2, 366.

Davidson, J. N., I. Leslie and C. Waymouth, (1949) Biochem. J., 44, 5.

Davidson, J. N. and C. Waymouth, (i943) Biochem. 7., 37, 27 I.

Davidson, J. N. and C. Waymouth, (1944) Quart. 7. Exptl. Physiol., 33, 19.

Davidson, J. N. and C. Waymouth, (I945) Biochem. F., 39, i88.

Davidson, J. N. and C. Waymouth, (1946) Biochem. F., 40, 568.

De Bruyn, W. M., (1949a) Anat. Record, Ioz, 22.

De Bruyn, W. M., (1949b) Bijdragen tot de Dierkunde, 28, 77.

De Bruyn, W. M., (1949c) Acta Unio Intern. contra Cancrum, 6, 621.

De Bruyn, W. M., R. Korteweg and E. K. van Waveren, (1949) Cancer Research, 9, 282.

Doljanski, L. and G. Goldhaber, (1945) Proc. Soc. Exptl. Biol. Med., 6o, 132.

Drew, A. H., (1923a) Brit. F. Exptl. Pathol., 4, 46.

Drew, A. H., (I923b) Lancet, i, 833.

EAGLe, H., (1955a) 7. Biol. Chem., 214, 839.

Eagle, H., (I955b) F. Exptl. Med., IO2, 37.

Eagle, H., V. I. Oyama, M. Levy, G. L. Horton and R. Fleischman, (i956) J. Biol. Chem., $218,607$.

Earle, W. R., (1927) Proc. Soc. Exptl. Biol. Med., 24, 6i i.

EARle, W. R., (1928) J. Exptl. Med., 48, 457;667;683.

EARLe, W. R., (1943) F. Natl. Cancer Inst., 4, I65.

Earle, W. R., J. G. Bryant and E. L. Schilling, (i954) Ann. N.Y. Acad. Sci., 58 , rooo.

Earle, W. R., J. C. Bryant, E. L. Schilling And V.J. Evans, (i956) Ann. N. Y. Acad. Sci., 63,666 .

Earle, W. R., V. J. Evans and E. L. Schilling, (1950) F. Natl. Cancer Inst., io, 943.

Earle, W. R., V.J. Evans, K. K. Sanford, J. E. Shannon and H. K. Waltz, (I95I) 7. Natl. Cancer Inst., 12, 563 .

Earle, W. R. and F. Highhouse, (1954) 7. Natl. Cancer Inst., 14, 84I.

Earle, W. R., K. K. Sanford, V. J. Evans, H. K. Waltz and J. E. Shannon, (i95 I) J. Natl. Cancer Inst., 12, 133 .

Earle, W. R., E. L. Schilling and J. C. Bryant, (1954) F. Natl. Cancer Inst., 14, 853

Earle, W. R., E. L. Schilling, J. C. Bryant and V. J. Evans, (1954) F. Natl. Cancer Inst., I4, I I 59.

Earle, W. R. and J. W. Thompson, (1930) Public Health Repts., Washington, 45, 2672.

Ebeling, A. H., (I9I4) 7. Exptl. Med., 20, I 30.

Ebeling, A. H., (1921) F. Exptl. Med., 34, 23 I.

Ehrmann, R. L. and G. O. Gey, (1953) F. Natl. Cancer Inst., I3, I099.

Ehrmann, R. L. and G. O. Gey, (1956) F. Natl. Cancer Inst., I6, i 375.

Enterline, H. T. and D. R. Coman, (1950) Federation Proc., 9, 329.

Ephrussi, B. And G. Teissier, (1932-33) Arch. exptl. Zellforsch. Gervebezücht., 13, I.

Evans, V.J., (1951) Methods in Med. Research, 4, 221.

Evans, V. J., J. G. Bryant, M. G. Fioramonti, W. T. McQuilkin, K. K. Sanford and W. R. Earle, (1956) Cancer Research, I6, 77.

Evans, V. J., J. G. Bryant, W. T. McQuilkin, M. G. Fioramonti, K. K. Sanford, B. B. Westfall and W. R. Earle, (I956) Cancer Research, I6, 87.

Evans, V. J. And W. R. Earle, (1947) 7. Natl. Cancer Inst., 8, io3.

Evans, V.J., W. R. Earle, K. K. Sanford, J. E. Shannon and H. K. Waltz, (195I) 7. Natl. Cancer Inst., II, 907.

Evans, V. J., W. R. Earle, E. P. Wilson, H. K. Waltz and C. J. Mackey, (1952) J. Natl. Cancer Inst., 12, 1245. 
Fell, H. B., (1936-37) Arch. exptl. Zellforsch. Gewebezücht., 19, 512.

Fell, H. B., (I95I) in G. H. Bourne, ed., Cytology and Cell Physiology, 2nd Ed., Oxford University Press, Oxford.

Fell, H. B. and R. G. Canti, (1934) Proc. Roy. Soc. (London), B, II6, 316.

Fell, H. B. And A. F. W. Hughes, (1949) Quart. 7. Microscop. Sci., 90, 355.

Fell, H. B. And E. Mellanby, (1952) J. Physiol. (London), II6, 320.

Fell, H. B. And E. Mellanby, (1953) F. Physiol. (London), 119, 470.

Fell, H. B. ANd R. Robison, (1929) Biochem. 7., 23, 767.

Firket, H., (1954) Compt. rend. soc. biol., I48, I685.

Fischer, A., (I923) 7. Exptl. Med., 38,667.

Fischer, A., (1926) Arch. exptl. Zellforsch. Gewebezücht., 2, 303.

Fischer, A., (1930) Gewebezüchtung. Handbuch der Biologie der Gewebezellen in vitro, Müller and Steinicke, München.

Fischer, A., (1946) Biology of Tissue Cells, Gyldendal, Copenhagen.

Fischer, A., (r948) Biochem. F., 43, 491.

Fischer, A. and R. G. Parker, (1928-29) Proc. Soc. Exptl. Biol. Med., $26,585$.

Fischer, A. And R. G. Parker, (1929) Arch. exptl. Zellforsch. Gewebezücht., 8, 297.

Frédéric, J., (1950) Proc. IV Congr. de l'assoc. intern. du cinéma scientif., Florence.

Frédéric, J., (I95I) Compt. rend. soc. biol., 145, I913.

Frédéric, J., (1952) Compt. rend. assoc. anat., XXXIX Réunion.

Frédéric, J., (1954a) Ann. N. Y. Acad. Sci., 58, I 246.

FRÉdÉRIC, J., (1954b) Compt. rend. soc. biol., I48, I678

Frédéric, J. and M. Ghèvremont, (1952) Arch. biol. Liège, 63, iog.

Frisch, A., (1952) Proc. Soc. Exptl. Biol. Med., 81, 545.

Gaillard, P. J., (1935) Protoplasma, 24, 384.

Gemmill, C. L., G. O. Gey and R. Austrian, (1940) Bull. Johns Hopkins Hosp., 66, i67.

Gerarde, H. W. and M. Jones, (1953) F. Biol. Chem., 201, 553.

Gerarde, H. W., M. Jones and T. Winnick, (I952a) F. Biol. Chem., 196, 5 I.

Gerarde, H. W., M. Jones and T. Winnick, (I952b) J. Biol. Chem., I96, 69.

GEY, G. O., (1956) Harvey Lectures, 50, 154.

Gey, G. O. And F. B. BAng, (I949) Cancer Research, 9, 729.

Gey, G. O., F. B. Bang And M. K. Gey, (1954) Texas Repts. Biol. and Med., 12, 805.

Gey, G. O., M. K. Gey, W. M. Firor and W. O. Self, (i949) Acta Unio Intern. contra Cancrum, 6, 706.

Gey, G. O., J. H. Hanks and R. Barrett, (1948) Growth, I2, 69.

Giese, A. C., (1950) Physiol. Revs., 30, 43 I.

Ginsberg, H. S., E. Gold and W. S. Jordan, (i955) Proc. Soc. Exptl. Biol. Med., 89, 66.

Goldblatt, H. and G. Cameron, (i953) F. Exptl. Med., 97, 525.

Goodrich, H. B. And J. A. Scott, (1922) Anat. Record, 24, 3 I 5.

Goossens, R., (I953) Compt. rend. soc. biol., I47, 999.

Goossens, R., (1954) Compt. rend. soc. biol., I $48,98 \mathrm{I}$.

Graham, A. F. and L. Siminovitch, (i 955 ) Proc. Soc. Exptl. Biol. Med., 89, 326.

Grobstein, C., (1953a) Science, II8, 52.

Grobstein, C., (1953b) 7. Morphol., 93, I9.

Grobstein, C., (I953c) F. Exptl. Zool., 124, 383 .

Grobstein, C., (I955) F. Exptl. Zool., 130, 319.

Halberstaedter, L. And L. DolJanski, (1937) Arch. exptl. Zellforsch. Gewebezücht., 19, 475

Hanks, J. H., (I948) 7. Cellular Comp. Physiol., 3I, 235.

Hanks, J. H., W. F. Scherer, D. W. Fawcett, J. Leighton and K. R. Porter, (i955) An Introduction to Cell and Tissue Culture, Burgess Publ. Co., Minneapolis.

Harris, H., (1955) Brit. J. Exptl. Pathol., 36, I 15.

Harris, H. and W. R. Barclay, (1955) Brit. 7. Exptl. Pathol., 36, 592.

Harrison, R. G., (igo6-07) Proc. Soc. Exptl. Biol. Med., 4, I40.

Harrison, R. G., (igo6-08) Anat. Record, I, i i 6.

Harrison, R. G., (igo8) Anat. Record, 2, 385.

Harrison, R. G., (1914) J. Exptl. Zool., I7, 52 I. 
Harrison, R. G., (i928) Arch. exptl. Zellforsch. Gewebezücht., 6, 4.

Harrison, R. G., (1940) in F. R. Moulton, ed., The Cell and Protoplasm, Princeton University Press, Princeton.

Healy, G. M., D. G. Fisher and R. G. Parker, (i954a) Can. F. Biochem. and Physiol., $32,319$.

Healy, G. M., D. C. Fisher and R. G. Parker, (1954b) Can. 7. Biochem. and Physiol., $32,327$.

Healy, G. M., D. G. Fisher and R. G. Parker, (i955) Proc. Soc. Exptl. Biol. Med., 89, 7 I.

Hollomon, J. H. and J. C. Fisher, (1950) Science, III, 489.

Holtfreter, J., (1947) J. Morphol., 8o, 57.

Hotchin, J. E., (I955) Nature, I75, 352.

Hughes, A., (1952) The Mitotic Cycle, Butterworth, London.

Hughes, A. F. W. and M. M'E. Preston, (ig49) 7. Roy. Microscop. Soc., 69, 121.

Hughes, A. F. W. And M. M. Swann, (I948) J. Exptl. Biol., 25, 45.

Huld, W. and P. L. Kirk, (1950a) 7. Gen. Physiol., 33, 327.

Hull, W. And P. L. Kirk, (1950b) 7. Gen. Physiol., 33, 335.

Hull, W. and P. L. Kirk, (I950c) F. Gen. Physiol., 33, 343.

JAcoBy, F., (1937) 7. Physiol. (London), 90, 23 P.

Lake, N. G., (1916) 7. Physiol. (London), 50, 364 .

LAKE, N. C., (I9I7) Lancet, ii, 557.

Lambert, R. A., (I9I2) Anat. Record, 6, 9 I.

Lambert, R. A., (1913) J. Exptl. Med., I8, 406.

Landschütz, C., (I953) Naturwissenschaften, 40, 444.

Landsteiner, K. and R. C. Parker, (1940) J. Exptl. Med., 7i, 23 I.

LASER, H., (1932) Biochem. Z., 25I, 2.

Lasnitzki, I., (1955) Exptl. Cell Research, 8, i2 I.

Leighton, J., (I95 I) J. Natl. Cancer Inst., I2, 545.

Leighton, J., (1954) Texas Repts. Biol. and Med., 12, 847.

Leighton, J. And I. Kline, (1954) Texas Repts. Biol. and Med., I2, 865.

Leighton, J., I. Kline, M. Belkin and Z. Tetenbaum, ( I956) F. Natl. Cancer Inst., i6, i 353. Lettré, H., (1954) Ann. N. Y. Acad.Sci., 58, 1264.

Levaditi, C. and S. Mutermilch, (I913a) Compt. rend. soc. biol., 74, 462.

Levaditi, C. and S. Mutermilch, (I9I3b) Compt. rend. soc. biol., 74, i i 80.

Lewis, M. R. and L. D. Felton, (1922) Bull. Johns Hopkins Hosp., 33, I 12.

Lewis, S. R., C. M. Pomerat and D. Ezell, (1949) Anat. Record, I04, 487.

Lewis, W. H., (1923) Anat. Record, 26, 15.

Lewis, W. H., (1930) Anat. Record, 45, 229.

Lewis, W. H., (1931 a) Bull. Fohns Hopkins Hosp., 48, 242.

Lewis, W. H., (193 I b) Bull. Johns Hopkins Hosp., 49, 17.

Lewis, W. H., (1936) Harvey Lectures 1935-36, 31, 2 I4.

Lewrs, W. H., (1936-37) Anat. Record, 67 (Suppl.3), 64 .

Lewis, W. H., (1937) Am. F. Cancer, 29, 666.

Lewis, W. H., (1939) Growth, Suppl. 3, 9.

Lewis, W. H., (1940) Anat. Record, 76 (Suppl.), 9 I.

Lewis, W. H., (I94Ia) Anat. Record, 80, 270.

Lewis, W. H., (I94I b) Anat. Record, 8o, 396.

Lewis, W. H., (I948) Anat. Record, Ior, 698.

Lewis, W. H., (1950) in P. Weiss, ed., Genetic Neurology, University of Chicago Press, Chicago.

Lewis, W. H. And M. R. Lewis, (1924) in E. V. Cowdry, ed., General Cytology, University of Chicago Press, Chicago.

Letwis, W. H. And M. R. Lewis, (I933) Arch. exptl. Zellforsch. Gewebezücht., I4, 346.

Lewis, W. H. And M. R. Lewis, (I94Ia) Anat. Record, 8o, 519.

LEwis, W. H. ANd M. R. Lewis, (194I Ib) in Cause and Growth of Cancer, University of Pernsylvania Bicentennial Conference, p. $4 \mathrm{I}$.

LipMann, F., (1933) Biochem. Z., 261, I57. 
Iipmann, F. ANd A. Fischer, (1932) Biochem. Z., 244, I87.

Longmuir, I. S. And W. ApRees, (1956) Nature, I77, 997.

Loofbourow, J. R., (1948) Growth, Suppl. 12, 77.

Lu, K. H. And T. Winnick, (1954a) Exptl. Cell Research, 6, 345.

Lu, K. H. And T. Winnick, (I954b) Exptl. Cell Research, 7, 238.

Lucké, B., L. Berwick and P. Nowell, (1953) J. Exptl. Med., 97, 505.

LudFord, R. J., (1940) Brit. Med. J., i, 20 I.

Ludford, R. J. And H. Barlow, (1944) Cancer Research, 4, 694.

Lumsden, C. E. And C. M. Pomerat, (195I) Exptl. Cell Research, 2, 103.

Luyet, B. J. and F. Gonzales, (195I) Biodynamica, 7, 6 I.

Markert, C. L., (ig55) Ann. N. Y. Acad. Sci., 6o, ioo3.

Mayer, E., (1939) Tabulae biologicae, 19 (Cellula, 65), 65.

Mayer, E. And H. Schreiber, (1934) Protoplasma, $21,34$.

Meier, R., (I93I) Biochem. Z., 231, 253.

MoOKeRJee, S., (I953) Nature, I7I, 796.

Morgan, J. F., H. J. Morton and R. G. Parker, (1950) Proc. Soc. Exptl. Biol. Med., 73, i.

Moscona, A., (1952) Exptl. Cell Research, 3, 535.

Moscona, A. and H. Moscona, (1952) F. Anat., 86, 287.

Moscona, A. and H. Moscona, (1953) Bull. Research Council of Israel, 3, 197.

Murray, M. R. and G. Kopech, (1953) A Bibliography of the Research in Tissue Culture, I884 to 1950, Academic Press, New York.

Neukomm, S. And M. Richard, (1952) Acta Anat., i6, i6o.

Neuman, R. E. And T. A. McCoy, (1955) Exptl. Cell Research, 9, 212.

Olivo, O. M., (1932) Arch. ital. anat. e embriol., 3o, 24 I.

Owens, O. von H., G. O. Gey and M. K. Gey, (1953) Proc. Am. Assoc. Cancer Research, $I, 4 \mathrm{I}$.

Owens, O. von H., G. O. Gey and M. K. Gey, (1954) Ann. N. Y. Acad. Sci., 58, 1039.

Owens, O. von H., M. K. Gey and G. O. Gey, (1956) Federation Proc., 15, 140.

Oyama, V. I. and H. Eagle (1956) Proc. Soc. Exptl. Biol. Med., 9I, 305.

PAFF, G. H., (I948) Anat. Record, IOO, 702.

Pannett, C. and A. Compton, (I924) Lancet, 206, 38 I.

PArker, R. C., (1936) J. Exptl. Med., 64, I21.

Parker, R. C., (1950) Methods of Tissue Culture, 2nd Ed., Cassell \& Co., Ltd., London.

Parker, R. C., (I955) in Begg, R. W., ed., Canadian Cancer Conference, Vol. I, Academic Press, New York.

Parker, R. G., G. M. Healy and D. C. Fisher, (1954) Can. F. Biochem. and Physiol., 32, 306.

Parshley, M. S. and R. A. Deterling, (1956) Am. F. Anat., 97, 359.

Parshley, M. S. and H. S. Simms, (1950) Am. J. Anat., 86, i63.

Paterson, E., (1942) Brit. F. Radiol., 15, 257.

Patt, H. M., (I955) Ann. N. Y. Acad. Sci., 59, 649.

Pearce, C. and E. Paterson, (1952) Brit. J. Cancer, 6, 93.

Perry, V. P., V.J. Evans and W. R. Earle, (1955) Science, i21, 805.

Pikovski, M. A., (1954) Expll. Cell Research, 7, 52.

Pincus, G. And A. Fischer, (i93 I) 7. Exptl. Med., 54, 323.

Pomerat, C. M., (195I) F. Nervous Mental Disease, II4, 430.

Pomerat, C. M., (1952) Texas Repts. Biol. and Med., IO, 2 I 7.

Pomerat, C. M., (1953a) U. S. Armed Forces Med. F., 4, I I.

Pomerat, C. M., (1953b) Exptl. Cell Research, 5, 191.

Pomerat, C. M., C. G. Lefeber and McD. Smith, (i954) Ann. N. T. Acad. Sci., 58 , i 3 I I.

Pomerat, C. M. And R. B. Lewis, (1953) Texas Repts. Biol. and Med., II, 307; 333.

Pomerat, C. M. and P. S. Moorhead, (1956) Texas Repts. Biol. and Med., 14, 237.

Price-Jones, C. And J. C. Mottram, (19I4) Arch. Middlesex Hosp., 33, 21. (Quoted by Stroud And Brues, 1954).

Prop, F. J. A., (1954) Exptl. Cell Research, 7, 303.

Puck, T. T. And P. I. Marcus, (1955) Proc. Natl. Acad. Sci., 4I, 432. 
Puck, T. T. and P. I. Marcus, (1956) 7. Exptl. Med., io3, 653.

Puck, T. T., P. I. Marcus and S. Cieciura, (1956) J. Exptl. Med., 103, 273.

Pulvertaft, R. J. V., (1952) 7. Pathol. Bacteriol., 64, 75.

Ray, R. D., R. Mosiman and J. Schmidt, (r954) F. Bone and Joint Surg., 36a, I 147.

Reid, T. R. And M. P. Gifford, (I952) F. Natl. Cancer Inst., I3, 43 I.

Rich, A. R., M. M. Wintrobe and M. R. Lewis, (1939) Bull. Johns Hopkins Hosp., 65, 3 I I.

Rienhoff, W. F., (ig22) Bull. Fohns Hopkins Hosp., 33, 392. (Quoted by Lewis And Lewis, 1924).

Rinaldini, L. M., (1953) J. Physiol. (London), I23, 20 P.

Rinaldini, L. M., (i954) Nature, I73, I I 34 .

Roffo, A. H., (1933) Prensa méd. argent., 20, 923.

Rous, P. And F. S. Jones, (1916) 7. Exptl. Med., 23, 549.

SANFORd, K. K., (I95I) Methods in Med. Research, 4, 2 I9.

Sanford, K. K., W. R. Earle, V. J. Evans, H. K. Waltz and J. E. Shannon, (I95 I) 7. Natl. Cancer Inst., II, 773.

Sanford, K. K., W. R. Earle and G. D. Likely, (1948) J. Natl. Cancer Inst., 9, 229.

Sanford, K. K., G. D. Likely and W. R. Earle, (I954) 7. Natl. Cancer Inst., 15, 2 I 5.

Scherer, W. F. and A. C. Hoogasian, (1955) 7. Cellular Comp. Physiol., 46, 477.

Scherer, W. F., J. T. Syverton and G. O. Gey, (r953) J. Exptl. Med., 97, 695.

Schilling, E. L., W. R. Earle and V. J. Evans, (1950) J. Natl. Cancer Inst., Io, 883.

Schleich, A., (1956) Ann. N. Y. Acad. Sci., 63, 849.

Signorotti, B., W. Hull and P. L. Kirk, (1950) 7. Gen. Physiol., 33, 315.

Sinnott, E. W., (1939) Science, 89, 4I.

Sмiтh, A. U., (1952) Exptl. Cell. Research, 3, 574.

Smith, A. U. ANd A. S. Parkes, (1954) in Giba Foundation Symposium on Preservation and Transplantation of Normal Tissues, G. E. W. Wolstenholme, ed., Little and Brown, Boston, p. 76 .

Sмyтн, H. F., (1914-15) 7. Med. Research, 3I, 256.

Spear, F. G., (1928) Arch. exptl. Zellforsch. Gewebezücht., 7, 484.

Spratt, N. T., (1948) 7. Exptl. Zool., I07, 39.

Stenger-Haffen, K., (1955) Compt. rend., 240 , 1018.

Stewart, D. C. And P. L. Kirk, (i952) 7. Cellular Comp. Physiol., 40, i83.

Stewart, D. C. And P. L. Kirk, (1954) Biol. Revs. Cambridge Phil. Soc., 29, I 19.

Strangeways, T. S. P., (1924a) Technique of Tissue Culture "in vitro", W. Heffer \& Sons, Ltd., Cambridge.

Strangeways, T. S. P., (1924b) Tissue Culture in Relation to Growth and Differentiation, W. Heffer \& Sons, Ltd., Cambridge.

Strangeways, T. S. P. and R. G. Canti, (I926) Brit. Med. F., ii, i 55.

Strangeways, T. S. P. and R. G. Canti, (1927-28) Quart. J. Microscop. Sci., 7I, I.

Stroud, A. N. And A. M. Brues, (1954) Texas Repts. Biol. and Med., I2, 931.

Syverton, J. T. And W. F. Scherer, (1954) Ann. N. Y. Acad. Sci., 58, 1056.

Taylor, A. C. and R. Gerstner, (i 955) 7. Cellular Comp. Physiol., 46, 477.

Tompkins, E. R., B. Cunningham and P. L. Kirk, (1947) 7. Cellular Comp. Physiol., zo, I.

Trowell, O. A., (1952) Exptl. Cell Research, 3, 79.

Trowell, O. A., (1954) Exptl. Cell Research, 6, 246.

Trowell, O. A., (1955) Exptl. Cell Research, 9, 258.

VanAllen, C. M., (1925) J. Am. Med. Assoc., 84, 202.

Warner, D., G. Hanawalt and F. Bischoff, (I949) J. Natl. Cancer Inst., io, 67.

Waymouth, C., (1949) Exptl. Cell Research, Suppl. I, 460.

Waymouth, C., (I95I) Methods in Med. Research, 4, 225.

Waymouth, C., (1952) in A. Hughes, The Mitotic Cycle, Butterworth, London.

Waymouth, C., (1954a) Biochem. 7., 56 , iv.

Waymouth, C., (I954b) Intern. Rev. Cytol., 3, I.

Waymouth, C., (I955) Texas Repts. Biol. and Med., I3, 522.

Waymouth, C., (I956a) 7. Natl. Cancer Inst., I7, 305.

Waymouth, C., (1956b) F. Natl. Cancer Inst., I7, 3 I 5. 
Weiss, L. P. and D. W. Fawcett, (1953) 7. Histochem. Cytochem., I, 47.

Weiss, P., (1939) Principles of Development, Henry Holt \& Co., New York.

Weiss, P., (1949a) in A. K. Parpart, The Chemistry and Physiology of Growth, Princeton

University Press, Princeton.

Weiss, P., (1949b) Exptl. Cell Research, Suppl. I, 475.

Weiss, P. and R. James, (1955) Exptl. Cell Research, Suppl. 3, 38 r.

Westfall, B. B., V. J. Evans, J. E. Shannon and W. R. Earle, (1953) J. Natl. Cancer Inst., I4, 655 .

White, P. R., (1946) Growth, Io, 23 I.

White, P. R., (1949) 7. Cellular Comp. Physiol., 34, 221.

White, P. R., (1954) The Cultivation of Animal and Plant Cells, Ronald Press, New York.

White, P. R., (1955) F. Natl. Cancer Inst., I6, 769.

Wilde, C. E., (1948) Proc. Soc. Exptl. Biol. Med., 69, 374.

Wilde, C. E., (1955) 7. Exptl. Zool., 130, 573.

Willmer, E. N., (1927) 7. Exptl. Biol., 4, 280.

Willmer, E. N., (1933a) F. Exptl. Biol., Io, 323.

Willmer, E. N., (I933b) 7. Expll. Biol., io, 340.

Willmer, E. N., (1942) J. Exptl. Biol., I8, 237.

Willmer, E. N., (1954) Tissue Culture, 2nd Ed., Methuen \& Co., London.

Willmer, E. N. and F. Jacoby, (1936) 7. Exptl. Biol. I3, 237.

Wilson, H., E. B. Jackson and A. M. Brues, (1942) 7. Gen. Physiol., 25, 689.

Winnick, T., (1952) Texas Repts. Biol. and Med., Io, 452.

Wirth, J. and C. Barski, ( I947) Ann. Inst. Pasteur, 73, 987.

Wolff, Em., (1955) Compt. rend., 240, 10 I6.

Wolff, Et. and K. Haffen, (1952) 7. Exptl. Zool., I19, 38 I.

Youngner, J. S., (1954) Proc. Soc. Exptl. Biol. Med., 85, 202.

Zwilling, E., (1954) Science, I20, 219. 


\title{
Chapter 7
}

\section{REGENERATION AND GROWTH}

\author{
A. E. Needham
}

\section{INTRODUCTION}

By regeneration is understood the restoration of the normal structure of a living organism following the loss of part or parts of it. For a uniform terminology "regenesis" would be a better term, implying a type of morphogenesis which restores the structure initially established in ontogenesis. It is one manifestation of "morphostatic" phenomena (Weisz, I954), the morphogenetic counterpart of the physiological servo-mechanisms of "homoeostasis" (Cannon, I932). Regeneration usually involves new growth, often as rapid as that of embryos and of malignant neoplasms (Christensen and Streicher, 1948). Like any other special case, it has certain advantages for the study of the general problem of growth.

One advantage is the short time-scale of regeneration. Others are the large size of many regenerates, and the great liberty to select the quantity and quality of the material to be regenerated, and the occasion. Further, adult tissues are generally much hardier than those of embryos. On the other hand regeneration is possibly more complicated than normal ontogenesis because of its dependence on the mature tissues to which the regenerate is attached, and because of the integrating control by nervous, hormonal and vascular systems (pp. 63I-647).

In the most common type of regeneration, "epimorphosis", virtually the whole of the part lost is restored by the growth of a bud or "blastema" of undifferentiated cells, which accumulates below the closed surface of amputation. By contrast, some organs, such as the vertebrate liver, after partial hepatectomy, regenerate by vigorous growth throughout the remainder, without restoration of the precise initial form of the organ. This "compensatory hypertrophy" sometimes occurs in an homologous organ topographically remote from the one lost, for example in the partner of a kidney, in a Vertebrate. Losses in sponges, hydroid coelenterates and other colonial animals are often "made good" by compensatory growth elsewhere, though it has not been shown generally that this is a specific response to the accident or to its sequelae, as it is in the vertebrate liver and kidney.

In some Protozoa (Summers, I94I), worms (Berrill, I952) and other animals, asexual reproduction by fission or fragmentation is followed by epimorphic regeneration of each daughter ("architomy"). Other animals, often quite closely related, regenerate the essential parts of the daughters before fission ("paratomy" or "neotomy"). Here regeneration is 
part of a spontaneous act of morphogenesis. "Physiological regeneration", the replacement of a relatively steady amount of "wear and tear", as among the cells of the mammalian epidermis, blood, etc., is often regarded as a normal growth-process but it is largely controlled by the extent of losses and is a true regeneration-process. Many Amphibia and Reptilia cast their superficial epidermis, en masse, periodically. Wear and tear is a recognised phenomenon at the molecular level (Morel, 194I ; Haldane, 1954); it is less rapid than the total rate of metabolic turnover revealed by studies using "tracer" atoms.

Like ontogenesis, regeneration involves not only growth but also the movements of cells, the differentiation of cells and their collective organisation into an integral structure. Indeed many sponges and coelenterates are able to regenerate from dissociated cells which initially reconstitute purely by aggregation and redistribution of the individual cells (Brien, I937; Beadle and Booth, I938; Von Bertalanffy and Rella, I94I). Growth occurs eventually (Brien, I937), but only after the essential processes of regeneration are complete. A number of animals regenerate largely or partly by "morphallaxis" (Morgan, Igor), another process involving relatively little growth, but mainly reorganisation within the parent portion of the body. However, the nature of morphallaxis is far from clear, and it may approximate to a disseminated epimorphosis of local blastemata throughout the body (Van Asperen, I 946). Planarian worms regenerate mainly by epimorphosis when well fed but by morphallaxis when starved (Chranova, 1938), and the sea squirt, Clavellina, by morphallaxis when young and by epimorphosis when mature (Millot, I93 I, p. I35), so that the distinction between the two methods is probably facultative rather than fundamental. Like epimorphosis, morphallaxis probably involves an initial process of dedifferentiation or regression ( $\mathrm{R}$-phase) followed by the progressive processes (P-phase) of growth and differentiation (p. 595).

This oscillation is probably characteristic of all types of true regeneration, following acute accidents, and probably distinguishes them from physiological regeneration and other types of response to chronic low-grade losses. The function of the R-phase is to provide cells capable of proliferation and growth (p. 596). In the lower metazoa cells not previously differentiated, "neoblasts" (Stolte, I935; Schotté, 1939) contribute to regeneration and the R-phase may be less extensive or less significant here. This method recalls a method of spontaneous asexual reproduction in the Parazoa and lower Metazoa by means of "gemmules" or "statoblasts",- - accumulations of "embryonic" cells. The "statoblasts" of some Polyzoa and other animals, however, are aggregates of dedifferentiated cells. These do not differ very fundamentally from the dedifferentiated remains of whole, starved animals of some groups of animals, which are capable of regenerating when refed (J. Needham, 1942). Starvation leads to increased fat-mobilisation (Frazer, 1954) as in the R-phase of regenerration (p. 6ig).

For the present purpose interest centres on the characteristic $\mathrm{R}-\mathrm{P}$ oscillation in metabolism during regeneration. The Protozoa show it not only during regeneration but also in the course of asexual and sexual reproductions, and in encystment, and there is evidence of it in the metamorphosis of amphibia (J. Needham, I942) and of insects (D. Needham, I93 I ; Wigglesworth, I954). The control of qualitative processes in regeneration will be considered only so far as it is related to growth-problems. Regeneration is viewed therefore as a re-accelerated growthprocess (Przibram, I 919; I 925; I 93I).

For further general information on regeneration as an independent study the following works may be consulted: Morgan (I90I), Davenport (1908), Przibram (I909), Korschelt 
(1927), Millot (1931), Abeloos (1932), A.E. Needham (1952). The subject is considered in relation to morphogenesis in general by Huxley and De Beer (1934), Weiss (1939), Child (194I), J. Needham (1942), Thompson (1942), Brachet (1950), Raven (I954) and Willier et al. (1955). Regeneration in specific groups of animals has been considered in the books of Summers and Calkins (I94I), Stephenson (1930), Wigglesworth (1950), Roeder (I953), Hyman (1940, 1951), Kükenthal and Krumbach (1926 onwards), and Graissé (1948 onwards). Recent symposia on the subject include those in the American Naturalist (1940, 74), Biological Symposia (J. Cattell). See also Bespoloe Razmnojenie i Regeneratsiya (M. A. Voronzova and L. D. Liosner, 1957, Moscow, Gosudarstvennoe Izdatelstvo).

\section{THE GENERAL NATURE OF REGENERATION}

\section{(a) The distribution of powers of regeneration among animals}

Some of the philosophical problems arising from this distribution (Needham, I 952) are not directly relevant here but it is important that powers of regeneration are very widespread and are generally related to powers of normal growth. Among Arthropods many Crustacea effectively grow throughout life (Needham, I95ob) and regenerate well their limbs, whereas the Copepoda have "determinate" growth and no power of regeneration in the adult. This is also true of insects, which often regenerate well as growing larvae. The Rotifera and Nematoda have determinate growth and poor powers of regeneration. At the other extreme are many Protozoa, Porifera, Coelenterata, Platyhelmia, Nemertinea and Annelida, which grow virtually indefinitely as individuals or through repeated fissions or other processes of asexual reproduction. These are able to regenerate a complete animal from almost any fragment larger than a critical size. A quantitative measure of their powers of regeneration is given by this minimal size of fragment (Table I). Many Echinodermata and Tunicata also have extensive powers of regenerating the whole body. These are all animals of simple organisation, and in general increasing differentiation of tissues, whether in phylogenesis or ontogenesis, is correlated with a decreasing power of growth and of regeneration. Mere increase in body-size is probably one aspect of differentiation and large oligochaetes and urodeles regenerate less well than species having smaller individuals. The Mollusca and the Vertebrata Craniata in general are large animals, highly differentiated also in other ways, and their powers of regenerating external organs are more limited. Their internal organs, however, retain remarkable powers. The rat can regenerate $2 / 3$ of its liver in a few days and although the adult Anuran Amphibia can no longer regenerate limbs, a transected gut rejoins and heals rapidly, even if the cut ends are divaricated. Even if one end is put out of reach in an external fistula, the other end opens into the side of the viscus, and reestablishes continuity in twelve days or so (Goodchild, r955).

There are apparent exceptions to the general rule that powers of regeneration have decreased progressively during evolution. For instance a number of Urodele Amphibia have far better powers of regeneration than those fishes which have been investigated. In this respect, as in their return to a more fully aquatic mode of life, the Urodela generally may show regressive evolution.

These generalisations are still based on observations form relatively few species, and they may hide considerable variation at lower taxonomic levels. Leeches, in contrast to most Annelida have virtually no power of regeneration. Some plana- 


\section{TABLE 1}

MINIMAL SIZE OF FRAGMENT CAPABLE OF REGENERATING A WHOLE ANIMAL

$\begin{array}{ccc}\text { Species and group } \quad \text { Minimal size } & \begin{array}{c}\text { Percentage of } \\ \text { parent-body }\end{array} & \text { Reference }\end{array}$

\begin{tabular}{|c|c|c|c|}
\hline Stentor (Ciliata) & $0.04 \mathrm{~mm}$ long & I.O & $\begin{array}{l}\text { Lillie, 1896; Weisz, I954; } \\
\text { Morgan, I901, p. } 56\end{array}$ \\
\hline Ephydatia (Porifera) & $\begin{array}{l}\text { less than I mm } \\
\text { diameter }\end{array}$ & 一 & Brien, 1937 \\
\hline Hydra (Coelenterata) & $0.2 \mathrm{~mm}$ long & 0.5 & $\begin{array}{l}\text { Peebles, I } 897 ; \text { Koelitz, 191 } ; \\
\text { Korschelt, I927; p. 581 }\end{array}$ \\
\hline Turbellaria & - & $1.0-0.36$ & Lillie, I 900 \\
\hline Turbellaria & - & $0.1-0.07$ & $\begin{array}{l}\text { Holmes, I9II (by repeated } \\
\text { section) }\end{array}$ \\
\hline Lineus (Nemertinea) & $\begin{array}{l}0.15 \mathrm{~mm} \text { long } \\
0.04 \mathrm{~mm} \text { wide }\end{array}$ & 0.0005 & $\begin{array}{l}\text { Coe, ig29 (by repeated } \\
\text { section) }\end{array}$ \\
\hline Lumbriculus & $\begin{array}{l}0.5^{-1} .0 \mathrm{~mm} \\
\text { (I segment) }\end{array}$ & $\mathrm{I}-2$ & C. Müller, I 908 \\
\hline
\end{tabular}

rians, for instance Procotyla, have very poor powers compared with typical species (Brondsted, I955) and there is a similar variety among rhabdocoele Turbellaria. These variations have yet to be compared with differences in normal growth rate and other relevant properties. The cestode and trematode Platyhelmia are said to have low powers of regeneration (Korschelt, 1927) notwithstanding their enormous powers of growth and of asexual reproduction. Other parasites also are reported to regenerate poorly (Morgan, I90 I, p. I46; Kocian, I930; Reinhard, 1952). In fact, as for certain species of Protozoa (Weisz, 1954) results obtained so far may show poor survival under experiment, rather than poor regeneration.

\section{(b) Regeneration and other growth processes}

Regeneration-rate, like normal growth-rate, declines with age (p. 6o7). Przibram (I9I7) found a correlation between the growth-rate of individual stickinsects and the rate of regeneration of their limbs. Growth-rate and regenerationrate show the same seasonal fluctuations (Otto, 192 I; Needham, I949a). Annelids regenerate best at cut surfaces near the hind end of the body where growth and asexual reproductive processes occur most rapidly and Protozoa regenerate best at surfaces near the normal fission-plane (Tartar, r 94I). The gonadal hormones (p. 632) promote the regeneration of those organs of which they promote the normal growth (Korschelt, I 927, p. 665). The gonopod of the fish Platypoecilus regenerates rapidly if amputated during its normal growth-phase, but slowly once it has entered the phase of differentiation (Grobstein, 1947). It seems doubtful if any agent affects regeneration in a way incompatible with its known effect on normal growth.

Since growth and regeneration are so similar, the two processes often compete, particularly where materials, etc., are limiting. In Hydra (Tardent, 1954) a regenerate and a normal asexual bud compete for the neoblast, interstitial cells which are raw materials for both. In the isopod Crustacean, Asellus, regeneration tends 
TABLE 2

INHIBITORY INTERACTION BETWEEN REGENERATION AND ASEXUAL REPRODUCTION

(a) Regeneration inhibited by asexual reproduction

\begin{tabular}{ll}
\hline \multicolumn{1}{c}{ Example } & \multicolumn{1}{c}{ Reference } \\
\hline Paramecium & Tartar, I94 I \\
Planarians & Vandel, I922 \\
Dero, (Oligochaeta) & Galloway, I899 \\
\hline
\end{tabular}

(b) Asexual reproduction inhibited by regeneration

\begin{tabular}{ll}
\hline \multicolumn{1}{c}{ Example } & \multicolumn{1}{c}{ Reference } \\
\hline Amoeba & Hartmann, I924; Phelps, I926 \\
Hydra & Goetsch, I92 I \\
Stenostomum (Rhabdocoela) & Hartmann, I922 \\
Nais (Oligochaeta) & Stolte, I922 \\
Syllid Polychaetes & Berrill, I952; pp. 428-9. \\
\hline
\end{tabular}

to inhibit growth competitively. In decapod Crustacea this inhibition is exercised through the hormones of the eyestalk-organs (Kamps, 1937) known to control growth and moulting more generally.

The competition is not necessarily severe, when conditions are not limiting. Tazelaar (1938) and Dixey (1938) detected no great effect of regenerating limbs of arthropods on the growth of intact limbs and in fact competition may be masked by a non-specific growth-stimulation by factors produced by the regenerate. There may be, in consequence, a complex interplay of inhibition and acceleration of normal growth (Przibram, I91 7; Huxley, 1932; Gabritchevsky, 1930) to which other factors also contribute (p. 6io). Accurate analysis demands a careful record of growth- and regeneration-rates throughout. The phenomenon of "rebound" acceleration of normal growth after inhibition also may contribute to the complexity; such rebound-effects have been recorded in regenerative growth also (Morgulis, I909; Du Noüy, I936, p. 92; Paton, 1955).

In rhabdocoele and triclad Turbellaria, in microdrile Oligochaeta and probably also in other groups, powers of regeneration are often closely correlated with powers of asexual reproduction by fission, etc. (Berrill, I95I, I952; Weisz, 1954). There is more evidence (Table 2) of competition between the two processes than between either and ontogenetic growth, because they are frequently evoked at the same time. Under different conditions the one or the other may have the advantage in the same animal (Goetsch, 1917, 1925; Boecker, 192I). In some Protozoa wounding may evoke either regeneration or fission, depending on conditions (Summers, I94I). In Planarians also (Vandel, I92 I) wounding may accelerate fission. Regeneration is slow immediately after fission in Protozoa and also after encystment (Calkins, I9I I Moore, I924) and improves progressively during a subsequent "physiological restoration". The change is attributed to cyclic changes in nucleo-cytoplasmic interaction during the cycle of fission (Bauer and Granowskaja, 1934); it seems possible that a similar cyclic change may contribute to the observed depression of regeneration-power during certain stages of a previous act of regeneration (Davidson and Berrill, i 948 ). By contrast regeneration is said to be better just after, than just before, an 
act of sexual reproduction in the ciliates (Calkins, I9I I; Moore, 1924). An act of regeneration is not always completed during a single cycle of fission (Tartar I94I) so that it is not "geared" completely to the mechanism which controls the latter.

In the oligochaete, Dero (Berrill, 1952), removal of the tip of the tail, during architomy (p. 588) in the middle of the body, does not inhibit fission, but it may inhibit subsequent head-regeneration by the decaudated posterior daughter. By contrast, if the polychaete, Procerastea, is transected in front of the fission plane at the time of incipient paratomy, a head regenerates at the anterad cut surface even if it is within one body segment of the developing head of the posterior daughter (Berrill, 195 I, 1952). These facts are consistent with rather precise chronological changes, during asexual reproduction and regeneration, in the amounts of the same promotor and inhibitor substances (p. 636 ).

Changes during a protozoon cycle of sexual reproduction are very different, if not completely inverse, and a commonly observed antagonism between regeneration and sexual reproduction therefore may be due to metabolic incompatibility rather than to direct competition between the two. However probably there is competition for materials in some animals, e.g. in Hydra, for neoblasts (Brien, I953); following transection of the body regeneration may be slower on the side bearing an ovary than on the opposite side (Goetsch, 1929) while under other conditions transection leads to regeneration, but to resorption of ovaries and testes (Goetsch, 1925). In Annelida, again, neoblasts probably are used for the production of both germinal and somatic tissues, (Korschelt, 1927; p. 199); a regenerate may even draw off cells from the gonad itself (ibid. p. 202). Similarly the testes and yolk glands of planarians are resorbed as material for regeneration (Steinmann, 1908). Such competition is inevitable in animals habitually operating near the limits of their productive metabolism.

Further analysis is necessary to establish particular instances as competitive or antagonistic interaction, for instance the retardation of sexual reproduction in the rhabdocoele Stenostomum, by simultaneous regeneration-activity (Hartmann, 1922). Some evidence does indicate an antagonism between the two processes. The sexual individuals or gonozooids of the coelenterate Hydractinia regenerate very poorly compared with the vegetative hydrozooids (Hyman, I940; p. 49 I) and earthworms regenerate badly from cut surfaces in the genital region. The gonad itself regenerates poorly in Annelida and Arthropoda (Korschelt, I927, p. 659, $660)$, though in fact animals as highly evolved as mice are able to regenerate an ovary, from new tissue, after complete spaying (Davenport, I925). It would be valuable to know if there is an $\mathrm{R}$-phase at any stage of the growth, maturation and development of the gametes and zygote of animals. The latter often has considerable powers of regeneration, in early stages by a morphallactic reorganisation called "regulation" (Huxley and De Beer, r934), and later by "postgeneration" (Morgan, I90I; Münch, I937), at first sight an epimorphic process, but in the light of recent work (Townes and Holtfreter, I 955; Feldman, I955) possibly a reconstitution process, that is a redistribution of cells rather than a proliferation. There follows a stage, which probably corresponds to the inflexible "mosaic" stage of the ontogenic processes themselves, during which regeneration does not occur readily (Harrison, I9 I 5; Lehmann, I 929; Holtfreter, I938; Murtasi, I 938; Murtasi and Sosnina, I943). This stage may also correspond to the stage during regeneration when re-regeneration cannot be induced (Davidson and Berrill, 1948). After the mosaic stage, embryos enter the definitive stage, of recovered regenerative power. The necessary local dedifferentiation and return to an embryonic condition again becomes possible. In the higher animals the final age- 
decline in regenerative power begins early, soon after the stage of I I-I 4 somites in the chick (Zwilling, I942).

The relationships and interactions between regeneration and abnormal cancerous growth have been studied. Skin regenerates more slowly near a grafted tumour than elsewhere (Zdriukovskaja, I942), but the presence of regeneration-

Fig. I. Parasagittal section through the anterior abdominal region of an adult male Asellus aquaticus showing the extent of fusion between a graft of tissues from an embryo and the adjacent normal and regenerating tissues of the host. The embryotissues are somewhat disorganised as a result of the procedure of grafting but there is no certain evidence of qualitative interaction between the three types of tissue. An infection of the host tissues (small dark spheres) has not affected those of the embryo, at any rate in the time available. There appears to be a tissue-barrier between embryo and host bodycavities.

D

A

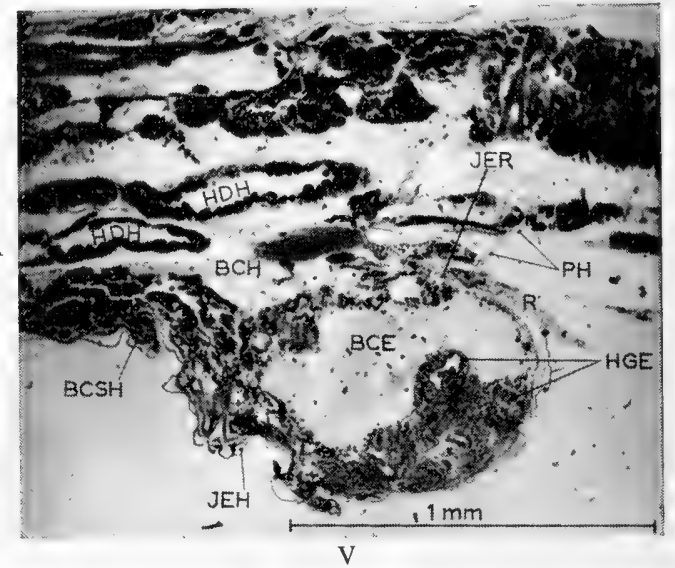

A : anterior, $\mathrm{AH}$ : abdomen of host, $\mathrm{BCE}$ : body cavity of embryo, $\mathrm{BCH}$ : body cavity of host, BCSH: base of copulatory style of host, $\mathrm{D}$ : dorsal, $\mathrm{HDH}$ : hepatic diverticula of host, HGE: hind-gut of embryo, JEH: junction between embryo-tissues and normal host tissues, JER: junction between embryo-tissues and regenerating tissues of host, P: posterior, $\mathrm{PH}$ : pleopods of host, $\mathrm{R}$ : regenerating anterior abdominal appendages of host, $\mathrm{V}$ : ventral.

activity in the liver of the rat facilitates the induction of tumours (Glinos et al., I950). The apparent paradox again may prove to depend on the relative timing of the two processes. These quantitative interactions seem to contrast with a number of records of qualitative, organisational indifference between the two types

TABLE 3

EVIDENGE CONCERNING QUALITATIVE INTERACTION BETWEEN TISSUES OF DIFFERENT MORPHOGENETIG TYPE, WHEN APPROXIMATED BY GRAFTING

Type of tissues approximated

Indifference recorded

Interaction recorded

Regenerating and cancerous

Regenerating and embryonic

Regenerating and mature
Peredelsky, I94 I

Breedis, I954

Ruben, I955

Emerson, 1940

Needham, I950a

Waddington, I940

Hubl, I95I

Ruben, 1955
Rose and Wallingford, I948

Umanski, I933

Schotté, 1937; Schotté and Hummel, 1939

Trampusch, I95I ;

Gebhardt, I926

Morphallaxis, generally 
of "growth" (Table 3). A similar indifference is often recorded between regenerative and embryonic "growths", and between regenerating and normal tissues (Table 3), even when grafted in close proximity (Needham, I950a). Two "organisers" in one embryo behave similarly. The qualitative interactions recorded by others (Table 3) usually have been slight and might be spontaneous reactions by each growth. In Asellus (Needham, I950a) the epidermis and exoskeleton of approximated normal, embryonic and regenerating tissues became continuous throughout, yet they remained a complete mosaic anatomically (Fig. I). In view of their contrasted metabolisms (p. 6og), some degree of independence between regenerating and local, parental tissues seems essential, and actual mechanical barriers have been recorded (Needham, I952 p. 22; Singer, I954).

\section{THE STAGES IN AN ACT OF REGENERATION}

For the present purpose a detailed analysis of the causal sequence of events in typical epimorphic regeneration (Needham, I 952) is not required, but only of the chronical and other relationships of growth-components to qualitative components and to the process as a whole. Particularly important is the biphasic nature of the whole process, an initial phase of regression (R-phase) involving demolition, dedifferentiation and segregation, followed by a P-phase of progressive processes, aggregation, cell-proliferation, growth and differentiation. The R-phase appears to be more than merely the clearing-up of damaged material, and is probably necessary to restore an "embryonic" condition favourable to new growth,_- "reculer pour mieux sauter",--particularly in the higher animals. Metabolism locally, and to some extent systemically, shows a corresponding biphasic change.

The cytolytic demolition of cells and intercellular material, damaged by injury and subsequently by foreign organisms and other secondary causes, is the first morphogenetic process and is often quite completed before the histolytic dedifferentiation of intact cells and material begins (Thornton, 1938). The latter is mainly a chemical process (Butler and Schotté, I 94I) as in the dedifferentiation of metamorphosis in insects, whereas phagocytosis plays a major role in demolition. Dedifferentiation does occasionally (Kamrin and Singer, I 955; Butler and Blum, I955) but not usually (Thornton, I954), occur without trauma, but it can then spread far from the site of injury. It is enhanced by a number of experimental procedures, e.g. denervation, irradiation and colchicine-treatment and halted by a regenerating nerve-supply and by a living, grafted blastema. It is not yet proved that all dedifferentiated cells are reused (Butler and Schotté I94I; Thornton, I953) and under certain experimental conditions certainly many are not.

However most of the cells of the blastema have a local origin, in Amphibia (Butler, I935; Butler and O'Brien, I942). In the lower Metazoa (Wolff and Dubois, I 947, I948; Evlakhova, I946) and to some extent in other Metazoa (Wolff and Wey-Schué, I953) immigrant, undifferentiated neoblasts contribute to the blastema, but there is at present no definite evidence that this affects the extent of dedifferentiation. Also there is no evidence that the extent of dedifferentiation is proportional to the amount of tissues to be regenerated: like nitrogen 
"flow" it seems much too sensitive to external factors (Butler and Schotté, I 94 I, 1944) to have any such precise quantitative significance and the production of a qualitative condition of regression is probably the extent of its significance. Dedifferentiation occurs also in regenerating Protozoa, so that it is not a reorganisational phenomenon which evolved only at the tissue-grade of structure. Presumably therefore it is a chemical rather than a cellular phenomenon, cytolytic in "spirit", though histolytic in appearance in metazoa. Cytolysis yields materials with potent inducing properties when injected into embryos (Holtfreter, I948; Weiss, 1950) and a similar mechanism of induction of regenerates seems probable; the complete cytolysis during demolition-processes perhaps induces a milder cytolytic change in intact cells which so become embryonic in character. The cells of the blastema are large, with large nuclei and nucleoli, and relatively little, clear cytoplasm (Horn, I 942) like the typical cells of a young embryo. Granules reappear in the cytoplasm later (Thornton, I938) probably after the phase of maximal proliferation.

It is evident enough that the blastema-cells, like cells in culture-media, and other appropriate conditions, are embryonic in their dedifferentiation and in their high powers of proliferation, but further evidence is necessary to prove that they return to a generally embryonic condition. Normal periosteal tissue of mammals does not readily proliferate in vitro but cells from a healing fracture do (Allgöwer and Rosin, I 953), so that there is little doubt also of the similarity between regeneration and culture in vitro. The movements of sodium and potassium between recently explanted cells and their medium (Davies, 1954) resemble these movements in regenerating tissues, and it would be valuable to know if a low $\mathrm{K} / \mathrm{Na}$ ratio characterises also the cells of young embryos. An uptake of water certainly renders cells more "embryonic" (J. Needham, I93I) and the glycolytic respiration of early regenerating tissues compares with that of many young embryos. The amount of the following substances normally diminishes with age, but increases again in regenerating tissues: hexosamine (Boas and Foley, I954); glucuronidase (Mills et al., 1953) cathepsins (Sokolova, I942) sulphydryl compounds, alkaline phosphatase (Junqueira, I 950). Yakovleva (I 943) found that the condition, as well as the amount, of pentosenucleic acids in regenerating tissues became more like that of embryo-tissues. An increased digestibility of the tissues (Orechovitch and Sokolova, 1940) is probably an embryonic property, though in general regenerating tissues are said to be less sensitive than embryo-tissues to chemical agents (Singer, I 952). Cells of regenerating tissues stain less readily than do mature cells (Singer, 1954) and this is probably true of the cells of early embryos and of the cells of lower invertebrates. However embryonic and regenerating tissues both are more sensitive than mature cells (Brunst, I938) to irradiation.

It is probably significant that the regenerative power in the limbs of Anuran Amphibia, which normally fails at metamorphosis, may be preserved by the repeated evocation of regeneration throughout metamorphosis (Schotté, I 939; Morosow, I94I; Polezhayev, 1946) and this has been seen in insects also (Przibram I 932). The "youth" of the cells is thereby preserved; metamorphosis itself is delayed (Polezhayev, I946).

Starvation of Planarians causes degrowth, dedifferentiation and an increase 
of regenerative powers (Abeloos, I930; Sivickis, I933). The number and size of cells decreases (Brondsted, I955) and oxygen-consumption decreases (Hyman, I919, I920) as in early regeneration. Repeated evocation of regeneration prolongs the life of Planarians (Chranova, I938). Rejuvenation in Protozoa has considerable similarities to the early phase of regeneration (Weisz, 1954).

The regeneration of nerves has been interpreted rather commonly as a return to an embryonic condition (Van Biervliet, I 900 ; May, I930; J. Needham, I942, p. 554; Bodian, 1947; Mannell and Rossiter, 1954).

In some animals very extensively (Wolff and Dubois, I947; 1948), and in most to some extent (Wolff and Wey-Schué, 1953) immigrant neoblasts contribute to the blastema. Cell-migration, whether local or over long distances, marks the "turn of the tide" in regeneration. As in vitro (Medawar, I940) migration is a necessary prelude to cell-proliferation (Abercrombie and Johnson, 1942, I 946), though Lüscher (1946), and Chalkley (I954), saw active cell-proliferation in the stump of the amputated limbs and tail of Amphibia, in the early stages, indicating that the onset of proliferation may precede that of migration. The daughter cells of this activity move into the "open" and later the maximal rate of proliferation is in the blastema itself, and moves progressively distalwards (Table 4). The epidermis behaves differently from the deeper tissues because of its role in healing the wound and because its cells move less individually, though they do show considerable shearing and jostling (Lehmann, personal communication). It is usually claimed (Schaxel, I92 I; Butler, I933; von Levetzow, I939; Waechter, I949; Paton, 1955) that there is no cell-division in the epidermis until after wound-closure, but again Manner (I953) saw mitosis in the stump-epidermis prior to cellmovements. After closure there is rapid proliferation in the covering cells, which become the epidermis of the regenerate, so that the epidermis probably behaves as do the other tissues, but somewhat ahead of them in phase.

Like the epidermis, the gut, blood vessels, and other epithelia regenerate in continuity in the Amphibia (Korschelt, 1927) and so do the non-epithelial tissues,-bone and muscle (Naville, I 922-24; Lüscher, I 946; Waechter, I949; Chalkley, 1954). Only organs completely removed, and connective-tissue, are regenerated from cells of uncertain, and possibly distant, origin. On the other hand in some animals neoblasts may enter tissues otherwise regenerating in continuity, and proliferate there (Korschelt, I 927, p. 298; Brondsted, I955; p. 75).

At first the growth of the blastema is almost entirely by cell-proliferation or "hyperplasia" (Litwiller, I939; Polezhayev and Ginsburg, I944; Singer and Craven, I948; Moment, I953) and only later by cell-enlargement or "hypertrophy". There is no evident differentiation at this stage though the blastematissues become "determined" qualitatively (Lüscher, I952; Needham, I952). Proliferation is maximal after one quarter of the total time of regeneration (Table 5), and corresponds to the steepest part of the curve of size/time. If proliferation is multiplicative then five to six cell-generations, each fifteen days long, would suffice for the complete regeneration of the limb of the newt studied by Litwiller (1939). The larvae of some species of newt regenerate four to five times as rapidly as this, however. Moreover the proximodistal progress of maximal activity implies an approximation to linear, arithmetic, rather than to geometric progression, so 
TABLE 4

DIRECTION OF PROGRESS OF REGENERATION: RECORDS AND INTERPRETATIONS (a) Centrifugal

\begin{tabular}{|c|c|c|c|}
\hline & Animal & Nature of regenerate & Reference \\
\hline (I) & $\begin{array}{l}\text { General } \\
\text { Tubularia } \\
\text { Planarians } \\
\text { Annelida } \\
\text { Sabella } \\
\text { Actinotrocha larva, } \\
\text { Phoronis } \\
\text { Asellus } \\
\text { Crustacea (Decapoda) } \\
\text { Dixippus } \\
\text { Coleoptera and } \\
\text { Hemiptera } \\
\text { Arachnida } \\
\text { Amphibia } \\
\text { Lizard }\end{array}$ & $\begin{array}{l}\text { hydranth } \\
\text { hind end of body } \\
\text { hind end of body } \\
\text { tentacles of crown } \\
\text { body } \\
\text { antenna } \\
\text { walking legs in general } \\
\text { antenna } \\
\text { walking legs } \\
\text { legs } \\
\text { legs } \\
\text { tail }\end{array}$ & $\begin{array}{l}\text { Berrill, I } 948 \\
\text { Brondsted, I } 955 \\
\text { Korschelt, I927; p. } 329 \\
\text { Berrill and Mees, } 1936 \\
\text { Schultz, I903 } \\
\text { Korschelt, I927, p. } 328 \\
\text { Przibram, I } 909, \text { p. } 99 \text {; } \\
\text { Hiatt, I948 } \\
\text { Stock, I937 } \\
\text { Millot, I } 93 \text { I ; p. } 63 ; \\
\text { Lüscher, I } 94^{8} \\
\text { Korschelt, I927, p. } 329 \\
\text { Korschelt, I } 927, \text { p. } 553 \\
\text { Korschelt, 1927, p. } 55^{2}\end{array}$ \\
\hline (2) & $\begin{array}{l}\text { Particular portions or tissues } \\
\text { Isopoda and Amphipoda }\end{array}$ & $\begin{array}{l}\text { pleoplods } \\
\text { limbs, mesodermal tissues } \\
\text { limbs, skin and connective } \\
\text { tissues, } \\
\text { epidermal organs }\end{array}$ & $\begin{array}{l}\text { Zeleny, I907; Haseman, } \\
\text { I907; Przibram, I909, p. } 99 \text {; } \\
\text { Paulain, I } 938 \\
\text { Przibram, I909, p. } 99 \\
\text { Child and Young, I903 } \\
\text { Tornier, I906, Schaxel, I92 I } \\
\text { Korschelt, I927, p. } 328\end{array}$ \\
\hline (3) & $\begin{array}{l}\text { Particular stages of the pro } \\
\text { Amphibia }\end{array}$ & $\begin{array}{l}\text { histogenetic induction } \\
\text { limb, inflation at eclosion }\end{array}$ & $\begin{array}{l}\text { Litwiller, I939; Lüscher, } \\
\text { I946; Chalkley, 1954 } \\
\text { Schotté, I939 } \\
\text { Needham, I } 943 \text {, p. } 5^{8}\end{array}$ \\
\hline
\end{tabular}

that the rate would be very high in the active region. Under these conditions proliferation continues until towards the end of regeneration, in contrast to the process in tail-regenerates of Annelids (Moment, I953), where it is completed very early, while the blastema is very small.

In Annelida the division-spindles are very regularly orientated relative to the axis of the body (Berrill, I952), as in early ontogenesis, but no great regularity has been described in other instances. A diurnal cycle of mitosis-rate has been recorded (Litwiller, I940; Jaffe, I954). As in normal growth (Bullough, I948) the maximal activity is in the late night or early morning. In Arthropoda proliferation is geared more or less closely with the moult-cycle (Paulain, I938; Charniaux-Legrand, I95I; O'Farrell and Stock, I954).

Ceil-number increases more rapidly than the absolute size of the regenerate and the cells become both smaller and more crowded (Litwiller, I939). However the 
Table 4 continued

DIRECTION OF PROGRESS OF REGENERATION: RECORDS AND INTERPRETATIONS

(b) Centripetal

\begin{tabular}{ccc}
\hline Animal & Nature of regenerate & Reference \\
\hline
\end{tabular}

(I) General

$\begin{array}{ll}\text { Tubularia } & \begin{array}{c}\text { whole hydranth and indi- } \\ \text { vidual tentacles } \\ \text { head-end of body }\end{array} \\ \text { Hydra } & \text { head-end of body } \\ \text { Planarians } & \text { head-end and tail-end } \\ \text { Annelida } & \text { arm } \\ \text { Asteroidea } & \end{array}$

Child, r $94^{\text {I }}$ p. 36

Abeloos, 1932, p. 208

Brondsted, 1955

Abeloos, 1932, p. 208

King, I898; Dawydoff, I 90 I;

Morgulis, I 912 ; Shapiro,

1914

Phoronis

adult, body

Schultz, I 903

Cambarus

chela and anterior walking legs

General

all regenerates

Haseman, I907

Abeloos, 1932, p. I 18

(2) Particular portions or tissues

Isopoda and

antenna, distal portion

Zeleny, I907; Haseman,

Amphipoda

Cambarus

Arthropoda

pleopods, distal portion

limb, epidermis

Amphibia

limb, skeleton

General

internal organs

I907; Paulain, 1938

Przibram, I909, p. 99

Child and Young, 1903

Tornier, I9o6; Schaxel, I92 I

Korschelt, I927, p. 328

(3) Particular stages of the process

General dedifferentiation

Asterias

arm, determination

various authors

Amphibia

limbs, determination and differentiation

Child, I 94 I ; p. 336

Guyenot and Schotté, I923

(see Abeloos, 1932, p. 206)

reduction in size is small by comparison with that of the blastomeres of some eggs, during cleavage, and true growth no doubt begins at the outset of the P-phase. Nevertheless experimental treatments appear to distinguish the phase of mainly proliferative growth from the later phase of hypertrophy (Paulain, I 938, p. 308).

In some Arthropoda, e.g. Leander (Paulain, 1938), regenerative growth of limbs is visibly continuous, as in animals of other phyla but often, as in Asellus, nothing is visible externally until after moulting, when the growing regenerate undergoes "eclosion" by distension with blood, a more dramatic counterpart of the normal "growth" of most Arthropods. The limb-regenerate of Carcinus grows continuously, though apparently slowly because it is very much folded up, and therefore it also shows a marked eclosive "growth". In the cockroach (O'Farrell and Stock, 1953, 1954), and probably in insects generally, cell-proliferation is restricted to a short period in the middle of each "stadium" or "intermoult". In Arthropods with long stadia this middle period is a much longer fraction of the whole, and the pre- and post-moult arrests of growth therefore are less evident.

The relationship between growth and differentiation needs further investigation (Needham, I 952). The results of differentiation become increasingly evident in the later stages of regeneration but so do the results of growth, though the growthrate is maximal very early. The early incidence of determination implies that 
differentiation may begin as early as growth, though serial culture of cells in vitro shows that it is held in check during rapid proliferation, possibly by a "dilution" mechanism, like the kappa particles of Paramecium (Sonneborn, 1948). Certainly there is considerable overlap between the processes of growth and differentiation, and the syncytial skeletal muscle-fibres of Vertebrates continue to grow and proliferate nuclei long after typical differentiation is evident. A good deal of macro- and micro-scopic differentiation in fact is simply differential growth. The amphibian limb-regenerate grows in length much more than in width (Singer and Craven, 1948) largely correlated with the elongation of the differentiating muscles. At the molecular level there are three possible modes of differentiation, by the rearrangement or by the chemical conversion of existing molecules or by the intussusception of new types of molecule, - this last a growth-process.

$\checkmark$ isible differentiation in a regenerate begins at a greater absolute size in a large than in a small individual (Paulain, I938) and if the growth of a regenerate is retarded then the regenerate may differentiate, normally, at an abnormally small size. These facts indicate some independence between differentiation-rate and growthrate, but if retardation is severe then differentiation also is abnormal (Paulain, I938; Needham, I950a) and the abnormality has a quantal aspect i.e. components either differentiate completely and normally or are completely lacking.

The role of quantitative, rate-differences in controlling differentiation, has been stressed by Child and his school (Child, I94I). The integrity and organisation of a regenerate depends on a system of differential control, proportional to a ratesystem, differential in space. Brøndsted (I 955) finds support for this interpretation. If the normally smooth gradient in rate from the mid-line to the lateral edges of a planarian body-regenerate is interrupted by an appropriate procedure, then two heads develop side by side at the anterad surface. Chandebois (I952) has thrown further light on the nature of this "dislocation" between right and left sides but her results essentially confirm the quantitative factor in both normal and abnormal differentiations. A piece cut from the lateral edge of a regenerate takes longer to re-regenerate than a piece from near the mid-line (Brøndsted, I955), again a quantitative manifestation. Chela-reversal during its regeneration in certain Crustacea (Huxley, I932; Darby, I 935, I939) and similar compensatory reversals (Abeloos, I 953; Ludwig and Ludwig, I 954) as well as the qualitative abnormalities known as heteromorphs (Przibram, I934) all seem to be controlled by essentially quantitative differentials.

Most heteromorphs are qualitative abnormalities along the long axis of the body. Some of these are relatively easily controlled by quantitative changes, for instance in an intrinsic gradient of electrical potential (Barth, I934; Marsh and Beams, I 952; Moment, I 953). One weakness of Child's theory (Needham, I 952) is the stress on a single quantitative morphogenetic gradient. The electrical (Moment, I954) and other manifestations (Huxley and De Beer, 1934) of quantitative gradients in the main axis of the annelid body in fact are amphiclinous. If it be admitted further that these gradients must be much more complex in local detail, and that some metabolic processes do, but others do not (Von Bertalanffy; I 942), coincide with morphogenetic gradients, then the application of the "quantitative theory" becomes reasonably clear. 
TABLE 5

TIME-TABLE OF EVENTS IN A TYPICAL ACT OF EPIMORPHIC REGENERATION

\begin{tabular}{lcc}
\hline \multicolumn{1}{c}{ Stage } & $\begin{array}{c}\text { Days from } \\
\text { the outset }\end{array}$ & $\begin{array}{c}\text { Percentage of total } \\
\text { time }\end{array}$ \\
\hline Epidermal overgrowth (closure) completed & 0.8 & 3.2 \\
Demolition completed & 0.8 & 3.2 \\
Onset of blastema-formation & 1.9 & 7.6 \\
Dedifferentiation complete & 2.6 & I I.4 \\
Cell-proliferation maximal & 5.8 & 23.2 \\
Gross differentiation first visible & 7.9 & 31.6 \\
Histological differentiation first visible & 16.3 & 65.2 \\
Regeneration complete & 24.5 & 100 \\
\hline
\end{tabular}

The work of Sengel (I 95I) showing that it is possible to induce partial regenerates in planarians, consisting only of organs from the pharynx backwards, shows that regeneration does not fail completely if the normal apex of the morphogenetic gradient is absent and indeed the production of micro- and acephalic regenerates by Child and others also illustrates this. Truncated anterior regenerates similarly are not uncommon, particularly in hydroid coelenterates (Child, I94I). This further stresses the qualitative effects of quantitative differences in morphogenetic potential, since only a quantitative, and no qualitative, change results if the end of a gradient is removed or suppressed. Amputation removes part of the intrinsic gradients (Brondsted, I955) in morphogenetic potential, but normally this is regenerated in the process of material regeneration (Abeloos, 1932, p. 175).

De Giorgi and Guyenot (I923) have shown that growth and differentiation can be dissociated in a regenerate, just as they appear to be in tissue-culture. If the leg-or tail-blastema of a urodele is grafted in an "indifferent" region of the body it grows to the size at which visible differentiation normally begins but does not differentiate. If reamputated it regrows to the same stage and again does not differentiate.

Differential growth continues for a long time after differentiation, particularly in whole-body regenerates of planarians (Dresden, I940). This no doubt is morphallactic change which always plays some part in whole-body regeneration and is active later than the epimorphic component (Abeloos, I932). In some instances, particularly when regenerates or supernumerary growths are induced by grafting, there may be extensive differential movements of parts (Goldsmith, I940), due largely to active resorption of tissues in one region and proliferation elsewhere (Santos, I93 ; Miller, r938).

The stages of regeneration overlap in time, at any point, and for each stage there is a spatio-temporal sequence along the regenerate (Table 4). Moreover there is asynchrony also between epidermis and deeper tissues at any point. Accurate time-tables for the process are not possible therefore. Again the relative durations of the various stages probably differ considerably in different animals. Table 5 gives average times from amputation to the main epochs in the process, computed from available results on whole-body and limb-regenerates, in the invertebrates and lower vertebrates. 


\section{QUANTITATIVE ASPECTS OF REGENERATION}

The maximal rate of regeneration in the mammalian liver approaches that of embryos and of malignant cancerous tissue (Christensen and Streicher, 1948). The maximal absolute rate probably varies relatively little throughout the animal kingdom (Table 6), though because the lower animals in general are small, they

TABLE 6

ABSOLUTE LINEAR RATES OF REGENERATION: REPRESENTATIVE EXAMPLES

\begin{tabular}{|c|c|c|c|}
\hline Animal & $\begin{array}{l}\text { Nature of } \\
\text { regenerate }\end{array}$ & $\begin{array}{l}\text { Average rate } \\
\text { in mon per day }\end{array}$ & Reference \\
\hline Podarke & body & $0.03-0.05$ & Morgulis, Igog \\
\hline $\begin{array}{c}\text { Allolobophora } \\
\text { caliginosa }\end{array}$ & tail-end & $\left\{\begin{array}{l}2.0 \text { (max.) } \\
0.2 \text { (average) }\end{array}\right.$ & auct. unpublished \\
\hline Asellus & $\operatorname{limb}$ & $0.15-0.30$ & Needham, ig49a \\
\hline Portunus & chela & 0.5 & Zeleny, I 908 \\
\hline Tenebrio & legs & O. I & Křiženecký, I 9 I 7 \\
\hline Amieurus & barbel & 0.04 & Kamrin and Singer, 1955 \\
\hline Rana, tadpole & tail & $0.23-0.4$ & Durbin, I9o9; Ellis, I9o9 \\
\hline Amphibia & skin-wound & $1.5-4.0$ & Braun and Orlova, 1945 \\
\hline fowl & feathers & $0.8-2.0$ & Juhn et al., 1931 \\
\hline $\operatorname{man}$ & skin & $0 . \mathrm{I}-0.2(\mathrm{app})$ & Du Noüy, I936 \\
\hline mammal & gastric mucosa & I.O & Janowitz et al., I955 \\
\hline rabbit & nerve (sciatic) & $2.5-4.5$ & Gutmann et al., I $94^{2}$ \\
\hline $\operatorname{man}$ & nerve & I.0-3.0 & Seddon et al., 1943 \\
\hline deer & antlers & up to 12 & Wislocki and Singer, I 946. \\
\hline
\end{tabular}

complete a regenerate of a particular relative size more rapidly than a larger animal (Table 7). Again the rate for intracellular regeneration, as in a nerve-fibre, is about equal to that for a cellular structure such as a featherbud.

The absolute rate of regeneration is not constant throughout one act, though it is sometimes approximately so (Paulain, I938), especially in Crustacean limbs; however there is always an initial "lag" period, corresponding to the regressivephase, and usually the cessation of growth is far from abrupt, the rate declining to zero according to an approximately exponential relation (Paulain, I938). Seddon et al. (1943) concluded that a number of very diverse algebraical relations fitted, with equal approximation, this part of the curve of length/time, for a regenerating mammalian nerve. A hyperbolic relation was most satisfactory on empirical grounds. Here, as in a number of the examples given by Paulain, the rate was virtually maximal once it did begin, after a long lag period. A relatively abrupt onset is indicated also by the results of Ellis (1909), for the tadpole's tail, of Du Noüy (1936) for human skin, and of Needham (1949a), giving a composite curve for limb-regeneration in a population of Asellus. Other instances show a much more gradual onset (Ellis, rgog Durbin, r 909; Paulain, I938; Singer and Craven, 1948) so that the complete curve is sigmoid (Fig. 2). In fact, no doubt, ail curves of regeneration and of other types of growth are essentially sigmoid since the transition from zero to maximal speed and back can never be instantaneous (see Thompson, 1942, p. 152). 
A number of factors may tend to make the pre-inflectional part of the curve more rounded, for instance if dedifferentiation continues after growth has begun, if the onset is not synchronous (Paulain, I938, p. 206) throughout the regenerate ( $c f$. Medawar, 1950), if there is an autocatalytic component of growth (Robertson, I923) or any other component which causes a geometric mode of increase. The growth of a population of microorganisms (Hinshelwood, 1946; Knaysi, 1951), and of an embryo, no doubt meets this last requirement, but regenerates probably often approximate to linear extrusions from the parent body-or to intrusions into it, in the case of whole-body regenerates of lower animals. In a regenerating nerve-fibre this is particularly true of the visible manifestation of growth; whatever the nature of the actual process of synthesis in the cyton, material flows into the growing axon or dendron at a linear rate (Young, 1942, 1945). Here, moreover, (Seddon et al., l.c.) the R-phase must be completely finished in the long period before growth begins. In limbregenerates, also, the gross increase may be virtually linear, because activity sweeps as a linear wave along the structure (Table 4 ), even if the activity of individual cells is geometric. It is quite probable (p. 641) that the linearly regenerating nerve determines the linear pattern of growth of the whole regenerate. The post-inflectional part of the curve is more rounded in this type of regenerate because some cell-division persists in the wake of the wave, and even more because growth by cell-hypertrophy which follows is not so sharply "quantised". Moreover, for each small cell proliferated in the pre-inflectional, a large cell ceases growth in the post-inflectional phase, so that the scale of the curve becomes magnified.

The curves relating to time, the total amount of various substances in the regenerating mouse-liver also are typically sigmoid (Tsuboi et al., 1954). The liver regenerates throughout its remainder and therefore probably geometrically, giving a rounded pre-inflectional portion to the curve.

Robertson (1923) pointed out the similarity between the sigmoid curve of growth and that for a monomolecular autocatalytic reaction, when the amount of the reagent is limited, and found that actual measurements of growth fitted very tolerably the algebraical relation for this chemical reaction. The resemblance might be explained if the curve of growth were determined by a particular "master reaction". It is doubtful if regeneration, or most other types of growth, are limited by exhaustion of materials, but a progressive retardation, due to a "deliberate" inhibitor which determines the limiting size, is consistent with a relation of the same form. There is in fact some evidence (p. 636) of an auto-accelerator, auto-inhibitor pair of substances, though probably no results yet obtained are sufficiently accurate to ascertain the exact mathematical expression of their action (Gray, I929).

Robertson derived his relation from its first differential: $\mathrm{d} x / \mathrm{d} t=k x(A-x)$, where $\mathrm{d} x / \mathrm{d} t$ is the rate of increase of $x, A$ is the initial amount of reagent and $k$ is a constant. On the modified hypothesis applied to regeneration, $A$ represents the definitive size and $(A-x)$ measures the effect of the progressively increasing inhibitor. An alternative interpretation of the relation above is that the instantaneous rate of regeneration, $\mathrm{d} x / \mathrm{d} t$, is related to the amount still to be regenerated, measured by $(A-x)$. This relation was tested by Przibram (I917). It follows as a corollary that the initial rate, the maximal rate, or any other suitable measure of regeneration-rate for any particular act, should be related to the amount amputated. This was verified qualitatively by Spallanzani and more quantitatively by many subsequent workers (Zeleny, 1905, 1907; Ellis, 1909; Durbin, 1909; Przibram, 1917; Paulain, I938; Needham, I947a; Lüscher, 1948). This relation, again, is sigmoid (Thompson, I 942 , p. 276), the response, like most physiological "mechanisms", being most sensitive over the middle of its normal range. Thompson plotted time, reciprocal of rate, against amount amputated, but the rate itself also gives a sigmoid curve. The results of Needham (1947a) on the limbs of Asellus also indicate a sigmoid relation. In the tadpole's tail (Ellis, 1909) further increase in the amount amputatated beyond that giving a maximal rate of regeneration results in a progressive decline in absolute rate which approaches zero when $75 \%$ of the tail is amputated. Paulain (1938) obtained a complex curve for the antenna of Gammarus which, after amputations distally, regenerates from material in the rest of the antenna, but from material of the body when amputation is near the base. Regenerationrate declines as the size of the residuum is decreased, up to the critical point at which bodymaterials begin to contribute, and then increases progressively, because the latter have a progressively shorter distance to travel.

Literature p. 649 
TABLE 7

TOTAL TIME FOR REGENERATION：REPRESENTATIVE EXAMPLES

\begin{tabular}{|c|c|c|c|}
\hline Example & $\begin{array}{c}\text { Type of regener- } \\
\text { ation }\end{array}$ & Time & Reference \\
\hline Coleps (Ciliata) & paratomic & $20-25$ mins & Korschelt, 1927, p. 30 \\
\hline Stentor (Ciliata) & $\begin{array}{l}\text { epimorphic and } \\
\text { morphallactic }\end{array}$ & 24 hours & Korschelt, 1927, p. 22 \\
\hline Ephydatia (Porifera) & reconstitution & 7 days & Brien, I937 \\
\hline Tubularia (Coelenterata) & morphallactic & $2-3$ days & Moore, I9io \\
\hline Hydra (Coelenterata) & & $4^{-6}$ days & Mattes, 1925 \\
\hline Mnemiopsis (Ctenophora) & epimorphic & 8-i I days & Coonfield, I 936 \\
\hline $\begin{array}{l}\text { Planarians } \\
\text { (Turbellaria) }\end{array}$ & $\begin{array}{l}\text { epimorphic and } \\
\text { morphallactic }\end{array}$ & 7-9 days & Lender, 1952, r 954 \\
\hline Maricolan Turbellaria & $\begin{array}{l}\text { epimorphic and } \\
\text { morphallactic }\end{array}$ & 20-35 days & Lüs, I926; Lloyd, I9I4 \\
\hline Lineus (Nemertinea) & epimorphic & $27-30$ days & Coe, 1929 \\
\hline Lumbriculus, Tubifex & epimorphic & $2 \mathrm{I}-40$ days & $\begin{array}{l}\text { Stone, I932; Turner, } \\
\text { I934; Stephan-Dubois, } \\
\text { I952 }\end{array}$ \\
\hline $\begin{array}{l}\text { Triops (Apus), Oniscus } \\
\text { Asellus, Leander }\end{array}$ & epimorphic & I6-28 days & $\begin{array}{l}\text { Przibram, I909, p. I I3; } \\
\text { Ost, I9o6; Auct.; } \\
\text { Paulain, I938 }\end{array}$ \\
\hline $\begin{array}{l}\text { Large decapod } \\
\text { Crustacea }\end{array}$ & epimorphic & up to 2 years & Przibram, I909, p. II 3 \\
\hline Octopus, Nassa (Mollusca) & epimorphic & 20-28 days & $\begin{array}{l}\text { Lange, 1920; Hanko, } \\
\text { 1914 }\end{array}$ \\
\hline Asteroid Echinodermata & epimorphic & $90-180$ days & $\left\{\begin{array}{l}\text { King, I898; Richters, } \\
\text { I912; Shapiro, I914 }\end{array}\right.$ \\
\hline Clavelina (Tunicata) & (see p. 589) & 12 days & Berrill and Cohen, I936 \\
\hline Larval urodeles & epimorphic & I4-23 days & various authors \\
\hline Adult urodeles & epimorphic & up to 100 days & various authors \\
\hline Mammalian cornea & epimorphic & $\frac{1}{2}-5$ days & various authors \\
\hline skin & epimorphic & up to 75 days & s various authors \\
\hline liver & $\begin{array}{l}\text { compensatory } \\
\text { hypertrophy }\end{array}$ & $4^{-21}$ days & various authors \\
\hline nerve & epimorphic & $100-200$ days & various authors \\
\hline
\end{tabular}

The first differential, i.e. the slope, of the curve of mean regeneration-rate relative to amount removed gives a measure of specific regeneration rate, i.e. rate per unit of tissue removed. This is maximal at the point of inflection of the original curve, which usually corresponds to the amputation of rather a small fraction. Consequently graphs of specific regeneration rate, relative to amount removed, for instance the "index of cicatrisation" of Du Noüy (1936), decline virtually throughout the range of the amount amputated. Anderson (1933) recorded no point of inflection in his curve of initial absolute rate, against amount removed from the carapace of Daphnia, probably because he did not study regeneration after very small losses. The curve of specific regeneration-rate derived from his results would resemble that of Du Noüy, likewise dealing with areas.

Du Noüy's index was found to be constant throughout a particular act of regeneration so that the instantaneous rate of regeneration at a point where an amount 
$x^{\prime}$ remains to be regenerated varies according to the amount $x$ initially removed, as shown also by the results of Ellis (I gog) and others. This is probably related to the fact (Abeloos, I930: Brondsted and Brondsted, 1952) that the rate of regeneration is specific to intrinsic properties of the tissues near the point of amputation rather than to the amount of loss itself. It should be noted, in parenthesis, that

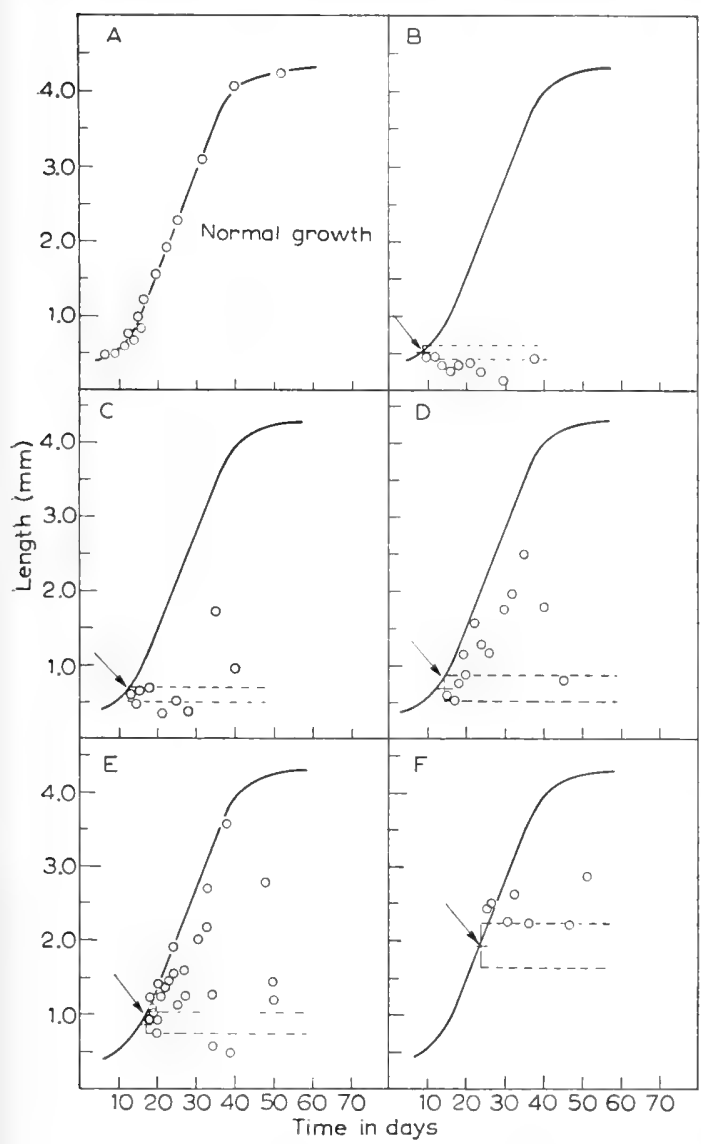

Fig. 2. Graphs of length/time for a regenerating limb of Triturus showing the normal curve of regenerative growth and the effect on this of denervating the limb at various stages of regeneration. The arrows mark the points of denervation and the dots show subsequent growth against the reference lines of the normal curve, and of the projections of the standard deviation for the length at the time of denervation (broken lines). (From Singer and Craven, 1948).

in the whole-body regeneration of planarians, studied by these last workers, this intrinsic power, as measured by the time for complete regeneration, and by the frequency of successful head-regeneration, is maximal near the anterior end, and so decreases disto-proximalwards, in contrast to the absolute initial rate in the various appendages considered above. However further careful quantitative work is required, since in the latter, also, the time required for regeneration is minimal when least is removed, and measures specific, not absolute, regeneration-rate.

The absolute amount regenerated per unit time increases also with the number removed, of a serially repeated organ, for instance the radial series of tentacles and arms of the medusa Cassiopea, and the brittle-star Ophioglypha (Zeleny, I905, I 907) and the linear series of limbs of arthropods. Zeleny found that even the specific 
replacement-rate, i.e. the rate per arm, increased with the number removed. As he pointed out, here the extent of injury as well as the amount of tissue removed increased in that order, whereas it increases little with increasing amount removed within one appendage. There is a mutual stimulation by the various wounds and blastemata. Between arthropod limb-regenerates this effect is smaller (Zeleny, l.c.; von Ubisch, I915; Needham, I946b) though probably significant (Przibram, I917; Huxley, I932). The curve of rate/number of organs removed again is sigmoid, i.e. sensitivity is maximal for an average degree of loss.

The regeneration-rate of different organs varies considerably, in a pattern which probably has biological significance (Needham, I952). Even within one organ (Needham, I947a; Lüscher, I 948) the detailed pattern, superimposed on the main gradient considered above, may be complex. Sharp discontinuities are evident in the gradient in the body of some Planarians (Brøndsted, I955) and other animals. Such sharply defined changes must depend on local intrinsic differences.

Age is one of the most important of natural factors affecting regeneration-rate, though there are few detailed quantitative studies of this (Zeleny, I 9o9; Przibram, I 909; Abeloos, I930; Curtis and Schultze, I934; Du Noüy, I936; Needham, i 949a). Zeleny's conclusions generally have been confirmed; most animals regenerate absolutely faster as they grow older and larger, but relatively more slowly, per unit of body-size, and per unit of tissue removed, just as for an increasing amount of loss in an individual of fixed age. It follows, therefore, that a larger animal replaces a loss of a particular size more rapidly than a smaller individual, and Anderson ( I 933) found this also for a two-dimensional wound in the carapace of Daphnia. Zeleny decided that even the relative rate of regeneration of the chela of the crab, Portunus, increases with age, but this was based on his conclusion that the duration of the stadium did not change with age, whereas in fact his records, when plotted graphically, or averaged, indicate a progressive lengthening of the stadium from eleven to twenty days; the rate per day, per unit of body-size, decreases with age as in other animals.

In the limbs of Asellus (Needham, I949a) the absolute regeneration-rate per day at first increases with body-size (age) but it is then constant over a considerable range of bodysize,-probably incidental evidence that regenerative growth is in arithmetic rather than in geometric progression (p. 603). The specific regeneration-rate, per unit of body size, therefore declines progressively with age (Fig.3), following a curve similar to that of Du Noüy's index of cicatrisation, as a function of amount lost. Like the specific rate of normal growth, the rate of decline itself decreases progressively (Medawar, 1945). Both the initial rate of decline, and the rate of slowing of this decline, are relatively greater for body-growth (Fig. 3) than for regeneration.

It follows that older individuals take longer than younger ones to regenerate the same percentage-loss. In the tail of frog-tadpoles (Ellis, I gog) usually less than $100 \%$ of the loss is ultimately regenerated, and this percentage, again, declines with age. The deficiency may be peculiar to larval Anura which cease to regenerate appendages at metamorphosis, but other indices of regenerative power also decline with age. In Planarians the frequency of successful head-regeneration so 
declines (Brondsted, I955). The quality, also, of a regenerate may change with age (Przibram, I93I) probably as a correlate of the decrease in specific growthrate. Abnormal regenerates in planarians are associated with slow regeneration (Child, I9 13, 192 I).

Some degree of correlation between specific regeneration-rate and the specific

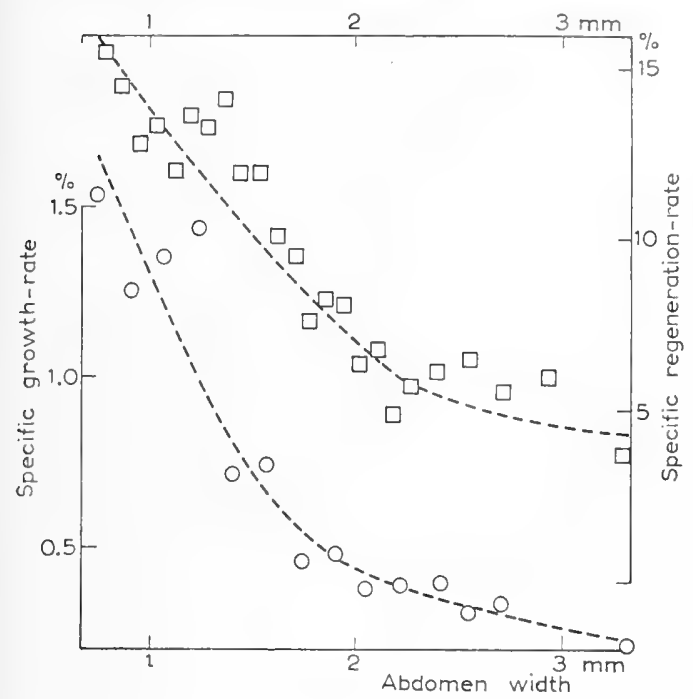

Fig. 3. The relationship between $(0)$ specific growth rate, ( $\square)$ specific regeneration rate per day, and body-size (abdomen-width) in Asellus aquaticus. Both specific rates expressed as a percentage of bodysize. The curves are approximately exponential or hyperboloid. The relative rate of decline, with increasing body-size, is at first greater for growth-rate than for regeneration-rate, and ultimately it is less. The specific regeneration-rate, throughout, is about ten times the normal growth-rate. In both curves there is an irregularity due to a temporary increase in rate, around the size $1.25 \mathrm{~mm}$ abdomen-width for growth-rate, and somewhat later for regeneration-rate. (From results of Needham, 1949a.).

rate of normal growth is to be expected (p. 59I). Apart from their age-decline (Fig. 3), their response to other variables is similar (Przibram, I9 I 7). This is indicated by a further analysis of the results of Needham (1947b), using individuals of a fairly narrow age (size)-band. When the individual results are grouped according to the amount of increase in body-size during the stadium following amputation, the amount of regeneration during that time increases approximately linearly from $1.35 \mathrm{~mm}$ in individuals making no body-growth, to $1.97 \mathrm{~mm}$ in those which grew a maximal amount of $0.27 \mathrm{~mm}$ in body-width. The increase in regenerationrate is therefore about twice that in growth-rate. However, the total excess of regeneration-, over growth-rate depends mainly on the high rate of regeneration pre-empted before there is any body-growth at all.

Przibram ( I 9 I 7) considered that his results were adequately fitted by a function which related regeneration-rate to both the normal growth-rate and the amount still to be regenerated (p. 603). The function was clumsier than necessary, but it shares with the work of Du Noüy ( 1936 ) the virtue of incorporating, simultaneously, more than one relevant variable into an algebraical definition of regenerationrate. Paulain (1938) and others also have stressed the complication of trying to measure regeneration-rate when the standard, body-size itself, is changing. In an animal which is not growing, the growth-parameter becomes a constant, and Przibram's relation for regeneration-rate simplifies and integrates to give one reasonably similar to those of Paulain (1938) and Du Noüy (1936).

Quantitative studies on regeneration-rate in relation to other variables are Literature p. 649 
scanty. Moore (IgIO) found that a sigmoid curve related it to temperature, but Florence Moog (1942) obtained a linear relation, in the same animal, Tubularia, though the size of the hydranth-primordium declined with temperature along a sigmoid curve. The rate/temperature relation described by her probably would be sigmoid over a wider temperature-range. The action of most other variables at present must be considered qualitatively only.

A word seems necessary on the measurement of regeneration-rate in Arthropoda since (a) the group is of critical value for accurate measurements and (b) the complication of ecdysis leads to conceptual difficulties. Although occasionally an individual is "caught" releasing an incompletely differentiated regenerate at ecdysis (O'Farrell and Stock, I953, 1954) usually eclosion is all-or-none and, up to a critical stage, ecdysis is delayed until an "all" eclosion is possible. This led Paulain (1938) and others to regard the stadium or intermoult as the true unit of time, rather than the clock and the calendar. In consequence he found that virtually no experimental factor significantly affected "regeneration rate" defined as the eclosion-size, though conceivably the intermoult may have been considerably prolonged or shortened by retardations and accelerations of the true rate of regeneration. The normal amount regenerated per day is relatively constant over a large range of bodysize (Needham, I949a) but on the other hand is sensitive to deviations from normal conditions.

By contrast the amount regenerated in one stadium ("eclosion-size") although increasing linearly with body-size (Needham, 1949a), is relatively insensitive to changes in conditions because the duration of the stadium in fact is increased or decreased proportionately to any retardation or acceleration of regeneration. The eclosion-length, therefore, is virtually invariant under all conditions, at a particular age and it is this spatial measurement, and not the time-measurement, which might be regarded as a fixed "unit" of regeneration. The relative eclosion-length, per unit of body-size (Needham, I949a), or as a fraction of the definitive length of the regenerate (Charniaux Legrand, I95I), of course is invariant also in age (body-size). Changes in phosphate-concentration and in $\mathrm{pH}$ affect eclosion-size very little, but eclosion-time, and therefore the specific regeneration-rate, very considerably (Needham, 1947b).

Paulain went further and used as the measure of regeneration-rate the constant $b$ of the relation $y=b x^{a}$, where $y$ is the eclosion-size, and $x$ is body-size, $b$ therefore being invariant not only under any variables affecting one stadium but probably also under those affecting body-growth and regeneration throughout life. His wealth of valuable quantitative results therefore have not yielded all possible information and it is unfortunate that more of the original measurements are not available. The power relation, $y=b x^{\alpha}$, is probably an unwarranted complication here. The observed values of $\alpha$ were not very different from unity, indicating that the linear relation, above, is adequate.

\section{METABOLIC CHANGES DURING REGENERATION}

In the R-phase (p. 595) there is locally an increase in acidity (Okunev, 1928, I929), in electropositivity (Crane, I950) and in reduction-potential (Okunev, 1932; Orechovitch, 1934). There is a decrease of oxygen uptake and increased glycolysis, with the production of lactic acid (Okunev, I933; Vladimirova, I934). Proteolytic activity increases, probably promoted by an increase in free SH-groups, which are largely responsible for the increased reduction-potential (J. Needham, I 942). Later another reducing agent, vitamin C, also increases in amount (Ryvkina. 1940). Pentose nucleic acid-concentration decreases (Hydén and Rexed, 1944; Hydén 1947; Novikoff and Potter, I948; Drochmans, I950). The amount 
of mucopolysaccharides in the connective tissues decreases, and similarly, the amount of calcium in broken bones. The water-content of the tissues increases (Paul et al., 1945) and there are probably significant changes in most metabolic processes. These trends are all reversed in the P-phase.

Systemic changes have been little studied in animals with high powers of regen-

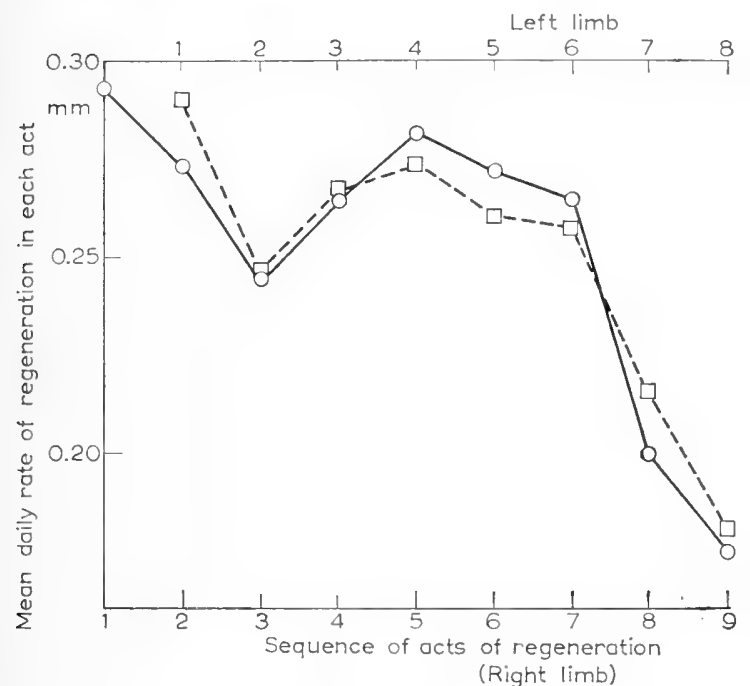

Fig. 4. Changes in gross regeneration-rate per day of a pair of limbs of Asellus, regenerating repeatedly, the rightlimb $(0)$ one repetition ahead of the left $(\square)$. The rate declines at first but recovers during further repetitions and finally declines progressively due to exhaustion, or to old age. The fluctuations for the left limb are forced into partial synchronisation with those for the right and are somewhat damped. (From results of Needham, I949b).

eration of external organs, but it seems probable (Needham, 1955) that there is a systemic nitrogen-flow comparable to that found in mammals, in the R-phase of liver-regeneration (Friedgood et al., 1950), and of skin- and bone-healing (Crofts and Peters, I945; Munro and Cummings, I 948; Cuthbertson, I 954; Campbell et al., I 954). This excess urinary nitrogen does not come mainly from local demolition, or from dedifferentiation which involves the destruction of extracellular protein, and of course urinary nitrogen represents protein lost to the body. It is possible therefore that these systemic events are not related specifically to the processes of regeneration, but rather to a non-specific "general alarm reaction" or "general adaptation syndrome" (GAS) following "stress" of any kind, traumatic or otherwise (Selye, I 946, I 948, I 955).

However, many components of the GAS resemble, or are consistent with, the local changes. There is an increase in acidity of the blood due, in part at least, to lactic acid and to increased glycolysis (Green and Stonor, I954). Water is retained by the body and movements of $\mathrm{Na}$ and $\mathrm{K}$ between cells and plasma may be both local and general. The GAS, like the local changes of the R-phase of regeneration, is in general regressive and catabolic in nature, and body-growth is inhibited during the GAS, as it is in the early stages of regeneration in the stick insect (p. 638). The changes associated with the GAS are subsequently reversed, just as the local changes. No doubt stresses of all kinds cause damage, often disseminated and perhaps inconspicuous morphologically, so that the sharp localisation of regeneration is only a special case. Selye (I 955) in fact refers to the local 
TABLE 8

EXAMPLES OF BICYGLIC FLUCTUATIONS DURING REGENERATION

Nature of fluctuation

Rate of mitosis in epidermis of newts' limb

Activity of alkaline phosphatase in newt's limb

Activity of alkaline phosphatase in mammalian skin

Activity of alkaline phosphatase in mammalian liver

Activity of acid phosphatase, mammalian nerve

Acetyl phosphatide concentration, rat liver

Acetyl choline activity

Ali esterase activity, mammalian nerve

Lactic acid, concentration, mammalian liver

Concentration of a number of constituents per cell, mammalian liver

Nitrogen-output, Carcinus limb

Activity of $\mathrm{N}$-corticoids
Reference

Manner, I953

| Ghiretti, 1950; Karczmar and

i Berg, I 95 I

(Danielli et al., I946;

1 Bourne, I 948

Norberg, 1950

Heinzen, 1947

Yarbro and Anderson, 1954

see p. 643

Cavanagh and Webster, 1955

Novikoff and Potter, I948

Tsuboi et al., 1954

Needham, I955

see p. 643

events as a local adaptation syndrome, and shows that the systemic response is necessary for the success of the local processes, during regeneration ( $c f$. Schotté and Chamberlain, I955). One characteristic of the GAS is that its progressive phase or "phase of resistance" improves on repeated evocation up to a certain limit and then progressively deteriorates ("phase of exhaustion"); this closely parallels changes in regenerative power (Fig. 4) on repeated evocation (Needham, I 949 b). Here there appears to be evidence of resemblance even to the temporary depression in response at the second evocation, before resistance develops (Selye, I 948). It seems probable that even the smallest stimulus, to the most obscure nerve, causes some degree of "stress", followed by recovery.

The precise significance of the excessive flow of nitrogen and other elements is still far from clear (Needham, I952), but it may be significantly linked with the processes of regeneration. Crofts and Peters (I945) and Williamson and Fromm ( 1955 ) concluded that it was necessary to supply adequate amounts of "key" amino acids, in particular of methionine (Localio, et al., I 948, I949; Bertolani et al., I 954), the other acids therefore being deaminated. Liver-methionine in fact is used to supply cystine for regenerating skin (Williamson and Fromm, I955) and muscleprotein is used to provide amino acids for regenerating liver (Man et al. I 946). The variation of flow with variations in protein intake however implies that it is not an invariable indispensable process.

The only evident contrast between local and systemic metabolism is that oxygenconsumption increases systemically during the R-phase (Cuthbertson, I944), after a brief fall associated with increased glycolysis (Malaguti and Vaccari, 1954). Perhaps differences of this kind necessitate the development of a barrier beween the regenerate and the rest of the body (Needham, 1952, p. 22; Singer et al., i954).

A simple oscillation in metabolism from R- to P-phases is not universal. A num- 
ber of components show two cycles of change (Table 8) and the present picture is probably an oversimplification. Nevertheless recent evidence seems to strengthen the general validity of the simple oscillation.

It now seems more certain (Fodor et al., 1955) that acid phosphatase is active in regressive processes. Of much wider significance is the demonstration by De Duve $e$ al. (I 955) that the enzyme is located on cytoplasmic particles, possibly the same particles ("lysosomes") as those containing cathepsins, deoxyribonuclease, ribonuclease, $\beta$-glucuronidase and other lytic enzymes. The lysosomes in fact may be complete batteries of R-phase enzymes, held inactive in normal healthy cells. $\beta$-Glucuronidase, one of the enzymes concerned in the breakdown of the mucopolysaccharides of the skeletal and connective tissues, is a recent addition to the enzymes known to become more active in the R-phase (Hollinger and Rossiter, I952; Mills et al., I953).

\section{(a) Protein metabolism}

There is an initial decrease in total nitrogen of regenerating liver (Price and Laird, I950; Harkness, I952) and in concentration of protein (Paul et al., 1945) from a normal $25.5 \%$ of the wet-weight to $12.5 \%$ on the third day. In regenerating skin it decreases from $4 \mathrm{I} .5 \mathrm{mg} / \mathrm{g}$ wet weight to $19.0 \mathrm{mg}$ (Williamson and Fromm, 1955). These decreases in protein-concentration, in fact, seem largely attributable to the increase in water-content (Paul et al., I945) but in sectioned nerves a genuine decrease in protein seems probable (see Caspersson, I950, p. I 30 ). Free amino acids and other breakdown-products of proteins accumulate locally (Orechovitch and Bromley, I934), including those from mucoproteins (Sylven, I94I; Balazs and Holmgren, I950) and the accumulation persists (Vladimirova, I935; Williamson and Fromm, I 955) presumably for re-use. In the regenerating liver most of the (protein) enzymes decrease in amount (Novikoff and Potter, I948; Rosenthal et al., I95 I). Proteolytic enzymes become more active, particularly 'cathepsins' intracellular proteases with a $\mathrm{pH}$-optimum at 4.7 (Orechovitch, I934; Orechovitch et al., I935; Striganova, I940; Maver et al., I952; Jensen et al., 1956). The regenerative power of tissues is proportional to their intrinsic proteolytic (autolytic) activity (Sokolova, I 942). The blood leaving an injured region also shows increased peptidase activity (Selye, I948). At the acid $\mathrm{pH}$ prevailing, cathepsins will digest even collagen (Sherry et al., 1954). Osteoclasts, (perhaps), and other cells (Menkin, I950), secrete proteases at this time. It is significant that cells transferred to culture-media also at first show increased proteolytic activity (Willmer, I942a) and other R-phase activities. Regressing cancerous growths also show increased proteolysis (Maver et al., I948; Fodor et al., I955). By contrast the tissues of embryos, which are probably in the P-phase throughout, never show high catheptic activity (Börger and Peters, I933).

At first more sensitive (Orechovitch and Sokolova, I940), regenerating tissues become less sensitive to catheptic digestion than normal during the P-phase (Orechovitch and Bromley, I934), since grafts of normal skin over the blastema are then digested. In consequence, perhaps, the young blastema is able to ensure adequate regression of tissues of the stump. On the other hand this production of proteases in the blastema may be associated with the converse activity of protein 
synthesis (Urbani, I 955). As in normal growth, there is no evidence of enzymes specific to the direction of synthesis. Pentose nucleic acid, PNA, appears to be concerned with the action of cathepsins in both directions (Maver et al., I952; Zacchei, I954). PNA is the major component of dipeptidases (Binkley, 1952) which also are very active during regeneration (Orechovitch, I936; Urbani, I955), and again probably in both phases. The pH-optimum for dipeptidases is between 7.4 and 7.9 so that they are probably more important in the P-phase than in the earlier, acid phase, but there is evidence also of earlier activity, during proteolysis (Duspiva, I942; Belfer et al., I 943; Zacchei, I954; Fleisher, I955). Dipeptidases can catalyse only the last stages of proteolysis and the first stages of synthesis, so that in the embryo they are active earlier than synthesizing proteases (Urbani, 1955). The cathepsin-bearing lysosomes, intermediate in size between the catabolising mitochondria and the smaller, synthesizing "microsomes", thus may be active in both catabolism and anabolism.

The use of amino acids for synthesis of proteins in regeneration has been adequately demonstrated. Their rate of turnover in regenerating skin is three to five times that in normal skin (Van Middlesworth, I953) and amino acids of the food rapidly accumulate in the regeneration blastemata and in the buds of Obelia (Hammett and Chapman, I938). The utilisation of methionine has been shown by various workers (p. 610). Amino acid-supplements, in physiological concentration, $10^{-5} \mathrm{M}$, accelerate regeneration (Lecamp, I 942, 1947) but a number of peptides have greater effects than the free amino acids, as also on growth in vitro (Fischer, 1946).

The synthesis of protein and the increase in total nitrogen in regenerating liver have been followed by Paul et al. (1947), Novikoff and Potter (1 948), Harkness ( I952), Tsuboi et al., (I954) and others, and those in healing skin by Williamson and Fromm (1955). In liver the increase begins as early as twenty four hours after partial hepatectomy, the R-phase being very short. Total nitrogen shows a single smooth curve of increase, both in liver and in skin, so that the material mobilised locally and that supplied systemically probably join a common pool. The liver-proteins normally supply some of the material for skin-healing (Williamson and Fromm, 1955) but dietary protein or amino acids are used freely (Hammett and Chapman, i 938; Localio et al., 1948, I 949; Bertolani et al., i 954; Baron and Allison, I 954; Williamson and Fromm, I952, I954). The precise interaction between dietary and body-protein needs clarification (p. 628).

\section{(b) Metabolism of nucleic acids}

The function of nucleic acids during the P-phase of regeneration is essentially the same as in other kinds of growth (Chapter 3) and needs no detailed treatment here. The synthesis of deoxyribosenucleic acid, DNA, runs roughly proportionally to that of protein (Tsuboi, et al., I 954). It is a very reliable index of the number of nuclei, and therefore of cells, proliferated, and so of growth itself (Brues et al., I 944; Davidson and Leslie, r 950). By contrast the maximal concentration of pentosenucleic acid, PNA, is reached earlier than that of DNA and protein (Hammarsten, I95 I ; Lombardo et al., I 953; Tsuboi et al., r 954) at the time when protein is being synthesised most rapidly (Novikoff and Potter, 1948), just as in 
TABLE 9

FUNCTIONS OF ALKALINE PHOSPHATASE DURING REGENERATION: SUMMARY OF RESULTS

Example Reference

(a) During regressive processes

\section{(i) Positive correlation}

Amphibian limb-regeneration

Mammalian wound-healing

Mammalian bone-regeneration

Mammalian nerve: Wallerian degeneration (histochemical evidence)

General adaptation response, produced by adrenal cortical hormones

Mammalian liver: atrophy during starvation

Embryonic development: cell-degeneration
Ghiretti, I950; Karczmar and Berg, I95 I Danielli et al., 1946; Balazs and Holmgren, I950; Utsunomiya et al., I952, I953; Valentine, I955

Bourne, $194^{8}$

Hollinger et al., 1952

( Kochakian and Bartlett, I948;

( Taleisnik, I953

I Wachstein, I945; Ely and Ross, I95I;

1 Rosenthal et al., 1950, I95 I

Yao, I950

\section{(ii) Negative correlation}

Enzyme inhibited by SH-compounds and / Fell and Danielli, 1944; Gould and other R-phase conditions

1 Gold, I951 ; Rosin, I952

(b) Association with cell-proliferation

(i) Positive correlation

Mammalian skin-regeneration

Mammalian liver-regeneration

Mammalian nerve-regeneration: Schwanncells

Tissue culture experimental wound

Tissue culture: normal proliferation

Mammalian liver: normal growth

Mouse tissues (nuclei): normal growth
Firket, 1950

Oppenheimer, I946

Hollinger et al., $195^{2}$

De Lustig and Sacerdote, I954

Willmer, I942b

Wachstein, I945

Brachet and Jeener, $194^{8}$

(ii) Negative correlation

Mammalian liver-regeneration

Taleisnik, I 953

(c) Association with differentiation

Eisenia: body-regeneration

Tunicates: regeneration and budding (ATP-ase)

Tadpole: tail regeneration

Amblystoma: limb-regeneration and ontogenesis

Mammalian skin-healing

Mammalian bone-healing

Ontogenesis (enzyme-activity high in later stages)

Powell, I95 I

Jaeger and Barth, $193^{8}$

Junquiera, $195^{\circ}$

Karczmar and Berg, I95 I

I Fell and Danielli, r944;

I Danielli et al., I946

McKelvie and Mann, 1948;

Fontaine et al., 1952; Buser,

( I948; Utsunomiya et al., I952, 1953

Yao, I950; Brachet, I950;

Krugelis et al., I952;

(Minganti, I954 
proliferating micro-organisms (Jeener, I952). This is good evidence that it is directly involved in the synthesis of cytoplasmic proteins. DNA may catalyse the synthesis of proteins of the nucleus (Allfrey et al., I955) but these are a small fraction of the total protein. Indirectly DNA is required also for synthesis in the cytoplasm (Allfrey et al., 1955), a conclusion in accord with classical evidence that the nucleus of Protozoa is indispensable for regeneration (Morgan, I90I; Needham, I952), but the turnover-rate of thymine (Roll et al., I950) and of adenine (Hammarsten, I95I), in DNA, is extremely low in the intact liver, by contrast with the rate during regeneration, whereas PNA-turnover is very rapid even in intact liver.

In addition to earlier examples (Needham, I952), new instances of the accumulation of PNA during the early P-phase of regeneration include those of Aquila ( $195^{2}$ ) for healing bone, of Barakina (I 95I) for the limbs of anuran tadpoles, and of Takata (1952) for the lens of the eye of Triturus. Barakina confirmed earlier observations that the maximal concentration in the epidermis is earlier than that in the deeper tissues, in accord with morphogenetic differences (p. 597). Augmentation of PNA, in physiological concentration, accelerates regeneration (Brondsted, 1953) but higher concentrations are inhibitory (Fischer, I 946, p. 219; Needham, $195^{2}$ p. 35; Thomas et al., 1952).

The coarse basophilic PNA-granules of mature cells break down into a fine dispersion during the R-phase (Jacovleva, I 945; Opie et al., i 946; Pirozynski and Von Bertalanffy, I952) and there may be little actual destruction of PNA during that phase (Needham, I952, p. 33-4), except in necrotic cells (Stowell and Lee, I950; Pirozynski and Von Bertalanffy, I952).

\section{(c) Activities of phosphatases}

The function of acid phosphatase in the R-phase is uncertain, and that of alkaline phosphatase remains even more obscure, probably because in fact it is concerned in many processes during regeneration. As a potential enzyme for the transfer of phosphate from organic monophosphates (Danielli, I 95 I Morton, I 955) it might be active wherever energy is required, at any stage, and available records, collectively, indicate that it is. It is often associated with the nucleic acids, the most important of organic phosphates (Ely and Ross, I95 I ; Rosin, I 952), or with their activity (Brachet and Jeener, I948) or with that of the chromosomes (Wachstein, I945), and cell-division may be one of the processes it promotes (Table 9). A large number of workers have found two major peaks in its activity during the course of regeneration (Table 8), and others (Norberg, I 950; Tsuboi et al., I 954) have found more complex fluctuations. This may indicate that it is mainly concerned in the "motor" activities of proteins (Danielli, I95I) and Gould and Gold (195I) concluded that it is not concerned in processes of synthesis. A summary of available records (Table 9) however indicates three main functions I) in dedifferentiation (2) in cell-division and (3) in differentiation, particularly of (r)llagen. At the molecular level de- and re-differentiation are probably motor processes not very different from muscular and amoeboid movements (Goldacre, 1952, Which again have considerable resemblances (Brachet, 1950, p. I84) to the mechanical processes of mitosis. 
In ontogenesis (Yao, I950; Karczmar and Berg, I95I ; Krugelis et al., 1952) the enzyme becomes maximally active only in later stages, and the correlation with differentiation therefore seems established (Needham, 1952). Nevertheless there remains much obsurity and conflict of evidence. Gould and Gold (195 I), administering the enzyme even to animals depleted of it, could detect no acceleration of regeneration, and regeneration was normal in the presence of inhibitors of alkaline phosphatase.

\section{(d) Metabolism of sulphur compounds}

Recent results (Christensen and Streicher, I948; Firket, I950; Layton, I950, I95I ; Frederic, I 952) support earlier evidence (Needham, I952) that there is an increase in number of free SH-groups during the R-phase. This is characteristic of denaturation-changes in proteins (Joly, I955), during which the SH-groups also become capable of activity in a more acid $\mathrm{pH}$-range, while the proteins become more sensitive to proteolytic and other agents. The R-phase therefore probably involves a reversible denaturation of proteins, locally, The $\mathrm{SH}$-concentration in the plasma declines during the first twenty-four h. after laparotomy (Schacter et al., I952), possibly owing to increased uptake by the wound-region, and that of the liver declines at this time (Beck and Linkenheimer, I952), for the same reason (Fromm, I955). Maloeuf (I936a) found the concentration of glutathione (GSH) to be below normal in a three weeks-old tail-regenerate of Rana clamitans, which is consistent with the results of Okunev (1932), Orechovitch (1934, 1936) and Ryvkina (1940) showing that the reduced form, SH, decreases in the later stages of regeneration, probably through reoxidation to the -SS-form.

That these SH-compounds play an important part in regeneration is proved by the accelerating, and the protein-sparing, effect of supplements of the sulphurcontaining amino-acids (Crofts and Peters, I 945; Williamson and Fromm, 1955). Regeneration-rate is probably more closely correlated with sulphur- than with nitrogen-metabolism, i.e. with these amino acids more than with any others (Williamson et al., I95I). This conclusion is confirmed by work on cells growing in vitro (Fischer, I946; Haffen and Wolff, I953). Morgulis and Green (1932) claimed that added sulphur compounds do not accelerate regeneration in the polychaete Podarke, but they did not use physiological SH-compounds. Their results with cystine, the oxidised dimer of cysteine, indicate a slight acceleration, which is to be expected,--in the P-phase. However, Gershbein and Labow (I 953) also have failed to detect acceleration of liver-regeneration in rats by cysteine, or by GSH, as $0.75 \%$ of the diet. Thiouracil did promote regeneration but this could have been indirectly, via its effect on the thyroid. Orechovitch (I936) showed that the catheptic activity of some regenerating tissues is not accelerated by $\mathrm{SH}$ compounds, probably because they are already fully activated by the autogenous increase in - $\mathrm{SH}$, and this may explain also the results of Gershbein and Labow.

Ryvkina (I940) has pointed out that the maximal concentration of SH-compounds is reached later than the maximum of proteolytic activity, and they probably also promote cell-division, as in other types of growth (Voegtlin and Chalkley, I930; Rapkine, 193 I ; Harris, I953). SH-groups are very abundant in many enzymes (Barron, I949) and therefore they probably also have a number of other functions in regeneration. In addition the disulphur, -SS-, bridges of cystine are essential structural components of synthesized keratin and other insoluble proteins 
TABLE 10

EVIDENCE FOR A DECREASE IN AEROBIC RESPIRATION DURING EARLY STAGES OF REGENERATION

\begin{tabular}{|c|c|c|}
\hline Nature of evidence & Material & Reference \\
\hline $\begin{array}{l}\text { Decrease in } \mathrm{O}_{2-}^{-} \\
\text {consumption }\end{array}$ & $\left\{\begin{array}{l}\text { Hydra } \\
\text { Tubularia } \\
\text { Turbellaria } \\
\text { Tubifex } \\
\text { axolotl } \\
\text { liver }\end{array}\right.$ & $\begin{array}{l}\text { Ajsupiet, I } 937 \\
\text { Barth, I937; Goldin and Barth, I941 } \\
\text { Lovtrup, I } 953 \text {; Von Levetzow, I } 939 \\
\text { Collier, I } 947 \\
\text { Ryvkina, I } 945 \\
\text { Van Bekkum and Peters, I95 I }\end{array}$ \\
\hline $\begin{array}{l}\text { Decrease in oxidation } \\
\quad \text {-reduction potential }\end{array}$ & axolotl & Okunev, I932 \\
\hline $\begin{array}{l}\text { Decrease in R.Q. and/or } \\
\text { increase in glycolysis }\end{array}$ & ( axolotl & $\begin{array}{l}\text { Ryvkina, I945; Okunev, I933; } \\
\text { Vladimirova, I934 } \\
\text { Rossiter and Clark, I } 944\end{array}$ \\
\hline $\begin{array}{l}\text { Increase in glycolysis } \\
\text { systemically }\end{array}$ & $\begin{array}{l}\text { mammalian } \\
\text { skin }\end{array}$ & $\left\{\begin{array}{l}\text { Malaguti and Vaccari, I954; } \\
\text { Green and Stonor, I954 }\end{array}\right.$ \\
\hline $\begin{array}{l}\text { Increase in aerobic } \\
\text { glycolysis }\end{array}$ & $\begin{array}{l}\text { mammalian } \\
\text { muscle }\end{array}$ & Pentimalli, 1927 \\
\hline $\begin{array}{l}\text { Decrease in concentration } \\
\text { of enzymes concerned in } \\
\text { aerobic respiration }\end{array}$ & $\left\{\begin{array}{l}\text { mammalian } \\
\text { liver } \\
\text { mammalian } \\
\text { nerve }\end{array}\right.$ & $\begin{array}{l}\text { Novikoff and Potter, 1948; Rosen- } \\
\quad \text { thal, et al., I95 I ; Harkness, I952 } \\
\text { Tsuboi, et al., I954 } \\
\text { Bodian, I947; Howe and Flexner, } \\
\quad \text { I947; } \\
\text { Howe and Mellors, I948 }\end{array}$ \\
\hline $\begin{array}{l}\text { Retardation of regeneration } \\
\text { by cytochrome } c\end{array}$ & $\begin{array}{l}\text { mammalian } \\
\text { liver }\end{array}$ & Drabkin et al., 1950 \\
\hline
\end{tabular}

(Williamson and Fromm, I 955). Again the sulphuric acid of mucopolysaccharides (Kent and Whitehouse, I955) possibly may be derived from the sulphur-containing amino-acids, inorganic sulphates being little absorbed from the gut. After laparotomy and after $\mathrm{X}$-irradiation there is an inverse correlation between plasma-SH and plasma-polysaccharide concentrations (Schacter et al., I952), which lends colour to this view. Methionine may be important mainly as a source of cysteine and of these other sulphur-compounds (Williamson and Fromm, I955), though Bertolani et al. (1954) find evidence that its action on regeneration is correlated with its well-known lipotropic action (McHenry and Patterson, 1944).

There is little doubt, however, that the SH-compounds are the most important sulphur-compounds here. They have key-functions at almost every stage of regeneration. GSH is highly concentrated in the adrenal cortex (Sayers, I950), and controls the action of corticotropin (Anon., I 955). Possibly for this reason stress causes a temporary depletion of SH-compounds from the liver (Beck and Linkenheimer, 1952 ), though during regeneration these may be mobilised also for specific use. Further, cortisone inhibits the uptake of sulphur into granulation-tissue (Layton, 
TABLE 11

EVIDENCE FOR ANINCREASEIN OXIDATION IN LATER STAGES OF REGENERATION

\begin{tabular}{|c|c|c|}
\hline Nature of evidence & Material & Reference \\
\hline Increase in $\mathrm{O}_{2}$-consumption & $\begin{array}{l}\text { Hydra } \\
\text { Tubularia } \\
\text { Turbellaria } \\
\text { Tubifex } \\
\text { Allolobophora } \\
\text { axolotl } \\
\text { mammalian } \\
\text { muscle } \\
\text { mammalian } \\
\text { skin }\end{array}$ & $\begin{array}{l}\text { Ajsupiet, I937 } \\
\text { Goldin, I942 } \\
\text { Von Levetzow, I939 } \\
\text { Collier, I } 947 \\
\text { O'Brien, I947 } \\
\text { Ryvkina, I945; Wolsky, I94I } \\
\text { Pentimalli, I927 } \\
\text { Groll, I927 }\end{array}$ \\
\hline $\begin{array}{l}\text { Regeneration accelerated by } \\
\text { increasing } \mathrm{O}_{2} \text {-supply }\end{array}$ & $\begin{array}{l}\text { Tubularia } \\
\text { Eisenia }\end{array}$ & $\begin{array}{l}\text { Loeb, I892; Torrey, I } 9 \text { I } 2 \text {; Goldin, } \\
\text { I } 942 \text {; Barth, I } 944 \\
\text { Zielinska, I9 I3 }\end{array}$ \\
\hline $\begin{array}{l}\text { Regeneration retarded by } \\
\text { restricting } \mathrm{O}_{2} \text {-supply }\end{array}$ & $\begin{array}{l}\text { Corymorpha } \\
\text { planarians }\end{array}$ & $\begin{array}{l}\text { Schechter, I94I } \\
\text { Schultz, I902 }\end{array}$ \\
\hline $\begin{array}{l}\text { Regeneration retarded by poi- } \\
\text { sons of the cytochrome system }\end{array}$ & Tubularia & $\begin{array}{l}\text { Bassina, 1937; Moog and Spiegel- } \\
\text { man, 1942 }\end{array}$ \\
\hline $\begin{array}{l}\text { Regeneration-rate varies with } \\
\text { variations in } \mathrm{O}_{2} \text {-consumption }\end{array}$ & $\begin{array}{l}\text { Tubularia } \\
\text { Allolobophora }\end{array}$ & $\begin{array}{l}\text { Barth, I } 937 \\
\text { O'Brien, I } 947\end{array}$ \\
\hline $\begin{array}{l}\text { Regeneration accelerated by } \\
\text { pyocyanin and dinitro-phenol } \\
\text { which speed oxygen-consump- } \\
\text { tion }\end{array}$ & $\begin{array}{l}\text { Hydroids } \\
\text { mammalian } \\
\text { skin }\end{array}$ & $\begin{array}{l}\text { Rulon, I938; Deotto, I939 } \\
\text { Finkelstein and Kovarskaja, I } 936\end{array}$ \\
\hline $\begin{array}{l}\text { Loss in body weight becomes } \\
\text { more rapid }\end{array}$ & Tubifex & Collier, I947 \\
\hline $\begin{array}{l}\text { Cell-products best accelerate } \\
\text { growth in the presence of } \\
\text { abundant } \mathrm{O}_{2}\end{array}$ & yeast & Loofbourow, I $94^{2}$ \\
\hline $\begin{array}{l}\text { Inhibition of } \mathrm{O}_{2} \text {-consumption } \\
\text { inhibits cell-movements }\end{array}$ & $\begin{array}{l}\text { Xenopus and I } \\
\text { mammalian liver }\end{array}$ & Lehmann et al., г 954 \\
\hline
\end{tabular}

I 95I) and the synthesis of mucopolysaccharides (Zoger, I952), and so restricts these syntheses to the P-phase. In turn, SH-compounds inhibit alkaline phosphatase (Fell and Danielli, I944; Gould and Gold, I95I) while the oxidised, -SS- form accelerates its activity (Fell and Danielli, 1944), thus restricting its activity to the later, differentiation processes of the P-phase. Oxidation of GSH in vivo is probably coupled with the reduction of dehydroascorbic acid to vitamin C. (Mapson and Goddard, I 95I ; Conn and Vennesland, I95I), which is associated with GSH, in the adrenal cortex, and which increases in amount, in regenerates, 
up to a late stage (Ryvkina, I940). It is well known (p. 629) to promote the differentiation of the connective tissues, in association with alkaline phosphatase (Needham, r952).

\section{(e) Respiration and carbohydrate-metabolism}

Recent work has substantiated in general the belief (Needham, I952) that respiration locally is extensively glycolytic in the $\mathrm{R}$-phase (Table $\mathrm{IO}$ ) and more fully aerobic again in the P-phase (Table II). The change probably does not occur until after cell-proliferation, which is favoured by reducing conditions. Antioxidants are extricable from proliferating plant-tissues (Van Fleet, I954), but pro-oxidants in the later stages of differentiation. The glycolytic phase appears to be no mere accident of disturbed blood-supply since lactic acid, and other products of incomplete oxidation, accumulate even in pure oxygen (Ryvkina, I 945). Glycolysis is favourable for proteolysis (Rubel, 1936). Systemically, glycolysis increases during the first is 2 . of traumatic shock (Malaguti and Vaccari, I954; Green and Stonor, I954), but it seems that here there is an increase in oxygenconsumption from an early stage (p.6ro) possibly because glycolytic conditions are intolerable for the healthy tissues of the body. Novikoff and Potter (1948) found little increase in lactic acid, even locally, during liver-regeneration, and more detailed knowledge is required.

The normal carbohydrates, glucose and glycogen, are no doubt the main sources of energy for regeneration (Weisz, I948; Novikoff and Potter, I948; Firket, I950 Frazer, I 953; Jacovleva, I 953; Malaguti and Vaccari, I954), though Jacovleva (I953), again (Needham, I 952) has found no evidence of extensive use of glycogen during the phase of growth; in vitro, also, there is little evident correlation between growth and carbohydrate-utilisation (Willmer, I942a).

On the other hand there is hyperglycaemia and glyconeogenesis in the R-phase (Green and Stonor 1954) which seems as excessive as protein-flow; the latter indeed may provide carbohydrate by glyconeogenesis, since it is "spared" by administered carbohydrate (Cuthbertson, et al., 1939). With such large quantities of carbohydrate mobilised systemically, local concentrations may be a false index of local utilisation-rate. Glucose is also the probable source of the amino-sugars and the glucuronic acid of new mucopolysaccharides but for this, again, may be supplied systemically and continuously.

Breakdown products of dedifferentiated mucoproteins (see Kent and Whitehouse, I 955, p. 35) are detected, in the R-phase, both locally (Boas and Foley, I 954; Baggi, I953) and systemically (Schacter et al., 1952). Under the acid conditions of the R-phase cathepsins probably initiate the mucolysis (Sherry et al., 1954), while $\beta$-glucuronidase catalyses one of the ultimate reactions. Free polysaccharides reach maximal concentration in the blood $24 \mathrm{~h}$. after laparotomy (Schacter et al., I 952), while locally in skin (Boas and Foley, I 954) and bone (Baggi I 953) the ultimate degradation-products, glucosamine and galactosamine, are detertable. A progressive increase in newly synthesized mucopolysaccharides during the P-phase also has been demonstrated (Sylven, I94I; Balazs and Holmgren, I 950).

D-Glucosamine inhibits further mucolysis (Lehmann, I 954a, b) and so when ad- 
ministered at an early stage it inhibits regeneration. Reciprocally the regenerated mucopolysaccharides may inhibit the earlier stages of cell-proliferation (Balazs and Holmgren, I950). They also inhibit the sprouting of regenerating nerve-fibres (Hoffmann, I 952), probably again by preventing lysis of the tough connective tissues of the innervated organs. Certain pyrogenic polysaccharides counteract this, possibly competitively, and induce the development of a delicate, easily penetrated stroma of connective tissue (Hoffmann, r 954; Bammer and Martini, I 953). For the same reason mucopolysaccharides prevent the migration of leucocytes (Meier et al., I 955), and of blastema-forming cells (Lehmann, I 954). The action of the pyrogenic polysaccharides recalls the anticoagulant action of the polysaccharide heparin, which in fact probably plays some part in the early stages of the GAS (Asboe-Hanson, I952; Bloom I952).

\section{(f) Fat-metabolism}

There is little knowledge of the metabolism of lipids during the regeneration of structures other than the liver and peripheral nerves of mammals; both are peculiar, the liver because of its function in the intermediate metabolism of fats and the nerve-fibre because of its thick lipoid myelin sheath. After partial hepatectomy there is a rapid increase in liver-fat (Ludewig et al., I939; Harkness, I 952; Johnson and Albert, I952; Yarbro and Anderson, I954; Tsuboi et al. 1954), from $3.3 \%$ of the wet-weight to $7.6 \%$ at $\mathrm{io} \mathrm{h}$, when all other constituents, except water, are decreasing in amount. This response is not peculiar to liver-regeneration (Frazer, I954; Gaunt, 1954). It is probably the counterpart (Selye, I948; Cuthbertson, i 954) of protein- and carbohydrate-flow. The fat comes mainly from the depots (Yarbro and Anderson, 1954). It is not required mainly as a source of energy. As a fuel, fat is certainly not more important than carbohydrate. Weisz (1949) found a depletion of glycogen in regenerating Protozoa but not of fat and this is probably true of cells growing in vitro (Willmer, I953). Fat is said to depress healing in mammals (Whipple, i 940) though Rogers et al. ( $195^{\circ}$ ) found that as much as $30 \%$ of fat in the diet was beneficial.

There is considerable evidence that lipids do play a relevant part in regeneration. Most lipid fractions show two cycles of abundance (Table 8), in regenerating liver, one of which may be part of the non-specific GAS and the other specific to regeneration. If hepatic trauma is induced chemically, by carbon tetrachloride (Tsuboi et al., r95I), the initial increase in lipids is delayed by one or two days, presumably associated with the greater demolition necessary than after surgery. The second maximum of fat-concentration, around the eighth day after hepatectomy, is associated with increased lipolytic activity (Bertolani et al ., I 954; Tsuboi et al., I 954). Gavanagh and Webster (I955) found two maxima in lipolytic (ali-esterase) activity in regenerating nerve so that the two cycles may be common (p. 6I I) among metabolic processes during regeneration.

The turnover of phospholipid in regenerating liver is maximal around the third day (Johnson and Albert, I952) at the time of maximal cell-proliferation Since the concentration of P-lipid is by then decreasing from its initial peak, it is probably being used for cell-growth or -proliferation. There is much evidence (Davidson and Leslie, I950; Hammarsten, I95 I ; Singal et al., I952) that phos- 
TABLE 12

EVIDENCE OF INCREASE IN HYDRATION IN THE EARLY STAGES OF REGENERATION

\begin{tabular}{|c|c|c|c|c|}
\hline Example & $\begin{array}{l}\text { Extent of } \\
\text { from }(\%)\end{array}$ & $\begin{array}{l}\text { ncrease } \\
\text { to }(\%)\end{array}$ & $\begin{array}{c}\text { Date of maximal } \\
\text { hydration }\end{array}$ & Reference \\
\hline Body of polyclads & - & - & 2nd -5 th day & Von Levetzow, I939 \\
\hline $\begin{array}{l}\text { Body of polychaete } \\
\text { Podarke }\end{array}$ & 76 & 79 & Ist-2nd week & Morgulis, I9I I \\
\hline Tail of axolotl & 75 & 82 & - & Wolsky, I94I \\
\hline Tail of salamander & 74 & 89 & $1 / 3$ of total time & Morgulis, I9I I \\
\hline Nerve of mammals & - & - & $5^{\text {th }}$ - roth day & Lumsden, I 952 \\
\hline Skin of mammal & 60 & 83 & 3 rd day & Paul et al., I 947 \\
\hline Liver of mammal & 61 & 70 & $4^{\text {th }}$ day & Tsuboi et al., I95 I \\
\hline
\end{tabular}

pholipid metabolism is linked with that of nucleic acids, in growth-processes.

The cholesterol content of regenerating liver (Tsuboi et al., 1954) and of the blood (Man et al., I 946) changes little, but such small changes may be significant in this precursor of important hormones. The wound-factor of certain plants (English et al., 1938) is a dicarboxylic fatty acid and lipid components are usual in the plant-growth hormones (Osborne and Wain, I951).

The fat-soluble vitamins probably affect regeneration in animals much as they do normal growth, though results to date have been conflicting (Arey, I936; Holmes, 1942). Vitamin A promotes bone-resorption during the remodellings of growth (Mellanby, 1944; Fell and Mellanby, 1953) and, consequently, may retard simple regeneration under experimental conditions (Barnicot, 1950). Vitamin D also retards regeneration, in Anura (Rose, 1944,) and keloid-formation in mammals (Marshall, 1952). Vitamin $\mathrm{K}_{3}$ has been found beneficial in skinhealing (Palladin, 1943).

Many workers, following Morgan (1901, p. 267), have been intrigued by the behaviour of a red carotinoid pigment in the regeneration of hydranths of Tubularia (Cohen, I952; Goldman, I953). It is most abundant during the early stages of regeneration (Goldman, 1953) and therefore may protect the regenerate, during its R-phase, against photo-activated oxidation, as in photosynthesizing organisms (Griffiths et al., 1955). The regeneration of many hydroids is sensitive to light. The high concentration of carotenoids in ova (Goodwin, 1950) probably has much the same significance as in regeneration.

\section{(g) Water-metabolism}

There is an increased hydration of the tissues locally during the first stages of regeneration (Table 12). This is not a passive waterlogging, through the wound, in aquatic animals (Von Levetzow, 1939) and occurs in unexposed organs and in terrestrial animals. The increase is both intracellular (Johnson and Albert, 1952) and intercellular (Menkin, 1950). Systemically, also, there is water-retention and a considerable "flow" of water from intact tissues, particularly the muscles (Courtice, 1954), comparable to protein, fat and glucose flows. Increased intercellular 
water facilitates the movements of cells in defence and demolition (Onwcleen, 19I7) and in blastema-formation. It probably also facilitates proteolysis, for the amount of hexosamine liberated from mucoproteins is proportional to the hydration of the tissue (Boas and Foley, r954). Intracellularly it restores a more youthful condition necessary for growth and proliferation.

Dilution of the external medium does increase the water-intake of aquatic animals, and it accelerates regeneration in brackish water Planarians (Lloyd, 1919; Steinmann, I932) and in the marine Coelenterates, Pennaria (Keil, I932) and Tubularia (Loeb, I892). Reciprocally an hypertonic medium depresses regeneration-rate (Morgulis, I 909), particularly in fresh,water animals (Sayles, I934; Davenport, 1899). Dilute alcohol increases tissue hydration and promotes regeneration whereas concentrated alcohol dehydrates, and retards regeneration (Morgulis, r 9o9; Bilski, 1926).

In the polychaete worm, Podarke (Morgulis, I909) and in the brine shrimp Artemia (Sciacchitano, 1925), accustomed to high salinities, regeneration is retarded by dilution of the medium. Concentrated salt-solutions, applied for a short time to the amputation-surface of the limbs of adult Anura, which normally show only very abortive regeneration, greatly improves the process (Rose, 1942, 1944; Polezhayev, 1946) but this is a very different phenomenon, - the result of damage by the concentrated salt. Saline improves the regenerative power of posterior pieces of planaria (Watanabe, I94I), again possibly through depressive action, - differentially on the anterior part of the parent body.

Lessona ( $c f$. Morgan, I $90 \mathrm{I}$, p. 93) found that terrestrial salamanders regenerate limbs more slowly than aquatic types, and adult Anura, which regenerate very poorly compared with adult Urodeles, in general are more terrestrial than the latter. A number of groups with poor powers of regeneration are terrestrial: insects, reptiles, birds and mammals. It might be concluded, therefore, that terrestrial conditions are inimical, owing to relative desiccation. However Goodwin (1946) found no significant difference in regenerative power between Urodeles of the same species regenerating in air and in water. Many terrestrial animals in fact regenerate well, not only moist-skinned types such as terricolan Turbellaria and earthworms but also insect-nymps, woodlice and centipedes. The terrestrial, pulmonate snails regenerate their shells much more rapidly than do the marine gastropods (Waage and Mittler, 1953). Even the mammals heal their skin very well and they regenerate the internal organs as rapidly as in any group. Any apparent correlation of poor regenerative power with the terrestrial habitat may be in fact a correlation with large body-size, with tissue-differentiation, which itself usually involves tissue-dehydration, or with determinate growth.

The movement of water into the cells, locally, during the R-phase is associated with an ingress of sodium and cgress of potassium (Anon., I 95 I). Tissues in general lose $\mathrm{K}$ and take in $\mathrm{Na}$ also after removal from the body to culture medium (Davies, I954). The trend is reversed when the cells begin to proliferate. The initial movements are favoured by anaerobic and the restoration by aerobic conditions, so that the latter are inhibited by respiratory poisons, and particularly those which uncouple phosphorylation from oxidation. Glutamic acid and its derivatives prevent the initial $\mathrm{K}$-loss; these ion-movements therefore are associated with fundamental components of metabolism. The resemblance between the whole sequence and that in nerve-conduction needs no further emphasis (p. 643). Cholinesterase affects the movements across the wall of other cells in the same way as across the nerve cell-membrane (Danielli, r954). 


\section{(h) Inorganic cations}

A correct balance of the physiologically important inorganic cations therefore is essential for normal regeneration (Davenport, I 899; Morgulis, I 909; Loeb, I 924; Korschelt, I 927; Beadle and Booth, I938; Von Bertalanffy and Rella, I94I). Any serious disturbance of the balance retards regeneration and causes qualitative abnormalities. Regeneration is accelerated also by the supplement of an inorganic element such as phosphorus, available in limited concentration (Needham, 1947b).

Calcium, like phosphorus, has a further function, as skeletal material in intercellular cement (Chambers, I942), in bone (Murray, I936; Roche and Morgue, I 939; Bohr and Sorensen, I950; Imanaga et al., I952; Schotté and Lamy, I953) and in the exoskeleton of molluscs and arthropods. Calcium is relatively easily demonstrated in situ, and estimated accurately, and its movements during regeneration are becoming well-known. An R-phase of mobilisation (Roche and Morgue, r 939) is followed by a P-phase of synthesis of insoluble calcium compounds. Mobilisation is most rapid locally at the site of fracture of a bone (Schotté and Lamy, I953) and grades away fairly sharply, little coming from other bones (Bohr and Sorensen, I950). However some is supplied systemically and there is an increased rate of exchange of Ca between plasma and bone (Bohr and Sorensen, I950). Injected $\mathrm{Ca}$ is taken up mainly by the regenerating bone but any excess is stored in the liver and kidney (Imanaga et al., I952). The normally growing epiphyses also take up a large amount, by contrast with intact parts of the diaphysis. In the regenerating limb of Triturus calcium increases almost from the outset and reaches maximal concentration by the tenth day, returning to normal at the end of regeneration, around the thirty-fifth day (Schotté and Lamy, I953). In the fractured limb-bone of the rat the maximal content, about three times normal, is reached at the end of the second week (Imanaga et al., I952).

VI. THE EFFECT OF EXTRINSIC FACTORS ON REGENERATIVE GROWTH

Most physical and chemicals agents which affect regeneration directly have the same action as on normal growth. Frequently, however, an external agent acts indirectly, triggering off a response appropriate to, and often counteracting the direct effect of, an external situation of which this agent is the most infallible signal. For instance, light acts as a trigger for the regeneration of the hydroids Eudendrium (Loeb, I 896; Goldfarb, I 906), Tubularia and Pennaria (Peebles, I899) and Syncoryne (Nakamura, I 94I); probably it is a signal of abundant food in the ultimate form of photosynthesising microorganisms. In other animals, by contrast (Marcus, I926; Coe, I929), and even in some other hydroids (Torrey and Martin, I910) light inhibits regeneration. In Sabella it inhibits the epimorphic component only, of regeneration. In the limbs of Carcinus the inhibitory action is indirect, via the sinus gland. However visible light reduces the direct inhibitory effect of shorter wave-radiations (Giese, I950). Light is surprisingly irrelevant to the regeneration of the eye of Crustacea (Korschelt, I927; p. 202) and of the lens of the urodele-eye (Rübsaamen, I950), for the ultimate function of which it is indispensable. 
A triggered response may give no direct information on the nature of the process. The direct effect of an increase in temperature is to speed regeneration, as it does most physiological processes, yet a rise in temperature causes the degeneration and casting of hydranths in Tubularia, and a fall in temperature induces regeneration (Moore, 1939; Berrill, 1948).

A second generalisation with a paradoxical quality is that low dosage of an inhibitory agent often accelerates regeneration, e.g. alcohol (Morgulis, I9o9; Bilski, I 926), colchicine (Lüscher, I 946; Havas, 1949), nitrogen-mustards (Supniewski, I949) and radiations (Schaper, 1904; Bardeen and Baetjer, I 904; Loofbourow, 1948). In most cases the acceleration is an indirect response to the slight damage caused, though both effects of alcohol are direct (p. 62I); similarly a progressive temperature-increase may directly speed the process at first and directly inhibit ultimately through heat-denaturation of enzymes.

\section{(a) Temperature}

Acceleration of regeneration by warmth was noticed by Trembley and other early workers ( $c f$. Morgan, I $90 \mathrm{I}$, p. 26). The regeneration of Hydra required 96-i 68 h. at I $2{ }^{\circ} \mathrm{C}$ and only $48 \mathrm{~h}$. at $26^{\circ} \mathrm{C}$ (Peebles, i 898 ; see also Moore, i 9 io); that of planarians 35 days at $5{ }^{\circ} \mathrm{C}$ and 4 days at $26^{\circ} \mathrm{C}$ (Wolff and Dubois, $1948 \mathrm{~b}$ ), I4 days at $10{ }^{\circ} \mathrm{C}$ and 8 days at ${ }^{\circ} 6^{\circ} \mathrm{C}$ (Vandel, I92 I). In a crocodile a certain area of skin healed in 29 days at $23{ }^{\circ} \mathrm{C}$ and in I I days at $38^{\circ} \mathrm{C}$ (Du Noüy, r 936, p. 99). Moore (I9Io) found a sigmoid relationship between regeneration-rate and temperature, but Spiegelman and Moog ( I944) obtained a virtually linear curve over the physiological range, and a constant temperature-coefficient.

Tadpoles cease to regenerate the tail at a lower limiting temperature of $14{ }^{\circ} \mathrm{C}$ (Ellis, I gog) considerably above the lower lethal temperature, but planarians show some regeneration down to $3{ }^{\circ} \mathrm{C}$ (Morgan, igo I). The upper limiting temperature for regeneration in poikilotherms is between $30^{\circ}$ and $35{ }^{\circ} \mathrm{C}$ (Korschelt, I 927 ; p. 695) and the maximal rate is reached $4-5^{\circ}$ below this. For mammals the normal body temperature is optimal, and further increase depresses regeneration (Hutchinson and Burdeaux, I95I). For some components of regeneration in mammals a subnormal temperature may be beneficial (Green and Stoner, I954; Moss and Weiss, I955), while during the defence-phase fever is beneficial (Menkin, I950) through differential action on the metabolisms of host and invading microorganisms. Pyrogenic polysaccharides accelerate regeneration in mammals but possibly not through their pyrogenic action.

Temperature-change may affect all components of regeneration proportionately to their normal rate (Nardi, I937; Weisz, I 948), but sometimes it acts differentially (Gross and Huxley, I935; Berrill and Mees, I936), and the quality of the regenerate is then affected. Cold increases the incidence of heteromorphic regenerates, for instance legs in place of antennae, in arthropods (Przibram, I934; Kenk, I935; Villee, I 942), while heat increases the frequency of replacement of halteres by wings in the normal ontogenesis of Diptera. Temperature-change may also affect the response to other agents, for instance (p. 627) it reverses that to lithium salts (Trampusch, I95I; Mifume, I 953). 


\section{(b) Short-wave radiation}

Low dosage of ultra violet (U.V.), X-rays or $\gamma$-rays, accelerates regeneration, but the direct effect is inhibitory. Doses of 5,000 to I0,000 may inhibit completely (Brunst, I950). Irradiation causes general damage to living material but this is reparable. Permanent inhibition of this, as of other types of growth, is due to inhibition of cell-division (Torraca, I914; Butler, I933; Pucket, I936; Horn, 1942; Wolff and Dubois, I 948a). The effect is entirely local (Butler and O'Brien, I 942; Wolff and Dubois, I948a) and in some animals regeneration is eventually resumed by immigrant neoblasts. An irradiated region, in fact, traps the neoblasts so that if amputation is performed distally to an irradiated zone, no neoblasts reach the site and there is no regeneration (Wolff and Dubois, I948a).

The effect of irradiation is proportional to the youth, rate of proliferation, and lack of differentiation of the cell. Cells of older animals, and of older regenerates, as proliferation-rate declines, become progressively less sensitive (Brunst, I950), whereas persistently proliferating cells of the gonads, bone marrow, lymphoid tissues, perichondrium, mesenchyme and epidermis remain very sensitive. The highly differentiated nerve-tissue is most resistant. The posterior growth-zone of annelids is the most sensitive region of the body (O'Brien, 1942), while in planarians the head-end is most active in growth and regeneration and most sensitive to radiations (Hinrichs, I924).

Chemically the only consistent effect of radiations (Thomson et al., I954) is a reduction in concentration, and in rate of turnover, of DNA. The more oxidised cytoplasmic form of nucleic acid, PNA, is less sensitive. SH-compounds, and other reducing agents, protect against irradiation (Bacq, I95I) which accelerates oxidation-processes and probably in this way inhibits the normal action of DNA in nuclear division.

Radioactive chemicals also inhibit regeneration; radiophosphorus completely inhibits that of the newt's leg at a dosage of I $66 \mu$-curies per gram of body weight, (Dent, I 949). Neutrons have the same effect as short-wave radiations, and are probably more potent; $62 \mathrm{n}$ causes complete inhibition in the urodele limb (Horn, I94I, I 942). The resemblance extends to a further effect, peculiar to regenerating tissues, - an excessive regression of the tissues of the stump. An established blastema prevents the induction of this regression even though its further growth may be completely inhibited (Butler, I 933).

There is here a close similarity to the responses to denervation (p.64I) and to colchicinetreatment (p. 627), the primary effect of which again is on cell-division. Regression is facilitated by amputation and by other kinds of wound (Thornton, I954), and only very high dosage of the inhibitor of mitosis will cause regression without this aid. There may be more than a simple quantitative summation of damage, therefore. A grafted blastema will halt regression already "under way", a demonstration of the incompatibility of R- and P-phase processes.

\section{(c) Electrical forces}

Electrical potential-differences are usually associated with activity of any kind, in the body (Lund, I 947), and regeneration is characterised by an oscillation in potential between active and uninjured regions (Crane, I950). The former is 
more electro-positive in the R-phase and more electronegative in the P-phase. Lund (I 947) finds growing regions in general negative to non-growing tissues. Imposed electrical potentials naturally affect regeneration (Davenport, I 899; Child, I94I; Lund, I 947), and the dosage has usually been adequate to show this, notwithstanding the reversal of intrinsic potential during the process, though this may explain some of the paradoxes reported. Recent work (Barth, I934; Marsh and Beams, I952; Dimmitt and Marsh, 1952; Moment, 1946-50) with physiological dosage, carefully controlled, has yielded more intelligible results, though the spontaneous intrinsic reversal of potential (Crane, I950) is still often ignored. The apparent sharp contrasts between closely related genera of hydroids (Barth, I 934; Child, I94I) possibly depends on this.

The ends of the intact body of the earthworm, Eisenia, (Moment, I 953, I 954) are electro-positive to the middle region, the posterior being more positive than the anterior end, so that SH-concentration (Perkins, I929; Maloeuf, I936b) is proportional to electronegativity. When either end is amputated the exposed surface becomes negative to the middle of the body; as it returns to the normal gradient, cell-poliferation ceases. There is no further change during hypertrophy and differentiation (Moment, I 953, I954). Crane's initial electropositive trend may be brief in these animals.

In Planarians and Hydroids the intrinsic gradient is probably simple, in both intact animals and isolated pieces, the head-end being the most electropositive. An imposed, adverse, direct current inhibits head-regeneration progressively, and induces increasingly vigorous regeneration of a head at the opposite end of an isolated piece (Marsh and Beams, I952). Rate of regeneration and the nature of the regenerate are both related to the intrinsic electrical field, in accordance with the views of Child (194I). In Tubularia and Pennaria, again, an adverse external field is inhibitory (Barth, I934); these hydroids are about three times as resistant as the Planarian Dugesia (Marsh and Beams, I952) as measured by the cur-

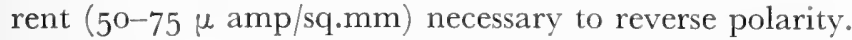

A current increasing the intrinsic potential does not accelerate regeneration appreciably, and indeed becomes inhibitory at about one tenth of the flow of an adverse current (Barth, I934). The adverse field retards regeneration but a concurrent field will accelerate the termination of the process (Moment, I953). Dimmitt and Marsh ( I 952) suggest that normal regeneration is possible only within a narrow range of gradients in ionic potential. They showed that the static potential is more important than the flow of current, the morphogenetic result of a particular potential-gradient being unchanged over a twelve-fold range in specific resistance and flow. This would seem to restrict effective changes to valencychanges, i.e. to oxidation-reduction processes, which is consistent with the SHgradient observed in earthworms.

\section{(d) Extraneous chemicals}

The action even of such completely unphysiological substances as beryllium salts (Needham, I94I ; Thornton, I 949, I 95 I; Tuchmann-Duplessis, I950, I 953) may help in the analysis of the process of regeneration, and may be very specific (Dubois et al., I949; Chèvremont and Firket, I949; Aldridge, r950). A compara- 
ble service in the analysis of ontogenesis is due to lithium salts (Lehmann, 1945). Some other agents used also are already known to affect normal growth, and include colchicine and nitrogen-mustards. Lehmann (1954) has initiated a more rational approach by the use of antagonists of specific metabolites and, in consequence perhaps, has produced effects with a high degree of specificity to particular stages of regeneration. Thus the amino-ketone, and other, analogues of the amino acids leucine, valine, tyrosine and histidine, inhibit relatively specifically the cell-migrations leading to formation of a blastema (Lüscher, 1946; Lehmann and Dettelbach, I952; Hadorn and Chen, I953; Lehmann, I954a). The subsequent stage of cell-proliferation is no longer sensitive (Dettelbach, I952). Cobalt salts, which form a complex with histidine, also inhibit at this stage (Lehmann, I 954a). Additional leucine does not prevent the action of its amino-ketone analogue (Lehmann, I949) possibly because peptides, rather than free amino acids, are the active agents (Lüscher, I952). Glucosamine, also, inhibits cell-migrations (Lehmann, i 954a), possibly as a partial analogue of amino acids or because, as a product of the breakdown of the mucoproteins, it inhibits the normal breakdown of these proteins in connective-tissue barriers. Tuchmann-Duplessis ( $195^{\circ}$ ) finds that beryllium salts also inhibit at this stage and this is consistent with the results of Thornton (I95I). Epidermal cells fail to cover the wound, which usually reopens (Thornton, I 95I), and causes a second phase of dedifferentiation. Further, mesodermal blastema-cells fail to accumulate (Thornton, I95I) possibly owing to the lack of a normal stimulus from active epidermis (Polezhayev and Favorina, I935; Lehmann, personal communication). Once the blastema is established, regeneration is no longer inhibited by beryllium which, in fact, must act within a few minutes of amputation, in Amphibia (Needham, I94I; Thornton, 1949). Epidermal cell-migration therefore is normally induced at a very early stage by the wound-factor (p. 637). Arthropods, by contrast, do not produce a woundfactor, and the stump of an amputated limb of the Isopod, Asellus, is much more resistant to the action of beryllium than that of an amphibian. To induce substantial retardation, - $45 \%$ of the rate of the partner control limb-by a brief exposure of $30 \mathrm{sec}$, it was necessary to use a $20 \%$ concentration of $\mathrm{BeCl}_{2}$, effectively an histological fixative. At this concentration it was not much more inhibitory than equimolar $\mathrm{HCl}(39 \%)$ or a saturated solution of lead acetate $(27 \%)$, though its action was more persistent.

Colchicine is fairly specific to cell-division (Thornton, I943; Lüscher, I946; Lehmann, I949), and so are nitrogen mustards (Supniewski, I 949; Skowron and Roguski, 1953), as in normal growth. Colchicine acts mainly on the divisionspindle and the mustards on the chromosomes. A number of basic dyes also are specific to cell-division (Steinmann and Wilhelmi, I950) and alizarin even to one cell, the osteoblast (Eksterowicz, I 950). Colchicine behaves very similarly (p. 624) to other antimitotic agents (Thornton, r 943). Some other aromatic ketones, structurally related to colchicine, also inhibit cell-division, in the tail-regenerate of Xenopus-tadpoles (Lehmann, I 954b).

Certain quinoxalins appear to inhibit at a stage between cell-proliferation and histodifferentiation (Lehmann and Dettelbach, 1952), presumably the stage of cell-hypertrophy, while other techniques (De Giorgi and Guyenot, I923) indicate 
that regeneration possibly may be inhibited also at a later stage, between growth and differentiation.

It may prove diagnostic of inhibitors specific to different stages, that their effects summate (Lehmann, I954a), whereas those of agents specific to the same stage do not. This may provide a valuable test since in fact the effects of all agents are usually apparent at several stages, probably because of the sequential effects of the initial action. Thus Thornton (I95I) found abnormalities of differentiation in beryllium-inhibited regenerates, probably because of deficiencies in the initial blastema, themselves due to the impediment of a thick dermal barrier: this is normally dissolved by overgrowing epidermis, previously activated by the woundfactor, which is the component specifically sensitive to beryllium.

One of the amino-ketones appears to inhibit not only cell-migration but also cell-division (Lehmann, I949) and it also inhibits the later growth of cells and nuclei (Dettelbach, I952). Colchicine affects the movements of cells to some extent (Hadorn and Chen, I 953; Lehmann, I 954b) and it also inhibits the sprouting of nerve-fibres (Hoffmann, I952), causing them to degenerate (Flinker and Sidman, I953), just as it causes regression of amputated limbs (Thornton, I943; Lüscher, I946). Short-wave radiations and denervation similarly both inhibit cell-division and promote regression. Colchicine has been found (Ebner and Strecker, I950) to affect nucleic acid-metabolism which again is very active in the functioning and regeneration of nerves (Bodian, 1947). All three groups of agent therefore may act primarily on the same stage. If so then their effects may be expected not to summate.

Lithium, which stands in the same chemical relationship to $\mathrm{Na}$ and $\mathrm{K}$ as beryllium does to the other two key physiological cations, Mg and Ca, retards regeneration at low, and accelerates at higher temperature (Trampusch, I95 I; Mifume i953). Rulon (i 948), also, observed paradoxical effects of lithium when applied to planarians before or after amputation. It induces supernumerary eyes in regenerating planarians (Brondsted, I942) which probably is the result of an initial inhibition (Butler and Blum, I955). Like beryllium (Dubois et al., i 949; Chèvremont and Firket, r949) it inhibits key reactions in phosphorus-metabolism (Runnström and Gustafson, I95I). Colchicine, also, acts upon P-metabolism (Ebner and Strecker, I950).

Anaesthetics and other depressant substances inhibit regeneration (Morgulis, I909; Bilski, I926). Paulain (1938) found atropine to have an inhibitory action, but his mathematical procedure when dealing with other depressants was not suitable (p. 608) to detect possible effects.

\section{INTERNAL FACTORS IN THE CONTROL OF REGENERATION}

\section{(a) Nutrition and regeneration}

Except in studies on the mammals, little fractionation into dietary components has been attempted, and it may be assumed provisionally that any observed effects of quantitative variations in intake are due mainly to the protein-constituent. Adequate nutrition is essential for regeneration in mammals (Anon., I953) 
and in other highly differentiated animals, e.g. in Anura (Bohn, igo4; Romeis, 1915; Schmalhausen, I925; Lüscher, I946) and in spiders (Friedrich, I906). Starved annelids regenerate slowly (Morgulis, I 9o9; Liebmann, I943). The less highly differentiated Metazoa, Coelenterates and Planarians, which are able to live on their own tissues to a remarkable degree, when fasting, can use the same material for regeneration, which persists to an advanced stage of inanition. Some cells and tissues are sacrificed to maintain and proliferate the more essential ones. Well-fed a Planarian regenerates mainly by epimorphosis, but when starved, mainly by morphallaxis (Chranova, I938), - a fact which also throws light on the nature and significance of morphallaxis itself. Even animals as highly differentiated as fishes are capable of living extensively on their muscle-tissues (Nardi, 1937) which, again, they can use for regeneration. Many of the lower Metazoa regenerate from mouthless and even gutless fragments.

Even in Planarians, however, prolonged fasting causes a progressive slowing of regeneration (Child, I9I I; Sivickis, I933-4; Wolsky, I935; Brondsted, I953, 1955) which, in Protozoa also, ceases before the stage of complete inanition (Weisz, I 954). Some Protozoa, including Paramecium, are very dependent on food (Tartar, I954), possibly because their normal metabolic activity is so high.

As opposed to starvation, brief fasting seems harmless or even beneficial to regeneration, even in mammals. Previously fasted rats regenerate the liver better than previously well-fed animals and in these rats continued fasting also is beneficial (Rosenthal et al., I 95I). Salamanders regenerate more rapidly after moderate fasting (Morgan, I go6) and similarly the Oligochaete, Criodrilus (Janda, I926), Planarians (Morgan, I go I Bardeen, I 90 I ; Lillie, I90o; Sivickis, I933) Coelenterates (Beutler, I926) and some Protozoa (Sokoloff, I924; Chajfec, I932).

Animals generally have a poor appetite during the early stages of regeneration (Bier, I9 17; Cuthbertson, I 946; Harkness, I952; Needham, I 955, p. 2 10), at the time when body-proteins are being mobilised and largely squandered as "nitrogen-flow". It is difficult to avoid the implication that excess protein is a sheer embarrassment at this time. The magnitude of nitrogen-flow is proportional to the protein-intake (Munro and Chalmers, 1945; Cuthbertson, 1946) and the negative balance (nitrogen output - intake), even, may be greater (Grossman et al., I 954). It seems very probable therefore that "flow" and reduced intake of nitrogen, are both beneficial. Anterior pituitary hormones will reduce $\mathrm{N}$-flow but this does not accelerate healing (Cuthbertson, I 954). In fact healing is retarded, whereas a low level of anterior pituitary hormone increases flow, but improves healing (Williamson and Newman, i 954).

The virtue of jettisoning excess protein may be to produce a more "youthful" condition of metabolism (p. 596), essential for regenerative growth. Mildly starved Planaria do become more youthful, metabolically as well as morphologically (Hyman, 1919, I920; Abeloos, 1930) and it is probably significant that in old Planarians regeneration is improved by fasting but not in young animals (Sivickis, I933 Brondsted, I955). In mammals, also, fasting is more deleterious to young than to old individuals (Guthbertson, 1944). Young Clavellina (p. 589) regenerate by morphallaxis (Millot, I93 I, p. I35) and this contrast holds also between fasted and fed Planarians.

There is some indication (Crofts and Peters, 1945; Williamson and Fromm, 1952) that nitrogen-flow reflects the destruction of most of the amino acids of proteins in order to pro- 
vide necessary quantities of a few essential amino acids, in particular of methionine, since an external supply of methionine reduces and even reverses the negative nitrogen-balance of the flow-period. However this mobilisation of methionine or other amino acids can scarcely be a critical requirement, since regeneration may be quite normal in fasting animals. The action of administered methionine may be more indirect, - for instance it tends to reverse the negative nitrogen-balance due to thyroid hormone (Gaunt, 1954; p. 205) (cf. also p. 616).

In the R-phase food also may impose a strain on metabolism. The gut of starved Planarians is less sensitive to KCN than that of well-fed animals (see Child, I94 I, p. I I4) and its oxygen-requirement is lower. This must be an advantage in the trend towards more reducing, anaerobic conditions. Fasting is accompanied by a decrease in oxygen-consumption (Hyman, I9I9, I920). This tends to reduce $\mathrm{pH}$ also and facilitates the breakdown of body-proteins, and the release of $\mathrm{SH}$ groups.

Fasting is beneficial also for the assertion of new individuations in regenerating Planarians (Child, I 94r, p. 350) i.e. it has qualitative effects.

In the P-phase, food-protein is beneficial (Clark, I9I9; Harvey and Howes, I930; Anon., I950; Calloway et al., I 955), and fasting retards cell-proliferation and growth (Schmalhausen, I925; Nardi, I937). Protein is then used with great economy (Needham, I952), as in the metamorphosis of insects (J. Needham, I942), and prior fasting may improve this economy, for animals previously well-fed show a more adverse nitrogen-balance during the $\mathrm{R}$-phase if intake is then reduced (Grossman et al., I 954); although they regenerate less well than fasting animals under any conditions, this inferiority is further increased if then they are made to fast (Rosenthal et al., I 95I).

The strange squandering of protein in the R-phase is probably not more puzzling than the high rate of excretion of indispensable vitamins and other substances which occurs even in the normal mammal.

Vitamins. The specific roles of dietary constituents other than the vitamins have been considered incidentally, in Section V. Little attention has yet been given to the action on regeneration of vitamins other than vitamin $\mathrm{C}$, ascorbic acid (Arey, I936; Holmes, I942), but it may be pointed out that the "maintenance" function for which they all seem essential, is a low-grade regenerationprocess. Acute regeneration, also, is promoted by all those water-soluble vitamins which have been tested.

Scurvy is the result of faulty maintenance-repair, through a deficiency of vitamin C. Lind, himself, noticed that men with scurvy also healed wounds slowly (Hunt, I94I) and it has been demonstrated experimentally that ascorbic acid is necessary for maintenance (Pirani and Levenson, I953) as well as for the initial regeneration (Bourne, I942; Murray and Kodicek, 1949) of the connective tissues in general. It improves regeneration of the mammalian cornea (Boyd, 1955), which is mesenchymal in origin. Its main function is in the synthesis of the mucopolysaccharides of the ground-substance (Bradfield and Kodicek, I95 I ; Kent and Whitehouse, I955) and of collagen (Murray and Kodicek, r949). Bone, a derivative connective tissue, similarly requires this vitamin (Bourne, I942; Murray and Kodicek, I949). It is thought also to control the number of polymorphs 
TABLE 13

ACTION OF ADRENAL CORTICOIDS ON REGENERATION

\begin{tabular}{|c|c|c|}
\hline Material & Corticoid & Reference \\
\hline \multicolumn{3}{|l|}{ (a) Promotion of regeneration } \\
\hline Triturus: $\operatorname{limb}$ & unspecified & $\begin{array}{l}\text { Schotté and Lindberg, I954; } \\
\text { Schottéand Chamberlain, I } 955\end{array}$ \\
\hline Mammal: liver & deoxycorticosterone & $\begin{array}{l}\text { Berman et al., 1947; Friedgood } \\
\text { et al., 1950 }\end{array}$ \\
\hline Mammal: liver & unspecified & $\begin{array}{l}\text { Taleisnik, I954; Giberti and } \\
\text { Bianchini, I954 }\end{array}$ \\
\hline Mammal: skin & unspecified & Wyburn-Mason, i $950 ;$ p. 246 \\
\hline \multicolumn{3}{|l|}{ (b) $\mathcal{N}$ o effect } \\
\hline Triturus: $\operatorname{limb}$ & cortisone & Bragdon and Dent, 1954 \\
\hline Mammal: bone & , & Key and Odell, I952 \\
\hline $\begin{array}{l}\text { Small gastric wounds of mam- } \\
\text { mal }\end{array}$ & , & Janowitz et al., 1955 \\
\hline $\begin{array}{l}\text { Mucin-production in gut of } \\
\text { mammal }\end{array}$ & , & Jennings and Florey, I954 \\
\hline \multicolumn{3}{|l|}{ (c) Inhibition } \\
\hline Triturus: limb & , & $\begin{array}{l}\text { Manner, } 1955 \\
\text { Billingham et al., I95 I ; Zoger, }\end{array}$ \\
\hline Mammal: skin & , & $\left\{\begin{array}{l}\text { I } 952 ; \text { Montgomery and Green, } \\
\text { I } 954 \text {; Gillman } \text { et al., I } 955\end{array}\right.$ \\
\hline Mammal: liver & , & $\begin{array}{l}\text { Perez-Tamayo, 1953; Einhorn } \\
\text { et al., } 1954\end{array}$ \\
\hline Mammal: bone & $\begin{array}{l}\text { cortisone and deoxycor- } \\
\text { ticosterone }\end{array}$ & Fontaine et al., 1952 \\
\hline & cortisone & Blunt et al., 1950 \\
\hline $\begin{array}{l}\text { Mammal: large gastric } \\
\text { wounds }\end{array}$ & , & Janowitz et al., I955 \\
\hline Mammal: nerve & , & $\begin{array}{l}\text { Lytton and Murray, i954; } \\
\text { Thomas, I954 }\end{array}$ \\
\hline $\begin{array}{l}\text { Mammal : reticuloendothelial } \\
\text { system }\end{array}$ & , & Mene, I953 \\
\hline
\end{tabular}

and macrophages in the demolition- and defence-period (Bourne, I948), and its depletion in this activity may explain the early, and temporary, decrease in amount of the vitamin, locally (Ryvkina, I940; Merezhinskii et al., I 954). This decrease is synchronous with an increase in free SH-groups and later both trends are reversed. The interaction between these two reducing agents, and their association with alkaline phosphatase in the synthesis (differentiation) of connective tissues has been indicated.

Thiamine normally increases locally throughout the process of healing of ratskin (Paul et al., I 945). It is depleted by infection, which retards healing,- - pos(ii) ly for this reason. Riboflavin accelerates regeneration in Planarians (Brondsted, I 955, p. I04) and in rats (Bosse and Axelrod, I 948). Biotin and pyridoxin also promote skin-healing in rats. Nicotinic acid speeds regeneration in Planarians 
(Owen et al., 1939) and pantothenic acid in the tail of Amphibia (Raunish, 1950). High concentrations of the latter have an inhibitory action. Vitamin $B_{12}$ promotes liver regeneration in rats (Schweigert et al., I 954), and its component, benzylimidazoline, the healing of fractures (Principe and Bellucci, 1953). Choline chloride, in $0.3 \%$ concentration, accelerates the regeneration of the newt's limbs (Lecamp, I 942).

\section{(b) Hormones in the control of regeneration}

In general the hormones of vertebrates affect regeneration in a manner consistent with their activity on normal growth and morphogenesis. Apparently paradoxical results are common because of the complexity of interactions between the various hormones. In addition, regeneration introduces its own peculiarities, due to the R-phase and to the subsequent reversal of metabolism. Moreover, the brevity and speed of the process may render it fatally sensitive to small changes in hormone-concentration, at critical periods.

(i) Steroid hormones. Probably the most equivocal results are those on the action of the hormones of the adrenal cortex though it seems clear from Table 13 that this is due mainly to the familiar contrast between the actions of the cortisone, or "S"-group of steroids, the gluco-corticoids, which are generally nitrogen-squandering and pro-catabolic, and the androgenic or " $\mathrm{N}$ " group, nitrogen-sparing and growth-promoting (Cope et al., I 943; Derbes and Weiss, I 95I). The mineralocorticoids resemble the latter group. The former might be expected to promote regeneration in the $\mathrm{R}$-phase and the latter in the $\mathrm{P}$-phase, and in fact cortisoneadministration does induce all essential components (Cuthbertson, 1954) of the GAS. Moreover these components are essential for regeneration (Schotté and Chamberlain, I 955). Deoxycorticosterone (DOCS), one of the mineralocorticoids, promotes the proliferation of connective tissue-cells (Selye, I955), increases the protein-content of the liver during its regeneration (Berman et al., 1947; Friedgood et al., 1950) and also of the serum (Roberts, I95I), which frequently reflects changes in the liver.

The promotion of regeneration directly, by extracts of the adrenal cortex, or indirectly by the adrenocorticotropic (ACTH) factor of the pituitary, seems to be limited to these early stages (Hall and Schotté, I95I; Schotté and Hall, I952; Schotté and Lindberg, I 954; Schotté and Chamberlain, I 955; Giberti and Bianchini, I 954). Wolffian lens-regeneration in the newt involves little R-phase activity and appears not to be affected by hypophectomy at any stage of regeneration (Schotté and Murphy, I953; Stone and Steinitz, I953). The presumption is that corticoids have little effect on the process in the P-phase, or possibly that any favourable effect of the N-group is counteracted by inhibitory action of the Sgroup, at that stage. Administered cortisone in fact does inhibit in the P-phase (Table 13), acting mainly on cell-division (Perez-Tamayo, I953; Mene, I953; Einhorn, et al., I954; Taleisnik, I 954; Thomas, I954; Manner, I955), and possibly also on cellmigration (Thomas, I 954). It also inhibits the growth of nerveaxons (Lytton and Murray, 1954),-altogether it shows a remarkable resemblance to the action of colchicine. The action of the adrenal S-corticoids, like the dedifferentiation caused by colchicine, by denervation and by irradiation, normally requires the additional stimulus of trauma (Selye, I955).

Literature p. 649 
TABLE 14

PROMOTION OF REGENERATION BY THYROID HORMONE

Tissue or organ

Mammalian skin

Mammalian bone

Mammalian liver

Mammalian muscle

Vertebrate nerve

Bird, feather

Triturus, limb

Triturus, tail

Axolotl, limb

Axolotl, tail

Rana, tadpole, limb
Reference

, Arey, 1936; Nettleship, I943;

+ Barclay et al., I944; Carlson, 1952

, Steinlin, Igoo; Bayon, I903; Fontaine

1 et al., 1952

Canzanelli et al., 1949

Kovalenko, 1954

, Wolff, I895; Walter, I9I I ; Diaz-Guerrero, I 1947

Belkin, 1946

Richardson, 1945

Kambara, 1953

Belkin, 1946

Jaques, I950; Hess and Kopf, I951

Peadon, 1953

In the R-phase of limb-regeneration in the newt, ACTH induces de-differentiation and the essential mobilisation of old collagen (pp. 6 I 9, 626) and of epidermis (Hall and Schotté, I95I). In the rabbit the same solution of dermal collagen and thinning of the epidermis is caused by cortisone (Gillman et al., I 955). Cortisone depresses P-phase activities in connective tissues (Zoger, I952; Kent and Whitehouse, I955, p. $35 \mathrm{ff}$ ), inhibiting the uptake of sulphur in the synthesis of mucoproteins. Extra ACTH administered to an animal with intact adrenals inhibits regeneration in the newt (Schotté and Chamberlain, I955), possibly by increasing abnormally, or differentially, the activity of the S-corticoids.

The action of the N-group and of the mineralocorticoids needs further, more specific study. DOCS has been found to depress bone-healing (Fontaine et al., I 952). On the other hand the androgens promote the regeneration of bone (Principe and Bellucci, I952) and of somatic tissues generally (Arey, I936; Hopper, I949) as they do somatic growth (Burrows, I949; Roberts and Szego, I953; Gaunt, I954). However the evidence is not unequivocal; Baxter et al. (1944), Guerner and Wrba (1953), and Brauchardt (1953) found no favourable effect on regeneration under various conditions ( $c f$. Korschelt, I927, p. 665). Androgens improve nitrogen-retention in regenerating animals (Cuthbertson, I944) as in normal animals, which shows that any favourable effect must be in the P-phase. With exceptions (Callan, I939), the gonadal hormones control the seasonal "regeneration" of male accessory and secondary sexual structures (Hopper, I949) but this is a more specific effect. Oestrogens similarly control the regeneration of the corresponding female structures, but they tend to inhibit general somatic growth (Burrows, I 949; Roberts and Szego, I953; Fishman, I951), and probably somatic regeneration also (Read, I954; Brauchard, I953), though a positive action on pancreatic islet-regeneration has been claimed (Foglia et al., I954). According to Lauber (1933) both male and female gonadal hormones promote somatic regeneration, but only (or particularly) in the appropriate sex. This 
seems plausible, since a serious disability in either sex would be biologically intolerable. However Needham (1949) found regeneration faster in the male Asellus than in a female of the same body-size. Emery (1937) failed to stimulate the regeneration (compensatory hypertrophy) even of the ovary itself, using oestrogens.

(ii) Thyroid. There is now a wealth of evidence that thyroid hormone is essential for healthy regeneration in vertebrates (Table I4). It is paradoxical that whereas the adrenal cortex, producing two types of factor, promotes only the R-phase of regeneration, the thyroid, with only one factor, assists both phases, just as it promotes all phases of ontogenesis (Brody, I 945, p. I68). It has been shown to improve various R-phase activities, - phagocytosis (Menkin, I940, p. 94), the proliferation of leucocytes (Ponder and Flint, I927), and nitrogen-flow (De Gribble and Peters, I95I). Methionine, which reduces nitrogen flow also prevents the loss of weight of hyperthyroid animals (Gaunt, I954). Thyroxin stimulates the activity of the adrenal cortex (Gaunt, I954) and may affect nitrogen-flow in this way. Thyroxin and the glucocorticoids both accelerate metamorphosis in the toad (Frieden and Naile, I955) a process with many similarities to regeneration (J. Needham, I 942). The androgenic corticoids, and androgens themselves, retard metamorphosis while the effect of oestrogens resembles that of " $\mathrm{S}$ " corticoids.

Peadon (1953) found that anti-thyroid agents inhibited mitosis but the general view is that thyroid itself retards rather than promotes cell-division (Peter, I945; Mittler and Herman, I950; Smelzer and Ozanics, 1954).

The hormone tends to accelerate regeneration but to reduce the final size (Korschelt, I 927) just as it does in normal growth (Brody, I945). It therefore promotes differentiation (Peadon, I 953; Hess and Kopf, I95I; Jaques, I950) rather than growth. Steinlin (I goo) found the hormone most essential for the later stages of regeneration. The phase-specificity therefore is essentially the same as that of the phosphatases and it may be significant that thyroxine increases the activities of acid and alkaline phosphatases and of adenosinetriphosphatase (Barker, I 95I) and that it depletes the liver of alkaline phosphatase (Kochakain and Bartlett, I948). Again it stimulates the turnover of the terminal phosphate of ATP (Venkataraman et al., 1950). It seems clear that thyroxin normally controls the coupling between oxidative reactions and the useful manipulation of energy through phosphorylation-processes. Antithyroid substances, such as the nitroanalogue, dinitrophenol (DNP) uncouple phosphorylation from oxidation (Glass, I952). Thyroxin itself in high concentration (Glass, I952; Klemperer, I955) acts as an uncoupler while low concentrations of DNP promote regeneration (Cuthbertson, I 944). This may explain the many claims that excess thyroid hormone retards regeneration (Pawlowsky, I923; Torrey, 1934; Richardson, I945; Drabkin et al., I950; Kambara, I953; Smelzer and Ozanics, I954). The indigenous thyroid is normally working at optimal concentration, or possibly sometimes rather above this level (Comsa, I952; Stone and Steinitz, I954). Excess thyroid causes much the same symptoms as a deficiency (Gaunt, I954). Both cause increased nitrogenloss (Carter and Thompson, I 953, p. 214) so that the physiological action is to retain rather than to squander nitrogen.

As part of its action on oxidative processes and the familiar stimulation of me- 
tabolic rate, thyroxin increases the activity of cytochrome $c$ (Drabkin et al., I 950) and of succinic oxidase (Barker, I95I). It inhibits lactic dehydrogenase, however, and therefore presumably promotes fat-oxidation, through formation of acetylcoenzyme A, rather than the terminal oxidation of carbohydrate. Thyroidectomy, indeed, causes fatty liver (Enteman et al., I 948). Thyroxin also accelerates the oxidation of vitamin $\mathrm{C}$ (Gemmill, 1952) and so increases the requirement for this vitamin and for the other antioxidant vitamins A and E (Drill, I 943), The action on vitamin $\mathrm{C}$ is probably related to the interaction of both with phosphatases and so with differentiation, in the connective tissues. Thyroid hormone would seem to pass from a synergistic action with adrenal $\mathrm{S}$-corticoids in the $\mathrm{R}$-phase to something diametrically opposed in the late P-phase.

As in normal growth (Marx et al., I942; Scow, I951; Gaunt, I954) and in amphibian metamorphosis, there is evidence of synergism between thyroid and anterior pituitary (Richardson, 1945) but this may depend on the ACTH factor rather than on the thyrotropic hormone or the pituitary growth-hormone, APGH, which is thought to antagonise thyroid here (Willier, I955).

(iii) Pituitary. Earlier results (Robertson, I923) showing that extracts of the anterior pituitary accelerate regeneration need re-investigation in view of the variety of action of its various factors. The ACTH and thyrotropic principles promote regeneration at appropriate time and concentration and this is probably true also (Selye, I955) of the growth-hormone (APGH). Thus Richardson (1945) and Ten Cate and Uyldert (I944) recorded acceleration of regeneration in Amphibia and Brues et al. ( 1936 ) in mammalian liver. Hypophysectomy prevents the regeneration of skull-bones and APGH restores this ability (Simpson, I953). It stimulates growth of the strengthening, connective tissues (Williamson and Neumann, 1954). Brues et al. ( I 936) observed a hyperplasia of all cells and an increase in water, ash and protein in liver cells, - a rejuvenation process (J. Needham, I 93 I). On the other hand, Astarabadi et al. (1 953) and Schotté's group (p. 63 I) detected no positive action on liver- and limb-regeneration. Cuthbertson et al. (I94I) found (p. 628) that the hormone reduced nitrogen excretion, as in intact animals (Li, I950; Gaunt, I 954) but nevertheless did not speed up regeneration. Williamson and Neumann (1954), in fact, found an inverse relation between the two: low concentrations of APGH $(0.3 \mathrm{mg} / \mathrm{rat}$, day) improved regeneration but decreased nitrogen-retention, while $1.0 \mathrm{mg} /$ day improved $\mathrm{N}$-retention but gave poorer healing than in controls.

It seems possible that ACTH normally controls the R-phase and APGH the $\mathrm{P}$-phase of regeneration and that the latter therefore inhibits if administered too early. The results of Schotté and Murphy (1953) and of Stone and Steinitz (1953) do indicate a slight effect of the pituitary in later stages. Even so, it is surprising that the familiar growth-promoting action of APGH, and of the N-corticoids, is not more evident during regeneration.

(iv) Thymus. The endocrine status of this gland is questionable (Anderson, I932) notwithstanding the correlations between its changes in size (? activity) and the processes of growth and maturation. Taken as a food it speeds regeneration (Korschelt, 1927, p. 654) perhaps through its content of nucleic acid. There are histological changes in the thymus of a guinea-pig while it is healing a fracture 
(Goldner, I925) and fat-soluble extracts of the "gland" accelerate the healing of bones in the rat (Eskelund and Plum, I953). Extracts of other lymphatic tissues, of spleen and of adrenals are inactive so that the effect is not simply that of a lymphoid tissue, stimulated in the normal way by the S-corticoids (p. 63I), during the G.A.S. (Selye, r948).

(v) Parathyroid. Because of its connexions with calcium metabolism, parathyroid activity naturally is essential for bone-healing (Greep, I949) and possibly more generally (Arey, I936), but there is little detailed knowledge of its action specifically during regeneration.

(vi) Insulin. Like APGH, insulin improves nitrogen-retention and normal growth (Fischer, I946; Sevag et al., I950; Gaunt, I954) but again a positive action on regeneration is not universally admitted (Arey, 1936), though healing is generally found to be slow in diabetics (Marshall, I 952). During the early stages of post-traumatic stress there are symptoms of diabetes (Green and Stonor, I954), associated with adrenal steroid-activity, and insulin in fact reduces that increase in amino acid-content of the blood following hepatectomy (Flock et al., I 953). Like APGH it is probably normally most active during the P-phase.

(vii) Hormones in invertebrates. The corpus allatum (Wigglesworth, 1954) is necessary for normal regeneration in larval insects; extirpation of the gland results in inhibition (Bodenstein, I953a) or in abnormal proliferations (Pflugfelder, I939). This might have been expected (p. 596) of the "juvenile" hormone. The corpus allatum seems to change its general action when the insect undergoes metamorphosis (Wigglesworth, I 954; Bodenstein, I 953b) and this applies also to its action on regeneration (Bodenstein, I955): extirpation of the gland in the imaginal cockroach now permits regeneration of amputated limbs. Regeneration occurs even in limbs amputated some time before allatectomy (Bodenstein, 1955), - a very elegant demonstration that a wound-factor is irrelevant to regeneration in Arthropoda (p. 626).

The prothoracic, "growth and differentiation", hormone of insects is believed to promote regenerative growth also (O'Farrell and Stock, I 954). In the cockroach, Blatella germanica, the prothoracic gland shows a sharp burst of mitoses in the middle of the stadium (p. 599) which is $5^{-6}$ days long. A burst of mitoses follows in the tissues of the body, the epidermis in particular, and the onset of ecdysis is then irreversibly determined. However if limbs are amputated before this critical period the next ecdysis is delayed by a period equal to that which has already elapsed between the previous inoult and amputation, so that a fully differentiated, and almost full-grown, regenerate is eclosed at the next moult. If amputation occurs after the critical period, then moulting is not retarded, and the regenerate appears only as a small blastema. Before the irreversible stage, therefore, amputation, or its consequences, is able to "put back the clock",-perhaps to delay activity in the prothoracic gland, - and so to cover the earlier R-phase of regeneration in time to benefit from this growth-promoting activity.

It might seem to follow from the precise amount of the delay induced that the normal arthropod growth-cycle in each stadium resembles the regeneration cycle, and it is perhaps significant that there is a period of nitrogen-flow after moulting and one of low N-output before moulting (Needham, I 957 and subsequent work). 
In effect the delay in moulting in the cockroach results in some retardation of normal growth, and in Crustacea the sinus gland (Hanström, I938; Scharrer, I955) similarly has been found to mediate a retardation of normal growth under the stimulus of regeneration-activity (Kamps, I937; Hanström, 1938).

The sinus gland also controls regeneration itself. Light stimulates the release of hormone from this eye-stalk organ and the hormone retards both normal growth and regeneration (Bliss, I 954a); both are accelerated in darkness, - until the hormone begins to overflow from the gland spontaneously (Bliss, I954b). The gland depresses N-flow (Needham, I955) and so presumably retards the early R-phase of the regeneration- and growth-cycles. It does not delay but, as might be expected, accelerates, the final P-phase of the moult-cycle (Carlisle and Dohrn, r953).

Certain large cells, probably incretory, in the brain of earthworms, multiply and grow after wounding of the body (Hubl, I 953). An endocrine function during regeneration has been suspected also for the cerebral organ of some annelids (Harms, I 947-9).

Claims that vertebrate hormones significantly affect regeneration in invertebrates, e.g. pituitary (Hankó, I912; Blumberg I940) and thyroid (Weimer et al., I938; Hanström, 1938) have been denied by others (Paulain, I938). It seems doubtful if the protein-hormones can survive, active, in taxonomically remote species, but recent careful work of Srinivasan et al., (I955) indicates that thyroxin, and therefore probably other simple active principles, may retain some relevant activity in invertebrates.

\section{(c) Chemical factors produced by regenerating tissues}

There is now much evidence that regenerating tissues themselves produce promotor and inhibitor substances, the former in early stages and the inhibitor in the later ones, so that they are probably components of an autoregulatory system. As in plants (Haberlandt, I922; English et al., I938), a promotor substance may be liberated by the damage of wounding (Needham, I94I; Thornton, I949), immediately and briefly, but indispensably for the whole subsequent train of events. Extracts of damaged cells induce supernumerary growths, in planarians (Loofbourow et al., 1939). Probably all damaging agents, including colchicine and short-wave radiation, accelerate regeneration in low dosage. Mechanical trauma stimulates growth even when inflicted on cells isolated in vitro (Fischer, I930; Suntzowa, I 944). The limbs of adult Anura have been induced to regenerate by excessive mechanical or chemical trauma (Rose, I942, I944, I949; Polezhayev, 1946, I 949). The effective stimulus for the release of this factor is damage, not the removal of tissue, since various types of trauma, without amputation, induce supernumerary "regenerates" (Goldsmith, I940; Fedotov, I946; Ruben, I955; Butler and Blum, I955). Such abnormal "growths" may be induced even in the unicellular Protozoa (Tartar, I954) so that the factor is not necessarily "spilled" from damaged cells and is probably bound to the proteins, locally. Although Arthropods appear not to produce a wound-factor (Needham, 1947a, I950a; Bodenstein, I955) quite frequently they develop supernumerary limbregenerates (Bateson, I894; Needham, 1950a; Wigglesworth, I954). 
Extracts of necrotic tissues also promote wound-healing (Arey, 1936; Palladina and Gudina, I953; Engley et al., I955; see also Chapter 8), and other regenerative growths (Fedotov, 1946; Levander, I949; Teir, I952; Weiss, 1952). Extraction-procedures in fact liberate such promotors from any cells (Loofbourow, 1948); extracts of most cells promote growth and regeneration (Laser, I933; Efimov, I943; Kerr and Werners, I 944; Margoliash and Doljansky, I950; Engley et al., I955). Regeneration-promoting extracts ("trephones") from leucocytes (Carrel, I922; Khruschev, I946) may have a more specific significance, since these cells are very active in the demolition-stage (Menkin, 1950). The extracts of tissues of embryos in general are probably more potent promotors than those from adults (Weiss, I 952, I 955) though weight for weight some adult tissues yield equally potent material (Laser, I933; Margoliash and Doljansky, I950). Embryo-extracts accelerate wound-healing (Arey, I936) and probably regeneration in Amphibia (Morosow, I935).

The wound-factor of Amphibia promotes the movement of cells to form a blastema, and in planarians also wounding stimulates the immigration of neoblasts (Goldsmith, I940; Wolff and Dubois, I 947, I 948). Curiously, irradiationdamage does not attract these cells, though an irradiated region traps neoblasts attracted into it by a normal type of trauma on the far side. In Annelids nutritive cells as well as neoblasts are attracted to the site of injury (Liebmann, I 946). In compensatory hypertrophy there is no cell-migration, and cell-proliferation is the first effect, on the orbital gland of the rat, of necrotic products from the homologous gland (Teir, I952).

It is uncertain if the wound-factor is always so evanescent as it appears to be in Amphibia but certainly this or other promotors are produced locally in subsequent stages (Nettleship, I 943; Neuman, I 946; Balazs and Holmgren, I 949, I 950; Auerbach, 1952; Engley et al., I955). The regressive processes in general probably release more of this wound-factor; thus although infection delays regeneration (Du Nouy, I936; Paulain, I938) it probably produces more wound-factor, since regeneration is more rapid once it does begin (Du Noüy, I936). A promotor factor escapes into the circulation (Auerbach and Doljansky, 1945; Engley et al., I955) and affects regenerates elsewhere (Nettleship, I943). It may be transfused to affect regeneration in another individual (Engley et al., I 955) and it persists long enough to affect later regenerates (Nettleship, I 943; Needham, I949b). Each injection of serum from a partially hepatectomised individual causes a burst of mitoses in the liver of a recipient (Friedrich-Freksa and Zaki, I955). The mutual stimulation of regeneration among the limbs of Crustacea etc., may be due to such a circulating factor, and similarly the improvement of regeneration on repeated evocation (Korschelt, I927; Chranova, I 938; Needham, I949b). A temporary depression, at the second and third evocation (Fig. 4 see p. 6o9), may be due to the inhibitor factor, which presumably diminishes progressively through the subsequent evocations.

A failure to detect any acceleration of regeneration due to active regeneration elsewhere (Taffel et al., I95I) might be due to subsequent neutralisation of the effect of the promotor by this inhibitor, which is produced later in the regeneration-cycle (Balazs and Holmgren, 1949, 1950; Auerbach, 1952; Steinberg, 1954). 
A regenerating nerve at first promotes the migration of Schwann cells (p. 64r) and later inhibits this (Abercrombie et al., I 949). Only the early stages of regeneration are sensitive to the inhibitor, so that self-inhibition is not possible, but reregeneration is inhibited (Davidson and Berrill, 1948) until the first act is complete, and much of the inhibitor eliminated (Weiss, 1955). Some of the inhibitor probably continues to circulate indefinitely and controls the equilibrium-size of the organ (Weiss, I955). There is probably an inhibitor, and a promotor, specific to each organ. The specific inhibitor of liver-growth is diluted by simple dilution of the serum which therefore permits resumption of growth (Glinos and Gey, I952; Warburton, I955). The circulating inhibitor, like the promotor, is active also on a parabiotic partner (Bucher et al., I95I) and on the homologous organ of the embryo (Rose, I 952).

A control of subsidiary levels by the most active region of a body-regenerate in planarians may depend, in part at least, on the production of inhibitor by the latter, since it regenerates fastest (Brondsted, I955). Subsidiary regions, when isolated take longer to recover the ability to re-regenerate than does the dominant region (Brondsted, I 954; Lender, 1954), which indicates that they must first recover from this inhibition. The action of a regenerate on normal growth appears to be converse to that on another regenerate, i.e. inhibitory by a young regenerate and promotor by later stages (Przibram, I9I7; Huxley, I932). The initial inhibition of normal growth may be due to simple competition, and the later acceleration to rebound, but it is also possible that the promotor is state-specific, and reflects the incompatibility between early regeneration and normal growth.

Nature of promotor and inhibitor. The release of the wound-factor by any kind of damage implies that it is probably a protein or one of its degradation-products, though masked lipid also may be released in this way, and the wound-factor of some plants has been identified (English et al., I 938) as a dicarboxylic fatty acid. Both water- and fat-soluble promotors of regeneration in animals have been recognized. Loofbourow ( $194^{8}$ ) found that virtually all of the essential nutrients in an extract of damaged cells,- - amino acids, nucleotides, vitamins, etc., contributed to its potency, and Fischer ( 1948 ) obtained a similar list for embryo-extracts. Harris (I953) and others also have stressed the heterogeneous nature of the promotors in the latter. At the other extreme Palladina and Gudina (I 953) concluded that the activity of extracts of necrotic tissues was due merely to $\left(\mathrm{NH}_{4}\right)_{2} \mathrm{CO}_{3}$, - an endproduct of protein-degradation.

The promotor released later by the regenerating tissues themselves has been identified with a component of the globulins of the serum at that time (Balazs and Holmgren, I949; Engley et al., 1955). There is an increase in the globulin/albumin ratio after wounding (Cuthbertson, I 954), and during stress in general (Selye, I948), and the globulin-fraction is more growth-promoting than the albumin (Balazs and Holmgren, I949). The increased globulin is associated with a nonspecific increase in all serological reactions and no specific regeneration-antigens have been detected (Engley et al., I 955). The antibody to normal axolotl-muscle inhibits muscle-regeneration in this animal (Striganova, 1949), indicating no change in muscle-protein during regeneration. Weiss (1952, I 955) also envisages the promotor as a normal typical protein of the tissues in question, possibly capable 
of promoting the synthesis of homologous protein autocatalytically. He further postulates that the inhibitor is related to it as antibody to antigen. In fact a promotor inhibitor (PI) complex has been detected by Marshak and Walker (1945) in mammalian liver, and by Teir (1952) in the orbital gland of the rat. In the orbital gland-extract the promotor is at first inactive or masked, possibly by the inhibitor. This masking no doubt would increase with the production of inhibitor in the later stages of regeneration. Wounding, like extraction techniques, probably frees the promotor from the inhibitor. Beryllium may inhibit the breakdown of the complex (p. 626).

If, as seems probable, there is a PI pair for every organ and tissue, then a very large number may be involved in any extensive regeneration, and a non-specific increase in serological reactions generally (p. 638) is to be expected. At present there is no evidence that when an organ is amputated, the non-specific tissues, connective tissues, muscle, etc.-are stimulated in non-homologous organs so that it may even be necesary to envisage a PI pair for every tissue of every organ, a detailed specificity which compares with that postulated by Sperry (I95 I) between each nerve and its end-organ.

Some of the inhibitor of the Tubularia hydranth diffuses out into the water (Rose and Rose, I 94 I ; Tardent, I955) and such washing-out may relieve regeneration in aquatic animals more generally. Ludwig and Ludwig (I954) have postulated such a process to explain compensatory reversal of opercular type in the serpulid, Hydrioides, - that is the growth of the vestigial operculum of one side of the body to replace the amputated, functional operculum of the other side.

Balazs and Holmgren (I949) found increasing amounts of a growth-inhibitor in the albumin-fraction of the serum in later stages of regeneration. Later (I950) they attributed its origin to basophilic material, probably mucopolysaccharides or mucoprotein of the differentiating dermis. Mucoprotein could be resolved into heat-labile protein and heat-stable, non-protein, prosthetic components, and it is conceivable that such a change in this type of factor may explain the discrepancy between the properties of the inhibitor of Tubularia, as described by Rose and Rose (I94I) and Tardent (I955), respectively. The inhibitors of the liver (Brues et al., I 940) and of the orbital gland (Teir, 1952) are heat-labile, while promotors are more heat-stable (Marshak and Walker, I945; Teir, r952) though both stable and labile components are demonstrable in embryo-extract (Fischer, I948; Margoliash and Doljanski, I950; Harris, 1953). Both promotor (Teir, 1952) and inhibitor (Rose and Rose, I941; Tardent, I955) are water-soluble but there are contradictory reports of the solubility-properties of the nerve-regeneration promotor (p. 643)

It is not clear at present how organ- or tissue-specific PI substances are related to the complex of direct metabolic controls, by SH-compounds, by muco-protein components, and probably by most active metabolites, though they presumably act at a higher level, on the process as a whole. In this respect, at least, they would be hormonal in nature. By contrast the direct metabolic controls are specific to particular reactions and on the other hand are not specific to organ or tissue. It would seem probable that the mucoprotein inhibitor of Balazs and Holmgren belongs to this latter group. 
(d) Nerve-supply and regeneration

It has been clear for some time (Korschelt, I 927) that in most Metazoa innervation of structures is necessary for their normal regeneration, and there is little doubt that it is essential in phyla, previously problematical (Korschelt, 1927; Abeloos, I932), the Platyhelminths, Annelids and Arthropods, (Lender, I954; Brondsted, I955; Janda, I930; Avel, I930; Zhinkin, I936; Harms, I944; Suster, I933; Needham, I945, I 946, I 953). Its importance is seen very clearly in some nemertines (Dawydoff, I gog; Coe, I 929, I 934) of which a very small fragment, containing the brain, regenerates a complete animal, whereas the whole of the rest of the worm, lacking the brain, does not regenerate the missing part.

Naturally Protozoa and sponges must regenerate without a nervous system, though the ciliate Protozoa have a functional analogue in the kinetosome-system (Lwoff, I 949) and this plays an analogous role also in regeneration (see Weisz, I 954). The anterior half of Paramecium, containing the "motorium", shows better regeneration than the posterior half (Peebles, I9I2). Reconstitution of hydroid coelenterates from masses of dissociated cells (p. 589) also is independent of innervation (Millot, I93 I ; Lehn, I 953). Even in animals so highly evolved as the Amphibia it is believed that regeneration is possible without innervation, if the latter is prevented initially, in ontogenesis (Yntema, I949; Singer, 1952). Innervation seems unnecessary for early ontogenetic development of limbs (Hamburger, I 928; Stone, I 93I), though required (tropic action) for subsequent maintenance (Huxley and De Beer, 1934) both of sense organs and of effectors (Lewis, 1937; Wyburn Mason, I 950). Some organs persist for a long time after denervation, e.g. the senseorgans of the lateral line of Anuran tadpoles for 140 days (Wright, I947), but eventually they undergo an atrophy. The local nerve-supply has been shown essential also for wound-healing in mammals (Gurevitsch, I 94 I) and any apparent exception, among animals possessing a nervous system, requires careful examination; it is extremely difficult to prevent re-innervation (Butler and Schotté, I 94 I, 1944).

Regenerating limbs of Amphibia become very richly innervated (Singer, I952; Thornton, I954) within the first few days (van Stone, I955) i.e. the nerves regenerate well ahead of other tissues. Their ability to do so depends on the normal dedifferentiation and "opening up" of the dermis (Thornton, 1954). The subsequent rate of regeneration of the blastema is proportional to the number of nervefibres per unit cross-sectional area of the limb (Singer, I952; van Stone, I955). Recently, Singer (I950) demonstrated that the normally non-regenerating limbs of adult Anura show considerable power if their nerve supply is increased. Singer (I952) concludes that the action depends on the quantity of nerve fibres and not on their qualitative neurological properties ( $c f$. Kamrin and Singer, I955), and it is perhaps equally significant that the action is only on the quantitative aspect of regeneration,- - on cell-proliferation, not on differentiation (Singer and Craven, 1948). The neurotropic action is not organ-specific (Singer, I952) or even speciesspecific (Lender, I 954; Harms, I 944).

The sensory nerve supply at one time seemed the most important component but this is probably because it is normally the largest component. In the limbs of newts one third to one half of the normal number of nerve fibres is adequate for regeneration but in adult Anura even the full number is inadequate. The number of nerve-fibres per unit cross-sec- 
tional area decreases with age (Van Stone, 1955) and this may underlie the normal agedecline in power of regeneration (Singer, 1952). A similar change may explain why the phylogenetic trend towards increased body-size involves a concomitant decline in regenerative power.

Denervation of the stump of an amputated limb results in regression of the stump (Schotté and Butler, I94I ; Thornton and Kraemer, I95I). This is perhaps a reversal of the growth-promoting and maintenance functions of intact nerve, though it is quite distinct from the slower process of atrophy which follows denervation of intact limbs (Schotté and Butler, I94I), and normally requires the additional stimulus of wound-factors. It is also different from normal dedifferentiation (Schotté and Butler, 1944). The injury need not be an amputation (Thornton, I953); if it is a crush-injury, not at the end of the denervated limb, then regression proceeds both ways from the crushed region. Denervated barbels of the catfish regress without amputation (Kamrin and Singer, I955). Regression halts as tissues are reinnervated and a blastema forms at a terminal surface of regression. Non-terminal regression however is not made good by regeneration (Thornton, l.c.) so that again factors other than neural also are involved. Regression can be halted by grafting an established blastema (Schotté and Butler, I94I, I944), too.

If a limb is denervated before amputation, regression is smaller (Karczmar, 1946) and regeneration is less retarded (Needham, I946a) than when denervation is simultaneous with amputation. Conversely both are enhanced if denervation is performed a few days after amputation. The P-phase begins as soon as the tip of the stump is innervated. Regression due to other causes (pp. 624, 627) is halted by nerve-regeneration.

Innervation is not necessary for the early stages of wound-healing ( $J$ anda, I930; Gorelova, I954; Roguski, I 954) but it appears to be essential for the cell-movements of blastema-formation (Schotté and Butler, I94I, I 944, I949; Thornton, r 954) in both epidermis and mesodermal tissues. In Planarians (Hyman, I95 I ; p. I88) and Annelids (Stone, I933), neoblasts migrate into the blastema alongside the ventral nerve cords. A regenerating nerve promotes the migration of its own Schwann-cells (Abercrombie et al., I949). Grafts of pieces of the central nervous system of Amphibia induce cell-migrations in the vicinity (Overton, I950). If the young blastema is denervated it regresses once more.

Cell-migration and cell-proliferation are intimately related (Medawar, I940) and the action of the peripheral nerve is probably primarily on the latter (Singer and Craven, I 948; Butler and Schotté, 1949). Denervation during the early stages of growth of the blastema (Fig. 2, p. 605) reduces the number of mitoses and retards or halts growth. As the interval between amputation and denervation is increased progressively the response of the regenerate changes from regression, through mere arrest and then decreasing retardation, to indifference; the later stages, of cell-hypertrophy and differentiation, appear to be virtually independent of innervation. No doubt it is significant that the kinetosome-system of ciliate Protozoa, and the corresponding structures in flagellates, are frequently connected to the centrosome and so, like the nervous system of Metazoa, control mitosis. Cell-division is a motor function with considerable similarity to muscular contraction (Brachet, I950, p. I 84) and other motor functions, so that its control by 
the nervous system seems natural enough. Grafts of nerve tissue also promote celldivision in neighbouring tissues (Overton, I950; Weiss, I950; Thornton, 1954); grafts of other tissues have no such action (Overton, 1950). Regenerating nerves stimulate cell-division among Schwann cells (Abercrombie and Johnson, r 946).

Although the action of the nervous system on regeneration is essentially nonspecific and quantitative it appears to have some qualitative effects. Thus in Platyhelminths and Annelids a particular cell is induced to develop as part of heador tail-regenerate according as transection is in front of it, or behind it (see Child, I94 I). A piece of nerve cord implanted into the flank of an Oligochaete induces the local tissues to regenerate a head or a tail depending on its orientation (Sayles, I942). Similarly the nerve-supply probably determines polarity in Vertebrate limbs and tail (Needham, 1952), and polarity-change in narrow transverse pieces of Planarians is believed to depend on the contained nerves (Huxley and De Beer, I934, p. 279).

It was believed that the homoeotic type of heteromorph in Arthropods, e.g. the regeneration of an antenna in place of an eye (Herbst, I896) was determined by the nervous system but this now seems improbable. S. Holter et al. (1954) found that the nerve-cord determined the typical pattern of the musculature of a tail-regenerate, but it did not affect limb-muscle and the apparent qualitative specificity needs further clarification. Experimental evidence in general continues to deny any influence of nerves on quality, other than polarity, in the regenerate (Needham, 1950a). The work of Sperry (I95I) and others indicates, on the contrary, that peripheral organs may determine the functional quality of any nerves which come to innervate them.

Recently Chandebois (1952) has shown that it is possible to inhibit differentially the two sides of the body in the triclad, Procerodes, by an inhibitor applied to the whole transverse section. It seems likely that such a sharp bilateral difference must depend on the bilaterally arranged nerve cords. The result,- - independent right and left heads, - is very similar to those of Brondsted (1955) when grafting together right and left halves of a Planarian, with their levels in the antero-posterior axis "staggered". It seems probable, therefore, that the instrinsic regeneration-rate at any level depends on a property of the nervous system at that level, a property graded through the body. In the normal body the combined activities of the nerve-cords results in a single regenerate with maximal activity in the mid-line of the body (Brondsted, 1955).

The asymmetry observed by Chandebois recalls an occasional bilateral asymmetry in the extent of morphallactic regeneration in Sabella (Gross and Huxley, 1934, 1935; Berrill and Mees, 1936). It is difficult to explain this except as the work of the nervous system, and likewise the observed progressive extension backwards of the morphallactic change, in response to repeated transections far away from the "front" of the change, or again an occasional tendency of the change to "skip" one or more segments. Bilateral independence is seen also in other Annelids (Berrill, I952, p. 429).

In the experiments of Chandebois the less inhibited side appeared to be dominating the other since, if it was reamputated, the other then took the lead. This strikingly resembles the phenomenon of chela-reversal in Crustacea and of operculum-reversal in Hydriodes, for which nervous control has been suggested by some (Huxley, I932) and denied by others (Abeloos, 1953; Ludwig and Ludwig, I 954). It also seems to show once more (pp. 636-639) both promotor- and inhibitor-actions, by the nervous system.

Regeneration of the limbs of Arthropods depends only on the peripheral nerve nid the local segmental ganglion (Needham, I953), and in the limbs of vertebrates the control is similarly local (Singer, 1952). The higher centres indeed may inhibit limb-regeneration (Jurand, I954; Maron, I954), during the early stages. The 
more extensive regeneration, of the whole body, in Platyhelminths and Annelids, involves the whole of the nervous system, and the power to promote regeneraton grades downwards from the brain in all directions (Brondsted, 1955). However, here also, higher levels of the nervous system inhibit regeneration at posterior levels in the body (Watanabe, I935).

It seems likely that there is a close relationship between the neurotropic action during regeneration and the tropic action of innervation on normal growth and on maintenance (Wyburn-Mason, I950), though clearly there are features peculiar to regeneration. More uncertain is the relationship between the tropic effects in general and the normal functional activity of the nervous system (Huxley and De Beer, I934; Needham, 1952). Innervation without actual conduction-activity is adequate for the maintenance of end-organs. On the other hand the latter further hypertrophy in response to function, and proportionately to it. Nerve-tissue itself shows functional hypertrophy (Eccles, 1953, p. 200-2) and this in its properties is distinct from the conduction processes, though causally dependent on them. An eye-regeneration factor from the brain of Planarians does not travel via the optic nerve (Lender, 1954). In sensory nerves the tropic effect must act antidromically (Lewis, 1937).

Nevertheless there is considerable evidence of significant similarities between the two activities of nerve tissue. Acetyl choline (ach) and other cholinergic drugs, e.g. pilocarpine, in low concentration, stimulate regeneration, and atropine retards (Morgulis, 1909; Paulain, I938; Welsh, 1946; Wyburn-Mason, r950; Lecamp, I 954). Reciprocally ephedrine, epinephrine and adrenalin,- - neurological antagonists of cholinergic drugs, - inhibit mitosis in the P-phase of regeneration (Friedwald and Buschke, I944).

Nerve-conduction and muscular contraction themselves, and no doubt response-mechanisms in general, resemble low-grade stress-reponses in their anaerobic, glycolytic initial phase, and their aerobic recovery-phase, during which protein and nucleic acids are synthezised (Hydén, I943). The influx of $\mathrm{Na}$ and efflux of $\mathrm{K}$ following trauma compare with their movements across the membrane of a conducting nerve-axon (Eccles, I953), and the growth of cells is accompanied by the reverse movements of these ions (Fenn, 1940). Cells removed from the body into culture-media regularly lose $\mathrm{K}$ and take up $\mathrm{Na}$ : and the movements are reversed when they begin to grow (p. 62I). Anaerobic conditions favour the first phase and aerobic the second.

Acetyl choline accelerates the inflow of $\mathrm{Na}$ into cells and atropine its extrusion (Ussing, 1954). This is in harmony with the fact that Ach induces shock (Sevag, I95I), the first phase of the GAS (p. 6o9). It also induces inflammation (Lecamp, 1954), as in the initial stages after trauma (Menkin, I950). The shock and countershock phases of the GAS are respectively cholinergic and adrenergic in type (Selye, I948). Adrenalin tends to act synergistically with the hormones of the cortex of the adrenal (Derbes and Weiss, 1951).

These two phases probably correspond to the "ebb" and "flow" periods following trauma (Cuthbertson, 1944; Needham, 1955), i.e. both are within the R-phase. Conceivably cholinergic drugs promote regeneration solely by virtue of this early action, but they generally promote anabolic, and adrenalin catabolic activities, so that it seems probable that there are two cholinergic phases, with an intervening adrenergic phase. Other bicyclic oscillations (Table 8, p. 610) may be related to this. The mineralocorticoids promote both inflammatory reactions and cell-proliferation (Selye, I955) and so possibly dominate activity both before and after the phase of maximal activity of the S-group.

The nature of the neurotropic factor(s) is still very uncertain. The factor of Overton ( 1950) which stimulates mitosis in surrounding tissues is resistant to freeze-drying and to alcohol and ether, but is inactivated by acetone. It is insoluble in alcohol-ether, and therefore could be a protein-derivative. Lender's "organisine" which induces the regeneration of eyes in Planarians is stable to $70 \%$ alcohol and to heat, which would destroy most proteins. It is not species-specific, but is readily diffusible, so that a relatively small, hydrophil molecule is probably indicated.

Factors which promote the regeneration of nerves themselves may be quite different from these "heterotropic" factors. The "NR" factor of Koechlin and Von Muralt (I946), 
and Lüscher and Von Muralt (1947), is a "homotropic" neural factor with a small, watersoluble molecule whereas the "neurocletin" of Hoffman (1950) and the factor of Edds (1953) are fat-soluble, probably originating from the lysis of myelin. Nucleoprotein, also, stimulates the sprouting of nerves (Cohen $e t$ al., I954), and these investigations clearly are still at an early stage.

\section{(e) The vascular system and regeneration}

Much of the early work on regeneration was by embryologists not accustomed to considering this system, but it is evident that the uses of the system have been fully exploited in adult morphogenetic processes. Completely devascularised tissues die, and partial devascularisation is compensated by new collateral vessels (Engley et al., I 955) and probably also by other means, so that specific experimental work in general has indicated a minor role for the vascular supply to a surviving regenerate (Korschelt, I927, p. 652; Janda, I931 ; Wyburn-Mason, I950; Goss, I954) though experimental restriction of agents known to be distributed by the blood stream shows clearly that it is important. Restriction of the blood-supply in fact has been shown to retard regeneration in a number of cases: the oligochaetes Rhynchelmis (Janda, I93 I) and Criodrilus (Zhinkin, I936), some Amphibia (Stone, 1944) and some mammals (Bowes, 1943). Reciprocally haemorrhage retards in Rhynchelmis (Janda, I93I), and probably in the isopod, Asellus (Needham, 1953). The membranes which form across the base of regenerates ( $p$. 595) allow the passage of vascular and nerve-supplies.

The vascular system is very important for the control of many processes of the defence- and demolition-period (Menkin, 1950), though inflammatory reactions probably do occur in avascular animals, and continue in isolated tissues, in vitro (Cooke et al., I 953). It brings nutrients and removes unwanted products. Cells for blastema-formation may travel this way, and injected cells are selectively taken up by the appropriate tissue (Andres and Weiss, 1952), though the neoblasts of oligochaetes are said to migrate alongside the nerve cord. It serves a mechanical function in the growth of the regenerate of Arthropods.

Many of these functions, the distribution of nutrients (Hammett and Chapman, I 938) and of cells, and the mechanical function (Hauschka, I 946; Berrill, I 948) are performed by the "gastrovascular" system in Coelenterates. This system also distributes the locally produced inhibitor-factor.

In the vascular metazoa the locally produced controlling factors travel in the blood stream and various active hormones throughout regeneration must be carried this way. Compensatory hypertrophy of some organs is induced by circulating, simpler metabolites, which accumulate and increase the "load" on the organs (Hartmann, I 933; Bollmann and Mann, I 935). These metabolites are usually substances used by the organ, and the accumulation is due to loss of part of the organ, so that the response is self-regulatory. For kidney-hypertrophy urea is the most potent factor, and for other organs correspondingly critical "raw materials". Size-adjustment of internally secreting organs may be controlled by the products of their activity rather than by the raw materials; those of many of the endocrine organs of vertebrates act on the pituitary gland, affecting its output of hormones, which act back on the "peripheral" organs (Gaunt, I954), a twostage control, therefore. 
More than one factor may be involved in vascular controls. The hypertrophy of a kidney after unilateral nephrectomy is slower and more prolonged than the rise in blood-urea (Warburton, I 955). This might be a simple inertia-phenomenon, but a kidney which atrophies because its ureter is ligatured, remains functional if, in addition, the partner-kidney is removed (Hinman and Butler, 1923). In a closely similar manner a lobe of the liver will atrophy if its portal blood-supply is ligatured, but not if the bile-outflow from the rest of the liver is obstructed (Rous and Larimore, I920). Compensatory hypertrophy of liver and kidney also depends on the amount of dietary protein in need of deamination (Rous and Larimore, I 920).

Most of the numerous systemic changes of the GAS depend on vascular control and many other examples, more peculiar to regeneration, have been cited in this chapter: for instance an increase in plasma-phosphatase during liver-regeneration (Taleisnik, 1954), a mobilisation of depot-fat (Yarbro and Anderson, 1954), and the transport of sulphydryl compounds from the liver to regenerating skin (Schacter et al., 1952; Beck and Linkenheimer, 1952; Williamson and Fromm, 1955). Peptidase activity is increased in the blood leaving an injured region (Selye, I948). Similarly neoplasms induce an increase in cathepsin activity throughout the body (Maver et al. I948). It is noteworthy, however, that methionine and cystine from the liver go only to regenerating skin and not to normal skin or to other organs (Williamson and Fromm, 1955). This unilateral type of vascular effect may prove to be more common. Hormones are well known to affect particular "target" organs (Gaunt, 1954).

\section{(f) Diffusing factors}

In such avascular Metazoa as the Platyhelminths this type of factor is to be expected (Lender, I 952; Brondsted, I 955) but even in vascularised animals diffusing factors seem common. Calcium for bone healing is mobilised mainly locally and in declining amount with distance from that point. Dedifferentiation, more generally, follows the same pattern (Thornton, I954) though it may "skip" resistant regions such as the head of a bone. There is a mutual stimulation of regeneration between hind-limb and tail-blastemata of Urodeles provided they are grafted within a critical distance of each other (Blacher et al., 1933); mechanical obstacles, as well as mere distance, reduce the effect. The interaction between regenerating arthropod-limbs similarly decreases with distance. Neoplasms inhibit regenerates in proportion to their nearness (Zdriukovskaja, 1942) and so do gonads, in Hydra (Goetsch, I929). Abeloos (1953) was unable to detect either nervous or vascular control of the phenomenon of reversal of operculum-types in Hydriodes, and Ludwig and Ludwig (1954) concluded that diffusing factors were involved.

Diffusion seems not only the primitive mode of coordination, but also the most efficient method of controlling the pattern of differentiation and differential growth, throughout the regenerate and the neighbouring stump-tissues. In planarians morphallactic changes throughout the body may be controlled in this way. The qualitative aspects are not strictly relevant here (p. 595). There is much evidence of interaction between the various local tissue-components, throughout the act of regeneration, and these in general must depend on diffusion-effects.

It was concluded (Needham, I952) that the epidermis controls the early stages 
of regeneration, and deeper tissues the later stages. A number of recent results indicate that the whole skin, rather than the epidermis alone, controls the early stages (Guyenot and Ponse, I930; Hellmich, I930; Kiortsis, 1953).

The epidermis has usually been found to induce regeneration and the dermis to determine its general nature. The general rule therefore is still valid that the deeper the tissue the later its dominance. The nervous system, controlling mainly cell-proliferation, might be said also to obey this rule. Each tissue is in control at the time of its own maximal activity (Needham, I952). Epidermis is necessary to induce dedifferentation (Polezhayev and Favorina, 1935; Gidge and Rose, I944). Dedifferentation begins in the dermis and without this regeneration is halted. In the Protozoa the ectoplasm probably plays a similar role to that of the epidermis of metazoa (Weisz, I954). The later, organising action of muscle tissue has been demonstrated by Liosner and Voronzova (1938), Thornton (1942) and by Trampusch (I 95I), that of cartilage and bone by Bischler (I926), Polezhayev (1949), Trampusch (I95I), and that of notochord by Morgan and Davis (1902), Marcucci (I 925), Lüscher (I946) and Santa (I95 I). The gut (endoderm) is relevant only to whole-body regeneration. Evidence of some controlling action by the gut is given by Krecker (1923) and Child (1941; p. 360).

In the reconstitution of hydroid Coelenterates (Beadle and Booth, I938; Von Bertalanffy and Rella, I94I) and of sponges (Huxley, I92 I ; Brien, I953) all or most cell-types from the parent must be present, and in fairly normal proportions, and this is true also of amphibian tail-regenerates (Lüscher, i 946). Each celltype therefore has its unique, indispensable contribution.

\section{(g) Local factors}

In the lower Metazoa vagrant neoblasts contribute cells to the regenerate but in Amphibia regeneration is inhibited by a very local inactivation of cells by radiations. Even in the Protozoa (Tartar, I94I) regeneration may be an extremely localised process. Not only the rate of regeneration but still more the quality of the regenerate is controlled by this very local slice of parental tissue (De Giorgi and Guyenot, 1923). Heteromorphic and more trivial abnormalities do occur, though infrequently under normal conditions. Heteromorphs show that the fate of regenerating tissues is susceptible to modification by the higher-grade, systemic type of controls already considered, and plasticity is shown also in the ability of tissues, at virtually all levels in the body of the lower Metazoa, to regenerate either anterior or posterior portions of the body. In some of these animals the parental tissues themselves are probably extensively modified by subsequent morphallactic processes and by grafts (Miller, I 938; Santos, I 931). Even in these simple animals, however, the modified cells only very gradually change their intrinsic character (Abeloos, I930). There are certainly limits to the plasticity of their tissues, and only a narrow antero-lateral region may be induced to regenerate eyes (Lender, I954). Very young blastemata, even in Amphibia and Arthropoda, may be determined placewise, after transplantation (Needham, I952, p. 7o): or at least may be changed in fate (Gebhardt, I 926 ), but they are normally determined, irreversibly, early in the process. Many tissues regenerate in continuity with the homologous tissues of the stump which determine their differen- 
tiation (De Giorgi and Guyenot, 1923). Promotor and inhibitor substances seem to be as localised in action as in origin, notwithstanding their systemic transport.

Quantitative powers of regeneration also vary locally (Polezhayev and Favorina, 1935), sometimes in great detail. The differences are largely, though perhaps not entirely, due to inherent local differences in the tissues. Thus the regenerationrate at a particular level of the Planarian body is independent of the size of the piece isolated (Brondsted, I954). Taxonomic differences in regenerative power likewise are intrinsic to the tissues; Anuran limbs grafted on to Urodeles lose the power to regenerate when the host metamorphoses, just as they do in situ, though the newt's own limbs retain the power. In general local differences in power, and species differences also, seem to be biologically adaptive (Needham, 1952), and are scarcely amenable to any other comprehensive explanation. Like other physiological "mechanisms", regenerative power is determined primarily by biological, and not by physical laws.

\section{GONCLUSION}

In most essentials, regenerative growth resembles other types of growth and competes with any occurring simultaneously in the same body. The nature of a regenerate depends more on local, slowly diffusing factors, and therefore there is little qualitative interaction with such other morphogenetic processes. To some extent the regenerate is an in vivo tissue-culture (Brøndsted, 1955, p. 76). The absolute rate of regeneration changes little during ontogenesis and has changed little during phylogenesis, wherever the power has persisted at all. It increases to a maximum soon after the onset of an act of regeneration and declines progressively as the limiting size is approached. The maximal rate similarly varies with the amount of material amputated.

Regenerative growth differs from normal growth most in the necessity for an initial regressive ( $\mathrm{R}-$ ) phase affecting tissue- and cell-structure, and metabolism, both locally and systemically. This R-phase produces rejuvenated cells, and the appropriate metabolic conditions for their subsequent proliferation, in the progressive (P-)phase. Some metabolic changes are bicyclic. Other peculiarities of regeneration also are probably associated with the special metabolism of the R-phase, for instance a squandering of protein, and other materials, and an indifference to the protein-sparing action of anterior pituitary (APGH) and other hormones. Anti-mitotic agents, of very varied nature, including denervation, all, with the aid of trauma, cause excessive regression; there is no neutral state between R- and P-phase activities. The quantitative, tropic action of the nervous system, therefore is more dramatically evident than in other types of growth.

There is evidence for an R-P oscillation in metabolism also in the intermoult growth-cycle of arthropods, in the metamorphosis of animals, in encystment and related processes, in starvation- and atrophy-phenomena, in the general adaptation-response to stresses in general, and in nerve-conduction and other workfunctions.

A young regenerate liberates a regeneration-promoting substance, and later 
stages an inhibitor. Both may be organ- or even tissue-specific. They are also stagespecific, and a number of extraneous agents, used experimentally, likewise show a degree of stage-specificity in action. Naturally occurring external agents tend to act as purely triggering stimuli, evoking a complete autogenous act of regeneration. In this, and in other properties, for instance the increase in rate of regeneration with increased amount being removed, regeneration shows a high degree of biological adaptation. 


\section{LITERATURE}

Abeloos, M, (1930) Bull. biol. France et Belg., 64, I.

Abeloos, M., (1932) La Régénération et les Problèmes de la Morphogenèse, Gauthier-Villars, Paris.

Abeloos, M., (1953) Bull. soc. zool. France, 77, 462.

Abercrombie, M. and M. L. Johnson, (1942) 7. Exptl. Biol., 19, 266.

Abercrombie, M. and M. L. Johnson, (1946) J. Anat., 8o, 37.

Abercrombie, M. et al., (1949) Proc. Roy. Soc. (London), B I36, 448.

Ajsupiet, M. P., (1937) Biol. Zhur., 6, 399.

Aldridge, W. N., (1950) Nature, 165, 772.

Allfrey, V. G. et al., (I955) Nature, I76, 1042.

Allgöwer, M. And A. Rosin, (I953) Bull. schweiz. Akad. med. Wiss., 9, 18I.

Anderson, B. G., (1933) Biol. Bull., 64, 70.

Anderson, D. H., (1932) Physiol. Revs., I2, I.

Andres, G. And P. Weiss, (1952) 7. Exptl. Zool., I21, 449.

Anon., (1950) Nutrition Revs., 8, 78.

Anon., (1951) Nutrition Revs., 9, 257.

Anon., (I953) Nutrition Revs., II, I24.

Anon., (1955) Nutrition Revs., I3, 202.

Aquila, N., (1952) Arch. sci. med., 94, 288.

Arey, L. B., (1936) Physiol. Revs., 16, 327.

Asboe-Hanson, G., (1952) Proc. Soc. Exptl. Biol. Med., 80, 677.

Astarabadi, T. B., et al., (1953) Endocrinology, 52, 652.

Auerbach, E., (1952) Proc. Soc. Exptl. Biol. Med., 8i, 294.

Auerbach, E. And K. Doljansky, (1945) Proc. Soc. Exptl. Biol. Med., 58 , i i i.

Avel, M., (1930) Compt. rend., 191, 78.

BAce, Z. M., (195I) Experientia, 7, II.

BAGGI, G. F., (I953) Boll. soc. med. chir. Modena, 53, 351.

Balazs, A. And H. Holmgren, (1949) Nature, I63, 488.

Balazs, A. And H. Holmgren, (1950) Exptl. Cell Research, I, 206.

Bammer, H. And V. Martini, (1953) Arch. ges. Physiol., Pflüger's, 257, 308.

Barakina, N. F., (I95I) Doklady Akad. Nauk. S. S. S. R., 8I, 293.

Barclay, T. H. G. et al., (1944) Quart. 7. Exptl. Psysiol., 32, 309.

Bardeen, C. R., (igoi) Am. F. Physiol., 5, i.

Bardeen, C. R. and F. H. Baetjer, (1904) 7. Exptl. Zool., I, I9I.

Barker, S. B., (I95I) Physiol. Revs. 31, 205.

Barnicot, N. A., (1950) 7. Anat., 84, 374 .

Baron, H. And J. B. Allison, (1954) Federation Proc., I3, 450.

Barron, E. S. G., (1949) in The Chemistry and Physiology of Growth, Ed. A. K. Parpart, University Press, Princeton.

Barth, L. G., (1934) Physiol. Zool., 7, 340.

Barth, L. G., (1937) Biol. Bull., 73, 381.

Barth, L. G., (1944) Physiol. Zool., I7, 355.

Bassina, J., (1937) Bull. biol. méd. exptl. U. R. S. S., 3, I84.

Bateson, W., (I894) Materials for the Study of Variation, Macmillan, London.

Bauer, E. S. and A. M. Granowskaja, (1934) Biol. Zhur., 3, 457.

Baxter, H. et al., (1944) Can. Med. Assoc. F., 50, 4 I I.

BAyon, G. P., (1903) see Korschelt, (1927), p. 678.

Beadle, L. C. And F. A. Booth, (1938) 7. Exptl. Biol., 15, 303.

Beck, L. V. and W. Linkenheimer, (1952) Proc. Soc. Exptl. Biol. Med., 81, 291.

Belfer, S. et al., (1943) J. Biol. Chem., 147, 345.

BeLkin, R. I., (1946) Compt. rend. acad. sci. U. R. S. S., 5 I, 477.

BERMAN, D. et al., (1947) Endocrinology, 4I, 258.

Berrill, N. J., (1948) F. Exptl. Zool., io7, 455.

Berrill, N. J., (I95I) Biol. Revs. Cambridge Phil. Soc., 26, 459. 
Berrill, N. J., (1952) Biol. Revs. Cambridge Phil. Soc., 27, 40.

Berrill, N. J. and A. Cohen, (1936) 7. Exptl. Biol., I3, 352.

Berrill, N. J. and D. Mees, (1936) 7. Exptl. Zool., 73, 67.

Bertolani, F. et al., (1954) Minerva med., 2, i i6o.

Beutler, R., (1926) Z. vergleich. Physiol., 3, 737.

Bier, A., (I9I 7) Deut. med. Wochschr., 43, 705, io25.

Billingham, R. E. et al., (195I) Brit. Med. F. (I), i 157.

Bilski, F., (1926) Arch. Entwicklungsmech. Organ., 107, 219.

Binkley, F., (1952) Exptl. Cell Research, 2, Suppl., 145.

Bischler, V., (1926) Rev. suisse zool., 33, 43 I.

Blacher, L. J. et al., (1933) Arch. Entwicklungsmech. Organ., 127, 370.

Buiss, D. E., (I954 a, b) Anat. Record, I20, 742, 799.

Bloom, F., (1952) Proc. Soc. Exptl. Biol. Med., 79, 65 I.

Blumberg, T. T., (1940) Proc. Soc. Exptl. Biol. Med., 44, i I7.

Blunt, J. W. et al., (1950) Proc. Soc. Exptl. Biol. Med., 73, 678.

Boas, N. F. And J. B. Foley, (1954) Proc. Soc. Exptl. Biol. Med., 86, 690.

Bodenstein. D., (i953a) see Roeder (i 953 ).

Bodenstein, D., (1953b) 7. Exptl. Zool., 123, 413.

Bodenstein, D., (1955) 7. Exptl. Zool., 129, 209.

Bodian, D., (1947) Symposia Soc. Exptl. Biol., I, 163.

Boecker, E., (I92 I) Biol. Zentr., 4I, II9.

Bohn, G., (1904) Compt. rend. soc. biol., 56, 661, 79 I.

Bohr, H. and A. H. Sorensen, (1950) 7. Bone and Joint Surg., 32A, 567.

Bollman, J. L. and F. C. Mann, (1935) A. M. A. Arch. Pathol., I9, 28.

Börger, G. and T. Peters, (I933) Z.physiol. Chem., Hoppe-Seyler's, 2I4, 9 I.

Bosse, M. D. And A. E. Axelrod, (1948) Proc. Soc. Exptl. Biol. Med., 67, 418.

Bourne, G. H., (1942) Quart. F. Exptl. Physiol., 3I, 3 I9.

Bourne, G. H., (1948) J. Anat., 82, 8 I.

Bowes, W. F., (I943) 7. Lab. Clin. Med., 28, 45 I.

Boyd, T. A., (1955) Brit. 7. Ophthalmol., 39, 204.

Brachet, J., (1950) Chemical Embryology, Interscience Publishers, New York.

Brachet, J. and R. Jeener, (1948) Biochim. et Biophys. Acta, 2, 423.

Bradfield, J. R. G. and E. Kodicek, (r95 I) Biochem. 7., 49, xvir.

Bragdon, D. E. and J. N. Dent, (1954) Proc. Soc. Exptl. Biol. Med., 87, 46o.

Brauchardt, G., (I953) Z. Vitamin-, Hormon- u. Fermentforsch., 5, 373.

Braun, A. A. and G. N. Orlova, (1945) Compt. rend acad. sci. U. R. S. S., 46, 340.

Breedis, C., (1954) Federation Proc., 13, 425.

Brien, P., (I937) Arch. biol. (Liège), 48, I85.

Brien, P., (i953) Biol. Revs. Cambridge Phil. Soc., 28, 308.

Brody, S., (1945) Bioenergetics and Growth, Reinhold Publishing Co., New York.

Brondsted, A. And H. V. Brondsted, (1952) Vidensk. Medd. dansk. naturh. Foren. Kbh., II 4,443 .

Brondsted, H. V., (1942) Arkiv. Zool., 34B, I.

Brondsted, H. V., (1953) 7. Embryol. Exptl. Morphol., I, 43.

Brondested, H. V., (1954) Colston Papers, Bristol, 7, I 2 I.

Brondsted, H. V., (1955) Biol. Revs. Cambridge Phil. Soc.,, $30,65$.

Brues, A. M. et al., (1936) A. M. A. Arch. Pathol., 22, 658.

Brues, A. M. et al., (1940) 7. Exptl. Med., 71, 423.

Brues, A. M. et al., (1944) J. Biol. Chem., 155,6ig.

Brunst, V. V., (1938) Bull. biol. méd. exptl. U.R. S. S., 6, 527, 530.

Brunst, V. V., (1950) Quart. Rev. Biol., 25, I.

Bucher, N. L. R. et al., (I95I) Cancer Research, II, 457.

Bullough, W. S., (1948) Proc. Roy. Soc. (London), B, I35, 2 1 2, 233.

Burrows, H., ( I 949) Biological Actions of Sex Hormones, 2nd Ed., University Press, Cambridge.

Buser, J., (I 948 ) Compt. rend. soc. biol., I I 8, 322.

Butler, E. Gr., (1933) F. Exptl. Zool., 65, 27. 
Butler, E. G., (1935) Anat. Record., 62, 295.

Butler, E. G. and H. F. Blum, (1955) F. Natl. Cancer Inst., 15, 877.

Butler, E. G., and J. P. O'Brien, (1942) Anat. Record, 84, 407.

Butler, E. G. And O. E. Schotté, (1941) 7. Exptl. Zool., 88, 307.

Butler, E. G. and O. E. Schotté, (1944) J. Exptl. Zool., 97, 95.

Butler, E. G. and O. E. Schotté, (1949) J. Expll. Zool., I12, 36 I.

Galkins, G. N., (I9I I) Biol. Bull., 21, 36.

Callan, H. G., (1939) Pubbl. staz. Zool. Napoli, I8, I5.

Calloway, D. H. et al., (1955) Surgery, 37, 935.

Campbell, R. M. et al., (1954) Brit. 7. Exptl. Pathol., 35, 566.

Cannon, W. B., (1932) The Wisdom of the Body, Kegan Paul \& Co., London.

Canzanell, A. et al., (1949) Am. J. Physiol., 157, 225.

Carlisle, D. B. And P. F. R. Dohrn, (1953) Pubbl. staz. zool. Napoli, 24, 69, 279, 285.

Carlson, A. J., (1952) in Cowdry's Problems of Ageing, Ed. A. I. Lansing, 3rd Ed., The Williams and Wilkins Co., Baltimore.

Carrel, A., (1922) 7. Exptl. Med., 36, 385.

Carter, C. W. and R. H. S. Thompson, (I953) Biochemistry in Relation to Medicine, 2nd Ed., Longmans, Green \& Co., London, New York, Toronto.

Caspersson, T. O., (1950) Cell Growth and Cell Function, Norton \& Co., Inc., New York; Chapman and Hall, London.

Cavanagh, J. B. and G. R. Webster, (1955) Quart. J. Exptl. Physiol., 4o, i2.

Chajfec, M., (I932) Acta Biol. Exptl. (Lodz), 7, i I 5.

Chalkley, D. W., (1954) F. Morphol., 94, 2 I.

Chanbers, R., (1942), Symposia on Quantitative Biology, Cold Springs Harbor, 8, I 44.

Chandebois, R., (1952) Bull. soc. zool. France, 76, 404.

Charniaux-Legrand, H., (195 I) Compt. rend., 232, 2 I 42.

Chèvremont, M. and H. Firket, (1949) Arch. biol. (Liège), 6o, $44 \mathrm{I}$.

Child, C. M., (I9II) Biol. Bull., 20, 309.

Child, C. M., (I913) Arch. Entwicklungsmech. Organ., 35, 598.

Child, G. M., (I92 I) F. Exptl. Zool., 33, 409.

Child, C. M., (194I) Patterns and Problems of Development, University Press, Chicago.

Child, G. M. And A. N. Young, (1903) Arch. Entwicklungsmech. Organ., 15, 543.

Chranova, A., (1938) Arch. Entwicklungsmech. Organ., I39, 65.

Christensen, H. N. and J. A. Streicher, (1948) F. Biol. Chem., 175, ioi.

Clark, A. H., (1919) Bull. Johns Hopkins Hosp., 30, i I 7.

Coe, W. R., (1929) 7. Exptl. Zool., 54, 4I I.

Coe, W. R., (1934) F. Exptl. Zool., 67, 283.

Cohen, A. I., (I952) Biol. Bull., IO2, 9 I.

Cohen, S. et al., (1954) Proc. Natl. Acad. Sci. U. S., 4o, Ior4.

Collier, J. G., (1947) Biol. Bull., 92, i67.

Comsa, J., (1952) Ann. endocrinol. (Paris), 13, 935.

Conn, E. E. And B. Vennesland, (I951) Nature, I67, 756.

Cooke, J. V. et al., (1953) F. Exptl. Med., 97, 65I.

Coonfield, B. R., (1936) Bioi. Bull., 7I, 421.

Cope, O. et al., (1943) Ann. Surg., II7, 939.

Courtice, F. G., (1954) Brit. Med. Bull., Io, 5.

Crane, E. E., (1950) in Progress in Biophysics, I, 85, Ed. J. A. V. Butler and J. T. Randall, Pergamon Press, London.

Crofts, P. B. And R. A. Peters, (1945) Lancet, 288, 266.

Curtis, W. C. And L. M. Schulze, (1934) 7. Morphol., 55, 477.

Cuthbertson, D. P., (1944) Med. Research Council Bull. War. Med., 4, 255.

Cuthbertson, D. P., (1946) Proc. Nutrition Soc. Engl. and Scotl., 4, I85.

Cuthbertson, D. P., (i954) Brit. Med. Bull., io, 35.

Cuthbertson, D. P. et al., (1939) Quart. F. Med., 25, 233.

Cuthbertson, D. P. et al., (I94I) J. Endocrinol., 2, 459, 468, 475 .

Danielli, J. F., (I95 I) Nature, I68, 464. 
Danielli, J. F., (1954) Symposia Soc. Exptl. Biol., 8, 508.

Danielli, J. F. et al., (1946) Proc. Nutrition Soc. Engl. and Scotl., 4, 197.

Darby, H. H., (1935) Papers Tortugas Lab., 29, I53, 349.

Darby, H. H., (1939) Papers Tortugas Lab., 32, 63.

Davenport, C. B., (1899) Experimental Morphology, The Macmillan Co., New York. Davenport, G. B., (1925) 7. Exptl. Zool., 42, I.

Davidson, J. N. And I. Leslie, (1950) Cancer Research, Io, 587.

Davidson, M. E. And N. J. Berrill, (1948) 7. Exptl. Zool., IO7, 465.

Davies, R. E., (1954) Symposia Soc. Exptl. Biol., 8, 453.

DAwydoff, C., (I901) Z. wiss. Zool., 69, 202.

Dawydoff, C., (1909) Bull. acad. imp. sci. (Petrograd), 3, 301.

De Duve, G., et al., (1955) Biochem. F., 59, 433, 438; 6o, 6o4.

De Giorgi, P. And E. Guyenot, (I 923 ) Compt. rend. soc. biol., 89, 488.

De Gribble, H. G. and R. A. Peters, (195I) Quart. F. Exptl. Physiol., 36, i 9.

De Lustig, E. S. and F. Sacerdote, (1954) Anat. Record, II8, 447.

Dent, J. N., (1949) Anat. Record, 105, 325.

Deotto, R., (1939) Pubbl. staz. zool. Napoli, 17, 206.

Derbes, V. J. And T. E. Weiss, (195I) Untoward Reactions of Cortisone and ACTH, Blackwell, Oxford.

Dettelbach, H. R., (I952) Rev. suisse zool., 59, 339.

Diaz-Guerrero, R. et al., (1947) Am. F. Physiol., 15I, 9 I.

Dimmitt, J. And G. Marsh, (I952) 7. Cellular Comp. Physiol., 40, i I.

Dixey, L. R., (1938) Proc. Zool. Soc. (London), Io8A, 257.

Drabkin, D. L. et al., (1950) 7. Biol. Chem., 182, 335.

Dresden, D., (1940) Acta Neerl. Morphol., 3, I40.

Drill, V. A., (1943) Physiol. Revs., 23, 355.

Drochmans, P., (1950) Arch. biol. (Liège), 6I, 475.

Dubois, K. P., et al., (1949) Science, IIO, 420.

Du Noüy, L., (1936) Biological Time, Methuen and Co., London.

Durbin, M. L., (1909) 7. Exptl. Zool., 7, 397.

Duspiva, F., (1942) Biol. Zentr., 62, 403.

Ebner, H. And H. Strecker, (1950) Experientia, 6, 388.

Eccles, J. G., (1953) The Neurophysiological Basis of Mind, Clarendon Press, Oxford.

Edds, M. V., (1953) Quart. Rev. Biol., 28, 260.

Efimov, M. I., (1943) Compt. rend. acad. sci. U. R. S. S., 4I, 406.

Einhorn, S. L. et al., (1954) 7. Gen. Physiol., 37, 559.

Eksterowicz, F. G., (1950) Anat. Record, 108, 45.

ElLIS, M. M., (1909) 7. Exptl. Zool., 7, 446.

ELy, J. O. AND M. H. Ross, (195I) Nature, I68, 323.

Emerson, H. S., (1940) J. Exptl. Zool., 83, 191.

Emery, F. E., (1937) Quart. F. Exptl. Physiol., 27, 17.

Engley, F. B. et al., (1955) Ann. N. Y. Acad. Sci., 59, 326.

English, J. et al., (1938) F. Am. Chem. Soc., 6r, 3434.

Enteman, C. et al., (1948) Endocrinology, 42, 210.

Eskelund, V. And G. M. Plum, (1953) Acta Endocrinol., I2, I 7 I,

Evlakhova, V. F., (1946) Compt. rend. acad. sci. U. R. S. S., 53, 369.

Fedotov, D. H., (1946) Nature, 158, 367.

Feldman, M., (1955) 7. Embryol. Exptl. Morphol., 3, 25 I.

Fell, H. B. and J. F. Danielli, (1944) Med. Research Council Bull. War. Med., 4, 253.

Fell, H. B. And E. Mellanby, (1950, I953) Brit. Med. F. II, 535; F. Physiol. (London), II9, 470 .

Fenn, W. O., (1940) Physiol. Revs., 20, 377

Finkelstein, E. A. and L. A. Kovarskaja, (1936) Eksptl. Med. (Moscoze), 7, 6 I.

FIRKET, H., (1950) Compt. rend. soc. biol., I44, 1718; Arch. biol. (Liège), 62, 335.

Fischer, A., (1930) Arch. path. Anat. u. Physiol., Virchow's, 279, 94.

Fischer, A., (1946) Biology of Tissue Cells, University Press, Cambridge. 
Fischer, A., (1948) Proc. Soc. Exptl. Biol. Med., 67, 40.

Fishman, W. H., (I951) Vitamins and Hormones, 9, 213.

Fleisher, G. A., (i955) Ann. N. Y. Acad. Sci., 59, 1012.

Flinker, D. And R. Sidman, (1953) Anat. Record., II7, 577.

Flock, E. V. et al., (1953) Federation Proc., ,12, 203.

Fodor, P. J. et al., (1955) Experientia, II, 206.

Foglia, V. G. et al., (1954) Compt. rend. soc. biol., I48, I656.

Fontaine, R. et al., (1952) Medizinische Welt, 899, 923.

Frazer, A. C., (1954) Symposia Soc. Exptl. Biol., 8, 490.

Frazer, R. G., (I953) Biol. Bull., 106, 39.

Frederic, J., (1952) Brit. J. Radiol., 25, 43.

Frieden, E. And B. Naile, (I955) Science, I21, 37.

Friedgood, G. E. et al., (1950) Am. F. Physiol., i63, 354.

Friedrich, P., (1906) Arch. Entwicklungsmech. Organ. 20, 469.

Friedrich-FreKsa, H. AND F. G. ZAKI, (I955) Z. Naturforsch., gb, 394.

Friedwald, C. S. and W. Buschke, (ig44) A. M. A. Arch. Ophthalmol., 32, 4 Io.

Fromm, H. J., (1955) Federation Proc., I4, 2 I6.

Gabritchevsky, E., (1930) Bull. biol. France et Belg., 64, 155.

Galloway, T. W., (1899) Bull. Mus. Comp. Zool., Harvard, 35, i 5.

Gaunt, R., (1954) in Dynamics of Growth Processes, Ed. E. J. Boell, University Press, Princeton.

Gebhardt, H., (1926) Arch. Entwicklungmech. Organ., I07, 695.

Gemmill, C. L., (1952) F. Biol. Chem., 192, 749.

Gershbein, L. L. And J. A. Labow, (1953) Am. F. Physiol., I73, 55.

Ghiretti, F., (1950) Experientia, 6, 98.

Giberti, A. And P. Bianchini, (1954) Riv. ist. sieroterap. ital., 29, 87.

Gidge, N. M. And S. M. Rose, (1944) F. Exptl. Zool., 97, 7I.

Giese, A. C., (1950) Physiol. Revs., 30, 43 I.

Gillman, T. et al., (1955) Nature, I76, 932.

Glass, B., (1952) in Phosphorus Metabolism, Editors W. D. McElroy and B. Glass, Johns Hopkins Press, Baltimore.

Guinos, A. D. et al., (1950) 7. Exptl. Med., 93, 313.

Glinos, A. D. and G. O. Gey, (I952) Proc. Soc. Exptl. Biol. Med.80, 42 I.

Goetsch, W., (I9I7) Biol. Zentr., 37, 465.

Goetsch, W., (I92 I) Biol. Zentr., 4I, 374.

Goetsch, W., (1925) Biol. Zentr., 45, I93.

Goetsch, W., (1929) Arch. Entwicklungsmech. Organ., II7, 2 II.

Goldacre, R. J., (1952) Symposia Soc. Exptl. Biol.6, 128.

Goldfarb, A. J., (Igo6) F. Exptl. Zool., 3, I 29.

Goldin, A., (I942) Biol. Bull., 82, 243, 340.

Goldin, A. And L. G. Barth, (I94I) Biol. Bull., 8I, I 77.

Goldman, A. S., (1953) Biol. Bull., 105, 450.

Goldner, J., (1925) Arch. Entwicklungsmech. Organ., 104, 72.

Goldsmith, E. D., (1940) Physiol. Zoöl., 13, 43.

Goodchild, G. G., (1955) Biol. Bull., 107, 298.

Goodwin, P. A., (1946) Growth, Io, 75.

Goodwin, T. W., (1950) Biol. Revs. Cambridge Phil. Soc., 25, 39 I.

Gorelova, L. P., (I954) Doklady Akad. Nauk. S. S. S. R., 96, 4 I 5.

Goss, R. J., (1954) Anat. Record, I18, 303.

Gould, B. S. And N. I. Gold, (195I) A. M. A. Arch. Pathol., 52, 413.

Graissé, P. P., (I948 onw.) Traité de Zoologie, Masson et Cie., Paris.

Gray, J., (Ig29) F. Exptl. Biol., 6, 248.

Green, H. N. and H. B. Stoner, (I954) Brit. Med. Bull., io, 38.

Greep, R. O., (1949) in The Hormones, Editors G. Pincus and K. V. Thimann, Vol. I, 255, Academic Press New York.

Griffiths, M. et al., (1955) Nature, I76, I2II. 
Grobstein, C., (1947) F. Morphol., 8o, I I 45.

Groll, H., (1927) Klin. Wochschr., 6, 30.

Gross, F. ANd J. S. Huxlex, (I934) Naturwissenschaften, 27, 456.

Gross, F. And J. S. Huxley, (1935) Arch. Entwicklungsmech. Organ., 133, 582.

Grossman, M. I. et al., (1954) Federation Proc., 13, 459.

Guerner, H. And H. Wrba, (1953) Z. Krebsforsch., 59, 564.

Gurevitsch, F. A., (I94I) Compt. rend. acad. sci., U.R.S.S., 3I, 400.

Gutmann, E. et al., (1942) J. Exptl. Biol., I9, I4.

Guyenot, E. and O. E. Schotté, (1923) Compt. rend, soc. biol., 89, $49 \mathrm{I}$.

Guyenot, E. And K. Ponse, (1930) Bull. biol. France et Belg., 64, 25 I.

Haberlandt, G., (1922) Biol. Zentr., 42, 145.

Hadorn, E. And P. S. Chen, (1953) Arch. Entwicklungsmech. Organ., 146, 515.

Haffen, K. And E. Wolff, (1953) Compt. rend., 237, 754.

Haldane, J. B. S., (1954) Biochemistry and Genetics, Allen and Unwin, London.

Hall, A. B. and O. E. Schotté, (I951) 7. Exptl. Zool., II8, 363.

Hamburger, V., (I928) Arch. Entwicklungsmech. Organ., II4, 272.

Hammarsten, E., (195I) Ciba Foundation Conference on Isotopes in Biochemistry, Ed. G. E. W.

Wolstenholme, p. 203.

Hammett, F. S. and S. S. Chapman, (1938) Growth, 2, 223.

Hankó, R., (igi2) Arch. Entwicklungsmech. Organ., 34, 477.

Hankó, R., (1914) Arch. Entwicklungsmech. Organ., 38, 447.

Hanström, B., (1938) Hormones in Invertebrates, Clarendon Press, Oxford.

Harkness, R. D., (1952) 7. Physiol. (London), II7, 267.

Harms, J. W., (1944) Biol. Zentr., 64, I.

Harms, J. W., (1947-9) Arch. Entwicklungsmech. Organ., I43, 332.

HARris, M., (1953) Growth, 17, I 47.

Harrison, R. G., (I9I5) Proc. Natl. Acad. Sci. U.S., I, 539.

Hartmann, F. W., (I933) 7. Exptl. Med., 58, 649

Hartmann, M., (1922) Biol. Zentr., 42, 364 .

Hartmann, M., (1924) Arch. Protistenk., 49, 447.

Harvey, C. B. And E. L. Howes, (1930) Ann. Surg., 9I, 64I.

Hasemann, J. D., (I907) Arch. Entwicklungsmech. Organ., 24, 6 I 7.

Hauschka, T., (1946) Growth, 8, 32 I.

Havas, L. J., (1949) Exptl. Cell Research, I, Suppl., 597.

Heinzen, B., (1947) Anat. Record, 98, 193.

Hellmich, W., (1930) Arch. Entwicklungsmech. Organ., 121, I35.

Herbst, C., (1896) Arch. Entwicklungsmech. Organ., 2, 544 .

Hess, G. And R. Kopf, (I95I) Z. ges. exptl. Med., II8, 7 I.

Hiatt, R. W., (1948) Pacific Sci., 2, 135.

Hinman, F. and O. W. Butler, (1923) 7. Am. Med. Assoc., 81, 2021.

Hinrichs, M. A., (1924) J. Exptl. Zool., 4I, 21.

Hinshelwood, C. N., (1946) The Chemical Kinetics of the Bacterial Cell, Clarendon Press, Oxford.

Hoffman, H., (1950) Australian 7. Exptl. Biol. Med. Sci., 28, 383.

Hoffmann, H., (1952) Australian 7. Exptl. Biol. Med. Sci., 30, 54 I.

Hofmann, H., (1954) J. Comp. Neurol., Ioo, 441.

Hollinger, D. M. and R. J. Rossiter, (1952) Biochem. 7., 52, 659.

Hollinger, D. M. et al., (1952) Biochem. J., 52, 652.

Holmes, A. D., (1942) New Engl. 7. Med., 227, 909.

Holmes, S. J., (I9I I) 7. Morphol., 22, 989.

Holter, S. et al., (1954) Biol. Bull., 107, 313.

Holtfreter, J., (1938) Arch. Entwicklungsmech. Organ., I38, I63, 522, 657.

Holtfreter, J., (1948) Symposia Soc. Exptl. Biol., 2, I 7.

Hopper, A. F., (I949) 7. Exptl. Zool., IIO, 299.

Horn, E. C., (I94I) Am. 7. Roentgenol. Radium Therapy, 46, 727.

Horn, E. C., (1942) 7. Morphol., 71, 185. 
Howe, H. A. and L. B. Flexner, (1947) 7. Biol. Chem., r67, 663.

Howe, H. A. and R. C. Mellors, (I948) \%. Exptl. Med., 8I, 489.

Hubl, H., (195I) Arch. Entwicklungsmech. Organ., I45, 267.

Hubl, H., (1953) Arch. Entwicklungsmech. Organ., I46, 42 I.

Hutchinson, W. J. and B. D. Burdeaux, (195I) F. Bone and Joint Surg., 33A, 155.

Huxley, J. S., (I921) Quart. 7. Microscop. Sci., 65, 293.

Huxley, J. S., (1932) Problems of Relative Growth, Methuen \& Co., London.

Huxley, J. S. AND G. R. DE Beer, (1934) Elements of Experimental Embryology, University Press, Cambridge.

Hydén, H., (1943) Arch. Physiol. Scand., 6, Suppl., I 7.

Hydén, H., (1947) Symposia Soc. Exptl. Biol., I, I52.

Hydén, H. ANd B. Rexed, (1944) Z. mikroskop.-anat. Forsch., 54, 352.

Hyman, L. H., (I919) Am. J. Physiol., 48, 340; 49, 377.

Hyman, L. H., (1920) Am. 7. Physiol., 53, 399.

Hyman, L. H., (1940) The Invertebrates, Vol. I, McGraw-Hill, New York.

Hyman, L. H., (I95I) The Invertebrates, Vol. 2, McGraw-Hill, New York.

Imanaga, H. et al., (1952) Nagoya J. Med. Sci., I5, I4I.

Jacovleva, T. M., (1943) Compt. rend. acad. sci. U. R. S. S., 4I, 286.

Jacovleva, T. M., (I945) Compt. rend. acad. sci. U. R. S. S., 46, 254.

Jacovleva, T. M., (1953) Doklady Akad. Nauk S. S. S. R., 92, 1035.

Jaeger, L. and L. G. Barth, (1938) F. Cellular Comp. Physiol., 32, 319.

JAfFe, J. J., (1954) Anat. Record, I20, 935.

JANDA, V., (1926) Arch. Entwicklungsmech. Organ., 107, 423.

Janda, V., (1930) Arch. Entreicklungsmech. Organ., I22, 432.

Janda, V., (I93I) Arch. Entwicklungsmech. Organ., I25, I48.

Janowitz, H. D. et al., (1955) Federation Proc., I4, 79.

JAQues, R., (1950) Experientia, 6, I 48.

Jeener, R., (I952) Biochim. et Biophys. Acta, 8, 270.

Jensen, P. K. et al., (1956) Helv. Physiol. Acta, I4, I 88.

Johnson, R. M. And S. Albert, (1952) Arch. Biochem. Biophys., 35, 340.

Joly, M., (1955) Progr. in Biophys. and Biophys. Chem., 5, I68.

Junquiera, L. C. U., (1950) 7. Anat., 84, 369.

Jurand, A. et al., (1954) Folia Biol. (Warsow), 2, 20 I.

Kambara, S., (1953) Annotationes, Zool. Japon., 26, 208.

KAMPS, L. F., (1937) Thesis, University of Groningen.

Kamrin, R. P. and M. Singer, (i955) J. Morphol. 96, i73; 7. Exptl. Zool., I28, 6 i I.

Karczmar, A. G., (1946) J. Exptl. Zool., 103, 40 I.

Karczmar, A. G. and G. G. Berg, (I951) J. Exptl. Zool., II7, I39.

KeIL, E. M., (1932) 7. Exptl. Zool., 63, 447 .

Kenk, R., (1935) Physiol. Zool., 8, 442 .

Kent, P. W. And M. W. Whitehouse, (1955) Biochemistry of the Amino-Sugars, Butterworth, London.

Kerr, A. B. And H. Werner, (1944) Brit. F. Surg., 32, .281.

Key, J. A. And R. T. Odell, (1952) F. Bone and Foint Surg., 34A, 665.

Khruschev, G. K., (1946) Am. Rev. Soviet Med., 4, I 75 (Rev. by E. Liebmann).

KING, H. D., (г898) Arch. Entwicklungsmech. Organ., 7, 351; 9, 724.

Kiortsis, V., (1953) Rev. suisse zool., 6o, 301.

Klemperer, F. W. et al., (1955) F. Biol. Chem., I87, I89.

Knaysi, G., (195I) Elements of Bacterial Cytology, 2nd ed., Constable \& Co, London.

Kochakain, C. D. and M. N. Bartlett, (1948) F. Biol. Chem., I76, 243.

Kocian, V., (1930) Arch. zool. exptl. et gén., Notes et Rev., 70, 23.

Koechlin, B. and A. Von Muralt, (1946) Helv. Chim. Acta, 3o, 519.

Koelitz, W., (19I I) Arch. Entwicklungsmech. Organ., 31, 423.

Korschelt, E., (1927) Regeneration und Transplantation, I. Band, Borntraeger, Berlin.

Kovalenko, T. M., (1954) Doklady Akad. Nauk S. S. S. R., 97, 353.

Krecker, F. H., (1923) F. Exptl. Zool., 37, 27. 
Křízeneckž, J., (I9I7) Arch. Entreicklungsmech. Organ., 42, 622.

Krugelis, E. J. et al., (1952) 7. Exptl. Zool., I2I, 489 .

Kükenthal, W. And T. Krumbach, (1923) Handbuch der Zoologie, Walter de Gruyter \& Co., Berlin and Leipzig.

LANGe, M. M., (1920) 7. Exptl., Zool., 3I, I.

LASER, E., (1933) Z. Krebsforsch., 39, 384.

Lauber, H. J., (1933) Bruns' Beitr. klin. Chir., 157, 244.

LAYTon, L. L., (1950) Cancer, 3, 725.

Layton, L. L., (I95I) Proc. Soc. Exptl. Biol. Med., 76, 596; Federation Proc., Io, 214.

LeCAMP, M., (1942) Compt. rend., 214, 330.

Lecamp, M., (1947) Compt. rend., 224, 363.

Lecamp, M., (1954) Compt. rend., 238, 955.

Lehmann, F. E., (1929) Arch. Entwicklungsmech. Organ., II7, 3 I2.

Lehmann, F. E., (1945) Einführung in die Physiologische Embryologie, Birkhauser, Basel.

Lehmann, F. E., (I949) Experientia, 5, 472.

Lehmann, F. E., (1954a) Rev. suisse zool., 61, 428.

Lemmann, F. E., (1954b) Arch. Julius Klaus-Stift. Vererbungs-Forsch. Sozialanthropol. u. Rassenhyg., 29, 359 .

Lehmann, F. E. And H. R. Dettelbach, (1952) Rev. suisse zool., 59, 253.

Lehmann, F. E. et al., (i 954) Helv. Physiol. Pharmacol. Acta, i 2, 147.

Lehn, H., (1953) Arch. Entrwicklungsmech. Organ., I46, 37 I.

Lender, T., (I952) Bull. biol. France et Belg., 85, 376.

Lender, T., (1954) Compt. rend. soc. biol., I48, 1859.

Levander, G., (1949) Acta Pathol. Microbiol. Scand., 26, I I3.

Lewis, T., (I937) Brit. Med. J., (I), 49 I.

Li, C. H., (1950) Harvey Lectures, 46, I8 I.

Liebmann, E., (1946) Growth, io, 29 I.

Lillie, F. R., (1896) 7. Morphol, 12, 239.

Lillie, F. R., (1900) Am. Naturalist, 34, I 73.

Liosner, L. D. and M. A. Voronzova, (1938) Bull. biol. méd. exptl. U. R. S. S., 6, 82.

Litwiller, R., (1939) 7. Exptl. Zool., 82, 273.

Litwiller, R., (1940) Growth, 4, I69.

Lloyd, D. J., (I9I4) Proc. Roy. Soc. (London), B, I87, 355.

Lloyd, D. J., (igra) Proc. Roy. Soc. (London), B, 88, I.

Localio, S. A. et al., (1948) Surg. Gynecol. Obstet., 86, 582.

Localio, S. A. et al., (1949) Surg. Gynecol. Obstet., 89. 69.

Loeb, J., (1892) Arch. ges. Physiol. Pfluger's, 63, 66.

Loeb, J., (1896) Arch, ges. Physiol. Pfuger's, 63, 273.

Loeb, J., (1924) Regeneration from a Physico-Chemical Viewpoint, McGraw-Hill, New York.

Lombardo, M. E. et al., (1953) 7. Biol. Chem., 202, 97.

Loofbourow, J. R., (1948) Growth, I2, Suppl., 77.

Loofbourow, J. R. et al., (1939) Nature, I44, 939.

Loofbourow, J. R. et al., (1942) Biochem. J., 36, 5 I3.

Lovtrup, E., (1953) 7. Exptl. Zool., I24, 427.

Ludewig, S. et al., (1939) Proc. Soc. Exptl. Biol. Med., 42, 158.

Ludwig, W. And H. W. Ludwig, (1954) Arch. Entwicklungsmech. Organ., I47, 259.

Lumsden, C. E., (1952) Quart. F. Exptl. Physiol., 37, 45.

Lund, E. J., (1947) Bioelectric Fields and Growth, University of Texas Press, Austin, Texas

Lüs, J., (1926) Arch. Entwicklungsmech. Organ., 108, 203.

Lüscher, M., (1946) Helv. Physiol. et Pharmacol. Acta, 4, 465.

Lüscher, M., (1948) J. Exptl. Biol., 25, 334.

Lüscher, M., (1952) Experientia, 8, 8I.

Lüscher, M. and A. Von Muralt, (1947) XVIIth internat. Cong. Physiol., Abst. of Papers, p. 203.

Lwoff, A., (1949) Growth, 13, Suppl.61.

Lytton, B. and J. G. Murray, (1954) 7. Physiol., 126, 627. 
McHenry, E. W. and J. M. Patterson, (1944) Physiol. Revs., 24, i 28.

McKelvie, A. M. And F. C. Mann, (1948) Proc. Staff Meetings Mayo Clinic, 23, 449.

Malaguti, G. and F. VacGari, (1954) Minerva chir., 9, I003.

Maloeuf, N. S. R., (1936a) Nature, I38, 75.

Maloeuf, N. S. R., (1936b) Biol. Zentr. $56,429$.

Man, E. B. et al., (1946) 7. Clin. Invest., 25, 701.

Mannell, W. A. And R. J. Rossiter, (1954) J. Exptl. Biol., 3I, 198.

Manner, H. W., (1953) J. Exptl. Zool., 122, 229.

Manner, H. W., (1955) Growth, 19, I6 I.

Mapson, D. W. and D. K. Goddard, (I95I) Nature, 167, 975.

Marcucci, E., (1925) Boll. soc. nat. Napoli, 37, I 26.

Marcus, E., (1926) Zool. Jahrb. Syst. Abt., 52, I.

Margoliash, E. And L. Doljansky, ( 1950 ) Growth, I4, 7.

Maron, K. et al., (1954) Folia Biol.(Warsow), 2, 77.

Marsh, G. and H. W. Beams, (1952) 7. Cellular Comp. Physiol., 39, I91.

Marshak, A. and A. C. Walker, (I945) Am. F. Physiol., I43, 226.

Marshall, W., (1952) Am. J. Surgery, 84, 675.

Marx, W. et al., (1942) Proc. Soc. Exptl. Biol. Med., 49, 594.

Mattes, O., (1925) Zool. Anz., 63, 34 .

Maver, M. E. et al., (1948) 7. Natl. Cancer Inst., 9, 39.

Maver, M. E. et al., (I952) F. Natl. Cancer Inst., I3, 687.

MAy, R. M., (1930) Bull. soc. chim. biol., 25, 447.

Medawar, P. B., (I940) Proc. Roy. Soc. (London), B, I29, 332.

Medawar, P. B., (I 945) in Essays on Growth and Form, Ed. W. E. LeGros Clark and P. B.

Medawar, Clarendon Press, Oxford.

Medawar, P. B., (1950) Proc. Roy. Soc. (London), B, 137, 474.

MeIER, R. et al., (I955) Experientia, II, I80.

Mellanby, E., (1944) Proc. Roy. Soc. (London), B, 132, 28.

Mene, G., (1953) Riv. ist. sieroterap. ital., 28, 203.

Menkin, V., (1940, I950) Dynamics of Inflammation (2nd ed. 1950), The Macmillan Co., New York.

Merezhinskir, M. F. et al., (1954) Ukraïn. Biokhim. Zhur., 26, 435.

Mifume, S., (1953) Annotationes Zool. Japon., 26, 32.

Miller, J. A., (I938) Physiol. Zool., I1, 214.

Millot, J., (193 I) Cicatrisation et Régénération, Alcan, Paris.

Mills, G. T. et al., (1953) Biochem. 7., 53, 245.

Minganti, A., (1954) Pubbl. staz. zool. Napoli, 25, 9.

Mittler, S. And R. H. Herman, (1950) Science, iII, $54^{8}$.

Moment, G. B., (1946-50) See Moment, i953.

Moment, G. B., (I953) Am. Naturalist, 87, I39.

Moment, G. B., (I954) Am. Naturalist, 88, i I 5.

Montgomery, P. O’ B. and C. Green, (1954) Proc. Soc. Exptl. Biol. Med., 86, 657.

Moog, F., (I942) Biol. Bull., 83, 29 I.

Moog, F. and S. Spiegelman, (1942) Proc. Soc. Exptl. Biol. Med., 49, 392.

Moore, A. R., (1910) Arch. Entwicklungsmech. Organ., 29, 145.

Moore, E. L., (1924) F. Exptl. Zool., 39, 249.

Moore, J. A., (1939) Biol. Bull., 76, i04.

Morel, M., (I94I) Ann. Inst. Pasteur, 67, 285.

Morgan, T. H., (I90I) Regeneration, Macmillan Co., New York.

Morgan, T. H., (1906) 7. Exptl. Zool., 3, 457.

Morgan, T. H. And S. E. Davis, (1902) Arch. Entwicklungsmech. Organ., I5, 3 I3.

Morgulis, S., (rgog) 7. Exptl. Zool., 7, 595.

Morgulis, S., (1911) 7. Exptl. Zool., Io, 321 .

Morgulis, S., (I9I2) Arch. ges. Physiol. Pflüger's, I43, 50 I.

Morgulis, S. and D. E. Green, (I932) Protoplasma, I4, i6 I.

Morosow, B. D., (1935) Arch. Entwicklungsmech. Organ., I33, 3 Io. 
Morosow, B. D., (1941) Compt. rend. acad. sci. U. R. S. S., 30, 675.

Morton, R. K., (1955) Biochem. F., 6I, 232.

Moss, W. G. And A. K. Weiss, (1955) Federation Proc., 14, I04.

Müller, C., (1908) Arch. Entreicklungsmech. Organ., 26, 209.

Münch, H., (1937) Arch. Entwicklungsmech. Organ., 137, 597.

Munro, H. N. and M. I. Chalmers, (1945) Brit. F. Exptl. Pathol., $26,396$.

Munro, H. N. and M. C. Cummings, (1948) Nature, I6I, 560.

Murray, P. D. F., (1936) Bones, University Press, Cambridge.

Murray, P. D. F. And E. Kodicek, (1949) 7. Anat., 83, 158, 205, 285.

Murtasi, F., (1938) Bull. Eksptl. Biol. Med., 6, 79.

Murtasi, F. and M. Sosnina, (I943) Compt. rend. acad. sci. U. R. S. S., 4I, 84.

Nakamura, N., (1941) Japan. J. Zoöl., 9, i85.

NARDI, F., (1937) Arch. Entwicklungsmech. Organ., I35, 298.

Naville, A., (1922-4) Arch biol. (Liège), 32, 5; 34, 235.

Needhair, A. E., (i94I) Proc. Zoöl. Soc. (London), A, III, 59.

Needham, A. E., (1943) Proc. Zoöl. Soc. (London), A, II3, 44.

Needham, A. E., (1945) J. Exptl. Biol., 21, I44.

Needham, A. E., (1946a) J. Exptl. Biol., 22, 107.

Needham, A. E., (1946b) Nature, I57, 339.

Needham, A. E., (1947a) 7. Exptl. Biol., 24, 220.

Needham, A. E., (1947b) J. Exptl. Zool., Io6, I81.

Needham, A. E., (I949a) J. Exptl. Zool., II2, 49.

Needham, A. E., (1949b) 7. Exptl. Zool., II2, 207.

Needham, A. E., (I950a) Quart. F. Microscop. Sci., 9I, 401.

Needham, A. E., (i95ob) 7. Gerontol., 5, 5.

Needham, A. E., (1952) Regeneration and Wound-Healing, Methuen \& Co., London.

Needham, A. E., (1953) J. Exptl. Biol., 30, I 5 I.

Needham, A. E., (1955) F. Embryol. Exptl. Morphol., 3, I89.

Needham, A. E., (1957) Physiol. Comparata et Oecol., 4, 209.

Needham, D. M., (I93 I) in: J. Needham, Chemical Embryology, Vol. 3, p. i685.

Needham, J., (I93 I) Chemical Embryology, University Press, Cambridge.

Needham, J., (1942) Biochemistry and Morphogenesis, University Press, Cambridge.

Nettleship, A., (1943) Am. 7. Clin. Pathol., 13, 349.

Neumann, I. M., (1946) Am. Rev. Soviet Med., 4, 257.

Norberg, B., (1950) Acta Physiol. Scand., 19, 246; 20, 180.

Novikoff, A. B. and V. R. Potter, (i948) F. Biol. Chem., I73, 223.

O'Brien, B. R. A., (1947) Proc. Linnean Soc. N. S. Wales, $72,367$.

O'Brien, J. P., (I942) Growth, 6, 203.

O'Farrell, A. F. And A. Stock, (I953) Austral. F. Biol. Sci., 6, 485.

O'Farrell, A. F. and A. Stock, (1954) Austral. 7. Biol. Sci., 7, 525.

Okunev, N., (1928) Biochem. Z., 195, 42.

Okunev, N., (1929) Biochem. Z., 208, 328.

Okunev, N., (1932) Biochem. Z., 255, 387.

Окunev, N., (1933) Biochem. Z., 257, 242.

Onweleen, J., (I9I 7) Archiv. ges. Physiol. Pflïger's, I68, 372.

Opie, E. L. th al., (1946) J. Exptl. Med., 84, 9 I.

Oppenheimer, M. J. (1946) Trans.5th Meeting Jos. Macy Found. N. Y. Conf. on Liver Injury

(Ed. F. W. Hoffbauer), p. 36.

Orechovitch, W. N., (1934) Z.physiol. Chem., Hoppe-Seyler's, 244, 6I.

Orechovitch, W. N., (I936) Biochem. Z., 286, 9 I, 248, 285.

Orechovitch, W. N. and N. W. Bromley, (1934) Compt. rend. acad. sci. U. R. S. S., 2, 249; Biol. Zentr., 54, 5 I 4 .

Orechovitch, W. N. and V. A. Sokolova, (1940) Compt.rend. acad. sci. U. R. S. S., $28,747$.

Orechovitch, W. N. et al., (1935) Biochem.Z., 277, i86.

Osborne, D. J. And R. L. Wain, (1951) Science, I14, 92.

Ost, J., (1906) Arch. Entwicklungsmech. Organ., 22, 289. 
Отто, F., (1921) Arch. Entwicklungsmech. Organ., 47, 399.

Overton, J., (1950) J. Exptl. Zool., 115, 521.

Owen, S. E. et al., (1939) Am. F. Cancer, 35, 424.

Palladin, A. V., (1943) Compl. rend. acad. sci.U.R. S. S., 4I, 8 I.

Palladina, L. I. And A. M. Gudina, (1953) Ukraïn. Biokhim. Zhur., 25, 97, 132.

Paton, R. T., (1955) Keratoplasty, McGraw-Hill, New York.

Paul, H. E. et al., (1945) Arch. Biochem., 7, 23 I.

PAul, H. E. et al., (1947) Arch. Biochem., I7, 269.

Paulain, R., (1938) Proc. Zoöl. Soc. (London), A, I08, 355.

Pawlowsky, E.N., (1923) Arch. Entwicklungsmech. Organ., 99, 620.

Peadon, A. M., (1953) Growth, I7, 21.

Peebles, F., (I897) Arch. Entwicklungsmech Organ., 5, 794.

Peebles, F., (1898) Zoöl. Bull., 2, 125.

Peebles, F., (1912) Biol. Bull., 23, 154.

Pentimalli, F., (1927) Z. Krebsforsch., 25, 337.

Peredelsky, A. A., (I94I) Compt. rend. acad. sci. U. R. S. S., 32, 448.

Perez-Tamayo, R. et al., (1953) A. M. A. Arch. Pathol., 56, 629.

Perkins, M., (1929) Nature, I24, 299.

Peter, K., (1945) Arch. Entwicklungsmech. Organ., I43, I.

Pflugfelder, O., (1939) Z. wiss. Zool., I52, I 59.

Phelps, L. A., (1926) Trans. Am. Microscop. Soc., 45, I33.

Pirani, C. L. and S. M. Levenson, (1953) Proc. Soc. Exptl. Biol. Med., 82, 95.

Pirozynski, W. J. and L. Von Bertalanffy, (i952) A. M. A. Arch. Pathol., 54, 340.

Polezhayev, L. W., (1946) Compt. rend acad sci. U. R. S. S., 48, 216 ; Biol. Rev., 2I, I4I.

Polezhayev, L. W., (1949) Compt. rend. acad. sci. U. R. S. S., 54, $28 \mathrm{I}$.

Polezhayev, L. W. and W. N. Favorina, (1935) Arch. Entwicklungsmech. Organ., I33, 70 I.

Polezhayev, L. W. And G. I. Ginsburg, (I944) Compt. rend. acad. sci. U. R. S. S., 43, 3 I 5.

Ponder, E. And K. N. Flint, (1927) Quart. F. Exptl. Physiol., 66, 393.

Powell, V. E., (I95I) Anat. Record, III, IoI.

Principe, U. And G. Belluca, (1952) Sperimentale, IO2, 266.

Principe, U. and G. Belluci, (I953) Sperimentale, 103, 62.

Prizbram, H., (Igo9) Experimental-Zoologie, 2, Regeneration, F. Deuticke, Leipzig and Vienna.

Przibram, H., (I9I 7 ) Arch. Entwicklungsmech. Organ., 43, I.

Przibram, H., (1919) Arch. Entwicklungsmech. Organ., 45, 52.

Przibram, H., (1925) 7. Exptl. Biol., 3, 3 I 3.

Przibram, H., (I93I) Connecting Laze's in Animal Morphology, University Press, London.

Przibram, H., (1932) Mitt. biol. Versuchs-Anstalt Akad. Wiss. (Wien) Zool., No. I89.

Przibram, H., (1934) Mitt. biol. Versuchs-Anstalt Akad. Wiss. (Wien) Zool. Akad. Anz., 2, No. $2 I O$.

Pucket, W. O., (1936) 7. Morphol., 59, i 72.

Rapkine, L., (I93 I) Ann. physiol. physicochim. biol., 7, 382.

Raunish, L., (1950) Ricerca sci., $20,1502$.

Raven, C. P., (I 954 ) An Outline of Developmental Physiology (Trans. L. De Ruiter), Pergamon Press, London.

Read, G., (1954) Am. 7. Anat., 2, I3.

Reinhard, E. G., (1952) Proc. Helminthol. Soc. Wash. D. C., 19, 105.

Richardson, D., (1945) F. Exptl. Zool., IOo, 417 .

Richters, C., (1912) Z. wiss. Zool., ioo, i 16.

Roberts, S., (I95I) Federation Proc., IO, 237.

Roberts, S. and C. M. Szego, (1953) Physiol. Revs., 33, 593.

Robertson, B. R., (1923) Chemical Basis of Growth and Senescence, Lippincott, Philadelphia. Roche, J. ANd M. Morgue, (1939) Bull. soc. chim. biol., 2I, I 43.

Roeder, K. P., (1953) Insect Physiology, John Wiley \& Sons, New York; Chapman and Hall, London.

Rogers, C. S. et al., (1950) Am. J. Physiol., 63, 347.

Roguski, H., (1954) Folia Biol. (Warsaw), 2, 215. 
Roll, P. M. et al., (1950) 7. Biol. Chem., 183, 25 I.

Roseis, B., (1915) Arch. Entwicklungsmech. Organ., 40, 571; 4I, 57.

Rose, S. M., (1942) Proc. Soc. Exptl. Biol. Med., 49, 408.

Rose, S. M., (1944) 7. Exptl. Zool., 95, I 49.

Rose, S. M., (1949) Exptl. Cell Research, I, 588.

Rose, S. M., (I952) Am. Naturalist, 86, 337; Anat. Record, II3, 527.

Rose, S. M. and F. C. Rose, (I94I) Physiol. Zool., I4, 328.

Rose, S. M. and H. M. Wallingford, (I948) Science, IO7, 457.

Rosenthal, O. et al., (1950) Federation Proc., 9, 220.

Rosenthal, O. et al., (1951) 7. Biol. Chem., I89, 831.

Rosin, A., (1952) Acta Anat., I6, 29.

Rossiter, R. J. and E. J. Clark, (1944) Med. Research Council Bull. War. Med., 4, 256.

Rous, P. and L. D. Larimore, (1920) 7. Exptl. Med., 3I, 609.

Rubel, W. M., (1936) Biochem.Z., 283, 180.

Ruben, L. N., (1955) F. Exptl. Zool., I28, 29.

Rübsaamen, H., (1950) Arch. Entwicklungsmech. Organ., 144, 355.

Rulon, O., (1938) Physiol. Zool., II, 203.

Rulon, O., (1948) Physiol. Zool., 2I, 232.

Runnstrom, J. And T. Gustafson, (195I) Ann. Rev. Physiol., 13, 57.

Ryvkina, D. E., (1940) Compt. rend., acad. sci. U. R. S. S., 27, 350.

Ryvkina, D. E., (I945) Compt. rend., acad. sci. U. R. S. S., 49, 457.

Santa, B., (I95I) Arch. zool. ital., 36, 105.

Santos, F. V., (I93I) Physiol. Zool., 4, i I I.

SAyers, G., (1950) Physiol. Revs., 30, $24 \mathrm{I}$.

Sayles, L. P., (1934) Biol. Bull., 66, 7.

SAyles, L. P., (1942) Biol. Bull., 82, 54.

Schacter, B. et al., (1952) Am. 7. Physiol., i69, 499.

Schaper, A., (rgo4) Anat. Anz., 25, 298, 326.

Scharrer, B., (1955) The Hormones, Vol., 3, 57, Eds. G. Pincus and K. V. Thimann, Academic Press Inc., New York.

Schaxel, J., (1921) Untersuchungen über die Formbildung der Tiere, Auffassungen und Erscheinungen der Regeneration, Borntraeger, Berlin.

Schechter, V., (1941) Physiol. Zool., I4, I 74 .

Schmalhausen, J., (1925) Arch. Entwicklungsmech. Organ., 105, 483.

Sсноттв́, O. E., (1937) Science, $85,438$.

Sснотте́, O. E., (1939) Growth, Suppl. 1, 5 I.

Schotté, O. E. And E. G. Butler, (1941) F. Exptl. Zool., 87, 279.

Schotté, O. E. And E. G. Butler, (1944) 7. Exptl. Zool., 97, 95.

Schotté, O. E. And E. G. Butler, (1949) F. Exptl. Zool., II2, $36 \mathrm{I}$.

Schotté, O. E. And J. L. Chamberlain, (I955) Rev. suisse zool., 62, Suppl., 253.

Sснотté, O. E. AND A. B. Hall, (1952) F. Exptl. Zool., I2I, $52 \mathrm{I}$.

Schotté, O. E. And K. P. Hummel, (1939) Jु. Exptl. Zool., 80, 13 I.

Schotté, O. E. and F. Lamy, (1953) F. Exptl. Zool., 122, 97.

Schotté, O. E. And D. A. B. Lindberg, (1954) Proc. Soc. Exptl. Biol. Med., 87, 26.

Schotté, O. E. And G. W. Murphy, (1953) F. Morphol., 93, 44I.

Schultz, E., (1902) Biol. Zentr., 22, 360.

Schultz, E., (1903) Z. wiss. Zool., 75, 391, 473.

Schweigert, B. S. et al., (1954) Am. F. Physiol., $r 78,338$.

Sciacchitano, J., (1923) see Korschelt, 1927.

Scow, R. O., (195I) Endocrinology, 49, 641.

Seddon, H. J. et al., (1943) 7. Physiol., 102, 191.

Selye, H., (1946) $\mathcal{J}$. Clin. Endocrinol., 6, i I 7.

Selye, H., (1948) Text book of Endocrinology, Acta Endocrinologia, Montreal.

Selye, H., (I955) Science, I22, 625.

Sengel, P., (195I) Bull. biol. France et Belg., 85, 376.

SevaG, M. G., (1951) Immuno-catalysis, C. C. Thomas, Springfield. 
Sevag, M. G. et al., (1950) in The Enzymes, Eds. J. B. Summer and K. Myrbach, Vol. I, Academic Press, New York.

Shapiro, J., (19I4) Arch. Entwicklungsmech. Organ., 38, 2 I0.

Sherry, S. et al., (1954) Proc. Soc. Exptl. Biol. Med., 87, 125.

Simpson, M. E., (1953) Anat. Record, 115, 615.

Singal, S. A. et al., (1952) Federation Proc., II, 287.

Singer, M., (I950) Anat. Record, I08, 5 I8.

Singer, M., (1952) Quart. Rev. Biol., 27, 169.

SINGER, M., (1954) 7. Exptl. Zool., 126, 419 .

Singer, M. and L. Craven, (1948) 7. Exptl. Zool., 108, 279.

Singer, M. et al., (1954) 7. Exptl. Zool., 128, 185.

Sivickis, P. B., (I933-4) See Brondsted, 1955.

Skowron, S. ANd H. Roguskr, (I953) Bull. acad. polon. sci., I, I 5.

Smelzer, G. K. and V. Ozanics, (1954) J. Cellular Comp. Physiol., 43, 107.

Sokoloff, B., (1924) Arch. Protistenk., 47, 143.

Sokolova, V. A., (1942) Compt. rend. acad. sci. U. R. S. S., 36, 247.

Sonneborn, T. M., (1948) Growth, II, Suppl. 29I; Am. Naturalist, 82, 26.

SPERry, R. W., (I95I) Growth, 15, Suppl.63.

Spiegelman, S. And F. Moog, (1944) Biol. Bull., 87, 227.

Srinivasan, V.et al., (1955) Science, I22, 644.

SteinberG, M. S., (1954) F. Exptl. Zool., I27, I.

Steinlin, M., (I9O0) See Korschelt, i927, p. 678.

Steinmann, P., (1908) Arch. Entwicklungsmech. Organ., 25, 523.

Steinmann, P., (1932) Rev. suisse zool., 39, 397.

Steinmann, P. and G. Wilhelmi, (1950) Arch. Entwicklungsmech. Organ., I44, 329.

Stephan-Dubois, F., (I952) Compt. rend. soc. biol., I46, I I27; I47, 890.

Stephenson, J., (1930) The Oligochaeta, Clarendon Press, Oxford.

Stock, A., (1937) Anz. Akad. Wiss. (Wien), No. 23.

Stock, A. And A. F. O'Farrell, (1954) Australian 7. Biol. Sci., 7, 302.

Stolte, H. A., (1922) Zool. Jahrb. Abt. Allgem. Zool. Physiol. Tiere, 39, I49.

Stone, J. H. van, (i955) F. Morphol., 97, 346.

Stone, L. S., (1931) Anat. Record, 48, Suppl.64.

Stone, L. S., (1944) Yale Sci. Mag., 18, 8, 32.

Stone, L. S. And H. Steinitz, (1953) Proc. Soc. Exptl. Biol. Med., 82, 763.

Stone, L. S. And H. Steinitz, (1954) 7. Exptl. Zool., I24, 469.

Stone, R. G., (1932) 7. Morphol., 53, 389.

Stone, R. G., (1933) 7. Morphol., 54, 303.

Stowell, R. E. And C. S. Lee, (1950) A. M. A. Arch. Pathol., 50, 5 I9.

Striganova, A., (1940) Compt. rend. acad. sci. U. R. S. S., 27, 385 .

Striganova, A., (1949) Doklady Akad. Nauk S. S. S. R., 69, 465.

Summers, F. M., (194I) Protozoa in Biological Research, Eds. G. N. Calkins and F. M.

Summers, Columbia University Press, New York.

Suntzowa, W., (1944) Compt. rend. acad. sci. U. R. S. S., 45, I33.

Supniewski, J., (1949) Bull. intern. acad. polon. sci., I/6, I3-17.

Suster, P. M., (1933) Zool. Jahrb. Abt. Physiol., 53, 41 .

Sylven, B., (1941) Acta Chir. Scand. Suppl., 66, I.

TAfFel, G. M., et al., (1951) Yale F. Biol. and Med., 23, 482.

TAKata, K., (1952) Experientia, 8, 2 I 7.

TAleisnik, S., (1953) Rev. soc. arg. biol., 29, 300.

Taleisnik, S., (1954) Am. F. Physiol., I78, 100.

TARdent, P., (1954) Arch. Entwicklungsmech. Organ., I46, 593.

Tardent, P., (1955) Rev. suisse zool., 62, 289.

TARTAR, V., (194I) Growth, 5, Suppl.2 2 .

Tartar, V., (1954) F. Exptl. Zool., 127, 5 I I ; J. Protozool., I, II.

Tazelaar, M. A., (1938) Proc. Zool. Soc. (London), A, 108, 257.

TEIR, H., (1952) Growth, I6, 85. 
Ten Cate, J. and I. E. Uyldert, (I944) Arch. néerl. physiol., 28 , i 24.

Thomas, G. A., (1954) 7. Embryol. Exptl. Morphol., 2, I I4.

Thomas, R. et al., (1952) Experientia, 8, I84.

Thompson, D' A. W., (1942) Growth and Form, University Press, Cambridge.

Thompson, J. F. et al., (I954) Radiation Research, I, I65.

Thornton, C. S., (1938) F. Morphol., 62, i 7.

Thornton, C. S., (1942) J. Exptl. Zool., 89, 374.

Thornton, C. S., (1943) 7. Exptl. Zool., 92, 28 I.

Thornton, C. S., (1949) F. Morphol., 84, 459.

Thornton, C. S., (195I) F. Exptl. Zool., II8, 467.

Thornton, C. S., (1953) F. Exptl. Zool., 122, I I9.

Thornton, C. S., (1954) F. Exptl. Zool., 127, 577.

Thornton, C. S. And D. W. Kraemer, (I95I) 7. Exptl. Zool., II7, 4I5.

Tornier, G., (1906) Arch. Entwicklungsmech. Organ., 22, 348.

Torraca, L., (I9I4) Intern. Monatsschr. Anat. u. Physiol., 3o, 297.

Torrey, H. B., (1934) Physiol. Zool., 7, 586.

Torrey, H. B. and L. Martin, (19I0) 7th Internat. Cong. Zool., Boston.

Townes, P. L. and J. Holtfreter, (1955) J. Exptl. Zool., 128, 53.

Trampusch, H. A. L., (I95 I) Abst. Paper, Nederl. Dierk. Veren. May. Igth.

Tsubor, K. K. et al., (I95I) Cancer Research, II, 87.

Tsubor, K. K. et al., (1954) Arch. Biochem. Biophys., 48, 275.

Tuchmann-Duplessis, H., (1950) Bull. histol. appl. et techn. microscop., 27, i I6.

Tuchmann-Duplessis, H., (1953) Compt. rend. soc. biol., I47, I 247.

Turner, C. D., (1934) F. Exptl. Zool., 68, 95.

Umanski, E., (1933) Zool. Anz., 104, i 19.

URBANi, E., (1955) Experientia, II, 209.

Ussing, H., (1954) Symposia Soc. Exptl. Biol., 8, 407.

Utsunomiya, S. And K. Yoshimura, (1952) Igaku to Seibutsugaku, 25, 302.

Utsunomiya, S. and T. Matsuda, (1953) Igaku to Seibutsugaku, 26, 213.

Valentine, W. N., (1955) Ann. N. Y. Acad. Sci., 59, I003.

Van Asperen, K., (1946) Koninkl. Ned. Akad. Wetenschap., Proc., 49, io83.

Van Bekrum, D. W. and R. A. Peters, (195I) Quart. J. Exptl. Physiol., 36, 127.

Van Biervliet, J., (igoo) Neuraxe, $1,33$.

VAndel, A., (192 I) Compt. rend., I72, 1072.

Vandel, A., (I922) Bull. biol. France et Belg., 55, 343.

Van Fleet, D. S., (1954) Dynamics of Growth Processes, Ed. E. J. Boell, University Press, Princeton.

Van Middlesworth, L., (1953) Arch. Biochem. Biophys., 43, 458.

Venkataraman, P. R. et al., (1950) J. Biol. Chem., I85, I75.

Villee, C. A., (I942) Am. Naturalist, $76,494$.

Vladimirova, E. A., (I934) Compt. rend. acad. sci. U. R. S. S., 3, 478.

Vladimirova, E. A., (1935) Trav.lab. zool. exptl. morphol. gén. (Moscow), 4, 163.

Voegthin, C. And H. W. Ghalkley, (i930) Public Health Repts. U. S., 45, 3041.

Von Bertalanffy, L., (1942) Biol. Generalis, I5, 295.

Von Bertalanffy, L. and M. Rella, (I94I) Arch. Entwicklungsmech. Organ., I4I, 98.

Von Levetzow, K. G., (I 939) Arch. Entwicklungsmech. Organ., I39, 780.

Von UBisch, L., (I915) Arch. Entwicklungsmech. Organ., 4I, 237.

Voronzova, M. A. And L. D. Liosner, (1957) Bespoloe Razmnojenie i Regeneratsiya, Gosudarstvennoe Izdatelstvo, Moscow.

Wachstein, M., (1945) A. M. A. Arch. Pathol., 40, 57.

Waddington, C. H., (1940) Organisers and Genes, University Press, Cambridge.

WAechter, H., (1949) Arch. Entwicklungsmech. Organ., I44, I32.

W'agge, L. A. And T. Mrttler, (1953) Nature, I7I, 528.

Walter, F. K., (I9I I) Arch. Entwicklungsmech. Organ., 3I, 9 I..

Warburton, F. E., (1955) Am. Naturalist, 89, I29.

Watanabe, Y., (1935) Physiol. Zool., 8, 374. 
Watanabe, Y., (1941) Physiol. Zool., 14, 316.

Weimer, B. R. et al., (1938) Physiol. Zool., II, 158.

Weiss, P., (1939) Principles of Development, Henry Holt \& Co., New York.

Weiss, P., (1950) 7. Exptl. Zool., 113, 397.

Weiss, P., (1952) Science, II5, 487.

Weiss, P., (1955) in Biological Specificity and Growth, Ed. G. Butler, University Press, Princeton.

Weisz, P. B., (1948) J. Exptl. Zool., Io9, 427.

Weisz, P. B., (1949) 7. Morphol., 84, 335.

Weisz, P. B., (1954) Quart. Rev. Biol., 29, 207.

Welsh, J. H., (1946) Anat. Record, 94, 42 I.

Whipple, A. O., (1940) Ann. Surg., II2, 48 I.

Wigglesworth, V. B., (1950) The Principles of Insect Physiology, $4^{\text {th }}$ ed., Methuen, London.

Wigglesworth, V.B., (I954) The Physiology of Insect Metamorphosis, University Press, Cambridge.

Williamson, M. B. And H. J. Fromm, (1952) Proc. Soc. Exptl. Biol. Med., 8o, 623.

Williamson, M. B. and H. J. Fromm, (1954) Federation Proc., I3, 322.

Williamson, M. B. and H. J. Fromm, (1955) 7. Biol. Chem., 212, 705.

Williamson, M. B. and G. L. Neumann, (I954) Federation Proc., 13, 322.

Williamson, M. B. et al., (i95 I) Proc. Soc. Exptl. Biol. Med., 77, 302.

Willier, B. H., P. Weiss and V. Hamberger, (i95j) Analysis of Deielopment, W. B. Saunders Co., Philadelphia and London.

Willmer, E. N., (1942a) 7. Exptl. Biol., 18, 237.

Willmer, E. N., (1942b) F. Exptl. Biol., 19, I I.

Willmer, E. N., (1953) Tissue Culture, 2nd ed., Methuen, London.

Wislocki, G. B. And M. Singer, (1946) J. Comp. Neurol., 85, I.

Wolff, E. And F. Dubois, (1947) Compt.rend., 224, I387; Compt. rend. soc. biol., I4I, $903,906$.

Wolff, E. And F. Dubors, (1948a) Rev. suisse zool., 55, 218; Experientia, 4, 273.

WolfF, E. And F. Dubois, (1948b) Compt. rend. soc. biol., r $422,533$.

Wolff, E. And M. Wey Schué, (1953) Arch. zool. exptl. et gén., 9I, Notes et Reu., 5-í.

Wolff, G., (1895) Arch. Entwicklungsmech. Organ., 1, 380.

Wolsky, A. A., (I935) Nature, I35, 102.

Wolsky, S. (A.), (I941) Allah Kozlen, 38, I 6.

Wright, M. R., (1947) F. Exptl. Zool., 105, 22 I.

Wyburn-Mason, R., (I950) Trophic Nerves, Henry Kimpton, London.

Yacovleva, T. M., (I943) see Jacovleva, T. M.

YAO, T., (I950) Quart. F. Microscop. Sci., 9I, 79.

Yarbro, C. L. And C. E. Anderson, (1954) Federation Proc., 13, 326.

Yntema, C. L., (1949) Anat. Record, 103, 524.

Young, J. Z., (1942) Physiol. Revs., 22, 318.

Young, J. Z., (I945) in Essays on Growth and Form, Eds. W. E. Le Gros Clark and P. B. Medawar, Oxford, Clarendon Press.

ZACCHEI, A. M., (1954) Ricerca sci., 24, I489

Zdriukovskaja, A. I., (1942) Compt. rend. acad sci. U. R. S. S., 35, 79.

Zeleny, C., (r905) J. Exptl. Zool., 2, 1.

Zeleny, C., (1907) Arch. Entwicklungsmech. Organ., 23, 324.

Zeleny, C., (1908) Papers Tortugas Lab., 2, 105.

Zhinkin, L., (I936) F. Exptl. Zool., 73, 43 .

Zielinska, J., (19I3) Arch. Entwicklungsmech. Organ., 38, 30.

Zoger, S., (1952) Yale J. Biol. and Med., 75, 202.

Zwilling, E., (1942) 7. Exptl. Zool., 9I, 453. 


\section{Chapter 8}

\section{WOUND HEALING AS A PROBLEM OF GROWTH ${ }^{1}$}

Wesley W. Washburn Fr.

\section{INTRODUCTION}

The creation of a wound initiates an immediate series of events directed toward closing the defect. No living organism will tolerate such an interruption of body continuity without attempting repair. The loss of epithelium and its subjacent components not only exposes the animal to bacterial invasion but also allows the escape of body fluids essential for its well being. Methods used to overcome this crisis are infinitely variable but can be summarized into two general processes. In the lower invertebrates, a simple redistribution of cells is common. This phenomenon, called morphallaxis, ultimately results in a change in body form (Needham, I952), however the most frequent mode of repair is epimorphysis, a direct regrowth of tissue at the wound site. The phylogeny of the organism seems to bear little relationship to its method of healing since many protozoa and coelenterates heal by epimorphysis similar to mammals. Furthermore, epimorphic healing is always associated with migration of cells and an increase in their number; hence morphallaxis, to a certain degree, is present in all healing processes.

Two distinctly different types of epimorphic healing can occur, and the final structure is entirely dependent upon which takes place. The terminology is confusing in this respect because much emphasis has been placed upon the similarity rather than upon the basic differences between these processes. In this chapter they will be designated as regeneration and as wound healing. The essential difference is the presence of pluripotent cells at one period during regeneration. The stages of regeneration are: wound closure, dedifferentiation, formation of a blastema, induction of the blastema and subsequent growth to form the missing part. On the other hand, the stages of wound healing are: clot formation, invasion of the area by phagocytic cells, proliferation of locally available differentiated tissue, epithelialization, and, ultimately, remodeling of the area to form a stable scar. Regeneration in the above sense is unknown in mammals; wound healing is the only recourse available. There is little evidence at present to suggest the existence of pluripotent cells during any stage of wound

1 Department of Surgery (Plastic and Maxillofacial), The University of Texas Medical Branch, Galveston, Texas. Supported by U. S. Army Contract DA-49-007-MD-447. 
healing. Levander (1950), however, believed the epithelium arose in part, at least, by differentiation from underlying granulation tissue, and Maximow ( I902) and more recently others (Allgöwer, 1956) have felt that the blood stream contains certain cells capable of differentiation into fibroblasts. The dilemma concerning the source of cells for either the blastema or granulation tissue is due to the fact that, so far, it has been impossible to identify individual or groups of cells for a sufficiently long period to follow their ultimate fate, as was done by Vogt (1925) in mapping the presumptive areas for amphibian embryos. Numerous experiments involving transplantation of whole blastemas and deletion of varying portions of the regenerate have definitely proven the presence of undifferentiated cells, but their source still remains obscure. It is conceivable that a solution to this problem may afford an entirely different approach to our understanding of wound healing.

The factors determining which type of epimorphysis occurs are: the position of the animal in the phylogenetic scale, its stage of development and the extent and location of the wound. In general, regeneration is restricted to certain amphibia and various lower forms. Species possessing the capacity to regenerate in certain areas, such as extremities and tail (Triturus), are able to undergo wound healing in other parts of the body.

It should be stressed that epimorphysis by either process is primarily a problem of cell movement with mitoses occurring later. In this respect, it bears strong resemblance to embryonic development, as pointed out by Medawar (1945). In regeneration the initial stages do not involve cell division, the epithelium migrates over the defect and dedifferentiation proceeds accompanied by phagocytosis. Cell division does, however, seem to be necessary to arrest dedifferentiation; the subsequent formation of a blastema is not accompanied by any sudden increase in mitotic rate (Needham, I952). Similarly, during wound healing epithelialization consists primarily of cell movement with mitoses at the wound edge occurring only later to make up the cell deficit. Such wounds initially manifest a large increase in the number of cellular elements which is not accompanied by cell division. This phenomenon was recognized by Maximow (I902) and represents a migration of phagocytic cells to the wound area via the vascular pathway. The subsequent formation of granulation tissue is brought about by local migration of endothelial and fibroblastic elements. In embryonic development migration of cell masses is a frequent occurrence, beginning with the initial invagination at the upper lip of the blastopore and continuing during the development of nearly all body organs. These mass movements are not accompanied by sudden rises in mitotic rate, hence formation of any bodily structure, whether primarily or secondarily, follows a similar pattern. Migration does not result from passive displacement but is an active process. The stimulus which initiates this mass movement of cells at certain critical periods in development or repair remains obscure. Menkin's (1940, I953, I956) work has done much to explain the force controlling specialized phagocytic cells and directing their movement to the site of injury. The factors controlling local migration are still unknown. It is obvious that in the adult organism migration of cell masses takes place only following the production of a defect. Weiss 
(I950, I 955) and his co-workers have emphasized this aspect and formulated the coaptation theory to explain such movements. This theory is not an answer to the problem but rather a scheme that categorizes the subsequent events. Undoubtedly the mechanism is of a highly complex physico-chemical nature; it occurs at the cell boundary, but its explanation is not known.

There are many questions to be solved in order to understand wound healing completely. These are not merely isolated problems restricted to a particular phase of an animal's biology but are intimately correlated with a basic understanding of cell populations and their reactions to environment during growth and repair. It is a fallacy to think that advances in our knowledge of wound healing, which will ultimately benefit the surgeon, must come from a study of mammals. All evidence strongly indicates that the cell mechanics involved in tissue formation and repair are essentially alike. It is difficult to believe that there are two sets of physico-chemical stimuli: one set regulating initial stages of development and another for repair.

In this chapter, the present concepts of mammalian wound healing and related studies that bear directly on its fundamental aspects are discussed. Particular emphasis has been placed on the recent literature concerning the origin of the cellular components and their chemical reactions during the healing process. An effort has been made to present both sides of the many controversial issues.

\section{THE STIMULUS FOR WOUND HEALING}

Wiesner, in 1892 , was the first to propose that injured cells might liberate substances capable of stimulating growth. In plant tissues this has been verified by English and Bonner (1937; English et al., I939), who isolated a dibasic acid, "traumatic acid", capable of promoting cell division in the bean mesocarp.

Marchand, in I9OI, and Bier, in I9I 7 , both suggested that the stimulus for mammalian wound healing might also result from products of cell degradation. Carrel (1922, I 924, 1930), one of the first investigators in this field, believed that growth-activating polypeptides, "trephones," were produced by the action of leukocytic enzymes at the wound site. In the superficial wound, where leukocytes are either absent or present in small numbers, he thought that damaged cells per se could produce such substances. According to Burrows, (I9 I 4, I924, I926a, I 926b, I927) accumulated waste products were the growth-stimulating substances. He noted that adequately nourished embryonic cells in tissue culture failed to thrive unless present in large numbers. Growth could be inhibited by washing the cells with serum or placing them in a large quantity of media.

Menkin (I940) has focused attention upon substances, e.g. leukotaxine, produced during the inflammatory reaction which invariably accompany healing. He demonstrated that injured cells at the site of a sterile abscess liberate growthrromoting factors into the inflammatory exudate (Menkin, r941, I956). Repeated sul)cuticular injections of sterile diffusible inflammatory exudate into rabbit ears eveintually produced proliferative activity of both the epithelium and underlying cartilage. Cameron (1935) had noted previously that cell growth in tissue 
cultures was stimulated by extracts of inflammatory tissue. A similar concentrated diffusate, when injected repeatedly into the nipple of non-pregnant rabbits, induced some growth response (Menkin, 1953, I955, 1956). The growthpromoting action of protein degradation products in vitro appears well established (Carrel and Baker, I926; Willmer and Kendal, I932; Davidson and Waymouth, I $944 \mathrm{a}, \mathrm{b})$. Embryo extracts which contain a large quantity of these substances also stimulate tissue culture growth, and Fisher (1930) has shown that when cells in culture are wounded, the remaining ones grow faster-an observation confirmed by Suntzowa (1944).

A number of references in the literature indicate that embryo juice has a favorable action on wound healing (Bergami, I925; Roulet, I926; Wallich, I926; Carnot and Terris, I926; Carrel and Baker, I926; Kiaer, I927; Bugliari, I927; Schloss, I928; Nakamura, I930; Nielsen, I939; Waugh, I940; Egorov, 1943). Many believe the active growth principle of embryo extract to be a pentosenucleoprotein (Fisher, I939, I940, I 942; Davidson and Waymouth, I944a, b) although others were unable to confirm this (Dvorak and Byram, I930; Auerbach and Doljanski, I944; Doljanski and Auerbach, I944). Fisher (1939, I940) claimed to have isolated an active principle from embryos, "embryonin", but this has not been confirmed by other investigators (Young et al., I 94I ; Botsford, I 94I ; Dann et al., I94I). At present there is no evidence that such embryonic extracts have any action other, than as nutrient materials (Davidson, 1943, 1945). The healing rate of a non-infected wound in a well-nourished individual is not increased by such products, and it is questionable whether they affect the healing rate under any circumstances. A substance with a specific catalytic effect on healing which would conform to the definition of a "wound hormone", has still to be demonstrated.

There are some facts indicating that the stimulus for wound healing may be of a humoral nature. Auerbach et al. (Auerbach and Doljanski, I944; Doljanski and Auerbach, I944), treating rat wounds with either adult or embryonic tissue extracts, found that control wounds on treated animals healed faster than control wounds on untreated animals. This could only be explained on the basis of a humoral mechanism. Furthermore, rats receiving intraperitoneal injections of saline-extracted adult chicken hearts, healed approximately $20 \%$ more rapidly than controls (Auerbach and Doljanski, 1945). Lorin-Epstein (1927) reported the presence of substances in the serum of wounded animals which accelerated healing, and Akamatsu (I922) demonstrated that plasma from such animals produced better growth in tissue culture than plasma from intact animals. Verification of a humoral substance which would directly stimulate healing is lacking, and clinical studies have not substantiated this concept.

The relationship between the healing rate of primary and secondary wounds has resulted in contradictory findings. If a secondary incision heals more rapidly than a distantly placed primary incision, a humoral mechanism can be inferred, as suggested by Lorin-Epstein (I927), Young, Fisher, and Young (I94I), and Sandblom (I949), however other investigators obtained negative results (Carnot and Terris, r926; Taffel et al., I950; Williams et al., I95 I; Engley et al., 1955). The bulk of evidence favors the carefully controlled studies of Billingham and 
Russell (1956), who were unable to detect any change in the healing rate as determined by decrease in area, and Savlov and Dunphy ( I 954b), who measured wound tensile strength. Sandblom and Muren (1954) believed that the interval between clipping the hair and wounding the skin is an important factor in such studies. Depilated areas heal more slowly, probably due to vasoconstriction and possibly to the release of inhibitory agents from the depilated site. The increased tensile strength in resutured wounds (Botsford, I94I; Williams et al., I95I; Savlov and Dunphy, I954) may be due to local effects, e.g. increased vascularity or concentration of fibroblasts, however, local excision of the primary wound prior to resuturing fails to abolish the effect completely, and secondary wounds when made at the primary site after the period of fibroplasia ( $15-40$ days), continue to heal more rapidly. Thus the factors affecting wound tensile strength appear to be related to the local environment, but so far have not been completely elucidated.

Another approach to the wound hormone theory was pursued by Loofbourow and his co-workers (I 948), who demonstrated that yeast cells subjected to ultraviolet radiation produced proliferation-promoting substances which are not products of autolysis but are normal cellular constituents, released as a result of increased permeability of the cell membrane or synthesized following injury. Their chief effect is to stimulate cellular respiration, as demonstrated by Adelstein et al. (1952), who found that oxygen consumption and carbon dioxide production of normal cells were increased upon exposure to the supernatant fluid of irradiated yeast cells. The oxygen consumption is closely associated with the growth curve; therefore, the effect is enhanced under aerobic conditions (Loofbourow et al., I 942). Anaerobiosis alone will produce some growth increase, perhaps resulting from greater permeability of the cell membrane. This type of reaction is not limited to yeast and has been demonstrated in bacteria (Loofbourow and Morgan, I940) and cultures of fibroblasts containing chick embryo extracts (Loofbourow et al., I $939 \mathrm{~b}$ ). The nature of this growth-promoting substance is unknown, however, in the supernatant, an increase in amino acids, vitamins of the B complex, nucleotides, and nucleosides was demonstrated (Webb and Loofbourow, I947; Loofbourow, 1947; Loofbourow et al., 1947). Most probably synthetic processes within the damaged cells are responsible for this effect. The substance produced, then diffuses through the cell wall, due to increased permeability. Although no such mechanism has been demonstrated in mammalian wound healing, certain evidence exists to indicate that it is possible. Since mechanical (Loofbourow et al., I939a) or chemical (Loofbourow and Dwyer, I939) damage to yeast cells will produce the same result as irradiation, it is conceivable that the mechanical damage incurred at a wound site might also result in the liberation of growth-stimulating products.

A relatively new and attractive concept with regard to the stimulus inducing wound healing can be developed from the "coaptation" theory of Weiss (1947, I $949 \mathrm{a}, \mathrm{b}, \mathrm{I95}$ ). Although the mechanics of cell reaction on a molecular level are as yet unknown, his concept coincides with present facts and lends itself to experimental verification. He has suggested that the affinity which exists between certain cells (Bronsted, I936; Holtfreter, I 939) may be due to specific stereochem- 
ical bonds (templates) produced at adjacent surfaces by the interlocking of macromolecules, similar to the lock-and-key theory of the antigen-antibody complex.

Epithelial growth would serve as a good example of the "coaptation" theory. In a one-cell layer each cell has specific coaptic relationships: laterally, the cell is in contact with an identical cell type; beneath, it is related to a basement membrane and the underlying dermal cells; above, it is in contact with the external environment. If the epithelium is damaged, those cells at the wound edge have a laterally exposed surface. The cell valences (of the template) on the exposed surface are unsaturated, and migration ensues. Movement continues until apposition is again established with a similar cell type. Upon contact, the lateral valences again become saturated and stability is restored. Chiakulas (1952) found that grafted flank epidermis in Amblystoma opacum and Triturus torosus fused equally well with epidermis from the tail, fin, fore-and-hind limbs, cornea, jaw, or head. Cells of endodermal origin, which normally are in coaptive relationship with the oral lining, also fused with the skin, however, gut and gall bladder epithelium were not compatible. Compatibility was manifested by a cessation of movement and fusion of the newly opposed edges. When incompatibility occurred the migrating epithelium either continued to move over the opposing edge or else the cells piled up and failed to fuse; hence, epithelial cells, even when transplanted, retained their type specificity and the ability to "distinguish" beween related and unrelated cell types. Further discussion of this concept as related to mammalian healing is presented under the section on epithelialization.

\section{WOUND GLOSURE}

There are essentially three processes by which wounds close: (I) contracture, (2) intussusceptive growth, and (3) growth from the wound edge. The role that each of these plays varies according to species and more particularly to the area of body surface involved.

\section{(a) Contracture}

Carrel (I9IO) was one of the first to study the influence of contracture on wound healing although this phenomenon had already been recognized earlier (Spallanzani, I 787 ; Born, I897; Minervini, r 904). In wounds on the trunk of dogs, cats, and guinea pigs, where skin is mobile, he found contraction was the primary method of closure. Loeb ( 1920 ) concluded from studies on guinea pig ear wounds, where skin is non-mobile, that closure was chiefly effected by epithelialization. The distinction between healing in mobile and non-mobile skin was postulated by Burrows (1924): mobile skin, characterized by a well developed layer of subdermal muscles (panniculus carnosus), e.g. the rat and guinea pig, heals chiefly by contraction. The initial granulation tissue and its overlying migrated epithelium have merely a temporary function and ultimately disappear. In non-mobile skin, contracture is minimal and granulation tissue is converted to a fibrous scar covered with epithelium. 
It is now established that the tensile forces controlling the degree of contraction are derived from granulating tissue and are not due to a sphincter type action of the wound perimeter (Hegemann, I950). Carrel (I920, I9I2) noted that the latent period for contracture was essentially the same as for granulation formation and assumed that it was due to shrinkage of this newly formed tissue. Granulation tissue has been aptly described by Billingham and Russell (1956) as an "organ of contracture," and its ultimate fate is directly dependent on the skin mobility of the area.

Two theories exist to explain the origin of tensile forces within the granulating base. The first states that it is derived from formation and subsequent shortening of collagen fibers (Gaza, I918: Kaufmann, I922). This viewpoint has been well presented by Lindquist (I946), who assumed that fibers may shorten or become kinked during maturation. The second theory is based upon evidence that connective tissue cells are the contractile mechanism. Abercrombie et al. (I954) demonstrated that collagen formation in the rat, when measured by hydroxyproline determinations, did not parallel the course of contraction. More recently they have shown (Abercrombie et al., I956) that wounds on scorbutic guinea pigs undergo normal contracture despite a failure to form collagen. These findings have led to the conclusion that connective tissue cells of granulation tissue play the important role in initiating tensile forces. Neither reticulin nor collagen fibers possess contractile ability under physiological conditions. This is a property of the cell, however, and can be demonstrated well in vivo with muscle or in tissue culture with a variety of cell types, including fibroblasts. The amount of granulation tissue seems to bear little relationship to the degree of contraction. Wounds that granulate excessively (e.g. burns) contract no more than excised wounds involving the same area. The degree of contracture appears to be related directly to mobility of the skin and its underlying tissues.

Attempts to measure the degree of wound contracture were first carried out by Carrel and Hartmann (I9I6) and Du Noüy (I916, I919, I932, I936). Since then the literature on this subject has become rather extensive (Lumière, I9I 7, I9I8a, b; Fauré-Frémiet and Vlès, ı 919 ; Brummelkamp, I9I9; Backman, I93 I Lindquist, I 946), but the results are confusing and difficult to evaluate. Burrows (I 924) reported that the speed of contraction progressively increases, while Du Noüy (1936) states the opposite view. This controversy has been re-evaluated recently by Billingham and Russell ( 1956 ), using standard-sized wounds in rabbits. They found that a linear relationship existed between the 6 th and 4 oth days when the greater part of closure took place. The initial wound always retracted because of elasticity of the adjacent skin, and contracture did not commence until approximately the $4^{\text {th }}$ day, when granulation tissue began to form (Loeb, I920; Burrows, I924; Fuke, I932; Lanber, I933; Eitel and Riecker, I936; Young et al., I94I). Similar results have also been reported by Van den Brenk (I956) who studied circular wounds on the flank of rabbits. He found that regardless of the size of the defect, there was a constant absolute linear rate of contraction for the major portion of the healing period. These recent reports confirm the previous results of Henshaw and Meyer (I944). In wounds excised to the level of the panniculus carnosus the latter served as a base for the contracting superficial 
layers and played no part in exerting a contractile force, as could be shown by the use of radiopaque markers (Billingham and Russell, 1956). Furthermore, no thickening of the panniculus was found. Circular wounds contract slower than triangular or rectangular ones. In the latter the corners remain stationary and the sides respond to increased tension by moving inward. Although tension is essentially equal throughout the wound a relatively greater amount is exerted at the sides because their length exceeds that of the corners. Circular wounds, on the other hand, collapse only slightly since the perimeter is under equal tension at all points.

\section{(b) Intussusceptive growth}

Intussusceptive growth, e.g. growth within the framework of pre-existing skin, has been demonstrated by Billingham and Medawar (1955). Islands of rabbit skin when surrounded by a wound surface ultimately expand. This expansion is due to the response of the fibrous corium to chronic tension. The hair follicles become further separated and histological sections reveal new collagen formation indicating true growth. Re-excision of the skin adjacent to the initial island resulted in immediate contraction followed by growth whose rate exceeds that of the first experiment.

\section{(c) Growth from the wound edge}

Many problems arise in accurately assessing dermal growth into a wounded area. An excellent review of the subject has been present by Van den Brenk ( $195^{6}$ ), and a large number of articles in the literature describe the growth of granulation tissue in vivo in transparent chamber preparations (Sandison, I928, I93 I ; Clark et al., I93I; Clark and Clark, I936; Ebert et al., I939; Williams, I954). Van den

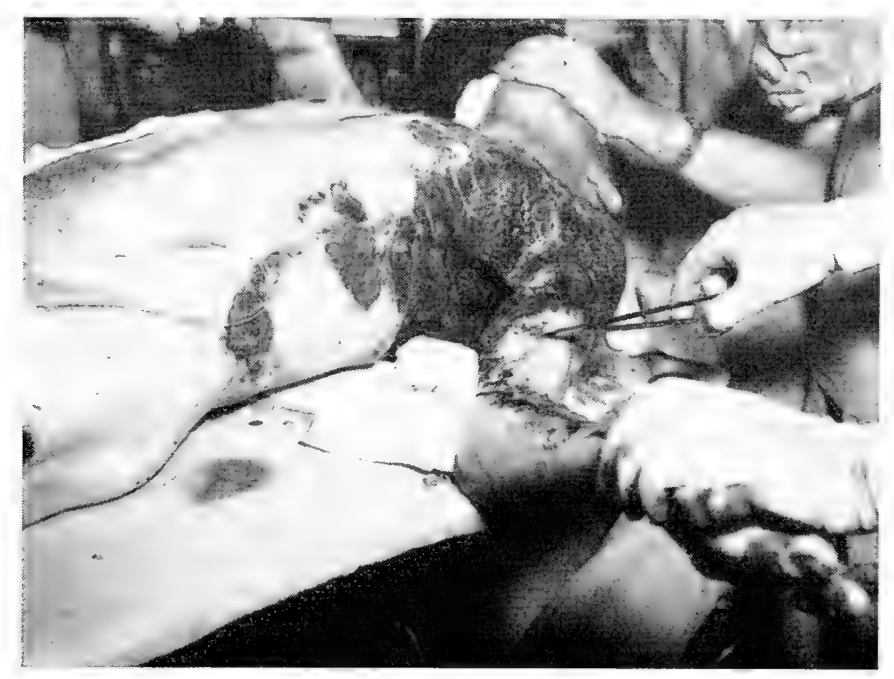

Fig. I. Third-degree burn of the left upper chest and arm at the time of initial debridement of the eschar. 


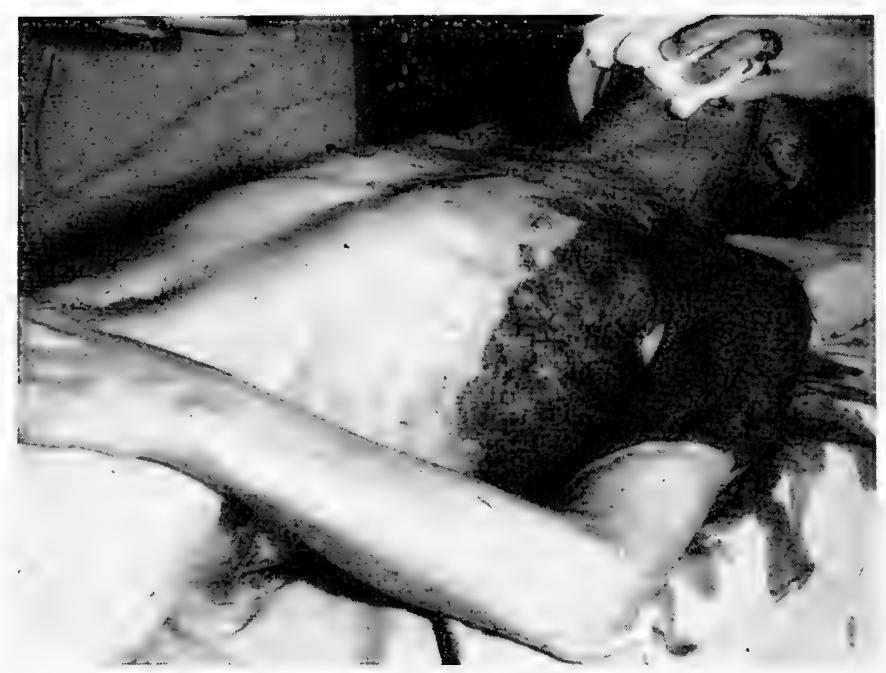

Fig. 2. The same area three weeks later showing the uniform formation of granulation tissue.

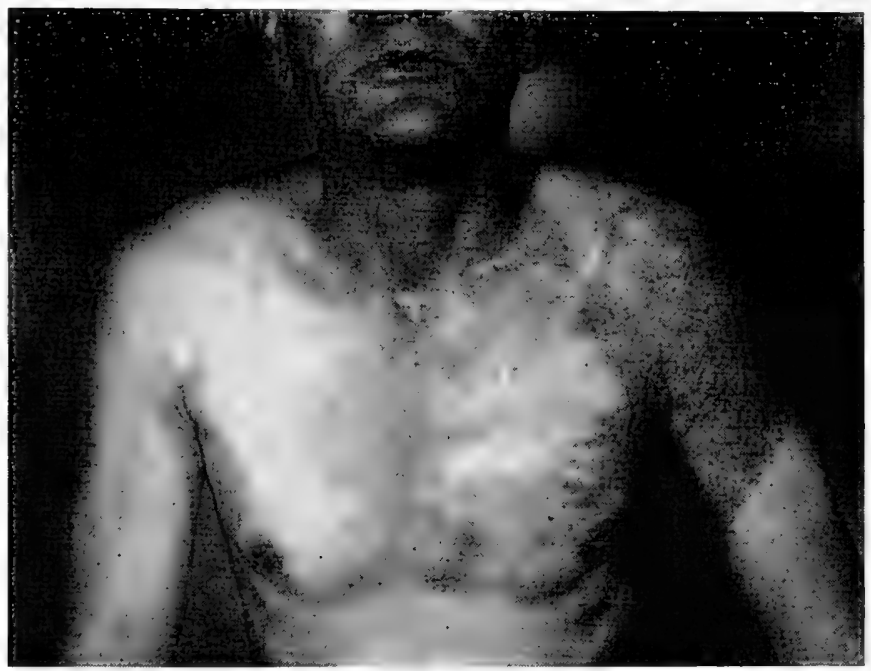

Fig. 3. The patient eight months following split-thickness skin grafting.

Brenk ( $195^{6}$ ) has used this technique to follow the complete growth pattern of granulation tissue in the rabbit ear. He found that after a latent period of approximately 8 days the growth curve maintained a linear relationship with time. The technique employed, however, possesses several shortcomings. Since the space occupied by the chamber is fixed and growth is limited to a lateral direction, it is unlikely that such a long latent period which appears to be dependent primarily upon vascularity of the area exists in the normal wound. If vascular loops 
are close to the table edge of the chamber then the latent period is short. Chambers located near a central artery or at the base of the ear in the neighborhood of large vessels also exhibit shorter latent periods. Furthermore, if tissue within the chamber is damaged, regrowth occurs within 24 hours from vessels already present.

It is generally accepted that the majority of ingrowth to a wound area occurs from the lateral edges, but clinical and histological evidence does not support such a view. An open wound, regardless of its size or depth, granulates uniformly from the base and not from the periphery, as may be demonstrated in extensive human burns, leg ulcers, etc. An excised area with soft tissue forming the base will ultimately granulate regardless of the depth of excision, and fat, fascia, and muscle are equally capable of forming granulation tissue. Exposed cortical bone, on the

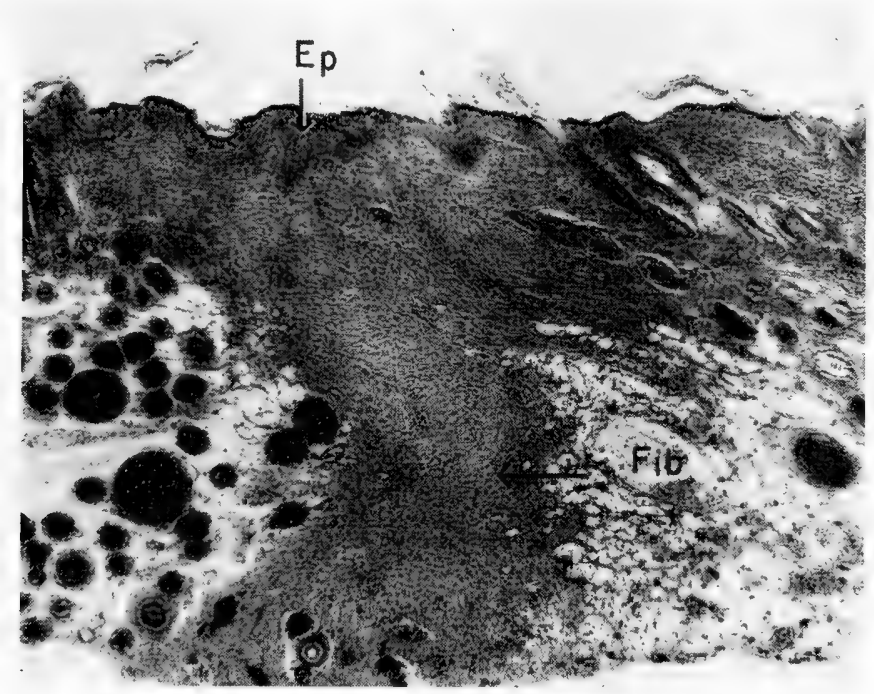

Fig. 4. Photomicrograph of a ten-day old rat burn showing the dense column of fibroblasts which extends down through the wound area into the underlying subcutaneous tissue (Van Gieson stain, $\times 48)$. Ep - epithelium; Fib - fibroblasts

other hand, fails to react in a similar manner and will sequestrate unless covered by soft tissue.

Histological sections of a wound invariably reveal a dense column of fibroblasts arising from the deeper layers with a sharp laterally demarkation between this column and the adjacent normal tissue. The base always extends to the area beneath the dermis, suggesting that fibroblasts originate from the lower rather than the peripheral aspects of the defect. Hartwell (I928, I929, I949, I950, I955) has long maintained that subcutaneous fat and fascia play a dominant role in this process. Gillman et al. ( 1956 ) verified his work and stated that there is also a downward growth from the adjacent subepithelial connective tissue. The reticulin pattern of the healing wound closely resembles that of the fibroblasts suggesting the fibers are derived in part at least from those cells. 
IV. EPITHELIALIZATION

The creation of a skin defect initiates an "effort" on the part of the adjacent epithelium to cover the denuded surface and the degree to which this is successful depends upon the size of the defect. Superficial abrasions involving only epidermis or upper portions of the dermis are rapidly healed by replacement from the basal and Malpighian layers or, in the case of dermal involvement (split-thickness skin graft donor sites), from epithelium of the glands and hair follicles in the dermis (Brown and McDowell, I942, I949; Pepper, I954; Gillman et al., I954).

Wounds, consisting of sharply incised edges with no loss of tissue, heal by re-approximation of contiguous borders. Gillman, Penn, Bronks, and Roux ( I 956)

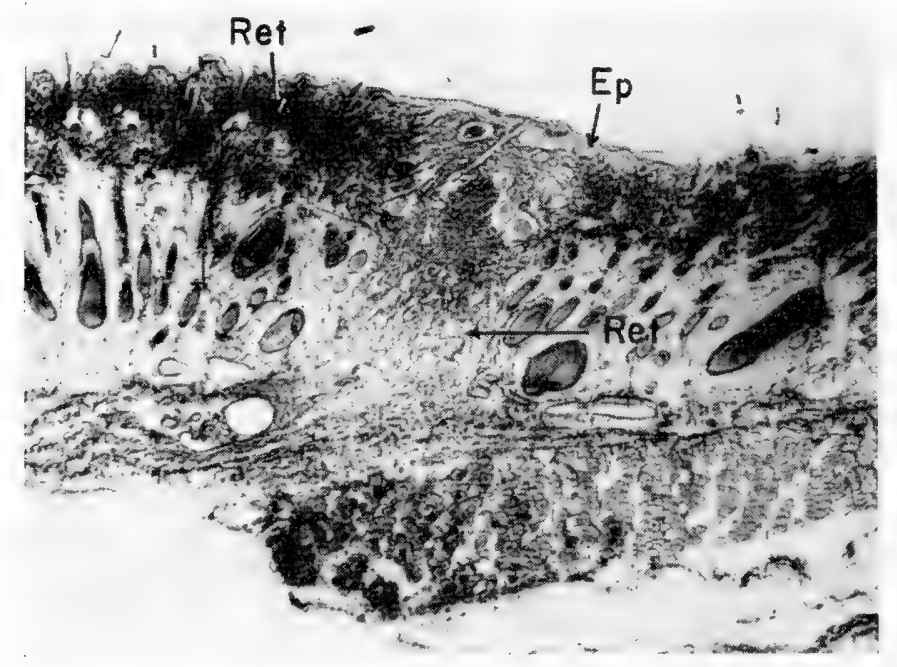

Fig. 5. Photomicrograph of an eight-day old rat burn showing the column of newly formed reticulin fibers which extends into the subcutaneous layers (Gomori reticulin stain, $\times 24$ ). Ret - reticulin; Ep — epithelium

have demonstrated that in such wounds epithelium inverts into the incision and migrates along the cut edges of the dermis. The subsequent growth of dermal elements results in detachment of many of these epithelial spurs from the overlying epithelium and ultimately the formation of keratinizing epithelial pearls. Eventually the migrating epidermal edges contact one another and fuse. The mechanism of this fusion is unknown, although some information now exists concerning the normal morphology of epithelial cell boundaries. Selby (1955) has demonstrated with the electron microscope that cell membranes of human skin are joined by a series of bridges separated by intercellular spaces. Cytoplasmic filaments, which most probably represent the keratin-like fibrous protein, "epidermin" of Rudall ( I952), do not cross these intercellular bridges but are connected by a series of rod-shaped granules of alternating density.

The following steps are required for epidermal healing: (a) Retraction of the 
epidermal edge; $(b)$ Detachment of the epidermis from the basement membrane; (c) Migration of epithelium over the wound surface; $(d)$ Cessation of cell movement; (e) Reattachment; and $(f)$ Remodeling of the wound.

\section{(a) Retraction of the epidermal edge}

Retraction of the epidermal edge occurs at the time of injury. It is distinct from that of the dermis and plays little role in mammalian skin, where epithelium is bound firmly to the underlying dermis. In amphibia, where the epidermis is loosely attached, it is quite marked, being 2 to $5 \%$ of the wound diameter (Lash, I955).

\section{(b) Detachment of the epidermis from the basement membrane}

The nature of the dermo-epidermal junction in mammals is not clearly understood, as has been pointed out in recent reviews by Montagna (I952), Medawar (1953), and Allen (1954). There is even some question as to whether a true basement membrane exists. Studies with the electron microscope have shed much light on this subject. In the adult human, human embryo, and rodent skin a submicroscopical membrane separates dermal connective tissue from the overlying epidermis (Selby, I955). This argyrophilic membrane is 300 to $400 \AA$ thick and is separated from the epidermis by $300 \AA$. It does not correspond to the classic basement membrane but possibly consists of a layer of reticulin fibers. The work of Bear (1952) and Gross (1950) suggests strongly that these reticulin filaments fuse to form the wider collagen fibers of the deeper dermal layers. This theory is in part substantiated by the fact that they possess the same periodicity and are considerably narrower in character than those of the upper layers.

The mechanism of attachment seems to be as follows: electron-dense areas composed of small, rod-shaped granules are spaced along the basal cell edge facing the connective tissue. They are converged upon by cytoplasmic filaments. Thus the junction consists of filaments from the dermal membrane which are attached to rod-shaped granules on the epithelial cell. Examination of a wound edge with the light microscope does not always show a separation between the epithelium and the basement membrane, as one would expect, if the epithelium is to migrate during wound healing. A study of this area with the electron microscope during the phase of migration should prove of interest.

Detachment in amphibia has been demonstrated by Lash (1955, I956). Here the epidermal surface adjacent to the basement lamella consists of a series of bobbin-shaped bodies. Weiss and Ferris (1954a) have postulated that these bodies play a role either in attachment or fibrogenesis. The adjacent basement membrane is a complex structure consisting of $20( \pm 2)$ layers of straight collagen fibers stacked crosswise (Porter, I954). The same pattern occurs in Anura and Urodela (Weiss and Ferris, I 954b). Following injury, detachment begins at the edge of the denuded surface and proceeds in a wave-like fashion, and migration does not begin until this stage is accomplished (Lash, I 955).

\section{(c) Migration of epithelium over the wound surface}

(i) Stimulus. After a lag phase of several hours epithelium begins to wander out over the wound surface. Although it was previously proposed that this movement 
resulted from an increased mitotic rate at the wound edge which forced the cells across the denuded surface (Eberth, I89I ; Von Bardeleben, I 9o I), Barfurth (I89I) demonstrated conclusively that such was not the case, and his work has been substantiated in numerous classes, including mammals. The stimulus which sets into motion active migration has not been elucidated, and attempts to explain it on the basis of a wound hormone have thus far failed. Weiss (1950) proposed that creation of a defect disturbs an existing equilibrium and permits cells to resume their inherent propensity of movement. The mechanism of such an equilibrium must rest on a molecular level and remains obscure at present. Furthermore, the manner in which such a stimulus is communicated to the cell and subsequently motion initiated, is unknown.

(ii) Source of energy for migration. Interesting findings are now available concerning the metabolic reactions of epithelium which ultimately result in cell movement. There is considerable evidence to indicate that basic reactions involving motility are similar in all cell types, and this subject has been thoroughly discussed by Weber (1955). Two different mechanisms, contraction and lengthening, are known to occur. Cell models have shown contraction to be a complex reaction involving contractile fibrillar proteins with ATP or ITP. Lengthening appears to be a reaction between a protein and ATP, but in this case inorganic polyphosphate can replace ATP. ATP or ITP, when bound to contractile proteins, makes them extensible. The splitting of ATP by contractile proteins and the subsequent contraction are dependent upon the presence of magnesium ions and free sulfhydryl groups on the protein. This requirement suggests that the energy is transferred to the protein by transphosphorylation and changed into mechanical energy by a conversion of the high energy phosphate bonds to low energy bonds as in muscle contraction (Weber, I955).

(iii) Rate of migration. Epithelialization of a wound produces a gradual decrease in the perimeter and area of the defect. It continues until the wound is completely healed, at which time lateral growth ceases. The lateral growth rate is influenced by many factors, such as the nutritional status of the animal, infection, presence of an eschar, etc. Attempts to elict information concerning this rate have yielded confusing data. It is difficult to separate the effects of contracture from those of epithelial migration, and in many early studies the differences were not recognized. Most investigators studied epithelialization using areas of non-mobile skin, e.g. the ear of rabbits and guinea pigs, assuming that contraction would be negligible. Another factor which hinders such a study is the presence of an overlying eschar. The migrating epidermal sheet is capable of undermining this eschar and indeed continues to do so until it is completely detached and sloughs. Unfortunately this process can be followed only with fixed histological sections since the new epithelium is hidden under the eschar. The properties of epidermis which permit this extensive undermining require further investigation. Clark and Clark ( I953) reported that advancing sheets of epithelium were able to dissolve fibrin in transparent chambers, indicating the presence of an epithelial fibrinolytic enzyme. The majority of measurements concerning healing rates have been based on successive 
planimeter determinations of the wound area (Carrel, 1910; Addison and Loeb, I91 3; Spain, I9I 5; Carrel and Hartmann, I9 16; Du Noüy, r 9 I6; Spain and Loeb,

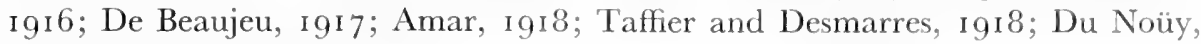
I9I9; Faurè-Fremiet and Vlès, r 919 ; Brownlee, i924; Dann et al., I94I). It has been concluded from numerous experiments by Carrel, Du Noüy and others that a greater area heals per unit time during the early phase of repair and that the rate is more rapid in large wounds. Lumière (1917, $1918 \mathrm{a}, \mathrm{b}$ ), on the other hand, measured epithelialization in terms of decrease in length and breadth of the wound and concluded that the healing rate is essentially constant. More recently Henshaw et al. (Henshaw and Meyer, I 944) compared both methods using the rabbit's ear as a test object. They found that results are similar and that length and breadth measurements are much easier to perform. These studies as well as those by Howes (1943) and Van den Brenk (1956) show that the growth curve for epithelium is essentially linear although three phases can be detected: ( I) lag phase, (2) main growth phase, and (3) reduced growth phase at the end of the healing process.

The lag phase which persists from $0-3$ days is less than half the lag phase required for contraction. During this time lateral migration is proceeding slowly and cannot be detected grossly. Microscopic sections of early wounds do reveal, however, that cell movement commences within a few hours after injury.

During the main growth phase the rate of lateral extension is essentially constant and the perimeter decreases as epithelialization occurs. Thus the area covered per unit of time is smaller. This observation verifies the seemingly conflicting statements of those who have measured the wound in terms of area: namely, that the rate of area coverage is greater in large wounds and during the early period of wound healing.

The reduced growth phase commences when epithelial elements from either side of the wound contact one another and continues until epithelialization is complete. Initially the new epithelium is considerably thicker than the surrounding normal skin, there are more cellular layers and an absence of rete pegs. Eventually it returns to normal thickness but without rete peg formation.

All wounds do not ultimately epithelialize. There appears to be a critical area beyond which healing fails to take place. The size of this area is variable and is affected by a number of factors. Gravity, for example, almost certainly exerts an influence on human wounds, probably by producing stasis. The lower leg ulcer is a classic example of this phenomenon. When individuals with such ulcers are put at bed rest and the involved extremity is elevated, partial healing occurs although the edge of the migrating epithelium becomes thickened and fails to advance beyond a certain stage. Full-thickness burns involving extensive areas also fail to become covered, although the epithelium begins to migrate under the periphery of the eschar within a few hours after injury. Such burns will not epithelialize even after prolonged periods and must ultimately be covered with skin grafts. The same phenomenon has also been observed in rabbit ear wounds with a diameter larger than $1.5 \mathrm{~cm}$ (Van den Brenk, 1956).

The reasons for this failure of complete epithelialization are not known and represent one of the most vexing problems in the study of wound healing. One can assume that somehow changes in either the cellular or chemical constituents of the 
granulation tissue preclude further epithelial growth. Van den Brenk (1956) believes that epithelial migration ceases when the vessels of the granulating area mature and the capillary loops at the advancing epidermal edge disappear, but his explanation fails to account for the fact that clean granulation tissue, regardless of its age, will readily accept split thickness skin grafts and the periphery of these grafts will migrate outward in a normal fashion. Connective tissue growth in vitro is definitely antagonistic to epithelium (Santesson, I935; Medawar, I 937; Parshley and Simms, I950), perhaps in relation to a difference in optimum pH requirements or to the liberation of inhibitory factors by the fibroblasts. The possibility that epidermis possesses only a limited capacity for migration in vivo cannot be ignored. If such is the case, then it must be related to cessation of cell divisions at the wound edge. Either the stimulus from the advancing sheet is no longer transmitted or else the cells are unable to acquire sufficient energy for continued mitotic activity. A study of the mitotic rate in extensive wounds, which fail to epithelialize, would be of interest in this respect, however histological sections from such wounds reveal that the migrating epithelium is thickened, indicating that sufficient cells are present and that the failure is due to cessation of migration.

(iv) Method of migration. The bulk of evidence regarding epidermal migration has been obtained from studies on amphibia and supported by similar findings in mammals. This subject has been thoroughly reviewed by Arey (1936). In amphibia the epidermis consists of an outer cuticular layer overlying large glandular and small epidermal cells. This relatively simple epithelium lends itself well to a study of individual cell movements. Although amphibian wound healing differs in many respects from mammals, the pattern of migration appears to be similar.

Peters in 1885 observed that creation of a skin defect resulted in rapid movement of adjacent epithelial cells over the area. According to Barfurth (I89I) such migration was independent of mitosis and was, he believed, the result of a combined active ameboid and mass movement involving all cells of the advancing sheet. His interpretation received considerable support (Klebs, I 875; Born, I896; Oppel, 1912; Poynter, I919; Arey, I932; Herrick, r932). On the other hand, the opinion of Rand (1905) was that individual movement of constituent cells is the chief means of movement of the whole sheet. Other investigators placed more stress on mass movement of cells (Oppel, I9I3; Osowski, I9I4), and Oppel (I9I3) introduced the term "Massenbewegung" to indicate this phenomenon. Arey ( 1932 ) observed the same mass movement in fish and salamanders as did Chiakulas (1952) in epithelial grafts of Triturus. It was also noted by Chiakulas (1952) that when grafts failed to fuse with adjacent skin, there was a piling up of epidermal cells similar to that reported by Arey and Cavode (1 943). Another explanation brought forth by Holmes (19I4) and Herrick (1932) was that peripheral cells of the advancing sheet pull those which lie further behind. This view seems unreasonable even though there can be no doubt that the initial stimulus for migration is mediated through cells at the wound edge. Lash (1955, I956) re-studied the froblem of migration in amphibia and concluded that its complexity precludes use of either the term "Massenbewegung" or "individual ameboid movement." He observed four distinct events: a) detachment from the underlying basement 
membrane; b) mobilization of epidermal cells in a wave-like fashion extending from the wound edge; c) emigration of the epidermal sheet; $d$ ) simultaneous cessation of movement when the wound was covered. Both detachment and mobilization begin at the wound margin and extend peripherally; cessation of cell migration, however, occurs simultaneously throughout the migrating sheet. The fact that some component cells are able to reverse direction when a closer border is available and others cells remain stationary is strong evidence against the theory that mass movement is the only important factor in this phenomenon.

(v) Layers involved in migration. Considerable confusion exists as to which cell layers are involved in migration. Eycleshymer (1907), for example, reported that all layers to Necturus larval epidermis take part, whereas Uhlenhuth (I9I4) found that it was limited chiefly to the basal cells. Cole ( I922) observed that the epidermis of frog wounds, which is initially one cell in thickness, later increased due to subsequent migration. Similar findings have been noted in skin grafts on frogs (Herrick, 1932).

Human skin has been studied both in vivo and in vitro. Matoltsy (r955) produced wounds in the center of cultured skin explants, which healed by migration of the basal cell and stratum germinativum layers. No cell divisions were found at the wound edge and the rete pegs became flattened, suggesting that their cells were used in migration. Bishop (1945) in studying forearm wounds at various depths concluded the Malpighian layer migrated while the basal layer remained stationary. Hartwell (1929, I955) has repeatedly emphasized that all cell layers are active in migration, although the majority of cells come from the middle and uppermost layers. He believes that the basal layer is formed by rounding and realignment of the lowermost cells which does not begin until migration ceases.

Present evidence indicates that migrating epithelial elements retain all of their morphological characteristics and do not dedifferentiate, as claimed by Eycleshymer (I907) and Poynter (I9I9). There is no support for Levander's theory ( $195^{\circ}$ ) that epithelialization is the result of differentiation of undifferentiated cells in the superficial portion of the granulation tissue.

(vi) The role of mitosis. The role of mitosis in epithelialization is now well understood. It was thought originally that the entire epidermal sheet arose as a result of increased cell division (Eberth, I89I; Von Bardeleben, r9o I), but it has been firmly established that mitoses only occur after initial migration is well underway (Oppel, I9I3; Osowski, I9I4; Poynter, I9I9; Gurwitsch, I926; Arey, I932; Herrick, I932; Arey and Cavode, 1943; Mann, I944; Pepper, 1954). An actual decline in mitotic rate may occur during the early stage of epithelialization (Loeb r898; Marchand, I90 I; Werner, r9o2; Arey and Cavode, r943; Bullough, I946). Akaiwa (rgrga, b) found that the mitotic rate increased rapidly at the wound margin in old epithelium as long as it was incompletely epithelialized. The nature of the stimulus for mitosis or the manner in which it is transmitted to the epithelial cells at the wound edge is obscure. It does not appear to be related to loss of contact with adjacent cells since this promotes migration rather than cell division.

The mitotic activity of cells in the migrating sheet is uniformly low or absent while movement is in progress. Hartwell (I 929, I955) believes that the onset of 
mitosis in the epithelial sheet is related to cessation of cell movement while Howes (1943) relates it to thickening of the migrating epithelium. It is interesting to note that mitotic activity in cultured skin parallels the findings of in vivo wound healing. Hsu (I952) observed that mitoses were extremely rare in the out-wandering epithelium and Washburn (I954) demonstrated that cell division was almost exclusively limited to the edge of the explant.

A relationship exists between cell division in old and new epithelium; it decreases in the former as it increases in the latter. Large wounds have a greater mitotic activity than small ones, and there is a sharp decline after epithelialization is completed. Blumenfeld (1943) demonstrated that at seven days the mitotic rate of the wound edge was twice as high as that of normal skin, and that the diurnal periodicity common to all species, including humans, was preserved (Cooper, I 939).

The energy source for mitosis of epidermal cells has been the subject of considerable investigation. It is now evident that there is a difference between the energy requirement for maintenance and for either migration or mitosis. Epithelium is unable to store large quantities of energy and the amount produced is sufficient for only one process or the other (Hartwell, i955; Bullough, I946).

The energy necessary for maintenance appears to be relatively small. Medawar ( 1947 ) demonstrated that pieces of skin can survive for at least one week in vitro without any oxygen source. Under such conditions mitosis is arrested, but when oxygen is again introduced, it will recur (Bullough and Johnson, I95 Ia, I95 Ib). The energy supply appears to be mediated through the Krebs cycle (Bullough, I952), and substrates such as glycogen, glucose, lactate, or pyruvate are all able to yield sufficient quantities for mitosis. In their absence cell division is inhibited both in vivo (Bullough, I949a, I949b) and in vitro (Bullough, I950a, b; Bullough and Johnson, I95I b). Intermediates of the Krebs cycle such as fumarate or citrate which are known to increase the rate of energy production also have a positive effect, when such substances are added in the presence of an adequate amount of glucose and oxygen there is a $25-30 \%$ increase in mitotic rate. Since cell division requires maximum energy production, any substance which interferes with glycolysis or the Krebs cycle, e.g. iodoacetate, will result in a reduced mitotic rate and ultimately cell death (Medawar, 1947).

The critical energy level must be reached in the antephase, the period immediately prior to prophase. Once a cell has started to divide, it appears to be independent of its environment. Mitosis goes to completion despite the presence of inhibitors of glycolyses, lack of glucose, oxygen, or even death of the animal (Bullough and Johnson, I95 Ia, b). These facts indicate that energy storage occurs during antephase and that the amount stored is sufficient to meet cell demands throughout mitosis. Substances which interfere with the Krebs cycle have no effect once this energy requirement is fulfilled. Little is known concerning the stored energy source; presumably it is creatine phosphate and adenosine triphosphate or similar compounds with a high energy phosphate bond.

\section{(d) Cessation of cell movement and reattachment}

Little emphasis has been placed on the events leading to cessation of migration (Young et al., I94I) and reattachment. When opposing epithelial surfaces contact 
one another in any species, they fuse and movement ceases. This phenomenon does not depend upon the mere presence of an obstruction. Chiakulas (1952) has demonstrated that in amphibians, migration ceases only when homologous cell contact is made. Epithelium from the intestinal tract, for example, fails to fuse with that of the skin. There is either a piling up of cells at this junction or else they migrate past one another. The presence of dead epithelium likewise fails to inhibit migration (Lash, I956). These findings are in complete accord with the coaptation theory of Weiss (1950, 1955). No experiments have been done to test this concept in mammals, however, clinical observations tend to support it. A mechanical obstruction such as fine-mesh gauze on a granulating surface, fails to block epithelial movement, and furthermore, epidermal cells are capable of

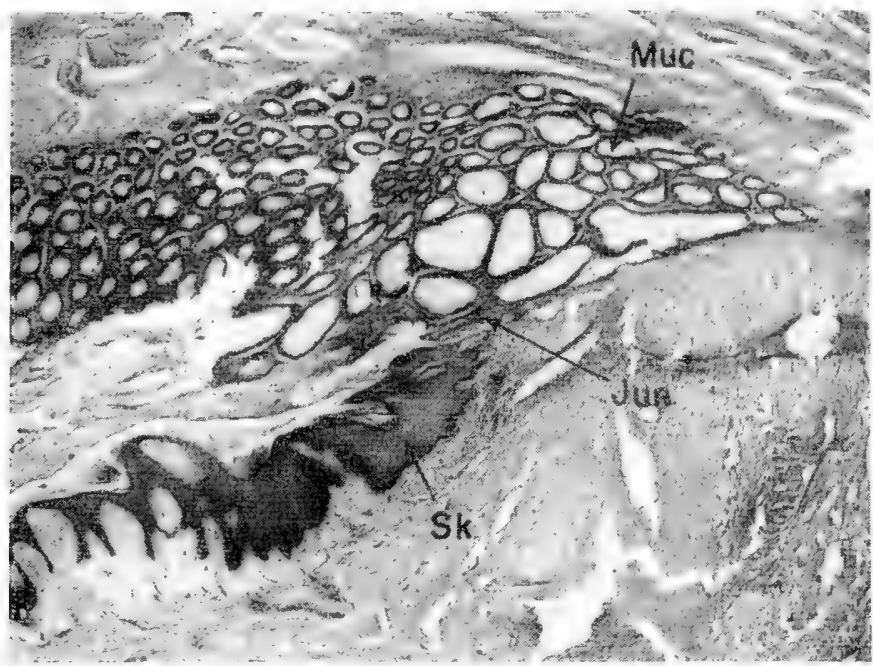

Fig. 6. Photomicrograph showing a typical junction area of abdominal skin and colon mucosa in an old colostomy. There is no true fusion and the abdominal epithelium is thickened indicating a state of instability. Sk - skin; Muc - mucosa; Jun - junction. (Hematoxylin and eosin, $x$ r6).

moving under an obstruction of dead cells such as a burn eschar. The extent of migration is dependent not only upon the lack of proper contact for the peripheral cells, but also upon an inherent limit beyond which epithelium, for reasons still obscure, fails to advance. Whether or not a homologous cell is the only type which will produce cessation of migration in mammals is a matter for conjecture.

Although this specific point has not been investigated, there are many reports in the literature indicating that mammalian epithelium reacts in a manner identical with that of amphibians. A colostomy would seem to be one instance where fusion of two entirely different epithelial cell types can occur; however, histological examination of 35 colostomy stumps has failed to reveal a true junction such as seen at the anal area (Washburn, I957). Intestinal segments transferred as a flap, and anastomosed to the urinary bladder exhibit overgrowth by the transitional bladder epithelium at the line of junction (McLean and Fais, 
I952; Barnes et al., I953; Hammer et al., I955; Sanders and Schein, I956). Splitthickness skin grafts react in the same manner when applied to a denuded bladder surface in dogs (Draper et al., I952; Draper and Stark, 1956). Similarly, when skin grafts are used to replace segments of the ureter (Horton and Politano, I955) there is an overriding at the junction. More recently Herbsman et al. (1957) observed the same phenomenon when grafts of jejunum, ileum, and colon were placed in the oral cavity or bladder.

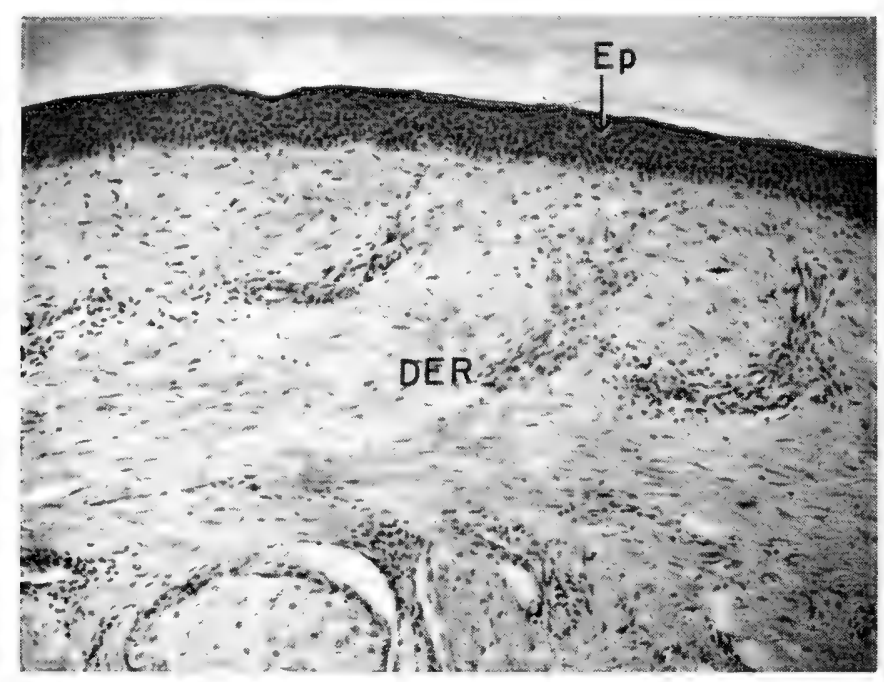

Fig. 7. Photomicrograph of a burn scar 18 months following epithelialization (Hematoxylin and Eosin, $\times 75)$. Ep - epithelium; Der - dermis.

Remodeling of the wound. All wounds involving the dermis inevitably leave a scar. A clean wound not situated in areas of excessive tension heals with a minimum of disfigurement. The gross and histological features associated with this remodeling process are relatively constant. Immediately following epithelialization the woundarea is elevated and erythematous for variable periods before it ultimately subsides, leaving the scar flattened and blanched. In wounds that have been healed for a long period, the epithelium is characterized by an absence of rete pegs and decreased pigmentation. The dermal layer becomes more cellular and there is no stratum papillaris, nor epithelial elements such as hair follicles and sweat glands. The time interval required for remodeling is affected by such factors as foreign bodies, radiation, and infection. In the presence of these disturbances healing is prolonged but ultimately achieved. The nature of the remodeling process is influenced by the site of the wound formation as well as by constitutional disturbances, e.g. keloid formation. The mechanics of scar hypertrophy have been discussed by $\mathrm{Ju}$ (195 I), as for example in flexor surface incisions perpendicular to the axis of joints or elsewhere parallel to lines of push-pull tension. Such hypertrophy can be largely obviated by incisions parallel to the joint axis or perpen- 
dicular to the direction of normal tension. The role of tension is also exemplified in extensive burns, the scars on non-mobile surfaces blanch and flatten much earlier than those situated in areas of motion. Hypertrophy continues until motion is overcome, and in many instances marked flexion and extension deformities are the result. Skoog (I 948) studied this process in Dupytren's contracture and concluded that active motion resulted in trauma and formation of microscopic hemorrhages in the fibrous tissue followed by further fibrosis and thickening of the scar.

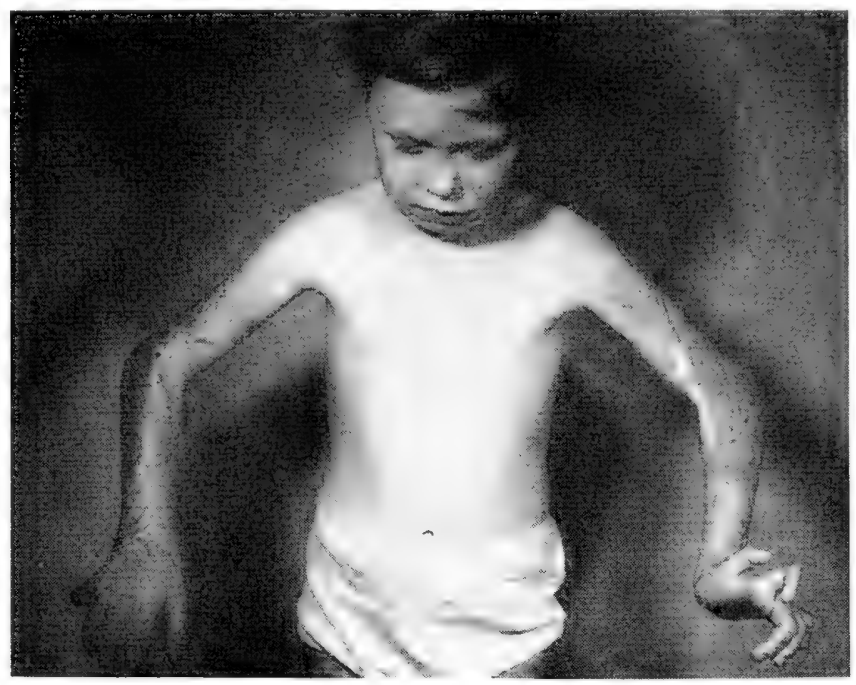

Fig. 8. Seven-year old girl one year following skin grafting of a third-degree burn. There is a severe contracture and scar hypertrophy involving the areas of "push-pull" tension.

Normal skin has been extensively investigated by both biochemical and histochemical techniques. These studies have proved complementary in many respects, the former serving as a quantitative, and the latter as a qualitative means of evaluating various substances. During wound healing, there is a variation from the normal chemical pattern, particularly with respect to glycogen, ribosenucleic acid, deoxyribosenucleic acid, acid and alkaline phosphatases, succinic dehydrogenase, and non-specific esterase.

The presence of glycogen in mammalian skin has been known since the description of Claude Bernard ( 1859 ). The quantity varies considerably, fetal skin is relatively rich in this carbohydrate (Lombardo, I907, 1934; Susakawa, 1921), in adult human epithelium it is limited to small amounts above the rete pegs in the stratum spinosum and about the orifices of pilosebaceous units (Unna and Golodetz, I 9o9; Unna, I926; Mancini, I948; Bolliger and McDonald, I949; Montagna et al., I95I; Montagna and Lobitz, I952). 
TABLE 1

RELATIVE QUANTITY OF SOME HISTOCHEMICALLY DETECTABLE SUBSTANGES IN MAMMALIAN EPITHELIUM DURING WOUND HEALING

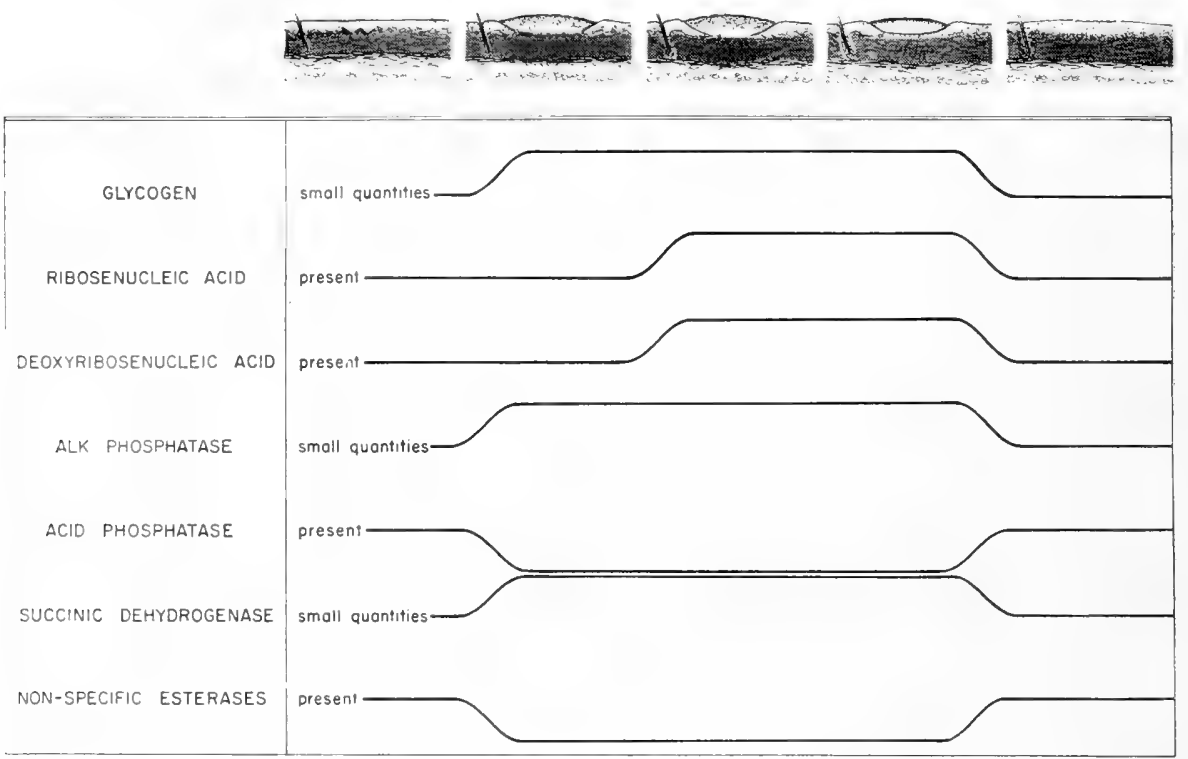

The production of a wound alters this pattern and epithelium suddenly becomes rich in glycogen. Bradfield (I95I) and Firket (I95I) studied the effect of carbon dioxide snow-induced wounds on the skin of guinea pigs, and found that large quantities of glycogen were present in all layers of the migrating epithelium with the exception of the basal and lower Malpighian stratum. Similar results have been obtained in human burns (Washburn, I954a), autografts (Scothorne and Scothorne, 1953), and homografts (Scothorne and Tough, I952). Epithelium within the body reacts in the same manner; wounds of the cat gall bladder (McMinn and Johnson, I957) and human oral mucosa (Washburn, I957), for example, show a similar increase in glycogen. When epithelialization is completed the cells containing glycogen gradually slough off and the newly formed cells contain little or none.

A similar glycogen distribution can be produced by application of methylcholanthrene to normal mouse skin (Argyris, I952). The reason for this accumulation in the upper layers of epithelium is unknown. Normally the amount of glycogen stored in the epidermis is extremely small and it is not certain that such cells are able to form glycogen and subsequently break it down to glucose, in a manner similar to liver and muscle tissue (Rothman, 1954). Recently, however, Wohnlich (1948, 1949) has shown that human skin is able to synthesize glycogen from lactic acid in vitro, the amount being approximately ten times as great as that produced by muscle under identical conditions. According to Cornbleet ( I 940) the shin glucose and glycogen content are unrelated since an intravenous injection of glucose causes a marked rise of this substance without affecting the glycogen level. 
Phlorhizin poisoning, on the other hand, produces a drop in skin glucose levels with no change in glycogen content.

Bradfield (I95I) has postulated that the wound environment is more anacrobic than that of normal skin. The migrating epithelium is greatly thickened; the underlying vessels have been destroyed by the wounding process; and, in addition, a heavy eschar overlies the entire area. Under these conditions, epithelial cells are forced to rely on anaerobic metabolic pathways to furnish an equivalent amount of energy in order to carry on their normal functions. A similar mechanism had previously been suggested by Dempsey and Wislocki (I944) to explain the presence of glycogen in the placenta. Scothorne et al. (Scothorne and Scothorne,

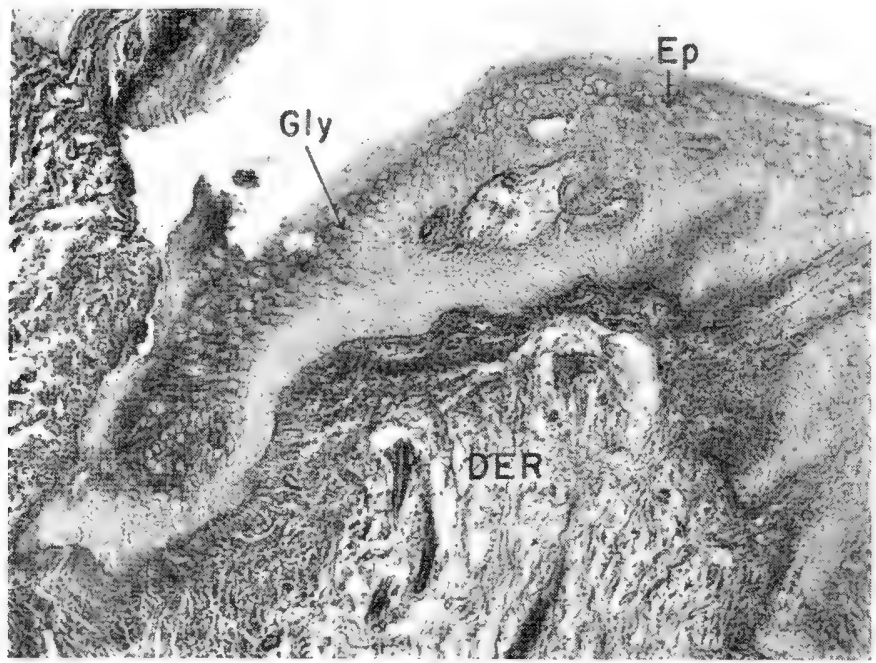

Fig. 9. Photomicrograph showing the wound edge of a threeweek old human burn. The epithelium contains glycogen in all layers except the basal and lower stratum germinativum

(Periodic Acid-Schiff stain, × 105). Gly - glycogen; Ep - epithelium; Der - dermis.

I953) believed that skin glycogen is a degenerative phenomenon caused by a reduced blood supply and resembling the accumulation in the rodent vagina at pro-oestrus.

When skin is cultured in vilro the edge of the explant resembles, in many ways, a wound margin. The epithelial layers are increased in number, as at a wound edge, and out-wandering cells of the explant have all the morphological characteristics of migrating epithelium in wounds (Lewis et al., 1949), thus providing an excellent opportunity to eliminate local environmental effects since the explant and its out-wandering cells have precisely the same environment. Under these circumstances, Washburn (I954b) found a glycogen pattern identical to that of an in vivo wound: namely, that the epithelium of the explant itself contains no glycogen while those cells at the border and in the out-wandering area do accumulate it.

If a change in local environment fails to explain deposition of glycogen, what 
other possibilities exist? The principal physiologic activities of epidermis are mitosis and keratinization. The only circumstance in which glycogen accumulates is when keratin production is absent, e.g. in fetal skin, in cells of the migrating margin of either wounds or tissue cultures and in areas where epithelium occurs without keratinization (soft palate). When keratinization starts, the glycogen disappears; this suggests an increased rate of glycolysis which may be related to greater energy requirements.

Cells undergoing active protein synthesis have been repeatedly shown to contain relatively large quantities of cytoplasmic ribonucleic acid (RNA). In normal epithelium, the activity of the lowermost cells is concerned with mitosis while

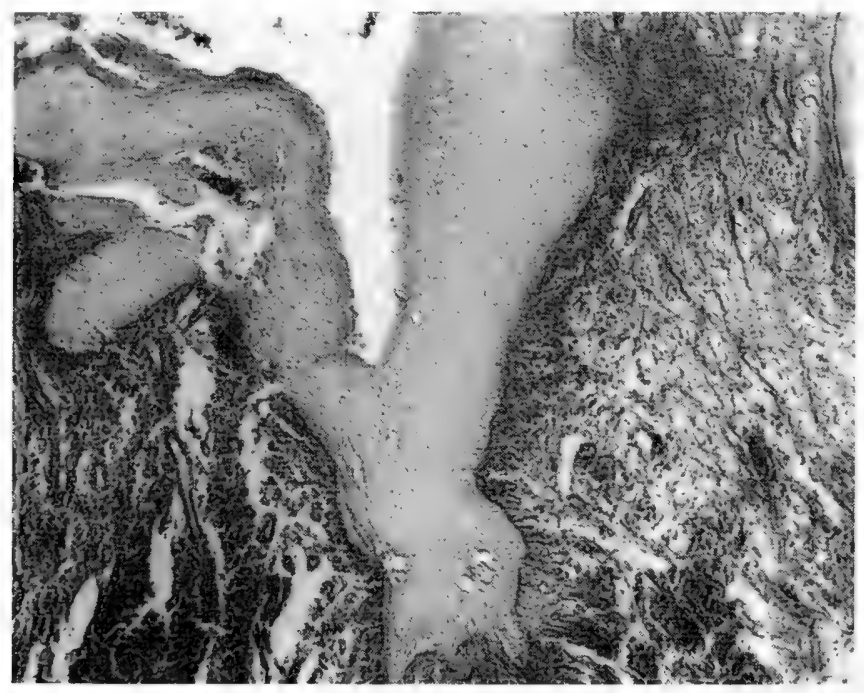

Fig. I0. Photomicrograph of same specimen as Fig. 9 showing absence of glycogen following digestion with diastase (Periodic Acid-Schiff stain, $x_{105}$ ).

that of the upper layers is related to keratinization. Brachet (I942) was the first to observe by histochemical methods that an RNA gradient existed in epithelium. He noted in amphibia that the cytoplasm of cells occupying the basal layer was relatively rich in RNA in contrast to cells located in the stratum corneum. This finding was confirmed by Hardy (1952) in mouse skin and by Nolte (1947) in the human toe. Biochemical assays in terms of nucleic acid phosphorus have also substantiated the presence of RNA in skin (Davidson and Waymouth, I944a).

During wound healing the epithelium initially migrates without the aid of increased mitotic activity and the RNA pattern resembles that of normal skin. When the mitotic rate increases, the cytoplasm of the cells contains a larger amount of RNA, as observed in mouse (Clement, I944), guinea pig (Firket, 195I), and rat wounds (Washburn, I954c), during wound healing, and with 
autografts (Scothorne and Scothorne, I 953). Methylcholanthrene-treated mouse skin (Biesele, 1944) and certain skin tumors (Stowell, 1947) follow a similar pattern.

The deoxyribosenucleic acid (DNA) content of the nuclei in normal epithelium presents a gradient similar to that of cytoplasmic RNA. Hardy (1952) observed in mouse skin that DNA is most concentrated in the nuclei of cells occupying the basal and lower stratum spinosum layers. Skin, proliferating in excess of the normal rate, contains larger quantities of DNA per unit volume and per cell. This has been shown by photometric measurements of the Feulgen reaction in methylcholanthrene-induced mouse cancer (Stowell, I942), in human epidermoid carcinoma, and in hyperplastic lesions (Stowell and Cooper, 1945). Although not measured by photometric means, the Feulgen reaction when applied to rat and human wounds seems to reflect the same direct relationship between mitosis and DNA concentration (Washburn, r954a, c).

The alkaline phosphatase content of normal skin is low. In epithelium it is restricted to the stratum granulosum and appears to be associated with the keratohyalin granules in mice (Hardy, I952), guinea pig (Bourne, I943), and human skin (Fisher and Glick, I 947; Pirilä and Eränkö, I950). Histochemical studies of this enzyme using the Gomori (1939) technique are difficult to evaluate because of the well known diffusion phenomenon. The subject has been thoroughly discussed by Goetsch et al. (1952) and Pearse (1953). Most evidence, both histochemical and biochemical, indicates that alkaline phosphatase is present only in the cytoplasm (Martin and Jacoby, I950; Palade, I95I).

When epithelium is stimulated either by application of methylcholanthrene (Biesele and Biesele, I944) or during wound healing (Fell and Danielli, I943), the quantity of epithelial alkaline phosphatase increases at the time when the underlying dermis is also rich in this enzyme; hence, diffusion is a possible explanation. More recently, the migrating epithelium has been examined following freeze-dry fixation and staining by both the Gomori (1939) and Seligman (Seligman and Manheimer, I949) techniques (Washburn, I955). There is an increased phosphatase activity especially in the lower layers of the epithelium, where it ordinarily does not exist. The role of this enzyme in the skin is not understood although it may be related to keratinization, which would account for its increase in migrating epithelium or in areas where cornification does not occur.

Acid phosphatase is present in the keratinized layers of normal epithelium. It can best be detected following the staining of frozen or frozen-dried specimens (Mescon, I950; Moretti and Mescon, 1956). During healing no increase is apparent at the wound edge and the migrating epithelium is completely devoid of this enzyme since no keratinization takes place.

There is an inverse relationship between succinic dehydrogenase and non-specific esterases in normal skin, dependent upon the degree of cell activity. In the resting phase of the hair cycle the esterase content is high while succinic dehydrogenase is low (Argyris, 1956a). According to Argyris, (1956b) during the growth phase of the hair cycle, in fetal skin, or in migrating epithelium following wounding, the pattern is reversed and succinic dehydrogenase is increased; he assumes that succinic dehydrogenase is related to the energy requirements of the cell. Areas 
where there is an increased mitotic rate would therefore contain greater amounts of the enzyme. No explanation for the corresponding decrease in non-specific esterase content has been proposed.

\section{SOURGE OF GRANULATION TISSUE}

The wounding of a skin surface induces a response not only from adjacent epithelium but also from the underlying dermis. Indeed the degree to which epithelialization is successful in covering a wound is largely dependent upon this dermal response. Although the histological sequence during formation of granulation tissue is well known, the origin of the cellular elements is still debated. Three theories exist: $(I)$ the granulation tissue is formed from foreign cells brought to the local site by the blood stream, (2) the cellular elements are formed entirely from local tissue adjacent to the wound area, (3) both local and distant elements are involved.

(a) The evidence in favor of blood stream elements forming the cellular constituents of granulation tissue has been presented by Allgöwer (1956). A brief summary of his review will suffice. Maximow in I 902 stated that any theory based upon local origin of granulation tissue fails to account for the rapid accumulation of new cells which takes place in the absence of an increased mitotic rate. In young wounds, an ameboid round cell with a large eccentric nucleus (polyblast), could be observed and he believed that polyblasts, lymphocytes, and clasmatocytes were actually transitional forms of this cell, the clasmatocyte ultimately being indistinguishable from a fibroblast in the healed wound. Evidence to support the contention that lymphocytes can be transformed to fibrocytes is lacking. Indeed, Hulliger ( 1956 ) found that cultures of "lymph buffy coat" failed to form a tissue network as did blood cultures (Allgöwer, I947).

Although lymphocytes probably play no role in granulation tissue formation, such a role may be assumed by other white cell elements (Danchakoff, I9I6). This theory has also been contested by Clark (1956) and others (Herzog, I923; Freund, I93I; Stearns, I940a), who have concluded that blood elements play no part in the formation of connective tissue. Ebert, Florey and Pullinger (1939), however, observed that india ink particles when injected into a wounded animal could be found in histocyte-like cells of the wound and they believed that the blood monocytes were most probably converted to histocytes in the granulating area (Ebert and Florey, I 939).

Allgöwer (1956), using the rabbit ear as a test object, reported a $50 \%$ to $70 \%$ reduction in the quantity of granulation tissue in animals that received sufficient total body irradiation to lower their leukocyte count from $7,000-10,000$ to $300-600$ per $\mathrm{mm}^{3}$. Interestingly enough, this occurred in shielded ear wounds as well as in those exposed to $\mathrm{x}$-ray, which is strong evidence for the hypothesis that leukocytes play a role in the granulating reaction. Needless to say, the effects of total body irradiation are profound. The possibility that some equally important factor (or factors) could also be influenced by irradiation has not been disproved.

In tissue culture the leukocytes assume a spindle shape and strongly resemble 
fibroblasts. Furthermore, in contrast to cultures of dermal and loose connective tissue, the cells have a tendency to spread and infiltrate the medium, forming new germinal centers apart from the original explant. Wound healing in cultured explants is retarded in both rate and degree (Ruth, I9II; Bentley, 1936) and no true granulation tissue is formed. Under such conditions the vascular pool is not available to the wound, but in view of numerous other environmental changes

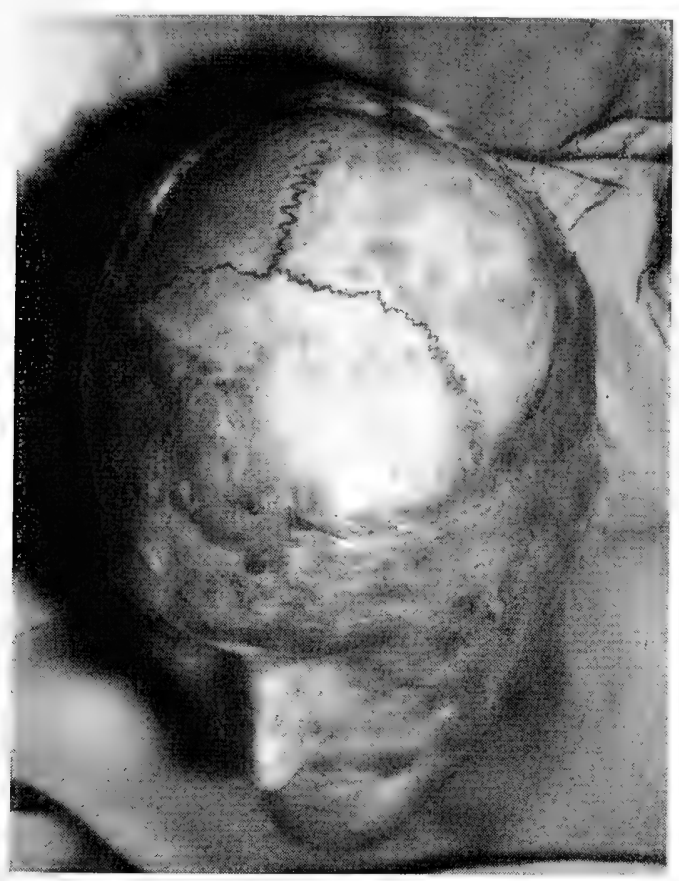

Fig. I I. Third-degree burn of the skull showing the exposed outer table. that occur in vitro it is difficult to evaluate such indirect evidence.

(b) There seems to be little question that a majority of cells which ultimately form granulation tissue arise from areas immediately adjacent to the wound. The initial stage of fibrin clot formation with its contained red blood cells, leukocytes, and mononuclear cells persists for only a short time, following which leukocytes rapidly degenerate and actual proliferation of mesenchymal elements begins. In vivo observation of wounds by means of transparent chambers have yielded considerable information concerning the manner in which granulation tissue invades the initial clot. ${ }^{1}$ Three distinct cell types are known to play an important role in this process: $a$ ) endothelial cells, $b$ ) reticuloendothelial cells, c) fibroblasts.

Capillary formation is essential for the production of granulation tissue, clinical evidence for which can be gained from observation of scalp wounds. If the outer table of the skull is chiseled off, multiple bleeding points are exposed. If the surface is kept moist and dressed frequently, it will form a profuse layer of granulation tissue which can be derived only from capillaries and their supporting structures or from blood elements. In transparent chambers, undamaged vessels adjacent to the defect can be seen to sprout solid endothelial buds. These buds move in the direction of the defect and ultimately develop a lumen. The advanced portion of the bud is freely permeable to erythrocytes and the latter form a hemorrhagic zone ahead of the capillaries. As pointed out by Van den Brenk (I956), mitosis is seldom seen in these buds, which indicates that either cell division occurs rapidly or that vascular penetration, like epithelialization, is chiefly a phenomenon of migration. There is no evidence to support the contention that capillaries

${ }^{1}$ For a thorough review of this subject, see Williams (1954). 


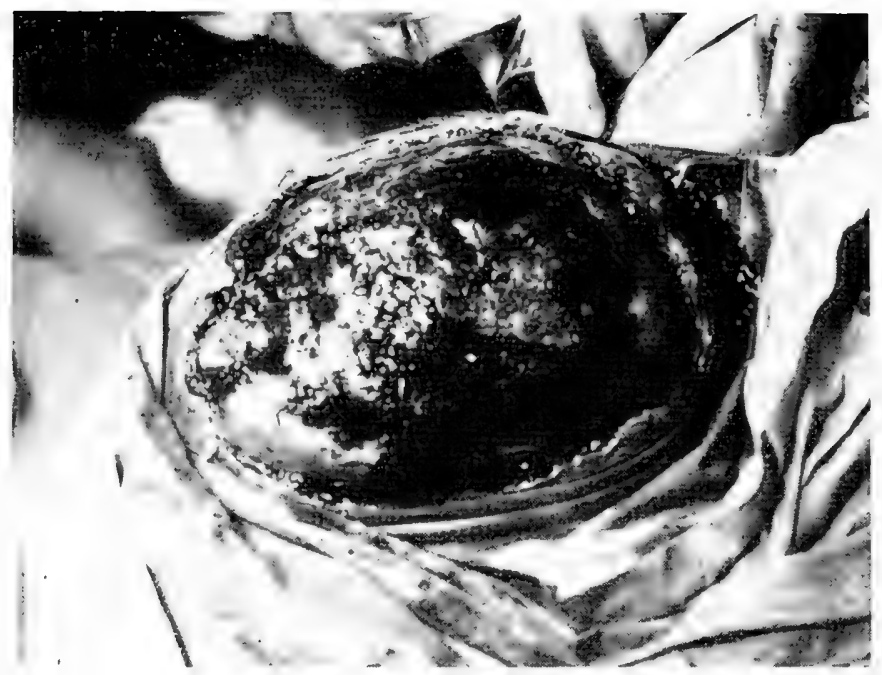

Fig. I2. The same patient three weeks following removal of the outer table of the skull showing the profuse granulations which form over the inner table.

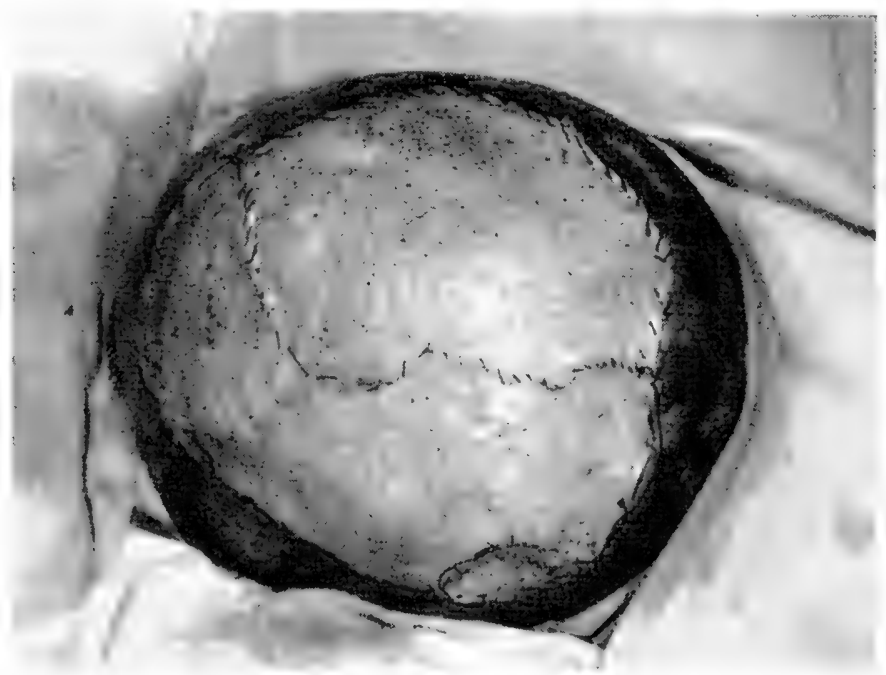

Fig. 13. The same patient following the application of split thickness skin grafts to the granulating area.

are derived from a resting or multipotent cell type, the new vascular system arises entirely from endothelial cells.

Concerning the role of reticulo-endothelial cells, there is an active migration of macrophages and round cells which always preceeds the formation of capillary buds. These cells can be identified in vivo using a transparent chamber; and in vitro, 
by time lapse cinephotography of fibroblast cultures; or in fixed histological sections of wounds. Some investigators (Ebert et al., 1939) believe that the monocytes are capable of transformation to macrophages and ultimately into fixed tissue histiocytes. The function of macrophage cells appears to be ingestion of erythrocytes and other clot elements. Certain cells of the reticulo-endothelial system remain undetected unless specifically stained by means of their cytoplasmic affinity for metallic salts (Marshall, I 946, I 948). These can be classified into two types. One consists of mononuclear cells which occur in increasing numbers during the period when leukocytes are degenerating. They enlarge and develop into ameboid macrophages, whose function is phagocytosis. The second type of metalophillic cell is elongated, either rod-shaped or with a slightly branching cytoplasm and can be found in decreasing numbers in the adjacent normal tissue. The close association with fibroblasts and presence in areas where reticulin and collagen are being formed has suggested that these cells become transformed into fibroblasts (Marshall, i 953).

Fibroblast migration into the wound area occurs relatively early and has been studied extensively by Stearns ( I939, I940a, b). Initially the cells are stellate in shape and irregularly oriented, but eventually they realign parallel to the new vessels (Howes, I957). The tension forces which guide their shape and alignment as well as theories concerning their source have been discussed in other parts of this chapter.

(c) Most probably both local and distant cells play an active role in wound healing. The initial response and subsequent phagocytic activity are undoubtedly brought about by cells of the vascular system, following which, adjacent fibroblasts and scattered fixed cells of the reticulo-endothelial system assume the role of forming granulation tissue. However, there is much about this latter process which remains obscure.

VII. FIBROBLASTIG PROLIFERATION

The immediate expansion and ultimate contraction of a wound (Billingham and Russell, 1956; Abercrombie and James, I957) exerts a profound influence upon stress patterns both within and without the defect. Fibroblastic proliferation as well as orientation of cells in adjacent normal skin are governed by these varying stress patterns. Initially the tensile forces are produced from without the wound. This phase persists for only I-2 days, hence its effects upon cell orientation are limited. The natural elasticity of skin causes a separation of the cut edges which in turn evokes forces within the clot, directed perpendicular to the margins. There is considerable evidence to indicate that tension within a clot can directly influence orientation of its contained cells.

The production of a wound produces a leakage of blood from the damaged vessels. The subsequent clotting of this blood leaves the defect filled with a matrix bound together by a dense meshwork of fibrin stands (Hudack and Blunt, I950). In clots formed by bovine fibrinogen and thrombin, the fibrinogen molecules are polymerized by the action of thrombin to form needle-shaped protofibrils; these 
in turn align into fiber strands by lateral association (Porter and Hawn, I949). The type of fiber formed depends upon the $\mathrm{pH}$ of the medium (Hawn and Porter, I947): in acid media the fibers are of a larger diameter.

This fibrin net serves as a framework into which the capillary buds and adjacent fibrocytes migrate. A similar phenomenon occurs in tissue culture where syneresis of the plasma clot results in alignment of fibrin filaments according to tension forces existing within the clot (Weiss, r 929), and these filaments in turn orient the outwandering cells (Weiss, I933). According to Weiss (1949a) the sequence is essentially as follows: tension produces an alignment of the matrix molecules into chains which in turn build up submicroscopic fibers and subsequently larger

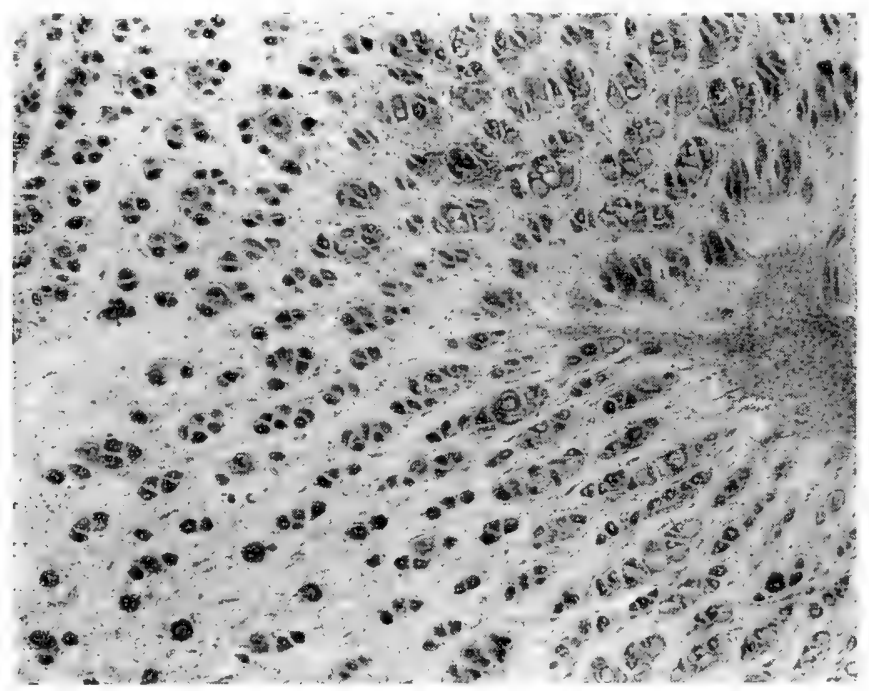

Fig. I4. Photomicrograph of a I2-day old rat burn. The wound is sectioned parallel to the skin surface and at the level of the lower portion of the dermis. The wound area is to the right of the figure and the fibrocytes of the adjacent normal skin are oriented in a radial fashion. (Hematoxylin and Eosin, $\times 24$ ).

units. The cytoplasmic filaments of cells already present and those invading the area assume a shape according to surrounding tension forces and extend along the surfaces of these fibers. Normally a single explant of fibroblasts grows in a radial manner, but when two such explants are placed close to one another an attraction field is formed. Katzberg (I95I), using polarized light, observed a birefringent pattern between two such explants and believed that syneresis of the plasma clot had developed tension forces which subsequently oriented the fibrin. He noted that fibroblasts of an explant, outside the attraction field, grew less rapidly than those within it. The cells in the field stopped growing when the gap was bridged; this also occurs in vivo, for example, between cut ends of healing tendons (Buck, I953). In liquid media cells migrate only after a fibrous exudate has been produced, and their orientation is govered by the fibrin strands (Weiss, 
1945). The mechanical force within the clot cannot act directly upon the cell but only upon the clotted medium. If no tension existed, cells would assume a rounded shape due to normally prevailing surface tension forces (Thompson, I942), as demonstrated with fibroblasts in the absence of fibrin (Huzella, I 929). It is most probable that tension within the clot acts upon the colloidal milieu of the cell substance and controls not only the rate and direction of movement but also the shape and direction of cell division (Weiss, r 949a).

The contraction phase of healing which is both prolonged and relatively constant produces a significant effect upon cellular orientation. Unlike the initial expansion phase, forces producing contraction are derived from within the wound (Abercrombie et al., I954; Abercrombie et al., 1956) and produce radial lines of tension extending into the adjacent normal tissue. Mature fibrocytes in these surrounding areas respond by re-orienting in the direction of the granulation tissue. This strong contraction force subsides when healing is completed and the entire area re-adjusts to the greater forces exerted by the surrounding parts.

VIII. THE CHEMISTRY OF GRANULATION TISSUE

The relationship between the appearance of fibroblasts in a wound area and the subsequent formation of collagen has been noted for years but only recently partially explained with the aid of electron microscope studies. In the past it has been thought that collagen was derived extracellularly from fibrin (Baitsell, i9 5 , I 92 I, I925; Nageotte and Guyon, I934); however, this is unlikely since the protein complex of collagen is of an entirely different nature (Astbury, i947). Careful observations of various tissue fibroblasts in culture suggests an extracellular origin of the fine fibrils which seem to form adjacent to those cells (Lewis, I9I7; Maximow, I928a, I929; McKinney, r 929; Maximow and Bloom, I929; Doljanski and Roulet, I933; Bloom and Sandstrom, 1935). Hass and McDonald (1940) noted that collagen production, in cultures of fibroblasts, follows a pattern similar to that of in vivo wound healing. The period of active fibroblastic proliferation is followed by one of relatively slow cell division during which collagen is formed. Once a certain level of collagen mass is reached, no more is laid down even though the fibroblasts are still viable. This impression has been substantiated in vivo with the transparent ear chamber (Stearns, I940a, b). Another view, first proposed by Mallory (1903), is that extracellular fibers originate from within the cell.

Porter (I95I) has suggested that the gelated layer beneath the cell membrane either forms collagen or else diffuses through the cell membrane and becomes polymerized into collagen fibrils extracellularly. Jackson's work (1953, I954, I 956) indicates that fibroblasts play a double role in collagen formation, first in connection with formation of the collagen fiber, and second, with elaboration of the ground substance. She observed that fibrogenic cells (fibroblasts, osteoblasts, osteocytes, and chondroblasts) contain intracellular cytoplasmic filaments and lamellae. In tendons these fibrils appear to be formed both intra- and extracellularly, although the method of extrusion is unknown. At first they are $80 \AA$ in diameter and later develop into typical collagen fibrils of about $750 \AA$ 
diameter and $640 \AA$ periodicity. Each fibril is surrounded by interfibrillary substance which probably contains either collagen molecules or components necessary for fibrillar enlargement. The fibrogenic cells also contain numerous cytoplasmic granules rich in mucopolysaccharides, alkaline phosphatase, and a protein component (Jackson, I955). These granules appear to be concerned with synthesis of intercellular material. Probably the cells secrete some substance, which then reacts with interstitial fluid to form intercellular material. The presence of fibrils within the cytoplasm of fibroblasts may be due either to absorption of interstitial fluid or extracellular fibrils.

The collagen of human skin has a cross-striated appearance with a periodicity of $620-660 \AA$ (Gross and Schmitt, I948). The basic unit is thought to be 2 Io $\AA$, three of which combine to form the regular period. Porter and Vanamee (I949) have found that unit fibers fuse to form larger strands, these small units are probably formed by a lateral association of several protofibrils and progressive deposition of molecular collagen on their surface, a process which can be reversed in vitro (Vanamee and Porter, I95I). Fibers treated with $0.01 \%$ acetic acid swell and dissociate into submicroscopic filaments, these in turn can be reconstituted by the addition of neutral salts or elevating the $\mathrm{pH}$, both of which directly influence the architecture of the resulting fiber. A similar mechanism may occur in vivo: Hass and McDonald ( 1940 ) have already shown that collagen production in cultures of fibroblasts is regulated by $\mathrm{pH}$. An acid environment ( $\mathrm{pH} 6.6)$ not only inhibits its production but also causes dissolution of previously formed fibers.

At present the majority of investigators believe that fibroblasts secrete a soluble protein procollagenous material (Tustanovsky, I947; Orekhovich et al., 1948), which in turn combines with a carbohydrate fraction of the ground substance to form insoluble collagen. Highberger et al. (195I) have shown that mucoprotein when placed with an extracted collagen solution in vitro will precipitate collagen fibers of normal periodicity, when an excess is added the so-called pro-collagenous fibers of 2000-3000 $\AA$ periodicity are formed. Various other substances, such as heparin, serum glycoproteins and plant gums, also yield a collagen precipitate indicating that this in vitro reaction is not specific (Gross et al., I952; Jackson and Randall, I 953). If the normal mucopolysaccharide content of fibroblast cultures is hydrolized with testicular hyaluronidase collagen formation is inhibited (Mancini and De Lustig, I950).

Mucopolysaccharides may also exert an indirect influence on collagen production. The dialized water-extracted material of granulation tissue, which is a rich source, inhibits the growth of fibroblasts in vitro (Balazs and Holmgren, I 949). This inhibition is especially pronounced during the period when the polysaccharide content of granulation tissue is high, i.e. the sixth to ninth day (Balazs and Homgren, I950). Under normal circumstances cell differentiation is inversely proportional to cell growth; perhaps mucopolysaccharides contribute to the role of fibroblasts in forming collagen, by inhibiting their excessive growth.

The source of mucoprotein in the wound area is still an open question. Some workers (Staemmler, I92 I; Sylvén, I94I; Asboe-Hansen, I953, I955) feel that it is secreted by the mast cells. Sylvén (I94I) based his conclusion on the observation that the number of mast cells as well as their content of granules is de- 
creased in areas where metachromasia is greatest, although this finding has not always been substantiated by others (Bunting and White, I950; Holczinger and Dévényi, I955; Taylor and Saunders, I957). Asboe-Hansen (1950a, b, 1954) found a definite relationship between number of these cells and amount of hyaluronic acid present in connective tissue, and mast cells seem to be present in large numbers wherever new formation of connective tissue takes place.

Wichmann (1955) made a detailed quantitative study of mast cells during wound healing in rats and found a decrease for the first $24 \mathrm{~h}$.; by the second day a definite rise was apparent, which reached a peak on the eighth to tenth day and gradually returned to normal by the 32 nd day. Mast cells release a large portion of their cytoplasmic granules whenever the water content of the surrounding tissue is increased (Benditt et al., I 954; Fawcett, I 955). Tyrode solution or physiological saline injected subcutaneously in hamster cheek pouches results in degranulation and release of mucopolysaccharide material to the intercellular substance (Wegelins and Asboe-Hansen, I 956). In wounds there is also an increased water content, this remains until the ioth to $5^{\text {th }}$ day, probably as an osmotic phenomenon, which further supports the idea that wound edema may be the factor causing mast cells to release their mucopolysaccharides. Asboe-Hansen has mentioned that the normal perivascular accumulation of mast cells is consistent with this view. $\mathrm{He}$ thinks that some hormonal influence causes mast cells to secrete the mesenchymal mucopolysaccharide, hyaluronic acid, via sulfonic precursors related to heparin. If such is the case, the secreted polysaccharide changes form because neither the mast cell metachromasia nor that of the granulation tissue is blocked by testicular hyaluronidase. Wichmann ( I 955), on the other hand, believes that the function of mast cells is probably to prevent clotting in newly formed capillaries by releasing heparin.

The majority of investigators Tustanovsky, I947; Orekhovich et al., I948; Klemperer, I 952; Bunting and Bunting, I 953; Glucksman et al., I 956) agree with Meyer (1947) that fibroblasts rather than mast cells produce the mucopolysaccharides. Taylor and Saunders (1957) claim this to be a function of immature fibroblasts, occurring for only a relatively short period of their normal physiologic activity. Curran and Kennedy ( I 955) recently upheld this idea by finding that the ${ }^{35} \mathrm{~S}$ uptake is high in fibroblasts surrounding a quartz focus. They believe it is fixed to the sulfated mucopolysaccharides, since mammals are unable to incorporate sulfate ions into sulfur-containing amino acids and the sulfur of cystine is formed by intestinal flora. In vitro experiments by Grossfeld et al., (1955) have shown that either the subcutaneous tissue of rats or human embryonic skin, both of which contain many fibroblasts, can form a mucin clot. Testicular or bacterial hyaluronidase inhibits this clot formation, indicating its mucopolysaccharide nature.

The mucopolysaccharide content of tissues may be studied by several methods. Histochemically these include metachromatic staining by Toluidine blue and other basic dyes; oxidation such as the P.A.S. method; iron absorption techniques, e.g. Hale's (1946) method; and Alcian blue staining ${ }^{1}$. Hexosamine determination, according to the method of Elson and Morgan (I 933) as modified by Blix (1948), is a satisfactory biochemical method for quantitating the mucopolysaccharide content. 
Bensley in 1934 first noted that the ground substance of tissues stains metachromatically. She also observed an increased metachromasia during fibrosis of the pancreas following ligation of its duct. Sylvén in I94 I found that increased extracellular metachromasia also occurs in the granulation tissue of healing wounds. This has been verified many times; the reaction is most intense at relatively early stages of wound healing, with a decrease between the third and tenth day, depending upon the type of wound produced (Penny and Balfour, I949; Bunting and White, r950; Campani and Reggianini, I950; Persson, I953; Dunphy and Udupa, 1955). This has suggested that the acid polysaccharide content of newly formed granulation tissue is elevated. In normal skin, metachromasia is limited to the papillary layer of the dermis, the stroma about hair follicles, and mast cells. Hydrolysis by various hyaluronidases has shed some light upon the type of polysaccharide involved. In vitro experiments show that streptococcal hyaluronidase affects only hyaluronic acid (Meyer et al., I938) while the testicular enzyme breaks down both chondroitin sulfate and hyaluronic acid in vitro (Meyer, I 947). Campani and Reggianini (1950) noted that the metachromasia of wounds varies in its response to hydrolysis by hyaluronidase. The metachromasia of the first four days (unlike the later period) disappears following enzyme treatment; this suggests that the initial reaction is due to hyaluronic acid which probably becomes more polymerized and hence resistant to hydrolysis at a later time.

The scorbutic state characterized by failure in formation of extracellular material, affords an interesting way to evaluate polysaccharides during wound healing (Hojer, I 924; Wolbach and Howe, I 926; Wolbach, I933). In animals with scurvy the preformed collagen content remains undisturbed (Elster, I 950) and fine collagen strands are formed; however, the amount of reticulin fails to diminish and collagen strands remain slender. Fibroblasts do not assume a mature morphology, and capillary proliferation is limited with many endothelial columns failing to form a lumen. This is associated with an almost complete absence of metachromasia (Penny and Balfour, I949). After injection of Vitamin C, metachromasia appears within 6-I2h. and the wound soon reverts to a normal appearance. In a subscorbutic state, Bunting and White (I950) observed increased metachromasia which was readily removed by testicular but not streptococcal hyaluronidase, indicating a sulfated mucopolysaccharide. Persson's studies (I 953) support the view that scurvy is characterized by an overproduction of incompletely polymerized ground substance, which stains readily by the P.A.S. reaction but is not highly metachromatic (Bradfield and Kodicek, I95I). Whether the lack of metachromasia is due to a depolymerized state, the absence of sulfate groups, or to an abnormal mucopolysaccharide is not known.

Persson (I953) believes that only highly polymerized hyaluronic acid produces significant metachromasia; in the depolymerized state it stains by the P.A.S. method. A reciprocal relationship seems to exist between these two staining reactions. Metachromatic substances are usually P.A.S. negative (Pearse, I953;

1 An excellent critical review of these techniques has been published recently by McMannus ( 1957 ). 
McManus, I 957); however, if the reaction is blocked with testicular hyaluronidase, they become positive (Hayashi et al., I955). The P.A.S. reaction depends upon free I, 2 glycol groups; if these are blocked by sulfation, the reaction becomes negative and metachromasia is positive (Kramer and Windrum, 1954). Whether metachromasia is dependent upon the state of polymerization (Lennox et al., 1952), the degree of sulfation, number and dissociation of the attached acidic radicals, or the solubility of the dye-substrate complex is not established (Walton and Ricketts, I954). Of interest is the fact that not only does the metachromatic reaction of Toluidine blue increase in granulation tissue but also the uptake of the dye itself (Balazs and Holmgren, I950). This uptake parallels that of metachromasia and is most probably due to the basic dye's becoming attached to acid (negative) groups such as high-molecular polysaccharides. Thus histochemical evidence strongly indicates that the mucopolysaccharide content of granulation tissue is elevated early in the course of wound healing and subsequently returns to normal. Direct estimations of hexosamine have verified this (Dunphy and Udupa, I955; Kodicek and Loewi, I955). Furthermore, there appears to be a qualitative change in the mucopolysaccharides during healing.

Recently this relationship between mucopolysaccharides and collagen content of wounds has been correlated by Dunphy and Udupa (I955). They used hexosamine and hydroxyproline determinations along with various histochemica methods. In sutured rat wounds the mucopolysaccharide content rose rapidly, and after the third day it gradually fell to normal levels. The curve of collagen content did not rise until the polysaccharide level commenced to fall and continued through the I2th-I 4 th day, at which time it began to form a plateau. Abercrombie and James (I957) investigated the collagen content of excised wounds for prolonged periods. They found the rate of deposition to be relatively slow until the $25^{\text {th }}$ day when it started to rise rapidly reaching normal levels by the 5 oth day. This was followed by a period of continued deposition until at the rooth day it surpassed normal skin in quantity per unit net weight and at 200 days, in quantity per unit area.

Tensile strength has long been recognized as a good index of wound healing (Paget, I853; Chlumsky, I899). This can be readily determined by tensiometer measurements of sutured skin wounds (Howes, Sooy, and Harvey, i 929) or by employing an intraluminal balloon to measure the bursting strength of gastric wounds (Harvey, I 929). The results of these investigations as well as many others (Sandblom, I944; Nelson and Dennis, I95I; Savlov and Dunphy, 1954a) have shown that tensile strength does not rise till after a lag period of four to seven days. This rise closely parallels the increase in collagen content, both of which reach normal levels at the same time (Abercrombie and James, I957). In view of our present knowledge concerning the early chemical changes in a wound, Dunphy and Udupa (I955) have reclassified wound healing into a "substrate" and a "productive phase." The "substrate stage" persists for the first four to five days during which time mucopolysaccharide and reticulin content are elevated and the groundwork laid for the subsequent "productive stage" when collagen is formed. This terminology is more realistic than the old classification in which healing was divided into an initial lag phase and subsequent period of fibroplasia. 
TABLE 2

RELATIVE CHARACTER OF THE CHANGE IN SOME CELLULAR AND NON-CELLULAR COMPONENTS OF MAMMALIAN DERMIS COMPARED WITH TENSILE STRENGTH DURING NORMAL WOUND HEALING

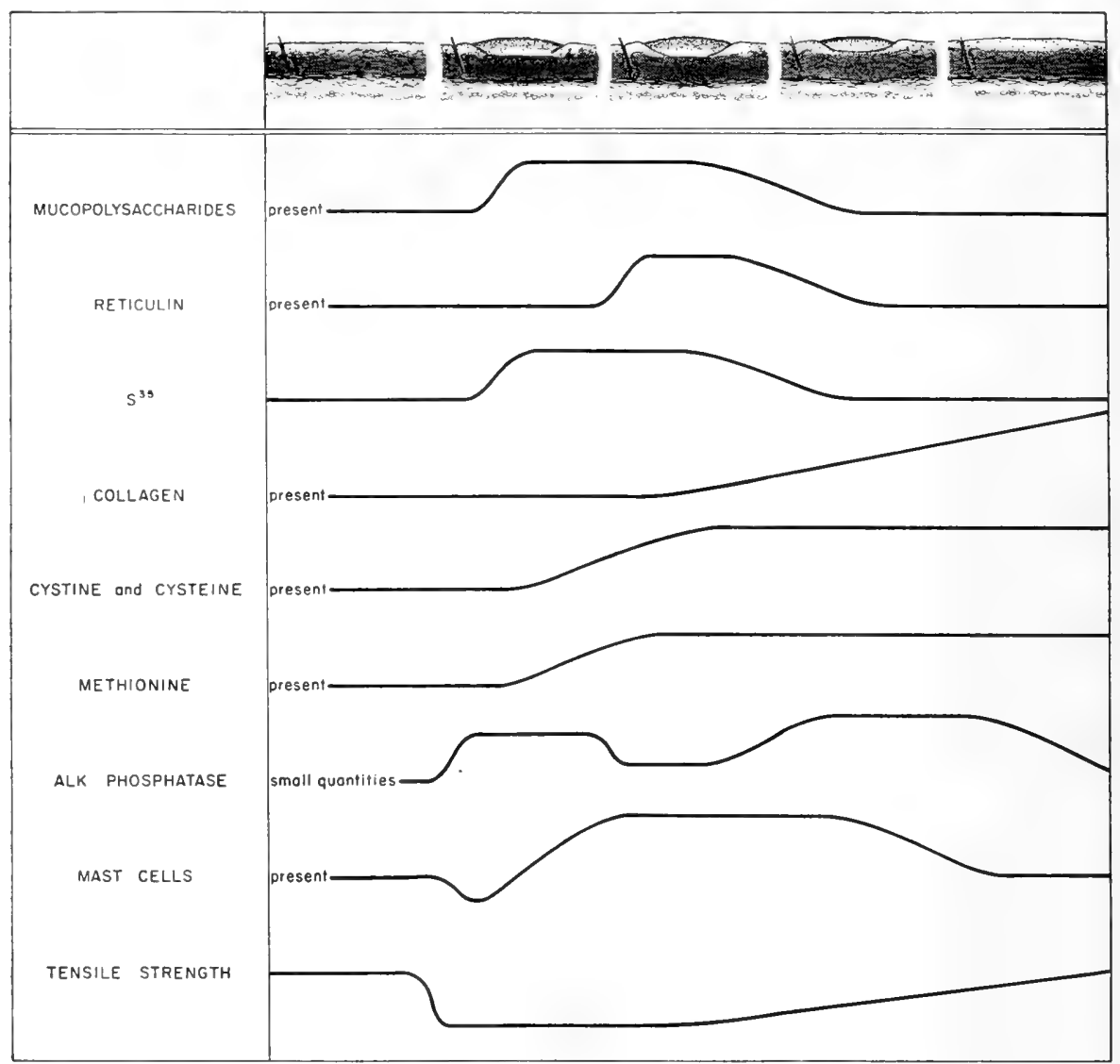

Factors which diminish collagen production will result therefore in a lowered tensile strength, e.g. Vitamin C deficiency (Taffel and Harvey, I939; Wolfer et al., I947) and protein deficiency (Harvey and Howes, I930; Kobak et al., I947; Localio et al., I948).

\section{ALKALINE PHOSPHATASE}

The presence of alkaline phosphatase in healing wounds was first detected by Fell and Danielli (I943), who observed two periods of maximum activity. The first was associated with the formation of an eschar and could be related to the concentration of polymorpho-nuclear leukocytes within the wound area. The second period occurred somewhat later, concomitant with collagen formation. 
The alkaline phosphatase was located in the cytoplasm of fibroblasts as well as in association with newly formed fibrils which has been verified by Firket (I95I) and Washburn (1955). According to Danielli et al., (I945) a direct relationship exists between phosphatase activity and the degree of collagen formation in scorbutic animals; however, Bunting and White (I950) were unable to substantiate this in partially scorbutic guinea pigs. Normally, mammalian dermis contains only small amounts of alkaline phosphatase limited to the outer sheath of hair follicles, sebaceous gland, and perivascular fibrocytes (Fisher and Glick, 1947). Phosphatase has been demonstrated in some areas of fibrous protein synthesis (Johnson et al., I 945; Johnson and Bevelander, I 946) but not in others (Marchant, I949; Robertson et al., I950). In view of the fact that freshly precipitated collagen fibers firmly bind alkaline phosphatase in vitro (Gold and Gould, 195I) it is questionable that this enzyme plays any role in collagen formation. Furthermore, in wounds the local concentration of phosphatase is independent of the serum level and there is no evidence that phosphatase inhibitors impair wound healing (Gould and Gold, I95I). There is considerable parallelism between the activity of alkaline phosphatase in mammalian wounds and the normal ontogeny of organisms. The enzyme appears to be most concentrated in areas of cell differentiation. Little is found in the early embryo (Brachet, I946; Moog, I944) or in well differentiated tissues except where it is related to specialized functions. During differentiation of amphibian limb buds or in regeneration of new limbs there is a marked increase in the mesenchymal cells. Karczmar and Berg (I95I) suggested that differentiating cells may pass through a transition phase rich in alkaline phosphatase. There appears to be, however, a direct relationship between epithelium and phosphatase. When epithelium is buried in the subcutaneous or muscle tissue of an animal a pronounced alkaline phosphatase reaction is detected about the newly formed connective tissue; control areas reveal no such reaction (French and Bendit, I 954). Gomori (I 943) has suggested the possibility that bladder epithelium, which evokes the same reaction when transplanted, may have a specific inductive power leading to the growth of phosphatase-producing fibrocytes. Normally, cultures of fibroblasts contain no alkaline phosphatase (Willmer, I942; Rodova, I948; Chevremont and Firket, I949); however, in old cultures when cells begin to degenerate the cytoplasm is rich in this enzyme (Henrichsen, I 956). It is of interest to note that alkaline phosphatase has also been detected in the granules of mast cells (Noback and Montagna, 1947). The reaction is limited to the mouse and rat and occurs most commonly in those situated perivascularly (Riley and Drennan, I 949). These authors believe that phosphatase is somehow concerned with elaboration of the metachromatic substance.

\section{ROLE OF SULFUR IN WOUND HEALING}

Studies using radioactive sulfur ${ }^{35} \mathrm{~S}$ have proved useful in gaining further information about the composition of the dermis under physiological conditions and during wound healing. If a tracer dose of ${ }^{35} \mathrm{~S}$ is given to an animal, the majority $(95 \%)$ is rapidly eliminated (five days) although a small portion remains in the 
tissues (Dziewiatkowski, I949). Autoradiographs of skin following such a dose reveal a low uptake in the cornified layer and a somewhat greater quantity evenly distributed throughout the dermal papilla and outer root sheaths of hair follicles and small vessels (Boström et al., I953; Montagna and Hill, I957). Boström and Gardell (I953) showed that the large majority of ${ }^{35} \mathrm{~S}$ is attached to the chondroitin sulfuric acid moiety which was first discovered by Meyer and Chaffee (I94I) in pigs and later in human skin by Pearce and Watson (1949). Dermal mast cells are foci of high activity, related most probably to ester sulfate groups of heparin (Jorpes et al., 1953; Asboe-Hansen, I 953). The uptake is low in most other sulfurcontaining compounds of skin. (See for review Boström, I 953; Boström and Jorpes, 1954; Boström, 1957.)

Layton (I950a, I95I) was the first to demonstrate the increased uptake of ${ }^{35} \mathrm{~S}$ in granulation tissue both in vitro and in vivo. This appears to be related to collagen formation since it is minimal around the immature fibroblasts of early wounds and later decreases when collagen deposition is completed (Upton et al., I 955). Glucksmann et al., (I 956) by means of autoradiographs have observed that ${ }^{35} \mathrm{~S}$ sulfate is incorporated into the fibroblasts prior to fiber formation. In older wounds when collagen formation is active, the main concentration of ${ }^{35} \mathrm{~S}$ is in the fiber bundles, which supports the conclusion of those who claim that fibroblasts are the source of mucopolysaccharides (Gersh and Catchpole, I947; Coon and Upton, I952). Kodicek and Loewi (I955) noted that the ${ }^{35} \mathrm{~S}$ uptake is greatest during the stage when polysaccharides are most concentrated in the wound. They suggested that sulfation of the mucopolysaccharide component may be a necessary prerequisite to collagen formation. In Vitamin C deficient aminals the uptake is markedly decreased though normal amounts of polysaccharide are present as shown by hexosamine determinations (Upton et al., I 955; Upton and Odell, I956; Kodicek and Loewi, 1955). The different staining reaction present in the scorbutic animal further supports the concept that the polysaccharides are abnormal (Penny and Balfour, 1949; Campani and Reggianini r 950; Persson, I 953; Bunting and White, I950). Kodicek and Loewi (I955) believe that either the polysaccharide cannot be sulfated because of this abnormality or else the absence of Vitamin $\mathrm{C}$ impairs some enzyme system necessary for this sulfation.

The uptake of sulfate ion, which plays a role in formation of the ground substance, must be distinguished from the uptake of sulfur in amino acid. Sulfate does not enter in large quantities into the sulfur-containing amino acids (Dziewiatkowski, I954; Tarver and Schmitt, I 939). The largest part of the non-sulfate sulfur appears to be bound to the protein of granulation tissue.

Harvey and Howes in 1930 showed that the level of protein intake markedly affects the healing rate of wounds, and their findings have since been verified many times (Thompson et al., I938; Localio et al., I943; Morris et al., I 945; Munroe and Chalmers, 1945; Chalkley et al., 1946; Abbot et al., 1946; Charney et al., 1947; Varco, I 947; Williamson et al., i 95 I a; Sandblom et al., I 953; Udupa et al., I956). Williamson and his co-workers (Williamson et al., I $95 \mathrm{Ib}$ ) established that it is the protein-sulfur rather than protein nitrogen component which is essential for this function. Further evidence of the importance of sulfur is its relative retention in granulation tissue, and this directly correlates with the healing index despite a negative nitrogen balance 
(Williamson et al., I95 Ia; Williamson and Fromm, I952, I953b; Williamson et al., I $95 \mathrm{ra}$ ). The ${ }^{35} \mathrm{~S}$ content of skin is doubled five days following wounding (Williamson and Fromm, 1953b) and there is a decrease in wound tensile strength when iodacetic acid, which blocks sulfhydryl groups, is given parenterally (Chassin and Localio, I949). The amino acids methionine and cystine have the same effect on healing as protein (Williamson et al., I95 Ia, b; Williamson and Fromm, I952, r 953a, r954b; Cuthbertson, I946; Localio et al., i 948; Peters, i 945; Croft and Peters, 1945). Normally, there is more methionine in tissue than cystine; however, in healing wounds the rate of cystine deposition is greater and appears directly related to the healing index (Williamson and Fromm, I953b). When radioactive ${ }^{35} \mathrm{~S}$ labeled $\mathrm{L}$-methionine is injected into protein-depleted animals the majority appears in granulation tissue as cystine ${ }^{35} \mathrm{~S}$. This conversion of methionine to cystine is irreversible (Binkley, I 944; Du Vigneaud et al., I 944), therefore, cystine is the limiting amino-acid in healing. Animals given ${ }^{35} \mathrm{~S}$ DL-cystine excrete more sulfate sulfur, the end product of sulfur metabolism, than animals receiving ${ }^{35} \mathrm{~S}$ DL-methionine. This is to be expected as methionine can be used per se or for conversion to cystine, whereas the latter is only available for incorporation into protein. Liver methionine normally acts as the source for cystine is this process (Williamson and Fromm, I955).

The tensile strength of wounds is correlated with the amount of cystine or methionine as well as the collagen content. These amino acids are almost entirely bound to the proteins of wound tissue; however, collagen, the principle protein, contains neither of these. This would indicate that collagen formation is related to these amino acids in a manner as yet unknown. There is a delayed production of both mucopolysaccharides and collagen in the wounds of protein depleted animals (Udupa et al., I 956), these substances are restored to normal upon addition of methionine to the diet. Although the action of methionine or cystine upon granulation tissue is obscure it has been postulated that these amino acids may increase the efficiency of protein metabolism (Forbes, I954), by stimulating the growth of fibroblasts (Morgan and Morton, I955) or in the case of polysaccharides by donating sulfate ions. Williamson's work (Williamson and Fromm, 1955) demonstrates that at least two different types of protein are produced in granulation tissue. During the early stage the protein contains a larger ratio of methionine than cystine, whereas later the cystine predominates. The scope of protein metabolism in healing has been recently presented by Williamson $\left(195^{6}\right)$ and will not be reviewed here.

\section{SUMMARY}

Wound healing is a complex process and many basic problems still remain unsolved. The over-all pattern consists of initial cell migration followed by an increase in cell numbers, however, the manner and rate varies considerably for the component tissues. The stimulus which initiates this chain of events is the interruption in cell continuity but the mechanism of this process is entirely unknown. No chemical substance which would fit the definition of a wound hormone has so 
far been isolated. More recently, Weiss has proposed that migration is linked to a disturbance in cellular relationship, a concept which coincides with the presently available facts and affords a fruitful field for further investigation.

The source of cellular components for epithelialization is well known; however, considerable confusion still exists concerning the origin of granulation tissue. The initial cell accumulation in the wound area is composed of phagocytes from the vascular system. Whether or not they remain at the site and differentiate into dermal elements is not known. Undoubtedly a large portion of the fibroblasts arise from the depths of the wound and enter the area along with invading capillary buds. Accurate evaluation of the role of various cells in the formation of granulation tissue must await means to identify individual cells for a period concommitent with that of wound healing.

Within the past decade the advent of reliable histochemical techniques supported by quantitative biochemical studies have made it evident that wound healing must be considered in a new light. Prior to this time healing was divided into a lag phase and subsequent period of fibroplasia. It is now known that the so-called lag phase is extremely important and sets the stage for the multiple series of events associated with clinical healing, in view of this, the term substrate phase appears more applicable. The subsequent period of fibroplasia associated with collagen formation has also proved a fruitful field for investigation. 


\section{LITERATURE}

Авbot, W. E., J. W. Hirshfeld, H. H. Williams and M. A. Pilling, (ig46) Surgery, 20, 284 .

Abercrombie, M., M. H. Flint and D. W. James, (1954) 7. Embryol. Exptl. Morphol., 2, 264

Abercrombie, M., M. H. Flint and D. W. James, (1956) 7. Embryol. Exptl. Morphol., 4, 167.

Abercrombie, M. and D. W. James, (1957) 7. Embryol. Exptl. Morphol., 5, 17 I.

Addison, W. H. F. And L. Loeb, (1913) Arch. Entwicklungsmech. Organ., 37, 635

Adelstein, S. J., F. B. Hershey, J. R. Loofbourow and I. W. Sizer, (1952) J. Cellular Comp. Physiol., 40, 269.

Akaiwa, H., (igiga) $\mathcal{f}$. Med. Research, 40, 3 I I.

Akaiwa, H., (I9I9b) 7. Med. Research, 40, 37 I.

Aкamatsu, N., (1922) Arch. path. Anat. u. Physiol., Virchow's, 240, 308.

Allen, A. G., (1954) Skin, A Clinicopathologic Treatise, C. V. Mosby Co., St. Louis.

Allgöwer, M., (1947) Schweiz. med. Wochschr., 77, I3I.

Allgöwer, M., (1956) The Cellular Basis of Wound Repair, C. C. Thomas, Springfield, Illinois.

Amar, J., (1918) Compt. rend., I66, 425.

ARey, L. B., (1932) Anat. Record, 5I, 299.

Arey, L. B., (1936) Physiol. Revs., 16, 327.

Arey, L. B. and W. M. Cavode, (1943) Anat. Record, 86, $75 \cdot$

Argyris, T. S., (1952) F. Natl. Cancer Inst., I2, II 59.

Argyris, T. S., (1956a) Anat. Record, 125, 105.

ArgYris, T. S., (1956b) Anat. Record, I26, I.

Asboe-Hansen, G., (1950a) Acta Dermato-Venereol., 30, 22 I.

Asboe-Hansen, G., (1950b) Ann. Rheumatic Diseases, 9, 449.

Asboe-Hansen, G., (1953) Cancer Research, 13, 587.

Asboe-Hansen, G., (I954) Intern. Rev. Cytol., 3, 415.

Asboe-Hansen, G., (I955) Humoral Effects on Connective Tissues, in Connective Tissues, Transactions at the Fifth Conference, 1954, Ed. C. A. RAGon, Josiah Macy Jr. Foundation, Princeton, New Jersey.

Asboe-Hansen, G., (1957) Connective Tissue in Health and Disease, Philosophical Library, Inc., New York, p. 159 .

Astbury, W. T., (1947) Exptl. Cell Research, Suppl. No. I, 234.

Auerbach, E. and L. Doljanski, (1944) Brit. F. Exptl. Pathol., 25, 38.

Auerbach, E. And L. Doljanski, (1945) Proc. Soc. Exptl. Biol. Med., 58 , i i i.

Aykroid, O. E. and S. Zuckerman, (1938) 7. Physiol. (London), 94, i3.

Backman, G., (I931) Ergeb. Physiol., 33, 883.

Baitsell, G. A., (1915) F. Exptl. Med., 21,455 .

Baitsell, G. A., (I92 I) Am. 7. Anat., $28,447$.

Baitsell, G. A., (1925) Quart. 7. Microscop. Sci., 69, 571.

Balazs, A. and H. J. Holmgren, (1949) Nature, $163,488$.

Balazs, A. And H. J. Holmgren, (1950) Exptl. Cell Research, I, 206.

Barfurth, D., (I89I) Arch. mikroskop. Anat. u. Entwicklungsmech., 37, 406.

Barnes, R. W., W. M. Wilson, R. J. Bergman, S. E. Farley and H. L. Hadley, (I953) 7. Urol., 69, 641.

Bear, R. S., (1952) Advances in Protein Chem., 7, 69.

Benditt, E. P., S. Bader, M. Arase, C. Corley and K. B. Lam, (1954) Federation Proc., I3, 422 .

Bensley, S. H., (1934) Anat. Record., 60, 93.

Bentley, F. H., (1936) 7. Anat., 70, 498.

Bergami, G., (1925) Rend. accad. nazl. Lincei, 2, I 40.

Bernard, C., (i 859 ) Compt. rend., 48, 673.

Bier, A., (igi 7a) Deut. med. Wochschr., 33, 833. 
Bier, A., (1917b) Deut. med. Wochschr., 33, 865.

Bier, A., (1917c) Deut. med. Wochschr., 33, 897.

Bier, A., (1917d) Deut. med. Wochschr., 33, 925.

Biesele, J. J., (1944) Cancer Research, 4, 737.

Biesele, J. J. And M. M. Biesele, (1944) Cancer Research, 4, $75 \mathrm{I}$.

Billingham, R. E. and P. B. Medewar, (1955) F. Anat., 89, i I4.

Billingham, R. E. and P. S. Russell (1956) Ann. Surg., I44, 96 I.

Binkley, F., (1944) F. Biol. Chem., 155, 39.

Bishop, G. H., (1945) Am. J. Anat., 76, 153.

Burx, G., (1948) Acta Chem. Scand., 2, 467.

Bloom, W., (1928) Folia Haematol., 36, 440.

Bloom, W. and R. H. Sandstrom (1935) Anat. Record, 64, 75.

Blumenfeld, C. M., (1943) A. M. A. Arch. Pathol., 36, 493.

Bolliger, A. And N. D. MaDonald, (1949) Australian 7. Exptl. Biol. Med. Sci., 27, 223.

Born, G., (1896) Arch. Entwicklungsmech. Organ., 4, 349.

Born, G., (1897) Arch. Entwicklungsmech. Organ., 4, 517.

Boström, H., (I953) Arkiv Kemi, 6, 43 .

Boström, H. (1957) Connective Tissue in Health and Disease, Ed. G. Asboe-Hansen, Philosophical Library, New York, p. 97.

Boström, H. and S. Gardell, (1953) Acta Chem. Scand., 7, 2 i 6.

Boström, H. ANd E. Jorpes, (I954) Experientia, Io, 392.

Boström, H., E. Odeblad and U. Friberg, (1953) Acta Pathol. Microbiol. Scand., 33, fasc. 4. Botsford, T. W., (I941) Surg. Gynecol. Obstet., 72, 690.

Bourne, G. H., (1943) Quart. 7. Exptl. Physiol., 32, I.

Brachet, J., (1942) Arch. biol. (Liège), 53, 207.

Brachet, J., (1946) Experientia, 2, 143.

Bradfield, J. R. G., (I95I) Nature, 167, 40.

Bradfield, J. R. G. And E. Kodicek, (1951) Biochem. 7., 49, I 7.

Bronsted, H. V., (1936) Acta Zool., 17, 75.

Brown, J. B. AND F. McDowell, (I942) Ann. Surg., II5, I I 66.

Brown, J. B. And F. McDowell, (1949) Skin Grafting, 2nd ed., J. B. Lippincott and Co., Philadelphia, Penn.

Brownlee, J., (1924) J. Exptl. Med., 39, 603.

Brummelkamp, R., (1929) Ned. Tijdschr. Geneesk., 73, 6055.

Buck, R. C., (1953) F. Pathol. Bacteriol., 66, I.

Bugliari, G. R., (1927) Ann. ital. chir., 6, 729.

Bullough, W. S., (I946) Phil. Trans. Roy. Soc. (London) Ser. B., 23I, 453.

Bullough, W. S., (1949a) J. Exptl. Biol., 26, 83.

Bullough, W. S., (1949b) Brit. F. Cancer, 3, 275.

Bullough, W. S., (1950a) Exptl. Cell Research, I, 4 Io.

Bullough, W. S., (1950b) F. Endocrinol., 6, 350.

Bullough, W. S. (1952) Biol. Revs. Cambridge Phil. Soc., 27, I33.

Bullough, W. S. and M. Johnson, (195Ia) Nature, 167, 488.

Bullough, W. S. And M. Johnson, (195I Ib) Proc. Roy. Soc. (London) Ser. B., I38, 562.

Bunting, H. and H. Bunting, (i953) A. M. A. Arch. Pathol., 55, 257.

Bunting, H. and R. F. White, (1950) A. M. A. Arch. Pathol., 49, 590.

Burrows, M. T., (1914) Anat. Record, II, 335.

Burrows, M. T., (1924) J. Med. Research, 44, 6 I 5 .

Burrows, M. T., (1926a) A. M. A. Arch. Internal Med., 37, 453.

Burrows, M. T., (1926b) Am. F. Anat., 37, 289.

Burrows, M. T., (1927) Am. J. Anat., 39, 83.

Cameron, G., (1935) Tissue Culture Technique, Farrar and Rinehart, New York.

Campani, M. and O. Reggianini, (1950) 7. Pathol. Bacteriol., 62, 563.

Carnot, P. And E. Terris, (1926) Compt. rend. soc. biol., 95, 655.

Carrel, A., (igio) F. Am. Med. Assoc., 55, 2148.

Carrel, A., (1912) 7. Exptl., Med., 15, 516. 
Carrel, A., (1922) 7. Exptl. Med., 36, 385 .

Carrel, A., (1924) 7. Am. Med. Assoc., 82, 255.

Carrel, A. (1930) Proc. Chicago Inst. Med., 8, 62.

Carrel, A. and L. E. Baker, (1926) J. Exptl. Med., 44, 387.

Carrel, A. And A. Hartmann, (I9I6) 7. Exptl. Med., 24, 429.

Chalkley, H. W., G. H. Algire and H. P. Morris, (1946) F. Nall. Cancer Inst., 6, 363.

Charney, J., M. B. Williamson and F. W. Bernhard, (1947) Science, 105, 396.

Chassin, J. L. and S. A. Localio, (1949) Bull. N. Y. Acad. Med., 25, 452.

Chèvremont, M. and H. Firket, (1949) Arch. biol. (Liège), 6o, 44I.

Chiakulas, J. J., (1952) F. Exptl. Zool., I2I, 383 .

Chlumsky, V., (1899) Bruns' Beitr. klin. Chir., 25, 539.

Clark, E. R., (1956) cited by Allgöwer, M., The Cellular Basis of Wound Repair, C. C. Thomas, Springfield, Illinois.

Clark, E. R. and E. L. Clark, (I936) Biol. Bull., 7I, 405.

Clark, E. R. and E. L. Clark, (1953) Am. 7. Anat., 93 , 7 I.

Glark, E. R., W. J. Hitschler, H. T. Krby-Smith, R. O. Rex and J. H. Smith, (i93i) Anat. Record, 50, 129.

Clement, H., (1944) Ann. soc. roy. zool. Belg., 75, 25.

Cole, W. H., (1922) J. Exptl., Zool., 25, 353.

Coon, W. W. And A. C. Upton, Surgical Forum (1951) Clinical Congress of American College of Surgeons, Published 1952, p. 493.

Cooper, Z. K., (1939) 7. Invest. Dermatol., 2, 289.

Cornbleet, T., (1940) Arch. Dermatol. and Syphilol., 4I, I93.

Croft, P. B. And R. A. Peters, (1945) Nature, I55, I 75.

Curran, R. G. and J. S. Kennedy, (1955) Nature, I75, 435.

Cuthbertson, D. P., (1946) Proc. Nutrition Soc. Engl. and Scotl., 4, 185.

Danchakoff, V., (19i6) Am. 7. Anat., 20, 255.

Danielli, J. F., H. B. Fell and E. Kodicek, (1945) Brit. J. Exptl. Pathol., $26,367$.

Dann, L., A. Glücksmann And K. Tansley, (I94I) Brit. F. Exptl. Pathol., 22, 70.

Davidson, J. N., (1943) Edinburgh Med. 7., 50, 70.

Davidson, J. N., (1945) Brit. Med. Bull., 3, 73.

Davidson J. N. And C. Waymouth, (1944a) Quart. 7. Exptl. Physiol., 33, 9.

Davidson, J. N. And C. Waymouth (1944b) Nutrition Abstr. E Reys., I4, I.

De Beaujeu, A. J., (1917) 7. Exptl. Med., 26, 81.

Dempsey, E. W. and G. B. Wislocki, (1944) Endocrinology, 35, 409.

Doljanski, L. And E. Auerbach, (i 944 ) Proc. Soc. Exptl. Biol. Med., 55, i i 2.

Doljanski, L. and F. Roulet, (1933) Arch. path. Anat. u. Physiol., Virchow's, 29I, 260.

Draper J. W. and R. B. Stark, (1956) Surgery, 39, 434.

Draper, J. W., R. B. Stark and M. W. Lau, (I952) Plast. Reconstr. Surg., 1o, 252.

Du Noüy, P. L., (1916) 7. Exptl. Med., 24, 46 I.

Du Noüy, P. L., (1919) J. Exptl. Med., 29, 329.

Du Noür, P. L., (1932) Compt. rend. soc. biol., IO9, 1227.

Du Noüy, P. L., (1936) Biological Time, Methuen, London.

Dunphy, J. E. And K. N. Udupa, (1955) Nere Engl. 7. Med., 253, 847.

Du Vigneaud, V., G. W. Kilmer, J. R. Rachele and M. Cohn, (ig44) J. Biol. Chem.y. I55, 645 .

Dvorak, H. J. and J. W. Byram, (1930) Proc. Soc. Exptl. Biol. Med., 27, 967.

Dziewiatkowski, D. D., (i949) 7 . Biol. Chem., I78, 197.

Dziewiatkowski, D. D., (1954) F. Biol. Chem., 207, i81.

Ebert, R. H. And H. W. Florey, (1939) Brit. F. Exptl. Pathol., 20, 342.

Ebert, R. H., H. W. Florey and B. D. Pullinger, (1939) F. Pathol. Bacteriol., $48,79$.

Eberth, G. J., (1891) Intern. Beitr. wiss. Med., 2, 75.

Egorov, N., (1943) Science, 97, 162.

Eitel, N. And O. E. Riecker, (1936) Bruns'Beitr. klin. Chir., 164, 69.

Elson, L. A. ANd W. T. J. Morgan, (1933) Biochem. F., 27, I824.

Eilster, S. K., (1950) 7. Biol. Chem., I86, 105. 
English, J. And J. Bonner, (1937) F. Biol. Chem., 12I, 79 I.

English, J., J. Bonner and A. J. HaAgen-Smit, (i939) 7. Am. Chem. Soc., 6i, 3434.

Engley, F. B., M. Allgöwer and G. D. Snyder, (1955) Ann. N. Y. Acad. Sci., 59, 326.

Eycleshymer, A. C., (1907) Am. 7. Anat., 7, 317.

Fauré-Frémiet, E. and F. Vlès, (igig) Compt. rend., i68, 363.

Fawcett, D. W., (1955) Anat. Record, I2I, 29.

Fell, H. B. and J. F. Danieliri, (1943) Brit. F. Exptl. Pathol., 24, 196.

Firket, H., (195I) Arch. biol. (Liège), 62, 335.

Fisher, A., (1930) Arch. Path. Anat. u. Physiol., Virchow's, 279, 94.

Fisher, A., (1939) Nature, I44, I I3.

Fisher, A., (1940) Chem. Prods., 3, 79.

Fisher, A., (1942) Acta Physiol. Scand., 4, 207

Fisher, A. And D. Glick, (1947) Proc. Soc. Exptl. Biol. Med., 66, 14.

Forbes, R. M., (1954) F. Nutrition, 53, 275.

French, J. E. And E. P. Benditt, (1954) A. M. A. Arch. Pathol., 57, 352.

Freund, F., (193I) Arch. path. Anat. u. Physiol., Virchow's 30, 279.

Fuke, T., (1932) Fapan. F. Obstet. Gynecol., I5, 234.

Gaza, W. V., (1918) Kolloid-Z., 23, r.

Gersh, I. and H. R. Catchpole, (1949) Am. J. Anat., 85, 457.

Gillman, T., J. Penn, D. Bronks and M. Roux, (1954) Brit. F. Plastic Surg., 6, I 53.

Gillman, T., J. Penn, D. Bronks and M. Roux, (I956) Brit. F. Surg., 43, I41.

Glücksmann, A., A. Howard and S. R. Pelc, (1956) J. Anat., 9o, 478.

Goetsch, J. B., P. M. Reynolds and H. Bunting, (1952) Proc. Soc. Exptl. Biol. Med., 8o, 7 I.

Gold, N. I. And B. S. Gould, (195I) Arch. Biochem. Biophys., 33, I 55.

Gomori, G., (1939) Proc. Soc. Exptl. Biol. Med., 42, 23.

Gomori, G., (1943) Am. J. Pathol., 19, 197.

Gould, B. S. And N. I. Gold, (195I) A. M. A. Arch. Pathol., 52, 413.

Gross, J. and F. O. Sснмitt, (1948) J. Exptl. Med., 88, 555.

Gross, J., (1950) J. Gerontol., 5, 343.

Gross, J., J. H. Highberger and F. O. Schmitt, (1952) Proc. Soc. Exptl. Biol. Med., 8o, 462.

Grossfeld, H., K. Meyer and G. Gudman, (i955) Proc. Soc. Exptl. Biol. Med., 88, 3 I.

Gurwitsch, A., (I926) Das Problem der Zellteilung physiologisch betrachtet, Springer, Berlin.

Hale, C. W., (I946) Nature, 157, 802.

Hammer, J. M., P. H. Seay, F. W. Prust, E. J. Hill and R. B. Campbell, (1955) $\mathcal{J}$.

Intern. Coll. Surgeons, 23, 500.

Hardy, M. H., (1952) Am. J. Anat., 9o, 285.

Hartwell, S. W., (1928) Proc. Mayo Clinic, 3, 344.

Hartwell, S. W., (1929) Proc. Mayo Clinic, 4, io6.

Hartwell, S. W., (I949) Arch. Surg., 19, 835.

Hartwell, S. W., (1950) Arch. Surg., 2I, 76.

Hartwell, S. W., (I955) The Mechanism of Healing in Human Wounds, C. C. Thomas, Springfield, Illinois.

Harvey, S. C., (I929) Arch. Surg., I8, 1227.

Harvey, S. C. and E. L. Howes, (1930) Ann. Surg., 9I, 641 .

Hass, G. and F. McDonald, (1940) Am. F. Pathol., I6, 525.

Hawn, C. V. Z. and K. R. Porter, (1947) 7. Exptl. Med., 86, 285.

Hayashi, H., K. Udaka, T. Funaki, and T. Inoue, (1955) Mie Med. F., 4, 159 (formerly 7. Mie Med. Coll.).

Hegemann, G., (1950) Bruns' Beitr. Klin. Chir., I80, 229.

Henrichsen, E., (1956) Exptl. Cell. Research, iI, i I5.

Henshaw, P. S. and H. L. Meyer, (1944) 7. Natl. Cancer Inst., 4, 35 I.

Herbsman, J. A., S. Rabson and W. E. Schatten, (1957) J. Plast. Reconstr. Surg., 20, 366.

Herrick, E. H., (1932) Biol. Bull., 63, 271.

Herzog, G., (1923) Klin. Wochschr., 2, 684.

Highberger, J. H., J. Gross and F. O. Sсhmitt, (1951) Proc. Natl. Acad. Sci. U. S., 37, 286.

Hojer, J. A., (1924) Acta Pediat., Suppl. 3. 
Holczinger, L. and I. Dévényi, (1955) Acta Morphol. Acad. Sci. Hung., 1, 37.

Holmes, S. J., (I914) 7. Exptl., Zool., t7, 28 I.

Holtfreter, J., (1939) Arch. expll. Zellforsch. Gewebezücht., 23, I69.

Horton, C. E. And V. Politano, (1955) F. Plast. Reconstr. Surg., 15, 26 I.

Howes, E. L., (1943) Surg. Gynecol. Obstet., 76, 738.

Howes, E. L., (1957) cited in G. Asboe-Hansen, Connective Tissue in Health and Disease, Philosophical Library, New York, p. I59.

Howes, E. L., J. Sooy and S. C. Harvey, (i 929) 7. Am. Med. Assoc., $92,42$.

Hsu, T. G., (1952) Texas Repts. Biol. and Med., Io, 336.

Hudack, S. S. and J. W. Blunt, (1950) Am. F. Surg., 8o, 680.

Hulliger, L., (1956) Cited by M. Allgöwer, The Cellular Basis of Wound Repair, C. C. Thomas, Springfield, Hllinois.

Huzella, T., (1929) Arch. Entwicklungsmech. Organ., II6, 430.

Jackson, S. F., (1953) Nature and Structure of Collagen, Ed. J. T. Randall, Butterworth, London, p. I 40 .

Jackson, S. F., (1954) Nature, I73, 950.

JACKson, S. F., (1955) Nature, 175, 39.

Jackson, S. F., (1956) Proc. Roy. Soc. (London), Ser. B., I44, 556.

Jackson, S. F. And J. T. Randall, ( 1953 ) Nature and Structure of Collagen, Ed. J. T. Randall, Butterworth, London, p. I8 I .

Johnson, P. L. And G. Bevelander, (1946) Anat. Record, 95, I93.

Johnson, P. L., E. O. Butcher and G. Bevelander, (I 945) Anat. Record, 93, 355.

Jorpes, E., E. Odeblad and H. Boström, (1953) Acta Haematol., 9, 273.

Ju, D. M., (195I) 7. Plast. Reconstr. Surg., 7, 343.

Karczmar, A. G. and G. G. BerG, (195I) F. Exptl. Zool., II7, i39.

Katzberg, A., (I95I) Science, II4, 43 I.

Kaufmann, E., (1922) Spezielle Pathologische Anatomie, De Gruyter and Co., Berlin and Leipzig.

Kiaer, S., (1927) Arch. klin. Chir. Langenbecks, I49, 146.

Klebs, E., (1875) Arch. exptl. Pathol. Pharmakol., Naunyn-Schmiedeberg's, 3, 124.

Klemperer, P., (1952) Bull. N. Y. Acad. Med., 28, 204.

Kobak,-M. W., E. P. Benditt, R. W. Wissler and C. H. Steffee, (1947) Surg. Gynecol., Obstet., 85, $75 \mathrm{I}$.

Kodicek, E. and G. Loewi, (1955) Proc. Roy. Soc. London, Ser. B., I44, 100.

Kramer, H. and G. M. Windrum, (I954) F. Histochem. Cytochem., 2, 196.

Lanber, H. J., (1933) Brims'Beitr. Klin. Chir., I58, 293.

Lash, J. W., (1955) 7. Exptl. Zool., I28, I3.

LAsh, J. W., (1956) J. Exptl. Zool., I3I, 239.

Layton, L. L., (1950a) Proc. Soc. Exptl. Biol. Med., 73, 570.

Layton, L. L., (I950b) Cancer, 2, Io89.

LAyton, L. L., (I95I) Cancer, 4, 198.

Lennox, B., A. G. E. Pearse and H. G. H. Richards, (1952) 7. Pathol. Bacteriol., 64, 865.

Levander, G., (1950) Acta Chir. Scand., Ioo, 637.

Lewis, M. R., (I9I 7) Carnegie Inst. Contribs. Embryol., 6, 45.

Lewis, S. R., C. M. Pomerat and D. Ezell, (1949) Anat. Record, io4, 487.

Lindeuist, G., (1946) Acta Chir. Scand., 94, Suppl. I07, I63.

Localio, A. S., W. Cassale and J. W. Hinton, (1943) Surg. Gynecol. Obstet., 77, 369.

Localio, A. S., M. E. Morgan and J. W. Hinton, (1948) Surg. Gynecol. Obstet., 86, 582.

Loeb, L., (1898) Arch. Entwicklungsmech. Organ., 6, 297.

Loeb, L., (1920) 7. Med. Research, 4I, 247.

Lombardo, C., (1907) Giorn. ital. malat. veneree ex. pelle, 48, 448.

Lombardo, C., (1934) Giorn. ital. dermatol. e sifilol., 75, 185.

Loofbourow, J. R. (1947) Biochem. 7., 4I, i 19.

Loofbourow, J. R., (1948) Growth, I2, 77.

Loofbourow, J. R. And C. M. Dwyer, (1939) Nature, I43, 725.

Loofbourow, J. R. and M. N. Morgan, (1940) F. Bacteriol., 39, 437. 
Loofbourow, J. R., E. S. Cook, S. M. Dwyer and M. J. Hart, (1939a) Nature, I44, 553.

Loofbourow, J. R., A. A. Gueto and M. M. Lane, (1939b) Arch. exptl. Zellforsch. Gewebezücht., 22, 607 .

Loofbourow, J. R., S. Oppenheim-Errera, D. G. Loofbourow and C. A. Yeats, (i947) Biochem. J., 4 I, 122.

Loofbourow, J. R., A. M. Webb, D. G. Loofbourow and R. K. Abramowitz, (1942) Biochem. F., 36, 5 I 3 .

Lorin-Epstein, M.J., (1927) Arch. klin. Chir. Langenbecks, I44, 632.

Lumière, A., (I9I7) Rev.chir. (Paris) 53, 656.

Lumière, A., (1918a) Rev.chir. (Paris), 54, I68.

Lumière, A., (1918b) Rev. chir. (Paris), 55, 549.

Mallory, F. B., (1903) 7. Med. Research, 10, 334.

Mancini, R. E., (I948) Anat. Record, IoI, I 49.

Mancini, R. E. and E. S. De Lustig, (1950) Rev. soc. arg. biol., 26, 234.

Mann, I., (1944) Brit. 7. Ophthalmol., 28, 26.

Marchand, F. J., (I go I) Der Prozess der Wundheilung mit Einschluss der Transplantation, XVI,

F. Enke, Stuttgart, Germany, $528 \mathrm{pp}$.

Marchant, J., (1949) 7. Anat., 83, 227.

Marshall, A. H. E., (1946) J. Pathol. Bacteriol., 58, 729.

Marshali, A. H. E., (1948) J. Pathol. Bacteriol., 6o, 5 I 5 .

Marshall, A. H. E., (1953) 7. Pathol. Bacteriol., 65, 29, 48.

Martin, B. F. and F. Jacoby, (1950) F. Anat., 84, 6 I.

Matoltsy, A. G., (1955) Anat. Record, I22, $58 \mathrm{I}$.

Maximow, A., (1902) Experimentelle Untersuchungen über die entzündliche Neubildung von Bindegewebe, G. Fischer, Jena.

Maximow, A., (1928a) Proc. Soc. Exptl. Biol. Med., 25, 439.

Maximow, A., (1928b) Arch. Exptl. Zellforsch. Gewebezücht., 5, I69.

Maximow, A., (1929) Zentr. allgem. Pathol. u. pathol. Anat., 43, I 45.

Maximow, A. And W. Bloom, (1929) Z. mikroskop-anat. Forsch., I7, 625.

McKinney, R. L., (1929) Arch. Exptl. Zellforsch. Gewebezücht., 9, 14.

Malean, D. W. and O. G. Fais, (1952) 7. Urol., 68, igo.

McManus, J. F. A., (1957) cited in G. Asboe-Hansen, Histochemistry of Connective Tissue, Connective Tissue in Health and Disease, Philosophical Library, New York, p. $3 \mathrm{I}$.

McMinn, R. M. H. and F. R. Johnson, (I957) Brit. J. Surg., 45, 76.

Medawar, P. B., (1937) Quart. 7. Exptl. Physiol., 27, 147.

Medawar, P. B., (1945) Brit. Med. Bull., 3, 70.

Medawar, P. B., (1947) Quart. 7. Microscop. Sci., 88, 27.

Medawar, P. B., (1953) Quart. 7. Microscop. Sci., 94, 48I.

Menkin, V., (1940) Dynamics of Inflammation, MacMillan and Co., New York.

Menkin, V., (194I) Cancer Research, I, 548.

Menkin, V., (1953) The Mechanism of Inflammation, Edited by G. Jasmin and A. Robert, Montreal, Acta Inc., 137.

Menkin, V., (1955) Proc. Am. Assoc. Cancer Research, 2, 34.

Menkin, V., (1956) Biological Mechanisms of Inflammation, C. C. Thomas, Springfield, Illinois, p. 3 I6.

Mescon, H., (1950) 7. Natl. Cancer Inst., io, 1365.

Meyer, K., (i947) Physiol. Revs., 27, 335.

Meyer, K. and E. Chaffee, (I941) F. Biol. Chem., I38, 49 I.

Meyer, K., E. Chaffee, G. L. Hobby and M. H. Dawson, (1938) 7. Exptl. Med., 73, 309.

Minervini, H., (1904) Arch. path. Anat. u. Physiol., Virchow's, 175, 238.

Montagna, W., (1952) Intern. Rev. Cytol., $1,265$.

Montagna, W., H. B. Ghase and J. B. Hamilton, (195I) F. Invest. Dermatol., I7, 147.

Montagna, W. and W. G. Lobitz, (I952) Anat. Record, II4, 23 I.

Montagna, W. and C. R. Hill, (1957) Anat. Record, 127, I63.

Moog, F., (1944) Biol. Bull., 86, 5 I.

Moretti, G. and H. Mescon, (1956) 7. Invest. Dermatol., 26, 347. 
Morgan, J. F. and H. J. Morton, (1955) Federation Proc., I4, 257.

Morris, H. P., G. S. Dubrik and T. B. Dunn, (1945) J. Natl. Cancer Inst., 5, 27 I.

Munro, H. N. and M. I. Chalmers, (1945) Brit. F. Exptl. Pathol., 26, 396.

Nageotte, J. And L. Guyon, (1934) Compt. rend. assoc. anat., 29, 408.

Nakamura, T., (1930) Compt. rend. soc. biol., io4, $19 \mathrm{I}$.

Needham, A. E., (1952) Regeneration and Wound Healing, Methuen, London.

Nelson, C. A. And C. D. Dennis, (1951) Surg. Gynecol. Obstet., 93, 46 I.

Nielsen, E., (1939) Ugeskrift Laeger, 37, 107 I.

Noback, C. R. and W. Montagna, (1947) Cancer Research, 7, 798.

Nolte, A., (1947) Naturforsch., 2B, 295.

Oppel, A., (I9I2) Arch. Entwicklungsmech. Organ., 35, 37 I.

Oppel, A., (1913) Anat. Anz., 45, I73.

Orekhovich, V. N., A. A. Tustanovsky, K. D. Orekhovich and N. E. Plotnikova, (1948) Biokhimiya, I3, 55.

Osowski, H. E., (19I4) Arch. Entwicklungsmech. Organ., 38, 547.

Paget, J., (1853) Lectures on Surgical Pathology, I, 27I.

Palade, G. E., (I95 I) 7. Exptl. Med., 94, 535.

Parshley, M. S. And H. S. Simms, (1950) Am. F. Anat., 86, I63.

Pearce, R. H. and E. M. Watson, (I949) Can. F. Research, 27, 43.

Pearse, A. G. E., (1953) Histochemistry, Theoretical and Applied, Churchill Ltd., London, p. 224 .

Penny, J. R. and B. M. Balfour, (1949) F. Pathol. Bacteriol., 6i, i 7 I.

Pepper, F. J., (1954) J. Morphol., 95, 47 I.

Persson, B. H., (1953) Acta Soc. Med. Upsaliensis, 58, 1.

Peters, A., (1885) Inaug. Diss. Univ. of Bonn, R. Georgi, Bonn, 32 pp.

Peters, R. A., (1945) Brit. Med. Bull., 3, 8I.

Pirilä, V. And O. Eränkö, (1950) Acta Pathol. Microbiol. Scand., 27, 650.

Porter, K. R. and R. Vanamee, (1949) Proc. Soc. Exptl. Biol. Med., 7I, 5 I3.

Porter, K. R. and C. V. Z. Hawn, (1949) 7. Exptl. Med., 90, 225.

Porter, K. R., (I95I) Conn. Tissues, 2, I 26.

Porter, K. R., (1954) Anat. Record, I18, 433.

Poynter, C. W. M., (I9I9) Anat. Record, I6, I.

Rand, H. W., (1905) Arch. Entwicklungsmech. Organ., 19, I6.

Riley, J. F. and J. M. Drennan, (1949) 7. Pathol. Bacteriol., 6I, 245.

Robertson, W., F. W. Dunihue and A. B. Novikoff, (I950) Brit. F. Exptl. Pathol., 3I, 545.

Rodova, H., (1948) 7. Anat., 82, 175.

Rothman, S., (1954) Physiology and Biochemistry of the Skin, Univ. of Chicago Press, Chicago, Ill.

Roulet, F., (I926) Compt. rend. soc. biol., 95, I340.

Rudall, K. M., (1952) Advances in Protein Chem., 7, 253.

Ruth, E. S., (I9II) 7. Exptl. Med., 13, 422.

Sandblom, P., (1944) Acta Chir. Scand., Suppl., 89.

Sandblom, P., (1949) Ann. Surg., 129, 305.

Sandblom, P. And A. Muren, (1954) Ann. Surg., 140, 449.

Sandblom, P., P. Petersen and A. Muren, (I953) Acta Chir. Scand., 105, 252.

Sanders, H. R. and C. J. Schein, (1956) F. Urol, 75, 659.

Sandison, J. C., (1928) Am. F. Anat., 4I, 447.

Sandison, J. C., (I931) Am. 7. Anat., 5o, 355.

Santesson, L., (1935) Acta Pathol. Microbiol. Scand., Suppl., 24.

Savlov, E. D. And J. E. Dunphy, (1954a) Surgery, 36, 362.

Savlov, E. D. ANd J. E. Dunphy, (1954b) Nere Engl. F. Med., 250, 1062.

Schloss, W., (1928) Arch. klin. Chir., Langenbecks, I4I, 701.

Scothorne, R. J. And A. W. Scothorne, (1953) F. Anat., 87, 22.

Scothorne, R. J. And J. S. Tough, (1952) Brit. F. Plastic Surg., 5, 16r.

Selby, C. C., (1955) 7. Biophys. Biochem. Cytol., I, 429.

Seligman, A. M. And L. H. Manheimer, (1949) F. Natl. Cancer Inst., 9, 427. 
Skoog, T., (1948) Acta Chir. Scand., 96, Suppl., I39.

SPAIN, K. C., (1915) 7. Exptl. Med., 21, I93.

Spain, K. C. and L. Loeb, (1916) F. Exptl. Med., 23; 107.

Spallanzani, ( 1787 ) Quoted by L. Du Noüy, Biological Time, London, Methuen, 1936.

Staemmler, M., (1921) Z. Frankfurt. Pathol., 25, 39 I.

Stearns, M. L., (r939) Anat. Record., 73, 49.

Stearns, M. L., (ig40a) Am. F. Anat., 66, i33.

Stearns, M. L., (ig4ob) Am. F. Anat., 67, 55.

Stowell, R. E., (1942) 7. Natl. Cancer Inst., 3, I I I.

Stowell, R. E., (1947) Symposia Soc. Exptl. Biol., Univ. Press, Cambridge, p. I9o.

Stowell, R. E. And Z. K. Cooper, (I945) Cancer Research, 5, 295.

Suntzowa, W., (1944) Compt. rend. acad. sci. U.R.S.S., 45, I33.

Susakaina, M., (I92 I) Arch. Dermatol. Syphilisa, I34, 418.

Sylvén, B., (1941) Acta Chir. Scand., 86, Suppl. 66, I.

Taffel, M. And S. C. Harvey, (1939) Proc. Soc. Exptl. Biol. Med., 38 , 5 I 8.

Taffel, M., A. J. Donovan and L. S. Lapinski, (1950) Tale 7. Biol. and Med., 23, 482.

Taffier, T. and R. Desmarres, (I918) 7. Exptl. Med., 27, i65.

Tarver, H. and C. L. A. Schmidt, (I939) 7. Biol. Chem., I30, 67.

Taylor, H. E. and A. M. Saunders, (1957) Am. 7. Pathol., 33, 525.

Thompson, D. A. W., (1942) On Growth and Form, Cambridge Univ. Press, Cambridge.

Thompson, W. D., I. S. Ravdin and I. L. Frank, (1938) Arch. Surg., 36, 500.

Tustanovsky, A. A., (1947) Biokhimiya, I2, 285.

Udupa, K. N., J. F. Woessner and J. E. Dunphy, (1956) Surg. Gynecol. Obstet., io2, 639.

Unna, P. G., (I926) Histochemie der Haut, Leipzig, Germany.

Unna, P. G. And L. Golodetz, (1909) Biochem. Z., 20, 469.

Uhlenhuth, E., (1914) J. Exptl. Med., 20,614.

Upton, A. C. And T. T. Odell, (I956) A. M. A. Arch. Pathol., 62, i94.

Upton, A. C., T. T. Odell and W. D. Gude, (1955) Federation Proc., I4, 421.

Vanamee, P. and K. R. Porter, (I951) F. Exptl. Med., 94, 255.

VAn den Brenk, H. A. S., (1956) Brit. J. Surg., 43, 525.

VArco, R. L., (1947) Surg. Gynecol. Obstet., 84, 6 I I.

Vogt, W., (1925) Arch. Entwicklungsmech. Organ., Io6, 542.

Von Bardeleben, H., (I901) Arch. pathol. Anat. u. Physiol., Virchow's, 163, 497.

Wallich, R., (1926) Compt. rend. soc. biol., 95, 1480.

Walton, K. W. and C. R. Ricketts, (1954) Brit. F. Exptl. Pathol., 35, 227.

Washburn, W. W., (1954a) Plast. Reconstr. Surg., I4, 393.

Washburn, W. W., (1954b) 7. Invest. Dermatol., 23, 79.

Washburn, W. W., (1954c) F. Invest. Dermatol., 23, 169.

Washburn, W. W., (1955) J. Invest. Dermatol., 24, 537.

Washburn, W. W., (1957) Unpublished data.

Waugh, W. G., (1940) Brit. Med. F., I, 249.

Webb, A. M. and J. R. Loofbourow, (1947) Biochem. J., 4I, I I4.

Weber, H. H., (1955) Fibrous Proteins and their Biological Significance, Symposia Soc. Exptl.

Biol., Ed. R. Brown and J. R. Danielli, Academic Press Inc., New York, p. 271.

Wegelins, O. And G. Asboe-Hansen, (1956) Exptl. Cell Research, II, 437.

Weiss, P., (1929) Arch. Entwicklungsmech. Organ., II6, 438.

Weiss, P., (1933) Am. Naturalist, 67, 322.

Weiss, P., (1945) 7. Exptl. Zool., ioo, 353.

Weiss, P., (1947) Tale 7. Biol. Med., 19, 235.

Weiss, P., (1949a) in Chemistry and Physiology of Growth, Princeton Univ. Press, Princeton, New Jersey.

Weiss, P., (1949b) Exptl. Cell Research, Suppl. I, 475.

Weiss, P., (1950) Quart. Rev. Biol., 25, I 77.

Weiss, P., (1955) in Biological Specificity and Growth, Ed. E. Butler, Princeton Univ. Press, Princeton, New Jersey, p. 195.

Weiss, P. And W. Ferris, (1954a) Proc. Nall. Acad. Sci. U. S., 4o, 528. 
Weiss, P. And W. Ferris (1954b) Exptl. Cell Research, 6, 546.

Werner, R., (1902) Bruns' Beitr. Klin. Chir., 34, I.

Wichmann, B. E., (1955) Acta Pathol. Microbiol. Scand., Suppl., ro8.

Wiesner, J., (1892) Elementarstructur und das Wachstum der lebenden Substanz, A. Holder, Vienna, p. 102.

Williams, R. G., (1954) Intern. Rev. Cytol., 3, 359.

Williams, R. W., L. B. Mason and H. H. Bradshaw, (I95I) Surg. Forum Clin. Congr. Am. Coll. Surg., W. B. Saunders, Philadelphia, p. 536.

Williamson, M. B., (1956) Clin. Chem., 2, I.

Williamson, M. B. And H. J. Fromm, (1952) Proc. Soc. Exptl. Biol. Med., 8o, 623.

Williamson, M. B. and H. J. Fromm, (I953a) Federation Proc., I2, 29 I.

Williamson, M. B. And H. J. Fromm, (1953b) Proc. Soc. Exptl. Biol. Med., 83, 329.

Williamson, M. B. and H. J. Fromm, (1954a) Federation Proc., 13, 322.

Williamson, M. B. and H. J. Fromm, (1954b) Proc. Soc. Exptl. Biol. Med., 87, 366.

Williamson, M. B. and H. J. Fromm, (I955) 7. Biol. Chem., 2I2, 705.

Williamson, M. B., T. H. McCarthy and H.J. Fromm, (i95ia) Proc. Soc. Exptl. Biol. Med., $77,302$.

Williamson, M. B., T. H. McCarthy and H. J. Fromm, (195 I b) Federation Proc, 10, 270.

Willmer, E. N., (I942) J. Exptl. Biol., I9, II.

Willmer, E. N. and L. P. Kendal, (1932) F. Exptl. Biol., 9, i 49.

Wohnlich, H., (1948) Arch. Dermatol. Syphilis, I87, 53.

Wohnlich, H., (1949) Arch. Dermatol. Syphilis, I88, I.

Wolbach, S. B., (1933) Am. J. Path., 9, 689.

Wolbach, S. B. And P. Howe, (1926) A. M. A. Arch. Pathol., I, I.

Wolfer, J. A., C. J. Farmer, W. W. Carroll and D. O. Manshardt, (1947) Surg. Gynecol. Obstet., 84, I.

Young, J. S., J. A. Fisher and M. Young, (1941) 7. Pathol. Bacteriol., 52, 225. 


\title{
Chapter 9
}

\section{NITROGEN METABOLISM AND GROWTH}

\author{
Hugh Clark
}

\section{INTRODUCTION}

The adult metazoan organism is an integrated composite of a great variety of cell types, similar in some respects but different in morphological and physiological qualities which identify them. One feature of individual distinctions is the complement of peculiar proteins, both enzymatic and nonenzymatic, which contribute in large measure to the identifiable individuality of the cell.

It is our purpose to examine the available data which describe, with respect to proteins, (I) the nature of the egg and sperm, (2) the pattern of acquiring diverse structural and enzymatic peculiarities, (3) where evidence is at hand, the mechanisms involved, including particularly energy requirements, and (4) a statement of residual problems. It should be unnecessary to add that details of all aspects of protein metabolism in all stages of development cannot be pursued with equal fidelity.

The author has included comprehensive and review papers in areas which have been particularly active and has cited original contributions which are germane to the main theme of the present review. Limitation of space forbids citation of all pertinent material and perhaps has resulted in serious omissions. It is hoped, however, that the most significant contributions and the most effective techniques of study have not been overlooked. For purposes of orientation, two aspects of the problem will be defined at some length, (A) growth and development, and (B) metabolism of nitrogen compounds.

\section{(a) Growth and development}

Phenomena of growth have appealed to the biologist since, and perhaps before, the dawn of recorded observations. The observations are varied and, certainly in many details, conflicting. The reasons are summarized by Weiss (I949): "Growth is not a simple and unitary phenomenon. Growth is a word, a term, a notion, covering a variety of diverse and complex phenomena. It is not even a scientific term with defined and constant meaning, but a popular label that varies with the accidental traditions, predilections and purposes of the individual or school using it. It has come to connote all and any of these: reproduction, increase in dimensions, linear increase, gain in weight, gain in organic mass, cell multiplication, mitosis, cell migration, protein synthesis and perhaps more." Earlier in the same paper Weiss proposes to "define growth in a more restricted sense, as the increase in that part of a molecular population of an organic system which is synthesized in that system". But even this refinement of concept does not eliminate the phenomena of 
chemical differentiation, or indeed, maintenance of the developing organism. A brief general outline of developmental processes is set down for purposes of orientation.

Development is an integrated complex of activities which are paradoxically meaningful and ill-defined: growth, differentiation and maintenance. Although each term resists precise definition, the superficial meaning of each is clear. Growth ordinarily refers to increase in mass of the organism or of some constituent selected for immediate consideration. Differentiation implies progressive embryonic modification, usually in a manner directed toward acquisition of adult form and function. Maintenance is the sum of basic metabolic activities at any instant, construed in such a way that growth and differentiation are excluded.

The last requirement, exclusion of growth and differentiation from purely maintenance activities, imposes the burden of impossibility on the problem of dissociation of the three fundamental developmental processes, at least at the present time. Schoenheimer (1942), whose work with isotopes of normal body constituents contributed so fruitfully to an understanding of metabolic processes, pointed out: "In general, every regeneration reaction involving an increase in free energy must be coupled with another process." More recent investigations of cellular physiology support and amplify the thesis of related chemical events within the cell. Great strides have been made in the understanding of cytochemistry, cyclic reactions, alternate metabolic pathways, relation of metabolic phenomena to the fine structure of the cell and many other aspects of protoplasmic behavior. Yet this continues to be one of the most active areas of inquiry in the field of biology. The volume and urgency of research in intracellular chemistry and physiology plainly describes the inadequacy of our understanding of the full meaning of maintenance metabolism. Confronted, then, with an incomplete picture of maintenance in adult cells, it would be patently premature to separate definitively the energy and mechanisms of maintenance in embryonic cells from those of growth or differentiation. Dissociability of the three phenomena requires distinction between mechanisms for provision of energy for work designated as growth and for work labeled differentiation. Let us suppose that synthesis of a protein initially, since it is new, is specified as a process of differentiation; repetition of the process, resulting in increase in quantity of the same protein, would then be considered growth. Allocation of energy to the one category or the other would be delicate at best. Continued submolecular modifications, certainly energy-requiring processes, would, however, be classified as maintenance. Attempts to discriminate between the processes, as reviewed by Needham (1942), have met with only partial and questionable success. When observations are interpreted as evidence for dissociation of growth from differentiation (Spratt, 1948) or maintenance from growth and differentiation (Boell and Poulson, I939; Moog, I944; Barth, 1946) the assumption is made that the defective embryo is normal, i.e. that it carries on metabolic activities unprejudiced by induced deficiencies. Such an assumption would appear to be unwarranted, in view of the subsequent fate of the embryos. Boell (1955) marshals rather cogent arguments for caution in interpretation of such experiments.

Against the background embodied in the foregoing statement rests the decision to refrain from parceling out the several facets of nitrogen metabolism, but rather to apply them to the totality of development, except in those rare instances when the developmental relevance of an observation can be pin-pointed. This aim requires a working interpretation of growth and differentiation.

Increase in organic mass results from multiplication of, or addition to, protoplasmic components. Let us consider the second item first. Some of the increase may result from the process of osmosis or diffusion through the limiting membrane of the cell; whether the accumulated substance be water or other diffusible molecule or ion, the cell membrane is selective. Selection and concentration, hence restriction as well, signifies work, and performance of work requires an energy source. Though the selective permeability of the 
cell membrane is a well known phenomenon whose analysis has resulted in a new concept of membrane physiology, namely active transport $(c f$. Symposium of Society for Experimental Biol., No. VIII, Active Transport and Secretion 1954) the problem with respect to embryonic development is more complex. Flexner and Flexner (1948, 1949) have shown that the apparent capacities of membranes of liver cells and of brain cells in the guinea pig change sharply at 42 days gestation age. The change may be due to structural modification of the membrane, though this is unlikely since their criteria include $\mathrm{Cl}^{-}$concentration and $\mathrm{Na}^{+}$concentration; it would appear that more probably the alteration of membrane selectivity is the result of acquisition of new mechanisms within the cells for altering concentration of the measured substances and energy necessary for doing it. Concentration of metabolites within the confines of a cell may be interpreted not only as evidence for modified membrane activity but also as the antecedent of further developmental phenomena, such as differential increase in enzyme activity. Adaptive enzyme formation is well established in microorganisms, though controversial in details (Spiegelman, 1948); but Gordon and Roder (1953) have supplied evidence for a similar phenomenon in development of the chick. They speculate that "it is not inconceivable that the known variations in the substrates on metabolites of the embryo, both qualitative and quantitative, are fundamental to and the cause of the variations in enzyme activity." Their viewpoint is supported by Jones, Featherstone and Bonting (1956) who demonstrated in vitro a stabilizing effect of added acetylcholine upon cholinesterase. It would hardly seem to be coincidence that, concomitant with acquisition of greater selectivity by liver and brain cells (Flexner and Flexner, 1948, 1949) is the appearance of new functional activities of these organs. It is too soon to generalize the applicability of the premise, but such "selective" growth should be given consideration as a means of promoting new activities in embryonic cells.

In addition to this aspect of cellular growth and its possible consequences, increase in organic mass requires the synthesis of complex molecules. Of special interest is the synthesis of structural proteins and enzymes. These problems will be considered in some detail below.

In passing, we must make note of the fact, however, that in metazoan animals the variety of processes of cellular growth is increased by cell division. Cell division is a mechanism for growth in metazoan animals (perhaps the result of growth in unicellular animals). Two exceptions will be noted. The earliest divisions of the egg, encompassing the period of cleavage, do not ordinarily result in a significant increase in total mass; cell division, in addition to providing for increase in mass in later development, provides also the basis for structural and functional diversity. Duplication of all parts of a cell, then, in later development is not always precise; or alternatively, if precise, requires during the intermitotic period of a cell alteration of its substance, and hence that of its progeny. The mechanisms responsible for perpetuation of identical protoplasmic components are those of growth; the mechanisms responsible for diversification are those of differentiation.

Differentiation asserts itself at the molecular and cellular level, but the consequences are more elaborate. The peculiar aptitudes conferred upon cells by their intrinsic qualities manifest themselves in a variety of ways (Weiss, i953a): migration, response to influence of their neighbors, secretory abilities, capacity to multiply and in the many specialized functions recognizable in adult tissues.

In summary, development consists of the epigenetic elaboration of the potential of the egg through mitosis, accretion, synthesis, differentiation, differential growth and migration of cells or cell aggregates. We shall be primarily concerned with 
events at the cellular level since it is here that metabolism of nitrogen compounds must be observed. Applications of nitrogen metabolism to higher levels of organization are not wanting, however, and these will be reported in their proper place. Ordinarily such application is invoked in terms of energy supply for developmental work. Proteins are involved as an energy source in two ways: first, by hydrolysis, deamination of constituent amino acids and oxidation of the carbohydrate skeleton, and secondly as agents in formation and use of high energy phosphate bonds (Barth and Barth, I954). With regard to the latter possibility, however, Lovtrup (1953) by rather conclusive calculations demonstrates that amphibian yolk phosphoproteins could supply not more than $0.15 \%$ of the required energy.

Of paramount importance in the concept of developmental phenomena is the organismic nature of the embryo. The processes concerned with growth, differentiation and maintenance are indissolubly intertwined. These, in turn, cannot be isolated from their environment; no embryo, even cleidoic, constitutes a closed thermodynamic system. Each has a direct, continuing and varying relationship with its surroundings in the present, and each has, through the germ line an extension into the past. Recent serological studies, which identify antigens of the egg with those of the adult and of the developing embryo, assign to the latter premise a tangible significance.

\section{(b) Metabolism of nitrogen compounds}

Nitrogen metabolism must include, because it constitutes prima facie evidence, excretion of nitrogenous waste. This implies a degradation of proteins, deamination and a failure of transamination or transamidation into protoplasmic nitrogen compounds of continuing service to the organism. As an immediate consequence ammonium salts are formed; these may be eliminated without change; free ammonia may be evolved, or, if the enzymatic machinery and the conditions necessary for its operation are present, the ammonium compounds may be diverted into more innocuous channels such as urea or uric acid formation (or in smaller quantities to other compounds). Nitrogen excretion may be construed as a measure of utilization of nitrogen compounds for purposes of providing energy for maintenance or development; it must, however, be inversely correlated with direct transformation of stored nitrogen compounds into the protoplasmic substance of the developing organism.

In addition, however, nitiogen metabolism must also include such anabolic processes as (a) synthesis of yolk, thus establishing a nutritional link between parent and offspring; (b) transfer of yolk protein to the embryo; $(c)$ synthesis of structural and enzymatic proteins of the embryo, with perhaps, a corollary of the acquisition of antigenic capacities; $(d)$ duplication of both nuclear and cytoplasmic components; $(e)$ factors related to the definitive structure of cell membranes and $(f)$ the functional interdependence of nucleus and cytoplasm.

Prior to I93I, as Needham's (I93I) magnificent compilation and synthesis clearly shows, chemical embryology leaned heavily on routine analyses for information regarding protein metabolism; such analyses, coupled with respiratory data constitute much of the basis for Needham's conclusions. Up to this time, 
and even later, embryologists were largely preoccupied with the infinite variety of questions related to the "organizer" and inductive phenomena. The methodology of these pursuits provided primarily information with regard to higher levels of organization: interrelationships between tissues, establishment of "fields" (Weiss, I939) and metabolic behavior of specific areas of the developing embryo (Child, I94I; Brachet, I950).

Although important work on behavior of tissues and larger cellular aggregates does, and must, continue, several factors have combined in focussing attention of embryologists on intracellular details during development. Among these must be included the search for the identity of "the organizer" of amphibia (see Brachet, $195^{\circ}$, for review) which inevitably led to the examination of intracellular behavior both in the inducing cells and in the responding cells; contemporaneously interest in physiological genetics grew rapidly (Goldschmidt, 1938) leading to pursuit of genic mechanisms for inheritance of metabolic patterns (see Wright, I94I; Beadle, I946; Wagner and Mitchell, I955), cytoplasmic inheritance (Sonneborne, I950; Beale, I952; Caspari, I948, I956), specific application of genetic methods to development through study of phenocopies (Landauer, 1948, I 952a, I 953) and lethal mutations (Gluecksohn-Waelsch, I954, review; Hadorn, I 948, I956), hybridization (Moore, I950) and exchange of nuclei (Briggs and King, I 955).

Technical advances, as well as conceptual orientation have contributed to more critical evaluation of the biochemical and physiological interpretation of development. Among these may be included metabolic studies with radioactive and stable isotopes, serological techniques, microbiological assay, tissue culture, histochemical and cytochemical methods, electrophoresis, immunophoresis, paper chromatography and the use of metabolic inhibitors and analogs. As a result of these, often used in combinations of various sorts, a basic appreciation of the problems of development at the cellular level is emerging.

The topic is complex; the investigative methods are varied; where interrelationships seem conclusive, we shall insist upon them; where discordant results cannot be reconciled we shall point them out. The delicately balanced and integrated systems of the developing organism will countenance few incongruities; their existence in the data, therefore, writes boldly the advice of unfinished business for the scientist.

To coordinate, in so far as possible, traditional morphological observations with metabolism of nitrogen compounds, the data will be presented with reference to gametogenesis and fertilization, cleavage and blastulation, gastrulation and organogenesis. The fact that certain of the data have a meaning which transcends artificial subdivisions of a continuous, organized process is expected; the difficulty 1, f reconciling information pertaining to the same stage in different animals may simply point up the fact that the designation of stages is semantically convenient, but biologically accurate in only a superficial way. 
(a) Gametogenesis and fertilization

The mechanics of fertilization are a perennially active field of inquiry. Several features of the process (approach of the sperm, attachment and penetration, responses of the egg) have been reviewed by Tyler (1955). He discusses four categories of substances related to the process of fertilization: fertilizins, antifertilizins from eggs, antifertilizins from sperm and lytic agents from sperm. Evidence is presented for the glycoprotein nature of fertilizin of Strongylocentrotus purpuratus, and for protein structure of antifertilizins of egg and sperm. Related to the gelatinous covering of fresh water and marine eggs and the cellular corona radiata of mammalian eggs is the presence of a lytic agent in the sperm. That too, both in aquatic forms, where investigated, and in mammalian sperm is protein in nature. The latter, because of its ability to effect the breakdown of hyaluronic acid is a hyaluronidase, one of a group of enzymes which act on intercellular binding materials in other situations as well (see Meyer and Rapport, I952). Rünnstrom (I 952) discusses the problem of fertilization principally from the point of view of the response of the egg.

Though it is known that sperm penetration is a trigger for completion of the

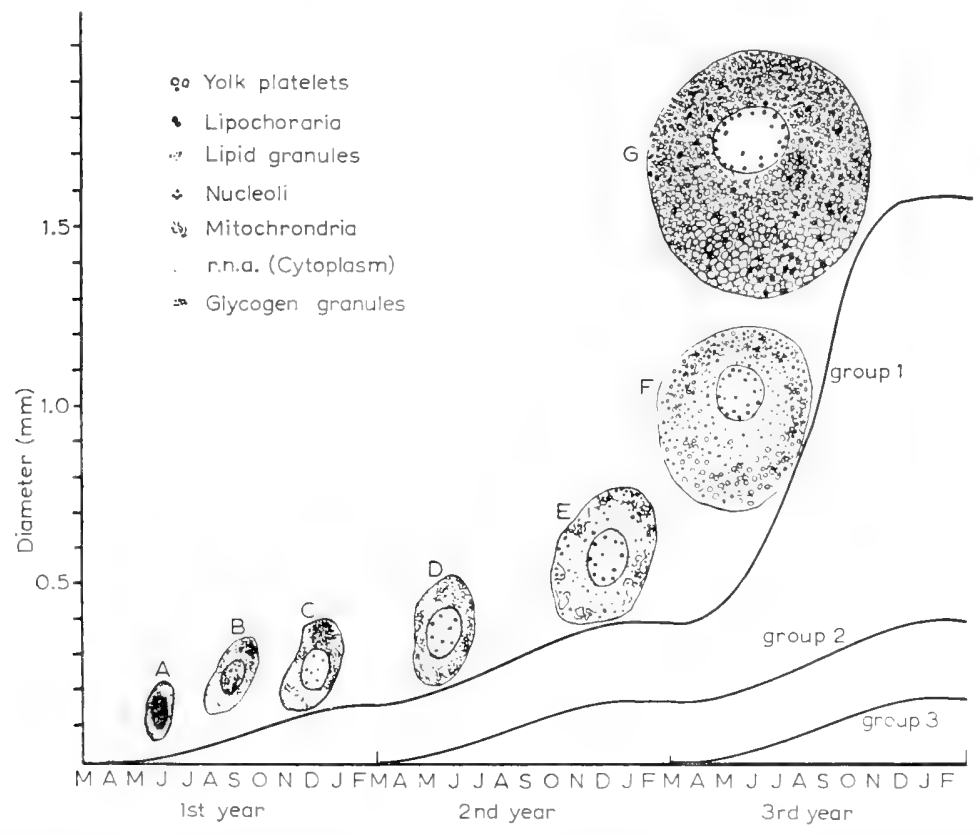

Fig. I. The cytological pattern for the seasonal growth of the oocyte of Rana temporaria, a European species of frog which requires three years to reach sexual maturity. During the first two years, the oocytes increase from $50 \mu$ to $300 \mu$ in diameter (A-E); synthetic activity occurs in the region of the nucleus but the newly formed products, lipid granules and mitochondria, move to the cortical area. During the third year (F, G) synthesis of yolk granules predominates; synthesis occurs principally in the cortical region and the products migrate toward the interior. Early provision of cytoplasm with mitochondria and RNA may point to their role in yolk synthesis, although other studies (e.g. Cooper, I 946, 1948, 1950) indicate incorporation of maternal proteins as well. (From Grant, 1953.) 
maturation of the ovum when incomplete, and for initiation of the cleavage process with attendant physiological changes, little is known of the chemistry involved. Lundblad (I950) reports strong but short-lasting proteolytic activity at fertilization in three species of sea urchin, disappearing after $30 \mathrm{~min}$. After $7 \mathrm{~h}$., proteolytic activity is resumed and continues to increase up to $30 \mathrm{~h}$. Lundblad's observation may be correlated with that of Rünnstrom (I949), who describes disintegration of cortical granules at the time of activation of the egg.

Because of the importance of the cortical and nutritional endowment of the egg, some attention is due the source of its dowry. Panijel (I950, I95 I) has followed the chemical development of the egg of the frog, Rana temporaria, in which he describes the early accumulation of RNA, glycogen and mitochondria and the final addition of yolk platelets. Grant (1953) has summarized Panijel's findings (Fig. I). The platelets are predominantly of two sizes (Panijel, I950): large $(35 \mu)$ which consist mainly of protein and lipid, and small $(2.0 \mu)$ containing relatively more RNA and enzymes. Similarly a mammalian egg has been followed through oogenesis (Alfert, I950); he concludes that, as far as the evidence goes, the ripe egg contains a store of proteins which is simply parceled out to the cells and nuclei resulting from the first few cleavage divisions.

Recently, Fauré-Frémiet, Ebel and Colas (I954) studied the accumulation of protein granules in Parascaris equorum, their migration to the surface and dispersal in the perivitelline space. They conclude that they constitute a source of nourishment for the developing embryo. This view differs from the observations of Panijel (I95I) who asserts that the sperm contributes nutritional material of a protein nature; however, he does not extend this conclusion to other invertebrates.

The chemical growth of the hen's egg has been more extensively studied both by chemical analysis and by experiment. Romanoff and Romanoff (1949) tabulate the average quantities of several groups of constituents of the egg: protein content is approximately $\mathrm{I} 2.8 \%$ of the total dry matter which is $26.4 \%$ of the entire egg content. At the time of hatching, the oocyte cytoplasm consists of water $(86 \%)$ and protein, and growth of the oocyte consists principally of addition of these substances. Marza and Marza (1935) correlate the transformation of the follicular epithelium and of the vitelline membrane with yolk formation. They describe three growth phases characterized by the type of material included in the yolk: $(I)$ formation of a peripheral fatty layer (prehatching); (2) addition of protein and phosphatides (from hatching to about two months of age); (3) deposition of phospholipids and yolk protein, principally ovovitellin.

In the chicken egg, it is of some interest to note that prematurity of ovulation, induced by desiccated male pituitary, does not impede embryonic development. Neher and Fraps (1946) found fertilizable 47 out of 53 prematurely ovulated eggs; of the $47,3^{8}$ hatched. When the prematurity was $22 \mathrm{~h}$. or less, all hatched; but $30 \mathrm{~h}$. prematurity ( 2 cases) apparently prevented hatching, but both embryos lived for a substantial part of the incubation period (one near hatching). Perhaps these data merely confirm the expected, for the last $30 \mathrm{~h}$. of the period of egg formation would not alter greatly the egg contents.

The work of Hevesy (1947) and of Chargaff (I942) with ${ }^{32} \mathrm{P}$ establishes quite 
clearly, at least for phosphorus compounds, that the principal site of synthesis of yolk material is the liver of the hen; such materials are then transported by the blood and selectively absorbed by the ovarian egg. An active role of the follicle cells and perhaps also of the vitelline membrane in the process is implicit in the failure of the uterine egg to incorporate ${ }^{32} \mathrm{P}$ into organic compounds. Telfer (1954) has demonstrated also that at least antigen 7 of the Cecropia silkworm is secreted into the blood by another tissue and is incorporated into the yolk by the ovary.

Kavanau (1954a), however, deduces from observations on amino acids of sea urchin eggs allowed to stand in sea water, that "the proteins of the egg are probably synthesized by the egg itself, the ovary merely supplying the free amino acids and small peptides". It is not possible to generalize along these lines for other isolecithal, nor indeed, for other invertebrate eggs.

The relationship between phosphatide deposition and protein deposition may be of functional significance; Smith (1952) feels that phosphatide fat of the trout may be important in transmission of protein from the yolk to the embryonic cells and "may be correlated with consumption of protein as an energy source".

The demonstration of adult proteins in the egg has created questions of two sorts: $(I)$ the method of utilization of the yolk proteins in embryo formation and (2) the extent to which adult proteins are represented in the yolk. Mystkowski (1936) examined the early chick embryo for cathepsin activity and found that the yolk sac activity was 15 to 20 times as great as that in the embryo proper. He was unable to demonstrate synthetic activity in vitro. He concludes with justifiable reserve that "the problem of utilization of egg proteins by the embryo is not yet satisfactorily solved". Goldstein and Guintsbourg (I 937) separated the embryo, yolk and each of the extraembryonic membranes and assayed extracts of each for proteolytic activity. With gelatin as a substrate, proteolytic activity is inexplicably low during the first days of incubation. It appears at 6-8 days with a maximum at $13^{-1} 4$ days.

Recently, Brierly and Hemmings (1956) demonstrated that hens actively immunized to Salmonella pullonum systematically incorporate antibodies in the yolk, so that the yolk titre is rather constantly in the ratio of $\mathrm{I}: 8$ to that of the adult serum. Not only is there a device for incorporating the antibodies into the yolk, but they are also selectively transported across the yolk sac membrane. Injection of fowl serum immune to $S$. pullorum or Brucella abortis gives rise to circulating titres in the embryo at hatching. Moreover, the yolk sac is selective, for immune rabbit, cow and horse sera do not give rise to circulating titres, and serum of the pigeon, though transmissible, has a lower titre. However, even homologous adult serum antibodies suffer extreme loss in the transmission, and the fate of the disappearing antibodies has not been ascertained.

Since the eutherian egg is essentially yolk-free, the question of incorporation of adult proteins into the egg is at once more difficult and less urgent. The work of Brambell and coworkers on transfer of large molecules to the embryo is, therefore, the more interesting. Rogers and Hemmings (1949) find that "the maternal plasma proteins pass in considerable quantities into the yolk sac cavity through the bilaminar omphalopleur of rabbit embryos of the $7^{\text {th }}$ and 8 th days post-coitum, that they retain their identity while doing so, and that there is 
convincing evidence of differential permeability of the membrane to the various kinds of protein involved". Examination of the blastocyst fluid showed albumen, $\alpha$-, $\beta$ - and $\gamma$-globulins in approximately the same proportions as in adult plasma. It was demonstrated that antibodies to Brucella abortus passed from the mother to the yolk sac cavity (Brambell, Hemmings and Rowlands, I948), and further (Brambell et al., I949) that "the allanto-chorionic placenta is an effective barrier to the passage of agglutinins in many, and probably in all, embryos". The absorption through the omphalomesenteric circulation and passage thence into the yolk sac cavity, they suggest, offers an experimental means of testing the introduction of proteins into the embryonic circulation without interfering with the maternal circulation. Brambell, Hemmings and Henderson (195I) have summarized their own as well as other pertinent studies and discussed the implications of the work.

Ranzi ( $195^{\mathrm{I}}$ ) describes the presence of fibrillar proteins (euglobulin a and b), a globular protein (euglobulin c) and a pseudoglobulin, having a folded fibrillar shape, in the eggs of sea urchins, fowl and frog eggs. The similarity of form of protein molecules in the three diverse forms is of interest, but of more pertinence is the identity of at least certain of the yolk proteins with those of the mother.

The serological similarity between proteins of the egg and serum seems well established (Schechtman, I947; Nace and Schechtman, 1948; Schechtman and Hoffman, I 952).

Nace (I953) found that livetin of yolk contains components representing each of the adult serum fractions and that each adult serum fraction contains yolk-like constituents. Antigenic determinants resembling those of serum albumin can be detected on or before the fifth day, those of $\alpha$ and $\beta$-globulins about the sixth day and those of $\gamma$-globulin between the ninth and twelfth day. "Just prior to the appearance of these constituents a vitelloid (yolk-like) antigen resembling each of them can be detected. It is suggested that the protein of the yolk forms a source of serum protein available to the embryo until its own synthetic mechanism is established, and secondly that such protein, originally derived intact from the maternal organism, may provide models (templates) for serum protein synthesis by the embryo." Independently Ranzi (I95I) identified at 6 days the appearance of a globular protein soluble in o. I $M \mathrm{KCl}$ and after the 9 th day a large synthesis of fibrillar or folded fibrillar proteins. These may be the same elements identified serologically by Nace (I953). Clayton (I 953) studied the same phenomenon in the newt and observed that "some adult antigens may be found as early as in the fertilized egg, and some of these may have been directly derived from the maternal organism".

Cooper (1946) found some evidence for identity of antigens of the egg, embryo and adult serum of Rana pipiens. Earlier literature (reviewed by Cooper) appeared to be negative or conflicting in this regard; the difficulties, she feels, were technical. When the technique of absorption by adult serum was used, saline extracts of developmental stages "failed to react or had lower titres in ring tests with anti-frog brain sera absorbed with frog serum than with anti-frog brain sera correspondingly diluted but unabsorbed". In a later paper (1948) Cooper is more positive: "eggs and young embryos certainly contain molecules capable of 
in ting the formation of antibodies, and although not all adult antigens and haptens are demonstrable in the egg by the usual techniques, at least some antigens (or their combining groups) of the egg are identical with or closely related to those of the adult." The discussion in this paper is particularly useful for review of problems concerning molecular differentiation as well as identity in successive stages of development. Using the Oudin (1948) technique, Cooper (1950) identified "5-7 antigens in the egg and neural plate stages which are represented by substances with immunologically related combining groups in adult frog serum".

In spite of the precision of antigen-antibody reactions, caution still must be exercised with regard to conclusions of serological identity of maternal and yolk proteins. Fevold (I95I) in a comprehensive review states that "no protein of egg yolk has been shown to have been isolated in pure form by the criteria currently applied". He describes the composition of vitellin, livetin and phosvitin, as well as other egg proteins, and points out the fact that livetin apparently contains the majority of enzymes of the egg. Lineweaver et al. (I 947) associated tributyrinase, esterase, amylase, peptidase, phosphatase and catalase activity with livetin.

\section{(b) Cleavage and blastulation}

During the period of cleavage, growth is slight. Protein metabolism studies may, therefore, be of interest in a negative sense. Brachet (I939) observed only a slight nitrogen excretion in the form of ammonia and urea through the neurulation stage (amphibia), and felt that this observation corroborated those of Bialaszewicz and Mincowna (192I). The fact that ammonia excretion increased under anaerobic conditions, he felt, favored the hypothesis that ammonia might be used in the presence of oxygen for synthesis of amino acids and proteins. Barth and Barth ( 1954) point out that "cleavage and blastulation of the frog egg are accompanied by a steadily increasing rate of respiration. As yet there is no conclusive evidence as to the nature of the compound oxidized. Direct chemical analyses of the lipid, carbohydrate and protein show no significant changes between fertilization and the beginning of gastrulation". They further conclude from analysis of the extensive study of Gregg and Ballentine (1946) that, since only traces of ammonia and urea are present, only traces of protein catabolism can occur. It should be added, however, that Gregg and Ballentine did not study excreta from the embryos, and much of the cogency of the Barths' position is thereby nullified. A similar confusion exists from the data of Wills (1936) which show, for different species of amphibia, a range from - $12.5 \%$ to $+13.3 \%$ in total nitrogen of the gastrula as compared with the egg.

In the sea urchin, careful studies have been made and indicate a somewhat greater activity of the nitrogen compounds. Kavanau (1953) describes a loss in protein nitrogen of $15.7 \%$ and of nonprotein nitrogen of $29.1 \%$ (See Fig. 2). The loss of total amino-acid nitrogen $(18.7 \%$ ) represents a conversion to other nitrogen fractions as well as combustion for energy. In the former connection, Abrams (I95I) confirmed conversion of glycine to purines. Similarly Gustafson and Hjelte ( $195 \mathrm{I}$ ) reported a conversion of $5.7 \%$ amino-acid nitrogen into other fractions prior to the $\mathrm{I} 8 \mathrm{-h}$. stage. 


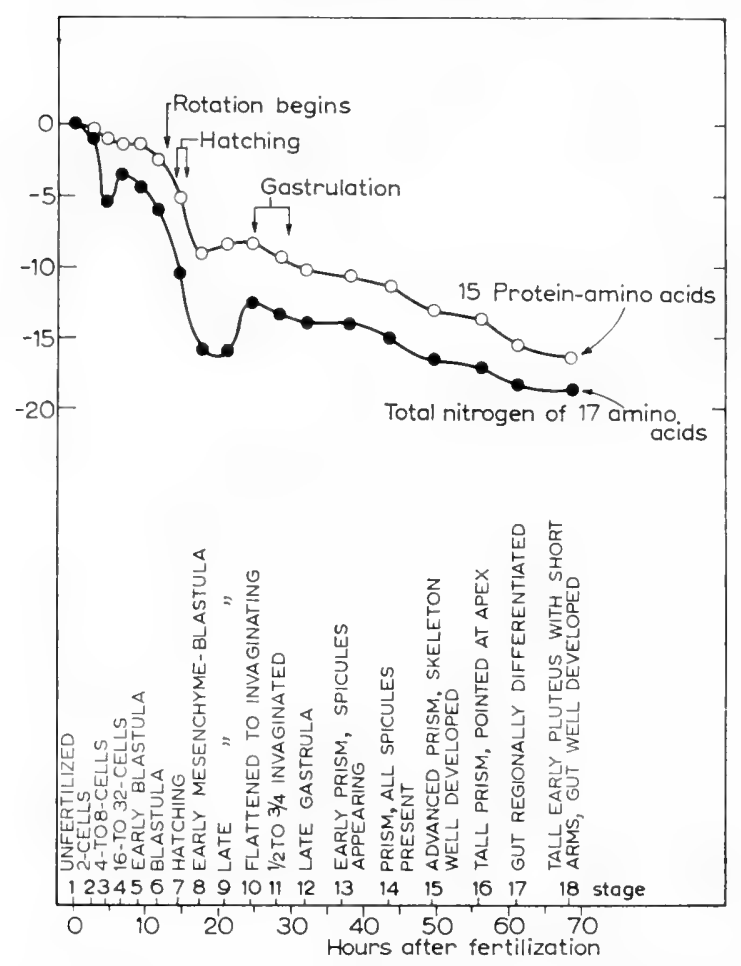

Fig. 2. Percentage changes of I $_{5}$ protein-contained amino acids and of total nitrogen of I 7 amino acids in early development of Strongylocentrotus purpuratus. Decline of amino acid content of both categories may be related to ammonia production during this period of development resulting from protein catabolism as an energy source. (From Kavanau, 1953.)

Kavanau (I 954b) describes 3 waves of protein synthesis: $(I)$ at midblastula, (2) at completion of hatching and (3) at midgastrulation. There are four periods of yolk protein breakdown: (I) at early cleavage, (2) at late blastula, (3) just before gastrulation and (4) late prism and early pluteus (see Fig. 3). Correlation between to the two processes is high. Kavanau further seeks to show a relationship between respiratory increase and the periods of augmentation of embryonic protein on the one hand, and yolk degradation on the other. He says that "oxidative degradation probably plays a major role in this net loss of amino acids to stage $3 "$. With regard to transformation of yolk protein to embryonic protein Kavanau (1954a) concludes that there is probably very little transfer of large intact polypeptide units from yolk to new protein, for many of the amino acids are found in only relatively small amounts in the peptide fraction. Banhidi and Kavanau (1956) find a correlation between pantothenate content (hence, probably coenzyme A) and protein synthesis in Paracentrotus lividus. They find cstraordinary concentrations of free niacin as well (Kavanau and Benhidi, I956) but establish no certain relationship between them and the synthesis of proteins.

Possible pertinence to embryonic development of the protein degradation and 
peptide formation, described by Kavanau, is apparent from the isolation of Lactobocillus casei growth factors, qualitatively different at different stages, by Gustafson, Hjelte and Hasselberg (1952).

Lundblad (1950) reported strong but ephemeral proteolysis at fertilization in

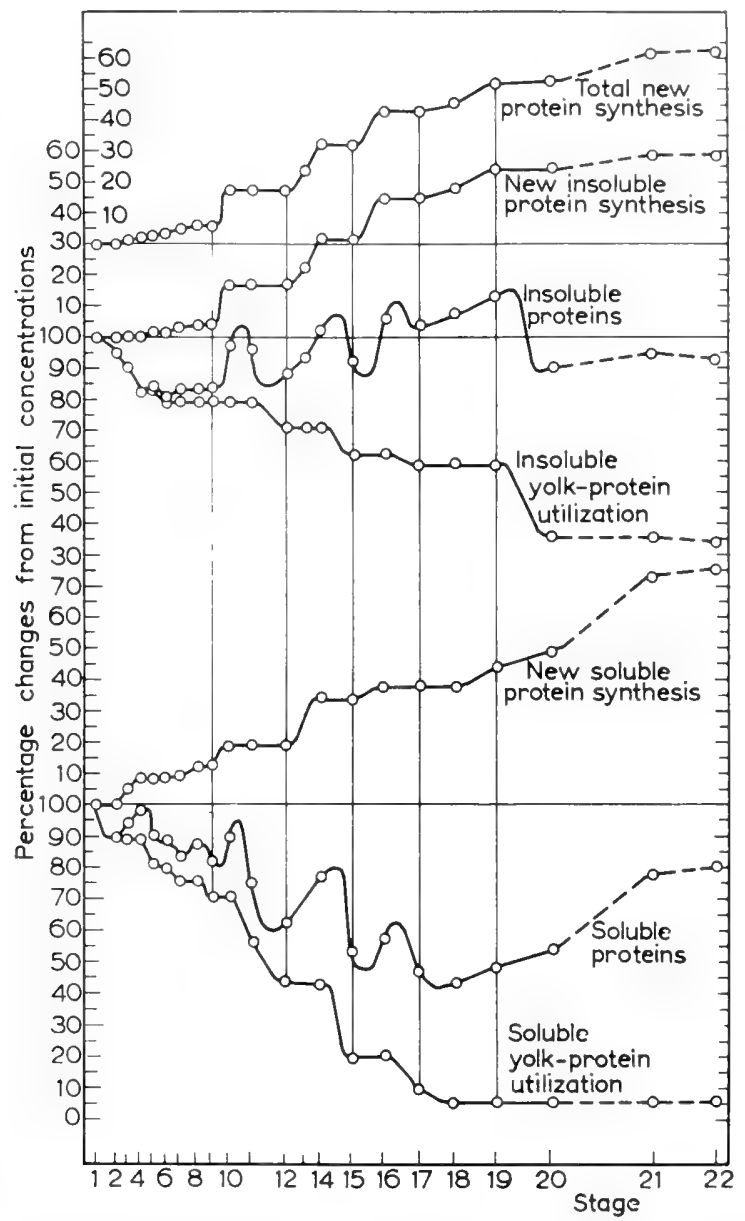

Fig. 3. Average percentage changes of the insoluble and soluble (o. I $M \mathrm{KCl}-0.0$ i $M \mathrm{HAc}$ ) protein-amino acids from their values in the unfertilized eggs of Paracentrotus lividus. Stages are described in Fig. 2. The top curve indicates a total new protein synthesis of $63 \%$ of that in the unfertilized egg. The next set of three curves indicates that $66 \%$ of the initial store of insoluble proteins is utilized, suggesting that the remaining $34 \%$ of insoluble proteins are structural and other non-yolk protein; during the same period, new insoluble proteins reach $58.8 \%$ of the initial store. The lower set of curves indicate that $95.1 \%$ of the initial soluble yolk protein is utilized, while new soluble protein increase amounts to $75.1 \%$ of that originally present, indicating that almost all soluble proteins in the unfertilized egg are yolk proteins, while those in the pluteus are new embryonic proteins. Depletion of soluble proteins at stage 18 may be responsible for the drop in insoluble yolk protein between stages 19 and 20. Note correlation of periods of protein synthesis with periods of yolk protein decrease. (From Kavanau, I954b.) 
three species of sea urchin, disappearing after 30 minutes. After seven hours proteolytic activity is resumed and continues to increase to the pluteus stage. Perlman and Gustafson (1948) imply that antigen appearance does not occur during the cleavage period. The pioneering study of Ephrussi and Rapkine (I928) suggested a continuous utilization of protein during early development. That not all of the disappearing protein is being diverted into other nitrogenous compounds is apparent from the study of Hutchens et al. (1942) who describe substantial amounts of ammonia production up to the pluteus stage in $A$. punctulata. Örström (1937, I94I) describes production of ammonia and oxidation of amino acids to keto acids.

Kavanau (r953) also investigated the effects of lithium vegetalization upon the free amino acids. He found that "the general effect is to damp or retard the changes which normally occur. This would be consistent with an inhibition of carbohydrate metabolism by $\mathrm{Li}^{+}$for it suggests a greater utilization of protein for energy".

Hultin (1950b), investigating protein precursors, found that ${ }^{15} \mathrm{NH}_{4} \mathrm{Cl}$ was taken up initially at the same rate by unfertilized and fertilized eggs. However, the unfertilized eggs became rapidly saturated, whereas the fertilized eggs continued to take up the salt throughout the duration of the experiment, to $5^{\circ} \mathrm{h}$. The highest rate of uptake occurred in insoluble proteins, probably including mitochondria and microsomes. Incorporation of the isotope occurred also in water soluble proteins continuously to $5^{0} \mathrm{~h}$., with a somewhat more rapid rate after $25 \mathrm{~h}$. This, he felt, might be correlated with antigen formation at this time as described by Perlman and Gustafson (I948). Less extensive experiments with ${ }^{15} \mathrm{~N}$-labeled glycine showed a distribution of the isotope during the same period of development approximately parallel with that resulting from ${ }^{15} \mathrm{NH}_{4} \mathrm{Cl}$ administration.

Although the interesting approach of Ranzi and his co-workers has application as well to later stages of development, some of their conclusions have a bearing on the processes of development prior to gastrulation. Ranzi has given attention to the form of protein molecules and its correlation with morphogenesis. His criteria for the alteration of the protein molecule consist of $(I)$ concentration of ammonium sulfate required for precipitation; (2) viscosity changes as measured by the Ostwald viscosimeter, (3) flow birefringence, (4) optical density, (5) diazo reaction, $\mathrm{OH}^{-}$reactions and change in immunological properties. Ranzi and Citterio (I955) describe five protein fractions related to different stages of development, of which four correspond to peaks in the salting-out curve of the adult frog $\left(0-25 \% ; 25-45 \% ; 45-70 \% ; 70-85 \%\right.$ saturation of $\left(\mathrm{NH}_{4}\right)_{2} \mathrm{SO}_{4}$; the fifth consists of proteins which remain in solution in ammonium sulfate of $85 \%$ saturation). The four precipitable fractions are fibrillar or folded filamentous particles; only the fraction which remains in solution in $85 \%$ saturation ammonium sulfate consists of globular particles. Ranzi (I95I) assigns to the change in form of protein molecules from fibrillar to globular a significant role in the "formation of intercellular spaces", presumably including blastulation. He also points out that $\mathrm{LiCl}$ vegetalization in both sea urchin and frog causes increased viscosity of proteins (euglobulin a and b), whereas KSCN, an animalizing agent, causes a 
decreased viscosity of the same proteins. Specifically, "good animalization is obtained for a decrease in viscosity above $14 \%$, and good vegetalization for an increase in viscosity above $28 \%$ ", from a variety of animalizing and vegetalizing agents. He asserts that "fibrillar shaped proteins preside over the embryonic determination", and generalizes that depolymerization of fibrillar proteins occurs during determination of the ectoderm of sea urchins and of the notochord of amphibia. Morphogenetic effects of change of $\mathrm{pH}$ which might be expected to produce similar effects on proteins can be reconciled with Ranzi's conclusions (Yamada, I950; Holtfreter, 1947). Needham (1955), however, feels that Ranzi's attempts to generalize are forced. Ranzi (I955) continues his analysis of lithium and CNS $^{-}$effects, and delegates a protein-stabilizing role to lithium, but feels that animalizing agents cause "the initial breakdown leading to further breakdowns", specifically by detaching "small fragments from the fibrillar molecules". "By applying all the above data to embryonic development, we may conclude that a strong breakdown of preexisting proteic structures is taking place in the areas hyperdeveloped by animalizing agents and inhibited by vegetalizing agents. This breakdown is necessary before new syntheses can occur."

Raven (1952) in a review of lithium in Limnaea points out, regarding increase in cytoplasmic density, that this is not due to dehydration but "must be due to a change in its submicroscopic structure, e.g. by increased polymerization of fibrillar macromolecules in the formation of cross connections between protoplasmic fibrils. This is in agreement with the classical observations of Rünnström in sea urchins and the more recent investigations of Ranzi and co-workers."

\section{(c) Observations of protozoa}

Although "development" of protozoa may be only tangentially related to our problem (Lwoff, 1950), we wish to include reference to some pertinent metabolic and experimental studies. Metabolic studies have been summarized by Kidder and Dewey (195I). They point out that glycine appears to be required by Tetrahymena for growth, and in addition, the same amino acids that are needed by the growing rat, namely, arginine, histidine, isoleucine, leucine, lysine, methionine, phenylalanine, threonine, tryptophan and valine. Glycine cannot be regarded as an essential amino acid, though in large concentrations it has a stimulating effect; serine and other hydroxyamino acids, however, have a more marked stimulatory effect when administered in smaller quantities. The essential quality of individual amino acids varies with the composition of the remainder of the medium: valine, for example, may be omitted if sufficient quantities of glycine, cystine and serine are present; conversely, unless these are present, valine is required.

Ammonium salts may also promote growth if the amino acid content of the medium is sufficiently low, and provided an accessory source of carbon is provided as, e.g. dextrose.

Dewey, Kidder and Parks (unpublished, cited in review) found an inhibition of their cultures by urea $(700 \mu \mathrm{g} / \mathrm{ml})$ and by a variety of urea analogs. Recently, however, Seaman (I954) observes that early in the growth of the colony urea is produced, but cannot be recovered even after a short time because of its destruc- 
tion by urease. Control of the $\mathrm{pH}$ of the medium to permit (or prevent) urease activity allows correlated measurements of urea production.

Protozoa have contributed information of another sort, namely, toward the processes related to events in the individual's growth prior to division. These have been made possible by a technique for obtaining dry weight of a protozoan under water (Zeuthen, I948) and by providing conditions which promote simultaneous divisions of a protozoan culture (Scherbaum and Zeuthen, I954; Mazia and Prescott, 1954). Mazia (1956) summarizes certain aspects of the individual cell growth: $(I)$ daughter cells are equivalent, (2) growth begins at once at the maximal rate, and (3) deceleration of growth occurs when each of the daughters has doubled its original mass, and as a corollary of this, growth does not occur during division, and (4) there is a time lag, following growth cessation and prior to actual division, amounting to about four hours in a 24-h. cycle. Using the colorimetric technique of Lowry et al. (I95I), Prescott demonstrated the virtual identity of the increase in protein with increase in dry weight. By causing an unequal division of a parent, daughter cells of different weight are produced; Prescott observed that, in such an instance, the cells did not simply double their starting weights, but that the two unequal cells arrived at the same end-point, suggesting a genetic control of size limitation. Further experiments of amputation of cytoplasm demonstrate that $(I)$ division can be postponed indefinitely (Hartman, I928) and (2) that the amputation may be performed so late in the life cycle of the cell that division is inevitable.

Several notions have been proposed to explain the triggering of cell division: (I) increase of cytoplasm to the point that a single nucleus cannot serve it, (2) completion of duplication of the genetic endowment of the cell, (3) ratio of mass to surface area, and (4) increase in $\mathrm{CO}_{2}$ content, perhaps related to the last postulate. It would seem from the experiments above that there is a triggering mechanism, and since this is related to the lag period after accumulation of definitive mass, that the genetic theory is now the most plausible. Prescott ( I 957) provides a thoughtful review of these problems.

Mazia and Dan (I952) succeeded in isolating the mitotic apparatus in quantity from sea urchin eggs. Their analyses indicate $(I)$ that it is made up of one kind of protein, part of which is combined with RNA, (2) that it constitutes about $12 \%$ of the total cellular protein (more than could be derived from the nucleus alone). Speculations with regard to the method of organization of the mitotic spindle and its activities after it has been formed are less conclusive.

From the foregoing discussion it is apparent that the role of proteins as energy source may be more important in the sea urchin egg than in amphibia. Pertinent information on the chick is wanting and certainly no more than the barest information is available on the mammal in this regard. There is evidence, cited above, that synthetic activity is high during early development of the sea urchin; that this may also be the case with amphibia is clear from the analyses of Kutsky et al. (1953). They describe a decrease in aspartic acid, glycine, histidine and threonine up to the midblastula period, and an increase in glutamic acid during this period which they feel "may be linked to the subsequent formation of specific proteins of the induction or neuralization process". Similarly, Clayton's (I 953) 
serological findings indicate synthesis of antigenic material prior to gastrulation. Although it is clear from both amphibian and chick studies that some adult proteins are included in the yolk complement of the egg, it is equally clear that not all adult proteins are represented at least in detectable quantities. This implies synthesis of new proteins by the embryo. This process begins early and may be correlated with the realization of developmental potential of the early blastomeres. However, Berg (1954) seeking a differential peptidase activity in the early blastomeres of Mytilus edulis was unable to find a significant difference. The pursuit of analyses of other details in individual blastomeres of this or other mosaic eggs would, none the less, appear to hold significant possibilities.

Although the direct application of studies of growth and mitosis of protozoa to the cleavage process has obvious shortcomings, the leads uncovered by such analyses have considerable merit. The mechanics of cell growth in metazoan tissues are by no means understood; it is reasonable, in the face of inadequate knowledge, to test the critical aspects of the protozoan studies for applicability to the problems of cellular growth and cessation of growth in higher animals as well.

\section{(d) Gastrulation}

The period of gastrulation has been recognized as a complex, and therefore, exciting one. The relation of the gastrulation process to inductive capacities has claimed the attention of embryologists since Spemann's definition of the role of the amphibian dorsal lip as "the organizer" (see Chapter 2). The findings of Brachet (I 94 I, I 942) of accumulation of nucleic acid in regions of active differentiation and the relation of this accumulation to protein synthesis (Brachet, I947; Caspersson, I947; Hultin, I950a) have provided a measure of integration of apparently diverse findings. Clayton (I953) found for the newt, Triturus alpestris, specific antigens in the gastrular ectoderm and archenteron roof. Brachet (I939), however, finds gastrulation "not especially interesting in light of excreted nitrogen". Brachet (I 950) and Boell (I 948) provide excellent reviews of respiratory details of the amphibian gastrula. Although much attention has been given to various phases of metabolism, to details of the composition of the organizer and to the process of induction, the relevance of protein to the process has been virtually neglected, except for the possible involvement of nucleoprotein by Brachet and his co-workers; even here the implication of the findings is that the protein moiety is relativcly unimportant.

\section{(e) Organogenesis}

We seek to cover by the term organogenesis the postgastrular phenomena which eventually produce the complete, integrated organism. The processes involved are induction of tissues, differential growth, migration of cells and tissues, and the multitude of changes which may be included under the heading chemodifferentiation, many of which are touched upon by Weiss (1953a). The latter's remarks transcend the significance of intracellular differentiation, for intracellular processes in sum constitute histogenetic changes.

From the point of view of energy requirements and building requirements the 
postgastrular period of development far overshadows the preceding stages. It is here, therefore, that we may expect to find more satisfying answers to a variety of questions. These may be outlined as follows:

I. Protein synthesis
a. Chemical limitations
b. Cytological mechanisms
c. Relationship with yolk storage

2. Growth Regulation
a. Differential growth
b. Cessation of growth
c. Genetic control

3. Energy Sources in Development
a. General
b. Evaluation of Needham's thesis
c. The Cleidoic Vertebrate Egg

\section{Protein Synthesis}

a. Chemical limitations. It is appropriate that we rely heavily on a mature and conservative assessment of the present status of this question by a biochemist keenly interested in the problem. Fruton (I 955) summarizes work on two fronts: chemical, largely by his own group, and biological. Working with cathepsins of animal material (Cathepsins A, B and C from beef spleen) and plant material (papain and ficin), Fruton has characterized some of the peculiarities of synthetic, transpeptidation reactions catalyzed by these enzymes. Important in the replacement reactions are the structure of both replacement reagent and the compound containing the sensitive $\mathrm{CO}-\mathrm{NH}$ bond, and the fact that the replacement reagent (amine) must be in uncharged form. Because transamidation reactions occur preferentially near $\mathrm{pH} 7$, Fruton suggests that "catalysis of replacement reactions may represent a major physiological role of these enzymes". In experiments with Cathepsin $\mathrm{C}$ from beef spleen, he was able, under carefully controlled conditions to recover a hexapeptide, octapeptide and decapeptide, the first of which appeared to be homogeneous, and the two latter mixtures of polypeptides of varying length.

Fruton has assembled information describing energy requirements in peptide bond syntheses and cites evidence for relating ATP in a variety of reactions: bacterial synthesis of CO-NH bond (Lipman, I954); glutathione (Snoke et al., I953); glutamine (Elliott, I 953); incorporation of isotopic amino acids into proteins of tissue preparations (Siekewitz, I 952). He suggests that energy yielding processes of the cell may be coupled with synthesis of peptide bonds of proteins through a relatively small number of amides or peptides such as glutamine and slutathione. Such hypotheses were advanced independently by Hanes et al. (1952) and Waelsch (1952). Rudnick, Mela and Waelsch (1954) correlate protein synthesis with glutamotransferase in the chick embryo. 
b. Cytological mechanisms. Biological studies have followed essentially three lines of approach: ( $I$ ) incorporation of labeled amino acids, (2) adaptive enzyme formation and, (3) nucleic acids in relation to protein synthesis. The evidence for association of RNA, particularly of microsomes, with synthesis of proteins seems overwhelming (Caspersson, I 947; 1950; Brachet, I952; Hultin, I 950a). Gale and Folkes (I955a) find that disrupted Staphylococcus aureus cells incorporate ${ }^{14} \mathrm{C}$ glutamic acid in the presence of ATP and hexosediphosphate as energy sources; the rate of incorporation is enhanced by nucleic acids. The same authors (1955 b) studying specifically enzyme formation show that when cells are partially depleted of nucleic acid, restoration of synthetic capacity can be accomplished by RNA or a mixture of purines and pyrimidines; however, when nucleic acids are severely depleted, DNA must be supplied, as well as RNA and purines and pyrimidines in order to restore maximal synthetic capacity. Brachet (I952) summarizes the apparent present status of the question as follows: protein synthesis may be due to the direct intervention of microsomes, and multiplication of these particles is under nuclear control. The role of the nucleolus, although probably significant, is not yet completely clear. The method by which the RNA of microsomes participates in protein synthesis is still a matter of speculation, whether by an indirect function or by serving as a "template".

Details of relationship between RNA and amino acid incorporation are becoming more clear by virtue of persistent work in several laboratories. Many features of this are summarized in a symposium of the Biophysical Society (Roberts, I958). The mechanism appears to be enzymatic amino acid activation in the presence of ATP, attachment of the activated amino acid to soluble, cytoplasmic RNA, transportation to microsomal RNA. Peptide linkage formation and separation from microsomal RNA are not as well understood, but these are the essential, final steps. (Cf. Zamecnik and Keller, I 954; Hoagland, Zamecnik and Stephenson, I957; Berg, I956).

c. Relationship with yolk storage. The problems raised by Fruton would appear to bear particularly on questions of maintenance; although the processes which he describes may not be eliminated from embryological consideration, almost certainly in yolk-bearing eggs they must be supplemented. We have reviewed above a substantial literature leading to the conclusion of identity of some adult and egg proteins, and have described a plausibility for the incorporation of intact protein molecules in the developing embryo. There is not yet experimental evidence to suggest that all adult proteins are deposited in the egg, and it would appear unnecessary to suggest such preparation of the egg. Such a view is at once prejudiced in favor of the notion of "template" activity, but rather than answer significant questions it raises new ones with respect to the assortment, or delegation of, peculiar proteins to their appropriate tissues.

It is an inevitability that limitations on the synthetic ability and selective permeability of the ovary require that only certain of the adult proteins become incorporated in the egg. But the epigenetic development of the embryo entails the appearance in its structure of some devices which have only local applicability, 
even as in the adult ovary; and further, the phenomena of growth, differentiation and maintenance, are the result of such local limitations.

Such a concept, perhaps unhappily, leaves essentially the same developmental problems which have tormented the minds of embryologists for three quarters of a century. Recent studies with immunological techniques and paper chromatography enable us to chart more successfully the milestones of progress by the embryo, and to a limited extent, the methods used by the embryo. It is in the latter connection that primary interest lies with respect to protein and nitrogen metabolism. Nitrogen metabolism may be related in two ways: $(I)$ in its relation to protein accumulation, both replication and differentiation, and (2) nitrogen compounds as energy sources for the reactions involved in $(I)$. The enzymatic maturation of the embryo then has both direct and indirect significance.

Enzymes are among the easiest proteins to characterize, because of specific relation to substrate. Moog (I952) has summarized the extensive literature on enzymes in the developing chick. She announces three major conclusions with supporting evidence: $(I)$ young embryonic tissue is enzymatically complex, (2) enzymogenesis proceeds at different rates for different enzymes, and (3) enzyme accumulation is an aspect of functional differentiation. She brings to bear on the first point evidence for the presence, during the first day, of amylase, lipase, protease, cytochrome oxidase, alkaline phosphatase and acid phosphatase, and during the second day, dipeptidase and aminopeptidase. In support of the second point, she has collected information from various sources about the rate of accumulation of the enzymes mentioned, and in addition, that of arginase. Of all enzymes studied, only the last shows a continuous trend (downward) and she feels that fluctuation in activity is a characteristic of each in early development. The last conclusion is supported $(I)$ by calculating different rates of accumulation of the same enzymes in different tissues, e.g., apyrase in brain, liver and muscle and (2) by relation of enzyme concentration to onset of functional activity in nervous tissue, muscle, kidney, intestinal epithelium and liver.

Boell (1955) extends the review to embryos other than the chick. He cautions against the conclusion of causal relationship between enzyme development and the process with which it is associated.

\section{Growth Regulation}

a. Differential growth. A large literature exists with regard to differential growth, for which Huxley (1932) offered, on the basis of a wide variety of examples the formulation of the heterogonic growth process as $y=b x^{k}$, in which $y$ is a measurement of one dimension of growth, $x$ is the corresponding measurement of the entire animal (or smaller unit of which $x$ is only a part, e.g. face and head), and $b$ and $k$ are constants. It is apparent that relationship between rates of change of two measurements may be expressed by the relationship between $b$ and $k$. Needham (I 934) substantiated the usefulness of the concept for "chemical "rrowth". Brody (I945) exploited a modification and refinement of this concept culculated to give, for any period of development, the instantaneous growth rate by use of a plot of the logarithm of the growth dimension against time. This 
technique has been used many times since (Boell, I948; Clark, I953a; Clark, Florio and Hurowitz, I 955; Clark and Sisken, I956). Levy (1952) makes a further modification, based on the concept that a logarithmic, rather than linear, expression of time is more appropriate for physiological events. He points out that such $\log -\log$ plots of various embryonic dimensions (weight, nitrogen, dipeptidase, aminopeptidase, cytochrome oxidase and diphosphopyridine nucleotides) have nearly identical time relations to each other and to the totality of development. A log-log plot results in a straight line with a change in slope corresponding to change in rate of accumulation of the units under consideration. The variables mentioned above have changes in rate between 4.3 and 4.5 days in the chick.

b. Cessation of growth. Conceptually, it might be easier to devise mechanisms for alteration in growth rate of a particular component of the embryo than to provide a device for stopping growth. For it is a common experience, at least in warmblooded animals that there is a constant species size of the organism and that, within the organism, the relative sizes of the several organs is constant $(e . g$. Latimer, I925, I947, I95 I, I $95^{2}$ and many other similar studies). Some experimental attempts have been made to provide explanation of a mechanism, apparently under genetic control, for stopping the growth process "at the right time". Weiss (1947) proposes such a mechanism. Its basic features are: $(I)$ Existence of intracellular "template" molecules, (2) proportionality of growth to the number of template molecules present, (3) confinement of template molecules to the cell, and (4) production by template molecules of antitemplates, which can escape the cellular barrier, (5) cessation of outward migration of antitemplates when equilibrium is reached across the cell membrane, and (6) fixation of intracellular antitemplates by templates and prevention, thereby, of further template production (growth). He supports this thesis (1952) by an in vitro experiment in which embryonic heart and kidney were cultured in extracts of whole embryos, or of whole embryos less the homologous organ. The results were as follows: (I) heart-of 333 in whole extract 2 pulsated after the fourth day; of 349 in deficient extract, I 29 pulsated; (2) of 1007 kidney explants 74 formed tubules in the growth zone in whole embryo extract, whereas of I006 explants in deficient extract, I 76 formed tubules; (3) on the other hand, injection of embryonic kidney "brei" into younger embryos caused a $50 \%$-increase in mitotic count in the host kidney, and removal of one embryonic kidney resulted in hyperplasia of the remaining kidney with more than $100 \%$ increase in mitotic activity after $4^{8} \mathrm{~h}$.

Rose (1952) makes a proposal along similar lines in which he conceives of growth limitation as a mass action phenomenon, whereby synthesis of a new product would be limited by the quantity of that product already formed. In support of his thesis he offers some data on the effect on amphibian differentiation when the embryos are reared in a medium to which specific extracts of adult organs have been added. Under such conditions he reports inhibition of brain growth by brain extract, heart and circulatory differentiation by heart extract. Shaver (1954) also submits data showing inhibition of nervous tissue in the frog by tissue fractions of the adult organ. 
The finding of Prescott (see above, Protozoa) may also have a bearing on this proposal. He finds that $(I)$ the adult, attained size in Amoeba proteus is constant, and that protein increase corresponds to total growth; (2) during the period of logarithmic increase of the cytoplasm, the nuclear volume increases only slightly, but sharply increases after the attainment of "adult" cytoplasmic volume. Although attainment of final cell volume seems to be inversely correlated with nuclear volume increase, it is apparent that the nucleus in some way governs the attainment of final cytoplasmic size (and protein content). It is possible that synthesis of cytoplasmic protein is governed by RNA content, that cessation of growth occurs by fixation of cytoplasmic RNA to or by protein, and that final impotence for further protein synthesis occurs when all cytoplasmic RNA is so fixed. Renewal of growth activities then could occur only by replenishing cytoplasmic RNA from the nucleus, as, e.g., following amputation of cytoplasm; the stimulus for migration of new RNA from the nucleus might simply be increased water uptake caused by the disturbance of the cortical area by the operation of amputation.

Ebert, in a series of reports has attempted to resolve doubts raised in the experiments described above. Preliminary results (Ebert, I95I) indicated from C-A grafts of adult chicken and mouse spleen that the stimulating effect of adult spleen on growth of embryonic spleen was real and class-specific, for mouse spleen elicited no such response in the chick. Certain other tissues of the adult chicken, however, did have stimulatory effects, viz., liver and thymus, but the spleen effect began only on the fourteenth day of incubation. He felt that common possession of certain antigen molecules by liver, spleen and thymus might be responsible for their common effect. The minimum number of antigens which appear on the I $4^{\text {th }}$ day in the spleen is three (Ebert, I952). He has not demonstrated that these are responsible for the growth stimulating effect, however. Later (Ebert, I 954) he showed that corresponding to a 4 -fold increase in weight of the host spleen, there is a 4 -fold increase in nitrogen content. By $\mathrm{C}$-A transplant of tissues from adult chickens and mice previously injected with ${ }^{35} \mathrm{~S}$ labeled methionine, he demonstrated again that mouse tissue components are not taken up selectively by homologous host organs, but chick spleen and kidney components are. By quantitative analysis of the radioactivity when incorporated in host tissue and when administered independently of the host tissue (yolk sac injections of ${ }^{35} \mathrm{~S}$ methionine, coupled with untreated C-A transplant of spleen), Ebert concludes that protein molecules or some portion thereof actually are incorporated into the host, and moreover, in such quantity that the hypothesis of a "template" or catalytic activity of a few molecules cannot be supported. Nor does he believe that whole cells are transferred from graft to host spleen. In a further set of experiments (Ebert, I 955) he reports no inhibition of spleen development when splenic transplants are made to the coelom of 66-72 h. chicks, nor when homogenates of fresh adult spleen are injected into the coelom.

Johnson and Leone (1955) observed inhibition of growth both in vivo and in itro at the primitive streak stage, when the embryos were grown in an environment of antiserum. Selective inhibition of heart differentiation, as well as general inhibition of growth, resulted from treatment of the embryo with anti-actomyosin. 
Antimyogen, on the other hand, generally stimulated growth and development. Antiactin and antiglobulin did not seriously affect the embryos.

We cannot resolve the dilemma created by the results of Ebert, Rose and Weiss. Since all three sets of data are exploratory, seeking to explain cellular events at a molecular level, it is too soon to expect a complete solution of the problem. It is apparent that Ebert's data indicate a selective up-take of homologous tissue proteins or protein constituents and that these have the effect of promoting growth selectively, presumably through increasing the mitotic rate. Rose's data seem to indicate a prevention of differentiation of embryonic tissues by homologous adult tissue extract. Though Rose suggests a theoretical mechanism to account for his results we cannot be certain that this mechanism operates, but almost certainly the mechanism suggested would not apply to the data of Ebert which are apparently concerned with the process of mitosis. Weiss, using a method different from that of either Ebert or Rose, adds still a different variable, namely, organization. His data appear to suggest that in tissue culture, absence of extract of homologous tissue favors differentiation; but his criteria for differentiation can hardly be equated: in the heart tissue, presumably we are concerned with the formation of contractile proteins, and in the kidney we are concerned with the orientation of cells with respect to each other (in addition to increase in mitotic rate, which almost certainly has a different explanation). Ebert, Rose and Weiss, then, have used different experimental materials and different methods; their data have application to different problems. Each of the contributions has merit which will be more fully appreciated when auxiliary and supplementary details of each system have been supplied.

c. Genetic control of growth. Because of its obvious relation to economics a wealth of data exists on genetic control of growth rate and final attained size in a variety of poultry and mammals. These are painstakingly and thoughtfully reviewed by Dickerson (1954). The data point to the fact that "growth is influenced by inherited differences both in the individual's own growth impulse and the direct nutritional influence of the mother, as well as by uncontrolled environmental factors". In short, Dickerson reiterates the conclusion of genetic relationship to growth, but in the face of the data is quite helpless to specify gene-related mechanisms. It is only a first approximation to assign from the collected data a role in "control of growth rate through effects on specific nutrient requirement, on endocrine activity, and on sensitivity of response to hormones". One is inclined to be impressed by the observation of Landauer (I952b): "It is important that we should not deceive ourselves. Our knowledge concerning the hereditary forces governing normal embryonic development, of the chick or any other vertebrate, is practically nil."

This view is probably unduly cynical. Development is the integration of processes of growth, differentiation and maintenance. The metabolic pathways in synthesis are at least partially known in view of data supplied above. What is actually sought, therefore, is confirmation of a genetic mechanism related to significant metabolic processes. There is no lack of such data. The preceding review has shown adequately that the three animals most often used for embryo- 
logical inquiry are the echinoderm, the amphibian and the chick. They serve different purposes. We are not reluctant to accept as developmental generalities the findings for each, yet we do appear to be somewhat hesitant in applying the principles of physiological genetics from Drosophila or microorganisms to our problem. It would seem unwise to neglect these basic findings.

The direct involvement of genetic control of metabolism in development of higher organisms is lacking, and probably must remain so. The complexity and interrelationships of metabolic cycles is sufficient indication of the virtual impossibility of genetic interference without resulting in death. It is for this reason that the mutations which have shed most light on development have been lethal ones, and it is likely the same background of reasoning which has given rise to the controversy over pleiotropy. Since qualitative genic effects are more easily noticed and more easily measured than quantitative effects which would be related to growth, it is these which have been stressed by developmental geneticists. Grueneberg (I95I) describes the multifactorial inheritance of absence of the third molar in mice as a case of maternal potentiation of the absence of the smallest teeth in a series. He refers to this (1952) as quasicontinuous variation and conceives of the threshold involved not as a quantity of dental lamina but of factors, related to maternal physiology, which may not be the same from litter to litter. Because, however, of the extreme likelihood that the same kind of mechanism is involved in continued synthesis of protein as in the synthesis of a new type of protein, the principles established in the study of differentiation must certainly also apply to growth.

The pertinent details of metabolic studies of Neurospora have been adequately. and lucidly reviewed by Wagner and Mitchell (I955); they have incorporated significant studies on other forms used in genetic metabolic studies, e.g., Drosophila and man. The studies of parthenogenetically stimulated animals are not without interest in the sense that successful development of haploid embryos implies either that the essential developmental processes are not under genetic control or that, if they are, the genes responsible are almost entirely homozygous. The latter is obviously not a remote possibility, particularly in light of the fact that literally hundreds of examples of gene-controlled metabolic processes have been studied. The interference with gastrulation by hybridization in Amphibia is consonant with such a notion (Moore, J., I941, '46, '47; Sze, I953; Gregg, I948). The experiments of Briggs and King on nuclear transfer point to a nuclear differentiation, suggesting on a genetic hypothesis, irrevocable nuclear (genic) inactivation with the course of time in development. Such a view is supported also by Brachet (I952) and by Waddington (I956). Moore (I950) feels that her results with androgenetic frog hybrids "suggest that the abnormal development and death of haploid amphibian embryos is due to both haploidy per se and to unmasked lethal and semilethal genes." Her review of the time of death of haploid amphibian embryos of different species raises interesting questions about the variability which presumably exists with regard to the nature of genetic control of similar processes in related animals.

That the genome does exert control over development is established by studies of Drosophila (Hadorn, I955; Poulson, I940, 1945) of the mouse (reviews by 
Gluecksohn-Waelsch, I954, I953), of the chick (Gluecksohn-Waclsch, 1953) and to a less extent of other forms. Most of these studies are conclusive only at the descriptive level, but valuable information is available from analysis of these studies. The time of primary genic action in the mouse, for example, varies from the preimplantation stages to the time of initiation of the metanephric kidney at about 12 days. Of particular interest, of course, is the syndrome of effects apparently caused by a single gene. For example, the effects on the urogenital and skeletal system result from a single dominant mutation ( $\mathrm{Sd}$ ) and here only when present against certain genetic backgrounds. Gluecksohn-Schoenheimer (1949) relates the action of Kink to "organizer phenomena" in the mouse. The literature on the genetic dependence of blood antigens is rich (see Irwin, I 955). Schechtman (1955) reviewing the nature of blood antigens particularly in the chick and mammals, assembles convincing data which reiterate genetic dependence and points out the epigenetic nature of development of blood antigens. He concludes that they are end-products of differentiation, and as such, are not mechanisms of differentiation. Telfer and Williams (I953) find that "the blood antigens of the Cecropia silkworm undergo changes which are closely correlated with the morphogenetic events comprising metamorphosis."

It is appropriate to close this section with a quotation from the father of physiological genetics, Richard Goldschmidt (1955): "The type of penetrating analysis of development and growth just reported leaves the geneticist where he was, specifically where I was in my book of 1927 : development consists of a series of hierarchical subdivisions of the embryonic material in regard to its intimate chemistry, a subdivision or stratification which is the consequence of the physical and chemical properties of the whole system and its subsystems. The stratification, an automatic outcome of the genically controlled syntheses of different substances and the direction, order, and place of the genically produced prompters of stratifications, provides the competent material for more and more restricted genic action, otherwise called the activation of the genes, which works mainly by influencing rate processes. Only then do the secondary effects under genic control by means of specific enzymes, hormones, and auxins, and also through contacts, set in. It does not help much if we express any or all of these steps in terms of plasmagenes, macromolecules, and templates. In view of our ignorance about what really happens, the old-fashioned general description is still adequate. This, however, does not mean that, within that frame, more specific notions cannot be developed. We are all anxious to see that done."

\section{Energy Sources of Development}

a. General. Bohr and Hasselbalch (1903) found a correspondence within 4\% between heat produced by the developing chick and heat loss expected from respiratory measurements. Smith (1946) in the trout found a precise balance between energy available in the egg and that available from the hatched embryo plus measured heat loss. As Weiss (1953b) points out "Developmental phenomena do not violate the laws of thermodynamics" and Butler (1946) after an analysis of the same question, concludes: "Thus there is no outstanding difficulty in 
accounting for the synthesis of living structures with a fairly modest expenditure of food."

Needham (I93I, I942), Tyler (I942) and Brachet (I950) have reviewed evidence and offered interpretations on theoretical grounds of the relative apportionment of energy among the several aspects of development (growth, differentiation, mechanical movements, organization and maintenance). Our earlier decision (pp. 7I3-7I5) that such segregation of processes is unprofitable, if not impossible, focuses attention on the question of energy sources for the manifold but integrated operations of development, and in particular the extent of protein utilization for such purposes.

Among the "provisional generalizations for chemical embryology" Needham (I93 I, III, pp. I656-I657) includes "a succession of sources of energy, carbohydrate preceding protein and protein preceding fat", and "cleidoic eggs possess metabolic peculiarities: (a) suppression of protein catabolism, $(b)$ an emphasis on fat catabolism, and $(c)$ election of uric acid as the main end-product of protein degradation".

Needham's monumental compilation of chemical data relating to development has been a fountain head of information for embryologists of the past generation, and his conclusions are widely quoted. If we accept the principle that nitrogenous waste products are evidence of catabolism of protein, serving primarily as an energy source, we must reexamine some of Needham's conclusions more critically.

b. Succession of energy sources. A sufficient body of information about development of both vertebrate and invertebrate animals has accumulated since I93 I that Needham's carbohydrate-protein-fat sequence of energy sources has lost its significance through contradictions. Hollett and Hayes (1946) find that $44.5 \%$ of the initially stored protein is used for energy production, accounting for $40 \%$ of the energy required up to the time of yolk sac absorption of the Atlantic salmon, Salmo salar. The sequence in this species appears to be fat-protein-fatprotein, and carbohydrate plays only a very minor role in energy supply. Smith (1946), studying the trout likewise finds a continuous increase in nitrogen excretion throughout development. In 1952, Smith reports that carbohydrate combustion is confined to $(I)$ a period immediately after gastrulation, (2) during hatching and (3) onset of starvation. He cannot substantiate peaks of carbohydrate, protein and fat combustion, but sees some evidence for an initial peak of protein + phosphatide fat and a later one of glyceride fat. He makes the interesting observation that combustion of phosphatide fats is conspicuous during yolk sac absorption, "and may be correlated with consumption of protein as an energy source." Gregg and Ballentine (I946) were able to find little evidence for protein breakdown early in frog development, though Brachet (I939) had found evidence for protein utilization in pregastrula embryos. Boell, Needham and Rogers (1939) observed that ammonia production of dorsal lip explants was three times as great as that of ventral ectoderm under similar conditions. Gregg and Ornstein ( I 952), however, find only traces of ammonia excreted by either type of explant and attribute the results of Boell et al. to liberation of ammonia from cytolizing cells. Carbohydrate utilization prior to gastrulation has not been unequivocally 
demonstrated in the frog, though Lovtrup (1953) after correlation of reduced weight, oxygen consumption and chemical analyses points out that there is a sequence of carbohydrate-protein-fat in A. mexicanum. Relative amounts used by the embryo during the period of observation were carbohydrate: 125 $\mu \mathrm{g}$; protein: Ig6 $\mu \mathrm{g}$; and fat $204 \mu \mathrm{g}$. The protein figure is uncertain; it "might have been lower if feeding had been allowed". Lovtrup points out that yolk phosphoprotein is not used prior to the tail bud stage and makes the curious observation that "yolk utilization is not directly related to protein combustion". Boell (1955) makes a more extended analysis of energy sources in amphibia and cites variability in size, hence protein content, of eggs as a possible explanation for conflicting results. If doubt exists with regard to the relative importance of protein as an energy source in early amphibian ontogenesis, none is apparently warranted with respect to alteration in protein structure during this period. Kutsky et al. (1953) have measured in amphibia quantitative changes in amino acids and find that arginine, leucine, lysine, methionine, threonine and tyrosine seem to increase slightly with minor fluctuations, while aspartic acid, glycine, histidine and threonine decrease to midblastula and increase through gastrulation to a maximum at midneurulation. Most clear-cut of their observations is the behavior of glutamic acid, which increases through cleavage, drops sharply at midgastrulation, increases in late gastrulation and drops again until neurulation. They feel that the increase during cleavage "may be linked to the subsequent formation of specific proteins of the induction or neuralization process".

Allusion has previously been made (pp. 723) to studies on protein metabolism of sea urchin embryos. The evidence appears to be consistent that continuous utilization of protein occurs during early development, and (Hayes, 1938) that protein combustion is supplemented by fat combustion. Indications of carbohydrate utilization during this period are based heavily on measurements of respiratory quotient. Such measurements are of some assistance in making a decision of substrate utilization but are not completely reliable. Compounding the difficulties attending the real meaning of respiratory quotients is the added confusion of conflicting data (see Boell, i955).

Needham's postulates (193I), though stated in general terms, are based heavily on data pertaining to chick development. The doubts with regard to general applicability of the carbohydrate-protein-fat succession are now extended to the chick and certain reptiles. In the black snake (Clark, 1953b) the R.Q. during the first week after egg laying is approximately 0.8 , but drops thereafter and remains consistently near 0.53 for the remainder of the incubation period of Io weeks. However, the rate of nitrogen excretion is highest during the earlier period; it would appear therefore, that, although carbohydrate may be burned during this period, certainly also protein contributes greatly to combustible food sources. The rate of protein destruction is 25 times greater at the beginning of development than at the end, with the sharpest drop coming during the second week. A similar phenomenon has been described for the garter snake, a placental species (Clark and Sisken, 1956), and for the alligator (Clark et al., 1957). The same kind of observation has also been made on the chick and the snapping turtle. These data are summarized in Fig. 4 . 
Careful study of the data compiled by Needham (I93I) indicates that the earliest period of development was uniformly neglected. Although the data presented in Fig. 4 do not deny the possibility of carbohydrate combustion during the earliest stages of development in the chick and various reptiles, they do focus attention on the hitherto neglected importance of protein as an energy source. Moreover, the peak of protein utilization in the chick at 8-9 days, described by Needham and related to protein catabolic events, is seen to disappear. With the curves of protein combustion presented in Fig. 4, one cannot be impressed by the 8-9 day peak. A word of possible explanation is offered. Fiske and Boyden ( 926 ), upon whose data Needham relies heavily, analyzed only the allantoic contents. Immediately after 7 days when uric acid synthesis proceeds rapidly, the evidence for burned protein would loom large. In actuality, both urea and uric acid are produced from the beginning of development; the findings with respect to ammonia are less certain for the reason that the amount found in the unincubated egg is already significant, and it is difficult, if not impossible, to distinguish between stored ammonium salts and newly created ammonia. No mechanism for the transfer of proteins to the embryo has been described for the chick, such as the possible "carrier" role of phosphatides as described by Smith (1952). Such a mechanism, however, would appear to warrant investigation.

c. Nitrogen metabolism in cleidoic eggs. The term cleidoic (Gr. I enclose) describes the essentially "closed box" eggs of terrestrial, non-viviparous vertebrates. The structure implies that the mother must include within the shell all non-gaseous materials necessary for the formation of an embryo. A further implication is that excretory waste must also be confined within the shell until hatching. In a careful study of the excreta of the chick embryo Fiske and Boyden (1926) point out that excretion of uric acid is virtually a necessity, since "a substance as soluble and diffusible as urea could not possibly replace it as an end-product when the organism and its excretions are confined to a closed system, the walls of which are only permeable to matter in the gaseous state". Needham (I93I) continuing in the same line of thought calculates that, if all the stored nitrogenous waste existed in the form of urea, the concentration of urea would be approximately I $65 \mathrm{mg} \%$, which "is undoubtedly of the pathological order of magnitude, and if the avian embryo had to suffer from a constant headache and other symptoms before hatching, natural selection would hardly have preserved it for our entertainment". The conclusions just stated, as well as those in the foregoing section, based largely on chick analyses were extrapolated to describe the situation for reptiles as well.

The unusual opportunity presented itself to make a study of nitrogen partition in embryos of the black snake. The findings on the black snake were sufficiently different from the "sauropsid pattern" commonly described, that I was led into similar studies of other reptiles and a reexamination of the chick. Since the results fall into a pattern whose meaning is substantially different from that heretofore accepted, they will be described at some length ${ }^{1}$.

${ }^{1}$ The work of the author herein described was supported in part by a grant ( $\mathrm{G} 3827$ ) from the Division of Research Grants, National Institute of Health. 


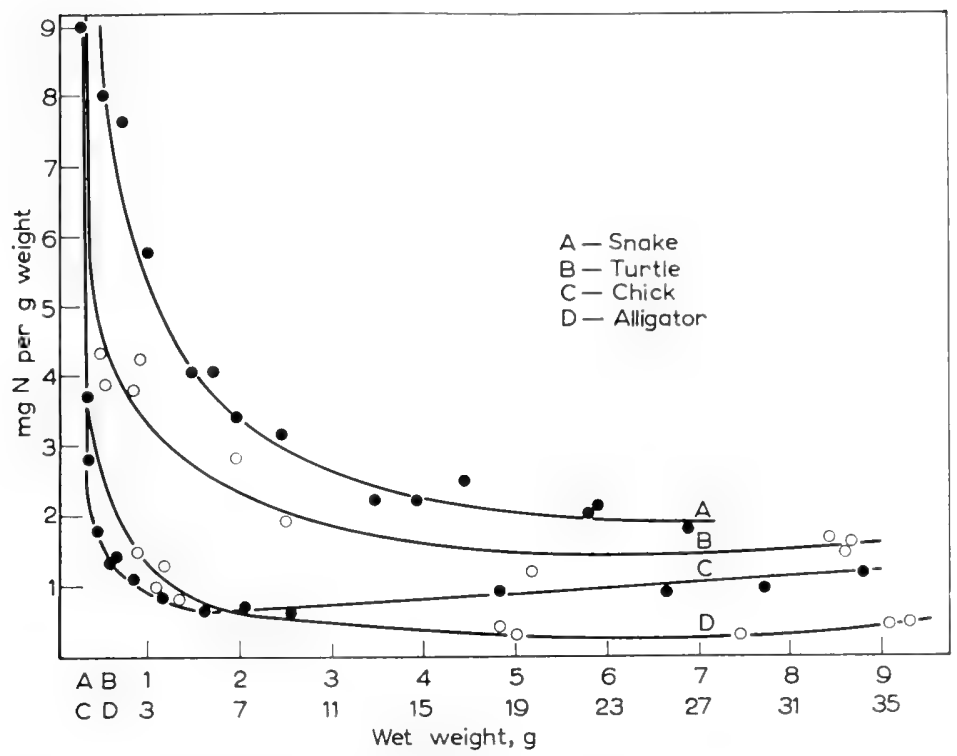

Fig. 4. Excreted nitrogen per gram wet weight throughout development in black snake, snapping turtle, chick and alligator embryos. The abscissa is calibrated in units of wet weight rather than time because of the similarity in hatching weights between the pairs as shown. Although the extremely high values at the beginning of development cannot be shown, the trend is apparent, and the relatively high rate of protein degradation early in development in each species is clear.

The most striking features of the initial observations on the black snake (Clark, I953a) were: $(I) 60 \%$ of the excreta in an incubation period of approximately two months appeared in the form of urea; the balance was evenly divided between ammonia, some of which escaped as a gas, and uric acid; (2) the total excreted nitrogen of the embryo (7.0 $\mathrm{g}$ at hatching) was greater than that atributed to the chick (approximately $40.0 \mathrm{~g}$ at hatching); (3) virtually all of the uric acid was accumulated during the last week of incubation, and coincident with its appearance there occurred a decline in urea content; (4) urease activity was demonstrated in the foetal liver and kidney at the time of urea decline, suggesting a mechanism for conversion of uric acid; (5) urea concentration in the allantoic fluid rises to a maximum of $500 \mathrm{mg} \%$.

TABLE 1

EXGRETED NITROGEN DURING EMBRYONIC DEVELOPMENT OF CERTAIN REPTILES AND THE CHICK

\begin{tabular}{lccc}
\hline \multicolumn{1}{c}{ Species } & Total Excreted $\mathcal{N}(m g)$ & Hatching Wt. $(g)$ & $m g \mathcal{N} / g$ Embryo \\
\hline Alligator & $\mathrm{I} 5.28$ & 36.0 & 0.42 \\
Gartner Snake & 2.52 & 1.5 & $\mathbf{1 . 6 8}$ \\
Black Snake & $\mathrm{I} 2.55$ & 7.0 & 1.80 \\
Snapping Turtle & $\mathrm{I} 5.49$ & 9.3 & $\mathbf{1 . 6 7}$ \\
Ghick & 39.00 & 39.00 & $\mathbf{1 . 0 0}$ \\
\hline
\end{tabular}


A summary of partition of nitrogen in the several species studied is shown in Fig. 5. From this it is clear that from these data no pattern of biochemical evolution is apparent. Each species excretes some of each of the three major excretory compounds. The enzymatic mechanism for uric acid synthesis, urea synthesis and ammonia production is therefore present. Only in the chick is the predominant excretory product uric acid, yet the egg of each species studied, except the viviparous garter snake, is cleidoic. No excreta are lost through the shell except gaseous ammonia, and this in relatively small amounts at the beginning of development.

The excretory "efficiency" of the several species (Table I) is of interest in terms of its variability. There is apparently no uniformly operative mechanism for the prevention of protein combustion among these cleidoic eggs. The low figure for the garter snake may be partially explained by loss of excreta through the placenta and by the transmission of amino acids to the embryo (Clark, Florio and Hurowitz, 1955). Such an explanation cannot, however, suffice for the alligator, described in detail by Clark et al. ( I 957).

One of the interesting features of the process of excretion by the reptiles and the chick is the mechanism responsible. The existence of urease activity in the black snake embryo concomitant with a rise in uric acid synthesis and decline in stored urea led to the further examination of the activity of this enzyme. Experiments were carried out on the garter snake (Clark and Sisken, 1956) to test the reality of its activity. Injection of ${ }^{15} \mathrm{~N}$ urea into the peritoneal cavity and yolk sac and examination of uric acid for ${ }^{15} \mathrm{~N}$ were made. These indicated that $22-56 \%$ of the ${ }^{15} \mathrm{~N}$ was recoverable in the adult uric acid after $48 \mathrm{~h}$. These results point rather clearly to an active, physiological role of urease in excretion of the

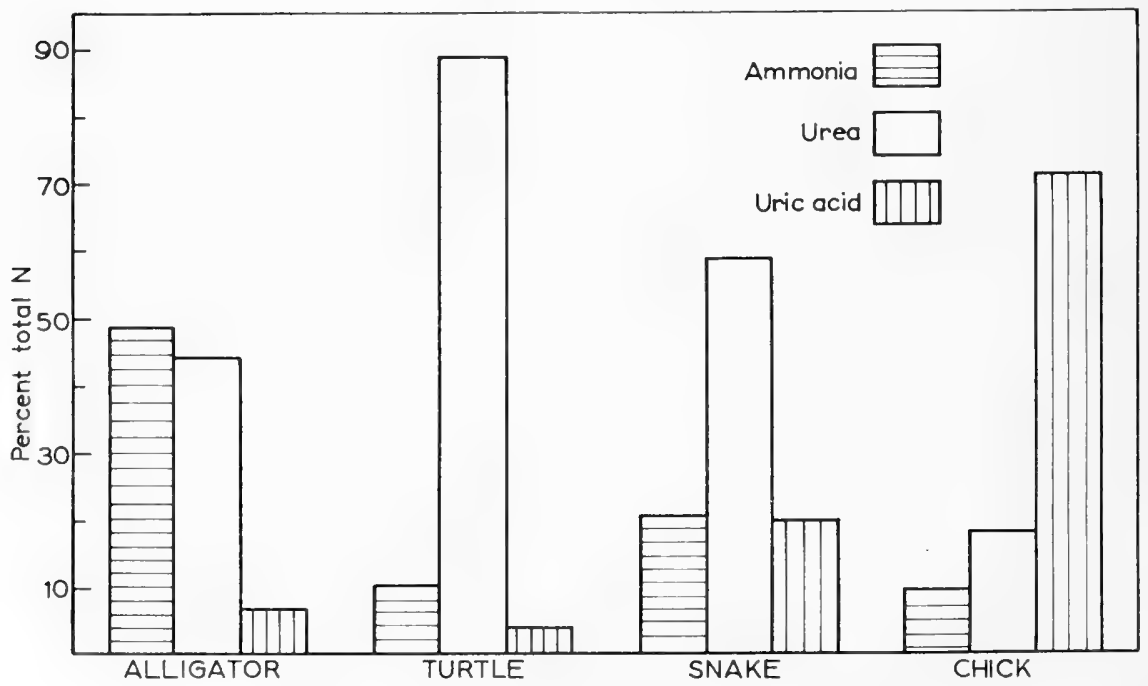

Fig. 5. Partition of excreted nitrogen among urea, ammonia and uric acid, shown as percentage of total nitrogen excreted, by embryos of alligator, snapping turtle, black snake and chick to hatching. There appears to be no systematic sequence. All eggs are cleidoic. 
adult and probably of the embryo as well. They point further to a possible concept of the evolution of the excretory mechanism. Excretion of ammonia and urea by amphibia is well established. It would appear that, genetically, the simplest explanation for the reptilian and avian devices would be transmission from primitive amphibia to reptiles and modern amphibia of a urea-synthesizing mechanism. Superimposed upon this device the mechanism for uric acid synthesis would be added; whether the latter appeared independently, or in response to the development of urease, must remain a moot question.

The presence of urease during a portion of the incubation period of the snapping turtle has been observed in our laboratory. It was not, however, detectable in the contents of the alligator egg. In the chick it was followed throughout development, increasing regularly but not so rapidly as arginase.

Goldie (1959) has followed the distribution of arginase in the chick embryo and extra embryonic membranes, its localization within the embryo, and its distribution between nucleus and cytoplasm. Although Needham, Brachet and Brown ( 1935$)$ deny the existence of the ornithine cycle in the chick, the basis for their conclusion seems inadequate. This question, as well as the role of urease, is currently under investigation.

If then, we have the demonstrated fact of maximal protein combustion early in development, what of the "advisability" of such a procedure from the point of view of the embryo? In the chick, for example, the question of toxicity is illusory. The total nitrogen excreted up to the eighth day in the chick requires the combustion of only $12 \mathrm{mg}$ protein; the available protein from the yolk alone is $8.0 \mathrm{~g}$. Since the accumulated waste is diffusible, and is indeed recoverable in the yolk, the concentration of either resultant ammonia or urea is of the order of $3.0 \mathrm{mg} \%$, obviously a harmless range.

In summary, it appears that reptiles have inherited the necessity of producing ammonia and the capacity of synthesizing urea from early amphibian ancestors. These capacities have been passed on to the chick (birds?), and superimposed upon urea synthesis has been the accessory production of uric acid, which may or may not have been accompanied by the evolutionary appearance of urease. Protein utilization as an energy source is highest very early in development and gradually is replaced by fat. Carbohydrate utilization may occur to a limited extent (certainly, even early in development, the capacity to burn carbohydrate is present), and might, indeed, be a requirement for protein utilization. There is no rational physiological impediment to the use of protein as an energy source early in development, when the absolute quantities are small. In the chick, uric acid is produced systematically from the beginning; evidence either from the chick, or from a study of the total performance of the several reptiles studied, of a biochemical recapitulation of excretory mechanism or products is wanting.

\section{GENERAL SUMMARY}

Progress in any field of science is determined by its conceptual orientation and the degree to which its concepts can be evaluated by the tools at hand. The 
integration of developmental phenomena in an organism and the delicate balance between the factors of growth, differentiation and maintenance have circumscribed the sphere of activity of developmental biologists. Organizational capacities and their interrelations have been explored at the levels of cells, tissues and organs. One can speak with reasonable assurance about the developmental prospects of areas of a developing embryo in terms of growth, differentiation, capacity for regeneration, metabolic requirements and the conditions for their realization (at least, in terms of a variety of conditions which prevent their realization).

It becomes apparent, however, that molecular details of development are essential to a complete understanding of the higher categories of organismic epigenesis. The need for such details has for a long time been recognized. Other groups of biologists have demonstrated similar interests by the continuous attention through the years to metabolic aspects of cellular behavior and the correlation of these with cytomorphological entities; by attention to the relations between metabolic activities and genic behavior on the one hand and phenotype on the other; by attention to analysis and synthesis of proteins; by attention to the ultramicroscopic structure particularly of proteins and the alteration of cellular behavior by delicate physical techniques. Confluence of the several streams of activity on developmental questions has enabled the developmental biologist to proceed intensively with the analysis of some molecular aspects of development, particularly with regard to protein. Hence work has been accomplished along the following lines: $(I)$ quantity of total protein and non-protein nitrogen of entire embryos and their parts, (2) use of total protein or specific proteins as an index of growth, (3) constitution of the non-protein nitrogen pool as an index of protein metabolism; (4) identification of proteins by virtue of specific immunological reactions; (5) the physical shape and size of protein molecules in relation to morphogenetic phenomena; (6) the permanence of the original store of protein and its use as an energy source, as determined by analysis both of residual protein and excreted nitrogen; ( 7 ) cytological mechanisms responsible for synthesis of proteins; (8) enzymatic studies concerned both with rate of accumulation and role in metabolic activities; (9) the behavior of proteins antigenically, either directly as in the events of fertilization or indirectly as may be involved in growth or its inhibition.

The investigations outlined above are carried out in a variety of animals; certain general similarities between developmental patterns in the diverse objects of study can acquire specific meaning only in light of events at the molecular level. Synthetic processes and the cytological mechanisms responsible for them may well be similar from organism to organism; the identification of the similarities of processes may clarify the major sources of difference between, say, alecithal and telolecithal eggs.

The most pressing residual questions for the developmental biologist are the degree of genetic control of development and the intracellular mechanisms responsible for it; the physiological and morphological characteristics of differentiation at the cellular level; the nature of the molecular population of individual cells as an index both of differentiation and its manner of influencing other cells. 
Some of the conclusive observations on these problems can be made now and are being made; others must await more specific direction by biological observation at a higher level of organization.

\section{ACKNOWLEDGEMENTS}

The author gratefully acknowledges permission by Dr. J. Lee Kavanau and the editors of 7. Exptl. Zool. and Exptl. Cell Research to use Figs. 2 and 3, and by Dr. Philip Grant to use Fig. I. I wish also to express thanks to Dr. C. Albert Kind and Dr. Arthur Chovnick for a critical reading of the manuscript and several valuable suggestions. 


\section{LITERATURE}

Abrams, R., (195I) Exptl. Cell Research, 2, 235.

Alfert, M., (1950) 7. Cellular Comp. Physiol., 36, 381.

Banhidi, Z. G. and J. L. Kavanau, (1956) Exptl. Cell Research, 10, 405.

BARTh, L., (1946) F. Exptl. Zool., 103, 463.

Barth, L., And L. G. Barth, (1954) The Energetics of Development, Columbia Univ. Press, New York.

BeAdle, G. W., (1946) Am. Scientist, 34, 3 I.

Beale, G. H., (1952) Genetics, 37, 62.

Berg, P. (1956) F. Biol. Chem., 222, I0I5.

Berg, W., (1954) Proc. Soc. Exptl. Biol. Med., 85, 606.

Bialascewicz, K. and M. Mincovna, (I92I) Trav. Lab. Physiol. Nencki, I, No. I I.

(Polish, cited by Needham, 1931).

Boell, E., (1948) Ann. N. Y. Acad. Sci., 49, 773.

Boell, E., (1955) Analysis of Development, Section VIII, Ed. B. Willier, P. Weiss and V. Hamburger, Saunders, Philadelphia.

Boell, E. and D. Poulson, (1939) Anat. Record, 75 Suppl., 65.

Boell, E., J. Needham and V. Rogers, (1939) Proc. Roy. Soc. (London), B, I27, 322.

Bohr, G. And K. A. Hasselbalch, (1903) Skand. Arch. Physiol., I4, 398 (cited by

Needham, I931).

Brachet, J., (I939) Arch. biol., (Paris), 50, 233.

BRAchet, J., (I94I) Enzymologia, I0, 87.

Brachet, J., (1942) Arch. biol., (Paris), 53, 207.

Brachet, J., (I947) Symp. Soc. Exptl. Biol., I, 207.

Brachet, J., (1950) Chemical Embryology, Interscience Publishers Inc., New York.

Brachet, J., (1952) Symp. Soc. Exptl. Biol., 6, i 73.

Brambell, F. W. R., W. A. Hemmings and M. Henderson, (1951) Antibodies and Embryos, Athlone Press, University of London.

Brambell, F. W. R., W. A. Hemmings, M. Henderson, H. J. Parry and W. T. Rowlands, (1949) Proc. Roy. Soc., (London), B, 136, I3I.

Brambell, F. W. R., W. A. Hemmings and W. T. Rowlands, (1948) Proc. Roy. Soc,, (London), B, I35, 390.

Brierly, J. and W. A. Hemmings, (1956) J. Embryol. Exptl. Morphol., 4, 34.

Briggs, R. And T. J. King, (I955) Biological Specificity and Growth, Ed. E. G. Butler, Princeton Univ. Press, Princeton, N. J.

Brody, S., (1945) Bioenergetics and Growth, Reinhold Publ. Corp., New York.

Butler, J. A. V., (I946) Nature, I58, I53.

Caspari, E., (1948) Advances in Genet., 2, I.

Caspari, E., (1956) Genetics, 4I, 107.

Caspersson, T., (1947) Symp. Soc. Exptl. Biol., I, 127.

Caspersson, T., (1950) Cell Growth and Cell Function, a Cytochemical Study, Norton, New York. Chargaff, E., (1942) F. Biol. Chem., 142, 505.

Child, C. M. (1941) Patterns and Problems of Development, Univ. of Chicago Press, Chicago.

Clark, H., (1953a) 7. Exptl. Biol., 30, 492.

Cllark, H., (1953b) 7. Exptl. Biol., 30, 502.

Clark, H. and B. Sisken, (1956) $\mathcal{7}$. Exptl. Biol., $33,3^{8} 4$.

Clark, H., B. Florio and R. Hurowitz, (i 955 ) Copeia, 9.

Clark H., B. Sisken and J. Shannon, (1957) F. Cellular Comp. Physiol., 50, 129.

Clayton, R. M., (I953) F. Embryol. Exptl. Morphol., I, 25.

CoOper, R., (1946) 7. Exptl. Zool., IOI, 143.

Cooper, R., (1948) 7. Exptl. Zool., 107, 397.

Cooper, R., (1950) 7. Exptl. Zool., 114, 403.

Dickerson, G. E. (1954) in Dynamics of Growth Processes, Ed. E. J. Boell, Princeton Univ. Press, Princeton, N.J.

Ebert, J., (195I) Physiol. Zoöl., 24, 20. 
Ebert, J., (1952) Ann. N. Y. Acad. Sci., 55, 67.

Ebert, J., (1954) Proc. Natl. Acad. Sci., U.S., 4o, 337.

Ebert, J., (1955) in Aspects of Synthesis and Order in Growth, Ed. D. Rudnick, Princeton Univ. Press, Princeton, N.J.

Elliott, W. H., (1953) 7. Biol. Chem., 20I, 66 I.

Ephrussi, B. ANd L. Rapkine, (1928) Ann. physiol. physiochim. biol., 4, 386.

Fauré-Frémiet, E., J. Ebel and J. Colas, (1954) Exptl. Cell Research, 7, 153.

Fevold, H. L., (I95I) Advances in Protein Chem., 6, I87.

Fiske, C. H. and E. A. Boyden, (1926) 7. Biol. Chem., 70, 535.

Flexner, J. B. And L. B. Flexner, (1948) J. Cellular Comp. Physiol., 3I, 3 I I.

Flexner, L. B. And J. B. Flexner, (i949) F. Cellular Comp. Physiol., 34, i 15.

Fruton J. S., (1955) in Aspects of Synthesis and Order in Growth, Ed. D. RudNick, Princeton Univ. Press, Princeton, N. J.

Gale, E. F. and Joan P. Folkes, (1955a) Biochem. J., 59, 66 I.

Gale, E. F. ANd Joan P. Folkes, (I955b) Biochem. F., 59, 675.

Gluecksohn-Schoenheimer, S., (1949) 7. Exptl. Zool., i1o, 47.

Gluecksohn-Waelsch, S., (i 953 ) Quart. Rev. Biol., 28, i 5 .

Gluegrohn-Waelsch, S., (1954) Cold Spring Harbor Symposia Quant. Biol., I9, 4I.

Goldie, M., (1959) Physiol. Zool., 32, 197.

Goldschmidt, R., (1938) Physiological Genetics, McGraw Hill Book Co., New York.

Goldschmidr, R., (1955) Theoretical Genetics, University of Calif. Press, Los Angeles and Berkeley.

Goldstein, B. And M. Guintsbourg, (1937) Enzymologia, r, 369.

Gordon, M. And M. Roder, (1953) F. Biol. Chem., 200, 859 .

Grant, P., (1953) 7. Exptl. Zool., I24, 513.

GreGG, J., (1948) 7. Exptl. Zool., Iog, i I9.

GregG, J. R. and R. Ballentine, (1946) 7. Exptl. Zool., Io3, i 43.

GregG, J. And N. Ornstein, (1952) Biol. Bull., IO2, 22.

Grueneberg, H., (195I) Proc. Roy. Soc., (London), B, $138,437$.

Grueneberg, H., (1952) Symposia Genetica, 3, 2 I 5.

Gustafson, T. and M.-B. HJelte, (195I) Exptl. Cell Research, 2, 474.

Gustafson, T., M.-B. HJelte and I. Hasselberg, (1952) Expil. Cell Research, 3, 275.

Hadorn, E., (1948) Symposia Soc. Expll. Biol., 2, I 77.

Hadorn, E., (1955) Letalfaktoren in Ihrer Bedeutung für Erbpathologie und Genphysiologie der Entwicklung, G. Thieme, Stuttgart.

Hadorn, E., (1956) Cold Spring Harbor Symposia Quant. Biol., 21, 363.

Hanes, C. S., F. J. R. Hird and F. A. Isherwood, (1952) Biochem. 7., 5 I, 25.

Hartman, M., (I928) Arch. Protistenk., 45, 973.

Hayes, F. R., (1938) Biol. Bull., 74, 267.

Hevesy, G., (1947) Advances in Enzymol., 7, I I I.

Hoagland, M. B., P. C. Zamecnik and M. L. Stephenson, (1957) Biochim. el Biophys. Acta, 24,215 .

Hollett, A. and F. R. Haxes, (1946) Can. J. Research, D, 24, 39.

Holtfreter, J. (1947) F. Expil. Zool., io6, I97.

Hultin, T., (1950a) Exptl. Cell Research, I, 376.

Hultin, T., (I950b) Exptl. Cell Research, I, 599.

Hutchens, J., A. K. Keltch, M. E. Krahl and C. H. A. Clowes, (1942) J. Gen.

Physiol., 25, 7 17.

Huxley, J., (1932) Problems of Relative Growth, The Dial Press, New York.

Irwin, M., (1955) in Biological Specificity and Growth, Ed. E. G. Butler, Princeton Univ. Press, Princeton, N. J.

Johnson, I. And C. A. Leone, (I955) 7. Exptl. Zool., I30, 515.

Jones, M., R. M. Featherstone and S. L. Bonting, (1956) J. Pharmacol. Exptl. Therap., II 6 , II 4 .

Kavanau, J. L., (1953) 7. Exptl. Zool., I22, 285.

Kavanau, J. L., (1954a) F. Exptl. Cell Research, 6, 563. 
Kavanau, J. L., (1954b) Exptl. Cell Research, 7, 530.

Kavanau, J. L. and Z. G. Banhidi, (1956) Exptl. Cell Research, io, 415.

Kidder, G. And V. C. Dewey, (I95I) in Biochemistry and Physiology of Prolozoa, Ed.

A. Lwoff, Academic Press, New York.

Kutsky, P., R. Eakin, W. Berg and J. KavanaU, (1953) 7. Exptl. Zool., 124, 263.

Landauer, W., (1948) Growth Symposia, I2, I 7 I.

Landauer, W., (1952a) J. Exptl. Zool., I20, 469.

Landauer, W., (1952b) Ann. N. Y. Acad. Sci., 55, I 72.

Landauer, W., (1953) J. Exptl. Zool., I22, 469.

Latimer, H., (1925) Anat. Record, 3I, 233.

Latimer, H., (1947) Growth, II, 6I.

Latimer, H., (I951) Anat. Record, III, 299.

Latimer, H., (1952) Anat. Record, I13, 247.

Levy, M., (1952) Ann. N. Y. Acad. Sci., 55, 5 I.

Lineweaver, H., H. J. Morris and R. S. Bean, (1947) Arch. Biochem., 16, 443.

Lipman, F., (1954) in The Mechanism of Enzyme Action, Eds. W. D. McElroy and B. Glass, Johns Hopkins Press, Baltimore.

Lovtrup, Soren, (1953) Compt. Rend. Lab. Carlsberg, Sér. chim., 28, 37 I.

Lowry, O. H., N. J. Rosebrough, A. L. Farr and R. J. Randall, (I95I) 7. Biol. Chem., 193,265 .

Lundblad, G., (1950) Exptl. Cell Research, I, 264.

Lwoff, A., (1950) Problems of Morphogenesis in Ciliates, John Wiley \& Sons, New York.

Marza, V. D. and E. Marza, ( I935) Quart. J. Microscop. Sci., 78, I34.

Mazia, D., (1956) Am. Scientist, 44, I.

Mazia, D. And K. Dan, (1952) Proc. Natl. Acad. Sci., U.S., 38, 326.

Mazia, D. and D. M. Prescott, (1954) Science, I20, 120.

Meyer, K. And M. M. Rapport, (1952) Advances in Enzymol., I3, 199.

Moog, F., (1944) 7. Cellular Comp. Physiol., 23, I31.

Moog, F., (г952) Ann. N. Y. Acad. Sci., 55, 57.

Moore, A.-B. C., (1950) Biol. Bull., 99, 88.

Moore, J., (1941) F. Exptl. Zool., 86, 405.

Moore, J., (1946) F. Exptl. Zool., IOI, I 73.

Moore, J., (1947) F. Exptl. Zool., 105, 349.

Mystrowski, E., (1936) Biochem. J., 30, 765.

NACE, G., (1953) 7. Exptl. Zool., 122, 423 .

Nace, G. And A. Schechtman, (1948) 7. Exptl. Zool., Io8, 217.

Needham, J., (I93I) Chemical Embryology, Cambridge Univ. Press, Cambridge, England.

Needham, J., (1934) Biol. Revs. Cambridge Phil. Soc., 9, 79.

Needham, J., (1942) Biochemistry and Morphogenesis, Cambridge Univ. Press, Cambridge, England.

Needham, J., (1955) Ann. Rev. Physiol., I7, 37.

Needham, J., J. Brachet and R. Brown, (1935) F. Exptl. Biol., I2, 321.

Neher, B. H. ANd R. M. Fraps, (I946) J. Exptl. Zool., IoI, 83.

Örström, ÅKe, (1937) Naturwissenschaften, 25, 300.

Örström, Åke, (1941) Z. physiol. Chem., Hoppe-Seyler's, 27I, I.

Oudin, J., (I948) Ann. Inst. Pasteur, 75, 30, Io9.

Panijel, J., (1950) Biochim. et Biophys. Acta, 5, 343.

Panijel, J. (195I) Métabolisme des Nucléoprotéines dans la Gamétogénèse et la Fécondation, (Actualités scientifiques et industrielles, I I 50) Hermann et Cie, Éditeurs, Paris.

Perlman, P. and T. Gustafson (1948) Experientia, 4, 48 I.

Poulson, D., (1940) 7. Exptl. Zool., 83, 271.

Poulson, D., (1945) Am. Naturalist, 79, $34^{\circ}$.

Prescott, D., (1957) Fifteenth Growth Symposium, Princeton Univ. Press, Princeton, N. J.

Ranzi, S., (1951) Experientia, 7, 169.

Ranzi, S., (I955) Le Macromolecule dei Viventi, Ist. Lombardo: Convegno Baselli.

Ranzi, S. And P. Citterio, (1955) Rev. suisse zool., 62, 275. 
Raven, C., (1952) Experientia, 8, 252.

Roberts, R. B., (1958) Microsomal Particles and Protein Synthesis, Pergamon Press, New York.

Rogers, F. W. R. and W. A. Hemmings, (1949) J. Physiol., 108, 177.

Romanoff, A. L. And A. J. Romanoff, (1949) The Avian Egg, John Wiley \& Sons, New York.

Rose, S. M., (1952) Am. Naturalist, 86, 337.

Rudnick, D., P. Mela and H. Waelsch, (1954) J. Exptl. Zool., I26, 297.

Rünnstrom, J., (1949) Advances in Enzymol., 9, 24I.

Rünnstrom, J., (1952) Symposia Soc. Exptl. Biol., 6, 39.

Schechtman, A., (1947) 7. Exptl. Zool., I05, 329.

Schechtman, A., (I955) in Biological Specificity and Growth. Ed. E. G. Butler, Princeton Univ. Press, Princeton, N. J.

Schechtman, A. And H. Hoffman, (1952) J. Exptl. Zool, 120, 375.

Scherbaum, O. and E. Zeuthen, (1954) Exptl. Cell Research, 6, 22 I.

Schoenheimer, R., (1942) The Dynamic State of Body Constituents, Harvard Univ. Press, Cambridge, Mass.

Seaman, G., (I954) 7. Protozool., $1,207$.

Shaver, J., (1954) Anat. Record, II8, 646.

Siekewitz, P., (1952) 7. Biol. Chem., 195, 549.

Sмiтн, S., (1946) 7. Exptl. Biol., 23, 357.

Sмiтн, S., (1952) F. Exptl. Biol., 29, 650 .

Snoke, J. E., S. Yanari and K. Bloch, (1953) J. Biol. Chem., 201, 573.

Sonneborne, T., (1950) Heredity, 4, II.

Spiegelman, S. (1948) Symposia Soc. Expll. Biol., II, Growth in Relation to Differentiation and Morphogenesis, Academic Press, New York.

Spratt, N. T., (1948) 7. Exptl. Zool., 107, 39.

Sze, L. C., (1953) 7. Exptl. Zool., 122, 577.

Telfer, W. H., (1954) F. Gen. Physiol., 37, 539.

Telfer, W. H. and G. Williams, (1953) 7. Gen. Physiol., 36, 389.

Tyler, A., (1942) Quart Rev. Biol., 17, 197; 339 .

Tyler, A., (1955) Analysis of Development, Sect. V, Ch. I. Ed. B. Willier, P. Weiss And V. Hamburger, Saunders, Philadelphia.

Waddington, G. H., (1956) Principles of Embryology, MacMillan, New York.

Waelsch, H., (1952) Advances in Enzymol., 13, 237.

Wagner, R. P. And H. K. Mitchell, (1955) Genetics and Metabolism, John Wiley \& Sons, New York.

Weiss, P., (1939) Principles of Development, Henry Holt and Co., New York.

Weiss, P., (1947) Yale 7. Biol. and Med., 19, 235.

Weiss, P., (1949) Chemistry and Physiology of Growth, Ed. K. Parpart, Princeton Univ. Press, Princeton, N. J.

Weiss, P., (1952) Science, II5, 487.

Weiss, P., (1953a) 7. Embryol. Exptl. Morphol., I, I8I.

Weiss, P., (I953b) Arch. néerl. zool., ro (suppl. I), i65.

Wills, I. A. (1936) 7. Exptl. Zool., 73, 481.

Wright, S., (1941) Physiol. Revs., 2I, 487.

Yamada, T., (1950) Biol. Bull., 98, 98.

Zamecnik, P. C. And E. G. Keller (i954) F. Biol. Chem., 209, 337.

Zeuthen, E., (1948) Compt. Rend. Lab. Carlsberg, Sér. chim., 26, 243. 


\title{
Chapter 10
}

\section{PLANT GROWTH ${ }^{1}$}

\author{
by Kenneth V. Thimann
}

In this chapter an attempt will be made to present as a whole the study of plant growth, principally that of higher plants, with primary emphasis on experimental analysis, and on the study of growth at the cellular and biochemical levels. Descriptive treatments of growth phenomena, especially in higher plants, may be found in many of the older German textbooks such as Goebel, Jost and Strasburger, who have presented extensive observations of great numbers of plant types. In recent times, many of the anatomical aspects of growth have been well treated in Esau's Plant Anatomy (1953); the Symposium on the Growth of Leaves (Milthorpe, ed., I957) is also valuable.

Much of the subject, especially that concerned with the action of auxin on growth, has been reviewed more than once. The books of Went and Thimann (1937), Thimann et al. (1952), Söding (1952), Audus (1953), Leopold (1955) and Thimann (1956a) as well as the Symposia held at Wisconsin (Skoog, ed., I95I) Brookhaven (I953) and Wye (Wain and Wightman, ed., I956) give extensive coverage and bibliographies. A number of reviews are also referred to in the text. Because of these publications, references to individual papers will only be made if their contents are discussed in detail or their data presented, or if for one reason or another they have been inadequately treated in the reviews and books. Papers of the last few years, especially of $195^{\circ}$ and later, will, of course, be cited freely. It must be made clear, however, that the aim has been to present a broad and balanced picture rather than a detailed bibliographic reference.

\section{GENERAL CHARAGTERISTIGS OF PLANT GROWTH}

The pattern of growth of plants differs from that of animals in four important respects.

In the first place, both for higher (flowering plants) and lower plants (ferns, mosses, fungi and algae) growth is typically indefinite. The plant body continues to

1 Support of the author's researches, over a period of years, by the Committee on Growth, acting for the American Cancer Society, and by the National Science Foundation, is gratefully acknowledged.

I wish to thank Dr. Bruce B. Stowe for valued criticism of the manuscript. 
grow throughout its life, or so long as conditions are favorable. The actual rate of growth may be slow, as in lichens growing on stones, or the Japanese dwarfed oak trees a hundred years old and still in flowerpots, but it does not stop, and when conditions are favorable it can always accelerate. Even the Sequoias of California, or the Eucalyptus trees of Australia, 2000 years old and 300 or more feet in height, continue to add a growth ring and to put out new twigs every year. Individual organs such as leaves, flowers or the fruiting bodies of mushrooms and other fungi have a determinate or finite growth, reaching a fixed size at the end of a given growth period, like a mammal. However, these are not the whole plant, and the stem and roots, or in the case of the fungi the mycelium spreading through soil or host, grow continuously and indefinitely.

Another characteristic of the pattern as a whole is the restriction of growth to certain localized areas. The local regions in which cells are rapidly dividing are referred to as meristems, and it is within, and particularly in the regions adjacent to, these meristems that the main enlargement takes place. The stems and roots of higher plants are long cylindrical organs, whose growth is at first essentially elongation. In the shoot, the meristem is at the apex, surrounded by young developing leaf primordia, and as the cells in it divide they enlarge. They continue to divide at first according to a characteristic pattern, but presently the cells furthest from the center of the meristem stop dividing, and continue to enlarge or perhaps even enlarge at an accelerated rate for a time. Most of this enlargement is along the axis, resulting in an elongated cylindrical stem. Elongation in the root is very similar, the meristem in this case being I to $2 \mathrm{~mm}$ behind the actual root tip, and giving rise to new cells both in front and behind it. The cells which it produces at the tip end become the root cap and are gradually sloughed off, while most of the new cells are on the side remote from the tip and these elongate to form the extending root.

In the leaf, the meristem at first occupies the whole primordium but very soon it is only the marginal cells which remain meristematic, first at the tip, and then gradually more and more towards the base, the tip of the leaf generally ceasing growth early. The meristematic cells in this case enlarge in two directions, to form a sheet or plate of cells, and in addition there are a few divisions perpendicular to the surface which give rise to the thickness of the leaf, perhaps a dozen or so cells in the typical case (Avery, I933). Growth of leaves is considered in more detail in section VII (p. 797).

In the algae and fungi a clearly defined meristem is not detectable, for the mass of tissue formed is commonly considerably less, but the general pattern of small cells dividing rapidly at or near the tip of the plant, and elongating for some time after division to produce the cylindrical or perhaps plate-like structure, is similar. Sometimes, however, in these plants only the nuclei may divide and the cytoplasm remain unified, enlarging to produce giant "cells" or coenocytes. This occurs in the Phycomycetes (except where fruiting bodies are formed) and the whole mycelium is a coenocyte with small nuclei throughout its length. Among the green algae the Siphonales, of which Oedogonium is a typical example, show the same habit. Nitella, in a subgroup of the green algae, has been much used for experiments on account of its very large cells, up to $\mathrm{so} \mathrm{cm}$ long and 3 or $4 \mathrm{~mm}$ 
wide, in the form of a long main "stem" with whorls of lateral (unicellular) branches. Here enlargement is not so localized but a whole series of "stem" and branch cells may enlarge together in length and girth (Green and Chapman, I955).

The other two differences from animal growth are at the cellular level, namely the rigid cell wall and the relatively large vacuole. Both have a marked influence on the growth process.

The rigid, elastic cell wall is characteristic of the vegetable kingdom from flowering plants to bacteria. Exceptions are found in the fungal group Myxomycetes (sometimes classed with the animals as Mycetozoa), in one group of Algae (the Eugleninae or Euglenophyta), and in the Myxobacteria and the Spirochaetes, among the bacteria. These have either flexible walls or a membrane around the cytoplasm (Thimann, 1955, Chaps. 2 and 3). All other plants have the characteristic rigid cellwalls, formed of various mixtures of polysaccharides and polyuronides. In most fungi other than some of the aquatic Phycomycetes the wall is in part chitin (a polymer of $\mathrm{N}$-acetyl-2-glucosamine) together with varying amounts of glucose polymers, though in yeasts the chitin appears to be present only in traces. In some algae, like the diatoms, the wall is heavily impregnated with silica and as a result the cells cannot enlarge at all but have to go through an occasional brief naked stage. In all land plants so far as known, in the green algae and in the Oömycetes among the fungi, the wall contains cellulose, the toughest and most inert of the fibermaterials formed out of polysaccharides.

Cell-walls of the land plants are divided into primary and secondary, according to whether they can still extend or not. Secondary walls are usually thickened by successive depositions of material from within. An example is given by the cells of wood, which are highly encrusted with lignin, interpenetrating the cellulose and other materials, and often filling up most of the lumen of the cell. Such cells are almost invariably dead. The elongated cell which comprises a cotton hair, on the other hand, has its secondary wall made up of almost pure $\alpha$-cellulose, which gives it its great tensile strength. Secondary walls are of most interest and importance for fiber and textile technology, but, from the viewpoint of growth studies, it is the primary walls that are of most interest. Analyses of the primary walls of the first shoot or coleoptile of corn (Zea mays) and oats (Avena sativa) and of the growing parts of two other seedlings are collected in Table 1 . The data must be considered quite rough since different investigators have used different material and different methods. It will be noted, however, that the cellulose, important as it is in principle, is not necessarily the largest fraction of the cellwall. Its role in growth will be taken up in section VI (p. 775).

The vacuole comprises a relatively thin liquid phase separated from the cytoplasm by a flexible membrane, the tonoplast. In small meristematic cells the vacuoles are numerous and minute filaments or globules, which may be really fragments of vacuolar membrane (tonoplast) with little or no liquid content. As the cell enlarges these primordia increase in volume and flow together, eventually forming a sap cavity which occupies most of the cell volume (Fig. I). In some cells, of which the stamen hairs of Tradescantia are a classical example, cytoplasmic threads penetrate the vacuole in many directions and the nucleus lies suspended in the center surrounded by a layer of cytoplasm and connected to the peripheral 
TABLE 1

COMPOSITION OF PUTATIVE ${ }^{1}$ GELL-WALls OF SEEDLings

All data are percent of the dry weight

\begin{tabular}{|c|c|c|c|c|c|}
\hline \multirow{3}{*}{ Fraction } & \multicolumn{2}{|c|}{ Corn Coleoptiles } & \multirow{2}{*}{$\begin{array}{l}\text { Sunflower } \\
\text { Epicotyls } \\
\text { Light-grown, } 2 \\
\text { weeks old }\end{array}$} & \multirow{2}{*}{$\begin{array}{c}\text { Oat Coleoptiles } \\
\text { Etiolated }\end{array}$} & \multirow{2}{*}{$\begin{array}{c}\text { Pea Stems } \\
\text { Etiolated } \\
(\text { apical } 20 \mathrm{~mm})\end{array}$} \\
\hline & $\begin{array}{c}\text { Etiolated } \\
(32 \mathrm{~mm} \text { long })\end{array}$ & Etiolated & & & \\
\hline & Wirth, 1946 & $\begin{array}{c}\text { Nahamura } \\
\text { and Hess, } \\
193^{8}\end{array}$ & Griffioen, $193^{8}$ & $\begin{array}{l}\text { Thimann and } \\
\text { Bonner, I933 }\end{array}$ & $\begin{array}{c}\text { Christiansen } \\
\text { and Thimann, } \\
\text { I950a, b }\end{array}$ \\
\hline Ether soluble ${ }^{2}$ & & 2.4 & - & - & 0.3 \\
\hline Pectic substances ${ }^{4}$ & 4.0 & - & 27.2 & 2.3 & 5.2 \\
\hline Protein $^{2}$ & - & - & - & $3 . \mathrm{I}$ & $3 \cdot 4$ \\
\hline Hemicelluloses & I 4.5 & - & 2.0 & II.O & I0.7 \\
\hline Cellulose $^{3}$ & 13.2 & IO.I & I 7.4 & I I.9 & 7.0 \\
\hline Lignin & - & - & 2.8 & - & - \\
\hline Total cell wall & - & 36.2 & $49 \cdot 4$ & 28.3 & 26.2 \\
\hline
\end{tabular}

${ }_{1}$ Material remaining after grinding and thorough extraction first with cold and then with hot water considered as cell-wall.

${ }^{2}$ Data only supplied for cell-wall plus water-soluble (cytoplasm).

${ }^{3}$ This figure includes such small amounts of lignin as may be present in the etiolated material.

4 The proportion of true polyuronides is probably lower than that given for pectic substances; in the case of oat coleoptiles not more than one half.

cytoplasm by the fine threads which are in constant streaming motion. In most large-vacuoled cells of land-plants, however, the nucleus is against the wall in the peripheral cytoplasm, which circulates around the whole vacuole ("cyclosis"). The cambium cells of many trees change their vacuolar type from the multipleglobule type in the fall and winter through rather unstable types containing changeable thread-like or "myelin" forms to the large central vacuole in the growing season (Bailey, I930; Fig. I).

The liquid or "sap" in the large vacuoles usually is a relatively dilute solution, though highly concentrated tannin-containing vacuoles are known. It often contains red or yellow pigments, the anthocyanins and anthoxanthins, in solution, and commonly it contains acidic and aromatic materials which stain intensely with Neutral Red. If the vacuole is acid it may even give a flocculent deeply colored precipitate with Neutral Red. Since it is relatively dilute and fills up so large a fraction of the cell volume, it follows that the increase in volume during growth is largely due to the intake of water into the vacuole. This consideration has dominated much of the work on plant growth.

\section{DEFINITIONS}

Before going further it will be convenient to define and discuss terms which will be used throughout this chapter.

Growth will be used in the sense of enlargement, either of a cell, a tissue, an 
organ or a whole plant. The definition is thus essentially irreversible increase in volume. It must be made clear that the term "growth by cell division" is inaccurate, for mere division of a cell does not in itself constitute growth. Usually division is accompanied by enlargement, so that the daughter cells rapidly reach the initial size of the parent, and commonly it is followed by much greater enlargement (see above). But cell enlargement may go on for days without any division, giving rise

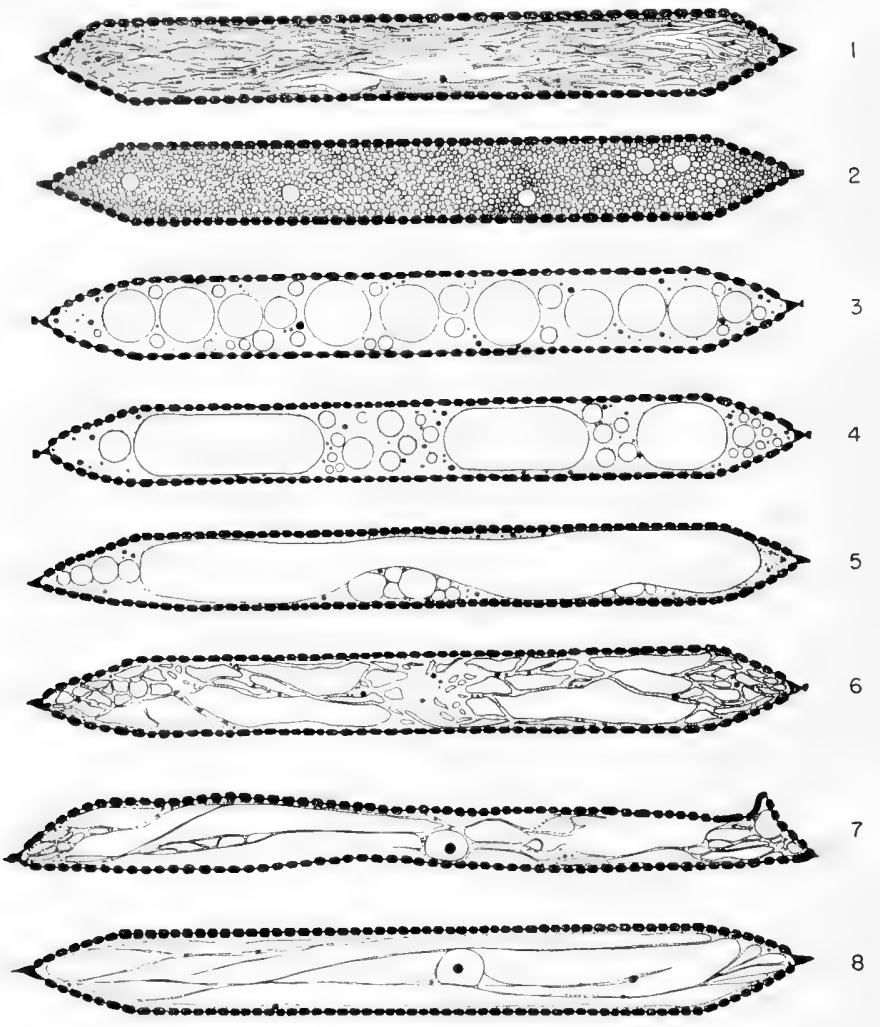

Fig. I. Examples of varied forms of the vacuole system. Fusiform initials of the cambium. I-7: Ash; (Fraxinus americana): I, "Myelin" form, transitional; 2-5, four different types from material collected in February; 6, transitional form; 7, highly vacuolated form; 8, similar cell from Robinia Pseudo-Acacia, growing season. Cytoplasm stippled, vacuole and nucleus not stippled. Originally from Bailey ( 1930); rearranged for "Contributions to Plant Anatomy";

I. W. Bailey, Waltham, Mass., The Chronica Botanica Company, I954.

to cells I oo times their initial size. It is this which constitutes the growth as ordinarily understood. Many phenomena, such as accumulation of solutes, synthesis of proteins and other polymers, and changes of physical properties may accompany growth without being an essential part of it. The problem of the nature of growth, indeed, is largely the problem as to which phenomena are essential to the growth process and which are not.

A growth hormone or phytohormone is an organic substance, produced naturally 
in higher plants, controlling growth at a site remote from its place of production, and active in minute amounts. This definition, proposed to minimize confusion (Thimann et al., I952) has been widely adopted and is parallel to similar definitions used for animal hormones. Similar growth hormones have not been demonstrated in fungi.

An auxin is an organic substance which promotes growth along the longitudinal axis, when applied in low concentrations to shoots of plants freed as far as practical from their own inherent growth-promoting substance. Auxins may, and generally do, have other properties, but this one is critical. Most auxins have a characteristic chemical structure (see section IV, p. 760) and are aromatic acids or their derivatives, but there are one or two aliphatic auxins. Furthermore the gibberellins, which are chemically very different, have many of the typical properties of auxins.

The definition, whose significance will be clearer after the discussion of auxins and their bioassay, excludes substances acting only to activate auxins already present, as for instance ordinary acids, causing marked growth effects in some green tissues, and also the ions of potassium and cobalt. It also excludes nutrients such as sucrose, which have to be supplied in relatively high concentrations.

Where action on roots is concerned, the effects of many synthetic auxins can be complex. In general auxins inhibit root elongation. Some auxin-like compounds antagonize the effects of true auxins. The resulting problems of definition, especially of the terms "auxin antagonist" and "root auxin", are taken up in section VII b (p. 8о I).

Kinins are substances of recent discovery which promote growth when acting in conjunction with auxin. The resulting growth is not typically along the longitudinal axis and the kinins are believed to act primarily by promoting cell division. Chemically they differ from the auxins, and are related to the purines (see section $\mathrm{V} \mathrm{d}, \mathrm{p} .773$ ). Both auxins and gibberellins also promote cell division in specific instances.

A meristem is a mass of undifferentiated tissue whose cells are actively dividing, or potentially capable of active division.

\section{THE MEASUREMENT OF GROWTH}

\section{(a) General}

In the i $9^{\text {th }}$ century the distribution of growth along a plant or a plant organ was measured by making a series of small ink marks at equal distances apart (say I $\mathrm{mm}$ ) and measuring their distances apart at the end of the growth period. In this way Sachs, in the I880's, demonstrated that the region of elongation in the seedling root is not at the tip but begins $\mathrm{I}-2 \mathrm{~mm}$ behind the tip and extends for several $\mathrm{mm}$ backwards. The extreme tip does not elongate appreciably, nor does the part $2 \mathrm{~cm}$ behind the tip. The intervening zone, especially its more apical part, may elongate several hundred per cent in $24 \mathrm{~h}$. The method is still in use for localization of growth zones. Some recent applications were by Went (1928) and Curry et al. (1956) for Avena coleoptiles, Van Overbeek and Went (I937) for isolated pea stem sections, and Gunckel et al. (r949) for leafy shoots of Ginkgo.

Another method much in favor was the attachment of the tip of a growing 
organ, usually a shoot, by a very light thread to a lever of large ratio, which then marked on a drum a magnified record of the growth. The device is called an auxanometer. This method does not give localization and is suitable only for relatively large organs. With its use several workers showed that shoots elongate more rapidly in the dark than in the light. In recent years more sensitive auxanometers have been devised for precise measurements on seedlings growing under controlled conditions. These depend on the closure of a contact by the growing shoot; this causes the contact to move away a fixed distance and the motion is recorded in some way. An example is the Koningsberger auxanometer, used by Dolk (1936) in studying how the growth rate alters when seedlings are placed horizontal. A more elaborate form was recently described by Idle ( $1955^{-56}$ ). These instruments can be operated in complete darkness.

More commonly, straight growth is measured by direct observation through a traveling microscope. The method is precise and the time periods can be short, though again the method does not give localization. Unfortunately, of course, illumination is required, although for many purposes a weak red or green light has little or no influence on the growth.

Growth can be measured by the increase in fresh weight, especially with isolated pieces of tissue, which can be readily blotted and weighed. This method has been used with tissue cultures ( $e . g$. by Heller, I 953) and with disks of tuber tissue (e.g. by Reinders, I 942 , and many later workers) and seems to have few disadvantages, beyond the difficulty of maintaining sterility during the transfers. It is, however, essential to avoid reversible gain or loss in weight by wetting or drying; in other words, the tissues have to be at their natural turgor.

Instead of the gain in weight of the tissue, the loss in weight or volume of the medium could be measured, but this has seldom been done. Cholodny (1933) measured the uptake of water by an isolated Avena coleoptile by following the movement of a water meniscus in a capillary tube connected to the base of the shoot.

Curvature often provides a sensitive growth response. A small difference in growth between the two sides of a shoot or root, or a fungus hypha, gives rise to an easily measurable curvature, the faster growing side becoming, of course, the convex side. In the Avena coleoptile a growth increment of $5^{\circ} \mu$ on one side appears as a curvature of about $10^{\circ}$. This can conveniently be measured with an error of $\pm I^{\circ}$. Such curvature methods have been widely used. The curvature can be produced either by applying the growth stimulant to one side only, as in the Avena test (Went and Thimann, 1937) and in its counterpart using lanoline (p. 756) or by applying it uniformly and making use of the inherent difference in response of different layers of tissue, as in the slit pea stem test (see below).

For unicellular algae the methods of bacteriology are applied-the hematocrit tube, turbidity measurements, or various types of counting plate. For fungi, most observers have washed the mycelium and determined its dry weight; this involves the assumption that the dry weight per unit volume is constant, or that assimilation of solutes does not proceed without enlargement. Neither of these assumptions appears to have troubled workers with fungal mycelia, and the error involved may not be very large. Indeed it is partly a matter of definition, since the growth of fungi (though not of green plants) might be defined in terms of gain in dry 
weight. The aerial sporangiophores of fungi can, of course, be treated like the shoots of higher plants; their elongation is limited to a very narrow zone. For example, in Phycomyces sporangiophores 60 or $70 \mathrm{~mm}$ long, the growing zone is less than $2 \mathrm{~mm}$ in length.

With these simple methods the facts of growth have gradually been uncovered, and work continues actively in many directions. Attempts to reduce growth phenomena to simple mathematical relations, popular in the I920's, have receded today, as it has become clear that they only describe the process and do not help in its analysis. "Autocatalytic" curves, the Compound Interest law, Huxley's "Heterogonic growth" formula, and the like, often approach closely to a representation of a given growth process but are seldom useful in analyzing it. Empirical relations are more informative, providing they are obtained under rigidly repeatable conditions.

\section{(b) The use and measurement of auxins}

The discovery of auxins and, more recently, of other plant growth factors, sharpened and gave meaning to growth measurements and made it possible to analyze them in causal terms. The history of the discovery of auxins has been presented several times and will not be repeated here. Full treatments are given in Went and Thimann, 1937, Chapter 2 and parts of Chapters 7,9 and Io, and in Boysen-Jensen, I 936, Chapter I. The important point to note is that the method by which it was finally proved that a diffusible growth substance exists and can be separated from the plant became the basis for a quantitative bioassay. For, in an attempt to isolate the growth substance whose presence was indicated by a great deal of indirect evidence, especially in the Avena coleoptile, Stark, in I92 I, extracted various tissues, mixed them with agar and applied the resulting agar, in little blocks, to one side of decapitated coleoptiles. Had they contained a growthpromoting substance, he reasoned, (from the behavior of isolated tips studied earlier by Paal) then the side to which the blocks were applied would have grown more than the opposite side, and a curvature would have resulted with the block on the convex side. Although Stark's attempt failed, this indeed was what was actually observed by Went (1928), after he placed living isolated coleoptile tips on agar blocks, so that they continued to excrete auxin into the block. Went then made the method quantitative by measuring the angle of curvature. This Avena test has been widely used, especially in the more botanical aspects of growth work. It has been modified by several workers and details of the final form need not be given here (Went and Thimann, I937, Chap. 3; Thimann et al., I952, Chap. 2 and Leopold, I955, Chap. 2). In essence the seedlings are grown to a standard age (about $74 \mathrm{~h}$. at $25^{\circ}$ ) in small glass holders in darkness or weak red light, the extreme tips of the coleoptiles removed, and the plants left $3 \mathrm{~h}$. for their internal auxin level to decrease to a minimum. Then a further $4 \mathrm{~mm}$ of the remaining coleoptile tissue is removed, the primary leaf inside is pulled partly out, and a small ( $c a$. Io $\mathrm{mm}^{3}$ ) block of $\mathrm{I} .5 \%$ agar containing the test substance is placed on the cut surface, resting against the primary leaf. The resulting curvature reaches its maximum at 90-I Io min. after applying the block; the plants are photographed or shadowgraphed and the curvatures measured at leisure with a simple goniometer. They are 
directly proportional to the auxin concentration, but only up to about $25^{\circ}$. Other variants of the test are described in the references cited.

A modification of the method is given by mixing the test substance with lanoline instead of agar and applying the resulting paste to the outside of intact coleoptiles. Other growing seedlings have also been used. After $24 \mathrm{~h}$. the lengths of the two sides of the (now curved) seedling are measured with a flexible scale, and the promotion or inhibition thus determined (Linser, I955, I956). Concentrations more than 1000 times as high as those in agar, and about roo times as high as those for straight growth in water (see below) are needed. However, the method has the advantage of being equally usable for growth-promoting and growthinhibiting substances, since the tip, which provides enough auxin for a moderate growth rate, is left intact.

In recent years straight growth measurements have come more into favor. They can be made by decapitating the coleoptiles, pulling out the primary leaf altogether and placing an agar block on top. The growth acceleration is measured directly on the plant with a traveling microscope, or on enlarged photographs. Controls treated with plain agar must be measured too, since growth only slows down after decapitation and by no means stops. In fact it accelerates again after $3 \mathrm{~h}$., due to production of auxin by the apical part of the remaining tissue ("regeneration of the physiological tip"); the duration of the test should therefore not exceed $2^{1} / 2 \mathrm{~h}$. Several other etiolated seedlings have been used in a similar way. Simpler straight growth measurements, however, can be made by floating sections of stems or coleoptiles on a test solution. These grow more slowly and are usually measured after i $8-24 \mathrm{~h}$. Sucrose $(2 \%)$ and a potassium salt, also occasionally manganese or better still cobalt, are added to the test solution (see section VIc). The elongation in this type of test is nearly proportional to the logarithm of the auxin concentration. There are many minor variations in this test in use in different laboratories. Descriptions are given by Thimann et al. (1952, Chap. 2, section 2), Smith et al. (1952) and in Leopold (1952, Chap. 2).

The mesocotyl or first internode, which bears the coleoptile at its apical end, is inhibited from elongating by the usual exposure to red light, but if the plants are kept in darkness it does elongate, and then sections cut from it can be used for auxin bioassay. Indeed they are more sensitive than coleoptile sections to low auxin concentrations (Nitsch and Nitsch, I956) and will determine quantities almost as small as in the curvature test with agar (see Table 2).

Of a different type are the tests based on slitting of a growing organ. Young stems, flower-stalks, petioles or coleoptiles, if slit lengthwise and held in air, curve outwards rapidly, due to the release of the longitudinal tension in the epidermis and outer layers of the cortex. If placed in water the curvature increases for several hours, probably due to swelling of the innermost thin walled layers. In pea stems, (Pisum satioum, var. Alaska), which have been most used since Went's discovery of the reaction, the curvature in water reaches a maximum of about - $160^{\circ}(-\operatorname{sign}$ signifies outwards) in 2-3 h. In hollow organs, such as Dandelion flower-stalks or onion leaves, the outward curvatures are so large that the slit stems roll up completely, but if they are cut off from the plant and placed in water for $24 \mathrm{~h}$. before slituing: the curvatures are greatly decreased (see Jost and Reiss, I936); evidently 
the reduced growth rate of the isolated organ enables the tissue tensions to be partly released.

Now if these slit organs are placed in auxin solution instead of in water the outward curvature in the more apical parts gradually becomes reversed and presently the two halves are curving inward. The extent of inward curvature reaches a maximum in about $28 \mathrm{~h}$. with etiolated pea stems (Thimann and Schneider, I938) and this maximum is roughly proportional to the logarithm of the auxin concentration. The inward curvature is greatly decreased by peeling the epidermis from the tissue, and it is increased by cutting the stems or coleoptiles into quarters instead of halves. It is deduced that the inward curvature is caused by greater elongation of the outer layers than of the inner, that is, greater response to a given auxin concentration (see section VIa, p. 775).

This type of curvature has one advantage over that due to placing auxin in agar on one side of a coleoptile, namely that it is not dependent on transport of the auxin down from a small cut surface because the whole organ is in the solution. Consequently many synthetic auxins which are almost inactive in the standard Avena test cause good curvatures on slit pea stems. The same is true for straight growth of coleoptile, mesocotyl or stem sections floating on solutions, although here there is (for reasons unknown) more specificity in Avena coleoptiles than in Pisum stems, and transport from the cut surface still plays some part.

Straight growth of stem sections from etiolated pea seedlings is a little more limited but otherwise similar to the growth of coleoptiles. Sections must be taken from near the growing apex since the elongating zone is quite limited. In Alaska peas, the procedure is to use the 3 rd internode when it is about two-thirds grown. This occurs at an age of 7 days at $25^{\circ}$, provided occasional weak red light is given; in complete darkness the internodes elongate more in situ and are less satisfactory as sections. If the sections are cut $20 \mathrm{~mm}$ long (as close as possible to the terminal "hook"), sucrose is not needed and they elongate in optimal concentrations of auxin solution about $60^{\circ}$, reaching their maximum growth in $\mathrm{i} 6 \mathrm{~h}$. at $25^{\circ}$ (Christiansen and Thimann, I950a, and literature there cited). Cobalt increases the growth a further $10-25 \%$, providing that sucrose is added (Miller, i 954; Thimann, I $956 \mathrm{~b})$. Gibberellin also increases the growth about $20 \%$, irrespective of the auxin concentration (Kato, I957).

Bioassays of auxins have also been based on other functions of these substances, such as the promotion of root development on stems, or of the growth of fruits without pollination (see section VIIc, p. 807), or even in some cases on the inhibition of growth.

One test based on inhibition has been widely used. If elongating roots are placed in auxin solutions their growth rate is greatly decreased, and the extent of decrease is roughly proportional, over a limited range of concentrations, to the logarithm of the concentration. Oat, wheat and flax roots are the most used (Wilske and Burström, I 950; Åberg, I 950). In many cases very low concentrations of auxins, about $\mathrm{IO}^{-11} \mathrm{M}$, slightly promote the elongation, but the effect is often elusive; this is most noticeable and reproducible with isolated sections of roots (Leopold and Guernsey, I953; Audus and Das, I955). Even without making use of this promotion, the root inhibition test is the most delicate of all tests, showing 
TABLE 2

SUMMARY OF METHODS FOR DETERMINATION OF AUXIN IN EXTRACTS

\begin{tabular}{|c|c|c|c|}
\hline Test & $\begin{array}{c}\text { Working } \\
\text { level } \\
\text { mg/l }\end{array}$ & $\begin{array}{l}\text { Volume } \\
\text { required } \\
\text { ml }\end{array}$ & $\begin{array}{c}\text { Minimum amount } \\
\text { determinable } \\
\mu g\end{array}$ \\
\hline I. Salkowski reaction in solution & 8 & 3 & 6 \\
\hline $\begin{array}{l}\text { 2. Avena coleoptile curvature } \\
\text { (lanoline-paste) }\end{array}$ & $20^{2}$ & I & 2 \\
\hline 3. Salkowski reaction on paper & 一 & 0.02 & 0.3 \\
\hline $\begin{array}{l}\text { 4. Pea stem curvature or straight } \\
\text { growth }\end{array}$ & 0.5 & $5^{-20}$ & 0.2 \\
\hline $\begin{array}{l}\text { 5. Avena straight growth; } \\
\text { coleoptiles } \\
\text { 6. Avena straight growth: }\end{array}$ & 0.2 & $\mathrm{I}-\mathbf{I O}$ & 0.03 \\
\hline $\begin{array}{l}\text { 6. Avena straight growth; } \\
\text { mesocotyls } \\
\text { 7. Avena coleoptile curvature }\end{array}$ & 0.1 & $0.5^{-2}$ & 0.005 \\
\hline (agar) & 0.06 & $0.06^{3}$ & 0.002 \\
\hline 8. Root inhibition tests & 0.05 & $4-100$ & $c a .0 .002$ \\
\hline
\end{tabular}

1 Upper limit about 10 times this level; lower limit about one quarter for nos. I and 7 , about one tenth for nos. 2-6.

${ }^{2}$ Would not in general be used with aqueous solutions.

${ }^{3}$ Mixed with an equal volume of $3 \%$ agar to make enough blocks for 12 plants.

a clear inhibition (about $20 \%$ ) at $\mathrm{IO}^{-9} M$ indoleacetic acid or about $0.2 \mu \mathrm{g}$ per liter of solution. However, because the roots require several $\mathrm{ml}$ of solution, the absolute amount of auxin detected, about $\mathrm{I} \cdot \mathrm{IO}^{-3} \mu \mathrm{g}$, is not much lower than with the Avena agar test, which detects about $2 \cdot \mathrm{IO}^{-3} \mu \mathrm{g}$, or the straight growth of Avena mesocotyl sections detecting about $5^{\cdot 10^{-3}} \mu \mathrm{g}$ (see section VIIb, p. 80 I for detailed discussion). Because biological extracts often contain non-specific inhibitors in addition to auxins, root inhibition is not satisfactory for bioassay of unknown extracts.

The sensitivities of several bioassays are compared with that of a chemical color reaction in Table 2.

Most of these tests have been used also for other growth factors and for growth inhibitors, including chemical enzyme poisons and naturally occurring inhibiting substances extracted from plants. The procedure in general is to add optimum concentrations of auxin and/or nutrients and study the inhibition of growth as function of concentration of inhibitor. Similar methods have been used to study the influence of external conditions such as temperature, light, salt solutions, etc.

\section{(c) Assays for other growth factors}

For kinins different methods are required, since kinetin and related kinins have little or no action on cell elongation as such. Though standardized methods have not jet been published, the most specific test would seem to rest on the ability of kinins to bring about the development of buds in undifferentiated tissue. Isolated 
pieces of the pith of tobacco stems (Nicotiana tabacum), for instance, grow well on a sugar-salts agar medium with the addition of auxin, to form a mass of callus, from which there soon differentiate roots, but no buds. If the auxin concentration is not too high - about $2 \mathrm{mg}$ indoleacetic acid per liter-then the addition of kinetin produces a profusion of buds (see Fig. 2). Up to kinetin levels of $\mathrm{I}-2 \mathrm{mg} / \mathrm{l}$,

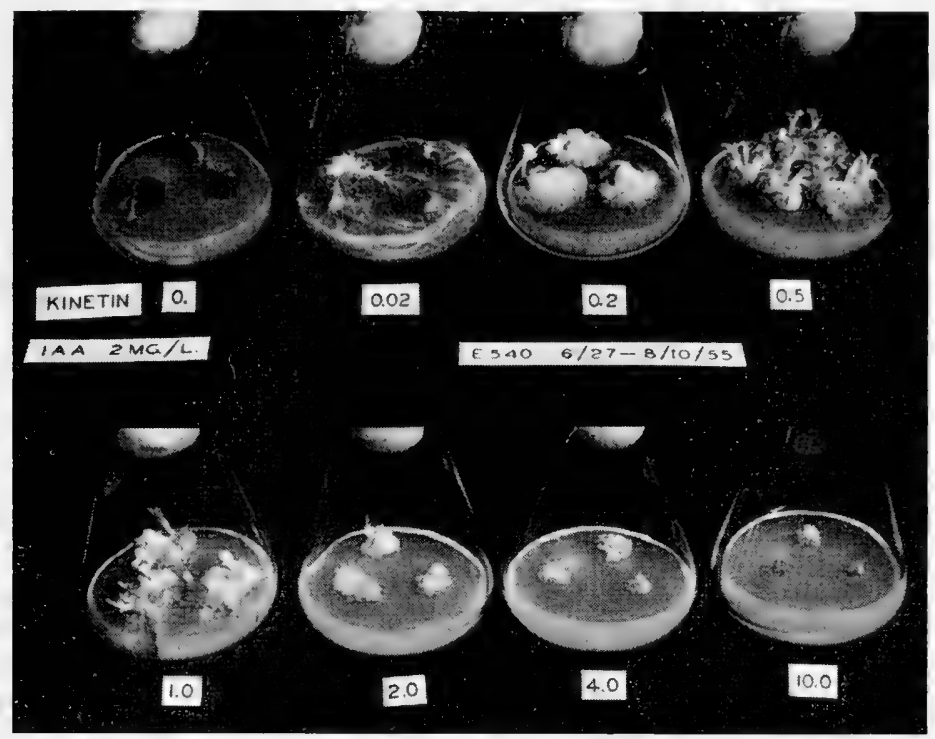

Fig. 2. Effect of kinetin concentration (in o-10 $\mathrm{mg} / \mathrm{l}$ range) on growth and organ formation of tobacco callus cultured on modified White's nutrient agar with $2.0 \mathrm{mg} / \mathrm{l}$ indoleacetic acid added to medium. Age of cultures 44 days. (From F. Skoog and C. O. Miller, Symp. Soc. Exptl. Biol., Cambridge University Press, 1957.)

the number of the buds appears to be roughly proportional to the logarithm of the kinetin concentration. The absolute concentrations, $0.2-\mathrm{Io} \mathrm{mg} / \mathrm{l}$, are similar to the effective auxin concentrations. Simple purines, especially adenine, have a similar effect but quantitatively are only about I/I 000 as active (Miller and Skoog, 1953). Increased auxin concentrations, in the presence of kinetin, suppress the bud development and lead to still larger masses of more or less undifferentiated callus. Thus the auxin and kinetin levels can be balanced against one another to give quantitative control over the formation of roots, buds and callus ( $c f$. p. 773).

In general where cell division is concerned, the requisite counts of cell number are laborious and inconvenient to make. This difficulty has been circumvented by the technique of maceration (Brown and Rickless, 1949). The tissue is shaken in a mixture of $5 \%$ chromic and $5 \%$ hydrochloric acids, which separates the cells from one another much as does strong $\mathrm{NaOH}$. Drawing the macerate in and out of a hypodermic needle increases the separation. Samples of the macerate are then placed on a hemocytometer slide and the cells counted in the same way as erythrocytes. A modification of this method has been extensively used in tissue 
culture work, especially that of Steward and coworkers on carrot tissue (Steward and Caplin, I954).

For gibberellins, the original bioassay, used in all the earlier work in Japan, rests on the excessive elongation of the leaves of rice (Oryza sativa). This was originally observed in plants infected with "bakanae" disease, caused by the fungus Gibberella fujikuroi, the commoner imperfect form of which is Fusarium moniliforme (see reviews of Stowe and Yamaki, I 957, I959). The assay was carried out by adding to the soil the gibberellin, or even the culture filtrate of the fungus, and observing the leaf elongations after varying numbers of days or weeks (Brian et al., I $954 ; c f$. p. 780$)$.

However, with the recent discovery of the powerful effects of gibberellins on the elongation of dwarf plants of several species, the more favored methods of assay make use of these. The gibberellin preparation is either added directly to the leaves, by spraying or as measured droplets, or else added to a nutrient solution in which the roots are immersed. The substance is rapidly transported through the plant and the resulting stem elongation can be measured in as little as $24 \mathrm{~h}$. The tests can be carried out in the light with light-grown plants, for the outstanding characteristic of gibberellin's action is that it is scarcely affected by light. In this it is quite unlike the action of auxin which, at least for stem elongation, is very greatly decreased in the light. In other respects it does have much in common with the auxins, and certainly qualifies as an auxin by definition (Brian and Hemming, I 955). As test plants, dwarf varieties of peas (Brian and Hemming, I955) or corn (Phinney, 1956) are very satisfactory.

Another possible assay would be based on the elongation of isolated sections of leaves, particularly young leaves of oats or barley (Hayashi and Murakami, I 954). The growth of this material is not promoted, and is even slightly inhibited, by auxin so that the test would be very specific. Since it is only in the last four years that the Western world has become aware of the extensive and thorough Japanese work on the gibberellins it may be expected that the methods used will be rapidly modified.

\section{CHEMISTRY OF THE GROWTH SUBSTANGES}

The most widely distributed of the auxins is indoleacetic acid I, (usually abbreviated as IAA). This has been isolated in pure form from corn seeds as well as from yeast, and from cultures of the fungus Rhizopus suimus. Its ethyl ester has been isolated from immature corn kernels, though the conditions of extraction were such that esterification with ethanol might have taken place. It has been identified (with varying degrees of certainty) by chromatographic and colorimetric methods in a great many plants, including both mono- and dicotyledons in many different families (Bentley, I958). It has been shown also to be present in plant tissue cultures (Table 3), where it is actually synthesized in good quantity (Kulescha, I 95 I ; Stowe, Thimann and Kefford, I 956), and in pollen, where it is formed in relatively large amounts from tryptophan (Lund, 1956). Some of these identifications rest on several properties, such as $R_{F}$ 's in different solvents (in paper chromatography), the Salkowski or other color reagent, molecular weight by diffusion 
TABLE 3

AUXIN CONTENT OF TISSUE GULTURES, EXPRESSED AS EQUIVALENT PARTS OF INDOLEACETIC ACID PER I ${ }^{8}$ PARTS OF FRESH WEIGHT

Data of Kulescha, 195I

\begin{tabular}{lcccc}
\hline & $\begin{array}{c}\text { Artichoke } \\
\text { (Helianthus } \\
\text { tuberosus) } \\
\text { tuber }\end{array}$ & $\begin{array}{c}\text { Grape } \\
\text { (Vitis vinifera) } \\
\text { stem }\end{array}$ & $\begin{array}{c}\text { Scorzonera } \\
\text { hispanica } \\
\text { root }\end{array}$ & $\begin{array}{c}\text { "Vigne-vierge" } \\
\text { (Parthenocissus } \\
\text { tricuspidata) } \\
\text { stem }\end{array}$ \\
\hline Freshly isolated & $\mathrm{I} .5$ & $4 . \mathrm{I}$ & 0.5 & $\mathrm{I} .9$ \\
After 4I-45 days' culture & $0.2-0.3$ & $\mathrm{I} . \mathrm{I}$ & $\mathrm{I} . \mathrm{I}-\mathrm{I} .2^{1}$ & 0.3 \\
Accoutumé tissue & - & 6.6 & $\mathrm{I} .5-2.3$ & $\mathrm{I} .8$ \\
Grown gall (in culture) & $\mathrm{I} .4-\mathrm{I} .5$ & $c a .5$ & $5.3-5.5$ & 4 \\
\hline
\end{tabular}

1 Probably due to buds formed

and the well known instability to hot acid. However, a number of the assumed identifications are less thorough and may require revision. Nevertheless, the reactions of indoleacetic acid are fairly characteristic, and there can be little doubt about its very widespread occurrence as a natural auxin.

In some plants a neutral material occurs which has little or no growth-promoting activity when freshly extracted but is rapidly converted by enzymes present in soil, milk or (in some cases) in test plants themselves, to an active auxin, which is an acid. This neutral material was first found in etiolated seedlings and therein tentatively identified by Larsen ( 1944, r 95 I b) as indoleacetaldehyde, II. The enzyme converting it to the acid, which of course is indoleacetic acid, I, is aldehyde dehydrogenase or Schardinger erizyme. The aldehyde occurs in pea and sunflower seedlings, in potato tubers, corn embryos and pineapple leaves. Probably, then, it is widespread, and this fact indirectly supports the importance of IAA as a general plant hormone. Aldehyde oxidases or dehydrogenases are, of course, universal and there is no reason to believe, at least as far as present evidence goes, that indoleacetaldehyde requires a specific dehydrogenase.

A third indole compound, indoleacetonitrile, III, has been isolated from cab-

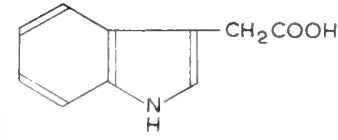

Indoleacetic acid I

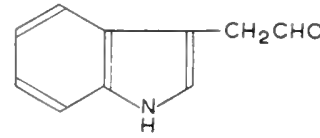

Indoleacetaldehyde

II<smiles>N#CCc1c[nH]c2ccccc12</smiles>

Indoleacetonitrile III

bage (Henbest et al., I 953) and its presence is virtually certain in several closely related vegetables. It has also been tentatively identified in several unrelated plants including tissue cultures. Unfortunately, the chromatographic behavior of this and other neutral compounds in most procedures is not highly characteristic, so that many of the identifications are as yet by no means certain. A remarkable characteristic of the nitrile is that, although more active then IAA on Avena cole- 
optile sections, it is almost completely inactive on pea sections under the same conditions (Bentley and Bickle, I952). On other test plants, notably lupine hypocotyls and corn coleoptiles, it shows only a low activity which is not proportional to concentration (Thimann, I 954a). This behavior has been taken to indicate that it is active only through conversion to indoleacetic acid, a process which would occur rapidly in Avena, slowly or to a strictly limited extent in other plants, and not at all in peas. The conversion in Avena has been proved by both chromatographic means and bioassay (Stowe and Thimann, 1954). It remains to be determined whether III has any true auxin activity of its own.

In addition to the above, indolepyruvic acid, IV, has been reported by chromatography and bioassay in corn (Stowe and Thimann, 1954). Similar materials are present in soybean and tobacco leaves (Vlitos and Meudt, I953-4) wheat roots and broad bean seedlings. Unfortunately, the pure substance is quite unstable, being rapidly converted in aqueous solution to a mixture of substances including indoleacetic acid. Whether it has true auxin activity of its own is thus, just as in the case of indoleacetonitrile, difficult to determine. Recently it has been shown that the chromatographic spot given by pure indolepyruvic acid is actually due to its conversion to indoleglycollic acid, $\mathrm{V}$, and other compounds so that the material in plants may really be indoleglycollic acid (Bentley et al., I 956). However, since the biological activity of this substance is extremely low, not over $0.3 \%$ of that of indoleacetic acid, it is not certain whether this interpretation can be consistent with all the facts. Apart from this, $\mathrm{V}$ has not been reported in plants, though it was tentatively identified among the products of the breakdown of indoleacetic acid by light (Fischer, I954). Evidence for other naturally occurring auxins, with and without the indole nucleus, has recently been reviewed by Bentley ( $\left.195^{8}\right)$.

The special significance of indolepyruvic acid would be, of course, as an intermediate in the conversion of tryptophan, VI, to auxin. This reaction, as carried

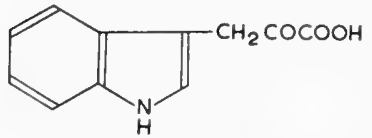

Indolepyruvic acid IV

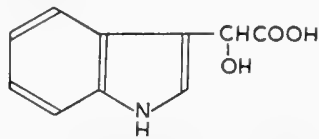

Indoleglycollic acid $\mathrm{V}$

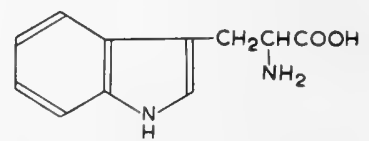

Tryptophan VI

out by bacteria (Stowe, I955) comprises two steps: (a) transamination to indolepyruvic acid, using $\alpha$-ketoglutaric or other $\alpha$-keto acids (but not pyruvic acid), together with pyridoxal-5-phosphate as co-enzyme, and (b) oxidation (or conceivably dismutation) of the resulting indolepyruvic to indoleacetic acid and $\mathrm{CO}_{2}$, a reaction which proceeds spontaneously to some extent and is probably accelerated by systems in the plant. The ready conversion of tryptophan to IAA by several bacteria and fungi, by spinach and pineapple leaves (see review of Gordon, I954) and by pollen tubes (Lund, I956) may proceed in this way or by direct oxidative deamination. However, some higher plants do not form an active auxin from tryptophan very readily. Avena coleoptiles show a delayed growth response to tryptamine, VII, which has been interpreted as due to its conversion to IAA. 
The various pieces of inconclusive evidence for tryptophan and tryptamine as precursors of IAA cannot be detailed here; the matter has been reviewed more than once (Larsen, I95 Ia; Gordon, I 954, I956).

A wide variety of synthetic substances have auxin activity. These are constructed on the general molecular plan of IAA, that is, with an aromatic ring and an aliphatic acid side-chain. Examples include indeneacetic acid, VIII, indolebutyric acid, IX, sometimes used for the rooting of cuttings, and naphthalene acetic acid,<smiles>NCCc1c[nH]c2ccccc12</smiles>

Tryptamine VII

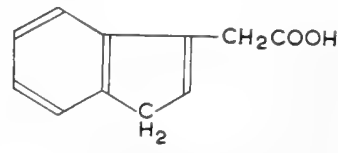

Indeneacetic acid VIII<smiles>O=C(O)CCCc1c[nH]c2ccccc12</smiles>

Indolebutyric acid IX

or NAA, X, widely used to inhibit the abscission of fruits in the orchard industry. Other substances will be discussed in various parts of this chapter.

High auxin concentrations are so toxic that synthetic auxins find their widest application as weed-killers. For this purpose NAA was first used, but now the most widely used substances are 2,4-dichlorophenoxyacetic acid or "2,4-D", $\mathrm{XI}$, the corresponding trichloro-derivative " $2,4,5-\mathrm{T}$ ", XII, and the 2-methyl-4chloroderivative "Methoxone", XIII.<smiles>O=C(O)Cc1cccc2ccccc12</smiles>

Naphthalene acetic acid $\mathrm{X}$<smiles>O=C(O)COc1ccc(Cl)cc1Cl</smiles>

2, 4-Dichlorophenoxyacetic acid $\mathrm{XI}$<smiles>O=C(O)COc1cc(Cl)ccc1Cl</smiles>

Trichloro-derivative of XI XII<smiles>Cc1cc(Cl)ccc1OCC(=O)O</smiles>

2-methyl-4-chloroderivative XIII

A large literature has grown up around the characteristic features of the molecule which are required for auxin activity (see Thimann, I95 Ia; Veldstra, I953, I956; Pybus et al., I 959). It was originally thought that an acid group, or a structure readily converted to an acid group, must be present, and that this must be held at a distance of one or more atoms from an unsaturated ring. Esters, amides and nitriles are examples of structures readily converted to carboxylic acids, and those active compounds which belong to these types may all owe their biological activity to conversion to the corresponding free acid. In a few cases, however, they show somewhat higher activity in one or another test than the free acid. For example, ethylindoleacetate is IO-IOO times more active than IAA in causing parthenocarpy, and indoleacetonitrile is IO-20 times more active than IAA on the growth of coleoptiles. Such behavior has been ascribed to the greater ease of entry of these neutral derivatives into the cells, but of course it might mean that they are active per se, and this is often difficult to disprove.

The requirement for distance between ring and acid group is exemplified by the inactivity of indole-carboxylic and benzoic acids, but it does not necessarily 
always hold. Exceptions are provided, among others, by the weak but real activity of I,2,3,4-tetrahydronaphthoic acid, XIV, and the quite strong activity of 2,3,6-trichlorobenzoic acid, XV, and some other chlorinated benzoic acids. An explanation for this latter case has been suggested, based on the idea that the acid<smiles>O=C(O)C1CCCc2ccccc21</smiles>

I, 2, 3, 4-Tetrahydronaphthoic acid XIV<smiles>O=C(O)c1ccc(Cl)c(Cl)c1Cl</smiles>

2, 3, 6-Trichlorobenzoic acid XV

group has to be spaced, not from the ring itself, but from specific combining positions on it. This is part of the general concept held by many workers that the auxin molecule combines with its substrate (whatever that may be) at several specific points; in other words, it is the shape of the molecule as a whole, as well as the location and reactivity of the main combining groups, that determines biological effectiveness. Strong support for this viewpoint is given by the differing activity of geometrical and optical isomers. In acids with unsaturated side-chains it is the cis-forms which are active, e.g. cinnamic acid, XVI, and several of its derivatives, all of which have real (though not very high) activity in the cis-form, while the trans-forms are inactive. In acids with an asymmetrical carbon atom in the sidechain it is the $\mathrm{D}$-forms which are the most active, e.g. $\alpha$-( 3 -indole)propionic acid, XVII. The L-forms do have some activity in most cases, but it is always less than the D, and it varies down almost to zero (Matell, I953).

Recently it has been shown that compounds without a ring may have weak but real activity, especially in the slit pea curvature test, which is usually the least specific. S-Carboxymethyl-N, N-dimethyldithiocarbamate, XVIII, was prepared in the course of a study of fungicides (Van der Kerk et al., I955) and shown to have

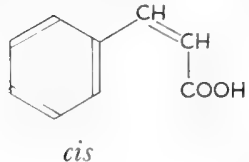

Cinnamic acid

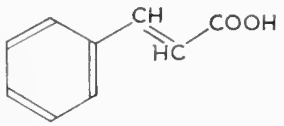

trans

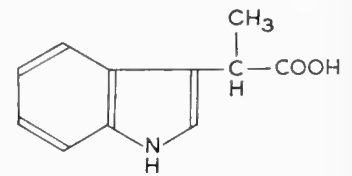

$\alpha$-(3-indole $)-$ propionic acid

XVII<smiles>CN(C)C(=S)SCC(=O)O</smiles>

S-Carboxymethyl$\mathrm{N}, \mathrm{N}$-dimethyldithiocarbamate

XVIII

auxin activity. It may be that resonating double bond structure gives the molecule a flat configuration similar to that conferred by a ring. Several related compounds are active also (Veldstra, I955; Fawcett et al., r 956a, b).

Many compounds with the general structure of auxins, but without actual growth-promoting activity, may still strongly affect the growth responses caused by auxins. In some cases, e.g. 2,4,6-trichlorophenoxyacetic acid, XIX or $\alpha$-indoleisobutyric acid, $\mathrm{XX}$, they act as antagonists, and there is evidence, based on kinetic 
analysis, that the antagonism is competitive $(c f$. section VIIb). In other cases, e.g. cyclohexaneacetic acid, XXI, or 2,3,5-triiodobenzoic acid, XXII, they act as<smiles>O=C(O)COc1ccccc1Cl</smiles>

2,4,6-Trichlorophenoxy acetic acid

XIX<smiles>CC(C)(C(=O)O)c1c[nH]c2ccccc12</smiles>

$\alpha$-Indoleisobutyric acid XX<smiles>O=C(O)C1CCCCC1</smiles>

Cyclohexaneacetic acid XXI<smiles>O=C(O)c1ccccc1</smiles>

2,3,5-Triiodobenzoic acid XXII

\section{ERRAT U M}

Chapter Io, page 765. The formula XXI should read:<smiles>O=C(O)C1CCCCC1</smiles>

\section{Cyclohexaneacetic acid XXI}

while gibberellin $\mathrm{A}_{2}$ of Sumiki and co-workers is $\mathrm{C}_{19} \mathrm{H}_{26} \mathrm{O}_{6}$. The crystalline product obtained from cultures of Fusarium moniliforme in the USA is a mixture of $\mathrm{A}_{1}$ and $\mathrm{A}_{3}$ (Stodola et al., I955). The original "gibberellin A", first isolated by Yabuta and Hayashi ( 1939) may have contained all three. So far as their potencies have been compared all three seem to be about equally active, but as yet the comparisons arc very incomplete. On the other hand the breakdown product allogibberic acid, (gibberellin $\mathrm{B}$ of Sumiki) XXIV, $\mathrm{C}_{18} \mathrm{H}_{20} \mathrm{O}_{3}$, may have lower activity, while its rearrangement product formed in hot dilute acid, gibberic acid, XXV, is apparently inactive. $\mathrm{A}_{3}$ has also been isolated from higher plants.

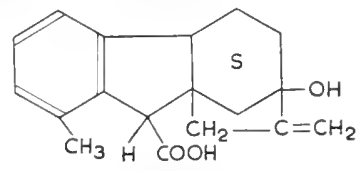

Allogibberic acid XXIV

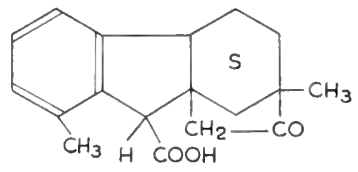

Gibberic acid XXV 
always hold. Exceptions are provided, among others, by the weak but real activity of I,2,3,4-tetrahydronaphthoic acid, XIV, and the quite strong activity of 2,3,6-trichlorobenzoic acid, XV, and some other chlorinated benzoic acids. An explanation for this latter case has been suggested, based on the idea that the acid<smiles>O=C(O)C1CCCc2ccccc21</smiles>

I, 2, 3, 4-Tetrahydronaphthoic acid XIV<smiles>O=C(O)c1ccc(Cl)c(Cl)c1Cl</smiles>

2, 3, 6-Trichlorobenzoic acid XV
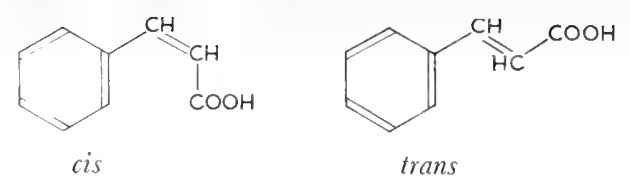

Cinnamic acid

\section{XVI}<smiles>CC(C(=O)O)c1c[nH]c2ccccc12</smiles><smiles>CN(C)C(=S)SCC(=O)O</smiles>

$\alpha$-(3-indole)propionic acid

XVII
S-Carboxymethyl$\mathrm{N}, \mathrm{N}$-dimethyldithiocarbamate XVIII

auxin activity. It may be that resonating double bond structure gives the molecule a flat configuration similar to that conferred by a ring. Several related compounds are active also (Veldstra, I955; Fawcett et al., I 956a, b).

Many compounds with the general structure of auxins, but without actual srowth-promoting activity, may still strongly affect the growth responses caused by auxins. In some cases, e.g. 2,4,6-trichlorophenoxyacetic acid, XIX or $\alpha$-indoleisobutyric acid, $\mathrm{XX}$, they act as antagonists, and there is evidence, based on kinetic 
analysis, that the antagonism is competitive ( $c f$. section VIIb). In other cases, e.g. cyclohexaneacetic acid, XXI, or 2,3,5-triiodobenzoic acid, XXII, they act as<smiles>O=C(O)COc1ccccc1Cl</smiles>

2,4,6-Trichlorophenoxy acetic acid XIX<smiles>CC(C)(C(=O)O)c1c[nH]c2ccccc12</smiles>

$\alpha$-Indoleisobutyric acid XX<smiles>O=C(O)C1CCCCC1</smiles>

Cyclohexaneacetic acid XXI

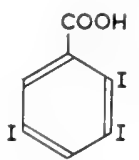

2,3,5-Triiodobenzoic acid XXII

synergists in the slit pea stem test, greatly increasing the apparent activity of added auxin.

High auxin concentrations, as mentioned above, inhibit growth and very high levels are toxic. Hence a curve plotting growth against auxin concentration necessarily passes through an optimum (see Fig. 7, p. 776). This phenomenon is discussed in detail in section VIb (p. 776).

The gibberellins are quite unrelated to the compounds described above, although in their physiological effects they have some properties in common (see section VId, p. 780). Gibberellic acid (also termed gibberellin $\mathrm{A}_{3}$ and gibberellin $\mathrm{X}$ ) is a lactone-acid derived from I,7-dimethylfluorene and has the tentative formula, XXIII, $\mathrm{C}_{19} \mathrm{H}_{22} \mathrm{O}_{6}$ :

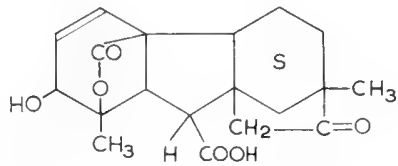

Gibberellic acid

XXIII

(Curtis and Cross, I 954; Stodola, I956). Gibberellin $A_{1}$ of Sumiki (Takahashi et al., I 955) has been termed gibberellin $\mathrm{A}$ in the USA and is probably $\mathrm{C}_{19} \mathrm{H}_{24} \mathrm{O}_{6}$, while gibberellin $\mathrm{A}_{2}$ of Sumiki and co-workers is $\mathrm{C}_{19} \mathrm{H}_{26} \mathrm{O}_{6}$. The crystalline product obtained from cultures of Fusarium moniliforme in the USA is a mixture of $\mathrm{A}_{1}$ and $\mathrm{A}_{3}$ (Stodola et al., I955). The original "gibberellin A", first isolated by Yabuta and Hayashi (1939) may have contained all three. So far as their potencies have been compared all three seem to be about equally active, but as yet the comparisons are very incomplete. On the other hand the breakdown product allogibberic acid, (gibberellin $\mathrm{B}$ of Sumiki) XXIV, $\mathrm{C}_{18} \mathrm{H}_{20} \mathrm{O}_{3}$, may have lower activity, while its rearrangement product formed in hot dilute acid, gibberic acid, $\mathrm{XXV}$, is apparently inactive. $\mathrm{A}_{3}$ has also been isolated from higher plants.

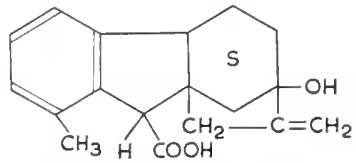

Allogibberic acid XXIV

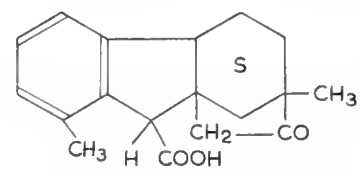

Gibberic acid $\mathrm{XXV}$ 


\section{GELL DIVISION}

Any consideration of growth at the cellular level must consider the two aspects of cell division and cell enlargement or "meresis" and "auxesis." In bacteria and many unicellular and filamentous algae, the cell divides only when it has reached a certain size. Thus division is preceded by enlargement. The pattern of growth in higher plants is generally the reverse, that is, division is followed by enlargement. The most active division in higher plants takes place in meristems, which it will be convenient to consider here. They were defined in section II; the only real criterion of a meristematic tissue is active cell division. However, in some cases the division is only potential and the meristem said to be resting or dormant. The location of the meristem, the cell types in it and the behavior of the cell after division may all vary widely.

\section{(a) Apical meristems}

Apical meristems are composed of cells dividing rapidly and enlarging slowly, thus forming a nest of small cells. Commonly they are rich in cytoplasm, and the vacuole is present only as a network of threads or globules which does not coalesce to a single sap-vacuole until the cells have enlarged (Fig. I). But sometimes, as in the apical cells of ferns or the cells near the top center of many other apical meristems, quite large vacuolated cells are present. Occasionally even cells with a high oil or tannin content may occur, though it is not certain that these are rapidly dividing. The typical vegetative meristem is most active on its flanks, where it gives rise to a series of leaf primordia; for a time these are continuous with the dividing meristem of the

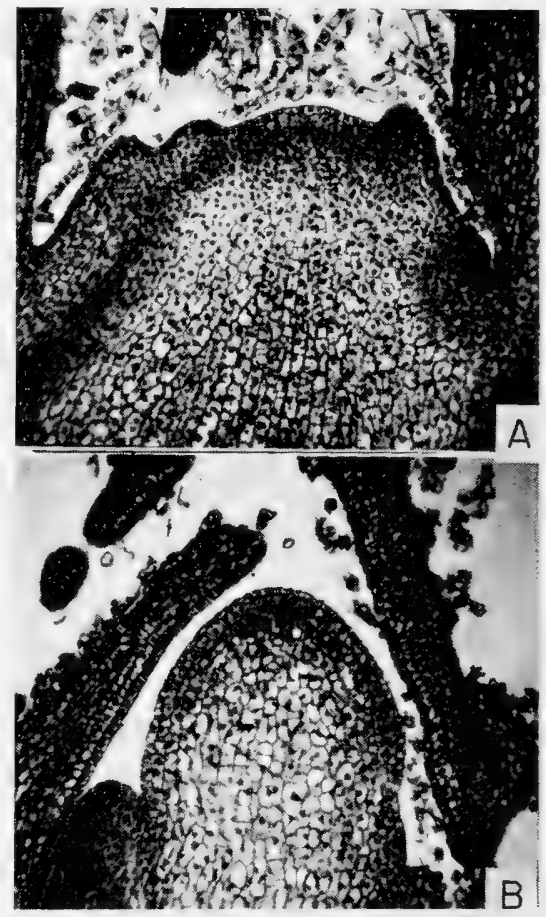

Fig. 3. Longitudinal sections of the apex of Xanthium pennsylvanicum (cocklebur). A: vegetative apex, growing on long days. B: apex becoming reproductive, after six 8-h. days. The vegetative apex has vacuolated non-dividing cells in the center and actively dividing cells forming leaf primordia on its flanks; the reproductive apex has changed shape, due to centrally located cell division and enlargement, and the non-dividing cells in the center have become active (Courtesy of Prof. R. H. Wetmore). apex, but then they get pushed away from it by the elongation of some of the cells that have divided to form the usually long and narrow leaf initial (see section VIIa, p. 797). Indeed the enlargement that goes on at the base of the meristem is also primarily elongation, so that the apex becomes pushed upwards at the top of an elongating cylindrical stem. Why the cells do not grow in all three dimensions is not known. A typical vegetative 
apex of a dicotyledon is shown in Fig. $3 \mathrm{~A}$, and the changes which precede its conversion to the reproductive phase are seen in Fig. $3 \mathrm{~B}$.

In the root, the extreme apex is made up of the root cap, which is continually being added to by division of cells of the meristem, which in this case is just behind the apex. The root-cap cells do not divide, nor do they enlarge much; they are sloughed off at the extreme tip as fast as they are added. The apical meristem, commonly about I/2-I mm long, comprises very small cells which elongate extensively and rapidly. There is no folding out at the flanks to produce anything corresponding to the leaf primordia. Lateral roots (see section VIIb, p. 8o I) appear at some distance from the apex and quite disconnected from it.

\section{(b) Secondary meristems}

Cells of the vascular cambium are quite different from those of the apical meristem; they are very long (up to $4 \mathrm{~mm}$ ) and narrow. They have a good-sized nucleus, usually in the center, and an elaborate system of vacuoles in fine threadlike channels, distinct globules or relatively large units (see Fig. I); these vacuoles change with the season in many plants and the patterns are often characteristic of the species, especially in trees (Bailey, r 930). Unlike apical meristematic cells, the walls of vascular cambium may be quite thick.

In general, division of these cells is longitudinal or oblique, and enlargement takes place transversely (giving rise to increased girth of the stem). Occasionally a long cell will divide up into short ones by short cross-walls; this is the unusual phenomenon of division without enlargement. It sometimes follows wounding, or the abnormal access of oxygen.

The cork cambium, which is active in stem cortex to cut off long and narrow sub-epidermal cells, resembles the vascular cambium in some ways, but the cells are not exceptionally long. They often contain fully differentiated chloroplasts. After transverse enlargement the cell-walls become heavily "suberized" i.e. coated internally with cork, and the contents die. It was the microscopic appearance of cork cells which historically gave rise to the cell theory.

\section{(c) Morphology of cell division}

Plant cell divisions differ from those in animal cells in two major respects: (a) no aster is formed, (b) the residues of the spindle fibers do not disappear after mitosis, but become incorporated into the cell plate or primary cell-wall initial.

The process of cell division is not easily studied in the meristems themselves since the cells are small. They are excellent for studying the nuclear phenomena, but for the role of the other parts of the cell, relatively large vacuolate cells are more informative. Such large dividing cells are found just below the meristem in the apex of the growing shoots, or can be induced in other more mature internodes by wounding nearby. In the latter case very large cells often divide; the cytoplasm and nucleus both increase in volume, the nucleus becomes spherical and thereafter the general process is like that for the sub-meristematic cells (see the review of Bloch, I94I).

The first stage in division appears to be the swelling of the nucleus from a flat spheroid into a sphere and its movement away from the cell wall. Cytoplasmic 
strands running to each wall appear. It is these which indicate the plane of division, for the one which lies in the position of the future wall becomes much thicker; this heavy persistent cytoplasmic strand is called the phragmosome (Sinnott and Bloch, I94I). The metaphase plate clearly lies in the plane of the phragmosome (Fig. ${ }_{4} \mathrm{~B}$ ).
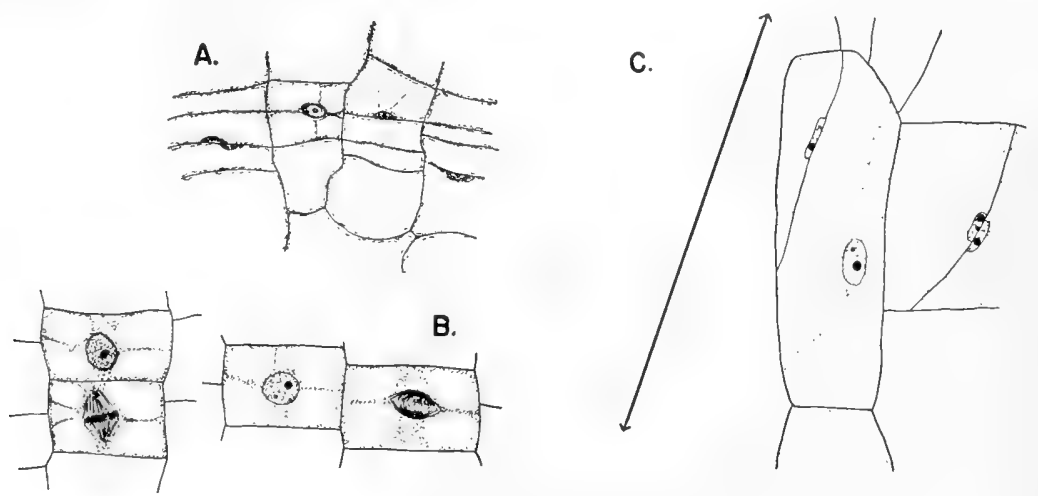

Fig. 4. Location of new cell-walls in meristematic tissue. A. Walls or phragmosomes opposite one another; transverse section through wounded petiole of Bryophyllum. B. Walls or phragmosomes avoiding the opposite position; longitudinal section through normal shoot tip of Polygonum. In the forked phragmosome (left) only one branch will persist. C. Walls and phragmosomes parallel to wounded surface (indicated by line at left); portion of wound meristem of Tradescantia. (All from Sinnott and Bloch, I94I).Camera lucida drawings.

The cell-wall initial, or phragmoplast, is apparently formed at first not from the spindle fibers themselves, but from droplets arising between the spindle fibers; however, parts of the spindle fibers become incorporated in it. As soon as it is formed, the phragmoplast expands laterally by adding new short fibrils at either end till it reaches the lateral walls. In cells below the apical meristem the plane of the new wall is, of course, in most cases transverse; in wounded tissue, on the other hand it lies parallel to the plane of the wound (Fig. $4 \mathrm{~A}$ and $\mathrm{C}$ ). These orientations have been ascribed to the diffusion gradient of auxin or of wound hormone, but with little factual support. The positions of the new walls are commonly opposite one another in wounded tissue, but alternate in normal meristems (Fig. 4).

In the cambium the plane of division is lengthwise in a long cell. The remarkable process of cell division in cambium cells of the Coniferae, such as white pine, has been fully described by Bailey (I9r9, I920). These cells, although uninucleate, may be several hundred times as long as they are wide. The mitotic figure is at angle of $20-40^{\circ}$ to the long axis of the cell. The spindle fibers expand laterally, after karyokinesis, by adding small fibrils peripherally; the expanding cell plate is thus oblique at first but it soon curves to become longitudinal. As it loses the fibers in the central part, to form the cell plate, it adds a mass of fibrils at its end (Fig. 5) and these move away from one another at equal rates towards the ends of the cell, which may be up to $4 \mathrm{~mm}$ away. In this way the divided nucleus is always at the center of the expanding cell plate. The process takes so long that the nuclei are 
fully returned to the "resting" state before the cell plate reaches the tip walls. No cytoplasmic strand to guide it, corresponding to the phragmosome mentioned above, was observed.

\section{(d) Physiology of cell division}

In spite of much work bearing to various extents on the subject, the physiology of cell division has seldom been visualized as a topic in itself, and as a result our knowledge of the conditions leading to division is fragmentary. Some of it has

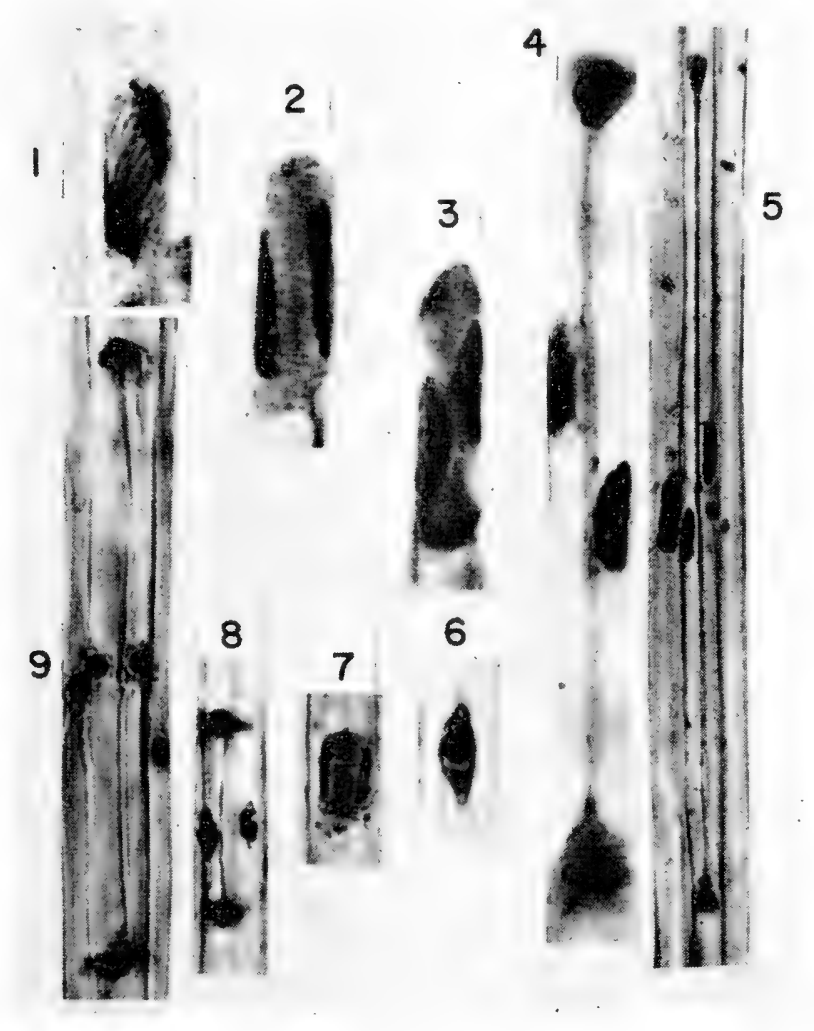

Fig. 5. Cell division in cambium. I-5, Pinus Strobus. (I) Early telophase axis diagonal to long axis of cell; (2) Widening of central spindle and beginning of cell plate formation; (3) Later stage, fibers disappearing in center; (4) Still later stage; cell plate extending, with fibers only at ends; (5) Still later stage, at lower magnification, with cell plate $350 \mu$ long and daughter nuclei returned to resting state. 6-9, Robinia Pseudo-acacia. (6) Resting nucleus; (7) Beginning of cell plate formation, axis perpendicular to long axis of cell; (8) Later stage; (9) Still later stage, with fibers only at ends. Magnifications: $1-4$ and $6-9,790 \times ; 5,180 \times$ (From Bailey, I920). been reviewed recently (Stern, I956).

The metabolic changes associated with cell division have long been uncertain. In the cambium cells discussed above, protoplasmic streaming (often considered a symbol of metabolic activity) continues actively during mitosis, but in that classical object, the stamen hairs of Tradescantia, it stops completely (see Hughes, I952). Roots were long thought to have more rapid respiration in the zone of cell division than in that of enlargement, but it is now clear that the reverse is the case (Kopp, I948; Goddard and Meeuse, I 950). In certain anthers meiosis and mitosis can be precisely timed, especially in relation to visible growth (Erickson, I947), and this makes it possible to correlate nuclear activity with metabolic changes. It has thus been shown, both in Lilium (Erickson, I948) and in Trillium, (Stern and Kirk, 1948-9) that the respiratory rate rises 
towards the beginning of prophase, then falls steeply, to remain low during cell division. Meiosis and mitosis both show this cycle. The concentration of ascorbic acid also shows a cyclic change, rising by nearly 50\% during mitosis (Stern and Timonen, 1954-5). The synthesis of deoxyribosenucleic acid (DNA), in these anthers, as evidenced by the incorporation of ${ }^{32} \mathrm{P}$ into it, goes on at specific periods, mainly just before the meiotic prophase, and in the interphases before mitosis; the amount of DNA measured microchemically doubles at each of these times. Synthesis of ribosenucleic acid (RNA), however, mainly follows that of DNA, coming in the interval between the end of DNA synthesis and the onset of prophase, which fits poorly with the concept of RNA as precursor of DNA (Taylor and McMaster, I954). All these changes suggest that energy is built up and used in synthesis, before the

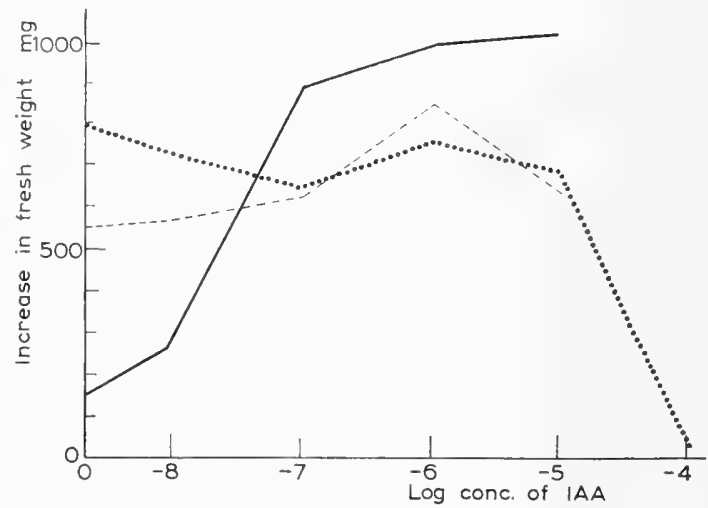

Fig. 6. Response of three types of Scorzonera tissue to indoleacetic acid. Solid line, normal tissue; dashed line, accoutumé tissue; dotted line, tissue from Crown gall.

(From Gautheret, I950). nuclear reorganization takes place, and that during the process-perhaps because mitochondria or other particles become involved as well,-it cannot so easily be provided. In other words, the dividing nucleus may be metabolically isolated. The evidence that the nucleus contains no cytochrome oxidase or cytochrome $\mathrm{G}$ reductase (although it can reduce DPN and TPN) suggests that little energy is available to the nucleus from its own sources (Stern and Timonen, I945).

Of the stimuli to cell division, three factors have been mainly studied: auxin, wounding, and special substances. A major part is played by auxin. Cambial division caused by auxin was demonstrated as early as 1935 by Snow in Helianthus and in 1936 by Söding in poplar twigs. The cell multiplication around a wound in trees is partly under the control of auxin, since it varies with the activity of buds higher up, and can be greatly stimulated by auxin application. In tissue cultures, parenchyma of many sorts are stimulated to divide by auxin, and Gautheret pointed out (1942-3) that in general low auxin concentrations cause division, higher ones promote root formation and still higher ones promote cell enlargement and tend to inhibit division. The optimum concentration of IAA for cell division in chicory root tissue cultures is about $\mathrm{O}$. I $\mathrm{mg} / \mathrm{l}$. Potato tuber is stimulated to divide by 2,4 -D but not by IAA; artichoke responds well to IAA. Indeed plant tissue cultures in general were only made possible by the use of auxins.

A remarkable change in the behavior of tissue cultures with respect to auxin was discovered in 1942 by Gautheret. After the culture has been maintained on an auxin-containing medium for a number of transfers, it becomes able to grow without auxin (see Gautheret, I946; Morel and Gautheret, I947). Carrot root, 
grape stem and artichoke tuber (Helianthus tuberosus) all behave in the same way. The modified, auxin-independent tissues were called accoutumé or adapted. Bioassay of extracts from them shows that they now contain auxin in appreciable amounts (Kulescha I95I). When these accoutumé tissues are cultured on media containing auxin, not only is their growth not promoted, but above about o. I mg/l IAA it is actually inhibited (see Fig. 6).

Special interest is attached to the comparable behavior of crown gall. The original galls, which contain the causative bacteria, Agrobacterium (Pseudomonas) tumefaciens, give rise to secondary galls free of bacteria; bacteria-free gall tissue can also be obtained by exposure to high temperatures (White and Braun, 1942). This bacteria-free gall tissue grows well on nutrient media free from auxin and shows no growth promotion, but rather some inhibition, by added auxin (Fig. 6). Bioassay shows it to contain considerable amounts of an auxin (probably IAA), indeed generally more than the accoutumé tissue (see Table $3, \mathrm{p} .76 \mathrm{I}$ ). Thus the change in the accoutumé tissue has been in the direction of the properties of the crown-gall, which are those of a tumor. The change is spontaneous and involves no marked increase in growth rate, only the acquired ability to dispense with the critical growth factor, auxin. Whether this means the gain of the ability to synthesize auxin, or the loss of the ability to destroy it, is not clear, since the destruction occurring in normal tissue may be quite slow (Platt, I 956).

It should be made clear that crown-gall is a complex tissue. It contains both large and small cells, cells with thickened and lignified walls and cells which are necrosing. In tissue culture the different galls become very similar ( $c f$. De Ropp, I 950) but in situ they retain considerable individuality (Kupila, I $95^{6}$ and literature there cited). Their growth cannot, therefore, be regarded as a measure of pure cell division.

Not uncommonly tissue cultures undergo multiplication of chromosome strands or even of whole chromosomes, without cell devision. Giant lobed nuclei result, which in one case were calculated to be I28-ploid (Therman, I95I). Binuclear cells, each nucleus of which is polyploid, also occur (Skoog, I 953). In tissue cultures of fern prothallia, by contrast, the chromosome number builds up gradually in successive transfers. Chromosome counts and microchemical DNA determinations agree in showing small, more or less random, increments reaching $3 \mu$ or $4 \mu$; along with these nuclear changes there is a morphological change away from typical prothallus tissue toward a tumor or callus type of growth (Partanen et al., I 955). Proihallia in which the nuclei remained normal continued to grow as recognizable prothallial tissue.

The formation of roots on stems, as in the rooting of cuttings, begins with cell division in the pericycle or phloem parenchyma, as was first shown by van Tieghem in 1888 and has more recently been demonstrated in several plants (Reano, I940; McDaniels, I955) and this process in the majority of plants is also stimulated by auxins. For woody tissues IAA, NAA or IBA at I oo $\mathrm{mg} / \mathrm{l}$ is applied to the base of the cutting for $\mathrm{I} 8 \mathrm{~h}$, while for soft green stems $\mathrm{I}-5 \mathrm{mg} / \mathrm{l}$ may be as much as can be applied without damage. Although too long a period of application may inhibit the subsequent elongation of the formed roots, there is no evidence that it inhibits the initiation process. The response of cuttings varies with the part of the 
tree and type of branch from which they are taken, with the age of the wood and season of the year, but, unfortunately, it is not known whether these influences control the initial cell-divisions or the subsequent elongation of the root initials. In addition to auxin, sucrose promotes outgrowth of the roots and a number of nitrogenous compounds increase the number of roots when the auxin treatment is optimal. There is reason to think that these act, at least in part, on the initiating cell-division. For etiolated pea-stems, the promoting factors include biotin, thiamine, adenine, nicotinamide and tryptophan; for green leafy cuttings, asparagine, adenine, guanine, arginine, vitamin $\mathrm{K}$ and even nitrate are effective, while woody cuttings in a few instances have responded to purines, pyridoxine and thiamine (see Burström, I953a and Torrey, I956b, for general reviews, and Thimann and Behnke-Rogers, I950, for the extensive literature on woody cuttings). The same group of nitrogenous substances seem to be essential for the initiation of lateral roots on the main root (section VIIb, p. 8oI).

Why wounding should stimulate cell division has not been satisfactorily explained, since, as a rule, auxin is destroyed by the wounded tissue. The wound response is very variable according to the tissue and its treatment. Potato slices, for example, which give 4 to 5 new cell divisions, parallel to the cut surface, within 7 days, fail completely to respond if they have been pre-stored at low temperatures and only recover after returning to room temperature for many weeks (Steward et al., I 943). Long ago, Haberlandt postulated a "wound hormone" liberated in crushed cells and causing cell division. Several of his students and colleagues worked out responsive systems that could be used for tests for this hormone. The formation of outgrowths or intumescences in the parenchyma tissue of the bean pod was one of the most sensitive (Wehnelt's test) and this has subsequently been used as assay for the wound hormone. In this way "traumatic acid" or $\Delta^{2}$-decene-I, Io-dicarboxylic acid, XXVI, has been isolated from crushed bean extract (English et al., I 939). A number of related compounds are also active. Traumatic acid requires co-factors, including glutamic acid, sucrose and phosphate, in order to cause the outgrowths (see Thimann et al., r 952, for review). The observed outgrowth results from cell enlargement as well as division. In other wound-reactions, however, traumatic acid has so far not been found to show any particular effect and it induces no cell division in the tobacco pith cultures which respond so powerfully to kinetin (below) ${ }^{1}$.

Tissue cultures are stimulated to divide by so many factors that it is evident that no single substance is in general the limiting factor. Endosperm, particularly when, as in some young fruits, it is in the liquid form, is particularly effective; coconut milk, corn in the milk stage, young walnuts and chestnuts and the tropical fruit Allenblackia have all been used in this way. Coconut milk is particularly useful and since its introduction by Van Overbeek et al. (I94I) has become a sine qua non for tissue culturists. The active compounds in coconut milk have recently been shown to include I,3-diphenylurea, XXVII, (Shantz and Sicward, I 955) while those from immature chestnuts include a leuco-anthocyanin, probably leucocyanidin monoglucoside, XXVIII (Steward and Shantz, I956).

\footnotetext{
${ }^{1}$ For wound hormones see Chapter 8.
} 
The latter is apparently active only in the glycosidic form. These last two have been evaluated on carrot tissue fragments (Steward and Shantz, I956) using a medium in which ions, sugar and casein hydrolysate are supplied. They give rise to large numbers of small cells, some of which remain attached while others float off into the solution and grow into new tissue masses. When synthetic auxins are

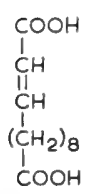

"Traumatic acid" XXVI<smiles>O=C(Nc1ccccc1)Nc1ccccc1</smiles>

I, 3-Diphenylurea XXVII

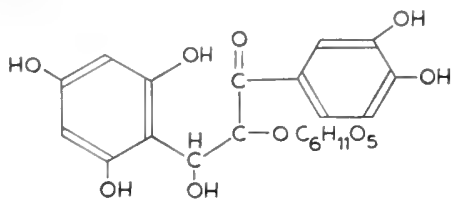

Leucocyanidin monoglucoside XXVIII

added, the cells enlarge. Without casein hydrolysate, the effects are much smaller.

While the material in coconut milk is present at all stages of the fruit's development, the corresponding material in Zea mays decreases rapidly 2 weeks after pollination (Steward and Caplin, I952). As far as its chemistry has been studied the active fraction appears to be nitrogenous and closely associated with the amino acids (Györffy et al., r955); its action is complicated by the large amount of auxin present ( $c f$. Fig. 12, p. 809).

A curious observation is that the synthetic auxin, benzothiazole oxyacetic acid, XXIX, has a very powerful effect on cell division in artichoke, even exceeding that of coconut milk (Steward and Shantz, 1956). It is much less effective on carrot tissue.

Probably the most powerful individual substance causing cell division so far isolated is kinetin, or 6-furfurylamino purine, $\mathrm{XXX}$. This compound is formed by<smiles>O=C(O)COc1nc2ccccc2s1</smiles>

Benzothiazole oxyacetic acid XXIX

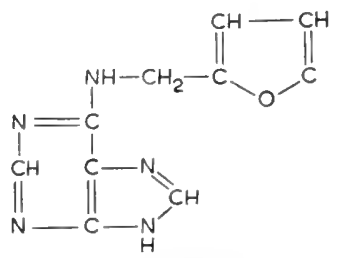

6-Furfurylamino purine (Kinetin) XXX

heating or autoclaving yeast nucleic acid (Miller et al., I 955a, b, I 956; Strong, I 958) and it (or a related compound) may be naturally present in traces in some samples of nucleic acids. So far it has not been demonstrated in plant tissues, though its occurrence there seems very probable, Its action on tobacco pith tissue cultures is very powerful ( $c f$. section IIIc, p. 759). On carrot tissue, in presence of IAA, it is about $20 \%$ as effective as whole coconut milk, but on artichoke tissue its activity is rather weak (Steward and Shantz, I956).

As in the above cases, its action is interrelated with that of auxin, and the effect depends on the balance between the concentrations of the two substances (see Fig. 2). Auxin alone causes root initiation in these cultures, kinetin added alone 
(doubtless in presence of traces of natural auxin) or at very low levels of IAA, produces large numbers of buds, and at higher concentrations the combination causes massive cell division to form unorganized callus (Strong, r 958).

In these experiments a clear distinction can be drawn between nuclear division and cell division (mitosis and cytokinesis). Tobacco pith treated with auxin alone exhibits a few mitoses (Naylor et al., 1954) which may perhaps be due to the presence of small amounts of natural kinins; these mitoses often do not lead to cell division, and as a result binucleate and polyploid cells were found. On the other hand, kinetin alone, in a wide range of concentrations, produced no mitoses (Das et al., I956). The combination (kinetin $0.05-1.6 \mathrm{mg} / \mathrm{l}$, IAA $0.2-2.0 \mathrm{mg} / \mathrm{l}$ ) causes onset of cell division after I-2 days, followed by a pause which is ascribed to the fact that all the cells ready to divide had been stimulated into doing so, while others had to "get ready." After another day or two, divisions occurred at a more or less constant rate, $2-3 \%$ of the cells being observed in mitosis at any one time. Practically all these mitoses were followed by cell division. Data not yet presented in detail show that either auxin or kinetin can cause an increase in DNA in the nuclei, but it is the subsequent chromosome apparatus and cytokinesis which requires both factors.

Skoog and Tsui had earlier shown (I95I) that adenine exerts the same effects on bud formation, but kinetin is active in concentrations about I ooo times as low as those of adenine. Several synthetic analogs, including especially 6-benzylaminopurine, are almost as active as kinetin. Perhaps, therefore, the observed small activity of adenine and other purines may be due to their conversion in vivo to a kinin. As stated in section II, the class of kinins, which includes kinetin, are considered to be primarily cell-division-promoting substances. Whether their action is limited to division is, however, not yet clear. Leaf sections floated on kinetin solution are caused to grow in thickness, as was long ago noted with adenine, but the important point here is that the growth results only from cell enlargement (Kuraishi and Okumura, I956). A more complex process is that in which kinetin causes the elongation of buds that were inhibited by auxin (see section VIId). This involves both division and enlargement. It is possible that kinetin, in conjunction with auxin, stimulates the necessary cell division, and that the subsequent enlargement of the newly divided cells is dependent upon auxin; however, it is also possible, and perhaps equally probable, that both substances act on some process in the cell at the molecular level (such as the DNA synthesis mentioned above) and that the subsequent effects may or may not go beyond cell division, depending on what other factors are limiting.

Interaction between auxin and kinetin may explain the production of tumors by crown-gall bacteria. On the one hand, auxin is essential, since many years ago inactive strains of bacteria were made tumorigenic by supplying IAA (see review of Riker et al., I 946) and, more recently, ionizing radiations have been shown to reduce growth of crown galls while added auxin reinstates it again (Klein and Vogel, I 956). For example, I $500 \mathrm{r}$ of X-rays reduced the tumor diameter to $8 \%$ of the control, while in presence of IAA it was $80 \%$ of the control. Neutrons and gamma rays had similar effects. Ionizing radiations are known to inactivate IAA both in vivo and in vitro. Isolated tobacco pith tissue, without auxin, inoculated with 
crown-gall bacteria produces no tumors. On the other hand, cell division must first occur in order for normal cells to become tumorigenic (Braun, 1953). Contact (3 days) with vascular tissue provided enough of a cell-division factor to allow division and the bacteria alone now produced a tumor. But if NAA ( $\mathrm{mg} / \mathrm{l}$ ) and an extract of tumors, or pure kinetin was supplied, then the tissue without inoculation grew into a tumor with "striking morphological and histological resemblance to crown gall" (Braun, I956). The inference that the kinetin provides the same stimulus as the bacteria, and that this is synthesized by growing tumors, is highly attractive. On this basis the "accoutumé" tissue cultures of Gautheret, which can grow without auxin, may be considered to form the necessary traces of kinetin and to have adaptively acquired the capacity to synthesize auxin, while crown-gall, in contrast, which can evidently form the necessary traces of auxin may be considered to have been influenced to synthesize kinetin. In a parallel way, cell-free extracts of crown-gall bacteria have caused carrot tissue, treated with auxin, to produce tumors (Klein and Knupp, I957) but here the effective substance has not yet been identified.

\section{CELL ENLARGEMENT}

\section{(a) The elongation of tissues in an axis}

Since the minimum working definition of growth (see section II) is Irreversible Increase in Volume, it follows that it is cell enlargement which fulfills the primary requirements of growth. Usually enlargement occurs directly after cell division, so that the small cells of a rapidly-dividing meristem either elongate in the direction of the axis (in a shoot or root), widen perpendicular to it (in the cambium), or enlarge isodiametrically (in pith, in tubers or in fruits).

When an axis elongates in this way, there is no special reason to believe that all cells respond at the same rate to the natural supply of auxins and other factors, nor that they inherently would elongate to the same extent. The firmness and continuity of the cell-wall holds them together and thus imposes a uniformity in the tissue which is very likely not present in the individual cells. This situation leads to the development of tissue tensions, or mechanical strains, in growing organs, which have been known since the time of Hofmeister in the I880's. Hollow stalks of dandelions, daffodils or onions show these very strongly; if they are slit lengthwise when growing, the two halves roll up, because of the tension in the epidermis or outer cell layers. The opposite reaction is shown by pithy organs like the petioles of rhubarb in which, when the outer layers are removed, the central tissues swell and elongate, showing that they had been under compression.

These tensions became prominent again when it was found that auxins cause an inward curvature in slit stems ( $c f$. section IIIb). In water alone, outward curvature occurs due to the tissue tension; in auxin solution, these same outer layers are caused to elongate more than the inner ones and hence an inward curvature results. Peeling off the epidermis, by removing one of the most responsive layers, greatly reduces the curvatures. Extensive analyses (Thimann and Schneider, I938; Jost, I938; Diehl et al., I939; Went, I939; Schneider, I942) show that the curvature is not due to differential auxin uptake on the two sides, but mainly to 
inherent differences in auxin sensitivity in the different layers, aided and abetted by an inhibition of extension caused by the wound itself. This latter effect is presumably minimal when coleoptiles or other hollow organs are slit, since the area of wound is very small. Besides, tissues wounded on all sides can still curve (Thimann and Schneider, I 938). More detailed discussion cannot be given here, but it is clear that the influence of the elongation of one cell on that of another is of importance in the growth of all kinds of plant tissue. It has the result that the elongation of the cells in an intact root or shoot is held to a certain uniformity.

From time to time it has been suggested that elongation of an organ includes "sliding growth" or slippage of the apex of one cell past another, as has been seen in the tips of long cambium initials. However, careful observation of the individual cells in the epidermis of growing roots, particularly by Sinnott and Bloch (I94I) offered no support for any such independence of action. More recently the idea that cells might be mainly elongating at their tips was revived by Mühlethaler's electron-microscope studies of the cell walls in young coleoptiles (I950). These showed that the wall at both the apical and basal tips of these elongating cells was much thinner than in the middle. Whatever the explanation, the difference in thickness does not appear to be due to tip growth since the application of fine copper oxide particles to the surface of a growing coleoptile shows clearly that at least in the epidermis the elongation is uniformly distributed along the length of each cell (Castle, I955).

For analysis of cell enlargement it will be convenient to consider in order the different factors which control the process, and then to try to visualize its essential character.

\section{(b) Control by auxin}

In shoot tissues, such as elongating stems, young leaves and buds, the auxin level, as determined by diffusion experiments, is of the order of $25^{-200 \mu \mathrm{g} / \mathrm{kg}}$ fresh weight. Reference to Table 2 ( $\left.\mathrm{p} .75^{8}\right)$ shows that this value is towards the low end of the range for cell enlargement; in other words, cell enlargement in growing tissues is commonly limited by the auxin available. This qualitative conclusion can also be drawn from the numerous instances where modification,

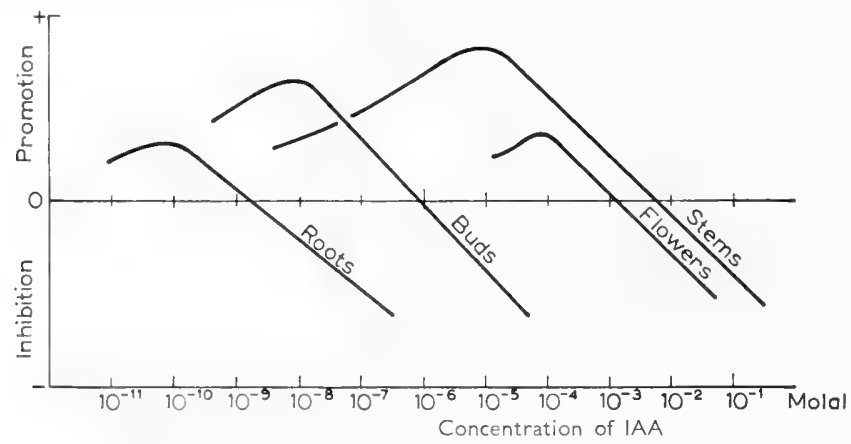

Fig. 7. Scheme of growth responses to auxin by different tissues. The curve for flowering refers to long-day plants growing under conditions close to the threshold for flower induction. The auxin concentrations are approximate only. From Leopold and Thimann, 1949. 
removal or supplementation of the auxin source has an immediate effect on growth. The tropisms, in which light or gravity acting from one side brings about curvature of the seedling, constitute the best example, since direct auxin determinations in the organs exposed show that the convex side receives, instead of $50 \%$ of the auxin, perhaps $65-70 \%$,- a relatively small change to be responsible for so major a change in the growth pattern.

The optimum levels of auxin for cell enlargement are of the order of ioo times greater than the above. In tests with isolated coleoptile or stem sections in indoleacetic acid solution the optimum concentration lies between 2 and $10 \mathrm{mg} / \mathrm{l}$. Up to the optimum, the elongation is very roughly proportional to the logarithm of the concentration (see Fig. 7). The same relationship holds for the inward curvatures of slit stems or coleoptiles (Thimann and Schneider, I938).

Growth of coleoptile tissues in auxin, in presence of certain buffers, is approximately linear for the first $12 \mathrm{~h}$., though pea stems and especially tuber tissues show a marked lag which may last from an hour to two days (see Hackett and Thimann, I 952a; Hanson and Bonner, 1955). Now the reciprocal of the growth rate of coleoptile sections during the first hours is roughly proportional to the reciprocal of the auxin concentration, and a parallel has been drawn between this relation and the Michaelis-Menten relation between enzyme concentration and reaction rate (McRae and Bonner, I953). Thus

$$
\frac{\mathrm{I}}{V} \propto \frac{\mathrm{I}}{S}+K
$$

where $V=$ reaction rate and $S=$ enzyme concentration. Here the auxin corresponds to the enzyme and the initial growth rate to the enzymatic reaction rate (see Bonner and Foster, I 956; Bennet-Clark, I 956b). There has been much inconclusive argument over this type of analysis, which logically would imply that growth is a single reaction. It may be worth while to point out that unless the range of concentrations used is very wide it is not easy to distinguish between a double reciprocal and a logarithmic relation. Thus over the range from $1.2-25,1 / \log n$ varies closely parallel to $K \cdot \mathrm{I} / n$, where $K=$ about Io. Then, too, the theory should apply only to the initial growth rate, but in most tissues there is a lag after application of auxin, before the growth rate reaches its full value, as noted above. Further, since initial growth rate and final length do not stand in any simple relationship to one another, and the initial rate especially is greatly altered by the sucrose concentration as well as that of other solutes (Bennet-Clark, I956b) the matter is indecisive.

Above the optimum the growth falls off drastically with relatively small increments of concentration. The $\mathrm{pH}$ of the solution determines the extent of depression; in unbuffered solutions, in which the auxin is in the free acid form, concentrations above $30 \mathrm{mg} / \mathrm{l}$ are strongly inhibitory and even toxic; the sections become flaccid within $\mathrm{ro}-20 \mathrm{~h}$. and the contents of the cells flow out into the solution. In pea stem sections it has been shown that the exudate contains reducing sugars, several aminoacids and other organic materials, which makes it probable that in general it is a sample of the cell contents (Christiansen, 1950). In other words the excess auxin has destroyed the normal retentiveness, or semipermeability, of the cell membrane. In less acid solutions, higher auxin concentrations are needed to exert the same effect. The combination of auxin with a grow th inhibitor such as iodoace- 
tate (see section VIf, p. 782) increases the toxicity. Similar toxic effects at high concentrations occur with tuber slices, tissue cultures and other material. The optimum curve has been explained as follows: the molecule has to combine with two receptors, e.g. an enzyme and a substrate, which it thus brings together. (The idea is somewhat similar to that of complement in immunology.) If there is an excess of auxin molecules present, one group will combine with all of the enzymes and another group with all of the substrates, so that, instead of bringing these two materials together, the auxin actually keeps them apart (Skoog et al., I 942). Recently this proposal has been revived and, though it has been claimed to be consistent with the kinetics of growth in coleoptile sections, there is disagreement in this regard. Several groups of workers find that high auxin concentrations actually accelerate the initial growth rate, for short times, and that thereafter a toxic effect becomes superimposed, while McRae, Foster and Bonner (I953) believe that there is true growth inhibition from the start, and ascribe considerable theoretical significance to this (see Bennet-Clark, I956b, for a critical discussion).

Another view of the optimum curve is that every auxin molecule has in it growthpromoting and growth-inhibiting properties (Linser, 1954). As a result, the optimum curve is analysable into two parts, of which that at the lower concentrations shows simple growth promotion, while that at higher concentrations can be imitated by a non-auxinic growth inhibitor such as eosin. Both these concepts rest upon mathematical analysis of growth curves, treating the growth phenomenon like a simple one-step enzymatic reaction. The extent to which they correspond with the undoubtedly complex biological facts cannot yet be deduced, pending detailed knowledge of the mode of action of auxins in causing growth, but it seems inherently unlikely that the simple treatment can be pressed very far. The action curves of many drugs and poisons show optima (see Thimann, i 956c, for discussion).

The toxic effect is in any case of great practical importance, since it is-as already mentioned - the basis of the popular weed-killing chemicals. Although naphthalene-acetic acid (p. 763) was used in the early experiments (Templeman and Sexton, I946) the chlorinated acids, 2,4-D, 2,4,5-T and Methoxone (XI, $\mathrm{XII}$ and XIII) were subsequently found more effective and are now universally used. Sprayed on leaves, they are rapidly absorbed, killing the leaf and the young shoot; they are also transported both upwards in the transpiration stream and downwards in the bark or phloem to kill most or all of the above-ground parts. Smaller quantities are transported into the roots, which often survive, especially in the case of trees and other woody plants. The latter are more effectively killed by treating the stems externally with an oil solution of $2,4,5-\mathrm{T}$ (XII) which is absorbed through the bark and which creeps down below ground level over the bark surface to be absorbed into the upper parts of the roots (see Hay, I956a).

The translocation of a toxic substance, in toxic amounts, through living tissues is a phenomenon that seems to require explanation, as pointed out by Crafts 1956), but it is clear that it takes place only for a very short time, and that thereafter the conducting system becomes immobilized. There is considerable destruction in the living tissues also, about $50 \%$ of the internally present 2,4-D being destroyed in bean seedlings in $24 \mathrm{~h}$. (Holley et al., I 950; Hay and Thimann, I 956, and literature there cited). Nevertheless, once in the xylem of woody plants, par- 
ticularly if in oil solution, some $2,4,5-\mathrm{T}$ can remain for months or into the next growing season to reenter the circulation in spring and kill or distort the young growth. Thus the herbicidal action of auxins is quite different from that of other toxic compounds (see the brief review by Crafts, I 956).

Auxins with long aliphatic side chains, such as 2,4-dichlorophenoxy-butyric acid and higher homologues, are subject to $\beta$-oxidation in the side chain and thus give rise to acids with two less carbon atoms (Wain and Wightman, I954; Fawcett et al., I $956 \mathrm{a}, \mathrm{b})$. In this way acids with an odd number of methylene groups eventually yield acetic derivatives and consequently have relatively high herbicidal activity, while those with an even number of methylene groups yield the phenol, which is essentially inactive or only weakly toxic:-

$$
\begin{aligned}
& \mathrm{R}-\mathrm{O}-\left(\mathrm{CH}_{2}\right)_{5} \mathrm{COOH} \rightarrow \mathrm{R}-\mathrm{O}-\left(\mathrm{CH}_{2}\right)_{3} \mathrm{COOH} \rightarrow \mathrm{R}-\mathrm{O}-\mathrm{CH}_{2} \mathrm{COOH} \\
& \mathrm{R}-\mathrm{O}-\left(\mathrm{CH}_{2}\right)_{4} \mathrm{COOH} \rightarrow \mathrm{R}-\mathrm{O}-\left(\mathrm{CH}_{2}\right)_{2} \mathrm{COOH} \rightarrow \mathrm{R}-\mathrm{OH}
\end{aligned}
$$

The ability to carry out the oxidation is not present in all plants and the enzymes concerned appear to differ for different $\mathrm{R}$ groups. It is hoped, therefore, that this behavior may be the basis for still more selectivity among auxin herbicides.

Some plants, especially the cereals and grasses, are quite resistant to high auxin concentrations and in this way dicotyledonous weeds can be killed in wheat, oats or sugar-cane without harm to the crop. Yet young seedlings of these same grasses are very sensitive, and within a given grass species, such as maize, varieties of at least moderate sensitivity have been observed. Some dicotyledons, too, including thistles and strawberries, are fairly resistant (Crafts, 1956). The basis for the resistance is still unknown. No attempt can be made to summarize here or even mention the flood of literature on synthetic auxins as herbicides; Audus (I953, Chap. 9) and Leopold (1955, Chap. I6) have given extensive treatments, while European practice is reviewed by Woodford and Kasasian ( 1956 ).

\section{(c) Control by oxygen}

The promotion of cell enlargement by auxin is aerobic. Sections of coleoptile or pea stem used in bioassays grow at a greatly reduced rate if immersed only a millimeter below the surface of the solution. Tuber slices grow best when breaking the surface of the solution or when shaken vigorously in shallow layers. Using such potato slices, the dependence of cell enlargement (in auxin solution) on oxygen tension has been determined (Hackett et al., I953). Enlargement increases linearly up to nearly $20 \%$ oxygen - an entirely different relationship from that shown by most oxidases which are saturated at oxygen tensions far below that of air. Since the oxidase controlling growth has the highest oxygen affinity of any of them (see p. 786) it follows that this curve is probably only measuring the rate of diffusion of oxygen into the tissue.

After a not too long period of partial anaerobiosis, e.g. in $7 \% \mathrm{O}_{2}$ and $93 \% \mathrm{~N}_{2}$, the sections recovered their previous growth rate completely on transference to air (Hackett and Thimann, I 952a). More complete, or more prolonged anaerobiosis usually has irreversible damaging effects and often causes outflow of cell contents ("exosmosis") like that due to supraoptimum auxin concentrations. These observations confer great importance on the intercellular spaces, stomata and 
lenticels as providing an essential system for the oxygenation of growing cells situated deep within plant tissues.

\section{(d) Control by gibberellin}

It is hardly possible as yet to discuss the action of gibberellins on growth in any detail. Gibberellin $\mathrm{A}_{3}$ or gibberellic acid is the compound most used, although the earlier work was all carried out with various mixtures.

Gibberellin, like auxin, acts powerfully to promote cell enlargement, as is seen most strikingly in the elongation of internodes of intact plants. Current researches in many laboratories, largely unpublished, have shown this effect in a variety of plants. Furthermore, in several cases gibberellin has caused flowering, which must involve cell division (Lang, I957). The formation of parthenocarpic fruit has also been reported. Elongation of plants out of the rosette condition, which has been reported in several instances, also involves initial cell division. Gibberellin differs from auxin, of course, in its completely non-polar transport, and also in the characteristic way in which its elongating action is almost wholly independent of light. It also differs specifically in inhibiting the formation of roots on stems, in somewhat promoting the outgrowth of lateral buds (see section VIId) and, curiously, in not influencing cell enlargement in potato tuber slices (Brian et al., r 955). When tested in the standard way on etiolated pea stem sections, the resulting elongation was additive to that caused by auxin and there was no sign of either

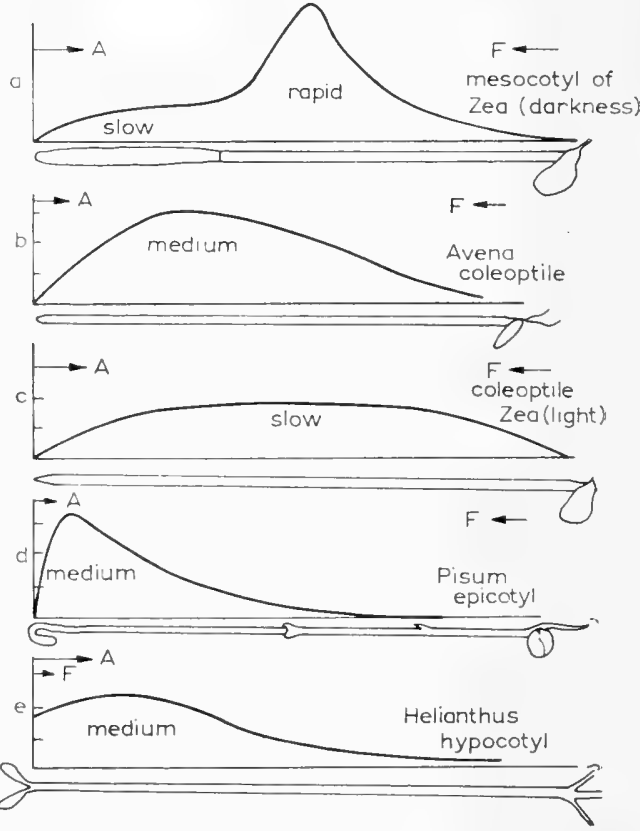

Fig. 8. Two-factor scheme of growth control in shoots of seedlings. Curves show the distribution of growth rate over the shoot. A, auxin; $\mathrm{F}$, food factor. Arrows indicate direction and intensity of flow; presumed rate of combination is noted beneath each curve. (From Went and Thimann, 1937).

synergistic or antagonistic action (Kato, I957, and several unpublished experiments by the writer). The action on coleoptile sections is very small, as is also that on some woody plants in light. Taking the data as a whole, it seems most probable that gibberellin is a second limiting factor for growth, independent of auxin and of food factors and that according to circumstances $(e . g$. in light or in presence of adequate nutrition) its effect may become dominant.

\section{e) The effects of mutrients and ions}

The role of what used to be called "plastic substances", that is, food materials, in cell enlargement cannot easily be assessed in the intact plant. In I937, Went and 
Thimann offered an explanation for the frequently observed fact that growth rate passes through an optimum in the middle parts of seedling shoots, and is low both near the apex and near the base. The explanation was based on the idea that auxin was produced at the apex while nutrients came from the base, i.e. the seed, the hypogeal cotyledons, or the root system. This 2 -factor scheme accounts for the different growth patterns in different seedlings by assigning different ratios of auxin to food factor (Fig. 8). In Avena coleoptiles, for instance, with a growth rate maximum near the middle, the two would be nearly equal, while in the pea seedling, with the growth rate maximum close to the apex, the food factor supply would be much greater than the auxin. Subsequent work with isolated sections has fitted in rather well with this concept, although it seems now something of a oversimplification.

Thus isolated sections of Avena coleoptiles do not respond much to auxin unless sugar is supplied. Sucrose is the most effective sugar, and its optimum concentration is about $2 \%$ or $0.06 \mathrm{M}$. Hexosephosphates are ineffective and are probably not taken in (Thimann and Marré, I 954). In presence of sugar, arginine (alone among aminoacids) further promotes the growth somewhat (Bonner, 1949). Organic acids, particularly malate, clearly improve the growth over that due to sucrose alone, their optimum concentration lying at about 0.00 I $M$. Corn coleoptiles and Avena mesocotyls also respond well to sucrose; sections from other seedlings have been inadequately studied. Sections of potato or artichoke tuber are well supplied with stored carbohydrates (starch and inulin respectively) and their response to auxin is, as might be expected, not improved by sugars or other nutrients.

In contrast to Avena, most sections of etiolated Pisum stems show no response to added sugars, though if the sections are limited to the apical $5 \mathrm{~mm}$ they may show a small response (see Galston and Hand, I949; Christiansen and Thimann, I 950a). Evidently, therefore, the pea stem is, except at the extreme apex, provided with sugars in non-limiting quantities (except in presence of cobalt, as noted below). Analysis shows, indeed, that $17 \%$ of the dry weight is reducing sugar (Christiansen and Thimann, I950a). Methyl linoleate (Stowe, I958) has been found to promote pea stem growth further. Also, with isolated pea epicotyls excised from the embryo, adenine, in addition to vitamins, markedly promotes the growth (Howell and Skoog, I 955, and earlier work there cited). A factor from coconut milk has additional growth promoting effect.

The organic requirements for the growth of root sections have not been extensively studied. For short periods, as with coleoptile sections, $2 \%$ sucrose gives good elongation (Robinson and Brown, I954). Whole roots grow well as isolated organs in culture, auxin not being necessary, and both cell division and cell enlargement continue in more or less normal balance, though in tomato roots the initial apex does not grow indefinitely and transfers have to be made from lateral root tips (Street et al., I952, 1953). For continuous culture sugars are again essential, sucrose being the best; glycine sometimes promotes growth slightly, and most roots require also thiamine, nicotinic acid, or pyridoxine for continued growth. In White's pioneer experiments on continuous culture of isolated roots (I 934, I 94 I) the vitamin requirement was supplied by yeast extract. In nature, these vitamins are supplied from the leaves, thus acting more or less as hormones (see Bonner 
and Bonner, 1948 , for review). Rye roots are exceptional in requiring IAA or a related compound formed by autoclaving tryptophan (Roberts and Street, I 955).

Sections of leaves differ from the above in that they require purines and amino acids in addition to sucrose for appreciable cell enlargement. Adenine and guanine are fairly effective, though kinetin brings about the same amount of growth at much lower concentration (see section VIIa). Arginine and asparagine have small growth-promoting effects in some cases. Leaves of higher plants do not grow for long in culture, though embryonic rye leaves in a sucrose-salts medium have enlarged to about 20 times their original size (De Ropp, I 945-47; see section VIIa, p. 798). Purines and vitamins had little effect on the growth. Fern leaves and young leaf primordia of Helianthus, however, seem to grow indefinitely but slowly on a complete nutrient medium in the light (Steeves and Sussex, I957, Steeves et al., I957), providing they are excised as young primordia.

Potassium ions greatly promote cell enlargement in most isolated tissues. The optimum concentration generally lies at about $0.005 M$. Growth of coleoptile sections in auxin plus sucrose may be increased up to $60 \%$ by optimum potassium (Thimann and Schneider, I938). Isolated roots in culture show a strong response to potassium, principally in cell enlargement. In tissue cultures, too, potassium has a large effect (Heller, r953).

Both manganese and cobalt further promote the growth of Avena coleoptile sections, cobalt being optimal at about $3 \cdot \mathrm{IO}^{-5} \mathrm{M}$ and $\mathrm{Mn}$ at $3 \cdot \mathrm{IO}^{-3} \mathrm{M}$. Pea stems respond equally well to cobalt but scarcely at all to manganese. There is considerable evidence that the two metals act in different ways (Thimann, I956b). Leaf tissue disks (Miller, I 952) and the tissue cultures of pea epicotyls mentioned also respond markedly to cobalt. The action of these metals is rather curious, since manganese is required for whole plants only in traces, and cobalt not at all. In leaf disks the effect of cobalt is interrelated with that of light (Miller, I952, I 954) but this seems not to be the case in coleoptile and pea segments (Thimann, I956b); rather the cobalt appears to act by "channelling" the metabolism towards growth. Thus its effect is exerted in presence of sucrose, but if acetate is supplied, cobalt acts only as an inhibitor. There are other interrelations with the energy supply.

Calcium ions strongly inhibit elongation, being at least 10 times more effective than could be accounted for by their osmotic concentration (Thimann et al., i 950). Magnesium, however, has little effect either in promoting or inhibiting elongation.

\section{(f) Specific inhibitors}

A great variety of more or less specific enzyme poisons have been shown to inhibit cell enlargement. From these effects it has been possible to deduce some of the enzyme systems participating in the process (see Thimann, 1956a, Chap. 6 for review).

The largest class are those compounds which react with sulfhydryl groups. So far, every such compound tested has proven to inhibit cell enlargement. Many enzymes of great importance in carbohydrate metabolism, including the dehydrogenases of phosphoglyceraldehyde, succinic acid and alcohol contain -SH groups which are essential for their action. Model reactions between cysteine on the one hand, and iodoacetate, triiodobenzoate and coumarin on the other, have been described by Leopold and Price (1957). Historically the first of these inhib- 
itors to be studied, and indeed one of the most effective, was iodoacetate. When added to Avena coleoptiles in a simple growth solution iodoacetate can inhibit growth down almost to zero. The concentration causing 50\% inhibition is about $3 \cdot \mathrm{IO}^{-5} \mathrm{M}$. This concentration has almost no effect on the respiration, so that the inhibition is not due to general damage but is specifically exerted on the elongation process. The response depends to some extent on the age of the coleoptiles and the $\mathrm{pH}$ of the solution. Strikingly, lower concentrations clearly promote growth, and below $10^{-5} \mathrm{M}$ promotions of $50 \%$ or so can be observed, though so great a promotion is not always easily duplicated, and values obtained by other workers are commonly not over $30 \%$.

Pea stem and potato tuber sections show a similar inhibition though with little or no promotion at low concentrations. Even Neurospora cultures respond similarly and the absolute iodoacetate levels effective are almost the same as in higher plant tissues (Ryan et al., I 944). Iodoacetamide has effects similar to those of the free acid.

The promotion of coleoptile growth by iodoacetate at $10^{-6} \mathrm{M}$ is completely prevented by lipoic acid (6,8-dithio- $n$-octanoic acid) at about $5 \cdot 10^{-6} \mathrm{M}$ (Slocum and Little, I956) but not by related dithio- or monothio- compounds. Similarly the inhibition by higher iodoacetate levels could be prevented by lipoic acid. Since lipoic acid is an intermediate in the oxidation of pyruvate, this relationship is interpreted in terms of a balance between two paths for pyruvate-to be oxidized, yielding energy for growth, or to be converted to sugars, yielding material for growth.

Organic mercurials, especially phenylmercuric salts and parachloromercuribenzoic acid, which are also powerful -SH reagents, inhibit cell enlargement in even lower concentrations than iodoacetate. They act on all tissues which have been investigated, and at nearly the same absolute concentration levels. Here also there is promotion at low concentrations, at least with coleoptiles (see Fig. 9).

Arsenite is a highly effective inhibitor which has been used a good deal in com-

Fig. 9. Examples of the action of enzyme inhibitors on cell enlargement in auxin solutions. A. Avena coleoptile sections cut from plants of four different ages (in h.) in $p$-chloromercuribenzoate. Arithmetical plot. Note clear growth promotion at low concentrations (the extent of which varies slightly with age). From Thimann and Bonner, 1949.

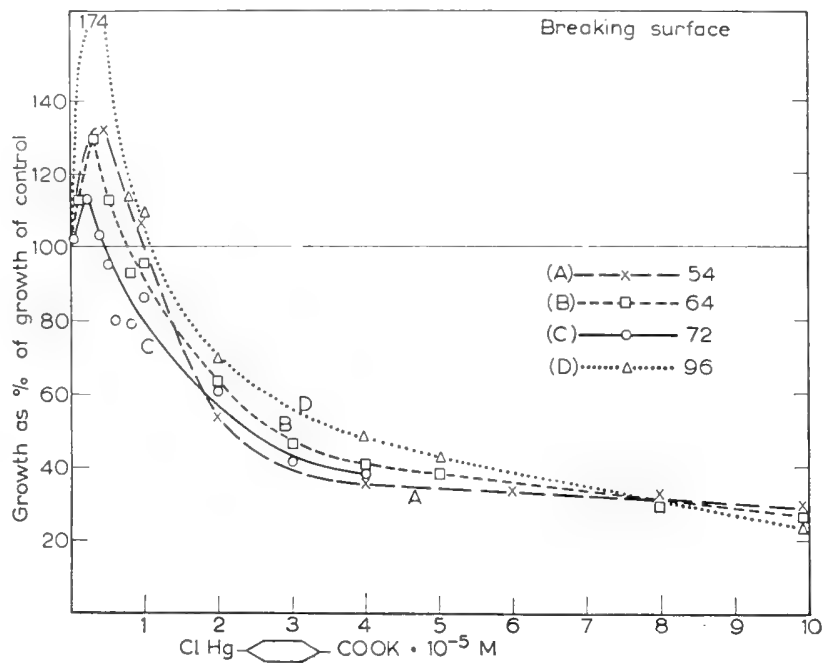


B
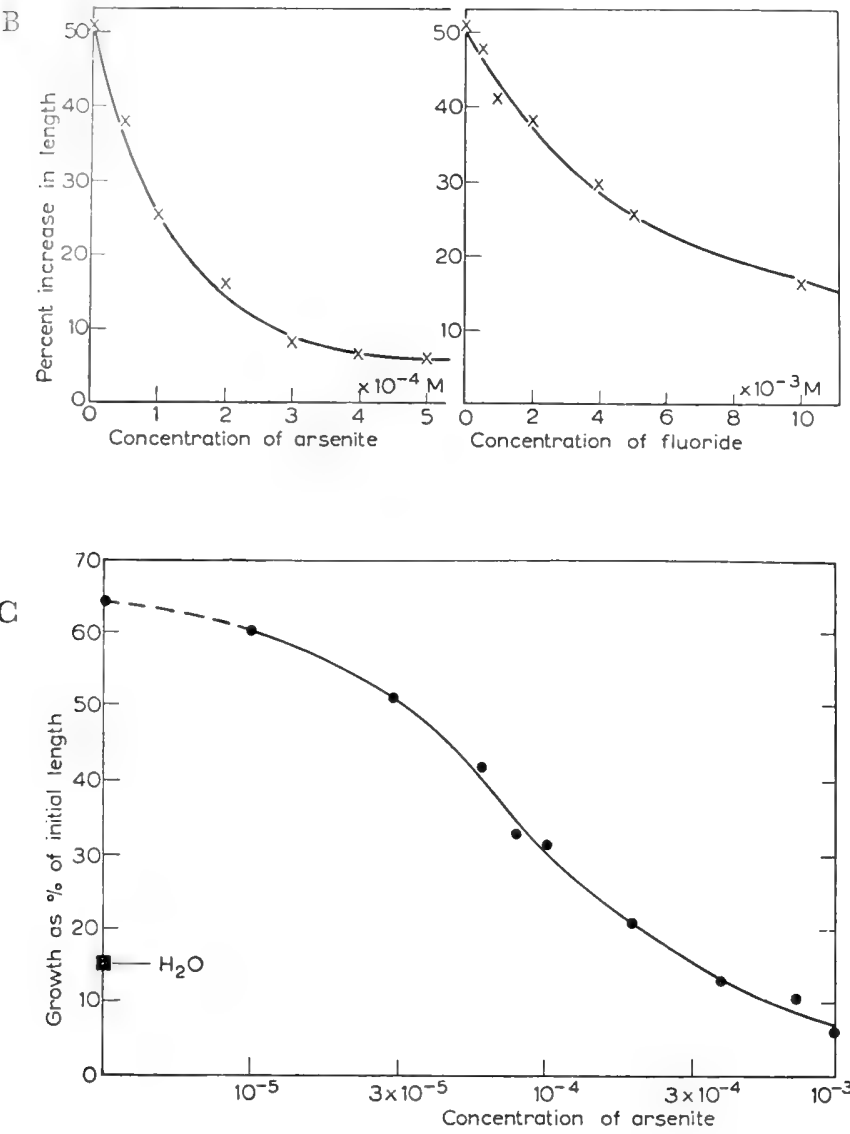

D

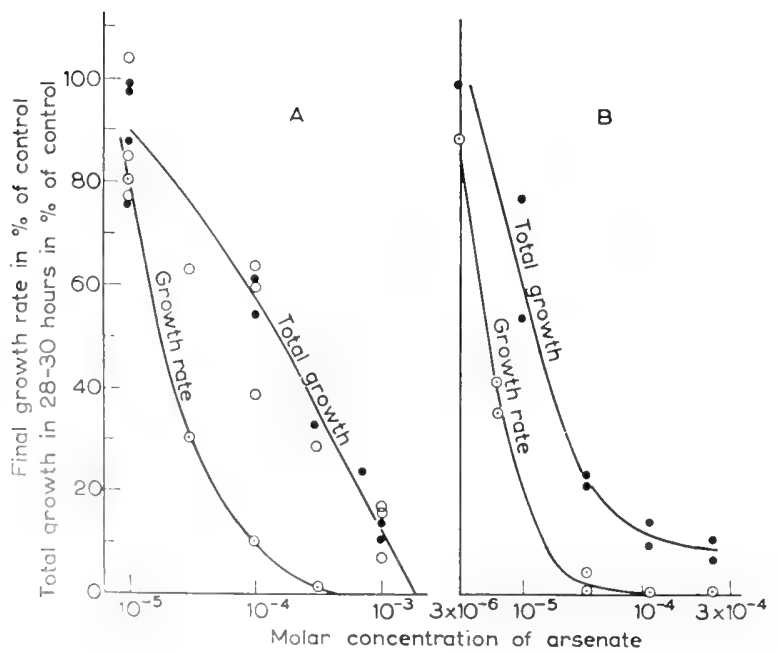

Fig. 9B. Pea stem sections in arsenite and fluoride. Arithmetical plot. Note no growth promotion at any concentration. From Christiansen and Thimann, I 950 a.

Fig. 9 C. Pea stem sections in arsenite. Logarithmic plot. Note almost linear proportionality. The point marked $\mathrm{H}_{2} \mathrm{O}$ at left shows elongation of control without auxin. From Thimann and Bonner, 1949.

Fig. 9D. Growth rates prevailing at the end of 24 hours compared with total elongation, in sodium arsenate. A. pea stem sections in IAA ( $\mathrm{I} \mathrm{mg} / \mathrm{l}$ ); white circles, with sucrose; black circles, no sucrose. B. pea root sections in sucrose.

From Audus, 1952. 
parative studies and in respiration. Cell enlargement is inhibited $50 \%$ at about $5 \cdot 10^{-5} M$ in many tissues (Thimann and Bonner, 1949). Although it is believed to act by combining with dithio compounds such as lipoic acid,

$$
\mathrm{HSCH}_{2} \mathrm{CH}_{2} \mathrm{CH}(\mathrm{SH})\left(\mathrm{CH}_{2}\right)_{4} \mathrm{COOH}
$$

the arsenite inhibition cannot be reversed or protected against by adding lipoic or other dithio acids.

Some years ago it was found that germination of seeds is inhibited by unsaturated lactones, such as parasorbic acid, coumarin, XXXI, and protoanemonin, XXXII.<smiles>O=c1ccc2ccccc2o1</smiles>

Coumarin XXXI<smiles>C=C1C=CC(=O)O1</smiles>

Protoanemonin XXXII

Both of these inhibit cell enlargement, XXXII being the more potent, and both cause very marked promotion at low concentrations. Although it has not been rigidly proved that these lactones do react with - $\mathrm{SH}$ groups in the plant (Price and Leopold, I957) it is clear that the growth inhibition of coleoptiles and pea stems can be prevented by treatment with certain sulfhydryl compounds such as 2,3-dimercaptopropanol (BAL). At about $3 \cdot \mathrm{IO}^{-4} M$ this dithiol appears to have few secondary effects and almost completely protects the plant tissue against both lactones (Thimann and W. Bonner, I 949b). Elongation of roots is inhibited by coumarin and other lactones too, the inhibition by coumarin being freely reversible on removing the coumarin (Audus, I 948). The inhibition caused by certain other lactones could not, however, be prevented by BAL (see section VIIb, p. 80I). An unknown germination inhibitor in oat hulls, on the other hand, had its inhibition largely removed by BAL or by glutathione (Elliott and Leopold, I953).

Perhaps comparable to the lactones are the maleimides. Maleic acid is known to be a powerful inhibitor of the - $\mathrm{SH}$ enzyme succinic dehydrogenase, and it does inhibit growth of sections. But the maleimides, perhaps due to higher lipoid solubility, are more effective than the acid, and $\mathrm{N}$-phenyl maleimide inhibits growth $50^{\circ} \%$ at $5^{\cdot 10^{-5}} \mathrm{M}$ (Van Overbeek et al., I955). Cysteine prevents the inhibition.

A second group of inhibitors comprises the compounds interfering with phosphorylation and phosphate transfer. 2,4-Dinitrophenol (DNP) is the most effective of these, acting powerfully on coleoptiles, pea stems, potato and artichoke tubers etc. It also inhibits protoplasmic streaming, a process which is correlated with cell enlargement (see section $\mathrm{VIj}$, p. 793). Although it may increase respiration by its uncoupling action, DNP was not found to increase growth at any level. At $5 \mathrm{mg} / \mathrm{l}$, respiration was promoted by $38 \%$ while elongation was inhibited $88 \%$ (J. Bonner, I949). Arsenate, which competes for phosphate in various metabolic systems, inhibits cell elongation clearly though more weakly, and the inhibition is dependent on the phosphate supply (J. Bonner, I950). It inhibits elongation of roots about as powerfully as that of shoots (Audus, I952; see Fig. 9D). 
Fluoride was first found to inhibit the enzyme enolase, which converts 2-phosphoglyceric to phosphopyruvic acid, but has since been found to inhibit phosphatases and also phosphoglucomutase. Perhaps for these reasons the poisoning of growth by fluoride appears to be complex. In coleoptile sections a careful study of the relations between fluoride concentration and inhibition yielded no evidence that enolase is involved but indicated that several enzymatic steps participating in cell enlargement are probably sensitive to fluoride (Bonner and Thimann, 1950). Curiously enough, it has little effect on root growth.

A third group would include substances directly inhibiting specific steps of oxidation. So far fluoracetate is the only effective one of this type to be studied; it interferes with the Krebs cycle, being converted therein to fluorocitrate. Fluoracetate inhibits cell enlargement in coleoptiles, pea stems, potato disks etc.; in young coleoptiles low concentrations give a very marked growth promotion which has not yet been explained (Bonner and Thimann, I950). A feature of the fluoracetate inhibition is its almost complete prevention by a slight excess of acetate or pyruvate. This provides good evidence of the role of Krebs cycle oxidations in the metabolism of cell enlargement.

All of these inhibitors show a similar relationship between their concentration and the resulting growth. At low levels there may or may not be a growth promotion; then in the effective range the growth is about linearly proportional to the logarithm of the inhibitor concentration (see Fig. 9G); finally at very high concentrations the inhibition flattens out, or else secondary effects supervene.

Of a different type are those inhibitors which interfere with the terminal oxidase. The first of these to be used was cyanide which Bonner in I 933 found to inhibit both growth and respiration of Avena coleoptile sections. Gyanide combines with, and thus inactivates, virtually all enzymes containing iron, copper or manganese, such as catalase $(\mathrm{Fe})$, peroxidase $(\mathrm{Fe})$, cytochrome oxidase $(\mathrm{Fe})$, polyphenol oxidase $(\mathrm{Cu})$ and ascorbic acid oxidase $(\mathrm{Cu})$. More specificity is provided by carbon monoxide, for although $\mathrm{CO}$ combines with several of these, only in the case of cytochrome oxidase is the $\mathrm{CO}$-compound dissociated by light, especially in the violet end of the spectrum at $43^{\circ} \mathrm{m} \mu$. It is of great importance, therefore, that the elongation of pea stem and Avena coleoptile sections is inhibited strongly by $\mathrm{CO}$ in the dark, while in light the elongation is nearly restored to normal (Hackett and Scheiderman I 952). The enlargement of potato tuber slices behaves similarly but in this case light reduces the growth by nearly $50 \%$ so that the restoration of growth in $\mathrm{CO}$ cannot be complete; it is, however, brought up to the level of the controls without $\mathrm{CO}$ in light (Hackett et al., r 953). Furthermore, a characteristic of cytochrome oxidase is that the inhibition by $\mathrm{CO}$ is dependent on the ratio between $\mathrm{CO}$ and $\mathrm{O}_{2}$; it can therefore be prevented by increased oxygen tensions. Such a competitive effect has been shown for all the three tissues mentioned. It can safely be concluded, therefore, that the terminal oxidase which functions in cell enlargement, i.e. the system through which electrons must pass in order to vield energy for cell enlargement, is cytochrome oxidase. Needless to say, other oxidases may be present, particularly in potato, which is a classical source of polyphenol oxidase, but evidently their function is not connected with cell enlargement. 
An inhibitor of a different type again is canavanine, which is an antagonist of arginine. This compound inhibits growth of coleoptiles at I $\mathrm{mg} / \mathrm{l}$ and above, which provides evidence that arginine is needed for growth (Bonner, I 949).

The evidence from the inhibitor studies can be summed up by the statement that cell enlargement is dependent on one or more sulfhydryl enzymes, probably more than one phosphate transfer, possibly some action of arginine, organic acid oxidation by way of the Krebs cycle and cytochrome oxidase.

\section{(g) Relation between cell enlargement and metabolism}

(i) Respiration. From the evidence just given, it is not surprising that a number of attempts have been made to elucidate the metabolic processes which accompany, or perhaps cause, cell enlargement, The relationships, however, are far from simple.

The application of auxin, in growth promoting concentration, to isolated shoot tissue nearly always increases oxygen consumption. Curiously enough, it is in the most-used tissue, Avena coleoptile sections, that the data are conflicting. The first researchers in this field agreed that IAA by itself did not stimulate oxygen uptake, and indeed Commoner and Thimann in I94I showed that only if the sections were pretreated with sucrose or potassium malate would there be an increase in respiration when auxin was added. Later, however, Bonner (1949; $f$. Ordin et al., I956) found, contrary to his own previous report, that IAA increased the respiration about $20 \%$, and Anker (I95I) obtained the same result in several different media with and without sucrose. At that time, however, the writer (in unpublished experiments) had no difficulty in duplicating his previous finding of no effect due to IAA added alone. Looking back on all these data it now appears that the positive results were all obtained in the presence of potassium salts or potassium-containing buffers; the negative results had no potassium. Since $\mathrm{K}^{+}$ions do have a large effect on growth, this difference is significant and it may mean that $\mathrm{K}^{+}$is required for auxin to exert its full effect on respiration.

In other shoot tissues there can be no doubt that added auxin increases oxygen consumption. In pea stem sections the increase is about $20 \%$; in potato slices it is about $40 \%$ but is delayed at least a day, while cell enlargement in this tissue shows a similar lag before the sections in auxin show an increase over the controls (Hackett and Thimann, I 953). NAA has to be used in order to obtain both effects in potato. In artichoke sections the increase is the most spectacular, averaging some $400 \%$ and taking place within a few h. (Hackett and Thimann, r 952b).

As might be expected, inhibitors of cell enlargement usually cause a decrease in respiration. This has been observed with iodoacetate, arsenite, dinitrophenol, organic mercury salts, cyanide and carbon monoxide. Fluoride, however, in growth-inhibiting concentrations, did not decrease the respiration of pea stem sections but even slightly increased them (Christiansen and Thimann, I950b), while arsenate had no effect on respiration of coleoptile sections (Bonner, I950; Audus, I952). Table 4 summarizes the data of a number of experiments in which the effects of the same inhibitor on respiration and on growth have been measured, under parallel conditions. It will be seen that the influence of the inhibitor on respiration is always much smaller than that on growth. An exception is provided 
TABLE 4

EFFECTS OF VARIOUS INHIBITORS ON CELL ENLARGEMENT AND RESPIRATION IN THE SAME TISSUE

\begin{tabular}{llcr}
\hline Tissue Inhibitor & Concn. needed for & Inhibition of respiration & Refer- \\
& $50 \%$ inhibition of & caused by this concn. of inhibitor & ence \\
& cell enlargement & &
\end{tabular}

\begin{tabular}{|c|c|c|c|c|}
\hline \multirow[t]{3}{*}{ Pea stems } & Iodoacetate & $6 \cdot 10^{-4} M$ & $26 \%$ & a \\
\hline & Arsenite & $\mathrm{I} \cdot \mathrm{IO}^{-4} \mathrm{M}$ & I $3 \%$ & a \\
\hline & Fluoride & $5^{\circ} \mathrm{IO}^{-3} \mathrm{M}$ & ca. $0 \%$ or slight promotion & a \\
\hline \multirow[t]{4}{*}{ Oat coleoptiles } & Iodoacetate & $4^{\cdot 10^{-5} M}$ & $10 \%$ & $\mathrm{~b}$ \\
\hline & Fluoride & $2.5^{\circ} \mathrm{IO}^{-3} \mathrm{M}$ & $9 \%$ & $\mathrm{c}$ \\
\hline & & $\begin{array}{c}c a .2 \mathrm{mg} / \mathrm{I} \\
\left(c a . \mathrm{Io}^{-5} M\right)\end{array}$ & $0 \%$ & d \\
\hline & $\begin{array}{l}\text { Dinitrophenol } \\
\text { at pH } 4.5\end{array}$ & $4 \mathrm{mg} / \mathrm{l}$ & $c a .0 \%$ or slight promotion & e \\
\hline Potato disks & monoxide & $5 \mathrm{CO}: \mathrm{IO}_{2}$ & ca. $25 \%$ & \\
\hline
\end{tabular}

References: a. Christiansen and Thimann (i950a)

b. Commoner and Thimann (194I)

c. W.D. Bonner and Thimann (1950) d. J. Bonner (1950) and Audus (1952)

e. J. Bonner (1949)

f. Hackett et al. (1953) and Thimann et al. (1954)

by cyanide, which inhibited growth and respiration of coleoptiles to the same extent (Bonner, I 936). An extreme case is shown by potato tissue, in which concentrations of dinitrophenol or arsenite which inhibit water uptake about $50 \%$ actually increase the respiration (Hackett and Thimann, I953). This tissue also shows a strong tendency for the inhibition of growth to disappear after 6 days, while the inhibition of respiration remains. Such phenomena confirm the indication of Table 4 that the relationship between respiration and growth is an indirect one. It is curious that mannitol, which inhibits elongation (see section below) largely prevents the rise in respiration caused by auxin, both in tuber and coleoptile tissue (Bonner et al., I953; Ordin et al., 1956). The explanation of this effect is not yet clear ( $c f$. Burström, r $953 \mathrm{~b}$ ).

In root tissues the principal inhibitor studied has been auxin itself. The data are less numerous but auxin appears to have a relatively small effect on respiration. At growth inhibiting levels of IAA $\left(c a . \mathrm{IO}^{-5} \mathrm{M}\right)$ maize roots showed a small reduction in oxygen consumption, but after 4 days it approached the control and, if calculated per mg of $\mathrm{N}$, even exceeded it (Kandler and Vieregg, I 953). The RQ went up to $\mathrm{I} .3$. Wheat roots in auxin behaved similarly when the respiration was calculated per mg $\mathrm{N}$, but the oxygen uptake per root was markedly affected parallel (1) the growth (Eliasson, I 955). Part of the explanation for the relative insensitivity to auxins on a nitrogen basis lies in the great increase in dry weight of roots, per unit length, when inhibited by IAA and the corresponding decrease when elongation is promoted by indoleisobutyric acid ( $c f$. the data on nitrogen, p. 792). Roots 
evidently change their geometry so greatly in auxins that data expressed only per unit length mean little.

The remarkable change in the relation between growth and respiration in potato slices deserves special mention. When freshly cut, the respiration of this tissue is inhibited by $\mathrm{CO}$ and $\mathrm{HCN}$, the former inhibition being controlled by the $\mathrm{CO}: \mathrm{O}_{2}$ ratio and reversed by light. Thus the respiration, like the growth, is mediated mainly by cytochrome oxidase. But $24 \mathrm{~h}$. later (at $25^{\circ}$, or 4 days later if the sections are kept at $14^{\circ}$ ) the respiration rate has spontaneously increased by $300-400 \%$, and now it is wholly insensitive to carbon monoxide and only slightly inhibited by cyanide. This new CO-insensitive oxidase has an oxygen affinity similar to that of cytochrome oxidase, and may therefore be a modified cytochrome system. Yet the cell enlargement remains light-reversibly CO-sensitive, i.e. controlled by a typical cytochrome oxidase, throughout (Thimann et al., I954). The mitochondria, in which the cytochrome oxidase is localized, do not seem to have significantly changed their sensitivity to inhibitors (Hackett, I956; Hackett and Haas, I 958). The change in respiration does not take place if the tissues are kept in $\mathrm{N}_{2}$ and thus it may perhaps be connected with the formation of a new protein.

(ii) Substrates. In most cases the substrates used in the metabolism that underlies cell enlargement can only be inferred. Tubers, as was mentioned, are rich in stored carbohydrates, and the often observed RQ of 1.0 which these tissues show is a good indication that carbohydrate is their main respiratory substrate. The marked decrease in starch in potato disks during cell enlargement, which has been observed by several workers since Reinders' experiments in I942, fits in with this finding. There is some indication that citrate promotes cell enlargement in artichoke disks (Hanson and Bonner, i955) but no real study of the substrates for growth in tubers has been made.

The growth substrate is consumed in metabolism in two ways: (a) for energy through the formation of adenosinetriphosphate (ATP) or similar materials, (b) for polysaccharides to be laid down as new cell wall. During growth new wall is laid down approximately proportional to the elongation, that is, the weight of wall material per unit length is the same in isolated sections growing in auxin solution as in comparable sections on the plant. Its composition $(c f$. the analyses in Table I) indicates content of several different polysaccharides and polyuronides.

The metabolism of pea stems during growth, however, is somewhat unexpected, since sugar does not appear to be the main substrate. Analyses show that after $24 \mathrm{~h}$. growth the reducing sugar content has fallen from the initial i $7 \%$ to about $12 \%$ (of the dry weight), but the important point is that the decrease is just as great in water as in auxin, although the elongation is only a third as much (Christiansen and Thimann, I950b). The explanation of this curious fact came from the observation that in presence of arsenite, fluoride or iodoacetate, in which the growth and respiration were inhibited, the reducing sugar did not accumulate but decreased even more. Changes in sucrose were too small to balance this. Evidently, therefore, the reducing sugar is not merely a reservoir of metabolizable material, but itself a product of metabolic processes. The respiratory quotient, initially I.o, falls quickly to about 0.84 , suggesting fat oxidation, and indeed direct analysis shows that the fat content does decrease during growth, from $9 \%$ initially 
to $6.2 \%$ after $24 \mathrm{~h}$. (based on initial dry weight). Furthermore, and most important, arsenite and other inhibitors prevent this decrease in fat. Correspondingly, they raise the $R Q$ back to 1 .o, or even above it. It was deduced that the major substrate for elongation (after the first few h.) is fat, that about equal quantities of fat and sugar are oxidized, and that some of the fat is continuously converted to sugar via processes poisoned by arsenite, fluoride or iodoacetate. Since these metabolic poisons can all act on several enzymes it is not surprising that they would all prevent the fat-sugar conversion, which involves combination with coenzyme A(requiring ATP), degradation to 2-carbon fragments, formation of pyruvate and thence of triose, followed by condensation to hexose.

In roots fat may also be a major metabolite, though perhaps not for growth. After wheat roots are cut off, the respiration decreases for some $h$. and simultaneously the RQ falls from a little above $\mathrm{I}$ to $0.7-0.8$. Added glucose prevents the fall. The RQ in the elongating zone, however, remains close to I for at least $6 \mathrm{~h}$., indicating that carbohydrates are, at least at first, the main substrate for elongation (Karlsson and Eliasson, I 955). The values 0.7-0.8 strongly indicate fat oxidation in the root as a whole but the authors do not draw this conclusion. The respiratory rate in the elongating zone is almost double that in the zone of cell division or that in the more basal maturing zones (Kopp, I 948; Goddard and Meeuse, I950).

(iii) Formation of enzymes and proteins. In many isolated tissues enzymes are formed during growth. Isolated sections of roots elongating in a simple solution (Robinson and Brown, I954) synthesized a slight amount of phosphatase, but lost invertase during elongation. However, since their growth is not promoted by auxin these root sections cannot be used to determine the effect of auxin on enzyme activity. The change in the terminal oxidase of potato slices, mentioned above, is another example but although this follows cell enlargement, it appears not to be connected with auxin action since the change occurs in water as well. Although auxin has not yet been proven to exert any recognizable effect on an enzyme system in vitro, there are at least three reports that after auxin has acted on a tissue, the enzyme content of the tissue has increased.

The first of these concerned ascorbic acid oxidase, which was found to be very greatly increased in tobacco pith tissue after it had been caused to grow by IAA (Newcomb, I 95I). The increase was of the order of nearly $400 \%$ and reached a maximum after 14 days. (The growth of this tissue is by no means pure cell enlargement, since numerous mitoses, including highly polyploid ones, have been reported in it [Skoog, I953]). The ascorbic oxidase was of a peculiar kind, being centrifuged down at low speeds with the cell-wall fragments. Most, but not all, workers with other tissues have found this enzyme to be soluble.

The connection with the cell-wall was still stronger in the second instance, when Bryan and Newcomb (I954) found pectin methylesterase increased in the same tissue after growth in IAA. Since pectin esters hydrolyze very readily under a variety of conditions, the significance of the enzyme is not wholly clear, though its relation to cell-wall metabolism is very suggestive. Indeed this observation led to a theory of the mode of auxin action, centering on the properties of the cell wall (see section $\mathrm{VIj}$, p. 793).

The third instance involves a quite unrelated enzyme, namely peroxidase 
(Jensen, I955). Here the stem or root tissue was incubated in IAA for a few $h$. and then tested, either after homogenizing or histochemically in the tissue. The exposure to IAA markedly increased peroxidase activity. The optimum IAA concentration for pea roots was $1 \mathrm{O}^{-8} \mathrm{M}$, for Vicia roots $\mathrm{IO}^{-7} \mathrm{M}$, both of which are high enough to inhibit elongation ( $c f$. section VIIb). The cells of the root cap, which are high in peroxidase, and those of the meristem, which are low in it, showed little or no change in the enzyme, but it was the cells of the elongating zone and those beginning to differentiate which responded, their peroxidase level being increased about 3 times. However, since the thin-walled parenchyma cells showed almost no peroxidase either before or after, while the protoxylem cells showed the biggest increase, the effect may be linked with differentiation rather than with cell enlargement (see Galston, I956). A similar increase of enzyme was earlier noted for "IAA-oxidase," the enzyme causing oxidative destruction of IAA (Galston and Dalberg, I 954). Here, however, other auxins not attacked by the enzyme, and even auxin antagonists, also increased the amount of enzyme, so that the effect is not specific. The destruction of IAA would of course not be expected to parallel the growth, and indeed Pilet and Galston (I955) found more of the enzyme in the non-growing than in the growing regions, of both pea stem and lentil root. In the older regions of the root it was the peroxide-generating capacity which increased, not the peroxidase itself; indeed the peroxidase itself seems to decrease with age of the cell. Whether this auxin-induced increase in auxin-destroying capacity is important in the control of growth remains to be seen, but is certainly possible. Unfortunately, the data showing the increase in oxidase in the lower parts of the pea stem are inconclusive, since they are presented per mg of nitrogen, and it is obvious that the nitrogen content would be highest in the young growing parts and relatively low in the mature and differentiated stem, with its secondary wall.

All in all, it may be said that some enzymes show an increase due to auxin, which may be related to growth, while others show increases which likely are quite unrelated. Such changes do raise the question of whether the action of auxin on growth involves a synthesis of enzymes or not. The broader, but closely related, question as to whether protein synthesis as a whole accompanies cell enlargement has been under discussion for some time. Whole coleoptiles, attached to the seed, do increase in protein content during growth, and so do the stems of whole plants swelling under the influence of high auxin concentrations. Protein is synthesized in root cells during elongation and far more than in the meristem (Clowes, I958). Invertase shows an increase of twenty-fold (Brown et al., I 952). On the other hand soluble nitrogen is of course flowing into these tissues and some of the changes may thus represent little more than nitrogen accumulation, unless the synthesis of protein and enzymes is clearly shown to take place at the expense of soluble nitrogen. Isolated pea stem sections, after growth in auxin, provide a more critical case, but here protein synthesis was found in early experiments and could not be repeated later (see Thimann and Loos, I 957, for review). Isolated corn coleoptile sections, when growing for 3 to $6 \mathrm{~h}$. in auxin solution, did not show any increase over auxin-free controls in their incorporation of labeled glycine or alanine into protein, but there was some incorporation, due to protein turnover, in both groups 
(Boroughs and Bonner i 953). Also the experiments were of short duration, coleoptile growth usually lasting at least $30 \mathrm{~h}$.

Roots caused to elongate by auxin antagonists (see section VIIb) have also been studied in this connection and it was concluded that no appreciable change in protein took place (Burström, I 95 I). Unfortunately the large increase in elongation $(c a .90 \%)$ was accompanied by only a small increase $(12-30 \%)$ in fresh weight, so that much of it must have been due to a change in geometry. The modest increases in fresh weight were in fact accompanied by increases in protein nitrogen of comparable magnitude $(4-30 \%$ in different experiments) but it would be hardly justified to draw a firm conclusion. A complication in the case of roots is that they form two proteolytic enzymes during elongation (Robinson, I956); these reach a maximum at the end of the growth zone. They do not increase when isolated root fragments are grown in sucrose, but they may be responsible for hydrolyzing protein that was formed during elongation.

Disks of potato tuber have given the most positive answer to this question at present. It was first shown in studies of salt uptake that they synthesize protein and it seems probable that some protein synthesis is necessary not only for the uptake of salt but for its subsequent retention (Steward and Preston, I94 I). Similar phenomena occur in growth. When the disks are aerated as for growth experiments, protein is synthesized rapidly for the first 2 days after cutting, and the soluble nitrogen (aminoacids) decreases correspondingly. Auxin clearly and reproducibly increases the protein synthesis, though only by some i $5 \%$ (Thimann and Loos, I 957). The water uptake was increased by $30-40 \%$, in the same auxin concentration. In artichoke tuber, which responds more strikingly to auxin, the protein increase was also much larger, - $400 \%$ in one experiment - though followed by some hydrolysis later. In both tissues protein synthesis mainly precedes the cell enlargement and cannot therefore be considered a result of growth. It may tentatively be deduced that auxin causes the formation of new protein in these tissues, and that this may well include one or more enzymes active in cell enlargement. Other enzymes not concerned with the process could well be synthesized too ( $c f$. above). If in some tissues the enzymes involved comprise only a small fraction of the total protein this would explain the lack of net protein synthesis therein.

\section{(h) Osmotic inhibition}

Cell enlargement can be stopped not only by inhibiting metabolism (section VIf) but also by reducing the availability of water. This was first shown in $193^{8}$ when Thimann and Schneider grew Avena coleoptile sections in auxin in presence of different concentrations of mannitol, which was chosen as being an essentially inert and relatively impermeable solute. Elongation was reduced in linear proportinnality to the mannitol concentration; at $0.3 \mathrm{I} M$ it was stopped altogether, though there was a slight recovery after $24 \mathrm{~h}$., no doubt because a small amount of mannitol enters the cells in time, reinstating an osmotic gradient. The suction force of the tissues, necessary for growth, is evidently $0.31 \times 22.4$ or some 7 atmospheres.

By making measurements every few h., Ordin et al. recently (I956) found a marked shrinkage at first in $0.3 M$ mannitol or higher, followed by recovery and 
slight elongation even up to concentrations of $0.5 \mathrm{M}$. Osmotic pressure measurements, by the method of incipient plasmolysis, showed that much of the solute had entered. This was evidently due to the provision of sucrose in the medium, since the data show that external sucrose causes marked increase of internal osmotic pressure. In the absence of sucrose only traces of mannitol enter, as the earlier data (above) showed. Recently Bennet-Clark (I956a) has done similar experiments using $\mathrm{KCl}$ as solute; it is much less satisfactory, since it enters the tissues even more rapidly, but the initial value of the suction force was found to be about 7 atmospheres, agreeing with the earlier data.

In these experiments, $\mathrm{MgCl}_{2}$ behaves like other solutes. $\mathrm{CaCl}_{2}$, on the other hand, inhibits much more strongly than other solutes. Concentrations as low as $2^{\cdot} \mathrm{IO}^{-3} \mathrm{M}$ give definite inhibition of elongation, both of coleoptile sections and of pea stems. It is evident that the calcium ion is acting as a specific inhibitor, and its mode of action will be discussed in the next section.

For pea stem sections placed in mannitol, the elongation is nearly proportional to the log. of the mannitol concentration; elongation ceases at about $0.4 M$, or some 9 atmospheres, and there is little or no evidence of recovery (Thimann et al., I950). In tuber tissues similar behavior occurs, but the recovery, i.e. presumably the entry of mannitol, is even more marked, and artichoke slices appear to come to equilibrium with mannitol concentrations approaching I $M$ (Burström, I953b). This entry of solute, as well as the uncertainty of the determinations of initial osmotic pressure, invalidates some earlier claims as to the action of auxin on growth and metabolism (Bonner et al., I 953). It remains true, however, that in mannitol, of a concentration high enough to prevent elongation, auxin causes no increase in respiration (Ordin et al., I 956).

Changes of the opposite type occur in tuber tissues given no external solute; these enlarge very well in auxin, and here it can be shown that the enlargement is accompanied by marked decrease of internal solute concentration. The decrease in osmotic content is indeed almost exactly proportional to the increase in volume which has occurred (Hackett, I952). Similar but much smaller decreases in osmotic pressure, accompanying growth, were recorded earlier by Ruge as long ago as $\mathbf{1 9 3 7}$, in elongating Helianthus hypocotyls. Some recent data for coleoptile sections which appear to show a decrease in osmotic pressure more than proportional to the volume increase in this tissue (Ordin et al., I956) are probably due to the metabolism of organic solutes.

Taking into account the complications due to solute entry and to dilution of the cell contents, it can be concluded that, provided energy is available, enlargement of tissues is governed directly by the water gradient. There is no evidence that cells can enlarge against a water gradient, i.e. that they can "secrete" water internally. But we have seen that oxidative metabolism is essential for enlargement. If the water enters only along its gradient, that is, it enters passively, then it must follow that the metabolic energy is needed for something else. Discussion of this problem follows.

\section{(j) Concepts of the mode of auxin action and the nature of cell enlargement}

In the thirties, after the role of auxin in growth had been discovered, numerous possible modes of its action were explored. It was shown, for example, that each 
molecule of auxin caused the deposition of more than one "molecule" of cellulose and indeed that not enough auxin was taken up to form a monomolecular layer over the surface of the newly-formed cell walls. Work on the physical and mechanical properties of the walls of growing organs, notably by Heyn and by Söding, did show that the plastic extensibility of the walls was increased by auxin action (see Went and Thimann, I 937; Heyn, I940, and Audus, I 949, for reviews). This led to the general concept that auxin causes cell enlargement by loosening the rigid structure of the wall, perhaps by enabling the cellulose micelles to separate from one another. Whether insertion or intussusception of new micelles between the old ones was a necessary accompaniment, or whether no more than a kind of passive stretching was involved, remained uncertain. From experiments with roots, Burström ( I 953a, c, I 954) came later to the view that both intussusception and stretching occurred, but in sequence, pure stretching constituting the first phase and wall deposition the second. Auxin was considered to promote the first phase in all concentrations but to inhibit the second at levels above $\mathrm{IO}^{-7} \mathrm{M}$, so that total growth of the root became inhibited in all but the very lowest concentrations.

These actions are envisaged as essentially physical. Other "physical" views have been put forward both before and subsequently.For example, Ruge in 1937 proposed that auxin becomes concentrated in the cell wall, increasing the acidity there and consequently (both by its acidity and by the specific effects of its anion) making the intermicellar colloids swell. Northen (I942, I 946 and other papers there cited) found that natural and synthetic auxins decreased the viscosity of the cytoplasm, as measured by high-speed centrifugation. This led him to the view that auxin causes the dissociation of complex proteins. As a result the viscosity would decrease, the protoplasmic swelling pressure would increase, and cell enlargement was considered to be a natural consequence. It was even suggested that the protein dissociation might liberate free -SH groups, which certainly participate in growth (Sect. VIf). Northen's observations of changes in viscosity may be another aspect of the increase in protoplasmic streaming rate caused by auxin (see below). Another physical view was based on the relation between chemical structure of the auxins and their growth-promoting activity and is largely due to Veldstra (see Veldstra, I 953, and Muir and Hansch, I 955, for reviews). The requirement for a ring and for one or more double bonds in it was ascribed by Veldstra to the necessity for the molecule to be adsorbed on a specific surface. The substitution of $\mathrm{Cl}$ atoms in the ring was supposed to enhance activity by increasing the lipophilic character of the ring and thus increasing its adsorption on to a lipoid phase. The well-known auxin activity of the cis-forms of cinnamic, $p$-methoxy cinnamic, $\alpha$-naphthaleneacrylic and tetralideneacetic acids, and the inactivity of their trans-isomers (p. 765), was ascribed to the necessity of holding the dipole of the carboxyl group out of the plane of the ring. Models show that this does occur readily in at least some of the cis-acids. Thus the auxin-molecule was considered to be adsorbed on a cell boundary, its ring in the lipoid phase of the membrane and its carboxyl group projecting out into the aqueous phase of the cytoplasm

see Fig. Io) In this state, Veldstra believed, the molecule would strongly influence the membrane potentials and by "opening" the tight packing of the membrane, 
also would increase the permeability to solutes. Parts of the chemical argument have been criticized (Thimann, I95Ia) and the views originally expressed have been somewhat modified, but Veldstra (1956) still interprets auxin action in essentially physical terms as acting "at a functioning macromolecular surface and/ or interfaces in the cell".

With the discovery that auxin had actions other than that on cell enlargement,

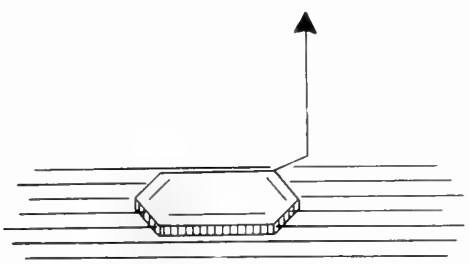

Fig. 1o. Veldstra's conception of the orientation of the ring of the auxin molecule in a lipoid membrane, and the projection of the carboxyl dipole at an angle to it. notably to promote cell division in cambial and other tissues, and to inhibit enlargement in lateral buds (section VIId) attention became focussed on some more fundamental action, a "master reaction" from which various observed effects might follow according to the nature of the cell acted on and the other materials available ( $c f$. Audus, I 954). This viewpoint became, indeed, essential when it developed that auxin acts on respiration, and now we know that many phases of metabolism may be influenced.

As far as cell enlargement is concerned, the argument in the preceding section has focused attention on the cell-wall. This might, of course, be considered as being extended passively by the entering water if it could be shown that auxin increases the osmotic concentration in the cell. However, we have seen above that the reverse is the case. The wall might also be extended passively if there were an active water-secreting mechanism which used metabolic energy to "pump" water into the cell. This possibility has been discussed before (Thimann, I95 Ib, I954a) and it is enough to say here that it would necessitate the "pump" competing with the natural permeability of the cell wall to water molecules. Recent experiments with isotopically labeled water virtually rule out this possibility. For example, using tritium water (Thimann and Samuel, I955) the water permeability of the walls of potato tuber tissue was found to be so great that the tritium water reaches $50 \%$ equilibrium with ordinary water throughout a $\mathrm{I} \mathrm{mm}$ thick section in less than a min. Furthermore, auxin treatment increases this permeability still further. Coleoptile sections, measured with deuterium water, show similar though less extreme behavior (Ketellapper, I952; Buffel, I952; Ordin and Bonner, I956); the permeability increases markedly with decreasing hydrogen ion concentration below pH 4.5. Ketellapper (1952) concluded that auxin did influence water permeablility, but this would let water out as much as in, and cannot therefore explain growth promotion. The critical point is that the permeability is already too high to be a limiting factor. It seems certain, therefore, that no pumping or secretion mechanism could operate against so "leaky" a membrane.

The possibility that water might be taken up by the same mechanism as that which absorbs ions, i.e. as $\mathrm{H}^{+}$and $\mathrm{OH}^{-}$ions, is eliminated by the mere fact that the respiratory energy is insufficient. During 4 days, $\mathrm{I} g$ of potato disks in auxin takes up 20 millimoles of $\mathrm{H}_{2} \mathrm{O}$ while the entire respiration uses only one half a 
millimole of $\mathrm{O}_{2}$, which could at most correspond to only 2 milliequivalents of $\mathrm{OH}$ ions (Hackett and Thimann, I953).

The remaining possibility is that the metabolic energy is used to modify the cell wall, reducing its resistance to enlargement. This would imply that the primary wall is not an inert excretion, but is under continuous metabolic control. There is some direct evidence for this mode of action: if auxin is applied to potato disks in presence of mannitol or other inert solutes, growth is reduced accordingly (as described above), but when the disks are afterwards transferred to water or plain auxin solution they enlarge rapidly, and essentially catch up with the controls which have been in plain auxin throughout (Thimann, 1954a). Evidently the auxin has been acting in presence of the mannitol to modify the cell-walls, even though water uptake was osmotically limited; on removal of the limitation, growth can then take place at maximum rate because the walls have already been modified.

In I95 I Kerr pointed out that the elastic properties of the cell-wall could best be explained by assuming that the protopectin forms a continuous phase in which the cellulose micelles are embedded. Growth would in this case be controlled by the pectin. Increases in pectin methylesterase after auxin treatment were mentioned above (p. 790). Operating along this line of thought, Ordin et al. (1955) have found that ${ }^{14} \mathrm{C}$ labeled methyl groups, from methionine added to coleoptile sections, yield ${ }^{14} \mathrm{C}$ activity in the hot-water-soluble fraction of the coleoptile cell wall after $4 \mathrm{~h}$. of auxin treatment, to a greater extent than in controls. The radioactivity incorporated into other fractions was much greater but showed no consistent response to auxin treatment, so that the results must be regarded as preliminary. The transfer of ${ }^{14} \mathrm{C}$ into the cell-wall from acetate or sucrose is not increased by auxin (Boroughs and Bonner, I953). The role of pectins for intercellular cementing, long known from cytological studies, has more recently been clearly shown by treating onion root-tips with pectinase (Brown, I95I). Not only did the cells separate, but the walls broke down enough so that free nuclei could be obtained. When whole barley roots were grown in solutions of pectinase (Cormack, 1955, 1956), the epidermal and other outer layers of cells separated completely from one another and grew in rows, attached only at their ends, and in various bizarre forms; on returning to water, or especially to solutions of calcium ions, the tissues slowly returned to normal. Growth was, however, not accelerated by the pectinase.

Bennet-Clark (1956a) has proposed that the growth-inhibiting effect of calcium (see p. 782) is due to its binding together in pairs the free $\mathrm{COOH}$-groups of pectic acids. Praseodymium, which is trivalent, was found to inhibit more strongly than calcium, while ethylenediaminetetracetic acid (EDTA, Sequestrene, Versene) which chelates with calcium and other metals, actually promotes growth 'Heath and Clark, I956), though its effects are small. In the writer's own experiments the effect of EDTA is shown on coleoptile sections but not on mesocotyl sections from the same plants, which makes it unlikely that it exerts a general effect on cell walls. Its effect is greatly increased in the presence of IAA.

In favor of an action of auxin on the cell wall is also the general observation that auxin operates only on plants with walls of the "higher plant" type, namely those 
consisting of cellulose, hemicellulose and pectins. Green algae with similar wall structure show growth promotion by auxin (Algeus, I946), but Euglena, with an elastic non-cellulosic membrane (unpublished data), and Phycomyces, with a chitinous wall, are clearly unaffected (Gruen, I 956).

The only difficulty with this view is that it does not allow for the other effects of auxin, such as promotion of cell division or of respiration. The latter might of course be indirect, but the action on cell division is direct and so are certain other effects. There is, for instance, a marked effect on the rate of cyclosis or cytoplasmic streaming in Avena, which appears within a few minutes after indoleacetic acid is applied (Sweeney and Thimann I 937, I 938; see Thimann et al., I 952, for brief review). Physiological concentrations are optimal; the effect is dependent upon oxygen and sugar, and is inhibited by iodoacetate and by dinitrophenol. The inhibition by iodoacetate can be prevented by malate. Furthermore, weaker auxins have a correspondingly weaker effect on streaming. The effect thus has remarkable parallelism with the effects on cell enlargement, except that it precedes them. Recently very similar effects in the staminal hairs of Tradescantia have been reported (Turner et al., I954) and extensive work from the author's laboratory has indicated the same process in the cambium of white pine (Pinus Strobus) (Thimann and Kaufman, I958).

Phenomena such as this suggest that the initial site of action of auxin may be in enzyme systems in the cytoplasm, and that only secondarily are enzymes which metabolize the cell wall affected. The role of auxin as "coenzyme" or "protective agent" in an enzyme system of fundamental importance, a "master reaction," seems more attractive than ever, but now it appears that this "master reaction" may be one which forms enzymes rather than merely acting upon them.

An unsolved question is whether auxin acts directly or only after conversion to some active form, like, e.g. indoleacetyl phosphate or indoleacetyl coenzyme A. It has been suggested (Thimann, I 956c) that the optimum curves of Fig. 7 depend on such conversion, the excess of unconverted IAA at high concentrations acting to antagonize the active form; however, as yet, no direct evidence exists to support this suggestion.

VII. GROWTH OF SPEGIFIC ORGANS

\section{(a) Growth of leaves}

The center of the vegetative apical meristem contains many non-dividing, vacuolate cells, and most of the activity is on the surface or tunica, particularly in a ring around the flanks (see Fig.3, p. 766) Leaf primordia arise as outgrowths of this ring on the flanks of the meristem, each forming a group of meristematic cells which curves up around the apex like a finger-nail. Underneath each growing primordium, the meristematic tissue of the apex begins to produce a line of elongated cells which form the procambium of a vascular strand, and quickly differentiate into xylem and phloem tissue which extend up into the leaf. As the primordium enlarges - more in length than in width at first-the basal part, in dicotyledonous plants, becomes less active and later forms the petiole; the upper part goes on to form the lamina. The most apical part of this in turn presently also becomes less 
active, so that in the half-grown leaf the continued enlargement is mainly from the middle and lower parts, particularly along the margins ("marginal meristem") where cell division continues until very late.

The change of form from a strap-shaped or finger-nail-shaped primordium to the elliptical or ovate leaf has been dealt with geometrically by D'Arcy Thompson and later by Huxley (see Jones, 1957), essentially on the basis that the rate of growth in width is an exponential function of the rate of growth in length, so that, if width be $y$ and length $x$ :

$$
y=a x^{b}
$$

where $a$ and $b$ are constants, the latter usually having a value between I and I.5 (see Chapter 2; $c f$. Avery, I933). Cast in the form:

$$
\log y=b \log x+\kappa
$$

the equation shows that a plot of $\log$ (width) against log (length) should give a straight line. In many cases it does (see Whaley and Whaley, i942, for a good example). The slope of the line is of course different for closely related species (Delisle, 1938) and even for different leaves on the same plant (Jones, I957). Deeply indented or compound leaves obviously cannot be treated in this way, and besides, their complexity often increases with successive nodes on the plant, or with changed external conditions.

The measurement of growth in area is experimentally more troublesome than that of length or breadth. Measurements have been made on a few leaves of relatively simple geometry, notably by Gregory (192 I) on cucumber leaves, who found that their enlargement follows a symetrically S-shaped autocatalytic curve. Similar curves were given by the cotyledons when grown at low temperatures, but as the temperature increased their growth curves became more and more asymmetrical and finally lost their S-shape altogether. The significance of an autocatalytic type of curve for growth of any organ is not quite clear. It could, of course, be said that any group of cells, each of which continuously divides into two, exhibits a kind of autocatalysis, but the increase in area is primarily due to cell enlargement; division is responsible only for creating cells which can subsequently enlarge. Furthermore, since cell division and enlargement are unevenly distributed in different parts of the growing leaf one can hardly hope that such mathematical analysis will yield much enlightenment as to the mechanism of the growth process.

One important point emerges from the general considerations of leaf enlargement, namely that the leaf primordium in dicotyledons must become physiologically differentiated very early, since expansion of the lamina is wholly dependent on light, while elongation of the petiole is not (see especially Gregory, I957). I) icotyledonous plants grown in complete darkness have virtually no leaf area. This effect of light seems not to be directly due to photosynthesis, for its temperature relations are different from those of photosynthesis, and besides, it exhibits an action spectrum somewhat different from that for photosynthesis and more nearly resembling that for the induction of flowering (Parker et al., I949), though the spectrum is not sharp enough to draw firm conclusions. However, Williams ( I957) has suggested that the action of light is exerted by promoting protein synthesis, a 
view which might unite many isolated pieces of information, such as the need of blade tissue for nitrogenous compounds, the fact that $\mathrm{CO}_{2}$ is essential for light to exert its leaf-forming action (Wiedow and Von Guttenberg, I953), and the stimulating effect of light on protein formation in isolated chloroplasts (Stephenson et al., I 956).

The leaves of monocotyledons differ in that they do grow in area in the dark; in fact the length of etiolated oat or barley leaves is about as great as that in the light, though the width is definitely less. In one or two cases, notably Narcissus, the leaves are actually longer in the dark than in the light. There is some anatomical evidence that in the early stages of development the leaf primordium of monocotyledons is homologous with that part of the primordium of dicotyledons which forms the petiole, i.e. the part which can elongate in darkness. Among the ferns some are said to expand their leaves in darkness, though the pinnae never reach the full size; others, however, behave like dicotyledons.

The elongation of leaf-veins is evidently controlled by factors different from those acting on the blade or lamina. This was seen early in the development of the auxin work when auxins were applied in lanoline paste to leaves. If applied to veins they would show growth-promotion by curving away from the applied auxin, but the laminar tissue would show no response at all. The differentiation is set up while in the late primordium stage, since when auxin is applied to the unopened bud the leaf laminae are afterwards found to be extended and sometimes grown together. A number of interesting abnormalities have been produced in this way (see Thimann et al., I952, for review). Another way in which the difference in growth control between the veins and lamina is seen is in the results of malnutrition and certain infections. "Frenching" of tobacco leaves, believed to be a virus disease, and "whiptail" of cauliflower, due to molybdenum deficiency, are both diseases in which development of the laminar tissue in the bud is inhibited, while the main vein grows more or less normally; as a result the leaves are shrunk to a narrow strip, which is not much more than the midrib. The gene "wiry" in tomato has the same effect.

Such differential response suggests that the more or less linear elongation of the vein tissue is under the control of auxin, while the plate-like enlargement of the lamina, with its indentations (or areas of no growth at all in compound leaves) would be under the control of other growth substances. This conclusion is in agreement with all the observations, though not rigidly proved. In the case of the long linear leaves of grasscs and other monocotyledons it is difficult to apply, since their elongation is not light-sensitive and yet apparently not controlled by auxin either. What are the controlling factors for leaf growth?

In the first place there is no doubt that auxin is produced in leaves, and transported polarly in them from the margins to the central veins and from apex to base therein. The production is dependent on light and the auxin content falls precipitously when the plants are placed in darkness. Correspondingly more auxin is formed, other conditions being equal, in long days than in short. The auxin yield per unit area, and sometimes per leaf, is greatest in the youngest leaves and decreases steadily with increasing age. On the other hand the role of this auxin in leaf growth is uncertain. Correlation needs to be made between the temperature 
characteristics of growth, or the action spectrum of the light effect, and the corresponding effects of temperature, or of different wavelengths of light, on production of auxin. Of late so much attention has been given to the role of light in destroying auxin, as a fancied explanation of the shortening of stem elongation by light (an explanation for which actually there is no direct evidence) that the role of light in the formation of auxin in leaves has been overlooked.

In the second place there is equally little doubt that auxin is not the major limiting factor in the growth of the lamina. Isolated disks or fragments of leaf tissue, when floated on auxin solution, usually do grow somewhat, depending on the plant used and the experimental conditions. In the author's experiments, sections cut from Pelargonium leaves, free from large veins, grew $21 \%$ in sucrose alone and $45 \%$ with IAA, I mg/l, added. In the work of Bonner et al. (I939), disks from radish leaves in sucrose showed no response to auxin, but when sources of nitrogen, such as adenine, proline, or asparagine were added, some growth was observed (up to $20 \%$ ). The diffusate from peas soaked in water was about twice as effective. However, not even this material caused appreciable growth of rye (Secale) leaf primordia attached to the stem apex; the elongation of these young leaves on a sucrose-salts medium was not increased by any of a large group of substances tried (De Ropp, I 945-47). Only when the tissue produced adventitious roots did the apex enlarge; it then produced 8 leaves. That the action of the roots could be due to increased uptake of salts or even of water was made unlikely by appropriate experiments, and it seems probable that some substance necessary for growth of rye leaves is synthesized in roots. Cabbage apices behaved in the same way, the leaves only developing appreciably after roots had been formed. Unfortunately in similar experiments with disks cut from cabbage leaves, roots did not have any such effect. It can only be concluded that the role of roots differs from plant to plant and perhaps also with the stage of development of the leaf.

Whole, rooted, bean plants grown in light with sugar but without $\mathrm{CO}_{2}$, make good experimental material since they form only minute leaves (Wiedow and Von Guttenberg, I953). These plants form leaves of moderate size when treated with a variety of substances, including thiamine, ascorbic acid, IAA, or yeast extract.

For monocotyledonous leaves an important growth factor is gibberellin (pp. 76o, $765)$. Leaves of rice plants infected with Fusarium moniliforme, or treated with gibberellin prepared from cultures of this fungus, elongate up to $50 \%$ more than normal (Brian et al., I 954 and Japanese literature there cited). Many, but not all, dwarf varieties of corn respond by elongating both leaves and internodes and as little as 0.00 I $\mu \mathrm{g}$ of gibberellin can be detected by placing it on a leaf (Phinney, r 956). Isolated sections of monocotyledonous leaves will also elongate in gibberellin. They will not generally elongate in auxin, and in fact, sections of the leaf sheaths of rice, whose elongation is promoted by sucrose, are inhibited by auxin (Murakami, 1956). It is curious that other members of the grass family, notably sugar-cane, although susceptible to the Bakanae infection, do not show the characteristic leaf recrerswth, and it may be that the gibberellin is already present in these plants in zirn-limiting concentrations. Indeed, gibberellin $A_{3}$ has been extracted from healthy plants (for review and discussion see Stowe and Yamaki, r957, I959), and a related substance from beans has been identified as gibberellin $\mathbf{A}_{5}$. 
Dicotyledonous leaves give no such marked response, and in most cases the very marked elongation of the stem which gibberellin brings about (Brian and Hemming, 1955) is accompanied by some reduction in leaf size, as well as by reduction in the chlorophyll density. Work in the writer's laboratory has shown that when the fronds of the duckweed Lemna are caused to expand by floating on gibberellic acid solution the chlorophyll density decreases so that the total chlorophyll per culture does not rise proportionately to the growth. With plants in soil this phenomenon can apparently be prevented by extra fertilization, and as a result some real, though small, increase in leaf area does result. On this account it is just possible that gibberellin may have some agricultural application for leafy crops. Root crops, too, whose yield has been shown to be strictly dependent on their leaf surface (Watson, I947) may prove to be benefited.

When the leaf becomes old, protein hydrolysis commonly occurs, leading to loss and fading of chloroplasts, migration of soluble ions out of the leaf, and other changes. It then falls off. This last process, "abscission," results from the division of cells at the base of the petiole (and sometimes also at the base of the blade) to form a line of new cell walls which crosses from one side to the other (see Plate 67 of Esau, I 953). These new walls differ from normal walls, or perhaps they contain pectinase, for they soon separate from one another, though the cells on either side remain intact. The separation eventually leaves the leaf hanging only by the conducting fibers, which soon break off, and it falls.

Almost the only fact that is understood about abscission is that it is inhibited by auxin and by conditions which promote auxin formation in the leaf. It is promoted by drought, ethylene and various chemical defoliants, as well as by a variety of injurious circumstances (see review by Addicott and Lynch, I 955). It is even, to a small extent, promoted by the presence of auxin in the stem below the leaf. Evidently the normal abscission of old leaves is a consequence of the cessation of auxin formation, and it was mentioned that auxin production in the leaf decreases with advancing age. In at least one fungus disease the leaf fall which results has been ascribed to auxin destruction by the fungus (Sequeira and Steeves, I954). A powerful enzyme for this, highly specific for IAA, is excreted externally by the fungus mycelium (Ray and Thimann, I956). It has been suggested that the determining factor in abscission is not merely the auxin coming from the leaf but rather the difference between this and the auxin in the stem, i.e. the auxin gradient across the abscission zone (Addicott and Lynch, I 955; Jacobs, I 955). It is difficult to conceive how cell division can be inhibited by a gradient. Direct inhibition by auxin would be a simpler concept, and it is therefore important that if very low auxin concentrations, such as those reaching the abscission zone from the stem, would slightly promote the process (Biggs and Leopold, I957).

\section{(b) Growth of roots}

The apex of the root differs from that of the shoot in two respects: (I) the meristem is not quite at the tip, but continually produces a small group of cells, the root-cap, distal to itself, and (2) no outgrow ths comparable to leaf primordia arise on its flanks. Lateral roots are indeed formed, but they appear at a considerable distance from the apex (see below). This second characteristic makes root develop- 
ment somewhat simpler than that of shoots and has led to considerable use of roots in varied physiological studies. It may be worth while to point out, though the work will not be discussed in detail here, that it was with roots that the important phenomenon of resistance to radiation damage was discovered. X-irradiation of roots in low oxygen tensions caused much less damage than in air (Thoday and Read, I 947-49) and the subsequent study of this phenomenon with bacteria has made it possible greatly to reduce radiation damage by treatment with ethanol, cysteine or other potential hydrogen donors (Hollaender and Stapleton, I953).

For present purposes, the main growth study to which roots have been put concerns the effect of auxins. In the last few years this work has been extensively developed in Sweden by $\AA$ berg, Burström and their collaborators. As stated, root elongation is inhibited by concentrations of auxin which promote the elongation of shoots. In the case of wheat roots, inhibition is exerted by $\mathrm{IO}^{-8} \mathrm{M} \mathrm{IAA}$, while NAA, 2,4-D or 6-chloroIAA have about the same activity (Burström, I95I ; Hansen, I954). Parallel with the inhibition of elongation of the root as a whole goes inhibited elongation of the epidermal cells, and in general also inhibition of their multiplication. Each of a large variety of substances operates over a different range of concentrations and the dose-response curve has to some extent different and characteristic shapes. Examples of the behavior of wheat roots, expressed in length and number of the

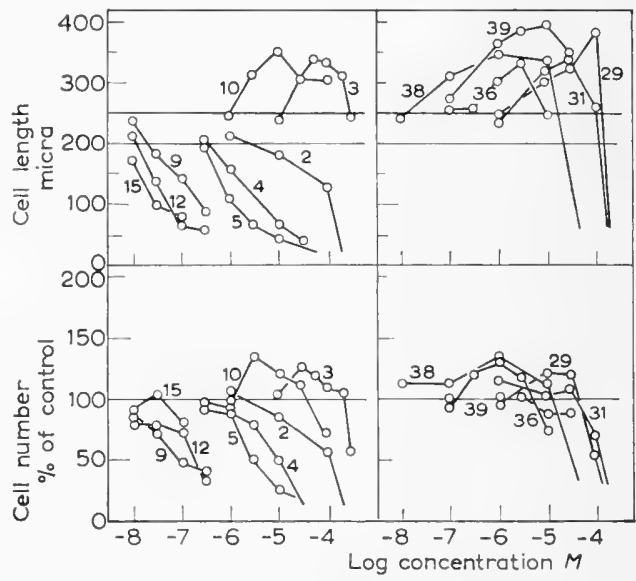

Fig. I 1. Elongation of wheat root epidermal cells, and cell numbers (calculated from root length divided by cell length). All data after 3 days in solutions.

Left: arylacetic acids:

2 Phenylacetic

3 Phenoxyacetic

4 2-methylphenoxyacetic

5 2,4-dimethylphenoxyacetic

9 2,4-dichlorophenoxyacetic $(2,4$-D)

Io 3,5-dichlorophenoxyacetic

I2 2-methyl, 4-chloro-phenoxyacetic

I 5 I-naphthaleneacetic (NAA)

Right: aryl-isobutyric acids:

$29 \alpha$-phenoxyisobutyric

3 I $\alpha$-(2,4-dimethylphenoxy)-isobutyric

$36 \alpha$-(2,4-dichlorophenoxy)-isobutyric

$3^{8} \alpha$-(2-methyl, 4,6-dichlorophenoxy)isobutyric

$39 \alpha$-3-indoleisobutyric

Selected from Hansen, I954. epidermal cells, are collected in Fig.I I. Each curve represents one substance.

The left-hand side of the figure shows a series of auxins (except nos. 3 and Io). They inhibit both elongation and multiplication, and their curves show rough parallelism. The right-hand side of the figure shows the effect of a series of arylisobutyric acids. These compounds were selected because they have very little auxin activity on shoot tissues, (though No. 39 is quite active in the slit pea stem test). In all cases these substances promote root cell extension over a wide range of concentrations (commonly three logarithmic units) and inhibit it sharply at 
around $\mathrm{IO}^{-4} \mathrm{M}$. The parallel with cell division is again noticeable though not quite so directly proportional. Compounds 3 and 10 , phenoxyacetic acid and its 3,5dichloro derivative, behave similarly; these two also have virtually no growthpromoting activity on shoots. Thus it appears that every auxin, as defined by growth promotion of shoots, inhibits growth of roots, while the compounds which promote growth of roots are those with little or no auxin activity on shoots. Furthermore, the most active growth-promoting compounds on shoots are in general the most active inhibitors on roots. Note that compound No. 2, phenylacetic acid, is certainly less active as an auxin on shoots than the other compounds (except Nos. 3 and 10 ) in that figure.

The special case of indoleacetonitrile (III p. 76I) may be mentioned. As shown in section IV the activity of this compound on shoots can be explained by its conversion to IAA, the extent of which differs in different plants. In tomato roots, however, its action differs from that of IAA in that it promotes formation of laterals and it does not decrease the survival of the meristem as does IAA (Street, I954; Street et al., I954) The conclusion that its mode of action is quite different from that of IAA is, however, unjustified. The concentrations of nitrile needed for $50 \%$ growth inhibition are 5000-10,000 times those of IAA which means that activity could be due to a conversion of $<0.1 \%$. The large excess of unconverted nitrile would not be wholly inactive but probably would weakly antagonize one or the other activity of IAA, just as it weakly synergizes with IAA on pea stems.

The exact activity of indoleacetic acid on roots is hard to estimate because this substance is rapidly oxidized in root tissue. Furthermore, as mentioned in VIg (p. 787), peroxidase increases in lentil roots exposed to IAA, and since peroxidase can oxidize IAA it follows that the ability of root tissue to destroy IAA would increase with time of immersion. Roots of flax and cress do not show this effect, while roots of oats (Bonner and Koepfli, I 939) and of wheat ( $\AA$ berg and Jönsson, 1955) destroy IAA very actively. A complication is that at subinhibitory concentrations, IAA reduces the survival of the main axis meristem (Street, I954).

At concentrations lower than the inhibiting level some true auxins cause a promotion of root growth. The effect is seldom more than $20 \%$ and is not obtained with all roots. Flax roots, studied by Åberg, for instance, do not show it at all, while wheat, oats and corn roots show clear promotions, 4-methyl-phenoxyacetic acid giving a $35 \%$ increase of the elongation of wheat roots ( $\AA$ berg, r $95^{6}$ ). In the older literature many workers have found IAA to cause increased elongation. Most of these results were obtained after one or more days, when presumably the IAA had been partially destroyed (see Table I in Larsen, I956, and Fig. 2 of Geiger-Huber and Burlet, I936).

When elongation is being inhibited by an auxin, the inhibition can be relieved by one of the substances on the right-hand side of Fig. I I which promote elongation. Such substances, which remove the inhibiting effect of auxins, are considered to be auxin antagonists. As a rule they inhibit the elongation of Avena coleoptile sections in presence of an auxin, which is of course another criterion of antagonistic activity. While in general they promote the growth of intact roots, especially wheat roots, the correspondence is not absolute, and in isolated roots the growth promotion depends upon the sucrose content of the medium (Street, 1955). For these 
reasons Hansen (I954) has suggested that root growth promoting compounds be called "root auxins," and only those compounds which relieve the inhibition due to externally applied auxin be considered true antagonists. The case is particularly complex with indoleisobutyric acid (XX, p. 765) which promotes root cell elongation by about $200 \mathrm{~m} \mu$ whether or no the root is being simultaneously inhibited by IAA (Burström and Hansen, I956). The IiBA is thus not really acting as an antagonist here, but as a simple root growth promoter, and the logical conclusion is that the two substances "act in two physiologically different systems, and that they do not simply compete in one single reaction mediating the cell elongation". (It may be noted that XX also acts as a true auxin on shoots).

Synthetic auxins with an aryl group in the $\alpha$ position of a propionic or butyric acid side-chain exist in $\mathrm{D}$ - and $\mathrm{L}$-forms. The $\mathrm{D}$-forms are invariably the ones with auxin activity, the L-forms having always less activity and sometimes none at all. Most of these $\mathrm{L}$-forms act as auxin antagonists, $\mathrm{L}-\alpha-(2$-naphthoxy)- $n$-butyric acid, XXXII, being particularly effective (Matell, I 953; Aberg, I 953). Some, however, are inactive even as antagonists.

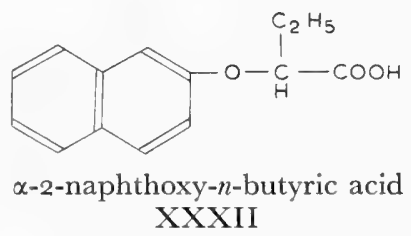

All these data have given rise to intensive theorizing. Many of the antagonisms are apparently competitive, i.e. the effects depend on the concentrations of both auxin and antagonist, and in a manner predictable from theory (Skoog et al., I 942; McRae et al., I 953; McRae and Bonner, I953; Linser, I954; Kaindl, I956). Similar competitive antagonism can often be shown in coleoptile sections. There are also compounds of an intermediate type, which act as auxins where the auxin concentration is low, but as antiauxins where it is high or when effective auxins are added (see Åberg, I 956). These compounds cause peculiar effects on shoot tissues such as coleoptiles, inhibiting growth at low concentrations and promoting it (or inhibiting it less) at higher ones. Evidently the relations are a little too complex to be expressed as yet in any simple theory (see Torrey, i956b, for review).

All the above considerations exemplify the opposite way in which roots react from shoots where auxin is concerned. The tropisms offer another example. Shoots curve upwards away from the earth (negative geotropism), roots downward (positive geotropism). Shoots curve towards high light intensities (the reaction to low light dosages being complex) while roots frequently curve away. In shoots the curvatures are well known to be due to differences in the concentration of auxin on the two sides, though the method by which this is achieved is still under discussion (see Went and Thimann, I937, and Brauner, I954, for reviews; Rufelt, I 954; Larsen, I 956; and Curry et al., I 956, for recent literature). In roots the proof of such a distribution is really not quite complete. For geotropism, an increase in auxin on the lower side of a horizontally-placed root has been shown by several workers in the past, and this would cause inhibition of the extension on the 
lower side, as required. However, geotropic curvature is not easy to imitate by local application of auxin, and besides, one must make a reservation for the case of those roots whose growth is promoted by low auxin concentrations; these ought to curve upwards. It may be that two components, positive and negative, interact in normal geotropism (Rufelt, I 954). Since some roots begin to show geotropic curvatures after $8 \mathrm{~min}$., while in general the promotions of growth due to auxin only occur after much longer times ( $c f$. above), the above reservation may not need to be made (Larsen, I 956), though it remains true that auxin applied for short times and then removed may cause marked growth accelerations, measurable within min., (Gast, I 942), an observation which seems to have been overlooked recently. It is interesting that some auxins do cause upward curvatures (Rufelt, I954). If the classical auxin theory of geotropism does prove to hold in roots then the contrast with shoots is complete.

Phototropism cannot be similarly compared, for lack of critical data on phototropism in roots.

A third sharp contrast with shoots is in the response to calcium, which has been known to be necessary for root growth since the work of Hansteen-Cranner in I 9 I O and of Mevius in the 20's. Calcium is not stored in roots in appreciable amounts but must be added to the nutrient solution. Not only is it necessary, it clearly promotes root growth; at the optimum concentration, which is $\mathrm{IO}^{-4} M$ for wheat roots, it increases the total root length by about $200 \%$, more or less, depending on the $\mathrm{pH}$ (Burström, I 952, I 954). The effect on cell length is greater than that on cell division, but even the latter is increased nearly $50 \%$. Burström found that on plasmolysis the root epidermal cells shrink more if they have been exposed to high calcium than do those of controls; this means that calcium has increased their elastic extensibility. A sample of the data is shown in Table 5. The results are explained in terms of the postulated two phases of growth $(c f \cdot p \cdot 794)$ :-the first, wall softening, promoted by auxin in roots as in shoots and independent of calcium; the second, deposition of new wall material, inhibited by auxin in roots, but promoted by calcium.

The action of calcium in promoting elongation of the root is in line with another well-known effect, namely that of promoting the outgrowth of root-hairs. The basis for this is not well understood (see Cormack, I 949, for review). Root hairs,

TABLE 5

ELASTIC TENSION OF WHEAT ROOT EPIDERMAL CELLS AS A FUNCTION OF CALGIUM CONTENT OF THE MEDIUM. FROM BURSTRÖM (I 954 )

\begin{tabular}{|c|c|c|c|}
\hline $\begin{array}{c}\text { Calcium concentration } \\
\qquad M\end{array}$ & $\begin{array}{c}\text { Initial length } \\
\mu\end{array}$ & $\begin{array}{l}\text { Length after } \\
\text { plasmolysis } \\
\qquad \mu\end{array}$ & $\begin{array}{c}\text { Elastic tension } \\
\%\end{array}$ \\
\hline $10^{-6}$ & 212 & 204 & 4 \\
\hline $10^{-5}$ & 247 & 219 & I 3 \\
\hline $10^{-4}$ & 335 & 294 & I 4 \\
\hline $10^{-3}$ & 327 & 282 & I 6 \\
\hline
\end{tabular}


which are outgrowths from the epidermal cells, are known to develop much more strongly in the air than in nutrient solution. Presumably their growth would require much new wall material and thus require both calcium and oxidative metabolism.

Thus the physiology of elongation in roots has at least three features opposite to those in shoots. On the other hand the general metabolism underlying the growth process has many points of similarity. For example, dinitrophenol and arsenate, which act on phosphorylation, and beryllium, believed to inhibit adenosinetriphosphatase, all inhibited elongation strongly (Hopkins, I952). Arsenite and iodoacetate, both sulfhydryl reagents, acted less powerfully, while fluoride had very little effect (Kandler, I953). Unsaturated lactones, especially coumarin and its derivatives, inhibit the elongation of oat (Goodwin and Taves, I950) and of wheat roots (Burström, I 954), but as yet it is not clear that this is due to their acting as sulfhydryl reagents, especially since Pollock et al. (I954) could not relieve the inhibition by adding BAL $(c f$. p. 785$)$. It appears also that coumarin and scopoletin operate in different ways since coumarin causes lateral swelling, which scopoletin does not, while scopoletin inhibits the formation and elongation of root hairs, which coumarin does not (Avers and Goodwin, 1956).

Isolated roots grown in culture require a complex nutrient medium, containing both macro- and micro-elements, sucrose, four or five B-vitamins and sometimes one or more other nitrogenous compounds. Correspondingly, antimetabolites for pyridoxin, $p$-aminobenzoic acid, folic acid, proline, arginine and guanine all inhibit the elongation of seedling roots and in many cases, but not all, these inhibitions can be overcome by adding the parent compound (Fries r 954; Torrey, I 956b). This demonstrates that roots on the plant are dependent on the supply (from the shoots or cotyledons) of the B-vitamins, some aminoacids and purines.

It only remains to consider the development of lateral roots on roots. These organs are not to be compared with lateral buds, for the lateral roots arise far from the apical meristem of the root, by division of cells which have ceased to elongate. The distance between the root tip and the first lateral roots is quite constant during normal growth (Geissbühler, I 953) and has been ascribed to inhibition of laterals by the main apex. An ether extract of the tip does inhibit lateral formation in the segments below by about 50\% (Torrey, I 956a), which supports this view.

Auxin strongly promotes the outgrowth of laterals in pea roots, but after a root has once responded in this way it cannot for some time produce a second crop of laterals on receiving a second auxin treatment (Torrey, I950). Evidently another factor is now limiting, a factor which was shown to be coming from the cotyledons. By using isolated segments, it was found that lateral root formation is promoted, in conjunction with auxin, by thiamine, nicotinic acid, adenine and a mixture of micro-elements (Torrey, I 956a). Antimetabolites for the three nitrogen compounds, iiz. pyrithiamine, 3-acetylpyridine and 2,6-diaminopurine, respectively inhibited the process and in each case the inhibition could be reversed by the parent compound. As was noted above, the same three nitrogen compounds are needed for the growth of normal roots, or act as stimulators for the growth of inhibited roots. A pH of 6 is optimal for lateral root formation in pea roots, and 2 to 3 days of treatment with IAA $\mathrm{IO}^{-5} \mathrm{M}(\mathrm{I} .75 \mathrm{mg} / \mathrm{l})$ was the optimum auxin treatment. This 
is a high concentration compared to those used in the elongation studies above; however, the isolated segments used were not elongating in these experiments.

The initiation of laterals evidently depends on periclinal cell divisions occurring in a specific layer, the pericycle, and the action of auxin is probably exerted in stimulating these divisions. In Torrey's work the laterals were counted as unelongated initials, still within the main root, so that the role of elongation was essentially excluded. The process thus offers another instance of the effect of auxin on cell division. Elongation of the formed laterals, just like elongation of the primary root, would be strongly inhibited by the auxin, but since 3 days suffices for initiation, the roots can be removed from the auxin solution and the laterals will then grow out. In these respects the formation of lateral roots on a root is comparable to the formation of roots on stem cuttings, which is also promoted by auxin and assisted by various nitrogenous compounds (p. 77I).

\section{(c) The growth of fruits}

In this limited space it would be quite impossible to discuss the entire topic of fruit growth. Both the physiology (Nitsch, I952, I953; Leopold, I955, Chaps. I I to I3) and the anatomy (Essau, I953 Chap. I9) have been well reviewed. Several short reviews were included also in the Wisconsin Symposium (Skoog, ed., I 95I). Only a few points will be made here.

In general, the fruit consists of the swollen ovary wall, receptacle, or other tissues surrounding or supporting the fertilized ovules. In cucurbits, peppers, and in berries generally, it is formed from the ovary wall and placentae, in the tomato mainly the placental tissue, while in the apple the fruit tissue derives from the floral tube, and in the strawberry it is the receptacle. The ovule, which on fertilization becomes the seed, is contained in the carpel. This is considered from the evolutionary viewpoint to be a spore-bearing leaf folded over in two and fused into a closed vessel (Bailey and Swamy, I95I). It is the carpel, with the ovule inside, which is the critical structure composing a fruit, and the succulent tissue is often quite secondary.

The growth of this tissue follows, and in good part results from, fertilization of the ovule. In almost all fruits, cell division occurs rapidly in the earliest stages, but comes to a stop either at the time the flowers open or shortly after fertilization has occurred. In the apple it lasts for 3 to 6 weeks. The visible growth of the fruit is, then, almost pure cell enlargement and in most cases the enlargement is roughly isodiametric. It is usually very extensive. The cells of the apple enlarge up to I oo times their initial size; in watermelon they even become visible to the naked eye. Large-fruited varieties may differ from small-fruited varieties in (a) prolongation of the period of cell division, (b) greater cell enlargement, or (c), as in the pumpkins and gourds, both (Sinnott, I 939). It must be understood that the apples, plums, berries, tomatoes and squashes of commerce have been selected and bred for maximum cell enlargement over a great many generations and that, as every gardener knows, full size is not achieved without generous fertilization. The factors essential for cell enlargement, then, are an adequate supply of water and nutrients, especially nitrogen (early in the season) and potassium. The nitrogen of the apple does not increase proportionally to its fresh weight, but rather to its surface; this means 
that in each cell the nitrogen increases proportional to the cell surface, and this in turn, it has been suggested, means that the enlarging cell maintains a layer of protoplasm of approximately constant thickness (Pearson and Robertson, I953). This relationship, which no doubt holds for other fruits, is similar to that of enlarging fragments of tubers (p. 792).

The sources of auxin for all this growth are by no means understood. Recent work indicates that no one single source is responsible and that several auxin sources succeed one another in making possible the whole process of fruit growth. That auxin is an essential and causative factor is certain, because the whole process of fertilization and seed development can be set aside, and normal-sized fruit still be obtained, if auxin is applied. This results in seedless, or parthenocarpic fruits. In a few cases, including tomatoes, gibberellins have a similar effect.

That parthenocarpic fruits are capable of more or less normal development has, of course, been known for a long time, because several fruits of commerce, including the banana and navel orange, are parthenocarpic. The work of Yasuda and of Gustafson, in the 3o's, was the first to show that petunia and tobacco fruits could be made to develop by extracts of pollen almost as well as by the pollen itself (see Gustafson I 942 and I95 I for reviews of early work). Later a variety of horticultural fruits have been made parthenocarpic,-including pears, squash, watermelons, peppers, strawberries and tomatoes, - the last even on a semi-commercial scale. The procedure is always the same, namely to spray the freshlyopened flowers with an auxin in suitable concentration. If it is desired to ensure complete seedlessness the stamens may be first removed with fine scissors. Auxin application after pollination leads to fruits with a reduced number of seeds (Fischnich and Lübbert, I955). However, the main advantage of commercial auxin treatment is not really seedlessness but an increase in the number of fruit that are set. The method is particularly valuable, therefore, in weather that is unfavorable for natural fruitsetting; e.g. greenhouse tomatoes in the winter months (Howlett, I 94I). The fruits are often rather small, but at least in some experiments normal-sized fruits have been obtained. Apparently the ethyl ester of IAA is the most effective auxin for the purpose, though 2,4-D (XI, p. 763) has been widely used on tomatoes and $\alpha$-2-naphthoxy-propionic acid seems the most effective on pears.

The natural supply of auxin to the ovary begins with the pollen, which several workers have shown to contain moderate quantities of auxin ( $c f$. above). However, the number of pollen grains falling on one stigma can be quite small and the quantity of auxin so transferred could hardly be enough for more than a minute amount of growth. This growth, however (or perhaps the act of fertilization) modifies the enzymes of the style and the ovary wall so that, in tobacco at least, they now can liberate an auxin from the ovary tissue (Muir, I942, I95 I). As a result the styles and ovary tissues soon contain more auxin than the pollen could have supplied. The flower petals behave in an oppositive way, since they fall off after pollination, presumably because of reduced auxin formation, and application of auxin will make them stay on, at least for a time (Fischnich and Lübbert, I955).

The second step is fertilization, which, in the apple, causes growth of the endosperm, first as free nuclei in a coenocyte ( $c f$. coconut milk) then as separate cells; thereafter it causes growth of the embryo. The seed appears to contain no free 
auxin for some 3 to 4 weeks after fertilization, then a "wave" of auxin production, demonstrable by ether extraction on successive days, coincides with the endosperm becoming cellular (Luckwill, i 953). A second and larger wave, reaching its peak some Io weeks after fertilization (the amount and the time depending on the variety of apple), was ascribed to the growing embryo itself, though it

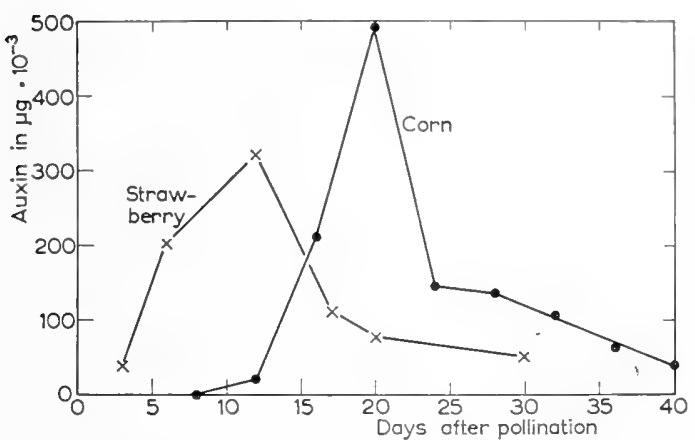

Fig. 12. "Waves" of formation of free auxin in the achenes of the strawberry (data of Nitsch, 1950) and in the grains of corn (data of Stehsel, r950, unpublished). Auxin determined by ether extraction and bioassay, without liberation of any in "bound" forms. (Replotted from Nitsch, I952.) may also be due to the seed-coat. Similar "waves" of auxin formation occur in strawberries, corn and other fruits (Fig. I2). In the apple variety Lane's Prince Albert some of the embryos abort, and this corresponds with even greater auxin production, presumably by the endosperm. Finally the fruit tissue, at least in apple and pear, is found to contain another, and a relatively unstable, auxin. It is when this auxin begins to disappear that the peduncle of the fruit begins to form an abscission layer (cf.p. 798) and hence the fruit soon falls. If the fruits are sprayed with NAA or $2,4-\mathrm{D}$ at this point, abscission can be delayed for some

weeks, and this practice is now general with orchardists.

Thus the auxin may be supplied from as many as 4 different sources during the growth and life of the fruit (assuming one may condense the partial findings on several different fruits into a single composite picture). The supply from the embryo is doubtless the most important, for if that part of very young crookneck squash fruits which contains the ovules is removed, growth virtually ceases. This growth could be reinstated by applying indolebutyric acid (Gustafson, I 95 I, and earlier work there cited). Similarly in the strawberry, where the "achenes" (seeds in their carpels) are on the outside and thus available to the experimenter, it is clear that if they are removed all growth stops (Nitsch, I950). If achenes are removed on only one side, growth on that side stops; hence the auxin supplied to the receptacle does not diffuse far. The receptacle appears to form no auxin itself, while the achenes produce it in a single "wave" with its steep peak about 12 days after fertilization (Nitsch, I 955; see Fig. I2).

There is evidence that these successive auxin sources may supply different substances. That in the first wave in the apple and in the black-currant is probably IAA (Luckwill, I954). The auxin in the apple seeds, has been thought to be the ethyl ester (Teubner, I953). The strawberry achenes contain 4 other auxins besides IAA (Nitsch, I955).

Analyses of developing bean pods showed that there is a "wave" in them at about 8 days from fertilization of a substance differing from auxin in being transported upward in test plants and causing excessive stem elongation (Mitchell et al., 
I 951 ). In the light of present knowledge, this is gibberellin $\mathbf{A}_{5}$ or a related compound. Its role in fruit growth is as yet unknown, but it can induce parthenocarpy.

As with the leaf, the abscission of the fruit depends on a cessation of the auxin supply. The fact that the auxin is formed in "waves," which do not necessarily overlap, may mean that there are temporary lacunae in the supply of auxin to the

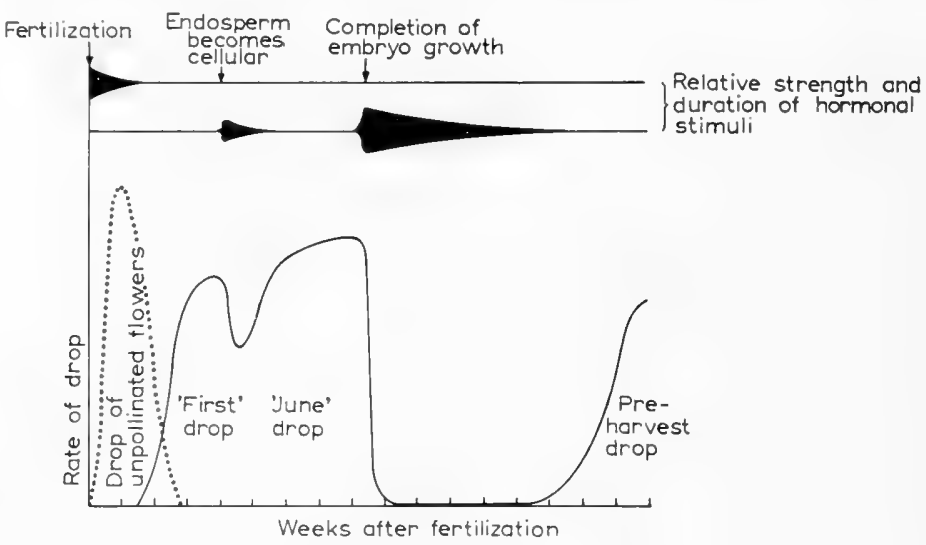

Fig. 13. Scheme of "hormone" production (at top) and fruit drop (below) in the apple. The hormone was determined by ether extraction and bioassay, using parthenocarpy of tomatoes as test; for this reason both auxins and gibberellins may be included in the activity.

From Luckwill, 1953.

peduncle. On this account some of the fruit may fall at specific times during the growing season. This is especially true of apples; almost all varieties suffer from "June drop" and some have a "first drop" period before that. These dropping periods have been correlated with the auxin production (Luckwill, I953) into a general picture which has a good deal of verisimilitude (Fig. I3). It is, of course, not certain that the abscission depends only on the auxin coming from the seed, but the data correspond fairly well. It is well known, too, that apples with exceptionally few seeds are likely to fall during the season.

Finally, it is interesting that the sex of flowers appears to be under hormonal control, auxin diverting it towards femaleness. In cucumbers the proportion of female flowers can be clearly increased by early treatment with IAA or NAA (Laibach and Kribben, I950). In acorn squash plants, which produce male flowers near the base and female flowers at about the 2oth node, treatment with NAA caused female flowers to appear at about the 9 th node. Most striking example of all is hemp (Cannabis sativa) in which the sexes are borne on separate male and female plants; male plants can be made to bear female flowers by auxin treatment. For this, $0.05 \%$ NAA in lanoline was used (Heslop-Harrison, I956). It is suggestire, too, that in the acorn squash which gradually becomes female, the last-formed fcmale flowers yield parthenocarpic fruits; there would seem, therefore, to be a continuous increase in the auxin supplied to the flower-buds, causing continuous change from male through female to parthenocarpic. The possible significance of these findings for horticulture may be very great. 
(d) Interrelations between buds; integration of the plant

On a typical dicotyledonous shoot there is a lateral bud in the axil of every leaf. Usually most of these do not develop, or develop only very slowly, but if the growing apex is killed or removed, a few of them develop at once. Such behavior implies that these lateral buds had been capable of development at all times, but were inhibited by the growing apex.

More than twenty years ago, in the "exploratory" phase of the study of plant growth hormones, this inhibition was shown to be exercised by auxin coming from the apical bud. If the apex was removed, then the lateral buds could be maintained in the inhibited state by applying auxin. Supplementary inhibitions are exercised by the much smaller amounts of auxin coming from the leaves in whose axils the lateral buds lie. (See Went and Thimann, I 937; and Söding, I 952, Chap. I I for reviews of the earlier experiments.)

It is evident that this system provides a major hormonal means for the integration of the growth of the plant. Its effectiveness varies, of course, from one plant to another. At one extreme are plants like the sunflower with a single axis and complete suppression of the lateral buds, a clear example of "apical dominance". At the other extreme are the much-branched dwarf composites with little or no dominance, bearing numbers of branches, both lateral and direct from the root crown, which develop simultaneously. Most dwarf plants, indeed, branch much more freely than their normal relatives. Monocotyledons are no exception, for in the grasses and cereals the lateral bud is the "tiller," of which one to several develop in a season and may bear additional heads; the development of these, which is greatest in dwarf forms, is also inhibited by auxin and released upon weakening or removal of the auxin source (Leopold, I949).

A feature of the inhibition is that it is dependent on continuity of the auxin supply. Thimann and Skoog in 1934 found that they had to apply fresh auxin in place of the terminal bud every $6 \mathrm{~h}$. in order to maintain complete inhibition; a gap of $12 \mathrm{~h}$. between applications led to development of the buds. Recently Libbert (1954a) has observed that, if the terminal bud of Pisum is removed and subsequently replaced intact, the break in auxin supply is enough to allow lateral buds to grow out, even though the terminal bud afterwards becomes grafted back in place. The growing lateral may then come to inhibit the further growth of the terminal. Correspondingly, any treatment interfering even slightly with the transport of auxin, or even a temporary fall in the rate of auxin formation (Hatcher, I948) may cause lateral buds to develop.

In a way the suppression of one meristem by another can be considered a general phenomenon. Bünning (I 952, I957) has proposed that all meristems fragment into smaller ones, called "meristemoids", which can only become active when removed from the inhibiting action of the parent or from one another. The formation of stomata and roothairs are offered as examples. Inhibition of the development of stomata by one another is responsible for the even pattern of their spacing on the leaf. The formation of stomata can also be inhibited by auxin through causing normal cell division of the epidermis instead (Bünning and Sagromsky, I948). Is was noted in section VIIb, too, that root tips inhibit the formation of lateral roots. It would be natural, therefore, to consider lateral bud apices as 
"meristemoids". A field of inhibition around each leaf and bud primordium in the apical meristem has been envisaged in a similar way (see e.g. Wardlaw, I952, especially Figs. 17 and 18 therein). Unfortunately the absence of concrete evidence for such a meristem inhibitor, other than auxin itself, limits the usefulness of the hypothesis at present.

A variant on the typical inhibition of lateral buds is provided by the "short shoot," a branch or axis which does not elongate but in which the leaves do develop, forming a rosette. Somewhat analogous is the behavior of biennial plants, which form a rosette in the first year and elongate in the second. Within the rosette, each leaf by its auxin production partially, but never completely, inhibits the next in order. In a few cases, notably the biennial Hyoscyamus, elongation of the short shoot is brought about by gibberellic acid and this may be followed by flowering (Lang, 1956). Or the normal requirement for light to cause elongation may be set aside by supplying gibberellic acid (Curry and Wassink, I 956). Thus, failure of the short shoot to elongate in these plants may be due to deficiency of gibberellins or physiologically similar growth factors.

In woody plants, especially in the larch (Larix) and the maidenhair (Ginkgo biloba), the short shoots will elongate if the part of the branch above them is removed; it is not enough to remove the apical bud alone. At least in young Ginkgo plants, if such decapitation is followed by application of auxin paste to the cut surface, the short shoots can be completely prevented from elongating (Gunckel et al., I949), though their leaves open normally. The short shoot bud is thus comparable to a lateral bud in which auxin inhibits the elongation but does not inhibit unfolding and expansion of the leaves. The necessity for removing part of the stem in order to relieve the inhibition receives its explanation from the absence of strict localization of the auxin production; this is not limited to the apical bud and indeed not even centered there, but in the growing long shoot most of the auxin is produced within the upper internodes of the stem itself (Gunckel and Thimann, 1949). Exactly the same situation appears to prevail in long shoots of the plum (Hatcher, I948). It may be connected with the development of a rib meristem.

In another short shoot system, that in Cercidiphyllum, an ornamental Japanese bush, the relations appear more complex, though they are the same in respect of the control over shoot elongation. Decapitation leads to outgrowth of many of the short shoots into long ones (providing they are not flower-bearing), while application of auxin to the cut surface keeps the shoots short (Titman and Wetmore, 1955): in this respect it is like Ginkgo. It differs, however, in that the terminal bud, together with the uppermost internode, abscisses in midsummer, when its auxin production is still high. The explanation for this odd behavior is probably that the lateral short shoot buds actually destroy auxin in the stem, for when they are removed the amount of auxin which diffuses from the stem cut surface increases, and correspondingly the terminal internode stays on. Apparently, this is a rare case of integrative action based on auxin destruction.

If the short shoots are lateral buds whose inhibition is only partial, the "anticipatory shoots" are lateral buds which are free from inhibition altogether. These are shoots derived from large buds close to the apex in several woody plants, especially birches (Champagnat, I954 and earlier notes) and they develop when the 
stem is still growing rapidly. Auxin diffusion determinations show that those apices which have anticipatory shoots nearby actually produce more auxin than those without such buds. It is always the most vigorously growing shoots that are concerned.

The polarity of auxin transport means that the inhibited buds are normally

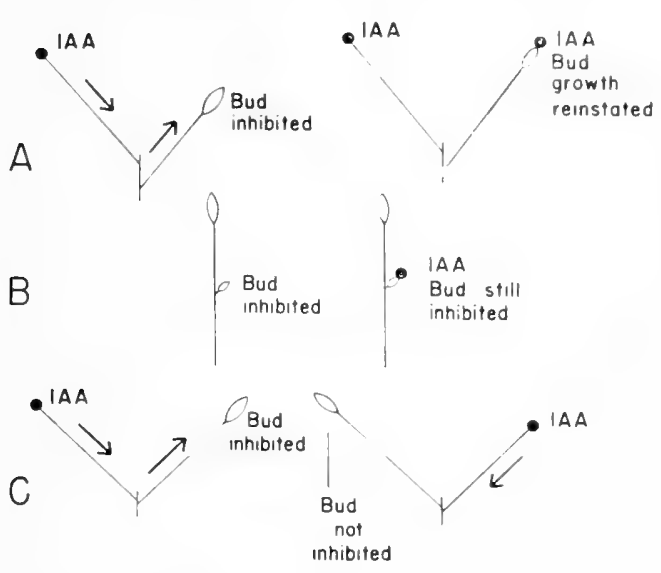

Fig. 14. Diagrams of some bud inhibition experiments. A, Libbert, I954 ( $c f$. also Bagda, I 956). B, Thimann, 1937. C, Bagda, 1956. below the source of auxin. But such polarity is evidently not absolute. Several workers have shown that small amounts of auxin can be transported in stems from base towards apex (see review of Thimann, I 954b). Also the transport can be interfered with by such substances as triiodobenzoic acid and 2,4-D (Kuse, r 953; Niedergang-Kamien and Skoog, I956; Hay, I 956b) and as a result apical dominance can be weakened or even prevented, axillary buds developing beneath the block in transport just as though the apical bud had been removed. Whether naturally occurring substances in the stem similarly modify polarity has not been proved, but the appearance of such polarity-modifying material has been reported in stems of Coleus when they enter the flowering state (Leopold and Guernsey, I 953). Buds have several times been observed to be inhibited by a source of auxin acting in the direction opposite to auxin polarity. A recently described example is the inhibition exerted by lilac leaves on the development of axillary buds situated above them on the stem (Champagnat, I 951). Long ago Snow observed that in pea plants with two equal shoots growing from the base, the axillary buds on one shoot were under (rather slight) inhibition from the terminal bud of the other. Now Libbert (I954a) and Bagda (I956) have shown that indoleacetic acid replacing the terminal bud of one shoot can exert clear inhibiting influence on such a distant bud situated up a side branch (Fig. I4A). Evidently polar transport in branched plants is not nearly as strict as was imagined. But if the shoots are unequal the polarities are unequal too, for auxin applied to the shorter of two shoots does not inhibit buds on the longer one (Fig. I4C; Bagda, I956). In general, however, it is clear that such anti-polar inhibition is not evidence against the role of auxin, as it has so often been interpreted to be, and thus the position of auxin as the inhibiting and therefore integrating agent is better established than before.

However, to explain how a growth hormone can inhibit the growth of lateral shoots while promoting that of the terminal shoot is no easier now than when it was discovered 22 years ago. Many of the theories were critically reviewed in detail by the writer in 1939 and it is unnecessary to go over them again here. One possibility is simply to deny that auxin could be the inhibitor, as has been attempted 
by several workers in recent years (see Thimann, I 954b, for review). Another is to implicate other parts of the plant. It has been suggested that the real inhibitor is a lactone formed in the roots (Libbert, I 954b), as witness the occurrence of scopoletin and related lactones in roots. In favor of this view is the fact that isolated stem tissue of Pisum can be partly inhibited from elongating by root tips or their ether extracts (Howell, 1954). Strongly against it is the fairly clear evidence that the concentration of lactones in roots is too low to exert an appreciable growth inhibition therein (Pollock et al., I954). A curious feature is that hydrolysis of the (crude) inhibitor preparation from roots is claimed to yield an auxin (Libbert, I955), which seems at variance with the idea that it is a lactone. Just how the stream of auxin would control the inhibitor, or its hydrolysis, has not been made clear.

New evidence does make it possible to set aside, at least tentatively, one theory, namely that the lateral buds are inhibited because the auxin concentration reaching them is supra-optimal (Thimann, 1937). For when in a two-shoot plant the apical bud of the first shoot is being inhibited by the apical bud of the second (or by auxin applied to the second apex) it has been found that auxin paste applied directly to the inhibited apical bud will cause it to elongate (Fig. I4A; Libbert, r954a). This means that its failure to elongate could not have been due to any excess of auxin in it. This evidence is somewhat weakened by the fact that similar application of auxin to inhibited lateral buds in situ does not cause their growth (Fig. I4B; Thimann, I937); even isolated lateral buds show inhibition by auxin, and in relatively low concentration, though the case is not quite identical. This theory had, however, been weakened much earlier through the demonstration by several workers, especially Ferman and Van Overbeek, that when lateral buds are released from inhibition their auxin content rises markedly and rapidly. While the situation is hard to understand, the sum of the evidence now makes it rather improbable that inhibition could be due to a simple excess of auxin in the buds.

Another new point, though unrelated to any of the proposed theories, is that cell divisions in the inhibited lateral bud show a sensitive response to auxin which could possibly be the initial effect. After decapitation of the terminal bud, the nuclei become deeply-staining, and mitoses soon begin, while these changes are prevented when auxin is applied (Naylor, cited by Skoog, I953).

Perhaps a more fruitful approach is concerned with the factors necessary for stem growth. To those already discussed in section VId should be added gibberellin, which clearly promotes the elongation of lateral buds in peas (Brian et al., 1955), being unlike auxin in this respect. It promotes branching and frond formation also in duckweeds (Spirodela), a process which is homologous with lateral bud development (author's unpublished data). Shoots in culture or buds growing on tissue cultures respond strongly (like roots) to adenine, particularly when they have rorsts attached. The combination of adenine with something synthesized in roots allowed isolated but rooted pea epicotyls to grow to $20 \mathrm{~mm}$ length in 46 days (Howell and Skoog, I955). Since coconut milk in addition brought the elongation up to $77 \mathrm{~mm}$ it is evident that many growth factors interact for normal growth. The growth promotion due to adenine and roots was, however, great 
enough to offset completely the inhibiting action of auxin, and in one experiment the combination of adenine and IAA gave even better elongation than adenine alone. Still more suggestive, however, is the powerful action of kinetin. Isolated pea stems bearing one bud and supplied only with sucrose showed the expected inhibition of growth of the bud by IAA, but when kinetin was added as well this inhibition was entirely removed and the buds elongated as fast as the controls (Wickson and Thimann, I956, I958). No interaction with roots or other growth factors was needed, and the concentrations of kinetin effective were closely comparable on a molar basis with those of auxin. These experiments could, of course, have the relatively simple explanation that kinetin somehow helps the tissue of the bud to destroy auxin (perhaps by promoting synthesis of an IAA-destroying enzyme), but they could, and with more attractiveness, be taken to mean that bud growth depends on a supply of kinetin and that it is with this supply that auxin interferes. For if auxin traveling down the stem were to combine with kinetin, perhaps on a mole-for-mole basis, then the buds would be deprived of kinetin which might well be essential for their cell division and hence for their growth. At any rate these findings open up new avenues for study of this still obscure problem.

\section{CONCLUSION}

This brief survey of plant growth has been conducted at four levels; those of chemical growth substances, of cells, of tissues and of organs. At all levels much knowledge has been accumulated and as a by-product valuable advances in applied plant science have accrued. Problems at the fifth level, that of the whole plant, remain elusive and difficult, although it is easy to believe that the recent discoveries of new growth substances will make some explanations possible. The similarities and differences between plant and animal growth, while fairly clear on the surface, remain none too clearly defined in physiological terms, and it may be that interfertilization between plant and animal sciences will be the most fruitful field for the future. 


\section{LITERATURE}

Áberg, B., (1950) Physiol. Plantarum, 3, 447.

Aberg, B., (1953) Ann. Roy. Agr. Coll. Sweden, 20, 24 I.

$\AA_{\text {berg, }}$ B., (1956) in R. L. Wain and F. Wightman, ed., (q.v.), 93.

Ǎberg, B. And E. Jönsson, (1955) Ann. Roy. Agr. Coll. Sweden, 21, 40 I.

Addicott, F. T. And R. S. Lynch, (1955) Ann. Rev. Plant Physiol., 6, 21 I.

Algeus, S., (1946) Botan. Notiser, 129.

Anker, L., (195I) Koninkl. Ned. Akad. Wetenschap., Proc., Ser. C, 54, 525.

Audus, L. J., (1948) New Phytologist, 47, 196; 48, 97.

Audus, L. J., (1949) Biol. Revs. Cambridge Phil. Soc., 24, 5 I.

Audus, L. J., (1952) J. Exptl. Botany, 3, 375.

Audus, L. J., (1953) Plant Growth Substances, Donald Hill Ltd., London, 465 pp.

Audus, L. J., (1954) Congr. intern. botan., 8e Congr. (Paris), Rapp. et comm., II, I 38 .

Audus, L. J. And N. Das, (1955) 7. Exptl. Botany, 6, 328.

Avers, G. J. And R. H. Goodwin, (I956) Am. J. Botany, 43, 6 I2.

Avery, G. S., (1933) Am. J. Botany, 20, 565 .

Bagda, H., (1956) Communs. fac. sci. univ. Ankara, Ser. C, 5, 38, 79.

Bailey, I. W., (1919) Proc. Natl. Acad. Sci. U.S., 5, 283.

Bailey, I. W.g (I920) Am. F. Botany, 7, 41 7.

Bailey, I. W., (1930) Z. Zellforsch. u. mikroskop. Anat., Io, $65 \mathrm{I}$.

Bailey, I. W., and B. G. L. Swamy, (i95i) Am. J. Botany, 38, 373.

Bennet-Clark, T. A., (i956a) in R. L. Wain and F. Wightman, ed., (q.v.), 284.

Bennet-Clark, T. A., (1956b) in R. L. Wain and F. Wightman, ed., (q.v.), 3 Io.

Bentley, J. A., (1958) Ann. Rev. Plant Physiol., 9, 47.

Bentley, J. A. And A. S. Bickle, (1952) F. Exptl. Botany, 3, 406.

Bentley, J. A., K. R. Farrar, S. Housley, A. F. Smith and W. G. Taylor, (1956) Biochem. 7., 64, 44.

Bigss, R. H. And A. C. Leopold, (ig57) Plant Physiol., 32, 626.

Bloch, R., (r94I) Botan. Rev., 7, i io.

Bonner, D. M., A. J. HaAgen Smit and F. W. Went, (i939) Botan. Gaz., ior, i28; cf.: Proc. Natl. Acad. Sci., U.S., 25, I84.

Bonner, J., (1936) J. Gen. Physiol., 20, I.

Bonner, J., (1949) Am. F. Botany, 36, 323, 429.

Bonner, J., (1950) Plant Physiol., 25, 181.

Bonner, J. AND H. Bonner, (I948) Vitamins and Hormones, 6, 225.

Bonner, J. and R. J. Foster, (i956) in R. L. Wain and F. Wightman, ed., (q.v.), 295.

Bonner, J. and J. B. Koepfli, (1939) Am. F. Botany, 26, 557.

Bonner, J., R. S. Bandurski and A. Millerd, (1953) Physiol. Plantarum, 6, 5 I I.

Bonner, W. D., Jr. and K. V. Thimann, (i950) Am. J. Botany, 37, 66.

Boroughs, H. And J. Bonner, (1953) Arch. Biochem. Biophys., 46, 279.

Boysen Jensen, P., (1936) Growth Hormones in Plants, Trans. and rev. by G. S. Avery And

P. R. Burkholder, McGraw Hill Book Co., New York and London, 268 pp.

Braun, A., (1956) Proc. Int. Congr. Tissue Culture, Woodstock, Vt., U.S.A., Oct. '56.

Braun, A. C., (I953) Botan. Gaz., II4, 36; also in Brookhaven National Lab., (I954) 22.

Brauner, L., (1954) Ann. Rev. Plant. Physiol., 5, i63.

Brian, P. W. and H. G. Hemming, (1955) Physiol. Plantarum, 8, 669; cf. P. W. Brian, H. G. Hemming and R. Radley, ibid., 899.

Brian, P. W., G. W. Elson, H. G. Hemming and M. Radley, (1954) J. Sci. Food Agric., 602. Brookhaven National Laboratory, (1954) Symposium on Normal and Pathological Plant Growth, Brookhaven Symposia in Biol., No. 6.

Brown, R., (I95I) Nature, I68, 94I.

Brown, R. and P. Rickless, (1949) Proc. Roy. Soc. (London), B, r36, i io.

Brown, R., W. S. Reith and E. Robinson, (1952) Symposia Soc. Exptl. Biol., 6, 329.

Bryax, W. H. and E. H. Newcomb, (1954) Physiol. Plantarum, 7, 290.

Befret, K., (1952) Mededel. Koninkl. Vlaam. Acad. Wetenschap. Belg., I4, No. 7. 
Bünning, E., (1952) in Survey of Biological Progress, G. S. Avery, ed., Academic Press, New York, 2, 105 .

Bünning, E., (1957) in F. L. Milthorpe, ed., (q.v.), i8.

Bünning, E. And SAGRomsky, (1948) Naturforsch., 3b, 203.

Burström, H., (195I) Physiol. Plantarum, 4, r99, 64 I.

Burström, H., (1952-1954) Physiol. Plantarum, 5, 391 (1952); 7, 332 (1954).

Burström, H., (1953a) Ann. Rev. Plant Physiol., 4, 237.

Burström, H., (1953b) Physiol. Plantanim, 6, 685.

Burström, H., (I953c) Physiol. Plantarum, 6, 262.

Burström, H., (I954) Physiol. Plantarum, 7, 548.

Burström, H. and B. A. M. Hansen, (I956) in R. L. Wain and F. Wightman, ed., (q.v.) I34.

Castle, E. S., (r955) Proc. Natl. Acad. Sci. U.S., 4I, 197.

Champagnat, P., (195I) Bull. Assoc. phil. d'Alsace et de Lorr., 9, 36, 54 .

Champagnat, P., (1954) Rev. cytol. et biol. végétales, 15, I.

Cholodny, N., (ig33) Planta, 20, 549.

Christiansen, G. S., (1950) Arch. Biochem., 29, 354.

Christiansen, G. S. and K. V. Thimann, (1950a) Arch. Biochem., 26, 230.

Chiristiansen, G. S. and K. V. Thimann, (1950b) Arch. Biochem., 26, 248.

Clowes, F. A. L., (1958) J. Exptl. Botany, 9, 229.

Commoner, B. and K. V. Thimann, (194I) J. Gen. Physiol., 24, 279.

Cormack, R. G. H., (1949) Botan. Rev., 15, 583.

Cormack, R. G. H., (I955) Science, I22, IOI9.

Cormack, R. G. H., (1956) Can. J. Botany, 34, 983.

Crafts, A. S., (1956) Am. 7. Botany, 43, 548.

Curry, G., K. V. Thimann and P. M. Ray, (1956) Physiol. Plantarum, 9, 429.

Gurry, G. M. and E. C. Wassink, (1956) Mededel. Landbouwhogeschool, Wageningen, 56, I.

Curtis, P. J. And B. E. Cross, (1954) Chem. E Ind. (London), io66.

Das, N. K., K. Patau and F. Skoog, (1956) Physiol. Plantarum, 9, 640.

Delisle, A. L., (1938) Am. J. Botany, 25, 420.

De Ropp, R. S., (1945-47) Ann. Botan., N.S., 9 (1945) 369; Io (1946) 31 ; Io (I946) 353; II (I947) 439.

De Ropp, R. S., (1950) Am. 7. Botany, 37, 352.

Diehl, J. M., G. J. Gorter, G. Van Herson Jr. and A. Kleinhoonte, (i939) Rec. trav. botan. néerl., 36, 7II.

Dolk, H. E., (1936) Rec. trav. botan. néerl., 33, 509.

Eliasson, L., (1955) Physiol. Plantarum, 8, 374.

Elliott, B. B. And A. C. Leopold, (1953) Physiol. Plantarum, 6, 66.

English, J. Jr., J. Bonner and A. J. HaAgen Smit, (1939) Proc. Natl. Acad. Sci. U.S., 25, 323; 7. Am. Chem. Soc., 61, 3434.

Erickson, R. O., (1947) Nature, 159, 275.

Erickson, R. O., (I948) Am. J. Botany, 35, 729.

Esau, K., (1953) Plant Anatomy, John Wiley and Sons, New York; Chapman and Hall, London, $735 \mathrm{pp}$. and 85 plates.

Fawcett, C. H., H. F. Taylor, R. L. Wain and F. Wightman, (i956a) in R. L. Wain and F. Wightman., ed., (q.v.) I87.

Fawcett, C. H., R. L. Wain and F. Wightman, (1956b) Nature, I78, 972.

Fischer, A., (1954) Planta, 43, 288.

Fischnich, O. And G. Lübbert, (1955) Beitr. Biol. Pflanz., 31, I 79.

Fries, N., (1954) Symbolae Botan. Upsalienses, 13, 83 pp.

Galston, A. W., (1956) in R. L. Wain and F. Wightman., ed., (q.v.) 2 r9.

Galston, A. W. and L. Dalberg, (1954) Am. F. Botany, 4 I, 373 .

Galston, A. W. and M. E. Hand, (1949) Am. F. Botany, 36, 85.

Gast, A., (1942) Ber. schweiz. botan. Ges., 52, 44 I.

Gautheret, R. J., (1942-I943) Rev. cytol. el cytophysiol. végétale, 6, 85.

Gautheret, R. J., (1946) Growth, Io, Suppl. (Sixth Growth Symposium) 2 I. 
Gattheret, R. J., (1950) Vierteljahresschr. naturforsch. Ges. Zürich, Beih., 95, 73.

Geiger-Huber, M. and E. Burlet, (1936) Jahrb. wiss. Botan., 84, 233.

Geissbühler, H., (1953) Ber. schweiz. botan. Ges., 63, 27.

Goddard, D. R. and B. J. D. Meeuse, (1950) Ann. Rev. Plant Physiol., I, 207.

Goodinin, R. H. and C. Taves, (1950) Am. F. Botany, 37, 224.

Gordon, S. A., (1954) Ann. Rev. Plant. Physiol., 5, 34 I.

Gordon, S. A., (I956) in R. L. Wain And F. Wightman, ed., (q.v.).

Green, P. B. and G. B. Chapman, (1955) Am. J. Botany, 42, 685.

Gregory, F. G., (I92 I) Ann. Botan., 35, 93.

Gregory, F. G., (I957) in F. L. Milthorpe, ed., (q.v.) I.

Griffioen, K., (1938) Rec. trav. botan. néerl., 35, 322; cf. Chem. Weekblad, 36, 8 1.

Gruen, H., (1956) Diss., Harvard Univ. (Sept. 1956).

Gunckel, J. E. and K. V. Thimann, (1949) Am. F. Botany, 36, i 45.

Gunckel, J. E., K. V. Thimann and R. H. Wetmore, (i949) Am. J. Botany, 36, 309.

Gustafson, F. G., (1942) Botan. Rev., 8, 599.

Gustafion, F. G., (I95I) in F. Skoog, ed., (q.v.) 35 I.

Györffy, B., G. Rédei and G. Rédei, (1955) Acta Botanica Acad. Sci. Hung., 2, 57.

Hackett, D. P., (1952) Plant Physiol., 27, 279.

Hackett, D. P., (I956) Proc. Am. Soc. Plant Physiol., (Storrs, Conn.) xl.

Hackett, D. P. and D. W. HaAs, (1958) Plant Physiol., 33, 27.

Hackett, D. P. and H. A. Schneiderman, (1952) Arch. Biochem. Biophys., 47, 190.

Hackett, D. P. and K. V. Thimann, (i952a) Am. J. Botany, 39, 553.

Hackett, D. P. and K. V. Thimann, (1952b) Proc. Natl. Acad. Sci. U.S., 38, 770.

Hackett, D. P. and K. V. Thimann, (1953) Am. F. Botany, 4o, 183.

Hackett, D. P., H. A. Schneiderman and K. V. Thimann, (I953) Arch. Biochem. Biophys., $47,205$.

Hansen, B. A. M., (1954) Botan. Notiser, 230, 3 I8.

Hanson, J. B. and J. Bonner, (r955) Am. J. Botany, 42, 4i I.

Hatcher, E. S. J., (1948) Ann. Rept. East Malling Research Sta. Kent., 1947, I 13.

HAY, J. R., (1956a) Weeds, 4, 218, 349.

Hay, J. R., (1956b) Plant Physiol., 3I, I 18.

Hay, J. R. and K. V. Thimann, (I956) Plant Physiol., 31, 382.

Hayashi, T. and I. Murakami, (1954) J. Agr. Chem. Soc. Japan, 28, 543; cf. ibid. (1953) 27,675 and 797 .

Heath, O. V. S. and J. E. Clark, (1956) Nature, I77, i I I8.

Heller, R., (1953) Thesis, Paris (Masson et Cie); Ann. sci. nat., Botan. et biol. végétale, I I e série, I.

Henbest, H. B., E. R. H. Jones and G. F. Smith, (i953) 7. Chem. Soc., 3796.

Heslop-Harrisson, J., (1956) Physiol. Plantarum, 9, 588.

Heyn, A. N. J., (1940) Botan. Rev., 6, 5 I 5.

Hollaender, A. and G. E. Stapleton, (1953) Physiol. Revs., 33, 77.

Holley, R. W., F. P. Boyle and D. B. Hand, (1950) Arch. Biochem., $27,143$.

Hopkins, H. T., (1952) Plant Physiol., 27, 526.

Howell, R. W., (1954) Plant Physiol., 29, 100.

Howell, R. W. And F. Skoog, (1955) Am. J. Botany, 42, 356.

Howlett, F. S., (1941) Ann. Rept., Vegetable Growers Assoc. Am., I 3 pp.

Hughes, A., (1952) The Mitotic Cycle, Butterworths Sci. Publ., London, 232 pp.

Idle, D. B., (1955-56) Nature (1955), I76, 778; J. Exptl. Botany, (1956), 7, 347.

Jacobs, W. P., (I955) Am. F. Botany, 42, 595.

Jexsen, W. A., (1955) Plant Physiol., 30, 426.

Jones, H., (1957) in F. L. Milthorpe, ed., (q.v.), 93.

Jost, L., (1938) Z. Botan., 33, 193.

Jost, L. And E. Reiss, (1936) Z. Botan., 30, 335.

KaINDL, K., (I956) in R. L. WAin AND F. Wightman, ed., (q.v.)

Kandler, O., (1953) Planta, 42, 304.

Kandler, O. And A. VieregG, (1953) Planta, 4I, 6 I 3. 
Karlsson, B. and L. Eliasson, (1955) Physiol. Plantarum, 8, 56 I.

Kato, J., (1957) Mem. Coll. Sci. Univ. Kyoto, Ser. B 24, I19; Science, I23, 1132.

KerR, T., (195I) in F. SKOOG, ed., (q.v.), 37.

Ketellapper, H. J., (1952) Thesis, Utrecht; Acta Botan. Neerl., 2, 388.

Klein, R. M. and J. L. Knupp, (1957) Proc. Natl. Acad. Sci., U.S., 43, 199.

Klein, R. M. And H. H. Vogel, (1956) Plant Physiol., 3I, 17.

Kopp, M., (1948) Ber. schweiz. botan. Ges., 58, 283.

Kulescha, Z., (I951) Thesis, Paris, I I4 pp.

Kupila, S., (1956) Arch. Soc. Zool. Botan. Fennicae, "Vanamo", Io, 38.

Kuraishi, S. And F. S. Okumura, (1956) Botan. Mag. (Tokyo), 69, 300.

Kuse, G., (1953) Mem. Coll. Sci. Univ. Kyoto, Ser. B 20, 207.

Laibach, F. and F. J. Kribben, (1950) Naturwiss., 5, i i4; Ber. deut. botan. Ges., 52, 53; 53, I19; Beitr. Biol. Pflanzen, 28, 64, I3 I.

Lang, A., (1956) Proc. Am. Soc. Plant Physiol., (Storrs, Conn.) xxxv; Naturwissenschaften, $43,257,284,544$.

Lang, A., (1957) Proc. Natl. Acad. Sci., U.S., 43, 709.

Larsen, P., (I944) Thesis, Copenhagen; Dansk. Botan. Arkiv, II, I I.

Larsen, P., (195ia) Ann. Rev. Plant Physiol., 2, I69.

Larsen, P., (195Ib) Plant Physiol., 26, 697.

Larsen, P., (1956) in R. L. Wain and F. Wightman, ed., (q.v.) 76.

Leopold, A. C., (1949) Am. F. Botany, 36, 437.

Leopold, A. C., (1955) Auxins and Plant Growth, Berkeley, Calif., Univ, of California Press, $354 \mathrm{pp}$.

Leopold, A. C. And F. S. Guernsey, (I953) Botan. Gaz., I15, 147.

Leopold, A. C. and C. A. Price, (i957) Plant Physiol., 32, 520.

Leopold, A. C. and K. V. Thimann, (1949) Am. J. Botany, 36, 342.

Libbert, E., (1954a) Flora (Jena), I4I, 27 I.

Libbert, E., (I954b) Planta, 44, 286.

Libbert, E., (1955) Planta, 46, 256, 405.

Linser, H., (1954) Monatsh. Chem., 85, 196.

Linser, H., (1955) Z. Pflanzenernähr. Düng. u. Bodenk., 69, 215.

Linser, H., (1956) in R. L. Wain and F. Wightman, ed., (q.v.) I 4 I.

Luckwill, L. G., (1953) J. Hort. Sci., 28, I4.

Luckwill, L. C., (1954) Congr. intern. botan., 8e Congr. (Paris), Rapp. et comm., II, 377.

Lund, H. A., (1956) Am. F. Botany, 43, 562.

MaDaniels, L. H., (i955) Cornell Univ. Exptl. Sta. Bull.

Marae, D. H. and J. Bonner, (1953) Physiol. Plantarum, 6, 685.

Mcrae, D. H., R. J. Foster and J. Bonner, (1953) Physiol. Plantarum, 28, 343.

Matell, M., (1953) Stereochemical Studies on Plant Growth Substances, Thesis, Uppsala.

Miller, C. O., (I952) Plant Physiol., 27, 408.

Miller, C. O., (1954) Plant Physiol., 29, 79.

Miller, C. O. And F. Skoog, (1953) Am. J. Botany, $40,768$.

Miller, C. O., F. S. Okumura, M. H. von Saltza and F. M. Strong, (I956) J. Am. Chem. Soc. 78, I375.

Miller, G. O., F. Skoog, M. H. von Saltza and F. M. Strong, (i955a) J. Am. Chem. Soc., 77, 1392.

Miller, C. O., F. Skoog, F. S. Okumura, M. H. von Saltza and F. M. Strong, (1955b) J.Am. Chem. Soc., 2662.

Milthorpe, F. L., ed., (1957) Symposium on Growth and Development of the Leaf, Butterworths Sci. Publ. Ltd., London; Academic Press, New York, 2 Io pp.

Mitchell, J. W., D. P. Skaggs and W. P. Anderson, (I95I) Science, 114, 159.

Morel, G. and R. J. Gautheret, (1947) Rev. Sci., 85, 649; cf. Compt. rend., 224, I 728.

Mühlethaler, K., (1950) Ber. schweiz. botan. Ges., 6o, 6i 4 .

Muir, R. M., (1942) Am. J. Botany, 29, 7 I6.

Muir, R. M., (I95I) in F. Skoog, ed., (q.v.) 357.

Muir, R. M. and C. Hansch, (1955) Ann. Rev. Plant Physiol., 6, I57. 
Murakami, Y., (i956) Botan. Mag. (Tokyo), 69, 258.

Nakamura, Y. and K. Hess, (I938) Ber. deut. chem. Ges., 7 I B, I 45

Naylor, J., G. Sander and F. Skoog, (1954) Physiol. Plantarum, 7, 25.

Newcomb, E. H., (I95 I) Proc. Soc. Exptl. Biol. Med., 76, 504.

Niedergang-Kamien, E. And F. Skoog, (1956) Physiol. Plantarum, 9, 60.

Nitsch, J. P., (1950) Am. J. Botany, 37, 2 I I.

Nitsch, J. P., (1952) Quart. Rev. Biol., 27, 33.

Nitsch, J. P., (1953) Ann. Rev. Plant Physiol., 4, 199.

Nitsch, J. P., (1955) Plant Physiol., 30, 33.

Nitsch, J. P. And G. Nitsch, (1956) Plant Physiol., 3I, 94.

Northen, H. T., (1942) Botan. Gaz., Io3, 668.

Northen, H. T., (1946) Plant Physiol., 2I, 148.

Ordin, L. And J. Bonner, (1956) Plant Physiol., 3I, 53.

Ordin, L., T. H. Applewhite and J. Bonner, (I956) Plant Physiol., 3I, 44.

Ordin, L., R. Cleland and J. Bonner, (1955) Proc. Natl. Acad. Sci. U.S., 4I, 1023.

Parker, M. W., S. B. Hendricks, H. A. Borthwick and F. W. Went, (i949) Am. $\mathcal{J}$. Botany, 36, 194.

Partanen, C. R., I. M. Sussex and T. A. Steeeves (1956) Am. J. Botany, 42, 245.

Pearson, J. A. and R. N. Robertson, (1953) Australian F. Biol. Sci., 6, i.

Phinney, B., (1956) Proc. Natl. Acad. Sci. U.S., 42, 185.

Pilet, P. E. and A. W. Galston, (i955) Physiol. Plantarum, 8, 888.

Platt, R., (1956) Année biol., 3o, 349.

Pollock, B. M., R. H. Goodwin and S. Greene, (i954) Am. J, Botany, 4I, 52 I.

Pybus, M. B., M. S. Smith, R. L. Wain and F. Wightman, (I959) Ann. Appl. Biol., $47,173$.

Ray, P. and K. V. Thimann, (1956) Arch. Biochem. Biophys., 64, 175.

Reano, B. C., (1940) Philippine Agriculturist, 29, 87.

Reinders, D. E., (1942) Rec. trav. botan. néerl., 19, I.

Riker, A. J., E. Spoerl and A. E. Gutsche, (I946) Botan. Rev., I2, 57.

Roberts, E. H. and H. E. Street, (1955) Physiol. Plantarum, 8, $23^{8}$.

Robinson, E., (1956) 7. Exptl. Botany, 7, 296.

Robinson, E. And Brown, R., (1954) 7. Exptl. Botany, 5, 7 I.

Rufelt, H., (1954) Physiol. Plantarum, 7, I4I.

Ryan, F. J., E. L. Tatum and A. C. Giese, (1944) 7. Cellular Comp. Physiol., $23,83$.

Schneider, C. L., (1942) Am. 7. Botan., 29, 20 I.

Sequeira, L. And T. A. Steeves, (1954) Plant Physiol., 29, i I.

Shantz, E. M. and F. C. Steward, (1955) 7. Am. Chem. Soc., 77, 635 I.

Sinnott, E. W., (1939) Am. 7. Botany, 26, i 79.

Sinnott, E. W. And R. Bloch, (I94I) Am. F. Botany, 28, 225.

Skoog, F., ed., (195I) Plant Growth Substances, Univ. of Wisconsin Press, Madison, Wis.

Skoog, F., (1953) In Brookhaven Symposium (q.v.) I.

Skoog, F. And C. O. Miller, (I957) Symposia Soc. Exptl. Biol., i I, i I8.

Skoog, F. And C. Tsui, (195 I) in F. Skoog, (ed.), (q.v.) 263.

Skoog, F., C. L. Schneider and P. Malan, (1942) Am. 7. Botany, 29, 563.

Slocum, O. H. and J. E. Little, (1956) Proc. Am. Soc. Plant Physiol., (Storrs, Conn) iv.

Smith, M. S., R. L. Wain and F. Wightman, (1952) Ann. Appl. Biol., 39, 295.

SöDing, H., (1952) Die Wuchsstoffehre; Ergebnisse und Probleme der Wuchsstoffforschung, Stuttgart, Georg Thieme Verlag, $305 \mathrm{pp}$.

Steeves, T. A. And I. M. Sussex, (1957) Am. 7. Botany, 44, 665.

Steeves, T. A., H. P. Gabriel and M. W. Steeves, (I957) Science, I26, 350.

Stephenson, M. L., K. V. Thimann and P. G. Zamecnik, (1956) Arch. Biochem. Biophys., 65,194 .

Stern, H., (1956) Ann. Rev. Plant Physiol., 7, 9 I.

Stern, H. And P. L. Kirk, (1948-49) F. Gen. Physiol., 3I, 243.

Stern, H. And S. Timonen, (1954-55) 7. Gen. Physiol., 38, 4 I.

Steward, F. C. and S. M. Caplin, (1952) Ann. Botan. N.S., I6, $49 \mathrm{I}$.

Steward, F. C. And S. M. Caplin, (1954) Année Biol., 30, 385, 395. 
Steward, F. C. And C. Preston, (ig4i) Plant Physiol., i6, 48 I.

Steward, F. C. and E. M. Shantz, (i 956) in R. L. Wain and F. Wightman, ed., (q.v.) i6 Steward, F. C., W. E. Berry, C. Preston and T. K. Ramamurti, (1943) Ann. Botan. N.S., 7, 221 .

Stodola, F. H., (1956) Paper given at Symposium of Am. Soc. Plant Physiol. (Storrs, Conn.) Aug. 28, 1956. (Obtainable from Agr. Res. Service, USDA, Peoria, Illinois); $c f$. Stodola F. H., Nelson And Spence, Arch. Biochem. Biophys., 66 (1957) 438.

Stodola, F. H., K. B. Raper, D. I Fennell, H. F. Conway, V. E. Sohns, C. T. Langford and R. W. Jackson, (1955) Arch. Biochem. Biophys., 54, 240.

Stowe, B. B., (1955) Biochem. F., GI, IX.

Stowe, B. B., (1958) Plant Physiol., 33, Suppl., xxxii; Science, I28, 42 I.

Stowe, B. B. And K. V. Thimann, (1954) Arch. Biochem. Biophys., 5I, 499.

Stowe, B. B. And T. Yamaki, (1957) Ann. Rev. Plant Physiol., 8, I 81.

Stowe, B. B. And T. Yamaki, (I959) Science, I29, 807.

Stowe, B. B., K. V. Thimann and N. P. Kefford, (1956) Plant Physiol., 3 I, 162.

Street, H. E., (1954) Physiol. Plantarum, 7, 2 I 2.

Street, H. E., (I955) Physiol. Plantarum, 8, 48.

Street, H. E., M. P. McGonagle and S. M. McGregor, (1952) Physiol. Plantarum, 5, 248.

Street, H. E., M. P. MaGonagle and E. H. Roberts, (1953) Physiol. Plantarum, 6, I.

Street, H. E., S. M. McGregor and I. M Sussex, (I954) F. Exptl. Botany, 5, 204.

Strong, F. M., (1958) Topics in Microbial Chemistry, John Wiley and Sons, New York.

Sweeney, B. M. and K. V. Thimann, (1937-38) F. Gen. Physiol., 21, 123, 439.

Takahashi, T., H. Kitamura, A. Kawarada, Y. Seta, M. Takai, S. Tumara and Y. Sumiki, (1955) 7. Agr. Chem, Soc. Japan, 19(4), 267.

TAylor, J. H. And R. D. McMaster, (1954) Chromosoma, 6, 489.

Templeman, W. G. and W. A. Sexton, (I946) Proc. Roy. Soc. (London), B, I33, 300.

Teubner, F. G., (1953) Science, 118, 418.

Therman, E., (195i) Ann. Acad. Sci. Fennicae, Ser. A iv, No. i6.

Thimann, K. V., (1937) Am. 7. Botany, 24, 407.

Thimann, K. V., (1951a) in F. Skoog, ed., (q.v.) 21.

Thimann, K. V., (195I Ib) Growth, Suppl. (Ioth Symp. Soc. Growth and Development) 5 .

Thimann, K. V., (1954a) Am. Scientist, 589; cf. Arch. Biochem. Biophys., 44 (1953) 242.

Thimann, K. V., (1954b) Congr. intern. botan., 8e Congr. (Paris), Rapp. et comm., II, 114.

Thimann, K. V., (1955) The Life of Bacteria, The MacMillan Co., New York.

Thimann, K. V., (1956a) L'Origine et les Fonctions des Auxines, Centre de Documentation Universitaire, Paris, V, $122 \mathrm{pp}$.

Thimann, K. V., (1956b) Am. J. Botany, 43, 241.

Thimann, K. V., (1956c) Am. Naturalist, 9o, I 45.

Thimann, K. V. and J. Behnke-Rogers, (1950) The Use of Auxins in the Rooting of Woody Cuttings, Cabot Foundation Publ. No. I, The Harvard Forest, Petersham, Mass.

Thimann, K. V. and J. Bonner, (1933) Proc. Roy. Soc. (London), B I13, I26.

Thimann, K. V. and W. D. Bonner, Jr., (1949a) Am. F. Botany, 36, 2 i 4.

Thimann, K. V. and W. D. Bonner Jr., (1949b) Proc. Natl. Acad. Sci. U. S., 35, 272.

Thmann, K. V. and D. Kaufman, (1958) The Physiology of Forest Trees, The Ronald Press, New York.

Thimann, K. V. and G. M. Loos, (1957) Plant Physiol., 32, 274.

Thimann, K. V. and E. Marré, (1954) Am. J. Botany, 4I, 556.

Thimann, K. V. and E. Samuel, (1955) Proc. Natl. Acad. Sci. U.S., 4I, 1029.

Thimann, K. V. And C. L. Schneider, (1938) Am. F. Botany, 25, 627; 26, 792.

Thimann, K. V., B. Scharrer and F. A. Brown, (1952) The Action of Hormones in Plants and Invertebrates, New York, Academic Press, Inc.

Thimann, K. V., R. R. Slater and G. S. Christiansen, (1950) Arch. Biochem., 28, izo.

Thimann, K. V., C. S. Yocum and D. P. Hackett, (1954) Arch. Biochem. Biophys., 53, 239.

Thoday, J. M. And J. Read, (1947-49) Nature, (1947) 160, 608; (1949) 163, 133.

Titman, P. W. and R. H. Wetmore, (i955) Am. 7. Botany, 42, 364.

Torrey, J., (1950) Am. J. Botany, 37, 257. 
Torrey, J., (r956a) Physiol. Plantarum, 9, 370.

Torrey, J., (1956b) Ann. Rev. Plant Physiol., 7, 237.

Turner, J. S., J. MacRae and P. G. Lipp, (1954) Congr. intern. botan., 8e Congr., (Paris), Rapp. et comm., II, I52.

Van der Kerk, G. J. M., M. H. Van Raalte, A. KaArs Sijpestijn and R. Van der VEEN, (I955) Nature, I76, 308.

Van Overbeek, J. and F. W. Went, (1937) Botan. Gaz., 99, 22.

Van Overbeek, J., R. Blondeau and V. Horne, (i955) Am. J. Botany, 42, 205.

Van Overbeek, J., M. Conklin and A. F. Blakeslee, (I94I) Am. F. Botany, $28,647$.

Veldstra, H., (1953) Ann. Rev. Plant Physiol., 4, I5I.

Veldstra, H., (I956) in R. L. Wain and F. Wightman, ed., (q.v.) i i 7.

Vlitos, A. J. And W. Meudt, (1953-54) Contribs. Boyce Thompson Inst., I7, 197, 413.

Wain, R. L. And F. Wightman, (1954) Proc. Roy. Soc. (London), B I42, 525.

Wain, R. L. and F. Wightman, ed., (I956) The Chemistry and Mode of Action of Plant Grozeth Substances, Symposium, Wye College, Fuly, 1955, Butterworths Sci. Publ., London.

Wardlaw, G. W., (1952) Morphogenesis in Plants, Methuen, Londen, Wiley \& Sons, New York, i $76 \mathrm{pp}$.

Watson, D. J., (1947) Ann. Botan., N.S., II, 4I, 375.

WENT, F. W., (1928) Rec. trav. botan. néerl., 25, I.

Went, F. W., (1939) Bull. Torrey Botan. Club, 66, $39 \mathrm{I}$.

Went, F. W. and K. V. Thimann, (1937) Phytohormones, MacMillan, New York, 294 pp.

Whaley, W. G. and C. I. Whaley, (1942) Am. J. Botany, 29, 195.

White, P. R., (1934) Plant Physiol., 9, 585.

White, P. R., (1941) Biol. Rev. Cambridge Phil. Soc., I6, 34.

White, P. R. and A. C. Braun, (I942) Cancer Research, 2, 597.

Wickson, M. and K. V. Thimann, (1956) Proc. Am. Soc. Plant. Physiol., (Storrs, Conn.) xxviii; Physiol. Plantarum, II (1958) 62.

Wiedow, H. L. And H. Von Guttenberg, (i953) Planta, 4I, 589.

Williams, W. T., (I957) in F. L. Milthorpe, ed., (q.v.) I27.

Wilske, C. and H. Burström, (1950) Physiol. Plantarum, 3, 58.

Wirth, P., (I946) Ber. schweiz. botan. Ges., 56, I 75.

Woodford, E. K. and L. Kasasian, ( 1956) Field Crop Abstracts, 9 (No. I) I.

Yabuta, T. and T. Hayashi, (1939) F. Agr. Chem. Soc. Japan, I5 (3), 257, 403. 


\section{Chapter 11}

\section{THE GARGINOGENIG STIMULUS}

\section{Arthur Kirschbaum $\dagger$}

Multiple factors have been demonstrated to be intimately involved in the genesis of certain neoplasms. Although combined action of specific forces may be necessary (Bittner, I942) the introduction of only a single factor (e.g., a chemical carcinogen) may induce a new growth (Berenblum and Shubik, I947).

Influences contributing to carcinogenesis are biological agents, such as hormones (Gardner, I953; Gardner et al., I953; Kirschbaum, I95 I, I956, I957) and viruses (Duran-Reynals, I953; Beard et al., I955), chemicals (Wolf, I952) such as palycyclic hydrocarbons, azo dyes, amino compounds, halogenated hydrocarbons, urethane and physical agents like ionizing radiations (Brues, 1954). Although the end results of their action is "autonomous" neoplastic growth, the mode of carcinogenesis might be different for each class of tumor-inciting agent. However, since chemical, hormonal and physical agents may operate in an additive or even synergistic fashion (Furth and Boon, I943; Kirschbaum et al., I953) it is probable that the carcinogenic agents involve a common pathway at some point in their action.

Certain agents are capable only of "initiating" and others of "promoting" carcinogenesis once initiated (Berenblum and Shubik, 1947). Some combine both properties (e.g. methylcholanthrene). Continuing neoplastic growth may or may not be dependent upon the action of the incitor (Furth, I953). Control of the growth of certain cancers may be influenced by the administration or withdrawal of agents involved in their genesis, as in the case of estrogenic hormone and breast cancer (Foulds, I 949).

Perhaps of greatest potential significance is the available information on biological agents (hormones, viruses) and carcinogenesis. These are apparently involved in the developmeni of certain "spontaneous" neoplasms (Bittner, I942). Specific chemical and physical agents may induce "environmental" cancer; animal experiments indicate that they may potentiate spontaneous (perhaps latent) carcinogenesis (Andervont, I938; Heston, I940; Shimkin, I940).

Regardless of the nature of the inciting agents, the host is a prime force in determining carcinogenesis. Within the species genetically controlled response may in one instance reveal the carcinogenic potential of an agent which is innocuous for genetically different members of the same species. Host response may be influenced by non-genetic changes in the "internal environment" effected during

$\dagger$ Deceased on May 28, 1958 , 
gestation, immediately after birth (Gross, I950) or later in life. Encouragement can be derived from alteration of host response to known cancer-producing agents, since otherwise genetic determination would appear to be inevitable. The nonappearance of neoplastic disease (e.g. leukemia) in members of inbred high-leukemia stocks indicates the importance of non-genetic influences, perhaps in altering the response of the host to the factors which induce neoplastic disease (MacDowell and Richter, I935).

\section{HORMONAL FACTORS IN GARCINOGENESIS}

The high incidence in the human population of cancer of the mammary and prostate glands, ovary and uterus suggests the involvement in carcinogenesis of hormones which stimulate normal tissue growth.

An approach has been made toward an experimental analysis of the hormonal etiology of neoplasms. Experimental neoplasms of the pituitary gland and its target tissues have been induced by altering the reciprocal hormonal relations between the pituitary gland and the organs sensitive to its trophic hormones. Induced neoplasms of the pituitary and other endocrine glands are considered below.

\section{(a) Pituitary and thyroid neoplasms induced by alteration in thyroid function}

Tumors of both the mouse (and rat) thyroid and pituitary have been induced by ${ }_{1}$ "mnsterng radioiodine (Figs. $\mathrm{I}-4$ ). If a relatively small amount is given, sufficient however, to interfere with thyroid function, then adenomata may develop (Purves and Griesbach, I946). Increased stimulation by pituitary thyrotrophic hormone (TSH) is probably a factor in the genesis of such thyroid adenomata. Thyroid carcinoma has developed in the non-stimulated rat thyroid gland following administration of ${ }^{131} \mathrm{I}$, suggesting the direct carcinogenic action of ionizing

Fig. I. Sequence of events in experimental pituitary tumorigenesis following hypothyroidism.

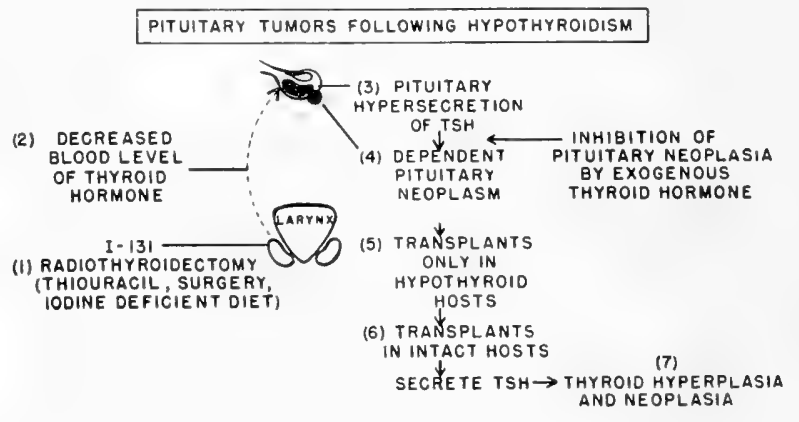

radiation (Goldberg and Chaikoff, 1952). If thyroidectomy is complete, then pituitary tumors develop after a latent period of several months (Gorbman, I956).

Radiothyroidectomy-induced pituitary tumors can be successfully transplanted rnly intn thyroidectomized recipients of appropriate genetic background (Furth, I953). Although the recipient satisfies the usual histocompatibility requirements for transplantation, pituitary tumor transplants will not grow unless the recipients are athyroid. The tumors attain the capacity to grow in intact mice (Fig. 4) after 
several passages through hosts without thyroid glands (Furth et al., 1952). Such transplants secrete thyrotrophic hormone, as evidenced by hyperplasia of the host thyroid gland (Fig. 4).

In this instance a factor necessary for induction has been found essential for the continued growth of a neoplasm. Such new growths have been termed "conditional" (Furth, I953; Foulds, 1954) or "dependent" (hormone responsive, Foulds). The pituitary adenomas induced by thyroidectomy are "dependent", but during transplantation "autonomy" is acquired, that is, progressive growth occurs in intact as well as thyroidectomized hosts.

The genesis of TSH-secreting pituitary neoplasms can be prevented by the administration of thyroid hormone after radiothyroidectomy (Gorbman, I952; Furth, 1955). Apparently hypothyroidism per se may under specified conditions lead to pituitary tumor formation. In support of this concept is the induction of tumors by propyl-thiouracil (Moore et al., 1953) or surgical thyroidectomy (Dent et al., I 955). In rats fed a low iodine diet both thyroid and pituitary tumors have developed on the basis of the induced hypothyroidism (Axelrad and LeBlond, I955; LeBlond et al., I957).

\section{THYROIO TUMORIGENESIS FOLLOWING HYPOTHYROIDISM}

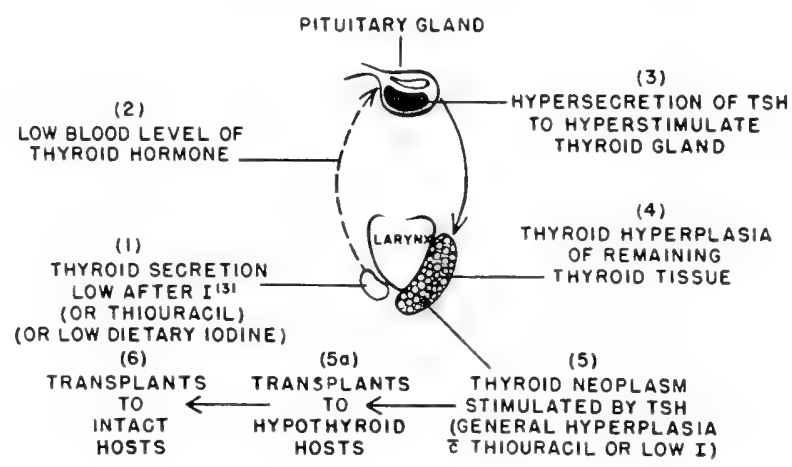

Fig. 2. Sequence of events in experimental thyroid tumorigenesis following hypothyroidism.

That factors superimposed on hypothyroidism may be importantly involved has been suggested (Gorbman, 1956). When mice were fed a low iodine diet so that a relatively small dose of radioiodine accomplished thyroidectomy, pituitary tumors were not induced. TSH-secreting tumors resulted, however, if added $\mathrm{X}$-irradiation, not necessarily to the pituitary gland, was given. Although hypothyroidism per se may induce pituitary tumor formation, it is likely that additional factors augment tumorigenesis.

Hypersecretion of the pituitary gland follows radiothyroidectomy. The increased secretory activity leads to adenoma formation which is followed by hormonally "independent" neoplasia (Gorbman, I956). The mechanism underlying this sequence of events is completely unknown. Apparently hypothyroidism is the stimulus to tumor formation in the anterior pituitary. These pituitary tumors are dependent, growing only in thyroidectomized hosts which duplicate the environment required for tumor development. The specificity of the altered interrelation, 

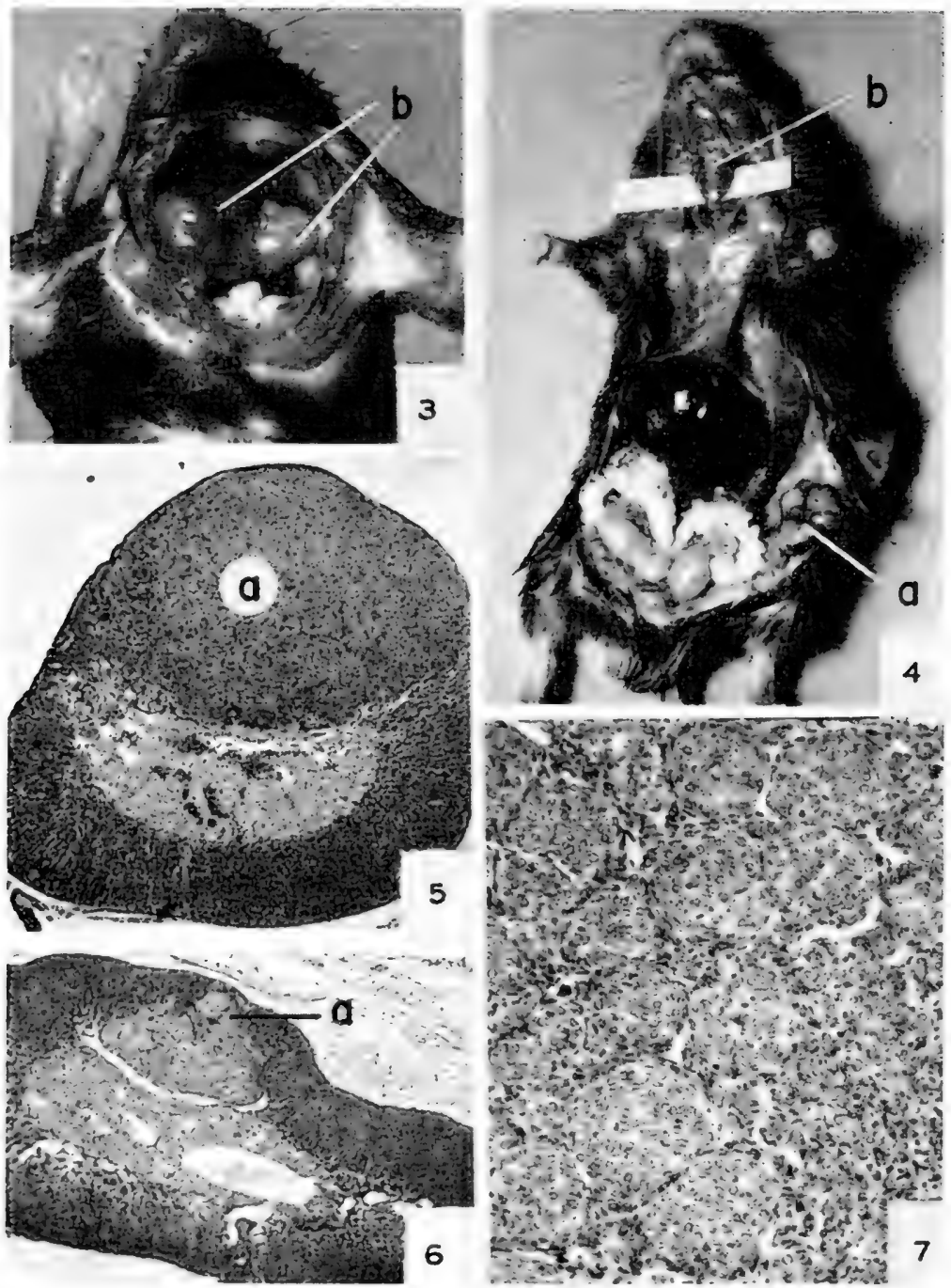

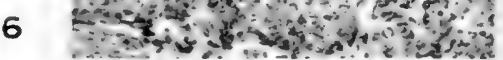

Fig. 3. TSH secreting pituitary tumor $(\mathrm{p})$ induced in $\mathrm{C}_{57} \mathrm{BL}$ mouse by administering radioiodine. The pituitary tumor developed as a response to the hypothyroid state. Latent period of induction several months.

Fig. 4. Mouse bearing autonomous TSH-secreting pituitary tumor transplant(a). Initially such pituitary tumors are transplantable only into hypothyroid hosts. Following several transplants these pituitary tumors grow in intact animals. Their secretion stimulates the normal thyroid gland (b) to hyperplastic activity.

Fig. 5. Post-castrational adrenal cortical adenoma (a). The adrenal cortex of certain strains of mice is susceptible, whereas in other strains the adrenal cortex resists the induction of such adenomas. $\times 50$

Fig. 6. Post-castrational adrenal cortical adenoma (a) with lipid-laden cells. Secretion of sex steroid is associated with the appearance of these cells. $\times 56$ 
in this case thyroid deficiency resulting in TSH-secreting pituitary tumors, does not seem to be invariable. Some ${ }^{131}$ I-induced pituitary tumors have gonadotrophic activity and ablation of the adrenal glands does not favor the induction of ACTH-secreting pituitary tumors, rather than gonadotrophic, in whole bodyirradiated mice (Furth, personal communication).

Thyroid tumors have been induced by the administration of goitrogens which create a hormonal imbalance involving the pituitary and thyroid glands (Bielschowsky, 1949; Griesbach et al., I 945; Morris, 1955). Propylthiouracil inhibits thyroid hormone output of the intact thyroid gland, and as a result secretion of pituitary TSH is increased, with the thyroid gland becoming hyperplastic. Dependent neoplasms arising from the hyperplastic thyroid tissues grow upon transplantation in thyroid-deficient but not intact animals (Bielschowsky, 1949; Fig. 61, p. 864). During the course of transplantation the tumors acquire autonomy, now growing in animals with functioning thyroid glands (Morris et al., I95 I). Thyroid tumors may occur in rats on low iodine diets; such hypothyroid animals may develop pituitary as well as thyroid tumors (Axelrad and LeBlond, 1955).

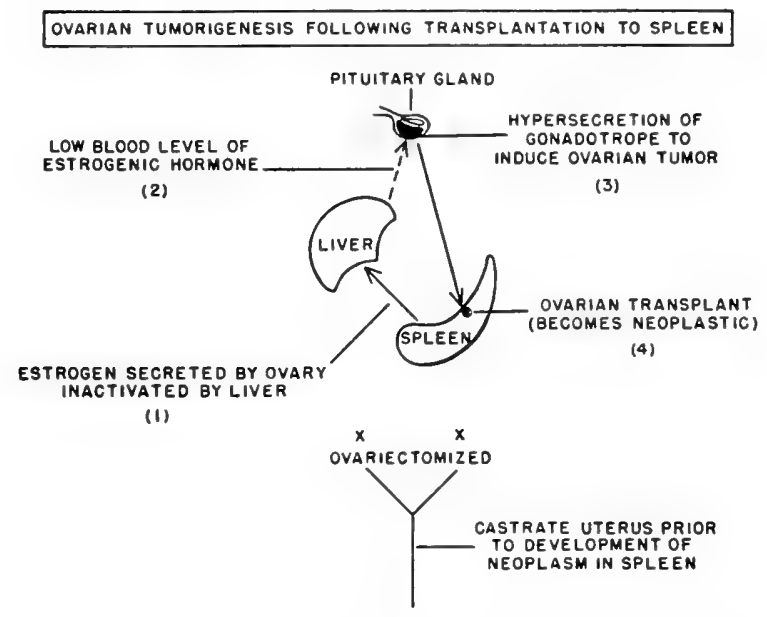

Fig. 8. Endocrine interrelationships involved in ovarian tumorigenesis following transplantation to the spleen.

The thyroid tumors resulting from a partial thyroid insufficiency have an origin associated with increased TSH blood levels. If hormonal imbalance per se is responsible for thyroid tumorigenesis, then the carcinogenic stimulus would be TSH. If other factors are involved then the thyroid growth resulting from the action of TSH merely facilitates their action. TSH would then be a "promotor" and not an "initiator" of carcinogenesis. It has been suggested that TSH merely accelerates the growth of latent tumor cells, and does not induce transformation of the thyroid cell from normal to neoplastic (Purves et al., I95 I).

Fig. 7. Undifferentiated tumor (carcinoma) of the adrenal cortex developing in a castrated Ce mouse. In the Ce strain post-castrational adenomas progress to carcinoma, whereas in other strains carcinomas develop only infrequently following post-castrational adenoma formation. 


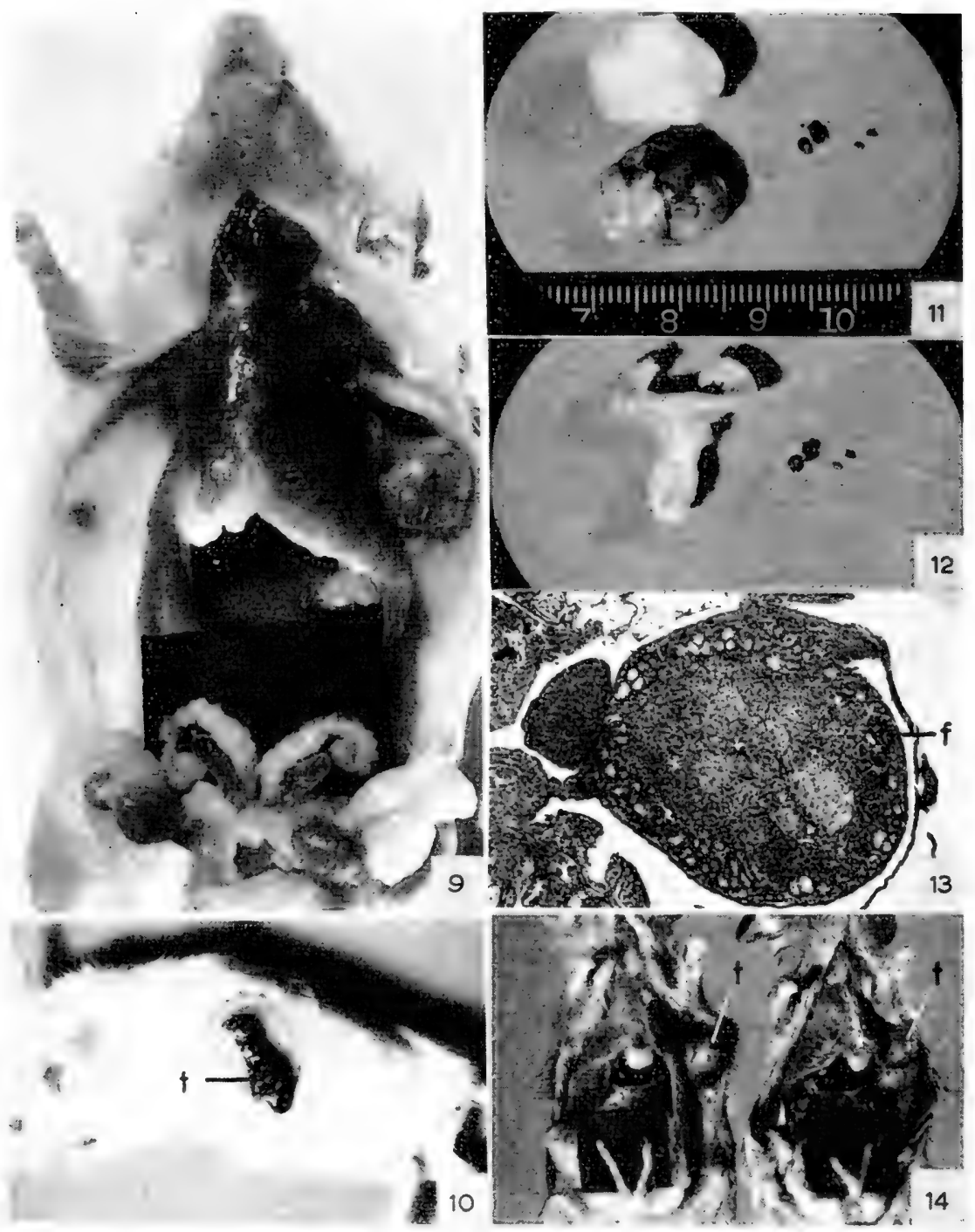

Fig. 9. Ovarian tumor ( $\mathrm{t}$ ) developing in an $\mathrm{X}$-irradiated ovary transplanted to a normal male of the same inbred strain. If such ovaries are transplanted to females, ovarian tumors do not develop, indicating that ovarian secretion suppresses tumorigenesis either directly, or indirectly via the pituitary gland.

Fig. Io. Ovarian tumor $(\mathrm{t})$ developing in an ovary transplanted to the spleen of an otherwise ovariectomized mouse.

Fig. Ir. Hormonal influence on X-ray induced ovarian tumors of mice. The tumor on the left was induced when one ovary was irradiated with $200 \mathrm{r}$ and the second ovary removed. The small ovary on the right is one which was irradiated with the same dose of X-rays, the second ovary (in the middle) remaining functional.

Fig. I2. Irradiation of a single mouse ovary with the second ovary remaining functional. As illustrated in Fig. I , here again irradiation of a single ovary was not tumorigenic if the 
(b) Hormonal imbalance per se as a factor in inducing neoplasia

Ovarian tumors can be induced by transplanting the normal rat or mouse ovary to the spleen (Figs. 8 and Io) if no functional ovary exists outside of the portal drainage system, or if adhesions do not form which allow for drainage from the spleen into the systemic circulation (Biskind and Biskind, 1944). Hosts with single ovaries successfully grafted to the spleen are physiologically castrate, ovarian hormone being inactivated in its passage through the liver. As a resu!t the gonadotrophic output of the pituitary gland is increased, and the grafted ovary is hyperstimulated, such hyperstimulation eventuating in neoplasia. Testicular tumors have been similarly induced by grafting testes into the spleen of castrates.

Some ovarian tumors induced by transplantation to the spleen are initially hormone-dependent, being transplantable only into the spleens of castrated hosts, an environment simulating that required for induction. Other neoplasms of the ovary, similarly induced, transplant readily to intact hosts (Furth and Sobel, I947; Greene, 1956).

A hormonal mechanism is apparently involved in the induction of ovarian tumors in mice by X-rays (Lick et al., I 949). Irradiation of a single ovary in mice of a highly susceptible strain does not yield ovarian tumor development if the other ovary is unirradiated and functional (Figs. I I-I 4). Removal of the functional ovary permits the development of a tumor in the irradiated ovary. Local irradiation of both ovaries causes bilateral tumorigenesis.

The pituitary gland becomes hyperactive in secreting gonadotrophin after $\mathrm{X}$-irradiation as a consequence of diminished ovarian hormone output by the irradiated ovaries (Vermande-Van Eck and Chang, i955). Tumor development would seem to result from stimulation by gonadotrophin of the remaining nonfunctional ovarian tissues. Such neoplasms may secrete sex steroids (Furth and Sobel, I947; Greene, I 956).

The role of gonadotrophins in ovarian tumor development has been further demonstrated by parabiosis experiments (Bielschowsky, I954). If a normal intact female rat is placed in parabiosis with an hypophysectomized female (Fig. 27, see p. 835), gonadotrophin from the latter crosses the parabiotic barrier to hyperstimulate the ovaries, for whose steroid secretion the parabiotic barrier is relatively impermeable. Consequently the ovaries are continuously bombarded by gonadotrophin from the pituitary gland of the castrated parabiont, and ovarian tumors

second ovary remained functional and non-irradiated. These ovaries were photographed sixteen months after exposure to X-rays at which time all irradiated female mice of this strain (Balb/c) have ovarian tumors. The uterus is of a size which indicates that the nonirradiated ovary was functional.

Fig. I 3. Histologic section of irradiated ovary shown in Fig. I2. The cortex contains anovular follicles (f). There is no evidence of tumor formation indicating that the non-irradiated functional ovary provided protection against irradiation-induced neoplasia. $\times 3^{8}$

Fig. I4. Ovarian tumorigenesis. The tumors in the left axillae of these mice developed from non-tumorous irradiated ovaries such as the one shown in Figs. 12 and I 3 when they were grafted into castrated females. Photograph 16 months after grafting. This demonstrates that tumorigenesis which had been suppressed for 16 months can proceed when a suitable tumorigenic environment is provided. 
are induced. Their estrogenic secretion may in turn excite adenomatous transformation of the pituitary of the intact parabiont; mammary gland stimulation results from the mammotrophic secretion of the pituitary tumor.

Although hormonal imbalance per se might be responsible in specific experimental situations for the development of ovarian tumors, it appears that excessive stimulation of the ovaries by gonadotrophin might not be necessary for carcinogenesis. There is no clinical evidence that hypo-ovarianism stimulating excessive output of pituitary gonadotrophins precedes human ovarian carcinogenesis.

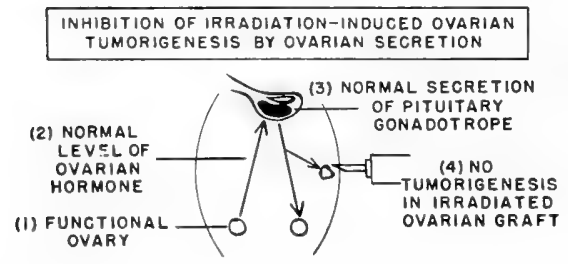

Fig. 15. Endocrine mechanism whereby functional ovaries inhibit tumorigenesis in an X-irradiated ovarian transplant.
Fig. I6. Mechanism of inhibition of ovarian tumorigenesis by functional ovarian transplants.
INHIBITION OF IRRADIATION-INDUCED OVARIAN TUMORIGENESIS BY OVARIAN GRAFTS

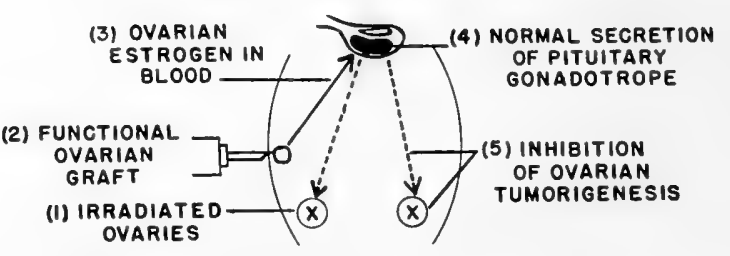

Carcinogenic hydrocarbons may induce ovarian tumors in intact female mice whose pituitary gonadotrophin output is probably within normal limits (Howell et al., I954).

If irradiated ovaries are transplanted to intact females (Fig. I 5) tumorigenesis is inhibited by ovarian secretion (Kaplan, I95ob). Functional ovarian grafts made following ovarian irradiation in situ (Fig. I6) also inhibit ovarian tumorigenesis (Kirschbaum et al., I956). Although androgenic hormone (exogenous) inhibits

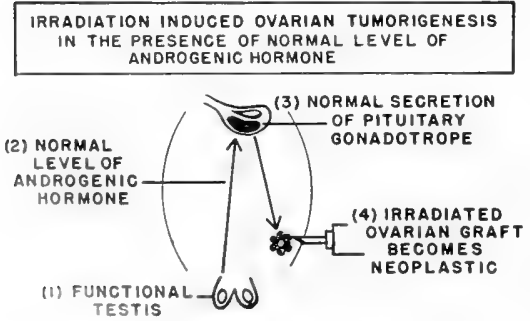

Fig. 17. X-ray induced ovarian tumorigenesis in grafts into intact male mice.

(1)arian umor development in splenic (ovarian) transplants, X-ray induced ovarian tumorigenesis is not similarly inhibited (Gardner, I950). When irradiated ovaries of inlored mice are transplanted to intact genetically related (histocompatible) 
males (Figs. 9, I 7), tumorigenesis proceeds in the transplants (Kirschbaum et al., I956).

In preventing X-ray induced ovarian tumor development either estrogen acts directly on the ovary, or the qualitative effect of estrogenic hormone on the pituitary is such that secretion of the tumor-inciting gonadotrophin is inhibited. Androgenic secretion would appear not to have a similar effect on the pituitary according to the latter interpretation.

Adrenal cortical tumors which secrete sex steroids can be induced by castrating male and female guinea pigs, mice, rats or hamsters (Spiegel, r 939; Woolley, I939; Houssay et al., I 954) (Figs. 5-7, see p. 826). Genetic factors are important in determining this response to gonadectomy (Figs. I9, 2I) since not all strains of mice respond to gonadectomy by cortical tumor development (Frantz and Kirschbaum, I949). The site of gene action determining tumorigenesis is in the adrenal cortex per se (Huseby and Bittner, I95I).

If sex steroids are administered following gonadectomy, post-castrational adenomas or carcinomas do not develop (Woolley and Little, I946). Since cortisone does not inhibit adenoma development (Monsen, I952), and since secretion of adenomas can be restored in hypophysectomized-gonadectomized rats by administration of gonadotrophin, but not ACTH, (Houssay et al., 1955), it appears that gonadotrophic rather than adrenocorticotrophic hormone influences development and secretion of these experimental post-castrational adrenal cortical overgrowths. Many of the carcinomas are hormone-dependent, growing earlier (Fig. 20) when transplanted into castrated rather than intact mice of the same inbred strain (Kirschbaum, unpublished; Browning, unpublished). Unlike the mouse and rat cortical neoplasms, sex-hormone secretion of at least certain human tumors of the adrenal cortex is influenced by ACTH (Gallagher, I957).

Certain hybrid mice develop pituitary as well as adrenal cortical tumors after castration (Dickie and Woolley, I949). Since the cortical tumor precedes the pituitary tumors, it has been suggested that the pituitary adenomas appear secondary to estrogenic stimulation (Figs. 22 and 23) arising from the cortical tumors (Houssay et al., I955).

The stimulus to adrenocortical tumor formation in mice appears to be pituitary gonadotrophin. In addition to the evidence cited above (Monsen, I952) parabiosis experiments (Fig. 24) suggest that preferential utilization of gonadotrophin by the functional testis and ovary may be a factor in inhibiting the genesis of these tumors in normal animals (Monsen and Kirschbaum, I954). When tumor-bearing mice were placed in parabiosis with intact females, the latter went into estrus due to ovarian stimulation, and the tumor-bearing mice acquired diestrus vaginal smears. With dilution of the gonadotrophin between two hosts the ovaries may have been stimulated preferentially, gonadotrophic but not estrogenic hormone crossing the parabiotic barrier.

\section{(c) Endocrine tumors following imbalance induced by estrogenic hormone}

Testicular interstitial cell tumors appear in mice of specific inbred strains as a result of administration of estrogenic hormone (Fig. 25) for relatively long periods of time (Hooker et al., I940; Shimkin et al., I941; Bonser, I942). Such tumors 

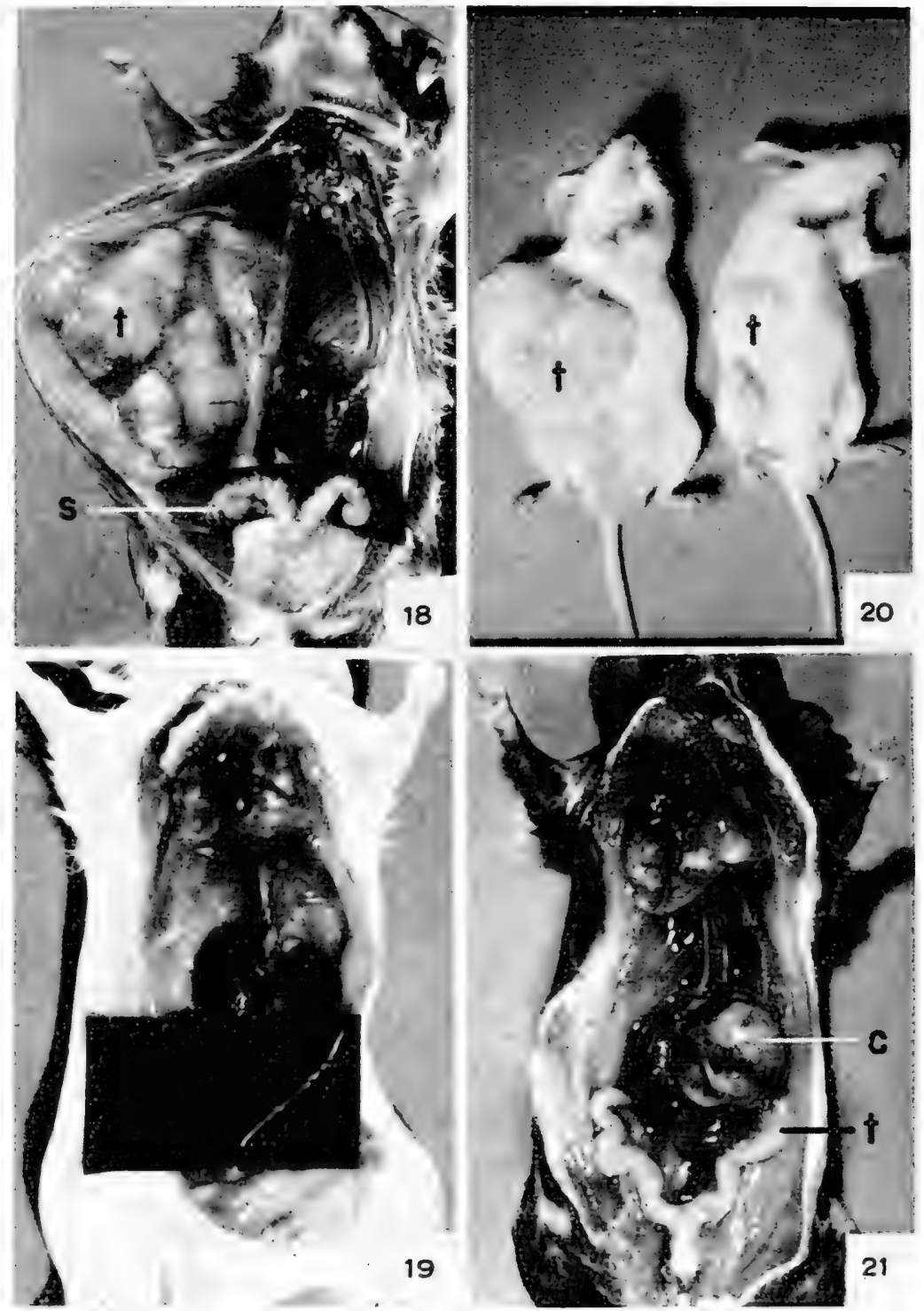

Fig. 18. Transplanted tumor ( $\mathbf{t})$ of the adrenal cortex. This tumor was induced in a Ce mouse by castration. The host was a castrated recipient. The stimulated seminal vesicles s) demonstrate androgenic secretion by the transplanted tumor. The adrenals of the host are non-tumorous.

Fig. 19. Effect of castration on the reproductive tract of strain A mice. This mouse had been ovariectomized one year before photography. The reproductive tract is atrophic. In other strains of mice castration provides the basis for development of neoplasia of the adrenal cortex (see Fig. 21).

Fig. 20. Acirenal cortical carcinoma transplants ( $t$ ) in castrated (left) and intact (right) male mice. Both transplants had been made at the same time several months before photo- 
Fig. 22. Endocrine factors in the production of adrenal cortical tumors by gonadectomy. Although the administration of cortisone suppresses the activity of the normal adrenal cortex, adrenal cortical tumors develop in cortisone-treated, gonadectomized mice, indicating that gonadotrophic rather than adrenocorticotrophic hormone is involved in the genesis of post-castrational tumors of the mouse adrenal cortex.

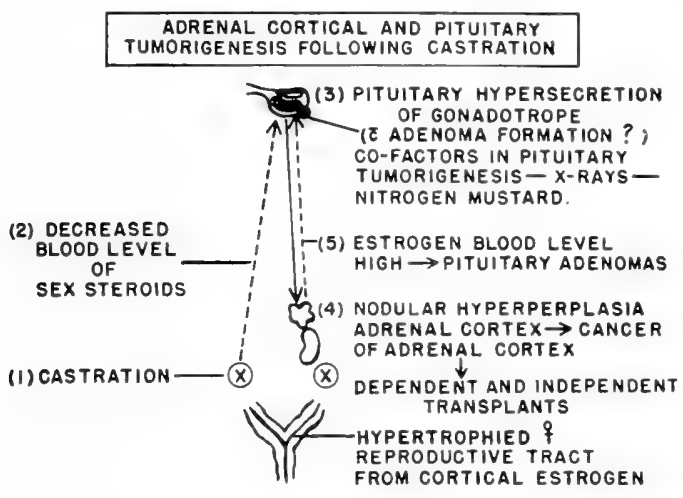

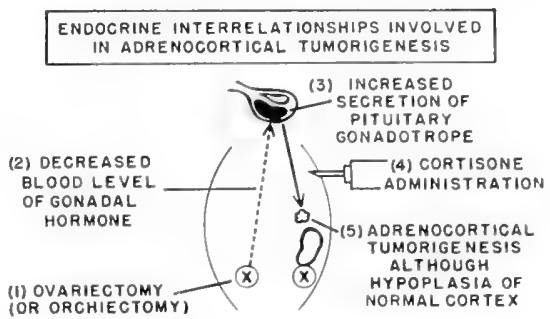

Fig. 23. Adrenal cortical and pituitary tumors following castration. Experimentally decreased blood level of sex steroids provides the stimulus to hypersecretion of pituitary gonadotrophin. Such hypersecretion may progress to pituitary adenoma formation which probably can be augmented by co-factors (X-rays, nitrogen mustard). Since exogenous estrogenic hormone may induce pituitary adenoma formation, it is possible that pituitary tumors may be induced by the endogenous estrogen secreted by adrenal cortical adenomas. Some tumors of the adrenal "take" best when transplanted into histocompatible, castrated recipients.

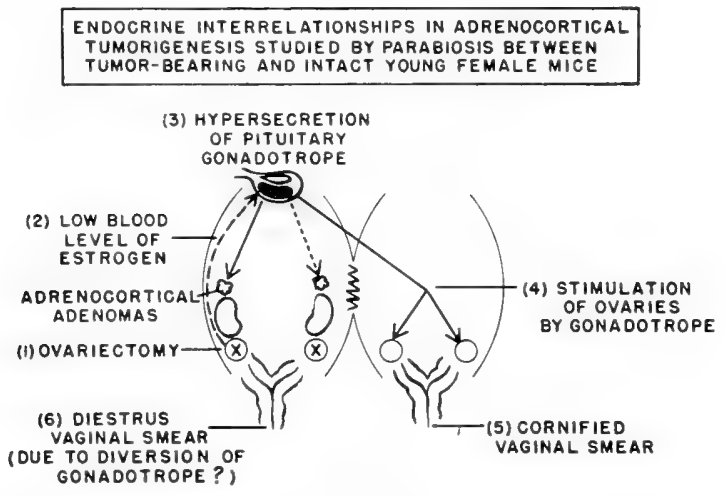

Fig. 24. Preferential utilization of pituitary gonadotrophic hormone as a factor in determining genesis and secretion of mouse tumors of the adrenal cortex. When castrated mice with adrenal cortical adenomas were placed in parabiosis with normal females, the gonadotrope crossed the parabiotic barrier tostimulate the ovaries; simultaneously the secretion of the tumors of the adrenal cortex was diminished as indicated by the diestrus vaginal smear of the tumor-bearing parabiont. This suggests preferential utilization of gonadotrope by the ovaries as compared with the adrenal cortical adenomas.

graphy. The transplant in the intact mouse began to grow later than in the castrated animal of the same strain. The larger tumor in the castrated mouse was secreting estrogenic hormone as indicated by the scrotal hernia (injection of estrogenic hormone induces scrotal hernia).

Fig. 21. Post-castrational adrenal cortical carcinoma (c). This mouse had been castrated one year before photography. The large carcinoma of the left adrenal was secreting estrogenic hormone as demonstrated by hypertrophy of the reproductive $\operatorname{tract}(\mathrm{t})$. 
also occur spontaneously, but only occasionally in inbred mice (Gardner, I 943b). Natural and synthetic estrogens have both been effective tumorigenic agents Gardner, r 943 a; Bonser, I942).

Fig. 25. Development of mouse testicular (interstitial) cell tumors following administration of estrogenic hormone. The induced tumors may grow only in estrogen-treated mice of the same inbred strain, indicative of estrogen-dependence.

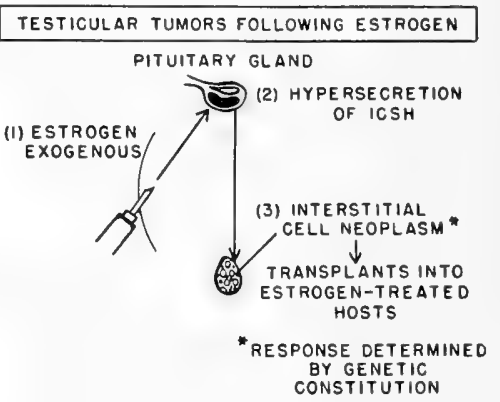

That the mechanism of induction involves the pituitary gland is suggested by the following experiments. As in the case of ovarian tumors, testicular tumors have developed in testes transplanted to the spleens (Fig. 26) of castrated animals (Biskind and Biskind, I945; Twombly et al., I949; Jones, I 955). The degree of success is less, however, than with ovarian grafts. Heterologous sera with large amounts of antigonadotrophin may inhibit the induction of testicular tumors by estrogenic hormone (Ely, 1953).

The administration of exogenous estrogen probably induces excessive secretion by the pituitary gland of a tumor-producing gonadotrophin (Fig. 25); response of the testicular target tissue is determined genetically (Fig. 26) (Trentin and Gardner, I957). Tumor induction initiated by the injection of estrogen may be completed by gonadotrophin although injection of exogenous gonadotrophin produces only interstitial cell hyperplasia (Pfeiffer and Hooker, I943).

Fig. 26. Sequences of events in testicular tumorigenesis when testis is grafted to the spleen.

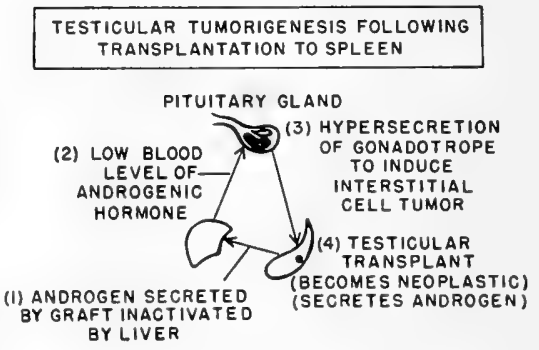

Transplantation of induced testicular tumors can be effected usually only by estrogenizing histocompatible recipients (Bonser, I942; Gardner, 1945). Andervont et al., (1957) have demonstrated that removal of the pellet of tumor-inducing estrogenic hormone is followed by progressive (independent) tumor growth in some cases, regression in others, and resumption of growth with a second hormonal stimulus. A similar mechanism of tumorigenesis may be involved in the induction 
of adrenal cortical neoplasms (Dunning et al., I 953) by estrogen, that is, pituitary secretion of tumorigenic gonadotrophin stimulated by the injection of estrogenic hormone.

Pituitary tumors have been induced in rats and mice by exogenous (Gardner and Strong, I940; Clifton and Meyer, r 956) and endogenous estrogen (Bielschowsky, I954). Genetic factors are very important in determining pituitary tumorigenesis of this type (Gardner and Strong, 1940). The tumors may be largely chromophobic (Gardner and Strong, I940) with some admixture of acidophiles (Bielschowsky, I954; Clifton and Meyer, I956); the hormone secreted by these tumors is mammotrophic (Bielschowsky, 1954). Although the acidophiles have been associated with secretion of this hormone, in one series of experiments stimulation of the mouse mammary gland occurred in mice bearing basophilic tumors (Dickie and Woolley, I949).

Mammotrophin-secreting pituitary tumors have also appeared in mice receiving heavy irradiation to the entire body (Furth, I955). The mechanism of induction remains obscure. Since the occurrence of ovarian tumors may precede pituitary

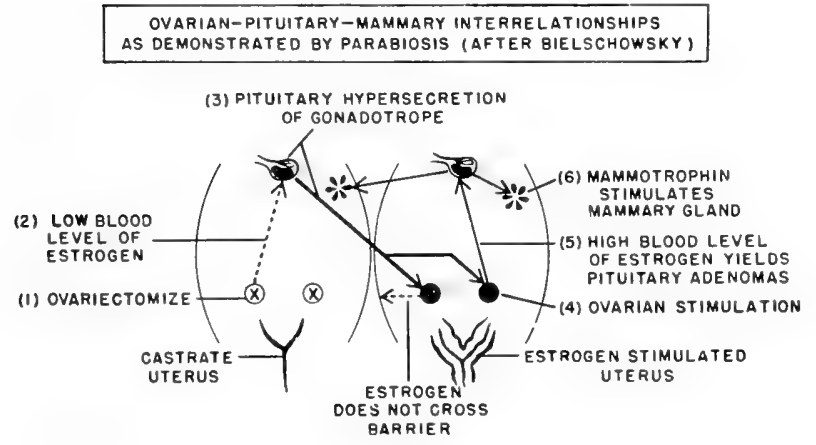

Fig. 27. Ovarian pituitary endocrine interrelationships involved in ovarian, pituitary and mammary tumorigenesis.

neoplasms (Furth and Butterworth, I936), estrogenic secretion may represent the stimulus for pituitary neoplasia in at least certain instances (Fig. 27). Secretion of mammotrophic hormone by the pituitary gland under the influence of estrogen may be an important factor in mammary tumorigenesis (Mühlbock, 1956). Bielschowsky et al., (1956) have studied an inbred strain in which acidophilic hyperplasia of the pituitary gland is associated with mammary hyperplasia and neoplasia.

\section{(d) Neoplasms of tissues whose normal growth is stimulated by estrogen}

The incidence of mammary cancer is much higher in human females than males, suggesting the probable importance of an endocrine status which characterizes females. In inbred mice spontaneous mammary cancer occurs only in females with the exception of one inbred strain in which testicular changes in the male suggest an hormonal imbalance (Athias and Furtado, I94 I). Similar alterations in the testis may be induced by exogenous estrogen.

Administered estrogen (Lacassagne, I 932) or ovarian (Fig. 32) transplantation 
(Aurray, I 928) induces mammary cancer in male mice of inbred strains in which a high incidence of mammary cancer occurs in the females. From the mammary cancers of high tumor strains, as well as from the normal mammary gland, there can be obtained a filterable agent which is important in influencing mammary carcinogenesis (Bittner, I942). Depriving female mice of this agent (by foster-

Fig. 28. This figure indicates the involvement and relationship of various factors in mouse mammary carcinogenesis. In certain inbred high mammary cancer strains (I) estrogenic hormone and a virus (mammary tumor agent) operate together, but only in mice of appropriate genetic constitution. The virus can be deleted by foster-nursing; if the foster-nursed mice are treated with a carcinogenic hydrocarbon (e.g., methylcholanthrene) mammary cancer may be induced (2). Such mammary cancers do not reveal the mammary tumor agent by bioassay. Certain sublines of foster-nursed high mammary cancer strains develop mammary cancer late in life, and no agent can be demonstrated biologically, raising the question concerning necessity of the mammary tumor agent for mammary tumorigenesis; administration of the mammary cancer agent accelerates the onset of mammary cancer. That hormonal factors may dominate in mammary carcinogenesis is demonstrated (4) by the effect of pituitary isografts on mammary tumor induction. Functional pituitary grafts will cause mammary cancer to appear in lines of mice presumably without the mammary tumor agent. The primary secretion of pituitary isografts is lactogenic hormone.

nursing) may remarkably depress the incidence of mammary cancer. Cell-free breast milk and mammary tumor extracts have been considered to contain a virus which is the etiologic agent in the appropriate genetic and hormonal environment (Bitner, 1942). Administration of cell-free extracts of mammary cancers may restore a high incidence of mammary cancer in lines of mice deprived of the agent by foster-nursing (Bittner, I 942).

That hormonal factors per se may induce mammary carcinogenesis independent of a viral agent has been suggested by a number of experiments (Fig. 28). The estrogen-induced mammary cancers of rats seem not to be influenced in their development by a filterable factor analogous to the mouse mammary tumor agent (Dunning and Curtis, I952). Certain lines of mice freed of the milk-agent by fosternursing develop mammary cancers, but relatively late in life (Heston et al., I950). When males of one agent-free subline of the $\mathrm{C}_{3} \mathrm{H}$ strain were injected with estrogenic hormone, mammary cancers appeared (Heston and Deringer, 1954). From these neoplasms a mammary tumor agent was not obtained utilizing methods which ordinarily reveal it (Heston and Deringer, r954; Mühlbock, I 956). Pituitary isografts may cause mammary cancer to develop in female mice lacking (by available tests) the mammary tumor agent (Mühlbock, I956). 
Thus by hormonal manipulation mammary cancers presumably lacking the milk-agent have been induced in agent-free mice. The role of the mammary tumor agent in spontaneous mammary neoplasia may be acceleration of onset of the disease; in the case of mammary cancer induced by pituitary isografts, cancer occurred earlier in mice given the mammary tumor agent (Mühlbock, 1956). Perhaps new methods may be devised, e.g. in tissue culture, for demonstrating a mammary tumor agent, presently not revealed by bioassay.

Cervical cancer has been induced in mice by estrogenic hormone administration (Allen and Gardner, I94I). Since carcinogenic effects are obtained by local application (Gardner and Frankenheuis, I 935), it appears that the tumor-inciting action of the hormone is in this case "direct", unlike the "indirect" action of estrogenic hormone in testicular (Fig. 25) and mammary tumorigenesis, where the pituitary gland is providing the more immediate tumor-inciting influence.

\section{(e) Neoplasms of "ton-target" tissues induced by estrogen}

Leukemia is induced in certain inbred strains of mice by relatively large doses of exogenous estrogen (Gardner et al., I940, I944); smaller doses augment the leukemogenic action of X-rays (Kirschbaum et al., 1953). Estrogenic hormone may overcome the protection against X-ray induced leukemogenesis afforded by thigh-shielding (Toch et al., I 955). Simultaneous administration of androgen may negate the leukemia-inciting action of estrogen (Gardner ct al., I 940).

In specific inbred strains of mice and their hybrids spontaneous leukemia occurs earlier in females (Murphy, I944; Liebelt, A.G., I 957) and the total incidence of the disease is also higher than in males. Castration increases the incidence of leukemia in males of certain high-leukemia strains (Murphy, I944). In another strain susceptibility to carcinogen-induced leukemia was increased by castration (Kirschbaum et al., I 955). The administration of androgen inhibited leukemogenesis in castrates. Although the genesis of lymphocytic neoplastic disease is augmented by estrogenic hormone, male mice are more sensitive than females to the induction of granulocytic leukemia by X-rays (Upton and Furth, I956).

The lymphoid neoplasms induced by methylcholanthrene and the estrogenic hormones are identical. If induction of leukemia is evidence of the carcinogenic potency of the carcinogenic hydrocarbons, by the same token estrogenic hormone is a carcinogen. The thymus appears to be the lymphoid organ primarily sensitive to the induction of neoplasia by estrogenic hormone (Gardner et al., I940), X-rays (Kaplan, I950a), or the carcinogenic hydrocarbons (Law and Miller, I950a). When methylcholanthrene is placed directly into the thymus, neoplasia results more readily than if similar local treatment is applied to lymph node; the action of methylcholanthrene may be considered local or "direct" (Rask-Nielsen, I950). Thymic radiation per se is not leukemogenic (Kaplan, I949); shielding of hemopoietic tissue is protective against the induction of leukemia (Kaplan and Brown, I 95 I) and secondary effects of irradiation are important in leukemogenesis (Kaplan et al., I 955, I 956). Similar data are not available for the leukemogenic activity of estrogens. The possible mediation of viral activity by estrogens will be discussed below.

Renal tumors have been induced in guinea pigs and hamsters by estrogenic 

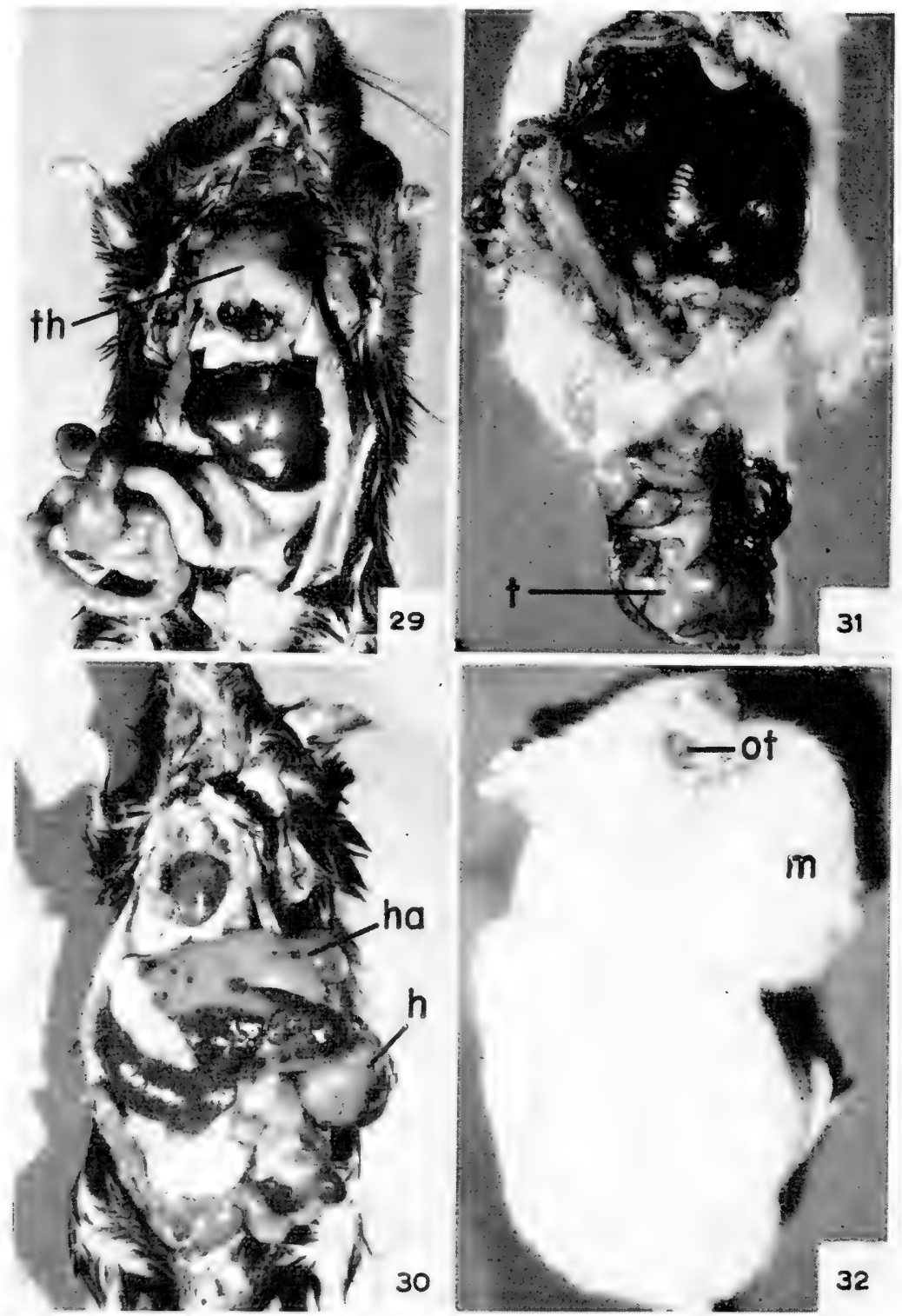

Fig. 29. Thymic lymphosarcoma (thymoma "th") development in a mouse injected with estrogenic hormone. Depending upon genetic constitution, similar neoplasms may be induced by carcinogenic hydrocarbons, X-rays or certain chemicals (e.g. urethane, thiotepa), either independently or in conjunction with other agents.

Fig. 30. Mouse with hepatoma (h) and hemangiomas (ha) of the liver. Hepatoma occurs more frequently in male than female mice, and the incidence in the sexes can be altered by administration of sex steroids. 
hormone (Kirkman and Bacon, I950). Upon transplantation such tumors are estrogen-dependent (Horning, I 956). The induction by estrogen of neoplasms in tissues other than those whose growth is "specifically" stimulated makes it appear doubtful that the stimulus to normal proliferation is identical with the carcinogenic stimulus (Figs. 29-3I). It has been suggested that carcinogenic agents may induce, within the tissues acted upon, "isocarcinogens" which are the more

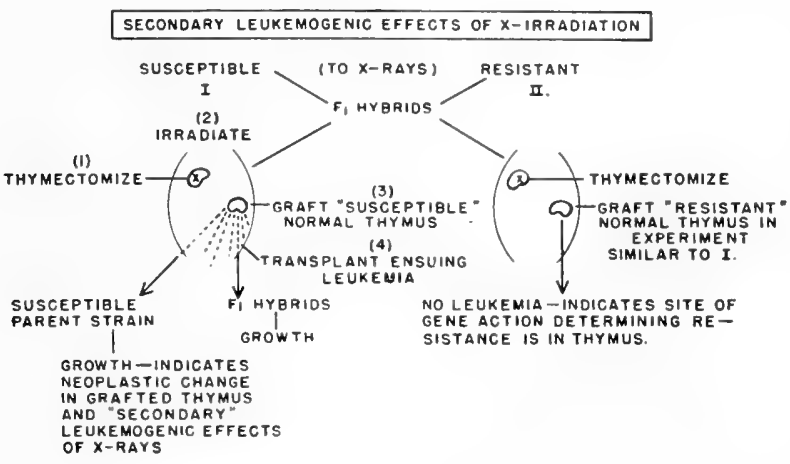

Fig. 33. Induction of mouse thymomas by $\mathrm{X}$-irradiation. If a strain susceptible (I) to the induction of thymomas by $\mathrm{X}$-rays is crossed with a resistant strain (II), the $F_{I}$ hybrids are susceptible. If the Fi hybrids are thymectomized, however, then this susceptibility is nullified since the thymus is the organ which is primarily susceptible. In these experiments of Kaplan et al., 1956 , the $\mathrm{C}_{57} \mathrm{BL}$ was the susceptible strain. If thymectomized mice received thymic grafts post-irradiation from the "susceptible" strain, then thymomas developed. Since the thymus itself was not irradiated, it would appear that the irradiation effect was "secondary". A similar result was not obtained if the thymic graft was from the "resistant" strain, demonstrating the genetic determination of susceptibility to this irradiation effect, such genetic susceptibility residing in the thymus per se. From the neoplastic thymus lymphocytes metastasize to the various organs of the body, yielding systemic leukemia.

immediate stimuli to carcinogenesis (Gardner, I 957). Such agents might be "viral" in nature. The "indirect" effects of irradiation (Kaplan et al., I 955, I 956c) suggest the likelihood that leukemogenic factors may arise in normal tissue post-irradiation (Fig. 33), and that direct action of ionizing irradiation on target tissues is not essential for leukemogenesis.

That estrogenic hormone was found to induce neoplastic growth experimentally in tissues (e.g. mammary, cervix) in which normal hyperplasia is hormonally stimulated was anticipated; but the leukemogenic action of estrogenic hormone and the induction of renal tumors are complete enigmas. Estrogenic and androgenic hormone and cortisone all cause hypoplasia of the thymus; of the three groups of compounds only the estrogenic induces neoplasia, the others inhibiting the development of thymic neoplasms of the mouse (Gardner et al., I 940; Kaplan, I948; Woolley and Peters, I953).

Fig. 3r. Testicular tumor (t) induced by exogenous estrogen. Only certain strains of mice are susceptible to the induction of interstitial cell tumors by estrogenic hormone.

Fig. 32. Mammary cancer $(\mathrm{m})$ in male mouse of a high mammary cancer strain. Mammary cancers appear spontaneously only in females of this strain. If males receive ovarian transplants as in this case (transplant "ot" is in the ear), or are injected with estrogenic hormone, or possess post-castrational estrogen-secreting adenomas of the adrenal cortex, then mammary cancer may appear. 
Transient exposure to hormonal action may make a permanent imprint on tissues, determining the ultimate development of cancer. Ovariectomy does not suppress mammary tumorigenesis in the susceptible gland once adequately stimulated by estrogenic hormone (Loeb, I940). The tumor-inducing hormone acts much as the chemical carcinogen which induces a skin-tumor following a latent period subsequent to a single skin painting.

\section{(f) Tumor-promoting activity of steroids other than estrogenic}

Although the development of the normal prostate gland is under androgenic influence, estrogen combined with methylcholanthrene was more carcinogenic for mouse prostatic tissue than methylcholanthrene alone or administered with androgen (Horning, I952). Hepatic tumors occur more frequently in male mice of strains in which the spontaneous incidence is high (Andervont, I 95ob); incidence in males is reduced by castration, increased in females by exogenous androgen (Agnew and Gardner, I952). Hepatic tumors are induced more readily in male than female rats by hepatic carcinogens (Morris and Firminger, I956). In two high-hepatoma strains of mice males with a high caloric intake induced by goldthioglucose, developed hepatomas early; in females hyperphagia did not have a comparable effect (Waxler, I 953; R. A. Liebelt, I957). Primary carcinoma of the human liver has been reported to appear more often in males, although this has been questioned (Davies, I955). With minimum doses of chemical carcinogens, male mice exhibited a higher incidence of cutaneous tumors than females (Leiter and Shear, I 943).

Apparently androgenic hormone is a factor in the genesis of prostatic cancer, but there is no conclusive evidence that androgens per se are carcinogenic. Cortical hormone has been implicated in the genesis of mouse leukemia (Upton and Furth, I 954; Silberberg and Silberberg, I 955), but, aside from these reports, the effects of cortical steroids appear to impede rather than promote leukemogenesis, the incidence of spontaneous and induced leukemia (by X-rays and carcinogenic hydrocarbons) being lower or the onset delayed in cortisone-treated mice (Woolley and Peters, 1953; Kirschbaum and Liebelt, unpublished)

\section{(g) Inhibition of tumorigenesis by steroid hormones}

Hepatic tumor incidence is lower in females than in males in both man and mouse, and hepatic tumorigenesis induced by liver carcinogens (Morris and Firminger, 1956) occurs less readily in female than in male rats. However, sex hormones apparently do not alter susceptibility to $o$-aminoazotoluene in mice (Andervont and Dunn, I947). In $\mathrm{C}_{3} \mathrm{H}$ mice receiving estrogenic hormone, the hepatic tumor incidence was decreased (Agnew and Gardner, 1952). Excessive quantities of either estrogen or androgen potentiated hepatic tumorigenesis by acetylaminoflourene in the rat (Cantarow et al., 1946).

Orarian tumorigenesis is inhibited by estrogenic hormone following either X-irradiation or ovarian transplantation to the spleen (Gardner, 1950). Although androgenic hormone inhibits the development of tumors in ovaries transplanted to the spleens of castrated mice, similar treatment with androgen did not prevent the induction of ovarian tumors by X-rays (Gardner, I950). Endogenous testic- 
ular androgen did not inhibit ovarian tumorigenesis in transplanted $\mathrm{X}$-irradiated ovaries (Kirschbaum et al., 1956).

Post castrational adenomas or carcinomas of the adrenal cortex do not develop if appropriate doses of either estrogen or androgen are administered, treatment beginning soon after gonadectomy (Woolley and Little, 1946). Such tumorsuppressing doses may alter neither the growth nor secretory activity of certain established cortical tumors (Monsen, 1952). The growth of transplanted "dependent" cortical tumors of the Ce strain, in which palpable transplants appear earlier in castrated than intact animals, may be delayed by either estrogen or androgen (Kirschbaum, unpublished; Browning, unpublished). These inhibiting effects are probably due to suppression of output of pituitary gonadotrophin. Although post-castrational adrenal cortical adenomas may secrete large amounts of estrogenic hormone, the adjustment of the pituitary is such that it is not suppressed in gonadotrophin output, continuing to stimulate the cortical adenomas to secrete sex hormones. Paradoxically, in the rat adrenal, cortical tumors have developed in response to the administration of estrogen (Dunning et al., I953).

The induction of post-castrational adrenal cortical tumors of mice is not inhibited by administering cortisone in doses large enough to suppress the normal adrenal cortex (Monsen, I 952). Studies on the secretion of sex steroids by human cortical neoplasms indicate dependence on ACTH (Gallagher, 1957), unlike the rat, where resumption of adrenal cortical sex steroid secretion after hypophysectomy could be induced by gonadotrophins but not ACTH (Houssay et al., I 955).

Pituitary tumors have been reported following castration coupled with either X-irradiation or nitrogen mustard (Gorbman and Edelmann, 1955). Since gonadectomy is essential for tumor development, sex steroids might be considered tumor-suppressive. Pituitary tumors have occurred in mice as a result of gonadectomy (Dickie and Woolley, I949); adrenal cortical neoplasms preceded the appearance of pituitary tumors and it is possible that estrogenic secretion of the cortical adenomas was responsible for pituitary tumorigenesis (Houssay et al., 1955).

Mammary carcinogenesis of mice is inhibited by exogenous androgen (Nathanson and Andervont, I 939). Lymphocytic leukemogenesis is suppressed by androgen, the spontaneous (Murphy, I944), X-ray (Gardner, I950) or methylcholanthreneinduced (Kirschbaum et al., 1955) incidence being lower in males of specific strains. Castration has increased susceptibility to spontaneous lymphocytic leukemia (Murphy, I944) and the leukemia-promoting effects of methylcholanthrene (Kirschbaum et al., I955). Androgen nullifies the leukemogenic activity of exogenous estrogens (Gardner et al., 1940). Androgen prevented X-ray induced lymphocytic leukemogenesis only when administered soon after exposure to X-rays; a delay of six weeks rendered it ineffective (Kaplan and Brown, I 95I). Apparently androgen may potentiate granulocytic leukemogenesis in the mouse (Upton and Furth, I956). Androgenic hormone (testosterone phenylacetate) delays lymphocytic leukemogenesis probably as effectively as cortisone in the high leukemia $\mathrm{C}_{5} 8$ strain of mice (Kirschbaum and Liebelt, unpublished).

Conflicting results have been obtained on the effects of cortisone on the carcinogenic induction of skin cancer. Cortisone has been observed either to inhibit 
(Schober, I953; Engelbreth-Holm and Asboe-Hansen, I953) or accelerate (Boutwell and Rusch, I 953; Sulzberger et al., I953; Spain et al., I 956) epidermal carcinogenesis. Diminished mast cell content of sulfomucopolysaccharide was observed in the mast cells infiltrating the region of cutaneous tumor development (Asboe-Hansen, I 954).

\section{(h) Effect of steroid hormones on tumor growth}

Certain "conditional" (hormone responsive) neoplasms of the mouse testis, pituitary and mammary gland are dependent upon estrogenic hormone for growth after transplantation (Foulds, I 954; Furth, I 953; Gardner, I 945; Foulds, I949; Kirkman, I957); the recipient must be estrogen-treated if implants are to grow progressively. Mammary cancers may be malignant, as judged by progressive growth, and still dependent (Foulds, I954). The "dependence" of experimental mammary and testicular tumors may actually be upon pituitary hormone secreted as a result of treatment with estrogen. The growth of specific transplanted adrenal cortical tumors may be suppressed by estrogen (Kirschbaum, unpublished) or androgen (Browning, unpublished). In this instance, and in the case of ovarian tumors which grow best in castrates, sex hormone is probably a suppressor of pituitary gonadotrophic secretion, upon which the neoplasm is dependent.

Human mammary and prostatic cancers may be hormone responsive (Haddow et al., I 944; Nathanson, I950; Pearson et al., I 955; Kennedy, I 956; Huggins and Hodges, I 94I). The effect of androgenic hormone on prostatic neoplastic tissue is considered to be "direct", but there is increasing evidence that the effects of estrogen on mammary cancer are probably via the pituitary gland.

Glucocorticoids inhibit normal lymphopoiesis (Dougherty, 1952) and restrain the proliferation of leukemic lymphocytes. Growth of certain transplanted mouse and rat leukemic cells is inhibited by cortisone (Burchenal et al., I 950); lymphoid tumors of chickens may be inhibited markedly, but temporarily, by ACTH or hydrocortisone (Lannek, I953). Adrenal cortical secretion profoundly influences lymphocytic mouse leukemogenesis, adrenalectomy favoring spontaneous development (Law et al., I 947) and X-ray induction (Kaplan et al., I95I); exogenous cortisone delays or suppresses the development of mouse leukemia (Woolley and Peters, I 953). Under certain circumstances adrenal cortical secretion apparently augments leukemogenesis (Upton and Furth, I954; Silberberg and Silberberg, I 955).

Ovariectomy provides palliation in metastatic human mammary cancer in probably 40 percent of premenopausal women (Pearson et al., I 955). Paradoxically the administration of estrogen is similarly beneficial in women several years postmenopausal (Nathanson, I950). Whether the benefits of androgen in breast cancer of young women are due only to suppression of ovarian activity has not been settled.

Adrenalectomy may in certain cases provide further remission (Pearson et al., 1955) after ovariectomy in young women and also cause temporary tumor regression in post-menopausal women. That the effects of adrenalectomy are those of estrogen withdrawal is suggested by the decline in calcium excretion (due to bony metastases) and the increase coincidental with the administration of estrogen. 
Similarly, adrenalectomy may affect the progression of prostatic cancer, in this instance the result being attributed to the withdrawal of androgen of adrenal cortical origin (Huggins and Bergenstal, r 952). Whether the effects of hypophysectomy on breast cancer (Luft and Olivecrona, 1955) are due to ovarian and adrenal cortical insufficiency is still not decided. Pituitary stalk section also has been reported

a
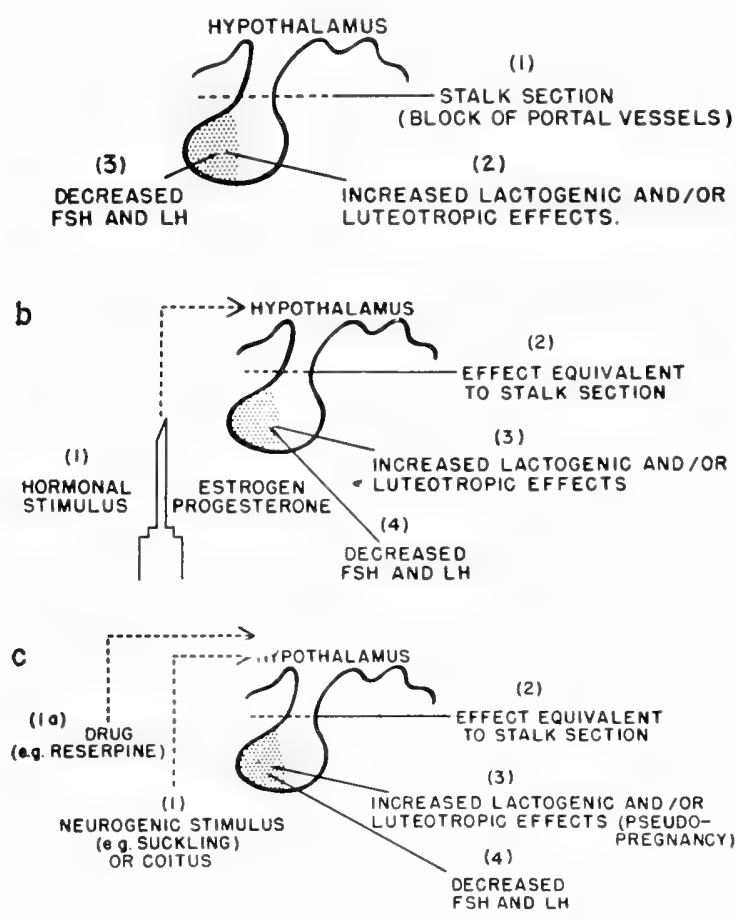

Fig. 34. Figure indicates role the hypothalamus may play in controlling certain pituitary functions. Following pituitary stalk section in the human female (a) output of gonadotrophic hormones (also ACTH, TSH and STH) is decreased (Eckles and Ehni, 1957) whereas lactogenic effects are increased. In the rat, luteotrophic and lactogenic activities are correlated. A similar reciprocal relationship between FSH and LH on the one hand, and lactogenic hormone on the other, may exist following the administration of appropriate amounts of estrogen and progesterone (b), suggesting that the ovarian steroids may influence the pituitary gland via the hypothalamus. That drugs (e.g., reserpine, Sawyer, I957) may act upon the hypothalamus to give an effect equivalent to stalk section is indicated by experimentally induced lactation in rabbits. The neurogenic stimulus provided by suckling induces pituitary lactogenic activity and the mating of female rats with vasectomized males results in pseudo-pregnancy (Rothschild, 1957). The lowermost chart (c) indicates the probability that chemical and neurogenic stimuli may act upon the hypothalamus to produce the same pituitary activities which result from stalk section. In the other charts which diagram reciprocal relations between the pituitary gland and target endocrine organs the hypothalamus has not been included. This figure indicates the likelihood that the hypothalamus may be conccined in the regulation of secretion of other pituitary trophic hormones which are involved in tumorigenesis.

to be associated with amelioration of human metastatic breast cancer (Ehni and Eckles, I 957); whether the benefits are mediated through the ovary and adrenal, or are due primarily to pituitary hormone withdrawal, or are associated with lactogenic effects (Fig. 34) has not been demonstrated. Although cortisone may dramatically ameliorate the signs (such as hypercalcemia) of breast cancer, the benefits have only rarely been associated objectively with tumor regression (Eckles, unpublished). Lactation occurs following pituitary stalk section; regression of metastatic breast cancer occurs coincidentally with the development of functional activity of the mammary gland. 
Cortisone may induce the regression of transplanted animal lymphosarcomas (Heilman and Kendall, I 944), not all tumors of this type responding, however. Both cortisone and testosterone independently induced regression of a transplanted lymphosarcoma in pyridoxine deficient rats (Stoerk, I950), but alone neither was as effective as pyridoxine deficiency per se. Remissions may be obtained in acute human leukemia with cortisone.

The effect of cortisone on certain non-lymphomatous transplanted tumors is similar to that observed for lymphosarcomas (Higgins and Woods, I950; Higgins et al., I 950), but many are non-responsive. The Walker tumor grows at a reduced rate in adrenalectomized rats (Talalay et al., I952; Ingle and Baker, I95I) and tumor size is increased by administering cortisone (Ingle et al., 1950). It has been suggested that the regression of non-lymphomatous tumors by cortisone results from suppression of adrenal cortical activity (Selye, I955).

According to Bloom (1952), cortisone induced regression of a canine mast cell tumor. Regression of a carcinogen-induced skin cancer of mice has resulted from administration of a cortical steroid (Zachariae and Asboe-Hansen, I 954).

The question arises as to whether the effect of adrenalectomy in breast cancer is due to sex hormone withdrawal or the action of specific corticoids of the adrenal cortex? Clinical evidence favors the concept that the benefits of adrenalectomy stem from the withdrawal of extragonadal sex steroid, estrogen or androgen. Experimentally the growth of mammary adenomas of rats has been inhibited by adrenalectomy (Huggins et al., I956). The growth of mouse mammary cancers arising in young females of the RIII strain is decellerated greater by adrenalectomy superimposed on ovariectomy than by ovariectomy alone (Liebelt and Eckles, 1957). The sex steroid secretion by the adrenals of this strain is not appreciable even in mice which have been castrated for several months.

By reducing the host's immune response to foreign tissue, prior treatment with cortisone has favored the growth of homologous (Allam et al., r 954; Foley, 1952) and heterologous (Toolan, I953) tumor grafts. Cortisone is not always effective in bridging this barrier and in one study was useful only when combined with ionizing radiation (Werder et al., I953). Natural resistance to transplantation is more easily overcome than an acquired immune state (Werder et al., I953).

Fig. 35. Influence of cortisone on metastases of transplanted mouse neoplasms.

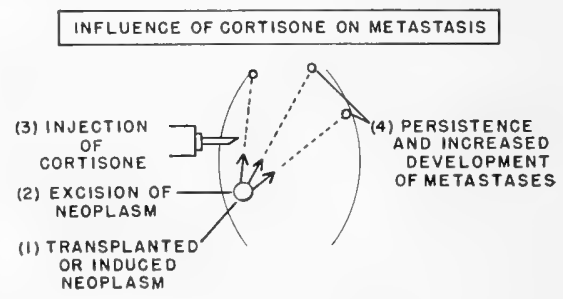

\section{(i) The infuence of the pituitary gland on the genesis, growth and secretion of tumors}

The development of experimental thyroid (Fig. 2, p. 825), adrenal cortical (Fig. 22 , p. 833) and gonadal neoplasms (Fig. 8, p. 827) seems to be dependent upon pituitary trophic hormonal stimuli. Suppression of pituitary secretory activity $(e . g$. by thyroid hormone) may inhibit tumorigenesis which would otherwise have 
resulted from the imbalance brought about by withdrawal of target organ secretion. Pituitary trophic hormones may be necessary for the growth of "hormone responsive" (e.g. adrenal) tumors. Change in the sensitivity of a target tissue (e.g. $\mathrm{X}$-irradiated transplanted ovary in intact male mouse) may result in tumorigenesis with normal levels of trophic hormone (Kirschbaum et al., I 956).

Mammotrophic hormone of the pituitary gland, secreted as a result of the action of estrogen on the pituitary, may be a factor as potent as any in mammary tumorigenesis (Mühlbock, I956).

Following hypophysectomy hepatic tumorigenesis did not result from feeding hepatic carcinogens (Griffin et al., r955). Susceptibility could be restored by administration of growth hormone, but not ACTH to hypophysectomized rats. Thyroidectomy had a similar effect on hepatic carcinogenesis when acetylaminofluorene was fed (Bielschowsky and Hall, i 953). Since acidophiles are absent from the hypophysis of thyroidectomized rats, growth hormone deficiency has been suggested as responsible for the results with thyroidectomy. The incidence of hepatic tumors was reduced when rats fed acetylaminofluorene were made hypothyroid with thiouracil (Paschkis et al., I948).

Initial experiments suggested that hepatic tumors can be induced in hypophysectomized rats receiving ACTH (Griffin et al., I 955); rats were made refractory to the tumor-inciting action of butter yellow by adrenalectomy (Symeonidis et al., I95I). The influence of sex steroids on hepatic tumor incidence has been discussed elsewhere. Whether the accelerating effect of androgenic hormone is via effects on other endocrine organs has not been determined.

Lymphatic tissue regenerates after irradiation in the absence of the pituitary gland. Hypophysectomy did not inhibit leukemogenesis, and the induction of leukemia was prevented in X-irradiated mice by shielding bone marrow (Nagareda and Kaplan, I955).

Pituitary growth hormone has been held responsible for the development of a variety of rat tumors (Moon et al., $195^{\circ}$ a, b, c, I 95 I, I952, I 955, I 956). Although the sarcogenic effects of methylcholanthrene were reported reduced by hypophysectomy (Moon et al., I955), other investigations indicate that initiation is either not altered or only delayed (Noble and Walters, I954); the subsequent growth rate of the neoplasms is reduced (Agate et al., I 955). Carcinogenesis by 9, 10dimethyl-1,2-benzanthracene occurred when hypophysectomized rats were given growth hormone, but not ACTH (Moon et al., I956).

Although growth hormone increased the growth rate of a transplanted tumor, the influence was comparable to that upon general body growth (Smith et al., 1952). Growth of two transplanted tumors was not influenced by purified growth hormone (Schulman and Greenberg, 1949). Transplants of the Walker sarcoma "took" in hypophysectomized rats (Talalay et al., I 952), but growth was inhibited by one-third (Rock et al., I 955). The seeding of mouse tumor cells was augmented after intravenous inoculation if the recipients were treated with growth hormone before and after tumor transfer (Wood et al., I955).

In all hypophysectomy experiments inanition effects must be differentiated from hormone withdrawal (Ball and Samuels, I938; Korteweg and Thomas, I949). The anabolic influence of growth hormone depends upon adequate food intake. In spite 
of the debilitating effects of hypophysectomy, spontaneous mammary tumors and hyperplastic nodules may grow progressively after pituitary removal (Gardner, I942), although the rate of growth is often diminished and many of the nodules regress.

Hypophysectomy has provided palliation in metastatic breast cancer of women (Luft and Olivecrona, I955). The mechanism underlying this response should be a fruitful field of investigation. Although eleven percent of spontaneous mouse mammary cancers in one series regressed following hypophysectomy, host survival was not prolonged (Martinez and Bittner, I954). The influence of hypophysectomy upon mouse mammary cancer has not been adequately tested in a sufficient number of strains.

\section{(j) Influence of thyroid secretion on genesis and growth of tumors}

Absolute hypothyroidism induces TSH-secreting pituitary tumors in inbred mice (Gorbman, I952); partial hypothyroidism results in the development of thyroid tumors (Purves et al., I95I).

Thyroidectomy inhibits the induction of hepatic tumors in rats by acetylaminofluorene (Bielschowsky and Hall, I953); similar effects were obtained by administering thiouracil (Paschkis et al., I 948). Only if rats were made hypothyroid prior to the feeding of acetylaminofluorene was the induction of liver tumors inhibited, suggesting interference with the initiation of carcinogenesis. Hyperthyroidism favored the induction of hepatic tumors by butter yellow (Miller and Baumann, I95I). The dye was taken up more readily by the livers of hyperthyroid rats; this could have resulted from increased intake of dye in the greater amount of food ingested. Since the hyperthyroid liver did not degrade the dye as readily as the normal liver, more dye could accumulate.

When a high leukemia strain of mice was made hypothyroid with a goitrogen, the development of spontaneous leukemia was favored; this effect was correlated with increase in body weight resulting from lowered metabolism (Grad et al., I 955) Radio-thyroidectomy, prior to treatment with methylcholanthrene remarkably reduced the incidence of methylcholanthrene-induced leukemia (Ida, unpublished). Similar effects on radiation-induced leukemogenesis have been obtained by radiothyroidectomy (Nagareda and Kaplan, I957). The results are similar to those obtained following thyroidectomy on hepatic tumor development with a carcinogen, acetylaminofluorene (Bielschowsky and Hall, i 953). Inhibition of leukemogenesis was greater in intact than castrated males. Whether radiothyroidectomy is effective after, rather than before, carcinogen treatment has not been determined to establish the influence of the procedure on metabolism of the carcinogen or on tissue response. The induction of hepatic reticulosarcoma (reticuloendothelioma-hepatic histocytoma) has also been prevented by hypothyroidism (Gillman et al., I955).

Hyperthyroidism increases metabolic rate and decreases body weight with a viven caloric intake. Increased metabolic rate with consequent decrease in body weisht, in spite of high caloric intake, results in reducing the incidence of spon1aner,us mammary and pulmonary tumors (Tannenbaum and Silverstone, I 949). IThis, increased metabolism may produce the same effect as caloric restriction upon tumorigenesis. 
Hyperthyroidism may depress tumorigenesis in ovaries transplanted to the spleens of castrated mice (Miller and Gardner, 1954). Although the post-castrational adrenal-cortical adenomas appearing in thiouracil-treated mice may be non-functional (neither estrogenic nor androgenic activitiy), hormone production was exhibited by the cortical adenomas of radiothyroidectomized-gonadectomized mice (Luckman and Kirschbaum, unpublished).

Results relative to the effect of thyroidectomy on the growth of transplanted tumors have been contradictory. The only reliable procedure for ablating the mouse thyroid gland is radiothyroidectomy. A transplanted fibrosarcoma grew equally well in normal and radiothyroidectomized mice (Sloviter, r95 I). Total thyroidectomy was reported to prolong significantly the interval between methylcholanthrene injection and the time of development of sarcoma in rats (Woyson. et al., 1956). The number of athyroid rats developing induced tumors was $86 \%$, compared with $100 \%$ tumor development in intact animals. Since animals were not pair-fed in this and other experiments, caloric intake has not been eliminated as a factor determining the difference in response of intact and athyroid animals.

\section{(k) Diabetes and the growth and development of neoplasms}

Sarcoma 37 grew equally well in normal and alloxan diabetic rats (Carrie and Ham, I 949). The diabetic state did not alter the susceptibility of rats to the induction of sarcoma by benzpyrene (Dunning et al., I949). It has been reported, however, that diabetic rats (Goranson et al., 1954) and mice (Jehl et al., 1955) do not support the growth of specific transplanted tumors as well as non-diabetic controls. Azo dye carcinogenesis was inhibited in alloxan-diabetic rats (Salzberg and Griffin, I952).

\section{IONIZING RADIATIONS AND CANCER}

There is clinical evidence that ionizing irradiations may be carcinogenic. Lung cancer in the Schneeberg miners (Hueper, 1942), skin cancer following heavy $\mathrm{X}$-irradiation (Hesse, I9II), malignant bone tumors after prolonged retention of radium in osseous tissue (Martland and Humphries, I 929), tumors of the bony pelvis after irradiation (Fournier, 1935), laryngeal tumors as a consequence of roentgen treatment of Graves disease (Petrov, 1932) and the increased incidence of leukemia among the survivors at Hiroshima (Moloney, I955) contribute to the conclusion that ionizing radiations represent a carcinogenic hazard in man.

\section{(a) Local action of ionizing radiations}

Experimentally, local irradiation has resulted in the induction of tumors of the skin and subcutaneous tissue. A high percentage of mice in ected with ${ }^{91} \mathrm{Y}$ phosphate or ${ }^{239} \mathrm{Pu}$ developed fibrosarcomas (Lisco et al., I $947 \mathrm{~b}$ ). Feeding of ${ }^{91} \mathrm{Y}$ induces adenocarcinoma of the colon after a relatively long latent period (Lisco et al., I947a). As little as one microgram of radium deposited in the human skeleton may be carcinogenic (Brues, I954). Bone tumors have been induced by radioactive ele- 
ments which deposit in bone. Lung tumors have resulted experimentally (Lisco and Finkel, r 949) from tracheal intubation of radioelements.

Unlike ultraviolet irradiation of the skin (Blum, I950), where repeated stimulation is necessary for carcinogenesis, a single exposure to ionizing radiations is sufficient for tumor development. The latent period of tumor induction is usually longer than for chemical carcinogenesis.

That ionizing radiations may induce the development of chemical carcinogens

Fig. 36. Endocrine factors implicated in the induction of ovarian and adrenal cortical tumors by X-rays.

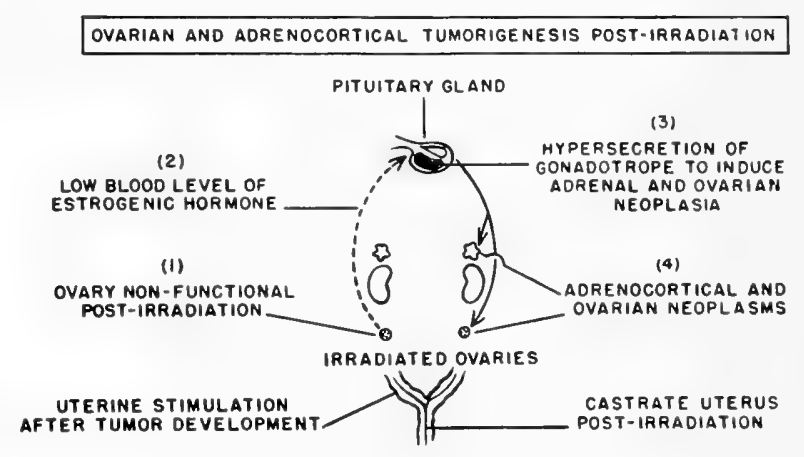

in the tissues is suggested by the work of Kaplan et al., (r954, r956a, b) and Carnes et al., (1956). The sequence of events in radiation-induced carcinogenesis invariably involves initial destruction or hypoplasia of irradiated tissue. In this regard the process is not unlike that involved in certain instances of chemically induced carcinogenesis (e.g., the induction of thymomas and leukemia by estrogenic hormone).

\section{(b) Somatic mutation and carcinogenesis}

The induction of germinal mutations (e.g., in Drosophila) by X-rays has led to speculation concerning somatic mutation and radiation-induced carcinogenesis (Brues, I 954). On the basis of histocompatibility studies, mouse mammary cancer cells have been considered genetically unlike homologous normal cells (Strong, I929). If carcinogenic agents are mutagenic, this property would seem, however, not to be specific for ionizing radiations.

\section{(c) Neoplasms following whole body irradiation}

After exposure of mice to total body X-irradiation, increased general tumor incidence has been observed. Lung tumors occur more frequently and earlier in irradiated, compared with genetically similar unirradiated mice (Lorenz, I950). Ovarian (Furth and Butterworth, I936) and hemopoietic neoplasms (Upton and Furth, I 956) are induced by whole body X-irradiation. That factors other than irracliation of the target tissue are important in influencing ovarian tumorigenesis and leukemogenesis is demonstrated as indicated below.

Although irradiation of ovaries alone or of a single ovary in unilaterally ovariectrmized mice results in tumor development, the presence of functional ovaries (Lick ct al., 1949), in situ or grafted, or the administration of estrogenic hormone 
(Gardner, 1950), inhibits tumor development in an irradiated ovary. It appears that irradiation creates an hormonal imbalance favorable to tumorigenesis in irradiated ovaries (Fig. 36). That testicular hormone does not inhibit irradiationinduced ovarian tumorigenesis has been demonstrated by grafting irradiated ovaries in intact male recipients (Kirschbaum et al., 1956), and by the administration of androgen post-irradiation (Gardner, I 950).

Partial body $X$-irradiation (lead-shielded portion of the body containing hemopoietic tissue) is ineffective in causing leukemia to appear in mice susceptible to the induction of leukemia by irradiation of the entire body (Kaplan, I95I). The sequence of events demonstrating that the protective agent resides in hemopoietic cells, or is the blood forming cell per se, follows. Thymectomy prevents the induction of leukemia in $\mathrm{C}_{57} \mathrm{BL}$ mice by $\mathrm{X}$-rays (Kaplan, I950a). Radiation of the thymus only or of the whole body except the thymus is not leukemogenic in either case (Kaplan, I949, I95I). Shielding of a thigh (containing hemopoietic marrow) during X-irradiation also prevents leukemogenesis (Kaplan, I95I). If the entire animal is irradiated, and bone marrow cells injected post-irradiation, protection is effected (Kaplan et al., I 953); protection against leukemogenesis can be correlated with thymic regeneration post-irradiation; in protected mice the thymus regenerates more rapidly (Kaplan et al., I 953). Protection against the leukemogenic effects of irradiation parallels protection against lethal effects, the same procedures being efficacious in both instances (Jacobson et al., I949; Lorenz et al., I95I).

Thus, irradiation of target tissue in itself may be non-leukemogenic. Furthermore, leukemogenic effects are obtained experimentally from irradiation of non-target tissue (Fig. 33, p. 839). Kaplan has demonstrated that grafted thymus undergoes neoplastic transformation if the recipient has been irradiated prior to transplantation of the normal thymus. Not all thymus is sensitive to these "secondary" leukemogenic effects of irradiation; the genetic constitution of the thymic cells determines their responsiveness. There is some indication that cells of the host which invade the thymic graft may become leukemic only in the thymic environment; otherwise they do not undergo leukemic transformation (Law, I952). The thymus involutes and then regenerates after grafting; this thymic regrowth may be important in determining susceptibility to neoplastic alteration.

\section{VIRUSES AND CANGER}

\section{(a) Chicken and rabbit tumors}

Although chicken leukemia was the first neoplasm transmitted by a filterable agent (Ellerman and Bang, Igo8), the passage of the Rous sarcoma (Rous, I9I I) by filtrates was more important to cancer biology. Prior to the discovery of a noncellular fibrosarcoma agent, which induces fibrosarcomas when inoculated into normal chickens, transmission of neoplasms to new hosts had been accomplished only by inoculation (transplantation) of viable cells into compatible hosts.

The first suggestion of the viral etiology of mammalian neoplasms came from the observations on the Shope papilloma (Shope, I933). The papilloma of the cottontail can be transmitted to wild rabbits of the same species by skin scarification and 
papilloma filtrates. It was thought at first that in the wild rabbit the lesion remains benign, but it has been demonstrated (Syverton et al., r950) that in some wild rabbits carcinomas develop from the initial benign lesion. Progression to a carcinoma occurs more readily in the domestic rabbit; from the metastatic cancer a virus can no longer be demonstrated (Friedewald and Kidd, I 939; Syverton et al., I950). Either the virus is "masked" in the carcinoma, or it has served only to initiate a precancerous lesion, the resultant carcinoma being virus-free. If the carcinoma is actually without virus, then the filterable agent cannot be considered necessary for the continuity of the neoplastic process.

The work on the Shope papilloma provides a basis for speculation concerning undiscovered tumor viruses. In the case of a neoplasm of known viral causation the carcinoma did not yield the virus. Perhaps in other neoplasms the apparent absence of a virus might then be explained on the basis of "masking", either by antibody, or by intimate combination with cellular constituents to the extent that separation by usual methods is not feasible.

\section{(b) Mouse mammary cancer}

That cancer viruses might easily escape detection is emphasized by the experiments which revealed the mouse mammary tumor agent (Bittner, I942). Mammary cancer of mice was thought to be influenced in its development primarily by hormonal factors. Early experiments indicated, however, that the incidence in $\mathrm{F}_{\mathbf{I}}$ hybrids between two high and low cancer strains ( $A$ and $C_{57}$ ) was very much higher when the mother was of the high rather than the low mammary cancer strain (Korteweg, I 934; Staff, Jackson Memorial Laboratory, I 933; Murray and Little, I936). Bittner (1936b) demonstrated by foster-nursing experiments that the "maternal influence" (tumor agent) is transmitted in the milk during suckling. High tumor strain A mice did not develop mammary cancer if foster-nursed immediately after birth by a low tumor $\mathrm{C}_{57}$ female. Ingestion of only relatively small amounts of milk from a high tumor (A) female made likely the development of mammary cancer.

Agent-free A mice give rise to progeny which are agent-free and consequently low-cancer. If the agent is reintroduced, either by foster-nursing or injection of tumor extracts, then the line again becomes "high tumor". The agent can be transferred to females by males of certain inbred mouse strains (Bittner, 1952).

Neither foster-nursed high tumor A mice, nor low cancer $\mathrm{C}_{57} \mathrm{BL}$ mice, develop mammary cancer if given estrogenic hormone. It appeared, then, that the hormonal factor, estrogen, acts only in conjunction with the mammary tumor agent or virus. The virus is the essential carcinogen, according to this concept, but only in hosts of appropriate and specific genetic constitution. The importance of the propagation of the virus has been demonstrated by Heston et al., (1956); passage through hosts of certain genetic constitution results in disappearance of the virus.

To recapitulate, it was postulated that hormonal, viral and genetic factors are interrelated in the genesis of spontaneous mammary carcinogenesis in mice of highly inbred strains, and that in the absence of any one the incidence of mammary cancer sinks to a negligible level. If the caloric intake is restricted, so that only two-thirds of the adult weight is attained, restricted mice do not breed, nor 
do they develop mammary cancer (Tannenbaum, I940). It was concluded that the altered hormonal status inhibits carcinogenesis (Huseby et al., 1945). Cystine deficient mice similarly fail to respond to otherwise adequate viral and genetic factors (White and Andervont, I 943), but exogenous estrogen may cause mammary carcinogenesis to proceed in one-half the mice fed inadequate amounts of cystine (White and White, I944).

That the mammary tumor agent is the etiological virus in the sense that infectious diseases are virus-caused is open to question on the basis of the following evidence. In one fosternursed high mammary tumor line $\left(\mathrm{C}_{3} \mathrm{Hf}\right)$ the incidence of mammary cancer in females is $38 \%$ (Heston et al., I950). The cancers appear significantly later in life than in genetically similar mice which possess the mammary tumor agent. $\mathrm{C}_{3} \mathrm{Hf}$ mammary cancers do not test positive for the agent; males of this line develop mammary cancer when given estrogenic hormone, and in such tumors no agent is demonstrable by the usual bioassay (Heston and Deringer, 1954). Similarly the agent has not been revealed in mammary tumors induced by methylcholanthrene in females of either pure lines made agent-free by foster-nursing (Bittner and Kirschbaum, I950) or of strains which do not possess the agent (Dmochowski and Orr, 1949; Figs. 37, 39-41). However, virus like particles have been observed by Bernhard and Oberling (1957) in electron micrographs of mouse mammary cancers which do not reveal the agent by biological methods.

Mühlbock (1956) has reared agent-free mice which were not only nursed by low tumor mothers, but which developed in their uteri (ovum transfer). One third of such agent-free females developed late mammary cancer. The incidence was increased to $100 \%$ if the animals carried functional pituitary isografts (from the same inbred strain). Such grafts cause hyperstimulation of the mammary gland.

With a favorable hormonal (functioning ovaries and pituitary isografts) and genetic status, favoring the development of mammary cancer ( $100 \%$ incidence), onset of the disease is accelerated by the introduction of the mammary tumor agent (Mühlbock, I956). The virus would, from these experiments, appear to be an accelerator of and not an essential etiological agent for mouse mammary cancer. It is possible, however, that the difference in the time of onset of mammary cancer is dependent upon amount of agent, and not presence or absence.

Multiple factors are involved in mouse mammary carcinogenesis. Under certain circumstances it appears that the agent may be deleted and still mammary cancer occurs (Heston et al., 1950). In other instances withdrawal of the agent results in an extremely low incidence of mammary cancer (Bittner, 1936b). The influence of the agent depends upon the potency of other factors, including a genetically determined environment favoring the propagation of the agent itself (Heston et al., 1956).

Apparently carcinogenic hydrocarbons may substitute for the mammary tumor agent in accelerating the onset of mammary cancer in agent-free mice (Kirschbaum et al., I946; Andervont and Dunn, I953). Such induced tumors usually show squamous metaplasia (Kirschbaum, I949). Since a mammary tumor agent could be extracted from neither these tumors, nor histologically similar tumors occurring in aged females of strains without the agent, it was suggested that this type of mammary histology might be correlated with agent-free tumorigenesis. Although agent-free tumors are characteristically variable microscopically, squamous metaplasia is not an essential structural feature (Heston et al., i950;

Literature p. 870 

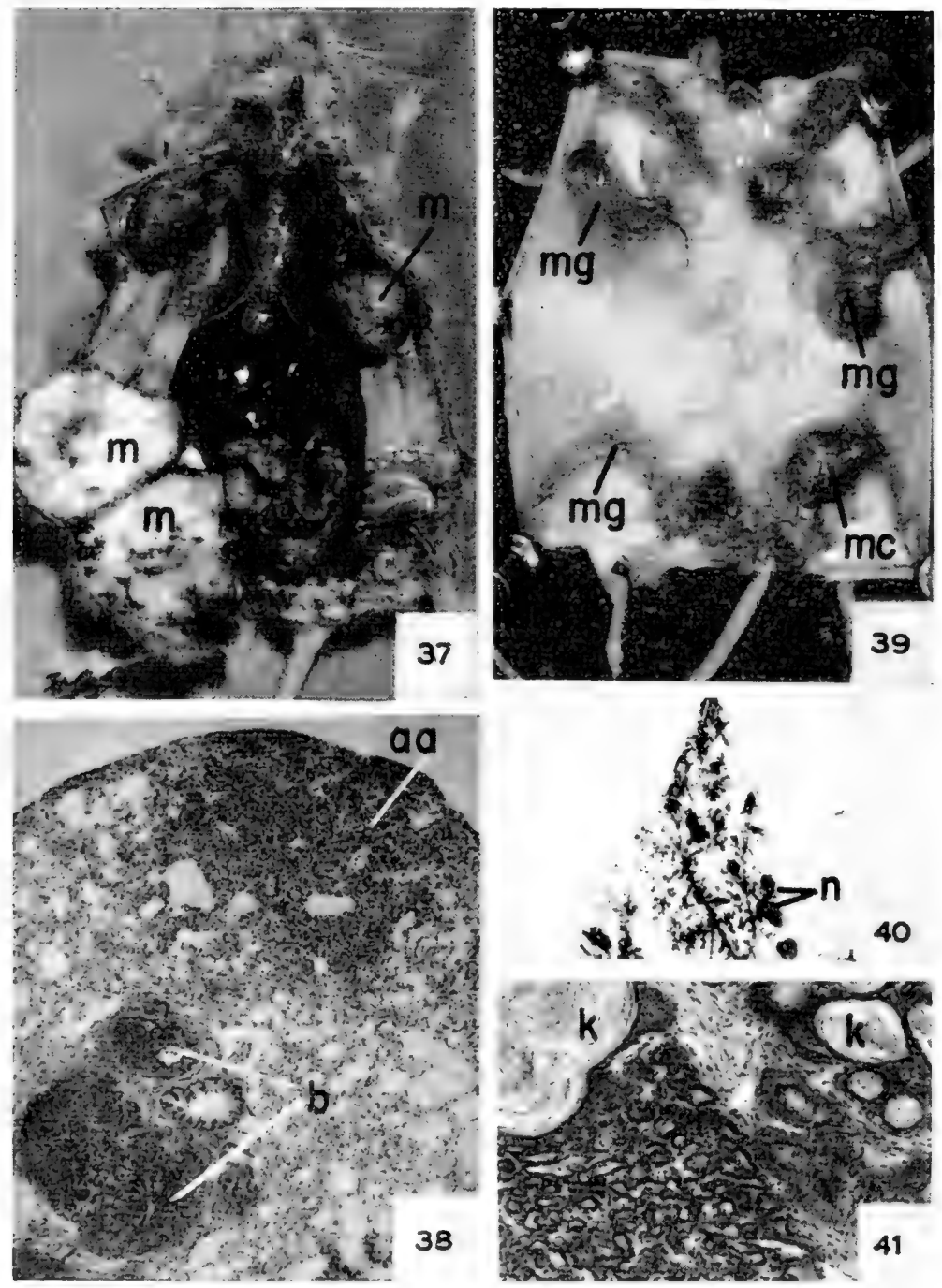

Fig. 37. Mammary cancers ( $\mathrm{m}$ ) occurring following the feeding of methylcholanthrene to a mouse which did not possess the mammary tumor agent. The agent has not been demonstrated in such tumors by bioassay.

Fig. 38. Mouse lung with a primary lung tumor (alveolar adenoma "as"). At (b) are metastases of a mammary cancer within blood vessels. Pulmonary alveolar adenomas of mice may be induced by carcinogenic hydrocarbons, urethane or nitrogen mustard, response being determined by genetic constitution. $\times 90$

Fig. 39. Whole mount of the skin with mammary glands $(\mathrm{mg})$ attached, to demonstrate the development of multiple mammary cancers $(\mathrm{mc})$ in a mouse fed methylcholanthrene. This animal did not have demonstrable mammary tumor agent.

Fig. 40. Portion of a stained dissected mammary gland to demonstrate hyperplastic nodules n) on the mammary tree of a high cancer strain female. These nodules are pre-cancerous lesions. Multiple nodules occur on the mammary tree of mice fed methylcholanthrene, but these nodules differ histologically from those occurring spontaneously. $\times 2$ 
Mühlbock, I956). In Mühlbock's studies the agent-free tumors occurring in mice bearing pituitary grafts were histologically similar to those associated with the agent.

Since agent-induced chicken tumors may pass through a non-filterable (masked) phase (Bryan et al., I955), it has been suggested that negative results do not constitute proof that a filterable agent is not involved (Beard et al., I 955). However, since a simple method provides evidence for the mammary tumor agent without difficulty, and since a "non-filterable" phase for the mammary tumor agent has not been demonstrated, it is reasonable to assume tentatively that these "agent-free" tumors lack an inciting agent which is present in other mammary neoplasms of the mouse.

\section{(c) Mouse leukemia}

Mouse leukemia occurs spontaneously in high leukemia strains (MacDowell and Richter, I935) and can be induced in certain "low-leukemia" stocks by the administration of either X-rays (Furth and Furth, I936, Kaplan, I947), estrogens (Gardner et al., I940) or carcinogenic hydrocarbons (Mider and Morton, I939) (Figs. 42-45). Gross (195I) reported the induction of leukemia in low-leukemia stocks by the injection into the newborns of extracts derived either from leukemic tissues or embryos of high leukemia strains. Age at time of inoculation, as well as genetic constitution is important in determining susceptibility to viral induction of leukemia.

The relationship of X-ray, estrogen and carcinogen-induced leukemogenesis to viral induction has not been elucidated. Although the $\mathrm{C}_{3} \mathrm{H}$ strain, which Gross has used primarily as a "low-leukemia strain" for induction, is considered to be virus-free, it is susceptible to the induction of leukemia by either X-rays (Kirschbaum and Mixer, I947; Gross, I957) or estrogenic hormone (Gardner et al., I940). Although the specific line of $\mathrm{C}_{3} \mathrm{H}$ mice may be important in considering leukemogenesis, it has been found that if $\mathrm{C}_{3} \mathrm{H}$ mice of the Bittner subline (used by Gross) are observed carefully throughout life, leukemia (and other lymphocytic neoplastic disease) occurs spontaneously after I 5 months of age (Ida et al., I957), when many investigators (Furth et al., 1956) terminate their experiments.

Is the "virus" of mouse leukemia an accelerating factor, operating in the same fashion as the mammary tumor agent? It appears that leukemia appears earlier when mice are inoculated with extracts of leukemic tissue at birth. The need for inoculating early in life has raised the question concerning the similarity of the leukemia agent to the "transforming principle" of Lederberg (1956). In a majority of cases, leukemia occurring in mice injected as newborns is genetically of the same type as the recipient (Gross, 1950; Furth et al., 1956; Ida et al., 1957). However, in some cases the genetic type is that of the donor whose leukemic tissue was extracted (Furth et al., I956), suggesting that a factor extracted from the leukemic tissue genetically altered cells of the inoculated newborn which not only developed leukemia, but acquired histocompatibility genic determinants of the donor.

Fig. 41. Section of mouse mammary cancer induced by methylcholanthrene. Centrally in this photo the histologic type is adenocarcinoma. In the upper left, and to the right, keratinized squamous epithelium $(\mathrm{k})$ is present. This type of epithelium is characteristic of portions of the carcinogen-induced mammary neoplasm. $\times 75$ 
The recent reports of Gross (1957) and Stewart et al., (1957) indicate that the activity of the leukemia and parotid tumor agents can be enhanced by frequent transfer in mice or in culture in vitro. Schwartz et al. (1957) have reported on the
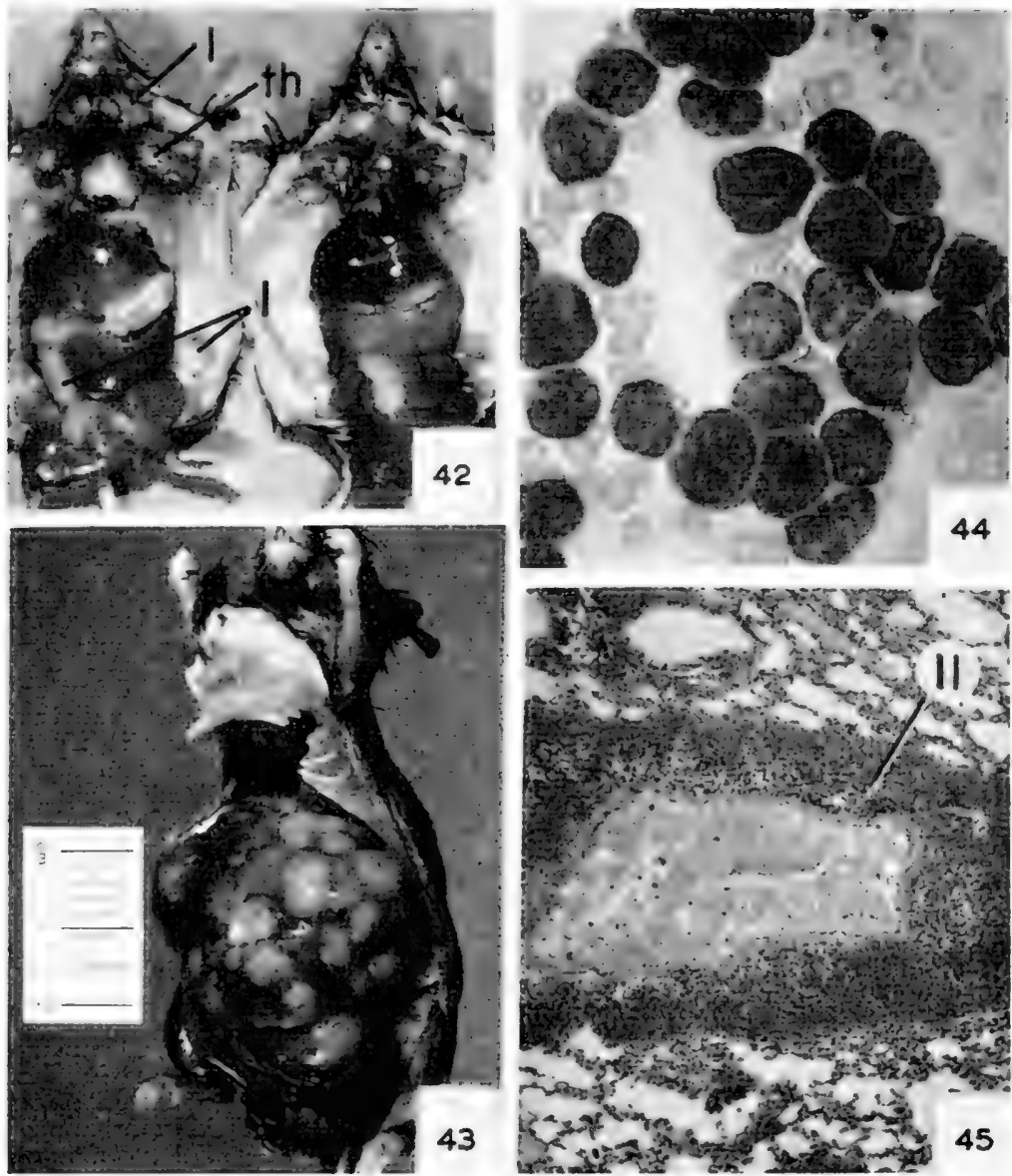

Fig. 42. Leukemic mice. Cervical, inguinal, axillary and mesenteric lymp nodes (I) and spleen are enlarged in both specimens. In the one on the left, the thymus (th) is very large also.

Fig. 43. Lymphomatous masses in the liver of a relatively old $\mathrm{C}_{3} \mathrm{H}$ mouse of a low-leukemia strain. Lymphomatous disease relatively infrequently appears spontaneously in these mice. If newborns are injected with leukemic extracts then leukemia appears early in life, and the incidence is probably higher than occurs spontaneously.

Fig. 44. Smear of peripheral blood of a mouse with lymphocytic leukemia. All cells are lymphoid. If these cells are inoculated into a genetically related animal they survive and proliferate giving rise to leukemia. $\times 250$

Fig. 45. Section of lung of leukemic mouse. The large blood vessel in the center of the field is surrounded by a thick collar of leukemic lymphocytes (I I ) $\times 70$ 
acceleration of onset of leukemia in AKR mice by the inoculation of cell-free filtrates of brain tissue extracts derived from leukemic mouse and human brains.

Maternal resistance factor. MacDowell and Taylor (1948) reported the presence of a "maternal resistance factor" in StoLi mice. If high leukemia $\mathrm{C}_{5} 8$ mice were foster-nursed by StoLi females the appearance of leukemia was delayed. Law (I954) confirmed MacDowell's work on the maternal resistance factor.

In reciprocal $\mathrm{F}_{\mathrm{I}}$ hybrids between the high leukemia $\mathrm{C}_{5} 8$ and several lowleukemia strains, leukemia appears later if the mothers are of the low rather than the high-leukemia strain. The incidence of leukemia is lower and the age of onset later in the offspring of older (as compared with younger less than 32 weeks) mothers (MacDowell, I955; Liebelt, A.G., 1957). Such differences in leukemia incidence are demonstrated best in females (Liebelt, A.G., 1957); androgenic hormone may delay leukemogenesis to such a degree that the influence of maternal age is not detected in males. In all FI hybrid mice between high- and lowleukemia strains, leukemia appears very much later than in the high-leukemia stock. Life expectance of $\mathrm{F}_{\mathrm{I}}$ hybrids is comparably greater.

Is the "maternal resistance factor" of MacDowell actually indicative of a low level of leukemia-inciting agent in female mice? With transfer of less agent it might appear that a positive resistance factor has been transmitted. Foster-nursing experiments have in the past employed low-leukemia nurses for high-leukemia newborn mice (Kirschbaum and Strong, I 942). High-leukemia nurses might provide "agent" to accelerate the development and increase the incidence of leukemia in low-leukemia strains; the investigations of Schwartz et al. (1957) are of interest in this regard.

\section{(d) Parotid and skin tumors of the mouse}

Neoplasms (carcinomas) of the parotid gland have appeared in $\mathrm{C}_{3} \mathrm{H}$ and AKR mice injected with leukemia extracts (Gross, I955, Stewart, I955). Such tumors have occurred spontaneously in one line of $\mathrm{C}_{3} \mathrm{H}$ mice (Law et al., 1955), and in $\mathrm{C}_{5} 8$ mice injected with cortisone (Woolley, I 954). But they do not occur in certain inbred stocks (Dulaney, 1956) unless the newborn is injected with mouse tissue extracts. In the experiments of Gross (1955), only infrequently did mice develop both parotid tumors and leukemia. It is his conclusion that a different virus is involved in each of these diseases.

Fibrosarcomas have devcloped at the site of inoculation of mouse tissue extracts (Gross, I955). It was assumed that these do not occur spontaneously in $\mathrm{C}_{3} \mathrm{H}$ mice, the recipient strain. Dunn et al. (I956) have reported the occurrence of similar tumors in aged $\mathrm{C}_{3} \mathrm{H}$ mice.

\section{(e) Nature of the tumor viruses}

The accumulated evidence suggests that the mouse tumor viruses may accelerate the onset of diseases which occur spontaneously (leukemia, mammary cancer, parotid carcinoma, fibrosarcoma of skin). Filterable factors derived from these neoplasms have caused the early appearance of these neoplasms in hosts which develop them later in life "spontaneously". In the case of leukemia the agent must 

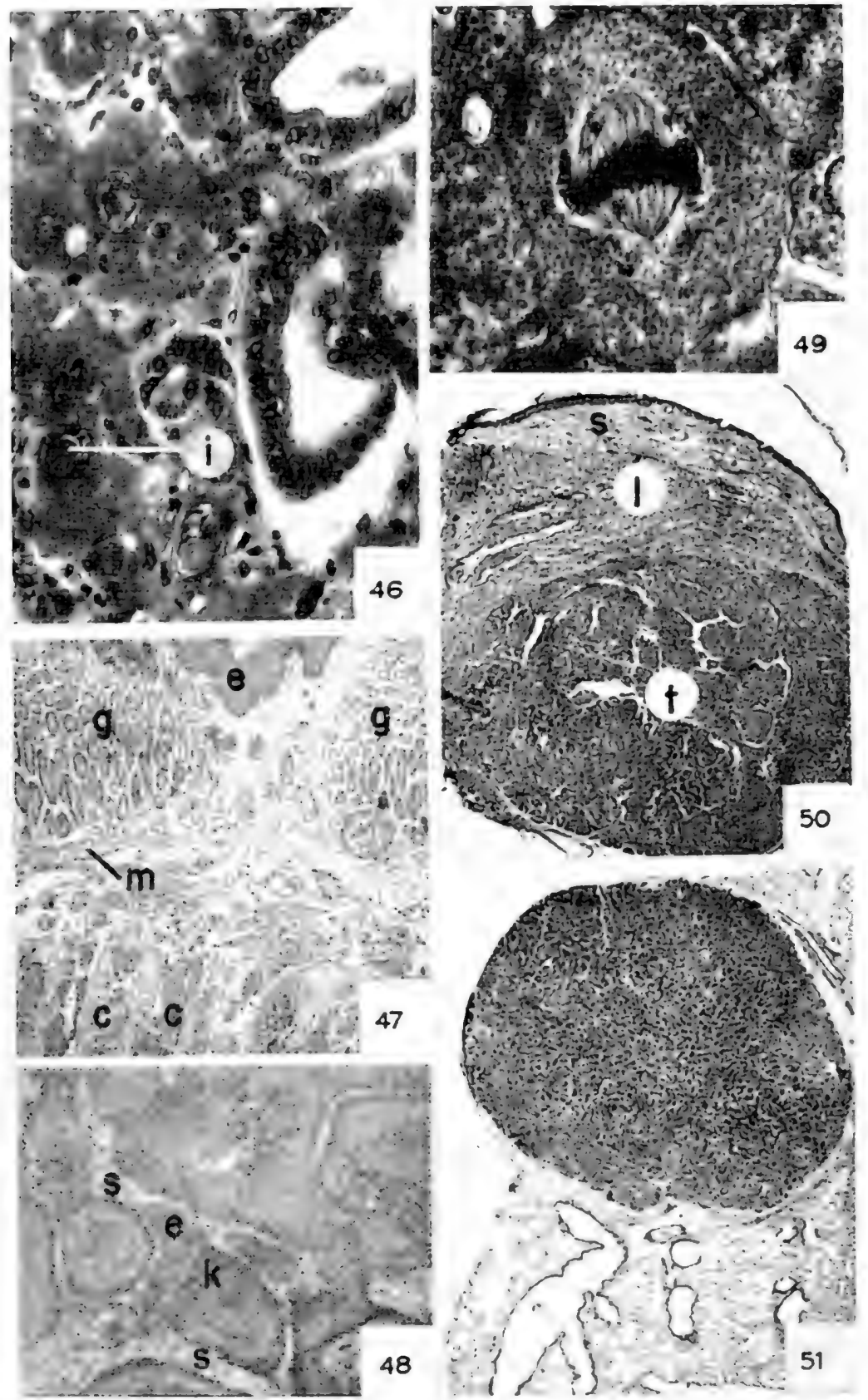

Fig. 46. Section of an hepatic neoplasm from a mouse treated with radiogold. The colloidal suspension of gold was administered intraperitoneally. The abnormal hepatic cells contain nuclei with eosinophilic inclusion bodies $(i) . \times 45$

Fig, $4 \bar{\gamma}$. Section of methylcholanthrene-induced carcinoma of the mouse forestomach. 
be introduced soon after birth, whereas for mammary cancer this is not so important, although relatively young mice are more susceptible than older animals.

No evidence has been obtained to demonstrate an immunologic basis for the resistance of older animals to mouse tumor agents (e.g. mammary tumor). Heterologous hosts develop antibodies which inactivate the Bittner agent (Andervont and Bryan, 1944), but antigenicity varies with genetic type of mouse host serving as the source of agent (Bittner and Imagawa, I 955), suggesting that a tissue antigen is associated with the agent. Similar observations have been made with chicken tumors (Beard et al., I955). Antisera have failed to inhibit mammary cancer development in high tumor strains which obtain the milk agent by nursing (Bittner, I948).

In the case of the chicken tumors, inactivating antibodies are generated by the host (Duran-Reynals, I940). That these are specific, however, is open to question, since they occur spontaneously in animals whose exposure to the agent is unlikely (Duran-Reynals, I953). Tissue antigens are separated from viral antigens with difficulty, if at all conclusively. There is cross immunization against the different chicken tumor viruses (Burmester and Belding, I947).

Duran-Reynals (I953) believed that the chicken tumor agents are analagous to the viruses responsible for the common infectious diseases. Newly-hatched chicks develop a hemorrhagic disease and old chickens neither hemorrhagic disease nor neoplasm when the virus is inoculated. The serum of chicks, in contrast to serum of old chickens, does not inactivate the virus of Rous sarcoma. The development of a neoplasm according to Duran-Reynals, is correlated with an intermediate immunologic status.

The immune bodies in the serum might be evidence of a latent infection, which may manifest itself by the appearance of a neoplasm. When chickens of flocks in which leukosis appears spontaneously have been isolated during hatching and rearing, they have remained free of the disease (Burmester, I957); those which develop leukosis may have obtained the infection by way of the egg. Tracheal and nasal washings of infected birds may transmit lymphomatosis. Birds reared in isolation do not have antibodies against the Rous virus, but possess them after immunization with lymphomatosis.

Cancerous cells (c) have invaded beneath the muscularis mucosae. (m). The squamous epithelium is designated by " $\mathrm{e}$ ", the normal glandular tissue by "g". $\times 45$

Fig. 48. Squamous cell carcinoma of the mouse skin. This tumor was induced by methylcholanthrene. Stroma is designated by "s", the keratinized epithelium by " $\mathrm{e}$ " and " $\mathrm{k}$ ". $\times 45$ Fig. 49. Mitotic figure in a hepatoma cell. $\times 600$

Fig. 50. Pulmonary tumor induced in lung grafted to the ear. "I" designates normal lung, " $t$ " the pulmonary tumor which is similar to that seen in Fig. 54, and "s" the skin of the ear within which the lung tissue graft had been made. Since pulmonary tissue from parent strains either susceptible or resistant to the induction of such tumors can be grafted into $F_{\text {I }}$ hybrids, which are susceptible, it is possible to test intrinsic susceptibility of the lung from "susceptible" and "resistant" strains within the same FI hybrid host to the action of the tumor-inducing agent, in this case urethane. $\times 40$

Fig. 51. Pulmonary tumor induced by the administration of urethane. Such tumors are of alveolar origin. $\times 40$ 
In chickens the "masked virus" is capable of inducing the hemorrhagic disease in newly hatched chicks (inoculation of tumor cells). Furthermore, chickens carring non-filterable tumors possess immune bodies against Rous sarcoma. The presence of antibodies against sarcoma or leukosis virus in chickens bearing chemically induced tumors has been used as evidence that these neoplasms possess an antigenic "masked" virus. However, the natural occurrence of such antibodies makes this a tenuous hypothesis.

Of interest is the adaptation of viruses from one species to another so that a chicken virus becomes "duck" or "turkey" virus, again adaptable to the chicken by inoculation of newly hatched birds (Duran-Reynals, I942, 1943).

Quoting from Duran-Reynals (I953), an extremist concerning viral etiology, "the viruses of chicken tumors and leukoses have an epidemiologic behavior, they exert both destructive and proliferative effects on cells, they are capable of varying or mutating, and they have the power to induce immune reactions conducive to solid resistance. These viruses, therefore, behave fundamentally like ordinary viruses. On the other hand, viruses of lesser virulence seem to be the cause of sarcomas developing after injection of chemical carcinogens. These viruses are generally masked and they have antigens in common with those causing the naturally occurring tumors or leukoses. Practically nothing is known concerning epithelial neoplasia, but the scant data available are not incompatible with a virus effect".

IV. CARCINOGENIC GHEMICALS

Chemical agents induce cancer in man and animals (Figs. 46-59). That coal tar may be tumor-inducing was a clinical observation which led ultimately to the extraction of carcinogenic-(polycyclic) hydrocarbons (Hieger, I930; Cook et al., I933); many more were subsequently synthesized to determine chemical configurations associated with carcinogenic potency. The phenanthrene structure of both the carcinogenic hydrocarbons and sex hormones, and the chemical similarity of bile acids and cancer-inducing hydrocarbons suggested that similar chemicals formed endogenously in man may be carcinogenic; the search for biologically synthesized carcinogenic hydrocarbons has not been successful up to the present.

\section{(a) Polycyclic hydrocarbons}

The polycyclic hydrocarbons are "complete" and "independent" carcinogens, since they may both "initiate" and "promote" the induction of neoplasms. The initiating action of the hydrocarbons may be completed by an agent, e.g. croton cil, which is not itself an initiator of carcinogenesis. According to Berenblum and Shubik (1949) the actual tumor yield is determined by the initiating agent, the latent period of carcinogenesis by the "promoting" agent, which may not itself be an initiator, but only a "cocarcinogen" (e.g., croton oil). The initiating process i- apparently irreversible, which suggests that the effect of the carcinogen may be mutagenic.

The carcinogenic hydrocarbons induce neoplasms of diverse tissues (Figs. 47, 48, $52,53,55,57,59)$, and "direct" contact of the tissue with the intact chemical seems 

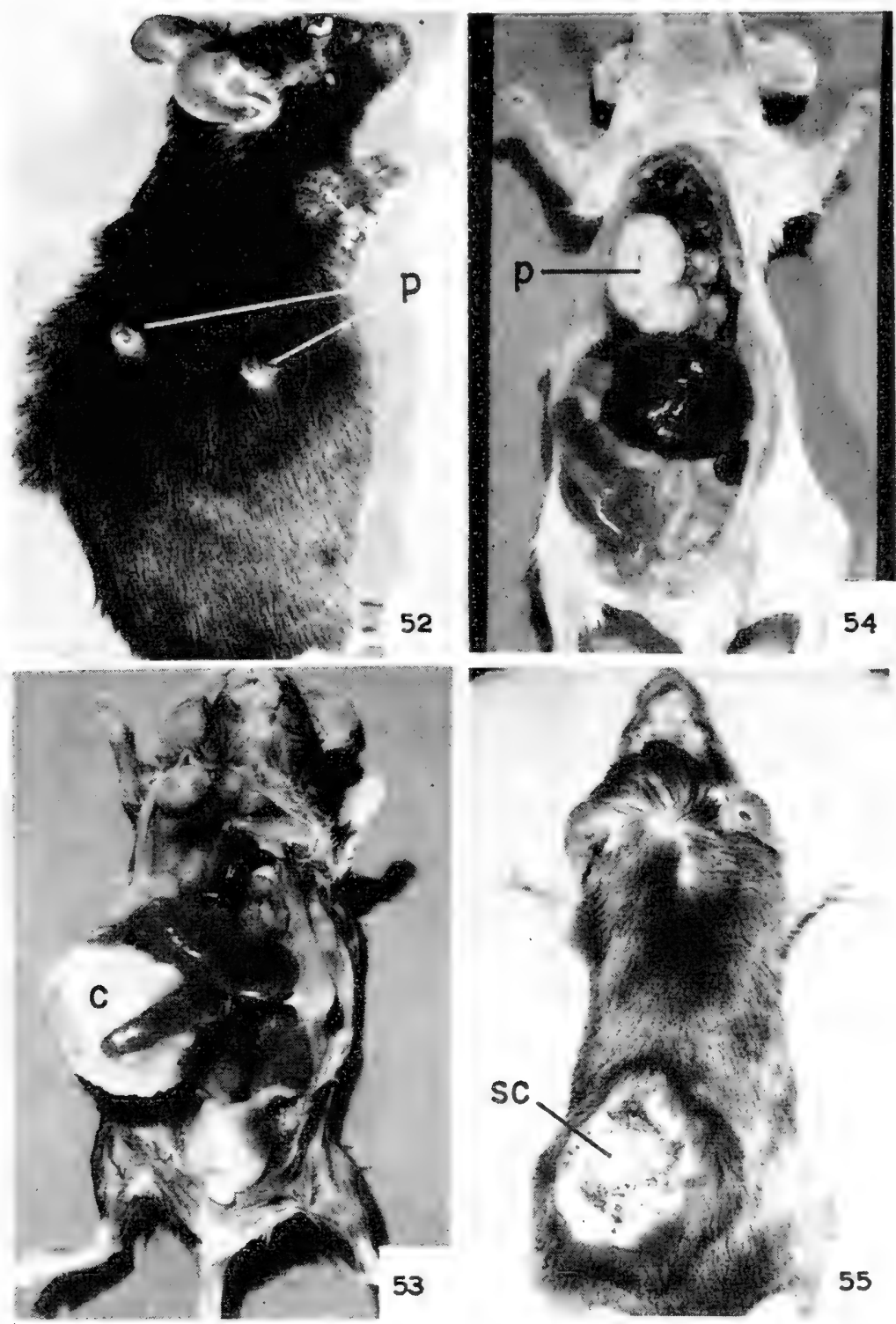

Fig. 52. Multiple skin papillomas (p) on a mouse painted with methylcholanthrene dissolved in benzene.

Fig. 53. Carcinoma of the fore-stomach $(\mathrm{c})$ of a $\mathrm{C}_{3} \mathrm{H}$ mouse fed methylcholanthrene (see Fig. 52 for microscopic section).

Fig. 54. Large pulmonary tumor $(p)$ in a mouse injected with urethane. Similar tumors occur spontaneously and are induced by carcinogenic hydrocarbons.

Fig. 55. Skin cancer (sc) in a mouse painted with methylcholanthrene (see Fig. 53). 

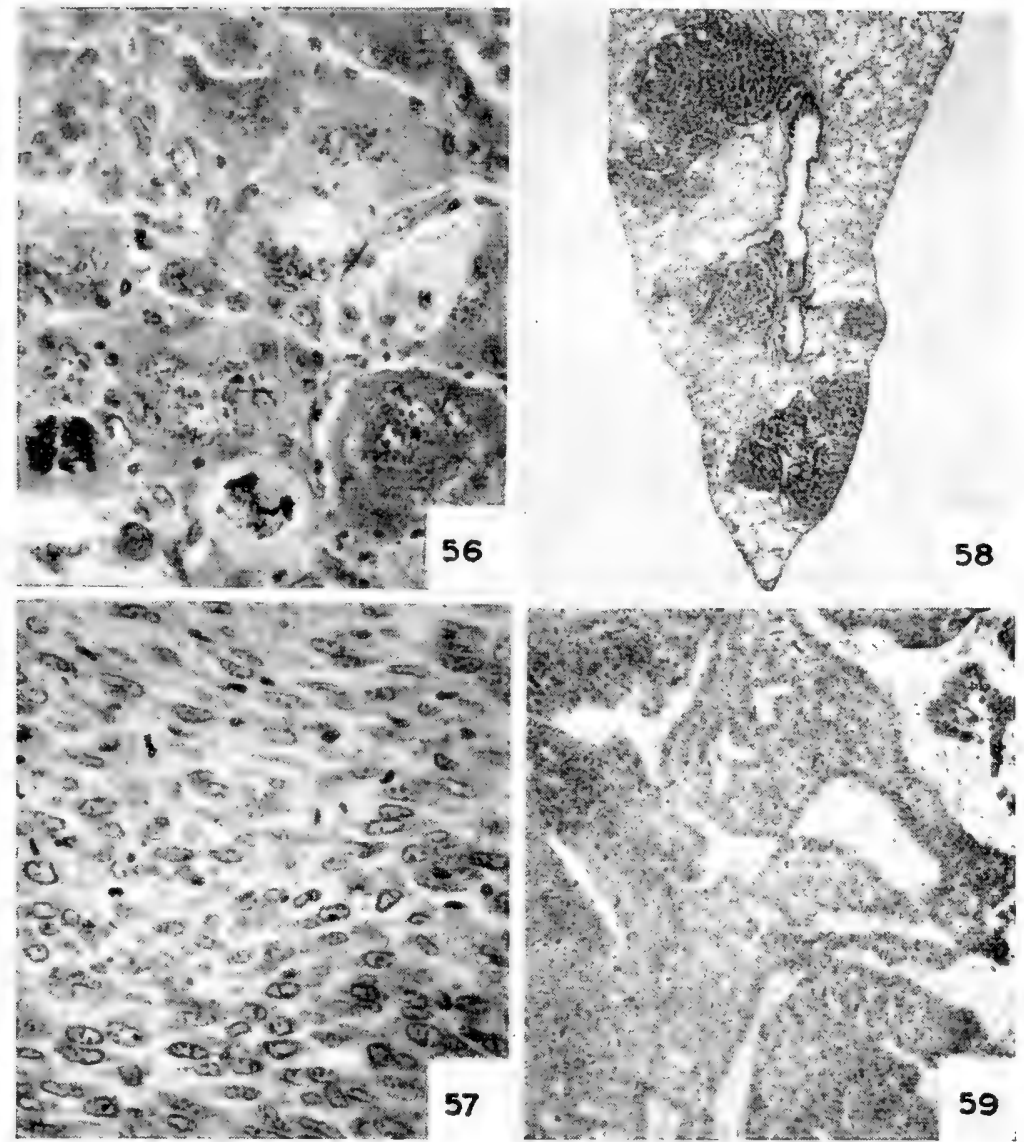

Fig. 56. Section of rhabdomyosarcoma induced by administration of peanut oil subcutaneously. Presumably this oil which is used as a vehicle for carcinogenic agents is not tumorinciting. However, since a certain number of neoplasms are induced by "non-carcinogenic" agent, either carcinogenic action is non-specific, or various materials contain carcinogens. 250

Fig. 57. Section of fibrosarcoma induced by the injection of methylcholanthrene in peanut oil into a mouse. Numerous mitotic figures present. $\times 200$

Fig. 58. Multiple pulmonary tumors in section of lung of a mouse fed methylcholanthrene. $\therefore 30$

Fig. 59. Section of epithelial neoplasm induced in the skin of a mouse by painting with methylcholanthrene. The arrangement of neoplastic cells simulates that seen in human basal cell carcinoma. $\times 45$ 
to be involved. The mechanism of carcinogenesis following entrance of the carcinogen into the cell is still unknown. There is evidence that there is "binding" of the carcinogen by cellular protein. (Wiest and Heidelberger, 1953a, b, c), similar to the "binding" of butter yellow by liver cells during hepatic carcinogenesis (Miller and Miller, I947). These findings have been interpreted as being of probable significance in elucidating the "enzyme deletion" hypothesis of Potter (I 944).

Crabtree (1946) has demonstrated that "anticarcinogens" (chemicals which inhibit carcinogenesis induced by polycyclic hydrocarbons) react with - $\mathrm{SH}$ groups which are present in cellular constituents (e.g. sulphur containing amino acids). These agents (anticarcinogens) which block carcinogenesis are excreted as mercapturic acids and deplete the sulphur content of the target tissue. The inhibitors probably combine preferentially with - $\mathrm{SH}$ groups. Although they probably react with - $\mathrm{SH}$ groups, the carcinogens are not excreted as mercapturates.

Species factors, as well as genetic factors within the species, are important in determining the carcinogenic action of the polycyclic hydrocarbons (Wolf, I952). In the case of mouse pulmonary (Shimkin, I955) and mammary tumors (Kirschbaum and Liebelt, unpublished) there is positive correlation between susceptibility to the spontaneous disease and induction by polycyclic carcinogenic hydrocarbons. This suggests that the cancer-inducing agents may augment a "spontaneous" process and that the same genes responsible for susceptibility to the spontaneous neoplasm also control the response to these tumor-inciting chemicals. Heston ( 195 I) believes that of the multiple factors involved in the genesis of mouse neoplasms, some may be deleted with carcinogenesis still occurring if others are sufficiently potent.

For mouse leukemia, however, high susceptibility to one leukemogenic agent (e.g., X-rays or estrogenic hormone in CBA mice) does not imply a similar degree of susceptibility to the hydrocarbons (Kirschbaum and Mixer, I947). The carcinogenic hydrocarbons may, however, be highly leukemogenic for other strains of mice (e.g., the Dba/2) (Mider and Morton, 1939). The $\mathrm{C}_{57} \mathrm{BL}$ strain is extremely susceptible to the leukemia (thymoma)-inciting action of X-rays (Kaplan, I947), but resistant to the induction of leukemia by either estrogenic hormone or methylcholanthrene (Kirschbaum and Mixer, I947).

Studies of the metabolites of the polycyclic hydrocarbons have yielded little information which might elucidate the mechanism of carcinogenesis. It has been suggested (Greenstein, I954) that new experimental biochemical approaches involving the interaction of carcinogens with tissue components might be of great value.

\section{(b) Azo dyes}

The azo dyes induce liver tumors although administered at extrahepatic sites. For example, oral ingestion of 4-dimethylaminoazobenzene with the appropriate diet may be carcinogenic for the liver (Kinosita, I940). Related compounds (e.g. o-aminoazotoluene) have also been demonstrated to be hepatic carcinogens (Andervont and Dunn, I947). The riboflavin content of the liver is an important 
factor in influencing the development of hepatic tumors; administration of riboflarin inhibits the tumor-inciting action of butter yellow and a riboflavin deficient diet favors the induction of liver tumors in rats by this compound (Kensler et al., I 940, I 941). Binding of the azo dye by liver proteins is inhibited by riboflavin and dre-binding can be correlated with hepatic tumor formation (Miller and Miller, I 947).

The studies of Miller and Miller (1947, I952) indicate that the azo dyes, which are hepatic carcinogens, are "bound" by nucleoprotein and soluble cytoplasmic proteins of the susceptible liver, but not by nucleic acids. Administration of methylcholanthrene (Richardson and Cunningham, I95I) or nitrogen mustard (Griffin et al., r95 Ib) simultaneously with the hepatic carcinogen reduces the number of induced tumors. Protein binding of the hepatic carcinogen is inhibited by methylcholanthrene.

According to the enzyme deletion hypothesis, certain enzymes are important in limiting the growth of normal cells, and if these are lost due to the action of a carcinogenic agent, then an irreversible change has occurred, the loss of growth restriction resulting in what is termed neoplastic growth (Potter, r944). Since this metabolic deviation is heritable, passed on to the progeny of altered cells, the alteration would presumably be genic with cytoplasmic enzymatic control lost as a result. The pattern for deviation of this type has been demonstrated in Neurospora by the classical investigations of Beadle and Tatum ( I945).

Hepatic nucleic acid is not bound by azo dyes (Miller and Miller, I952). If the nuclear genes which are rich in deoxyribonucleic acid do not react with the chemical carcinogen, then the reproducible alteration (resulting in neoplasia) must go via cytoplasmic genic material. Such "plasmagenes" have been postulated by Spiegelman ( 1946 ).

\section{(c) Amino compounds}

Certain amino compounds (e.g., acetylaminofluorene) are of particular interest because of the diversity of their carcinogenic effects (e.g., induction of hepatic, mammary, urinary bladder, thyroid and ear-duct tumors). As in the case of azo dyes, the carcinogen is bound by protein of the liver for which it is carcinogenic (Weisburger et al., I953). Following the ingestion of acetylaminofluorene, the compound is apparently metabolized so that a breakdown product (or products) is tumor-provoking. Local application of acetylaminofluorene yields no neoplasm (Bielschowsky, I944a). In the case of 2-naphthylamine, which induces urinary bladder tumors, a carcinogenic metabolite, 2-amino-I-naphthol has been identified (Bonser et al., I95I) in the urine.

Haddow (I 953) has suggested that certain of the amino compounds (e.g., alkylating agents such as nitrogen mustard) affect genic components of the cell. These agents are both radiomimetic and mutagenic, and have also been demonstrated to be carcinogenic (Heston, I 949; Griffin et al., I 950; Boyland and Horning, I949). Haddow, proposes that the primary step in carcinogenesis induced by these agents is the "inhibition of certain fundamental processes of genetic synthesis", followed by the "generation of a new self-duplicating fiber or template chemically, and hence genetically, modified". This first step may be the primary 
and irreversible change referred to by Berenblum and Shubik for the polycyclic hydrocarbons (I 949). Gross chromosomal abnormalities result from the administration of these agents, but the carcinogenic alterations are considered to be more subtle, probably occurring during the "resting" stage of mitosis (Haddow).

Mutagenic agents are not necessarily carcinogenic, and in Drosophila, although methylcholanthrene appears not to be mutagenic, a melanotic atypical growth occurs more frequently following methylcholanthrene treatment (Burdette, I 955). Strong ( $1945 \mathrm{a}, \mathrm{b}$ ) has described germinal mutations in mice succeeding the injection of methylcholanthrene and thinks that such mutations are correlated with somatic (mutagenic) effects which result in cancer formation.

\section{(d) Halogenated hydrocarbons}

That chemically unrelated compounds (and physical or hormonal agents, e.g. in leukemia) may induce neoplasms of the same target tissue, is one of the perplexing problems of carcinogenesis. The halogenated aliphatic hydrocarbons which damage the liver, and which are chemically unrelated to the azo dyes, induce hepatic tumors (Edwards, I94I, Eschenbrenner, 1945). Apparently hepatic necrosis is not essential for tumorigenesis, since non-necrotizing doses of carbon tetrachloride may induce hepatic tumors. Thus, the sequence of histologic injury followed by regeneration seems not to be necessarily causally related (Eschenbrenner, I 945 ).

\section{(e) Urethane}

Urethane has a simple structure and until recently had been thought to be carcinogenic specifically for the lungs (Nettleship and Henshaw, I 943). However, there is evidence that urethane can contribute to experimental skin carcinogenesis (Salaman and Roe, 1953) and to leukemogenesis (Kirschbaum and Kawamoto, 1957). The phases of carcinogenesis may be divided into initiation, promotion and growth in light of the work of Berenblum and others (1949). Agents which complete carcinogenesis (e.g. croton oil), after initiation by a carcinogen (e.g. methylcholanthrene), may be incapable of initiating the process. Apparently urethane may initiate skin carcinogenesis, the supplementary action of a cocarcinogen (e.g. croton oil) being necessary to complete tumor development. Although urethane per se may not be leukemogenic for $\mathrm{C}_{57} \mathrm{BL}$ mice, it potentiates the leukemogenic action of X-rays or estrogenic hormone, being in this case a cocarcinogen (coleukemogen).

Recently Burnett (1956) has suggested that the carcinogenic effect on normal cells is an alteration in antigenicity, perhaps by deletion of "self-markers" so that the controlling influences or "recognition units" are no longer available. The inhibitory effects on tumor growth of chemicals simulating carcinogenic structure could result from such alterations of cellular proteins so that new antigens are formed, which stimulate the formation of antibodies; thus the control of growth would be by host response. Successful immunization against early transplants of carcinogen-induced tumors within the strain of origin (Foley, r953) suggests that the induced tumors contain antigens against which the host may react positively from the standpoint of growth inhibition. These results seem to be inconsistent with 

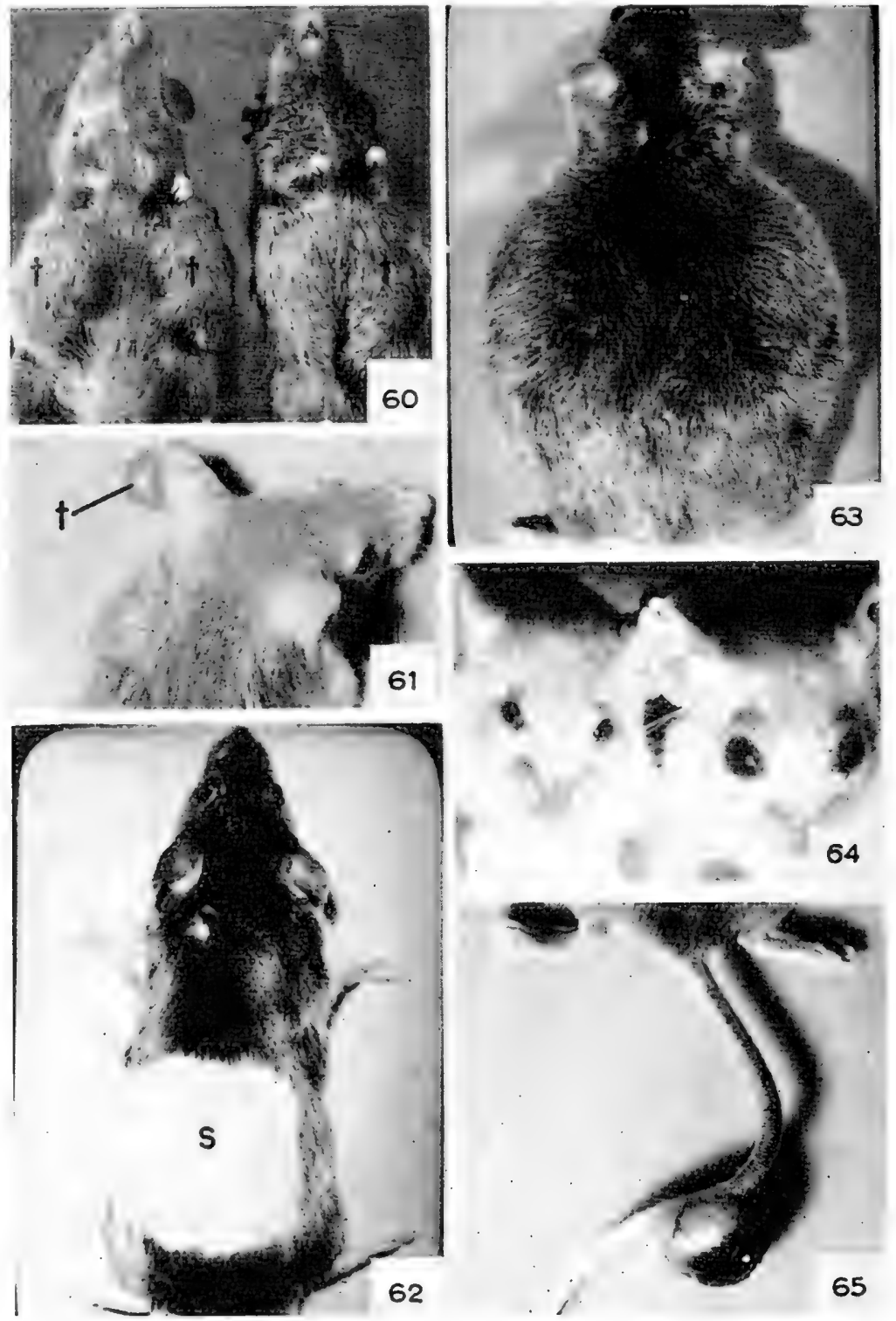

64

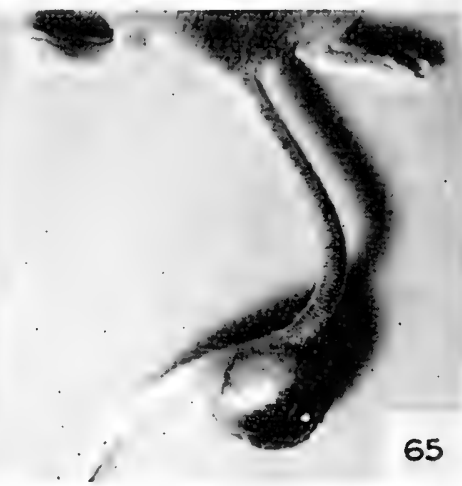

Fig. 6o. Subcutaneous transplants of a mouse lymphosarcoma. The animal on the left has bi-lateral transplants ( $t$ ) which were made simultaneously. The mouse on the right also received two transplants, but the second was made ten days after the first. This mouse developed an immune reaction as evidenced by the resistance of the second transplant made into the right side. Immune reactions of this type have been demonstrated against transplanted but not spontaneous mouse neoplasms.

Fig. 6I. Transplant of thyroid tissue ( $\mathrm{t}$ ) into the ear of a mouse. This tissue was hyperplastic thyroid from a mouse fed thiouracil. Such transplants grew only in hypothyroid histocom- 
Burnett's suggestion that altered antigenicity, which decreases immunologic ability of the host to control tissue growth, is a factor in carcinogenesis.

Not only do diverse agents produce neoplasms of one tissue, but certain agents are more or less tissue specific in their carcinogenic action. For example, butter yellow is carcinogenic for only the liver, and then only when the experimental animals are placed on a B-vitamin deficient diet. By its "independent" and unaided action, urethane induces tumors only of the lung. The basis for such specificity is presently unknown.

An analogy was attempted between the development of malignancy (Wolf, 1952) and Lederberg's (1946) findings on adaptative mutation in Neurospora. A mutant strain of this organism may lose the ability to synthesize an amino acid (e.g. leucine) essential for its metabolism. This strain can then survive only if the nutrient, capacity for the biosynthesis of which has been lost, is supplied in the medium. When the nutrient environment becomes unfavorable a new mutation occurs, with a regaining of the ability to synthesize the essential amino acid. The cancer cell is analogous to the Neurospora which, as a result of mutation, is less dependent on its environment, by virtue of mutation having acquired the enzyme systems essential for independence. Haddow (I947) thus "correlates the new and adaptive growth properties of the cancer cell, especially its power of continued and unregulated growth, with the acquisition of a genetic property to synthesize an essential growth factor, or factors, previously supplied from an external source".

If the effect of carcinogens is cytoplasmic and heritable, "plasmagenes" of the cytoplasm would be acted upon, resulting in chemical (enzymatic) alterations leading to uncontrolled growth. Carcinogens might affect plasmagenes in situ and thus hereditarily alter the course of cellular enzyme synthesis and hence growth and differentiation. Cancer viruses might be mutated plasmagenes which can be transmitted from cell to cell.

\section{NUTRITION AND CANGER}

Caloric restriction and other dietary alterations (amino acid deficiency, vitamin deficiency) have been reported as altering the induction of specific neoplasms.

patible recipients demonstrating that factors other than genetic determine growth of tissue transplants. In this case the endocrine status of the host was very important.

Fig. 62. Skin graft into an Fi hybrid mouse. One parent strain was albino. Skin from this strain (s) served as donor for the graft. It is possible to graft either normal or neoplastic tissue from inbred parent stocks into Fr hybrids. Dominant histocompatibility genes determine such tissue compatibility.

Fig. 63. Mouse with ascites tumor. The abdominal cavity is distended with fluid within which the neoplastic cells are individually suspended, growing in the fluid medium without stroma. Fig. 64. Anterior chamber intraocular transplants into mice. The transplants of the tumor shown in the eyes of the animal on the right grow temporarily in a foreign strain and regress if the host has been previously untreated. If the host has resisted a previous transplant it is immune to this particular tumor, as demonstrated by the absence of tumor in the eyes of the mouse on the left.

Fig. 65. Tumor transplant in subcutaneous tissue of tail. Such transplants are valuable for ready amputation of a transplanted tumor (following which immunization occurs in the case of some), or irradiation of a tumor transplant. 
Methionine deficiency per se induced by feeding ethionine (Popper et al., I953) and choline deficiency (Copeland and Salmon, 1946) may be responsible for the induction of hepatic neoplasms. These are also cirrhotogenic agents; the correlation of cirrhosis with hepatic carcinogenesis in man is well known. Low riboflavine content of the liver can be associated with the induction of liver tumors by azo dyes: maintenance of a high riboflavine content is tumor-inhibitory (Griffin and Baumann, I 948). Dye-binding is facilitated by riboflavine deficiency.

Mice on a cystine-deficient diet resist the leukemogenic action of methylcholanthrene (White et al., I 947); the body growth of mice on a lysine-deficient diet is similarly inhibited, but susceptibility to the induction of leukemia is not altered, suggesting that cystine-deficiency per se is a potent inhibiting influence.

Caloric restriction may inhibit and high fat diet favor the development of certain types of neoplasms. Tannenbaum (1953) has done crucial experiments to demonstrate the stage of carcinogenesis affected by diet. Apparently initiation proceeds, but the "developmental" or "promotional" stage is inhibited by caloric restriction.

\section{GENETIC STUDIES}

The possibility that genic alteration is basic to carcinogenesis has been discussed above. Since cancer cells pass on the abnormality responsible for uncontrolled proliferation to their progeny, it seems reasonable to regard cancer as a heritable cellular anomaly, heritable as used here in the "somatic" sense, since the neoplasm might arise in an individual whose germ cells are not similarly deviated. Upon transplantation, cancer cells may proliferate in a histocompatible normal (non-cancerous) host (Figs. 60, 62-65). Carcinogenic agents might act either by entering or entrance in to cells altering cellular constituents, or by altering the cellular environment. Cellular mutation might result from either change.

Certain inbred strains of mice are susceptible and others resistant to the induction of tumors by specific agents. Susceptibility is determined by dominant genes; F I hybrids between susceptible and resistant strains exhibit susceptibility to carcinogenic induction of neoplasms. If the target tissue for the carcinogenic agent is grafted into FI hybrids, parent stock tissue serving as donors, and if the F I hybrid is treated with the carcinogen, tumors appear in the FI hybrid and grafted susceptible parent tissue, but not in the grafted tissue derived from the resistant parent. This phenomenon of control of tumorigenesis by target tissue applies to mammary (Prehn, I953), pulmonary (Heston and Dunn, I95I; Shapiro and Kirschbaum, I 95I), thymic (Kaplan et al., 1956a; Law andMiller, I 95ob) testicular (Trentin and Gardner, I957) and adrenal cortical (Huseby and Bittner, I95I) tissue of mice (Fig. 66).

When gonadectomized-adrenalectomized Fi hybrids between stocks susceptible and resistant to the induction of post-castrational cortical carcinomas icreived adrenal grafts from both, castration-induced carcinomas developed only in the adrenal cortices supplied by the susceptible donors (Huseby and Bittner, $195 \mathrm{~J})$. Since the same trophic hormonal stimulus acted upon both adrenals in the common host, the adrenal cortex per se determined tumorigenesis. Similarly, when 
testicular grafts were made into estrogen-treated $\mathrm{F}_{\mathrm{I}}$ hybrids betwecn susceptible and resistant strains, tumors developed only in the grafts derived from the susceptible strain (Trentin and Gardner, 1957). Pulmonary tissue has been grafted into F I hybrids treated with either urethane (Shapiro and Kirschbaum, I95 I) or carcinogenic hydrocarbon (Heston and Dunn, I95I), mammary tissue to deter-

GENETIC DETERMINATION OF TUMORIGENESIS IN TARGET TISSUE

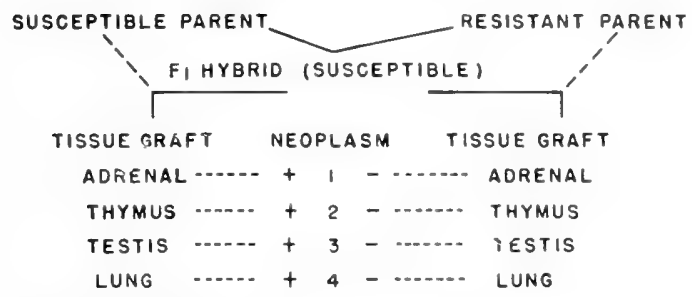

1. CASTRATION - INDUCED

2. IRRADIATION, CARCINOGEN-INOUCED OR SPONTANEOUS

3. ESTROGEN - INDUCED

4. URETHANE OR CARCINOGENIC HYDROCARBON-INDUCED
Fig. 66. Table illustrating results on genic determination of susceptibility to the induction of neoplasms. In the case of indcution of neoplasms by various means, it appears that the site of gene action is in the target tissue. F I hybrids between susceptible and resistant strains demonstrate susceptibility. By grafting untreated tissue into F I hybrids, the susceptibility of so-called "susceptible" and "resistant" tissue can be determined within a common $\mathrm{F}_{\mathrm{I}}$ hybrid host.

mine a similar effect for mammary tumorigenesis (Prehn, I953) and thymic tissue for irradiation-induced neoplasia (Kaplan et al., I956a).

Thus, target tissues of certain hosts may exhibit a neoplastic response to stimuli innocuous to the same, but genetically different tissues of the same species. There is evidence, however, that host factors may modify the intrinsic tissue response (Huseby and Bittner, I 95I ; Heston and Dunn, I95I). In some situations, such as induction of pituitary tumors by radio-thyroidectomy, tumorigenesis is not strainlimited. Degree of response varies, however, from strain to strain. For example, the application of methylcholanthrene results in the development of skin tumors in all strains of mice, but some are more susceptible than others.

That the host is not necessary for malignant transformation is demonstrated by the development in vitro of sarcoma cells from fibroblasts (Earle, I943; Earle and Nettleship, I943; Nettleship and Earle, I 943). These studies suggest that the environment of tissue culture may provide a carcinogenic stimulus. With the recent developments on clones derived from single cells and propagated on artificial media, precise studies in this area may be anticipated. Since viruses may be propagated in cell culture, their possible relation to malignant transformation in vitro remains to be determined.

\section{SUMMARY}

Viruses, hormones, chemical carcinogens and ionizing radiations are responsible for the induction of various types of cancer in different species of animals. The wide variety of carcinogenic agents proposes the perplexing problem of common 


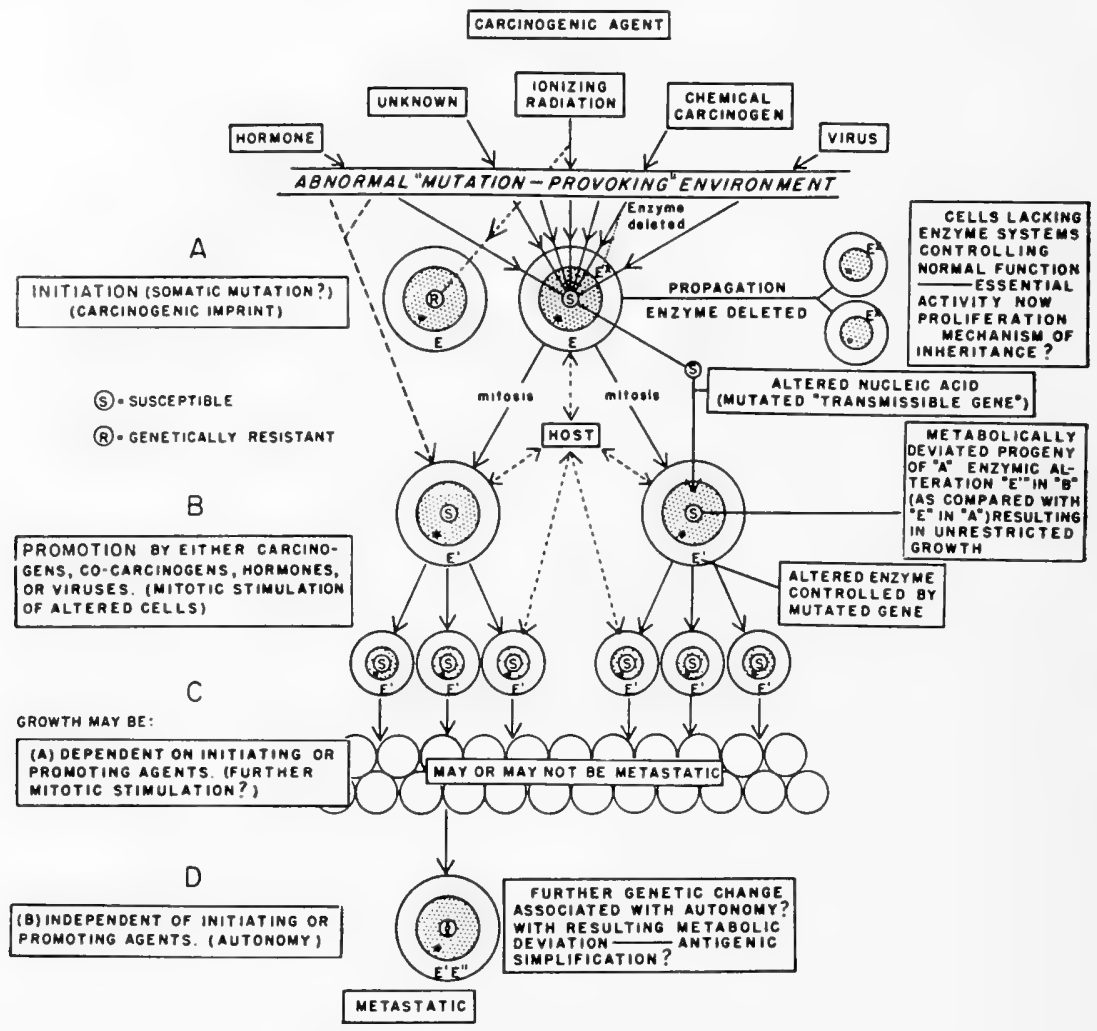

COMPOSITE SCHEME OF CARCINOGENESIS

Fig. 67. Chart illustrating a composite scheme of carcinogenesis.

factors for all if such exist. Neoplasms of different tissues have different "remote" causes even if the change from normal to malignant in all cases is precipitated by a mechanism common to all. Whether or not carcinogenic agents augment a spontaneously occurring process or set into motion a new chain of events is still unknown, although certain induced neoplasms occur spontaneously very rarely. The host influences the response to carcinogenic agents; within individuals of the same species genetic factors may determine divergence of response as great as between species.

Carcinogenic chemicals may combine with or act upon cellular constituents (genes, plasmagenes, gene-enzyme complex) so that the heritable cellular physiology is altered in the direction of uncontrolled proliferation, The mechanism remains obscure, although there are suggestions concerning pathway (e.g. combination with - SH groups). Ionizing radiations might produce similar cytologic alterations, cither by direct action on nuclear constituents, or through chemicals (indirect effects) originating from tissue, post-irradiation. It is possible that the 
tissue environment created by carcinogenic agents contributes towards carcinogenesis by I) creating an unfavorable environment which stimulates genic alteration "initiation" and 2) damaging surrounding tissue to contribute a non-specific stimulus to "promotion".

The subtle differential factors (genetic) in target tissue (lung, thymus, etc.) which determine the cancerous response have not been demonstrated. There is no evidence that abnormal metabolites are carcinogenic. Normal secretion (e.g., hormones) may, however, be carcinogenic even when secreted at normal levels, indicating that the reaction of the altered target tissue is primary in the carcinogenic response.

Hormones may merely "promote" carcinogenesis by inducing the multiplication of cells upon which the carcinogenic imprint (initiation) has already been made. This could apply, however, only to the tissues whose proliferation is normally stimulated by hormones. Or they may cause the growth of normal tissue, preparing it for the action of carcinogenic agents. In other instances (e.g., the induction of leukemia) carcinogenesis by hormones is unrelated to their proliferative stimulus. The neoplasm in certain cases appears to be an outcome of sustained proliferation (e.g., thyroid tumors). The absence of circulating hormone may serve as a carcinogenic stimulus (e.g., pituitary tumors induced by thyroidectomy).

Cancer viruses may be of the same character as those inducing infectious diseases. However, in the induction of certain cancers, the virus might be an accelerant, and not essential for carcinogenesis if other factors are sufficiently potent. If alteration in genic material by carcinogens determines tumorigenesis, viruses may be transmissible "plasmagenes", providing cells with the genic factors altering cellular metabolism via enzymatic derangement. In the "masked" form viruses may have attached themselves to cells so intimately that they are transmitted only during cell (nuclear-mitotic) division.

Adding confusion to the picture is the fact that "cocarcinogens" augment carcinogenesis, and these may be difficult to detect because independently they do not induce neoplasms. Various inorganic chemicals (Hueper, I953) have been associated with the induction of cancer (e.g., beryllium, arsenic, nickel, etc.). The role of these and agents such as plastics (Oppenheimer et al., I952, 1955) in tumorigenesis demonstrates the wide variety of agents whose tumor inducing properties are still to be reconciled with the various well documented modes of carcinogenesis involving viruses, hormones, radiation and specific chemicals (Fig. 67). Inflammatory tissue response is still an unclarified and nebulous factor in carcinogenesis.

In conclusion, cancer is induced by a variety of agents which may be either "initiators" or "promoters" of the process. Continuing growth may or may not be dependent upon the factors necessary for genesis. Presently there is no one hypothesis which can be used to explain satisfactorily the various modes of carcinogenesis. The definition of "cancer" may be changed as more information is acquired. 


\section{LITERATURE}

Agate, Jr., F. J., W. Antopos, S. Glaubach, F. Agate and S. Graff, (1955) Cancer Research, $15,6$.

Agnew, L. R. C. And W. U. Gardner, (1952) Cancer Research, 12, 757.

Allam, M. W., L. S. Lombard, E. L. Stubbs and J. F. Shirer, (1954) Cancer Research, I4, 734 .

Allen, E. and W. U. Gardner, (194I) Cancer Research, I, 359.

Andervont, H. B., (I938) Public Health Repts., (U.S.), 53, 232.

Andervont, H. B., (i950a) 7. Natl. Cancer Inst., II, 73.

Andervont, H. B., (I950b) F. Natl. Cancer Inst.. iI, 58 I,

Andervont, H. B. and W. R. Bryan, (1944) F. Natl. Cancer Inst., 5, I43.

Andervont, H. B. And T. B. Dunn, (I947) 7. Natl. Cancer Inst., 7, 455.

Andervont, H. B. and T. B. Dunn, (1953) F. Natl. Cancer Inst., I4, 329.

Andervont, H. B., M. B. Shimkin and H. Y. Cantes, (1957) F. Natl. Cancer Inst., I8, i.

Asboe-Hansen, G., (1954) Cancer Research, 14, 94.

Athias, M. and D. M. T. Furtado, (194I) Arquiv. patol., 13, 38 I.

Axelrad, A. A. and C. P. LeBlond, (1955) Cancer, 8, 339.

Ball, H. A. and L. T. Samuels, (1938) Am. F. Cancer, 32, 50.

Beadle G. W. and E. L. Tatum, (1945) Am. 7. Botany, 32, 678.

Beard, J. W., D. G. Sharp and E. A. Eckert, (1955) in Advances in Virus Research, Academic Press, New York, p. I 49.

Berenblum, I. and P. Shubik, (1947) Brit. F. Cancer, $1,3^{8} 3$.

Berenblum, I. and P. Shubik, (1949) Brit. J. Cancer, 3, iog.

Bernhard, W. and C. Oberling, (i957) Proc. Can. Cancer Research Conf., 2nd Conf., 2, 59.

Bielschowsky, F., (1944a) Brit. F. Exptl. Pathol., 25, I.

Bielschowsky, F., (1944b) Brit. 7. Exptl. Pathol., 25, 90.

Bielschowsky, F., (1949) Brit. F. Cancer, 3, 547.

Bielschowsky, F., (1954) Brit. F. Cancer, 8, I54.

Bielschowsky, F. and W. H. Hall, (i953) Brit. F. Cancer, 7, 358.

Bielschowsky, F., M. Bielschowsky and D. Lindsay, (ig56) Brit. F. Cancer, io, 688.

Biskind, M. S. and G. Biskind, (i944) Proc. Soc. Exptl. Biol. Med., 55, i 76.

Biskind, M. S. and G. Biskind, (i945) Proc. Soc. Exptl. Biol. Med., 59, 408.

Bittner, J. J., (1936a) Proc. Soc. Exptl. Biol. Med., 34, 42.

Bittner, J. J., (i936b) Science, 84, 162.

Bittner, J. J., (1942) Cancer Research, 2, 7 I0.

Bittner, J. J., (1948) Cancer Research, 8, 625.

Bittner, J. J., (1952) Cancer Research, I2, 388.

Bittner, J. J. and A. Kirschbaum, (1950) Proc. Soc. Exptl. Biol. Med., 74, 19 I.

Bittner, J. J. and D. T. Imagawa, (1955) Cancer Research, 15, 464.

Bloom, F., (1952) Proc. Soc. Exptl. Biol. Med., 79, 65I.

Blum, H. F., (1950) 7. Natl. Cancer Inst., II, 463.

Bonser, G., (1942) J. Pathol. Bacteriol., 54, 149.

Bonser, G., D. B. Clayson and J. W. Jull, (195I) Lancet, 2, 286.

Boutwell, R. K. And H. P. Rusch, (1953) Proc. Am. Assoc. Cancer Research, I, 9.

Boyland, E. and E. S. Horning, (i949) Brit. J. Cancer, 3, i i 8.

Browning, H., unpublished.

Brues, A. M., (1954) in Advances in Cancer Research, Academic Press, New York, II, p. I77.

Bryan, W. R., D. Calnan and J. B. Moloney, (1955) 7. Natl. Cancer Inst., i6, 3 I 7.

Burchenal, J. H., G. C. Stock and C. P. Rhoads, (1950) Cancer Research, Io, 209.

Burdette, W., (I955) Cancer Research, 15, 20 I.

Burmester, B. R. and D. U. M. Belding, (1947) Am. J. Vet. Research, 8, i 28.

Burmester, B. R., (1957) Personal communication.

Burnett, F. M., (I956) Enzyme Antigen and Virus, Cambridge University Press, London.

Cantarow, A., K. E. Paschisis, J. Stasney and M. S. Rothenberg, (ig46) Cancer Research, 6, 610. 
Carnes, W. H., H. S. Kaplan, M. B. Brown and B. B. Hirsch, (1956) Cancer Research, I6, 429 .

Carrie, A. W. and A. W. Ham, (1949) Cancer Research, 9, 629.

Clifton, K. H. and R. K. Meyer, (1956) Anat. Record, 125, 65.

Cook, J. W., C. L. Hewett and I. Heiger, (I932) Nature, (London), $130,926$.

Copeland, D. H. and W. D. Salmon, (1946) Am. J. Pathol., 22, 1059.

Crabtree, H. G., (1946) Cancer Research, 6, 553.

Davies, J. N. P., (1955) J. Natl. Cancer Inst., 15, 1637.

Dent, J. N., E. L. Gadsden and J. Furth, (1955) Cancer Research, 15, 70.

Dickie, M. M. and G. W. Woolley, (1949) Cancer Research, 9, 372.

Dmochowski, L. and J. W. OrR, (1949) Brit. 7. Cancer, 3, 520.

Dougherty. T. F., (1952) Physiol. Rev., 32, 379.

Dulaney, A. D., (1956) Cancer Research, I6, 877.

Dunn, T. B., W. E. Heston and M. K. Deringer, (1956) 7. Natl. Cancer Inst., I7, 639.

Dunning, W. F. and M. R. Curtis, (1952) Cancer Research, I2, 702.

Dunning, W. F., M. R. Curtis and C. Friedgood, (1949) Cancer Research, 9, 546.

Dunning, W. F., M. R. Gurtis and A. Segaloff, (i953) Cancer Research, i 3, 147.

Duran-Reynals, F., (i940) Tale F. Biol. and Med., I3, 6I.

Duran-Reynals, F., (I942) Cancer Research, 2, 343.

Duran-Reynals, F., (1943) Cancer Research, 3, 569.

Duran-Reynals, F., (I953) in Physiopathology of Cancer, ed. Homberger and Fishman, Hoeber-Harper, New York, p. 298.

EArle, W. R., (1943) J. Natl. Cancer Inst., 4, I65.

Earle, W. R. And A. Nettleship, (1943) $\mathcal{F}$. Natl. Cancer Inst., 4, 213.

ECKLEs, N. E., Unpublished.

Eckles, N. E. and G. Ehni, (1958) Proc. Am. Assoc. Cancer Research, 2, 494.

Edwards, J. E. (I94I) 7. Natl. Cancer Inst., 2, I97.

Ehni, G. And N. E. Eckles, (1957) Surg. Forum, 7, 449.

Ellerman, V. and O. Bang, (I908) Zentr. Bakteriol. Parasitenk., 46, 595.

Ely, C. A., (1953) Proc. Soc. Exptl. Biol. Med., 84, 501 .

Engelbreth-Holm, J. and G. Asboe-Hansen, (1953) Acta Pathol. Microbiol. Scand., $32,564$.

Eschenbrenner, A. B., (1945) 7. Natl. Cancer Inst., 5, 25 I.

Foley, E. J., (1952) Proc. Soc. Exptl. Biol. Med., 8o, 669.

Foley, E. J., (1953) Cancer Research, 13, 835.

Foulds, L., (1949) Brit. F. Cancer, 3, 345.

Foulds, L., (1954) Cancer Research, 14, 327.

Fournier, R., (1935) Rev. franç. gynécol., 3o, 445.

Frantz, M. J. and A. Kirschbaum, (1949) Cancer Research, 9, 257.

Friedewald, W. F. and J. G. Kidd, (1939) Proc. Soc. Exptl. Biol. Med., 41, 2 I8.

Furth, J., (1953) Cancer Research, I3, 477.

Furth, J., (1955) Recent Progr. in Hormone Research, II, 221.

FurTh, J., personal communication.

Furth, J. and O. Furth, (1936) Am. 7. Cancer, 28, 54 .

Furth, J. and J. S. Butterworth, (1936) Am. 7. Cancer, 28, 66.

Furth, J. AND M. Boon, (1943) Science, 98, I38.

Furth, J. And H. Sobel, (1947) Cancer Research, 7, 246.

Furth, J., E. L. Gadsden and W. T. Burnett, Jr., (1952) Proc. Soc. Exptl. Biol. Med., 8o, 4.

Furth, J., W. T. Burnett, Jr. And E. L. Gadsden, (1953) Cancer Research, 13, 298.

Furth, J., R. F. Buffett, M. Banasiewicz-Rodriguez and A. G. Upton, (1956) Proc. Soc. Exptl. Biol. Med., 93, I65.

Gallagher, T. F., (1957) Cancer Research, 17, 520.

Gardner, W. U., (1942) Cancer Research, 2, 476.

Gardner, W. U., (I943a) Cancer Research, 3, 99.

Gardner, W. U., (1943b) Cancer Research, 3, 757.

Gardner, W. U., (1945) Cancer Research, 5, 497.

Gardner, W. U., (1950) Proc. Soc. Exptl. Biol. Med., 75, 434. 
Gardner, W. U., (1953) in Advances in Cancer Research, ed. L. W. LAw, Academic Press, New York, I, p. I73.

Gardner, W. U., (1957) Proc. Can. Cancer Research Conf., 2nd Conf. 2, 207.

Gardner, W. U., T. F. Dougherty and W. L. Williams, (I944) Cancer Research, 4, 73.

Gardner, W. U. and B. Frankenheuis, (1955) Proc, Am. Assoc. Cancer Research, 2(i), i8.

Gardner, W. U., A. Kirschbaum and L. C. Strong, (i940) A.M.A. Arch. Pathol., 29, i.

Gardner, W. U., C. A. Pfieffer, J. J. Trentin and J. T. Wolstenholme, (1953) in Physiopathology of Cancer, ed. Homberger and Fishman, Hoeber-Harper, New York, p. 225 .

Gardner, W. U. and L. C. Strong, (1940) Tale F. Biol. and Med., 12, 543.

Gillman, J., C. Gilbert and L. Spence, (I955) Experientia, i I, 157.

Goldberg, R. C. And I. L. Chaikoff, (1952) A.M.A. Arch. Pathol., 53, 22.

Goranson, E. S., F. Botham and M. Williams, (1954) Cancer Research, 14, 730.

Gorbman, A., (1952) Proc. Soc. Exptl. Biol. Med., 8o, 538.

Gorbman, A., (1956) Cancer Research, I6, 99.

Gorbman, A. and A. Edelman, (1955) Proc. Am. Assoc. Cancer Research, 2, 13.

Grad, B., J. Berenson and L. Caplan, (1955) Proc. Am. Assoc. Cancer Research, 2, 20.

Greene, J. A., (1956) Proc. Am. Assoc. Cancer Research, 2(2), I I I.

Greenstein, J., (1954) Biochemistry of Cancer, Academic Press, New York.

Griesbach, W. E., T. H. Kennedy and H. V. Purves, (1945) Brit. J. Exptl. Pathol., 26, 18. Griffin, A. C. and C. A. Baumann, (1948) Cancer Research, 8, 279.

Griffin, A. C., E. L. Brandt and V. Setter, (i95ia) Cancer Research, il, 868.

Griffin, A. C., E. L. Brandt and E. L. Tatum, (195I b) Cancer Research, II, 253.

Griffin, A. C., E. L. Brandt and E. L. Tatum, (I950) 7. Am. Med. Assoc., I44, 57 I.

Griffin, A. C., H. D. Richardson, C. H. Robertson, M. A. O'Neal and J. D. Spain, (1955) 7. Natl. Cancer Inst., 15, I623.

Gross, L., (1950) Proc. Soc. Exptl. Biol. Med., 73, 246.

Gross, L., (195 I) Proc. Soc. Exptl. Biol. Med., 76, 27.

Gross, L., (1955) Proc. Soc. Exptl. Biol. Med., 88, 362.

Gross, L., (1957) Cancer Research, 2(3), 209.

Gross, L., (1957) Proc. Soc. Exptl. Biol. Med., 94, 767.

Haddow, A., (1947) Brit. Med. Bull., 4, 33 I.

Haddow, A., (I 953 ) in Physiophathology of Cancer, ed. Homberger and Fishman, HoeberHarper, New York, p. 44I.

Haddow, A., J. M. Watkinson, E. Paterson and P. C. Koller, (1944) Brit. Med. 7., 2, 393.

Heilman, F. R. and E. C. Kendall, (1944) Endocrinology, 34, 4 I6.

Hesse, O., (I9I I) Fortschr. Gebiete Röntgenstrahlen, 42, 82.

Heston, W. E., (1940) F. Natl. Cancer Inst., I, 105.

Heston, W. E., (1949) J. Natl. Cancer Inst., Io, i 25.

Heston, W. E., (I95I) Growth, 15, 23.

Heston, W. E. and M. K. Deringer, (1954) Proc. Soc. Exptl. Biol. Med., 82, 73 I.

Heston, W. E. And T. B. Dunn, (i95 I) J. Natl. Cancer Inst., iI, 1057.

Heston, W. E., M. K. Deringer, T. B. Dunn and M. D. Levillam, (i950) J. Natl. Cancer Inst., IO, I 139.

Heston, W. E., M. K. Deringer and T. B. Dunn, (1956) J. Natl. Cancer Inst., i6, izog.

Hieger, I., (1930) Biochem. 7., 24, 505.

Higgins, G. M. And K. A. Woods, (1950) Anat. Record, ro6, 204.

Higgins, G. M., K. A. Woods and W. A. Bennett, (I950) Cancer Research, Io, 203.

Hooker, W., W. U. Gardner and C. A. Pfeiffer, (i940) J. Am. Med. Assoc., II5, 443.

Horning, E. S., (1952) Brit. 7. Cancer, 6, 80.

Horning, E. S., (1956) Brit. 7. Cancer, 10, 678.

Howell, J. S., J. Marchant and J. W. Orr, (1954) Brit. 7. Cancer, 8, 636.

Hotssay, B. A., F. A. Cardeza, V. G. Foglia, A. B. Houssay and R. Printo, (1954) Compt. rend. soc. biol., I48, 918.

Hotssay, B. A., A. B. Houssay, A. F. Cardeza and R. Printo, (1955) Schweiz. med. Wochschr., 85, 29 I. 
Hueper, W. C., (1942) Occupational Tumors and Allied Diseases, Charles C. Thomas, Springfield, Illinois.

Hueper, W. C., (1953) in Physiopathology of Cancer, ed. Homberger and Fishman, HoeberHarper, New York, p. 730 .

Huggins, C. and D. M. Bergenstal, (1952) Cancer Research, I2, 134.

Huggins, C. And C. V. Hodges, (I94I) Cancer Research, I, 293.

Huggins, G., Y. Torralba and K. Mainzen, (1956) J. Exptl. Med., 104, 525.

Huseby, R. A., Z. B. Ball and M. B. Visscher, (1945) Cancer Research, 5, 40.

Huseby, R. A. And J. J. Bittner, (I95I) Cancer Research, II, 954.

IDA, N., (I957) Unpublished.

Ida, N., A. Kirschbaum and H. G. Taylor, (1957) Proc. Am. Assoc. Cancer Research, 2(3), $2 \mathrm{I} 6$.

Ingle, D. J. AND B. L. BAKer, (I95I) Endocrinology, 48, 3 I 3.

Ingle, D. J., M. C. Prestrud and K. L. Rice, (1950) Endocrinology, 46, 510.

Jacobson, L. O., E. K. Marks, M. J. Robson, E. O. Gaston and R. E. Zirkle, (I949) 7. Lab. Clin. Med., 34, I 538 .

Jehl, J., G. Mayer and R. W. McKee, (I955) Cancer Research, 15, 341.

Jones, A., (1955) Brit. J. Cancer, 9, 640.

Kaplan, H. S., (1947) Cancer Research, 7, 14 I.

Kaplan, H. S., (1948) F. Natl. Cancer Inst., 8, I9I.

Kaplan, H. S., (1949) 7. Natl. Cancer Inst., io, 267.

Kaplan, H. S., (I95Oa) J. Natl. Cancer Inst., II, 83.

Kaplan, H. S., (I950b) 7. Natl. Cancer Inst., II, I 25.

Kaplan, H. S., (195I) Cancer Research, II, 26I.

Kaplan, H. S. And M. B. Brown, (I95I) Cancer Research, II, 706.

Kaplan, H. S. ANd M. B. Brown, (1954) Science, II9, 439.

Kaplan, H. S., M. B. Brown and S. N. Marder, (I95I) Cancer Research, II, 629.

Kaplan, H. S., M. B. Brown, B. Hirsch and W. H. Carnes, (1955) Proc. Am. Assoc. Cancer Research, 2(I), 27.

Kaplan, H. S., M. B. Brown and J. Paull, (1953) F. Natl. Cancer Inst., I4, 303.

Kaplan, H. S., W. A. Carnes, M. B. Brown and B. B. Hirsch, (1956a) Cancer Research, I 6,422 .

Kaplan, H. S., M. B. Brown, B. B. Hirsch and W. H. Carnes, (1956b) Cancer Research, 16, 426.

Kaplan, H.S., B. B. Hirsch and M. B. Brown, (1956c) Cancer Research, 16, 434.

KenNedy, B. J., (1956) Am. F. Med., 2I, 72I.

Kensler, C. J., K. Suguira and C. P. Rhoades, (I940) Science, 9I, 623.

Kensler, C. J., K. Suguira, N. F. Young, C. R. Holter and C. P. Rhoades, (194i) Science, 93, 308.

Kinosita, R., (1940) Yale 7. Biol. and Med., 12, 287.

Kirkman, H., (1957) Lancet, Io, 757.

Kirkman, H. and R. L. Bacon, (I950) Cancer Research, io, 122.

Kirkman, H. and R. L. Bacon, (1952) F. Natl. Cancer Inst., 13, 745.

Kirkman, H. And R. L. Bacon, (I952) J. Natl. Cancer Inst., I3, 757.

Kirschbaum, A., unpublished.

Kirschbaum, A., (1949) Cancer Research, 9, 93.

Kirschbaum, A., (I95I) Cancer Research, II, 74I.

Kirschbaum, A., (1956) Am. 7. Med., 2I, 659.

Kirschbaum, A., (1957) Cancer Research, I7, 432.

Kirschbaum, A. And S. Kawamoto, (1957) Proc. Am. Assoc. Cancer Research, 2(3), 222.

Kirschbaum, A. and A. G. Liebelt, (1957) unpublished.

Kirschbaum, A., A. G. Liebelt and N. G. Falls, (i955) Cancer Research, I5, 685.

Kirschbaum, A., A. G. Liebelt and G. H. Fletcher, (1956) Proc. Soc. Exptl. Biol. Med., $92,221$.

Kirschbaum, A. and H. W. Mrxer, (1947) 7. Lab. Clin. Med., 32, 720.

Kirschbaum, A., J. R. Shapiro and H. W. Mrxer, (I953) Cancer Research, I3, 262. 
Kirschbaum, A. and L. C. Strong, (1942) Proc. Soc. Exptl. Biol. Med., 5I, 404. Kirschbaum, A., W. L. Williams and J. J. Bittner, (i946) Cancer Research, 6, 354. Kortetveg, R., (1934) Ned. Tijdschr. Geneesk., 78, 240.

Korteineg, R. and F. Thomas, (1949) Am. F. Cancer, 37, 36.

Lacassagne, A.g (1932) Compt. rend., 195, 630.

Lannek, N., (1953) Brit. 7. Cancer, 6, 369.

Law, L. W., (1952) 7. Natl. Cancer Inst., 12, 789.

LAw, L. W., (r 954 ) Advances in Cancer Research, Academic Press, New York, Vol. II, p. 28 I.

Law, L. W., L. E. Bunker Jr. and B. A. Norris, (1947) F. Natl. Cancer Inst., 8, i 57.

Law, L. W., T. B. Dunn and P. J. Doyle, (1955) F. Natl. Cancer Inst., I6, 495.

Law, L. W. and J. H. Miller, (I950a) J. Natl. Cancer Inst., II, 253.

Law, L. W. and J. H. Miller, (I950b) F. Natl. Cancer Inst., II, 425.

LeBlond, G. P., H. Isler and A. Axelrad, (1957) Proc. Can. Cancer Research Conf., 2nd Conf., 2, 248.

LederberG, J., (I946) Science, I04, 428.

Lederberg, J., (1956) Am. Scientist, 44, 264.

Leiter, J. and M. J. Shear, ( 1943 ) J. Natl. Cancer Inst., 3, 455.

Liebelt, A. G., (1957) Proc. Am. Assoc. Cancer Research, 2(3), 226.

Liebelt, R. A., (1957) Proc. Am. Assoc. Cancer Research, 2(3), 226.

Liebelt, R. A. And N. Eckles, (i957) Proc. Am. Assoc. Cancer Research, 2 (3), 227.

Lick, L., A. Kirschbaum and H. W. Mixer, (i949) Cancer Research, 9, 535.

Lisco, H. And M. Finkel, (1949) Federation Proc. 8, 360.

Lisci, H., M. Finkel and A. M. Brues, (1947) Radiology, 49, 36r.

Lisco, H., A. Brues, M. Finkel and W. Grundhauser, (1947) Cancer Research, 7, 721.

Loeb, L., (1940) 7. Natl. Cancer Inst., I, I69.

Lorenz, E., (1950) Am. J. Roentgenology Radium Therapy, 63, i 76.

Lorenz, E., D. Uphoff, R. T. Reid and E. Shelton, (I95 I) J. Natl. Cancer Inst., I2, i97.

Luckman, C. and A. Kirschbaum, Unpublished.

Luft, R. and H. Olivecrona, (I955) Cancer, 8, 26 r.

MacDowell, E. C., (I955) Cancer Research, 15, 23.

MacDowell, E. C. And M. N. Richter, (I935) A.M.A. Arch. Pathol., $20,709$.

MacDowell, E. C. And M. H. Taylor, (1948) Proc. Soc. Exptl. Biol. Med., 68, 57 I.

Martinez, C. and J. J. Bittner, (i954) Proc. Soc. Exptl. Biol. Med., 86, 92.

Martland, H. S. and R. E. Humphries, (1929) A.M.A. Arch. Pathol., 7, 406.

Mider, G. B. And J. J. Morton, (1939) Am. F. Cancer, 37, 355.

Miller, W. L. and C. A. Baumann, (I95 I) Cancer Research, iI, 634.

Miller, O. J. and W. U. Gardner, (1954) Cancer Research, 14, 220.

Miller, E. G. And J. A. Miller, (1947) Cancer Research, 7, 468.

Miller, E. C. And J. A. Miller, (1952) Cancer Research, 12, 557.

Moloney, W. C., (1955) New Engl. F. Med., 253, 88.

Monsen, H., (1952) Cancer Research, I2, 284.

Monsen, H. And A. Kirschbaum, (i 954) Proc. Am. Assoc. Cancer Research, I (2), 33.

Moon, H. D. And M. E. Simpson, (1955) Cancer Research, 15, 403.

Moon, H. D., C. H. Li And M. E. Simpson, (I956) Cancer Research, I6, i I I.

Moon, H. D., M. E. Simpson, C. H. Li and H. M. Evans, (I950a) Cancer Research, 10, 297.

Moon, H. D., M. E. Simpson, C. H. Li and H. M. Evans, (i950b) Cancer Research, Io, 364.

Moon, H. D., M. E. Simpson, C. H. Li and H. M. Evans, (1950c) Cancer Research, Io, 549.

Moon, H. D., M. E. Simpson, C. H. Li and H. M. Evans, (I95I) Cancer Research II, 535.

Moon, H. D., M. E. Simpson, C. H. Li and H. M. Evans, (1952) Cancer Research, I2, 448.

Moore, G. E., E. L. Brackney and F. Bock, (i953) Proc. Soc. Exptl. Biol. Med., 82, 643.

Morris, H. P., (1955) Advances in Cancer Research, 3, 5 I.

Morris, H. P., A. Dalton and G. Green, (I95i) J. Clin. Endocrinol. and Metabolism, i I, I $28 \mathrm{I}$.

Morris, H. P. and H. I. Firminger, (1956) J. Natl. Cancer Inst., 16, 927.

Nïrilbock, O., (1956) Advances in Cancer Research, 4, 37 I.

Murphy, J. B., (r 944 ) Cancer Research, 4, 622. 
Murray, W. S., (1928) Cancer Research, I2, I8.

Murray, W. S. and C. C. Little, (1936) Am. F. Cancer, 27, 516.

Nagareda, C. S. and H. S. Kaplan, (1955) F. Natl. Cancer Inst. 16, 139.

Nagareda, C. S. and H. S. Kaplan, (I957) Presented at Fifth Annual Meeting of Radiation Research Society (Rochester, N.Y.)

Nathanson, I. T., (1950) Med. Clin. N. Am., 34, I904.

Nathanson, I. T. and H. B. Andervont, (1939) Proc. Soc. Exptl. Biol. Med., 40, 421.

Nettleship, A. and W. R. Earle, (1943) 7. Natl. Cancer Inst., 4, 229.

Nettleship, A. and P. S. Henshaw, (1943) 7. Natl. Cancer Inst., 4, 309.

Noble, R. L. And J. H. Walters, (1954) Proc. Am. Assoc. Cancer Research, I, 35.

Oppenheimer, B. S., E. T. Oppenhemer and A. Stout, (1952) Proc. Soc. Exptl. Biol. Med., $79,366$.

Oppenheimer B. S., E. T. Oppenheimer, A. Stout, I. Danischevsky and F. R. Eirich, (1955) Cancer Research, I5, 333.

Paschis, K. E. A. Cantarow and J. Stasney, (1948) Cancer Research, 8, 257.

Pearson, O. H., M. C. Li, J. P. Maclean, M. B. Lipsett and C. W. West, (1955) 7. Am. Med. Assoc., I59, I701.

Petrov, N. and N. Erotkin, (I932) Z. Krebsforsch., 38, 249.

Pfeiffer, C. A. And C. W. Hooker, (1943) Cancer Research, 3, 762.

Popper, H., J. De la Huerga and C. Yesmick, (I953) Science, i I 8, 80.

Potter, V. R., (1944) Advances in Enzymology, 4, 201.

Prehn, R. T., (I953) F. Natl. Cancer Inst., I3, 859.

Purves, H. D. and W. E. Griesbach, (1946) Brit. 7. Exptl. Pathol., 27, 294.

Purves, H. D., W. E. Griesbach and T. H. Kennedy, (I95I) Brit. F. Cancer, 5, 30 I.

Rask-Nielsen, R., (1950) Brit. F. Cancer, 4, 108.

Richardson, H. L. And L. Cunningham, (195I) Cancer Research, II, 274.

Rock, T and G. C. Rabotti, (1955) Tumori, 4I, 289.

Rothschild, I., (1957) Proceedings Endocrine Soc., 22.

Rous, P., (igi i) 7. Am. Med. Assoc., 56, 198.

Sawyer, C. H., (1957) Anat. Record, 127, 362.

Salaman, M. H. and J. C. Roe, (r953) Brit. 7. Cancer, 7, 472.

Salzberg, D. A. And A. C. Criffin, (I952) Cancer Research, I2, 294.

Schoвer, R., (1953) Z. Krebsforschung, 59, 28.

Schulman, P. P. and D. M. Greenberg, (1949) Proc. Soc. Exptl. Biol. Med., 72, 676.

Schwartz, S. O., H. M. Schoolman, P. B. Szanto and W. Spurrier, (1957) Cancer Research, $17,218$.

SElye, H., (1955) Cancer Research, 15, 26.

Shapiro, J. R. And A. Kirschbaum, (195I) Cancer Research, II, 644.

Shimkin, M. B., (1940) A.M.A. Arch. Pathol., 29, 229.

Shimkin, M. B., (I955) Advances in Cancer Research, 3, 223.

Shimkin, M. B., H. G. Grady and H. B. Andervont, (ig4i) F. Natl. Cancer Inst., 2, 65. Shope, R., (I933) 7. Exptl. Med., 58,607.

Silberberg, M. And R. Silberberg, (I955) Cancer Research, I5, 29 I.

Sloviter, H. A., (I95I) Cancer Research, II, 447.

Smith, M. C., P. A. Slatter, M. B. Shimkin, C. H. Li, R. Lee, J. C. Clarke and W. R. Lyone, (1952) Cancer Research, 12, 59.

Spain, D. M., M. Molomut and A. B. Novikoff, (1956) Cancer Research, I6, i 38.

Spiegel, A., (1939) Arch. path. Anat. u. Physiol., Virchow's, 303, 367.

Spiegelman, S., (1946) Quant. Biol., II, 265.

Staff, Jackson Memorial Laboratory, (1933) Science, 78, 465.

Stewart, S. E., (1955) J. Natl. Cancer Inst., I5, r39r.

Stewart, S. E., B. E. Eddy, A. M. Gochenour, N. G. Borgese and G. E. Grubbs, (1957) Virology, 3,380 .

Stoerk, H. C., (1950) Proc. Soc. Exptl. Biol. Med., 74, 798.

Strong, L. C., (1929) 7. Cancer Research, I3, I03.

Strong, L. C., (1945a) A.M.A. Arch. Pathol., 39, 232. 
Strrong, L. C., (I945b) Proc. Natl. Acad. Sci. U. S., 3I, 290.

Sulzberger, M. B., F. Herrman, R. Piccagli and L. Frank, (1953) Proc. Soc. Exptl. Biol. Med., 82, 673 .

Symeonidis, A., A. S. Mulas and F. H. Burgoyne, (I95I) Cancer Research, II, 285.

Syverton, J. T., H. E. Dascomb, J. Kooman, E. B. Wells and G. P. Berry, (i950) Cancer Research, IO, 379 .

Talalay, P., G. M. U. Takano and G. Huggins, (1952) Cancer Research, 12, 838.

Taylor, S. G., M. C. Li, N. E. Eckles, D. P. Slaughter and J. H. MaDonald, (1953) Cancer, 6, 997.

Tannenbaum, A., (1940) A.M.A. Arch. Pathol., $30,509$.

Tannenbaum, A., (1953) in Physiopatholog $y$ of Cancer, ed. Homberger and Fishman, HoeberHarper, New York, p. 392.

Tannenbaum, A. And H. Silverstone, (i 949 ) Cancer Research, 9, 403.

Toch, P., B. Hirsch, M. P. Brown and H. S. Kaplan, (1955) Cancer Research, 2(I), 5 I.

Toolan, H. W., (1953) Cancer Research, 13, 389.

Trentin, J. J. and W. U. Gardner, (I958) Cancer Research, i8, i io.

Twombley, G. H., D. Meisel And A. P. Stout, (1949) Cancer, 2, 884.

Upton, A. C. And J. Furth, (1954) Blood, 9, 686.

Upton, A. C. And J. Furth, (1956) Proc. Am. Assoc. Cancer Research, 2(2), I 54.

Vermande-VanEck, G. J. and C. H. Chang, (1955) Cancer Research, I5, 280.

Waxler, S. H. And P. TABAR, (1953) Stanford Med. Bull., II, 272.

Werder, A. A., J. Friedman, E. C. MacDowell and J. T. Syverton, (1953) Cancer Research, 13, 389.

Weisburger, J. H., E. K. Weisburger, H. P. Morris and H. A. Sober, (1953) Cancer Research, 12, 305.

Wiest, W. E. and G. Heidelberger, (1953a) Cancer Research, I3, 246.

Wiest, W. E. and C. Heidelberger, (1953b) Cancer Research, I3, 250.

Wiest, W. E. And C. Heidelberger, (I953C) Cancer Research, I3, 255.

White, J. and H. B. Andervont, (I943) J. Natl. Cancer Inst., 3, 449.

White, F. R. AND J. White, (1944) F. Natl. Cancer Inst., 4, 4I3.

White, J., F. R. White and G. B. Mider, (1947) 7. Natl. Cancer Inst., 7, 199.

Wolf, G., (1952) Chemical Induction of Cancer, Harvard University Press, Cambridge, Mass. Wood, J. S., Jr. And E. D. Holyoke, (1955) Bull. Johns Hopkins Hosp., 96, 93.

Woolley, G. W., (r939) Proc. Natl. Acad. Sci. U.S., 25, 277.

Woolley, G. W., (I954) Proc. Am. Assoc. Cancer Research, I (2), 53.

Woolley, G. W. And C. C. Little, (1946) Cancer Research, 6, 49 I.

Woolley, G. W. and B. A. Peters, (1953) Proc. Soc. Exptl. Biol. Med., 82, 286.

Woyson, S. L., J. Drake and A. H. Bass, (1956) Growth, 20, I9.

Zachariae, L. and G. Asboe-Hansen, (1954) Cancer Research, I4, 488. 


\title{
Chapter 12
}

\section{THE METABOLISM OF THE GANGER GELL ${ }^{1}$}

\author{
A. Clark Griffin
}

\section{INTRODUCTION}

A metabolic or compositional pattern, unique for the malignant cell, has not been firmly established. Nevertheless, we must recognize that cancer cells do differ in certain fundamental aspects from normal cells. Such differences may occur at the metabolic level or the differences may exist at higher levels of cellular activity. The many classes of tumors appear to be remarkably constant in their overall metabolic patterns. At this time, however, there is insufficient evidence to assume that this general pattern is specific for tumors. There is increasing evidence that the biosynthetic pathways involving amino acid, protein and nucleic acid formation, as well as the energy forming reactions, are common for normal and malignant cells. Certainly, quantitative differences do exist and in many instances it has been observed that tumors rely to a large extent on a certain metabolic sequence or pathway for a given function. This same pathway may also be present in normal tissues but operative under normal circumstances to a limited extent. Generally, these quantitative differences provide the basis for the present approach to the chemotherapy of cancer. The chances for success in this area would be increased if some unique metabolic feature of cancerous cells could be discovered.

Every effort has been extended in order to provide the reader with a review of the studies made on the metabolism and composition of cancerous tissues. These findings obviously must be compared with the normal tissue patterns. The selection of a normal pattern for comparison has presented many difficulties since each normal tissue possesses more or less characteristic features. Also, the normal values are dependent upon species, strain, age, sex, nutritional status and many other factors. Malignant tissues may also vary in their metabolic or compositional patterns depending upon the homogeneity of sample, extent of necrosis and other factors. In general, comparisons have to be made on the basis of the normal or the cancerous "ranges". The advent of such powerful techniques as sequential and preferential blocking in Veurospora and other organisms, chromatography,

${ }^{1}$ The author acknowledges with sincere thanks the invaluable assistance of M. A. O'Neal in the editorial preparation of this Chapter and Mrs. Beth B. Rafferty in the typing and assembly of the manuscript. Also, the author wishes to thank Dr Van R. Potter for the opportunity to use his valuable charts in this Chapter. 
and almost unlimited availability of labeled precursors has made it possible to study metabolic pathways in both normal and malignant tissues. Sooner or later we will have definite proof that tumor cells do possess characteristic metabolic features or that they do not differ appreciably from normal cells.

In order to facilitate the solving of certain of the current problems we should evaluate the existing data as a guide to the most profitable approaches. If sufficient data are not available to provide an answer, further studies will have to be made. Alternatively we should seek new approaches to explain the malignant behavior. Perhaps this behavior may be associated with the response of the normal or malignant cell to inherent regulatory factors or to the hormonal factors elaborated by the endocrine or other glands. Hormones must exist, intracellularly or systemically, that regulate or control the metabolic reactions involved in the mobilization of nucleic acids and proteins for cellular division. Finally, the answer to malignancy may possibly depend upon further elucidation of the composition and function of the macromolecular deoxy- and ribonucleoproteins.

Metabolic studies made on tumors or in tumor bearing individuals extend well beyond the start of the present century. Müller (I 889) and Klemperer (I889) studied nitrogen balance in cancer patients. From the findings of these and subsequent investigators the concept has developed that a negative balance may be associated with malignancy. More than fifty years ago, Blumenthal and Wolff (I 905), Neuberg and Ascher (I 906) and Blumenthal et al. (I gog) investigated the proteolytic enzymes in a group of tumors. During the $1920(\mathrm{~s})$ Warburg and his colleagues (Warburg, I930) made extensive studies of the oxidative behavior of many normal and malignant tissues. It is obviously impossible to cover all of the metabolic studies related to cancer that have been made over this long period of time or to give credit to the many deserving investigators. Review articles of recent years which cover most of the many diversified areas of tumor metabolism and composition are presented in Table $I$. In the current review the author has emphasized the coverage of journals publishing studies on the composition or metabolism of tumors from the beginning of 1953 to mid-year I956. It has also been necessary to include references before 1953 in order to obtain and develop the full significance of a concept. In describing the patterns of malignant tissues, it is imperative that we always refer to some normal counterpart. Chapter I has served as a basis for many of the critical comparisons that have been made between normal and malignant tissues. Some mention must be made of the inerent difficulty encountered in evaluation of tumor data. Such factors as homogeneity of the tumor sample being studied, methodology, expressions of units of concentration or activity, degree of malignancy, species and other influencing factors will be considered whenever these data have been presented in the original publication.

One of the foremost problems in preparing this chapter was that of a logical crganization of the large number of components involved in the overall aspects () metabolism. The great advances that have been made in the area of intermediary metabolism during recent years makes it difficult to consider the carbohydrates, lipids ()r any other group individually. A complete synthetic or degradative pathway may involve carbohydrates, lipids, amino acids, inorganic elements and all the 
TABLE 1

SUMMARY OF REVIEW ARTIGLES ON VARIOUS ASPECTS OF METABOLISMAND COMPOSITION OF TUMORS

\begin{tabular}{|c|c|c|}
\hline Subject & Reviewer $(s)$ & Year \\
\hline $\begin{array}{l}\text { Organic constituents including: carbohy- } \\
\text { drates, proteins, nucleoproteins, lipids, } \\
\text { etc. }\end{array}$ & Stern and Willheim & 1943 \\
\hline Metabolism & $\begin{array}{l}\text { Stern and Willheim } \\
\text { Kit and Griffin }\end{array}$ & $\begin{array}{l}1943 \\
195^{8}\end{array}$ \\
\hline Chemistry of tumors & $\begin{array}{l}\text { Winzler } \\
\text { Greenstein } \\
\text { Heidelberger }\end{array}$ & $\begin{array}{l}\text { I } 953 \\
\text { I } 954, \text { I } 956 \\
\quad \text { I } 956\end{array}$ \\
\hline Oxidative metabolism & $\begin{array}{l}\text { Weinhouse } \\
\text { Schmidt }\end{array}$ & $\begin{array}{l}1955 \\
\text { I } 955\end{array}$ \\
\hline Carbohydrate metabolism & $\begin{array}{l}\text { Olson } \\
\text { Beck and Valentine }\end{array}$ & $\begin{array}{l}1951 \\
\text { I953 }\end{array}$ \\
\hline Nucleic acids, nucleoproteins & $\begin{array}{l}\text { Davidson and Leslie } \\
\text { Schmidt } \\
\text { Leslie }\end{array}$ & $\begin{array}{l}\text { I } 950 \\
\text { I } 953 \\
\text { I } 955\end{array}$ \\
\hline Deoxyribosides & Schneider & I 955 \\
\hline Proteins & $\begin{array}{l}\text { Toennies } \\
\text { Biserte }\end{array}$ & $\begin{array}{l}\text { I } 947 \\
\text { I } 953\end{array}$ \\
\hline Plasma proteins & Winzler & I953a \\
\hline Enzymes & $\begin{array}{l}\text { Fishman } \\
\text { Giarelli } \\
\text { Lemon et al. }\end{array}$ & $\begin{array}{l}\text { I } 953 \\
\text { I } 954 \\
\text { I } 954 \mathrm{a}\end{array}$ \\
\hline Cholesterol & Glement & I954 \\
\hline Metabolism of thyroid tumors & Morris & 1955 \\
\hline Application of radioisotopes & $\begin{array}{l}\text { Heidelberger } \\
\text { Greenberg }\end{array}$ & $\begin{array}{l}\text { I953 } \\
\text { I955 }\end{array}$ \\
\hline
\end{tabular}

accompanying enzymes and their respective co-factors. Several methods for presentation of the subject were attempted. However, the most logical comparison of the metabolism and composition of normal and neoplastic tissues may be made by reference to the major biochemical groupings. Accordingly, the discussion is presented on this basis, but with the full realization that the function of a cell or tissue depends upon the reactions and interreactions of all the component groups. 
II. CARBOHYDRATES AND LIPIDS

\section{(a) Composition}

There are insufficient data at this time to provide any evidence for significant differences between the carbohydrate or lipid composition of normal and malignant tissues. With respect to these components, the overall pattern observed in tumors as a group largely resembles, or certainly falls within the normal range. Specific alterations in the lipid composition of some tumors have been reported. Johnson and Dutch (1952) found that the total fat of mouse mammary carcinoma was only $1-2 \%$ of the amount present in normal resting mammary tissues. Nearly all of this differential in concentration could be attributed to neutral fat since the total cholesterol and total phospholipids of the two tissues were almost identical. In contrast, the content of both free and esterified cholesterol of rat hepatoma is considerably higher than in normal liver. The phosphatide and fatty acid content of the liver tumor is lower than in normal liver. Generally, the phospholipid fraction may account for approximately half of the total lipid in the tumor (Johnson and Dutch, r952; Boyd and McEwen, r952). Polli and Ratti (r953) obtained the values for the various lipoids of human myeloid leukemic, lymphatic leukemic and normal leukocytes (Table 2).

These findings indicate that there is no lipid pattern that would characterize the leukemic cell. In addition these investigators reported that there were no significant differences in the iodine number of the fats isolated from the normal or leukemic leukocytes. The nuclei of both lymphatic and myeloid leukemic cells contained considerably less phospholipid than the nuclei of normal human leukocytes. Ackerman et al. (I95I) reported that Hodgkins cells contain more lipid and mucopolysaccharide granules than reticulum cells. Seibert et al. (I954) have also made comparisons of the chemical composition of alcoholic extracts obtained from rat sarcoma or lymphoma with similar extracts obtained from normal skeletal muscle.

TABLE 2

(after Polli and Ratti, 1953)

LIPOID CONTENT OF LEUKEMIC AND NORMAL LEUKOCYTES in $\mathrm{mg} \%$

\begin{tabular}{lccccccc}
\hline & $\begin{array}{c}\text { Total } \\
\text { Fat }\end{array}$ & $\begin{array}{c}\text { Total } \\
\text { Fatty } \\
\text { acids }\end{array}$ & $\begin{array}{c}\text { Neutral } \\
\text { fat }\end{array}$ & $\begin{array}{c}\text { Total } \\
\text { Choles- } \\
\text { terol }\end{array}$ & $\begin{array}{c}\text { Choles- } \\
\text { terol } \\
\text { esters }\end{array}$ & $\begin{array}{c}\text { Free } \\
\text { Chol- } \\
\text { esterol }\end{array}$ & $\begin{array}{c}\text { Phospho- } \\
\text { lipid }\end{array}$ \\
\hline $\begin{array}{c}\text { Human myeloid } \\
\text { leukemia }\end{array}$ & 1978 & II 70 & 204 & 329 & 57 & 272 & 1463 \\
$\begin{array}{c}\text { Human lymphatic } \\
\text { leukemia }\end{array}$ & 1002 & 625 & 236 & 200 & 35 & 165 & 59 I \\
$\begin{array}{c}\text { Human normal } \\
\text { leukocytes }\end{array}$ & 1447 & 830 & 209 & 320 & 82 & 238 & 896 \\
\hline
\end{tabular}


The significance of the lipid content or concentration upon the origin or growth of malignant tissue is not apparent at this time. Perhaps more extensive qualitative studies on the fatty acid composition and the steroid composition or the phospholipids of larger number of tumors of varying types may reveal some importance of these structures in the malignant processes. Medes et al. (1953) have found that neoplastic tissues cannot synthesize sufficient fatty acids to meet the lipid needs of the rapidly growing tissue. This aspect will be considered in further detail in a following section.

The interesting observation has been made by Kandutsch and Baumann (I954) that administration of carcinogenic hydrocarbons greatly reduces the concentration of $\Delta^{7}$-cholestenol in mouse skin. The concentration of this sterol in a series of tumors was essentially the same as that in most normal tissues. It would appear that sterol metabolism may be involved in the process of cancer induction, however there are not sufficient data to define a sterol pattern that may be associated with the growth or maintenance of the malignant cell. Lipoids in serum were administered by Rondoni (1954) into tumor bearing animals; the growth of the tumor was subsequently impeded which was attributed to antibody formation. Rondoni also found a sulphophospholipid on both human and experimental tumors.

The Rous fowl sarcoma is rich in hyaluronic acid (Harris et al., 1954). This hyaluronate was similar to the hyaluronic acids of normal mammalian tissues. Certain polysaccharides of bacterial origin possess considerable tumor necrotizing activity (See Greenstein, Chapter viII, I954). Takashi et al., (I954) have reported that polysaccharide fractions, extracted, from ascites carcinoma, placenta, Yoshida sarcoma or beef liver, exert an inhibitory action on cell mitosis.

\section{(b) Respiration and glycolysis}

The observations made by Warburg and associates almost 30 years ago still retain the greatest importance in the search for a specific metabolic pattern for malignant tissues. These investigators established that the cancer cell has the capacity to produce large quantities of lactic acid from glucose and that this capacity wâs not lost in the presence of oxygen. In order to reemphasize the importance of these lasting observations the following quotation has been taken from the translation of Warburgs' collected papers on tumor metabolism. "Whilst, however, normal cells die if they glycolyse aerobically, the glycolysing tumor cell not only continues to exist, but is even able to grow to an unlimited extent, turning to account the chemical energy of the glycolysis. The aerobic glycolysis of the tumour cell is derived in any case from a disturbance of the respiration. As a rule, the respiration of the tumour cell is small, but in recent years tumour cells with a large respiration have also been found" (Warburg, 1930). Currently there is a considerable difference of opinion amongst some of our foremost scientists as to the interpretation of the above Warburg observations. Certainly this area of cancer investigation is of the greatest importance and merits considerable attention. Accordingly, an attempt will be made to thoroughly review all findings to present the views of the qualified participants in this controversy.

Most of Warburg's original as well as subsequent findings reveal a high aerobic and anaerobic glycolysis that is characteristic of cancer cells. The respiration 
TABLE 3

CRITERION OF MALIGNANCY

(from Burk, 1939)

\begin{tabular}{|c|c|c|c|}
\hline & & Malignant range & Normal range \\
\hline$Q_{\mathrm{O}_{2}}$ & & $2-10$ & $2-20$ \\
\hline$Q_{\mathrm{A}}^{\mathrm{O}_{2}}$ & & O-I 5 & $\begin{array}{l}\text { less I with excep- } \\
\text { tion noted above }\end{array}$ \\
\hline$Q_{\mathrm{A}}^{\mathrm{N}_{2}}$ & & $8-20$ & $2-8$ \\
\hline $3\left(Q_{\mathrm{A}}^{\mathrm{N}_{2}}-Q_{\mathrm{A}}^{\mathrm{O}_{2}}\right) / Q_{\mathrm{O}_{2}}$ & Meyerhof oxidation quotient & $3^{-6}$ & $0.5^{-2.0}$ \\
\hline$Q_{\mathrm{A}}^{\mathrm{N}_{2}-2} Q_{\mathrm{O}_{2}}$ & (fermentation excess) & -5 to +25 & -2 to -30 \\
\hline$Q_{\mathrm{CO}_{2}} / Q_{\mathrm{O}_{2}}$ & respiratory quotient & $0.75-0.9$ & over I.o \\
\hline
\end{tabular}

rates of most normal and cancer tissues are of approximately the same magnitude, $3^{-20} \mathrm{ml}$ of oxygen per milligram of dry tissue per $\mathrm{h}$. $\left(\mathrm{Qo}_{2}\right)$. A high anaerobic metabolism (usual range of $5^{-40} \mathrm{ml}$ of lactic acid equivalent per milligram dry tissue per h.) is observed in most neoplastic tissues. With the exception of embryonic, placental, retinal and brain cortex, most tissues exhibit an anaerobic glycolytic metabolism of $10-20^{\circ}$ that of the cancer tissues. In the presence of oxygen, glycolysis is reduced in cancer tissues to approximately $50 \%$ of that observed anaerobically while in most normal tissues glycolysis is abolished in the presence of oxygen. This difference in oxidative metabolism led Burk to postulate a criterion of malignancy (Table 3 ).

Thus, in general, it may be observed that tumors as a group do possess a characteristic glycolytic metabolism. On the basis of these oxidative differences, Burk and Schade (See Weinhouse et al., 1956) and Burk and Woods (1956) have proposed a general test for malignancy. Ten mg. of tissue is inoculated in serum medium in a Warburg flask. Glucose is added to the medium and the reaction is carried out in the presence of $5.5 \% \mathrm{CO}_{2}$ in oxygen. All tumor tissues will exhibit a positive manometric pressure while all normal and embryonic tissues give a negative pressure. Overlapping between the normal and neoplastic ranges does occur and consequently there has been a tendency to deemphasize the importance of the general Warburg concepts. Nevertheless, the convergence of most malignant tissues in the direction of a characteristic and uniform metabolism still constitutes the most consistent metabolic difference yet observed between normal and neoplastic tissues.

The Warburg school accounts for this aspect of the metabolism of tumors on a basis of a faulty respiration or a respiratory impairment. Many investigators have opposed some of the Warburg concepts. It would appear however, that most scientists are in agreement as to the importance and the validity of the differences that generally exist in the overall oxidative metabolism of normal tissues and 
TABLE 4

(After Warburg - See Weinhouse et al., 1956)

\begin{tabular}{lccc}
\hline Cells & $\begin{array}{c}Q_{\mathrm{O}_{2}} \\
\left(\mathrm{O}_{2} \text { consumed) }\right.\end{array}$ & $\begin{array}{c}Q_{\mathrm{M}}^{\mathrm{O}_{2}} \\
\text { (lactic produced } \\
\text { aerobically) }\end{array}$ & $\begin{array}{c}Q_{\mathrm{M}}^{\mathrm{N}_{2}} \\
\text { (lactic produced } \\
\text { anaerobically) }\end{array}$ \\
\hline Ascites cancer cells & -7 & 30 & 70 \\
$\begin{array}{l}\text { Earle's cancer cells } \\
\text { low malignancy }\end{array}$ & -7 & 30 & 70 \\
Chorion of young embryos & $-\mathrm{I} 3$ & $\mathrm{I0}$ & 25 \\
\hline
\end{tabular}

cancerous tissues. The real point of contention has been in the interpretation of the underlying causes for these oxidative differences. Weinhouse and others do not accept the concept that this glycolytic activity of tumors does result from a faulty respiration (Weinhouse, I956). Some of the more recent findings and views will be presented in further detail.

Since the original methodology and the observations of Warburg dealing with the respiration and glycolysis of embryonic, retinal, normal body and cancerous cells are adequately presented (Warburg, I930; Greenstein, I954) reference will be made only to the recent findings in this area. Warburg (see Weinhouse et al., I956) has referred to the findings presented in Table 4 as further proof that the degree of malignancy may be correlated with an increased fermentation and a decrease in respiration. Woods and associates (Woods et al., I953, I955) on the basis of their results strongly adhere to the Warburg concepts. Mice bearing S9I melanoma were exposed to increased environmental temperatures $\left(35^{\circ}-40^{\circ} \mathrm{C}\right)$. Growth of the $35^{\circ}-40{ }^{\circ} \mathrm{C}$ tumor was inhibited by continued exposure to the increased temperature. The in vitro anaerobic glycolysis was lowered in slices of the excised tumor. However, insulin restored the $Q_{\mathrm{CO}_{2}}^{\mathrm{N}_{2}}$ of slices from heat treated tumors to a level equal to that of slices from the tumors of mice maintained at lower temperatures.

Certain of the steroid sex hormones also inhibited anaerobic tumor glycolysis and in some instances this inhibition could be reversed by insulin. While many factors are undoubtedly involved, it would appear that the high glycolytic rate of melanoma and other tumors may depend upon the hexokinase phosphorylation system and insulin activity. Factors that tend to disturb glycolytic capacity may be directly involved in malignant transformation as well as in the maintenance of the glycolytic metabolism characteristic of the malignant cell (Burk and Woods, 1956). Actually, Warburg (1955) has postulated that carcinogenesis occurs in two stages. An oxygen deficiency may result in irreversible damage to cellular respiration. In the second stage, the cell types that have succeeded in replacing their lost respiratory energy by fermentative energy become predominant. Rondoni (1955) is of the opinion that cancer formation represents a failure in protein 
synthesis and that no decisive importance may be attached to the glycolytic mechanism.

It would appear that the glycolytic activity does play an important role in malignant tissue metabolism. While Warburg and his colleagues still favor the hypothesis of a faulty or impaired respiration, the overall effect may be controlled by several factors. Since Warburg's original observations, our knowledge of the respiratory process has been greatly increased with the advances made in electron transport mechanisms, phosphorylation and in the further establishment of the cycles involved in the oxidation of glucose and fatty acids (see Chapter $\mathrm{r}$ ). Burk and Woods (I956) are of the opinion that the impaired respiration of tumors may involve any or all of the following: ( I) a high ratio of glycolysis to respiration, (2) a low absolute value for $\mathrm{O}_{2}$ consumption, (3) a deficient or uncoupled respiration, (4) a low paraphenylenediamine oxidative response.

Weinhouse (1955, I956) in referring to Warburg's concept of a disturbed respiration in tumor tissues believes that it would have been more accurate to state that the anaerobic glycolysis of tumor slices is sufficiently high that a normal respiration and a normal Pasteur effect are incapable of eliminating it. This, according to Weinhouse would place the emphasis on the high glycolytic rate of tumors rather than on the respiration or the Pasteur effect in the neoplastic cells. A similar view has also been taken by Schmidt (1955). Weinhouse (1955) in reviewing the work of LePage (1948, I950) agrees that the Embden-Meyerhof scheme of phosphorylative glycolysis and all of the phosphorylated intermediates found in normal tissues are present in many primary as well as in transplanted tumors. Weinhouse states "a highly significant feature of LePage's results is that potential rates of glycolysis of normal tissues are as great as, or greater than, those of tumor tissues, and that the low rate of glycolysis displayed by normal tissue slices are not due to lack of enzymes for the reactions involved".

There is some evidence to suggest that the high aerobic glycolysis of tumors may have its origin in the pathways of electron transport. The following values are reported for the comparative activities of oxidized and reduced diphosphopyridine nucleotides in normal and neoplastic tissues (Weinhouse, I 955).

Oxidized diphosphopyridine

nucleotide $(D P \mathcal{N})$

$\begin{array}{lrr}\text { Normal tissue range } & \text { I02-630 } & \text { I6-264 } \\ \text { Neoplastic tissue range } & 89-297 & \text { 0-6 I }\end{array}$

Also, there has been some indication of a relative deficiency of cytochrome c and cytochrome oxidase in tumor tissues. Chance and Castor (1952) employing spectrophotometric methods studied ascites tumor cells by measuring the optical density of the cytochrome $\mathrm{a}, \mathrm{b}, \mathrm{c}$, and $\mathrm{a}_{3}$ when respiration was stopped by oxygen cxhaustion. Their findings indicated that the cytochrome oxidase activity of the neoplastic cells was within the ranges for normal cells. Many investigators have established that glucose is metabolized via the Embden-Meyerhof scheme in malignant tissues. Emmelot and associates (1955) compared the ${ }^{14} \mathrm{C}$ recoveries in 
${ }^{14} \mathrm{CO}_{2}$ after incubation of mouse tumor slices with glucose- ${ }^{14}{ }^{14} \mathrm{C}$ or glucose-6${ }^{14} \mathrm{C}$ in Krebs-Ringer phosphate. The $\mathrm{CO}_{2}$ from the labeled glucose is initially more active from $\mathrm{C}_{1}$ than $\mathrm{C}_{6}$ if the oxidative pathway is in operation. Carbon atoms I and 6 appear at equal rates if the glycolytic scheme is operative. The ratio $R_{6} / R_{1}$ (when $R=$ radioactivity as $\mathrm{CO}_{2}$ ) was found to be 0.7 for mouse liver, I. I for diaphragm, 0.47 for mammary carcinoma, 0.35 for hepatoma, 0.69 for ovarian tumor and 0.65 for sarcoma. From the lactic acid isolated from the medium it could be calculated that approximately $70 \%$ of the glucose- ${ }^{14}{ }^{14} \mathrm{C}$ molecules giving rise to the lactic acid has followed the oxidative pathway in mammary carcinoma and $50 \%$ in ovarian tumors and sarcoma.

Wenner et al. ( $195^{6}$ ) employing glucose labeled with ${ }^{14} \mathrm{C}$ in all positions, in carbon ${ }^{1}$ or carbon ${ }^{6}$, estimated the pathway of respiration and glycolysis in solid and ascites tumors. They reported that $77-94 \%$ of the glucose catabolized in tumors was converted into lactic acid and the remainder into $\mathrm{CO}_{2}$. Most of the respiratory $\mathrm{CO}_{2}$ arose via the Embden-Meyerhof pathway and the citric cycle. Holzer et al. (I955) also reported that cells of the Ehrlich ascites tumor metabolize glucose via the Embden-Meyerhof scheme. Fructose may also be directly phosphorylated to fructose-6-phosphate and enter the pathway through fructose-I,6-diphosphate. The Embden-Meyerhof pathway is active in the glucose metabolism of normal liver. In transplantable hepatocarcinoma in $\mathrm{C}_{57}$ mice the hexose monophosphate shunt may account for $25-50 \%$ of the fatty acids and lactate derived from glucose (Abraham et al., I 955, I956). In contrast, normal rat or mouse mammary tissue is characterized by a high hexosemonophosphate shunt activity, which is greatly reduced in adenocarcinoma of the mammary gland. Tumor tissues never exceed an aerobic oxidative rate of $Q_{\mathrm{O}_{2}}{ }^{20}-25$ according to Reif et al. (I953) in either the malic or succinoxidase systems. However, the oxidative capacity of certain normal tissues greatly exceeds that of tumors. These investigators developed a system for aerobic glycolysis of fructose- I,6-diphosphate by tissue homogenates. A gradation of oxidative capacity was demonstrated from high values for normal to the lowest range for tumor tissues. The addition of brilliant cresyl blue doubled the oxygen uptake of tumors but did not affect the normal tissues, suggesting a limited DPNcytochrome c reductase in tumors. They also found that tumor homogenates produced lactic acid from glucose. Brain homogenates carried out this reaction anaerobically but other normal tissues were unable to produce lactic acid in the absence of other substrates.

Both normal and leukemic myeloid cells are characterized by high aerobic glycolysis while lymphatic leukemic lymphocytes and blasts forms have low aerobic glycolytic rates (Beck and Valentine, I952, I953; Beck, I955). Leukemic cells require more cytochrome c for a maximal uptake of oxygen than normal cells. McKinney and Rundles (1956) have also noted that leukocytes from patients with either chronic myelocytic or lymphatic leukemia produced less lactic acid aerobically than anaerobically. The leukemic leukocytes produced less lactic acid than normal leukocytes. From the findings of Steinberg et al. (1952) it would appear that the oxidative capacity is low for all lymphoid tissues, normal or malignant. On a basis of oxidative activity these investigators could not distinguish between normal and malignant lymphoid tissues. Kit ( $\left.195^{6}\right)$ incubated suspensions 
of rat thymus, mouse spleen, appendix, Ehrlich and Gardner lymphosarcomas or lymphatic leukemia with glucose- $\mathrm{I}-{ }^{14} \mathrm{C}$, glucose- $2-{ }^{14} \mathrm{C}$, glucose- $6-{ }^{14} \mathrm{C}$ or uniformly labeled glucose. It was observed that the hexose monophosphate shunt was active in intact tumor and spleen cells. All of the tissues utilized glycolysis to the extent of $60-80 \%$. On the basis of the differential oxidation of glucose- $-{ }^{14} \mathrm{C}$ and glucose-6- ${ }^{14} \mathrm{C}$ to ${ }^{14} \mathrm{CO}_{2}$ it was calculated that the intact tumor cells formed two to five times as much pentose from the glucose as did the normal lymphatic cells. The growth of implanted tumors was suppressed in severely diabetic rats and these animals excreted less than half of the glucose excreted by force-fed, nontumor bearing diabetic controls (Ingle, I 956).

Picco and Dogliotti (1952) in studies on human tumors have provided further information that tumors contain more glucose, pyruvic acid, and lactic acid than homologous normal tissues. The original demonstration of a lowered $\mathrm{pH}$ in animal tumors (Greenstein, I954) has been confirmed and extended by Eden et al. (1955). Using large numbers of several varieties of rat tumors they reported an average pH of 6.99 for the neoplastic tissues. Following the injection of glucose, the $\mathrm{pH}$ dropped to 6.55 within three to four hours. Normal muscle and hepatic tissues did not exhibit any pH decrease. Naeslund and Swensen (1953) and Naeslund (1954) attempted to find compounds that would precipitate at this lowered pH and thereby accumulate in the malignant tissue. Benzoylsulphamethylpyrimidine has a solubility three times as high at $\mathrm{pH} 7.5$ than at $\mathrm{pH} 6.5^{\mathrm{I}}$.

The addition of glucose, mannose, or fructose inhibits the respiration of ascites tumor cells (Brin and McKee, I956). Within 2-3 hour this inhibition is released. Just prior to the release of inhibition the glucose has disappeared and the lactate concentration is maximal. The addition of inorganic phosphate reduced this inhibition, indicating that the diffusion or entry of inorganic phosphate into the tumor cell may not be adequate to support the metabolic requirements. McKee et al. (1953) also found that glucose added in amounts greater than I.5 millimoles inhibited oxidation in mouse ascites carcinoma cells. They concluded that a shunting of the metabolism from oxidative to glycolytic may be of importance in the abnormal growth and the non-differentiation of the tumor cells. Roitt (1956) has observed an inhibition of carbohydrate metabolism in ascites tumor cells by the ethyleneimines. The analog, 2-deoxy-D-glucose inhibits aerobic and anaerobic glycolysis in slices of rat tumors, rat brain and diaphragm. This compound did not inhibit endogenous respiration in any of the tissues (Woodward and Hudson, I954). Tri- and tetra-iodothyroacetic acid accelerated glycolysis in several ascites tumors (Heimberg et al., I 955). Lettré (1952, I 952a) reported that pyocanin decreased considerably the number of mitoses in cultures of fibroblasts and of mouse ascites tumor. This effect was attributed to the stimulatory effect of pyocanin on respiratory metabolism. A relationship was proposed between mitosis and the glycolytic metabolic state. The anaerobic consumption of glucose and also lactate production was stimulated by dinitrocresol in ascites tumor cells (Clowes and Keltch, I 954). However, the consumption of fructose and mannose was not stimulated by this compound, suggesting that the amounts and types of hexrkinase in the ascites cells may differ from that of brain and other normal cells. El'Tsina (1953), in ascertaining the role of glycolytic and oxidative processes in 
the formation of protein in Ehrlich carcinoma cells, reported that the oxidative metabolism was blocked by $\mathrm{KCN}$ or $\mathrm{NaN}_{3}$, while the glycolytic process was blocked by the elimination of glucose. When the respiratory processes alone were active the rate of assimilation of radioactive methionine into the proteins was approximately half of that observed when the glycolytic process was active.

These observations regarding respiration and glycolysis in neoplastic cells represent a small proportion of the investigations that have been carried out in this important area. From the seemingly unending list of publications the impression may be gained that an explanation for the high glycolytic activity of tumors should be readily available. Upon closer examination of these experimental results it soon becomes apparent that a really concerted effort is lacking. The investigations encompass a wide range of tumors-animal and human, ascites and solid types, variations in methods and other variables, making the task of critical correlation or proper evaluation extremely difficult. Indeed, many of these findings appear to be completely isolated. A thorough understanding of the methods and the particular systems employed is an essential prerequisite for the planning of studies to establish or to build upon existing concepts.

The literature generally supports the concept of a high anaerobic and aerobic glycolysis in cancerous cells. Also there seems to be little doubt that the glycolytic pathway in tumors follows the established Embden-Meyerhof scheme for normal tissues. Most investigators are in agreement that this glycolytic pathway may account for $60-90 \%$ of the $\mathrm{CO}_{2}$ that is derived from the glucose utilized in tumors. A majority of the findings point to a rather uniform metabolism for malignant tissues. Certainly this glycolytic pattern is of significance in the origin or the subsequent growth of tumor cells. In attempting to account for or explain this metabolic difference in too general terms, much of the real significance of these important findings has been overshadowed. The concept of a faulty or impaired respiration in tumor cells has little meaning in terms of specific reactions or components of the cells. The available evidence would indicate that all of the enzymes as well as the intermediate components involved in the complete breakdown of glucose are present in the cancerous cell. Factors that may account for this difference include: disturbance in electron transport, differences in membrane permeability between normal and cancerous cells thereby limiting the exchange and availability of essential metabolites, relative differences in the concentration of specific enzymes or cofactors in tumor cells such as cytochrome, cytochrome oxidase etc., the relation of lipid oxidation to the overall metabolic pattern, and finally, the possibility of the inherent response of malignant cells to the hormones of the pituitary, adrenal, pancreas, and thyroid glands which virtually control the various steps of carbohydrate metabolism. Further discussion of these important areas of glucose and lipid metabolism will appear in subsequent sections.

Recently, Potter (1956) has proposed a concept of respiratory enzyme balance to replace that of a faulty respiration. The respiration of the tumor is geared to the synthesis of the many essential constituents of growth. This includes the many phosphorylative reactions and the formation of the constituents of the nucleic acids. Potter relates respiratory balance to the biosynthesis of deoxyribonucleic and and ribonucleic acids as presented in Fig. $\mathbf{I}$. 

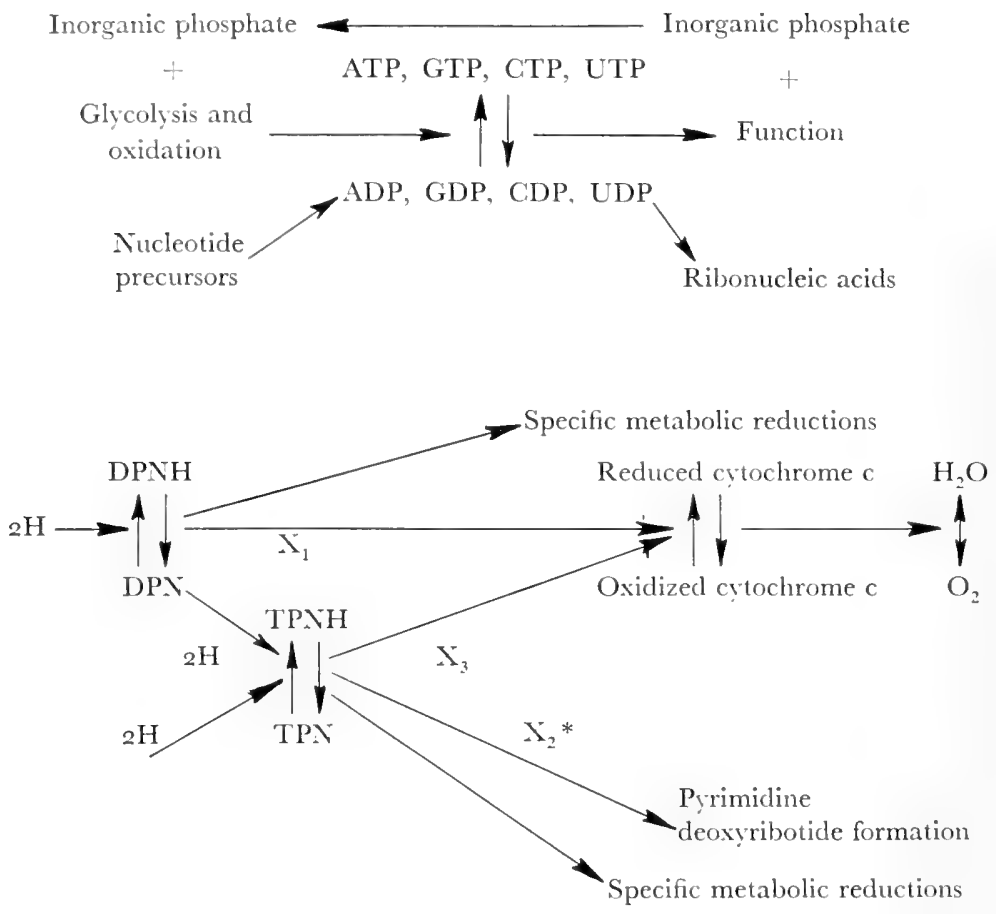

Fig. I. Relation between respiratory balance and DNA or RNA synthesis. * Enzyme $\mathrm{X}_{2}$ is speculative, the other enzymes are known to exist. (From Potter, 1956).

These speculative charts illustrate how alterations in respiratory enzyme balance may shift the metabolism in the direction of deoxyribonucleic acid production, ribonucleic acid formation or towards other metabolic functions.

\section{(c) Citric cycle}

The excellent compilations of Greenstein (1954, I956) provide a most comprehensive review of the concentrations and activities of the components and enzymes involved in the oxidation of pyruvate. It is evident from a qualitative standpoint that tumors do contain all of the known enzymes involved in the citric cycle. Quantitatively there are wide ranges in the activities of these enzymes in various normal tissues. Generally, the activities of these enzymes in tumors approximate the lowest normal values, the tumors showing a uniform behavior in this respect. Pyruvate, oxalacetate, citrate and ketoglutarate, succinate, fumarate and malate are readily oxidized by tumor homogenates fortified with DPN (Weinhouse, I95I, I955; Wenner and Weinhouse, I953). Weinhouse (I955) 
found that several transplanted tumors are capable of carrying on the condensation of acetyl coenzyme A with oxalacetate to form citrate at rates comparable to normal tissues. From studies involving the incorporation of isotope from labeled acetate, propionate, octanoate, pyruvate and glucose, Brown et al. (1956) concluded that the citric cycle was operative in liver tumor.

Several investigators have demonstrated that the succinoxidase activity is considerably lower in malignant tissues than in the normal tissue of origin (Schneider and Hogeboom, I950; Dmochowski and Stickland, I 953; Mor, I 955). Schneider and Potter (1 943) reported that the succinoxidase activity of a group of rat tumors ranged from 9-26 $\mathrm{ml}$ of oxygen consumed per $\mathrm{mg}$ dry weight per hour with succinate as substrate. The values for normal tissues ranged from i 8 for lung to 2 I 9 for heart. These comparative values are quite representative of the relative enzyme activities of normal and neoplastic tissues. Several investigators determined the incorporation of radioactivity from acetate $-\mathrm{I}-{ }^{14} \mathrm{C}$ into the succinate pools induced by malonate in normal and neoplastic tissues in rats bearing FlexnerJobling carcinoma (Busch and Potter, I952, I952a, 1953; Busch, I953; Busch and Baltrush, 1954). A maximum incorporation into the succinate pool of normal tissues occurred within $2-4 \mathrm{~min}$. after the intraperitoneal injection of the labeled acetate. The total radioactivity in the succinate pool accounted for $30 \%$ of the total radioactivity within the kidney and approximately $10 \%$ in other normal tissues. In the carcinoma, however, a high percentage of the radioactivity remained volatile for $30 \mathrm{~min} /$. after the injection of the acetate. This low incorporation of ${ }^{14} \mathrm{C}$ into the succinate pool within the tumor almost completely differentiates this tissue from heart, spleen, lung and the other normal tissues.

The anaerobic metabolism of pyruvate in homogenates of normal and neoplastic rat tissues has been investigated by Groth and LePage (I 954). Under these conditions, glycolyzing, homogenates of both normal tissue and Flexner-Jobling carcinoma metabolized pyruvate at a rapid rate to yield components other than lactic acid. One of the major products of the anaerobic metabolism was I,2-propanediol-I-phosphate in both tissues. In homogenates of the normal tissues the decarboxylation of pyruvate to yield a two carbon fragment and $\mathrm{CO}_{2}$ was also observed. Emmelot and Bosch (I955) studied the relative rates of pyruvate and acetate incorporation into $\mathrm{CO}_{2}$, amino acids, cholesterol and fatty acids in mouse tumors in vitro. The ability of tumors to utilize pyruvate relative to acetate was as follows: hepatomas, ovarian tumors of granulosa cell type and ovarian tumors of the sarcomatoid type in increasing order.

An important study on the metabolism of pyruvate in tumor bearing mice has been made by Busch (1955). One $\mathrm{mg}$ of pyruvate- $2-{ }^{14} \mathrm{C}$ was injected into rats with Flexner-Jobling carcinoma, Walker $25^{6}$ carcinoma, or Jensen sarcoma. The experiments were terminated from $\mathrm{I} 5 \mathrm{sec}$. to $8 \mathrm{~min}$. following the injection of the labeled pyruvate. In the normal tissues, $30-80 \%$ of the isotope was converted into alanine, glutamic acid and aspartic acid within one min. after injection of the radioactive compound. Adenine accounted for approximately $30 \%$ of the total isotope in liver, testes, muscle and intestine after injection of the pyruvate. Tumors were slow to form labeled amino acids. At short time intervals following the isotope administration a considerable quantity of ${ }^{14} \mathrm{C}$ was found 

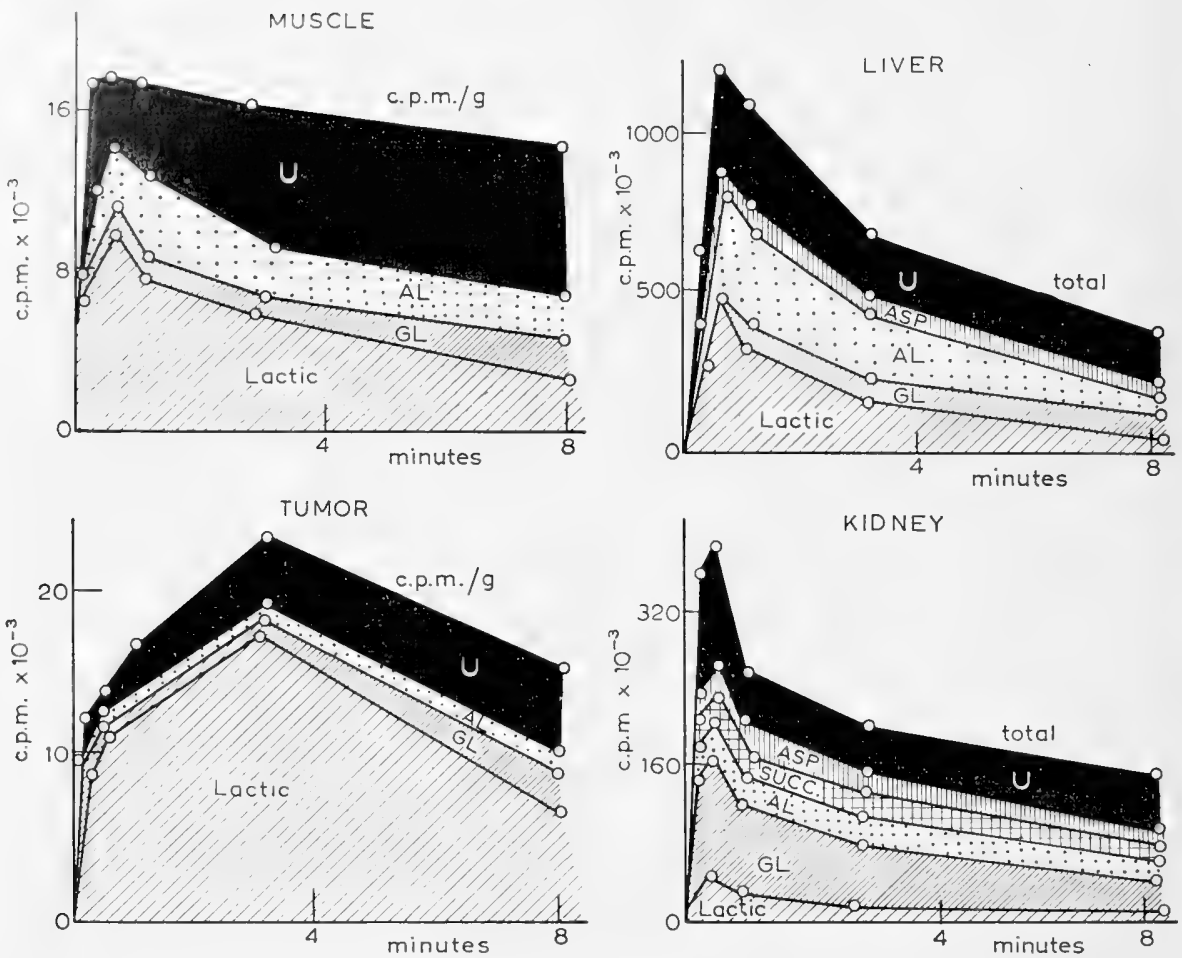

Fig. 2. Metabolic patterns for pyruvate-2- ${ }^{14} \mathrm{C}$ in various tissues. (Busch, 1955). The upper line represents the total isotope in the whole tissue (total) or the isotope/g of tissue (counts $/ \mathrm{min} / \mathrm{g}$ ). The weights for various tissues were, approximately: liver, $8 \mathrm{~g}$; kidney, $3 \mathrm{~g}$; spleen, $4 \mathrm{~g}$; testis, $5 \mathrm{~g}$; heart, $\mathrm{I} .5 \mathrm{~g}$; brain, $3 \mathrm{~g}$. The shaded areas are percentages of the total isotope in the particular compound at the various time points and are labeled as follows: lactic acid, LActic; Glutamic acid, GL; alanine, AL; aspartic, ASP; succinic acid, SUCC; and unknowns, U. Maximal recoveries of the isotope in tissues ranged from 75 to Ioo per cent.

in lactate in the tissues, but was reduced to $3-20 \%$ for non-tumor tissues by eight min. In the tumors $50 \%$ of the total radioactivity remained in lactate after this same eight minute interval. These interesting findings are further illustrated in Fig. 2 (Busch, 1955). An extension of the studies involved the metabolism of pyruvate-2- ${ }^{14} \mathrm{C}$ in in vitro systems containing slices of Walker 256 carcinoma, Jensen sarcoma, uterine carcinoma, liver, kidney, spleen and brain (Busch et al., I 956). The tumor slices incorporated approximately $14 \%$ of the ${ }^{14} \mathrm{C}$ as $\mathrm{CO}_{2}, 64 \%$ into lactate and $2 \mathrm{I} \%$ into amino acids. Addition of glucose to the medium resulted in the following incorporation: $3 \%, 92 \%$ and $5 \%$ into the $\mathrm{CO}_{2}$, lactate and :mino acids respectively. The addition of glucose to kidney or liver slices did not significantly alter the metabolic pattern. Of interest, in view of the metabolism of brain tissues was the varying distribution of ${ }^{14} \mathrm{C}$ in different mediums. In the unsupplemented medium, brain slices transferred $66 \%$ of the ${ }^{14} \mathrm{C}$ to amino acids. 
Addition of glucose resulted in a shift towards lactate while addition of glutamate increased the labeling of adenine.

The findings of Busch and colleagues point up the fact that neoplastic tissues are more or less characterized by a uniform but not an entirely unique metabolism. Lactate is readily formed from pyruvate, especially in the presence of an increased glucose concentration. The lactate formed is only slowly converted into other metabolites. Several of the normal tissues have the capacity to convert pyruvate into lactate, amino acids, or adenine, depending somewhat on the tissue involved or the presence of other substrates. This would suggest that respiratory enzyme balances as proposed by Potter may be the deciding factor in meeting the needs of the particular tissue. Tumor tissues may depend upon their glycolytic pattern to provide the energy and the metabolites that are required for mitotic division and rapid growth. The significance of the high aerobic, anaerobic glycolysis and the formation of relatively large quantities of lactate during the growth of neoplastic tissues is not entirely clear at this time.

\section{(d) Phosphorylation}

The available evidence would indicate that tumors are capable of obtaining energy from the phosphorylative reactions associated with the various stages of oxidation within the citric acid cycle. Under carefully controlled conditions, Kielley (1952) demonstrated that tumor mitochondrial preparations do have the capacity to readily synthesize ATP during the oxidation of $\alpha$-ketoglutarate and succinate. The question has been posed by Weinhouse (1955) as to whether the differences in glycolytic metabolism between normal and tumor tissues may occur at the hexokinase level where ATP and glucose react to give glucose-6phosphate. There is no definite evidence that would provide an answer to this proposal. Morelli et al. (1953) in studying aerobic and anaerobic phosphorylation in leukemic leucocytes did not detect any significant differences in the inorganic phosphate between normal and leukemic cells. The level of phosphate was decreased and the lactic acid increased in the blood of cancer patients injected with fructose (Reymond and Wild, I 953). In mouse mammary tumors and in rat hepatoma the phosphorylase, according to Goranson et al. (1954), was inactive without the addition of adenylic acid. With added adenylic acid no significant differences were found between the phosphorylase in the tumors or in normal liver.

Glucose-6-phosphate and 6-phosphogluconate dehydrogenase activities were determined by Glock and McLean (1954) in several experimentally induced and also spontaneous tumors. These enzyme activities were highest in lymphomas and lowest in sarcomas. However, the activities fell within the normal range. Weber and Cantero (I955) reported that the glucose-6-phosphatase activity was lower in fetal liver and in hepatoma than in normal or regenerating liver. Phosphoglucomutase was found to be absent in the Novikoff hepatoma (Weber and Cantero, 1956). Glucose-6-phosphate dehydrogenase was increased in the hepatoma. Schlief and Schmidt (I955) reported that hexokinase and aldolase of mouse ascites tumors were independent of the rate of growth of the tumors. Adenosinetriphosphatase, however, was considerably increased in the rapidly growing tumors. It has been previously reported that the ATPase activities of various nor- 
mal and neoplastic tissues are nearly equal (Potter and Liebl, 1945). The acid phosphatase was not altered significantly during the different stages of growth or regression of the Flexner-Jobling carcinoma. The alkaline phosphatase values were considerably elevated in the growing tumors (Foder et al., 1955).

Williams-Ashman and Kennedy (1952), in studying oxidative phosphorylation, prepared mitochondrial fractions from tumors. Succinate was added as substrate and fructose hexokinase as the terminal phosphorus acceptor system. By use of ${ }^{32} \mathrm{P}$ it was observed that there was considerable incorporation of phosphorus into the lipids, nucleic acid and residual protein fractions. The dependence of this process upon the substrate as well as inhibition by DPN led these investigators to conclude that glycolytic mechanisms could not account for the observed synthesis. They further indicated that tumor cytoplasmic fractions possess the same aerobic mechanisms for the generation of high energy phosphate components as are operative in other mammalian tissues. Lindberg et al., (1953) reported that both respiration and phosphorylation were low in mitochondria prepared from Ehrlich ascites tumor, the rate of phosphorus uptake in micromoles of phosphorus per $\mathrm{mg}$ of nitrogen per min. being between 0.05 and 0.3 with various substrates. A value of two was obtained for normal mouse liver and four for rabbit heart. The $\mathrm{P} / \mathrm{O}$ ratios were also depressed in the tumor mitochondrial preparations. ATPase from tumor mitochondria was capable of destroying the ATP required for the formation of the acyl coenzyme A bond, according to Emmelot and Bos (1955).

Siekevitz and Potter (1953), and Wenner and Weinhouse (I955) have studied the effects of dinitrophenol and fluoride on oxidation in normal and tumor tissues. The former investigators added both of these factors to several different tumor tissues and all gave somewhat different patterns of oxidative response. Wenner and Weinhouse reported that dinitrophenol stimulated glucose oxidation in neoplastic and normal tissues at low concentrations and inhibited at higher concentrations. Their conclusions are summarized as follows: "The neoplastic cell does not differ in any fundamental respect from non-neoplastic tissues in mechanisms of phosphorylation, and dephosphorylation associated with glucose metabolism". Clowes and Keltch (1952) also observed that by adjustment of chloride concentration, the preponderance of glycolytic over oxidative phosphorylation in Walker 256 carcinoma was considerably enhanced. Phosphorylation in this tumor appeared to differ from that in normal tissues mostly on a quantitative rather than on a qualitative basis.

The effect of diethylstilbestrol and benzoquinone on the interrelationships between respiratory, phosphorylative and mitotic activities in Ehrlich ascites tumor cells was studied by Shacter ( 1956 ). Both of these compounds in increasing concentrations inhibited cellular respiration and altered the acid soluble components. At higher concentrations the permeability of cellular membranes was also affected.

\section{(e) Electron transport}

Weinhouse (1955) gave an excellent summary of the present status of the electrron transport system. Greenstein (I954) has also provided a compilation of the contents and activities of the aerobic enzymes and cofactors that are present in many normal and neoplastic tissues. As has already been mentioned, there is a 
general contention that tumors may be characterized by their relatively low activities of cytochrome c, cytochrome oxidase, riboflavin and several other of the oxidative enzymes. Nevertheless, the level of these enzymes in neoplastic tissues is apparently sufficient to maintain the oxidative processes at normal rates.

Lenta and Riehl (1952) studied the ability of extracts of normal and neoplastic tissues to oxidize reduced DPN. The DPN was reduced enzymatically and the oxidase activity was then determined spectrophotometrically. The oxidative system was analyzed for diaphorase, cytochrome c reductase, and cytochrome oxidase. The considerable reduction in the coenzyme I oxidase system of mouse hepatoma 98/15, transplanted sarcoma and adenosarcoma was attributed to the reduction or failure of cytochrome $c$ and cytochrome oxidase. Ascites tumors do not appear to follow the pattern of a lowered cytochrome $\mathrm{c}$ and cytochrome oxidase activity as has been noted for other tumors. Chance and Castor (1952) measured the spectra due to cytochrome activity in ascites tumor cells and found a pattern for cytochromes a and $\mathrm{a}_{3}$ similar to that of normal muscle tissue or yeast cells. Cytochrome b was low in the ascites cells while cytochrome c was abnormally high. Actually the ratio of cytochrome $\mathrm{c}$ to $\mathrm{b}$ in the tumor was over four times as great as in normal heart muscle. Subsequently Chance (I953) reported that in suspensions of ascites tumor cells, spectrophotometric observations revealed that cytochrome c, flavoprotein and reduced DPN were present in normal amounts. Schmidt and Schlief (I 955) also concluded that the cytochrome c content and cytochrome oxidase of ascites tumor are similar to that of liver. The presence of peroxidase in the Walker carcinoma and other growing tissues led Neufeld et al. ( 1955 ) to postulate that this enzyme may form part of the respiratory system which would partially replace the conventional cytochrome system.

The oxidation of pyruvate, citrate, $\alpha$-keto glutarate, fumarate and malate was increased by factors of from two to ten fold by the addition of DPN to mitochondria obtained from mouse hepatoma $7 \mathrm{~A}_{77}$ (Wenner and Weinhouse, I953). With the addition of DPN, the oxidative response was similar to that obtained with mitochondrial preparations from normal mouse liver. The addition of DPN increased the oxidative and phosphorylative activity of the tumor to the level of normal liver (Kielley, r952; Wenner and Weinhouse, I953). This requirement of hepatoma and other tumors for additional DPN cannot be fully explained. The high DPNase activity of tumor mitochondrial preparations was mentioned previously (Williams-Ashman and Kennedy, I952). Wenner and Weinhouse (1953) also proposed that the mitochondria of tumor do not bind DPN as strongly as normal tissues and that there may be a correspondingly higher level of DPN in the tumor cytoplasm. This would provide a possible explanation for the relatively high glycolytic rate of tumors. Kertesz and Albano (I955) postulated that the high glycolytic activity of tumor may be attributed to some defect between reduced DPN and oxygen. This being the case, the addition of $o$-diphenol and polyphenoloxidase to reoxidize reduced DPN or TPN should inhibit the high glycolysis and also increase the respiration of tumors. In fact, these investigators did observe that aerobic glycolysis was inhibited and respiration was increased by the addition of diphenol and polyphenoloxidase to the Ehrlich adenocarcinoma system.

An assay of oxidized and reduced DPN in normal and neoplastic tissues was 
carried out by Jedeikin and Weinhouse (1955). The ratios of DPN:DPNH in the neoplastic tissues extended over the relatively narrow range of $2 \cdot 5-4 \cdot 5$. The normal range was $1.2-20$. The total content of DPN was generally lower in tumor than in normal tissues although certain normal tissues, i.e., brain and spleen were low in DPN. Some consideration should be given the fact that tumors have a low mitochondrial count. Any significant change in DPN content or the ability of tumor mitochondria to concentrate this factor may exert a considerable influence on the oxidative capacity of these tissues. Weinhouse ( I 955) suggested the possibility that the permeability of membranes of tumor mitochondria to DPN may be increased.

\section{(f) Fatty acid metabolism}

Interesting quantitative differences occur in the lipid metabolism in tumors (Greenstein, I954; Weinhouse, I955). Tumor cells have the capacity to oxidize fatty acids, usually at a reduced rate when compared with normal cells. Most of the investigations in this area have been carried out in normal or cancerous livers and our knowledge of lipid metabolism has been largely obtained from these organs. Compared to normal liver, hepatoma has a greatly reduced capacity to produce acetoacetate from fatty acids (Weinhouse et al. I953), however, the capacity to oxidize acetoacetate is enhanced. The observed rates of lipid synthesis in some tissues are too slow to account for the fat present, indicating that the tumor has to obtain at least a portion of its lipid from the host (Medes et al., I953; Wenner and Weinhouse, I953). Olson (I95 I), Zamecnik et al. (I 95I), and Medes et al. (I 953) have reported that hepatomas and also other tumors incorporate the carbon of radioactive acetate and glucose into fatty acids. As mentioned, the findings would indicate that the process is too slow to supply the lipid needs of rapidly growing tissues, and that the tumor may have to obtain its lipids preformed from the host.

A rate differential in the utilization of acetate by tumors has been established by Busch and Potter (1952; I 952a; I 953; Busch I953, Bush and Baltrush 1954). The utilization of acetate, which occurs readily in the majority of normal tissues, is markedly diminished in several tumors which were tested. Acetate $-\mathrm{I}-{ }^{14} \mathrm{C}$ was injected intravenously into normal rats and into rats bearing the Flexner-Jobling carcinoma, Jensen carcinoma, or Walker 256 carcinoma: the half-time for disappearance of the radiolabeled acetate in most non-tumor tissues was from 6- 5 sec., while the half-time in liver was $48 \mathrm{sec}$, but $4^{1 / 2} \mathrm{~min}$. for tumors (Busch and Baltrush, I954). This inability to utilize acetate readily constitutes an important metabolic difference between neoplastic and normal tissues.

Chapman et al. (1954) studied incorporation of the labeled carbon of octanoate$\mathrm{I}-{ }^{14} \mathrm{C}$, octanoate- $7-{ }^{-14} \mathrm{C}$ and butyrate $-\mathrm{I}-{ }^{14} \mathrm{C}$ into acetate, and $\mathrm{CO}_{2}$ by liver slices from normal and tumor-bearing mice, and cell suspensions of mouse hepatoma. These investigators concluded that the distribution of carbon ${ }^{14}$ in the two positions of acetracetate, as represented by the $\mathrm{C} * \mathrm{O}: \mathrm{C}^{*} \mathrm{OOH}$ ratio was compatible with the concept of a normal $\beta$-oxidation-cleavage-condensation in the tumor. They also studied the incorporation of labeled carbon from acetate- $\mathrm{I}-{ }^{14} \mathrm{C}$, acetate $-2-{ }^{14} \mathrm{C}$, propionate- $\mathrm{I}-{ }^{14} \mathrm{C}$, octanoate- $\mathrm{I}-{ }^{14} \mathrm{C}$, pyruvate- $2-{ }^{14} \mathrm{C}$ and uniformly labeled glucose 
into $\mathrm{CO}_{2}$ and other metabolites in slices of mouse liver or mouse hepatoma C954. The tumor was capable of incorporating isotope into tri- and dicarboxylic acids, glutamate and aspartate. While normal liver readily incorporated isotope from labeled 2-carbon fragments into glucose, the hepatoma failed to carry out this incorporation. Certain quantitative differences were observed in the pattern of labeling of various intermediates when the above components were incubated with slices of liver or hepatoma. The overall conclusion was reached, however, that the citric cycle was operative in the tumor (Brown et al., 1956).

A series of recent publications has contributed to our knowledge of the metabolism of neoplastic tissues. (Emmelot and Bos, I955, I955a, I955b; Emmelot and Bos, I955a, I955b; Emmelot et al., 1956). A marked stimulation of fatty acid and cholesterol synthesis from labeled acetate was found after addition of glucose to surviving slices of transplanted mouse tumor. This fatty acid synthesis appeared to be directly related to glycolysis in tumor tissues (Emmelot and Bos, I 955b). The ATPase from tumor mitochondria was also capable of destroying the ATP that is required for the formation of acyl coenzyme A and further fatty acid metabolism (Emmelot and Bos, I 955, I 955a). Further evidence was obtained that increased ATPase and DPNase activities may be an inherent feature of tumor mitochondria. This could account for the inhibition of fatty acid oxidation that is present in tumor tissues (Emmelot et al. 1956; Emmelot and Bos, 1956).

Tumors of adrenal origin utilize acetate- $\mathrm{I}^{-14} \mathrm{C}$ for the synthesis of cholesterol (Smith and Werthessen, I953). Medes et al. (1956) found that the in vitro conversion of labeled acetate to $\mathrm{CO}_{2}$ and cholesterol was essentially the same in normal rat liver, in the precancerous liver of rats fed diets containing 4-dimethylaminoazobenzene, or in the resulting hepatomas. The synthesis of cholesterol from labeled acetate in transplanted mouse tumors was also investigated by Emmelot and Bos (1955a), who found that the total activity incorporated into the cholesterol of the tumors was at least equal to that of liver. A much higher rate of cholesterol synthesis occurred in hepatoma than in ovarian tumor, indicating that all tumors do not behave quantitatively in this respect.

Berliner et al. (I 956) studied the chemical transformation of cortisol- $4-^{-14} \mathrm{C}$ by normal and leukemic lymphatic tissues of mice. The latter cells converted $10-20 \%$ of the cortisol to six or more metabolites, i.e., cortisone, substances $\mathrm{E}$ and $\mathrm{U}$ (Reichstein). No conversion was obtained with the normal lymphatic tissues. The significance of this effect of the leukemic tissues upon corticoid metabolism must await further studies. Extracts of ultraviolet irradiated methyl lineolate and methyl linolenate, according to Shuster (I 955), inhibited respiratory and glycolytic activities of Ehrlich ascites tumor cells. Since ascites cells contain unsaturated fatty acids this investigator assumed that a mechanism must be present within the cell with prevents the oxidation of these unsaturated fatty acids, since the fatty acid oxidation products would inhibit cellular activity. 
III. PROTEINS AND AMINO AGIDS

\section{(a) Composition}

The readers attention is directed to the following reviews relative to protein and amino acid composition of malignant tissues: Toennies (I947), Stern and Willheim, chapter II (1943), Greenstein (1954, 1956) and Biserte (1953). The compositional pattern of the proteins that constitute an integral part of all cells or tissues undoubtedly play an important role in the function and destiny of the organism. Up to this time, however, the biochemist has been unable to discover any such pattern of amino acids in proteins or any molecular features of these macromolecules that would in any way characterize the proteins of normal or neoplastic tissues. We may reasonably expect that the complex nucleoproteins may be involved in malignant transformation and in the behavior of the cancerous cell. This aspect will be further considered in a subsequent section. Also, some important advances have been made in studying the immunological properties of proteins derived from normal and neoplastic tissues (see Chapter I I).

A striking resemblence of overall amino acid composition of a variety of normal and neoplastic tissues was noted by Sauberlich and Baumann (I95I). Included in this study were analyses for the total concentration of each of 12 amino acids of many normal tissues from different species and also of fibrosarcomas, carcinoma and hepatomas. This approach did not reveal either quantitative or qualitative differences that may occur in the individual proteins of these tissues.

However, the application of end group and sequential amino acid analysis, as employed by Sanger, Du Vigneaud and others, may reveal significant compositional differences between protein components of normal and neoplastic tissues.

Hydrolysates of mouse tumor and embryo were analyzed for amino acid content by Boman (1952). Lysine, taurine and tyrosine were reported to be present in carcinoma but not in embryonic tissue. Also, more glycine, valine, serine and alanine were found in carcinoma than in the embryonic tissue. Amino acid analyses were carried out by starch column and paper chromatography on unhydrolyzed and hydrolyzed alcoholic extracts of sarcomas and skeletal tissues of rats (Seibert et al., I 954a). Several differences were noted between the normal and neoplastic tissue extracts, of which the most significant was the free amino nitrogen which constituted approximately $60 \%$ of the total nitrogen in extracts of tumor tissue and $20 \%$ of the total nitrogen in the extracts from normal tissue.

Several investigations have been made on the free amino acid patterns in normal and neoplastic tissues. Lymphosarcomas have higher concentrations of alanine, glycine and proline than normal lymphatic tissues (Kit and Awapara, I953), but aspartic acid and glutamine were lower in tumor than in the normal tissues. Smith and Rossi (I 954) also found that lymphosarcomas have a higher concentration of alanine and glycine. Taurine was also present in high concentration in tumors, however proline, aspartic acid, serine and threonine occurred in lower concentrations. Taurine, alanine and triiodothyronine, according to Christensen ct al. (1954) were present in high concentrations in Ehrlich mouse ascites carcinoma frollowing in vitro incubation. Roberts and Borges (1955), and Roberts and Tanaka ( 1956 ) concluded that the pattern of free amino acids in Yoshida ascites 
tumor was similar to that of other ascites tumors and tumors undergoing regression. Human tumors contained more total free amino acids than normal tissues, and Kotake and Ohsuka (1953) indicated that this difference was probably due to the presence of trypsin-like enzymes of higher activity in the tumor tissues.

Several attempts have been made to resolve the extractable proteins of normal and neoplastic tissues by electrophoresis and in the ultra centrifuge (Greenstein, 1956). Thus far the results have been difficult to interpret because of the complexity of the mixtures involved, though, some indication of differences have been obtained. Miller et al. (1950), in screening the electrophoretic patterns of mouse muscle and rhabdomyosarcoma, found three major components in the muscle and seven in the tumor extract. The individual components of the tumor extract all appeared to be quite distinct from the muscle components. Toennies ( I 952) has also pointed out that there is a definite difference between the electrophoretic behavior of proteins of normal and tumor tissues. Transplantation of tumors did not change protein composition (Gigante et al., 1954).

It is apparent that the experimental findings up to the present time have not revealed any characteristic compositional pattern in tumors with respect to amino acids or proteins. However, differences have been noted that may be of significance in the overall problem. New techniques for the isolation and purification of individual proteins should greatly increase the potential for future success in this area. By the use of column chromatography, gel electrophoresis and other methods, it is now possible to obtain workable quantities of highly purified protein fractions. These individual components may then be subjected to further rigid characterization by various physical methods and by amino acid composition and possible differences in protein composition or behavior of normal and tumor tissue may then be firmly established.

\section{(b) Protein metabolism}

It is obviously very difficult to discuss protein and amino acid metabolism separately. An attempt has been made to include under protein metabolism those studies in which the effort is directed towards the formation or biosynthesis of proteins.

An uptake of the lower peptides, glycylglycine, glycylglycylglycine and $\alpha-\mathrm{L}-$ glutamyl-L-glutamic acid was observed by Christensen and Rafn (1952) in suspensions of cells of ascites carcinoma, but not by erythrocytes. Busch and Greene (I955) injected into normal and tumor-bearing rats, glycine-2- ${ }^{14} \mathrm{C}$, D L-lysine${ }^{2-}{ }^{14} \mathrm{C}$ or plasma proteins containing the same radioactive amino acids. They found that the uptake of the labeled amino acids by the tumor proteins was not significantly different from that of the normal tissues. The important observation was made, however, that the rate of increase of ${ }^{14} \mathrm{C}$ in tumor proteins was two to ten times that of the proteins of normal tissues following the injection of labeled plasma. In a subsequent study, Busch et al. (1956) isolated the radioactive plasma albumin and globulin from rats injected with ${ }^{14} \mathrm{C}$-lysine. These protein fractions were injected into rats with Walker $25^{6}$ carcinoma and three hours later the following specific activities of proteins in various tissue homogenates were recorded: (Albumin injections) tumor- 10.5 ; liver-3.4; kidney-3.8; spleen -3.6 ; testes-3.6; brain -0.7 ; and lungs - 8.9, counts $/ \mathrm{min} / \mathrm{mg}$. (Globulin 
injection) tumor - I 0.6 ; liver -7.7 ; kidney -5.5 ; lung -5.4 , counts per $\mathrm{min} / \mathrm{mg}$. These findings indicate that the tumor does utilize the labeled plasma albumin more effectively than most normal tissues. The globulin was utilized by the tumor and also by several normal tissues to approximately the same extent.

Babson and Winnick (1954) have also demonstrated that tumors may utilize plasma protein for the synthesis of tumor protein. They believe that the liver hypertrophy in rats bearing transplanted tumors is a result of the tumors' requirement for protein (Babson, I954). Radioactive plasma or labeled albumin or globulin was infused into normal rats or rats with Walker $25^{6}$ carcinoma and the animals were sacrificed at intervals up to 20 hours (Babson, I956). The specific activity of plasma protein fractions is illustrated in Figure 3. These findings indicate that the albumin and globulin disappeared from the plasma of the tumor bearing rats at a rate almost twice that of the controls. Campbell et al. (1956) also obtained evidence that the plasma protein may serve as a precursor of the proteins of hepatoma. The above observations all point strongly to an extensive utilization of plasma albumin by some malignant tissues. Campbell ( 1958 ) has written an excellent review on protein composition and synthesis in normal and abnormal growth.

Transplants of Flexner-Jobling carcinoma grew as rapidly in fasting as in fed rats, according to LePage et al. (1952). After administration of radioactive glycine it was found that the radioactivity in the

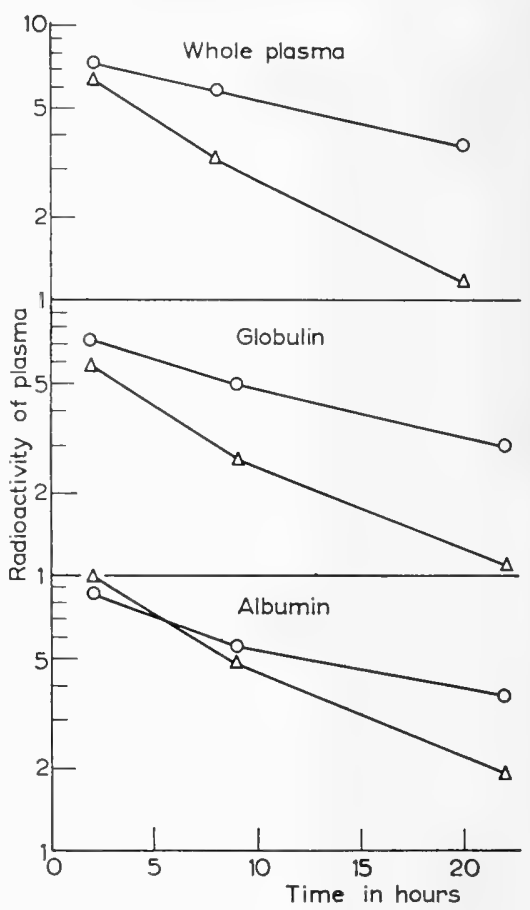

Fig. 3. The disappearance of infused ${ }^{14} \mathrm{C}$ labeled plasma, globulins and albumin from the circulation of normal and tumorbearing rats. The circles represent normal rats, and the triangles, tumorbearing rats. The radioactivity is expressed as the ratio of the radioactivity of the plasma to the radioactivity of the corresponding infusion $\times \mathrm{IO}^{-3}$. normal tissues of the fasting animals diminished with time, whereas that of tumor tissue increased. These investigators concluded that the proteins of the tumor were not available to the fasted animal for its metabolic requirements.

Several references have been made in previous sections to the relation between ylucose and protein metabolism. Campbell (I955) incubated rat liver and also hepatoma in the presence of uniformly labeled glucose. After four hours the radioactivity of the hepatoma proteins was from five to ten times greater than that of the proteins from liver tissue. Glucose proved to be more effectively utilized for synthesis of proteins in hepatoma than in normal liver tissues. From studies involving the carbohydrate and protein metabolism in rat liver and hepatoma, 
Zamecnik et al. (195I) concluded that there were no qualitative differences in the metabolic pathway followed by neoplastic and normal tissues. Their findings did indicate that the synthetic activities in tumors were directed more towards protein formation and cellular growth rather than glycogen formation or storage.

Studies involving the uptake of ${ }^{32} \mathrm{P}$ have indicated that a phosphoprotein fraction may be involved in the metabolism of normal and malignant cells (Griffin et al., I95I; McIndoe and Davidson, 1952; Albert and Johnson, I954). Kennedy and Smith (1954) incubated Ehrlich ascites cells with inorganic ${ }^{32} \mathrm{P}$. The specific activity of the phosphoprotein residue as obtained by the Schneider procedure did not differ appreciably from the acid insoluble fraction. Phosphoserine, isolated from the hydrolyzed phosphoprotein fraction, did have a very high specific activity. This high activity may be indicative of the utilization by the protein of the phosphate energy of ATP.

\section{(c) Amino acid metabolism}

The attention of the reader is again directed to the chapter on intermediary metabolism (Chapter I). This provides an excellent background for the known metabolic pathways involving the amino acids. Extensive investigations have been made in this area and the present discussion will of necessity be limited to those studies involving malignant tissues or cells. Mention should be made of the widespread application of radioisotopes and it will become increasingly apparent that most investigators have utilized labeled amino acids or related metabolites in the course of their studies.

Busch and Baltrush (1954) injected acetate- ${ }^{14}{ }^{14} \mathrm{C}$ intravenously into normal rats and rats bearing carcinoma. One min. after injection of the labeled acetate the glutamate, aspartate and succinate accounted for $33-75 \%$ of the total radioactivity present in the normal body tissues and only $4-6 \%$ of the activity present in the carcinoma tissue. Kit (I955) has carried out studies on the conversion of acetate- $-2-{ }^{14} \mathrm{C}$ to glycine by cell suspensions of Gardner lymphosarcomas and several normal tissues. The total radioactivity of glycine in tumor tissues exceeded that of normal tissues and also several other tumors by a factor of eight. The Gardner lymphosarcoma utilized this pathway to a considerably greater extent than other studied tissues. In a subsequent study, Kit and Graham (1956) incubated spleen or lymphosarcoma with glucose $-{ }^{14} \mathrm{C}$ or acetate- $2-{ }^{14} \mathrm{C}$. The free serine and glycine of the tumor were highly labeled while the corresponding amino acids of spleen contained little radioactivity. The formation of these two amino acids was also investigated with glycerol-1- ${ }^{14} \mathrm{C}$. After incubation with this labeled compound, the free and protein bound serine and glycine of lymphosarcoma were three to five times as radioactive as that of the splenic cells. The free and protein bound aspartate and glutamate of the spleen were 2-6 times as radioactive as that of tumors. At least $80 \%$ protein radioactivity of the tumor was due to glycine and serine and less than $10 \%$ to glutamate and aspartate. Only $35 \%$ protein radioactivity of the spleen was due to glycine and serine and approximately $50 \%$ to aspartate and glutamate. These results are in agreement with those of Busch and Baltrush (1954) and indicate the general inability of tumors to synthesize aspartate and glutamate. 
Further evidence has been presented by Rabinowitz et al. (I955) that glycolysis can supply energy for the incorporation of amino acids into the proteins of tumors. The incorporation of radioactive leucine, valine, lysine, phenylalanine and methionine into proteins of Ehrlich ascites carcinoma was supported anaerobically under conditions of active glycolysis. Nyhan and Busch (1956) added labeled glutamate to systems containing slices of liver, kidney or Walker 256 carcinoma. Specific activities of tumor, kidney and liver proteins were 63,39 and $27 \mathrm{CPM} / \mathrm{mg}$, respectively, in unsupplemented systems; I I 7,39 and $24 \mathrm{CPM} / \mathrm{mg}$ respectively, when these systems were supplemented with $0.006 \mathrm{M}$ glucose. In systems containing $\mathrm{L}-\mathrm{lysine}-\mathrm{U}-{ }^{14} \mathrm{C}$ the ratio of specific activities of tumor, kidney and liver was 7.5: I.7: I compared with ratios of 2.4:I.4:I with labeled glutamate. The oxidation of glutamate in the tumor was suppressed by glucose, but enhanced the protein labeling. The uptake of histidine is coupled with the glycolytic splitting of glucose according to Negelein (1952). Slices transplanted rat tumor had an uptake of L-histidine of almost ten times that of normal liver. D-Histidine was taken up at only $20 \%$ of that observed for the L-isomer. The in vitro formation of amino acids from labeled glucose in normal mouse tissues, rat thymus, rabbit appendix, several lymphosarcomas, Ehrlich ascites tumor and mouse melanoma was investigated by Kit and Graham (1956a). High levels of radioactivity were attained in the free glycine, serine and alanine of the lymphomas and the ascites tumor. Also of interest was the relatively high activity of the alanine and the low activity of serine in the melanomas. Radioactivity of the glycine and serine of the normal tissues was considerably below that of the tumors. Kit (1955a) has proposed the following mechanism for the biosynthesis of glycine and serine in lymphosarcomas:

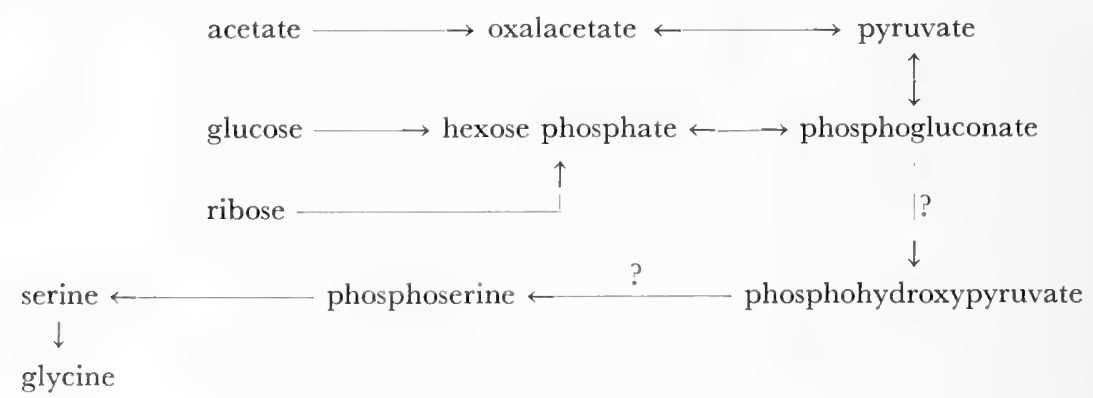

The conversion of acetate- $2-{ }^{14} \mathrm{C}$ to glycine was inhibited by glucose, serine, fluoride and malonate. Some labeling of glycine was obtained when ribose- ${ }^{14} \mathrm{C}$ was added to the system.

Further observations have been made with respect to the uptake of amino acids by normal and cancerous tissues (Roberts and Tanaka, 1956; Christensen and Henderson, I952; Heinz, I 954; Riggs et al., I 954; Rutman et al., I 954; Reid et al., I 752 . Minller (I 953) found that transplanted rat tumors exhibited high uptake of histidine, serine and asparagine. Intermediate uptakes were noted for glycine, alanine, threonine and phenylalanine while glutamic acid and arginine were 
not utilized at all. Cell suspensions of rabbit appendix, rat thymus, mouse spleen and two mouse lymphosarcomas were injected with radiolabeled glutamate or aspartate by Kit (1 954). The respiration and the incorporation of labeled carbon into the $\mathrm{CO}_{2}$ and into the cellular proteins were measured. The conclusion was reached that the low endogenous concentration of free aspartate in lymphosarcomas could be in part attributed to the high glycolysis of the tumor. Glutamine was readily taken up and metabolized by Yoshida sarcoma cells when injected intraperitoneally into rats bearing this tumor (Roberts et al., I 956). There was a limited permeability of the cells to glutamic acid. Eagle and associates (1956) have studied the growth response of a mouse fibroblast and the human carcinoma cell (HeLa) to L-glutamic acid and L-glutamine. Both of these cells required L-glutamine for survival and growth in tissue culture. Glutamic acid was approximately one tenth as active as glutamine in the HeLa cell and was non-active in the fibroblasts. Further evidence was obtained as to the impermeability of these cells to glutamic acid.

The occurrence of D-glutamic acid in the proteins of tumor tissues has been given considerable attention for many years (Miller, I950). Boulanger and Osteux (1954) isolated and purified glutamic acid from hydrolysates prepared by the method of Kögl. No significant differences were observed between the contents of D-glutamic acid in normal and neoplastic tissues. These same investigators also incubated tumor and intestinal homogenates in the presence of pyruvic acid (Boulanger and Osteux, I953) and a D-amino acid, possibly D-alanine, was found. Muscular, renal and hepatic tumors, however did not produce this acid. D-leucine, according to Vescia et al. (1952), inhibited the hydrolysis of glycyl-L-leucine in normal and in pathological non-neoplastic tissues. In a large number of malignant tumors the D-leucine activated this peptidase activity.

L-cysteine, selenium cystine and phenyl selenium cysteine were effective in

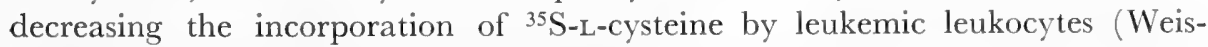
berger et al., I 956). Melchior and Goldkamp (1953) determined the incorporation of ${ }^{35} \mathrm{~S}$ labeled methionine in vitro by the tissues of $\mathrm{C}_{3} \mathrm{H}$ mice. The mammary tumor had a considerably higher rate of incorporation of methionine than the normal tissues.

The reactions leading to melanin formation have been established by Fitzpatrick et al. (1950) and Lerner and Fitzpatrick (1950). Markert (1955) also found that tyrosine or its oxidative products were the only substrates used by melanoblasts in the synthesis of melanin. The labeled side chains of tyrosine or dihydroxyphenylalanine did not serve as melanin precursors as was shown by radioautographs. This finding would cast some doubt upon the accepted scheme for melanogenesis. Poppe and Fraedrick (1954) reported a selective uptake of tyrosine$3^{-14} \mathrm{C}$ in superficial melanotic lesions. Mice bearing Harding-Passey melanoma were injected with single or repeated doses of tyrosine-2 $-{ }^{14} \mathrm{C}$ and sacrificed at varying time intervals (Robertson et al., 1955). No significant differences were noted between the normal or malignant tissues in the incorporation of the labeled tyrosine. No differences were found between the normal and melanotic tissues following administration of tyrosine- $2-{ }^{14} \mathrm{C}$ in a patient with malignant melanoma. 


\section{(a) Composition}

A most comprehensive compilation of the purine and pyrimidine content of normal and neoplastic tissues appears in the two volume treatise on Nucleic Acids, edited by Chargaff and Davidson (Chargaff, I955; Magasanik, I955; Vendrely, I955; Leslie, I 955). Greenstein (I954; Chapter VIII), has reviewed many of the studies on the concentration of nucleic acids and nucleoproteins in neoplastic tissues. In general, it would appear that some quantitative differences between normal and cancerous tissues do occur. There is no consistent purine or pyrimidine pattern, however, that would characterize the nucleic acids of malignant tissues.

(i) Purines and pyrimidines. Chargaff and Lipshitz (1953) found that the purine and pyrimidine composition of DNA from normal human liver and carcinomatous human liver were almost identical. These investigators also established that the sugar in each of the DNA fractions was 2-deoxyribose. DNA, prepared from nuclei by salt extraction, was hydrolyzed with formic acid and the bases separated by chromatography (Khouvine, I954). Certain differences were reported in the purine and pyrimidine composition of the DNA from normal and cancerous tissues. The adenine content of an ovarian epithelioma was low compared to normal tissues. Khouvine and Hirsch (1952) observed that the RNA extracted from cancerous epithelial tissues of rats was high in guanine and cytosine, and relatively low in uracil.

Woodhouse (r954) prepared DNA from several spontaneous and induced animal tumors and also from human tumors. Following perchloric acid hydrolysis the purines and pyrimidines were separated and determined by ultraviolet spectroscopy. The findings are shown in Table 5. Ratios of adenine to guanine and thymine to cytosine were greater than unity in normal as well as in the cancerous tissues. Certain tumor preparations of DNA exhibited some deviation in the base content i.e., a low value for thymine in mouse sarcoma. In all instances the various normal tissues were remarkably constant. A much wider range of values was noted

TABLE 5

$$
\text { MOLAR RATIOS OF BASES IN DNA }
$$

(From Woodhouse 1954)

All values expressed on a molar basis relative to adenine $=\mathrm{I} . \mathrm{O}$

\begin{tabular}{|c|c|c|c|c|c|}
\hline & $\mathrm{G}$ & $\mathrm{C}$ & $\mathrm{T}$ & $\mathrm{A} / \mathrm{G}$ & $\mathrm{T} / \mathrm{C}$ \\
\hline \multicolumn{6}{|l|}{ Io different tumors } \\
\hline human and animal & $0.68-0.82$ & $0.68-0.84$ & $0.82-1.20$ & I.1 $2-1.47$ & I. I 7-I.5 I \\
\hline Calf thymus & 0.85 & $0.7 \mathrm{I}$ & 0.99 & 1.01 & I. 40 \\
\hline Rat liver & 0.86 & 0.73 & 0.93 & I. 16 & 1.27 \\
\hline Human spleen & 0.88 & 0.75 & 0.93 & 1.14 & 1.24 \\
\hline
\end{tabular}

$\mathrm{A}=$ Adenine $\mathrm{G}=$ Guanine $\mathrm{C}=$ Gytosine $\mathrm{T}=$ Thymine 
TABLE 6

DEOXYRIBOSIDE CONTENT OF ANIMAL TISSUES

(Schneider, I955)

\begin{tabular}{|c|c|c|c|}
\hline Species & Strain & Tissue & Deoxyriboside content ${ }^{1}$ \\
\hline \multirow[t]{15}{*}{ Mouse } & $\mathrm{C}_{3} \mathrm{H}$ & Liver & $0.8 \mathrm{I}^{2}, 0.8 \mathrm{r}, 0.80,0.66,0.80$ \\
\hline & dba &, & $0.5^{8}$ \\
\hline & A & , & 0.88 \\
\hline & $\mathrm{C}_{3} \mathrm{H}$ & Brain & 0.67 \\
\hline & , & Kidney & 1.23 \\
\hline & , & Lung & I.3 I \\
\hline & ", & Spleen & $15.2,9.7$ \\
\hline & ," & Blood & 1.0, $0.83^{3}, 0.93^{3}$ \\
\hline & , & Hepatoma 37 & $2.5,5.4,3.1,3.8$ \\
\hline &, &,,$\quad 98 / 15$ & $5.1,5.0$ \\
\hline & dba & Thymoma & $42.6,22.6$ \\
\hline & $\mathrm{C}_{3} \mathrm{H}$ & Mammary carcinoma & 5.7 \\
\hline & A & Lymphoma I cells & $24.4,26.9$ \\
\hline & " & fluid I ascitic & I I. 5, I 3.4 \\
\hline & & Sarcoma 37 & 2.6 \\
\hline \multirow[t]{8}{*}{ Rat } & Holtzman & Liver & $\begin{array}{l}\text { I } 4.8, \text { I } 2.7, \text { I } 4.3^{4}, \text { I } 2.4, \text { I } 2 . I^{5}, \text { I I.2, } \\
\quad \text { I I. } 4^{2}, \text { I } 2.8, \text { I I. } 5,{ }^{4} \text { I } 6.5\end{array}$ \\
\hline & ", & Lung & 8.7 \\
\hline & ," & Brain & 3.2 \\
\hline & , & Spleen & $17.3,30.7$ \\
\hline & 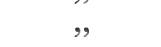 & Kidney & I2. I, 20.9 \\
\hline &, & Blood & 1 $3.6,10.6^{6}, 7.5^{6}, 10.4,12.7$ \\
\hline & , & Blood plasma & 14.1 \\
\hline & , & Novikoff hepatoma & I 2.3, I 0.1, I 4.9 \\
\hline
\end{tabular}

${ }^{1}$ Expressed as thymidine.

2 Animals decapitated without anesthesia.

${ }^{3}$ Hepatoma 37 -bearing animals.

\footnotetext{
${ }^{4}$ Animals frozen in liquid air.

5 Removed 2 min. after death of animal.

${ }^{6}$ Hepatoma-bearing animals
}

in the purine and pyrimidine composition of the DNA isolated from tumors. However, the normal range of values was usually encompassed by the tumor values.

(ii) Nucleotides and nucleosides. The free deoxyriboside content of several normal and neoplastic tissues were determined by microbiological assay (Schneider, I955) Table 6.

It may be noted that the mouse tumors contained more deoxyribosides than the normal tissues. All normal rat tissues were also higher than the corresponding mouse tissues. The hepatoma was no higher than the liver or other rat tissues in deoxyribosides. Schneider ascertained that most of the deoxy components measured were pyrimidine deoxyribosides, and that these compounds were localized in the soluble fraction of the tissues. Of interest was the discovery in rat liver of deoxyuridine which may be a precursor of thymidine. Further observations in 
this area may reveal the more specific function of the deoxy components in the biosynthesis and mobilization of DNA required for cellular divisions.

Studies on the isolation and determination of the free nucleotides have been carried out by Schmitz (I954, I 954a). Values were obtained for the 5'-mono-, diand triphosphates of adenosine, guanosine, cytidine and uridine from perchloric acid extracts of mouse ascite tumors (Fig. 4).

The ratio of diphosphopyridine nucleotides to AMP in ascites tumor was $\mathrm{I}: \mathrm{I}$, while in solid tumors the values ranged from $I: 3$ to I :5, AMP being present in the highest concentration. The composition of the RNA of the ascites cells was quite similar to that of other tumors (Schmitz et al., I 955). DeLamirande et al. (i955) reported the following variation in hepatic guanylic acid from normal liver: nuclear fraction + I $2.2 \%$, mitochondrial fraction +22 ; microsomal fraction- 1.9 , supernatant fraction $+51.7 \%$.

(iii) Nucleic acids and nucleoproteins, A comprehensive analytical study has been completed by Caspersson and associates (Caspersson, I 950) as to the role of nucleic

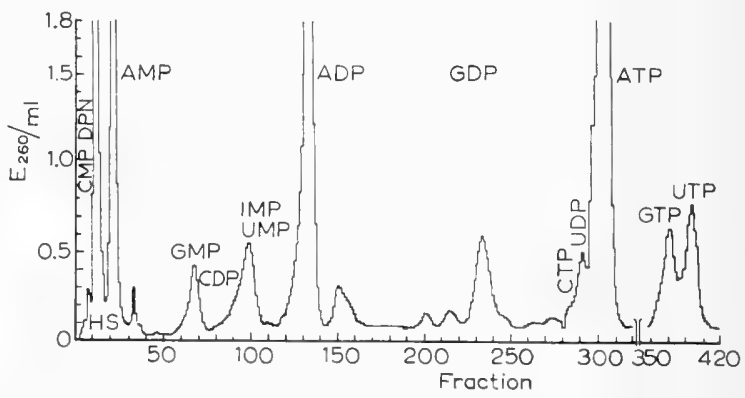

Fig. 4. The ordinate is the UV absorption at $260 \mu(d=$ I $\mathrm{mm}$ ) of the fractions plotted on the abscissa. The fractions were separated by means of an automatic fractioncollector. Each fraction had a volume of $4 \mathrm{ml}$. The fractions were eluted with gradually increasing concentrations of $\mathrm{O} \rightarrow 4_{4} \mathcal{N} \mathrm{HCOOH}(\mathrm{I}-\mathrm{I} 4 \mathrm{O})$ and of $4 \mathcal{N} \mathrm{HCOOH} \rightarrow 4_{4} \mathcal{N}$ $\mathrm{HCOOH}+\mathrm{I} \mathcal{N} \mathrm{HCOONH}_{4}$ (I 4 I-end). The following abbreviations have been used: MP, monophosphate; DP, diphosphate; TP, triphosphate; $\mathrm{A}$, adenosine; $\mathrm{G}$, guanosine; C, cytidine; $U$, uridine; I, inosine; DPN, diphosphopyridinenucleotide. The extinction values beyond the scale are: DPN: 1.9, 4.05, 2.68; AMP: 4.16, 5.77, 2.19; ADP: $2.06,2.70,3.07,2.83,2.07$; ATP: $1.89,2.22,2.60$, $2.70,2.80,2.60,2.40,1.935$ (Schmitz, 1954, from his original paper). acids and nucleoproteins in the process of cellular reduplication. Their findings would indicate that the appearance of nucleolar substances and the formation of cytoplasmic proteins are closely related (see Chapter 3 ). The nucleoli and the cytoplasmic nucleotides are conspicuous in the pancreas where the cell has the capacity to produce its own weight in protein during a $24 \mathrm{~h}$. period. Caspersson believes that the chromatin associated with the nucleolus or the heterochromatin is involved in the production of proteins in the cell. Deoxyribonucleotides, according to Caspersson, participate in the reproduction of the gene proteins in the chromosomes while the ribonucleotides are involved in the formation of proteins of the cytoplasm. These investigators carried out microspectrographic studies on a large number of human tumors. The tumor cell, they believe, has the same systems for protein formation in the nucleus and the cytoplasm as are present in normal cells. In the cancerous cell the endocellular inhibitory mechanism which controls protein formation is lost or altered in some way. 
Many other investigators have employed microspectrophotometric methods in order to determine the nucleic acid contents of malignant cells. Mellors et al. ( I 952) reported the following values for the total nucleic acids of the nuclei of interphase cells:

Normal epithelial cells $=9 \cdot 10^{-12} \mathrm{~g} /$ nucleus

Squamous carcinoma cells $=35 \cdot 10^{-12} \mathrm{~g} /$ nucleus

The latter group could be divided into three subgroups with average values of I 7, $3^{8}$ and $67 \cdot 10^{-12} \mathrm{~g} /$ nucleus. In a subsequent study Mellors et al. (I954) found that 13 types of mouse tumors had an average content of nucleic acid per nucleus or a chromosome number that was approximately twice that of normal diploid somatic nuclei. Lymphoma, lymphatic leukemia and a sarcoma had normal contents of nuclear nuclei acid. Petrakis and Folstad (I954) also found that the DNA content of a lymphoma (designated number two) was close to the normal diploid value $(2 \mathrm{~N})$. Lymphoma number one had an average of DNA per cell of $4 \mathrm{~N}$ to $8 \mathrm{~N}$. Since this latter lymphoma presumably contains a tetraploid number of chromosomes, this would indicate a premitotic build-up of DNA in the presence of aneuploid chromosome numbers. Attention may also be directed to the investigations of Ludford (I954) on modifications of nuclear structure in tumors; the histochemical studies on the ribonucleic acids in basocellular and spinocellular carcinoma (Prunieras, I 954): the findings of Villa et al. (1955) concerning the nucleic acids of normal and leukemic blood cells; and Ackerman et al. (I95I) who have reported that Hodgkin's cells contain more ribonucleoprotein than the reticulum cells of origin.

The nuclei of fowl tumor cells contain considerably more RNA than the nuclei of cells from several normal tissues (McIndoe and Davidson, 1952) which has been generally observed by other investigators. Several compositional and metabolic studies have been carried out on the nuclei isolated from normal or cancerous cells (McIndoe and Davidson, I952; Magasanik, 1955). The nuclear RNA has a different purine and pyrimidine composition than the RNA of the cytoplasmic fractions. As mentioned above, tumor cell nuclei may contain more RNA than most normal cell nuclei; however, there is no evidence to suggest that the nuclear RNA of the malignant tissues differs in qualitative aspects from the RNA of normal cell nuclei. Attention is called to the critical comparison made by Kay et al. (I956) of cell nuclei isolated by aqueous and non-aqueous procedures.

Cerecedo et al. (I955) found that the DNA and RNA decreased in concentration as the tumor (Sarcoma 256) increased in size. Azo dye induced hepatomas have a much higher DNA potassium ratio than the liver of the control rats (Rodriques et al., I955) The DNA content of nuclei was determined in normal and tumorous tissue of brain by Heller and Elliott (1954). The nuclear density, as determined by these investigators, was higher in the brain tumor than in the normal brain tissues. Levy et al. (I 953) determined the RNA and the DNA per tumor cell following the inoculation of an ascites thymoma in mice. Both RNA per cell and DNA increased up to 6-8 days following the inoculation of the tumor and then declined.

Irradiation of $\mathrm{C}_{57} \mathrm{Bl}$ mice resulted in a large increase in the RNA per thymic cell (Weymouth et al., I 955). If these animals were thigh shielded during irradia- 
tion the RNA value promptly returned to normal. The unshielded mice which eventually develop a high incidence of thymus lymphosarcomas maintained high thymic RNA values. The RNA per cell of a transplantable lymphosarcoma was also found to be many times higher than the RNA of the thymus cell which served as the control. This increase noted in the RNA per cell of lymphosarcoma does not hold for all tumors when compared with their tissues of origin. Several tumors, f.e. hepatoma, have a decreased cytoplasmic nuclear ratio relative to normal tissues of origin and a net decrease in RNA expressed either on a content or concentration basis. The morphological characteristics of the cell have a direct bearing upon the composition or metabolism of the cell and should be considered whenever biochemical comparisions are made of normal and neoplastic tissues.

Two DNA fractions were isolated from normal or cancerous tissues of the rat. The fraction designated $\mathrm{DNA}_{1}$ was precipitated by a $0.87 \%$ salt solution while a second fraction, $\mathrm{DNA}_{2}$ remained in solution. Rats bearing tumors were given ${ }^{32} \mathrm{P}$ and the specific activities of the two DNA fractions from several normal tissues, as well as the tumors, were measured. The $\mathrm{DNA}_{1}, \mathrm{DNA}_{2}$ ratio was higher in the tumors than in the normal tissues (Backmann and Harbers, I955). These findings, if extended, may reveal that there are DNA components within the tumor that may be more or less characteristic of the rapidly growing tissue. Evidence for the heterogeneity of tissue DNA has been obtained by the chromatographic fractionation on base-substituted cellulose anion exchange columns (Bendick et al., 1956). Butler et al. ( I 956) observed that DNA prepared from tumors of the rat and mouse contained varying amounts of RNA. However, the same methods of preparation in normal tissues give a DNA that was free of RNA. These investigators suggested that the RNA of tumors may differ from that of normal tissues. Some evidence was presented that tumor RNA preparations did have an abnormally high adenine and guanine content.

A deoxyribonucleohistone has been isolated from transplantable mouse lymphosarcoma (Shack and Thompsett, I 953; Shack et al., I 953). Viscosity, solubility and spectrophotometric titration behaviour indicated that the DNA component exists in a macromolecular state. The degree of hydrogen bonding was the same as was observed with DNA of calf thymus nucleohistone.

\section{(b) Metabolism}

In recent years a most intensive effort has been extended in the study of nucleic acid metabolism in both normal and neoplastic tissues. A comprehensive review, however, of all the findings in the important area would extend beyond the space limitations of this chapter. The present status of purine and pyrimidine, nucleoside and nucleotide biosynthetic pathways are adequately presented in the chapter on intermediary metabolism (Chapter I). Also, the entire subject has been reviewed prior to 1954 by Reichard, Schlenk, Brown, Roll, Smellie, Hotchkiss, Brachet and others in the two volume treatise on the nucleic acids (Chargaff and Davidson, 1955). The present review will be largely restricted to the more recent findings concerned with nucleic acid metabolism in abnormal growth. An informactive chart compiled by Potter is presented in Fig. 5, in order to facilitate this discussion. 

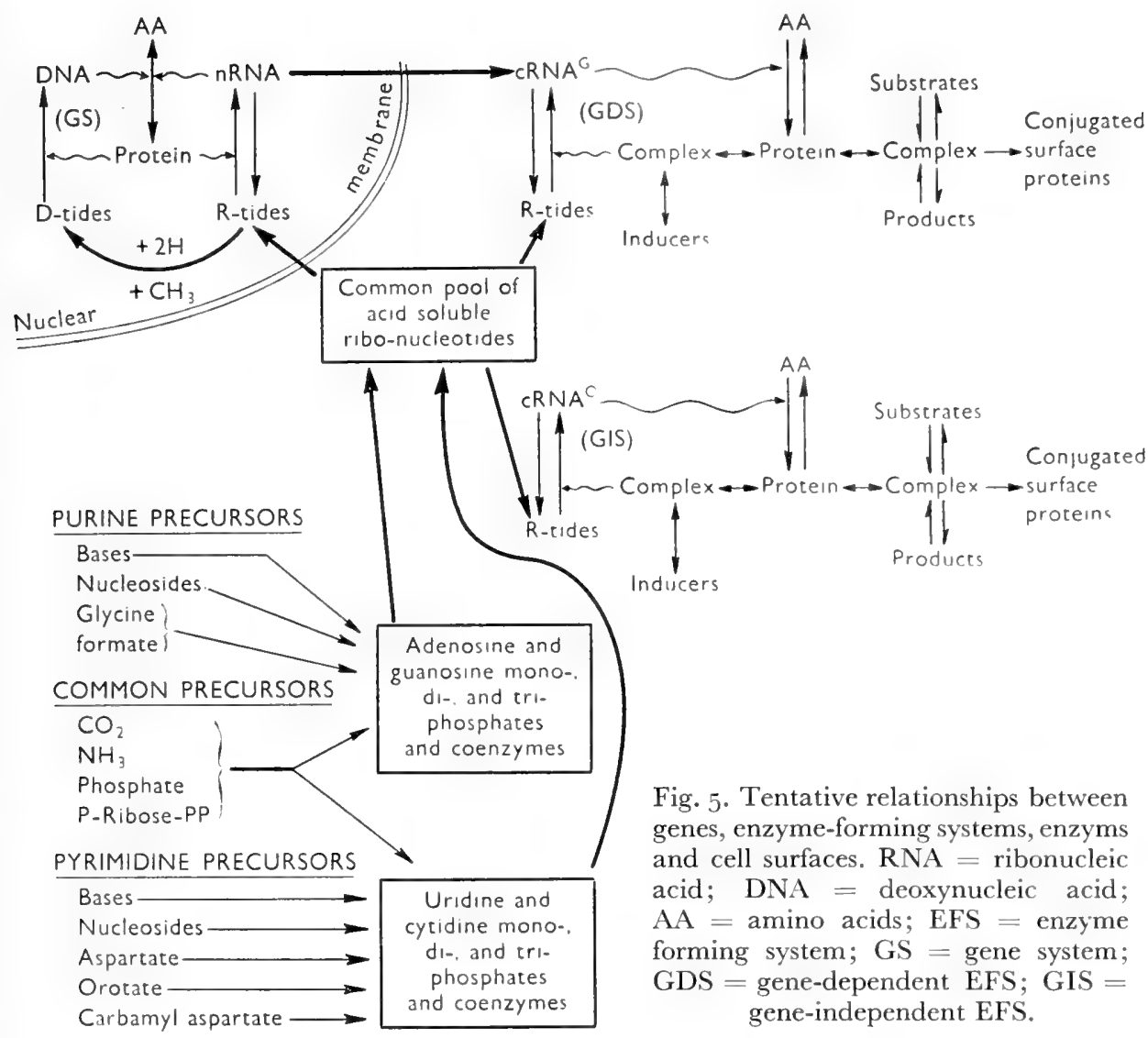

(i) Purines and pyrimidines. Ehrlich ascites tumor can synthesize RNA pyrimidine from the same precursors as are utilized by liver (Lagerkvist et al., I954). In these studies it was observed that uracil ${ }^{15} \mathrm{~N}$ was incorporated into the RNA pyrimidines at approximately the same rate as orotic acid- ${ }^{15} \mathrm{~N}$. The utilization of uracil- $2-{ }^{14} \mathrm{C}$ in rat liver and in acetylaminofluorine induced hepatomas was investigated by Rutman et al. (1 954a) and Cantarow et al. (1955). Exogenous uracil was utilized by the hepatomas, but not in the normal rat liver. Uracil served as a precursor of uridylic acid but not of the purine nucleotides or of the deoxypentose pyrimidine nucleotides. This enhanced utilization of uracil in the hepatomas may reflect the rapid growth that is occurring in these tissues. Proliferative activity in normal tissues may also be accompanied by a measurable incorporation of uracil. Eidinoff et al. (1956) injected ureidosuccinic acid- ${ }^{14} \mathrm{C}$, a pyrimidine precursor, into rats bearing human tumor transplants. Seven hours after administration of this compound the specific activity of thymine was highest in the tumor, followed by in- 
testine, spleen and liver in decreasing order. The specific activity of the DNA cytosine was approximately the same as the DNA thymine.

Purine metabolism in neoplastic tissues has been extensively investigated by LePage (1953). In Ehrlich carcinoma cells the antibiotic isomer of chloramphenicol inhibited the incorporation of glycine-2- ${ }^{14} \mathrm{C}$ into proteins and nucleic acid purines. The inactive isomer inhibited the glycine incorporation into purines but not into protein. Both isomers were equally effective in inhibiting the incorporation into both the proteins and purines in lymphosarcoma cells (LePage, I953a). In a subsequent study, Edmonds and LePage (I955) investigated the in vivo incorporation of glycine-2- ${ }^{14} \mathrm{C}$ into the acid soluble purine nucleotides and into the nucleic acids of rat liver and Flexner-Jobling carcinoma. More ${ }^{14} \mathrm{C}$ was incorporated into the tumor than into the liver nucleotides. Soon after administration of the labeled glycine, the acid soluble nucleotides were isolated from the liver and tumor. IMP had a higher activity than AMP, ADP or ATP in both tissues. GMP, GDP and GTP were more highly labeled than the corresponding adenine nucleotides in the tumor. Further studies in purine metabolism were also carried out in Ehrlich carcinoma cells. (Edmonds and LePage, r 956). These cells were unable to convert deoxy-AMP to the corresponding inosine compound, thus accounting for the ineffectiveness of deoxy-AMP in diluting the radioactivity of glycine- $2-{ }^{14} \mathrm{C}$ that is incorporated into the purines. The deoxyadenosine was converted to hypoxanthine or deoxyinosine. These investigators also found that AMP need not be converted to inosinic acid prior to its incorporation into nucleic acid adenine. These tumor cells utilized AMP more effectively than adenosine.

De novo synthesis of nucleic acids from precursors such as glycine or adenine occurs readily in intestines, liver or in mouse sarcoma i 8o. (Barclay and Garfinkel, I 955). Balis et al. (I955) reported that preformed purines were incorporated to a smaller extent into a human tumor explant in hamsters than they were in several of the normal tissues of the animal. Little guanine was utilized by the tumor, while glycine was used to a considerable extent. Azaserine inhibits the incorporation of glycine-2 $-{ }^{14} \mathrm{C}$ into acid soluble and nucleic acid purines in mouse ascites tumor and in the spleen. 6-Mercaptopurine did not affect the incorporation of glycine $-2-{ }^{14} \mathrm{C}$ or adenine-8- ${ }^{14} \mathrm{C}$ into nucleic acid compounds (Fernandes et al., I956). Azaserine was shown by Greenlees and LePage (1956) to react with a tumor cell constituent either in vivo or in vitro and to inhibit the de novo synthesis of purines. An intermediate, glycineamide ribotide (GAR), accumulated in the ascites tumor cells, indicating that these tumor cells are inhibited at the point where formylation of GAR occurs. In pigeon liver systems the reaction involving the conversion of \%-N-formyl glycineamide ribotide to the corresponding amidine is the most sensitive to azaserine. Whether or not this difference in the point of action of azaserine is general for tumor cells and normal mammalian cells still must be ascertained.

Studies on the incorporation of guanine-2- ${ }^{14} \mathrm{C}, 2,6$-diaminopurine- $2-{ }^{14} \mathrm{C}$, adenine-8 $-{ }^{14} \mathrm{C}$ and formate ${ }^{14} \mathrm{C}$ into polynucleotides of animal tumors and normal tissues were initiated by Bennett et al. (I 955). Relative to the control tissues, all tumors investigated incorporated guanine poorly. The tumors utilized 2,6diaminopurine as a polynucleotide guanine source as well as did intestine or liver. A comparable study was also carried out on the incorporation of these and other 
precursors into nucleic acid purines in heterologus implants of human tumors and in the livers and intestines of hamsters and rats treated with cortisone (Bennett et al., 1956). The human tumor explants exhibited de novo synthesis of nucleic acid purines. Guanine and hypoxanthine were not utilized to any significant extent. The tumors incorporated adenine to approximately the same extent as normal tissues. Diaminopurine was found to be a good precursor of nucleic acid guanine in the tumors, relative to normal tissues.

(ii) Nucleosides and nucleotides. Nucleotides were isolated from the acid-soluble fraction of normal and tumor tissues in animals previously injected with glucose${ }^{1-}{ }^{14} \mathrm{C}$ (Schmitz et al., I 954). Also, the mono-, di- and triphosphates of cytidine, guanosine, uridine and adenosine were isolated from the acid soluble fraction of Flexner-Jobling carcinoma (Schmitz et al., 1955). These studies did not indicate the presence of a different metabolic or compositional pattern for tumor tissues. Daoust and Cantero (I955) determined the specific activity of the acid soluble nucleotides in rat liver, intestinal mucosa, and hepatoma following the administration of ${ }^{32} \mathrm{P}$. The incorporation of the ${ }^{32} \mathrm{P}$ into the nucleotides was rapid, but occurred at approximately the same rate in the liver, liver tumor or intestinal mucosa. Etinghof and Balyasnaya (1953) have investigated the metabolism of inosinic acid in ascites tumor cells.

Glycine-2 ${ }^{14} \mathrm{C}$ and ${ }^{32} \mathrm{P}$ were injected simultaneously by the intraperitoneal route into normal rats and rats bearing Flexner-Jobling carcinoma. These animals were sacrificed at various time intervals and the livers and tumors were fractionated by the sucrose method. Nucleic acids from the various fractions were hydrolyzed and the nucleotides separated by ion exchange chromatography (Tyner et al., 1953). The phosphorus specific activity was measured, the purine nucleotides were further hydrolyzed and the ${ }^{14} \mathrm{C}$ specific activity of the purines was ascertained. The specific activity of the DNA and RNA nucleotides in tumors was higher at all times than in the corresponding liver nucleotides. Specific activities of the ${ }^{32} \mathrm{P}$ and ${ }^{14} \mathrm{C}$ in the purine nucleotides from DNA were of the same magnitude. However, the specific activity of the ${ }^{32} \mathrm{P}$ in the RNA purine nucleotides of liver was higher than that of ${ }^{14} \mathrm{C}$. This would indicate that the phosphorus was incorporated into an intermediate precursor earlier than was the glycine. The nuclear RNA of both liver and tumors contained more radioactivity than the cytoplasmic fraction at early time intervals following the administration of the labeled components. This high metabolic activity of the nuclear RNA confirms the observations of several other investigators. In general, Tyner et al. ( I 953) were of the opinion that the nucleic acid metabolism of tumors was qualitatively similar but quantitatively more rapid than that of liver. Quantitative differences in the extent of labeling of nucleic acids of liver or hepatoma were observed by Werkheiser and Visser (1955). Following the addition of formate- ${ }^{14} \mathrm{C}$, the purine nucleotides from hepatoma RNA contained several times more ${ }^{14} \mathrm{C}$ than the corresponding fraction from normal liver. The uridylic acid from the tumor was approximately twice as active as the liver uridylic acid after the injection of carbamyl aspartate- ${ }^{14} \mathrm{C}$. The extent of labeling of the cytidylic acid was considerably lower than that of the uridylic acid in both tissues. Aminouridine greatly 
inhibited the incorporation of these labeled precursors into both purines and prrimidines of liver and only mildly inhibited their incorporation into the purines and pyrimidines of hepatoma. These findings would indicate that alternative biosynthetic pathways may be active in tumors.

(iii). Vucleic acids and nucleoproteins. Many investigators have made comparisons of the incorporation or uptake of labeled precursors into the nucleic acids of normal or neoplastic tissues. Schmitz et al. (1954a) injected glucose- $\mathrm{I}^{14} \mathrm{C}$ into rats bearing Flexner-Jobling carcinoma. Within $\mathrm{I}-5 \mathrm{~h}$. there was considerable activity in the RNA of the tumor while the liver RNA had little activity. The ${ }^{14} \mathrm{C}$ of the tumor RNA was found almost entirely in the ribose. DNA of the tumor was also active within a few hours while the DNA of the liver did not contain any ${ }^{14} \mathrm{C}$ after this same period. ${ }^{32} \mathrm{P}$ incorporation studies into the DNA and RNA of epithelioma and of rat liver have been reported by Khouvine and Mortreuil (1954, 1954a). The rate of uptake of labeled cell constituents into the cells of mouse ascites tumors was as follows: inorganic ${ }^{32} \mathrm{P}$, phosphatide, mitochondria, nuclei, plasma, homogenate, nucleic acid and supernatant fluid in decreasing order (Lettré et al., 1955). Inorganic ${ }^{32} \mathrm{P}$ and nucleotide components entered the nucleus very rapidly. It is difficult to ascertain if structural units maintained their integrity as they entered the ascites cells. The investigators suggested that these particles may be utilized as structural units in the tumor. Amethopterin had no effect on ${ }^{32} \mathrm{P}$ incorporation into phospholipids or nucleic acids of mouse leukemia or of normal tissues, indicating that direct interference with nucleic acid formation is not associated with the action of folic acid antagonists (Williams et al., 1954).

Some interesting observations relative to nucleic acid biosynthesis in rapidly growing tissue have been reported by Nygaard and Rusch (I955). The incorporation of ${ }^{32} \mathrm{P}$ into nucleic acids of regenerating rat liver was investigated. The labeled phosphate was injected $20 \mathrm{~h}$. after partial hepatectomy since it has been previously observed that there was little evidence of nucleic acid formation as measured by phosphorus incorporation during this period. Specific activity of the acid soluble phosphorus fraction and the nuclear RNA were high within two h. following administration of the labeled phosphate. At this same time interval the activity of the DNA fraction was low. Approximately $24 \mathrm{~h}$. following the injection of the ${ }^{32} \mathrm{P}$ the specific activities of the acid soluble fraction and the nuclear RNA had decreased and the DNA had increased so that all were approximately equal. Several conclusions with respect to the role of nucleic acid metabolism in cellular growth energed from these findings. The relatively long induction period between hepatectomy and the initiation of nucleic acid synthesis would suggest that a supply of precursors must be mobilized before actual nucleic acid synthesis or cellular proliferation may proceed. Lettré et al. (I955) are also of the opinion that stimulation of mitosis in the tumor cell is controlled by the supply of nucleic acid 1) nilding material. The findings of Nygaard and Rusch (I955) further confirm the rapid incorporation of ${ }^{32} \mathrm{P}$ or other precursors into the nuclear RNA of proliferating cells. The specific function of this nuclear RNA has not been established. Balis ' 1956$)$ studied the effects of regenerating liver on the synthesis of nucleic acids in human tumor explants in the hamster. Labeled adenine or glycine was 
injected in these tumor bearing animals $24 \mathrm{~h}$. after partial hepatectomy. The uptake was increased almost threefold in the tumors of the operated animals.

A most important contribution towards the mechanism of nucleic acid formation has been made by Ochoa and coworkers (Grunberg-Manago et al., I955; Ochoa, I956; Heppel et al., 1956). An enzyme isolated from the organism, Azotobacter vinelandii catalyzes the synthesis of polyribonucleotides from 5 -nucleoside diphosphates. This polynucleotide phosphorylase is widely distributed in microorganisms. Recently, Kornberg et al. (1956) have reported the formation of a thymidylic acid polynucleotide following incubation of $E$. coli extracts with ${ }^{14} \mathrm{C}$ thymidine and ATP. These observations do not, as yet, provide an explanation for the mechanism of synthesis of the polydeoxyribonucleotide of the nucleus or of the role of these structures in cellular division. The discovery of enzyme systems capable of catalyzing the synthesis of the polynucleotides provides a stimulus for further studies in this area, and further elucidation of the mechanism of nucleic acid biosynthetic pathways will undoubtedly appear in the near future. Whitfeld ( 1954) has established a method whereby the sequence of nucleotides in unbranched polyribonucleotides may be ascertained.

Enzymatic depolymerization of deoxyribonucleoproteins by tumor cells has been investigated by Kutscher and Eggers (1954). They concluded that the nuclear enzymes, deoxyribonuclease, cathepsin, peptidases etc., are not involved in mitosis, but are involved in other physiological functions. The rate of ribonuclease activity was found by Maver and Greco ( I 956) to be considerably higher in fractions obtained from hepatomas than in corresponding fractions from normal rat liver.

The virus studies of Cohen and associates (Cohen, 1955, I956), while not bearing directly on the cancer problem, may provide a basis for a more complete understanding of malignant transformation and the growth of cancerous cells. In studying the virus that infects $E$. coli it was found that this organism contained 5-hydroxymethyl cytosine (HMC) in the DNA. This pyrimidine was not found in the DNA of the host. A pathway for the conversion of cytosine in the host DNA to the HMC of the T virus is presented in Fig. 6.

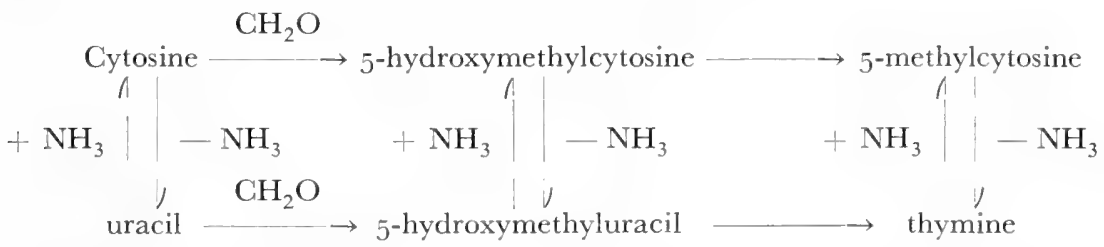

Fig. 6. The biosynthesis of 5-hydroxymethylcytosine (after Cohen, I955).

The deoxyribosides of HMC or hydroxymethyluracil do not fulfill the pyrimidine requirements in organisms requiring deoxyribosides of cytosine or of uracil. Thus the hydroxymethylation appears irreversible. Cohen concluded that this type of hydroxymethylation does not exist in normal cells.

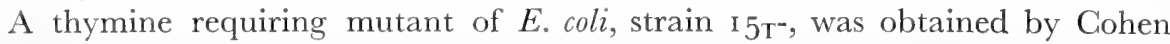
and associates. This organism did not respond to the hydroxy derivatives mentioned above. Exhaustion of thymine from the medium did not produce an abrupt 
cessation of growth as measured by turbidity increase. Incubation of the ${ }^{1} 5 \mathrm{~T}^{-}$ strain in the absence of thymine resulted in an increase in turbidity, considerable enlargement of the cell in girth and length, a doubling of the RNA and a significant increase in DNA before the death of the organisms. It was also found that development of a thymine deficiency in other organisms also produces death of the cell. When the thymineless mutant was incubated in the presence of uniformly labeled glucose it was observed that RNA components were synthesized but that DNA synthesis was reduced nearly to zero (Cohen and Barner, I954, I955; Barner and Cohen, I954, I 955, I956; Cohen, 1956a).

Bromouracil, a competitive antagonist for thymine in lactobacilli and $E$. coli is incorporated into the nucleic acid fraction (Weygand and Wacker, I952). Cohen and associates also found that this antagonist partially replaced the thymine requirement in the $15 \mathrm{~T}^{-}$mutant, permitting some increase in the number of viable cells and also DNA and RNA synthesis. The growth fell off markedly indicating that the DNA that had incorporated the bromouracil was unsatisfactory for the continued survival of this organism. Cohen has thus established a concept of unbalanced growth wherein the cell is unable to survive because of the inability to synthesize deoxyribonucleic acid. He furthermore has speculated that cytoplasmic synthesis might be inhibited without inhibition of nuclear synthesis. A block in the biosynthesis of cytosine riboside, but not of the cytosine deoxyriboside, might produce this condition. Burton ( 1955) has reported that after infection of the host cell with bacteriophage $T_{2}$ there must be a synthesis of protein before the synthesis of the phage DNA is initiated. When these proteins have been formed, the synthesis of the DNA is independent of the further synthesis of proteins.

Leuchtenberger et al. (I954) have made further studies on the relationships between hereditary pituitary dwarfism in mice and the formation of multiple DNA classes. Dwarf mice with a recessive anterior pituitary hypoplasia lack the multiple DNA classes that are present in the tissues of their normal litter males. Treatment of these dwarfs with pituitary growth hormone completely restored the DNA classes, establishing the anterior pituitary as a factor in the formation of these classes. Further evidence has been obtained that there is a basic DNA content or multiple thereof in normal human cells (Leuchtenberger et al., I 954a). Malignant cells usually lack this uniformity, which may be attributed to the mitotic processes. Zamenhof (1956) has reviewed the relation of the transforming principle of microorganisms to the problem of growth. This principle, which appears to be DNA, may be readily inactivated by mutagenic agents such as ultraviolet light, mustard compounds etc. Other agents which have carcinogenic and some mutagenic properties are without effect on this principle, i.e., methylcholanthrene, urethane, and azo dyes. Nucleic acid metabolism, as related to the transformation to resistance or dependence in leukemia cells of the mouse, has been extensively studied by Law (1954). Incubation of a homogeneous solution of normal rat liver or sarcoma with RNA resulted in a transformation of this acid into DNA. Heating for I 5 minutes at $\mathrm{I}$ oo ${ }^{\circ} \mathrm{C}$ destroyed this reaction (Cruz Coke et al., I953). Apparently this observation has not been extended, nor has it received further confirmation.

Several important approaches to the synthesis and mobilization of DNA prior 
to cellular division have appeared recently. Colchicine, in spite of its action in blocking mitosis, does not inhibit the synthesis of DNA (Bloch, I953). During a $26 \mathrm{~h}$. period following the addition of colchicine to cultured rat fibroblasts, DNA synthesis proceeded at a normal rate. However, this agent caused a progressive increase in the number of cells belonging to the higher DNA classes. Stevens et al. (1953, r953a) demonstrated that approximately twice as much DNA was synthesized in a group of normal cells prior to cell division as had previously been present. This was interpreted as evidence that the DNA of the cell is replaced by newly synthesized DNA before mitosis occurs. The findings of Kelly (1956) would indicate that the Ehrlich ascites cell, following irradiation, synthesizes DNA until it reaches the premitotic content of DNA (octaploid). These cells are then arrested since they are unable to go through mitosis.

An intriguing study of the distribution of the newly synthesized DNA in mitotic division has been carried out by Plaut and Mazia (1956, I956a). A partial answer was obtained as to whether in the course of division, the components of the old chromosome are partitioned equally between the two daughter cells or whether the old chromosome remains and a new unit is formed. Cells of the plant, Crepis capillaris were exposed to radiolabeled precursors of DNA for a period sufficiently long for cells to proceed from interphase synthesis to anaphase or telephase and then fixed. It was reasoned that the radioactivity in the whole of the anaphase or telophase cell is a measure of DNA synthesis in the preceeding interphase. Therefore the distribution of radioactivity between the two halves of this cell makes it possible to ascertain the mitotic division of the newly synthesized DNA. Radioautographic techniques were employed, and it was demonstrated that this newly synthesized DNA was not distributed equally between the products of the nuclear division. The extension of these important observations may result in some new concepts of the mechanism of chromosomal reduplication.

V. OTHER METABOLIC OR COMPOSITIONAL FEATURES OF NEOPLASTIC TISSUES

(a) Enzymes

The most extensive compilation of the activities of enzyme, or enzyme co-factors in neoplastic tissues has been made by Greenstein (1954, I956). Only the more recent findings will be included in this discussion.

Burstone (1956) employing histochemical techniques has studied the proteolytic activity in human tumors. The connective tissue stroma adjacent to the tumors exhibited an intense staining reaction for aminopeptidase. Stromal elements at greater distances from the tumor had less activity or none at all. Peripheral tumor areas adjacent to normal muscle or skin were found to exhibit higher dipeptidase and cathepsin activities than the central areas of the tumor (Sylven and Malmgren, I 955). Homogenates of regenerating liver or hepatoma had higher specific catheptic activities than normal rat liver. The nuclear activity of cathepsin was higher in some hepatomas than in normal liver nuclei (Maver et al., I952). Fiore (1952) reported that there was less lysozyme in benign tumors than in malignant mammary neoplasms. Also the metastatic lesions had more lysozyme activity than the 
primary tumor. The enzyme present in normal endocrine tissues which catalyzes the oxidation of $\Delta^{5}$ unsaturated steroids with a $3-\beta$ alcohol group to the corresponding $\alpha, \beta$ unsaturated $\Delta^{3}$-4-ketone was also found in tumors arising from mouse adrenal cortex and testicular interstitial cells (Huseby et al., I 954).

Rhodanese, the enzyme which catalyzes the conversion of hydrocyanic acid and thiosulfate into thiocyanate and bisulfite, was present in much lower concentration in rat hepatoma than in liver. However, the activity was still higher in the tumor than in any other normal tissue (Rosenthal, I955). It was suggested that the high aerobic glycolysis of normal and neoplastic tissue deficient in rhodanese may be attributed to the inhibition of the Pasteur reaction by the minute quantities of cyanide formed in the intermediary metabolism of the cells. Intact mammalian cells and slices of normal and tumor tissues utilize ribose-5-phosphate to form sedoheptulose, fructose and lactid acid. Fixation of $\mathrm{CO}_{2}$ into the carbonyl group of lactic acid occurred during anaerobic conditions suggesting that these tissues may possess phosphoribulokinase and carboxydesmutase (Guzman Barron et al., I955). Ehrlich ascites tumor cells do not exhibit cholinesterase activity according to Holler and Leonhartsberger (I 954).

Rat hepatomas and normal tissues have the same acid and alkaline ribonuclease activity when expressed on a fresh weight basis, but different activities when based on the mitochondrial fraction. Mitochondria isolated from the rat liver tumor possessed the same acid phosphatase activity and deoxyribonucleodepolymerase activity as normal liver mitochondria (Allard, I955). An association of acid and alkaline phosphomonesterase with cytoplasmic particles from fowl sarcoma has been noted by Harris (1954). The tissue concentration of acid phosphatase was the same in benign human tumors and inflammatory lesions. Adenocarcinoma of the colon had a higher tissue acid phosphatase than was present in the adjacent normal colon. Similar increases of tissue acid phosphatase were found in other types of adenocarcinomas and in some epidermoid and sarcomatous tumors compared to homologous normal tissues of origin (Lemon et al., I954). A large number of surgical specimens of adenocarcinoma of the colon and rectum were found to have increased contents of adolase (Sibley and Fleisher, 1955). This finding is in agreement with the general tumor pattern of higher levels of the enzyme of glycolysis.

The phenol-oxidizing system of human melanomas was studied by Kertesz (1954). Dopa and tyrosine oxidase activity of these tumors varied considerably. It was reported that this mammalian enzyme system was bound to the particulate elements of the tumor homogenates and is highly specific for dopa and tyrosine as opposed to the low specificity of the plant polyphenoloxidases. Humm and Clark (I955) investigated the oxidative metabolism of melanomas in platyfishswordtail hybrids. Some evidence was obtained that the tyrosinase system may be involved in hydrogen transfer in these tissues as an adjunct to the usual respiratory mechanism. The question as to whether or not the melanin system may be essential to the origin or growth of malignant melanoma has not been definitely answered. While some melanomas are low in tyrosinase activity and also in the degree of pigmentation, this does not provide conclusive proof that tyrosine metabolism is completely inhibited. The tyrosine or dopa may proceed through 
several stages of the oxidative sequence but not to the stage of the formation of the melanin complex. New developments may be expected in the near future that will clarify the entire mechanism of melanization as well as the function of the tyrosinase system in the metabolism of malignant melanoma.

Shapiro and associates ( $195^{6}$ ) have continued their investigations on the quantitative differences in various components of the tumor and host. Total riboflavin, flavin adenine dinucleotide, mononucleotide and free riboflavin were measured in the adenocarcinoma and host tissues of $\mathrm{C}_{57}$ mice. Riboflavin was lower in tumor than any other tissue analyzed except muscle. This general pattern has been observed with many of the vitamins in that the normal tissue concentrations will cover a wide range while the values for tumor are usually near the lowest normal values. This group of investigators (Shils et al., I956) measured the thiamine, coenzyme A, riboflavin, pyridoxine in mammary adenocarcinoma and in several normal tissues. Heart, kidney and tumor were all high in these components. The tumor was relatively low but the in same general range as muscle, lung, testes, brain, intestine and stomach.

\section{(b) Minerals}

Several minerals, calcium, copper, iron and zinc are present in lower concentration in methylcholanthrene induced carcinoma than in normal mouse skin (Cowdry, I953). Much of the older literature relative to the chemical composition of tumor has been reviewed by Greenstein (1954, Chapter 8). DeLong et al. (1950) have discussed the significance of the generally low calcium and high potassium contents in neoplastic tissues. Spectrographic methods were developed by Smith et al. (1953) for determining the mineral content of normal human tissues and neoplasms.

A large number of human neoplasms were analyzed by Waterhouse et al. ( I 955) for water, nitrogen and electrolyte content. A high total water content was observed while the total nitrogen, based on a dry weight basis, was in the normal tissue range. A high intracellular concentration of potassium and phosphorus was noted in most tumors. Injections of zinc salts, according to Chahovitch (I955) accumulated in sarcoma tissue and also accelerated the rate of growth of this tumor. Several trace elements were studied spectrographically in the livers of patients with cancer. Iron, zinc, chromium and cobalt were elevated in the liver liver in instances of acute lymphatic leukemia with hepatic involvement (Olson et al., i 954).

\section{(c) Other metabolic features}

The highest levels of acetyl choline were found in the peripheral parts of tumors. The level of this component was roughly proportional to mitotic activity in the tumors (Svec and Hlavayovā, i 953). Furth et al. (r 953) have obtained transplantable pituitary tumors capable of secreting ACTH. Crystalline protoporphyrin was obtained from an extract of chloroma (Schulz and Schwartz, I956). Administration of glycine- $2-{ }^{14} \mathrm{C}$ resulted in a greater uptake of ${ }^{14} \mathrm{C}$ on porphyrin of the tumor than in hemes of the non-tumorous tissue, indicating the de novo synthesis of porphyrin by this neoplasm. 
In 12 cases of clinical cancer the oxygen content of venous blood draining the tumor was higher than that in other parts of the body. This was interpreted as being the result of an increased blood flow through these tumors (Bierman et al., I952). Tumor tissues, as has been shown by Warburg and others usually have a low oxygen tension. Recently Urbach (1956) has demonstrated that tumors have a very low oxygen tension as compared to that of the immediate adjacent tissues. This decrease of tissue oxygen tension may be due to a combination of diminished capillary flow and an increase in the oxygen consumption of the tumor.

Two types of transplantable tumors were found in female mice exposed to. whole body ionizing irradiation: adrenotrophic and mammatrophic (Furth et al., I956, I956a). Hyperestrogenization may be the growth stimulant for the latter type. The mammotrophic tumor induces mammary gland hyperplasia and also some somatrophic effects. These investigators attribute both the mammotrophic and somatrophic effects to a single hormone secreted by the tumor. Newman and McCoy (1956) have recently reported that Walker $25^{6}$ carcinoma cells, in vitro have a dual requirement for both aspargine and glutamine and do not require the corresponding amino acids. This unusual requirement does not occur in any known living system.

\section{SUMMARY}

There is no conclusive evidence, as yet, that malignant cells may differ qualitatively from normal cells either in their metabolic or compositional patterns. The same precursors, enzymes and metabolic pathways are present and operative in normal as well as in cancerous tissues. Many quantitative differences in the concentration of the enzymes or the intermediary metabolites have been reported for individual tumors. In some respects, tumors may be characterized or classified as a group by the concentration or activities of the metabolic constituents. A good example of this is the generally lowered concentration of the B vitamins and many of the oxidative enzymes in neoplastic tissues. However, no consistent pattern has been found that would distinguish malignant cells from the many normal cellular types. Since malignant cells differ from normal cells in other aspects such as morphology, rate of division, lack of response to inherent growth regulatory mechanisms, metastatic tendencies, etc, we must attribute these differences either to an altered intermediary metabolism or to other levels of cellular activity. The possibility does exist that the same metabolites and pathways present in normal tissues may undergo quantitative rearrangements that would give rise to a malignant prototype. We have no definite proof that this is the case.

All of the enzymes and the various intermediates involved in the breakdown of glucose and the operation of the citric acid cycle are apparently present in malignant cells. However, these cells are generally characterized by high aerobic and anaerobic glycolytic rates. This metabolic feature has been attributed to one or a combination of these factors; (a) disturbances in the electron transport system, (b) differences in membrane permeability between normal and cancerous cells limiting the exchange and availability of essential metabolites, (c) relative differ- 
ences in the concentration of enzymes or cofactors, (d) the relation of lipid oxidation to the overall metabolic pattern. Weinhouse and others have observed that many tumors are not self sufficient in the biosynthesis of fatty acids. A further elucidation of this respiratory behavior of the malignant cell will contribute greatly to our knowledge of the origin and growth of these abnormal cells.

An important contribution to this problem has been made by Busch and associates. Tumors appear to have a lowered capacity to utilize acetate. Labeled acetate is quickly incorporated into the succinate pool in normal tissues, while in carcinoma this conversion is delayed to a considerable extent. Also, tumors do not convert added pyruvate into amino acids as rapidly as most normal tissues. Kit has shown that tumors of lymphatic origin have an unusual capacity to synthesize glycine and serine and a very limited capacity for the production of aspartic and glutamic acids. Since many quantitative variations occur in various normal cells we cannot say whether the metabolic pattern of a tumor is concerned with the malignant nature of the cell or is quite incidental to this process. The need for further studies in this area which would include a wide spectrum of tumors in several species and more control tissues is obvious.

Several investigators have found that following administration of labeled plasma proteins, the specific activity of proteins in cancerous tissues is higher than in proteins of the normal tissues. This apparent extensive utilization of plasma proteins by malignant tissues would appear highly significant. Several quantitative differences in the incorporation of precursors into the nucleic acids of tumors and normal tissues have been reported. These differences in the biosynthesis of nucleic acids in normal and cancerous cells are largely responsible for the action of many of the antimetabolites that have been applied in the chemotherapy of cancer. The establishment of greater quantitative differences or of some unique qualitative differences would provide a basis for a greater success in this area. As the various intermediates are established for the different pathways of nucleic acid formation we may find that such differences do exist. LePage and coworkers have observed that azaserine reacts with constituents of the Ehrlich ascites tumor cell to inhibit purine biosynthesis. An intermediate, glycineamideribotide accumulates, indicating that these tumor cells are inhibited at a point where formylation of this compound occurs. In liver systems, azaserine blocks this reaction sequence at a different stage. The significance of this and related studies in the eventual control of the metabolic functions of malignant cells cannot be overemphasized.

Whether or not the metabolism has any direct relationship to malignant behavior as mentioned above has not been established. Many different métabolic patterns exist according to the specific tissues or cells involved and tumors may not differ in this respect. Accordingly, we should attempt to explain malignant behavior, or control of the metabolism of tumors at another level. Factors of either intracellular or extracellular origin may control the mobilization of nucleoproteins and associated processes of cellular reduplication. The nature of these regulatory growth factors is as yet unknown. This regulatory activity may reside within the complex deoxyribonucleoproteins, in simpler structures, or in a combination of both. Thus, we may theorize that malignant cells, because of altera- 
tions in deoxyribonucleoprotein are no longer under the control of normal extracellular factors that regulate cellular division. Also, the possibility exists that the tumor cell has elaborated new factors which are responsible for the unrestrained wrowth. The further elucidation of the mechanism which regulates mobilization of the nucleic acids and proteins associated with mitotic activity may be of more importance to the understanding of malignant behavior than metabolism per se. 


\section{LITERATURE}

Abraham, S., R. Hill and I. L. Chaikoff, (1955) Cancer Research, I5, i 77.

Abraham, S., P. Cady and I. L. Chaikoff, (1956) Proc. Am. Assoc. Cancer Research, 2, 89. Ackerman, G. A., R. A. Knouff and H. A. Hoster, (1951) 7. Natl. Cancer Inst., 12, 465. Albert, S. And R. M. Johnson, (1954) Cancer Research, 14, 27 I.

Allard, C., (1955) 7. Natl. Cancer Inst, , 5 , I607.

Babson, A. L., (1954) Cancer Research, 14, 89.

Babson, A. L. (1956) Biochim. et Biophys. Acta, 20, 418.

Babson, A. L. AND T. Winnick, (1954) Cancer Research I4, 606.

Backmann, R. and E. Harbers, (I955) Biochim. et Biophys. Acta, i6, 604.

Balis, M. E., (1956) Proc. Am. Assoc. Cancer Research, 2, 92.

Balis, M. E., D. V. Praag and G. B. Brown, (I955) Cancer Research, 15, 673.

Barclay, R. K. and E. Garfinkel, (1955) 7. Biol. Chem., 212, 397.

Barner, H. D. and S. S. Cohen, (I954) 7. Bacteriol., 68, 80.

Barner, H. D. and S. S. Cohen, (I955) Federation Proc., I4, 177.

Barner, H. D. and S. S. Cohen, (1956) 7. Bacteriol., 7I, I49.

BECK, W. S., (1955) 7. Biol. Chem., 216, 333.

Beck, W. S. And W. N. Valentine, (1952) Cancer Research, I2, 823.

Beck, W. S. And W. N. Valentine, (I953) Cancer Research, I3, 309.

Bendich, A., H. B. Pahl And S. M. Beiser (1954) Cold Springs Harbor Symposia Quant. Biol., II, 3I.

Bennett, L. L., Jr., H. E. Skipper, C. C. Stock and C. P. Rhoads, (i955) Cancer Research, I5, 485 .

Bennett, L. L., Jr., H. E. Skipper, H. W. Toolan and C. P. Rhoads, (ig56) Cancer Research, $16,262$.

Berliner, M. L., D. L. Berliner and T. F. Dougherty, (i956) Proc. Am. Assoc. Cancer Research, 2, 94.

Bierman, H. R., K. H. Kelly and G. Singer, (i952) 7. Natl. Cancer Inst., I2, 701.

Biserte, G., (I953) Cancérologie (Lille), I, 3 I.

Bloch, D. P., (1953) Proc. Soc. Exptl. Biol. Med., 84, 34 I.

Blumenthal, F. and H. Wolff, (1905) Med. Klin. (Münich), I, 166.

Blumenthal, F., E. Jacoby and C. Neuberg, (Igog) Med. Klin. (Mïnich), 5, 1595.

Boman, T. J., (1952) Indian 7. Med. Sci., 6, 623.

Boulanger, P. And R. Osteux, (1953) Compt. rend., $236,2177$.

Boulanger, P. And R. Osteux, (1954) Cancérologie (Lille), I, I83.

Boyd, E. M. and H. D. McEwen, (1952) Can. F. Med. Sci., zo, 163.

BRin, M. AND R. W. McKee, (I956) Cancer Research, I6, 364.

Brown, G. W. Jr., J. Katz and I. L. Ghaikoff, (1956) Cancer Research, 16, 509.

Burk, D., (1939) Cold Spring Harbor Symposia Quant. Biol., 7, 420.

Burk, D. and M. Woods, (1956) Proc. Am. Assoc. Cancer Research, 2, 98.

Burstone, M. S., (I956) 7. Natl. Cancer Inst., i6, I I 49.

Burton, K., (1955) Biochem. 7., 6I, 473.

Busch, H., (1953) Cancer Research, 13, 789.

Busch, H., (1955) Cancer Research, 15, 365.

Busch, H. And H. A. Baltrush, (I954) Cancer Research, I4, 448.

Busch, H. and H. S. N. Greene, (1955) Fale 7. Biol. and Med., 27, 339.

Busch, H. And V. R. Potter, (1952) Cancer Research, 12, 66o.

Busch, H. and V. R. Potter, (i952a) F. Biol. Chem., I98, 7 I.

Busch, H. and V. R. Potter, (1953) Cancer Research, 13, i68.

Busch, H., M. H. Goldberg and D. C. Anderson, (1956) Cancer Research, i6, i 75.

Busch, H.. S. Simbonis, D. C. Anderson and H. S. N. Greene, (1956) Proc. Am. Assoc. Cancer Research, 2, 98.

Butler, J. A. V., E. W. Johns, J. A. Lucy and P. Simson, (i956) Brit. F. Cancer, io, 202.

Campbell, P. N., (1955) Biochem. J., 6r, 496. 
Campbell, P. N., (1958) in Adrances in Cancer Research, Vol. V, Academic Press, New York. Cavpbell, P. N., H. E. H. Jones and N. E. Stone, (1956) Nature, I77, 138.

Cantarow, A., K. E. Paschkis and R. J. Rutman, (1955) 7. Natl. Cancer Inst., 15, i6 I5. Casperson, T. O., (1950) Cell Growth and Cell Function, W. W. Norton and Company, New York.

Cerecedo, L. R., P. T. McGarthy, E. J. Singer and E. T. McGuinness, (1955) Exptl. Med. Surg., 13, 85 .

Chahovitch, X., (1955) Glas Srpske Akad. Nauk. (Belgrade), 215, I43.

Chance, B., (1953) Trans. N. Y. Acad. Sci., i6, 74 .

Chance, B. and L. N. Castor, (i952) Science, II6, 200.

Chapman, D. D., G. W. Brown Jr., I. L. Chaikoff, W. G. Dauben and N. O. Fansah, (1954) Cancer Research, I4, 372.

Chargaff, E., (i 955 ) in The Nucleic Acids, Vol. I, Academic Press, New York.

Chargaff, E. And J. N. Davidson, (1955) The Nucleic Acids, 2 Volumes, Academic Press New York.

Chargaff, E. and R. Lipshitz, (i953) 7. Am. Chem. Soc., 75, 3658.

Ghristensen, H. N. And M. E. Henderson, (1952) Cancer Research, 12, 229.

Christensen, H. N. And M. L. Rafn, (1952) Cancer Research, 12, 495.

Christensen, H. N., B. Hess and T. R. Riggs, (1954) Cancer Research, I4, 124.

Clement, G., (1954) Bull. assoc. franç. étude cancer, 4I, 65 .

Clowes, G. H. A. And A. K. Keltch, (1952) Proc. Soc. Exptl. Biol. Med., 8I, 356.

Clowes, G. H. A. And A. K. Keltch, (I954) Proc. Soc. Exptl. Biol. Med., 86, 629.

Conen, S. S., (1955) Aspects of Synthesis and Order in Growth, Ed. D. Rudnick, Princeton University Press, New Jersey.

Cohen, S. S., (1956) Essays in Biochemistry, Ed. Graff, John Wiley and Sons, New York.

Cohen, S. S., (I956a) Science, I23, 653.

Cohen, S. S. and H. D. Barner, (1954) Federation Proc., I3, 193.

Cohen, S. S. and H. Barner, (I955) $\mathcal{H}$. of Bacteriol., 69, 59.

Cowdry, E. V., (1953) in Advances in Cancer Research, Vol. I, Academic Press, New York.

Cruz Coke, E., M. Plaza De los Reyes, J. Martens, J. Del Nido and J. Araya E.

Ines Bradford, (1953) Bol. soc. biol. Santiago, Chile, 1o, 46.

Daoust, R. And A. Cantero, (1955) Cancer Research, 15, 734.

Davidson, J. N. and I. Leslie, (I950) Cancer Research, IO, 587.

Delamirande, G., C. Allard and A. Cintero, (1955) Cancer Research, 15, 329.

DeLong, R. P., D. R. Coman and I. Zeidman, (i950) Cancer, 3, 718.

Dmochowski, L. and L. H. Stickland, (i953) Brit. Cancer, 7, 250.

Eagle, H., V. I. Oyama, M. Levy, C. L. Horton and R. Fleischman, (i956) 7. Biol. Chem., 218,607 .

Eden, M., B. Haines and H. Kahler, (1955) F. Natl. Cancer Inst., I6, 54 I.

Edmonds, M. P. And G. A. LePage, (I955) Cancer Research, I5, 93.

Edmonds, M. and G. A. LePAGe, (1956) Cancer Research, I6, 222.

Eidinoff, M. L., J. E. Knoll and B. J. Marano, (1956) Proc. Am. Assoc. Cancer Research, $2,107$.

EL'Tsina, N. V., (1953) Doklady Akad. Nauk. S.S.S.R., gI, 6о I.

Emmelot, P. and C. J. Bos, (I 955) Biochim. et Biophys. Acta, i6, 620.

Emmelot, P. And C. J. Bos, (1955a) Rec. trav. chim., 74, I343.

Emmelot, P. And C. J. Bos, (1955b) Biochim. et Biophys. Acta, 18, 28 I.

Emmelot, P. And C. J. Bos, (1956) Biochim. et Biophy's. Acta, 19, 565.

Emmelot, P. and L. Bosch, (1955) Brit. 7. Cancer, 9, 344.

Emmelot, P. And L. Bosch, (1955a) Brit. 7. Cancer, 9, 327.

Emmelot, P. And L. Bosch, (r 955b) Brit. 7. Cancer, 9, 339.

Eminelot, P., C. J. Bos and P. J. Brombacher, (1956) Brit. F. Cancer, io, i88.

Emmelot, P., L. Bosch and G. H. van Vals, (1955) Biochim. et Biophys. Acta, 17, 45 I.

Etinghof, R. N. And A. I. Balyasnaya, (1953) Biokhimia, I8, 538.

Fernandes, J. F., G. A. LePage and A. Lindner, (i956) Cancer Research, i6, i54.

FIORE, M., (1952) Tumori, 38, 57. 
Fishman, W. H., (I953) The Physiopathology of Cancer, Ed. F. Homburger and W. H. Fishman Hoeber-Harper, New York.

Fitzpatrick, T. B., S. W. Becker, A. B. Lerner and H. Montgomery, (i950) Science, II2, 223.

Fodor, P. J., C. Funk and P. Tomashevsky, (I955) Experientia (Basel), II, 266.

Furth, J., E. L. Gadsen and A. C. Upton, (I953) Proc. Soc. Exptl. Biol. Med., 84, 253.

Furth, J., E. L. Gadsen, K. H. Glifton and E. Anderson, (1956) Cancer Research, 16, 600.

Furth, J., K. H. Clifton, E. L. Gadsen and R. F. Buffett, (I956a) Cancer Research, i6, 608.

Giarelli, L. (1954). Arch. ital. anat. $e$ istol. patol., 28, 103.

Gigante, D., M. Capone, G. F. Marinoni and A. Rossi-Espagnet, (i954) Bull. assoc. frang. étude cancer, 4 I, 498.

Glock, G. E. and P. McLean, (1954) Biochem. 7., 56, i 7 I.

Goranson, E. S., J. McBride and G. Weber, (I954) Cancer Research, I4, 227.

Greenberg, D. M., (I955) Cancer Research, 15, 42 I.

Greenlees, J. and G. A. LePage, (1956) Cancer Research, 16, 808.

Greenstein, J. P., (I954) Biochemistry of Cancer, 2nd Ed., Academic Press, New York.

Greenstein, J. P., (1956) Cancer Research, I6, 641.

Griffin, A. C., L. Cunningham, E. L. Brandt and D. W. Kupke, (i95I) Cancer, 4, 4io.

Groth, D. P. and G. A. LePage, (I954) Cancer Research, I4, 837.

Grunberg-Manago, M., P. J. Ortiz and S. Ochoa, (I955) Science, I22, 907.

Guzman-Barron, E. S., M. Villadicencio and D. W. King, Jr., (1955) Arch. Biochem. Biophys., 58, 500 .

Harris, R. J. C., (I954) Brit. 7. Cancer, 8, 714.

Harris, R. J. C., H. Malmgren and B. Sylven, (i954) Brit. F. Cancer, 8, 14 I.

Heidelberger, C., (1953) Advances in Cancer Research, Vol. I, Academic Press, New York, p. 274 .

Heidelberger, C., (1956) Ann. Rev. Biochem., 25, 573.

Heimberg, M., J. H. Park, A. Isaacs and R. Pitt-Rivers, (1955) Endocrinology, 57, 756. Heinz, E., (1954) 7. Biol. Chem., 211, 781.

Heller, I. H. And K. A. C. Elliott, (1954) Can. 7. Biochem. and Physiol., 32, 584.

Heppel, L. A., P. J. Ortiz and S. Ochoa, (1956) Science, 123, 415.

Holler, H. and F. Leonhartsberger, (I954) Krebsarzt, 9, 233.

Holzer, H., J. HaAn and S. Schneider, (I 955) Biochem. Z., 326, 45 I.

Humm, D. G. and E. E. Clark, (1955) 7. Natl. Cancer. Inst., I6, 741.

Huseby, R. A., L. T. Samuels and M. L. Helmreich, (i954) Proc. Soc. Exptl. Biol. Med., 86,580 .

INGLE, D. J., (1956) Endocrinology, 59, 259.

Jedeikin, L. A. And S. Weinhouse, (I955) 7. Biol. Chem., 213, 27 I.

Johnson, R. M. And P. H. Dutch, (1952) Arch. Biochem. Biophys., 40, 239.

Kandutsch, A. A. and C. A. Baumann, (1954) Cancer Research, I4, 667.

Kay, E. R. M., R. M. S. Smellie, G. F. Humphrey and J. N. Davidson, (i956) Biochem. 7., 62, I60.

Kelly, L. S., (1956) Federation Proc., 15, 108.

Kennedy, E. P. and S. W. Smith, (1954) F. Biol. Chem., 207 , I53.

Kertesz, D., (1954) 7. Natl. Cancer Inst., I4, I 08 I.

Kertesz, D. And A. Albano, (1955) Cancer Research, 15, 394.

Khouvine, Y., (1954) Compt. rend., 239, 782.

Khouvine, Y. And M. L. Hirsch, (1952) Bull. soc. chim. biol., 34, 305.

Khouvine, Y. And M. Mortreuil, (I954) Compt. rend. soc. biol., I48, 1534.

Khouvine, Y. And M. Mortreull, (I954a) Compt. rend., 238, 2129.

Kielley, R. K., (1952) Cancer Research, 12, 124.

KIT, S., (1954) Cancer Research, I4, 397.

KIT, S., (I955) 7. Biol. Chem., 2I2, 3 I I.

KIт, S., (1955a) Cancer Research, 15, 7 I 5.

Kit, S., (1956) Cancer Research, 16, 70.

Kit, S. and J. Awapara, (1953) Cancer Research, 13, 694. 
Kit, S. And O. L. Graham, (1956) Proc. Am. Assoc. Cancer Research, 2, i 25.

Kit, S. and O. L. Graham, (1956a) Cancer Research, I6, i I 7 .

Kit, S. And A. C. Griffin, (1958) Gancer Research, I8, 621.

Klemperer, G., (1889) Z. klin. Med., I6, 550.

Kornberg, A., I. R. Lehman and E. S. Simms, (1956) Federation Proc., I5, 29 I.

Kotake, M. And A. Ohsuka, (i 953) Proc. Fapan. Acad., 29, 59.

Kutscher, W. And K. EgGers, (1954) Z. Krebsforsch., 6o, 66.

Lagerkvist, U., P. Reichard, B. Carlsson and J. Grabosz, (i 954) Cancer Research, 15, i64.

Law, L. W., (1954) 7. Natl. Canc. Inst., I5, 8 I 7.

Lemon, H. M., M. M. Davison and I. Asimov, (1954) Cancer, 7, 92.

Lemon, H. M., B. S. Walker, M. M. D. Reynolds and H. H. Wotiz, (1954a) New Engl. 7. Med., 25I, 975 .

Lenta, M. P. And M. A. Riehl, (1952) Cancer Research, I2, 498.

LePage, G. A., (1948) 7. Biol. Chem., I76, ioog.

LePage, G. A., (1950) Cancer Research, $10,77$.

LePAge, G. A., (1953) Cancer Research, 13, I 78.

LePage, G. A., (1953a) Proc. Soc. Exptl. Biol. Med., 83, 724.

LePage, G. A., V. R. Potter, H. Busch, C. Heidelberger and R. B. Hurlbert, (1952) Cancer Research, I2, I53.

Lerner, A. B. and T. B. Fitzpatrick, (1950) Physiol. Revs., 30, 91.

Leslie, I., (1955) in The Nucleic Acids, Vol. 2, Academic Press, New York.

LetTré, H., (1952) Naturwissenschaften, 39, 483.

Letró, H., (1952a) Z. Krebsforsch., 58,62 r.

Lettré, H., H. Wrba and E. Seidler, (1955) Z. Krebsforsch., 6o, 294.

Leuchtenberger, C., H. F. Helweg-Larsen and L. Murmanis, (1954) Lab. Invest., 3, 245.

Leuchtenberger, C., R. Leuchtenberger and A. M. Davis, (1954a) Am. F. Pathol., 30,65 .

Levy, H. B., H. M. Davidson, R. W. Reinhart and A. L. Schade, (i953) Cancer Research, I3, 716 .

Lindberg, O., M. Ljungaren, L. Ernster and L. Revesz, (1953) Exptl. Cell Research, $4,243$.

Ludford, R. J., (1954) Brit. 7. Cancer, 8, i I 2.

Magasanik, B., (I955) in The Nucleic Acids, Vol. I, Academic Press, New York.

Markert, C. L., (1955) Ann. N. Y. Acad. Sci., 6o, ioo3.

Maver, M. E. And A. E. Greco, (1956) Proc. Am. Assoc. Cancer Research, 2, 132.

Maver, M. E., A. E. Greco, E. Lovtrup and A. J. Dalton, (1952) F. Natl. Cancer Inst., I3, 687 .

McIndoe, W. M. and J. N. Davidson, (i952) Brit. 7. Cancer, 6, 200.

McKee, R. W., K. Lonberg-Holm and J. A. Jehl, (1953) Cancer Research, 13, 537.

McKinney, G. R. and R. W. Rundles, (1956) Cancer Research, i6, 67.

Medes, G., B. Friedmann and S. Weinhouse, (1956) Cancer Research, i6, 57.

Medes, G., A. Thomas and S. Weinhouse, (1953) Cancer Research, 13, 27.

Melchior, J. B. and A. H. Goldkamp, (1953) Cancer Research, I3, 798.

Mellors, R. G., J. F. Keane Jr. and G. N. Papanicolaou, (I952) Science, i I6, 265.

Mellors, R. G., J. Hlinka, A. Kupfer and K. Sugiura, (i 954 ) Cancer, 7, 779.

Miller, J. A., (1950) Cancer Research, 10, 65.

Miller, G. L., E. U. Green, E. E. Miller and J. J. Kolb, (I950) , Cancer Research, io I 48 .

Mor, F., (1955) Rass. ital. chir. e med., 4, 227.

Morelli, A., F. D'Abramo and P. Gullino, (1953) Boll. soc. ital. biol. sper., 29, 1445.

Morris, H. P., (1955) Advances in Cancer Research, 3, 52.

Müller, F., (1889) Z. klin. Med., I6, 496.

Muller, G., (1953) Arch. Geschwulstforsch., 6, 3 I.

Naeslund, J., (1954) Gynécol. prat., 5, 243.

Naeslund, J. And K. E. Swenson, (1953) Acta Obstet. Gynecol. Scand., 32, 359.

Negelein, E., (1952) Biochem.Z., 323, 2 I4. 
Neuberg, C. And E. Ascher, (i go6) Arb. pathol. Inst. Bern, 593,

Neufeld, H. A., F. V. Lucas, A. P. Martin and E. Stotz, (1955) Cancer Research, 15, 550.

Newman, R. E. And T. A. McCoy, (1956) Science, I24, 124.

NygaARd, O. and H. P. Rusch, (1955) Cancer Research, 15, 240.

Nyhan, W. L. and H. Busch, (1956) Proc. Am. Assoc. Cancer Research, 2, 136.

Ochoa, S., (1956) Federation Proc., 15, 832.

Orson, R. E., (195I) Cancer Research, II, 57 I.

Olson, K. B., G. Heggen, C. F. Edwards and L. W. Gorham, (1954) Science, 119, 77 I.

Petrakis, N. L. and L. J. Folstad, (1954) 7. Natl. Cancer Inst., 15, 63.

Pigco, A. and G. Dogliotti, (1952) Tumori, $38,85$.

Plaut, W. And D. Mazia, (1956) Reports Annl. Beriner Symposizm, Univ. of Texas, M. D.

Anderson Hospital and Tumor Institute.

Plaut, W. and D. Mazia, (1956a) J. Biophys. Biochem. Cytol., in the press.

Polli, E. and G. Ratti, (1953) Biochem. Z. 323, 546.

Poppe, H. And A. Fraedrick, (1954) Arch. klin. Chir. Langenbecks, 218, 50.

Potter, V. R., (1956) Cancer Research, I6, 658.

Potter, V. R, and G. J. Liebl, (1945) Cancer Research, 5, I8.

Prunieras, M., (1954) 7. méd. Lyon, 35, 62 I.

Rabinovitz, M., M. E. Olson and D. M. Greenberg, (1955) 7. Biol. Chem. 213 , i.

Reid, J. C., M. O. Landefeld and J. L. Simpson, (i 952$)$ 7. Natl. Cancer Inst., I2, 929.

Reif, A. E., V. R. Potter and G. A. LePage, (I953) Cancer Research, 13, 807.

Reymond, C. And C. Wild, (1953) Oncologia, 6, I48.

Riggs, T. R., B. A. Coyne and H. N. Christensen, (I954) F. Biol. Chem., 209, 395.

Roberts, E. And P. R. F. Borges, (1955) Cancer Research, 15, 697.

Roberts, E. and T. TAnaka, (I956) Cancer Research, i6, 204.

Roberts, E., F. W. Sayre, D. G. Simonsen and K. Kano, (1956) Proc. Am. Assoc. Cancer Research, 2, I42.

Robertson, C. H., A. C. Griffin, W. O. Russell, J. W. Gurrie, and E. E. Neu, (1955) Texas Repts. Biol. and Med., I3, 689 .

Rodriguez, N. M., M. T. Bolton and L. R. Cerecedo, (i955) Biochim. et Biophys. Acta, I6, 444 .

Roitt, I. M., (1956) Biochem. 7., 63, 300.

Rondoni, P., (1954) Boll. oncol., 28, 221.

Rondoni, P., (1955) Bull. schweiz. Akad. med. Wiss., II, 274.

Rosenthal, O., (1955) 7. Natl. Cancer. Inst., 15, I6I I.

Rutman, R. J., A. Cantarow and K. E. Paschkis, (I954) Cancer Research, I4, I I 5.

Rutman, R. J., A. Cantarow and K. E. Paschkis, (I954a) Cancer Research, I4, i 19.

Sauberlich, H. E. and C. A. Baumann, (I95I) Cancer Research, II, 67.

Schlief, H. And C. G. Schmidt, (I955) Naturwissenschaften, 42, I04.

Schmidt, C. G., (1955) Klin. Woch., 33, 409.

Schmidt, C. G. And H. Schlief, ( I955) Naturwissenschaften, 42, 105.

Schmid, G., (I953) in The Physiopathology of Cancer, Ed. F. Homburger and W. H. FishMan, Hoeber-Harper, New York.

Schmitz, H., (1954) Biochim. et Biophys. Acta, I4, I60.

Schmitz, H., (1954a) Biochem. Z., 325, 555.

Schmitz, H., W. Hart and H. Ried, (I955) Z. Krebsforsch., 6o, 30 I.

Schmitz, H., V. R. Potter, R. B. Hurlbert and D. M. White, (i954) Cancer Research, I4, 66.

Schmitz, H., V. R. Potter And R. B. Hurlbert, (i954a) Cancer Research, 14, 58.

Schmitz, H., V. R. Potter and R. B. Hurlbert, (I955) Z. Krebsforsch., 6o, 4 i 9.

Schneider, W. C., (1955) 7. Biol. Chem., 216, 287.

Schneider, W. G. And G. H. Hogeboom, (1950) 7. Natl. Cancer Inst., ro, 969.

Schneider, W. C. And V. R. Potter, (1943) Cancer Research, 3, 353.

Schultz, J. And S. Schwartz, (I956) Cancer Research, I6, 565.

Seibert, F. B., E. Soto-Figueroa, E. E. Miller, M. V. Seibert, P. M. Aptekman ani) M. R. Lewis, (1954) Growth, I 8 , I. 
Setbert, F. B., E. Soto-Figueroa, E. E. Miller and M. V. Seibert, (i954a) Growth, I8, I 45 .

Shack, J., R. J. Jenkins and J. M. Thompsett, (I953) 7. Natl. Cancer Inst., I3, I 435.

Shack, J. And J. M. Thompsett, (I953) 7. Natl. Cancer Inst., 13, 1425.

Shacter, B., (I956) 7. Natl. Cancer Inst., i6, I 453.

Shapiro, D. M., L. S. Dietrich and M. E. Shils, (1956) Cancer Research, 16, 575.

Shils, M. E., I. M. Friedland, A. S. Fine and D. M. Shapiro, (i956) Cancer Research, I6, $58 \mathrm{I}$.

Shuster, C. W., (r955) Proc. Soc. Exptl. Biol. Med., 9o, 423.

Sibley, J. A. And G. A. Fleisher, (I955) Cancer Research, I5, 609.

Siekevitz, P. And V. R. Potter, (1953) Cancer Research, I3, 5 I3.

Smith, I. L., N. Kaufman, E. Yeager, T. D. Kinney and F. Hovorka, (I 953) Lab. Invest., 2,284 .

Smith, L. C. and F. M. Rossi, (1954) Proc. Soc. Exptl. Biol. Med., 87, 643.

Smith, L. L. And N. T. Werthessen, (1953) Endocrinology, 53, 506.

Steinberg, M. A., K. G. Stern and A. Rottino, (1952) 7. Natl. Cancer Inst., 12, 97 I.

Stern, K. And R. Willmeim, (1943) The Biochemistry of Malignant Tumors, Reference Press, Brooklyn.

Stevens, G. E., R. Daoust and C. P. Leblond, (1953) Can. 7. Research E, 3i, 263.

Stevens, G. E., R. Daoust and C. P. Leblond, (ig53a) 7. Biol. Chem., 202, 177.

Svec, F. and E. Z. Hlavayovā, (1953) Časopis lékáru českých, 92, 210.

Sylven, B. and H. Malmgren, (1955) Exptl. Cell Research, 8, 575.

Takashi, S., H. Matso, H. Ishida and T. Miyaji, (1954) Gann, 2, 552.

Toennies, G., (1947) Cancer Research, 7, I93.

Toennies, G., (1952) Tex. Repts. Biol. and. Med., ro, 254.

Tyner, E. P., C. Heidelberger and G. A. LePage, (I953) Cancer Research I3, 86.

Urbach, F., (1956) Proc. Soc. Exptl. Biol. Med., 92, 644.

Vendrely, R., (1955) in The Nucleic Acids, Vol. II, Academic Press, New York.

Vescia, A., A. Albano and A. Iacono, (1952) Nature, I70, 804.

Villa, L., E. Polli, G. Semenza, G. P. Di Maiorca and G. D'Amico, (i955) Ricerca sci., 25,59 .

Warburg, O., (1930) The Metabolism of Tumours, Constable and Co. Ltd., London.

WARBurg, O., (I955) Naturwissenschaften, 42, 40I.

Waterhouse, C., A. R. Terepka and C. D. Sherman Jr., (i955) Cancer Research, I5, 544

Weber, G. and A. Cantero, (1955) Cancer Research, I5, 679.

Weber, G. And A. Cantero, (1956) Proc. Am. Assoc. Cancer Research, 2, I 56.

Weinhouse, S., (I95I) Cancer Research, II, 585.

Weinhouse, S., (1955) Advances in Cancer Research, Vol. 3, Academic Press, New York.

Weinhouse, S., (1956) Cancer Research, I6, 654.

Weinhouse, S., A. Allen and R. H. Millington, (1953) Cancer Research, 13, 367.

Weinhouse, S., O. Warburg, D. Burk and A. L. Schade, (1956) Science, 124, 267.

Weisberger, A. S., L. G. Suhrland and J. Seifter, (1956) Blood, ir, I.

Wenner, C. E. And S. Weinhouse, (1953) Cancer Research, I3, 2 I.

Wenner, C. E. And S. Weinhouse, (1955) Cancer Research, I5, 497.

Wenner, C. E., L. Bloch-Frankenthal and S. Weinhouse, (i956) Proc. Am. Assoc. Cancer Research, 2, 156 .

Werkheiser, W. C. And D. W. Visser, (1955) Cancer Research, 15, 644.

WEYGand, F. AND A. WACKeR, (1952) Naturforsch., 7b, 26.

Weymouth, P. P., N. E. Delfel, R. J. Doell, H. L. Sternbock and H. S. Kaplan, (1955) 7. Natl. Cancer Inst., $15,98 \mathrm{I}$.

Whitheld, P. R., (1954) Biochem. F., 58, 390.

Williams, A. D., R. J. Winzler and L. W. Law, (1954) Cancer Research, I4, 135.

Wilinams-Ashman, H. G. And E. P. Kennedy, (1952) Cancer Research, I2, 4I5.

Wixzler, R. J., (1953) The Physiopathology of Cancer, Ed. F. Homberger and W. H. FishMAN, Hoeber-Harper, New York. 
Winzler, R. J., (I953a) Advances in Cancer Research, Vol. I, Academic Press, New York.

Woodhouse, D. L., (1954) Biochem. J., 56, 349.

Woods, M., J. Hunter and D. Burk, (1955) 7. Natl. Cancer Inst., 16, 35 I.

Woods, M., K. Wight, J. Hunter and D. Burk, (1953) Biochim. et Biophys. Acta, I2, 329. Woodward, G. E. And M. T. Hudson, (1954) Cancer Research, 14, 599.

Zamecnik, P. C., R. B. Loftfield, M. L. Stephenson and J. M. Steele, (1951) Cancer Research, II, 592 .

Zamenhof, S., (1956) Progr. in Biophys. and Biophys. Chem., 6, 85. 


\title{
Chapter 13
}

\section{ACTION OF GERTAIN ANTIMETABOLITES AS MITOTIG POISONS}

\author{
by John 7. Biesele
}

\section{INTRODUCTION}

Many substances deleterious to living systems injure cell division. When this effect is especially evident, the agent is commonly labeled a "mitotic poison". Efforts have been made to maintain the usefulness of this term by restricting its application to substances acting specifically on dividing cells (Eigsti and Dustin, 1955), to agents interrupting mitosis in concentrations without visible effect on interphasic cells (Hughes, I952a), to agents effective against mitosis by direct action as demonstrated in vitro (Lettré, I 952), and to substances that make mitosis abnormal without killing the cell (Cornman, I954).

However, almost any substance may influence cell division in some manner under appropriate conditions (Ludford, I 953). Cell division depends on an integration of many orderly processes and is consequently sensitive to a wide variety of substances, which may include not only foreign material but also certain materials native to the cell's metabolism when present in abnormal concentrations. A substance that influences a cell adversely may also interfere in some sense with the reproduction of the cell. This may occur through a number of different mechanisms. Specificity must be borne in mind, and it is not too unusual to find that a substance that poisons mitosis for one cell type does not necessarily exert the same effect on another cell type. Ludford (I 953) preferred that a "mitotic poisoning action" be attributed to a substance only with conditions and biological test system specified and the form of effect described. A relative specificity is evident with certain antimetabolites, which in some respects exert mitotic poisoning actions, and it is the fact of their selective activity that is one basis for hope in experimental cancer chemotherapy (Rhoads, I 954).

It is the purpose of this chapter to discuss the effects on mitosis of structural analogues of normal metabolites. To some extent antimetabolite actions become more intelligible in the light of the activities on mitosis of excesses or deficiencies of normal metabolites. The following sections consider mitotic poisoning actions that (I) are initiated before visible mitosis begins, (2) are expressed in damage to chromosomes, or (3) result in inhibition of spindle formation or function. 


\section{PREPROPHASIC INHIBITION}

Certain syntheses must occur before the visible mitotic stages in order to prepare a cell for its division into two viable daughter cells. Poisons that interfere with mitosis by acting during the period of essential synthesis before the prophase are known as preprophase poisons (D'Amato, 1949a, 1949b). The sensitive period before prophase has also been termed the antephase; poisons acting then need not interfere with mitoses already in progress (Bullough and Johnson, I95I).

Of the greatest importance for the mitosis itself and for the daughter cells is the reproduction of the hereditary material, which appears to be either deoxyribonucleic acid or deoxyribonucleoproteins. It is now established that the reproduction of the hereditary material in ordinary somatic cells occurs during interphase and not during any strictly mitotic phase. This conclusion has been reached by means of a number of complementary techniques, including photometric determination of the amount of stain combined with nucleus or chromosomes after the Feulgen nucleal reaction (Alfert, I950; Seshachar, r950; Patau and Swift, I953; Roels, I 954), studies of ultraviolet absorption (Walker and Yates, I 952; Walker, 1953; Davies, Deeley, Richards and Walker, r 954; Richards, Walker and Deeley, I956), and radioautographic determination of the uptake of ${ }^{32} \mathrm{P}$ (Howard and Pelc, 195I ; Howard and Pelc, I953; Marshak, I 955) or of ${ }^{14} \mathrm{C}$-labeled thymidine into DNA (Plaut and Mazia, I 956).

Chromosomal histone is also doubled when DNA is synthesized before cell division (Bloch and Godman, I955; Alfert, I955). This finding is related to that made by Vendrely ( 1954 ) of a constant relation between the amounts of arginine and DNA in nuclei of a number of animal species. Inorganic radiosulfur is incorporated into chromosomes, probably into their proteins, at about the same time before division as phosphorus is taken up into new DNA (Pelc and Howard, I 952). It may be necessary that histone or other protein be reproduced in order to permit reduplication of the DNA, for it has been suggested (Friedrich-Freksa, I940; Kacser, I956; Mazia, I956) that protein intervenes as a mold in the synthesis of each strand of DNA. Perhaps other materials must also be synthesized in preparation for cell division. The lipid content of rapidly growing cells, for example, may be high, in agreement with their nucleotide content (Berg, I 95 I).

Although the reduplication of all the chromosomal constituents appears to be essential to cell division, it still may not be sufficient. Perhaps the old and the new strands of DNA or nucleoprotein must be separated by an uncoiling process (Schwartz, I 955). It seems that synthesis of DNA is not alone sufficient to trigger cell division (Moses and Taylor, I955; Mazia, I 956). Nevertheless, the metabolic processes which Potter ( $195^{6}$ ) sees as the prerequisites of mitosis are: the formation of thymine and 5-methylcytosine by methylation of the pyrimidine ring, as well as the formation of deoxyribotides by a reductive process.

Some of the syntheses preparatory to cell division make use of energy derived from carbohydrate metabolism. This is obligatorily aerobic respiration for some cells, such as those of mouse ear epidermis (Bullough, I952). Some eggs require oxygen for cleavage, while others can act as facultative anaerobes (Brachet, I 947). In general, the metabolism may be partly aerobic and partly anaerobic (Boy- 
land, 1950); the yield of high-energy phosphate bonds may be used in the synthesis of nucleic acids and proteins. Adenosine triphosphate enters repeatedly into the series of reactions leading from thymidine and the deoxynucleosides of adenine, guanine, and cytosine through their triphosphates to DNA-like polynucleotides in the enzymatic synthesis studied by Kornberg, Lehmann, Bessman, and Simms (1956). Similarly, Grumberg-Manago, Ortiz and Ochoa (I955) have found that RNA-like polynucleotides are enzymatically fashioned from nucleoside diphosphates. Phosphorylation is also involved in several hypotheses of protein synthesis on RNA templates (Dounce, r952; Dounce, Work and Campbell, i 953; Zamecnik and Keller, I954; Borsook, I956).

In the soluble protein fraction of rat liver homogenates, Zamecnik, Keller, Littlefield, Hoagland and Loftfield (1956) have found that amino acids in the presence of ATP and soluble enzymes are activated by conversion to aminoacyladenosine monophosphate compounds, which are built into proteins in microsomal ribonucleoprotein particles with the assistance of guanosine polyphosphate.

Protein synthesis may depend on ribonucleic acid, because ribonuclease abolishes protein synthesis in disrupted staphylococcal cells (Gale and Folkes, I953), in onion root cells (Brachet, I954), and in rat liver microsomes (Zamecnik and Keller, I954). Addition of purines and pyrimidines to staphylococcal cells in a medium containing glucose and amino acids enhances protein synthesis and permits a concomitant synthesis of nucleic acids; if amino acids are absent, no nucleic acid is synthesized; and if nucleic acid content falls too low, protein synthesis ceases (Gale and Folkes, 1953). Specific nucleotide patterns on the RNA template govern the synthesis of proteins with specific amino acid sequences (Dounce, I952; Gamow, I 954; Dounce, Gamow, Spiegelman, Newmark, Harker and Soodak, I 956; Gale and Folkes, I955). 8-Azaguanine, 2,6-diaminopurine, and to some extent benzimidazole, inhibit formation of certain bacterial enzymes, probably because the incorporation of these purine analogues into RNA interferes in its action as a template in protein synthesis (Creaser, I955a, I 955 b). The experiments of Pardee (I 954) suggest the necessity of continued synthesis of RNA for maintaining the synthesis of $\beta$-galactosidase in uracil-requiring mutants of $E$. coli, because the enzyme is produced only when uracil is present in the medium. Formation of this enzyme is inhibited in an adenine-requiring mutant of $E$. coli by 5-hydroxyuridine, which presumably has the initial effect of inhibiting RNA synthesis (Spiegelman, Halvorson and Ben-Ishai, 1955). The evidence indicated to Borsook (1956) that new RNA must continually be synthesized in order to maintain protein synthesis because of a continuing denaturation of RNA.

All this is pertinent to the analysis of mitotic inhibition by purine and pyrimidine analogues. Their incorporation into DNA may interfere in the reduplication of the DNA and in its direction of the syntheses implied in the gene-enzyme concept. Incorporation of the analogues into RNA may interfere in the functions of this substance (or substances) in mitosis, including synthesis of special proteins. The presence of the analogues may prevent the autosynthesis of nucleic acids. Mitotic inhibition by amino acid analogues may also be considered here, for protein synthesis on the nucleic acid template may be regarded as impossible unless all the necessary amino acids are present at the same time (Borsook, 1956). Inhibitory 
analogues of leucine, methionine, and phenylalanine prevent the net incorporation into protein not only of their homologues but also of all other amino acids. Finally, stimulation or inhibition of the synthesis of nucleic acid or of protein tends to have a similar effect on synthesis of the other (Borsook, 1956).

The effect of ribonuclease on mitosis illuminates some of these considerations. In onion roots, ribonuclease produces mitotic inhibition and abnormalities (Kaufman and Das, 1954). The effect on living chick fibroblasts and myoblasts has been studied by Firket, Chèvremont-Comhaire, and Chèvremont (I955). Measurement of Feulgen stain in intermitotic cell nuclei showed that a greater proportion of nuclei in treated cultures have doubled amounts of DNA or amounts approaching that needed for division. Mitoses become less common in treated cultures, and they are held at prophase. Evidently the action of ribonuclease as a prophasic mitotic inhibitor in these cells is not exerted through an interference in DNA synthesis. Ribonucleic acid, however, is in some way essential for mitosis.

Brachet (1955) suggested that the soluble pentose nucleic acid of the cell sap is concerned with cell division. Of the several classes of cytoplasmic ribonucleoprotein macromolecules (Petermann, Hamilton and Mizen, I954), one class becomes especially prominent when liver regenerates after partial hepatectomy (Petermann, Mizen and Hamilton, I 953). Perhaps a special class of cytoplasmic ribonucleoprotein is required to synthesize proteins of the mitotic apparatus, for example.

In summary, it is seen that inhibitors of various phases of intermediary metabolism or of the metabolism of nucleic acids or proteins may reduce the incidence of mitosis in some cell types and in that sense act as preprophasic mitotic poisons.

There are numerous examples of such inhibitors. Among them are many antimetabolites that interfere in the metabolism of nucleic acids, nucleotides, or proteins. Certain groups of these are considered below.

\section{(a) Antifolic acids}

Folic acid is important as a precursor of coenzymes of one-carbon transfers, including those involved in synthesis of purines and thymine leading to nucleic acids (Schlenk, I955). Analogues of folic acid are consequently inhibitors of growth, and their effect may be seen in a diminution of mitotic activity. The effects of such antifolics as aminopterin (4-aminopteroylglutamic acid) in arresting cells in the metaphase of mitosis is discussed in a later section (see p. 942). Arrest in metaphase may contribute to the overall suppression of mitotic activity, and it is of interest that Hughes ( $195^{\circ}$ ) noted no preprophasic inhibition of chick fibroblasts in vitro early in the exposure to concentrations of aminopterin and a-methopterin that cause metaphase arrest. Longer exposure to the antifolic, however, may lead to general mitotic inhibition. Mouse leukemia cells sensitive to a-methopterin showed no specific phase inhibition after 24 -hour exposure to the drug in vitro, and it was suggested that the general suppression of mitotic activity by doses as small as $0.0 \mathrm{I} \mathrm{mg} / \mathrm{ml}$ (about $0.02 \mathrm{mM}$ ) indicated an interference in metabolic reactions concerned with the initiation of mitosis (Kieler and Kieler, I 954).

Mitotic incidence is also decreased in mouse tissue cultures by exposure for $24 \mathrm{~h}$. to 5 different antifolic acids (Biesele, I 954b). Mitosis is more readily suppressed 
in cells of mouse sarcoma 180 than in cells of embryonic mouse skin by aminopterin, a-methopterin, 4-aminopteroylthreonine, and 4-aminopteroylalanine in concentrations from $0.2-1.0$ or $5.0 \mathrm{~m} M$. 4-Aminopteroyltryptophan is more inhibitory to cells of embryonic mouse skin.

\section{(b) Antiglutamines}

The amide group of glutamine furnishes the nitrogen for positions 3 and 9 of the purine skeleton ( $c f$. Reichard, 1955), and this is the basis for an additional set of antimetabolites. Azaserine, or O-diazoacetyl-L-serine, inhibits certain tumors (Stock, Reilly, Buckley, Clarke and Rhoads, I954) and interferes with the role of glutamine in purine biosynthesis, causing the accumulation of certain acyclic ribotide precursors of inosinic acid (Hartman, Levenberg and Buchanan, I 956). Azaserine thus inhibits incorporation of formate (Skipper, Bennett and Schabel, I954) and of glycine (Greenlees and LePage, I956) into nucleic acid.

Azaserine strongly inhibits mitosis in mouse tissue cultures (Biesele, I958 a). Exposure for $24 \mathrm{~h}$. to o.I $\mathrm{m} M$ azaserine depresses mitotic incidence in mouse sarcoma I 80 cells to about io percent of the control value but in mouse embryonic skin cells to only about 70 percent of the control. A partial reversal of the mitotic inhibition from azaserine can be achieved with L-histidine, but L-glutamine is completely effective (Biesele, I $95^{8} \mathrm{~b}$ ). Similar effects are achieved with $\gamma$-glutamyl hydrazide. It is somewhat less toxic than azaserine but also suppresses mitosis differentially in sarcoma i 80 cells. The mitotic suppression can be reversed in mouse tissue cultures and in cultures of certain human carcinoma cell strains with glutamine (Biesele, $195^{8} \mathrm{~b}$ ).

Azaserine at $0.2 \mathrm{mg} / \mathrm{kg}$ decreases the mitotic index in 4 ascites tumors of the mouse for 12 hours after injection; at the same time, it inhibits incorporation of glycine-2- ${ }^{14} \mathrm{C}$ into their acid-soluble and nucleic acid purines (Fernandes, LePage and Lindner, I956).

\section{(c) Purine and pyrimidine analogues}

Structural analogues of the physiological purines are often inhibitory antimetabolites. Many of them depress mitotic incidence, as is illustrated by Kihlmann's results (1952) with 8-ethoxycaffeine. Pea roots were suspended in $7.5 \mathrm{mM}$ 8-ethoxycaffeine for $3 \mathrm{~h}$. and then returned to tap water. Mitotic incidence, which had been about $6 \%$, fell to less than i percent in the first i 2 hours but then returned to the original level by 24 hours of the recovery period.

Mouse embryonic skin cells in culture suffer a greater depression of mitosis from exposure to $2.0 \mathrm{~m} M$ 2-ethoxycaffeine for 2 days than do mouse sarcoma I 80 cells (Biesele, Berger, Clarke and Weiss, I952). Caffeine and 8,8'-ethylenediaminoditheophylline behave in the reverse fashion.

Studies of the reversal of the in hibitions caused by substituted purines and purine analogues have indicated generally complex situations. Kidder and Dewey (1949) concluded that the methylxanthines inhibit more than one enzyme system in the ciliate, Tetrahymena geleii, because their inhibition can only be partly reversed with guanine or other purines. Caffeine inhibition of conidial growth of the ascomycete, Ophiostoma multiannulatum, is likewise only partially reversed with 
adenine (Hultgren, Kihlman and Fries, I955); antagonism of caffeine by means of tryptophan, aniline, or procaine suggests that caffeine does not necessarily inhibit by competing for purine-specific enzymes.

Benzimidazole is also conventionally regarded as an antagonist of adenine (Woolley, 1952). Benzimidazole at $300 \mathrm{ppm}$ inhibits mitosis in onion roots, not only preventing nuclei with doubled DNA from entering mitosis but also preventing new DNA synthesis in younger nuclei. These inhibitions can be blocked for some hours with 800 ppm adenine sulfate (Duncan and Woods, 1953). However, Slonimski ( I 954) concluded that the reversal by salts of adenine or guanine of the benzimidazole-induced inhibition of yeast growth results from a lowering of the $\mathrm{pH}$ and consequent movement of benzimidazole from the cells into the medium.

8-Azaguanine furnishes an example of a more specific relation between physiological purine (guanine) and antagonist (Kidder and Dewey, 1949). 8-Azaguanine specifically inhibits entry into mitosis of cells of the Brown-Pearce tumor in rabbits but does not affect mitosis in jejunal crypts and testes (Shapiro, Weiss and Gellhorn, I950). In the mouse, 8-azaguanine restricts mitotic rates in carcinomas 755 and $\mathrm{EO}_{77} \mathrm{I}$ but not in the tumors $\mathrm{C}_{954}, \mathrm{C}_{1} 300, \mathrm{~S}_{1} 80$, and $\mathrm{S}_{91}$, nor in the jejunum, ileum, or testis (Woodside, Kidder, Dewey, and Parks, I953). The differential susceptibility of various tumors to 8-azaguanine inhibition is inversely related to their ability to deaminate 8-azaguanine to noninhibitory 8-azaxanthine (Hirschberg, Kream and Gellhorn, I952). Tumor EO77 I gives anomalous results.

8-Azaguanine may inhibit because of its incorporation into ribonucleic acid (Kidder, Dewey, Parks and Woodside, I 949). However, more azaguanine was found to be incorporated into visceral RNA than into tumor RNA (Mitchell, Skipper and Bennett, I 950). No solution to this dilemma was seen by Parks ( I 950). Nevertheless, RNA of the susceptible mouse leukemia Li2 io incorporates 8azaguanine- $2-{ }^{14} \mathrm{C}$ at $\mathrm{I}$ oo times the rate of its incorporation into RNA of the derivative azaguanine-dependent leukemia (Bennett, Skipper and Law, I953).

The incorporation of 8-azaguanine probably makes RNA defective. Mandel ( 1955$)$ considered the action of 8-azaguanine as an antimetabolite to lie at the polynucleotide level rather than at the free purine level. 8-Azaguanine seems to occupy positions in tobacco mosaic virus normally held by guanine (Matthews, I954). 8-Azaguanylic acid can be recovered from the RNA of such virus (Matthews, I953) much as thiouridylic acid can be recovered from TMV grown in the presence of thiouracil (Jeener and Rosseels, I953). When one-fifth of the RNA uracil in Bacillus megatherium is replaced with thiouracil, the growth rate changes from exponential to linear (Hamers, I 956). Incorporation may well proceed by way of the riboside of thiouracil, which Strominger and Friedkin (I954) made with the aid of phosphorylase. This and a similarly catalyzed reaction between 8-azaguanine and ribose-I-phosphate or deoxyribose-I-phosphate (Friedkin, 1954) supported the suggestion made by Kalckar (r953) that unnatural purines and pyrimidines might trap ribose or deoxyribose.

It is the fate of 8-azaguanine to be incorporated into the ribonucleic acids of various microorganisms and animal tissues but not into their deoxyribonucleic acids (Lasnitzki, Matthews and Smith, I954). These authors pointed out that a defective bonding between 8-azaguanine and cytosine might be expected in RNA, 
if the guanine residues in RNA, by analogy with the Watson and Crick (1953) double-helix model of DNA structure, are hydrogen-bonded to cytosine residues. Creaser ( $1955^{\mathrm{a}}$, I 955 b) found that the inhibition of enzyme formation by 8-azaguanine in certain bacteria can be reversed with guanine, xanthine, or hypoxanthine. A similar effect of 2,6-diaminopurine is partly reversed by hypoxanthine. Although these effects may be related to inadequate function of RNA containing the analogues, another mechanism may be suggested for the inhibition of formate incorporation into the nucleic acids of mouse viscera by 8-azaguanine (Skipper, Mitchell, Bennett, Newton, Simpson and Eidson, I95I), because this guanine analogue partly inhibits transformation of folic acid into citrovorum factor (Doctor and Trunnell, I955). It is of interest that 8-azaguanine inhibits respiration of sarcoma 37 in mice (Finkelstein, Winters, Thomas, Davidson and Smith, I 95 I) over the same period in which it depresses mitosis (Finkelstein and Thomas, I95I).

2,6-Diaminopurine has shown little selectivity toward neoplastic cells with respect to mitotic inhibition, although it appears to be grossly rather more toxic to sarcoma i 80 cells than to skin fibroblasts in tissue culture of mouse embryo (Biesele, Berger, Wilson, Hitchings and Elion, I95I). Mitosis is inhibited in both cell types by about o. I $\mathrm{m} M$ 2,6-diaminopurine (Biesele, I954b). The agent is too toxic to the host animal to be therapeutically effective against mouse sarcoma I80 (Stock, I950). The mitotic inhibition of cultured mouse cells caused by 2,6diaminopurine is readily reversed by adenine sulfate at the same or one-fourth the concentration (Biesele, Berger, Clarke and Weiss, I952).

There are several examples of adenine-reversed inhibition of growth in plants by 2,6-diaminopurine (e.g., Nickell, I 955). The inhibition of cell division in tobacco callus cultures by diaminopurine is reversed by adenine and slightly by guanine and hypoxanthine (Miller, 1953). Vicia faba root cells are prevented from entry into prophase by $0.096 \mathrm{~m} M$ 2,6-diaminopurine, but a competitive reversal is achieved by adenine with an inhibition index of 0.5 , perhaps because there is a need for free adenine to initiate mitosis in this plant (Setterfield and Duncan, 1955). This may be related to the observation that adenine sulfate promotes growth of pea epicotyls and reverses inhibition by the plant hormone, indole acetic acid (Howell and Skoog, I955).

2,6-Diaminopurine also appears to prevent the onset of DNA synthesis while permitting that underway to continue, for treated Vicia meristem contains nuclei with both the diploid and the doubled amounts of DNA, but not with intermediate quantities (Setterfield and Duncan, I955). The inhibition of DNA synthesis is reversed by adenine, adenosine, or deoxyadenosine, indicating that the interference may proceed by way of nucleoside or nucleotide metabolism.

2,6-Diaminopurine is a precursor of nucleic acid purines and is utilized in a manner paralleling the utilization of guanine (Bendich and Brown, I948; Brown, 1955). A small portion of it may be incorporated as such into mouse nucleic acids (Skipper, I953). If 2,6-diaminopurine is a normal metabolite, it is possible that physiologically excessive concentrations are inhibitory (Brown, I955). However, diaminopurine probably competes with adenine because they are incorporated by the same pathways into similar but antagonistic derivatives, which are more likely to be simpler nucleosides or nucleotide coenzymes than to be nucleic acids. 
It is known that the nucleoside, 2,6-diamino-9- $\beta$-D-ribofuranosylpurine, which inhibits mitosis in mouse tissue cultures at $2 \mathrm{mM}$ (Biesele, Berger, Clarke and Weiss, I 952), is enzymatically phosphorylated to 2-aminoadenosine triphosphate (Kornberg and Pricer, I95I). In the intact mouse, 2,6-diaminopurine- $2-{ }^{14} \mathrm{C}$ is rapidly incorporated into a phosphorylated derivative of the riboside, probably also 2-aminoadenosine triphosphate (Wheeler and Skipper, 1953).

2,6-Diaminopurine is also known to affect adversely the de novo synthesis of nucleic acid purine. It blocks conversion of folic acid to citrovorum factor (Doctor and Trunnell, I955) and inhibits the incorporation into nucleic acid of labeled formate (Skipper, Mitchell, Bennett, Newton, Simpson and Eidson, I95I), 4-amino-5-imidazolecarboxamide (Miller and Warren, I953), and radiophosphate (Visser, I 955).

Several adenine analogues with changes at the 2-position act somewhat like 2,6-diaminopurine on mouse tissue cultures. Sarcoma 180 cells are damaged selectively, and few mitoses occur in concentrations of $0.05 \mathrm{~m} M$ of 2-chloroadenine or higher (Biesele, Berger, Clarke and Weiss, I952) or 2-azaadenine (Biesele, I 952). Both these compounds differ from 2,6-diaminopurine and adenine in their failure to break chromosomes.

Another adenine analogue that selectively inhibits mitosis in certain neoplastic cells is 4-aminopyrazolo-(3,4-d)pyrimidine (Hsu, Robins and Cheng, 1956). This agent inhibits mitoses or renders them pathological in cultures of some neoplasms, including the human cancer strain HeLa, and causes pyknosis of resting nuclei. It appears to have little effect on the normal mouse and human cells tested.

Unsubstituted purine at about o. I $\mathrm{m} M$ differentially suppresses mitosis in cells of mouse sarcoma I8o in culture (Biesele, Slautterback and Margolis, I955). Adenine reverses the mitotic inhibition more readily in the skin cells than in the sarcoma cells. Various adenine nucleotides also show blocking ability, especially for the embryonic mouse skin cells.

The 6-substituted purines include some agents that inhibit the growth of certain neoplasms (Clarke, Philips, Sternberg, Stock, Elion and Hitchings, I953). Some also affect the growth of certain normal tissues. Tentacle-regeneration in hydra, for example, can be inhibited with 6-(phenylalkyl) aminopurines (Ham, Eakin, Skinner, and Shive, I956).

Both 6-mercaptopurine and 6-chloropurine at concentrations of $\mathrm{I} \operatorname{m} M$ and higher cause a differential partial inhibition of cell division in cultures of mouse sarcoma I80 (Biesele, I954c, I957). Much the same effect is produced by 6methylmercaptopurine. 6-Methylpurine is far more toxic and suppresses mitosis completely at o.OI $\mathrm{m} M$. Thioguanine is of intermediate toxicity but causes no differential suppression of mitosis of sarcoma cell in culture (Biesele, I958a).

Several other 6-substituted purines have been studied. One of these, 6-furfurylaminopurine or kinetin, increases mitotic rate greatly in plant tissue cultures (Miller, Skoog, Okumura, Von Saltza and Strong, 1955), but it has little effect on animal cells. Lettré and Endo (1956) found kinetin neither to stimulate nor to inhibit normal and malignant animal and human cells in culture, but the derivative, 6- $\beta$-indolylethylaminopurine, was found to inhibit mitosis at concentrations of Io $\mu \mathrm{g} / \mathrm{ml}$ and above. Biesele (I957) found that kinetin at the high con- 
centration of I $\mathrm{m} M$ produces only moderate inhibition of mitosis in sarcoma I 80 cells but not in cultivated mouse skin fibroblasts.

Much study has been devoted to the mechanism of action of 6-mercaptopurine as an anti-cancer agent. Some of its actions can be explained on the basis of interference in nucleic acid metabolism. Various physiological purines, nucleosides, and nucleotides block its toxic effects (Elion, Singer and Hitchings, I 954; Goldin, Venditti, Humphreys, Dennis, Mantel and Greenhouse, I 954).6-Mercaptopurine inhibits de novo synthesis of nucleic acid (Skipper, I954) and is itself incorporated into nucleic acid in mice (Elion, Bieber and Hitchings, I 954). 6-Mercaptopurine decreases the synthesis of purines in E. coli (Mandel, Inscoe, Maling and Smith, I956), but does not affect the incorporation of exogenous adenine into the nucleic acid of mouse tissues (Fernandes, LePage and Lindner, 1956).

The partial mitotic inhibition caused in mouse tissue cultures by $1.0 \mathrm{~m} M$ 6-mercaptopurine is relieved to different extents by equimolar concentrations of various physiological purines, nucleosides, and nucleotides (Biesele, I 954c, 1958). These substances protect mouse embryonic skin cells more readily than sarcoma I 80 cells against 6-mercaptopurine.

Coenzyme $\mathrm{A}$ is a nucleotide that produces the opposite effect (Biesele, I954C, I 955). This coenzyme at both 0.2 and $0.02 \mathrm{~m} M$ concentrations prevents the mitotic inhibition of sarcoma i 80 cells caused by г.o $\mathrm{m} M$ 6- mercaptopurine, but in the case of embryonic skin cells higher concentrations are needed. 6-Mercaptopurine and coenzyme $\mathrm{A}$ also have antagonistic effects on lipogenesis and mitochondrial morphology in tissue culture (Biesele, I 955). Therefore mitotic suppression by 6mercaptopurine may result, in part, from its action as an antagonist of coenzyme $\mathrm{A}$, possibly with respect to functions of coenzyme $\mathrm{A}$ in the tricarboxylic acid cycle and the oxidative phosphorylation (formation of high-energy phosphoric bond) ${ }^{1}$. Perhaps 6-mercaptopurine interferes in a coenzyme A-regulated synthesis of a hypothetical lipid essential for mitosis. 6-Mercaptopurine and thioguanine both block acetylation of sulfanilamide in a coenzyme A system (Garattini, Morpurgo, and Passerini, I955).6-Mercaptopurine acts as a preprophase poison in inhibiting liver regeneration for the first day after partial hepatectomy of rats (Garattini, Pacilli and Settimi, I955), and it also decreases the rate of cholesterol increase in the regenerating liver (Garattini, Mor and Pacilli, I955). 6-Mercaptopurine inhibits the incorporation of acetate into lipid at lower concentrations than it interferes in nucleic acid synthesis in E. coli (Mandel and Bolton, I956).

There are other indications that 6-mercaptopurine might influence purine coenzymes. This conclusion was drawn, for instance, from the depression by 6 mercaptopurine of the incorporation of glycine-2- ${ }^{14} \mathrm{C}$ into acid-soluble purines but not into nucleic acid purines of the susceptible mouse tumors $\mathrm{TA}_{3}$ and sarcoma I80 (Fernandes, LePage and Lindner, I956). 6-Mercaptopurine also inhibits the increased synthesis of diphosphopyridine nucleotide that normally follows injection of nicotinamide in mice (Kaplan, Goldin, Humphreys, Ciotti and Stolzenbach, I 956). Adenylic acid partly reverses this inhibition.

The mitotic inhibition of mouse cells in culture by 6-mercaptopurine is po-

1 See Chapter $\mathbf{I}$. 
tentiated by insulin and by $\alpha$-lipoic acid (Biesele, I958a). Insulin is known to increase the consumption of glucose and oxygen and the synthesis of nucleic acid and protein in tissue cultures (Paul and Leslie, I954), as well as the incorporation of exogenous labeled adenine into nucleic acids (Lu and Winnick, I 954). Hence, insulin perhaps increases incorporation of 6-mercaptopurine into nucleic acids, and the resultant defective nucleic acids could account for some of the toxicity and mitotic inhibition. An additional indication of the uptake of 6-mercaptopurine into chromosomal nucleic acid is furnished by the increased frequency of chromosomal breakage when insulin or lipoic acid is added with 6-mercaptopurine to the medium (Biesele, r958a).

\section{(d) Nucleosides}

The more toxic among 28 purine nucleosides studied in cultures of mouse tissues corresponded closely in structure to adenosine (Biesele, Berger and Clarke, I952). Ribofuranosyl compounds are more likely to show activity than are glycopyranosyl compounds.

Adenosine itself may influence mitosis adversely. Thus it has been found to inhibit cultivated chick cells from entering prophase (Hughes, I952c). Adenosine at $1.5 \mathrm{~m} M$ progressively decreases the number of mitoses in mouse sarcoma 180 cultures over two days (Biesele, Berger, Clarke and Weiss, 1952).

Several substituted adenosines have interesting effects on mitosis in mouse tissue cultures (Biesele, Berger, Clarke and Weiss, 1952). Crotonoside, or 2hydroxyadenosine, at $0.2 \mathrm{mM}$ selectively inhibits mitosis in embryonic skin cultures, but 2-methyladenosine is more inhibitory to divisions of sarcoma 180 cells than to embryonic skin cells at 4 and I $\mathrm{m} M$. Two days exposure to $2 \mathrm{~m} M$ 2-aminoadenosine eliminates mitoses from both embryonic skin cultures and sarcoma I 80 cultures. This agent also inhibits Ophiostoma growth at 0.003 to $0.03 \mathrm{mM}$ concentrations, but adenosine at 0.5 or $2.0 \mathrm{~m} M$ blocks this inhibition (Fries, I 955)

The nucleoside of unsubstituted purine, or $9-\beta$-D-ribofuranosylpurine, is highly active (Biesele, Slautterback and Margolis, I955). Two days exposure to as little as $0.0025 \mathrm{~m} M$ results in a mitotic incidence only one third of normal in mouse tissue cultures, with sarcoma i 80 cells showing greatest sensitivity and embryonic skin epithelial cells showing greatest resistance. By contrast, 9- $\beta$-D-ribopyranosylpurine is almost innocuous (Biesele, I 957).

In antagonism studies, the mitotic inhibition and nuclear degeneration caused by o.o I $\mathrm{m} M$ 9- $\beta$-D-ribofuranosylpurine in mouse tissue cultures were found to be blocked in part by concentrations about one hundred times greater of adenosine or physiological substances containing the adenosine- 5 '-phosphate structure (Biesele, Slautterback and Margolis, 1955). This may be related to the formation of mono-, di-, and triphosphates of this nucleoside after its injection into rats (Gordon and Brown, I956). These authors suggested that the toxicity of $9-\beta$-Dribofuranosylpurine in vivo probably results from the formation of coenzyme analogues containing it. Gordon and Brown (1956) also found this nucleoside gives rise to polynucleotide adenine and guanine when injected into rats but is not incorporated unchanged into nucleic acid.

The 6-substituted purine nucleosides are in general more toxic than their free 
purines and sometimes display a different selectivity of action against different cell types (Biesele, I957). The ribofuranosyl derivative of 6-chloropurine has much the same pattern of relative inhibition of mitosis in sarcoma 180 cells and the fibroblasts and epithelial cells of mouse skin as the free purine. The ribofuranosyl derivative of 6-methylpurine is even more inhibitory than the very toxic free base, and the sensitivity of fibroblasts is increased to equal that of the sarcoma I 80 cells. 6-Furfurylamino- 9 - $\beta$-D-ribofuranosylpurine is hundreds of times more inhibitory than its free base, kinetin, and especially so to fibroblasts. A similar effect is seen with the ribofuranosyl derivative of 6-methylmercaptopurine.

In general, purine nucleosides are not as effective precursors of nucleic acid as are the free purines or their nucleotides, but the reverse is often true for pyrimidine nucleosides compared with the free pyrimidines ( $c f$. Schlenk, I955). Consequently, the nucleosides of abnormal pyrimidines are better antimetabolites than the free bases (Visser, I 955). In mouse tissue cultures, pyrimidine nucleosides are more likely to exhibit activity than are the pyrimidines themselves (Biesele, unpublished). With some, such as 5 -bromocytidine, a differential mitotic suppression occurs in mouse embryo skin cells rather than in sarcoma 180 cultures.

The four nucleosides of ribonucleic acid inhibit growth in vitro of Trichomonas vaginalis, apparently by acting as antimetabolites against the free bases and the ribonucleotides. In a sense, the ribonucleosides act as metabolic regulators of cell division in this organism (Sprince, Goldberg, Kucker and Lowy, I953). In other cells, the nucleotides may serve this purpose.

\section{(e) Nucleotides}

Despite their incorporability into nucleic acids (Roll, Weinfeld and Brown, I954; Brown and Roll, I955; Goldwasser, I955), the natural ribonucleotides individually suppress growth in some systems. Adenylic acids prevent chick cells in vitro from entering prophase (Hughes, I 952c). Adenylic, guanylic, and cytidylic acids inhibit mitosis in the germinal epithelium of rat ovaries cultured in vitro, but uridylic acid is without effect (Berrian and Dornfeld, r950). Growth of certain mouse tumors is inhibited by adenylic acid and guanylic acid and promoted by uridylic acid, while cytidylic acid has little effect (Parsons, Gulland and Barker, 1947). Perhaps these inhibitory effects are related to the upset of a certain balance in availability of the nucleotides needed for orderly nucleic acid synthesis.

\section{(f) Amino acids and analogues}

This concept of balance among precursors is seen to possess special validity for the amino acids needed for protein synthesis. Kieler (1954b) has pointed out not only that the necessary amino acids must be present in order to promote mitotic activity in chick fibroblast cultures, but also that they should be present in the correct relative concentrations. Using dialyzed medium, Kieler (I953a) has found that addition of arginine, histidine, or lysine to a medium deficient in that amino acid stimulates mitosis. Prophases accumulate in lysine-deficient medium. Excesses of any of these three amino acids depress mitotic activity (Kicler, I.953b). Glutamic and aspartic acids greatly stimulate mitosis in deficient 
media. Aspartic acid produces chromosomal abnormalities and delays in metaphase (Kieler, I953c). Methionine and tryptophan also stimulate cell division, but not cystine and proline.

The presence of an effective amino acid analogue may be equivalent to a deficiency of the corresponding normal amino acid and prevent the incorporation of the latter into protein and of other amino acids as well (Halvorson and Spiegelman, 1952). Francis and Winnick (1953) observed that fluorophenyl alanines, $\beta$ - 3 -thienylalanine, and ethionine inhibit protein synthesis, nucleic acid synthesis, and growth in cultures of chick heart cells. The syntheses of protein and the corresponding ribonucleic acid may be closely related. In pea extracts (Webster, 1956), amino acid antagonists inhibit ribonucleic acid formation as well as protein synthesis, and nucleic acid antimetabolites also inhibit protein synthesis. It is of interest that the more active analogues of physiological amino acids studied in tissue culture by Biesele and Jacquez (I954) produce mitotic aberrations similar to those caused by some purines. In cultures of mouse sarcoma $\mathrm{T}_{24} \mathrm{I}$ and newborn mouse heart cells, $\beta$ - $(p$-fluorophenyl)-DL-alanine at I.o $\mathrm{m} M$ for $24 \mathrm{~h}$. completely suppresses mitosis, but $0.25 \mathrm{~m} M$ permits a few cell divisions. The inhibition is partly reversed by L- phenylalanine. In cultures of mouse sarcoma $\mathrm{I} 8 \mathrm{o}$ and mouse embryo skin, mitotic inhibitions greater than $5^{\circ}$ percent are caused by $0.5 \mathrm{mM}$ of the para isomer of fluorophenylalanine, by $1.0 \mathrm{~m} M$ of the ortho, and by $2.0 \mathrm{~m} M$ of the meta isomer.

III. THE POISONING OF CHROMOSOMES

Among the antimetabolites that hinder the syntheses necessary to permit a cell to enter mitosis are some whose adverse effect is also expressed in damage to the chromosomes of cells that do manage to divide. Perhaps the chromosomal damage is mediated by errors in reduplication of nucleoprotein.

Although the structure of chromosomes is not fully understood, it is known that they contain, in addition to deoxyribonucleic acids, their quota of ribonucleic acids, histones or protamines, and more complex proteins. The last vary in amount in accordance with metabolic activity (Mirsky, 1947). It is perhaps impossible to assign a primary role in chromosome structure to any one component (Kaufmann, McDonald and Bernstein, 1955), although histone is less important than the combination of DNA and residual protein, which may include RNA, in maintaining morphological integrity of isolated chromosomes (Mirsky and Ris, I 95I). It is therefore perhaps incorrect to ascribe all chromosome breaks and other aberrations to a primary effect on the integrity or reproduction of DNA alone.

Thus the production of "stickiness" in chromosomes by treatment with ribonuclease (Kaufmann and Das, I954), which has been compared to the primary effects of radiation (Kaufmann, McDonald and Bernstein, I955), may result from a dissociation of nucleoprotein by the ribonuclease. Chromosomal "stickiness" may occur "spontaneously" (D'Amato, I 948), as in mitotic cells near the necrotic center of tumors, whose chromosomes probably agglutinate because of toxic materials liberated by dying cells (Koller, I949). Some tissue extracts have 
similar effects; thus extracts of dried bulb scales cause "sticky" bridges in onion root mitoses in anaphase (Resende, I95I). The toxic materials causing chromosomal agglutination may be nucleic acids or their degradation products, for Woll (1953) has found that ribonucleic acid clumps chromosomes in root tip mitoses. Adenine causes "sticky" anaphase bridges in mouse tissue cultures at $1.5 \mathrm{mM}$ concentration (Biesele, Berger, Clarke and Weiss, I952), and the 8-ethers and 8-thioethers of caffeine produce much chromosomal "stickiness" in onion root tips (Kihlman, I 952).

Understanding of chromosomal breakage presents several difficulties. One of these lies in the extension of theoretical concepts of an event at the molecular level in a poorly understood structure to the complex organization of the chromosome. The individual mitotic chromosome is moderately polytene, the unit microfibrils being perhaps I Oo-200 $\AA$ in diameter (Ambrose, Cuckow and GopalAyengar, 1955). Prophase chromosomes in staminate hair cells of Tradescantia are each made up of as many as 64 small strands of some i2 $5 \AA$ diameter (Kaufmann and De, I 956). Unit microfibrils about $200 \AA$ wide have been reported in chromosomes of various organisms by $\mathrm{Ris}$ (1956), who suggested that the microfibril consists of a core of DNA surrounded by a protein sheath. Breakage in such multimicrofibrillar chromosomes would appear to require multiple ruptures across the entire structure. There is the possibility of only partial breakage across the chromatid, however, such as the subchromatid errors observed by Kihlman (I 955a) in onion root tip cells treated with 8-ethoxycaffeine during prophase. An error or interruption in synthesis in a chromosomal subunit might not be immediately evident, but it might result in the deferred appearance of breaks some cell generations later, as McLeish ( 1954 ) observed with maleic hydrazide and as Ambrose, Cuckow and Gopal-Ayengar (I955) suggested, might occur with carcinogens.

\section{(a) Amino acids}

The necessity of a balanced supply of amino acids for normal growth has been discussed already, as well as the interrelationships of protein synthesis and nucleic acid synthesis. It is accordingly to be expected that chromosomal damage shoud result from deficiencies or excesses of given amino acids or from the presence of inhibitory analogues. Chromosomal damage has been reported in chick tissues cultured in media deficient in lysine (Kieler, I953b), glutamic acid (Kieler, I953c), and tryptophan (Kieler, I954a). Excess of aspartic stimulates mitosis and causes displacement and breakage of chromosomes (Kieler, I 953c). Chromosomal bridging and fragmentation become more frequent in cultures of mouse sarcoma I 80 treated with the three isomeric fluorophenylalanines (Biesele and Jacquez, 1954). Azaserine, which Hemerly and Demerec ( 955 ) found to be one of two substances with outstanding mutagenic effect on bacteria tested in a series of 28 agents of cancer chemotherapy, is not highly active in increasing the frequency of chromosomal aberrations in mouse tissue cultures (Biesele, I958a). However, it ureatly potentiates the action of 6-mercaptopurine in this respect (Biesele, 1958b). This same effect is also more marked when adenine is added to the gammahydrazide of L-glutamic acid (Biesele, r958b). 


\section{(b) Antifolic acids}

These agents cause breakage of chromosomes in certain tissues of some animals. In the intestinal epithelium of the mouse, various 4 -aminofolic acids inhibit mitosis and cause a cellular hypertrophy followed by abnormal mitoses with injured chromosomes (Dustin, I949, I 95ob, I950c). Aminopterin, a-methopterin, and aminoanfol break chromosomes in abnormal normoblast mitoses in man (Thiersch, I949). Citrovorum factor administered before aminopterin to mice blocks chromosomal rupture and the other observed effects of the aminopterin (Dustin, 1952). Antifolic acids selectively damage intestinal epithelium, bone marrow, the red pulp of spleen and spermatogenic cells, but they do not damage mitoses in lymphoid tissue (Dustin, 1953). Chromosomal breakage has not been reported for cells in tissue culture treated with antifolic agents.

\section{(c) Purines and pyrimidines}

The discovery of chromosome breakage by caffeine in onions by Kihlman and Levan (I949) was followed by an extensive study of related xanthine derivatives and other purines (Kihlman, I949). The members of one group of purines, of which 8-ethoxycaffeine is an example, were found to act rapidly, to be more lipoid soluble, and to be able to penetrate the lipid enveloping the resting nuclei of the onion root tip. The members of the other group, including caffeine and I, 3, 7,9tetramethyluric acid, have a greater solubility in water and gain access to the chromosomes only when mitosis exposes them (Kihlman, I95I). In later work, Kihlman (1952) found that unlike methylxanthines the ethylxanthines do not break chromosomes. The "radiomimetic" activity of the purines tested is correlated with their solubilizing power and with their surface activity. Kihlman also suggested an indirect effect by way of enzyme inhibition, perhaps involving nucleotide coenzymes.

Studies with the physiological purines and with substances more closely analogous to them lend this suggestion an increased interest. Adenine itself causes structural chromosome changes in onion root tips (Kihlman, I950a). With an induction of abnormalities in about 20 percent of anaphase figures by concentrations of 20 or $40 \mathrm{~m} M$, adenine is less active than 8-ethoxycaffeine, which at ro $\mathrm{m} M$ can produce up to $75 \%$ abnormal anaphases in onion roots (Kihlman, I950b).

The closely related agent, 2,6-diaminopurine, breaks chromosomes in intestinal mitoses and also damages lymphoid cells of the mouse (Dustin, I 950a). It is also effective in mouse tissue cultures (Biesele, Berger, Clarke and Weiss, I 952). With both mouse sarcoma I 80 and embryonic mouse skin in cultures, the incidence of chromosomal bridging and fragmentation produced by treatment with 2,6-diaminopurine lactate can be decreased by simultaneous treatment with adenine sulfate. However, the chromosome-breaking proclivities of adenine are also displayed in this system, and there results an optimal relative blocking concentration of adenine. If the concentration of 2,6-diaminopurine just exceeds the threshold, too much as well as too little adenine makes for a higher incidence of chromosomal aberrations. With higher concentrations of diaminopurine, aberrations also increase despite maintenance of the otherwise optimal equimolar blocking ratio with adenine. In mouse tissue cultures, chromosomal damage begins with about 
$0.5 \mathrm{~m} M$ adenine sulfate or with about $0.05 \mathrm{~m} M$ diaminopurine. The purines in these concentrations individually produce about $30-40 \%$ abnormal postmetaphasic figures after two days exposure.

Adenine often causes chromosomal "erosion", with occasional segments poorly stained or poorly coiled along the chromosome (Kihlman, I 952). Maleic hydrazide, which can be construed to be structurally analogous to uracil, also "erodes" chromosomes and causes other cytological effects resembling those of adenine. It is considerably more active than 8-ethoxycaffeine. The question of whether maleic hydrazide acts as an antimetabolite antagonist of pyrimidines is unresolved, however. Damage by maleic hydrazide to vetch chromosomes is not blocked by uracil, thymine, or orotic acid (Loveless, I953). Uracil blocks neither maleic hydrazide action on onion chromosomes (Deysson and Deysson, I953) nor their fragmentation by barbital (Deysson and Rollen, I 95 I). Uracil itself breaks onion chromosomes (Deysson, I 952), and this effect is not blocked by thymine (Deysson, I954). Although thymine also fails to block chromosomal "erosion" in onion roots by 5 -aminouracil (Duncan and Woods, I953), cytidine sulfate does antagonize this effect (Martinez Pico and Duncan, r 953).

Various other analogues of physiological purines have also been tested on chromosomes. 2-Aminopurine at I and $4 \mathrm{~m} M$ concentrations causes chromosomal bridging and fragmentation in cultivated mouse cells. Although 7-methyladenine also has some activity, neither 2-methyladenine, 9-methyladenine, nor 2-chloroadenine appear to have any (Biesele, Berger, Clarke and Weiss, I952).

Unsubstituted purine increases the incidence of chromosomal breaks and bridges in sarcoma 180 cultures, and is blocked by adenine in low concentrations (Biesele, Slautterback and Margolis, I955). Thioguanine also causes chromosomal breakage, and 6-chloropurine causes agglutination and occasional failure of anaphasic separation of the chromosomes in addition, but 6-mercaptopurine causes little obvious damage to the chromosomes unless it is supplemented with insulin or lipoic acid, provided that coenzyme $\mathrm{A}$ is not added (Biesele, I958a). These results have been interpreted to mean that chromosomal breakage occurs in the presence of 6-mercaptopurine when the conditions provoke an increased uptake of exogenous purines into nucleic acid (Biesele, I958a). However, Kihlman (1955a) has objected to the hypothesis that chromosome breakage by purines results from an effect on nucleic acid synthesis because of biochemical antagonism between caffeine and such apparently unrelated substances as tryptophan, procaine, or aniline and because of the failure of 8-azaguanine to break chromosomes in onions. It may be mentioned, however, that 8-azaguanine incorporation appears to be primarily into RNA, not DNA (Lasnitzki, Matthews and Smith, I954).

Chromosome breakage by 8-ethoxycaffeine shows an interesting parallel to that by X-rays (Kihlman, I955b). 8-Ethoxycaffeine does not break onion root chromosomes in the absence of oxygen, air, or hydrogen peroxide, and under aerobic conditions activity is suppressed by cyanide, azide, or dinitrophenol. Hence the conclusion was drawn that compounds such as adenosine triphosphate containing energy-rich phosphate bonds may be involved in the breakage of chromosomes by 8-ethoxycaffeine. Moreover, chromosomal stickiness is affected in the same manner as breakage (Kihlman, I955c). 
An analogous situation has been found by Novick (1956) in the decreased mutagenicity of free purines to bacteria under anaerobic conditions, which make for higher concentrations of antimutagenic nucleosides in the bacteria. Moreover, Gersh (cited by Novick, I 956) has observed that the breakage is decreased in the presence of guanosine.

However, some nucleosides do contribute to chromosome breakage. 2,6-Diamino-9- $\beta$-D-ribofuranosylpurine breaks chromosomes in mouse sarcoma 180 cultures (Biesele, Berger, Clarke and Weiss, I952); and 9- $\beta$-D-ribofuranosylpurine breaks chromosomes in onions (Kihlman, cited by Löfgren and Lüning, 1953) and in sarcoma I8o cells (Biesele, Slautterback and Margolis, 1955).

\section{POISONING OF THE SPINDLE}

There are several points in the formation and functioning of the mitotic spindle at which antimetabolites like those under consideration in this chapter might intervene. These points may be made evident in a short discussion of the composition and behavior of the mitotic spindle as now understood.

The fact that colchicine, the best-known spindle poison, has a nearly universal influence on mitosis, indicates a fundamental uniformity in composition of the spindle in diverse organisms susceptible to attack by this compound. The essential part of the colchicine effect, according to Eigsti and Dustin (I955), is that it converts the mitotic spindle from a polarized fibrous structure to an amorphous hyaline globule (Gaulden and Carlson, I 95I) no longer capable of supporting the anaphasic movement of chromosomes.

There are only two electrophoretically demonstrable oriented fibrous proteins of the spindle (Schmidt, I 937; Swann, I 952a, I 952b; Inoué, 1952). This has been shown by Mazia (1956) and on the isolated mitotic apparatus of the sea urchin egg by Mazia and Dan (1952). The two components do not differ in their amino acid composition (Mazia, I 955), but do differ in that one is combined with ribonucleic acid (Mazia, I 956). Other evidence for the occurrence of ribonucleic acid on the spindle is also available. Kaufmann, McDonald and Gay (I95I) found the mitotic spindle of onion root tip cells to contain both ribonucleic acid and protein. Stich (I95I) noted an acumulation of RNA in the nuclear sap of the Cyclops egg after the increase of polysaccharide in prophase. Perhaps synthesis of the spindle protein occurs on a ribonucleic acid template, in which case antimetabolites of nucleic acid as well as amino acid analogues, might be expected to disturb the synthesis.

Analogues of nucleic acid components might change the spindle in other ways, in particular by interfering in nucleotide metabolism, if the hypothesis of Brachet ( 1947 ) correctly names adenosine triphosphate as the source of energy for contraction of the mitotic spindle. The view was supported by Lettré's finding (1950) that ATP antagonizes the effect of colchicine on the spindle. However, this finding has met with criticism (Benitez, Murray and Chargaff, 1954). A manifold participation of ATP in cell division is indicated by the experiments of HoffmannBerling (I954a, I954b, I954c) with dividing cells extracted with cold aqueousglycerol. In these so-called cell models, adenosine triphosphate evokes elongation 
of the spindle, equatorial constriction in telophase, cytokinesis, and perhaps contraction of the chromosomal fibers of the spindle in the early anaphasic separation of sister chromosomes. Contraction of the cell models depends on a splitting of ATP, but anaphasic elongation of the stembody of the spindle, which pushes the separating groups of chromosomes even farther apart (Belar, I929), depends on a plasticizing effect of unsplit ATP or other polyphosphates (Weber, I955). Spindlebody elongation is thus equivalent to the simple relaxation of most contractile structures. The asters also become larger in anaphase (Hughes and Swann, 1948). The orientation of some of the cell organelles during mitosis can be interpreted as contributing to the provision of polyphosphate to the desired structures in the proper sequence in time and space. Mitochondria, the sites of aerobic ATP production, assume characteristic positions in some grasshopper cell divisions, lining up in the equatorial cytoplasm outside the spindle and elongating parallel to the spindle axis. Nakahara (1952) has suggested that the mitochondria may participate in induction of the cleavage furrow and has observed their elongation in this region despite inhibition of anaphasic chromosome movement by caffeine or acriflavine. The mitochondria behave abnormally in cells whose cleavage is inhibited by podophyllin components (Momma, 1953) or auramin (Kawamura, I 953). Perhaps the mitochondria provide adenosine triphosphate to the equatorial cell cortex, or possibly to a substance responsible for triggering anaphasic movement of the chromosomes.

Material released from the chromosomes in anaphase and staining in a manner similar to ribonucleic acid has occasionally been described. Jacobson and Webb (I952) noted that material presumed to be ribonucleoprotein is released from anaphasic chromosomes into the cytoplasm between the chromosome groups. This material is not released from the chromosomes of metaphases blocked by antifolic acids (Jacobson, I 952). The chromosomes leave a high concentration of ribose nucleoprotein behind them at the equatorial plate after metaphase in the lily (Swift, I 953). Similar observations have been made on living chick fibroblasts in vitro with ultraviolet light (Davies, I952). This may be the material described as flowing from the kinetochore onto the spindle fibers (Belar, I929; Kupka and Seelich, I 949).

Perhaps ribonucleoprotein normally released from anaphasic chromosomes carries adenosine triphosphate, which is involved in contraction of the chromosomal fibers of the spindle, or polyphosphate groups that serve to relax the stembody. However, the concept of phosphorylated pentose nucleic acid is uncertain (Brachet, I955).

It was suggested by Swann (1952a, I952b) and by Mitchison (1952) that nucleic acid or individual nucleotides might be related to the "structural agents" postulated to be released from chromosomes and to be responsible for the changes in structure of the mitotic apparatus after metaphase, especially for the decrease in birefringence of the spindle as the chromosomes move poleward in anaphase. Mitchison (I 952) suggested that when Swann's structural agent reaches the polar cytoplasm of the cell it causes the cell membrane to expand and bubble there, and Boss (1955) has proposed that this membrane activity is caused by ribonucleoprotein released from the chromosomes. 
In summary, it would appear that antimetabolites capable of interfering in the synthesis of protein, of ribonucleic acid, or of high-energy phosphate bonds might interfere with proper spindle assemblage or in its function, which seems to be in part subject to chromosomal control.

\section{(a) Antifolics}

The tendency of antifolics to arrest mitosis in metaphase has already been mentioned. That this is probably related to an influence on nucleic acid or nucleotide metabolism is evident from what is known concerning the role in Icarbon transfer played by some derivative of folic acid, such as the citrovorum factor, or N-5-formyl-5,6,7,8-tetrahydropteroylglutamic acid ( $c f$. Schlenk, I 955), or the possibly dinucleotide coenzyme formed from the citrovorum factor and ATP (Nichol et al., I 955). A relevant finding is that of Skipper, Morgan and Bennett (1952), who showed that a-methopterin decreases the incorporation of labeled formate and glycine into adenine of adenosine triphosphate in mice with about the same order of activity as it inhibits their incorporation into nucleic acid adenine. Actions of folic acid analogues, of folic acid itself and of analogues of the precursors of folic acid or folinic acid may be considered here.

Metaphase arrest was reported in onion roots as an effect of sulfanilamide (Berger, I 944). This effect as well as the preprophasic inhibition caused by o.I or $0.5 \%$ sulfanilamide can be reversed with $p$-aminobenzoic acid, which is a precursor of folic acid (Hindmarsh, I949). The analogue, aminopterin, causes mitotic aberrations in plants (Himes and Leuchtenberger, I950; Jakowska, Nigrelli and Goldsmith, I950).

Folic acid itself injected in a dose of about $200 \mathrm{mg} / \mathrm{kg}$ into mice causes metaphases to accumulate in Ehrlich ascites tumor cells, with chromosomal clumping (Lettré and Landschütz, I947). Folic acid in such a massive dose might well act as an antagonist of its functional derivative. Folic acid has also been described as being more strongly inhibitory to growth in onion root tips than 5 -aminouracil (Duncan and Woods, r 953).

Antifolic acids have been studied in tissue culture by several investigators. Hughes (1950) found that $0.5 \mathrm{mM}$ aminopterin completely arrests mitosis in chick cells in metaphase, while $5.5 \mathrm{mM}$ a-methopterin causes only partial metaphase arrest. On rat fibroblasts, the optimum concentration of aminopterin for metaphase blocking is $0.2 \mathrm{mM}$ (Benitez, Murray and Chargaff, I 954). Metaphase arrest has been said to set in within a few min. (Hughes, I952b), I 5 min. (Jacobson, I 954a), or $20 \mathrm{~min}$. (Benitez, Murray and Chargaff, I 954) but to disappear some hours after antifolics are added to the medium.

.Metaphase arrest was seen even after $24 \mathrm{~h}$. in cultures of mouse embryonic skin and sarcoma 180 exposed to 4 -aminopteroylglutamic acid and its analogues containing threonine or alanine in place of the glutamic acid moiety, but not in cultures exposed to 4-aminopteroyltryptophan (Biesele, I 954a). These analogues are also antifolics (Oleson, I950).

Antagonism studies showed that folic acid applied even in ten times the concentration of aminopterin does not block mitotic arrest by the latter in cultures of chick osteoblasts and fibroblasts (Jacobson, I 954b). Citrovorum factor does block 
this effect of aminopterin, but only if it is applied $24 \mathrm{~h}$. beforehand. Hence the antagonism may depend on conversion of the citrovorum factor to an active agent. The antagonism succeeds with chick osteoblasts and acute leukemic cells of the mouse, but not with normal bone marrow of the mouse. It was concluded that the citrovorum factor or a derivative of it contributes significantly to the early anaphasic separation of sister chromosomes. The antifolics may be held to displace this active factor, which participates in the synthesis of the RNA-like material that is given off from the chromosomes at anaphase and that may be concerned in contraction of the chromosomal fibers, elongation of the spindle-body, polar bubbling, and equatorial cleavage.

\section{(b) Nucleic acid components and analogues}

The methylated xanthines were the first purines investigated for their effects on the second half of cell division. Gosselin ( 1940 ) treated rootlets of peas and oats with I: I, ooo caffeine or theophylline for I-6 days and observed that binucleate cells were sometimes produced because cell walls were not formed. Some of the polyploid cells resulting from nuclear fusion had non-functional giant spindles. Mangenot and Carpentier ( I 944) also found that caffeine and theophylline at 2 and Io $\mathrm{m} M$ concentrations do not arrest mitosis in the manner of colchicine in plant root tips, but that a single irregular mitotic figure may form from the two nuclei of a binucleate cell if the nuclei were joined by chromatin bridges from the previous mitosis. Nakahara (1952) observed that caffeine tends to inhibit separation of chromosomes in early anaphase but not to affect elongation of the spindle in grasshopper germ cells.

The suppression of plant cell wall formation by caffeine and theophylline was confirmed by Kihlman and Levan (I 949). The N-alkylated oxypurines also do this (Kihlman, I 949). In addition, clear tendencies to a colchicine-like metaphase arrest were found with caffeine and theophylline in such strong concentrations as I \% (Kihlman and Levan, I949). These tendencies were pronounced in substituted xanthines having the methyl groups replaced by larger alkyl groups, which decreased the water-solubility. The results were therefore in agreement with the Levan and Ostergren ( 1943 ) theory of narcosis.

Adenine also causes metaphase arrest at 20 and $40 \mathrm{~m} M$ concentrations in onion root tips (Kihlman, r95oa). However, the low relative lipoid-solubility of ad enine suggests that this effect may be more specific than a simple narcosis (Kihlman, 1952).

With less extreme concentrations on chick tissue cultures, Hughes (I952C) found that adenine, 2,6-diaminopurine, and benzimidazole prevent cleavage and tend to inhibit anaphasic movement of chromosomes. Concentrations as low as Io $\mu \mathrm{g} / \mathrm{ml}$ of 6 - $\beta$-indolylethylaminopurine have a similar effect (Lettré and Endo, 1956). The adenine analogue, 4-aminopyrazolo-(3,4-d) pyrimidine, at o. I $\mathrm{m} M$ and up causes abortive metaphases in cultures of the human carcinoma, HeLa (Hsu, Robins and Cheng, i 956).

Adenine produces this effect in Vicia root tips at concentrations of 2 and $5 \mathrm{mM}$ (Woll, I953). Metaphase arrest also results from treatment with I and $4 \%$ solutions of sodium ribose nucleate. Von Olah (1952) also observed this in onion roots. 
A similar interpretation may be given to the mitotic figures regarded as somatic reduction divisions observed in root meristems treated with sodium ribose nucleate by Kodani (1948) and Huskins (1948). Adenosine did not show this activity (Woll, I953).

However, $2 \mathrm{~m} M$ concentration of adenosine was found to antagonize the suppression of mitosis caused by its toxic analogue, 2-aminoadenosine, at the same concentration in cultures of mouse sarcoma 180 and then to potentiate or to cause an extensive metaphase arrest, followed either by chromosomal agglutination and cell death or by nuclear reconstruction. In the latter case, a nucleus of doubled size is formed in toroid shape because of the original disposition of the chromosomes in a hollow ring in the arrested metaphase. Guanosine alone at $4 \mathrm{mM}$ causes a few such figures in sarcoma i 80 cultures, however they have not been observed in mouse embryo skin cultures (Biesele, Berger, Clarke and Weiss, 1952).

Amino acids. The prominence of protein in the composition of the mitotic apparatus, which seems to be largely synthesized anew for each division, offers a ready basis for metaphase arrest with amino acid analogues. It must not be forgotten, of course, that protein enzymes instrumental in providing the energy for synthesis and function of the apparatus may also be influenced. The amino acid analogues to be discussed here are analogues of phenylalanine, which is a minor constituent of the protein of the mitotic apparatus of isolated sea urchin egg, (Mazia, 1955).

When mice bearing the Lewis sarcoma T24I are treated with massive doses of $\beta$-(2-thienyl)-DL-alanine, some cells in the shrinking tumor become highly polyploid and exhibit up to 20-polar mitotic figures (Jacquez, Stock and Barclay, I 953). Treatment of mice with $\beta$-( $p$-fluorophenyl $)$-DL-alanine raises the proportion of metaphases among the mitotic figures in the tumor from about $20 \%$ to near $60 \%$ (Biesele and Jacquez, I 954). Many of the metaphases are blocked and revert directly to the resting state.

More information was gained on metaphasic delay through tissue culture studies with the fluorophenylalanines (Biesele and Jacquez, I 954). The prolongation of metaphase caused by 0.25 and $0.5 \mathrm{mM} \beta$-( $p$-fluorophenyl)-DL-alanine in cultures of newborn mouse heart cells and sarcoma T24 I cells is prevented by equimolar L-phenylalanine. The fluorophenylalanines do not evoke a pronounced response in cells of sarcoma 180 and of embryonic mouse skin, although some effect can be produced in sarcoma 180 cells with $\beta$-( $m$-fluorophenyl)-DL-alanine. Some of the blocked metaphases revert to toroid resting nuclei, much as with 2-aminoadenosine plus adenosine. The metaphasic blocking with this meta isomer can be increased by adding adenosine triphosphate to the cultures. This might make for a greater incorporation of the phenylalanine analogue into protein of the spindle or the kinetochore, which could thus become defective.

Sarcoma 180 cells are also blocked in metaphase by $\beta$ - $(p$-fluorophenyl $)$-DLalanine if liver explants are cultured in the same vessel. Tripolar mitoses also occur in sarcoma 180 cultures as a result of treatment with any of the three fluorophenylalanine isomers if liver explants are present, but not otherwise. This suggests that perhaps the fluorophenylalanines are converted to more active derivatives. 


\section{GONCLUSION}

This review has emphasized the mitotic poisoning effects of agents presumably acting on the nucleic acids and nucleoproteins of the chromosomes on one hand, and on the proteins and nucleoproteins of the mitotic apparatus on the other. Many substances which are known to be antimetabolites or which are structurally analogous to precursors of nucleotides, nucleic acids, or proteins may be said to possess a mitotic poisoning action in that they prevent, delay, or disrupt the divisions of cells that would otherwise proliferate unimpeded. Various of these antimetabolites and related compounds poison the several stages of mitosis and produce morphological aberrations in manners reminiscent of the actions of the several groups of better-known, classical mitotic poisons.

Events in cellular life leading to proliferation and continuing through mitosis may be considered to be complex sets of interlocking phenomena, delicately regulated and easily upset. The presence of an antimetabolite is in some respects equivalent to absence or deficiency of the corresponding metabolite. An antimetabolite may not merely inhibit some synthesis, but it may also be incorporated into consequently defective products, whose failure to function properly may be expressed in mitotic inhibitions or aberrations.

Mitotic poisons may be exogenous or endogenous for given cells. Analysis of the mitotic poisoning action of introduced antimetabolites may provide clues to the normal control of cell division in individual cells and in multicellular organisms. Endogenous mitotic poisons, products of the cell's own metabolism, may include spontaneous mutagens and chromosome-breaking agents (D'Amato and Hoffmann-Ostenhof, I956) such as putrescine (Marquardt, I949) or a purine (Kihlman, I950a; Novick, I956).

For use in experimental cancer chemotherapy, antimetabolites possess one possible advantage over the classical mitotic poisons. In particular with antinucleic acid antimetabolites, the heterogeneity of nucleic acid anabolism (Bendich, 1952) makes it likely that differential effects on various cell types may be achieved. Mitotic poisoning action could be used as an indicator of inhibitory effect. A few amino acid analogues evince similar promise. The most useful to date, however, such as azaserine, probably act through their interference in nucleic acid metabolism. 


\section{LITERATURE}

Alfert, M., (1950) 3. Cellular Comp. Physiol., 36, 38 I.

Alfert, M., (1955) in Symposium on Fine Structure of Cells, Interscience Publishers, New York.

Ambrose, E. J., F. W. Cuckow and A. R. Gopal-Ayengar, (1955) Symposium on Fine Structure of Cells, Interscience Publishers, New York,

Belar, K., (1929) Arch. Entwicklungsmech. Organ., II8, 359.

Bendich, A., (1952) Exptl. Cell Research, Suppl. 2, i8r.

Bendich, A. And G. B. Brown, (1948) 7. Biol. Chem., I 76, 147 I.

Benitez, H. H., M. R. Murray and E. Chargaff, (1954) Ann. N. Y. Acad. Sci., 58 , i 288.

Bennett, L. L., Jr., H. E. Skipper and L. W. Law, (1953) Federation Proc., I2, 300.

Berg, N. O., (I95I) Acta Pathol. Microbiol. Scand., Suppl. go, I.

Berger, C. A., (1944) Torreya, 44, 4I.

Berrian, J. H. ANd E. J. Dornfeld, (1950) 7. Exptl. Zool., II5, 513.

Biesele, J. J., (1952) Cancer, 5, 787 .

Biesele, J. J., (1954a) Anat. Record, II8, 444.

Biesele, J. J., (1954b) Ann. N. Y. Acad. Sci., 58, i I29.

Biesele, J. J., (1954c) Ann. N. Y. Acad. Sci., 6o, 228.

Biesele, J. J., (1955) F. Biophys. Biochem. Cytol., I, i I9.

Biesele, J.J., ( 1957) Proc. Natl. Cancer Conf., 3rd Conf., 1956, J. B. Lippincott Co., Philadelphia.

Biesele, J. J., (1958a) Frontiers in Cytology, ed. S. L. PAlay, Yale University Press, New Haven.

Biesele, J. J., (i 958b) Ann. N. Y. Acad. Sci., 7I, ro54.

Biesele, J. J., R. E. Berger and M. Clarke, (I952) Cancer Research, I2, 399.

Biesele, J. J., R. E. Berger, M. Glarke and L. Weiss, (1952) Exptl. Cell Research, Suppl. 2, 279.

Biesele, J. J., R. E. Berger, A. Y. Wilson, G. H. Hitchings and G. B. Elion, (i95i) Cancer, 4, 186.

Biesele, J. J. and J. A. Jacquez, (r954) Ann. N. Y. Acad. Sci., 58, i 276.

Biesele, J. J., M. C. Slautterback and M. Margolis, (i955) Cancer, 8, 87.

Bloch, D. P. and G. C. Godman, (1955) J. Biophys. Biochem. Cytol., r, i 7.

Borsook, H., (1956) 7. Cellular Comp. Physiol., 47, Suppl. I, 35.

Boss, J., (1955) Exptl. Cell Research, 8, I81.

Boyland, E., (1950) Biochim. et Biophys. Acta, 4, 293.

Brachet, J., (1947) Embryologie chimique, Masson \& Cie, Paris.

BRACHet, J., (1954) Nature, I74, 876.

Brachet, J., (1955) in The Nucleic Acids, ed. E. Chargaff and J. N. Davidson, Academic Press, New York.

Brown, G. B., (I955) in Antimetabolites and Cancer, ed. C. P. Rhoads, Am. Assoc. Adv. Sci., Washington.

Brown, G. B. and P. M. Roll, (1955) in The Nucleic Acids, ed. E. Cihargaff and J. N. Davidson, Academic Press, New York.

Bullough, W. S., (1952) Biol. Revs. Cambridge Phil. Soc., 27, 133.

Bullough, W. S. And M. Johnson, (195I) Nature, I67, 488.

Glarke, D. A., F. S. Philips, S. S. Sternberg, C. C. Stock, G. B. Elion and G. H. Hitchings, (1953) Cancer Research, I3, 593.

Cornman, I., (1954) Internat. Rev. Cytol., 3, 113 .

Creaser, E. H., (I955a) Nature, I75, 899.

CReaser, E. H., (I 955 b) Nature, 176,556 .

D'Aмato, F., (1948) Rend. accad. nazl. Lincei, 4, 94.

D’Aмato, F., (1949a) Caryologia, I, Iog.

D'Aмato, F., (I949b) Caryologia, I, 327.

D'Amato, F. and O. Hoffman-Ostenhof, (1956) Advances in Genet., 8, I.

Davies, H. G., (1952) Exptl. Cell Research, 3, 453.

Davies, H. G., E. M. Deeley, B. M. Richards and P. M. B. Walker, (i954) abstract in Excerpta Med., Sect. I, 8, 419. 
Deysson, M., (1952) Bull. soc. botan. France, 99, 5 I.

Deysson, M., (1954) Compt. rend., 238, 145.

Deysson, G. and M. Deysson, (1953) Bull. soc. chim. biol., 35, i 209.

Deysson, G. And A. Rollen, (I95 I) Compt. rend., 232, 548.

Doctor, V. M. and J. B. Trunnell, (1955) Proc. Am. Assoc. Cancer Research, 2, I3.

Dounce, A. L., (1952) Enzymologia, 15, 25 I.

Dounce, A. L., G. Gamow, S. Spiegelman, P. Newmark, D. Harker and M. Soodak, (1956) 7. Cellular Comp. Physiol., 47, Suppl. I, 103.

Dounce, A. L., T. S. Work and P. N. Campbell, (1953) Nature, I72, 54 I.

Duncan, R. E. and P. S. Woods, (i953) Chromosoma, 6, 45.

Dustin, P., Jr., (1949) Compt. rend. soc. biol., 143, I609.

Dustin, P.: Jr., (i950a) Compt. rend. soc. biol., 144, 586.

Dustin, P., JR., (1950b) Compt. rend. soc. biol., I44, I 297.

Dustin, P., Jr., (I950c) Rev. hématol., 5, 603.

Distin, P., Jr., (1952) Rev'. belge pathol. et méd. exptl., 22, 55.

Dustin, P., Jr., (I953) Acta Unio Intern. contra Cancrum, 9, 739.

Eigsti, O. J. And P. Dustin, JR., (1955) Colchicine in Agriculture, Medicine, Biology and Chemistry, Iowa State College Press, Ames.

Elion, G. B., S. Bieber and G. H. Hitchings, (i954) Ann. N. Y. Acad. Sci., 6o, 297.

Elion, G. B., S. Singer and G. H. Hitchings, (i954) Ann. N. Y. Acad. Sci., 6o, 200.

Fernandes, J. F., G. A. LePage and A. Lindner, (1956) Cancer Research, i6, I54.

Finkelstein, M. and P. A. Thomas, (I95I) Cancer Research, iI, 80I.

Finkelstein, M., W. D. Winters, P. A. Thomas, C. Davidson and P. K. Smith, (r95I) Cancer Research, II, 807.

Firket, H., S. Chèvremont-Comhaire and M. Chèvremont, (1955) Nature, i 76, 1075.

Francis, M. D. And T. Winnick, (1953) J. Biol. Chem., 202, 273.

Friedkin, M., (I954) 7. Biol. Chem., 209, 295.

Friedrich-Freksa, H., (I940) Naturwissenschaften, 28, 376.

Fries, N., (1955) Acta Chem. Scand., 9, ro20.

Gale, E. F. and J. P. Folkes, (1953) Biochem. J., 55, xi.

Gale, E. F. And J. P. Folkes, (1955) Nature, I75, 592.

Gamow, G., (1954) Nature, I73, 318,

Garattini, S., C. Mor and N. Pacilli, (I955) Giorn. ital. chemioterap., 2, I94.

Garattini, S., C. Morpurgo and N. Passerini, (1955) Giorn. ital chemioterap., 2, 29.

Garattini, S., N. Pacilli and A. Settimi, (I955) Giom. ital. chemioterap., 2, i 99.

Gaulden, M. E. and J. G. Carlson, (195I) Exptl. Cell Research, 2, 4I6.

Goldin, A., J. M. Venditti, S. R. Humphreys, D. Dennis, N. Mantel and S. W. Greenhouse, (1954) Ann. N. Y. Acad.Sci., 6o, $25 \mathrm{I}$.

Goldwasser, E., (1955) J. Am. Chem. Soc., 77, 6083.

Gordon, M. P. And G. B. Brown, (1956) J. Biol. Chem., 220, 927.

Gosselin, A., (1940) Compt. rend., 210, 544.

Greenlees, J. and G. A. LePage, (I956) Cancer Research, i6, 808.

Grunberg-Manago, M., P. J. Ortiz and S. Ochoa, (1955) Science, I22, 907.

Halvorson, H. O. And S. Spiegelman, (1952) 7. Bacteriol., 64, 207.

Ham, R. G., R. G. Eakin, C. G. Skinner and W. Shive, (i956) 7. Am. Chem. Sic., 78, 2648. Hamers, R., (1956) Biochim. et Biophys. Acta, 2I, 170.

Hartman, S. C., B. Levenberg and J. M. Buchanan, (1956) F. Biol. Chem., 221 , 1057. Hemmerly, J. And M. Demerec, (1955) Cancer Research, I5 (suppl. 3), 69.

Himes, M. and C. Leuchtenberger, (i950) Genetics, 35, i I 4.

Hindmarsh, M. M., (1949) Nature, 163,6 io.

Hirschberg, E., J. Kream and A. Gellhorn, (1952) Cancer Research, I2, 524.

Hoffmann-Berling, H., (I954a) Biochim. et Biophys. Acta, I4, I82.

Hoffmann-Berling, H., (1954b) Biochim. et Biophys. Acta, I5, 226

Hoffmann-Berling, H., (1954C) Biochim. et Biophys. Acta, I5, 332.

Howard, A. And S. R. Pelc, (195I) Exptl. Cell Research, 2, i 78.

Howard, A. and S. R. Pelc, (1953) Heredity, 6, Suppl., 26 I. 
Howell, R. W. and F. Skoog, (I955) Am. F. Botany, 42, 356.

Hisu, T. C., R. K. Robins and C. C. Cheng, (1956) Science, 123, 848.

Hughes, A. F. W., (1950) Quart. 7. Microscop. Sci., 91, 25 I.

Hughes, A., (1952a) Symposia Soc. Exptl. Biol., 6, 256.

Hughes, A., (1952b) The Mitotic Cycle, The Cytoplasm and Nucleus during Interphase and Mitosis, Academic Press, New York.

Hughes, A., (1952c) Exptl. Cell Research, 3, 108.

Hughes, A. and M. M. Swann, (1948) 7. Exptl. Biol. 25, 45.

Hultgren, B., B. Kihlman and N. Fries, (1955) Physiol. Plantarum, 8, 493.

Huskins, C. L., (1948) 7. Heredity, 39, 311.

Inoué, S., (1952) Exptl. Cell Research, 2, Suppl., 305.

Jacobson, W., (1952) J. Pathol. Bacteriol., 64, 245.

Jacobson, W., (1954a) 7. Physiol., I23, 603.

Jacobson, W., (1954b) 7. Physiol., I23, 6 I 8.

Jacobson, W. and M. Webb, (1952) Exptl. Cell Research, 3, i63.

Jaceuez, J. A., C. C. Stock and R. K. Barclay, (1953) Cancer, 6, 828.

Jakowska, S., F. R. Nigrelli and E. D. Croldsmith, (i950) Caryologia, 3, I.

Jeener, R. And Rosseels, (I953) Biochim. et Biophys. Acta, iI, $43^{8}$.

KaCSER, H., (1956) Science, 124, I 5 I.

Kalgkar, H. M., (1953) Biochim. et Biophys. Acta, I2, 250.

Kaplan, N. O., A. Goldin, S. R. Humphreys, M. M. Giotti and F. E. Stolzenbach, (1956) 7. Biol. Chem., 219, 287.

Kaufmann, B. P. And N. K. Das, (1954) Proc. Natl. Acad.Sci. U. S., 40, 1052.

Kaufmann, B. P. and D. N. De, (1956) J. Biophys. Biochem. Cytol., 2 (Suppl.), 419.

Kaufmann, B. P., M. R. McDonald and M. H. Bernstein, (i955) Ann. N. Y. Acad. Sci., 59,553 .

Kaufmann, B. P., M. R. McDonald and H. Gay, (I95I) F. Cellular Comp. Physiol., 38 , (Suppl. I), 7I.

Kawamura, K., (1953) Japan. 7. Genetics, $28,8$.

Kidder, G. W. And V. Dewey, (I949) F. Biol. Chem., I79, I8I.

Kidder, G. W., V. G. Dewey, R. W. Parks, Jr. and G. L. Woodside, (1949) Science, IOg, 5 I I .

Kieler, J., (1953a) Acta Pathol. Microbiol. Scand., 33, 337.

Kieler, J., (I953b) Acta Pathol. Microbiol. Scand., 33, 350.

Kieler, J., (1953c) Acta Pathol. Microbiol. Scand., 33, 36 I.

Kieler, J., (1954a) Acta Pathol. Microbiol. Scand., 34, I.

Kieler, J., (1954b) Acta Pathol. Microbiol. Scand., 34, i I.

Kieler, J. ANd E. Kieler, (I954) Cancer Research, I4, 428.

Kihlman, B., (1949) Hereditas, 35, 393.

Kinlman, B., (I950a) Hereditas, 36, I03.

Kinlman, B., (1950b) Exptl. Cell Research, I, I35.

Kinlman, B., (I95I) Symbolae Botan. Upsalienses, II (2), I.

Kinlman, B., (1952) Symbolae Botan. Upsalienses, II (4), I

Kinlman, B., (1955a) Exptl. Cell Research, 8, 345.

Kinlman, B., (1955b) Exptl. Cell Research, 8, 404.

Kinlman, B., (I955c) Hereditas, 4I, 384 .

Kihlman, B. And A. Levan, (1949) Hereditas, 35, Iog.

Kihlman, B. And A. Levan, (I 95 I) Hereditas, 37, 382.

Kodani, M., (I948) J. Heredity, 39, 327.

Koller, P. C., (I949) Exptl. Cell Research, Suppl. I, 85.

Kornberg, A., I. R. Lehman, M. J. Bessman and E. S. Simms, 1956) Biochim. et Biophys. Acta, 21, 197 .

Kornberg, A. and W. E. Pricer, Jr., (i95I) J. Biol. Chem., 193, 48 I.

Kupka, E. And F. Seelich, (I949) Chromosoma, 3, 302.

Lasnitzki, I., R. E. F. Matthews and J. D. Smith, (1954) Nature, I73, 346.

Lettré, H., (1950) Ergeb. Physiol. biol. Chem. u. exptl. Pharmakol., 46, 379. 
Lettré, H., (1952) Cancer Research, I2, 847.

Lettré, H. And H. Endo, (1956) Naturwissenschaften, 43, 84.

Lettré, H. And C. LAndschütZ, (1947) Naturwissenschaften, 34, 245.

Levan, A. And G. Óstergren, (1943) Hereditas, 29, 38 I.

Löfgren, N. And B. Lüning, (1953) Acta Chem. Scand., 7, 225.

Loveless, A., (I953) Heredity, 6 (Suppl.), 293.

Lu, K. H. And T. Winnick, (1954) Exptl. Cell Research, 7, 238.

Ludford, R. J., (1953) 7. Roy. Microscop. Soc., 73, I.

Mandel, H. G., (1955) in Antimetabolites and Cancer, ed. C. P. Rhoads, Am. Assoc. Adv. Sci., Washington.

Mandel, H. G. and E. T. Bolton, (1956) Proc. Am. Assoc. Cancer Research, 2, i3 I.

Mandel, H. G., J. K. Inscoe, H. M. Maling and P. K. Smith, (1956) Federation Proc., I5, 454.

Mangenot, G. and S. Carpentier, (i944) Compt. rend. soc. biol., i38, 232.

Marquardt, H., (1949) Experientia, 5, 401.

Marshak, A., (1955) Trans. N. Y. Acad. Sci., Ser. II, $17,506$.

Martinez Pico, L. M. and R. E. Duncan, (i953) Records Genetics Soc. Am., 22, 86.

Matthews, R. E. F., (i953) Nature, I7I, I065.

Matthews, R. E. F., (1954) 7. Gen. Microbiol., 10, 52 I.

Mazia, D., (1955) Symposia Soc. Exptl. Biol., 9, 335.

Mazia, D., (1956) Am. Scientist, 44, I.

Mazia, D. And K. Dan, (1952) Proc Natl. Acad. Sci. U. S., 38, 826.

McLeish, J., (I954) Heredity, 8, 385 .

Miller, C., (1953) Proc. Soc. Exptl. Biol. Med., 83, 56 i.

Miller, C. O., F. Skoog, F. S. Okumura, M. H. Von Saltza and F. M. Strong, (r955) J. Am. Chem. Soc., 77, 2662.

Miller, Z. and L. Warren, (1953) F. Biol. Chem. 205, 33 I.

Mirsky, A. E., (1947) Symposia on Quantitative Biology, Cold Springs Harbor, 12, 143.

Mirsky, A. E. and H. Ris, (I95 I) F. Gen. Physiol., 34, 475.

Mitchell, J. H., H. E. Skipper and L. L. Bennett, Jr., (1950) Cancer Research, 10, 647.

Mitchison, J. M., (1952) Symp. Soc. Exptl. Biol., 6, 105.

Momma, E., (1953) F. Fac. Sci. Hokkaido Univ., Ser. VI, II, 502.

Moses, M. J. and J. H. Taylor, (1955) Exptl. Cell Research, 9, 474.

Nakahara, H., (I952) Cytologia, I7, I68.

Nichol, C. A., A. H. Anton and S. F. Zakrzewski, (1955) Science, I2I, 275.

Nickell, L. G., (1955) in Antimetabolites and Cancer, ed. C. P. Rhoads, Am. Assoc. Adv. Sci., Washington.

Novick, A., (1956) Brookhaven Symposia on Biol., 8, 20 I.

Oleson, J. J., (1950) Trans. N. Y. Acad. Sci., Ser. II, 12, I 18.

Pardee, A. B., (1954) Proc. Natl. Acad. Sci. U. S., 4o, 263.

Parks, R. E., Jr., (1955) in Antimetabolites and Cancer, ed. C. P. Rhoads, Am. Assoc. Adv. Sci., Washington.

Parsons, L. D., J. M. Gulland and G. R. Barker, (1947) Symposia Soc. Exptl. Biol., I, i 79.

Patau, K. And H. H. Swift, (1953) Chromosoma, 6, I 49.

Paul, J. And I. Leslie, (I954) Anat. Record, II8, 452.

Pelc, S. R. And A. Howard, (1952) Exptl. Cell Research, Suppl. 2, 269.

Petermann, M. L., M. G. Hamilton and N. A. Mizen, (i954) Cancer Research, 14, 360.

Petermann, M. L., N. A. Mizen and M. G. Hamilton, (1953) Cancer Research, 13, 372.

Plaut, W. and D. Mazia, (1956) 7. Biophys. Biochem. Cytol., 2, 573.

Potter, V. R., (1956) Cancer Research, I6, 658.

Rerchard, P., (1955) in The Nucleic Acids, ed. E. Chargaff and J. N. Davidson, Academic Press, New York.

Resende, F., (195I) Bol. soc. portug. cienc. nat., 3, r8I.

Rhoads, C. P., (1954) Science, I19, 77.

Richards, B. M., P. M. B. Walker and E. M. Deeley, (1956) Ann. N. Y. Acad. Sci., 63, 831 . 
Ris, H., (1956) 7. Biophys. Biochem. Cytol., 2, Suppl., 385.

Roels, H., (1954) Nature, 173, I039.

Roll, P. M., H. Weinfeld and G. B. Brown, (1954) Biochim. et Biophys. Acta, 13, I4I.

Schlenk, F., (1955) in The Nucleic Acids, ed. E. Chargaff and J. N Davidson, Academic Press, New York.

Sснмidt, W. J., (1937) Die Doppelbrechung von Karyoplasma, Zytoplasma, und Metaplasma, Borntraeger, Berlin.

Schwartz, D., (1955) 7. Cellular. Comp. Physiol., 45, Suppl. 2, i 7 I.

Seshachar, B. R., (I950) Nature, I65, 848.

Setterfield, G. and R. E. Duncan, (1955) F. Biophys. Biochem. Cytol., I, 399.

Shapiro, D. M., R. Weiss and A. Gellhorn, (1950) Cancer, 3, 896.

SKipPER, H. E., (I953) Cancer Research, I3, 545.

Skipper, H. E., (1954) Ann. N. Y. Acad. Sci., 60, 315.

Skipper, H. E., L. L. Bennett, Jr. and F. M. Schabel, (i954) Federation Proc., I3, 298.

Skipper, H. E., J. H. Mitchell, Jr., L. L. Bennett, Jr., M. A. Newton, L. Simpson and M. EIDSON, (I95I) Cancer Research, II, I45.

Skipper, H. E., C. Morgan and L. L. Bennett, Jr., (I952) Cancer Research, I2, $4^{1} 3$.

Slonimski, P. P., (1954) Ann. Inst. Pasteur, 87, 404.

Spiegelman, S., H. O. Halvorson and R. Ben-Ishai, (1955) in Amino Acid Metabolism, ed. W. D. McElroy and B. Glass, Johns Hopkins Press, Baltimore.

Sprince, H., R. Goldberg, G. Kucker and R. S. Lowy, (1953) Ann. N. Y. Acad. Sci., 56 , IOI6.

Sтісн, H., (195I) Z. Naturforsch., 6b, 259.

Sтоск, C. C., (1950) Am. F. Med., 8, 658 .

Stock, C. C., H. C. Reilly, S. M. Buckley, D. A. Cllarke and C. P. Rhoads, (i954) Nature, $173,7 \mathrm{I}$.

Strominger, D. B. And M. Friedkin, (i954) J. Biol. Chem., 208, 663.

Swann, M. M., (I952a) Intern. Rev. Cytol., I, I95.

Swann, M. M., (1952b) Symposia Soc. Exptl. Biol., 6, 89.

Swift, H., (I953) Texas Repts. Biol. and Med., II, 755.

Thiersch, J. B., (1949) Cancer, 2, 877.

Vendrely, R. And C. M. J. Vendrely, (1954) abstract in Excerpta Med. Sect. I, 8, 4I9.

Visser, D. W., (r955) Antimetabolites and Cancer, ed. C. P. Rhoads, Am. Assoc. Adv. Sci., Washington.

Von Olah, L., (1952) Lilloa, 25, 615.

Walker, P. M. B., (1953) Heredity, 6, Suppl., 275.

Walker, P. M. B. and H. B. Yates, (1952) Symposia Soc. Exptl. Biol., 6, 265.

Watson, J. D. and F. H. C. CRick, (I953) Nature, I7I, 737.

Weber, H. H., (I955) Symposia Soc. Exptl. Biol., 9, 27I.

Webster, G. C., (1956) Federation Proc., I5, 380.

Wheeler, G. P. and H. E. Skipper, (1953) Federation Proc., 12, 289.

Woll, E., (I953) Chromosoma, 5, 39I.

Woodside, G. L., G. W. Kidder, V. C. Dewey and R. E. Parks, Jr., (1953) Cancer Research, 13, 289.

Woolley, D. W., (1952) A Study of Antimetabolites, John Wiley \& Sons, New York.

Zamecnik, P. G. AND E. B. Keller, (1954) F. Biol. Chem., 209, 337.

Zamecnik, P. G., E. B. Keller, J. W. Littlefield, M. B. Hoagland and R. B. Loftfield, (1956) 7. Cellular Comp. Physiol., 47, Suppl. I, 81. 


\section{Chapter 14}

\section{GROWTH AND THE AGING PROCESS}

\section{by Warren Andrew}

It is perhaps natural in attempting any philosophical contemplation of the aging process to ask ourselves, "How is growth related to aging?" The intimate relation of the metabolic processes to so many of the features of the life of the organism must come into consideration both in relation to growth and to aging. It seems clear, for example, that for growth to occur, the rate of anabolism must be higher than the rate of catabolism, in order that the complicated living substance may increase in amount by the transformation of assimilated foodstuffs. Must the reverse be true in the aging process? Must the rate of breakdown in old age, or the period of decline, be more rapid than the rate of building up? This question is not as easy to answer, and we shall have more to say about it. Certainly this question would seem to be one of critical importance in determining the relationship between growth and the aging process.

The problem which is posed here might also be expressed as follows: "Is senescence a continuing part of the same general processes constituted by growth and development? Is it truly the downward slope of a continuous curve? Do growth and senescence constitute, as Flourens has said, an "uninterrupted chain"?

To answer these questions we must attempt to see the processes of growth and senescence in their relation to the pattern of the total life-history. First, we must examine the chronological relationship of the two processes; second, we must seek to find whether they may be occurring simultaneously or whether they are mutually incompatible; and third, we must examine the characteristics-biological, physical and chemical一of each.

The growth of a living organism frequently has been compared with that of a crystal, and the two usually have been contrasted, the statement being made that the crystal grows by accretion, the acquisition of material by adding it to its surfaces, the organism by intussusception, the taking in of new materials and the transformation of them within the organism into living substance. On closer study, the contrast in growth of the organism and of the crystal does not seem as sharp as at first survey. Thus certain liquid crystals can grow by intussusception. The restoration of a part of a crystal resembles to a certain extent the regenerative processes in a plant or animal. Nor does a crystal always grow equally in all directions but, like an organism, has its gradients of growth.

One great contrast between the growth of the non-living crystal and the living 


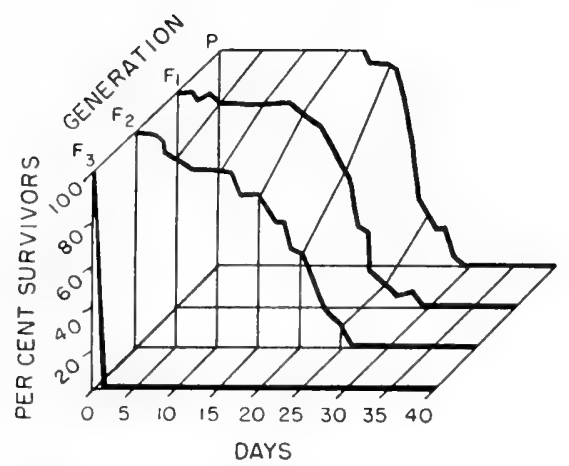

Fig. I. Three dimensional diagram to show the changing survival curve properties in an "old" orthoclone. The $\mathrm{F}_{2}$ data show significant changes and the $\mathrm{F}_{3}$ is essentially nonviable. (Redrawn from Lansing, 1952).

organism does stand out-this is the inherent limitation of growth of the organism. There is no such characteristic for crystals,although their growth can be limited by external factors.

This definite limitation of the growth process makes us think of another definitely limited characteristic of an organism - the duration of life. The life of man is as subject to this limitation as that of other living beings. "The days of our years are threescore and ten", says the psalmist, "and if by reason of strength they be fourscore, still is that strength labor and sorrow, for it is soon cut off and we fly away".

Just as the amount of growth and the time for growth, at least under given environmental conditions, is limited in each species, so is the life span of a fixed duration. Here, then, we have a possible clue to the riddle of the life-history. It is almost as though the period of growth and the duration of life could be considered as two factors in an algebraic equation. A third factor is needed to complete the equation. Is this the factor of senescence?

Almost immediately those who have concentrated on the study of the human organism may raise the objection that here growth ceases long before the proces-

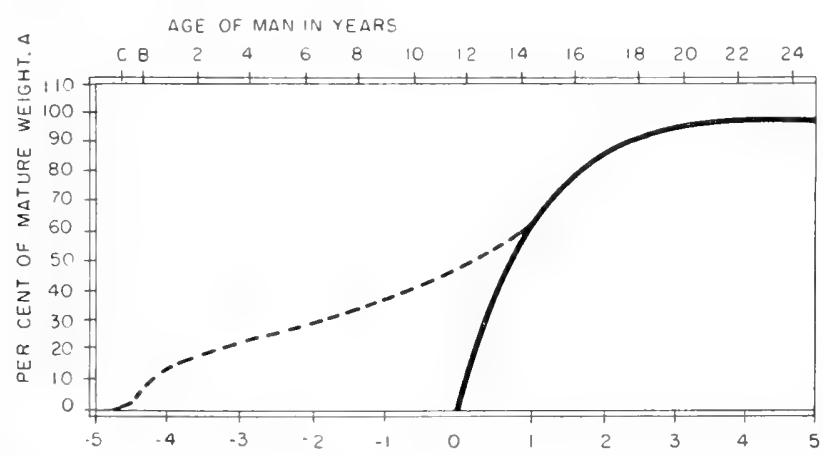

Fig. 2. Comparison of the growth curves of man (dotted line continued into solid line) with curve made from a composite of a number of species of animals (solid line). The growth curves of many warm-blooded animals investigated are so alike that they can be made to coincide. Man differs from other species in having a long juvenile period (age 3 to 13 years). In other species the end of weaning is almost coincidental with the attainment of sexual maturity, so that they have little or no juvenile period.

(Redrawn after Brody, 1945). 
ses of decline, with which we associate senescence, begin to appear. This objection, however, may be more apparent than real. The line of division between the upWard slope of growth and the downward one of senescence may not be a sharp one. Perhaps the cessation of growth, while the actual initiating factor in senescent change, cannot make its effect felt except over a period of some time, varying in different species.

If we turn to the lower animals, we find that very cogent evidence has been obtained that some sort of "aging potential" does begin to accumulate at the time of cessation of growth (Lansing, r 947a). This "aging potential" of the organism is demonstrable in an indirect manner. The organism studied was the rotifer, a minute but multicellular animal, with a reproductive process which often is parthenogenic through many generations. Lansing found that the offspring from parents who were beyond the growth period showed a life-span of shorter duration if the parents were older at the time of production of the egg. Rotifers still actively growing appeared to be completely free of this aging potential. Of the results of these studies, Lansing says (I 947b, p. 333): "The evidence obtained by Lansing supported the view that aging does not, as commonly believed, begin in the fertilized ovum; on the contrary, aging appears to be a by-product of changes which occur in the cell at the time of growth cessation".

The term orthoclone is used by Lansing to designate a series of generations with constant parental age through the generations. Thus a "senile orthoclone" is one in which the eggs used for rearing the individuals are taken always from old mothers.

In such an orthoclone the mean life span of each successive generation can be decreased until the point of extinction is reached (Fig. I). This trend can be stopped and actually reversed by altering the selection technique, simply choosing young mothers rather than old.

In contrast, the mean life span of successive generations increases progressively in a young or adolescent orthoclone. In one experiment which lasted through 49 generations the span was lengthened to I oo days as compared with 24 days for the ancestral stock.

It should be stressed that the "aging potential", as measured by these experiments, is present not only in senile rotifers but in all maternal animals which are not actively growing.

Rate of sexual maturation bears an inverse relation to longevity. As longevity decreases in an "old" orthoclone, the age of sexual maturation, measured by the beginning of egg-laying, becomes progressively less; while as longevity increases in a "young" orthoclone, the age of sexual maturation becomes increasingly greater. Thus an intimate relation exists between growth and longevity and between rate of sexual maturation and longevity.

Do similar relationships occur in animals other than the relatively primitive rotifer? A good deal of evidence exists that they do, and this evidence extends even to the mammals (MacDowell and Taylor, I948; Strong, I948; and Strong, 1950).

The growth curves of a number of different species of warm-blooded animals bear a surprising resemblance to each other and in fact can be made to coincide. Man differs from other species in showing a very long "juvenile period" between 


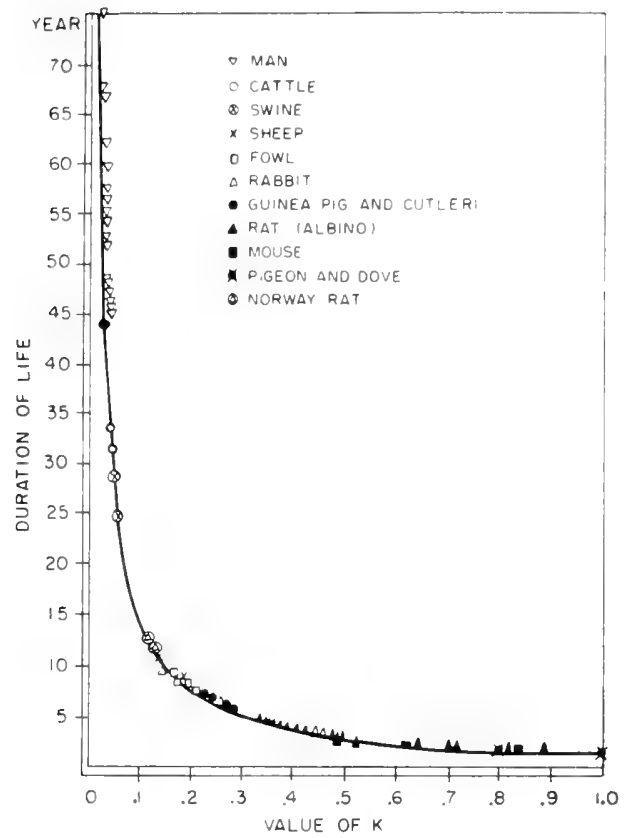

Fig. 3. Diagram showing that in a number of animal species the duration of life is directly proportional to the length of time to reach mature size and therefore inversely proportional to the speed of maturation (value of $\mathrm{K}$ ).

(Redrawn after Brody, I945).

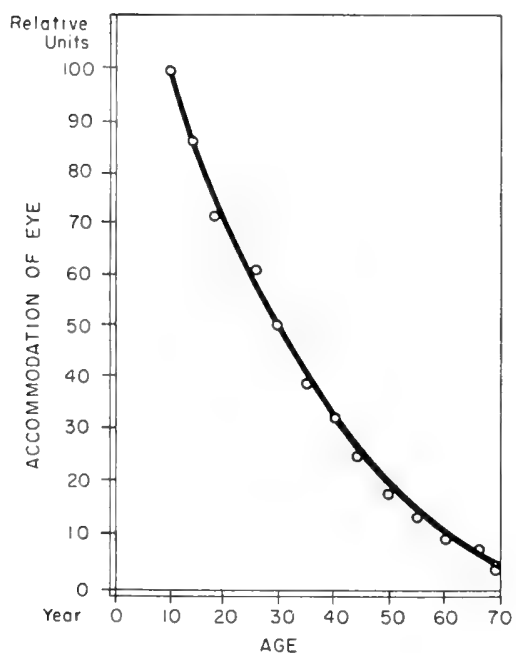

Fig. 4

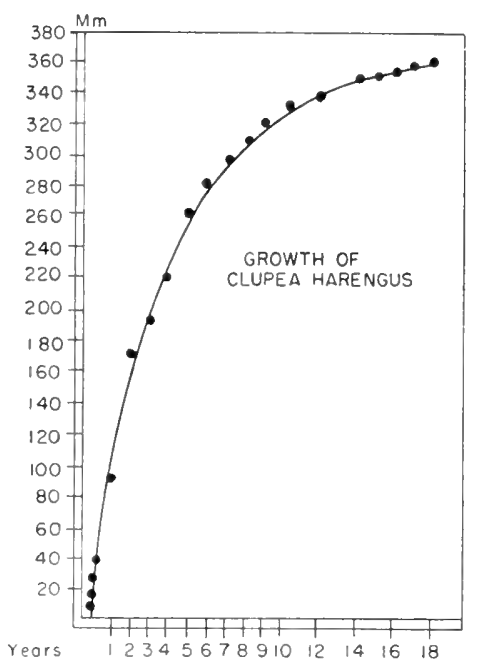

Fig. 5

Fig. 4. Curve to show the rapid decline in power of accommodation of the eye at age Io years. This phenomenon tends to substantiate Minot's view that the rate of aging is greatest in earliest years. While the lens continues to grow, it is at a declining rate, thus bringing about a decrease in the amount of young tissues with increasing age.

(Redrawn after Brody, I945).

Fig. 5. Typical growth curve of a fish, Clupea harengus. The long continuation of growth is paralleled, too, by increasing egg production. (Redrawn after Bourlière, I953). 
ages 3 to 13 . In other mammalian species the end of the weaning period is very close to the onset of sexual maturity, and they may be said to have little or no juvenile period (Fig. 2).

Data for a large number of species of mammals and birds (Brody, I 945) indicate that in each case the duration of life is directly proportional to the length of time to reach mature size and inversely proportional to speed of maturation (Fig. 3).

There are specific instances where the rate of "aging", as measured by change in a particular characteristic of a tissue or an organ, can be shown to run parallel with the rate of decrease in the growth curve. An interesting example of this phenomenon is seen in decline in accomodation of the eye (Fig. 4).

The growth characteristics of cold-blooded vertebrates are different from those of birds and mammals. They are examples of indeterminate growth (Backman, I938). In fishes growth appears to continue throughout life, only slowing down as the animal becomes older (Fig. 5). By means of the scale method of age determination in fishes of temperate waters, it has been possible to show also that the fertility of female fish, as measured by rate of egg production, actually increases with age. This is shown in data from Raitt (1932) on haddocks of the North Sea, as follows:

\begin{tabular}{cl}
\hline Number of eggs & Tear of life \\
\hline 31,000 & second \\
I 00,000 & third \\
I 59,000 & fourth \\
224,000 & fifth \\
278,000 & sixth \\
\hline
\end{tabular}

Bourlière (1953) has obtained evidence for a similar continuing growth in snakes and a continuing increase in fertility. Thus the evidence is that in cold-blooded vertebrates, where growth is indeterminate, the decrease or loss of fertility seen in warm-blooded females is not encountered. This is not to say that some evidences of senile decline cannot be found in cold-blooded forms, but it does indicate that where no marked termination of growth occurs, senile decline is a much less clearcut process.

There is, then, a definite chronological relationship between growth and senescence. Thus, the first task of our three tasks of comparison has been accomplished.

Is senescence compatible with growth? Robertson (1923), in a penetrating work, stressed the nature of aging as an antithesis of growth. The upholding of this view, of course, must presuppose to some extent that we understand what we mean by senescence. We must realize that the precipitating cause of death in old organisms is not an actual part of the process. Thus the rupture of a bloodvessel in a vital organ such as the heart or brain may terminate the life of the organism. "Senescence" was involved here perhaps in the subtle tissue changes which led to the greater fragility of the wall. Yet even this change may be more of a pathological nature and not a part of a "natural" senescence any more than is the invasion of pneumococci in an elderly individual who has been hospitalized with a fractured 
hip. Here again the truly "senescent" factors involved in the train of events probably were (I) the increasing feebleness which was a factor in the accidental fall and (2) the increased brittleness of the bone (Cobb, I952). On the basis of what he considers the processes which represent true senescence, Robertson describes the latter as incompatible with growth.

This means that in a given mass of protoplasm the two processes cannot be occurring in the same location at the same time. Obviously, in a large organism growth processes in individual cells, in tumor masses, or even in separate organs, may be occurring although the organism as such is senescent, and on the downward slope of the life curve.

We may inquire now concerning the physical, chemical and biological characteristics of the process of growth upon the one hand and of senescence upon the other. While no one is more fundamental than the others, we may consider first the physical aspects.

If growth is an increase in amount of living matter, is senescence a decrease? Or is it simply the reaching of a plateau of quantity of protoplasm, the mere cessation of increase then setting in motion forces of change, not necessarily involving loss of protoplasm but nevertheless leading inevitably onward to degeneration. Such a process might well be compared to the cessation of flow of a rapidly coursing stream as it settles first into a slowly running one and ends finally as a series of stagnant pools or a patch of marshland. While the waters leap and sparkle among the rocks, they are clean, pure, and "living". As the flow (growth) is slowed and when it finally ceases altogether, new processes are initiated, the cleanliness and purity are lost, and the waters become choked by foreign growths. While this analogy should not be carried too far, it may correspond in not too inaccurate a way to the dynamics of growth and senescence.

There is, in fact, not a great deal of evidence of loss of living material as such in the senescent organism. More important, the loss which occurs may well be accounted for by degenerative changes brought about by cessation of growth, not as an orderly process (such as that of growth itself), but rather as a series of varied phenomena in different organs or tissues, very probably as the individual reaction of those organs and tissues to the process of "stagnation". We shall return to the consideration of these varied reactions in discussing the biological characteristics of growth and senescence.

Our comparison of the physical characteristics of these processes is hampered to some extent by our lack of knowledge of the physical organization of protoplasm. We do know enough, however, to describe protoplasm as a colloid. Many penetrating attempts have been made to compare the aging of protoplasm with the aging of "other colloids" (Dhar, I932; Ružička, I 922). If such a comparison could be considered valid, it would be of great interest and importance, since it probably would concern the really fundamental nature of the age process. The difficulties are considerable here, however, since one is comparing a rather artificial colloidal system in a laboratory tube with the mysterious living system of the cell which, while it may have ceased to "grow", still is dynamic in the sense of presenting an interchange of molecules between itself and its environment. There is probably a constant shifting of molecules within its body from nucleus to cytoplasm, 
TABLE 1

VARIATION DURING HUMAN FETAL DEVELOPMENT IN PHASE RELATIONS AND CELL WATER CONTENTS

(after Iob and Swanson, r934)

\begin{tabular}{|c|c|c|c|c|c|c|c|c|c|}
\hline \multirow[t]{2}{*}{$\begin{array}{c}\text { Age, } \\
\text { lunar } \\
\text { months }\end{array}$} & Weight & $\begin{array}{l}\text { Fat- } \\
\text { free* } \\
\text { solids }\end{array}$ & Bone* $\neq$ & $\mathrm{Cl}+$ & $\kappa+$ & $\begin{array}{l}\text { Cell+ } \\
\text { phase }\end{array}$ & $\begin{array}{l}\text { Cell }+\S \\
\text { solids }\end{array}$ & $\begin{array}{c}\text { Cell } \\
\text { solids }\end{array}$ & $\kappa$ \\
\hline & $g$ & $g / k g$ & $g / k g$ & $\begin{array}{c}\text { mequiv. } \\
/ \mathrm{kg}\end{array}$ & $\begin{array}{l}\text { mequiv. } \\
/ \mathrm{kg}\end{array}$ & $g / k g$ & $g / k g$ & $\begin{array}{l}\mathrm{g} / \mathrm{kg} \\
\text { cells }\end{array}$ & $\begin{array}{l}\text { mequiv. } \\
\mid \mathrm{kg} \text { cells }\end{array}$ \\
\hline 3.2 & 23.9 & $6 o$ & 4.0 & 21.2 & 8.2 & 716 & 54 & 75 & I I \\
\hline $3 \cdot 7$ & $59 \cdot 4$ & 70 & $4 \cdot 4$ & 56.9 & 28.0 & 505 & $6 r$ & 121 & 55 \\
\hline $4 \cdot 3$ & I I 4.5 & 107 & 7.7 & 78.1 & $43 \cdot 3$ & 320 & 93 & 290 & 135 \\
\hline 5.1 & 259 & 120 & I 2.0 & 77.5 & 43.8 & 325 & 102 & 307 & 135 \\
\hline $5 \cdot 4$ & 335 & 117 & I I. 5 & $75 \cdot 3$ & 36.1 & 334 & IOO & 299 & I I I \\
\hline $5 \cdot 7$ & 490 & II 8 & I 6.6 & 70.7 & $37 \cdot 7$ & 385 & 97 & 252 & $9^{8}$ \\
\hline 6.2 & 590 & I35 & I6.4 & 72.0 & 40.7 & 374 & 114 & 305 & 109 \\
\hline 6.4 & 570 & I 34 & $14 . \mathrm{I}$ & 74.2 & 31.3 & 354 & I I 5 & 325 & 88 \\
\hline 7.2 & IOIO & I 26 & I 2.3 & 64.2 & 39.8 & $44^{2}$ & I09 & 247 & 90 \\
\hline 7.6 & 960 & 150 & I 7.8 & 64.2 & $3^{8.6}$ & $44^{2}$ & 128 & 289 & 87 \\
\hline $7 \cdot 7$ & 1205 & I 45 & I $4 \cdot 3$ & 71.2 & 49. I & 380 & 127 & 335 & 129 \\
\hline 8.2 & I 555 & 164 & 15.5 & 68.6 & 46.0 & 403 & I 45 & 359 & I I 4 \\
\hline 8.2 & 1545 & I 49 & 14.8 & 67.7 & 45.7 & $4^{12}$ & I 30 & $3^{16}$ & I I I \\
\hline 8.4 & 1615 & 157 & I 5.3 & 66.8 & 47.1 & 419 & I 38 & 329 & 112 \\
\hline 10.0 & 2915 & I9I & 22.4 & 56.1 & 47.2 & $5^{12}$ & I 68 & 326 & 92 \\
\hline
\end{tabular}

* Calculated on the basis of total fat-free tissue

+ Calculated on the basis of fat-free, bone-free tissue.

₹ Bone calculated from calcium by formula: Bone $(\mathrm{g})=0.102(\mathrm{~m} M \mathrm{Ca}-5)$.

$\S$ Corrected for bone and for extracellular salts $=\mathrm{I} \%$ of extracellular fluid.

from cytoplasm to nucleus, and from one region to another within each of these cell elements.

On the physical side, then, senescence does not show any orderly and recognizable features such as that which characterizes growth. While there are weight changes in various organs, a decrease in weight is not found in all organs nor in those where it usually occurs is it seen in all senescent individuals.

The chemical aspects of growth and senescence include the changes in the relative quantities of the various elements and compounds in the organism as a whole and the shifts in their distribution. During the process of growth, from a very early embryonic stage and until maturity is reached, there is a progressive decrease in the amount of water present relative to the weight of the organism. The majority of authors who have written concerning this decrease have given the impression that it represents an actual partial "desiccation" of the protoplasm. A number of recent writers, however (Needham, I931; Shohl, I939; Stearns, I939) have indicated that by far the greater part of the decrease in amount of water is due to a decrease of extracellular fluid rather than a "dehydration" of the cells themselves. Thus Needham (193I) mentioned the possibility that the decrease in water con- 
TABLE 2

EFFECT OF GROWTH ON MUSCLE, LIVER, AND BRAIN OF CATS

(Yannet and Darrow, 1938)

$A=$ cats weighing $300-800 \mathrm{~g}$ (approximately $\mathrm{I}-2$ months of age)

$B=$ cats weighing $800-2500 \mathrm{~g}$ (2-6 months of age and older)

\begin{tabular}{|c|c|c|c|c|c|c|}
\hline \multirow{4}{*}{ Group } & \multicolumn{2}{|c|}{ Muscle } & \multicolumn{2}{|c|}{ Liver } & \multicolumn{2}{|c|}{ Brain } \\
\hline & $A$ & $B$ & $A$ & $B$ & $A$ & $B$ \\
\hline & \multicolumn{6}{|c|}{ Number of animals } \\
\hline & II & 9 & II & 9 & 9 & 8 \\
\hline \multicolumn{7}{|l|}{ Observed data } \\
\hline Water- $\mathrm{g} / \mathrm{kg}$ total tissue & 785 & $77^{\circ}$ & $73^{\circ}$ & 707 & 846 & 808 \\
\hline Fat - $\mathrm{g} / \mathrm{kg}$ total tissue & 20 & 25 & 25 & 60 & 30 & 60 \\
\hline Chloride-mequiv./kg total tissue & 28.2 & $15 \cdot 1$ & $3^{1.0}$ & 27.7 & 43.2 & $39 \cdot 0$ \\
\hline Sodium-mequiv. $/ \mathrm{kg}$ total tissue & 36.8 & $23 \cdot 3$ & $3^{8.6}$ & $33 \cdot 3$ & $55 \cdot \mathrm{I}$ & 52.5 \\
\hline $\begin{array}{l}\text { Potassium-mequiv. } / \mathrm{kg} \text { total tis- } \\
\text { sue }\end{array}$ & 80.0 & 90.4 & 80.1 & $73 \cdot 7$ & 87.0 & 88.0 \\
\hline $\begin{array}{l}\text { Phosphorus- } \mathrm{m} M / \mathrm{kg} \text { total tis- } \\
\text { sue }\end{array}$ & $59 \cdot 0$ & 66.4 & 75.6 & 90.3 & 76.9 & 96.3 \\
\hline Nitrogen $-\mathrm{g} / \mathrm{kg}$ total tissue & $24 \cdot 7$ & 29.1 & 23.7 & $27 \cdot 4$ & 14.7 & I6.8 \\
\hline \multicolumn{7}{|l|}{ Derived data } \\
\hline $\begin{array}{l}\text { Extracellular fluid - } \mathrm{g} / \mathrm{kg} \text { total } \\
\text { tissue }\end{array}$ & 220 & I I 8 & 244 & 214 & 342 & $30 \mathrm{I}$ \\
\hline Cell water $-\mathrm{g} / \mathrm{kg}$ cells & 746 & 762 & 677 & $68 \mathrm{I}$ & 808 & 798 \\
\hline Potassium-mequiv. $/ \mathrm{kg}$ cell water & 139 & 137 & I6I & 145 & I67 & I66 \\
\hline Phosphorus- $\mathrm{m} M / \mathrm{kg}$ cell water & 104 & 102 & I 55 & 184 & $(160) *$ & $(\mathbf{1} 89)^{*}$ \\
\hline Nitrogen-g/kg cell water & 43.6 & 44.6 & 47.6 & $55 \cdot 7$ & $(29.1)^{*}$ & $(33.1)^{*}$ \\
\hline
\end{tabular}

* The presence of an undetermined amount of extracellular nitrogen and phosphorus in brain makes these figures uncertain.

tent may be actually an index of the decreasing amount of "primitive connective tissue" which contains great quantities of intercellular fluid.

The decrease in sodium and in chloride during human development is taken by Shohl (1939) and Stearns (1939) as a consequence of the decrease of intercellular fluid. Murray, ( I 926) had shown that in the chick, between the sixth and the eighteenth days while the dry body weight (fat-free, feather-free, bone-free) increases to 2 I 7 per cent of its starting point, there is a drop of about 25 per cent in chloride content. Such a decrease in extracellular fluid probably is a phenomenon common to vertebrate embryos.

Similar evidence of a decrease in extracellular fluid is seen in studies by Iob and Swanson (1934) on a series of fetuses ranging from 3.2 to ro lunar months (Table I). In this series there is a progressive fall in chloride, at least from 3.7 . months to Io months, and a fairly steady rise in potassium. The changes in these constituents have been interpreted to signify a progressive rise in the proportion of cells, from a value of $320 \mathrm{~g} / \mathrm{kg}$ of body weight at 4.3 months to $5^{12} \mathrm{~g} / \mathrm{kg}$ at term. The concentration of solids in the cells after 4.3 months fails to show any consistent trend with age. 
T A B L E 3

DISTRIBUTION OF CELLS, WATER, AND POTASSIUM IN THE LEFT CARDIAG VENTRICLE OF YOUNG AND ADULT DOGS

All figures corrected for blood and fat

\begin{tabular}{|c|c|c|c|c|c|}
\hline & \multicolumn{2}{|c|}{5 Young dogs* } & \multicolumn{2}{|c|}{ I6 Adult dogs } & \multirow{2}{*}{$\begin{array}{c}\text { Change in } \\
\text { ventricle, \% }\end{array}$} \\
\hline & Serum & Ventricle & Serum & Ventricle & \\
\hline \multicolumn{6}{|l|}{ Observed data } \\
\hline Water- $\mathrm{g} / \mathrm{kg}$ tissue & $934 \cdot 5$ & 802.8 & $925 \cdot 4$ & 785.9 & -2.1 \\
\hline Chloride-mequiv./kg tissue & 108.8 & 31.6 & I 10.8 & $25 \cdot 4$ & -20 \\
\hline Potassium-mequiv./kg tissue & & $75 \cdot 4$ & & 88.2 & +17 \\
\hline \multicolumn{6}{|l|}{ Derived data } \\
\hline Cells $-\mathrm{g} / \mathrm{kg}$ tissue & & 739 & & $79^{8}$ & +8 \\
\hline Extracellular fluid - $\mathrm{g} / \mathrm{kg}$ tissue & & 261 & & 202 & -23 \\
\hline Cell water - $\mathrm{g} / \mathrm{kg}$ cells & & 736 & & 734 & -0.3 \\
\hline Potassium-mequiv. $/ \mathrm{kg}$ cell water & & I 39 & & I 50 & +8 \\
\hline
\end{tabular}

* Average age $=$ I 87 days.

Prior to the work of Iob and Swanson, other workers had studied the chloride content and dry weight, but this had not always been done for the same fetuses. Aron (1927), however, summarized these results, and they agree in general with the later study of Iob and Swanson. They indicate that at a fetal weight of $250 \mathrm{~g}$, the quantity of cells per kilogram of "net" tissue (with corrections for fat, bone, and extracellular solids) is only about $325 \mathrm{~g}$, while at term this proportion has risen to $480-580 \mathrm{~g}$. It continues to rise after birth to reach the adult level of approximately $700 \mathrm{~g}$ of cells per kilogram of the "net" tissue. These figures support the concept that cells vary in their abundance or concentration during growth without much change in the water content of the individual cells.

Not only for the body as a whole have such data been obtained, but also for several individual tissues. Hines and Knowlton (I939) studied the water content of skeletal muscle of the rat, using animals ranging from $15-336$ days of age. They found very constant figures for the water present within individual fibers but a decreasing proportion of water outside of the fibers (extracellular fluid). Yannet and Darrow (1938) found a similar change in the skeletal muscle of the cat (Table 2). Their histological observations showed an increase in size of the individual muscle fibers and a relative decrease in size of the spaces between the fibers. Apparently the new cytoplasm added to the fibers is of composition similar to that already present. Similar but much less marked decrease in extracellular fluid seems to occur in liver and brain of the cat, without change in the fluid content "per cell".

A study on cardiac muscle tissue of the dog (Hastings, Blumgart, Lowry and Gilligan, 1939) showed that here, too, there is a decrease in amount of water in the tissue and that the water content of the muscle fibers themselves remains essentially constant. The proportion of fibers apparently rose from $739 \mathrm{~g} / \mathrm{kg}$ in the case of puppies to $798 \mathrm{~g} / \mathrm{kg}$ in the case of adult dogs (Table 3 ). 
T A B L E 4

EMBRYONIC GROWTH OF THE CEREBRAI CORTEX OF THE GUINEA PIG

(Flexner and Flexner r $950 \mathrm{a}, \mathrm{b}-$ recalculated)

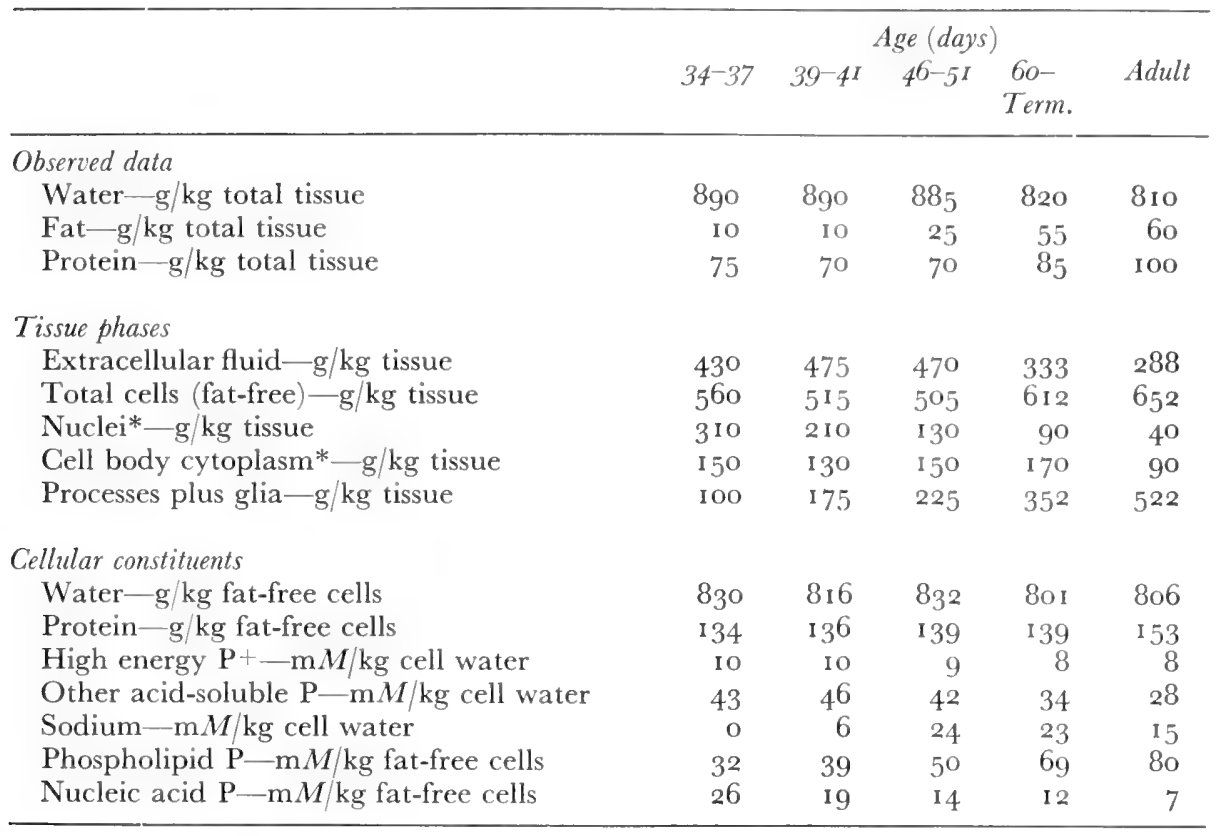

* From histological measurements.

+ From phosphocreatine, adenosine triphosphate, and adenosine diphosphate.

In order that the picture seen in muscle tissue should not be considered as possibly exceptional and specific for that type of tissue, we may cite in more detail the findings of Yannet and Darrow (1938) on postnatal changes in the liver. With corrections made for fat, no significant change was seen in the concentration of water in the hepatic cells in older as compared with younger cats. The livers of the group of older animals did show a 12 per cent average decrease in extracellular fluid.

These authors also found a 12 per cent decrease in extracellular fluid in the brains of the older group. Their calculations indicated that the water content of the brain cells remains essentially constant.

Detailed studies by Flexner and Flexner (I 949, I 950a, I950b) and by Peters and Flexner (1950) on the fetal development of the cerebral cortex of the guinea pig show that extracellular fluid, again calculated from chloride, rises slightly at an early period, when the spaces between the nerve cells are becoming wider, and then undergoes a marked fall of about $5^{\circ}$ per cent by the time that the adult stage is reached (Table 4 ).

We see, then, that the opinion that cells during the growth of the body are undergoing a progressive dehydration is not supported by the data obtained in a 
T A B L E 5

CHANGES INDUGED BY ATROPHY IN THE GASTROGNEMIUS MUSCLE OF THE R A T

(Hines and Knowlton, I937)

\begin{tabular}{|c|c|c|c|c|c|c|c|c|}
\hline & $\begin{array}{l}\text { Weight } \\
\text { lost by } \\
\text { muscle }\end{array}$ & $\begin{array}{l}\text { Water } \\
\text { content }\end{array}$ & $\begin{array}{l}\text { Extra- } \\
\text { cell. } \\
\text { water }\end{array}$ & $\begin{array}{l}\text { Cell* } \\
\text { water }\end{array}$ & $\kappa$ & $\begin{array}{l}\text { Acid- } \\
\text { sol. } P\end{array}$ & $\left(K^{\prime}\right) c$ & $\begin{array}{l}\text { Acid- } \\
\text { sol.P }\end{array}$ \\
\hline & & $\begin{array}{l}g / k g \\
\text { tissue }\end{array}$ & $\begin{array}{l}g / k g \\
\text { tissue }\end{array}$ & $\begin{array}{l}g / k g \\
\text { cells }\end{array}$ & $\begin{array}{l}\text { mequiv. } \\
\mathrm{kg} \\
\text { tissue }\end{array}$ & $\begin{array}{c}m M / k g \\
\text { tissue }\end{array}$ & $\begin{array}{c}\text { mequiv. } \\
\mid \mathrm{kg} \text { cell } \\
\mathrm{H}_{2} \mathrm{O}\end{array}$ & $\begin{array}{c}m M / k g \\
\text { cell } \\
\mathrm{H}_{2} \mathrm{O}\end{array}$ \\
\hline Normal & & $75^{8}$ & I08 & $75^{\mathrm{I}}$ & 94 & $5^{\mathbf{I}}$ & 145 & 78 \\
\hline Denervated 3 days & 6.7 & 765 & $13^{8}$ & $75^{8}$ & $9^{\text {I }}$ & & I 46 & \\
\hline Denervated 7 days & 28.2 & 762 & I $5^{8}$ & $75^{2}$ & 79 & $5^{\circ}$ & I 3 I & 82 \\
\hline Denervated I4 days & $49 \cdot 9$ & 768 & 212 & $75^{6}$ & 78 & $4^{2}$ & 140 & 75 \\
\hline Denervated 28 days & 72.3 & 772 & 298 & 756 & 72 & $3^{I}$ & I 5 I & \\
\hline Tenotomy Io days & 27.9 & 770 & I 59 & 762 & & & & \\
\hline Fasting 8 days & 26 & 771 & I 46 & 764 & & & & \\
\hline Fasting and denervation & & 773 & 198 & 765 & & & & \\
\hline
\end{tabular}

* Assuming extracellular phase originally contained $20 \%$ solids.

fairly large number of studies both on the whole organism and on various individual tissues. It seems, rather, that a decrease of extracellular fluid occurs and that the water content of the protoplasm of the cells or, in the case of muscle, of the syncytial fibers, remains essentially constant. The present concept is that the decrease in extracellular fluid is due primarily to an increase in the proportion of cells, e.g. of protoplasm, to the total mass of the tissue. These findings indicate the need for studies on individual cells as by the methods of histochemistry, rather than on masses of tissue, if conclusions are to be drawn concerning chemical changes during growth within the cells.

What, now, is the picture concerning the proportion of the intracellular and extracellular phases of fluid content during the process of senescence as compared with that during the process of growth? Does a comparison bear out the idea that senescence may be the antithesis of growth?

Lowry et al. (1942) have studied the chemical make-up of skeletal and cardiac muscle in senile rats (988 days) compared with those of middle age (6o3 days). They found an increase in water, sodium, and chloride and a decrease in acidsoluble phosphorus and potassium. On a histochemical interpretation, this means that the extracellular fluid has almost doubled, while no essential change has taken place in the water-content of the fibers. There has been a moderate increase in concentration of lipid in the tissue, a change which, like that of the extracellular fluid, is the reverse of the change seen with growth.

The increase in extracellular fluid with aging may be considered as due probably to histological changes occurring during growth. In other words, loss of individual fibers and atrophy of fibers may account for this increase.

An experimental study by Hines and Knowlton ( I 937) on the effects of atrophy 
on the chemical composition of skeletal muscle gives an interesting comparison with the effects of aging. They produced atrophy of the gastrocnemius muscle of the rat by three methods: starvation, tenotomy, and denervation. The water content of the fibers appeared to remain relatively constant, the total amount of protoplasm to decrease markedly, and the extracellular mass of tissue to decrease only slightly (Table 5 ).

There appears to be very little change with senescence in heart, liver, and brain of the rat in relation either to the amount or composition of the intracellular and extracellular phases. The heart shows a slight increase in amount of extracellular fluid and an increase in lipid phosphorus such as was found for skeletal muscle. In both brain and heart there is a slight displacement of intracellular potassium by sodium.

On the other hand, data on total solids in the human brain do indicate a consistent decline with senescence, amounting to from 8-I5 per cent (Strobel, I 939). Histological studies, as we shall see later, indicate a loss of cells in old age both in the rat and man.

The rat kidney shows an increase in extracellular fluid when the middle-aged (6o3-day) is compared with the young mature animal (60-day) (Lowry and Hastings, 1952); and in the senile animal (988 days) there was a slight further increase. The data suggest to the authors a decrease in the proportionate cell mass in this organ.

The picture of intracellular and extracellular phases of fluid in many tissues, then, is of an increasing "hydration" of the tissue with advancing age, a sort of extracellular edema. On the other hand, the fluid content of the protoplasm appears to remain essentially constant. As in almost all studies on the aging process, it is a difficult task to separate out the factor of "pure" senescence from pathological conditions, including systemic disorders, which may operate to further this type of change.

Comparison of the biological characteristics of growth and of senescence involves the study of the appearance and activities of the units of all living things, the cells. That the majority of cell types show definite biological features during periods of growth, particularly of rapid growth, is the opinion of Caspersson ( 1950 ). In the process of synthesis of protein, the nucleolus and the nucleolus-associated chromatin play a central rôle. The increase of nucleolar masses is a most conspicuous phenomenon during cytoplasmic protein synthesis. During periods of intense growth the nucleolus often becomes very large and shows large quantities of ribose nucleotides and of proteins rich in diamino acids. The great increase of nucleotides in the cytoplasm is seen in a marked basophilia and can be detected easily by means of the absorption of ultraviolet light at $2600 \AA$.

Caspersson presents figures of the cells of embryonic liver, kidney, and other organs showing the large nucleoli and nucleotide-rich cytoplasm of these rapidly growing cells. Cells of the regenerating liver, after partial hepatectomy, also show the large nucleoli and the high concentration of nucleotides in the cytoplasm.

In actively growing tissues another feature is, of course, the presence of large numbers of mitotic figures, for with cell size as a fairly sharply limited characteristic, the attainment of such a size is marked by cell division. 
In relation to the problems of protein synthesis it is interesting to note that one type of cell, the neuron, while not actively "growing", still shows evidence of constant production and using up of protein during its functional activity. This has been shown to be true not only for cells which have been undergoing exhaustive activity (Hydén, I 943, I 944, 1945, 1947) but also for those carrying out entirely normal function (Hydén and Hamberger, I 945). The organization of the nerve cell seems to be determined by the necessity for its being ready to produce protein rapidly whenever called upon to do so. While these recent studies have made excellent use of the quantitative methods of photoelectric microspectrography, much credit should be given to the early studies by Dolley (rgoga, b,

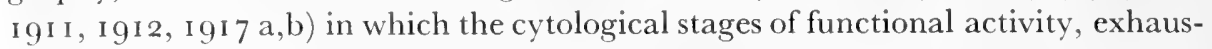
tion, and recovery of the nerve cell were worked out in great detail.

By the time that growth has to all effects ceased in the multicellular organism the histological appearance of the tissues is considerably different from that seen in the rapidly growing phase. The concentration of nucleotides is much reduced and the nucleoli, except in certain types of cells where rapid protein synthesis is a part of normal function, have become much less conspicuous. Mitotic figures are very scarce in the tissues of the mature body. To return to our analogy, we may say that from this time on through middle age the stream of life has reached a relatively stagnant condition. The results of this stagnation upon the individual tissues and cells begin to appear at the inception of senescence.

This is not the place to present a detailed description of the changes found in the tissues of senile organisms. We have attempted to summarize the work on this subject in a monograph (Andrew, I952) and in recent papers (Andrew, 1956a and $1956 \mathrm{~b}$ ). However it is pertinent to consider here how the microscopic appearance of the tissues in the rapidly growing phase, in the mature condition, and in senescence compare with one another. We have mentioned the visible indications of rapid growth in embryonic and in regenerating tissues. In the mature organism the general picture is one of stability, of a retention of the "typical" histological picture of the organ, whether it be liver, kidney, salivary gland, thyroid gland, spleen, lymph node, or brain.

In the senescent organism we have found changes in all of these organs, consistent in type for any one organ, but differing considerably with the type of organ. Thus, in the liver the appearance of numerous scattered large hepatic cells with enormous nuclei, a much increased number of nucleoli, and often intranuclear inclusions, is conspicuous (Andrew, Brown and Johnson, r943) (Fig. 6). In the kidney accumulation of colloid in the tubules with considerable atrophy of lining epithelium is seen (Andrew, I955b). In the parotid gland great masses of the secreting parenchyma undergo fatty degeneration (Andrew, 1949) (Fig. $7 \mathrm{~A}$ ).

In the thyroid gland a marked deterioration of follicles occurs and fibrosis is a prominent feature (Fig. 8A and B). In the spleen and lymph nodes there is a loss of germinal centers (Andrew, I946) and in the lymph nodes an appearance of fluid-filled cysts or a replacement of lymphoid tissue by adipose or fibrous tissue (Andrew, 1948). In the brain we have found changes in the individual cells in many areas (Andrew, r936, 1937, Andrew and Cardwell, 1940) (Figs. 9 and Io) 
and have confirmed the observation by a number of earlier workers that there is a considerable loss of nerve cells in old age.

While the nature of the changes in old age is generally "degenerative", with loss of the essential tissues of the organ, there are a number of instances where

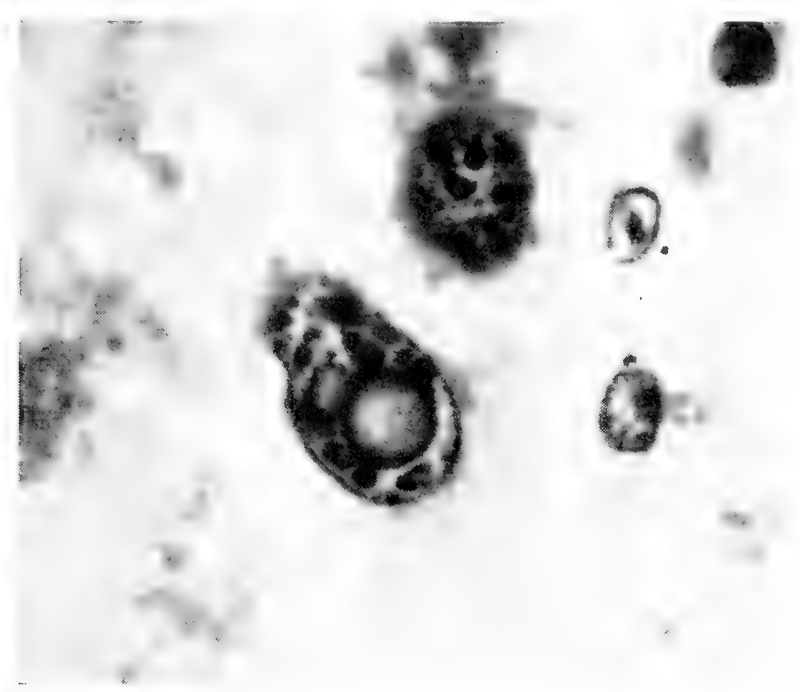

Fig. 6. Hypertrophied liver cell nucleus, with inclusion bodies, from a mouse of 8 I I days. $\times$ I500. (After Andrew, 1956b).

proliferation of cells occurs (Andrew, I 944; Korenchevsky, Paris and Benjamin, I950). The proliferation, however, is that of duct cells and tends to hasten rather than retard the degeneration of the functioning cells.

In a number of organs aberrant forms of cells, which in general have been called "oncocytes", have been described in old age (Hamperl, I937) (Fig. 7B). These cells frequently show an amitotic division of the nucleus. This process we have interpreted (Andrew, I955a) as indicating a "defensive" reaction of the cell by which the surface area of the interface between nucleus and cytoplasm is increased and the cell is enabled to survive. Recently Rudzinska (1 955) has shown such amitotic division in senile individuals of a protozoan, Tokophyra infusionum (Fig. I I).

These senile changes in tissues and cells are varied and certainly are not the reverse of the changes occurring during growth. Still, in the complicated biological features of the two processes, we would not expect a reversal of growth changes. We do see, however, that the stabilization of the tissues, the cessation of growth, leads sooner or later to the onset of senile change. Senescence is not synonymous with the cessation of growth but appears to be an inevitable result of such cessation.

We have dealt with the chemical, physical, and biological characteristics of 
growth and senescence. We may ask ourselves whether, if such an important relation exists between these two processes, the one may be altered experimentally by altering the other. The evidence in this regard is extensive and, it would seem, conclusive. Northrop (I9I7) showed that prolongation of the period of

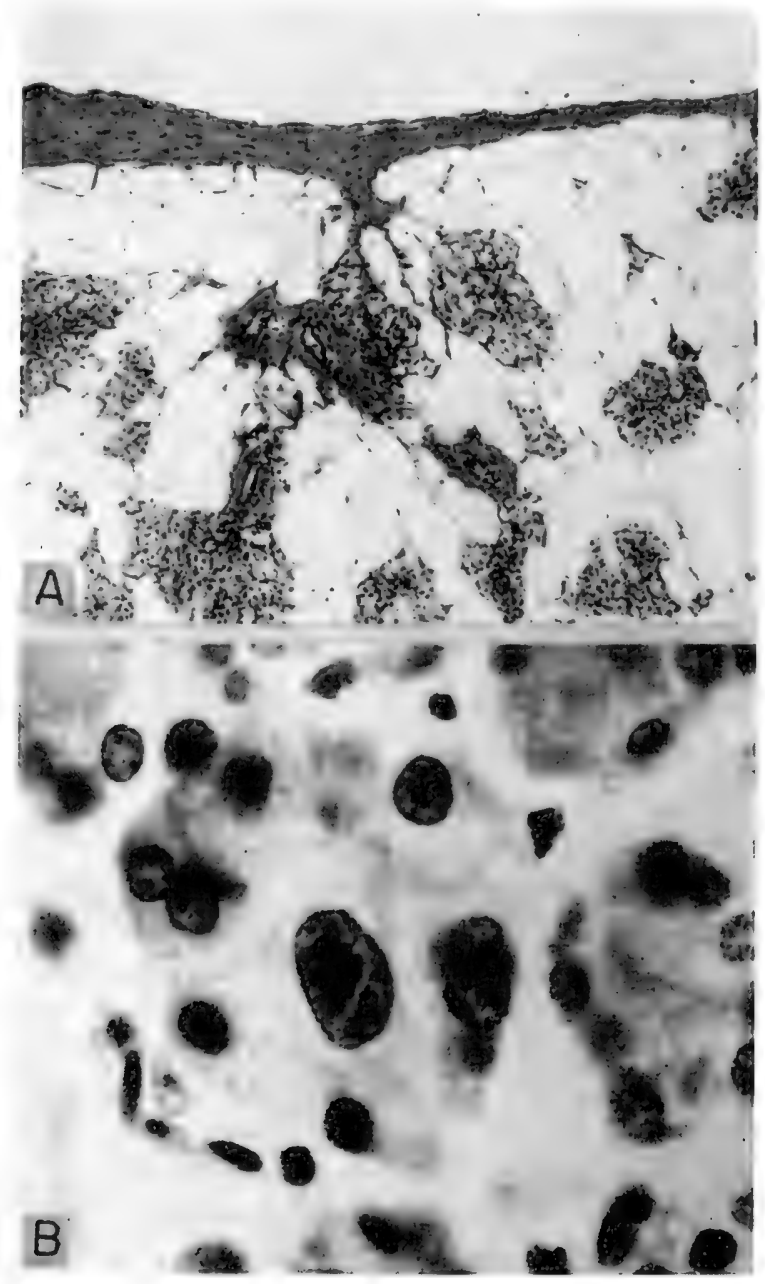

Fig. 7A. Fatty degeneration in the parotid gland of a senile rat, a 70o-day old female. $\times$ 106.

Fig. 7 B. Peculiar large cell or "oncocyte" in the parotid gland of a senile rat, a Iooo-day male. $\times$ ro 14. (Figs. $7 \mathrm{~A}$ and $7 \mathrm{~B}$ after Andrew, 1956).

growth in Drosophila resulted in prolongation of the life span. Jennings and Lynch (I 928a,b) and Ingle (I 933) showed that semi-starvation of Cladocera prolongs the length of life. Kopec (I928) was able to generalize that in vertebrate 
and invertebrate forms the life span is prolonged by extension of the growth period. Since that time a number of investigators have shown that in many different zoological forms a diminution in food intake increases longevity. Thus Rudzinska (195I) has shown this to be true for Protozoa; Tannenbaum (1947) for mice; and Carlson
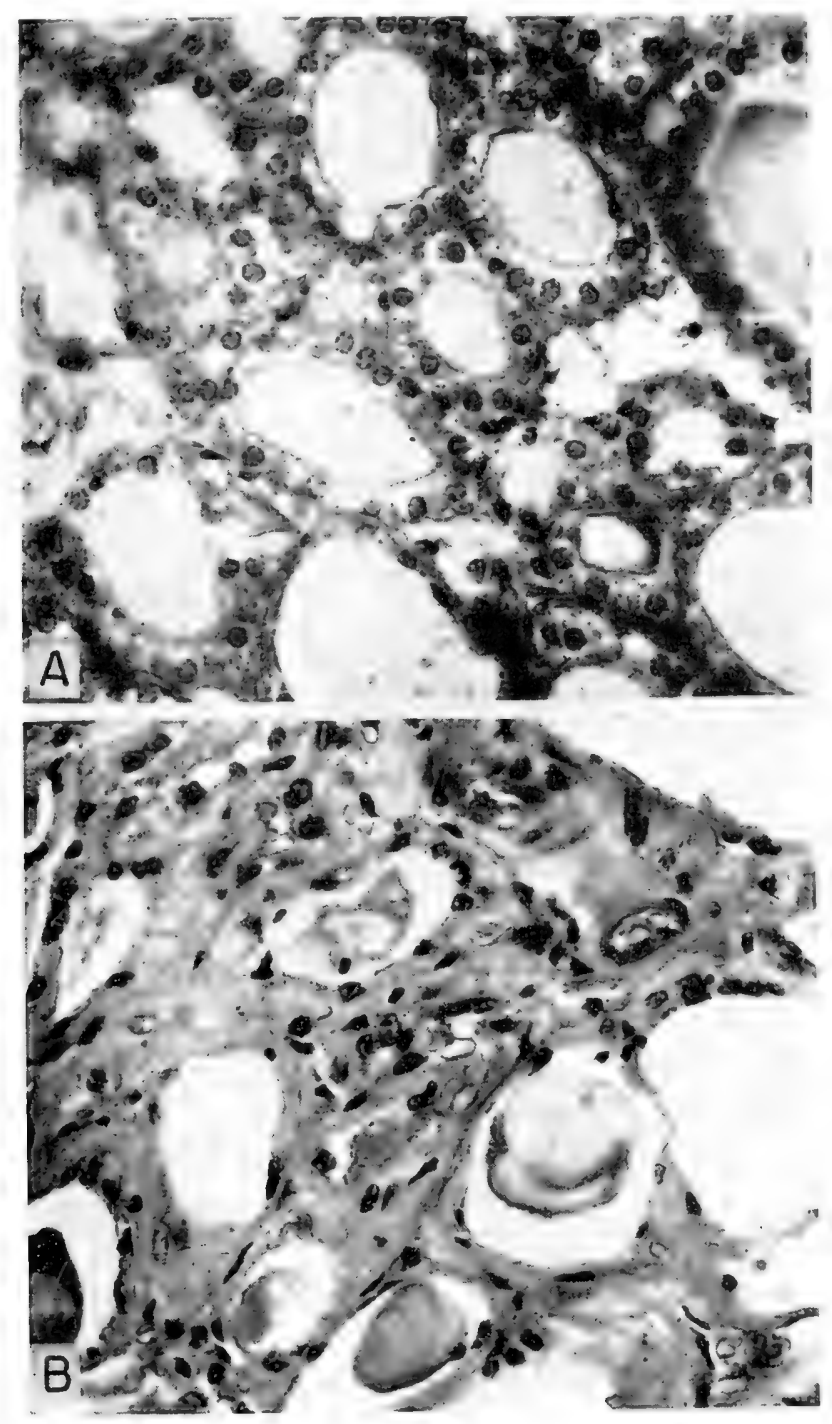

Fig. 8A. Thyroid gland of a young mouse, a 213 -day female. There is little connective tissue between the follicles. The epithelium is of simple cuboidal type. $\times 427$.

Fig. 8B. Thyroid gland of a senile mouse, an $8 \mathrm{II}$-day female. The epithelium in many follicles is flattened and atrophic, while the colloid appears very dense. Fibrous connective tissue is abundant. $\times 427$. (Figs. $8 \mathrm{~A}$ and $8 \mathrm{~B}$ after Andrew, 1956). 
Fig. 9. Giant cell of Betz from the motor cortex of a 29 year old man. The Niss I material is abundant. The karyoplasm is clear and the nucleolus stains deeply. $\times$ I 500 .

(After Andrew, 1956a).

Fig. 10. Giant cell of Betz from the motor cortex of a 78 year old woman. The Nissl material is scanty. The karyoplasm is lightly basophilic and the nucleolus is pale. $\times 1500$.

(After Andrew, 1956a). 
and Hoelzel (1946), Templeton and Ershoff (1949) and McCay (1942) for rats.

The relation of regeneration, the replacement of lost parts or even of very large portions of the body, to the process of senescence also has been investigated. The great work of Child many years ago (I9I5) had pointed out the rejuvenative

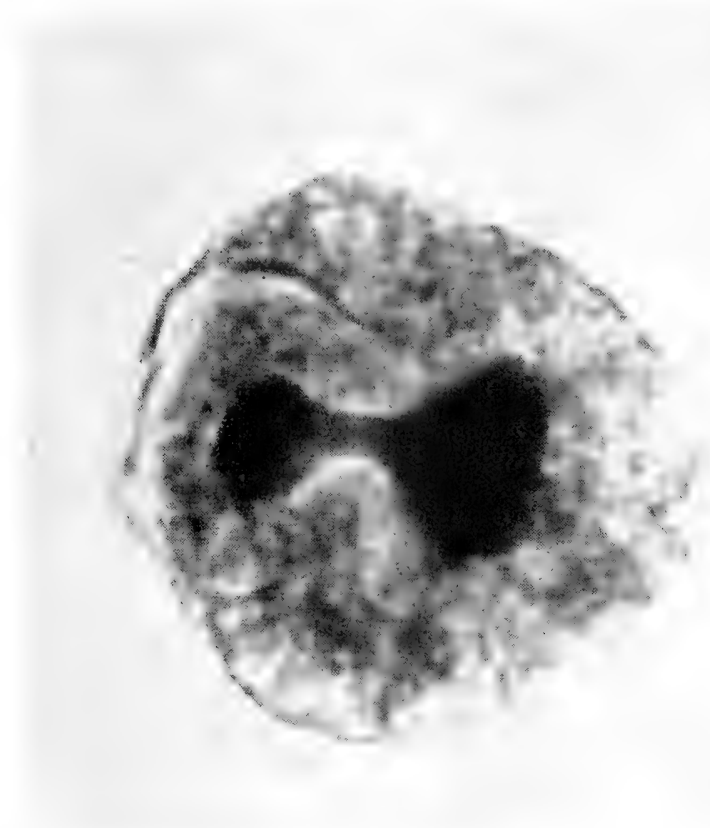

Fig. II. Amitotic division of the nucleus in an old specimen of the protozoan Tokophyra infusionum. $\times$ 1692. (Courtesy of Dr. M. A. Rudzinska).

effects on the organism of the stimulus to regeneration. Sonneborn (I930) showed that in the worm Stenostomum there is a distinct difference in the fate of lines which are maintained from the anterior half of the worms and from the posterior halves. Those from the posterior halves, where there is need for a great deal of regeneration and differentiation, continue to remain vigorous. Those from the anterior halves, where there is need for relatively little regeneration, soon "age" and die out. Indeed, the lines arising by regeneration from the posterior halves may be said to be essentially immortal! Again we see the close relation between the processes of growth, "regeneration" in this instance, and of senescence.

\section{CONCLUSION}

In its broad sense, taken to mean an increase in living matter, growth probably lasts as long as life; that is, there are some parts of the organism, some cells throughout the body, which are building up new protoplasm even though the organism as 
a whole is showing a loss or deficit. Studies with radioactive isotopes indicate that living tissue is constantly exchanging some of the amino acids, its building blocks, for new ones acquired from the intake of food and resynthesis. In this sense, growth continues its struggle throughout the life span and finally "sinks outwearied rather than o'ercome"*.

* Byron-The Corsair, Canto the Second, VI. 


\section{LITERATURE}

Andrew, W., (1936) Z. Zellforsch. u. mikroskop. Anat., 25, 583.

ANDREw, W., (1937) Z. Zellforsch.u. mikroskop. Anat., 27, 534.

Andrew. W., (1944) Am. J. Anat., 74, 97.

Andrew, W., (1946) Am. 7. Anat., 79, I.

Andrew, W., (1948) Am. 7. Anat., 82, 105.

Andrew, W., (1949) Am. 7. Anat., 85, 157.

Andrew, W., (1952) Cellular Changes with Age, Charles C. Thomas, Springfield, Illinois.

ANDREW, W., (1955a) 7. Gerontol., IO, I.

ANDREW, W., (1955b) 7. Gerontol., Io, 466.

Andrew, W., (1956a) 7. Chronic Diseases, 3, 575.

Andrew, W., (1956b) Federation Proc., 15, 942.

Andrew, W. and E. S. Cardwell, (i940) A. M. A. Arch. Pathol., 29, 400.

Andrew, W., H. M. Brown and J. B. Johnson, (1943) Am. J. Anat., 72, 199.

Aron, H., (1927) Biochemie des Wachstums des Menschen und der höheren Tiere, in OppenHeIMer's Handb. d. Biochemie, 7, I 52.

Backman, G., (1938) Lunds Univ. Arsskr., N. F. Avd. 2, 34, 5.

Bourlière, F., (1953) The Role of Comparative Physiology in Studies of Aging in Problems of Aging, Transactions of the Fifteenth Conference, The Josiah Macy Jr. Foundation, New York.

Brody, S., (1945) Bioenergetics and Growth, Reinhold Publishing Corp., New York.

Carlson, A. J. and F. J. Hoelzel, (I946) F. Nutrition, 3I, 363 .

Caspersson, T. O., (1950) Cell Growth and Cell Function, W. W. Norton, Inc., New York.

Child, C. M., (I9I5) Senescence and Rejuvenescence, University of Chicago Press, Chicago.

Совв, W. M., (1952) The Skeleton, Chapter 30 in Cowdry's Problems of Ageing, 3rd Ed., The Williams and Wilkins Co., Baltimore.

Dhä, N. R., (1932) Quart. Rev. Biol., 7, 68.

Dolley, D. H., (1909a) Am. F. Physiol., 25, i56.

Dolley, D. H., (1909b) F. Med. Research, 2I, 95.

Dolley, D. H., (I9II) 7. Med. Research, 24, 309.

Dolley, D. H., (I912) F. Med. Research, 25, 285.

Dolley, D. H., (1917a) 7. Comp. Neurol., 27, 299.

Dolley, D. H., (1917b) F. Comp. Neurol., 28, 465 .

Flexner, L. B. (1950) Anat. Record, Io6, 413.

Flexner, L. B. And J. B. Flexner, (1949) 7. Cellular Comp. Physiol., 34, I 15.

Flexner, L. B. And J. B. Flexner, (1950a) Anat. Record, 106, 413.

Flexner, J. B. and L. B. Flexner, (i950b) 7. Cellular Comp. Physiol., 36, 351.

Flourens, cited from Preface to Levi, G., (1946) Accrescimento e Senescenza, La Nuova Italia, Florence, Italy.

Hamberger, C. A. and H. Hydén, (1947) Acta Oto-Laryngol., 35, 479.

Hamperl, H., (1937) Arch. path. Anat. u. Physiol., Virchow's, 298, 327.

Hastings, A. B., H. L. Blumgart, O. H. Lowry and D. R. Gilligan, (i939) Trans. Assoc. Am.Physicians, 54, 237.

Hines, H. M. and G. C. Knowlton, (1937) Am. F. Physiol. 120, 719.

Hines, H. M. And G. C. Knowlton, (I939) Proc. Soc. Exptl. Biol. Med., 42, I 33.

HYdén, H., (1943) Z. mikroskop-anat. Forsch., 54, 96.

Hyó́n, H., (1944) Nord. Med., 22, 904.

Hydén, H., (1945) Nord. Med., 25, 90.

Hydén, (1947) Symposia Soc. Exptl. Biol., I, 450.

Hydén, H. and C. A. Hamberger, (1945) Acta Oto-Laryngol., 6I, Suppl. LXI.

INGLE, L., (I933) Science, 78, 5 I I.

Iob, V. And W. W. Swanson, (1934) Am. 7. Diseases Children, 47, 302.

Jennings, H. S. and R. S. Lynch, (1928a) 7. Exptl. Zool., 5o, 345.

Jennings, H. S. And R. S. Lynch, (1928b) 7. Exptl. Zool., 5I, 339.

Kopec, S., (1928) 7. Exptl. Biol., 5, 204.

Korenchevsky, V., S. K. Paris and B. Benjamin, (1950) J. Gerontol., 5, i 20. 
LANSING, A. I., (1947a) 7. Gerontol., 2, 228.

LANSing, A. I., (1947b) 7. Gerontol., 2, 327.

Lansing, A. I., (1952) General Physiology, Chapter I in Cowdry's Problems of Ageing, 3 rd Ed., The Williams and Wilkins Company, Baltimore, Maryland.

Lowry, O. H. And A. B. Hastings, (1932) Chapter 5 in Cowdry's Problems of Ageing, 3rd Ed., The Williams \& Wilkins Comp., Baltimore, Maryland, p. I05.

Lowry, O. H., A. B. Hastings, T. Z. Hull and A. N. Brown, (ig42) J. Biol. Chem., I43, 271.

McGay, C. M., (1942) in Cowdry's Problems of Ageing, 2nd Ed., The Williams and Wilkins Co., Baltimore.

MacDowell, E. C. and M. J. Taylor, (1948) Proc. Soc. Exptl. Biol. Med., 68, 57 I.

Murray, H. A., (1926) J. Gen. Physiol., 9, 789.

Needham, J., (193I) Chemical Embryology, 3 vols., University Press, Cambridge.

Northrop, J. H., (1917) F. Biol. Chem., 32, 123.

Peters, V. B. and L. B. Flexner, (1950) Am. 7. Anat., 86, 133.

RaItT, D. S., (I932) The Fecundity of the Haddock, Fishery Board for Scotland, Scientific Investigations, No.r.

Robertson, T. B., (1923) The Chemical Basis of Growth and Senescence, Lippincott, Philadelphia.

Rudzinska, M. A., (1951) Science, I13, 10.

Rudzinska, M. A., (1955) Personal communication.

RužrčKa, V., (1922) Deut. med. Wochschr., 48, 93 I.

Shohl, A. T., (1939) Mineral Metabolism, Am. Chem. Soc. Monograph, Reinhold, New York.

Sonneborn, T. M., (1930) 7. Exptl. Zool., 57, 57.

Stearns, G., (1939) Physiol. Revs., 19, 415.

Strobel, T., (1939) Z. ges. Neurol. Psychiat., i66, i6 I.

Strong, L., (1948) Science, IO8, 688.

Strong, L., (1950) Brit. J. Cancer, 4, 3 I5.

Tannenbaum, A., (1947) Ann. N. Y. Acad. Sci., 49, 6.

Templeton, H. A. And B. A. Ershoff, (1949) Am. 7. Physiol., I59, 33.

Yannet, H. and D. C. Darrow, (1938) J. Biol. Chem., I23, 295. 


\section{SUBJECT INDEX}

Abramis, 185, 190

absolute growth rate, 168

Acanthoscelides, $3 \mathrm{I} 5$

accoutumé tissue (auxin independent), 771

accretion, 7 I 4,952

accumulation of colloid in tubulus with considerable atrophy of lining epithelium in senecent kidney, 964

Acetabularia mediterranea, 273, 5I5

acetaldehyde, I I

acetalphosphatides, 332

acetate, utilization of - for RNA synthesis, 524

acetic acid, II

acetoacetate production from fatty acids in hepatoma, 894

acetoacetic acid, I I

acetoacetyl-CoA, 7, I I, 29

Act tobacter suboxydans, I 8

$\alpha$-acetolactate, io

acetylaminofluorene as carcinogen, 862

acetylaminofluorene inducing hepatic tumors, 840

acetyl-choline, accelerating $\mathrm{Na}$-inflow in cells, 643

- in peripheral parts of tumors, 915

acetyl-CoA, I I

-, oxidation of pyruvate to, 5

acetyl ornithinase in Escherichia coli extracts, 79

3-acetylpyridine as anti-metabolite, 806 acidity change in regeneration, 608 acid phosphatase, 46I, 525

- concentration in adenocarcinoma of the colon, 914

-, localisation of - - in rat egg, $33 \mathrm{I}$

acrencephalon, 367

—, secondary, (ventral), $3^{8} 4$

acriflavine inhibiting anaphasic chromo-

some movement, 942

acrogenesis, 368,444

-, obtained with a water-soluble hydrocarbon, 433

-, regular shifting from notogenesis to, 446

acromegalia, 245

acromerit, 368 acro-organizin, 478

ACTH, 2 I9

- function in regeneration, 631

- influence on sex-hormone secretion of human adrenal cortex tumors, 83 I

- -secreting pituitary tumors, 827

actin, cardiac, 349

Actinotrocha larvae, 598

activating contacts, $39 \mathrm{I}$

activating action of the implant, 403

activating reactions of energy-rich phosphate bonds, 36

activation, 520

- of cholic acid, 40

- of fatty acids, 40

- of sulfate, 41

- reactions in nucleic acid synthesis, 44

active $\mathrm{CO}_{2}, 43$

active dihydroxyacetone donors, 49

active glycolaldehyde donor, 62

active glycolaldehyde donors and acceptors

in transketolase reactions, 48

active methionine, 46

active transport by cell membrane, 7 I 4

actomyosin, 523, 526

acyl-coenzyme A bond, 892

acyl-dehydrogenase, 6, 2 I

- in mitochondria, 14

adaption, $55^{2}$

adaption syndrome, general, 609

adenine, 261, 759

- causing "sticky" anaphase bridges, 938

- causing structural chromosome changes, 939

- deoxyribose phosphate phosphorylation to di- and triphosphate, 103

-, incorporation of - in RNA, 276

adenocarcinoma, 9 I 5

- of mammary gland, reduced hexosemonophosphate shunt in, 885

- of the colon, acid phosphatase concentration in, 914

— of the colon, ${ }^{91} \mathrm{Y}$ induced, 847

adenosine, 75

- decreasing mitose incidency in mouse sarcoma- I80, 935 
adenosine diphosphate, $96 \mathrm{I}$, see also ADP

- monophosphate, see AMP

- monophosphate and triphosphate in unfertilized eggs, 349

- $-3^{\prime}$-phosphate-5'-phosphosulfate, 4 I

- thiomethylriboside in methionine synthesis by yeast, 75

- triphosphatase, 525

- triphosphate, 961

- triphosphate activity in growing tumors, $89 \mathrm{I}$

- triphosphate as stored energy source for mitosis, 680

adenyloctanoate, 35

adenylate kinase, $\mathrm{I} 02$

adenyl- $\mathrm{CO}_{2}, 43$

adenylic acid, inhibiting mitosis in germinal epithelium of rat ovaries, 936

- in mouse mammary tumor and rat hepatoma necessary for phosphorylase, 891

- preventing prophase of chick cells, 936

- system, 150

adenylmethionine, 35

adenylpantoate, 35

adolescent spurt, 247

ADP competition between glycolytic

enzymes and mitochondria, 32

adrenal cortex, adenomas and carcinoma, post-castrational, $84 \mathrm{I}$

- tumors, ACTH influence on sexhormone secretion of human _ _, $83 \mathrm{I}$

— tumors secreting sex steroids, 83 I

adrenal cortical, adenoma, postcastrational, 826

- and ovarian tumorigenesis, postirradiation, 848

- carcinoma, post-castrational, 826

- neoplasms induction by estrogen, 835

- tumors following castration, 833

adrenal corticoids action on regeneration, 630

adrenalectomy, affecting progression of prostatic cancer, 843

- favorising lymphocytic leukemogenesis in mice, 842

adrenalin inhibiting mitosis in P-phase of regeneration, 643

adrenal S-corticoids in regeneration, action of, 63 I

adrenotrophic plantable tumors, 916

Aerobacter aerogenes, Io, 65, I I 9

aerobic, glycolysis of tumors, 54, $88 \mathrm{I}$

- phosphorylation, 3 I

- phosphorylation in plant tissues, 33

aging, potential, 954

- process and growth, 952 agglutinins, 720

Agrobacterium tumefaciens, $77 \mathrm{I}$

AICA (aminoimidazolecarboxamide), accumulating in sulfanilamide inhibited Escherichia coli, 94

AICAR (aminoimidazole carboxamide ribotide), 93

AIR (5-aminoimidazoleribotide) alanine, 68 alanine, anaerobic incorporation of labelled - into microsome protein, 45

—, in lymphosarcoma, 896

$\beta$-alanine, conversion of uracil to, i 8

-, source, II 8

alarm reaction, general, 609

albumen and $\alpha-, \beta$ - and $\gamma$-globulines in blastocyst fluid, 720

alcohol dehydrogenase, 782

alcohol, enzymes oxidizing, ro

— in low dosage accelerating regeneration, 623

aldehyde dehydrogenase, 761

- oxidases, II, 26

aldehydes as substrate in reaction between ornithine and glyoxylate, 84

aldolase content of adenocarcinoma of colon and rectum, 914

-, muscle - catalyzing xylulose phosphate synthesis, 49

aldosterone, 12

Algae, 749

alicethal eggs, $74^{2}$

ali-esterase, 6 I 9

alizarin, 626

alkaline phosphatase, 289, 596

- activity during phase of DNA accumulation, 525

- activity in growing tumors, 892

- content of normal skin, 687

- during regeneration, functions of, 6 I2

- in early stages of rat embryo, 364

— in healing wounds, 698

- in mast cell granules, 699

- synthesis in rat embryo, 365

- synthesis in induced epithelium, 457

allanto-chorionic placenta as barrier to

passage of agglutinins in embryos, 720

allelocatalysis, $55^{\mathrm{I}}$

Allenblackia, 772

Allen's rule, 243

alligator eggs, 737

allogibberic acid, 765

Allolobophora caliginosa, 602

allometric equation, 142, 224

- —, physiological processes and, 178

- growth of the brain, $25 \mathrm{I}$

- regression, I 42 
allometry, chemical, 232

- coefficient, 227

- constant, I $4 \mathrm{I}$

-, evolutionary, 228, $24^{\circ}$

-, interspecific, 24I

-, intraspecific, 24 I

- mathematical formulations of

evolutionary - , 25 I

—, morphological applications, 229

-, negative, 240

-, ontogenetic, 228

-, positive, 240

-, - ontogenic, 24I

alloxan, 426

allylglycine, vegetalizing effect, 505

alveolar adenoma, 852

Ambystoma, 409, 4 I9

Ambystoma mexicanum, 525

Amblystoma opacum, 669

a-methopterin, 9 I 0

- as chromosome breaking agent, 939

amiboid movements, 34 I

amide group of glutamide, transfer, 85

Amieurus, 602

D-amino acid, 13

L-amino acid, I 3

amino acid, activation of - prior to fixation in templates, 285

- analogues, 936

- analogues, mitotic inhibition by, 928

- in animal tissue, transfer of $\mathrm{N}$-atoms of, 84

- antagonists inhibiting ribonucleic acid formation, 937

-, biosynthesis, 53

-, biosynthesis of aromatic _ , 66

—, building blocks with free - 284

- compounds affecting genic components of the cell, 862

- compounds as carcinogens, 862

- determination of $-\mathrm{s}$ necessary for the maintenance of nitrogen equilibrium, 55

-, distribution of free - in tissues, 53

- enzymatic incorporation of labelled into proteins of liver homogenates, 44

- essential $-\mathrm{s}, 55$

- , free - pattern in Yoshida ascites tumor, 896

-, free - pool of the cell, 285

- incorporated into reticulocytes protein, 296

- incorporation of - into the mitochondrial proteins, 513

—, intracellular pools of, 53

— key -s, 6 io

-, maintenance of the - pool of the cell, 530 amino acid metabolism in tumors, 899

-, peptide bond formation in the incorporation of - into protein, 44

- as precursors in PFS, $49^{8}$

-, protein precursors larger than -, 497

- quantitative changes in - in amphibia

blastula, gastrula and neurula, 737

- relationships, 54

- synthesis of the carbon skeleton, 56

— synthesis, organic oxime intermediates, 82

- synthesis, transamination and, 86

- of tumors, patterns of the free -, 54

-, uptake of labelled - in chick embryo, 500

-, uptake in tumors of labelled — or related metabolites, 899

-, utilizing of - in embryos, $49^{6}$

aminoacyl-adenosine monophosphate compounds, 928

2-aminoadenosine, as mitose inhibitor, 935

- suppressing mitosis in mouse sarcoma I 80 cultures, 945

— triphosphate, 933

$\alpha$-aminoadipic acid as intermediate in

lysine synthesis in Neurospora, 76

aminoadipic acid pathway of synthesis, 2,76

aminoamfol as chromosome breaking agent, 939

o-aminoazotoluene inducing hepatic tumors, 840,86 I

$p$-aminobenzoic acid, 94, I I 9, see also PABA

$\gamma$-aminobutyric acid levels in brain tissue, 54

aminofumaric acid as precursor of

pyrimidine, 98

aminoimidazolecarboxamide, 94 , see also AICA

4-amino-5-imidazolecarboxamide, 933

5-aminoimidazolecarboxamide ribotide,

57,94 , see also AICAR

5-aminoimidazole ribotide, 94

5-amino-4-imidazole ribotide synthesis, 87

- $\mathrm{N}$-succinylo-carboxamide ribotide, $4 \mathrm{I}$

aminoketones, effects of - as analogs of leucine, 502

$\delta$-aminolevulinic acid, protoporphyrin synthesis from, 90

2-amino-I-naphthol carcinogenic metabolite in urinary bladder tumors, 862

$\alpha$-amino nitrogen, aspartic acid and the transfer of, 87

amino nitrogen, free - in extract of tumor tissue, 896

m-aminophenylsulfate, $4 \mathrm{I}$

aminopterin, arresting metaphase of mitosis, 929

— as chromosome breaking agent, 939 
4-aminopteroylglutamic acid

(aminopterin), 929

4 -aminopteroylthreonine as

antimitotic agent, 930

4-aminopyrazolo- $\left(3,4^{-}-d\right)$-pyrimidine, caus-

ing abortive metaphases in cultures of

human HeLa cells, 944

- inhibiting mitosis in human HeLa cells, 933

amino transfer reactions and

transaminations, 83

amino-uridine inhibitor of purine and pyri-

midine synthesis in hepatoma, 909, 910

amitotic division of cell nucleus in oncocytes, 965

Amoeba proteus, 280, 592, 732

amoebae, incorporation of phenylalanine into, 282

ammonia, increased - excretion under anaerobic conditions, $72 \mathrm{I}$

- fixation, 44,82

—, liberation of - from cytolizing cells, 736

- utilization and nitrogen transfer reactions, 88

- utilization of - for protein synthesis, 222

ammonium salts promoting growth at low amino acid content of medium, 725

amniotic groove in gastrula, 324

Amniots, 459

-, kinematics in, 347

AMP (adenosine-5-phosphate), 45

-, formation of - - from iosinic acid, 94

amphetamine, I2

amphiclinous manifestations, 600

Amphioxus, 325

amylase, $72 \mathrm{I}$

- activity during DNA accumulation, 525 anabolic influence of growth hormone, 845

- processes, permeation as regulating

factor, 216

- - , resorbing surfaces, I8o

anabolism and catabolism, animal growth

as result of counteraction of processes of, I 78

- of building materials, 180

Anadonta, 200

anaerobic glycolysis, I6 I

- - dinitrocresol stimulation of - in ascites tumor cells, 886

_ - of tumor cells, 88 I

- incorporation of labelled alanine into microsome protein, 45

- tumor glycolysis inhibition by steroid sex hormones, 883

Anamniota, 342

anaphase, 3 I8 anaphase, "sticky" — bridges caused by adenine, 938

anaphasic movement of the chromosomes interrupted by spindle poisons, $94^{\mathrm{I}}$

- separation of the chromosomes, 6-chloropurine causing failure of the, $94^{\circ}$

androgen, exogenous, 840

- inhibition of mammary carcinogenesis in mice, $84 \mathrm{I}$

- potentiating granulocytic

leukemogenesis in mice, $8_{4} \mathrm{I}$

- suppression of lymphocytic

leukemogenesis, 84 I

androgenetic frog hybrids, 734

androgenic hormone delaying lymphocytic leukemogenesis, $84 \mathrm{I}$

- steroid hormones, 219

androgens promoting regeneration of bone, 632

-, effects of - on regeneration, 632

androsterone, 12

anhydroleucovorum, I2 I

animal body, polarity of the, 233

animal growth, quantitative theory, 177

animalization, 52 I, 537

animalizing $\mathrm{NaCNS}, 35^{\circ}$

animal pole, 3 I I 322

Annelida, 590

Anodonta cygnaea, I84

antephase, 157

anthranilic acid, 36

antiactin, 733

anti-actomyosin, 732

antibody, 535

antibodies against leukosis virus, $85^{8}$

anticarcinogens, 86 $\mathrm{I}$

anticipatory shoots, 812

antifertilizines from egg and sperm, 7I 7

antifolic acids, 929, 939, 943

-, selective damage of intestinal

epithelium, bone marrow, red pulp of

spleen and spermatogenic cells by, 939

antigen, 535

antigen-antibody complex, 535

_ - lock-and-key theory, 669

antigenicity, carcinogenic effect on normal cell is alteration in - 863

antigenic material, synthesis of - prior to gastrulation, 727

antiglobulin, 733

antiglutamines, 930

antigonadotrophin, 834

antimetabolites as mitotic poisons, 926

antimitotic activity of ribonuclease, 282

antimycin A, 20

-, acting between cytochrome $\mathrm{b}$ and 
cytochrome c, 30

antimyogen, 733

anti-oxidants, 6 I 8

antipole, 3 I I

antitemplate hypothesis, 2 Io

antitemplates, 209, 731

antithyroid substances, 633

Anura, 675

Anuran egg, 410

Anurans, 378, 590

APGH (pituitary growth-hormone), effect

of - on regeneration, 634

apical, cap, 456

- dominance in plants, 8 I I

- meristems, 766

— ridge, 457

apoenzymes catalizing transaminase reactions, 85

apyrase, 527

- content in amphibian gastrula, $34^{8}$

Arachnida, 598

Arbacia $p, 33^{6}$

archencephalon, 367

archenteric roof, 355

- _ inducing effect, 400

archenteron, pouch, 392

- roof, induction by - - 443

- roof specific ——, antigen in Triturus alpestris, 727

architomy, 588

archusia, 55 I

area pellucida, 324

arginase, distribution in chick embryo, 74 I

arginine, 41,78

- biosynthesis, 80

- conversion of ornithine to, 80

argininosuccinate, 80

argyrophylic membrane, 675

arista, 525

aristapedia, 525

Armadillidium pallassii, 183

aromatic, amino acids, biosynthesis, 66

- polyauxotrophs, 65

arsenic as cancer inductor, 869

Artemia salina, I85, 62 I

Arthobacter, 10

Arthropods, 590

Arthropods, limb regeneration of, 642

arylacetic acid, 802

Ascaris, 166

Ascaris lumbricoides, 184

Ascidians, 339

Ascidiella scabra, 333, 337

ascites, cancer cells, $88_{3}$

- cells, hexokinase amount in, 886

- tumor cells, I 8 ascites tumor cells, inhibition of carbohydrate metabolism by ethyleneimines in, 886

- in mice, 865

L-ascorbate, II

ascorbate, postulated precursors of, II I

ascorbic acid, I I I

- - glutathione respiratory pathway, 25

- oxidase, 786

— - copper as prosthetic group in — in plants, 25

- as respiratory catalyst, 25

Asellus aquaticus, I85, 59I, 594, 606, 644

Ashbya gossypii, 5 I

asparagine, as amino donor in

transamination reactions, 85

- synthesis, 83

-, uptake in transplanted rat tumors, 900 aspartase, 83

aspartate, 72

-, tumor inability to synthesize -, 899

—, $\propto$-decarboxylation, I I 8

_, relationship between — and threonine in $E$. coli, 73

aspartic acid in amphibian morula, 348

- delaying metaphase, 937

-, conversion of - to homoserine, 72

- , incorporation of labelled — into nucleic acid pyrimidines, 98

- oxalacetic acid transamination to, 72

- producing chromosomal abnormalities, 937

- and the transfer of $\alpha$-amino nitrogen, 87

aspartic- $\beta$-semialdehyde, I I

$\beta$-aspartyl phosphate, I I

Aspergillus, 13,62

- nidulans, 64

-, thiosulfateless strains, 64

assimilation, I 37

assimilative stimulus at distance, 462

Astacus astacus, 185

Asterias, 599

Asteroidea, 599

asymmetry, characteristical, $45^{6}$

asynchrony between epidermis and deeper tissues in regeneration, $60 \mathrm{I}$

ATP, 33, 285

-, as energy source, 729

-, utilization, 37

$\mathrm{ATP}^{3}, 34 \mathrm{I}$

ATPase activity, 3

- and DPNase activity in tumor mitochondria, 895

- from tumor mitochondria, 892

atropine, $\mathrm{Na}$-extrusion from cells accelerated by, 643 
atrophy, induced - by starvation, tenotony and denervation, 963

auromerit, 368

auto-accelerator in regeneration, 603 autocatalytic reaction, monomolecular, 603 autogenesis, 247

auto-inductors, $44^{8}$

auto-inhibitor in regeneration, 603

autolytic activity, regenerative power of

tissues proportional to intrinsic, 6 I I

auto-oxidizable flavoproteins, 2 I

auxanometer, Koningsberger, 754

auxesis, 766

auxin, 753

-, mode of - action and nature of cell enlargement, 793

- adapted tissue, 77 I

- and kinetin, interaction, 774

- antagonist, 753

-, role of - as coenzyme, 797

- -independent tissue, $77 \mathrm{I}$

- influencing waterpermeability of plant cell wall, 795

- modifying induction process, 425

— production in seeds, 809

-, role of light in - formation in leaves, 800

-, root, 753

-, synthetic, 773

- theory of geotropism, 805

- transport in plant stem, 8 i 3

-, use and measurement, 755

auxocytes, 307, 467

auxotroph of Escherichia coli accumulating

keto analogs of valine and isoleucine, 69

Avena agar test, $75^{8}$

Avena sativa coleoptiles, $75^{\circ}$

axial chondrogenesis, 462

- gradients, 233

- organ stabilization, 353

Axolotl, 6 i 6

- gastrula, 392

- neurula, 404

2-aza-adenine as antimitotic agent, 933

azaguanine, 275

- -dependent leukemia, 93 I

8 -azaguanine, differential susceptibility of

various tumors to, 931

- inhibiting formation of bacterial enzymes, 928

azaserine, 92

- as antagonist of glutamine in GAR synthesis, $9^{2}$

- depressing mitotic incidence in mouse sarcoma I 80 cells, 930

- inhibiting de novo synthesis of purines in tumor cells, 908 azaserine as tumor inhibitor, 930

azo dyes as carcinogens, 86 I

Azotobacter vinelandii, 24, I 03, 91 I

Bacillus cereus, 532

- licheniformis, 23

- megatherium, $16 \mathrm{I}$

- subtilis, 23, 535

Backman's formula, I 74

bacteria, filamentous, 294

bacterial transformations by DNA, 268

- xeno-inductions, 439

bacteria, mechanism of spore formation in, 537

Bacterium linens, II 7

BAL (2,3-dimercaptopropanol), I9, 785

balance of growth, 569

— of organs, 230

Barnea candida, 3 I 8

basal cell carcinoma, human, 860

- epithelial cells, I63

basement membrane, detachment of the epidermis from the, 675

basocellular carcinoma, ribonucleic acids in, 905

basophilic tumors, 835

basophily in rat embryo in first week of development, 326

Bauplan, 229

benzimidazole as adenine antagonist, 93 I

benzopyrene modifying induction process, 425

benzoquinone, I3, 32, 892

benzothiazole oxyacetic acid, 773

benzoylsulphamethylpyrimidine, 886

benzpyrene induction of sarcoma, 847

benzylimidazoline, effect of - on regener. ation, 63 I

Bergmann rule, 2 I 6

beryllium, as cancer inductor, 869

- basic acetate as specific inhibitor of the phosphatases, 460

— inhibiting root elongation, 806

- salts, 626

betaine, I I

- aldehyde, I I

- transmethylase, 74

bicyclicy of metabolic changes, 647

bilateral organization, 3 I 5

binuclear plant cells, $77 \mathrm{I}$

biochemical modification of inductors and reactors, 4I9

biological effectiveness of auxines determined by shape of the molecule as a whole, 764

— similarity, principle, 227 
biomass, 137

—, biologically complete -, $13^{8}$

-, functionally complete - , I 38

bionomogenesis, 247

biosynthesis of amino acids, 53

- of aromatic amino acids, 66

- of isoleucine, 70

- of leucine, 69

- of valine, 69

biosynthetic potentialities, I

biotin, 42, I 14

- as cell division promoting factor in plants, 772

- promoting skin-healing in rats, 630

blastema, 588

-, mesonephric, 466

Blastocladiella, 537

blastocoele, 326,360

-, method to insert implant in the, 377

blastocyst, 326

- fluid, albumen, $\alpha$-, $\beta$ - and $\gamma$-globulines in, 720

-, mammalian, 342

blastodisc, 347

blastomers, 306, 326

blastoporal groove, 376

blastopore, localization of the - in

Anamniots, 343

—, secondary, 386

- stages, semicircular and circular, $39^{8}$

blastula, 345

- stage, rapid DNA synthesis in late, 287

blastulation and nitrogen metabolism, 722

Blatella germanica, 635

Blatta orientalis, 185,197

blood antigens, epigenetic nature of development of, 734

body mass, active, 208

-, differentiated, 208

-, essential, 208

-, generative, 208

—, inert, 208

--, metabolically active, 208

body-size, absolute, 142

-, dependence of metabolic rate on, I8 I

—, interspecifically relation between metabolic rate and, $\mathrm{I} 8 \mathrm{I}$

body, surfaces of the, I 42

body weight, increase of, I 37

Bombyx mori, 188

bone organizing action in regeneration, 646

boric acid effects on morphochoresis, 422

brain, allometric growth of the, 25I

- and body, relation between the size, 250

-, evolution, $25^{\circ}$ brain, interspecific increase of the - size in mammals in relation to body size, 250 -, loss of nerve cells in — in senescence, 965

- morphogenesis, 407

- parts, constriction zones between the five basic -, 408

- tumor, nuclear density in, 905

- weight in mammals, 250

breast cancer, effects of hypophysectomy, 843

-, hypophysectomy palliative in human metastatic - 846

brittleness increasing - of the bone in senescence, 957

Broca's center, development of, 252

5 -bromocytidine, differential mitose suppression by, 936

bromouracil as antogonist of thymine in lactobacilli and E. coli, 912

Brontotherium, 239

Brown-Pearce tumor in rabbits, 931

Brucella abortus, 719

Bryophyllum, 768

buds of plants, interrelation, 8 I I

building blocks with free amino acids, 284

- materials, anabolism, I80

- - of the organism, I 37

- - - turnover of, 147

burns, 670

butter yellow binding by liver cells during hepatic carcinogenesis, $86 \mathrm{I}$

— - inducing hepatic tumors, 846

- - riboflavin as anticarcinogen of - 862

butyryl-CoA, 6, I I, 3o, 40

caffeine inhibition of conidial growth of ascomycetes, $93^{\circ}$

- as mutagenic agent for bacteria, $27 \mathrm{I}$

calcium content of methylcholanthrene induced carcinoma, 9 I 5

- decrease in broken bones, 609

- as growth inhibitor, 796

- ions inhibiting elongation of plant cells, 782

- promoting elongation of the root, 805

Camaesura anguina, 324

Cambarus, 598

cambium, $75^{2}$

-, cork, 767

-, vascular, 767

canaliculi, 352

cancer, ribonuclease in chemotherapy of, 282

— and viruses, 849

cancer diagnosis, cytological, I 59 
cancer, environmental, 823

- experimental, chemotherapy, 926

- induction by beryllium, nickel, arsenic and plastics, 869

_ - , sterol metabolism in _ —, 88I

- and ionizing radiation, 847

—, metabolism of the - cell, 877

- and nutrition, 865

cancerous growth, increased proteolysis in, 6 I I

- response, genetic factors in target tissues determining ——, 869

Cannabis sativa, 8 го

capillary formation, 689

carbamyl phosphate, $35,4^{\text {I }}$

- synthesis, 83

carbohydrate, respiration and - metabo-

lism in regeneration, $6 \mathrm{I} 8$

-, conversion of fat to - by plants, 123

- - protein-fat sequence of energy sources, 736

-, proteinsparing effect of, 222

carbohydrates in cancer cell metabolism, 880

$\beta$-carbon, conversion of - of serine to the methyl groups of choline and thymine, 73 carbon skeleton of amino acids, synthesis, 56 carbontetrachloride inducing hepatic tumors, 863

carboxydesmutase, $9 \mathbf{I} 4$

carboxylase, oxalacetic, $4{ }^{\mathrm{I}}$

carboxylation of ribulose diphosphate, 43

carcinogens, absorption of - in Urodeles embryos, 425

—, amino compounds as, 862

-, binding of - by cellular protein, $86 \mathrm{I}$

—, halogenated hydrocarbons as, 863

-, ionizing radiation inducing development of chemical, 848

- virus, 850

carcinogenesis, agents initiating, 823

—, agents promoting, 823

- by 9, Io-dimethyl-1,2-benzanthracene, 845

-, hormonal factors in, 824

-, host as prime force in determining, 823

- , initiating and promoting agents in, $85^{8}$

-, phases of, 863

- and somatic mutation, 848

—, Warburg's phases of, 883

carcinogenic chemicals, 858

- hydrocarbons inducing ovarian tumors, 830

— - phenanthrene structure of, 858

- - reducing concentration of $\Delta^{7}$-cholestenol in mouse skin, $88 \mathrm{I}$ carcinogenic induction of skin cancer, $84 \mathrm{I}$ carcinoma, DNA in - human epidermoid, 687

—, Flexner-Jobling, 889

—, human basal cell, 860

-, lysine, taurine and tyrosine in, 896

-, mammary, 49

- of the parotid gland in $\mathrm{C}_{3} \mathrm{H}$ and $\mathrm{AKR}$ mice, 855

—, squamous cell — of the mouse skin, 857

Carcinus, 622

cardiac actin, 349

cardiomyoblasts, pulsating, 353

Carnot-Clausius principle, I 47

carotenoids concentration in ova, 620

cartilage organizing action in regeneration, 646

cartilages, induction of, $46 \mathrm{I}$

cartilaginous capsule of the ear, formation of the, 453

caryclastic agents, $5 \mathrm{I} 7$

Cassiopea, 605

castration, adrenal cortical and pituitary tumors following, 833

catabolic constant, I 99

- loss of generative mass, 209

catabolism, I 45

-, rate of - calculated from growth data, 200

- of glucose and fatty acids, 3

-, nitrogenous waste products of protein -, 736

catalase, 22, 275, 786

- activity of livetin, 721

catechol, 13

cathepsins, 506, 596, 61 I , 728

cathepsin and dipeptidase activities in central areas of the tumor, 913

catheptic activity; 344

cauliflower, whiptail of, 799

Cavia cobaya, 244

C-distribution in tumors, 894

CDP-choline (cytidine diphosphocholine) as coenzyme, 107

CDP-choline formation, 107

CDP-ethanolamine (cytidine diphosphoethanolamine) as coenzyme, I07

Cecropia, antigen 7 of, 719

-, blood antigens of, 734

cells, adhesiveness or non-adhesiveness of, $34^{2}$

cell autonomy and interdependence, 549

- clones, single, 549

- constancy, I66

- counts in tissue cultures, 572

- dissociation, 320 
cell differentiation, relation of proliferation and, 523

— - respiration rates in plant, 770

— - triggering, explanation, 726

- division, metabolic changes associated with, 769

- -, morphology of plant, 767

- -, plant, 766

_ —, physiology of plant, 769

- - promoting factors in plant, 772

- enlargement, 775

- - nature of, 793

- - plant, 766

- growth, limitation of, 163

—- mathematical theory, I6 1

- - and multiplication, 156

- loss in old age, 963

- — and cell renewal, harmonization between, 165

- membrane, active transport through -, 714

- - , selective permeability of the, 7I 3

- migration in regeneration, 597, 626

- mitosis, inhibitory action of polysaccharide fractions extracted from ascites carcinoma and Yoshida sarcoma on, 88 I

- multiplication, I39

- and growth, ${ }_{5} 5$

-, postmitotic, 163

- proliferation and differentiation, relation of, 523

- renewal, i63

- - , harmonization between cell loss and, 165

- respiration, 150

- size, principle of constant, 162

—, social behaviour, $55^{\circ}$

- water content, variation during human fetal development in phase relation and - - - $95^{8}$

cellular feedback, 2 I I

- localization of nucleic acid, 260

- orientation in wound healing, 693

- process, growth as, I40

cellulose, 750

$\alpha$-cellulose, 750

cellulose micelles, 796

cell-wall formation, suppression by caffeine and theophylline, 944

- initial in plant cell division, 768

-, primary and secondary plant -, $75^{\circ}$

centrifugation, effects on amphibian embryos, 4I 3

centriole, 313

centro-Golgi complex, 3 I 5

centrosomes, interference of ribonuclease with - and spindle development, 283

Cepaea vindobonensis, 184

cephalic mesoblast, $37 \mathrm{I}$

- placodes, 449

cephalization, $25^{\circ}$

cephalo-caudal, hierarchy of the organs, $35^{\circ}$

- stretching in gastrulation, 324

cephalogenesis, 397

Cercidiphyllium, 8 1 2

cervical cancer induced by estrogenic

hormone in mice, 837

cessation of growth, 73 I

- - degenerative changes brought

about by, 957

- of migration, 680

Chaetopterus, 332, 336

chalaziae, torsion of the, 324

chela-reversal during regeneration of certain Crustacea, 600

chemical, allometry, 232

-, balance of organs and of - constituents, 214

-, changes in the - composition of the animal body during development, 232

- groundplan, 232

- growth, 730

chemodifferentiation of cells, 727

chemotrypsin, 434

chicken tumor viruses, cross immunization against, 857

$\mathrm{C}_{5} 8$ high leukemia mice, 855

Chilopods, 315

chimpanzee, development of the - in

comparison with man, 249

Chironomus, 185

chitin, $75^{\circ}$

2-chloradenine as antimitotic agent, 933

chloramphenicol, inhibitor of incorporation of glycine- $2-{ }^{14} \mathrm{C}$ into proteins in Ehrlich carcinoma cells, 908

Chlorella, 90

chloride decrease in human development, 959

chloroma extract, crystalline protoporphyrin from, 915

$p$-chloromercuribenzoate, 783

p-chloromercuribenzoic acid as plant growth inhibitor, 783

chlorophyl density, 80 I

chloroplasts, 32

-, differentiated - in sub-epidermal cells of cork cambium, 767

—, isolated, 799

- phosphorylation system, 33

-, photolysis of water by, $3^{2}$ 
chloroplasts, photosynthesis by, 33

6-chloropurine inhibiting mitosis in cultures of mouse sarcoma 18o, 933

$\Delta^{7}$-cholestenol, concentration of - in mouse skin reduced by carcinogenic hydrocarbons, 88 I

cholestenone, 12

cholesterol, structure, i io

- synthesis, 26

cholic acid, activation, 40

choline biosynthesis from serine and ethanolamine, io6

- , effect of - chloride on regeneration, 631

- formation, I 06

- kinase, $3^{8}$

-, oxidation, 21

- oxidation by rat liver mitochondria, 2 I cholinesterase, $525,533,62$ I

cholyl-CoA, condensation with taurine to taurocholic acid, $4^{\circ}$

chondroblasts, 693

chondrogenesis, 453

-, axial, 462

chondrogenic influence of chick notochord, $46 \mathrm{I}$

chondroitin sulfuric acid, 534

chorda, 342

chordal islets in the neuroepithelium, $4^{1} 3$, 477

chordalization of neuroepithelium as result of centrifugation of frog blastula, 4 I 4

chordamesoderm, 538

Chordates, 468

chordencephalon, 367

chordomesoblast, 319

- graft, 385

-, inductive action, $39^{\mathrm{I}}$

-, selective induction of, 445

chordomesoderm, explanted, 29 I

chorion, 322

-, zona striata in the, $3 \mathrm{I} 4$

chorionallantoic membrane of chick embryo, injection of ethionine into the, 503

choroid, formation of the, 452

chromatin, RNA in, 264

Chromobacterium, $44^{2}$

chromophobic pituitary tumors, 835

chromosomal abnormalities, aspartic acid producing, 937

- agglutination caused by toxic materials, 938

- bridging and breaking in cultures of mouse sarcoma 180 by fluorphenylalanines, 938

chromosomal erosion caused by adenine

and maleic hydrazide, $94^{\circ}$

- histone, 927

- proteins, modifications in, 518

- reduplication, concepts of the

mechanism of, 9 I 3

chromosomes, 307

-, adenine causing structural - changes, 939

-, breakage by aminopterin,

a-methopterin, aminoamfol, 939

— - by 2,6-diamino-9- $\beta$-D-

ribofuranosylpurine, $94^{\mathrm{I}}$

- - by 8-ethoxycaffeine and X-rays, 940

—, poisoning of, 937

-, polytene giant, $15^{8}$

- reduplication and genetic activity, 260

—, "stickness" in, 937

chromotrope granules as mobilisers of metabolic resources, $34^{\circ}$

_ - relationship of the _ _ of the egg to initiation of development and beginning of cleavage, $34^{\circ}$

chymotrypsin, 478

- as inhibitor of induction, 290

cicatrisation, index of, 604

Ciliata 59 I

cinnamic acid, 764

cirrhosis, correlation of - with hepatic carcinogenesis in man, 866

cirrhotogenic agents, 866

citrate as intermediate of Krebs cycle, 680 citric acid, 572

- cycle, see tricarboxylic acid cycle

— - in liver tumor, 889

citrovorum factor, 93

- - 2,6-diaminopurine blocking conversion of folic acid to, 933

citrulline, 4 I

Cladocera, 966

clasmatocytes, 688

Clavelina, 604, 628

cleavage and morphochoretic pattern, 325

- and nitrogen metabolism, 7I6, 721

cleidoic eggs, nitrogen metabolism in, 738

- embryo, 7 I 5

clonal lines of cells, 549

closed system, I 44

Clostridium enzymes, $5^{8}$

Clostridia, heterotrophe, 8 I

Clostridium HF, I2 I

Clostridium Kluyveri, I I

Clupea harengus, growth curve of, 955

coadaptation, 245

coal tar as tumor-inducing agent, 858 
cobalamine, 73

cobalt, as plant growth promoting factor, 782

- salts, 626

cocarcinogen, 858

codeine, 12

Coelenterata, 590

coenocytes, 749

coenzyme A, 40

- content of mammary adenocarcinoma, 9 I 5

- as inhibitor of mitosis in sarcoma 180 cells, 934

- as antagonist of lipogenesis, 934

—, formation of - from pantothenate, I 8

- synthesis, I 17

-, functions of - in the tricarboxylic acid cycle, 934

coenzyme factor, 19

coenzyme, pyridoxal-5-phosphate as, 762

—, role of auxin as, 797

-, vitamin A as, 52 I

coenzymes, B-vitamins as precursors of metabolically active,- 555

-, folic acid derivatives as - in formimino group transfers, 57

-, folic acid as precursor of - of onecarbon transfers, 929

一, $Q_{6}-Q_{10}, 123$

-, synthesis, 37

cofactors of traumatic acid, 772

$\mathrm{CO}$ inhibiting plant cell elongation, 786

$\mathrm{CO}_{2}$ fixation, $4 \mathrm{I}$

$\mathrm{CO}_{2}$ formation, 8

$\mathrm{CO}_{2}$ respiration in tumor tissue, 885

collagen, 5 I 8

- formation, role of fibroblasts in, 693

-, relationship between mucopolysaccharides and - content of wounds, 697

- synthesis, 499

colchicine, 165,913

- in low dosage accelerating regeneration, 623

- as spindle poison, 94I

cold, extra-liminal, 4 I 6

Coleoptera, 598

coleoptile, 750

Coleps, 604

Coleus, 8 1 3

coleukemogen, urethane as, 863

compensatory growth, 210

- hyperplasia, 2 Iо

compensation, 246

complementary induction, 398

compound $\mathrm{X}, 99$

compound $Z_{1}, 65$ coaptation theory of Weiss, $68 \mathrm{r}$

conditional neoplastic growth, 825

$\mathrm{CO}-\mathrm{NH}$ bond, bacterial synthesis of, 728

conjugates, intermediate, 507

connective tissue, as contractile mechanism in wound closing, 670

-, hyaluronic acid in - 695

constriction zones between the five basic

brainparts, 408

constructive genes, 246

contact-inhibition, positive, $55^{\circ}$

Contarinia, 243

continuing growth in snakes, 956

continuing increase in fertility, 956

contracting phase of wound healing, 693

contracture, Dupuytren's, 683

- in wounds, $66 \mathrm{~g}$

control of tumorigenesis by target tissues, 866

Coppepoda, 590

copper, content of methylcholanthrene induced carcinoma, 915

- as prosthetic group in ascorbic acid oxidases in plants, 25

cork cambium, 767

cormogenesis, 368

cormomerit, 368

cormoneuron, 368

corpus allatum, 635

cortex, stabilizing influence of the, 3 I 8

- of unfertilized egg, 316

cortical layer, 3 I 6

corticosterone, 12

cortisol, 12

cortisone, I 2, 2 I 9, 499

- induced regression of canine mast cell tumor, 844

- skin cancer in mice, 844

- influence on metastasis, 844

Corynebacterium, 442

- diphtheriae, 52

coumarin as seed germination

inhibitor, 785

countershock phase of the GAS, 643

cover all concepts, I 7 I

cranial ganglia, 404

cranio-ventral ectoblast, presumptive, 390

creatine, 33

- formation, 80

- kinase, 33

- phosphate, guanidine phosphate bond of, 37

- - as stored energy source for mitosis, 680

- synthesis, guanidoacetic acid

intermediate in, 80 
creatine synthesis and transimidation, 80

Crepis capillaris, $9 \mathbf{I} 3$

Criodrillus, 628, 644

crista, $45^{\mathrm{I}}$

Crocker mouse sarcoma, 55 I

crotonase, 7

crotoniside (2-hydroxyadenosine) as mitose

inhibitor, 935

croton oil as cocarcinogen, 858

crotonyl-CoA, I I, 30

cross immunization against chicken

tumor viruses, 857

crown gall, 771

- , ionizing radiation reducing growth of, 774

Crustacea, 590

Ctenophora, 243

${ }^{14} \mathrm{C}$ in tumor proteins, rate of increase of, 897

cupro-oxidase, 25

cutaneous tumors, 840

cyanide inactivating enzymes containing $\mathrm{Cu}, \mathrm{Mn}$ and $\mathrm{Fe}, 786$

- inhibiting growth and respiration in plant cells, 786

cyclosis, 75I, 797

cyclic compounds, oxidation by enzymes, Io

cyclohexaneacetic acid, 765

cyclophorase, 15 I

Cyclops egg, 94I

Cyprinus, 185, 200

cystathionine, 63,73

- formed by condensation of homoserine with cysteine, 73

cysteine, 12, 63

- bridges as structural components of synthesized keratin, 6 I 5

-, conversion of sulfate to -, 63

-, cystathionine forming by condensation of homoserine with - 73

- deposition rate in healing wounds, $70 \mathrm{I}$

-, metabolism of — sulfinic acid, 64

-, methionine as source of - 6 I 6

-, reduction of - sulfinic acid to cysteine, 64

- as reductor for neuralization of ventral explants, 426

- -S-sulfonate as intermediate in cysteine synthesis, 83

- synthesis, methionine as sulfur source for $-\ldots, 63$

cytidine, conversion to the deoxycytidine and thymidine of DNA, 105

-, deamination, Ioo

- diphosphocholine as coenzyme, ro7 cytidine diphosphoethanolamine as coenzyme, 107

- phosphorylase activity, I I I

- phosphorylation, 102

- triphosphate formation, 83

cytidylic acid inhibiting mitosis in germinal epithelium of rat ovaries, 936

cytochrome $\mathrm{a}_{3}, \mathrm{~b}, \mathrm{c}$, e and $\mathrm{m}, \mathrm{I} 7$

- c, 634

- $\mathrm{c}$ and cytochrome oxidase deficiency in tumor tissues, 884

- $\mathrm{m}, 26$

— oxidase, 513,786

- - activity, 20, 187

- c-reductase, I 7

- m-reductase, 26

- reductase, 527

- reductase activity, 20

- system, I6

cytodifferentiation, 320

cytokinesis, 536

- in plant cells, 774

cytolytic, agents, 422

- demolition of cells and intercellular material, 595

cytolizing cells, liberation of ammonia from, 736

cytological, mechanism of nitrogen metabolism, 729

- stages of functional activity, exhaustion and recovery of the nerve cell, 964

cyton, 603

cytoplasm, amputation of, 726

- of the gametes, 306

-, interaction between nucleus and, $15^{8}$

cytoplasmic bridges, 354

- displacements in the egg, 354

- granules of fibrogenic cells, 694

- organelles, $34^{\mathrm{r}}$

- particles, distribution of, 289

- reticulum, 307

- streaming in plant cells, 797

cytosine, $26 \mathrm{I}$

-, amination of uracil compounds to, 100

- riboside biosynthesis, block in, 912

- in RNA from epithelial rat tumors, 902

—, utilization, 99

Dandelion, 756

Daphnia, carapace of, 604

Daphnia pulex, 185

dealkylating enzymes, 12

deaminating enzymes, 12

deamination of cytidine, 100

-, direct oxidative, 762 
decapeptide, 728

decarboxylation of diaminopimelic acid to lysine, 77

decaying exponential function for growth curves, I 75

$\Delta^{2}$-decene-1, 10-dicarboxylic acid, 772

decrease of extracellular fluid in senescence, $95^{8}$

- in sodium and in chloride during

human development, 959

dedifferentiation, $562,589,665$

- - epidermis necessary to induce, 646

—, initial process of, 589

deficiency of cytochrome c and cytochrome oxydase in tumor tissue, 884

degeneration, harmonization between protein synthesis and, 166

degenerative cell changes in senescence, 965

- changes brought about by cessation of growth, 957

degradative processes, I 79

Degusia, 625

dehydroascorbic acid, plant enzymes catalyzing reduction of - to reduced glutathione, 25

- - , reduction of - to vitamin $\mathrm{C}, 6 \mathrm{I} 7$

- reductase, 25

$\Delta$-7-dehydrocholesterol, i io

dehydrogenase, enzymes of the hexose monophosphate shunt, 47

-, hemiacetal, i I

- leading to unsaturation, II

- reaction, glyceraldehyde phosphate, 27

- of the soluble cytoplasm in the energy economy of the cell, 29

5 -dehydroquinic acid, 10

dehydroshikimic acid, 65

5-dehydroshikimic acid, Io

delayed inductions, 454

deoxycholyl-CoA, 40

deoxycorticosterone (DOCS) promoting proliferation of connective tissue-cells, 63 I deoxycytidine, 9 I

- deaminase, Ior

-, deoxyuridine as intermediate in conversion to thymidine, 104

- diphosphate, 35

deoxycytidylic acid, $9^{\text {I }}$

deoxypentose, 288

deoxyribonuclease, 6 I I

deoxyribonucleic acid, 26o, 509, 6 I 2

deoxyribonucleohistone from transplantable mouse lymphosarcoma, 906

deoxyribonucleoprotein, enzymatic depolymerization of - by tumor cells, 9I I deoxyribonucleoprotein, phosphorus measurements in growing tissue cultures, 573 deoxyribonucleoside metabolism and

DNA-thymine synthesis, 104

$d$-deoxyribose, $26 \mathrm{I}$

deoxyribose, aldolase, 52

-, de novo synthesis of, 53

—, ribose as - precursor, 52

- synthesis from two and three carbon

compounds, $5^{2}$

deoxyriboside nucleotides, 9 I

deoxyribosides, formation of - by trans $-\mathrm{N}$ glycosidation, IOI

deoxyuridine, 91

- in rat liver, 903

dephospho-CoA, 35

dephospho-CoA kinase, 118

dephosphorylation, contractile proteins coupled with energetic system

responsible for, $34^{\mathrm{I}}$

depolymerization, of DNA by X-radiation, 272

-, enzymatic - of deoxyribonucleoproteins by tumor cells, 9 I I

depot material, $13^{8}$

dermomyotome, 503

Dero, $59^{2}$

desaminase enzymes, 97

dessication, partial - of the protoplasma, $95^{8}$

desthiobiotin, I I 4

determinants, 172

deuterencephalon, 367

deutomerit, 368

- territory of young neurulae, 390

deutogenesis, 368

deutoplasm, 313

devascularisation, 644

developing cells, protein forming system of, 495

developmental inductors, 445

- kinetics, 34 ${ }^{\mathrm{I}}$

development, changes in the chemical composition of the body during -, 232

-, effects of abnormal nuclear composition on -, 292

—, energy sources of - -734

-, nitrogen metabolism in relation to -, 716

-, urea and uric acid formation during $73^{8}$

DHO, 99

diabetes, growth and development of neoplasms, 847

diabetic animals, aerobic formation of lactate in liver of -, 49 
diacetylmorphine, 12

diamine, 13

diaminopimelic acid, decarboxylation of to lysine, 77

- as lysine precursor in Escherichia coli, 76

- pathway of Escherichia coli, 77

- pathway of synthesis, 2

2,6-diaminopurine as antimetabolite, 806

- inhibiting enzyme formation, $93^{2}$

- inhibiting formation of bacterial enzymes, 928

- lactate as chromosome bridging and chromosome breaking agent, 939

- as precursor of nucleic acid purines, 932

2,6-diamino-9- $\beta$-D-ribofuranosylpurine inhibiting mitosis, 933

diaphorase activity, 19

—, Straub's, 20

I, 2,5,6-dibenzanthracene-endosuccinate modifying induction process, 425

dicarboxylic acids, synthesis of - from

pyruvate and phosphoenolpyruvate, 8

- fatty acid as wound factor in plants, 620

2,4-dichlorophenoxyacetic acid $(2,4$-D), 763,802

3,5-dichlorophenoxyacetic acid, 802

2,6-dichlorophenolindophenol, 2 I

$x$-(2,4-dichlorophenoxy)-isobutyric acid, 802

dicotyledons, 799

dicumarol as depressor of aerobic phosphorylation, 3 I

- inhibiting cytochrome $\mathrm{c}$ reduction, I9

- - menadione reductase, 23

2,4-D (2,4-dichlorophenoxyacetic acid), 763,802

diencephalon, 393

diethylstilbestrol, 892

differential growth, 730

differentiated cells, protein forming system of, 507

differentiation, 137, 139

-, antagonism between proliferation and -, 2 I I

- feedback term, 209

- , irreversibility of, 534

-, progressive, I 4 I

-, relationship of - and proliferation in early embryogenesis, 524

- visible - in a regenerate, 600

diffusing factors in regeneration, 645

diffusion as regulating factor in anabolic processes, 216

- - thermo effect, 153 7,8-dihydrofolic acid, II

dihydroorotic acid, I I

- - conversion of ureidosuccinic acid to - - 99

- dehydrogenase, 99

dihydrothymine, I I

dihydroxyacetone, active, 49

- phosphate, Io

- - condensation with glycolaldehyde

phosphate to xylulose diphosphate, 49

dihydrouracil, I I, I 8

dilution mechanism in regeneration, 600

2,3-dimercaptopropanol (BAL), I9, 785

2,3-dimethoxy-5-methylbenzoquinone

compounds, 123

4-dimethylaminoazobenzene, 895

- as hepatic carcinogen, 86 I

9,10-dimethyl-I, 2-benzanthracene as

carcinogen, 845

I,7-dimethylfluorene, 765

dimethylglycine, 74

2,4-dimethylphenoxyacetic acid, 802

$\alpha$-(2,4-dimethylphenoxy)-isobutyric acid, 802

dimorphism, sexual, I99

dinitrocresol stimulating anaerobic glycolysis in ascites tumor cells, 886

2,4-dinitrophenol (DNP), 785

dinitrophenol action, 32

- as antithyroid substance, 633

- inhibiting root elongation, 806

- as metabolic poison, 286

- uncoupling phosphorylation, 30

Diodon, 239

dipeptidase, 289

- and cathepsin activities in central areas of the tumor, $9^{1} 3$

dipeptides formation, $15 \mathrm{I}$

I,3-diphenylurea as active compound of coconut milk, 772, 773

I,3-diphosphoglycerate, 16

1,3-diphosphoglyceric acid, I I

diphosphopyridine, -AMP ratio in ascites tumors and solid tumors, 904

- nucleotide $\left(\mathrm{DPN}^{+}\right), 9$

— nucleotides, 73 I

disaggregating agents, 422

Discoglossus, 347, 454

- gastrula, 380

- pictus, 379

dismutation reactions and pyridine nucleotide dehydrogenases, I 5

Distaplia, 524

distribution coefficient, 227

division, physical prerequisites of, 163

Dixippus, $59^{8}$ 
Dixippus morosus, $183, \mathbf{1} 85$

DNA, 260, 509, 612

-, bacterial transformations by, 268

- content in gastrula of Rana pipiens, $3^{62}$

— - of lymphoma, 905

— - in nuclei, 157

- - of tumor cells, 157

- depolymerization by nitrogen mustard, 27 I

- depolymerization of - by X-radiation, 272

-, distribution of newly synthesized — in mitotic division, 913

-, doubling of the amount of - immediately after telophase, 340

-, Feulgen reaction for, 262

-, genetic activity of, 268

- gradient in epithelium, 687

- and growth, 265

-, metabolic stability of, 266

- in methylcholanthren induced mouse cancer, human epidermoid carcinoma and hyperplastic lesions, 687

- as organizer for RNA synthesis, 278

- in plant cell nuclei caused by auxin or kinetin, 774

- potassium ration in azo dye induced hepatomas, 905

- precursor, 340

- precursors in unfertilized eggs, 288

-, purine and pyrimidine composition of of cancerous tissues, 902

-, relationships between hereditary pituitary dwarfism and formation of multiple - classes, 912

- or RNA synthesis, relation between respiratory balance and, 888

- and RNA in tumor cell nuclei, 904

- as self-duplicating mechanism, 262

-, specific activity of - and RNA nucleotides in tumors, 909

- structure, Watson and Crick's, 26 I

- synthesis, rapid _ - in late blastula stage, 287

- - in blocked embryos, 293

- - dissociation between - - and development, 293

- - effects of physical and chemical agents, $27 \mathrm{I}$

- - immediate inhibition of - - by ultraviolet light, 294

— - independence of _ — - and protein synthesis, 293

— - during mitosis, 269

- - in first part of interphase, 270
DNA, synthesis of - and RNA during development, 285

-, ultraviolet radiation modifications, 271

$\mathrm{DNA}_{1}: \mathrm{DNA}_{2}$ ration in tumors, 906

DNAP, 576

DNase, 295

DNA-T (DNA-thymine), 103

- synthesis, mechanism, 103

- - , deoxyribonucleoside metabolism and - - 104

DNP (2,4-dinitrophenol), 785

DOC (deoxycortisone), 12

DOCS (deoxycorticosterone), 63 I

Donaldson's tables, 235

DOPA, 25

- activity of human melanomas, 9 I4

dorsal convergence, 342

dorsalization exerted by ammonium on the ectoblast, 422

dorso-ventral hierarchy of the organs, 350

DPN-cytochrome $c$ reductase in tumors, 885

-, tumor requirement for additional 893

-, quinone mediated oxidation of, 24

$\mathrm{DPN}^{+}$(diphosphopyridine nucleotide) 9 , I I 5

- DPNH, ratios in tissues, 18

- _ in neoplastic tissues, 894

- mechanism of oxidation of - by lactic acid, I 4

- or $\mathrm{TPN}^{+}$reduction by plant

chloroplasts, 33

- in tumors, oxidized and reduced, 884

DPNase and ATPase activity in tumor mitochondria, 895

DPNH, I 2

DPNH-cytochrome c reductase, I9

$\mathrm{DPNH}_{2}$, oxidizing enzyme system, 20

-., reoxidation, 19,20

$\mathrm{DPNH}_{2}$ or $\mathrm{TPNH}_{2}$ reoxidation by plant mitochondria, 33

Dreissensia polymorpha, 184

Drosophila, 27 I, 315, 499, 515, 734, 848, 863

- larvae, salivary glands of, 273

Dubois' cephalization factor, $25 \mathrm{I}$

Dubois formula, I 8 I

duck virus, 858

ductus endolymphaticus, 395

Dufour effect, I 53

Dugesia gonocephala, 184

Dupuytren's contracture, 683

duration of life, 953

dyad, 328

dynamic state of the organism, I 47 
ear-duct tumors induced by carcinogenic amino compounds, 862

Earle's cancer cells, 883

ecdysis, 608

Echinodermata, 590

echinoid rudiments, 320

eclosion, 599

- size, 608

- time, 608

ectoblast, 3 I 9

-, activation, $39^{1}$

-, autonomous neuralization of - 479

-, differentiation of - into neuroblasts or pigmentocytes or myoblasts, 408

-, dorsalization exerted by ammonium on the - 422

- induction of - in epiblast, 460

- influence of age on the reacting ability of the - $4^{\text {I I }}$

-, maintenance of competence of isolated -, 4 II

-, realization of a dorsal — fold, 388

-, structure of - and neurectoblast in normal Pleurodeles embryo, 359

-, presumptive cranioventral, 390

-, young indifferent, 426

ectoderm, preinduced, 533

ectogenesis, 247

ectomesenchyme, chemical processes of differentiation, 455

-, pigmented, 454

ectomesoblast, 389

ectoplacental trophoblast, 463

ectoplasmic layer, 316

ectophyll, 3 I 9

--, gradient field of RNA in the, 476

EDTA (ethylenediaminetetracetic acid), $79^{6}$

egg cell, polarization of, 3 I I

eggs acting as facultative anaerobes, 927

Ehrlich, protein formation in - carcinoma cells, 887

-, taurine, alanine and triiodothyronine in - mouse ascites carcinoma, 896

- tumor cells, 25, 97, 104

Eisenia, 6 I 3

- foetida, 184

Einsteckungsmethode, Mangold's, 367

electrical forces in regeneration, 624

-, quantitative changes in an intrinsic gradient of - potential, 600

electrolyte, content of human tumors, 9 I 5

electron transfer via pyridine nucleotide transhydrogenase, 30

- transport in aerobic glycolyse in tumors, 884 electron transport chain, i8

- - disturbance in — - in cancerous cells, 887

- - particle, 19

- - phosphorylation, 29

- - system, mitochondrial, 29

_ - - terminal hydrogen and, 15

— - in tumor cells, 892,893

electropositivity change in regeneration, 608

elongation at base of meristem, primary, 766

- of roots, physiology, 806

Embden-Meyerhof glycolytic sequence, 3

- pathway of glucose oxidation, 49

- scheme for phosphorylative glycolysis, 884

embryo, cleidoic, 715

-, enzymatic maturation, $73^{\circ}$

- extracts promoting wound-healing, 637

—, haploid, 734

- juice action on wound healing, 667

--, organismic nature, $7 \mathbf{I} 5$

-, protein utilization in pregastrula, 736

- structural and enzymatic - protein synthesis as anabolic process of nitrogen metabolism, 715

-, transfer of large molecules to the, 719

embryogenesis, relationship of proliferation and differentiation in early -, 524

embryonic area, 347

- cells, protein forming system of, 507

_- , qualitative aspects of protein formation in, 522

- development, nucleic acids and, 285

-, effect of - extract on migratory activity, 567

- fields, 354

- growth, 223

- - of the cerebral cortex of the guinea pig, 961

-, permeability of - cells to large molecules, 497

embryonin, 554, 667

encystment, 589

endoblast, 3 19, 347

endocrine relationships involved in adrenocortical tumorigenesis, 833

- tumors following imbalance induced by estrogenic hormone, 83 I

endoderm organizing action in regeneration, 646

endogenous mitotic poisons, 946

endo-mesoblast, foreward migration of the prechordal - 473

endomitosis, 158,266 
endophyll, 319

- layer, $34^{2}$

-, migrating, 326

endoplasmic reticulum, 344, 53 I

endothelial cells in granulation tissue, 689

energy intake, 194

- output, 195

- requirements, of the maintenance of the steady state of the organism, 151

— - of protein synthesis, 149

- stored in tissues, 194

- sources of mitosis, I59

— - of development, 734

enolase, 786

enoyl-hydrase enzymes, 7

Enriques' equation, I 74

enteron, 339

enteropneust, 327

entropy, 143

- function of Prigogine, 152

- and the living world, I 55

- in open systems, 152

Entwicklungsmechanik, $45^{\circ}$

enzymatic, depolymerization of deoxyribonucleoproteins by tumor cells, 9 I I

- derangement, 869

- incorporation of labelled amino acids into proteins of liver homogenates, 44

- maturation of the embryo, 730

enzyme, and enzyme co-factors activities in neoplastic tissues, 9 I 3

— forming system (EFS), 907

- - gene complex, 868

— induction in bacteria, kinetics of, 532

$--\mathrm{P}, 8_{3}$

- preparations reducing nitrate, 23

- poisons inhibiting cell enlargement, 782

-, Schardinger, $76 \mathrm{I}$

- synthesis, by reticulocytes, 296

- - induced, 285, 294

- systems, catalyzing synthesis of polynucleotides, 9 I I

- - of the heart, 109

- - inhibited by methylxanthine, 930

enzymes, dealkylating, 12

-, deaminating, 12, 97

-, delation hypothesis of Potter, 861

-, epithelial fibrinolytic -, 676

-, formation of adaptive -, 7 I 4

- , formation of - and proteins in plant cells, 790

-, hydroxylating, 12

- of kidney, 103

-, kinase, 97

-, localization of - within cells, 25 enzymes, lytic, 6 I I

-, muscle, I03

—, mutase, 96

- oxidizing, alcohols, Io

- cyclic compounds, Io

- hydroxyacids, Io

- of photosynthetic phosphorylation, 33

- of Proteus vulgaris, 64

- of reductive carboxylation, 33

- -, synthesis of induced - 275

-, thimidine phosphorylase -, 100

Eohippus, 239

Eotitanops, 239

ephedrine in regeneration, 643

Ephestia, 499

Ephydatia, 591, 604

epiblast, 319

-, action of retinal layer on the - $45^{\circ}$

- derivatives, 459

- induction of ectoblast in - 460

- , induction of the olfactory pits from the

- 37 I

-, stomodoeal, 454

epiboly, 342

epicotyls, $75^{I}$

epidermal edge, retraction, 675

-, emigration of the - sheet in wound healing, 679

- epithelium, keratinization, 564

epidermis necessary to induce dedifferentiation, 646

-, way-back induction by, 460

epidermoid, human - carcinoma, 687

- tumors, 914

epigenesis, 322,356

一, biochemical, 349

-, organismic, 742

-, trophic, 453

epimorphic component, 6or

- healing, 664

epimorphosis, 588

-, disseminated - of local blastemata, 589

epimorphysis, 664

epinephrine in regeneration, 643

epithelial cells, basal, I63

- pearls, keratinizing, 674

epithelialization, 664, 674

-, chemical changes associated with, 683

—, role of mitosis in, 679

epithelioma, ${ }^{32} \mathrm{P}$ incorporation into DNA and RNA of -, 910

epithelium, DNA gradient in, 687

- growth curve, 677

-, RNA gradient in, 686

-, migration of - over the wound surface, 675 
Epiwocklumeria applanata, 215

equatorial translocation in blastula or gastrula, $38 \mathrm{I}$

equifinality, 146,162

- as general characteristic of open systems, 213

- of growth, 213

equilibrium, I 44

- of the organs, 229

Equus caballus, 240

ergastoplasm, 158

— of secretory cells, 307

ergastoplastic structures, 307

ergosterol, I I O

erythroblasts, differentiation of the, $35^{2}$

erythrocyte enzymes, 90

erythrocyte, polyochromatic, 528

erythrocytes, ingestion of - by macrophage cells, 69 I

D-erythroimidazoleglycerol phosphate, 57

erythrose phosphate, 5

L-erythrulose, Io

eschar, 676

Escherichia coli, 271, 294, 442, 510, 53 I, 9 I I

-, diaminopimelic pathway of, 77

- enzyme catalizing reduction of dehydroshikimic acid to shikimic acid, 65

- extract dehydrating 5 -dehydroquinic acid to 5-dehydroshikimic acid, 65

-, histidineless mutant of, 57

-, hydrogen transport system of, 23

- mutant extract converting fructose-6phosphate to dehydroshikimic acid, 67

_ - phenylalanineless, 67

-, serineless mutant of, 6 I

- -, synthesis of $\beta$-galactosidase in uracil requiring mutants of - 928

—, T phages of, 276

-, thymine requiring mutant of, 294

-, tryptophaneless mutant of, 67

Esox, 200

esterases, non-specific, in skin, 687

estradiol, 12

estrogen inducing, cervical cancer in mice, 837

- neoplasms in "non-target" tissues, 837

-, leukogenic activity of, 837

-, neoplasms of tissues whose normal growth is stimulated by - 835

estrogenic hormone inducing hormonal imbalance causing endocrine tumors, 831

—_ — thymus neoplasia, 839

- stimulation, appearance of pituitary adenomas secondary to $\_-, 83$ I
ETF (electron transferrring flavoprotein), 13, 2 I

$\mathrm{ETFH}_{2}, \mathrm{I} 3$

ethanol, 16, 424

ethanolamine, formation, Io6

- in transmethylase reactions, 75

- kinase, $3^{8}$

ethionine, 502

- inhibiting protein synthesis, 937

-, vegetalizing effect, 505

8-ethoxycaffeine as antimitotic agent, 930

ethylenediaminetetracetic acid (EDTA), 796

ethyleneimines inhibiting carbohydrate metabolism in ascites tumor cells, 886 ethylindoleacetate, 763

Eucalyptus trees, 749

euchromatin, 267

Eudendrium, 622

Euglena, 797

Eugleninae, $75^{\circ}$

Euglenophyta, $75^{\circ}$

euglobin, a, b, c, 720

eutheria egg, 719

evolution, 215

- of the brain, $25^{\circ}$

-, growth and, 238

- of inductive processes, 468

evolutionary allometry, 228,240

- - , mathematical formulations, 25I

- constraint, 247

- progress, 141

excessive formations, 246

excretion of ammonia and urea in

amphybian embryo, 74I

excretory efficiency in eggs, $74^{\circ}$

exhaustion, phase of - of GAS, 6 Io

exosmosis, 779

explantations, $35^{8}$

explantation of Urodele dorso-marginal material, 397

exponential functions for growth curves, I 75

extracellular covering layer, $34^{\mathrm{I}}$

- fluid in senescence, decrease of, $95^{8}$

extra-liminal temperatures, $4_{1} 6$

extrinsic morphochoresis, 319

- _, neurogenic induction as main aspect of - - 357

FAD I 7, II 4

- cytochrome c-reductase, I 7

- xanthine oxidase, 17

- - reductase, I 7

$\mathrm{FADH}_{2}, \mathbf{I} 3$ 
false start, 146

fasting, 216

fat, conversion of - to carbohydrate by plants, 123

fat-mobilisation caused by starvation, 589

fat-sugar conversion in plant cells, 790

fatty acids, activation, 40

-, biosynthesis in tumors, 917

-, catabolism, 3

-, oxidation, 2 I

- oxidation reactions localized in mitochondria, 29

- - inhibition of - - in tumor tissues, 895

- spiral, 6

fatty degeneration of secreting parenchyma of parotid gland in senescence, 964

feebleness in senescence, increasing, 957

feedback, cellular, 2 I I

- mechanism, negative, I22

— term, differentiation, 209

- - generative, 209

feeder cells, immobilized, 553

female pronucleus, 287

fermentation, 150

- excess as criterion of malignancy, 882

ferroflavoprotein enzyme, 2 I

fertilization and drop of RNA content, 286

- and morphochoretic pattern, $32 \mathrm{I}$

- and nitrogen metabolism, 7 I 7

- of the plant ovule, 807

fertilized egg, field-gradient system of the, 347

fertilizins, 322, 717

fetalization, 249

Feulgen reaction, 687

_ - for DNA, 262

FGAM ( $\alpha-\mathrm{N}$ formylglycinamidine ribotide), 85

-, as intermediate in conversion of FGAR to AIR, 94

FGAR (formylglycinamide ribotide), synthesis of FGAM from - 85

fibrinolytic enzyme, epithelial, 676

fibroblast in collagen formation, role of, 693

- culture, pyocanin decreasing mitosis in, 886

- migration into wound area, 69I

fibroblastic proliferation, $69 \mathrm{I}$

fibroblasts in granulation tissue, 689

-, malignant, 565

- as source of mucopolysaccharides, 700

fibrocytes, migration of, 692

fibrogenesis, 675 fibrinogen, polymerization of - molecules by thrombin, 691

fibrogenic cells, 693

fibroplasia, 697

fibrosarcomas, in mice induced by inoculation of mouse tissue extracts, 855

-, ${ }^{91} \mathrm{Y}$ induced, 847

ficin, 728

Flagellates, kinetosome system of, 641

flavin adeninedinucleotide, as coenzyme, I 14

- - content of adenocarcinoma, 915

flavin enzymes, 26

- mononucleotide, I4, II 4

flavoproteins, autooxidizable, 2 I

-, metal, 536

Flexner-Jobling carcinoma, 889

Fliessgleichgewicht, I 44

fluoracetate, inhibiting oxidation in plant cells, 786

fluorocitrate, 786

fluorphenyl alanines, inhibiting protein synthesis, 937

$\beta$ - $(p$-fluorphenyl $)$-DL-alanine, as metaphase blocking agent, 945

— as mitose suppressor, 937

fluxes, I 53

flying coverslips, 549

FMN, I 9, I I 4

$\mathrm{FMNH}_{2}, 23$

folic acid, 74

- compounds, II9

- conversion to citrovorum factor blocked by 2,6-diaminopurine, 933

- derivatives as coenzymes in formimino group transfers, 57

- polyglutamates as catalyzers, 59

- as precursor of coenzymes of one-carbon transfers, 929

Foraminifera, 2 I 6

fore-brain, secondary, 386

foreign tissues, as xeno-inductors, fresh, 427

_ — as xeno-inductors, killed, 428

formaldehyde, I I

formalin reducing proteolytic enzymes activity by combining with amino groups, 422

formation rate of new cells in tissue cultures, $57 \mathrm{I}$

formative movements in growth, I 40

formic acid, II

formiminoaspartic acid, I 2

formiminoglycine intermediate in purine degradation, $5^{8}$

formimino, folic acid derivatives as coenzymes in - group transfer reactions, 57 
formylase, is 5

formylglycineamidine ribotide, 85

formylkynurenine, I 16

$\mathrm{N} 10$-formyl-tetrahydrofolic acid, Io

- as active formyl group donor, 59

Fourier's law, I 53

Fraxinus americana, cambium, 752

free amino acids pattern in Yoshida ascites tumor, 896

free amino nitrogen in extract of tumor tissue, 896

frog hybrids, androgenetic, 734

Frontonia, 216

fructose-6-phosphate, 4,5

fructosylamine-6-phosphate isomerizing to glucosamine-6-phosphate, 83

fruits, growth, 807

fruiting bodies, 749

fumarate as intermediate of Krebs cycle, 680

fumaric acid, 13

functions for growth curves, I 75

Fundulus, 453

- embryos, 46 I

Fungi, 749

fungus hypha, 754

furfural, 286

6-furfurylamino purine, 773

6-furfurylamino- 9 - $\beta$-D-ribofuranosylpurine as mitose inhibitor, 936

Fusarium molyniforme, $760,765,800$

(G) acyl-CoA $\left(\mathrm{C}_{4}-\mathrm{C}_{8}\right), \mathrm{I} 3$

galactosamine, $6 \mathrm{I} 8$

galactosidase, 510,532

$\beta$-galactosidase, 275, 532

galactowaldenase, 39

galactozymase synthesis, direct nuclear

control of induced — - 294

Gallileo's law, i 70

gametes cytoplasm, 306

gametogenesis, 305

- and nitrogen metabolism, 7 I 7

GAR (glycineamide ribotide), 908, 9 I 7

Gardner lymphosarcoma, 899

- tumor, 97

garter snake, 737

GAS (general adaption syndrome), 609

gastrula, phosphor balance of

amphibian -, 349

gastrular, morphochoresis, 387

-, specific - ectoderm antigen in Triturus alpastris, 727

gastrulation, I 40, 288

-, cephalo caudal stretching in, 324

- , as initial stage of new protein synthesis, 286 gastrulation and nitrogen metabolism, 722

-, synthesis of antigenic material prior to - 727

-, yolk packet as prerequisite for - 343

GDP, 908

GDP-mannose synthesis from

mannose- I-phosphate, 38

gemmules, 589

gene action, Groldschmidt's theory, 525

-, pleiotropic, 246

gene-dependent, enzyme forming system

(GDS), 907

- hormones, I4I

gene-enzyme complex, 868

gene-independent enzyme forming

system (GIS), 907

genes, constructive, 246

-, dominant histocompatibility, 865

general adaption syndrome, 609

general alarm reaction, 609

general system theory, 194

generation of energy-rich phosphate

bonds, 27

generative body mass, 208

- feedback term, 209

genetical determination of specific

capacity of organs, 228

genetic activity, chromosome

reduplication and - - 260

— - of DNA, 268

- control of growth, 733

- - of metabolism 734

- determination of susceptibility to induction of neoplasms, 867

- - of tumorigenesis in target tissue, 867

- factors in target tissues determining cancerous response, 869

-, importance of - factors in response to gonadectomy, $83 \mathrm{I}$

- mechanism related to metabolic processes, 733

- nature of development of blood antigens, 734

- relationship to growth, 733

genital ridge, 466

$\mathrm{G}$ enzyme (a cuproflavoprotein) in mitochondria, I4

geotropism, 804

germinal localizations, 356

giant cells, $5 \mathrm{I} 7$

- forms in glacial periods, 244

- nuclei in malignant cells, 159

Gibberella fujikuroi, 760

gibberellic acid, 765

gibberellins, 753

gibberellin $\mathrm{A}_{1}, 765$ 
gibberellin $\mathrm{A}_{2}, 765$

gibberellin B, 765

gibberellin control on growth of plants, 780 gibberic acid, 765

Ginkgo, 753, 81 2

globin, 528

$\alpha$-, $\beta$ - and $\gamma$-globulines and albumen in

blastocyst fluid, 720

glucocorticoids inhibiting normal

lymphopoiesis, 842

glucocorticosteroids, 2 I 9

L-gluconate, I0, I I

gluconeogenesis, 42

gluconokinase, 276

gluconolactone, I I

D-glucorono- $\gamma$-lactone, Io

glucosamine, 6 I 8

- inhibiting cell-migrations, 626

-, synthesis of the - moiety of hyaluronic acid, 87

-, transfer of amide group of glutamine in - synthesis, 87

glucosamine-6-phosphate, 85

- synthesis, 83

D-glucosamine inhibitor, of mucolyse, 6 I 8

- - of regeneration, 6 I 9

glucose, 6 r 8

- breakdown inhibition by oxidative cellular processes, 32

- catabolism, 3

- degradation via the phosphogluconate pathway, 52

- -6-P-dehydrogenase, I 5

- metabolism, direct oxidative pathway, 9, 5 I

- oxidation, Embden-Meyerhof pathway, 49

— - , phosphogluconate pathway, 49

- as principal source of energy for the cells, 555

- -6-phosphatase activity in tumors, $89 \mathrm{I}$

- -6-phosphate, 4, 5

- - activity in hepatoma, $89 \mathrm{I}$

- - dehydrogenase activity in thymus cell nuclei, 25

glucozymase, 275

glucuronidase, 596

glutamate, tumor inability to synthesize 899

glutamic acid, 78

- - in amphibian morula, 348

- —, anaerobic disappearance, 84

- - as cofactor of traumatic acid, 772

- - conversion of - - to proline, 79

- - preventing initial $\mathrm{K}$-loss in regeneration, 62 I
L-glutamic acid, HeLa cell growth response to - - and L-glutamine, $90 \mathrm{I}$

D-glutamic acid in tumor proteins, $90 \mathrm{I}$

glutamic dehydrogenase, 78,82

glutamine, 506, 728

- as amino donor in conversion of uridine nucleotides to cytidine nucleotides by rat tumor enzymes, I23

- as amino donor in transamination reactions, 85

- metabolizing by Yoshida sarcoma, 90 I

- pool in growing tumor tissues, 54

- synthesis inhibition by methionine sulfoxide, 87

- synthetase, 82

- as transaminating agent, 82

-, transfer of the amide group of,- 85

glutaminic acid in amphibian gastrula, $34^{8}$

glutamohydroxamate, 83

glutamotransferase, 506, 728

glutamylcysteine, 47

glutamyl transfer reaction with

hydroxylamine, 83

$\alpha$-L-glutamyl-glutamic acid, 897

$\gamma$-glutamyl hydrazide as antimitotic agent, 930

glutathione, 728

- reductase, 25

- as respiratory catalyst, 25

- synthesis from glutamylcysteine and glycine, 46

glyceraldehyde- $-\mathrm{P}, 5$

glyceraldehyde-P-dehydrogenase, I6

glyceraldehyde phosphate, 4 , I I

- - dehydrogenase reaction, mechanism,

27

- - oxidation of - - to 3-phosphoglyceric acid, 27

D-glycerate as precursor of serine, 6 I

$d$-glyceric acid, I I

glycerolphosphorylethanolamine in tissue extracts, 54

glycineamide ribotide (GAR), 908, 9 I 7

glycine, biosynthesis, 58,60

-, formation of - from pentose, $6 \mathbf{I}$

- incorporated into reticulocytes RNA, 296

- interconversion, $5^{8}$

- labelled with ${ }^{15} \mathrm{~N},{ }_{4} 8$

— in lymphosarcoma, 896

—, ribulose phosphate as precursor of, 62

- pool in tumor tissue, 54

- synthesis in lymphosarcomas, 900

-, tracer, 50 I

glycolaldehyde, active, $4^{8}$

- donor, active, 62 
glycocholic acid, 40

glycogen, 6r 8, 683

- in mammalian skin, 683

- synthesis from lactic acid in human skin, 684

glycolate, 22

- as hydrogen carrier, 22

glycolic acid, I0, I3

- oxidase-FAD, 22

- reductase, 22

glycolysis, 2, I 59

-, anaerobic, I6I

-, Embden-Meyerhof scheme for phosphorylative -, $88_{4}$

- increase after traumatic shock, 6 I 8

— of tumors, aerobic, 54

- in tumor cells, 88I

glycolytic respiration of early regenerating tissues, 596

- - in R-phase of regeneration, 618

glycolytic sequence, 27

— - of Embden-Meyerhof, 3

glyconeogenesis in R-phase of regeneration, 6 I 8

glycopyranosyl compounds as mitose inhibitors, 935

glycylglycine uptake in suspension of cells of ascites carcinoma, 897

glyoxylate, 22

-, aldehydes as substrate in reaction between - and ornithine, 84

- cyclus, I23

- from malate, 62

glyoxylic acid, io

GMP (guanylic acid) synthesis, 95, 908

goitrogens, 827,846

Golgi apparatus, 338

— system, 307

- vesicles, 307,338

Gompertz equation, 176

gonadal hormones, effects of - - on regeneration, 632

gonads, organogenesis of the, 467

gonadectomy, importance of genetic

factors in response to, 831

gonadic inductions, 466

gonadotrophin, 829

gonocytes, 306, 467

gonopod, 59 I

GP-uridyl transferase, 39

graafian follicle, primary, 326

grafting experiments, $35^{2}$

gramicidin uncoupling phosphorylation, 30

granulating base of wound, tensil forces

within, 670

granulation tissue, 665 granulation tissue, chemistry, 693

- - increased uptake of $\mathrm{S}$ in, 700

— - , source of, 688

granules, chromotrope, 340

-, metachromatic, $33^{8}$

$\beta$-granules, 334

granulocytic leukemia induced by X-rays, 837

- leukemogenesis potentiated by androgen in mice, 84 I

gravity, influence of - on wounds, 677

grey crescent, 323

growth and aging process, 952

- of albino rat, $22 \mathrm{I}$

-, allometric - of the brain, 25 I

- - the Bertalanffy - equations in fishery biology, 193

- as cellular process, I 40

-, cessation of, $73 \mathrm{I}$

- , changes of the organism-as-a-whole as - problem, 245

—, chemical, $73^{\circ}$

-, compensatory, 2 Iо

-, constants of the - equation, 198

- curves, I67

- - cycles, 2 I9

- - , functions used for describing, I 75

- - human, 247

— - of intelligence, 248

- - linear, 180

— - of mammals, 219

- - segments, 2 I9

- - weight, I80

- data, methods to calculate, 192

-, definition, I37

-, deductive derivation of the system of - equations, 200

- and development, 712

-, differential, 730

- and differentiation, I4I

- and DNA, 265

—, embryonic, 223

-, equifinality, 2 I 3

- and evolution, 238

-, exponential, I6I

-, factors, 140

- (first growth type), limited, 236

- formulas, empirical, I 74

— of fruits, 807

- of fruits without pollination, 757

--, general considerations on models and laws of -, I 70

—, genetic control, 733

- gradients, 233

-, hereditary changes of - processes, 245 
growth and hormones, 219

- hormone, anabolic influence of, 845

— - plant, 752

- as identical reproduction, I40

-, indeterminate, 206

- inherent limitation of - of the organism, 953

— -inhibiting components, 209

- - effect of glucocorticosteroids and ACTH, 2 I9

- - mesenchym, 408

- of leaves, 797

-, limitations, I4I

-, limited, 200

- of mammals, 2 I 8

- in man, mental, 248

_- , somatic, 248

-, mathematical theory of cell - , I6r

- , measurements of plant,- 753

—, metabolism, I94

- model, discussion, 204

- - of Weiss and Kavanau, 208

- as morphogenetic process, 140

-, multiplicative, I6I

- and nitrogen metabolism, 7 I2

-, normal, 222

-, nuclear, 156

-, nucleic acids and regulation of - 297

—, optimal, 222

- of organs in time, 234

-, peptides with — promoting activity, 498

- and polarization of the oocytes, 307

-, postembryonic, $2 \mathrm{I} 8$

- potency of tissues and organs, I89

-, pre- and post-moult arrest of growth, 599

-, proportional, I69

-, quantitative theory of animal -, I 77

- rate, absolute, I68

- - , proportionality of RNA/protein ratio to - -, 275

- - specific, 168

- and regeneration, 588

- regulation, 730

-, relationship between - and senescence, 956

-, relative, 224

- ring, 749

-, and RNA, 272

- , role of the cell nucleus in -, 295

- of roots, $80 \mathrm{I}$

- (second growth type), unlimited, 236

- - second rise of - - at puberty, 248

-, sexual differentiation, 218

— stimulating hormones, 529

_, suboptimal, 222 growth substances, chemistry, 760

-, supernormal, 222

-, supernumerary, 601

- as synthesis of species-specific

substances, 140

- and temperature, 216

-, terminate, 206

- in time of total organism, $\mathbf{1 6 8}$

— of tissues, 163

- in tissue culture, 546

— — - effects of environment, 549

- — , effects of temperature, $55^{6}$

— — - mathematical theory, 166

— — - residual, 167

- - _ stimulating effect of RNA and nucleoproteins on, 298

- types, 180

-, uniqueness of man, 247

-, white mouse, 22 I

-, yeast mutants requiring purines for 509

Gryllus domesticus, 188

Gryphaea, $33^{2}$

- aculeata, 309

2 GSH, 12

GSH (glutathione), 6 15

GSSG, 12

GTP, 37, 38, 908

guanidine phosphate bond of creatine phosphate, 37

guanidoacetic acid, 80

- as intermediate in creatine synthesis, 80

- in transmethylase reactions, 75

guanine, 26 I

- in RNA from epithelial rat tumors, 902

guanylic acid, 95

- inhibiting mitosis in germinal epithelium of rat ovaries, 936

- synthesis, $85,95,908$

guanylic transferase, $3^{8}$

hair follicles, 682

Haller's rule, 243,315

halogenated hydrocarbons as carcinogens, 863

hamster, oocytes of the, 308

hanging drop cultures, $54^{8}$

haploid embryos, 734

haploidy, 292

hapten, $53^{8}$

head organizer, Spemann's, 376

head-regeneration in Planarians, 606

head, secondary, 386

head and trunk-tail organizers, opposition between, 398 
healing index, 70I

heart enzyme system, I09

- myosin, 533

heat economy in homoiotherm animals, 216

HeLa cells, 56, 446, 553

HeLa cell growth response to L-glutamic acid and L-glutamine, 901

Helianthus tuberosus, 761,771

Helicella candicans, 184

Helicidae, I 75

hemangiomas, 838

hematoblasts, $163,35^{\circ}$

hemiacetal dehydrogenases, I I

hemicelluloses of plant cell wall, 751

hemin, 528

Hemiptera, 598

hemoglobin, 522

- synthesis by reticulocytes, 296

hemopoietic neoplasms induced by whole body $\mathrm{X}$-irradiation, 848

heparin, 694

-

hepatic carcinogens, 840

- carcinogenesis, binding of butter yellow by liver cells during, 86 I

- - in man, correlation with cirrhosis, 866

- cells in senescence, 964

- neoplasms induction by methionine deficiency, 866

- reticulosarcoma induction prevented by hypothyroidism, 846

- tumors, 840

- tumorigenesis by acetylaminofluorene, 840

- tumor induction by butter yellow, 846

_ _ by carbontetrachloride, 863

hepatocarcinoma, transplantable, $88_{5}$

hepatoma, activity of uridylic acid from, 909

-, aminouridine inhibitor of purine and pyrimidine synthesis in -, 909, 910

-, production of acetoacetate from fatty acids in -, 894

hepatomas, 49,838

-, rate of ribonuclease activity in -, 911

heriditary changes, of growth processes, 245

— - of the hormon balance, 245

heteroauxin modifying induction process, 425

heterochromatin, 267

heteromorphs, 600

heteroplastic insertions in the blastocoele, 387 hexapeptide, 728

hexokinase, 4,31

- phosphorylation, 883

hexosamine, 596

- determination, 695

- liberated from mucoproteins in regeneration, 62 I

hexosediphosphate as energy source, 729

hexose isomerase, 4

- monophosphate shunt, 4,47

— — - reduced — - - activity in adenocarcinoma of mammary gland, 885

Hieracium, I 72

hind-brain, secondary, 386

hirsutism, 245

L-histidinal, Io

histidine, 55

-, uptake in transplanted rat tumors, 900

- biosynthesis, 56

—, imidazole nitrogen of, 87

L-histidine, II

histidineless mutant of Escherichia coli, 57

histidinol, 56

L-histidinol, ro

histocompatibility, dominant - genes, 865

histocytes, 688

histolytic dedifferentiation of intact cells and intercellular material, 595

histones, 298

—, chromosomal, 927

-, replacement of - by protamines during spermatogenesis, 298

HMC (5-hydroxymethylcytosine), biosynthesis, 9 I I

Homarus, 200

— vulgaris, 185

homocysteine, 63

-, methionine formation from - 73

homoeogenetic inductor, 477

homoeostasis, 588

homogentisic acid, 12

homoiothermic animals, heat economy, 2 I6

- - , thermoregulation, 187

homoserine, I I, 63

- condensation with cysteine forming cystathionine, 73

-, conversion of - to $\alpha$-ketobutyric, 75

- conversion of - to methionine, 73

HOQNO (2-heptyl-4-hydroxyquinoline-Noxide) inhibiting electron transport, 23

hormonal factors in carcinogenesis, 824

- imbalance inducing neoplasms, 829

hormone, hereditary changes of the balance, 245

- linkage of - synthesis stimulation and increase of RNA, 273 
hormone responsive (dependent) neoplastic growth, 825

hormones, in invertebrates, action on regeneration -, 635

- , in the control of regeneration, 631

-, gene-dependent, I4I

-, growth stimulating, 529

host, regional influence of the, $43 \mathrm{I}$

human, development, decrease in sodium and in chloride during —,- 959

--, glycogen synthesis from lactic acid in skin, 684

- primary follicle, 309

- growth curve, 247

-, variation during - fetal development in phase relation and cell water content, $95^{8}$

hyaline layer of sea urchin eggs, 34I

hyaloplasm, 332

hyaluronic acid, 696, 717

- in connective tissue and mast cells, 695

- in Rous sarcoma, 88 $\mathrm{r}$

- synthesis, $3^{8}$

hyaluronidase, 7 I 7

hybridization, 292, 71 6

Hydatina senta, I 66

Hydria, 59 I, 6 I 6

Hydractinia, 593

hydration, increasing - of tissues with advancing age, 963

- of tissues in regeneration, $62 \mathrm{I}$

$\gamma$-hydrazide of L-glutamic acid, $93^{8}$

Hydrioides, 639

-, operculum-reversal in regeneration of, 642

hydrocarbons, polycyclic, 858

Hydrochoerus capybara, 244

hydrogen acceptor, nitrate as, 22

— - nitrite as, 23

- carrier, glycolate as, 22

-, terminal - and electron transportsystem, I 5

hydrolysis of a nucleic acid, 26I

hydroquinone, I3, 32

hydroxyacids, enzymes oxidizing -, Io

$\beta$-hydroxy-acyl-dehydrogenase, 6

hydroxyamino acids, 725

p-hydroxyamphetamine, 12

$\beta$-hydroxybutyrate oxidation by intact mitochondria, 29

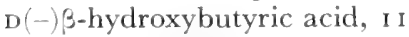

63-hydroxyestradiol, I2

5-hydroxyindole acetaldehyde, I I

- acetic acid, II

$\beta$-hydroxyisobutyric acid, Io

hydroxyisovaleryl carboxylase, 43
$\beta$-hydroxyisovaleryl-CoA, 43

3-hydroxykynurenine, 12

hydroxylamine, 13

-, glutamyl transfer reaction with, $8_{3}$

- reductase, 82

- - in $\mathrm{NH}_{3}$ requiring Neurospora

mutants, 82

- reduction of - to ammonia, 23

-, reduction of nitrite to, 23

hydroxylating enzymes, 12

$\beta$-hydroxy- $\beta$-methylbutyryl-CoA, I I

5-hydroxymethylcytosine (HMC), 9 I I

$\mathrm{N}^{10}$-hydroxymethyltetrahydrofolic acid, 59

p-hydroxyphenylpyruvic acid, I2, 66

I 7 -hydroxyprogesterone, 12

hydroxyproline, 78,499

-, determination, 670

hydroxypyruvate and alanine transamination to serine and pyruvate, $6 \mathrm{I}$

- as direct precursor of serine, $6 \mathrm{I}$

hydroxypyruvic acid, I I

$3 \alpha$-hydroxysteroid, 10

$3 \beta$-hydroxysteroid, io

5-hydroxy-uridine as inhibitor of RNA synthesis, 510,928

Hyla regilla, $49^{8}$

Hynobius, gastrular ectoblast of, 449

Hyoscyamus, elongation of short shoot by gibberellic acid, 8 I 2

hypercalcemia, 843

hyperchromasia, I 59

hyperestrogenization as growth stimulant of mammatrophic tumor, 916

hyperglycaemia in R-phase of regeneration, 6 I 8

hypermorphic chemicals, 35 I

hypermorphose, $35^{\text {I }}$

hyperphagia, 840

hyperplasia, 597

-, compensatory, I4I, 210

hyperplastic lesions, DNA in, 687

hyperthyroidism favoring induction of hepatic tumors by butter yellow, 846

hypertonicity, 560

hypertrophy, 597

-, compensatory, 588

- of nerve-tissue, functional, 643

hypomorphic chemicals, 35 I

- embryos, 290

hypomorphosis, $35 \mathrm{I}$

hypo-ovarianism, 830

hypophysectomized animals, i 89

hypophysectomy, effects of - on breast cancer, 843

-, palliation by - in human metastatic breast cancer, 846 
hypotaurine, 63

hypothalamus, role of - in controlling pituitary functions, 843

hypothyroidism, pituitary tumors following -, 824

- preventing induction of hepatic reticulosarcoma, 846

hypotonic solutions, 559

hypoxanthine, 96

- as opponent of azaguanine, 289

Hyracotherium, 240

hystiocytes, tissue, 691

IAA (indolacetic acid), 761,803

IAA-oxidase, 79 I

I-cells, indifferentiated, I65, 234

identical reproduction, growth as, 40

iditol, ro

Ilyanassa, 332

imaginal discs, 320

imidazole acetic acid, 12

- nitrogen of histidine, 87

immigration, 342

- process in gastrulation, 324

immune bodies against Rous sarcoma, 858

immunological specificities, 563

immunoproteins, uptake of - in cells of neoplastic tissues, 497

I MP, 94, 908

implant, activition by the, 403

—, method to insert - in the blastocoele, 377

increasing feebleness in senescence, 957

incubation of inductors with ribonuclease, 434

indeneacetic acid, 763

indeterminate growth, 956

individuation, 470

indolacetic acid, 758

- formed from tryptophan in pollen, 760

- as growth inhibitor, 932

indolacetic aldehyde, $76 \mathrm{I}$

indolacetonitrile, 761

indolacetyl-CoA, 797

- phosphate, 797

indole-anthranilic pathway, 68

indolebutyric acid, 763

indole-3-glycerol phosphate, 36

indoleglycolic acid, 762

$\alpha$-indoleisobutyric acid, 765

$x$-3-indoleisobutyric acid, 802

$x$-(3-indole)-propionic acid, 764

indolepyruvic acid, 762

6-3-indolylethylaminopurine preventing cell cleavage, 944
6- $\beta$-indolylethylaminopurine inhibiting mitosis, 933

index of cicatrisation, 604

induced atrophy by starvation, tenotomy

and denervation, 963

inducing agents, nature, 474

- effect of the archenteric roof, 400

induction, 5 I 9

- by archenteron roof, 443

- of cartilages and somites, $46 \mathrm{r}$

-, complementary, $39^{8}$

-, conception of a general - field, 353

-, delayed, 454

- of ectoblast in epiblast, 460

-, gonadic, 466

- of granulocytic leukemia by X-rays, 837

-, intradermic, 35I, 466

—, minor, $44^{8}$

-, morphogenetic effects of - 469

-, neurogenic, $35 \mathbf{I}$

-, notogenic, $44^{2}$

- with nucleoprotein and protein

extracts, 434

-, primary, 357

-, reciprocal, 455

- role of parachordal and prechordal mesoblast on rhombencephalon, optic vesicle and telencephalic lobes, 407

-, secondary, $357,44^{8}$

—, selective - of (chordo) mesoblast, 445

一, tertiary, 357

-, typical — of an acrencephalon by the PT inductor, $43^{8}$

-, way back, 460

inductive ability of the prechordal plate, 35 I

- correlation between spinal cord and vertebral column, 462

- processes, evolution of, 468

inductors, abnormal, 29I

—, auto-, $44^{8}$

—, coordinated - systems, 378

-, developmental, 445

-, homoeogenetic, 477

-, incubation of - with ribonuclease, 434

- to proteolytic digestion, 29 I

— and reactor, 357

— —, adherence between, 376

_- , biochemical modifications of, 419

inert body mass, 208

inflexion, 175

-, point of, 204

influence of pituitary gland on genesis, growth and secretion of tumors, 844 - of thyroid secretion on genesis and growth of tumors, 846 
influenza virus, 284

infundibulum, 395

inhibition, immediate - of DNA synthesis

by ultraviolet light, 294

- of tumorigenesis by steroid hormones, $84^{\circ}$

- of X-ray induced ovarian

tumorigenesis by ovarian secretion, 830

inhibitors, 209

-, natural, 298

-, nature of - and promotor in regeneration, 638

-, deliberate - in regeneration, 603

inhibitory effects of protamins and histones on cell division, 298

initiating agents in carcinogenesis, 858

inner nuclear division, 158

innervation as essential condition for regeneration, 528

inorganic cations in regeneration, 622

-, fixation of - nitrogen and transfer, 81

inosinic acid, 10, 92

-, acyclic ribotide precursors of - $93^{\circ}$

insertions, heteroplastic, 387

insulin, 883

- action on regeneration, 635

integration of the plant, $8 \mathrm{ir}$

intercellular spaces in plant tissue, 779

interconversion of glycine and serine, $5^{8}$

intermediate conjugates, 507

intermicellar colloids in plant tissue, 794

intermitosis, duration, 571

intermitotic cells, 163

intermoult, 599

interphase, DNA synthesis in first part of, 270

intracellular, cytoplasmic filaments and

lamellae of fibrogenic cells, 693

- template molecules, $73 \mathrm{I}$

intradermic inductions, $35 \mathrm{I}, 466$

intraliminal temperatures, 4 I 9

intramolecular dehydration of serine in Neurospora, 68

intranuclear inclusions in liver cells in senescence, 964

intrinsic morphochoresis, 319

- - , kinetic and causative aspects, 34I

- - mechanisms, 354

intussusception, $95^{2}$

intussusceptive growth, 669

- — in wound healing, 671

invagination, 140,342

invertase in plant root cells, $79 \mathrm{I}$

involution of warts, 230

iodoacetamide as plant growth inhibitor, 783 iodoacetate as plant growth inhibitor, 783

iodoacetic acid decreasing wound tensile

strength, 701

ionic equilibrium, 419

ionizing radiation, and cancer, 847

- _, effects of - on tissue cultures, $55^{8}$

- - inducing development of chemical

carcinogens, 848

— - local action, 847

- - reducing growth of crown gall, 774

iron content of methylcholanthrene induced carcinoma, 915

iron-porphyrin pigments, 16

irreversibility of regeneration, 534

Isidora proteus, $\mathbf{I} 84$

isocarcinogens, 838

D-isocitric acid, II

isocitric lysase, 62, I 23

- - reaction, 62

isolation experiments, $35^{2}$

isolated system, I44

isoleucine, 68

isomerase, 43

- enzymes, 4

isoprene-like precursor compound, Io8

isotope competition method of establishing

biochemical relationships, 55

isovaleryl-CoA, 43

ITP, 37

Jensen sarcoma, 889

juvenile hormone, 635

—, long — period of man, 954

Kamptoclymenia endogena, 215

- trivaricata, 2 I 5

karyokinesis. 768

KCN blocking oxidative metabolism in Ehrlich carcinoma cells, 887

$\mathrm{K}$-efflux and Na-influx following trauma, 643

keloid formation, 682

kephalin, enzymatic synthesis, 107

keratin, cystine bridges as structural components of synthesized,- 6 I 5

keratinization of epidermal epithelium, 564

keratohyalin granulus, 687

$\beta$-ketobutyrate in isoleucine synthesis, 70

$\alpha$-ketobutyric acid, conversion of homoserine to - - 75

2-keto-3-deoxy-7-phospho-D-glucoheptonic acid, 66

2-keto-3-deoxy-6-phosphogluconate intermediate in 6-phosphogluconate metabolism, 53

$\alpha$-ketoglutarate, 8 
$x$-ketoglutarate as amino acceptor in transamination reactions, 84

- oxidation in tumor mitochondrial preparations, 891

-, phosphorylation coupled to the oxidation of,- 28

- regeneration by $\beta$-sulfinylpyruvate, 65

$\alpha$-ketoglutaric acid, I I, 762

2-ketoinositol, Io

$x$-ketoisocaproic acid, 22

$\alpha$-ketoisovalerate, 10,68 , I I 7

ketopantoate, I I 7

I I-ketoprogesterone, I2

3-ketosteroid, Io

$x$-ketothiolase, 6

key amino acids, 6 Io

kidney, accumulation of colloid in tubulus with atrophy of lining epithelium in in senescence, 964

kinase enzymes, 97

kinematic regulation in newt gastrula and Discoglossus egg, 379

kinetic aspects of intrinsic morphochoresis, 34 I

kinetics, dominants of, 342

- of open systems, I 45

kinetin, 272, 758, 933

- and auxin interaction, 774

kinetosome system of ciliate Protozoa and Flagellates, 64 I

kinetosomes, 534

kinin, 753

$\mathrm{K}^{+}$ions, effect of - on plant growth, 787

Knudsen effect, 154

kombinative Einheitsleistung, Lehmann's, 357

Krebs cycle, see tricarboxylic acid cycle

kynureninase, I I 6

kynurenine, I 16

L-kynurenine, I2

\section{Lacerta, 185}

\section{- agilis, 427}

lactate metabolizing through tricarboxylic acid cycle to oxalacetate, 7 I

lactic acid production, by leukemic leukocytes, 885

— - by rabbit macrophages, 575

L-lactic acid, Io

lactic dehydrogenase, 16

- - activity increased by thyroxin, 634

Lactobacillus, $9^{\text {I }}$

— arabinosus, 43,78

- bulgaricus, I00

- casei. I20

_- . growth factor, 723
Lactobacillus helveticus, IO I

- pentosus, 48

- plantarum, 5 S, 120

lag phase of epithelium growth curve, 677

Lamellibranchiae, 180

lanoline, 754

lanosterol, I2, I I0

Larix, 8 I 2

lateral plate, $35^{\circ}$

leaf primordia, 749

Leander, 599

leaves, growth, 797

least-square method to calculate growth data, 192

Lebistes, 244, 309, 3 I 5

- reticulatus, 185, I89, 199, 2 18

Le Chatelier, principle, I 45

lecithin, enzymatic synthesis, 107

lecithocoele, 326

Lemna, 8 o I

lens induction, $45 \mathrm{I}$

- organizin, 478

- proteins, 518

- - , production of - - by iris cells, 520

- - , synthesis of specific - - $45^{\circ}$

lenticells, 780

leptomeninx, 454

lethal Rana combinations, 292

leucine, 22, 68

-, conversion of - to $\beta$-methylcrotonyl-

CoA, 108

-, effects of aminoketones as analogs of 502

-, oxidation of - by amino acid oxidase,

22

- synthesis by condensation of acetate with ketoisovalerate, 7 I

D-leucine activating peptidase activity in malignant tumors, 90 I

$d$-leucine, vegetalizing effect, 505

leucoanthocyanin, 772,773

leucocytes, leukemic, 82

- , concentration of polymorphonuclear within wound area, 698

leucovorin, 94

leucocyadinin monoglucoside, 772, 773

leukemia incidence increased by castration, 837

- induction by exogenous estrogen, 837

-, spontaneous, 837

leukemic leukocytes, 82

- - , lipoids of human myeloid and

lymphatic — - 880

_- aerobic and anaerobic

phosphorylation in - - $89 \mathrm{I}$

$\ldots$, production of lactic acid by _-,- 885 
leukemogenesis, granulocytic — potentiated by androgen in mice, $84 \mathrm{I}$

- inhibition in castrates by androgen administration, 837

-, lymphocytic — suppression by androgen, 84 I

leukogenic activity of estrogens, 837

-, secondary — effects of X-rays, 839

leukosis virus, antibodies, 858

Lewis sarcoma T 24I, 945

light, crescent, 325

- , stimulating effect of - on protein formation in isolated chloroplasts, 799

Ligia oceanica, 185

lignin of plant cell wall, $75 \mathrm{I}$

Lillium, 769

limb buds, 320,456

- regenerates, 603

— - arthropod, 606

-, regeneration of Arthropods, 642

limitations of growth, I4I

limited growth (first growth type), 236

Limnea, 3I I, 419, 725

Linaeus, 591

lines, pure - of cells, 549

lipid cofactor, 123

lipochondria, 312

lipogenesis, antagonistic effects of coenzyme

$\mathrm{A}$ and 6-mercaptopurine on -, 934

lipoic acid, 5,783

-, oxidized, 12

-, reduced, 12

lipoids of human myeloid and lymphatic

leukemic leukocytes, 880

lipolytic activity in regenerating nerve, 6 I 9

lipomas, 51

lipothiamide pyrophosphate, 5

lithium chloride, 286

-, effect of - on morphochoresis, 423

-, vegetalizing effect, $35^{\circ}$

lithium ion, hypomorphic effect, 423

lithium salts, 626

lithocholyl-CoA, 40

Lithoglyphus, I84

Lithopterna, 24I

liver cells in senescence, intranuclear inclusions in, 964

- enzymes, 7

- microsomes, 12

- mitochondria, oxidative metabolism, I8

—, regenerates, 603

- tumor, citric cycle in, 889

— —, phospholipid fractions, 880

livetin, 720

living mass, 232
Ln. citrovorum factor, 120

Ln. mesenteroides P-60, 120

local action of ionizing radiations, 847

- factors in regeneration, 646

lock-and-key theory of antigen-antibody complex, 669

logistic function for growth curves, 176

Lumbriculus, $59^{\mathrm{I}}$

Lumbricus sp., 184

lung tumors, increased incidence by whole body irradiation, 848

Lycoria, 243

Lymnaea, 183

- auricularia, 184

- stagnalis, 184

lymphatic leukemic leukocytes, aerobic glycolyte rate, 885

_ _ - lipoids of human, 880

- tissues, mammalian, 44

- tumors, 49

lymph nodes in senescence, 964

lymphoblasts MB III, 56 I

lymphocytic leukemogenesis favorised by adrenalectomy, 842

- - suppression by androgen, $84 \mathrm{I}$

- neoplastic disease, genese, 837

lymphoma, DNA content, 905

lymphomatosis, 857

lymphoid neoplasms induced by methylcholanthrene, 837

lymphopoiesis, normal - inhibited by glucocorticoids, 842

lymphosarcoma, 864

lymphosarcomas, alanine, glycine and proline concentration in, 896

- , biosynthesis of glycine and serine in, 900

-, mouse, 565

—, thymic, 838

lysase, $\alpha$-aminoadipic acid as intermediate in - synthesis in Neurospora, 76

— biosynthesis, 76

- in carcinoma, 896

-, decarboxylation of diaminopimelic acid to,- 77

-, diaminopimelic acid as - precursor in E. coli, 76

—, isocitric, 62

—, isocitric — reaction, 62

-, prophase accumulation in - deficient medium, 936

- requiring mutants of Neurospora, 78

lysosomes, 340, 6I I

lysozyme, 278

- activity of metastatic lesions of primary tumor, 9i 3 


\section{Lytechinus, 34 I}

lytic enzymes, 6 I I

maceration technique, 759

macrophages, 565

macula utriculi, $45^{\text {I }}$

main growth phase of epithelium growth curve, 677

maintenance metabolism, I94

malate, glyoxylate from, 62

- promoting plant growth, $78 \mathrm{I}$

maleic acid as inhibitor of succinic

dehydrogenase, 785

- hydrazide and adenine causing

chromosal erosion, 940

L-malic acid, Io

malic apoenzyme, 43

- dehydrogenase, 42, 527

- enzyme, 8, I5, 4I

- - and oxalacetic enzyme, relationship, 42

malignancies, polyploidy in, 157

malignancy, 563

-., development of, 865

- Meyerhof quotient as criterion of, 882

malignant cells, giant nuclei in, I59

- - - inherent response to hormones controlling carbohydrate metabolism, 887

- fibroblasts, 565

- growth, 2 II, 478

— - , susceptibility to, I66

- tissues, succinoxidase activity in, 889

- transformation, complex nucleoproteins involved in, 896

- tumors, D-leucine activating peptidase activity in, 90 I

Malpighian layers, 674

mammalian blastocyst, 342

-- lymphatic tissues, 44

- skin, glycogen in, 683

- tumors, 44

- wound healing, 666

mammals, growth, 2 I 8

-, growth curves, 219

mammary adenocarcinoma, 915

-, association of pituitary acidophilic

hyperplasia with - hyperplasia and neoplasms, 835

- cancer, human, 835

- - in mice, induced, 836

- - ovariectomy palliative in human metastatic - -, 842

- carcinogenesis, hormonal domination in, 836 mammary carcinogenesis inhibition by exogenous androgen in mice, 84 I

-, hormone responsivity of human - and prostatic cancers, 842

mammotrophic pituitary hormone potentiating mammary tumorigenesis, 845

- transplantable tumors, 9 I 6

mammotrophin secreting pituitary tumors, 835

manganese as plant growth promoting factor, 782

mannanprotein, disulfide bonds in cell-wall, 537

mannitol, 792

D-mannitol-I-phosphate, ro

marginal phenomenon, 167

— zone, 347

masked virus, 858

mass movement of cells in wound

healing, 678

mast cells, 694

—- hyaluronic acid in, 695

- - mucopolysaccharides, 695

- - perivascular accumulation, 695

- - tumor, cortisone induced regression of canine - - - 844

master reaction, in forming plant enzymes, 797

- - in regeneration, 603

maternal resistance factor in

leukemogenesis, 855

mature body, scarceness of mitotic figures in tissues of the - - , 964

measurements of growth in tissue cultures, 569

medullary plate in gastrula, 324

Meeh formula, i 8 i

Megaceros, 245

meiosis, 307,770

melanin, 452

melanocytes, 404, 564

melanogenesis at animal pole, 3 I I

melanoma, dopa and tyroxine oxidase

activity of human -, 9I4

--, glycolytic rate, 883

-, phenol-oxidizing system of human 914

melanophores, $44^{1}$

melanotic atypical growth in Drosophila following methylcholanthrene treatment, 863

membrane permeability in cancerous cells, 887

membranae tectoriae, 407

menadione, 13,32

- quinone, I3 
mental growth in man, 248

meperidine, 12

6-mercaptopurine, as anti-cancer agent, 934

- as antagonist of lipogenesis, 934

- inhibiting mitosis in cultures of mouse sarcoma, I 80,933

meresis, 766

meristem, 749

—, secondary, 767

-, suppression of one by another, 8 I I

meristemoids, 8 I I

meristic segmentation, 353

merogonic hybrids, 516

Merychippus, 240

mesenchyme, and apical epiblast, interrelations, 458

- blastula stage, 5 I 4

- in differentiation of epithelium, 464

-, growth-inhibiting, 408

-, thoracic, 523

mesoblast, 347

-, segmentation of the, 468

mesoblastic layer of the archenteric roof, 355

mesocotyl, $75^{6}$

mesodermal, activating factors, $52 \mathrm{I}$

- induction with bonemarrow extracts, 521

-, primordium, 520

mesoderm, transforming principle, $52 \mathrm{I}$

mesonephros, 47 I

mesonephric blastema, 466

mesothelium, 563

metabolically active body mass, 208

metabolic, acid phosphomonoesterase in processes in the cell, $34^{\circ}$

- changes in regeneration, 608

- contagion, 479

- cycles, interrelationship, 734

-, genetic mechanism related to - processes, 733

- genic mechanism for inheritance of patterns, 7 I 6

- gradients, 233

- measurements of tissue cultures, 575

- poisons, 286

- rate and body size, interspecifically relation, I 8 I

- _ explanations of the size-dependence, 185

- stability of DNA, 266

- types, 180

- - connection of - - with types of

respiratory apparatus, $\mathbf{I} 88$

metabolism, genetic control, 734 metabolism, growth, 194

-, maintenance, 194

- of nitrogen compounds, 715

-, oxidative - of liver mitochondria, 18

-, reproductive, 194

-, selfregulation, 145

- of sulphur compounds in regeneration, 615

- of thyroid tumors, 879

-, working, 194

metabolizing systems, energetics, 155

metachromasia, 329

metachromatic granules, 338

- plasm, 340

metamorphosis and thyroxin, 633

metaphase, arrest by 4-aminopteroylglutamic acid, 4-aminopteroylthreonine and 4-aminopteroylalanine, 943

- arrest by sulfanilamide, 943

- blocking by aminopterin, 943

- blocking by $\beta$-( $p$-fluorphenyl)-DLalanine, 945

- delaying by aspartic acid, 937

- of first maturation spindle, 3 I 8

- of mitosis blocking by a-methopterin, 929

- plate in plant cell division, 768

metaplasm, diffuse, 339

metastatic lesions, lysozyme activity, 913

Metazoa, 468

Metazoa, lower, 589

metanephric tubules, formation of definite - - $44^{6} 5$

metanephros, 47 I

methadone, I 2

methionine, $46,63,74,87,505,6$ I

-, active, 46

- , adenosine thiomethylriboside in synthesis in yeast, 75

-, conversion of homocysteine to, 73

- - - of homoserine to, 73

- deficiency responsibility for induction of hepatic neoplasms, 866

- incorporation of - into proteins, 296

_, methyl group transfer from - 74

- as source of cysteine synthesis, 63,616

- sulfoxide inhibiting glutamine

synthesis, 87

- sulfoxide, vegetalizing effect, 505

- as sulfur source for cysteine synthesis, 63

- synthesis of - from homocysteine and formaldehyde, 74

methods to calculate growth data, 192

a-methopterin arresting metaphase of mitosis, 929

$p$-methoxy cinnamic acid, 794 
2-methyladenosine as mitose inhibitor, 935

3-methyl-4-aminoazobenzene, I 2

methylamphetamine, 12

methylaniline, I2

methylated xanthines effect on second

half of cell division, 944

2-methyl-4-chlorophenoxyacetic acid, 763 , 802

methylcholanthrene induced carcinoma, calcium, copper, iron and zinc content, 9I5

- - mouse cancer, DNA in, 687

— inducing lymphoid neoplasms, 837

- modifying induction process, 425

$\beta$-methylcrotonyl-CoA, conversion of leucine to -, 108

5 -methylcytosine, 26 I

$x$-(2-methyl-4,6-dichlorophenoxy)isobutyric acid, 802

methyl groups, neogenesis, 73

- group transfer from methionine, 74

$\alpha$-methylmalonic semialdehyde, io

6-methylmercaptopurine as mitotic inhibitor, 933

3-methyl-4-monomethylaminoazobenzene, I 2

2-methylphenoxyacetic acid, 802

6-methylpurine inhibiting mitosis, 933

methylxanthines inhibiting enzyme systems, 930

mevalonic acid, I 23

Meyerhof oxidation quotient as criterion of malignancy, 882

migration, cessation, 680

- of epithelium, over the wound surface, 675

- - energy source, 676

- of fibrocytes, 692

-, layers involved in - 679

-, local - of endothelial and fibroblastic elements in wound healing, 665

- of Malpighian layer in wound healing, 679

- of phagocytic cells to wound area by vascular pathway, 665

- rate in wound healing, 676

migratory activity, effect of embryonic extract, 567

milieu extérieure, 549

- extra-organique, 549

- intra-organique, 549

- intérieur, 465

micrencephaly, 423

Micrococcus, I 3

Micrococcus lysodeikticus, 103

micromeres, 327 micromeres, vegetalizing activity, 339

microphysics, I 73

microplacodes, 449

microscopic reversibility, principle, 154

microsomal protein synthesis, I 23

- ribonucleoprotein particles, 928

microsomes, 150, 307, 501

-, protein formation in, $5 \mathrm{I} 2$

-, protein synthesis by, 290

microvilli, zone of, 312

minor inductions, $44^{8}$

Minot's formula of specific growth rate, 168

mitochondria, 307

-, DPNH oxidase enzymes

reoxidizing $\mathrm{DPNH}_{2}, 28$

-, $\mathrm{DPNH}_{2}$-oxidase, 29

-, electron transport system, 29

-, oxidative phosphorylation in, I50

-, protein formation in, $5 \mathrm{I} 2$

-, respiratory chain of, 29

- , selective permeability of the membrane, 29

mitosis, 157,265

-, abnormal, 159

-, duration, $57 \mathrm{I}$

— in epithelialization, 679

—, energy sources of, I 59

-, energy storage during antephase of 680

— suppression by $\beta$-( $p$-fluorophenyl)DL-alanine, 937

—, DNA synthesis during —, 269

mitotic abberations in plants caused by aminopterin, 943

- activity in skin cultures, 680

- figures in tissues of mature body, 964

— index, $57 \mathrm{I}$

- inhibition by amino acid analogues, 928

- poisons, antimetabolites as, 926

— - preprophasic, 929

- rate, determination, 165

- rhythm, 57I

—, X-radiation as - inhibitor, 272

Mnemiopsis, 604

modulating contacts, $39 \mathrm{I}$

modulation, limits, 230

- loss of some actual functions without change of potencies, 563

Mollusca, 590

monoamine, 13

monocotyledons, 799

monomethylethanolamine, 106

mononucleotide content of

adenocarcinoma, 915

morphallaxis, 589,664 
morphallaxis, regeneration by - in young Clavellina, 628

morphochoresis, experimental modifications, 4 I I

-, extrinsic, 3 I 9

-, gastrular, $3^{87}$

-, intrinsic, 3 I9

-, mechanisms of intrinsic - 354

-, participation of yolk in - 476

-, secondary fields, 320

-, ultraviolet irradiation effect on - , 412 morphochoretic pattern and cleavage, 325

- - and fertilization, $32 \mathrm{I}$

- intrinsic mechanism of the movements, 34I

morphogenesis, arrest of - by purines and pyrimidines, 289

- and RNA, 288

morphogenetic abnormalities produced by $\mathrm{LiCl}, 286$

- effect of urea, 423

- - of induction, 469

- factors, selforganizing systems, 354

- gradient, 233

- gradient, quantitative, 600

- potential, $343,387,466$

- - lowering of the, 35 I

- process, growth as ——, I 40

morphological applications of allometry, 229

morphology, dynamic, 156

morphostatic phenomena, 588

morula, aspartic and glutamic acid in amphibian - 348

mosaic eggs, 33 I

- stage of the ontogenetic processes, 593 motorium of Paramecium, 640

mouse cells, strain L, $55^{2}$

- fibroblasts, strain L, 56 I

- leukemia, 853

- liver epithelium clone, 469, 56 I

- lymphosarcoma, 565

- mammary cancer, 850

- - tumor, 565

- — - viral agent, 850

- sarcoma, Crocker, 55 I

- - producing hyperplasia of sympathetic ganglia, $29 \mathrm{I}$

MPS, 329

mucolysis in R-phase of regeneration, 6 I 8 mucopolysaccharides, 307

- combined synthesis of acid - and acid phosphatase, $34^{\circ}$

- and collagen content of wounds, 697

- decrease in regeneration, 609

-, distribution, 329 mucopolysaccharides, fibroblasts as source of - 700

- , released from mast cells by hormonal influences, 695

mucoproteins, 6 I I

Mullerian duct, 467

multienzyme systems, 32

muscle tissue organizing action in regeneration, 646

mutagenic agents as carcinogens, 863

mutant, isolation of - clones from large population of cells, 553

mutase, 39

- enzymes, 96

mutation, somatic — and carcinogenesis, $84^{8}$

mutations, lethal, 716

mycelium, yeast filamentous - 536

Mycetozoa, 750

myelin, 751

myeloid, lipoids of human - leukemic leukocytes, 880

myogenic cells, $35^{2}$

- plasm, 337

myoinositol, IO, I I 5

myo-organizin, 478

myosin ATPase, 534

myotoms, $35^{\circ}$

Mytilus edulis, 727

Myxobacteria, $75^{\circ}$

Myxomycetes, $75^{\circ}$

Naa (naphthalene acetic acid), 763, 802

N-acetylglucosamide-I - phosphate, 39

N-acetylglucosamine-6-phosphate, 83

$\mathrm{N}$-acetyl glutamic semialdehyde, intermediate in ornithine synthesis, 79

$\mathrm{NaCNS}$, animalizing, $35^{\circ}$

Na-inflow, acceleration by acetylcholine of - in cells, 643

$\mathrm{Na}$-influx and $\mathrm{K}$-efflux following trauma, 643

Nais, 592

N-alkylated oxypurines suppressing cell wall formation in mitosis, 944

$\mathrm{NaN}_{3}$ blocking oxidative metabolism in

Ehrlich carcinoma cells, 887

naphthalene acetic acid, 763, 802

$\alpha$-naphthaleneacrylic acid, 794

$\alpha$-2-naphthoxy- $n$-butyric acid, 804

2-naphthylamine inducing urinary bladder tumors, 862

nasal placodes, 385,393

Nassa, 604

natural inhibitors, 298

Neanderthal man, $25^{\circ}$ 
erotic tissues extract promoting

wound-healing, 637

Vecturus larvae, 679

Vematoda, 590

Nemertinea, 590

neoblasts, 589

neogenesis of methyl groups, 73

neoplasms, experimental - of the pituitary, 824

- following whole body irradiation, 848

- hormonal imbalance per se as factor

in inducing - 829

- of non-target tissues induced by estrogen, 837

-, spontaneous, 823

- of tissues whose normal growth is stimulated by estrogen, 835

neoplastic growth, autonomous, 823

- - conditional and hormone responsive - - 825

-, uptake of immunoproteins and serum proteins in - tissues, 497

neotomy, 588

nephelometry, 575

nephrotomes, $342,35^{2}$

nerve cell, cytological stages of functional activity, exhaustion and recovery of the - - 964

- supply and regeneration, 640

- tissue, functional hypertrophy, 643

neural crest, 404

— - and derivatives, 454

- field, 389

- plate, $34^{\circ}$

- tube, 367

- _ effects of neighboring tissues on shape and differentiation, 406

- - tissue and somite mesoderm, interaction, 520

neuralization of ectoblast, autonomous,

479

- of mesoblastic material, direct, 398

neurarcuals, 462

neurectoblast, presumptive activated - of

Urodele gastrula, 39 I

-, structure of — in normal Pleurodeles

embryo, 359

neuroblast, 3 r9

neuro-epithelial, zones of intense - growth, 407

neuro-epithelium, chordal islets in -, 413, 477

-, explanted, 454

- influence of neighboring tissue on

differentiation of -, 405

neurogenic induction, $35 \mathrm{I}$ neurogenic induction as main aspect of extrinsic morphochoresis, 357

neuron, constant production and using up of protein during functional activity of -, 964

neuro-organizin, 478

Neurospora, 2, 23, 63, 71, 76, 81, 98, I I 5

- enzym catalizing transamination between $\alpha$-amino-adipic acid and $\alpha$-ketogluturate, 77

—, hydrogen transport system, 23

-, lysine requiring mutants, 78

- mutants accumulating protocatechuic acid, 67

neurotropic factor, 643

neurulation, 288, 3 I9

$\mathrm{N}$-excretion, size dependence, 203

$\alpha$-N-formyl glycineamide ribotide, 908

$\mathrm{N}$-formyl glycineamidine ribotide, 85

Nio-formyl-THFA, I i 9

$\mathrm{N}^{5}$-formyl-5,6,7,8-tetrahydropteroylglutamic acid (citrovorum factor), 943

N10-hydroxymethyl-THFA, I o, I I 9

niacin, 5, I 15

nickel as cancer inductor, 869

Nicotiana tabacum, 759

nicotinamide, 36

- as cell division promoting factor in plants, 772

nicotinic acid, conversion of tryptophane to -, I I 6

-, effect of - in regeneration, 630

- synthesis, 1 I 5

nidation stage in rat embryo, 364

nidiculous mammals, $25^{\circ}$

nidifugous mammals, $25^{\circ}$

Nitella, 749

nitrate as a hydrogen acceptor, 22

- capacity of plants and microorganisms to reduce - and nitrite, $8 \mathrm{I}$

- and nitrite as hydrogen acceptors, 23

- reductase, 82

- - system of microorganisms, 26

- reduction, 22

nitrite reductase, 82

- and hydroxylamine reductase in $\mathrm{NH}_{3}$ requiring Neurospora mutants, 82

—, reduction of - - to hydroxylamine, 23

nitrogen, amino acids necessary for - equi-

librium, 55

- compounds, metabolism, 715

—, content of human tumors, 9 I 5

_, fixation and transfer of inorganic - $8 \mathrm{I}$

- flow in regeneration, 633

- metabolism in cleidoic eggs, 738

— _, cytological mechanism, 729 
nitrogen metabolism and growth, 7 I 2

—... in relation to development, 716

—- relationship of _ - and yolk storage, 729

- mustard, 517

_- D DNA depolymerization by —-, 271

- in low dosage accelerating regeneration, 623

-, pathways of - assimilation, $8 \mathrm{I}$

- transfer reactions and $\mathrm{NH}_{3}$ utilization, 88

nitrogenous waste products, evidence of catabolism of proteins, 736

$\mathrm{N}$-methylnicotinamide in transmethylase reactions, 75

non-reproductive structures, I 39

noradrenaline in transmethylase

reactions, 75

$d$-norleucine, vegetalizing effect, 505

normal growth, 222

normoblast, abnormal - mitosis in man, 939

normogenesis, 3 I9

notochord, $340,347,35^{\circ}$

- organizing action in regeneration, 646

notogenesis, 368,427

-, regular shifting from - to acrogenesis, $44^{6}$

notogenic induction, 443

notomerit, 368

Notonacta, 236

notoneuron, 368

noto-organizin, 478

$\mathrm{N}$-phenyl maleimide as plant growth inhibitor, 785

nuclear composition, abnormal, 292

- counts in tissue cultures, 572

- density of brain tumor, 905

- division, inner, $15^{8}$

- - unequal, I 72

- growth, 156

- series, 156

nucleases inhibiting protein synthesis, 278

nuclei, giant — in malignant cells, I 59

nucleic acid, absorption spectrum, 294

- antimetabolites inhibiting protein synthesis, 937

- autosynthesis inhibition by analogues, 928

—, cellular localization, 260

- components and analogues as antimitotic agents, 944

- , de novo synthesis of - from precursors in mouse sarcoma- I80, 908

- formation, mechanism, 9 I I

- inhibition of — de novo synthesis by 6-mercaptopurine, 934 nucleic acid metabolism, of tumor cells, 906 - - in tumors, quantitative rapidity, 909 - synthesis, 44, 289

- inhibited by fluorphenyl alanines, $\beta$-3-thienylalanine and ethionine, 937

nucleic acids, effects of abnormal nuclear composition, 292

- and embryonic development, 285

-, metabolism, 6 I $2_{2}$

- and nucleoproteins, in tumor cells, 902

- - in tumor metabolism, 910

- and regulation of growth, 297

— in relation to protein synthesis, 729

nucleo-cytoplasmatic, interactions related to protein formation, 5 I 4

- ratio, 159

nucleoprotein, dissociation by ribonuclease, 937

- as inductor, 434

-, necessity of - synthesis for protein synthesis, 276

nucleoproteins, $13^{8}$

- and nucleic acids in tumor cells, 902

—, self-reproduction of structured -, 139

nucleoside, diphosphate kinase, IO2

- diphosphates as RNA precursors, 103

- monophosphate kinase, 102

- phosphorylase, 96

— - enzymes, 96

- - in erythrocytes and brain tissue, $9^{6}$

nucleosides and nucleotides in tumor cells, 909

nucleotide, synthesis, $9 \mathrm{I}$

— transfer reactions, $3^{8}$

- transphosphorylation reaction, 28

nucleotides, conversion of purine

ribosides to - 97

- and nucleosides in tumor tissues, 903

nucleus, and cytoplasm, interaction, 158

-, protein metabolism in -, 297

nutrients, effect of - and ions on plant growth, 780

nutrition, 554

— and cancer, 865

- and regeneration, 627

Obelia, 6 I 2

octapeptide, 728

octanoate, CoA independent acyl

activation of,$- 4^{\circ}$

Octopus, 604

odontoblasts, future - of the mesenchyme mounts, 459

Oedogonium, 749

oestrogens, effects of - on regeneration, 632 old age, cell loss in - , 963 
whactory pits, induction of - from the piblast, 37 I

ilfactory placodes, $393,45^{\circ}$

Oligochaeta, 592

oligonucleotides, 285

oligosaccharides of the hyaluronic

acid chain, 39

omphalopleur, bilaminar, 719

omphalomesenteric circulation, 720

oncocytes, 965

Oniscus, 604

- asellus, 185

Onsager coefficients, 154

- reciprocal relations, I53

ontogenesis, 588

ontogenetic allometry, 228

- changes, I40

OOA carboxylase, 42

oocyte, preexisting polarity, 3 I 8

oocytes, growth and polarization, 307

- of the hamster, 308

oogenesis, symmetrization during - 315

oogonia, 306

Oömycetes, $75^{\circ}$

ooplasmic segregation, 33 I

open system, the organism as an -, I 43

open systems, entropy, I 52

- - equifinality as characteristic, 2 I 3

_- kinetics, 145

- - thermodynamics, I5I

operculum-reversal in regeneration of Hydriodes, 642

Ophioglypha, 605

Ophiostoma multiannulatum, 930, 935

optic vesicles, 385,407

optimal growth, 222

Organ Anlagen, I 40, 2 I 4

Organisationszentrum, 357

organisine, 387,478

- inducing regeneration of Planarian eyes, 643

organism, building materials, 137

- as an open system, I43

organismic epigenesis, 742

- nature of the embryo, 715

organization, 152

-, bilateral, 3 I 5

- of a regenerate, 600

organizing action in regeneration of muscle tissue, cartilage, bone, endoderm and notochord, 646

organizer, 35 I, 5 I 9

- of amphibia, 716

-., effect of ultraviolet irradiation, $4 \mathbf{1 2}$

—, fundamental — experiment, $35^{\text {I }}$

- graft of - with reversal of the cephalo- caudal axis, 386

organizer territory, 346

- transplantation, Spemann's, 352

organogenesis of the gonads, 467

- and nitrogen metabolism, 727

organogenetic areas, 354

organs, balance of - and chemical constituents, 2 I 4

organ-specific protein, $45^{\circ}$

organ stabilization, axial _- -353

ornithine, 78

- aldehydes as substrate in reaction between - and glyoxylate, 84

- - citrulline-arginine cycle, 80

-, conversion to arginine, 80

- cycle in the chick embryo, 741

- as proline precursor in mammals, fungi and bacteria, 78

- synthesis, 79

orotic acid, I I

$-.15 \mathrm{~N}, 907$

-, biosynthesis, $9^{8}$

- utilization, 99

Orthagoriscus, 239

orthoclone, 954

-, senile, 954

orthogenesis, 245

Orthoptera, 180

Oryza sativa, 760

osmotic gradient in plant cells, 792

- inhibition of plant cell enlargement, 792

- pressure, 793

osteoblasts, 693

osteoclasts, protease secreting, 6I I

osteocytes, 693

otocyst, $45^{2}$

--, ectopic, 453

ourogenesis, 368,397

ouroneuron, 368

outgrowth, 567

ovarian, and adrenocortical tumorigenesis, post-irradiation, 848

- hormonal influence on X-ray induced - tumor, 828

- neoplasms induced by whole body Xirradiation, 848

- pituitary endocrine relationships involved ovarian, pituitary and mammary tumorigenesis, 835

- tumors, induced, 829

- tumorigenesis, 840

_ _, endocrine interrelationship involved in _ - following transplantation in the spleen, 827

- - inhibition by functional ovarian transplants, mechanism, 830 
ovarian tumorigenesis, inhibition of X-ray induced - - by ovarian secretion, 830 ovariectomy palliative in human metastatic mammary cancer, 842

overshoot, I 46, 2 I I

Overton factor, mitosis stimulating, 643 ovovitellin, 7 I 8

oxalacetic acid transamination to aspartic acid, 72

- carboxylase, 8, 4I, 6I

- - and malic enzyme, relation, 42

oxalosuccinic acid, 8, I I

oxidase-FAD, glycolic —, 22

oxidase, mitochondria $\mathrm{DPNH}_{2}--, 29$

oxidases, aldehyde - I I

oxidative phosphorylation, 27, 33

oxidative shunt, direct - - 4

oxidation of pyruvate to acetyl-CoA, 5

oxime compounds, organic, $8 \mathrm{I}$

— - organic _ - as intermediates in amino acid synthesis, 82

oxygen-consumption, dimunition of - in early regeneration, 597

oxygen, low - tension of tumors, 9 I 6

PABA ( $p$-aminobenzoic acid), 94, I 19

- metabolic antagonist of sulfanilamide, 94

Pachygrapsus crassipes, 185

Palade bodies, 338

- granules, 307

Paludina fasciata, I 84

- vivipara, 184

panchresta, I 7 I

paniculus carnosis, 669

Pan panicus, 3 I 7

pantetheine, 34

pantoate, i 7

- dependent exchange reactions, 44

- synthesis from valine, I I 7

pantothenate, formation of coenzyme A from -, I I 8

-, peptide bond of - 444

pantothenic acid, 5

-, effect of - on regeneration, 63 I

papain, 728

parabiotic barrier, 829

Paracentrotus, $333,33^{8}$

- lividus, 341, 722

parachordal, induction role of - and prechordal mesoblast on rhomencephalon, optic vesicle, telencephalic lobes, 407

Paraclymenia abeli, 2 I 5

paragenesis, 3 I 9,409

Paramecium, 216, 592, 628

_, kappa particles, 600

-, motorium, 640 paraphysis, 393

paraplasm, 3 I 3

Parascaris equorum, 328, 718

parathyroid action on regeneration, 635

paratomy, 588

Parawocklumeria paradoxa, 2 I 5

Parazoa, 589

parenchyme, 770

parotid, fatty degeneration of secreting parenchyma of - gland in senescence, 964

- tumors of the mouse, 855

parthenocarpy, 763

parthenocarpic fruits, 808

Parthenocissus tricuspidata, 761

parthenogenesis, 305,734

P.A.S. reaction, 696

passage cultures, 570

Pasteur effect, $88_{4}$

PC-cytidyl transferase, 107

peanut oil as tumor inducing agent, 860

Pearl's formula, I 74

pectic substances of plant cell wall, $75^{1}$

pectinase, 796

pectin methylesterase, 790

Pelargonium, 8 oo

penicillase, 532

Pennaria, 622

pentose epimerase, 48

- formation, 47

_- via direct oxidative pathway by

lipomas, 5 I

-, formation of glycine from - 6 I

- - transketolase-transaldolase pathway, 50

pentose isomerase, 48

- nucleic acid (PNA), 596, 6I2, see also PNA

- - decrease in regeneration, 608

- nucleoprotein, 44I

peptidase, increased - activity of blood, leaving injured region, 6 I I, 645

-, D-leucine activating _ in malignant tumors, $90 \mathrm{I}$

peptidases, high activity of - in centers of active growth, 506

peptide bond formation in the incorporation of amino acids into protein, 44

- linkage formation, 729

peptides with growth promoting

activity, 498

peptine chain formation, 506

perfusion chamber for tissue culture, 549

periblast, 347

pericycle, $77 \mathrm{I}$

perinodal area, 4 Io 
inuclear process of secondary

vitellogenesis, 315

eriophthalmus, 245

perivitelline space, 7 I 8

-, selective - of the cell membrane, 7 I 3

permeability of embryonic cells to large

molecules, 497

- selective - of the mitochondrial mem-

brane, 29

permeation as regulating factor in anabolic processes, 216

peroxidase, 22

- in plant cells, 790

- in Walker carcinoma, 893

petioles, 756

PFS (protein forming system), 496

PFS of differentiated and embryonic cells, 507

PGA, 120

$\mathrm{pH}$, lowered - in animal tumors, 886

$\mathrm{pH}$, effects of - on tissue culture, 559

phage $T_{1}, 298$

phagocytosis, 633

pharyngeal endoblast, 371

- - as secondary inductor for the heart, 453

phenanthrene structure of carcinogenic

hydrocarbons and sex hormones, $85^{8}$

phenocopies, 7 I 6

phenol oxidases as terminal respiratory

catalysts in plants, 24

phenol-oxidizing system of human

melanomas, 914

phenomenological laws, I 52

phenotypical compensation, 246

phenoxyacetic acid, 802

$\alpha$-phenoxyisobutyric acid, 802

phenylacetic acid, 802

phenylalanine, I2, 65, 280, 455

-, incorporation of - in amoebae, 282

- -less E. coli mutant, 67

- as precursor of protein, 296

$p$-phenylenediamine oxidative response in tumor cells, 884

phenyllactic acid, vegetalizing effect, 505

phenylmercuric salts as plant growth

inhibitors, 783

phloem parenchyma, 77 I

phlorizin poisoning, 685

Phoronis, 598, 599

phosphatase, acid, 525

- activity, in regeneration, 614

— - relation of _ _ to yolk platelet

utilization, 499

-, alkaline, 525

phosphatases, 683 phosphatases, beryllium basic acetate as a specific inhibitor of,- 460

phosphate acceptors in mitochondrial oxidation, 3 I

- bonds, activating reactions of energyrich — - 36

- - generation of energy-rich — - 27

— - , high-energy, I50

- -, storage of high-energy - - 33

- esters, free energy of hydrolysis, $3^{8}$

phosphatidic acid formation, I07

phosphoarginine, 37

phosphocholine, 35

phosphocreatine, 37, 96 I

phosphoenolpyruvate, 8,28

-, synthesis of dicarboxylic acids from pyruvate and - 8

phosphoglucomutase of yeast and muscle, 97

phosphogluconate pathway, 52

- - of glucose oxidation, 49

6-phosphogluconate, I5

- dehydrogenase activity in tumors, $89 \mathrm{I}$

- metabolism with 2-keto-3-deoxy-6phosphogluconate as intermediate, 53

- metabolism of Pseudomonas saccharophila, 53

6-phosphogluconic acid, 5, I I

phosphogluconic dehydrogenase pathway,

53

phosphoglucomutase, 786

phosphoglyceraldehyde dehydrogenase, 782

2-phosphoglyceric acid, 786

3-phosphoglyceric acid, oxidation of glyceraldehyde phosphate to -, 27

phosphohydroxypyruvate, conversion of to serine, 6 I

phosphoketopentose epimerase, 48

phospholipid biosynthesis, I 66

- fractions of liver tumor, 880

- synthesis, 38

- turnover in regenerating liver, 619

phosphomonoesterase, 333

- acid, $34^{\circ}$

phosphopantetheine, 34

phosphoprotein-phosphatase activity and yolk platelet utilization, relation, 476

phosphopyruvic acid, 786

phosphor balance of gastrula, 349

phosphoribose isomerase, $4^{8}$

- mutase, catalyzing synthesis of nucleosides, 96

phosphoribosylamine, 85

5-phosphoribosyl-I-pyrophosphate (PRPP) as donor of two carbon atoms, 68 phosphoribulokinase, 914 
phosphoribulose kinase, 44

phosphor, tracer, 7 I 9

phosphorylase, I 77

-, polynucleotide, 36, I 03

phosphorylated sugars, 33

phosphorylation, and activation of $\mathrm{RNA}$

by ATP, 285

-, aerobic, 3 I

-, aerobic and anaerobic — in leukemic leukocytes, 89 I

- coupled to the oxidation of $\alpha$-ketogluturate, 28

-, electron transport - , 29

-, oxidative, $27,33,15^{\circ}$

-, photosynthetic, 27,32

-, substrate, 27

- in tumor cells, $89 \mathrm{I}$

phosphorylative glycolysis, 884

phosvitin, 72 I

photolysis of water, $3^{2}$

photosynthesis, 798

- by isolated chloroplasts, 33

photosynthetic phosphorylation, 27, 32

- - enzymes, 33

phototropism, 805

phragmoplast, 768

phragmosome, 768

Phycomyces, 797

- blakesleeanus, 109

- sporangiophores, 755

Phycomycetes, $749,75^{\circ}$

phylogenesis, $59^{\circ}$

phylogenetic changes, 140

physiological competition, 230

- gradients, 233

-.. processes and allometric equation, I 78

- regeneration, 589

- regeneration of - tip, 756

- restoration, 592

physiology of root elongation, 806

phytohormone, 752

picolinic acid, I 16

pigmentocytes, 408

pigments, iron-porphyrin, I 6

pimelic acid, I 14

pinocytosis, 579

Pinus strobus, 769

Pisum sativum, 756

Pithecanthropos, 25

pituitary, appearance of - adenomas secondary to estrogenic stimulation, 83 I

- - association of - acidophilic hyperplasia with mammary hyperplasia and neoplasia, 835

- deficiency, 530

- dwarf, mice, I 89 pituitary, effect of - growth-hormone (APGH) on regeneration, 624

- gland, $45^{\circ}$

- gonadotropin stimulus to adrenocortical tumor formation in mice, $83 \mathrm{I}$

-, hereditary - dwarfism, 912

- hypersecretion of TSH, 824

- neoplasia in hypothyroidism inhibited by exogenous thyroid hormone, 824

- neoplasms, experimental, 824

- - induced by alteration of thyroid function, 824

- stalk section and amelioration of human metastatic breast cancer, 843

-, stimulation of - secretion of tumorigenic gonadotropin by injection of estrogenic hormone, 835

- following castration, 833

- tumors following hypothyroidism, 824

- - secreting ACTH, 827

- thyrotrophic hormone (TSH), 824

placodes, 364

planimeter determinations of the wound area, 677

Planaria, 183, 589, 599

- maculata, 217

Planarians, head-regeneration in -, 606

-, whole-body regenerates of,- 601

Planorbidae, 180

Planorbis corneus, 184

- spec., 183, 199

plant, cell enlargement and metabolism, 787

- growth factors, 755

- - , general characteristics, $74^{8}$

- respiration, and quinone reductase, 24

-, role of RNA in - virus multiplication, 276

plasma, tumors utilizing labelled albumin, 898

plasmagenes, 534,862

plasma-SH, inverse correlation between and plasma-polysaccharide concentrations after $\mathrm{X}$-irradiations, 6 I 6

plasm, metachromatic, $34^{\circ}$

plasmolemma, 317

plasmolysis, osmotic pressure measurement by method of incipient -, 793

plastics as cancer inductors, 869

Plathelmynta, 590

Platipoecilus, 591

pleiotropic gene action, 246

pleiotropy, 734

pleomorphism, I 59 
odeles, $283,37 \mathrm{I}$

imbryo, 392

gastrula, $35^{8}$

larvae, 449

ieuronectes, 200

platessa, 2 I 7

Pliohippus, 240

pluteus, 339

PNA (pentose nucleic acid), 596, 6 I 2

- , basophilic - granules of mature cells, 6 I 4

Podarke, 602, 6i5

podophyllin components inhibiting

anaphasic chromosome movement, 942

polar bodies, 328

polarity, of the animal body, 233

- axis, 3 I I

-, pre-existing - of the oocyte, 3 I 8

polarization of the egg cell, 3 I I

- of the oocytes, and growth, 307

polyauxotrophs, aromatic, 65

polyblast, 688

polycyclic hydrocarbons as complete and

independent carcinogenes, 858

polyglutamyl-folic acid derivatives cata-

lyzing conversion of serine to glycine, I 2 I

Polygonum, 768

polymers, synthesis of high - 150

polynucleotide phosphorylase, 36, I 03

- in microorganisms, 9I I

- reactions, 37

polynucleotides, enzyme systems catalyzing synthesis of - 9 I I

-, synthesis, 37

Polyodon, 225

polypeptide chain formation on

ribonucleoprotein particulates of microsomes, 45

polypeptides, equilibrium concentrations, I 50

polyphenol oxidase, 786

- - increasing tumor respiration, 893

polyploidy, I 57, 292

polyploid nucleus, $77 \mathrm{I}$

polysaccharides, of plant cell wall, $75^{\circ}$

-, pyrogenic, 6 I 9

-, tumor necrotizing activity of bacterial —, 88 I

polytene giant chromosomes, 158

Polytomella coeca, 274

polyuronides of plant cell wall, $75^{\circ}$

Polyzoa, 589

Porcellio scaber, 185

Porifera, 590

porphobilinogen, 90

- synthesis by human erythrocyte enzymes, 9o

porphyrin synthesis, 88

Portunus, 602, 606

post-castrational, adenomas and carcinoma of the adrenal cortex, $84^{\mathrm{I}}$

- adrenal cortical adenoma, 826

postembryonic growth, 2 I 8

postgeneration, 593

postmitotic cells, 163

Potamobius torrentium, I 85

potassium see also $\mathrm{K}$

potassium ions promoting cell enlargement

in isolated plant tissues, 782

$\mathrm{P}$-phase in regeneration, 595

PP-uridyltransferase, 39

PRA, 85

- as intermediate in GAR synthesis, 92

praseodymium as plant growth inhibitor, 796

prebone condition, 534

prechordal, differentiated - territory, 372

- foreward migration of the - endomesoblast, 473

-, grafts of - neuroepithelium, 370

- inductive ability of the - plate, $35 \mathrm{I}$

-, inductive action of the - mesoblast, 396

-, mesoblast, 407

—, pre-existing — plate, 384

—, pre-existing — primordium, 383

precursors, amino acids as - in PFS, 498

-, utilization of small molecular — in

formation of proteins, 499

predispositions, 347

pre-existing skin, 67 I

preformism, biochemical, 349

pregastrula embryos, protein utilization in - - 736

pregastrular kinematics, 347

pregastrulation, 3 I 9

pregnancy, 49

premuscle condition, 534

prephenic acid, 67

prepigment granules, 314

prepituitary, 463

preprophasic, inhibition by sulfanilamide, 943

- mitotic poisons, 929

prevention of differentiation of embryonic

tissues by homologous adult tissue

extract, 733

previtellogenesis, 3 I 0

Prigogine's theorem, I 54

primary, follicle, 308

- induction, 366

- plant cell wall, $75^{\circ}$

primitive streak, 463 
primitive streak stage in rat embryo, 364 proamniotic crescent in gastrula, 324

Procerastea, 593

Procerodes, 642

pro-collagenous fibers, 694

Procotyla, 59 I

proctodeum, 469

productive phase of wound healing, 697

pro-encephalic structures, development, 520

progesterone, 12

prognathism, 245

proliferation and differentiation,

antagonism between - - 2 I I

_- , relationship of - - in early

embryogenesis, 524

-, senescent — of duct cells, 965

proline, I I, 78

- concentration in lymphosarcoma, 896

-, conversion of glutamic acid to - 79

-., ornithine as - precursor in mammals,

fungi and bacteria, 78

promoting agents in carcinogenesis, 858

pronephros, $343,35^{\circ}$

pronephric duct activating mesonephric

blastema, 466

pronucleus, female, 287

pro-oxidants, 6 I 8

I ,2-propanediol- I-phosphate, 889

prophase, accumulation in lysine

deficient medium, 936

- arrest in Vicia faba roots by

2,6-diaminopurine, 932

propionyl-CoA, 36, 42

propyl-thiouracil, induction of pituitary

tumors by $\longrightarrow, 825$

PRPP reactions, 36,96

prosencephalic differentiation tendencies,

liberation of - - $39 \mathrm{I}$

prosencephalon, 393

prostatic hormone responsivity of human -

and mammary cancers, 842

promotor and inhibitor in regeneration, nature of, 638

protagonist, contact between the - tissues,

474

protamines, 298

protease activity in amphibian gastrula,

$34^{8}$

proteases, intracellular, 6 I I

protective agent, role of auxin as - - 797

proteinases, intracellular, 506

protein, anabolism in nucleus, 297

- , changes in - and RNA content relatively

to DNA content during gastrulation in

Rana pipiens, 362

-, compositional differences between - components of normal and neoplastic tissues, 896

protein extracts as inductors, 434

- formation in Ehrlich carcinoma cells, 887

- - in microsomes and mitochondria, 512

_- nucleo-cytoplasmatic interactions related to - - 5 I 4

- - qualitative aspects of - - in embryonic cells, 522

- - stimulating effect of light on _- in isolated chloroplasts, 799

- forming system, see PFS

— forming system of developing cells, 495

- metabolism, remote control of nucleus on - - 297

- in tumor tissues, 897

- precursors, larger than amino acids, 497

—- pathways of - - utilization, 505

- - utilization of - _ - in the differentiating cell, 497

- sparing effect of carbohydrates, 222

- synthesis, energy requirements, 149

- - gastrulation as initial stage of new - 286

— - harmonization between _ - and degeneration, I 66

- - inhibited by fluorphenyl alanines,

$\beta-3$-thienylalanine and ethionine, 937

— - inhibition of - - - by nucleases, 278

- - inhibition of - - in ribonucleasetreated roots, 280

_ _ linkage of _ - - and RNA synthesis during spermatogenesis, 273

- _, mechanisms, 284

- - in microsomes, 26, I23, 290

- - necessity of nucleoprotein synthesis for - - 276

- - and nitrogen metabolism, 728

- - in plant root cells during elongation,

79 I

- - and RNA, 272

— - role of the cell nucleus in _ -, 295

— - stimulation of _ — with testosterone in seminal glands of rats, 273

— - utilization of ammonia for — - 222

- turnover, I 48, I 65, 222

- utilization in pregastrula embryos, 736

proteins and amino acids in tumor tissues, 896

-, contractible, $34^{\text {I }}$

-, formation of - and enzymes in plant cells, 790

- incorporation of methionine into - 296

-, mechanism of formation of specific 284 
proteins, modifications in chromosomal -, 318

non-centrifugable, non-particulate 514

-, non-sedimentable, 5 I 4

-, organ-specific, $45^{\circ}$

-, peptide bond formation in the incorporation of amino acids into - 44

- $\mathrm{pH}_{5.2-}-45$

-, phenylalanine as precursor of - 296

-, role of - as energy source, 726

-, SH-groups linked to - 289

- in tissue cultures, measurement of total - 577

proteolysis, increased - in regressing cancerous growth, 6I I

proteolytic activity in human tumors, 9 I 3

- - increase in regeneration, 608

- degradation of implants, 290

- enzymes, 6 I I

- activity reduced by formalin, 422

- _ , correlation between content of - protein turnover and growth capacity, I 66

- - in tumors, 878

- - , use of - - for separation of tissues, 537

- inductors to - digestion, 29 I

Proteus, 442

- vulgaris enzymes, 64

prothallus, 771

prothoracic hormone of insects, 635

protoanemonin as seed germination

inhibitor, 785

protocatechuic acid, accumulated in

Neurospora mutants, 67

protochordates, 327

protofibrils, 69 I, 694

protopectin, 796

protoplasm, I $3^{8}$

-, undifferentiated, I4I

protoporphyrin, 88

-, crystalline - from chloroma extract, 915

- synthesis from $\delta$-aminolevulinic acid, 90

Protozoa, 590

- , cell size and temperature in -, 217

-, kinetosome system of ciliate -, 641

-, nitrogen metabolism, 725

proximo-distal axis of the limb, $45^{8}$

Pseudemys, 466

Pseudomonas, 10,62

- aeruginosa, 24

- reduction of nitrite in - 23

- saccharophila, metabolism of

6-phosphogluconate by —-, 53 pteroglutamic acid, 120

PT inductor, typical induction of an acrencephalon by the - 438

pubertas praecox, 245

puberty, second rise of growth rates at -, 248

Pugettia producta, 185

pulmonary tumor induced by the administration of urethane, 857

Pulvertaft-Harris method, 553

purine degradation, formimino-glycine intermediate in $-\cdots, 58$

- metabolism in neoplastic cells, 908

- analogues, 930

- composition of DNA in tumor tissue, 902

-, conversion of - ribosides to nucleotides, 97

- synthesis, de novo, $9 \mathrm{I}$

purines, 729

- , yeast mutants requiring — for growth, 509

-, labelled, 95

-, utilization of free - 95

putrescine, 946

pyknosis, I 59

pyocanin decreasing mitosis in

fibroblast culture, 886

pyridine nucleotide dehydrogenase, 7

- - dehydrogenases in amino acid oxidation and sterol synthesis, 9

- - and dismutation reactions, I5

- linked glutathione reductase enzymes in plants and animal tissues, 25

- linked reductases of Neurospora, 23

- - transhydrogenase, electron transfer via - - -30

- nucleotides, 33

- _, reoxidation of reduced _ - via the cytochrome system, 16

pyridoxal phosphate, 84 , I I 4

- as co-factor in glycine and serine interconversion, 59

- as co-factor in transamination

reactions, 84

- and folic acid derivatives in serine

and glycine interconversion, 58

pyridoxal-5-phosphate as co-enzyme, 762

pyridoxamine, 34,84

- as cofactor in transamination reactions, 84

- phosphate, 34

pyridoxine content of adenocarcinoma, 9 I 5

- promoting skin-healing in rats, 630

pyrimidine, 729 
pyrimidine, aminofumaric acid as precursor of,- 98

- composition of DNA in tumor tissue, 902

- nucleoside phosphorylase, Ioo

- analogues, 930

- synthesis, de novo, 98

pyrithiamine as antimetabolite, 806

pyrogenic polysaccharides, 6 I 9

pyrophosphate transfer, reactions, 35

- - to acceptor compounds, 37

pyrrole synthesis, succinyl-CoA and glycine condensation in -,- 89

pyrroline-5-carboxylic acid, I I

pyruvate metabolism in tumor tissues, 889

-, oxidation of - to acetyl-CoA, 5

pyruvate-2-14 $\mathrm{C}$ metabolic patterns for - in various tissues, 890

quantitative theory of morphogenetic

gradients, 600

quinacrine, 12

quinic acid, to

quinolinic acid, I I 6

quinone, 13

- reductase, 24

quinoxalins, 626

radioactive chemicals inhibiting regeneration, 624

radiothyroidectomy-induced pituitary tumors, 824

Rana clamitans, 6 I 5

- esculenta, 292

- fusca, 292

-, lethal - combinations, 292

- pipiens, 292, 498, 5I5, 720

- temporaria, 379, 4I4

- temporaria, seasonal growth of oocyte of - - 717

reacting, influence of age on the - ability of the ectoblast, 4 I I

reaction rates, principle of harmonized - - 228

reactions, reversible, 144

reactor and inductor, 357

-, intimate changes, 479

-, receptive, 368

-, Urodele ectoblast as -, 444

reactors, biochemical modifications of 419

reaggregation, $42 \mathrm{I}$

reattachment of epithelium, 680

reciprocal induction, 456

- phenomena, I53

reconstitution process, 593

redistribution of cells, 593 redox potential, maintenance of the - of the cell, 9

redox potentials, 9,17

reductase, cytochrome $m, 26$

-, dehydroascorbic, 25

-, glutathione, 25

-, glycolic, 22

reduction-potential increase in regeneration, 608

reductive carboxylation, enzymes of the 一, 33

regenerate, organization of a - 600

$\ldots$, visible differentiation in a - 600

regenerating nerve-fiber, 603

- tissues, chemical factors produced by -, 636

regeneration, 214

-, acceleration of - - , by warmth, 623

-, action of adrenal corticoids on -, 630

—, bone androgens promoting -, 632

-, diffusing factors in -, 645

-, distribution of powers of - , among animals, 590

—, effect of gonadal hormones, 632

-, effect of oestrogens, 632

- function of alkaline phosphatase during -, $6 \mathrm{I} 3$

-, d-glucosamine inhibitor of - 619

- glucose and glycogen energy sources for,- 6 I 8

- and growth, 588

-, hexosamine liberated from mucoproteins in - $62 \mathrm{I}$

-, hormones in the control of - $63 \mathrm{I}$

- , hydration of tissues in - 62 I

-, hyperglycaemia and glyconeogenesis in R-phase of - , 6 I 8

- increasing of free $\mathrm{SH}$-groups during R-phase of -, 6 I 5

— inhibiting chemicals, 626,627

- inhibition by radioactive chemicals, 624

- , initial rate, 603

—, inorganic cations in - 622

-, innervation as essential condition, 528

-, instantaneous rate, 603

- internal factors in the control of - 627

-, local factors, 646

-, metabolic changes during -, 608

—, metabolism of sulphur compounds in -, 6 I 5

- by morphallaxis in young Clavellina, 628

- and nerve supply, $64^{\circ}$

- and nutrition, 627

-, phosphatase activity in -, 6I4

—, physiological, 21 5,589

- of the physiological tip, $75^{6}$ 
regeneration of planarians, stimulating effect of RNA, 298

-, promotion of -, by thyroid hormone, 632

-, quantitative aspects, 602

-, respiration and carbohydrate metabolism, 6 I 8

一, restitutive, 215

- and temperature, 623

-, unit, 608

-, upper limiting temperature - in poikilotherms, 623

- and vascular system, 644

-, water metabolism in -, 620

regenerative organizing action of muscle, cartilage, bone, notochord and endoderm, 646

- potencies, I4I

- powers, local variation of quantitative - -647

- - taxonomic differences, 647

regenesis, 588

regression, 589

-, allometric, 142

regulation, 593

- of growth, and nucleic acids, 297

-, phenomena, 214

rejuvenation in Protozoa, 597

relative, eclosion length, 608

- growth, 224

remodelling of the wound, 682

renal tumors induced by estrogenic

hormones, 837

replacement-rate, specific, 606

reproduction, growth as identical -, 140

re-regeneration, 593

resorbing surfaces in anabolic processes, I 80

resistance, phase of - of GAS, 6ro

respiration, and anabolic processes, 180

—, impaired — of tumors, 884

- in tumor cells, $88 \mathrm{I}$

- of isolated tissues, I 85

— in plant cells, 787

- rates in plant cell division, $77^{\circ}$

respiratory apparatus, connection of metabolic types with types of - - , I88

- balance and DNA or RNA synthesis, 888

- catalysts, 25

- chain of mitochondria, 29

— inhibitors, 576

- quotient as criterion of malignancy, $88_{2}$

retardation, 248

rete pegs, 677

reticulin, 670

-, layer of - fibers, 675 reticulocytes, glycine incorporated into

RNA of - 296

- protein, amino acids incorporated into - - 296

reticulo-endothelial cells in granulation tissue, 689

reticulo-endothelioma-hepatic histocytoma, 846

retina, so

retinal layer, action of - - on the epiblast, $45^{\circ}$

- - , formation, $45^{2}$

retinene, io

reversibility, principle of microscopic - , 154

rhabdomyosarcoma, induced by administration of peanut oil subcutaneously, 860

rhizopterin, I 19

Rhizopus, 62

- suinus, 760

rhodanase, 527

— in rat hepatoma, $9^{\mathrm{I}} 4$

Rhodospirillum, 40

rhombencephalon, 375, 407

-, presumptive, 389

Rhynchelmus, 644

ribitol, io

riboflavin, II 3

- accelerating regeneration, 630

- content of adenocarcinoma, 9I5

— - of mammary adenocarcinoma, 9 I 5

- inhibitor of tumor-inciting action of

butter yellow, 862

ribofuranosyl derivative of 6-methylpurine as mitose inhibitor, 936

— - of 6-methylmercaptopurine, as mitose inhibitor, 936

ribofuranosyl as mitose inhibitor, 935

9- $\beta$-D-ribofuranosylpurine, as chromosome breaking agent, $94^{\mathrm{I}}$

- as mitose inhibitor, 935

ribonuclease, $263,278,6$ I I

-, antimitotic activity, 282

-, biochemical effect of - on various cells, 279

— in chemotherapy of cancer, 282

- concentration in resting and growing cells, 298

- incubation of inductors with - 434

- , inhibition of protein synthesis in treated roots, 280

—, interference of - with centrosomes and spindle development, 283

- interference with virus multiplication, 284

-, rate of - activity in hepatomas, 9 I I

ribonucleic acid, see also RNA 
ribonucleic acid synthesis, 103

- in basocellular and spinocellular carcinoma, 905

- formation inhibited by amino acid antagonists, 937

- purines of Ehrlich tumor cells, 97

ribonuclein ( $\mathrm{RNP}$ ), 434

ribonucleoprotein particles, microsomal, 928

ribose as a deoxyribose precursor, 52

- diphosphate, 96

$d$-ribose, $26 \mathrm{I}$

ribose nucleic acid, see ribonucleic acid and RNA

ribose-5-phosphate, 5

ribulose diphosphate, carboxylation of - 43

ribulose phosphate as precursor of glycine, 62

ribulose-5-phosphate, 5, I I

Ringer's solution, 559

RNA, 26o, 508, 683, 732

- accumulation in nuclear sap of Cyclops egg after increase of polysaccharide in prophase, $94^{\mathrm{I}}$

- in antipolar plasm, 3 I 2

_- _ cephalo-caudal and dorso-ventral - during gastrulation and neurulation, 288

- content after fertilization, 286

- - in gastrula of Rana pipiens, 362

- of tumor cells, 157

- - in Cyclops eggs, $33 \mathrm{I}$

- and DNA in tumor cell nuclei, 905

- in the ectophyll, 476

-, glycine incorporated into - of reticulocytes, 296

- gradient in epithelium, 686

- granules, 344

- and growth, 272

-, guanine and cytosine in - from epithelial rat tumors, 902

-, incorporation of adenine in - 276

- increase and hormone synthesis stimulation, 273

- , liberation of parts of - nucleosides by ribonuclease, 438

- and morphogenesis, 288

-, pattern of basophily due to - corpuscules in rat embryo in first week of development, 326

-, phosphorylation and activation of by ATP, 285

- in plant-virus multiplication, 276

- and protein synthesis, 272

- and protein synthesis during spermatogenesis, 273
RNA, release of - to the cytoplasmic granules, $36 \mathrm{I}$

- stimulating effect of - and nucleoproteins on growth of tissue cultures, 298

-, stimulating effects of - on regeneration of planarians, 298

- synthesis of DNA and - during development, 285

RNA synthesis, blocked by metabolic poisons, 286

_- - DNA as organizer, 278

- in plant, 770

- - utilization of acetate, 524

— values in thymus lymphosarcoma, 906

RNAP, 576

$\mathrm{RNA} /$ protein ratio, proportionality of - to growth rate, 275

RNase, 5 I 5

Robinia pseudacacia, 752

roller tube tissue cultures, 577

root auxins, 753,804

- elongation, physiology, 806

- inhibition test, 757

roots, growth, 80 I

Rotifera, 590

Rous sarcoma, 39,849

- extracts synthesizing oligosaccharides of the hyaluric acid chain, 39

— - , hyaluronic acid in _ -,$- 88 \mathrm{I}$

_- immune bodies against _- -, $85^{8}$

R-phase, 589

-, glytolytic respiration in - of regeneration, 6 r 8

-, hyperglycaemia and glyconeogenesis in - of regeneration, 618

2 RSH, I 2

RSSR, I 2

Rubner's physiological rule, 2 I 6

rudimentation, 246

Sabella, 598, 622, 642

Saccharomyces cerevisiae, 50, 205

Saccoglossus kow, 327

S-adenosylmethionine, 46, 74

S-adenosylhomocysteine, cleavage by crude liver enzyme extracts of - to homocysteine and adenosine, 75

Salamandra atra, 292

- liver as xenoinductor of lens, 427

Salkowski reaction for auxin determination, $75^{8}$

Salmo salar, 736

Salmonella pullorum, 7 I 9

Salvelinus fontinalis, 205

sarcoma, 37, 55 I, 847

- agent, non-cellular, 849 
sarcoma, Jensen, 889

-, Yoshida, 88r

sarcomatous tumors, acid phosphatase content of - - 914

sarcosine, 73

S-carboxymethyl-N,N-dimethyldithiocarbamate, 764

scar forming, 664

Scendesmus, 6 I

Schardinger enzyme, 76 I

Schwann cells, 64 I

sclerotome, $5^{02}$

scopoletin inhibiting formation and elongation of root hairs, 806

Scorpaena, 185

Scorzonera hispanica, 76I

Secale, 800

secondary inductions, $44^{8}$

- meristems, 767

- plant cell wall, $75^{\circ}$

secretory cells, ergastoplasm of - - , 307

sedoheptulose-P, 3

sedoheptulose-7-P, 5

segregation, 140

self-accelerating phase, 175

self-inhibiting phase, 176

self-organizing systems of

morphogenetic factors, 354

self-reproduction of structured

nucleoproteins, I 39

self-regulation of metabolism, I 45

senescence, 952

senescent proliferation of duct cells, 965

senile orthoclone, 954

sensory placodes, 446

sequestrene as plant growth inhibitor, 796

Sequoias, 749

serine, 725

- biosynthesis, 58,60

-, conversion of phosphohydroxypyruvate to,- 6 I

— family, 55

-, hydroxypyruvate and D-glycerate as direct precursors of,$- 6 \mathrm{I}$

- interconversion of -, $5^{8}$

- intramolecular dehydration of - in Neurospara, 68

-, polyglutamyl-folic acid catalyzing conversion of - to glycine, I 21

-- synthesis in lymphosarcomas, 900

- synthesis from phosphoglycerate, 59

- transamination of hydroxypyruvate and alanine to - and pyruvate, 6 I

- uptake in transplanted rat tumors, 900 serineless mutant of $E$. coli, 6 I

serum proteins, uptake of - in cells of neoplastic tissues, 497

sex differentiation, 529

sex hormones, ACTH influence on - secretion of human adrenocortical tumors, 831

- - phenanthrene structure, 858

- - tumor suppression by — - $84 \mathrm{I}$

sexual differentiation in growth, 2 I 8

- dimorphism, 199

- hormones, 467

—, inverse relation of - maturation to longevity, 954

$\mathrm{SH}$-groups, increase of free - in regeneration, 608

- increase of free - during R-phase of regeneration, 6 I 5

- linked to proteins, 289

shikimate pathway, 65

shikimic acid, Io, 65

shock phase of the GAS, 643

Shope papilloma, 849

short wave radiation in regeneration, 624

$\mathrm{SH}$-protein complexes, 360

sinus gland, hormonal function of - of Crustacea in regeneration, 635

Siphonales, 749

size, dependence of $\mathrm{N}$-excretion, 203

-, limiting, I6 $\mathrm{I}$

skin cancer, carcinogenic induction of - 84 I

— - , cortison induced - - in mice, 844

—, mitotic activity in - cultures, 680

—, multiple — papillomas, 859

-, pre-existing, $67 \mathrm{I}$

- tumors of the mouse, 855

slime molds, 526

small molecular precursors of proteins, utilization, 499

snapping turtle egg, $74^{\circ}$

sodium, see also $\mathrm{Na}$

sodium, azide dissociating metabolism of maintenance from metabolism of differentiation and growth, 422

— decrease during human development, 959

- linoleate, 425

- oleate, 425

- versenate, 573

somatic growth in man, 248

- mutation and carcinogenesis, 848

somatoblast, 339

somatopleural epithelium, 467

- material of frog embryo, 461

somatotropin, 157, 219

somite genesis, 46 I

- mesoderm, interactions with neural tube tissue, 520 
somites, induction, 46 I

- presumptive, $35^{2}$

sorbitol, Io

D-sorbitol, IO

sorbose, Io

Soret effect, 153

sorting out in intermingled epiblast and

neuroepithelial cells in Amphibian neurula, 32 I

Spemann's organizer transplantation, $35^{2}$

specific, growth rate, 168

- proteins, mechanism of formation, 284

species-specific, constant, 157

- substances, growth as synthesis of - -

I 40

spermatic track, 323

-, development of the - aster, 322

spermatides, 163

spermatocytes, 163

spermatogenesis, 298

-, linkage of RNA and protein synthesis

during -, 273

spermatogonia, 163,306

spermatozoon, 308

sperm entrance point, 323

sphincter type action of wound perimeter, 670

spina bifida, 382

spinal ganglia, 404

spindle poisoning, 94I

- protein synthesis, 94I

spino-caudal structures, 367

spinocellular carcinoma, ribonucleic acids in - - , 905

Spiralia, 318, 327

Spirochaetes, 750

Spirodella, 8 I 4

Spisula, 338

spleen, loss of germinal centers in - and

lymph nodes in senescence, 964

spontaneous leukemia, 837

sporangiophores, aerial - of fungi, 755

spore formation in bacteria, mechanism, 537

sporulation, suppression, 535

Sprinson and Rittenberg expression, 149

squalene, 12, I09

- synthesis, I23

squamous cell carcinoma of the mouse skin, 857

Staphylococcus, $44^{2}$

- aureus, 83, 729

- muscae, 275

statoblasts, 589

steady state, 144

- - energy requirements of the maintenance of - of the organism, I5I
Stenostomum, 592

Stentor, 59 I, 604

steroid hormones, effect on tumor growth, 842

- inhibiting tumorigenesis, 840

— sex hormones inhibiting anaerobic tumor glycolysis, 883

steroids, biosynthesis, 43

sterol metabolism in process of cancer induction, $88_{\mathrm{I}}$

StiLi mice, maternal resistance factor of - - 855

stomata, 779

stomodoeal epiblast, 454

stomodeum, 469

stratum corneum, 686

- germinativum, 679

- granulosum, 687

- papillaris, 682

— spinosum, glycogen in _ —, 683

Straub's diaphorase, 20

streptococcal hyalurodinase 696

Streptococcus faecalis, I 9, 52, 78

- hemolyticus, 87

- lactis $\mathrm{R}$ factor, 120

stress, 609

- patterns governing orientation of cells and fibroblastic proliferation, 69 I

striated muscle, 454

Strongylocentrotus, fertilizin of,- 717

- purpuratus, 722

struggle of the parts, 230

Stylonychia, 2 I 6

subchromatid errors caused by

8-ethoxycaffeine during prophase, $93^{8}$

suboptimal growth, 222

substrate phase of wound healing, 697

- phosphorylation, 27

succinate, oxidation, 2 I

- pool in tumor tissue, 889

succinic acid, I3

— dehydrogenase, $14,525,683,782$

— - in mitochondria, I4

- oxidase, 634

succinoxidase, 527

- activity in malignant tissues, 889

succinyl-CoA, 7

- generating from acetoacetyl-CoA by a thiotransferase reaction, 29

sucrose as cofactor of traumatic acid, 772

sugar phosphorylation to sugar phosphate, 37

sugars, phosphorylated, 33

sulfanilamide arresting metaphase in

mitosis, 943

sulfate, activation, $4^{\text {I }}$ 
iulfate, conversion of - to cysteine, 63

-sulfinylpyruvate, 63

3-sulfinylpyruvic acid, 63

sulphophospholipid in human and experimental tumors, 88I

sulphur compounds, metabolism of - - in regeneration, 6 I 5

— in wound healing, 699

sulphydryl compounds, 596

supernormal growth, 222

supernumerary growth, 6o I

surface properties of cells, 474

- rule of Rubner, $18 \mathrm{I}$

- specialization of cells, 357

sweat glands, 682

symmetrization during oogenesis, 3 I 5

Syncoryne, 622

syneresis of plasma clot in wound healing, 692

synthesis of the carbon skeletons of amino acids, 56

- of coenzymes, 37

- of complex molecules in cellular growth, 7I4

- of polynucleotides, 37

tail buds, 320

target tissues, control of tumorigenesis by - - 866

- - genetic determination of tumorigenesis in — - 867

Tarichia ectoblast, 363

taurine, 40

- in carcinoma, 896

taurocholic acid, 40

Taylor series, I 74

telencephalic lobes, 407

telencephalon, 393

telolecithal eggs, $74^{2}$

telophase, 3 I 8

- of first cleavage, 328

temperatures, intra-liminal, 419

template-antitemplate theory of Weiss, 73 I

template hypothesis, 284

-, intracellular - molecules, $73 \mathrm{I}$

templates, 209, 669, 729

tendency toward perfection, 247

Tenebrio, 602

- molitor, 185

tensile forces within granulating base of wound, 670

- strength as index of wound healing, 697

tenso-active substances, 425

terminal hydrogen and electron transport system, 15

terpenes, biosynthesis, 43 testicular hyaluronidase, 694

_, interstitial cell tumors in mice induced by estrogenic hormone, 831,834

- tumorigenesis following transplantation to spleen, 834

- tumor induced by exogenous estrogen, 839

testosterone, 12

- phenylacetate, 84 I

tetrahydrofolic acid, 93, see also THFA

- derivatives, cofactors in glycine and serine interconversion, 59

$5,6,7,8$-tetrahydrofolic acid, II

I,2,3,4-tetrahydronaphthoic acid, 764

I,3,7,9-tetramethyluric acid as chromosome breaking agent, 939

Tetrahymena, 9o, 95, 515, 930

- requiring glycine for growth, 725

tetralideneacetic acid, 794

Tetrapods, 459

Theobaldia, 243

theophylline producing binucleate cells, 944

- as mutagenic agent for bacteria, 27I

Thermobacterium, $44^{2}$

— acidophilus, 294

thermodiffusion, 153

thermodynamics, irreversible, 152

- of open systems, I 5 I

thermoosmosis, 154

thermoregulation in homoiothermic animals, 187

THFA (tetrahydrofolic acid) required for FGAR synthesis, 93

thiamine, 5, II 2

- content of mammary adenocarcinoma, $9^{1} 5$

- pyrophosphate, 5

- in regeneration, 630

- synthesis, proposed pathways, I 12

thiazole, I 12

thienylalanine, 502

-, vegetalizing effect, 505

$\beta$-2-thienyl-DL-alanine causing polyploid cells in Lewis sarcoma, 241, 945

$\beta$-3-thienylalanine inhibiting protein synthesis, 937

Thiobacillus denitrificans, 44

8-thioethers of caffeine causing chromosome stickyness, $93^{8}$

thiokinase, 6

thiomalic acids as reductors for neuralization of ventral explants, 426

thiophorase, 7

thiourea, 529

thoracic mesenchyme, development, 523

threitol, Io 
threonine, aldolase reaction, 63

-, conversion of - to $\alpha$-ketobutyrate, 76

thrombin, 69I

thymidylic acid, $9^{1}$

- - polynucleotide, formation, $9^{11}$

thymidine phosphorylase enzymes, roo

- triphosphate, polymerization, 106

thymine, II, 26 I, 288

-, degradation, I 8

- requiring mutant of E. coli, 294

-, utilization, 99

thymoma, 838

thymoma-inciting action of X-rays, 86 I

thymus, effect of - on regeneration, 634

-, hypoplasia caused by estrogenic and androgenic hormones and cortisone, 839

— lymphosarcoma, 838

- neoplasia induced by estrogenic hormones, 839

- RNA values in thymus lymphosarcoma, 906

thyroid adenomata, 824

- carcinoma, 824

- hormone promoting regeneration, 632

— —, effects of _ — on regeneration, 633

- neoplasma induced by alteration of

thyroid function, 824

- secretion influence on genesis and growth of tumors, 846

- in senescence, detoriation of follicles and fibrosis, 964

- tumorigenesis following hypothyroidism, 825

- tumors, metabolism, 879

thyroidectomy, 824

thyrotrope hormone, effect of - on regeneration, 634

thyroxin, 219

-, activity increase of cytochrome $\mathrm{c}$ and succinic oxidase by - 634

-, adrenal cortex stimulation by -, 633

- increasing lactic dehydrogenase activity, 634

time axiom, I 55

tissue compatibility, 865

- culture, effect of ionizing radiations, $55^{8}$

- - effect of $\mathrm{pH}, 559$

- - effect of temperature on growth in - -, 556

- - effect of tonicity, 559

- - effect of ultraviolet irradiation, $55^{8}$

- - , effect of visible light, $55^{8}$

— - , formation rate of new cells

in - - 57 I

- - growth in - - 546

_- mathematical theory of growth, 166 tissue culture, measurements of growth, 567

- - metabolic measurements, 575

— - organized, 549

— - roller tube, 577

— - volume, 574

- water-content increase in regeneration, 609

tissues growth, 163

Titanotheria, 239

tobacco mosaic virus, $284,290,437,93$ I

- RNA isolated from crystalline -, 262

Tokophyra infusionum, 965

tonicity, effects of - on tissue culture, 559

Torulopsis kefyr, 205, 216

- utilis, 55, 76

T phages of $E$. coli, 276

$\mathrm{TNP}^{+}$(triphosphopyridine nucleotide), 9, I 5

$\mathrm{TNP}^{+}$or $\mathrm{DNP}^{+}$reduction by plant chloroplasts, 33

TPNH, I 2

TPNH-nitrate reductase of Neurospora, 23

$\mathrm{TPNH}_{2}$ oxidation coupled with

phosphorylation, 30

- or $\mathrm{DPNH}_{2}$ reoxidation by plant

mitochondria, 33

tracer adenine, 576

- alanine, $5^{13}$

- C, 796, 885

- glycine, 5 O I

- iodine, 824

- methionine, 504

- nitrogen, $5 \mathrm{I} 8,740$

- phosphor, 576

- - uptake, 507

- sulfur, 699, 700

- urea, 740

Tradescantia, staminal hairs of - -797

- wound meristem, 768

Tragulidae, $25^{1}$

transaldolase, 4,5

- reaction, donors and acceptors

in - - 49

transamidation, 728

transaminase, 78

- apoenzymes in E. coli and Neurospora, 85

-, apoenzymes catalyzing - reactions, 85

transaminating agent, glutamine

as — - 82

transamination, 86

- reactions, $\alpha$-ketoglutarate as amino acid acceptor in - - 84

- - pyridoxamine and pyridoxal

phosphate as cofactors in,-- 84 
transfer of large molecules to the embryo, 719

- of nitrogen atoms of amino acids in animal tissue, 84

- reactions, nucleotide - - , 38

- - XMP, 35

- -, pyrophosphate, 35

transformation, 238

-, harmonious — of the organism-as-awhole, 245

transforming principle, 520

— - of the mesoderm, $52 \mathrm{I}$

transformation process, 390

transhydrogenase, I2

transketolase, 4,5

transketolase-transaldolase pathway, 48

- - of pentose formation, 50

transmethylase, 74

- reactions, 75

trans-N-glycosidation, IO I

transpeptidation reactions, 728

transphosphorylation, 676

- reaction, nucleotide - —, 28

traumatic acid, 772

trephones, 554, 637, 666

tributyrinase, 721

tricarboxylic acid cycle, 2, 7, 8, 29, I $60,786,885,889$

— - - synthesis of - catalysts, $4^{\mathrm{I}}$

- - intermediates, 680

2,3,6-trichlorobenzoic acid, 764

2,4,5-trichlorophenoxyacetic acid, 763

2,4,6-trichlorophenoxyacetic acid, 765

Trichomonas vaginalis, 936

triiodobenzoate, 782

2,3,5-triiodobenzoic acid, 765

triiodothyronine in Ehrlich mouse ascites carcinoma, 896

Trillium, 769

Triops, 604

tripeptidase, 525

triphosphopyridine nucleotide $\left(\mathrm{TPN}^{+}\right)$, see TPN

tritomerit, 368

tritoneuron, 368

Triton palmatus, 292

- alpestris, 498

- helveticus, 355

Triturus, 665

- alpestris, 511,727

- cristatus, 399,516

- palmatus, 5 I 6

- pyrrhogaster, 375, 398, 425

- rivularis, 409

- tigrinum, 477

- toeniatus, 399
Triturus torosus, 477, 669

-, lens of eye of - 614

- neurula, 353

trophoblast, 327

trunk organizer, Spemann's, 376

trypsic dissociations of the caudal bud, 461

trypsin, $5^{\mathrm{I}} 8$

- dispersed tissue cultures, 573

- as inhibitor of induction, 290

tryptophanase, II 4

tryptophane, 65,760

-, conversion of - to IAA by direct oxidative desamination, 762

-, metabolic pathway of conversion of to nicotinic acid, I 6

- peroxydase, I I 6

-, terminal steps in - synthesis, 67

tryptophaneless mutant of E. coli, 67

tryptamine, 763

TSH (pituitary thyrotrophic hormone), 824

T'TP, го6

Tubifex, 6о4, 6 I 6

-, polarized changes of form in the egg of - $3{ }^{1} 8$

Tubularia, 16

-, inhibitor of the - hydranth formation, 639

Tunicata, 590

Turbellaria rhabdocoele, 59I

tumbling tube cultures of mouse lymphosarcoma, 574

tumor, ATPase and DPNase activity in mitochondria, 895

- cells, DNA and RNA content, I57

- - enzymatic depolymerization of deoxyribonucleoproteins, 9 I I

- - glycolysis and respiration, $88 \mathrm{I}$

_- , nucleic acid metabolism, 906

_ - nucleic acids and nucleoproteins, 902

-, differential diagnosis of certain types, 579

-, dipeptidase and cathepsin activities in central areas, $9 \mathrm{I} 3$

--, DNA and RNA in - cell nuclei, 904

—, DNP:DNPH ratios in - tissues, 894

_-, genetically determined - producing gonadotrophin response of testicular tissue, 834

- growth, effects of steroid hormones, 842

- - influence of dietetic factors, $23 \mathrm{I}$

- inability to synthesize aspartate and glutamate, 899

— inciting agents, 823

- induced by the administration of urethane, 857 
tumor, influence of pituitary gland on genesis, growth and secretion, 844

- mitochondrial preparations utilizing ATP during oxidation of $\alpha$-ketoglutarate and succinate, 89 I

- necrotizing activity of bacterial polysaccharides, 88 I

- producing capacity of tissue cultures, 565

- promoting activity of steroids other than estrogenic, 840

- proteins, D-glutamic acid in - - 901

- _ , rate of increase of ${ }^{14} \mathrm{C}, 897$

- requirement for additional DNP, 893

- respiration increased by polyphenyloxidase, 893

- suppression by sex hormones, 84 I

- tissue, deficiency of cytochrome $\mathrm{c}$ and cytochrome oxydase, 884

- - enzyme and co-enzyme activities, 913

- - , free amino nitrogen in extract of -

- 896

- - glutamine pool, 54

— - glycine pool, 54

- - inhibition of fatty acid oxidation in,-- 895

— - nucleotides and nucleosides, 903

- - proteins and amino acids, 896

-..., protein metabolism, 897

- - purine and pyrimidine composition of DNA, 902

- - pyruvate metabolism, 889

_- , succinate pool, 889

-, thyroid secretion influence on - genesis and growth, 846

- viruses, 855

- - cross immunization against chicken, 857

tumors, acetyl choline in peripheral parts of - 9 , 95

-, adenosinetriphosphate and alkaline phosphatase activity in growing -, 89I, 892

-, aerobic glycolysis, 54

-, amino acid metabolism, 899

—, C-distribution, 894

-, cutaneous, 840

- , $\mathrm{DNA}_{1}-\mathrm{DNA}_{2}$ ratios in -, 906

--, electron transport in aerobic glycolyse, 884

- , hepatic, 840

- high uptake of histidine, serine and asparagine in transplanted rat -, 900

—, impaired respiration, 884

-, low oxygen tension, 9 I 6

_, lowered pH in animal _-, 886 tumors, mammalian, 44

- - oxidized and reduced DPN in - 884

-.., patterns of free amino acids of - 54

_-, 6-phosphogluconate dehydrogenase and glucose-6-phosphatase activity, 891

-, proteolytic activity in human -, 913

-, - enzymes, 878

-, quantitative rapidity of nucleic acid metabolism, 908

-, specific activity of DNA and RNA nucleotides, gog

-, sulphophospholipid in human and experimental - 881

-, unusual capacity to synthesize glycine and serine in - of lymphatic origin, 917

--, uptake of labelled amino acids or related metabolites in $\longrightarrow, 899$

- utilizing labelled plasma albumin, 898

-, water, nitrogen and electrolyte content of human -, 9 I 5

tumorigenesis inhibition by steroid hormones, 840

-, control of - by target tissues, 866

-, genetic determination of - in target tissues, 867

turkey virus, 858

turnover of building materials of the organism, I 47

Tyrode's solution, 559

tyrosine, I2, 65, 455

- in carcinoma, 896

-, changes in - activity in frog egg, $34^{8}$

- forming from dietary phenylalanine in animal tissues, 67

- oxidase activity of human melanomas, 9 I 4

UDP, 99

UDP-acetylglucosamine phosphate in hen oviduct, 9 I

UDPG, 35, 38, 99

UDP-galactose, io

UDP-glucose, io

UDP-glucoronic acid, 10

UDP-xylose, 35

ultramicrosomes, 332

ultraviolet irradiation, of the dorso-marginal zone, 4 I 2

- effect on morphochoresis, 412

- _, effect of —— of organizer on conformation and differentiation of neural organ, 412

—- effects of - - on tissue culture, 558

- modifications of DNA, 27 I

- light, immediate inhibition of DNA synthesis by - - 294 
L 1 P , 99

undifferentiated protoplasm, I4I

unlimited growth (second growth type), 236

Lnna procedure, 360

uracil, I I, 26 I

uracil $15 \mathrm{~N}, 907$

uracil, conversion of - to $\beta$-alanine, roo

- compounds, amination to cytosine, Ioo uracil-2-14 C as DNA-T precursor in

Ehrlich tumor cells, 104

uracil deoxyriboside, 288

-, utilization, 99

urea, 715

- formation during development, 738

_, morphogenetic effect, 423

urease, 726

- activity in black snake embryo, 740

_, evolutionary appearance, 74I

- presence of — during incubation period of snapping turtle, 741

$\beta$-ureidopropionate, I 8

ureidosuccinic acid, $4 \mathrm{I}$

- - , conversion to dihydroorotic acid by liver homogenates, 99

- ${ }^{14} \mathrm{C}$ as pyrimidine precursor, 907

urethane as carcinogen, 863

- as coleukemogen, 863

- inducing pulmonary tumor, 857

uric acid, 13, 92

- formation, 7 I 5

- formation during development, 738

uridylic acid, activity of - from hepatoma, 909

uridine nucleotides, glutamine as amino donor in conversion of - - to cytidine nucleotides by rat tumor enzymes, 123

- phosphorylase, IOI

- phosphorylation, 102

uridyl transferase reactions, 38

urinary bladder tumors induced by

carcinogenic amino compounds, 862

-, excess - nitrogen in regeneration, 609

urogenital cords, 467

uropygial gland, 46 I

Urodele ectoblast as reactor, 444

- egg, 410

Urodeles, 358, 378, 590, 604, 675

USA, 99

usnic acid as metabolic poison, 286

U'TP, 37, 99

vacuoles of plant cell, $75^{\circ}$

vacuolizing effect of Nile blue, 367

vagocytic cells, migration of $-\frac{1}{-}$ to the wound area, 665

valine, 68,348 valine, pantoate synthesis from - I I 7

Van 't Hoff's rule, 216

vascular cambium, 767

- penetration, 689

- system and regeneration, 644

vegetal pole, 322

vegetative pole, 3 I I

vegetalizing activity of micromeres, 339

- effect of several amino acid analogs, 505

- LiCl, $35^{\circ}$

ventral marginal zone of gastrula, 35 I

versene as plant growth inhibitor, 796

Vicia faba, 932

Vinis vinera, $76 \mathbf{1}$

viral agents of carcinogenesis, 839

— induction of mouse leukemia, 853

virus, inhibition of - multiplication, 277

—, masked, 858

- - ribonuclease interference with multiplication, 284

viruses and cancer, 849

vitamin $A$, Io

- as coenzyme, $52 \mathrm{I}$

- , effect of - on tissue, 564

B-vitamins as precursors of metabolically active coenzymes, 555

vitamin $B_{11}$ promoting liver regeneration in rats, 631

vitamin $\mathrm{B}_{12}, 53,74$

vitamin biosynthesis, 108

vitamin $\mathrm{C}$, reduction of dehydroascorbic acid to,- 617

vitamin $\mathrm{D}$, I 10

vitamin $\mathbf{K}$, antagonist, I9

- as cell division promoting factor in plants, 772

vitamins in regeneration, 629

vitalism, I 47

vitelline cells, 3 I 5

- membrane, 718

vitellogenesis, 467

-, secondary, 313

vitelloid antigen, 720

Vogt's Bahnung, 347

Von Baer's law, 2 I 4, 324

Walker tumor, 844

Warburg's theory, I59

warmth, extra-liminal, 416

warts, involution of -, 230

water content of human tumors, 915

water metabolism in regeneration, 620

way-back induction by epiderm, 460

wear and tear, 589

wearing out quota, I 79

weed killers, 763 
Wehnelt's test, 772

weight-growth curve, I8o

Weismann's theory of determinants, I 72

Weiss' coaptation theory, 68 I

whole-body, neoplasms following -

$\mathrm{X}$-irradiation, 848

- X-irradiation inducing ovarian and hemopoietic neoplasms, 848

-, regenerates of Planarians, 601

Wocklumeria sphaeroides, 215

Wolffian duct, 463

- regeneration, 534

wound area, planimeter determination, 677

- closure, 669

- contracture, 669

- _, degree of, 670

- edema, 695

- edge, growth from - - 67 I

- healing, 664

- - contraction phase, 693

- _ favorable embryo juice action on

$-\cdots, 677$

- - mammalian, 666

- - productive phase, 697

_- role of sulfur, 699

- - stimulus, 666

—_, substrate phase, 697

- - syneresis of plasma clot, 692

- - tensile strength as index of - 697

- hormone, 666

- in plants, 768

-, sphincter type action of - perimeter, 670

_-, remodelling, 682

- tensile strength, decreased by iodoacetic acid, 70 I

wounding stimulating cell division in plants, 771

wounds, influence of gravity, 677

Xanthium pennsylvanicum, 766

xanthine oxidase, 26

-, oxidation, 2 I

xanthosinic acid, I o, 36

xanthylic acid, 95

Xenopus, 23I, 342, 459, 6 I 7

- embryo, 504

- oocytes, 3 I0

xeno-inductions, 427

-, bacterial, 439

- biochemical nature of agents

causing - 447

xeno-inductors, $35^{8}$

-, fractionation, 433
Xiphophorus, 244

XMP, 95

- transfer reactions, 35

$\mathrm{X}$-irradiation, depolymerization of DNA by - 272

- as mitotic inhibitor, 272

XMP (xanthylic acid), 95

$\mathrm{X}$-ray induced ovarian and adrenocortical tumorigenesis, 848

$\mathrm{X}$-rays, thymoma-inciting action, 861

XRPP, Iо3

xylitol, 10

xylose isomerase, synthesis, 294

xylulose, muscle aldolase catalizing phosphate synthesis, 49

xylulose-5-P, 5

Y-acyl-CoA-dehydrogenase, I 7

yeast, 6 I 7

- filamentous mycelium, 536

- mutant, divisionless, 536

- mutants requiring purines for growth, 509

- nucleic acids, 95

" $Y_{1}$ " enzyme, 6

"Y $\mathrm{Y}_{2}$ " enzyme, 6

yolk degradation, 499

- , breakdown of the - platelets in chordomesoblast during invagination, $36 \mathrm{I}$

- nucleus, $3 \mathbf{I} 4$

-, participation of - in morphochoresis, 476

- platelets, 310,322

- proteins degradation to amino acids, 53 I

-, relation of phosphatase activity to platelet utilization, 499

_, relationship of nitrogen metabolism with — storage, 729

-_, small - plug stage, 390

- synthesis as anabolic process of nitrogen metabolism, 7 I 5

- wall, inner, 322

Yoshida ascites tumor, of the rat, 565

_ _ - free amino acids pattern, 896

- sarcoma, 88 I

- - glutamine metabolism, gor

young orthoclone, 954

Zea mais, $75^{\circ}$

zinc content of methylcholanthrene induced carcinoma, 915

zinc salts as cause of expansion of the ectoblast cytoplasm, 422

zona striata in the chorion, 314

Zymobacterium oroticum, 99

zymosterol, I Io 



\section{.}






\section{A}

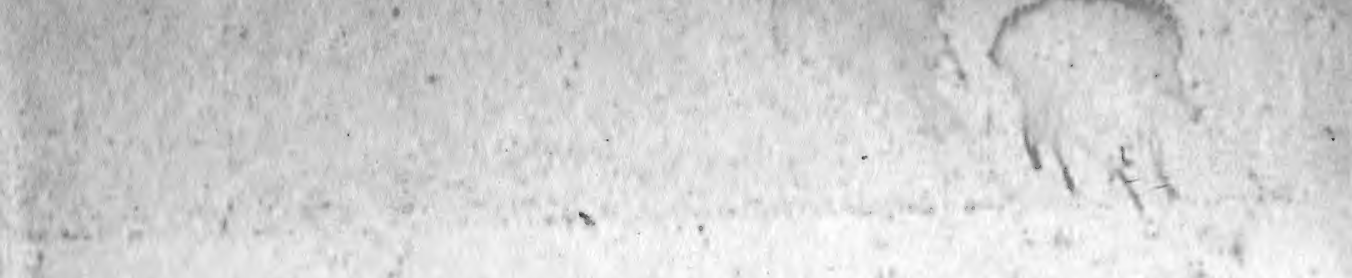

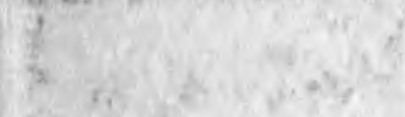

X.

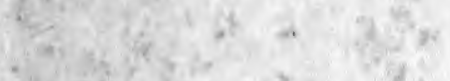

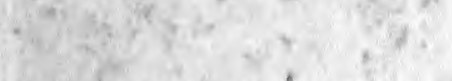

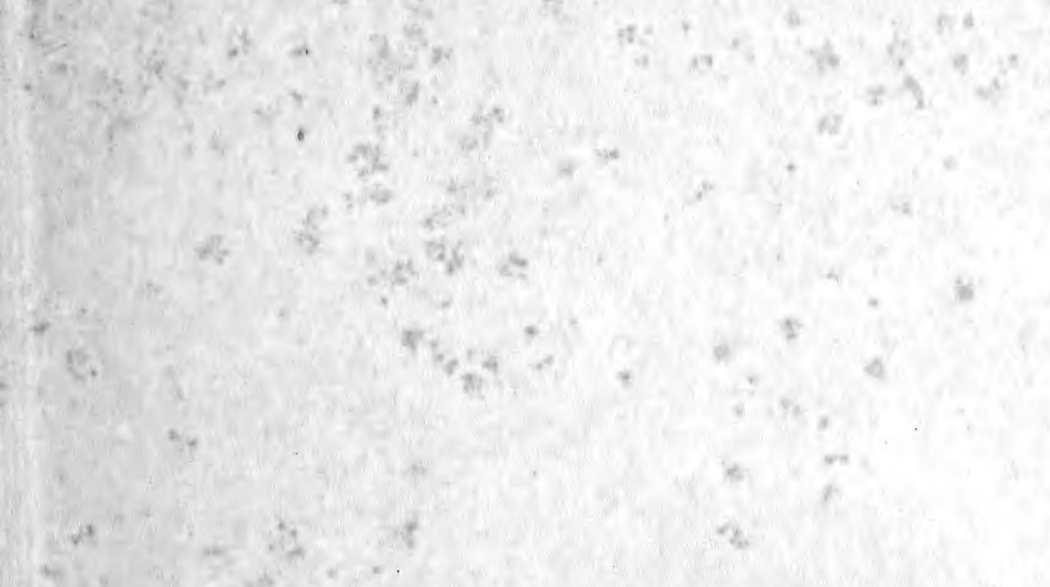

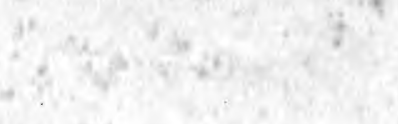

(4)

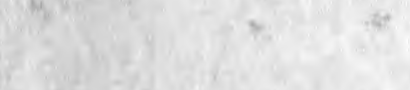

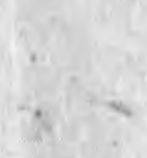

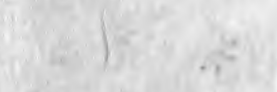

is

X

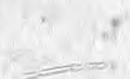

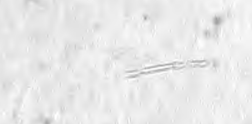


